Prepared for the U.S. Department of Energy under Contract DE-AC05-76RL01830

\title{
Glass Property Data and Models for Estimating High-Level Waste Glass Volume
}

JD Vienna

A Fluegel

DS Kim

P Hrma

October 2009

\section{Pacific Northwest}

NATIONAL LABORATORY

Proudly Operated by Battelle Since 1965 


\title{
DISCLAIMER
}

This report was prepared as an account of work sponsored by an agency of the United States Government. Neither the United States Government nor any agency thereof, nor Battelle Memorial Institute, nor any of their employees, makes any warranty, express or implied, or assumes any legal liability or responsibility for the accuracy, completeness, or usefulness of any information, apparatus, product, or process disclosed, or represents that its use would not infringe privately owned rights. Reference herein to any specific commercial product, process, or service by trade name, trademark, manufacturer, or otherwise does not necessarily constitute or imply its endorsement, recommendation, or favoring by the United States Government or any agency thereof, or Battelle Memorial Institute. The views and opinions of authors expressed herein do not necessarily state or reflect those of the United States Government or any agency thereof.

\author{
PACIFIC NORTHWEST NATIONAL LABORATORY \\ operated by \\ BATTELLE \\ for the \\ UNITED STATES DEPARTMENT OF ENERGY \\ under Contract DE-ACO5-76RL01830
}

Printed in the United States of America
Available to DOE and DOE contractors from the Office of Scientific and Technical Information,
P.O. Box 62, Oak Ridge, TN 37831-0062;
ph: (865) 576-8401
fax: (865) 5765728
email: reports@adonis.osti.gov

\author{
Available to the public from the National Technical Information Service, \\ U.S. Department of Commerce, 5285 Port Royal Rd., Springfield, VA 22161 \\ ph: (800) 553-6847 \\ fax: (703) 605-6900 \\ email: orders@nits.fedworld.gov \\ online ordering: http://www.ntis.gov/ordering.htm
}




\section{Glass Property Data and Models for Estimating High-Level Waste Glass Volume}

JD Vienna

A Fluegel

DS Kim

P Hrma

October 2009

Prepared for the U.S. Department of Energy under Contract DE-AC05-76RL01830

Pacific Northwest National Laboratory

Richland, Washington 99354 



\begin{abstract}
This report describes recent efforts to develop glass property models that can be used to help estimate the volume of high-level waste (HLW) glass that will result from vitrification of Hanford tank waste. The compositions of acceptable and processable HLW glasses need to be optimized to minimize the waste-form volume and, hence, to save cost. A database of properties and associated compositions for simulated waste glasses was collected for developing property-composition models. This database, although not comprehensive, represents a large fraction of data on waste-glass compositions and properties that were available at the time of this report. Glass property-composition models were fit to subsets of the database for several key glass properties. These models apply to a significantly broader composition space than those previously publised. These models should be considered for interim use in calculating properties of Hanford waste glasses.
\end{abstract}





\section{Summary}

Efforts are being made to increase the efficiency and decrease the cost of vitrifying radioactive waste stored in tanks at the U.S. Department of Energy's Hanford Site. The compositions of acceptable and processable high-level waste (HLW) glasses need to be optimized to minimize the waste-form volume and, hence, to save cost. A database of properties and associated compositions for simulated waste glasses was collected at Pacific Northwest National Laboratory for developing property-composition models. The database includes waste-glass compositions and properties, such as Product Consistency Test (PCT) response, viscosity $(\eta)$, electrical conductivity $(\varepsilon)$, Toxicity Characteristic Leach Procedure (TCLP) response, density $(\rho)$, one-percent crystal temperature $\left(T_{1 \%}\right)$, and liquidus temperature $\left(T_{L}\right)$, that are important for processability and product performance. Data from Pacific Northwest National Laboratory, West Valley Demonstration Project, Savannah River National Laboratory, Vitreous State Laboratory at the Catholic University of America, Idaho National Engineering and Environmental Laboratory, and several other institutions were reviewed and compiled into a single, easy-to-use database. This database, although not comprehensive, represents a large fraction of data on waste-glass compositions and properties that were available at the time of this report. Because of the size of the database, two versions of this report were printed, one version with the database attached as Appendix $A$ and one version without the database attached.

Glass property-composition models were fit to subsets of the database for several key glass properties. Models were generated for normalized boron, sodium, and lithium release in the PCT $\left(P C T_{B}, P C T_{N a}\right.$, and $P C T_{L i}$, respectively); $T_{1 \%}$ in the spinel ([Fe,Ni, Mn] $\left.[\mathrm{Fe}, \mathrm{Cr}]_{2} \mathrm{O}_{4}\right)$ phase region; $T_{L}$ in the zircon $\left(\mathrm{ZrSiO}_{4}\right)$ primary phase field; $\eta$ at 950,1150 , and $1250^{\circ} \mathrm{C}\left(\eta_{950}, \eta_{1150}\right.$, $\left.\eta_{1250}\right) ; \varepsilon$ at $1000,1100,1150$, and $1200^{\circ} \mathrm{C}\left(\varepsilon_{1000}, \varepsilon_{1100}, \varepsilon_{1150}, \varepsilon_{1200}\right)$; and the specific volume (SpV).

The models are of the form:

$$
\mathrm{g}_{\alpha}\left(p_{\alpha}\right)=\sum_{i=1}^{N} b_{\alpha i} x_{i}+\text { Selected }\left\{\sum_{i=1}^{N} b_{\alpha i i}\left(x_{i}\right)^{2}+\sum_{i}^{N-1} \sum_{<j}^{N} b_{\alpha i j} x_{i} x_{j}\right\}
$$

where $g_{\alpha}=\alpha^{\text {th }}$ predicted transformed property

$p_{\alpha}=\alpha^{\text {th }}$ property

$N=$ number of terms in the model

$b_{a i}=$ the $i$-th component coefficient for the $g_{\alpha}$ transformed property

$x_{i}=$ the $i$-th component mass fraction in glass.

Note that $\sum_{i=1}^{q} x_{i}=1$, so that the models of the form in Equation (S.1) are classified as mixture models.

Tables S.1 through S.6 summarize the results and validity regions for these models. The results (shown on the left side of the tables) include model coefficients estimated by least squares regression from subsets of the database, model goodness-of-fit statistics $\left(R^{2}, R_{A}^{2}, R^{2}\right.$, $\mathrm{R}^{2} \mathrm{v}$, and RMSE), and other summary statistics. The model validity regions (shown on the right side of the tables) are the lower and upper bounds of glass compositions for which the models 
are valid. In addition to single-component concentration constraints, the models are valid only over the range of predicted property values for which they were fitted (min and max values on left side of the tables). Note that although the model validity constraints are listed in wt $\%$ of components, the coefficients are based on mass fractions.

Table S.1. Viscosity Model Coefficients and Validity Regions (in In[Pa-s] and wt\%)

\begin{tabular}{|c|c|c|c|c|c|c|c|c|c|c|}
\hline Term & $\ln \left[n_{950}\right]$ & $\ln \left[n_{1150}\right]$ & $\ln \left[\eta_{1250}\right]$ & Comp & \multicolumn{2}{|c|}{$\ln \left[n_{950}\right]$} & \multicolumn{2}{|c|}{$\ln \left[\eta_{1150}\right]$} & \multicolumn{2}{|c|}{$\ln \left[n_{1250}\right]$} \\
\hline$\overline{\mathrm{Al}_{2} \mathrm{O}_{3}}$ & 18.13541 & 10.6085 & 8.94357 & & Min & Max & Min & Max & Min & Max \\
\hline $\mathrm{B}_{2} \mathrm{O}_{3}$ & -12.1316 & -9.37529 & -7.66615 & $\mathrm{Al}_{2} \mathrm{O}_{3}$ & 0 & 20.4 & 0 & 26.6 & 0 & 25.9 \\
\hline $\mathrm{BaO}$ & -2.69662 & -3.41816 & -3.89304 & $\mathrm{~B}_{2} \mathrm{O}_{3}$ & 0 & 20 & 0 & 20.2 & 0 & 20 \\
\hline $\mathrm{Bi}_{2} \mathrm{O}_{3}$ & & & -3.54929 & $\mathrm{BaO}$ & 0 & 4.7 & 0 & 4.7 & 0 & 4.7 \\
\hline $\mathrm{CaO}$ & -4.6401 & -6.9328 & -7.96797 & $\mathrm{Bi}_{2} \mathrm{O}_{3}$ & & & 0 & 7.4 & 0 & 16.4 \\
\hline $\mathrm{F}$ & -13.7453 & -12.3445 & -12.3147 & $\mathrm{CaO}$ & 0 & 15 & 0 & 18.2 & 0 & 15 \\
\hline $\mathrm{Fe}_{2} \mathrm{O}_{3}$ & 2.542852 & & -1.29457 & $\mathrm{~F}$ & 0 & 6 & 0 & 2.4 & 0 & 4.7 \\
\hline $\mathrm{K}_{2} \mathrm{O}$ & -3.46383 & -3.82491 & -3.45232 & $\mathrm{Fe}_{2} \mathrm{O}_{3}$ & 0 & 18.4 & 0 & 20 & 0 & 24.3 \\
\hline $\mathrm{La}_{2} \mathrm{O}_{3}$ & -1.48037 & -4.96954 & -4.70143 & $\mathrm{~K}_{2} \mathrm{O}$ & 0 & 10 & 0 & 10 & 0 & 10 \\
\hline $\mathrm{Li}_{2} \mathrm{O}$ & -54.8992 & -39.0249 & -34.7926 & $\mathrm{La}_{2} \mathrm{O}_{3}$ & 0 & 5 & 0 & 5 & 0 & 5 \\
\hline $\mathrm{MgO}$ & 0.785951 & -3.23141 & -4.85407 & $\mathrm{Li}_{2} \mathrm{O}$ & 0 & 9 & 0 & 8.9 & 0 & 8.9 \\
\hline $\mathrm{MnO}$ & -4.34993 & -6.88677 & -7.14845 & $\mathrm{MgO}$ & 0 & 8 & 0 & 8.2 & 0 & 8.2 \\
\hline $\mathrm{Na}_{2} \mathrm{O}$ & -9.52319 & -9.63275 & -8.81239 & $\mathrm{MnO}$ & 0 & 8 & 0 & 8 & 0 & 8 \\
\hline $\mathrm{P}_{2} \mathrm{O}_{5}$ & 8.837872 & 5.305007 & & $\mathrm{Na}_{2} \mathrm{O}$ & 2.5 & 25.9 & 3.4 & 25.5 & 2.5 & 25.5 \\
\hline $\mathrm{PbO}$ & -22.2775 & -23.1436 & -35.1899 & $\mathrm{Nd}_{2} \mathrm{O}_{3}$ & & & 0 & 8.6 & 0 & 8.6 \\
\hline $\mathrm{SiO}_{2}$ & 13.09017 & 9.368089 & 7.719283 & $\mathrm{P}_{2} \mathrm{O}_{5}$ & 0 & 5 & 0 & 5 & & \\
\hline $\mathrm{SrO}$ & -3.84188 & -4.35052 & -6.42168 & $\mathrm{SiO}_{2}$ & 32.3 & 60.4 & 25 & 62.8 & 26 & 64.1 \\
\hline $\mathrm{TiO}_{2}$ & & & -2.96399 & $\mathrm{SrO}$ & 0 & 10.3 & 0 & 10.3 & 0 & 10.3 \\
\hline $\mathrm{UO}_{3}$ & 5.952491 & 2.151455 & 1.061988 & $\mathrm{ThO}_{2}$ & & & 0 & 6.8 & 0 & 7.8 \\
\hline $\mathrm{ZnO}$ & 0.664542 & -2.69626 & -2.91064 & $\mathrm{TiO}_{2}$ & & & 0 & 10 & 0 & 9.6 \\
\hline $\mathrm{ZrO}_{2}$ & 15.1512 & 7.14044 & & $\mathrm{UO}_{3}$ & 0 & 6.5 & 0 & 7 & 0 & 15.6 \\
\hline Others & 3.523275 & -0.09027 & 2.681183 & ZnO & 0 & 5.8 & 0 & 5.8 & 0 & 9.8 \\
\hline $\mathrm{B}_{2} \mathrm{O}_{3} \times \mathrm{B}_{2} \mathrm{O}_{3}$ & 44.83921 & 24.59262 & 17.33823 & $\mathrm{ZrO}_{2}$ & 0 & 15.5 & 0 & 15.5 & 0 & 15.5 \\
\hline $\mathrm{Na}_{2} \mathrm{O} \times \mathrm{B}_{2} \mathrm{O}_{3}$ & -23.3502 & -26.9571 & -25.4993 & Remaining & 0 & 7.5 & 0 & 7.1 & 0 & 9.4 \\
\hline $\mathrm{Li}_{2} \mathrm{O} \times \mathrm{Li}_{2} \mathrm{O}$ & 140.7392 & 47.35918 & 35.43191 & & & & & & & \\
\hline $\mathrm{Na}_{2} \mathrm{O} \times \mathrm{Al}_{2} \mathrm{O}_{3}$ & & 17.51718 & 14.98563 & & & & & & & \\
\hline $\mathrm{Na}_{2} \mathrm{O} \times \mathrm{Fe}_{2} \mathrm{O}_{3}$ & -9.58328 & & & & & & & & & \\
\hline $\mathrm{B}_{2} \mathrm{O}_{3} \times \mathrm{Al}_{2} \mathrm{O}_{3}$ & -17.9433 & & & & & & & & & \\
\hline $\mathrm{CaO} \times \mathrm{Al}_{2} \mathrm{O}_{3}$ & & -8.13474 & & & & & & & & \\
\hline Statistic & & & & & & & & & & \\
\hline $\mathrm{R}^{2}$ & 0.953 & 0.9619 & 0.9678 & & & & & & & \\
\hline $\mathrm{R}_{\mathrm{A}}^{2}$ & 0.9513 & 0.9610 & 0.9669 & & & & & & & \\
\hline $\mathrm{R}_{\mathrm{P}}^{2}$ & 0.9491 & 0.9586 & 0.9649 & & & & & & & \\
\hline$R^{2} v$ & 0.9491 & 0.9619 & 0.9649 & & & & & & & \\
\hline RMSE & 0.1832 & 0.1632 & 0.1612 & & & & & & & \\
\hline Minimum & 0.8643 & -0.4588 & -1.055 & & & & & & & \\
\hline Mean & 3.75 & 1.673 & 1.007 & & & & & & & \\
\hline Maximum & 5.775 & 4.599 & 4.159 & & & & & & & \\
\hline $\mathrm{n}$ & 673 & 967 & 817 & & & & & & & \\
\hline $\mathrm{N}$ & 25 & 24 & 24 & & & & & & & \\
\hline
\end{tabular}


Table S.2. Electrical Conductivity Model Coefficients and Validity Regions (in $\ln [\mathrm{S} / \mathrm{m}]$ and wt\%)

\begin{tabular}{|c|c|c|c|c|c|c|c|c|c|c|c|c|c|}
\hline Term & $\ln \left[\varepsilon_{1000}\right]$ & $\ln \left[\varepsilon_{1100}\right]$ & $\ln \left[\varepsilon_{1150}\right]$ & $\ln \left[\varepsilon_{1200}\right]$ & Comp & \multicolumn{2}{|c|}{$\ln \left[\varepsilon_{1000}\right]$} & \multicolumn{2}{|c|}{$\ln \left[\varepsilon_{1100}\right]$} & \multicolumn{2}{|c|}{$\ln \left[\varepsilon_{1150}\right]$} & \multicolumn{2}{|c|}{$\ln \left[\varepsilon_{1200}\right]$} \\
\hline$\overline{\mathrm{Al}_{2} \mathrm{O}_{3}}$ & -0.234445 & 0.0821118 & 0.3447447 & 0.3312382 & & Min & $\operatorname{Max}$ & Min & $\operatorname{Max}$ & Min & Max & Min & $\operatorname{Max}$ \\
\hline $\mathrm{B}_{2} \mathrm{O}_{3}$ & 0.8170189 & 1.7269964 & 2.2131363 & 2.380146 & $\mathrm{Al}_{2} \mathrm{O}_{3}$ & 0 & 25.9 & 0 & 25.9 & 0 & 26.6 & 0 & 25.9 \\
\hline $\mathrm{CaO}$ & -0.812738 & 0.4816237 & 1.1386022 & 1.7033775 & $\mathrm{~B}_{2} \mathrm{O}_{3}$ & 0 & 20 & 0 & 20 & 0 & 20.2 & 0 & 20 \\
\hline $\mathrm{Fe}_{2} \mathrm{O}_{3}$ & 1.7770346 & 2.3177094 & 2.4167485 & 2.5149398 & $\mathrm{Bi}_{2} \mathrm{O}_{3}$ & 0 & 16.4 & 0 & 16.4 & 0 & 16.4 & 0 & 16.4 \\
\hline $\mathrm{Li}_{2} \mathrm{O}$ & 37.351081 & 35.619913 & 35.108383 & 33.714638 & $\mathrm{CaO}$ & 0 & 11.9 & 0 & 13.9 & 0 & 18.2 & 0 & 13.9 \\
\hline $\mathrm{MgO}$ & -1.153956 & 0.1206499 & 0.2090895 & 0.9980765 & $\mathrm{Fe}_{2} \mathrm{O}_{3}$ & 0 & 15 & 0 & 15 & 0 & 15 & 0 & 15 \\
\hline $\mathrm{Na}_{2} \mathrm{O}$ & 16.053203 & 15.435281 & 15.226886 & 14.801322 & $\mathrm{~K}_{2} \mathrm{O}$ & 0 & 5.4 & 0 & 5.4 & 0 & 6.1 & 0 & 5.4 \\
\hline $\mathrm{NiO}$ & -4.78784 & -3.347901 & 0.284527 & -1.239448 & $\mathrm{Li}_{2} \mathrm{O}$ & 0 & 7.9 & 0 & 7.9 & 0 & 7.9 & 0 & 7.9 \\
\hline $\mathrm{SiO}_{2}$ & -0.649396 & -0.067465 & 0.1665476 & 0.534619 & $\mathrm{MgO}$ & 0 & 8 & 0 & 8 & 0 & 9.6 & 0 & 9.6 \\
\hline $\mathrm{SrO}$ & 0.564366 & 1.8585572 & 2.2279737 & 2.7731117 & $\mathrm{MnO}$ & 0 & 8 & 0 & 8 & 0 & 8 & 0 & 8 \\
\hline $\mathrm{ZnO}$ & 4.7824589 & 6.0673766 & 6.4620647 & 6.5598526 & $\mathrm{Na}_{2} \mathrm{O}$ & 2.5 & 23 & 2.5 & 23 & 2.5 & 23 & 2.5 & 23 \\
\hline $\mathrm{ZrO}_{2}$ & 0.2999368 & 0.9202707 & 1.0944233 & 1.4700505 & $\mathrm{Nd}_{2} \mathrm{O}_{3}$ & 0 & 7.8 & 0 & 8.6 & 0 & 8.6 & 0 & 8.6 \\
\hline Others & 1.9602265 & 2.4486435 & 2.6305908 & 2.8441506 & $\mathrm{NiO}$ & 0 & 1.8 & 0 & 2 & 0 & 2.1 & 0 & 2 \\
\hline $\mathrm{Na}_{2} \mathrm{O} \times$ & -111.9769 & -101.2064 & -100.2325 & -91.29459 & $\mathrm{P}_{2} \mathrm{O}_{5}$ & 0 & 4 & 0 & 4 & 0 & 9 & 0 & 9 \\
\hline & & & & & $\mathrm{PbO}$ & 26 & 60 & 0 & 5.2 & 0 & 8.6 & 0 & 5.2 \\
\hline Statistic & Value & Value & Value & Value & $\mathrm{SiO}_{2}$ & 0 & 10.3 & 26 & 60 & 19.4 & 60 & 26 & 60.1 \\
\hline$R^{2}$ & 0.8962 & 0.8856 & 0.8802 & 0.8770 & $\mathrm{SrO}$ & 0 & 6 & 0 & 10.3 & 0 & 10.3 & 0 & 10.3 \\
\hline $\mathrm{R}^{2}{ }_{\mathrm{A}}$ & 0.8936 & 0.8828 & 0.8774 & 0.8740 & $\mathrm{ThO}_{2}$ & 0 & 6.5 & 0 & 6 & 0 & 6 & 0 & 6 \\
\hline$R_{P}^{2}$ & 0.8899 & 0.8789 & 0.8735 & 0.8704 & $\mathrm{UO}_{3}$ & 0 & 5.8 & 0 & 6.5 & 0 & 6.5 & 0 & 6.5 \\
\hline $\mathrm{R}^{2} \mathrm{v}$ & 0.8917 & 0.8793 & 0.8708 & 0.8708 & ZnO & 0 & 15.5 & 0 & 5.8 & 0 & 5.8 & 0 & 5.8 \\
\hline RMSE & 0.1732 & 0.1673 & 0.1668 & 0.1600 & $\mathrm{ZrO}_{2}$ & 0.3 & 6.4 & 0 & 15.5 & 0 & 15.5 & 0 & 16 \\
\hline Min & 0.84 & 1.53 & 1.70 & 2.13 & Others & 0.3 & 6.4 & 0.3 & 6.1 & 0.3 & 6.4 & 0.3 & 6.1 \\
\hline Mean & 2.854 & 3.3 & 3.47 & 3.65 & & & & & & & & & \\
\hline Max & 4.058 & 4.42 & 4.58 & 4.73 & & & & & & & & & \\
\hline$n$ & 527 & 542 & 575 & 558 & & & & & & & & & \\
\hline $\mathrm{N}$ & 14 & 14 & 14 & 14 & & & & & & & & & \\
\hline
\end{tabular}


Table S.3. Product Consistency Test Model Coefficients and Validity Regions (in $\mathrm{In}\left[\mathrm{g} / \mathrm{m}^{2}\right]$ and wt $\%)$

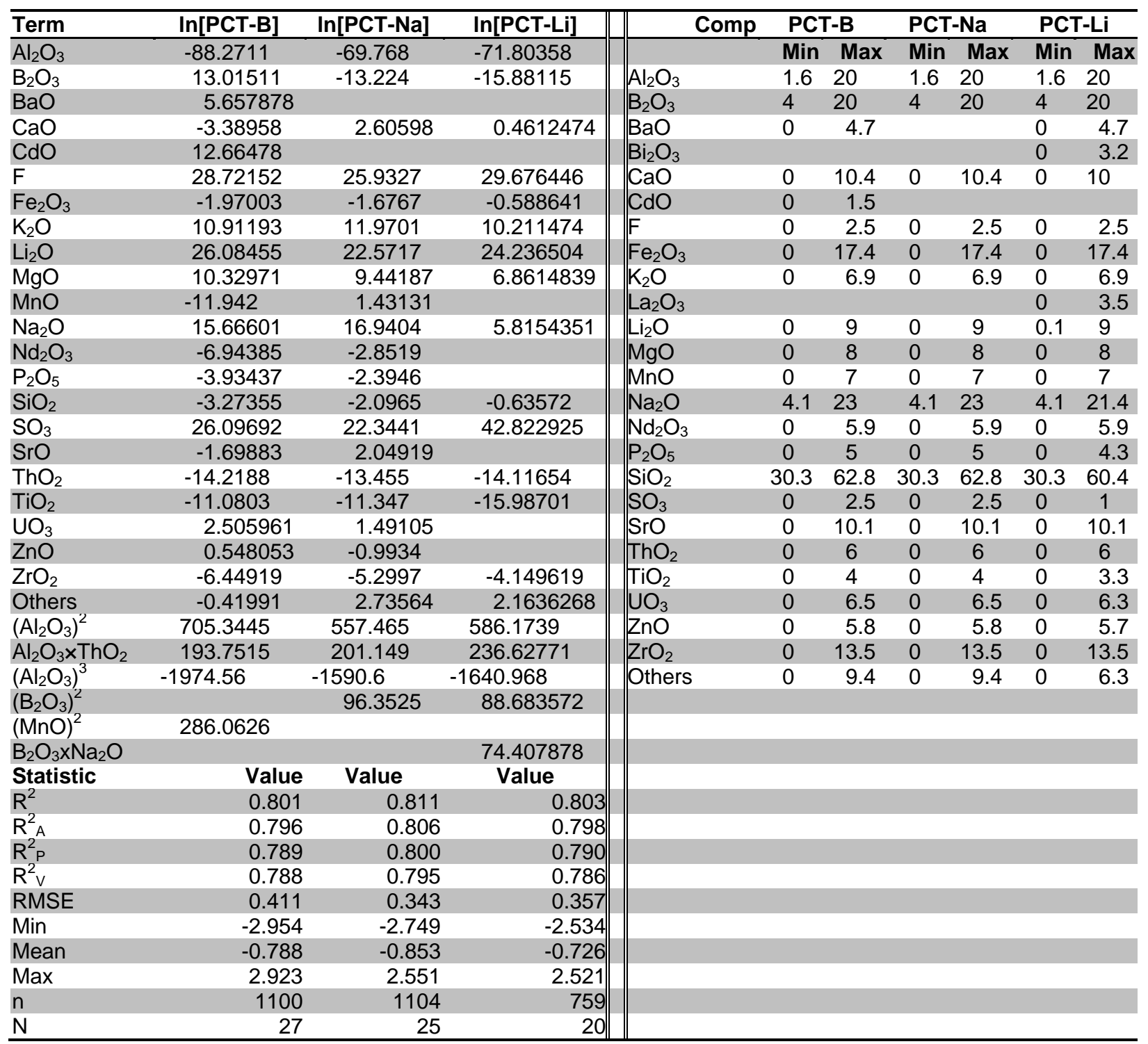


Table S.4. Toxicity Characteristic Leaching Procedure Model Coefficients and Validity Regions (in $\ln [\mathrm{mg} / \mathrm{L}]$ and $w \mathrm{t} \%$ )

\begin{tabular}{|c|c|c|c|c|}
\hline Term & $\ln \left[\mathrm{N}_{\mathrm{TCLP}}\right]$ & Comp & Min & $\operatorname{Max}$ \\
\hline$\overline{\mathrm{Al}_{2} \mathrm{O}_{3}}$ & -0.524992 & $\mathrm{Al}_{2} \mathrm{O}_{3}$ & 0 & 16.3 \\
\hline $\mathrm{B}_{2} \mathrm{O}_{3}$ & 12.003262 & $\mathrm{~B}_{2} \mathrm{O}_{3}$ & 2 & 21 \\
\hline $\mathrm{CaO}$ & 11.147865 & $\mathrm{BaO}$ & 0 & 4.5 \\
\hline $\mathrm{Fe}_{2} \mathrm{O}_{3}$ & -1.209342 & $\mathrm{Bi}_{2} \mathrm{O}_{3}$ & 0 & 2.4 \\
\hline $\mathrm{Li}_{2} \mathrm{O}$ & 20.870752 & $\mathrm{CaO}$ & 0 & 13.9 \\
\hline $\mathrm{Na}_{2} \mathrm{O}$ & 15.242625 & CdO & 0 & 4 \\
\hline $\mathrm{SiO}_{2}$ & -2.004927 & $\mathrm{~F}$ & 0 & 1.5 \\
\hline $\mathrm{ThO}_{2}$ & 2.468589 & $\mathrm{Fe}_{2} \mathrm{O}_{3}$ & 1.9 & 18.7 \\
\hline $\mathrm{ZrO}_{2}$ & -0.661848 & $\mathrm{~K}_{2} \mathrm{O}$ & 0 & 8.7 \\
\hline \multirow[t]{2}{*}{ Others } & \multirow[t]{2}{*}{9.2805424} & $\mathrm{Li}_{2} \mathrm{O}$ & 0 & 7.4 \\
\hline & & $\mathrm{MgO}$ & 0 & 5 \\
\hline Statistic & Value & $\mathrm{MnO}$ & 0 & 7.6 \\
\hline$R^{2}$ & 0.8941 & $\mathrm{Na}_{2} \mathrm{O}$ & 2.9 & 20 \\
\hline $\mathrm{R}_{\mathrm{A}}^{2}$ & 0.8907 & $\mathrm{NiO}$ & 0 & 3 \\
\hline$R_{p}^{2}$ & 0.8840 & $\mathrm{P}_{2} \mathrm{O}_{5}$ & 0 & 3.9 \\
\hline$R^{2} v$ & 0.8853 & $\mathrm{PbO}$ & 0 & 8.6 \\
\hline RMSE & 0.308 & $\mathrm{SiO}_{2}$ & 29.4 & 55 \\
\hline Min & 2.624 & $\mathrm{SO}_{3}$ & 0 & 2.1 \\
\hline Mean & 3.896 & SrO & 0 & 14.2 \\
\hline Max & 7.712 & $\mathrm{ThO}_{2}$ & 0 & 6 \\
\hline $\mathrm{n}$ & 291 & $\mathrm{TiO}_{2}$ & 0 & 2 \\
\hline \multirow[t]{4}{*}{$N$} & \multirow[t]{4}{*}{10} & $\mathrm{UO}_{3}$ & 0 & 8.4 \\
\hline & & $\mathrm{ZnO}$ & 0 & 4.9 \\
\hline & & $\mathrm{ZrO}_{2}$ & 0 & 10.4 \\
\hline & & Others & 0 & 5 \\
\hline
\end{tabular}


Table S.5. One Percent Crystal and Liquidus Temperature Model Coefficients and Validity Regions (in ${ }^{\circ} \mathrm{C}$ and wt\%)

\begin{tabular}{|c|c|c|c|c|c|c|c|}
\hline Term & $T_{1 \%}-s p$ & $\mathrm{~T}_{\mathrm{L}}-\mathrm{ZS}$ & Comp & \multicolumn{2}{|c|}{$T_{1 \%}$-sp } & \multicolumn{2}{|c|}{$T_{L}-\mathbf{Z S}$} \\
\hline$\overline{\mathrm{Al}_{2} \mathrm{O}_{3}}$ & 2835.11 & 3193.36 & & Min & Max & Min & $\operatorname{Max}$ \\
\hline $\mathrm{B}_{2} \mathrm{O}_{3}$ & -201.41 & 651.397 & $\mathrm{Al}_{2} \mathrm{O}_{3}$ & 1.9 & 20 & 0 & 17.2 \\
\hline $\mathrm{Cr}_{2} \mathrm{O}_{3}$ & 12468.2 & & $\mathrm{~B}_{2} \mathrm{O}_{3}$ & 3 & 20 & 2 & 20 \\
\hline $\mathrm{Fe}_{2} \mathrm{O}_{3}$ & 3328.76 & & $\mathrm{Bi}_{2} \mathrm{O}_{3}$ & 0 & 7 & 0 & 10 \\
\hline${ }_{2} \mathrm{O}$ & -409.94 & & $\mathrm{CaO}$ & 0 & 7 & 0 & 10 \\
\hline $\mathrm{Li}_{2} \mathrm{O}$ & -735.44 & -1904.42 & $\mathrm{CdO}$ & 0 & 2 & & \\
\hline $\mathrm{LN}_{2} \mathrm{O}_{3}$ & & 2156.41 & $\mathrm{Cr}_{2} \mathrm{O}_{3}$ & 0 & 1.2 & & \\
\hline $\mathrm{MgO}$ & 3927.07 & & $\mathrm{~F}$ & 0 & 2 & & \\
\hline $\mathrm{MnO}$ & 2618.91 & & $\mathrm{Fe}_{2} \mathrm{O}_{3}$ & 4 & 20 & 0 & 10.5 \\
\hline $\mathrm{la}_{2} \mathrm{O}$ & -717.5 & -1947.71 & $\mathrm{~K}_{2} \mathrm{O}$ & 0 & 6 & & \\
\hline $\mathrm{NiO}$ & 11916.1 & & $\mathrm{Li}_{2} \mathrm{O}$ & 0 & 6 & 0 & 9 \\
\hline NM & 14871.5 & & $\mathrm{LN}_{2} \mathrm{O}_{3}$ & & & 0 & 3.9 \\
\hline $\mathrm{SiO}_{2}$ & 424.651 & & $\mathrm{MgO}$ & 0 & 6 & 0 & 4 \\
\hline $\mathrm{SrO}$ & 421.296 & 13011.9 & $\mathrm{MnO}$ & 0 & 8 & 0 & 1.9 \\
\hline $\mathrm{ThO}_{2}$ & 897.152 & & $\mathrm{Na}_{2} \mathrm{O}$ & 3.7 & 25 & 4 & 15 \\
\hline $\mathrm{ZnO}$ & 3103.31 & & $\mathrm{NiO}$ & 0 & 3 & & \\
\hline $\mathrm{ZrO}_{2}$ & 1933.8 & 3747.42 & NM & 0 & 0.5 & & \\
\hline Others & 735.728 & 1259.22 & $\mathrm{P}_{2} \mathrm{O}_{5}$ & 0 & 2.5 & 0 & 5 \\
\hline Statistic & Value & Value & $\mathrm{SiO}_{2}$ & 28 & 53 & 39.7 & 59.6 \\
\hline $\mathrm{R}^{2}$ & 0.772 & 0.9069 & $\mathrm{SrO}$ & 0 & 10.3 & 0 & 1.2 \\
\hline $\mathrm{R}_{\mathrm{A}}^{2}$ & 0.761 & 0.8962 & $\mathrm{ThO}_{2}$ & 0 & 6 & 0 & 6 \\
\hline $\mathrm{R}_{\mathrm{P}}^{2}$ & 0.744 & 0.8693 & $\mathrm{TiO}_{2}$ & 0 & 3.1 & & \\
\hline$R^{2} v$ & 0.739 & 0.8718 & $\mathrm{UO}_{3}$ & 0 & 6.5 & 0 & 1 \\
\hline RMSE & 60.69 & 26.2 & $\mathrm{ZnO}$ & 0 & 4 & 0 & 1.2 \\
\hline Min & 635.7 & 897 & $\mathrm{ZrO}_{2}$ & 0 & 9.6 & 4 & 16.5 \\
\hline Mean & 950.3 & 1079 & Others & 0 & 4.5 & 0.3 & 3.3 \\
\hline Max & 1279 & 1298 & & & & & \\
\hline$n$ & 350 & 69 & & & & & \\
\hline & 17 & & & & & & \\
\hline
\end{tabular}


Table S.6. Specific Volume Model Coefficients and Validity Regions (in $\mathrm{cm}^{3} / \mathrm{g}$ and wt\%)

\begin{tabular}{|c|c|c|c|c|}
\hline Term & $v_{i}^{(a)}$ & Statistic & $\rho^{(b)}$ & $v$ \\
\hline $\mathrm{Al}_{2} \mathrm{O}_{3}$ & 0.35634 & Minimum & 2.41 & 0.3175 \\
\hline $\mathrm{B}_{2} \mathrm{O}_{3}$ & 0.50877 & Mean & 2.656 & 0.377 \\
\hline $\mathrm{BaO}$ & 0.16153 & Maximum & 3.15 & 0.4149 \\
\hline $\mathrm{Bi}_{2} \mathrm{O}_{3}$ & 0.09932 & $\mathrm{R}^{2}$ & 0.8231 & 0.8121 \\
\hline $\mathrm{CaO}$ & 0.33202 & $\mathrm{R}_{\mathrm{A}}^{2}$ & 0.8227 & 0.8116 \\
\hline $\mathrm{Fe}_{2} \mathrm{O}_{3}$ & 0.23186 & RMSE & 0.0431 & 0.00616 \\
\hline $\mathrm{K}_{2} \mathrm{O}$ & 0.45147 & $\mathrm{n}$ & 411 & 111 \\
\hline $\mathrm{Li}_{2} \mathrm{O}$ & 0.48306 & $\mathrm{~N}$ & 2 & 2 \\
\hline $\mathrm{MgO}$ & 0.34608 & & & \\
\hline $\mathrm{MnO}$ & 0.20998 & & $a$ & $b$ \\
\hline $\mathrm{Na}_{2} \mathrm{O}$ & 0.36235 & Coeff. & 1.33999 & 0.81725 \\
\hline $\mathrm{P}_{2} \mathrm{O}_{5}$ & 0.41608 & & & \\
\hline $\mathrm{SiO}_{2}$ & 0.39547 & & & \\
\hline $\mathrm{SrO}$ & 0.20425 & & & \\
\hline $\mathrm{ThO}_{2}$ & 0.1055 & & & \\
\hline $\mathrm{UO}_{3}$ & 0.13134 & & & \\
\hline $\mathrm{ZnO}$ & 0.17329 & & & \\
\hline $\mathrm{ZrO}_{2}$ & 0.20873 & & & \\
\hline (a) & \multicolumn{4}{|c|}{$\begin{array}{l}\text { Shannon (1976), there is a coefficient for all } \\
\text { elements in glass. Only selected coefficients are } \\
\text { shown here for example. }\end{array}$} \\
\hline (b) & \multicolumn{4}{|c|}{$\begin{array}{l}\text { The fit statistics are calculated based on specific } \\
\text { volume }(v) \text { and on density }(\rho) \text {. }\end{array}$} \\
\hline
\end{tabular}

The models in Tables S.1 to S. 6 are applicable over significantly broader composition spaces than those in use at the Hanford Tank Waste Treatment and Immobilization Plant (WTP) (Piepel et al. 2008a). They are for predicting glass volumes over broad waste and glasscomposition spaces and therefore are less precise than those needed to operate a plant within a relatively narrow composition space.

Ordinary "good practices" quality assurance (QA) practices were followed, but the QA was not compliant with RW-0333P (DOE-RW 2008) or Nuclear Quality Assurance Requirements and Descriptions requirements (Barnes 2000). Future updates of models could be developed to tighter QA requirements when required.

These models should be considered for interim use in calculating properties of Hanford waste glasses. The models presented here represent updates of the models developed by Vienna et al. (2002) and appear to perform as well or better than those models; they cover broader composition regions with comparable or higher $\mathrm{R}^{2}$ values, especially for validation datasets. However, as we discuss below, the models should be updated when the database is updated. 



\section{Quality Assurance}

Ordinary "good laboratory practices" quality assurance (QA) was performed in developing the database and models. Formal QA documentation, such as would be required for Nuclear Quality Assurance Requirements and Descriptions, was not performed because of the interim nature of the work. 



\section{Abbreviations}

\begin{tabular}{|c|c|}
\hline ASTM & American Society for Testing and Materials \\
\hline BNFL & British Nuclear Fuels, Limited \\
\hline $\mathrm{CCC}$ & canister centerline cooled \\
\hline CUA & Catholic University of America \\
\hline CVS & composition variation study \\
\hline DF & decontamination factor \\
\hline DOE & U.S. Department of Energy \\
\hline DWPF & Defense Waste Processing Facility \\
\hline EA & environmental assessment \\
\hline EC & electrical conductivity \\
\hline EDS & energy dispersive spectroscopy \\
\hline HAW & high-activity waste \\
\hline HLP & Hanford LAW product acceptance \\
\hline HLW & high-level waste \\
\hline HWVP & Hanford Waste Vitrification Plant \\
\hline INEEL & Idaho National Engineering and Environmental Laboratory \\
\hline INTEC & Idaho Nuclear Technology and Engineering Center \\
\hline ISV & in situ vitrification \\
\hline LAW & low-activity waste \\
\hline LLW & low-level waste \\
\hline MCC & Materials Characterization Center \\
\hline MS & melter study \\
\hline NCAW & neutralized current acid waste \\
\hline OM & optical microscopy \\
\hline ORP & Office of River Protection \\
\hline PCT & Product Consistency Test \\
\hline PNNL & Pacific Northwest National Laboratory \\
\hline PQM & partial quadtatic mixture \\
\hline Q & quenched (glasses) \\
\hline QA & quality assurance \\
\hline RCRA & Resource Conservation and Recovery Act \\
\hline RPP & River Protection Project \\
\hline RMSE & root mean squared error \\
\hline RSD & relative standard deviation \\
\hline SBW & sodium-bearing waste \\
\hline SBS & submerged bed scrubber \\
\hline SEM & scanning electron microscopy \\
\hline SG & Savannah River Glass \\
\hline SP & spinel (study) \\
\hline SpV & specific volume \\
\hline SRL & Savannah River Laboratory \\
\hline
\end{tabular}




$\begin{array}{ll}\text { SRS } & \text { Savannah River Site } \\ \text { S/V } & \text { surface area-to-volume ratio } \\ \text { TCLP } & \text { Toxicity Characteristic Leaching Procedure } \\ \text { TEM } & \text { transmission electron microscopy } \\ \text { THERMO } & \text { Thermodynamic Hydration Energy Reaction Model } \\ \text { TRU } & \text { transuranic } \\ \text { TWRS } & \text { Tank Waste Remediation System } \\ \text { VSL } & \text { Vitreous State Laboratory at the Catholic University of America } \\ \text { VFT } & \text { Vogel -Fulcher-Tamman } \\ \text { WAPS } & \text { Waste Acceptance Product Specifications } \\ \text { WESP } & \text { wet electrostatic precipitator } \\ \text { WQR } & \text { Waste Form Qualification Report } \\ \text { WTP } & \text { Hanford Tank Waste Treatment and Immobilization Plant } \\ \text { WV } & \text { West Valley } \\ \text { WVDP } & \text { West Valley Demonstration Project } \\ \text { WVNS } & \text { West Valley Nuclear Services } \\ \text { XRD } & \text { X-ray diffraction }\end{array}$




\section{Acknowledgments}

We acknowledge the U.S. Department of Energy (DOE), Office of Environmental Management for continued support of data collection and $\mathrm{CH} 2 \mathrm{M}$ Hill Hanford Group and Washington River Protection Solutions (WRPS) for model fitting and reporting support. Our thanks also go to Wayne Cosby (Pacific Northwest National Laboratory, PNNL) for formatting and technical editing of the documents, John McCloy (PNNL), David Peeler (Savannah River National Laboratory, SRNL), Albert Kruger (DOE Office of River Protection), Wing Kot (The Catholic University of America, CUA), Rod Gimpel (Hanford Tank Waste Treatment and Immobilization Plant, WTP) for technical review of the document, and Greg Piepel (PNNL) for statistical advice, counseling, and review of the document.

Paul Certa (WRPS) was instrumental in the conduct of this work. He encouraged the work to be performed, set the requirements, consulted on application of the models, and reviewed the models and report.

We would like to thank those who generously supplied data for this effort. In particular, we acknowledge David Peeler, Kevin Fox, Fabienne Raszewski, Carol Jantzen, and Irene Reamer at SRNL; Ian Pegg, Wing Kot, Isabelle Muller, and Keith Matlack at CUA; Vijay Jain at WTP; Albert Kruger at ORP; Brad Scholes and Bruce Staples, formerly of Idaho National Engineering and Environmental Laboratory; and the many past and present researchers at PNNL. 



\section{Table of Contents}

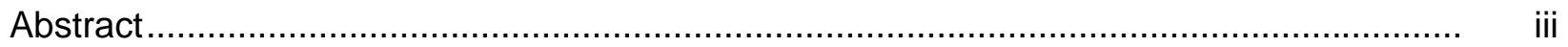

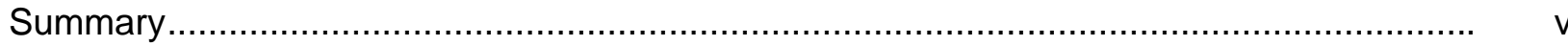

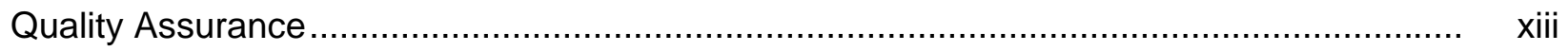

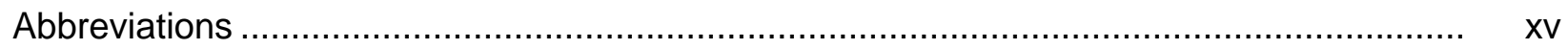

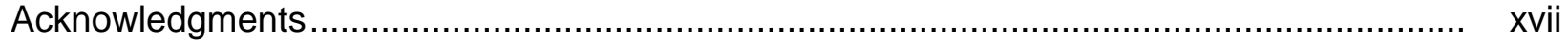

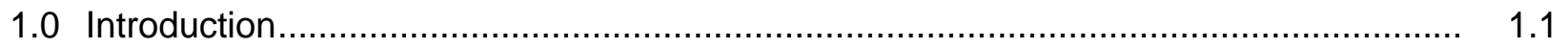

1.1 The Role of Property-Composition Models .................................................... 1.2

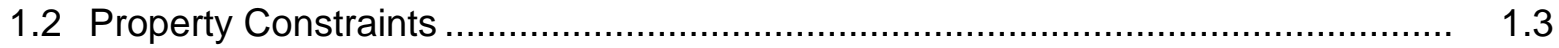

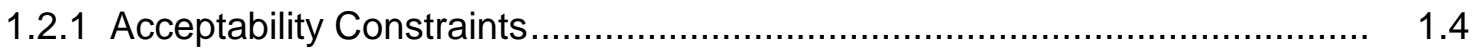

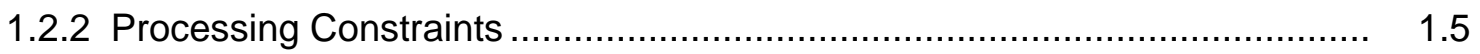

1.2.3 Economic Constraints .................................................................. 1.6

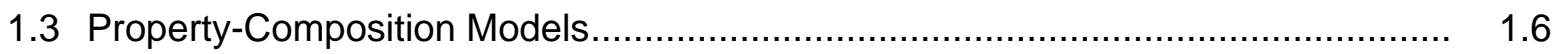

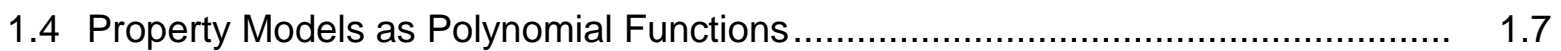

1.5 Property Models in Composition and Temperature ....................................... 1.9

1.6 Model Fit and Validation Statistics ..................................................... 1.10

1.7 Comment on Significant Figures........................................................... 1.11

1.8 Need for Property-Composition Data ..................................................... 1.11

1.9 Existing Property-Composition Models for Hanford HLW Glass ......................... 1.13

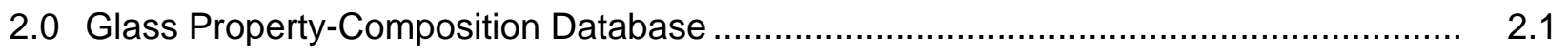

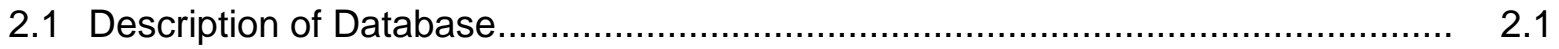

2.2 Data Sets Used in Model Development .................................................. 2.2

3.0 Glass Property-Composition Models ................................................................ 3.1

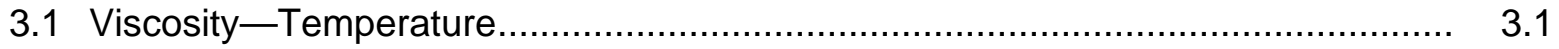

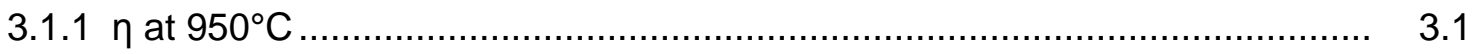

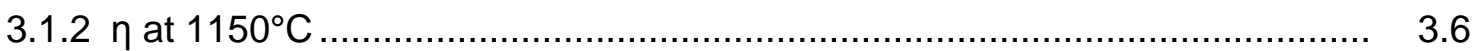

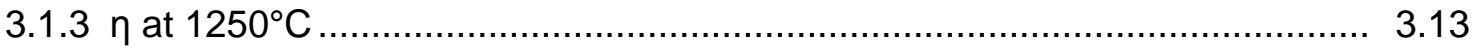

3.1.4 Viscosity as a Function of Temperature ........................................... 3.18

3.2 Electrical Conductivity-Temperature .................................................... 3.21

3.2.1 Initial Data Screening and Evaluation ............................................... 3.21

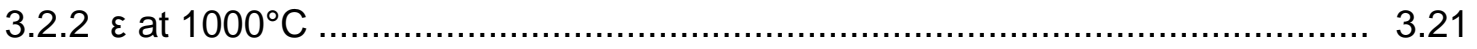

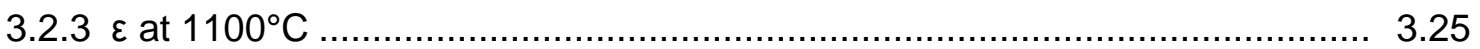

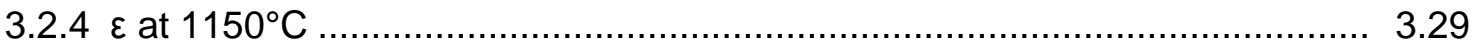

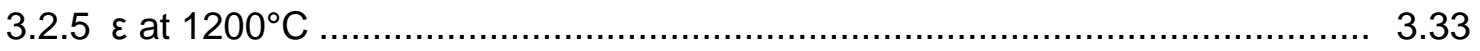

3.2.6 Electrical Conductivity as a Function of Temperature .............................. 3.37

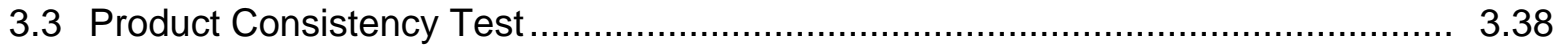

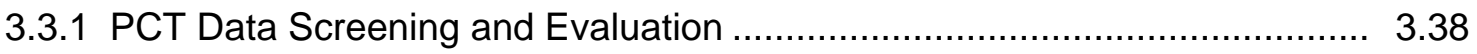




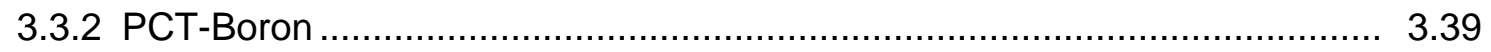

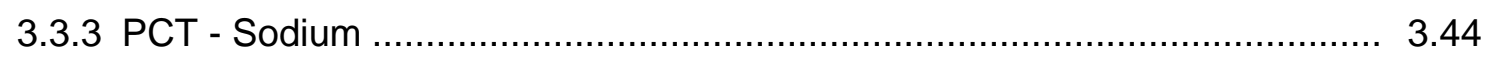

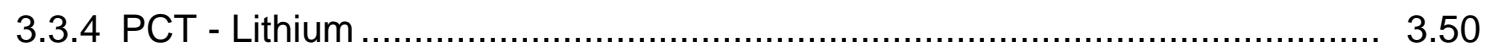

3.4 Toxicity Characteristic Leaching Procedure ................................................... 3.54

3.4.1 Data Conversion, Screening, and Evaluation .......................................... 3.54

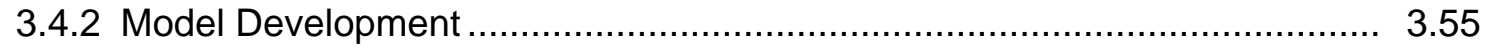

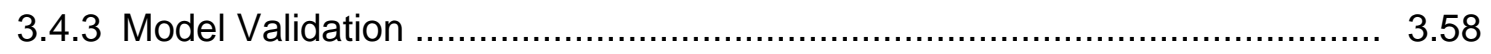

3.4.4 Application of $\operatorname{In}\left[N_{T C L P}, \mathrm{mg} / \mathrm{L}\right]$ Model to Regulatory Compliance .................. 3.59

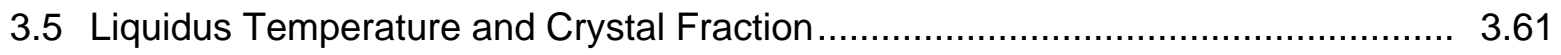

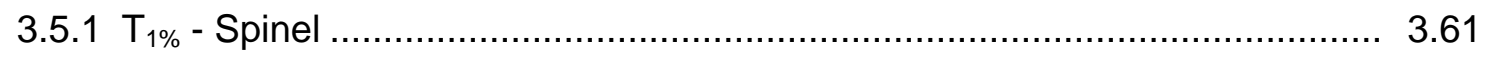

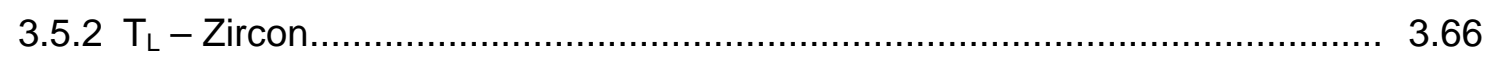

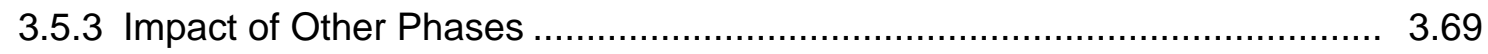

3.6 Glass Density (Specific Volume).................................................................. 3.69

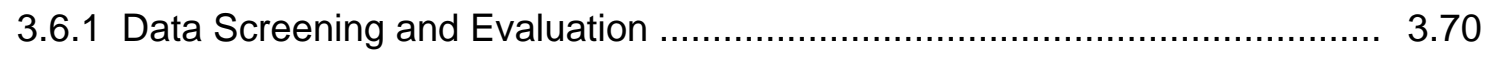

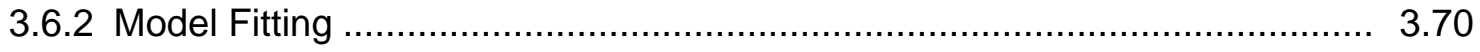

4.0 Glass Composition Constraints ……............................................................ 4.1

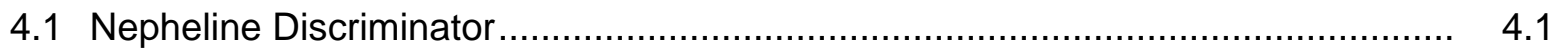

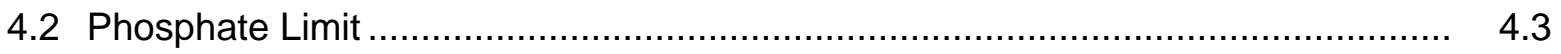

4.3 Chromium Oxide and Alumina Limits........................................................ 4.4

4.4 Sulfate and Salt Separation Limits ............................................................ 4.5

4.5 Other Composition Limits...................................................................... 4.6

5.0 Elemental Losses During Melting ................................................................... 5.1

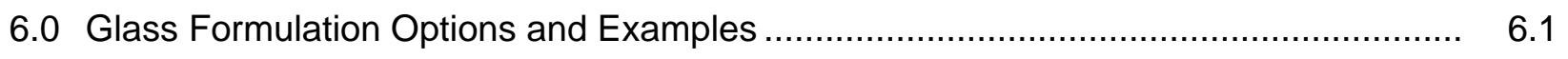

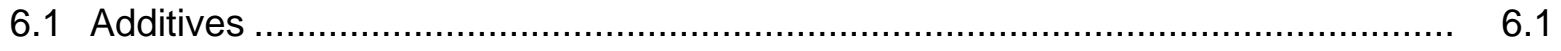

6.2 Property and Composition Constraints …………........................................ 6.2

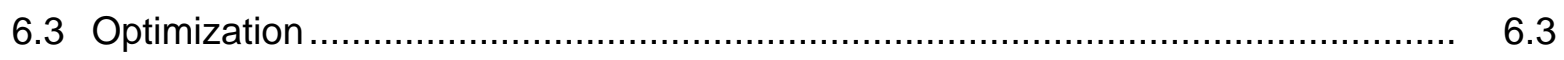

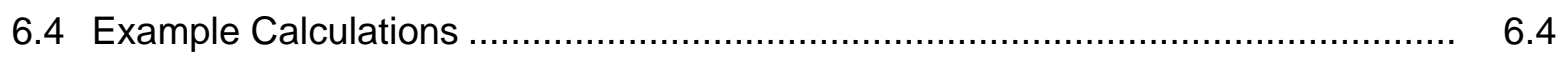

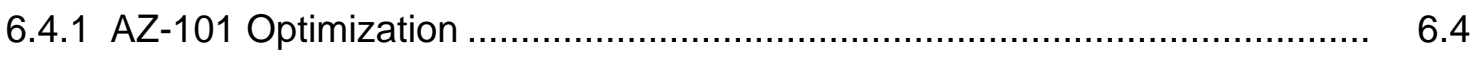

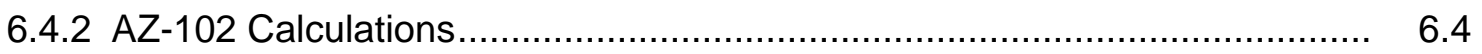

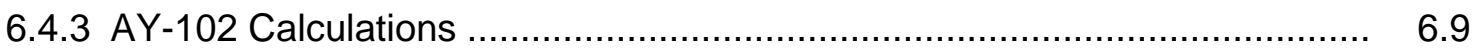

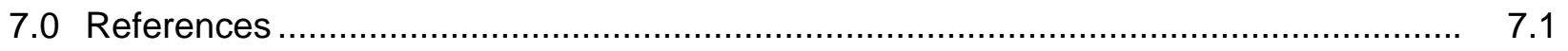

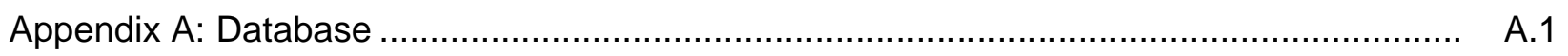

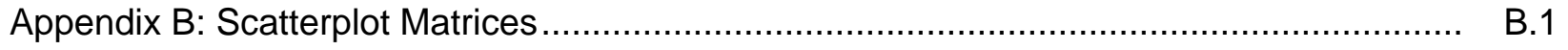

Appendix C: Variance-Covariance Matrices ………….............................................. C.1 


\section{Figures}

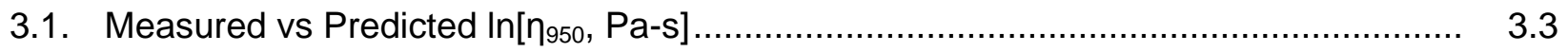

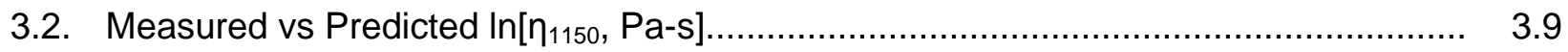

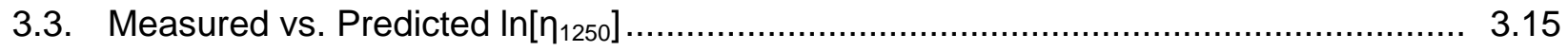

3.4. $\ln \left[\eta_{T}\right]$ Model Coefficient Comparison .......................................................... 3.20

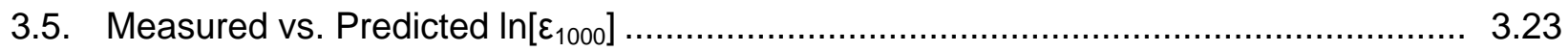

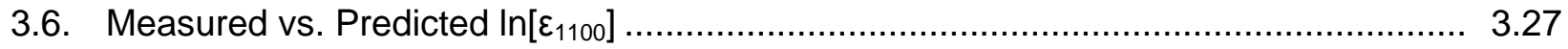

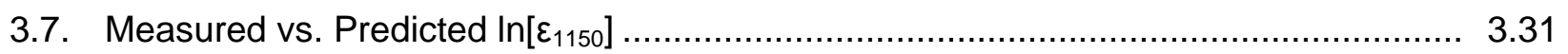

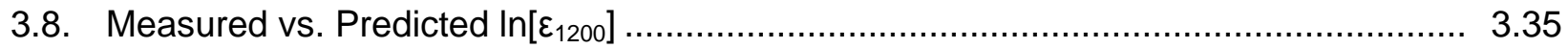

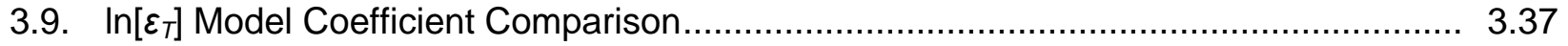

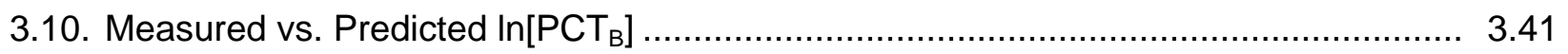

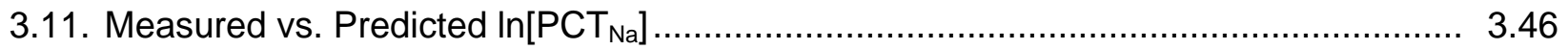

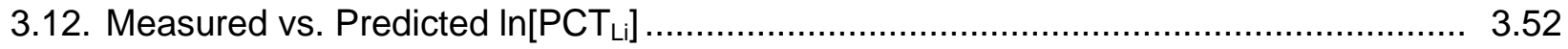

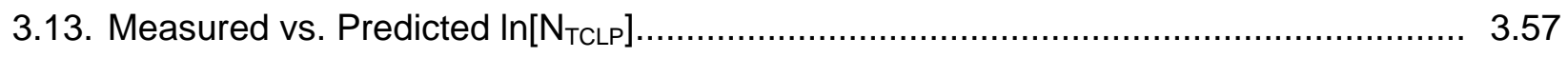

3.14. $\ln \left(r_{i}\right)$ Versus $\ln \left(\mathrm{r}_{\mathrm{B}}\right)$ for (a) Ba, Cd, Ni, and $\mathrm{Zn}$ and (b) $\mathrm{Ag}, \mathrm{As}, \mathrm{Cr}, \mathrm{Pb}, \mathrm{Se}$, and $\mathrm{Tl}$ in the Glasses Studied by Kot and Pegg (2001) from Kim and Vienna (2003) .............. 3.60

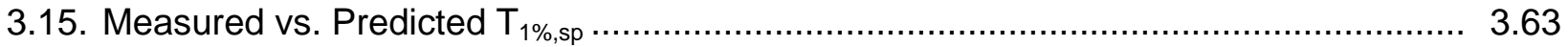

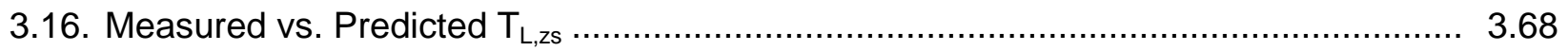

3.17. Predicted vs Measured Glass Density ......................................................... 3.73

4.1. CCC Curves for (a) DWPF HLW Glass (Marra and Jantzen 1993) and (b) WTP HLW Glass

4.2. CCC Glass Nepheline Content and PCT-B Response vs. Normalized Silica Discriminator.

4.3. Summary of WTP Phosphate Phase Separation Data 


\section{Tables}

S.1. Viscosity Model Coefficients and Validity Regions (in $\ln [\mathrm{Pa}-\mathrm{s}]$ and $\mathrm{wt} \%$ ) .................. vi

S.2. Electrical Conductivity Model Coefficients and Validity Regions (in $\ln [S / m]$ and $w t \%$ ). vii

S.3. Product Consistency Test Model Coefficients and Validity Regions (in $\mathrm{In}\left[\mathrm{g} / \mathrm{m}^{2}\right]$ and $w t \%)$

S.4. Toxicity Characteristic Leaching Procedure Model Coefficients and Validity Regions (in $\ln [\mathrm{mg} / \mathrm{L}]$ and $w \mathrm{t} \%$ ).

S.5. One Percent Crystal and Liquidus Temperature Model Coefficients and Validity Regions (in ${ }^{\circ} \mathrm{C}$ and $w t \%$ )

S.6. Specific Volume Model Coefficients and Validity Regions (in $\mathrm{cm}^{3} / \mathrm{g}$ and $\mathrm{wt} \%$ )

1.1. Advantages and Disadvantages of Two Approaches for Exploring a GlassComposition Region of Interest

2.1. Summary of Number of Glasses Tested and Properties Measured in Each Data Set

3.1. $\ln \left[\eta_{950}\right]$ Model Coefficients ....

3.2. Summary of $\ln \left[\eta_{950}\right]$ Model Fit Statistics

3.3. $\ln \left[\eta_{950}\right]$ Model Validity Region (wt\%)

3.4. Correlation Matrix for the $\ln \left[\eta_{950}\right]$ Model Dataset

3.5. Summary of $\ln \left[\eta_{950}\right]$ Model Validation

3.6. Composition Extreme Outliers

3.7. $\ln \left[\eta_{1150}\right]$ Model Coefficients

3.8. Summary of $\ln \left[\eta_{1150}\right]$ Model Fit Statistics ...

3.9. $\ln \left[\eta_{1150}\right]$ Model Data and Validity Regions (wt\%)

3.10. Correlation Matrix for $\ln \left[\eta_{1150}\right]$ Model Data.

3.11. Summary of $\ln \left[\eta_{1150}\right]$ Model Validation....

3.12. $\ln \left[\eta_{1250}\right]$ Model Coefficients

3.13. Summary of $\ln \left[\eta_{1250}\right]$ Model Fit Statistics

3.14. $\ln \left[\eta_{1250}\right]$ Model Validity Region (wt\%).

3.15. Correlation Matrix for the $\ln \left[\eta_{1250}\right]$ Model Dataset

3.16. Summary of $\ln \left[\eta_{1250}\right]$ Model Validation.

3.17. $\ln \left[\eta_{T}\right]$ Model Coefficient Summary ....

3.18. $\ln \left[\varepsilon_{1000}\right]$ Model Coefficients.

3.19. Summary of $\ln \left[\varepsilon_{1000}\right]$ Model Fit Statistics.

3.20. $\ln \left[\varepsilon_{1000}\right]$ Model Validity Region (wt\%)

3.21. Correlation Matrix for the $\ln \left[\varepsilon_{1000}\right]$ Model Dataset 


\section{Tables}

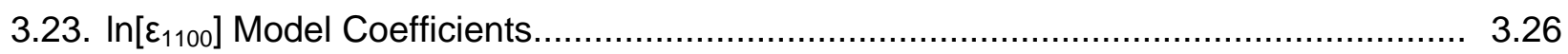

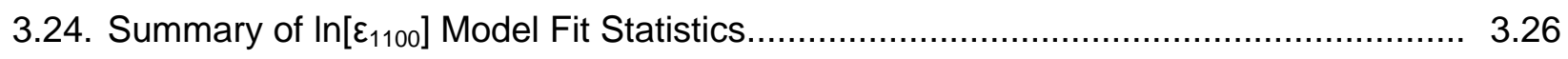

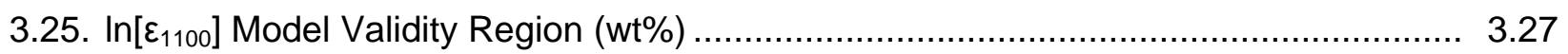

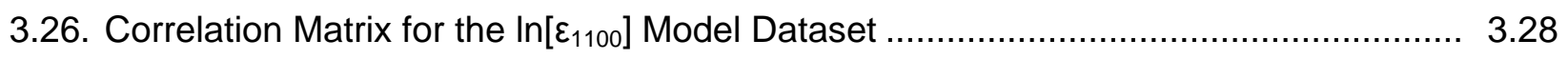

3.27. Summary of $\ln \left[\varepsilon_{1100}\right]$ Model Validation .............................................................. 3.28

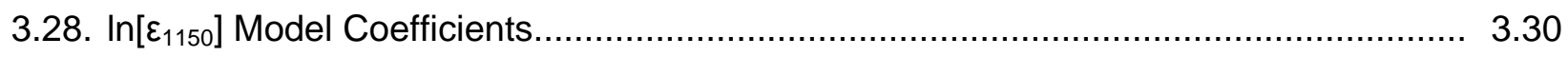

3.29. Summary of $\ln \left[\varepsilon_{1150}\right]$ Model Fit Statistics.......................................................... 3.30

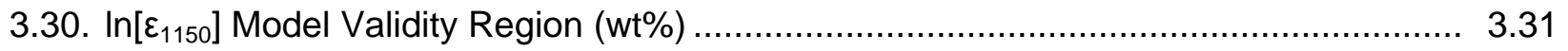

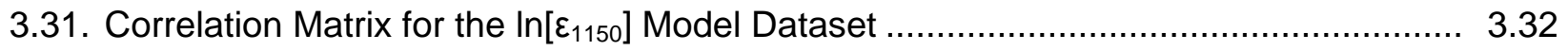

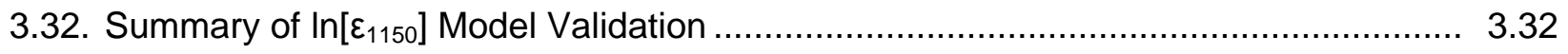

3.33. $\ln \left[\varepsilon_{1200}\right]$ Model Coefficients............................................................................ 3.34

3.34. Summary of $\ln \left[\varepsilon_{1200}\right]$ Model Fit Statistics............................................................... 3.34

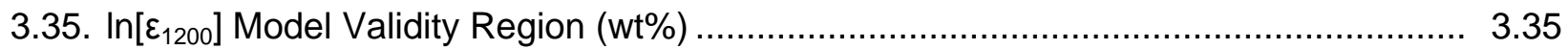

3.36. Correlation Matrix for the $\ln \left[\varepsilon_{1200}\right]$ Model Dataset .................................................. 3.36

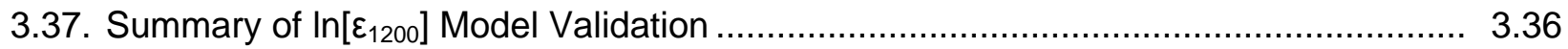

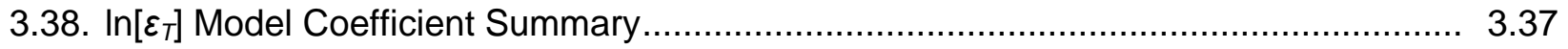

3.39. Summary of Data Screening for $\operatorname{In}[\mathrm{PCT}]$ Model Development ................................. 3.39

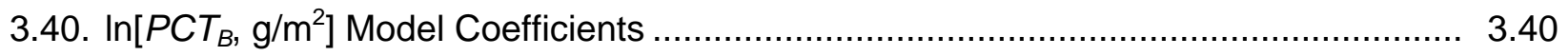

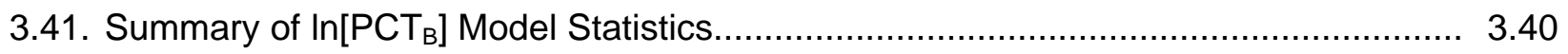

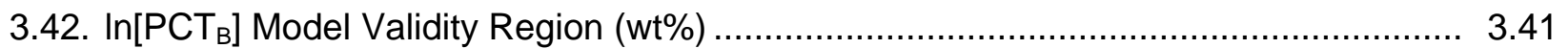

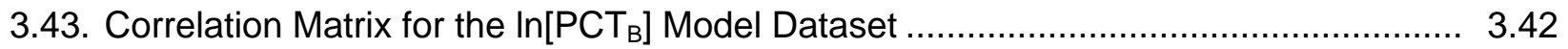

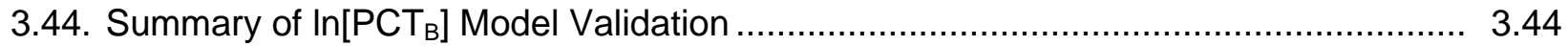

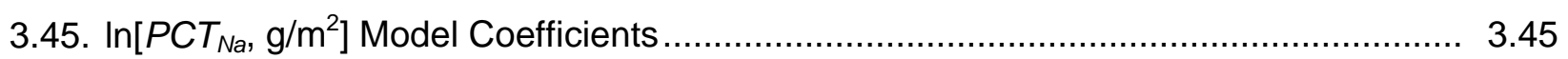

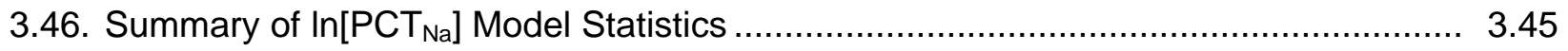

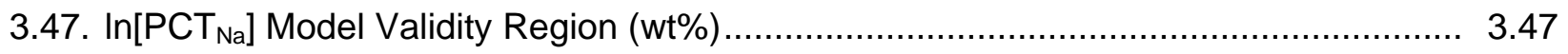

3.48. Correlation Matrix for the $\ln \left[\mathrm{PCT}_{\mathrm{Na}}\right]$ Model Dataset ............................................. 3.48

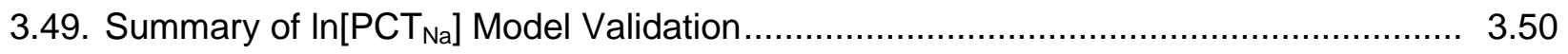

3.50. $\ln \left[P C T_{L i}, \mathrm{~g} / \mathrm{m}^{2}\right]$ Model Coefficients .............................................................. 3.51

3.51. Summary of $\ln \left[\mathrm{PCT}_{\mathrm{Li}}\right]$ Model Statistics ............................................................ 3.51

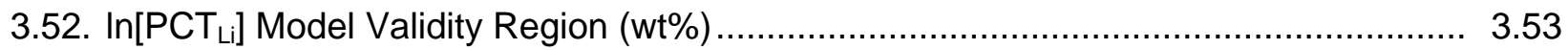

3.53. Correlation Matrix for the $\ln \left[\mathrm{PCT}_{\mathrm{L}]}\right]$ Model Dataset ................................................ 3.53

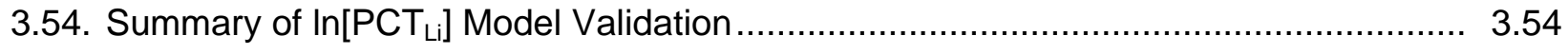

3.55. Summary of Data Screening for In[TCLP] Model ............................................... 3.55

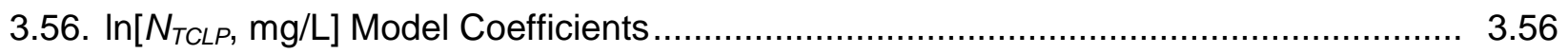




\section{Tables}

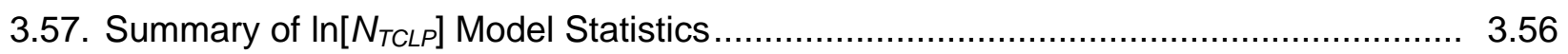

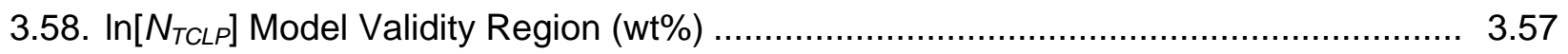

3.59. Correlation Matrix for the $\ln \left[N_{T C L P}\right]$ Model Dataset.............................................. 3.58

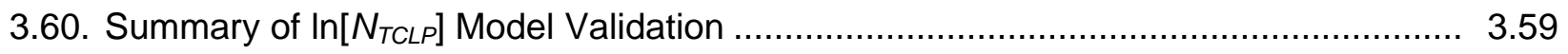

3.61. RCRA Toxicity Limits and WTP Delisting Petition Limits by TCLP (mg/L) ................. 3.61

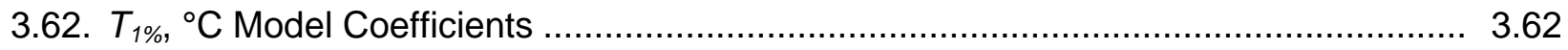

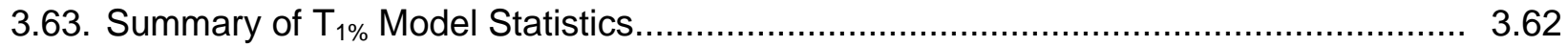

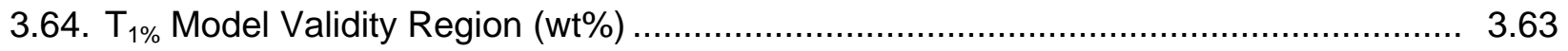

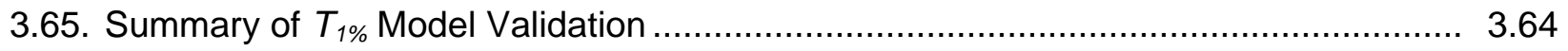

3.66. Correlation Matrix for the $T_{1 \%}$ Model Dataset …................................................. 3.65

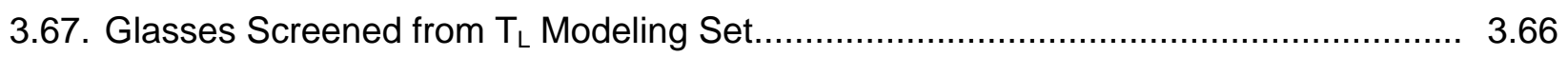

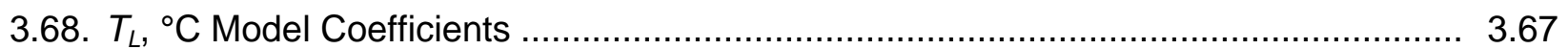

3.69. Summary of $T_{L}$ Model Statistics...................................................................... 3.67

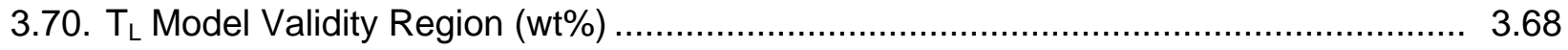

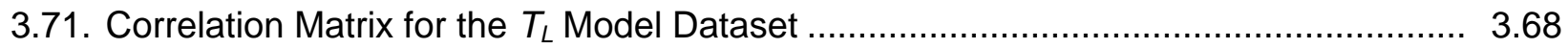

3.72. Summary of $T_{L, z S}$ Model Validation ................................................................... 3.69

3.73. Partial-Specific Volume-Related Values........................................................... 3.71

3.74. $\rho$ and $v$ Model Fit Summary ......................................................................... 3.72

4.1. Summary of $\mathrm{Cr}_{2} \mathrm{O}_{3}$ and $\mathrm{Al}_{2} \mathrm{O}_{3}$ Model Validity Constraints (mass fraction) .................. 4.5

4.2. Summary of $\mathrm{SO}_{3}$ Concentrations in Melter Testing ............................................... 4.6

4.3. Trace Elements of Concern (levels in wt\% of glass) ............................................ 4.7

5.1. Estimated Melter DF and $v$ (from Jain 2008) ..................................................... 5.1

6.1. Additive List for Glass Formulation .................................................................. 6.2

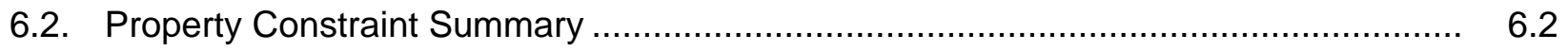

6.3. Property Model Validity Limits (wt\%) ............................................................. 6.3

6.4. Chemical Composition of Pretreated HLW Samples (wt \%) ..................................... 6.5

6.5. Initial AZ-101 Glass Calculation (wt \%) …......................................................... 6.6

6.6. Calculated Glass Properties for Initial Calculation................................................ 6.7

6.7. Second AZ-101 Glass Calculation (wt\%) ……............................................... 6.7

6.8. Calculated Glass Properties for Second Calculation ............................................... 6.8

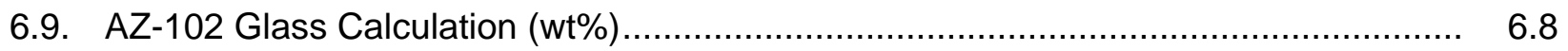

6.10. Calculated Glass Properties for AZ-102 Calculation ............................................ 6.9

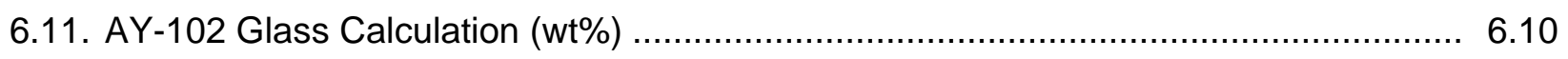

6.12. Calculated Glass Properties for AY-102 Calculation ............................................ 6.11 


\subsection{Introduction}

This report discusses interim property-composition models developed for Hanford highlevel tank waste (HLW) glasses. This effort is aimed at improving the understanding of composition effects on glass properties over the broad composition space of expected Hanford $\mathrm{HLW}$ glasses. With this increased understanding, the ability to more accurately estimate glass volumes, cleanup cost and schedule, and the impacts of changing property constraints and flowsheet options will be possible.

The effort described in this report is an update of that performed by Vienna et al. (2002). The differences between the work performed previously and this effort include the following: 1) the amount of additional data collected since 2002 are substantial, 2) the composition region of HLW glass property data has expanded in several important directions-with the design and construction of the Hanford Tank Waste Treatment and Immobilization Plant (WTP) progressing, the constraints for HLW glasses have evolved, and 4) models developed in 2002 were based on mole fraction of components while the mass fractions used here are easier to implement.

The interim models were developed from a glass property-composition database compiled for this purpose. Because the compiled database covers a large fraction of the region of HLW glass compositions considered potentially of interest for Hanford, the resulting interim property-composition models are considered global models (Piepel et al. 1998). Global models are useful when it is necessary to make property predictions over a wide HLW glasscomposition region. Global models are likely to yield less precise property predictions (compared to local models near specific waste-glass compositions), but still can be useful. Eventually, when target compositions and composition-variation regions around target compositions for specific waste types ${ }^{(a)}$ are better defined, it is advisable to develop local models to predict HLW glass properties over the composition-variation region corresponding to a target composition for a given waste type. Local models, because they cover smaller composition regions centered near the glass composition of interest, are typically more precise (see Piepel et al. 2008a, for example).

The glass property-composition models presented in this report are referred to as interim models for several reasons. The most obvious reason is that property-composition models will continue to be developed as more property-composition data become available and better information about HLW compositions ${ }^{(b)}$ and corresponding waste-glass compositions become available. However, the models should also be considered interim because of limitations in the data used to develop them. Ideally, to provide good support for developing propertycomposition models, a waste-glass-composition region of interest should be defined and covered with property-composition data specifically designed to systematically cover a composition region of interest. The interim models presented in this report were developed from a compilation of existing data. The data were screened so that only relevant data (i.e., data in the appropriate composition region) were used. However, the coverage of the waste-glass-

(a) A waste type is defined in the Waste Acceptance Product Specifications (WAPS) (DOE 1996) as "the waste material fed to each vitrification plant, the composition and properties of which will remain relatively constant over an extended period of time during waste-form production."

(b) It is expected that waste-feed compositions will be updated as tank characterization, retrieval/blending scenarios, and definition of chemical impacts from separations and pretreatment processes are improved. 
composition region(s) of interest was not as extensive as desired. Hence, the quality of the property-composition models discussed in this report may be affected by the limitations in the available data upon which they were based. Further, the time to develop, evaluate, and validate the interim-property models was limited. The available time did not permit the use of many statistical regression diagnostic methods (Draper and Smith 1998, Montgomery and Peck 1992) to identify outlying or influential data points and to identify the subset of waste-glass components that should be included in the interim model for a given property. Statistical modelvalidation methods (Montgomery and Peck 1992, Chapter 10) were not applied. Instead, informal model-validation methods were applied to indicate the prediction uncertainties of these models. In summary, although the interim property-composition models presented in this report have certain limitations, they should be useful for the intended purpose. These models are expected to give improved predictions of glass properties over those previously developed (Vienna et al. 2002) for Hanford HLW waste glasses and over broader composition regions.

The following subsections in this introduction address the role of property-composition models, property constraints implemented through models, the need for property-composition data to develop models, the forms of property-composition models, model fitting and reduction, and previous work to develop property models for Hanford HLW glasses. Section 2.0 presents a property-composition database compiled from previous studies to provide a basis for developing HLW property-composition models. Section 3.0 discusses the interim propertycomposition models developed for Hanford HLW glasses, the data used to develop the models, and the glass-composition regions over which the models are valid for predicting glass properties. Section 4.0 discusses the use of property-composition models and mathematically constrained optimization methods to develop glass formulations and estimate waste-glass volumes.

\subsection{The Role of Property-Composition Models}

The processability and acceptability of a waste glass is specified in terms of its properties. To make waste glass, the waste must be mixed with glass-forming additives in proportions to obtain a target glass composition that must have properties within prescribed limits. HLW glass compositions vary as a result of the changing composition of waste and optimization objectives of the formulation. The economic aspect of vitrification requires that waste-glass composition should reduce the expense. An optimum glass will consider that expense along with other aspects of the glass and process.

It is practically impossible to develop an optimized waste glass for each waste composition on a purely experimental basis. Mathematical, statistical, and optimization methods are extremely useful in developing optimal glass compositions. Therefore, the task of developing optimum glass compositions must be addressed within a mathematical framework (Vienna and Kim 2008). An indispensable element of such a framework is a set of propertycomposition models. These models should be developed for waste glasses covering both the glass composition region of interest and a sufficiently broad range of values for glass properties of interest.

Property-composition models applicable to Hanford waste glasses have been developed at Pacific Northwest National Laboratory (PNNL) for roughly two decades. Initially, only one HLW waste stream was considered that would be processed in a certain type of melter without any attempt for optimization (Chick et al. 1981, Hrma et al. 1994). However, the need for property-composition models broadened over time because the waste-composition estimates 
changed, new waste streams were taken into consideration, new types of melters were tested, and economic considerations became more important. Thus, it became clear that the composition region of waste glass for the development of property-composition models was too narrow, and the ranges of existing property-composition models were not broad enough for practical applications.

The composition region of waste glass is determined by compositions and concentrations of waste and additives. These, in turn, are determined by the range of glass properties that define acceptability for waste-form storage, transport, and disposal; the processing technology related constraints; and the applicable economic considerations. These factors are not fixed; all are frequently changing, and some are interdependent. Estimated waste compositions change with new samples and chemical analyses, pretreatment methods, retrieval strategies, and blending options. The acceptability conditions for HLW glass have been relatively stable for the last decade (DOE 1996), but, the methods of meeting some of those constraints have changed (for example, see Blumenkranz 2006). Opinions vary as to how much crystallinity should be allowed in the glass and whether the glass can include immiscible amorphous phase-separation. Different melter operating strategies require different sets of glass-property values for processability. Economic considerations are often assessed in terms of waste loading in glass and processing rates. Maximizing waste loading and processing rates generally minimizes the costs of producing and disposing of the waste glass.

With each change, whether in:

- the estimated composition of waste in the tanks currently considered for vitrification

- the group of tanks being considered for processing

- the key glass components resulting from pretreatment or

- the melter operating strategy,

the set of property-composition models needs to be updated.

Extrapolation beyond the composition region on which the models are established can lead to misleading results. Hence, models should be updated if new waste glass compositions outside the current database region are considered.

There is another reason for periodic updating of property-composition models. As more glasses are being tested to support various programs, more and more glass-property data accumulate. These data can be used to validate the existing property-composition models and ultimately to update these models. The development of property-composition models must respond to continuous changes. Consequently, developing property-composition models for waste glass is a continuous process.

\subsection{Property Constraints}

Three kinds of constraints on waste-glass properties exist: acceptability constraints, processing constraints, and economic constraints. Each of these kinds of constraints is discussed below. ${ }^{(a)}$

(a) In addition to property constraints, constraints are added to confine property predictions to the range of data over which the models are valid. These are referred to as model validity constraints. 


\subsubsection{Acceptability Constraints}

Acceptability constraints are concerned with the acceptability of the final product. Roughly, waste glass should have sufficiently high chemical durability ${ }^{(a)}$ and should retain this durability over thousands of years. Although the engineered barriers in the repository and the geology of the repository itself are designed and selected to prevent the spreading of radioactive elements into the environment, the glass itself should have good resistance against corrosion by water. The benchmark test for U.S. HLW glass is the product consistency test (PCT) (ASTM 2008). DOE (1996) states:

For acceptance, the mean concentrations of lithium, sodium and boron in the leachate, after normalizing for the concentrations in the glass, shall each be less than those of the benchmark glass described in the Environmental Assessment for selection of the [Defense Waste Processing Facility] DWPF waste form.

As our knowledge of glass behavior increases, glasses are routinely formulated that surpass the environmental assessment (EA) constraint by nearly an order of magnitude. However, to implement this constraint, various uncertainties must be accounted for in 1) PCT measurement $\mathrm{s}, 2$ ) release predictions from models that are functions of glass composition, and 3) glass compositions used in predicting PCT release (see Vienna and Kim 2008).

The waste glass must be shown to not be hazardous under Resource Conservation and Recovery Act (RCRA) regulation. As stated by the U.S. Department of Energy (DOE 2008):

The Producer shall perform the appropriate tests and procedures, as described in 40CFR261.20 through 40CFR261.24 [6], using samples from production runs or prototypical specimens to determine if the waste that will be received by DOE/RW, for transportation and disposal, has hazardous characteristics. Any waste that is shown to have hazardous characteristics shall be treated to remove such characteristics.

As Hanford tank waste is a listed hazardous waste, a delisting action is required. A draft petition for delisting specifies that the toxicity characteristic leaching procedure (TCLP) (EPA 1997) response of glasses will be below a certain limit (Blumenkranz 2006). This requires that a TCLP model be developed.

HLW glass properties may strongly depend on the temperature history of the glass. Slowly cooled glass is prone to amorphous phase separation and crystallization. Crystals are usually durable, which means that they remove components from the glass phase that endow glass with durability. Thus, the crystallization of certain minerals, such as nepheline $\left(\mathrm{NaAlSiO}_{4}\right)$, may produce non-durable glass. Therefore, PCT performance should be studied and modeled for glass that is quickly cooled, such as quenched glass, and glass with the slowest rate of cooling, such as canister-centerline-cooled; see Marra and Jantzen (1993), Kim et al. (1995), and Riley et al. (2001). Although the primary consideration in waste-form acceptance is its performance, the formation of additional phases impacts the ability to predict the performance,

(a) Chemical durability is typically defined as resistance to degradation caused by chemical attack. In the case of waste glasses, chemical durability can be considered the capability of the glass to withstand attack from an aqueous medium without the release of hazardous or radioactive components. 
such as PCT release. This imposes a further restriction on glass composition by increasing the error margin in PCT release for which an account must be given.

\subsubsection{Processing Constraints}

The second type of constraint is added to make sure of adequate processability of glass in the melter. The acceptable ranges for processability-related glass properties differ for different melter types and operating strategies. Generally, glass is processable when its viscosity $(\eta)$ is lower than 8 to $15 \mathrm{~Pa} \cdot \mathrm{s}$ - the lower the viscosity, the higher the rate of processing. However, when $\eta$ is below 1 to $2 \mathrm{~Pa} \cdot \mathrm{s}$, glass becomes more corrosive to those materials that are in contact with the melt (e.g., refractories, electrodes, and bubblers), and steam excursions ${ }^{(a)}$ become more likely. Therefore, between 1 and $2 \mathrm{~Pa} \cdot \mathrm{s}$ is generally considered the lower limit for melt viscosity, although lower viscosities may be acceptable.

An often limiting property of HLW glass is its liquidus temperature $\left(T_{L}\right)$, the highest temperature at which a solid phase can exist in the melt at equilibrium. ${ }^{(b)}$ With increasing waste loading, the $T_{L}$ increases until it reaches and exceeds the melter operating temperature. When this happens, solids can precipitate in the melter. If these solids are not removed from the melter as the glass is poured, they accumulate and can eventually obstruct melter operation. To prevent a premature shutdown of these melters, the $T_{L}$ is historically constrained to be lower than a certain temperature appropriate for the melter. Typically, a $100^{\circ} \mathrm{C}$ or more safety margin is used so that $T_{L} \leq T_{M}-100^{\circ} \mathrm{C}$, where $T_{M}$ is the nominal melter operating temperature. It has long been known that a certain amount of crystals can be tolerated within a melter. However, the exact amount of crystals tolerated by a melter was not defined nor was a technique to control the melter and model crystal accumulation. The WTP has adopted a constraint to limit the equilibrium volume percent of crystals at $950^{\circ} \mathrm{C}$ to less than $1 \%\left(\mathrm{~T}_{1 \%}\right)$ (Vienna and Kim 2008) based on the work of Kot and Pegg (2001). Hrma et al. (2003) proposed a method to control crystal settling in melters; however, there is currently insufficient data to adopt this constraint in the form of mathematical models. More recently, higher crystal contents have been processed successfully, but there are not currently sufficient data to model this behavior.

Another property that has been required to be within certain limits is the electrical conductivity $(\varepsilon)$ of the melt. Because glass is heated by an electric current passing through it, the glass conductivity must be substantially higher than that of the melter walls and sufficiently low to prevent electrode damage or thermal instability. The permitted range of $\varepsilon$ of molten glass is wide, usually 10 to $70 \mathrm{~S} / \mathrm{m}$ (Vienna and Kim 2008). With this wide range, $\varepsilon$ is unlikely to affect the formulation of HLW glasses that meet viscosity, $\mathrm{T}_{1 \%}$, and PCT constraints.

(a) Steam excursions may occur when water from the melter feed is intimately mixed with a hot glass or salt melt. The heat from the melt is quickly transferred to the water, which abruptly transforms the liquid into a steam. This is only likely if the water and hot melt are allowed to quickly mix as might be the case with an exceedingly low-viscosity melt.

(b) Frequently, the presence of melt insolubles from the waste, such as noble metals and their oxides, are ignored. This is consistent with the definition of $T_{L}$ since these components are not considered part of the melt (or thermodynamic system) and therefore are more like the crucible in which the melt is held. However, for practical purposes, the presence of melt insolubles is an important consideration in glass processing. 


\subsubsection{Economic Constraints}

The cost of producing HLW glass depends on the total volume of glass to be produced and the required processing time. By increasing the waste loading of HLW glass (i.e., the fraction of HLW incorporated in the glass), the volume of HLW glass will be reduced, reducing the storage, transportation, and disposal costs. By increasing the waste throughput (waste loading times glass-production rate) the mission life will be reduced if HLW glass production is rate limiting (not currently the best estimate). Thus, the cost will be reduced by decreasing the time required to process the waste. Hence, constraints may be placed on the waste loading of HLW glass to control the cost of producing the glass. For example, it is often desirable to optimize glass composition to have the maximum waste loading while meeting other product acceptability and processability constraints.

Acceptability constraints and processing constraints are typically placed on waste-glass properties, whereas waste-loading (economic) constraints are placed directly on the wasteglass composition itself. For glass development and qualification work, property-composition models provide a way to implement property constraints as functions of glass composition.

\subsection{Property-Composition Models}

Property-composition models are constitutive equations of the form:

$$
p_{\alpha}=\mathrm{f}_{\alpha}(\mathbf{x})
$$

where $p_{\alpha}$ is the $\alpha$-th property, $\mathbf{x}$ is the composition vector, and $f_{\alpha}$ denotes the functional form of the model. The composition is defined as $\mathbf{x}=\left(x_{1}, x_{2}, \ldots, x_{N-1}\right)$, where $x_{i}$ is the $i$-th component mass or mole fraction, and $N$ is the number of components. Only $N-1$ components are independent because the mass and mole fractions must sum to 1 :

$$
\sum_{i=1}^{N} x_{i}=1
$$

Typically, the functional form $f_{\alpha}$ involves parameters or coefficients that are independent of state variables, such as $\mathbf{x}$. Values of these coefficients must be determined by measurement. Models in which all coefficients are estimated from data are called empirical models. Models in which some coefficients are derived from fundamental principles of physics and chemistry, while other coefficients are estimated from data, are called semi-empirical models. Models that are developed from the fundamental laws of physics (e.g., quantum and statistical mechanics), without the use of any experimental data are called first-principle models. First-principle models $^{(a)}$ are not applicable for predicting multi-component waste-glass properties but may be useful in understanding fundamental relationships that guide model development.

(a) A good working definition of first principles was given by Cohen (1994): "In a first principles approach, one uses fundamental quantum physics to obtain energetics and the static and dynamic properties of a system, without fitting any experimental data." 


\subsection{Property Models as Polynomial Functions}

A simple but very useful property-composition model form is given by

$$
p_{\alpha}=\mathrm{f}_{\alpha}\left(\sum_{i=1}^{N} b_{\alpha i} x_{i}\right)
$$

or, more conveniently,

$$
\mathrm{g}_{\alpha}\left(p_{\alpha}\right)=\sum_{i=1}^{N} b_{\alpha i} x_{i}
$$

where $b_{\alpha i}$ is the $i$-th component coefficient for $\alpha$-th property, and $g_{\alpha}$ is the inverse of $f_{\alpha}$. Note that the mathematical transformation, $g_{\alpha}$, could be the identity transformation (i.e., no transformation). Also note that the sum runs from 1 to $N$, and thus not all $x_{i}$ are independent. This form enabled us to write Equation (1.4) without a constant (i.e., an intercept term) because, by Equation (1.2),

$$
X_{N^{\prime}}=1-\sum_{i=1}^{N^{\prime}} X_{i}
$$

where N' represents the number of components in a subset of glass components.

Models of the form (1.4) are often referred to as first-order or linear, mixture models (Cornell 2002) because the functional form is first-order in composition (i.e., linear). If individual components in waste glasses are confined to sufficiently narrow ranges of concentrations, nonlinear functions of composition may be approximated as linear with acceptable error. As components vary over wider ranges, linear functions of composition may not adequately approximate the underlying nonlinear relationship. In such cases, nonlinear approximating functions may be required. In polynomial models (which can be thought of as polynomial expansions of the properties in composition), the second-order model form includes quadratic and cross-products terms, such as:

$$
\mathrm{g}_{\alpha}\left(p_{\alpha}\right)=\sum_{i=1}^{N} b_{\alpha i} x_{i}+\text { Selected }\left\{\sum_{i=1}^{N} b_{\alpha i i}\left(x_{i}\right)^{2}+\sum_{i}^{N-1} \sum_{<j}^{N} b_{\alpha i j} x_{i} x_{j}\right\}
$$

where $b_{\text {oii }}$ is the coefficient for the $i$-th component squared for $\alpha$-th property, and $b_{\text {oij }}$ is the coefficient for the cross-product of the $i$-th and $j$-th components for $\alpha$-th property. Piepel et al. (2002) discuss models of the form (1.6), referred to as partial quadratic mixture (PQM) models.

Glass composition (the $\mathbf{x}$ vector) can be expressed in three basic ways. Glass composition is traditionally expressed as a mixture of single metal oxides such as $\mathrm{SiO}_{2}, \mathrm{Fe}_{2} \mathrm{O}_{3}$, $\mathrm{SO}_{3}, \mathrm{Na}_{2} \mathrm{O}$, etc. and halogens (e.g., F). Multivalent oxides are all represented with the most prevalent oxidation state. Though the oxygen content in the glass is not counted accurately, the composition is uniquely defined and is probably the most suitable for technological and engineering applications, especially when mass fractions are used. Accounting for the true oxidation-reduction states of all components is not practicable because this state depends on 
glass-making conditions such as the selection of raw materials, temperature, and atmosphere during glass making. However, models have been previously developed that account for variations in redox conditions by including separate terms for $\mathrm{FeO}$ and $\mathrm{Fe}_{2} \mathrm{O}_{3}$ and other multivalent oxides (see Hrma et al. 2002, for example).

Glass can be viewed as a mixture of electronegative elements, such as $\mathrm{O}$ and $\mathrm{F}$, and electropositive elements, such as $\mathrm{Si}, \mathrm{B}, \mathrm{Fe}, \mathrm{Na}$, etc. This may be advantageous for semiempirical models that include fundamental properties of ionic species, such as the size or charge of the ions.

Glass composition can be resolved into simple silicates and borates (e.g., see Jantzen 1992). This representation is not unique and can be accomplished in different ways for different properties, dependent on which structural units are associated with different transport properties, hydration reactions, or crystallization behavior. This may be advantageous for modeling the effects of elements that fill multiple structural roles in glass, depending on composition. This method has not been applied to models in this report. However, this method may be useful in developing future models.

Mass fractions of single-metal oxides and halogens were used in developing models in this study. Mole fractions of electropositive elements were used in the previous study (Vienna et al. 2002), and fractions of multi-metal oxides were used by Jantzen et al. (1995) and Jantzen and Brown (2007).

Empirical models have been the most successful models to date in predicting waste glass properties. The disadvantage of an empirical model, such as Equations (1.4) and (1.6), is that a large number of coefficients must be estimated from data. Waste glass can have up to 80 elements, and some of these elements can be in multiple oxidation states. It would be difficult to cover a large composition region with enough compositions to estimate 80 or more coefficients. However, in practice, most glass properties are only significantly affected by "major" glass components (e.g., those appearing at mass fractions greater than 0.005). Hence, the number of components for which $b_{a i}$ coefficients in Equation (1.4) must be estimated is usually much smaller than the total number of glass components.

The problem of estimating a large number of component coefficients in Equation (1.4) can be resolved if the coefficients are related to some basic atomic or thermodynamic characteristic of the components. If such relationships are simple, the number of empirical coefficients can drastically decrease. Suppose that

$$
b_{\alpha i}=n_{\alpha}+m_{\alpha} L_{i}
$$

where $L_{i}$ is some independently known atomic or thermodynamic characteristic of the $i$-th component, and $n_{\alpha}$ and $m_{\alpha}$ are empirical coefficients for $\alpha$-th property. Combining Equations (1.4) and (1.7), we obtain a semi-empirical relationship

$$
\mathrm{g}_{\alpha}\left(p_{\alpha}\right)=n_{\alpha}+m_{\alpha} \sum_{i=1}^{N} L_{i} x_{i}
$$

Because Equation (1.8) has only two empirical coefficients $\left(n_{\alpha}\right.$ and $\left.m_{\alpha}\right)$, it will generally yield less accurate property predictions than the fully empirical Equations (1.4) and (1.6). However, 
Equation (1.8) may yield better results if $b_{\alpha i}$ coefficients are not known or cannot be estimated empirically from data for some influential components. A semi-empirical relationship such as Equation (1.8) is easier to develop and use, and $N$ can be larger than for an empirical relationship represented by Equations (1.4) and (1.6). Comparing empirical and semi-empirical $b_{a i}$ values can identify shortcomings in the assumptions inherent in Equation (1.8); see, for example, Piepel et al. (1996 and 1997).

\subsection{Property Models in Composition and Temperature}

Some glass properties, such as $\eta$ and $\varepsilon$, are functions of temperature $(T)$ as well as composition. For such properties, models are of the form

$$
p_{\alpha}=\mathrm{f}_{\alpha}(\mathbf{x}, T)
$$

For a given waste glass, the temperature dependence of a property, such as viscosity, is often approximated by the Vogel-Fulcher-Tammann (VFT) equation ${ }^{(a)}$

$$
\eta=\exp \left(A+\frac{B}{T-T_{0}}\right)
$$

or, in a narrow temperature interval, by the Arrhenius equation

$$
\eta=\exp \left(E+\frac{F}{T}\right)
$$

where $A, B, E, F$, and $T_{0}$ are temperature-independent coefficients.

In any of these equations, the parameters $A, B, T_{0}, E$, and $F$ can be expressed as functions of composition to also capture the dependence of the property on composition. Expanding the parameters in Equations (1.10) and (1.11) as linear functions of composition (i.e., linear mixture models), yields:

$$
\ln (\eta)=\sum_{i=1}^{N} A_{i} x_{i}+\frac{\sum_{i=1}^{N} B_{i} x_{i}}{T-\sum_{i=1}^{N} T_{0, i} x_{i}}
$$

and

(a) See H. Vogel, Phys. Z. 22, 645-646 (1921), G. S. Fulcher, J. Am. Ceram. Soc. 8, 339-366 (1925), G. S. Fulcher, J. Am. Ceram. Soc. 8, 789-794 (1925), and G. Tammann and W. Hesse, Z. Anorg. Allg. Chem., 156, 245-257 (1926). 


$$
\ln (\eta)=\sum_{i=1}^{N} E_{i} x_{i}+\frac{\sum_{i=1}^{N} F_{i} x_{i}}{T} .
$$

Coefficients $A, B, T_{0}, E$, and $F$ can be treated as composition-dependent properties, and thus they may be reparameterized as in Equations (1.4) or (1.6). The $A$ coefficient of the VFT model is only moderately composition dependent. Hrma (2008) successfully modeled waste glass viscosity assuming $A$ to be a composition-independent variable. When fitting models of the forms (1.12) and (1.13) to data, care must be taken to use the correct regression methods.

Alternatively, the $\ln [\eta]$ (or $\ln [\varepsilon]$ ) can be measured or estimated as three fixed temperatures using Equation (1.10). The $\ln [\eta]$ can be expanded in composition using Equations (1.4) or (1.6). The viscosity (or conductivity) of the melt can then be estimated at any temperature by predicting the viscosities at the three fixed temperatures and interpolating using Equation (1.10) (see Fluegel 2007, for example).

\subsection{Model Fit and Validation Statistics}

The "goodness" of models is at least partially judged by model fit and validation statistics. A good reference for the meaning and application is given by Hrma et al. (1994). $R^{2}$ estimates the fraction of the variability in the property data accounted for by the fitted model. $\mathrm{R}^{2}$-adjusted $\left(R_{A}^{2}\right)$ adjusts $R^{2}$ to the number of terms in the model and the number of data points used to fit the model. It is useful for comparing models fitted with a different number of model terms. $R^{2}$-predicted $\left(R_{P}^{2}\right)$ is the $R^{2}$ where each data point is left out of the fit in evaluating how well the model predicts that data point (one-point-at-a-time). It is useful in estimating the fraction of variability that would be explained in predicting new observations drawn from the same model composition region. $R^{2}$-validation $\left(R_{V}^{2}\right)$ is the $R^{2}$ calculated using a validation data set, one not used to fit the model. In this report, it is taken as the average $R_{V}^{2}$ from five individual $R_{V}^{2}$ values calculated from five separate modeling and validation subsets of the model data. Each modeling and validation subset of data was obtained by taking every fifth data point out of the model fit data set and using them for model validation. The model error or root-meansquare-error (RMSE) is an averaged square root for model-fit data prediction and can be taken as $R M S E$-predicted $\left(R M S E_{P}\right)$ or $R M S E_{V}$ if desired. The equations for these statistics are listed below:

$$
\begin{gathered}
R^{2}=1-\frac{\sum_{i=1}^{n}\left(\hat{y}_{i}-y_{i}\right)^{2}}{\sum_{i=1}^{n}\left(y_{i}-\bar{y}_{i}\right)^{2}} \\
R_{A}^{2}=1-\frac{\sum_{i=1}^{n}\left(\hat{y}_{i}-y_{i}\right)^{2} /(n-N)}{\sum_{i=1}^{n}\left(y_{i}-\bar{y}_{i}\right)^{2} /(n-1)}
\end{gathered}
$$




$$
\begin{gathered}
R_{P}^{2}=1-\frac{\sum_{i=1}^{n}\left(\hat{y}_{(i)}-y_{i}\right)^{2}}{\sum_{i=1}^{n}\left(y_{i}-\bar{y}_{i}\right)^{2}} \\
R M S E=\sqrt{\frac{\sum_{i=1}^{n}\left(\hat{y}_{i}-y_{i}\right)^{2}}{n-N}} \\
R M S E_{V}=\sqrt{\frac{\sum_{i=1}^{n}\left(\hat{y}_{i}-y_{i}\right)^{2}}{n_{V}}}
\end{gathered}
$$

where $\quad n=$ number of data points used to fit the model

$N=$ number of coefficients or terms in the model

$y_{i}=$ the measured property value of the $i$-th data point

$\hat{y}_{i}=$ predicted property value of the $i$-th data point made using the model $\begin{aligned} \hat{y}_{(i)}= & \text { predicted property value of the } i \text {-th data point made using a model fitted to } \\ & \text { all data points except the } i \text {-th }\end{aligned}$

$\bar{y}=$ average (mean) of the measured property values.

\subsection{Comment on Significant Figures}

Throughout this document, a number of model coefficients and other values are reported with a higher number of figures than are significant. Ideally, the appropriate number of figures to report should be evaluated in detail. However, no such evaluation was performed. We therefore suggest using all reported figures in the model coefficients for consistency. The reader has sufficient data within this report to reproduce the calculations and to use only those figures that are deemed significant.

\subsection{Need for Property-Composition Data}

Property coefficients in Equations (1.4) and (1.6) can only be measured or estimated from data. The temperature dependence of a property, such as $\eta$, can be determined by 1) measuring the property at a series of constant temperatures or 2) continuously measuring the property while the temperature is gradually increasing or decreasing. To establish a $\eta-T$ relationship in analytical terms, an empirical model is fitted to measured data. Such a relationship or model ${ }^{(a)}$ is ready for use in applications, for example, in the Navier-Stokes equation for fluid flow (see Fung 1994 for example) with a variable temperature. It can also be

(a) The terms "model" and "relationship" are interchangeable in this report, though their connotations are different in materials science and statistics. 
used for computing viscosity at a given temperature if such a number is needed for understanding a more complex behavior, such as the settling of solid particles.

With empirical models, it is important to be aware of uncertainties, such as the uncertainty of 1) measured data, 2) the empirical model form (i.e., how closely it represents or approximates the true behavior), and 3) model input values (e.g., the composition of the glass or the degree of uniformity of the melter feed). To determine the empirical coefficients in propertycomposition models, a set of property-composition data is needed. Historical data sets can be compiled for this purpose, or a test matrix of compositions can be designed and the properties measured. Unlike temperature, which is expressed by one number, composition is expressed as a vector with $\mathrm{N}-1$ dimensions. The goal in compiling property-composition data to develop property-composition models is to adequately cover the $(N-1)$-dimensional glass-composition region with a manageable number of compositions.

Several approaches can be used to select a test matrix to explore an ( $N$-1)-dimensional composition region. Two common approaches are to 1 ) vary each component one-at-a-time (i.e., adding or removing a single component to or from a baseline composition with the remaining components adjusting for the change while maintaining constant proportions), or replacing one component with another and 2) changing the fractions of several (up to $N-1$ ) components-at-a-time. Table 1.1 summarizes some advantages and disadvantages of these two approaches.

Table 1.1. Advantages and Disadvantages of Two Approaches for Exploring a GlassComposition Region of Interest

\begin{tabular}{|c|c|c|}
\hline \multirow{3}{*}{\begin{tabular}{l}
\multicolumn{1}{c}{ Approach } \\
One-at-a-time \\
variations from \\
baseline \\
composition or \\
replacing one \\
component with \\
another.
\end{tabular}} & Advantages & Disadvantages \\
\hline & $\begin{array}{l}\text { Component effects can be graphically } \\
\text { visualized. }\end{array}$ & $\begin{array}{l}\text { Results may depend on the baseline } \\
\text { composition. }\end{array}$ \\
\hline & $\begin{array}{l}\text { The linearity or nonlinearity of } \\
\text { component effects is immediately } \\
\text { apparent. } \\
\text { Does not require sophisticated software }\end{array}$ & $\begin{array}{l}\text { Does not provide information about } \\
\text { non-linear blending (i.e., "interaction" } \\
\text { effects of the components). }\end{array}$ \\
\hline & $\begin{array}{l}\text { for design or evaluation. } \\
\text { Component replacement is useful if one } \\
\text { component makes up the majority of the } \\
\text { glass. }\end{array}$ & $\begin{array}{l}\text { Inefficient way to cover composition } \\
\text { space and generate data for property- } \\
\text { composition models. }\end{array}$ \\
\hline $\begin{array}{l}\text { Many-at-a-time } \\
\text { variations within } \\
\text { a defined glass } \\
\text { composition } \\
\text { region. }\end{array}$ & $\begin{array}{l}\text { Provides information about nonlinear } \\
\text { blending (i.e., "interaction" effects of the } \\
\text { components). } \\
\text { Provides information about linear effects } \\
\text { of components over the region. } \\
\text { Provides for best coverage of the } \\
\text { composition region. }\end{array}$ & $\begin{array}{l}\text { Requires statistical optimal } \\
\text { experimental design methods and } \\
\text { software to implement. } \\
\text { Requires models to assess whether } \\
\text { components have linear or non-linear } \\
\text { (i.e., curvilinear or interaction blending } \\
\text { effects). }\end{array}$ \\
\hline
\end{tabular}

The large number of components in Hanford HLW glass means that there are seldom enough data to develop highly accurate constitutive equations over the entire glass-composition region in question. Moreover, the dependence of waste-glass properties on glass composition is fundamentally nonlinear, and thus the linear representation in Equations 1.4 or 1.8 has limited validity. There is sufficient knowledge of glass composition effects on composition to rule out the impacts of many minor components. In addition, the "true," but unknown, propertycomposition relationships are generally expected to be smooth or piecewise smooth as functions of composition. For smooth functions of composition, first- and second-order functions 
provide satisfactory approximations over sufficiently small portions of the composition space. Luckily, in many-component HLW glasses, the range of interest for each component is likely to be sufficiently narrow so that a second-order function provides a reasonable approximation to the "true" property-composition relationship.

Generally, the models reported here cover a broad enough of composition space that a small number of non-linear terms were required. The previous effort focused solely on linear terms that resulted in a reduction of the composition region over which the models were valid (Vienna et al. 2002).

\subsection{Existing Property-Composition Models for Hanford HLW Glass}

Historically, simple relations between properties and composition have been developed for commercial glasses for nearly a century. The book by Scholtze (1990) provides a good review of property-composition relationships for simple glasses. A similar approach became a necessity for HLW glass to deal with the large composition region of the wastes and resulting glasses. Before the Hanford experience, a semi-empirical equation, originally developed by Paul for assessing glass durability (Paul 1977), was applied to Savannah River HLW glasses (Jantzen 1992).

The Hanford HLW composition region is not fully known, but reasonable estimates of its boundaries have been made based on HLW sample analyses and process inventories. Several years ago, a glass-composition region for neutralized current acid waste (NCAW) was assessed and represented by more than 100 compositions, for which several properties were measured (Hrma et al. 1994, Piepel et al. 1995). Property-composition models were then developed from these data (Hrma et al. 1994, 1995a, 1995b).

The basic processing properties measured and modeled (Hrma et al. 1995b, Vienna et al. 1996b) were $\eta, \varepsilon$, and $T_{L}$. Other properties were also characterized and modeled: glasstransition temperature, thermal-expansion coefficients of solid and molten glass, and density (Hrma et al. 1994). Considerable attention focused on PCT response (Hrma et al. 1995a, Vienna et al. 1996a, Kot and Pegg 2001). Other studies for Hanford HLW glass have focused attention on TCLP (EPA 1997) to a lesser extent (Vienna et al. 1998, Kot and Pegg 2001, Jantzen et al. 2000). These outcomes of specific tests are, strictly speaking, not properties, but can be treated as such because they are reproducible results of well-defined experimental procedures.

The form of the function $g_{\alpha}$ in Equations (1.4), (1.6), and (1.8) is the natural logarithmic function for $\eta, \varepsilon$, and normalized PCT elemental releases. For other properties, $g_{\alpha}$ is the identity function. The transport properties, $\eta$ and $\varepsilon$, are also functions of temperature, as discussed in Section 1.3. For $\eta$, Hrma et al. (1995a) developed property-composition-temperature models of the form (1.12) and (1.13) as presented in Section 1.3. For $\varepsilon$, they developed models of the form (1.13). Models for $\eta$ and $\varepsilon$ at a constant temperature were also developed. Hrma et al. (1995b) also used a model of the form (1.8) for PCT-normalized elemental releases. A semiempirical model based on the hydration energies of silicate, borate, and oxide components of glass (Jantzen et al. 1995) did not work well for Hanford HLW glass (Piepel et al. 1996, 1997). Feng et al. $(1990,1996)$ developed a semi-empirical model for viscosity and PCT release based on the heat of formation of oxide components in glass. Jantzen (1991) developed a model for $T_{L}$ based on the free energies of formation of three mineral phases. Jantzen (1991) also 
developed a model for viscosity based on a calculation of the number of non-bridging oxygen atoms.

Hrma et al. (2001) developed a database of glass compositions and properties from the literature. This database was used to fit interim glass-property models for use in predicting the properties of projected Hanford HLW glasses. However, several shortcomings of the database used to develop the models were identified: 1) the data developed in support of the DOE Office of River Protection's (ORP's) WTP project were not included in the database because of the timing of data release, 2) there was insufficient coverage of the expected composition regions for many properties, primarily $T_{L}$, and 3 ) several datasets were inputted to the database, but were not verified and tested for consistency with other data, and therefore could not be used in model development. Vienna et al. (2002) updated these models to address some of these concerns. However, additional shortcomings of the work of Vienna et al. (2002) were identified, including the following: 1) a significant amount of additional data have been collected since 2002 , 2) the composition region of interest has significantly expanded, 3) additional properties are needed for HLW glass, including TCLP response and $T_{1 \%}$, and 4) the linear model form was forced, which caused a reduction in validity region. In the study reported here, we take the next logical step in the series of required studies to develop and refine the models for Hanford HLW glass properties. 


\subsection{Glass Property-Composition Database}

This section describes the waste-glass property-composition database that was compiled to support developing property-composition models. Section 2.1 briefly describes the structure and contents of the database. Section 2.2 lists the major sources of data incorporated in the database and used for model development. Appendix A lists the data summarized in this section. The subsets of these data used for each model are described in the modeling sections that follow.

The database certainly does not contain all HLW glass property-composition data, but an attempt was made to include as much data as practical. Some available data sets were not included because of incomplete documentation to confirm the accuracy of the data. More data will be added as necessary after checking for accuracy or when new reports become available after this report is issued to validate and improve property-composition models. As mentioned is Section 1.0, this report is intended to document the most recent step in a continuing process.

\subsection{Description of Database}

The current database was compiled in an Excel spreadsheet with individual glasses in rows and compositions and properties in columns as described below.

Study, Data Source, and Glass ID: Each set of data is distinguished by a study name (i.e., the names for a group or groups of data), and the reference (source) for the data. The same Glass IDs are used as given in the original Data Source where possible.

Glass Composition (mass fraction): Target (-t) and analyzed (-a) compositions are entered in separate columns in alphabetical order for 61 components. The "Other" component is used in the database only when there is no information available to separate "Others" into individual component concentrations, and the sum of oxide components in the target composition did not equal one.

Melting Temperature $\left(T_{M}\right.$ in $\left.{ }^{\circ} \mathrm{C}\right)$ : The melting temperatures are the reported glass-fabrication temperatures where available. When a glass was melted two or three times, only the final melting temperature was entered.

Liquidus Temperature $\left(T_{L}\right.$ in $\left.{ }^{\circ} \mathrm{C}\right)$ : The $T_{L} \mathrm{~s}$ measured by a gradient-furnace method and by a uniform-temperature method are given in separate columns. The primary phase was also recorded in a column when available.

Crystallinity and Homogeneity: The information on the crystalline and amorphous phase separation was given for quenched, canister centerline cooled (CCC), and heat-treated glasses. The results from visual inspection/optical (light) microscopic (OM) observation, electron microscopy (i.e., scanning electron microscopy/electron-dispersive spectroscopy [SEM/EDS] or transmission electron microscopy [TEM]), and X-ray diffraction (XRD) were recorded in separate columns.

Density $\left(\rho\right.$ in $\left.\mathrm{g} / \mathrm{cm}^{3}\right)$ : Density data are available for a limited number of glasses. 
Viscosity ( $n$ inPa.s): The coefficients for VFT and Arrhenius equations; the calculated viscosity at $1150^{\circ} \mathrm{C}$; and the temperatures at 2, 5, and $10 \mathrm{~Pa} \cdot \mathrm{s}$, based on these coefficients, were entered as provided in the references. The $T_{n}$ and $V_{n}(n \equiv$ data point number $=1$ to 14$)$ columns are for the viscosity-at-temperature data.

PCT Normalized Releases $\left(r_{j}\right.$ in $\left.g / m^{2}\right)$ : Normalized ${ }^{(a)}$ elemental releases of $j=\mathrm{B}, \mathrm{Li}, \mathrm{Na}$, and Si and final $\mathrm{pH}$ values from standard 7-day $\mathrm{PCT}$ at $90^{\circ} \mathrm{C}$ and target surface area-to-volume ratio $(\mathrm{S} / \mathrm{V})$ of $2000 \mathrm{~m}^{-1}$ are given for quenched (Q) and CCC glasses. The releases are all normalized to $S / V$ in addition to the element concentration in glass, giving $r_{j}$ values in $\mathrm{g} / \mathrm{m}^{2}$.

TCLP Response (in $\mathrm{mg} / \mathrm{L}$ or $\mathrm{ppm}$ ): The TCLP data are available for a limited number of glasses.

The database compiled in this report contains glasses designed for the vitrification of HLW and low-activity waste (LAW) that were used for the model development. Although many LAW glasses are distinct from HLW compositions, the overlap was sufficient to include LAW glasses and expand the composition regions. The impacts of adding LAW glasses to the model datasets were not quantitatively evaluated.

\subsection{Data Sets Used in Model Development}

This subsection provides a list of studies represented in the database with a table that contains the number of glasses characterized for each property along with the references for the documentation of the data and the unique numbers assigned to the data in the database. Table 2.1 summarizes these studies in terms of the number of glasses tested, their compositions, and properties provided. The figures in parentheses in the "property" column indicate the number of glasses for which the property data are provided.

(a) PCT responses were normalized by dividing the elemental responses to the concentration of those elements in the glass. This gives an indication of the amount of glass in solution if release is congruent. Normalizations were based on target glass compositions when available, and the composition is analyzed when target values are not reported. 
Table 2.1. Summary of Number of Glasses Tested and Properties Measured in Each Data Set ${ }^{(a)}$

\begin{tabular}{|c|c|c|c|c|c|c|c|c|c|c|c|c|c|}
\hline Study & Reference & $\mathbf{t}$ & $\mathbf{a}$ & $\eta$ & $\varepsilon$ & $\mathrm{PCT}_{\mathrm{Q}}$ & $\mathbf{P C T}_{\mathrm{C}}$ & $T_{L}$ & $\rho$ & $\mathrm{C}_{\mathrm{c}}$ & $\mathbf{C}_{\mathrm{T}}$ & TCLP & Dbase \# \\
\hline INEEL $^{(\mathrm{b})}$ CVS Phase 1 & Staples et al. 1999 & 44 & 44 & 36 & - & 44 & - & 28 & & 8 & - & - & $1-44$ \\
\hline INEEL CVS Phase 2 & Staples et al. 2000 & 37 & 37 & 36 & - & 37 & - & 26 & 37 & 15 & - & - & $45-81$ \\
\hline INEEL CVS Phase 3 & Scholes et al. 2000 & 30 & 30 & 30 & - & 30 & - & 24 & 30 & 28 & - & - & $82-111$ \\
\hline Hanford CVS 1 \& 2 & Hrma et al. 1994 & 146 & 92 & 122 & 122 & 146 & 146 & 122 & 122 & 146 & - & - & $112-257$ \\
\hline Hanford CVS 3 & Vienna et al. 1996c & 40 & - & 39 & 39 & 40 & 40 & 31 & - & 38 & - & - & 258-297 \\
\hline$T_{R U^{(c)}}$ Study & Crum et al. 1997 & 44 & - & - & - & - & - & 42 & - & - & - & - & $298-341$ \\
\hline Zr Study & Vienna et al. 1999 & 29 & - & - & - & 28 & - & 29 & - & - & - & - & $342-370$ \\
\hline SP $^{(d)}$ Study & Mika et al. 1997 & 41 & - & - & - & - & - & 41 & - & - & 14 & - & $371-411$ \\
\hline $\mathrm{MS}^{(\mathrm{e})}$ Study a & Hrma 1999 & 13 & - & - & - & - & - & 13 & - & - & 28 & - & $412-424$ \\
\hline MS Study b & Wilson et al. 2002 & 15 & - & - & - & - & - & 15 & - & - & - & - & $425-439$ \\
\hline SG $^{(t)}$ Study & Hrma et al. 1999 & 63 & - & - & - & - & - & 63 & - & - & - & - & $440-502$ \\
\hline $\mathrm{SP} 3, \mathrm{SP} \times 4, \mathrm{Misc}$ & Vienna et al. 2001a & 50 & - & - & - & - & - & 50 & - & - & - & - & $503-552$ \\
\hline DWPF PCT Model & Jantzen et al. 1995 & 177 & 177 & - & - & 177 & - & - & - & - & - & - & $553-729$ \\
\hline Plutonium Vit & Bulkley and Vienna 1997 & 45 & - & 40 & - & 39 & - & - & - & - & - & 28 & $730-774$ \\
\hline Comp vs. Prop Study & Chick et al. 1981 & - & 102 & 102 & - & - & - & - & - & 44 & - & - & $775-876$ \\
\hline WV ${ }^{(g)}$ HLW Form. & Chick et al. 1984 & 103 & 103 & 96 & - & - & - & - & - & 103 & $103^{*}$ & - & $877-979$ \\
\hline HWVP $85^{(\mathrm{h})}$ & Bates 1985 & 1 & - & 1 & - & - & - & - & - & - & - & - & 980 \\
\hline WV Glasses by VSL ${ }^{(i)} \&$ PNL & Johnston et al. ${ }^{(j)}$ & 128 & - & - & - & $128^{*}$ & - & - & - & - & - & - & $981-1108$ \\
\hline WVDP $^{(k)}$ Support & Olson 1993, 1994 & 20 & 20 & 20 & - & 20 & - & - & - & - & - & - & $1109-1128$ \\
\hline WV CVS Glasses PCT & Olson et al. 1994 & - & 44 & - & - & 44 & - & - & - & - & - & - & $1129-1172$ \\
\hline West Valley WQR ${ }^{(I)}$ & Barnes 1995 & 48 & - & 10 & - & 48 & - & - & - & - & - & - & $1173-1220$ \\
\hline
\end{tabular}

(a) $C_{C}$ represents crystallinity after canister centerline cooling; $C_{T}$ represents crystallinity after isothermal heat treatments.

(b) INEEL = Idaho National Engineering and Environmental Laboratory.

(c) TRU = transuranics.

(d) $\mathrm{SP}=$ spinel (study)

(e) MS = melter study.

(f) SG = Savannah River Glass.

(g) $\mathrm{WV}=$ West Valley

(h) HWVP = Hanford Waste Vitrification Plant.

(i) $\mathrm{VSL}=$ Vitreous State Laboratory at the Catholic University of America.

(j) Johnston JW, GF Piepel, and BA Pulsipher. 1990. Evaluation of Empirical Models for Glass Durability. Letter Report Prepared for West Valley Nuclear Services, Pacific Northwest National Laboratory, Richland, WA.

(k) WVDP = West Valley Demonstration Project.

(l) WQR = Waste Form Qualification Report. 
Table 2.1 (contd)

\begin{tabular}{|c|c|c|c|c|c|c|c|c|c|c|c|c|c|}
\hline Study & Reference & $\mathbf{t}$ & $\mathbf{a}$ & $\eta$ & $\varepsilon$ & $\mathrm{PCT}_{\mathrm{Q}}$ & $\mathrm{PCT}_{\mathrm{C}}$ & $\mathbf{T}_{\mathrm{L}}$ & $\rho$ & $\mathrm{C}_{\mathrm{c}}$ & $\mathbf{C}_{\mathrm{T}}$ & TCLP & Dbase \# \\
\hline Canonical Correlation & Oksoy et al. 1994 & 30 & 30 & - & - & 30 & - & - & - & - & - & - & $1221-1250$ \\
\hline TWRS LAW Form. 2 & Ferrara et al. 1998 & 3 & 3 & - & - & - & - & - & - & - & - & 3 & $1302-1304$ \\
\hline TWRS Env D HLW Glass & Crawford et al. 1998 & 1 & 1 & - & - & 1 & - & - & - & - & - & - & 1309 \\
\hline $\mathrm{SRS}^{(\mathrm{C})} \mathrm{M}$ area Mixed Waste & Fu et al. 1997 & 6 & 6 & - & - & - & - & - & - & - & - & 6 & $1310-1315$ \\
\hline Kinetics of Spinel Cryst. & Reynolds and Hrma 1997 & 1 & - & - & - & - & - & 1 & - & - & 1 & - & 1316 \\
\hline INEEL CVS Phase 2a & Edwards et al. 2000 & 18 & - & 6 & - & 18 & - & - & - & - & - & - & $1396-1413$ \\
\hline $\mathrm{SRL}^{(\mathrm{d})} 165$ & Pye 1985 & 3 & - & 3 & - & - & - & - & 3 & - & - & - & $1414-1416$ \\
\hline RPP $^{(e)}$-WTP HLW Form. & Kot and Pegg 2001 & 181 & 140 & 23 & 23 & 26 & & 142 & 20 & & $115^{\star}$ & 140 & $1417-1597$ \\
\hline RPP-WTP LAW Form. & Muller et al. 2001 & 106 & 65 & 62 & 62 & 66 & - & - & 18 & - & $72^{*}$ & 4 & $1598-1703$ \\
\hline DP Glasses for INEEL HLW & Pittman et al. 2001 & 27 & 27 & - & - & 27 & 27 & - & - & 27 & - & - & $1704-1730$ \\
\hline Waste Loading Max & Bailey and Hrma 1995 & 9 & - & - & - & 9 & - & - & - & - & - & - & 2003-2011 \\
\hline Nepheline precip. & Li et al. 1997b & 20 & 0 & - & - & 11 & 20 & - & - & 20 & - & - & $2012-2031$ \\
\hline CUA Data & Peters et al. 1993 & - & 79 & - & - & 55 & - & - & - & - & - & - & 2032-2110 \\
\hline HWVP & Bates 1987 & 9 & 43 & 52 & - & - & - & - & - & - & - & - & 2111-2162 \\
\hline West Valley Studies & Bowen et al. 1985 & - & - & 7 & - & - & - & - & - & - & - & - & 2163-2169 \\
\hline HWVP Comp Vari. Scope & Brouns et al. 1988 & 4 & - & 4 & - & - & - & - & - & - & - & - & $2170-2173$ \\
\hline Comp. Vari. Study & Brouns and Powel 1988 & 24 & 16 & - & - & 24 & - & - & - & - & - & - & 2174-2197 \\
\hline Italian HLW & Cantale et al. 1991 & - & 2 & 1 & - & - & - & - & 2 & - & - & - & 2198-2199 \\
\hline Low Leach Rate Glasses & Chick and Buckwalter 1980 & 9 & - & 5 & - & - & - & - & - & - & - & - & $2200-2208$ \\
\hline Evaluation of HLW Form & Chick et al. 1986 & - & 1 & 1 & - & - & - & - & - & - & - & - & 2209 \\
\hline
\end{tabular}

(a) TWRS = Tank Waste Remediation System.

(b) ISV = in situ vitrification.

(c) SRS = Savannah River Site.

(d) $\mathrm{SRL}=$ Savahhan River Laboratory.

(e) RPP = River Protection Project.

(f) $\mathrm{HLP}=$ Hanford low-activity waste product acceptance

(g) $\mathrm{LLW}=$ low-level waste.

(h) SBW = sodium-bearing waste. 
Table 2.1 (contd)

\begin{tabular}{|c|c|c|c|c|c|c|c|c|c|c|c|c|c|}
\hline Study & Reference & $\mathbf{t}$ & $\mathbf{a}$ & $n$ & $\varepsilon$ & $\mathrm{PCT}_{\mathrm{Q}}$ & $\mathrm{PCT}_{\mathrm{C}}$ & $\mathbf{T}_{\mathrm{L}}$ & $\rho$ & $\mathbf{C}_{\mathrm{c}}$ & $\mathbf{C}_{\mathrm{T}}$ & TCLP & Dbase \# \\
\hline TWRS LAW Formulation & Matlack et al. 1997a & 21 & 16 & 3 & - & 11 & - & - & - & - & - & 10 & $2242-2262$ \\
\hline Savannah River & Plodinec 1978 & 19 & - & 19 & - & - & - & - & - & - & - & - & $2297-2315$ \\
\hline WV Production Glass & Reimus et al. 1988 & 11 & 34 & - & - & 5 & 6 & - & - & 6 & 22 & - & 2316-2349 \\
\hline Pamela Testing & Scheiwer 1986 & 4 & - & 4 & - & - & - & - & - & - & - & - & $2350-2353$ \\
\hline Savannah River Site & Shade 1991 & 2 & - & - & - & 2 & - & - & - & - & - & - & 2354-2355 \\
\hline BNFL HLW98 & Fu and Pegg 1998 & 62 & - & 14 & 13 & 17 & - & - & 6 & - & - & 38 & $2486-2547$ \\
\hline Fernald Glasses & Gimpel 1996 & 7 & 6 & - & 6 & - & - & 6 & 6 & - & - & 6 & $2548-2554$ \\
\hline DWPF Nepheline 1 & Peeler et al. 2005 & 12 & - & - & - & - & 12 & - & - & - & - & - & $2555-2566$ \\
\hline DWPF Nepheline 2 & Peeler et al. 2006 & 28 & - & - & - & - & 28 & - & - & - & - & - & $2567-2594$ \\
\hline DWPF Nepheline 3 & Fox et al. 2006 & 16 & - & - & - & - & 16 & - & - & - & - & - & $2595-2610$ \\
\hline HLW Comparison Study & Kot et al. $2005 a$ & 11 & 7 & 1 & 1 & 7 & 4 & 1 & - & - & 1 & 6 & 2611-2621 \\
\hline HLW06 Spinel Matrix & Kot et al. $2005 b$ & 35 & - & 5 & 5 & 35 & - & - & - & - & 35 & - & $2762-2796$ \\
\hline WTP HLW98 & Piepel et al. $2008 a$ & 45 & 4 & 16 & 15 & 21 & 6 & 11 & 12 & - & - & 16 & 2797-2841 \\
\hline Round Robin Standards & many ${ }^{\text {(a) }}$ & 8 & 9 & 1 & - & 3 & - & 1 & 4 & - & - & - & $2842-2850$ \\
\hline Russian High Alumina Study & Fox et al. 2008 & 93 & - & - & - & 54 & 54 & - & - & 54 & - & - & 2851-2943 \\
\hline $\mathrm{V}$ and $\mathrm{T}$ Series & Kot et al. 2003b & 39 & - & 3 & 3 & 6 & - & 18 & 18 & - & - & 8 & 2944-2982 \\
\hline ORP Enhanced HLW & Matlack et al. 2007 & 91 & - & 4 & 20 & 22 & 2 & - & - & 21 & 47 & 22 & 2983-3073 \\
\hline WTP Actual LAW & many ${ }^{(b)}$ & 9 & 8 & - & - & 2 & 7 & - & 1 & - & - & - & $3074-3082$ \\
\hline WTP LAW CrP & Muller et al. 2006b & 14 & 14 & - & 5 & 7 & 7 & - & - & 14 & - & 4 & 3083-3096 \\
\hline HLW Algorithm Testing & Kot et al. $2005 a$ & 40 & 40 & - & 40 & 40 & 10 & - & - & 10 & 37 & - & $3097-3136$ \\
\hline WTP Phase II HLW Matrix & Kot et al. 2006 & 40 & 40 & - & 40 & 40 & - & - & - & - & 39 & - & $3137-3176$ \\
\hline WTP LAW & many $^{(\mathrm{c})}$ & 154 & 25 & - & 123 & 145 & 3 & - & 2 & - & - & 4 & $3177-3330$ \\
\hline WTP LAW Augmentation & Muller et al. $2006 a$ & 21 & - & - & 10 & 21 & - & - & - & - & - & - & $3331-3351$ \\
\hline WTP LAW Comparison & Muller et al. 2005 & 22 & 17 & - & 2 & 12 & 8 & - & - & - & - & 1 & $3352-3373$ \\
\hline
\end{tabular}

(a) Ebert and Wolf 1999, Edwards et al. 2004, Jantzen et al. 1993, Mellinger and Daniel 1984, Smith 1993, Wald 1985, Wald and Daniel 1986.

(b) Smith et al. 2000, Urie et al. 1999, Brooks et al. 2000, Fiskum et al. 2000, TRPT-W375-00-00017, Urie et al. 2002a, 2002b, 2002c, and Crawford et al. 2000a, 2000b

(c) Muller et al. 2001, Kot et al. 2003a, Muller and Pegg 2003a, 2003b, Muller et al. 2005, Rielley et al. 2004. 


\subsection{Glass Property-Composition Models}

Models were developed for 1$) \eta$ at three different temperatures (which enables the calculation of the VFT coefficients [Section 3.1]), 2) $\varepsilon$ at three temperatures (which enables the calculation of VFT coefficients [Section 3.2]), 3) normalized releases of $\mathrm{B}, \mathrm{Na}$, and Li by PCT (Section 3.3), 4) TCLP (Section 3.4), 5) $T_{L}$ and $T_{1 \%}$ for glasses in the spinel primary phase field, and 6) $T_{L}$ for melts forming zirconium-containing crystals (Section 3.5). For each of these properties, linear or PQM models of glass-component mass fractions were fitted to the available data described in Section 2.0. The subsections begin with a description of initial data evaluation and screening, final model fitting, and model validation.

\subsection{Viscosity-Temperature}

Hrma (2008) convincingly showed that a VFT model with a constant "preexponential" term has several advantages over other methods for modeling temperature and composition effects on waste glass viscosity. However, for the purposes of this application, the viscosity at fixed temperatures, in particular $1150^{\circ} \mathrm{C}$, is to be used in glass formulations using the Hanford Tank Waste Optimization Simulator (HTWOS). Viscosities at other temperatures were available and are likely of use for other applications, so the $\ln [\eta]$ was estimated at three fixed temperatures $\left(950,1150\right.$, and $\left.1250^{\circ} \mathrm{C}\right)$ for each test glass with sufficient data. The $\ln \left[\eta_{\mathrm{T}}\right]$ were then fitted to composition using linear or PQM models with a small number of non-linear terms (not more than five non-linear terms in any case). Equation (1.6) is rewritten as:

$$
\ln \left(\eta_{T}\right)=\sum_{i=1}^{N} b_{\eta_{T} i} x_{i}+\text { Selected }\left\{\sum_{i=1}^{N} b_{\eta_{T} i i}\left(x_{i}\right)^{2}+\sum_{i}^{N-1} \sum_{<}^{N} b_{\eta_{T} i j} x_{i} x_{j}\right\}
$$

where $T$ is $950^{\circ} \mathrm{C}$ (as described in Section 3.1.1), $1150^{\circ} \mathrm{C}$ (as described in Section 3.1.2), and $1250^{\circ} \mathrm{C}$ (as described in Section 3.1.3).

\subsection{1 $\eta$ at $950^{\circ} \mathrm{C}$}

Initial Data Screening and Evaluation

The data listed in Appendix A were first screened before use in viscosity modeling. Of the 3406 glasses in the database, 1500 contained some viscosity data. These glasses were screened according to the following procedure:

1. fit the VFT Equation (1.10) to $\eta-T$ data

2. tabulate minimum $\left(T_{\min }\right)$ and maximum temperatures $\left(T_{\max }\right)$ for data for each glass

3. keep only glasses with $\mathrm{T}_{\min } \leq 950^{\circ} \mathrm{C} \leq \mathrm{T}_{\max }$

4. remove glasses that were found to be "inhomogeneous" or multiphasic

5. substitute the analyzed compositions for target compositions for those glasses with only analyzed compositions

6. remove nine glasses with incomplete chemical compositions 
7. remove three glasses with a high deviation between target and analyzed compositions (9978, IG3-29, LAWPC7)

8. convert multivalent oxides to preferred oxidation states for Hanford HLW glasses $\mathrm{Fe}_{2} \mathrm{O}_{3}$, $\mathrm{As}_{2} \mathrm{O}_{5}, \mathrm{Ce}_{2} \mathrm{O}_{3}, \mathrm{CoO}, \mathrm{MnO}, \mathrm{MoO}_{3}, \mathrm{Pr}_{2} \mathrm{O}_{3}, \mathrm{Re}_{2} \mathrm{O}_{7}, \mathrm{Rh}_{2} \mathrm{O}_{3}, \mathrm{Sb}_{2} \mathrm{O}_{3}, \mathrm{SnO}_{2}, \mathrm{Tl}_{2} \mathrm{O}$, and $\mathrm{UO}_{3}$.

The resulting data set included 687 glasses for $\ln \left[\eta_{950}\right]$ model development. A scatterplot matrix of these data is given in Appendix $B$.

\section{Model Development}

Preliminary model development was performed using stepwise regression methods allowing only first-order terms. Those components with sufficient data to allow for coefficient estimates were included in the stepwise process. ${ }^{\text {(a) }}$ Stepwise regression comparing the coefficient to the value of zero while maintaining the intercept near the average response of $3.75 \ln [\mathrm{Pa} \cdot \mathrm{s}]$ was used to reduce the influential components to:

$\mathrm{SiO}_{2}, \mathrm{Na}_{2} \mathrm{O}, \mathrm{B}_{2} \mathrm{O}_{3}, \mathrm{Al}_{2} \mathrm{O}_{3}, \mathrm{Fe}_{2} \mathrm{O}_{3}, \mathrm{CaO}, \mathrm{Li}_{2} \mathrm{O}, \mathrm{K}_{2} \mathrm{O}, \mathrm{ZrO}_{2}, \mathrm{MgO}, \mathrm{P}_{2} \mathrm{O}_{5}, \mathrm{SrO}, \mathrm{ZnO}$,

$\mathrm{BaO}, \mathrm{MnO}, \mathrm{PbO}, \mathrm{UO}_{3}, \mathrm{~F}$, and $\mathrm{La}_{2} \mathrm{O}_{3}$

To generate a suitable $\mathrm{PbO}$ coefficient $\left(b_{\eta 950, P b 0}\right)$ (e.g., one that was not erratic), 14 glasses with $x_{P b O}>0.0050$ (mass fraction) were removed. This left 673 glasses for model fitting.

Attempts were made to improve the fit of the model data by adding a limited number (up to 5) second-order terms. The terms found to most significantly improve the fit to model data were:

$$
\mathrm{Na}_{2} \mathrm{O} \times \mathrm{B}_{2} \mathrm{O}_{3}, \mathrm{Na}_{2} \mathrm{O} \times \mathrm{Fe}_{2} \mathrm{O}_{3}, \mathrm{~B}_{2} \mathrm{O}_{3} \times \mathrm{B}_{2} \mathrm{O}_{3}, \mathrm{~B}_{2} \mathrm{O}_{3} \times \mathrm{Al}_{2} \mathrm{O}_{3} \text {, and } \mathrm{Li}_{2} \mathrm{O} \times \mathrm{Li}_{2} \mathrm{O}^{(b)}
$$

Table 3.1 lists the coefficients and the standard errors (i.e., deviations) of the coefficients for the final $\ln \left[\eta_{950}\right]$ model. This model was fit to all 673 glasses remaining after glass screening described above. The statistics for the model fit are summarized in Table 3.2. The $R^{2}$ coefficient of $>0.95$ suggests that the data are well represented by the model. The $R^{2}$-adjusted value suggests that there are not too many coefficients, or the data are not over-fit. The $R^{2}$ predicted being so close to the $R^{2}$ value suggests that there are not any very influential data points, and the model is likely to predict similarly for new data as it does for the model development data.

Figure 3.1 compares the predicted and measured $\ln \left[\eta_{950}\right]$ values. The data appear to be well represented by the model with no trends to over or under prediction. No single group of data (e.g., study) was found to be misrepresented by the model.

(a) A number of methods were used to determine if a component had sufficient variation to include in the model development. Chief among them are the visual examination of pairwise concentration plots in the form of a scatterplot matrix. The absolute maximum concentrations of the components along with the number of glasses containing the component over $10 \%$ of the maximum concentration were also considered.

(b) This process for reducing linear mixture models is not the most efficatious and in theory could inappropriately include or exclude components from the reduced linear model. However, this process generally works well in practice. The method discussed by Piepel and Cooley (2008) is more directly appropriate for reducing linear mixture models, but requires special software and is often not necessary. So, it was not implemented in this study. 


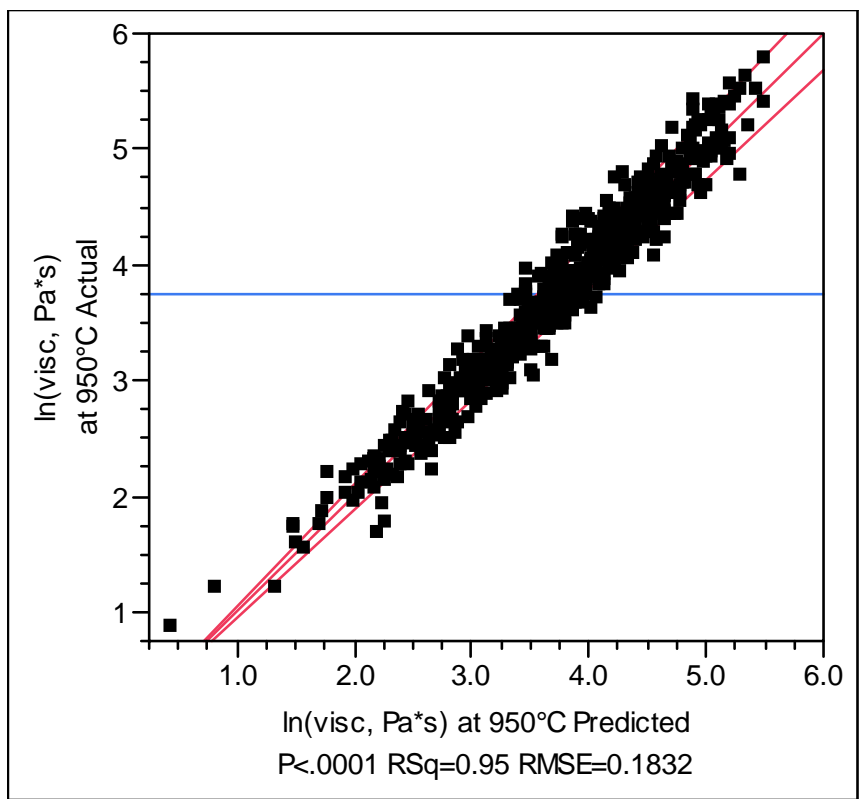

Figure 3.1. Measured vs Predicted $\ln \left[\mathrm{n}_{950}, \mathrm{~Pa}-\mathrm{s}\right]$

Table 3.1. $\ln \left[\eta_{950}\right]$ Model Coefficients

\begin{tabular}{lcc}
\hline Term $(\boldsymbol{i})$ & Coefficient $\left(\boldsymbol{b}_{\boldsymbol{\eta} 950, \boldsymbol{i}}\right)$ & Std Error \\
\hline $\mathrm{Al}_{2} \mathrm{O}_{3}$ & 18.13541298 & 0.576 \\
$\mathrm{~B}_{2} \mathrm{O}_{3}$ & -12.13160541 & 1.31 \\
$\mathrm{BaO}$ & -2.69661986 & 1.24 \\
$\mathrm{CaO}$ & -4.640096602 & 0.279 \\
$\mathrm{~F}$ & -13.74526627 & 1.74 \\
$\mathrm{Fe}_{2} \mathrm{O}_{3}$ & 2.542852069 & 0.474 \\
$\mathrm{~K}_{2} \mathrm{O}$ & -3.46383498 & 0.446 \\
$\mathrm{La}_{2} \mathrm{O}_{3}$ & -1.48036958 & 1.66 \\
$\mathrm{Li}_{2} \mathrm{O}$ & -54.89924359 & 1.27 \\
$\mathrm{MgO}_{\mathrm{MnO}}$ & 0.785950916 & 0.585 \\
$\mathrm{Na}_{2} \mathrm{O}$ & -4.349934915 & 0.677 \\
$\mathrm{P}_{2} \mathrm{O}_{5}$ & -9.523194498 & 0.454 \\
$\mathrm{PbO}_{\mathrm{SiO}}$ & 8.837872224 & 1.11 \\
$\mathrm{SrO}_{2}$ & -22.27753547 & 9.70 \\
$\mathrm{UO}_{3}$ & 13.09016798 & 0.142 \\
$\mathrm{ZnO}_{\mathrm{ZrO}}$ & -3.841881893 & 0.576 \\
$\mathrm{Others}$ & 5.952490873 & 0.868 \\
$\mathrm{Na}_{2} \mathrm{O} \times \mathrm{B}_{2} \mathrm{O}_{3}$ & 0.664542357 & 0.571 \\
$\mathrm{Na}_{2} \mathrm{O} \times \mathrm{Fe}_{2} \mathrm{O}_{3}$ & 15.15119702 & 0.334 \\
$\mathrm{~B}_{2} \mathrm{O}_{3} \times \mathrm{B}_{2} \mathrm{O}_{3}$ & 3.523275465 & 0.465 \\
$\mathrm{~B}_{2} \mathrm{O}_{3} \times \mathrm{Al}_{2} \mathrm{O}_{3}$ & -23.35021548 & 4.00 \\
$\mathrm{Li}_{2} \mathrm{O} \times \mathrm{Li}_{2} \mathrm{O}$ & -9.583283843 & 3.41 \\
\hline & 44.83921231 & 4.95 \\
\hline & -17.94328251 & 4.83 \\
\hline & 140.7392045 & 16.5 \\
\hline
\end{tabular}


Table 3.2. Summary of $\ln \left[\eta_{950}\right]$ Model Fit Statistics

\begin{tabular}{lc}
\hline Statistic & Value \\
\hline$R^{2}$ & 0.9530 \\
$R_{A}^{2}$ & 0.9513 \\
$R_{P}^{2}$ & 0.9491 \\
RMSE & 0.1832 \\
Minimum & 0.8643 \\
Mean & 3.750 \\
Maximum & 5.775 \\
$\mathrm{~N}$ & 673 \\
$\mathrm{~N}$ & 25 \\
\hline
\end{tabular}

The $\ln \left[\eta_{950}\right]$ model is based on data spanning a specific glass composition region and therefore should only be relied upon within that region. The model validity region is defined as the region of data used to develop the model (including property-value ranges) with three exceptions:

1. $\mathrm{Na}_{2} \mathrm{O}$ ranges up to $35.0 \mathrm{wt} \%$, but only three glasses contain more than $25.9 \mathrm{wt} \%$.

2. Others ranges to $10.5 \mathrm{wt} \%$, but only six glasses exceed $7.5 \mathrm{wt} \%$ with a large fraction of the Others being made up of $\mathrm{ThO}_{2}, \mathrm{Bi}_{2} \mathrm{O}_{3}, \mathrm{Nd}_{2} \mathrm{O}_{3}$, and $\mathrm{TiO}_{2}$.

3. Three glasses contain $24 \mathrm{wt} \%$ of $\mathrm{Fe}_{2} \mathrm{O}_{3}$ while the next highest value is $18.4 \mathrm{wt} \%$.

Table 3.3 lists the resulting model validity region. Table 3.4 lists the correlation coefficients calculated for the model data (a matrix of correlation coefficients that summarizes the strength of the linear relationships between each pair of independent variables). Only SrO$\mathrm{MnO}$ have a correlation of 0.5 or above.

Table 3.3. $\operatorname{In}\left[n_{950}\right]$ Model Validity Region (wt\%)

\begin{tabular}{lrr}
\hline \multicolumn{1}{c}{ Component } & \multicolumn{1}{c}{ Min } & \multicolumn{1}{c}{ Max } \\
\hline $\mathrm{Al}_{2} \mathrm{O}_{3}$ & 0.0 & 20.4 \\
$\mathrm{~B}_{2} \mathrm{O}_{3}$ & 0.0 & 20.0 \\
$\mathrm{BaO}$ & 0.0 & 4.7 \\
$\mathrm{CaO}$ & 0.0 & 15.0 \\
$\mathrm{~F}$ & 0.0 & 6.0 \\
$\mathrm{Fe}_{2} \mathrm{O}_{3}$ & 0.0 & 18.4 \\
$\mathrm{~K}_{2} \mathrm{O}$ & 0.0 & 10.0 \\
$\mathrm{La}_{2} \mathrm{O}_{3}$ & 0.0 & 5.0 \\
$\mathrm{Li}_{2} \mathrm{O}$ & 0.0 & 9.0 \\
$\mathrm{MgO}_{\mathrm{MnO}}$ & 0.0 & 8.0 \\
$\mathrm{Na}_{2} \mathrm{O}$ & 0.0 & 8.0 \\
$\mathrm{P}_{2} \mathrm{O}_{5}$ & 2.5 & 25.9 \\
$\mathrm{PbO}_{\mathrm{SiO}}$ & 0.0 & 5.0 \\
$\mathrm{SrO}$ & 0.0 & 0.5 \\
$\mathrm{UO}$ & 32.3 & 60.4 \\
$\mathrm{ZnO}_{\mathrm{ZrO}}$ & 0.0 & 10.3 \\
$\mathrm{Remaining}$ & 0.0 & 6.5 \\
\hline & 0.0 & 5.8 \\
\hline
\end{tabular}


Table 3.4. Correlation Matrix for the $\ln \left[\eta_{950}\right]$ Model Dataset

\begin{tabular}{|c|c|c|c|c|c|c|c|c|c|c|c|c|c|c|c|c|c|c|c|c|}
\hline & $\mathrm{SiO}_{2}$ & $\mathrm{Na}_{2} \mathrm{O}$ & $\mathrm{B}_{2} \mathrm{O}_{3}$ & $\mathrm{Al}_{2} \mathrm{O}_{3}$ & $\mathrm{Fe}_{2} \mathrm{O}_{3}$ & $\mathrm{CaO}$ & $\mathrm{Li}_{2} \mathrm{O}$ & $\mathrm{K}_{2} \mathrm{O}$ & $\mathrm{ZrO}_{2}$ & $\mathrm{MgO}$ & $\mathbf{P}_{2} \mathrm{O}_{5}$ & SrO & Zno & $\mathrm{BaO}$ & MnO & $\mathrm{PbO}$ & $\mathrm{UO}_{3}$ & $F$ & $\mathrm{La}_{\mathbf{2}} \mathrm{O}_{3}$ & Others \\
\hline $\mathrm{SiO}_{2}$ & 1.00 & & & & & & & & & & & & & & & & & & & \\
\hline $\mathrm{Na}_{2} \mathrm{O}$ & -0.31 & 1.00 & & & & & & & & & & & & & & & & & & \\
\hline $\mathrm{B}_{2} \mathrm{O}_{3}$ & -0.06 & -0.28 & 1.00 & & & & & & & & & & & & & & & & & \\
\hline $\mathrm{Al}_{2} \mathrm{O}_{3}$ & -0.22 & 0.08 & 0.01 & 1.00 & & & & & & & & & & & & & & & & \\
\hline $\mathrm{Fe}_{2} \mathrm{O}_{3}$ & -0.24 & -0.04 & -0.11 & -0.33 & 1.00 & & & & & & & & & & & & & & & \\
\hline $\mathrm{CaO}$ & -0.03 & -0.11 & -0.06 & 0.01 & -0.26 & 1.00 & & & & & & & & & & & & & & \\
\hline $\mathrm{Li}_{2} \mathrm{O}$ & 0.36 & -0.67 & 0.06 & 0.06 & -0.09 & -0.11 & 1.00 & & & & & & & & & & & & & \\
\hline $\mathrm{K}_{2} \mathrm{O}$ & -0.23 & 0.11 & -0.05 & 0.17 & -0.07 & -0.01 & -0.19 & 1.00 & & & & & & & & & & & & \\
\hline $\mathrm{ZrO}_{2}$ & -0.16 & -0.06 & -0.03 & -0.27 & -0.24 & -0.07 & 0.13 & -0.04 & 1.00 & & & & & & & & & & & \\
\hline $\mathrm{MgO}$ & 0.16 & -0.10 & 0.01 & -0.10 & -0.22 & 0.20 & 0.00 & -0.14 & -0.09 & 1.00 & & & & & & & & & & \\
\hline $\mathrm{P}_{2} \mathrm{O}_{5}$ & -0.21 & 0.12 & -0.12 & 0.12 & 0.02 & -0.10 & -0.10 & 0.34 & 0.02 & -0.15 & 1.00 & & & & & & & & & \\
\hline $\mathrm{SrO}$ & -0.26 & -0.17 & -0.15 & -0.08 & 0.16 & -0.20 & 0.06 & -0.18 & 0.09 & -0.22 & -0.03 & 1.00 & & & & & & & & \\
\hline $\mathrm{ZnO}$ & -0.11 & 0.00 & -0.10 & -0.11 & -0.06 & 0.31 & -0.28 & -0.12 & 0.02 & 0.31 & -0.17 & -0.06 & 1.00 & & & & & & & \\
\hline $\mathrm{BaO}$ & -0.01 & 0.06 & 0.00 & 0.22 & -0.03 & -0.15 & -0.02 & 0.23 & -0.18 & -0.19 & 0.15 & -0.07 & -0.26 & 1.00 & & & & & & \\
\hline $\mathrm{MnO}$ & -0.21 & -0.15 & -0.06 & -0.20 & 0.34 & -0.36 & 0.06 & -0.18 & 0.05 & -0.27 & -0.03 & 0.56 & -0.10 & 0.01 & 1.00 & & & & & \\
\hline $\mathrm{PbO}$ & -0.15 & -0.03 & -0.14 & -0.07 & 0.37 & -0.33 & 0.01 & -0.16 & 0.01 & -0.30 & -0.02 & 0.37 & -0.03 & 0.10 & 0.49 & 1.00 & & & & \\
\hline $\mathrm{UO}_{3}$ & -0.19 & -0.15 & -0.07 & -0.15 & 0.09 & -0.21 & 0.03 & -0.11 & 0.16 & -0.17 & -0.01 & 0.41 & -0.04 & -0.03 & 0.35 & 0.26 & 1.00 & & & \\
\hline $\mathrm{F}$ & -0.11 & 0.08 & -0.14 & 0.20 & -0.29 & 0.15 & -0.04 & 0.18 & 0.15 & -0.10 & 0.20 & 0.04 & -0.21 & 0.10 & -0.15 & -0.10 & -0.10 & 1.00 & & \\
\hline $\mathrm{La}_{2} \mathrm{O}_{3}$ & -0.05 & -0.10 & -0.03 & 0.01 & -0.12 & -0.01 & 0.17 & -0.17 & 0.21 & -0.08 & -0.02 & 0.21 & -0.25 & -0.07 & 0.01 & 0.00 & 0.03 & 0.39 & 1.00 & \\
\hline Others & -0.12 & -0.15 & -0.14 & -0.33 & 0.18 & -0.17 & 0.04 & -0.12 & 0.04 & 0.05 & -0.04 & 0.19 & 0.00 & -0.14 & 0.19 & 0.11 & 0.38 & -0.10 & 0.14 & 1.00 \\
\hline
\end{tabular}

A variance-covariance matrix is required to calculate the measurement plus prediction uncertainties of this model. This matrix is defined as:

$$
\mathbf{V}_{\alpha}=\operatorname{RMSE}^{2}\left[\mathbf{X}_{\alpha}^{\mathrm{T}} \mathbf{X}_{\alpha}\right]^{-1}
$$

where $\mathbf{V}_{\alpha}$ is the variance-covariance matrix for the $\alpha$-th property model, and $\mathbf{X}_{\alpha}$ is the "model matrix" for the model-fit data set. The model matrix is developed from the model-fit data set, with the columns of the matrix corresponding to the terms in the model. The use of $\mathbf{V}_{\alpha}$ is explained in detail elsewhere (e.g., Cornell 2002, Hrma et al. 1994, Piepel et al. 2008a, Vienna and Kim 2008). The $\mathbf{V}_{\ln [n 950]}$ is given in Appendix $C$ with the other variance-covariance matrices.

\section{Model Validation}

To validate the model, data not used in model fitting must be obtained. Because all appropriate data within the desired composition region were used in model fitting, subsets of the model data were used to validate the model. The data were sorted by $\ln \left[\eta_{950}\right]$ value. The data were then numbered $1,2,3,4,5,1,2, \ldots$ to split them into five roughly representative groups. The model was then refit to subsets 2 to 5 and used to predict data in subset 1 . Then the model was fit to each group of four subsets and used to predict the remaining subset in sequence.

Table 3.5 summarizes the results of the model validation. The coefficients are reasonably close with a relative standard deviation (RSD) of less than $25 \%$ with the exceptions of $\mathrm{ZnO}, \mathrm{BaO}$, $\mathrm{La}_{2} \mathrm{O}_{3}$, and $\mathrm{Na}_{2} \mathrm{O}^{*} \mathrm{Fe}_{2} \mathrm{O}_{3}$. Only the $\mathrm{La}_{2} \mathrm{O}_{3}$ coefficients show $>40 \% \mathrm{RSD}$.

The model fit $R^{2}$ values are all close to each other at approximately 0.95 . The $R_{V}^{2}$ are also close to 0.95 , and their average is almost identical to the $R_{P}^{2}$ value of 0.9491 . This model is well validated and should give predictions of unknown data within the model-validity region nearly as well as for the model-fit data. 
Table 3.5. Summary of $\ln \left[n_{950}\right]$ Model Validation

\begin{tabular}{|c|c|c|c|c|c|c|c|}
\hline Term & All Data & Set 1 & Set 2 & Set 3 & Set 4 & Set 5 & \%RSD \\
\hline $\mathrm{SiO}_{2}$ & 13.090 & 13.127 & 13.073 & 13.127 & 13.055 & 13.078 & 0.3 \\
\hline $\mathrm{Na}_{2} \mathrm{O}$ & -9.523 & -10.114 & -9.202 & -10.114 & -9.519 & -9.331 & -4.5 \\
\hline $\mathrm{B}_{2} \mathrm{O}_{3}$ & -12.132 & -12.137 & -11.854 & -12.137 & -11.850 & -11.767 & -1.5 \\
\hline $\mathrm{Al}_{2} \mathrm{O}_{3}$ & 18.135 & 18.689 & 17.629 & 18.689 & 18.551 & 17.652 & 3.0 \\
\hline $\mathrm{Fe}_{2} \mathrm{O}_{3}$ & 2.543 & 2.066 & 2.950 & 2.066 & 2.352 & 2.641 & 15.8 \\
\hline $\mathrm{CaO}$ & -4.640 & -4.689 & -4.607 & -4.689 & -4.810 & -4.489 & -2.5 \\
\hline $\mathrm{Li}_{2} \mathrm{O}$ & -54.899 & -55.231 & -55.252 & -55.231 & -54.824 & -55.164 & -0.3 \\
\hline $\mathrm{K}_{2} \mathrm{O}$ & -3.464 & -3.478 & -3.233 & -3.478 & -3.614 & -3.323 & -4.3 \\
\hline $\mathrm{ZrO}_{2}$ & 15.151 & 14.838 & 15.054 & 14.838 & 15.159 & 15.526 & 1.9 \\
\hline $\mathrm{MgO}$ & 0.786 & 0.744 & 1.050 & 0.744 & 0.642 & 0.823 & 19.2 \\
\hline $\mathrm{P}_{2} \mathrm{O}_{5}$ & 8.838 & 9.283 & 8.306 & 9.283 & 8.336 & 8.906 & 5.5 \\
\hline SrO & -3.842 & -3.880 & -3.519 & -3.880 & -4.052 & -4.387 & -8.0 \\
\hline $\mathrm{ZnO}$ & 0.665 & 0.811 & 0.210 & 0.811 & 0.805 & 0.693 & 39.0 \\
\hline $\mathrm{BaO}$ & -2.697 & -3.605 & -3.443 & -3.605 & -2.463 & -1.551 & -30.9 \\
\hline MnO & -4.350 & -3.905 & -4.395 & -3.905 & -4.230 & -4.431 & -6.1 \\
\hline $\mathrm{PbO}$ & -22.278 & -25.420 & -19.118 & -25.420 & -28.269 & -16.024 & -22.2 \\
\hline $\mathrm{UO}_{3}$ & 5.952 & 7.201 & 5.164 & 7.201 & 6.154 & 5.155 & 16.5 \\
\hline $\mathrm{F}$ & -13.745 & -12.909 & -13.745 & -12.909 & -15.059 & -12.821 & -7.1 \\
\hline $\mathrm{La}_{2} \mathrm{O}_{3}$ & -1.480 & -1.690 & -1.686 & -1.690 & -0.396 & -1.832 & -41.0 \\
\hline Others & 3.523 & 3.825 & 3.395 & 3.825 & 3.523 & 3.438 & 5.8 \\
\hline $\mathrm{Na}_{2} \mathrm{O} \times \mathrm{B}_{2} \mathrm{O}_{3}$ & -23.350 & -18.956 & -24.704 & -18.956 & -24.082 & -26.663 & -15.5 \\
\hline $\mathrm{Na}_{2} \mathrm{O} \times \mathrm{Fe}_{2} \mathrm{O}_{3}$ & -9.583 & -5.929 & -13.186 & -5.929 & -8.148 & -9.759 & -35.3 \\
\hline $\mathrm{B}_{2} \mathrm{O}_{3} \times \mathrm{B}_{2} \mathrm{O}_{3}$ & 44.839 & 43.660 & 43.230 & 43.660 & 44.516 & 44.111 & 1.1 \\
\hline $\mathrm{B}_{2} \mathrm{O}_{3} \times \mathrm{Al}_{2} \mathrm{O}_{3}$ & -17.943 & -21.398 & -14.193 & -21.398 & -20.661 & -15.864 & -18.3 \\
\hline $\mathrm{Li}_{2} \mathrm{O} \times \mathrm{Li}_{2} \mathrm{O}$ & 140.739 & 145.416 & 146.730 & 145.416 & 142.422 & 141.086 & 1.6 \\
\hline $\mathrm{R}^{2}$ & 0.9530 & 0.9534 & 0.9532 & 0.9534 & 0.9523 & 0.9557 & \\
\hline $\mathrm{R}_{\mathrm{A}}^{2}$ & 0.9513 & 0.9512 & 0.9510 & 0.9512 & 0.9501 & 0.9537 & \\
\hline RMSE & 0.1832 & 0.1824 & 0.1835 & 0.1824 & 0.1862 & 0.1795 & \\
\hline Mean & 3.750 & 3.752 & 3.750 & 3.752 & 3.750 & 3.749 & \\
\hline Obs, $\mathrm{n}$ & 673 & 538 & 538 & 538 & 539 & 539 & \\
\hline $\mathrm{R}^{2} \mathrm{v}$ & 0.9491 & 0.9484 & 0.9498 & 0.9564 & 0.9529 & 0.9379 & \\
\hline
\end{tabular}

\subsection{2 $\eta$ at $1150^{\circ} \mathrm{C}$}

Model development and validation for the $\ln \left[n_{1150}\right]$ follows the same methods described in Section 3.1.1 for $\ln \left[\eta_{950}\right]$ model development.

\section{Initial Data Screening and Evaluation}

The data listed in Appendix A were first screened before use in viscosity modeling. Of the 3406 glasses in the database, 1500 contained some viscosity data. These glasses were screened according to the following procedure:

1. fitting the VFT Equation (1.10) to $\eta-T$ data

2. tabulating minimum $\left(T_{\min }\right)$ and maximum temperatures $\left(T_{\max }\right)$ for data for each glass

3. keeping only glasses with $T_{\min } \leq 1150^{\circ} \mathrm{C} \leq T_{\max }$

4. removing 94 glasses that were found to be "inhomogeneous" or multiphasic

5. substituting analyzed compositions for target compositions for those glasses with only analyzed compositions 
6. removing nine glasses with incomplete chemical compositions

7. removing three glasses with a high deviation between target and analyzed compositions (99-78, IG3-29, LAWPC7)

8. converting multivalent oxides to preferred oxidation states for Hanford HLW glasses $\mathrm{Fe}_{2} \mathrm{O}_{3}$, $\mathrm{As}_{2} \mathrm{O}_{5}, \mathrm{Ce}_{2} \mathrm{O}_{3}, \mathrm{CoO}, \mathrm{MnO}, \mathrm{MoO}_{3}, \mathrm{Pr}_{2} \mathrm{O}_{3}, \mathrm{Re}_{2} \mathrm{O}_{7}, \mathrm{Rh}_{2} \mathrm{O}_{3}, \mathrm{Sb}_{2} \mathrm{O}_{3}, \mathrm{SnO}_{2}, \mathrm{Tl}_{2} \mathrm{O}$, and $\mathrm{UO}_{3}$.

The resulting data set included 1069 glasses for $\ln \left[\eta_{1150}\right]$ model development.

\section{Model Development}

Preliminary model development was performed with stepwise regression methods, allowing only first-order terms. Those components with sufficient data to allow for coefficient estimates were included in the stepwise process:

$\mathrm{SiO}_{2}, \mathrm{Na}_{2} \mathrm{O}, \mathrm{B}_{2} \mathrm{O}_{3}, \mathrm{Al}_{2} \mathrm{O}_{3}, \mathrm{Fe}_{2} \mathrm{O}_{3}, \mathrm{CaO}, \mathrm{Li}_{2} \mathrm{O}, \mathrm{K}_{2} \mathrm{O}, \mathrm{ZrO}_{2}, \mathrm{MgO}, \mathrm{P}_{2} \mathrm{O}_{5}, \mathrm{SrO}, \mathrm{ZnO}$,

$\mathrm{BaO}, \mathrm{TiO}_{2}, \mathrm{NiO}, \mathrm{MnO}, \mathrm{PbO}, \mathrm{ThO}_{2}, \mathrm{UO}_{3}, \mathrm{Nd}_{2} \mathrm{O}_{3}, \mathrm{Bi}_{2} \mathrm{O}_{3}, \mathrm{Ce}_{2} \mathrm{O}_{3}, \mathrm{~F}$, and $\mathrm{La}_{2} \mathrm{O}_{3}$

Outliers were identified during the initial model fitting as summarized in Table 3.6. In addition, 63 glasses containing $x_{P b O}>0.0050$ (mass fraction) were removed because of unrealistic $\mathrm{PbO}$ and "Others" coefficients. This left 991 data points for model fitting.

Stepwise regression comparing the coefficient to the value of zero while maintaining the intercept near the average response of $1.67 \ln [\mathrm{Pa} \cdot \mathrm{s}]$ was used to reduce the influential components to the same set used in the final $\ln \left[\eta_{1150}\right]$ model except $\mathrm{Fe}_{2} \mathrm{O}_{3}$ - $\mathrm{SiO}_{2}, \mathrm{Na}_{2} \mathrm{O}, \mathrm{B}_{2} \mathrm{O}_{3}, \mathrm{Al}_{2} \mathrm{O}_{3}, \mathrm{CaO}, \mathrm{Li}_{2} \mathrm{O}, \mathrm{K}_{2} \mathrm{O}, \mathrm{ZrO}{ }_{2}, \mathrm{MgO}, \mathrm{P}_{2} \mathrm{O}_{5}, \mathrm{SrO}, \mathrm{ZnO}, \mathrm{BaO}, \mathrm{MnO}$, $\mathrm{PbO}, \mathrm{UO}_{3}, \mathrm{~F}$, and $\mathrm{La}_{2} \mathrm{O}_{3}$.

Attempts were made to improve the fit of the model data by adding a limited number (up to 5) second-order terms. The terms found to most significantly improve the fit to model data were:

$$
\mathrm{Na}_{2} \mathrm{O} \times \mathrm{B}_{2} \mathrm{O}_{3}, \mathrm{Na}_{2} \mathrm{O} \times \mathrm{Al}_{2} \mathrm{O}_{3}, \mathrm{~B}_{2} \mathrm{O}_{3} \times \mathrm{B}_{2} \mathrm{O}_{3}, \mathrm{CaO} \times \mathrm{Al}_{2} \mathrm{O}_{3} \text {, and } \mathrm{Li}_{2} \mathrm{O} \times \mathrm{Li}_{2} \mathrm{O}
$$

Through this process, 24 additional glasses were found to be outliers. These all contained extreme concentrations of the second-order terms listed above. The final model was then fit to

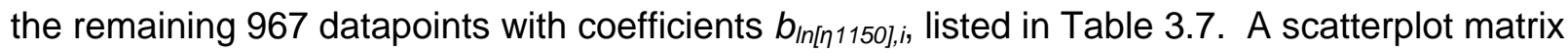
of these data is given in Appendix $B$. 
Table 3.6. Composition Extreme Outliers

\begin{tabular}{ll}
\hline \multicolumn{1}{c}{ Glass ID } & \multicolumn{1}{c}{ Composition Extreme } \\
\hline $\mathrm{HLW}$ 98-96B & glass containing $\mathrm{HfO}_{2}$ \\
SRC-Eu-1 & glass containing $\mathrm{Eu}_{2} \mathrm{O}_{3}$ \\
LAWA46 & glass containing $\mathrm{Ga}_{2} \mathrm{O}_{3}$ above $0.6 \%$ \\
$\mathrm{HLW} 99-15$ & high $\mathrm{NiO}$ concentration with strong influence on model \\
W-Fe/159 & high NiO concentration with strong influence on model \\
W-Fe/151 & high NiO concentration with strong influence on model \\
W-Fe/145 & high NiO concentration with strong influence on model \\
W-Fe/143 & high NiO concentration with strong influence on model \\
W-Fe/156 & high NiO concentration with strong influence on model \\
W-Fe/152 & high NiO concentration with strong influence on model \\
W-Fe/160 & high NiO concentration with strong influence on model \\
W-Fe/158 & high NiO concentration with strong influence on model \\
W-Fe/147 & high NiO concentration with strong influence on model \\
W-Fe/154 & high NiO concentration with strong influence on model \\
W-Fe/146 & high NiO concentration with strong influence on model \\
\hline
\end{tabular}

Table 3.7. $\ln \left[\eta_{1150}\right]$ Model Coefficients

\begin{tabular}{|c|c|c|}
\hline Term (i) & Coefficient $\left(b_{\ln [\eta 1150], i}\right)$ & Std Error \\
\hline $\mathrm{Al}_{2} \mathrm{O}_{3}$ & 10.60850109 & 0.3922 \\
\hline $\mathrm{B}_{2} \mathrm{O}_{3}$ & -9.375292157 & 0.6860 \\
\hline $\mathrm{BaO}$ & -3.418158325 & 0.9650 \\
\hline $\mathrm{CaO}$ & -6.932801478 & 0.3012 \\
\hline $\mathrm{F}$ & -12.34448744 & 1.559 \\
\hline $\mathrm{K}_{2} \mathrm{O}$ & -3.824906136 & 0.3447 \\
\hline $\mathrm{La}_{2} \mathrm{O}_{3}$ & -4.969542588 & 1.227 \\
\hline $\mathrm{Li}_{2} \mathrm{O}$ & -39.02491029 & 0.8721 \\
\hline $\mathrm{MgO}$ & -3.231407331 & 0.4429 \\
\hline $\mathrm{MnO}$ & -6.886774142 & 0.4695 \\
\hline $\mathrm{Na}_{2} \mathrm{O}$ & -9.632750972 & 0.2954 \\
\hline $\mathrm{P}_{2} \mathrm{O}_{5}$ & 5.305006777 & 0.6427 \\
\hline $\mathrm{PbO}$ & -23.14362222 & 6.139 \\
\hline $\mathrm{SiO}_{2}$ & 9.368088941 & 0.07994 \\
\hline $\mathrm{SrO}$ & -4.350515691 & 0.3299 \\
\hline $\mathrm{UO}_{3}$ & 2.151455253 & 0.4839 \\
\hline $\mathrm{ZnO}$ & -2.696255253 & 0.4011 \\
\hline $\mathrm{ZrO}_{2}$ & 7.140440337 & 0.2299 \\
\hline Remaining & -0.090267559 & 0.1220 \\
\hline $\mathrm{Na}_{2} \mathrm{O} \times \mathrm{B}_{2} \mathrm{O}_{3}$ & -26.95708996 & 2.706 \\
\hline $\mathrm{Na}_{2} \mathrm{O} \times \mathrm{Al}_{2} \mathrm{O}_{3}$ & 17.5171818 & 2.625 \\
\hline $\mathrm{B}_{2} \mathrm{O}_{3} \times \mathrm{B}_{2} \mathrm{O}_{3}$ & 24.5926202 & 2.669 \\
\hline $\mathrm{CaO} \times \mathrm{Al}_{2} \mathrm{O}_{3}$ & -8.134744021 & 2.930 \\
\hline $\mathrm{Li}_{2} \mathrm{O} \times \mathrm{Li}_{2} \mathrm{O}$ & 47.35918258 & 12.11 \\
\hline
\end{tabular}

The statistics for the model fit are summarized in Table 3.8. The $R^{2}$ coefficient of $>0.95$ suggests that the data are well represented by the model. The $R^{2}$-adjusted value suggests that there are not too many coefficients, or the data are not over-fit. The $R^{2}$-predicted being so close to the $R^{2}$ value suggests that there are not any very influential data points, and the model is likely to predict similarly for new data as it does for the model development data. 
Figure 3.2 compares the predicted and measured $\ln \left[\eta_{1150}\right]$ values. The data appear to be well represented by the model with no trends to over or under prediction. No single group of data (e.g., study) was found to be misrepresented by the model.

Table 3.8. Summary of $\ln \left[\eta_{1150}\right]$ Model Fit Statistics

\begin{tabular}{lc}
\hline Statistic & Value \\
\hline$R^{2}$ & 0.9619 \\
$R_{A}^{2}$ & 0.9610 \\
$R_{P}^{2}$ & 0.9586 \\
RMSE & 0.1632 \\
Minimum & -0.4588 \\
Mean & 1.673 \\
Maximum & 4.599 \\
$\mathrm{~N}$ & 967 \\
$\mathrm{~N}$ & 24 \\
\hline
\end{tabular}

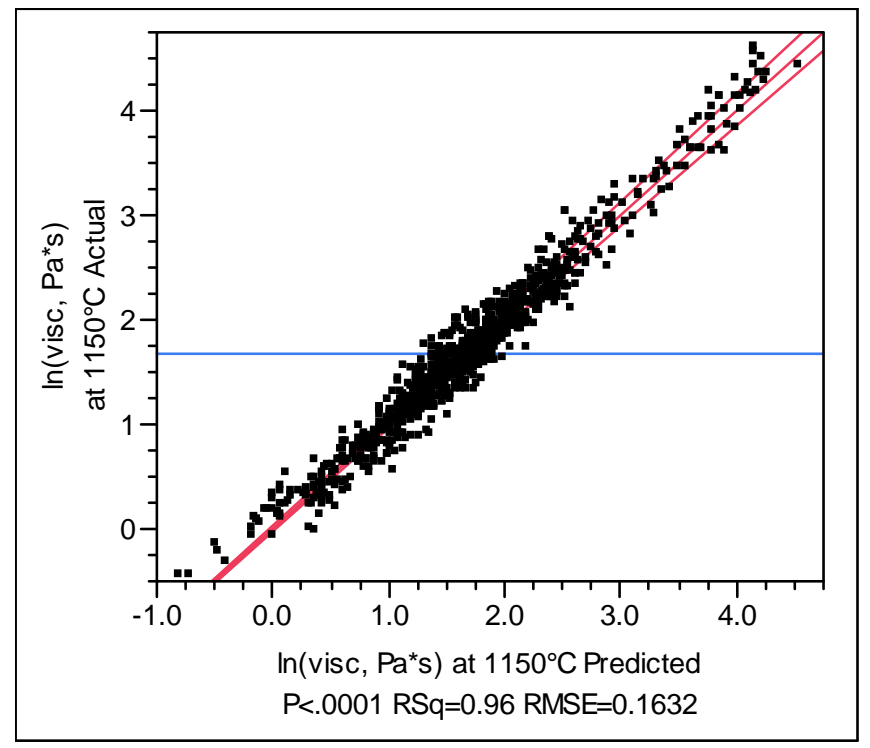

Figure 3.2. Measured vs Predicted $\ln \left[\eta_{1150}\right.$, Pa-s]

This model is based on data spanning a specific glass composition region and therefore should only be relied upon within that region. The validity region is defined as the range of data within the model with 10 exceptions:

1. $\mathrm{Al}_{2} \mathrm{O}_{3}$ ranges to $30.3 \mathrm{wt} \%$ in one glass but up to $26.6 \mathrm{wt} \%$ with continuous data.

2. Three glasses increase the range of $F$ from 2.4 to $4.7 \mathrm{wt} \%$.

3. Only two glasses increase the range of $\mathrm{MnO}$ from 8 to $13.6 \mathrm{wt} \%$.

4. Only four glasses reduce the lower level of $\mathrm{Na}_{2} \mathrm{O}$ from 3.4 to $1 \mathrm{wt} \%$.

5. Seven glasses increase the upper level of $\mathrm{Na}_{2} \mathrm{O}$ from 25.5 to $35.0 \mathrm{wt} \%$.

6. Only three glasses increase the range of $\mathrm{P}_{2} \mathrm{O}_{5}$ from 5 to $6.7 \mathrm{wt} \%$.

7. Four glasses reduce the lower $\mathrm{SiO}_{2}$ level from 25 to $19.4 \mathrm{wt} \%$.

8. Only two glasses increase the range of SrO from 10.3 to $29.9 \mathrm{wt} \%$. 
9. The $\mathrm{UO}_{3}$ upper bound is increased from 7 to $9.6 \mathrm{wt} \%$ by two glasses.

10. Only one glass increases the range of $\mathrm{ZnO}$ from 5.8 to $9.8 \mathrm{wt} \%$.

The Others component ranges from 10.0 to $33.3 \mathrm{wt} \%$, being dominated by $\mathrm{Fe}_{2} \mathrm{O}_{3}$ ( 0 to $24.3 \mathrm{wt} \%$ ), $\mathrm{ThO}_{2}$ (0 to $6.8 \mathrm{wt} \%$ ), $\mathrm{Bi}_{2} \mathrm{O}_{3}$ (0 to $16.4 \mathrm{wt} \%$ ), $\mathrm{Nd}_{2} \mathrm{O}_{3}$ (0 to $8.6 \mathrm{wt} \%$ ), and $\mathrm{TiO}_{2}$ (0 to $10 \mathrm{wt} \%$ ). Only five glasses increase the range of $\mathrm{Fe}_{2} \mathrm{O}_{3}$ from 20 to $24.3 \mathrm{wt} \%$, and two glasses increase the range of $\mathrm{Bi}_{2} \mathrm{O}_{3}$ from 7.4 to $16.4 \mathrm{wt} \%$. Excluding those seven glasses, one high Others glass, and excluding the five major contributors to Others yields an Others component that ranges from 0 to $7.1 \mathrm{wt} \%$. It is recommended that the five major contributors to Others be used as separate components when defining the model validity region.

Table 3.9 lists the model validity range while Table 3.10 lists the correlation coefficients calculated for the model data. Only SrO-MnO have a correlation of 0.5 or above.

Table 3.9. $\ln \left[\eta_{1150}\right]$ Model Data and Validity Regions (wt\%)

\begin{tabular}{|c|c|c|c|c|}
\hline & \multicolumn{2}{|c|}{ Model Data } & \multicolumn{2}{|c|}{ Validity Region } \\
\hline & Min & $\operatorname{Max}$ & Min & $\operatorname{Max}$ \\
\hline $\mathrm{Al}_{2} \mathrm{O}_{3}$ & 0.0 & 30.3 & 0 & 26.6 \\
\hline $\mathrm{B}_{2} \mathrm{O}_{3}$ & 0.0 & 20.2 & 0 & 20.2 \\
\hline $\mathrm{BaO}$ & 0.0 & 4.7 & 0 & 4.7 \\
\hline $\mathrm{Bi}_{2} \mathrm{O}_{3}$ & 0.0 & 16.4 & 0 & 7.4 \\
\hline $\mathrm{CaO}$ & 0.0 & 18.2 & 0 & 18.2 \\
\hline $\mathrm{F}$ & 0.0 & 4.7 & 0 & 2.4 \\
\hline $\mathrm{Fe}_{2} \mathrm{O}_{3}$ & 0.0 & 24.3 & 0 & 20 \\
\hline $\mathrm{K}_{2} \mathrm{O}$ & 0.0 & 10.0 & 0 & 10 \\
\hline $\mathrm{La}_{2} \mathrm{O}_{3}$ & 0.0 & 5.0 & 0 & 5 \\
\hline $\mathrm{Li}_{2} \mathrm{O}$ & 0.0 & 8.9 & 0 & 8.9 \\
\hline $\mathrm{MgO}$ & 0.0 & 8.2 & 0 & 8.2 \\
\hline $\mathrm{MnO}$ & 0.0 & 13.6 & 0 & 8 \\
\hline $\mathrm{Na}_{2} \mathrm{O}$ & 1.0 & 35.0 & 3.4 & 25.5 \\
\hline $\mathrm{Nd}_{2} \mathrm{O}_{3}$ & 0.0 & 8.6 & 0 & 8.6 \\
\hline $\mathrm{P}_{2} \mathrm{O}_{5}$ & 0.0 & 6.7 & 0 & 5 \\
\hline $\mathrm{SiO}_{2}$ & 19.4 & 62.8 & 25 & 62.8 \\
\hline $\mathrm{SrO}$ & 0.0 & 29.9 & 0 & 10.3 \\
\hline $\mathrm{ThO}_{2}$ & 0.0 & 6.8 & 0 & 6.8 \\
\hline $\mathrm{TiO}_{2}$ & 0.0 & 10.0 & 0 & 10 \\
\hline $\mathrm{UO}_{3}$ & 0.0 & 9.6 & 0 & 7 \\
\hline $\mathrm{ZnO}$ & 0.0 & 9.8 & 0 & 5.8 \\
\hline $\mathrm{ZrO}_{2}$ & 0.0 & 15.5 & 0 & 15.5 \\
\hline Remainer & 0.0 & 12.0 & 0 & 7.1 \\
\hline
\end{tabular}

The $\mathbf{V}_{\operatorname{In}[n 1150]}$ is given in Appendix $\mathbf{C}$ with the other variance-covariance matrices. 
Table 3.10. Correlation Matrix for $\ln \left[\eta_{1150}\right]$ Model Data

\begin{tabular}{|c|c|c|c|c|c|c|c|c|c|c|c|c|c|c|c|c|c|c|c|}
\hline $\ln \left[\mathrm{n}_{1150}\right]$ & $\mathrm{SiO}_{2}$ & $\mathrm{Na}_{2} \mathrm{O}$ & $\mathrm{B}_{2} \mathrm{O}_{3}$ & $\mathrm{Al}_{2} \mathrm{O}_{3}$ & $\mathrm{CaO}$ & $\mathrm{Li}_{2} \mathrm{O}$ & $\mathrm{K}_{2} \mathrm{O}$ & $\mathrm{ZrO}_{2}$ & $\mathrm{MgO}$ & $\mathrm{P}_{2} \mathrm{O}_{5}$ & SrO & $\mathrm{ZnO}$ & $\mathrm{BaO}$ & $\mathrm{MnO}$ & $\mathrm{PbO}$ & $\mathrm{UO}_{3}$ & $F$ & $\mathrm{La}_{2} \mathrm{O}_{3}$ & Others \\
\hline $\mathrm{SiO}_{2}$ & 1.00 & & & & & & & & & & & & & & & & & & \\
\hline $\mathrm{Na}_{2} \mathrm{O}$ & -0.07 & 1.00 & & & & & & & & & & & & & & & & & \\
\hline $\mathrm{B}_{2} \mathrm{O}_{3}$ & -0.23 & -0.34 & 1.00 & & & & & & & & & & & & & & & & \\
\hline $\mathrm{Al}_{2} \mathrm{O}_{3}$ & -0.35 & 0.10 & 0.10 & 1.00 & & & & & & & & & & & & & & & \\
\hline $\mathrm{CaO}$ & -0.02 & -0.03 & -0.10 & 0.11 & 1.00 & & & & & & & & & & & & & & \\
\hline $\mathrm{Li}_{2} \mathrm{O}$ & 0.15 & -0.69 & 0.17 & 0.02 & -0.13 & 1.00 & & & & & & & & & & & & & \\
\hline $\mathrm{K}_{2} \mathrm{O}$ & -0.20 & 0.06 & 0.05 & 0.04 & -0.05 & -0.14 & 1.00 & & & & & & & & & & & & \\
\hline $\mathrm{ZrO}_{2}$ & -0.10 & -0.12 & -0.04 & -0.27 & -0.08 & 0.14 & -0.02 & 1.00 & & & & & & & & & & & \\
\hline $\mathrm{MgO}$ & 0.12 & -0.14 & 0.10 & -0.13 & 0.15 & 0.01 & -0.07 & -0.05 & 1.00 & & & & & & & & & & \\
\hline $\mathrm{P}_{2} \mathrm{O}_{5}$ & -0.26 & 0.06 & -0.03 & 0.04 & -0.06 & -0.15 & 0.31 & -0.09 & -0.10 & 1.00 & & & & & & & & & \\
\hline SrO & -0.24 & -0.20 & -0.07 & -0.06 & -0.19 & 0.10 & -0.18 & 0.13 & -0.18 & -0.10 & 1.00 & & & & & & & & \\
\hline $\mathrm{ZnO}$ & -0.06 & -0.03 & 0.00 & -0.14 & 0.19 & -0.11 & -0.09 & 0.11 & 0.30 & -0.24 & -0.02 & 1.00 & & & & & & & \\
\hline $\mathrm{BaO}$ & -0.10 & 0.00 & 0.05 & 0.10 & -0.12 & -0.01 & 0.19 & -0.13 & -0.15 & 0.12 & -0.01 & -0.20 & 1.00 & & & & & & \\
\hline $\mathrm{MnO}$ & -0.20 & -0.18 & -0.07 & -0.14 & -0.35 & 0.11 & -0.17 & 0.05 & -0.24 & -0.07 & 0.57 & -0.10 & 0.02 & 1.00 & & & & & \\
\hline $\mathrm{PbO}$ & -0.35 & -0.10 & 0.01 & 0.11 & -0.17 & 0.04 & -0.15 & 0.01 & -0.24 & 0.00 & 0.27 & -0.01 & 0.16 & 0.35 & 1.00 & & & & \\
\hline $\mathrm{UO}_{3}$ & -0.25 & -0.11 & -0.10 & -0.18 & -0.20 & 0.00 & -0.12 & 0.13 & -0.19 & 0.03 & 0.26 & -0.08 & 0.07 & 0.33 & 0.28 & 1.00 & & & \\
\hline $\mathrm{F}$ & -0.12 & 0.04 & -0.16 & 0.18 & 0.20 & -0.05 & 0.21 & 0.13 & -0.09 & 0.15 & 0.00 & -0.16 & 0.13 & -0.15 & 0.04 & -0.03 & 1.00 & & \\
\hline $\mathrm{La}_{2} \mathrm{O}_{3}$ & -0.06 & -0.13 & -0.01 & -0.04 & -0.01 & 0.13 & -0.14 & 0.21 & -0.08 & -0.04 & 0.13 & -0.17 & -0.01 & 0.03 & 0.04 & 0.10 & 0.43 & 1.00 & \\
\hline Others & -0.30 & -0.17 & -0.15 & -0.35 & -0.32 & 0.00 & -0.03 & -0.14 & -0.13 & 0.20 & 0.09 & -0.12 & 0.02 & 0.27 & 0.28 & 0.30 & -0.21 & -0.02 & 1.00 \\
\hline
\end{tabular}




\section{Model Validation}

To validate the model, data not used in model fitting must be obtained. Because all appropriate data within the desired composition region were used in model fitting, subsets of the model data were used to validate the model. The data were sorted by $\ln \left[\eta_{1150}\right]$ value. The data were then numbered $1,2,3,4,5,1,2, \ldots$ to split them into five roughly representative groups. The model was then refit to subsets 2 to 5 and used to predict data in subset 1 . Then the model was fit to each group of four subsets and used to predict the remaining subset in sequence. Table 3.11 summarizes the results of the model validation. The coefficients are reasonably close with an RSD of less than $25 \%$ with the exceptions of $\mathrm{UO}_{3}$ and Others.

The model fit $R^{2}$ values are all close to each other at 0.96. The $R_{V}^{2}$ are also close to 0.96 , and their average is close to the $R_{P}^{2}$ value of 0.9586 . This model is well validated and should give predictions of unknown data within the model validity region nearly as well as model fit data.

Table 3.11. Summary of $\ln \left[\eta_{1150}\right]$ Model Validation

\begin{tabular}{|c|c|c|c|c|c|c|c|}
\hline Term & Full & Set 1 & Set 2 & Set 3 & Set 4 & Set 5 & $\%$ RSD \\
\hline $\mathrm{SiO}_{2}$ & 9.3681 & 9.3364 & 9.4406 & 9.3553 & 9.3931 & 9.3146 & 0.5 \\
\hline $\mathrm{Na}_{2} \mathrm{O}$ & -9.6328 & -9.5071 & -9.7526 & -9.7104 & -9.7022 & -9.4382 & -1.5 \\
\hline $\mathrm{B}_{2} \mathrm{O}_{3}$ & -9.3753 & -8.4168 & -9.8816 & -9.8190 & -9.6537 & -8.8802 & -7.0 \\
\hline $\mathrm{Al}_{2} \mathrm{O}_{3}$ & 10.6085 & 10.2879 & 10.9346 & 10.5060 & 10.4918 & 10.6676 & 2.3 \\
\hline $\mathrm{CaO}$ & -6.9328 & -7.2417 & -6.9281 & -6.8793 & -6.7513 & -6.8827 & -2.6 \\
\hline $\mathrm{Li}_{2} \mathrm{O}$ & -39.0249 & -39.3440 & -38.9421 & -38.9438 & -39.2712 & -38.8607 & -0.6 \\
\hline $\mathrm{K}_{2} \mathrm{O}$ & -3.8249 & -3.9651 & -4.0004 & -3.8227 & -3.3992 & -3.8991 & -6.4 \\
\hline $\mathrm{ZrO}_{2}$ & 7.1404 & 7.0472 & 6.9700 & 7.1554 & 7.3700 & 7.0909 & 2.1 \\
\hline $\mathrm{MgO}$ & -3.2314 & -3.3789 & -3.7320 & -2.8759 & -2.7723 & -3.3797 & -12.3 \\
\hline $\mathrm{P}_{2} \mathrm{O}_{5}$ & 5.3050 & 5.4761 & 5.2643 & 4.6221 & 5.2025 & 5.7383 & 7.9 \\
\hline SrO & -4.3505 & -3.9248 & -4.2216 & -4.6062 & -4.4388 & -4.7077 & -7.2 \\
\hline $\mathrm{ZnO}$ & -2.6963 & -3.0052 & -2.4468 & -2.6692 & -2.8656 & -2.5553 & -8.4 \\
\hline $\mathrm{BaO}$ & -3.4182 & -3.5772 & -3.2042 & -2.7007 & -4.1152 & -3.6735 & -15.4 \\
\hline $\mathrm{MnO}$ & -6.8868 & -7.2545 & -6.7956 & -6.6870 & -6.6422 & -7.0921 & -3.9 \\
\hline $\mathrm{PbO}$ & -23.1436 & -22.5627 & -29.3399 & -22.8896 & -21.1889 & -17.9551 & -18.2 \\
\hline $\mathrm{UO}_{3}$ & 2.1515 & 1.4359 & 1.6532 & 2.4394 & 2.5022 & 2.6310 & 25.6 \\
\hline F & -12.3445 & -14.1716 & -11.4438 & -10.6373 & -12.2919 & -12.5777 & -10.9 \\
\hline $\mathrm{La}_{2} \mathrm{O}_{3}$ & -4.9695 & -5.0992 & -4.3789 & -5.3426 & -5.3613 & -4.9734 & -7.9 \\
\hline Others & -0.0903 & -0.1109 & -0.0650 & 0.0188 & -0.1140 & -0.1845 & -82.1 \\
\hline $\mathrm{Na}_{2} \mathrm{O} \times \mathrm{B}_{2} \mathrm{O}_{3}$ & -26.9571 & -29.6713 & -24.6098 & -25.5386 & -27.7063 & -28.5939 & -7.7 \\
\hline $\mathrm{Na}_{2} \mathrm{O} \times \mathrm{Al}_{2} \mathrm{O}_{3}$ & 17.5172 & 19.8134 & 15.3487 & 17.9607 & 18.5592 & 16.5910 & 9.8 \\
\hline $\mathrm{B}_{2} \mathrm{O}_{3} \times \mathrm{B}_{2} \mathrm{O}_{3}$ & 24.5926 & 20.8049 & 25.6446 & 27.0574 & 26.6569 & 22.8969 & 10.9 \\
\hline $\mathrm{Al}_{2} \mathrm{O}_{3} \times \mathrm{CaO}$ & -8.1347 & -5.8228 & -8.4772 & -8.6202 & -8.9364 & -8.9493 & -16.2 \\
\hline $\mathrm{Li}_{2} \mathrm{O} \times \mathrm{Li}_{2} \mathrm{O}$ & 47.3592 & 52.7654 & 41.3129 & 50.1104 & 49.8875 & 45.3391 & 9.5 \\
\hline $\mathrm{R}^{2}$ & 0.9619 & 0.9619 & 0.9634 & 0.9630 & 0.9604 & 0.9625 & \\
\hline $\mathrm{R}_{\mathrm{A}}^{2}$ & 0.9610 & 0.9608 & 0.9622 & 0.9618 & 0.9591 & 0.9614 & \\
\hline RMSE & 0.1632 & 0.1631 & 0.1599 & 0.1619 & 0.1674 & 0.1626 & \\
\hline Mean & 1.6732 & 1.6735 & 1.6723 & 1.6748 & 1.6734 & 1.6720 & \\
\hline $\mathrm{n}$ & 967 & 773 & 773 & 774 & 774 & 774 & \\
\hline$R_{v}^{2} v$ & 0.9619 & 0.9531 & 0.9546 & 0.9539 & 0.9674 & 0.9638 & \\
\hline
\end{tabular}




\subsection{3 $\quad \eta$ at $1250^{\circ} \mathrm{C}$}

Model development and validation for the $\ln \left[\eta_{1250}\right]$ follows the same methods described in Section 3.1.1 for $\ln \left[\eta_{950}\right]$ and Section 3.1.2 for $\ln \left[\eta_{1150}\right]$ model development.

\section{Initial Data Screening and Evaluation}

The data listed in Appendix A were first screened before use in viscosity modeling. Of the 3406 glasses in the database, 1500 contained some viscosity data. These glasses were screened according to the following procedure:

1. fitting the VFT Equation (1.10) to $\eta-T$ data

2. tabulating minimum $\left(T_{\min }\right)$ and maximum temperatures $\left(T_{\max }\right)$ for data for each glass

3. keeping only glasses with $\mathrm{T}_{\min } \leq 1250^{\circ} \mathrm{C} \leq \mathrm{T}_{\max }$

4. removing glasses that were found to be "inhomogeneous" or multiphasic

5. substituting the analyzed compositions for target compositions for those glasses with only analyzed compositions

6. removing nine glasses with incomplete chemical compositions

7. removing three glasses with a high deviation between target and analyzed compositions (99-78, IG3-29, LAWPC7)

8. converting multivalent oxides to preferred oxidation states for Hanford HLW glasses $\mathrm{Fe}_{2} \mathrm{O}_{3}$, $\mathrm{As}_{2} \mathrm{O}_{5}, \mathrm{Ce}_{2} \mathrm{O}_{3}, \mathrm{CoO}, \mathrm{MnO}, \mathrm{MoO}_{3}, \mathrm{Pr}_{2} \mathrm{O}_{3}, \mathrm{Re}_{2} \mathrm{O}_{7}, \mathrm{Rh}_{2} \mathrm{O}_{3}, \mathrm{Sb}_{2} \mathrm{O}_{3}, \mathrm{SnO}_{2}, \mathrm{Tl}_{2} \mathrm{O}$, and $\mathrm{UO}_{3}$.

The resulting data set included 892 glasses for $\ln \left[\eta_{1250}\right]$ model development. A scatterplot matrix of these data is given in Appendix $B$.

\section{Model Development}

Preliminary model development was performed using stepwise regression methods allowing only first-order terms. Those components with sufficient data to allow for coefficient estimates were included in the stepwise process. Stepwise regression comparing the coefficient to the value of zero while maintaining the intercept near the average response of $1.01 \ln [\mathrm{Pa} \cdot \mathrm{s}]$ was used to reduce the influential components to:

$\mathrm{SiO}_{2}, \mathrm{Na}_{2} \mathrm{O}, \mathrm{B}_{2} \mathrm{O}_{3}, \mathrm{Al}_{2} \mathrm{O}_{3}, \mathrm{Fe}_{2} \mathrm{O}_{3}, \mathrm{CaO}, \mathrm{Li}_{2} \mathrm{O}, \mathrm{K}_{2} \mathrm{O}, \mathrm{ZrO}_{2}, \mathrm{MgO}, \mathrm{P}_{2} \mathrm{O}_{5}, \mathrm{SrO}, \mathrm{ZnO}$,

$\mathrm{BaO}, \mathrm{TiO}_{2}, \mathrm{NiO}, \mathrm{MnO}, \mathrm{PbO}, \mathrm{ThO}_{2}, \mathrm{UO}_{3}, \mathrm{Nd}_{2} \mathrm{O}_{3}, \mathrm{Bi}_{2} \mathrm{O}_{3}, \mathrm{Ce}_{2} \mathrm{O}_{3}, \mathrm{Gd}_{2} \mathrm{O}_{3}, \mathrm{~F}$, and $\mathrm{La}_{2} \mathrm{O}_{3}$

Three outliers were identified during the initial model fitting (HLW98-96B, SRC-Eu-1, and LAWA46). In addition, 53 glasses with $x_{P b O}>0.0050$ (mass fraction) were removed, leaving 836 data points for model fitting.

Attempts were made to improve the fit of the model data by adding a limited number (up to 5) second-order terms. The terms found to most significantly improve the fit to model data were:

$$
\mathrm{Na}_{2} \mathrm{O} \times \mathrm{B}_{2} \mathrm{O}_{3}, \mathrm{Na}_{2} \mathrm{O} \times \mathrm{Al}_{2} \mathrm{O}_{3}, \mathrm{~B}_{2} \mathrm{O}_{3} \times \mathrm{B}_{2} \mathrm{O}_{3} \text {, and } \mathrm{Li}_{2} \mathrm{O} \times \mathrm{Li}_{2} \mathrm{O}
$$


Through this process, 19 additional glasses were found to be outliers. These all contained extreme concentrations of the second-order terms listed above. The final model was then fit to the remaining 817 datapoints with coefficients $b_{\ln [\eta 1250], i}$, listed in Table 3.12.

The statistics for the model fit are summarized in

Table 3.13. The $R^{2}$ coefficient of $>0.95$ suggests that the data are well represented by the model. The $R^{2}$-adjusted value suggests that there are not too many coefficients, or the data are not over-fit. The $R^{2}$-predicted being so close to the $R^{2}$ value suggests that there are not any very influential data points, and the model is likely to predict similarly for new data as it does for the model development data.

Table 3.12. $\ln \left[\eta_{1250}\right]$ Model Coefficients

\begin{tabular}{lcc}
\hline Term $(i)$ & Coefficient $\left(b_{\eta 1250, i}\right)$ & Std Error \\
\hline $\mathrm{Al}_{2} \mathrm{O}_{3}$ & 8.94357 & 0.377517 \\
$\mathrm{~B}_{2} \mathrm{O}_{3}$ & -7.66615 & 0.714886 \\
$\mathrm{BaO}$ & -3.89304 & 0.96681 \\
$\mathrm{Bi}_{2} \mathrm{O}_{3}$ & -3.54929 & 0.790447 \\
$\mathrm{CaO}$ & -7.96797 & 0.206528 \\
$\mathrm{~F}$ & -12.3147 & 1.607114 \\
$\mathrm{Fe}_{2} \mathrm{O}_{3}$ & -1.29457 & 0.153089 \\
$\mathrm{~K}_{2} \mathrm{O}$ & -3.45232 & 0.362315 \\
$\mathrm{La}_{2} \mathrm{O}_{3}$ & -4.70143 & 1.297667 \\
$\mathrm{Li}_{2} \mathrm{O}$ & -34.7926 & 0.888125 \\
$\mathrm{MgO}$ & -4.85407 & 0.437357 \\
$\mathrm{MnO}$ & -7.14845 & 0.508207 \\
$\mathrm{Na}_{2} \mathrm{O}$ & -8.81239 & 0.28903 \\
$\mathrm{PbO}_{\mathrm{SiO}}$ & -35.1899 & 7.331512 \\
$\mathrm{SrO}_{2}$ & 7.719283 & 0.077338 \\
$\mathrm{TiO}_{2}$ & -6.42168 & 0.416158 \\
$\mathrm{UO} \mathrm{O}_{3}$ & -2.96399 & 0.655929 \\
$\mathrm{ZnO}_{\mathrm{Others}}$ & 1.061988 & 0.436435 \\
$\mathrm{Na}_{2} \mathrm{O} \times \mathrm{B}_{2} \mathrm{O}_{3}$ & -2.91064 & 0.413253 \\
$\mathrm{Na}_{2} \mathrm{O} \times \mathrm{Al}_{2} \mathrm{O}_{3}$ & 2.681183 & 0.164159 \\
$\mathrm{~B}_{2} \mathrm{O}_{3} \times \mathrm{B}_{2} \mathrm{O}_{3}$ & -25.4993 & 2.681469 \\
$\mathrm{Li}_{2} \mathrm{O} \times \mathrm{Li}_{2} \mathrm{O}$ & 14.98563 & 2.744072 \\
\hline
\end{tabular}

Table 3.13. Summary of $\ln \left[\eta_{1250}\right]$ Model Fit Statistics

\begin{tabular}{lc}
\hline Statistic & Value \\
\hline$R^{2}$ & 0.9678 \\
$R_{A}^{2}$ & 0.9669 \\
$R_{P}^{2}$ & 0.9649 \\
$\mathrm{RMSE}$ & 0.1612 \\
Minimum & -1.055 \\
Mean & 1.007 \\
Maximum & 4.159 \\
$\mathrm{n}$ & 817 \\
$\mathrm{~N}$ & 24 \\
\hline
\end{tabular}


Figure 3.3 compares the predicted and measured $\ln \left[\eta_{1250}\right]$ values. The data appear to be well represented by the model with no trends to over or under prediction. No single group of data (e.g., study) was found to be misrepresented by the model.

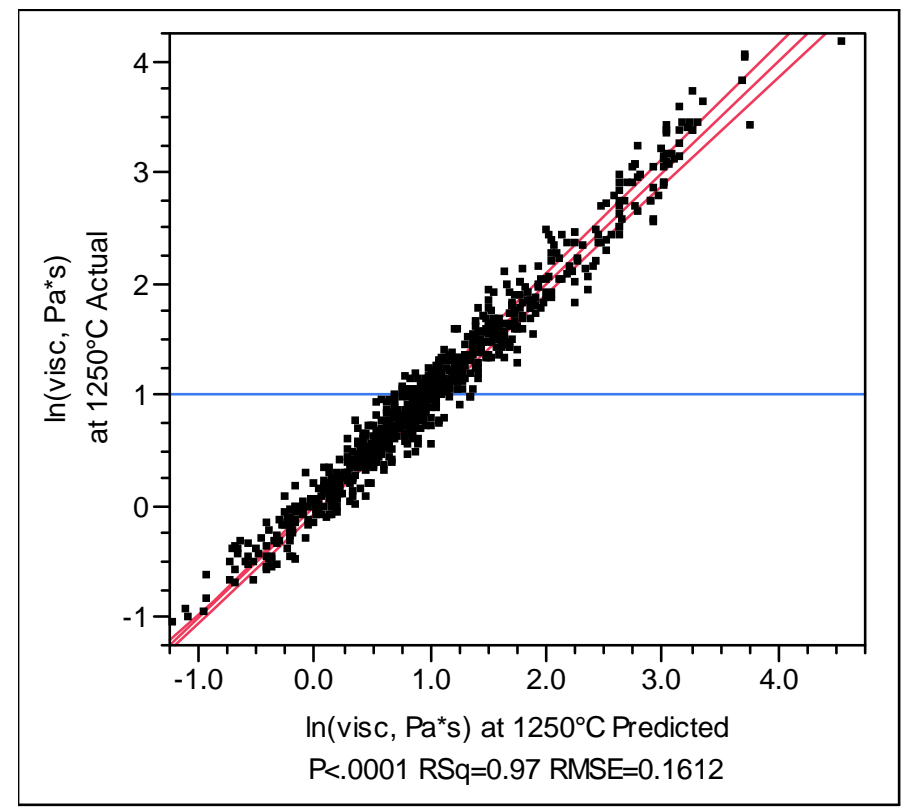

Figure 3.3. Measured vs. Predicted $\ln \left[\eta_{1250}\right]$

This model is based on data spanning a specific glass composition region and therefore should only be relied upon within that region. The validity region is defined as the composition region of data used to develop the model with three exceptions:

1. $\mathrm{Al}_{2} \mathrm{O}_{3}$ ranges to $30.3 \mathrm{wt} \%$, but only one glass contains more than $25.9 \mathrm{wt} \%$.

2. Eight glasses increase the upper bound of $\mathrm{Na}_{2} \mathrm{O}$ from 25.5 to $30.0 \mathrm{wt} \%$.

3. The Others component ranges up to $22 \mathrm{wt} \%$ with most of Others composed of $\mathrm{Nd}_{2} \mathrm{O}_{3}, \mathrm{ThO}_{2}$, and $\mathrm{ZrO}_{2}$. These three components should be taken as separate limits. Then one glass increases the "Others" component concentrations from 9.4 to $12.3 \mathrm{wt} \%$.

Table 3.14 lists the model validity region while Table 3.15 lists the correlation coefficients calculated for the model data. Only SrO-MnO have a correlation of 0.5 or above. 
Table 3.14. $\ln \left[\eta_{1250}\right]$ Model Validity Region (wt $\%$ )

\begin{tabular}{lrr}
\hline Component & Min & Max \\
\hline $\mathrm{Al}_{2} \mathrm{O}_{3}$ & 0.0 & 25.9 \\
$\mathrm{~B}_{2} \mathrm{O}_{3}$ & 0.0 & 20.0 \\
$\mathrm{BaO}_{\mathrm{Bi}} \mathrm{O}_{3}$ & 0.0 & 4.7 \\
$\mathrm{CaO}$ & 0.0 & 16.4 \\
$\mathrm{~F}$ & 0.0 & 15.0 \\
$\mathrm{Fe}_{2} \mathrm{O}_{3}$ & 0.0 & 4.7 \\
$\mathrm{~K}_{2} \mathrm{O}$ & 0.0 & 24.3 \\
$\mathrm{La}_{2} \mathrm{O}_{3}$ & 0.0 & 10.0 \\
$\mathrm{Li}_{2} \mathrm{O}$ & 0.0 & 5.0 \\
$\mathrm{MgO}_{\mathrm{MnO}}$ & 0.0 & 8.9 \\
$\mathrm{Na}_{2} \mathrm{O}$ & 0.0 & 8.2 \\
$\mathrm{Nd}_{2} \mathrm{O}_{3}$ & 0.0 & 8.0 \\
$\mathrm{PbO}_{\mathrm{SiO}}$ & 2.5 & 25.5 \\
$\mathrm{SrO}$ & 0.0 & 8.6 \\
$\mathrm{ThO}_{2}$ & 0.0 & 0.5 \\
$\mathrm{TiO}_{2}$ & 26.0 & 64.1 \\
$\mathrm{UO}$ & 0.0 & 10.3 \\
$\mathrm{ZnO}_{\mathrm{ZnO}}$ & 0.0 & 7.8 \\
$\mathrm{Remaining}_{2}$ & 0.0 & 9.6 \\
\hline & 0.0 & 15.6 \\
\hline & 0.0 & 9.8 \\
\hline
\end{tabular}

A variance-covariance matrix is required to calculate the measurement plus prediction uncertainties of this model. The $\mathbf{V}_{\ln [n 1250]}$ is given in Appendix $\mathbf{C}$ with the other variancecovariance matrices.

\section{Model Validation}

To validate the model, data not used in model fitting must be obtained. Because all appropriate data within the desired composition region were used in model fitting, subsets of the model data were used to validate the model. The data were sorted by $\ln \left[\eta_{1250}\right]$ value. The data were then numbered $1,2,3,4,5,1,2, \ldots$ to split them into five roughly representative groups. The model was then refit to subsets 2 to 5 and used to predict data in subset 1 . Then the model was fit to each group of four subsets and used to predict the remaining subset in sequence. Table 3.16 summarizes the results of the model validation. The coefficients are reasonably close with an RSD of less than $25 \%$ with the exception of $\mathrm{UO}_{3}$.

The model fit $R^{2}$ values are all close to each other at just under 0.97 . The $R_{V}^{2}$ are also close to the 0.965 , and their average is almost identical to the $R_{P}^{2}$ value of 0.9649 . This model is well validated and should give predictions of unknown data within the model validity region nearly as well as model fit data. 
Table 3.15. Correlation Matrix for the $\ln \left[\eta_{1250}\right]$ Model Dataset

\begin{tabular}{|c|c|c|c|c|c|c|c|c|c|c|c|c|c|c|c|c|c|c|c|c|}
\hline & $\mathrm{SiO}_{2}$ & $\mathrm{Na}_{2} \mathrm{O}$ & $\mathrm{B}_{2} \mathrm{O}_{3}$ & $\mathrm{Al}_{2} \mathrm{O}_{3}$ & $\mathrm{Fe}_{2} \mathrm{O}_{3}$ & $\mathrm{CaO}$ & $\mathrm{Li}_{2} \mathrm{O}$ & $\mathrm{K}_{2} \mathrm{O}$ & $\mathrm{MgO}$ & $\mathrm{SrO}$ & $\mathrm{ZnO}$ & $\mathrm{BaO}$ & $\mathrm{TiO}_{2}$ & $\mathrm{MnO}$ & $\mathrm{PbO}$ & $\mathrm{UO}_{3}$ & $\mathrm{Bi}_{2} \mathrm{O}_{3}$ & $\mathrm{~F}$ & $\mathrm{La}_{2} \mathrm{O}_{3}$ & Others \\
\hline $\mathrm{SiO}_{2}$ & 1.00 & & & & & & & & & & & & & & & & & & & \\
\hline $\mathrm{Na}_{2} \mathrm{O}$ & -0.11 & 1.00 & & & & & & & & & & & & & & & & & & \\
\hline $\mathrm{B}_{2} \mathrm{O}_{3}$ & -0.19 & -0.33 & 1.00 & & & & & & & & & & & & & & & & & \\
\hline $\mathrm{Al}_{2} \mathrm{O}_{3}$ & -0.25 & 0.16 & 0.01 & 1.00 & & & & & & & & & & & & & & & & \\
\hline $\mathrm{Fe}_{2} \mathrm{O}_{3}$ & -0.29 & -0.11 & -0.09 & -0.33 & 1.00 & & & & & & & & & & & & & & & \\
\hline $\mathrm{CaO}$ & -0.01 & -0.11 & -0.10 & 0.09 & -0.26 & 1.00 & & & & & & & & & & & & & & \\
\hline $\mathrm{Li}_{2} \mathrm{O}$ & 0.13 & -0.68 & 0.18 & -0.07 & 0.02 & -0.14 & 1.00 & & & & & & & & & & & & & \\
\hline $\mathrm{K}_{2} \mathrm{O}$ & -0.24 & 0.09 & 0.05 & 0.12 & -0.08 & 0.01 & -0.12 & 1.00 & & & & & & & & & & & & \\
\hline $\mathrm{MgO}$ & 0.05 & -0.15 & 0.10 & -0.11 & -0.09 & 0.18 & -0.01 & -0.07 & 1.00 & & & & & & & & & & & \\
\hline $\mathrm{SrO}$ & -0.29 & -0.18 & -0.05 & -0.08 & 0.18 & -0.21 & 0.12 & -0.16 & -0.19 & 1.00 & & & & & & & & & & \\
\hline $\mathrm{ZnO}$ & -0.15 & -0.07 & 0.09 & -0.12 & -0.04 & 0.23 & -0.12 & -0.04 & 0.33 & -0.06 & 1.00 & & & & & & & & & \\
\hline $\mathrm{BaO}$ & -0.12 & -0.01 & 0.07 & 0.14 & 0.05 & -0.12 & 0.02 & 0.22 & -0.16 & -0.02 & -0.22 & 1.00 & & & & & & & & \\
\hline $\mathrm{TiO}_{2}$ & -0.09 & 0.09 & 0.09 & -0.03 & -0.08 & 0.11 & -0.22 & 0.05 & 0.26 & -0.15 & 0.44 & -0.11 & 1.00 & & & & & & & \\
\hline $\mathrm{MnO}$ & -0.21 & -0.16 & -0.03 & -0.19 & 0.32 & -0.35 & 0.14 & -0.18 & -0.25 & 0.53 & -0.09 & 0.03 & -0.17 & 1.00 & & & & & & \\
\hline $\mathrm{PbO}$ & -0.29 & -0.07 & -0.02 & -0.09 & 0.40 & -0.29 & 0.07 & -0.08 & -0.22 & 0.37 & -0.05 & 0.16 & -0.18 & 0.47 & 1.00 & & & & & \\
\hline $\mathrm{UO}_{3}$ & -0.21 & -0.11 & -0.13 & -0.17 & 0.15 & -0.20 & 0.01 & -0.12 & -0.18 & 0.28 & -0.11 & 0.03 & -0.10 & 0.30 & 0.28 & 1.00 & & & & \\
\hline $\mathrm{Bi}_{2} \mathrm{O}_{3}$ & 0.03 & -0.05 & -0.23 & -0.06 & 0.03 & -0.06 & 0.01 & -0.07 & -0.08 & -0.02 & -0.11 & 0.03 & -0.06 & 0.02 & -0.02 & 0.06 & 1.00 & & & \\
\hline $\mathrm{F}$ & -0.12 & 0.04 & -0.17 & 0.15 & -0.20 & 0.15 & -0.03 & 0.28 & -0.07 & 0.03 & -0.19 & 0.12 & -0.16 & -0.14 & -0.02 & -0.04 & 0.03 & 1.00 & & \\
\hline $\mathrm{La}_{2} \mathrm{O}_{3}$ & -0.09 & -0.14 & 0.02 & -0.04 & -0.06 & 0.00 & 0.16 & -0.13 & -0.09 & 0.19 & -0.20 & -0.03 & -0.15 & 0.03 & 0.05 & 0.09 & 0.02 & 0.45 & 1.00 & \\
\hline Others & -0.20 & -0.16 & -0.21 & -0.31 & -0.01 & -0.19 & 0.16 & -0.05 & -0.16 & 0.18 & -0.19 & -0.10 & -0.26 & 0.18 & 0.12 & 0.31 & 0.23 & 0.14 & 0.23 & 1.00 \\
\hline
\end{tabular}


Table 3.16. Summary of $\ln \left[\eta_{1250}\right]$ Model Validation

\begin{tabular}{|c|c|c|c|c|c|c|c|}
\hline Term & Full & Set 1 & Set 2 & Set 3 & Set 4 & Set 5 & \%RSD \\
\hline $\mathrm{SiO}_{2}$ & 7.7193 & 7.6468 & 7.6838 & 7.7524 & 7.8208 & 7.6923 & 0.9 \\
\hline $\mathrm{Na}_{2} \mathrm{O}$ & -8.8124 & -8.4085 & -8.6725 & -8.9225 & -9.0827 & -8.9652 & -3.1 \\
\hline $\mathrm{B}_{2} \mathrm{O}_{3}$ & -7.6662 & -7.2424 & -7.5826 & -8.1540 & -8.0485 & -7.4374 & -5.1 \\
\hline $\mathrm{Al}_{2} \mathrm{O}_{3}$ & 8.9436 & 8.9317 & 9.8818 & 8.7561 & 8.6773 & 8.6629 & 5.7 \\
\hline $\mathrm{Fe}_{2} \mathrm{O}_{3}$ & -1.2946 & -1.3477 & -1.2258 & -1.3273 & -1.2697 & -1.2780 & -3.8 \\
\hline $\mathrm{CaO}$ & -7.9680 & -8.1592 & -8.0171 & -7.8551 & -7.8166 & -8.0409 & -1.8 \\
\hline $\mathrm{Li}_{2} \mathrm{O}$ & -34.7926 & -34.3975 & -35.6451 & -34.5208 & -35.0070 & -34.3351 & -1.6 \\
\hline $\mathrm{K}_{2} \mathrm{O}$ & -3.4523 & -3.8026 & -3.5126 & -3.1419 & -3.4487 & -3.4771 & -6.8 \\
\hline $\mathrm{MgO}$ & -4.8541 & -5.1009 & -4.7712 & -5.0280 & -4.9118 & -4.3786 & -5.9 \\
\hline SrO & -6.4217 & -6.5340 & -6.6174 & -6.0932 & -6.6025 & -6.4128 & -3.4 \\
\hline $\mathrm{ZnO}$ & -2.9106 & -3.0286 & -2.9079 & -2.9571 & -2.6950 & -2.9849 & -4.5 \\
\hline $\mathrm{BaO}$ & -3.8930 & -3.4883 & -4.0765 & -3.8516 & -4.5016 & -3.6186 & -10.3 \\
\hline $\mathrm{TiO}_{2}$ & -2.9640 & -2.2435 & -3.3046 & -2.8394 & -3.2405 & -2.9019 & -14.5 \\
\hline $\mathrm{MnO}$ & -7.1484 & -7.1929 & -7.1408 & -6.9119 & -7.2598 & -7.1166 & -1.8 \\
\hline $\mathrm{PbO}$ & -35.1899 & -36.8197 & -33.2197 & -35.0035 & -32.0734 & -38.1779 & -7.1 \\
\hline $\mathrm{UO}_{3}$ & 1.0620 & 0.7868 & 1.3071 & 0.7843 & 0.9721 & 1.3121 & 25.6 \\
\hline $\mathrm{Bi}_{2} \mathrm{O}_{3}$ & -3.5493 & -3.5797 & -3.8360 & -3.4960 & -3.3885 & -3.5428 & -4.6 \\
\hline $\mathrm{F}$ & -12.3147 & -10.3063 & -13.2017 & -12.1572 & -12.9962 & -12.0347 & -9.4 \\
\hline $\mathrm{La}_{2} \mathrm{O}_{3}$ & -4.7014 & -6.8186 & -4.4621 & -4.4748 & -4.1042 & -4.3040 & -23.2 \\
\hline Others & 2.6812 & 2.7828 & 2.6951 & 2.5956 & 2.5905 & 2.7601 & 3.3 \\
\hline $\mathrm{Na}_{2} \mathrm{O} \times \mathrm{B}_{2} \mathrm{O}_{3}$ & -25.4993 & -28.5896 & -25.8705 & -22.6264 & -23.4713 & -26.6182 & -9.5 \\
\hline $\mathrm{Na}_{2} \mathrm{O} \times \mathrm{Al}_{2} \mathrm{O}_{3}$ & 14.9856 & 13.9477 & 10.2240 & 15.1635 & 16.3217 & 18.2907 & 20.4 \\
\hline $\mathrm{B}_{2} \mathrm{O}_{3} \times \mathrm{B}_{2} \mathrm{O}_{3}$ & 17.3382 & 16.9739 & 16.3538 & 18.3905 & 18.0544 & 17.2305 & 4.7 \\
\hline $\mathrm{Li}_{2} \mathrm{O} \times \mathrm{Li}_{2} \mathrm{O}$ & 35.4319 & 32.1499 & 39.9658 & 36.6515 & 38.0167 & 27.7052 & 14.2 \\
\hline $\mathrm{R}^{2}$ & 0.9678 & 0.9678 & 0.9686 & 0.9685 & 0.9680 & 0.9678 & \\
\hline $\mathrm{R}_{\mathrm{A}}^{2}$ & 0.9669 & 0.9666 & 0.9674 & 0.9674 & 0.9668 & 0.9666 & \\
\hline RMSE & 0.1612 & 0.1614 & 0.1593 & 0.1606 & 0.1620 & 0.1622 & \\
\hline Mean & 1.0074 & 1.0076 & 1.0060 & 1.0094 & 1.0079 & 1.0060 & \\
\hline $\mathrm{n}$ & 817 & 653 & 653 & 654 & 654 & 654 & \\
\hline$R^{2} v$ & 0.9649 & 0.9606 & 0.9607 & 0.9664 & 0.9673 & 0.9697 & \\
\hline
\end{tabular}

\subsubsection{Viscosity as a Function of Temperature}

The preceding subsections discussed the prediction of $\ln \left[\eta_{T}, \mathrm{~Pa}-\mathrm{s}\right]$ where $\mathrm{T}$ is given as 950, 1150 , and $1250^{\circ} \mathrm{C}$. The models are summarized in Table 3.17. The models are similar in many respects. The fit and validation statistics are similar, and the coefficients are similar with the exception of the high $\mathrm{B}_{2} \mathrm{O}_{3} \times \mathrm{B}_{2} \mathrm{O}_{3}$ and $\mathrm{Li}_{2} \mathrm{O} \times \mathrm{Li}_{2} \mathrm{O}$ coefficients of the $\ln \left[\eta_{950}\right]$ model as shown in Figure 3.4. 
Table 3.17. $\ln \left[\eta_{T}\right]$ Model Coefficient Summary

\begin{tabular}{|c|c|c|c|}
\hline Term & $\ln \left[\eta_{950}\right]$ & $n\left[n_{1150}\right]$ & $\ln \left[\eta_{1250}\right]$ \\
\hline $\mathrm{Al}_{2} \mathrm{O}_{3}$ & 18.14 & 10.61 & 8.94 \\
\hline $\mathrm{B}_{2} \mathrm{O}_{3}$ & -12.13 & -9.38 & -7.67 \\
\hline $\mathrm{BaO}$ & -2.70 & -3.42 & -3.89 \\
\hline $\mathrm{Bi}_{2} \mathrm{O}_{3}$ & & & -3.55 \\
\hline $\mathrm{CaO}$ & -4.64 & -6.93 & -7.97 \\
\hline $\mathrm{F}$ & -13.75 & -12.34 & -12.31 \\
\hline $\mathrm{Fe}_{2} \mathrm{O}_{3}$ & 2.54 & & -1.29 \\
\hline $\mathrm{K}_{2} \mathrm{O}$ & -3.46 & -3.82 & -3.45 \\
\hline $\mathrm{La}_{2} \mathrm{O}_{3}$ & -1.48 & -4.97 & -4.70 \\
\hline $\mathrm{Li}_{2} \mathrm{O}$ & -54.90 & -39.02 & -34.79 \\
\hline $\mathrm{MgO}$ & 0.79 & -3.23 & -4.85 \\
\hline $\mathrm{MnO}$ & -4.35 & -6.89 & -7.15 \\
\hline $\mathrm{Na}_{2} \mathrm{O}$ & -9.52 & -9.63 & -8.81 \\
\hline $\mathrm{P}_{2} \mathrm{O}_{5}$ & 8.84 & 5.31 & \\
\hline $\mathrm{PbO}$ & -22.28 & -23.14 & -35.19 \\
\hline $\mathrm{SiO}_{2}$ & 13.09 & 9.37 & 7.72 \\
\hline SrO & -3.84 & -4.35 & -6.42 \\
\hline $\mathrm{TiO}_{2}$ & & & -2.96 \\
\hline $\mathrm{UO}_{3}$ & 5.95 & 2.15 & 1.06 \\
\hline $\mathrm{ZnO}$ & 0.66 & -2.70 & -2.91 \\
\hline $\mathrm{ZrO}_{2}$ & 15.15 & 7.14 & \\
\hline Others & 3.52 & -0.09 & 2.68 \\
\hline $\mathrm{Na}_{2} \mathrm{O} \times \mathrm{B}_{2} \mathrm{O}_{3}$ & -23.35 & -26.96 & -25.50 \\
\hline $\mathrm{Na}_{2} \mathrm{O} \times \mathrm{Al}_{2} \mathrm{O}_{3}$ & & 17.52 & 14.99 \\
\hline $\mathrm{Na}_{2} \mathrm{O} \times \mathrm{Fe}_{2} \mathrm{O}_{3}$ & -9.58 & & \\
\hline $\mathrm{B}_{2} \mathrm{O}_{3} \times \mathrm{B}_{2} \mathrm{O}_{3}$ & 44.84 & 24.59 & 17.34 \\
\hline $\mathrm{Al}_{2} \mathrm{O}_{3} \times \mathrm{CaO}$ & & -8.13 & \\
\hline $\mathrm{B}_{2} \mathrm{O}_{3} \times \mathrm{Al}_{2} \mathrm{O}_{3}$ & -17.94 & & \\
\hline $\mathrm{Li}_{2} \mathrm{O} \times \mathrm{Li}_{2} \mathrm{O}$ & 140.74 & 47.36 & 35.43 \\
\hline $\mathrm{R}^{2}$ & 0.953 & 0.962 & 0.968 \\
\hline $\mathrm{R}_{\mathrm{A}}^{2}$ & 0.951 & 0.961 & 0.967 \\
\hline RMSE & 0.183 & 0.163 & 0.161 \\
\hline Mean & 3.750 & 1.673 & 1.007 \\
\hline$n$ & 673 & 967 & 817 \\
\hline$R^{2} v$ & 0.949 & 0.959 & 0.965 \\
\hline
\end{tabular}




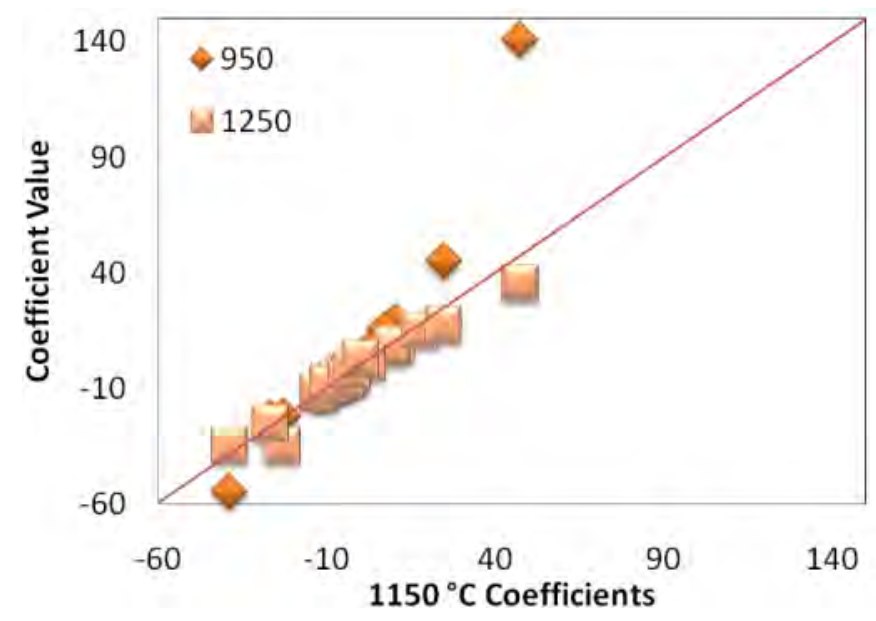

Figure 3.4. $\ln \left[\eta_{T}\right]$ Model Coefficient Comparison

To estimate the viscosity at temperatures between 950 and $1250^{\circ} \mathrm{C}$, the $\ln [\eta]$ is predicted at each of the three temperatures. Then using Equation (1.10), the $\ln [\eta]$ is interpolated:

$$
\ln \left[\eta_{T}, \mathrm{~Pa}-\mathrm{s}\right]=A+\frac{B}{T-T_{0}}
$$

replacing for the predicted properties

$$
\ln \left[\eta_{950}\right]=A+\frac{B}{950-T_{0}} ; \ln \left[\eta_{1150}\right]=A+\frac{B}{1150-T_{0}} ; \ln \left[\eta_{1250}\right]=A+\frac{B}{1250-T_{0}}
$$

and solving for $\mathrm{A}, \mathrm{B}$, and $\mathrm{T}_{0}$; we get

$$
\begin{gathered}
A=\frac{3 \cdot \ln \left[\eta_{950}\right] \cdot \ln \left[\eta_{1250}\right]-\ln \left[\eta_{1150}\right]\left(2 \cdot \ln \left[\eta_{950}\right]+\ln \left[\eta_{1250}\right]\right)}{-3 \cdot \ln \left[\eta_{1150}\right]+\ln \left[\eta_{950}\right]+2 \cdot \ln \left[\eta_{1250}\right]}, \\
B=\frac{-600 \cdot\left(\ln \left[\eta_{1150}\right]-\ln \left[\eta_{950}\right]\right) \cdot\left(\ln \left[\eta_{1150}\right]-\ln \left[\eta_{1250}\right]\right) \cdot\left(\ln \left[\eta_{950}\right]-\ln \left[\eta_{1250}\right]\right)}{\left(-3 \cdot \ln \left[\eta_{1150}\right]+\ln \left[\eta_{950}\right]+2 \cdot \ln \left[\eta_{1250}\right]\right)^{2}}, \text { and } \\
T_{0}=\frac{50\left(69 \cdot \ln \left[\eta_{1150}\right]-19 \cdot \ln \left[\eta_{950}\right]-50 \cdot \ln \left[\eta_{1250}\right]\right)}{3 \cdot \ln \left[\eta_{1150}\right]-\ln \left[\eta_{950}\right]-2 \cdot \ln \left[\eta_{1250}\right]}
\end{gathered}
$$

These values for $A, B$, and $T_{0}$ are then substituted into Equation (3.3) to obtain viscosity at any temperature between 950 and $1250^{\circ} \mathrm{C}$. 


\subsection{Electrical Conductivity-Temperature}

Model development and validation for the $\ln \left[\varepsilon_{T}\right]$ follow the same methods described in Section 3.1 for $\ln \left[\eta_{\mathrm{T}}\right]$.

\subsubsection{Initial Data Screening and Evaluation}

The data listed in Appendix A were first screened before use in electrical conductivity modeling. Of the 3406 glasses in the database, 591 contained some electrical conductivity data. These glasses were screened according to the following procedure:

1. fitting the VFT Equation (1.10) to $\varepsilon$-T data

2. tabulating minimum $\left(T_{\min }\right)$ and maximum temperatures $\left(T_{\max }\right)$ for data for each glass

3. substituting the analyzed compositions for target compositions for those glasses with only analyzed compositions

4. converting multivalent oxides to preferred oxidation states for Hanford HLW glasses $\mathrm{Fe}_{2} \mathrm{O}_{3}$, $\mathrm{As}_{2} \mathrm{O}_{5}, \mathrm{Ce}_{2} \mathrm{O}_{3}, \mathrm{CoO}, \mathrm{MnO}, \mathrm{MoO}_{3}, \mathrm{Pr}_{2} \mathrm{O}_{3}, \mathrm{Re}_{2} \mathrm{O}_{7}, \mathrm{Rh}_{2} \mathrm{O}_{3}, \mathrm{Sb}_{2} \mathrm{O}_{3}, \mathrm{SnO}_{2}, \mathrm{Tl}_{2} \mathrm{O}$, and $\mathrm{UO}_{3}$.

\subsection{2 $\varepsilon$ at $1000^{\circ} \mathrm{C}$}

\section{Model Development}

The data set included 531 glasses with $\ln \left[\varepsilon_{1000}\right]$ for model development. Preliminary model development was performed using stepwise regression methods allowing only first-order terms. Those components with sufficient data to allow for coefficient estimates were included in the stepwise process. ${ }^{(a)}$

$\mathrm{Al}_{2} \mathrm{O}_{3}, \mathrm{~B}_{2} \mathrm{O}_{3}, \mathrm{Bi}_{2} \mathrm{O}_{3}, \mathrm{CaO}, \mathrm{F}, \mathrm{Fe}_{2} \mathrm{O}_{3}, \mathrm{~K}_{2} \mathrm{O}, \mathrm{La}_{2} \mathrm{O}_{3}, \mathrm{Li}_{2} \mathrm{O}, \mathrm{MgO}, \mathrm{MnO}, \mathrm{Na} \mathrm{O}_{2} \mathrm{Nd} \mathrm{O}_{2} \mathrm{O}_{3}$, $\mathrm{NiO}, \mathrm{P}_{2} \mathrm{O}_{5}, \mathrm{PbO}, \mathrm{SiO}_{2}, \mathrm{SrO}, \mathrm{ThO}_{2}, \mathrm{UO}_{3}, \mathrm{ZnO}$, and $\mathrm{ZrO}_{2}$

Stepwise regression comparing the coefficient to the value of zero while maintaining the intercept near the average response of $2.85 \ln [\mathrm{S} / \mathrm{m}]$ was used to reduce the influential components to:

$\mathrm{Al}_{2} \mathrm{O}_{3}, \mathrm{~B}_{2} \mathrm{O}_{3}, \mathrm{CaO}, \mathrm{Fe}_{2} \mathrm{O}_{3}, \mathrm{Li}_{2} \mathrm{O}, \mathrm{MgO}, \mathrm{Na}_{2} \mathrm{O}, \mathrm{NiO}, \mathrm{SiO}_{2}, \mathrm{SrO}, \mathrm{ZnO}$, and $\mathrm{ZrO}_{2}$

Attempts were made to improve the fit of the model data by adding a limited number (up to 5) second-order terms. The term found to most significantly improve the fit to model data was $\mathrm{Na}_{2} \mathrm{O} \times \mathrm{Li}_{2} \mathrm{O}$. The final set of terms was exactly the same as other electrical conductivity (EC) models for different temperatures.

Four glasses were found to be outliers for both the first-order and first-order plus $\mathrm{Na}_{2} \mathrm{O} \times \mathrm{Li}_{2} \mathrm{O}$ models: CVS2-81, LAWM30, HLW07-28, and HLW07-40. These data points were removed from the model fitting dataset, leaving 527 datapoints. A scatterplot matrix of these data is given in Appendix $B$.

(a) A number of methods were used to determine if a component had sufficient variation to include in the model development. Chief among them are the visual examination of pairwise concentration plots in the form of a scatterplot matrix. The absolute maximum concentrations of the components along with the number of glasses containing the component over $10 \%$ of the maximum concentration were also considered. 
Table 3.18 lists the coefficients and the standard errors of the coefficients for the final $\ln \left[\varepsilon_{1000}\right]$ model. This model was fit to the 527 glasses remaining after the glass screening described above. The statistics for the model fit are summarized in Table 3.19. The $\mathrm{R}^{2}$ coefficient of $>0.89$ suggests that the data are well represented by the model. The $R^{2}{ }_{A}$ value suggests that there are not too many coefficients, or the data are not over-fit. The $R_{P}^{2}$ being so close to the $R^{2}$ value suggests that there are not any very influential data points, and the model is likely to predict similarly for new data as it does for the model development data.

Table 3.18. $\operatorname{In}\left[\varepsilon_{1000}\right]$ Model Coefficients

\begin{tabular}{lcl}
\hline Term $(i)$ & Coefficient $\left(b_{\varepsilon 1000, i}\right)$ & Std Error \\
\hline $\mathrm{Al}_{2} \mathrm{O}_{3}$ & -0.234445 & 0.252654 \\
$\mathrm{~B}_{2} \mathrm{O}_{3}$ & 0.8170189 & 0.218903 \\
$\mathrm{CaO}$ & -0.812738 & 0.314498 \\
$\mathrm{Fe}_{2} \mathrm{O}_{3}$ & 1.7770346 & 0.223832 \\
$\mathrm{Li}_{2} \mathrm{O}$ & 37.351081 & 1.017349 \\
$\mathrm{MgO}$ & -1.153956 & 0.519692 \\
$\mathrm{Na}{ }_{2} \mathrm{O}$ & 16.053203 & 0.26576 \\
$\mathrm{NiO}$ & -4.78784 & 2.762766 \\
$\mathrm{SiO}$ & -0.649396 & 0.108254 \\
$\mathrm{SrO}$ & 0.564366 & 0.386148 \\
$\mathrm{ZnO}$ & 4.7824589 & 0.561489 \\
$\mathrm{ZrO}_{2}$ & 0.2999368 & 0.306204 \\
$\mathrm{Others}$ & 1.9602265 & 0.229278 \\
$\mathrm{Na}_{2} \mathrm{O} \times \mathrm{Li}_{2} \mathrm{O}$ & -111.9769 & 7.452845
\end{tabular}

Table 3.19. Summary of $\ln \left[\varepsilon_{1000}\right]$ Model Fit Statistics

\begin{tabular}{lc}
\hline Statistic & Value \\
\hline$R^{2}$ & 0.8962 \\
$R_{A}^{2}$ & 0.8936 \\
$R_{P}^{2}$ & 0.8899 \\
$\mathrm{RMSE}$ & 0.1732 \\
Minimum & 0.840 \\
Mean & 2.854 \\
Maximum & 4.058 \\
$\mathrm{n}$ & 527 \\
$\mathrm{~N}$ & 14 \\
\hline
\end{tabular}

Figure 3.5 compares the predicted and measured $\ln \left[\varepsilon_{1000}\right]$ values. The data appear to be well represented by the model with no trends to over or under prediction. No single group of data (e.g., study) was found to be misrepresented by the model. 


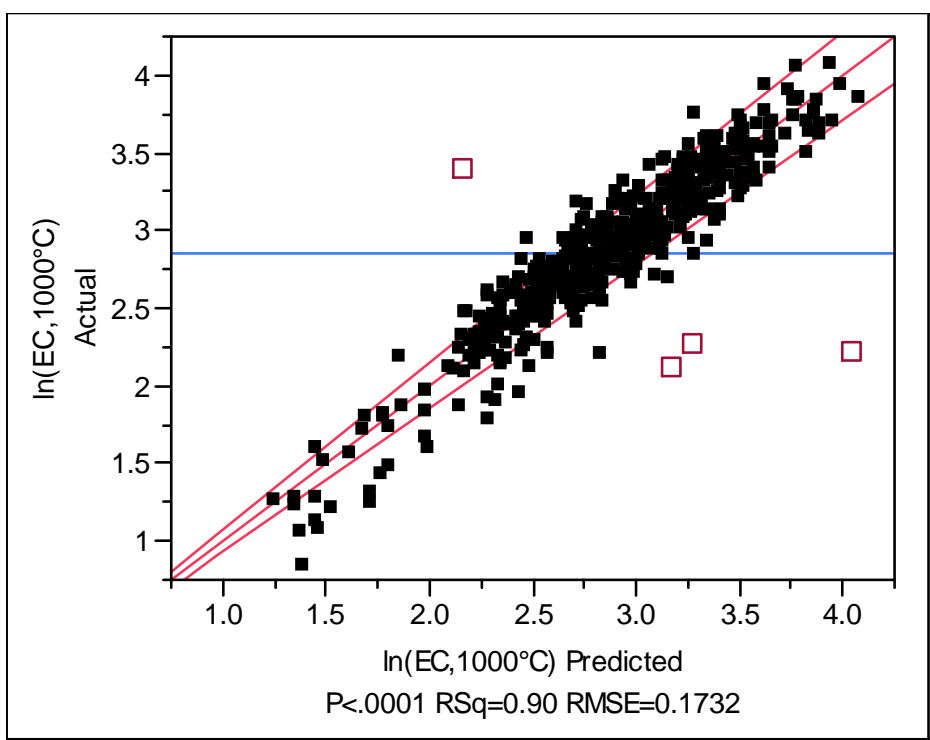

Figure 3.5. Measured vs. Predicted $\ln \left[\varepsilon_{1000}\right]$

This model is based on data spanning a specific glass composition region and therefore should only be relied upon within that region. The validity region, defined as the composition region of data used to develop the model, is listed in Table 3.20. Table 3.21 lists the correlation coefficients calculated for the model data. Only $\mathrm{Na}_{2} \mathrm{O}-\mathrm{Li}_{2} \mathrm{O}$ and $\mathrm{NiO}-\mathrm{ZnO}$ have a correlation of 0.5 or above.

Table 3.20. $\ln \left[\varepsilon_{1000}\right]$ Model Validity Region (wt\%)

\begin{tabular}{|c|c|c|}
\hline Component & Min & $\operatorname{Max}$ \\
\hline $\mathrm{Al}_{2} \mathrm{O}_{3}$ & 0.0 & 25.9 \\
\hline $\mathrm{B}_{2} \mathrm{O}_{3}$ & 0.0 & 20.0 \\
\hline $\mathrm{Bi}_{2} \mathrm{O}_{3}$ & 0.0 & 16.4 \\
\hline $\mathrm{CaO}$ & 0.0 & 11.9 \\
\hline $\mathrm{Fe}_{2} \mathrm{O}_{3}$ & 0.0 & 15.0 \\
\hline $\mathrm{K}_{2} \mathrm{O}$ & 0.0 & 5.4 \\
\hline $\mathrm{Li}_{2} \mathrm{O}$ & 0.0 & 7.9 \\
\hline $\mathrm{MgO}$ & 0.0 & 8.0 \\
\hline $\mathrm{MnO}$ & 0.0 & 8.0 \\
\hline $\mathrm{Na}_{2} \mathrm{O}$ & 2.5 & 23.0 \\
\hline $\mathrm{Nd}_{2} \mathrm{O}_{3}$ & 0.0 & 7.8 \\
\hline $\mathrm{NiO}$ & 0.0 & 1.8 \\
\hline $\mathrm{P}_{2} \mathrm{O}_{5}$ & 0.0 & 4.0 \\
\hline $\mathrm{SiO}_{2}$ & 26.0 & 60.0 \\
\hline $\mathrm{SrO}$ & 0.0 & 10.3 \\
\hline $\mathrm{ThO}_{2}$ & 0.0 & 6.0 \\
\hline $\mathrm{UO}_{3}$ & 0.0 & 6.5 \\
\hline ZnO & 0.0 & 5.8 \\
\hline $\mathrm{ZrO}_{2}$ & 0.0 & 15.5 \\
\hline Remaining & 0.3 & 6.4 \\
\hline
\end{tabular}


Table 3.21. Correlation Matrix for the $\ln \left[\varepsilon_{1000}\right]$ Model Dataset

\begin{tabular}{|c|c|c|c|c|c|c|c|c|c|c|}
\hline Corr & $\mathrm{Al}_{2} \mathrm{O}_{3} \quad \mathrm{~B}_{2} \mathrm{O}_{3}$ & $\mathrm{CaO}$ & $\mathrm{Fe}_{2} \mathrm{O}_{3} \mathrm{Li}_{2} \mathrm{O}$ & $\mathrm{MgO}$ & $\mathrm{Na}_{2} \mathrm{O}$ & $\mathrm{NiO}$ & $\mathrm{SiO}_{2}$ & SrO & $\mathrm{ZnO}$ & $\mathrm{ZrO}_{2}$ Others \\
\hline$\overline{\mathrm{Al}_{2} \mathrm{O}_{3}}$ & 1.000 & & & & & & & & & \\
\hline $\mathrm{B}_{2} \mathrm{O}_{3}$ & 0.2451 .000 & & & & & & & & & \\
\hline $\mathrm{CaO}$ & $0.191 \quad 0.155$ & 1.000 & & & & & & & & \\
\hline $\mathrm{Fe}_{2} \mathrm{O}_{3}$ & $-0.275-0.195$ & -0.325 & 1.000 & & & & & & & \\
\hline $\mathrm{Li}_{2} \mathrm{O}$ & $0.028 \quad 0.001$ & -0.030 & -0.0371 .000 & & & & & & & \\
\hline $\mathrm{MgO}$ & -0.0690 .106 & 0.236 & $-0.256-0.046$ & 1.000 & & & & & & \\
\hline $\mathrm{Na}_{2} \mathrm{O}$ & $-0.037-0.157$ & -0.244 & $-0.014-0.575$ & -0.078 & 1.000 & & & & & \\
\hline $\mathrm{NiO}$ & $-0.102-0.377$ & -0.322 & $0.208 \quad 0.121$ & -0.240 & -0.058 & 1.000 & & & & \\
\hline $\mathrm{SiO}_{2}$ & $-0.493-0.242$ & -0.086 & -0.0920 .237 & 0.161 & -0.201 & 0.062 & 1.000 & & & \\
\hline SrO & $-0.066-0.149$ & -0.296 & 0.2210 .016 & -0.253 & -0.158 & 0.105 & -0.305 & 1.000 & & \\
\hline $\mathrm{ZnO}$ & -0.1300 .061 & 0.343 & $-0.139-0.210$ & 0.155 & 0.026 & -0.506 & -0.040 & -0.11 & 1.000 & \\
\hline $\mathrm{ZrO}_{2}$ & $-0.350-0.212$ & -0.176 & -0.0790 .033 & -0.140 & 0.007 & 0.129 & -0.091 & 0.116 & -0.029 & 1.000 \\
\hline Others & $-0.098-0.442$ & -0.325 & $0.088-0.098$ & -0.309 & -0.055 & 0.489 & -0.267 & 0.369 & -0.247 & 0.2121 .000 \\
\hline
\end{tabular}

A variance-covariance matrix is required to calculate the measurement plus prediction uncertainties of this model. This matrix is defined as:

$$
\mathbf{V}_{\alpha}=\operatorname{RMSE}^{2}\left[\mathbf{X}_{\alpha}^{\mathrm{T}} \mathbf{X}_{\alpha}\right]^{-1}
$$

The use of $\mathbf{V}_{\alpha}$ is explained in detail elsewhere (e.g., Cornell 2002, Hrma et al. 1994, Piepel et al. 2008a, Vienna and Kim 2008). The $\mathbf{V}_{\ln [\varepsilon 1000]}$ is given in Appendix $C$ with the other variancecovariance matrices.

\section{Model Validation}

To validate the model, data not used in model fitting must be obtained. Because all appropriate data within the desired composition region were used in model fitting, subsets of the model data were used to validate the model. The data were sorted by $\ln \left[\varepsilon_{1000}\right]$ value. The data were then numbered $1,2,3,4,5,1,2, \ldots$ to split them into five roughly representative groups. The model was then refit to subsets 2 to 5 and used to predict data in subset 1 . Then the model was fit to each group of four subsets and used to predict the remaining subset in sequence.

Table 3.22 summarizes the results of the model validation. The coefficients are reasonably close with an RSD of less than $25 \%$ with the exceptions of $\mathrm{Al}_{2} \mathrm{O}_{3}, \mathrm{MgO}$, SrO, and $\mathrm{ZrO}_{2}$ which are all very near zero and $\mathrm{NiO}$ that has a high standard error.

The model fit $R^{2}$ and $R_{P}^{2}$ values are all close to each other at 0.89 . The $R_{V}^{2}$ are all between 0.88 and 0.90 . This model is well validated and should give predictions of unknown data within the model validity region nearly as well as model fit data. 
Table 3.22. Summary of $\ln \left[\varepsilon_{1000}\right]$ Model Validation

\begin{tabular}{|c|c|c|c|c|c|c|c|}
\hline Term & All Data & Set 1 & Set 2 & Set 3 & Set 4 & Set 5 & \%RSD \\
\hline $\mathrm{Al}_{2} \mathrm{O}_{3}$ & -0.23 & -0.23 & -0.14 & -0.35 & -0.31 & -0.16 & -38.2 \\
\hline $\mathrm{B}_{2} \mathrm{O}_{3}$ & 0.82 & 0.91 & 0.78 & 0.75 & 0.83 & 0.76 & 8.3 \\
\hline $\mathrm{CaO}$ & -0.81 & -1.02 & -0.74 & -0.62 & -0.85 & -0.77 & -18.3 \\
\hline $\mathrm{Fe}_{2} \mathrm{O}_{3}$ & 1.78 & 1.63 & 1.82 & 2.04 & 1.59 & 1.75 & 10.1 \\
\hline $\mathrm{Li}_{2} \mathrm{O}$ & 37.35 & 37.08 & 37.00 & 37.24 & 37.98 & 37.72 & 1.1 \\
\hline $\mathrm{MgO}$ & -1.15 & -1.23 & -1.66 & -0.77 & -1.11 & -0.96 & -29.0 \\
\hline $\mathrm{Na}_{2} \mathrm{O}$ & 16.05 & 16.08 & 15.87 & 16.09 & 16.23 & 16.06 & 0.8 \\
\hline $\mathrm{NiO}$ & -4.79 & -0.19 & -6.39 & -5.93 & -4.51 & -6.61 & -55.6 \\
\hline $\mathrm{SiO}_{2}$ & -0.65 & -0.66 & -0.58 & -0.69 & -0.66 & -0.67 & -6.5 \\
\hline $\mathrm{SrO}$ & 0.56 & 1.00 & 0.23 & 0.34 & 0.67 & 0.61 & 53.8 \\
\hline $\mathrm{ZnO}$ & 4.78 & 5.58 & 4.63 & 4.40 & 4.56 & 4.66 & 9.7 \\
\hline $\mathrm{ZrO}_{2}$ & 0.30 & 0.31 & 0.52 & 0.34 & 0.05 & 0.32 & 55.9 \\
\hline Others & 1.96 & 1.72 & 2.02 & 1.99 & 1.92 & 2.15 & 8.0 \\
\hline $\mathrm{Na}_{2} \mathrm{O} \times \mathrm{Li}_{2} \mathrm{O}$ & -112 & -111 & -111 & -109 & -115 & -114 & -2.3 \\
\hline $\mathrm{R}^{2}$ & 0.8962 & 0.8933 & 0.8960 & 0.8990 & 0.8940 & 0.8993 & \\
\hline $\mathrm{R}_{\mathrm{A}}^{2}$ & 0.8936 & 0.8899 & 0.8927 & 0.8958 & 0.8906 & 0.8961 & \\
\hline $\mathrm{R}_{\mathrm{P}}^{2}$ & 0.8899 & 0.8855 & 0.8879 & 0.8912 & 0.8860 & 0.8911 & \\
\hline RMSE & 0.1732 & 0.1744 & 0.1739 & 0.1717 & 0.1762 & 0.1720 & \\
\hline Mean & 2.8533 & 2.8540 & 2.8548 & 2.8535 & 2.8521 & 2.8505 & \\
\hline $\mathrm{n}$ & 527 & 421 & 422 & 422 & 422 & 422 & \\
\hline$R_{v}^{2}$ & 0.8917 & 0.9003 & 0.8937 & 0.8807 & 0.9030 & 0.8808 & \\
\hline
\end{tabular}

\subsection{3 $\varepsilon$ at $1100^{\circ} \mathrm{C}$}

\section{Model Development}

The data set included 548 glasses with $\ln \left[\varepsilon_{1100}\right]$ for model development. Preliminary model development was performed using stepwise regression methods allowing only first-order terms. Those components with sufficient data to allow for coefficient estimates were included in the stepwise process. ${ }^{(a)}$

$\mathrm{Al}_{2} \mathrm{O}_{3}, \mathrm{~B}_{2} \mathrm{O}_{3}, \mathrm{Bi}_{2} \mathrm{O}_{3}, \mathrm{CaO}, \mathrm{F}, \mathrm{Fe}_{2} \mathrm{O}_{3}, \mathrm{~K}_{2} \mathrm{O}, \mathrm{La}_{2} \mathrm{O}_{3}, \mathrm{Li}_{2} \mathrm{O}, \mathrm{MgO}, \mathrm{MnO}, \mathrm{Na}_{2} \mathrm{O}, \mathrm{Nd}_{2} \mathrm{O}_{3}$, $\mathrm{NiO}, \mathrm{P}_{2} \mathrm{O}_{5}, \mathrm{PbO}, \mathrm{SiO}_{2}, \mathrm{SrO}, \mathrm{ThO}_{2}, \mathrm{UO}_{3}, \mathrm{ZnO}$, and $\mathrm{ZrO}_{2}$

Stepwise regression comparing the coefficient to the value of zero while maintaining the intercept near the average response of $3.29 \ln [\mathrm{S} / \mathrm{m}]$ was used to reduce the influential components to:

$$
\mathrm{Al}_{2} \mathrm{O}_{3}, \mathrm{~B}_{2} \mathrm{O}_{3}, \mathrm{CaO}, \mathrm{Fe}_{2} \mathrm{O}_{3}, \mathrm{Li}_{2} \mathrm{O}, \mathrm{MgO}, \mathrm{Na}_{2} \mathrm{O}, \mathrm{NiO}, \mathrm{SiO}_{2}, \mathrm{SrO}, \mathrm{ZnO} \text {, and } \mathrm{ZrO}_{2}
$$

Attempts were made to improve the fit of the model data by adding a limited number (up to 5) second-order terms. The term found to most significantly improve the fit to model data was $\mathrm{Na}_{2} \mathrm{O} \times \mathrm{Li}_{2} \mathrm{O}$. The final set of terms was exactly the same as other EC models.

(a) A number of methods were used to determine if a component had sufficient variation to include in the model development. Chief among them are the visual examination of pairwise concentration plots in the form of a scatterplot matrix. The absolute maximum concentrations of the components along with the number of glasses containing the component over $10 \%$ of the maximum concentration were also considered. 
Six glasses were found to be outliers in both first-order and first-order plus $\mathrm{Na}_{2} \mathrm{O} \times \mathrm{Li}_{2} \mathrm{O}$ models: CVS2-81, LAWM30, HLW07-28, HLW07-40, C4D-04, and C4D-15. ${ }^{\text {(a) }}$ These data points were removed from the model fitting dataset, leaving 542 datapoints. A scatterplot matrix of these data is given in Appendix B.

Table 3.23 lists the coefficients and the standard errors of the coefficients for the final $\ln \left[\varepsilon_{1100}\right]$ model. This model was fit to the 527 glasses remaining after glass screening described above. The statistics for the model fit are summarized in Table 3.24. The $R^{2}$ coefficient of $>0.88$ suggests that the data are well represented by the model. The $R^{2}$-adjusted value suggests that there are not too many coefficients, or the data are not over-fit. The $R^{2}$-predicted of $\sim 0.88$ being so close to the $R^{2}$ value suggests that there are not any very influential data points, and the model is likely to predict similarly for new data as it does for the model development data.

Table 3.23. $\ln \left[\varepsilon_{1100}\right]$ Model Coefficients

\begin{tabular}{lcl}
\hline Term $(i)$ & Coefficient $\left(b_{\varepsilon 1100, i}\right)$ & Std Error \\
\hline $\mathrm{Al}_{2} \mathrm{O}_{3}$ & 0.0821118 & 0.240141 \\
$\mathrm{~B}_{2} \mathrm{O}_{3}$ & 1.7269964 & 0.200007 \\
$\mathrm{CaO}$ & 0.4816237 & 0.2933 \\
$\mathrm{Fe}_{2} \mathrm{O}_{3}$ & 2.3177094 & 0.21044 \\
$\mathrm{Li}_{2} \mathrm{O}$ & 35.619913 & 0.964786 \\
$\mathrm{MgO}$ & 0.1206499 & 0.488016 \\
$\mathrm{Na}$ & 15.435281 & 0.251401 \\
$\mathrm{NiO}$ & -3.347901 & 2.468248 \\
$\mathrm{SiO}$ & -0.067465 & 0.100487 \\
$\mathrm{SrO}$ & 1.8585572 & 0.362634 \\
$\mathrm{ZnO}$ & 6.0673766 & 0.530546 \\
$\mathrm{ZrO}_{2}$ & 0.9202707 & 0.286307 \\
$\mathrm{Others}$ & 2.4486435 & 0.213806 \\
$\mathrm{Na}_{2} \mathrm{O} \times \mathrm{Li}_{2} \mathrm{O}$ & -101.2064 & 7.176916 \\
\hline
\end{tabular}

Table 3.24. Summary of $\ln \left[\varepsilon_{1100}\right]$ Model Fit Statistics

\begin{tabular}{ll}
\hline Statistic & \multicolumn{1}{c}{ Value } \\
\hline$R^{2}$ & 0.8856 \\
$R_{A}^{2}$ & 0.8828 \\
$R_{P}^{2}$ & 0.8789 \\
$\mathrm{RMSE}$ & 0.1673 \\
Minimum & 1.53 \\
Mean & 3.30 \\
Maximum & 4.42 \\
$\mathrm{n}$ & 542 \\
$\mathrm{~N}$ & 14 \\
\hline
\end{tabular}

Figure 3.6 compares the predicted and measured $\ln \left[\varepsilon_{1100}\right]$ values. The data appear to be well represented by the model with no trends to over or under prediction. No single group of data (e.g., study) was found to be misrepresented by the model.

(a) The first four glasses were also outliers for $\ln \left[\varepsilon_{1000}\right]$ model development while the last two glasses did not contain $\ln \left[\varepsilon_{1000}\right]$ data. 


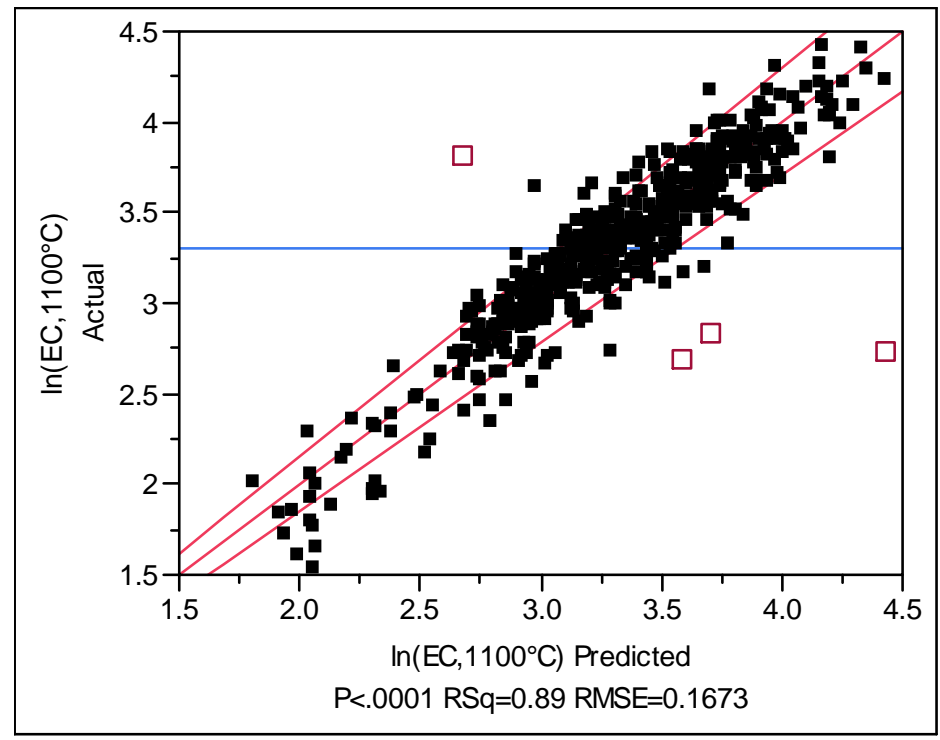

Figure 3.6. Measured vs. Predicted $\ln \left[\varepsilon_{1100}\right]$

This model is based on data spanning a specific glass composition region and therefore should only be relied upon within that region. The validity region, defined as the composition region of data used to develop the model, is listed in Table 3.20. Table 3.26 lists the correlation coefficients calculated for the model data. Only $\mathrm{Na}_{2} \mathrm{O}-\mathrm{Li}_{2} \mathrm{O}$ and $\mathrm{NiO}-\mathrm{ZnO}$ have a correlation of 0.5 or above.

Table 3.25. $\ln \left[\varepsilon_{1100}\right]$ Model Validity Region (wt\%)

\begin{tabular}{lrr}
\hline Component & \multicolumn{1}{c}{ Min } & Max \\
\hline $\mathrm{Al}_{2} \mathrm{O}_{3}$ & 0.0 & 25.9 \\
$\mathrm{~B}_{2} \mathrm{O}_{3}$ & 0.0 & 20.0 \\
$\mathrm{Bi}_{2} \mathrm{O}_{3}$ & 0.0 & 16.4 \\
$\mathrm{CaO}$ & 0.0 & 13.9 \\
$\mathrm{Fe}_{2} \mathrm{O}_{3}$ & 0.0 & 15.0 \\
$\mathrm{~K}_{2} \mathrm{O}$ & 0.0 & 5.4 \\
$\mathrm{Li}_{2} \mathrm{O}$ & 0.0 & 7.9 \\
$\mathrm{MgO}$ & 0.0 & 8.0 \\
$\mathrm{MnO}$ & 0.0 & 8.0 \\
$\mathrm{Na}_{2} \mathrm{O}$ & 2.5 & 23.0 \\
$\mathrm{Nd}_{2} \mathrm{O}_{3}$ & 0.0 & 8.6 \\
$\mathrm{NiO}$ & 0.0 & 2.0 \\
$\mathrm{P}_{2} \mathrm{O}_{5}$ & 0.0 & 4.0 \\
$\mathrm{PbO}_{\mathrm{SiO}}$ & 0.0 & 5.2 \\
$\mathrm{SrO}_{2}$ & 26.0 & 60.0 \\
$\mathrm{ThO}_{2}$ & 0.0 & 10.3 \\
$\mathrm{UO}_{3}$ & 0.0 & 6.0 \\
$\mathrm{ZnO}_{\mathrm{ZrO}}$ & 0.0 & 6.5 \\
$\mathrm{Remaining}_{2}$ & 0.0 & 5.8 \\
\hline & 0.0 & 15.5 \\
\hline
\end{tabular}


Table 3.26. Correlation Matrix for the $\ln \left[\varepsilon_{1100}\right]$ Model Dataset

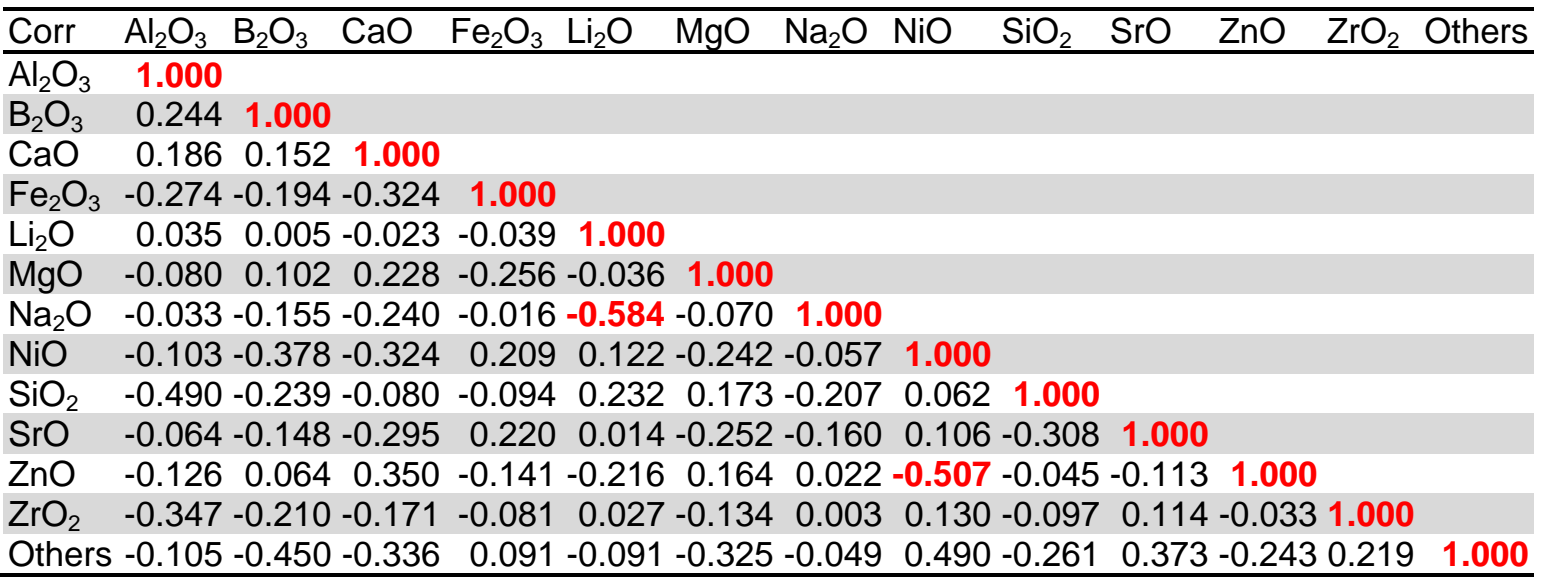

The $\mathbf{V}_{\ln [\varepsilon 1100]}$ is given in Appendix $\mathbf{C}$ with the other variance-covariance matrices.

\section{Model Validation}

Table 3.27 summarizes the results of the model validation. The coefficients are reasonably close with an $\mathrm{RSD}$ of less than $25 \%$ with the exceptions of $\mathrm{Al}_{2} \mathrm{O}_{3}, \mathrm{CaO}, \mathrm{MgO}, \mathrm{SiO}_{2}$, and $\mathrm{ZrO}_{2}$, which are all very near zero, and $\mathrm{NiO}$ that has a high standard error.

The model fit $R^{2}$ and $R_{P}^{2}$ values are all close to each other at 0.88 . The $R_{V}^{2}$ are all between 0.86 and 0.89 . This model is well validated and should give predictions of unknown data within the model validity region nearly as well as the model fit data.

Table 3.27. Summary of $\ln \left[\varepsilon_{1100}\right]$ Model Validation

\begin{tabular}{|c|c|c|c|c|c|c|c|}
\hline Term & All Data & Set 1 & Set 2 & Set 3 & Set 4 & Set 5 & \%RSD \\
\hline $\mathrm{Al}_{2} \mathrm{O}_{3}$ & 0.08 & 0.24 & 0.01 & -0.05 & 0.20 & 0.01 & 153.2 \\
\hline $\mathrm{B}_{2} \mathrm{O}_{3}$ & 1.73 & 1.74 & 1.90 & 1.67 & 1.70 & 1.63 & 6.0 \\
\hline $\mathrm{CaO}$ & 0.48 & 0.81 & 0.45 & 0.31 & 0.21 & 0.66 & 51.2 \\
\hline $\mathrm{Fe}_{2} \mathrm{O}_{3}$ & 2.32 & 2.34 & 2.35 & 2.26 & 2.22 & 2.41 & 3.3 \\
\hline $\mathrm{Li}_{2} \mathrm{O}$ & 35.62 & 35.48 & 35.86 & 34.95 & 35.25 & 36.37 & 1.6 \\
\hline $\mathrm{MgO}$ & 0.12 & 0.19 & -0.11 & 0.02 & 0.17 & 0.35 & 144.6 \\
\hline $\mathrm{Na}_{2} \mathrm{O}$ & 15.44 & 15.40 & 15.49 & 15.42 & 15.35 & 15.48 & 0.4 \\
\hline $\mathrm{NiO}$ & -3.35 & -4.73 & -3.01 & -4.40 & -0.74 & -3.99 & -48.1 \\
\hline $\mathrm{SiO}_{2}$ & -0.07 & -0.10 & -0.14 & 0.02 & -0.02 & -0.11 & -97.6 \\
\hline $\mathrm{SrO}^{-}$ & 1.86 & 2.02 & 1.55 & 1.79 & 2.01 & 1.94 & 10.7 \\
\hline $\mathrm{ZnO}$ & 6.07 & 5.67 & 6.15 & 5.81 & 6.62 & 6.11 & 6.1 \\
\hline $\mathrm{ZrO}_{2}$ & 0.92 & 0.92 & 0.89 & 1.05 & 0.80 & 0.94 & 9.7 \\
\hline Others & 2.45 & 2.64 & 2.53 & 2.43 & 2.24 & 2.41 & 6.1 \\
\hline $\mathrm{Na}_{2} \mathrm{O} \times \mathrm{Li}_{2} \mathrm{O}$ & -101.21 & -100.79 & -100.09 & -97.58 & -100.18 & -105.47 & -2.8 \\
\hline $\mathrm{R}^{2}$ & 0.8856 & 0.8841 & 0.8914 & 0.8831 & 0.8858 & 0.8869 & \\
\hline $\mathrm{R}^{2} \mathrm{~A}$ & 0.8828 & 0.8805 & 0.8881 & 0.8795 & 0.8822 & 0.8834 & \\
\hline $\mathrm{R}_{\mathrm{P}}^{2}$ & 0.8789 & 0.8760 & 0.8834 & 0.8746 & 0.8772 & 0.8782 & \\
\hline RMSE & 0.1673 & 0.1677 & 0.1627 & 0.1700 & 0.1684 & 0.1679 & \\
\hline Mean & 3.2979 & 3.2991 & 3.2976 & 3.2988 & 3.2975 & 3.2963 & \\
\hline $\mathrm{n}$ & 542 & 433 & 433 & 434 & 434 & 434 & \\
\hline$R^{2} v$ & 0.8793 & 0.8871 & 0.8607 & 0.8917 & 0.8808 & 0.8761 & \\
\hline
\end{tabular}




\subsection{4 $\varepsilon$ at $1150^{\circ} \mathrm{C}$}

\section{Model Development}

The data set included 583 glasses with $\ln \left[\varepsilon_{1150}\right]$ for model development. Preliminary model development was performed using stepwise regression methods allowing only first-order terms. Those components with sufficient data to allow for coefficient estimates were included in the stepwise process. ${ }^{(a)}$

$\mathrm{Al}_{2} \mathrm{O}_{3}, \mathrm{~B}_{2} \mathrm{O}_{3}, \mathrm{Bi}_{2} \mathrm{O}_{3}, \mathrm{CaO}, \mathrm{F}, \mathrm{Fe}_{2} \mathrm{O}_{3}, \mathrm{~K}_{2} \mathrm{O}, \mathrm{La}_{2} \mathrm{O}_{3}, \mathrm{Li} 2 \mathrm{O}, \mathrm{MgO}, \mathrm{MnO}, \mathrm{Na}_{2} \mathrm{O}, \mathrm{Nd}_{2} \mathrm{O}_{3}$, $\mathrm{NiO}, \mathrm{P}_{2} \mathrm{O}_{5}, \mathrm{PbO}, \mathrm{SiO}_{2}, \mathrm{SrO}, \mathrm{ThO}_{2}, \mathrm{UO}_{3}, \mathrm{ZnO}$, and $\mathrm{ZrO}_{2}$

Stepwise regression comparing the coefficient to the value of zero while maintaining the intercept near the average response of $3.46 \ln [\mathrm{S} / \mathrm{m}]$ was used to reduce the influential components to:

$$
\mathrm{Al}_{2} \mathrm{O}_{3}, \mathrm{~B}_{2} \mathrm{O}_{3}, \mathrm{CaO}, \mathrm{Fe}_{2} \mathrm{O}_{3}, \mathrm{Li}_{2} \mathrm{O}, \mathrm{MgO}, \mathrm{Na}_{2} \mathrm{O}, \mathrm{NiO}, \mathrm{SiO}_{2}, \mathrm{SrO}, \mathrm{ZnO} \text {, and } \mathrm{ZrO}_{2}
$$

Attempts were made to improve the fit of the model data by adding a limited number (up to 5) second-order terms. The term found to most significantly improve the fit to model data was $\mathrm{Na}_{2} \mathrm{O} \times \mathrm{Li}_{2} \mathrm{O}$. The final set of terms was exactly the same as other EC models.

Eight glasses were found to be outliers in both first-order and first-order plus $\mathrm{Na}_{2} \mathrm{O} \times \mathrm{Li}_{2} \mathrm{O}$ models: CVS1-10, CVS2-81, LAWM30, HLW07-28, HLW07-40, C4D-04, C4D-15, and HLW-EAl-06. ${ }^{b}$ These data points were removed from the model fitting dataset leaving 575 datapoints. A scatterplot matrix of these data is given in Appendix B.

Table 3.28 lists the coefficients and the standard errors of the coefficients for the final $\ln \left[\varepsilon_{1150}\right]$ model. This model was fit to the 575 glasses remaining after glass screening described above. The statistics for the model fit are summarized in Table 3.29. The $R^{2}$ coefficient of 0.88 suggests that the data are well represented by the model. The $R^{2}$-adjusted value suggests that there are not too many coefficients, or the data are not over-fit. The $R^{2}$-predicted of 0.87 being so close to the $R^{2}$ value suggests that there are not any very influential data points, and the model is likely to predict similarly for new data as it does for the model development data.

(a) A number of methods were used to determine if a component had sufficient variation to include in the model development. Chief among them are the visual examination of pairwise concentration plots in the form of a scatterplot matrix. The absolute maximum concentrations of the components along with the number of glasses containing the component over $10 \%$ of the maximum concentration were also considered.

(b) The outlier glasses were also outliers for $\ln \left[\varepsilon_{1000}\right]$ and $\ln \left[\varepsilon_{1100}\right]$ model development with the exception of four glasses not in the $\varepsilon_{1000}$ model set and two glasses not in the $\varepsilon_{1100}$ model set. 
Table 3.28. $\ln \left[\varepsilon_{1150}\right]$ Model Coefficients

\begin{tabular}{lcl}
\hline Term $(i)$ & Coefficient $\left(b_{\varepsilon 1150, i}\right)$ & Std Error \\
\hline $\mathrm{Al}_{2} \mathrm{O}_{3}$ & 0.3447447 & 0.193725 \\
$\mathrm{~B}_{2} \mathrm{O}_{3}$ & 2.2131363 & 0.1869 \\
$\mathrm{CaO}$ & 1.1386022 & 0.265163 \\
$\mathrm{Fe}_{2} \mathrm{O}_{3}$ & 2.4167485 & 0.198137 \\
$\mathrm{Li}_{2} \mathrm{O}$ & 35.108383 & 0.931871 \\
$\mathrm{MgO}$ & 0.2090895 & 0.451666 \\
$\mathrm{Na}_{2} \mathrm{O}$ & 15.226886 & 0.243131 \\
$\mathrm{NiO}$ & 0.284527 & 2.280322 \\
$\mathrm{SiO}$ & 0.1665476 & 0.0909 \\
$\mathrm{SrO}$ & 2.2279737 & 0.35226 \\
$\mathrm{ZnO}$ & 6.4620647 & 0.514638 \\
$\mathrm{ZrO}$ & 1.0944233 & 0.27449 \\
$\mathrm{Others}$ & 2.6305908 & 0.20218 \\
$\mathrm{Na}_{2} \mathrm{O} \times \mathrm{Li}_{2} \mathrm{O}$ & -100.2325 & 6.978406 \\
\hline
\end{tabular}

Table 3.29. Summary of $\ln \left[\varepsilon_{1150}\right]$ Model Fit Statistics

\begin{tabular}{ll}
\hline Statistic & Value \\
\hline$R^{2}$ & 0.8802 \\
$R_{A}^{2}$ & 0.8774 \\
$R_{P}^{2}$ & 0.8735 \\
RMSE & 0.1668 \\
Minimum & 1.70 \\
Mean & 3.47 \\
Maximum & 4.58 \\
$\mathrm{n}$ & 575 \\
$\mathrm{~N}$ & 14 \\
\hline
\end{tabular}

Figure 3.7 compares the predicted and measured $\ln \left[\varepsilon_{1150}\right]$ values. The data appear to be well represented by the model with no trends to over or under prediction. No single group of data (e.g., study) was found to be misrepresented by the model. 


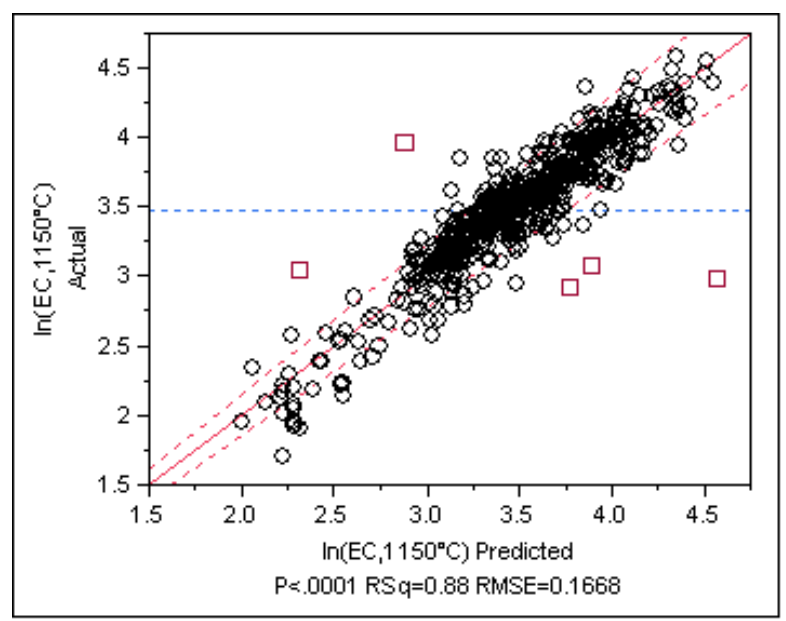

Figure 3.7. Measured vs. Predicted $\ln \left[\varepsilon_{1150}\right]$

This model is based on data spanning a specific glass composition region and therefore should only be relied upon within that region. The validity region, defined as the composition region of data used to develop the model, is listed in Table 3.30. Table 3.31 lists the correlation coefficients calculated for the model data. Only $\mathrm{Na}_{2} \mathrm{O}-\mathrm{Li}_{2} \mathrm{O}$ has a correlation of 0.5 or above.

Table 3.30. $\ln \left[\varepsilon_{1150}\right]$ Model Validity Region (wt $\left.\%\right)$

\begin{tabular}{lrr}
\hline Component & Min & Max \\
\hline $\mathrm{Al}_{2} \mathrm{O}_{3}$ & 0.0 & 26.6 \\
$\mathrm{~B}_{2} \mathrm{O}_{3}$ & 0.0 & 20.2 \\
$\mathrm{Bi}_{2} \mathrm{O}_{3}$ & 0.0 & 16.4 \\
$\mathrm{CaO}$ & 0.0 & 18.2 \\
$\mathrm{Fe}_{2} \mathrm{O}_{3}$ & 0.0 & 15.0 \\
$\mathrm{~K}_{2} \mathrm{O}$ & 0.0 & 6.1 \\
$\mathrm{Li}_{2} \mathrm{O}$ & 0.0 & 7.9 \\
$\mathrm{MgO}$ & 0.0 & 9.6 \\
$\mathrm{MnO}$ & 0.0 & 8.0 \\
$\mathrm{Na}_{2} \mathrm{O}$ & 2.5 & 23.0 \\
$\mathrm{Nd}_{2} \mathrm{O}_{3}$ & 0.0 & 8.6 \\
$\mathrm{NiO}$ & 0.0 & 2.1 \\
$\mathrm{P}_{2} \mathrm{O}_{5}$ & 0.0 & 9.0 \\
$\mathrm{PbO}_{\mathrm{SiO}}$ & 0.0 & 8.6 \\
$\mathrm{SrO}$ & 19.4 & 60.0 \\
$\mathrm{ThO}_{2}$ & 0.0 & 10.3 \\
$\mathrm{UO}$ & 0.0 & 6.0 \\
$\mathrm{ZnO}_{2 r O}$ & 0.0 & 6.5 \\
$\mathrm{ZrO}_{2}$ & 0.0 & 5.8 \\
$\mathrm{Remaining}$ & 0.0 & 15.5 \\
\hline & 0.3 & 6.4 \\
\hline
\end{tabular}

The $\mathbf{V}_{\operatorname{In}[\varepsilon 1150]}$ is given in Appendix $\mathbf{C}$ with the other variance-covariance matrices. 
Table 3.31. Correlation Matrix for the $\ln \left[\varepsilon_{1150}\right]$ Model Dataset

\begin{tabular}{|c|c|c|c|c|c|c|c|c|c|c|c|c|c|}
\hline Corr & $\mathrm{Al}_{2} \mathrm{O}_{3}$ & $\mathrm{~B}_{2} \mathrm{O}_{3}$ & $\mathrm{CaO}$ & $\mathrm{Fe}_{2} \mathrm{O}_{3}$ & $\mathrm{Li}_{2} \mathrm{O}$ & $\mathrm{MgO}$ & $\mathrm{Na}_{2} \mathrm{O}$ & $\mathrm{NiO}$ & $\mathrm{SiO}_{2}$ & SrO & $\mathrm{ZnO}$ & $\mathrm{ZrO}_{2}$ & Others \\
\hline $\mathrm{Al}_{2} \mathrm{O}_{3}$ & 1.000 & & & & & & & & & & & & \\
\hline $\mathrm{B}_{2} \mathrm{O}_{3}$ & 0.284 & 1.000 & & & & & & & & & & & \\
\hline $\mathrm{CaO}$ & 0.166 & 0.141 & 1.000 & & & & & & & & & & \\
\hline $\mathrm{Fe}_{2} \mathrm{O}_{3}$ & -0.279 & -0.207 & -0.334 & 1.000 & & & & & & & & & \\
\hline $\mathrm{Li}_{2} \mathrm{O}$ & 0.054 & -0.008 & -0.015 & -0.043 & 1.000 & & & & & & & & \\
\hline $\mathrm{MgO}$ & -0.071 & 0.086 & 0.234 & -0.260 & -0.043 & 1.000 & & & & & & & \\
\hline $\mathrm{Na}_{2} \mathrm{O}$ & -0.017 & -0.170 & -0.237 & -0.018 & -0.595 & -0.076 & 1.000 & & & & & & \\
\hline $\mathrm{NiO}$ & -0.137 & -0.335 & -0.329 & 0.224 & 0.145 & -0.231 & -0.046 & 1.000 & & & & & \\
\hline $\mathrm{SiO}_{2}$ & -0.495 & -0.215 & -0.047 & -0.093 & 0.241 & 0.187 & -0.215 & 0.029 & 1.000 & & & & \\
\hline $\mathrm{SrO}^{-}$ & -0.058 & -0.169 & -0.302 & 0.220 & 0.010 & -0.258 & -0.164 & 0.124 & -0.312 & 1.000 & & & \\
\hline $\mathrm{ZnO}$ & -0.112 & 0.034 & 0.362 & -0.147 & -0.229 & 0.155 & 0.014 & -0.496 & -0.033 & -0.122 & 1.000 & & \\
\hline $\mathrm{ZrO}_{2}$ & -0.355 & -0.194 & -0.166 & -0.083 & 0.028 & -0.132 & 0.003 & 0.115 & -0.112 & 0.121 & -0.026 & 1.000 & \\
\hline Others & -0.118 & -0.437 & -0.336 & 0.095 & -0.085 & -0.320 & -0.044 & 0.478 & -0.291 & 0.384 & -0.233 & 0.214 & $1.00 \mathrm{C}$ \\
\hline
\end{tabular}

\section{Model Validation}

Table 3.32 summarizes the results of the model validation. The coefficients are reasonably close with an RSD of less than $25 \%$ with the exceptions of $\mathrm{Al}_{2} \mathrm{O}_{3}, \mathrm{MgO}$, and $\mathrm{SiO}_{2}$, which are all very near zero and $\mathrm{NiO}$ that has a high standard error.

The model fit $R^{2}$ and $R_{P}^{2}$ values are all close to each other at 0.87 to 0.88 . The $R_{V}^{2}$ are all between 0.845 and 0.88 . This model is well validated and should give predictions of unknown data within the model validity region nearly as well as model fit data.

Table 3.32. Summary of $\ln \left[\varepsilon_{1150}\right]$ Model Validation

\begin{tabular}{|c|c|c|c|c|c|c|c|}
\hline Term & All Data & Set 1 & Set 2 & Set 3 & Set 4 & Set 5 & \%RSD \\
\hline $\mathrm{Al}_{2} \mathrm{O}_{3}$ & 0.34 & 0.43 & 0.32 & 0.45 & 0.23 & 0.21 & 31.9 \\
\hline $\mathrm{B}_{2} \mathrm{O}_{3}$ & 2.21 & 2.23 & 2.30 & 2.05 & 2.47 & 2.04 & 8.1 \\
\hline $\mathrm{CaO}$ & 1.14 & 1.04 & 1.24 & 1.31 & 1.26 & 0.85 & 17.0 \\
\hline $\mathrm{Fe}_{2} \mathrm{O}_{3}$ & 2.42 & 2.48 & 2.41 & 2.42 & 2.25 & 2.48 & 3.9 \\
\hline $\mathrm{Li}_{2} \mathrm{O}$ & 35.11 & 35.06 & 34.97 & 35.09 & 34.80 & 35.61 & 0.9 \\
\hline $\mathrm{MgO}$ & 0.21 & 0.63 & 0.05 & 0.28 & 0.12 & -0.03 & 126.4 \\
\hline $\mathrm{Na}_{2} \mathrm{O}$ & 15.23 & 15.29 & 15.17 & 15.29 & 15.13 & 15.25 & 0.5 \\
\hline $\mathrm{NiO}$ & 0.28 & 1.56 & 0.77 & 2.03 & 0.94 & -4.73 & 968 \\
\hline $\mathrm{SiO}_{2}$ & 0.17 & 0.12 & 0.15 & 0.15 & 0.19 & 0.25 & 30.8 \\
\hline SrO & 2.23 & 2.30 & 2.06 & 1.92 & 2.41 & 2.46 & 10.4 \\
\hline $\mathrm{ZnO}$ & 6.46 & 6.85 & 6.49 & 6.47 & 6.27 & 6.10 & 4.3 \\
\hline $\mathrm{ZrO}_{2}$ & 1.09 & 0.91 & 1.20 & 1.32 & 0.83 & 1.22 & 19.5 \\
\hline Others & 2.63 & 2.58 & 2.71 & 2.61 & 2.68 & 2.60 & 2.1 \\
\hline $\mathrm{Na}_{2} \mathrm{O} \times \mathrm{Li}_{2} \mathrm{O}$ & -100.23 & -100.36 & -98.94 & -102.42 & -96.98 & -102.03 & -2.2 \\
\hline $\mathrm{R}^{2}$ & 0.8802 & 0.8806 & 0.8830 & 0.8772 & 0.8795 & 0.8854 & \\
\hline $\mathrm{R}_{\mathrm{A}}^{2}$ & 0.8774 & 0.8771 & 0.8796 & 0.8736 & 0.8760 & 0.8821 & \\
\hline $\mathrm{R}_{\mathrm{P}}^{2}$ & 0.8735 & 0.8726 & 0.8743 & 0.8684 & 0.8709 & 0.8776 & \\
\hline RMSE & 0.1668 & 0.1662 & 0.1653 & 0.1695 & 0.1679 & 0.1640 & \\
\hline Mean & 3.4745 & 3.4771 & 3.4755 & 3.4745 & 3.4733 & 3.4720 & \\
\hline $\mathrm{n}$ & 575 & 460 & 460 & 460 & 460 & 460 & \\
\hline $\mathrm{R}^{2} \mathrm{v}$ & 0.8708 & 0.8751 & 0.8674 & 0.8874 & 0.8784 & 0.8455 & \\
\hline
\end{tabular}




\subsection{5 $\varepsilon$ at $1200^{\circ} \mathrm{C}$}

\section{Model Development}

The data set included 565 glasses with $\ln \left[\varepsilon_{1200}\right]$ for model development. Preliminary model development was performed using stepwise regression methods allowing only first-order terms. Those components with sufficient data to allow for coefficient estimates were included in the stepwise process. ${ }^{(a)}$

$\mathrm{Al}_{2} \mathrm{O}_{3}, \mathrm{~B}_{2} \mathrm{O}_{3}, \mathrm{Bi}_{2} \mathrm{O}_{3}, \mathrm{CaO}, \mathrm{F}, \mathrm{Fe}_{2} \mathrm{O}_{3}, \mathrm{~K}_{2} \mathrm{O}, \mathrm{La}_{2} \mathrm{O}_{3}, \mathrm{Li}_{2} \mathrm{O}, \mathrm{MgO}, \mathrm{MnO}, \mathrm{Na}_{2} \mathrm{O}, \mathrm{Nd}_{2} \mathrm{O}_{3}$, $\mathrm{NiO}, \mathrm{P}_{2} \mathrm{O}_{5}, \mathrm{PbO}, \mathrm{SiO}_{2}, \mathrm{SrO}, \mathrm{ThO}_{2}, \mathrm{UO}_{3}, \mathrm{ZnO}$, and $\mathrm{ZrO}_{2}$

Stepwise regression comparing the coefficient to the value of zero while maintaining the intercept near the average response of $3.64 \ln [\mathrm{S} / \mathrm{m}]$ was used to reduce the influential components to:

$$
\mathrm{Al}_{2} \mathrm{O}_{3}, \mathrm{~B}_{2} \mathrm{O}_{3}, \mathrm{CaO}, \mathrm{Fe}_{2} \mathrm{O}_{3}, \mathrm{Li}_{2} \mathrm{O}, \mathrm{MgO}, \mathrm{Na}_{2} \mathrm{O}, \mathrm{NiO}, \mathrm{SiO}_{2}, \mathrm{SrO}, \mathrm{ZnO} \text {, and } \mathrm{ZrO}_{2}
$$

Attempts were made to improve the fit of the model data by adding a limited number (up to 5) second-order terms. The term found to most significantly improve the fit to model data was $\mathrm{Na}_{2} \mathrm{O} \times \mathrm{Li}_{2} \mathrm{O}$. The final set of terms was exactly the same as other $\mathrm{EC}$ models.

Seven glasses were found to be outliers in both first-order and first-order plus $\mathrm{Na}_{2} \mathrm{O} \times \mathrm{Li}_{2} \mathrm{O}$ models: CVS1-10, CVS2-81, LAWM30, HLW07-28, HLW07-40, C4D-04, and C4D$15{ }^{(b)}$ These data points were removed from the model fitting dataset, leaving 558 datapoints. A scatterplot matrix of these data is given in Appendix B.

Table 3.33 lists the coefficients and the standard errors of the coefficients for the final $\ln \left[\varepsilon_{1200}\right]$ model. This model was fit to the 527 glasses remaining after the glass screening described above. The statistics for the model fit are summarized in Table 3.34. The $R^{2}$ coefficient of $\sim 0.88$ suggests that the data are well represented by the model. The $R^{2}$-adjusted value suggests that there are not too many coefficients, or the data are not over-fit. The $R^{2}$ predicted of 0.87 being so close to the $R^{2}$ value suggests that there are not any very influential data points, and the model is likely to predict similarly for new data as it does for the model development data.

(a) A number of methods were used to determine if a component had sufficient variation to include in the model development. Chief among them are the visual examination of pairwise concentration plots in the form of a scatterplot matrix. The absolute maximum concentrations of the components along with the number of glasses containing the component over $10 \%$ of the maximum concentration were also considered.

(b) All seven outliers were found to be outliers at all temperatures for which they had existing data. 
Table 3.33. $\ln \left[\varepsilon_{1200}\right]$ Model Coefficients

\begin{tabular}{lcl}
\hline Term $(i)$ & Coefficient $\left(b_{\varepsilon 1200, i}\right)$ & Std Error \\
\hline $\mathrm{Al}_{2} \mathrm{O}_{3}$ & 0.3312382 & 0.219805 \\
$\mathrm{~B}_{2} \mathrm{O}_{3}$ & 2.380146 & 0.181394 \\
$\mathrm{CaO}$ & 1.7033775 & 0.281154 \\
$\mathrm{Fe}_{2} \mathrm{O}_{3}$ & 2.5149398 & 0.195437 \\
$\mathrm{Li}_{2} \mathrm{O}$ & 33.714638 & 0.921843 \\
$\mathrm{MgO}$ & 0.9980765 & 0.44056 \\
$\mathrm{Na}_{2} \mathrm{O}$ & 14.801322 & 0.237223 \\
$\mathrm{NiO}$ & -1.239448 & 2.278039 \\
$\mathrm{SiO}$ & 0.534619 & 0.092159 \\
$\mathrm{SrO}$ & 2.7731117 & 0.342327 \\
$\mathrm{ZnO}$ & 6.5598526 & 0.505838 \\
$\mathrm{ZrO}$ & 1.4700505 & 0.261058 \\
Others & 2.8441506 & 0.198924 \\
$\mathrm{Na}_{2} \mathrm{O} \times \mathrm{Li}_{2} \mathrm{O}$ & -91.29459 & 6.898661 \\
\hline
\end{tabular}

Table 3.34. Summary of $\ln \left[\varepsilon_{1200}\right]$ Model Fit Statistics

\begin{tabular}{lc}
\hline Statistic & Value \\
\hline$R^{2}$ & 0.8770 \\
$R_{A}^{2}$ & 0.8740 \\
$R_{P}^{2}$ & 0.8704 \\
RMSE & 0.1600 \\
Minimum & 2.13 \\
Mean & 3.65 \\
Maximum & 4.73 \\
$\mathrm{n}$ & 558 \\
$\mathrm{~N}$ & 14 \\
\hline
\end{tabular}

Figure 3.8 compares the predicted and measured $\ln \left[\varepsilon_{1200}\right]$ values. The data appear to be well represented by the model with no trends to over or under prediction. No single group of data (e.g., study) was found to be misrepresented by the model.

This model is based on data spanning a specific glass composition region and therefore should only be relied upon within that region. The validity region, defined as the composition region of data used to develop the model, is listed in Table 3.35.

Table 3.36 lists the correlation coefficients calculated for the model data. Only $\mathrm{Na}_{2} \mathrm{O}$ $\mathrm{Li}_{2} \mathrm{O}$ and $\mathrm{NiO}-\mathrm{ZnO}$ have a correlation of 0.5 or above. 


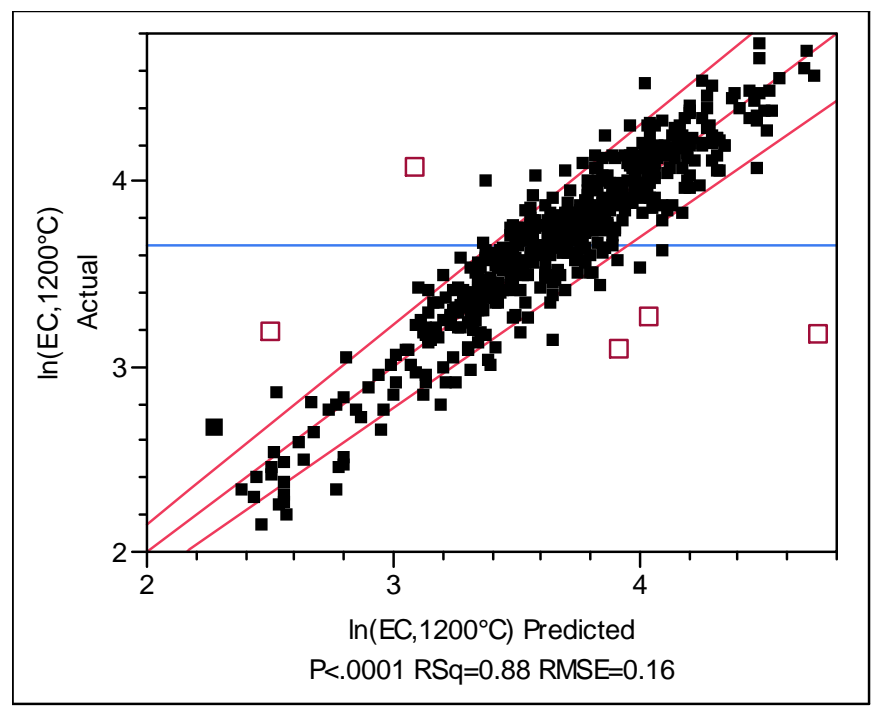

Figure 3.8. Measured vs. Predicted $\ln \left[\varepsilon_{1200}\right]$

Table 3.35. $\ln \left[\varepsilon_{1200}\right]$ Model Validity Region (wt\%)

\begin{tabular}{lrr}
\hline Component & Min & Max \\
\hline $\mathrm{Al}_{2} \mathrm{O}_{3}$ & 0.0 & 25.9 \\
$\mathrm{~B}_{2} \mathrm{O}_{3}$ & 0.0 & 20.0 \\
$\mathrm{Bi}_{2} \mathrm{O}_{3}$ & 0.0 & 16.4 \\
$\mathrm{CaO}$ & 0.0 & 13.9 \\
$\mathrm{Fe}_{2} \mathrm{O}_{3}$ & 0.0 & 15.0 \\
$\mathrm{~K}_{2} \mathrm{O}$ & 0.0 & 5.4 \\
$\mathrm{Li}_{2} \mathrm{O}$ & 0.0 & 7.9 \\
$\mathrm{MgO}$ & 0.0 & 9.6 \\
$\mathrm{MnO}$ & 0.0 & 8.0 \\
$\mathrm{Na}_{2} \mathrm{O}$ & 2.5 & 23.0 \\
$\mathrm{Nd}_{2} \mathrm{O}_{3}$ & 0.0 & 8.6 \\
$\mathrm{NiO}$ & 0.0 & 2.0 \\
$\mathrm{P}_{2} \mathrm{O}_{5}$ & 0.0 & 9.0 \\
$\mathrm{PbO}_{\mathrm{SiO}}$ & 0.0 & 5.2 \\
$\mathrm{SrO}_{2}$ & 26.0 & 60.1 \\
$\mathrm{ThO}_{2}$ & 0.0 & 10.3 \\
$\mathrm{UO}_{3}$ & 0.0 & 6.0 \\
$\mathrm{ZnO}_{\mathrm{ZrO}}$ & 0.0 & 6.5 \\
$\mathrm{Remaining}_{2}$ & 0.0 & 5.8 \\
\hline & 0.0 & 16.0 \\
\hline
\end{tabular}

The $\mathbf{V}_{\ln [\varepsilon 1200]}$ is given in Appendix $\mathbf{C}$ with the other variance-covariance matrices.

\section{Model Validation}

Table 3.37 summarizes the results of the model validation. The coefficients are reasonably close with an $\mathrm{RSD}$ of less than $25 \%$ with the exception of $\mathrm{Al}_{2} \mathrm{O}_{3}$, which is all very near zero, and $\mathrm{NiO}$ that has a high standard error. 
Table 3.36. Correlation Matrix for the $\ln \left[\varepsilon_{1200}\right]$ Model Dataset

\begin{tabular}{|c|c|c|c|c|c|c|c|c|c|c|c|c|c|}
\hline Corr & $\mathrm{Al}_{2} \mathrm{O}_{3}$ & $\mathrm{~B}_{2} \mathrm{O}_{3}$ & $\mathrm{CaO}$ & $\mathrm{Fe}_{2} \mathrm{O}_{3}$ & $\mathrm{Li}_{2} \mathrm{O}$ & $\mathrm{MgO}$ & $\mathrm{Na}_{2} \mathrm{O}$ & $\mathrm{NiO}$ & $\mathrm{SiO}_{2}$ & SrO & $\mathrm{ZnO}$ & $\mathrm{ZrO}_{2}$ & Others \\
\hline $\mathrm{Al}_{2} \mathrm{O}_{3}$ & 1.000 & & & & & & & & & & & & \\
\hline $\mathrm{B}_{2} \mathrm{O}_{3}$ & 0.125 & 1.000 & & & & & & & & & & & \\
\hline $\mathrm{CaO}$ & 0.082 & 0.098 & 1.000 & & & & & & & & & & \\
\hline $\mathrm{Fe}_{2} \mathrm{O}_{3}$ & -0.275 & -0.184 & -0.372 & 1.000 & & & & & & & & & \\
\hline $\mathrm{Li}_{2} \mathrm{O}$ & 0.029 & -0.031 & -0.031 & -0.041 & 1.000 & & & & & & & & \\
\hline $\mathrm{MgO}$ & -0.033 & 0.117 & 0.283 & -0.265 & -0.050 & 1.000 & & & & & & & \\
\hline $\mathrm{Na}_{2} \mathrm{O}$ & 0.029 & -0.135 & -0.198 & -0.019 & -0.600 & -0.075 & 1.000 & & & & & & \\
\hline $\mathrm{NiO}$ & -0.165 & -0.385 & -0.366 & 0.219 & 0.171 & -0.243 & -0.057 & 1.000 & & & & & \\
\hline $\mathrm{SiO}_{2}$ & -0.293 & -0.124 & 0.068 & -0.148 & 0.295 & 0.161 & -0.291 & 0.069 & 1.000 & & & & \\
\hline $\mathrm{SrO}$ & -0.011 & -0.137 & -0.309 & 0.216 & 0.015 & -0.266 & -0.175 & 0.122 & -0.396 & 1.000 & & & \\
\hline $\mathrm{ZnO}$ & -0.013 & 0.118 & 0.428 & -0.167 & -0.224 & 0.154 & -0.001 & -0.521 & -0.134 & -0.137 & 1.000 & & \\
\hline $\mathrm{ZrO}_{2}$ & -0.320 & -0.162 & -0.157 & -0.106 & 0.034 & -0.156 & -0.014 & 0.146 & -0.211 & 0.098 & -0.077 & 1.000 & \\
\hline Dthers & -0.131 & -0.467 & -0.348 & 0.099 & -0.066 & -0.327 & -0.055 & 0.473 & -0.333 & 0.391 & -0.243 & 0.236 & 1.000 \\
\hline
\end{tabular}

The model fit $R^{2}$ and $R_{P}^{2}$ values are all close to each other at 0.87 . The $R_{V}^{2}$ are quite scattered (between 0.835 and 0.90 ) with set 3 being notably low. This model is well validated and should give predictions of unknown data within the model validity region nearly as well as model fit data.

Table 3.37. Summary of $\ln \left[\varepsilon_{1200}\right]$ Model Validation

\begin{tabular}{|c|c|c|c|c|c|c|c|}
\hline Term & All Data & Set 1 & Set 2 & Set 3 & Set 4 & Set 5 & \%RSD \\
\hline$\overline{\mathrm{Al}_{2} \mathrm{O}_{3}}$ & 0.33 & 0.40 & 0.52 & 0.31 & 0.22 & 0.23 & 37.9 \\
\hline $\mathrm{B}_{2} \mathrm{O}_{3}$ & 2.38 & 2.32 & 2.29 & 2.41 & 2.44 & 2.43 & 2.9 \\
\hline $\mathrm{CaO}$ & 1.70 & 1.54 & 2.06 & 1.52 & 1.79 & 1.60 & 13.3 \\
\hline $\mathrm{Fe}_{2} \mathrm{O}_{3}$ & 2.51 & 2.55 & 2.65 & 2.47 & 2.39 & 2.52 & 3.9 \\
\hline $\mathrm{Li}_{2} \mathrm{O}$ & 33.71 & 33.82 & 33.00 & 33.88 & 33.52 & 34.26 & 1.4 \\
\hline $\mathrm{MgO}$ & 1.00 & 1.37 & 0.73 & 1.08 & 0.97 & 0.83 & 24.8 \\
\hline $\mathrm{Na}_{2} \mathrm{O}$ & 14.80 & 14.81 & 14.62 & 14.87 & 14.75 & 14.91 & 0.8 \\
\hline $\mathrm{NiO}$ & -1.24 & -0.74 & -1.68 & -1.84 & -1.02 & -1.29 & -36.6 \\
\hline $\mathrm{SiO}_{2}$ & 0.53 & 0.53 & 0.56 & 0.52 & 0.56 & 0.53 & 3.7 \\
\hline $\mathrm{SrO}$ & 2.77 & 2.74 & 2.86 & 2.79 & 2.80 & 2.65 & 2.9 \\
\hline $\mathrm{ZnO}$ & 6.56 & 6.64 & 6.42 & 6.52 & 6.47 & 6.73 & 2.0 \\
\hline $\mathrm{ZrO}_{2}$ & 1.47 & 1.59 & 1.91 & 1.37 & 1.33 & 1.15 & 19.8 \\
\hline Others & 2.84 & 2.89 & 2.65 & 2.91 & 2.85 & 2.91 & 3.9 \\
\hline $\mathrm{Na}_{2} \mathrm{O} \times \mathrm{Li}_{2} \mathrm{O}$ & -91.29 & -93.64 & -86.49 & -92.16 & -86.55 & -97.20 & -5.1 \\
\hline $\mathrm{R}^{2}$ & 0.8770 & 0.8726 & 0.8782 & 0.8874 & 0.8714 & 0.8789 & \\
\hline $\mathrm{R}_{\mathrm{A}}^{2}$ & 0.8740 & 0.8688 & 0.8745 & 0.8840 & 0.8675 & 0.8753 & \\
\hline $\mathrm{R}_{\mathrm{P}}^{2}$ & 0.8704 & 0.8642 & 0.8701 & 0.8794 & 0.8626 & 0.8708 & \\
\hline RMSE & 0.1600 & 0.1625 & 0.1592 & 0.1532 & 0.1649 & 0.1602 & \\
\hline Mean & 3.6511 & 3.6526 & 3.6514 & 3.6501 & 3.6514 & 3.6502 & \\
\hline $\mathrm{N}$ & 558 & 446 & 446 & 446 & 447 & 447 & \\
\hline$R^{2} v$ & 0.8708 & 0.8909 & 0.8654 & 0.8352 & 0.8976 & 0.8646 & \\
\hline
\end{tabular}




\subsubsection{Electrical Conductivity as a Function of Temperature}

The preceding subsections discussed the prediction of $\ln \left[\varepsilon_{T}, \mathrm{~S} / \mathrm{m}\right]$ where $\mathrm{T}$ is given as $1000,1100,1150$, and $1200^{\circ} \mathrm{C}$. The models are summarized in Table 3.38. The models are similar in many respects. The fit and validation statistics are similar, and the coefficients are similar as shown in Figure 3.9.

Table 3.38. $\operatorname{In}\left[\varepsilon_{T}\right]$ Model Coefficient Summary

\begin{tabular}{lcccc}
\hline \multicolumn{1}{c}{ Term } & $\ln \left[\varepsilon_{1000}\right]$ & $\ln \left[\varepsilon_{1100}\right]$ & $\ln \left[\varepsilon_{1150}\right]$ & $\ln \left[\varepsilon_{1200}\right]$ \\
\hline $\mathrm{Al}_{2} \mathrm{O}_{3}$ & -0.23 & 0.34 & 0.08 & 0.33 \\
$\mathrm{~B}_{2} \mathrm{O}_{3}$ & 0.82 & 2.21 & 1.73 & 2.38 \\
$\mathrm{CaO}$ & -0.81 & 1.14 & 0.48 & 1.70 \\
$\mathrm{Fe}_{2} \mathrm{O}_{3}$ & 1.78 & 2.42 & 2.32 & 2.51 \\
$\mathrm{Li}_{2} \mathrm{O}$ & 37.35 & 35.11 & 35.62 & 33.71 \\
$\mathrm{MgO}$ & -1.15 & 0.21 & 0.12 & 1.00 \\
$\mathrm{Na}_{2} \mathrm{O}$ & 16.05 & 15.23 & 15.44 & 14.80 \\
$\mathrm{NiO}$ & -4.79 & 0.28 & -3.35 & -1.24 \\
$\mathrm{SiO}$ & -0.65 & 0.17 & -0.07 & 0.53 \\
$\mathrm{SrO}_{2}$ & 0.56 & 2.23 & 1.86 & 2.77 \\
$\mathrm{ZnO}_{\mathrm{ZrO}}$ & 4.78 & 6.46 & 6.07 & 6.56 \\
$\mathrm{ZrO}_{2}$ & 0.30 & 1.09 & 0.92 & 1.47 \\
$\mathrm{Others}_{\mathrm{Na}}$ & 1.96 & 2.63 & 2.45 & 2.84 \\
$\mathrm{Na}_{2} \mathrm{O} \times \mathrm{Li}_{2} \mathrm{O}$ & -112 & -100 & -101 & -91 \\
$\mathrm{R}^{2}$ & 0.896 & 0.880 & 0.886 & 0.877 \\
$\mathrm{R}^{2}{ }_{\mathrm{A}}$ & 0.894 & 0.877 & 0.883 & 0.874 \\
$\mathrm{R}_{\mathrm{P}}^{2}$ & 0.890 & 0.873 & 0.879 & 0.870 \\
$\mathrm{RMSE}_{\mathrm{Mean}}$ & 0.173 & 0.167 & 0.167 & 0.160 \\
$\mathrm{Mea}_{\mathrm{N}}$ & 2.85 & 3.47 & 3.30 & 3.65 \\
$\mathrm{R}^{2} \mathrm{v}$ & 527 & 575 & 542 & 558 \\
\hline & 0.892 & 0.871 & 0.879 & 0.871 \\
\hline
\end{tabular}
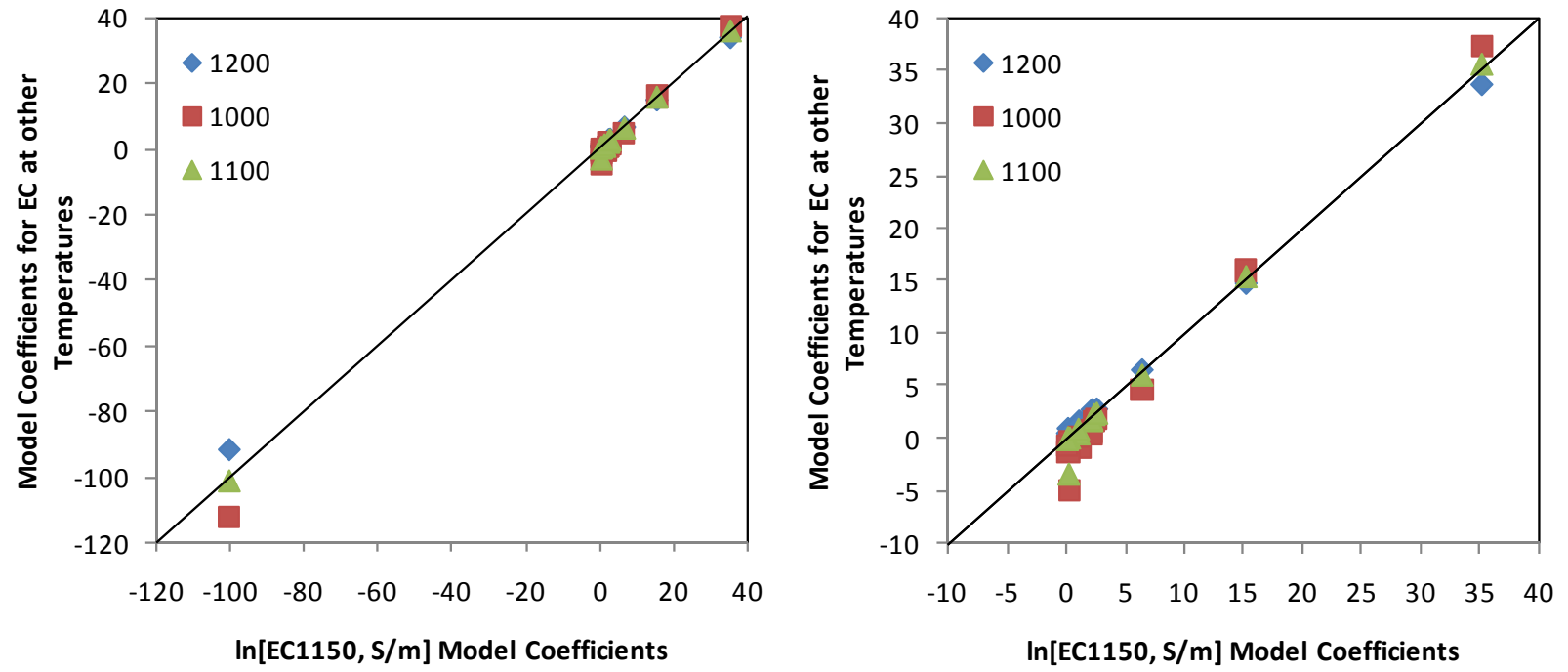

Figure 3.9. $\operatorname{In}\left[\varepsilon_{T}\right]$ Model Coefficient Comparison 
To estimate the electrical conductivity at any temperature between 1000 and $1200^{\circ} \mathrm{C}$, the $\operatorname{In}[\varepsilon]$ is predicted at three temperatures $\left(1000,1100\right.$, and $\left.1200^{\circ} \mathrm{C}\right)$. The $\ln [\varepsilon]$ is interpolated in much the same manner as $\ln [n]$ described in Section 3.1.4:

$$
\ln \left[\varepsilon_{T}, \mathrm{~Pa}-\mathrm{s}\right]=A+\frac{B}{T-T_{0}}
$$

replacing for the predicted properties

$$
\ln \left[\varepsilon_{1000}\right]=A+\frac{B}{1000-T_{0}} ; \ln \left[\varepsilon_{1100}\right]=A+\frac{B}{1100-T_{0}} ; \ln \left[\varepsilon_{1200}\right]=A+\frac{B}{1200-T_{0}}
$$

and solving for $A, B$, and $T_{0}$; we get

$$
\begin{gathered}
A=\frac{2 \cdot \ln \left[\varepsilon_{1000}\right] \cdot \ln \left[\varepsilon_{1200}\right]-\ln \left[\varepsilon_{1100}\right]\left(\ln \left[\varepsilon_{1000}\right]+\ln \left[\varepsilon_{1200}\right]\right)}{-2 \cdot \ln \left[\varepsilon_{1100}\right]+\ln \left[\varepsilon_{1000}\right]+\ln \left[\varepsilon_{1200}\right]} \\
B=\frac{-200 \cdot\left(\ln \left[\varepsilon_{1100}\right]-\ln \left[\varepsilon_{1000}\right]\right) \cdot\left(\ln \left[\varepsilon_{1100}\right]-\ln \left[\varepsilon_{1200}\right]\right) \cdot\left(\ln \left[\varepsilon_{1000}\right]-\ln \left[\varepsilon_{1200}\right]\right)}{\left(-2 \cdot \ln \left[\varepsilon_{1100}\right]+\ln \left[\varepsilon_{1000}\right]+\ln \left[\varepsilon_{1200}\right]\right)^{2}} \text { and } \\
T_{0}=\frac{-200\left(11 \cdot \ln \left[\varepsilon_{1100}\right]-5 \cdot \ln \left[\varepsilon_{1000}\right]-6 \cdot \ln \left[\varepsilon_{1200}\right]\right)}{-2 \cdot \ln \left[\varepsilon_{1100}\right]+\ln \left[\varepsilon_{1000}\right]+\ln \left[\varepsilon_{1200}\right]}
\end{gathered}
$$

These values for $A, B$, and $T_{0}$ are then substituted into Equation (3.9) to obtain viscosity at any temperature between 1000 and $1200^{\circ} \mathrm{C}$.

\subsection{Product Consistency Test}

\subsubsection{PCT Data Screening and Evaluation}

Of the initial 3406 glasses in the database (Appendix A), 1938 contained PCT responses on quenched glass. These data were screened for use in $\ln [\mathrm{PCT}]$ model development in the order listed in Table 3.39. After this data screening, 1127 glasses were available for model development. Different releases have a different number of glasses remaining in this set:

PCT-B dataset contains 1119 glasses

PCT-Na dataset contains 1127 glasses

PCT-Li dataset contains 770 glasses. 
Table 3.39. Summary of Data Screening for $\operatorname{In}[\mathrm{PCT}]$ Model Development

\begin{tabular}{|c|c|c|}
\hline \# & Process & Glasses \\
\hline 1 & Start with all glasses containing quenched glass PCT data & 1938 \\
\hline 2 & Removed 147 inhomogeneous (multiphasic) glasses & 1791 \\
\hline 3 & Removed 110 glasses with incomplete compositions & 1681 \\
\hline 4 & $\begin{array}{l}\text { Removed } 5 \text { glasses with substantial differences between target and measured } \\
\text { compositions }\end{array}$ & 1676 \\
\hline 5 & Removed 55 glasses with incongruence between PCT-B, -Na, and -Li > 4x & 1621 \\
\hline 6 & Removed 10 glasses with PCT-B > $30 \mathrm{~g} / \mathrm{m}^{2}$ & 1611 \\
\hline 7 & Removed 262 glasses with extreme compositions & 1349 \\
\hline 8 & $\begin{array}{l}\text { Removed } 11 \text { glasses reported by Matlack et al. }(1997 a, b) \text { due to systematic } \\
\text { offset }\end{array}$ & 1338 \\
\hline 9 & Removed 15 "BNFL HLW" glasses due to systematic offset & 1321 \\
\hline 10 & $\begin{array}{l}\text { Removed } 33 \text { additional glasses with }>5 \mathrm{wt} \% \text { differences between target and } \\
\text { measured component concentration }\end{array}$ & 1288 \\
\hline 11 & Removed 23 glasses because of highly reduced conditions & 1263 \\
\hline 12 & Removed SBW1-38B because of excessive foaming & 1262 \\
\hline 13 & $\begin{array}{l}\text { Removed glasses reported by Bulkley and Vienna (1997) because of extreme } \\
\text { composition }\end{array}$ & 1223 \\
\hline 14 & Removed INEEL CVS Phase 2a glasses because of systematic offset & 1207 \\
\hline 15 & Removed data from Johnston et al. ${ }^{(a)}$ because of systematic offset & 1152 \\
\hline 16 & Removed the 25 "PX" study glasses due to systematic offset & 1127 \\
\hline
\end{tabular}

\subsubsection{PCT-Boron}

\section{Model Development}

Initial $\ln \left[\mathrm{PCT}_{\mathrm{B}}, \mathrm{g} / \mathrm{m}^{2}\right]$ models were fit with the 1119 data using stepwise regression methods allowing only first-order terms. Those components with sufficient data to allow for coefficient estimates were included in the stepwise process. Stepwise regression comparing the coefficient to the value of zero while maintaining the intercept near the average response of $-0.76 \ln \left[\mathrm{g} / \mathrm{m}^{2}\right]$ was used to reduce the influential components to:

$$
\begin{aligned}
& \mathrm{Al}_{2} \mathrm{O}_{3}, \mathrm{~B}_{2} \mathrm{O}_{3}, \mathrm{BaO}, \mathrm{Bi}_{2} \mathrm{O}_{3}, \mathrm{CaO}, \mathrm{CdO}, \mathrm{F}, \mathrm{Fe}_{2} \mathrm{O}_{3}, \mathrm{~K}_{2} \mathrm{O}, \mathrm{La}_{2} \mathrm{O}_{3}, \mathrm{Li}_{2} \mathrm{O}, \mathrm{MgO}, \mathrm{MnO}, \\
& \mathrm{Na}_{2} \mathrm{O}, \mathrm{Nd}_{2} \mathrm{O}_{3}, \mathrm{P}_{2} \mathrm{O}_{5}, \mathrm{SiO}_{2}, \mathrm{SO}_{3}, \mathrm{SrO}, \mathrm{ThO}_{2}, \mathrm{TiO}_{2}, \mathrm{UO}_{3}, \mathrm{ZnO} \text {, and } \mathrm{ZrO}_{2}
\end{aligned}
$$

Further narrowing removed the coefficients for $\mathrm{Bi}_{2} \mathrm{O}_{3}$ and $\mathrm{La}_{2} \mathrm{O}_{3}$ as insignificant, while removing 19 glasses found to be outliers left 1100 data points for model fitting.

Attempts were made to improve the fit of the model data by adding a limited number (up to 5) second- and third-order terms. The terms found to most significantly improve the fit to model data were:

$$
\mathrm{Al}_{2} \mathrm{O}_{3} \times \mathrm{Al}_{2} \mathrm{O}_{3}, \mathrm{ThO}_{2} \times \mathrm{Al}_{2} \mathrm{O}_{3}, \mathrm{MnO} \times \mathrm{MnO} \text {, and } \mathrm{Al}_{2} \mathrm{O}_{3} \times \mathrm{Al}_{2} \mathrm{O}_{3} \times \mathrm{Al}_{2} \mathrm{O}_{3}
$$

A scatterplot matrix of these data is given in Appendix $B$. The final model was then fit to the

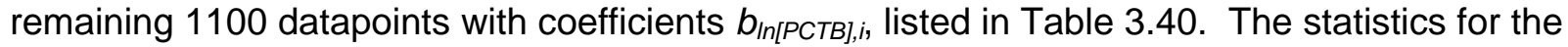
model fit are summarized in

(a) JW Johnston, GF Piepel, and BA Pulsipher. 1990. Evaluation of Empirical Models for Glass Durability. Letter Report Prepared for West Valley Nuclear Services, Pacific Northwest National Laboratory, Richland, WA. 
Table 3.41. The $R^{2}$ coefficient of 0.81 suggests that the data are well represented by the model. The $R^{2}$-adjusted value (0.80) suggests that there are not too many coefficients, or the data are not over-fit. The $R^{2}$-predicted being so close to the $R^{2}$ value $(0.79)$ suggests that there are not any very influential data points, and the model is likely to predict similarly for new data as it does for the model development data.

Table 3.40. $\ln \left[P C T_{B}, \mathrm{~g} / \mathrm{m}^{2}\right]$ Model Coefficients

\begin{tabular}{lcc}
\hline Term & Coefficient & Std Error \\
\hline $\mathrm{Al}_{2} \mathrm{O}_{3}$ & -88.27106 & 4.449629 \\
$\mathrm{~B}_{2} \mathrm{O}_{3}$ & 13.015106 & 0.404034 \\
$\mathrm{BaO}$ & 5.6578777 & 2.777042 \\
$\mathrm{CaO}$ & -3.389582 & 0.737565 \\
$\mathrm{CdO}$ & 12.664775 & 4.860392 \\
$\mathrm{~F}$ & 28.721522 & 5.02168 \\
$\mathrm{Fe}_{2} \mathrm{O}_{3}$ & -1.970029 & 0.406756 \\
$\mathrm{~K}_{2} \mathrm{O}$ & 10.911932 & 0.919621 \\
$\mathrm{Li}_{2} \mathrm{O}$ & 26.084546 & 0.910804 \\
$\mathrm{MgO}$ & 10.329709 & 1.321522 \\
$\mathrm{MnO}$ & -11.942 & 2.927281 \\
$\mathrm{Na}_{2} \mathrm{O}$ & 15.666007 & 0.380501 \\
$\mathrm{Nd}_{2} \mathrm{O}_{3}$ & -6.943853 & 2.309245 \\
$\mathrm{P}_{2} \mathrm{O}_{5}$ & -3.934373 & 1.820589 \\
$\mathrm{SiO}_{2}$ & -3.273545 & 0.186615 \\
$\mathrm{SO}_{3}$ & 26.096922 & 6.217899 \\
$\mathrm{SrO}$ & -1.698829 & 0.965119 \\
$\mathrm{ThO}_{2}$ & -14.21879 & 2.322881 \\
$\mathrm{TiO}_{2}$ & -11.0803 & 1.970779 \\
$\mathrm{UO}_{3}$ & 2.5059609 & 1.659739 \\
\hline $\mathrm{ZnO}_{\mathrm{ZrO}_{2}}$ & 0.5480525 & 1.272039 \\
$\mathrm{Others}_{\mathrm{Al}_{2} \mathrm{O}_{3} \times \mathrm{Al}_{2} \mathrm{O}_{3}}$ & -6.449192 & 0.623514 \\
$\mathrm{Al}_{2} \mathrm{O}_{3} \times \mathrm{ThO}_{2}$ & -0.419909 & 1.626648 \\
$\mathrm{MnO}_{2} \times \mathrm{MnO}_{\mathrm{Al}_{2} \mathrm{O}_{3} \times \mathrm{Al}_{2} \mathrm{O}_{3} \times \mathrm{Al}_{2} \mathrm{O}_{3}}$ & -105.34448 & 52.89769 \\
\hline & 193.75149 & 31.02577 \\
\hline & 286.06257 & 54.14021 \\
\hline
\end{tabular}

Table 3.41. Summary of $\ln \left[\mathrm{PCT}_{\mathrm{B}}\right]$ Model Statistics

\begin{tabular}{lr}
\hline Statistic & Value \\
\hline $\mathrm{R}^{2}$ & 0.801 \\
$\mathrm{R}^{2}{ }_{\mathrm{A}}$ & 0.796 \\
$\mathrm{R}^{2}$ & 0.789 \\
$\mathrm{RMSE}$ & 0.411 \\
Min & -2.954 \\
Mean & -0.788 \\
Max & 2.923 \\
$\mathrm{n}$ & 1100 \\
$\mathrm{~N}$ & 27 \\
\hline
\end{tabular}

Figure 3.10 compares the predicted and measured $\ln \left[\mathrm{PCT}_{\mathrm{B}}\right]$ values. The data appear to be well represented by the model with no trends to over or under prediction. No single group of data (e.g., study) was found to be misrepresented by the model. 


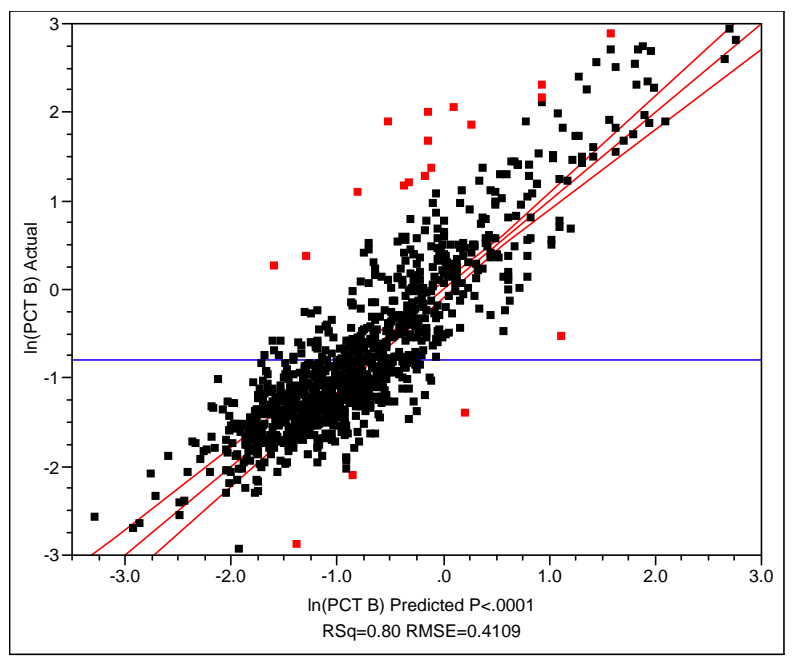

Figure 3.10. Measured vs. Predicted $\ln \left[\mathrm{PCT}_{\mathrm{B}}\right]$

This model is based on data spanning a specific glass composition region and therefore should only be relied upon within that region. The validity region is defined as the composition region of data used to develop the model.

Table 3.42 lists the model validity region while Table 3.43 lists the correlation coefficients calculated for the model data. Only $\mathrm{Li}_{2} \mathrm{O}-\mathrm{Na}_{2} \mathrm{O}, \mathrm{CaO}-\mathrm{SO}_{3}, \mathrm{Nd}_{2} \mathrm{O}_{3}$-Others, and $\mathrm{ThO}_{2}-\mathrm{UO}_{3}$ have a correlation of 0.5 or above.

Table 3.42. $\operatorname{In}\left[\mathrm{PCT}_{\mathrm{B}}\right]$ Model Validity Region (wt\%)

\begin{tabular}{lrr}
\hline Comp & Min & Max \\
\hline $\mathrm{Al}_{2} \mathrm{O}_{3}$ & 1.6 & 20.0 \\
$\mathrm{~B}_{2} \mathrm{O}_{3}$ & 4.0 & 20.0 \\
$\mathrm{BaO}$ & 0.0 & 4.7 \\
$\mathrm{CaO}$ & 0.0 & 10.4 \\
$\mathrm{CdO}$ & 0.0 & 1.5 \\
$\mathrm{~F}$ & 0.0 & 2.5 \\
$\mathrm{Fe}_{2} \mathrm{O}_{3}$ & 0.0 & 17.4 \\
$\mathrm{~K}_{2} \mathrm{O}$ & 0.0 & 6.9 \\
$\mathrm{Li}_{2} \mathrm{O}$ & 0.0 & 9.0 \\
$\mathrm{MgO}$ & 0.0 & 8.0 \\
$\mathrm{MnO}_{\mathrm{Na}} \mathrm{O}$ & 0.0 & 7.0 \\
$\mathrm{Nd}_{2} \mathrm{O}_{3}$ & 4.1 & 23.0 \\
$\mathrm{P}_{2} \mathrm{O}_{5}$ & 0.0 & 5.9 \\
$\mathrm{SiO}_{2}$ & 0.0 & 5.0 \\
$\mathrm{SO}_{3}$ & 30.3 & 62.8 \\
$\mathrm{SrO}$ & 0.0 & 2.5 \\
$\mathrm{ThO}_{2}$ & 0.0 & 10.1 \\
$\mathrm{TiO}_{2}$ & 0.0 & 6.0 \\
$\mathrm{UO}_{3}$ & 0.0 & 4.0 \\
$\mathrm{ZnO}_{2 \mathrm{O} O}$ & 0.0 & 6.5 \\
$\mathrm{ZrO}_{2}$ & 0.0 & 5.8 \\
$\mathrm{Remaining}$ & 0.0 & 13.5 \\
\hline & 0.0 & 9.4 \\
\hline
\end{tabular}


Table 3.43. Correlation Matrix for the $\ln \left[\mathrm{PCT}_{\mathrm{B}}\right]$ Model Dataset

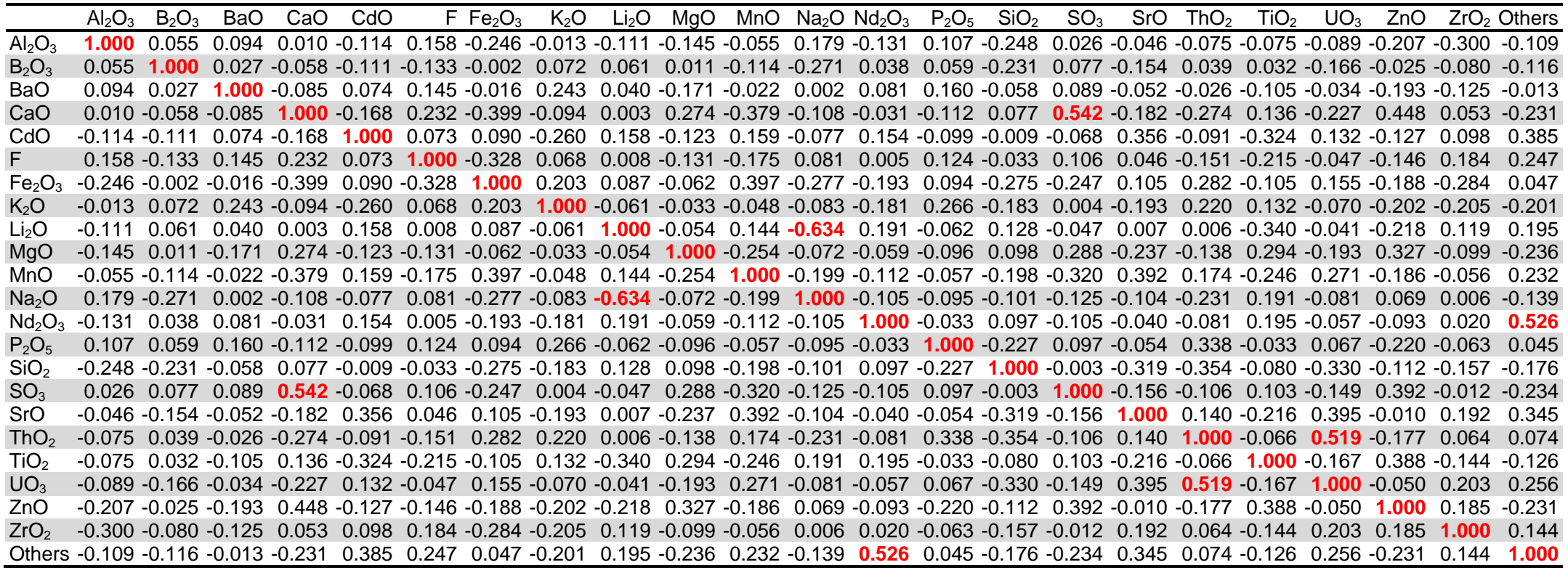


A variance-covariance matrix is required to calculate the measurement plus prediction uncertainties of this model. This matrix is defined as:

$$
\mathbf{V}_{\alpha}=\operatorname{RMSE}^{2}\left[\mathbf{X}_{\alpha}^{\mathrm{T}} \mathbf{X}_{\alpha}\right]^{-1}
$$

The use of $\mathbf{V}_{\alpha}$ is explained in detail elsewhere (e.g., Cornell 2002, Hrma et al. 1994, Piepel et al. 2008a, Vienna and Kim 2008). The $\mathbf{V}_{\mathrm{In}[\text { РСтв] }}$ is given in Appendix $C$ with the other variancecovariance matrices.

\section{Model Validation}

To validate the model, data not used in model fitting must be obtained. Because all appropriate data within the desired composition region were used in model fitting, subsets of the model data were used to validate the model. The data were sorted by $\ln \left[\mathrm{PCT}_{\mathrm{B}}\right]$ value. The data were then numbered $1,2,3,4,5,1,2, \ldots$ to split them into five roughly representative groups. The model was then refit to subsets 2 to 5 and used to predict data in subset 1 . Then the model was fit to each group of four subsets and used to predict the remaining subset in sequence. Table 3.44 summarizes the results of the model validation. The coefficients are reasonably close with an RSD of less than $25 \%$ with the exceptions of:

- $\quad \mathrm{CdO}$, which has a low value for set 4

- $\mathrm{P}_{2} \mathrm{O}_{5}$, which has a low value for set 1 and a high value for set 3

- $\mathrm{SrO}$

- $\mathrm{UO}_{3}$, which has a low value for set 3 and a high value for set 1

- ZnO with values near zero

- Others with values near zero.

The model fit $R^{2}$ values are all close to each other at 0.80 . The $R_{V}^{2}$ are also close to the 0.785 , and their average (0.788) is close to the $R_{P}^{2}$ value of 0.789 . This model is well validated and should give predictions of unknown data within the model validity region nearly as well as model fit data. 
Table 3.44. Summary of $\operatorname{In}\left[\mathrm{PCT}_{\mathrm{B}}\right]$ Model Validation

\begin{tabular}{|c|c|c|c|c|c|c|c|}
\hline Term & Full & Set 1 & Set 2 & Set 3 & Set 4 & Set 5 & $\%$ RSD \\
\hline $\mathrm{Al}_{2} \mathrm{O}_{3}$ & -88.27 & -91.27 & -86.33 & -88.10 & -89.28 & -87.84 & -2 \\
\hline $\mathrm{B}_{2} \mathrm{O}_{3}$ & 13.02 & 13.09 & 13.31 & 12.80 & 13.04 & 12.95 & 1 \\
\hline $\mathrm{BaO}$ & 5.66 & 6.11 & 4.96 & 5.32 & 5.11 & 7.26 & 17 \\
\hline $\mathrm{CaO}$ & -3.39 & -3.15 & -3.35 & -3.39 & -3.44 & -3.49 & -4 \\
\hline $\mathrm{CdO}$ & 12.66 & 13.78 & 13.14 & 16.24 & 5.67 & 14.75 & 32 \\
\hline $\mathrm{F}$ & 28.72 & 25.61 & 29.09 & 29.35 & 28.97 & 30.68 & 7 \\
\hline $\mathrm{Fe}_{2} \mathrm{O}_{3}$ & -1.97 & -2.10 & -2.26 & -1.89 & -1.79 & -1.77 & -11 \\
\hline $\mathrm{K}_{2} \mathrm{O}$ & 10.91 & 10.94 & 11.28 & 10.33 & 10.79 & 11.00 & 3 \\
\hline $\mathrm{Li}_{2} \mathrm{O}$ & 26.08 & 25.90 & 25.67 & 26.75 & 26.06 & 25.95 & 2 \\
\hline $\mathrm{MgO}$ & 10.33 & 10.23 & 10.21 & 10.04 & 9.54 & 12.42 & 11 \\
\hline $\mathrm{MnO}$ & -11.94 & -12.21 & -11.40 & -11.30 & -11.79 & -13.67 & -8 \\
\hline $\mathrm{Na}_{2} \mathrm{O}$ & 15.67 & 15.53 & 15.62 & 15.83 & 15.90 & 15.42 & 1 \\
\hline $\mathrm{Nd}_{2} \mathrm{O}_{3}$ & -6.94 & -7.38 & -7.97 & -7.54 & -6.07 & -5.77 & -14 \\
\hline $\mathrm{P}_{2} \mathrm{O}_{5}$ & -3.93 & -5.64 & -3.59 & -2.45 & -3.85 & -4.36 & -30 \\
\hline $\mathrm{SiO}_{2}$ & -3.27 & -3.09 & -3.34 & -3.35 & -3.19 & -3.34 & -4 \\
\hline $\mathrm{SO}_{3}$ & 26.10 & 27.57 & 21.53 & 27.79 & 27.71 & 24.33 & 11 \\
\hline $\mathrm{SrO}$ & -1.70 & -2.02 & -2.54 & -1.50 & -1.14 & -1.35 & -33 \\
\hline $\mathrm{ThO}_{2}$ & -14.22 & -13.59 & -13.38 & -12.70 & -18.06 & -12.74 & -16 \\
\hline $\mathrm{TiO}_{2}$ & -11.08 & -10.89 & -11.20 & -9.86 & -11.34 & -12.19 & -8 \\
\hline $\mathrm{UO}_{3}$ & 2.51 & 5.39 & 2.16 & -0.26 & 3.52 & 2.21 & 83 \\
\hline $\mathrm{ZnO}$ & 0.55 & -0.36 & 0.62 & 1.46 & 0.29 & 0.46 & 120 \\
\hline $\mathrm{ZrO}_{2}$ & -6.45 & -6.98 & -6.27 & -6.56 & -6.29 & -6.26 & -5 \\
\hline Others & -0.42 & 0.09 & 0.08 & -0.56 & -1.18 & -0.49 & -126 \\
\hline $\mathrm{Al}_{2} \mathrm{O}_{3} \times \mathrm{Al}_{2} \mathrm{O}_{3}$ & 705.3 & 758.2 & 681.6 & 691.4 & 706.8 & 709.1 & 4 \\
\hline $\mathrm{Al}_{2} \mathrm{O}_{3} \times \mathrm{ThO}_{2}$ & 193.8 & 180.9 & 177.5 & 195.9 & 235.1 & 173.0 & 13 \\
\hline $\mathrm{MnO} \times \mathrm{MnO}$ & 286.1 & 285.5 & 278.7 & 283.3 & 282.6 & 313.4 & 5 \\
\hline $\mathrm{Al}_{2} \mathrm{O}_{3} \times \mathrm{Al}_{2} \mathrm{O}_{3} \times \mathrm{Al}_{2} \mathrm{O}_{3}$ & -1974.6 & -2217.4 & -1889.5 & -1896.0 & -1963.1 & -1989.7 & -7 \\
\hline $\mathrm{R}^{2}$ & 0.8007 & 0.7984 & 0.8041 & 0.8026 & 0.8031 & 0.8024 & \\
\hline $\mathrm{R}_{\mathrm{A}}^{2}$ & 0.7959 & 0.7922 & 0.7982 & 0.7965 & 0.7971 & 0.7963 & \\
\hline $\mathrm{R}_{\mathrm{P}}^{2}$ & 0.7889 & 0.7834 & 0.7894 & 0.7879 & 0.7883 & 0.7874 & \\
\hline RMSE & 0.4109 & 0.4153 & 0.4092 & 0.4106 & 0.4094 & 0.4092 & \\
\hline$R_{v}^{2}$ & 0.7879 & 0.7977 & 0.7829 & 0.7850 & 0.7856 & 0.7881 & \\
\hline
\end{tabular}

\subsubsection{PCT - Sodium}

\section{Model Development}

Initial $\ln \left[\mathrm{PCT}_{\mathrm{Na}}, \mathrm{g} / \mathrm{m}^{2}\right]$ models were fit with the 1127 data using stepwise regression methods allowing only first-order terms. Those components with sufficient data to allow for coefficient estimates were included in the stepwise process. Stepwise regression comparing the coefficient to the value of zero while maintaining the intercept near the average response of $-0.818 \mathrm{ln}\left[\mathrm{g} / \mathrm{m}^{2}\right]$ was used to reduce the influential components to:

$$
\begin{aligned}
& \mathrm{Al}_{2} \mathrm{O}_{3}, \mathrm{~B}_{2} \mathrm{O}_{3}, \mathrm{BaO}, \mathrm{Bi}_{2} \mathrm{O}_{3}, \mathrm{CaO}, \mathrm{CdO}, \mathrm{F}, \mathrm{Fe}_{2} \mathrm{O}_{3}, \mathrm{~K}_{2} \mathrm{O}, \mathrm{La}_{2} \mathrm{O}_{3}, \mathrm{Li} \mathrm{i}_{2} \mathrm{O}, \mathrm{MgO}, \mathrm{MnO}, \\
& \mathrm{Na}_{2} \mathrm{O}, \mathrm{Nd}_{2} \mathrm{O}_{3}, \mathrm{P}_{2} \mathrm{O}_{5}, \mathrm{SiO}_{2}, \mathrm{SO}_{3}, \mathrm{SrO}, \mathrm{ThO}_{2}, \mathrm{TiO}_{2}, \mathrm{UO}_{3}, \mathrm{ZnO} \text {, and } \mathrm{ZrO}_{2}
\end{aligned}
$$

Further narrowing removed the coefficients for $\mathrm{BaO}, \mathrm{Bi}_{2} \mathrm{O}_{3}, \mathrm{CdO}$, and $\mathrm{La}_{2} \mathrm{O}_{3}$ as insignificant, while removing 23 glasses found to be outliers left 1104 data points for model fitting.

Attempts were made to improve the fit of the model data by adding a limited number (up to 5) second- and third-order terms. The terms found to most significantly improve the fit to model data were: 


\section{$\mathrm{Al}_{2} \mathrm{O}_{3} \times \mathrm{Al}_{2} \mathrm{O}_{3}, \mathrm{~B}_{2} \mathrm{O}_{3} \times \mathrm{B}_{2} \mathrm{O}_{3}, \mathrm{ThO}_{2} \times \mathrm{Al}_{2} \mathrm{O}_{3}$, and $\mathrm{Al}_{2} \mathrm{O}_{3} \times \mathrm{Al}_{2} \mathrm{O}_{3} \times \mathrm{Al}_{2} \mathrm{O}_{3}$}

A scatterplot matrix of these data is given in Appendix $B$. The final model was then fit to the remaining 1104 datapoints with coefficients $b_{\ln [P C T N a], i}$ listed in Table 3.45. The statistics for the model fit are summarized in Table 3.46. The $R^{2}$ coefficient of 0.81 suggests that the data are well represented by the model. The $R^{2}$-adjusted value $(0.806)$ suggests that there are not too many coefficients, or the data are not over-fit. The $R^{2}$-predicted being so close to the $R^{2}$ value (0.8) suggests that there are not any very influential data points, and the model is likely to predict similarly for new data as it does for the model development data.

Table 3.45. $\operatorname{In}\left[P C T_{N a}, \mathrm{~g} / \mathrm{m}^{2}\right]$ Model Coefficients

\begin{tabular}{lcc}
\hline $\mathrm{Term}$ & Estimate & Std Error \\
\hline $\mathrm{Al}_{2} \mathrm{O}_{3}$ & -69.768 & 3.734 \\
$\mathrm{~B}_{2} \mathrm{O}_{3}$ & -13.224 & 1.781 \\
$\mathrm{CaO}$ & 2.60598 & 0.605 \\
$\mathrm{Fe}_{2} \mathrm{O}_{3}$ & -1.6767 & 0.344 \\
$\mathrm{~K}_{2} \mathrm{O}$ & 11.9701 & 0.74 \\
$\mathrm{Li} 2 \mathrm{O}$ & 22.5717 & 0.766 \\
$\mathrm{MgO}$ & 9.44187 & 1.064 \\
$\mathrm{MnO}$ & 1.43131 & 0.989 \\
$\mathrm{Na}_{2} \mathrm{O}$ & 16.9404 & 0.315 \\
$\mathrm{Nd}_{2} \mathrm{O}_{3}$ & -2.8519 & 1.891 \\
$\mathrm{P}_{2} \mathrm{O}_{5}$ & -2.3946 & 1.482 \\
$\mathrm{SiO}_{2}$ & -2.0965 & 0.18 \\
$\mathrm{SrO}_{\mathrm{ThO}}$ & 2.04919 & 0.788 \\
$\mathrm{ThO}_{2}$ & -13.455 & 1.934 \\
$\mathrm{TiO}_{2}$ & -11.347 & 1.61 \\
$\mathrm{UO}_{3}$ & 1.49105 & 1.382 \\
$\mathrm{ZnO}_{\mathrm{ZrO}}$ & -0.9934 & 1.083 \\
$\mathrm{Zr}_{2}$ & -5.2997 & 0.527 \\
$\mathrm{~F}$ & 25.9327 & 4.127 \\
$\mathrm{SO}_{3}$ & 22.3441 & 5.087 \\
$\mathrm{Other}_{\mathrm{Al}_{2} \mathrm{O}_{3} \times \mathrm{Al}_{2} \mathrm{O}_{3}}$ & 2.73564 & 1.109 \\
$\mathrm{~B}_{2} \mathrm{O}_{3} \times \mathrm{B}_{2} \mathrm{O}_{3}$ & 557.465 & 44.34 \\
$\mathrm{Al}_{2} \mathrm{O}_{3} \times \mathrm{ThO}_{2}$ & 96.3525 & 8.35 \\
$\mathrm{Al}_{2} \mathrm{O}_{3} \times \mathrm{Al}_{2} \mathrm{O}_{3} \times \mathrm{Al}_{2} \mathrm{O}_{3}$ & -1590.6 & 25.98 \\
\hline & & 154.3 \\
\hline
\end{tabular}

Table 3.46. Summary of $\operatorname{In}\left[\mathrm{PCT}_{\mathrm{Na}}\right]$ Model Statistics

\begin{tabular}{lc}
\hline Statistic & Value \\
\hline $\mathrm{R}^{2}{ }^{2}$ & 0.8105 \\
$\mathrm{R}^{2}{ }_{\mathrm{A}}$ & 0.8062 \\
$\mathrm{R}_{\mathrm{P}}{ }_{\mathrm{P}}$ & 0.7996 \\
$\mathrm{RMSE}$ & 0.3431 \\
Min & -2.749 \\
Mean & -0.8525 \\
Max & 2.551 \\
$\mathrm{n}$ & 1104 \\
$\mathrm{~N}$ & 25 \\
\hline
\end{tabular}


Figure 3.11 compares the predicted and measured $\ln \left[\mathrm{PCT}_{\mathrm{Na}}\right]$ values. The data appears to be well represented by the model with no trends to over or under prediction. No single group of data (e.g., study) was found to be misrepresented by the model.

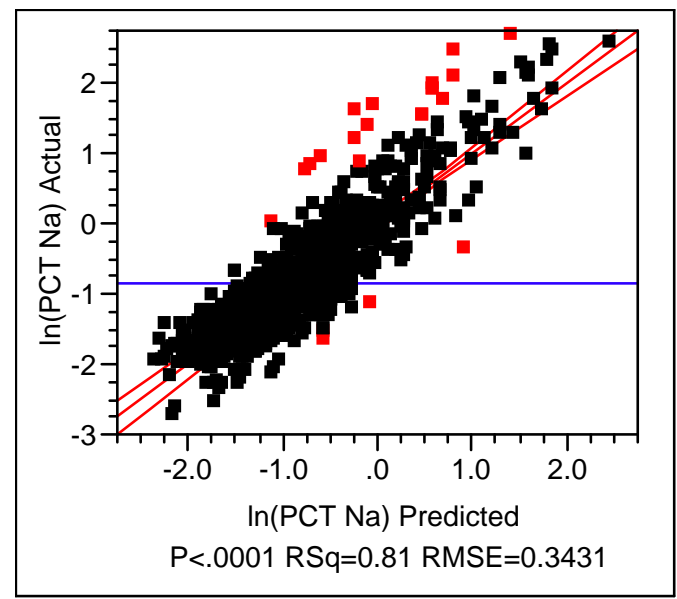

Figure 3.11. Measured vs. Predicted $\ln \left[\mathrm{PCT}_{\mathrm{Na}}\right]$

This model is based on data spanning a specific glass composition region and therefore should only be relied upon within that region. The validity region is defined as the composition region of data used to develop the model.

Table 3.47 lists the model validity region while Table 3.48 lists the correlation coefficients calculated for the model data. Only $\mathrm{Li}_{2} \mathrm{O}-\mathrm{Na}_{2} \mathrm{O}, \mathrm{CaO}-\mathrm{SO}_{3}$, and $\mathrm{ThO}_{2}-\mathrm{UO}_{3}$ have a correlation of 0.5 or above.

A variance-covariance matrix is required to calculate the measurement plus prediction uncertainties of this model. The $\mathbf{V}_{\mathrm{In}[\mathrm{PCTNa}]}$ is given in Appendix $\mathbf{C}$ with the other variancecovariance matrices. 
Table 3.47. $\operatorname{In}\left[\mathrm{PCT}_{\mathrm{Na}}\right]$ Model Validity Region (wt\%)

\begin{tabular}{lrr}
\hline Comp & Min & Max \\
\hline $\mathrm{Al}_{2} \mathrm{O}_{3}$ & 1.6 & 20.0 \\
$\mathrm{~B}_{2} \mathrm{O}_{3}$ & 4.0 & 20.0 \\
$\mathrm{CaO}$ & 0.0 & 10.4 \\
$\mathrm{~F}$ & 0.0 & 2.5 \\
$\mathrm{Fe}_{2} \mathrm{O}_{3}$ & 0.0 & 17.4 \\
$\mathrm{~K}_{2} \mathrm{O}$ & 0.0 & 6.9 \\
$\mathrm{Li} 2$ & 0.0 & 9.0 \\
$\mathrm{MgO}$ & 0.0 & 8.0 \\
$\mathrm{MnO}$ & 0.0 & 7.0 \\
$\mathrm{Na}_{2} \mathrm{O}$ & 4.1 & 23.0 \\
$\mathrm{Nd}_{2} \mathrm{O}_{3}$ & 0.0 & 5.9 \\
$\mathrm{P}_{2} \mathrm{O}_{5}$ & 0.0 & 5.0 \\
$\mathrm{SiO}_{2}$ & 30.3 & 62.8 \\
$\mathrm{SO}_{3}$ & 0.0 & 2.5 \\
$\mathrm{SrO}_{\mathrm{ThO}}$ & 0.0 & 10.1 \\
$\mathrm{TiO}_{2}$ & 0.0 & 6.0 \\
$\mathrm{UO}_{3}$ & 0.0 & 4.0 \\
$\mathrm{ZnO}_{2 r O}$ & 0.0 & 6.5 \\
$\mathrm{ZrO}$ & 0.0 & 5.8 \\
$\mathrm{Other}$ & 0.0 & 13.5 \\
\hline
\end{tabular}


Table 3.48. Correlation Matrix for the $\ln \left[\mathrm{PCT}_{\mathrm{Na}}\right]$ Model Dataset

\begin{tabular}{|c|c|c|c|c|c|c|c|c|c|c|c|c|c|c|c|c|c|c|c|c|c|}
\hline & $\mathrm{Al}_{2} \mathrm{O}_{3}$ & ${ }_{2} \mathrm{O}_{3}$ & & & $\mathrm{O}$ & & & & & & & & & & 1102 & $\mathrm{UO}_{3}$ & $\angle \mathrm{nO}$ & $\mathrm{ZrO}_{2}$ & 1 & $\mathrm{SO}_{3}$ & \\
\hline $\mathrm{I}_{2} \mathrm{O}_{3}$ & & 0.061 & & & & & & 0.059 & & & & & & & & & & & & & \\
\hline${ }_{2} \mathrm{O}_{3}$ & 061 & 1.000 & -0.049 & 0.009 & 0.070 & 0.065 & & & -0.279 & .039 & 0.0 & & & P 044 & & & & & -0.137 & & \\
\hline $\mathrm{aO}$ & 0.011 & -0.049 & 1.000 & -0.406 & -0.101 & .009 & & & & .029 & & - & & -0.273 & & & & 0.058 & 0.235 & 0.542 & -0.256 \\
\hline $\mathrm{e}_{2} \mathrm{O}_{3}$ & -0.259 & 0.009 & -0.406 & 1.000 & 0.218 & .086 & & & & & & & 0.101 & 0.278 & & & & & -0.329 & 52 & 0.057 \\
\hline${ }_{2} \mathrm{O}$ & -0.024 & 0.070 & -0.101 & 0.218 & 1.000 & & & & & & 0 & & & 218 & & & & & 6 & 02 & 43 \\
\hline $\mathrm{i}_{2} \mathrm{O}$ & 0.105 & 0.065 & 0.009 & 0.086 & -0.056 & & & 0 & & 0.188 & & & 0.008 & 08 & & & & 112 & 0.00 & -0.046 & 0.213 \\
\hline gO & & 0.000 & 0.256 & & & -0.0 & & & & & & & & & & & & & & & -0.281 \\
\hline nO & & & & & & & & & & & & & & & & & & & & & 0.222 \\
\hline $\mathrm{a}_{2} \mathrm{O}$ & & & & & & & & & & & & & & & & & & & & & -0.134 \\
\hline${ }_{2} \mathrm{O}_{5}$ & 0.086 & 0 & 120 & 108 & 277 & & & & & & & & & 324 & & & $3 x+2$ & & 114 & .085 & 0.067 \\
\hline & 243 & .241 & 072 & -0.2 & 80 & 121 & & & & & & & & & & & & 65 & 31 & 000 & \\
\hline & & & & & & & & & & & & & & & & & & & 48 & & \\
\hline $\mathrm{hO}_{2}$ & 0.075 & 044 & -0.273 & 0.278 & 0.218 & & & & & & & & 40 & & 67 & & .75 & 63 & & 105 & 0.033 \\
\hline $\mathrm{iO}_{2}$ & -0.075 & 0.036 & & -0.104 & & & & & & & & & & & & & & & 15 & 3 & 208 \\
\hline $\mathrm{O}_{3}$ & -0.089 & -0.1 & -0.226 & & & & & & & & & & & & & 00 & & & 145 & 48 & 0.232 \\
\hline nO & 0.202 & -0.022 & 0.451 & -0.1 & & & & & & & & & & & & 49 & 100 & 185 & 144 & 0.395 & -0.285 \\
\hline $\mathrm{rO}_{2}$ & -0.300 & -0.085 & 0.058 & -0.274 & -0.200 & 0.112 & -0.09 & & 0.007 & .018 & & & & D63 & & 203 & 185 & 1.000 & 0.184 & 0.012 & 0.103 \\
\hline & 0.164 & -0.137 & 0.235 & -0.329 & 0.061 & .009 & & & $0.0 \varepsilon$ & & & & & & & -0.045 & & 84 & 1.000 & .106 & 0.265 \\
\hline the & -0081 & & -0.256 & 0.057 & & & -0.281 & & & & & & & 0.033 & & 0.232 & -0.285 & 0.103 & 0.265 & -0.184 & 1.000 \\
\hline
\end{tabular}




\section{Model Validation}

To validate the model, data not used in model fitting must be obtained. Because all appropriate data within the desired composition region were used in model fitting, subsets of the model data were used to validate the model. The data were sorted by $\ln \left[\mathrm{PCT}_{\mathrm{Na}}\right]$ value. The data were then numbered $1,2,3,4,5,1,2, \ldots$ to split them into five roughly representative groups. The model was then refit to subsets 2 to 5 and used to predict data in subset 1 . Then the model was fit to each group of four subsets and used to predict the remaining subset in sequence. Table 3.49 summarizes the results of the model validation. The coefficients are reasonably close with an RSD of less than $25 \%$ with the exceptions of:

- MnO with low values for sets 4 and 5

- $\mathrm{P}_{2} \mathrm{O}_{5}$ with a high value for set 3

- $\mathrm{UO}_{3}$ with a low value for set 1

- ZnO with low values for sets 2 and 4 .

The model fit $R^{2}$ values are all close to each other at 0.81 . The $R_{V}^{2}$ are also close with values near 0.79 , and their average $(0.795)$ is close to the $R_{P}^{2}$ value for the full model of 0.80 . This model is well validated and should give predictions of unknown data within the model validity region nearly as well as model fit data. 
Table 3.49. Summary of $\operatorname{In}\left[\mathrm{PCT}_{\mathrm{Na}}\right]$ Model Validation

\begin{tabular}{|c|c|c|c|c|c|c|c|}
\hline Term & Full & $1 \mathrm{st}$ & 2nd & 3rd & 4th & 5th & $\%$ RSD \\
\hline $\mathrm{Al}_{2} \mathrm{O}_{3}$ & -69.77 & -77.75 & -67.71 & -72.47 & -66.90 & -67.18 & 6.7 \\
\hline $\mathrm{B}_{2} \mathrm{O}_{3}$ & -13.22 & -12.25 & -12.41 & -12.88 & -13.09 & -15.46 & 9.8 \\
\hline $\mathrm{CaO}$ & 2.61 & 3.35 & 2.69 & 2.58 & 2.78 & 1.74 & 22.2 \\
\hline$F$ & 25.93 & 23.72 & 27.88 & 24.83 & 24.32 & 28.69 & 8.7 \\
\hline $\mathrm{Fe}_{2} \mathrm{O}_{3}$ & -1.68 & -1.50 & -1.97 & -1.89 & -1.43 & -1.66 & 14.2 \\
\hline $\mathrm{K}_{2} \mathrm{O}$ & 11.97 & 12.47 & 11.82 & 12.34 & 11.44 & 11.94 & 3.5 \\
\hline $\mathrm{Li}_{2} \mathrm{O}$ & 22.57 & 21.98 & 22.93 & 22.79 & 21.96 & 23.31 & 2.7 \\
\hline $\mathrm{MgO}$ & 9.44 & 9.10 & 8.98 & 10.22 & 9.22 & 10.10 & 6.2 \\
\hline $\mathrm{MnO}$ & 1.43 & 1.72 & 1.84 & 2.30 & 0.38 & 0.89 & 54.2 \\
\hline $\mathrm{Na}_{2} \mathrm{O}$ & 16.94 & 16.93 & 16.97 & 16.95 & 16.89 & 17.03 & 0.3 \\
\hline $\mathrm{Nd}_{2} \mathrm{O}_{3}$ & -2.85 & -2.58 & -2.95 & -2.62 & -2.79 & -3.33 & 10.8 \\
\hline $\mathrm{P}_{2} \mathrm{O}_{5}$ & -2.39 & -2.77 & -3.21 & -0.76 & -3.11 & -2.03 & 42.4 \\
\hline $\mathrm{SiO}_{2}$ & -2.10 & -1.96 & -2.15 & -2.03 & -2.21 & -2.07 & 4.8 \\
\hline $\mathrm{SO}_{3}$ & 22.34 & 19.53 & 23.71 & 22.96 & 23.86 & 21.43 & 8.2 \\
\hline SrO & 2.05 & 2.08 & 1.65 & 2.32 & 2.59 & 1.75 & 19.2 \\
\hline $\mathrm{ThO}_{2}$ & -13.46 & -13.57 & -11.37 & -15.14 & -13.43 & -13.86 & 10.1 \\
\hline $\mathrm{TiO}_{2}$ & -11.35 & -10.69 & -12.22 & -10.60 & -11.60 & -11.55 & 6.0 \\
\hline $\mathrm{UO}_{3}$ & 1.49 & 0.05 & 1.58 & 2.15 & 2.14 & 1.75 & 58.1 \\
\hline $\mathrm{ZnO}$ & -0.99 & -0.74 & -1.33 & -0.85 & -1.65 & -0.25 & 54.5 \\
\hline $\mathrm{ZrO}_{2}$ & -5.30 & -4.84 & -6.38 & -4.90 & -5.09 & -5.28 & 11.9 \\
\hline Other & 2.74 & 2.70 & 2.62 & 2.40 & 2.94 & 3.20 & 11.2 \\
\hline $\mathrm{Al}_{2} \mathrm{O}_{3} \times \mathrm{Al}_{2} \mathrm{O}_{3}$ & 557.47 & 666.14 & 531.94 & 577.49 & 526.27 & 528.67 & 10.7 \\
\hline $\mathrm{B}_{2} \mathrm{O}_{3} \times \mathrm{B}_{2} \mathrm{O}_{3}$ & 96.35 & 94.41 & 91.43 & 95.79 & 94.78 & 106.09 & 5.8 \\
\hline $\mathrm{Al}_{2} \mathrm{O}_{3} \times \mathrm{ThO}_{2}$ & 201.15 & 204.95 & 181.94 & 223.30 & 193.02 & 206.25 & 7.7 \\
\hline $\mathrm{Al}_{2} \mathrm{O}_{3} \times \mathrm{Al}_{2} \mathrm{O}_{3} \times \mathrm{Al}_{2} \mathrm{O}_{3}-$ & -1590.64 & -2017.15 & -1511.68 & -1621.11 & -1478.58 & -1494.93 & 14.2 \\
\hline $\mathrm{R}^{2}$ & 0.8105 & 0.8098 & 0.8119 & 0.8142 & 0.8108 & 0.8136 & \\
\hline $\mathrm{R}_{\mathrm{A}}^{2}$ & 0.8062 & 0.8045 & 0.8066 & 0.8090 & 0.8055 & 0.8084 & \\
\hline $\mathrm{R}_{\mathrm{P}}^{2}$ & 0.7996 & 0.7969 & 0.7984 & 0.8003 & 0.7973 & 0.8003 & \\
\hline RMSE & 0.3431 & 0.3450 & 0.3426 & 0.3399 & 0.3428 & 0.3432 & \\
\hline$R_{v}^{2}$ & 0.7949 & 0.7943 & 0.7972 & 0.7892 & 0.8028 & 0.7908 & \\
\hline
\end{tabular}

\subsubsection{PCT - Lithium}

\section{Model Development}

Initial $\ln \left[\mathrm{PCT}_{\mathrm{Li}}, \mathrm{g} / \mathrm{m}^{2}\right]$ models were fit with the 770 data using stepwise regression methods allowing only first-order terms. Those components with sufficient data to allow for coefficient estimates were included in the stepwise process. Stepwise regression comparing the coefficient to the value of zero while maintaining the intercept near the average response of $-0.72 \mathrm{ln}\left[\mathrm{g} / \mathrm{m}^{2}\right]$ was used to reduce the influential components to:

$$
\begin{aligned}
& \mathrm{Al}_{2} \mathrm{O}_{3}, \mathrm{~B}_{2} \mathrm{O}_{3}, \mathrm{BaO}, \mathrm{Bi}_{2} \mathrm{O}_{3}, \mathrm{CaO}, \mathrm{CdO}, \mathrm{F}, \mathrm{Fe}_{2} \mathrm{O}_{3}, \mathrm{~K}_{2} \mathrm{O}, \mathrm{La}_{2} \mathrm{O}_{3}, \mathrm{Li}_{2} \mathrm{O}, \mathrm{MgO}, \mathrm{MnO}, \\
& \mathrm{Na}_{2} \mathrm{O}, \mathrm{Nd}_{2} \mathrm{O}_{3}, \mathrm{P}_{2} \mathrm{O}_{5}, \mathrm{SiO}_{2}, \mathrm{SO}_{3}, \mathrm{SrO}, \mathrm{ThO}_{2}, \mathrm{TiO}_{2}, \mathrm{UO}_{3}, \mathrm{ZnO} \text {, and } \mathrm{ZrO}_{2}
\end{aligned}
$$

Further narrowing removed the coefficients for $\mathrm{BaO}, \mathrm{Bi}_{2} \mathrm{O}_{3}, \mathrm{CdO}, \mathrm{La}_{2} \mathrm{O}_{3}, \mathrm{MnO}, \mathrm{Nd}_{2} \mathrm{O}_{3}, \mathrm{P}_{2} \mathrm{O}_{5}$, $\mathrm{SrO}, \mathrm{UO}_{3}$, and $\mathrm{ZnO}$ as insignificant, while removing 11 glasses found to be outliers left 759 data points for model fitting. 
Attempts were made to improve the fit of the model data by adding a limited number (up to 5) second- and third-order terms. The terms found to most significantly improve the fit to model data were:

$$
\mathrm{Al}_{2} \mathrm{O}_{3} \times \mathrm{Al}_{2} \mathrm{O}_{3}, \mathrm{~B}_{2} \mathrm{O}_{3} \times \mathrm{B}_{2} \mathrm{O}_{3}, \mathrm{~B}_{2} \mathrm{O}_{3} \times \mathrm{Na}_{2} \mathrm{O}, \mathrm{ThO}_{2} \times \mathrm{Al}_{2} \mathrm{O}_{3} \text {, and } \mathrm{Al}_{2} \mathrm{O}_{3} \times \mathrm{Al}_{2} \mathrm{O}_{3} \times \mathrm{Al}_{2} \mathrm{O}_{3}
$$

A scatterplot matrix of these data is given in Appendix $B$. The final model was then fit to the remaining 759 datapoints with coefficients $b_{\ln [P C T L i], i}$, listed in Table 3.50. The statistics for the model fit are summarized in Table 3.51. The $R^{2}$ coefficient of 0.803 suggests that the data are well represented by the model. The $R^{2}$-adjusted value $(0.798)$ suggests that there are not too many coefficients, or the data are not over-fit. The $R^{2}$-predicted being so close to the $R^{2}$ value (0.79) suggests that there are not any very influential data points, and the model is likely to predict similarly for new data as it does for the model development data.

Table 3.50. $\ln \left[P C T_{L i}, \mathrm{~g} / \mathrm{m}^{2}\right]$ Model Coefficients

\begin{tabular}{|c|c|c|}
\hline Term & Estimate & Std Error \\
\hline $\mathrm{Al}_{2} \mathrm{O}_{3}$ & -71.80358 & 4.065619 \\
\hline $\mathrm{B}_{2} \mathrm{O}_{3}$ & -15.88115 & 2.518614 \\
\hline $\mathrm{CaO}$ & 0.4612474 & 1.045549 \\
\hline $\mathrm{F}$ & 29.676446 & 4.684815 \\
\hline $\mathrm{Fe}_{2} \mathrm{O}_{3}$ & -0.588641 & 0.427145 \\
\hline $\mathrm{K}_{2} \mathrm{O}$ & 10.211474 & 0.931885 \\
\hline $\mathrm{Li}_{2} \mathrm{O}$ & 24.236504 & 0.983968 \\
\hline $\mathrm{MgO}$ & 6.8614839 & 1.409214 \\
\hline $\mathrm{Na}_{2} \mathrm{O}$ & 5.8154351 & 0.961167 \\
\hline $\mathrm{SiO}_{2}$ & -0.63572 & 0.262725 \\
\hline $\mathrm{SO}_{3}$ & 42.822925 & 8.399326 \\
\hline $\mathrm{ThO}_{2}$ & -14.11654 & 2.001598 \\
\hline $\mathrm{TiO}_{2}$ & -15.98701 & 2.544938 \\
\hline $\mathrm{ZrO}_{2}$ & -4.149619 & 0.613619 \\
\hline Others & 2.1636268 & 0.416881 \\
\hline $\mathrm{Al}_{2} \mathrm{O}_{3} \times \mathrm{Al}_{2} \mathrm{O}_{3}$ & 586.1739 & 49.16406 \\
\hline $\mathrm{Al}_{2} \mathrm{O}_{3} \times \mathrm{Al}_{2} \mathrm{O}_{3} \times \mathrm{Al}_{2} \mathrm{O}_{3}$ & -1640.968 & 172.1203 \\
\hline $\mathrm{Al}_{2} \mathrm{O}_{3} \times \mathrm{ThO}_{2}$ & 236.62771 & 28.38881 \\
\hline $\mathrm{B}_{2} \mathrm{O}_{3} \times \mathrm{B}_{2} \mathrm{O}_{3}$ & 88.683572 & 9.676586 \\
\hline $\mathrm{B}_{2} \mathrm{O}_{3} \times \mathrm{Na}_{2} \mathrm{O}$ & 74.407878 & 10.18339 \\
\hline
\end{tabular}

Table 3.51. Summary of $\ln \left[\mathrm{PCT}_{\mathrm{Li}}\right]$ Model Statistics

\begin{tabular}{lc}
\hline Statistic & Value \\
\hline $\mathrm{R}^{2}$ & 0.8034 \\
$\mathrm{R}^{2}{ }_{\mathrm{A}}$ & 0.7983 \\
$\mathrm{R}_{\mathrm{P}}$ & 0.7895 \\
$\mathrm{RMSE}$ & 0.3565 \\
Min & -2.534 \\
Mean & -0.726 \\
Max & 2.521 \\
$\mathrm{n}$ & 759 \\
$\mathrm{~N}$ & 20 \\
\hline
\end{tabular}

Figure 3.12 compares the predicted and measured $\operatorname{In}\left[\mathrm{PCT}_{\mathrm{L}}\right]$ values. The data appear to be well represented by the model with no trends to over or under prediction. No single group of data (e.g., study) was found to be misrepresented by the model. 


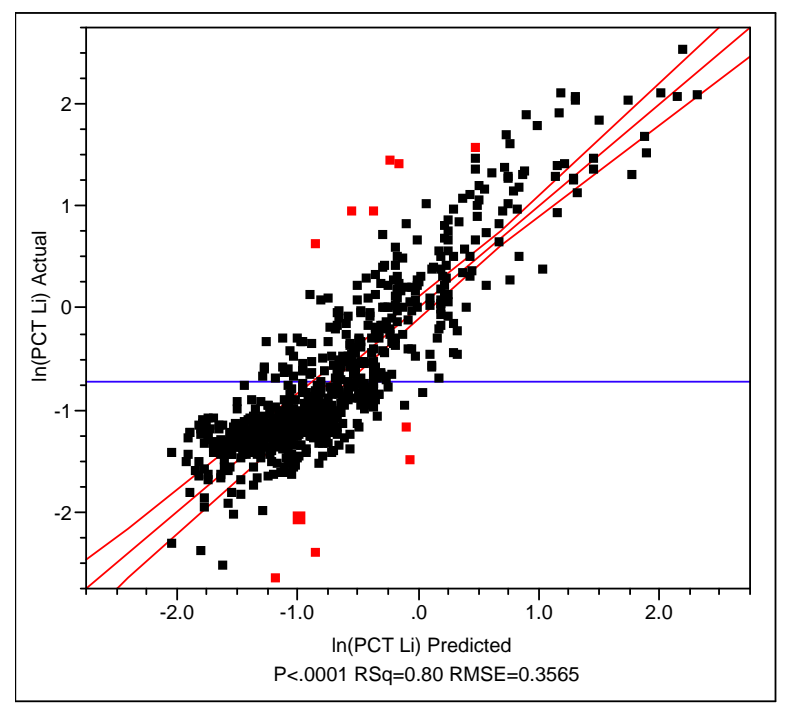

Figure 3.12. Measured vs. Predicted $\ln \left[\mathrm{PCT}_{\mathrm{Li}}\right]$

This model is based on data spanning a specific glass composition region and therefore should only be relied upon within that region. The validity region is defined as the composition region of data used to develop the model. Note that due to the relatively large concentrations of "Others" in model glasses, additional components were broken out of "Others" in defining the model validity region ( $\mathrm{BaO}, \mathrm{Bi}_{2} \mathrm{O}_{3}, \mathrm{MnO}, \mathrm{P}_{2} \mathrm{O}_{5}, \mathrm{SrO}, \mathrm{UO}_{3}$, and $\mathrm{ZnO}$ ).

Table 3.52 lists the model validity region while Table 3.53 lists the correlation coefficients calculated for the model data. Only CaO-F have a correlation of 0.5 or above.

A variance-covariance matrix is required to calculate the measurement plus prediction uncertainties of this model. The $\mathbf{V}_{\mathrm{In}[\mathrm{PCTL}]}$ is given in Appendix $\mathrm{C}$ with the other variancecovariance matrices. 
Table 3.52. $\operatorname{In}\left[\mathrm{PCT}_{\mathrm{Li}}\right]$ Model Validity Region (wt\%)

\begin{tabular}{lrr}
\hline Comp & Min & Max \\
\hline $\mathrm{Al}_{2} \mathrm{O}_{3}$ & 1.6 & 20.0 \\
$\mathrm{~B}_{2} \mathrm{O}_{3}$ & 4.0 & 20.0 \\
$\mathrm{BaO}$ & 0.0 & 4.7 \\
$\mathrm{Bi}_{2} \mathrm{O}_{3}$ & 0.0 & 3.2 \\
$\mathrm{CaO}$ & 0.0 & 10.0 \\
$\mathrm{~F}$ & 0.0 & 2.5 \\
$\mathrm{Fe}_{2} \mathrm{O}_{3}$ & 0.0 & 17.4 \\
$\mathrm{~K} 2 \mathrm{O}$ & 0.0 & 6.9 \\
$\mathrm{La}_{2} \mathrm{O}_{3}$ & 0.0 & 3.5 \\
$\mathrm{Li}_{2} \mathrm{O}$ & 0.1 & 9.0 \\
$\mathrm{MgO}$ & 0.0 & 8.0 \\
$\mathrm{MnO}_{\mathrm{Na}}$ & 0.0 & 7.0 \\
$\mathrm{Na}_{2} \mathrm{O}$ & 4.1 & 21.4 \\
$\mathrm{Nd}_{2} \mathrm{O}_{3}$ & 0.0 & 5.9 \\
$\mathrm{P}_{2} \mathrm{O}_{5}$ & 0.0 & 4.3 \\
$\mathrm{SiO}_{2}$ & 30.3 & 60.4 \\
$\mathrm{SO}_{3}$ & 0.0 & 1.0 \\
$\mathrm{SrO}_{\mathrm{ThO}_{2}}$ & 0.0 & 10.1 \\
$\mathrm{TiO}_{2}$ & 0.0 & 6.0 \\
$\mathrm{UO}_{3}$ & 0.0 & 3.3 \\
$\mathrm{ZnO}_{\mathrm{ZrO}}$ & 0.0 & 6.3 \\
$\mathrm{Others}_{2}$ & 0.0 & 5.7 \\
\hline
\end{tabular}

Table 3.53. Correlation Matrix for the $\ln \left[\mathrm{PCT}_{\mathrm{Li}}\right]$ Model Dataset

\begin{tabular}{|c|c|c|c|c|c|c|c|c|c|c|c|c|c|c|c|}
\hline & $\mathrm{Al}_{2} \mathrm{O}_{3}$ & $\mathrm{~B}_{2} \mathrm{O}_{3}$ & $\mathrm{CaO}$ & $\mathrm{F}$ & $\mathrm{Fe}_{2} \mathrm{O}_{3}$ & $\mathrm{~K}_{2} \mathrm{O}$ & $\mathrm{Li}_{2} \mathrm{O}$ & $\mathrm{MgO}$ & $\mathrm{Na}_{2} \mathrm{O}$ & $\mathrm{SiO}_{2}$ & $\mathrm{SO}_{3}$ & $\mathrm{ThO}_{2}$ & $\mathrm{TiO}_{2}$ & $\mathrm{ZrO}_{2}$ & Others \\
\hline $\mathrm{Al}_{2} \mathrm{O}_{3}$ & 1.000 & & & & & & & & & & & & & & \\
\hline $\mathrm{B}_{2} \mathrm{O}_{3}$ & 0.089 & 1.000 & & & & & & & & & & & & & \\
\hline $\mathrm{CaO}$ & 0.156 & -0.088 & 1.000 & & & & & & & & & & & & \\
\hline $\mathrm{F}$ & 0.164 & -0.124 & 0.550 & 1.000 & & & & & & & & & & & \\
\hline $\mathrm{Fe}_{2} \mathrm{O}_{3}$ & -0.249 & -0.049 & -0.415 & -0.418 & 1.000 & & & & & & & & & & \\
\hline $\mathrm{K}_{2} \mathrm{O}$ & 0.000 & 0.089 & 0.008 & 0.045 & 0.203 & 1.000 & & & & & & & & & \\
\hline $\mathrm{Li}_{2} \mathrm{O}$ & -0.088 & -0.092 & 0.098 & 0.005 & -0.286 & -0.102 & 1.000 & & & & & & & & \\
\hline $\mathrm{MgO}$ & -0.084 & 0.003 & -0.061 & -0.098 & 0.106 & 0.084 & 0.048 & 1.000 & & & & & & & \\
\hline $\mathrm{Na}_{2} \mathrm{O}$ & 0.204 & -0.260 & 0.045 & 0.131 & -0.165 & -0.202 & -0.236 & -0.143 & 1.000 & & & & & & \\
\hline $\mathrm{SiO}_{2}$ & -0.379 & -0.230 & 0.063 & -0.061 & -0.160 & -0.131 & 0.321 & 0.135 & -0.258 & 1.000 & & & & & \\
\hline $\mathrm{SO}_{3}$ & 0.214 & 0.132 & 0.202 & 0.274 & -0.136 & 0.193 & -0.174 & -0.056 & 0.048 & -0.141 & 1.000 & & & & \\
\hline $\mathrm{ThO}_{2}$ & -0.081 & 0.026 & -0.231 & -0.185 & 0.189 & 0.234 & -0.219 & -0.025 & -0.203 & -0.362 & 0.069 & 1.000 & & & \\
\hline $\mathrm{TiO}_{2}$ & 0.009 & 0.137 & -0.102 & -0.236 & 0.080 & 0.289 & -0.072 & 0.066 & -0.225 & -0.128 & -0.034 & 0.157 & 1.000 & & \\
\hline $\mathrm{ZrO}_{2}$ & -0.276 & -0.097 & 0.051 & 0.216 & -0.396 & -0.260 & 0.142 & -0.182 & 0.079 & -0.095 & -0.113 & 0.044 & -0.280 & 1.000 & \\
\hline Others & -0.163 & -0.242 & -0.176 & -0.005 & -0.004 & -0.321 & -0.180 & -0.238 & -0.030 & -0.370 & -0.100 & 0.179 & 0.091 & 0.198 & 1.000 \\
\hline
\end{tabular}

\section{Model Validation}

To validate the model, data not used in model fitting must be obtained. Because all appropriate data within the desired composition region were used in model fitting, subsets of the model data were used to validate the model. The data were sorted by $\ln \left[\mathrm{PCT}_{\mathrm{Li}}\right]$ value. The data were then numbered $1,2,3,4,5,1,2, \ldots$ to split them into five roughly representative groups. The model was then refit to subsets 2 to 5 and used to predict data in subset 1 . Then the model was fit to each group of four subsets and used to predict the remaining subset in sequence. 
Table 3.54 summarizes the results of the model validation. The coefficients are reasonably close with an RSD of less than $25 \%$ with the exceptions of:

- $\quad \mathrm{CaO}$ with values near zero

- $\mathrm{Fe}_{2} \mathrm{O}_{3}$ with a high value for set 3

- $\mathrm{SiO}_{2}$ with values near zero.

The model fit $R^{2}$ values are all close to each other at 0.805 . The $R_{V}^{2}$ are also close with values near 0.79 (except set 3), and their average $(0.786)$ is close to the $R_{P}^{2}$ value for the full model of 0.79 . This model is well validated and should give predictions of unknown data within the model validity region nearly as well as model fit data.

Table 3.54. Summary of $\ln \left[\mathrm{PCT}_{\mathrm{Li}}\right]$ Model Validation

\begin{tabular}{|c|c|c|c|c|c|c|c|}
\hline Term & Full & $1 \mathrm{st}$ & 2nd & 3rd & 4th & 5th & \%RSD \\
\hline $\mathrm{Al}_{2} \mathrm{O}_{3}$ & -71.80 & -70.75 & -72.01 & -72.60 & -70.12 & -74.38 & 2.3 \\
\hline $\mathrm{B}_{2} \mathrm{O}_{3}$ & -15.88 & -16.82 & -14.08 & -19.00 & -16.39 & -14.64 & 12.3 \\
\hline $\mathrm{CaO}$ & 0.46 & 0.01 & 0.40 & 1.66 & -0.85 & 1.09 & 210.2 \\
\hline $\mathrm{F}$ & 29.68 & 28.73 & 30.93 & 30.29 & 32.07 & 27.06 & 6.6 \\
\hline $\mathrm{Fe}_{2} \mathrm{O}_{3}$ & -0.59 & -0.69 & -0.93 & 0.29 & -0.76 & -0.72 & 82.1 \\
\hline $\mathrm{K}_{2} \mathrm{O}$ & 10.21 & 10.57 & 10.66 & 9.48 & 10.21 & 10.19 & 4.5 \\
\hline $\mathrm{Li}_{2} \mathrm{O}$ & 24.24 & 24.97 & 24.27 & 24.69 & 24.18 & 23.08 & 3.0 \\
\hline $\mathrm{MgO}$ & 6.86 & 7.26 & 8.63 & 7.18 & 6.18 & 5.28 & 18.3 \\
\hline $\mathrm{Na}_{2} \mathrm{O}$ & 5.82 & 5.76 & 6.81 & 4.44 & 5.03 & 6.87 & 18.5 \\
\hline $\mathrm{SiO}_{2}$ & -0.64 & -0.70 & -0.83 & -0.44 & -0.45 & -0.62 & 26.1 \\
\hline $\mathrm{SO}_{3}$ & 42.82 & 37.28 & 44.75 & 46.56 & 45.51 & 40.47 & 9.1 \\
\hline $\mathrm{ThO}_{2}$ & -14.12 & -15.35 & -13.92 & -13.42 & -15.39 & -11.79 & 10.6 \\
\hline $\mathrm{TiO}_{2}$ & -15.99 & -16.94 & -16.55 & -13.66 & -16.33 & -16.16 & 8.1 \\
\hline $\mathrm{ZrO}_{2}$ & -4.15 & -4.00 & -4.01 & -4.19 & -4.32 & -4.12 & 3.2 \\
\hline Others & 2.16 & 2.41 & 2.06 & 2.20 & 2.24 & 2.03 & 7.1 \\
\hline $\mathrm{Al}_{2} \mathrm{O}_{3} \times \mathrm{Al}_{2} \mathrm{O}_{3}$ & 586.17 & 576.65 & 584.91 & 596.19 & 566.90 & 617.41 & 3.3 \\
\hline $\mathrm{Al}_{2} \mathrm{O}_{3} \times \mathrm{Al}_{2} \mathrm{O}_{3} \times \mathrm{Al}_{2} \mathrm{O}_{3}$ & -1641.0 & -1621.9 & -1619.9 & -1658.8 & -1588.0 & -1752.1 & 3.9 \\
\hline $\mathrm{Al}_{2} \mathrm{O}_{3} \times \mathrm{ThO}_{2}$ & 236.63 & 251.70 & 234.04 & 235.78 & 247.73 & 205.99 & 7.6 \\
\hline $\mathrm{B}_{2} \mathrm{O}_{3} \times \mathrm{B}_{2} \mathrm{O}_{3}$ & 88.68 & 89.65 & 86.81 & 97.65 & 88.74 & 86.83 & 5.1 \\
\hline $\mathrm{B}_{2} \mathrm{O}_{3} \times \mathrm{Na}_{2} \mathrm{O}$ & 74.41 & 80.55 & 59.57 & 89.05 & 80.46 & 65.07 & 16.4 \\
\hline $\mathrm{R}^{2}$ & 0.8034 & 0.8048 & 0.8060 & 0.8119 & 0.8015 & 0.8017 & \\
\hline $\mathrm{R}_{\mathrm{A}}^{2}$ & 0.7983 & 0.7985 & 0.7997 & 0.8058 & 0.7951 & 0.7953 & \\
\hline $\mathrm{R}_{\mathrm{P}}^{2}$ & 0.7895 & 0.7883 & 0.7894 & 0.7943 & 0.7830 & 0.7844 & \\
\hline RMSE & 0.3565 & 0.3568 & 0.3552 & 0.3495 & 0.3574 & 0.3613 & \\
\hline$R_{v}^{2}$ & 0.7864 & 0.7903 & 0.7809 & 0.7572 & 0.8031 & 0.8005 & \\
\hline
\end{tabular}

\subsection{Toxicity Characteristic Leaching Procedure}

\subsubsection{Data Conversion, Screening, and Evaluation}

Of the initial 3406 glasses in the database (Appendix A), 313 contained TCLP responses on quenched glass. The data were first converted into a normalized TCLP response by taking those responses that are known to release at the same rate as the glass (see Kim and Vienna 2004)—Ba, Cd, and B according to: 


$$
N_{\text {TCLP }}=\frac{\sum_{i=B, B a, C d}^{R} \frac{r_{i}(\mathrm{mg} / \mathrm{L})}{g_{i}(\mathrm{~g} / \mathrm{g})}}{R}
$$

where $N_{T C L P}=$ normalized TCLP response

$r_{i}=i$-th component TCLP response

$g_{i}=i$-th component mass fraction in glass

$R=$ number of components for which reliable TCLP response is available from the three components evaluated.

In most cases, $r_{B} g_{B} \approx r_{B a} g_{B a} \approx r_{C d} g_{C d}$. In those cases where two of the $r_{i} / g_{i}$ values closely matched and the third did not, the average of the two was used (this occurred for 71 of the 313 data points). It was the $N_{T C L P}$ values that represented the rate of glass release in TCLP conditions that were modeled.

The compositions of glasses contained several different species for multivalent elements (in most cases, these were self inconsistent [e.g., oxidized $\mathrm{Mn}$ and reduced U]). The multivalent elements were converted to one set of assumed states consistent with those used by WTP (Vienna and $\mathrm{Kim}$ 2008) $\rightarrow \mathrm{As}_{2} \mathrm{O}_{5}, \mathrm{Ce}_{2} \mathrm{O}_{3}, \mathrm{Fe}_{2} \mathrm{O}_{3}, \mathrm{MnO}, \mathrm{MoO}_{3}, \mathrm{Re}_{2} \mathrm{O}_{7}, \mathrm{RuO}_{2}, \mathrm{Sb}_{2} \mathrm{O}_{3}, \mathrm{Tl}_{2} \mathrm{O}$, and $\mathrm{UO}_{3}$.

The resulting data were screened for use in $\ln \left[N_{T C L P}\right]$ model development. Five glasses were removed from the model dataset as shown in Table 3.55. After this data screening, 308 glasses were available for model development.

Table 3.55. Summary of Data Screening for In[TCLP] Model

\begin{tabular}{cl}
\hline Glass ID & \multicolumn{1}{c}{ Reason } \\
\hline HLW98-79 & no TCLP value \\
HLW98-V06 & no TCLP value \\
Envelope D & $\mathrm{K}_{2} \mathrm{O}$ (target) $=0.0086 ; \mathrm{K}_{2} \mathrm{O}$ (analyzed) $=0.0845$ \\
HLW99-55 & $\mathrm{Na}_{2} \mathrm{O}$ (target) $=0.2 ; \mathrm{Na}_{2} \mathrm{O}$ (analyzed) $=0.1612$ \\
HLW98-96C & $\mathrm{HfO}_{2}>0$ \\
\hline
\end{tabular}

\subsubsection{Model Development}

Initial $\ln \left[\mathrm{N}_{\mathrm{TCLP}}, \mathrm{mg} / \mathrm{L}\right]$ models were fit with the 308 data using stepwise regression methods allowing only first-order terms. Those components with sufficient data to allow for coefficient estimates were included in the stepwise process. Stepwise regression comparing the coefficient to the value of zero while maintaining the intercept near the average response of $1.39 \mathrm{In}[\mathrm{mg} / \mathrm{L}]$ was used to reduce the influential components to:

$\mathrm{Al}_{2} \mathrm{O}_{3}, \mathrm{~B}_{2} \mathrm{O}_{3}, \mathrm{BaO}, \mathrm{Bi}_{2} \mathrm{O}_{3}, \mathrm{CaO}, \mathrm{CdO}, \mathrm{Fe}_{2} \mathrm{O}_{3}, \mathrm{~K}_{2} \mathrm{O}, \mathrm{La}_{2} \mathrm{O}_{3}, \mathrm{Li}_{2} \mathrm{O}, \mathrm{MgO}, \mathrm{MnO}, \mathrm{Na}_{2} \mathrm{O}$, $\mathrm{Nd}_{2} \mathrm{O}_{3}, \mathrm{NiO}, \mathrm{P}_{2} \mathrm{O}_{5}, \mathrm{PbO}, \mathrm{SiO}_{2}, \mathrm{SO}_{3}, \mathrm{SrO}, \mathrm{ThO}_{2}, \mathrm{TiO}_{2}, \mathrm{UO}_{3}, \mathrm{ZnO}$, and $\mathrm{ZrO}_{2}$

Further narrowing removed the coefficients to $\mathrm{Al}_{2} \mathrm{O}_{3}, \mathrm{~B}_{2} \mathrm{O}_{3}, \mathrm{CaO}, \mathrm{Fe}_{2} \mathrm{O}_{3}, \mathrm{Li}_{2} \mathrm{O}, \mathrm{Na}_{2} \mathrm{O}, \mathrm{SiO}_{2}, \mathrm{ThO}_{2}$, and $\mathrm{ZrO}_{2}$ as insignificant, while removing 17 glasses found to be outliers left 291 data points for model fitting. A scatterplot matrix of these data is given in Appendix $B$. 
Attempts were made to improve the fit of the model data by adding a limited number (up to 5) second- and third-order terms. No significant improvements were made.

The final model was then fit to the remaining 291 datapoints with coefficients $b_{\ln [N T C L P], i}$ listed in Table 3.56. The statistics for the model fit are summarized in Table 3.57. The $R^{2}$ coefficient of 0.89 suggests that the data are well represented by the model. The $R^{2}{ }_{\mathrm{A}}$ value (0.89) suggested that there are not too many coefficients, or the data are not over-fit. The $R_{\mathrm{P}}^{2}$ value $(0.88)$ being so close to the $R^{2}$ value (0.89) suggests that there are not any very influential data points, and the model is likely to predict similarly for new data as it does for the model development data.

Table 3.56. $\ln \left[N_{T C L P}, \mathrm{mg} / \mathrm{L}\right]$ Model Coefficients

\begin{tabular}{lcc}
\hline Term & Estimate & Std Error \\
\hline $\mathrm{Al}_{2} \mathrm{O}_{3}$ & -0.524992 & 0.628355 \\
$\mathrm{~B}_{2} \mathrm{O}_{3}$ & 12.003262 & 0.448488 \\
$\mathrm{CaO}$ & 11.147865 & 1.033483 \\
$\mathrm{Fe}_{2} \mathrm{O}_{3}$ & -1.209342 & 0.519293 \\
$\mathrm{Li}_{2} \mathrm{O}$ & 20.870752 & 1.184471 \\
$\mathrm{Na}_{2} \mathrm{O}$ & 15.242625 & 0.526148 \\
$\mathrm{SiO}_{2}$ & -2.004927 & 0.221359 \\
$\mathrm{ThO}_{2}$ & 2.468589 & 1.323125 \\
$\mathrm{ZrO}_{2}$ & -0.661848 & 0.821379 \\
Others & 9.2805424 & 0.22558 \\
\hline
\end{tabular}

Table 3.57. Summary of $\ln \left[N_{T C L P}\right]$ Model Statistics

\begin{tabular}{ll}
\hline Statistic & Value \\
\hline $\mathrm{R}^{2}$ & 0.8941 \\
$\mathrm{R}^{2}{ }_{\mathrm{A}}$ & 0.8907 \\
$\mathrm{R}_{\mathrm{P}}$ & 0.8840 \\
$\mathrm{RMSE}$ & 0.308 \\
Min & 2.624 \\
Mean & 3.896 \\
Max & 7.712 \\
$\mathrm{n}$ & 291 \\
$\mathrm{~N}$ & 10 \\
\hline
\end{tabular}

Figure 3.13 compares the predicted and measured $\ln \left[\mathrm{N}_{\mathrm{TCLP}}\right]$ values. The data appear to be well represented by the model with no trends to over or under prediction. No single group of data (e.g., study) was found to be misrepresented by the model.

This model is based on data spanning a specific glass composition region and therefore should only be relied upon within that region. The validity region is defined as the composition region of data used to develop the model.

Table 3.58 lists the model validity region while Table 3.59 lists the correlation coefficients calculated for the model data. Only pairs containing $\mathrm{Li}_{2} \mathrm{O}, \mathrm{Na}_{2} \mathrm{O}$, and $\mathrm{SiO}_{2}$ have a correlation of 0.5 or above. 


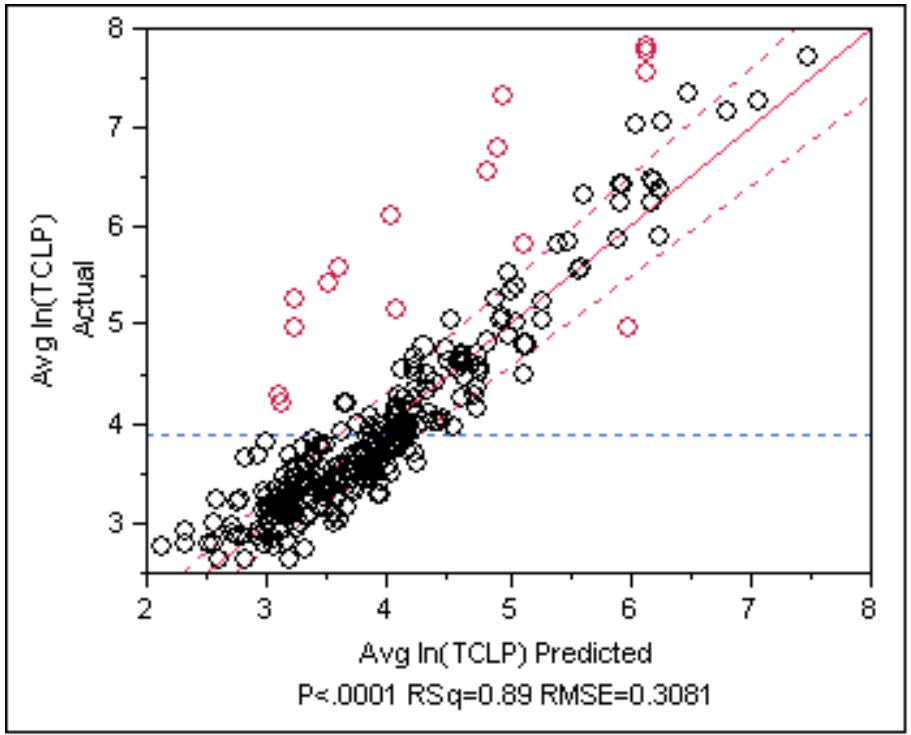

Figure 3.13. Measured vs. Predicted $\ln \left[\mathrm{N}_{\mathrm{TCLP}}\right]$

Table 3.58. $\ln \left[N_{T C L P}\right]$ Model Validity Region (wt\%)

\begin{tabular}{lrr}
\hline Comp & Min & \multicolumn{1}{c}{ Max } \\
\hline $\mathrm{Al}_{2} \mathrm{O}_{3}$ & 0.0 & 16.3 \\
$\mathrm{~B}_{2} \mathrm{O}_{3}$ & 2.0 & 21.0 \\
$\mathrm{BaO}_{\mathrm{Bi}}$ & 0.0 & 4.5 \\
$\mathrm{BaO}$ & 0.0 & 2.4 \\
$\mathrm{CaO}$ & 0.0 & 13.9 \\
$\mathrm{CdO}$ & 0.0 & 4.0 \\
$\mathrm{~F}$ & 0.0 & 1.5 \\
$\mathrm{Fe}_{2} \mathrm{O}_{3}$ & 1.9 & 18.7 \\
$\mathrm{~K}_{2} \mathrm{O}$ & 0.0 & 8.7 \\
$\mathrm{Li}_{2} \mathrm{O}$ & 0.0 & 7.4 \\
$\mathrm{MgO}$ & 0.0 & 5.0 \\
$\mathrm{MnO}_{\mathrm{Na}}$ & 0.0 & 7.6 \\
$\mathrm{NiO}_{2} \mathrm{O}$ & 2.9 & 20.0 \\
$\mathrm{NiO}_{2}$ & 0.0 & 3.0 \\
$\mathrm{P}_{2} \mathrm{O}_{5}$ & 0.0 & 3.9 \\
$\mathrm{PbO}_{\mathrm{SiO}}$ & 0.0 & 8.6 \\
$\mathrm{SO}_{3}$ & 29.4 & 55.0 \\
$\mathrm{SrO}_{\mathrm{ThO}}$ & 0.0 & 2.1 \\
$\mathrm{TiO}_{2}$ & 0.0 & 14.2 \\
$\mathrm{UO}{ }_{3}$ & 0.0 & 6.0 \\
$\mathrm{ZnO}_{\mathrm{ZrO}}$ & 0.0 & 2.0 \\
$\mathrm{Remaining}_{2}$ & 0.0 & 8.4 \\
\hline & 0.0 & 4.9 \\
\hline & 0.0 & 10.4 \\
\hline
\end{tabular}


Table 3.59. Correlation Matrix for the $\ln \left[N_{T C L P}\right]$ Model Dataset

\begin{tabular}{|c|c|c|c|c|c|c|c|c|c|c|}
\hline & $\mathrm{Al}_{2} \mathrm{O}_{3}$ & $\mathrm{~B}_{2} \mathrm{O}_{3}$ & $\mathrm{CaO}$ & $\mathrm{Fe}_{2} \mathrm{O}_{3}$ & $\mathrm{Li}_{2} \mathrm{O}$ & $\mathrm{Na}_{2} \mathrm{O}$ & $\mathrm{SiO}_{2}$ & $\mathrm{ThO}_{2}$ & $\mathrm{ZrO}_{2}$ & Others \\
\hline $\mathrm{Al}_{2} \mathrm{O}_{3}$ & 1.000 & & & & & & & & & \\
\hline $\mathrm{B}_{2} \mathrm{O}_{3}$ & -0.190 & 1.000 & & & & & & & & \\
\hline $\mathrm{CaO}$ & -0.164 & -0.126 & 1.000 & & & & & & & \\
\hline $\mathrm{Fe}_{2} \mathrm{O}_{3}$ & -0.037 & -0.125 & 0.320 & 1.000 & & & & & & \\
\hline $\mathrm{Li}_{2} \mathrm{O}$ & -0.218 & -0.032 & 0.155 & -0.042 & 1.000 & & & & & \\
\hline $\mathrm{Na}_{2} \mathrm{O}$ & -0.256 & -0.022 & 0.023 & -0.059 & 0.534 & 1.000 & & & & \\
\hline $\mathrm{SiO}_{2}$ & -0.103 & -0.209 & -0.192 & -0.469 & -0.516 & -0.538 & 1.000 & & & \\
\hline $\mathrm{ThO}_{2}$ & 0.218 & 0.175 & 0.099 & 0.192 & -0.126 & -0.045 & -0.205 & 1.000 & & \\
\hline $\mathrm{ZrO}_{2}$ & 0.157 & -0.150 & 0.073 & 0.107 & -0.275 & -0.328 & 0.026 & -0.269 & 1.000 & \\
\hline Others & 0.007 & -0.089 & -0.300 & -0.004 & -0.197 & -0.140 & -0.037 & -0.066 & -0.039 & 1.000 \\
\hline
\end{tabular}

A variance-covariance matrix is required to calculate the measurement plus prediction uncertainties of this model. This matrix is defined as:

$$
\mathbf{V}_{\alpha}=\operatorname{RMSE}^{2}\left[\mathbf{X}_{\alpha}^{\mathbf{T}} \mathbf{X}_{\alpha}\right]^{-1}
$$

The use of $\mathbf{V}_{\alpha}$ is explained in detail elsewhere (e.g., Cornell 2002, Hrma et al. 1994, Piepel et al. 2008a, Vienna and Kim 2008). The $\mathbf{V}_{\ln [\mathrm{NTCLP}]}$ is given in Appendix $\mathbf{C}$ with the other variancecovariance matrices.

\subsubsection{Model Validation}

To validate the model, data not used in model fitting must be obtained. Because all appropriate data within the desired composition region were used in model fitting, subsets of the model data were used to validate the model. The data were sorted by the $\ln \left[N_{T C L P}\right]$ value. The data were then numbered $1,2,3,4,5,1,2, \ldots$ to split them into five roughly representative groups. The model was then refit to subsets 2 to 5 and used to predict data in subset 1 . Then the model was fit to each group of four subsets and used to predict the remaining subset in sequence. Table 3.60 summarizes the results of the model validation. The coefficients are reasonably close with an RSD of less than $25 \%$ with the exceptions of $\mathrm{Al}_{2} \mathrm{O}_{3}$ and $\mathrm{ZrO}_{2}$ that are both close to zero.

The model fit $R^{2}$ values are all close to each other at $\sim 0.89$. The $R_{V}^{2}$ are also close to each other, and their average (0.885) is close to the $R_{P}^{2}$ value of 0.884 . This model is well validated and should give predictions of unknown data within the model validity region nearly as well as model fit data. 
Table 3.60. Summary of $\operatorname{In}\left[N_{T C L P}\right]$ Model Validation

\begin{tabular}{lcccccrr}
\hline Term & Full & Set 1 & Set 2 & Set 3 & Set 4 & Set 5 & \%RSD \\
\hline $\mathrm{Al}_{2} \mathrm{O}_{3}$ & -0.52 & -0.63 & -0.89 & -0.32 & -0.23 & -0.45 & -49.9 \\
$\mathrm{~B}_{2} \mathrm{O}_{3}$ & 12.00 & 11.97 & 12.18 & 11.87 & 12.05 & 11.88 & 1.1 \\
$\mathrm{CaO}$ & 11.15 & 11.86 & 9.55 & 10.82 & 11.87 & 11.74 & 9.0 \\
$\mathrm{Fe}_{2} \mathrm{O}_{3}$ & -1.21 & -1.35 & -1.47 & -1.12 & -1.21 & -0.91 & -17.7 \\
$\mathrm{Li}_{2} \mathrm{O}$ & 20.87 & 21.86 & 20.57 & 20.63 & 20.07 & 21.07 & 3.2 \\
$\mathrm{Na}_{2} \mathrm{O}$ & 15.24 & 15.15 & 15.45 & 15.42 & 15.11 & 15.05 & 1.2 \\
$\mathrm{SiO}_{2}$ & -2.00 & -2.00 & -1.93 & -2.06 & -1.93 & -2.08 & -3.4 \\
$\mathrm{ThO}_{2}$ & 2.47 & 1.72 & 2.85 & 2.68 & 2.63 & 2.50 & 17.8 \\
$\mathrm{ZrO}_{2}$ & -0.66 & -1.25 & -1.40 & -0.43 & -0.34 & -0.02 & -91.2 \\
$\mathrm{Others}^{2}$ & 9.28 & 9.33 & 9.47 & 9.31 & 9.09 & 9.21 & 1.5 \\
$\mathrm{R}^{2}$ & 0.8941 & 0.8897 & 0.9047 & 0.8897 & 0.8919 & 0.8985 & \\
$\mathrm{R}^{2}$ & 0.8907 & 0.8853 & 0.9009 & 0.8852 & 0.8876 & 0.8944 & \\
$\mathrm{R}_{\mathrm{p}}^{2}$ & 0.8840 & 0.8766 & 0.8933 & 0.8757 & 0.8783 & 0.8862 & \\
$\mathrm{RMSE}$ & 0.3081 & 0.3097 & 0.2967 & 0.3178 & 0.3136 & 0.3029 & \\
$\mathrm{Mean}$ & 3.8957 & 3.8894 & 3.9024 & 3.8987 & 3.8960 & 3.8918 & \\
$\mathrm{~N}$ & 291 & 232 & 233 & 233 & 233 & 233 & \\
$\mathrm{R}^{2} \mathrm{v}$ & 0.8853 & 0.9036 & 0.8381 & 0.9115 & 0.8991 & 0.8741 & \\
\hline
\end{tabular}

\subsubsection{Application of $\operatorname{In}\left[N_{T C L P}, \mathrm{mg} / \mathrm{L}\right]$ Model to Regulatory Compliance}

The normalized TCLP response is an indication of the amount of glass released per unit volume if all elements are released congruently. Kim and Vienna (2004) found that the releases of $\mathrm{Ba}, \mathrm{Cd}, \mathrm{Ni}, \mathrm{Zn}$, and $\mathrm{B}$ release more-or-less congruently while other RCRA hazardous metals were released at concentrations below those of the congruent components. The slightly high releases of some metals at low boron release are due to detection limits being reported for lessthan-detection-limit results as these components are generally limited by solubility. The TCLP response of each RCRA element $(c r, \mathrm{mg} / \mathrm{L})$ is estimated by the relation:

$$
C_{r}=N_{T C L P} f_{r}=f_{r} \exp \left[\sum_{i=1}^{N} b_{N T C L P, i} x_{i}\right]
$$

where $f_{r}$ is the mass fraction of $r$-th element in glass. As shown in Figure 3.14b, this estimate is conservative for solubility-limited metals such as $\mathrm{Ag}, \mathrm{As}, \mathrm{Cr}, \mathrm{Pb}, \mathrm{Se}$, and $\mathrm{Tl}$. 

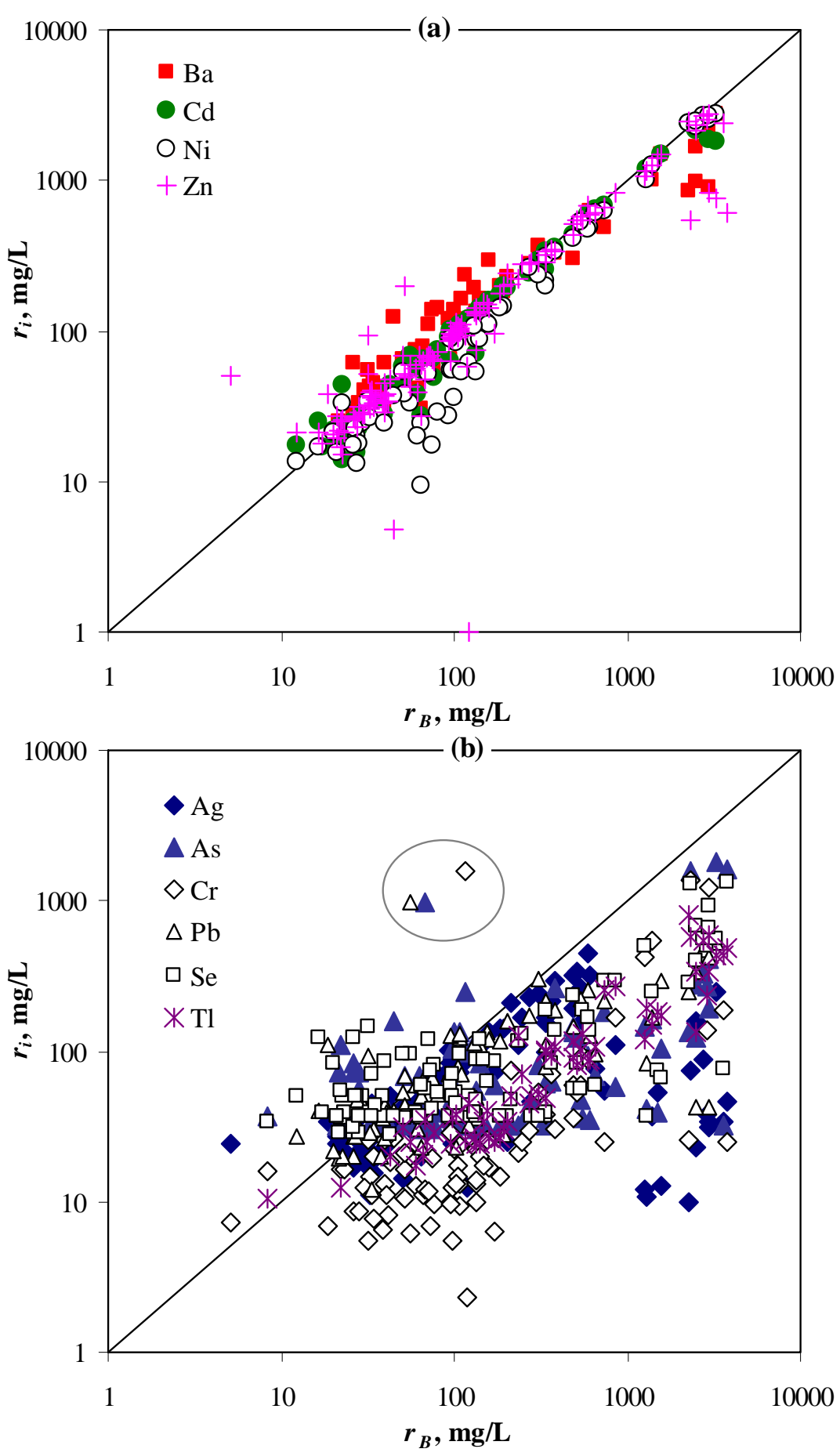

Figure 3.14. $\ln \left(r_{i}\right)$ Versus $\ln \left(\mathrm{r}_{\mathrm{B}}\right)$ for (a) $\mathrm{Ba}, \mathrm{Cd}, \mathrm{Ni}$, and $\mathrm{Zn}$ and (b) $\mathrm{Ag}, \mathrm{As}, \mathrm{Cr}, \mathrm{Pb}$, Se, and $\mathrm{Tl}$ in the Glasses Studied by Kot and Pegg (2001) from Kim and Vienna (2004) 
The TCLP response values $\left(c_{r}\right)$ can be compared to regulatory limits as shown in Table 3.61. Also listed in the table are the limits established for delisting Hanford HLW glasses per Blumenkranz (2006).

Table 3.61. RCRA Toxicity Limits and WTP Delisting Petition Limits by TCLP (mg/L)

\begin{tabular}{|c|c|c|c|c|c|c|c|c|c|c|c|c|c|c|}
\hline Limit & $\mathrm{Ag}$ & As & $\mathrm{Ba}$ & $\mathrm{Be}$ & $\mathrm{Cd}$ & $\mathrm{Cr}$ & $\mathrm{Cu}$ & $\mathrm{Hg}$ & $\mathrm{Ni}$ & $\mathrm{Pb}$ & $\mathrm{Sb}$ & $\mathrm{Se}$ & $\mathrm{TI}$ & $\mathrm{Zn}$ \\
\hline $\begin{array}{l}\text { RCRA } \\
\text { Toxicity }\end{array}$ & 5 & 5 & 100 & - & 1 & 5 & - & 0.2 & - & 5 & - & 1 & - & - \\
\hline $\begin{array}{l}\text { RCRA } \\
\text { UTS }\end{array}$ & 0.14 & 5 & 21 & - & 0.11 & 0.6 & - & 0.025 & 11 & 0.75 & - & 5.7 & - & - \\
\hline $\begin{array}{l}\text { WTP } \\
\text { Delisting }\end{array}$ & 3.07 & 0.616 & 100 & 1.33 & 0.48 & 4.95 & 200 & 0.2 & 22.6 & 5 & 0.659 & 1.0 & 0.282 & 225 \\
\hline
\end{tabular}

\subsection{Liquidus Temperature and Crystal Fraction}

\subsection{1 $\quad \mathrm{T}_{1 \%}$ - Spinel}

\section{Data Screening and Evaluation}

Of the initial 3406 glasses in the database (Appendix A), only 371 contained $T_{1 \%}$ values with spinel as a dominant phase. These data were all used in $\mathrm{T}_{1 \%}$ model development.

\section{Model Development}

Initial $\mathrm{T}_{1 \%}\left({ }^{\circ} \mathrm{C}\right)$ models were fit with the 371 data using stepwise regression methods allowing only first-order terms. Those components with sufficient data to allow for coefficient estimates were included in the stepwise process:

$\mathrm{Al}_{2} \mathrm{O}_{3}, \mathrm{~B}_{2} \mathrm{O}_{3}, \mathrm{Cr}_{2} \mathrm{O}_{3}, \mathrm{Bi}_{2} \mathrm{O}_{3}, \mathrm{CaO}, \mathrm{CdO}, \mathrm{F}, \mathrm{Fe}_{2} \mathrm{O}_{3}, \mathrm{~K}_{2} \mathrm{O}, \mathrm{Li}_{2} \mathrm{O}, \mathrm{MgO}, \mathrm{MnO}, \mathrm{Na}_{2} \mathrm{O}$, $\mathrm{NiO}, \mathrm{P}_{2} \mathrm{O}_{5}, \mathrm{SiO}_{2}, \mathrm{SrO}, \mathrm{ThO}_{2}, \mathrm{TiO}_{2}, \mathrm{UO}_{3}, \mathrm{ZnO}$, and $\mathrm{ZrO}_{2}$

Stepwise regression comparing the coefficient to the value of zero while maintaining the intercept near the average response of $951^{\circ} \mathrm{C}$ was used to reduce the influential components to:

$\mathrm{Al}_{2} \mathrm{O}_{3}, \mathrm{~B}_{2} \mathrm{O}_{3}, \mathrm{Cr}_{2} \mathrm{O}_{3}, \mathrm{Fe}_{2} \mathrm{O}_{3}, \mathrm{~K}_{2} \mathrm{O}, \mathrm{Li}_{2} \mathrm{O}, \mathrm{MgO}, \mathrm{MnO}, \mathrm{Na}_{2} \mathrm{O}, \mathrm{NiO}, \mathrm{SiO}_{2}, \mathrm{SrO}, \mathrm{ThO}_{2}$, $\mathrm{ZnO}$, and $\mathrm{ZrO}_{2}$

In addition, a combined noble-metals component ( $\left.\mathrm{NM}=\mathrm{PdO}+\mathrm{Rh}_{2} \mathrm{O}_{3}+\mathrm{RuO}_{2}\right)$ was found to significantly improve the fit. This is most likely due to the participation of $\mathrm{Rh}_{2} \mathrm{O}_{3}$ in the spinel structure (Annamalia et al. 2004). During this process, 21 glasses were found to be outliers (studentized residuals above 3 ):

SPA-27, HLW02-10, HLW-ALG-20(c), HLW07-07, HLW03-12, WTP-TL-19, HLW03-05, HLW03-43, HLW-ALG-05, HLW03-06, HLW07-32, HLW03-17, HLW07-16, HLW07-11, HLW07-24, HLW06-25, HLW-ALG-36, HLW-ALG-32, HLW07-05, EM07-Cr-02, SPA-02

Many of these glasses were found to be outliers in previous studies (Vienna et al. 2002, Vienna 2002, Piepel et al. 2008a). A scatterplot matrix of these data is given in Appendix B.

Attempts were made to improve the fit of the model data by adding a limited number (up to 5) second- and third-order terms. No higher order terms were found to significantly improve 
the fit, and the two most dominant terms decreased the model validation statistics. As a result, only first-order terms are used in the final model.

The final model was then fit to the remaining 350 datapoints with coefficients $b_{T 1 \%, i}$, listed in Table 3.62. The statistics for the model fit are summarized in Table 3.63. The $R^{2}$ coefficient of 0.772 suggests that the data are well represented by the model, but not as well as those models for previous properties. The $R^{2}$ A value $(0.761)$ suggests that there are not too many coefficients, or the data are not over-fit. The $R^{2}$ p being so close to the $R^{2}$ value $(0.744)$ suggests that there are not any very influential data points, and the model is likely to predict similarly for new data as it does for the model development data.

Table 3.62. $T_{1 \%},{ }^{\circ} \mathrm{C}$ Model Coefficients

\begin{tabular}{lcc}
\hline Term & Coefficient & Std Error \\
\hline $\mathrm{Al}_{2} \mathrm{O}_{3}$ & 2835.11 & 115.3 \\
$\mathrm{~B}_{2} \mathrm{O}_{3}$ & -201.41 & 101.8 \\
$\mathrm{Cr}_{2} \mathrm{O}_{3}$ & 12468.2 & 1509 \\
$\mathrm{Fe}_{2} \mathrm{O}_{3}$ & 3328.76 & 116.9 \\
$\mathrm{~K}_{2} \mathrm{O}$ & -409.94 & 333.6 \\
$\mathrm{Li}_{2} \mathrm{O}$ & -735.44 & 256.8 \\
$\mathrm{MgO}$ & 3927.07 & 803.2 \\
$\mathrm{MnO}$ & 2618.91 & 207.1 \\
$\mathrm{Na} \mathrm{O}$ & -717.5 & 94.61 \\
$\mathrm{NiO}$ & 11916.1 & 748.9 \\
$\mathrm{SiO}$ & 424.651 & 48.04 \\
$\mathrm{SrO}_{2}$ & 421.296 & 147.1 \\
$\mathrm{ThO}_{2}$ & 897.152 & 284.5 \\
$\mathrm{ZnO}$ & 3103.31 & 425.4 \\
$\mathrm{ZrO}$ & 1933.8 & 165 \\
$\mathrm{NM}$ & 14871.5 & 3248 \\
$\mathrm{Others}$ & 735.728 & 145 \\
\hline
\end{tabular}

Table 3.63. Summary of $T_{1 \%}$ Model Statistics

\begin{tabular}{lc}
\hline Statistic & Value \\
\hline $\mathrm{R}^{2}$ & 0.772 \\
$\mathrm{R}^{2}{ }_{\mathrm{A}}$ & 0.761 \\
$\mathrm{R}^{2}{ }_{\mathrm{P}}$ & 0.744 \\
$\mathrm{RMSE}$ & 60.69 \\
Min & 635.7 \\
Mean & 950.3 \\
Max & 1279 \\
$\mathrm{n}$ & 350 \\
$\mathrm{~N}$ & 17 \\
\hline
\end{tabular}

Figure 3.15 compares the predicted and measured $\mathrm{T}_{1 \%, \mathrm{sp}}$ values. The data appear to be well represented by the model with no trends to over or under prediction. No single group of data (e.g., study) was found to be misrepresented by the model.

This model is based on data spanning a specific glass composition region and therefore should only be relied upon within that region. The validity region is defined as the composition region of data used to develop the model. 


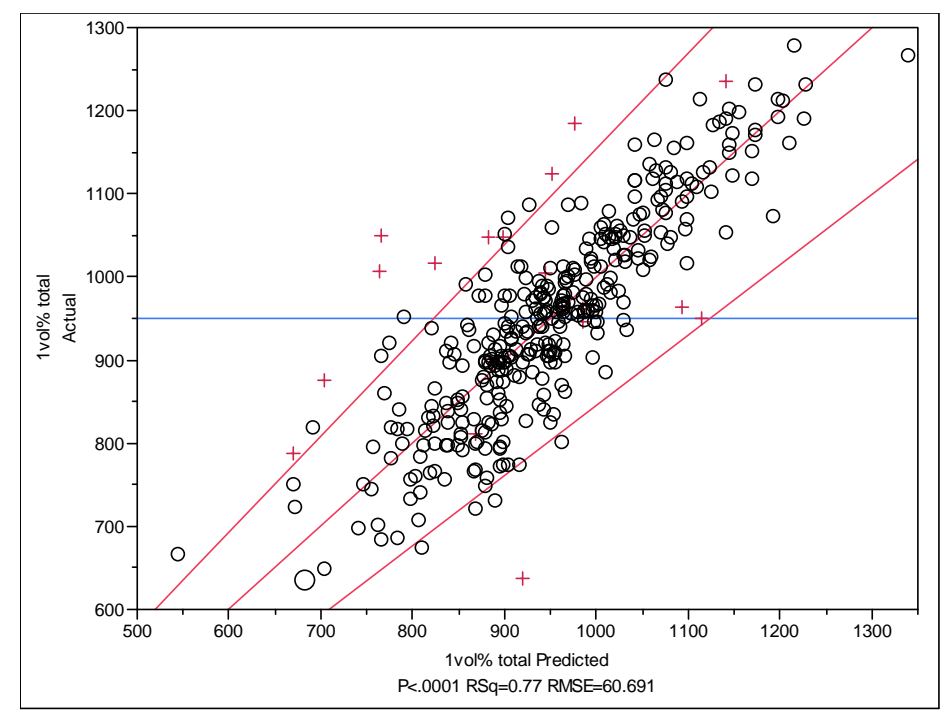

Figure 3.15. Measured vs. Predicted $\mathrm{T}_{1 \%, \mathrm{sp}}$

Table 3.64 lists the model validity region while Table 3.66 lists the correlation coefficients calculated for the model data. Only the $\mathrm{Al}_{2} \mathrm{O}_{3}-\mathrm{Na}_{2} \mathrm{O}$ pair has a correlation coefficient of 0.5 or above.

Table 3.64. $\mathrm{T}_{1 \%}$ Model Validity Region (wt $\%$ )

\begin{tabular}{lrr}
\hline Comp & Min & Max \\
\hline $\mathrm{Al}_{2} \mathrm{O}_{3}$ & 1.9 & 20.0 \\
$\mathrm{~B}_{2} \mathrm{O}_{3}$ & 3.0 & 20.0 \\
$\mathrm{Bi}_{2} \mathrm{O}_{3}$ & 0.0 & 7.0 \\
$\mathrm{CaO}$ & 0.0 & 7.0 \\
$\mathrm{CdO}$ & 0.0 & 2.0 \\
$\mathrm{Cr}_{2} \mathrm{O}_{3}$ & 0.0 & 1.2 \\
$\mathrm{~F}$ & 0.0 & 2.0 \\
$\mathrm{Fe}_{2} \mathrm{O}_{3}$ & 4.0 & 20.0 \\
$\mathrm{~K}_{2} \mathrm{O}$ & 0.0 & 6.0 \\
$\mathrm{Li}_{2} \mathrm{O}$ & 0.0 & 6.0 \\
$\mathrm{MgO}$ & 0.0 & 6.0 \\
$\mathrm{MnO}$ & 0.0 & 8.0 \\
$\mathrm{Na}_{2} \mathrm{O}$ & 3.7 & 25.0 \\
$\mathrm{NiO}$ & 0.0 & 3.0 \\
$\mathrm{NM}$ & 0.0 & 0.5 \\
$\mathrm{P}_{2} \mathrm{O}_{5}$ & 0.0 & 2.5 \\
$\mathrm{SiO}_{2}$ & 28.0 & 53.0 \\
$\mathrm{SrO}$ & 0.0 & 10.3 \\
$\mathrm{ThO}_{2}$ & 0.0 & 6.0 \\
$\mathrm{TiO}_{2}$ & 0.0 & 3.1 \\
$\mathrm{UO}_{3}$ & 0.0 & 6.5 \\
$\mathrm{ZnO}_{\mathrm{ZrO}}$ & 0.0 & 4.0 \\
$\mathrm{Remaining}_{2}$ & 0.0 & 9.6 \\
\hline & 0.0 & 4.5 \\
\hline
\end{tabular}

A variance-covariance matrix is required to calculate the measurement plus prediction uncertainties of this model. This matrix is defined as: 


$$
\mathbf{V}_{\alpha}=\operatorname{RMSE}^{2}\left[\mathbf{X}_{\alpha}^{\mathbf{T}} \mathbf{X}_{\alpha}\right]^{-1}
$$

The use of $\mathbf{V}_{\alpha}$ is explained in detail elsewhere (e.g., Cornell 2002, Hrma et al. 1994, Piepel et al. $2008 \mathrm{a}$, Vienna and Kim 2008). The $\mathbf{V}_{\mathrm{T} 1 \%}$ is given in Appendix $\mathrm{C}$ with the other variancecovariance matrices.

\section{Model Validation}

To validate the model, data not used in model fitting must be obtained. Because all appropriate data within the desired composition region were used in model fitting, subsets of the model data were used to validate the model. The data were sorted by $T_{1 \%}$ value. The data were then numbered $1,2,3,4,5,1,2, \ldots$ to split them into five roughly representative groups. The model was then refit to subsets 2 to 5 and used to predict data in subset 1 . Then the model was fit to each group of four subsets and used to predict the remaining subset in sequence. Table 3.65 summarizes the results of the model validation. The coefficients are reasonably close with an RSD of less than $25 \%$ with the exceptions of $\mathrm{B}_{2} \mathrm{O}_{3}$ and $\mathrm{ThO}_{2}$, which have extreme values for set 3 .

The model fit $R^{2}$ values are all close to each other at 0.775 . The $R_{V}^{2}$ are also close to each other, and their average (0.739) is close to the $R_{P}^{2}$ value of 0.74 . This model is well validated and should give predictions of unknown data within the model validity region nearly as well as model fit data in the $0.74 \mathrm{R}^{2}$ range with RMSEs near $61^{\circ} \mathrm{C}$.

Table 3.65. Summary of $T_{1 \%}$ Model Validation

\begin{tabular}{|c|c|c|c|c|c|c|c|}
\hline Term & Full & Set 1 & Set 2 & Set 3 & Set 4 & Set 5 & \%RSD \\
\hline$\overline{\mathrm{Al}_{2} \mathrm{O}_{3}}$ & 2,835 & 2,816 & 2,776 & 2,994 & 2,805 & 2,775 & 3 \\
\hline $\mathrm{B}_{2} \mathrm{O}_{3}$ & -201 & -200 & -125 & -336 & -172 & -177 & -40 \\
\hline $\mathrm{Cr}_{2} \mathrm{O}_{3}$ & 12,468 & 12,633 & 13,024 & 12,853 & 11,884 & 11,844 & 4 \\
\hline $\mathrm{Fe}_{2} \mathrm{O}_{3}$ & 3,329 & 3,400 & 3,427 & 3,288 & 3,205 & 3,320 & 3 \\
\hline $\mathrm{K}_{2} \mathrm{O}$ & -410 & -459 & -412 & -334 & -373 & -481 & -15 \\
\hline $\mathrm{Li}_{2} \mathrm{O}$ & -735 & -743 & -816 & -905 & -459 & -828 & -24 \\
\hline $\mathrm{MgO}$ & 3,927 & 3,815 & 3,818 & 3,592 & 3,873 & 5,507 & 20 \\
\hline $\mathrm{MnO}$ & 2,619 & 2,618 & 2,545 & 2,762 & 2,611 & 2,616 & 3 \\
\hline $\mathrm{Na}_{2} \mathrm{O}$ & -717 & -782 & -739 & -767 & -612 & -677 & -10 \\
\hline $\mathrm{NiO}$ & 11,916 & 11,897 & 11,672 & 12,579 & 11,896 & 11,479 & 3 \\
\hline $\mathrm{SiO}_{2}$ & 425 & 427 & 409 & 453 & 416 & 422 & 4 \\
\hline SrO & 421 & 507 & 342 & 310 & 451 & 454 & 20 \\
\hline $\mathrm{ThO}_{2}$ & 897 & 1,066 & 789 & 1,320 & 674 & 610 & 33 \\
\hline $\mathrm{ZnO}$ & 3,103 & 2,995 & 3,148 & 3,842 & 2,598 & 3,005 & 15 \\
\hline $\mathrm{ZrO}_{2}$ & 1,934 & 1,998 & 2,027 & 1,760 & 1,901 & 1,977 & 6 \\
\hline NM & 14,871 & 14,559 & 14,391 & 14,353 & 15,884 & 14,738 & 4 \\
\hline Others & 736 & 723 & 747 & 609 & 739 & 828 & 11 \\
\hline $\mathrm{R}^{2}$ & 0.772 & 0.789 & 0.77 & 0.777 & 0.777 & 0.765 & \\
\hline $\mathrm{R}_{\mathrm{A}}^{2}$ & 0.761 & 0.776 & 0.756 & 0.763 & 0.763 & 0.751 & \\
\hline$R_{P}^{2}$ & 0.744 & 0.754 & 0.732 & 0.746 & 0.741 & 0.727 & \\
\hline RMSE & 60.69 & 58.77 & 61.41 & 60.53 & 60.39 & 61.97 & \\
\hline$R^{2} v$ & 0.739 & 0.696 & 0.77 & 0.716 & 0.735 & 0.777 & \\
\hline
\end{tabular}


Table 3.66. Correlation Matrix for the $T_{1 \%}$ Model Dataset

\begin{tabular}{|c|c|c|c|c|c|c|c|c|c|c|c|c|c|c|c|c|c|}
\hline Corr & $\mathrm{Al}_{2} \mathrm{O}_{3}$ & $\mathrm{~B}_{2} \mathrm{O}_{3}$ & $\mathrm{Cr}_{2} \mathrm{O}_{3}$ & $\mathrm{Fe}_{2} \mathrm{O}_{3}$ & $\mathrm{~K}_{2} \mathrm{O}$ & $\mathrm{Li}_{2} \mathrm{O}$ & $\mathrm{MgO}$ & $\mathrm{MnO}$ & $\mathrm{Na}_{2} \mathrm{O}$ & $\mathrm{NiO}$ & $\mathrm{SiO}_{2}$ & SrO & $\mathrm{ThO}_{2}$ & $\mathrm{ZnO}$ & $\mathrm{ZrO}_{2}$ & NM & Others \\
\hline $\mathrm{Al}_{2} \mathrm{O}_{3}$ & 1.000 & & & & & & & & & & & & & & & & \\
\hline $\mathrm{B}_{2} \mathrm{O}_{3}$ & -0.352 & 1.000 & & & & & & & & & & & & & & & \\
\hline $\mathrm{Cr}_{2} \mathrm{O}_{3}$ & 0.105 & -0.057 & 1.000 & & & & & & & & & & & & & & \\
\hline $\mathrm{Fe}_{2} \mathrm{O}_{3}$ & 0.129 & 0.046 & -0.034 & 1.000 & & & & & & & & & & & & & \\
\hline $\mathrm{K}_{2} \mathrm{O}$ & -0.150 & 0.029 & -0.149 & -0.026 & 1.000 & & & & & & & & & & & & \\
\hline $\mathrm{Li}_{2} \mathrm{O}$ & -0.270 & 0.086 & -0.088 & -0.142 & 0.022 & 1.000 & & & & & & & & & & & \\
\hline $\mathrm{MgO}$ & -0.120 & 0.108 & -0.022 & 0.013 & -0.022 & 0.069 & 1.000 & & & & & & & & & & \\
\hline $\mathrm{MnO}$ & -0.065 & -0.071 & -0.066 & 0.051 & -0.066 & 0.014 & 0.101 & 1.000 & & & & & & & & & \\
\hline $\mathrm{Na}_{2} \mathrm{O}$ & -0.503 & 0.208 & -0.111 & -0.455 & 0.047 & 0.428 & 0.058 & -0.038 & 1.000 & & & & & & & & \\
\hline $\mathrm{NiO}$ & 0.115 & 0.039 & -0.093 & 0.062 & -0.031 & -0.081 & -0.027 & 0.022 & -0.291 & 1.000 & & & & & & & \\
\hline $\mathrm{SiO}_{2}$ & -0.096 & -0.449 & -0.062 & -0.403 & 0.066 & -0.413 & -0.170 & -0.060 & -0.233 & -0.117 & 1.000 & & & & & & \\
\hline $\mathrm{SrO}$ & -0.245 & 0.182 & -0.040 & -0.205 & -0.039 & 0.248 & 0.085 & -0.316 & 0.224 & 0.055 & -0.048 & 1.000 & & & & & \\
\hline $\mathrm{ThO}_{2}$ & 0.043 & 0.108 & -0.009 & 0.172 & 0.092 & -0.092 & 0.094 & 0.124 & 0.005 & 0.136 & -0.138 & -0.096 & 1.000 & & & & \\
\hline $\mathrm{ZnO}$ & 0.199 & -0.188 & 0.073 & 0.099 & -0.066 & -0.107 & 0.070 & 0.046 & -0.015 & 0.045 & -0.122 & -0.020 & 0.009 & 1.000 & & & \\
\hline $\mathrm{ZrO}_{2}$ & 0.344 & -0.119 & 0.044 & 0.218 & 0.089 & -0.289 & -0.061 & -0.013 & -0.291 & -0.083 & -0.124 & -0.210 & -0.207 & 0.080 & 1.000 & & \\
\hline NM & 0.126 & 0.052 & 0.216 & -0.113 & 0.083 & 0.009 & -0.155 & -0.230 & -0.014 & 0.100 & -0.092 & -0.172 & -0.146 & -0.459 & 0.006 & 1.000 & \\
\hline Others & -0.025 & -0.041 & -0.232 & 0.034 & -0.321 & 0.006 & 0.050 & -0.058 & -0.069 & -0.107 & -0.002 & -0.029 & -0.252 & -0.120 & -0.032 & -0.220 & 1.000 \\
\hline
\end{tabular}




\subsection{2 $\quad \mathrm{T}_{\mathrm{L}}-$ Zircon}

Besides spinel, zircon $\left(\mathrm{ZrSiO}_{4}\right)$ is the only primary phase for which there is sufficient data to fit models for $T_{L}$. Several attempts were made to fit models to combined zirconia containing phases as was done by Crum et al. 1997. However, the validation of these models showed unsatisfactory results. Furthermore, while there is sufficient data to fit a $T_{L}$ model to glasses precipitating zircon, there are not enough data to fit a $\mathrm{T}_{1 \%}$ model.

\section{Initial Data Screening and Evaluation}

There are 77 zircon precipitating glasses with $T_{L}$ in the database. Four of these glasses have compositions that depart from the bulk of the data by a significant amount as summarized in Table 3.67. The remaining 73 glasses were used in model fitting.

Table 3.67. Glasses Screened from $T_{L}$ Modeling Set

\begin{tabular}{ll}
\hline Glass ID & Composition Extreme \\
\hline CVS1-8 & $\mathrm{Fe}>10.5, \mathrm{Mg}>4$ \\
CVS2-3 & $\mathrm{Zr}<4$ \\
\hline CVS2-39 & $\mathrm{Mg}>4$ \\
WTP-TL-22 & $\mathrm{Fe}>10.5$ \\
\hline
\end{tabular}

\section{Model Development}

Initial $T_{L}\left({ }^{\circ} \mathrm{C}\right)$ models were fit with the 73 data using stepwise regression methods allowing only first-order terms. Those components with sufficient data to allow for coefficient estimates were included in the stepwise process:

$\mathrm{Al}_{2} \mathrm{O}_{3}, \mathrm{~B}_{2} \mathrm{O}_{3}, \mathrm{Bi}_{2} \mathrm{O}_{3}, \mathrm{CaO}, \mathrm{Fe}_{2} \mathrm{O}_{3}, \mathrm{Li}_{2} \mathrm{O}, \mathrm{LN}_{2} \mathrm{O}_{3}, \mathrm{MgO}, \mathrm{MnO}, \mathrm{Na}_{2} \mathrm{O}, \mathrm{Nd}_{2} \mathrm{O}_{3}, \mathrm{P}_{2} \mathrm{O}_{5}$, $\mathrm{SiO}_{2}, \mathrm{SrO}, \mathrm{ThO}_{2}, \mathrm{UO}_{3}, \mathrm{ZnO}$, and $\mathrm{ZrO}_{2}$

where $\mathrm{Ln}_{2} \mathrm{O}_{3}$ is a combined lanthanide oxide $\left(=\mathrm{Y}_{2} \mathrm{O}_{3}+\mathrm{La}_{2} \mathrm{O}_{3}+\mathrm{Ce}_{2} \mathrm{O}_{3}+\mathrm{Pr}_{2} \mathrm{O}_{3}+\mathrm{Nd}_{2} \mathrm{O}_{3}+\mathrm{Sm}_{2} \mathrm{O}_{3}\right.$ $+\mathrm{Gd}_{2} \mathrm{O}_{3}$ on a mass fraction basis). Stepwise regression comparing the coefficient to the value of zero while maintaining the intercept near the average response of $1091^{\circ} \mathrm{C}$ was used to reduce the influential components to:

$$
\mathrm{Al}_{2} \mathrm{O}_{3}, \mathrm{~B}_{2} \mathrm{O}_{3}, \mathrm{Li}_{2} \mathrm{O}, \mathrm{Ln}_{2} \mathrm{O}_{3}, \mathrm{Na}_{2} \mathrm{O}, \mathrm{SrO} \text {, and } \mathrm{ZrO}_{2}
$$

During this process, two glasses were found to be outliers (studentized residuals above 3 ) TRU-Na-1 and Zr-27; and two glasses were found to highly influential on model fit (Cook's D Influence > 0.3) - CVS3-21 and HLW07-23. A scatterplot matrix of these data is given in Appendix B.

Attempts were made to improve the fit of the model data by adding a limited number (up to 5) second- and third-order terms. No higher order terms were found to significantly improve the fit, and the two most dominant terms decreased the model validation statistics. As a result, only first-order terms are used in the final model.

The final model was then fit to the remaining 69 datapoints with coefficients $b_{T L, i}$, listed in Table 3.68. The statistics for the model fit are summarized in Table 3.69. The $R^{2}$ coefficient of 0.91 suggests that the data are well represented by the model. The $R^{2}$ A value $(0.896)$ suggests 
that there are not too many coefficients, or the data are not over-fit. The $R^{2}{ }_{\mathrm{P}}$ of 0.869 being somewhat below the $R^{2}$ value (0.907) suggests that there may be influential data, and the model may not adequately validate.

Table 3.68. $T_{L},{ }^{\circ} \mathrm{C}$ Model Coefficients

\begin{tabular}{lcc}
\hline Term & Coefficient & Std Error \\
\hline $\mathrm{Al}_{2} \mathrm{O}_{3}$ & 3193.3628 & 192.0059 \\
$\mathrm{~B}_{2} \mathrm{O}_{3}$ & 651.39721 & 96.32151 \\
$\mathrm{Ln}_{2} \mathrm{O}_{3}$ & 2156.4074 & 473.657 \\
$\mathrm{Li}_{2} \mathrm{O}$ & -1904.417 & 240.8539 \\
$\mathrm{Na}_{2} \mathrm{O}$ & -1947.711 & 163.0475 \\
$\mathrm{SrO}$ & 13011.909 & 1447.658 \\
$\mathrm{ZrO}$ & 3747.4241 & 182.0445 \\
Others & 1259.2233 & 32.29355 \\
\hline
\end{tabular}

Table 3.69. Summary of $T_{L}$ Model Statistics

\begin{tabular}{lc}
\hline Statistic & Value \\
\hline $\mathrm{R}^{2}$ & 0.9069 \\
$\mathrm{R}^{2}{ }_{\mathrm{A}}$ & 0.8962 \\
$\mathrm{R}_{\mathrm{P}}$ & 0.8693 \\
$\mathrm{RMSE}$ & 26.2 \\
Min & 897 \\
Mean & 1079 \\
Max & 1298 \\
$\mathrm{n}$ & 69 \\
$\mathrm{~N}$ & 8 \\
\hline
\end{tabular}

Figure 3.16 compares the predicted and measured $\mathrm{T}_{1 \%, \mathrm{sp}}$ values. The data appear to be well represented by the model with no trends to over or under prediction. No single group of data (e.g., study) was found to be misrepresented by the model.

This model is based on data spanning a specific glass composition region and therefore should only be relied upon within that region. The validity region is defined as the composition region of data used to develop the model.

Table 3.70 lists the model validity region while Table 3.71 lists the correlation coefficients calculated for the model data. Only the $\mathrm{ZrO}_{2}-\mathrm{Li}_{2} \mathrm{O}$ pair has a correlation coefficient of 0.5 or above.

The $\mathbf{V}_{\mathrm{TL}}$ is given in Appendix $\mathbf{C}$ with the other variance-covariance matrices. 


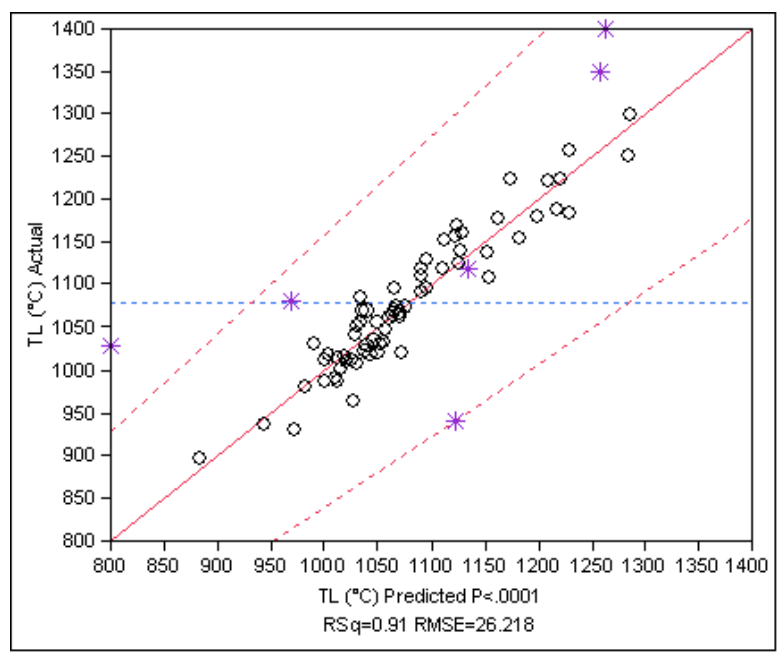

Figure 3.16. Measured vs. Predicted $T_{L, z s}$

Table 3.70. $T_{L}$ Model Validity Region ( $\left.w t \%\right)$

\begin{tabular}{lrr}
\hline Comp & Min & Max \\
\hline $\mathrm{Al}_{2} \mathrm{O}_{3}$ & 0.0 & 17.2 \\
$\mathrm{~B}_{2} \mathrm{O}_{3}$ & 2.0 & 20.0 \\
$\mathrm{Bi}_{2} \mathrm{O}_{3}$ & 0.0 & 10.0 \\
$\mathrm{CaO}$ & 0.0 & 10.0 \\
$\mathrm{Fe}_{2} \mathrm{O}_{3}$ & 0.0 & 10.5 \\
$\mathrm{Li}_{2} \mathrm{O}$ & 0.0 & 9.0 \\
$\mathrm{Ln}_{2} \mathrm{O}_{3}$ & 0.0 & 3.9 \\
$\mathrm{MgO}$ & 0.0 & 4.0 \\
$\mathrm{MnO}$ & 0.0 & 1.9 \\
$\mathrm{Na}_{2} \mathrm{O}$ & 4.0 & 15.0 \\
$\mathrm{Nd}_{2} \mathrm{O}_{3}$ & 0.0 & 2.2 \\
$\mathrm{P}_{2} \mathrm{O}_{5}$ & 0.0 & 5.0 \\
$\mathrm{SiO}_{2}$ & 39.7 & 59.6 \\
$\mathrm{SrO}$ & 0.0 & 1.2 \\
$\mathrm{ThO}_{2}$ & 0.0 & 6.0 \\
$\mathrm{UO}_{3}$ & 0.0 & 1.0 \\
$\mathrm{ZnO}_{\mathrm{ZrO}}$ & 0.0 & 1.2 \\
$\mathrm{ZrO}_{2}$ & 4.0 & 16.5 \\
$\mathrm{Remaining}$ & 0.3 & 3.3 \\
\hline
\end{tabular}

Table 3.71. Correlation Matrix for the $T_{L}$ Model Dataset

\begin{tabular}{lrrrrrrrr}
\hline Corr & $\mathrm{Al}_{2} \mathrm{O}_{3}$ & $\mathrm{~B}_{2} \mathrm{O}_{3}$ & $\mathrm{Ln}_{2} \mathrm{O}_{3}$ & $\mathrm{Li}_{2} \mathrm{O}$ & $\mathrm{Na}_{2} \mathrm{O}$ & $\mathrm{SrO}$ & $\mathrm{ZrO}_{2}$ & Others \\
\hline $\mathrm{Al}_{2} \mathrm{O}_{3}$ & 1.000 & & & & & & & \\
$\mathrm{~B}_{2} \mathrm{O}_{3}$ & -0.314 & 1.000 & & & & & & \\
$\mathrm{Ln}_{2} \mathrm{O}_{3}$ & 0.490 & -0.428 & 1.000 & & & & & \\
$\mathrm{Li}_{2} \mathrm{O}$ & -0.580 & 0.106 & -0.258 & 1.000 & & & & \\
$\mathrm{Na}_{2} \mathrm{O}$ & -0.433 & 0.501 & -0.348 & 0.430 & 1.000 & & & \\
$\mathrm{SrO}^{2}$ & -0.131 & -0.166 & 0.089 & 0.295 & 0.028 & 1.000 & & \\
$\mathrm{ZrO}_{2}$ & 0.512 & -0.372 & 0.497 & -0.747 & -0.564 & -0.036 & 1.000 & \\
Others & -0.059 & -0.377 & -0.254 & -0.048 & -0.550 & -0.156 & -0.196 & 1.000 \\
\hline
\end{tabular}




\section{Model Validation}

Table 3.72 summarizes the results of the model validation. The coefficients are reasonably close with an RSD of less than $25 \%$.

The model fit $R^{2}$ values are all close to each other ranging from 0.90 to 0.92 . The $R_{V}^{2}$ are also close to each other, and their average (0.87) is close to the $R_{P}^{2}$ value of 0.87 . This model is well validated and should give predictions of unknown data within the model validity region nearly as well as model fit data in the $0.87 \mathrm{R}^{2}$ range with RMSEs near $29^{\circ} \mathrm{C}$.

Table 3.72. Summary of $T_{L, z S}$ Model Validation

\begin{tabular}{lcccccrc}
\hline Term & Full & Set 1 & Set 2 & Set 3 & Set 4 & Set 5 & \%RSD \\
\hline $\mathrm{Al}_{2} \mathrm{O}_{3}$ & 3193 & 3234 & 3288 & 3232 & 3110 & 3145 & 2 \\
$\mathrm{~B}_{2} \mathrm{O}_{3}$ & 651 & 699 & 669 & 621 & 638 & 624 & 5 \\
$\mathrm{Ln}_{2} \mathrm{O}_{3}$ & 2156 & 1857 & 2798 & 2134 & 1886 & 2136 & 18 \\
$\mathrm{Li}_{2} \mathrm{O}$ & -1904 & -2005 & -2108 & -1981 & -1799 & -1800 & -7 \\
$\mathrm{Na}_{2} \mathrm{O}$ & -1948 & -1962 & -1943 & -2116 & -1837 & -1939 & -5 \\
$\mathrm{SrO}$ & 13012 & 12468 & 14976 & 11561 & 12321 & 13423 & 10 \\
$\mathrm{ZrO}_{2}$ & 3747 & 3745 & 3984 & 3774 & 3663 & 3686 & 3 \\
$\mathrm{Others}^{2}$ & 1259 & 1266 & 1216 & 1293 & 1260 & 1264 & 2 \\
$\mathrm{R}^{2}$ & 0.9069 & 0.8964 & 0.9240 & 0.9163 & 0.9050 & 0.9089 & \\
$\mathrm{R}^{2} \mathrm{~A}$ & 0.8962 & 0.8810 & 0.9127 & 0.9039 & 0.8909 & 0.8956 & \\
$\mathrm{R}^{2}$ & 0.8693 & 0.8376 & 0.8970 & 0.8508 & 0.8438 & 0.8614 & \\
$\mathrm{RMSE}$ & 26.218 & 28.036 & 24.077 & 25.200 & 26.174 & 27.173 & \\
$\mathrm{Mean}$ & 1079 & 1081 & 1079 & 1078 & 1076 & 1079 & \\
$\mathrm{n}$ & 69 & 55 & 55 & 55 & 55 & 56 & \\
$\mathrm{R}^{2} \mathrm{~V}$ & 0.8718 & 0.9381 & 0.7820 & 0.8519 & 0.9011 & 0.8860 & \\
\hline
\end{tabular}

\subsubsection{Impact of Other Phases}

Applying the $T_{1 \%, s p}$ and $T_{L, z s}$ constraints helps to avoid crystal precipitation during melting. Many other phases were found to form at and near $T_{L}$ in Hanford HLW glasses. However, simultaneous application of the two models with uncertainties (90\% confidence intervals) predicts higher $\mathrm{T}_{1 \%, \mathrm{sp}}$ or $\mathrm{T}_{\mathrm{L}, \mathrm{zs}}$ than $99.2 \%$ of the measured data for glasses within the composition region of validity, independent of phase. Further constraints to avoid other phases do not appear to be necessary at this time, unlike the "non-spinel phase rule" applied by Vienna and Kim (2008).

As no measured data with spinel as a primary (or secondary) phase had $\mathrm{ZrO}_{2}$ concentrations higher than $9.6 \mathrm{wt} \%$, it is appropriate to include the $\mathrm{T}_{\mathrm{L}, \mathrm{zs}}$ upper limit for $\mathrm{ZrO}_{2}$ and the $\mathrm{T}_{1 \%, \mathrm{sp}}$ lower limit for $\mathrm{ZrO}_{2}$.

\subsection{Glass Density (Specific Volume)}

The prediction of glass density proceeds by a distinctly different process that the other properties described above. Specifically, specific or molar volumes of the constituent oxides are additive for ideal mixtures. Ionic radii from constituent cations and anions are then used to estimate the partial-specific volumes of glass oxides and halogens. Two constants used to 
account for non-ideality are fitted to experimental data on waste glasses in the same manner as described in Vienna et al. (2002). The experimental data are first screened in Section 3.6.1, and the model fitting is described in Section 3.6.2.

\subsubsection{Data Screening and Evaluation}

Of the initial 3406 glasses in the database (Appendix A), 459 contained density data. Multi-valent oxides were converted to consistent sets of oxides, including $\mathrm{Fe}_{2} \mathrm{O}_{3}, \mathrm{As}_{2} \mathrm{O}_{5}, \mathrm{Ce}_{2} \mathrm{O}_{3}$, $\mathrm{CoO}, \mathrm{MnO}, \mathrm{MoO}_{3}, \mathrm{Pr}_{2} \mathrm{O}_{3}, \mathrm{Re}_{2} \mathrm{O}_{7}, \mathrm{Rh}_{2} \mathrm{O}_{3}, \mathrm{Sb}_{2} \mathrm{O}_{3}, \mathrm{SnO}_{2}, \mathrm{Tl}_{2} \mathrm{O}$, and $\mathrm{UO}_{3}$. Target and analyzed compositions were then compared. Those glasses with more than one major component (e.g., > 2 wt\% of the glass) or more than 20 minor components with a more than 20 relative percent difference between target and analyzed compositions were removed from the modelfitting dataset. Thirty glasses (IG2-10, IG3-01, IG3-04, IG3-05, IG3-06, IG3-07, IG3-08b, IG309, IG3-11, IG3-13, IG3-14, IG3-16, IG3-17, IG3-18, IG3-19, IG3-20, IG3-21, IG3-22, IG3-23, IG3-24, IG3-25, IG3-26, IG3-27, IG3-28, IG3-29, IG3-30, CVS2-14, CVS2-51, HLW98-61, and HLP-56) were removed from the dataset because of differences between target and measured compositions. As crystalline phases may impact glass density, the 14 multiphase glasses were removed from the model fitting dataset (IG2-01, IG2-03, IG2-05, IG2-06, IG2-09, IG2-17, IG218, IG2-22, IG2-27, IG2-34, IG3-03, HLW99-11, HLW99-12, and SBW1-33A). This left 415 glasses for model fitting. A scatterplot matrix of these data is given in Appendix $B$.

\subsubsection{Model Fitting}

The modeling approach taken is to estimate the partial-specific volume of oxides using published ionic radii from Shannon (1976). From ionic radii, the partial-specific volume can be estimated by:

$$
v_{i}=a \frac{4}{3} \pi r_{O}^{3} \frac{N_{i}^{\text {OperOx }}}{M W_{i}}+b \frac{4}{3} \pi r_{i}^{3} \frac{N_{i}^{\text {MperOx }}}{M W_{i}}
$$

where $\quad v_{i}=$ partial-specific volume of the $i^{\text {th }}$ glass component

$$
\begin{aligned}
a & =\text { a fit parameter to account for non-ideality in oxygen volume } \\
r_{O} & =\text { ionic radius of oxygen }(1.28 \mathrm{pm}) \text { from Shannon }(1976) \\
N_{i}^{\text {OperOx }} & =\text { number of oxygen atoms in the } i^{\text {th }} \text { oxide } \\
M W_{i} & =\text { molecular weight of the } i^{\text {th }} \text { oxide } \\
r_{i} & =\text { ionic radius of the metal in the } i^{\text {th }} \text { oxide from Shannon }(1976) \\
N_{i}^{\text {MperOx }} & =\text { number of metal atoms in the } i^{\text {th }} \text { oxide. }
\end{aligned}
$$

Table 3.73 lists the parameters of Equation (3.19) for the components in the 415 model glasses. These values were then used to estimate the fit coefficients $a$ and $b$. The density data from the 415 glasses were converted to specific volumes $(v)$ according to:

$$
\nu\left(\frac{\mathrm{cm}^{3}}{g}\right)=\frac{1}{\rho\left(\frac{g}{c m^{3}}\right)}
$$

where $\rho$ is the glass density reported in Appendix A. The fit coefficients $a$ and $b$ were fitted to minimize the RMSE between measured and estimated $v$ according to: 


$$
v=\sum_{i=1}^{N} v_{i} x_{i}
$$

where $x_{i}$ is the mass fraction of the $i^{\text {th }}$ component in glass and $\mathrm{N}$ is the total number of glass components. While fitting the model parameters, four glasses were found to be outliers (IG214, CVS2-86, HLWMS-11, and SBW1-36). The fit statistics are listed in Table 3.74, and the resulting densities are plotted against measured values in Figure 3.17.

Table 3.73. Partial-Specific Volume-Related Values

\begin{tabular}{|c|c|c|c|c|c|c|}
\hline ele & r, pm & Oxide & $N_{i}^{\text {MperOx }}$ & $N_{i}^{\text {OperOx }}$ & $r^{3}$ & $v_{i}$ \\
\hline$\overline{A C}$ & 1.26 & $\mathrm{Ac}_{2} \mathrm{O}_{3}$ & 2 & 3 & 2.000376 & 0.097617 \\
\hline $\mathrm{Ag}$ & 1.29 & $\mathrm{Ag}_{2} \mathrm{O}$ & 2 & 1 & 2.146689 & 0.114219 \\
\hline $\mathrm{Al}$ & 0.53 & $\mathrm{Al}_{2} \mathrm{O}_{3}$ & 2 & 3 & 0.148877 & 0.356339 \\
\hline Am & 1.115 & $\mathrm{Am}_{2} \mathrm{O}_{3}$ & 2 & 3 & 1.3861959 & 0.084523 \\
\hline As & 0.475 & $\mathrm{As}_{2} \mathrm{O}_{5}$ & 2 & 5 & 0.1071719 & 0.259265 \\
\hline B & 0.25 & $\mathrm{~B}_{2} \mathrm{O}_{3}$ & 2 & 3 & 0.015625 & 0.508767 \\
\hline $\mathrm{Ba}$ & 1.56 & $\mathrm{BaO}$ & 1 & 1 & 3.796416 & 0.161534 \\
\hline $\mathrm{Be}$ & 0.41 & $\mathrm{BeO}$ & 1 & 1 & 0.068921 & 0.480061 \\
\hline $\mathrm{Bi}$ & 1.17 & $\mathrm{Bi}_{2} \mathrm{O}_{3}$ & 2 & 3 & 1.601613 & 0.09932 \\
\hline $\mathrm{Br}$ & 1.82 & $\mathrm{Br}$ & 1 & 0 & 6.028568 & 0.258279 \\
\hline $\mathrm{Ca}$ & 1.26 & $\mathrm{CaO}$ & 1 & 1 & 2.000376 & 0.332023 \\
\hline $\mathrm{Cd}$ & 1.24 & $\mathrm{CdO}$ & 1 & 1 & 1.906624 & 0.142497 \\
\hline $\mathrm{Ce}$ & 1.15 & $\mathrm{Ce}_{2} \mathrm{O}_{3}$ & 2 & 3 & 1.520875 & 0.139312 \\
\hline $\mathrm{Cl}$ & 1.67 & $\mathrm{Cl}$ & 1 & 0 & 4.657463 & 0.449721 \\
\hline $\mathrm{Cm}$ & 1.11 & $\mathrm{Cm}_{2} \mathrm{O}_{3}$ & 2 & 3 & 1.367631 & 0.083489 \\
\hline Co & 0.885 & $\mathrm{CoO}$ & 1 & 1 & 0.6931541 & 0.188757 \\
\hline $\mathrm{Cr}$ & 0.755 & $\mathrm{Cr}_{2} \mathrm{O}_{3}$ & 2 & 3 & 0.4303689 & 0.251727 \\
\hline Cs & 1.88 & $\mathrm{Cs}_{2} \mathrm{O}$ & 2 & 1 & 6.644672 & 0.203202 \\
\hline $\mathrm{Cu}$ & 0.87 & $\mathrm{CuO}$ & 1 & 1 & 0.658503 & 0.176319 \\
\hline Dy & 1.052 & $\mathrm{Dy}_{2} \mathrm{O}_{3}$ & 2 & 3 & 1.1642526 & 0.116045 \\
\hline Eu & 1.206 & $\mathrm{Eu}_{2} \mathrm{O}_{3}$ & 2 & 3 & 1.7540498 & 0.134467 \\
\hline $\mathrm{F}$ & 1.19 & $\mathrm{~F}$ & 1 & 0 & 1.685159 & 0.303646 \\
\hline $\mathrm{Fe}$ & 0.63 & $\mathrm{Fe}_{2} \mathrm{O}_{3}$ & 2 & 3 & 0.250047 & 0.231855 \\
\hline $\mathrm{Ga}$ & 0.61 & $\mathrm{Ga}_{2} \mathrm{O}_{3}$ & 2 & 3 & 0.226981 & 0.196685 \\
\hline Gd & 1.193 & $\mathrm{Gd}_{2} \mathrm{O}_{3}$ & 2 & 3 & 1.6979361 & 0.129486 \\
\hline $\mathrm{Hf}$ & 0.85 & $\mathrm{HfO}_{2}$ & 1 & 2 & 0.614125 & 0.121834 \\
\hline $\mathrm{Hg}$ & 1.33 & $\mathrm{Hg}_{2} \mathrm{O}$ & 2 & 1 & 2.352637 & 0.066827 \\
\hline I & 2.06 & I & 1 & 0 & 8.741816 & 0.235813 \\
\hline K & 1.65 & $\mathrm{~K}_{2} \mathrm{O}$ & 2 & 1 & 4.492125 & 0.451472 \\
\hline La & 1.3 & $\mathrm{La}_{2} \mathrm{O}_{3}$ & 2 & 3 & 2.197 & 0.154555 \\
\hline $\mathrm{Li}$ & 0.73 & $\mathrm{Li}_{2} \mathrm{O}$ & 2 & 1 & 0.389017 & 0.483063 \\
\hline $\mathrm{Mg}$ & 0.86 & $\mathrm{MgO}$ & 1 & 1 & 0.636056 & 0.34608 \\
\hline $\mathrm{Mn}$ & 0.97 & $\mathrm{MnO}$ & 1 & 1 & 0.912673 & 0.209981 \\
\hline Mo & 0.73 & $\mathrm{MoO}_{3}$ & 1 & 3 & 0.389017 & 0.25459 \\
\hline $\mathrm{Na}$ & 1.16 & $\mathrm{Na}_{2} \mathrm{O}$ & 2 & 1 & 1.560896 & 0.362348 \\
\hline $\mathrm{Nb}$ & 0.78 & $\mathrm{Nb}_{2} \mathrm{O}_{5}$ & 2 & 5 & 0.474552 & 0.233644 \\
\hline $\mathrm{Nd}$ & 1.249 & $\mathrm{Nd}_{2} \mathrm{O}_{3}$ & 2 & 3 & 1.9484412 & 0.144597 \\
\hline $\mathrm{Ni}$ & 0.83 & $\mathrm{NiO}$ & 1 & 1 & 0.571787 & 0.1838 \\
\hline $\mathrm{Np}$ & 1.01 & $\mathrm{NpO}_{2}$ & 1 & 2 & 1.030301 & 0.100612 \\
\hline $\mathrm{P}$ & 0.31 & $\mathrm{P}_{2} \mathrm{O}_{5}$ & 2 & 5 & 0.029791 & 0.416076 \\
\hline $\mathrm{Pb}$ & 1.43 & $\mathrm{PbO}$ & 1 & 1 & 2.924207 & 0.097588 \\
\hline
\end{tabular}


Table 3.73 (contd)

\begin{tabular}{lrlccrr}
\hline ele & $\mathbf{r}, \mathbf{p m}$ & Oxide & $N_{i}^{\text {MperOx }}$ & $N_{i}^{\text {OperOx }}$ & $\mathbf{r}^{3}$ & $\boldsymbol{v}_{\boldsymbol{i}}$ \\
\hline $\mathrm{Pd}$ & 1 & $\mathrm{PdO}^{2}$ & 1 & 1 & 1 & 0.124118 \\
$\mathrm{Pr}$ & 1.266 & $\mathrm{Pr}_{2} \mathrm{O}_{3}$ & 2 & 3 & 2.0290891 & 0.149193 \\
$\mathrm{Pu}$ & 1 & $\mathrm{PuO}_{2}$ & 1 & 2 & 1 & 0.099468 \\
$\mathrm{Rb}$ & 1.75 & $\mathrm{Rb}_{2} \mathrm{O}$ & 2 & 1 & 5.359375 & 0.259259 \\
$\mathrm{Re}$ & 0.67 & $\mathrm{Re}_{2} \mathrm{O}_{7}$ & 2 & 7 & 0.300763 & 0.174351 \\
$\mathrm{Rh}$ & 0.805 & $\mathrm{Rh}_{2} \mathrm{O}_{3}$ & 2 & 3 & 0.5216601 & 0.153206 \\
$\mathrm{Ru}$ & 0.76 & $\mathrm{RuO}_{2}$ & 1 & 2 & 0.438976 & 0.188211 \\
$\mathrm{Sb}$ & 0.74 & $\mathrm{Sb}_{2} \mathrm{O}_{3}$ & 2 & 3 & 0.405224 & 0.130656 \\
$\mathrm{Se}$ & 0.42 & $\mathrm{SeO}_{2}$ & 1 & 2 & 0.074088 & 0.214457 \\
$\mathrm{Si}$ & 0.4 & $\mathrm{SiO}_{2}$ & 1 & 2 & 0.064 & 0.395468 \\
$\mathrm{Sm}$ & 1.219 & $\mathrm{Sm}_{2} \mathrm{O}_{3}$ & 2 & 3 & 1.8113865 & 0.13683 \\
$\mathrm{Sn}$ & 0.83 & $\mathrm{SnO}_{2}$ & 1 & 2 & 0.571787 & 0.169198 \\
$\mathrm{~S}$ & 0.26 & $\mathrm{SO}_{3}$ & 1 & 3 & 0.017576 & 0.441816 \\
$\mathrm{Sr}$ & 1.4 & $\mathrm{SrO}_{\mathrm{T}}$ & 1 & 1 & 2.744 & 0.204254 \\
$\mathrm{Ta}$ & 0.88 & $\mathrm{Ta}_{2} \mathrm{O}_{5}$ & 2 & 5 & 0.681472 & 0.143749 \\
$\mathrm{Tc}$ & 0.7 & $\mathrm{TC}_{2} \mathrm{O}_{7}$ & 2 & 7 & 0.343 & 0.276953 \\
$\mathrm{Te}$ & 0.8 & $\mathrm{TeO}_{2}$ & 1 & 2 & 0.512 & 0.158491 \\
$\mathrm{Th}$ & 1.08 & $\mathrm{ThO}_{2}$ & 1 & 2 & 1.259712 & 0.105495 \\
$\mathrm{Ti}$ & 0.56 & $\mathrm{TiO}_{2}$ & 1 & 2 & 0.175616 & 0.302252 \\
$\mathrm{Tl}$ & 1.73 & $\mathrm{Tl}_{2} \mathrm{O}_{2}$ & 2 & 1 & 5.177717 & 0.111169 \\
$\mathrm{U}$ & 0.87 & $\mathrm{UO}_{3}$ & 1 & 3 & 0.658503 & 0.131343 \\
$\mathrm{~V}$ & 0.68 & $\mathrm{~V}_{2} \mathrm{O}_{5}$ & 2 & 5 & 0.314432 & 0.335433 \\
$\mathrm{~W}$ & 0.74 & $\mathrm{WO}_{3}$ & 1 & 3 & 0.405224 & 0.158296 \\
$\mathrm{Y}$ & 1.159 & $\mathrm{Y}_{2} \mathrm{O}_{3}$ & 2 & 3 & 1.5568627 & 0.20359 \\
$\mathrm{Zn}$ & 0.88 & $\mathrm{ZnO}_{\mathrm{Zn}}$ & 1 & 1 & 0.681472 & 0.173291 \\
$\mathrm{Zr}$ & 0.86 & $\mathrm{ZrO}_{2}$ & 1 & 2 & 0.636056 & 0.208725 \\
\hline & & & & & & \\
\hline
\end{tabular}

Table 3.74. $\rho$ and $v$ Model Fit Summary

\begin{tabular}{lcc}
\hline & $\rho$ & $v$ \\
\hline Minimum & 2.410 & 0.3175 \\
Mean & 2.656 & 0.3770 \\
Maximum & 3.150 & 0.4149 \\
$\mathrm{R}^{2}$ & 0.8231 & 0.8121 \\
$\mathrm{R}^{2} \mathrm{~A}$ & 0.8227 & 0.8116 \\
$\mathrm{RMSE}$ & 0.0431 & 0.00616 \\
Count & 411 & 411 \\
\multicolumn{1}{c}{$\boldsymbol{a}$} & $\boldsymbol{b}$ \\
Coefficients & 1.339987 & 0.817249 \\
\hline
\end{tabular}




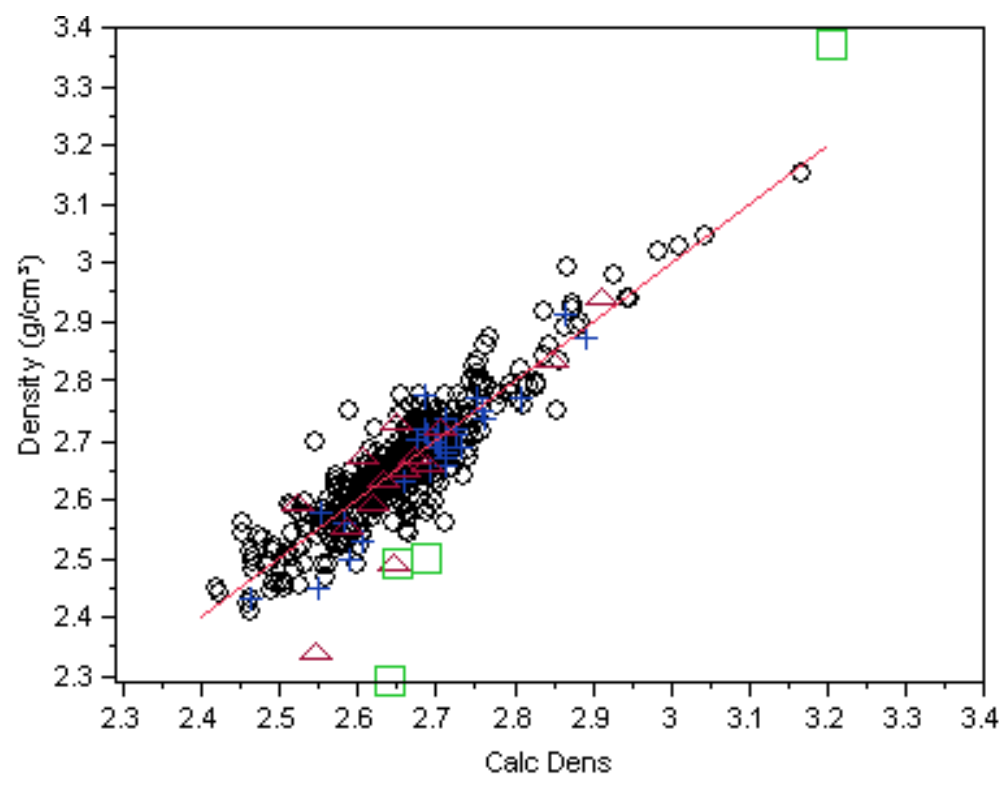

Figure 3.17. Predicted vs Measured Glass Density (black circles are model fit data, green squares are outliers, red triangles are multiphase glasses, blue crosses are data with differences between target and analyzed compositions) 


\subsection{Glass Composition Constraints}

In addition to the property constraints discussed in Section 1.2 and the model validity constraints reported in Section 3.0, there are several constraints placed on glass to facilitate successful glass processing and adequate quality. These constraints are typically set as single and multiple component constraints that limit composition space to reduce the risk of an adverse effect for which adequate data and models are unavailable.

This section describes these constraints in the context of the event that they aim to avoid and the data available to support them. As more data become available, these constraints may change and thereby change the predictions of glass volume to be produced at Hanford.

\subsection{Nepheline Discriminator}

Models that relate TCLP and PCT to composition are largely based on glasses that have been fast cooled from the melting temperature. This cooling schedule represents a fraction of the canistered glass that contacts the canister wall and cools to below the glass transition temperature $\left(T_{g}\right)$ relatively quickly. However, a portion of the glass in the center of the canister cools significantly slower and may be reheated by hot glass being poured on top. The timetemperature profile of the extreme ends of the cooling-rate spectra were measured for DWPF canisters (Marra and Jantzen 1993) and WTP canisters. ${ }^{\text {(a) }}$ As seen in Figure 4.1, it requires between 25 and $30 \mathrm{~h}$ for glass in the center of the canister to cool to below $400^{\circ} \mathrm{C}$ (a conservative estimate of $\mathrm{T}_{\mathrm{g}}$ ). Several glasses have been tested for the impact of different heattreatment schedules on product quality (Marra and Jantzen 1993, Kim et al. 1995, Li et al. 1997b, Vienna et al. 2001b, Riley et al. 2001, Peeler et al. 2006, Fox et al. 2007, Kim et al. 2008 , etc.). The overwhelming conclusion of these studies is that the cooling has an insignificant impact on durability so long as gross crystallization does not occur. Once crystallization occurs, then the impact on glass durability is given by the impact of the crystals on residual glass composition. Some crystalline phases, such as transition metal spinels, $[\mathrm{Ni}, \mathrm{Fe}, \mathrm{Mn}, \mathrm{Zn}][\mathrm{Fe}, \mathrm{Cr}]_{2} \mathrm{O}_{4}$, have almost no impact on PCT response. Nepheline, $\mathrm{NaAlSiO}_{4}$, however, significantly degrades PCT response.

(a) L Petkus. 2003. "Canister Centerline Cooling Data, Revision 1, CCN 074851." Memo to CA Musick, River Protection Project, Waste Treatment and Immobilization Plant, Richland, Washington. 


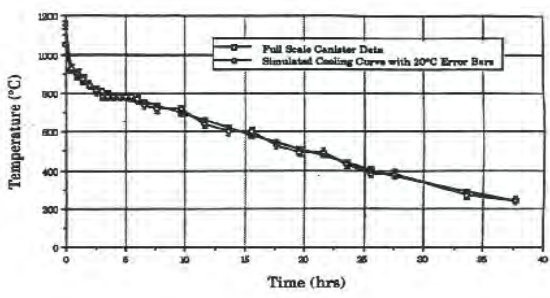

(a) DWPF

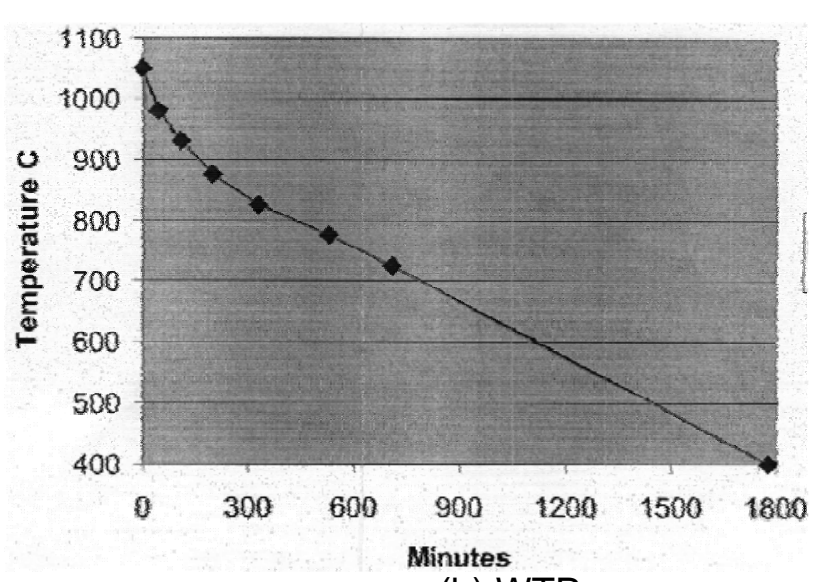

(b) WTP

Figure 4.1. CCC Curves for (a) DWPF HLW Glass (Marra and Jantzen 1993) and (b) WTP HLW Glass ${ }^{(a)}$

To avoid the impact of nepheline on slow-cooled HLW glasses, a nepheline discriminator is employed. The original discriminator developed by Li et al. (1997b) is given as:

$$
N_{S i}=\frac{x_{\mathrm{SiO}_{2}}}{x_{\mathrm{Al}_{2} \mathrm{O}_{3}}+x_{\mathrm{Na}_{2} \mathrm{O}}+x_{\mathrm{SiO}_{2}}} \geq 0.62
$$

where $N_{S i}$ is the normalized silica concentration, and $x_{i}$ is the $i$-th component mass fraction in glass. This rule is based on avoiding the low-silica end of the $\mathrm{Na}_{2} \mathrm{O}-\mathrm{Al}_{2} \mathrm{O}_{3}-\mathrm{SiO}_{2}$ phase diagram where $\mathrm{NaAlSiO}_{4}$ is prevalent. A number of recent studies have found this constraint to be conservative in that glasses meeting the $N_{S i} \geq 0.62$ do not contain nepheline on slow cooling while many glasses with $N_{S i}<0.62$ also do not contain nepheline (see Figure 4.2). The presence of boron has been clearly shown to reduce the tendency toward nepheline formation (Li et al. 2003), and CaO may also reduce the tendency. However, there is no clear explanation for the $\mathrm{CaO}$ effect nor is there a quantitative model for the effect of $\mathrm{B}_{2} \mathrm{O}_{3}$. It is very likely that other components have a strong impact on nepheline precipitation; however, until sufficient data and predictive models are available, it is recommended that the discriminator given in Equation (4.1) be used.

(a) L Petkus. 2003. "Canister Centerline Cooling Data, Revision 1, CCN 074851." Memo to CA Musick, River Protection Project, Waste Treatment and Immobilization Plant, Richland, Washington. 

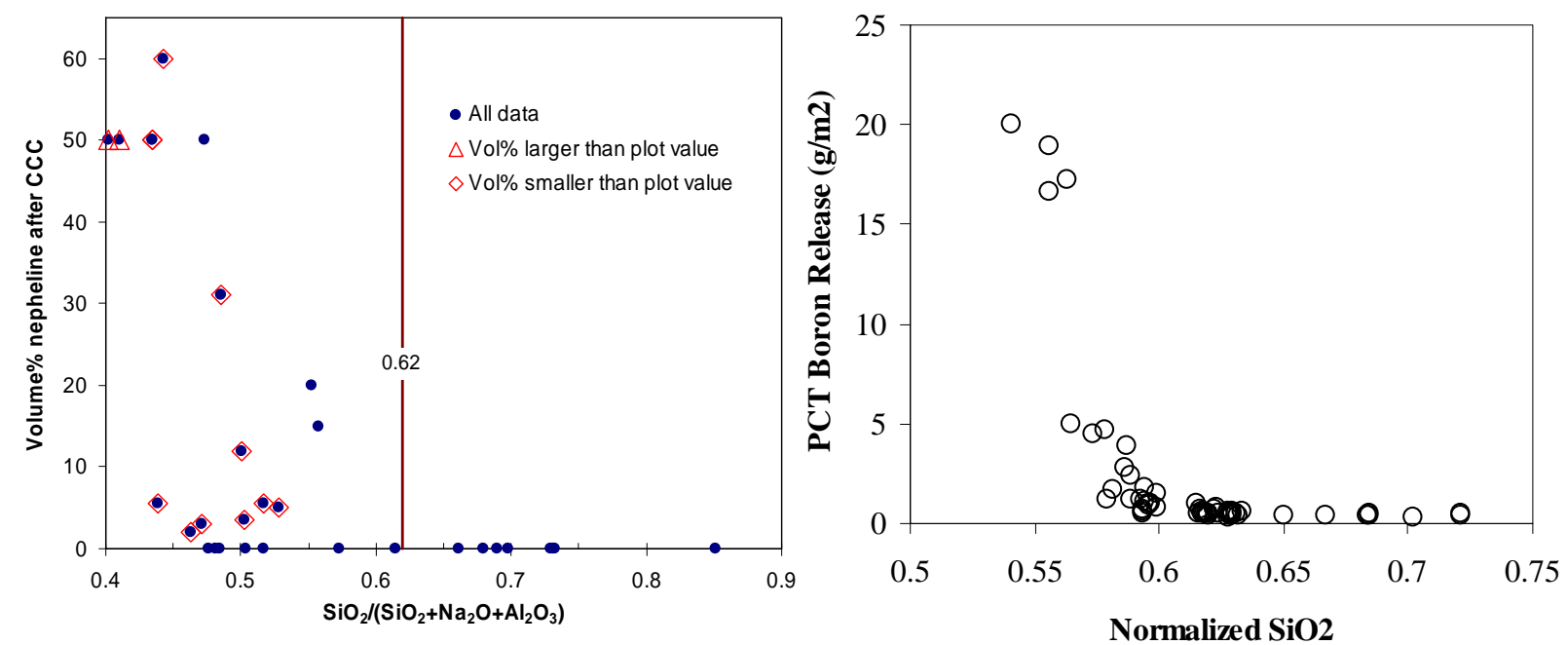

Figure 4.2. CCC Glass Nepheline Content and PCT-B Response vs. Normalized Silica Discriminator (after Kim et al. 2008)

\subsection{Phosphate Limit}

An excessive quantity of $\mathrm{P}_{2} \mathrm{O}_{5}$ in an alkali-silicate glass will promote immiscible liquid separation in the melt (for examples, see Bunnell 1988, Jantzen et al. 2000, Kot et al. 2007, Li et al. 1995a, 1995b, 1995c, 1995d, 1997a, 1997b, 1997d, and Li 1998; Langowski 1996 and Langowski et al. 1997). If this liquid contains high-alkaline earth oxides (CaO, MgO, etc.), then a refractory scum may form at the melt surface impeding the melting process. If this liquid contains high-alkali oxide concentrations $\left(\mathrm{Na}_{2} \mathrm{O}, \mathrm{Li}_{2} \mathrm{O}\right.$, etc. $)$, then a water-soluble phase will result and will likely contain significant radionuclide $\left({ }^{137} \mathrm{Cs},{ }^{99} \mathrm{Tc}\right.$, etc. $)$ and hazardous $(\mathrm{Cr}, \mathrm{Pb}$, etc.) components.

To avoid the deleterious effects of phosphate-based phase separation, controls are put in place on the phosphate concentration in the target glass. Vienna and Kim (2008) found that limiting the concentrations of $\mathrm{P}_{2} \mathrm{O}_{5}, \mathrm{CaO}$, and $\mathrm{Li}_{2} \mathrm{O}$ according to the following rules would greatly reduce the chance of phosphate-based phase separation in Hanford HLW glasses:

$$
\begin{aligned}
& x_{P 2 O 5} \leq 0.045 \\
& x_{C a O} \cdot x_{P 2 O 5}<6.5 \times 10^{-4} \\
& x_{L i 2 O} \leq 0.060
\end{aligned}
$$

where $x_{i}$ is the $i$-th oxide mass fraction in glass. These rules are shown graphically in Figure 4.3. 

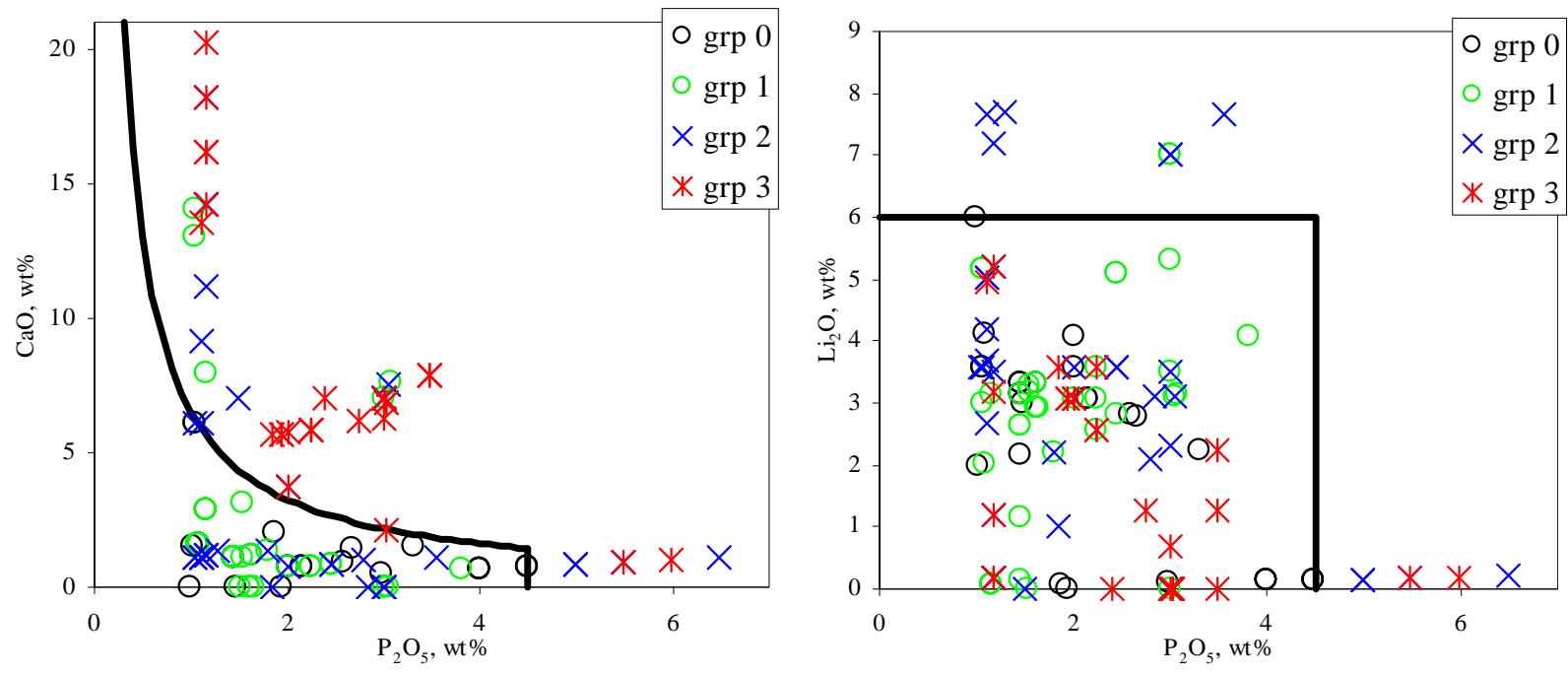

\begin{tabular}{clcc}
\hline Grp & Description & $\#$ & $\mathrm{P}_{2} \mathrm{O}_{5}$ Range, wt\% \\
\hline 0 & pass all constraints and show no adverse effect of $\mathrm{P}_{2} \mathrm{O}_{5}$ & 33 & $1.00-4.49$ \\
1 & fail at least one constraint that is clearly unrelated to $\mathrm{P}_{2} \mathrm{O}_{5}$ & 36 & $1.05-3.81$ \\
2 & fail at least one constraint, the role of $\mathrm{P}_{2} \mathrm{O}_{5}$ is uncertain & 40 & $1.05-6.48$ \\
3 & fail a constraint that is clearly due to $\mathrm{P}_{2} \mathrm{O}_{5}$ & 26 & $1.11-5.98$ \\
\hline
\end{tabular}

Figure 4.3. Summary of WTP Phosphate Phase Separation Data (from Vienna and Kim 2008)

These rules were applied to a broader region of glasses from Appendix $A$ and found to equally well screen those glasses prone to phosphate phase separation. Therefore, it is recommended that they be applied when estimating glass volumes.

\subsection{Chromium Oxide and Alumina Limits}

The amounts of $\mathrm{Cr}_{2} \mathrm{O}_{3}$ and $\mathrm{Al}_{2} \mathrm{O}_{3}$ have a significant influence on the $\mathrm{HLW}$ glass volume to be produced at Hanford. These components are limited primarily because of their influence on crystal formation in the melter. In addition, $\mathrm{Cr}_{2} \mathrm{O}_{3}$ can be oxidized to chromate $\left(\mathrm{CrO}_{4}{ }^{2-}\right)$ and promote/participate in salt segregation in the melter. However, $\mathrm{Al}_{2} \mathrm{O}_{3}$ will promote crystallization of the melt during canister cooling as described in Section 4.1. The primary function of caustic and oxidative leaching to be performed at the WTP is to remove $\mathrm{Al}_{2} \mathrm{O}_{3}$ and $\mathrm{Cr}_{2} \mathrm{O}_{3}$, respectively, to minimize HLW glass volume.

The single-component limits have traditionally been used to limit the concentrations of these two components and thereby reduce the risk of their deleterious effects on glass melting and properties. This report documents models to avoid crystallization within the melter (Section 3.5), nepheline formation during slow cooling (Section 4.1), and salt separation within the melter (Section 4.4). Therefore, no additional limits should be placed on $x_{\mathrm{Cr}_{203}}$ and $x_{A 12 O 3}$ in target glass compositions. It should be noted, though, that the model-validity constraints for both $x_{\mathrm{Cr} 2 \mathrm{O} 3}$ and $x_{\mathrm{Al} 2 \mathrm{O} 3}$ and the nepheline constraint are quite restrictive and will likely still impact HLW glass volumes (see Table 4.1). As TCLP and electrical conductivity are initially to be calculated as characteristics of glasses formulated with other limits, the range of glass compositions will extend to $20 \mathrm{wt} \%$ for $x_{\mathrm{Al} 2 \mathrm{O} 3}$ and $1.2 \mathrm{wt} \%$ for $x_{\mathrm{Cr} 2 \mathrm{O} 3}$. 
Table 4.1. Summary of $\mathrm{Cr}_{2} \mathrm{O}_{3}$ and $\mathrm{Al}_{2} \mathrm{O}_{3}$ Model Validity Constraints (mass fraction)

\begin{tabular}{lcc}
\hline \multicolumn{1}{c}{ Modeled Property } & $x_{\text {CI2O3 }}$ & $x_{\text {Al2O3 }}$ \\
\hline $\ln \left[\eta_{950}\right]$ & - & 0.204 \\
$\ln \left[\eta_{1150}\right]$ & - & 0.266 \\
$\ln \left[\eta_{1250}\right]$ & - & 0.259 \\
$\ln \left[\varepsilon_{1000}, \varepsilon_{1100}, \varepsilon_{1200}\right]$ & - & 0.259 \\
$\ln \left[\varepsilon_{1150}\right]$ & - & 0.266 \\
$\operatorname{In}[\mathrm{PCT}-\mathrm{B},-\mathrm{Na},-\mathrm{Li}]$ & - & 0.200 \\
$\ln \left[\mathrm{N}_{\mathrm{TCLP}}\right]$ & - & 0.163 \\
$\mathrm{~T}_{1 \%}$, spinel & 0.012 & 0.200 \\
$\mathrm{~T}_{\mathrm{L}}$, zircon & - & 0.172 \\
\hline
\end{tabular}

\subsection{Sulfate and Salt Separation Limits}

Glass melts with relatively high concentrations of certain salt components (e.g., $\mathrm{SO}_{4}{ }^{2-}, \mathrm{F}^{-}$, $\mathrm{Cl}^{-}, \mathrm{CrO}_{4}{ }^{2-}$, etc.) are prone to the accumulation of a separated molten salt on the melt surface. If the salt layer persists on the surface of the melt, it will significantly accelerate the corrosion of bubblers, thermowells, level probes, and potentially melt-line refractories. These components are likely to drop to the melter bottom and reduce melter life by the accumulation of conductive metals on the melter floor. If persistent for long times, melt-line refractory corrosion may cause premature and potentially catastrophic melter failure.

Since there is currently no method of detection for accumulated salt layers on the melt surface, feed composition control must be used to avoid the deleterious effects of salt on HLW melter operation. This section describes the collection and evaluation of available data on the effect of $\mathrm{SO}_{3}$ concentration on $\mathrm{HLW}$ glass processing and the establishment of preliminary $\mathrm{SO}_{3}$ constraints to prevent potential processing problems associated with salt accumulation.

The allowable sulfur in the feed is determined by a combination of thermodynamic and kinetic aspects of sulfate incorporation into the silicate-based melts (Pegg et al. 2000, Hrma et al. 2003, Hrma et al. 2004). Therefore, scaled melter testing is the most certain way of determining the allowable sulfur level for a given feed. Since the melter operating parameters (e.g., melting rate, raw material source, bubbling rate) impact the sulfur tolerance, scaled Hanford HLW testing should be used in setting the constraint on sulfur loading.

The amount of sulfur that the typical HLW melter feeds can sustain has not been systematically tested, and therefore information required to determine if salt accumulation in $\mathrm{HLW}$ is a problem and in what compositions (e.g., concentrations of $\mathrm{SO}_{3}, \mathrm{Na}_{2} \mathrm{O}, \mathrm{Li}_{2} \mathrm{O}, \mathrm{K}_{2} \mathrm{O}$, $\mathrm{CaO}, \mathrm{MgO}, \mathrm{Cr}_{2} \mathrm{O}_{3}, \mathrm{P}_{2} \mathrm{O}_{5}, \mathrm{Cl}$, and $\mathrm{F}$ ) is very limited. The concentrations of $\mathrm{SO}_{3}$ in various melter feeds reported in the melter test reports are listed in Table 4.2. These results suggest that the tolerable $\mathrm{SO}_{3}$ could be as low as $0.19 \mathrm{wt} \%$ (HLW04-09) or as high as $0.7 \mathrm{wt} \%$ (HLW02-46). This result plus that of Darab et al. (2001) suggest that with increased scale, the tolerance to sulfur increases. Therefore, the $0.19 \mathrm{wt} \%$ salt accumulation in the DM100 can be ignored in favor of the DM1200 test with the same feed. This leaves two data points of value in setting a limit: HLW98-86 (0.44 wt\% $\left.\mathrm{SO}_{3}\right)$ and HLW02-46 (0.7 wt\% $\left.\mathrm{SO}_{3}\right)$. Based on such limited data, it 
is difficult to justify any limit different from that traditionally applied (DOE 2000) of $0.5 \mathrm{wt} \%$ as $\mathrm{SO}_{3}$ in the melter feed.

Table 4.2. Summary of $\mathrm{SO}_{3}$ Concentrations in Melter Testing

\begin{tabular}{ccccl}
\hline Glass ID & $\mathrm{SO}_{3}$, wt $\%$ & Scale & $\begin{array}{c}\text { Salt } \\
\text { Accumulation }\end{array}$ & Reference \\
\hline HLW98-31 & 0.25 & DM100, DM1200 & No, No & Matlack et al. 2001 and 2002 \\
HLW98-77 & 0.08 & DM1200 & No & Matlack et al. 2003a \\
HLW98-80 & 0.04 & DM1200 & No & Matlack et al. 2003b \\
HLW04-09 & 0.19 & DM100, DM1200 & Yes, No & Matlack et al. 2005a and 2005b \\
HLW98-86 & $0.44,0.5$ & DM10 & No, Yes & Matlack and Pegg 2008 \\
HLW02-46 & $0.7,0.9$ & DM10 & No, Yes & Matlack and Pegg 2008 \\
\hline
\end{tabular}

\subsection{Other Composition Limits}

Other components that are not typically large fractions of Hanford tank waste may limit loading in glass. These components and estimates of their limits are described below.

- $\mathrm{MoO}_{3}$ forms immiscible liquid or solid phases when present at too high a concentration in alkali alkaline-earth silicate glasses. It is estimated that a limit between 1 and $3.5 \mathrm{wt} \% \mathrm{MoO}_{3}$ will result from thorough studies of Mo solubility in Hanford waste glasses. For the purpose of completeness, a limit of $1.5 \mathrm{wt} \%$ should be adopted until sufficient data are available.

- Fluorine may promote salt accumulation in the melter (in concert with sulfate, chromate, phosphate, molybdate, etc.) or crystallize as alkali or alkaline-earth fluorides. With model validity regions spanning maximum $\mathrm{F}$ contents of 1.5 to $4.7 \mathrm{wt} \%$, the $2 \mathrm{wt} \%$ limit associated with the spinel $\mathrm{T}_{1 \%}$ model should be taken as the maximum $\mathrm{F}$ concentration to make certain that phase separation problems do not occur.

- Noble metals $\left(\mathrm{RuO}_{2}, \mathrm{Rh}_{2} \mathrm{O}_{3}, \mathrm{PdO}\right.$, and $\left.\mathrm{Ag}_{2} \mathrm{O}\right)$ are sparingly soluble in silicate melts. For the most part, they will form an insoluble phase in the melter that will act as a nucleating agent to help crystallization approach its equilibrium state within the melter and reduce crystal sizes. If they exceed some critical concentration, they will agglomerate and accumulate at the melter bottom. Experience at the West Valley Nuclear Services (WVNS) suggests that less than $0.15 \mathrm{wt} \%$ of $\mathrm{RuO}_{2}, \mathrm{Rh}_{2} \mathrm{O}_{3}$, and $\mathrm{PdO}$ can be effectively removed from that melter. The WTP melter is designed to tolerate higher concentrations, but prolonged operation at concentrations well above this limit are still expected to shorten melter life. For the time being, the WTP contract limit of $0.25 \mathrm{wt} \%$ should be used as a limit until more operating experience with noble metals in a WTP-like melter is available.

- Elements that are traditionally found in Hanford tank waste in only small concentrations could also have an impact if their concentrations exceed traditional data concentrations. Although no limit will be placed on those components at this time, those that should cause concern, including the levels at which they would cause a concern, are listed in Table 4.3. 
Table 4.3. Trace Elements of Concern (levels in wt $\%$ of glass)

\begin{tabular}{|c|c|}
\hline Element & Concentration of concern, wt $\%$ \\
\hline $\mathrm{Be}$ & 1 \\
\hline $\mathrm{Cl}$ & 0.5 \\
\hline Sc & 1 \\
\hline $\mathrm{Ti}$ & 5 \\
\hline V & 5 \\
\hline Co & 1 \\
\hline $\mathrm{Cu}$ & 0.1 (lower with $\mathrm{Ag}$ ) \\
\hline $\mathrm{Ga}$ & 5 \\
\hline $\mathrm{Ge}$ & 5 \\
\hline As & 1 \\
\hline $\mathrm{Se}$ & 0.5 \\
\hline $\mathrm{Br}$ & 0.5 \\
\hline $\mathrm{Rb}$ & 1 \\
\hline $\mathrm{Nb}$ & 1 \\
\hline Tc & 0.1 \\
\hline In & 1 \\
\hline Sn & 1 \\
\hline $\mathrm{Sb}$ & 1 \\
\hline $\mathrm{Te}$ & 0.1 \\
\hline I & 1 (will be lost to off-gas) \\
\hline Cs & 1 \\
\hline $\mathrm{Ba}$ & 5 \\
\hline La-Lu & 5 (total lanthanides) \\
\hline $\mathrm{Hf}$ & 1 \\
\hline $\mathrm{Ta}$ & 1 \\
\hline W & 0.5 \\
\hline $\mathrm{Re}$ & 0.1 \\
\hline Os & 0.1 \\
\hline Ir & 0.1 \\
\hline $\mathrm{Pt}$ & 0.1 \\
\hline $\mathrm{Au}$ & 0.1 \\
\hline $\mathrm{Hg}$ & 0.5 (will be lost to off-gas) \\
\hline $\mathrm{Tl}$ & 0.1 (RCRA) \\
\hline $\mathrm{Pb}$ & 5 \\
\hline Po & 0.5 \\
\hline At & 1 \\
\hline $\mathrm{Fr}$ & 1 \\
\hline $\mathrm{Ra}$ & 1 \\
\hline Ac-Lr & 0.1 \\
\hline
\end{tabular}




\subsection{Elemental Losses During Melting}

During the melting process, components are lost to the off-gas treatment system. In the current WTP baseline process, those components that are captured within the submerged bed scrubber (SBS) or the wet electrostatic precipitator (WESP) will be recycled back to the head end of the pretreatment process while those that escape the SBS/WESP will be purged from the process as secondary wastes or through the stack.

Two primary mechanisms are typically credited for the loss of components to the off-gas treatment system-entrainment and volatility. For the purpose of this report, these mechanisms will not be distinguished. However, it should be noted that melter processing parameters such as operating temperature, redox state, bubbling rate, feed composition, etc. will impact loss to the off-gas. The extent to which these parameters will impact loss will be different for the different mechanisms and are currently not well understood.

An estimate of melter decontamination factors (DFs) was recently generated by Jain (2008). These DFs were converted to fractions of the elements retained in glass $(v)$ in Table 5.1 according to:

$$
v=1-\frac{1}{D F}
$$

As a large fraction of the losses will be recycled back to the WTP, the use of these loss factors must be considered carefully. If the flowsheet model accounts for the return of mass to the WTP, then these should be removed from the glass, and a fraction of the loss will then be returned. If no recycle loop is made, then those elements that are not purged should be left in the glass.

Table 5.1. Estimated Melter DF and $v$ (from Jain 2008)

\begin{tabular}{lrl}
\hline Element & Est DF & \multicolumn{1}{c}{ Est $v$} \\
\hline $\mathrm{Ag}$ & 160 & 0.99375 \\
$\mathrm{Al}$ & 152 & 0.993434012 \\
$\mathrm{Am}$ & 59 & 0.983050847 \\
$\mathrm{As}$ & 28 & 0.964285714 \\
$\mathrm{~B}$ & 92 & 0.989130435 \\
$\mathrm{Ba}$ & 137 & 0.992673993 \\
$\mathrm{Be}$ & 88 & 0.988636364 \\
$\mathrm{Bi}$ & 170 & 0.994117647 \\
$\mathrm{C}$ & 1 & 0 \\
$\mathrm{Ca}$ & 87 & 0.988545246 \\
$\mathrm{Cd}$ & 21 & 0.951923077 \\
$\mathrm{Ce}$ & 88 & 0.988636364 \\
$\mathrm{Cl}$ & 2.3 & 0.565217391 \\
$\mathrm{Co}$ & 88 & 0.988636364 \\
$\mathrm{Cr}$ & 84 & 0.988066826 \\
$\mathrm{Cr}$ & 84 & 0.988066826 \\
$\mathrm{Cs}$ & 33 & 0.96978852 \\
\hline
\end{tabular}


Table 5.1 (contd)

\begin{tabular}{|c|c|c|}
\hline Element & Est DF & Est $v$ \\
\hline $\mathrm{Cu}$ & 161 & 0.993804213 \\
\hline $\mathrm{Eu}$ & 88 & 0.988636364 \\
\hline $\mathrm{F}$ & 1.6 & 0.375 \\
\hline $\mathrm{Fe}$ & 121 & 0.991708126 \\
\hline $\mathrm{H}$ & 1 & 0 \\
\hline $\mathrm{Hg}$ & 1 & 0 \\
\hline 1 & 1.2 & 0.166666667 \\
\hline $\mathrm{K}$ & 40 & 0.975062344 \\
\hline $\mathrm{La}$ & 88 & 0.988636364 \\
\hline $\mathrm{Li}$ & 221 & 0.995473065 \\
\hline $\mathrm{Mg}$ & 61 & 0.983471074 \\
\hline $\mathrm{Mn}$ & 282 & 0.996458924 \\
\hline Mo & 84 & 0.988095238 \\
\hline $\mathrm{N}$ & 1 & 0 \\
\hline $\mathrm{Na}$ & 110 & 0.990900819 \\
\hline $\mathrm{Nd}$ & 88 & 0.988636364 \\
\hline $\mathrm{Ni}$ & 140 & 0.992836676 \\
\hline $\mathrm{P}$ & 68 & 0.985294118 \\
\hline $\mathrm{Pb}$ & 84 & 0.988123515 \\
\hline $\mathrm{Pd}$ & 79 & 0.987341772 \\
\hline $\mathrm{Pr}$ & 88 & 0.988636364 \\
\hline $\mathrm{Pu}$ & 88 & 0.988636364 \\
\hline $\mathrm{Ra}$ & 4 & 0.736842105 \\
\hline $\mathrm{Rb}$ & 33 & 0.96969697 \\
\hline $\mathrm{Rh}$ & 53 & 0.981132075 \\
\hline $\mathrm{Ru}$ & 4 & 0.756097561 \\
\hline $\mathrm{S}$ & 3 & 0.666666667 \\
\hline $\mathrm{Sb}$ & 168 & 0.994044074 \\
\hline $\mathrm{Se}$ & 2 & 0.5 \\
\hline $\mathrm{Si}$ & 251 & 0.996012759 \\
\hline $\mathrm{Sr}$ & 138 & 0.99275887 \\
\hline $\mathrm{Ta}$ & 88 & 0.988636364 \\
\hline Tc & 2 & 0.375 \\
\hline $\mathrm{Te}$ & 13 & 0.921259843 \\
\hline Th & 88 & 0.988636364 \\
\hline $\mathrm{Ti}$ & 50 & 0.980119284 \\
\hline TI & 4 & 0.736842105 \\
\hline U & 88 & 0.988636364 \\
\hline V & 59 & 0.983050847 \\
\hline W & 88 & 0.988636364 \\
\hline Y & 88 & 0.988636364 \\
\hline $\mathrm{Zn}$ & 123 & 0.991856678 \\
\hline $\mathrm{Zr}$ & 202 & 0.995047053 \\
\hline
\end{tabular}




\subsection{Glass Formulation Options and Examples}

The models discussed in Section 3.0 and the additional constraints described in Section 4.0 are used to formulate glasses from waste compositions. The precise methods for estimating glass composition and waste loading are described in detail by Vienna and Kim (2008). This process can be summarized as:

1. For a given waste, the glass composition is moved into a composition region where all the glass property models are valid (that is to say, the region where we have experimental experience). This is done by adding various amounts of wastes and additives.

2. The additives are altered until all the properties are within acceptable regions, including prediction and composition uncertainties. This forces the composition toward the region of more experimental data, because the uncertainties increase rapidly as the composition approaches the boundaries of data.

3. If there are degrees of freedom left after meeting all the property constraints, then the composition is further moved within the "acceptable glass processing window" toward points that are more favorable (i.e., away from boundaries). This optimization includes five key factors:
a. viscosity toward $50 \mathrm{P}$ at $1150^{\circ} \mathrm{C}$
b. PCT below $4 \mathrm{~g} / \mathrm{L}$
c. $\mathrm{T}_{1 \%}$ below $850^{\circ} \mathrm{C}$
d. waste loading away from the boundaries (min acceptable to max possible)
e. composition closer to those processed successfully in the DM1200 pilot melter.

This process is aimed at formulating glass composition during operations and is relatively high in computational effort. For the purpose of estimating $\mathrm{HLW}$ glass volume to be produced, a less precise and less computationally heavy method would be appropriate.

\subsection{Additives}

Glass-forming additives will be added to a waste to form a waste glass composition. The typical list of $\mathrm{HLW}$ glass-forming additives includes $\mathrm{B}_{2} \mathrm{O}_{3}, \mathrm{Li}_{2} \mathrm{O}, \mathrm{Na}_{2} \mathrm{O}, \mathrm{SiO}_{2}$, and $\mathrm{ZnO}$. The WTP currently has the capability to also add $\mathrm{Al}_{2} \mathrm{O}_{3}, \mathrm{CaO}, \mathrm{Fe}_{2} \mathrm{O}_{3}, \mathrm{MgO}, \mathrm{TiO}_{2}$, and $\mathrm{ZrO}_{2}$. These additives are currently in the form of: borax, lithium carbonate, sodium carbonate, fume silica, zincite, kyanite, wollastonite, hematite, olivine, rutile, and zircon, respectively. However, the impacts of impurities in these chemical sources are relatively inconsequential. So, for the purposes of evaluating glass volumes, the mineral sources are not necessary. As many of the property model validity regions list minimum values for $\mathrm{Al}_{2} \mathrm{O}_{3}$ and $\mathrm{Fe}_{2} \mathrm{O}_{3}$, it is recommended that they be used in glass formulations for the purpose of minimizing glass volume. Two sets of additives should be considered the base set and the expanded set (first six plus additional five) as listed in Table 6.1. 
Table 6.3. Property Model Validity Limits (wt\%)

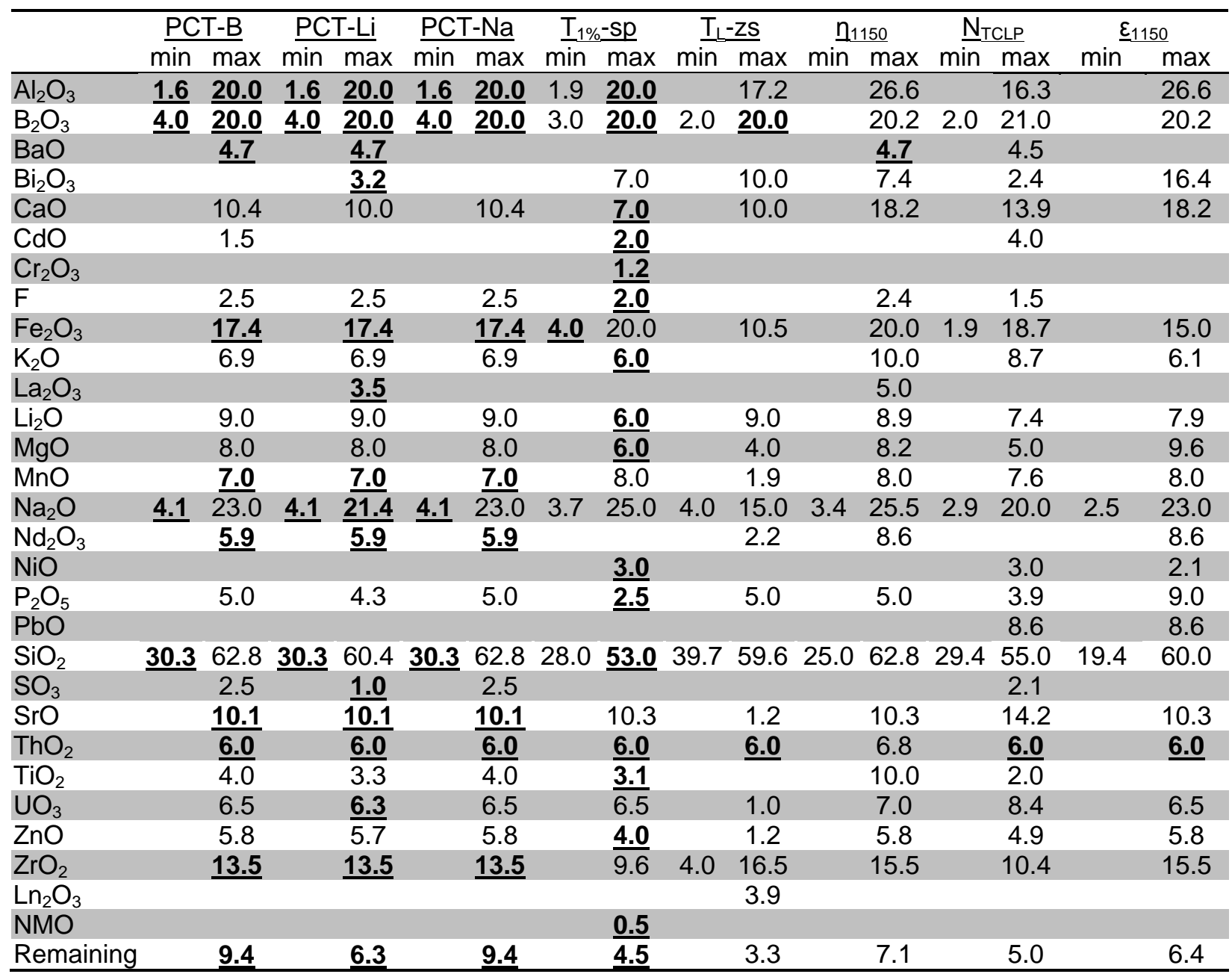

Additional composition constraints are added for noble metals $\left(=\mathrm{PdO}+\mathrm{Rh}_{2} \mathrm{O}_{3}+\mathrm{RuO}_{2}\right)$ at $0.25 \%$ as described in Section 4.5 and $\mathrm{SO}_{3}$ at $0.5 \mathrm{wt} \%$ as described in Section 4.4. Other single-component constraints are less restrictive than those of model-validity.

In addition to these constraints, it is appropriate to limit the concentration of waste and each additive to between 0 and $100 \mathrm{wt} \%$ to avoid non-physical solutions to the optimization calculations.

\subsection{Optimization}

The most common optimization criterion is to maximize waste loading, which will yield the minimum glass mass for a given waste. By adjusting the ratios of waste oxides and each of the additives, the waste loading can be maximized until all property and composition constraints are attained.

Other potential optimal criteria can be considered, such as: 
- maximum robustness (furthest distance from all constraints)

- highest chemical durability (lowest PCT and TCLP responses)

- lowest waste loading (for highest glass production rate)

- similarity to glasses demonstrated in pilot scale melter testing (for highest confidence)

These optimization criteria can be applied as needed.

\subsection{Example Calculations}

To demonstrate the use of these glass property models and optimize maximum waste loading, examples are given in this subsection. The calculations use measured compositions of recent tank waste samples shown in AZ-101 Optimization in Table 6.4. An Excel ${ }^{\circledR}$ spreadsheet was developed to optimize for maximum waste loading within the constraints listed above.

\subsubsection{AZ-101 Optimization}

Glasses were formulated using the composition of $A Z-101$ listed in Table 6.4. The first calculation was performed with the base set of additives $\left(\mathrm{Al}_{2} \mathrm{O}_{3}, \mathrm{~B}_{2} \mathrm{O}_{3}, \mathrm{Fe}_{2} \mathrm{O}_{3}, \mathrm{Li}_{2} \mathrm{O}, \mathrm{Na}_{2} \mathrm{O}\right.$, and $\mathrm{SiO}_{2}$ ). The resulting glass is summarized in Table 6.5. In this calculation, the maximum waste loading was $37.66 \mathrm{wt} \%$. The additives were $0.00 \mathrm{wt} \% \mathrm{Al}_{2} \mathrm{O}_{3}, 31.82 \mathrm{~B}_{2} \mathrm{O}_{3}, 0.00 \mathrm{Fe}_{2} \mathrm{O}_{3}, 1.63 \mathrm{Li}_{2} \mathrm{O}$, $13.45 \mathrm{Na}_{2} \mathrm{O}$, and $53.09 \mathrm{SiO}_{2}$. If the loading is not limited by a single-component constraint for a waste component, then there must be as many constraints as there are additive components. Otherwise, loading could be increased by changing the concentrations of the additives and loading. For this first example, the limiting constraints include $\eta_{1150}=4 \mathrm{~Pa}-\mathrm{s}, \mathrm{N}_{\mathrm{SiO} 2}=62 \mathrm{wt} \%$, $\mathrm{B}_{2} \mathrm{O}_{3}=20 \mathrm{wt} \%$, and $\mathrm{T}_{1 \%}-\mathrm{sp}=950^{\circ} \mathrm{C}$. The calculated glass properties are summarized in Table 6.6.

The next calculation used the same (AZ-101) waste and the expanded set of additives. The resulting glass is summarized in Table 6.7. In this calculation, the maximum waste loading was $38.16 \mathrm{wt} \%$. The additives were $0.00 \mathrm{wt} \% \mathrm{Al}_{2} \mathrm{O}_{3}, 0.00 \mathrm{CaO}, 31.82 \mathrm{~B}_{2} \mathrm{O}_{3}, 0.00 \mathrm{Fe}_{2} \mathrm{O}_{3}, 1.63$ $\mathrm{Li}_{2} \mathrm{O}, 0.00 \mathrm{MgO}, 13.45 \mathrm{Na}_{2} \mathrm{O}, 53.09 \mathrm{SiO}_{2}, 0.00 \mathrm{TiO}_{2}, 0.00 \mathrm{ZnO}$, and $0.00 \mathrm{ZrO}_{2}$. For this first example, the limiting constraints include $\eta_{1150}=4 \mathrm{~Pa}-\mathrm{s}, \mathrm{N}_{\mathrm{SiO} 2}=62 \mathrm{wt} \%, \mathrm{~T}_{1 \%}-\mathrm{sp}=950^{\circ} \mathrm{C}$, and $\mathrm{T}_{\mathrm{L}^{-}}$ $\mathrm{zs}=1050^{\circ} \mathrm{C}$. The calculated glass properties are summarized in Table 6.8.

\subsubsection{AZ-102 Calculations}

The third example uses the AZ-102 waste composition with the standard set of additives. The resulting glass is summarized in Table 6.9. In this calculation, the maximum waste loading was $35.99 \mathrm{wt} \%$. In this case, the loading is limited by a single-component concentration constraint- $\mathrm{CdO}=1.5 \mathrm{wt} \%$. Therefore, the additives and thus glass properties are not unique for this waste. An infinite number of glass compositions can achieve the same loading. The calculated glass properties for this particular set of additives are summarized in Table 6.10. 
Table 6.4. Chemical Composition of Pretreated HLW Samples (wt\%)

\begin{tabular}{|c|c|c|c|}
\hline Oxide & AZ-101 & AZ-102 & AY-102 \\
\hline $\mathrm{Ag}_{2} \mathrm{O}$ & 0.118 & 0.055 & 0.200 \\
\hline $\mathrm{Al}_{2} \mathrm{O}_{3}$ & 23.103 & 22.949 & 13.575 \\
\hline $\mathrm{B}_{2} \mathrm{O}_{3}$ & 0.431 & 0.060 & 0.000 \\
\hline $\mathrm{BaO}$ & 0.208 & 0.112 & 0.140 \\
\hline $\mathrm{Bi}_{2} \mathrm{O}_{3}$ & 0.020 & 0.000 & 0.000 \\
\hline $\mathrm{CaO}$ & 1.342 & 1.426 & 1.030 \\
\hline $\mathrm{CdO}$ & 2.028 & 4.168 & 0.030 \\
\hline $\mathrm{Ce}_{2} \mathrm{O}_{3}$ & 0.750 & 0.167 & 0.360 \\
\hline $\mathrm{Cl}$ & 0.176 & 0.148 & 0.000 \\
\hline $\mathrm{CoO}$ & 0.000 & 0.018 & 0.020 \\
\hline $\mathrm{Cr}_{2} \mathrm{O}_{3}$ & 0.450 & 0.276 & 0.600 \\
\hline $\mathrm{Cs}_{2} \mathrm{O}$ & 0.000 & 0.030 & 0.000 \\
\hline $\mathrm{CuO}$ & 0.093 & 0.086 & 0.090 \\
\hline$F$ & 0.050 & 0.037 & 0.000 \\
\hline $\mathrm{Fe}_{2} \mathrm{O}_{3}$ & 35.385 & 36.265 & 37.164 \\
\hline $\mathrm{K}_{2} \mathrm{O}$ & 0.429 & 0.079 & 0.000 \\
\hline $\mathrm{La}_{2} \mathrm{O}_{3}$ & 0.833 & 0.896 & 0.180 \\
\hline $\mathrm{Li}_{2} \mathrm{O}$ & 0.030 & 0.000 & 0.160 \\
\hline $\mathrm{MgO}$ & 0.312 & 0.357 & 0.320 \\
\hline MnO & 1.038 & 2.754 & 5.832 \\
\hline $\mathrm{MoO}_{3}$ & 0.011 & 0.000 & 0.130 \\
\hline $\mathrm{Na}_{2} \mathrm{O}$ & 10.826 & 8.891 & 15.596 \\
\hline $\mathrm{Nd}_{2} \mathrm{O}_{3}$ & 0.612 & 0.625 & 0.000 \\
\hline $\mathrm{NiO}$ & 1.558 & 2.289 & 1.090 \\
\hline $\mathrm{P}_{2} \mathrm{O}_{5}$ & 1.262 & 1.387 & 1.190 \\
\hline $\mathrm{PbO}$ & 0.233 & 0.281 & 1.320 \\
\hline $\mathrm{PdO}$ & 0.323 & 0.000 & 0.000 \\
\hline $\mathrm{Rh}_{2} \mathrm{O}_{3}$ & 0.077 & 0.000 & 0.000 \\
\hline $\mathrm{RuO}_{2}$ & 0.242 & 0.000 & 0.000 \\
\hline $\mathrm{SiO}_{2}$ & 3.808 & 1.900 & 17.056 \\
\hline $\mathrm{SO}_{3}$ & 0.000 & 0.064 & 0.000 \\
\hline $\mathrm{SnO}_{2}$ & 0.000 & 0.494 & 1.891 \\
\hline $\mathrm{SrO}$ & 0.493 & 4.454 & 0.570 \\
\hline $\mathrm{TiO}_{2}$ & 0.036 & 0.028 & 0.080 \\
\hline $\mathrm{UO}_{3}$ & 2.928 & 5.139 & 1.244 \\
\hline $\mathrm{V}_{2} \mathrm{O}_{5}$ & 0.000 & 0.000 & 0.050 \\
\hline $\mathrm{Y}_{2} \mathrm{O}_{3}$ & 0.000 & 0.046 & 0.000 \\
\hline $\mathrm{ZnO}$ & 0.042 & 0.124 & 0.080 \\
\hline $\mathrm{ZrO}_{2}$ & 10.742 & 4.388 & 0.000 \\
\hline
\end{tabular}


Table 6.5. Initial AZ-101 Glass Calculation (wt\%)

\begin{tabular}{|c|c|c|c|}
\hline Oxide & AZ-101 & Additive & Glass \\
\hline $\mathrm{Ag}_{2} \mathrm{O}$ & $0.12 \%$ & & $0.04 \%$ \\
\hline $\mathrm{Al}_{2} \mathrm{O}_{3}$ & $23.10 \%$ & $0.00 \%$ & $8.70 \%$ \\
\hline $\mathrm{B}_{2} \mathrm{O}_{3}$ & $0.43 \%$ & $31.82 \%$ & $20.00 \%$ \\
\hline $\mathrm{BaO}$ & $0.21 \%$ & & $0.08 \%$ \\
\hline $\mathrm{Bi}_{2} \mathrm{O}_{3}$ & $0.02 \%$ & & $0.01 \%$ \\
\hline $\mathrm{CaO}$ & $1.34 \%$ & & $0.51 \%$ \\
\hline $\mathrm{CdO}$ & $2.03 \%$ & & $0.76 \%$ \\
\hline $\mathrm{Ce}_{2} \mathrm{O}_{3}$ & $0.75 \%$ & & $0.28 \%$ \\
\hline $\mathrm{Cl}$ & $0.18 \%$ & & $0.07 \%$ \\
\hline $\mathrm{Cr}_{2} \mathrm{O}_{3}$ & $0.45 \%$ & & $0.17 \%$ \\
\hline $\mathrm{Cs}_{2} \mathrm{O}$ & $0.00 \%$ & & $0.00 \%$ \\
\hline $\mathrm{CuO}$ & $0.09 \%$ & & $0.04 \%$ \\
\hline $\mathrm{F}$ & $0.05 \%$ & & $0.02 \%$ \\
\hline $\mathrm{Fe}_{2} \mathrm{O}_{3}$ & $35.38 \%$ & $0.00 \%$ & $13.33 \%$ \\
\hline $\mathrm{K}_{2} \mathrm{O}$ & $0.43 \%$ & & $0.16 \%$ \\
\hline $\mathrm{La}_{2} \mathrm{O}_{3}$ & $0.83 \%$ & & $0.31 \%$ \\
\hline $\mathrm{Li}_{2} \mathrm{O}$ & $0.03 \%$ & $1.63 \%$ & $1.03 \%$ \\
\hline $\mathrm{MgO}$ & $0.31 \%$ & & $0.12 \%$ \\
\hline $\mathrm{MnO}$ & $1.04 \%$ & & $0.39 \%$ \\
\hline $\mathrm{MoO}_{3}$ & $0.01 \%$ & & $0.00 \%$ \\
\hline $\mathrm{Na}_{2} \mathrm{O}$ & $10.83 \%$ & $13.45 \%$ & $12.46 \%$ \\
\hline $\mathrm{Nd}_{2} \mathrm{O}_{3}$ & $0.61 \%$ & & $0.23 \%$ \\
\hline $\mathrm{NiO}$ & $1.56 \%$ & & $0.59 \%$ \\
\hline $\mathrm{P}_{2} \mathrm{O}_{5}$ & $1.26 \%$ & & $0.48 \%$ \\
\hline $\mathrm{PbO}$ & $0.23 \%$ & & $0.09 \%$ \\
\hline $\mathrm{PdO}$ & $0.32 \%$ & & $0.12 \%$ \\
\hline $\mathrm{Rh}_{2} \mathrm{O}_{3}$ & $0.08 \%$ & & $0.03 \%$ \\
\hline $\mathrm{RuO}_{2}$ & $0.24 \%$ & & $0.09 \%$ \\
\hline $\mathrm{SiO}_{2}$ & $3.81 \%$ & $53.09 \%$ & $34.53 \%$ \\
\hline $\mathrm{SO}_{3}$ & $0.00 \%$ & & $0.00 \%$ \\
\hline SrO & $0.49 \%$ & & $0.19 \%$ \\
\hline $\mathrm{TiO}_{2}$ & $0.04 \%$ & & $0.01 \%$ \\
\hline $\mathrm{UO}_{3}$ & $2.93 \%$ & & $1.10 \%$ \\
\hline $\mathrm{Y}_{2} \mathrm{O}_{3}$ & $0.00 \%$ & & $0.00 \%$ \\
\hline $\mathrm{ZnO}$ & $0.04 \%$ & & $0.02 \%$ \\
\hline $\mathrm{ZrO}_{2}$ & $10.74 \%$ & & $4.05 \%$ \\
\hline Total & $99.99 \%$ & $100.00 \%$ & $100.00 \%$ \\
\hline Loading & $37.66 \%$ & $62.34 \%$ & \\
\hline
\end{tabular}


Table 6.6. Calculated Glass Properties for Initial Calculation

\begin{tabular}{lcc}
\hline Property & Value & Limit \\
\hline PCT-B, g/m & 0.66 & $\leq 4$ \\
PCT-Li, g/m & 0.98 & $\leq 4$ \\
PCT-Na, g/m & 0.68 & $\leq 4$ \\
$\mathrm{~T}_{1 \%}-\mathrm{Sp},{ }^{\circ} \mathrm{C}$ & 950 & $\leq 950$ \\
$\mathrm{~T}_{\mathrm{L}}-\mathrm{Zs},{ }^{\circ} \mathrm{C}$ & 1004 & $\leq 1050$ \\
$\eta_{1150}, \mathrm{~Pa} . \mathrm{S}$ & 4.00 & $\geq 4, \leq 6$ \\
$\mathrm{Si} /(\mathrm{Si}+\mathrm{Al}+\mathrm{Na})$ & $62.0 \%$ & $\geq 0.62$ \\
$\varepsilon_{1150}, \mathrm{~S} / \mathrm{m}$ & 23.52 & not constrained \\
density, g/cm & 2.69 & not constrained \\
\hline $\mathrm{TCLP}, \mathrm{mg} / \mathrm{L}$ & 63.19 & not constrained \\
\hline
\end{tabular}

Table 6.7. Second AZ-101 Glass Calculation (wt\%)

\begin{tabular}{|c|c|c|c|}
\hline Oxide & AZ-101 & Additive & Glass \\
\hline $\mathrm{Ag}_{2} \mathrm{O}$ & $0.12 \%$ & & $0.04 \%$ \\
\hline $\mathrm{Al}_{2} \mathrm{O}_{3}$ & $23.10 \%$ & $0.00 \%$ & $8.70 \%$ \\
\hline $\mathrm{B}_{2} \mathrm{O}_{3}$ & $0.43 \%$ & $31.82 \%$ & $20.00 \%$ \\
\hline $\mathrm{BaO}$ & $0.21 \%$ & & $0.08 \%$ \\
\hline $\mathrm{Bi}_{2} \mathrm{O}_{3}$ & $0.02 \%$ & & $0.01 \%$ \\
\hline $\mathrm{CaO}$ & $1.34 \%$ & $0.00 \%$ & $0.51 \%$ \\
\hline $\mathrm{CdO}$ & $2.03 \%$ & & $0.76 \%$ \\
\hline $\mathrm{Ce}_{2} \mathrm{O}_{3}$ & $0.75 \%$ & & $0.28 \%$ \\
\hline $\mathrm{Cl}$ & $0.18 \%$ & & $0.07 \%$ \\
\hline $\mathrm{Cr}_{2} \mathrm{O}_{3}$ & $0.45 \%$ & & $0.17 \%$ \\
\hline $\mathrm{CuO}$ & $0.09 \%$ & & $0.04 \%$ \\
\hline $\mathrm{F}$ & $0.05 \%$ & & $0.02 \%$ \\
\hline $\mathrm{Fe}_{2} \mathrm{O}_{3}$ & $35.38 \%$ & $0.00 \%$ & $13.33 \%$ \\
\hline $\mathrm{K}_{2} \mathrm{O}$ & $0.43 \%$ & & $0.16 \%$ \\
\hline $\mathrm{La}_{2} \mathrm{O}_{3}$ & $0.83 \%$ & & $0.31 \%$ \\
\hline $\mathrm{Li}_{2} \mathrm{O}$ & $0.03 \%$ & $1.63 \%$ & $1.03 \%$ \\
\hline $\mathrm{MgO}$ & $0.31 \%$ & $0.00 \%$ & $0.12 \%$ \\
\hline $\mathrm{MnO}$ & $1.04 \%$ & & $0.39 \%$ \\
\hline $\mathrm{MoO}_{3}$ & $0.01 \%$ & & $0.00 \%$ \\
\hline $\mathrm{Na}_{2} \mathrm{O}$ & $10.83 \%$ & $13.45 \%$ & $12.46 \%$ \\
\hline $\mathrm{Nd}_{2} \mathrm{O}_{3}$ & $0.61 \%$ & & $0.23 \%$ \\
\hline $\mathrm{NiO}$ & $1.56 \%$ & & $0.59 \%$ \\
\hline $\mathrm{P}_{2} \mathrm{O}_{5}$ & $1.26 \%$ & & $0.48 \%$ \\
\hline $\mathrm{PbO}$ & $0.23 \%$ & & $0.09 \%$ \\
\hline $\mathrm{PdO}$ & $0.32 \%$ & & $0.12 \%$ \\
\hline $\mathrm{Rh}_{2} \mathrm{O}_{3}$ & $0.08 \%$ & & $0.03 \%$ \\
\hline $\mathrm{RuO}_{2}$ & $0.24 \%$ & & $0.09 \%$ \\
\hline $\mathrm{SiO}_{2}$ & $3.81 \%$ & $53.09 \%$ & $34.53 \%$ \\
\hline $\mathrm{SO}_{3}$ & $0.00 \%$ & & $0.00 \%$ \\
\hline $\mathrm{SrO}$ & $0.49 \%$ & & $0.19 \%$ \\
\hline $\mathrm{TiO}_{2}$ & $0.04 \%$ & $0.00 \%$ & $0.01 \%$ \\
\hline $\mathrm{UO}_{3}$ & $2.93 \%$ & & $1.10 \%$ \\
\hline $\mathrm{V}_{2} \mathrm{O}_{5}$ & $0.00 \%$ & & $0.00 \%$ \\
\hline $\mathrm{Y}_{2} \mathrm{O}_{3}$ & $0.00 \%$ & & $0.00 \%$ \\
\hline $\mathrm{ZnO}$ & $0.04 \%$ & $0.00 \%$ & $0.02 \%$ \\
\hline $\mathrm{ZrO}_{2}$ & $10.74 \%$ & $0.00 \%$ & $4.05 \%$ \\
\hline Total & $99.99 \%$ & $100.00 \%$ & $100.00 \%$ \\
\hline Loading & $37.66 \%$ & $62.34 \%$ & \\
\hline
\end{tabular}


Table 6.8. Calculated Glass Properties for Second Calculation

\begin{tabular}{lcc}
\hline Property & Value & Limit \\
\hline PCT-B, g/m & 0.58 & $\leq 4$ \\
PCT-Li, g/m & 0.45 & $\leq 4$ \\
PCT-Na, g/m & 0.43 & $\leq 4$ \\
$\mathrm{~T}_{1 \%}-\mathrm{Sp},{ }^{\circ} \mathrm{C}$ & 950 & $\leq 950$ \\
$\mathrm{~T}_{\mathrm{L}}-\mathrm{Zs},{ }^{\circ} \mathrm{C}$ & 1050 & $\leq 1050$ \\
$\eta_{1150}, \mathrm{~Pa} . \mathrm{S}$ & 4.00 & $\geq 4, \leq 6$ \\
$\mathrm{Si} /(\mathrm{Si}+\mathrm{Al}+\mathrm{Na})$ & $62.0 \%$ & $\geq 0.62$ \\
$\varepsilon_{1150}, \mathrm{~S} / \mathrm{m}$ & 23.70 & not constrained \\
density, g/cm & 2.72 & not constrained \\
TCLP, $\mathrm{mg} / \mathrm{L}$ & 54.31 & not constrained \\
\hline
\end{tabular}

Table 6.9. AZ-102 Glass Calculation (wt $\%)$

\begin{tabular}{|c|c|c|c|}
\hline Oxide & AZ-102 & Additive & Glass \\
\hline $\mathrm{Ag}_{2} \mathrm{O}$ & $0.05 \%$ & & $0.02 \%$ \\
\hline $\mathrm{Al}_{2} \mathrm{O}_{3}$ & $22.95 \%$ & $0.00 \%$ & $8.26 \%$ \\
\hline $\mathrm{B}_{2} \mathrm{O}_{3}$ & $0.06 \%$ & $17.93 \%$ & $11.50 \%$ \\
\hline $\mathrm{BaO}$ & $0.11 \%$ & & $0.04 \%$ \\
\hline $\mathrm{Bi}_{2} \mathrm{O}_{3}$ & $0.00 \%$ & & $0.00 \%$ \\
\hline $\mathrm{CaO}$ & $1.43 \%$ & & $0.51 \%$ \\
\hline $\mathrm{CdO}$ & $4.17 \%$ & & $1.50 \%$ \\
\hline $\mathrm{Ce}_{2} \mathrm{O}_{3}$ & $0.17 \%$ & & $0.06 \%$ \\
\hline $\mathrm{Cl}$ & $0.15 \%$ & & $0.05 \%$ \\
\hline $\mathrm{CoO}$ & $0.02 \%$ & & $0.01 \%$ \\
\hline $\mathrm{Cr}_{2} \mathrm{O}_{3}$ & $0.28 \%$ & & $0.10 \%$ \\
\hline $\mathrm{Cs}_{2} \mathrm{O}$ & $0.03 \%$ & & $0.01 \%$ \\
\hline $\mathrm{CuO}$ & $0.09 \%$ & & $0.03 \%$ \\
\hline$F$ & $0.04 \%$ & & $0.01 \%$ \\
\hline $\mathrm{Fe}_{2} \mathrm{O}_{3}$ & $36.27 \%$ & $0.00 \%$ & $13.05 \%$ \\
\hline $\mathrm{K}_{2} \mathrm{O}$ & $0.08 \%$ & & $0.03 \%$ \\
\hline $\mathrm{La}_{2} \mathrm{O}_{3}$ & $0.90 \%$ & & $0.32 \%$ \\
\hline $\mathrm{Li}_{2} \mathrm{O}$ & $0.00 \%$ & $0.72 \%$ & $0.46 \%$ \\
\hline $\mathrm{MgO}$ & $0.36 \%$ & & $0.13 \%$ \\
\hline $\mathrm{MnO}$ & $2.75 \%$ & & $0.99 \%$ \\
\hline $\mathrm{Na}_{2} \mathrm{O}$ & $8.89 \%$ & $20.22 \%$ & $16.14 \%$ \\
\hline $\mathrm{Nd}_{2} \mathrm{O}_{3}$ & $0.63 \%$ & & $0.23 \%$ \\
\hline $\mathrm{NiO}$ & $2.29 \%$ & & $0.82 \%$ \\
\hline $\mathrm{P}_{2} \mathrm{O}_{5}$ & $1.39 \%$ & & $0.50 \%$ \\
\hline $\mathrm{PbO}$ & $0.28 \%$ & & $0.10 \%$ \\
\hline $\mathrm{Rh}_{2} \mathrm{O}_{3}$ & $0.00 \%$ & & $0.00 \%$ \\
\hline $\mathrm{RuO}_{2}$ & $0.00 \%$ & & $0.00 \%$ \\
\hline $\mathrm{SiO}_{2}$ & $1.90 \%$ & $61.13 \%$ & $39.82 \%$ \\
\hline $\mathrm{SO}_{3}$ & $0.06 \%$ & & $0.02 \%$ \\
\hline $\mathrm{SnO}_{2}$ & $0.49 \%$ & & $0.18 \%$ \\
\hline SrO & $4.45 \%$ & & $1.60 \%$ \\
\hline $\mathrm{TiO}_{2}$ & $0.03 \%$ & & $0.01 \%$ \\
\hline $\mathrm{UO}_{3}$ & $5.14 \%$ & & $1.85 \%$ \\
\hline $\mathrm{Y}_{2} \mathrm{O}_{3}$ & $0.05 \%$ & & $0.02 \%$ \\
\hline $\mathrm{ZnO}$ & $0.12 \%$ & & $0.04 \%$ \\
\hline $\mathrm{ZrO}_{2}$ & $4.39 \%$ & & $1.58 \%$ \\
\hline Total & $99.99 \%$ & $100.00 \%$ & $100.00 \%$ \\
\hline Loading & $35.99 \%$ & $64.01 \%$ & \\
\hline
\end{tabular}


Table 6.10. Calculated Glass Properties for AZ-102 Calculation

\begin{tabular}{lcc}
\hline Property & Value & Limit \\
\hline PCT-B, g/m & 0.36 & $\leq 4$ \\
PCT-Li, g/m & 0.28 & $\leq 4$ \\
PCT-Na, g/m & 0.30 & $\leq 4$ \\
$\mathrm{~T}_{1 \%}-\mathrm{Sp},{ }^{\circ} \mathrm{C}$ & 916 & $\leq 950$ \\
$\mathrm{~T}_{\mathrm{L}}-\mathrm{Zs},{ }^{\circ} \mathrm{C}$ & 1050 & $\leq 1050$ \\
$\mathrm{\eta}_{1150}, \mathrm{~Pa} . \mathrm{S}$ & 6.00 & $\geq 4, \leq 6$ \\
$\mathrm{Si} /(\mathrm{Si}+\mathrm{Al}+\mathrm{Na})$ & $62.0 \%$ & $\geq 0.62$ \\
$\varepsilon_{1150}, \mathrm{~S} / \mathrm{m}$ & 31.00 & not constrained \\
density, g/cm & 2.80 & not constrained \\
TCLP, $\mathrm{mg} / \mathrm{L}$ & 44.23 & not constrained \\
\hline
\end{tabular}

\subsubsection{AY-102 Calculations}

The final example uses the AY-102 waste composition and the standard group of additives. The resulting glass is summarized in Table 6.11. In this calculation, the maximum waste loading was $45.41 \mathrm{wt} \%$. The additives were $0.00 \mathrm{wt} \% \mathrm{Al}_{2} \mathrm{O}_{3}, 7.33 \mathrm{~B}_{2} \mathrm{O}_{3}, 0.00 \mathrm{Fe}_{2} \mathrm{O}_{3}, 3.09 \mathrm{Li}_{2} \mathrm{O}$, $24.39 \mathrm{Na}_{2} \mathrm{O}$, and $65.19 \mathrm{SiO}_{2}$. For this first example, the limiting constraints include $\eta_{1150}=4$ $\mathrm{Pa}-\mathrm{s}, \mathrm{N}_{\mathrm{SiO} 2}=62 \mathrm{wt} \%, \mathrm{~T}_{1 \%}-\mathrm{sp}=950^{\circ} \mathrm{C}$, and $\mathrm{B}_{2} \mathrm{O}_{3}=4.0 \mathrm{wt} \%$. The calculated glass properties are summarized in Table 6.12. 
Table 6.11. AY-102 Glass Calculation (wt\%)

\begin{tabular}{|c|c|c|c|}
\hline Oxide & AY-102 & Additive & Glass \\
\hline $\mathrm{Ag}_{2} \mathrm{O}$ & $0.20 \%$ & & $0.09 \%$ \\
\hline $\mathrm{Al}_{2} \mathrm{O}_{3}$ & $13.57 \%$ & $0.00 \%$ & $6.16 \%$ \\
\hline $\mathrm{B}_{2} \mathrm{O}_{3}$ & $0.00 \%$ & $7.33 \%$ & $4.00 \%$ \\
\hline $\mathrm{BaO}$ & $0.14 \%$ & & $0.06 \%$ \\
\hline $\mathrm{Bi}_{2} \mathrm{O}_{3}$ & $0.00 \%$ & & $0.00 \%$ \\
\hline $\mathrm{CaO}$ & $1.03 \%$ & & $0.47 \%$ \\
\hline $\mathrm{CdO}$ & $0.03 \%$ & & $0.01 \%$ \\
\hline $\mathrm{Ce}_{2} \mathrm{O}_{3}$ & $0.36 \%$ & & $0.16 \%$ \\
\hline $\mathrm{Cl}$ & $0.00 \%$ & & $0.00 \%$ \\
\hline $\mathrm{CoO}$ & $0.02 \%$ & & $0.01 \%$ \\
\hline $\mathrm{Cr}_{2} \mathrm{O}_{3}$ & $0.60 \%$ & & $0.27 \%$ \\
\hline $\mathrm{Cs}_{2} \mathrm{O}$ & $0.00 \%$ & & $0.00 \%$ \\
\hline $\mathrm{CuO}$ & $0.09 \%$ & & $0.04 \%$ \\
\hline $\mathrm{F}$ & $0.00 \%$ & & $0.00 \%$ \\
\hline $\mathrm{Fe}_{2} \mathrm{O}_{3}$ & $37.16 \%$ & $0.00 \%$ & $16.87 \%$ \\
\hline $\mathrm{K}_{2} \mathrm{O}$ & $0.00 \%$ & & $0.00 \%$ \\
\hline $\mathrm{La}_{2} \mathrm{O}_{3}$ & $0.18 \%$ & & $0.08 \%$ \\
\hline $\mathrm{Li}_{2} \mathrm{O}$ & $0.16 \%$ & $3.09 \%$ & $1.76 \%$ \\
\hline $\mathrm{MgO}$ & $0.32 \%$ & & $0.15 \%$ \\
\hline $\mathrm{MnO}$ & $5.83 \%$ & & $2.65 \%$ \\
\hline $\mathrm{MoO}_{3}$ & $0.13 \%$ & & $0.06 \%$ \\
\hline $\mathrm{Na}_{2} \mathrm{O}$ & $15.60 \%$ & $24.39 \%$ & $20.40 \%$ \\
\hline $\mathrm{Nd}_{2} \mathrm{O}_{3}$ & $0.00 \%$ & & $0.00 \%$ \\
\hline $\mathrm{NiO}$ & $1.09 \%$ & & $0.50 \%$ \\
\hline $\mathrm{P}_{2} \mathrm{O}_{5}$ & $1.19 \%$ & & $0.54 \%$ \\
\hline $\mathrm{PbO}$ & $1.32 \%$ & & $0.60 \%$ \\
\hline $\mathrm{PdO}$ & $0.00 \%$ & & $0.00 \%$ \\
\hline $\mathrm{Rh}_{2} \mathrm{O}_{3}$ & $0.00 \%$ & & $0.00 \%$ \\
\hline $\mathrm{RuO}_{2}$ & $0.00 \%$ & & $0.00 \%$ \\
\hline $\mathrm{SiO}_{2}$ & $17.06 \%$ & $65.19 \%$ & $43.34 \%$ \\
\hline $\mathrm{SO}_{3}$ & $0.00 \%$ & & $0.00 \%$ \\
\hline $\mathrm{SnO}_{2}$ & $1.89 \%$ & & $0.86 \%$ \\
\hline $\mathrm{SrO}$ & $0.57 \%$ & & $0.26 \%$ \\
\hline $\mathrm{TiO}_{2}$ & $0.08 \%$ & & $0.04 \%$ \\
\hline $\mathrm{UO}_{3}$ & $1.24 \%$ & & $0.56 \%$ \\
\hline $\mathrm{V}_{2} \mathrm{O}_{5}$ & $0.05 \%$ & & $0.02 \%$ \\
\hline $\mathrm{Y}_{2} \mathrm{O}_{3}$ & $0.00 \%$ & & $0.00 \%$ \\
\hline $\mathrm{ZnO}$ & $0.08 \%$ & $0.00 \%$ & $0.04 \%$ \\
\hline $\mathrm{ZrO}_{2}$ & $0.00 \%$ & $0.00 \%$ & $0.00 \%$ \\
\hline Total & $100.00 \%$ & $100.00 \%$ & $100.00 \%$ \\
\hline Loading & $45.41 \%$ & $54.59 \%$ & \\
\hline
\end{tabular}


Table 6.12. Calculated Glass Properties for AY-102 Calculation

\begin{tabular}{lcc}
\hline Property & Value & Limit \\
\hline $\mathrm{PCT}-\mathrm{B}, \mathrm{g} / \mathrm{m}^{2}$ & 0.39 & $\leq 4$ \\
$\mathrm{PCT}-\mathrm{Li}, \mathrm{g} / \mathrm{m}^{2}$ & 0.34 & $\leq 4$ \\
$\mathrm{PCT}-\mathrm{Na}, \mathrm{g} / \mathrm{m}^{2}$ & 0.88 & $\leq 4$ \\
$\mathrm{~T}_{1 \%}-\mathrm{Sp},{ }^{\circ} \mathrm{C}$ & 950 & $\leq 950$ \\
$\mathrm{~T}_{\mathrm{L}}-\mathrm{Zs},{ }^{\circ} \mathrm{C}$ & 677 & $\leq 1050$ \\
$\mathrm{\eta}_{1150}, \mathrm{~Pa} . \mathrm{S}$ & 4.00 & $\geq 4, \leq 6$ \\
$\mathrm{Si} /(\mathrm{S}+\mathrm{A}+\mathrm{Al}+\mathrm{Na})$ & $62.0 \%$ & $\geq 0.62$ \\
$\varepsilon_{1150}, \mathrm{~S} / \mathrm{m}$ & 62.07 & not constrained \\
density, $\mathrm{g} / \mathrm{cm}^{3}$ & 2.85 & not constrained \\
TCLP, $\mathrm{mg} / \mathrm{L}$ & 34.92 & not constrained \\
\hline
\end{tabular}




\subsection{References}

American Society for Testing and Materials (ASTM). 2008. Standard Test Methods for Determining Chemical Durability of Nuclear Waste Glasses: Product Consistency Test (PCT). ASTM-C-1285, 2008 Annual Book of ASTM Standards, West Conshohecken, Pennsylvania.

Annamalia S, H Gan, M Chaudhary, WK Kot, and IL Pegg. 2004. "Spinel Crystallization in HLW Glass Melts: Cation Exchange Systematics and the Role of $\mathrm{Rh}_{2} \mathrm{O}_{3}$ in Spinel Formation." In: Ceramic Transactions, Vol. 155, 279-288.

Bailey AW and P Hrma. 1995. "Waste Loading Maximization for Vitrified Hanford HLW Blend." Ceram. Trans. 61:549-556.

Barnes SM. 1995. WVDP Waste Form Qualification Report. WVDP-186, Rev 2, West Valley Nuclear Services, West Valley, New York.

Barnes BO. 2000. Nuclear Quality Assurance Requirements and Description (NQARD), PNNLQAP-EMG-01, Pacific Northwest National Laboratory, Richland, Washington.

Bates SO. 1985. Issue a Recommended HWVP Glass Composition for the NCAW-CRW Stream for Future Work. HWVP Baseline Milestone 020202B, Pacific Northwest National Laboratory, Richland, Washington.

Bates SO. 1987. Report on FY87 Glass Variability Testing Conducted for the Hanford Waste Vitrification Program, Interim Milestone HWVP-87-V110202C, Pacific Northwest Laboatory, Richland, Washington.

Blumenkranz D. 2006. Petition to Delist Immobilized High-Level Waste Generated at the Hanford Tank Waste Treatment and Immobilization Plant. 24590-WTP-RPT-ENV-06-001, Rev. 0, River Protection Project, Waste Treatment Plant, Richland, Washington.

Bowen BW, et al. 1985. Glass Melting, Composition Development, and Phase Stability In the System Glass Formers Zeolite West Valley Waste. DOE/NE/44139-39, Catholic University of America, Washington, D.C.

Brooks KP, PR Bredt, SK Cooley, GR Golcar, LK Jagoda, KG Rappe, and MW Urie. 2000. Characterization, Washing, Leaching, and Filtration of AZ-102 Sludge. PNWD-3045, BattellePacific Northwest Division, Richland, Washington.

Brouns, RA, CR Allen, and JA Powell. 1988. LFCM Vitrification Technology Quarterly Progress Report April-June 1987. Pacific Northwest Laboratory, Richland, Washington.

Brouns RA and JA Powell. 1988. Nuclear Waste Treatment Program Annual Report for FY 1987. PNL-6686, Pacific Northwest Laboratory, Richland, Washington.

Bulkley SA and JD Vienna. 1997. "Composition Effects on Viscosity and Chemical Durability of Simulated Plutonium Residue Glasses." Mat. Res. Soc. Symp. Proc. 465:1243-1250. 
Bunnell LR. 1988. Laboratory Work in Support of West Valley Glass Development. PNL-6539, Pacific Northwest Laboratory, Richland, Washington.

Cantale C, S Castelli, A Donato, DM Traverso, P Colombo, and G Scarinci. 1991. "A Borosilicate Glass for the Italian High Level Waste: Characterization and Behaviour." Radioactive Waste Management and the Nuclear Fuel Cycle 16(1):25-47.

Carter JG, SS Koegler, and SO Bates. 1988. Process Performance of the Pilot Scale ISV of a Simulated Waste Disposal Site at ORNL. PNL-6530, Pacific Northwest Laboratory, Richland, Washington.

Chick LA and CQ Buckwalter. 1980. Low Leach Rate Glasses for Immobilization of Nuclear Wastes. PNL-3522, Pacific Northwest Laboratory, Richland, Washington.

Chick LA, GF Piepel, GB Mellinger, RP May, WJ Gray, and CQ Buckwalter. 1981. The Effects of Composition on Properties in an 11-Component Nuclear Waste Glass System. PNL-3188, Pacific Northwest Laboratory, Richland, Washington.

Chick LA, WM Bowen, RO Lokken, JW Wald, LR Bunnell, and DM Strachan. 1984. West Valley High-Level Nuclear Waste Glass Development: A Statistically Designed Mixture Study. PNL 4992, Pacific Northwest Laboratory, Richland, Washington.

Chick LA, LR Bunnell, DM Strachan, HE Kissinger, and FN Hodges. 1986. Evaluation of LeadIron-Phosphate Glass as a High-Level Waste Form. PNL-5878, Pacific Northwest Laboratory, Richland, Washington.

Cornell JA. 2002. Experiments with Mixtures: Designs, Models, and the Analysis of Mixture Data. Third Edition, John Wiley \& Sons, New York.

Crawford CL, DM Ferrara, BC Ha, and NE Bibler. 1998. "Production of a High-Level Waste Glass from Hanford Waste Samples." In: Proceedings of the International Conference on Decommissioning and Decontamination and on Nuclear Hazardous Waste Management. Volume 1, American Nuclear Society, La Grange Park, Illinois.

Crawford CL, DM Ferrara, RF Schumacher, and NE Bibler. 2000a. Crucible-Scale Active Vitrification Testing Envelope C, Tank 241-AN-102 (U). WSRC-TR-2000-00371, Rev. 0, Westinghouse Savannah River Company, Aiken, South Carolina.

Crawford CL, DM Ferrara, RF Schumacher, and NE Bibler. 2000b. Crucible-Scale Active Vitrification Testing Envelope A, Tank 241-AN-103 (U). WSRC-TR-2000-00322, Rev. 1, Westinghouse Savannah River Company, Aiken, South Carolina.

Crum JV, MJ Schweiger, P Hrma, and JD Vienna. 1997. "Liquidus Temperature Model for Hanford High-Level Waste Glasses with High Concentrations of Zirconia." In: Mat. Res. Soc. Symp. Proc., 465:79-85.

Darab JG, DD Graham, BD Maclsaac, RL Russell, DK Peeler, HD Smith, and JD Vienna. 2001. Sulfur Partitioning During Vitrification of INEEL Sodium Bearing Waste: Status Report.

PNNL-13588, Pacific Northwest National Laboratory, Richland, Washington.

DOE (see U.S. Department of Energy) 
Draper NR and H Smith. 1998. Applied Regression Analysis. Third Edition, John Wiley \& Sons, New York.

Ebert WL and SF Wolf. 1999. Round-Robin Testing of a Reference Glass for Low-Activity Waste Forms. ANL-99/22, Argonne National Laboratory, operated by the University of Chicago, Argonne, Illinois.

Edwards TB, DK Peeler, IA Reamer, GF Piepel, JD Vienna, and H Li. 2000. Phase $2 b$ Experimental Design for the INEEL Glass Composition Variation Study. WSRC-TR-99-00224, Rev. 0, Westinghouse Savannah River Company, Aiken, South Carolina.

Edwards TB, JV Crum, DE Smith, RJ Workman, MJ Schweiger, RF Schumacher, JD Vienna, DK Peeler, and RL Russell. 2004. "DWPF Startup Frit Viscosity Measurement Round Robin Results." Pacific Northwest National Laboratory, Richland, Washington.

EPA (see U.S. Environmental Protection Agency)

Feng X, EE Saad, and IL Pegg. 1990. "A Model for the Viscosity of Multicomponent Glass Melts." Ceram. Trans. 9:457-468.

Feng X, PR Hrma, JH Westsik, NR Brown, MJ Schweiger, H Li, JD Vienna, G Chen, GF Piepel, DE Smith, BP McGrail, SE Palmer, DS Kim, Y Peng, WK Hahn, AJ Bakel, WL Ebert, DK Peeler, and C Chang. 1996. Glass Optimization for Vitrification of Hanford Site Low-Level Tank Waste. PNNL-10918, Pacific Northwest National Laboratory, Richland, Washington.

Ferrara DM, CL Crawford, BC Ha, and NE Bibler. 1998. "Vitrification of Three Low-Activity Radioactive Waste Streams from Hanford." In: Proceedings of the International Conference on Decommissioning and Decontamination and on Nuclear and Hazardous Waste Management. 1:706-713.

Flinn JE, SP Henslee, and PV Kelsey Jr. 1981. TRU Waste Form Studies With Special Reference to Iron-Enriched Basalt: 1980. Annual Report, EGG-FM-5366, EG\&G Inc. Idaho Falls, Idaho.

Fiskum SK, PR Bredt, JA Campbell, LR Greenwood, OT Farmer, GJ Lumetta, GM Mong, RT Ratner, CZ Soderquist, RG Swoboda, MW Urie, and JJ Wagner. 2000. Inorganic, radioisotopic and organic analysis of 241-AP-101 tank waste. PNWD-3046, Battelle-Pacific Northwest Division, Richland, Washington.

Fluegel A. 2007. "Glass viscosity calculation based on a global statistical modelling approach." Glass Technol.: Eur. J. Glass Sci. Technol. A, 48(1):13-30.

Fox KM, TB Edwards, Peeler DK, DR Best, IA Reamer, and RJ Workman. 2006. Nepheline Formation Study for Sludge Batch 4 (SB4): Phase 3 Experimental Results. WSRC-TR-200600093, Savannah River National Laboratory, Aiken, South Carolina.

Fox KM, JD Newell, TB Edwards, DR Best, IA Reamer, and RJ Workman. 2007. Refinement of the Nepheline Discriminator: Results of a Phase I Study. WSRC-STI-2007-00659, Savannah River National Laboratory, Aiken, South Carolina. 
Fox KM, DK Peeler, TB Edwards, DR Best, IA Reamer, RJ Workman, JC Marra, BJ Riley, JD Vienna, JV Crum, J Matyas, AB Edmondson, JB Lang, NM Ibarra, A Fluegel, A Aloy, AV Trofimenko, and R Soshnikov. 2008. International Study of Aluminum Impacts on Crystallization in U.S. High Level Waste Glass. SRNS-STI-2008-00057, Savannah River National Laboratory, Aiken, South Carolina.

Fu SS and IL Pegg. 1998. Glass Formulation and Testing with TWRS HLW Simulants. VSL Final Report, the Catholic University of America, Washington, D.C.

Fu SS, H Gan, IS Muller, IL Pegg, and PB Macedo. 1997. "Optimization of Savannah River MArea Mixed Waste for Vitrification.” In: MRS Symposium Proceedings 465:139-146.

Fung YC. 1994. A First Course in Continuum Mechanics, for Physical and Biological Engineers and Scientists, $3^{\text {rd }}$ Ed. Prentice Hall, Englewood Cliffs, New Jersey.

Gimpel R. 1996. Operable Unit 4 Vitrification Plant, Phase I Interim Treatability Study Report. 40110-WP-0002-Campaign 2, Rev. 1, prepared by Fluor Daniel Fernald, Fernald, Ohio.

Hrma P. 1999. "Modeling of Spinel Settling in Waste Glass Melter." In: Science to Support DOE Site Cleanup. PNNL-12208, Pacific Northwest National Laboratory, Richland, Washington.

Hrma P. 2008. "Arrhenius Model for High-Temperature Glass-Viscosity with a Constant Preexponential Factor." Journal of Non-Crystalline Solids 354:1962-1968.

Hrma P, GF Piepel, MJ Schweiger, DE Smith, DS Kim, PE Redgate, JD Vienna, CA LoPresti, DB Simpson, DK Peeler, and MH Langowski. 1994. Property/Composition Relationships for Hanford High-Level Waste Glasses Melting at $1150^{\circ} \mathrm{C}$. PNL-10359, Vol. 1 and 2, Pacific Northwest Laboratory, Richland, Washington.

Hrma P, GF Piepel, JD Vienna, PE Redgate, MJ Schweiger, and DE Smith. 1995a. "Prediction of Nuclear Waste Glass Dissolution as a Function of Composition." Ceram. Trans. 61:497-504.

Hrma P, GF Piepel, PE Redgate, DE Smith, MJ Schweiger, JD Vienna, and DS Kim. 1995b. "Prediction of Processing Properties for Nuclear Waste Glasses." Ceram. Trans. 61:505-513.

Hrma P, JD Vienna, M Mika, JV Crum, and GF Piepel. 1999. Liquidus Temperature Data for DWPF Glass. PNNL-11790, Pacific Northwest National Laboratory, Richland, Washington.

Hrma P, GF Piepel, JD Vienna, SK Cooley, DS Kim, and RL Russell. 2001. Database and Interim Glass Property Models for Hanford HLW Glasses. PNNL-13573, Pacific Northwest National Laboratory, Richland, Washington.

Hrma P, P Izak, JD Vienna, GM Irwin, and ML Thomas. 2002. "Partial Molar Liquidus Temperatures of Multivalent Elements in Multicomponent Borosilicate Glass." Phys. Chem. Glasses 43 (2):128-136.

Hrma, P., J.D. Vienna, and J.S. Ricklefs. 2003. "Mechanism of Sulfate Segregation during Glass Melting." In: Scientific Basis for Nuclear Waste Management XXVI, pp. 147-152, Materials Research Society, Warrendale, Pennsylvania. 
Hrma, P., J.D. Vienna, W.C. Buchmiller, and J.S. Ricklefs. 2004. "Sulfate Retention during Waste Glass Melting." In: Ceramic Transactions 155:239-248, American Ceramic Society, Westerville, Ohio.

Hrma et al. 2008

Jain VJ. 1990. "Viscosity Data on the Compositions Processed during FACTS Campaigns between December 1984 and December 1989." Vitrification Laboratory Report, To SM Barnes From VJ Jain, Letter number EK:90:0231 August 1, 1990; Transmitted via TA-1062 dtd 6/28/91.

Jain A. 2008. Reconciliation of HLW and LAW Melter Decontamination Factors for Flowsheet Bases, Assumptions, and Requirements Document, Rev. 4 Update, CCN: 160525, Memorandum to Saunders, Lee, and Lenski, dtd. April 16, 2008, Hanford Tank Waste Treatment and Immobilization Plant, Richland, Washington.

Jantzen CM. 1991. "Relationship of Glass Composition to Glass Viscosity, Resistivity, Liquidus Temperature, and Durability: First-Principle Process Product Models for Vitrification of Nuclear Waste." Ceram. Trans. 23:37-51.

Jantzen CM. 1992. "Thermodynamic Approach to Glass Corrosion." In: Corrosion of Glass, Ceramics and Ceramic Superconductors. DE Clark and K Ziotos (Eds.), Noyes, Park Ridge, New Jersey.

Jantzen CM, NE Bibler, DC Beam, CL Crawford, MA Pickett. 1993. Characterization of the Defense Waste Processing Facility (DWPF) Environmental Assessment (EA) Glass Standard Reference Material (U). WSRC-TR-92-346, Rev. 1, Westinghouse Savannah River Co., Savannah River Site, Aiken, South Carolina.

Jantzen CM, JB Pickett, KG Brown, TB Edwards, and DC Beam. 1995. Process/Product Models for the Defense Waste Processing Facility (DWPF): Part I. Predicting Glass Durability from Composition Using a THermodynamic Hydration Energy Reaction MOdel (THERMO). WSRC-TR-93-0672, Westinghouse Savannah River Company, Aiken, South Carolina.

Jantzen CM, JB Pickett, and I Joseph. 2000. "Toxic Characteristic leaching Procedure (TCLP) Testing of Waste Glass and K-3 Refractory: Revisited." Ceramic Transactions 107:271-280.

Jantzen CM and KG Brown. 2007. "Predicting the Spinel-Nepheline Liquidus for Application to Nuclear Waste Glass Processing. Part I: Primary Phase Analysis, Liquidus Measurement, and Quasicrystalline Approach." J. Am. Ceram. Soc. 90(6):1866-1879.

Kim DS and J.D. Vienna. 2004. "Glass Composition-TCLP Response Model for Waste Glasses." In: Ceramic Transactions, 155, pp. 297-308, American Ceramic Society, Westerville, Ohio.

Kim DS, Peeler DK, and Hrma P. 1995. "Effects of Crystallization on the Chemical Durability of Simulated Nuclear Waste Glasses." In: Environmental Issues and Waste Management Technologies, Ceramic Transactions 61:177-185.

Kim DS, WK Kot, I Joseph, and IL Pegg. 2008. Product Consistency and Phase Stability for Immobilized High Level Waste Product Qualification. 24590-HLW-RPT-RT-08-001-03, Rev A, 
River Protection Project, Hanford Tank Waste Treatment and Immobilization Plant Project, Richland, Washington.

Kot WK and IL Pegg. 2001. Glass Formulation and Testing with RPP-WTP HLW Simulants Final Report. VSL-01R2540-2, Vitreous State Laboratory, The Catholic University of America, Washington D.C.

Kot WK and IL Pegg. 2004. HLW Glass Formulation to Support C-106/AY-102 Actual Waste Testing, Final Report. VSL-04R4770-1, Rev. 0, Vitreous State Laboratory, The Catholic University of America, Washington D.C.

Kot WK, K Klatt, IS Muller, CN Wilson, and IL Pegg. 2003a. Regulatory Spike Testing of RPP. WTP LAW and HLW Glasses for Compliance with Land Disposal Restrictions. VSL-03R3760-1, Rev. 1, prepared for Duratek, Inc. and Bechtel National, Inc., Vitreous State Laboratory, The Catholic University of America, Washington, D.C.

Kot WK, K Klatt, and IL Pegg. 2003b. Glass Formulation to Support Melter Runs with HLW Simulants. VSL-03R3760-2, Rev. 0, Vitreous State Laboratory, The Catholic University of America, Washington D.C.

Kot WK, K Klatt, H Gan, and IL Pegg. 2004. Regulatory Testing of RPP-WTP HLW Glasses to Support Delisting Compliance, Final Report. VSL-04R4780-1, Rev. 0, Vitreous State Laboratory, The Catholic University of America, Washington D.C.

Kot WK, I Joseph, and IL Pegg. 2005a. Comparison of HLW Simulant, Actual Waste, and Melter Glasses, Final Report. VSL-05R5760-1, Rev. 0, Vitreous State Laboratory, The Catholic University of America, Washington D.C.

Kot WK, H Gan, and IL Pegg. 2005b. Preparation and Testing of Glasses to Support Development of WTP IHLW Formulation Algorithm, Final Report. VSL-06R1240-1, Rev. 0, Vitreous State Laboratory, The Catholic University of America, Washington D.C.

Kot WK, H Gan, and IL Pegg. 2006. Preparation and Testing of HLW Matrix Glasses to Support Development of WTP Phase 2 Property-Composition Models, Final Report.

VSL-06R6780-2, Rev. 0, Vitreous State Laboratory, The Catholic University of America, Washington D.C.

Kot WK, L Meyers, and IL Pegg. 2007. Baseline HLW Glass Formulations for Bismuth Phosphate Wastes. VSL-07R1240-2, Rev. 0, Vitreous State Laboratory at The Catholic University of America, Washington, D.C.

Langowski MH. 1996. The Incorporation of $\mathrm{P}, \mathrm{S}, \mathrm{Cr}, \mathrm{F}, \mathrm{Cl}, \mathrm{I}, \mathrm{Mn}, \mathrm{Ti}, \mathrm{U}$, and Bi into Simulated Nuclear Waste Glasses: Literature Study. PNNL-10980, Pacific Northwest National Laboratory, Richland, Washington.

Langowski MH, H Li, P Hrma, MJ Schweiger, and DE Smith. 1997. "The Effect of Phosphate on Crystallization, Viscosity, and Chemical Durability of Simulated Hanford Site High-Level Radioactive Waste Glasses." In: Ceramic Transactions 72:291-298, Am. Ceram. Soc., Westerville, Ohio. 
Li H. 1998. "Phosphate in Borosilicate Glasses for Immobilization of Radioactive Tank Wastes." In: Proceedings of XVIII International Congress on Glass, Am. Ceram. Soc., Westerville, Ohio.

Li H, JG Darab, PA Smith, X Feng, and DK Peeler. 1995a. "Chemical Durability of Low-Level Waste Glasses Containing High Concentrations of Minor Components." In: Proceedings, $36^{\text {th }}$ Institute for Nuclear Materials Management Annual Meeting, p. 460-465, INMM, Pittsburgh, Pennsylvania.

Li H, JG Darab, PA Smith, MJ Schweiger, DE Smith, and P Hrma. 1995b. "Effect of Minor Components on Vitrification of Low-Level Simulated Nuclear Waste Glasses." In: Proceedings, $36^{\text {th }}$ Institute of Nuclear Materials Management Annual Meeting, p. 466-471, INMM, Pittsburgh, Pennsylvania.

Li H, MH Langowski, and PR Hrma. 1995c. "Segregation of Sulfate and Phosphate in the Vitrification of High-Level Wastes." In: Ceramic Transactions 61:195-202, Am. Ceram. Soc., Westville, Ohio.

Li H, MH Langowski, P Hrma, MJ Schweiger, JD Vienna, and DE Smith. 1995d. Minor Component Study for Simulated High-Level Nuclear Waste Glasses. PNNL-10996, Pacific Northwest Laboratory, Richland, Washington.

Li H, JD Vienna, Y Chen, L Wang, and J Liu. 1997a. "Phase Separation in Simulated Plutonium Glasses with Phosphate and Fluorine and the Effect on Glass Corrosion in Water." In: Scientific Basis for Nuclear Waste Management XX, pp. 277-283, Materials Research Society, Pittsburgh, Pennsylvania.

Li H, JD Vienna, P Hrma, DE Smith, and MJ Schweiger. 1997b. "Nepheline Precipitation in High-Level Waste Glasses: Compositional Effects and Impact on the Waste Form Acceptability." In: Scientific Basis for Nuclear Waste Management XX, pp. 261-268, Materials Research Society, Pittsburgh, Pennsylvania.

Li H, JD Vienna, Y Chen, L Wang, and J Liu. 1997d. "Phase Separation in Simulated Plutonium Glasses with Phosphate and Fluorine and the Effect on Glass Corrosion in Water." In: Scientific Basis for Nuclear Waste Management XX, pp. 277-283, Materials Research Society, Pittsburgh, Pennsylvania.

Li H, P Hrma, JD Vienna, M Qian, Y Su, and DE Smith. 2003. Effects of $\mathrm{Al}_{2} \mathrm{O}_{3}, \mathrm{~B}_{2} \mathrm{O}_{3}, \mathrm{Na}_{2} \mathrm{O}$, and $\mathrm{SiO}_{2}$ on Nepheline Formation in Borosilicate Glasses: Chemical and Physical Correlations, in J. Non-Cryst. Sol. 331(1-3):202-216.

Marra SL and CM Jantzen. 1993. Characterization of Projected DWPF Glasses Heat Treated to Simulate Canister Centerline Cooling (U). WSRC-TR-92-142, Rev. 1, Westinghouse Savannah River Company, Aiken, South Carolina.

Matlack KS and IL Pegg. 2008. Data Summary Report - Small Scale Melter Testing of HLW Algorithm Glasses: Matrix 2 Tests. VSL-07S1400-1, the Vitreous State Laboratory at The Catholic University of America, Washington, D.C.

Matlack KS, IS Muller, and IL Pegg. 1997a. Results of Melter Tests Using TWRS LAW Envelope C Simulants. VSL Final Report, August, 1997, Vitreous State Laboratory at The Catholic University of America, Washington, D.C. 
Matlack KS, IS Muller, SS Fu, and IL Pegg. 1997b. Results of Melter Tests Using TWRS LAW Envelope C Simulants. VSL Final Report, October 24, 1997, Vitreous State Laboratory at The Catholic University of America, Washington, D.C.

Matlack KS, WK Kot, and IL Pegg. 2001. Melter Tests with AZ-101 HLW Simulant Using a Duramelter 100 Vitrification System. VSL-01R10N0-1, Rev 1, the Vitreous State Laboratory at The Catholic University of America, Washington, D.C.

Matlack KS, WK Kot, T Bardakci, W Gong, NA D'Angelo, TR Schatz, and IL Pegg. 2002. Tests on the Duramelter 1200 HLW Pilot Melter System with AZ-101 HLW Simulants.

VSL-02R1000-2, Rev 1, the Vitreous State Laboratory at The Catholic University of America, Washington, D.C.

Matlack KS, W Gong, T Bardakci, NA D'Angelo, WK Kot, and IL Pegg. 2003a. DM1200 Tests with AZ-101 HLW Simulants. VSL-03R3800-4, Rev 0, the Vitreous State Laboratory at The Catholic University of America, Washington, D.C.

Matlack KS, W Gong, T Bardakci, NA D'Angelo, WK Kot, and IL Pegg. 2003b. Integrated DM1200 Melter Testing of AZ-102 Composition Using Bubblers. VSL-03R3800-2, Rev 0, the Vitreous State Laboratory at The Catholic University of America, Washington, D.C.

Matlack KS, W Gong, and IL Pegg. 2005a. Duramelter 100 HLW Simulant Validation Tests with C-106/AY-102 Feeds. VSL-05R5710-1, Rev 0, the Vitreous State Laboratory at The Catholic University of America, Washington, D.C.

Matlack KS, W Gong, T Bardakci, NA D'Angelo, M Brandys, WK Kot, and IL Pegg. 2005b. Integrated DM1200 Melter Testing Using AZ-102 and C-106/AY-102 HLW Simulants: HLW Simulant Verification. VSL-05R5800-1, Rev 0, the Vitreous State Laboratory at The Catholic University of America, Washington, D.C.

Matlack KS, H Gan, W Gong, IL Pegg, CC Chapman, and I Joseph. 2007. High Level Waste Vitrification System Improvements. VSL-07R1010-1, Rev. 0, Vitreous State Laboratory at The Catholic University of America, Washington, D.C.

McPherson D, I Joseph, A Mathur, C Capozzi, S Armstrong, and LD Pye. 1987. The Influence of Waste Variability on the Properties and Phase Stability of the West Valley Reference Glass. NOE/NE/44139-29, West Valley Nuclear Services Co. Inc., State Univ. of New York, Alfred, New York.

Mellinger GB and JL Daniel. 1984. Approved Reference and Testing Materials for Use in Nuclear Waste Management Research and Development Programs. PNL-4955-2, Pacific Northwest Laboratory, Richland, Washington.

Mika M, MJ Schweiger, JD Vienna, and P Hrma. 1997. "Liquidus Temperature of Spinel Precipitating High-Level Waste Glasses." Mat. Res. Soc. Symp. Proc. 465:71-78.

Montgomery DC and EA Peck. 1992. Introduction to Linear Regression Analysis, Second Edition. John Wiley \& Sons, New York. 
Muller IS and IL Pegg. 1998. Glass Formulation and Testing with TWRS LAW Simulants. Final Report for GTS Durateck Inc. and BNFL Inc., The Catholic University of America, Washington D.C.

Muller IS, AC Buechele, and IL Pegg. 2001. Glass Formulation and Testing with RPP-WTP LAW Simulants - Final Report. VSL-01R3560-2, Vitreous State Laboratory, The Catholic University of America, Washington D.C.

Muller IS and IL Pegg. 2003a. LAW Glass Formulation to Support Melter Runs with Simulants. VSL-03R3460-2, Vitreous State Laboratory, The Catholic University of America, Washington D.C.

Muller IS and IL Pegg. 2003b. Baseline LAW Glass Formulation Testing. VSL-03R3460-1. Vitreous State Laboratory, the Catholic University of America, Washington D.C.

Muller IS, I Joseph, and IL Pegg. 2005. Comparison of LAW Simulant, Actual Waste, and Melter Glasses. VSL-05R5460-1, Vitreous State Laboratory, The Catholic University of America, Washington, DC.

Muller IS, I Joseph, and IL Pegg. 2006a. Preparation and Testing of LAW High-Alkali Correlation and Augmentation Matrix Glasses. VSL-06R6480-3, Vitreous State Laboratory, The Catholic University of America, Washington, D.C.

Muller IS, I Joseph, and IL Pegg. 2006b. Preparation and Testing of LAW High Phosphorus and High-Chromium Glasses. VSL-06R6480-2, Vitreous State Laboratory, The Catholic University of America, Washington, D.C.

Musick CA, BA Scholes, RD Tillotson, DM Bennert, JD Vienna, JV Crum, DK Peeler, IA Reamer, DF Bickford, JC Marra, and NL Waldo. 2000. Technical Status Report: Vitrification Technology Development Using INEEL Run 78 Pilot Plant Calcine. INEELIEXT-2000-00110, Idaho National Engineering and Environmental Laboratory, Idaho Falls, Idaho.

Oksoy DL, D Pye, DF Bickford, and WG Ramsey. 1994. "Canonical Correlation of Waste Glass Compositions and Durability, Including pH." Ceram. Trans. 39:365-380.

Olson KM. 1993. Fabrication and Leaching of West Valley Demonstration Project Glasses: Ten Quarter 2 and Ten Quarter 3 Glasses. Pacific Northwest National Laboratory, Richland, Washington.

Olson KM. 1994. Viscosity Testing of 30 WVDP Glasses. WVSP 94-16, Pacific Northwest National Laboratory, Richland, Washington.

Olson KM, GF Piepel, SC Marschman, and GK Whiting. 1994. Product Consistency Testing of West Valley Compositional Variation Glasses. PNL-10191, Pacific Northwest National Laboratory, Richland, Washington.

Paul A. 1977. "Chemical Durability of Glasses a Thermodynamic Approach." J. Mat. Sci. 12:2246-2268. 
Peeler DK, TB Edwards, IA Reamer, and RJ Workman. 2005. Nepheline Formation Study for Sludge Batch 4 (SB4): Phase 1 Experimental Results. WSRC-TR-2005-00371, Savannah River National Laboratory, Aiken, South Carolina.

Peeler DK, TB Edwards, DR Best, IA Reamer, and RJ Workman. 2006. Nepheline Formation Study for Sludge Batch 4 (SB4): Phase 2 Experimental Results. WSRC-TR-2006-00006, Savannah River National Laboratory, Aiken, South Carolina.

Pegg IL, H Gan, IS Muller, DA McKeown, and KS Matlack. 2000. Summary of Preliminary Results on Enhanced Sulfate Incorporation During Vitrification of LAW Feeds, VSL-00R3630-1, Vitreous State Laboratory, The Catholic University of America, Washington D.C.

Peters RD, CC Chapman, JE Mendel, and CG Williams. 1993. Database for Waste Glass Composition and Properties. PNL-SA-22529, Pacific Northwest Laboratory, Richland, Washington.

Piepel GF and SK Cooley. 2008. Automated Method for Reducing Scheffe Linear Mixture Experiment Models. PNNL-SA-7469, Rev. 2, Pacific Northwest National Laboratory, Richland, Washington.

Piepel GF, PE Redgate, and P Hrma. 1995. "Statistical Experimental Design of a Waste Glass Study." Ceram. Trans. 61:489-496.

Piepel GF, PE Redgate, and P Masuga. 1996. Mixture Models Versus Free Energy of Hydration Models for Waste Glass Durability. PNL-10823, Pacific Northwest Laboratory, Richland, Washington.

Piepel GF, PE Redgate, and P Masuga. 1997. "Comparison of Mixture Models and Free Energy of Hydration Models for Waste Glass Releases." Glass Tech. 38:210-215.

Piepel GF, P Hrma, and JD Vienna. 1998. "Glass Chemistry Development Strategy for Hanford High Level Waste (HLW)." In: Science and Technology for Disposal of Radioactive Tank Wastes, pp. 393-402, Plenum Press, New York.

Piepel GF, JM Szychowski, and JL Loeppky. 2002. "Augmenting Scheffe Linear Mixture Models with Squared and/or Crossproduct Terms." Journal of Quality Technology 34:297-314.

Piepel GF, SK Cooley, IS Muller, H Gan, I Joseph, and IL Pegg. 2007. ILAW PCT, VHT, Viscosity and Electrical Conductivity Model Development. VSL-07R1230-1, Rev. 0, Vitreous State Laboratory, The Catholic University of America, Washington D.C.

Piepel GF, SK Cooley, A Heredia-Langner, SM Landmesser, WK Kot, H Gan, and IL Pegg. 2008a. IHLW PCT, Spinel $T_{1 \%}$, Electrical Conductivity, and Viscosity Model Development. VSL-07R1240-4, Rev. 0, Vitreous State Laboratory, The Catholic University of America, Washington D.C.

Piepel GF, A Heredia-Langner, and SK Cooley. 2008b. "Property-Composition-Temperature Modeling of Waste Glass Melt Data Subject to a Randomization Restriction." Journal of the American Ceramic Society 91(10):3222-3228. 
Pittman DJ, IA Reamer, DK Peeler, and TB Edwards. 2001. Property-Composition Relationships for the DP Glasses: Effect of Crystallization on Durability (U). WSRC-TR-200100166, Westinghouse Savannah River Company, Aiken, South Carolina.

Plodinec MJ. 1978. Viscosity of Glasses containing Simulated Savannah River Plant Waste. DP-1507, Savannah River Laboratory, Aiken, South Carolina.

Pye LD. 1985. The Physical and Thermal Properties of Simulated Nuclear Waste Glasses and Their Melts. DPST-85-397, New York State College of Ceramics, Alfred University, Alfred, New York.

Reimus MAH, GB Mellinger, GF Piepel, and LR Bunnell. 1988. West Valley Glass Product Qualification Durability Studies, FY 1987-1988: Effects of Composition, Redox State, Thermal History, and Groundwater. PNL-6723, Pacific Northwest Laboratory, Richland, Washington.

Reynolds JG and P Hrma. 1997. "The Kinetics of Spinel Crystallization from a High-Level Waste Glass." In: MRS Symposium Proceedings 465:65-69.

Rielley E, IS Muller, and IL Pegg. 2004. Preparation and Testing of LAW Matrix Glasses to Support WTP Property-Composition Model Development. VSL-04R4480-1, Vitreous State Laboratory, The Catholic University of America, Washington, DC.

Riley BJ, JA Rosario, and P Hrma. 2001. Impact of HLW Glass Crystallinity on the PCT Response. PNNL-13491, Pacific Northwest Laboratory, Richland, Washington.

Scheiwer. 1986. "The Borosilicate Glass for 'Pamela'." Radioactive Waste Management and the Nuclear Fuel Cycle 7:121-138.

Scholes BA, DK Peeler, and JD Vienna. 2000. The Preparation and Characterization of INTEC Phase 3 Composition Variation Study Glasses. INEEL/EXT-2000-01566, Idaho National Engineering and Environmental Laboratory, Idaho Falls, Idaho.

Scholes BA, JD Vienna, DK Peeler, and TB Edwards. 2002. The Preparation and Characterization of INTEC Sodium Bearing Waste Phase 1 Composition Variation Study Glasses. INEEL/EXT-02-00386, Idaho National Engineering and Environmental Laboratory, Idaho Falls, Idaho.

Scholtze H. 1990. Glass Nature, Structure, and Properties. Springer, New York.

Shade JW and GF Piepel. 1991. Evaluation of Experimental Factors that Influence the Application and Discrimination Capability of the Product Consistency Test. PNL-7530, Pacific Northwest Laboratory, Richland, Washington.

Shannon RD. 1976. "Revised Effective Ionic Radii and Systematic Study of Interatomic Distances in Halides and Chalcogenides." Acta Cryst. A32:751-767.

Smith GL. 1993. Characterization of Analytical Reference Glass-1 (ARG-1). PNL-8992, Pacific Northwest Laboratory, Richland, Washington. 
Smith GL, LR Greenwood, GF Piepel, MJ Schweiger, HD Smith, MW Urie, and JJ Wagner. 2000. Vitrification and Product Testing of AW-101 and AN-107 Pretreated Waste.

PNNL-13372, Pacific Northwest National Laboratory, Richland, Washington.

Soper PD, GJ Roberts, LF Lightner, DD Walker and MJ Plodinec. 1982. Optimization of Glass Composition for the Vitrification of Nuclear Waste at the Savannah River Plant. DP-MS-81-108, El du Pont de Nemours \& Co., Inc., Savannah River Laboratory, Aiken, South Carolina.

Staples BA, DK Peeler, JD Vienna, BA Scholes, and CA Musick. 1999. The Preparation and Characterization of INTEC HAW Phase 1 Composition Variation Study Glasses. INEEL/EXT98-00970, Idaho National Engineering and Environmental Laboratory, Idaho Falls, Idaho.

Staples BA, BA Scholes, DK Peeler, LL Torres, JD Vienna, CA Musick, and BR Boyle. 2000. The Preparation and Characterization of INTEC Phase $2 b$ Composition Variation Study Glasses. INEEL/EXT-99-01322, Idaho National Engineering and Environmental Laboratory, Idaho Falls, Idaho.

Urie MW, JJ Wagner, LR Greenwood, OT Farmer, SK Fiskum, RT Ratner, and CZ Soderquist. 1999. Inorganic and Radiochemical Analysis of AW-101 and AN-107 Tank Waste.

PNWD-2462, Battelle-Pacific Northwest Division, Richland, Washington.

Urie MW, JA Campbell, OT Farmer, SK Fiskum, LR Greenwood, EW Hoppe, GM Mong, CZ Soderquist, RG Swoboda, MP Thomas, and JJ Wagner. 2002a. Chemical Analysis and Physical Property Testing of 241-AN-102 Tank Waste-Supernatant and Centrifuged Solids. PNWD-3229, Battelle-Pacific Northwest Division, Richland, Washington.

Urie, MW, PR Bredt, JA Campbell, OT Farmer, SK Fiskum, LR Greenwood, EW Hoppe, LK Jagoda, GM Mong, AP Poloski, RD Scheele, CZ Soderquist, RG Swoboda, MP Thomas, and JJ Wagner. 2002b. Chemical Analysis and Physical Property Testing of 241-AZ-101 Tank Waste-Supernatant and Centrifuged Solids. PNWD-3215, Battelle-Pacific Northwest Division, Richland, Washington.

Urie, MW, JA Campbell, OT Farmer, SK Fiskum, LR Greenwood, EW Hoppe, GM Mong, CZ Soderquist, RG Swoboda, MP Thomas, and JJ Wagner. 2002c. Chemical Analysis and Physical Property Testing of 241-AN-102 Tank Waste-Supernatant and Centrifuged Solids, PNWD-3173, Battelle-Pacific Northwest Division, Richland, Washington.

U.S. Department of Energy (DOE). 1996. Waste Acceptance Product Specifications for Vitrified High-Level Waste Forms. EM-WAPS Rev. 02, Office of Environmental Management, Washington, D.C.

U.S. Department of Energy (DOE). 2000. Design, Construction, and Commissioning of the Hanford Tank Waste Treatment and Immobilization Plant. Contract Number: DE-AC2701RV14136, As Amended, U.S. Department of Energy, Office of River Protection, Richland Washington.

U.S. Department of Energy-Civilian Radioactive Waste Management (DOE-RW). 2008. Waste Acceptance System Requirements Document, Rev. 5, ICN 01, U.S. Department of EnergyCivilian Radioactive Waste Management, Washington, D.C. 
U.S. Environmental Protection Agency (EPA). 1997. SW-846, Test Methods for Evaluating Solid Waste, Physical/Chemical Methods. Office of Solid Waste and Emergency Response, Washington, D.C.

Vienna JD. 2002. The Effects of Temperature and Composition on the Solubility of Chromium in Multi-component Alkali-borosilicate Glass Melts. Ph.D. Dissertation, Washington State University, Pullman, Washington.

Vienna JD and DS Kim. 2008. Preliminary IHLW Formulation Algorithm Description. 24590HLW-RPT-RT-05-001, Rev. 0, River Protection Project, Hanford Tank Waste Treatment and Immobilization Plant, Richland, Washington.

Vienna JD, P Hrma, MJ Schweiger, and MH Langowski. 1996a. "Compositional Dependence of Elemental Release from HLW Glasses by the Product Consistency Test: One Component at a Time Study." Ceram. Trans. 72:307-316.

Vienna JD, P Hrma, DS Kim, MJ Schweiger, and DE Smith. 1996b. "Compositional Dependence of Viscosity, Electrical Conductivity, and Liquidus Temperature of Multicomponent Borosilicate Glasses." Ceram. Trans. 72:427-436.

Vienna JD, PR Hrma, MJ Schweiger, MH Langowski, PE Redgate, DS Kim, GF Piepel, DE Smith, CY Chang, DE Rinehart, SE Palmer, and H Li. 1996c. Effect of Composition and Temperature on the Properties of High-Level Waste (HLW) Glass Melting Above $1200^{\circ} \mathrm{C}$. PNNL-10987, Pacific Northwest National Laboratory, Richland, Washington.

Vienna JD, RP Thimke, GF Piepel, JV Crum, ML Elliott, RK Nakaoka, and GW Veazey. 1998. Glass Development for Treatment of Evaporator Bottoms Waste. PNNL-11865, Pacific Northwest National Laboratory, Richland, Washington.

Vienna JD, DK Peeler, RL Plaisted, TJ Plaisted, IA Reamer, RD Tillotson, JV Crum, CA Musick, and TL James. 1999. Glass Formulation for Idaho National Engineering and Environmental Laboratory Zirconia Calcine High-Activity Waste. PNNL-12202, Pacific Northwest National Laboratory, Richland, Washington.

Vienna JD, P Hrma, JV Crum, and M Mika. 2001a. "Liquidus Temperature-Composition Model for Multi-Component Glasses in the Fe, Cr, Ni, and Mn Spinel Primary Phase Field." J. NonCryst. Sol. 292:1-24.

Vienna JD, P Hrma, A Jiricka, DE Smith, TH Lorier, IA Reamer, and RL Schulz. 2001b. Hanford Immobilized LAW Product Acceptance Testing: Tanks Focus Area Results. PNNL-13744, Pacific Northwest National Laboratory, Richland, Washington.

Vienna JD, WC Buchmiller, JV Crum, DD Graham, DS Kim, BD Maclsaac, MJ Schweiger, DK Peeler, TB Edwards, IA Reamer, and RJ Workman. 2002. Glass Formulation Development for INEEL Sodium-Bearing Waste. PNNL-14050, Pacific Northwest National Laboratory, Richland, Washington.

Wald JW. 1985. Fabrication and Characterization of MMC Approved Testing Material - ATM-1 Glass. PNL-557-1, Pacific Northwest Laboratory, Richland, Washington. 
Wald JW and JL Daniel. 1986. Fabrication and Characterization of MCC Approved Testing Material - ATM-11 Glass. PNL-5577-11, Pacific Northwest Laboratory, Richland, Washington.

Wilson BK, P Hrma, J Alton, TJ Plaisted, and JD Vienna. 2002. "The Effect of Composition on Spinel Equilibrium and Crystal Size in High-Level Waste Glass." J. Matl. Sci. 37(24):53275331. 
Appendix A

\section{Database}




\begin{tabular}{|c|c|c|c|c|c|c|}
\hline \# Glass ID & Study & Reference & Al2O3-t & B2O3-t & CaO-t & $\mathrm{Fe} 2 \mathrm{O}-\mathrm{t}$ \\
\hline 1 IG1-01 & INEEL CVS Phase 1 & Staples et al. 1999 & 0.0552 & 0.0947 & 0.0007 & 0.0005 \\
\hline $\begin{array}{l}2 \text { IG1-02 } \\
3 \text { | IG1-03 }\end{array}$ & $\begin{array}{l}\text { INEEL CVS Phase } 1 \\
\text { INEEL CVS Phase }\end{array}$ & $\begin{array}{l}\text { Staples et al. } 1999 \\
\text { Staples et }\end{array}$ & $\begin{array}{l}0.0656 \\
0.0000\end{array}$ & $\begin{array}{l}0.1312 \\
0.0438\end{array}$ & $\begin{array}{l}0.0007 \\
0.0007\end{array}$ & $\begin{array}{l}0.0005 \\
0.0005\end{array}$ \\
\hline 4 IG1-04 & INEEL CVS Phase 1 & Staples et al. 1999 & 0.0000 & 0.0437 & 0.0007 & 0.0005 \\
\hline 5 IG1-05 & INEEL CVS Phase 1 & Staples et al. 1999 & 0.0657 & 0.1313 & 0.0007 & 0.0005 \\
\hline 6 IG1-06 & INEEL CVS Phase 1 & Staples et al. 1999 & 0.0000 & 0.1501 & 0.0008 & 0.0005 \\
\hline 7 IG1-07 & INEEL CVS Phase 1 & Staples et al. 1999 & 0.1501 & 0.1501 & 0.0008 & 0.0005 \\
\hline $\begin{array}{l}8 \text { IGG1-18 } \\
9 \text { |G1-09 }\end{array}$ & $\begin{array}{l}\text { INELE CVS Phase } \\
\text { INEEL CVS Phase }\end{array}$ & $\begin{array}{l}\text { Staples et al. } 1999 \\
\text { Staples et }\end{array}$ & $\begin{array}{l}0.1312 \\
0.0360\end{array}$ & $\begin{array}{l}0.0437 \\
0.1312\end{array}$ & $\begin{array}{l}0.0007 \\
0.0007\end{array}$ & $\begin{array}{l}0.0005 \\
0.0005\end{array}$ \\
\hline 10 IG $1-10$ & $\begin{array}{l}\text { INE } \\
\text { INEEL CVS P Pase } 1\end{array}$ & $\begin{array}{l}\text { Staples et al. } 1999 \\
\text { Staples et al. } 1999\end{array}$ & $\begin{array}{l}0.0360 \\
0.0000\end{array}$ & $\begin{array}{l}0.1312 \\
0.0654\end{array}$ & 0.0007 & 0.0005 \\
\hline 11 IG1-11 & INEFL CVS Phase 1 & Staples et al. 1999 & 0.1464 & $\begin{array}{l}0.0054 \\
0.0500\end{array}$ & 0.0008 & $\begin{array}{l}0.0005 \\
0.0006\end{array}$ \\
\hline 12 IG1-12 & INEEL CVS Phase 1 & Staples et al. 1999 & 0.0317 & 0.1501 & 0.0008 & 0.0005 \\
\hline 13 IG1-13 & INEEL CVS P Pase 1 & Staples et al. 1999 & 0.0759 & 0.0501 & 0.0008 & 0.0006 \\
\hline 14 IG1-14 & INEEL CVS Phase 1 & Staples et al. 1999 & 0.1087 & 0.0724 & 0.0007 & 0.0006 \\
\hline 15 IG1-15 & INEEL CVS Phase 1 & Staples et al. 1999 & 0.0644 & 0.0677 & 0.0007 & 0.0005 \\
\hline 16 IG1-16 & INEEL CVS Phase 1 & Staples et al. 1999 & 0.0725 & 0.0725 & 0.0007 & 0.0006 \\
\hline $\begin{array}{l}17 \text { IG1-17 } \\
18 \text { IG1-18 }\end{array}$ & $\begin{array}{l}\text { INEEL CVS P P } 1 \\
\text { INEEL CVS Pase }\end{array}$ & $\begin{array}{l}\text { Staples et al. } 1999 \\
\text { Staples et al } 1999\end{array}$ & $\begin{array}{l}0.0677 \\
0.0684\end{array}$ & $\begin{array}{l}0.0678 \\
0.1129\end{array}$ & 0.0007 & 0.0005 \\
\hline $\begin{array}{l}18 / G 1-18 \\
19 / G 1-19\end{array}$ & INEEL CVS P P 1 & $\begin{array}{l}\text { Staples et al. } 1999 \\
\text { Staples et al } 1999\end{array}$ & $\begin{array}{l}0.0684 \\
0.0683\end{array}$ & 0.1129 & 0.0007 & 0.0005 \\
\hline $20161-20$ & INEFL CVS Phase 1 & Staples et al 1999 & $\begin{array}{l}0.06839 \\
0.0449\end{array}$ & 0.1129 & 0.0007 & $\begin{array}{l}0.0005 \\
0.0005\end{array}$ \\
\hline 21 IG1-21 & INEEL CVS Phase 1 & Staples et al. 1999 & 0.0890 & 0.1207 & 0.0007 & 0.0005 \\
\hline 22 IG1-22 & INEEL CVS Phase 1 & Staples et all. 1999 & 0.1013 & & 0.0007 & 0.0005 \\
\hline 23 IG1-23 & INEEL CVS Phase 1 & Staples et al. 1999 & 0.0979 & 0.0677 & 0.0007 & 0.0005 \\
\hline 24 IG1-24 & INEEL CVS Phase 1 & Staples et al. 1999 & 0.0863 & 0.1207 & 0.0007 & 0.0006 \\
\hline 25 IG1-25 & INEEL CVS Phase 1 & Staples et al. 1999 & 0.0724 & 0.0724 & 0.0007 & 0.0006 \\
\hline 26 IG1-26 & INEEL CVS P P 1 & Staples et al. 1999 & 0.0552 & 0.0947 & 0.0007 & 0.0005 \\
\hline 27 IG1-27 & INEEL CVS P P 1 & 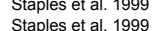 & 0.1312 & 0.0437 & 0.0007 & 0.0005 \\
\hline $\begin{array}{l}29161-20 \\
29\end{array}$ & INEEL CVS Phase 1 & Staples et etal. 1999 & $\begin{array}{l}0.1312 \\
0.1400\end{array}$ & $\begin{array}{l}0.0437 \\
0.0839\end{array}$ & $\begin{array}{l}0.0007 \\
0.0000\end{array}$ & $\begin{array}{l}0.0005 \\
0.0200\end{array}$ \\
\hline 30 IG1-30 & INEEL CVS Phase 1 & Staples et al. 1999 & 0.0203 & 0.0607 & 0.0042 & $\begin{array}{l}0.020051 \\
0.005\end{array}$ \\
\hline 31 IG1-31 & INEL CVS Phase 1 & Staples et al. 1999 & 0.1500 & 0.1500 & 0.0009 & 0.0005 \\
\hline 32 IG1-32 & INEEL CVS Phase 1 & Staples et al. 1999 & 0.1500 & 0.0500 & 0.0009 & 0.0005 \\
\hline 33 IG1-33 & INEEL CVS Phase 1 & Staples et al. 1999 & 0.0000 & 0.0500 & 0.0009 & 0.0005 \\
\hline 34 IG1-34 & INEEL CVS Phase 1 & Staples et al. 1999 & 0.0000 & 0.0701 & 0.0009 & 0.0005 \\
\hline 35 IG1-35 & INEEL CVS Phase 1 & Staples et al. 1999 & 0.0750 & 0.1500 & 0.0009 & 0.0005 \\
\hline $\begin{array}{l}36 \text { IG1-36 } \\
37 \text { IG1-37 }\end{array}$ & INEEL CVS P P 1 & $\begin{array}{l}\text { Staples et al. } 1999 \\
\text { Staples et }\end{array}$ & $\begin{array}{l}0.0750 \\
0.0762\end{array}$ & $\begin{array}{l}0.0500 \\
0.013\end{array}$ & 0.0009 & 0.0005 \\
\hline $\begin{array}{l}38 \text { IG1-38 } \\
38 \text { IG1 }\end{array}$ & INEEL CVS Phase 1 & $\begin{array}{l}\text { Staples et al. } 1999 \\
\text { Stapeles et }\end{array}$ & $\begin{array}{l}0.0762 \\
0.0375\end{array}$ & $\begin{array}{l}0.1013 \\
0.1250\end{array}$ & $\begin{array}{l}0.0009 \\
0.0009\end{array}$ & $\begin{array}{l}0.0005 \\
0.0005\end{array}$ \\
\hline 39 IG1-39 & INEEL CVS Phase 1 & Staples et al. 1999 & 0.0751 & 0.1250 & 0.0009 & $\begin{array}{l}0.0005 \\
0.0005\end{array}$ \\
\hline 40 IG1-40 & INEEL CVS Phase 1 & Staples et al. 1999 & 0.0546 & 0.1022 & 0.0009 & 0.0005 \\
\hline 41 IG1-41 & INEEL CVS Phase 1 & Staples et al. 1999 & 0.0513 & 0.0750 & 0.0009 & 0.0005 \\
\hline 42 IG1-42 & INEEL CVS Phase 1 & Staples et al. 1999 & 0.1084 & 0.0750 & 0.0009 & 0.0005 \\
\hline 43 IG1-43 & INEEL CVS Phase 1 & Staples et al. 1999 & 0.0704 & 0.1250 & 0.0009 & 0.0005 \\
\hline $\begin{array}{lll}44161-44 \\
4\end{array}$ & INEEL CVS Phase 1 & $\begin{array}{l}\text { Staples et al. } 1999 \\
\end{array}$ & 0.0000 & 0.0701 & 0.0009 & 0.0005 \\
\hline 年 & INEEE CVS Phase 2 & $\begin{array}{l}\text { Staples et al. } 2000 \\
\text { Staples et } 200\end{array}$ & 0.1986 & 0.0496 & 0.0000 & 0.0000 \\
\hline 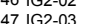 & INEEC CVS Phase 2 & $\begin{array}{l}\text { Staples et al. } 2000 \\
\text { Staples } 2000\end{array}$ & $\begin{array}{l}0.1986 \\
0.0348\end{array}$ & $\begin{array}{l}0.1787 \\
0.1790\end{array}$ & 0.1791 & 0.0000 \\
\hline 48 IG2-04 & INEEL CVS Phase 2 & Staples et al. 2000 & $\begin{array}{l}0.0348 \\
0.1986\end{array}$ & $\begin{array}{l}0.1790 \\
0.1787\end{array}$ & $\begin{array}{l}0.0000 \\
0.0000\end{array}$ & $\begin{array}{l}0.0196 \\
0.0000\end{array}$ \\
\hline 49 IG2-05 & INEL CVS Phase & $\begin{array}{l}\text { Staples et al } 2000 \\
\text { Sal }\end{array}$ & 0.0347 & 0.0496 & 0.1191 & 0.0794 \\
\hline 50 IG2-06 & INEEL CVS P Pase 2 & Staples et al. 2000 & 0.0645 & 0.1787 & 0.0000 & 0.0794 \\
\hline 51 |G2-07 & INEEL CVS Phase 2 & Staples et al. 2000 & 0.0794 & 0.0496 & 0.1191 & 0.0000 \\
\hline 52 IG2-08 & INEELL CVS Phase 2 & Staples et al. 2000 & 0.0347 & 0.1787 & 0.1191 & 0.0000 \\
\hline (5) & INEEL CVS Phase 2 & Staples et al. 2000 & 0.1986 & 0.0496 & 0.1191 & 0.0794 \\
\hline $5476-10$ & INEEL CVS Phase 2 & $\begin{array}{l}\text { Staples et al. } 2000 \\
\end{array}$ & 0.0347 & 0.0496 & 0.0000 & 0.0794 \\
\hline 56 IG2-12 & $\begin{array}{l}\text { INEEL } \\
\text { INEEL CVS Pase }\end{array}$ & $\begin{array}{l}\text { Staples et ala. } 2000 \\
\text { Staples et al. } 2000\end{array}$ & $\begin{array}{l}0.0347 \\
0.0347\end{array}$ & $\begin{array}{l}0.0496 \\
0.1787\end{array}$ & $\begin{array}{l}0.0146 \\
0.0000\end{array}$ & $\begin{array}{l}0.00000 \\
0.0000\end{array}$ \\
\hline 57 IG2-13 & INEEL CVS Phase 2 & Staples et al. 2000 & 0.0485 & 0.0706 & 0.0000 & 0.0000 \\
\hline 58 IG2-14 & INEEL CVS Phase 2 & Staples et al. 2000 & 0.1986 & 0.0613 & 0.0000 & 0.0794 \\
\hline 59 IG2-15 & INEEL CVS Phase 2 & Staples et al. 2000 & 0.0794 & 0.0596 & & 0.0050 \\
\hline 60 IG2-16 & INEEL CVS Phase 2 & Staples et al. 2000 & 0.1092 & 0.1191 & 0.0397 & 0.0050 \\
\hline 61 IG2-17 & INEEL CVS Phase 2 & $\begin{array}{l}\text { Staples et al. } 2000 \\
\end{array}$ & 0.0794 & 0.0596 & 0.0794 & 0.0050 \\
\hline $\begin{array}{l}62 \text { IG2-18 } \\
63 \text { IG-19 }\end{array}$ & $\begin{array}{l}\text { INELL CVS P } 2 \\
\text { INEEL CVS Pase }\end{array}$ & $\begin{array}{l}\text { Staples et al. } 2000 \\
\text { Stapeles }\end{array}$ & $\begin{array}{l}0.1191 \\
0.0794\end{array}$ & $\begin{array}{l}0.0596 \\
0.0596\end{array}$ & $\begin{array}{l}0.0397 \\
0.0397\end{array}$ & $\begin{array}{l}0.0298 \\
0.0050\end{array}$ \\
\hline $\begin{array}{l}63 \mid G 2-19 \\
64 \mathrm{IG}-20\end{array}$ & INEEL CVS Phase 2 & $\begin{array}{l}\text { Staples et ala. } 2000 \\
\text { Staples et al. } 2000\end{array}$ & 0.0794 & $\begin{array}{l}0.0596 \\
0.0596\end{array}$ & 0.0397 & $\begin{array}{l}0.0050 \\
0.0050\end{array}$ \\
\hline 65 IG2-21 & $\begin{array}{l}\text { INEEL CVS Phase } 2 \\
\text { IN }\end{array}$ & Staples et al. 2000 & 0.0794 & $\begin{array}{l}0.0596 \\
0.0596\end{array}$ & 0.0794 & 0.0199 \\
\hline 66 IG2 & INEEL CVS Phase 2 & Staples et al. 2000 & 0.1134 & 0.0651 & 0.0375 & 0.0048 \\
\hline 67 IG2-23 & INEEL CVS Phase 2 & Staples et al. 2000 & 0.1050 & 0.1191 & 0.0397 & 0.0050 \\
\hline 68 IG2- & INEEL CVS Phase 2 & Staples et al. 2000 & 0.1191 & 0.0596 & 0.0794 & 0.005 \\
\hline 69 IG2-25 & INEEL CVS Phas & Staples et al. 2000 & 0.1191 & 0.0596 & 0.0794 & 0.0050 \\
\hline 70 IG & INEEL CVS P Pase 2 & Staples et al. 2000 & 0.0817 & 0.0611 & 0.0257 & 0.0051 \\
\hline $\begin{array}{l}71 \text { IG2-27 } \\
72 \text { IG2-28 }\end{array}$ & INEEL CVS Phase 2 & $\begin{array}{l}\text { Staples et al. } 2000 \\
\text { Staples et }\end{array}$ & $\begin{array}{l}0.0981 \\
0.0794\end{array}$ & $\begin{array}{l}0.0596 \\
0.0596\end{array}$ & $\begin{array}{l}0.0794 \\
0.0735\end{array}$ & \\
\hline $\begin{array}{l}72 \text { IG2-28 } \\
73 \text { IG2-29 }\end{array}$ & $\begin{array}{l}\text { INELE CVS } \\
\text { INEEL CVS Pase }\end{array}$ & $\begin{array}{l}\text { Staples et al. } 2000 \\
\text { Staples et al } 2000\end{array}$ & $\begin{array}{l}0.0984 \\
0.0987\end{array}$ & $\begin{array}{l}0.0596 \\
0.0915\end{array}$ & $\begin{array}{l}0.0735 \\
0.0489\end{array}$ & $\begin{array}{l}0.025 \\
0.025\end{array}$ \\
\hline $\begin{array}{l}74 \mathrm{IG} 2-30 \\
74 \mathrm{I}\end{array}$ & INEEL CVS Phase 2 & Staples et al. 2000 & 0.1503 & 0.1503 & 0.0008 & 0.000 \\
\hline & INEEL CVS Pha & Staples et al. 2000 & & & 0.0009 & 0.00 \\
\hline 76162 & INEEL CVS Pha & Staples et al. 20 & 0.0 & 0.0908 & 0.0485 & 0.02 \\
\hline 77 IG2-33 & INEEL CVS Phase 2 & Staples et al. 2000 & 0.0980 & 0.0908 & 0.0485 & 0.02 \\
\hline 78 IG2-34 & INEEL CVS Phase 2 & Staples et al. 2000 & 0.0960 & 0.0890 & 0.0476 & 0.02 \\
\hline 79 IG2-35 & INEEL CVS Phase 2 & al. 2000 & 0.0975 & 0.0904 & 0.0483 & \\
\hline & INEEL CVS Phase 2 & & 0.0960 & 0.0890 & 0.0476 & 0.0249 \\
\hline 81 IG2-37 & INEEL CVS Phase 2 & Staples et al. 2000 & 0.0940 & 0.0872 & 0.0466 & 0.0244 \\
\hline 82 |G3-01 & INEEL CVS Phase 3 & Scholes et al. 2000 & 0.0850 & 0.0925 & 0.0487 & \\
\hline
\end{tabular}

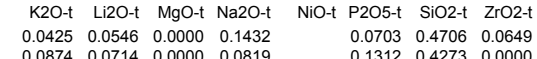

$\begin{array}{lllllll}0.0874 & 0.0714 & 0.0000 & 0.0819 & 0.1319 & 0.4273 & 0.06000 \\ 0.0875 & 0.0010 & 0.0000 & 0.131 & 0.1312 & 0.3971 & 0.1224\end{array}$

$\begin{array}{llll}0.0000 & 0.0787 & 0.0000 & 0.0953 \\ 0.0000 & 0.0014 & 0.0000 & 0.2131\end{array}$

$\begin{array}{lll}0.1310 & 0.5248 & 0.1225 \\ 0.1310 & 0.4537 & 0.0000\end{array}$

$\begin{array}{llll}0.0000 & 0.0845 & 0.0000 & 0.0500\end{array}$

$\begin{array}{llll}0.0875 & 0.0722 & 0.0000 & 0.0819\end{array}$

$\begin{array}{llll}0.0000 & 0.09000 & 0.0000 & 0.1560\end{array}$

$\begin{array}{llll}0.0000 & 0.0511 & 0.0000 & 0.2000 \\ 0.1001 & 0.0000 & 0.0000 & 0.1530\end{array}$

$\begin{array}{llll}0.1001 & 0.0000 & 0.0000 & 0.1530 \\ 0.1001 & 0.0709 & 0.0000 & 0.1720\end{array}$

$\begin{array}{llll}0.0242 & 0.0507 & 0.0000 & 0.1674 \\ 0.0677 & 0.0600 & 0.0000 & 0.1086\end{array}$

$\begin{array}{llll}0.0242 & 0.0464 & 0.0000 & 0.1674 \\ 0.0226 & 0.0386 & 0.0000 & 0.1763 \\ 0.0226 & 0.0564 & 0.000 & 0.186\end{array}$

$\begin{array}{lllll}0.0222 & 0.0386 & 0.0000 & 0.174 \\ 0.0226 & 0.0563 & 0.0000 & 0.1086 \\ 0.0226\end{array}$

$\begin{array}{llll}0.0226 & 0.0564 & 0.0000 & 0.1086 \\ 0.0226 & 0.0528 & 0.0000 & 0.1086\end{array}$

$\begin{array}{lll}0.0000 & 0.5722 & 0.075 \\ 0.0000 & 0.5308 & 0.0301\end{array}$

$\begin{array}{lll}0.1312 & 0.4221 & 0.0262 \\ 0.1312 & 0.3411 & 0.1215\end{array}$

$\begin{array}{llll}0.0000 & 0.5441 & 0.1401 \\ 0.0000 & 0.5479 & 0.000\end{array}$

$\begin{array}{lll}0.0000 & 0.4206 & 0.1401 \\ 0.0000 & 0.5266 & 0.0000\end{array}$

$\begin{array}{lll}0.0000 & 0.5266 & 0.0000 \\ 0.0362 & 0.5023 & 0.0338\end{array}$

$\begin{array}{lll}0.1016 & 0.4943 & 0.0316 \\ 0.0362 & 0.4752 & 0.1014\end{array}$

$\begin{array}{lll}0.0362 & 0.4752 & 0.1014 \\ 0.1016 & 0.4266 & 0.0948\end{array}$

$\begin{array}{lll}0.1016 & 0.4941 \quad 0.0316\end{array}$

$\begin{array}{lll}0.1016 & 0.4944 & 0.0316 \\ 0.1016 & 0.4578 & 0.0948\end{array}$

$\begin{array}{llll}0.0226 & 0.0528 & 0.0000 & 0.1086 \\ 0.0724 & 0.0652 & 0.0000 & 0.0950 \\ 0.022 & 0.050 & 0.000 & 0.136\end{array}$

$\begin{array}{llll}0.1016 & 0.4578 & 0.0948\end{array}$

$\begin{array}{llll}0.0226 & 0.0610 & 0.00000 & 0.1316 \\ 0.0663 & 0.0610 & 0.0000 & 0.1086\end{array}$

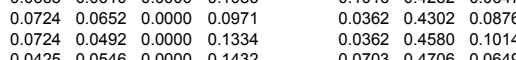

$\begin{array}{llll}0.1016 & 0.4282 & 0.0615 \\ 0.0647 & 0.404\end{array}$

$\begin{array}{llll}0.0875 & 0.0721 & 0.0000 & 0.0819 \\ 0.0875 & 0.0722 & 0.0000 & 0.0819\end{array}$

$0.1312 \quad 0.422$

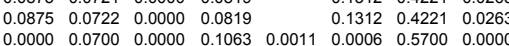

$\begin{array}{llllllllll}0.0000 & 0.0707 & 0.0012 & 0.1833 & 0.0094 & 0.0049 & 0.4598 & 0.1112\end{array}$

$\begin{array}{llllllll}0.0000 & 0.0900 & 0.0000 & 0.1187 & 0.0000 & 0.00000 & 0.4565 & 0.0300 \\ 0.1000 & 0.0836 & 0.0000 & 0.0500 & 0.0000 & 0.0500 & 0.5116 & 0.0000\end{array}$

$\begin{array}{llllllll}0.1000 & 0.0836 & 0.0000 & 0.0500 & 0.0000 & 0.0500 & 0.5116 & 0.0000 \\ 0.1000 & 0.0116 & 0.0000 & 0.2000 & 0.0000 & 0.0000 & 0.4936 & 0.1400\end{array}$

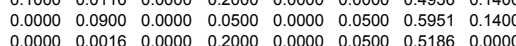

$\begin{array}{llllllll}0.0000 & 0.0016 & 0.0000 & 0.2000 & 0.0000 & 0.0500 & 0.5186 & 0.0000 \\ 0.1000 & 0.0900 & 0.0000 & 0.1174 & 0.0000 & 0.0000 & 0.5628 & 0.0000\end{array}$

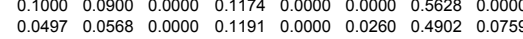

$\begin{array}{llllllll}0.0250 & 0.0631 & 0.0000 & 0.0875 & 0.0000 & 0.0125 & 0.5396 & 0.105 \\ 0.0750 & 0.0675 & 0.0000 & 0.0883 & 0.0000 & 0.0125 & 0.4469 & 0.1049\end{array}$

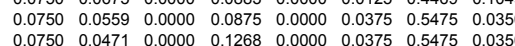

$\begin{array}{llllllll}0.0734 & 0.0675 & 0.0000 & 0.0875 & 0.0000 & 0.0375 & 0.4743 & 0.0716\end{array}$

$\begin{array}{llllllll}0.0250 & 0.0249 & 0.0000 & 0.1625 & 0.0000 & 0.0375 & 0.4449 & 0.1050 \\ 0.0000 & 0.0900 & 0.0000 & 0.0500 & 0.0000 & 0.0500 & 0.5951 & 0.1400\end{array}$

$\begin{array}{llllllll}0.0000 & 0.0900 & 0.0000 & 0.0500 & 0.0000 & 0.0500 & 0.5951 & 0.1400 \\ 0.0000 & 0.0000 & 0.0021 & 0.1986 & 0.0149 & 0.0000 & 0.4715 & 0.0000\end{array}$

$\begin{array}{llllllll}0.0000 & 0.0000 & 0.0021 & 0.0496 & 0.0000 & 0.0298 & 0.4170 & 0.0000\end{array}$

$\begin{array}{llllllll}0.0000 & 0.0000 & 0.0021 & 0.0497 & 0.0149 & 0.0000 & 0.5969 & 0.0000 \\ 0.0993 & 0.0894 & 0.0021 & 0.0496 & 0.0149 & 0.0000 & 0.3474 & 0.0149\end{array}$

$\begin{array}{llllllll}0.0993 & 0.0894 & 0.0021 & 0.0496 & 0.0149 & 0.0000 & 0.3474 & 0.0149 \\ 0.0099 & 0.0000 & 0.0021 & 0.1986 & 0.0149 & 0.0000 & 0.3476 & 0.1390\end{array}$

$\begin{array}{llllllll}0.0000 & 0.0894 & 0.0021 & 0.0496 & 0.0149 & 0.0298 & 0.3445 & 0.1390\end{array}$

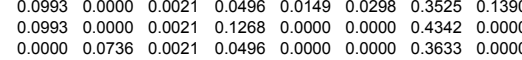

$\begin{array}{llllllll}0.0993 & 0.0834 & 0.0021 & 0.0496 & 0.0000 & 0.0298 & 0.5074 & 0.0000 \\ 0.0000 & 0.0894 & 0.0021 & 0.1588 & 0.0149 & 0.0298 & 0.5410 & 0.0000\end{array}$

$\begin{array}{lllllllll}0.0000 & 0.0052 & 0.0021 & 0.1986 & 0.00000 & 0.0000 & 0.4044 & 0.1116\end{array}$

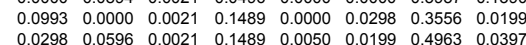

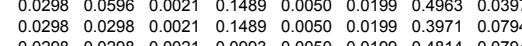

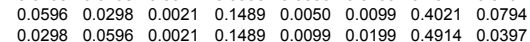

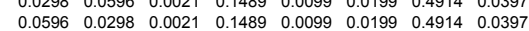

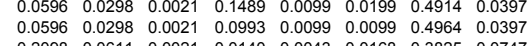

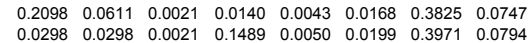

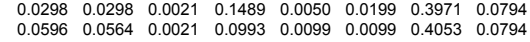

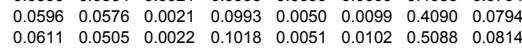

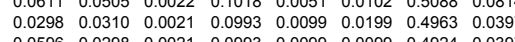

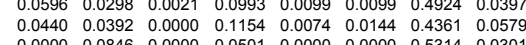

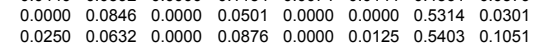

$\begin{array}{lllllllll}0.0437 & 0.0389 & 0.0021 & 0.1146 & 0.0073 & 0.0143 & 0.4331 & 0.0575\end{array}$

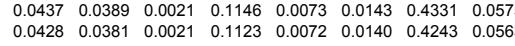

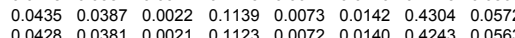

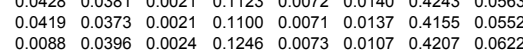

Cdo-t Ce203-t CeO2-t Cl-

$\begin{array}{lll}0.0000 & 0.0006 & 0.0000 \\ 0.0000 & 0.0006 & 0.0000\end{array}$

0.0000

0.0000
0.0000

0.0000
0.0000

0.0000
0.0000
0.0000

0.00000
0.0000

0.0000

0.0000
0.0000
0.0000

0.0000

0.0000

0.0000
0.0000
0.0000

0.00000

0.0000

0.0000
0.0000
0.0000
0

0.0000
0.0000
0.00000

0.0000
0.0000
0

0.0000
0.0000
0

0.0000

0.0000
0.0000
0

0.0000

0.0000

0.0000
0.0000
0.0000

0.0000

0.0000
0.0000

0.00000

0.0000
0.0000

0.0000

0.0000
0.0000

0.0000
0.0000
0

0.0000
0.0000

0.00000

0.0000
0.0000
0.0000

0.0000
0.0000

0.0000

0.0000
0.00000

0.0000
0.0000
0.0000
0

0.00000
0.0000
0.0000

0.0000
0.0000
0

0.0000
0.0000
0.0000

0.0000
0.0000

0.0000

0.0000
0.0000
0.0000

0.0000
0.0000
0.0000
.0000

0.0000
0.0000
0.0000

$0.0000 \quad 0.000$

$\begin{array}{lll}0.0006 & 0.0000 \\ 0.0006 & 0.0000 \\ 0\end{array}$

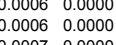

$0.0007 \quad 0.0000$

$0.0006 \quad 0.0000$

$\begin{array}{ll}.0007 & 0.0000 \\ .0007 & 0.0000\end{array}$

$0.0007 \quad 0.0000$

$\begin{array}{ll}0.006 & 0.0000 \\ 0.006 & 0.0000\end{array}$

$0.0007 \quad 0.0000$

$\begin{array}{ll}0.0006 & 0.000 \\ 0.0006 & 0.0000\end{array}$

$\begin{array}{lll} & 0.006 & 0.0000 \\ 0.0006 & 0.0000 \\ 0.0006 & 0.000\end{array}$

$0.0006 \quad 0.0000$

$0.0006 \quad 0.0000$

0.0060
0.00006
0.000000
0.0003
0.0000

$\begin{array}{ll}0.0003 & 0.0000 \\ 0.0025 & 0.0000\end{array}$

$\begin{array}{ll}0.0007 & 0.0000 \\ 0.0007 & 0.0000\end{array}$

0.00070 .0000

0.0007
0.0007
0.00000
0.0007 0.0000

0.00070 .0000

$\begin{array}{ll}0.0007 & 0.0000 \\ 0.0007 & 0.0000 \\ 0.0007 & .0000\end{array}$

$\begin{array}{ll}0.0007 & 0.0000 \\ 0.0007 & 0.0000 \\ 0.0007 & 0.0000\end{array}$

$0.0007 \quad 0.0000$

$\begin{array}{lll}0.0000 & 0.0003 \\ 0.0001 & 0.0003\end{array}$

$0.0000 \quad 0.0000$

$0.0000 \quad 0.0003$

$\begin{array}{lll}0.0000 & 0.0003 \\ 0.0000 & 0.0003\end{array}$

0.00000 .0003

0.0000
0.0000
0.000003
0.0000

$0.0000 \quad 0.000$

0.00000 .0003

$0.0000 \quad 0.0003$

0.00000
0.0000
0.00003
0.0000

$\begin{array}{lll}0.0000 & 0.0003 \\ 0 & 0.000 & 0.0003\end{array}$

$\begin{array}{lll}0.0000 & 0.0003 \\ 0.0000 & 0.0003\end{array}$

.00010 .0003

0.00000 .0003

$0.0000 \quad 0.0000$

$\begin{array}{lll}0.00006 & 0.00000 \\ 0.0000 & 0.0000\end{array}$

.0000 0.0003

$\begin{array}{lll}0.0000 & 0.0003 \\ 0.0001 & 0.0003\end{array}$

$\begin{array}{lll}0.0000 & 0.0003 \\ 0.0000 & 0.0003\end{array}$ 


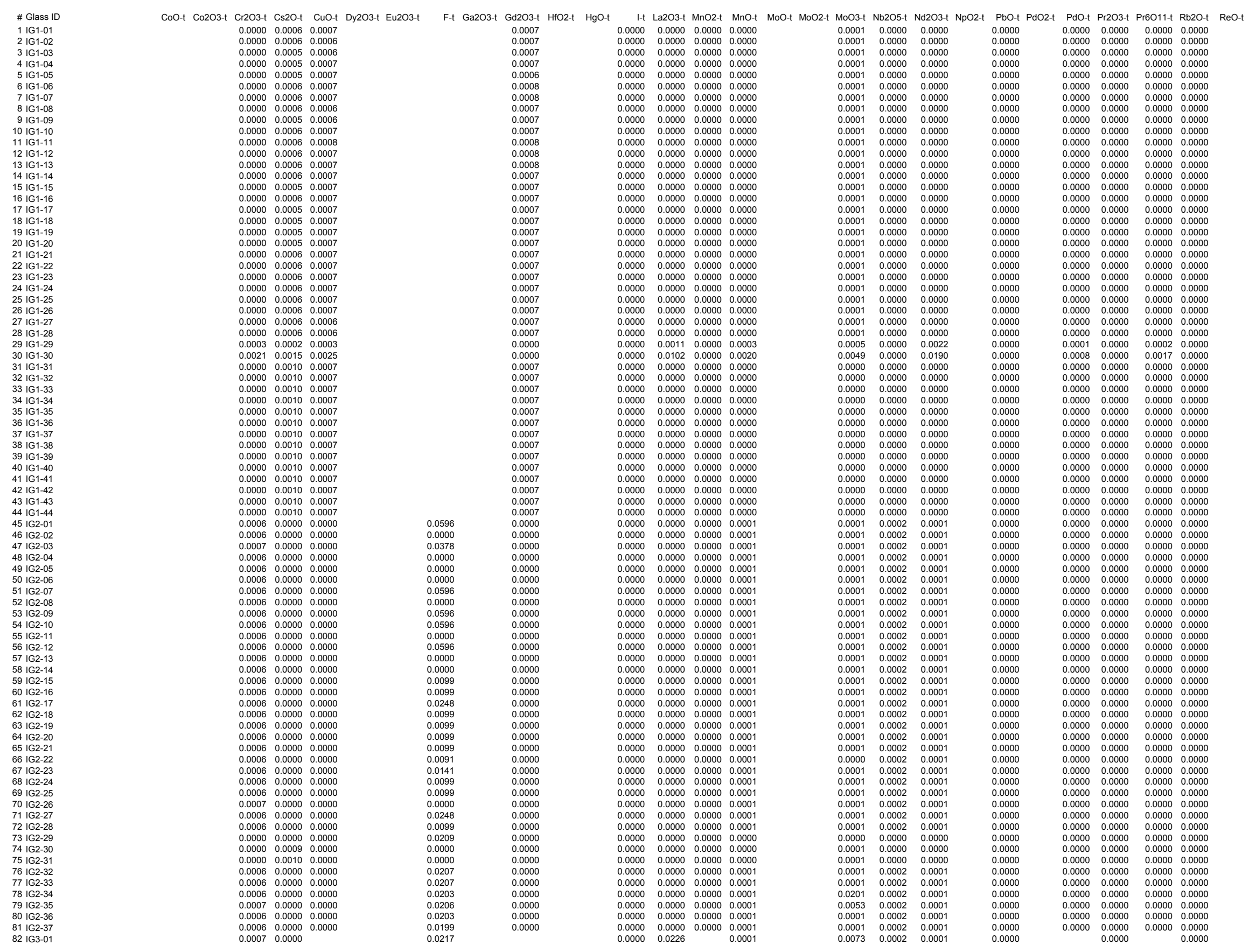




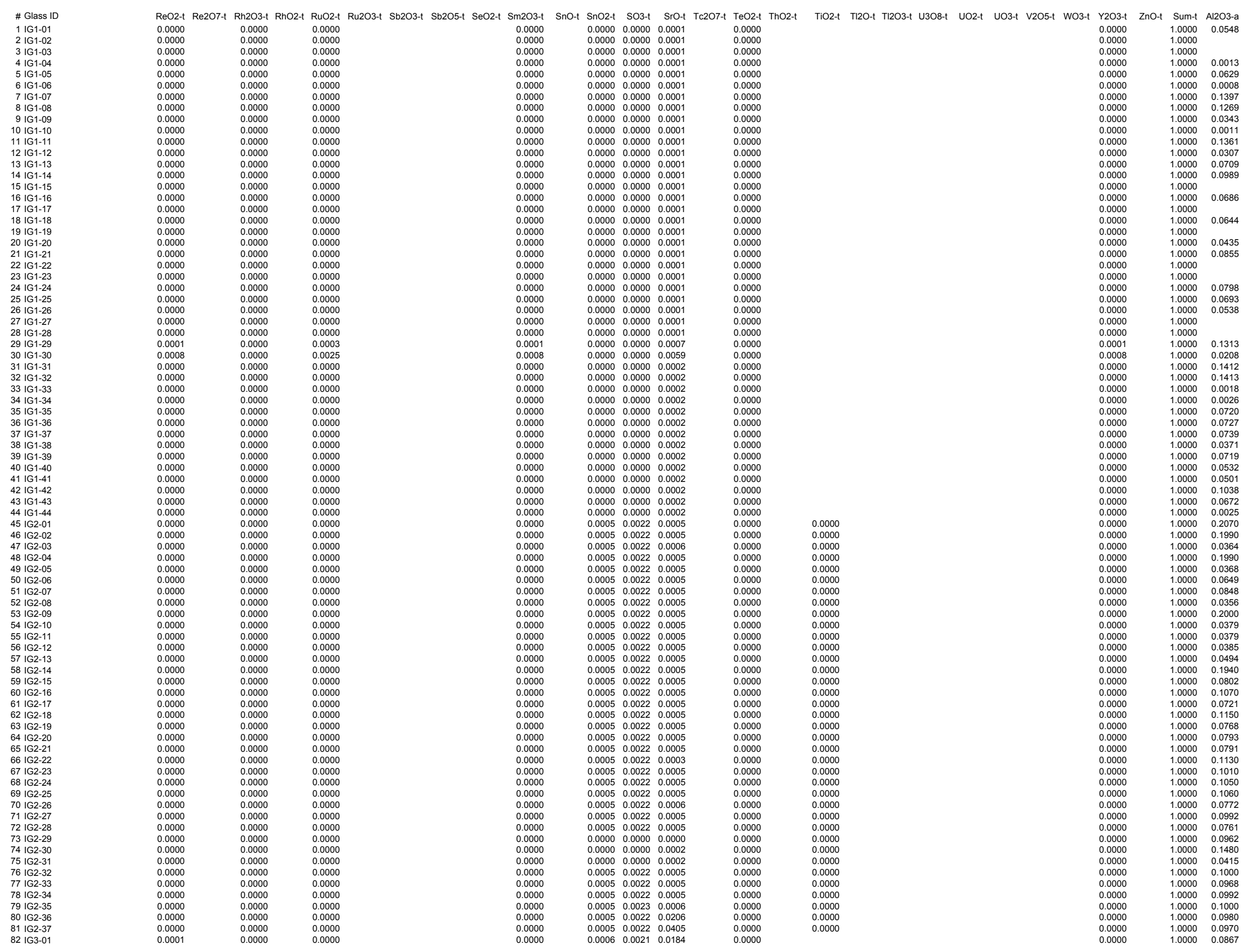




\section{$\begin{array}{ll}0.0009 & 0.0659 \\ 0.0009 & 0.0664\end{array}$} $\begin{array}{ll}0.0008 & 0.0833 \\ 0.0884 & 0.0789\end{array}$ 0.00050 .0017 0.09140 .0867 0.02290 .0601 $\begin{array}{ll}0.0645 & 0.0629 \\ 0.0658 & 0.0544\end{array}$ $\begin{array}{lll}0.0669 & 0.0463\end{array}$ $0.0227 \quad 0.0242$ 0.00060 .0868 $0.0000 \quad 0.0000$ $0.0000 \quad 0.0000$ 0.13000 .0931 $0.0000 \quad 0.1067$ 0.11100 .0000 0.00000 .0911 0.00000 .0964

0.10900 .0000 0.03130 .0294 $\begin{array}{lll}0.0320 & 0.0306 \\ 0.0607 & 0.0309\end{array}$ 0.03110 .0631 0.06970 .0326 0.20900 .0609 0.06620 .0589 0.0554
0.0329 0.03270 .0338 $0.0480 \quad 0.0390$ $0.0000 \quad 0.0970$ $0.0439 \quad 0.0424$ 0.04310 .0370 0.04890 .0360 


$$
1
$$


$\begin{array}{ll}0.9763 & 1150 \\ 0.0000 & 1150 \\ 0.0000 & 1150 \\ 0.9679 & 1150 \\ 0.9653 & 150\end{array}$

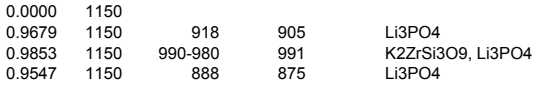

$\begin{array}{lll}0.9547 & 1150 \\ 0.00000 & 1150 \\ 0.0900 & 1150\end{array}$

$\begin{array}{lll}.0 .9005 & 1150 & 1090-1080 \\ 0.8813 & 1150 & 1135-1125 \\ 0 & 1150\end{array}$

$\begin{array}{ll}0.8813 & 1150 \\ 0.9708 & 1150 \\ 0.054 & 1150 \\ 0.090 & 1150\end{array}$

$\begin{array}{ll}0.9644 & 1150 \\ 0.9999 & 1450 \\ 0.9655 & 1150 \\ 0.9694 & 1150\end{array}$

$\begin{array}{ll}0.9655 & 1150 \\ 0.9694 & 1150 \\ 0.9647 & 1150 \\ 0.9747 & 1150\end{array}$ 


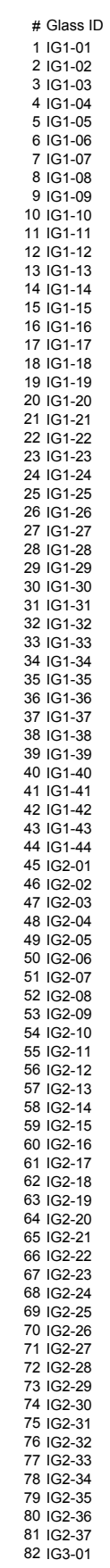

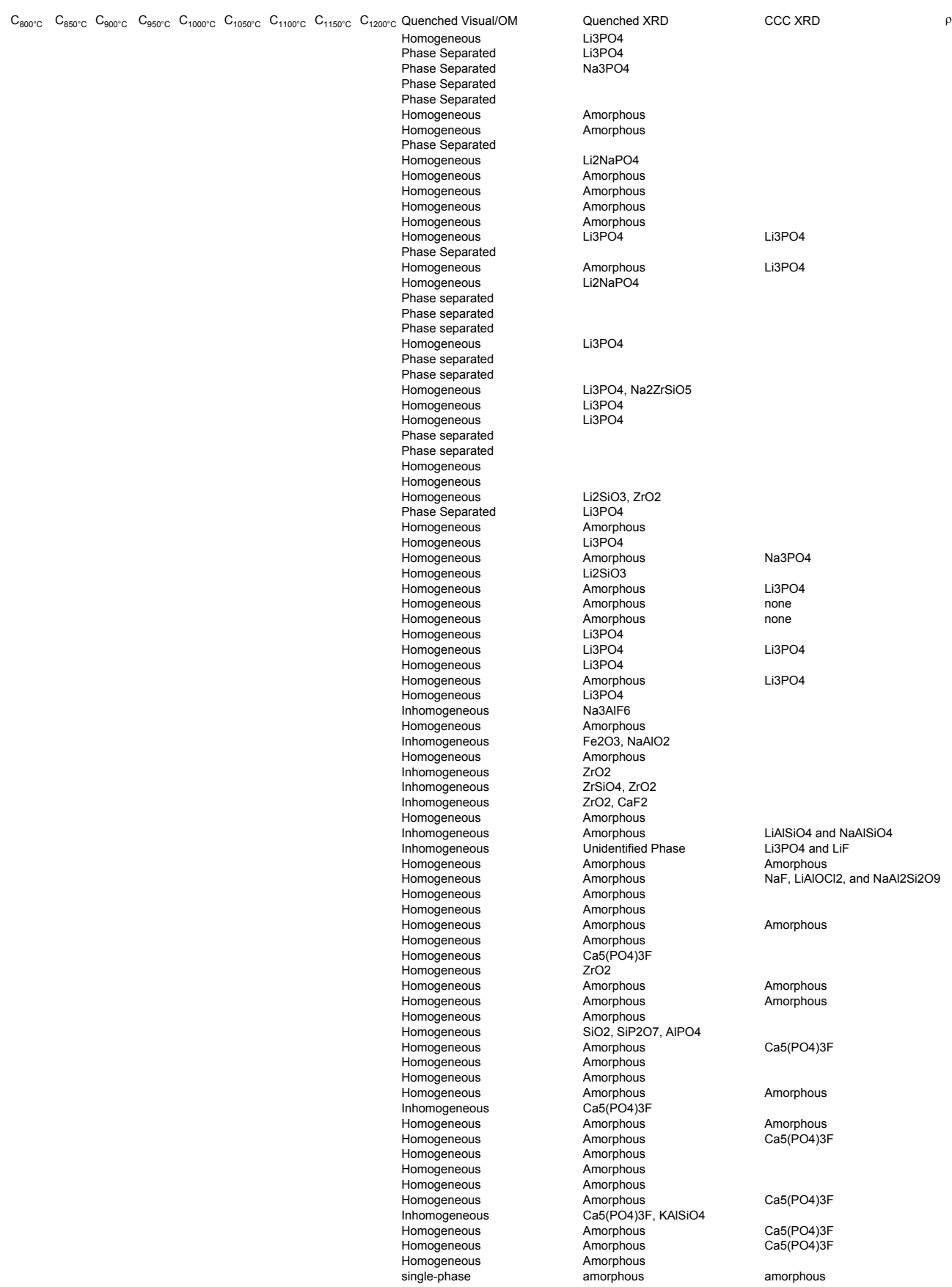

$\rho\left(g / \mathrm{cm}^{3}\right)$ Fulc Visc A Fulc Visc B Fulc Visc To Arrh Visc E Arrh Visc F T1 ( $\left.{ }^{\circ} \mathrm{C}\right)$ V1 (Pa:s) $\begin{array}{llllllr}-5.879 & 6252.6 & 296.5 & -11.624 & 18626.3 & 1160.0 & 3.800 \\ -3.589 & 2400.8 & 584.2 & -10.903 & 16498.7 & 1162.2 & 1.7\end{array}$

\begin{tabular}{|c|c|c|c|c|c|}
\hline 5.934 & 6419.0 & 337.5 & -12.931 & 21236.6 & 1156.1 \\
\hline & 6470 & 264.4 & -10.218 & & 1159.5 \\
\hline $\begin{array}{l}-1.154 \\
.7 .255\end{array}$ & & $\begin{array}{l}849.2 \\
3.537\end{array}$ & & & 1159 \\
\hline $\begin{array}{l}-7.025 \\
-7.334\end{array}$ & $\begin{array}{l}6030.0 .0 \\
7607.1\end{array}$ & $\begin{array}{l}353.7 \\
2429\end{array}$ & $\begin{array}{l}-14.109 \\
-12854\end{array}$ & $\begin{array}{l}20894.0 \\
1092232\end{array}$ & $\begin{array}{l}11155.9 \\
1119.9\end{array}$ \\
\hline $\begin{array}{l}-.334 \\
-4.821\end{array}$ & $\begin{array}{l}66007.1 \\
6807.0\end{array}$ & $\begin{array}{l}242.9 \\
216.5\end{array}$ & $\begin{array}{l}-212.8544 \\
-9.233\end{array}$ & $\begin{array}{l}19828.4 .4 \\
16682.8\end{array}$ & $\begin{array}{l}\begin{array}{l}111959.1 \\
1158.6\end{array}\end{array}$ \\
\hline 9.621 & $\begin{array}{c}100796.0 \\
0\end{array}$ & 209 & $\begin{array}{r}9-2.233 \\
-16.359\end{array}$ & $\begin{array}{l}16082.8 \\
25957.7\end{array}$ & $\begin{array}{l}1158.6 .6 \\
158.7\end{array}$ \\
\hline & & & -9.104 & 14234.8 & 1156.0 \\
\hline & 6601.5 & 255.8 & -10.419 & 17754.0 & 1157.6 \\
\hline
\end{tabular}

$\begin{array}{lllllll}-5.572 & 6781.3 & 305.4 & -12.405 & 20626.9 & 1162.1 & 7.050 \\ -6.077 & 6626.8 & 338.1 & -13.320 & 21964.5 & 1159.9 & 7.25\end{array}$

$\begin{array}{lllllll}-5.475 & 6233.2 & 325.6 & -10.958 & 18584.3 & 1160.8 & 7.23\end{array}$

$\begin{array}{lllllll}-6.114 & 6360.0 & 285.1 & -11.656 & 18383.0 & 1158.0 & 3.240\end{array}$

$\begin{array}{rrrrrrr}-5.842 & 6380.9 & 337.0 & -11.773 & 19632.8 & 1158 & 6.88 \\ -5.732 & 5834.4 & 311.3 & -11.364 & 17948.6 & 1162.4 & 3.060 \\ -5330 & 63.2 & 30.9 & -12.74 & 2063.5 & 11580 & 5378\end{array}$

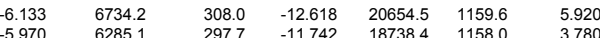

$\begin{array}{lllllll}-1.237 & 1166.9 & 848.6 & -13.892 & 23590.1 & 1159.9 & 1.780 \\ -1.875 & 1522.3 & 811.1 & -14.667 & 24669.0 & 1158.1 & 12.17 \\ -5011 & 05949.1 & 425.7 & -1153 & 17273 & 11553 & 1710\end{array}$

$\begin{array}{rrrrrrr}-4.061 & 6594.3 & 238.9 & -9.329 & 16959.1 & 1163.2 & 11.740 \\ -5.011 & 4049.1 & 425.7 & -11.533 & 17273.8 & 1155.3 & 1.710\end{array}$

$\begin{array}{rrrrrrr}-5.816 & 6034.3 & 220.1 & -9.786 & 14901.3 & 1157.3 & 1.870 \\ -4.920 & 6656.4 & 273.1 & -10.040 & 18112.8 & 1157.1 & 13.75\end{array}$

$\begin{array}{lllll}6799.9 & 373.1 & -13.818 & 23769.5 & 1158\end{array}$

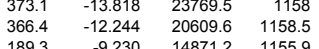

$\begin{array}{lllll}286.7 & -11.618 & 1987.2 & 1155.9 & 3.250 \\ 2897 & -11575 & 5450\end{array}$

$-5.849$

-5.529
-6.331
-5320

$-5.220$

2.490
2.450

-6.053
-5.938
-3.682 


\begin{tabular}{|c|c|c|c|c|c|c|c|c|c|c|c|c|c|c|c|c|c|}
\hline $\begin{array}{l}1105.9 \\
11066\end{array}$ & $\begin{array}{l}11.210 \\
13\end{array}$ & 1056 & 20.0 & 1107.9 & 10.9 & 1157.5 & 6.6 & 1206.1 & 4.3 & 1255.3 & 2.9 & 1155.2 & 7.0 & 1006.7 & 39.2 & 957.2 & 83.1 \\
\hline $\begin{array}{l}11066.6 \\
1107.9\end{array}$ & $\begin{array}{r}33.830 \\
27.35\end{array}$ & $\begin{array}{l}1055.6 \\
11586\end{array}$ & $\begin{array}{r}22.6 \\
1248\end{array}$ & $\begin{array}{l}1107.6 \\
12072\end{array}$ & $\begin{array}{l}13.6 \\
814\end{array}$ & $\begin{array}{r}1158.0 \\
1256\end{array}$ & $\begin{array}{r}8.9 \\
5.31\end{array}$ & $\begin{array}{l}1206.1 \\
11562\end{array}$ & $\begin{array}{r}6.1 \\
137\end{array}$ & 1254.9 & 4.3 & 1154.4 & 9.2 & 1005.9 & 39.3 & 955.9 & 73.0 \\
\hline 1105.5 & $\begin{array}{r}2.35 \\
2.7\end{array}$ & $\begin{array}{l}1008.6 \\
1055.6\end{array}$ & $\begin{array}{r}12.48 \\
4.77\end{array}$ & $\begin{array}{l}1207.2 \\
1105.9\end{array}$ & $\begin{array}{l}8.14 \\
2.71\end{array}$ & $\begin{array}{r}1256 \\
1155.2\end{array}$ & $\begin{array}{l}5.31 \\
1.65\end{array}$ & $\begin{array}{l}1156.2 \\
1204.1\end{array}$ & $\begin{array}{r}13.76 \\
1.06\end{array}$ & 1252.9 & 0.72 & 1154.6 & 1.69 & 1006 & 9.31 & 956.9 & 19.38 \\
\hline 1106.4 & 4.470 & 1055.8 & 7.7 & 1107.3 & 4.4 & 1157.0 & 2.7 & 1206.1 & 1.8 & 1256.0 & 1.2 & 1155.0 & 2.8 & 1006.1 & 14.1 & 956.8 & 27.4 \\
\hline 1105.4 & 17.350 & 1055.4 & 27.3 & 1107.2 & 17.0 & 1156.6 & 11.3 & 1205.8 & 7.8 & 1254.7 & 5.5 & 1154.0 & 11.8 & 1006.1 & 45.2 & 956.6 & 78.5 \\
\hline $\begin{array}{r}1108 \\
11051\end{array}$ & 10.800 & 1057.9 & 21.3 & $\begin{array}{l}1109.5 \\
1105\end{array}$ & 10.6 & 1158.8 & 5.8 & 1207.6 & 3.3 & 1255.8 & 2.0 & 1156.2 & 6.6 & 1007.9 & 50.7 & 958.6 & 120.8 \\
\hline $\begin{array}{l}1105.1 \\
1106.6\end{array}$ & $\begin{array}{r}3.350 \\
11.300\end{array}$ & $\begin{array}{r}1055 \\
1056.3\end{array}$ & $\begin{array}{c}4.9 \\
183\end{array}$ & $\begin{array}{l}1105.3 \\
1106.7\end{array}$ & $\begin{array}{r}3.4 \\
11.3\end{array}$ & $\begin{array}{l}1154.8 \\
1156.2\end{array}$ & $\begin{array}{l}.4 .4 \\
7.3\end{array}$ & $\begin{array}{l}17040.0 \\
12052\end{array}$ & $\begin{array}{l}1.7 \\
50\end{array}$ & $\begin{array}{l}1253.1 \\
12542\end{array}$ & $\begin{array}{l}1.3 \\
3.5\end{array}$ & $\begin{array}{l}1154.1 \\
1155.4\end{array}$ & 2.4 & 1005.2 & 7.7 & 956.1 & 12.1 \\
\hline & & & & & & & & & & & 0.0 & & & 1006.4 & 32.0 & (301.0 & 57.8 \\
\hline $\begin{array}{r}1109 \\
1107.4\end{array}$ & $\begin{array}{r}12.030 \\
12.77\end{array}$ & $\begin{array}{l}10588.4 \\
1056.5\end{array}$ & $\begin{array}{r}21.3 \\
23.31\end{array}$ & $\begin{array}{l}1109.5 \\
1107.6\end{array}$ & $\begin{array}{l}12.0 \\
12.61\end{array}$ & $\begin{array}{l}1158.8 .8 \\
1157.1\end{array}$ & $\begin{array}{r}7.3 \\
7.63\end{array}$ & $\begin{array}{l}1207.7 \\
1206.2\end{array}$ & $\begin{array}{r}4.7 \\
4.75\end{array}$ & $\begin{array}{l}1256.4 \\
1255.2\end{array}$ & $\begin{array}{l}3.1 \\
3.1\end{array}$ & $\begin{array}{l}1156.6 \\
1155.4\end{array}$ & $\begin{array}{r}7.5 \\
7.76\end{array}$ & $\begin{array}{l}1008.1 \\
1006.5\end{array}$ & $\begin{array}{r}40.4 \\
47.37\end{array}$ & $\begin{array}{l}958.9 \\
957.2\end{array}$ & $\begin{array}{r}80.7 \\
100.39\end{array}$ \\
\hline 1108.5 & 11.95 & 1057.6 & 21.06 & 1108.9 & 11.94 & 1158.3 & 7.42 & 1207.1 & 4.93 & 1255.8 & 3.43 & 1156.1 & 7.74 & & & & \\
\hline 1105.8 & 5.200 & 1055.4 & 8.6 & 1107.9 & 5.1 & 1157.3 & 3.3 & 1206.1 & 2.2 & 1255.0 & 1.5 & 1154.8 & 3.4 & 1005.9 & 15.2 & 956.7 & 28.4 \\
\hline 1107 & 11.53 & 1056.8 & 20.36 & 1107.2 & 11.55 & 1156.5 & 7.01 & 1205.5 & 4.52 & 1254.4 & 3 & 1155.7 & 7.19 & & & & \\
\hline 1109.8 & 4.780 & 1059 & $\begin{array}{r}7.8 \\
7.0\end{array}$ & 1110.2 & 4.8 & 1159.5 & 3.1 & 1208.3 & 2.2 & 1256.9 & 1.6 & 1157.3 & 3.3 & 1008.7 & 14.2 & 959.6 & 26.4 \\
\hline $\begin{array}{l}1107.2 \\
1117\end{array}$ & 10.090 & $\begin{array}{l}1056.5 \\
10571\end{array}$ & 17.8 & $\begin{array}{l}1107.9 \\
11071\end{array}$ & 10.0 & $\begin{array}{l}1157.4 \\
1156\end{array}$ & 6.1 & 1206.3 & 3.9 & 1255.1 & 2.6 & $\begin{array}{l}1155.3 \\
1150\end{array}$ & 6.3 & 1006.5 & 34.3 & 957.3 & 68.3 \\
\hline $\begin{array}{l}1107 \\
1109\end{array}$ & $\begin{array}{l}6.030 \\
25.34\end{array}$ & $\begin{array}{l}1057.1 \\
1158.5\end{array}$ & $\begin{array}{r}10.0 \\
12.54\end{array}$ & $\begin{array}{l}1107.1 \\
1107.6\end{array}$ & $\begin{array}{l}6.1 \\
27\end{array}$ & $\begin{array}{l}1156.9 \\
1158.8\end{array}$ & $\begin{array}{r}3.9 \\
11.91\end{array}$ & $\begin{array}{l}1206.0 \\
1207.5\end{array}$ & $\begin{array}{l}2.6 \\
7.6\end{array}$ & $\begin{array}{l}1255.0 \\
1256.2\end{array}$ & $\begin{array}{r}1.8 \\
5.11\end{array}$ & $\begin{array}{l}1156.2 \\
1156.2\end{array}$ & $\begin{array}{r}3.9 \\
13.9\end{array}$ & 1007.0 & 18.3 & 957.6 & 34.7 \\
\hline 1107 & $\begin{array}{l}25.34 \\
26.29\end{array}$ & $\begin{array}{l}1106.5 \\
106.2\end{array}$ & $\begin{array}{r}12.54 \\
27.59\end{array}$ & 1156.4 & $\begin{array}{r}27 \\
12.02\end{array}$ & 1205.4 & 7.27 & $\begin{array}{l}1207.5 \\
1254.3\end{array}$ & $\begin{array}{r}7.6 \\
4.92\end{array}$ & $\begin{array}{l}1256.2 \\
1155.5\end{array}$ & $\begin{array}{r}5.11 \\
12.76\end{array}$ & 1156.3 & & & & & \\
\hline 1110.3 & 18.220 & 1059.5 & 29.0 & $\begin{array}{l}1110.4 \\
110.9\end{array}$ & 18.2 & 1160.0 & $\begin{array}{l}1.27 \\
12.1\end{array}$ & $\begin{array}{l}1208.9 \\
120.9\end{array}$ & $\begin{array}{r}4.92 \\
8.4\end{array}$ & 1258.0 & $\begin{array}{r}2.10 \\
6.0\end{array}$ & 1158.1 & 12.5 & 1009.4 & 49.4 & 960.2 & 86.9 \\
\hline 1104.1 & 2.550 & 1055.8 & 4.1 & 1107.4 & 2.5 & 1157.0 & 1.7 & 1206.1 & 1.2 & 1255.2 & 0.9 & 1155.6 & 1.7 & 1006.9 & 7.3 & 957.1 & 13.5 \\
\hline 1106.6 & 2.700 & 1056.2 & 4.1 & 1106.7 & 2.7 & 1156.1 & 1.9 & 1205.4 & 1.4 & 1254.2 & 1.0 & 1155.4 & 1.9 & 1006.2 & 6.4 & 956.9 & 10.7 \\
\hline 1106.1 & 21.69 & 1055.9 & 35.77 & 1106.4 & 21.65 & 1155.8 & 13.83 & 1204.8 & 9.23 & 1253.8 & 6.35 & 1155 & 14.05 & 1006.1 & 63.75 & & \\
\hline 1106.9 & 29.34 & 1056.5 & 57.34 & 1106.7 & 29.46 & 1156.1 & 16.34 & 1205.1 & 9.7 & 1254.1 & 6.06 & 1155.2 & 16.67 & 1006.1 & 124.1 & & \\
\hline 1107.4 & 13.590 & 1057.1 & 23.9 & 1107.4 & 13.7 & 1156.9 & 8.5 & 1205.8 & 5.7 & 1254.7 & 3.9 & 1155.8 & & 1006.8 & 48.2 & 957.5 & 101.6 \\
\hline 1106 & 4.660 & 1056.1 & 7.0 & 1106.7 & 4.6 & 1156.1 & 3.2 & 1205.3 & 2.3 & 1254.2 & 1.7 & 1155.4 & 3.3 & 1006.4 & 11.0 & 957.1 & 17.7 \\
\hline 1106.8 & 8.810 & 1056.7 & 14.8 & 1107.0 & 8.8 & 1156.5 & 5.6 & 1205.5 & 3.7 & 1254.4 & 2.5 & 1155.6 & 5.7 & 1006.7 & 26.9 & 957.6 & 51.2 \\
\hline 1106.7 & 11.310 & 1056.4 & 20.0 & 1106.6 & 11.3 & 1156.1 & 6.9 & 1205.2 & 4.4 & 1254.2 & 3.0 & 1155.3 & 7.0 & 1006.3 & 38.4 & 956.9 & 78.9 \\
\hline $\begin{array}{l}1107.7 \\
111067\end{array}$ & $\begin{array}{r}4.170 \\
11.090\end{array}$ & $\begin{array}{l}1057.1 \\
10565\end{array}$ & $\begin{array}{r}6.9 \\
185\end{array}$ & $\begin{array}{l}1107.4 \\
1107\end{array}$ & $\begin{array}{r}4.2 \\
111\end{array}$ & $\begin{array}{l}1156.7 \\
115 .\end{array}$ & 2.7 & $\begin{array}{l}1205.6 \\
105\end{array}$ & 1.8 & $\begin{array}{l}1254.4 \\
\end{array}$ & 1.3 & 1155.5 & 2.7 & 1006.6 & 12.5 & 957.3 & 23.4 \\
\hline $\begin{array}{l}1106.7 \\
1106.9\end{array}$ & $\begin{array}{l}\begin{array}{l}11.090 \\
11.320\end{array}\end{array}$ & $\begin{array}{l}1056.5 \\
1056.5\end{array}$ & $\begin{array}{l}18.5 \\
18.6\end{array}$ & $\begin{array}{l}1107.0 \\
1106.9\end{array}$ & $\begin{array}{l}11.1 \\
11.4\end{array}$ & $\begin{array}{l}1156.5 \\
1156.2\end{array}$ & $\begin{array}{l}7.2 \\
7.3\end{array}$ & $\begin{array}{l}12205.5 \\
1205.3\end{array}$ & $\begin{array}{l}4.8 \\
5.0\end{array}$ & $\begin{array}{l}1254.4 \\
1254.1\end{array}$ & $\begin{array}{l}3.4 \\
3.5\end{array}$ & $\begin{array}{l}1155.6 \\
1155.2\end{array}$ & $\begin{array}{l}7.3 \\
7.6\end{array}$ & $\begin{array}{l}1006.8 \\
1006.3\end{array}$ & $\begin{array}{l}34.3 \\
33.4\end{array}$ & $\begin{array}{l}957.4 \\
956.9\end{array}$ & $\begin{array}{l}65.2 \\
62.3\end{array}$ \\
\hline 1107.2 & $\begin{array}{l}11.32510 \\
1251\end{array}$ & 1056.7 & $\begin{array}{l}18.6 \\
21.5\end{array}$ & $\begin{array}{l}1106.9 \\
1107.2\end{array}$ & $\begin{array}{l}11.4 \\
12.6\end{array}$ & $\begin{array}{l}1156.2 \\
1156.6\end{array}$ & $\begin{array}{l}.3 \\
7.8\end{array}$ & $\begin{array}{l}1205.3 \\
1205.5\end{array}$ & $\begin{array}{l}5.0 \\
5.1\end{array}$ & $\begin{array}{l}1254.1 \\
1254.5\end{array}$ & $\begin{array}{l}3.5 \\
3.5\end{array}$ & $\begin{array}{l}11555.2 \\
1155.5\end{array}$ & $\begin{array}{l}7.6 \\
8.0\end{array}$ & $\begin{array}{l}10066.3 \\
10067\end{array}$ & $\begin{array}{l}33.4 \\
39.8\end{array}$ & $\begin{array}{l}956.9 \\
957.5\end{array}$ & $\begin{array}{l}62.3 \\
76.9\end{array}$ \\
\hline 1107.2 & 11.980 & 1057.1 & 21.9 & 1107.8 & 11.9 & 1157.7 & 7.1 & 1206.2 & 4.5 & $\begin{array}{l}1255.2 \\
\end{array}$ & 2.9 & 1155.5 & 7.4 & 1006.3 & 43.9 & 958.8 & 92.8 \\
\hline 1107.5 & 28.44 & 1057 & 55.43 & 1107.3 & 28.61 & 1156.6 & 15.92 & 1205.5 & 9.42 & 1254.4 & 5.88 & 1155.5 & 16.16 & 1006.5 & 119.96 & & \\
\hline 1105.7 & 46.490 & 1055.4 & 76.2 & 1006.5 & 130.9 & 956.6 & 237.9 & 1155.6 & 31.0 & 1204.6 & 21.8 & 1254.5 & 15.8 & 1303.8 & 11.3 & 1353.8 & 8.6 \\
\hline 1205 & 42.210 & 1155.5 & 81.0 & 1105.8 & 170.1 & 1056.1 & 415.4 & 1156.6 & 84.6 & 1205.9 & 42.0 & 1255.0 & 23.5 & 1304.5 & 13.9 & 1354.7 & 9.4 \\
\hline 1106.2 & 2.350 & 1056.1 & 3.5 & 1006.4 & 5.4 & 956.8 & 8.6 & 1156.5 & 1.7 & 1205.6 & 1.2 & 1255.7 & 0.9 & 1156.9 & 1.7 & & \\
\hline 1205.8 & 1.980 & 1156.1 & 3.2 & 1106.7 & 5.8 & 1254.7 & 1.0 & 1304.2 & 0.7 & 1254.6 & 1.2 & & & & & & \\
\hline 958 & 5.770 & 1157.4 & 0.9 & 1206.7 & 0.6 & 1256.1 & 0.5 & & 0.9 & & & & & & & & \\
\hline 1057.2 & 749.130 & 1157.4 & 99.8 & 1206.4 & 29.2 & 1255.2 & 7.2 & 1304.5 & 2.9 & 1354.0 & 1.9 & 1255.8 & 8.9 & & & & \\
\hline $\begin{array}{l}1107.4 \\
1108\end{array}$ & 2.110 & $\begin{array}{l}1057.3 \\
10757\end{array}$ & 3.5 & 1007.5 & 6.2 & $\begin{array}{l}957.7 \\
\end{array}$ & 12.4 & & 3.4 & $\begin{array}{l}1157.9 \\
1159\end{array}$ & 1.4 & 1206.7 & 1.0 & $\begin{array}{l}1255.6 \\
\end{array}$ & 0.7 & $\begin{array}{l}1156.8 \\
11556\end{array}$ & 1.4 \\
\hline $\begin{array}{l}108.1 \\
1007.7\end{array}$ & $\begin{array}{r}2.100 \\
11730\end{array}$ & $\begin{array}{l}1057.7 \\
9568 .\end{array}$ & $\begin{array}{r}3.4 \\
21.7\end{array}$ & $\begin{array}{r}1007.9 \\
906.8\end{array}$ & $\begin{array}{r}6.0 \\
468\end{array}$ & $\begin{array}{r}958.3 \\
10573\end{array}$ & $\begin{array}{r}13.3 \\
73\end{array}$ & $\begin{array}{l}1059.1 \\
11068\end{array}$ & $\begin{array}{l}3.9 \\
4.3\end{array}$ & $\begin{array}{l}1158.2 \\
1156 ?\end{array}$ & $\begin{array}{l}1.4 \\
2.9\end{array}$ & & $\begin{array}{l}1.0 \\
20\end{array}$ & $\begin{array}{l}1256.6 \\
\end{array}$ & $\begin{array}{l}0.7 \\
15\end{array}$ & $\begin{array}{l}1157.6 \\
11557\end{array}$ & 1.5 \\
\hline $\begin{array}{l}1007.1 \\
1007.4\end{array}$ & $\begin{array}{r}11 / . / 30 \\
4.880\end{array}$ & $\begin{array}{l}956.8 \\
956.9\end{array}$ & $\begin{array}{r}21.7 \\
8.1\end{array}$ & $\begin{array}{l}906.8 \\
906.9\end{array}$ & $\begin{array}{l}46.8 \\
14.2\end{array}$ & $\begin{array}{r}1057.3 \\
857.1\end{array}$ & $\begin{array}{r}7.3 \\
27.5\end{array}$ & $\begin{array}{r}1106.8 \\
957.5\end{array}$ & $\begin{array}{l}4.3 \\
8.0\end{array}$ & $\begin{array}{l}1156.2 \\
1057.1\end{array}$ & $\begin{array}{l}2.9 \\
3.2\end{array}$ & $\begin{array}{l}1205.5 \\
1106.7\end{array}$ & $\begin{array}{l}2.0 \\
2.2\end{array}$ & $\begin{array}{l}1254.8 \\
1156.3\end{array}$ & $\begin{array}{l}1.5 \\
1.5\end{array}$ & $\begin{array}{l}1155.7 \\
1205.7\end{array}$ & $\begin{array}{l}3.0 \\
1.1\end{array}$ \\
\hline 957.6 & $\begin{array}{r}4.8000 \\
18.060\end{array}$ & $\begin{array}{l}900.9 \\
907.3\end{array}$ & $\begin{array}{r}8.1 \\
42.0\end{array}$ & 857.3 & $\begin{array}{l}14.2 \\
112.6\end{array}$ & $\begin{array}{l}80.1 .1 \\
958.1\end{array}$ & $\begin{array}{l}18.8 \\
18.8\end{array}$ & $\begin{array}{r}95.5 \\
1007.3\end{array}$ & $\begin{array}{l}8.0 \\
9.3\end{array}$ & $\begin{array}{l}105 \% .1 \\
1057.4\end{array}$ & $\begin{array}{l}3.2 \\
4.8\end{array}$ & $\begin{array}{l}1706 . / \\
1106.9\end{array}$ & $\begin{array}{l}2.2 \\
2.9\end{array}$ & $\begin{array}{l}11566.3 \\
11563\end{array}$ & $\begin{array}{l}1.5 \\
1.8\end{array}$ & $\begin{array}{l}\text { T200.7. } \\
1205.5\end{array}$ & $\begin{array}{l}1.1 \\
1.3\end{array}$ \\
\hline 1204.7 & 12.190 & 1155.2 & 20.7 & 1105.6 & 36.9 & 1055.7 & 71.2 & 1156.1 & $\begin{array}{l}.9 .3 \\
20.7\end{array}$ & 1205.5 & $\begin{array}{r}.8 .8 \\
12.4\end{array}$ & 1254.9 & $\begin{array}{l}2.9 \\
7.7\end{array}$ & 1304.4 & $\begin{array}{l}1.8 \\
5.0\end{array}$ & $\begin{array}{r}1205.5 \\
1354.3\end{array}$ & $\begin{array}{l}1.3 \\
3.4\end{array}$ \\
\hline 1204.9 & 68.020 & 1155.4 & 120.9 & 1205.1 & 66.9 & 1254.3 & 39.1 & 1303.8 & 24.6 & 1403.0 & 9.2 & 1452 & 6.1 & 1353.7 & 15.5 & 1254.7 & 44.2 \\
\hline 1106.4 & 5.900 & 1056.1 & 9.5 & 956.4 & 28.4 & 1057.4 & 9.4 & 1156.6 & 3.9 & 1205.7 & 2.8 & 1254 & 2.0 & 1155.6 & 4.0 & & \\
\hline 1105.3 & 7.140 & 1054.8 & 12.3 & 1004.9 & 22.3 & 955.2 & 44.1 & 105 & 12.2 & 1155.3 & 4.5 & 1204 & 3.0 & 1254.2 & 2.0 & 1155.4 & 4.6 \\
\hline 1204.1 & 7.510 & 1154 & 12.4 & 110. & 21.6 & 105 & 40.1 & & 12.6 & 1204.5 & $\begin{array}{r}7.6 \\
70 .\end{array}$ & & 4.9 & & 3.3 & 1253.9 & 5.1 \\
\hline $\begin{array}{l}1205.1 \\
1105.1\end{array}$ & $\begin{array}{l}4.42 \\
552\end{array}$ & & 7.11 & & 11.9 & $\begin{array}{l}105 \\
0.5\end{array}$ & 20.7 & & 39.0 & & 79.5 & & 20.4 & & 6.9 & & 2.9 \\
\hline $\begin{array}{l}10105.6 \\
1107.3\end{array}$ & $\begin{array}{r}5.520 \\
10.930\end{array}$ & $\begin{array}{l}1055.5 \\
1056.9\end{array}$ & $\begin{array}{r}8.8 \\
18.4\end{array}$ & $\begin{array}{l}1007.0 \\
1006.9\end{array}$ & $\begin{array}{l}\begin{array}{l}44.8 \\
326\end{array} \\
3\end{array}$ & $\begin{array}{l}957.3 \\
9572\end{array}$ & $\begin{array}{l}26.4 \\
62.0\end{array}$ & & $\begin{array}{l}8.7 \\
18.5\end{array}$ & $\begin{array}{l}1157.3 \\
1157\end{array}$ & $\begin{array}{l}3.6 \\
7.0\end{array}$ & & $\begin{array}{l}2.5 \\
46\end{array}$ & & $\begin{array}{l}1.8 \\
31\end{array}$ & $\begin{array}{l}11156.6 \\
111566\end{array}$ & $\begin{array}{l}3.7 \\
7.3\end{array}$ \\
\hline 1108 & 17.950 & $\begin{array}{l}1056.9 \\
1057.7\end{array}$ & $\begin{array}{l}18.4 \\
31.6\end{array}$ & $\begin{array}{l}1006.9 \\
1007.5\end{array}$ & $\begin{array}{l}32.6 \\
59.6\end{array}$ & $\begin{array}{l}957.2 \\
957.6\end{array}$ & $\begin{array}{l}62.0 \\
120.8\end{array}$ & $\begin{array}{l}1058.2 \\
1058.8\end{array}$ & $\begin{array}{l}18.5 \\
31.5\end{array}$ & 1158.1 & $\begin{array}{r}71.0 \\
11.1\end{array}$ & $\begin{array}{l}1206 \\
1207\end{array}$ & $\begin{array}{l}4.6 \\
7.2\end{array}$ & $\begin{array}{l}1255.8 \\
1256.1\end{array}$ & $\begin{array}{l}3.1 \\
4.7\end{array}$ & & 7.3 \\
\hline 11167 & 9290 & 1058.4 & 18.5 & 10076 & 35.8 & 957.9 & 75.1 & $\begin{array}{l}10593 \\
1059\end{array}$ & $\begin{array}{l}19.2 \\
\text {. }\end{array}$ & 1158.4 & 64 & & 38 & & 25 & 1157.0 & 6.3 \\
\hline 1107.5 & 5.680 & 105 & 9.8 & 100 & 17.7 & 957 & 34.5 & & 9.7 & & 3.6 & 1206 & 2.4 & & 1.6 & 1156.8 & 3.6 \\
\hline 1108.9 & 5.850 & 105 & 10.0 & 1008 & 17.9 & & 33.4 & & 9.7 & & 3.6 & & 2.3 & & 1.6 & & 3.7 \\
\hline 1108.3 & 6.150 & 1058.2 & 10.5 & 1008 & 18.7 & 958 & 34.1 & 105 & 10.0 & 115 & 3.8 & 1207. & 2.5 & 1256 & 1.7 & 1157.3 & 3.9 \\
\hline $\begin{array}{l}1106.6 \\
111068\end{array}$ & 18.270 & 1056 & 32.3 & 100 & 60.4 & 957 & 121.5 & & 31.7 & $\begin{array}{l}1157.3 \\
1156\end{array}$ & 11.0 & 1206.5 & 7.2 & 1255.7 & 4.8 & 1156.3 & 11.2 \\
\hline $\begin{array}{l}106 \\
1107\end{array}$ & $\begin{array}{l}22.34 \\
1207\end{array}$ & $\begin{array}{l}105 \\
105\end{array}$ & 43.4 & & 13.5 & & $\begin{array}{r}8.3 \\
76.5\end{array}$ & & 5.4 & & 13.2 & & & & & & \\
\hline $\begin{array}{l}11107 . \\
1107 .\end{array}$ & $\begin{array}{r}12.070 \\
6560\end{array}$ & $\begin{array}{l}1056.7 \\
10563\end{array}$ & $\begin{array}{l}20.9 \\
110\end{array}$ & $\begin{array}{l}100 \\
100\end{array}$ & $\begin{array}{l}38.4 \\
192\end{array}$ & $\begin{array}{l}957 \\
956\end{array}$ & $\begin{array}{l}\begin{array}{r}66.5 \\
35.9\end{array} \\
3\end{array}$ & $\begin{array}{l}1057 \\
1057\end{array}$ & $\begin{array}{l}20.6 \\
10.9\end{array}$ & $\begin{array}{l}1157.0 \\
1156\end{array}$ & $\begin{array}{l}7.2 \\
4.3\end{array}$ & $\begin{array}{l}1206.2 \\
12056\end{array}$ & $\begin{array}{l}4.7 \\
2.9\end{array}$ & $\begin{array}{l}1255.3 \\
12548\end{array}$ & $\begin{array}{l}3.3 \\
20\end{array}$ & $\begin{array}{l}1156.2 \\
1155.7\end{array}$ & $\begin{array}{l}7.4 \\
4.5\end{array}$ \\
\hline 1107.7 & $\begin{array}{l}0.000 \\
15.800\end{array}$ & $\begin{array}{l}1057.6 \\
1050.3\end{array}$ & $\begin{array}{r}11.0 \\
258\end{array}$ & $\begin{array}{l}1007.4 \\
1007.9\end{array}$ & $\begin{array}{l}19.2 \\
44.4\end{array}$ & $\begin{array}{l}950.5 \\
958.2\end{array}$ & $\begin{array}{l}35.9 \\
82.8\end{array}$ & 1059 & $\begin{array}{l}20.9 \\
25.5\end{array}$ & $\begin{array}{l}1150.6 \\
1158.4\end{array}$ & $\begin{array}{c}4.3 \\
10.3\end{array}$ & 1207 & $\begin{array}{l}.9 \\
7.0\end{array}$ & $\begin{array}{l}1254 \\
1255\end{array}$ & $\begin{array}{l}.0 \\
4.9\end{array}$ & $\begin{array}{l}1150.1 \\
1156.8\end{array}$ & $\begin{array}{r}4.5 \\
10.5\end{array}$ \\
\hline 1107.1 & 12.900 & 1056.9 & 22.8 & 100 & 43.1 & 958.5 & 88.5 & & 22.5 & & 7.9 & & 5.1 & & 3.5 & & 8.1 \\
\hline 1108.7 & 7.310 & & 12.3 & & 21.9 & & 41.7 & & 12.4 & & 4.7 & & 3.2 & & & & 4.9 \\
\hline 66.4 & 5.690 & 1056 & 9.5 & & 16.9 & 956 & 32.0 & & 9.6 & & 3.6 & 120 & 2.5 & & 1.7 & 115 & 3.8 \\
\hline 1106 & 7.380 & 1056.6 & 12.5 & & 22.3 & 956.9 & 42.8 & & 12.3 & & 4.7 & & 3.1 & 1255.3 & 2.2 & 1156.0 & 4.9 \\
\hline 1106.7 & 6.560 & 1056.8 & 10.9 & & 19.2 & 957.2 & 36.2 & & 10.7 & 1157.5 & 4.2 & & 2.8 & & 2.0 & & 4.4 \\
\hline $\begin{array}{l}\begin{array}{l}1107.4 \\
1107.3\end{array}\end{array}$ & $\begin{array}{l}6.260 \\
6.690\end{array}$ & $\begin{array}{l}1057.1 \\
1057.4\end{array}$ & $\begin{array}{l}10.4 \\
11.2\end{array}$ & $\begin{array}{l}100 \\
100\end{array}$ & $\begin{array}{l}18.4 \\
19.8\end{array}$ & & $\begin{array}{l}34.7 \\
37.6\end{array}$ & & $\begin{array}{l}10.4 \\
11.0\end{array}$ & & $\begin{array}{l}4.1 \\
4.3\end{array}$ & & $\begin{array}{l}2.8 \\
2.9\end{array}$ & & $\begin{array}{l}2.0 \\
2.0\end{array}$ & $\begin{array}{l}1155.8 .8 \\
1157.4\end{array}$ & $\begin{array}{l}4.3 \\
4.5\end{array}$ \\
\hline $\begin{array}{l}1107.3 \\
1106\end{array}$ & $\begin{array}{l}6.680 \\
3.860\end{array}$ & $\begin{array}{r}1057.4 \\
1056\end{array}$ & $\begin{array}{r}11.2 \\
64\end{array}$ & $\begin{array}{l}1007.6 \\
10062\end{array}$ & $\begin{array}{l}19.8 \\
112\end{array}$ & $\begin{array}{l}955.8 \\
956.6\end{array}$ & $\begin{array}{l}37.6 \\
21.5\end{array}$ & $\begin{array}{l}10558.8 \\
10573\end{array}$ & 6.3 & $\begin{array}{l}1158.0 \\
1156.4\end{array}$ & 25 & $\begin{array}{l}1207.3 .3 \\
1205.5\end{array}$ & 17 & 12546 & $\begin{array}{l}2.0 \\
1.2\end{array}$ & $\begin{array}{l}1155.4 \\
1155.7\end{array}$ & $\begin{array}{l}4.5 \\
2.6\end{array}$ \\
\hline & & & & & & & & & & & & & & & & & \\
\hline
\end{tabular}




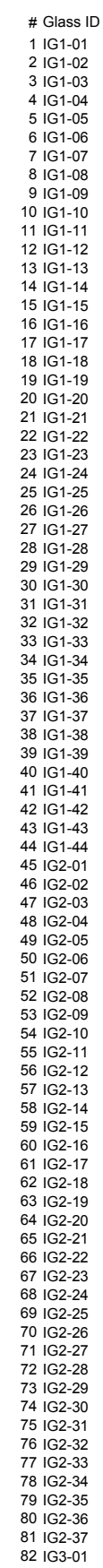

TCLP Se TCLP Zn TCLPAI TCLP B TCLP Sb TCLP TI TCLPV QPCT B $\left(g / \mathrm{m}^{2}\right)$ Q PCT Li $\left(g / \mathrm{m}^{2}\right)$ Q PCT Na $\left(g / \mathrm{m}^{2}\right)$ Q PCT Si $\left(g / \mathrm{m}^{2}\right)$ Q PCT pH

CCC PCT B $\left(g / \mathrm{m}^{2}\right) \operatorname{CCC~PCT~Li~}\left(g / \mathrm{m}^{2}\right) \operatorname{CCC~PCT~Na~}\left(\mathrm{g} / \mathrm{m}^{2}\right) \operatorname{CCC~PCT~Si~}\left(\mathrm{g} / \mathrm{m}^{2}\right) \operatorname{CCC~PCT~pH~T1~}\left({ }^{\circ} \mathrm{C}\right)$

$\begin{array}{llll}2.790 & 1.636 & 2.279 & 0.670 \\ 3.546 & 0.877 & 2.094 & 0.426\end{array}$

\begin{tabular}{|c|c|c|c|c|}
\hline 3.546 & 0.877 & 2.984 & 0.423 & \\
\hline 21.404 & 6.168 & 16.491 & 2.569 & \\
\hline $\begin{array}{l}0.384 \\
4.061\end{array}$ & 1.686 & & & \\
\hline & & 2.330 & 0.401 & \\
\hline 9.634 & 9.516 & 8.923 & 1.583 & \\
\hline 0.282 & 0.362 & 0.063 & 0.258 & \\
\hline $\begin{array}{l}0.183 \\
2865\end{array}$ & 0.283 & 0.339 & 0.127 & \\
\hline $\begin{array}{r}2.8655 \\
14.658\end{array}$ & $\begin{array}{r}1.115 \\
10665\end{array}$ & $\begin{array}{l}2.292 \\
95512\end{array}$ & $\begin{array}{l}0.250 \\
5635\end{array}$ & \\
\hline $\begin{array}{r}0.050 \\
0.291\end{array}$ & $\begin{array}{r}10.665 \\
0.38\end{array}$ & $\begin{array}{l}.512 \\
0.938\end{array}$ & $\begin{array}{l}5.635 \\
0.283\end{array}$ & \\
\hline 4.605 & & 3.999 & 0.155 & \\
\hline 30.051 & $\begin{array}{l}23.368 \\
\end{array}$ & 29.596 & 20.071 & \\
\hline & & 0.650 & 0.195 & \\
\hline $\begin{array}{l}0.493 \\
0.353\end{array}$ & 0.395 & 0.584 & 0.203 & \\
\hline 0.202 & $\begin{array}{l}0.276 \\
0.066\end{array}$ & $\begin{array}{l}.0 .594 \\
0.335\end{array}$ & 0.119 & \\
\hline 0.318 & 0.290 & 0.353 & 0.136 & \\
\hline 0.279 & 0.251 & 0.323 & 0.125 & \\
\hline 0.254 & 0.219 & 0.282 & 0.110 & \\
\hline 0.905 & 0.736 & 0.738 & 0.130 & \\
\hline $\begin{array}{l}0.396 \\
0.176\end{array}$ & $\begin{array}{l}0.223 \\
0.207\end{array}$ & $\begin{array}{l}0.396 \\
0.328\end{array}$ & $\begin{array}{l}0.129 \\
0.112\end{array}$ & \\
\hline $\begin{array}{l}1.042 \\
1.042\end{array}$ & $\begin{array}{l}0.207 \\
0.725\end{array}$ & $\begin{array}{l}0.328 \\
0.750\end{array}$ & 0.112 & \\
\hline 0.450 & $\begin{array}{l}0.4250 \\
0.40\end{array}$ & 0.632 & 0.145 & \\
\hline 3.143 & 1.812 & 2.495 & & \\
\hline 0.151 & 0.293 & 0.336 & 0.118 & \\
\hline 0.181 & 0.315 & 0.357 & 0.130 & \\
\hline 0.227 & 0.357 & 0.175 & 0.205 & \\
\hline 12.142 & 8.152 & 8.975 & 3.428 & \\
\hline $\begin{array}{r}1.512 \\
0\end{array}$ & $\begin{array}{l}1.270 \\
0\end{array}$ & $\begin{array}{l}0.783 \\
0.62\end{array}$ & 0.265 & \\
\hline $\begin{array}{l}0.194 \\
5.822\end{array}$ & $\begin{array}{l}0.289 \\
9968\end{array}$ & $\begin{array}{r}0.268 \\
1061\end{array}$ & $\begin{array}{l}0.150 \\
4288\end{array}$ & \\
\hline $\begin{array}{l}5.8222 \\
0.543\end{array}$ & $\begin{array}{l}0.058 \\
0.541\end{array}$ & $\begin{array}{r}0.615 \\
0.119\end{array}$ & $\begin{array}{l}\text {.4.825 } \\
0.257\end{array}$ & \\
\hline 0.440 & 18.427 & 0.358 & $\begin{array}{l}.237 \\
0.152\end{array}$ & \\
\hline 8.858 & 8.973 & 8.924 & 4.634 & \\
\hline 7.792 & 0.057 & 6.358 & 0.484 & \\
\hline 1.225 & 1.108 & 0.737 & 0.315 & \\
\hline 1.694 & 1.396 & 1.080 & 0.170 & \\
\hline $\begin{array}{r}4.115 \\
\end{array}$ & $\begin{array}{l}2.835 \\
887\end{array}$ & $\begin{array}{r}3.623 \\
16.8\end{array}$ & 1.076 & \\
\hline 20.179 & 8.174 & 16.802 & & \\
\hline 0.202 & 0.264 & 0.307 & 0.126 & \\
\hline 0.725 & 0.355 & 0.552 & 0.120 & \\
\hline 0.580 & 0.573 & 0.125 & 0.275 & \\
\hline 0.226 & & 0.213 & 0.131 & 8.500 \\
\hline $\begin{array}{c}0.099 \\
12.404\end{array}$ & & $\begin{array}{l}5.1049 \\
554\end{array}$ & 0.0414 & $\begin{array}{l}7.680 \\
6780\end{array}$ \\
\hline 2.884 & 2601 & 1.597 & 0.250 & $\begin{array}{r}6.790 \\
10.880\end{array}$ \\
\hline 0.490 & & 1.429 & 0.163 & 11.270 \\
\hline 2.067 & 1.661 & 0.525 & 0.184 & 9.500 \\
\hline 0.307 & & 0.351 & 0.072 & 9.340 \\
\hline 0.440 & & 0.694 & 0.208 & 10.110 \\
\hline 0.100 & 0.223 & 0.087 & 0.059 & 9.670 \\
\hline $\begin{array}{l}1.397 \\
1.987\end{array}$ & 1.377 & 1.427 & 0.246 & 10.440 \\
\hline $\begin{array}{l}1.981 \\
11\end{array}$ & 10.00 & $\begin{array}{l}2.2066 \\
7\end{array}$ & $\begin{array}{l}1.376 \\
\end{array}$ & $\begin{array}{r}11.720 \\
8660\end{array}$ \\
\hline $\begin{array}{r}0.673 \\
0.289\end{array}$ & 0.433 & 0.023 & $\begin{array}{l}. .123 \\
0.210\end{array}$ & $\begin{array}{r}8.600 \\
10.190\end{array}$ \\
\hline 0.425 & & 0.593 & 0.185 & 10.440 \\
\hline 0.739 & 1.016 & 1.139 & 0.269 & \\
\hline 0.766 & 0.639 & 0.546 & 0.086 & 10.010 \\
\hline 0.213 & 0.378 & 0.335 & 0.086 & 9.900 \\
\hline 0.391 & 0.478 & 0.783 & 0.156 & 10.780 \\
\hline 0.729 & 0.959 & $\begin{array}{l}1.102 \\
\end{array}$ & 0.261 & 11.230 \\
\hline $\begin{array}{l}0.6222 \\
0.253\end{array}$ & $\begin{array}{l}0.846 \\
0.526\end{array}$ & $\begin{array}{l}0.946 \\
0.428\end{array}$ & $\begin{array}{l}0.233 \\
0.103\end{array}$ & $\begin{array}{l}11.130 \\
1020\end{array}$ \\
\hline 0.542 & $\begin{array}{l}1.160 \\
1.0\end{array}$ & $\begin{array}{l}.420 \\
0.756\end{array}$ & $\begin{array}{l}.103 \\
0.186\end{array}$ & $\begin{array}{l}10.260 \\
113.300\end{array}$ \\
\hline 0.911 & 0.735 & 0.786 & 0.115 & 10.230 \\
\hline 0.379 & 0.72 & 0.563 & 0.095 & 10 \\
\hline 0.431 & 0.811 & 0.619 & 0.050 & 10.830 \\
\hline 0.303 & 0.533 & 0.481 & 0.144 & \\
\hline 0.287 & 0.500 & 0.371 & 0.106 & 10.050 \\
\hline 0.380 & 0.730 & 0.727 & 0.110 & 10.540 \\
\hline 0.481 & 0.503 & 0.549 & 0.121 & 10.270 \\
\hline 0.558 & 0.647 & 0.040 & 0.360 & 9.230 \\
\hline 1.617 & 1.506 & 0.942 & 0.320 & 9.990 \\
\hline 0.566 & 0.681 & 0.653 & 0.136 & 10.110 \\
\hline 0.501 & $0.54 \%$ & 0.581 & 0.131 & 10.260 \\
\hline 0 & 0.007 & 0.074 & & $\begin{array}{r}10.220 \\
0.220\end{array}$ \\
\hline 0.408 & 0.410 & 0.493 & 0.122 & \\
\hline 0.378 & 0.461 & 0.466 & 0.119 & 10.140 \\
\hline 0.449 & 0.590 & 0.473 & 0.125 & 10.180 \\
\hline 0.542 & 0.572 & & & \\
\hline
\end{tabular}

2 1 $163-01$ 
15 IG1-15

$7161-17$

$19161-19$

$20161-20$

$22161-22$

$23161-23$
$2416-24$

$26161-26$

27 IG1-27

$28161-28$
$29161-29$
$316-190$

I0 I11-30

31 IG1-31

$3161-33$
$34 \mid 161-34$

34 III-34
$3516-135$
$\quad 5$

$36161-36$
$37161-37$

38 IG1-38

$39161-39$
$\quad 4161-19$

$40161-40$
$41 / 61-41$
$4216-12$

42 IG1-42

$43161-43$

44 IG1-44
45 IG2-01

46 162-02

$47162-03$
48 162-04
4

$50162-06$

$51 \mid 2-07$
$551202-28$

$3162-09$

54 IG2-10

(5)

列

162-17

$4162-20$
$5162-21$
6

6 IG2-22

$67162-23$
$68162-24$
6016225

9 IG2-25

$70162-26$

721622.28

$72162-28$
$73162-29$

$74162-30$
755623

$76162-32$

77 IG2-33

71025.35

$80162-26$
$81162-37$

31 162-37
$82163-01$ 


\begin{tabular}{|c|c|c|c|c|c|c|}
\hline \# Glass ID & Study & Reference & Al2O3-t & B203-t & CaO-t & $\mathrm{Fe} 2 \mathrm{O}-\mathrm{t}$ \\
\hline 83 IG3-02b & INEEL CVS Phase 3 & Scholes et al. 2000 & 0.0450 & 0.1625 & 0.1057 & 0.0046 \\
\hline 84 IG3-03 & INEEL CVS Phase 3 & Scholes et al. 2000 & 0.0400 & 0.0500 & 0.0000 & 0.0000 \\
\hline $\begin{array}{l}85 \text { IG33-04 } \\
86 \text { IG-05 }\end{array}$ & $\begin{array}{l}\text { INEEE CVS Phase } 3 \\
\text { INEL CVS Phase }\end{array}$ & $\begin{array}{l}\text { Scholes et al. } 2000 \\
\text { Scholose }\end{array}$ & $\begin{array}{l}0.0350 \\
0.0350\end{array}$ & 0.1800 & 0.0000 & $\begin{array}{l}0.0300 \\
0.0000\end{array}$ \\
\hline 87 IG3-06 & INEEL CVS Phase 3 & $\begin{array}{l}\text { Scholese et al. } 2000 \\
\text { Scholes et al. } 2000\end{array}$ & $\begin{array}{l}0.0350 \\
0.0400\end{array}$ & $\begin{array}{l}0.0500 \\
0.0500\end{array}$ & $\begin{array}{l}0.1200 \\
0.1200\end{array}$ & $\begin{array}{l}0.00000 \\
0.0000\end{array}$ \\
\hline 88 IG3-07 & INEEL CVS Phase 3 & Scholes et al. 2000 & 0.1690 & 0.1800 & 0.0000 & 0.0800 \\
\hline 89 IG3-08b & INEEL CVS Phase 3 & Scholes et al. 2000 & 0.0535 & 0.1538 & 0.0146 & 0.0628 \\
\hline $\begin{array}{l}90 \text { IG3-09 } \\
91 / \mathrm{I} 3-10 \mathrm{~b}\end{array}$ & $\begin{array}{l}\text { INEEL CVS Phase } 3 \\
\text { INEEL CVS Phase }\end{array}$ & $\begin{array}{l}\text { Scholes et all. } 2000 \\
\text { Scholes et al. } 2000\end{array}$ & $\begin{array}{l}0.0350 \\
0.0490\end{array}$ & $\begin{array}{l}0.0500 \\
0.1625\end{array}$ & $\begin{array}{l}0.0000 \\
0.0097\end{array}$ & $\begin{array}{l}0.0800 \\
0.0457\end{array}$ \\
\hline 92 IG3-11 & INEEL CVS Phase 3 & $\begin{array}{l}\text { Scholes et alal. } 2000 \\
\text { Scholes et al. } 2000\end{array}$ & $\begin{array}{l}0.0490 \\
0.1205\end{array}$ & $\begin{array}{l}0.1625 \\
0.0500\end{array}$ & $\begin{array}{l}.0 .097 / \\
0.1200\end{array}$ & $\begin{array}{l}0.0457 \\
0.0800\end{array}$ \\
\hline 93 IG3-12 & INEEL CVS Phase 3 & Scholes et al. 2000 & 0.0350 & 0.1800 & 0.0899 & $\begin{array}{l}0.0800 \\
0.0300\end{array}$ \\
\hline 94 IG3-13 & INEEL CVS P P 3 & Scholes et al. 2000 & 0.0400 & 0.0754 & 0.0000 & 0.0800 \\
\hline 95 IG3-14 & INEEL CVS Phase 3 & Scholes et al. 2000 & 0.0800 & 0.0600 & 0.0400 & 0.0300 \\
\hline 96 IG3-15 & INEEL CVS Phase 3 & Scholes et al. 2000 & 0.1000 & 0.0600 & 0.0800 & 0.0050 \\
\hline 97 IG3-16 & INEEL CVS Phase 3 & Scholes et al. 2000 & 0.0989 & 0.0751 & 0.0800 & 0.0050 \\
\hline $\begin{array}{l}98 \text { IG3-17 } \\
99 \text { IG3-18 }\end{array}$ & INEEL CVS Phase 3 & Scholes et al. 2000 & 0.0893 & 0.0600 & 0.0800 & 0.0300 \\
\hline $\begin{array}{l}100 \text { IG3-19 } \\
100\end{array}$ & $\begin{array}{l}\text { INEEE CVS Phase } 3 \\
\text { INEL CVS Phase }\end{array}$ & $\begin{array}{l}\text { Scholes et al. } 20000 \\
\text { Sccles ental } 2000\end{array}$ & $\begin{array}{l}0.1200 \\
0.1200\end{array}$ & $\begin{array}{l}0.0600 \\
0.0600\end{array}$ & $\begin{array}{l}0.0440 \\
0.0800\end{array}$ & $\begin{array}{l}0.0050 \\
0.0150\end{array}$ \\
\hline 101 IG $3-20$ & INEEL CVS Phase 3 & 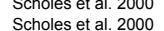 & 0.0800 & $\begin{array}{l}0.0600 \\
0.1200\end{array}$ & 0.0800 & $\begin{array}{l}0.0150 \\
0.0300\end{array}$ \\
\hline 102 IG $3-21$ & INEEL CVS Phase 3 & Scholes et al. 2000 & & & & \\
\hline 103 IG3-22 & INEEL CVS P P 3 & Scholes et al. 2000 & 0.1000 & 0.0600 & 0.0400 & 0.0300 \\
\hline 104 IG3-23 & INEEL CVS Phase 3 & Scholes et al. 2000 & 0.1200 & & 0.0400 & 0.0050 \\
\hline 105 IG3-24 & INEEL CVS Phase 3 & Scholes et al. 2000 & 0.1200 & 0.0600 & 0.0400 & 0.0050 \\
\hline 106 IG3-25 & INEEL CVS Phase 3 & Scholes et al. 2000 & 0.1200 & 0.0600 & 0.0400 & 0.0050 \\
\hline 107 IG3-26 & INEEL CVS Phase 3 & Scholes et al. 2000 & 0.1200 & 0.0843 & 0.0800 & 0.0150 \\
\hline $10863-27$ & INEEL CYS Phase 3 & $\begin{array}{l}\text { Shcholes et al. } 20000 \\
\text { Schela }\end{array}$ & $\begin{array}{l}0.0843 \\
0.0850\end{array}$ & 0.0917 & $\begin{array}{l}0.0483 \\
0.047\end{array}$ & $\begin{array}{l}0.0226 \\
0.028\end{array}$ \\
\hline $\begin{array}{l}1 \\
1101033-20 \\
103\end{array}$ & $\begin{array}{l}\text { INEFE CVS Phase } \\
\text { INES Pase }\end{array}$ & 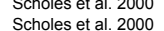 & 0.0850 & 0.0925 & $\begin{array}{l}0.0487 \\
0.0008\end{array}$ & $\begin{array}{l}0.0228 \\
0.0005\end{array}$ \\
\hline $1111 \mathrm{IG} 3-30$ & INEFL CVS Phase 3 & Scholes et al. 2000 & 0.0375 & 0.1250 & 0.0009 & $\begin{array}{l}0.0005 \\
0.0005\end{array}$ \\
\hline 112 CVS1-1 & Hanford CVS 1 & Hrma et al. 1994 & 0.0636 & 0.1142 & 0.0275 & 0.0568 \\
\hline 113 CVS1-2 & Hanford CVS 1 & Hrma et al. 1994 & 0.1500 & & 0.1000 & 0.0200 \\
\hline 114 CVS1-3 & Hanford CVS 1 & Hrma et al. 1994 & 0.1400 & 0.2000 & 0.0000 & 0.0200 \\
\hline 115 CVS1-4 & Hanford CVS 1 & Hrma et al. 1994 & 0.0000 & 0.2000 & 0.0200 & 0.0200 \\
\hline 1116 CVS1-5 & Hanford CVS 1 & Hrma et al. 1994 & 0.0800 & 0.0500 & 0.0000 & 0.1500 \\
\hline The & Hantord CVS 1 & Hrma et al. 1994 & 0.0000 & 0.2000 & 0.0000 & 0.0200 \\
\hline 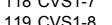 & $\begin{array}{l}\text { Hanford CVS } 1 \\
\text { Hanford CVS } 1\end{array}$ & Hrme etal. T994 & 0.0000 & 0.0500 & $\begin{array}{l}0.1000 \\
0.0000\end{array}$ & $\begin{array}{l}0.0336 \\
0.1500\end{array}$ \\
\hline 120 CVS1-9 & Hanford CVS 1 & Hrma et al. 1994 & 0.0000 & 0.1962 & 0.0000 & 0.1400 \\
\hline 121 CVS1-10 & Hanford CVS 1 & Hrma et al. 1994 & 0.1200 & 0.0851 & 0.0000 & 0.0200 \\
\hline 122 CVS1-11 & Hanford CVS 1 & Hrma et al. 1994 & 0.1400 & 0.1549 & 0.1000 & 0.0200 \\
\hline 123 CVS1-12 & Hanford CVS 1 & Hrma et al. 1994 & 0.0000 & 0.1764 & 0.1000 & 0.1500 \\
\hline 124 CVS1-13 & Hanford CVS 1 & Hrma et al. 1994 & 0.0038 & 0.2000 & 0.0000 & 0.0200 \\
\hline 125 CVS1-14 & Hanford CVS 1 & Hrma et al. 1994 & 0.0238 & 0.2000 & 0.0000 & 0.0200 \\
\hline $\begin{array}{l}126 \mathrm{CVS}-15 \\
120116\end{array}$ & $\begin{array}{l}\text { Hantord CVS } 1 \\
\text { Hofrd }\end{array}$ & Hrma et ala. 1994 & 0.0000 & 0.0500 & 0.0000 & 0.0200 \\
\hline 1. & 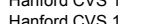 & 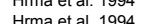 & 0.1442 & 0.0500 & 0.0000 & $\begin{array}{l}0.0858 \\
0.1400\end{array}$ \\
\hline 129 CVS1-18 & Hanford CVS 1 & Hrma et al. 1994 & 0.0000 & $\begin{array}{l}0.0500 \\
0.0500\end{array}$ & $\begin{array}{l}0.10200 \\
0.0200\end{array}$ & $\begin{array}{l}0.1400 \\
0.0200\end{array}$ \\
\hline 130 CVS1-19 & Hanford CVS 1 & Hrma et al. 1994 & 0.0636 & 0.1142 & 0.0275 & 0.0568 \\
\hline 131 CVS1-20 & Hanford CVS 1 & Hrma et al. 1994 & 0.0636 & 0.1142 & 0.0275 & 0.0568 \\
\hline 132 CVS1-21 & Hanford CVS 1 & Hrma et al. 1994 & 0.0000 & 0.2000 & 0.0200 & 0.0200 \\
\hline 133 CVS1-22 & Hanford CVS 1 & Hrma et al. 1994 & 0.0000 & 0.0500 & 0.0000 & 0.1500 \\
\hline 134 CVS1-23 & Hanford CVS 1 & Hrma et al. 1994 & 0.0456 & 0.0956 & 0.0289 & 0.1179 \\
\hline $135 \mathrm{cVSS} 2-1$ & Hantord CVS 2 & Hrma et al. 1994 & 0.0800 & 0.0874 & 0.0000 & 0.0400 \\
\hline 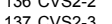 & 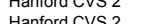 & Hirma ex al. 19944 & 0.1100 & 0.0700 & 0.07700 & 0.0450 \\
\hline 138 CVS2-4 & $\begin{array}{l}\text { Hanford CVS } \\
\text { Hanford CVS } 2\end{array}$ & $\begin{array}{l}\text { Hrma et al. } \\
\text { Hrma et al. } 1994\end{array}$ & $\begin{array}{l}0.0632 \\
0.0619\end{array}$ & $\begin{array}{l}0.1320 \\
0.1095\end{array}$ & 0.0700 & $\begin{array}{l}0.0450 \\
0.0400\end{array}$ \\
\hline $\begin{array}{l}139 \text { CVS2-5 } \\
139\end{array}$ & Hanford CVS 2 & Hrma et al. 1994 & 0.0800 & & 0.0348 & 0.0400 \\
\hline 140 CVS2-6 & Hanford CVS 2 & Hrma et al. 1994 & 0.0159 & 0.0700 & 0.0700 & \\
\hline 141 CVS2-7 & Hanford CVS 2 & Hrma et al. 1994 & 0.0953 & 0.1700 & 0.0094 & 0.0400 \\
\hline 142 CVS2-8 & Hanford CVS 2 & Hrma et al. 1994 & 0.0610 & 0.0700 & 0.0000 & 0.1140 \\
\hline 143 CVS2-9 & Hanford CVS 2 & Hrma et al. 1994 & 0.0138 & 0.0944 & 0.0000 & 0.0712 \\
\hline & Hantord C & Hrma et al. 1994 & 0.1043 & & 0.0000 & 0.0669 \\
\hline $145 \cos 2-17$ & 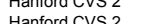 & Hirma ex al. 1994 & 0.0100 & 0.0751 & 0.0700 & 0.0400 \\
\hline 147 CVS2-13 & Hanford CVS 2 & Hrma et al. 1994 & $\begin{array}{l}0.0901 \\
0.0896\end{array}$ & $\begin{array}{l}0.1700 \\
0.0700\end{array}$ & $\begin{array}{l}.0 .0300 \\
0.0300\end{array}$ & $\begin{array}{l}0.0400 \\
0.0400\end{array}$ \\
\hline 148 CVS2-14 & Hanford CVS 2 & Hrma et al. 1994 & & 0.1312 & 0.0500 & 0.0400 \\
\hline 149 CVS2-15 & & Hrma et al. 1994 & 0.0756 & & 0.0601 & 0.0400 \\
\hline 150 CVS2-16 & Hanford CVS 2 & Hrma et al. 1994 & 0.0231 & 0.1053 & 0.0083 & 0.07 \\
\hline 151 CVS2-17 & Hanford CVS 2 & Hrma et al. 1994 & 0.0636 & 0.1142 & 0.0275 & 0.05 \\
\hline 152 CVS2-18 & Hanford CVS 2 & & 0.0231 & 0.1053 & 0.0083 & 0.07 \\
\hline & & Hrma et al. 1994 & & 0.1048 & 0.0082 & 0.0733 \\
\hline $154 \mathrm{CV}$ & & & 0.0100 & 0.0500 & 0.0000 & 0.0600 \\
\hline $155 \mathrm{cVs}$ & Hanford CVS 2 & Hrma et al. 1994 & 0.0686 & 0.131 & 0.0000 & 0.0200 \\
\hline 156 CVS2-22 & Hanford CVS 2 & Hrma et al. 1994 & 0.0365 & 0.0500 & 0.0000 & 0.022 \\
\hline & Hanford CVS 2 & $\begin{array}{ll}\text { Hrma et al. } 1994 \\
H\end{array}$ & 0.0578 & 0.0522 & 0.0800 & 0.022 \\
\hline $\begin{array}{l}158 \mathrm{CVS} 2-24 \\
159 \text { CVS } 225\end{array}$ & $\begin{array}{l}\text { Hanford CVS } \\
\text { Hanford CVS } 2\end{array}$ & $\begin{array}{l}\text { Him et al. } \\
\text { Hrma et al. } 1994\end{array}$ & $\begin{array}{l}0.0961 \\
0.0033\end{array}$ & 0.2000 & $\begin{array}{l}0.0000 \\
0.0800\end{array}$ & 0. \\
\hline 160 cVs2-26 & Hanford CVS 2 & Hrma et al. 1994 & $\begin{array}{l}0.0257 \\
0.0035\end{array}$ & 0.2000 & 0.0800 & $\begin{array}{l}0.15 \\
0.02\end{array}$ \\
\hline 161 CVS2-27 & Hanford CVS 2 & Hrma et al. 1994 & 0.0782 & 0.0500 & 0.0000 & 0.0200 \\
\hline & Hanford CVS 2 & Hrma et al. 1994 & 0.0555 & 0.0500 & 0.0000 & \\
\hline Evs2-29 & Hanford CVS 2 & Hrm et al. 1994 & 0.0000 & 0.2000 & 0.0800 & \\
\hline 164 CVS2-30 & Hanford CVS 2 & Hrma et al. 1994 & 0.0458 & 0.2000 & 0.0000 & 0.1320 \\
\hline
\end{tabular}

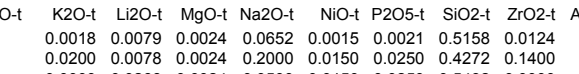

$\begin{array}{llllllll}0.0200 & 0.0078 & 0.0024 & 0.2000 & 0.0150 & 0.0250 & 0.4272 & 0.1400 \\ 0.0000 & 0.0268 & 0.0024 & 0.0500 & 0.0150 & 0.0250 & 0.5432 & 0.0000\end{array}$

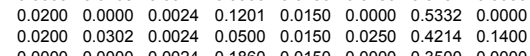

$\begin{array}{llllllll}0.0000 & 0.0000 & 0.0024 & 0.1860 & 0.0150 & 0.0000 & 0.3500 & 0.0000 \\ 0.0026 & 0.0119 & 0.0024 & 0.1190 & 0.0127 & 0.0032 & 0.4086 & 0.1167 \\ 0.0200 & 0.0900 & 0.024 & 0.0568 & 0.0000 & 0.0250 & 0.5626 & 0.0000\end{array}$

$\begin{array}{llllllll}0.0200 & 0.0900 & 0.0024 & 0.0568 & 0.0000 & 0.0250 & 0.5626 & 0.0000 \\ 0.0178 & 0.0390 & 0.0024 & 0.0649 & 0.0135 & 0.0021 & 0.3641 & 0.1244\end{array}$

$\begin{array}{lllllllllll}0.0000 & 0.0146 & 0.0024 & 0.2000 & 0.0000 & 0.0000 & 0.3649 & 0.0000\end{array}$

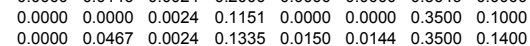
$\begin{array}{llllllll}0.0000 & 0.0467 & 0.0024 & 0.1335 & 0.0150 & 0.0144 & 0.3500 & 0.1400 \\ 0.0100 & 0.0600 & 0.0024 & 0.1000 & 0.0050 & 0.0150 & 0.4925 & 0.0400\end{array}$ $\begin{array}{lllllllll}0.0100 & 0.0425 & 0.0024 & 0.1000 & 0.0100 & 0.0050 & 0.4000 & 0.0800\end{array}$ $\begin{array}{llllllll}0.0097 & 0.0600 & 0.0024 & 0.1000 & 0.0050 & 0.0150 & 0.44000 & 0.08000\end{array}$ $\begin{array}{llllllllll}0.01100 & 0.0580 & 0.0024 & 0.1500 & 0.0100 & 0.0145 & 0.4000 & 0.0600\end{array}$ $\begin{array}{llllllll}0.0050 & 0.0305 & 0.0024 & 0.1000 & 0.0050 & 0.0050 & 0.4120 & 0.04400 \\ 0.0050 & 0.0600 & 0.0224 & 0.1000 & 0.050 & 0.0050 & 0.4075 & 0.0800\end{array}$ $\begin{array}{llllllll}0.0050 & 0.0600 & 0.0024 & 0.1000 & 0.0050 & 0.0050 & 0.4075 & 0.0800 \\ 0.0050 & 0.0538 & 0.0024 & 0.1500 & 0.0050 & 0.0050 & 0.4000 & 0.0800\end{array}$ $\begin{array}{lllllllll}0.0050 & 0.0300 & 0.0024 & 0.1497 & 0.0100 & 0.0050 & 0.4478 & 0.0400\end{array}$

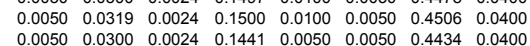
$\begin{array}{llllllll}0.0050 & 0.0300 & 0.0024 & 0.1441 & 0.0050 & 0.0050 & 0.4434 & 0.0400 \\ 0.0050 & 0.0300 & 0.0024 & 0.1000 & 0.0050 & 0.0150 & 0.4147 & 0.0600\end{array}$ $\begin{array}{llllllll}0.0050 & 0.0300 & 0.0024 & 0.1000 & 0.050 & 0.050 & 0.4434 & 0.0400 \\ 0.0087 & 0.0393 & 0.0024 & 0.1237 & 0.0072 & 0.0106 & 0.4173 & 0.0600 \\ 0 & 0.0617\end{array}$ $\begin{array}{llllllll}0.0088 & 0.0396 & 0.0024 & 0.1246 & 0.0073 & 0.0107 & 0.4207 & 0.0622 \\ 0.0000 & 0.0845 & 0.0014 & 0.0500 & 0.0000 & 0.0000 & 0.5308 & 0.0301\end{array}$

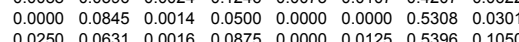
$\begin{array}{lllllllllll}0.0000 & 0.0376 & 0.0363 & 0.1003 & 0.0042 & 0.0007 & 0.4801 & 0.0429 & 0.0000 & 0.0000\end{array}$

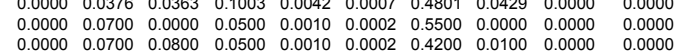

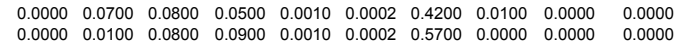

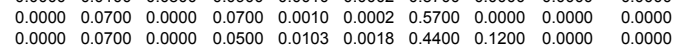
$\begin{array}{llllllllll}0.0000 & 0.0100 & 0.0000 & 0.0964 & 0.0010 & 0.0002 & 0.5750 & 0.1300 & 0.0000 & 0.0000 \\ 0.0000 & 0.0100 & 0.0800 & 0.0837 & 0.0010 & 0.0002 & 0.5363 & 0.0800 & 0.0000 & 0.0000\end{array}$

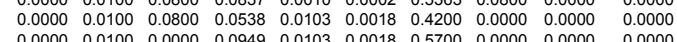

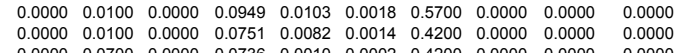
$\begin{array}{llllllllll}0.0000 & 0.0100 & 0.0000 & 0.1862 & 0.0010 & 0.0002 & 0.5700 & 0.0000 & 0.0000 & 0.00000\end{array}$

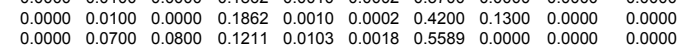

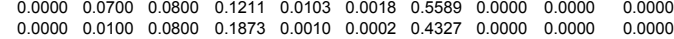
$\begin{array}{llllllllll}0.00000 & 0.0100 & 0.0000 & 0.1455 & 0.0103 & 0.0018 & 0.4545 & 0.0000 & 0.0000 & 0.00000 \\ 0.0000 & 0.0700 & 0.0800 & 0.1186 & 0.0093 & 0.0016 & 0.4214 & 0.1300 & 0.0000 & 0.0000\end{array}$

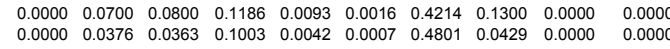

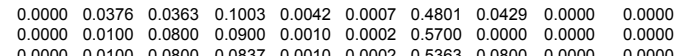

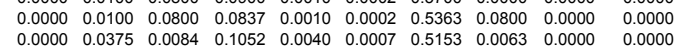
$\begin{array}{lllllllllll}0.0000 & 0.0375 & 0.0084 & 0.1052 & 0.0040 & 0.0007 & 0.5153 & 0.0063 & 0.0000 & 0.0000 \\ 0.0000 & 0.0600 & 0.0500 & 0.0700 & 0.0082 & 0.0014 & 0.5226 & 0.0100 & 0.0000 & 0.0000\end{array}$ $\begin{array}{llllllllll}0.0000 & 0.0600 & 0.0000 & 0.0883 & 0.0026 & 0.0004 & 0.5017 & 0.0300 & 0.0000 & 0.0000 \\ 0.0000 & 0.0435 & 0.0100 & 0.0700 & 0.0026 & 0.0004 & 0.4645 & 0.0368 & 0.0000 & 0.0000\end{array}$ $\begin{array}{llllllllll}0.0000 & 0.0536 & 0.0000 & 0.070 & 0.0026 & 0.0004 & 0.5660 & 0.0100 & 0.00000 & 0.0000\end{array}$

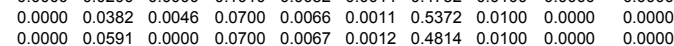
$\begin{array}{llllllllll}0.0000 & 0.0600 & 0.0500 & 0.0985 & 0.00026 & 0.0004 & 0.5145 & 0.0100 & 0.00000 & 0.0000 \\ 0.0000 & 0.0600 & 0.0000 & 0.0924 & 0.0026 & 0.0004 & 0.05432 & 0.1000 & 0.0000 & 0.0000\end{array}$

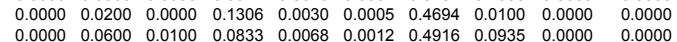
$\begin{array}{llllllllll}0.0000 & 0.0600 & 0.0100 & 0.0833 & 0.0068 & 0.0012 & 0.4916 & 0.0935 & 0.0000 & 0.0000 \\ 0.0000 & 0.0466 & 0.0100 & 0.0700 & 0.0026 & 0.0004 & 0.4683 & 0.0100 & 0.0000 & 0.0000\end{array}$

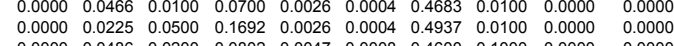
$\begin{array}{llllllllll}0.0000 & 0.0486 & 0.0200 & 0.0802 & 0.0047 & 0.0008 & 0.4600 & 0.1000 & 0.0000 & 0.0000 \\ 0.0000 & 0.0214 & 0.0000 & 0.1700 & 0.0082 & 0.0014 & 0.4729 & 0.0100 & 0.0000 & 0.0000\end{array}$ $\begin{array}{llllllllll}0.0000 & 0.0214 & 0.0000 & 0.1700 & 0.0082 & 0.0014 & 0.4779 & 0.0100 & 0.00000 & 0.0000 \\ 0.0000 & 0.0375 & 0.0084 & 0.1125 & 0.0061 & 0.0011 & 0.5353 & 0.0385 & 0.0000 & 0.00000\end{array}$ $\begin{array}{llllllllll}0.0000 & 0.0376 & 0.0363 & 0.1003 & 0.0042 & 0.0007 & 0.4801 & 0.0429 & 0.00000 & 0.0000 \\ 0.0000 & 0.0375 & 0.0084 & 0.1125 & 0.0061 & 0.0011 & 0.5353 & 0.0385 & 0.0000 & 0.0000\end{array}$

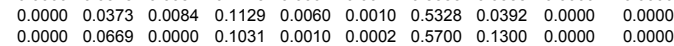

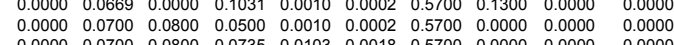
$\begin{array}{llllllllll}0.0000 & 0.0700 & 0.0800 & 0.0735 & 0.0103 & 0.0018 & 0.5700 & 0.0000 & 0.0000 & 0.0000 \\ 0.0000 & 0.0100 & 0.0000 & 0.2000 & 0.0010 & 0.0002 & 0.5700 & 0.0000 & 0.0000 & 0.0000\end{array}$

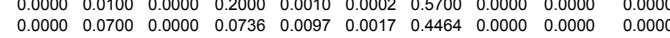

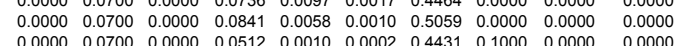

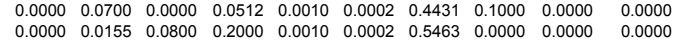

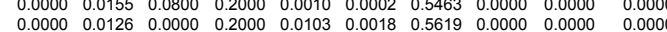

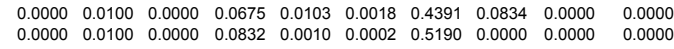

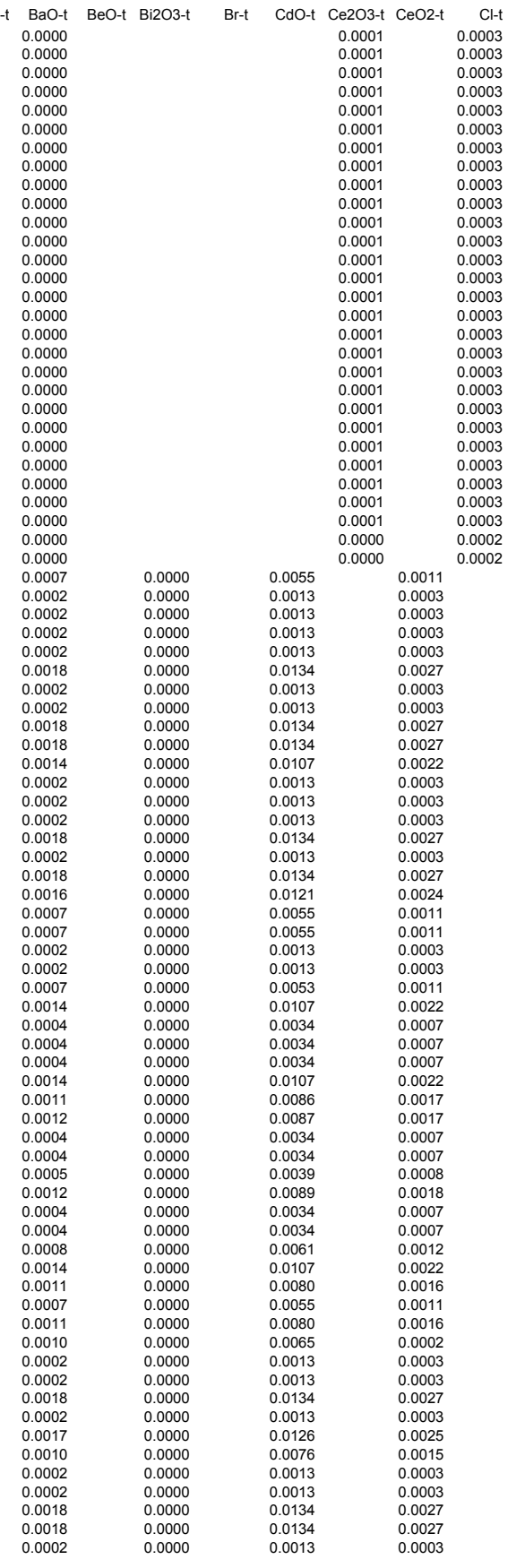




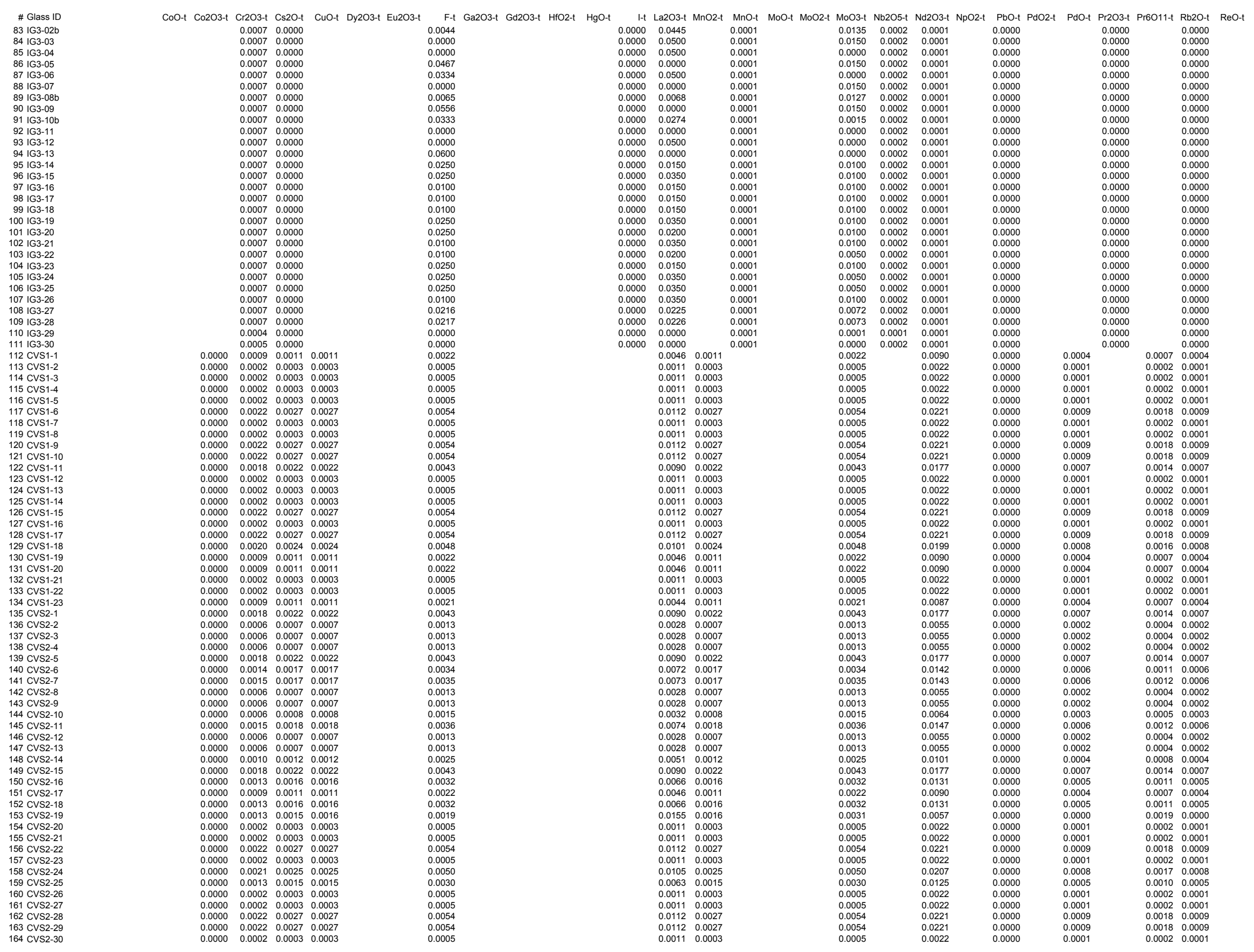




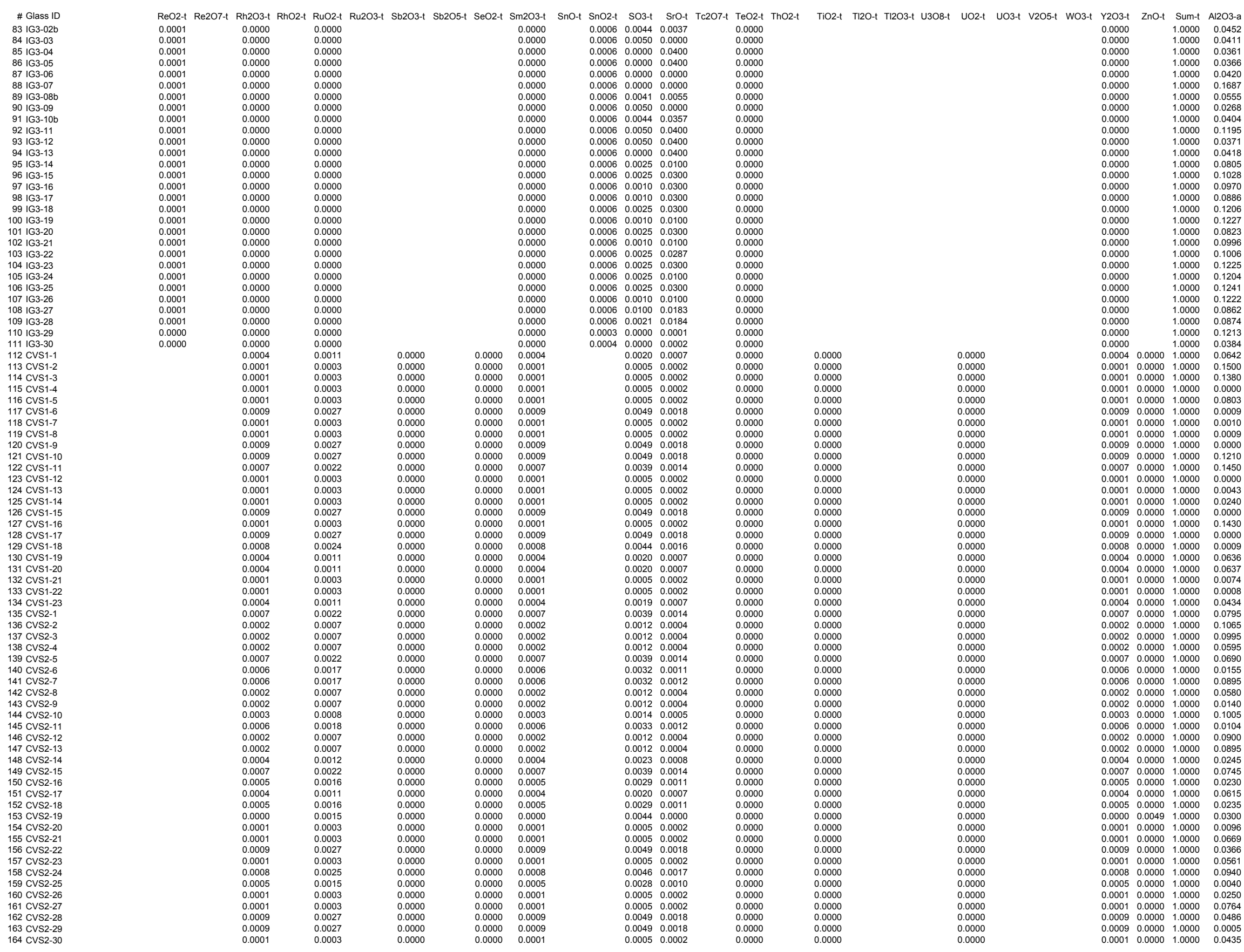




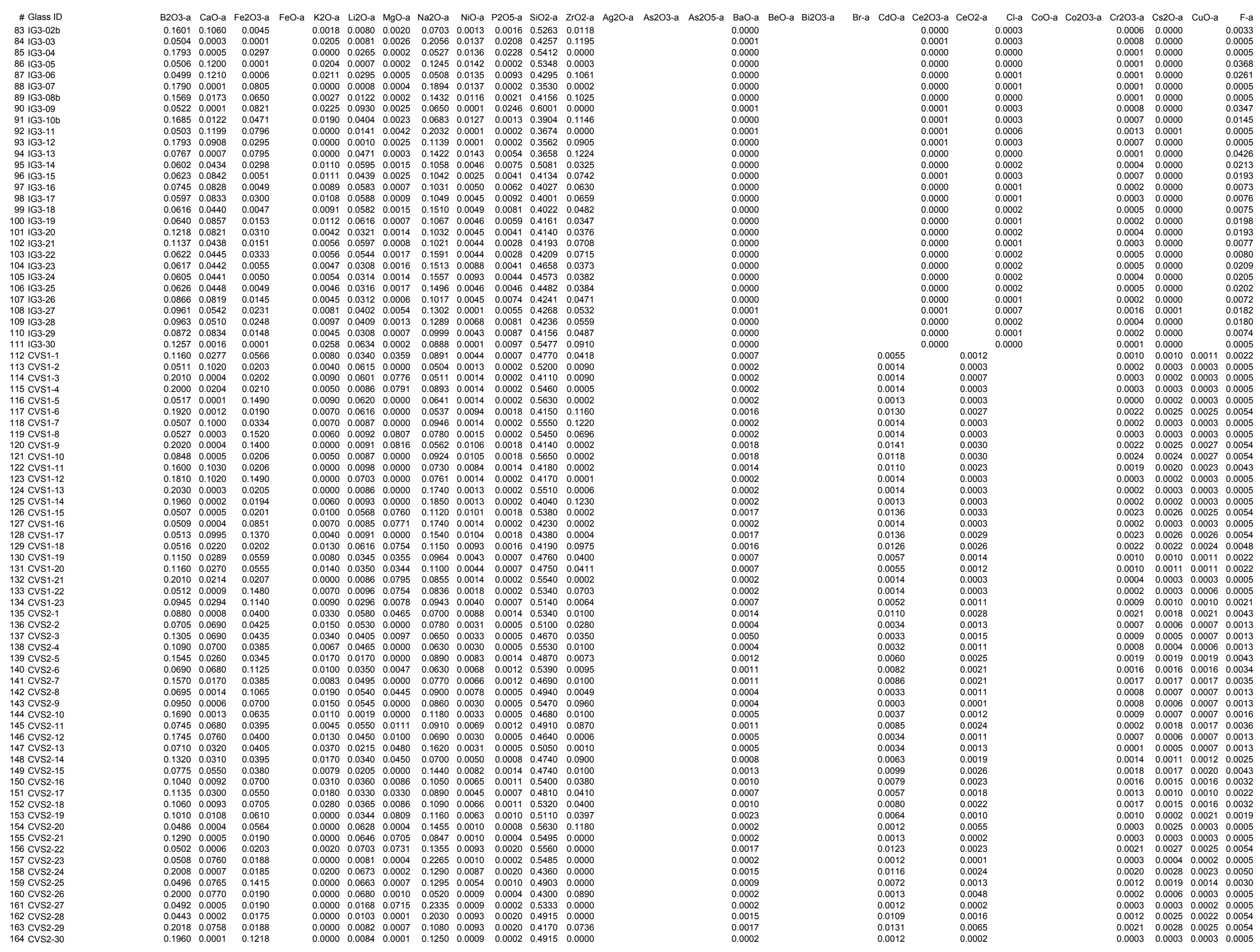




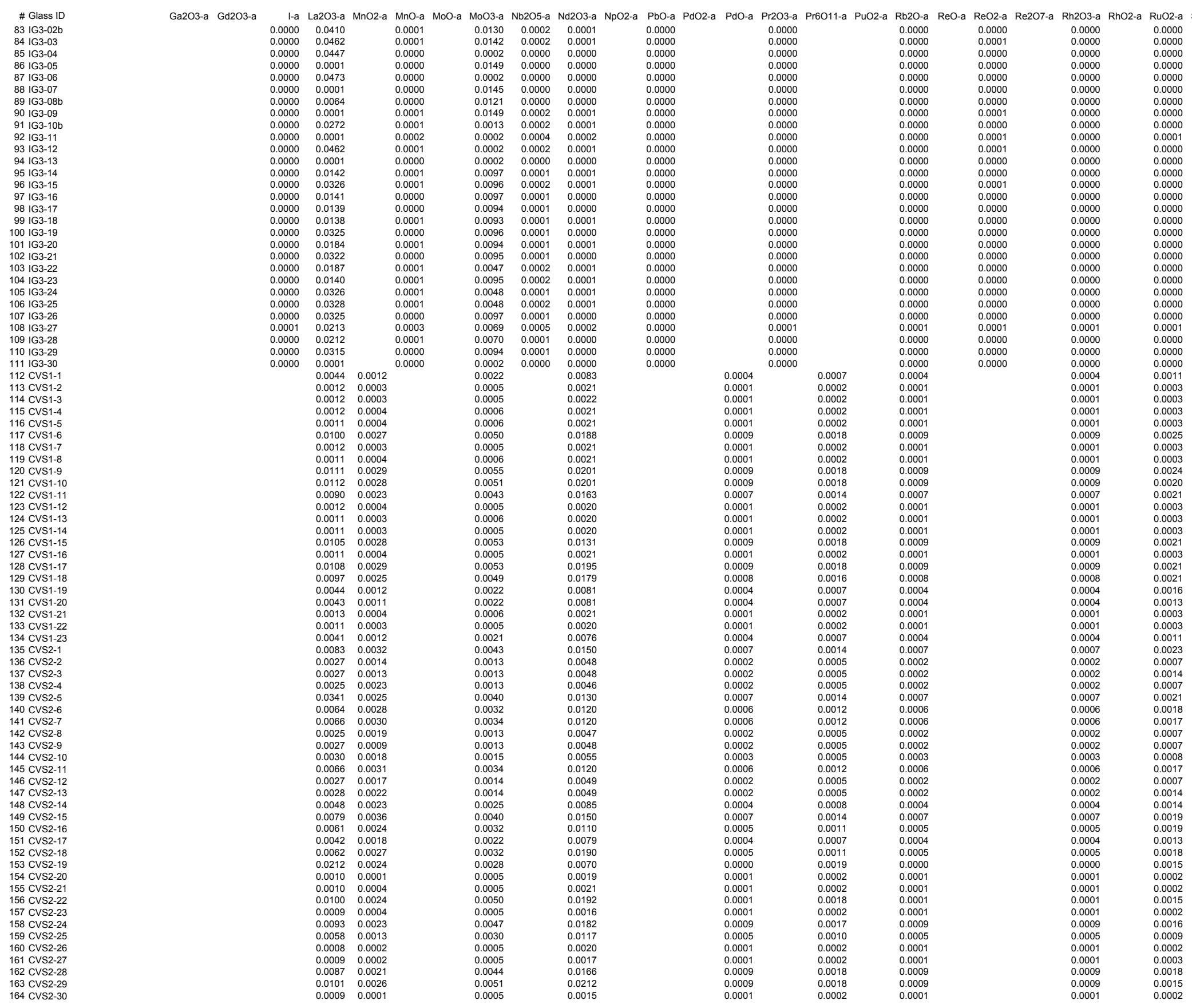




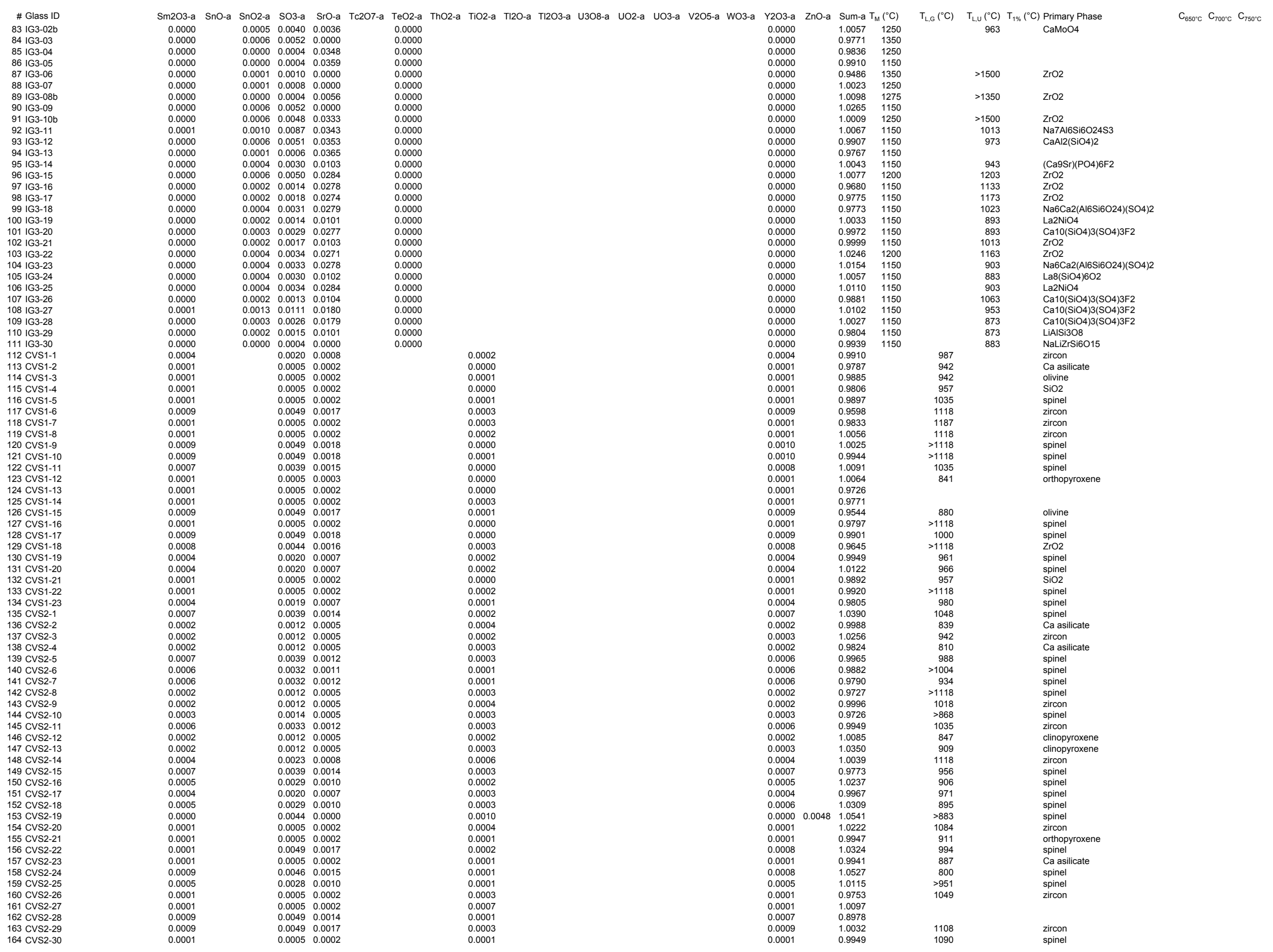




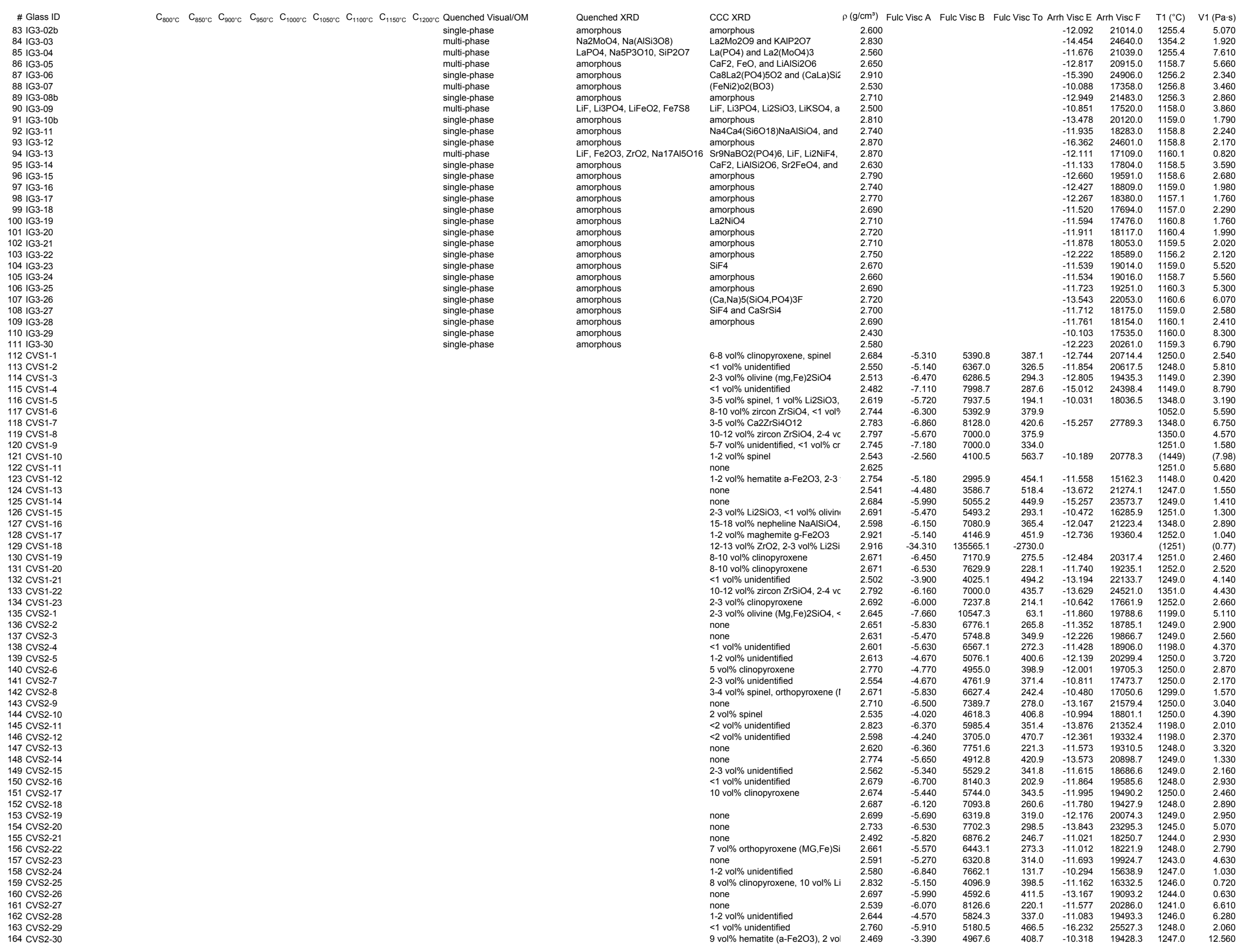




\begin{tabular}{|c|c|c|c|c|c|c|c|c|c|c|c|c|c|c|}
\hline 13.1 & 1106.0 & 23.2 & 1056.1 & 44.8 & 1156.4 & 13.1 & 1254.7 & 5.2 & 1304.0 & & 1539 & & 1545 & \\
\hline 5.0 & 1205.3 & 8.7 & 1156.0 & 16.4 & 1106.6 & 33.6 & 1255.5 & 5.0 & $\begin{array}{l}1354.2 \\
\end{array}$ & 2.0 & 1403.8 & 1.3 & 1453.4 & 0.9 \\
\hline $\begin{array}{l}21.0 \\
175\end{array}$ & 1106.5 & 36.3 & 1056.6 & 66.2 & $\begin{array}{l}1156.9 \\
1\end{array}$ & 20.6 & $\begin{array}{l}1255.7 \\
1157\end{array}$ & 7.8 & 1305.0 & 5.2 & 1355.8 & 3.9 & 1256.7 & 7.7 \\
\hline $\begin{array}{c}17.5 \\
81\end{array}$ & $\begin{array}{l}1007.1 \\
1157.8\end{array}$ & $\begin{array}{r}33.8 \\
78\end{array}$ & $\begin{array}{r}957.5 \\
1256.0\end{array}$ & $\begin{array}{r}71.8 \\
23\end{array}$ & $\begin{array}{l}1058.3 \\
1305.5\end{array}$ & $\begin{array}{r}17.3 \\
1.3\end{array}$ & $\begin{array}{l}1157.6 \\
13548\end{array}$ & $\begin{array}{l}5.8 \\
10\end{array}$ & $\begin{array}{l}1206.5 \\
1255.4\end{array}$ & $\begin{array}{l}3.8 \\
2.6\end{array}$ & 1256.0 & 2.6 & 1157.0 & 6.3 \\
\hline $\begin{array}{l}8.1 \\
7.7\end{array}$ & $\begin{array}{l}1107.8 \\
\end{array}$ & $\begin{array}{l}1.8 \\
12.0\end{array}$ & $\begin{array}{l}1057.2 \\
1056\end{array}$ & $\begin{array}{l}2.3 \\
19.9\end{array}$ & $\begin{array}{l}1305.5 \\
1157.6\end{array}$ & $\begin{array}{l}7.5 \\
7.7\end{array}$ & $\begin{array}{l}1354.8 \\
1256.0\end{array}$ & $\begin{array}{l}1.0 \\
3.5\end{array}$ & $\begin{array}{l}1255.4 .4 \\
1305.3\end{array}$ & $\begin{array}{l}2.6 \\
2.5\end{array}$ & 1354.8 & 1.8 & 1255.7 & 3.6 \\
\hline 7.7 & 1107.3 & 13.7 & 1057.2 & 26.1 & 1157.7 & 7.9 & $\begin{array}{l}1256.3 \\
12055\end{array}$ & 3.0 & 1305.5 & 2.0 & $\begin{array}{l}1354.8 \\
1145 .\end{array}$ & 1.4 & 1255.5 & 3.0 \\
\hline $\begin{array}{l}9.7 \\
552\end{array}$ & $\begin{array}{l}1006.3 \\
\end{array}$ & 16.8 & $\begin{array}{l}956.4 \\
0571\end{array}$ & 32.6 & 115 & 4.0 & $\begin{array}{l}1205.9 \\
1157\end{array}$ & 2.8 & , 1255.1 & 2.0 & $\begin{array}{l}1155.7 \\
1525\end{array}$ & 4.1 & & \\
\hline $\begin{array}{l}5.2 \\
5.9\end{array}$ & $\begin{array}{l}10066.9 \\
10069\end{array}$ & $\begin{array}{r}9.6 \\
10.5\end{array}$ & $\begin{array}{l}957.1 \\
957.1\end{array}$ & $\begin{array}{l}18.7 \\
202\end{array}$ & $\begin{array}{l}1057.9 \\
1058\end{array}$ & $\begin{array}{l}4.8 \\
5.9\end{array}$ & $\begin{array}{l}1157.0 \\
151572\end{array}$ & $\begin{array}{l}1.7 \\
23\end{array}$ & 1206.1 & $\begin{array}{l}1.1 \\
1.1\end{array}$ & $\begin{array}{l}1255.4 \\
11555\end{array}$ & 0.8 & $\begin{array}{l}1156.0 \\
1156.0\end{array}$ & $\begin{array}{l}1.8 \\
2.3\end{array}$ \\
\hline $\begin{array}{l}5.9 \\
7.7\end{array}$ & $\begin{array}{l}10067.9 \\
1000\end{array}$ & $\begin{array}{l}10.5 \\
17.2\end{array}$ & 957.8 & $\begin{array}{l}20.2 \\
44.5\end{array}$ & $\begin{array}{l}1058.0 \\
\end{array}$ & $\begin{array}{l}5.9 \\
7.6\end{array}$ & $\begin{array}{l}1157.2 \\
1157.7\end{array}$ & $\begin{array}{l}2.3 \\
2.2\end{array}$ & $\begin{array}{l}1206.2 .2 \\
1206.7\end{array}$ & $\begin{array}{l}1.5 \\
1.4\end{array}$ & $\begin{array}{l}12555.5 \\
1255.9\end{array}$ & $\begin{array}{l}1.1 \\
0.9\end{array}$ & $\begin{array}{l}11166.3 \\
1156.4\end{array}$ & $\begin{array}{l}2.3 \\
2.3\end{array}$ \\
\hline 2.1 & 1008.2 & 3.5 & 958.6 & 5.9 & & 2.1 & $\begin{array}{l}1158.2 \\
1157\end{array}$ & 0.9 & 1207.3 & 0.6 & 1256.3 & 0.4 & 1157.0 & 0.9 \\
\hline $\begin{array}{l}9.3 \\
77\end{array}$ & $\begin{array}{l}1007.2 \\
1071\end{array}$ & $\begin{array}{l}16.0 \\
141\end{array}$ & $\begin{array}{l}957.6 \\
957.6\end{array}$ & $\begin{array}{l}29.4 \\
277\end{array}$ & $\begin{array}{l}1058.5 \\
10779\end{array}$ & 9.2 & $\begin{array}{l}1157.8 \\
1157\end{array}$ & $\begin{array}{l}3.6 \\
2.8\end{array}$ & 1206.8 & 2.5 & $\begin{array}{l}1255.9 \\
12554\end{array}$ & 1.8 & $\begin{array}{l}1156.6 \\
11159\end{array}$ & 3.8 \\
\hline $\begin{array}{l}.17 \\
5.4\end{array}$ & $\begin{array}{l}100.1 .1 \\
1006.8\end{array}$ & $\begin{array}{r}14.1 \\
9.6\end{array}$ & $\begin{array}{l}957.3 \\
957.0\end{array}$ & $\begin{array}{l}27.7 \\
18.8\end{array}$ & $\begin{array}{l}1057.9 \\
1057.8\end{array}$ & $\begin{array}{l}7.5 \\
5.4\end{array}$ & $\begin{array}{l}1157.0 \\
1157.2\end{array}$ & $\begin{array}{l}2.8 \\
2.1\end{array}$ & $\begin{array}{l}12 \\
12 \\
12\end{array}$ & $\begin{array}{l}1.8 \\
1.4\end{array}$ & $\begin{array}{l}1255.4 \\
12554\end{array}$ & $\begin{array}{l}1.2 \\
0.9\end{array}$ & $\begin{array}{l}11155.9 \\
11560\end{array}$ & $\begin{array}{l}2.9 \\
2.1\end{array}$ \\
\hline $\begin{array}{l}5.44 \\
4.7\end{array}$ & 1005.8 & $\begin{array}{l}8.0 \\
8.3\end{array}$ & $\begin{array}{l}956.0 \\
956\end{array}$ & $\begin{array}{l}15.0 \\
15.4\end{array}$ & $\begin{array}{l}1056.0 \\
1056\end{array}$ & $\begin{array}{l}4.4 \\
4.6\end{array}$ & 1155.8 & $\begin{array}{l}1.1 \\
1.8\end{array}$ & 1205.3 & $\begin{array}{l}1.4 \\
1.2\end{array}$ & $\begin{array}{l}1255.4 \\
1255.4\end{array}$ & 0.8 & 55.6 & 1.8 \\
\hline 5.9 & 1005.8 & 10.2 & $\begin{array}{l}956.0 \\
958.0\end{array}$ & 18.8 & 1056.4 & 5.8 & 1155.9 & 2.3 & & 1.6 & 1254.7 & 1.1 & 1155.2 & 2.4 \\
\hline $\begin{array}{l}4.5 \\
5.5\end{array}$ & $\begin{array}{l}1008.2 \\
1\end{array}$ & $\begin{array}{l}7.7 \\
9.4\end{array}$ & $\begin{array}{r}958.3 \\
958.3\end{array}$ & $\begin{array}{l}14.4 \\
178\end{array}$ & $\begin{array}{l}1059.5 \\
10595\end{array}$ & $\begin{array}{l}4.4 \\
52\end{array}$ & $\begin{array}{l}1158.6 \\
1158\end{array}$ & 1.8 & $\begin{array}{l}1207.4 \\
107075\end{array}$ & 1.3 & $\begin{array}{l}1256.5 \\
1256\end{array}$ & 0.9 & $\begin{array}{l}11575.1 \\
11175.1\end{array}$ & $\begin{array}{l}1.9 \\
2.1\end{array}$ \\
\hline $\begin{array}{l}5.2 \\
5.3\end{array}$ & 1006.8 & $\begin{array}{l}9.4 \\
9.3\end{array}$ & $\begin{array}{l}958.3 \\
957.0\end{array}$ & $\begin{array}{l}17.8 \\
17.5\end{array}$ & $\begin{array}{l}1059.5 \\
1058.0\end{array}$ & $\begin{array}{l}5.2 \\
5.2\end{array}$ & 1157.1 & $\begin{array}{l}2.1 \\
2.1\end{array}$ & $\begin{array}{l}1207.5 \\
1206.1\end{array}$ & $\begin{array}{l}1.4 \\
1.4\end{array}$ & $\begin{array}{l}1256.6 \\
1255.2\end{array}$ & $\begin{array}{l}1.0 \\
1.0\end{array}$ & $\begin{array}{l}1155.7 \\
115.7\end{array}$ & $\begin{array}{l}2.1 \\
2.1\end{array}$ \\
\hline $\begin{array}{l}5.0 \\
5.7\end{array}$ & $\begin{array}{l}1006.2 \\
100\end{array}$ & $\begin{array}{l}50.1 \\
10.1\end{array}$ & 956.5 & 19.1 & 1057.7 & 5.6 & $\begin{array}{l}1157.0 \\
\end{array}$ & 2.2 & 1205.8 & $\begin{array}{l}1.4 \\
1.4\end{array}$ & $\begin{array}{l}1254.9 \\
\text { a }\end{array}$ & $\begin{array}{l}1.0 \\
\text {. }\end{array}$ & 1155.5 & 2.2 \\
\hline $\begin{array}{l}15.3 \\
153\end{array}$ & $\begin{array}{l}1007.3 \\
1\end{array}$ & $\begin{array}{l}27.2 \\
275\end{array}$ & $\begin{array}{r}957.5 \\
95659\end{array}$ & $\begin{array}{l}52.5 \\
5.2\end{array}$ & 1058.6 & $\begin{array}{l}15.6 \\
155\end{array}$ & $\begin{array}{l}1157.6 \\
1157\end{array}$ & 5.6 & & 3.7 & 1255.3 & 2.6 & $\begin{array}{l}1156.0 \\
111556\end{array}$ & 5.9 \\
\hline $\begin{array}{l}15.3 \\
14.8\end{array}$ & $\begin{array}{l}1006 . / . \\
1008.6\end{array}$ & $\begin{array}{l}27.5 \\
26.9\end{array}$ & $\begin{array}{l}\begin{array}{l}556.9 \\
958.9\end{array}\end{array}$ & ${ }_{5}^{2}$ & $\begin{array}{l}105 \\
105\end{array}$ & $\begin{array}{l}\begin{array}{l}15.5 \\
15.2\end{array} \\
15\end{array}$ & $\begin{array}{l}1517.1 \\
11588\end{array}$ & $\begin{array}{l}5.7 \\
5.4\end{array}$ & $\begin{array}{l}12 \\
12\end{array}$ & $\begin{array}{l}3.8 \\
3.6\end{array}$ & $\begin{array}{l}1254.9 \\
12565\end{array}$ & $\begin{array}{l}2.7 \\
2.5\end{array}$ & $\begin{array}{l}\begin{array}{l}11155.6 \\
11573\end{array} \\
\end{array}$ & $\begin{array}{l}6.0 \\
5.8\end{array}$ \\
\hline $\begin{array}{l}14.0 \\
19.5\end{array}$ & 1008.3 & $\begin{array}{l}2.9 \\
38.6\end{array}$ & $\begin{array}{l}930.9 \\
958.6\end{array}$ & 84.9 & $\begin{array}{l}1059.7 \\
\end{array}$ & 20.1 & $\begin{array}{l}1150.0 \\
1158.0\end{array}$ & $\begin{array}{l}0.4 \\
6.3 \\
\end{array}$ & 1207.3 & $\begin{array}{l}5.0 \\
3.9\end{array}$ & $\begin{array}{l}1256.0 \\
\text { a }\end{array}$ & $\begin{array}{l}2.5 \\
2.6\end{array}$ & 1156.9 & $\begin{array}{l}5.0 \\
6.4\end{array}$ \\
\hline 6.8 & 1007.6 & 12.0 & 958.0 & 22.4 & & 6.8 & & 2.7 & & 1.8 & $\begin{array}{l}1255.5 \\
\end{array}$ & 1.3 & 1156.3 & 2.7 \\
\hline 6.3 & $\begin{array}{l}1007.9 \\
\end{array}$ & $\begin{array}{l}11.1 \\
358\end{array}$ & $\begin{array}{l}958.1 \\
95884\end{array}$ & 21.4 & $\begin{array}{l}1059.1 \\
10995\end{array}$ & 6.2 & $\begin{array}{l}1158.1 \\
1158\end{array}$ & $\begin{array}{l}2.5 \\
8.5\end{array}$ & $\begin{array}{l}1207.0 \\
107\end{array}$ & $\begin{array}{l}1.7 \\
5.7\end{array}$ & $\begin{array}{l}1256.0 \\
25.0\end{array}$ & 1.2 & 1156.9 & 2.6 \\
\hline $\begin{array}{l}20.9 \\
19.3\end{array}$ & $\begin{array}{l}10088.1 \\
1008.0\end{array}$ & $\begin{array}{l}35.8 \\
36.1\end{array}$ & $\begin{array}{l}958.4 \\
958.4\end{array}$ & $\begin{array}{l}65.9 \\
73.7\end{array}$ & $\begin{array}{l}1059.5 \\
10594\end{array}$ & $\begin{array}{l}20.8 \\
19.1\end{array}$ & $\begin{array}{l}1158.3 \\
11583\end{array}$ & $\begin{array}{l}8.5 \\
6.8\end{array}$ & $\begin{array}{l}1207.0 \\
1207.0\end{array}$ & $\begin{array}{l}5.8 \\
4.4\end{array}$ & $\begin{array}{l}12555.6 \\
12557\end{array}$ & $\begin{array}{l}4.1 \\
3.0\end{array}$ & $\begin{array}{l}\begin{array}{l}111656.6 \\
1156.5\end{array} \\
\end{array}$ & $\begin{array}{l}8.7 \\
7.0\end{array}$ \\
\hline $\begin{array}{l}19.3 \\
16.7\end{array}$ & 949.0 & $\begin{array}{l}36.1 \\
72.4\end{array}$ & & & & & & & & & & & & \\
\hline $\begin{array}{l}38.3 \\
218\end{array}$ & $\begin{array}{l}951.0 \\
(852)\end{array}$ & $\begin{array}{r}156.5 \\
112181\end{array}$ & & & & & & & & & & & & \\
\hline $\begin{array}{l}21.8 \\
140.5\end{array}$ & (852) & )(121.81) & & & & & & & & & & & & \\
\hline $\begin{array}{r}13.2 \\
71.56)\end{array}$ & 1050.0 & 35.1 & 950.0 & 118.9 & & & & & & & & & & \\
\hline & & & & & & & & & & & & & & \\
\hline & & & & & & & & & & & & & & \\
\hline & 1150.0 & $\begin{array}{r}83.5 \\
(2782\end{array}$ & & & & & & & & & & & & \\
\hline 2.3 & (852) & $(10.47)$ & (752) & (131.67) & & & & & & & & & & \\
\hline 9.8 & 949.0 & 46.8 & & & & & & & & & & & & \\
\hline & 950.0 & $\begin{array}{l}61.3 \\
17.8\end{array}$ & & $(79.16)$ & & & & & & & & & & \\
\hline $\begin{array}{r}5.9 \\
18.0\end{array}$ & $\begin{array}{l}951.0 \\
(1049)\end{array}$ & $\begin{array}{r}117.8 \\
(113.81)\end{array}$ & (851) & $(79.16)$ & & & & & & & & & & \\
\hline $\begin{array}{r}5.8 \\
12.23)\end{array}$ & 953.0 & 23.1 & & & & & & & & & & & & \\
\hline 16.4 & 951.0 & 64.5 & & & & & & & & & & & & \\
\hline $\begin{array}{r}15.3 \\
28.12)\end{array}$ & 953.0 & 1388 & & & & & & & & & & & & \\
\hline $\begin{array}{r}28.12) \\
36.0\end{array}$ & 950.0 & 138.8 & & & & & & & & & & & & \\
\hline 13.9 & 953.0 & 44.7 & & & & & & & & & & & & \\
\hline 6.1 & $\begin{array}{l}958.0 \\
1149.0\end{array}$ & $\begin{array}{l}7.8 \\
6.2\end{array}$ & 1147.0 & & 1100.0 & 9.7 & & 10.4 & 1047.0 & & 999.0 & & 948.0 & \\
\hline 55 & 1148.0 & $\begin{array}{r}5.6 \\
595\end{array}$ & 1147.0 & 5.9 & 1100.0 & 8.8 & 1096.0 & 9.7 & 1048.0 & 15.7 & 998.0 & 30.1 & 949.0 & 61.9 \\
\hline $\begin{array}{l}17.4 \\
7.9\end{array}$ & $\begin{array}{r}948.0 \\
1150.0\end{array}$ & $\begin{array}{r}59.5 \\
8.1\end{array}$ & 11470 & & 1097.0 & 136 & 1100.0 & 13.7 & 1047. & 238 & 9990 & 46.3 & 948.0 & \\
\hline 6.2 & 1149.0 & $\begin{array}{l}6.1 \\
6.2\end{array}$ & & 6.4 & & $\begin{array}{l}10.0 \\
10.0\end{array}$ & & 10.0 & & 17.3 & & $\begin{array}{l}40.5 \\
33.3\end{array}$ & & $\begin{array}{l}98.4 \\
68.4\end{array}$ \\
\hline 2 & 1148.0 & 4.4 & 1149.0 & 4.4 & 1099.0 & 6.5 & & 6.6 & & 10.7 & & & & 35.5 \\
\hline & 1150.0 & 4.3 & 1148.0 & 4.4 & & 4.6 & & 6.6 & & 7.1 & 1046 & 11.2 & & 9.9 \\
\hline & 1150.0 & 7.2 & 1149.0 & 7.3 & 1148.0 & 7.4 & 1123.0 & 9.6 & & 12.0 & 1099 & 12.4 & & 22.1 \\
\hline 8.7 & 1150.0 & 8.7 & 1147.0 & 9.4 & 1100.0 & 14.2 & 1098.0 & 14.5 & 1048.0 & 24.1 & 999.0 & 44.8 & 949.0 & 88.8 \\
\hline & $\begin{array}{l}94 \mathrm{q} \\
94\end{array}$ & $\begin{array}{l}38.8 \\
35.6\end{array}$ & & & & & & & & & & & & \\
\hline 7.0 & & 7.2 & & 7.7 & & 11.8 & & 12.5 & & 20.5 & & 39 & & \\
\hline 2.9 & 1150.0 & 2.9 & & 3.0 & & 5.0 & & 5.0 & & 8.9 & & & & 38.4 \\
\hline & $\begin{array}{l}1150.0 \\
1148.0\end{array}$ & $\begin{array}{l}4.4 \\
6.7\end{array}$ & & $\begin{array}{l}4.7 \\
6.7\end{array}$ & & $\begin{array}{l}7.0 \\
6.9\end{array}$ & & $\begin{array}{l}7.2 \\
8.4\end{array}$ & & $\begin{array}{l}11.7 \\
10.6\end{array}$ & & & & $\begin{array}{l}42.4 \\
18.6\end{array}$ \\
\hline & $\begin{array}{l}1148 \\
1149\end{array}$ & $\begin{array}{l}6.7 \\
5.6\end{array}$ & $\begin{array}{l}114 \\
114\end{array}$ & $\begin{array}{l}6.7 \\
5.7\end{array}$ & & $\begin{array}{l}6.9 \\
8.5\end{array}$ & & $\begin{array}{l}8.4 \\
8.9\end{array}$ & & $\begin{array}{l}10.6 \\
14.9\end{array}$ & $\begin{array}{l}1097 \\
999 \\
\end{array}$ & $\begin{array}{l}11.2 \\
27.4\end{array}$ & & $\begin{array}{l}18.6 \\
57.7\end{array}$ \\
\hline & 1150.0 & $\begin{array}{l}5.6 \\
6.5\end{array}$ & $\begin{array}{l}114 \\
114\end{array}$ & $\begin{array}{l}.1 .1 \\
6.8\end{array}$ & & & & & & $\begin{array}{l}14.9 \\
17.8\end{array}$ & & & & $\begin{array}{l}51.1 \\
66.8\end{array}$ \\
\hline & 1150.0 & 6.8 & & 7.1 & & & & & & 18.7 & & & & 7.0 \\
\hline 12 & 1144.0 & 13.0 & & 13.1 & & 23.5 & & 23.5 & & & & & & \\
\hline & 1146.0 & 6.2 & 114 & 6.4 & & 9.7 & & 9.9 & & 16 & & & & 5.6 \\
\hline & 114 & 6.1 & 114 & 6.2 & & & & 9.4 & & & & & & 1.3 \\
\hline 10.2 & 1145.0 & 10.5 & 114 & 10.6 & & 17.0 & & 17.0 & & 29.4 & & 53.7 & & 117.2 \\
\hline & $\begin{array}{l}1148.0 \\
11470\end{array}$ & $\begin{array}{l}2.0 \\
1.4\end{array}$ & & $\begin{array}{l}2.1 \\
1.4\end{array}$ & & 3.0 & & 3.1 & & $\begin{array}{l}4.7 \\
3.7\end{array}$ & & 7. & & 13.1 \\
\hline & $\begin{array}{l}747.0 \\
1145.0\end{array}$ & $\begin{array}{l}1.4 \\
1.3\end{array}$ & 114 & $\begin{array}{l}1.4 \\
1.3\end{array}$ & & $\begin{array}{l}2.0 \\
2.1\end{array}$ & & $\begin{array}{l}2.0 \\
2.1\end{array}$ & & 5 & & & & 3.7 \\
\hline 15 & 1144.0 & $\begin{array}{l}15.1 \\
15.1\end{array}$ & 1143.0 & $\begin{array}{l}15.9 \\
15.9\end{array}$ & & 24.9 & & 25.1 & & $\begin{array}{r}32.9 \\
42.9\end{array}$ & & 88.7 & & \\
\hline 13.3 & 1148.0 & 13.7 & & 14.5 & & 21.7 & & 22.0 & & 37.5 & & 75. & & .2 \\
\hline & 1149.0 & 5.4 & 1149.0 & 5.5 & 1099.0 & 9.8 & 1098.0 & 9.9 & 1048.0 & 20.4 & 998.0 & 46.6 & 949.0 & 124.8 \\
\hline
\end{tabular}




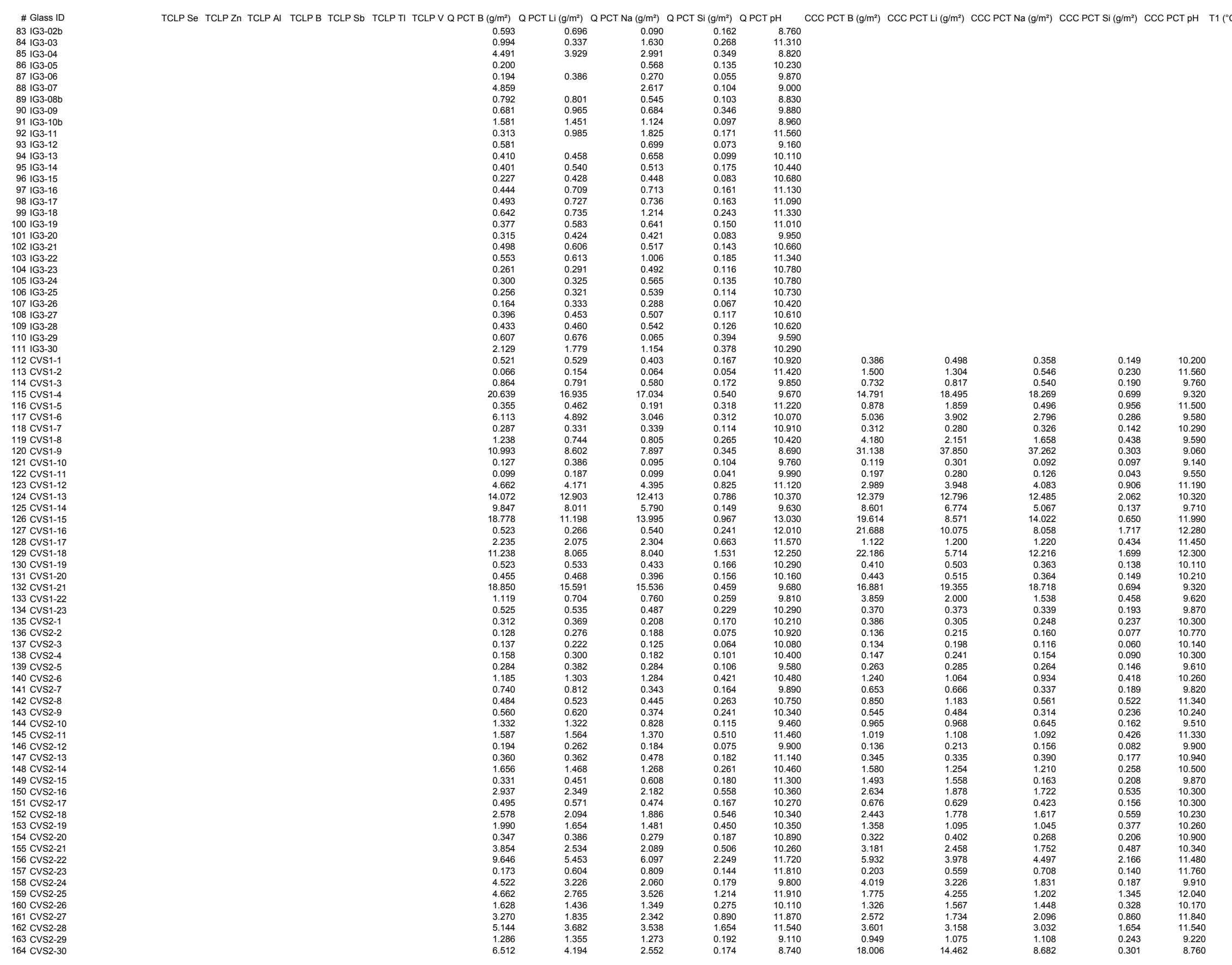


$97163-16$
$98163-17$

(1)

$101163-20$

$1021163-21$
10316322

$103163-22$
$104163-23$
1

$105163-24$

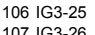

1071 IG3-26
108 IG3-27

109 IG3-28

$110,163-29$
$111163-30$

112 CVS1-1

113 CVS1-2

114 CVS1-3
115 CVS1-4

116 CVS1-5

117 CVS1-6

118 CVS1-7
119 cVs1-8

120 CVS1-9
121 CVS1 10

122 CVS1-11
123 CVS1 12

$122 \mathrm{CVSS1}-1$
$125 \mathrm{CVS} 1 \mathrm{1}$

26 CVS1-1
127 CVS 11.17

28 CVS1-17

129 CVS1-18

130 CVS1-19
131 cVS1-20

132 CVS1-21

133 CVS1-22

134 CVS2-1

36 CVS2-2

117 CVS2-3
138 CVS2-4

138 CVS2-4
139 CVS2 25

140 CVS2-6

141 CVS2-7
142 CVS2 -8

142 CVS2-8
143 CVS2-9

144 CVS2-10

145 CVS2-11
146 CVS2-12

146 CVS2-12
147 CVS2 -13

148 CVS2-14

149 CVS2-15

150 CVS2-16
151 CVS2-17

152 CVS2-18
153
$15352-19$

154 CVS2-20
155 CVS2-21

156 CVS2-22

157 CVS2-23
158 CVS2-24

159 CVS2-25

160 cVS2-2

161 CVS2-27
112 CSS2-28

1633 CVS2-29
164 CVS2-30 


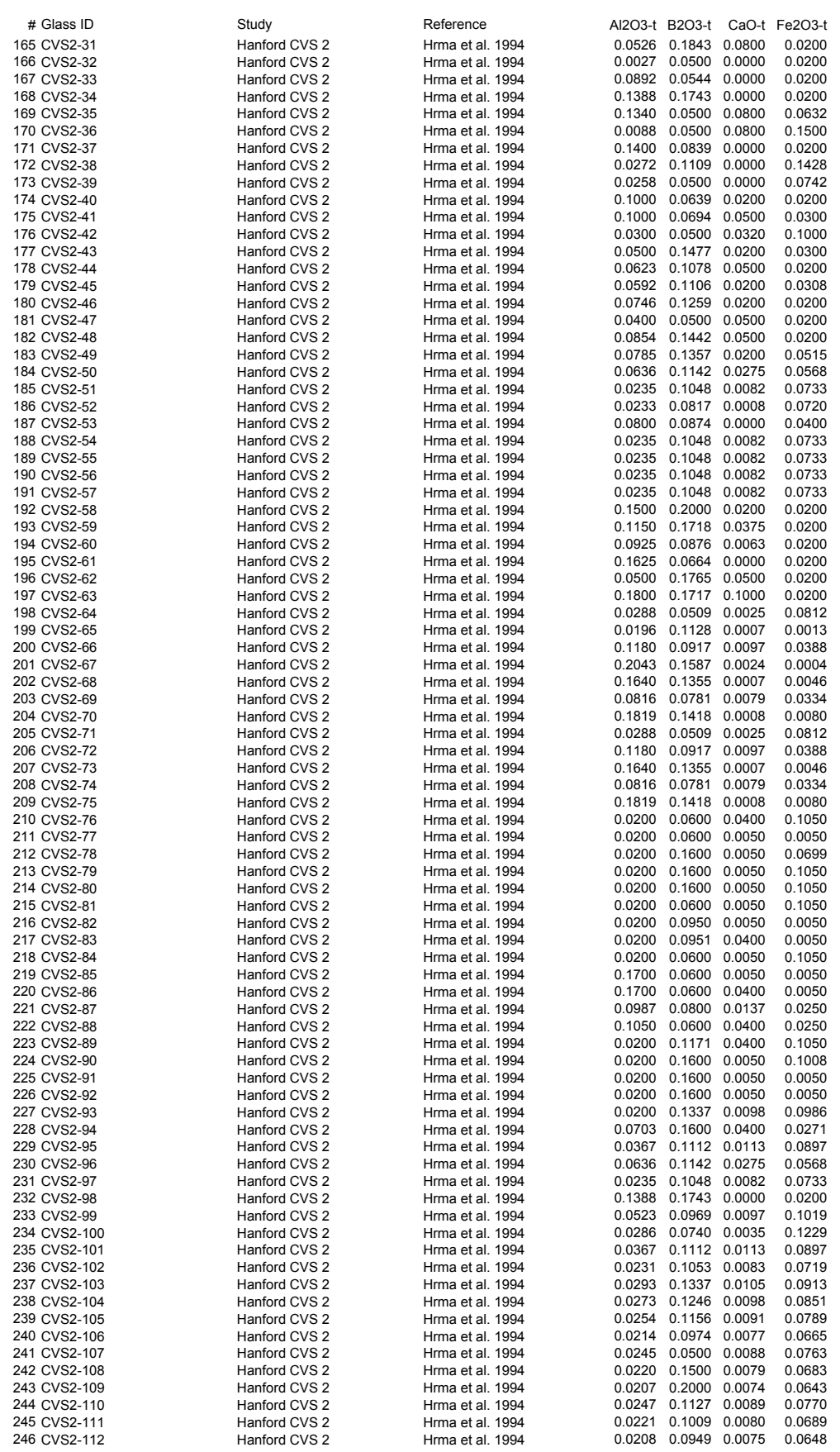

K2O-t Li2O-t MgO-t Na2O-t NiO-t P2O5-t SiO2-t Zro2-t Ag2O-t As2O3-tAs2O5$\begin{array}{llllllllll}0.0000 & 0.0331 & 0.0000 & 0.0500 & 0.0010 & 0.0002 & 0.5700 & 0.0000 & 0.0000 & 0.0000 \\ 0.0000 & 0.0428 & 0.0000 & 0.2000 & 0.0010 & 0.0002 & 0.5445 & 0.1300 & 0.0000 & 0.0000\end{array}$ $\begin{array}{llllllllll}0.00000 & 0.0364 & 0.0800 & 0.2000 & 0.0103 & 0.0018 & 0.4200 & 0.0000 & 0.00000 & 0.00000\end{array}$

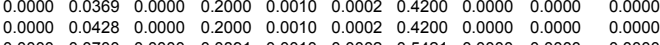

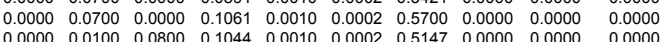

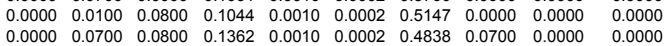
$\begin{array}{llllllllll}0.0000 & 0.0421 & 0.0500 & 0.1500 & 0.0031 & 0.0005 & 0.5040 & 0.02000 & 0.0000 & 0.00000\end{array}$ $\begin{array}{llllllllll}0.0000 & 0.0700 & 0.02200 & 0.0781 & 0.0031 & 0.0005 & 0.5325 & 0.0200 & 0.00000 & 0.00000\end{array}$ $\begin{array}{llllllllll}0.0000 & 0.0700 & 0.0380 & 0.0625 & 0.0031 & 0.0005 & 0.5675 & 0.02200 & 0.0000 & 0.0000 \\ 0.0000 & 0.0653 & 0.0300 & 0.0500 & 0.0031 & 0.0005 & 0.5070 & 0.0700 & 0.0000 & 0.0000\end{array}$

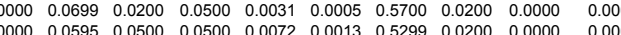

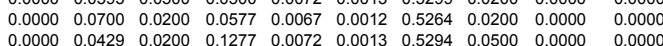

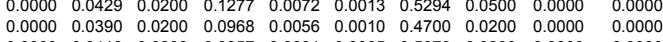

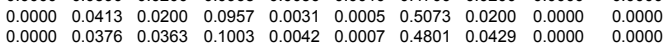
$\begin{array}{lllllllllll}0.0000 & 0.0376 & 0.0363 & 0.1003 & 0.0042 & 0.0007 & 0.4801 & 0.0429 & 0.0000 & 0.0000 \\ 0.0000 & 0.0373 & 0.0084 & 0.1129 & 0.0060 & 0.0010 & 0.5328 & 0.0392 & 0.0000 & 0.0000\end{array}$

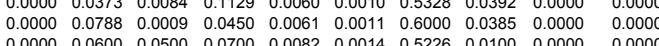

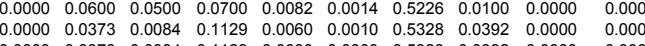
$\begin{array}{llllllllll}0.0000 & 0.0373 & 0.0084 & 0.1129 & 0.0000 & 0.0000 & 0.5328 & 0.0392 & 0.0000 & 0.0000 \\ 0.0000 & 0.0373 & 0.0084 & 0.1129 & 0.0000 & 0.0000 & 0.5328 & 0.0392 & 0.0000 & 0.0000\end{array}$ $\begin{array}{llllllllll}0.0000 & 0.0373 & 0.0084 & 0.1129 & 0.0000 & 0.0000 & 0.5328 & 0.0392 & 0.0000 & 0.0000 \\ 0.0000 & 0.0700 & 0.0800 & 0.0500 & 0.0010 & 0.0002 & 0.3900 & 0.0100 & 0.0000 & 0.0000\end{array}$ $\begin{array}{llllllllll}0.0000 & 0.0700 & 0.0800 & 0.0500 & 0.0010 & 0.0002 & 0.3900 & 0.0100 & 0.0000 & 0.0000 \\ 0.0000 & 0.0727 & 0.0005 & 0.1268 & 0.0011 & 0.0002 & 0.4380 & 0.0075 & 0.0000 & 0.0000\end{array}$ $\begin{array}{lllllllllllll}0.0000 & 0.0743 & 0.0005 & 0.1725 & 0.0011 & 0.0002 & 0.5281 & 0.0075 & 0.0000 & 0.0000\end{array}$ $\begin{array}{llllllllll}0.0000 & 0.0730 & 0.0000 & 0.1200 & 0.0013 & 0.0002 & 0.5281 & 0.0175 & 0.0000 & 0.0000 \\ 0.0000 & 0.0156 & 0.0005 & 0.1125 & 0.0010 & 0.0002 & 0.5579 & 0.0075 & 0.0000 & 0.0000\end{array}$

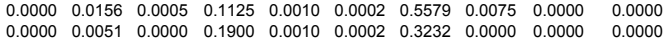

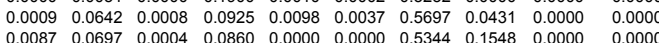
$\begin{array}{llllllllll}0.0087 & 0.0697 & 0.0004 & 0.0860 & 0.0000 & 0.0000 & 0.5344 & 0.1548 & 0.0000 & 0.0000 \\ 0.0000 & 0.0523 & 0.0061 & 0.1211 & 0.0052 & 0.0000 & 0.5175 & 0.0026 & 0.0000 & 0.0000\end{array}$ $\begin{array}{llllllllll}0.0000 & 0.0523 & 0.0061 & 0.1211 & 0.0052 & 0.0000 & 0.5175 & 0.0026 & 0.0000 & 0.0000 \\ 0.0000 & 0.0583 & 0.0001 & 0.1086 & 0.0000 & 0.0049 & 0.4596 & 0.0000 & 0.0000 & 0.0000\end{array}$ $\begin{array}{lllllllllll}0.0016 & 0.0696 & 0.0002 & 0.0797 & 0.0000 & 0.0000 & 0.5040 & 0.0001 & 0.0000 & 0.0000\end{array}$ $\begin{array}{llllllllll}0.0000 & 0.0713 & 0.0032 & 0.0664 & 0.0000 & 0.0326 & 0.5660 & 0.0005 & 0.0000 & 0.0000 \\ 0.0000 & 0.0691 & 0.0008 & 0.0812 & 0.0008 & 0.0007 & 0.4854 & 0.0005 & 0.0000 & 0.0011 \\ 0.0000 & 0.0642 & 0.0008 & 0.0925 & 0.0068 & 0.0012 & 0.5697 & 0.0431 & 0.0000 & 0.0000\end{array}$ $\begin{array}{llllllllll}0.0000 & 0.0523 & 0.0061 & 0.1211 & 0.0043 & 0.0008 & 0.5175 & 0.0026 & 0.0000 & 0.0000 \\ 0.0000 & 0.0696 & 0.0002 & 0.0797 & 0.0043 & 0.0007 & 0.5040 & 0.0001 & 0.0000 & 0.0000 \\ 0\end{array}$ $\begin{array}{llllllllll}0.0000 & 0.0713 & 0.0032 & 0.0664 & 0.0094 & 0.0016 & 0.5660 & 0.0005 & 0.0000 & 0.0000\end{array}$

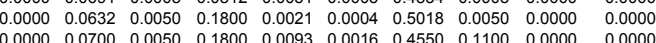

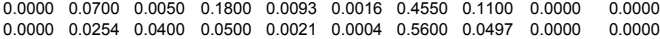
$\begin{array}{llllllllll}0.0000 & 0.0254 & 0.0400 & 0.0500 & 0.0021 & 0.0004 & 0.5600 & 0.0497 & 0.0000 & 0.0000 \\ 0.0000 & 0.0121 & 0.0050 & 0.0500 & 0.0093 & 0.0016 & 0.5479 & 0.0050 & 0.0000 & 0.0000\end{array}$ $\begin{array}{llllllllll}0.0000 & 0.0176 & 0.0400 & 0.0500 & 0.0021 & 0.0004 & 0.5074 & 0.0750 & 0.0000 & 0.0000\end{array}$ $\begin{array}{llllllllll}0.0000 & 0.0700 & 0.0400 & 0.1734 & 0.0084 & 0.0015 & 0.4400 & 0.0050 & 0.0000 & 0.0000 \\ 0.0000 & 0.0700 & 0.0400 & 0.1800 & 0.0021 & 0.0004 & 0.5600 & 0.0050 & 0.0000 & 0.0000\end{array}$ $\begin{array}{llllllllll}0.0000 & 0.0700 & 0.0400 & 0.1800 & 0.0021 & 0.0004 & 0.5600 & 0.0050 & 0.0000 & 0.0000 \\ 0.0000 & 0.0699 & 0.0050 & 0.1800 & 0.0093 & 0.0016 & 0.4900 & 0.0050 & 0.0000 & 0.0000\end{array}$ $\begin{array}{llllllllll}0.0000 & 0.0700 & 0.0050 & 0.1800 & 0.0021 & 0.0004 & 0.4550 & 0.0800 & 0.0000 & 0.0000 \\ 0.0000 & 0.0700 & 0.0200 & 0.1800 & 0.0046 & 0.0008 & 0.4400 & 0.0050 & 0.0000 & 0.0000\end{array}$ $\begin{array}{llllllllll}.0000 & 0.0136 & 0.0050 & 0.1800 & 0.0046 & 0.0008 & 0.4764 & 0.0050 & 0.0000 & 0.0000\end{array}$ $\begin{array}{llllllllll}0.0000 & 0.0700 & 0.0050 & 0.1403 & 0.0021 & 0.0004 & 0.4597 & 0.0750 & 0.0000 & 0.0000 \\ 0.0000 & 0.0100 & 0.0050 & 0.1800 & 0.0021 & 0.0004 & 0.4400 & 0.0629 & 0.0000 & 0.0000\end{array}$ $\begin{array}{lllllllllll}0.0000 & 0.0700 & 0.0050 & 0.0542 & 0.0021 & 0.00004 & 0.5600 & 0.00050 & 0.00000 & 0.00000\end{array}$ $\begin{array}{llllllllll}0.0000 & 0.0700 & 0.0400 & 0.1000 & 0.0093 & 0.0016 & 0.4400 & 0.0700 & 0.0000 & 0.0000 \\ 0.0000 & 0.0700 & 0.0050 & 0.1279 & 0.0093 & 0.0016 & 0.4400 & 0.0050 & 0.0000 & 0.0000\end{array}$ $\begin{array}{llllllllll}0.0000 & 0.0700 & 0.0050 & 0.1279 & 0.0093 & 0.0016 & 0.4400 & 0.0050 & 0.0000 & 0.0000 \\ 0.0000 & 0.0526 & 0.0050 & 0.1800 & 0.0021 & 0.0004 & 0.4400 & 0.0050 & 0.0000 & 0.0000\end{array}$

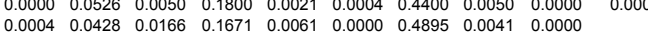
$\begin{array}{llllllllll}0.0000 & 0.0376 & 0.0363 & 0.1003 & 0.0042 & 0.0007 & 0.4801 & 0.0429 & 0.0000 & 0.0000\end{array}$

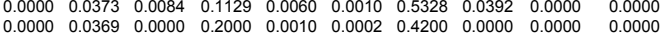

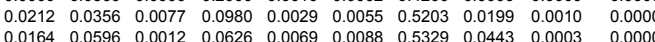
$\begin{array}{lllllllllll}0.0004 & 0.0428 & 0.0166 & 0.1671 & 0.0061 & 0.0000 & 0.4895 & 0.0041 & 0.0000 & & 0.0000\end{array}$

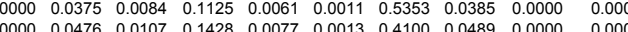
$\begin{array}{llllllllll}0.0000 & 0.0444 & 0.0099 & 0.1332 & 0.0072 & 0.0013 & 0.4500 & 0.0446 & 0.0000 & 0.00000 \\ 0.0000 & 0.0412 & 0.0092 & 0.1235 & 0.0067 & 0.0012 & 0.4900 & 0.0423 & 0.0000 & 0.0000\end{array}$

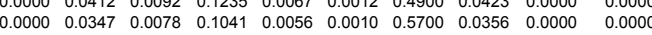

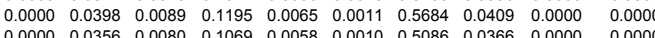
$\begin{array}{llllllllll}0.0000 & 0.0356 & 0.0080 & 0.1069 & 0.0058 & 0.0010 & 0.5086 & 0.0366 & 0.0000 & 0.0000 \\ 0.0000 & 0.0335 & 0.0075 & 0.1006 & 0.0055 & 0.0009 & 0.4786 & 0.0344 & 0.0000 & 0.0000\end{array}$ $\begin{array}{lllllllllll}0.0000 & 0.0335 & 0.0075 & 0.1006 & 0.0055 & 0.0009 & 0.4786 & 0.0344 & 0.0000 & 0.0000 \\ 0.0000 & 0.0401 & 0.0090 & 0.0500 & 0.0065 & 0.0011 & 0.5730 & 0.0412 & 0.0000 & 0.0000\end{array}$ $\begin{array}{llllllllll}0.0000 & 0.0359 & 0.0081 & 0.1500 & 0.0058 & 0.0010 & 0.5127 & 0.0369 & 0.0000 & 0.0000 \\ 0.0000 & 0.0338 & 0.0076 & 0.2000 & 0.0055 & 0.0010 & 0.4825 & 0.0347 & 0.0000 & 0.0000\end{array}$

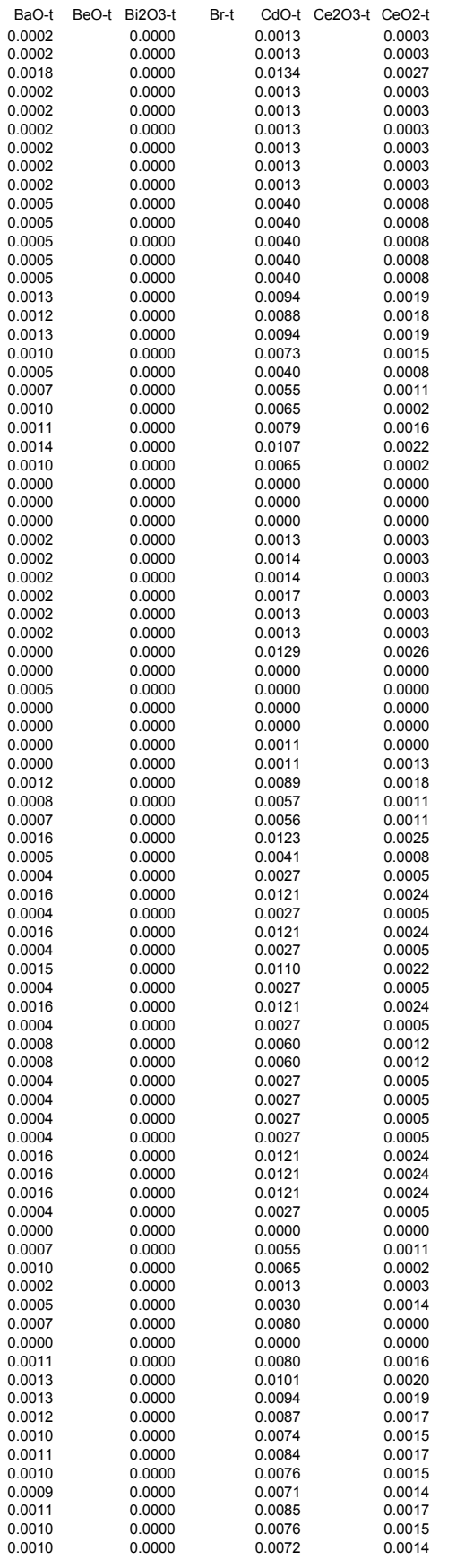




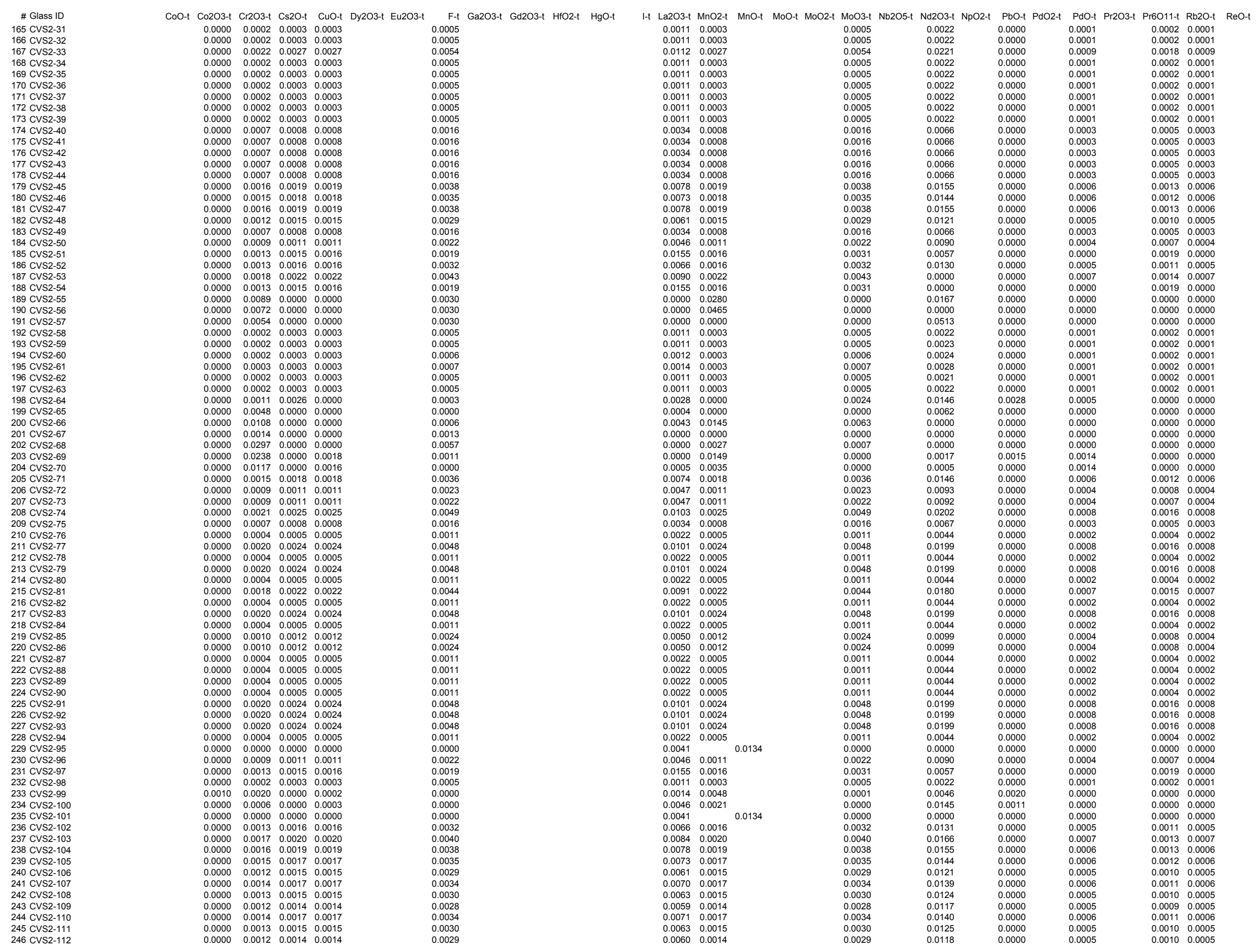




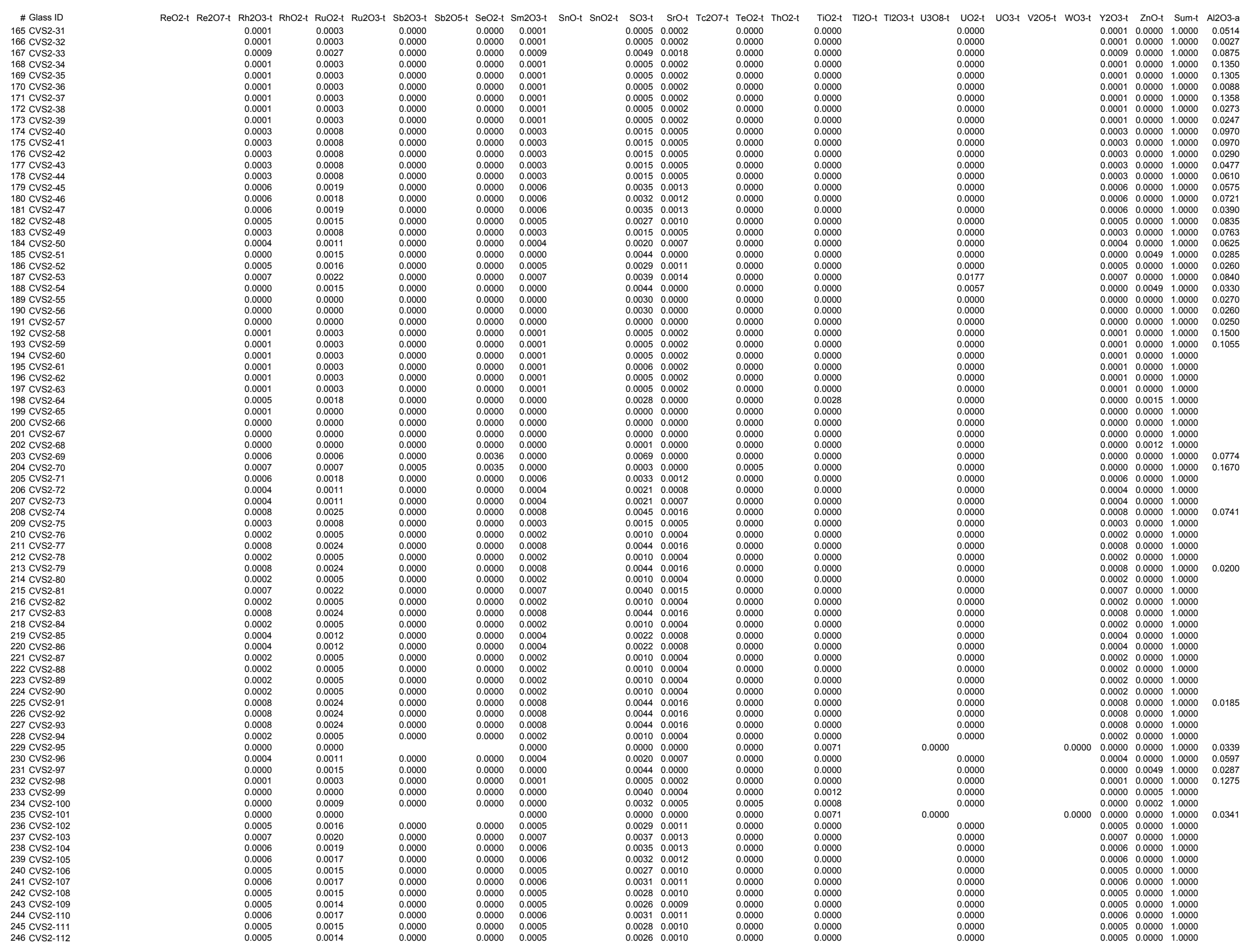


B203-a CaO-a Fe2O3-a FeO-a K2O-a Li2O-a MgO-a Na2O-a NiO-a P2O5-a SiO2-a ZrO2-a Ag2O-a As203-a As2O5-a BaO-a BeO-a Bi2O3-a B $\begin{array}{lllllllll}0.0000 & 0.0692 & 0.0005 & 0.1395 & 0.0009 & 0.0002 & 0.5338 & 0.0000\end{array}$ $\begin{array}{llllllll}0.0000 & 0.0643 & 0.0690 & 0.1725 & 0.0009 & 0.0005 & 0.4551 & 0.0601\end{array}$ $\begin{array}{lll}0.1895 & 0.0757 & 0.0193 \\ 0.0491 & 0.0005 & 0.194 \\ 0.940 & 0.005 & 0.960\end{array}$

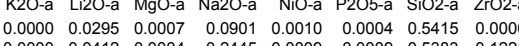
$\begin{array}{lll}0.0491 & 0.0005 & 0.0194 \\ 0.0540 & 0.0005 & 0.0190\end{array}$ $\begin{array}{lll}0.1700 & 0.0005 & 0.0195 \\ 0.0493 & 0.0776 & 0.0597\end{array}$ $\begin{array}{lll}0.0497 & 0.0780 & 0.1443 \\ 0.0830 & 0.0002 & 0.0190\end{array}$ $0.1100 \quad 0.0002 \quad 0.1338$ $0.0483 \quad 0.00050 .0675$ $0.0680 \quad 0.0480 \quad 0.0285$ $\begin{array}{lll}0.0490 & 0.0310 & 0.0930 \\ 0.1418 & 0.0209 & 0.0283 \\ 0.1100 & 0.190 & 0.0290\end{array}$ $0.1050 \quad 0.0470 \quad 0.0190$ $\begin{array}{lll}0.1100 & 0.0190 & 0.0290 \\ 0.1225 & 0.0196 & 0.0194\end{array}$ $\begin{array}{lll}0.1225 & 0.0196 & 0.0194 \\ 0.0495 & 0.0490 & 0.0190\end{array}$ $\begin{array}{lll}0.1300 & 0.0490 & 0.0190 \\ 0.1325 & 0.0191 & 0.0494\end{array}$ $\begin{array}{lll}0.1150 & 0.0270 & 0.0535 \\ 0.0975 & 0.0099 & \end{array}$ $\begin{array}{lll}0.09875 & 0.0099 & 0.057 \\ 0.0890 & 0.0012 & 0.0755\end{array}$ $\begin{array}{lll}0.0910 & 0.0018 & 0.0360 \\ 0.1100 & 0.0094 & 0.0660 \\ 0.1150 & .0069 & 0.065\end{array}$ $0.1150 \quad 0.0069 \quad 0.0765$ $\begin{array}{lll}0.1100 & 0.0066 & 0.0760 \\ 0.1100 & 0.0067 & 0.0755\end{array}$ $\begin{array}{lll}0.2050 & 0.0190 & 0.0210 \\ 0.1620 & 0.0331 & 0.0173\end{array}$

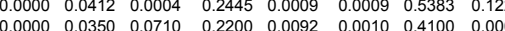
$\begin{array}{llllllll}0.0000 & 0.0350 & 0.0002 & 0.2100 & 0.0009 & 0.0001 & 0.4000 & 0.0009 \\ 0.0000 & 0.0420 & 0.0006 & 0.2450 & 0.0009 & 0.0002 & 0.3978 & 0.0000\end{array}$ $\begin{array}{llllllll}0.0000 & 0.0642 & 0.0000 & 0.1405 & 0.0009 & 0.0004 & 0.5508 & 0.0000\end{array}$ $\begin{array}{llllllll}0.0000 & 0.0660 & 0.0180 & 0.1660 & 0.0028 & 0.0005 & 0.4900 & 0.0160 \\ 0\end{array}$ $\begin{array}{llllllll}0.0000 & 0.0660 & 0.0335 & 0.0660 & 0.0029 & 0.0006 & 0.5500 & 0.017\end{array}$ $\begin{array}{lllllllll}0.0000 & 0.0560 & 0.0445 & 0.0520 & 0.0065 & 0.0010 & 0.4700 & 0.0170\end{array}$ $\begin{array}{lllllllll}0.0000 & 0.0632 & 0.0181 & 0.0927 & 0.0065 & 0.0010 & 0.5060 & 0.015\end{array}$

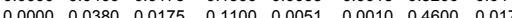
$\begin{array}{lllllllll}0.0000 & 0.0368 & 0.0176 & 0.1280 & 0.0031 & 0.0006 & 0.4800 & 0.0167\end{array}$ $\begin{array}{llllllll}0.0000 & 0.0370 & 0.0317 & 0.1100 & 0.0037 & 0.0007 & 0.4400 & 0.037\end{array}$ $\begin{array}{lllllllll}0.0000 & 0.0830 & 0.0001 & 0.0680 & 0.0000 & 0.0020 & 0.5800 & 0.0390\end{array}$ $\begin{array}{llllllll}0.0000 & 0.0630 & 0.0440 & 0.0650 & 0.0077 & 0.0020 & 0.5000 & 0.0099 \\ 0.0000 & 0.0390 & 0.0075 & 0.1000 & 0.0056 & 0.0010 & 0.5100 & 0.035\end{array}$ $\begin{array}{lllllllll}0.0000 & 0.0405 & 0.0071 & 0.1100 & 0.0000 & 0.0004 & 0.5200 & 0.0390\end{array}$ $\begin{array}{lllllll}0.0405 & 0.0069 & 0.0940 & 0.0000 & 0.0004 & 0.5000 & 0.0370\end{array}$

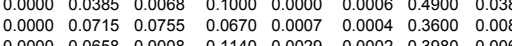

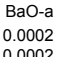

0.0002
0.0002
0.0017

0.0017
0.0002
0.0002

0.0002
0.0002
0.002

0.0002

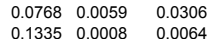

$\begin{array}{lll}0.0751 & 0.0070 \quad 0.0293\end{array}$

$\begin{array}{lll}0.1565 & 0.0046 \quad 0.0999\end{array}$

\subsection{0}

0.000

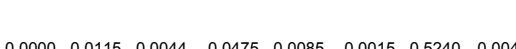

$\begin{array}{cc}\text { Bra } & \begin{array}{c}\text { CdO-a } \\ 0.0013\end{array} \\ 0.0012 \\ 0.0125 \\ 0.0012 \\ 0.0012 \\ 0.0012 \\ 0.0012 \\ 0.0013 \\ 0.0012 \\ 0.0038 \\ 0.0039 \\ 0.0037 \\ 0.0037 \\ 0.0004 \\ 0.0088 \\ 0.0081 \\ 0.0088 \\ 0.0069 \\ 0.0038 \\ 0.0051 \\ 0.0059 \\ 0.0075 \\ 0.0100 \\ 0.0062 \\ 0.0000 \\ 0.0000 \\ 0.0000 \\ 0.0012 \\ 0.0018\end{array}$

CdO-a
0.0013
0.0012
0.0125
0.0012
0.0012
0.0012
0.0012
0.0013
0.0012
0.0038
0.0039
0.0037
0.0037
0.0004
0.0088
0.0081
0.0088
0.0069
0.0038
0.0051
0.0059
0.0075
0.0100
0.0062
0.0000
0.0000
0.0000
0.0012
0.0018
Cl-a CoO-a Co2O3-a Cr2O3-a Cs2O-a CuO-a F-a 0.0002 0.0055

$\begin{array}{llll}0.0001 & 0.0005 & 0.0003 & 0.0005 \\ 0.0002 & 0.000\end{array}$ $\begin{array}{lllll}0.0002 & 0.0003 & 0.0002 & 0.0005 \\ 0.0021 & 0.0028 & 0.0025 & 0.0054\end{array}$ $\begin{array}{llll}0.0002 & 0.0006 & 0.0003 & 0.0005 \\ 0.0002 & 0.0002 & 0.0002 & 0.0005\end{array}$ $\begin{array}{llll}0.0002 & 0.0002 & 0.0002 & 0.0005\end{array}$ $\begin{array}{llll}0.0003 & 0.0003 & 0.0003 & 0.00005\end{array}$ $\begin{array}{lllll}0.0002 & 0.0004 & 0.0002 & 0.0005\end{array}$ $\begin{array}{lllll}0.0006 & 0.0011 & 0.0008 & 0.0016\end{array}$ $\begin{array}{llll}0.0006 & 0.0009 & 0.0007 & 0.0016\end{array}$ $\begin{array}{llll}0.0007 & 0.0012 & 0.0008 & 0.0016 \\ 0.0006 & 0.0010 & 0.0008 & 0.0016\end{array}$ $\begin{array}{lllll}0.0014 & 0.0022 & 0.0018 & 0.0038\end{array}$ $\begin{array}{llll}0.0014 & 0.0024 & 0.0017 & 0.0035\end{array}$ $\begin{array}{llll}0.0017 & 0.0022 & 0.0017 & 0.0038 \\ 0.0011 & 0.0017 & 0.0014 & 0.0029\end{array}$ $\begin{array}{lllll}0.0001 & 0.0113 & 0.0008 & 0.0016\end{array}$ $\begin{array}{llll}0.0008 & 0.0012 & 0.0001 & 0.0219\end{array}$ $\begin{array}{lllll}0.00013 & 0.0016 & 0.0019 & 0.0019 \\ 0.0013 & 0.0016 & 0.0015 & 0.0032\end{array}$ $\begin{array}{lllll}0.0017 & 0.0022 & 0.0020 & 0.0043\end{array}$ $\begin{array}{lllll}0.0013 & 0.0015 & 0.0015 & 0.0019\end{array}$ $\begin{array}{llll}0.0082 & 0.0015 & 0.0015 & 0.043 \\ 0.0066 & 0.0000 & 0.0006 & 0.0030\end{array}$ $\begin{array}{llll}0.0049 & 0.0000 & 0.0000 & 0.0030 \\ 0.0002 & 0.0003 & 0.0003 & 0.0005\end{array}$

\begin{tabular}{|c|c|c|c|c|c|c|c|c|c|c|c|c|c|c|c|c|c|c|c|}
\hline 0.1545 & 0.0044 & 0.0036 & 0.0000 & 0.0091 & 0.0342 & 0.0999 & 0.0081 & 0.0020 & 0.4895 & 0.0050 & 0.0000 & 0.0015 & 0.0102 & 0.0021 & 0.0000 & 0.0019 & 0.0024 & 0.0022 & 0.0048 \\
\hline 0.1055 & 0.0107 & 0.0833 & 0.0000 & 0.0407 & 0.0140 & 0.1600 & 00055 & 0.006 & 04630 & 0,003 & 0,0000 & 00006 & 0.0042 & 0.0008 & 00001 & 00007 & 0,0008 & & \\
\hline 0.1120 & 0.0247 & 0.0527 & 0.0000 & 0.0347 & 0.0317 & 0.0994 & 0.0036 & 0.0007 & 0.4460 & 0.0398 & 0.0000 & 0.0007 & 0.0047 & 0.0010 & 0.0000 & 0.0009 & 0.0011 & 0.0010 & 0.0022 \\
\hline 0.0994 & & 0.0590 & 0.0000 & 0.0328 & 0.0078 & 0.1140 & 0.0060 & 0.0015 & 0.4850 & 0.0367 & 0.0000 & 0.0023 & 0.0060 & 0.0002 & 0.0004 & 0.0011 & 0.0015 & 0.0020 & 0.0019 \\
\hline 0.1645 & 0.0008 & 0.0172 & 0.0000 & 0.0333 & 0.0000 & 0.1840 & 0.0008 & 0.0002 & 0.3840 & 0.0001 & 0.0000 & 0.0002 & 0.0011 & 0.0003 & 0.0000 & 0.0003 & 0.0003 & 0.0003 & 0.0005 \\
\hline 0.1055 & 0.0100 & 0.0850 & 0.0000 & 0.0415 & 0.0155 & 0.1650 & 0.0054 & & 0.4610 & 0.0043 & & & 0.0001 & & 0.0001 & & & & \\
\hline
\end{tabular}




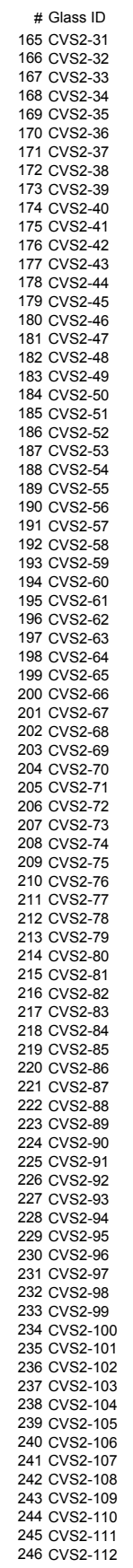

Ia La203-a MnO2-a MnO-a MoO-a MoO3-a Nb205-a Nd203-a NpO2-a PbO-a PdO2-a $0.0010 \quad 0.0004$ $\begin{array}{ll}0.0010 & 0.0002 \\ 0.0101 & 0.0024\end{array}$

$\begin{array}{ll}0.0011 & 0.0003 \\ 0.0009 & 0.0001\end{array}$ $\begin{array}{ll}0.0009 & 0.0002 \\ 0.0009 & 0.0003\end{array}$

\begin{tabular}{lll}
0.0009 & 0.0003 \\
0.0010 & 0.0003 \\
\hline & 0.0002 & 0.002
\end{tabular}

\begin{tabular}{ll}
0.0008 & 0.0002 \\
0.0029 & 0.0007 \\
\hline 0.0030 & 0.007
\end{tabular}

$\begin{array}{ll}0.0029 & 0.0027 \\ 0.0033 & 0.0007 \\ 0.0030 & 0.0007 \\ 0.0028 & 0.0007\end{array}$

$\begin{array}{ll}0.0030 & 0.0007 \\ 0.0028 & 0.0008\end{array}$

$\begin{array}{ll}0.0029 & 0.0007 \\ 0.0071 & 0.0017 \\ 0 & 0.009\end{array}$

$0.0066 \quad 0.0018$

$\begin{array}{ll}0.0069 & 0.0018 \\ 0.0057 & 0.001\end{array}$

$\begin{array}{ll}0.0031 & 0.0009 \\ 0.0038 & 0.0010 \\ 0.0255 & 0.0021\end{array}$

$\begin{array}{ll}0.0038 & 0.0010 \\ 0.0205 & 0.0021 \\ 0.001 & 0.016\end{array}$

$\begin{array}{ll}0.0001 & 0.0011 \\ 0.0081 & 0.0021 \\ 0.0000 & 0.025\end{array}$

$\begin{array}{ll}0.0140 & 0.0016 \\ 0.0000 & 0.0250 \\ 0.0000 & 0.0000\end{array}$

$\begin{array}{ll}0.0000 & 0.038 \\ 0.0000 & 0.0000\end{array}$

$\begin{array}{lll}0.0010 & 0.0003 \\ 0.0010 & 0.0004 & 0.0000\end{array}$

0.0005
0.0005
0.0044
0.0005
0.0005
0.0005
0.0005
0.0005
0.0005
0.0014
0.0015
0.0014
0.0014
0.0014
0.0035
0.0034
0.0035
0.0029
0.0015
0.0021
0.0028
0.0032
0.0042
0.0030
0.0000
0.0000
0.0000
0.0005
0.0006

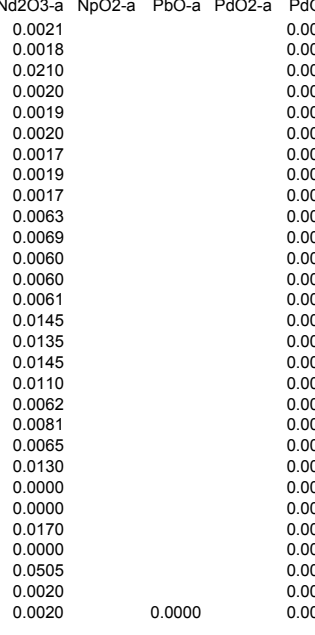

\begin{tabular}{|c|c|c|c|}
\hline & Pr2O3-a & Pr6011-a PuO2-a & \\
\hline $\begin{array}{l}0.0001 \\
0.0001\end{array}$ & & $\begin{array}{l}0.0002 \\
0.0002\end{array}$ & $\begin{array}{l}0.0001 \\
0.0001\end{array}$ \\
\hline & & & 0.0009 \\
\hline 0.0009 & & 0.0002 & 0.0009 \\
\hline $\begin{array}{l}0.0001 \\
0.001\end{array}$ & & 0.0002 & 0.0001 \\
\hline & & $\begin{array}{l}0.0002 \\
0.0002\end{array}$ & $\begin{array}{l}0.0001 \\
0.0001\end{array}$ \\
\hline 0.0001 & & $\begin{array}{l}0.0002 \\
0.0002\end{array}$ & $\begin{array}{l}0.0001 \\
0.0001\end{array}$ \\
\hline 0.0001 & & & \\
\hline 0.0003 & & 0.0005 & 0.0003 \\
\hline & & 0.0005 & 0.0003 \\
\hline 0.003 & & 0.0005 & 0.0003 \\
\hline 0000 & & 0.0005 & 0.0003 \\
\hline & & $\begin{array}{l}0.0005 \\
0.0013\end{array}$ & 0.0003 \\
\hline & & 0.0012 & 0.0006 \\
\hline & & 0.0013 & 0.0006 \\
\hline & & 0.0010 & 0.0005 \\
\hline & & 0.0005 & 0.0003 \\
\hline & & 0.0007 & 0.0004 \\
\hline & & 0.0019 & 0.0000 \\
\hline & & 0.0011 & 0.0005 \\
\hline & & 0.0014 & 0.0007 \\
\hline & & 0.0000 & 0.0000 \\
\hline 0.0000 & & 0.0000 & 0.0000 \\
\hline & & 0.0000 & 0.0000 \\
\hline & & 0.0018 & 0.0001 \\
\hline
\end{tabular}

Rh203-a RhO2-a RuO2-a Sb203-a Sb205-a SeO2-a

\begin{abstract}
.
\end{abstract}

0.0000
0.0000

$\begin{array}{llll}0.0000 & 0.0142 & 0.0000 & 0.0000 \\ 0.0004 & 0.0033 & 0.0000 & 0.0000 \\ & & & \\ 0.0084 & 0.0022 & 0.0000 & 0.0044 \\ & & & \\ & & & \\ & & \end{array}$

\begin{tabular}{|c|c|c|c|c|}
\hline $\begin{array}{l}0.0013 \\
0.0004\end{array}$ & $\begin{array}{l}0.0020 \\
0.0000\end{array}$ & $\begin{array}{l}0.0014 \\
0.0014\end{array}$ & $\begin{array}{l}0.0000 \\
0.0000\end{array}$ & $\begin{array}{l}0.0000 \\
0.0000\end{array}$ \\
\hline 0.0177 & 0.0000 & 0.0008 & 0.0016 & 0.0008 \\
\hline
\end{tabular}

$\begin{array}{lll}0.0083 & 0.0022 & 0.0000\end{array}$

0.0043

0.0000
0.0000
0.0000
0.0000

$\begin{array}{lll}0.0035 & 0.0125 & 0.0000 \\ 0.0039 & 0.0011 & 0.0000 \\ 0.0007 & 0.0003 & 0.0000\end{array}$

0.0017
0.0020
0.0029
0.0005

$0.0069 \quad 0.0000$

0.000

0.0016

0.0008

0.0008

$0.0018 \quad 0.0000$

0.0000

$\begin{array}{lll}0.0007 & 0.0003 & 0.00000\end{array}$

0.0077
0.0066
0.0015

0.0000
0.0000
0.0000
0.0000

0.0003
0.0004
0.0000
0.0001

0.0006
0.0007
0.0019

0.0003
0.0004

$\begin{array}{lll}0.0038 & 0.0153 & 0.0000\end{array}$

(1)




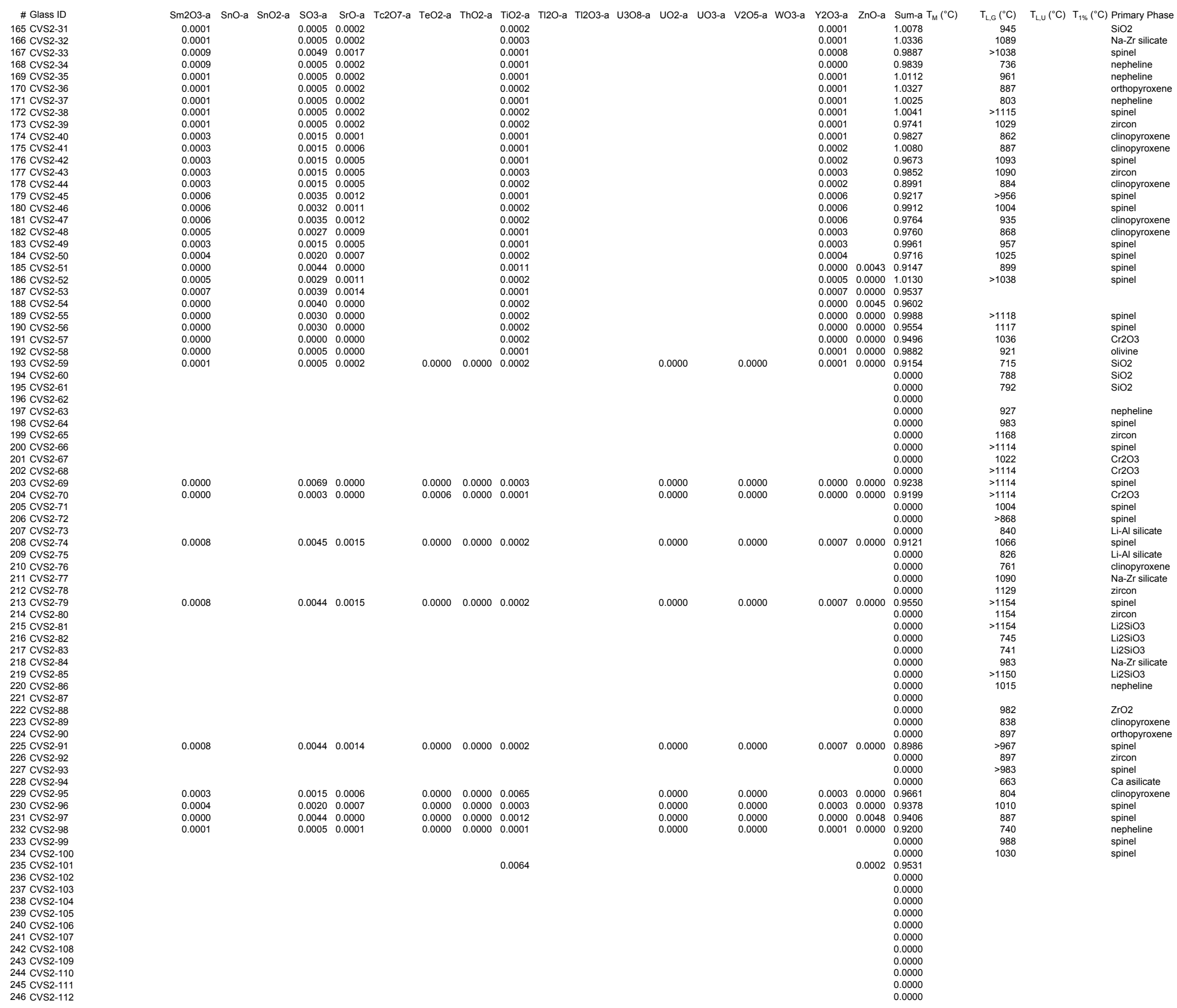




-7.670
-6.660
-.870
-5.490
-5.930
-4.800
-5.260
-7.060
-.750
-5.500
-5.740
-4.860
-5.650
-5.460
-4.810
-5.900
-5.060
-4.990
-6.130
-4.930

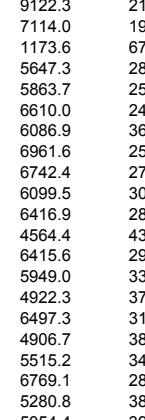

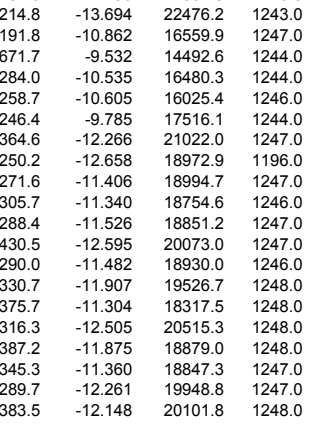




\begin{tabular}{|c|c|c|c|c|c|c|c|c|c|c|c|c|c|c|c|c|c|c|}
\hline \# Glass ID & $\mathrm{T} 2\left({ }^{\circ} \mathrm{C}\right)$ & V2 (Pa:s) & $\mathrm{T} 3\left({ }^{\circ} \mathrm{C}\right)$ & V3 (Pa:s) & $\mathrm{T} 4\left({ }^{\circ} \mathrm{C}\right)$ & V4 (Pa:s) & $\mathrm{T} 5\left({ }^{\circ} \mathrm{C}\right)$ & V5 (Pa-s) & $\mathrm{T} 6\left({ }^{\circ} \mathrm{C}\right)$ & V6 (Pa:s) & $\mathrm{T} 7\left({ }^{\circ} \mathrm{C}\right)$ & V7 (Pa:s) & $\mathrm{T} 8\left({ }^{\circ} \mathrm{C}\right)$ & V8 (Pa:s) & T9 $\left({ }^{\circ} \mathrm{C}\right)$ & V9 (Pa:s) & $\mathrm{T} 10\left({ }^{\circ} \mathrm{C}\right)$ & V10 (Pa:s) TCLP Ag TCLPAs TCLP Ba TCLP Cd TCLP Cr TCLP Ni TCLP Pb \\
\hline 165 CVS2-31 & 1195 & 6.820 & 1146 & 10.5 & 1146.0 & 10.8 & 1146.0 & & 1096.0 & & 1096.0 & 17.8 & 1047.0 & 30.0 & 996.0 & 64.2 & 947.0 & 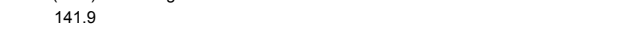 \\
\hline 166 CVS2-32 & 1193 & 5.270 & 1142 & 8.6 & 1143.0 & 8.7 & 1143.0 & 8.8 & 1093.0 & 15.1 & 1092.0 & 15.7 & 1043.0 & 28.2 & 994.0 & 57.5 & 943.0 & 128.6 \\
\hline 167 CVS2-33 & 1198 & 1.520 & 1148 & 2.1 & 1149.0 & 2.2 & 1148.0 & 2.2 & 1099.0 & 3.2 & 1098.0 & 3.3 & 1048.0 & 5.2 & 999.0 & 8.7 & 948.0 & 15.5 \\
\hline $\begin{array}{l}168 \text { CVS2-34 } \\
169 \text { CVS2-35 }\end{array}$ & $\begin{array}{l}1195 \\
1193\end{array}$ & $\begin{array}{l}1.440 \\
2004\end{array}$ & 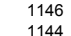 & $\begin{array}{l}2.0 \\
2.9\end{array}$ & $\begin{array}{l}1145.0 \\
1144.0\end{array}$ & 2.0 & $\begin{array}{l}1145.0 \\
11430\end{array}$ & 2.0 & $\begin{array}{l}1097.0 \\
1040\end{array}$ & 2.8 & $\begin{array}{l}1095.0 \\
10930\end{array}$ & 2.8 & $\begin{array}{l}1046.0 \\
1\end{array}$ & 4.1 & $\begin{array}{l}996.0 \\
9930\end{array}$ & 6.6 & 945.0 & 11.3 \\
\hline $\begin{array}{l}169 \text { CVS22-35 } \\
170 \text { CVS2-36 }\end{array}$ & $\begin{array}{l}1193 \\
1196\end{array}$ & $\begin{array}{l}2.040 \\
1.380\end{array}$ & $\begin{array}{l}1144 \\
1145\end{array}$ & $\begin{array}{l}2.9 \\
2.0\end{array}$ & $\begin{array}{l}1144.0 \\
1146.0\end{array}$ & $\begin{array}{l}3.0 \\
2.0\end{array}$ & $\begin{array}{l}1143.0 \\
1145.0\end{array}$ & $\begin{array}{l}3.0 \\
2.0\end{array}$ & $\begin{array}{l}1094.0 \\
1096.0\end{array}$ & $\begin{array}{l}4.4 \\
2.9\end{array}$ & $\begin{array}{l}1093.0 \\
1095.0\end{array}$ & $\begin{array}{l}4.5 \\
2.9\end{array}$ & $\begin{array}{l}1043.0 \\
10450\end{array}$ & $\begin{array}{l}7.1 \\
4.7\end{array}$ & $\begin{array}{l}993.0 \\
995.0\end{array}$ & $\begin{array}{r}12.1 \\
7.5\end{array}$ & $\begin{array}{l}943.0 \\
945.0\end{array}$ & $\begin{array}{l}21.7 \\
13.7\end{array}$ \\
\hline 171 CVS2-37 & 1195 & 8.700 & 1146 & 12.4 & $\begin{array}{l}1146.0 \\
1146.0\end{array}$ & $\begin{array}{l}2.0 \\
12.7\end{array}$ & 1146.0 & $\begin{array}{c}2.0 \\
13.1\end{array}$ & $\begin{array}{l}1096.0 \\
1097.0\end{array}$ & $\begin{array}{l}2.9 \\
19.5\end{array}$ & $\begin{array}{l}10955.0 \\
1095.0\end{array}$ & $\begin{array}{l}20.9 \\
20.1\end{array}$ & $\begin{array}{l}1045.0 \\
1046.0\end{array}$ & $\begin{array}{c}4.1 \\
31.8\end{array}$ & $\begin{array}{l}995.0 \\
997.0\end{array}$ & $\begin{array}{r}7.5 \\
54.7\end{array}$ & $\begin{array}{l}945.0 \\
947.0\end{array}$ & $\begin{array}{c}13.1 \\
103.0\end{array}$ \\
\hline 172 CVS2-38 & 1197 & 7.750 & 1147 & 12.2 & 1147.0 & 12.4 & 1147.0 & 12.4 & 1096.0 & 21.2 & 1097.0 & 21.3 & 1047.0 & 38.8 & $\begin{array}{l}998.0 \\
998\end{array}$ & 77.1 & 1097.0 & $\begin{array}{r}103.0 \\
21.3\end{array}$ \\
\hline 173 CVS2-39 & 1147 & 2.020 & 1143 & 2.1 & 1145.0 & 2.1 & 1097.0 & 3.2 & 1095.0 & 3.3 & 1047.0 & 5.5 & 994.0 & 9.6 & 944.0 & 20.0 & & \\
\hline 174 CVS2-40 & 1197 & 4.720 & 1148 & 6.8 & 1147.0 & 6.8 & 1147.0 & 7.2 & 1098.0 & 11.2 & 1097.0 & 11.4 & 1048.0 & 18.8 & 998.0 & 35.1 & 947.0 & 68.1 \\
\hline 175 CVS2-41 & 1197 & 4.160 & 1147 & 6.3 & 1147.0 & 6.4 & 1147.0 & 6.5 & 1098.0 & 10.0 & 1097.0 & 10.2 & 1047.0 & 16.8 & 998.0 & 29.6 & 947.0 & 61.7 \\
\hline 176 CVS2-42 & 1197 & 3.680 & 1147 & 5.7 & 1148.0 & 5.7 & 1147.0 & 5.8 & 1098.0 & 8.9 & 1096.0 & 9.1 & 1047.0 & 15.0 & 998.0 & 26.7 & 948.0 & 54.5 \\
\hline 177 CVS2-43 & $\begin{array}{l}1197 \\
1197\end{array}$ & 2.940 & 1147 & 4.6 & 1148.0 & 4.6 & 1147.0 & 4.7 & 1097.0 & 7.2 & 1097.0 & 7.3 & 1047.0 & 12.4 & 997.0 & 25.2 & 947.0 & 53.3 \\
\hline $\begin{array}{l}178 \text { CVS22-44 } \\
179 \text { CVS2-45 }\end{array}$ & $\begin{array}{l}1197 \\
1198\end{array}$ & $\begin{array}{l}4.120 \\
4.090\end{array}$ & $\begin{array}{l}1147 \\
1148\end{array}$ & $\begin{array}{l}6.2 \\
6.1\end{array}$ & $\begin{array}{l}1148.0 \\
1149.0\end{array}$ & $\begin{array}{l}6.2 \\
6.1\end{array}$ & $\begin{array}{l}1147.0 \\
11490\end{array}$ & $\begin{array}{l}6.3 \\
6.3\end{array}$ & $\begin{array}{l}1098.0 \\
11000\end{array}$ & $\begin{array}{l}9.8 \\
96\end{array}$ & $\begin{array}{l}1097.0 \\
1098.0\end{array}$ & $\begin{array}{c}10.0 \\
97\end{array}$ & $\begin{array}{l}1047.0 \\
1049.0\end{array}$ & $\begin{array}{l}16.6 \\
164\end{array}$ & $\begin{array}{l}998.0 \\
9980\end{array}$ & $\begin{array}{l}30.4 \\
326\end{array}$ & $\begin{array}{l}948.0 \\
948.0\end{array}$ & $\begin{array}{l}60.0 \\
64.2\end{array}$ \\
\hline $\begin{array}{l}1 / 9 \text { CVS2-45 } \\
180 \text { CVS2 }\end{array}$ & $\begin{array}{l}7198 \\
1199\end{array}$ & $\begin{array}{l}4.0990 \\
3.270\end{array}$ & $\begin{array}{l}71448 \\
1148\end{array}$ & $\begin{array}{l}6.1 \\
4.8\end{array}$ & $\begin{array}{l}1149.0 \\
1150.0\end{array}$ & $\begin{array}{l}6.1 \\
4.8\end{array}$ & $\begin{array}{l}1149.0 \\
1148.0\end{array}$ & $\begin{array}{l}6.3 \\
4.9\end{array}$ & $\begin{array}{l}11100.0 \\
1100.0\end{array}$ & $\begin{array}{l}9.6 \\
7.2\end{array}$ & $\begin{array}{l}1098.0 \\
1097.0\end{array}$ & $\begin{array}{l}9.7 \\
7.3\end{array}$ & $\begin{array}{l}1049.0 \\
1048.0\end{array}$ & $\begin{array}{l}16.4 \\
123\end{array}$ & $\begin{array}{l}998.0 \\
9980\end{array}$ & 32.6 & $\begin{array}{l}948.0 \\
948.0\end{array}$ & $\begin{array}{l}64.2 \\
44.4\end{array}$ \\
\hline 181 CVS2-47 & 1198 & $\begin{array}{l}3.2720 \\
4.320\end{array}$ & $\begin{array}{l}1148 \\
1148\end{array}$ & $\begin{array}{l}.8 . \\
6.6\end{array}$ & 1148.0 & $\begin{array}{l}4.8 \\
6.8\end{array}$ & 1149.0 & $\begin{array}{l}4.9 \\
7.1\end{array}$ & 1098.0 & $\begin{array}{l}11.2 \\
11.0\end{array}$ & 1100.0 & $\begin{array}{l}11.0 \\
11.0\end{array}$ & $\begin{array}{l}1048.0 \\
1048.0\end{array}$ & $\begin{array}{l}12.3 \\
19.3\end{array}$ & $\begin{array}{l}998.0 \\
999.0\end{array}$ & $\begin{array}{l}22.4 \\
37.3\end{array}$ & $\begin{array}{l}948.0 \\
948.0\end{array}$ & $\begin{array}{l}44.4 \\
80.6\end{array}$ \\
\hline 182 CVS2-48 & 1198 & 2.690 & 1148 & 3.9 & 1149.0 & 4.0 & 1148.0 & 4.1 & 1099.0 & 6.2 & 1098.0 & 6.3 & 1048.0 & 10.5 & $\begin{array}{l}999.0 \\
999.0\end{array}$ & 19.6 & $\begin{array}{l}940.0 \\
948.0\end{array}$ & $\begin{array}{l}0.0 \\
39.7\end{array}$ \\
\hline 183 CVS2-49 & 1198 & 4.350 & 1147 & 6.6 & 1147.0 & 6.7 & 1147.0 & 6.7 & 1098.0 & 10.3 & 1098.0 & 10.5 & 1048.0 & 17.6 & 998.0 & 31.3 & 948.0 & 64.9 \\
\hline 184 CVS2-50 & 1197 & 3.780 & 1147 & 5.9 & 1147.0 & 5.9 & 1147.0 & 6.0 & 1098.0 & 9.5 & 1096.0 & 9.6 & 1047.0 & 16.5 & 998.0 & 31.3 & 948.0 & 63.5 \\
\hline 185 CVS2-51 & 1198 & 4.750 & 1149 & 7.0 & 1149.0 & 7.0 & 1148.0 & 7.3 & 1099.0 & 11.6 & 1099.0 & 11.7 & 1048.0 & 20.0 & 999.0 & 39.6 & 948.0 & 82.2 \\
\hline 186 CVS2-52 & 1198 & 6.230 & 1148 & 9.2 & 1149.0 & 9.4 & 1149.0 & 9.6 & 1099.0 & 14.7 & 1098.0 & 14.8 & 1048.0 & 25.9 & 999.0 & 48.6 & 949.0 & 100.7 \\
\hline $\begin{array}{l}187 \text { CVS2-53 } \\
188 \text { CVS2-54 }\end{array}$ & & & & & & & & & & & & & & & & & & \\
\hline 189 cVS2-55 & 1197 & 4.180 & 1147 & 6.4 & 1147.0 & 6.5 & 1147.0 & 6.5 & 1097.0 & 10.4 & 1096.0 & 10.6 & 1046.0 & 17.8 & 997.0 & 33.9 & 946.0 & 70.3 \\
\hline 190 CVS2-56 & 1197 & 4.120 & 1146 & 6.2 & 1147.0 & 6.3 & 1147.0 & 6.4 & 1097.0 & 10.1 & 1096.0 & 10.1 & 1047.0 & 17.1 & 996.0 & 32.5 & 946.0 & 65.9 \\
\hline 191 CVS2-57 & $\begin{array}{l}1197 \\
1195\end{array}$ & $\begin{array}{l}4.490 \\
1310\end{array}$ & $\begin{array}{l}1147 \\
1146\end{array}$ & 6.9 & 1147.0 & 7.0 & 1147.0 & 7.0 & 1097.0 & 11.3 & 1096.0 & & 1046.0 & 19.9 & & 38.6 & 945.0 & 82.6 \\
\hline $\begin{array}{l}192 \text { CVS22-58 } \\
193 \text { CVS2-59 }\end{array}$ & $\begin{array}{l}1195 \\
1196\end{array}$ & $\begin{array}{l}1.310 \\
0.890\end{array}$ & $\begin{array}{l}1146 \\
1147\end{array}$ & $\begin{array}{l}1.9 \\
1.2\end{array}$ & $\begin{array}{l}1146.0 \\
1147.0\end{array}$ & $\begin{array}{l}1.9 \\
1.2\end{array}$ & $\begin{array}{l}1146.0 \\
1147.0\end{array}$ & $\begin{array}{l}1.9 \\
1.2\end{array}$ & $\begin{array}{l}1097.0 \\
1098.0\end{array}$ & $\begin{array}{l}2.9 \\
16\end{array}$ & $\begin{array}{l}1096.0 \\
1097.0\end{array}$ & $\begin{array}{l}3.0 \\
17\end{array}$ & $\begin{array}{l}1046.0 \\
1048.0\end{array}$ & $\begin{array}{l}4.8 \\
2.4\end{array}$ & $\begin{array}{l}997.0 \\
998.0\end{array}$ & 8.1 & $\begin{array}{l}946.0 \\
947.0\end{array}$ & $\begin{array}{r}15.3 \\
6.8 \\
\end{array}$ \\
\hline 194 CVS2-60 & $\begin{array}{l}1196 \\
1196\end{array}$ & $\begin{array}{l}.8 .920 \\
1.920\end{array}$ & $\begin{array}{l}7147 \\
1147\end{array}$ & $\begin{array}{l}1.2 \\
2.6\end{array}$ & 1147.0 & $\begin{array}{l}1.2 \\
2.6\end{array}$ & $\begin{array}{l}1147.0 \\
1147.0\end{array}$ & $\begin{array}{l}1.2 \\
2.7\end{array}$ & $\begin{array}{l}1998.0 \\
1098.0\end{array}$ & $\begin{array}{l}1.6 \\
3.8\end{array}$ & 1098.0 & $\begin{array}{l}1.7 \\
3.9\end{array}$ & $\begin{array}{l}1048.0 \\
1048.0\end{array}$ & $\begin{array}{l}2.4 \\
5.7\end{array}$ & $\begin{array}{l}998.0 \\
998.0\end{array}$ & $\begin{array}{l}3.8 \\
9.1\end{array}$ & 947.0 & $\begin{array}{l}0.8 \\
15.2\end{array}$ \\
\hline 195 CVS2-61 & 1197 & 8.780 & 1148 & 13.1 & 1147.0 & 13.1 & 1147.0 & 13.6 & 1098.0 & $\begin{array}{r}5.0 \\
20.4\end{array}$ & 1097.0 & 20.6 & 1047.0 & 33.6 & $\begin{array}{l}998.0 \\
998.0\end{array}$ & 60.1 & 948.0 & $\begin{aligned} & 111.0 \\
& 111.0\end{aligned}$ \\
\hline 196 CVS2-62 & 1195 & 6.700 & 1147 & 9.8 & 1147.0 & 10.4 & 1146.0 & 10.6 & 1097.0 & 17.0 & 1097.0 & 17.1 & 1047.0 & 30.7 & 997.0 & 65.3 & 946.0 & 149.7 \\
\hline 197 CVS2-63 & 1197 & 1.650 & 1147 & 2.4 & 1147.0 & 2.4 & 1147.0 & 2.5 & 1098.0 & 3.7 & 1097.0 & 3.8 & 1047.0 & 6.1 & 998.0 & 10.9 & 947.0 & 20.9 \\
\hline 198 CVS2-64 & 1199 & 5.790 & 1149 & 8.9 & 1149.0 & 8.9 & 1149.0 & 9.0 & 1100.0 & 14.6 & 1099.0 & 14.8 & 1049.0 & 24.8 & 999.0 & 45.7 & 948.0 & 91.6 \\
\hline 199 CVS2-65 & 1196 & 5.060 & 1146 & 8.5 & 1146.0 & 8.6 & 1146.0 & 8.7 & 1096.0 & 15.1 & 1096.0 & 15.3 & 1046.0 & 29.6 & 996.0 & 62.6 & 945.0 & 149.1 \\
\hline $\begin{array}{l}200 \text { CVS2-66 } \\
201 \text { cVS2-67 }\end{array}$ & $\begin{array}{l}1200 \\
1195\end{array}$ & $\begin{array}{r}5.550 \\
6.370\end{array}$ & $\begin{array}{l}1150 \\
1146\end{array}$ & 8.1 & 1150.0 & 8.3 & $\begin{array}{l}1150.0 \\
11460\end{array}$ & 8.4 & $\begin{array}{l}1100.0 \\
10960\end{array}$ & 12.7 & $\begin{array}{l}1100.0 \\
10960\end{array}$ & $\begin{array}{l}12.9 \\
14.3\end{array}$ & 1050.0 & 20.7 & 1000.0 & 37.3 & 949.0 & 70.7 \\
\hline $\begin{array}{l}201 \text { CVS22-67 } \\
202 \text { CVS2-68 }\end{array}$ & $\begin{array}{r}1195 \\
(1201)\end{array}$ & $\begin{array}{r}6.370 \\
(1139)\end{array}$ & $\begin{array}{r}1146 \\
(1151)\end{array}$ & $\begin{array}{r}9.2 \\
(16.13)\end{array}$ & $\begin{array}{l}1146.0 \\
(1150)\end{array}$ & $\begin{array}{r}9.3 \\
(17.68\end{array}$ & $\begin{array}{l}1146.0 \\
(1151)\end{array}$ & $\begin{array}{r}9.4 \\
(1783)\end{array}$ & $\begin{array}{l}1096.0 \\
(1101)\end{array}$ & $\begin{array}{r}14.2 \\
(23.9)\end{array}$ & $\begin{array}{l}1096.0 \\
(1101)\end{array}$ & $\begin{array}{r}14.3 \\
(24.29\end{array}$ & $\begin{array}{l}1046.0 \\
(1051)\end{array}$ & $\begin{array}{r}22.4 \\
(3977)\end{array}$ & $\begin{array}{r}996.0 \\
(1000)\end{array}$ & $\begin{array}{r}39.4 \\
(74.27\end{array}$ & $\begin{array}{l}946.0 \\
950\end{array}$ & $\begin{array}{r}72.6 \\
(106.71)\end{array}$ \\
\hline $\begin{array}{l}202 \text { CVS22-68 } \\
203 \text { CVS2-69 }\end{array}$ & $\begin{array}{l}(1201) \\
(1201)\end{array}$ & $\begin{array}{c}(11.139) \\
(13.6)\end{array}$ & $\begin{array}{l}(1151) \\
(1151)\end{array}$ & $\begin{array}{l}(16.13) \\
(1977)\end{array}$ & $\begin{array}{l}\left(\begin{array}{l}(150) \\
(1150)\end{array}\right. \\
\text { (n) }\end{array}$ & $\begin{array}{l}(17.68) \\
(21.97)\end{array}$ & $\begin{array}{l}(1151) \\
(1151)\end{array}$ & $\begin{array}{l}(17.83) \\
(22.56)\end{array}$ & $\begin{array}{l}(1101) \\
(1101)\end{array}$ & $\begin{array}{r}(236.9) \\
(36.91)\end{array}$ & $\begin{array}{l}(11011) \\
(1101)\end{array}$ & $\begin{array}{l}(24.29) \\
(36.91)\end{array}$ & $\begin{array}{l}(1051) \\
(1051)\end{array}$ & $\begin{array}{l}(39.77) \\
(66.83)\end{array}$ & $\begin{array}{l}(1000) \\
(1000)\end{array}$ & $\begin{array}{l}(14.27) \\
(105.67)\end{array}$ & (950) & (106.71) \\
\hline 204 CVS2-70 & 1204 & 7.390 & 1154 & 10.0 & 1153.0 & 10.3 & 1154.0 & $\begin{array}{r}(2.2 .5) \\
10.5\end{array}$ & 1105.0 & $\begin{array}{l}(0.9) \\
16.0\end{array}$ & 1104.0 & (0.9) & 1053.0 & $\begin{array}{r}(0.00) \\
27.2\end{array}$ & 1003.0 & 43.5 & 953.0 & 79.6 \\
\hline 205 CVS2-71 & 1200 & 5.490 & 1150 & 8.3 & 1150.0 & 8.6 & 1150.0 & 8.8 & 1100.0 & 13.9 & 1100.0 & 13.9 & 1050.0 & 23.4 & 1000.0 & 43.3 & 950.0 & 88.0 \\
\hline 206 CVS2-72 & 1200 & 5.280 & 1150 & 7.7 & 1150.0 & 7.8 & 1150.0 & 8.0 & 1101.0 & 11.9 & 1100.0 & 12.2 & 1050.0 & 19.3 & 1000.0 & 34.4 & 950.0 & 63.4 \\
\hline 207 CVS2-73 & 1199 & 5.980 & 1149 & 8.7 & 1149.0 & 8.8 & 1149.0 & 8.8 & 1100.0 & 13.4 & 1098.0 & 13.6 & 1049.0 & 21.5 & 999.0 & 38.4 & 949.0 & 69.7 \\
\hline 208 CVS2-74 & 1201 & 6.390 & 1151 & 9.3 & 1151.0 & 9.5 & 1151.0 & 9.7 & 1102.0 & 14.7 & 1101.0 & 14.7 & 1050.0 & 24.3 & 1001.0 & 44.2 & 950.0 & 85.0 \\
\hline 209 CVS2-75 & 1200 & 5.930 & 1150 & 8.5 & 1150.0 & 8.7 & 1150.0 & 8.9 & 1100.0 & 13.2 & 1100.0 & 13.4 & 1050.0 & 21.3 & 1000.0 & 37.2 & 950.0 & 69.7 \\
\hline $\begin{array}{l}210 \text { CVS2-76 } \\
211 \text { CVS2-77 }\end{array}$ & $\begin{array}{l}1198 \\
1199\end{array}$ & $\begin{array}{l}0.880 \\
1040\end{array}$ & $\begin{array}{l}1148 \\
1150\end{array}$ & $\begin{array}{l}1.2 \\
1.5\end{array}$ & $\begin{array}{l}1148.0 \\
1148.0\end{array}$ & $\begin{array}{l}1.2 \\
1.6\end{array}$ & $\begin{array}{l}1148.0 \\
11490\end{array}$ & $\begin{array}{l}1.2 \\
1.6\end{array}$ & $\begin{array}{l}1098.0 \\
10990\end{array}$ & $\begin{array}{l}1.7 \\
24\end{array}$ & $\begin{array}{l}1098.0 \\
1100.0\end{array}$ & $\begin{array}{l}1.7 \\
25\end{array}$ & $\begin{array}{l}1048.0 \\
10490\end{array}$ & $\begin{array}{l}2.5 \\
4.1\end{array}$ & $\begin{array}{l}999.0 \\
9990\end{array}$ & $\begin{array}{l}3.4 \\
77\end{array}$ & $\begin{array}{l}947.0 \\
948.0\end{array}$ & $\begin{array}{r}6.1 \\
153\end{array}$ \\
\hline $\begin{array}{l}211 \text { CVS2-77 } \\
212 \text { cVs2-78 }\end{array}$ & $\begin{array}{l}1199 \\
1200\end{array}$ & $\begin{array}{l}1.040 \\
16.790\end{array}$ & $\begin{array}{l}11100 \\
1149\end{array}$ & $\begin{array}{r}1.5 \\
26.9\end{array}$ & $\begin{array}{l}1148.0 \\
1149.0\end{array}$ & $\begin{array}{r}1.6 \\
27.3\end{array}$ & $\begin{array}{l}1149.0 \\
1150.0\end{array}$ & $\begin{array}{r}1.6 \\
28.3\end{array}$ & $\begin{array}{l}1099.0 \\
1099.0\end{array}$ & $\begin{array}{r}2.4 \\
47.6\end{array}$ & $\begin{array}{l}\begin{array}{l}1100.0 \\
1100.0\end{array}\end{array}$ & $\begin{array}{r}2.5 \\
54.9\end{array}$ & $\begin{array}{l}1049.0 \\
1050.0\end{array}$ & $\begin{array}{r}4.1 \\
97.6\end{array}$ & $\begin{array}{l}999.0 \\
999.0\end{array}$ & $\begin{array}{r}187.6 \\
187.6\end{array}$ & 948.0 & 15.3 \\
\hline 213 CVS2-79 & (1152) & (32.87) & (1203) & $(34.75)$ & 1151.0 & $\begin{array}{l}27.7 \\
69.7\end{array}$ & 1151.0 & 71.6 & 1101.0 & $\begin{array}{l}41.0 \\
121.3\end{array}$ & 1102.0 & $\begin{array}{c}54.9 \\
124.2\end{array}$ & (1002) & $\begin{array}{l}(199.92) \\
(190)\end{array}$ & 1051.0 & $\begin{array}{l}231.0 \\
235.8\end{array}$ & & \\
\hline 214 CVS2-80 & 1200 & $\begin{array}{l}(37.620 \\
27.620\end{array}$ & 1150 & $\begin{aligned} & 34.8 \\
& 54.8\end{aligned}$ & 1150.0 & $\begin{array}{l}57.9 \\
57.9\end{array}$ & (1149) & $\begin{array}{l}(110.88) \\
\text { (1) }\end{array}$ & 1101.0 & $\begin{array}{l}128.2 \\
128.2\end{array}$ & 1100.0 & $\begin{array}{l}124.2 \\
136.9\end{array}$ & & & & & & \\
\hline 215 CVS2-81 & 1198 & 0.540 & 1150 & 0.7 & 1149.0 & 0.7 & 1149.0 & 0.8 & (1099) & $(0.99)$ & (1099) & (1) & $(1050)$ & (1.4) & 1000.0 & 3.9 & 949.0 & 7.9 \\
\hline 216 CVS2-82 & (1196) & (1.26) & 1146 & 1.5 & 1146.0 & 1.5 & 1147.0 & 1.6 & 1098.0 & 2.2 & 1097.0 & 2.3 & 1047.0 & 3.3 & 997.0 & 5.3 & 946.0 & 9.2 \\
\hline 217 CVS2-83 & 1196 & 0.560 & 1147 & 0.8 & 1148.0 & 0.8 & 1148.0 & 0.8 & 1097.0 & 1.1 & 1098.0 & & 1048.0 & 1.6 & 999.0 & 2.8 & 948.0 & 5.0 \\
\hline 218 CVS2-84 & 1201 & $\begin{array}{l}0.820 \\
2700\end{array}$ & 1152 & 1.2 & 1152.0 & 1.2 & 1152.0 & 1.2 & 1101.0 & 1.8 & 1102.0 & 1.8 & 1051.0 & 2.7 & 1002.0 & 4.6 & 951.0 & 8.6 \\
\hline $\begin{array}{l}219 \text { CVS2-85 } \\
220 \text { CVS2-86 }\end{array}$ & $\begin{array}{l}1198 \\
1199\end{array}$ & $\begin{array}{r}2.790 \\
18.490\end{array}$ & $\begin{array}{l}1148 \\
1150\end{array}$ & $\begin{array}{r}4.1 \\
29.8\end{array}$ & $\begin{array}{l}1148.0 \\
11500\end{array}$ & $\begin{array}{r}4.1 \\
302\end{array}$ & $\begin{array}{l}1148.0 \\
11490\end{array}$ & $\begin{array}{r}4.2 \\
308\end{array}$ & $\begin{array}{l}1099.0 \\
11100\end{array}$ & $\begin{array}{r}6.2 \\
499\end{array}$ & $\begin{array}{l}1098.0 \\
11000\end{array}$ & $\begin{array}{r}6.2 \\
502\end{array}$ & $\begin{array}{l}1049.0 \\
1490\end{array}$ & 9.7 & 999.0 & $\begin{array}{r}16.2 \\
1858\end{array}$ & 948.0 & 29.1 \\
\hline $\begin{array}{l}220 \text { CVS2-86 } \\
221 \text { cVS2-87 }\end{array}$ & $\begin{array}{l}1199 \\
1198\end{array}$ & $\begin{array}{l}18.490 \\
11.130\end{array}$ & $\begin{array}{l}1150 \\
1149\end{array}$ & $\begin{array}{l}29.8 \\
17.9\end{array}$ & $\begin{array}{l}1150.0 \\
1150.0\end{array}$ & $\begin{array}{l}30.2 \\
18.0\end{array}$ & $\begin{array}{l}1149.0 \\
1150.0\end{array}$ & $\begin{array}{l}30.8 \\
18.1\end{array}$ & $\begin{array}{l}11100.0 \\
1100.0\end{array}$ & $\begin{array}{l}49.9 \\
30.9\end{array}$ & $\begin{array}{l}1100.0 \\
1100.0\end{array}$ & $\begin{array}{l}50.2 \\
31.4\end{array}$ & $\begin{array}{l}1049.0 \\
1050.0\end{array}$ & $\begin{array}{l}90.3 \\
56.6\end{array}$ & $\begin{array}{l}999.0 \\
999.0\end{array}$ & $\begin{array}{l}185.8 \\
114.0\end{array}$ & 949.0 & 247.7 \\
\hline 222 CVS2-88 & 1200 & 2.320 & $\begin{array}{l}1149 \\
1152\end{array}$ & 3.5 & 1151.0 & & 1150.0 & $\begin{array}{r}18.1 \\
36\end{array}$ & 11020 & $\begin{array}{r}30.9 \\
56\end{array}$ & 11020 & $\begin{array}{r}31.4 \\
56\end{array}$ & $\begin{array}{l}1050.0 \\
1052.0\end{array}$ & $\begin{array}{r}50.0 \\
9.5\end{array}$ & $\begin{array}{l}9990.0 \\
10010\end{array}$ & $\begin{array}{l}114.0 \\
177\end{array}$ & $\begin{array}{l}949.0 \\
951.0\end{array}$ & $\begin{array}{r}24.1 .1 \\
353\end{array}$ \\
\hline 223 CVS2-89 & 1199 & 1.850 & 1149 & $\begin{array}{l}2.0 \\
2.8\end{array}$ & 1149.0 & 2.8 & 1149.0 & $\begin{array}{l}2.0 \\
2.8\end{array}$ & 1099.0 & 4.5 & 1100.0 & 4.5 & 1049.0 & 7.9 & 999.0 & $\begin{array}{l}17.7 \\
14.7\end{array}$ & 949.0 & $\begin{array}{l}31.3 \\
31.5\end{array}$ \\
\hline 224 CVS2-90 & 1199 & 2.580 & 1149 & 3.6 & 1149.0 & 3.7 & 1149.0 & 3.7 & 1099.0 & 5.5 & 1100.0 & 5.5 & 1049.0 & 8.8 & 999.0 & 15.1 & 948.0 & 28.5 \\
\hline 225 CVS2-91 & 1200 & 9.140 & 1150 & 13.9 & 1150.0 & 14.3 & 1150.0 & 14.8 & 1100.0 & 23.9 & 1100.0 & 23.9 & 1049.0 & 44.3 & 1000.0 & 97.2 & 949.0 & 219.9 \\
\hline 226 CVS2-92 & 1197 & 0.700 & 1149 & 1.0 & 1148.0 & 1.0 & 1148.0 & 1.0 & 1099.0 & 1.5 & 1099.0 & 1.5 & 1049.0 & 2.4 & 999.0 & & 949.0 & 7.7 \\
\hline 227 CVS2-93 & 1198 & 0.480 & 1148 & 0.6 & 1148.0 & 0.6 & 1148.0 & 0.7 & 1099.0 & 0.9 & 1099.0 & 0.9 & 1049.0 & 1.3 & $\begin{array}{l}3999.0 \\
\end{array}$ & 2.0 & 949.0 & 3.4 \\
\hline 228 CVS2-94 & $\begin{array}{l}1148 \\
1202\end{array}$ & $\begin{array}{l}0.810 \\
1180\end{array}$ & $\begin{array}{l}1148 \\
1152\end{array}$ & 0.8 & 1099.0 & 1.1 & $\begin{array}{l}1098.0 \\
11520\end{array}$ & 1.2 & 1048.0 & 1.6 & 11020 & & 10510 & & & & & \\
\hline 229 CVS2-95 & $\begin{array}{l}1202 \\
1199 \\
1199\end{array}$ & $\begin{array}{l}1.180 \\
3.600\end{array}$ & $\begin{array}{l}1152 \\
1150\end{array}$ & $\begin{array}{l}1.6 \\
5.5\end{array}$ & $\begin{array}{l}1152.0 \\
11490\end{array}$ & $\begin{array}{l}1.6 \\
5.6\end{array}$ & $\begin{array}{l}1152.0 \\
11490\end{array}$ & $\begin{array}{l}1.6 \\
556\end{array}$ & $\begin{array}{l}1102.0 \\
10990\end{array}$ & $\begin{array}{l}2.3 \\
9.1\end{array}$ & $\begin{array}{l}1102.0 \\
11000\end{array}$ & $\begin{array}{l}2.3 \\
9.2\end{array}$ & $\begin{array}{l}3051.0 \\
10990\end{array}$ & $\begin{array}{l}3.4 \\
15.9\end{array}$ & $\begin{array}{l}1002.0 \\
999.0\end{array}$ & $\begin{array}{r}5.6 \\
312\end{array}$ & $\begin{array}{l}951.0 \\
948.0\end{array}$ & $\begin{array}{l}10.0 \\
66.2\end{array}$ \\
\hline $\begin{array}{l}230 \text { CVS2-96 } \\
231 \text { cys2-97 }\end{array}$ & $\begin{array}{l}1199 \\
1200\end{array}$ & $\begin{array}{l}3.600 \\
4.720\end{array}$ & 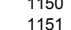 & $\begin{array}{l}5.5 \\
71\end{array}$ & $\begin{array}{l}1449.0 \\
11510\end{array}$ & $\begin{array}{l}5.6 \\
7.2\end{array}$ & $\begin{array}{l}1149.0 \\
1150.0\end{array}$ & $\begin{array}{l}5.6 \\
7.5\end{array}$ & $\begin{array}{l}1999.0 \\
1101.0\end{array}$ & $\begin{array}{r}9.1 \\
115\end{array}$ & $\begin{array}{l}1100.0 \\
1100.0\end{array}$ & $\begin{array}{c}9.2 \\
11.7\end{array}$ & $\begin{array}{l}1049.0 \\
1051.0\end{array}$ & $\begin{array}{l}15.9 \\
20.0\end{array}$ & $\begin{array}{r}990.0 \\
10010\end{array}$ & $\begin{array}{l}31.2 \\
39.3 \\
39 .\end{array}$ & $\begin{array}{l}948.0 \\
950.0\end{array}$ & $\begin{array}{l}66.2 \\
80.5\end{array}$ \\
\hline $\begin{array}{l}231 \text { CVS2-97 } \\
232 \text { CVS2-98 }\end{array}$ & $\begin{array}{l}1200 \\
1196\end{array}$ & $\begin{array}{l}4.220 \\
1.430\end{array}$ & 1146 & $\begin{array}{l}1.1 \\
1.9\end{array}$ & 1147.0 & $\begin{array}{l}.2 .2 \\
2.0\end{array}$ & 1147.0 & $\begin{array}{l}.5 \\
2.0\end{array}$ & 1098.0 & $\begin{array}{r}11.5 \\
2.7\end{array}$ & 1096.0 & $\begin{array}{l}21.1 \\
2.8\end{array}$ & 1047.0 & $\begin{array}{l}20.0 \\
42\end{array}$ & 997.0 & $\begin{array}{r}39.3 \\
6.5\end{array}$ & $\begin{array}{l}950.0 \\
947.0\end{array}$ & $\begin{array}{l}80.5 \\
112\end{array}$ \\
\hline 233 CVS2-99 & 1198 & 5.670 & 1148 & 8.7 & 1146.0 & 8.8 & 1146.0 & 8.8 & 1098.0 & 14.1 & 1096.0 & 14.3 & 1045.0 & 24.5 & 997.0 & 45.7 & 946.0 & 93.9 \\
\hline 234 CVS2-100 & 1197 & 4.610 & 1147 & 6.9 & 1148.0 & 7.0 & 1145.0 & 7.3 & 1098.0 & 11.2 & 1097.0 & 11.3 & 1047.0 & 19.1 & 998.0 & 36.9 & 947.0 & 74.4 \\
\hline 235 CVS2-101 & 1202 & 1.090 & 1153 & 1.5 & 1152.0 & 1.5 & 1152.0 & 1.5 & 1103.0 & 2.1 & 1103.0 & 2.1 & 1053.0 & 3.2 & 1002.0 & 5.1 & 952.0 & 8.8 \\
\hline $\begin{array}{l}236 \text { CVS2-102 } \\
237 \text { CVS-103 }\end{array}$ & & & & & & & & & & & & & & & & & & \\
\hline 238 CVS2-104 & & & & & & & & & & & & & & & & & & \\
\hline 239 CVS2-105 & & & & & & & & & & & & & & & & & & \\
\hline $\begin{array}{l}240 \text { CVSS-106 } \\
241 \text { CVSS-107 }\end{array}$ & & & & & & & & & & & & & & & & & & \\
\hline $\begin{array}{l}241 \text { CVS2-107 } \\
242 \text { CVS2-108 }\end{array}$ & & & & & & & & & & & & & & & & & & \\
\hline $\begin{array}{l}243 \text { CVS2-109 } \\
24 \text { CVSS2-10 }\end{array}$ & & & & & & & & & & & & & & & & & & \\
\hline $\begin{array}{l}244 \text { CVSS-110 } \\
245 \text { CVS2-111 }\end{array}$ & & & & & & & & & & & & & & & & & & \\
\hline 246 CVS2-112 & & & & & & & & & & & & & & & & & & \\
\hline
\end{tabular}




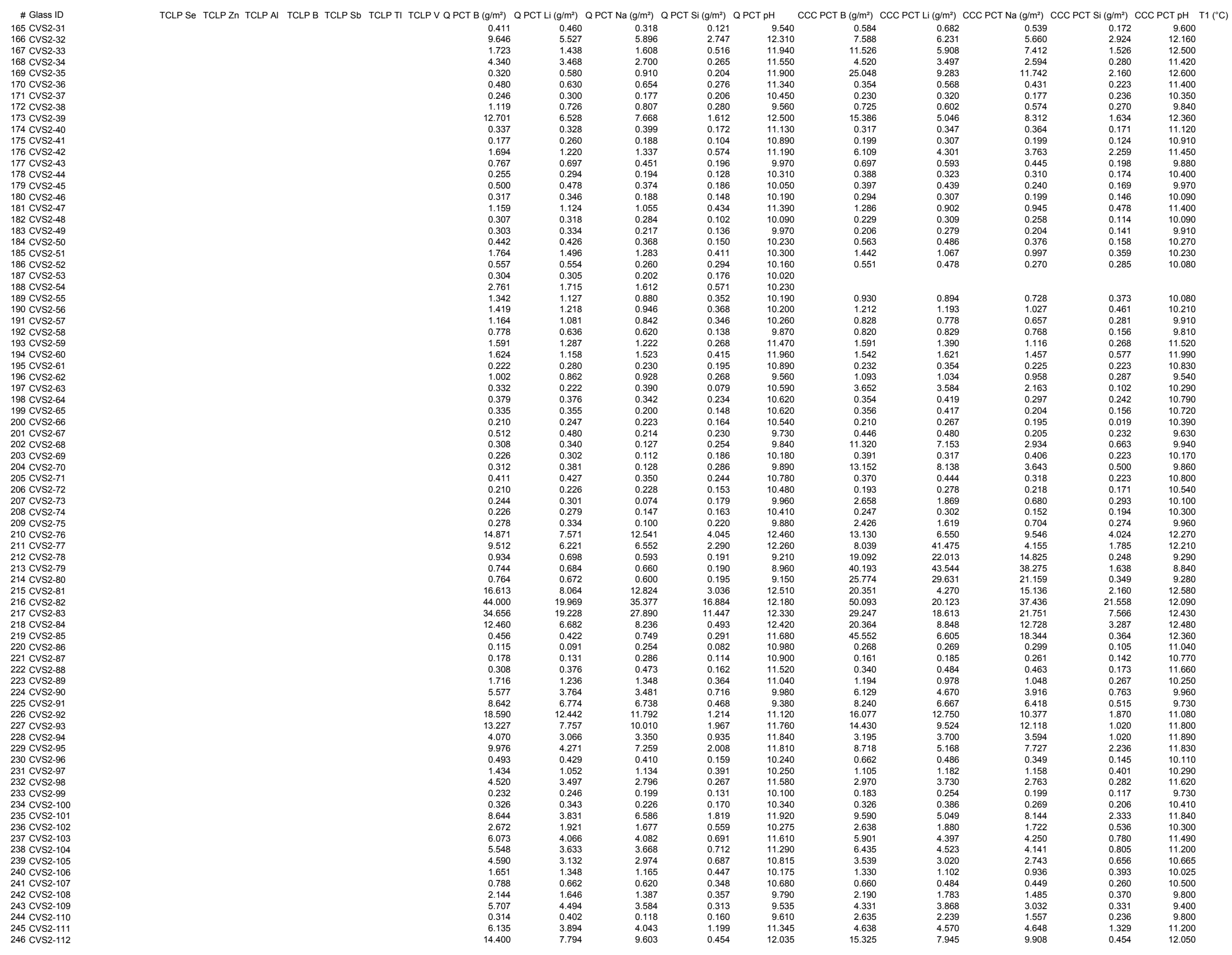




\begin{tabular}{|c|c|c|c|c|c|c|}
\hline \# Glass ID & Study & Reference & $\mathrm{Al} 2 \mathrm{O} 3-\mathrm{t}$ & B2O3-t & CaO-t & $\mathrm{Fe} 2 \mathrm{O} 3-\mathrm{t}$ \\
\hline 247 CVS2-113 & Hanford CVS 2 & Hrma et al. 1994 & 0.0238 & 0.1083 & & \\
\hline 248 CVS2-114 & Hanford CVS 2 & Hrma et al. 1994 & 0.0226 & 0.1028 & 0.0081 & 0.0702 \\
\hline 249 CVS2-115 & Hanford CVS 2 & Hrma et al. 1994 & 0.0223 & 0.1017 & 0.0080 & 0.0695 \\
\hline $\begin{array}{l}250 \text { CVS2-116 } \\
251 \text { CVS2-117 }\end{array}$ & $\begin{array}{l}\text { Hanford CVS } 2 \\
\text { Hanford CVS }\end{array}$ & Hrma et al. 1994 & 0.0228 & 0.1041 & 0.0200 & 0.0711 \\
\hline $\begin{array}{l}251 \text { CVS2-117 } \\
252 \text { CVS2-118 }\end{array}$ & $\begin{array}{l}\text { Hanford CVS } 2 \\
\text { Hanford CVS 2 }\end{array}$ & $\begin{array}{l}\text { Hrma et al. } 1994 \\
\text { Hrma et al. } 1994\end{array}$ & $\begin{array}{l}0.0233 \\
0.0228\end{array}$ & $\begin{array}{l}0.1062 \\
0.1041\end{array}$ & $\begin{array}{l}0.0084 \\
0.0082\end{array}$ & $\begin{array}{l}0.0275 \\
0.0711\end{array}$ \\
\hline $\begin{array}{l}252 \text { CVS2-118 } \\
253 \text { CVS2-119 }\end{array}$ & $\begin{array}{l}\text { Hanford CVS } 22 \\
\text { Hanford CVS } 2\end{array}$ & $\begin{array}{l}\text { Hrma et al. } 19944 \\
\text { Hrrme et al. } 1994\end{array}$ & $\begin{array}{l}0.0228 \\
0.0000\end{array}$ & $\begin{array}{l}0.1041 \\
0.1078\end{array}$ & $\begin{array}{l}0.0082 \\
0.0085\end{array}$ & $\begin{array}{l}0.0711 \\
0.0736\end{array}$ \\
\hline 254 CVS2-120 & Hanford CVS 2 & Hrma et al. 1994 & 0.0500 & 0.1024 & 0.0081 & $\begin{array}{l}0.0699 \\
0.069\end{array}$ \\
\hline 255 CVS2-121 & Hanford CVS 2 & Hrma et al. 1994 & 0.1000 & 0.0970 & 0.0077 & 0.0662 \\
\hline 256 CVS2-122 & Hanford CVS 2 & Hrma et al. 1994 & 0.1500 & 0.0916 & 0.0072 & 0.0626 \\
\hline 257 CVS2-123 & Hanford CVS 2 & Hrma et al. 1994 & 0.0235 & 0.1048 & 0.0082 & 0.0733 \\
\hline 258 CVS3-1 & Hanford CVS 3 & Vienna et al. 1996 & 0.0487 & 0.0005 & 0.0126 & 0.0680 \\
\hline 259 CVS3-2 & Hanford CVS 3 & Vienna et al. 1996 & 0.0417 & 0.0004 & 0.0108 & 0.0583 \\
\hline 261 CVS3-3 & Hantord CVS 3 & $\begin{array}{l}\text { Vienna et al. } 1996 \\
\text { Vienna et }\end{array}$ & $\begin{array}{l}0.05 / 4 \\
0.0626\end{array}$ & 0.00006 & $\begin{array}{l}0.0148 \\
0.0162\end{array}$ & $\begin{array}{l}0.0801 \\
0.0874\end{array}$ \\
\hline 262 CVS3-5 & Hanford CVS 3 & Vienna et al. 1996 & $\begin{array}{l}0.0026 \\
0.0657\end{array}$ & 0.0006 & 0.0170 & $\begin{array}{l}0.0884 \\
0.0918\end{array}$ \\
\hline 263 CVS3-6 & Hanford CVS 3 & Vienna et al. 1996 & 0.0446 & 0.0837 & 0.0116 & $\begin{array}{l}0.0918 \\
0.0623\end{array}$ \\
\hline 264 CVS3-7 & Hanford CVS 3 & Vienna et al. 1996 & 0.0468 & 0.0400 & 0.0121 & 0.0653 \\
\hline 265 CVS3-8 & Hanford CVS 3 & Vienna et al. 1996 & 0.0430 & 0.0004 & 0.0111 & 0.0600 \\
\hline 266 CVS3-9 & Hanford CVS 3 & Vienna et al. 1996 & 0.0527 & 0.0005 & 0.0137 & 0.0737 \\
\hline 267 CVS3-10 & Hanford CVS 3 & Vienna et al. 1996 & 0.0465 & 0.0004 & 0.0120 & 0.0650 \\
\hline 268 CVS3-11 & Hanford CVS 3 & Vienna et al. 1996 & 0.0476 & 0.0005 & 0.0123 & 0.0665 \\
\hline 20 & $\begin{array}{l}\text { Hanford CVS } 3 \\
\text { Hanford CVS } 3\end{array}$ & Vienna et al. 1996 & 0.0501 & $\begin{array}{l}0.0005 \\
0.0004\end{array}$ & 0.0130 & $\begin{array}{l}0.0700 \\
0.1500\end{array}$ \\
\hline $\begin{array}{l}271 \text { CVS3-14 } \\
274\end{array}$ & Hanford CVS 3 & Vienna et al. 1996 & $\begin{array}{l}0.0444 \\
0.0465\end{array}$ & $\begin{array}{l}.00004 \\
0.0004\end{array}$ & 0.0120 & $\begin{array}{l}0.1500 \\
0.1100\end{array}$ \\
\hline 272 CVS3-15 & Hanford CVS 3 & Vienna et al. 1996 & $\begin{array}{l}0.04652 \\
0.0522\end{array}$ & $\begin{array}{l}.0 .0004 \\
0.0005\end{array}$ & 0.0135 & $\begin{array}{l}0.1100 \\
0.0000\end{array}$ \\
\hline 273 CVS $3-16$ & Hanford CVS 3 & Vienna et al. 1996 & 0.2500 & 0.0004 & 0.0099 & 0.0536 \\
\hline 274 CVS3-17 & Hanford CVS 3 & Vienna et al. 1996 & 0.1800 & 0.0004 & 0.0109 & 0.0586 \\
\hline 275 CVS3-18 & Hanford CVS 3 & Vienna et al. 1996 & 0.1300 & 0.0004 & 0.0115 & 0.0622 \\
\hline 276 CVS3-19 & Hanford CVS 3 & Vienna et al. 1996 & 0.0000 & 0.0005 & 0.0132 & 0.0715 \\
\hline 277 CVS3-20 & Hanford CVS 3 & Vienna et al. 1996 & 0.0428 & 0.0004 & 0.0111 & 0.0597 \\
\hline 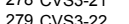 & $\begin{array}{l}\text { Hantord CVS } 3 \\
\text { Hanfor CVS } 3\end{array}$ & Vienna et al. 1996 & 0.0448 & 0.0004 & 0.0116 & 0.0626 \\
\hline $\begin{array}{l}218 \text { CVS } 33.23 \\
23\end{array}$ & Hanford CVS 3 & Vienna et al. 1996 & $\begin{array}{l}0.0468 \\
0.0509\end{array}$ & $\begin{array}{l}.0 .004 \\
0.0005\end{array}$ & $\begin{array}{l}0.012132 \\
0.0132\end{array}$ & $\begin{array}{l}0.0654 \\
0.0711\end{array}$ \\
\hline 281 CVS3-24 & Hanford CVS 3 & $\begin{array}{l}\text { Vienna et al. } 19960 \\
\text { Vien }\end{array}$ & 0.0453 & 0.0004 & 0.0117 & 0.0633 \\
\hline 282 CVS $3-25$ & Hanford CVS 3 & Vienna et al. 1996 & 0.0524 & 0.0005 & 0.0136 & 0.0732 \\
\hline 283 CVS3-26 & Hanford CVS 3 & Vienna et al. 1996 & 0.0235 & 0.1048 & 0.0082 & 0.0733 \\
\hline 284 CVS3-27 & Hanford CVS 3 & Vienna et al. 1996 & 0.0444 & 0.0004 & 0.0115 & 0.1500 \\
\hline 285 CVS3-28 & Hanford CVS 3 & Vienna et al. 1996 & 0.0472 & 0.0005 & 0.0122 & 0.0659 \\
\hline 286 CVS3-29 & Hanford CVS 3 & Vienna et al. 1996 & 0.0462 & 0.0005 & 0.0120 & 0.0645 \\
\hline (2010 & $\begin{array}{l}\text { Hanford CVS } 3 \\
\text { Hanfor }\end{array}$ & Vienna et al. 1996 & 0.0437 & 0.0005 & 0.0113 & 0.0611 \\
\hline $\begin{array}{l}208 \text { CVS } 35.32 \\
298\end{array}$ & Hantord CVS 3 & $\begin{array}{l}\text { Vienna et al. } 1996 \\
\text { Vienna et }\end{array}$ & $\begin{array}{l}0.0413 \\
0.0452\end{array}$ & $\begin{array}{l}.0 .004 \\
0.0004\end{array}$ & $\begin{array}{l}0.0107 \\
0.0117\end{array}$ & $\begin{array}{l}0.0566 \\
0.0631\end{array}$ \\
\hline $\begin{array}{l}290 \text { CVS3-33 } \\
20\end{array}$ & Hanford CVS 3 & Vienna et al. 1996 & 0.0481 & 0.0005 & 0.0124 & $\begin{array}{l}.0 .0637 \\
0.0672\end{array}$ \\
\hline 291 CVS3-34 & Hanford CVS 3 & Vienna et al. 1996 & 0.0467 & 0.0004 & 0.0121 & 0.0652 \\
\hline 292 CVS3-35 & Hanford CVS 3 & Vienna et al. 1996 & 0.0438 & 0.0004 & 0.0113 & 0.0611 \\
\hline & Hanford CVS 3 & Vienna et al. 1996 & 0.0423 & 0.0004 & 0.0110 & 0.0591 \\
\hline 294 CVS3-37 & Hanford CVS 3 & Vienna et al. 1996 & 0.0476 & 0.0005 & 0.0123 & 0.0665 \\
\hline 295 CVS3 38 & Hanford CVS 3 & Vienna et al. 1996 & 0.0466 & 0.0005 & 0.0121 & 0.0651 \\
\hline $\begin{array}{l}2966 \mathrm{CVSS3}-39 \\
{ }_{207}\end{array}$ & Hanford CVS 3 & Vienna et al. 1996 & 0.0456 & 0.0005 & 0.0118 & 0.0637 \\
\hline 297 CVS3-40 & Hanford CVS 3 & Vienna et al. 1996 & $\begin{array}{l}0.0447 \\
0.0230\end{array}$ & 0.0005 & 0.0116 & $\begin{array}{l}0.0624 \\
0.0435\end{array}$ \\
\hline $\begin{array}{l}298 \text { TRUUBL-1 } \\
299 \text { TRUAZ-1 }\end{array}$ & $\begin{array}{l}\text { IRU Study } \\
\text { TRU Study }\end{array}$ & $\begin{array}{l}\text { Crum et al. } 199 / \\
\text { Crum et }\end{array}$ & $\begin{array}{l}0.0230 \\
0.0300\end{array}$ & $\begin{array}{l}0.0815 \\
0.0803\end{array}$ & $\begin{array}{l}0.0098 \\
0.0096\end{array}$ & $\begin{array}{l}0.0435 \\
0.0429\end{array}$ \\
\hline 300 TRU-NL-1 & TRU Study & Crum et al. 1997 & 0.0235 & 0.0834 & 0.0100 & 0.0446 \\
\hline 301 TRU-AB-1 & TRU Study & Crum et al. 1997 & 0.0400 & 0.1200 & 0.0092 & 0.0408 \\
\hline 302 TRU-NB-1 & TRU Study & Crum et al. 1997 & 0.0234 & 0.1000 & 0.0099 & 0.0444 \\
\hline 303 TRU-ZN-1 & TRU Study & Crum et al. 1997 & 0.0221 & 0.0785 & 0.0094 & 0.0419 \\
\hline 304 TRU-ZB-1 & TRU Study & Crum et al. 1997 & 0.0215 & 0.1200 & 0.0091 & 0.0408 \\
\hline 305 TRU-ANZ-1 & TRU Study & Crum et al. 1997 & 0.0400 & 0.0744 & 0.0089 & 0.0397 \\
\hline 306 TRU-ABZ-1 & $\begin{array}{l}\text { TRU Study } \\
\text { TRU Stury }\end{array}$ & Crum et al. 1997 & $\begin{array}{l}0.0400 \\
0.0227\end{array}$ & 0.1000 & 0.0092 & $\begin{array}{l}0.0409 \\
0.0431\end{array}$ \\
\hline 307 TRU-NBZ-1 & $\begin{array}{l}\text { TRU Study } \\
\text { TRU Study }\end{array}$ & $\begin{array}{l}\text { Crum et al. } 1997 \\
\text { Crum et al. } 1997\end{array}$ & $\begin{array}{l}0.0227 \\
0.0600\end{array}$ & 0.0678 & 0.0094 & $\begin{array}{l}.0 .0431 \\
0.0419\end{array}$ \\
\hline $\begin{array}{l}308 \text { TRUU-Al-1 } \\
309 \text { TRUAl- }\end{array}$ & TRU Study & Crum et al. 1997 & 0.0400 & 0.0801 & $\begin{array}{l}0.00964 \\
0.0096\end{array}$ & 0.0428 \\
\hline 310 TRU-B-1 & TRU Study & Crum et al. 1997 & 0.0250 & 0.0000 & 0.0106 & 0.0474 \\
\hline 311 TRU-B-2 & TRU Study & Crum et al. 1997 & 0.0245 & 0.0200 & 0.0104 & 0.0465 \\
\hline 312 TRU-B-3 & TRU Study & Crum et al. 1997 & 0.0238 & 0.0500 & 0.0101 & 0.0450 \\
\hline 313 TRU-B-4 & TRU Study & Crum et al. 1997 & 0.0220 & 0.1200 & 0.0094 & 0.0417 \\
\hline $\begin{array}{l}314 \text { TRU-B-B5 } \\
\text { TR }\end{array}$ & TRU Study & Crum et al. 1997 & 0.0248 & 0.0100 & 0.0105 & 0.0469 \\
\hline 315 TRU-Bi-1 & TRU Study & $\begin{array}{l}\text { Crum et al. } 1997 \\
\text { Coum et }\end{array}$ & 0.0243 & 0.0082 & 0.0103 & 0.0460 \\
\hline $\begin{array}{l}316 \text { TRU-Bi-2 } \\
317 \text { TRU-Bi-3 }\end{array}$ & $\begin{array}{l}\text { TRU Study } \\
\text { TRU Study }\end{array}$ & $\begin{array}{l}\text { Crum et al. } 1997 \\
\text { Coum }\end{array}$ & $\begin{array}{l}0.0237 \\
0.0225\end{array}$ & $\begin{array}{l}0.08840 \\
0.0797\end{array}$ & $\begin{array}{l}0.0101 \\
0.0096\end{array}$ & $\begin{array}{l}0.0449 \\
0.0426\end{array}$ \\
\hline $\begin{array}{l}317 \text { TRUU-Bi-3 } \\
318 \text { TRU-Bi-4 }\end{array}$ & $\begin{array}{l}\text { TRU Study } \\
\text { TRU Study }\end{array}$ & Crum et al. 1997 & $\begin{array}{l}0.0229 \\
0.0219\end{array}$ & 0.0776 & 0.0093 & $\begin{array}{l}.0 .0414 \\
0.0414\end{array}$ \\
\hline 319 TRU-Ce-1 & TRU Study & Crum et al. 1997 & 0.0226 & 0.0803 & 0.0096 & 0.0429 \\
\hline 320 TRU-Ce-2 & TRU Study & Crum et al. 1997 & 0.0223 & 0.0791 & 0.0095 & 0.0422 \\
\hline 321 TRU-Ce-3 & TRU Study & Crum et al. 1997 & 0.0219 & 0.0778 & 0.0093 & 0.0416 \\
\hline 322 TRU-Li-1 & TRU Study & Crum et al. 1997 & 0.0241 & 0.0854 & 0.0102 & 0.0456 \\
\hline 323 TRU-Li-2 & TRU Study & Crum et al. 1997 & 0.0237 & 0.0841 & 0.0101 & 0.0450 \\
\hline 324 TRU-Li-3 & $\begin{array}{l}\text { TRU Study } \\
\text { TPS }\end{array}$ & Crum et al. 1997 & 0.0234 & 0.0829 & 0.00999 & 0.0443 \\
\hline 325 TRU-Li-4 & TRU Study & Crum et al. 1997 & 0.0226 & 0.08038 & 0.0096 & \\
\hline 326 TRU-Na-1 & TRU Study & Crum et ala. 1997 & 0.0248 & 0.0878 & 0.0105 & 0.0469 \\
\hline $\begin{array}{l}327 \text { TRUUNa-2 } \\
328 \text { TRUNa-Na- }\end{array}$ & $\begin{array}{l}\text { TRU Study } \\
\text { TRU Stur }\end{array}$ & $\begin{array}{l}\text { Crum et al. } 19997 \\
\text { Crum et al. } 1997\end{array}$ & $\begin{array}{l}0.02341 \\
0.0235\end{array}$ & $\begin{array}{l}0.0855 \\
0.0832\end{array}$ & $\begin{array}{l}0.0102 \\
0.0100\end{array}$ & $\begin{array}{l}0.0457 \\
0.0445\end{array}$ \\
\hline
\end{tabular}

K2O-t Li2O-t MgO-t Na2O-t NiO-t P2O5-t SiO2-t ZrO2-t Ag2O-t As2O3-tAs2O5$\begin{array}{llllllllll}0.0000 & 0.0100 & 0.0086 & 0.1157 & 0.0063 & 0.0011 & 0.5506 & 0.0396 & 0.0000 & 0.0000 \\ 0.0000 & 0.0600 & 0.0082 & 0.1099 & 0.0060 & 0.0010 & 0.5228 & 0.0376 & 0.0000 & 0.0000\end{array}$

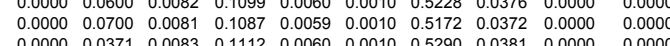
$\begin{array}{llllllllll}0.0000 & 0.0371 & 0.0083 & 0.1112 & 0.0060 & 0.0010 & 0.5290 & 0.0381 & 0.0000 & 0.0000 \\ 0.0000 & 0.0378 & 0.0000 & 0.1135 & 0.0061 & 0.0011 & 0.5398 & 0.0388 & 0.0000 & 0.0000\end{array}$ $\begin{array}{lllllllllllll}0.0000 & 0.0384 & 0.0086 & 0.1152 & 0.0062 & 0.0011 & 0.5480 & 0.0394 & 0.0000 & 0.00000\end{array}$

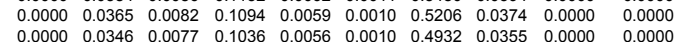
$\begin{array}{lllllllllll}0.0000 & 0.0326 & 0.0073 & 0.0979 & 0.0053 & 0.0000 & 0.4658 & 0.0335 & 0.00000 & 0.0000\end{array}$

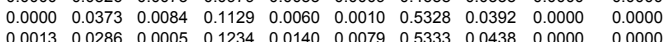

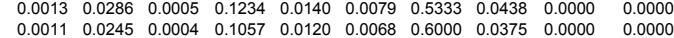

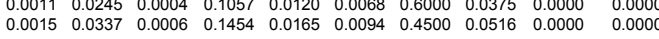
$\begin{array}{llllllllll}0.0017 & 0.0367 & 0.0006 & 0.1586 & 0.0180 & 0.0102 & 0.4000 & 0.0563 & 0.0000 & 0.0000\end{array}$ $\begin{array}{llllllllll}0.0018 & 0.0386 & 0.0006 & 0.1665 & 0.0189 & 0.0107 & 0.3700 & 0.0591 & 0.0000 & 0.0000 \\ 0.0012 & 0.0262 & 0.0004 & 0.1131 & 0.0129 & 0.0073 & 0.4889 & 0.0401 & 0.0000 & 0.0000\end{array}$

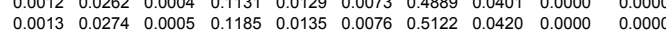
$\begin{array}{llllllllll}0.0012 & 0.0252 & 0.0004 & 0.2258 & 0.0124 & 0.0070 & 0.4710 & 0.0387 & 0.0000 & 0.00000 \\ 0.0014 & 0.0309 & 0.0005 & 0.0500 & 0.0152 & 0.0086 & 0.5779 & 0.0474 & 0.0000 & 0.0000\end{array}$ $\begin{array}{llllllllll}0.0014 & 0.0309 & 0.0005 & 0.0500 & 0.0152 & 0.0086 & 0.5779 & 0.0474 & 0.0000 & 0.00000 \\ 0.0013 & 0.0716 & 0.0005 & 0.1179 & 0.0134 & 0.0076 & 0.5097 & 0.0418 & 0.0000 & 0.0000\end{array}$ $\begin{array}{lllllllllll}0.0013 & 0.0500 & 0.0005 & 0.1206 & 0.0137 & 0.0078 & 0.5215 & 0.0428 & 0.0000 & 0.0000\end{array}$ $\begin{array}{llllllllll}0.0014 & 0.0000 & 0.0005 & 0.1270 & 0.0144 & 0.0082 & 0.5490 & 0.0451 & 0.0000 & 0.0000 \\ 0.0012 & 0.0260 & 0.0004 & 0.1125 & 0.0128 & 0.0072 & 0.4864 & 0.0399 & 0.0000 & 0.0000\end{array}$

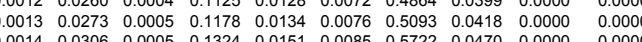
$\begin{array}{llllllllll}0.0010 & 0.0225 & 0.0004 & 0.0972 & 0.0111 & 0.0063 & 0.4204 & 0.0345 & 0.0000 & 0.0000 \\ 0.0011 & 0.0246 & 0.0004 & 0.1063 & 0.0121 & 0.0068 & 0.4597 & 0.0377 & 0.0000 & 0.0000\end{array}$ $\begin{array}{lllllllllll}0.0011 & 0.0246 & 0.0004 & 0.1063 & 0.0121 & 0.0068 & 0.4597 & 0.0377 & 0.0000 & 0.0000 \\ 0.0012 & 0.0261 & 0.0004 & 0.1128 & 0.0128 & 0.0073 & 0.4877 & 0.0400 & 0.0000 & 0.0000\end{array}$ $\begin{array}{lllllllllllll}0.0014 & 0.0300 & 0.0005 & 0.1297 & 0.0148 & 0.0084 & 0.5606 & 0.0460 & 0.0000 & 0.0000\end{array}$ $\begin{array}{llllllllll}0.0012 & 0.0251 & 0.0004 & 0.1084 & 0.0123 & 0.0070 & 0.4685 & 0.1600 & 0.0000 & 0.0000 \\ 0.0012 & 0.0263 & 0.0004 & 0.1135 & 0.0129 & 0.0073 & 0.4908 & 0.1200 & 0.0000 & 0.0000\end{array}$ $\begin{array}{llllllllll}0.0012 & 0.0263 & 0.0004 & 0.1135 & 0.0129 & 0.0073 & 0.4908 & 0.1200 & 0.0000 & 0.0000 \\ 0.0013 & 0.0275 & 0.0005 & 0.1187 & 0.0135 & 0.0076 & 0.5131 & 0.0800 & 0.0000 & 0.0000\end{array}$ $\begin{array}{llllllllll}0.0014 & 0.0299 & 0.00005 & 0.1290 & 0.0147 & 0.0083 & 0.5577 & 0.0000 & 0.0000 & 0.0000 \\ 0.0019 & 0.0266 & 0.0004 & 0.1149 & 0.0199 & 0.0113 & 0.4966 & 0.0408 & 0.0000 & 0.0000\end{array}$ $\begin{array}{lllllllllll}0.0007 & 0.0307 & 0.0005 & 0.1328 & 0.0075 & 0.0042 & 0.5742 & 0.0471 & 0.0000 & 0.0000\end{array}$ $\begin{array}{lllllllllll}0.0012 & 0.0260 & 0.0004 & 0.1125 & 0.0128 & 0.0072 & 0.4864 & 0.0399 & 0.0000 & 0.0000\end{array}$ $\begin{array}{lllllllllll}0.0013 & 0.0277 & 0.0005 & 0.1196 & 0.0136 & 0.0077 & 0.5170 & 0.0424 & 0.0000 & 0.00000 \\ 0.0012 & 0.0271 & 0.0005 & 0.1171 & 0.0133 & 0.0075 & 0.5062 & 0.0416 & 0.0000 & 0.0000\end{array}$ $\begin{array}{lllllllllll}0.0011 & 0.0242 & 0.0004 & 0.1046 & 0.0119 & 0.0067 & 0.4522 & 0.0371 & 0.0000 & 0.0000\end{array}$ $\begin{array}{llllllllll}0.0012 & 0.0287 & 0.00004 & 0.1146 & 0.0130 & 0.0074 & 0.4522 & 0.03707 & 0.0000 & 0.00000 \\ 0.0013 & 0.0305 & 0.0005 & 0.1219 & 0.0138 & 0.0078 & 0.5644 & 0.0432 & 0.00000 & 0.00000\end{array}$ $\begin{array}{llllllllll}0.0013 & 0.0305 & 0.0005 & 0.1219 & 0.0138 & 0.0078 & 0.5644 & 0.0432 & 0.0000 & 0.0000 \\ 0.0013 & 0.0296 & 0.0005 & 0.1182 & 0.0134 & 0.0076 & 0.5475 & 0.0420 & 0.0000 & 0.0000\end{array}$ $\begin{array}{llllllllll}0.0012 & 0.0277 & 0.0004 & 0.1190 & 0.0126 & 0.0071 & 0.5136 & 0.0394 & 0.00000 & 0.0000\end{array}$ $\begin{array}{llllllllll}0.0011 & 0.0268 & 0.0004 & 0.1072 & 0.0122 & 0.0069 & 0.4966 & 0.0381 & 0.0000 & 0.0000 \\ 0.0013 & 0.0279 & 0.0005 & 0.1206 & 0.0137 & 0.0300 & 0.5214 & 0.0428 & 0.0000 & 0.0000\end{array}$ $\begin{array}{llllllllll}0.0013 & 0.0279 & 0.0005 & 0.1206 & 0.0137 & 0.0300 & 0.5214 & 0.0428 & 0.0000 & 0.0000 \\ 0.0013 & 0.0274 & 0.0005 & 0.1181 & 0.0134 & 0.0500 & 0.5107 & 0.0419 & 0.0000 & 0.0000\end{array}$ $\begin{array}{llllllllll}0.0012 & 0.0268 & 0.0004 & 0.1156 & 0.0132 & 0.0700 & 0.4999 & 0.0410 & 0.0000 & 0.0000 \\ 0.0012 & 0.0262 & 0.0004 & 0.1132 & 0.0129 & 0.0900 & 0.4892 & 0.0402 & 0.0000 & 0.0000\end{array}$

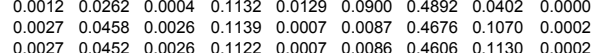

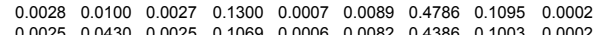

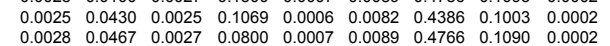

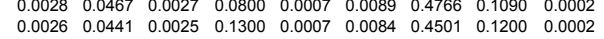

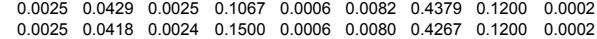

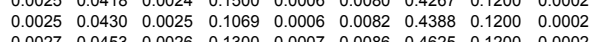
$\begin{array}{lllllllll}0.0026 & 0.0441 & 0.0025 & 0.1096 & 0.0007 & 0.0084 & 0.4499 & 0.1029 & 0.0002 \\ 0.0027 & 0.0450 & 0.0026 & 0.1119 & 0.0007 & 0.0086 & 0.4595 & 0.1051 & 0.0002\end{array}$

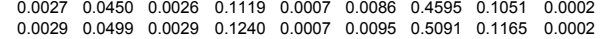

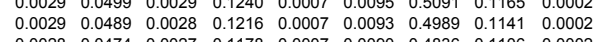
$\begin{array}{lllllllll}0.0028 & 0.0474 & 0.0027 & 0.1178 & 0.0007 & 0.0090 & 0.4836 & 0.1106 & 0.0002 \\ 0.0026 & 0.0439 & 0.0025 & 0.1091 & 0.0007 & 0.0083 & 0.4480 & 0.1025 & 0.0002\end{array}$ $\begin{array}{lllllllll}0.0026 & 0.0439 & 0.0025 & 0.1091 & 0.0007 & 0.0083 & 0.4480 & 0.1025 & 0.0002 \\ 0.0029 & 0.0494 & 0.0028 & 0.1228 & 0.0007 & 0.0094 & 0.5040 & 0.1153 & 0.0002\end{array}$ $\begin{array}{lllllllll}0.0029 & 0.0485 & 0.0028 & 0.1204 & 0.0007 & 0.0092 & 0.4944 & 0.1131 & 0.0002 \\ 0.0028 & 0.0473 & 0.0027 & 0.1174 & 0.0007 & 0.0090 & 0.4820 & 0.1103 & 0.0002\end{array}$ $\begin{array}{llllllllll}0.0026 & 0.0448 & 0.0026 & 0.1144 & 0.0007 & 0.0085 & 0.452 & 0.113 & 0.1046 & 0.0002\end{array}$

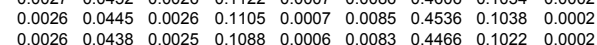
$\begin{array}{llllllllll}0.0022 & 0.0438 & 0.0025 & 0.108 & 0.0006 & 0.0083 & 0.4466 & 0.1022 & 0.0002 \\ 0.028 & 0.0000 & 0.0028 & 0.1194 & 0.0007 & 0.0091 & 0.4901 & 0.1121 & 0.0002\end{array}$

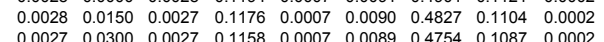
$\begin{array}{lllllllll}0.0027 & 0.0300 & 0.0027 & 0.1158 & 0.0007 & 0.0089 & 0.4754 & 0.1087 & 0.0002 \\ 0.0027 & 0.0600 & 0.0026 & 0.1122 & 0.0007 & 0.0086 & 0.4607 & 0.1054 & 0.0002\end{array}$

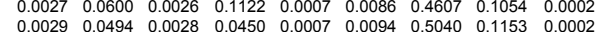

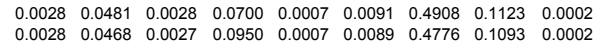

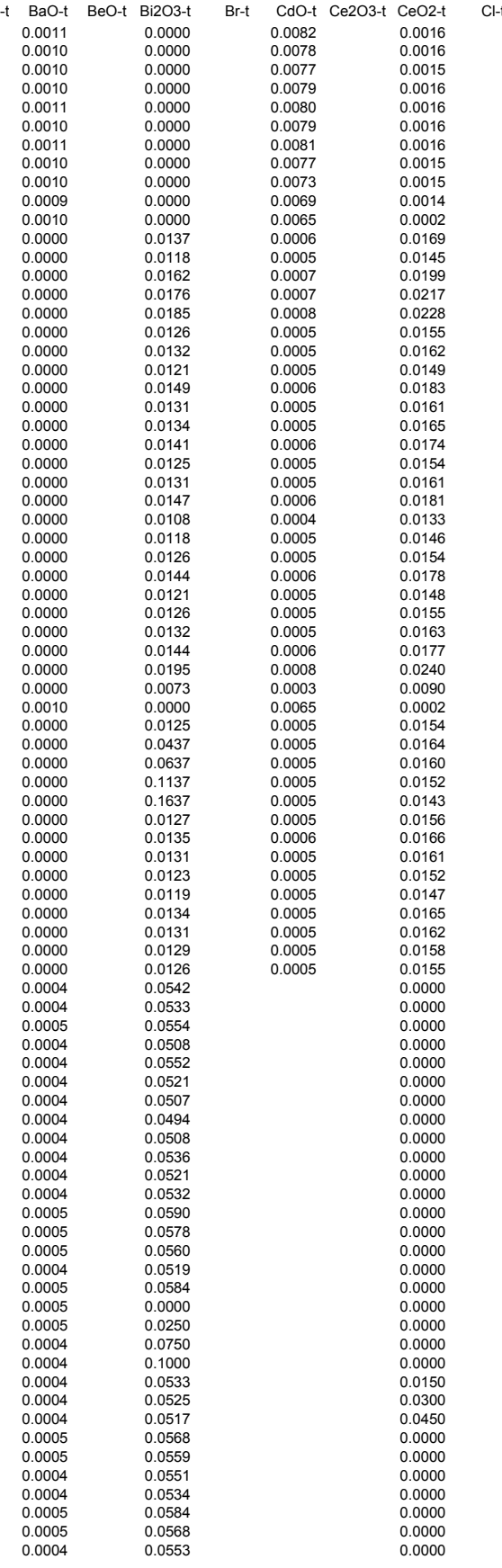




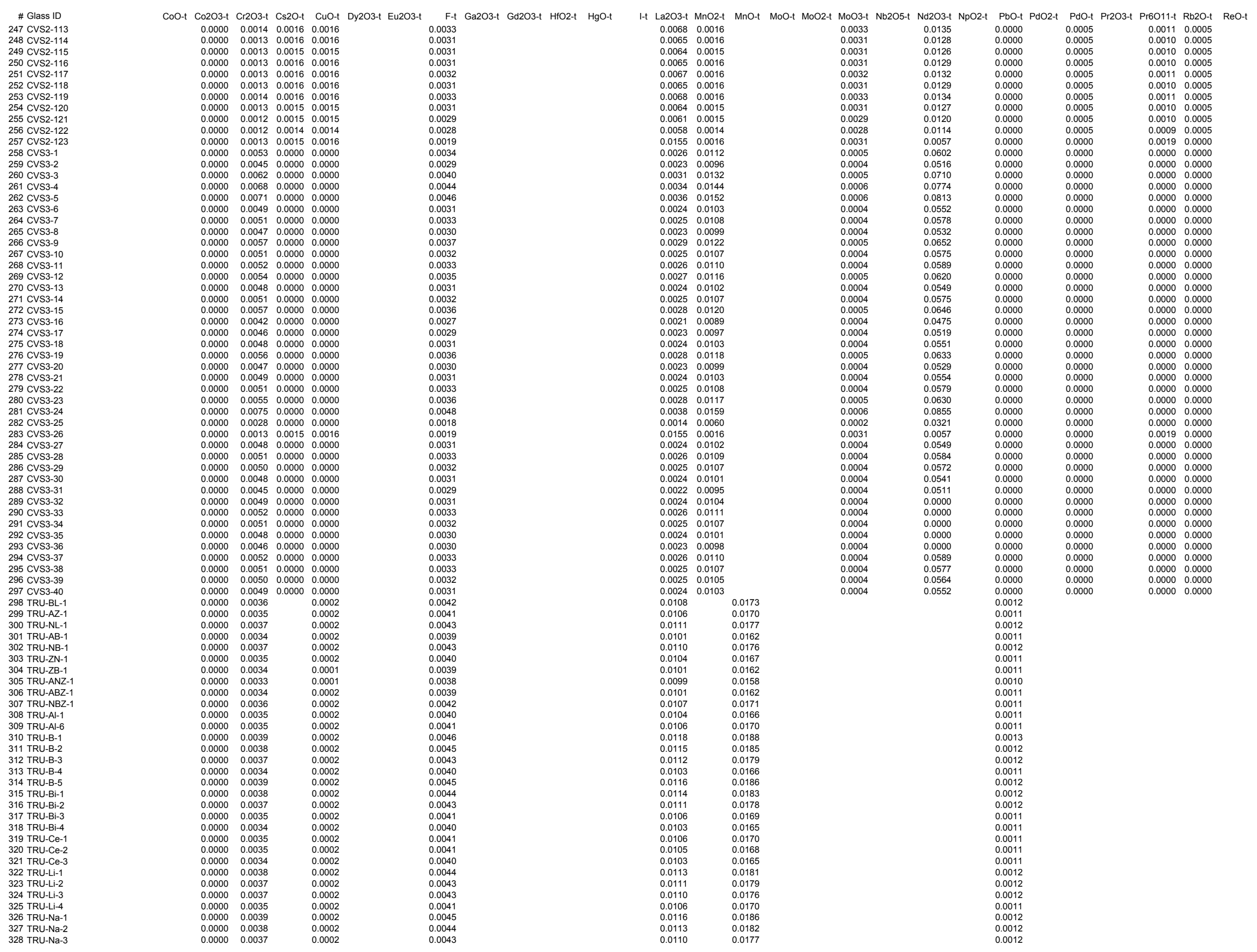




\begin{tabular}{|c|c|c|c|c|c|c|c|c|c|c|c|c|c|c|c|c|c|c|c|}
\hline \# Glass ID & ReO2-t Re207-t & Rh2O3-t RhO2-t & RuO2-t Ru2O3-t & $\mathrm{sb} 203-\mathrm{t} \mathrm{Sb} 205-\mathrm{t}$ & $\mathrm{SeO} 2-\mathrm{t}$ & Sm203-t & SnO-t SnO2-t & so3-t & Sro-t Tc207-t & TeO2-t ThO2-t & TiO2-t & T12O-t T12O3-t U3O8-t & UO2-t & UO3-t V2O5-t & wO3-t & Y2O3-t & Zno-t & Sum-t & Al2O3-a \\
\hline $\begin{array}{l}247 \text { CVS2-113 } \\
248 \text { CVS2-114 }\end{array}$ & & 0.0005 & $\begin{array}{l}0.0016 \\
0.0016\end{array}$ & 0.0000 & 0.0000 & 0.0005 & & 0.0030 & 0.0011 & 0.0000 & 0.0000 & & 0.0000 & & & 0.0005 & 0.0000 & 1.0000 & \\
\hline 249 CVS2-115 & & 0.0005 & 0.0015 & 0.0000 & 0.0000 & 0.0005 & & 0.0028 & 0.0010 & $\begin{array}{l}0.00000 \\
0.0000\end{array}$ & 0.0000 & & $\begin{array}{l}0.0000 \\
0.0000\end{array}$ & & & $\begin{array}{l}.0 .0005 \\
0.0005\end{array}$ & $\begin{array}{l}0.00000 \\
0.0000\end{array}$ & $\begin{array}{l}1.0000 \\
1.000\end{array}$ & \\
\hline 250 CVS2-116 & & 0.0005 & 0.0016 & 0.0000 & 0.0000 & 0.0005 & & 0.0029 & 0.0010 & 0.0000 & 0.0000 & & 0.0000 & & & 0.0005 & 0.0000 & 1.0000 & \\
\hline 251 CVS2-117 & & 0.0005 & 0.0016 & 0.0000 & 0.0000 & 0.0005 & & 0.0029 & 0.0011 & 0.0000 & 0.0000 & & 0.0000 & & & 0.0005 & 0.0000 & 1.0000 & \\
\hline 252 CVS2-118 & & 0.0005 & 0.0016 & 0.0000 & 0.0000 & 0.0005 & & 0.0029 & 0.0010 & 0.0000 & 0.0000 & & 0.0000 & & & 0.0005 & 0.0000 & 1.0000 & \\
\hline $\begin{array}{l}253 \text { CVS2-119 } \\
254 \text { CVS2-120 }\end{array}$ & & $\begin{array}{l}0.0005 \\
0.0005\end{array}$ & $\begin{array}{l}0.0016 \\
0.0015\end{array}$ & $\begin{array}{l}0.0000 \\
0.0000\end{array}$ & 0.0000 & 0.0005 & & 0.0030 & 0.0011 & 0.0000 & 0.0000 & & 0.0000 & & & 0.0005 & 0.0000 & 1.0000 & \\
\hline $\begin{array}{l}254 \text { CVS2-120 } \\
255 \text { CVS2-121 }\end{array}$ & & $\begin{array}{l}0.0005 \\
0.0005\end{array}$ & $\begin{array}{l}0.0015 \\
0.0015\end{array}$ & $\begin{array}{l}0.0000 \\
0.0000\end{array}$ & $\begin{array}{l}0.0000 \\
0.0000\end{array}$ & $\begin{array}{l}0.0005 \\
0.0005\end{array}$ & & $\begin{array}{l}0.0028 \\
0.0027\end{array}$ & $\begin{array}{l}0.00010 \\
0.0010\end{array}$ & $\begin{array}{l}0.0000 \\
0.0000\end{array}$ & $\begin{array}{l}0.0000 \\
0.0000\end{array}$ & & $\begin{array}{l}0.0000 \\
0.0000\end{array}$ & & & $\begin{array}{l}0.0005 \\
0.0005\end{array}$ & $\begin{array}{l}0.0000 \\
0.0000\end{array}$ & $\begin{array}{l}1.0000 \\
1.0000\end{array}$ & \\
\hline 256 CVS2-122 & & 0.0005 & 0.0014 & 0.0000 & $\begin{array}{l}0.00000 \\
0.0000\end{array}$ & $\begin{array}{l}0.00005 \\
0.0005\end{array}$ & & $\begin{array}{l}0.0027 \\
0.0025\end{array}$ & 0.0009 & 0.0000 & $\begin{array}{l}0.0000 \\
0.0000\end{array}$ & & $\begin{array}{l}0.0000 \\
0.0000\end{array}$ & & & $\begin{array}{l}.0 .0005 \\
0.0005\end{array}$ & 0.0000 & $\begin{array}{l}1.0000 \\
1.0000\end{array}$ & \\
\hline 257 CVS2-123 & & 0.0000 & 0.0015 & 0.0000 & 0.0000 & 0.0000 & & 0.0044 & 0.0000 & 0.0000 & 0.0000 & & 0.0000 & & & 0.0000 & 0.0049 & 1.0000 & \\
\hline 258 CVS3-1 & & 0.0000 & 0.0000 & 0.0000 & 0.0000 & 0.0000 & & 0.0020 & 0.0000 & 0.0000 & 0.0000 & & 0.0000 & & 0.0011 & 0.0000 & 0.0000 & 1.0000 & \\
\hline 259 CVS3-2 & & 0.0000 & 0.0000 & 0.0000 & 0.0000 & 0.0000 & & 0.0017 & 0.0000 & 0.0000 & 0.0000 & & 0.0000 & & 0.0009 & 0.0000 & 0.0000 & 1.0000 & \\
\hline 260 CVS3-3 & & 0.0000 & 0.0000 & 0.0000 & 0.0000 & 0.0000 & & 0.0024 & 0.0000 & 0.0000 & 0.0000 & & 0.0000 & & 0.0013 & 0.0000 & 0.0000 & 1.0000 & \\
\hline 261 CVS3-4 & & 0.0000 & 0.0000 & 0.0000 & 0.0000 & 0.0000 & & 0.0026 & 0.0000 & 0.0000 & 0.0000 & & 0.0000 & & 0.0014 & 0.0000 & 0.0000 & 1.0000 & \\
\hline 262 CVS3-5 & & 0.0000 & 0.0000 & 0.0000 & 0.0000 & 0.0000 & & 0.0028 & 0.0000 & 0.0000 & 0.0000 & & 0.0000 & & 0.0015 & 0.0000 & 0.0000 & 1.0000 & \\
\hline $\begin{array}{l}263 \text { CVSS3-6 } \\
264 \text { CVS3 }\end{array}$ & & $\begin{array}{l}0.00000 \\
0.0000\end{array}$ & $\begin{array}{l}0.0000 \\
0.0000\end{array}$ & $\begin{array}{l}0.0000 \\
0.0000\end{array}$ & $\begin{array}{l}0.0000 \\
0.0000\end{array}$ & $\begin{array}{l}0.0000 \\
0.0000\end{array}$ & & $\begin{array}{l}0.0019 \\
0.0020\end{array}$ & $\begin{array}{l}0.0000 \\
0.0000\end{array}$ & $\begin{array}{l}0.0000 \\
0.0000\end{array}$ & $\begin{array}{l}0.0000 \\
0.0000\end{array}$ & & $\begin{array}{l}0.0000 \\
0.0000\end{array}$ & & $\begin{array}{l}0.0010 \\
0.0010\end{array}$ & $\begin{array}{l}0.0000 \\
0.0000\end{array}$ & $\begin{array}{l}0.0000 \\
0.0000\end{array}$ & $\begin{array}{l}1.0000 \\
1.0000\end{array}$ & \\
\hline $\begin{array}{l}264 \text { CVS3 3-7 } \\
265 \text { CVS3-8 }\end{array}$ & & $\begin{array}{l}0.0000 \\
0.0000\end{array}$ & $\begin{array}{l}0.0000 \\
0.0000\end{array}$ & $\begin{array}{l}.0 .0000 \\
0.0000\end{array}$ & $\begin{array}{l}0.00000 \\
0.0000\end{array}$ & $\begin{array}{l}0.00000 \\
0.0000\end{array}$ & & $\begin{array}{l}0.0020 \\
0.0018\end{array}$ & $\begin{array}{l}.0 .0000 \\
0.0000\end{array}$ & $\begin{array}{l}0.0000 \\
0.0000\end{array}$ & $\begin{array}{l}.0 .0000 \\
0.0000\end{array}$ & & $\begin{array}{l}.0 .0000 \\
0.0000\end{array}$ & & $\begin{array}{l}0.0010 \\
0.0009\end{array}$ & $\begin{array}{l}0.00000 \\
0.0000\end{array}$ & $\begin{array}{l}0.0000 \\
0.0000\end{array}$ & $\begin{array}{l}1.0000 \\
10000\end{array}$ & \\
\hline 266 CVS3-9 & & 0.0000 & 0.0000 & 0.0000 & 0.0000 & 0.0000 & & 0.0022 & 0.0000 & 0.0000 & 0.0000 & & 0.0000 & & 0.0012 & 0.0000 & 0.0000 & $\begin{array}{l}1.00000 \\
1.000\end{array}$ & \\
\hline 267 CVS3-10 & & 0.0000 & 0.0000 & 0.0000 & 0.0000 & 0.0000 & & 0.0020 & 0.0000 & 0.0000 & 0.0000 & & 0.0000 & & 0.0010 & 0.0000 & 0.0000 & 1.0000 & \\
\hline 268 CVS3-11 & & 0.0000 & 0.0000 & 0.0000 & 0.0000 & 0.0000 & & 0.0020 & 0.0000 & 0.0000 & 0.0000 & & 0.0000 & & 0.0011 & 0.0000 & 0.0000 & 1.0000 & \\
\hline 269 CVS3-12 & & 0.0000 & 0.0000 & 0.0000 & 0.0000 & 0.0000 & & 0.0021 & 0.0000 & 0.0000 & 0.0000 & & 0.0000 & & 0.0011 & 0.0000 & 0.0000 & 1.0000 & \\
\hline 270 cVS3-13 & & 0.0000 & 0.0000 & 0.0000 & 0.0000 & 0.0000 & & 0.0019 & 0.0000 & 0.0000 & 0.0000 & & 0.0000 & & 0.0010 & 0.0000 & 0.0000 & 1.0000 & \\
\hline 271 CVS3-14 & & 0.0000 & 0.0000 & 0.0000 & 0.0000 & 0.0000 & & 0.0019 & 0.0000 & 0.0000 & 0.0000 & & 0.0000 & & 0.0010 & 0.0000 & 0.0000 & 1.0000 & \\
\hline $\begin{array}{l}272 \text { CVS3-15 } \\
273 \text { cyS3 } 16\end{array}$ & & $\begin{array}{l}0.0000 \\
0.000\end{array}$ & 0.0000 & 0.0000 & 0.0000 & 0.0000 & & 0.0022 & $\begin{array}{l}0.0000 \\
0.000\end{array}$ & 0.0000 & 0.0000 & & 0.0000 & & 0.0012 & 0.0000 & 0.0000 & 1.0000 & \\
\hline $\begin{array}{l}273 \text { CVS3-16 } \\
274 \text { CVS3-17 }\end{array}$ & & $\begin{array}{l}0.0000 \\
0.0000\end{array}$ & $\begin{array}{l}0.0000 \\
0.0000\end{array}$ & $\begin{array}{l}0.0000 \\
0.0000\end{array}$ & $\begin{array}{l}0.0000 \\
0.0000\end{array}$ & $\begin{array}{l}0.0000 \\
0.0000\end{array}$ & & $\begin{array}{l}0.0016 \\
0.0018\end{array}$ & $\begin{array}{l}0.0000 \\
0.0000\end{array}$ & $\begin{array}{l}0.0000 \\
0.0000\end{array}$ & $\begin{array}{l}0.0000 \\
0.0000\end{array}$ & & $\begin{array}{l}0.0000 \\
0.0000\end{array}$ & & 0.0008 & $\begin{array}{l}0.0000 \\
0.0000\end{array}$ & $\begin{array}{l}0.0000 \\
0.0000\end{array}$ & $\begin{array}{l}1.0000 \\
1.0000\end{array}$ & \\
\hline 275 CVS3-18 & & $\begin{array}{l}0.00000 \\
0.0000\end{array}$ & $\begin{array}{l}0.0000 \\
0.0000\end{array}$ & $\begin{array}{l}0.0000 \\
0.0000\end{array}$ & $\begin{array}{l}0.0000 \\
0.0000\end{array}$ & $\begin{array}{l}0.0000 \\
0.0000\end{array}$ & & $\begin{array}{l}0.0018 \\
0.0019\end{array}$ & $\begin{array}{l}0.0000 \\
0.0000\end{array}$ & $\begin{array}{l}0.0000 \\
0.0000\end{array}$ & $\begin{array}{l}0.0000 \\
0.0000\end{array}$ & & $\begin{array}{l}0.00000 \\
0.0000\end{array}$ & & $\begin{array}{l}0.0009 \\
0.0010\end{array}$ & $\begin{array}{l}0.0000 \\
0.0000\end{array}$ & $\begin{array}{l}0.0000 \\
0.0000\end{array}$ & $\begin{array}{l}1.0000 \\
1.0000\end{array}$ & \\
\hline 276 CVS3-19 & & 0.0000 & 0.0000 & 0.0000 & 0.0000 & 0.0000 & & 0.0021 & 0.0000 & 0.0000 & 0.0000 & & 0.0000 & & 0.0011 & 0.0000 & 0.0000 & 1.0000 & \\
\hline 277 CVS3-20 & & 0.0000 & 0.0000 & 0.0000 & 0.0000 & 0.0000 & & 0.0018 & 0.0000 & 0.0000 & 0.0000 & & 0.0000 & & 0.0009 & 0.0000 & 0.0000 & 1.0000 & \\
\hline 278 CVS3-21 & & 0.0000 & 0.0000 & 0.0000 & 0.0000 & 0.0000 & & 0.0019 & 0.0000 & 0.0000 & 0.0000 & & 0.0000 & & 0.0010 & 0.0000 & 0.0000 & 1.0000 & \\
\hline 279 cVS3-22 & & 0.0000 & 0.0000 & 0.0000 & 0.0000 & 0.0000 & & 0.0020 & 0.0000 & 0.0000 & 0.0000 & & 0.0000 & & 0.0010 & 0.0000 & 0.0000 & 1.0000 & \\
\hline 280 CVS3-23 & & 0.0000 & 0.0000 & 0.0000 & 0.0000 & 0.0000 & & 0.0021 & 0.0000 & 0.0000 & 0.0000 & & 0.0000 & & 0.0011 & 0.0000 & 0.0000 & 1.0000 & \\
\hline $\begin{array}{l}281 \text { CVS3-24 } \\
282 \text { CVS }-25\end{array}$ & & $\begin{array}{l}0.0000 \\
0.000\end{array}$ & 0.0000 & $\begin{array}{l}0.0000 \\
0.000\end{array}$ & $\begin{array}{l}0.0000 \\
0.000\end{array}$ & 0.0000 & & 0.0029 & 0.0000 & 0.0000 & 0.0000 & & $\begin{array}{l}0.0000 \\
0.000\end{array}$ & & 0.0015 & 0.0000 & 0.0000 & 1.0000 & \\
\hline $\begin{array}{l}282 \text { CVS3 } 25 \\
283 \text { CVS3-26 }\end{array}$ & & $\begin{array}{l}0.0000 \\
0.0000\end{array}$ & $\begin{array}{l}0.0000 \\
0.0015\end{array}$ & $\begin{array}{l}0.0000 \\
0.0000\end{array}$ & $\begin{array}{l}0.0000 \\
0.0000\end{array}$ & $\begin{array}{l}0.0000 \\
0.0000\end{array}$ & & $\begin{array}{l}0.0011 \\
0.0044\end{array}$ & $\begin{array}{l}0.0000 \\
0.0000\end{array}$ & $\begin{array}{l}0.0000 \\
0.0000\end{array}$ & $\begin{array}{l}0.0000 \\
0.0000\end{array}$ & & $\begin{array}{l}0.0000 \\
0.0000\end{array}$ & & 0.0006 & $\begin{array}{l}0.0000 \\
0.0000\end{array}$ & $\begin{array}{l}0.0000 \\
0.0049\end{array}$ & $\begin{array}{l}1.0000 \\
1.0000\end{array}$ & \\
\hline 284 CVS3-27 & & 0.0000 & 0.0000 & 0.0000 & 0.0000 & 0.0000 & & $\begin{array}{l}0.0019 \\
0.0019\end{array}$ & 0.0000 & 0.0000 & 0.0000 & & 0.0000 & & 0.0010 & 0.0000 & 0 & $\begin{array}{l}l_{1.0000} \\
1.0000\end{array}$ & \\
\hline 285 CVS $3-28$ & & 0.0000 & 0.0000 & 0.0000 & 0.0000 & 0.0000 & & 0.0020 & 0.0000 & 0.0000 & 0.0000 & & 0.0000 & & 0.0010 & 0.0000 & 0.0000 & 1.0000 & \\
\hline 286 CVS $3-29$ & & 0.0000 & 0.0000 & 0.0000 & 0.0000 & 0.0000 & & 0.0019 & 0.0000 & 0.0000 & 0.0000 & & 0.0000 & & 0.0010 & 0.0000 & 0.0000 & 1.0000 & \\
\hline 287 CVS3-30 & & 0.0000 & 0.0000 & 0.0000 & 0.0000 & 0.0000 & & 0.0018 & 0.0000 & 0.0000 & 0.0000 & & 0.0000 & & 0.0010 & 0.0000 & 0.0000 & 1.0000 & \\
\hline 288 CVS3-31 & & 0.0000 & 0.0000 & 0.0000 & 0.0000 & 0.0000 & & 0.0017 & 0.0000 & 0.0000 & 0.0000 & & 0.0000 & & 0.0009 & 0.0000 & 0.0000 & 1.0000 & \\
\hline 289 CVS3-32 & & 0.0000 & 0.0000 & 0.0000 & 0.0000 & 0.0000 & & 0.0019 & 0.0000 & 0.0000 & 0.0000 & & 0.0900 & & 0.0010 & 0.0000 & 0.0000 & 1.0000 & \\
\hline 290 CVS3-33 & & 0.0000 & 0.0000 & 0.0000 & 0.0000 & 0.0000 & & 0.0020 & 0.0000 & 0.0000 & 0.0000 & & 0.0320 & & 0.0011 & 0.0000 & 0.0000 & 1.0000 & \\
\hline 291 CVS3-34 & & 0.0000 & 0.0000 & 0.0000 & 0.0000 & 0.0000 & & 0.0019 & 0.0000 & 0.0000 & 0.0000 & & 0.0610 & & 0.0010 & & 0.0000 & 1.0000 & \\
\hline 292 CVS3-35 & & 0.0000 & 0.0000 & 0.0000 & 0.0000 & 0.0000 & & 0.0018 & 0.0000 & 0.0000 & 0.0000 & & 0.1190 & & 0.0010 & 0.0000 & 0.0000 & 1.0000 & \\
\hline 293 CVS3-36 & & 0.0000 & 0.0000 & 0.0000 & 0.0000 & 0.0000 & & 0.0018 & 0.0000 & 0.0000 & 0.0000 & & 0.1480 & & 0.0009 & & 0.0000 & 1.0000 & \\
\hline 294 CVS3-37 & & 0.0000 & 0.0000 & 0.0000 & 0.0000 & 0.0000 & & 0.0020 & 0.0000 & 0.0000 & 0.0000 & & 0.0000 & & 0.0011 & 0.0000 & 0.0000 & 1.0000 & \\
\hline $\begin{array}{l}295 \text { CVSS3-38 } \\
296 \text { CVS } 3399\end{array}$ & & $\begin{array}{l}0.0000 \\
0.0000\end{array}$ & & $\begin{array}{l}0.0000 \\
0.0000\end{array}$ & $\begin{array}{l}0.0000 \\
0.0000\end{array}$ & $\begin{array}{l}0.0000 \\
0.0000\end{array}$ & & $\begin{array}{l}0.0020 \\
0.0019\end{array}$ & $\begin{array}{l}0.0000 \\
0.0000\end{array}$ & $\begin{array}{l}0.0000 \\
0.0000\end{array}$ & $\begin{array}{l}0.0000 \\
0.0000\end{array}$ & & $\begin{array}{l}0.0000 \\
0.0000\end{array}$ & & $\begin{array}{l}0.0010 \\
0.0010\end{array}$ & 0.0000 & 0.0000 & $\begin{array}{l}1.0000 \\
1.000\end{array}$ & \\
\hline $\begin{array}{l}296 \\
297 \text { CVS } 3-39\end{array}$ & & $\begin{array}{l}0.00000 \\
0.0000\end{array}$ & $\begin{array}{l}0.0000 \\
0.0000\end{array}$ & $\begin{array}{l}0.0000 \\
0.0000\end{array}$ & 0.00000 & 0.00000 & & $\begin{array}{l}0.0019 \\
0.0019\end{array}$ & $\begin{array}{l}0.0000 \\
0.0000\end{array}$ & 0.00000 & 0.00000 & & $\begin{array}{l}0.00000 \\
0.0000\end{array}$ & & 0.0010 & 0.00000 & 0.0000 & 1.0000 & \\
\hline 298 TRU-BL-1 & & & & & & & & & 0.0006 & & & & & & & & 0.0005 & 10000 & \\
\hline 299 TRU-AZ-1 & & & & & & & & & 0.0006 & & & & & & & & 0.0005 & 1.0000 & \\
\hline 300 TRU-NL-1 & & & & & & & & & 0.0006 & & & & & & & & 0.0005 & 1.0000 & \\
\hline 301 TRU-AB-1 & & & & & & & & & 0.0006 & & & & & & & & 0.0005 & 1.0000 & \\
\hline $\begin{array}{l}302 \text { TRU-NB-1 } \\
303 \text { TRU-ZN-1 }\end{array}$ & & & & & & & & & $\begin{array}{l}0.0006 \\
0.0006\end{array}$ & & & & & & & & 0.0005 & 1.0000 & \\
\hline $\begin{array}{l}303 \text { TRUUZN-1 } \\
304 \text { TRU-ZB-1 }\end{array}$ & & & & & & & & & $\begin{array}{l}0.0006 \\
0.0006\end{array}$ & & & & & & & & 0.0005 & $\begin{array}{l}1.0000 \\
1.0000\end{array}$ & \\
\hline $\begin{array}{l}304 \text { TRU-ZB-1 } \\
305 \text { TRU-ANZ-1 }\end{array}$ & & & & & & & & & 0.0005 & & & & & & & & $\begin{array}{l}0.0005 \\
0.0005\end{array}$ & $\begin{array}{l}f_{1.0000} \\
1.0000\end{array}$ & \\
\hline 306 TRU-ABZ-1 & & & & & & & & & 0.0006 & & & & & & & & 0.0005 & $\begin{array}{l}1.00000 \\
1.0000\end{array}$ & \\
\hline 307 TRU-NBZ-1 & & & & & & & & & 0.0006 & & & & & & & & 0.0005 & 1.0000 & \\
\hline 308 TRU-Al-1 & & & & & & & & & 0.0006 & & & & & & & & 0.0005 & 1.0000 & \\
\hline 309 TRU-Al-6 & & & & & & & & & 0.0006 & & & & & & & & 0.0005 & 1.0000 & \\
\hline 310 TRU-B-1 & & & & & & & & & 0.0007 & & & & & & & & 0.0006 & 1.0000 & \\
\hline 311 TRU-B-2 & & & & & & & & & 0.0006 & & & & & & & & 0.0006 & 1.0000 & \\
\hline 312 TRU-B-3 & & & & & & & & & 0.0006 & & & & & & & & 0.0005 & 1.0000 & \\
\hline $\begin{array}{l}313 \text { TRU-B-B-4 } \\
314 \text { TRUB-5 }\end{array}$ & & & & & & & & & 0.0006 & & & & & & & & 0.0005 & 1.0000 & \\
\hline $\begin{array}{l}314 \text { TRUU-B-5 } \\
315 \text { TRU-Bi-1 }\end{array}$ & & & & & & & & & $\begin{array}{l}0.0006 \\
0.0006\end{array}$ & & & & & & & & $\begin{array}{l}0.0006 \\
0.0005\end{array}$ & $\begin{array}{l}1.0000 \\
1.0000\end{array}$ & \\
\hline 316 TRU-Bi-2 & & & & & & & & & 0.0006 & & & & & & & & 0.0005 & $\begin{array}{l}1.100000 \\
1.0000\end{array}$ & \\
\hline 317 TRU-Bi-3 & & & & & & & & & 0.0006 & & & & & & & & 0.0005 & 1.0000 & \\
\hline 318 TRU-Bi-4 & & & & & & & & & 0.0006 & & & & & & & & 0.0005 & 1.0000 & \\
\hline 319 TRU-Ce-1 & & & & & & & & & 0.0006 & & & & & & & & 0.0005 & 1.0000 & \\
\hline 320 TRU-Ce-2 & & & & & & & & & 0.0006 & & & & & & & & 0.0005 & 1.0000 & \\
\hline 321 TRU-Ce-3 & & & & & & & & & 0.0006 & & & & & & & & 0.0005 & 1.0000 & \\
\hline $\begin{array}{l}322 \text { TRU-Li-1 } \\
323 \text { TRUL-2-2 }\end{array}$ & & & & & & & & & 0.0006 & & & & & & & & 0.0005 & $\begin{array}{l}1.0000 \\
1.000\end{array}$ & \\
\hline 324 TRU-Li-3 & & & & & & & & & 0.0006 & & & & & & & & $\begin{array}{l}0.0005 \\
0.0005\end{array}$ & $\begin{array}{l}1.00000 \\
1.000\end{array}$ & \\
\hline & & & & & & & & & & & & & & & & & 0.0005 & 1.0000 & \\
\hline 326 TRU-Na-1 & & & & & & & & & 0.0006 & & & & & & & & 0.0006 & 1.0000 & \\
\hline 327 TRU-Na-2 & & & & & & & & & 0.0006 & & & & & & & & 0.0005 & 1.0000 & \\
\hline 328 TRU-Na-3 & & & & & & & & & 0.0006 & & & & & & & & 0.0005 & 1.0000 & \\
\hline
\end{tabular}


248 CVS2-114

250 CVS2-116
251 cVS2-117

253 c VS2-119

254 CVS2-120

256 CVS2-12

25 CVS3-1

259 CVS3-2
260 CVS3-3

261 CVS3-4
262 CVS3-5

263 CVS3-6
264 CVS

265 CVS3-8

266 CVS3-9

267 CVS3-1
268 CVS3-1

2269 CVS3-12

270 CVS3 313
271 CVS -14

272 CVS3 -15
273 CVS3 -16

274 CVS3-17
275 CVS3 18

275 CVS3-18
276 CVS3 -19

277 CVS3-20

278 CVS3-21

280 CVS3-23
281 cVss-24

282 CVS3-25
283 CVS3-26

284 CVS3-27

285 CVS3 28

CVS3-30

289 CVS3-32

290 CVS3 33

292 CVS3-35

293 CVS3-36

294 CVSS-37
225 CVSS3-38

296 CVS3-39

297 CVS3 30

${ }_{299}^{298}$ TRU-BL-A

300 TRU-NL-1

301 TRU-AB-

303 TRU-ZB-

304 TRU-ZB-1

305 TRU-ANZ-1

306 TRU-ABZ-1
307 TRU-NBZ-1

308 TRU-A-1 1

309 TRU-Al- 6

310 TRU-B-1
311 TRU-B-2

312 TRU-B-3

313 TRU-B- 4

315 TRU-Bi-1

316 TRU-Bi-2

(18 TRUBi-

$19 \mathrm{TRU}-\mathrm{Ce}-1$

320 TRU-Ce-2

政

323 TRU-LL-2

324 TRU-Li- 3

255 TRU-Li-4
26 TRU-Na-1

TRU-Na-2

$328 \mathrm{TRU}-\mathrm{Na}-3$ 
248 CSS2-114

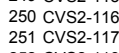

252 CVS2-118

254 CVS2-120

255 C CSS2-121
256 CVS-122
257

258 CVS3-1

259 CVS3-2
260 CVS3 3
2655

261 CVS3-4

262 CVS3.5
263 CVS 5.6

264 CVS3 37

265 CVS3 -8
266 CVSS3-9
258 CS

267 CVS3-10
268 CVS3-11

269 CVS3 -12

270 CVS3 313
271 CVS -14

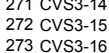

274 CVS3-17

275 CVS3-18
276 CVS3-19

277 CVS3-20

278 CVS3-21

280 CVS3-23
281 cVss-24

282 CVS3 25
283
CYS -26

284 CVS3-27

285 CVS3 28
286
$285-29$

286 CVS3-29
287 CVS 3039

288 CVS3-31

289 CVS3 332

291 CVSS-34
292 CVS3 35

293 CvV3 -36
2045 CS3

294 CVS3-37
295 CVS3 38

296 CVS3-39

297 CVS3 30

298 TRU-BL-

300 TRU-NL-1

301 TRU-AB-

303 TRU-ZN-

304 TRU-ZB-1

305 TRU-ANZ-1

306 TRU-ABZ-1

308 TRU-A-1

309 TRU-AA-6

310 TRU-B-1
311 TRUB--2

312 TRU-B-3

313 TRU-B- 4

315 TRU-Bi-1

316 TRU-Bi-2

(18 TRUBi-

19 TRU-Ce-1

320 TRU-Ce-2

政

323 TRU-LL-2

324 TRU-Li-3

325
326 TRUU-Na-

27 TRU-Na-2

328 TRU-Na-3 


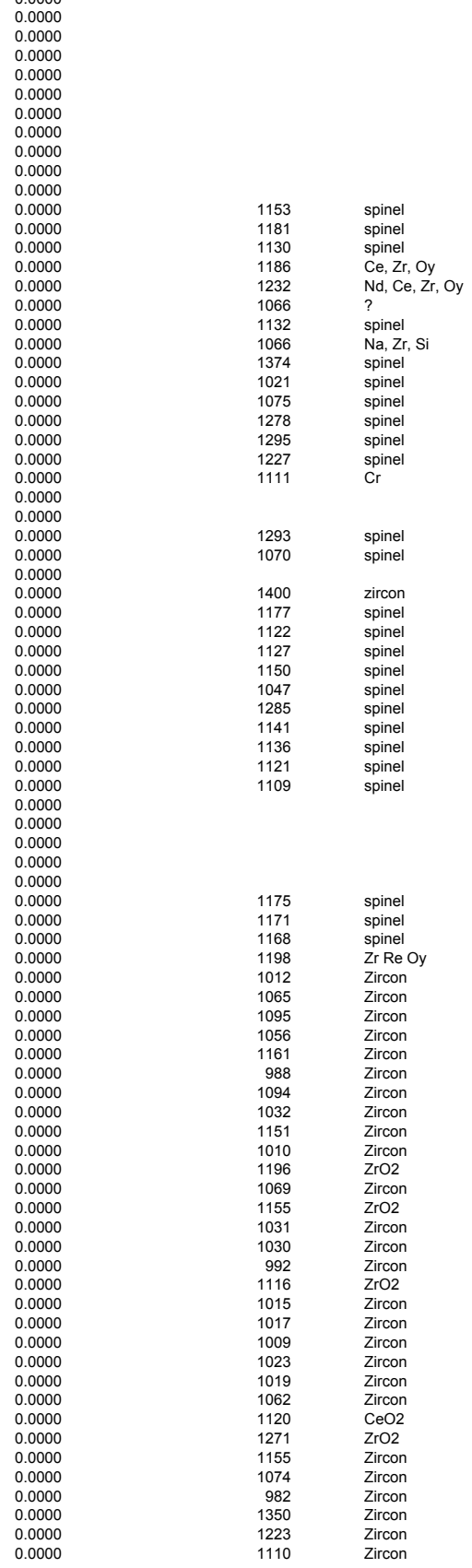




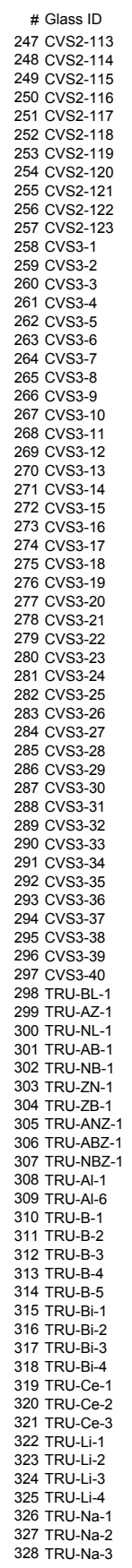

(n)

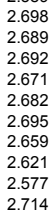

NoX XL
NoX $X L$

Amorphous

$\begin{array}{rrrrrrr}-7.170 & 10073.0 & 492.1 & -12.040 & 22335.1 & 1095.0 & 76.079 \\ -2.610 & 3388.7 & 979.1 & -11.320 & 227749 & 11940 & 7409\end{array}$

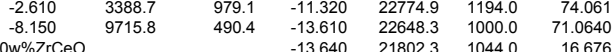

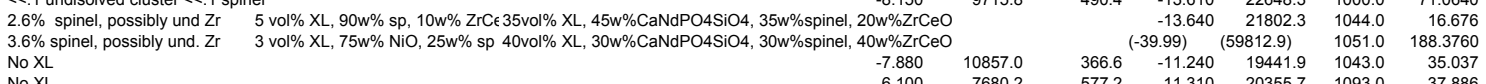
No X XL
No XL No XL very small particles
No XL

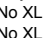

very small particles

No XL

10 vol\% Spinel

11477.0

$\begin{array}{lllll}366.6 & -11.240 & 19441.9 & 1043.0 & 35.037 \\ 577.2 & -11.310 & 20355.7 & 1093.0 & 37.886 \\ 332.8 & -11.950 & 19755.5 & 992.0 & 40.279\end{array}$

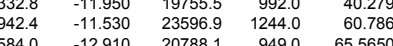
\begin{tabular}{rrrrr}
501.4 & -12.050 & 20895.7 & 992.0 & 93.903 \\
977.9 & -12.580 & 25798.1 & 1245.0 & 92.087 \\
\hline & $(-12.45$ & $(22264.1$ & 10930 & 42938
\end{tabular}

${ }_{3.8 \%} \mathrm{XL}$, inho

C.

$\begin{array}{ll}-6.850 & 8702.9 \\ -3.330 & 4247.6\end{array}$

$3.8 \% \mathrm{XL}$, inhomo

10 vol\% XL, 75 w\% sp, $25 \mathrm{w}$

very small particles similar to $3-17$

3.1\% XL inhomo, und Zr and oth $10 \%$ ZrO2, YZrO type

$.32 \%$ same as $3-20$

25 vol\% XL, 50w\% NaNdSi, 35w\% sp

-8.240
-6.900

11874.0

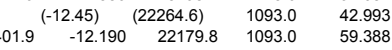

No XL

amorphous $\quad 5 \% \mathrm{Sp}$

$15 \% \mathrm{ZrO} 2, \mathrm{Sp}$
10 vol\% XL, 60w\% Zro2, 40w\% sp

-4.170
-6.220

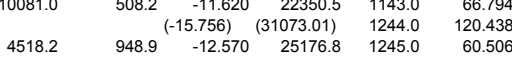

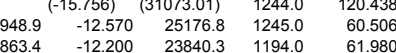

\begin{tabular}{lllll}
3.4 & -12.200 & 23840.3 & 1194.0 & 60.506 \\
\hline & -11.660 & 20994.0 & 110950 & 42.114
\end{tabular}

$\begin{array}{lrrr}(-16.606) & (31488.601) & 1194.0 & 94.237 \\ -14.540 & 26355.5 & 1144.0 & 73.108 \\ -13330 & 24485 & 10940 & 10.899\end{array}$

scattered spinel

-6.410
-6.130

7887.1

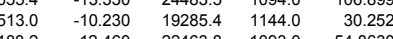

scattered spine
No $X L$
No $X L$
No $X L$

10vol\% XL, 70wt\% Spinel, 30wt\% Ca5((Si,P,S)O4)

Amorphous
Amorphous

amorphous

Amorphors

Amorphous
Amorphous

\section{small amt of $\mathrm{Al} 2 \mathrm{O}, \mathrm{cp}$}

No XL

amorphous

mall amt of $\mathrm{Ce} 2 \mathrm{O} 3$ and $\mathrm{cp}$

No $X L$
No $X L$
No $X L$

amorphous

17 vol\% XL, Na3Nd(PO4)2, Li3PO4, NdPO

22 vol\% XL, Na3Nd(PO4)2, Li3PO4, NdPO

$-10.550$

$\begin{array}{llllll}7624.7 & 513.0 & -10.230 & 19285.4 & 1144.0 & 30.252 \\ 1718.0 & 188.2 & -12.460 & 24243.8 & 1993.0 & 54.8630 \\ 9108.6 & 547.0 & -10.890 & 21058.0 & 1193.0 & 33.2710 \\ & & -12300 & 220122 & 10940 & 43412\end{array}$

$\begin{array}{lllllll}3 & & & -12.300 & 22012.2 & 1094.0 & 43.412 \\ -5.280 & 6550.7 & 671.7 & -11.730 & 21601.3 & 1093.0 & 63.952 \\ -5.730 & 7242.1 & 623.2 & -11.620 & 21281.6 & 1093.0 & 55.661 \\ -5150 & 60623 & 6770 & -12090 & 21496.7 & 10430 & 760666\end{array}$

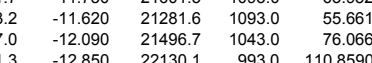

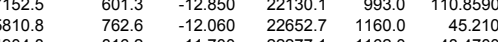

$\begin{array}{lllllll}4904.8 & 816.2 & -11.700 & 22277.1 & 1162.0 & 48.4700 \\ 2504.3 & 1035.1 & -12320 & 23248.5 & 1160.0 & 568020\end{array}$

$\begin{array}{lrrrrr}3717.8 & 917.3 & -12.530 & 23315.2 & 1136.5 & 6.50 .795 \\ 1563.1 & 1154.7 & -10.750 & 20346.7 & (1136.5) & (94.1288\end{array}$

$\begin{array}{rrrrrr}1563.1 & 1154.7 & -10.750 & 20346.7 & (1136.5) & (94.1288) \\ 8214.7 & 606.9 & -11.860 & 22236.4 & 1144.0 & 48.668 \\ & & & -11.00 & & \end{array}$

$\begin{array}{lll}8599.9 & 590.0 & -11.900 \\ 8593 & -120\end{array}$

3 vol\% XL, NaNdPO4
7 vol\% XL, NaNdPO4

7434.1

$\begin{array}{lllll}5.1 & -12.000 & 22833.0 & 1144.0 & 66.2050 \\ 7.1 & -12.190 & 23294.0 & 1194.0 & 42.0180\end{array}$ 
$\begin{array}{llll}23.2 & 1244.0 & 14.1 & 12450 \\ 23.4 & 1344.0 & 14.8 & 1340\end{array}$

$\begin{array}{rrrr}4.4 & 1294.0 & 8.9 & 1294.0\end{array}$

$\begin{array}{rrrrr}9.1 & 1295.0 & 9.1 & 1344.0 \\ 10.3 & 1395.0 & 105 & 14450 \\ 4 & 1250 & & 9480 & 1300.0\end{array}$

$\begin{array}{lll}5.9 & 1395.0 & 4.0 \\ 7.2 & 1497.0 & 5.5 \\ 2.2 & 1350.0 & 1.5\end{array}$

$\begin{array}{rrrrr}6.2 & 1144.0 & 5.8 & 1193.0 \\ 21.3 & 1126.0 & 7.9 & 1200.0\end{array}$

$\begin{array}{ll}11.8 & 1195.0 \\ 127 & 12440\end{array}$

$\begin{array}{ll}7.9 & 1244.0 \\ 7.9 & 1244.0 \\ 7 & \end{array}$

$\begin{array}{rl}12.2 & 1144.0 \\ 20.3 & 1394.0 \\ 2.7 & 1140\end{array}$

$\begin{array}{rl}7.2 & 1194.0 \\ 12.9 & 1394.0 \\ 5.1 & 11990\end{array}$

$\begin{array}{rl}8.7 & 1149.0 \\ 24.9 & 1143.0\end{array}$

$\begin{array}{rl}5.1 & 1199.0 \\ 14.3 & 1193.0 \\ 16.6 & 1394.0 \\ 1.4 & 124.0\end{array}$

18.51244 .0

$\begin{array}{ll}16.6 & 1394.0 \\ 10.4 & 1244.0 \\ 11.4 & 1245.0\end{array}$

$\begin{array}{rrrr}13.6 & 1244.0 & 11.4 & 1245.0 \\ 21.6 & 1294.0 & 13.7240 & 1294 .\end{array}$

$\begin{array}{lllll}38.8 & 1395.0 & 18.5 & 1395.0 \\ 19.9 & 1395.0 & 112 . & 1395.0 \\ 193 & 1340 & 12.0 & \end{array}$

$\begin{array}{rrrrr}13.6 & 1244.0 & 8.5 & 1244.0\end{array}$

$\begin{array}{llll}21.0100 & 1345.0 & 24.3 & 1345.0 \\ 21.6 & 1294.0 & 12.4 & 1295.0 \\ 27.6 & 1249\end{array}$

$\begin{array}{rrrrr}11.6 & 1244.0 & 7.6 & 1244.0 \\ 17.4 & 1244.0 & 10.4 & 1244.0 \\ 12.7 & 1340 & & \end{array}$

$\begin{array}{rr}7.6 & 1294.0 \\ 10.4 & 1244.0 \\ 8.2 & 1344.0\end{array}$

$\begin{array}{llll}16.4 & 1244.0 & 9.7660 & 1245.0\end{array}$

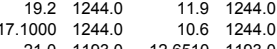

$\begin{array}{llll}27.4 & 1142.0 & 15.4 & 1143.0\end{array}$

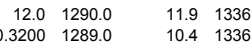

$\begin{array}{rrrr}9.7 & 1290.0 & 9.7 & 1336.0 \\ 15.6 & 1294.0 & 10.1 & 1295.0\end{array}$

$\begin{array}{rrrr}17.3640 & 1294.0 & 11.1 & 12950\end{array}$

$\begin{array}{rrrrr}5.8 & 1235.0 & 8.840 & 1355.0 \\ 33 & 1194.0 & 36 & 1194.0\end{array}$

$\begin{array}{llrr}4.1 & 1235.0 & 3.9480 & 1300.0 \\ 3.2 & 1244.0 & 20 & \end{array}$

$4.7 \quad 12450$

$\begin{array}{llll}3.2 & 1294.0 & 3.2 & 1295.0 \\ 53 & 1295 & 5\end{array}$

$\begin{array}{rrrrrr}4.9 & 1294.0 & 5.1 & 1294.0 & & \\ 4.5 & 1194.0 & 4.5 & 1243.0 & 2.8910 & 1244.0\end{array}$

13.41444 .0

$\begin{array}{ll}8.6 & 1193.0 \\ 17.2 & 1444.0\end{array}$

$\begin{array}{ll}10.7 & 1294.0 \\ 11.0 & 1294.0\end{array}$

$\begin{array}{ll}13.9 & 1343.0 \\ 20.5 & 1445.0\end{array}$

$\begin{array}{ll}20.5 & 1445.0 \\ 12.4 & 1444.0 \\ 12.3 & 1394.0\end{array}$

$\begin{array}{rr}8.6 & 1294.0 \\ 23.2 & 1395.0\end{array}$

$\begin{array}{rrrr}1.2 & 135.0 & 11.7 & 13950 \\ 1.9 & 1340 & 6.220 & 1350\end{array}$

$\begin{array}{lllll}9.2 & 12950 & 6.7220 & 1345.0\end{array}$

$\begin{array}{rrrrr}7.8 & 1344.0 & 5.2110 & 1344.0 \\ 10.6 & 1294.0 & 6.4 & 1295.0\end{array}$

\begin{tabular}{rr}
9.4 & 1295 \\
12.0 & 1293 \\
\hline .8
\end{tabular}

$\begin{array}{ll}6.4 & 1295.0 \\ 5.6 & 1394.0\end{array}$

$\begin{array}{ll}12.8 & 1243.0 \\ 15.1 & 1192.0 \\ 7.4 & 133.0\end{array}$

$\begin{array}{ll}5.5 & 1295.0 \\ 7.6 & 1294.0\end{array}$

$\begin{array}{ll}4 & 1444.0 \\ & \end{array}$

$\begin{array}{rlr}10.7 & 1445.0 \\ 58 & 1290 \\ 7.0 & 2950\end{array}$

1445.0

$\begin{array}{rl}7.8 & 1396.0 \\ 5.7 & 1295.0 \\ 103 & 1390.0\end{array}$

$\begin{array}{rl}5.7 & 1295.0 \\ 10.3 & 1396.0 \\ 72 & 13460\end{array}$

$\begin{array}{ll}7.2 & 1346.0 \\ 5.9 & 1345.0\end{array}$

$\begin{array}{ll}5.9 & 1345.0 \\ 5.4 & 1345.0 \\ 6.6 & 1295.0 \\ 5.7 & 139.0\end{array}$

$\begin{array}{ll}6.6 & 1295.0 \\ 5.7 & 1394.0\end{array}$

$\begin{array}{rrrrrr}5.1 & 1192.0 & 9.3 & 1193.0 & 9.2 & 1194.0 \\ 7.4 & 1338.0 & 7.4 & 1338.0 & 7.5 & 1424.0 \\ 8.8 & 1329.0 & 9.2 & 1330.0 & 99 . & 140.10 \\ 7.7 & 1337.0 & 7.9670 & 1338.0 & 9.2520 & 1425.0\end{array}$

$\begin{array}{rrrrrrr}6.9 & 1337.0 & 6.8 & 1338.0 & 9.2520 & 1425.0 \\ 6.9 & 133190 & 1424.0\end{array}$

$\begin{array}{ll}6.3 & 1339.0 \\ 6.3 & 1345.0 \\ 7.0 & 1345.0\end{array}$

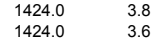

$\begin{array}{rrrr} & & & \\ 1244.0 & 3.6 & 1344.0 & 2.2 \\ 1245.0 & 3.0 & 1294.0 & 2.6 \\ & 6.0 & 2.0\end{array}$

3 TRU-ZN-1

304 TRU-ZB-1

306 TRU-ABZ-1

307 TRU-NBZ-1
308 TRU-Al-1

309 TRU-Al-C

310 TRU-B-1
311 TRU-B-2

312 TRU-B-3

314 TRU-B- 5

316 TRU-Bi-2

317 TRU-Bi-3

319 TRU-Ce-1

320 TRU-Ce-2
321 TRU-Ce-3

322 TRU-Li-1

324 TRU-Li-3

325
326 TRUU--Na-1

328 TRU-Na-3 
CCC PCT B $\left(g / \mathrm{m}^{2}\right)$ CCC PCT Li $\left(\mathrm{g} / \mathrm{m}^{2}\right)$ CCC PCT Na $\left(g / \mathrm{m}^{2}\right)$ CCC PCT Si $\left(\mathrm{g} / \mathrm{m}^{2}\right) \quad$ CCC PCT pH T1 ( $\left({ }^{\circ} \mathrm{C}\right)$ 249 CVS2-11 250 CVS2-116 253 CVS2-119 254 CVS2-120 255 CVS2-12 257 CVS2-123 259 CVS3-2

261 CVS3-4

262 CVS3 -5

264 CVS3-7

265 CVS3-8

267 CVS3-11

269 CVS3-12

270 CVS3-1 1

271 CVS3-14

273 CVS3-1
274 CVS3-17

275 CVS3-1
276 CVS3-19

277 CVS3-20

279 CVS3-22

281 CVS3-24

282 CVS3-25

285 CVS3-28

286 CVS3-29

288 CVS3-31

290 CVS3-33

292 CVS $3-35$

293 CVS3-36

295 CVS3-38

297 CVS3-40

298 TRU-BL-1

300 TRU-NL-1

301 TRU-AB-

303 TRU-ZN-

304 TRU-ZB-1

305 TRU-ANZ-1

307 TRU-NBZ-1

308 TRU-Al-1

309 TRU-AI-

311 RRU-B-2

312 TRU-B-3

314 TRU-B-5

316 TRU-Bi-2

317 TRU-Bi-3

319 TRU-Ce-1

320 TRU-Ce-2

322 TRU-Li-1

323 TRU-Li-2
324 TRU-Li-3
325 TRU-Li-4

325 TRU-Li-4
326 TRU-Na-1

327 TRU-Na-2

\begin{tabular}{|c|c|c|c|c|c|c|c|c|c|}
\hline 0.612 & 0.630 & 0.510 & 0.216 & 9.630 & 0.558 & 0.515 & 0.437 & 0.202 & 9.630 \\
\hline 7.116 & 4.928 & 4.599 & 1.860 & 11.410 & 7.038 & 4.498 & 4.170 & 1.758 & 11.350 \\
\hline 9.406 & 5.837 & 5.765 & 1.942 & 11.550 & 9.472 & 5.704 & 5.611 & 1.827 & 11.550 \\
\hline $\begin{array}{l}3.012 \\
1.590\end{array}$ & $\begin{array}{l}2.203 \\
1.337\end{array}$ & $\begin{array}{r}2.030 \\
1090\end{array}$ & $\begin{array}{l}0.606 \\
0.45\end{array}$ & $\begin{array}{l}10.425 \\
10.1\end{array}$ & $\begin{array}{l}2.754 \\
1600\end{array}$ & $\begin{array}{r}2.019 \\
1.36\end{array}$ & 1.922 & 0.570 & $\begin{array}{l}10.400 \\
1020\end{array}$ \\
\hline $\begin{array}{l}\begin{array}{l}1.590 \\
3.630\end{array}\end{array}$ & $\begin{array}{l}1.337 \\
2.609\end{array}$ & $\begin{array}{l}1.099 \\
2.394\end{array}$ & $\begin{array}{l}0.445 \\
0.606\end{array}$ & $\begin{array}{l}10.190 \\
10.395\end{array}$ & $\begin{array}{r}1.600 \\
2.808\end{array}$ & $\begin{array}{l}1.366 \\
2.106\end{array}$ & $\begin{array}{l}1.141 \\
1.968\end{array}$ & $\begin{array}{l}0.436 \\
0.555\end{array}$ & $\begin{array}{l}10.200 \\
10.300\end{array}$ \\
\hline 3.803 & 2.688 & 2.574 & 0.829 & 10.320 & 3.593 & 3.014 & 2.815 & 0.827 & 10.340 \\
\hline 0.291 & 0.324 & 0.231 & 0.154 & 10.096 & 0.291 & 0.306 & 0.216 & 0.138 & 10.090 \\
\hline $\begin{array}{l}0.199 \\
0.193\end{array}$ & 0.311 & 0.137 & $\begin{array}{l}0.129 \\
0\end{array}$ & 10.055 & 0.175 & 0.270 & 0.135 & 0.119 & $\begin{array}{r}10.005 \\
9\end{array}$ \\
\hline $\begin{array}{l}0.193 \\
1.473\end{array}$ & $\begin{array}{l}0.363 \\
1.125\end{array}$ & $\begin{array}{l}0.089 \\
1.075\end{array}$ & $\begin{array}{l}0.128 \\
0.391\end{array}$ & $\begin{array}{r}9.910 \\
10220\end{array}$ & $\begin{array}{l}0.213 \\
1.352\end{array}$ & $\begin{array}{l}0.309 \\
1.054\end{array}$ & $\begin{array}{l}0.089 \\
1.006\end{array}$ & $\begin{array}{l}0.116 \\
0.356\end{array}$ & $\begin{array}{r}9.920 \\
10200\end{array}$ \\
\hline $\begin{array}{l}0.473 \\
0.422\end{array}$ & 0.383 & 0.400 & 0.160 & 11.200 & $\begin{array}{l}. .252 \\
0.294\end{array}$ & $\begin{array}{l}0.054 \\
0.361\end{array}$ & $\begin{array}{l}.000 \\
0.354\end{array}$ & 0.1520 & $\begin{array}{l}10.200 \\
11.145\end{array}$ \\
\hline 4.803 & 0.360 & 0.306 & 0.132 & 10.800 & $\begin{array}{l}0.294 \\
0.262\end{array}$ & $\begin{array}{l}0.321 \\
0.328\end{array}$ & $\begin{array}{l}0.354 \\
0.286\end{array}$ & $\begin{array}{l}0.132 \\
0\end{array}$ & $\begin{array}{l}11.1404 \\
10.770\end{array}$ \\
\hline 0.450 & 0.447 & 0.609 & 0.202 & 11.610 & 0.450 & 0.477 & 0.606 & 0.206 & 11.625 \\
\hline 0.654 & 0.595 & 0.907 & 0.254 & 11.875 & 0.645 & 0.882 & 1.356 & 0.359 & 12.045 \\
\hline 0.627 & 0.620 & 1.174 & 0.271 & 12.030 & 1.216 & 1.300 & 2.237 & 0.479 & 12.295 \\
\hline 0.309 & 0.346 & 0.296 & 0.151 & 10.230 & 0.275 & 0.306 & 0.268 & 0.144 & 10.170 \\
\hline $\begin{array}{l}0.264 \\
1264\end{array}$ & $\begin{array}{l}0.326 \\
1643\end{array}$ & $\begin{array}{l}0.307 \\
2625\end{array}$ & $\begin{array}{l}0.150 \\
0.585\end{array}$ & $\begin{array}{l}11.725 \\
1244\end{array}$ & 0.241 & $\begin{array}{l}0.295 \\
1.475\end{array}$ & 0.278 & 0.137 & $\begin{array}{l}10.585 \\
12415\end{array}$ \\
\hline $\begin{array}{l}1.266 \\
0.299\end{array}$ & $\begin{array}{l}1.643 \\
0.355\end{array}$ & $\begin{array}{l}2.625 \\
0.034\end{array}$ & $\begin{array}{l}0.585 \\
0.100\end{array}$ & $\begin{array}{l}12.445 \\
10.235\end{array}$ & $\begin{array}{l}1.548 \\
0.627\end{array}$ & $\begin{array}{l}1.477 \\
0.345\end{array}$ & $\begin{array}{l}2.462 \\
0.045\end{array}$ & $\begin{array}{l}0.580 \\
0.101\end{array}$ & $\begin{array}{l}12.415 \\
10.21\end{array}$ \\
\hline $\begin{array}{l}0.209 \\
.0703\end{array}$ & $\begin{array}{l}0.355 \\
0.865\end{array}$ & $\begin{array}{l}0.034 \\
0.824\end{array}$ & $\begin{array}{l}0.100 \\
0.334\end{array}$ & $\begin{array}{l}10.235 \\
11845\end{array}$ & & $\begin{array}{l}0.345 \\
1324\end{array}$ & $\begin{array}{l}0.045 \\
0.732\end{array}$ & $\begin{array}{l}0.101 \\
0.319\end{array}$ & $\begin{array}{l}10.2101 \\
11.790\end{array}$ \\
\hline 0.029 & 0.540 & 0.564 & $\begin{array}{l}0.34 \\
0.224\end{array}$ & $\begin{array}{r}11.845 \\
11.575\end{array}$ & & $\begin{array}{l}.324 \\
0.844\end{array}$ & 0.502 & 0.229 & 11.470 \\
\hline 0.100 & 0.000 & 0.314 & 0.113 & 10.785 & 0.268 & & 0.299 & 0.114 & 10.640 \\
\hline 0.804 & 0.380 & 0.336 & 0.162 & 11.020 & 0.583 & 0.364 & 0.332 & 0.160 & 11.045 \\
\hline 0.301 & 0.356 & 0.346 & 0.155 & 11.060 & 0.503 & 0.554 & 0.614 & 0.200 & 11.505 \\
\hline 0.466 & 0.529 & 0.585 & 0.187 & 11.535 & 0.306 & 0.333 & 0.320 & 0.139 & 11.135 \\
\hline 0.268 & 0.511 & 0.079 & $\begin{array}{l}0.120 \\
\end{array}$ & 10.345 & 1.250 & 0.418 & 0.037 & 0.117 & $\begin{array}{r}9.460 \\
0\end{array}$ \\
\hline $\begin{array}{l}0.523 \\
0.643\end{array}$ & $\begin{array}{l}0.422 \\
0.304\end{array}$ & $\begin{array}{l}0.129 \\
0.199\end{array}$ & $\begin{array}{l}0.118 \\
0.111\end{array}$ & $\begin{array}{l}10.640 \\
10.740\end{array}$ & $\begin{array}{l}0.563 \\
0.302\end{array}$ & $\begin{array}{l}0.476 \\
0.301\end{array}$ & $\begin{array}{l}0.126 \\
0.199\end{array}$ & $\begin{array}{l}0.160 \\
0.112\end{array}$ & $\begin{array}{l}10.475 \\
10.695\end{array}$ \\
\hline $\begin{array}{l}0.643 \\
2.022\end{array}$ & $\begin{array}{l}0.304 \\
1.640\end{array}$ & $\begin{array}{l}0.1999 \\
1.717\end{array}$ & $\begin{array}{l}0.111 \\
0.842\end{array}$ & $\begin{array}{l}10.740 \\
11.430\end{array}$ & $\begin{array}{l}0.302 \\
2.320\end{array}$ & $\begin{array}{l}0.301 \\
1398\end{array}$ & $\begin{array}{l}0.199 \\
1.470\end{array}$ & $\begin{array}{l}0.1122 \\
0.743\end{array}$ & $\begin{array}{l}10.695 \\
11300\end{array}$ \\
\hline 0.181 & $\begin{array}{l}.640 \\
0.331\end{array}$ & 0.298 & $\begin{array}{l}0.180 \\
0.110\end{array}$ & $\begin{array}{l}11.450 \\
11.030\end{array}$ & $\begin{array}{l}2.220 \\
0.804\end{array}$ & $\begin{array}{l}1.398 \\
0.396\end{array}$ & $\begin{array}{l}.440 \\
0.364\end{array}$ & $\begin{array}{l}0.143 \\
0.135\end{array}$ & $\begin{array}{l}11.300 \\
1.175\end{array}$ \\
\hline 0.196 & 0.301 & 0.254 & 0.097 & 11.025 & 0.530 & 0.359 & 0.322 & 0.124 & 11.140 \\
\hline 0.324 & 0.310 & 0.303 & 0.119 & 11.070 & 0.432 & 0.330 & 0.303 & 0.118 & 10.965 \\
\hline 0.402 & $\begin{array}{l}0.502 \\
0.355\end{array}$ & 0.562 & 0.232 & $\begin{array}{l}11.280 \\
1100\end{array}$ & 0.515 & 0.481 & 0.528 & 0.228 & 11.170 \\
\hline 0.261 & 0.355 & 0.380 & 0.151 & 11.070 & 0.362 & 0.356 & 0.342 & 0.155 & 10.970 \\
\hline 0.209 & 0.384 & 0.420 & 0.161 & 11.260 & 0.338 & 0.368 & 0.382 & 0.156 & 11.175 \\
\hline 0.342 & 0.358 & 0.329 & 0.157 & 10.975 & 0.704 & 0.395 & 0.391 & 0.159 & 11.145 \\
\hline 0.305 & 0.382 & 0.400 & 0.160 & 11.210 & 1.094 & 0.365 & 0.366 & 0.157 & 11.085 \\
\hline 0.241 & 0.411 & 0.432 & 0.189 & 11.220 & 0.531 & 0.376 & 0.381 & 0.155 & 11.155 \\
\hline 0.338 & 0.397 & 0.420 & 0.183 & $\begin{array}{l}11.230 \\
1120\end{array}$ & $\begin{array}{l}0.463 \\
2311\end{array}$ & 0.385 & 0.379 & 0.159 & $\begin{array}{l}11.030 \\
11.030\end{array}$ \\
\hline $\begin{array}{l}0.261 \\
0.487\end{array}$ & $\begin{array}{l}0.451 \\
0.370\end{array}$ & $\begin{array}{l}0.465 \\
0.326\end{array}$ & $\begin{array}{l}0.202 \\
0.159\end{array}$ & $\begin{array}{l}11.250 \\
1085\end{array}$ & $\begin{array}{l}2.311 \\
0.42\end{array}$ & $\begin{array}{l}0.382 \\
0.367\end{array}$ & $\begin{array}{l}0.376 \\
0.289\end{array}$ & $\begin{array}{l}0.156 \\
0.155\end{array}$ & $\begin{array}{l}11.045 \\
10.840\end{array}$ \\
\hline $\begin{array}{l}0.487 \\
0.403\end{array}$ & 0.338 & $\begin{array}{l}0.320 \\
0.335\end{array}$ & 0.149 & 10.950 & $\begin{array}{l}0.412 \\
0.368\end{array}$ & 0.334 & $\begin{array}{l}0.289 \\
0.299\end{array}$ & 0.144 & 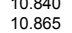 \\
\hline 0.411 & 0.357 & 0.331 & 0.154 & 10.890 & 0.394 & 0.369 & 0.297 & 0.151 & 10.875 \\
\hline 0.422 & 0.395 & 0.325 & 0.163 & 10.780 & 0.441 & 0.468 & 0.343 & 1.740 & 10.940 \\
\hline 0.543 & 0.393 & 0.304 & 0.165 & 10.800 & 0.523 & 0.458 & 0.313 & 0.174 & 10.880 \\
\hline 0.547 & 0.188 & 0.370 & 0.151 & 10.520 & 0.294 & 0.354 & 0.361 & 0.152 & 10.955 \\
\hline 0.418 & $\begin{array}{l}0.292 \\
0.234\end{array}$ & 0.327 & 0.147 & $\begin{array}{l}11.030 \\
1081\end{array}$ & 0.370 & $\begin{array}{l}0.326 \\
0.681\end{array}$ & 0.283 & 0.120 & $\begin{array}{l}10.900 \\
10890\end{array}$ \\
\hline 0.209 & 0.234 & $\begin{array}{l}0.252 \\
0.248\end{array}$ & $\begin{array}{l}0.121 \\
0.014\end{array}$ & 10.871 & 0.242 & 0.081 & 0.2860 & $\begin{array}{l}0.130 \\
0.16\end{array}$ & $\begin{array}{l}10.8909 \\
10.720\end{array}$ \\
\hline 0.225 & & & & & & & & & 10.720 \\
\hline
\end{tabular}


248 CVS2-114
249 CVS2-115

250 CVS2-116
251 cVS2-117

253 c cus2-119

254
255 CVSS2-120

256 CVS2-12
257 CVS2-123

255 CVS3-1

250 CVS3 $32-3$

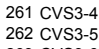

263 CVS3-6
$264 \mathrm{CVS}^{-7}$

265 CVS3-8

266 CVS3-9

267 CVS3 -10
268 CVS -11

269 cvs3 -12

270 CVS3 313
271 CVS -14

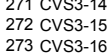

274 CVS3-17

275 CVS3-18
276 CVS3-19

277 CVS3-20

278 CVS3 321

280 CVS3-23
281 CVS3-24

282 CVS3-25

284 CVS3-27

285 CVS -28

286 CVS3-29
287 CVS 3031

288 CVS3-31
289 CVSS -32

290 CVVS 33
201
CVS 35.34

292 CVS3-35

293 CVS3-36
204 CVS 37

294 CVSS-37
225 CVSS3-38

296 CVS3-39

297 CVS3 30

2989 TRU-BL-1

300 TRU-NL-1

301 TRU-AB-

303 TRU-ZN-1

304 TRU-ZB-1

305 TRU-ANZ-1

306 TRU-ABZ-1

308 TRUA-A-1

3 310 TRQB-B-1
311 TRU-B-2

312 TRU-B-3

313 TRU-B- 4

315 TRU-Bi-1

316 TRU-Bi-2

(18 TRUBi-

19 TRU-Ce-1

320 TRU-Ce-2

(25)

323 TRU-LL-2

324
325 TRUUL-Li-

325
326 TRUU-Na-

27 TRU-Na-2

328 TRU-Na-3 


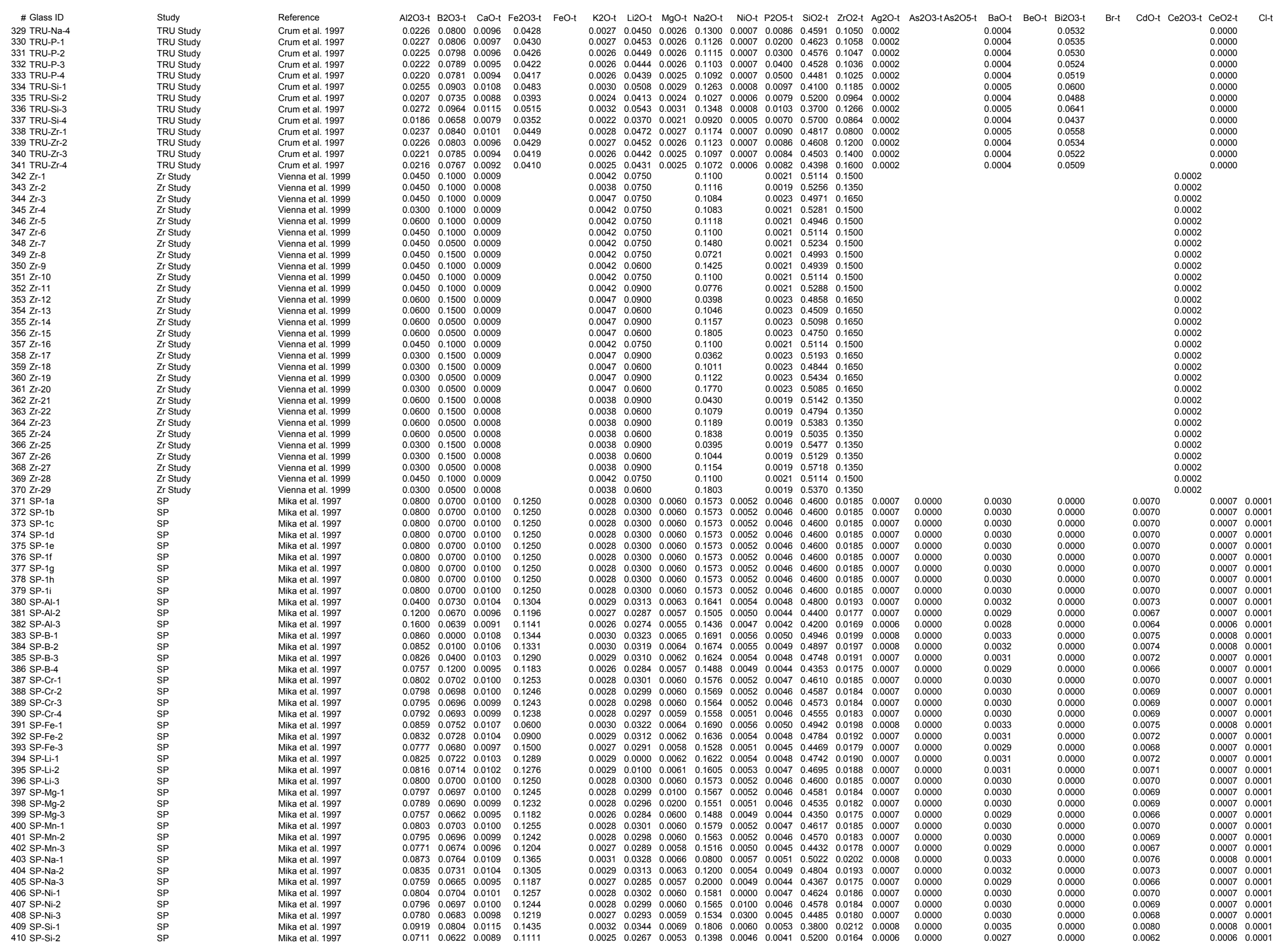




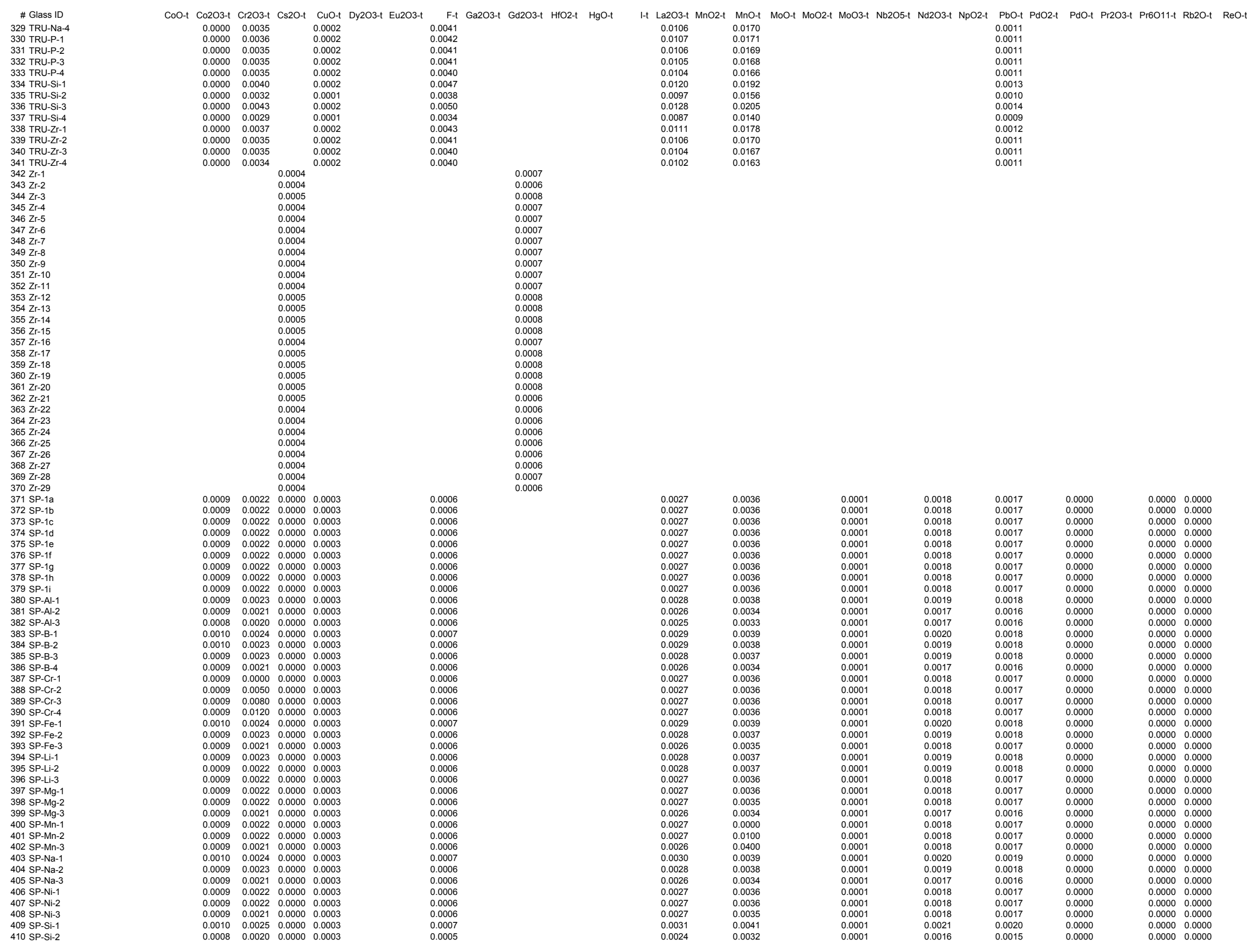




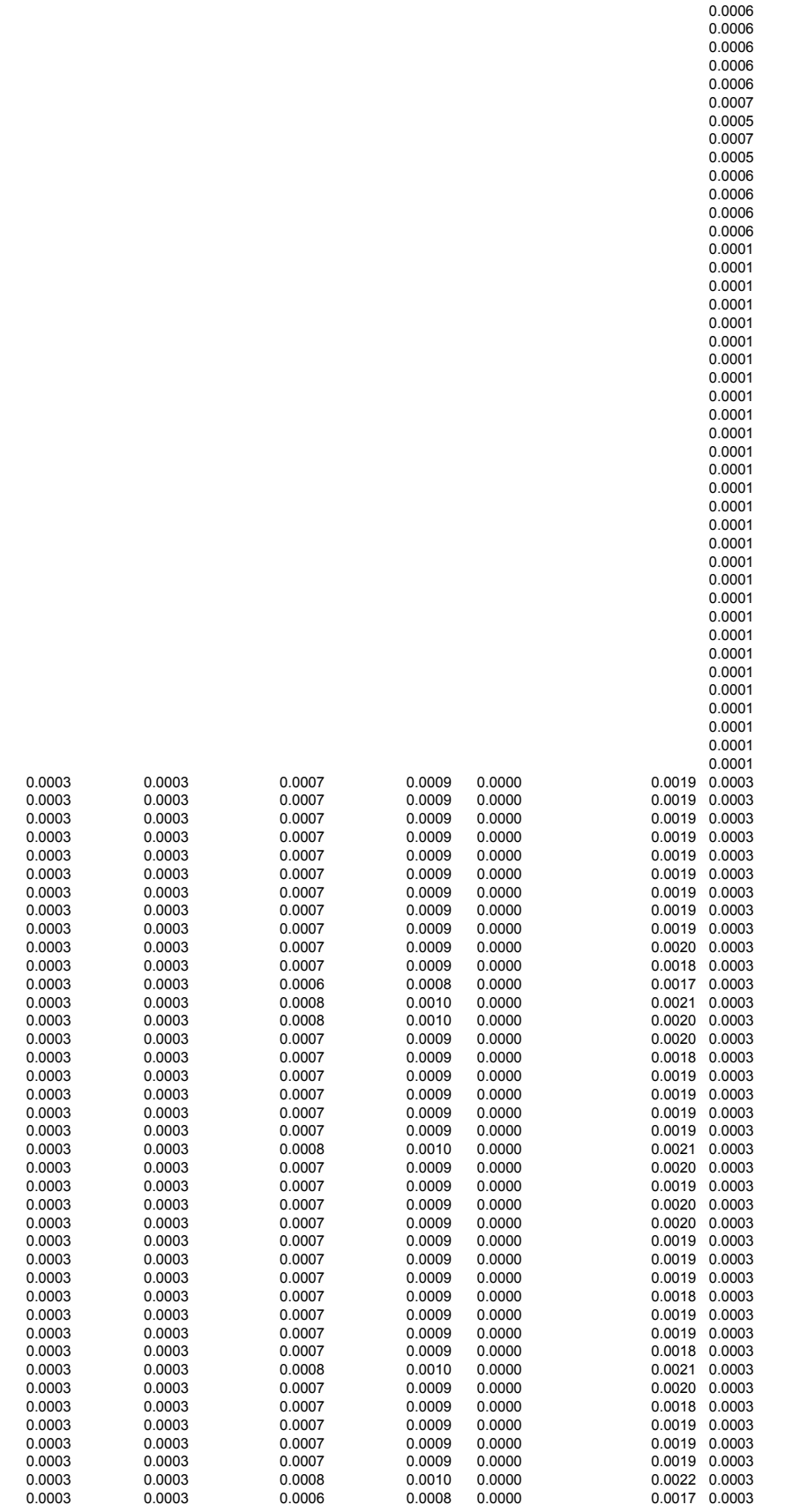

0.0000
0.0000
0.0000
0.0000
0.0000
0.0000
0.0000
0.0000
0.0000
0.0000
0.0000
0.0000
0.0000
0.0000
0.0000
0.0000
0.0000
0.0000
0.0000
0.00000
0.0000
0.0000
0.0000
0.0000
0.00000
0.0000
0.0000
0.0000
0.00000
0.0000
0.0000
0.0000
0.0000
0.0000
0.0000
0.0000
0.00000
0.0000
0.00000
0.0000

$\begin{aligned} & 0.003 \\ & 0.0003 \\ & 0.0003 \\ & 0.0003 \\ & 0.0003 \\ & 0.0003 \\ & 0.0003 \\ & 0.0003 \\ & 0.0003 \\ & 0.0003 \\ & 0.0003 \\ & 0.0003 \\ & 0.0003 \\ & 0.0003 \\ & 0.0003 \\ & 0.003 \\ & 0.0003 \\ & 0.0003 \\ & 0.0003 \\ & 0.0003 \\ & 0.0003 \\ & 0.0003 \\ & 0.0003 \\ & 0.0003 \\ & 0.0003 \\ & 0.0003 \\ & 0.0003 \\ & 0.0003 \\ & 0.0003 \\ & 0.0003 \\ & 0.0003 \\ & 0.0003 \\ & 0.0003 \\ & 0.0003 \\ & 0.0003 \\ & 0.0003 \\ & 0.0003 \\ & 0.0003 \\ & 0.0003 \\ & 0.0003 \\ & 0.00003\end{aligned}$
0.000

$\begin{array}{llll}0.0000 & 0.0000 & 0.0004 & 1.00000 \\ 0.0000 & 0.0000 & 0.0004 & 1.0000\end{array}$ $\begin{array}{llll}0.0000 & 0.0000 & 0.0004 & 1.00000 \\ 0.0000 & 0.0000 & 0.0004 & 1.0000\end{array}$

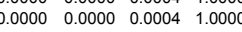
$\begin{array}{llllll}0.0000 & 0.0000 & 0.0004 & 1.0000\end{array}$ $\begin{array}{llll}0.0000 & 0.0000 & 0.00004 & 1.0000\end{array}$ $\begin{array}{llll}0.0000 & 0.0000 & 0.0004 & 1.0000\end{array}$ 0.0000
0.00000
0.000004
0.00000 0.00000
0.00000
0.0000
0.0000
0.00004 0.0000
0.00000
0.00000

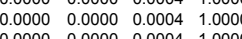

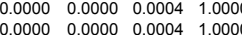
0.00000
0.0000
0.00000
0.0000004 0.0000
0.000000
0.0000004

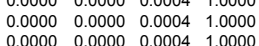
$\begin{array}{llll}0.00000 & 0.00000 & 0.0004 & 1.00000 \\ 0.0000 & 0.0000 & 0.0004 & 1.0000 \\ 0 & 0.0000\end{array}$

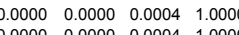

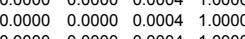
$\begin{array}{lll}0.00000 & 0.00000 & 0.0004 \\ 0 & 0.0000 & 0.00000 \\ 0 & 0.0000 & 0.0004\end{array}$

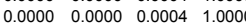
0.00000
0.00000
0.00000
0.000000004
0.00004 D. 0.0000
0.00000
0.0000
0.00000
0.00004
0.0004
0.00000
0.0000

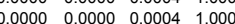

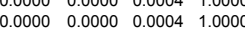

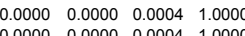

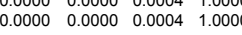
0.00000
0.000000
0.0000 
329 TRU-Na-4

331
332 TRUU-P-P-3
32

333 TRU-P-A

335 TRU-Si- 2

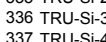

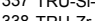

339 TRU-Zr-2

340 TRU-Zr-3

$341 \mathrm{TRU}-\mathrm{Z}$
$342 \mathrm{ZT}-1$

$343 \mathrm{z}-2$

$344 \mathrm{Z}-\mathrm{r}-3$
$345 \mathrm{r}-4$
$3.36-5$

$346 \mathrm{Z}-5$
$347 \mathrm{Z}-6$

$348 \mathrm{Zz}-7$
$349-8$

$349 \mathrm{Zr}-8$
$350 \mathrm{Z}-9$

$351 \mathrm{zr}-10$

$352 \mathrm{rz}-11$

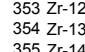

$356 \mathrm{z}-15$

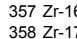

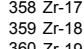

360
$362 \mathrm{Zr}-19$
$302-20$

362
363
$\mathrm{Z} r-22$

364
$365 \mathrm{Zr}-23$
$36 \mathrm{Z}-24$

366
$367 \mathrm{Zr}-25$
$3 \mathrm{r}-26$

$368 \mathrm{Zr}-27$

$370 \mathrm{Zr}-29$

371 SP-1a

372 SP-11
373 SP-10

3745 PP.1d
375 SP-1e

376 SP.-1f
377 SP-19

378 SP-1h

379 SP-11

380 SP-AA-1
381 SP-A-2

382 SP-Al-3

338 SPP.B- 1
384 SP.- 2

385 SPP-B-3
386 SP-B.-4

387 SP-Cr-1

388 SPPC-Cr-2
389 SP-Cr-3

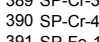

391 SP-Fe-1

392 SP-FE-2
393 SP-Fe-3

394 SP-Li-1

395 SPPLL-2-2
396 SP-Li-3

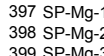

399 SP-Mg-3
400 SP-Mn-1

401 SP-Mn-2

402 SP-Mn-3

403 SP-Na-1
404 SP-Na-2

405 SP-Na-3

406 SP-N-Ni-1

407 SPPN-2
408 SPN-3

400
410 SP-SPSi-1
41 
329 TRU-Na-4

331 TRU-P-2

333 TRU-P.-

335 TRU-Si-2 2

336 TRU-Si:-3

${ }_{338}^{3}$ TRU-ZIr-4

339 TRU-Zr-2

340 TRU-Zr-3

$341 \mathrm{TRU}-\mathrm{Z}$
$342 \mathrm{Zr}-\mathrm{Z}$

$343 \mathrm{Zr}-2$

$344 \mathrm{Z}-\mathrm{r}-3$
$345 \mathrm{r}-4$
3.46
$-34-5$

$346 \mathrm{Z}-5$
$347 \mathrm{Z}-6$

$348 \mathrm{Z} \mathrm{Z}-\mathrm{r}$
$34 \mathrm{r}$
$349 \mathrm{Z}-8$

$349 \mathrm{Zr}-8$
$350 \mathrm{Zr}-9$

351 zr-10

$352 \mathrm{rz}-11$

$353 z-12$
$354 \mathrm{Z}-13$
$355-13$
$35-14$

$356 \mathrm{z}-15$

$3557 \mathrm{Zr}-16$
358
$\mathrm{Z} r-17$

$359 \mathrm{Zr}-18$

$360 \mathrm{zr}-1$

$362 \mathrm{r}$
$362-21$
$363 \mathrm{r}-22$

$364 \mathrm{Zr}-23$

$366 \mathrm{Zr}-25$

$367 \mathrm{Zr}-26$
$368 \mathrm{Zr}-27$

$370 \mathrm{Zr}-29$

371 SP-1a

372 SP- -16
373 SP-10

374 SP-1d
375 SP-1e

376 SP.-1f
377 SP-19

378 SP-1h

379 SP-11

380 SP-AA-1
381 SP-A-2

382 SP-Al-3

338 SPP.B- 1
384 SP.- 2

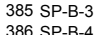

387 SP-Cr-1

388 SP-CC-2
389 SP-Cr-3

390 SP-Cr-4

391 SP-Fe-1

392 SP-Fe-2

394 SP-Li-1

395 SPPLL-2-2
396 SP-Li-3

39 SP-Mg-1
398 SPP-Mg-2
3999 SP-MG-3

399 SP-Mg-3
400 SP-Mn-1

401 SP-Mn-2

402 SP-Mn-3

403 SP-Na-1
404 SP-Na-2

405 SP-Na-3

406 SP-N-Ni-1

407 SPPN-2
408 SPN-3

400
410 SP-SPSi-1
41 
330 TRU-P-1

332 TRU-P- 3

333 TRU-P-4

335 TRU-Si-2

335 TRU-Si-2
337 TRU-Si-3
337 TRU-Si-4

338 TRU-Si-4

339 TRU-Zr-2

340 TRU-Zr-3

341 TRU-Z-Z

$343 \mathrm{Zr}-2$

$344 \mathrm{Zr}-3$
$345 \mathrm{Zr}-4$
$346-5$

$346 \mathrm{Zr}-5$

$348 \mathrm{Zr}-7$

$349 \mathrm{Zr}-8$
$350 \mathrm{Zr}-9$
351

$352 \mathrm{Zr}-11$

$353 \mathrm{Zr}-12$

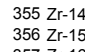

$357 \mathrm{Zr}-16$

$359 \mathrm{Zr}-18$

$360 \mathrm{Zr}-19$
$36 \mathrm{Z}-20$
$362 \mathrm{r}-21$

$362 Z-22$
$3635 \mathrm{r}-22$
5

$3044 \mathrm{r}-23$
$305 \mathrm{z} 25$

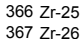

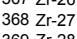

370 Zr-29

371 SP-1a

372 SP-11
373 SP-1C

374 SP-1d
375 SP-1e

376 SP-11
377 SP-19

378 SP-1h

379 SP-1i

380 SP-Al-1
381 SP-A-2

382 SP-Al-3

383 SP-B-1
384 SP $-B-2$

385 SP-B-3

387 SP-Cr-1

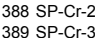

390 SP-Cr-4

391 SP-Fe-1

392 SP-Fe-2

394 SP-Li-1

395 SP-Li-2
396 SP-Li-3

397 SP-Mg-1

398 SP-Mg-2

399 SP-Mg-3

401 SP-Mn-2

402 SP-Mn-3

403 SP-Na-1

405 SP-Na-3

406 SP-Ni-1

407 SP-N-N 2

409 SP-Si-1
410 SP-Si-2

410 SP-Si-2 
329 TRU-Na-4

332 TRU-P- 3

333 TRU-P-4

335 TRU-Si-2

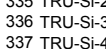

337 TRU-Si-4

339 TRU-Zr-2

340 TRU-Zr-3

344 TRU-Zr
$342 \mathrm{zr}-\mathrm{Z}$

$343 \mathrm{Zr}-2$

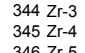

$346 \mathrm{Z}-5$
$347 \mathrm{Z}-6$

$348 \mathrm{Zr}-7$

$349 \mathrm{zr}-8$
$350 \mathrm{Zr}-9$

351 zr-10

$352 \mathrm{rz}-11$

$353 \mathrm{Z}-\mathrm{Z}-12$
$354 \mathrm{Z}-13$
$355 \mathrm{Z}-14$

$356 \mathrm{z}-15$

$3557 \mathrm{zr}-16$
358
$\mathrm{Z} r-17$

$359 \mathrm{Zr}-18$

$360 \mathrm{zr}-19$

362
$362 \mathrm{r}-21$
$36 \mathrm{r}-22$

$364 \mathrm{zr}-22$

$366 \mathrm{Zr}-25$

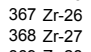

$370 \mathrm{Zr}-29$

$3702-29$
37 SPP. 19

372 SP- -16
373 SP-10

3745 PP.1d
375 SP-1e

376 SP.-1f
377 SP-19

378 SP-1h

379 SP-11

380 SP-AA-1
381 SP-A-2

382 SP-A- 3

338 SPP.B- 1
384 SP.- 2.2

385 SPP.B-3
386 SP-B.-4

386 SPP-B-4
387 SP-Cr-1

388 SPPCC.-2
389 SP-Cr-3

390 SP-Cr-4

391 SP-Fe-1

392 SP-FE-2
393 SP-Fe-3

394 SP-Li-1

395 SPPLL-L-2
396 SP-Li-3

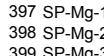

3999 SP-Mg-3
400 SP-Mn-1

401 SP-Mn-2

402 SP-Mn-3

403 SP-Na-1
404 SP-Na-2

4005 SP-Na-3

406 SP-N-Ni-1

407 SPPN-2
408 SPN-3

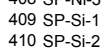




$\begin{array}{lll}0.239 & 0.338 & 0.241 \\ 0.277 & 0.364 & 0.269 \\ 0.231 & 0.352 & 0.242 \\ 0.301 & 0.383 & 0.268 \\ 0.228 & 0.327 & 0.234 \\ 0.2251 & 0.361 & 0.259 \\ 0.290 & 0.492 & 0.810 \\ 0.443 & 0.451 & 0.127 \\ 0.293 & 0.295 & 0.383 \\ 0.247 & 0.336 & 0.240 \\ 0.237 & 0.356 & 0.127 \\ 0.220 & 0.310 & 0.020 \\ 0.420 & 0.485 & 0.205 \\ 0.210 & 0.300 & 0.970 \\ 0.185 & 0.340 & 0.230 \\ 0.265 & 0.330 & 0.020 \\ 0.660 & 0.750 & 0.335 \\ 0.275 & 0.540 & 0.495 \\ 0.490 & 0.505 & 1.175 \\ 0.300 & 0.360 & 0.035 \\ 1.030 & 0.965 & 0.525 \\ 0.255 & 0.515 & 0.545 \\ 0.295 & 0.410 & 1.160 \\ 0.405 & 0.450 & 0.065 \\ 0.610 & 0.620 & 0.315 \\ 0.595 & 0.735 & 0.710 \\ 0.240 & 0.340 & 0.260 \\ 3.890 & 2.715 & 3.085\end{array}$


330 TRU-P-1

332 TRU-P-3

333 TRU-P-4

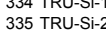

336 TRU-Si: 3

338 TRU-ST-4

339 TRU-Zr-2

340
341 TRUU-ZZ- $\mathrm{T}-4$

${ }_{343}^{342 \mathrm{Zr}-2}$

344
$345-3-3$

$345 \mathrm{Zr}-4$
$346 \mathrm{Z}-5$
3

$347 \mathrm{Zr}-6$
$348 \mathrm{r}-\mathrm{T}$

$349 \mathrm{Zr}-8$
$350 \mathrm{Zr}-9$

351 zr-10

$352 \mathrm{rz}-11$

$353 \mathrm{Z}-\mathrm{Z}-12$
$354 \mathrm{Z}-13$
$355 \mathrm{Z}-14$

$356 \mathrm{z}-15$

$357 \mathrm{z}-16$
$358 \mathrm{r}-17$

$358 z \mathrm{z}-17$
$359 \mathrm{rr}-18$
$360 \mathrm{rr}-19$

360 Zr-19

362
$362-21$
363
3

$364 \mathrm{Zr}-23$

$366 \mathrm{Zr}-25$

$367 \mathrm{Zr}-26$
$368 \mathrm{Zr}-27$

$370 \mathrm{Zr}-29$

371 SP-1a

372 SP-11
373 SP -10

374 SP-1d
375 SP-1e

376 SP.-1f
377 SP-19

378 SP-1h

379 SP-11

380 SPPA-A-1
381 SP-A-2

382 SP-Al-3

(303 SP-B-1

385 SPPB-B
386
SP $-B-4-4$

387 SP-Cr-1

388 SPPCC-2
389 SP-Cr-3

390 SP-Cr-4

392 SP-Fe-2
303

394 SP-Li-1

395 SPPLL-2-2
396 SP-Li-3

397 SP-MG-1

3988 SP-Mg-2

399 SP-Mg--3
400 SP-Mn-1

401 SP-Mn-2

402 SP-Mn-3

403 SP-Na-1
404 SP-Na-2

4005 SP-Na-3

406 SP-Ni-1

407 SPPN-2
408 SPN-3

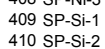




\begin{tabular}{|c|c|c|c|c|c|c|}
\hline \# Glass ID & Study & Reference & Al2O3-t & B2O3-t & CaO-t & $\mathrm{Fe} 2 \mathrm{O}-\mathrm{t}$ \\
\hline 411 SP-Si-3 & SP & Mika et al. 1997 & 0.0593 & 0.0519 & 0.0074 & 0.0926 \\
\hline 412 MS-1a & MS & Hrma 1999 & 0.0800 & 0.0700 & & 0.1300 \\
\hline $\begin{array}{l}413 \text { MS-1b } \\
414 \mathrm{MS}-2 \mathrm{a}\end{array}$ & MS & $\begin{array}{l}\text { Hrma } 1999 \\
H\end{array}$ & 0.0800 & $\begin{array}{l}0.0700 \\
0.070\end{array}$ & & $\begin{array}{l}0.1300 \\
0.1450\end{array}$ \\
\hline $\begin{array}{l}\begin{array}{l}414 \text { MS-2a } \\
415 \text { MS-2b }\end{array}\end{array}$ & $\begin{array}{l}\text { MS } \\
\text { MS }\end{array}$ & $\begin{array}{l}\text { Hrma } 19999 \\
\text { Hrma } 1999\end{array}$ & $\begin{array}{l}0.0800 \\
0.0800\end{array}$ & $\begin{array}{l}0.0700 \\
0.0700\end{array}$ & & $\begin{array}{l}0.1450 \\
0.1450\end{array}$ \\
\hline $\begin{array}{l}416 \text { MS-3a } \\
41\end{array}$ & MS & $\begin{array}{l}\text { Hirma 1999 } \\
\text { Hrma } 1999\end{array}$ & 0.0800 & 0.0700 & & $\begin{array}{l}0.1450 \\
0.1150\end{array}$ \\
\hline $417 \mathrm{MS}-3 \mathrm{~b}$ & MS & Hrma 1999 & 0.0800 & 0.0700 & & 0.1150 \\
\hline 418 MS-4a & MS & Hrma 1999 & 0.0800 & 0.0700 & & 0.0889 \\
\hline 419 MS-4b & MS & Hrma 1999 & 0.0800 & 0.0700 & & 0.0889 \\
\hline 420 MS-5 & MS & Hrma 1999 & 0.0800 & 0.0700 & & 0.1150 \\
\hline 421 MS-6 & MS & Hrma 1999 & 0.0800 & 0.0700 & & 0.1250 \\
\hline $\begin{array}{l}422 \mathrm{MS}-7 \\
423 \mathrm{MS} 8\end{array}$ & MS & Hrma 1999 & 0.0800 & 0.0700 & & 0.1150 \\
\hline $\begin{array}{l}423 \text { MS-8 } \\
424 \text { MS-9 }\end{array}$ & $\begin{array}{l}\text { MS } \\
\text { MS }\end{array}$ & $\begin{array}{l}\text { Hrma } 1999 \\
\text { Hrma } 1999\end{array}$ & $\begin{array}{l}0.0800 \\
0.0800\end{array}$ & $\begin{array}{l}0.0700 \\
0.0700\end{array}$ & & $\begin{array}{l}0.1250 \\
0.1100\end{array}$ \\
\hline $\begin{array}{l}\text { 424 MS-9 } \\
425 \text { MS-7 H-Al }\end{array}$ & $\begin{array}{l}\text { MS } \\
\text { MS }\end{array}$ & $\begin{array}{l}\text { Hrma } 1999 \\
\text { Wilson et al. } 2001\end{array}$ & $\begin{array}{l}0.0800 \\
0.1100\end{array}$ & $\begin{array}{l}0.0700 \\
0.0677\end{array}$ & & $\begin{array}{l}0.1100 \\
0.1113\end{array}$ \\
\hline $426 \mathrm{MS}-7 \mathrm{~L}-\mathrm{Al}$ & MS & Wilson et al. 2001 & 0.0500 & 0.0723 & & 0.1180 \\
\hline $427 \mathrm{MS}-7 \mathrm{H}-\mathrm{Cr}$ & MS & Wilson et al. 2001 & 0.0789 & 0.0699 & & 0.1148 \\
\hline 428 MS-7 L-Cr & MS & Wilson et al. 2001 & 0.0802 & 0.0701 & & 0.1152 \\
\hline $429 \mathrm{MS}-7 \mathrm{H}-\mathrm{Fe}$ & MS & Wilson et al. 2001 & 0.0768 & 0.0672 & & 0.1500 \\
\hline 430 MS-7 L-Fe & MS & Wilson et al. 2001 & 0.0832 & 0.0728 & & 0.0800 \\
\hline $431 \mathrm{MS}-7 \mathrm{H}-\mathrm{Li}$ & MS & Wilson et al. 2001 & 0.0788 & 0.0689 & & 0.1132 \\
\hline $\begin{array}{l}432 \mathrm{MS}-7 \text { L-Li } \\
433 \text { MS-7 H-M }\end{array}$ & MS & $\begin{array}{l}\text { Wilson et al. } 2001 \\
\text { Wiston et al } 2001\end{array}$ & $\begin{array}{l}0.0813 \\
0.0781\end{array}$ & $\begin{array}{l}0.0711 \\
0.0833\end{array}$ & & $\begin{array}{l}0.1169 \\
0.1122\end{array}$ \\
\hline $\begin{array}{l}433 \text { MS-7 H-Mg } \\
434 \mathrm{MS}-7 \mathrm{~L}-\mathrm{Mg}\end{array}$ & $\begin{array}{l}\text { MS } \\
\text { MS }\end{array}$ & $\begin{array}{l}\text { Wilson et ala. } 2001 \\
\text { Wilson et al. } 2001\end{array}$ & $\begin{array}{l}0.0781 \\
0.0805\end{array}$ & $\begin{array}{l}0.0683 \\
0.0704\end{array}$ & & $\begin{array}{l}0.1122 \\
0.1157\end{array}$ \\
\hline $\begin{array}{l}434 \mathrm{MS}-\mathrm{L}-\mathrm{Lg} \\
435 \mathrm{MS}-7 \mathrm{H}-\mathrm{Na}\end{array}$ & $\begin{array}{l}\text { MS } \\
\text { MS }\end{array}$ & $\begin{array}{l}\text { Wilson et al. } 2001 \\
\text { Wilson et } 201\end{array}$ & $\begin{array}{l}0.0774 \\
0.0705\end{array}$ & $\begin{array}{l}0.0704 \\
0.0678\end{array}$ & & 0.1113 \\
\hline 436 MS-7 L-Na & MS & Wilson et al. 2001 & 0.0831 & 0.0727 & & 0.1195 \\
\hline $437 \mathrm{MS}-7 \mathrm{VH}-\mathrm{Ni}$ & MS & Wilson et al. 2001 & 0.0783 & 0.0685 & & 0.1126 \\
\hline $438 \mathrm{MS}-7 \mathrm{H}-\mathrm{Ni}$ & MS & Wilson et al. 2001 & 0.0793 & 0.0694 & & 0.1140 \\
\hline 439 MS-7 L-Ni & MS & Wilson et al. 2001 & 0.0805 & 0.0705 & & 0.1158 \\
\hline 440 SG01 & SG & Hrma et al. 1999 & 0.0250 & 0.0999 & 0.0200 & 0.1499 \\
\hline $\begin{array}{l}441 \text { SG02 } \\
442 \mathrm{SGO3}^{2}\end{array}$ & SG & Hrma et al. 1999 & 0.0250 & $\begin{array}{l}0.0500 \\
0.0876\end{array}$ & $\begin{array}{l}0.0200 \\
0.0158\end{array}$ & 0.0599 \\
\hline $\begin{array}{l}442 \text { SG03 } \\
443 \text { SG04 }\end{array}$ & $\begin{array}{l}\text { SG } \\
\text { SG }\end{array}$ & $\begin{array}{l}\text { Hrma et al. } 19999 \\
\text { Hrma et al. } 1999\end{array}$ & $\begin{array}{l}0.0390 \\
0.0799\end{array}$ & $\begin{array}{l}0.0876 \\
0.0500\end{array}$ & $\begin{array}{l}0.01158 \\
0.030\end{array}$ & $\begin{array}{l}0.1202 \\
0.1499\end{array}$ \\
\hline 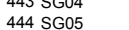 & $\begin{array}{l}S G \\
\text { SG }\end{array}$ & $\begin{array}{l}\text { Hrma et al. } \\
\text { Hrma et al. } 1999\end{array}$ & $\begin{array}{l}0.0799 \\
0.0530\end{array}$ & $\begin{array}{l}0.0500 \\
0.0752\end{array}$ & $\begin{array}{l}.0 .030 \\
0.0115\end{array}$ & $\begin{array}{l}0.1499 \\
0.1052\end{array}$ \\
\hline 445 SG06 & SG & Hrma et al. 1999 & 0.0799 & 0.0500 & 0.0200 & 0.1499 \\
\hline 446 SG07 & SG & Hrma et al. 1999 & 0.0799 & 0.0999 & 0.0030 & 0.0599 \\
\hline 447 SG08 & SG & Hrma et al. 1999 & 0.0390 & 0.0626 & 0.0158 & 0.1275 \\
\hline $448 S G 09$ & SG & Hrma et al. 1999 & 0.0799 & 0.0999 & 0.0200 & 0.1499 \\
\hline 449 SG10 & SG & Hrma et al. 1999 & 0.0390 & 0.0626 & 0.0073 & 0.0825 \\
\hline $\begin{array}{l}450 \text { SG11 } \\
451 \text { SG12 }\end{array}$ & SG & Hrma et al. 1999 & 0.0390 & 0.0876 & 0.0073 & $\begin{array}{l}0.0825 \\
0.1499\end{array}$ \\
\hline 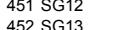 & $\begin{array}{l}\text { SG } \\
\text { SG }\end{array}$ & $\begin{array}{l}\text { Hrma et al. } 1999 \\
\text { rrma et al. } 1999\end{array}$ & $\begin{array}{l}0.0250 \\
0.0250\end{array}$ & $\begin{array}{l}0.0500 \\
0.0999\end{array}$ & $\begin{array}{l}0.00030 \\
0.030\end{array}$ & $\begin{array}{l}0.1499 \\
0.0874\end{array}$ \\
\hline $453 \mathrm{SG} 14$ & SG & $\begin{array}{l}\text { Hrma et al. } 1999 \\
\text { rrma et al. } 1999\end{array}$ & $\begin{array}{l}0.0250 \\
0.0250\end{array}$ & $\begin{array}{l}0.0999 \\
0.0999\end{array}$ & 0.0030 & $\begin{array}{l}0.08 / 4 \\
0.1499\end{array}$ \\
\hline 454 SG15 & SG & Hrma et al. 1999 & 0.0250 & 0.0999 & 0.0200 & 0.0599 \\
\hline 455 SG16 & SG & Hrma et al. 1999 & 0.0664 & 0.0626 & 0.0158 & 0.0825 \\
\hline $456 \mathrm{SG} 17$ & SG & Hrma et al. 1999 & 0.0390 & 0.0725 & 0.0158 & 0.1275 \\
\hline 457 SG18a & SG & Hrma et al. 1999 & 0.0250 & 0.0999 & 0.0030 & 0.1499 \\
\hline 458 SG18b & SG & Hrma et al. 1999 & 0.0250 & 0.0999 & 0.0030 & 0.1499 \\
\hline 459 SG18c & SG & Hrma et al. 1999 & 0.0250 & $\begin{array}{l}0.0999 \\
0.099\end{array}$ & 0.0030 & 0.1499 \\
\hline 461 SG18d & SG & $\begin{array}{l}\text { Hrma et al. } 1999 \\
\text { Horma }\end{array}$ & 0.0250 & $\begin{array}{l}0.0999 \\
0.0999\end{array}$ & $\begin{array}{l}0.0030 \\
0.030\end{array}$ & $\begin{array}{l}0.1499 \\
0.1499\end{array}$ \\
\hline 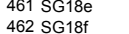 & $\begin{array}{l}\text { SG } \\
\text { SG }\end{array}$ & $\begin{array}{l}\text { Hrma et al. } 19999 \\
\text { Hrma et al. } 1999\end{array}$ & $\begin{array}{l}0.0250 \\
0.0250\end{array}$ & $\begin{array}{l}0.0999 \\
0.099\end{array}$ & $\begin{array}{l}0.00030 \\
0.030\end{array}$ & $\begin{array}{l}0.1499 \\
0.1499\end{array}$ \\
\hline $463 \mathrm{SG} 18 \mathrm{~g}$ & SG & Hrma et al. 1999 & 0.0250 & $\begin{array}{l}0.09999 \\
0.0999\end{array}$ & 0.0030 & 0.1499 \\
\hline 464 SG19 & SG & Hrma et al. 1999 & 0.0799 & 0.0999 & 0.0030 & 0.0599 \\
\hline 465 SG20 & SG & Hrma et al. 1999 & 0.0799 & 0.0500 & 0.0200 & 0.0599 \\
\hline 466 SG21 & SG & Hrma et al. 1999 & 0.0390 & 0.0876 & 0.0158 & 0.0825 \\
\hline $467 \mathrm{SG} 22$ & SG & Hrma et al. 1999 & 0.0664 & 0.0626 & 0.0158 & 0.1275 \\
\hline 468 SG23 & SG & Hrma et al. 1999 & 0.0417 & 0.0626 & 0.0158 & $\begin{array}{l}0.0825 \\
01194\end{array}$ \\
\hline $\begin{array}{l}469 \text { SG24 } \\
470 \text { SG25 }\end{array}$ & SG & Hrma et al. 1999 & 0.0250 & $\begin{array}{l}0.0500 \\
0.0999\end{array}$ & $\begin{array}{l}0.00030 \\
0.0030\end{array}$ & $\begin{array}{l}0.1194 \\
0.1499\end{array}$ \\
\hline $\begin{array}{l}471 \text { SG26 } \\
471\end{array}$ & SG & $\begin{array}{l}\text { Arma etal. } 19999 \\
\text { Hrma et al. } 1999\end{array}$ & 0.0390 & 0.0626 & $\begin{array}{l}0.0073 \\
0.0030\end{array}$ & 0.1275 \\
\hline 472 SG27 & SG & Hrma et al. 1999 & 0.0664 & 0.0876 & 0.0158 & 0.1109 \\
\hline 473 SG28 & SG & Hrma et al. 1999 & 0.0250 & 0.0999 & 0.0200 & 0.1499 \\
\hline 474 SG29 & SG & Hrma et al. 1999 & 0.0799 & 0.0500 & 0.0030 & 0.0599 \\
\hline $475 \mathrm{SG} 30$ & SG & Hrma et al. 1999 & 0.0799 & 0.0500 & 0.0200 & 0.0599 \\
\hline 476 SG31 & SG & Hrma et al. 1999 & 0.0799 & 0.0999 & 0.0200 & 0.1494 \\
\hline 477 SG32 & SG & Hrma et al. 1999 & 0.0799 & 0.0999 & 0.0030 & 0.1499 \\
\hline $\begin{array}{l}478 \text { SG33 } \\
479 \mathrm{SG} 34\end{array}$ & $S G$ & Hrma et al. 1999 & 0.0799 & $\begin{array}{l}0.0999 \\
\end{array}$ & 0.0200 & $\begin{array}{l}0.0599 \\
0.1454\end{array}$ \\
\hline $\begin{array}{l}479 \text { SG } 34 \\
480 \text { SG } 35\end{array}$ & SG & $\begin{array}{l}\text { Hrma et al. } 1999 \\
\text { Prma }\end{array}$ & $\begin{array}{l}0.0799 \\
0.0799\end{array}$ & $\begin{array}{l}0.0999 \\
0.050\end{array}$ & 0.0200 & $\begin{array}{l}0.1454 \\
0.1449\end{array}$ \\
\hline $\begin{array}{l}480 \text { SG } 35 \\
481 \mathrm{SG} 36\end{array}$ & SG & Hrma et al. 1999 & $\begin{array}{l}0.0250 \\
0.0259\end{array}$ & $\begin{array}{l}0.0500 \\
0.0999\end{array}$ & 0.0200 & $\begin{array}{l}0.1449 \\
0.0599\end{array}$ \\
\hline 482 SG37 & SG & Hrma et al. 1999 & 0.0250 & 0.0999 & 0.0200 & 0.0599 \\
\hline $483 \mathrm{SG} 38$ & SG & Hrma et al. 1999 & 0.0250 & 0.0999 & 0.0030 & 0.1464 \\
\hline $484 \mathrm{SG} 39$ & SG & Hrma et al. 1999 & 0.0250 & 0.0500 & 0.0200 & 0.1499 \\
\hline $485 \mathrm{SG} 40$ & SG & Hrma et al. 1999 & 0.0799 & 0.0999 & 0.0030 & 0.0599 \\
\hline 486 SG41 & SG & Hrma et al. 1999 & 0.0799 & 0.0999 & 0.0200 & 0.1499 \\
\hline $\begin{array}{ll}487 \\
48 G 442\end{array}$ & SG & Hrma et al. 1999 & 0.0449 & 0.08866 & 0.0073 & 0.1275 \\
\hline 488 SG 43 & 56 & Hrma et al. 1999 & 0.0664 & 0.0876 & . & 0.0825 \\
\hline $\begin{array}{l}489 \mathrm{SG} 44 \\
490 \mathrm{SG} 45\end{array}$ & SG & Hrma et al. 1999 & 0.0250 & 0.0999 & 0.0200 & 0.0599 \\
\hline 491 SG46 & SG & Hrma et al al. 1999 & 0.0250 & 0.0500 & 0.0030 & 0.1499 \\
\hline 492 SG47 & SG & Hrma et al. 1999 & 0.0250 & 0.0500 & 0.0200 & 0.1499 \\
\hline
\end{tabular}

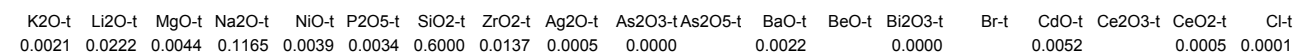

\begin{tabular}{|c|c|c|c|c|c|}
\hline & & & & & \\
\hline 0450 & 0.0060 & 0.1570 & 0.0200 & 0.4500 & 0.0300 \\
\hline & & & & & \\
\hline & 0.0060 & 0.1530 & 0.0120 & 0.4240 & \\
\hline & 0.0060 & $\begin{array}{l}0.1530 \\
0.1130\end{array}$ & $\begin{array}{l}0.0120 \\
0.0120\end{array}$ & $\begin{array}{l}0.4240 \\
0.4540\end{array}$ & 0.0600 \\
\hline & $\begin{array}{l}0.0060 \\
0.0060\end{array}$ & $\begin{array}{l}0.1530 \\
0.1530\end{array}$ & $\begin{array}{l}0.0120 \\
0.0120\end{array}$ & $\begin{array}{l}0.4540 \\
0.4540\end{array}$ & $\begin{array}{l}0.0600 \\
0.0600\end{array}$ \\
\hline & 0.0060 & $\begin{array}{l}0.1530 \\
0.1530\end{array}$ & 0.0120 & $\begin{array}{l}0.4746 \\
0.4746\end{array}$ & $\begin{array}{l}0.06000 \\
0.0600\end{array}$ \\
\hline & & & 0.0120 & 0.4746 & \\
\hline & 0.0060 & & & & \\
\hline 0.0300 & 0.0060 & 0.1573 & 0.0140 & 0.4600 & \\
\hline 0.0454 & 0.0060 & 0.1530 & 0.0095 & 0.4531 & 0.0600 \\
\hline 0.0300 & 0.0060 & 0.1573 & 0.0075 & 0.4600 & 0.0571 \\
\hline & & & & & \\
\hline 0.0439 & 0.0058 & $\begin{array}{l}0.1480 \\
0\end{array}$ & $\begin{array}{l}0.0092 \\
0.0098\end{array}$ & 0.4383 & 0.0580 \\
\hline 0.0453 & $\begin{array}{l}0.0060 \\
0.006\end{array}$ & $\begin{array}{l}.0 .1527 \\
0\end{array}$ & $\begin{array}{l}0.0095 \\
0.0095\end{array}$ & 0.4522 & $\begin{array}{l}0.0599 \\
0.0599\end{array}$ \\
\hline & & 0.1533 & 0.0095 & 0.4540 & 0.0601 \\
\hline 0.0436 & 0.0058 & $\begin{array}{l}0.1469 \\
0.1191\end{array}$ & 0.0091 & 0.4352 & 0.0576 \\
\hline 0.04 & $\begin{array}{l}.0 .0622 \\
0.0059\end{array}$ & $\begin{array}{l}0.11991 \\
0.1507\end{array}$ & $\begin{array}{l}0.0099 \\
0.0094\end{array}$ & $\begin{array}{l}0.4 / 10 \\
0.4462\end{array}$ & $\begin{array}{l}0.0624 \\
0.0591\end{array}$ \\
\hline 0.0300 & $\begin{array}{l}0.0061 \\
0.006\end{array}$ & 0.1555 & 0.0097 & 0.4604 & 0.0610 \\
\hline 0843 & & 0.1493 & 0.0093 & 0.4422 & 0.0586 \\
\hline 045 & 0000 & 0.1539 & 0.0096 & $0.455 \varepsilon$ & 0.06 \\
\hline & & 0.1800 & 0.0092 & & 0.0581 \\
\hline & 0062 & 0.1200 & $0.0 c$ & & 0.0623 \\
\hline & & 0.1498 & 0.03 & & 0.0588 \\
\hline & & & & & 0.0595 \\
\hline
\end{tabular}

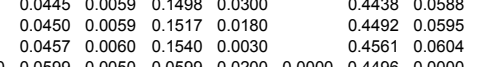

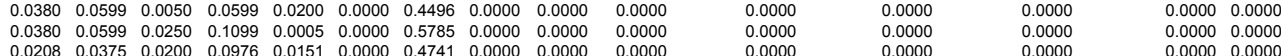

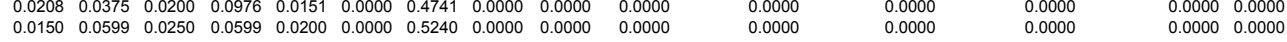

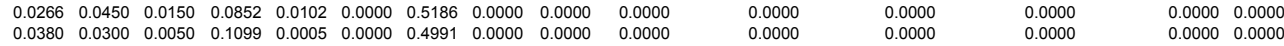

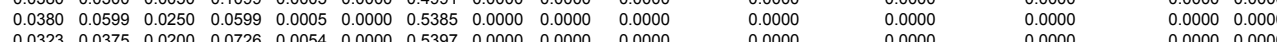
$\begin{array}{lllllllllllllll}0.0150 & 0.0599 & 0.0050 & 0.0599 & 0.0005 & 0.0000 & 0.4396 & 0.0000 & 0.0000 & 0.0000 & 0.0000 & 0.0000 & 0.00000 & 0.0000 & 0.0000\end{array}$

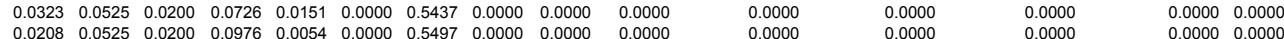

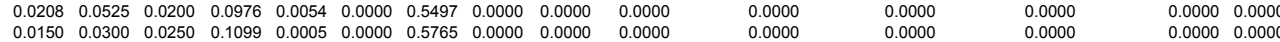

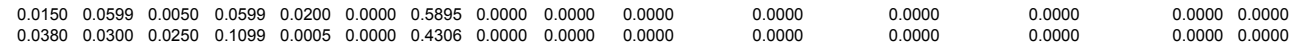

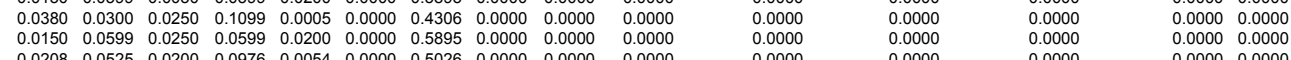
$\begin{array}{lllllllllll}0.0323 & 0.0525 & 0.0100 & 0.0976 & 0.0151 & 0.0000 & 0.4741 & 0.0000 & 0.0000 & 0.0000 & 0.0000\end{array}$

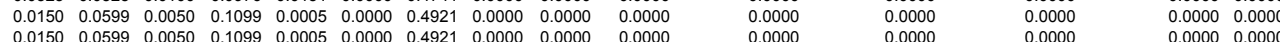

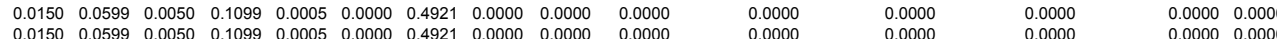

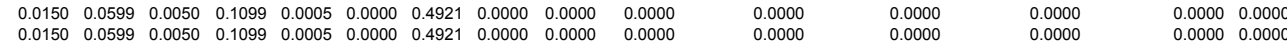

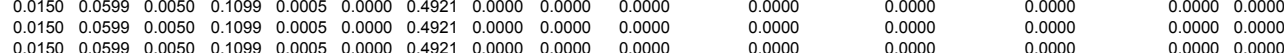

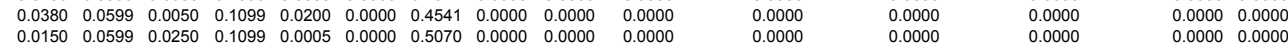

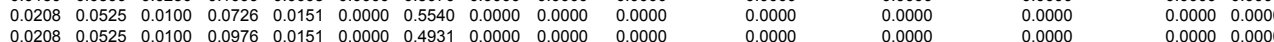

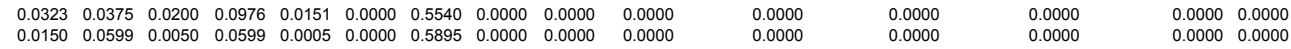

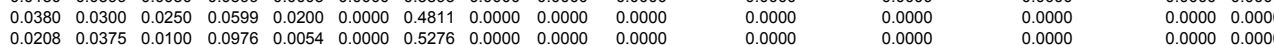

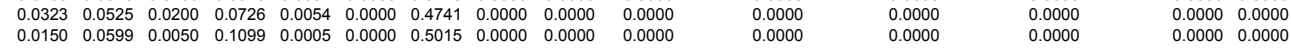

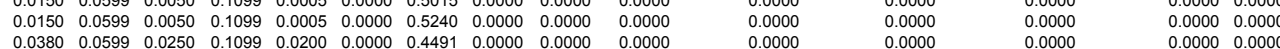

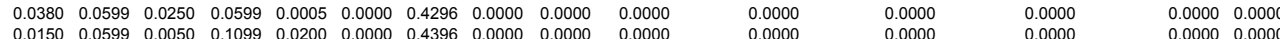

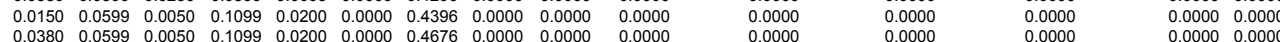

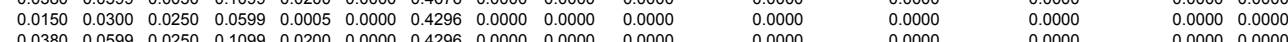

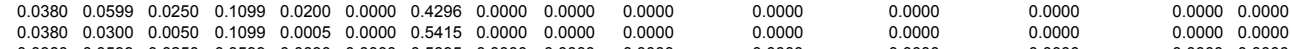

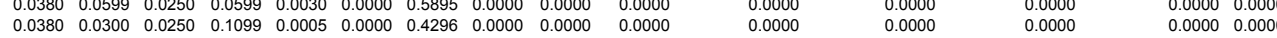

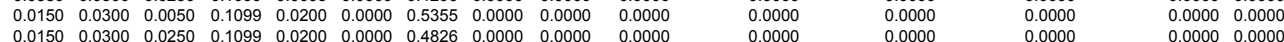

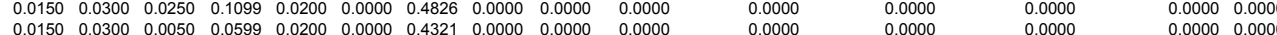

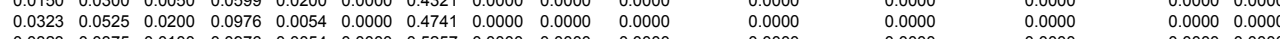

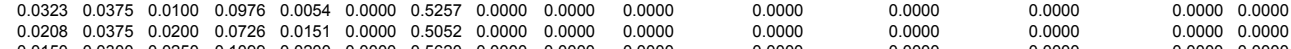

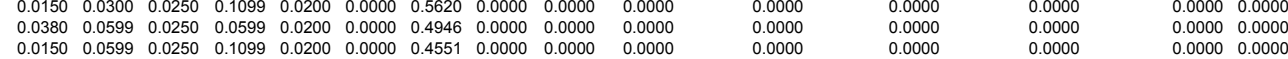




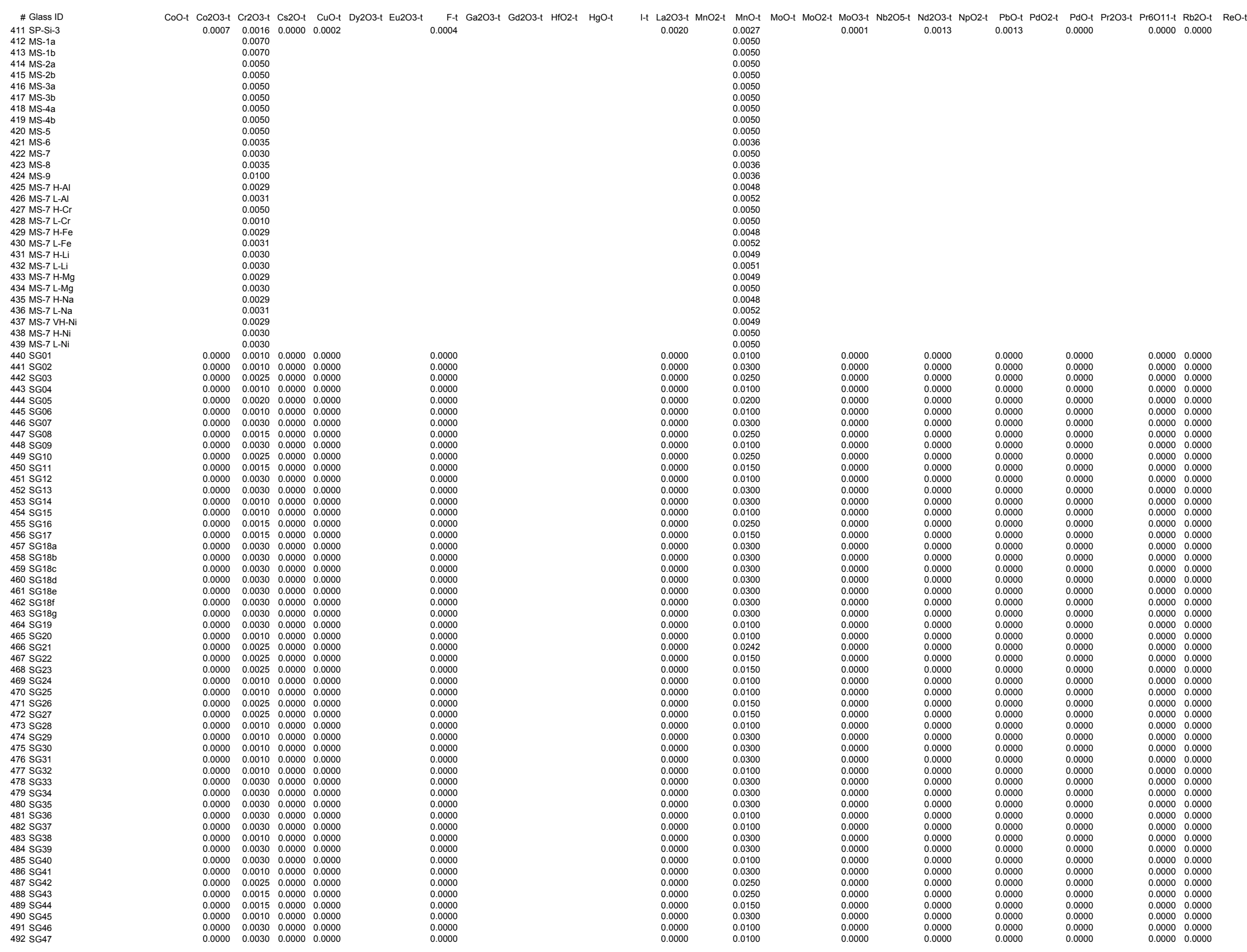




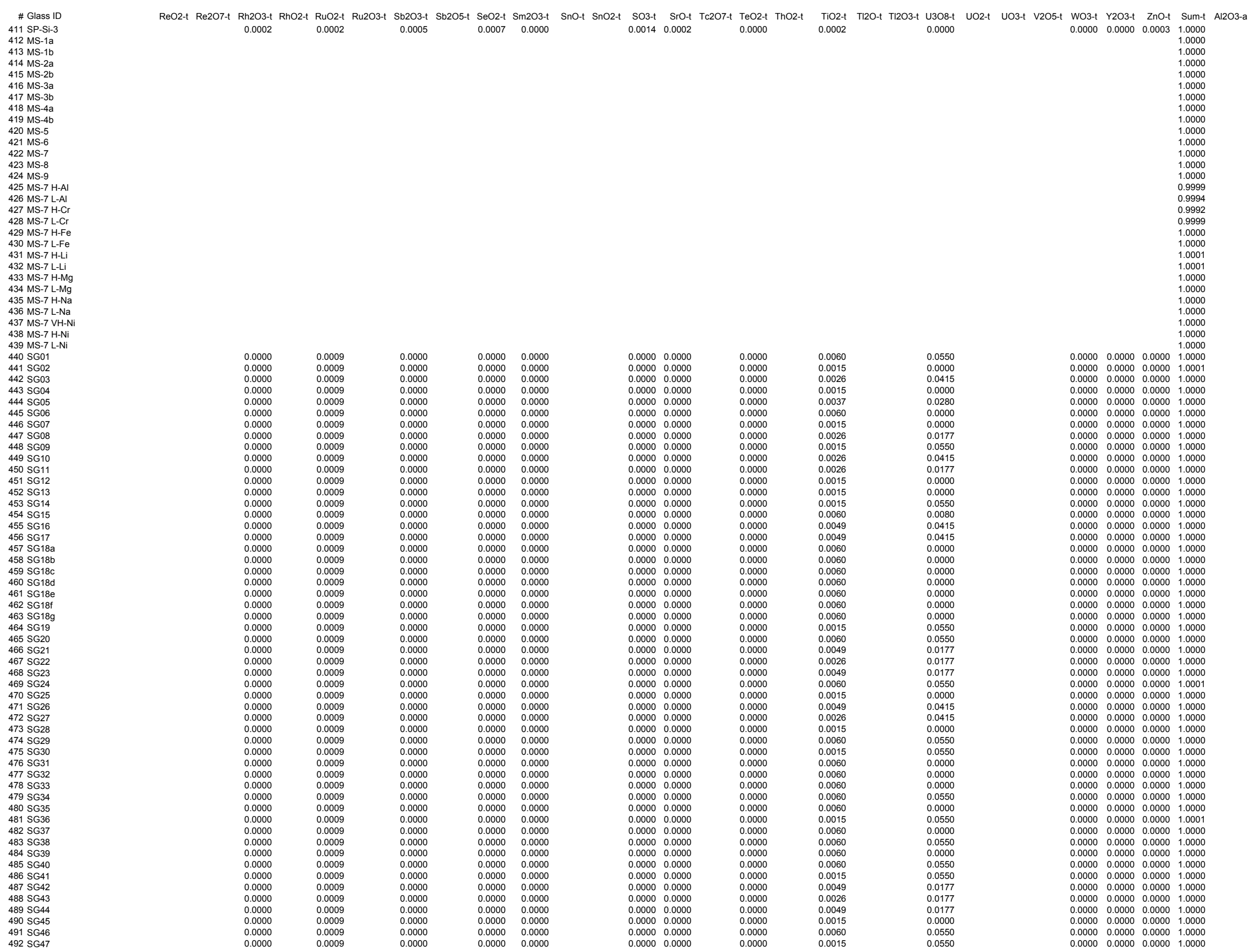


412 MS-1a
413 MS-16

$414 \mathrm{MS}-2 \mathrm{a}$
$415 \mathrm{MS}-2 \mathrm{~b}$

$417 \mathrm{MS}-3 \mathrm{~b}$

$417 \mathrm{MS}-3 \mathrm{sb}$
$418 \mathrm{MS}-4 \mathrm{a}$

419 MS-4b
420 MS -5

421 MS-6

$422 \mathrm{MS}-7$

$423 \mathrm{MS}-8$
$424 \mathrm{MS}-9$

425 MS-7 H-AA
426 MS- 7 LAI

$427 \mathrm{MS}-7 \mathrm{H}-\mathrm{C}$

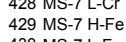

$430 \mathrm{MS}-7 \mathrm{~L}-\mathrm{Fe}$

431 MS-7 7 -LL
432 MS- $7 L-L i$

$433 \mathrm{MS}-7 \mathrm{H}-\mathrm{Mg}$

$434 \mathrm{MS}-7 \mathrm{~L}-\mathrm{Mg}$

$435 \mathrm{MS}-7 \mathrm{H}$ H.Na
$436 \mathrm{MS}-\mathrm{T}-\mathrm{Na}$

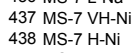

$440 \mathrm{SGO1}$
$441 \mathrm{SG}(\mathrm{SO} 2$

$442 \mathrm{SG60}$
$443 \mathrm{SG04}$

$443 \mathrm{SG04}$
$444 \mathrm{SSO05}$

445606
4465007

$4465 \mathrm{SGO}$
4475508

448 SG09

4489609
4495610

$450 \mathrm{SS} 11$
$451 \mathrm{GS12}$

$4525 \mathrm{SG} 13$

454561
$455 \mathrm{SG} 16$

$456 \mathrm{SG17}$

$4558 \mathrm{SG} 18 \mathrm{Bb}$
$456 \mathrm{~s}$

460 SG18d

4661 SG
$468 \mathrm{e}$

$462 \mathrm{SGG} 18 \mathrm{f}$
$463 \mathrm{SG} 18 \mathrm{~g}$

${ }_{464 \mathrm{SG} 19}^{469}$

4665621

$467 \mathrm{SG22}$

$469 \mathrm{SG} 22$
$470 \mathrm{~S} 25$

$470 \mathrm{SG} 25$
$471 \mathrm{~S} 2626$

$472 \mathrm{SG27}$

$473 \mathrm{SG28}$
$474 \mathrm{SG} 29$

$474 \mathrm{SG} 29$
$475 \mathrm{SG} 30$

$476 \mathrm{SG} 31$

$477 \mathrm{SG} 32$
$478 \mathrm{SG} 33$

$479 \mathrm{SG} 34$

$480 \mathrm{SG} 35$
$481 \mathrm{sG}$

$482 \mathrm{SG} 37$

$4823 \mathrm{GG} 37$
$483 \mathrm{SG} 38$

$485 \mathrm{SG} 40$

$486 \mathrm{SG} 41$
$487 \mathrm{SG} 42$

$488 \mathrm{SG} 43$

$4899 \mathrm{SS} 44$
$495 \mathrm{~S} 45$
4915946

491 SG46
4925647 
412 MS-1a

$414 \mathrm{MS}-2 \mathrm{a}$

$415 \mathrm{MS}-2 \mathrm{~b}$

$417 \mathrm{MS}-3 \mathrm{~b}$
$418 \mathrm{MS}-4 \mathrm{a}$

49 MS.4b

421 MS-6

$422 \mathrm{MS}-7$

$423 \mathrm{MS}-8$
424 MS -9

425 MS-7 H-AA
426 MS-7 LAI

427 MS-7 H-C
428 MS- $7 \mathrm{~L}-\mathrm{C}$

$429 \mathrm{MS}-7 \mathrm{H}-\mathrm{Fe}$

$430 \mathrm{MS}-7 \mathrm{~L}-\mathrm{Fe}$

$431 \mathrm{MS}-7 \mathrm{H}-\mathrm{L}$
$432 \mathrm{MS}-7 \mathrm{~L}-\mathrm{Li}$

433 MS- 7 H-Mg

$43 \mathrm{MS}-7 \mathrm{~L}-\mathrm{Mg} g$
$435 \mathrm{MS}-7 \mathrm{Hg}$

435 MS-7 H H Na
436 MS-7 -Na

$437 \mathrm{TS}-7 \mathrm{TH}-\mathrm{N} / \mathrm{N}$
$438 \mathrm{MS}-\mathrm{TH}-\mathrm{Ni}$

$440 \mathrm{SGO1}$
$441 \mathrm{SGO}$

$442 \mathrm{SG0}$
$443 \mathrm{SG04}$

4435604
$444 \leq 505$

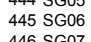

44565607
4475608

4485609

4489609
4495610

$450 \mathrm{SG} 11$
$451 \mathrm{GS12}$

$4525 \mathrm{SG} 13$
$453 \mathrm{SG} 14$

454561
$455 \mathrm{SG} 16$

$456 \mathrm{SG} 17$

$457 \mathrm{SG} 18 \mathrm{a}$
$458 \mathrm{~S} 188 \mathrm{~b}$

$4585 \mathrm{SG18b}$
$459 \mathrm{SG} 18 \mathrm{c}$

$4605 \mathrm{SG} 18 \mathrm{~d}$
$461 \mathrm{SG} 18 \mathrm{e}$

$462 \mathrm{SGG}$
$463 \mathrm{SG} 18 \mathrm{f}$

${ }_{464 \mathrm{SG} 19}^{4039}$

4655620

$467 \mathrm{SG} 222$
$468 \mathrm{~S} S 23$

$4685 \mathrm{SG23}$
$469 \mathrm{SS24}$
$470 \mathrm{~s} 25$

$470 \mathrm{~S} 622$
$471 \mathrm{~S} 222$
4

4725627

$473 \mathrm{SG28}$
$474 \mathrm{SG} 29$

$474 \mathrm{SG29}$
$475 \mathrm{SG} 30$

$476 \mathrm{SG} 31$

$477 \mathrm{SG} 32$
$478 \mathrm{SG} 33$

$479 \mathrm{SG} 34$

$480 \mathrm{SG} 35$
$481 \mathrm{sG36}$

482563

$483 \mathrm{SG} 38$

$485 \mathrm{SG} 40$

$487 \mathrm{TSG42}$

$488 \mathrm{SG} 43$

$4899 \mathrm{SS} 44$
$495 \mathrm{~S} 45$
4915946

$491 \mathrm{SG} 4$
$492 \mathrm{SG} 4$ 


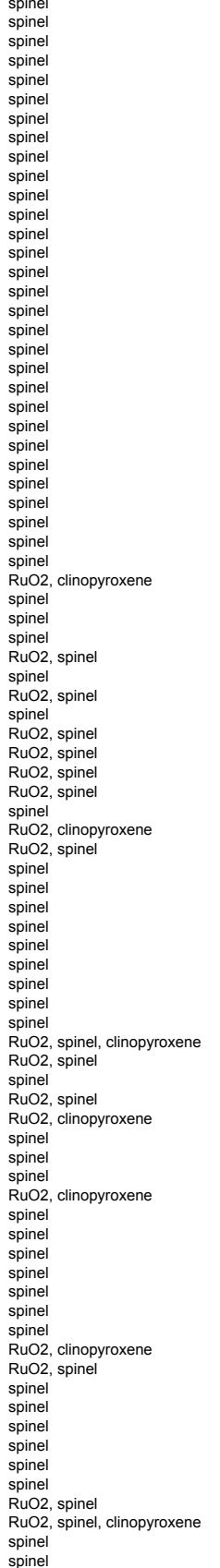


$44 \mathrm{MS}-2 \mathrm{a}$

$415 \mathrm{MS}-2 \mathrm{~b}$

$417 \mathrm{MS}-3 \mathrm{~b}$
$418 \mathrm{MS}-4 \mathrm{a}$

$419 \mathrm{MS}-4 \mathrm{~B}$
$420 \mathrm{MS}$

421 MS-6 6

$422 \mathrm{MS}-7$

$423 \mathrm{MS}-8$
$424 \mathrm{MS}-9$

425 MS-7 H-AA
426 MS-7 - LAl

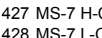

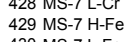

$430 \mathrm{MS}-7 \mathrm{~L}-\mathrm{Fe}$

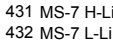

433 MS- 7 H-Mg

$434 \mathrm{MS}-7 \mathrm{~L}-\mathrm{Mg}$

435 MS-7 H H Na
436 MS-7 -Na

$437 \mathrm{TS}-7 \mathrm{TH}-\mathrm{N} /$
$438 \mathrm{MS}-\mathrm{TH}-\mathrm{Ni}$

$440 \mathrm{SGO1}$
$441 \mathrm{SGO}$

$4425 \mathrm{SG03}$
$443 \mathrm{~S} 604$

4435604
$444 \leq 505$

4445605
445606
4456907

4465507
4475608

$447 \mathrm{SG08}$
$448 \mathrm{SG} 09$

4489609
4495610

$450 \mathrm{SG} 11$
$451 \mathrm{GS12}$

$4525 \mathrm{SG} 13$
$453 \mathrm{SG} 14$

454561
$455 \mathrm{SG} 16$

$455 \mathrm{SG} 16$
$456 \mathrm{SG17}$

$457 \mathrm{SG} 18 \mathrm{a}$
$458 \mathrm{SG} 18 \mathrm{~b}$

$459 \mathrm{SG} 18 \mathrm{c}$
$460 \mathrm{SG} 18 \mathrm{~d}$

461 SG18e

$462 \mathrm{SGG}$
$463 \mathrm{SG} 18 \mathrm{f}$

$463 \mathrm{SG} 189$
$464 \mathrm{SG} 19$

$466 \mathrm{SG} 21$

$467 \mathrm{SG22}$

$469 \mathrm{SG} 22$
$470 \mathrm{~S} 25$

$470 \mathrm{SG} 25$
$471 \mathrm{~S} 2626$

4725627

$474 \mathrm{SG29}$
$475 \mathrm{~S} 3030$

$476 \mathrm{SG} 31$

$477 \mathrm{SG} 32$
$478 \mathrm{SG} 33$

$479 \mathrm{SG} 34$

$4801 \mathrm{SG} 35$

$482 \mathrm{SG} 37$

$4823 \mathrm{GG} 37$
$483 \mathrm{SG} 38$

$485 \mathrm{SG} 40$

$486 \mathrm{SG} 41$
$487 \mathrm{SG} 42$

4509045

$490 \mathrm{SG} 4$

$492 \mathrm{SG} 4$ 
$415 \mathrm{MS}-2 \mathrm{~b}$
$416 \mathrm{MS}$

417 MS-3b

418 MS-4a

419 MS-4b

421 MS-6 6

$422 \mathrm{MS}-7$

$423 \mathrm{MS}-8$
$424 \mathrm{MS}-9$

425 MS-7 H-AA
426 MS-7 LAI

$427 \mathrm{MS}-\mathrm{T}$ - $\mathrm{-C}$

$42 \mathrm{MS}-7 \mathrm{LCC}$
$429 \mathrm{MS}-7 \mathrm{LHE}$

$430 \mathrm{MS}-7 \mathrm{~L}-\mathrm{Fe}$

431 MS-7 H-L

433 MS-7 7 -Mg

$434 \mathrm{MS}-7 \mathrm{~L}-\mathrm{Mg}$

435 MS-7 H H Na
436 MS-7 -Na

$437 \mathrm{TS}-7 \mathrm{TH}-\mathrm{N} / \mathrm{N}$
$438 \mathrm{MS}-\mathrm{TH}-\mathrm{Ni}$

$440 \mathrm{SGO1}$
$441 \mathrm{SGO}$

$442 \mathrm{SGO3}_{4}$
$443 \mathrm{SGO4}$

4435604
$444 \leq 505$

4455606
4465007

44565607
4475608

$447 \mathrm{SG08}$
$448 \mathrm{SG} 09$

44485609
$445 \mathrm{SG} 11$
$455 \mathrm{SG11}$

$450 \mathrm{SG} 11$
$451 \mathrm{GS12}$

$4525 \mathrm{SG} 13$
$453 \mathrm{SG} 14$

454561
$455 \mathrm{SG} 16$

$456 \mathrm{SG} 17$

$457 \mathrm{SG} 18 \mathrm{a}$
$458 \mathrm{~S} 188 \mathrm{~b}$

$4585 \mathrm{SG18b}$
$459 \mathrm{SG} 18 \mathrm{c}$

$4605 \mathrm{SG} 18 \mathrm{~d}$
$461 \mathrm{SG} 18 \mathrm{e}$

$462 \mathrm{SGG}$
$463 \mathrm{SG} 18 \mathrm{f}$

${ }_{464 \mathrm{SG} 19}^{4039}$

$465 \mathrm{SG} 20$

$466 \mathrm{SG2}$

$468 \mathrm{SG} 23$

$470 \mathrm{SG} 25$

4725627

$473 \mathrm{SG28}$
$474 \mathrm{SG} 29$

$474 \mathrm{SG29}$
$475 \mathrm{SG} 30$

$476 \mathrm{SG} 31$

$4775 \mathrm{SG} 32$
$478 \mathrm{SG} 33$

$479 \mathrm{SG} 34$

$480 \mathrm{SG} 35$
$481 \mathrm{sG36}$

482563

$4823 \mathrm{GG} 37$
$483 \mathrm{SG} 38$

$485 \mathrm{SG} 40$

486
$487 \mathrm{SG} 44$

4509045

$490 \mathrm{SG} 4$

$492 \mathrm{SG} 47$ 
$414 \mathrm{MS}-2 \mathrm{a}$

$415 \mathrm{MS}-2 \mathrm{~b}$

$417 \mathrm{MS}-3 \mathrm{sb}$
$418 \mathrm{MS}-4 \mathrm{a}$

$419 \mathrm{MS}-4 \mathrm{~B}$
$420 \mathrm{MS}$

421 MS-6 6

$422 \mathrm{MS}-7$

$423 \mathrm{MS}-8$
$424 \mathrm{MS}-9$

$425 \mathrm{MS}-7 \mathrm{H}-\mathrm{Al}$
$426 \mathrm{MS}-7 \mathrm{LAl}$

$427 \mathrm{MS}-7 \mathrm{~T}-\mathrm{C}$

429 MS-7 H H.Fe

$430 \mathrm{MS}-7 \mathrm{~L}-\mathrm{Fe}$

431 MS-7 H-LL
432 MS- $\mathrm{LLLI}$

433 MS-7 7 -Mg

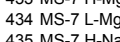

435 MS-7 H H Na
436 MS-7 -Na

437 MS-7 TH-N

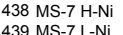

$4405 \mathrm{SG} 01$
$441 \mathrm{SG} 02$

$442 \mathrm{SGO3}_{4}$
$443 \mathrm{SGO4}$

44335604
4445605

4445605
4455606
4456507

44565607
4475608

$447 \mathrm{SG08}$
$448 \mathrm{SG} 09$

4489609
4495610

$450 \mathrm{SG} 11$
$451 \mathrm{GS12}$

$452 \mathrm{SG13}$
$453 \mathrm{SG} 14$

454561
$455 \mathrm{SG} 16$

$455 \mathrm{SG} 16$
$456 \mathrm{SG17}$

$457 \mathrm{SG} 18 \mathrm{a}$
$458 \mathrm{~S} 188 \mathrm{~b}$

$4585 \mathrm{SG18b}$
$459 \mathrm{SG} 18 \mathrm{c}$

$4605 \mathrm{SG} 18 \mathrm{~d}$
$461 \mathrm{SG} 18 \mathrm{e}$

$462 \mathrm{SG} 18 \mathrm{f}$
$463 \mathrm{SG} 18 \mathrm{~g}$

${ }_{464 \mathrm{SG} 19}^{4039}$

$465 \mathrm{SG} 20$

$466 \mathrm{SG2}$

$468 \mathrm{SG} 23$

$470 \mathrm{SG} 25$

$472 \mathrm{SG} 27$

$473 \mathrm{SG28}$
$474 \mathrm{SG} 29$

$474 \mathrm{SG29}$
$475 \mathrm{SG} 30$

$476 \mathrm{SG} 31$

$477 \mathrm{SG} 32$
$478 \mathrm{SG} 33$

$479 \mathrm{SG} 34$

$480 \mathrm{SS} 35$
$481 \mathrm{GG} 36$

482563

$4823 \mathrm{GG} 37$
$483 \mathrm{SG} 38$

$485 \mathrm{SG} 40$

$487 \mathrm{SG} 42$

4509045

$490 \mathrm{SG} 4$

$492 \mathrm{SG} 47$ 
412 MS-1a
413 MS

$414 \mathrm{MS}-2 \mathrm{a}$

$415 \mathrm{MS} 2 \mathrm{2b}$
$416 \mathrm{MS}-3 \mathrm{a}$
$417 \mathrm{MS} 23$

$417 \mathrm{MS}-3 \mathrm{~b}$
$418 \mathrm{MS}-4 \mathrm{a}$

$419 \mathrm{MS}-4 \mathrm{~B}$
$420 \mathrm{MS}$

421 MS-6 6

$422 \mathrm{MS}-7$

$423 \mathrm{MS}-8$
$424 \mathrm{MS}-9$

$425 \mathrm{SS}-7 \mathrm{H}$ HAI
$426 \mathrm{MS}-\mathrm{TLAl}$

$427 \mathrm{MS}-7 \mathrm{H}-\mathrm{Cr}$
$428 \mathrm{MS}-7 \mathrm{LC}-\mathrm{Cr}$

$429 \mathrm{MS}-7 \mathrm{H}-\mathrm{Fe}$

$430 \mathrm{MS}-7 \mathrm{~L}-\mathrm{Fe}$

431 MS-7H H.Li
432 MS- 7 LLL

433 MS- 7 H-Mg

$43 \mathrm{MS}-7 \mathrm{~L}-\mathrm{Mg} g$
$435 \mathrm{MS}-7 \mathrm{Hg}$

435 MS-7 H H Na
436 MS-7 -Na

$437 \mathrm{TS}-7 \mathrm{TH}-\mathrm{N} / \mathrm{N}$
$438 \mathrm{MS}-\mathrm{TH}-\mathrm{Ni}$

$4405 \mathrm{SG} 01$
4415602

$442 \mathrm{SGO3}_{4}$
$443 \mathrm{SGO4}$

4435604
4445605

4445605
445606
4456907

44565607
4475608

$447 \mathrm{SG08}$
$448 \mathrm{SG} 09$

4485509
4495610

$450 \mathrm{SG} 11$
$451 \mathrm{GS12}$

$4525 \mathrm{SG} 13$
$453 \mathrm{SG} 14$

454561
$455 \mathrm{SG} 16$

$456 \mathrm{SG} 17$

$458 \mathrm{SG} 186$
456
45956189

$460 \mathrm{SG} 18 \mathrm{~d}$
$461 \mathrm{SG} 18 \mathrm{e}$
$462 \mathrm{SG}$

${ }_{463}^{462} \mathrm{SGG} 18 \mathrm{fg}$

${ }_{464 \mathrm{SG} 19}^{4039}$

4665621

4675622
$468 \mathrm{~S} 223$

$469 \mathrm{SG} 24$

$470 \mathrm{SG} 25$
$471 \mathrm{SG} 26$

47525627
4735628

$474 \mathrm{SG} 29$
$475 \mathrm{SG} 30$

$476 \mathrm{SG} 31$

$477 \mathrm{SG} 32$
$478 \mathrm{SG} 33$

$479 \mathrm{SG} 34$

$480 \mathrm{SG} 35$
$481 \mathrm{sG36}$

$482 \mathrm{SG} 37$

$4823 \mathrm{GG} 37$
$483 \mathrm{SG} 38$

$485 \mathrm{SG} 40$

$486 \mathrm{SG} 41$
$487 \mathrm{SG} 42$

489 SG4

$490 \mathrm{SG} 4$

$492 \mathrm{SG} 4$ 


\begin{tabular}{|c|c|c|}
\hline 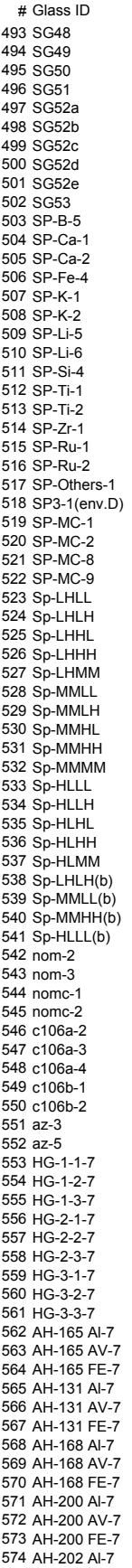 & 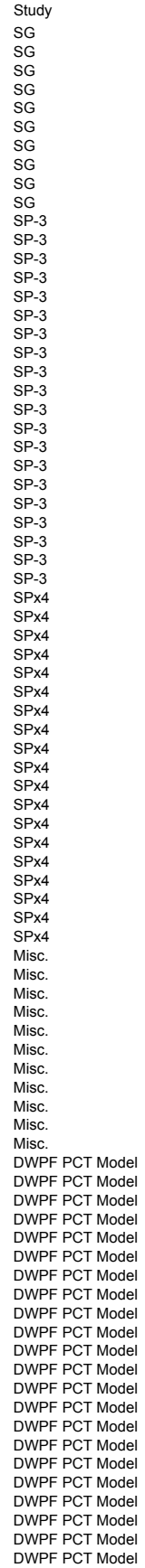 & 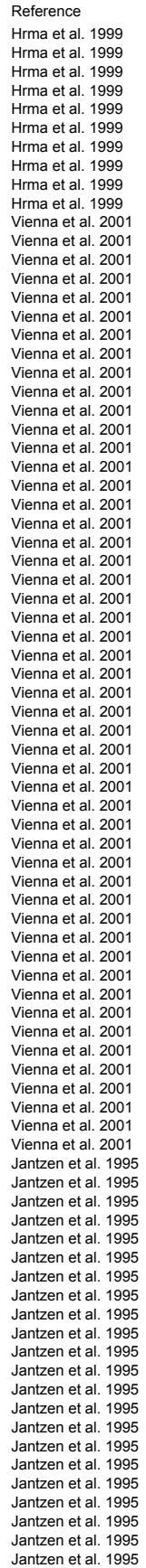 \\
\hline
\end{tabular}

A1203-t B2O3-t CaO-t Fe2O3-t FeO-t

K2O-t Li2O-t MgO-t Na2O-t NiO-t P2O5-t SiO2-t ZrO2-t Ag2O-t As2O3-tAs2O

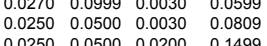
$\begin{array}{llll}0.0799 & 0.0500 & 0.0200 & 0.1499 \\ 0.0250 & 0.0999 & 0.0030 & 0.1499\end{array}$ $\begin{array}{llll}0.0250 & 0.0999 & 0.0030 & 0.1499\end{array}$ $\begin{array}{llll}0.0250 & 0.0999 & 0.0030 & 0.1499 \\ 0.0250 & 0.0999 & 0.0030 & 0.1499\end{array}$ $\begin{array}{llll}0.0250 & 0.0999 & 0.0030 & 0.1499 \\ 0.0530 & 0.0752 & 0.0115 & 0.1052\end{array}$

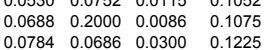
$\begin{array}{llll}0.0784 & 0.0686 & 0.0300 & 0.1225 \\ 0.0768 & 0.0672 & 0.0500 & 0.1199\end{array}$ $\begin{array}{llll}0.0786 & 0.0616 & 0.0088 & 0.2300 \\ 0 & 0.0688 & 0.0098 & 0.1228\end{array}$ $\begin{array}{llll}0.0770 & 0.0674 & 0.0096 & 0.1203 \\ 0.0788 & 0.0689 & 0.0098 & 0.1231\end{array}$ $\begin{array}{llll}0.0788 & 0.0689 & 0.0098 & 0.1231 \\ 0.0763 & 0.0668 & 0.0095 & 0.192\end{array}$ $\begin{array}{llll}0.1037 & 0.0907 & 0.0130 & 0.1620 \\ 0.0780 & 0.0683 & 0.0098 & 0.1219\end{array}$

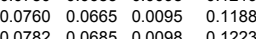

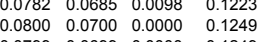
$\begin{array}{llll}0.0799 & 0.0699 & 0.0000 & 0.1249 \\ 0.0777 & 0.0680 & 0.0097 & 0.1215\end{array}$ $\begin{array}{llll}0.0777 & 0.0680 & 0.0097 & 0.1215 \\ 0.1014 & 0.0699 & 0.0404 & 0.2297 \\ 0.0000 & 0.1500 & 0.0000 & 0.0500\end{array}$ $\begin{array}{llll}0.1600 & 0.1500 & 0.0000 & 0.0500 \\ 0.0000 & 0.1500 & 0.0000 & 0.0500\end{array}$ $\begin{array}{llll}0.0057 & 0.0000 & 0.0500 & 0.0500 \\ 0.0573 & 0.1500 & 0.0500 & 0.0500\end{array}$ $\begin{array}{llll}0.0500 & 0.0706 & 0.0000 & 0.1260 \\ 0.0500 & 0.0688 & 0.0000 & 0.1228\end{array}$ $\begin{array}{llll}0.0500 & 0.0688 & 0.0000 & 0.1228 \\ 0.0500 & 0.0700 & 0.0000 & 0.1251\end{array}$ $\begin{array}{llll}0.0500 & 0.0683 & 0.00000 & 0.1225 \\ 0.0500 & 0.0700 & 0.00000 & 0.1219 \\ 0.1250 & 0.000\end{array}$ $0.0800 \quad 0.0706 \quad 0.0000 \quad 0.1260$

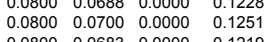
$\begin{array}{llll}0.0800 & 0.0683 & 0.0000 & 0.1219 \\ 0.0800 & 0.0700 & 0.0000 & 0.1250 \\ 0.1100 & 0.0688 & 00000 & 0.1228\end{array}$ $\begin{array}{llll}0.1100 & 0.0706 & 0.00000 & 0.1260 \\ 0.1100 & 0.0700 & 0.0700 & 0.1250\end{array}$ $\begin{array}{llll}0.1100 & 0.0688 & 0.0000 & 0.1228 \\ 0.1100 & 0.0700 & 0.0000 & 0.1251 \\ 0.1100 & 0.070 & 0.000 & 0.1250\end{array}$ $\begin{array}{llll}0.1100 & 0.0683 & 0.0000 & 0.1219 \\ 0.1100 & 0.0700 & 0.0000 & 0.1250\end{array}$ $\begin{array}{llll}0.0500 & 0.0688 & 0.0000 & 0.1228 \\ 0.0800 & 0.0706 & 0.0000 & 0.1260\end{array}$ $\begin{array}{llll}0.0800 & 0.0706 & 0.00000 & 0.1228 \\ 0.0800 & 0.0683 & 0.0000 & 0.1219 \\ 0.11000 & 0.00706 & 0.00000 & 0.1260\end{array}$ $\begin{array}{lllllllllllllll}0.0681 & 0.0982 & 0.0086 & 0.1076 & 0.0000 & 0.0024 & 0.0600 & 0.0051 & 0.0913 & 0.0045 & 0.0039 & 0.5011 & 0.0155 & 0.0006 & 0.00003 \\ 0.0808 & 0.1093 & 0.0102 & 0.1278 & 0.0000 & 0.0029 & 0.0100 & 0.0060 & 0.1733 & 0.0053 & 0.0046 & 0.4113 & 0.0184 & 0.0007 & 0.0004\end{array}$

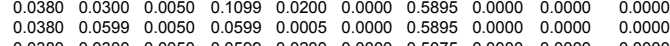

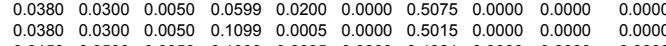

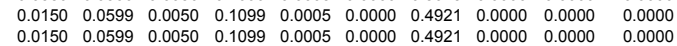

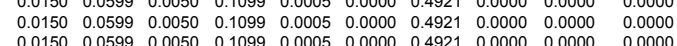

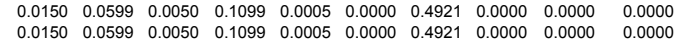

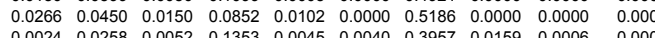

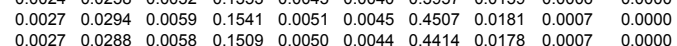

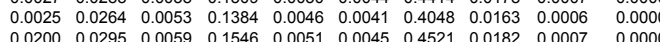
$\begin{array}{llllllllll}0.0400 & 0.0289 & 0.0058 & 0.1514 & 0.0050 & 0.0045 & 0.4428 & 0.0178 & 0.0007 & 0.00000 \\ 0.0028 & 0.0450 & 0.0059 & 0.1549 & 0.0051 & 0.0046 & 0.4529 & 0.0182 & 0.0007 & 0.0000\end{array}$ $\begin{array}{llllllllll}0.0028 & 0.0450 & 0.0059 & 0.1549 & 0.0051 & 0.0046 & 0.4529 & 0.0182 & 0.0007 & 0.0000 \\ 0.0027 & 0.0750 & 0.0057 & 0.1500 & 0.0050 & 0.0044 & 0.4387 & 0.0176 & 0.0007 & 0.0000\end{array}$

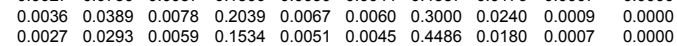

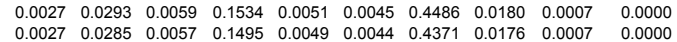
$\begin{array}{llllllllll}0.0027 & 0.0293 & 0.0059 & 0.1539 & 0.0051 & 0.0045 & 0.4499 & 0.0400 & 0.0007 & 0.0000 \\ 0.0000 & 0.0300 & 0.0060 & 0.1572 & 0.0052 & 0.0059 & 0.4597 & 0.0234 & 0.0009 & 0.0000\end{array}$ $\begin{array}{llllllllll}0.0000 & 0.0300 & 0.00600 & 0.1572 & 0.0052 & 0.0059 & 0.4596 & 0.0234 & 0.0009 & 0.00000 \\ 0.0027 & 0.0292 & 0.0058 & 0.1529 & 0.0051 & 0.0085 & 0.4470 & 0.0180 & 0.0013 & 0.0000\end{array}$

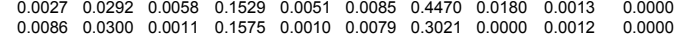

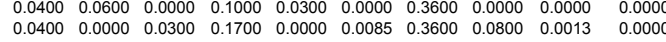

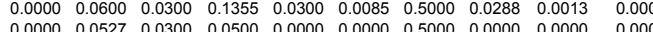

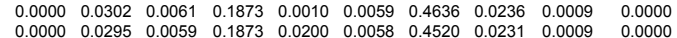
$\begin{array}{llllllllll}0.0000 & 0.0295 & 0.0059 & 0.1873 & 0.0200 & 0.0058 & 0.4520 & 0.0231 & 0.0009 & 0.0000 \\ 0.0000 & 0.0300 & 0.0060 & 0.1873 & 0.0010 & 0.0059 & 0.4602 & 0.0234 & 0.0009 & 0.0000\end{array}$

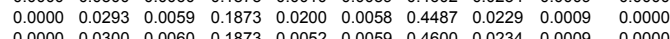

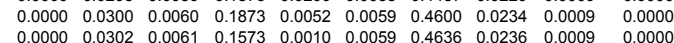
$\begin{array}{lllllllllll}0.0000 & 0.0295 & 0.0059 & 0.1573 & 0.0200 & 0.0058 & 0.4520 & 0.0231 & 0.0009 & 0.0000\end{array}$ $\begin{array}{llllllllll}0.00000 & 0.0300 & 0.0060 & 0.1573 & 0.0010 & 0.0059 & 0.4602 & 0.0234 & 0.0009 & 0.0000 \\ 0.0293 & 0.0059 & 0.1573 & 0.0200 & 0.0058 & 0.4487 & 0.0229 & 0.0009 & 0.0000\end{array}$ $\begin{array}{llllllllll}0.0000 & 0.0300 & 0.0060 & 0.1573 & 0.0052 & 0.0059 & 0.4600 & 0.0234 & 0.0009 & 0.0000 \\ 0.0000 & 0.0302 & 0.0061 & 0.1273 & 0.0010 & 0.0059 & 0.4636 & 0.0236 & 0.0009 & 0.0000\end{array}$ $\begin{array}{llllllllll}0.0000 & 0.0295 & 0.0059 & 0.1273 & 0.0200 & 0.0058 & 0.4520 & 0.0231 & 0.0009 & 0.0000 \\ 0.0000 & 0.0300 & 0.0060 & 0.1273 & 0.0010 & 0.0059 & 0.4602 & 0.0234 & 0.0009 & 0.0000\end{array}$

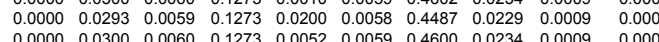

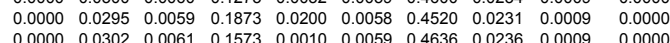

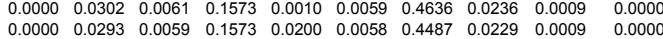

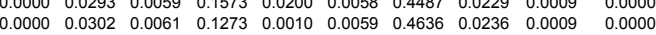
$\begin{array}{lllllllllllllll}0.0493 & 0.0500 & 0.0119 & 0.1500 & 0.0000 & 0.0034 & 0.0100 & 0.0071 & 0.1883 & 0.0062 & 0.0019 & 0.4535 & 0.0216 & 0.00008 & 0.0005 \\ 0.0412 & 0.0588 & 0.0100 & 0.1254 & 0.0000 & 0.0028 & 0.0521 & 0.0059 & 0.1177 & 0.0052 & 0.0015 & 0.5221 & 0.0181 & 0.0007 & 0.0004\end{array}$

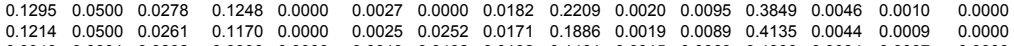

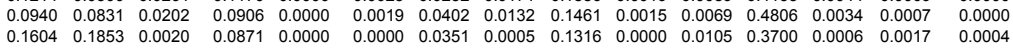
$\begin{array}{lllllllllllllll}0.1440 & 0.1094 & 0.0018 & 0.0781 & 0.0000 & 0.0000 & 0.0599 & 0.0005 & 0.1311 & 0.0000 & 0.0094 & 0.4501 & 0.0005 & 0.0015 & 0.0003 \\ 0.0664 & 0.0500 & 0.0031 & 0.1397 & 0.0000 & 0.0032 & 0.0100 & 0.0011 & 0.2114 & 0.0079 & 0.0027 & 0.4296 & 0.0264 & 0.0005 & 0.0006\end{array}$

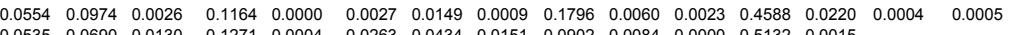
$\begin{array}{lllllllllllll}0.0499 & 0.0672 & 0.0104 & 0.1160 & 0.0004 & 0.0297 & 0.0448 & 0.0147 & 0.0849 & 0.0076 & 0.0004 & 0.5349 & 0.0009 \\ 0.0489 & 0.0681 & 0.0108 & 0.1149 & 0.0014 & 0.0254 & 0.0453 & 0.0148 & 0.0866 & 0.0073 & 0.0004 & 0.5394 & 0.0000\end{array}$

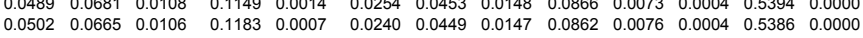
$\begin{array}{lllllllllllll}0.0502 & 0.0665 & 0.0106 & 0.1183 & 0.0007 & 0.0244 & 0.0449 & 0.0147 & 0.0862 & 0.0076 & 0.0004 & 0.5386 & 0.0000 \\ 0.0535 & 0.0594 & 0.0108 & 0.1254 & 0.0007 & 0.0177 & 0.0432 & 0.0135 & 0.1015 & 0.0075 & 0.0007 & 0.5255 & 0.0021\end{array}$ $\begin{array}{lllllllllllll}0.0527 & 0.0592 & 0.0108 & 0.1235 & 0.0007 & 0.0177 & 0.0436 & 0.0135 & 0.1021 & 0.0075 & 0.0000 & 0.5308 & 0.0008 \\ 0.0549 & 0.0602 & 0.0112 & 0.1226 & 0.0051 & 0.0197 & 0.0426 & 0.0134 & 0.0988 & 0.0078 & 0.0004 & 0.5221 & 0.0017\end{array}$

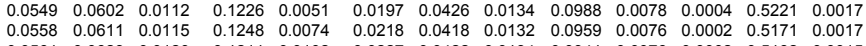
$\begin{array}{lllllllllllll}0.0561 & 0.0623 & 0.0120 & 0.1211 & 0.0102 & 0.0227 & 0.0422 & 0.0134 & 0.0941 & 0.0076 & 0.0002 & 0.5182 & 0.0015 \\ 0.1351 & 0.0740 & 0.0052 & 0.0462 & 0.0024 & 0.0000 & 0.0424 & 0.0067 & 0.1068 & 0.0068 & 0.0000 & 0.5403 & 0.0079\end{array}$

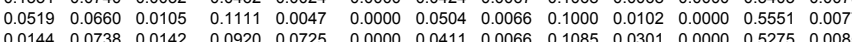

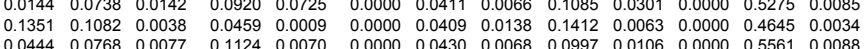
$\begin{array}{lllllllllllll}0.0444 & 0.0768 & 0.0077 & 0.1124 & 0.0070 & 0.0000 & 0.0430 & 0.0068 & 0.0997 & 0.0106 & 0.0000 & 0.5561 & 0.0088 \\ 0.0229 & 0.0745 & 0.0103 & 0.0774 & 0.0895 & 0.0000 & 0.0412 & 0.0067 & 0.1108 & 0.0261 & 0.0000 & 0.5224 & 0.0088\end{array}$

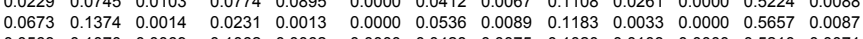

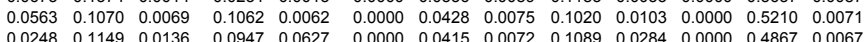
$\begin{array}{lllllllllllll}0.0248 & 0.1149 & .0136 & 0.0947 & 0.0627 & 0.00000 & 0.0415 & 0.0072 & 0.1089 & 0.0284 & 0.0000 & 0.4867 & 0.0067 \\ 0.1347 & 0.1026 & 0.0055 & 0.0441 & 0.0007 & 0.0313 & 0.0266 & 0.0125 & 0.1066 & 0.0061 & 0.0000 & 0.4867 & 0.0004 \\ 0.0517 & 0.1037 & 0.0063 & 0.1155 & 0.0039 & 0.0321 & 0.0270 & 0.0125 & 0.0984 & 0.0103 & 0.00000 & 0.4986 & 0.0002\end{array}$

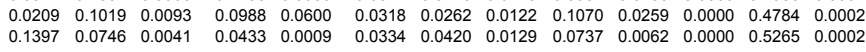

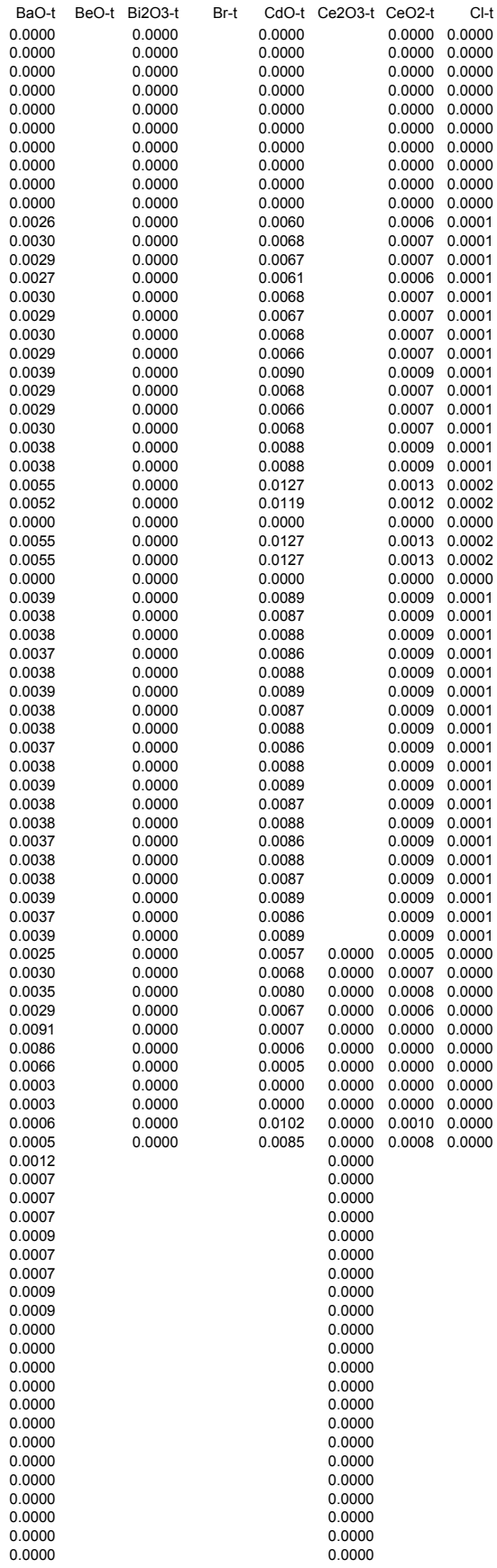




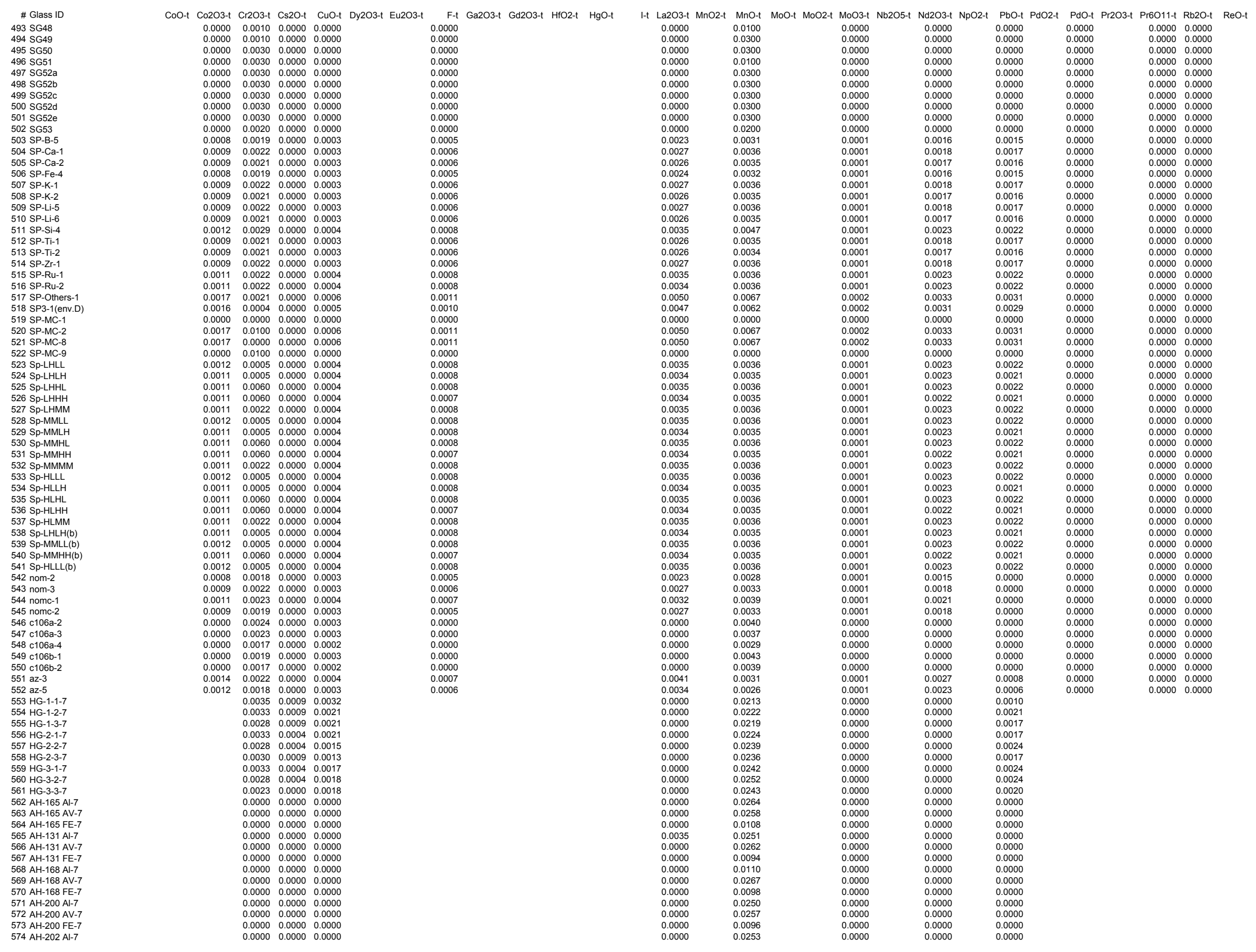




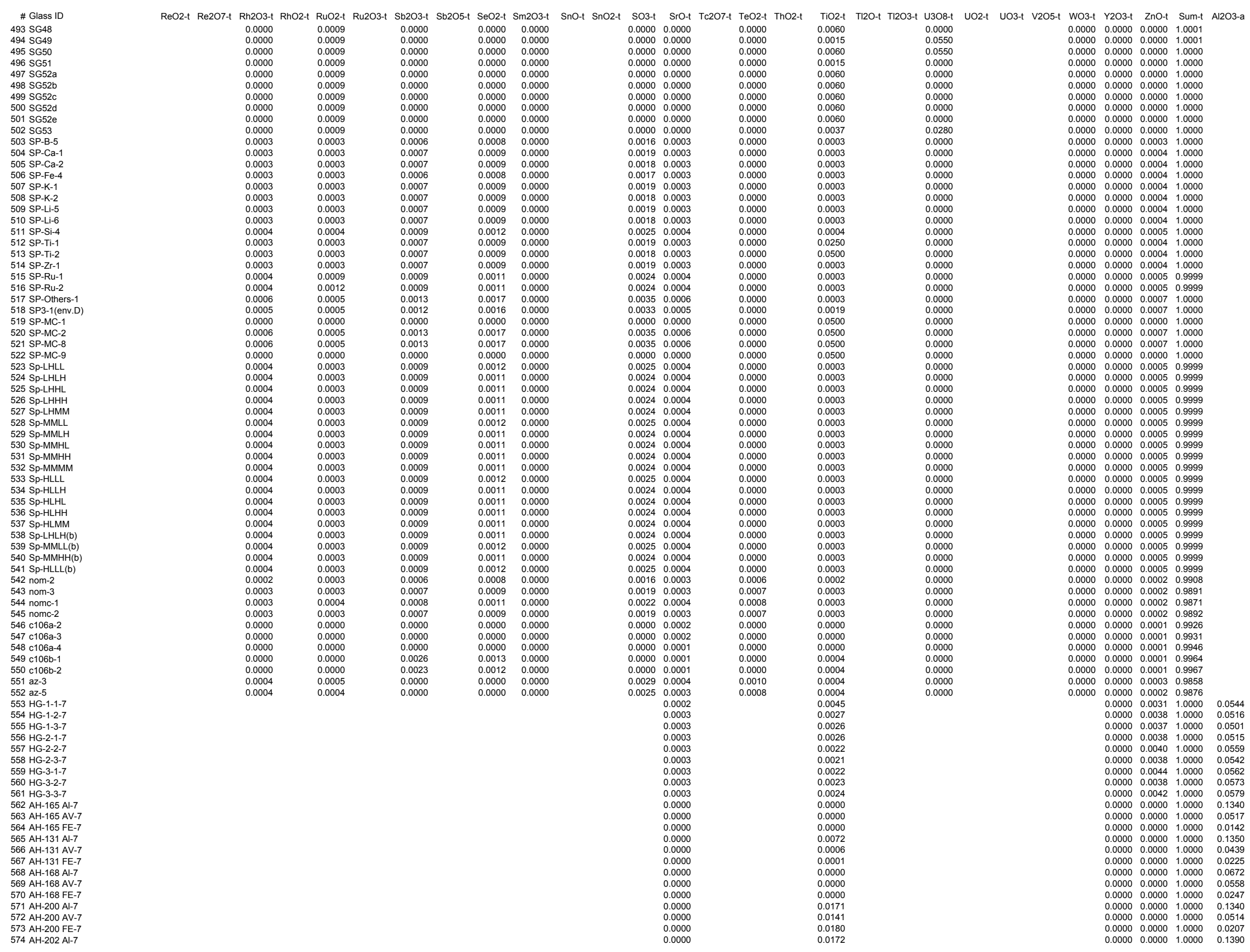


542 nom-2
543 nom-3
54 nom-

54 nomc- 1
545 nomc-2

545 nomc-2
546 c106a-2

$547 \mathrm{c} c 106 \mathrm{a}-3$
$548 \mathrm{c} 1002-4$

548 c c106a-4
549 c106-1

$549 c 1066-1$
550 c1066-2

551 az-3

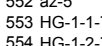

$554 \mathrm{HG}-1-2.27$
$555 \mathrm{HG}-1-3.7$

$556 \mathrm{HG}-2-1.7$

$557 \mathrm{HG}-2-2.27$
$558 \mathrm{HG}-2.3 .7$

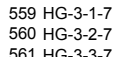

562 AH-165 AL-7

563 AH-165 AV-7
564 AH-165 FE-7

565 AA-131 A-7

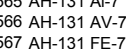

567 AH-131 FE-7
568 AH-168 A-7

569 AH-168 AV-7

570 AH-168 EE-T
571 A

57 AH-200 AA-7
572 AH-200 AV- 7

573 AH-200 FE-7

$\begin{array}{ll}0.0217 & 0.0011 \\ 0.0229 & 0.0020 \\ 0.0225 & 0.0018 \\ 0.0230 & 0.019 \\ 0.0249 & 0.0025 \\ 0.0243 & 0.029 \\ 0.0248 & 0.0023 \\ 0.0259 & 0.023 \\ 0.0250 & 0.0232 \\ 0.0262 & \end{array}$

0.0036 
543 nom-3
544 nomc- 1

545 nomc-2
546 c $106 \mathrm{a}-2$

$547 \mathrm{c} 106 \mathrm{a}-3$

$548 \mathrm{cc} 106 \mathrm{a}-\mathrm{A}$
$549 \mathrm{c} 106 \mathrm{C}-1$

$549 \mathrm{c} 106 \mathrm{~b}-1$
$550 \mathrm{c} 106 \mathrm{~b}-2$

551 az-3

552 az-5
553 HG-1-1-7
554 HG-1-2-7

554 HG-1-2-7

556 HG-2-1-7
557 HG-2-2-7
558 HG-2-3-7

558 HG-2-3-7

560 HG-3-2-7

562 AH-165 Al-7
563 AH-165 AV-7

565 AH-131 Al-7

566 AH-131 AV-7

567 AH-131 $\mathrm{FE}-7$

569 AH-168 AV- 7

570 AH-168 FE-7

571 AH-200 Al-7
572 AH-200 AV-7

573 AH-200 FE-7
574 AH-202 Al-7

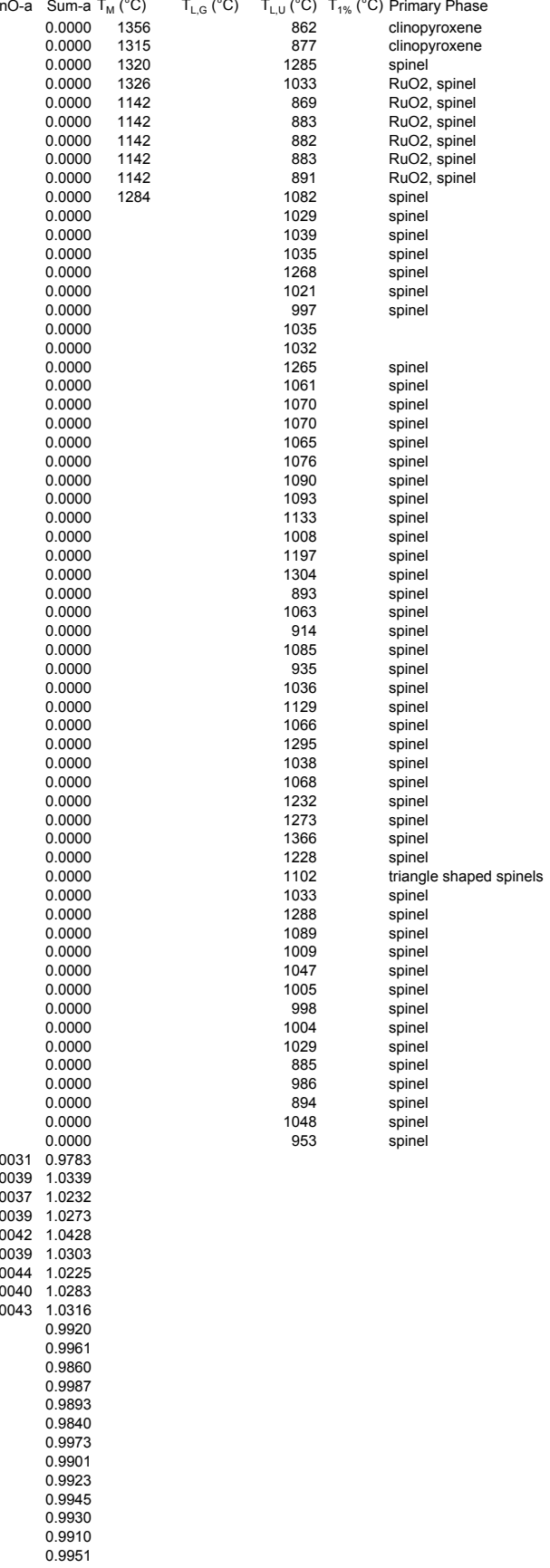

$\begin{array}{ll}0.0001 & 0.0046 \\ 0.0003 & 0.0028 \\ 0.0003 & 0.0026 \\ 0.0003 & 0.0026 \\ 0.0003 & 0.0023 \\ 0.0003 & 0.0022 \\ 0.0003 & 0.0023 \\ 0.0003 & 0.0024 \\ 0.0003 & 0.0025 \\ & \\ & 0.0072 \\ & 0.0006 \\ & 0.0001 \\ & \\ & 0.0170 \\ & 0.0141 \\ & 0.0178 \\ & 0.0171\end{array}$

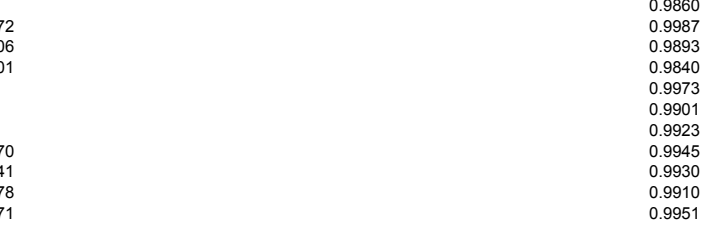


531 SP-MMHH

533 Sp-HLLL

S5 Sp-HLH.

536 SS-HLH

538 Sp 1 -LLLH(b)
539 SML(b)

5930 Sp-MLLL(b)

540 Sp-MMHH(b)
541 Sp-HLLL(b)

542 nom-2

542 non-
543 nom-3

544 nomc- 1
545 nomc-2

546 c106a-2

$547 c 106-2-3$
$548 c 106-4$
549 c106-1 -1

550 c c106b-2
551060

551 az-3

552 az-5

$555 \mathrm{HG}-1-3.7$

$556 \mathrm{HG}-2-1.7 .7$
$557 \mathrm{HG}-2.2-2.7$

558 HG-2-3.-7

$559 \mathrm{HG}-3-3-1.7$
$560 \mathrm{HG}-3-2.7$

$560 \mathrm{HG}-3-2.7$
$561 \mathrm{HG}-3-3-7$

562 AH-165 $5 \mathrm{AL}-7$
$563 \mathrm{AH}-165 \mathrm{AV}-\mathrm{T}$

565 AH-131 A-7

566 AH-13iAV-T

567 AHA-131 E E-T
$568 \mathrm{AH}-168 \mathrm{Al}-\mathrm{T}$

569 AH-168 AV-T

570 AH-168 EE-T
571 A

571 AH 12000 AA-7
572 A A-200 AV-

573 AH-200 FE-7 
528 Sp-MMLL
529 Sp-MMLH

530 Sp-MMHL
531 Sp-MMHH
532 Sp-MMMM

5322 Sp-MMMM
53 SOHLLL

534 Sp-HLLH

535 Sp-HLLHL

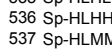

538 Sp-LLLH(b)
539 S-MLLLL(b)
540 Sp-MMHH(b)

540 Sp-MMHH(b)
541 Sp-HLLL(b)

542 nom-2

542 nom-2
543 nom-3

54 nomc-1
545 nomc-2

546 c106a-2

547 c1066-3
$548 \mathrm{c} c 1062-4$

$548 \mathrm{c} 106 \mathrm{a}-4$
$549 \mathrm{c} 106 \mathrm{~b}-1$

550 c106b-2

551 az-3

552 az-5

$555 \mathrm{HG}-1-3-7$

$556 \mathrm{HG}-2-1.7$
$557 \mathrm{HG}-2 \cdot 2.7$

$558 \mathrm{HG}-2-2-3-7$

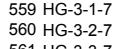

562 AH-165 A-7

563 AH-165 AV-7

565 AH-131 A-7.7
566 AH-131 AV-7
567 AH-131 FE-7

567 AH-131 EE-7
568 AH-168 Al- 7

569 AH-168 AV- 7

570 AH-168 FE-7

57 AH-200 AA-7
572 AH-200 AV- 7

573 AH-200 FE-7

574 AH-202 Al-7 
54 nombc-1
545 nomc-2
54 cos

546 c106a-2

547 c1066-3
$548 \mathrm{c} c 1062-4$

548 c c106a-4
549 c106-1

550 c c106b-2
551060

551 az-3

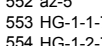

$554 \mathrm{HG}-1-2.27$
$555 \mathrm{HG}-1-3.7$

$556 \mathrm{HG}-2-1-7$

$557 \mathrm{HG}-2-2.27$
$558 \mathrm{HG}-2.3 .7$

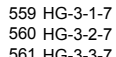

62 AH-165 A- 7

$563 \mathrm{AH}-165 \mathrm{AV}-\mathrm{T}$
564 AH-165 FE-7

56 AH-131 A-7

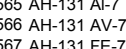

$567 \mathrm{AH}-131 \mathrm{FE}-7$
$568 \mathrm{AH}-168 \mathrm{Al}-\mathrm{T}$

569 AH-168 AV-T

570 AH-168 EE-T
571 A

57 AH-200 AA-7
572 AH-200 AV- 7

573 AHA-200 EE-7
574 A-202 A-7

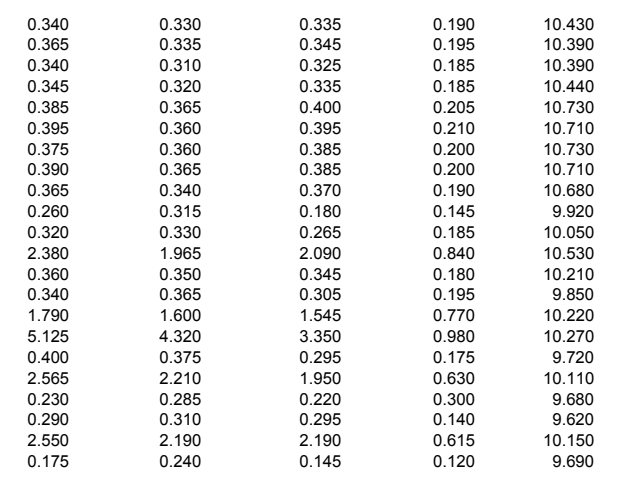


$494 \mathrm{SG} 49$

$495 \mathrm{SG50}$
$496 \mathrm{SG51}$

$4975652 a$
$498 \mathrm{SG} 520$

$4995652 c$
$500 \mathrm{SG} 52 \mathrm{~d}$

501 SG52e
$502 \mathrm{SG} 53$

503 SP-B-5

504 SP-Ca-1

505 SP-Ca-2
506 SP-Fe- 4

507 SP-K- -1
508 SP-K- 2

509 SP-LL-5

511 SP-Si-4

512 SP-Ti-1

514 PP.

515 SP-Ru-

516 SP-Ru-2
517 SP-others-1

518 SP3-1(env. D)
519 SP-MC-1

$520 \mathrm{SP}-\mathrm{MC}-2$

521 SP-MC-8

523 sp-LHLL

524 Sp-LHLH

525 Sp-LHHL

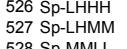

528 Sp-MMLL

530 Sp-MMH

531 Sp-MMHH
523 Sp-MMMM

534 Sp-HLLH

535 Sp-HLLH

536 SP-HLHH
537 SP.HLMM

538 Sp-LHLHH(b)

539 SP-MMLL(b)

540 Sp-MMHH(b)
541 Sp-HLLL(b)

542 nom-2

542
543 nom--3

54 nomc- 1
545 nomc-2

546 c106a-2

547 c c106a-3
548 c106-4

548 c106a-4
549 c c106b-1

550 c106b-2

551 az-3

552 az-5

$555 \mathrm{HG}-1-3-7$

$556 \mathrm{HG}-2-1.7$
$557 \mathrm{HG}-2-2.27$

558 HG-2-2-3

$559 \mathrm{H} G-3-3-17$
$560 \mathrm{GG}-3.2-7$

562 AH-165 Al-

563 AH-165 AV-7

565 AH-131 A-7

56 AH-131 AV- 7

567 AH-131 FE-7

569 AH-168 AV-T

577 AH-168 EA-7
571 A-200

57 AH-200 AA-7
572 AH-200 AV- 7

$573 \mathrm{AH}-200 \mathrm{FE}-7$

574 AH-202 Al-7 


\begin{tabular}{|c|c|c|}
\hline \\
\hline \multicolumn{3}{|l|}{$\begin{array}{l}\text { \# Glass ID } \\
575 \text { AH-202-AV-7 }\end{array}$} \\
\hline 577 АH-1-7 & DWPF PCT Model & Jantzen et al. 1995 \\
\hline 578 AH-2-2 & DWPF PCT Model & Jantzen et al. 1995 \\
\hline $\begin{array}{l}5 / 9 \text { AH-4-7 } \\
580 \text { AH-5-7 }\end{array}$ & $\begin{array}{l}\text { DWPF PCT Model } \\
\text { DWPF PCT Model }\end{array}$ & $\begin{array}{l}\text { Jantzen et al. } 1995 \\
\text { Jantzen et al. } 1995\end{array}$ \\
\hline 581 AH-6-7 & $\begin{array}{l}\text { DWPF PCT Model } \\
\text { DWF }\end{array}$ & $\begin{array}{l}\text { Janzen et al. } \\
\text { Jantzen et al. } 19995\end{array}$ \\
\hline 582 AH-7-7 & DWPF PCT Model & Jantzen et al. 1995 \\
\hline 583 АН-8-7 & DWPF PCT Model & Jantzen et al. 1995 \\
\hline 584 AH-9-7 & DWPF PCT Model & Jantzen et al. 1995 \\
\hline 585 АН-10-7 & DWPF PCT Model & Jantzen et al. 1995 \\
\hline $\begin{array}{l}586 \text { AA-11-7 } \\
587 \text { AH-12-7 }\end{array}$ & $\begin{array}{l}\text { DWPF PCT Model } \\
\text { DWP PCT Model }\end{array}$ & $\begin{array}{l}\text { Jantzen et al. } 1995 \\
\text { Janten et }\end{array}$ \\
\hline $\begin{array}{l}587 \text { AA-12-7 } \\
58 \text { AH-13-7 }\end{array}$ & $\begin{array}{l}\text { WWPF PCT Moddel } \\
\text { DWWF PCT Model }\end{array}$ & $\begin{array}{l}\text { Jantzen et al. } 1995 \\
\text { Jantzen et al. } 1995\end{array}$ \\
\hline $\begin{array}{l}588 \text { AA-13-7 } \\
589 \text { AH-14-7 }\end{array}$ & $\begin{array}{l}\text { DWPF PCT Model } \\
\text { DWPF PCT Model }\end{array}$ & $\begin{array}{l}\text { Jantzen et al. } 1995 \\
\text { Jantzen et al. } 1995\end{array}$ \\
\hline 590 AH-15-7 & DWPF PCT Model & $\begin{array}{l}\text { Janzene et a. } \\
\text { Jantzen et al. } 19955\end{array}$ \\
\hline 591 AH-16-7 & $\begin{array}{l}\text { DWPF PCT Model } \\
\text { DW }\end{array}$ & Jantzen et al. 1995 \\
\hline 592 AH-17-7 & DWPF PCT Model & Jantzen et al. 1995 \\
\hline 593 SFRIT1 & DWPF PCT Model & Jantzen et al. 1995 \\
\hline 594 SFRIT2 & DWPF PCT Model & Jantzen et al. 1995 \\
\hline 595 SFRIT3 & DWPF PCT Model & Jantzen et al. 1995 \\
\hline $596202 \mathrm{P}$ w/o Mn-7 & DWPF PCT Model & Jantzen et al. 1995 \\
\hline $\begin{array}{l}5972026 \text { W/o Mn-7 } \\
598008-7\end{array}$ & $\begin{array}{l}\text { DWPF PCT Model } \\
\text { DWPFE PCT }\end{array}$ & Jantzen et al. 1995 \\
\hline & & Jantzen et al. 1995 \\
\hline $\begin{array}{l}599 \text { NAS SRM 623-7 } \\
600165 \text { CGW STD-7 }\end{array}$ & DWPF PCT Model & Jantzen et al. 1995 \\
\hline 601 ARM-1-7 (4/88) & $\begin{array}{l}\text { DWPF PCT Model } \\
\text { DWPF PCT Mdel }\end{array}$ & $\begin{array}{l}\text { Jantzen et al. } 1995 \\
\text { Jantzen et }\end{array}$ \\
\hline $\begin{array}{l}602 \text { ARM-1-7 } \\
6(5 / 89)\end{array}$ & $\begin{array}{l}\text { DWPF PCT Model } \\
\text { DWF }\end{array}$ & $\begin{array}{l}\text { Jantzen et at. } 1.1995 \\
\text { Jantzen et al. } 1995\end{array}$ \\
\hline 603 ARM-1-7 (7/90) & DWPF PCT Model & $\begin{array}{l}\text { Janzzen et al. } 1995 \\
\text { Jantzen et al. } 1995\end{array}$ \\
\hline 604 ARM-1-7 (12/90) & $\begin{array}{l}\text { DWPF PCT Model } \\
\text { DCF }\end{array}$ & $\begin{array}{l}\text { Janzene et al. } 1995 \\
\text { Jantzen et al. } 1995\end{array}$ \\
\hline 605 ARM-1-7 (5/91) & $\begin{array}{l}\text { DWPF PCT Model } \\
\text { DCT }\end{array}$ & Jantzen et al. 1995 \\
\hline 606 ARM-1-7 (10/91) & DWPF PCT Model & Jantzen et al. 1995 \\
\hline 607 ARM-1-7 (10/92) & DWPF PCT Model & Jantzen et al. 1995 \\
\hline 608 ARM-1-7 (4/93) & DWPF PCT Model & Jantzen et al. 1995 \\
\hline 609 ARM-1-7 $(6 / 93)$ & DWPF PCT Model & Jantzen et al. 1995 \\
\hline $\begin{array}{l}610 \text { ARM-1-7 }(8 / 93) \\
611 \text { ARM-1-7 }\end{array}$ & $\begin{array}{l}\text { WWPF PCT Moddel } \\
\text { DWWF PCT Model }\end{array}$ & $\begin{array}{l}\text { Jantzen et al. } 1995 \\
\end{array}$ \\
\hline 612 T-ARM-1 & $\begin{array}{l}\text { DWPF PCT Model } \\
\text { DWPF }\end{array}$ & $\begin{array}{l}\text { Jantzen et al. } 1995 \\
\text { Jantzen et al a. } 1995\end{array}$ \\
\hline 613 SS-ARM-1 & $\begin{array}{l}\text { DWPF PCT Model } \\
\text { DWF }\end{array}$ & $\begin{array}{l}\text { Janzene et al. } 1995 \\
\text { Jantzen et al. } 1995\end{array}$ \\
\hline 614 SS-ARM-1 & DWPF PCT Model & Jantzen et al. 1995 \\
\hline 615 EA-1-7 & DWPF PCT Model & Jantzen et al. 1995 \\
\hline 616 EA-1-7 & DWPF PCT Model & Jantzen et al. 1995 \\
\hline 617 EA-2-7 & DWPF PCT Model & Jantzen et al. 1995 \\
\hline 618 EA-7 & DWPF PCT Model & Jantzen et al. 1995 \\
\hline 619 T-EA & DWPF PCT Model & Jantzen et al. 1995 \\
\hline 620 SS-EA-19 & DWPF PCT Model & Jantzen et al. 1995 \\
\hline 621 SS-EA-15 & DWPF PCT Model & Jantzen et al. 1995 \\
\hline 622 SS-EA-1-7 & DWPF PCT Model & Jantzen et al. 1995 \\
\hline 623 SS-EA-2-7 & DWPF PCT Model & Jantzen et al. 1995 \\
\hline $\begin{array}{l}624 \text { SRS-SEA-A-I } \\
625 \text { SRS-SEA-B-7 }\end{array}$ & $\begin{array}{l}\text { DWPF PCT Model } \\
\text { DWPP PCT Model }\end{array}$ & $\begin{array}{l}\text { Jantzen et al. } 1995 \\
\text { Jantzen et al. } 1995\end{array}$ \\
\hline 626 CUASEA-A-7 & $\begin{array}{l}\text { DWF PCT Model } \\
\text { DWPF PCT Model }\end{array}$ & \\
\hline 627 CUASEA-B-7 & $\begin{array}{l}\text { DWPF PCT Model } \\
\text { DWF }\end{array}$ & Jantzen et al. 1995 \\
\hline 628 131-TDS-EA-7 & $\begin{array}{l}\text { DWPF PCT Model } \\
\text { DW }\end{array}$ & Jantzen et al. 1995 \\
\hline 629 131-TDS-3A-SOPER-7 & DWPF PCT Model & Jantzen et al. 1995 \\
\hline 630 BLEND 1-7 & & Jantzen et al. 1995 \\
\hline 631 BLEND 1-7 (2) & DWPF PCT Model & Jantzen et al. 1995 \\
\hline 632 BLEND 1.6-7 & DWPF PCT Model & \\
\hline $\begin{array}{l}633 \text { BACH 1-7 } \\
634 \text { BATCH 1-7 (2) }\end{array}$ & $\begin{array}{l}\text { DWPF PCT Model } \\
\end{array}$ & $\begin{array}{l}\text { Jantzen et al. } 1995 \\
\end{array}$ \\
\hline 635 BATCH 1-1.6 & $\begin{array}{l}\text { DWPF PCT Model } \\
\text { DWPF PCT Model }\end{array}$ & $\begin{array}{l}\text { Jantzen et al. } 1995 \\
\text { Jantzen et al. } 1995\end{array}$ \\
\hline 636 BATCH 2-7 & $\begin{array}{l}\text { DWPF PCT Model } \\
\text { DWF }\end{array}$ & $\begin{array}{l}\text { Janzen eet al. } 1995 \\
\text { Jantzen et al. } 1995\end{array}$ \\
\hline 637 BATCH 2-7 (2) & DWPF PCT Model & Jantzen et al. 1995 \\
\hline 638 BATCH 2-1.6 & DWPF PCT Model & Jantzen et al. 1995 \\
\hline 639 ВАTCH 3-7 & DWPF PCT Model & Jantzen et al. 1995 \\
\hline 640 BATCH 3-7 (2) & DWPF PCT Model & Jantzen et al. 1995 \\
\hline 641 BATCH 3-7 (3) & DWPF PCT Model & Jantzen et al. 1995 \\
\hline 642 BACH 4-7 & $\begin{array}{l}\text { DWPF PCT Model } \\
\end{array}$ & $\begin{array}{l}\text { Jantzen et al. } 1995 \\
\end{array}$ \\
\hline $\begin{array}{l}\text { (6) } \\
644 \text { BATCH 4-7 (3) }\end{array}$ & & $\begin{array}{l}\text { Jantzen et al. } 1995 \\
\text { Jantzen et al. } 1995\end{array}$ \\
\hline $\begin{array}{l}644 \mathrm{HM}-1-7 \\
64-(3)\end{array}$ & $\begin{array}{l}\text { DWPF PCT Model } \\
\text { DWF }\end{array}$ & $\begin{array}{l}\text { Janzen eet a. } 1995 \\
\text { Jantzen et al. } 1995\end{array}$ \\
\hline $646 \mathrm{HM}-1-7$ (2) & $\begin{array}{l}\text { DWPF PCT Model } \\
\text { DWF }\end{array}$ & Jantzen et al. 1995 \\
\hline $647 \mathrm{HM}-1.6-7$ & DWPF PCT Model & Jantzen et al. 1995 \\
\hline 648 PUREX 1-7 & DWPF PCT Model & Jantzen et al. 1995 \\
\hline 649 PUREX 1-7 & DWPF PCT Model & Jantzen et al. 1995 \\
\hline 650 PUREX 1.6- & DWPF PCT Model & Jantzen et al. 1995 \\
\hline $\begin{array}{l}651 \text { PUREX SRSS } 1.6 \\
652 \text { PUREX SRST-4 }\end{array}$ & $\begin{array}{l}\text { DWPF PCT Model } \\
\text { DWWF PCT Model }\end{array}$ & $\begin{array}{l}\text { Jantzen et al. } 1995 \\
\text { Jantzen et al } 1995\end{array}$ \\
\hline 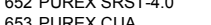 & DWPF PC Model & 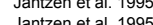 \\
\hline & $\begin{array}{l}\text { DWPF PCT Model } \\
\text { DWPF }\end{array}$ & Jantzen et al. 1995 \\
\hline $\begin{array}{l}654 \text { BLEND 1-345/ } \\
655 \text { BLEND 1-3479 }\end{array}$ & $\begin{array}{l}\text { DWPF PCT Model } \\
\text { DWP }\end{array}$ & Jantzen et al. 1995 \\
\hline 656 BLEND 1-3498 & DWPF PCT Model & Jantzen et al. 1995 \\
\hline
\end{tabular}

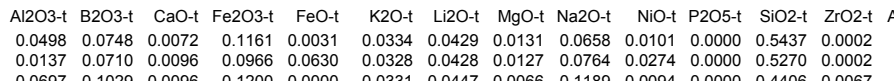

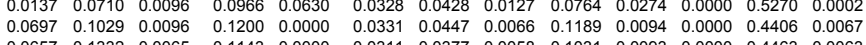

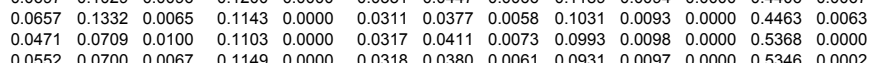

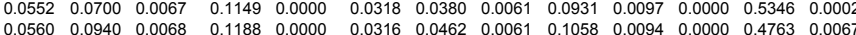

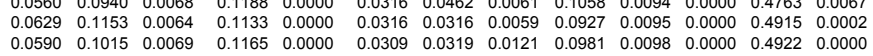

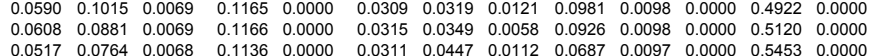
$\begin{array}{llllllllllllllll}0.0576 & 0.1214 & 0.0065 & 0.1113 & 0.0000 & 0.0303 & 0.0366 & 0.0112 & 0.0649 & 0.0094 & 0.0000 & 0.5099 & 0.0000\end{array}$ $\begin{array}{lllllllllllll}0.0608 & 0.0881 & 0.0069 & 0.1166 & 0.0000 & 0.0315 & 0.0349 & 0.0058 & 0.0926 & 0.0098 & 0.0000 & 0.5121 & 0.0000 \\ 0.0660 & 0.0653 & 0.0127 & 0.1386 & 0.0000 & 0.0311 & 0.0338 & 0.0050 & 0.0896 & 0.0116 & 0.0000 & 0.4989 & 0.0002\end{array}$

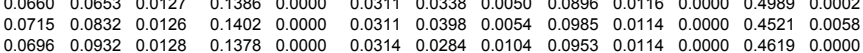
$\begin{array}{llllllllllllll}0.0696 & 0.0932 & 0.0128 & 0.1378 & 0.0000 & 0.0314 & 0.0284 & 0.0104 & 0.0953 & 0.0114 & 0.0000 & 0.4619 & 0.0000 \\ 0.0643 & 0.0728 & 0.0127 & 0.1354 & 0.0000 & 0.0310 & 0.0411 & 0.0101 & 0.0661 & 0.0111 & 0.0000 & 0.5077 & 0.0000\end{array}$ $\begin{array}{llllllllllllll}0.0578 & 0.0820 & 0.0066 & 0.1151 & 0.0000 & 0.0309 & 0.0473 & 0.0058 & 0.0667 & 0.0092 & 0.00000 & 0.5298 & 0.00066\end{array}$ $\begin{array}{lllllllllllll}0.0462 & 0.0855 & 0.0148 & 0.1428 & 0.00000 & 0.0271 & 0.0426 & 0.0085 & 0.1158 & 0.0111 & 0.00000 & 0.4813 & 0.0011 \\ 0.0462 & 0.0855 & 0.0148 & 0.1428 & 0.0000 & 0.0271 & 0.0326 & 0.0085 & 0.1158 & 0.0111 & 0.0000 & 0.4813 & 0.0011\end{array}$ $\begin{array}{lllllllllllll}0.0462 & 0.0855 & 0.0148 & 0.1428 & 0.0000 & 0.0271 & 0.0326 & 0.0085 & 0.1158 & 0.0111 & 0.0000 & 0.4813 & 0.0011 \\ 0.0462 & 0.0855 & 0.0148 & 0.1428 & 0.0000 & 0.0271 & 0.0326 & 0.0085 & 0.1158 & 0.0111 & 0.0000 & 0.4813 & 0.0011\end{array}$

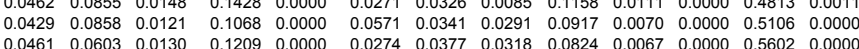
$\begin{array}{lllllllllllll}0.0461 & 0.0603 & 0.0130 & 0.1209 & 0.0000 & 0.0274 & 0.0377 & 0.0318 & 0.0824 & 0.0067 & 0.0000 & 0.5602 & 0.0000 \\ 0.0478 & 0.0979 & 0.0152 & 0.1272 & 0.0000 & 0.0362 & 0.0311 & 0.0129 & 0.1375 & 0.0055 & 0.0000 & 0.4666 & 0.0000\end{array}$

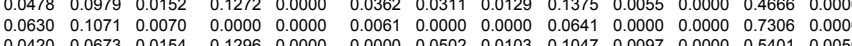

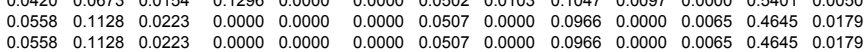

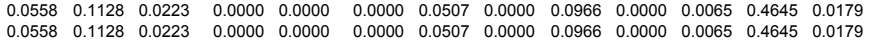

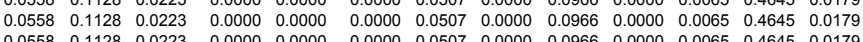

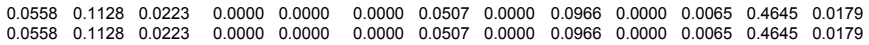
$\begin{array}{llllllllllllllll}0.0558 & 0.1128 & 0.0223 & 0.0000 & 0.0000 & 0.0000 & 0.0507 & 0.0000 & 0.0966 & 0.0000 & 0.0065 & 0.4645 & 0.0179\end{array}$

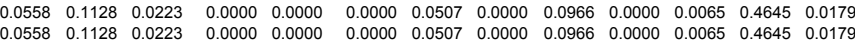
$\begin{array}{lllllllllllll}0.0558 & 0.1128 & 0.0223 & 0.0000 & 0.0000 & 0.0000 & 0.0507 & 0.0000 & 0.0966 & 0.0000 & 0.0065 & 0.4645 & 0.0179 \\ 0.0558 & 0.1128 & 0.0223 & 0.0000 & 0.0000 & 0.0000 & 0.0507 & 0.0000 & 0.0966 & 0.0000 & 0.0065 & 0.4645 & 0.0179\end{array}$ $\begin{array}{lllllllllllll}0.0558 & 0.1128 & 0.0223 & 0.00000 & 0.0000 & 0.0000 & 0.0507 & 0.0000 & 0.0966 & 0.0000 & 0.0065 & 0.4645 & 0.0179\end{array}$ $\begin{array}{lllllllllllll}0.0558 & 0.1128 & 0.0223 & 0.0000 & 0.0000 & 0.0000 & 0.0507 & 0.0000 & 0.0966 & 0.0000 & 0.0065 & 0.4645 & 0.0179 \\ 0.0558 & 0.1128 & 0.0223 & 0.0000 & 0.0000 & 0.0000 & 0.0507 & 0.0000 & 0.0966 & 0.0000 & 0.0065 & 0.4645 & 0.0179\end{array}$ $\begin{array}{llllllllllllll}0.0370 & 0.1130 & 0.0112 & 0.0738 & 0.0145 & 0.0005 & 0.0426 & 0.0172 & 0.1681 & 0.0057 & 0.0000 & 0.4873 & 0.0045\end{array}$

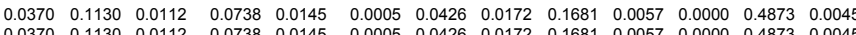

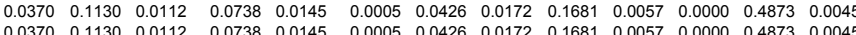
$\begin{array}{lllllllllllll}0.0370 & 0.1130 & 0.0112 & 0.0738 & 0.0145 & 0.0005 & 0.0426 & 0.0172 & 0.1681 & 0.0057 & 0.00000 & 0.4873 & 0.0045 \\ 0.0370 & 0.1130 & 0.0112 & 0.0738 & 0.0145 & 0.0005 & 0.0426 & 0.0172 & 0.1681 & 0.0057 & 0.0000 & 0.4873 & 0.0045\end{array}$ $\begin{array}{lllllllllllll}0.0370 & 0.1130 & 0.0112 & 0.0738 & 0.0145 & 0.0005 & 0.0426 & 0.0172 & 0.1681 & 0.0057 & 0.0000 & 0.4873 & 0.0045 \\ 0.0370 & 0.1130 & 0.0112 & 0.0738 & 0.0145 & 0.0005 & 0.0426 & 0.0172 & 0.1681 & 0.0057 & 0.0000 & 0.4873 & 0.0045\end{array}$ $\begin{array}{lllllllllllll}0.0370 & 0.1130 & 0.0112 & 0.0738 & 0.0145 & 0.0005 & 0.0426 & 0.0172 & 0.1681 & 0.0057 & 0.0000 & 0.4873 & 0.0045 \\ 0.0370 & 0.1130 & 0.0112 & 0.0738 & 0.0145 & 0.0005 & 0.0426 & 0.0172 & 0.1681 & 0.0057 & 0.0000 & 0.4873 & 0.0045\end{array}$ $\begin{array}{lllllllllllll}0.0370 & 0.1130 & 0.0112 & 0.0738 & 0.0145 & 0.0005 & 0.0426 & 0.0172 & 0.1681 & 0.0057 & 0.0000 & 0.4873 & 0.0045 \\ 0.0370 & 0.1130 & 0.0112 & 0.0738 & 0.0145 & 0.0005 & 0.0426 & 0.0172 & 0.1681 & 0.0057 & 0.0000 & 0.4873 & 0.0045\end{array}$ $\begin{array}{lllllllllllll}0.0370 & 0.1130 & 0.0112 & 0.0738 & 0.0145 & 0.0005 & 0.0426 & 0.0172 & 0.1681 & 0.0057 & 0.0000 & 0.4873 & 0.0045 \\ 0.0370 & 0.1110 & 0.0112 & 0.0738 & 0.0145 & 0.0005 & 0.0426 & 0.0172 & 0.1681 & 0.0057 & 0.0000 & 0.4873 & 0.0045 \\ 0.0370 & 0.1130 & 0.0112 & 0.0738 & 0.0145 & 0.0005 & 0.0426 & 0.0172 & 0.1681 & 0.0057 & 0.00000 & 0.4873 & 0.0045\end{array}$ $\begin{array}{lllllllllllll}0.0571 & 0.1259 & 0.0091 & 0.1418 & 0.0000 & 0.0004 & 0.0479 & 0.0152 & 0.1447 & 0.0143 & 0.0000 & 0.3947 & 0.0043 \\ 0.0548 & 0.1073 & 0.0091 & 0.1133 & 0.0000 & 0.0004 & 0.0418 & 0.0140 & 0.1361 & 0.153 & 0.0000 & 0.4590 & 0.0034\end{array}$

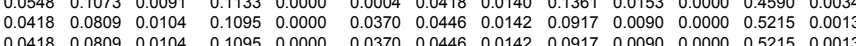

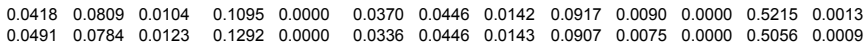

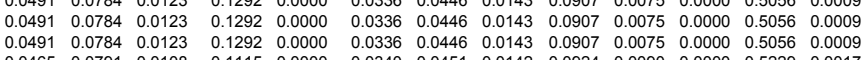
$\begin{array}{lllllllllllll}0.0465 & 0.0791 & 0.0108 & 0.1115 & 0.0000 & 0.0340 & 0.0451 & 0.0142 & 0.0924 & 0.0090 & 0.0000 & 0.5229 & 0.0017 \\ 0.0465 & 0.0791 & 0.0108 & 0.1115 & 0.0000 & 0.0340 & 0.0451 & 0.0142 & 0.0924 & 0.0090 & 0.0000 & 0.5229 & 0.0017\end{array}$ $\begin{array}{lllllllllllll}0.0465 & 0.0791 & .0108 & 0.1115 & 0.0000 & 0.0344 & 0.0451 & 0.0142 & 0.024 & 0.00990 & 0.00000 & 0.5229 & 0.0017 \\ 0.04656 & 0.0791 & 0.0108 & 0.1115 & 0.0000 & 0.0340 & 0.0451 & 0.0142 & 0.0924 & 0.0090 & 0.0000 & 0.5229 & 0.0017\end{array}$ $\begin{array}{lllllllllllll}0.0346 & 0.0775 & 0.0100 & 0.1181 & 0.0000 & 0.0343 & 0.0455 & 0.0143 & 0.0908 & 0.0106 & 0.0000 & 0.5303 & 0.0011\end{array}$ $\begin{array}{lllllllllllll}0.0346 & 0.0775 & 0.01100 & 0.1181 & 0.0000 & 0.0343 & 0.0455 & 0.0143 & 0.0908 & 0.0116 & 0.0000 & 0.5303 & 0.0011 \\ 0.0345 & 0.0819 & 0.0085 & 0.1180 & 0.0000 & 0.0388 & 0.0432 & 0.0144 & 0.0922 & 0.0107 & 0.00000 & 0.5042 & 0.0023\end{array}$ $\begin{array}{llllllllllllll}0.0345 & 0.0819 & 0.0085 & 0.1180 & 0.0000 & 0.0388 & 0.0432 & 0.0144 & 0.0922 & 0.0107 & 0.0000 & 0.5042 & 0.0023\end{array}$

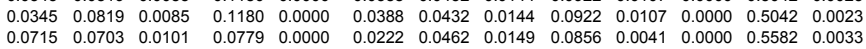
$\begin{array}{lllllllllllll}0.0715 & 0.0703 & 0.0101 & 0.0779 & 0.0000 & 0.0222 & 0.0462 & 0.0149 & 0.0856 & 0.0041 & 0.0000 & 0.5582 & 0.0033\end{array}$

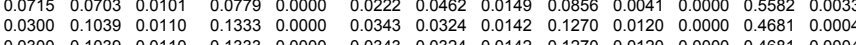
$\begin{array}{lllllllllllllllllllll}0.0300 & 0.1039 & 0.0110 & 0.1333 & 0.0000 & 0.0343 & 0.0324 & 0.0142 & 0.1270 & 0.01220 .00000 & 0.4681 & 0.0004\end{array}$ $\begin{array}{lllllllllllllll}0.0300 & 0.1039 & 0.0110 & 0.1333 & 0.0000 & 0.0343 & 0.0324 & 0.0142 & 0.1270 & 0.0120 & 0.00000 & 0.4681 & 0.00004\end{array}$ $\begin{array}{lllllllllllll}0.0300 & 0.1039 & 0.0110 & 0.1333 & 0.0000 & 0.0343 & 0.0324 & 0.0142 & 0.1270 & 0.0120 & 0.0000 & 0.4681 & 0.0004\end{array}$

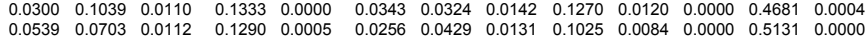

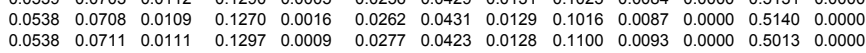

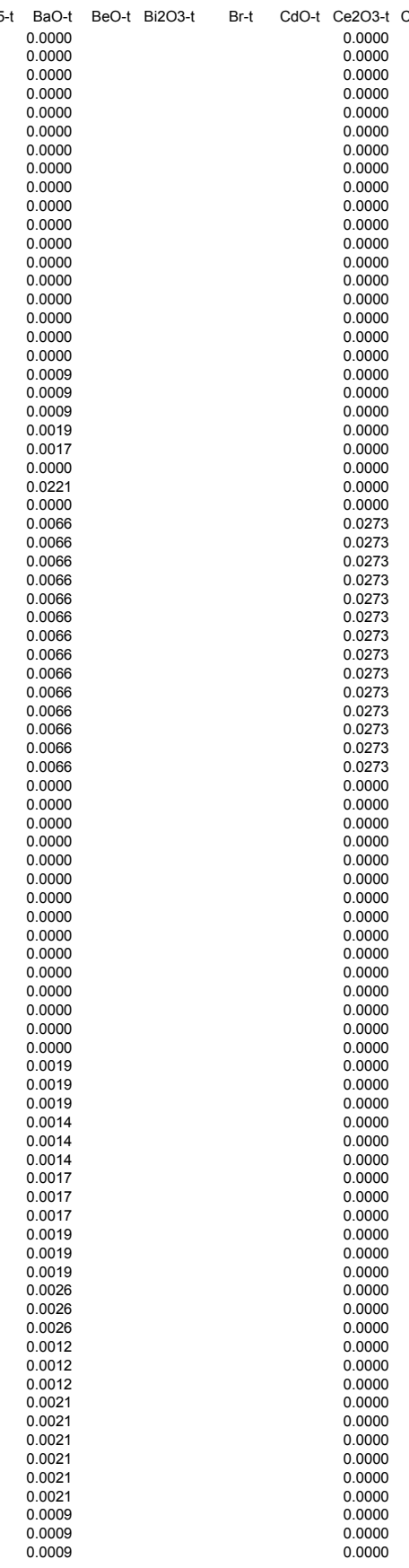


\# Glass ID 575 AH-202-AV-7
576 AH-202 FE-7

578 AH-2-7

579 AH-4-7

580 AH-5-7
581 AH-6-7
582 AH-7-7

583 AH-8-7

584 AH-9-7

585 AH-10-7

$587 \mathrm{AH}-12-7$

588 AH-13-7

590 AH-15-7

591 AH-16-7
592 AH-17-7

593 SFRIT1
594 SFRIT2

595 SFRIT3

$596202 \mathrm{P}$ w/o Mn-7
5972026 w/o Mn-7

$598200 R-7$
599 NBS SRM 623-7

599 NBS SRM 623-7

$\begin{array}{ll}601 \text { ARM-1-7 } & (4 / 88) \\ 602 \text { ARM-1-7 } & (5 / 89) \\ 603 \text { ARM-17 } & (1290)\end{array}$

\begin{tabular}{ll}
602 ARM-1-7 & $(5 / 89)$ \\
603 ARM-1-7 & $(7 / 90)$ \\
\hline 05 ARM &
\end{tabular}

$\begin{array}{ll}604 \text { ARM-1-7 } & (12 / 90) \\ 605 \text { ARM-1.7 } & (5 / 91)\end{array}$

(1)

607 ARM-1-7 (10/9)

609 ARM-1-7 $(6 / 93)$

612 T-ARM-1

613 SS-ARM-1
614 SS-ARM-1

$615 \mathrm{EA}-1-7$
$616 \mathrm{EA}-1-7$

616 EA-1-7
618 EA-7
$618-7$

618 EA-7
619 T-EA

620 SS-EA-19

622 SS-EA-1-7

624 SRS-SEA-A-7
625 SRS-SEA-B-7

625 SRS-SEA-B-7

626 CUASEA-A-7
627 CUASEA-B-7

628 131-TDS-EA-7

630 BLEND 1-7

631 BLEND 1-7
632 BLEND 1.6-7

633 BATCH $1-7$
634 BATCH $1-7(2)$

635 BATCH 1-7 1.6

636 BATCH 2-7
637 BATCH 2-7 (2)

638 BATCH 2-1.6
639 BATCH 3-7

640 BATCH 3-7 (2)
641 BATCH 3-7 (3)
642 BATCH $4-7$

643 BATC 4-7 (2)

645 HM-1-7

$646 \mathrm{HM}-1-7$
$647 \mathrm{HM}-16-7$

648 PUREX $1-7$
649 PUREX $1-7$

649 PUREX 1-7 (2)

651 PUREX SRSS 1.6
652 PUREX SRST-4.0

653 PUREX CUA

655 BLEND 1-3479
656 BLEND 1-3498
CoO-t Co2O3-t Cr2O3-t Cs2O-t CuO-t Dy203-t Eu2O3-t F-t Ga203-t Gd2O3-t HiO2-t HgO-t $\begin{array}{lll}0.0000 & 0.0000 & 0.0000 \\ 0.0000 & 0.0000 & 0.0000\end{array}$

$0.0002 \quad 0.0008 \quad 0.0000$

$\begin{array}{lll}0.0002 & 0.0004 & 0.0000 \\ 0.0005 & 0.0004 & 0.0000\end{array}$

$\begin{array}{lll}0.0000 & 0.00000 & 0.0000 \\ 0.0000 & 0.0004 & 0.0000\end{array}$

$0.0000 \quad 0.0000 \quad 0.0000$

$\begin{array}{lll}0.0002 & 0.0004 & 0.0000 \\ 0.0000 & 0.0004 & 0.0000\end{array}$

$\begin{array}{llll}0.0002 & 0.0004 & 0.0000 \\ 0 & 0.0000 & 0.0004 & 0.0000\end{array}$

$\begin{array}{llll}0.0000 & 0.0004 \quad 0.0000 \\ 0.0000 & 0.000 & 0.000\end{array}$

$\begin{array}{lll}0.0000 & 0.0004 & 0.00000 \\ 0.0000 & 0.00008 & 0.0000 \\ .0002 & 0.0004 & 0.000\end{array}$

$\begin{array}{llll}0.0002 & 0.0004 & 0.0000 \\ 0.0007 & 0.0004 & 0.0000\end{array}$

$.00090 .0004 \quad 0.0000$

0.00090 .00000 .0000

$\begin{array}{llll}0.0007 & 0.00013 & 0.00066 \\ 0.0007 & 0.0004 & 0.0040\end{array}$

$\begin{array}{llll}0.0000 & 0.0004 & 0.0040 \\ 0.0000 & 0.0000 & 0.0000 \\ 0 & 0.0000 & 0.0000 & 0.000\end{array}$

$\begin{array}{lll}0.0000 & 0.0000 & 0.0000 \\ 0.0000 & 0.0000 & 0.0000\end{array}$

$\begin{array}{lll}0.0000 & 0.0117 & 0.0000 \\ 0.0000 & 0.0117 & 0.0000\end{array}$

$\begin{array}{lll}0.0000 & 0.0117 & 0.0000 \\ 0.0000 & 0.0117 & 0.0000\end{array}$

$0.0000 \quad 0.01170 .0000$

$\begin{array}{lll}0.0000 & 0.0117 & 0.0000\end{array}$

$\begin{array}{llll}0.0000 & 0.0117 & 0.0000\end{array}$

$\begin{array}{lll}0.0000 & 0.0117 & 0.0000 \\ 0.0000 & 0.0117 & 0.0000\end{array}$

$\begin{array}{lll}0.0000 & 0.0117 & 0.0000 \\ 0.0000 & 0.0117 & 0.0000\end{array}$

$\begin{array}{llll}0.0000 & 0.0117 & 0.0000\end{array}$

$\begin{array}{llll}0.0000 & 0.0000 & 0.00000 \\ 0.0000 & 0.00000 & 0.0000\end{array}$

$\begin{array}{llll}0.0000 & 0.0000 & 0.0000 \\ 0.0000 & 0.0000 & 0.000000\end{array}$

$\begin{array}{lll}0.0000 & 0.0000 & 0.0000 \\ 0.0000 & 0.0000 & 0.0000\end{array}$

$\begin{array}{lll}0.0000 & 0.0000 & 0.00000 \\ 0.0000 & 0.0000 & 0.0000\end{array}$

$0.0000 \quad 0.0000 \quad 0.0000$

$\begin{array}{llll}0.0000 & 0.0000 & 0.0000 \\ 0.000 & 0.0000 & 0.000\end{array}$

$\begin{array}{llll}.0000 & 0.0000 & 0.0000 \\ .0000 & 0.0000 & 0.0000 \\ .0000 & 0.0000 & 0.0000\end{array}$

$\begin{array}{llll}0.0005 & 0.0000 & 0.0000\end{array}$

$\begin{array}{lll}0.0012 & 0.00000 & 0.0000 \\ 0.0014 & 0.0009 & 0.0044\end{array}$

$\begin{array}{ccc}0.0014 & 0.0009 & 0.0044 \\ 0.014 & 0.0009 & 0.0044 \\ 0.002 & 0.004 & 0.040\end{array}$

$\begin{array}{llll}0.0012 & 0.0004 & 0.0040 \\ 0.0012 & 0.0004 & 0.0040\end{array}$

$\begin{array}{llll}0.0012 & 0.0004 & 0.0040 \\ 0.0012 & 0.0004 & 0.0040\end{array}$

$\begin{array}{llll}0.0014 & 0.0000 & 0.0042 \\ 0.0014 & 0.0000 & 0.0042\end{array}$

$\begin{array}{llll}0.0014 & 0.0000 & 0.0042 \\ 0.0014 & 0.0004 & 0.004 \\ 0.0044 & 0.004 & 0.0041\end{array}$

$\begin{array}{llll}0.0014 & 0.0004 & 0.0041 \\ 0.0014 & 0.0004 & 0.0041\end{array}$

$\begin{array}{llll}0.0014 & 0.0004 & 0.0041\end{array}$

$\begin{array}{llll}0.0014 & 0.0009 & 0.0045\end{array}$

$\begin{array}{llll}0.0014 & 0.0009 & 0.0045 \\ 0.0010 & 0.0004 & 0.0025 \\ 0.010 & 0.004 & 0.025\end{array}$

$\begin{array}{lll}0.0010 & 0.00004 & 0.0025 \\ 0.0010 & 0.0004 & 0.025\end{array}$

$\begin{array}{llll}0.0016 & 0.0004 & 0.0042 \\ 0 & 0.0046 & 0.004 & 0.0042\end{array}$

$\begin{array}{llll}0.0016 & 0.0004 & 0.0042 \\ 0.0016 & 0.0004 & 0.0042\end{array}$

$0.0016 \quad 0.0004 \quad 0.0042$

$\begin{array}{llll}0.0016 & 0.0004 & 0.0042 \\ 0.0016 & 0.0004 & 0.0042 \\ 0.0023 & 0.000 & 0.022\end{array}$

$\begin{array}{lll}0.0023 & 0.0000 & 0.0018 \\ 0.0023 & 0.0000 & 0.0019\end{array}$
I-t La203-t MnO2-t MnO-t MoO-t MoO2-t MoO3-t Nb205-t Nd2O3-t NpO2-t PbO-t PdO2-t PdO-t Pr203-t Pr6011-t Rb2O-t ReO-t

$\begin{array}{lllll}0.0000 & 0.0261 & 0.0000 & 0.0000 & 0.0000 \\ 0.0000 & 0.0095 & 0.0000 & 0.000 & 0.0000\end{array}$

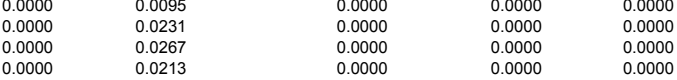

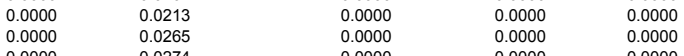

$\begin{array}{ll}0.0000 & 0.0274 \\ 0.0000 & 0.0262\end{array}$

0.0000

0.0000

$\begin{array}{ll}0.0000 & 0.033 \\ 0.0000 & 0.033 \\ 0 & 0.0025\end{array}$

0.0000
0.0000
0.0000

0.0000

$\begin{array}{ll}0.0000 & 0.0000 \\ 0.0000 & 0.0220 \\ 0\end{array}$

0.00000

$\begin{array}{ll}0.0000 & 0.0000 \\ 0.0000 & 0.0000 \\ 0 & 0.0000\end{array}$

$\begin{array}{ll}0.00000 & 0.0000 \\ 0.0000 & 0.0000 \\ 0 & 00000\end{array}$

$\begin{array}{ll}0.0000 & 0.0000 \\ 0.0000 & \end{array}$

$\begin{array}{ll}0.00000 & 0.0000 \\ 0.0000 & 0.0000 \\ 0 & 00000\end{array}$

$\begin{array}{ll}0.00000 & -0.0000 \\ 0.0000 & 0.0000\end{array}$

0.0000

$\begin{array}{ll}0.0000 & 0.0000 \\ 0.0000 & 0.0000 \\ 0 & 0.0004\end{array}$

0.0004

0.0042

0.0042

0.00042
0.042
0.042

0.0042

0.0004
0.002
0.002

$\underset{\substack{0.0042 \\ 0.0042}}{0.042}$

..0039

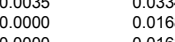

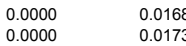

$\begin{array}{ll}0.0000 & 0.0173 \\ 0 & 0.0000\end{array}$

$\begin{array}{ll}0.0000 & 0.014 \\ 0.0000 & 0.014 \\ 0.014 & 0.000\end{array}$

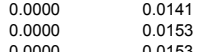

0.0000
0.0000
0.0000

$\begin{array}{ll} & 00000 \\ 0.0000 & 0.0255 \\ 0.0000 & 0.2255 \\ 0 & 0.0255\end{array}$

0.0000
0.0000
0.0000

0.0000
0.0000
0.0000

0.00000

0.00000

0.0000
0.0000

0.0000

0.0176
0.0176

0.0176
0.0170

0.0170
0.0170

0.0170
0.0170
0.0216

0.0220
0.0228

0.0000
0.0000

$\begin{array}{ll}0.0000 & 0.0000 \\ 0.0000 & 0.0000\end{array}$

00000 


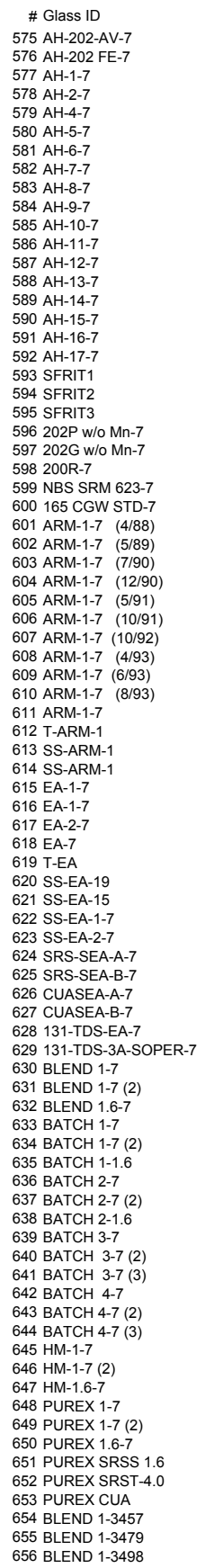

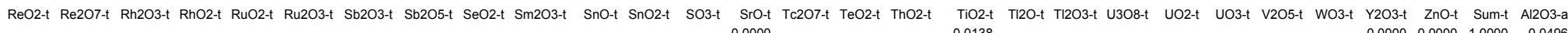

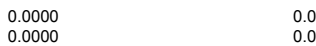

\begin{tabular}{|c|c|c|c|c|}
\hline & 0.0000 & 0.0000 & 1.0000 & 0.0136 \\
\hline 0.0133 & & & & \\
\hline 0.0130 & 0.0000 & 0.0000 & 1.0000 & 0.0655 \\
\hline $\begin{array}{l}0.0130 \\
0.0132\end{array}$ & 0.0000 & 0.0000 & 1.0000 & 0.0469 \\
\hline $\begin{array}{l}0.0132 \\
0.0141\end{array}$ & $\begin{array}{l}0.00000 \\
0.00000\end{array}$ & $\begin{array}{l}0.0000 \\
0.0000\end{array}$ & & $\begin{array}{l}0.0548 \\
0.0556\end{array}$ \\
\hline $\begin{array}{l}0.0129 \\
0.01\end{array}$ & $\begin{array}{l}0.00000 \\
0.0000\end{array}$ & $\begin{array}{l}0.0000 \\
0.0000\end{array}$ & $\begin{array}{l}1.00000 \\
1.0000\end{array}$ & $\begin{array}{l}0.05566 \\
0.0627\end{array}$ \\
\hline & & & & \\
\hline 0.0134 & & 0.0000 & & 0.0604 \\
\hline 0.0130 & 0.0000 & 0.0000 & 1.0000 & $\begin{array}{l}.000044 \\
0.0514\end{array}$ \\
\hline 0.0131 & 0.0000 & 0.0000 & 1.0000 & 0.0570 \\
\hline 0.0134 & 0.0000 & 0.0000 & 1.0000 & 0.0604 \\
\hline 0.0131 & 0.0000 & 0.0000 & 1.0000 & 0.0648 \\
\hline $\begin{array}{l}0.01133 \\
0.0136\end{array}$ & $\begin{array}{l}0.0000 \\
0.00000\end{array}$ & $\begin{array}{l}0.0000 \\
0\end{array}$ & 1.0000 & 0.0708 \\
\hline $\begin{array}{l}0.0116 \\
0.0132\end{array}$ & $\begin{array}{l}0.00000 \\
0.0000\end{array}$ & $\begin{array}{l}0.00000 \\
0.00000\end{array}$ & $\begin{array}{l}1.00000 \\
1.0000\end{array}$ & $\begin{array}{l}0.0687 \\
0.0636\end{array}$ \\
\hline & $\begin{array}{l}0.00000 \\
0.0000\end{array}$ & $\begin{array}{l}0.0000 \\
0.0000\end{array}$ & $\begin{array}{l}1.00000 \\
1.0000\end{array}$ & $\begin{array}{l}.00636 \\
0.0572\end{array}$ \\
\hline 0.0119 & 0.0000 & 0.0000 & 1.0000 & $\begin{array}{l}.000760 \\
0.047\end{array}$ \\
\hline 0.0119 & 0.0000 & 0.0000 & 1.0000 & \\
\hline 0.0119 & 0.0000 & 0.0000 & 1.0000 & \\
\hline 0.0124 & 0.0000 & 0.0000 & 1.0000 & 0.0430 \\
\hline 0.0068 & 0.0000 & & 1.0000 & 0.0469 \\
\hline 0.00000 & 0.0000 & 0.0000 & $\begin{array}{l}1.0000 \\
1\end{array}$ & 0.0462 \\
\hline $\begin{array}{l}0.00000 \\
0.0000\end{array}$ & $\begin{array}{l}0.00000 \\
0.0000\end{array}$ & $\begin{array}{l}0.0000 \\
0.0000\end{array}$ & $\begin{array}{l}1.00000 \\
1.0000\end{array}$ & $\begin{array}{l}0.0630 \\
0.0419\end{array}$ \\
\hline 0.0321 & 0.0000 & 0.0146 & 1.0000 & 0.0559 \\
\hline 0.0321 & 0.0000 & $\begin{array}{l}0.0146 \\
0.0146\end{array}$ & $\begin{array}{l}1.0000 \\
1\end{array}$ & \\
\hline $\begin{array}{l}0.0321 \\
0.0321\end{array}$ & $\begin{array}{l}0.00000 \\
0.0000\end{array}$ & $\begin{array}{l}0.0146 \\
0.0146\end{array}$ & $\begin{array}{l}1.00000 \\
1.0000\end{array}$ & \\
\hline 0.0321 & 0.0000 & 0.0146 & 1.0000 & \\
\hline 0.0321 & 0.00000 & 0.0146 & 1.0000 & \\
\hline 0.0321 & 0.0000 & & $\begin{array}{r}1.0000 \\
1.0000\end{array}$ & \\
\hline $\begin{array}{l}0.0321 \\
0.0321\end{array}$ & $\begin{array}{l}0.00000 \\
0.0000\end{array}$ & $\begin{array}{l}0.0146 \\
0.0146\end{array}$ & $\begin{array}{l}1.00000 \\
1.0000\end{array}$ & \\
\hline $\begin{array}{l}0.0321 \\
0.031\end{array}$ & & $\begin{array}{l}.0 .0140 \\
0.0146\end{array}$ & & \\
\hline 0.0321 & & 0.0146 & $\begin{array}{l}1.00000 \\
\end{array}$ & \\
\hline 0.0321 & 0.0000 & 0.0146 & 1.0000 & \\
\hline 0.0321 & & 0.0146 & 1.0000 & \\
\hline 0.0321 & 0.0000 & 0.0146 & 1.0000 & \\
\hline 0.0070 & 0.0000 & 0.0000 & 1.0000 & 0.0370 \\
\hline $\begin{array}{l}0.0070 \\
0.0770\end{array}$ & 0.0000 & 0.0000 & 1.0000 & \\
\hline 0.0070 & & $\begin{array}{l}0.0000 \\
0.0000\end{array}$ & $\begin{array}{l}1.00000 \\
1.0000\end{array}$ & \\
\hline 0.0070 & 0.0000 & $\begin{array}{l}0.0000 \\
0.0000\end{array}$ & $\begin{array}{l}1.1 .00000 \\
1.0000\end{array}$ & \\
\hline 0.0070 & 0.0000 & 0.0000 & $\begin{array}{l}1.00000 \\
1.0000\end{array}$ & \\
\hline 0.0070 & 0.0000 & 0.0000 & 1.0000 & \\
\hline 0.0070 & 0.0000 & 0.0000 & 1.0000 & \\
\hline 0.0070 & 0.0000 & 0.0000 & 1.0000 & \\
\hline 0.0070 & 0.0000 & 0.0000 & 1.0000 & \\
\hline 0.0070 & 0.0000 & & 1.0000 & \\
\hline $\begin{array}{l}0.0000 \\
0.0070\end{array}$ & $\begin{array}{l}0.00000 \\
0.0000\end{array}$ & $\begin{array}{l}0.0000 \\
0.0000\end{array}$ & $\begin{array}{l}1.00000 \\
1.0000\end{array}$ & \\
\hline $\begin{array}{l}0.0092 \\
0.009\end{array}$ & 0.00000 & $\begin{array}{l}.0 .00000 \\
0.000\end{array}$ & $\begin{array}{l}1.000000 \\
\end{array}$ & 0.0577 \\
\hline & & & 1.0000 & \\
\hline 0.0090 & 0.0000 & 0.0000 & 1.0000 & 0.0416 \\
\hline 0.0090 & 0.0000 & 0.0000 & 1.0000 & \\
\hline 0.0090 & 0.0000 & 0.0000 & 1.0000 & \\
\hline 0.0068 & 0.0000 & 0.0000 & 1.0000 & 0.0488 \\
\hline 0 & 0.00000 & 0.00000 & 1.0000 & \\
\hline $\begin{array}{l}0.0008 \\
0.0069\end{array}$ & 0.00000 & 0.0000 & $\begin{array}{r}1.0000 \\
1000\end{array}$ & \\
\hline $\begin{array}{l}0.0069 \\
0.009\end{array}$ & 0.0000 & 0.0000 & 1.0000 & \\
\hline 0.0069 & 0.0000 & & & \\
\hline 0.0069 & 0.0000 & 0.0000 & 1.0000 & 0.0344 \\
\hline 0.0069 & 0.0000 & & & \\
\hline 0.0069 & 0.0000 & 0.0000 & 1.0000 & \\
\hline 0.0104 & 0.0000 & 0.0000 & 1.0000 & 0.0343 \\
\hline 0.0104 & 0.0000 & 0.0000 & $\begin{array}{l}1.0000 \\
1\end{array}$ & \\
\hline $\begin{array}{l}0.0104 \\
0.0056\end{array}$ & $\begin{array}{l}0.00000 \\
0.0000\end{array}$ & $\begin{array}{l}0.0000 \\
0.0000\end{array}$ & $\begin{array}{l}1.00000 \\
1.0000\end{array}$ & 0.0715 \\
\hline 0.0056 & 0.0000 & 0.0000 & 1.0000 & \\
\hline 0.0056 & 0.0000 & & & \\
\hline 0.0068 & 0.0000 & 0.0000 & 1.0000 & 0.0299 \\
\hline $\begin{array}{l}0.0068 \\
0.0068\end{array}$ & 0.0000 & 0.0000 & 1.0000 & \\
\hline $\begin{array}{l}0.00088 \\
0.0068\end{array}$ & $\begin{array}{l}0.00000 \\
0.0000\end{array}$ & $\left\{\begin{array}{l}0.0000 \\
0.0000\end{array}\right.$ & $\begin{array}{l}1.00000 \\
1.0000\end{array}$ & \\
\hline 0.0068 & 0.0000 & 0.0000 & 1.0000 & \\
\hline 0.0068 & 0.0000 & 0.0000 & 1.0000 & \\
\hline 0.0024 & 0.0000 & 0.0000 & & \\
\hline 0.0023 & 0.0000 & 0.0000 & 1.0000 & 0.0515 \\
\hline & & & & \\
\hline
\end{tabular}


\# Glass ID

575 AH-202-AV-7

577 AH-1-17

578 AH-2-7

579 AH-4-7
580 AH-5-7

581 AH- $-6-7$

583 AH-8-7

584 AH-9-7

585 AH-10-7
586 AH-11-7
587 AH-127

$587 \mathrm{AH}-12-7$

589 AH-14-7

590 AH-15-7

592 AH-17-7
593 SFRIT1

594 SRRIT2

$596202 \mathrm{P}$ w/o Mn-7
$50202 \mathrm{G}$ w/o Mn-7

$9200 \mathrm{R}-7$
9

599 NBS SRM 623-7

001 ARM-1-7 (4/88)

602 ARM-1-7 (5/89)
603 ARM-1-7
$67190)$

604 ARM-1-7 (12/90)

$\begin{array}{ll}605 \text { ARM-1-7 } & (5 / 91) \\ 006 \text { ARM-1-7 } & (10 / 91)\end{array}$

606 ARM-1-7 (10/9)
007 ARM-1-7 (10/92)

600 ARM-1-7 (10/92)

609 ARM-1-7
610 ARM-1/93)
$(8 / 93)$

12 T-ARM- 1

613 SS-ARM-1

EA-1-7
6 EA-1-7

617 EA-2-7

618 EA-7

620 SS-EA-19
621 SS-EA-15

621 SS-EA-15

622 SS-EA-1-7

624 SRS-SEA-A-7

625 SRS-SEA-B-7

627 CUASEA-B-7
628 131-TDS-EA-7

629 131-TDS-3A-SOPER-7

31 BLEND 1-7

632 BLEND 1.6-7

33 BATCH 1-7

34 BATCH 1-7 (2)

636 BATCH 2-7
337 BATCH 2-7 (2)

38 BATCH 2-1.6

40 BATCH 3-7 (2)

641 BATCH 3-7 $(3)$
642 BATCH $4-7$

43 BATCH 4-7 (2)

644 BATCH 4-7 (3)

$645 \mathrm{HM}-1-7$
$646 \mathrm{HM}-17$

647 HM-1. $6-7$

648 PUREX 1-7
649 PUREX 1-7 (2)

649 PUREX 1-7 (2)
650 PUREX 1.6-7

651 PUREX SRSS 1.6
652 PUREX SRST-4.0

653 PURE C CUA

655 BLEND 1-3479
B2O3-a CaO-a Fe2O3-a FeO-a K2O-a Li2O-a MgO-a Na2O-a NiO-a P2O5-a SiO2-a Zro2-a Ag2O-a As2O3-a As205-a BaO-a BeO-a Bi2O3-a Br-a CdO-a Ce2O3-a CeO2-a Cl-a CoO-a Co203-a Cr2O3-a Cs2O-a CuO-a F-a $\begin{array}{lllllllll}0.0744 & 0.0072 & 0.1156 & 0.0031 & 0.0333 & 0.0427 & 0.0130 & 0.0655 & 0.0100 \\ 0.0708 & 0.0096 & 0.0962 & 0.0628 & 0.0328 & 0.0427 & 0.0126 & 0.0762 & 0.0273\end{array}$ $\begin{array}{ll}0.5410 & 0.000 \\ 0.5255 & 0.0002\end{array}$

$0.03110 .03760 .0058 \quad 0.1030 \quad 0.0093$

$0.0695 \quad 0.0066 \quad 0.1140 \quad 0.03160 .04100 .0073 \quad 0.09890 .0097$

$\begin{array}{llllllll}0.0933 & 0.0068 & 0.1180 & 0.0314 & 0.0459 & 0.0060 & 0.1050 & 0.0094\end{array}$

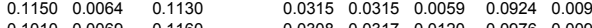

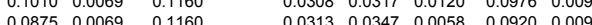

$\begin{array}{llllllllll}0.0759 & 0.0068 & 0.1130 & & 0.0309 & 0.0444 & 0.0111 & 0.0683 & 0.0096\end{array}$

$\begin{array}{lllllllll}0.1200 & 0.0065 & 0.1100 & & 0.0299 & 0.0362 & 0.0111 & 0.0642 & 0.0093 \\ 0.0875 & 0.0069 & 0.1160 & & 0.0313 & 0.0347 & 0.0058 & 0.0920 & 0.0097\end{array}$

$0.06410 .00125 \quad 0.1360$

$\begin{array}{lllll}0.0308 & 0.0394 & 0.0053 & 0.0976 & 0.0113 \\ 0.0310 & 0.0280 & 0.0102 & 0.0941 & 0.0113\end{array}$

$\begin{array}{lllll}0.0310 & 0.0280 & 0.0102 & 0.0941 & 0.0113 \\ 0.0306 & 0.0406 & 0.0100 & 0.0654 & 0.0110\end{array}$

$\begin{array}{llllll}0.0306 & 0.0469 & 0.0058 & 0.0661 & 0.0092 \\ 0.0270 & 0.0325 & 0.0084 & 0.1153 & 0.011\end{array}$

0.44550 .0063

$\begin{array}{lll}53308 & 0.000 \\ 4 & 0.001 \\ 0.430 & 0.0067 \\ 0.000 & 0.002\end{array}$

0.49000 .0001

$0.5420 \quad 0.0001$

0.50400 .0001

0.49000 .0003

$0.4480 \quad 0.0058$

0.45600 .0001

$\begin{array}{lll}0.5250 & 0.006 \\ 0.4790 & 0.001\end{array}$

$\begin{array}{lll}0.0812 & 0.0065 & 0.1140 \\ 0.0851 & 0.0147 & 0.1420\end{array}$

$\begin{array}{lllll}0.0573 & 0.0342 & 0.0292 & 0.0920 & 0.0070\end{array}$

0.5120
0.5700

$\begin{array}{lll}0.08600 & 0.0122 & 0.1070 \\ 0.0613 & 0.0132 & 0.1230 \\ 0.0946 & 0.0147 & 0.1230 \\ 0.067 & 0.130\end{array}$

$\begin{array}{lll}0.0946 & 0.0147 & 0.1230 \\ 0.1070 & 0.0070 & \end{array}$

$0.06710 .0154 \quad 0.1292$

$\begin{array}{lllll}0.0573 & 0.0342 & 0.0292 & 0.0920 & 0.0070 \\ 0.0278 & 0.0383 & 0.0324 & 0.0838 & 0.0068 \\ 0.0349 & 0.0301 & 0.0125 & 0.1329 & 0.0063 \\ 0.0060 & 0 & 0.0640 & 0.0053\end{array}$

0.4510

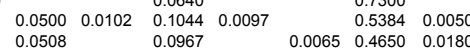

0.0220

0.0066

$\begin{array}{ll}0.0003 & 0.0007 \\ 0.0002 & 0.0005 \\ 0.0005 & 0.0006\end{array}$

0.000

$0.0002 \quad 0.0004$

$\begin{array}{ll}0.0001 & 0.0006 \\ 0.0001 & 0.0006 \\ 0.0001 & 0.0006\end{array}$

$\begin{array}{ll}0.0001 & 0.0005 \\ 0.0001 & 0.0006 \\ 0.0001 & 0.0005 \\ 0.000 & 0.003\end{array}$

$\begin{array}{ll}0.0001 & 0.0005 \\ 0.0008 & 0.0003\end{array}$

0.0009

$\begin{array}{lll}0.0007 & 0.0015 & 0.0063 \\ 0.0008 & 0.0005 & 0.0038\end{array}$

0.0116

$\begin{array}{lllllllll}0.1130 & 0.0112 & 0.0738 & 0.0145 & 0.0004 & 0.0426 & 0.0172 & 0.1680 & 0.0057\end{array}$

$0.4873 \quad 0.0046$

\begin{tabular}{|c|c|c|c|c|c|c|c|c|c|c|}
\hline $\begin{array}{l}0.1270 \\
0.1080\end{array}$ & $\begin{array}{l}0.0092 \\
0.0092\end{array}$ & $\begin{array}{l}0.1430 \\
0.1140\end{array}$ & $\begin{array}{l}0.0004 \\
0.0004\end{array}$ & $\begin{array}{l}0.0483 \\
0.0421\end{array}$ & $\begin{array}{l}0.0153 \\
0.0141\end{array}$ & $\begin{array}{l}0.1460 \\
0.1370\end{array}$ & $\begin{array}{l}0.0144 \\
0.0153\end{array}$ & $\begin{array}{l}0.3980 \\
0.4620\end{array}$ & $\begin{array}{l}0.0043 \\
0.0034\end{array}$ & \\
\hline & & & & 0.0444 & 0.0141 & 0.0913 & & 0.5190 & 0.0014 & 0.0018 \\
\hline 0.0778 & 0.0122 & 0.1284 & 0.0333 & 0.0443 & 0.0142 & 0.0900 & 0.0075 & 0.5020 & 0.0010 & 0.0015 \\
\hline 0.0788 & 0.0108 & 0.1112 & 0.0338 & 0.0450 & 0.0142 & 0.0921 & 0.0090 & 0.5210 & 0.0017 & 0.0016 \\
\hline 0.0769 & 0.0099 & 0.1171 & 0.0340 & 0.0451 & 0.0142 & 0.0901 & 0.0105 & 0.5260 & 0.0012 & 0.0018 \\
\hline 0.0814 & 0.0084 & 0.1171 & 0.0386 & 0.0429 & 0.0143 & 0.0916 & 0.0106 & 0.5010 & 0.0022 & 0.0025 \\
\hline 0.0703 & 0.0101 & 0.0778 & 0.0221 & 0.0462 & 0.0149 & 0.0856 & 0.0041 & 0.5580 & 0.0033 & 0.0011 \\
\hline 0.1033 & 0.0109 & 0.1325 & 0.0341 & 0.0322 & 0.0141 & 0.1262 & 0.0119 & 0.4650 & 0.0005 & 0.0020 \\
\hline & & & 0.0247 & & 0.0126 & 0.0987 & 0.0081 & 0.4935 & & \\
\hline $\begin{array}{l}0.0688 \\
0.0682\end{array}$ & $\begin{array}{l}0.0104 \\
0.0107\end{array}$ & $\begin{array}{ll}0.1216 & 0.00006 \\
0.1243 & 0.0008\end{array}$ & $\begin{array}{l}0.0251 \\
0.0265\end{array}$ & 0.0406 & $\begin{array}{l}0.0124 \\
0.0122\end{array}$ & $\begin{array}{l}0.09 / 4 \\
0.1054\end{array}$ & $\begin{array}{l}0.00839 \\
0.0089\end{array}$ & $\begin{array}{l}0.4925 \\
0.4806\end{array}$ & & $\begin{array}{l}0.0010 \\
0.0010\end{array}$ \\
\hline
\end{tabular}

0.0004
0.0012
0.0013

$\begin{array}{lll}0.0012 & 0.0008 & 0.0042\end{array}$

$0011 \quad 0.0006 \quad 0.0038$

$0.0013 \quad 0.0002 \quad 0.0040$

$0.0014 \quad 0.0006 \quad 0.0038$

$0.0014 \quad 0.0009 \quad 0.0043$

$0.0009 \quad 0.0006 \quad 0.0024$

$0.0015 \quad 0.0006 \quad 0.0040$

$\begin{array}{ll}0.0023 & 0.0020 \\ 0.0022 & 0.0017 \\ 0.0023 & 0.0019\end{array}$ 
\# Glass ID

575 AH-202-AV-7
576 AH-202 FE-7

578 AH-2-7

579 AH-4-7

580 AH-5-7

582 AH-7-7 7

583 AH-8-7
584 AH-9-7

585 AH-10-7

$587 \mathrm{AH}-12-7$

589 AH-14-7

590 AH-15-7

591 AH-16-7

593 SFRIT1

595 SFRIT3

$596202 \mathrm{P}$ w/o Mn-7

$598200 R-7,7$

600165 CGW STD - 7

602 ARM-1-7
603 (5RM-1-7
6090

604 ARM-1-7 (12109)

$\begin{array}{ll}605 \text { ARM-1-7 } & (5 / 91) \\ 606 \text { ARM-1-7 } & (10 / 91)\end{array}$

607 ARM-1-7 $(10 / 92)$
608 ARM-1-7 $(4 / 93)$

609 ARM-1-7 (6/93)

6910 ARM-1-7
611 ARM-1-7
$6 / 993)$

612 T-ARM-1

613 SS-ARM-1

$615 \mathrm{EA}-1-7$
$616 \mathrm{EA}-1-7$

617 EA-2-7

618 EA-7
619 T-EA

620 SS-EA-19

622 SS-EA-1-7

624 SRS-SEA-A-7

625 SRS-SEA-B-7
626 CUASEA-A-7

627 CUASEA-7 7

628 131-TDS-EA-7
629 131-TDS-3A-SOPER-7

629 131-TDS-3A-SOPER-7

631 BLEND 1-7 (2)

632 BLEND 1.6-7

633 BATCH 1-7

634 BATCH 1-7 (2)

636 BATCH 2-7

637 BATCH 2-7 (2)
638 BATCH 2-1.6

639 BATCH 3-7

640 BATCH $3-7$ (2)

641 BATCH $3-7(3)$

643 BATCH 4-7 (2)

64 BATCH $4-7$ (3)

645 HM-1-7

646 HM-1-7 (2)
647 HM-1.6-7
648 PUREX

648 PUREX 1-7
649 PUREX 1-7 (2)

649 PUREX 1-7 (2)
650 PUREX 1.6-7

651 PUREX SRSS 1.6
652 PUREX SRST-4.

653 PUREX CUA
654 BLEND 1-3457

655 BLEND 1-3479

656 BLEND 1-3498
Ga2O3-a Gd2O3-a

I-a La2O3-a MnO2-a MnO-a MoO-a MoO3-a Nb2O5-a Nd2O3-a NpO2-a PbO-a PdO2-a PdO-a Pr2O3-a Pr6011-a PuO2-a Rb2O-a ReO-a ReO2-a Re207-a Rh203-a RhO2-a RuO2-a Sb203-a Sb205-a SeO2-a 0.0259
0.0095

ocose

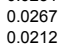

0.0264
0.0272

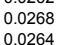

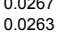

${ }_{0.034}^{0.0325}$

0.0322

0.021

0.0256

0.0167

0.0596

0.0042

0.0134

$\begin{array}{ll}0.0037 & 0.0317 \\ 0.0035 & 0.0336 \\ & 0.0167\end{array}$

$\begin{array}{lll}0.0336 & & \\ 0.0167 & 0.0015 & 0.0022\end{array}$

$\begin{array}{lll}0.0172 & 0.0011 & 0.0015\end{array}$

$\begin{array}{lll}0.0141 & 0.0017 & 0.0026\end{array}$

$\begin{array}{lll}0.0153 & 0.0012 & 0.0017\end{array}$

$\begin{array}{lll}0.0254 & 0.0020 & 0.0039\end{array}$

$\begin{array}{lll}0.0175 & 0.0022 & 0.0055\end{array}$

$\begin{array}{lll}0.0169 & 0.0008 & 0.0006\end{array}$

0.0207
0.0211
0.0219
0.0020 
$577 \mathrm{AH}-1-7$

578 AH-2-7

580 AH-5-7

582 AH-7-7 7

583 AH- $-8-7$

585 AH-10-7

$586 \mathrm{AH}-11-7$

587 AH-12-7

$589 \mathrm{AH}-14-7$

590 AH-15-7

591 AH-16-7

593 SFRII1
594 SRRTI2

S95 SFRIT3

$596202 \mathrm{P}$ w/ Mn-7
$59202 \mathrm{~W} / \mathrm{Mn}-7$

$598200 R-7$ NBS SRM $623-7$

600165 CGW STD-7
601 ARM-1-7 (4/88)

$\begin{array}{ll}602 \text { ARM-1-7 } & (5 / 89) \\ 603 \text { ARM-1.7 } & (7190)\end{array}$

604 ARM-1-7 (12/90)

605 ARM-1-7
606 ARM-1-7 (10191)
$60191)$

607 ARM-1-7 (10/92)

$\begin{array}{ll}609 \text { ARM-1-7 } & (6 / 93) \\ 610 \text { ARM-1-7 } & (8 / 93) \\ & \end{array}$

611 ARM-1-7
612 T-ARM-1

613 SS-ARM-1
614 SS-ARM-1

614 SS-ARM-1
615 EA-1-7

$616 \mathrm{EA}-1-7$
$617 \mathrm{EA}-2-7$

618 EA-7

620 SS-EA-19

621 SS-EA-19

622 SS-EA-1-7

624 SRS-SEA-A-7

626 CUASEA-A-A
627 CUASEA-B-7

6278 UA1-TDA-BA-7
629 131-TDS-3A-SOPER-7

629 131-TDS-3A-SOPER-7

631 BLEND 1-7 (2)

632 BLEND 1.6-7

633 BATCH 1-7
634 BATCH 1-7 (2)

633 BATCH 1-1.
636 BATCH 2.6

637 BATCH 2-7 (2)

639 BATCH 3-7

640 BATCH 3-7 (2)
641 BATCH 3-7 (3)

641 BATCH $3-7(3)$

643 BATCH 4-7 (2)

645 HM-1-7

$646 \mathrm{HM}-1-7$
$647 \mathrm{HM}-1.6-7$

648 PUREX 1-7

649 PUREX 1-7 (2)

651 PUREX SRSS 1.6

653 PURE C CUA

65 BLEND D-13479
656 BLEND 1-3498
Sum-a
0.9953
0.9970
1.0004

0.0137
0.0172

0.0172
0.0133
0.0130

0.0130

0.0140

0.0132

0.0130

0.0133

0.0132

0.0130
0.0130

0.0130
0.0118

0.0124
0.0070

0.0321

0.0004

0.0045

0.9983

0.9925
0.9932
0.932

0.995

0.9886

0.9823

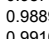

0.9952
0.0000
10000

1.0172
0.0669
0.969

0.0146

0.0070

0.0000

0.0000

0.0000
0.0000
0

0.0000
0.0000
0.0099
0.009

0.0000
0.0000
0.0000

0.0000
0.0000
0.0000

0.00000
0.0000

0.0000
0.0000
0.0000

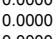

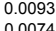

0.0074
0.0089

0.0069

0.0068

0.0103

0.0056

0.0068

0.0024
0.0022
0.0019
$\mathrm{C}_{650^{\circ} \mathrm{C}} \mathrm{C}_{700^{\circ} \mathrm{C}} \mathrm{C}_{750^{\circ} \mathrm{C}}$ 
578 AH-2.-7

580 AH-5.5-7
581 A-6-6

582 AH- -7.7
5 A -7.7

583 AH-8-7
584 AH-9-7

585 AH-10-7

586 AH-11-7

588 AH-12-7

589 AH-14-7

59 AH-16-7

593 SFRIT1

594 SFRIT2

$5972022 \mathrm{w}$ w/o Mn-7

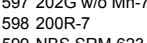

599 NBS SRM 623-7
600165 CGW STD-

601 ARM-1-7 (4/88)

602 ARM-1-7 (5/89)
603 ARM-1.7

604 A A MM-1.7 (12190)

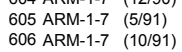

607 ARM-1-7 (10/92)
608 ARM-1-7 (4/93)

609 ARM-1-7.
610 ARM-193)
$61093)$

612 T-ARM-1

613 SS-ARM-1
614 SS-ARM-1
615 EA-1-7

616 EA-1-7
617 EA-2-7

618 EA-7

620 SS-EA-19

621 SS-EA-19
621 SS-EA-15
0.225 S

622
$6 S S-E A-1.7$
623
$6 S-E A-2-7$

624 SRS-SEA-A-7

625 SRS-SEA-B-7

626 CUASEA-A-7

628 131-TDS-EA-7

629 131-TDS-3A-SOPER-7

630 BLEND $1-7$
631 BLEND $1-7$ (2)

632 BLEND 1.6-7

633 BATCH 1-7

$634 \mathrm{BATCH} 1-7(2)$
635 BATCH $1-1.6$

636 BATCH $2-7$

637 BATCH 2-7 (2)
638 BATCH 2-1.6

638 BACH $2-1.6$
639 BATCH $3-7$

640 BATCH 3-7 (2)

641 BATCH $3-7(3)$

643 BATCH 4-7 (2)

644 BATC 4-7 (3)

$645 \mathrm{HM}-1-7$
$646 \mathrm{HM}-17$

$646-1 \mathrm{M}-1-7.7(2)$
$647 \mathrm{MM}-1.6-7$

648 PUREX 1-7
649 PUREX $1-7$ (2)

649 PUREX 1-7 (2)

655 PUER XSRSS 1.6
652 PUREX SRST

653 UUREX CUA

654 BLEND 1-3457

6555
656 BLEND $1-3498$ 
578 AH-2-7

580 AH-5.-7
581 AH-6-7

582 AH-7-7

583 AH-8-7
584 AH-9-7

585 AH-10-7

586 AH-11-7

587 AH-12-7

589 AH-14-7

590 AH-15-7

591 AH-16-7
592 AH-17-7

593 SFRIT1

594 SFRIT2

$5962022 \mathrm{~W} / \mathrm{Mn}-7$
5972026 W/o Mn-7

$5982000-7$

599 NBS SRM 623-7

601 ARM-1-7 (4/88)

602 ARM-1-7 (5/89)
603 ARM-1.7

604 A A MM-1.7 (12190)

605 ARM-1-7 $(5919)$
606 ARM-1-7 (1091)

607 ARM-1-7 (10/92)
608 ARM-1-7 (4/93)

609 ARM-1-7 (61933)

610 ARM-1-7
611 ARM-1-7
$(8 / 9)$

612 T-ARM-1

613 SS-ARM-1
614 SS-ARM-1

615 EA-1-7
616 EA-1-7
$612-7$

617 EA-2-2

618 EA-7
619 T-EA

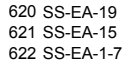

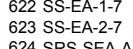

624 SRS-SEA-A-

625 SRS-SEA-B-7

626 CUASEA-A-7

628 131-TDS-EA-7

629 131-TDS-3A-SOPER-7
630 BLEND 1-7

631 BLEND $1-7$

632 BLEND 1.6-7

633 BATCH 1-7

634 BATCH 1-7 (2)

636 BATCH $2-7$

633 BATCH 2-1 16

638 BACH $2-1.6$
639 BATCH $3-7$

640 BATCH 3-7 (2)

641 BATCH $3-7(3)$

643 BATCH 4-7 (2)

644 BATC 4-7 (3)

$645 \mathrm{HM}-1-7$
$646 \mathrm{HM}-17$

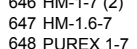

648 PUREX X-7
649 PUREX $1-7$ (2)

649 PUREX 1-7 (2)

655 PUER XSRSS 1.6
652 PUREXSRST-40

652
653 PUREXX CRSA
65 CUA

655 BLEND 1-3479

656 BLEND 1-3498 
582 AH-7-7 7

583 AH-8-7
584 AH-9-7
585 AH-10-7

584 AH-9-7
586 AH-10-7
$587-7$

$586 \mathrm{AH}-11-7$

587 AH-12-7
588 AH-13-7

588 AH-14-7
590 AH-15-7

$590 \mathrm{AH}-15-7$
$591 \mathrm{AH}-16-7$

592 AH-17-7

593 SFRIT1
594 SFRIT2
595 SFRIT3

$596202 \mathrm{P}$ w/ Mn-7
5972026 w/O Mn-7

$598200 R-7$

599 NBS SRM 623-7

$\begin{array}{ll}601 \text { ARM-1-7 } & (4 / 88) \\ 602 \text { ARM-1-7 } & (5 / 89) \\ \end{array}$

602 ARM-1-7 (5/89)

604 ARM-1-7 (12/90)

$\begin{array}{ll}605 \text { ARM-1-7 } & (5 / 91) \\ 606 \text { ARM-1-7 } & (10 / 91)\end{array}$

607 ARM-1-7 $(10 / 92)$
608 ARM-1-7 (4/93)

$\begin{array}{ll}609 \text { ARM-1-7 } & (6 / 93) \\ 610 \text { ARM-1-7 } & (8 / 93) \\ & \end{array}$

611 ARM-1-7

613 SS-ARM-1

614 SS-ARM-1
615 EA-1-7

616 EA-1-7
617 EA-2-7

618 EA-7

620 SS-EA-19

621 SS-EA-15

622 SS-EA-1-7

624 SRS-SEA-A-7
625 SRS-SEA-B-7

626 CUASEA-A-7

628 131-TDS-EA-7
629 131-TDS-3A-SOPER-7

629 131-TDS-3A-SOPER-

631 BLEND 1-7 (2)

632 BLEND 1.6-7

633 BATCH 1-7
633 BATCH 1-7 (2)

635 BATCH 1-1.6

636 BATCH 2-7
637 BATCH 2-7 (2)

638 BATCH 2-1.6

640 BATCH 3-7 (2)

641 BATCH 3.7 (3)

643 BATCH 47 (2)

644 BATCH 4-7 (3)

$646 \mathrm{HM}-1.7$

647 HM-1.6-7

648 PUREX $1-7$
649 PUREX $1-7$

652 PUREX SRST -4.

653 PUREX CUA

655 BLEND 1-3479
656 BLEND 1-3498

\begin{tabular}{|c|c|c|c|c|}
\hline & & & & \\
\hline 1.580 & 1.275 & 1.355 & 0.555 & 10.120 \\
\hline 0.290 & 0.355 & 0.365 & 0.220 & \\
\hline 0.475 & 0.475 & 0.435 & 0.120 & 9.950 \\
\hline 0.265 & 0.305 & 0.315 & 0.190 & 10.430 \\
\hline 0.210 & $\begin{array}{l}0.260 \\
0365\end{array}$ & $\begin{array}{l}0.245 \\
0350\end{array}$ & 0.150 & $\begin{array}{r}10.350 \\
0\end{array}$ \\
\hline $\begin{array}{l}0.345 \\
0.220\end{array}$ & $\begin{array}{l}0.365 \\
0.245\end{array}$ & $\begin{array}{l}0.350 \\
0.225\end{array}$ & $\begin{array}{l}0.195 \\
0.125\end{array}$ & $\begin{array}{c}10.610 \\
9.90\end{array}$ \\
\hline $\begin{array}{l}0.220 \\
0.250\end{array}$ & $\begin{array}{l}0.245 \\
0.275\end{array}$ & $\begin{array}{l}0.225 \\
0.245\end{array}$ & $\begin{array}{l}0.125 \\
0.150\end{array}$ & $\begin{array}{r}9.970 \\
10.050\end{array}$ \\
\hline & $\begin{array}{l}0.225 \\
0.255\end{array}$ & $\begin{array}{l}0.245 \\
0.230\end{array}$ & 0.140 & \\
\hline 0.215 & 0.260 & 0.215 & 0.155 & 10.060 \\
\hline 0.235 & 0.285 & 0.225 & 0.135 & 9.800 \\
\hline 0.425 & 0.305 & 0.365 & 0.095 & 10.120 \\
\hline 0.215 & 0.260 & 0.235 & 0.145 & 10.330 \\
\hline 0.260 & 0.290 & 0.305 & 0.155 & 10.660 \\
\hline $\begin{array}{l}0.215 \\
0.210\end{array}$ & $\begin{array}{l}0.240 \\
0.240\end{array}$ & $\begin{array}{l}0.225 \\
0.220\end{array}$ & $\begin{array}{l}0.120 \\
0.140\end{array}$ & $\begin{array}{l}10.180 \\
10\end{array}$ \\
\hline $\begin{array}{l}0.270 \\
0.180\end{array}$ & $\begin{array}{l}0.240 \\
0.225\end{array}$ & $\begin{array}{l}0.220 \\
0.185\end{array}$ & $\begin{array}{l}0.140 \\
0.145\end{array}$ & $\begin{array}{l}10.120 \\
10.090\end{array}$ \\
\hline $\begin{array}{l}0.100 \\
0.375\end{array}$ & & $\begin{array}{l}.100 \\
0.355\end{array}$ & $\begin{array}{l}0.145 \\
0.095\end{array}$ & $\begin{array}{r}10.0000 \\
9.740\end{array}$ \\
\hline 0.365 & 0.350 & 0.350 & 0.175 & 10.090 \\
\hline 0.380 & 0.410 & 0.435 & 0.215 & 10.210 \\
\hline 0.320 & 0.255 & 0.370 & 0.195 & 9.950 \\
\hline 0.195 & 0.195 & 0.260 & 0.175 & 9.790 \\
\hline 0.575 & 0.485 & 0.505 & 0.230 & 10.540 \\
\hline $\begin{array}{l}0.055 \\
0.420\end{array}$ & $\begin{array}{l}0.005 \\
0.365\end{array}$ & $\begin{array}{l}0.075 \\
0.410\end{array}$ & $\begin{array}{l}0.045 \\
0.265\end{array}$ & $\begin{array}{r}8.480 \\
8\end{array}$ \\
\hline $\begin{array}{l}0.4290 \\
0.290\end{array}$ & $\begin{array}{l}0.365 \\
0.375\end{array}$ & $\begin{array}{l}0.410 \\
0.345\end{array}$ & $\begin{array}{l}0.265 \\
0.175\end{array}$ & $\begin{array}{l}10.320 \\
10.130\end{array}$ \\
\hline & 0.280 & & & \\
\hline 0.240 & 0.285 & 0.255 & 0.140 & $\begin{array}{r}9.640 \\
10.220\end{array}$ \\
\hline 0.250 & 0.295 & 0.260 & 0.140 & 10.520 \\
\hline 0.270 & 0.315 & 0.270 & 0.145 & 10.420 \\
\hline 0.255 & 0.300 & 0.270 & 0.140 & 10.330 \\
\hline 0.220 & 0.250 & 0.225 & 0.130 & 10.160 \\
\hline & 0.290 & 0.250 & 0.135 & 9.900 \\
\hline 0.235 & 0.260 & 0.240 & 0.130 & \\
\hline 0.205 & 0.235 & 0.215 & 0.120 & 10.240 \\
\hline 0.255 & 0.300 & 0.255 & 0.135 & 9.380 \\
\hline 0.245 & 0.300 & 0.265 & 0.135 & 10.320 \\
\hline 0.265 & 0.375 & 0.275 & 0.145 & 10.320 \\
\hline & 0.320 & 0.280 & 0.145 & 10.360 \\
\hline 7.000 & 3.925 & 5.760 & 2.330 & 11.800 \\
\hline 8.395 & 5.345 & 7.245 & 2.330 & 11.800 \\
\hline 7.605 & $\begin{array}{r}4.715 \\
4.945\end{array}$ & $\begin{array}{l}6.390 \\
6.945\end{array}$ & 2.005 & 11.870 \\
\hline $\begin{array}{l}8.860 \\
7.375\end{array}$ & $\begin{array}{l}4.945 \\
4.265\end{array}$ & $\begin{array}{l}6.9455 \\
5.96\end{array}$ & $\begin{array}{l}2.040 \\
1.710\end{array}$ & $\begin{array}{l}12.010 \\
118\end{array}$ \\
\hline 8.205 & $\begin{array}{l}4.500 \\
4.560\end{array}$ & 6.545 & $\begin{array}{l}1.750 \\
1.70\end{array}$ & \\
\hline 8.395 & 4.870 & 6.800 & 1855 & 11.950 \\
\hline 9.250 & 5.140 & 7.165 & 2.090 & 11.780 \\
\hline 8.880 & 4.885 & 6.860 & 2.005 & 11.750 \\
\hline 8.225 & 4.670 & 6.425 & 1.895 & 11.780 \\
\hline 8.495 & 4.560 & 6.755 & 1.960 & 11.780 \\
\hline $\begin{array}{l}8.425 \\
8.230\end{array}$ & $\begin{array}{r}4.835 \\
4605\end{array}$ & $\begin{array}{l}6.475 \\
6770\end{array}$ & $\begin{array}{l}1.935 \\
1.965\end{array}$ & $\begin{array}{l}11.770 \\
1177\end{array}$ \\
\hline $\begin{array}{l}8.230 \\
2.215\end{array}$ & $\begin{array}{l}4.6055 \\
1.925\end{array}$ & $\begin{array}{l}6.710 \\
2.140\end{array}$ & $\begin{array}{l}\text {. } 1.965 \\
1.190\end{array}$ & $\begin{array}{l}11.770 \\
10590\end{array}$ \\
\hline 1.405 & 1.225 & 1.150 & 0.445 & 10.570 \\
\hline 0360 & 0405 & 0390 & 0.20 & $\begin{array}{l}10.0220 \\
102\end{array}$ \\
\hline & 0.400 & 0.395 & 0.210 & 10.450 \\
\hline 0.360 & 0.380 & 0.375 & & 10.170 \\
\hline 0.365 & 0.405 & 0.400 & 0.210 & 10.310 \\
\hline 0.365 & 0.380 & 0.375 & 0.205 & 10.470 \\
\hline 0.355 & 0.380 & 0.370 & 0.215 & 10.190 \\
\hline $\begin{array}{l}0.330 \\
0.335\end{array}$ & $\begin{array}{l}0.385 \\
0.360\end{array}$ & $\begin{array}{l}0.365 \\
0.345\end{array}$ & $\begin{array}{l}0.200 \\
0.200\end{array}$ & 10.180 \\
\hline $\begin{array}{l}0.335 \\
0.320\end{array}$ & $\begin{array}{l}0.360 \\
0.355\end{array}$ & $\begin{array}{l}0.345 \\
0.335\end{array}$ & $\begin{array}{l}0.200 \\
0.200\end{array}$ & $\begin{array}{l}10.420 \\
10.090\end{array}$ \\
\hline 0 & 0 & 0 & 0.230 & $\begin{array}{l}10.099 \\
10310\end{array}$ \\
\hline 0.445 & 0.425 & 0.425 & 0.235 & 10.370 \\
\hline 0.425 & 0.425 & 0.415 & 0.240 & 10.110 \\
\hline 0.485 & 0.500 & 0.500 & 0.250 & 10.200 \\
\hline 0.450 & 0.445 & 0.435 & 0.230 & 10.430 \\
\hline 0.470 & 0.470 & 0.470 & 0.250 & 10.240 \\
\hline 0.230 & 0.310 & 0.245 & $\begin{array}{l}0.150 \\
0.150\end{array}$ & $\begin{array}{ll}10.090 \\
1\end{array}$ \\
\hline $\begin{array}{l}0.230 \\
0.215\end{array}$ & $\begin{array}{l}0.285 \\
0.280\end{array}$ & $\begin{array}{l}.220 \\
0.220\end{array}$ & $\begin{array}{l}0.150 \\
0.155\end{array}$ & $\begin{array}{r}10.280 \\
9.870\end{array}$ \\
\hline 1.095 & 0.930 & 1.045 & 0.405 & 10.600 \\
\hline 0.985 & 0.825 & 0.880 & 0.360 & 10.640 \\
\hline 2.230 & 1.625 & 2.130 & 0.585 & 10.880 \\
\hline 1.190 & 1.020 & 1.040 & 0.430 & 10.630 \\
\hline 1.130 & 0.980 & 0.995 & 0.425 & 10.620 \\
\hline 1.425 & 1.185 & 1.210 & 0.480 & 10.750 \\
\hline 0.300 & 0.335 & 0.325 & & 10.220 \\
\hline 0 & 0.020 & 0.73 & 0.100 & 10.260 \\
\hline
\end{tabular}


578 AH-2-7

580 AH-5-7
581 AH-6-7

581 AH- $6-7$
582 A-7-7

583 AH-8-7

585 AH-10-7

$586 \mathrm{AH}-11-7$

587 AH-12-7

589 AH-14-7

590 AH-15-7

591 AH-16-7

593 SFRIT1

594 SFRIT2

$5962022 \mathrm{~W} / \mathrm{Mn}-7$
5972026 W/o Mn-7

$5982000-7$

599 NBS SRM 623-7

601 ARM-1-7 (4/88)

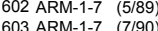

$\begin{array}{ll}603 \text { ARM-1-7 } & (7 / 190) \\ 604 \text { ARM- }-1-7 & (12 / 90)\end{array}$

$\begin{array}{ll}605 \text { ARM-1-7 } & (5 / 91) \\ 606 \text { ARM-1-7 } & (10 / 91)\end{array}$

607 ARM-1-7
608 ARM-1 1 (10/92)
$(4 / 93)$

609 ARM-1-7 (6/93)

6910 ARM-1-7
611 ARM-1-7
$6 / 993)$

612 T-ARM-1

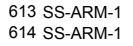

614 SS-ARM-1

616 EA-1-7
617 EA-2-7

618 EA-7

620 SS-EA-19

620 SS-EA-19
621 SS-EA-15
622 SS-EA-1-7

622 SS-EA-1-7

624 SRS-SEA-A-7

625 SRS-SEA-B-7

626 CUASEA-A-7

628 131-TDS-EA-7

$629131-$ TDS-3A-SOPER-7
630 BLEND $1-7$

630 BLEND 1-7

632 BLEND 1.6-7

633 BATCH 1-7

634 BATCH 1-7 (2)
635 BATCH 1-1.6

636 BATCH 2-7

637 BATCH 2-7 (2)
638 BATCH 2-1.6

638 BATCH 2-1.6
639 BATCH $3-7$

640 BATCH 3-7 (2)

641 BATCH $3-7(3)$

643 BATCH 4-7 (2)

64 BATCH $4-7$ (3)

$645 \mathrm{HM}-1-7$

647 HM-1. $6-7$

648 PUREX $1-7$

649 PUREX 1-7 (2)

651 PUREX SRSS 1

652 PUREX SRST -4.

653 PUREX CUA
654 BLEND 1-3457

655 BLEND 1-3479

656 BLEND 1-3498 


\begin{tabular}{|c|c|c|}
\hline \multirow{2}{*}{\multicolumn{2}{|c|}{$\begin{array}{l}\text { \# Glass ID } \\
657 \text { BLEND 1-3510 }\end{array}$}} & \\
\hline & -3510 & DWPF PCT Model \\
\hline 658 BLEND $1-$ & $1-3526$ & DWPF PCT Model \\
\hline 659 BLEND 2- & -3611 & DWPF PCT Model \\
\hline & & DWPF PCT Model \\
\hline 661 BLEND $2-$ & $\begin{array}{l}-3635 \\
-3635\end{array}$ & DWPF PCT Model \\
\hline 662 BLEND 2- & $\begin{array}{l}-3654 \\
-3656\end{array}$ & DWPF PCT Model \\
\hline $\begin{array}{l}663 \text { BLEND 2- } \\
664 \text { BLNDD 2- }\end{array}$ & $\begin{array}{l}-36666 \\
-3676\end{array}$ & $\begin{array}{l}\text { DWPF PCT Model } \\
\text { DWPF PCT Model }\end{array}$ \\
\hline $\begin{array}{l}664 \text { BLEND 2- } \\
665 \text { BLEND 3- }\end{array}$ & $\begin{array}{l}-3676 \\
-3768\end{array}$ & $\begin{array}{l}\text { DWPFF PCT Model } \\
\text { DWPF PCT Model }\end{array}$ \\
\hline $\begin{array}{l}6666 \text { BLEND 3- } \\
665-1\end{array}$ & -3789 & $\begin{array}{l}\text { DWFF PCT Model } \\
\text { DWPF PCT Mod }\end{array}$ \\
\hline 667 BLEND 3- & -3793 & DWPF PCT Model \\
\hline 668 BLEND 3- & $3802 \mathrm{~B}$ & DWPF PCT Model \\
\hline 669 HM 1-382 & & DWPF PCT Model \\
\hline 670 HM 1-382. & & DWPF PCT Model \\
\hline 671 HM 1-385 & & DWPF PCT Model \\
\hline 672 HM 1-3855 & 55 & DWPF PCT Model \\
\hline 677 HM-2-1 & $\begin{array}{l}\text { (3979C) } \\
(309 \mathrm{~A})\end{array}$ & DWPF PCT Model \\
\hline $\begin{array}{l}67 / 4 \mathrm{HM}-2-2 \\
675 \mathrm{HM}-2-3\end{array}$ & 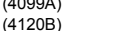 & DWPF PCT Model \\
\hline $676 \mathrm{HM}-3-1$ & 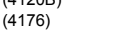 & $\begin{array}{l}\text { DWPF PCT Model } \\
\text { DWPF PCT Model }\end{array}$ \\
\hline $677 \mathrm{HM}-3-2$ & (4225) & DWPF PCT Model \\
\hline $678 \mathrm{HM}-3-3$ & $(4357)$ & DWPF PCT Model \\
\hline 679 HM-4-1 & & DWPF PCT Model \\
\hline $680 \mathrm{HM}-4-2$ & (5641) & DWPF PCT Model \\
\hline $681 \mathrm{HM}-4-3$ & (5748) & DWPF PCT Model \\
\hline 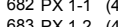 & (4643) & DWPF PCT Model \\
\hline $\begin{array}{l}6838 \times 1-2 \\
684 \mathrm{PX}^{2}-3\end{array}$ & $\begin{array}{l}(4726) \\
(4776)\end{array}$ & $\begin{array}{l}\text { DWPF PCT Model } \\
\text { DWPF PCT Model }\end{array}$ \\
\hline $685 \mathrm{PX}_{2-1}$ & (4455) & $\begin{array}{l}\text { DWPF PCT Model } \\
\text { DWPF PCT Mol }\end{array}$ \\
\hline 686 PX 2-2 & (4509) & DWPF PCT Model \\
\hline $687 \mathrm{PX} 2-3$ & $(4566)$ & DWPF PCT Model \\
\hline $688 \mathrm{PX} 3-1 \quad 5$ & (5780) & DWPF PCT Model \\
\hline $689 \mathrm{PX} 3-2 \quad(5$ & (5818) & DWPF PCT Model \\
\hline $\begin{array}{l}690 \mathrm{PX} 3-3 \\
691 \mathrm{PX} 4-1\end{array}$ & (5880) & $\begin{array}{l}\text { DWPF PCT Model } \\
\text { PWPF PCT Medel }\end{array}$ \\
\hline $\begin{array}{l}691 P X 4-1 \\
692 P \times 4-2\end{array}$ & $\begin{array}{l}(6390) \\
6634)\end{array}$ & DWPF PCT Model \\
\hline $\begin{array}{l}692 \mathrm{PX} 4-2 \\
693 \mathrm{PX} 4-3\end{array}$ & $\begin{array}{l}(6434) \\
6458)\end{array}$ & $\begin{array}{l}\text { DWPF PCT Model } \\
\text { DWPF PCT Mol }\end{array}$ \\
\hline 694 PX 5-1 6 & $\begin{array}{l}(64887) \\
(6787)\end{array}$ & DWPF PCT Model \\
\hline 695 PX 5-2 & $(6795)$ & DWPF PCT Model \\
\hline 696 PX 5-3 $\quad 6$ & (6812) & DWPF PCT Model \\
\hline 697 PX 5-4 & (6820) & DWPF PCT Model \\
\hline $698 \mathrm{PX} 5-5 \quad($ & (6839) & DWPF PCT Model \\
\hline 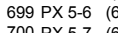 & (6862) & $\begin{array}{l}\text { DWPF PCT Model } \\
\text { PWPF PCT Medel }\end{array}$ \\
\hline 700 PX 5-7 & & \\
\hline 701 PX 5-8 & (6884) & DWPF PCT Model \\
\hline 702 PX 5-9 & $(6960)$ & DWPF PCT Model \\
\hline $\begin{array}{ll}703 \mathrm{PX} 5-10 \\
704 \mathrm{PX} 6-1\end{array}$ & (6972) & DWPF PCT Model \\
\hline 704 PX 6-1 & (7340) & DWPF PCT Model \\
\hline 705 ВАТСН 1 & ISTUD & DWPF PCT Model \\
\hline 706 BATCH 1 & $\begin{array}{l}1 \text { STUD } \\
\text { STUD }\end{array}$ & DWPF PCT Model \\
\hline $\begin{array}{l}707 \text { BATCH } 1 \\
708 \text { H-GLAS-0 }\end{array}$ & & $\begin{array}{l}\text { DWPF PCT Model } \\
\text { DWPF PCT Model }\end{array}$ \\
\hline 709 H-GLAS-0 0 & & DWPF PCT Model \\
\hline 710 H-GLAS-0 & & DWPF PCT Model \\
\hline 711 H-GLAS-0 & 0244 & DWPF PCT Model \\
\hline 712 H-GLAS-0 & 0254 & DWPF PCT Model \\
\hline $\begin{array}{l}713 \text { H-GLAS- } 0 \\
714 \text { H-GSAS- } 0\end{array}$ & 0278 & DWPF PCT Model \\
\hline $\begin{array}{l}114 \text { H-GASA- } 0 \\
715 \text { H-GLAS- } 0\end{array}$ & 0293 & DWPF PCT Model \\
\hline 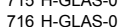 & 0.0334 & $\begin{array}{l}\text { DWPF Model } \\
\text { DWPF Pal }\end{array}$ \\
\hline 717 H-GLAS- 0 & 0352 & DWPF PCT Model \\
\hline 718 H-GLAS-0 0 & & DWPF PCT Model \\
\hline 719 H-GLAS-0 0 & & DWPF PCT Model \\
\hline 720 H-GLAS-0 & 0466 & DWPF PCT Model \\
\hline 721 FRIT-202- & 2-CLEAR & DWPF PCT Model \\
\hline 722 FRIT-202-T & -INT & DWPF PCT Model \\
\hline 723 FRIT-202- & 2-WHITE & DWPF PCT Model \\
\hline 724 FRIT-165- & & DWPF PCT Model \\
\hline 725 FRIT-131- & & DWPF PCT Model \\
\hline $\begin{array}{l}7766 \mathrm{MG} 9-7 \\
727\end{array}$ & & DWPF PCT Model \\
\hline $\begin{array}{l}727 \text { MG } 18-18 \\
728 \text { MG 25-7 }\end{array}$ & & $\begin{array}{l}\text { DWPF PCT Model } \\
\text { DWPF PCT Mol }\end{array}$ \\
\hline $729 \mathrm{MG} 28-7$ & & DWPF PCT Model \\
\hline 730 PBG3/Ce & & Plutonium Vitrification \\
\hline 731 PBG3/0x & & Plutonium Vitrification \\
\hline PBG3.5C & & Plutonium Vitrification \\
\hline SRC-Al-1 & & Plutonium Vitrification \\
\hline SRC-Al-2 & & onium Vitrification \\
\hline SRC-B-1 & & \\
\hline 736 SRC-B-2 & & Dlutonium Vitrification \\
\hline $\begin{array}{l}737 \text { SRC-B-3 } \\
738 \text { SRC-Ca-1 }\end{array}$ & & $\begin{array}{l}\text { Plutonium Vitrification } \\
\text { Plutonium Vitrification }\end{array}$ \\
\hline & & \\
\hline
\end{tabular}

Reference
Jantzen et al. 1995
Jantzen et al. 1995

Jantzen et al. 1995
Jantzen et al. 1995

Jantzen et al. 1995
Jantzen et al. 1995

Jantzen et ala. 1995
Jantzen et al. 1995

Jantzen et al. 1995

Jantzen et al. 1995

Jantzen et al. al. 1995

Jantzen et al. 1995
Jantzen et al. 1995

Jantzen et al. 1995

Jantzen et al. 1995

en et al. 1995

Jantzen et al. 1995
Jantzen et al. 1995

Jantzen et al. 1995
Jantzen et al. 1995

Jantzen et al. 1995
Jantzen et al. 1995

Jantzen et al. 1995
Jantzen et al. 1995

Jantzen et al. 1995
Jantzen et al. 1995

Jantzen et al. 1995
Jantzen et al. 1995

Jantzen et al. 1995
Jantzen et al. 1995

Jantzen et al. 1995

Jantzen et al. 1995

Jantzen et al. 1995

Jantzen et al. 1995

Jantzen et al. 1995

Jantzen et al. 1995

Jantzen et al. 1995
Jantzen et al. 1995

Jantzen et al. 1995

Jantzen et al. 1995
Jantzen et al. 1995

Jantzen et al. 1995

Jantzen et al. 1995

Janten et al.
Jantzen et al. 1995
J 1995

Jantzen et al. 1995
Jantzen et al. 1995

Jantzen et al. 1995
Jantzen et al. 1995

Jantzen et al. 1995

Jantzen et al. 1995

Jantzen et al. 1995
Jantzen et al. 1995

Jantzen et al. 1995

antzen et al. 1995

nntzen et al. 1995

Jantzen et al. 1995
Jantzen et al. 1995

Jantzen et al. 1995

Jantzen et al. 1995
Jantzen et al. 1995 Bulkey and Vienna 1997
Bukley and Vienna 1997 Bulkley and Vienna 1997
Bulkley and Vienna 1997 Bukley and Vienna 1997 Bukley and Vienna 1997
Bukley and Vienna 1997 Bukley and Vienna 1997
Bulkley and Vienna 1997
A203-t B2O3-t CaO-t Fe203-t FeO-t K2O-t Li2O-t MgO-t Na2O-t NiO-t P2O5-t SiO2-t ZrO2-t Ag2O-t As2O3-tAs2O5$\begin{array}{lllllllllllll}0.0544 & 0.0705 & 0.0111 & 0.1313 & 0.0009 & 0.0303 & 0.0421 & 0.0127 & 0.1091 & 0.0091 & 0.0000 & 0.4986 & 0.0000\end{array}$ $\begin{array}{lllllllllllll} & \end{array}$ $\begin{array}{lllllllllllll}0.0508 & 0.0700 & 0.0092 & 0.1201 & 0.0000 & 0.0241 & 0.0438 & 0.0133 & 0.1044 & 0.0097 & 0.0000 & 0.5264 & 0.0000 \\ 0.0512 & 0.0696 & 0.0093 & 0.1174 & 0.0000 & 0.0247 & 0.0440 & 0.0134 & 0.1051 & 0.0095 & 0.0000 & 0.5276 & 0.0000\end{array}$ $\begin{array}{lllllllllllll}0.0499 & 0.0694 & 0.0090 & 0.1181 & 0.0000 & 0.0250 & 0.0436 & 0.0132 & 0.1073 & 0.0098 & 0.0000 & 0.5264 & 0.0000 \\ 0.0502 & 0.0701 & 0.0090 & 0.1191 & 0.0000 & 0.0238 & 0.0442 & 0.0135 & 0.1061 & 0.0097 & 0.0000 & 0.5256 & 0.0000\end{array}$

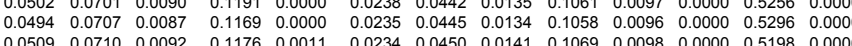

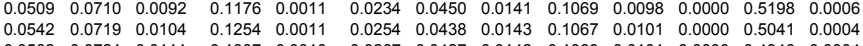

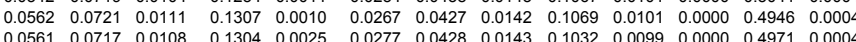
$\begin{array}{lllllllllllll}0.0561 & 0.0717 & 0.0108 & 0.1304 & 0.0025 & 0.0277 & 0.0428 & 0.0143 & 0.1032 & 0.0099 & 0.0000 & 0.4971 & 0.0004 \\ 0.0576 & 0.0696 & 0.0094 & 0.1091 & 0.0022 & 0.0194 & 0.0458 & 0.0163 & 0.0993 & 0.0079 & 0.0000 & 0.5327 & 0.0002\end{array}$ $\begin{array}{lllllllllllll}0.0576 & 0.0666 & 0.0094 & 0.1091 & 0.0022 & 0.0194 & 0.0458 & 0.01463 & 0.0993 & 0.00079 & 0.0000 & 0.5327 & 0.0002 \\ 0.0615 & 0.0680 & 0.0092 & 0.1102 & 0.0019 & 0.0170 & 0.0457 & 0.0164 & 0.0953 & 0.0079 & 0.0000 & 0.5348 & 0.00004\end{array}$ $\begin{array}{lllllllllllll}0.0624 & 0.0669 & 0.0094 & 0.1073 & 0.0018 & 0.0145 & 0.0456 & 0.0166 & 0.1012 & 0.0078 & 0.0000 & 0.5342 & 0.0004\end{array}$ $\begin{array}{lllllllllllll}0.0664 & 0.0662 & 0.0086 & 0.0990 & 0.0021 & 0.0139 & 0.0461 & 0.0165 & 0.1021 & 0.0069 & 0.0000 & 0.5397 & 0.0002 \\ 0.0750 & 0.0664 & 0.0075 & 0.0992 & 0.0000 & 0.0159 & 0.0439 & 0.0154 & 0.1017 & 0.0068 & 0.0000 & 0.5289 & 0.0008\end{array}$ $\begin{array}{lllllllllllll}0.0769 & 0.0666 & 0.0072 & 0.0989 & 0.0000 & 0.0182 & 0.0435 & 0.0151 & 0.1037 & 0.00066 & 0.00000 & 0.5235 & 0.00006\end{array}$ $\begin{array}{lllllllllllll}0.0748 & 0.0674 & 0.0070 & 0.0938 & 0.0000 & 0.0194 & 0.0463 & 0.0153 & 0.1052 & 0.0060 & 0.0000 & 0.5270 & 0.0008 \\ 0.0774 & 0.0646 & 0.0068 & 0.0951 & 0.0000 & 0.0217 & 0.0416 & 0.0148 & 0.1082 & 0.0058 & 0.0000 & 0.5281 & 0.0000\end{array}$ $\begin{array}{lllllllllllll}0.0774 & 0.0646 & 0.0068 & 0.0951 & 0.0000 & 0.0217 & 0.0416 & 0.0148 & 0.1082 & 0.0058 & 0.0000 & 0.5281 & 0.0006 \\ 0.0858 & 0.0669 & 0.0074 & 0.1001 & 0.0000 & 0.0221 & 0.0426 & 0.0154 & 0.1057 & 0.0059 & 0.0000 & 0.5093 & 0.0006\end{array}$ $\begin{array}{lllllllllllll}0.08858 & 0.0669 & 0.0074 & 0.1001 & 0.0000 & 0.0221 & 0.0426 & 0.4154 & 0.1057 & 0.0059 & 0.0000 & 0.50933 & 0.0000 \\ 0.0907 & 0.0670 & 0.0078 & 0.1041 & 0.0000 & 0.0226 & 0.0421 & 0.0158 & 0.1008 & 0.0060 & 0.0000 & 0.5027 & 0.0008\end{array}$ $\begin{array}{lllllllllllll}0.0528 & 0.0996 & 0.0050 & 0.0845 & 0.0000 & 0.0235 & 0.0446 & 0.0088 & 0.0946 & 0.0062 & 0.0000 & 0.5414 & 0.0119 \\ 0.0626 & 0.0919 & 0.0050 & 0.0794 & 0.0000 & 0.0269 & 0.0431 & 0.0105 & 0.0928 & 0.0098 & 0.0002 & 0.5396 & 0.0069\end{array}$ $\begin{array}{lllllllllllll}0.0566 & 0.09880 & 0.00049 & 0.0794 & 0.0000 & 0.0269 & 0.0431 & 0.015 & 0.0928 & 0.0098 & 0.0002 & 0.53396 & 0.0069 \\ 0.0727 & 0.0000 & 0.0279 & 0.0444 & 0.0117 & 0.0934 & 0.0052 & 0.0000 & 0.5631 & 0.0045\end{array}$

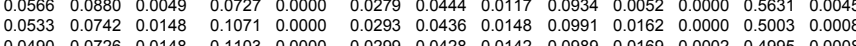

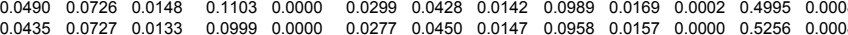

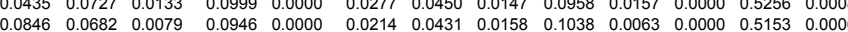
$\begin{array}{lllllllllllll}0.0114 & 0.1100 & 0.0000 & 0.0248 & 0.0432 & 0.0155 & 0.0955 & 0.0118 & 0.0000 & 0.5020 & 0.0000\end{array}$ $\begin{array}{lllllllllllll}0.0601 & 0.0744 & 0.0146 & 0.1095 & 0.0000 & 0.0303 & 0.0431 & 0.0151 & 0.0989 & 0.0152 & 0.0000 & 0.4907 & 0.0008 \\ 0.0409 & 0.0793 & 0.0066 & 0.0875 & 0.0000 & 0.0234 & 0.0463 & 0.0138 & 0.0851 & 0.0071 & 0.0002 & 0.5762 & 0.0070\end{array}$ $\begin{array}{lllllllllllll}0.0374 & 0.0770 & 0.0070 & 0.0953 & 0.0000 & 0.0221 & 0.0464 & 0.0142 & 0.0817 & 0.0076 & 0.0002 & 0.5765 & 0.0070\end{array}$ $\begin{array}{lllllllllllll}0.0310 & 0.0729 & 0.0072 & 0.0989 & 0.0000 & 0.0229 & 0.0474 & 0.0149 & 0.0886 & 0.0085 & 0.0000 & 0.5723 & 0.0089 \\ 0.0291 & 0.0819 & 0.0085 & 0.1063 & 0.0000 & 0.0268 & 0.0501 & 0.0169 & 0.0898 & 0.0096 & 0.0007 & 0.5423 & 0.0085\end{array}$

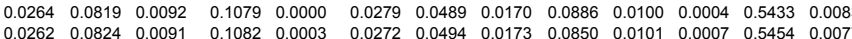
$\begin{array}{lllllllllllll}0.0358 & 0.0798 & 0.0092 & 0.1124 & 0.0000 & 0.0286 & 0.0447 & 0.0155 & 0.1164 & 0.0135 & 0.0000 & 0.4998 & 0.0095 \\ 0.0342 & 0.0801 & 0.0096 & 0.1197 & 0.0000 & 0.0333 & 0.0432 & 0.0149 & 0.1323 & 0.0131 & 0.0000 & 0.4761 & 0.0075\end{array}$ $\begin{array}{llllllllllllll}0.0304 & 0.0775 & 0.0093 & 0.1092 & 0.0000 & 0.0330 & 0.0437 & 0.0152 & 0.1373 & 0.0123 & 0.0000 & 0.4881 & 0.0093\end{array}$ $\begin{array}{lllllllllllll}0.0283 & 0.0806 & 0.0083 & 0.0955 & 0.0000 & 0.0287 & 0.0463 & 0.0157 & 0.12291 & 0.0116 & 0.0000 & 0.5166 & 0.0008\end{array}$

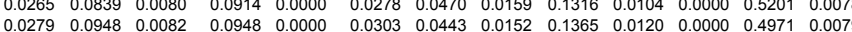
$\begin{array}{lllllllllllll}0.0268 & 0.1087 & 0.0076 & 0.0916 & 0.0000 & 0.0294 & 0.0438 & 0.0145 & 0.1364 & 0.0112 & 0.0000 & 0.4928 & 0.0075\end{array}$ $\begin{array}{lllllllllllll}0.0266 & 0.1095 & 0.0075 & 0.0872 & 0.0000 & 0.0279 & 0.0439 & 0.0145 & 0.1354 & 0.0111 & 0.0000 & 0.5000 & 0.0072 \\ 0.0207 & 0.0863 & 0.0084 & 0.0991 & 0.0000 & 0.0281 & 0.0487 & 0.0159 & 0.1090 & 0.0114 & 0.0000 & 0.5336 & 0.0101\end{array}$

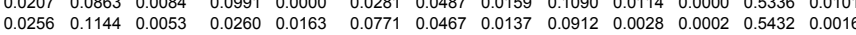

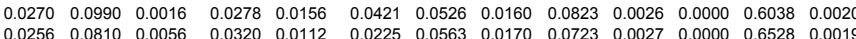
$\begin{array}{lllllllllllll}0.0256 & 0.0810 & 0.0056 & 0.0320 & 0.0112 & 0.0225 & 0.0563 & 0.0170 & 0.0723 & 0.0027 & 0.0000 & 0.6528 & 0.0019 \\ 0.0407 & 0.0826 & 0.0133 & 0.0984 & 0.0000 & 0.0159 & 0.0462 & 0.0136 & 0.0938 & 0.0145 & 0.0007 & 0.5295 & 0.0062\end{array}$ $\begin{array}{lllllllllllll}0.0407 & 0.0826 & 0.0133 & 0.0984 & 0.0000 & 0.0159 & 0.0462 & 0.0136 & 0.0938 & 0.0145 & 0.0007 & 0.5295 & 0.0062 \\ 0.0386 & 0.0945 & 0.0126 & 0.0945 & 0.0006 & 0.0162 & 0.0471 & 0.0116 & 0.0887 & 0.0131 & 0.0009 & 0.5374 & 0.0062\end{array}$ $\begin{array}{lllllllllllll}0.0324 & 0.1161 & 0.0078 & 0.0859 & 0.0002 & 0.0082 & 0.0479 & 0.0069 & 0.0799 & 0.0100 & 0.0009 & 0.5458 & 0.0261 \\ 0.0435 & 0.0940 & 0.0100 & 0.1102 & 0.0004 & 0.0144 & 0.0453 & 0.0100 & 0.0928 & 0.0091 & 0.0007 & 0.5236 & 0.0133\end{array}$

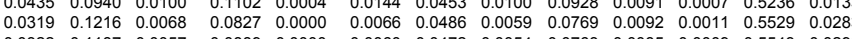

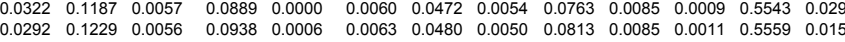
$\begin{array}{lllllllllllll}0.0310 & 0.1264 & 0.0058 & 0.0841 & 0.0002 & 0.0051 & 0.0503 & 0.0046 & 0.0791 & 0.0101 & 0.0011 & 0.5417 & 0.0323\end{array}$

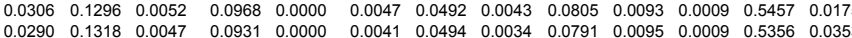

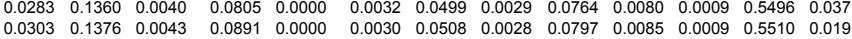

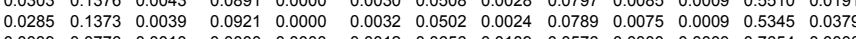

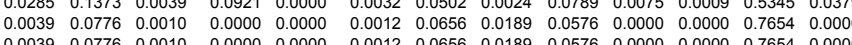

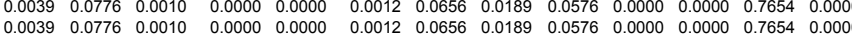
$\begin{array}{llllllllllllllll}0.0000 & 0.0939 & 0.0000 & 0.0000 & 0.0000 & 0.0000 & 0.0701 & 0.0100 & 0.1224 & 0.00000 & 0.0000 & 0.6937 & 0.0099\end{array}$

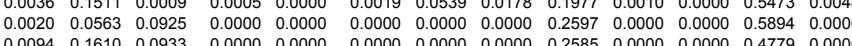

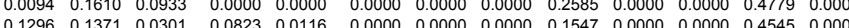
$\begin{array}{lllllllllllll}0.1463 & 0.0923 & 0.00000 & 0.1119 & 0.0215 & 0.0000 & 0.0000 & 0.0000 & 0.1685 & 0.00000 & 0.00000 & 0.4594 & 0.00000\end{array}$ $\begin{array}{lllllllllllllll}0.0500 & 0.0600 & 0.0490 & 0.0160 & & 0.0300 & 0.0400 & 0.0200 & 0.1000 & 0.0050 & 0.0030 & 0.4500 & 0.0300\end{array}$ $\begin{array}{llll}0.0500 & 0.0600 & 0.0490 & 0.0160 \\ 0.0500 & 0.0600 & 0.0490 & 0.0160\end{array}$

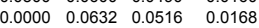
$\begin{array}{llll}0.1000 & 0.0568 & 0.0464 & 0.0152 \\ 0.0532 & 0.0000 & 0.0521 & 0.0170\end{array}$

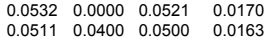

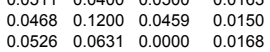
$\begin{array}{llllllll} & 0.000 \\ 030 & 0.0400 & 0.0200 & 0.1000 & 0.0050 & 0.0030 & 0.4500 & 0.030\end{array}$ $\begin{array}{lllllllll}0.0284 & 0.0379 & 0.0189 & 0.0947 & 0.0047 & 0.0028 & 0.4263 & 0.028\end{array}$ $\begin{array}{lllllllll}0.0319 & 0.0426 & 0.0213 & 0.1064 & 0.00053 & 0.0032 & 0.4787 & 0.0319\end{array}$

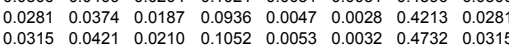

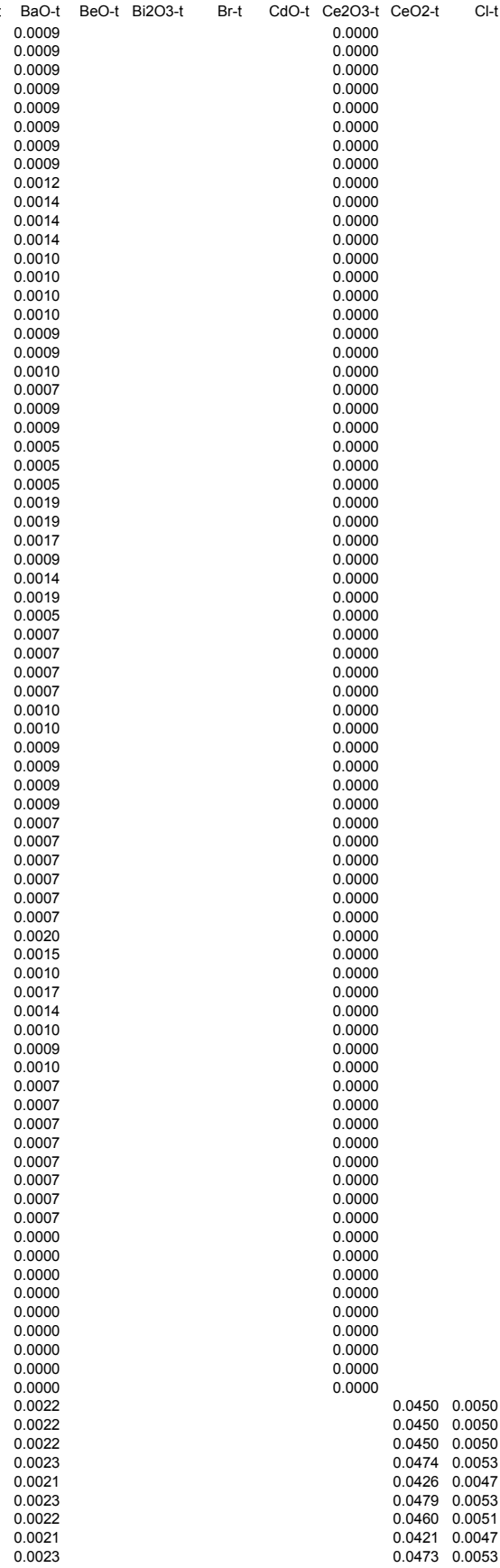




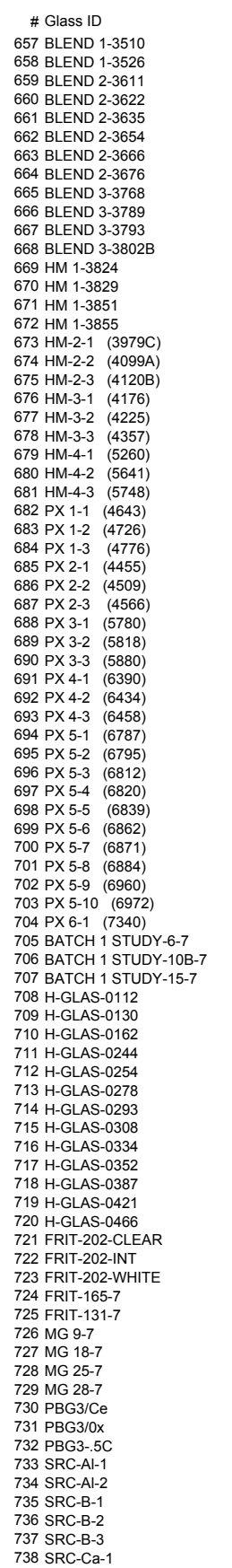

CoO-t Co2O3-t Cr2O3-t Cs2O-t CuO-t Dy2O3-t Eu2O3-t $\begin{array}{lll}0.0023 & 0.0000 & 0.0019 \\ 0.0021 & 0.0000 & 0.0018\end{array}$

$\begin{array}{llll}0.0026 & 0.0000 & 0.0017\end{array}$

$\begin{array}{lll}0.0023 & 0.0000 & 0.0017 \\ 0.0023 & 0.0000 & 0.0017\end{array}$

$\begin{array}{lll}0.0023 & 0.0000 & 0.0017 \\ 0.0026 & 0.0000 & 0.0017\end{array}$

$\begin{array}{lll}0.0026 & 0.0000 & 0.0017 \\ 0.0024 & 0.0000 & 0.0017\end{array}$

$\begin{array}{lll}0.0031 & 0.0000 & 0.0020 \\ 0.0026 & 0.0000 & 0.0021\end{array}$

$\begin{array}{llll}0.0025 & 0.0000 & 0.0023\end{array}$

$\begin{array}{lll}0.0025 & 0.0000 & 0.0022 \\ 0.0024 & 0.0000 & 0.0015\end{array}$

$\begin{array}{lll}0.024 & 0.0000 & 0.0015 \\ 0.024 & 0.0000 & 0.0015 \\ 0.021 & 0.0000 & 0.014\end{array}$

$\begin{array}{lll}0.0024 & 0.0000 & 0.0015 \\ 0.0021 & 0.0000 & 0.0014 \\ 0.0049 & 0.0000 & 0.0014\end{array}$

$0.0047 \quad 0.0000 \quad 0.00$

$0.0040 \quad 0.0000 \quad 0.0014$

$\begin{array}{llll}0.0040 & 0.0000 & 0.0015\end{array}$

$0.042 \quad 0.0000 \quad 0.0016$

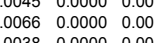

$\begin{array}{llll}0.0038 & 0.0000 & 0.0001 \\ 0.0047 & 0.0000 & 0.0031\end{array}$

$\begin{array}{lll}0.0054 & 0.0000 & 0.0032 \\ 0.0052 & 0.0000 & 0.0028\end{array}$

$0.0040 \quad 0.00000 .0016$

$0.0047 \quad 0.0000 \quad 0.0028$

$\begin{array}{lll}0.0034 & 0.0000 & 0.0019 \\ 0.0034 & 0.0000 & 0.0019 \\ 0.0031 & 0.000 & 0.019\end{array}$

$\begin{array}{lll}0.0031 & 0.0000 & 0.0019 \\ 0.036 & 0.0000 & 0.0029\end{array}$

$\begin{array}{llll}0.0036 & 0.0000 & 0.0029 \\ 0.0036 & 0.0000 & 0.0034\end{array}$

$\begin{array}{lll}0.0041 & 0.0004 & 0.0030\end{array}$

$\begin{array}{lll}0.0037 & 0.0004 & 0.0039\end{array}$

$\begin{array}{lll}0.0035 & 0.0009 & 0.0044 \\ 0.0042 & 0.0009 & 0.0043\end{array}$

$0.0038 \quad 0.0004 \quad 0.0042$

$\begin{array}{llll}0.0043 & 0.0004 & 0.0036 \\ 0 & 0.0034 & 0.0004 & 0.0036\end{array}$

$\begin{array}{llll}0.0034 & 0.0004 & 0.0036 \\ 0.0050 & 0.0004 & 0.0037\end{array}$

$\begin{array}{llll}0.0040 & 0.0004 & 0.0036\end{array}$

$\begin{array}{lll}0.0045 & 0.0004 & 0.0035 \\ 0.0038 & 0.0000 & 0.0028\end{array}$

$\begin{array}{lll}0.0005 & 0.0014 & 0.003\end{array}$

$\begin{array}{lll}0.0003 & 0.0005 & 0.0021 \\ 0.0045 & 0.0000 & 0.0026 \\ 0.0023 & 0000 & 0.021\end{array}$

$\begin{array}{llll}0.0043 & 0.0000 & 0.001\end{array}$

$\begin{array}{lll}0.0037 & 0.0000 & 0.0017 \\ 0.0038 & 0.0000 & 0.0011\end{array}$

$\begin{array}{llll}0.0038 & 0.0000 & 0.0012\end{array}$

$\begin{array}{lll}0.0038 & 0.0000 & 0.0010 \\ 0.0052 & 0.0000 & 0.0010\end{array}$

$\begin{array}{lll}0.0035 & 0.0000 & 0.0010 \\ 0.0045 & 0.0000 & 0.0007 \\ 0.0004 & 0.0000 & 0.0007\end{array}$

$\begin{array}{llll}0.0040 & 0.0000 & 0.0006\end{array}$

$\begin{array}{llll}0.0038 & 0.00000 & 0.0006\end{array}$

$0.0000 \quad 0.00890 .0000$

$\begin{array}{lll}0.0000 & 0.0089 & 0.0000 \\ 0.0000 & 0.0089 & 0.0000 \\ 0 & 0.0000 & 0.0000\end{array}$

$\begin{array}{lll}0.0000 & 0.0000 & 0.0000\end{array}$

$\begin{array}{llll}0.0000 & 0.0000 & 0.0000\end{array}$

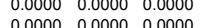

$\begin{array}{llll}0.0000 & 0.0000 & 0.0000\end{array}$

$\begin{array}{lll}0.0040 & 0.0100 & 0.0004 \\ 0.0040 & 0.0100 & 0.0004\end{array}$

$\begin{array}{lll}0.0040 & 0.0100 & 0.0004 \\ 0.0042 & 0.0105 & 0.0004\end{array}$

$\begin{array}{lll}0.0038 & 0.0095 & 0.0004\end{array}$

$\begin{array}{llll}0.0041 & 0.0102 & 0.0004\end{array}$

$\begin{array}{llll}0.0042 & 0.0105 & 0.0004\end{array}$

F-t Ga2O3-t Gd2O3-t HfO2-t HgO-t

It La203-t MnO2-t MnO-t MoO-t MoO2-t MoO3-t Nb205-t Nd203-t NpO2-t PbO-t PdO2-t PdO-t Pr2O3-t Pr6011-t Rb2O-t ReO-t

$\begin{array}{lll}0.0000 & 0.0229 & 0.0000\end{array}$

0.0000

0.0000

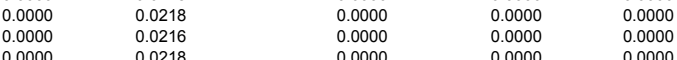

$\begin{array}{ll}0.0000 & 0.0218 \\ 0.0000 & 0.0220 \\ 0.0000 & 0.0215\end{array}$

0.0000

0.0000
0.0000
0.0000

0.0000

0.0000
0.0000

0.0000

0.0280

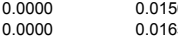

0.0000
0.0000

0.0000
0.0000
0.0000
.0000

$\begin{array}{lll}0 & 0.022 \\ 0.00000 & 0.028\end{array}$

$\begin{array}{ll}0.0000 & 0.0326 \\ 0.0000 & 0.0167 \\ & \end{array}$

0.0000

0.0000
0.0000
0.000

0.0000

0.0000
0.0000
0.0000

0.0000

0.0000
0.0000
0.0000
0.0000

0

0.0077

0.00005
0.0000
0.000
0.000

0.0025
0.0010
0.025

0.0025
0.0025
.0025

$0.025 \quad 0.00100$
0.0025

$0.0025 \quad 0.009$

$\begin{array}{ll}0.0025 & 0.0079 \\ 0.0020 & 0.0070 \\ 0.0020 & 0.0070 \\ 0 & 0.0005\end{array}$

$\begin{array}{ll}0.00025 & 0.0006 \\ 0.0000 & 0.0000 \\ 0 & 0.0000\end{array}$

$\begin{array}{ll}0.0000 & 0.0000 \\ 0.0000 & 0.0000 \\ 0 & 0.0000\end{array}$

$\begin{array}{ll}0.0000 & 0.0000 \\ 0.0000 & 0.0000 \\ 0.0000 & 0.0015\end{array}$

$\begin{array}{ll}0.0000 & 0.001 \\ 0.0000 & 0.0000 \\ 0.0000 & 0.0000 \\ 0.0000 & 0.0000 \\ 0.0000 & 0.000\end{array}$

$\begin{array}{ll}0.0010 & 0.0450 \\ 0.0010 & 0.0450 \\ 0.0010 & 0.0450 \\ 0.0011 & 0.0474 \\ 0.0009 & 0.0426 \\ 0.0011 & 0.0479 \\ 0.0010 & 0.0460 \\ 0.0009 & 0.0421 \\ 0.0011 & 0.0473\end{array}$


\# Glass ID

657 BLEND 1-3510

659 BLEND 2-3611

660 BLEND 2-3622

662 BLEND 2-3654

664 BLEND 2-3676

665 BLEND 3-3768

667 BLEND 3-37893

669 HM 1-3824
670 HM 1-3829

671 HM 1-3851

672 HM 1-3855

673 HM-2-1 (3979C)
674 HM-2-2
$(40999)$

$\begin{array}{lll}675 \mathrm{HM}-2-3 & \text { (4120B) } \\ 676 \mathrm{HM}-3-1 & (4176)\end{array}$

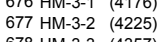

$\begin{array}{lll}678 \text { HM-3-3 } & (4357) \\ 679 H M-4-1 & (5260)\end{array}$

$680 \mathrm{HM}-4-2$
681
681

681 HM- $4-3$
682 PX $1-1(474843$

683 PX 1-2 (4726)

$\begin{array}{lll}684 \mathrm{PX} 1-3 & (4776) \\ 685 \mathrm{PX} 2-1 & (4455)\end{array}$

$\begin{array}{lll}685 & \text { PX 2-1 } & (4455) \\ 686 \text { PX 2-2 } & (4509)\end{array}$

687 PX 2-3 (4566)

$\begin{array}{lll}688 & \\ 6 \times 3-1 & (5780) \\ 689 & \text { PX } 32 & (5818)\end{array}$

$\begin{array}{lll}689 & \text { PX 3-2 (5818) } \\ 690 & \text { PX 3-3 } & (5880) \\ 9 & \text { PX } & \end{array}$

691 PX 4-1 (6390)

$\begin{array}{lll}692 & \text { PX 4-2 } & (6434) \\ 693 & \text { PX 4-3 } & (6458)\end{array}$

694 PX 5-1 (6787)

695 PX 5-2 (6795)

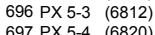

698 PX 5-5 (6839)

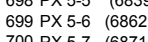

700 PX 5-7 (6871)

702 PX 5-9 $(6960)$
703 PX 5-10
$(6972)$

704 PX 6-1 (7340)

706 BATCH 1 STUDY-10B-7

707 BATCH 1 STUDY-15-7

708 H-GLAS-0112

710 H-GLAS-0162

712 H-GLAS-02254

713 H-GLAS-0278

714 H-GLAS-20293
715 H-GLAS-0308
716 HG

717 H-GLAAS-0334
717 -GLAS2
710 HGLAS-387

719 H-GLAS-0421

720 H-GLAS-0466
721 FRIT-202-CIEAR

722 FRIT-202-INT

723 FRITT-202-WHITE
724 RPIT-W

724 FRITT-165-7
725 FRIT-131-7

$726 \mathrm{MG} 9-7$

727 MG 18-7
728 MG 25-7

729 MG 25-7

$730 \mathrm{PBG} 3 / \mathrm{Ce}$

$731 \mathrm{PBG} 3 / 0 \mathrm{X}$

733 SRC-Al-1

734 SRC-Al-2
735 SRC-B-1

735 SRC-B-1

738 SRC-Ca-1
B2O3-a CaO-a Fe2O3-a FeO-a K2O-a Li2O-a MgO-a Na2O-a NiO-a P205-a SiO2-a Zro2-a Ag2O-a As2O3-a As205-a BaO-a BeO-a Bi203-a Br-a CdO-a Ce2O3-a CeO2-a Cl-a CoO-a Co203-a Cr203-a Cs2O-a CuO-a $\begin{array}{lllllllll}0.0674 & 0.0106 & 0.1256 & 0.0008 & 0.0291 & 0.0403 & 0.0122 & 0.1044 & 0.0087\end{array}$

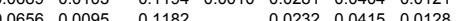

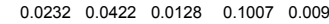

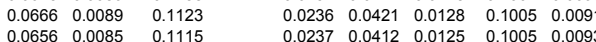
$\begin{array}{lllllllll}0.0670 & 0.0086 & 0.1137 & & 0.0228 & 0.0422 & 0.0129 & 0.1013 & 0.0092\end{array}$ $\begin{array}{llllllll}0.0676 & 0.0083 & 0.1119 & 0.0225 & 0.0426 & 0.0128 & 0.1012 & 0.0092\end{array}$

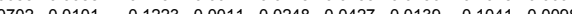
$\begin{array}{llllllllll}0.0701 & 0.0108 & 0.1269 & 0.0010 & 0.0260 & 0.0415 & 0.0138 & 0.1039 & 0.0098\end{array}$ $\begin{array}{lllllllll}0.0688 & 0.0104 & 0.1253 & 0.0024 & 0.0266 & 0.0411 & 0.0137 & 0.0992 & 0.0095\end{array}$

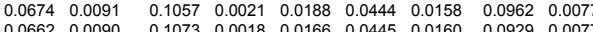
$\begin{array}{llllllllll}0.0651 & 0.0091 & 0.1045 & 0.0017 & 0.0142 & 0.0444 & 0.0162 & 0.0985 & 0.0076\end{array}$ $\begin{array}{lllllllll}0.0649 & 0.0084 & 0.0971 & 0.0021 & 0.0136 & 0.0452 & 0.0162 & 0.1001 & 0.0068\end{array}$ $\begin{array}{lllll}0.0185 & 0.0443 & 0.0154 & 0.1038 & 0.0070\end{array}$

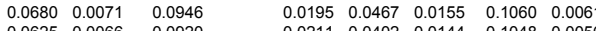

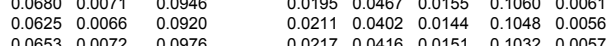
$\begin{array}{llllllllll}0.0661 & 0.0077 & 0.1027 & 0.0223 & 0.0415 & 0.0155 & 0.1095 & 0.0057\end{array}$ $\begin{array}{llllllll}0.1005 & 0.0050 & 0.0854 & 0.0237 & 0.0450 & 0.0090 & 0.0955 & 0.0062\end{array}$ $\begin{array}{lll}0.0952 & 0.0051 & 0.0823 \\ 0.0912 & 0.0051 & 0.0753\end{array}$ $\begin{array}{lll}0.0729 & 0.0146 & 0.1051\end{array}$ $\begin{array}{lll}0.0736 & 0.0150 & 0.1117 \\ 0.0725 & 0.133 & 0.0996\end{array}$ $\begin{array}{lll}0.0725 & 0.0133 & 0.0996 \\ 0.0687 & 0.0079 & 0.0952\end{array}$ $\begin{array}{lll}0.0719 & 0.0114 & 0.1099\end{array}$

$\begin{array}{lll}0.0740 & 0.0145 & 0.1090 \\ 0.021 & 0.0068 & 0.098\end{array}$

$\begin{array}{lll}0.0821 & 0.0068 & 0.0908 \\ 0.0806 & 0.0074 & 0.0999\end{array}$

$0.0690 \quad 0.0069 \quad 0.0938$

$\begin{array}{llll}0.0789 & 0.0081 & 0.1026\end{array}$

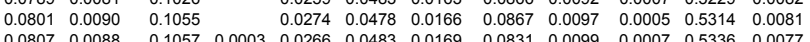
$\begin{array}{llllllllll}0.0807 & 0.0082 & 0.0951 & 0.0226 & 0.0488 & 0.0169 & 0.0338 & 0.0112 & 0.007439 & 0.0066\end{array}$ $\begin{array}{lllllllllll}0.0774 & 0.0090 & 0.1090 & & 0.0278 & 0.0434 & 0.0150 & 0.1129 & 0.0131 & 0.4847 & 0.0092\end{array}$

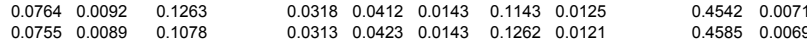
$\begin{array}{lllllllllll}0.0762 & 0.0091 & 0.1073 & 0.0325 & 0.0430 & 0.0150 & 0.1351 & 0.0121 & 0.4797 & 0.0092\end{array}$

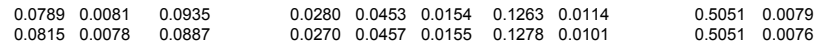
$\begin{array}{llll}0.0437 & 0.0149 & 0.1346 & 0.0119\end{array}$

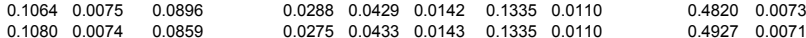

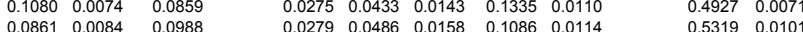
$\begin{array}{llllllllllll}0.0796 & 0.0152 & 0.1012 & 0.0372 & 0.0481 & 0.0366 & 0.0122 & 0.0952 & 0.0084 & 0.0003 & 0.4353 & 0.0048\end{array}$ $\begin{array}{llllllllllll}0.0970 & 0.0055 & 0.0120 & 0.0247 & 0.0413 & 0.0515 & 0.0157 & 0.0805 & 0.0025 & 0.0001 & 0.5910 & 0.0020\end{array}$

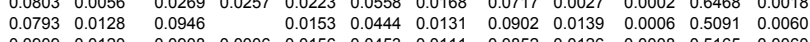
$\begin{array}{lllllllllllll}0.1104 & 0.0074 & 0.0818 & 0.0003 & 0.0078 & 0.0456 & 0.0065 & 0.0761 & 0.0095 & 0.0009 & 0.5195 & 0.0248\end{array}$ $\begin{array}{llllllllllll}0.0907 & 0.0096 & 0.1057 & 0.0004 & 0.0138 & 0.0436 & 0.0096 & 0.0890 & 0.0088 & 0.0007 & 0.5038 & 0.0129\end{array}$ $\begin{array}{llllllllllll}0.1132 & 0.0054 & 0.0845 & 0.0057 & 0.0450 & 0.0052 & 0.0726 & 0.0081 & 0.0008 & 0.5273 & 0.0279\end{array}$ $\begin{array}{llllllllllll}0.1143 & 0.0052 & 0.0874 & 0.0005 & 0.0059 & 0.0446 & 0.0047 & 0.0756 & 0.0079 & 0.0011 & 0.5168 & 0.0148 \\ 0.1218 & 0.0055 & 0.0811 & 0.0002 & 0.0050 & 0.0485 & 0.0045 & 0.0762 & 0.0097 & 0.0010 & 0.5217 & 0.0312 \\ 0.1241 & 0.0049 & 0.0925 & & 0.0045 & 0.0471 & 0.0041 & 0.0771 & 0.0089 & 0.0009 & 0.5223 & 0.167\end{array}$ $\begin{array}{lllllllllll}0.1265 & 0.0048 & 0.0854 & 0.0039 & 0.0473 & 0.0036 & 0.0761 & 0.0087 & 0.0010 & 0.5173 & 0.0338\end{array}$

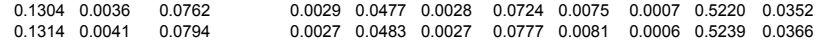

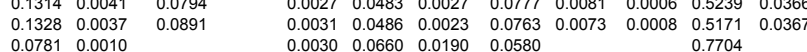

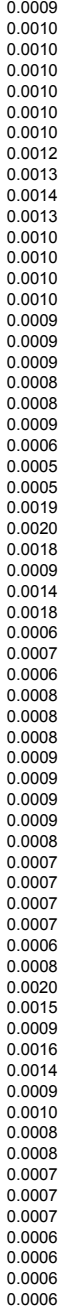

$\begin{array}{ll}0.6889 & 0.0099 \\ 0.5206 & 0.0046\end{array}$

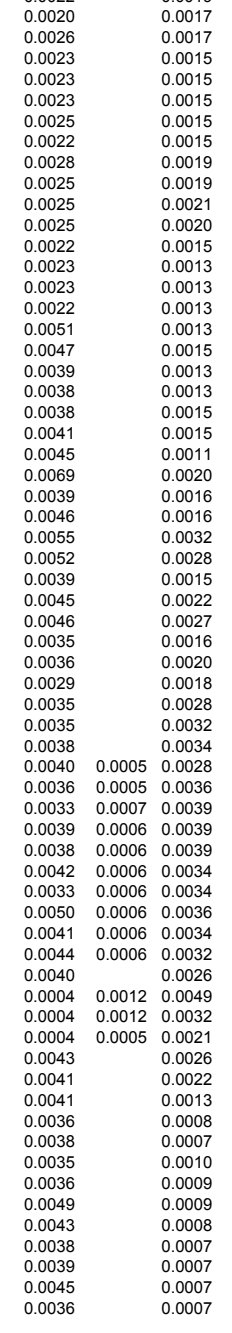


\# Glass ID 657 BLEND 1-3510 659 BLEND 2-3611 660 BLEND 2-3622 661 BLEND 2-3635
662 BLEND 2-3654 663 BLEND 2-3666 664 BLEND 2-3676 665 BLEND 3-3768 667 BLEND 3-3793 668 BLEND 3-3802 669 HM 1-3824
670 HM 1-3829

671 HM 1-3851

672 HM 1-3855
673 HM-2-1 (3979 $)$
674 HM-2-2 (4099)

675 HM-2-2
676 HM-3
$6774120 \mathrm{~B})$

$676 \mathrm{HM}-3-1$
$677 \mathrm{HM}-3-2$

678 HM-3-3 (4357)

680 HM-4-2 (5641)

$\begin{array}{ll}683 \mathrm{PX} 1-2 & (4726) \\ 684 \mathrm{PX} 1-3 & (4776)\end{array}$

685 PX 2-1 (4455)

687 PX 2-3 (4566)

$\begin{array}{lll}688 & P X 3-1 & (5780) \\ 689 & P X 3-2 & (5818)\end{array}$

690 PX 3-3 (5880)

691 PX 4-1 (6390)

$\begin{array}{lll}692 \mathrm{PX} & 4-2 & (6434) \\ 693 \mathrm{PX} 4-3 & (6458)\end{array}$

694 PX 5-1 (6787)

695 PX 5-2 (6795)

$\begin{array}{lll}696 & \text { PX 5-3 } & (6812) \\ 697 \text { PX 5-4 } & (6820)\end{array}$

698 PX 5-5 (6839)

699 PX 5-6 (6862)

$700 \times 195(6871)$

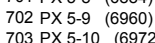

704 PX 6-1 (7340)

706 BATCH 1 STUDY-10B-

707 BATCH 1 STUDY-15-7

708 H-GLAS-0112

711 H-GLAS-0162

712 H-GLAS-0254

713 H-GLAS-0278
711 H-GLAS-0293

715 H-GLAS-0308

17 H-GLAS-0352

718 H-GLAS-0037

720 H-GLAS-0466

722 FRIT-202-INT

723 FRIT-202-WHITE
724 FRIT-165-7

725 FRIT-131-7

$726 \mathrm{MG} 9-7$

$727 \mathrm{MG} 18-7$
$728 \mathrm{MG} 25-7$

728 MG 25-7
729 MG 28-7

$730 \mathrm{PBG} 3 / \mathrm{Ce}$

731 PBG $3 / 0 \mathrm{X}$
$732 \mathrm{PBG}-5 \mathrm{C}$

733 SRC-Al-1

734 SRC-Al-2

735 SRC-B- 1

737 SRC-B-3
Ga203-a Gd2O3-a

I-a La2O3-a MnO2-a MnO-a MoO-a MoO3-a Nb2O5-a Nd2O3-a NpO2-a PbO-a PdO2-a PdO-a Pr2O3-a Pr6011-a PuO2-a Rb2O-a ReO-a ReO2-a Re207-a Rh203-a RhO2-a RuO2-a Sb203-a Sb205-a SeO2-a

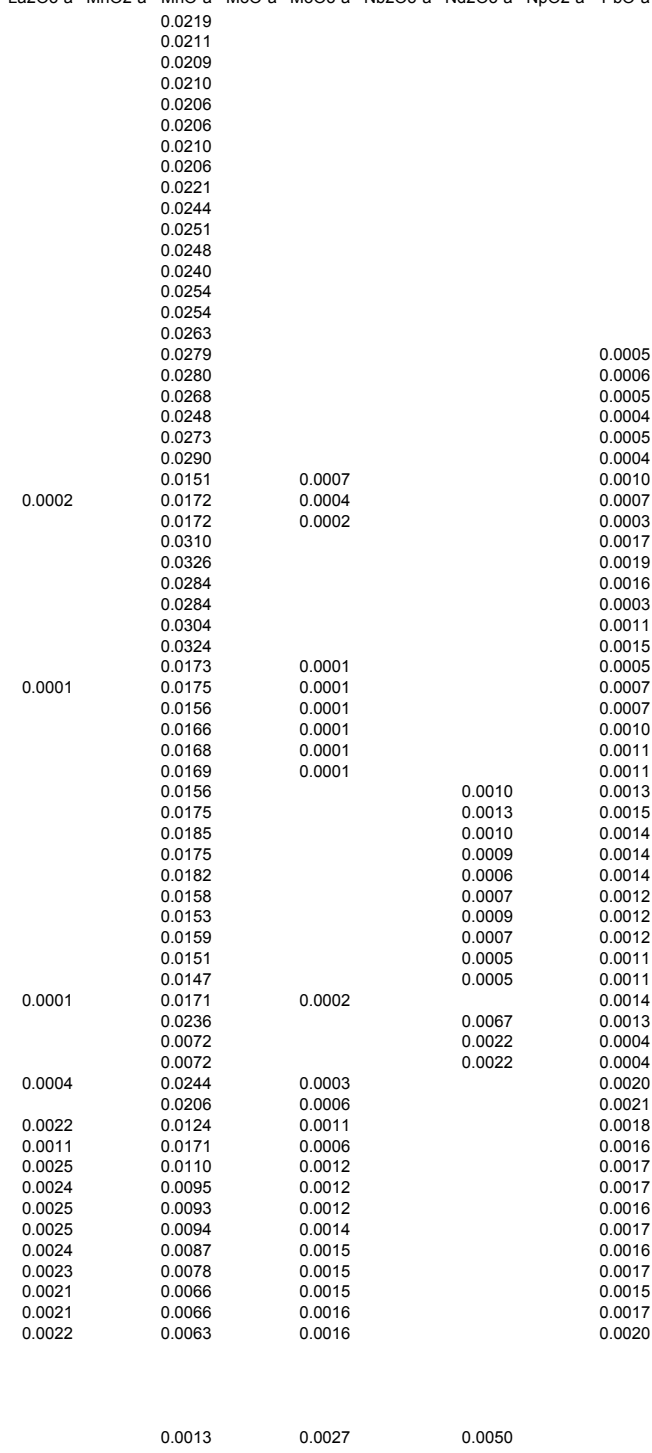


712 H-GLAS-0254

714 H-GLAS-0293

716 H-GLAS-0334
717 -GLAS-0352

717 H-GLAS-0352

720 H-GLAS-0466

722 RRIT-202-NT

723 FRIT-202-WHITE
724 FRIT-165-7

725 FRIT-131-7

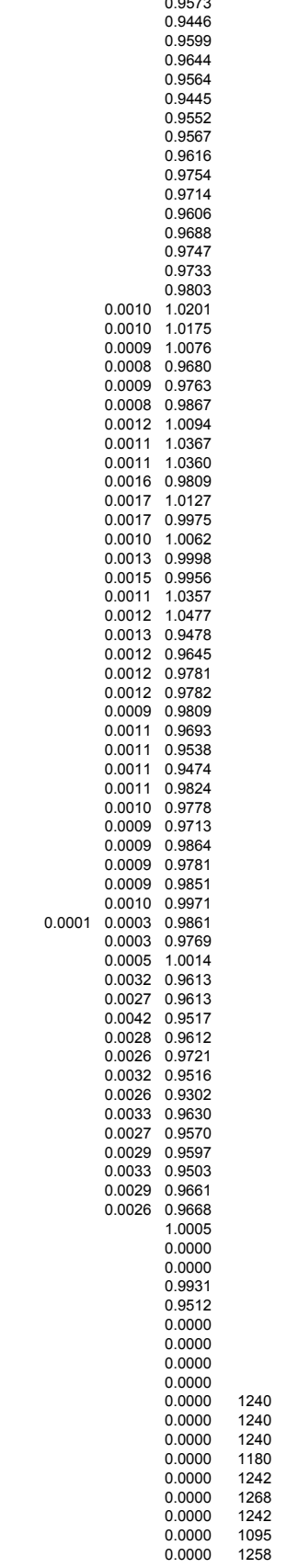


CCC PCT B $\left(g / \mathrm{m}^{2}\right)$ CCC PCT Li $\left(g / \mathrm{m}^{2}\right) \operatorname{CCC}$ PCT Na $\left(g / \mathrm{m}^{2}\right) \quad$ CCC PCT Si $\left(g / \mathrm{m}^{2}\right)$ CCC PCT pH T1 ( ${ }^{\circ} \mathrm{C}$ 657 BLEND 1-3510 659 BLEND 2-3611 660 BLEND 2-3622 661 BLENN 2-3635
662 BLEND 2-3654 663 BLEND 2-3666

664 BLEND 2-3676 665 BLEND 3-3768 667 BLEND 3-3793 668 BLEND 3-3802 669 HM 1-3824 671 HM 1-3851 672 HM 1-3855 $673 \mathrm{HM}-2-1$
$674 \mathrm{HM}-2-2$$(3979 \mathrm{C})$ $\begin{array}{ll}675 \mathrm{HM}-2-3 & (4120 \mathrm{~B}) \\ 676 \mathrm{HM}-3-1 & (4176)\end{array}$ $677 \mathrm{HM}-3-3-2(4176)$ $678 \mathrm{HM}-3-3.3(4357)$ $679 \mathrm{HM}-4-1$
$680 \mathrm{HM}-4-2$
$(56260)$
$6811)$ $\begin{array}{ll}680 \mathrm{HM}-4-2 & (5641) \\ 681 \mathrm{HM}-4-3 & (5748)\end{array}$ 682 PX 1-1 (4643) $\begin{array}{ll}683 & \mathrm{PX} 1-2 \\ 684 & (4726) \\ \mathrm{PX} 1-3 & (4776)\end{array}$ $\begin{array}{lll}684 \mathrm{PX} 1-3 & (4776) \\ 685 \mathrm{PX} 2-1 & (4455)\end{array}$ 686 PX 2-2 (4509) $\begin{array}{ll}687 \mathrm{PX} 2-3 & (4566) \\ 688 \mathrm{PX} 3-1 & (5780)\end{array}$ 690 PX 3-3 (5880) 691 PX 4-1 (6390) $\begin{array}{lll}692 \mathrm{PX} & 4-2 & (6434) \\ 693 \mathrm{PX} 4-3 & (6458)\end{array}$ 694 PX 5-1 (6787) 695 PX 5-2 (6795) 696 PX 5-3 (6812) 698 PX 5-5 (6839) 699 PX 5-6 (6862) $700 \times 195(6871)$ 702 PX 5-9
703 PX 5-10
$(6960)$ 704 PX 6-1 (7340) 706 BATCH 1 STUDY-10B-7 707 BATCH 1 STUDY-15-7 709 H-GLAS-0112 710 H-GLAS-0162 711 H-GLAS-0244 713 H-GLAS-0278 715 H-GLAS-0308 717 H-GLAS-0352 718 H-GLAS-0387 719 H-GLAS-042 721 FRIT-202-CLEAR 722 FRIT-202-INT 723 FRIT-202-WHIT
724 FRIT-165-7 725 FRIT-131-7 726 MG 9-7 $727 \mathrm{MG} 18-7$ 728 MG 25-7
729 MG 28-7 $730 \mathrm{PBG} 3 / \mathrm{Ce}$ 731 PBG $3 / 0 \mathrm{X}$ 733 SRC-Al-1 734 SRC-Al-2

\begin{tabular}{|c|c|c|c|c|}
\hline & & & & \\
\hline 0.335 & 0.370 & 0.360 & 0.210 & 10.280 \\
\hline 0.305 & 0.335 & 0.335 & 0.195 & $\begin{array}{l}10.210 \\
1.2240\end{array}$ \\
\hline 0.305 & 0.330 & 0.330 & 0.195 & 10.240 \\
\hline 0.305 & 0.325 & 0.330 & 0.195 & 10.140 \\
\hline $\begin{array}{l}0.300 \\
0.300\end{array}$ & $\begin{array}{l}0.325 \\
0.325\end{array}$ & $\begin{array}{l}0.320 \\
0\end{array}$ & 0.195 & $\begin{array}{r}10.230 \\
10\end{array}$ \\
\hline $\begin{array}{l}0.300 \\
0.295\end{array}$ & $\begin{array}{l}0.325 \\
0.320\end{array}$ & $\begin{array}{l}0.320 \\
0.315\end{array}$ & $\begin{array}{l}0.195 \\
0.195\end{array}$ & $\begin{array}{l}10.100 \\
10.070\end{array}$ \\
\hline $\begin{array}{l}0.295 \\
0.300\end{array}$ & $\begin{array}{l}0.320 \\
0.320\end{array}$ & $\begin{array}{l}0.315 \\
0.330\end{array}$ & $\begin{array}{l}0.195 \\
0.185\end{array}$ & $\begin{array}{r}10.070 \\
9.990\end{array}$ \\
\hline 0.315 & 0.330 & 0.350 & $\begin{array}{l}.1100 \\
0.185\end{array}$ & $\begin{array}{c}9.990 \\
10.140\end{array}$ \\
\hline 0.315 & 0.330 & 0.355 & 0.190 & 10.130 \\
\hline 0.350 & 0.355 & 0.385 & 0.195 & 10.180 \\
\hline 0.295 & 0.315 & 0.310 & 0.180 & 9.980 \\
\hline 0.285 & 0.315 & 0.305 & 0.175 & 9.960 \\
\hline 0.260 & 0.300 & 0.275 & 0.180 & 9.980 \\
\hline $\begin{array}{l}0.265 \\
0.265\end{array}$ & $\begin{array}{l}0.300 \\
0.305\end{array}$ & $\begin{array}{l}0.275 \\
0.270\end{array}$ & $\begin{array}{l}0.170 \\
0\end{array}$ & $\begin{array}{l}9.990 \\
9560\end{array}$ \\
\hline $\begin{array}{l}0.265 \\
0.275\end{array}$ & $\begin{array}{l}0.305 \\
0.315\end{array}$ & $\begin{array}{l}.2270 \\
0.280\end{array}$ & $\begin{array}{l}0.185 \\
0.180\end{array}$ & $\begin{array}{l}9.560 \\
9.600\end{array}$ \\
\hline 0.265 & 0.310 & 0.270 & 0.180 & $\begin{array}{l}9.600 \\
9.220\end{array}$ \\
\hline 0.280 & 0.325 & 0.265 & 0.180 & $\begin{array}{l}5.220 \\
9.220\end{array}$ \\
\hline 0.275 & 0.320 & 0.260 & 0.180 & 9.220 \\
\hline 0.255 & 0.295 & 0.265 & 0.175 & 9.200 \\
\hline 0.375 & 0.380 & 0.320 & 0.175 & 9.290 \\
\hline 0.265 & 0.285 & 0.260 & 0.150 & $\begin{array}{r}9.320 \\
9700\end{array}$ \\
\hline $\begin{array}{l}0.350 \\
0.360\end{array}$ & $\begin{array}{l}0.355 \\
0.390\end{array}$ & $\begin{array}{l}0.325 \\
0.365\end{array}$ & $\begin{array}{l}0.170 \\
0210\end{array}$ & $\begin{array}{l}9.700 \\
9460\end{array}$ \\
\hline $\begin{array}{l}0.360 \\
0.355\end{array}$ & $\begin{array}{l}0.390 \\
0.380\end{array}$ & $\begin{array}{l}.3 .365 \\
0.360\end{array}$ & $\begin{array}{l}0.210 \\
0.215\end{array}$ & $\begin{array}{l}9.460 \\
9.360\end{array}$ \\
\hline 0.395 & 0.415 & $\begin{array}{l}0.360 \\
0.385\end{array}$ & $\begin{array}{l}0.215 \\
0.230\end{array}$ & $\begin{array}{l}9.3600 \\
9.380\end{array}$ \\
\hline 0.240 & 0.285 & 0.240 & 0.165 & 9.320 \\
\hline 0.290 & 0.325 & 0.305 & 0.185 & 9.350 \\
\hline 0.365 & 0.390 & 0.365 & 0.205 & 9.490 \\
\hline 0.420 & 0.410 & 0.335 & 0.210 & 9.680 \\
\hline 0.540 & 0.505 & 0.425 & 0.245 & $\begin{array}{r}9.700 \\
\end{array}$ \\
\hline $\begin{array}{l}0.675 \\
1.135\end{array}$ & $\begin{array}{l}0.605 \\
0.985\end{array}$ & $\begin{array}{l}0.520 \\
0.920\end{array}$ & $\begin{array}{l}0.315 \\
0.465\end{array}$ & $\begin{array}{r}9.620 \\
10050\end{array}$ \\
\hline $\begin{array}{l}1.1355 \\
2.270\end{array}$ & $\begin{array}{l}0.985 \\
1.815\end{array}$ & $\begin{array}{l}.9920 \\
1.800\end{array}$ & $\begin{array}{l}0.465 \\
0.780\end{array}$ & $\begin{array}{l}\begin{array}{l}10.050 \\
10.520\end{array}\end{array}$ \\
\hline 2.18 & $\begin{array}{l}1.710 \\
1.745\end{array}$ & $\begin{array}{l}1.800 \\
1.825\end{array}$ & $\begin{array}{l}0.760 \\
0.765\end{array}$ & \\
\hline 0.535 & 0.525 & 0.530 & $\begin{array}{l}0.175 \\
0.250\end{array}$ & $\begin{array}{l}10.420 \\
10.360\end{array}$ \\
\hline 4.580 & 3.095 & 4.085 & 0.995 & 11.610 \\
\hline 9.600 & 5.305 & 8.235 & 1.965 & 12.040 \\
\hline 10.500 & 5.645 & 8.940 & 2.225 & 12.010 \\
\hline 10.515 & 5.700 & 8.485 & 2.330 & 11.990 \\
\hline $\begin{array}{r}9.485 \\
92905\end{array}$ & $\begin{array}{l}5.405 \\
55210\end{array}$ & $\begin{array}{r}7.560 \\
7.215\end{array}$ & $\begin{array}{r}2.285 \\
2.315\end{array}$ & $\begin{array}{l}11.840 \\
11.840\end{array}$ \\
\hline $\begin{array}{r}9.290 \\
10.790\end{array}$ & $\begin{array}{l}5.210 \\
5.500\end{array}$ & $\begin{array}{l}7.215 \\
8.575\end{array}$ & $\begin{array}{l}2.315 \\
2.350\end{array}$ & $\begin{array}{l}11.840 \\
11870\end{array}$ \\
\hline $\begin{array}{r}10.790 \\
8570\end{array}$ & $\begin{array}{l}5.900 \\
4.790\end{array}$ & $\begin{array}{l}8.575 \\
6.850\end{array}$ & $\begin{array}{l}2.350 \\
2.195\end{array}$ & $\begin{array}{l}\begin{array}{l}11.870 \\
11.780\end{array}\end{array}$ \\
\hline 10.950 & $\begin{array}{l}4.900 \\
4.745\end{array}$ & $\begin{array}{l}.8 .850 \\
8.685\end{array}$ & $\begin{array}{l}2.195 \\
1.615\end{array}$ & 11.730 \\
\hline 7.560 & 4.805 & 8.415 & 1.950 & 11.650 \\
\hline 8.595 & 6.810 & 7.725 & 2.270 & 11.340 \\
\hline 5.265 & 4.495 & 4.765 & 1.565 & 10.900 \\
\hline 0.830 & 0.760 & 0.710 & 0.360 & 10.350 \\
\hline 0.440 & $\begin{array}{l}0.415 \\
0.515\end{array}$ & $\begin{array}{l}0.375 \\
0.460\end{array}$ & $\begin{array}{r}0.220 \\
\end{array}$ & $\begin{array}{r}9.830 \\
9630\end{array}$ \\
\hline $\begin{array}{l}0.580 \\
1005\end{array}$ & $\begin{array}{l}0.515 \\
0.835\end{array}$ & $\begin{array}{l}0.460 \\
0.690\end{array}$ & $\begin{array}{l}0.245 \\
0.320\end{array}$ & $\begin{array}{l}9.630 \\
9.690\end{array}$ \\
\hline $\begin{array}{l}1.005 \\
1.475\end{array}$ & $\begin{array}{l}\text {. } 1.085 \\
1.085\end{array}$ & $\begin{array}{l}0.690 \\
0.690\end{array}$ & $\begin{array}{l}0.320 \\
0.365\end{array}$ & $\begin{array}{l}9.690 \\
9.890\end{array}$ \\
\hline $\begin{array}{l}1.050 \\
1.050\end{array}$ & 0.910 & 0.715 & 0.320 & $\begin{array}{l}9.890 \\
9.790\end{array}$ \\
\hline 1.250 & 1.060 & 0.770 & 0.350 & 9.820 \\
\hline 1.540 & 1.290 & 0.925 & 0.380 & 9.840 \\
\hline 1.010 & 0.865 & 0.660 & 0.300 & 9.730 \\
\hline 1.205 & 1.075 & 0.775 & 0.325 & 9.760 \\
\hline 1.330 & $\begin{array}{l}1.180 \\
1155\end{array}$ & 0.835 & 0.340 & 9.620 \\
\hline $\begin{array}{l}1.290 \\
1.1140\end{array}$ & $\begin{array}{l}1.155 \\
1.400\end{array}$ & $\begin{array}{l}0.785 \\
06885\end{array}$ & $\begin{array}{l}0.330 \\
0.305\end{array}$ & $\begin{array}{l}9.670 \\
9600\end{array}$ \\
\hline $\begin{array}{l}1.140 \\
1395\end{array}$ & $\begin{array}{l}1.040 \\
10205\end{array}$ & $\begin{array}{l}0.685 \\
0.830\end{array}$ & $\begin{array}{l}0.305 \\
0.330\end{array}$ & $\begin{array}{l}9.620 \\
9690\end{array}$ \\
\hline $\begin{array}{l}1.095 \\
15.980\end{array}$ & 14.445 & $\begin{array}{l}0.050 \\
15.190\end{array}$ & $\begin{array}{l}0.330 \\
5.755\end{array}$ & \\
\hline $\begin{array}{l}15.980 \\
21.950\end{array}$ & $\begin{array}{l}14.445 \\
18.635\end{array}$ & 19.540 & $\begin{array}{r}5.175 \\
10.485\end{array}$ & \\
\hline 31.535 & $\begin{array}{l}29.130 \\
2.050\end{array}$ & $\begin{array}{l}28.720 \\
2.720\end{array}$ & $\begin{array}{l}1.055 \\
\end{array}$ & \\
\hline 40.160 & 42.155 & 37.080 & 30.515 & \\
\hline 25.150 & 24.300 & 21.565 & 14.830 & \\
\hline 35.120 & & 28.210 & 17.005 & $\begin{array}{r}12.630 \\
\end{array}$ \\
\hline 28.910 & & $\begin{array}{r}23.885 \\
\end{array}$ & 8.340 & $\begin{array}{l}12.010 \\
10.050\end{array}$ \\
\hline $\begin{array}{l}0.415 \\
0.315\end{array}$ & & $\begin{array}{l}0.395 \\
0.360\end{array}$ & $\begin{array}{l}0.120 \\
0.160\end{array}$ & $\begin{array}{l}10.050 \\
10.650\end{array}$ \\
\hline & & & & \\
\hline 0.48 & 0.554 & 0.554 & 0.197 & \\
\hline \multirow{3}{*}{0.226} & 4.909 & 4.961 & 1.536 & \\
\hline & 0.306 & 0.291 & 0.104 & 10.950 \\
\hline & 0.627 & 0.603 & 0.197 & 11.530 \\
\hline .34 & $\begin{array}{l}0.306 \\
167\end{array}$ & 0.292 & 0.101 & \\
\hline 0.334 & 0.411 & $\begin{array}{l}1.541 \\
0.394\end{array}$ & $\begin{array}{l}0.449 \\
0.173\end{array}$ & 10.900 \\
\hline
\end{tabular}


658 BLEND 1-3526

659 BLEND 2-3611
660 BLEND 2-3622

661 BLEND 2-3635
662 BLEND 2-3654

662 BLEND 2-3654

664 BLEND 2-3676

665 BLEND 3-3768
666 BLEND $3-3789$

667 BLEND 3-3793

608 BLEND 3-3802

670 HM 1-3829

673 HM-2-1
674 HM-2-2 (3979C)

$\begin{array}{ll}675 \mathrm{HM}-2-3 & (4120 \mathrm{~B}) \\ 676 \mathrm{HM}-3-1 & (4176)\end{array}$

$677 \mathrm{HM}-3-2(4225)$

678 MM-3-3
679 HM-4-1
63260

680 HM-4-2 $2(5641)$
681 HM-4-3
$(5748)$

$681 \mathrm{HM}-4-3$
$682 \mathrm{PX} 1-1(4748)$

683 PX 1-2 (4726)

$684 \mathrm{PX} 1-3 \quad$ (4776)

686 PX 2-2 (4509)

687 PX 2-3 (4566)

$\begin{array}{lll}688 & \text { PX 3-1 } & (5780) \\ 689 & \text { PX } 3-2 & (5818)\end{array}$

$\begin{array}{lll}690 & \text { PX } 3-3 & (5880)\end{array}$

691 PX 4-1 (6390)

$\begin{array}{lll}692 & \text { PX 4-2 } & (6434) \\ 693 & \text { PX 4-3 } & (6458) \\ 6\end{array}$

694 PX 5-1 (6787)

695 PX 5-2 (6795)

696 PX 5-3 (6812)

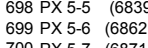

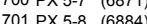

702 PX 5-9 $(6960)$
703 PX 5-10
$60972)$

704 PX 6-1 (7340)

706 BATCH 1 STUDY-10B-

707 BATCH 1 STUDY-15-7

708 H-GLAS-0112

710 H-GLAS-0162

711 H-GLAS-0244

713 H-GLAS-0278

714 H-GLAS-0293
715 H-GLAS-0308

717 H-GLAS-0352

718 H-GLAS-0387

719 H-GLAS-0421

721 FRIT-202-CLEAR

722 FRIT-202-INT

723 FRIT-202-WHITE
724 FRIT-165-7

725 FRIT-131-7

726 MG 9-7

727 MG 18-7

728 MG 25-7
729 MG 28-7

$729 \mathrm{MG} 28-7$
$730 \mathrm{PBG} 3 / \mathrm{Ce}$

731 PBG $3 / 0 x$
732 PBG $3.5 \mathrm{C}$

733 SRC-Al-1

734 SRC-Al-2

735 SRC-B-1
736 SRC-B-2

737 SRC-B-3

738 SRC-Ca-1 


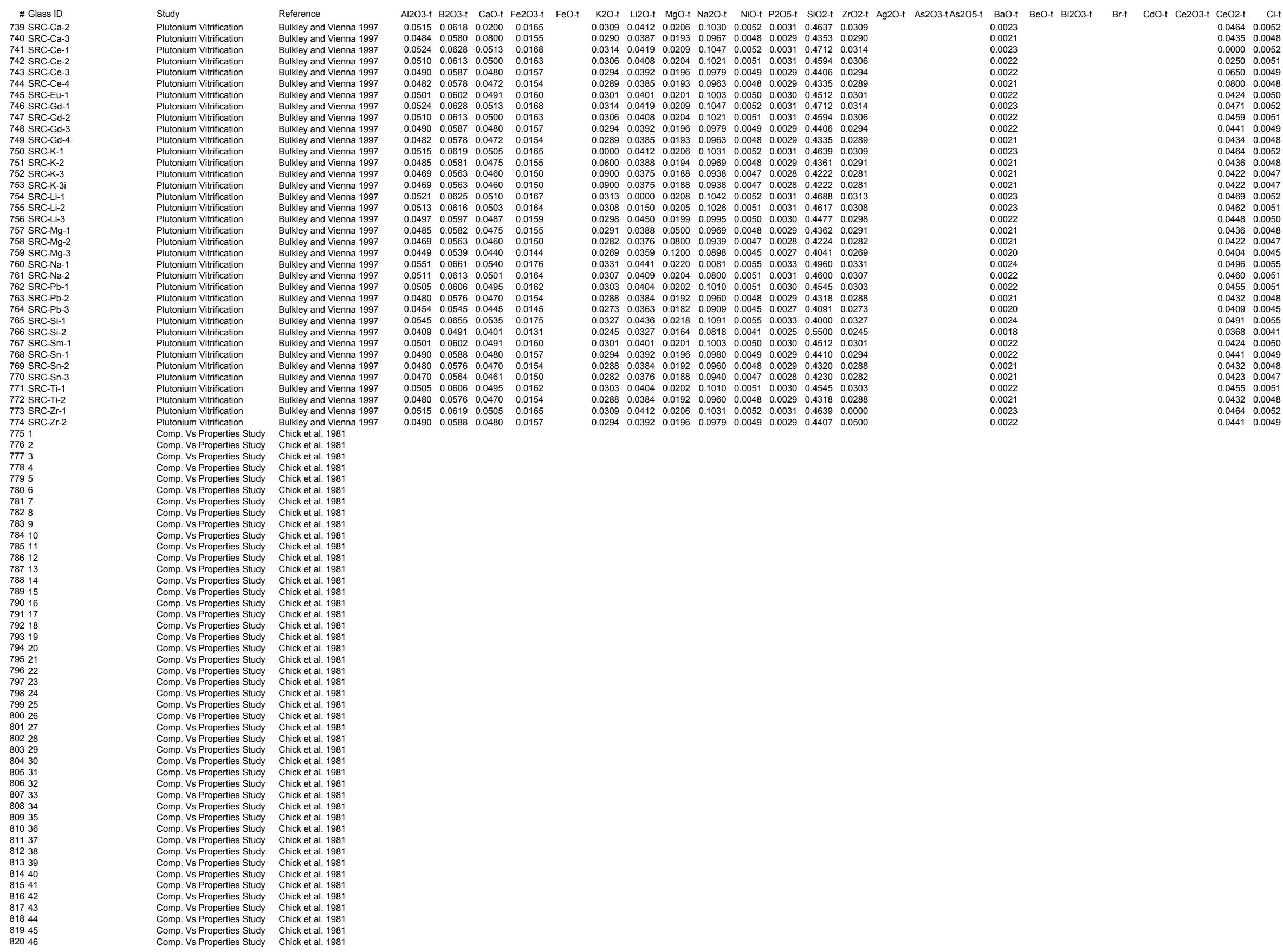


\# Glass ID
739 SRC-Ca-

740 SRC-Ca-3
741 SRC-Ce-1

742 SRC-Ce-2
743 SRC-Ce-3

744 SRC-Ce-

746 SRC-Gd-1

747 SRC-Gd-2
748 SRC-G-3

749 SRC-Gd-A

751 SRC-K- -2
752 SRC-K-

753 SRC-K-Ki
754 SRC-Li-1

755 SRC-Li-2

757 SRC-Mg- 1

757 SRC-Mg-1
758 SRC-Mg-2

759 SRC-Mg-3

761 SRC-Na-2

763 SRC-Pb-2

765 SRC-Si-1
766 SRC-Si-2

767 SRC-Sm-1

769 SRC-Sn-2

770 SRC-Sn-
771 SRC-Ti-1

772 SRC-Ti-2

773 SRC-Zr-1
774 SRC-Zr-2

7751

7773
7784
7795

7795

7806

7828
7839

78410

78612
78713

78814
78915
79016

79011
79117

79218
79319

79420

79522
79622
7924

79824
79025

79925
80026

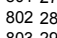

80322
8043
8

80032

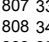

8103

811

8133
814
814

815
CoO-t Co203-t Cr2O3-t Cs2O-t CuO-t Dy203-t Eu2O3-t $\begin{array}{lll}0.0041 & 0.0103 & 0.0004 \\ 0.0039 & 0.0097 & 0.0004 \\ 0.0042 & 0.0105 & 0.0004\end{array}$ $\begin{array}{llll}0.0039 & 0.0097 & 0.0004 \\ 0.0042 & 0.0105 & 0.0004\end{array}$

$\begin{array}{lll}0.0041 & 0.0102 & 0.0004 \\ 0.0039 & 0.0098 & 0.0004 \\ 0.0040 & 0.0000 & 0.004\end{array}$

$\begin{array}{llll}0.0039 & 0.0096 & 0.0004 \\ 0 & 0.040 & 0.004 & 0.004 \\ 0 & 0.0100 & 0.004\end{array}$

$\begin{array}{llll}0.0042 & 0.0105 & 0.0004\end{array}$

$\begin{array}{lll}0.0039 & 0.0098 & 0.0004 \\ 0.039 & 0.0096 & 0.004\end{array}$

$\begin{array}{lll}0.0041 & 0.0103 & 0.0004 \\ 0 & 0.039 & 0.0097 \\ 0\end{array}$

$\begin{array}{llll}0.0039 & 0.0097 & 0.0004 \\ 0.0038 & 0.0094 & 0.0004 \\ 0.0038 & 0.0094 & 0.0004\end{array}$

$\begin{array}{llll}0.0038 & 0.0094 & 0.0004\end{array}$

$\begin{array}{lll}0.0042 & 0.0104 & 0.0004 \\ 0.041 & 0.0103 & 0.0004\end{array}$

$\begin{array}{lll}0.0099 & 0.0004 \\ 0.0097 & 0.0004 \\ 0.0094 & 0.004\end{array}$

$\begin{array}{llll}0.0039 & 0.0097 & 0.00004 \\ 0.038 & 0.0094 & 0.0004 \\ 0.004 & 0.0910 & 0.0004\end{array}$

.

$\begin{array}{lll}0.0041 & 0.0102 & 0.0004 \\ 0.0040 & 0.0101 & 0.0004\end{array}$

$\begin{array}{llll}0.0038 & 0.0096 & 0.0004 \\ 0.0036 & 0.0091 & 0.0004\end{array}$

$\begin{array}{llll}0.0044 & 0.0109 & 0.0004 \\ 0.0033 & 0.0082 & 0.0003\end{array}$

$\begin{array}{llll}0.0040 & 0.0100 & 0.0004 \\ 0.0039 & 0.0098 & 0.0004\end{array}$

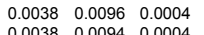

$\begin{array}{llll}0.0040 & 0.0101 & 0.0004\end{array}$

$\begin{array}{llll}0.0038 & 0.0096 & 0.0004 \\ 0.0041 & 0.0103 & 0.0004\end{array}$

$\begin{array}{lll}0.0041 & 0.0103 & 0.0004 \\ 0.0039 & 0.0098 & 0.0004\end{array}$

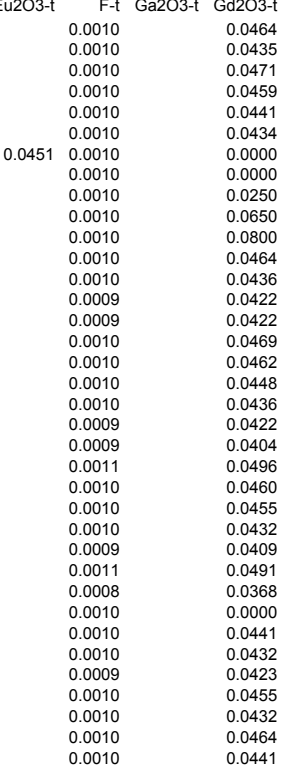

I-t La2O3-t MnO2-t MnO-t MoO-t MoO2-t MoO3-t Nb205-t Nd203-t NpO2-t PbO-t PdO2-t PdO-t Pr2O3-t Pr6011-t Rb2O-t ReO-t

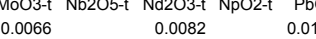

\begin{tabular}{|c|c|c|}
\hline & & \\
\hline & 0.0077 & 0.0097 \\
\hline 0.0067 & 0.0084 & 0.0105 \\
\hline 0.0065 & 0.0082 & 0.0102 \\
\hline 0 & 0.007 & 0.0098 \\
\hline 0 & 0.0080 & $\begin{array}{l}0.0096 \\
0.0100\end{array}$ \\
\hline 0.0067 & 0.0084 & \\
\hline 0.0065 & 0.0082 & 0.0102 \\
\hline 0.0063 & 0.0078 & 0.0098 \\
\hline 0.0062 & 0.0077 & \\
\hline 0.0066 & 0.0082 & 0.0103 \\
\hline 0.0062 & 0.0078 & 0.0097 \\
\hline 0.0060 & 0.0075 & 0.0094 \\
\hline 0.0060 & 0.0075 & 0.0094 \\
\hline 0.0066 & $\begin{array}{l}0.0083 \\
0.0082\end{array}$ & $\begin{array}{l}0.0104 \\
0.0103\end{array}$ \\
\hline & $\begin{array}{l}0.0008 \\
0.008\end{array}$ & $\begin{array}{l}0.0103 \\
0.0099\end{array}$ \\
\hline 0.0062 & 0.0078 & 0.0097 \\
\hline & 0.0075 & 0.0094 \\
\hline 0.0057 & 0.0072 & 0.0090 \\
\hline 0.0071 & 0.0088 & 0.0110 \\
\hline 0.0065 & 0.0082 & 0.0102 \\
\hline 0.0055 & 0.0081 & 0.0000 \\
\hline $\begin{array}{l}0.0606 \\
0.0058\end{array}$ & $\begin{array}{l}0.00073 \\
0.007\end{array}$ & $\begin{array}{l}0.0500 \\
0.1000\end{array}$ \\
\hline & & 0.0109 \\
\hline 0.0052 & 0.0065 & 0.0082 \\
\hline 0.0064 & 0.0080 & 0.0100 \\
\hline 0.0063 & $\begin{array}{l}0.0078 \\
0.0077\end{array}$ & $\begin{array}{l}0.0098 \\
0.0096\end{array}$ \\
\hline 0.0061 & $0.007 / 7$ & $\begin{array}{l}0.0096 \\
0.0094\end{array}$ \\
\hline 0.00065 & 0.0081 & $\begin{array}{l}0.0094 \\
0.0101\end{array}$ \\
\hline 0.0061 & 0.0077 & \\
\hline 0.0066 & 0.0082 & 0.0103 \\
\hline 0.0063 & 0.0078 & 0.0098 \\
\hline
\end{tabular}




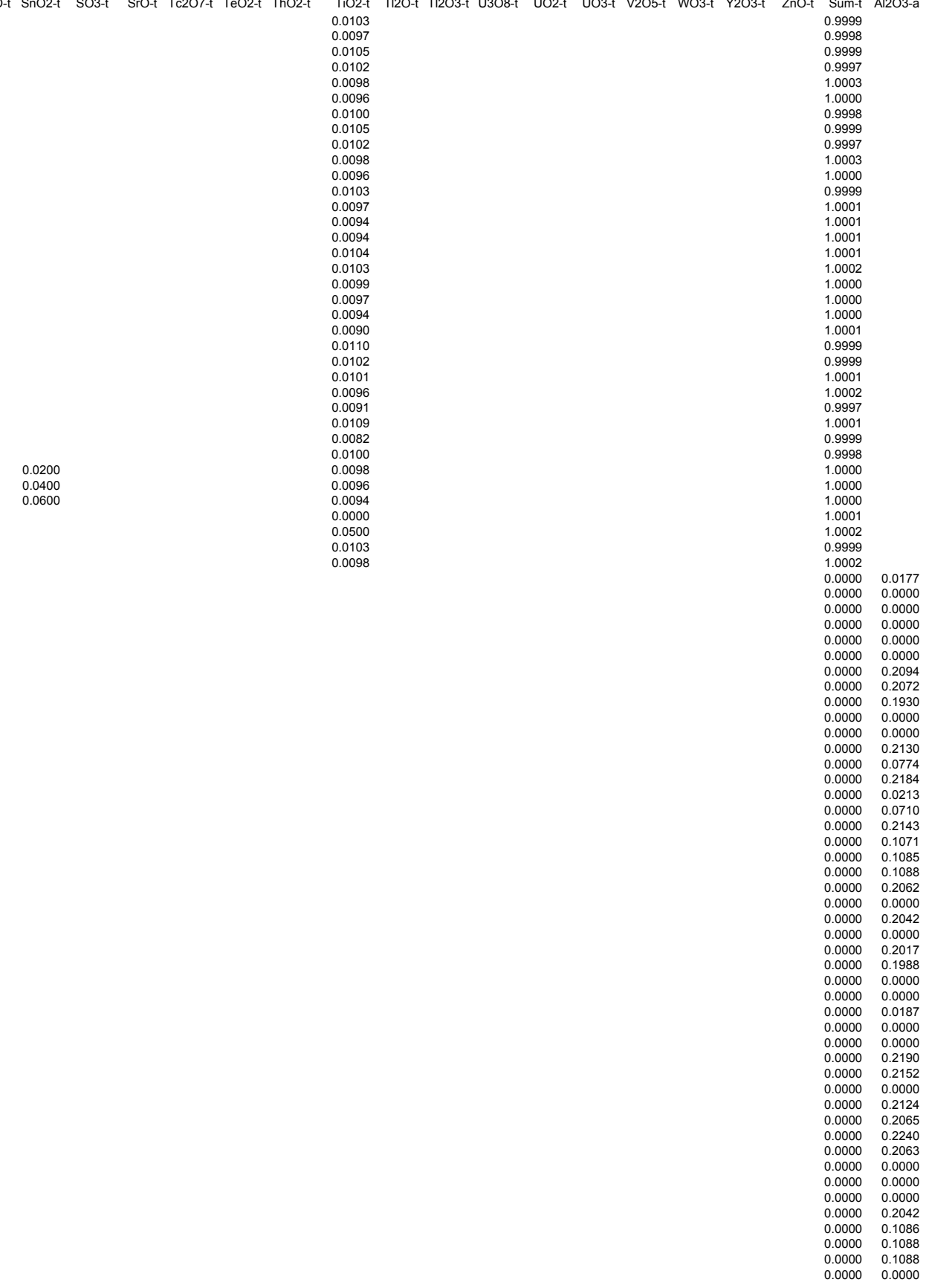




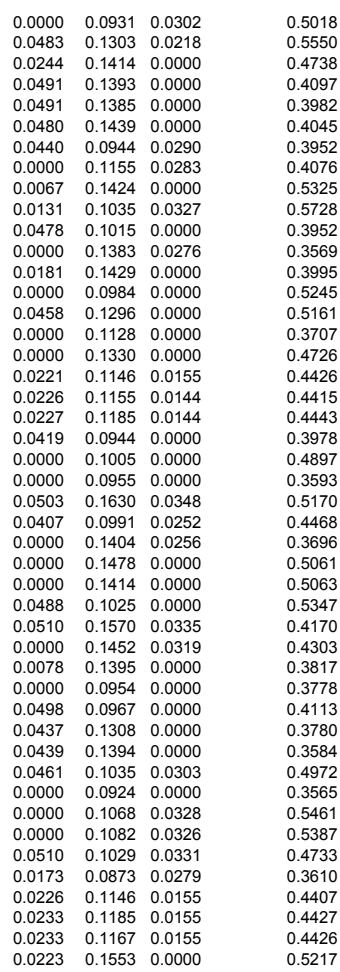


740 SRC-Ca-3
711 SPRC -1

741 SRCCC-C-1
724 SRC-Ce-2

743 SRC-CC-3

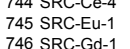

746 SRC-Gd-1
747 SRC-Gd-2

748 SRC-Gd-3
749 SRC-Gd-4

7790 SRC-Cd-A -1
751 SRC-K-1

751 SRCK-K-2
752 SRC-K-3

753 SSCCK-.3i
754 SRC-Li-1

754 SRCLL-Li-1
755 SRC-LL-2

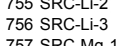

757 SRC-Mg-1
758 SRC-Mg-2

759 SRC-Mg-3

761 SRC-Na-2

762 SRC-PD- 1

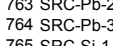

765 SRC-Si-1
766 SRC-Si-2

767 SRC-SM-1
768 SRC-Sn-1

769 SRC-Sn-2

770 SRC-Sn-3
771 SRC-Ti-1

771 SRC-TIT-1

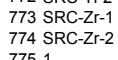

7751
77762
773

7778

7817

7828

78390
78511
785

78612
78713
78914

78713
788
789
75

79016
79117

79218
79319

79420
79521
7

79622
797
77924

79824
79925

80127

80228
80329

80431
80532

80633

80834
80935

81137

81238
81339

81642

81743
81844

81945
82046 
$\begin{array}{llll}-12.000 & 21104.0 & 1388.0 & 2.059 \\ -12.300 & 19914.0 & 1238.0 & 2.393 \\ -1200 & 1392.0 & 1280 & 2.939\end{array}$

$\begin{array}{rrrr}-11.800 & 18771.0 & 1239.0 & 1.901 \\ -12.700 & 19628.0 & 1196.0 & 2.033 \\ -11.000 & 11682.0 & 12390 & 1.584\end{array}$

$\begin{array}{llll}-11.300 & 17621.0 & 1239.0 & 1.033 \\ -1.566 & 1.700\end{array}$

$\begin{array}{rrrr}-11.000 & 16823.0 & 1239.0 & 1.184 \\ -11.100 & 20166.0 & 1386.0 & 2.737 \\ -1200 & 204720 & 12390 & 1.839\end{array}$

$\begin{array}{rrrr}-11.800 & 18947.0 & 1240.0 & 1.947 \\ -12.900 & 20472.0 & 1239.0 & 1.839 \\ -14200 & 22304.0 & 1239.0 & 1.769\end{array}$

$\begin{array}{llll}-11.700 & 18672.0 & 1239.0 & 1.898 \\ -12.800 & 19802.0 & 1239.0 & 1.329 \\ -10.700 & 17113.0 & 1239.0 & 1.822 \\ - & & \end{array}$

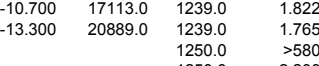

\begin{tabular}{|c|c|}
\hline & $\begin{array}{l}2.200 \\
2.400\end{array}$ \\
\hline 1250.0 & $\begin{array}{l}.4 .300 \\
.300\end{array}$ \\
\hline 250.0 & $\begin{array}{l}1.800 \\
1200\end{array}$ \\
\hline 250.0 & $>580$ \\
\hline 1250.0 & 74.000 \\
\hline 1250.0 & 97.000 \\
\hline $\begin{array}{l}1250.0 \\
1250.0\end{array}$ & $\begin{array}{l}1.8000 \\
6.000\end{array}$ \\
\hline 1250.0 & 10.500 \\
\hline 1250.0 & 35.000 \\
\hline & \\
\hline $\begin{array}{l}1250.0 \\
1250.0\end{array}$ & \\
\hline 1250.0 & 82.000 \\
\hline $\begin{array}{c}1250.0 \\
12500\end{array}$ & 33.000 \\
\hline & \\
\hline $\begin{array}{l}1250.0 \\
1250.0\end{array}$ & \\
\hline 1250.0 & $\begin{array}{l}1.200 \\
\end{array}$ \\
\hline & \\
\hline $\begin{array}{l}1250.0 \\
1250.0\end{array}$ & \\
\hline 1250.0 & \\
\hline 1250.0 & 4.400 \\
\hline 1250. & \\
\hline $\begin{array}{l}1250.0 \\
1250.0\end{array}$ & \\
\hline 1250.0 & \\
\hline 1250.0 & \\
\hline & \\
\hline $\begin{array}{l}1250.0 \\
1250.0\end{array}$ & $\begin{array}{l}0.620 \\
>5580\end{array}$ \\
\hline $\begin{array}{l}1250.0 \\
1250.0\end{array}$ & \\
\hline 1250.0 & 140.000 \\
\hline 1250.0 & \\
\hline $\begin{array}{l}1250.0 \\
1250.0\end{array}$ & \\
\hline $\begin{array}{l}1250 \\
1250\end{array}$ & \\
\hline & \\
\hline 50 & \\
\hline & \\
\hline & \\
\hline
\end{tabular}

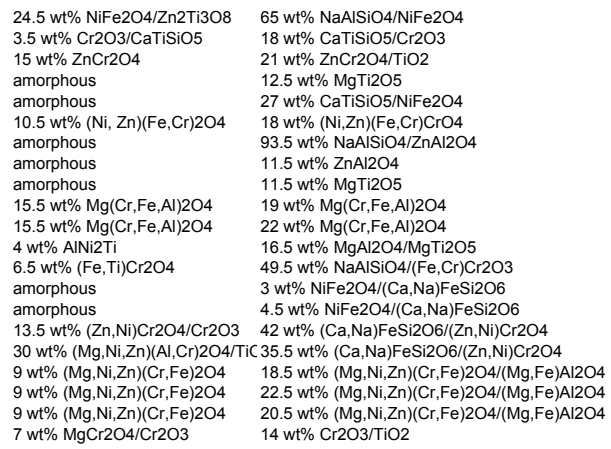


\# Glass ID

739 SRC-Ca-2

741 SRC-Ce-1
742 SRC-Ce 2

743 SRC-Ce- 3

744 SRC-Ce-4
745 SRC-Eu-1
746 SRC-Gd-1

746 SRC-Cd-1
747 SRC-Gd-2

748 SRC-Gd-3

750 SRC-K- 1

751 SRC-K-2
752 SRC-K-3

753 SRC-K- 3

755 SRC-Li-2

757 SRC-Mg-1
758 SRC-Mg-2

759 SRC-Mg-3

763 SRC-Pb-2

765 SRC-Si-1

766 SRC-SI-2

8 SRC-Sn-1

770 SRC-Sn-3

S72 SRC-Ti-2

773 SRC-Zr-1
774 SRC-Zr-2

775

7773

7817

7839
784

78410
78511
78511

78713
78814
759

7889
78915
79016
7916

79117
79218
7018

79319

79522

79723
79824
79925

80026
80127

80228
80329

80430
8053
80632
632

80733
80834

81036

8113

1440

8154

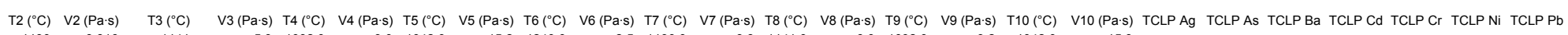

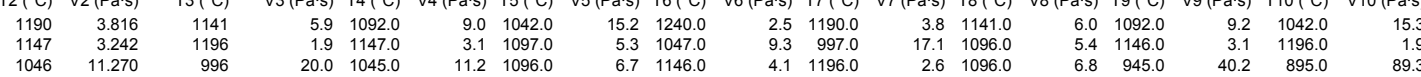

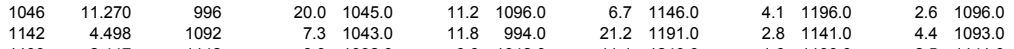

$\begin{array}{llllllll}3.9 & 1092.0 & 6.6 & 1042.0 & 11.1 & 1240.0 & 1.6 & 1190.0 \\ 5.3 & 1093.0 & 9.2 & 1043.0 & 17.0 & 12390 & 1.5 & 1190.0\end{array}$

$\begin{array}{llllll}4.7 & 1092.0 & 7.9 & 1043.0 & 128 & 1240.0\end{array}$

$\begin{array}{lllllll}4.3 & 1092.0 & 7.0 & 1042.0 & 11.2 & 1239.0 \\ 46 & 10920 & 7.5 & 10420 & \end{array}$

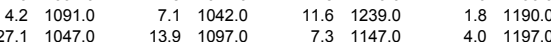

$\begin{array}{llll}3.1 & 1141.0 & 4.9 & 1092.0 \\ 2.0 & 1148.0 & 33 & 10980\end{array}$

$\begin{array}{ll}7.6 & 1489.0 \\ 5 & 1289.0 \\ 5 & 10480\end{array}$

$\begin{array}{rr}1.5 & 1240.0 \\ 9.4 & 998.0\end{array}$

$\begin{array}{ll}2.4 & 1141.0 \\ 3.0 & 141.0\end{array}$

$\begin{array}{ll}3.0 & 1142.0 \\ 2.7 & 1141.0 \\ 3.0 & 1141.0\end{array}$

$\begin{array}{lll}3.0 & 1141.0 \\ 2.7 & 1141.0 \\ 2.2 & 1097.0\end{array}$

$\begin{array}{ll}2.2 & 11097.0 \\ 2.1 & 1190.0 \\ 1.7 & 1097.0\end{array}$

7.21043 .0

$\begin{array}{ll}4.1 & 1092.0 \\ 4.0 & 1092.0 \\ 4.7 & 1093.0\end{array}$

$\begin{array}{ll}4.7 & 1093.0 \\ 4.3 & 1092.0 \\ 4 & -1\end{array}$

$\begin{array}{ll}7.3 & 1043 . \\ 7.3 & -1020 \\ 7.5 & 10420\end{array}$

$\begin{array}{lllll}4.3 & 1092.0 & 7.5 & 1042.0 & 11.3 \\ 7.1 & 1042.0 & 12.3 \\ 3 & & \end{array}$

$\begin{array}{rrr}1047 & 13.543 & 997 \\ 239 & 2.049 & 1190 \\ 148 & 3.370 & 1198\end{array}$

$\begin{array}{llll}7.7 & 1240.0 & 12.6 & 1189.0\end{array}$

$\begin{array}{ll}21.1 & 1388.0 \\ 13.7 & 1338.0\end{array}$

$\begin{array}{ll}3.5 & 1339.0 \\ 23 & 1289.0 \\ 9 & 9970\end{array}$

$\begin{array}{rr}5.4 & 1289.0 \\ 3.6 & 1239.0 \\ 3.7 & 109.0\end{array}$

$\begin{array}{lll}3.2 & 1141.0 \\ 5.6 & 1147.0\end{array}$

$\begin{array}{rr}7.4 & 897.0 \\ 5.0 & 1092.0 \\ 3 & 1197.0\end{array}$

$\begin{array}{rrrrr}7.9 & 1240.0 & 13.0 & 1189.0 & 21.8 \\ 5.6 & 1190.0 & 8.3 & 1140.0 & 14.1 \\ 56 & 1146.0 & 33 . & 1197.0 & 2.1\end{array}$

$\begin{array}{rrrr}6.3 & 1042.0 & 11.4 & 994.0\end{array}$

$\begin{array}{rr}5.4 & 1047.0 \\ 21.4 & 1190.0\end{array}$

2.11141 .0

$\begin{array}{lll}16.7 & 1096.0 \\ 3.7 & 1092.0\end{array}$

$\begin{array}{rrrr}6.3 & 1091.0 & 5.6 & 1042.0\end{array}$

(19.0.0

$\begin{array}{ll}6.2 & 1042.0 \\ 3.3 & 1091.0\end{array}$

$\begin{array}{rr}11.1 & 994.0 \\ 5.7 & 1042.0\end{array}$

\begin{tabular}{ll}
4 & 1240.0 \\
.2 & 1090.0 \\
\hline 4 & 1091.0
\end{tabular}

$\begin{array}{ll}6.0 & 1090.0 \\ 4.4 & 1091.0\end{array}$

$\begin{array}{rr}7.4 & 1190.0 \\ 10.0 & 1040.0 \\ 7.3 & 1041.0 \\ 9.0 & 960\end{array}$

$\begin{array}{ll}17.3 & 1238.0 \\ 11.9 & 1240.0 \\ 164 & 1196.0 \\ 15 & 11200\end{array}$

$\begin{array}{ll}2.1 & 1339.0 \\ 2.4 & 1188.0 \\ 1.8 & 1190.0 \\ 2.0 & 1116.0\end{array}$

$\begin{array}{ll}3.1 & 1289.0 \\ 3.9 & 1139.0 \\ 2.8 & 1141.0 \\ 3.3 & 10960\end{array}$

$\begin{array}{lll}4.4 & 1091.0 \\ 5.4 & 10460\end{array}$

$\begin{array}{ll}5.4 & 1046.0 \\ 3.3 & 1091.0\end{array}$

-1
-1.8

$\begin{array}{lllll}1.5 & 1142.0 & 2.3 & 1092.0 \\ 2.8 & 1338.0 & 4.3 & 1289.0 \\ 20 & 1190.0 & 3 . & 141.0\end{array}$

$\begin{array}{llll}1.2 & 1190.0 & 1.5 & 1142.0\end{array}$

$\begin{array}{llll}2.9 & 1142.0 & 4.7 & 1092.0 \\ 1.8 & 1190.0 & 29 & 1140.0\end{array}$

$\begin{array}{lll}8.0 & 1042.0 \\ 5.0 & 1091.0\end{array}$

$\begin{array}{cc}9.6 & 1190.0 \\ 8.0 & 1042.0\end{array}$

$\begin{array}{rrrrr}4.7 & 1092.0 & 7.3 & 1189.0 \\ 4.8 & 1042.0\end{array}$

$\begin{array}{ll}4.6 & 1050.0 \\ 4.9 & 1091.0\end{array}$

8.51042 .0

15.71239 .0

4.51091 .0

$\begin{array}{rr}8.9 & 1042.0\end{array}$

$\begin{array}{ll}4.4 & 1091.0 \\ 3.2 & 1092.0 \\ 4.0 & 1092.0\end{array}$

$\begin{array}{ll}7.3 & 1042.0 \\ 5.3 & 1042.0 \\ 6.3 & 1042.0 \\ 7.6 & 1042.0\end{array}$

$\begin{array}{rr}11.9 & 1240.0 \\ 9.2 & 1189.0 \\ 9.7 & 1240.0 \\ 13.7 & 1239.0\end{array}$

$\begin{array}{ll}1.0 & 1140.0 \\ 1.8 & 1190.0 \\ 1.7 & 11890\end{array}$

$\begin{array}{ll}3.2 & 11992.0 \\ 2.7 & 1140.0 \\ 2.7 & 114.0\end{array}$

$\begin{array}{ll}5.4 & 1042.0 \\ 4.0 & 1092.0 \\ 4.5 & 10910\end{array}$

$\begin{array}{ll}7.5 & 1042.0 \\ 9.4 & \end{array}$

$\begin{array}{ll}9.4 & \\ 6.4 & 1042.0 \\ 7.7 & 1041.0\end{array}$ 
\# Glass ID

739 SRC-Ca-2

741 SRC-Ce--
742 SRC-Ce-2

742 SRC-Ce-2
743 SRC-Ce-3

745 SRC-E- 1

746 SRC-Ed-1

747 SRC-Gd-2
748 SRC-Gd-3

749 SRC-Gd-4

750 SRC-K-1

753 SRC-K- -31

754 SRC-Li-1

755 SRC-Li-2

57 SRC-Mg-1

758 SRC-Mg-1

(1)

761 SRC-Na-2

$763 \mathrm{SRC}^{2}$

764 SRC-Pb-3

765 SRC-Si- -1

767 SRC-Sm-1

769 SRC-Sn-2

770 SRC-Sn- 3

772 SRC-Ti-2

773 SRC-Zr-1
774 SRC-Zr-2
7751

775

7784

781

7828
7839
784

785

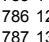

78814
79915
79015

79016
79117
709

79218
79319
79319

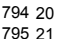

79622
79923

79824
79925
79025

8026
80127
50127

80228
80329

80539
80032

8006
80733
8

${ }_{809}^{808}$

811

81238
81238

814

81541
81642
8

81743
81844
6846

82046

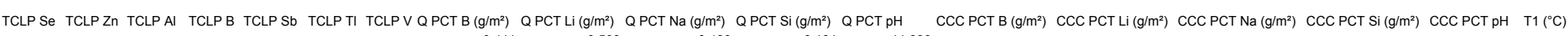

$0.411 \quad 0.503 \quad 0.191 \quad 11.090$

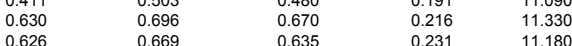

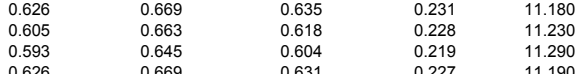

$\begin{array}{lllll}0.626 & 0.669 & 0.631 & 0.227 & 11.190 \\ 0.718 & 0.773 & 0.731 & 0.269 & 11.260\end{array}$

$\begin{array}{ll}0.718 & 0.73 \\ 0.342 & 0.431\end{array}$

$\begin{array}{lllll}0.558 & 0.554 & 0.541 & 0.201 & 11.180 \\ 0.580 & 0.562 & 0.579 & 0.216 & 11.190 \\ 0.620 & 0.588 & 0.202 & 11.180\end{array}$

$\begin{array}{lllll}0.472 & 0.504 & 0.481 & 0.182 & 11.100 \\ 0.602 & 0.675 & 0.639 & 0.222 & 11.31\end{array}$

$\begin{array}{lllll}0.253 & & 0.314 & 0.108 & 10.260 \\ 0.315 & 0.361 & 0.363 & 0.130 & 10.610 \\ 0.617 & 0.668 & 0.639 & 0.243 & 11300\end{array}$

$\begin{array}{lllll}0.617 & 0.668 & 0.639 & 0.243 & 11.300 \\ 1.270 & 1.187 & 1.116 & 0.320 & 11.550 \\ 4.042 & 3.419 & 3.306 & 0.651 & 11.980\end{array}$

$\begin{array}{lllll}0.140 & 0.239 & 0.165 & 0.088 & 10.160 \\ 0.422 & 0.484 & 0.454 & 0.170 & 10.920 \\ 0.542 & 0.918 & 0.571 & 0.193 & 11210\end{array}$

$\begin{array}{lllll}0.542 & 0.618 & 0.571 & 0.200 & 11.240 \\ 0.498 & 0.573 & 0.534 & 0.193 & 11.210 \\ 0.465 & 0.568 & 0537 & 0.19 & 11.52\end{array}$

$\begin{array}{lllll}0.935 & 0.990 & 0.537 & 0.191 & 11.200 \\ 0.275 & 0.359 & 0.940 & 0.285 & 11.550 \\ 0.332 & 0.826 & 0.735 & 0.151 & 11350\end{array}$

$\begin{array}{lllll}0.792 & 0.826 & 0.795 & 0.290 & 11.380 \\ 0.541 & 0.615 & 0.568 & 0.202 & 11.170\end{array}$

$\begin{array}{lllll}0.550 & 0.616 & 0.576 & 0.199 & 11.270 \\ 0.483 & 0.538 & 0.493 & 0.188 & 11.110 \\ 0.52 & 0.570 & 0.39 & 0.168 & 11230\end{array}$

$\begin{array}{lllll}0.483 & 0.538 & 0.493 & 0.188 & 11.110 \\ 0.582 & 0.670 & 0.631 & 0.235 & 11.240 \\ 0.462 & 0.538 & 0.498 & 0.168 & 11.230 \\ & & & & \end{array}$




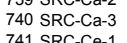

741 SRC-Ce-1

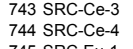

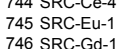

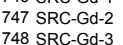

748 SRC-Gd-3

750 SSC KC- -1
751 SRC-K-

752 SRC-K- 3

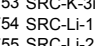

755 SRCLL-Li-
756 SRC-Li-3

157 SRC-Mg-1

758 SRC-Mg--

160 SRC-Na-1

761 SRC-Na-2

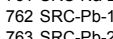

763 SRC.PD-2

765 SRCCS-Si-1
766 SRC-Si-2

767 SRC-Sm-1

769 SRC-Sn-2

770 SRC-Sn-3
7711 SRC-T-1-1

772 SRC-Ti-2

773 SRC-ZZ-1
774 SRC-Zr-2

7751

777

7784
7795

7806
7817

7828
7839

7839
78410

78512
78713

78713
78814
78915

79016
79117
793

\begin{tabular}{l}
79218 \\
79319 \\
\hline 79420
\end{tabular}

79420
79521

79622
797
77924

79824
79925

80127

80228
80329

80431
80532

80733

80935

81137

81238
88339
8

81541

81642

81844

82046 

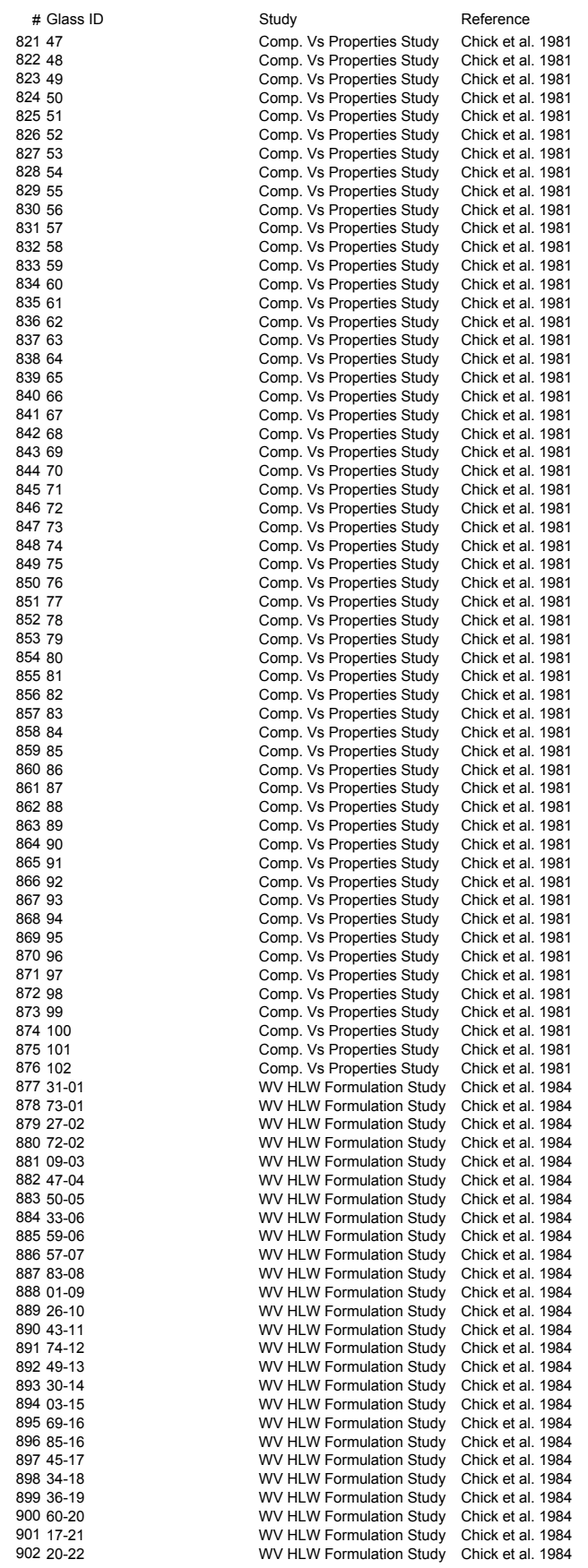

$0.0800 \quad 0.0000 \quad 0.1500 \quad 0.0000$ $\begin{array}{llll}0.0800 & 0.0000 & 0.1500 & 0.0000 \\ 0.0800 & 0.0200 & 0.0000 & 0.0000 \\ 0.08000 & 0.0200 & 0.000 & 0.000\end{array}$ $\begin{array}{llll}0.0800 & 0.0200 & 0.00000 & 0.00000 \\ 0.0000 & 0.0000 & 0\end{array}$ $\begin{array}{llll}0.0000 & 0.0600 & 0.0000 & 0.0000 \\ 0.0000 & 0.0000 & 0.0000 & 0.0000\end{array}$ $0.0800 \quad 0.2000 \quad 0.0000 \quad 0.0000$ $0.0000 \quad 0.0000 \quad 0.0400 \quad 0.2400$ $0.00000 .00000 .0000 \quad 0.2400$ $0.0000 \quad 0.2000 \quad 0.0000 \quad 0.0800$

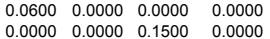
$\begin{array}{llll}0.0000 & 0.00000 & 0.1500 & 0.0000 \\ 0.0000 & 0.2000 & 0.1500 & 0.0000\end{array}$ $\begin{array}{llll}0.0800 & 0.0000 & 0.1500 & 0.00000 \\ 0.0000 & 0.0000 & 0.0000 & 0.2400\end{array}$ $\begin{array}{llll}0.0000 & 0.0000 & 0.0000 & 0.2400 \\ 0.0800 & 0.0000 & 0.1500 & 0.2400\end{array}$ $\begin{array}{llll}0.0000 & 0.1500 & 0.2400 \\ 0800 & 0.0000 & 0.0000 & 0.0000\end{array}$ $\begin{array}{lllll}0.0800 & 0.2000 & 0.0000 & 0.0000\end{array}$ $\begin{array}{lllll}0.0800 & 0.0000 & 0.0000 & 0.2400 \\ 0.0800 & 0.2000 & 0.0000 & 0.2400\end{array}$ $\begin{array}{llll}0.0000 & 0.1100 & 0.0000 & 0.2400 \\ 0.0000 & 0.0000 & 0.0000 & 0.2400\end{array}$

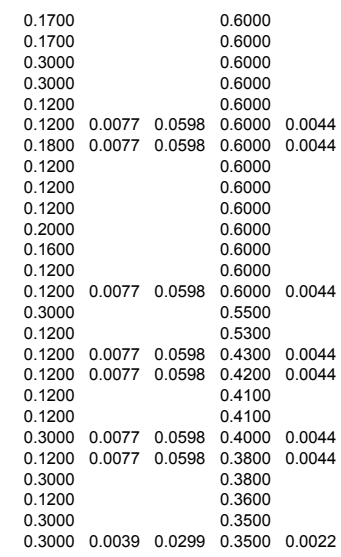


$\begin{array}{ll}0.0163 & 0.0033 \\ 0.0163 & 0.0033\end{array}$

$0.0163 \quad 0.0033$

$\begin{array}{ll}0.0163 & 0.0033 \\ 0.0163 & 0.0033\end{array}$

$\begin{array}{ll}0.0163 & 0.0033 \\ 0.0163 & 0.0033\end{array}$

$0.0081 \quad 0.0017$
0.0101

0.0101
0.0101

0.0101
0.0101

0.0051
0.0042
0.0042

0.0042

0.0042

0.0042
0.0042

0.002
0.0040
0.0040

0.0040

0.0040
0.0040

0.0040
0.0040

0.0020 


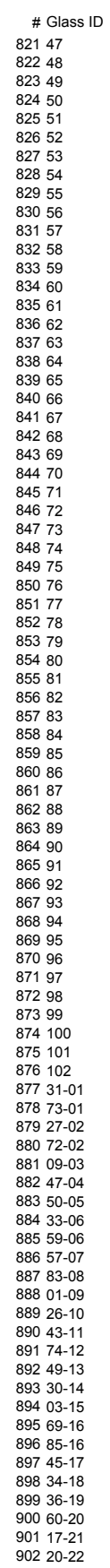

B2O3-a CaO-a Fe2O3-a FeO-a K2O-a Li2O-a $\begin{array}{lll}0.0714 & 0.0989 & 0.0650 \\ 0.064 & 0.0357 & 0.046\end{array}$

$\begin{array}{lll}0.0604 & 0.0359 & 0.0476 \\ 0.159 & 0.0000 & 0.0267\end{array}$

$\begin{array}{lll}0.1359 & 0.0000 & 0.0000 \\ 0.1344 & 0.0000 & 0.0000 \\ 0.0739 & 0.000 & 0.073\end{array}$

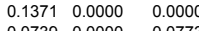

$\begin{array}{llll}0.0615 & 0.0557 & 0.0573\end{array}$

$\begin{array}{lll}0.1272 & 0.0081 & 0.0000 \\ 0.1422 & 0.1119 & 0.0000 \\ 0.000\end{array}$

$\begin{array}{lll}0.0638 & 0.0000 & 0.0000 \\ 0.146 & 0.0000 & 0.0000\end{array}$

$\begin{array}{lll}0.1346 & 0.0000 & 0.0000 \\ 0.0701 & 0.0976 & 0.0000\end{array}$

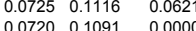

$\begin{array}{lll}0.1278 & 0.0292 & 0.0656 \\ 0.1446 & 0.1122 & 0.0743\end{array}$

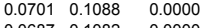

$\begin{array}{lll}0.0687 & 0.1082 & 0.0000 \\ 0.0687 & 0.1090 & 0.0000\end{array}$

$\begin{array}{llll}0.1274 & 0.0009 & 0.0000 \\ 0 & 0.1317 & 0.1013 & 0.0000\end{array}$

$\begin{array}{lll}0.0713 & 0.0093 & 0.0000 \\ 0.0975 & 0.0646 & 0.0419\end{array}$

$\begin{array}{llll}0.0764 & 0.0393 & 0.0268\end{array}$

$\begin{array}{lll}0.1484 & 0.0468 & 0.0309\end{array}$

$\begin{array}{lll}0.1017 & 0.0664 & 0.0442 \\ 0.0768 & 0.0401 & 0.0252\end{array}$

$\begin{array}{llll}0.0919 & 0.0000 & 0.0417\end{array}$

$\begin{array}{lll}0.0867 & 0.1141 & 0.0291 \\ 0.0889 & 0.0578 & 0.0394 \\ 0.0962 & 0.058 & 0.031\end{array}$

$\begin{array}{llll}0.0891 & 0.0504 & 0.0341\end{array}$

$\begin{array}{lll}0.0902 & 0.0586 & 0.0376 \\ 0.0875 & 0.0503 & 0.0334\end{array}$

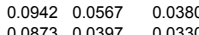

$\begin{array}{llll}0.0933 & 0.0584 & 0.0404\end{array}$

$\begin{array}{lll}0.0843 & 0.0488 & 0.0330 \\ 0.0946 & 0.0576 & 0.0386 \\ 0.0927 & 0.569 & 0.000\end{array}$

$\begin{array}{lll}0.0778 & 0.0505 & 0.0324 \\ 0.0927 & 0.0569 & 0.0000\end{array}$

$\begin{array}{lll}0.0867 & 0.0487 & 0.0786\end{array}$

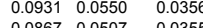

$\begin{array}{lll}0.0504 & 0.0318 & 0.0793 \\ 0.0532 & 03330 & 0.0000\end{array}$

$\begin{array}{llll}0.0287 & 0.0327 & 0.0000\end{array}$

$\begin{array}{lll}0.1204 & 0.0899 & 0.0764\end{array}$

$\begin{array}{lll}0.1538 & 0.0189 & 0.0955 \\ 0 & 0.194 & 0.1176 \\ 0\end{array}$

$\begin{array}{lll}0.1384 & 0.1176 & 0.0000 \\ 0.1194 & 0.1115 & 0.0805\end{array}$

$\begin{array}{lll}0.1183 & 0.0363 & 0.0000 \\ 0.1320 & 0.0391 & 0.0824 \\ 0.0922 & .0 .87 & 0.520\end{array}$

$\begin{array}{lll}0.1320 & 0.0391 & 0.0824 \\ 0.0982 & 0.1087 & 0.0520\end{array}$

$\begin{array}{lll}0.0000 & 0.1496 & 0.0000 \\ 0.0000 & 0.1530 & 0.0000\end{array}$

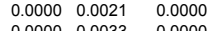

$\begin{array}{lll}0.0000 & 0.0033 & 0.0000 \\ 0.0000 & 0.0000 & 0.1719\end{array}$

$\begin{array}{lll}0.0601 & 0.0015 & 0.0000 \\ 0.0000 & 0.0013 & 0.0000 \\ 0.1925 & 0.014 & 0.000\end{array}$

$\begin{array}{lll}0.1922 & 0.0014 & 0.0000 \\ 0.1945 & 0.0016 & 0.0000\end{array}$

$\begin{array}{lll}0.0000 & 0.0423 & 0.2002 \\ 0.1947 & 0.0025 & 0.0000 \\ 0.1937 & 0.020 & 0.063\end{array}$

$\begin{array}{lll}0.1944 & 0.0025 & 0.0000 \\ 0.0000 & 0.0039 & 0.2043 \\ 0.0000 & 0.0031 & 0.0000\end{array}$

$0.19310 .0020 \quad 0.0680$

$\begin{array}{lll}0.0000 & 0.0031 & 0.0000 \\ 0.0000 & 0.1492 & 0.0000 \\ 0.0000 & 0.1548 & 0.000\end{array}$

$\begin{array}{lll}0.2005 & 0.1511 & 0.0000 \\ 0.0000 & 0.1548 & 0.0000\end{array}$

$\begin{array}{lll}0.0000 & 0.0031 & 0.2086 \\ 0.0000 & 0.1568 & 0.2037 \\ 0.0000 & 0.1007 & 0.000\end{array}$

$\begin{array}{lll}0.0000 & 0.1568 & 0.2037 \\ 0.0000 & 0.1584 & 0.2000 \\ 0.1092 & 0.0020 & 0.000\end{array}$

$\begin{array}{lll}0.0000 & 0.0007 & 0.0000 \\ 0.1992 & 0.0020 & 0.0000 \\ 0.000\end{array}$

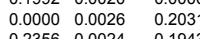

$\begin{array}{lll}0.2356 & 0.0024 & 0.1943 \\ 0.1126 & 0.0022 & 0.2050 \\ 0.0000 & 0.0039 & 0.2085\end{array}$

\begin{tabular}{lll} 
MgO-a & Na2O-a & NiO-a \\
\hline 0.0511 & 0.1009 & 0.0270 \\
0.0000 & 0.1336 & 0.0192 \\
\hline 0050 & 0.171 &
\end{tabular}

$\begin{array}{lll}0.0000 & 0.1471 & 0.0000 \\ 0.0533 & 0.0896 & 0.0000\end{array}$

$\begin{array}{llll}0.0488 & 0.0883 & 0.000 \\ 0.0508 & 0.0867 & 0.0000 \\ 0.054 & 0.1413 & 0.031\end{array}$

$\begin{array}{llll}0.0554 & 0.1413 & 0.0315\end{array}$

$\begin{array}{llll}0.0000 & 0.0966 & 0.0000 \\ 0.0464 & 0.1445 & 0.0226\end{array}$

$\begin{array}{lll}0.0000 & 0.1229 & 0.0225\end{array}$

$\begin{array}{lll}0.0498 & 0.1429 & 0.0000 \\ 0.0000 & 0.1022 & 0.0308\end{array}$

$\begin{array}{lll}0.0000 & 0.1590 & 0.0000 \\ 0.0517 & 0.1130 & 0.0000\end{array}$

$\begin{array}{lll}0.0000 & 0.0790 & 0.0000\end{array}$

$\begin{array}{llll}0.0519 & 0.0923 & 0.0290 \\ 0.0000 & 0.1405 & 0.0288\end{array}$

$\begin{array}{lll}0.0000 & 0.1332 & 0.0286\end{array}$

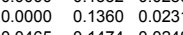

$0.0000 \quad 0.0979 \quad 0.025$

$\begin{array}{llll}0.0000 & 0.1270 & 0.028 \\ 0.0265 & 0.1338 & 0.0174\end{array}$

\begin{tabular}{lllll}
0.0178 & 0.1124 & 0.0125 \\
\hline & 0.224 & 0.120 & 0.1750
\end{tabular}

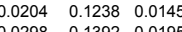

$\begin{array}{lll}0.0298 & 0.1392 & 0.0195 \\ 0.0167 & 0.1091 & 0.0107\end{array}$

$\begin{array}{llll}0.0196 & 0.1233 & 0.0147\end{array}$

$\begin{array}{llll}0.0000 & 0.1287 & 0.0174 \\ 0.0541 & 0.1256 & 0.0148\end{array}$

$\begin{array}{lll}0.0243 & 0.0849 & 0.0165 \\ 0.0205 & 0.1557 & 0.0145 \\ 0.0246 & 0.129 & 0.0250\end{array}$

$\begin{array}{lll}0.0246 & 0.1291 & 0.0200 \\ 0.0220 & 0.1235 & 0.0154\end{array}$

$\begin{array}{lll}0.0264 & 0.1319 & 0.018\end{array}$

$\begin{array}{llll}0.0214 & 0.1207 & 0.0143 \\ 0.0255 & 0.1310 & 0.0169\end{array}$

$\begin{array}{llll}0.0255 & 0.1310 & 0.0169 \\ 0.0211 & 0.11151 & 0.0152\end{array}$

$\begin{array}{lll}0.0244 & 0.1285 & 0.017 \\ 0.0228 & 0.1274 & 0.015\end{array}$

$\begin{array}{llll}0.0233 & 0.1280 & 0.0000 \\ 0.0227 & 0.1241 & 0.0377\end{array}$

$\begin{array}{llll}0.0000 & 0.0871 & 0.0360\end{array}$

$\begin{array}{lll}0.0000 & 0.0929 & 0.0000 \\ 0.0000 & 0.0873 & 0.0000\end{array}$

$\begin{array}{llll}0.00593 & 0.1654 & 0.0000 \\ 0.055 & 0.000\end{array}$

$\begin{array}{llll}0.0557 & 0.0972 & 0.0394 \\ 0.0000 & 0.1527 & 0.0161\end{array}$

$\begin{array}{llll}0.0000 & 0.1231 & 0.0000 \\ 0.0000 & 0.1326 & 0.0000\end{array}$

$\begin{array}{llll}0.0000 & 0.1326 & 0.0000 \\ 0.0388 & 0.1336 & 0.0000\end{array}$

0.3878
0.3526

5028

0.5395
.5406

0.5432
0.4486

0.3605
0.3958
0.3732

0.3732
0.3754
0.5265

0.5456

0.3565

0.4024
0.3863
0.3822

0.3822
0.3814
0.366

0.3576
0.3676
0.5394

0.5394
0.3690
0.5514

0.5514
0.4427
0.4290

0.4290
0.4676
0.4181

0.4409

0.4315
0.4388

0.4388

0.4512
0.4421
0.4460

0.4421
0.4460
0.4371
0.4464

0.4464
0.4274

0.4274
0.4433
0.4385

0.4538

0.4453

0.5115
0.5210

0.5263

0.5252
0.3507
0.4184

0.3798
0.3645

.5080
.5460

.4193
0.625

$\begin{array}{ll}0.1336 & .0000 \\ 0.1665 & 0.6025 \\ 0.1740 & 0.5900\end{array}$

$\begin{array}{ll}0.2996 & 0.6145 \\ 0.3084 & 0.6047 \\ 0.1200 & 0.0267\end{array}$

$\begin{array}{llllll}0.0008 & 0.1328 & 0.0069 & 0.0533 & 0.6055 & 0.0039 \\ 0.0008 & 0.1816 & 0.0070 & 0.0547 & 0.6053 & 0.0040\end{array}$

$\begin{array}{ll}0.0145 & 0.0029 \\ 0.0149 & 0.0030\end{array}$

0.0008

0.1193

0.6043
0.6014
0.6237

$\begin{array}{ll}0.1227 & 0.6237 \\ 0.2103 & 0.58\end{array}$

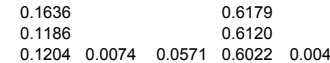

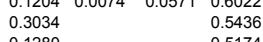

$\begin{array}{llllll}0.0009 & 0.1272 & 0.0075 & 0.0580 & 0.4167 & 0.0043 \\ 0.0008 & 0.1239 & 0.0074 & 0.0574 & 0.4398 & 0.0042\end{array}$

$\begin{array}{lll}.4398 & 0.0042 \\ 0.4226 & & \\ 0.4274 & 0.0042\end{array}$

$\begin{array}{llllll}0.0008 & 0.2984 & 0.0074 & 0.0576 & 0.4063 & 0.0042 \\ 0.0008 & 0.1215 & 0.0071 & 0.0553 & 0.3892 & 0.0041\end{array}$

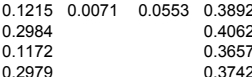

$\begin{array}{lllll}0.2956 & 0.0038 & 0.0292 & 0.3740 & 0.0022\end{array}$ 


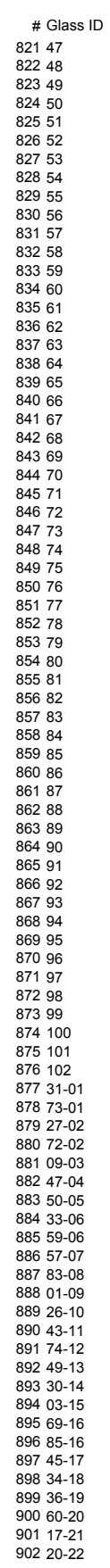

Sm203-a SnO-a SnO2-a SO3-a SrO-a Tc207-a TeO2-a ThO2-a TiO2-a T12O-a T1203-a U308-a U02-a UO3-a V2O5-a wO3-a Y2O3-a ZnO-a Sum-a $T_{M}\left({ }^{\circ} \mathrm{C}\right) \quad T_{L, G}\left({ }^{\circ} \mathrm{C}\right) \quad T_{L, U}\left({ }^{\circ} \mathrm{C}\right) T_{1 \%}\left({ }^{\circ} \mathrm{C}\right)$ Primary Phase 0.0855 $\begin{array}{lll}0.0000 & 1.0000 & 1250 \\ 0.0000 & 1.0000 & 1300\end{array}$ $\begin{array}{lll}0.0000 & 1.0000 & 1300 \\ 0.0780 & 1.0000 & 1200\end{array}$

$\begin{array}{lll}0.0751 & 1.0000 & 1200 \\ 0.0723 & 1.0000 & 1200\end{array}$

$\begin{array}{llll}0.0775 & 1.0000 & 1200\end{array}$

$\begin{array}{lll}0.0000 & 1.0000 & 1250 \\ 0.0000 & 1.0000 & 1250\end{array}$

$\begin{array}{lll}0.0000 & 1.0000 & 1250 \\ 0.0737 & 1.0000 & 1050\end{array}$

$0.0668 \quad 1.0000 \quad 1150$

$\begin{array}{lll}0.0000 & 1.0000 & 1150 \\ 0.0000 & 1.0000 & 1300\end{array}$

$\begin{array}{lll}0.0000 & 1.0000 & 1050\end{array}$

$\begin{array}{lll}0.0000 & 1.0000 & 1250\end{array}$

$\begin{array}{lll}0.0647 & 1.0000 & 1300 \\ 0.0744 & 1.0000 & 1150\end{array}$

$\begin{array}{lll}0.0735 & 1.0000 \quad 1200\end{array}$

$\begin{array}{lll}0.0719 & 1.0000 & 1200 \\ 0.0731 & 1.0000 & 1200\end{array}$

$\begin{array}{lll}0.0000 & 1.0000 & 1400 \\ 0.0772 & 1.0000 & 1400 \\ 0.004\end{array}$

$\begin{array}{lll}0.0404 & 1.0000 & 1280 \\ 0.0261 & 1.0000 & 1570\end{array}$

$0.0385 \quad 1.0000 \quad 1350$

$\begin{array}{lll}0.0303 & 1.0000 & 1250 \\ 0.0426 & 1.0000 & 1150\end{array}$

$\begin{array}{lll}0.0413 & 1.0000 & 1450\end{array}$

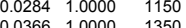

$\begin{array}{lll}0.0366 & 1.0000 & 1150 \\ 0.0322 & 1.0000 & 1250\end{array}$

$0.0383 \quad 1.0000 \quad 1450$

$\begin{array}{lll}0.0316 & 1.0000 & 1210 \\ 0.0000 & 1.0000 & 1250 \\ 0.0758 & 1.000 & 1250\end{array}$

$\begin{array}{lll}0.0745 & 1.0000 \quad 1250\end{array}$

$\begin{array}{lll}0.0388 & 1.0000 & 1250 \\ 0.0324 & 1.0000 & 1350\end{array}$

$\begin{array}{lll}0.0368 & 1.0000 & 1250 \\ 0.0331 & 1.0000 & 1550 \\ 0 & \end{array}$

$\begin{array}{lll}0.0370 & 1.0000 & 1350\end{array}$

$\begin{array}{lll}0.0330 & 1.0000 & 1300 \\ 0.0351 & 1.0000 & 1410\end{array}$

$\begin{array}{lll}0.0338 & 1.0000 & 1250\end{array}$

$0.0000 \quad 1.0000 \quad 1610$

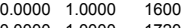

$0.0000 \quad 1.0000 \quad 1600$

$\begin{array}{lll}0.0733 & 1.0000 & 1450\end{array}$

$\begin{array}{lll}0.0886 & 1.0000 & 1050 \\ 0.0000 & 1.0000 & 1350\end{array}$

$\begin{array}{lll}0.0000 & 1.0000 & 1350 \\ 0.0762 & 1.0000 & 1450\end{array}$

$\begin{array}{lll}0.0624 & 1.0000 & 1400 \\ 0.0079 & 1.0000 & 1250\end{array}$

0.0000

1.0001
1.0000

1.0000
1.0000

0.0029
0.0030

${ }_{0.0232}^{0.025}$

0.0696
0.0715

1.0000

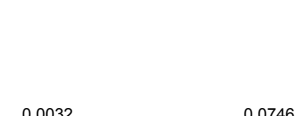

1.0000
1.0000
1.0000

0.0032

0.0746

0.0242

$\begin{array}{ll}0.0032 & 0.0757 \\ 0.0032 & 0.0750\end{array}$

0.0245
0.0243

0.0032
0.0031

0.0753
0.0722

${ }_{0.0234}^{0.0244}$

0.0016

0.0382 


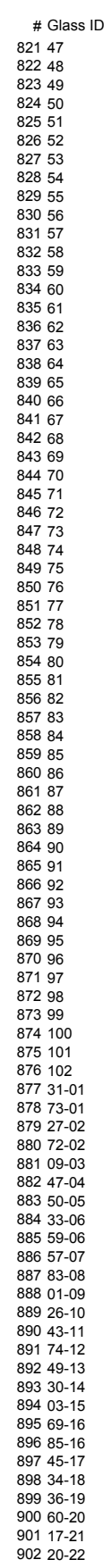

$\mathrm{C}_{800^{\circ} \mathrm{C}} \mathrm{C}_{850^{\circ} \mathrm{C}} \mathrm{C}_{900^{\circ} \mathrm{C}} \mathrm{C}_{950^{\circ} \mathrm{C}} \mathrm{C}_{1000^{\circ} \mathrm{C}} \mathrm{C}_{1050^{\circ} \mathrm{C}} \mathrm{C}_{1100^{\circ} \mathrm{C}} \mathrm{C}_{1150^{\circ} \mathrm{C}} \mathrm{C}_{1200^{\circ} \mathrm{C}}$ Quenched Visual//OM
Quenched XRD Nt\% Ni(Fe,Ti)2O4 2 wt\% ( $\mathrm{Ca}$ Fe $\mathrm{Mg}) \mathrm{SiO} 3 \mathrm{CaT}$

$18.5 \mathrm{wt} \%(\mathrm{Mg}, \mathrm{Zn}) \mathrm{Cr} 2 \mathrm{O} 4$

w.5 wi\% (Mg, Zn) Cr2O

(9w\% (Mg,Zn)Cr2O4

$20 \mathrm{wt} \%(\mathrm{Mg}, \mathrm{Zn}) \mathrm{Cr} 2 \mathrm{C} 4 \quad 18.5 \mathrm{wt} \%(\mathrm{Mg}, \mathrm{Zn}) \mathrm{Cr} 2 \mathrm{O}$

$9.5 \mathrm{wt} \%(\mathrm{Cr}, \mathrm{Fe}) \mathrm{CrO} 32.4 \mathrm{wt} \%(\mathrm{Cr}, \mathrm{Fe}) \mathrm{CrO} 3 /(\mathrm{Na}, \mathrm{Ca}) \mathrm{Al} 3 \mathrm{SS} 5 \mathrm{~S}$

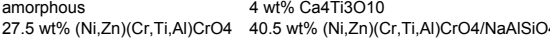
$\begin{array}{ll}\text { amorphous } & 15.5 \mathrm{wt} \% \text { NaAlSiO4/MgTi2O5 } \\ 11.5 \text { wt\% Cr203TiO2 } & 195 \mathrm{wt} \% \text { Cr2O3/CaTiSiO }\end{array}$

amorphous

21 wi\% NaALSOO4/CAAl2SiO

16 wt\% Fe2TiO5/ZnAl204

30 wt\% (Ca,Fe,Mg)SiO3/NiFe2O4

6 wt\% NaALSiO4/Ca2A2SiOZ

18.5 wi\% (Mg,Ni)(Al,Cr)CrO4

78 wt\% NaAlSiO4/Ca2Al2SiO

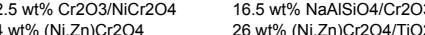

$14 \mathrm{wt} \%$ spinel

$5.9 \mathrm{wt} \%$ spine

$9.4 \mathrm{wt} \%$ spinel

$13.9 \mathrm{wt} \%$ spine

$7.2 \mathrm{w} \%$ spinel

$11.3 \mathrm{wt} \%$ spinel

$9.4 \mathrm{wt} \%$ spinel
$12.9 \mathrm{wt} \%$ spinel

$12.7 \mathrm{wt} \%$ spinel

$10.2 \mathrm{w} \%$ spinel

amorphous

$10.7 \mathrm{wt} \%$ spine

$12.8 \mathrm{w} \%$ spine

15.5 wt\% spinel

2 wt\% unknown
18.4 wt\% spine

10.9 wt\% Cr2O3

amorphous
amorphous
amorphous

amorphous

amorphous

0.05 crystallinity

amorphous
amorphous

amorphous

amorphous
0.16 crystallinity

amorphous

amorphous

amorphous

amorphous
0.14 crystallinity

amorphous

amorphous
0.12 crystallinity

0.71 crystallinity
$12500 \quad 80000$

$\begin{array}{rr}1250.0 & 2.300 \\ 1250.0 & 52300\end{array}$

1250.0
1250.0000

$\begin{array}{ll}1250.0 & 1.300 \\ 1250.0 & >580\end{array}$

$\begin{array}{rr}1250.0 \\ 1250.0 \\ 12500 & 0.390 \\ 7580 & -500 \\ 12500 & 7.400\end{array}$

$\begin{array}{lr}1250.0 & 7.400 \\ 12500 & 29300\end{array}$

$\begin{array}{rr}1250.0 & 29.300 \\ 1250.0 & 3.700 \\ 12500 & 13000\end{array}$

\begin{tabular}{rr}
1250.0 & 13.000 \\
1250.0 & 9.000 \\
\hline
\end{tabular}

$\begin{array}{ll}1250.0 & 0.900 \\ 1250.0 & 5.100\end{array}$

\begin{tabular}{ll}
1250.0 & 6.800 \\
1250.0 & 5.700 \\
\hline
\end{tabular}

$12500 \quad 68.000$
1250

$\begin{array}{ll}1250.0 & 15.000 \\ 1250.0 & 11.500 \\ 12500 & 23000\end{array}$

$\begin{array}{ll}1250.0 & 23.000 \\ 1250.0 & 25.000\end{array}$

\begin{tabular}{ll}
1250.0 & 6.100 \\
1250.0 & 4.600 \\
\hline &
\end{tabular}

$\begin{array}{lr}1250.0 & 59.000 \\ 1250.0 & 72.000\end{array}$

$\begin{array}{rr}1250.0 & 2.800 \\ 1250.0 & 18.000\end{array}$

$\begin{array}{ll}1250.0 & 13.000 \\ 1250.0 & 35.000\end{array}$

$\begin{array}{ll}1250.0 & 7.000 \\ 12500 & 10.000\end{array}$

$12500 \quad 14.000$

$1250.0 \quad 15.000$

\begin{tabular}{rr}
1250.0 & 8.200 \\
1250.0 & 2.700 \\
\hline
\end{tabular}

$\begin{array}{lr}1250.0 & 8.600 \\ 12500 & 21.000\end{array}$

\begin{tabular}{rr}
1250.0 & 6.100 \\
1250.0 & 64.000 \\
\hline
\end{tabular}

$\begin{array}{ll}1250.0 & 64.0000 \\ 1250 . & \end{array}$

$\begin{array}{rr}1250.0 & 230.000 \\ 1250.0 & 19.000 \\ 1250.0 & 0.130\end{array}$

\begin{tabular}{lr}
12550.0 & 4.000 \\
12500 & 11.000 \\
\hline
\end{tabular}

\begin{tabular}{rr}
$\begin{array}{rr}1255.0 \\
1250.0\end{array} \quad \begin{aligned} 16.000 \\
1.600\end{aligned}$ \\
\hline 1250 & 3000
\end{tabular}

$\begin{array}{ll}1250.0 & 0.450 \\ 1540 & 3000\end{array}$

$1460.0 \quad 3.000$

$\begin{array}{ll}1431.0 & 3.000 \\ 1332.0 & 3.000 \\ 14000 & 3000\end{array}$

3.000
3.000

$\begin{array}{ll}1522.0 & 3.000 \\ 15030 & 3.000\end{array}$

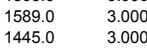

$\begin{array}{ll}1156.0 & 3.0000 \\ 15030 & 3000\end{array}$

3.000

\begin{tabular}{ll}
$\begin{array}{ll}1806.0 \\
1176.0\end{array} \quad 3.000$ \\
\hline .000
\end{tabular}

3.000

$1399.0 \quad 3.000$

$\begin{array}{ll}\begin{array}{ll}1307.0 \\ 1330.0\end{array} & 3.000 \\ 3.000 & \end{array}$

$\begin{array}{ll}1343.0 & 3.000 \\ 12490 & 3.000\end{array}$

$\begin{array}{rr}1258.0 & 3.000 \\ 870.0 & 3.000\end{array}$

$\begin{array}{ll}870.0 & 3.000 \\ 073.0 & 3.000\end{array}$ 


$$
\mid
$$




$$
\mid
$$




$$
\square
$$




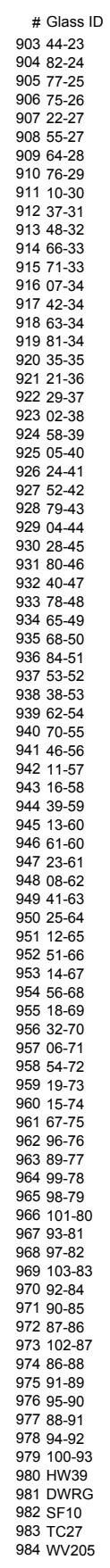

$\begin{array}{ll}\text { Study } & \text { Reference } \\ \text { WV HLW Formulation Study } & \text { Chick et al. } 198\end{array}$ WV HLW Formulation Study Chick et al 1984 WV HLW Formulation Study Chick et al. 1984 WV HLW Formulation Study Chick et al. 1984 WV HLW Formulation Study Chick et al. 1984 WV HLW Formulation Study Chick et al. 1984 WV HLW Formulation Study
Chick et al. 1984
WV HLW Formulation Study
Chick et al 1984 WV HLW Formulation Study Chick et al. 1984 WV HLW Formulation Study Chick et al. 1984 WV HLW Formulation Study Chick et al. 1984 WV HLW Formulation Study Chick et al. 1984 WV HLW Formulation Study Chick et al. 1984 WV HLW Formulation Study Chick et al. 1984 WV HLW Formulation Study Chick et al. 1984 WV HLW Formulation Study Chick et al. 1984 WV HLW Formulation Study
Chick et al. 1984
WV HLW Formulation Study Chick ta. 1984 WV HLW Formulation Study Chick et al. 1984 WV HLW Formulation Study Chick et al. 1984 WV HLW Formulation Study
Chick et al. 1984
WV HLW Formulation Study
Chick et al. 1984 WV HLW Formulation Study Chick et al. 1984 WV HLW Formulation Study Chick et al. 1984 WV HLW Formulation Study Chick et al. 1984
WV HLW Formulation Study Chick et al 1984 WV HLW Formulation Study Chick et al. 1984 WV HLW Formulation Study Chick et al. 1984 WV HLW Formulation Study Chick a 1984 WV HLW Formulation Study Chick et al. 1984 WW HLW Fomulation Sludy Chick et al. 1984 WV HLW Formulation Study Chick et al. 1984
WV HLW Formulation Study
Chick et al 1984 WV HLW Formulation Study Chick et al. 1984 WV HLW Formulation Study Chick et al. 1984
WV HLW Formulation Study Chick et al 1984 WV HLW Formulation Study Chick et al. 1984 $\begin{array}{ll}\text { WV HLW Formulation Study } & \text { Chick et al. } 1984 \\ \text { WV HLW Formulation Study } & \text { Chick et al. } 1984\end{array}$ WV HLW Formulation Study Chick et al. 1984 WV HLW Formulation Study Chick et al. 1984 WV HLW Formulation Study Chick at. 1984 WV HLW Formulation Study Chick et al. 1984 WV HLW Formulation Study Chick et al. 1984 WV HLWW Formulation Study Chick et al. 1984 WV HLW Formulation Study Chick et al. 1984 WV HLW Formulation Study Chick et al. 1984 WV HLW Formulation Study Chick tal. 1984 WV HLW Formulation Study Chick et al. 1984 WV HLW Formulation Study Chick et al. 1984 WV HLW Formulation Study Chick et al. 1984 WV HLW Formulation Study Chick et al. 1984 WV HLW Formulation Study Chick et al. 1984 WV HLW Formulation Study Chick et al. 1984 WV HLW Formulation Study Chick et al. 1984 WV HLW Formulation Study Chick et al. 1984 WV HLW Formulation Study Chick et 1984 WV HLW Formulation Study Chick et al. 1984 WV HLW Formulation Study Chick et al. 1984 WV HLW Formulation Study Chick et al. 1984 WV HLW Formulation Study Chick et al. 1984 WV HLW Formulation Study Chick et al. 1984 WV HLW Formulation Study Chick et al. 1984 HWVP 85 Bates 1985 WV Glasses by CUA and PNL Johnston et al. 1990 WV Glasses by CUA and PNL Johnston et al. 1990
Al2O3-t B2O3-t CaO-t Fe2O3-t FeO-t K2O-t Li2O-t MgO-t Na2O-t NiO-t P2O5-t SiO2-t ZrO2-t Ag2O-t As2O3-tAs205-t BaO-t BeO-t Bi2O3-t Br-t CdO-t Ce203-t CeO2-t

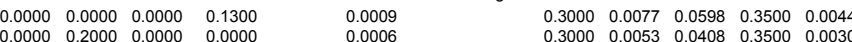
$\begin{array}{llll}0.000 & 0.2000 & 0.0000 & 0.0000 \\ 8000 & 0.2000 & 0.1500 & 0.1000\end{array}$

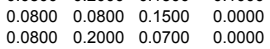
$\begin{array}{lllll}0.0800 & 0.2000 & 0.0700 & 0.0000\end{array}$ $\begin{array}{llll}0.0000 & 0.2000 & 0.0900 & 0.2400\end{array}$ $\begin{array}{llll}0.0000 & 0.2000 & 0.1100 & 0.0000 \\ 0.0000 & 0.0000 & 0.1500 & 0.2000\end{array}$ $\begin{array}{llll} & 0\end{array}$ $\begin{array}{llll}0.0000 & 0.2000 & 0.1500 & 0.0000\end{array}$ $\begin{array}{llll}0.0000 & 0.2000 & 0.1500 & 0.0000 \\ 0.0374 & 0.0809 & 0.0630 & 0.0926\end{array}$ $\begin{array}{lllll}0.0374 & 0.0809 & 0.0630 & 0.0926\end{array}$ $\begin{array}{llll}0.0374 & 0.0809 & 0.0630 & 0.0926 \\ 0.0374 & 0.0809 & 0.0630 & 0.0926\end{array}$ $\begin{array}{lll}0.0986 & 0.0768 & 0.1128 \\ 0.0521 & 00406 & 0.0596\end{array}$ $\begin{array}{llll}0.0241 & 0.0521 & 0.0406 & 0.0596 \\ 0.0441 & 0.0000 & 0.0743 & 0.1093\end{array}$ $\begin{array}{llll}0.0287 & 0.0621 & 0.0483 & 0.10711\end{array}$ $\begin{array}{llll}0.0424 & 0.0918 & 0.0000 & 0.1051 \\ 0.0304 & 0.0658 & 0.1500 & 0.0753\end{array}$ $\begin{array}{llll}0.0000 & 0.0870 & 0.0678 & 0.0996 \\ 0 & 0.0976 & 0.0946\end{array}$ $\begin{array}{llll}0.0800 & 0.0739 & 0.0576 & 0.0846 \\ 0.0453 & 0.0980 & 0.0763 & 0.0000\end{array}$ $\begin{array}{llll}0.0248 & 0.0536 & 0.0418 & 0.2400 \\ 0.0448 & 0.0968 & 0.0754 & 0.1108\end{array}$ $\begin{array}{llll}0.0262 & 0.0566 & 0.0441 & 0.0648 \\ 0.0000 & 0.0000 & 0.1500 & 0.2000\end{array}$ $\begin{array}{llll}0.0000 & 0.0000 & 0.1550 & 0.20000 \\ 0.0000 & 0.0000 & 0.1500 & 0.0000\end{array}$

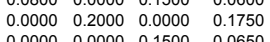

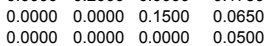
$\begin{array}{llll}0.0400 & 0.2000 & 0.1500 & 0.0000\end{array}$ $\begin{array}{lllll}0.0400 & 0.2000 & 0.0000 & 0.1100\end{array}$ $\begin{array}{llll}0.0800 & 0.0000 & 0.0750 & 0.2400 \\ 0.0400 & 0.0900 & 0.0000 & 0.0000\end{array}$ $\begin{array}{llll}0.1200 & 0.0000 & 0.1300 & 0.0600\end{array}$ $\begin{array}{llll}0.0000 & 0.0900 & 0.0200 & 0.1550 \\ 0.0000 & 0.0900 & 0.0200 & 0.1550\end{array}$ $\begin{array}{llll}0.0000 & 0.0900 & 0.0200 & 0.1550 \\ 0.0000 & 0.0650 & 0.0100 & 0.1550 \\ 0.1200 & 1500\end{array}$ $\begin{array}{llll}0.0550 & 0.1050 & 0.0100 & 0.1350 \\ 0.1200 & 0.1050 & 0.0100 & 0.0700 \\ 0.1200 & 0.120 & 0.000 & 0.000\end{array}$ $\begin{array}{llll}0.1200 & 0.1050 & 0.0100 & 0.0700 \\ 0.1200 & 0.1200 & 0.0000 & 0.0000\end{array}$ $\begin{array}{llll}0.1200 & 0.1200 & 0.0000 & 0.0000 \\ 0.0000 & 0.1200 & 0.0000 & 0.1200\end{array}$ $\begin{array}{llll}0.0600 & 0.1200 & 0.0000 & 0.0600 \\ 0.000 & 0.0900 & 0.000 & 0.2400\end{array}$ $\begin{array}{llll}0.00000 & 0.0900 & 0.0000 & 0.24400 \\ 0.0000 & 0.0000 & 0.11100 & 0.0000 \\ 0.1200 & 0.0000 & 0.0000 & 0.000\end{array}$ $\begin{array}{llll}0.1200 & 0.2000 & 0.0000 & 0.0000 \\ 0.1200 & 0.0000 & 0.1500 & 0.0800 \\ 0.12200 & 0.200 & 0.150 & 0.000\end{array}$

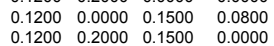
$\begin{array}{llll}0.1200 & 0.0900 & 0.1500 & 0.00000 \\ 0 & 0.1500\end{array}$ $\begin{array}{llll}0.1200 & 0.2000 & 0.0000 & 0.2400 \\ 0.1200 & 0.0000 & 0.0000 & 0.2350 \\ 0 & 0000 & 0.130 & 0.130\end{array}$ $\begin{array}{llll}0.0000 & 0.0000 & 0.1330 & 0.0000\end{array}$ $\begin{array}{llll}0.0000 & 0.0000 & 0.1330 & 0.0000 \\ 0.0710 & 0.1770 & 0.0000 & 0.2120 \\ 0.0000 & 0.1700 & 0.0000 & 0.1130\end{array}$ $\begin{array}{llll}0.0000 & 0.1000 & 0.0000 & 0.2180 \\ 0.0000 & 0.0000 & 0.0000 & 0.1130\end{array}$

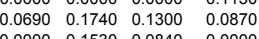
$\begin{array}{llll}0.0000 & 0.0000 & 0.1150 & 0.0000 \\ 0.0000 & 0.1450 & 0.1080 & 0.0000 \\ 0.013 & 0.067 & .0 .27\end{array}$ $\begin{array}{llll}0.0313 & 0.0677 & 0.0527 & 0.0775\end{array}$ $\begin{array}{llll}0.0730 & 0.0000 & 0.11360 & 0.1650 \\ 0.12000 & 0.050 & 0.1300\end{array}$ $\begin{array}{llll}0.1090 & 0.1820 & 0.0000 & 0.0000 \\ 0.1200 & 0.0000 & 0.000 & 0.0000\end{array}$ $\begin{array}{llll}0.1200 & 0.1000 & 0.0000 & 0.1200 \\ 0.12000 & 0.0000 & 0.1000 & 0.1230\end{array}$ $\begin{array}{llll}0.0000 & 0.0600 & 0.1500 & 0.0300\end{array}$ $\begin{array}{llll}0.0300 & 0.0600 & 0.0000 & 0.0000 \\ 0.0400 & 0.0000 & 0.0000 & 0.1280\end{array}$ $\begin{array}{llll}0.0430 & 0.0960 & 0.0290 & 0.1110\end{array}$

$\begin{array}{llllll}0.0009 & 0.1200 & 0.000 & 0.3500 & \\ & 0.1200 & 0.0077 & 0.0598 & 0.3500 & 0.0044\end{array}$

0.3000
0.3000

0.3500
0.3500
0.3500

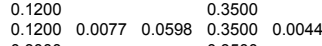

0.0009

0.0009

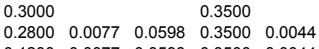

$\begin{array}{lllll}0.2800 & 0.0077 & 0.0598 & 0.3500 & 0.0044 \\ 0.1200 & 0.0077 & 0.0598 & 0.3500 & 0.0044 \\ 0.3000 & & 0.3500 & \end{array}$

$\begin{array}{llllll}0.0003 & 0.1939 & 0.0031 & 0.0237 & 0.4450 & 0.0017 \\ 0.0003 & 0.1939 & 0.0031 & 0.0237 & 0.4450 & 0.0017 \\ 0.0003 & 0.1939 & 0.0031 & 0.0237 & 0.4450 & 0.0017\end{array}$ $\begin{array}{lllll}0.1939 & 0.0031 & 0.0237 & 0.4450 & 0.0017\end{array}$

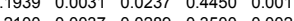
$\begin{array}{llllll}0.1676 & 0.0020 & 0.0152 & 0.5999 & 0.0011\end{array}$ $\begin{array}{lllll}0.2072 & 0.0036 & 0.0280 & 0.4621 & 0.00021\end{array}$ $\begin{array}{llllllll}0.3000 & 0.0023 & 0.0182 & 0.4229 & 0.0013\end{array}$ $\begin{array}{lllll}0.2039 & 0.0035 & 0.0269 & 0.42578 & 0.002\end{array}$ $\begin{array}{lllll}0.1995 & 0.0033 & 0.0255 & 0.4522 & 0.0014\end{array}$ $\begin{array}{lllll}0.1875 & 0.0028 & 0.0217 & 0.4368 & 0.0016\end{array}$ $\begin{array}{lllll}0.2095 & 0.0037 & 0.0287 & 0.4651 & 0.0021 \\ 0.1690 & 0.0020 & 0.0157 & 0.4130 & 0.0012\end{array}$ $\begin{array}{lll}0.2085 & 0.4637\end{array}$ $\begin{array}{lllll}0.1717 & 0.0077 & 0.0598 & 0.4165 & 0.0044 \\ 0.1200 & 0.0063 & 0.0489 & 0.3500 & 0.0036 \\ 0.1200 & 0.0025 & 0.0421 & 0.3500 & 0.0031\end{array}$ $\begin{array}{lllll}0.1200 & 0.0063 & 0.0489 & 0.3500 & 0.0036 \\ 0.3000 & 0.0021 & 0.0163 & 0.3500 & 0.0012 \\ 0.1200 & 0.0023 & 0.177 & 0.0000 & 0.0013\end{array}$ $\begin{array}{lllll}0.1200 & 0.0054 & 0.0421 & 0.3500 & 0.0031 \\ 0.1200 & 0.0023 & 0.0177 & 0.6000 & 0.0013\end{array}$

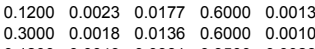
$\begin{array}{lllll}0.3000 & 0.0047 & 0.0367 & 0.3500 & 0.0027\end{array}$ $\begin{array}{lllll}0.1200 & 0.0047 & 0.0367 & 0.3500 & 0.0027 \\ 0.3000 & 0.0077 & 0.0598 & 0.3500 & 0.0044 \\ 0.1900 & 0.074 & 0.040\end{array}$ $\begin{array}{lllll}0.0700 & 0.0021 & 0.0163 & 0.5600 & 0.0012 \\ 0.100 & 0.0040 & 0.0313 & 04600 & 0.0023\end{array}$ $\begin{array}{lllll}0.1600 & 0.0040 & 0.0313 & 0.4600 & 0.0023 \\ 0.1600 & 0.0040 & 0.0313 & 0.4600 & 0.0023\end{array}$ $\begin{array}{lllll}0.1600 & 0.0040 & 0.0313 & 0.4600 & 0.0023 \\ 0.1350 & 0.0040 & 0.0313 & 0.5200 & 0.0023\end{array}$ $\begin{array}{ll}0.1650 & 0.0040 \\ 0.1650 & 0.5300 \\ 0.16300 & 0.5300\end{array}$

$\begin{array}{ll}0.1650 & 0.5300 \\ 0.1650 & 0.5300 \\ 0.1600 & 0.6000 \\ 0.1600 & 0.6000 \\ 0.1600 & 0.6000 \\ 0.000 & 0.6000\end{array}$

0.1600

$\begin{array}{rrr}0.0700 & 0.6000 \\ 0.0700 & 0.6000\end{array}$

$\begin{array}{llllll}0.0009 & 0.0700 & 0.0077 & 0.0598 & 0.60000 & 0.0044 \\ 0.0009 & 0.3000 & 0.0077 & 0.0598 & 0.3600 & 0.0044\end{array}$ $\begin{array}{llllll}0.1100 & 0.0077 & 0.0598 & 0.3500 & 0.0044\end{array}$ 0.3000 0.35000 .0044

$\begin{array}{llllll} & 0.1800 & 0.0009 & 0.3500 & & 0.0000 \\ 0.0700 & 0.0077 & 0.0598 & 0.3500 & 0.0044 & 0.0026\end{array}$ $\begin{array}{ll}0.0900 & 0.3500 \\ 0.2950 & 0.3500 \\ 0.270 & 0.6000\end{array}$ $\begin{array}{ll}0.2670 & 0.6000 \\ 0.1200 & 0.4200 \\ 0.2720 & 0.000\end{array}$ $\begin{array}{lllll}0.0009 & 0.2720 & & 0.4100 & \\ 0.2610 & 0.0067 & 0.0519 & 0.4350 & 0.0038 \\ 0 & 0.1200 & 0.4200 & 0.0000\end{array}$

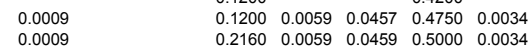

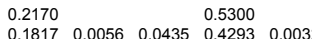

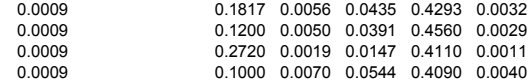

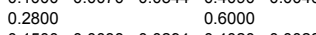

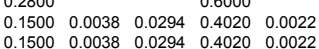
$\begin{array}{llllll}0.0004 & 0.1500 & 0.0038 & 0.0294 & 0.4020 & 0.0022 \\ 0.0004 & 0.1500 & 0.0038 & 0.0294 & 0.4020 & 0.0022 \\ 0.0009 & 0.1800 & 0.0021 & 0.0163 & 0.5200 & 0.0012 \\ 0.0009 & 0.1800 & 0.0074 & 0.0571 & 0.5200 & 0.0042\end{array}$ $\begin{array}{lllllll} & & 0.1000 & 0.0039 & 0.0299 & 0.5420 & 0.0022 \\ 0.0380 & 0.0080 & 0.1040 & 0.0060 & 0.5120 & 0.0060\end{array}$ 


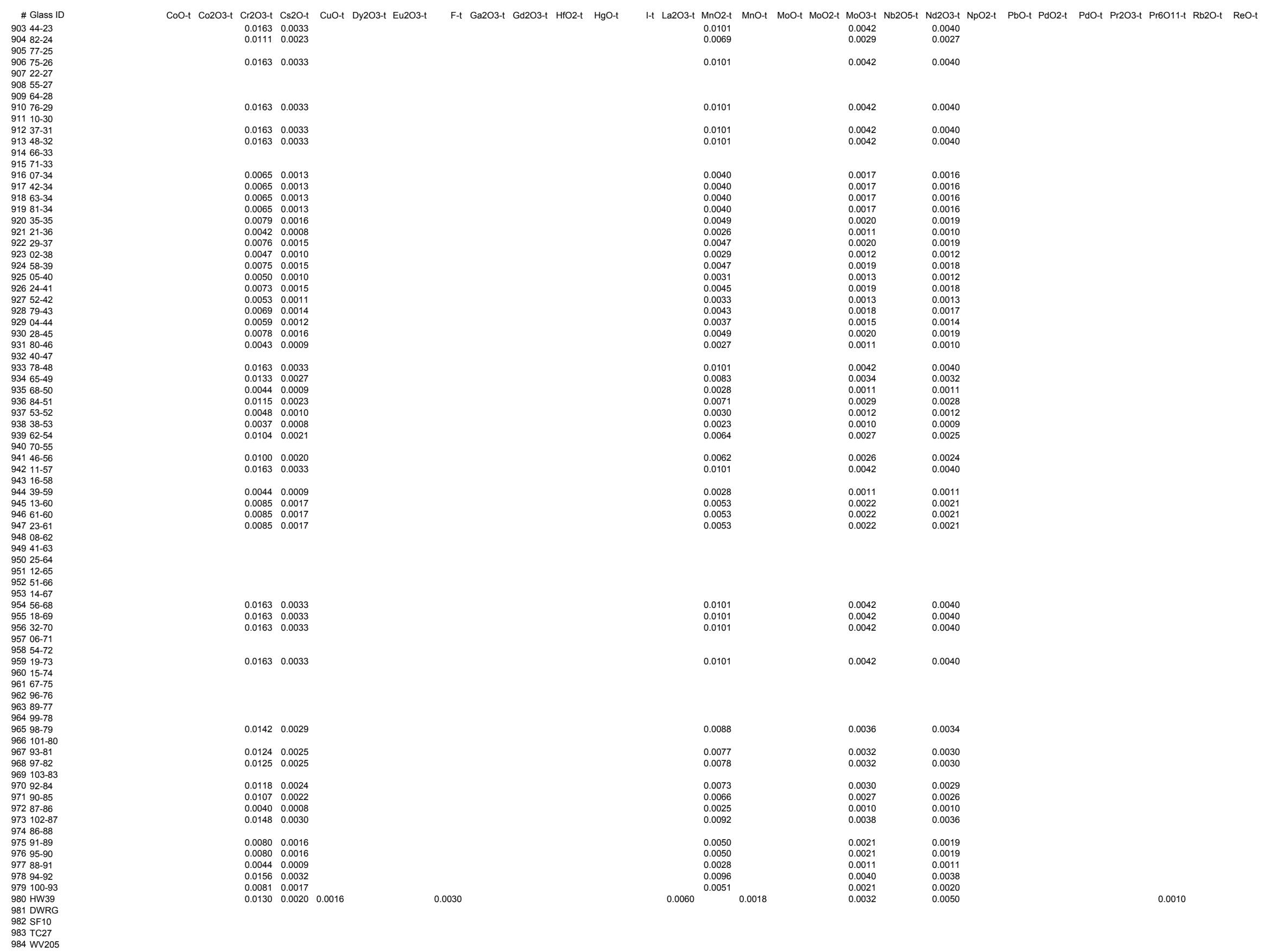




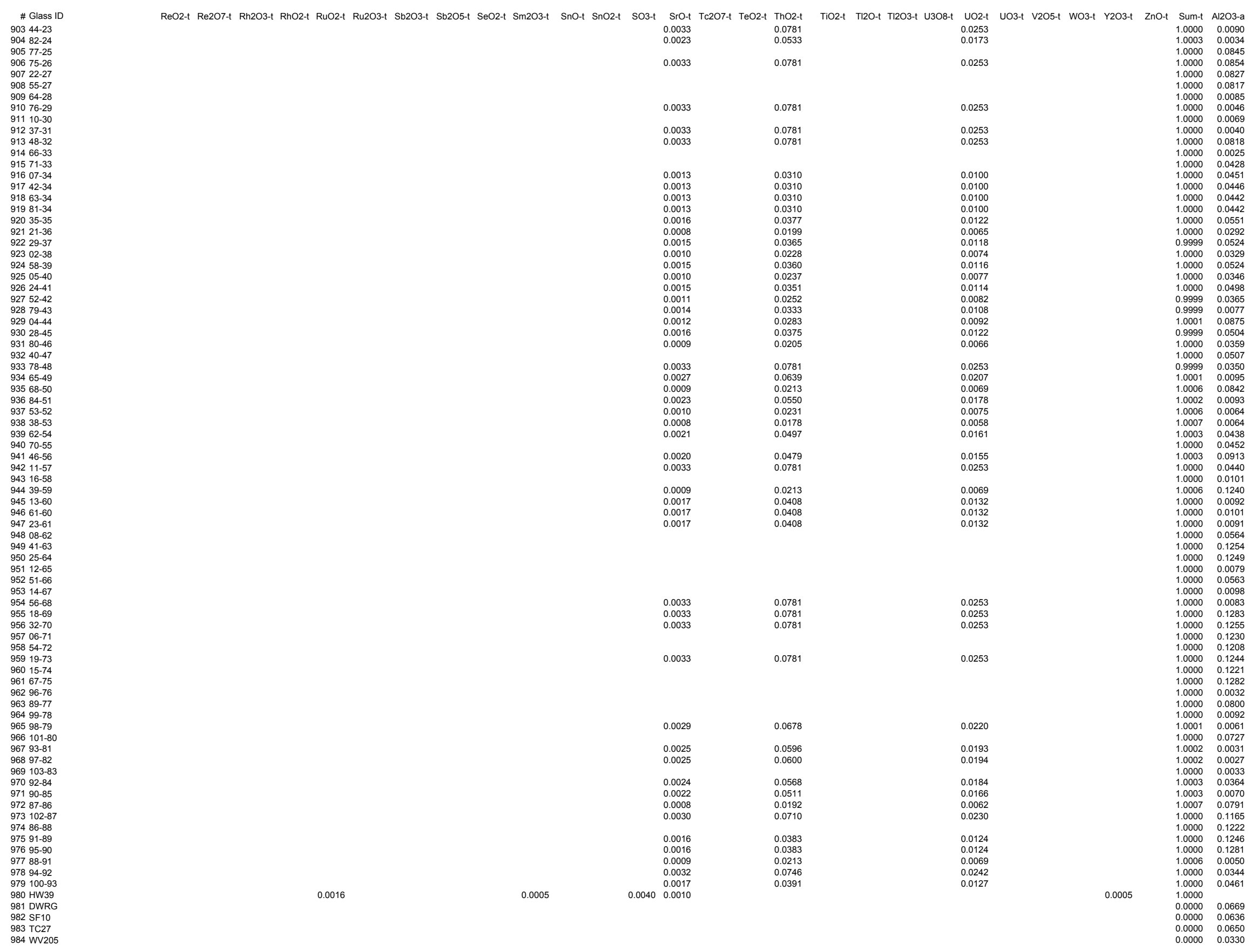




\begin{tabular}{|c|c|c|c|c|c|c|c|c|c|c|}
\hline \# Glass ID & B203-a & CaO-a & Fe2O3-a & $\mathrm{FeO}-\mathrm{a}$ & K2O-a Li2O-a MgO-a & Na2O-a & NiO-a & P205-a & SiO2-a & ZrO2-a \\
\hline $90344-23$ & 0.0000 & 0.0016 & 0.1151 & & 0.0008 & 0.3013 & 0.0073 & 0.0570 & 0.3634 & 0.0042 \\
\hline $90482-24$ & 0.1909 & 0.0000 & $\begin{array}{l}0.0000 \\
-0.045\end{array}$ & & & 0.2933 & 0.0062 & & 0.3381 & 0.0035 \\
\hline $\begin{array}{l}905 / 1-25 \\
906-26\end{array}$ & $\begin{array}{l}0.1976 \\
0.0830\end{array}$ & $\begin{array}{l}0.1558 \\
0.1502\end{array}$ & $\begin{array}{l}0.0845 \\
0.0000\end{array}$ & & 00008 & 0.1253 & 00072 & 00558 & 0.3534 & 0,0041 \\
\hline $907 \quad 22-27$ & 0.1982 & 0.0746 & 0.0000 & & 0.0008 & 0.2891 & 0.0072 & 0.0558 & $\begin{array}{l}0.3333 \\
0.3555\end{array}$ & 0.0041 \\
\hline $90855-27$ & 0.1962 & 0.0707 & 0.0000 & & & 0.3038 & & & 0.3476 & \\
\hline $\begin{array}{l}90964-28 \\
91076-29\end{array}$ & 0.1997 & 0.0918 & 0.2017 & & & 0.1251 & & & 0.3732 & \\
\hline $\begin{array}{l}91076-29 \\
91110-30\end{array}$ & $\begin{array}{l}0.1925 \\
0.0000\end{array}$ & $\begin{array}{l}0.1112 \\
0.1527\end{array}$ & $\begin{array}{l}0.0000 \\
0.1701\end{array}$ & & 0.0009 & $\begin{array}{l}0.1221 \\
0.3003\end{array}$ & 0.0075 & 0.0581 & $\begin{array}{l}0.3359 \\
0.3700\end{array}$ & 0.0043 \\
\hline $912 \quad 37-31$ & 0.0000 & 0.1531 & 0.0000 & & 0.0009 & 0.2813 & 0.0075 & 0.0581 & 0.3478 & 0.0043 \\
\hline $91348-32$ & & 0.0040 & 0.1749 & & 0.0008 & 0.1097 & 0.0069 & 0.0532 & 0.4336 & $\begin{array}{l}0.0039 \\
0.0039\end{array}$ \\
\hline $91466-33$ & 0.1918 & 0.1535 & 0.0000 & & & 0.3069 & & & 0.3453 & \\
\hline $91571-33$ & 0.0911 & 0.0666 & 0.0000 & & & 0.2745 & & & 0.3426 & \\
\hline $916 \quad 07-34$ & 0.0841 & 0.0625 & 0.0810 & & 0.0003 & 0.1917 & 0.0029 & 0.0224 & 0.4532 & 0.0016 \\
\hline $91742-34$ & 0.0810 & 0.0633 & 0.0800 & & 0.0003 & 0.1973 & 0.0027 & 0.0209 & 0.4569 & 0.0015 \\
\hline $\begin{array}{l}918 \text { 63-34 } \\
91981-34\end{array}$ & 0.0802 & 0.0627 & 0.0792 & & 0.0003 & 0.1984 & 0.0029 & 0.0225 & 0.4524 & 0.0016 \\
\hline $\begin{array}{l}91981-34 \\
920-35\end{array}$ & $\begin{array}{l}0.0802 \\
0.1044\end{array}$ & $\begin{array}{l}0.0637 \\
0.0805\end{array}$ & $\begin{array}{l}0.0791 \\
0.0580\end{array}$ & & $\begin{array}{l}0.0003 \\
0.0004\end{array}$ & $\begin{array}{l}0.1963 \\
0.2193\end{array}$ & $\begin{array}{l}0.0000 \\
0.033\end{array}$ & $\begin{array}{l}0.0229 \\
0.0254\end{array}$ & $\begin{array}{l}0.4523 \\
03894\end{array}$ & $\begin{array}{l}0.0016 \\
0.0018\end{array}$ \\
\hline $92121-36$ & 0.0514 & 0.0423 & 0.0524 & & 0.0002 & 0.1633 & 0.0021 & $\begin{array}{l}0.0264 \\
0.0161\end{array}$ & $\begin{array}{l}.0 .6094 \\
0.6020\end{array}$ & $\begin{array}{l}0.0018 \\
0.0012\end{array}$ \\
\hline 922 29-37 & 0.0000 & 0.0768 & 0.0956 & & 0.0004 & 0.2095 & 0.0032 & 0.0251 & 0.4735 & 0.0018 \\
\hline $92302-38$ & 0.2044 & 0.0462 & 0.0596 & & 0.0002 & 0.1736 & $\begin{array}{l}0.0022 \\
0.022\end{array}$ & $\begin{array}{l}0.0169 \\
0.01\end{array}$ & 0.4212 & 0.0012 \\
\hline 924 58-39 & 0.0929 & 0.0709 & 0.0919 & & 0.0004 & 0.1237 & 0.0031 & 0.0244 & 0.4785 & 0.0018 \\
\hline $92505-40$ & 0.0631 & 0.0508 & 0.0620 & & 0.0003 & 0.2873 & 0.0028 & 0.0218 & 0.4220 & 0.0016 \\
\hline $92624-41$ & 0.0916 & 0.0060 & 0.0906 & & 0.0004 & 0.2010 & 0.0034 & 0.0267 & 0.4628 & 0.0020 \\
\hline $92752-42$ & 0.0657 & 0.1511 & 0.0646 & & 0.0002 & 0.1814 & 0.0021 & 0.0166 & 0.4399 & 0.0012 \\
\hline $\begin{array}{l}92879-43 \\
92904-44\end{array}$ & 0.0870 & $\begin{array}{l}0.0686 \\
0.0590\end{array}$ & 0.0849 & & 0.0004 & $\begin{array}{l}0.2077 \\
0.1872\end{array}$ & $\begin{array}{l}0.0031 \\
0.026\end{array}$ & $\begin{array}{l}0.0244 \\
0.0201\end{array}$ & 0.4544 & 0.0018 \\
\hline $93028-45$ & 0.0977 & 0.0793 & 0.0000 & & 0.0004 & $\begin{array}{l}0.1812 \\
0.2082\end{array}$ & $\begin{array}{l}.0 .0032 \\
0.032\end{array}$ & $\begin{array}{l}0.0221 \\
0.0251\end{array}$ & $\begin{array}{l}0.4416 \\
0.4721\end{array}$ & $\begin{array}{l}0.0015 \\
0.0018\end{array}$ \\
\hline $93180-46$ & 0.0553 & 0.0430 & 0.1998 & & 0.0002 & 0.1742 & 0.0021 & 0.0167 & & 0.0012 \\
\hline $93240-47$ & 0.0954 & 0.0775 & 0.0974 & & & 0.2078 & & & 0.4712 & \\
\hline $93378-48$ & 0.0577 & 0.0433 & 0.0566 & & 0.0008 & 0.1792 & 0.0072 & 0.0557 & 0.4233 & 0.0041 \\
\hline $93465-49$ & 0.0000 & 0.1545 & 0.1713 & & 0.0008 & 0.1251 & 0.0056 & 0.0438 & 0.3784 & 0.0032 \\
\hline $93568-50$ & 0.0000 & 0.1500 & 0.0503 & & 0.0009 & 0.3010 & 0.0021 & 0.0166 & 0.3534 & 0.0012 \\
\hline $93684-51$ & 0.1974 & 0.0020 & 0.1490 & & 0.0009 & 0.1234 & 0.0054 & 0.0416 & 0.3659 & 0.0031 \\
\hline $93853-52$ & $\begin{array}{l}0.0000 \\
0.0000\end{array}$ & $\begin{array}{l}0.1489 \\
0.017\end{array}$ & 0.0557 & & $\begin{array}{l}0.0009 \\
0.0009\end{array}$ & $\begin{array}{l}0.1205 \\
0.2944\end{array}$ & $\begin{array}{l}0.0023 \\
0.0018\end{array}$ & 0.0176 & $\begin{array}{l}0.6036 \\
0.6038\end{array}$ & 0.0013 \\
\hline $\begin{array}{l}938 \\
939 \\
982-53\end{array}$ & $\begin{array}{l}0.0000 \\
0.2004\end{array}$ & $\begin{array}{l}.0 .0017 \\
0.1545\end{array}$ & $\begin{array}{l}0.0427 \\
0.0000\end{array}$ & & $\begin{array}{l}0.0009 \\
0.0008\end{array}$ & $\begin{array}{l}0.2944 \\
0.1232\end{array}$ & $\begin{array}{l}0.0018 \\
0.0043\end{array}$ & $\begin{array}{l}0.0119 \\
0.0335\end{array}$ & $\begin{array}{l}0.6038 \\
0.3549\end{array}$ & $\begin{array}{l}0.00010 \\
0.0025\end{array}$ \\
\hline $94070-55$ & $\begin{array}{l}0.1977 \\
0.1977\end{array}$ & 0.0025 & 0.0933 & & & 0.3101 & & & 0.03512 & \\
\hline $94146-56$ & 0.0000 & 0.0810 & 0.2020 & & 0.0009 & 0.1231 & 0.0047 & & $\begin{array}{l}0.03692 \\
0.3692\end{array}$ & 0.0027 \\
\hline $94211-57$ & 0.0911 & 0.0021 & 0.0000 & & 0.0008 & 0.2977 & 0.0072 & 0.0558 & 0.3598 & 0.0041 \\
\hline $943 \quad 16-58$ & 0.0717 & 0.1515 & 0.2121 & & & 0.1818 & & & 0.3727 & \\
\hline 944 39-59 & 0.0000 & 0.1370 & 0.0430 & & 0.0008 & 0.0700 & 0.0020 & 0.0152 & 0.5699 & 0.0011 \\
\hline 945 13-60 & 0.0898 & 0.0225 & 0.1348 & & 0.0005 & 0.1613 & 0.0037 & 0.0290 & 0.4757 & 0.0021 \\
\hline $94661-60$ & 0.0891 & $\begin{array}{l}0.0246 \\
0.0131\end{array}$ & 0.1281 & & 0.0005 & 0.1629 & 0.0040 & 0.0309 & $\begin{array}{l}0.4714 \\
\end{array}$ & 0.0023 \\
\hline $94808-62$ & 0.0637 & $\begin{array}{l}0.01111 \\
0.0123\end{array}$ & $\begin{array}{l}0.1344 \\
0.1163\end{array}$ & & 0.0005 & 0.1344 & & & $\begin{array}{l}0.5355 \\
0.5410\end{array}$ & 0.0022 \\
\hline 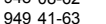 & 0.0973 & $\begin{array}{l}.00123 \\
0.0120\end{array}$ & $\begin{array}{l}0.0602 \\
0.0602\end{array}$ & & & $\begin{array}{l}0.1639 \\
0.1625\end{array}$ & & & $\begin{array}{l}.5 .5410 \\
0.5426\end{array}$ & \\
\hline $95025-64$ & 0.1159 & 0.0042 & 0.0000 & & & 0.1563 & & & 0.5988 & \\
\hline 951 12-65 & 0.1186 & 0.0000 & 0.1090 & & & 0.1561 & & & 0.6083 & \\
\hline $95251-66$ & 0.1031 & 0.0053 & 0.0473 & & & 0.1454 & & & 0.6057 & \\
\hline 953 14-67 & 0.0859 & 0.0040 & 0.2063 & & & 0.0689 & & & 0.6250 & \\
\hline $95456-68$ & 0.0000 & 0.1094 & 0.0000 & & 0.0009 & 0.0733 & 0.0077 & 0.0597 & 0.5894 & 0.0044 \\
\hline $95518-69$ & $\begin{array}{l}0.0000 \\
0.9983\end{array}$ & $\begin{array}{l}0.0030 \\
0.020\end{array}$ & $\begin{array}{l}0.0000 \\
0.0000\end{array}$ & & 0.0008 & 0.2921 & 0.0074 & 0.0553 & 0.3658 & 0.0042 \\
\hline $\begin{array}{llll}957 & 06-71\end{array}$ & $\begin{array}{l}0.1983 \\
0.0000\end{array}$ & $\begin{array}{l}0.0021 \\
0.1516\end{array}$ & $\begin{array}{l}0.0000 \\
0.0697\end{array}$ & & 0.0000 & $\begin{array}{l}0.1123 \\
03022\end{array}$ & 0.0071 & 0.0555 & $\begin{array}{r}0.3577 \\
0.3555\end{array}$ & 0.0041 \\
\hline $95854-72$ & $\begin{array}{l}0.0000 \\
0.1974\end{array}$ & $\begin{array}{l}.1 .1516 \\
0.1511\end{array}$ & $\begin{array}{l}0.0000 \\
0.000\end{array}$ & & & 0.033 & & & $\begin{array}{l}.3 .3555 \\
0.3474\end{array}$ & \\
\hline $959919-73$ & 0.1455 & 0.0943 & 0.0000 & & 0.0008 & 0.0752 & 0.0073 & 0.0563 & 0.3533 & 0.0041 \\
\hline $96015-74$ & 0.2096 & 0.0000 & 0.1974 & & & 0.0926 & & & 0.3784 & 0.0041 \\
\hline $96167-75$ & 0.0000 & 0.0019 & 0.1968 & & & 0.3048 & & & 0.3684 & \\
\hline $96296-76$ & 0.0000 & 0.1297 & 0.0000 & & & 0.2654 & & & 0.6017 & \\
\hline 963 89-77 & 0.1666 & 0.0016 & 0.1798 & & & 0.1219 & & & 0.4501 & \\
\hline $96499-78$ & 0.0000 & $\begin{array}{l}0.1217 \\
0\end{array}$ & 0.1454 & & 80009 & $\begin{array}{l}0.1279 \\
0.2658\end{array}$ & & & 0.4889 & \\
\hline $\begin{array}{l}9656101-19 \\
966101\end{array}$ & $\begin{array}{l}0.0000 \\
0.1707\end{array}$ & $\begin{array}{l}0.00044 \\
0.1309\end{array}$ & $\begin{array}{l}0.0964 \\
0.0747\end{array}$ & & 0.0009 & $\begin{array}{l}0.2658 \\
0.1196\end{array}$ & 0.0065 & 0.0503 & 0.4424 & 0.0037 \\
\hline $96793-81$ & 0.1749 & 0.0838 & 0.0000 & & 0.0007 & 0.1288 & 0.0047 & 0.0365 & $\begin{array}{l}0.4754 \\
0.4754\end{array}$ & 0.0027 \\
\hline $96897-82$ & 0.0000 & 0.1176 & 0.0000 & & 0.0009 & 0.2270 & 0.0059 & 0.0460 & 0.4834 & 0.0034 \\
\hline $969103-83$ & 0.1456 & 0.1084 & 0.0000 & & & 0.2210 & & & 0.5216 & \\
\hline $97092-84$ & 0.0673 & 0.0512 & 0.0664 & & 0.0008 & 0.1871 & 0.0052 & 0.0407 & 0.4417 & 0.0030 \\
\hline $97190-85$ & 0.0000 & 0.1228 & 0.1264 & & 0.0011 & 0.1218 & 0.0060 & 0.0468 & 0.4497 & 0.0034 \\
\hline $97287-86$ & 0.0000 & 0.1384 & 0.0462 & & 0.0010 & 0.2811 & 0.0021 & 0.0161 & 0.3959 & 0.0012 \\
\hline 973 102-87 & 0.1689 & 0.0019 & 0.0000 & & 0.0008 & 0.0997 & 0.0065 & 0.0504 & 0.4276 & 0.0037 \\
\hline $\begin{array}{l}97591-80 \\
97599\end{array}$ & 0.0961 & 0.0019 & 0.1014 & & 0.0004 & $\begin{array}{l}0.1508 \\
0.1513\end{array}$ & 0.0038 & 0.0293 & 0.4170 & 0.0022 \\
\hline 976 95-90 & 0.0000 & 0.0997 & 0.1027 & & 0.0004 & 0.1525 & 0.0038 & 0.0297 & 0.4077 & 0.0022 \\
\hline $97788-91$ & 0.0592 & 0.1517 & 0.0256 & & 0.0009 & 0.1819 & 0.0021 & 0.0166 & 0.5154 & 0.0012 \\
\hline $97894-92$ & 0.0587 & 0.0025 & 0.0000 & & 0.0009 & 0.1875 & 0.0072 & & & 0.0041 \\
\hline 979 100-93 & 0.0000 & 0.0781 & 0.1086 & & 0.0004 & 0.1026 & 0.0039 & 0.0301 & 0.5541 & 0.0022 \\
\hline $980 \mathrm{HW} 3$ & & & & & & & & & & \\
\hline 981 DWRG & 0.0663 & & 0.1040 & & & & & 0.0020 & $0.5 \mathrm{C}$ & \\
\hline $\begin{array}{l}982 \text { SF10 } \\
983 \text { TC27 }\end{array}$ & $\begin{array}{l}0.0970 \\
0.0973\end{array}$ & & $\begin{array}{l}0.1145 \\
0.1176\end{array}$ & & & & & 0.0271 & $\begin{array}{l}0.4676 \\
0.474\end{array}$ & \\
\hline 984 WV205 & 0.0996 & & 0.1180 & & & & & 0.0250 & $\begin{array}{l}0.4522 \\
0.4522\end{array}$ & \\
\hline
\end{tabular}

CeO2-a

0.0025

0.0026

0.0026
0.0024

0.0009
0.0009
0.0010
0.00011

0.0010

0.0007
0.0011

0.0001

0.0011
0.0010
0.0012
0.0007

0.0007

0.0009
0.0011

0.0025

0.0025
0.0019
0.0007
0.0018

0.0007
0.0018
0.0008
0.0006
0.0015

0.0016
0.0025

0.0007
0.0013
0.0014
0.0013

0.0026
0.0025
0.0024

0.0025

0.0022

0.0016
0.0020

0.0018
0.0021
0.0007
0.0022

0.0007
0.0022

0.0013
0.0013
0.0007
0.0025
Cl-a CoO-a Co2O3-a Cr2O3-a Cs2O-a CuO-a F-a $\begin{array}{ll}0.0155 & 0.0031 \\ 0.0129 & 0.0027\end{array}$

$0.0152 \quad 0.003$

$0.0158 \quad 0.0032$

$\begin{array}{ll}0.0158 & 0.0032 \\ 0.0145 & 0.0029\end{array}$

$\begin{array}{ll}0.0061 & 0.0012 \\ 0.0057 & 0.0011 \\ 0 & 0.002\end{array}$

$\begin{array}{ll}0.0062 & 0.0012 \\ 0.0063 & 0.0013\end{array}$

$0.0044 \quad 0.0009$

$\begin{array}{ll}0.0068 & 0.0014 \\ 0.0046 & 0.0009\end{array}$

$\begin{array}{ll}0.00066 & 0.0013 \\ 0.0059 & 0.0012\end{array}$

$\begin{array}{ll}0.0059 & 0.0012 \\ 0.0073 & 0.0015 \\ 0.00075 & 0.0009\end{array}$

$\begin{array}{ll}0.0045 & 0.0009 \\ 0.0067 & 0.0013\end{array}$

$\begin{array}{ll}0.0055 & 0.0011 \\ 0.0068 & 0.0014 \\ 0.0055 & 0.009\end{array}$

$\begin{array}{ll}0.0152 & 0.0031 \\ 0.0119 & 0.0024 \\ 0.0045 & 0.0009\end{array}$

$0.0113 \quad 0.0023$

$\begin{array}{ll}0.0048 & 0.0010 \\ 0.0038 & 0.0008\end{array}$

$0.0099 \quad 0.0020$

$0.0152 \quad 0.003$

$\begin{array}{ll}0.0042 & 0.0008 \\ 0.0079 & 0.0016\end{array}$

$\begin{array}{ll}0.0084 & 0.0017 \\ 0.0081 & 0.0016\end{array}$

$\begin{array}{ll}0.0163 & 0.0033 \\ 0.0156 & 0.0032\end{array}$

$0.0151 \quad 0.003$

$0.0154 \quad 0.0031$

$0.0137 \quad 0.002$

$\begin{array}{ll}0.0099 & 0.0020 \\ 0.0125 & 0.0025\end{array}$

$0.0111 \quad 0.0022$

$\begin{array}{ll}0.0044 & 0.0009 \\ 0.0137 & 0.0028\end{array}$

$\begin{array}{ll}0.0080 & 0.0016 \\ 0.0081 & 0.0016\end{array}$

$\begin{array}{ll}0.0045 & 0.0000 \\ 0.0152 & 0.0031 \\ 0.0082 & 0.0017\end{array}$ 


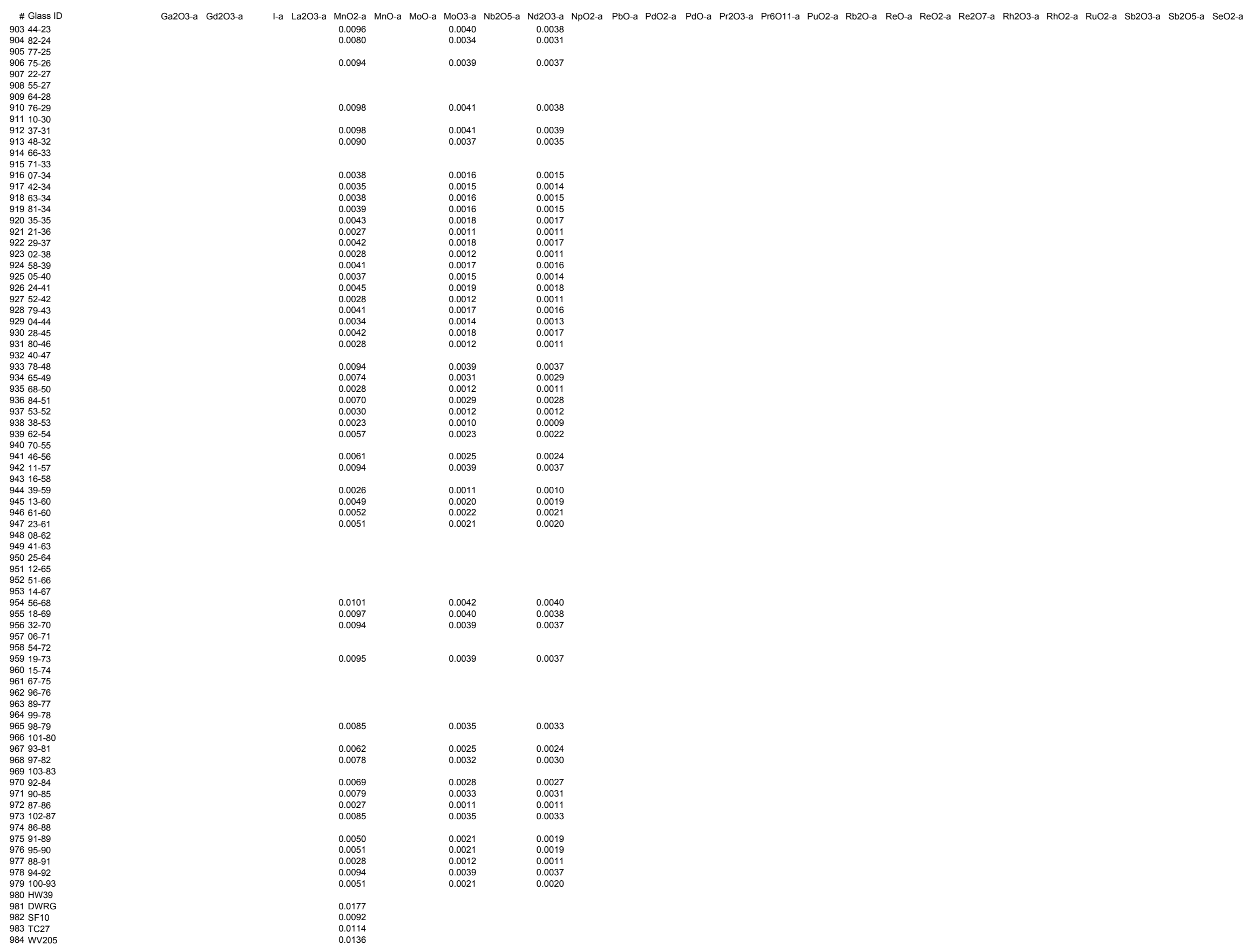




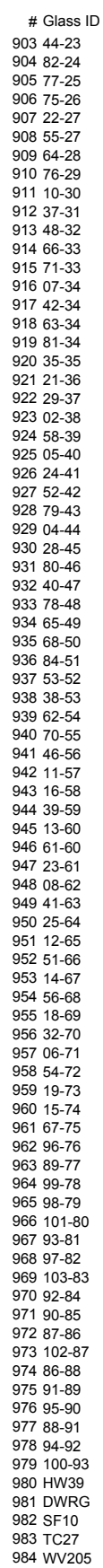

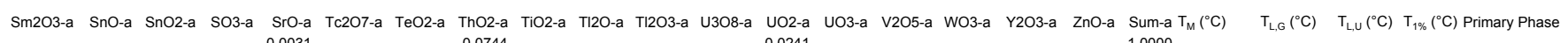

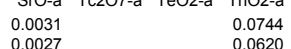
0.0241

0.0032

0.0246

$\begin{array}{ll}0.0032 & 0.0759 \\ 0.0029 & 0.0695\end{array}$

0.0246
0.0225

$\begin{array}{ll}0.0012 & 0.0293 \\ 0.0011 & 0.027\end{array}$

0.0011
0.0012
0.0013
0.0014

0.0014
0.0009

0.0014

0.0009
0.0013
0.0012

0.0015

0.0009

0.0013
0.0011
0.0014

$\begin{array}{ll}0.0009 & 0.0218 \\ 0.0031 & 0.02727\end{array}$

0.0031
0.0024
0.0009

0.0009
0.0023

0.0023
0.0010
0.0008
0.0018

0.0020

0.0031

0.0008
0.0016
0.0017
0.0016

0.0273
0.0295
0.0299
0.0331

0.0331
0.0210
0.0327
0.0228

0.0220
0.0318
0.0284

0.0284
0.0349
0.0216

0.0216
0.0263
0.0328
0.0218

0.0727
0.0572

0.0217

0.0544
0.0230
0.0181
0.0437

0.0473

0.0199
0.0379

0.0390

$\begin{array}{ll}0.0033 & 0.0780 \\ 0.0032 & 0.0748 \\ 0.0031 & 0.0724\end{array}$

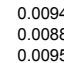

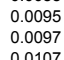

0.0107
0.0068
0.0106
0.060

0.0071
0.0103
0.010

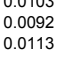

0.0070
0.0103
0.0003

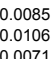

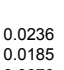

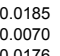

0.0176
0.075
0.0059
0.0142

0.0153

0.0065
0.0122
0.0131

0.0131

$0.0031 \quad 0.073$

0.0253
0.0242
0.0235

0.0238

0.0028

0.0656

0.0020
0.0025

0.0476
0.0601

0.0022
0.0026

0.0026
0.0009
0.0028

0.0028

0.0016
0.0016
0.0009

0.0031
0.0017

0.0532
0.0612

0.0612
0.0211
0.0658

0.0382
0.0388
0.0217

0.0217
0.0728
0.0393

0.0000
0.0000
0.0000

0.0000
0.0000

0.0213
0.0154
0.0195
0.0172
0.0198
0.0068
0.0213
0.0124
0.0125
0.0070
0.0236
0.0127
0.0301
0.0000
0.0000
0.0000

0.0154
0.0195

0.0172
0.0198
0.0068

0.0213

0.0124
0.0125
0.0070
0.0236

0.0236
0.0127

0.0000
0.0000
0.0000 


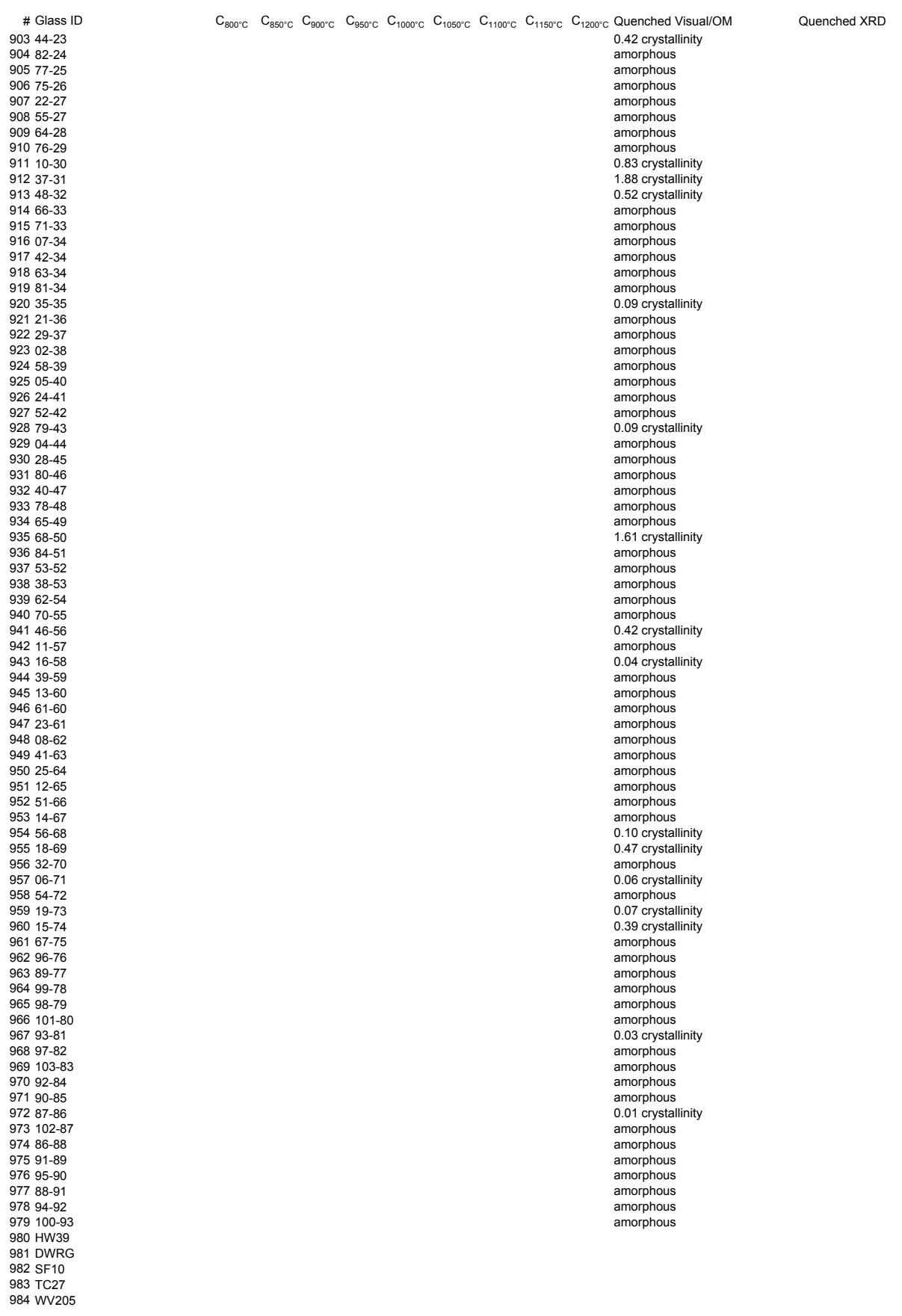




$$
\mid
$$


956 32-70

$95706-7$

$958554-72$
959
$99-73$

960 15-74

$96167-75$

$96296-70$
963 89-77

$96499-78$

965 98-79

9667 93-81

968 97-82

$9699103-83$
9070284

970
$92-84$
$97190-85$

$97287-86$
$973702-87$

$97486-88$
975
$91-89$

$97591-89$
$97955-90$

97778891
$9789-92$

$97894-922$
$970-93$

$980 \mathrm{HW} 39$

981 DWRG
982 SFF

983 TC27
984 W 205 
956 32-70

$95706-7$

95854.72
959
$99-73$

960 15-74

$96167-5$

$962296-76$
$96389-77$

964 99-78
965 98-79

966 101-80

$96793-81$
968
$97-82$

969 103-83
$97092-84$

$97092-84$
$971100-85$

$97287-86$
$973702-87$

$97486-88$
975
$91-89$

$97591-89$
$976-90$

97788.91
978 94.92

$97894-92$
979
$900-93$

979000.93
980

98 DWRG
982 SF10
983 TC27

984 WV205 
986 WVCM44 WV Glasses by CUA and PNL Johnston et al. 1990

WV Glasses by CUA and PNL Johnston et al. 1990

WV Glasses by CUA and PNLJohnston et al. 1990

WV Glasses by CUA and PNL Johnston et al 1990

WV Glasses by CUA and PNL Johnston et al. 1990

WV Glasses by CUA and PLohnston et al. 1990

WV Glasses by CUA and PNL Johnston et al. 1990

WV Glasses by CUA and PNL Johnston et al. 1990

WV Glasses by CUA and PNLJohnston et al. 1990

WV Glasses by CUA and PNL Johnston et al. 1990

WV Glasses by CUA and PNL Johnston et al. 1990

WV Glasses by CUA and PN Johnsto et al. 1990

WV Glasses by CUA and PNL Johnston et al. 1990

WV Glasses by CUA and PNL Johnston et al. 1990

WV Glasses by CAA asses by CUA and PNL J J

WV Glasses by CUA and PNL Johnston et al. 1990 WV Glasses by CUA and PNL Johnston et al. 1990

WV Glasses by CAA and PNLJohnston et al. 1990

WV Glasses by CUA and PNLJohnston et al. 1990 WV Glasses by CUA and PNL Johnston et al. 1990 WV Glasses by CUA and PNes by CUA and PNL Johnston et al. 1990 WV Glasses by CUA and PNL Johnston et al. 1990 WV Glasses by CUA and PNL Johnston et al. 1990 WV Glasses by CUA and PNL Johnston et ta. 1990 WV Glasses by CUA and PNL Johnston et al. 1990 WV Glasses by CUA and PNL Johnston et al. 1990 WV Glasses by CUA and PNL Johnston et al 1990 WV Glasses by CUA and PNL Johnston et al. 1990 WV Glasses by $\mathrm{CA}$ and PNLJohnston et al. 1990 WV Glasses by CUA and PNL Johnston et al 1990 WV Glasses by CUA and PNL Johnston et al. 1990 WV Glasses by CUA and PNL Jonton al. 1990 WV Glasses by CUA and PNL Johnston et al. 1990 WV Glasses by CUA and PNL Johnston et al. 1990 WV Glasses by CUA and PLL Johnston et al. 1990 WV Glasses by CUA and PNL Johnston et al. 1990 WV Glasses by CUA and PNL Johnston et al. 1990 WV Glasses by CUA and PNL Johnston et al 1990 WV Glasses by CUA and PNL Johnston et al. 1990 WV Glasses by CUA and PNLJohnston et al. 1990 WV Glasses by CUA and PNL Johnton et al 1900 WV Glasses by CUA and PNL Johnston et al. 1990 WV Glasses by CUA and PNL Johnston et al. 1990 WV Glasses by CHA and PNN J WV Glasses by CUA and PNL Johnston et al. 1990 WV Glasses by CUA and PNLJohnston et al. 1990 WV Glasses by CUA and PNL Johnston et al 1990 WV Glasses by CUA and PNL Johnston et al. 1990 WV Glasses by CUA and PNL Johnston et al. 1990 WV Glasses by CUA and PNL Johnston et al 1990 WV Glasses by CUA and PNL Johnston et al. 1990 WV Glasses by CUA and PNLJohnston et al. 1990 WV Glasses by CUA and PNL Johnston et al 1990 WV Glasses by CUA and PNL Johnston et al. 1990 WV Glasses by CAA and PNLJohnston et al. 1990 WV Glasses by CUA and PNL Johnston et al. 1990 WV Glasses by CUA and PNL Johnston et al. 1990 WV Glasses by CUA and PNLJohnston et al. 1990 WV Glasses by CUA and PNLJohnston et al. 1990 WV Glasses by CUA and PNL Johnston et al. 1990 
1014 WVUTH35

1015 WVUTH36

1016 WVUTH37
1017 WVUTH38

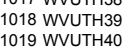

1020 WVUTH 41
1021 WVTH4

1022 WVUTHA8

1023 WVUTH49

1024 WVUTH51
1025 WVTTH 52

1026 WVUTH5 53

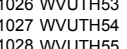

1028 WWUTH55

1030 WVUTH57

1031 WVUTH5s

1032 WVUTH59
1033 WVUTHG1

1034 WUUTH62
1035 WVTHH63

1036 WUTH64
1037 WUUTH65

1038 WVUTH66

1039 WVUTH67

1041 WVUTHE9

1042 WUUTH70

1043 WVUTH71

1045 WVUTH73

1046 WVUTH74

1047 WUUTH75

1049 WVUTH78

1050 WVUTH79

1051 WVUTH80

1053 WVUTH82

1055 WVUTH 83

1055 WUTH 84

1057 WVUTH90

1057 WWUTH90
1058 WVUTH91

1059 WVUTH92

1061 WVUTH94

1062 WWUTTH96

1063 WVUTH98
1064 WVUTH99

1005 WVUTH 100

1066 WVUTH 102 


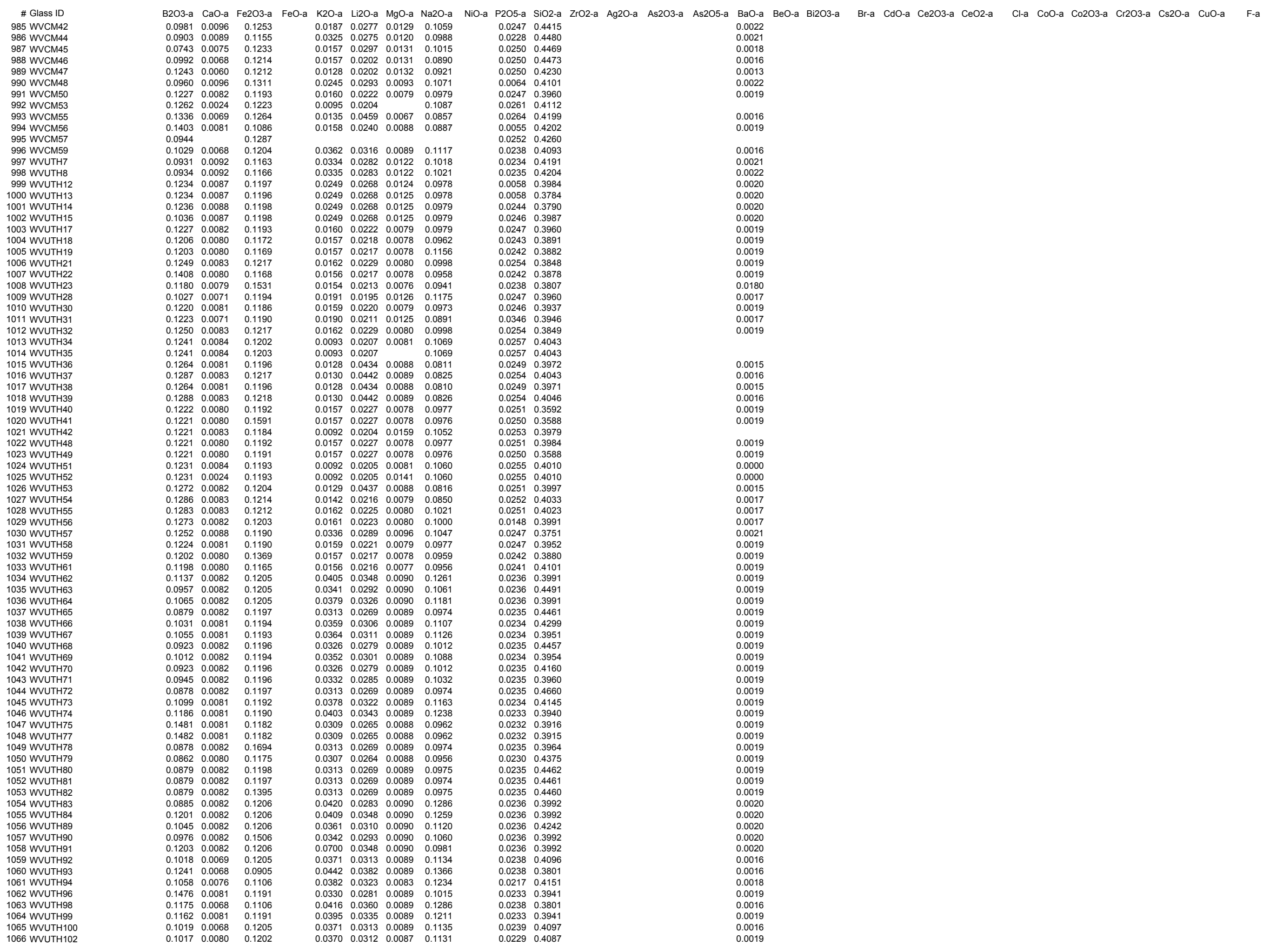




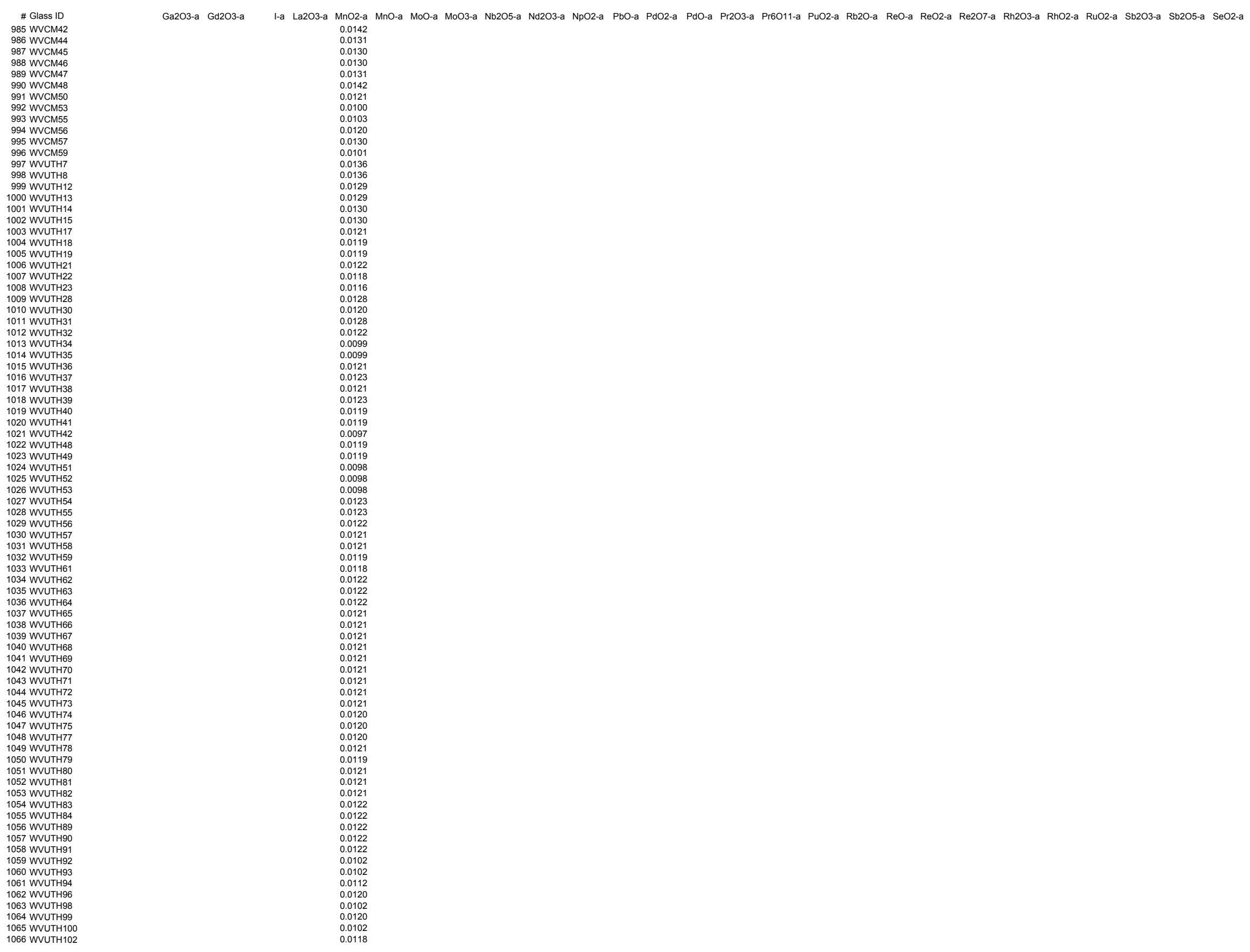


1014 WVUTH35

1015 WVUTH36

1016 WVUTH37
1017 WVUTH38

1017 WVUTH 39

1019 WVUTH40

1020 WVUTH41
1021 WVUTHA2

1022 WUTH48
1023 WVUTH49

1024 WVUTH51

1026 WVUTH53

1027 WVUTH54

1028 WWUTH55

1030 WUUTH57
1031 WVTHH58

1032 WVUTH59
1033 WUTHH61

1034 WVUTH62
1035 WVUTH63
103 W

1036 WVUTH64
1037 WUTHH65

1038 WVUTH66

1039 WVUTH67

1041 WVUTHE9

1042 WUUTH70

1043 WVUTH71

1045 WVUTH73

1046 WVUTH74

1047 WUUTH75

1049 WVUTH78

1050 WVUTH79

1051 WUUTH80
1055 WVUTH81
105 WUTH2

1053 WUTHB22
1054 WVUTH83

1055 WVUTH84

1057 WVUTH90

1057 WWUTH90
1058 WVUTH91

1059 WVUTH92
1060 WVUTH93

1061 WVUTH94

1062 WWUTTH96

1063 WVUTH98
1064 WVUTH99

11065 WUTHT1100
1066 WVUTH102 
1014 WVUTH35

1015 WVUTH36

1016 WVUTH37
1017 WVUTH38

1018 WVUTH399
1019 WVUTH40

1019 WUUTH4
1020 W WUTH4
1021 WVUTH4
1

1022 WVUTH48

1023 WUUTHA9
1024 WUUTH51

1026 WVUTH53

1026 WUUTH53
1027 WUTHH4

1028 WVUTH55
1029 WVUTH56

1030 WVUTH57

1031 WVUTH5s

1032 WVUTH59
1033 WVUTHG1

1034 WUUTH62
1035 WVTHH63

1036 WUTH64
1037 WUUTH65

1038 WVUTH66

1039 WVUTH67

1041 WVUTHE9

1042 WUUTH70

1043 WVUTH71

1045 WVUTH73

1046 WVUTH74

1048 WVUTH77

1049 WVUTH78

1050 WVUTH79

1051 WVUTH80

1053 WVUTH82

1055 WVUTH 83

1055 WUTH 84

1057 WVUTH90

1058 WWUTH91

1059 WVUTH92

1061 WVUTH94

1062 WWUTTH96

1063 WUTTH98
1064 WWUTH999

1065 WVUTH100

1066 WVUTH 102 


$$
\|
$$


1014 WVUTH35

1015 WVUTH36

1016 WVUTH37
1017 WVUTH38

1018 WVUTH39

1019 WVUTH40

1020 WVUTH 41
1021 WVUTH42

1022 WUTH48
1023 WVUTH49

1022 WVUTH51
1025 WVUTH52

1026 WVUTH5 53

1027 WVUTHTH4

1028 WVUTH55
1029 WVUTH 56

1030 WUUTH57
1031 WWUTH58
1023 WUTH59

1032 WVUTH59
1033 WUTHH61

1034 WUUTH62
1035 WUTHH3

1036 WVUTH64
1037 WUTHH65

1038 WVUTH66

1039 WVUTH67

1041 WVUTHE9

1042 WUUTH70

1043 WVUTH71

1045 WVUTH73

1046 WVUTH74

1047 WVUTH75

1049 WVUTH78

1050 WVUTH79

1051 WUUTH80
1055 WVUTH81
105 WUTH2

1053 WVUTH82
1054 WVTHB83

1055 WVUTH8 4

1057 WVUTH90

1058 WWUTH91

1059 WVUTH92

1061 WVUTH94

1062 WWUTTH96

1063 WVUTH98
1064 WVUTH99

11065 WUTHT1100
1066 WVUTH102 


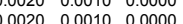

$\begin{array}{lll}0.0020 & 0.0010 & 0.00000 \\ 0.0220 & 0.0010 & 0.0000 \\ 0 & 0.020\end{array}$

$0.0020 \quad 0.00100 .00000$

$0.0020 \quad 0.0010 \quad 0.0000$

$\begin{array}{lll}0.0022 & 0.0010 & 0.0000 \\ 0.0020 & 0.0010 & 0.00000 \\ 0 & 0.020 & 0.0000\end{array}$

$\begin{array}{lll}0.0022 & 0.0010 & 0.00000 \\ 0020 & 0.0010 & 0.0000 \\ 0 & 0\end{array}$

0.0020
0.020010
0.0200000

$\begin{array}{llllll}0.0410 & 0.0010 & 0.0000\end{array}$

$\begin{array}{llll}0.0590 & 0.0010 & 0.0000 \\ 0 & 02020 & 0.00010 & 0.0000\end{array}$

$0.0020 \quad 0.00100 .0000$

0.02020
0.001010 0.0200

$0020 \quad 0.0010 \quad 0.0000$

$\begin{array}{llll}0.0020 & 0.0010 & 0.0000 \\ 0.0020 & 0.0010 & 0.0000\end{array}$

1132 WVDG-4

1134 WVDG-6

1136 WVDG- 8

1137 WVDG-1R
1138 WVDG-12R

1139 WVDG-13R

1140 WVDG-14R

114 WDDG-15

1143 WVDG-17

1144 WVDG-18

1145 WVDG-19
1146 WDDG-20

1147 WDG-21
1148 WVDG-22

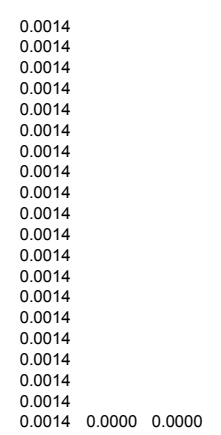

$\begin{array}{lll}0.00000 & 0.0140 \\ 0.0000 & 0.0130\end{array}$

0.00000 .01410

0.00000 .0140

0.00000 .0140

0.0000
0.0000
0.0000
0

$\begin{array}{lll}0.0000 & 0.0140 \\ 0.0000 & 0.0140 & 0\end{array}$

$\begin{array}{ll}0.0000 & 0.0140 \\ 0.0000 & 0.0130\end{array}$

$\begin{array}{lll}0.00000 & 0.0130 \\ 0 & 0.0000 & 0.140\end{array}$

$\begin{array}{lll}0.00000 & 0.0130 \\ 0.00000 & 0.0130 \\ 0.0130\end{array}$

0.00000 .0140

0.00000 .0130

$\begin{array}{lll}0.00000 & 0.0140 \\ 0.0000 & 0.130 \\ 0 & 0.000 & 0.30\end{array}$

0.00000 .0130

0.00000 .0140

0.000000 .0130

0.00000
0.0000
0.00140
0.0200
0.0140

$\begin{array}{ll}0.0200 & 0.0140 \\ 0.0570 & 0.0130\end{array}$

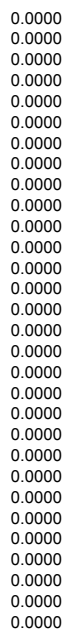

0.0000

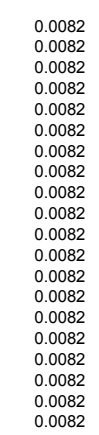


1070 cuoj

1072 COOL

1073 CUOB

1075 CUOE

1077 CUOC

1077 CUOG
$1078 \mathrm{CUOH}$
1079 CUOO

1080 CUOP

1081 CUOR
1082 CUOU

1083 CUOV

1085 CU31
1086 CU33

1087 CU34

1088 CU35

1090 CU37

1091 CU38

1093 CU40

1094 CU4 1

1096 Cu43

1097 CU44

1098 CU4

1100 Cu49

1101 CU50

1102 CU52
1103 CU53

1105 Cu55

1109 PNL 1

1110 PNL 2

1113 PNL

1114 PNL 6

1116 PNL 8

(117) PNL9

1120 Alkal

(122 Akl14

(125 Alkall

1127 Alkali9

1129 WVDG-

$0.0000 \quad 0.0000$

0.0010
0.0010
0.0010
0.0010
0.0010
0.0010
0.0010
0.0010
0.0010
0.0010
0.0010
0.0010
0.0010
0.0010
0.0010
0.0010
0.0010
0.0010
0.0010
0.0010
0.0010
0.0010
0.0010
0.0010
0.0010
0.0010
0.0010
0.0010
0.0010
0.0010

$\begin{array}{ll}0.0000 & 0.0100 \\ 0.0000 & 0.0090 \\ 0\end{array}$

$\begin{array}{lll}0.0000 & 0.0090 \\ 0.0000 & 0.0100 \\ 0.0000 & 0.0090\end{array}$

$\begin{array}{ll}0.0000 & 0.0090 \\ 0.0000 & 0.01100 \\ 0.0000 & 0.000\end{array}$

\begin{tabular}{ll}
.00000 & 0.0100 \\
0.0000 & 0.0100 \\
\hline
\end{tabular}

$\begin{array}{ll}0.00000 & 0.00100 \\ 0.00000 & 0.0100 \\ 0.0000 & 0.0100\end{array}$

$\begin{array}{ll}0.0000 & 0.0100 \\ 0.0000 & 0.0100\end{array}$

$\begin{array}{ll}0.00000 & 0.0100 \\ 0.0100 & 0.0000 \\ 0 & 0.0100\end{array}$

\begin{tabular}{ll}
0.0000 & 0.0100 \\
0.0000 & 0.0100 \\
\hline 0.0000 & 0.0090
\end{tabular}

\begin{tabular}{ll}
0.0000 & 0.0300 \\
\hline & .00000
\end{tabular}

$\begin{array}{ll}0.0000 & 0.0480 \\ 0.0000 & 0.0660\end{array}$

$\begin{array}{ll}0.0000 & 0.0100 \\ 0.0000 & 0.0100\end{array}$

$0.0000 \quad 0.0090$

$\begin{array}{ll}0.0000 & 0.0100 \\ 0.0000 & 0.0100\end{array}$

\begin{tabular}{ll}
$0.0000 \quad 0.0100$ \\
\hline
\end{tabular}

$0.0000 \quad 0.00100$

\begin{tabular}{ll}
0.0000 & 0.0090 \\
0.0000 & 0.0100 \\
\hline
\end{tabular}

$0.0000 \quad 0.0602$

$\begin{array}{ll}0.0000 & 0.0321 \\ 0.0000 & 0.0648 \\ 0.0000 & 0.0312\end{array}$

$\begin{array}{lll}0.000 & 1.0010 \quad 0.0668\end{array}$

$\begin{array}{lll}0.0000 & 1.0010 & 0.0309 \\ 0.0000 & 1.0010 & 0.0321\end{array}$

$\begin{array}{lll}0.0000 & 1.0000 & 0.0323\end{array}$

$\begin{array}{lll}0.0000 & 1.0000 & 0.0312 \\ 0.0000 & 0.9990 & 0.0325\end{array}$

$\begin{array}{lll}0.0000 & 1.0010 \\ 0.0000 & 1.0000\end{array}$

$\begin{array}{ll}0.0000 & 1.0010 \\ 0.0000 & 1.0000\end{array}$

$0.0000 \quad 1.0010$

0.00001 .0000

0.00001 .0020

0.00001 .0000

$\begin{array}{lll}0.0200 & 1.0000 \\ 0.0390 & 1.0020\end{array}$

$.0570 \quad 1.0000$

0.00001 .0000

0.00001 .0000

$\begin{array}{lll}0.0000 & 0.9990 \\ 0.0000 & 1.0000\end{array}$

$\begin{array}{lll}0.00000 & 1.0000 \\ 0.0000 & 1.0000\end{array}$

$\begin{array}{ll}0.0023 & 0.0025 \\ 0.0023 & 0.0025\end{array}$

$\begin{array}{ll}0.0178 & 0.0080 \\ 0.0178 & 0.0080\end{array}$

0.0063

$\begin{array}{ll}0.00000 & 0.0330 \\ 0 & 0.0304\end{array}$

$\begin{array}{lll}0.0023 & 0.0025 \\ 0.0023 & 0.0025 \\ 0 & 0.023 & 0.025\end{array}$

$\begin{array}{ll}0.0023 & 0.0025 \\ 0.0023 & 0.0025\end{array}$

$\begin{array}{lll}0.0023 & 0.0025 \\ 0.0023 & 0.0025\end{array}$

$0.0023 \quad 0.0025$

$\begin{array}{ll}0.0023 & 0.0025 \\ 0.0023 & 0.0025 \\ 0.0023 & 0.025\end{array}$

$0.0023 \quad 0.0025$

$\begin{array}{lll}0.0023 & 0.0025 \\ 0.023 & 0.025\end{array}$

$\begin{array}{lll}0.0023 & 0.0025 \\ 0.0023 & 0.0025 \\ 0 & \end{array}$

$\begin{array}{lll}0.0023 & 0.0025 \\ 0 & 0.023 & 0.025\end{array}$

$\begin{array}{ll}0.0023 & 0.0025 \\ 0.0023 & 0.0025 \\ 0.023 & 0.0025\end{array}$

$\begin{array}{ll}0.0178 & 0.0080 \\ 0.0178 & 0.0080 \\ 0.0178 & 0.0080 \\ 0.0178 & 0.0080 \\ 0.0178 & 0.0080 \\ 0.0534 & 0.0080 \\ 0.0534 & 0.0080 \\ 0.0534 & 0.0080 \\ 0.0534 & 0.0080 \\ 0.0400 & 0.0080 \\ 0.0402 & 0.0080 \\ 0.0330 & 0.0080 \\ 0.0370 & 0.0080 \\ 0.03855 & 0.0080 \\ 0.0345 & 0.0080 \\ 0.0413 & 0.0080 \\ 0.0394 & 0.0080 \\ 0.0419 & 0.0080 \\ 0.0356 & 0.0080\end{array}$

1132 WVDG-4

1133 WVDG-5

135 WVDG-7

1136 WVDG-8

1137 WVDG-11R

1139 WVDG-13R

1140 WVDG-14R

1142 WVDG-16

1143 WVDG-17

1144 WVDG-18

1146 WVDG-20

1147 WVDG-21

$\begin{array}{lll}0.0026 & 1.0000 & 0.0824 \\ 0.0026 & 1.000 & 0.0469\end{array}$ $\begin{array}{lll}0.0026 & 1.0000 & 0.0448 \\ 0.0026 & 1.0000 & 0.0453 \\ 0.0026 & 1.0000 & 0.0086\end{array}$ $0.00261 .0000 \quad 0.0806$ $\begin{array}{lll}0.0026 & 1.0000 & 0.0481\end{array}$ $\begin{array}{lll}0.0026 & 1.0000 & 0.0818 \\ 0.0026 & 1.0000 & 0.0487\end{array}$ $\begin{array}{lll}0.0026 & 1.0000 & 0.0487 \\ 0.0026 & 1.0000 & 0.0654\end{array}$ $\begin{array}{lll}0.0026 & 1.0000 & 0.0600 \\ 0.0026 & 1.0000 & 0.0607\end{array}$ $0.0026 \quad 1.0000 \quad 0.0626$ $\begin{array}{lll}0.0026 & 1.0000 & 0.0654 \\ 0.0026 & 1.0000 & 0.0587\end{array}$ $\begin{array}{lll}.0026 & 1.0000 & 0.0569\end{array}$ $\begin{array}{llll}0.0000 & 0.0026 & 1.0000 & 0.066 \\ & 1.0000 & 0.062\end{array}$ $\begin{array}{ll}0.0000 & 0.0874 \\ 0.0000 & 0.0681 \\ 0.0000 & 0.0716 \\ 0.0000 & 0.0838 \\ 0.0000 & 0.0690 \\ 0.0000 & 0.0855 \\ 0.0000 & 0.0884 \\ 0.0000 & 0.0901 \\ 0.0000 & 0.1046 \\ 0.0000 & 0.1052 \\ 0.0000 & 0.1058 \\ 0.0000 & 0.1058 \\ 0.00000 & 0.0960 \\ 0.0000 & 0.0943 \\ 0.00000 & 0.0943 \\ 0.0000 & 0.0947 \\ 0.0000 & 0.1223 \\ 0.0000 & 0.1224 \\ 0.0000 & 0.1251 \\ 0.0000 & 0.1250\end{array}$ 
\# Glass ID

1067 WVUTH103

1068 CUOD
1069 CUOI

1070 CUO

1071 CUOK

1073 CUOB

1074 CUOC

$1077 \mathrm{CUOG}$
$1078 \mathrm{CUOH}$

1079 CUOO

1081 CUOR

1082 CUOU

1085 CU31
1086 CU33

1087 CU34

1089 CU36

1090 CU37

1092 CU3

1095 CU42

1097 CU44

1101 Cu50

1103 CU5

1105 CU55

1106 CU56

1108 CU58

109 PNL

1110 PNL

1112 PNL 4

1114 PNL 6

1117 PNL 9

1118 PNL 10

1120 Alkali2

121 Alkali3

1123 Alkalis

124 Alkalir

1126 Alkali8

1128 Ref6Qtr2

1129 WVDG-

1131 WVDG-3

1132 WVDG-4

134 WVDG-6

136 WVDG-

1137 WVDG-11R
1138 WVDG-12R

1139 WVDG-13R

1140 WVDG-14R

1141 WVG-15

143 WVDG-17

1144 WVDG-18

146 WVDG-20

1147 WVDG-21 B2O3-a CaO-a Fe2O3-a FeO-a K2O-a Li2O-a MgO-a Na2O-a NiO-a P2O5-a SiO2-a ZrO2-a Ag2O-a As2O3-a As2O5-a BaO-a BeO-a Bi2O3-a Br-a CdO-a Ce2O3-a CeO2-a Cl-a CoO-a Co2O3-a Cr2O3-a Cs2O-a CuO-a F-a

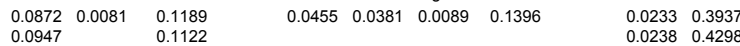

0.1127

$\begin{array}{ll}0.0970 & 0.1149 \\ 0.0950 & 0.1125 \\ 0.0904 & 0.1142\end{array}$

$0.0964 \quad 0.1142$

$0.0933 \quad 0.1105$

$\begin{array}{ll}0.0942 & 0.1117 \\ 0.0980 & 0.1160\end{array}$

$\begin{array}{ll}0.0942 & 0.1117 \\ 0.0980 & 0.1160\end{array}$

$\begin{array}{ll}0.0949 & 0.1125 \\ 0.0942 & 0.1115 \\ 0.0923 & 0.114\end{array}$

$\begin{array}{ll}0.0923 & 0.11094 \\ 0.0993 & 0.1112 \\ 0.0959 & 0.1131 \\ 0.095 & 0\end{array}$

$\begin{array}{lll}0.0996 & 0.1180 \\ 0.1656 & 0.0051 & 0.1611 \\ 0.166 & 0.055 & 0.0989\end{array}$

$0.1656 \quad 0.0055 \quad 0.0943$

$\begin{array}{lll}0.0922 & 0.0058 & 0.1633 \\ 0.1657 & 0.0061 & 0.0919 \\ 0.0922 & 0.0052 & 0.090\end{array}$

$09220.0052 \quad 0.0910$

$\begin{array}{lll}0.1652 & 0.0062 & 0.1605\end{array}$

$\begin{array}{lll}0.0922 & 0.0068 & 0.1615 \\ 0.0922 & 0.0049 & 0.0928\end{array}$

$\begin{array}{lll}0.0922 & 0.0049 & 0.0928 \\ 0.1050 & 0.0085 & 0.1271\end{array}$

$\begin{array}{lll}0.1460 & 0.0088 & 0.1269 \\ 0.1330 & 0.0073 & 0.1351\end{array}$

$\begin{array}{lll}0.1110 & 0.0085 & 0.1195 \\ 0.1450 & 0.0073 & 0.1151 \\ 0.160 & 0.0075 & 0.147\end{array}$

$\begin{array}{lll}0.1180 & 0.0075 & 0.1407 \\ 0.1390 & 0.0084 & 0.1211 \\ 0.1251 & 0.008 & 0\end{array}$

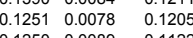

$\begin{array}{lll}0.1289 & 0.0064 & 0.1250 \\ 0.1215 & 0.0068 & 0.1174\end{array}$

$\begin{array}{lll}0.1215 & 0.0068 & 0.1174 \\ 0.1217 & 0.0083 & 0.1172\end{array}$

$\begin{array}{lll}0.0933 & 0.0063 & 0.1081\end{array}$

$\begin{array}{lll}0.1177 & 0.0068 & 0.1106 \\ 0.1241 & 0.0068 & 0.1191\end{array}$

$\begin{array}{lll}0.1241 & 0.0068 & 0.1191 \\ 0.1215 & 0.0069 & 0.1155 \\ 0.1215 & 0.0055 & 0.1155\end{array}$

$\begin{array}{lll}0.1215 & 0.0005 & 0.1155\end{array}$

$\begin{array}{lll}0.0751 & 0.0067 & 0.1229 \\ 0.1097 & 0.0062 & 0.1190 \\ 0.1078 & 0.006 & 0.1120\end{array}$

$\begin{array}{lll}0.1078 & 0.0061 & 0.1190\end{array}$

$\begin{array}{lll}0.1098 & 0.0061 & 0.1199 \\ 0.1258 & 0.0136 & 0.0880\end{array}$

$\begin{array}{lll}0.1258 & 0.0136 & 0.0880 \\ 0.1261 & 0.0137 & 0.1442\end{array}$

$\begin{array}{lll}0.0978 & 0.0143 & 0.0887\end{array}$

$\begin{array}{lll}0.1062 & 0.0054 & 0.1444 \\ 0.1008 & 0.0131 & 0.1420 \\ 0.1062 & 0.014 & 0.075\end{array}$

$\begin{array}{lll}0.1008 & 0.0131 & 0.1420 \\ 0.1062 & 0.0134 & 0.0875\end{array}$

$\begin{array}{lll}0.1005 & 0.0050 & 0.1467 \\ 0.1157 & 0.0050 & 0.1403\end{array}$ $\begin{array}{lll}0.0238 & 0.4298 \\ 0.0239 & 0.4320\end{array}$

$\begin{array}{ll}0.0244 & 0.4501 \\ 0.0238 & 0.4473 \\ 0.0237 & 0.4468\end{array}$

0.02420 .4378

$0.0234 \quad 0.4235$

0.02370 .4846

$\begin{array}{ll}0.0246 & 0.4447 \\ 0.0245 & 0.4425\end{array}$

$\begin{array}{ll}0.0237 & 0.4846 \\ 0.0246 & 0.4644\end{array}$

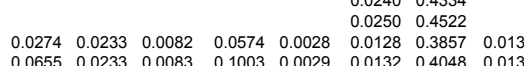

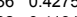
$\begin{array}{lllllllll}0.0466 & 0.0079 & 0.1119 & 0.0027 & 0.0105 & 0.3385 & 0.0125\end{array}$ $\begin{array}{llllllll}0.0623 & 0.0222 & 0.0078 & 0.0564 & 0.0027 & 0.0137 & 0.4614 & 0.0131 \\ 0.0279 & 0.0487 & 0.0080 & 0.0551 & 0.0028 & 0.0130 & 0.4669 & 0.0140 \\ 0.025 & 0.003\end{array}$ $\begin{array}{llllllll}0.0622 & 0.0415 & 0.0080 & 0.0988 & 0.0028 & 0.0120 & 0.4712 & 0.0133\end{array}$ $\begin{array}{llllllll}0.0302 & 0.0221 & 0.0080 & 0.1031 & 0.0027 & 0.0119 & 0.3385 & 0.0133\end{array}$

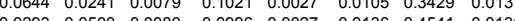

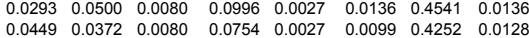
$\begin{array}{lllllllll}0.0436 & 0.0424 & 0.0082 & 0.0818 & 0.0027 & 0.0103 & 0.3792 & 0.0119\end{array}$ $\begin{array}{llllllll}0.0470 & 0.0365 & 0.0082 & 0.0823 & 0.0028 & 0.0084 & 0.3984 & 0.0090\end{array}$ $\begin{array}{lllllllllll}0.0429 & 0.0385 & 0.0081 & 0.0810 & 0.028 & 0.0106 & 0.4393 & 0.019\end{array}$ $\begin{array}{llllllll}0.0501 & 0.0405 & 0.0081 & 0.0785 & 0.0027 & 0.0114 & 0.3933 & 0.0112 \\ 0.0517 & 0.0421 & 0.0080 & 0.0807 & 0.0027 & 0.0120 & 0.3831 & 0.0137\end{array}$ $\begin{array}{llllllll}0.0544 & 0.0352 & 0.0082 & 0.0889 & 0.0027 & 0.0112 & 0.3995 & 0.0121\end{array}$ $\begin{array}{llllllll}0.0499 & 0.0392 & 0.0080 & 0.0797 & 0.0028 & 0.0117 & 0.4022 & 0.0109\end{array}$

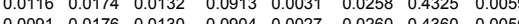
$\begin{array}{lllllllll}0.0192 & 0.0234 & 0.0170 & 0.0982 & 0.0036 & 0.0241 & 0.4546 & 0.0064\end{array}$ $\begin{array}{llllllll}0.0171 & 0.0171 & 0.0129 & 0.1147 & 0.0027 & 0.0254 & 0.4194 & 0.0054\end{array}$

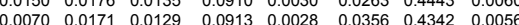
$\begin{array}{lllllllll}0.0127 & 0.0171 & 0.0128 & 0.0868 & 0.0028 & 0.0251 & 0.4303 & 0.0062\end{array}$ $\begin{array}{llllllll}0.0171 & 0.0184 & 0.0139 & 0.0938 & 0.0030 & 0.0268 & 0.4583 & 0.0057 \\ 0.0267 & 0.0263 & 0.0125 & 0.0952 & 0.0031 & 0.0248 & 0.4061 & 0.0046\end{array}$ $\begin{array}{llllllll}0.0285 & 0.0257 & 0.0125 & 0.0958 & 0.0029 & 0.0252 & 0.4016 & 0.0054\end{array}$ $\begin{array}{llllllll}0.0287 & 0.0259 & 0.0126 & 0.0938 & 0.0031 & 0.0250 & 0.4018 & 0.0052 \\ 0.194 & 0.0261 & 0.0125 & 0.0951 & 0.0029 & 0.0250 & 0.4020 & 0.0073\end{array}$ $\begin{array}{llllll}0.0164 & 0.0273 & 0.0127 & 0.1017 & 0\end{array}$ $\begin{array}{lllllllll}0.0207 & 0.0234 & 0.0129 & 0.1057 & 0.0058 & 0.0124 & 0.3957 & 0.0075\end{array}$ $\begin{array}{llllllll}0.0175 & 0.0244 & 0.0044 & 0.1114 & 0.0017 & 0.0155 & 0.4320 & 0.0022\end{array}$

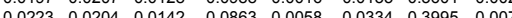
$\begin{array}{llllllll}0.0123 & 0.0231 & 0.0053 & 0.1046 & 0.0017 & 0.0338 & 0.3754 & 0.0022 \\ 0.0184 & 0.0182 & 0.0048 & 0.0831 & 0.0057 & 0.0143 & 0.3738 & 0.0073\end{array}$

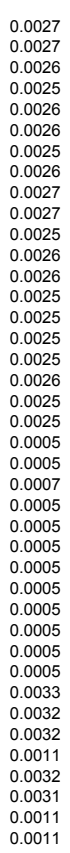

0.0031
0.0031
0.0031
0.0031
0.0031
0.0031
0.0031
0.0031
0.0031
0.0031
0.0031
0.0031
0.0031
0.0031
0.0031
0.0031
0.0031
0.0031
0.0031
0.0031

0.0007
0.0007
0.0010
0.0007
0.0009
0.0005
0.0007
0.0010
0.0007
0.0007
0.0007
0.0007
0.0124
0.038
0.0136
0.039
0.0038
0.0135
0.0039
0.0132

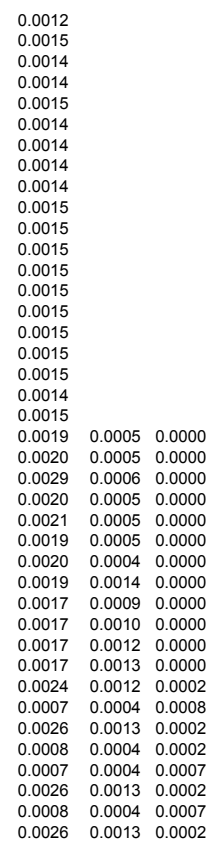

$\begin{array}{lll}0.0026 & 0.0013 & 0.0002\end{array}$ 


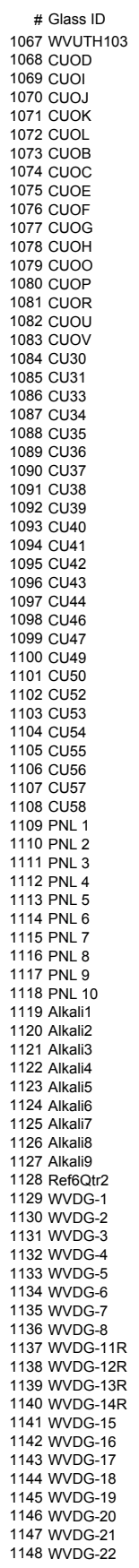

I-a La2O3-a MnO2-a MnO-a MoO-a MoO3-a Nb205-a Nd2O3-a NpO2-a PbO-a PdO2-a PdO-a Pr2O3-a Pr6011-a PuO2-a Rb2O-a ReO-a ReO2-a Re207-a Rh203-a RhO2-a RuO2-a Sb203-a Sb205-a SeO2-a $0_{0.0132}^{0.0120}$

0.0132
0.0131
0.0134

0.0134

0.0135
0.0131
0.0136

0.0136

0.0131

.0132

0.0131
0.0129
0.0111
0.0133

0.0136

0.0087

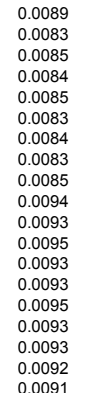

$\begin{array}{ll}0.0004 & 0.0130 \\ 0.0004 & 0.0132\end{array}$

$\begin{array}{ll}0.0004 & 0.0132 \\ 0.0005 & 0.0176 \\ 0.0004 & 0.130\end{array}$

$\begin{array}{ll}0.0004 & 0.0130 \\ 0.0004 & 0.0136\end{array}$

$\begin{array}{ll}0.0004 & 0.0130 \\ 0.0004 & 0.0129\end{array}$

$\begin{array}{ll}0.0004 & 0.0129 \\ 0.0004 & 0.0137 \\ 0.0003 & 0.0132\end{array}$

$\begin{array}{ll}0.0003 & 0.0132 \\ 0.0003 & 0.0131 \\ 0.0003 & 0.0131\end{array}$

$\begin{array}{ll}0.0003 & 0.0131 \\ 0.0003 & 0.0131 \\ 0.0003 & 0.0131\end{array}$

$\begin{array}{ll}0.0003 & 0.0131 \\ 0.0007 & 0.0251\end{array}$

$\begin{array}{ll}0.0007 & 0.0251 \\ 0.0002 & 0.0223 \\ 0.0008 & 0.249\end{array}$

$\begin{array}{ll}0.0008 & 0.0223 \\ 0.0002 & 0.0054 \\ 0.002 & 0.050\end{array}$

$\begin{array}{ll}0.0002 \quad 0.0050 \\ 0007 & 0.052\end{array}$

$\begin{array}{ll}0.0002 & 0.0250 \\ 0.0007 & 0.0246\end{array}$

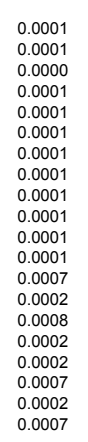

$\begin{array}{ll}0.0000 & 0.0000 \\ 0.0001 & 0.0008\end{array}$

$\begin{array}{ll}0.0001 & 0.0008 \\ 0.0001 & 0.0007\end{array}$

$\begin{array}{ll}0.0001 & 0.0007 \\ 0.0000 & 0.0000 \\ 0.0000 & 0.000\end{array}$

$\begin{array}{lll}0.00000 & 0.00000 \\ 0.0000 & 0.0000\end{array}$

$\begin{array}{ll}0.0000 & 0.0000 \\ 0.0000 & 0.0000 \\ 0.0000 & \end{array}$

$\begin{array}{ll}0.0001 & 0.0008 \\ 0.0001 & 0.0008\end{array}$

$\begin{array}{ll}0.0001 & 0.0008 \\ 0.0001 & 0.0008\end{array}$ 
\# Glass ID

1067 WVUTH10

1068 CUO
1069 CUO

1070 CUOJ
1071 CUOK

1072 CUOL

1074 CUOC

1075 CUOE

1077 CUOG

1077 CUOG
1079 CUOO
1079

$1080 \mathrm{CUOP}$

1081 CUOR
1082 CUOU

1083 CUOV

1085 CU31

1086 CU33

1087 CU34
1088 CU35

1089 CU36

(091

1091 CU38

1093 CU40

1094 CU4

$1095 \mathrm{CU} 42$
$1096 \mathrm{CU} 43$

1097 CU44

1098 CU4

100 CU49

1101 CU50

1102 CU52

1105 Cu55

1109 PNL 1

111 PNL

1113 PNL 5

1114 PNL

1117 PNL

1118 PNL 10

1120 Alkali2

1121 Alkal 3

1122 Alkali4

1124 Alkalí

126 Alkali8

1128 Ref6Qtr2

1130 WVDG-2

1132 WVDG-4

134 WVDG-

1136 WVDG-8

1137 WVDG-11R
1138 WVDG-12R

1139 WVDG-13R

1140 WVDG-14R

1142 WVDG-16

1143 WVDG-17

1144 WVDG-18

1146 WVDG-20

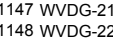

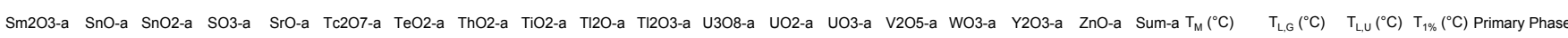

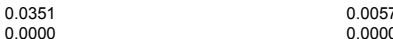

0.0057
0.0000
0.0000

0.9689
0.7418
0.732

0.741
0.7372
0

0.7566

0.7524

0.7515

0.7503

$\begin{array}{rl}0.0000 & 0.0000 \\ 0.0000 & 0.0000\end{array}$

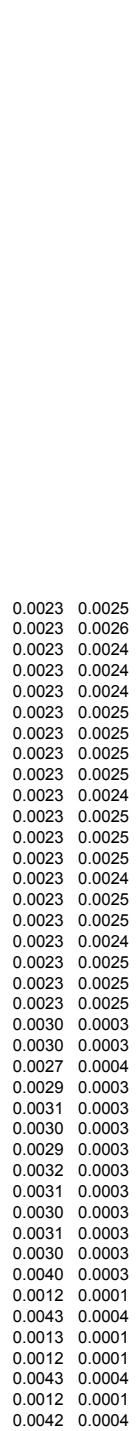

0.0000
0.0000
0.0000
0.0000
0.0000

$\begin{array}{lll}0.00178 & 0.0076 \\ 0.0078 & 0.0079\end{array}$

$\begin{array}{lll}0.0178 & 0.0079 \\ 0.0178 & 0.0079 \\ 0.0178 & 0.074\end{array}$

$0.0178 \quad 0.0074$

$\begin{array}{lll}0.0178 & 0.0075\end{array}$

0.05340 .0074

$0.0534 \quad 0.0074$

$\begin{array}{lll}0.0534 & 0.0074\end{array}$

$\begin{array}{lll}0.0534 & 0.0076 \\ 0.0400 & 0.0076 \\ 0.0402 & 0.0076\end{array}$

$0.0402 \quad 0.0076$

$\begin{array}{lll}0.0330 & 0.0078 \\ 0.0370 & 0.0076\end{array}$

0.03850 .0076

$\begin{array}{ll}0.0345 & 0.0077 \\ 0.0413 & 0.0076\end{array}$

$\begin{array}{lll}0.0394 & 0.0078 \\ 0 & 0.0419 & 0.0077\end{array}$

$0.0356 \quad 0.0076$

$\begin{array}{lll}0.0286 & 0.0096 \\ 0.0280 & 0.0096\end{array}$

$\begin{array}{lll}0.0280 & 0.0096 \\ 0.0273 & 0.0122\end{array}$

$0.0294 \quad 0.0090$

$0.0275 \quad 0.0093$

0.03830 .0096

0.02750 .0101

$0.0220 \quad 0.0095$

0.02480 .0096

0.02590 .0096

$0.0160 \quad 0.0044$

0.04690 .0158

$0.0196 \quad 0.0046$

$\begin{array}{lll}0.0524 & 0.0044 \\ 0.0187 & 0.0157\end{array}$

$\begin{array}{ll}0.0159 & 0.0046 \\ 0.0175 & 0.0154\end{array}$

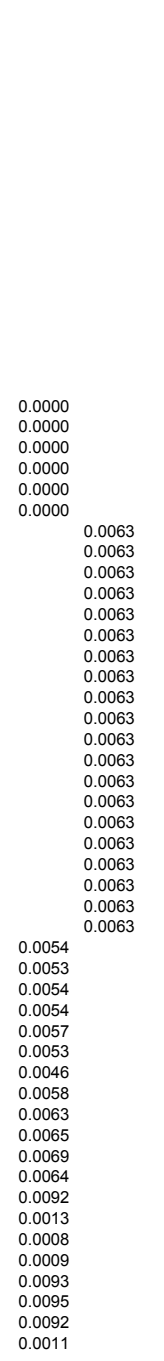

$0.0026 \quad 1.0000$

$0.027 \quad 1.0000$

$\begin{array}{ll}0.0026 & 1.0000 \\ 0.0028 & 1.0000 \\ 0.026 & 1.000\end{array}$

$\begin{array}{ll}0.0026 & 1.0000 \\ 0.0027 & \end{array}$

$\begin{array}{ll}0.0027 & 1.0000 \\ 0.0027 & 1.0000\end{array}$

$0.0028 \quad 1.0000$

0.00271 .0000

$.0027 \quad 1.0000$
0.027
0.027

$\begin{array}{ll}0.0226 & 1.000 \\ 0.0027 & 1.0000\end{array}$

$\begin{array}{ll}.0028 & 1.0000 \\ .0028 & 1.0000 \\ 0.000 & 0.007\end{array}$

$\begin{array}{lll}0.0002 & 0.0000 & 0.9987\end{array}$

$\begin{array}{llll}0.0003 & 0.0000 & 0.9983\end{array}$

$\begin{array}{llll}0.0002 & 0.00000 & 0.9990\end{array}$

$\begin{array}{llll}0.0002 & 0.0000 & 0.9992\end{array}$

$\begin{array}{lll}0.0002 & 0.0005 & 0.9999 \\ 0.0002 & 0.0002 & 0.9994\end{array}$

$\begin{array}{llll}0.0001 & 0.0006 & 0.9997 \\ 0.0002 & 0.0000 & 0.9989\end{array}$

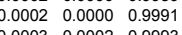

$\begin{array}{lll}0.0001 & 0.0002 & 1.0005 \\ 0.0004 & 0.0006 & 0.9996 \\ 0.001 & 0.006 & 0.096\end{array}$

$\begin{array}{lll}0.0001 & 0.0000 & 0.9997 \\ 0.0001 & 0.0000 & 0.9902\end{array}$

$\begin{array}{lll}0.0001 & 0.0006 & 1.0008 \\ 0.0004 & 0.0002 & 0.998\end{array}$ 
Hor Cu5

1103 C C55
11044 Cus

1104 C C54
1105 C U55

11106 Cu55
1107 C157

1108 CU58

1108 CUS8
1109 PNL

1110 PNL2

1112 PNL4
1113 PNL 5

1114 PNL

1116 PNL8

1117 PNL 9

1118 PNL 10

1120 Alkalir

1121 Akall

1122 Alkalii
1123 Akali5

1124 Akkali
1125 Akkail

1126 Akalis
1127 Akkio

1128 Refrolit 2
1129

1119 WVQG-1

1130 WVDG-2

1132 WVDG-4

1133 WVDG-5

135 WVDG-7

1136 WVGG-8

1137 WVGG-11R
1138 WVDG-12R

1139 WVDG-13R

140 WVDG-14R

1142 WVDG-16

1143 WVDG-17

144 WVDG-18

1145 WVGG-19
1146 WVDG-20

1147 WVDG-21
1148 WVDG-22

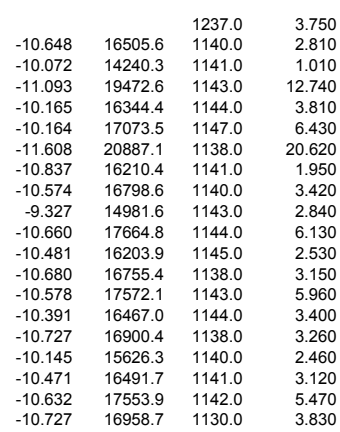


1125 Alkalii

1127 Alkali9
1128 Refíatri

1129 WVDG-

1130 WVDG-2
1131 WVDG-3

1132 WVDG-4

1134 WVDG-6

\begin{tabular}{|c|c|c|c|c|c|c|c|}
\hline $\begin{array}{l}1415 \\
1051\end{array}$ & $\begin{array}{r}7.210 \\
0.07\end{array}$ & & & & & & \\
\hline 1052 & $\begin{array}{l}6.0900 \\
1.920\end{array}$ & $\begin{array}{l}12399 \\
1237\end{array}$ & & $\begin{array}{l}1147.0 \\
1177.0\end{array}$ & $\begin{array}{l}2.6 \\
1.0\end{array}$ & $\begin{array}{l}945.0 \\
945\end{array}$ & $\begin{array}{r}18.6 \\
55\end{array}$ \\
\hline & (15.9) & 1241 & & 1147.0 & 13.6 & $\begin{array}{l}943.0 \\
944.0\end{array}$ & $\begin{array}{c}5.1 \\
140.2\end{array}$ \\
\hline & & & 2.0 & & 3.8 & 950.0 & \\
\hline 1051 & 13.890 & 1147 & 6.2 & $\begin{aligned} 1242.0 \\
\end{aligned}$ & 3.2 & 948.0 & $\begin{array}{l}49.0 \\
270\end{array}$ \\
\hline $\begin{array}{l}1238 \\
1046\end{array}$ & $\begin{array}{r}10.690 \\
4.1 .9\end{array}$ & $\begin{array}{l}1145 \\
1237\end{array}$ & $\begin{array}{r}22.0 \\
0.9\end{array}$ & $\begin{array}{l}1046.0 \\
11460\end{array}$ & $\begin{array}{r}66.3 \\
17\end{array}$ & 946.0 & $\begin{array}{l}272.0 \\
11\end{array}$ \\
\hline $\begin{array}{l}1246 \\
1241\end{array}$ & 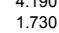 & $\begin{array}{l}1237 \\
1148\end{array}$ & $\begin{array}{l}0.9 \\
3.7\end{array}$ & $\begin{array}{l}1144.0 \\
1042.0\end{array}$ & $\begin{array}{l}1.7 \\
8.7\end{array}$ & $\begin{array}{l}946.0 \\
946.0\end{array}$ & $\begin{array}{l}11.9 \\
25.5\end{array}$ \\
\hline & 6.110 & $\begin{array}{r}1140 \\
1239\end{array}$ & $\begin{array}{l}3.1 \\
1.5\end{array}$ & $\begin{array}{l}1042.0 \\
1148.0\end{array}$ & $\begin{array}{l}8.1 \\
5.8\end{array}$ & $\begin{array}{l}946.0 \\
945.0\end{array}$ & $\begin{array}{l}25.5 \\
20.0\end{array}$ \\
\hline & 2.870 & 1148 & $\begin{array}{l}5.0 \\
5.7\end{array}$ & $\begin{array}{l}11840 \\
1047.0\end{array}$ & $\begin{array}{r}3.8 \\
14.4\end{array}$ & $\begin{array}{l}945.0 \\
945.0\end{array}$ & $\begin{array}{l}20.0 \\
48.7\end{array}$ \\
\hline 1054 & 5.530 & 1242 & 1.3 & 1148.0 & 2.5 & 947.0 & 16.8 \\
\hline 1050 & 6.900 & 1236 & 1.6 & 1147.0 & 3.2 & 946.0 & 22.3 \\
\hline 1050 & 13.870 & 1240 & 3.0 & 1147.0 & 6.1 & 945.0 & 49.6 \\
\hline 1053 & 7.270 & 1242 & 1.7 & 1148.0 & 3.3 & 947.0 & 23.1 \\
\hline 1051 & $\begin{array}{l}7.220 \\
53550\end{array}$ & $\begin{array}{l}1237 \\
1238\end{array}$ & $\begin{array}{l}1.7 \\
1.7\end{array}$ & $\begin{array}{l}1145.0 \\
1137\end{array}$ & $\begin{array}{r}3.3 \\
2.5\end{array}$ & 946.0 & $\begin{array}{l}24.3 \\
15.3\end{array}$ \\
\hline & $\begin{array}{l}5.350 \\
6.930\end{array}$ & $\begin{array}{l}72838 \\
1241\end{array}$ & $\begin{array}{l}1.6 \\
1.6\end{array}$ & $\begin{array}{l}1148.0 \\
\end{array}$ & & $\begin{array}{l}943.0 \\
945.0\end{array}$ & $\begin{array}{l}25.4 \\
22.4\end{array}$ \\
\hline & 12.870 & 1241 & 2.8 & 1149.0 & 5.6 & 945.0 & 46.4 \\
\hline 1049 & 7.920 & 1131 & 3.8 & 1237.0 & 1.7 & 948.0 & 24.4 \\
\hline
\end{tabular}


$1070 \mathrm{CUO}$

1072 cyol

1073 CUOB

1074 CUOC

$1076 \mathrm{CUOF}$

1078 cuor

1079 cuoo

1081 CUOR

1082 CuOU

1083 CUOV
1084 CU30

1085 CU31

1086 CU33

1088 CU35

1089 CU36

1091 CU38

1094 CU41

1096 Cu43

1097 CU44

1098 CU46

1100 CU49

1101 CU50

1102 CU52

CU55

1109 PNL

1110 PNL

1113 PNL

114 PNL 6
1115 PNL?

1116 PNL 8

1117 PNL9

1120 Alkal

1122 Akalis

123 Alkali5

1125 Akal

126 Alkali8

1128 Ref6Qtr

1130 WVDG

1131 WVDG-3

1132 WVDG-4

1134 WVDG

136 WVDG

1137 WVG-11R

1139 WVDG-13R

1140 WVDG-14R

1142 WVDG-16

1143 WVDG-17

1144 WVDG-18

146 WVDG-20

1147 WVDG-21

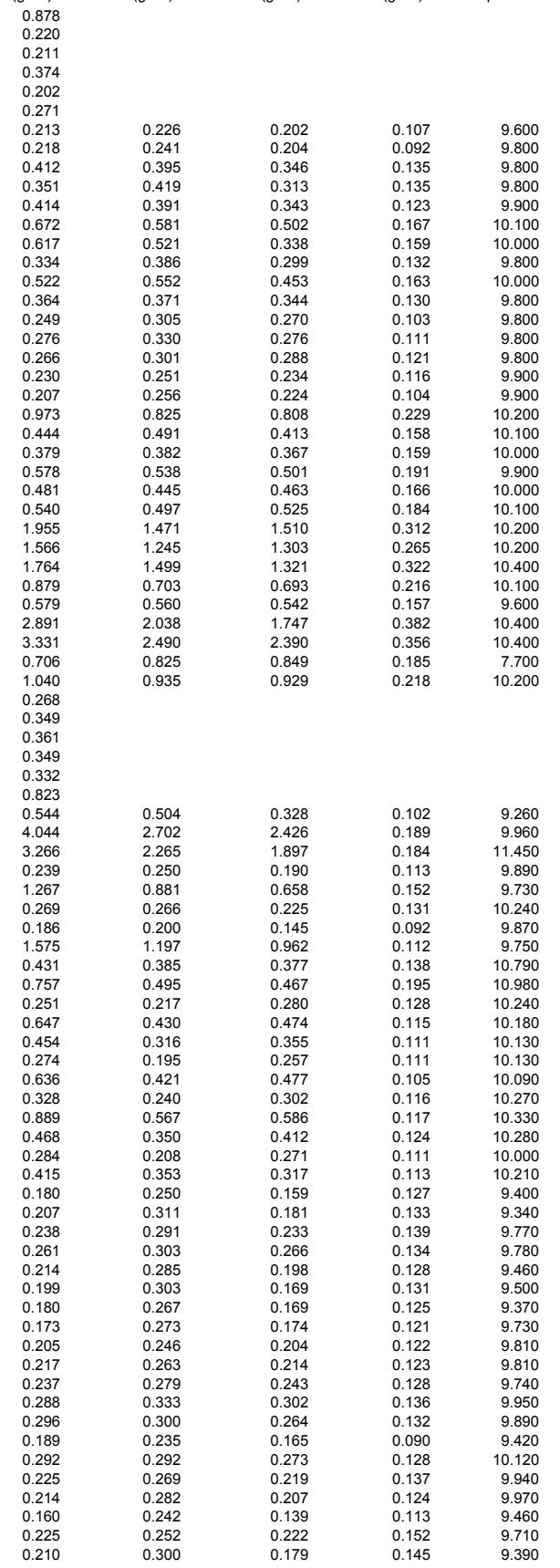


1073 CUOB
1074 CUOC

1075 CUOE

1077 cuoG

1078 CUOH

1079 cuoo
1000 cuop

1081 CUOR
1082 CUOO

$1083 \mathrm{CUOV}$
$1084 \mathrm{CU}$
1030

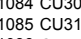

1086 CU33

1087 C C34
1088 Cu35

1088 CU35
1089 CU36

1089 C Cu36
1090 CU37
10911038

1091 CU38
1092 Cu39

1093 CU40

1094 CU41

$1095 \mathrm{CU} 42$
$1096 \mathrm{CU} 33$

1097 CU44

1098 CU46
1099
109047

1099 CU47
1100 CU49

1101 Cu50

1102 CU52

1104 CU54

1105 Cu55

1106 CU56
1107 Cu57

1108 Cus8

1119 PNL 1

1113 PNL5

1114 PNL6 6
115 PN 7
116 .

1116 PNL8

1117 PNL 9

1118 PNL 10
1119 Alkali

1120 Alkali2

1121 Alkali3

1123 Akalis

124 Alkallo

126 Akkali8

127
1128 Refífolt2

11292 WVQG-1
1130 WVGG-2

1130 WVDG-2
1131 WVDG-3

1132 WVDG-4

1133 WVDG-5

135 WVDG-7

1136 WVGG-8

1137 WVDG-11R

1139 WVDG-13R

140 WVDG-14R

1142 WVDG-16

143 WVDG-17

1144 WVDG-18

1145 WVDG-19
1146 WVDG-20

1147 WVDG-22

1148 WVDG-22 
\# Glass ID

1149 WVDG-23

1151 WVDG-25

1153 WVDG-27

1155 WVDG-29

1156 WVDG-30

1157 WVDG-33

1159 WVDG-3

1160 WVDG-36

1164 WVDG-40

165 WVDG-

167 WVDG-43

1168 WVDG-44

1170 WVDG-4

1172 WVDG-48

1173 WVDG-40

$1175 \mathrm{FY} 2 \# 5$
1176 FY92\#6

1777 FY92\#7

1179 FY92\#10

181 Ratio2

1182 Ratio

1183 Ratios

1185 LoT

$1187 \mathrm{HiFe2}$

1188 HiFe3
1189 HiFe4

1900 PNL190

191 FY93\#1

192 FY93\#2

$1194 \mathrm{FY} 93 \# 4$
$1195 \mathrm{FY} 93 \# 5$

196 FY93\#6

198 FY93\#8

199 FYst9

201 FY94\#1

202 FY94\#2

(204 FYO4t

1206 FY94\#6

1208 FY94\#8
1209
12909 FY4t\#9

$1210 \mathrm{FY} 94 \# 110$

211 Sigma1

213 Sigma3 3

1214 Sigma4

215 Sigma5
1216 Sigma6

1218 Sigma8
1219 Sigma9

1222

(1223

12256

12289

122910
Study

Reference

West Valley CVS Glasses PC' Olson et al. 1994

West Valley CVS Glasses PC'Olson et al 1994

West Valley CVS Glasses PC' Olson et al. 1994

West Valley CVS Glasses PC OIson et al. 1994

West Valley CVS Glasses PC' Olson et al. 1994

West Valley CVS Glasses PC' Olson et al. 1994

West Valley CVS Glasses PC. OIson et al. 1994

West Valley CVS Glasses PC' Olson et al. 1994

West Valley CVS Glasses PC' OIson et al. 1994

West Valley CVS Glasses PC. Ots et. 1994

West Valley CVS Glasses PC' OIson et al. 1994

West Valley CVS Glasses PC' Olson et al. 1994

West Valley CVS G

West Valley CVS Glasses PC O Olson et al. 1994

West Valley CVS Glasses PC Olson et al. 1994

West Vlley CVS Glases PC. Oton 1994

West Valley CVS Glasses PC' Olson et al. 1994

West Valley CVS Glasses PC Olson et al. 1994

West Valley WOR
West Valley WOR

WVNS 1995
WVNS 1995

$\begin{array}{ll}\text { West Valley WQR } & \text { WVS } 1995 \\ \text { West Valley WQR } & \text { WWNS } 1995\end{array}$

West Valley WQR

$\begin{array}{ll}\text { West Valley WOR } & \text { WVNS } 1995 \\ \text { West Valley WQR } & \text { WNS 1995 } \\ \text { West Valley WOR } & \text { WWNS 1995 }\end{array}$

West Valley WQR

West Valley WQR

WVNS 1995

WVNS 1995

West Valley WOR

WWNS 1995

West Valley WQR

WVNS 1995

West Valley WQR
West Valley WOR

WVNS 1995

列 Valley WOR

West Valley WQR
West Valley WQR

WVNS 1995
WVNS 1995

st valley WQR

WNS 1995

West Valley WQR

West Valley WQR
West Valley WQR
West Valley WQR

WVNS 1995

WVNS 1995

West Valley WQR
West Valley WQR

West Valley WQR

WVNS 1995

WVNS 1995

West Valley WQR
West Valley WQR

WVNS 1995

West Valley WQR

WVNS 1995

West Valley WOR

WWNS 1995

WVNS 1995

West Valley WQR

West Valley WOR

WVNS 1995

WVNS 1995
WVNS 1995

WUNS 1995

West Valley wa

WVNS 1995

West Valley WQR
West Valley WQR

WVNS 1995

West Valley WQR
West Valley WQR

WWNS 1995

Canonical Correlation (Ramse Oksoy et al. 1994 Canonical Correlation (Ramse osoy et al. 1994

Canonical Correlation (Ramse Oksoy et al. 1994

Canonical Correlation (Ramse Oksoy et al. 1994

Canonical Corration (Rams Orsy el al. 1994

Canonical Correlation (Ramse Oksoy et al. 1994

Canonical Correlation (Ramse Oksoy et al. 1994 $\begin{array}{llll}0.1545 & 0.1583 & 0.0000 & 0.0000\end{array}$

$\begin{array}{llll}0.1578 & 0.0539 & 0.0868 & 0.0000 \\ 0.1563 & 0.0534 & 0.0000 & 0.0000\end{array}$

$\begin{array}{llll}0.0000 & 0.1701 & 0.0913 & 0.0000 \\ 0.0000 & 0.1455 & 0.0000 & 0.2225\end{array}$

$\begin{array}{llll}0.0000 & 0.1685 & 0.0000 & 0.0000\end{array}$

$\begin{array}{llll}0.0000 & 0.0490 & 0.0000 & 0.2249 \\ 0.1555 & 0.1592 & 0.0855 & 0.0000\end{array}$

$\begin{array}{ll}0.1409 & 0.5463 \\ 0.1438 & 0.5578 \\ 0.2376 & 0.5527 \\ 0.1514 & 0.5872 \\ 0.1296 & 0.5024 \\ 0.2500 & 0.5816 \\ 0.1320 & 0.5120 \\ 0.2555 & 0.5546 \\ 0.2182 & 0.5078 \\ 0.1418 & 0.4581\end{array}$


1119 WVGG-23

1151 WDGG-25

1153 WVDG-22

1155 WVGG-29

157 WDOG-33

1159 WVDG- 5

1160 WVGG-36

1162 WVDG

1163 WVDG-39

1164 WVDG-40

1166 WVDG-

1167 WVDG-43

1168 WVGG-44

1170 WVDG-

1171 WVDG-47

1172 WVDG-48

1174 WVDGG

1175 FY9245

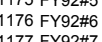

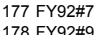

1179 F F924110
1180 FYg2Ref5

1181 Ratio2

1182 Ratio4
1183 Ratio5

1184 Loth2

1185 LoTh4
1186 LoTh5

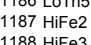

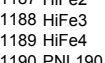

1190 PNL190
1191 FY93:11

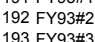

1194 FY93\#4

$1195 \mathrm{FYY3}+45$
$11165 \mathrm{FY}$

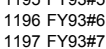

1198 FY93\#\#

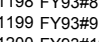

$1200 \mathrm{FY} Y 3 \#+10$
1201
FY944t

1202 FY94\#2

1203 FYY4\#3
1205 FYA4t

$1204 \mathrm{FY} Y 4444$
$1205 \mathrm{FY} 9445$

1205 FY9445
1206 FY94t66

1207 FY94\$7
1208 FY944

1208 FY9448
1209 FY944t9

1209 FY 9449
1210 FY994t10

${ }_{211}^{21}$ sigma 1

1212 Sigma2
1213 sigma3

214 Sigma4

1215 Sigmas
1214 Sigmas

1217 Sigma7

1218 sigmma
1219 sigma9
1220 sigma10
1222

12211
12222

12234

12245
12256

\begin{tabular}{l}
1226 \\
1226 \\
\hline
\end{tabular}

12278
12289
1229
1290

122910
123011 
1150 WVDG-24

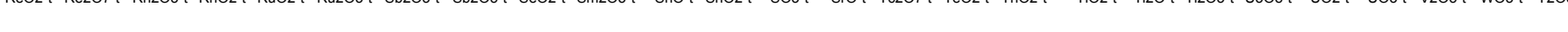

152 WVDG-26 27

1153 WVDG-27

1155 WVGG-29

1157 WVDG-33

159 WVDG-35

1160 WVGG-36

1161 WVDG-37

1163 WVDG-39

1164 WVDG-40

1166 WVDG-12
1167 WVDG-

1168 WVGG-44

170 WVDG-46

1171 WVGG-

1173 WVDG-40

1174 WVDG-
1175 FY92:5

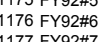

1177 FY9247
1178 FY9249

1179 Fre24110
1180 FYg2Ref5

1181 Ratio2

1182 Ratio4

1183 Ratios
1184 Loth2

1187 Hifer

1189 HiFe4

1190 PNL190
1191 FY93:11

1192 FY93\#\#
1193 FY93

1194 FY93\#4

1196 FY93\#t6

197 FY93\#7
198 FYa3\#8

1199 FY93\#9

$1200 \mathrm{FY} 93 \$ 10$
1201 FY94411

202 FY94\#2

$1203 \mathrm{FY} 9443$

1204 FY944t4
1205 FY944t5

1206 FY94\#6

1207 FY944t?

1208 FY9448
1209 FY944t9

1209 FY9449
1210 Frg4t11

121 Sigma 1

1212 Sigma2
1213 sigma3

1214 Sigma4 4

1216 Sigma6
1217 Sigmaz

1218 Sigmaa

1219 Sigma9
1220 Sigma 10

12222

1223
1224
1228

12245
12256

12267

12278
12289
1230

122910
123011

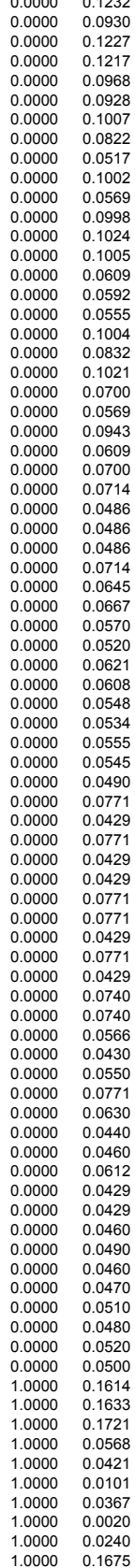




\begin{tabular}{|c|c|c|c|c|c|c|c|c|c|c|c|c|}
\hline \# Glass ID & B2O3-a & CaO-a & Fe2O3-a & FeO-a & K2O-a & Li2O-a & MgO-a & Na2O-a & NIIU-a & P2O5-a & SiO2-a & Zro2-a \\
\hline 1149 WVDG-23 & 0.1248 & 0.0050 & 0.1424 & & 0.0186 & 0.0180 & 0.0054 & 0.0823 & 0.0018 & 0.0330 & 0.3931 & 0.0024 \\
\hline $\begin{array}{l}1150 \text { WVDG-24 } \\
1151 \text { WVDG-25 }\end{array}$ & $\begin{array}{l}0.1322 \\
0.1090\end{array}$ & $\begin{array}{l}0.0048 \\
0.0050\end{array}$ & $\begin{array}{l}0.0867 \\
0.0867\end{array}$ & & $\begin{array}{l}0.0252 \\
0.0202\end{array}$ & $\begin{array}{l}0.0205 \\
0.0203\end{array}$ & $\begin{array}{l}0.0056 \\
0.0053\end{array}$ & $\begin{array}{l}0.0996 \\
0.0909\end{array}$ & $\begin{array}{l}0.0017 \\
0.0019\end{array}$ & $\begin{array}{l}0.0141 \\
0.0313\end{array}$ & $\begin{array}{l}0.4210 \\
0.4500\end{array}$ & $\begin{array}{l}0.00022 \\
0.024\end{array}$ \\
\hline 1152 WVDG-26 & 0.1338 & 0.0134 & 0.0867 & & 0.0264 & & 0.0135 & 0.0909 & 0.0019 & 0.0162 & 0.4095 & $\begin{array}{l}.0 .0024 \\
0.0025\end{array}$ \\
\hline 1153 WVDG-27 & 0.1258 & & 0.0868 & & 0.0245 & 0.0228 & 0.0062 & 0.1014 & 0.0062 & 0.0348 & 0.3672 & 0.0080 \\
\hline 54 WVDG-28 & 0.1066 & 0.0048 & 0.1211 & & 0.0135 & 0.0205 & 0.0062 & 0.0886 & 0.0059 & 0.0153 & 0.4338 & 0.0077 \\
\hline 1155 WVDG-29 & 0.1145 & 0.0133 & 0.0874 & & 0.0184 & 0.0257 & 0.0136 & 0.1074 & 0.0019 & 0.0327 & 0.4468 & 0.0024 \\
\hline 1156 WVDG-30 & 0.1188 & 0.0084 & 0.1148 & & 0.0181 & 0.0210 & 0.0101 & 0.0963 & 0.0035 & 0.0242 & 0.4006 & 0.0045 \\
\hline 1157 WVDG-33 & 0.0903 & 0.0040 & 0.1145 & & 0.0344 & 0.0291 & 0.0098 & 0.1051 & 0.0029 & 0.0247 & 0.4250 & 0.0037 \\
\hline $\begin{array}{l}1158 \text { WVDG-34 } \\
1159 \text { WVDG-35 }\end{array}$ & $\begin{array}{l}0.1081 \\
0.0715\end{array}$ & $\begin{array}{l}0.0018 \\
0.0070\end{array}$ & 0.0977 & & 0.0362 & 0.0307 & $\begin{array}{l}0.0045 \\
0.0744\end{array}$ & 0.1107 & 0.0082 & 0.0187 & 0.4000 & 0.0106 \\
\hline $\begin{array}{l}1159 \text { WVDG-35 } \\
110 \text { WVDG-36 }\end{array}$ & $\begin{array}{l}0.0715 \\
0.1080\end{array}$ & $\begin{array}{l}0.0070 \\
0.0016\end{array}$ & $\begin{array}{l}0.1448 \\
0.1466\end{array}$ & & $\begin{array}{l}0.0371 \\
0.0254\end{array}$ & $\begin{array}{l}0.0314 \\
0.0215\end{array}$ & $\begin{array}{l}0.0174 \\
0.0040\end{array}$ & $\begin{array}{l}0.1134 \\
0.0775\end{array}$ & $\begin{array}{l}0.0014 \\
0.0081\end{array}$ & $\begin{array}{l}0.0006 \\
0.0059\end{array}$ & $\begin{array}{l}0.3800 \\
0.110\end{array}$ & 0.0018 \\
\hline 1161 WVDG-37 & $\begin{array}{l}0.0778 \\
0.078\end{array}$ & 0.0014 & $\begin{array}{l}0.1460 \\
0.0977\end{array}$ & & $\begin{array}{l}0.0254 \\
0.0390\end{array}$ & $\begin{array}{l}0.0715 \\
0.0330\end{array}$ & $\begin{array}{l}.0 .0040 \\
0.036\end{array}$ & 0.1191 & 0.0080 & $\begin{array}{l}0.0059 \\
0.0050\end{array}$ & 0.4200 & $\begin{array}{l}0.00104 \\
0.0103\end{array}$ \\
\hline 1162 WVDG-38 & 0.0758 & 0.0016 & 0.0967 & & 0.0326 & 0.0276 & 0.0039 & 0.0995 & 0.0083 & 0.0060 & 0.4090 & 0.0107 \\
\hline 1163 WVDG-39 & 0.1122 & 0.0066 & 0.0928 & & 0.0362 & 0.0306 & 0.0164 & 0.1104 & 0.0022 & 0.0381 & 0.3800 & 0.0028 \\
\hline 1164 WVDG-40 & 0.0753 & 0.0072 & 0.1535 & & 0.0317 & 0.0269 & 0.0178 & 0.0968 & 0.0018 & 0.0060 & 0.4436 & 0.0023 \\
\hline 1165 WVDG-41 & 0.0726 & 0.0020 & 0.0948 & & 0.0375 & 0.0317 & 0.0051 & 0.1144 & 0.0021 & 0.0380 & 0.4500 & 0.0027 \\
\hline 1166 WVDG-42 & 0.0675 & 0.0068 & 0.0953 & & 0.0347 & 0.0294 & 0.0017 & 0.1060 & 0.0080 & 0.0368 & 0.4000 & 0.0103 \\
\hline 1167 WVDG-43 & 0.1243 & 0.0020 & 0.0953 & & 0.0268 & 0.0227 & 0.0049 & 0.0817 & 0.0018 & 0.0239 & 0.4200 & 0.0023 \\
\hline $\begin{array}{l}1168 \text { WVDG-44 } \\
1169 \text { WVDG-45 }\end{array}$ & $\begin{array}{l}0.1150 \\
0.0713\end{array}$ & $\begin{array}{l}0.0019 \\
0.016\end{array}$ & $\begin{array}{l}0.1581 \\
0.1551\end{array}$ & & $\begin{array}{l}0.0353 \\
0.0384\end{array}$ & $\begin{array}{l}0.0299 \\
0.025\end{array}$ & 0.0048 & 0.1079 & 0.0017 & 0.0062 & 0.3800 & 0.0022 \\
\hline 1170 WVDG-46 & $\begin{array}{l}0.0173 \\
0.1223\end{array}$ & $\begin{array}{l}.0 .0016 \\
0.0068\end{array}$ & $\begin{array}{l}0.151 \\
0.1039 \\
0.03\end{array}$ & & $\begin{array}{l}0.0384 \\
0.0337\end{array}$ & $\begin{array}{l}0.0325 \\
0.0286\end{array}$ & $\begin{array}{l}.0 .040 \\
0.0167\end{array}$ & $\begin{array}{l}0.1713 \\
0.1030\end{array}$ & $\begin{array}{l}.0 .0018 \\
0.0016\end{array}$ & $\begin{array}{l}0.0390 \\
0.0059\end{array}$ & $\begin{array}{l}0.3800 \\
0.4500\end{array}$ & $\begin{array}{l}0.0023 \\
0.0021\end{array}$ \\
\hline 1171 WVDG-47 & 0.1121 & 0.0016 & 0.1494 & & 0.0267 & 0.0226 & 0.0040 & 0.0818 & 0.0015 & $\begin{array}{l}0.0393 \\
0\end{array}$ & 0.4500 & 0.0020 \\
\hline 1172 WVDG-48 & 0.1056 & 0.0068 & 0.0924 & & 0.0268 & 0.0227 & 0.0167 & 0.0818 & 0.0088 & 0.0428 & 0.3800 & 0.0114 \\
\hline 1173 WVDG-40 & 0.0753 & 0.0072 & 0.1535 & & 0.0317 & 0.0269 & 0.0178 & 0.0968 & 0.0018 & 0.0060 & 0.4436 & 0.0023 \\
\hline 1174 WVDG-46 & 0.1223 & 0.0068 & 0.1039 & & 0.0337 & 0.0286 & 0.0167 & 0.1030 & 0.0016 & 0.0059 & 0.4500 & 0.0021 \\
\hline 1175 FY92\#5 & 0.1044 & 0.0048 & 0.1430 & & 0.0688 & 0.0510 & 0.0089 & 0.0875 & 0.0025 & 0.0120 & 0.3688 & 0.0132 \\
\hline $\begin{array}{l}1176 \text { FY92\#\# } \\
11777 \text { F992377 }\end{array}$ & 0.1044 & 0.0048 & 0.0974 & & 0.0688 & 0.0232 & 0.0089 & 0.1028 & 0.0025 & 0.0120 & 0.4497 & 0.0132 \\
\hline $\begin{array}{l}11777 \text { FY92247 } \\
1178 \text { FY92\#9 }\end{array}$ & $\begin{array}{l}0.1044 \\
0.1044\end{array}$ & $\begin{array}{l}0.0048 \\
0.0048\end{array}$ & 0.1430 & & 0.0688 & 0.0232 & 0.0089 & $\begin{array}{l}0.0572 \\
\end{array}$ & 0.0025 & 0.0120 & 0.4497 & 0.0132 \\
\hline 1179 FY92\#10 & $\begin{array}{l}0.1044 \\
0.1534\end{array}$ & $\begin{array}{l}.0 .048 \\
0.0048\end{array}$ & $\begin{array}{l}0.1430 \\
0.0974\end{array}$ & & $\begin{array}{l}0.0313 \\
0.0688\end{array}$ & $\begin{array}{l}0.0010 \\
0.0232\end{array}$ & $\begin{array}{l}0.0089 \\
0.0089\end{array}$ & $\begin{array}{l}0.05 / 2 \\
0.0572\end{array}$ & $\begin{array}{l}.0 .025 \\
0.0025\end{array}$ & $\begin{array}{l}0.0120 \\
0.0120\end{array}$ & $\begin{array}{l}0.4416 \\
0.4057\end{array}$ & $\begin{array}{l}0.0132 \\
0.0132\end{array}$ \\
\hline 1180 FY92Ref5 & 0.1289 & 0.0068 & 0.1202 & & 0.0318 & 0.0271 & 0.0089 & 0.0982 & 0.0025 & 0.0237 & 0.4116 & 0.0032 \\
\hline 1181 Ratio2 & 0.1100 & 0.0048 & 0.1606 & & 0.0550 & & 0.0089 & 0.0880 & 0.0025 & & 0.3900 & 0.0120 \\
\hline 1182 Ratio4 & 0.1120 & 0.0048 & 0.1008 & & 0.0560 & 0.0410 & 0.0089 & 0.0720 & 0.0025 & 0.0120 & 0.4340 & 0.0190 \\
\hline 1183 Ratio5 & 0.1460 & 0.0048 & 0.1298 & & 0.0500 & 0.0410 & 0.0089 & 0.0900 & 0.0025 & 0.0120 & 0.3880 & 0.0100 \\
\hline 1184 LoTh2 & 0.1170 & 0.0048 & 0.1300 & & 0.0450 & 0.0392 & 0.0089 & 0.0890 & 0.0025 & 0.0120 & 0.4250 & 0.0135 \\
\hline 1185 LoTh4 & 0.1380 & 0.0048 & 0.1050 & & 0.0480 & 0.0330 & 0.0089 & 0.0890 & 0.0025 & 0.0120 & 0.4310 & 0.0120 \\
\hline $\begin{array}{l}1186 \text { LoTh5 } \\
1187 \text { HiFe2 }\end{array}$ & 0.1433 & $\begin{array}{l}0.0048 \\
0.048\end{array}$ & $\begin{array}{l}0.1380 \\
0.1550\end{array}$ & & 0.0437 & 0.0325 & 0.0089 & 0.0700 & 0.0025 & 0.0120 & 0.4170 & 0.0115 \\
\hline $\begin{array}{l}1187 \text { Hife2 } \\
1188 \text { HiFe3 }\end{array}$ & $\begin{array}{l}0.1100 \\
0.1123\end{array}$ & $\begin{array}{l}0.0048 \\
0.0048\end{array}$ & $\begin{array}{l}0.1550 \\
0.1470\end{array}$ & & 0.0540 & 0.0400 & $\begin{array}{l}0.0089 \\
0.0089\end{array}$ & $\begin{array}{l}0.0880 \\
0.920\end{array}$ & $\begin{array}{l}0.0025 \\
0.025\end{array}$ & 0.0120 & $\begin{array}{l}0.3926 \\
0.3879\end{array}$ & 0.0114 \\
\hline $\begin{array}{l}1188 \text { HiFes } \\
1189 \text { HiFe4 }\end{array}$ & 0.1470 & $\begin{array}{l}.0 .0048 \\
0.0048\end{array}$ & $\begin{array}{l}0.1470 \\
0.1360\end{array}$ & & 0.0435 & $\begin{array}{l}.0424 \\
0.0415\end{array}$ & $\begin{array}{l}.0 .0089 \\
0.089\end{array}$ & $\begin{array}{l}0.0920 \\
0.0830\end{array}$ & $\begin{array}{l}.0 .0025 \\
0.025\end{array}$ & 0.0120 & 0.3880 & $\begin{array}{l}0.0112 \\
0.0093\end{array}$ \\
\hline 1190 PNL190 & 0.1040 & 0.0048 & 0.0970 & & 0.0688 & 0.0510 & 0.0089 & 0.1030 & 0.0025 & 0.0120 & 0.4040 & 0.0130 \\
\hline 1191 FY93\#1 & 0.1656 & 0.0048 & 0.1545 & & 0.0313 & 0.0232 & 0.0089 & 0.0572 & 0.0025 & 0.0120 & 0.3949 & 0.0132 \\
\hline 1192 FY93\#2 & 0.1656 & 0.0048 & 0.0859 & & 0.0688 & 0.0232 & 0.0089 & 0.1028 & 0.0025 & 0.0120 & 0.4146 & 0.0132 \\
\hline 1193 FY93\#3 & 0.1656 & 0.0048 & 0.0859 & & 0.0688 & 0.0510 & 0.0089 & 0.1028 & 0.0025 & 0.0120 & 0.3526 & 0.0132 \\
\hline 1194 FY93\#4 & 0.0922 & 0.0048 & 0.1545 & & 0.0688 & 0.0232 & 0.0089 & 0.0572 & 0.0025 & 0.0120 & 0.4650 & 0.0132 \\
\hline 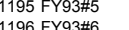 & 0.1656 & $\begin{array}{l}0.0048 \\
0.048\end{array}$ & $\begin{array}{l}0.0859 \\
0.0859\end{array}$ & & 0.0313 & 0.0510 & 0.0089 & 0.0572 & 0.0025 & 0.0120 & 0.4699 & 0.0132 \\
\hline $\begin{array}{l}11966 \text { F99336 } \\
1197 \text { FY93\#7 }\end{array}$ & $\begin{array}{l}0.0922 \\
0.0922\end{array}$ & $\begin{array}{l}0.0048 \\
0.0048\end{array}$ & $\begin{array}{l}0.0859 \\
0.0859\end{array}$ & & $\begin{array}{l}0.0313 \\
0.0688\end{array}$ & $\begin{array}{l}0.0432 \\
0.0232\end{array}$ & $\begin{array}{l}0.0089 \\
0.0089\end{array}$ & $\begin{array}{l}0.1028 \\
0.0572\end{array}$ & $\begin{array}{l}0.0025 \\
0.025\end{array}$ & 0.0120 & 0.4713 & 0.0132 \\
\hline 1198 FY93\#\# & 0.1652 & $\begin{array}{l}0.0048 \\
0.048\end{array}$ & $\begin{array}{l}0.1545 \\
0.089\end{array}$ & & $\begin{array}{l}0.0388 \\
0.0313\end{array}$ & $\begin{array}{l}0.0232 \\
0.0232\end{array}$ & 0.0089 & $\begin{array}{l}0.05 / 2 \\
0.1028\end{array}$ & $\begin{array}{l}.0 .0025 \\
0.025\end{array}$ & 0.0120 & $\begin{array}{l}0.4368 \\
0.3483\end{array}$ & $\begin{array}{l}0.0132 \\
0.0132\end{array}$ \\
\hline 1199 FY93\#9 & $\begin{array}{l}0.0922 \\
0.0922\end{array}$ & 0.0048 & 0.1545 & & 0.0688 & 0.0245 & 0.0089 & 0.1028 & 0.0025 & 0.0120 & 0.3483 & 0.0132 \\
\hline $\begin{array}{l}1200 \mathrm{FY} 93 \# 10 \\
0\end{array}$ & 0.0922 & 0.0048 & 0.0859 & & 0.0313 & 0.0510 & 0.0089 & 0.1028 & 0.0025 & 0.0120 & 0.4621 & 0.0132 \\
\hline 1201 FY94\#1 & 0.0900 & 0.0048 & 0.0661 & & 0.0600 & 0.0280 & 0.0089 & 0.0840 & 0.0025 & 0.0120 & 0.4774 & 0.0073 \\
\hline 1202 FY94\#2 & 0.0709 & 0.0048 & 0.1743 & & 0.0590 & 0.0400 & 0.0089 & 0.0520 & 0.0025 & 0.0120 & 0.4300 & 0.0073 \\
\hline 1203 FY94\#3 & 0.1869 & 0.0048 & 0.1100 & & 0.0460 & 0.0280 & 0.0089 & 0.1100 & 0.0025 & 0.0120 & 0.3700 & 0.0073 \\
\hline & 0.1710 & & 0.1695 & & 0.0530 & 0.0460 & & 0.0910 & 0.0025 & 0.0120 & 0.3450 & 0.0073 \\
\hline 1205 FY94\#地 & 0.0709 & 0.0048 & 0.0661 & & 0.0660 & 0.0445 & 0.0089 & 0.1070 & 0.0025 & 0.0120 & 0.4770 & 0.0173 \\
\hline $\begin{array}{l}12007 \text { FY94\$\#7 } \\
1209\end{array}$ & 0.1753 & $\begin{array}{l}.0048 \\
0.0048\end{array}$ & $\begin{array}{l}0.1743 \\
0.0661\end{array}$ & & 0.0400 & 0.0240 & $\begin{array}{l}.00089 \\
0.0089\end{array}$ & $\begin{array}{l}0.0000 \\
0.0660\end{array}$ & $\begin{array}{l}.0 .0025 \\
0.025\end{array}$ & 0.0120 & $\begin{array}{l}.3 .4350 \\
0.4350\end{array}$ & $\begin{array}{l}0.0191 \\
0.0184\end{array}$ \\
\hline 1208 F94t: & 0.1800 & $\begin{array}{ll}0.048 \\
0.048\end{array}$ & 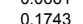 & & 0.0380 & 0.0380 & 0.0089 & 0.085 & 0.025 & & 0.3540 & 0.019 \\
\hline 1209 FY94\$f & 0.1350 & 0.0048 & 0.1400 & & 0.0640 & 0.0340 & 0.0089 & 0.0612 & 0.0025 & 0.0120 & 0.4126 & 0.0180 \\
\hline 1210 FY94: & 0.1869 & 0.0048 & 0.0661 & & 0.0313 & 0.0480 & 0.0089 & 0.0780 & 0.0025 & 0.0120 & 0.4200 & 0.0073 \\
\hline 1211 Sigma1 & 0.1869 & 0.0048 & 0.1168 & & 0.0688 & 0.0510 & 0.0089 & 0.1100 & 0.0025 & 0.0120 & 0.3422 & 0.0073 \\
\hline 1212 Sigma2 & 0.1869 & 0.0048 & 0.0741 & & 0.0688 & 0.0510 & 0.0089 & 0.1100 & 0.0025 & 0.0120 & 0.3422 & 0.0073 \\
\hline 1213 Sigr & 0.0761 & 0.0 & 0.1743 & & 0.0670 & 0.0490 & 0.0089 & 0.1090 & 0.0025 & 0.0120 & 0.3672 & 0.0078 \\
\hline $\begin{array}{l}1214 \text { Sigma44 } \\
1215 \text { SSigmas }\end{array}$ & 0.1850 & 0.0048 & 0.1117 & & 0.0640 & 0.0500 & 0.0089 & 0.1030 & $\begin{array}{l}0.0025 \\
0.025\end{array}$ & $\begin{array}{l}0.0120 \\
0.0120\end{array}$ & $\begin{array}{l}0.3550 \\
0.3500\end{array}$ & 0.0081 \\
\hline $\begin{array}{l}1215 \text { Sigmab } \\
1216 \text { siga6 }\end{array}$ & $\begin{array}{l}0.1800 \\
0.1750\end{array}$ & $\begin{array}{l}0.0048 \\
0.0048\end{array}$ & $\begin{array}{l}0.1288 \\
0.1036\end{array}$ & & 0.0650 & 0.0480 & 0.0089 & $\begin{array}{l}0.1000 \\
0.1010\end{array}$ & $\begin{array}{l}.00025 \\
0.025\end{array}$ & 0.0120 & 0.3650 & $\begin{array}{l}0.0000 \\
0.0082\end{array}$ \\
\hline 1217 Sigma7 & 0.1700 & 0.0048 & $\begin{array}{l}0.1125 \\
0.1125\end{array}$ & & 0.0660 & 0.0495 & 0.0089 & 0.1070 & 0.025 & 0.0120 & 0.3600 & 0.078 \\
\hline 1218 Sigma8 & 0.1600 & 0.0048 & 0.1299 & & 0.0630 & 0.0485 & 0.0089 & 0.0970 & 0.0025 & 0.0120 & 0.3700 & 0.0084 \\
\hline 1219 sigma9 & 0.1550 & 0.0048 & 0.0938 & & 0.0610 & 0.0475 & 0.0089 & 0.0990 & 0.0025 & 0.0120 & 0.3750 & 0.0085 \\
\hline 1220 Sigma 10 & 0.1580 & 0.0048 & 0.0845 & & 0.0620 & 0.0470 & 0.0089 & 0.0950 & 0.0025 & 0.0120 & 0.3800 & 0.0083 \\
\hline & 0.1482 & 0.0000 & 0.0000 & 0.0000 & & & & 0.1406 & & & 0.5498 & \\
\hline 12222 & 0.0533 & 0.0895 & 0.0000 & 0.0000 & & & & 0.1463 & & & 0.5475 & \\
\hline $\begin{array}{l}12234 \\
12245\end{array}$ & 0.0456 & 0.0000 & 0.0000 & 0.0000 & & & & 0.2319 & & & 0.5504 & \\
\hline 12256 & $\begin{array}{l}0.1514 \\
0.1271\end{array}$ & $\begin{array}{l}0.00599 \\
0.0000\end{array}$ & $\begin{array}{l}0.0000 \\
0.1937\end{array}$ & $\begin{array}{l}0.0256 \\
0.0256\end{array}$ & & & & $\begin{array}{l}0.1420 \\
0.1214\end{array}$ & & & $\begin{array}{l}0.5698 \\
0.490\end{array}$ & \\
\hline 1226 & 0.1618 & & 0.0000 & 0.0000 & & & & $\begin{array}{l}0.12546 \\
0.2546\end{array}$ & & & $\begin{array}{l}0.5734 \\
0.5734\end{array}$ & \\
\hline & 0.0469 & 0.0748 & 0.1886 & 0.0297 & & & & 0.1260 & & & & \\
\hline & 0.0564 & 0.0926 & 0.0000 & & & & & 0.2597 & & & & \\
\hline 2910 & 0.0408 & 0.0000 & 0.1814 & 0.0302 & & & & 0.2270 & & & 0.49 & \\
\hline & 0.1628 & 0.0884 & 0.0000 & .0000 & & & & 0.1425 & & & 0.4388 & \\
\hline
\end{tabular}
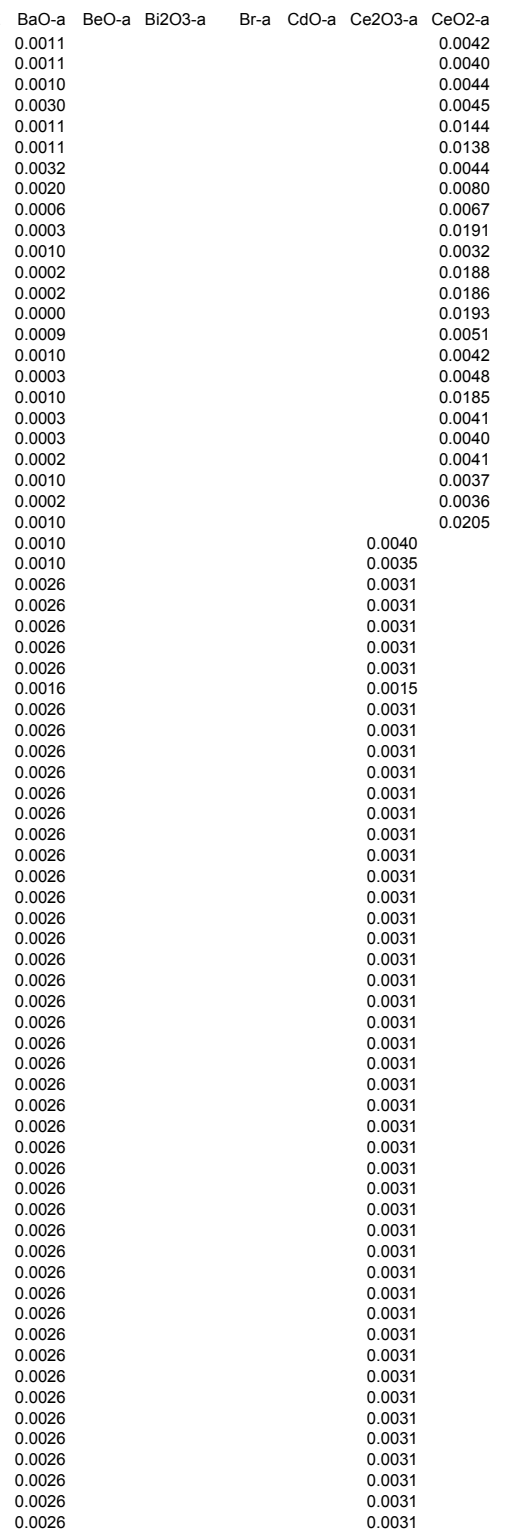

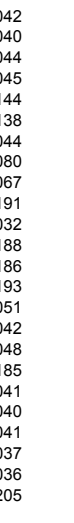

CoO-a Co2O3-a Cr2O3-a Cs2O-a CuO-a $\begin{array}{lll}0.0008 & 0.0004 & 0.0000 \\ 0.0008 & 0.0004 & 0.002\end{array}$ $\begin{array}{llll}0.0008 & 0.0004 & 0.0002\end{array}$ $\begin{array}{llll}0.0009 & 0.0004 & 0.0003 \\ 0.0028 & 0.0014 & 0.0008\end{array}$ $\begin{array}{llll}0.0027 & 0.0013 & 0.0008\end{array}$ $\begin{array}{lll}0.0008 & 0.0004 & 0.0002 \\ 0.0016 & 0.0008 & 0.0004 \\ 0.0013 & 0.0007\end{array}$ $\begin{array}{llll}0.0013 & 0.0007 & 0.0004 \\ 0 & 0.0004\end{array}$ $\begin{array}{lll}0.0006 & 0.0003 & 0.0002 \\ 0.0037 & 0.0018 & 0.0010 \\ 0.00036 & 0.0019 & 0.010\end{array}$ $\begin{array}{lll}0.0037 & 0.0018 & 0.0010 \\ 0.0036 & 0.0018 & 00010\end{array}$ $\begin{array}{lll}0.0036 & 0.0018 & 0.0010 \\ 0.0037 & 0.0019 & 0.0011\end{array}$ \begin{tabular}{lll}
0.0010 & 0.0005 & 0.0003 \\
\hline
\end{tabular} $\begin{array}{llll}0.0008 & 0.0004 & 0.0002 \\ 0 & 0.0096 & 0.005 & 0.003\end{array}$ $\begin{array}{lll}0.0009 & 0.0005 & 0.0003 \\ 0.0036 & 0.0018 & 0.0010\end{array}$ $\begin{array}{llll}0.0008 & 0.0004 & 0.0002\end{array}$ $\begin{array}{llll}0.0008 & 0.0004 & 0.0002 \\ 0 & 0.008 & 0.0004 & 0.002\end{array}$ $\begin{array}{llll}0.0008 & 0.0004 & 0.0002 \\ 0.0007 & 0.0004 & 0.0002 \\ 0.0007 & 0.0004 & 0.0002\end{array}$ $\begin{array}{llll}0.0007 & 0.0004 & 0.0002 \\ 0.0040 & 0.0020 & 0.0011\end{array}$ $\begin{array}{llll}0.0008 & 0.0004 & 0.0002\end{array}$ $\begin{array}{llll}0.0014 & 0.0000 & 0.00000\end{array}$ $\begin{array}{llll}0.0014 & 0.0000 & 0.0000 \\ 0 & 00000 & 0.0000 & 0.0000\end{array}$ $\begin{array}{llll}0.0014 & 0.0000 & 0.0000\end{array}$ $\begin{array}{llll}0.0014 & 0.0000 & 0.0000 \\ 0 & 0.0000 & 0.000 & 0.003\end{array}$

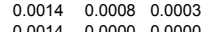
$\begin{array}{llll}0.0014 & 0.0000 & 0.0000 \\ 0.0014 & 0.0000 & 0.0000 \\ 0.0044 & 0.0000 & 0.0000\end{array}$ $\begin{array}{lll}0.0014 & 0.0000 & 0.0000 \\ 0.0014 & 0.0000 & 0.0000\end{array}$ 0.0000 $\begin{array}{llll}0.0014 & 0.0000 & 0.0000\end{array}$ $\begin{array}{lll}0.0014 & 0.0000 & 0.0000 \\ 0.0014 & 0.0000 & 0.0000 \\ 0.0014 & 0.0000 & 0.0000\end{array}$ $\begin{array}{llll}0.0014 & 0.0000 & 0.00000\end{array}$ 0.0014

0.0014

0.0014
0.0014
0.0014

0.0014

0.0014
0.0014

$\begin{array}{llll}0.0014 & & & \\ 0.0014 & 0.0000 & 0.0000\end{array}$ $\begin{array}{lll}0.0014 & 0.0000 & 0.00000 \\ 0.0004 & 0.0000 & 0.0000\end{array}$ $\begin{array}{lll}0.0014 & 0.0000 & 0.0000 \\ 0.0014 & 0.0000 & 0.0000\end{array}$ $\begin{array}{lll}0.00014 & 0.00000 & 0.0000 \\ 0.0014 & 0.0000 & 0.0000 \\ 0.000 & 0.0000\end{array}$ $\begin{array}{lll}0.0014 & 0.0000 & 0.0000 \\ 0.0014 & 0.0000 & 0.0000\end{array}$ $\begin{array}{llll}0.0014 & 0.0000 & 0.0000 \\ 0.0014 & 0.0000 & 0.0000\end{array}$ $\begin{array}{lll}0.0014 & 0.0000 & 0.0000 \\ 0.0014 & 0.0000 & 0.0000\end{array}$ $\begin{array}{llll}0.0014 & 0.0000 & 0.0000 \\ 0.0014 & 0.0000 & 0.0000\end{array}$ $\begin{array}{llll}0.0014 & 0.0000 & 0.0000 \\ 0 & 0.0014 & 0.0000 & 0.0000\end{array}$ $\begin{array}{lll}0.0014 & 0.0000 & 0.0000 \\ 0.0014 & 0.0000 & 0.0000\end{array}$ $\begin{array}{llll}0.0014 & 0.0000 & 0.0000\end{array}$ $\begin{array}{lll}0.0014 & 0.0000 & 0.0000 \\ 0.0014 & 0.0000 & 0.0000 \\ 0.014 & 0.0000 & 0.000\end{array}$ 
\# Glass ID

1149 WVDG-23

1151 WVGG-25

1152 WVDG-26
1153 WVDG-27

1154 WVDG-28

1156 WVDG-30

1158 WVDG-34

1160 WVDG-36

1161 WVDG-37

1163 WVDG-39

1164 WVDG-40

1167 WVDG-43

1169 WVDG-45

1170 WVDG-4

1172 WVDG-4

1744 WVDG-46

1176 FY92\#6

1177 FY92\#7

1179 FY92\#10

181 Ratio2

1182 Ratio

1183 Ratio
1184 LoTh

1186 LoTh5

1187

191 FY93\#1

192 FY93\#2

$1194 \mathrm{FY} 93 \# 4$
$1195 \mathrm{FY} 93+5$

1966 FY93\#6
1197 FY93\#7

198 FY93\#8

1199 FY93\#\#

1201 FY94\#1
1202 FYast

203 FY94\#3

204 FY94\#4

1206 FY94\#6

1208 FY94\#8

1210 FY94\#10

211 Sigma1

1214 Sigma4
1215 Sigma5

1218 Sigma8
1219 Sigma9
1220 Sigma10

1222

1223

12256

12278

122910
123011
Ga2O3-a Gd2O3-a

I-a La203-a MnO2-a MnO-a MoO-a MoO3-a Nb2O5-a Nd2O3-a NpO2-a PbO-a PdO2-a PdO-a Pr2O3-a Pr6011-a PuO2-a Rb2O-a ReO-a ReO2-a Re207-a Rh203-a RhO2-a RuO2-a Sb203-a Sb205-a SeO2-a $\begin{array}{rr}0.0002 & 0.0051 \\ 0.0002 & 0.0244\end{array}$

$\begin{array}{ll}0.0002 & 0.0244 \\ 0.0002 & 0.0240 \\ 0.003 & 0.055\end{array}$

$\begin{array}{ll}0.0003 & 0.0055 \\ 0.0008 & 0.0051\end{array}$

$\begin{array}{ll}0.0008 & 0.0052 \\ 0.0002 & 0.0050\end{array}$

$\begin{array}{ll}0.0002 & 0.0050 \\ 0.0004 & 0.0120 \\ 0.0004 & 0.0128\end{array}$

$\begin{array}{ll}0.0004 & 0.0128 \\ 0.0011 & 0.0198 \\ 0.0002 & 0.0195\end{array}$

$\begin{array}{ll}0.0011 & 0.0198 \\ 0.0002 & 0.0195 \\ 0.0010 & 0.012\end{array}$

$0.0010 \quad 0.0013$

$\begin{array}{ll}0.0011 & 0.016 \\ 0.0003 & 0.0011 \\ 0.0003 & 00012\end{array}$

$0.0002 \quad 0.0012$

$\begin{array}{lll}0.0003 & 0.0012 \\ 0.0010 & 0.019\end{array}$

$\begin{array}{ll}0.0002 & 0.0194 \\ 0.0002 & 0.0013 \\ 0.0002 & 0.0201\end{array}$

$\begin{array}{ll}0.0002 & 0.0201 \\ 0.0002 & 0.0196 \\ 0.0002 & 0.0194\end{array}$

$\begin{array}{ll}0.0002 & 0.0194 \\ 0.0011 & 0.0012\end{array}$

$\begin{array}{ll}0.00002 & 0.0012 \\ 0.0002 & 0.0010\end{array}$

$\begin{array}{ll}0.0010 \\ 0.0002 & 0.0160 \\ 0.0000 & 0.0082\end{array}$

0.0000

0.0000
0.0000

0.0000
0.0004

0.0000

0.0000
0.0000
0.0000

0.0000
0.0000

0.0000

0.0000
0.0000
0.0000

0.0082
0.0082
0.0082

0.0082
0.0082
0.0082
0.0082

0.0082
0.0082
0.0082

0.0082
0.0082

0.0082

0.0082
0.0082
0.0082
0.0082

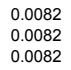

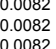

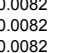

0.0082

0.0082
0.0082

$\begin{array}{lll}0.0000 & 0.0082 & \\ 0.0082 & 0.0000\end{array}$

$\begin{array}{lll}0.0000 & 0.0082 & 0.0000 \\ 0.0000 & 0.0082 & 0.00000 \\ 0.00000 & 0.008 & 0.000\end{array}$

$\begin{array}{lll}0.0000 & 0.0082 & 0.00000 \\ 0.0000 & 0.0082 & 0.0000\end{array}$

0.0000

0.00000

0.0082
0.0082

0.0000
0.0000
0.0000

0.0000
0.0000
0.0000

$\begin{array}{ll}0.0082 & 0.0000 \\ 0.0082 & 0.0000 \\ 0 & 0.0082\end{array}$

$\begin{array}{ll}0.0082 & 0.0000 \\ 0.0082 & 0.0000 \\ 0.0082 & 0.0000\end{array}$

$\begin{array}{ll}0.0082 & 0.0000 \\ 0.0082 & 0.0000 \\ 0 & 0.0082\end{array}$

$\begin{array}{ll}0.0082 & 0.000 \\ 0.0082 & 0.0000 \\ 0.0082 & 0.0000\end{array}$

0.0082
0.0082
0.0082
0.0082

0.0000
0.0000
0.0000

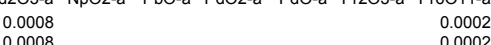

0.0008
0.0009
0.0028

0.0027

0.0008
0.0016

$\begin{array}{ll}0.0013 & 0.0000\end{array}$

0.0000

0.0000
0.0000

0.0000
0.0000

0.0000
0.0000
0.0000

0.0000

0.0000

0.0000
0.0000
0.0000
0.0000
0.0000
0.0000
0.0000
0.0003
0.0000
0.0000
0.0000
0.0000
0.00000
0.0000
0.00000
0.0000
0.0000
0.0000

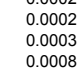

.

0.0004
0.0004
0.0002

0.0011
0.0002
0.0010

0.0010

0.0011
0.0003
0.0002
0.0010

0.0003
0.0010
0.0002

0.0002
0.0002
0.0002

0.0002
0.0002
0.0002
0.0002

0.0002
0.0011
0.0002
0.0000

0.0002
0.0002
0.0000
0.0000

0.0000
0.0000

0.0000
0.0000
0.0000

0.0004

0.0000
0.0000
0.0000

0.0000
0.0000
0.0000

0.0000
0.0000
0.0000

0.0000
0.0000
0.0000

$\begin{array}{ll}0.0000 & 0.0000 \\ 0.0000 & 0.0000 \\ 0.0000 & 0.0000\end{array}$

$\begin{array}{ll}0.0000 & 0.0000 \\ 0.0000 & 0.0000\end{array}$

$\begin{array}{ll}0.0000 & 0.0000 \\ 0.0000 & 0.0000 \\ 0.0000 & 0.0000\end{array}$

$0.0000 \quad 0.0000$

$\begin{array}{ll}0.0000 & 0.0000 \\ 0.0000 & 0.0000\end{array}$

$0.0000 \quad 0.0000$

$\begin{array}{ll}0.0000 & 0.0000 \\ 0.0000 & 0.0000\end{array}$

$0.0000 \quad 0.0000$

$0.0000 \quad 0.0000$

$\begin{array}{lll}0.0000 & 0.0000 \\ 0 & 0.000 & 0.0000\end{array}$

$\begin{array}{ll}0.0000 & 0.0000 \\ .0000 & 0.0000 \\ 0.002 & 0.000\end{array}$

$0.0002 \quad 0.0008$

$\begin{array}{ll}0.0000 & 0.0000 \\ 0.0000 & 0.0000 \\ 0.0000 & 0.0000\end{array}$

\begin{tabular}{ll}
0.0000 & 0.0000 \\
0.0000 & 0.0000 \\
\hline & 0.0000
\end{tabular}

$0.0000 \quad 0.0000$

$0.0000 \quad 0.0000$

$\begin{array}{ll}0.0000 & 0.0000 \\ 0.0000 & 0.0000\end{array}$

$\begin{array}{ll}0.0000 & 0.0000 \\ 0.0000\end{array}$

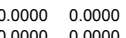

\begin{tabular}{ll}
0.0000 & 0.0000 \\
0.0000 & 0.0000 \\
\hline
\end{tabular}

$\begin{array}{ll}0.0000 & 0.0000 \\ 0.0000 & 0.0000\end{array}$

$\begin{array}{ll}0.0000 & 0.0000 \\ 0.0000 & 0.0000\end{array}$

$0.0000 \quad 0.0000$

$0.0000 \quad 0.0000$

$0.0000 \quad 0.0000$

$\begin{array}{ll}0.0000 & 0.0000 \\ 0.0000 & 0.0000 \\ 0.000 & 0.000\end{array}$

$\begin{array}{ll}0.0000 & 0.0000 \\ 0.0000 & 0.0000 \\ 0.0000 & 0.000\end{array}$

$0.0000 \quad 0.0000$

$\begin{array}{ll}0.0000 & 0.0000 \\ 0.0000 & 0.0000 \\ 0.0000 & 0.0000\end{array}$

$0.0000 \quad 0.0000$ 
\# Glass ID

1149 WVDG-2

1151 WVDG-25
1152 WVDG-2

1153 WVDG-27

1155 WVGG-29
1156 WVDG-30

1157 WVDG-33

1159 WVDG-35

1160 WVDG-36

164 WVG-39-40

1165 WVDG-4

1167 WVDG-43

1169 WVDG-44

1170 WVDG-4

1172 WVDG-48

174 WVDG-46

$1175 \mathrm{FY} 2 \# 5$
1176 FY92\#6

1177 FY2\#7
1178 FY92\#9

1179 FY92\#10

181 Ratio2

1182 Ratio 4

1184 LoT 2

1186 LoTh5

1187 HiFe2

189 HiFe4

191 FY93\#1

192 FY93\#2

1194 FY93\#4

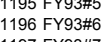

198 FY93\#8

1199 FY93\#9

1200 FY93\#10
1201 FY94\#1

202 FY94\#2

(204 FYO4t

205 FY94\#5

1206 FY94\#6
1207 FY94\#7

208 FY94\#8

1210 FY94\#10

211 Sigma 1

212 Sigma2

1214 Sigma4
1215 Sigma5

1218 Sigma8
1219 Sigma9

1222

(2234

12256

12278

122910
123011

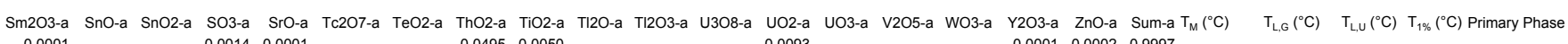
0.00010 .00020 .9997

$\begin{array}{lll}0.0495 & 0.0050 \\ 0.0188 & 0.0047\end{array}$

0.0093

$\begin{array}{lll}0.0001 & 0.0002 & 1.0001 \\ 0.0004 & 0.0006 & 1.0000\end{array}$

$\begin{array}{llll}0.0001 & 0.0002 & 0.9996\end{array}$

$\begin{array}{lll}0.0002 & 0.0003 & 1.0000 \\ 0.0002 & 0.0003 & 1.0002\end{array}$

$\begin{array}{llll}0.0002 & 0.0003 & 1.0002 \\ 0.0005 & 0.0008 & 1.0000\end{array}$

$\begin{array}{llll}0.0001 & 0.0001 & 0.9946\end{array}$

$\begin{array}{lll}0.0005 & 0.0008 & 0.9999 \\ 0.0005 & 0.0008 & 1.0000\end{array}$

$\begin{array}{lll}0.0005 & 0.0008 & 0.9963 \\ 0.0001 & 0.0002 & 0.999\end{array}$

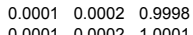

$\begin{array}{lll}0.0005 & 0.0008 & 0.9850 \\ 0.0001 & 0.0002 & 0.999\end{array}$

$0.00010 .0002 \quad 0.9997$

$\begin{array}{lll}0.0001 & 0.0002 & 1.0001\end{array}$

$\begin{array}{lll}0.0001 & 0.0002 & 1.0002 \\ 0.0006 & 0.0009 & 1.0000\end{array}$

$\begin{array}{lll}0.0001 & 0.0002 & 1.0001 \\ 0.0001 & 0.0002 & 0.9965\end{array}$

$\begin{array}{lll}0.0000 & 0.0026 & 0.9999\end{array}$

$0.0000 \quad 0.0026 \quad 0.9999$

$\begin{array}{lll}0.0000 & 0.0026 & 0.9999 \\ 0.0000 & 0.0026 & 0.9999\end{array}$

$\begin{array}{lll}0.0002 & 0.0002 & 1.0004 \\ 0.0000 & 0.0026 & 0.9974\end{array}$

$\begin{array}{llll}0.0000 & 0.0026 & 0.9999\end{array}$

$\begin{array}{llll}0.0000 & 0.0026 & 0.9999\end{array}$

$0.0000 \quad 0.0026 \quad 0.9999$

$\begin{array}{llll}0.0000 & 0.0026 & 0.9999\end{array}$

$\begin{array}{llll}0.0000 & 0.0026 & 0.9999\end{array}$

0.00260 .9999

$\begin{array}{ll}.0026 & 0.9999 \\ 0.026 & 0.999\end{array}$

0.00260 .9999

$0.0026 \quad 0.9999$

0.00260 .9999

$\begin{array}{lll}0.0026 & 0.9999 \\ 0.0026 & 0.9999\end{array}$

$\begin{array}{lll}0.0000 & 0.0026 & 0.9999\end{array}$

$\begin{array}{llll}0.0000 & 0.0026 & 0.9999\end{array}$

$\begin{array}{llll}0.0000 & 0.0026 & 0.9999\end{array}$

$\begin{array}{lll}0.0000 & 0.0026 & 0.9999 \\ 0.0000 & 0.0026 & 0.9999\end{array}$

$\begin{array}{lll}.0000 & 0.0026 & 0.9999 \\ .0000 & 0.0026 & 0.9999\end{array}$

$\begin{array}{lll}0.0000 & 0.0026 & 0.9999\end{array}$

$0.0000 \quad 0.0026 \quad 0.9999$

$\begin{array}{lll}0.0000 & 0.0026 & 0.9999\end{array}$

$\begin{array}{lll}0.0000 & 0.0026 & 0.9999 \\ 0.0000 & 0.0026 & 0.9999 \\ 0.000 & 0.026\end{array}$

$\begin{array}{llll}0.0000 & 0.0026 & 0.9999\end{array}$

$\begin{array}{llll}0.0000 & 0.0026 & 0 . \\ 0.0000 & 0.0026 & 0 .\end{array}$

$0.0000 \quad 0.0026$

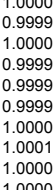


1150 WVDG-24

1152 WVGG 26
1153 WDDG-27

1154 WVDG-22

1156 WVGG 30
1157 WWDG -33

1158 WVGG-33

1159 WVVG-35
1160 WVDG-36

1161 WVDG

1163 WVDG-39

1164 WVDG-40

1166 WVDG-4

1168 WVDG-43

1169 WVDG-45

1170 WVDG-46

1172 WDGG 18 -48

1174 WVDG-46
1175 FY92

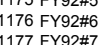

1177 FY9247
1178 FY9249

1179 F F924110
1180 FYg2Ref5

1181 Ratio2

1182 Ratio4
1183 Ratio5

1184 LoTh

1185 LoTh4
1186 LTTh5

1187 Hifee 2

1188 HiFe
1189 HiFed

190 PNL190

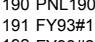

1192 FY93\#\#
1193 FY93

1194 FY93\#4

$1195 \mathrm{FYY3} 345$

1196 FY93\#t
1197 FY93"t

1198 FY93\#\#

1199 FY93\$9

$1200 \mathrm{FY} 93 \# 10$
$1201 \mathrm{FY} 94+1$

1202 FY94\#2

1203 FY94\#3

1204 FY944t4
1205 FY944t5

1205 FY94\#5
1206 FY94Af

1207 FY94\#t

1208 FY9448
1209 FY944t9

1209 FY 9449
1210 FY994t10

1211 sigma

1212 sigma2
1213 sigma3

1214 Sigma4

1215
1215 sigmas

1217 Sigma

1218 Sigmaz
1219 Sigma9

1220 sigma10

${ }_{12222}^{1221}$

1225

12267
12278
1229

12289
122910
123011

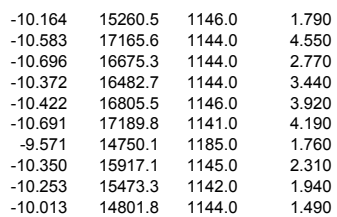


1150 WVDG-24

1153 WVDG-22

155 WVDG-29

1156 WVDG-30

1157 WVDG-33

1159 WVDG-35

1116 WVDG

163 WDDG-39

165 WVDG-4

167 WVDG-43

1168 WVDG-4

1169 WVDG-4

1.7rWWDat

1173 WVDG-40

1174 WVDG-46
1175 FY92455

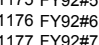

1177 FY9247
1178 FY9249

1179 F F92410
1180 FY92Ref5

1181 Ratio2

1182 Ratio4
1183 Ratio5

1184 LoTh

1186 LoTh

1187 HiFe?

1189 HiFe4

1190 PNL 190
1191 FY93\#1

$1192 \mathrm{FY} Y 332$
1193
$\mathrm{FY} Y 3 * 3$

1194 FY93\$4

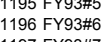

$1197 \mathrm{FY} 93 \# 7$
$1198 \mathrm{FY} 93 \# 8$

1199 FY93:9

$1200 \mathrm{FY} Y 3 \#+10$
1201
FY944t

1202 FY94\#2

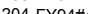

1205 FY9445

1208 FY9

1209 FY94\$S

1210 FY94\#110

1212 Simal

1217 Sigem

1218 Sigmaz
1219 Signa9

12211
12222
1

12234
12245

12245
12256
1227

1226

12278
12289
12209
1220

122910
123011

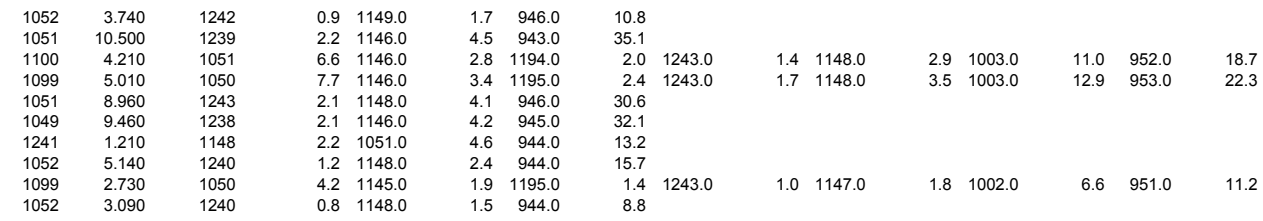


\# Glass ID

1149 WVDG-23

1151 WVDG-25

1152 WVDG-26

154 WVDG-28

1155 WVDG-29
1156 WVDG-30

1157 WVDG-3

1159 WVDG-35

1160 WVDG-36

1162 WVDG-37

1163 WVDG-39

1164 WVDG-40

1168 WVDG-44

1170 WVDG

1171 WVDG-47

1172 WVDG-48

1176 FY92\#6

1177 FY92\#7

1179 FY92\#10

1181 Ratio2

1182 Ratio4

1184 Loth

1186 LoTh

1188 HiFe3

190 PNL 190

(191 FY3\#1

$1194 \mathrm{FY} 93 \# 4$

1966 FY93\#6
1197 FY93\#7

198 FY93\#8

1199 FY93\#9

1201 FY94\#1

1203 FY94\#3

1204 FY94\#4

1206 FY94\#6

1208 Fr944t:

1210 FY94\#110

211 Sigma1

1214 Sigma4
1215 Sigma5

1218 Sigma8

1220 sigma10

12211
12222

1225

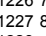

122910
123011

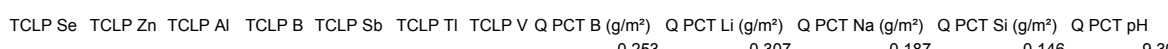

\begin{tabular}{|c|c|c|c|c|}
\hline 0.253 & 0.307 & 0.187 & 0.146 & \\
\hline & 0.286 & 0.173 & 0.124 & 9.450 \\
\hline & & 0.164 & 0.131 & 9.580 \\
\hline $\begin{array}{l}0.185 \\
0.309\end{array}$ & $\begin{array}{l}0.257 \\
0.327\end{array}$ & $\begin{array}{l}0.156 \\
0243\end{array}$ & $\begin{array}{l}0.105 \\
0.154\end{array}$ & $\begin{array}{l}9.480 \\
9555\end{array}$ \\
\hline $\begin{array}{l}0.3099 \\
0.201\end{array}$ & $\begin{array}{l}0.327 \\
0.289\end{array}$ & $\begin{array}{l}0.243 \\
0.169\end{array}$ & $\begin{array}{l}0.154 \\
0.139\end{array}$ & $\begin{array}{l}9.550 \\
9.510\end{array}$ \\
\hline 0.217 & 0.018 & $\begin{array}{l}0.21149 \\
0.0\end{array}$ & $\begin{array}{l}.0139 \\
0.120\end{array}$ & $\begin{array}{l}9.750 \\
.790\end{array}$ \\
\hline 0.209 & 0.007 & 0.196 & 0.126 & 9.630 \\
\hline 0.247 & 0.263 & $\begin{array}{l}0.245 \\
0\end{array}$ & 0.138 & $\begin{array}{l}10.170 \\
1\end{array}$ \\
\hline $\begin{array}{l}0.411 \\
0.400\end{array}$ & $\begin{array}{l}0.388 \\
0.394\end{array}$ & $\begin{array}{l}0.363 \\
0.399\end{array}$ & $\begin{array}{l}0.168 \\
0.185\end{array}$ & $\begin{array}{l}10.140 \\
10.830\end{array}$ \\
\hline 0.244 & 0.251 & 0.164 & 0.134 & 9.680 \\
\hline 0.231 & 0.267 & 0.267 & 0.148 & $\begin{array}{l}10.380 \\
\end{array}$ \\
\hline 0.197 & $\begin{array}{l}0.240 \\
0240\end{array}$ & $\begin{array}{l}0.197 \\
0.247\end{array}$ & $\begin{array}{l}0.133 \\
0\end{array}$ & $\begin{array}{r}10.230 \\
0\end{array}$ \\
\hline 0.305 & $\begin{array}{l}0.249 \\
0.305\end{array}$ & $\begin{array}{l}0.247 \\
0.289\end{array}$ & $\begin{array}{l}.0128 \\
0.154\end{array}$ & $\begin{array}{r}9.900 \\
10.320\end{array}$ \\
\hline 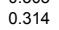 & 0.299 & $\begin{array}{l}0.289 \\
0.369\end{array}$ & $\begin{array}{l}0.154 \\
0.161\end{array}$ & 10.520 \\
\hline & 0.355 & 0.364 & 0.173 & 10.440 \\
\hline & 0.257 & 0.138 & 0.138 & 9.350 \\
\hline & 0.358 & 0.331 & 0.152 & 10.290 \\
\hline 260 & 0.265 & 0.300 & 0.155 & 10.570 \\
\hline & 0.317 & 0.299 & 0.145 & 10.010 \\
\hline & $\begin{array}{l}0.233 \\
0.226\end{array}$ & $\begin{array}{l}0.150 \\
0.129\end{array}$ & $\begin{array}{l}. .140 \\
0.123\end{array}$ & $\begin{array}{l}9.300 \\
9320\end{array}$ \\
\hline & 0.206 & 0.129 & 0.123 & 9.320 \\
\hline
\end{tabular}

CCC PCT B $\left(g / \mathrm{m}^{2}\right)$ CCC PCT Li $\left(g / \mathrm{m}^{2}\right) \quad C C C$ PCT Na $\left(g / \mathrm{m}^{2}\right) \quad C C C$ PCT Si $\left(g / \mathrm{m}^{2}\right)$ CCC PCT pH T1 $\left({ }^{\circ} \mathrm{C}\right.$ 9.480 9.630 140 380 320 440

0.290

0.529
0.267

0.208

0.569

0.909

0.297

0.487
0.385

0.385
0.498
0.382
0.572

0.572
1.014

0.504
2.702
2.265

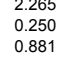

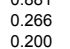

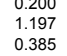

0.095

2.253

${ }_{0.495}^{2.296}$

$\frac{0.066}{0.093}$

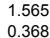

2.151
4.920
.5929

${ }_{3.55}^{5.42}$

3.951
3.930
3.683

3.326

2.559
2.485
2.913

$\begin{array}{lll}2.913 & & \\ 0.239 & 0.093 & 9.050\end{array}$

$\begin{array}{lll}0.187 & 0.045 & 10.920 \\ 0.685 & 0.187 & 11.760 \\ 0.364 & 0.179 & 110530\end{array}$

$\begin{array}{rrr}0.564 & 0.179 & 11.00 \\ 0.512 & 0.162 & 9.530 \\ 25.044 & 15.029 & 11.390\end{array}$

$\begin{array}{rrr}0.597 & 0.108 & 11.450 \\ 27.827 & 16.036 & 12.630 \\ & 0.710 & 1.30\end{array}$

$\begin{array}{lll}1.286 & 0.047 & 11.950\end{array}$ 
11150 WDOG-23

1151 WVG-25

1153 WVGG-27

1155 WVDG-29
1156 WVDG-30

1157 WVOG-33

1159 WVOG-35

1161 WVDG-37

163 WVDG-39

1164 WVDG-40

1166 WVDG-4

1167 WVDG 43

1169 WVDG-45

1170 WVDG-46
1171 WVDG-4t

1172 WVDG-48

1173 WVDG-40

1175 FY9245

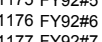

1177 FY9247
1178 FY92 49

1179 Fre24110
1180 FYg2Ref5

1181 Ratio2
11122 Ratios

1182 Ratio4
1183 Ratio5

1184 LoTh

1185 LoTh4
1186 LoTh5

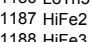

1188 HiFe3
11199 HiFe4
1190 PN 1900

1190 PNL1900
11191 F 93311

$1192 \mathrm{FY} Y 332$
1193
$\mathrm{FY} Y 3 * 3$

194 FY93\#4

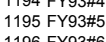

1196 FY93\#t
1197 FY93\#?

1198 FY93\#\#

1199 FY93\$9

1200 FYY3A1
1201 FY94t1

1202 FY94\#2

1203 FYY4\#3
1205 FYA4t

$1204 \mathrm{FY} Y 4444$
$1205 \mathrm{FY} 9445$

1205 FY94\#5
1206 FY94Af

1207 FY94\$7
1208 FY944

$1208 \mathrm{FY} Y 448$
1209
FY944t9

1209 FY 9449
1210 FY994t10

121 Sigma 1

1212 Sigma2
1213 sigma3

1213 Sigma3
1214 sigma4
1215

1215 Sigmas
1214 Sigmas

1217 sigma7

1218 Sigmaz
1219 sigma9

1220 sigma10

12211
12222

1223

12245
12256

12267
12278
1228

12289
122910
123011 


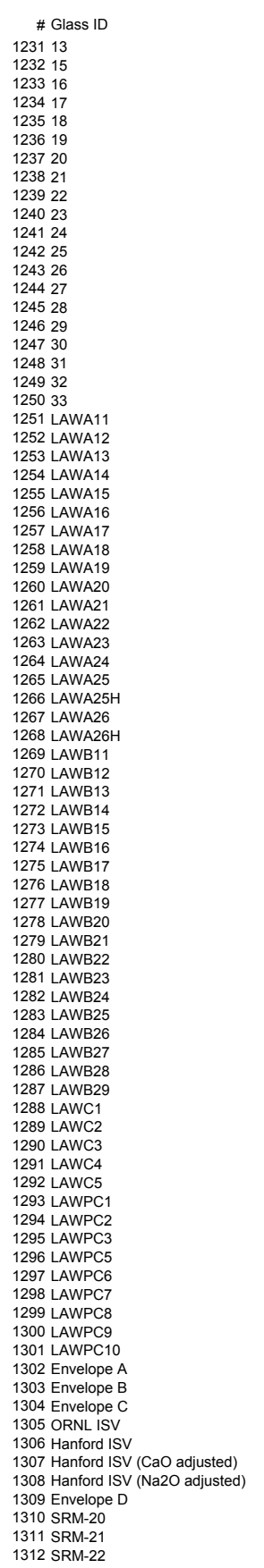

$0.0034 \quad 0.0000 \quad 0.0000$

$\begin{array}{llll}0.0000 & 0.0034 & 0.0000 & 0.0000 \\ 0.0000 & 0.0034 & 0.0000 & 0.0000\end{array}$

$0.00000 .0034 \quad 0.0000 \quad 0.0000$

$0.00000 .0034 \quad 0.0000 \quad 0.0000$

$\begin{array}{llll}0.0000 & 0.0034 & 0.0000 & 0.0000 \\ 0.0244 & 0.0034 & 0.0000 & 0.0000\end{array}$

$\begin{array}{llll}0.0244 & 0.0034 & 0.0000 & 0.0000 \\ 0.0000 & 0.0034 & 0.0000 & 0.0244\end{array}$

$0.0000 \quad 0.0034 \quad 0.0000 \quad 0.0000$

$\begin{array}{llll}0.0000 & 0.0034 & 0.0000 & 0.0000 \\ 0.0000 & 0.0001 & 0.0000 & 0.0000\end{array}$

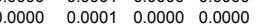

$\begin{array}{llll}0.0000 & 0.0034 & 0.0000 & 0.0000 \\ 0.0000 & 0.0039 & 0.0000 & 0.0000\end{array}$

$\begin{array}{llll}0.0000 & 0.0039 & 0.0000 & 0.0000\end{array}$

$\begin{array}{llll}0.0000 & 0.0039 & 0.0000 & 0.0000\end{array}$

$\begin{array}{lllll}0.0000 & 0.0037 & 0.0000 & 0.0000\end{array}$

$\begin{array}{lll}0.0037 & 0.0000 & 0.0000 \\ 0.0037 & 0.0000 & 0.0000\end{array}$

$\begin{array}{llll}.0000 & 0.0037 & 0.0000 & 0.0000 \\ 0.000 & 0.0037 & 0.0000 & 0.0000\end{array}$

$\begin{array}{llll}0.0000 & 0.0037 & 0.0000 & 0.00000 \\ 0.0000 & 0.0037 & 0.0000 & 0.0000\end{array}$

$\begin{array}{llll}0.0000 & 0.0037 & 0.0000 & 0.0000\end{array}$

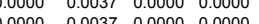

$\begin{array}{llll}0.0037 & 0.0000 & 0.0000\end{array}$

$\begin{array}{llll}0.0000 & 0.0037 & 0.0000 & 0.0000\end{array}$

$\begin{array}{lllll}0.0000 & 0.0037 & 0.0000 & 0.0000\end{array}$

$\begin{array}{llllll}0.0000 & 0.0037 & 0.0000 & 0.0000 \\ 0.0000 & 0.0037 & 0.000 & 0.0000\end{array}$

$\begin{array}{llll}0.0000 & 0.0037 & 0.0000 & 0.0000\end{array}$

$\begin{array}{llll}0.0000 & 0.0037 & 0.0000 & 0.0000\end{array}$

$\begin{array}{llll}0.0000 & 0.0010 & 0.0000 & 0.0000\end{array}$

$\begin{array}{llll}0.0000 & 0.0034 & 0.0000 & 0.0000 \\ 0.0000 & 0.0034 & 0.0000 & 0.0000\end{array}$

$\begin{array}{lllll}0.0000 & 0.0034 & 0.0000 & 0.00000\end{array}$

$\begin{array}{llll} & 0.0031 & 0.0000 & 0.0000 \\ 0.000 & 0.0034 & 0.0000 & 0.0000\end{array}$

$\begin{array}{lllll}0.0000 & 0.0025 & 0.0008 & 0.0000\end{array}$

$\begin{array}{lllll}.00000 & 0.0025 & 0.0008 & 0.00000\end{array}$

$\begin{array}{lllll}0.0000 & 0.0025 & 0.0008 & 0.0198\end{array}$

$\begin{array}{llllll}0 & 0 & 0.0000 & 0.025 & 0.0008 & 0.0000\end{array}$

$\begin{array}{lll}0.0001 & 0.0000 & 0.0000\end{array}$

$\begin{array}{llll}0.0000 & 0.0025 & 0.0008 & 0.0000 \\ 0.0000 & 0.0001 & 0.0000 & 0.0000\end{array}$

0.0001
0.0010
0.0001

0.0004
0.0002

0.0002
0.0002
0.0002

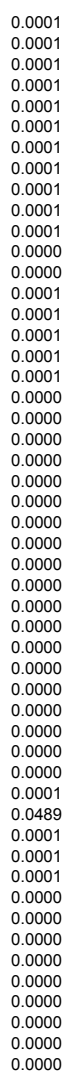

0.0010
0.0010
0.0010
0.0010
0.0010
0.0010
0.0010
0.0010
0.0010
0.0010
0.0010
0.0000
0.0000
0.0010
0.0011
0.0011
0.0011
0.0011
0.0004
0.0004
0.0004
0.0004
0.0004
0.0004
0.0004
0.0004
0.0004
0.0004
0.0004
0.0004
0.0004
0.0004
0.0004
0.0004
0.0004
0.0004
0.0000
0.0010
0.0010
0.0010
0.0009
0.0010
0.0007
0.0007
0.0007
0.0007
0.0007
0.0002
0.0002
0.0007
0.00002

0.0041

0.0019

0.0000 


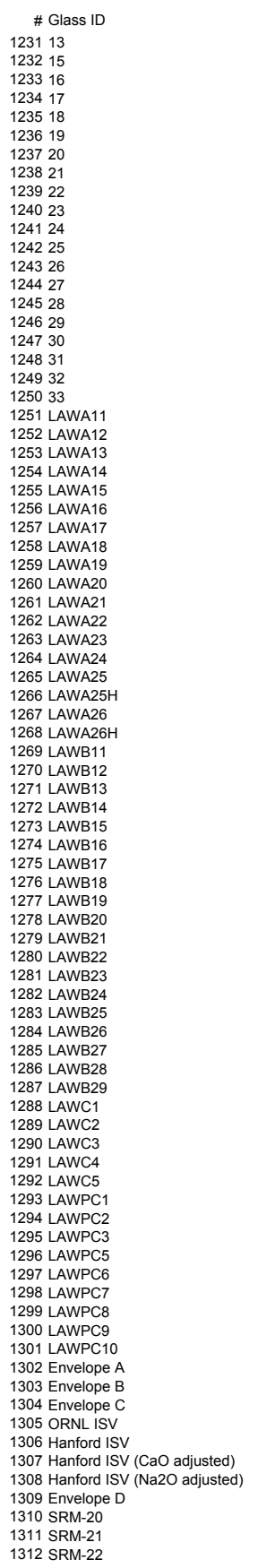

ReO2-t Re207-t Rh203-t RhO2-t RuO2-t Ru203-t Sb203-t Sb205-t SeO2-t Sm203-t SnO-t SnO2-t SO3-t Sro-t Tc207-t TeO2-t ThO2-t TiO2-t T12O-t T1203-t U308-t UO2-t U03-t V205-t wO3-t Y203-t ZnO-t Sum-t Al203-a $\begin{array}{rr}1.0000 & 0.1826 \\ 1.000 & 0.175 \\ 1.000 & 0.1484\end{array}$ $\begin{array}{ll}1.0000 & 0.175 \\ 1.0000 & 0.1484 \\ 1.0000 & 0.145\end{array}$ $\begin{array}{ll}1.0000 & 0.1145 \\ 1.0000 & 0.0094\end{array}$ $\begin{array}{ll}1.0000 & 0.0094 \\ 1.0000 & 0.0368 \\ 1.0000 & 0.1126\end{array}$ $\begin{array}{ll}1.0000 & 0.0096 \\ 1.0000 & 0.1126\end{array}$ $1.0000 \quad 0.1843$ $\begin{array}{ll}1.0000 & 0.226 \\ 1.0000 & 0.0742\end{array}$ $1.0000 \quad 0.1296$ $\begin{array}{ll}1.0000 & 0.1116 \\ 1.0000 & 0.1450\end{array}$ $\begin{array}{ll}1.0000 & 0.1463 \\ 1.0000 & 0.1148\end{array}$ 1.00000 .1070 $1.0000 \quad 0.1634$

$\begin{array}{llll}0.0000 & 0.9998 & 0.0509\end{array}$

$\begin{array}{lll} & 0.9998 & 0.055 \\ 005 & 0.9997 & 0.1410\end{array}$

$\begin{array}{lll}0.0305 & 0.9996 & 0.116 \\ 0.0305 & 0.9996 & 0.115 \\ 0.0305 & 0.9997 & 0.142\end{array}$

$\begin{array}{lll}.0305 & 0.9997 & 0.1412 \\ .0000 & 0.9998 & 0.0899\end{array}$

$\begin{array}{lll}0.0000 & 0.0050 \\ 0.0000 & 0.0050\end{array}$

$\begin{array}{ll}0.0000 & 0.0050 \\ 0.0000 & 0.0004 \\ 0.0000 & 0.0004\end{array}$

$\begin{array}{lll}.0000 & 0.005 \\ 0.0000 & 0.0057\end{array}$

$\begin{array}{lll}0.00000 & 0.0029 \\ 0.0000 & 0.0257 & 0\end{array}$

$.0000 \quad 0.0029$

$\begin{array}{lll}0.0000 & 0.0136 \\ 0 & 0.0000 & 0.0136\end{array}$

$\begin{array}{lll}0.0000 & 0.0136 \\ 0.0000 & 0.0136\end{array}$

$\begin{array}{ll}0.0000 & 0.0136 \\ 0.0000 & 0.0136\end{array}$

$\begin{array}{lll}0.0000 & 0.0136 \\ 0.0000 & 0.0136 & 0.0000 \\ 0.0000 & 0.136 & 0.0000\end{array}$

$\begin{array}{lll}0.0000 & 0.0136 & 0.0000 \\ 0.0000 & 0.0136 & 0.0000 \\ 0.0000 & 0.0136 & 0.0000 \\ 0.0000 & 0.0136 & 0.000\end{array}$

$\begin{array}{lll}0.0000 & 0.0000 & 0.0000 \\ 0.0000 & 0.0000 & 0.0000\end{array}$

$0.0000 \quad 0.0000$ $\begin{array}{llll}0.0000 & 0.0136 & 0.0000\end{array}$

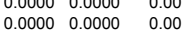

.

$\begin{array}{lll}0.0000 & 0.0000 & 0.0000 \\ 0.0000 & 0.0000 & 0.0000\end{array}$

$\begin{array}{lll}0.0000 & 0.0136 & 0.0000 \\ 0.0000 & 0.0035 & 0.0000\end{array}$

$\begin{array}{lll}0.0000 & 0.0000 & 0.0000 \\ 0.0000 & 0.0000 & 0.0000 \\ 0\end{array}$

$\begin{array}{llll}0.0000 & 0.0069 & 0.0000\end{array}$

$\begin{array}{lll}0.0000 & 0.00000 & 0.00000 \\ 0.0000\end{array}$

.

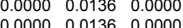

$\begin{array}{llll}0.0000 & 0.0000 & 0.0000 \\ 0.0000 & 0.0000 & 0.000\end{array}$

$\begin{array}{lll}0.0000 & 0.0000 & 0.0000 \\ 0.0000 & 0.0000 & 0.0000\end{array}$

$\begin{array}{llll}0.0000 & 0.0050 & 0.0007\end{array}$

$\begin{array}{lll}0.0000 & 0.0000 & 0.0000\end{array}$

$\begin{array}{llll}0.0000 & 0.0050 & 0.0007 \\ 0.0488 & 0.0050 & 0.0007\end{array}$

$\begin{array}{lll}0.0046 & 0.0006 \\ 0 & 0.0050 & 0.0007\end{array}$

$\begin{array}{llll}0.0000 & 0.0006 & 0.006 \\ 0.0000 & 0.0037 & 0.0000 \\ 0.0000 & 0.0037 & 0.000\end{array}$

$\begin{array}{lll}.0000 & 0.0037 & 0.0008 \\ 0.0000 & 0.0037 & 0.0008 \\ 0.0000 & 0.037 & 0.008\end{array}$

$\begin{array}{lll}0.0000 & 0.0037 & 0.0008 \\ 0.0000 & 0.0037 & 0.0008\end{array}$

$\begin{array}{lll}0.0000 & 0.0038 & 0.0000 \\ 0.0000 & 0.0076 & 0.0008\end{array}$

$\begin{array}{lll}0.0000 & 0.0000 & 0.0000\end{array}$

$\begin{array}{lll}0.0000 & 0.00000 & 0.0000 \\ 0.0000 & 0.0000 & 0.0000\end{array}$

$\begin{array}{llll}0.0000 & 0.0000 & 0.0000 \\ 0 & 0.0000 & 0.0000 & 0.0000\end{array}$

$\begin{array}{lll}0.0000 & 0.00000 & 0.00000 \\ 0.0000 & 0.0000 & 0.0000 \\ 0.000 & 0.000 & 0.000\end{array}$

$\begin{array}{lll}0.0000 & 0.0000 & 0.0000 \\ 0.0000 & 0.0000 & 0.0000\end{array}$

$\begin{array}{lll}0.0000 & 0.0000 \\ 0.0000 & 0.0000 \\ 0\end{array}$

0.00000 .0000

$0.0000 \quad 0.0000$

0.00000 .0000

$\begin{array}{lll}0.0000 & 0.0000 \\ 0.0000 & 0.0000\end{array}$

$0.0000 \quad 0.0000$ $\begin{array}{ll}0.0000 & 0.0000 \\ 0.0000 & 0.0000\end{array}$

$0.0000 \quad 0.0000$

$\begin{array}{lll}0.0328 & 0.9998 & 0.0957 \\ 0.0328 & 0.0998 & 0.0978\end{array}$

$\begin{array}{lll}0.0305 & 0.9996 & 0.1124 \\ 0.0278 & 0.9999 & 0.1024 \\ 0.2838 & 0.9998 & 0.193\end{array}$

$\begin{array}{lll}.0283 & 0.9998 & 0.0983 \\ 0.0278 & 0.999 & 0.093\end{array}$

$\begin{array}{lll}0.0283 & 0.9998 & 0.1093\end{array}$

$\begin{array}{lll}0.0000 & 0.9997 & 0.0584 \\ 0 & 0000 & 0.0997\end{array}$

$\begin{array}{lll}0.0000 & 0.9997 & 0.060 \\ 0.0407 & 0.9997 & 0.0598\end{array}$ $\begin{array}{lll}0.0000 & 0.9997 & 0.0565\end{array}$ $\begin{array}{lll}0.0978 & 0.9997 & 0.0619 \\ 0.0407 & 0.9996 & 0.0621\end{array}$ $\begin{array}{lllll}0.0000 & 0.0000 & 0.9589 & 0.0610\end{array}$

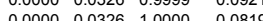
$\begin{array}{lllll}0.0000 & 0.0326 & 1.0000 & 0.0872\end{array}$ $\begin{array}{lllll}0.0000 & 0.0326 & 1.0000 & 0.0856\end{array}$ $\begin{array}{lll}0.0000 & 0.0331 & 1.0001 \\ 0.0000 & 0.0329 & 1.0000\end{array}$

$\begin{array}{lll}0.0000 & 0.0329 & 1.0000 \\ 0.0000 & 0.0328 & 1.0000\end{array}$

$\begin{array}{lllll}0.0000 & 0.0407 & 0.9998 & 0.0771\end{array}$ $\begin{array}{lllll}0.0000 & 0.0407 & 0.9999 & 0.074\end{array}$ $\begin{array}{lllll}0.0000 & 0.0399 & 0.9998 & 0.084\end{array}$ $\begin{array}{lll}0.0305 & 0.9999 & 0.0941 \\ 0.0305 & 0.9999 & 0.0941\end{array}$ $\begin{array}{llll}0.0000 & 0.9987 & 0.0920\end{array}$ $\begin{array}{lll}0.0287 & 0.9999 & 0.0862 \\ 0.0330 & 0.9999 & 0.0938\end{array}$ $\begin{array}{llll}0.0000 & 0.0237 & 0.999 & 0.0852 \\ 0.0000 & 0.0330 & 0.9999 & 0.0948\end{array}$ $\begin{array}{lllll}0.0198 & 0.0330 & 0.9999 & 0.0951\end{array}$ $\begin{array}{llll}0.0000 & 0.0330 & 0.9999 & 0.0909 \\ 0 & 0.000 & 0.0332 & 0.9997\end{array}$ $\begin{array}{lllll}0.0000 & 0.0332 & 0.9997 & 0.0872\end{array}$ $\begin{array}{lllll}0.0000 & 0.0328 & 1.0000 & 0.0935 \\ 0 & 03000 & 0332 & 0.0997 & 0.095\end{array}$ $0.0000 \quad 0.0000$ $\begin{array}{lll}0.0000 & 0.0000 \\ 0.0000 & 0.0000\end{array}$ $0.0000 \quad 0.0000$ $\begin{array}{ll}0.0000 & 0.0000 \\ 0.0000 & 0.0000\end{array}$

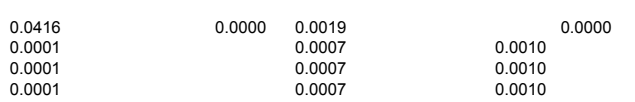
$\begin{array}{lll}0.0330 & 0.9981 & 0.0940 \\ 0.0400 & 0.9977 & 0.0770 \\ 0 & 0.070 & 0.1360\end{array}$ \begin{tabular}{lll}
0.0400 & 0.9977 & 0.0770 \\
.0330 & 0.9978 & 0.0910 \\
\hline & 0.0000 & 0.1360
\end{tabular} $\begin{array}{ll}0.0000 & 0.1220 \\ 0.0000 & 0.1020\end{array}$ $\begin{array}{lll} & 0.0000 & 0.1030 \\ 0.0001 & 0.9993 & 0.098\end{array}$ $\begin{array}{ll}1.0053 & 0.1000 \\ 1.0053 & 0.1000 \\ 1.0053 & 0.1000\end{array}$ 


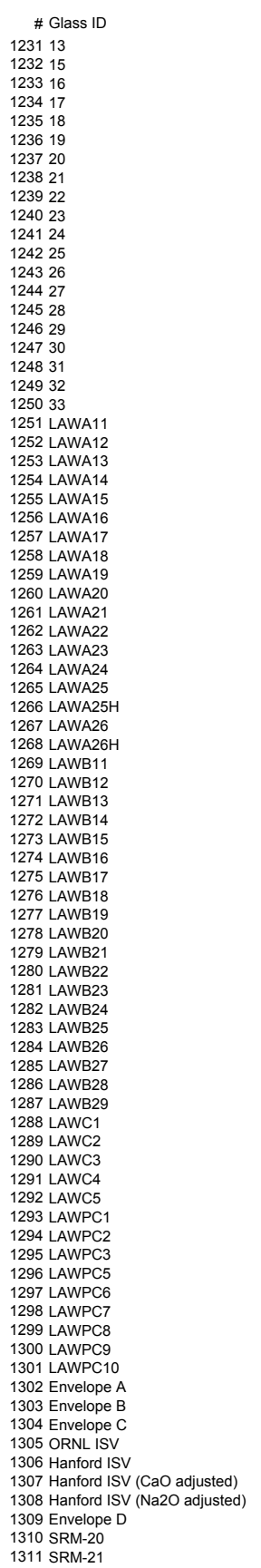

1311 SRM-21
1312 SRM-22

B203-a CaO-a Fe2O3-a FeO-a K2O-a Li2O-a MgO-a Na2O-a NiO-a P2O5-a SiO2-a ZrO2-a Ag2O-a As203-a As205-a BaO-a BeO-a Bi203-a Br-a CdO-a Ce2O3-a CeO2-a Cl-a CoO-a Co203-a Cr203-a Cs2O-a CuO-a F-a

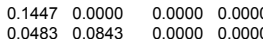

$\begin{array}{llll}0.048311 & 0.00000 & 0.0000 & 0.0000 \\ 0.1875 & 0.0218\end{array}$

$\begin{array}{llll}0.1297 & 0.0696 & 0.1582 & 0.0338 \\ 0.1611 & 0.0931 & 0.0000 & 0.0000\end{array}$

$0.0487 \quad 0.0786 \quad 0.2200 \quad 0.0136$

$\begin{array}{llll}0.0880 & 0.0316 & 0.0877 & 0.0084 \\ 0.047 & 0.0416 & 0.090 & 0\end{array}$

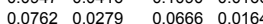

$\begin{array}{lllll}0.1049 & 0.0457 & 0.1225 & 0.0173\end{array}$

$\begin{array}{llll}0.1372 & 0.0300 & 0.0823 & 0.0116\end{array}$

$\begin{array}{llll}0.0889 & 0.0768 & 0.0778 & 0.0129 \\ 0.0922 & 0.0000 & 0.1119 & 0.0215\end{array}$

$\begin{array}{llll}0.0735 & 0.0266 & 0.1725 & 0.0298 \\ 0.1227 & 0.0552 & 0.0000 & 0.0000\end{array}$

$\begin{array}{llll}0.0784 & 0.0299 & 0.0803 & 0.0113 \\ 0.030 & 0.0430 & 0.1001 & 0.028\end{array}$

$\begin{array}{llll}0.0871 & 0.0343 & 0.0847 & 0.02885\end{array}$

$\begin{array}{llllllll}0.0520 & 0.0006 & 0.0006 & 0.1580 & 0.1902 & 0.0023 & 0.0172 & 0.4381 \\ 0.4305 & 0.0412\end{array}$

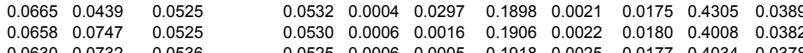

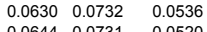

$\begin{array}{lll}0.0644 & 0.0731 & 0.052 \\ 0.1035 & 0.0432 & 0.0496\end{array}$

$\begin{array}{lll}0.0675 & 0.0241 & 0.0524 \\ 0.0667 & 0.0246 & 0.0473\end{array}$

$\begin{array}{lll}0.0663 & 0.0252 & 0.0466\end{array}$

$\begin{array}{lll}0.0490 & 0.0244 & 0.0561 \\ 0.0338 & 0.1066 & 0.0578 \\ 0.031 & 0430\end{array}$

$\begin{array}{lll}0.0311 & 0.0430 & 0.0712 \\ 0 & 0.474 & 0.0440\end{array}$

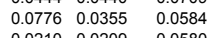

$\begin{array}{lll}0.0210 & 0.0209 & 0.058 \\ 0.0239 & 0.0208 & 0.0632\end{array}$

$\begin{array}{lll}0.0016 & 0.0203 & 0.0437\end{array}$

$\begin{array}{lll}0.1038 & 0.1091 & 0.0877 \\ 0.1366 & 0.0578 & 0.0908\end{array}$

$\begin{array}{lll}0.1036 & 0.0578 & 0.0908 \\ 0.1076 & 0.0586 & 0.0907\end{array}$

$\begin{array}{lll}0.1084 & 0.1037 & 0.0847 \\ 0.1365 & 0.1093 & 0.0839\end{array}$

$\begin{array}{lll}0.1365 & 0.1093 & 0.0839 \\ 0.1047 & 0.0067 & 0.0895\end{array}$

$\begin{array}{lll}0.0805 & 0.0555 & 0.0810 \\ 0.0643 & 0.0389 & 0.0932\end{array}$

$\begin{array}{lll}0.0651 & 0.0383 & 0.0914 \\ 0.0659 & 0.0418 & 0.0623 \\ 0.0659 & 0.0414 & 0.0671\end{array}$

$\begin{array}{lll}0.0879 & 0.0515 & 0.0696 \\ 0.0872 & 0.0520 & 0.0752\end{array}$

$\begin{array}{lll}0.0918 & 0.0508 & 0.0688 \\ 0.0787 & 0.0726 & 0.0730\end{array}$

$\begin{array}{lll}0.06600 & 0.0243 & 0.0997 \\ 0.0661 & 0.0237 & 0.0540 \\ 0.065 & 0.52 & 0.059\end{array}$

$\begin{array}{llll}0.0675 & 0.0250 & 0.0539\end{array}$

$\begin{array}{llll}0.0316 & 0.0759 & 0.0670\end{array}$

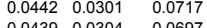

$0.04370 .0295 \quad 0.0716$

$\begin{array}{lll}0.0106 & 0.0300 & 0.0749 \\ 0.0205 & 0.0289 & 0.0719\end{array}$

$\begin{array}{lll}0.0285 & 0.0442 \quad 0.0640\end{array}$

$\begin{array}{lll}0.0446 & 0.0409 & 0.0667 \\ 0.0340 & 0.0511 & 0.0704 \\ 0.0250 & 0.650 & 0.087\end{array}$

$\begin{array}{lll}0.0420 & 0.0440 & 0.0810\end{array}$

$\begin{array}{lll}0.0300 & 0.0460 & 0.0690\end{array}$

$\begin{array}{lll}0.0016 & 0.1390 & 0.0378 \\ 0 & 0.009 & 0.0454\end{array}$

$\begin{array}{lll}0.0000 & 0.0454 & 0.0357 \\ 0.0008 & 0.1810 & 0.0550\end{array}$

$\begin{array}{lll}0.0393 & 0.0559 \\ 0.0590 & 0.0550 & 0.2165 \\ 0.1600 & 0.0050 & 0.035\end{array}$

$\begin{array}{lll}0.1300 & 0.0050 & 0.0030 \\ 0.1600 & 0.0050 & 0.0030 \\ 0.2000 & 0.0050 & 0.030\end{array}$

0.2235
0.2289
0.1941
0.1154
0.2586
0.2347
0.2155
0.1699
0.1601
0.1580
0.1721
0.1547
0.1761
0.1570
0.1687
0.1400
0.1957
0.2070
0.1231
0.1580

0.4492

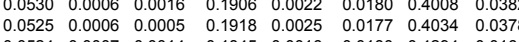

$\begin{array}{llllllll}0.0543 & 0.0004 & 0.1920 & 0.0017 & 0.0195 & 0.4345 & 0.0169\end{array}$

$\begin{array}{llllllll}0.0527 & 0.0004 & & 0.1910 & 0.0022 & 0.0185 & 0.3764 & 0.0231 \\ 0.0538 & 0.0004 & & 0.1852 & 0.0016 & 0.0192 & 0.3827 & 0.0303\end{array}$

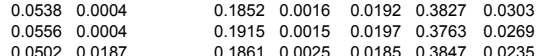

0.0187

$\begin{array}{llllllll}0.0501 & & & 0.1928 & 0.0016 & 0.0195 & 0.4187 & 0.0183 \\ 0.0308 & 0.0219 & 0.0187 & 0.1832 & 0.0010 & 0.0018 & 0.4395 & 0.0257\end{array}$

$\begin{array}{lllllll}0.1803 & 0.0001 & 0.0015 & 0.4299 & 0.0245\end{array}$

$\begin{array}{llllllll}0.0499 & 0.0009 & 0.0008 & 0.1809 & 0.0024 & 0.0209 & 0.3835 & 0.0282 \\ 0.0376 & 0.0015 & 0.0111 & 0.2456 & 0.0021 & 0.0213 & 0.4176 & 0.0169\end{array}$

$\begin{array}{llllllll}0.0380 & 0.0015 & 0.0120 & 0.2369 & 0.0027 & 0.0124 & 0.4255 & 0.0166 \\ 0.0382 & 0.0135 & 0.0003 & 0.2419 & 0.0018 & 0.0218 & 0.4460 & 0.0234\end{array}$

$\begin{array}{llllllll}0.0410 & 0.0143 & & 0.2540 & 0.0023 & 0.0111 & 0.4439 & 0.0161\end{array}$

$\begin{array}{llllllll}0.0204 & 0.0008 & 0.0314 & 0.0727 & 0.0015 & 0.0227 & 0.4395 & 0.0338 \\ 0.0199 & 0.0000 & 0.0005 & 0.0708 & 0.0015 & 0.0220 & 0.4504 & 0.0311 \\ 0 & 0.0280\end{array}$

$\begin{array}{llllllll}0.0228 & 0.0006 & 0.0790 & 0.0731 & 0.0006 & 0.0229 & 0.4113 & 0.0221\end{array}$

$\begin{array}{llllllll}0.0234 & 0.0178 & 0.0354 & 0.0701 & 0.0006 & 0.0242 & 0.4013 & 0.0264\end{array}$

$\begin{array}{lllllll}0.0406 & 0.0320 & 0.0700 & 0.0007 & 0.0233 & 0.4417 & 0.0351\end{array}$

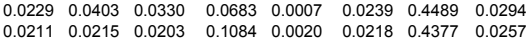

$\begin{array}{lllllllll}0.0213 & 0.0323 & 0.0210 & 0.1154 & 0.0024 & 0.0231 & 0.4273 & 0.026\end{array}$

$\begin{array}{llllllll}0.0215 & 0.0326 & 0.0203 & 0.1379 & 0.0007 & 0.0248 & 0.4310 & 0.029 \\ 0.0212 & 0.0012 & 0.0204 & 0.1644 & 0.0007 & 0.0243 & 0.4349 & 0.0273\end{array}$

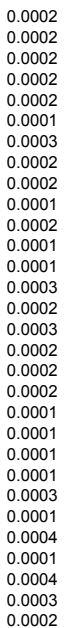

0.0033
0.0035
0.0034
0.0033
0.0033
0.0036
0.0035

0.0050
0.0060

$\begin{array}{lll} & 0.0049 & \\ & 0.0045 & \\ & 0.0046 & \\ & 0.0052 & \\ & 0.0038 & \\ 0.0000 & 0.0033 & \\ 0.0000 & 0.0044 & 0.0000 \\ 0.0257 & 0.00000 \\ 0.0003 & 0.0058 & 0.0001 \\ & 0.0053 & 0.0226 \\ & 0.0053 & \end{array}$

$\begin{array}{llllllll}0.0210 & 0.0412 & 0.0211 & 0.0766 & 0.0018 & 0.0217 & 0.4514 & 0.0249 \\ 0.0228 & 0.0336 & 0.0223 & 0.1012 & 0.0020 & 0.0223 & 0.4174 & 0.0296\end{array}$

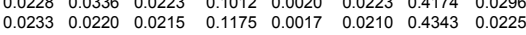
$\begin{array}{llllllll}0.0050 & 0.0398 & 0.0287 & 0.0993 & 0.0000 & 0.0018 & 0.4511 & 0.0214\end{array}$ 0.055

0.0533 $\begin{array}{lllll}0.1912 & 0.0021 & 0.0187 & 0.3757 & 0.0251 \\ 0.1934 & 0.0021 & 0.0182 & 0.3769 & 0.0216\end{array}$ $\begin{array}{llllll}0.1906 & 0.0024 & 0.0185 & 0.3875 & 0.0184 \\ 0.1731 & 0.0018 & 0.0096 & 0.4290 & 0.0199\end{array}$

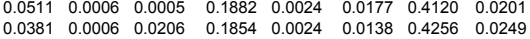
$\begin{array}{lllllllll}0.0372 & 0.0004 & 0.0206 & 0.1861 & 0.0023 & 0.0143 & 0.3986 & 0.0215\end{array}$ $\begin{array}{llllllll}0.0386 & 0.0003 & 0.0203 & 0.1973 & 0.0021 & 0.0136 & 0.4128 & 0.0252\end{array}$ $\begin{array}{llllllll}0.0386 & 0.0004 & 0.0199 & 0.1935 & 0.0022 & 0.0141 & 0.4232 & 0.025 \\ 0.0374 & 0.0219 & 0.0196 & 0.1943 & 0.0024 & 0.0138 & 0.4276 & 0.0244 \\ 0\end{array}$ $\begin{array}{lllllllll}0.0026 & 0.0340 & 0.0228 & 0.1439 & 0.0015 & 0.0013 & 0.5335 & 0.0228\end{array}$

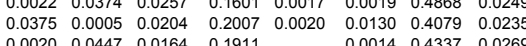

$\begin{array}{llllllllll}0.0220 & 0.0210 & 0.0200 & 0.2000 & 0.0014 & 0.00007 & 0.4100 & 0.0310 & 0.0000 & 0.0000\end{array}$ $\begin{array}{llllllllll}0.0038 & 0.04400 & 0.0280 & 0.1300 & 0.0016 & 0.0002 & 0.4300 & 0.03300 & 0.0000 & 0.0000 \\ 0.0017 & 0.0390 & 0.0280 & 0.1200 & 0.0017 & 0.0006 & 0.4100 & 03330 & 0.0000 & 0.0000\end{array}$ $\begin{array}{llllllllll}0.0017 & 0.0390 & 0.0280 & 0.2200 & 0.0017 & 0.0006 & 0.4100 & 0.0330 & 0.00\end{array}$ $\begin{array}{lllllll}0.0190 & 0.0221 & 0.0313 & 0.00005 & 0.06160 & 0.0004 \\ 0.0150 & 0.0005 & 0.0192 & 0.0301 & 0.0025 & 0.5110 & 0.00002\end{array}$ $\begin{array}{lllllllll}0.0090 & 0.0003 & 0.0183 & 0.1780 & 0.0004 & 0.5220 & 0.0003 & \\ 0.0845 & 0.0245 & 0.0022 & 0.1355 & 0.0025 & 0.0054 & 0.2570 & 0.0022\end{array}$

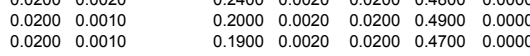

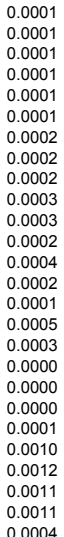

0.0000

$\begin{array}{ll}0.0000 & 0.0058 \\ 0.0000 & 0.0027\end{array}$

0.0021
0.0001
0.0037
0.0043
0.0008
0.0039
0.0056
0.0032
0.0001
0.0032
0.0024
0.024
0.0039
0.0037
0.0033
0.0059
0.0071
0.00035
0.0037

$\begin{array}{llll}0.0056 & & & \\ 0.0067 & & & \\ 0.0052 & & & \\ 0.0010 & & & \\ 0.0049 & & & \\ 0.0044 & & & \\ 0.0043 & & & \\ 0.0060 & & & \\ 0.0052 & & & \\ 0.0049 & & & \\ 0.0052 & & 0.0312 & \\ 0.0043 & & 0.0001 & \\ 0.0051 & & 0.0208 & \\ 0.0057 & & 0.0001 & \\ 0.0024 & & & \\ 0.0027 & & & \\ 0.0040 & & & \\ 0.0024 & & & \\ 0.0022 & 0.0017 & 0.0006 & 0.0003 \\ 0.0 .035 & 0.0016 & 0.0013 & 0.0000 \\ 0.0020 & 0.0008 & 0.0008 \\ & 0.0196 & & \\ & 0.0218 & & \\ 0.00008 & 0.0195 & & \\ 0.0037 & 0.0215 & & \end{array}$




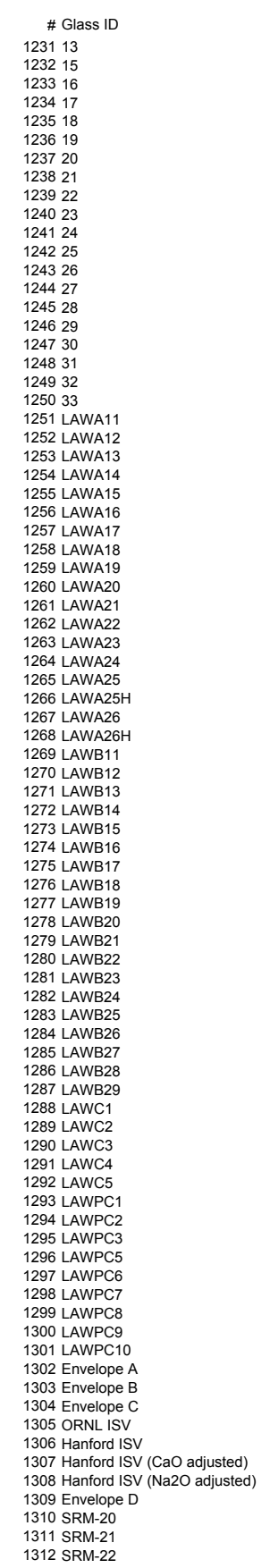

0.0001

0.0001

0.0001

0.0015
0.0013
0.0013
0.0012
0.0017
0.0014
0.0012
0.014
0.0013
0.0011
0.0014
0.0003
0.0002
0.0014
0.0014
0.0014
0.0013
0.0013
0.0008
0.0007
0.0007
0.0007
0.0008
0.0006
0.0007
0.0007
0.0006
0.0006
0.0006
0.0006

0.0002

0.0007

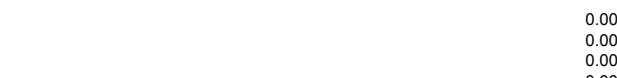

0.000
0.001
0.001

0.0011
0.0012
0.0012

0.0012

0.0012

0.0011

0.0010
0.0011
0.0003

0.0004

$\begin{array}{ll}0.0000 & 0.0002 \\ 0.0000 & 0.0003 \\ 0.0000 & 0.0003 \\ & 0.0013 \\ & 0.0014 \\ & 0.0012 \\ & 0.0012 \\ 0.0003 & 0.0051\end{array}$

0.0002

0.0000
0.0000

0.0001
0.0001

0.0037

0.0002 


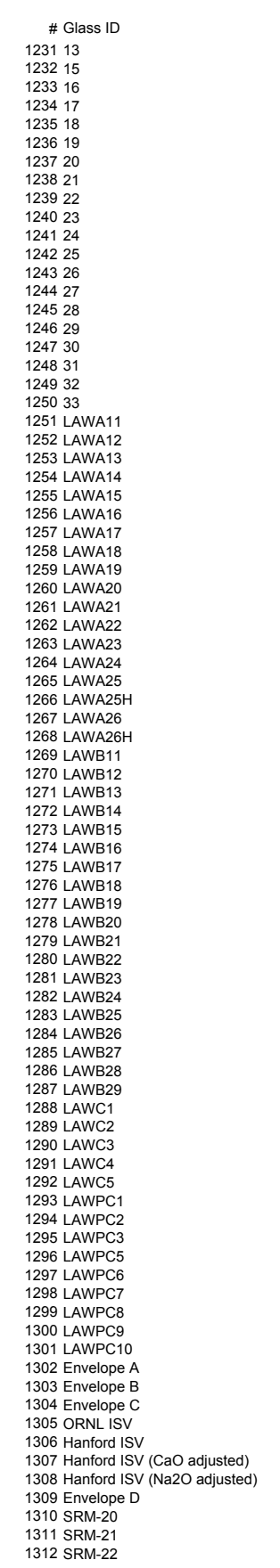

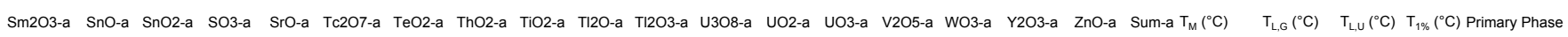

\begin{tabular}{|c|c|c|c|}
\hline $\begin{array}{l}1.0000 \\
1.000\end{array}$ & & & \\
\hline & & & \\
\hline & 1.0000 & & \\
\hline & 1.0000 & & \\
\hline & $\begin{array}{l}1.0000 \\
1.0000\end{array}$ & & \\
\hline & & & \\
\hline & 1.0000 & & \\
\hline & $\begin{array}{l}1.0000 \\
1.0001\end{array}$ & & \\
\hline & 1.0000 & & \\
\hline & 1. 1.0001 & & \\
\hline & 0.9999 & & \\
\hline & $\begin{array}{l}1.0000 \\
1.0000\end{array}$ & & \\
\hline & 0.9999 & & \\
\hline & 1.0001 & & \\
\hline & 1.0000 & & \\
\hline 0.0001 & 1.0041 & & $<950$ \\
\hline 0.0001 & 1.0059 & & $<950$ \\
\hline 0.0001 & 1.0064 & & $<950$ \\
\hline 0.0301 & 1.0072 & & $<950$ \\
\hline $\begin{array}{l}0.0003 \\
0.0001\end{array}$ & $\begin{array}{l}1.0044 \\
1.038\end{array}$ & & $\begin{array}{l}<950 \\
<950\end{array}$ \\
\hline $\begin{array}{l}0.0327 \\
0.0327\end{array}$ & $\begin{array}{l}1.0038 \\
1.0039\end{array}$ & & $\begin{array}{r}<950 \\
950-1050\end{array}$ \\
\hline 0.0328 & 1.0059 & & \\
\hline 0.0311 & 1.0057 & & $<950$ \\
\hline 0.0289 & 1.0045 & & - 1050 \\
\hline & 1.0031 & & $\sim 950$ \\
\hline 0.0316 & 0.9992 & & $<950$ \\
\hline 0.0317 & 0.9993 & & $<950$ \\
\hline $\begin{array}{l}0.0335 \\
0.0266\end{array}$ & $\begin{array}{l}1.0008 \\
1.0031\end{array}$ & & $<950$ \\
\hline $\begin{array}{l}0.0301 \\
0.0301\end{array}$ & 0.9988 & & $<950$ \\
\hline 0.0294 & 1.0029 & & \\
\hline & 0.9981 & & $<950$ \\
\hline 0.0003 & 1.0039 & & 950-1050 \\
\hline 0.0001 & 0.9279 & & $950-1050$ \\
\hline 0.0408 & 1.0020 & & -1050 \\
\hline 0.0000 & 1.0020 & & 950-1050 \\
\hline 0.0000 & $\begin{array}{l}1.0021 \\
1.027\end{array}$ & & $\begin{array}{r}\sim 950 \\
>>1050\end{array}$ \\
\hline $\begin{array}{l}0.0067 \\
0.0407\end{array}$ & $\begin{array}{l}1.00037 \\
1.0052\end{array}$ & & $\begin{array}{r}>1050 \\
950-1050\end{array}$ \\
\hline & $\begin{array}{l}1.0062 \\
0.9669\end{array}$ & & $\begin{array}{l}950-1050 \\
9950\end{array}$ \\
\hline 0.0322 & 1.0020 & & $\begin{array}{l}<950 \\
-1050\end{array}$ \\
\hline 0.0328 & 1.0023 & & $\sim 950$ \\
\hline 0.0322 & 1.0024 & & $<950$ \\
\hline 0.0348 & 1.0027 & & $\sim 950$ \\
\hline & 0.0000 & & \\
\hline & 0.0000 & & \\
\hline 0.0404 & $\begin{array}{l}1.0017 \\
0\end{array}$ & & -950 \\
\hline 0.0424 & 0.9989 & & $<950$ \\
\hline 0.0359 & 1.0022 & & $<950$ \\
\hline 0.04300 & 0.9990 & & $\begin{array}{c}<950 \\
<950\end{array}$ \\
\hline $\begin{array}{l}0.03315 \\
0.0309\end{array}$ & $\begin{array}{l}1.00033 \\
1.0056\end{array}$ & & $\begin{array}{l}-950 \\
-950 \\
-950\end{array}$ \\
\hline 0.0276 & $\begin{array}{l}1.0045 \\
1.0045\end{array}$ & & $\begin{array}{r}\sim 9500 \\
>1050\end{array}$ \\
\hline 0.0003 & 1.0042 & & $\sim 1050$ \\
\hline 0.0292 & 1.0055 & & $<950$ \\
\hline 0.0329 & 0.9996 & & $<950$ \\
\hline 0.0319 & 0.9990 & & $<950$ \\
\hline 0.0328 & 0.9993 & & - 950 \\
\hline $\begin{array}{l}0.0337 \\
0.231\end{array}$ & $\begin{array}{l}1.00000 \\
\end{array}$ & & $\begin{array}{r}-950 \\
-9500\end{array}$ \\
\hline 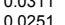 & . & & $\begin{array}{l}<950 \\
<950\end{array}$ \\
\hline $\begin{array}{l}0.0291 \\
0.021\end{array}$ & 0 & & $<950$ \\
\hline 0.0325 & 1.0032 & & $<950$ \\
\hline 0.0317 & 0.9968 & & $<950$ \\
\hline 0.0320 & 1.0072 & & \\
\hline 0.0370 & 1.0175 & & \\
\hline 0.0300 & 1.0119 & & \\
\hline & 1.04141 & & \\
\hline & 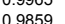 & & \\
\hline & 0.9974 & & \\
\hline 0.0010 & 1.0001 & & \\
\hline & 1.0030 & 1150 & \\
\hline & 1.0020 & & \\
\hline & & 1150 & \\
\hline
\end{tabular}




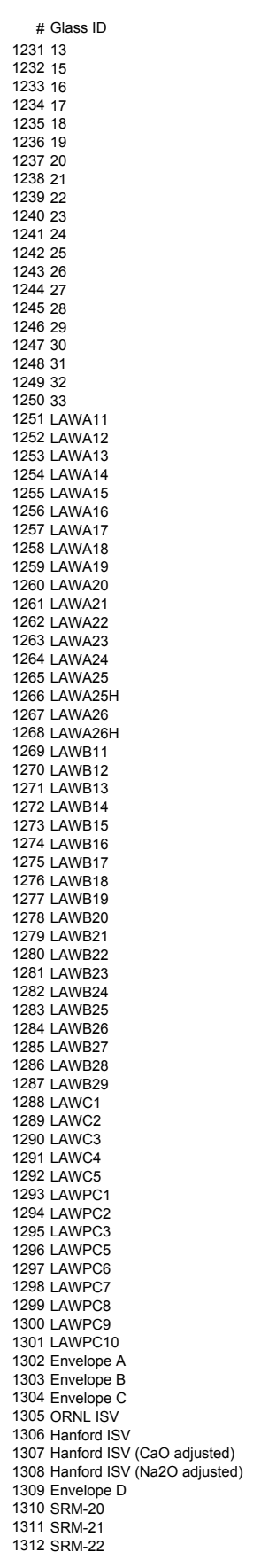

\section{12412}

1244
1245
1228

$$
\begin{aligned}
& 12462 \\
& 1243 \\
& 12433 \\
& \hline 2432
\end{aligned}
$$$$
125033
$$

1251 LAWA 1

1252 LAWA12

1255 LAWA15

1257 LAWA 17

2258 LAWA

$\begin{array}{llll}-12.372 & 20653.8 & 950.0 & 94.700\end{array}$

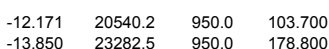

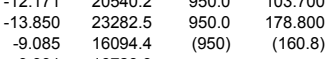

$\begin{array}{rl}-10.744 & 17686.3 \\ -1.252 & \end{array}$

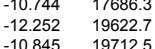

$\begin{array}{rl}-10.845 & 19712.5 \\ -11.695 & 18746.8 \\ -12152 & 2075\end{array}$

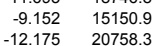

$\begin{array}{llll}-12.175 & 20758.3 & & \\ -12495 & 20530 & 9500 & 71200\end{array}$

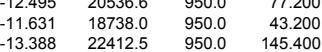

2.727

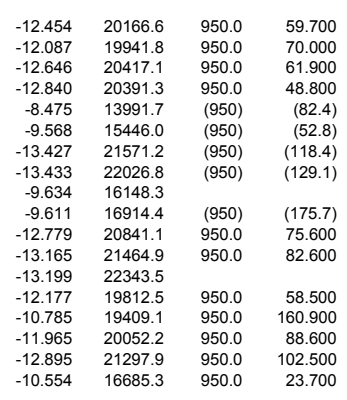




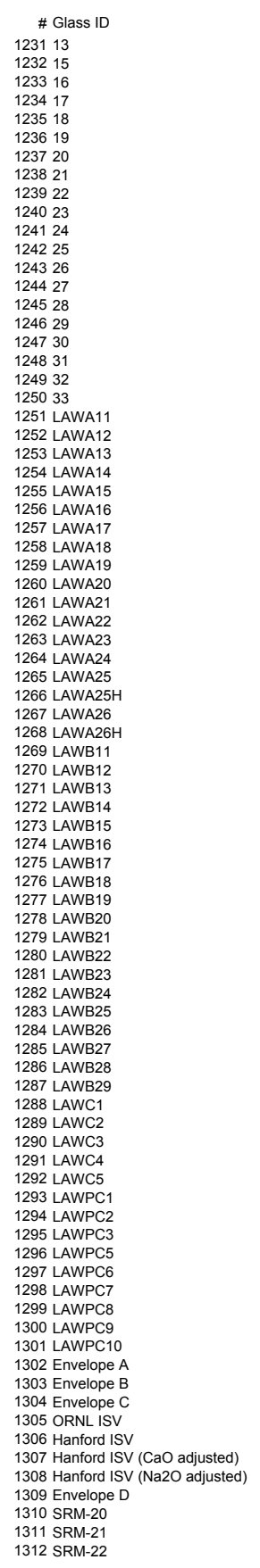

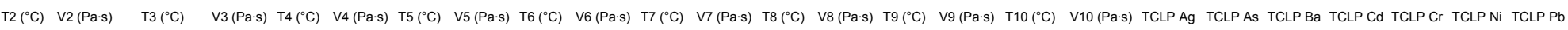

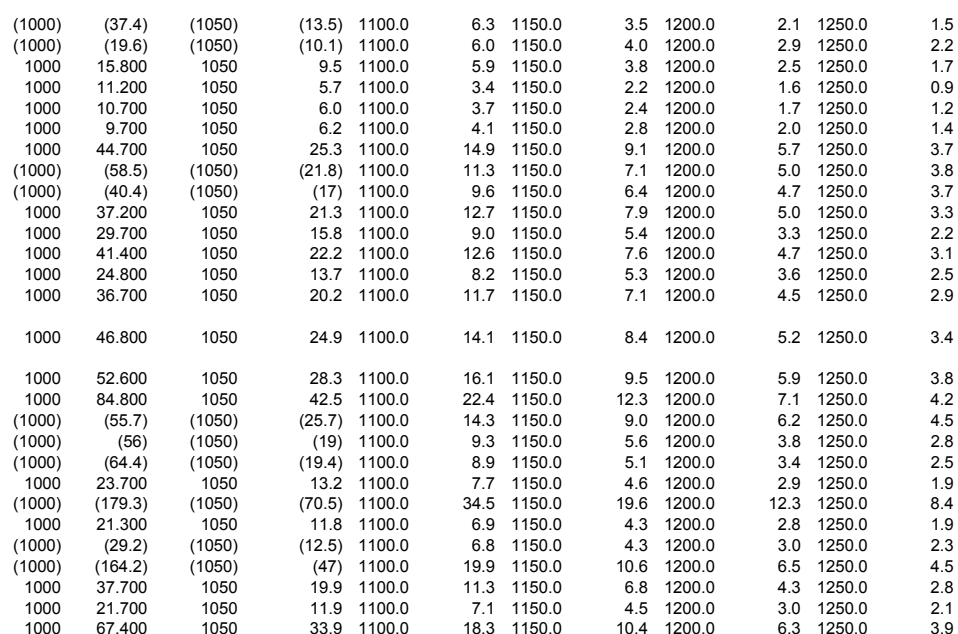

\begin{tabular}{|c|c|c|c|c|c|c|c|c|c|c|c|}
\hline 1000 & 29.300 & 1050 & 15.7 & 1100.0 & & 1150.0 & 5.4 & 1200.0 & $\begin{array}{l}3.5 \\
\end{array}$ & 1250.0 & 2.3 \\
\hline & 35.600 & 1050 & & & & & & 12000.0 & & 1250.0 & 2.8 \\
\hline $\begin{array}{l}1000 \\
1000\end{array}$ & $\begin{aligned} 29.400 \\
23.800\end{aligned}$ & $\begin{array}{l}1050 \\
1050\end{array}$ & $\begin{array}{l}15.4 \\
126\end{array}$ & $\begin{array}{l}1100.0 \\
1100\end{array}$ & 8.7 & $\begin{array}{l}1150.0 \\
11150\end{array}$ & 5.3 & 1200.0 & $\begin{array}{l}3.4 \\
\end{array}$ & $\begin{array}{l}1250.0 \\
1250\end{array}$ & $\begin{array}{l}2.3 \\
1.8\end{array}$ \\
\hline & $\begin{array}{r}23.800 \\
(22.8)\end{array}$ & $\begin{array}{r}1050 \\
(1050)\end{array}$ & $\begin{array}{l}\begin{array}{l}12.6 \\
(10)\end{array} \\
\text { (1) }\end{array}$ & $\begin{array}{l}11100.00 \\
1100.0\end{array}$ & $\begin{array}{l}.2 .2 \\
5.7\end{array}$ & 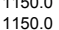 & $\begin{array}{l}4.3 \\
3.8\end{array}$ & $\begin{array}{l}12200.0 \\
1200.0\end{array}$ & $\begin{array}{l}2.8 \\
2.7\end{array}$ & $\begin{array}{l}1250.0 \\
1250\end{array}$ & $\begin{array}{l}1.8 \\
2.1\end{array}$ \\
\hline & (19.7) & (1050) & $(9.5)$ & 1100.0 & $\begin{array}{l}5.1 \\
5.5\end{array}$ & 1150.0 & & 1200.0 & 2.5 & 1250.0 & \\
\hline & & 1050 & & $(1100)$ & $(42.1)$ & 1150.0 & 5.3 & 1200.0 & 3.3 & & \\
\hline & 51.800 & 1050 & & 1100.0 & & 1150.0 & 72 & 1200.0 & 4.6 & 1250.0 & \\
\hline & $(38.8)$ & (1050) & (16.2) & 1100.0 & 8.6 & 1150.0 & 54 & 1200.0 & & & \\
\hline & (61.9) & (1050) & (28.3) & 11000.0 & 15.4 & 1150.0 & & 1200.0 & 6.3 & 1250.0 & \\
\hline & 35.900 & $\begin{array}{l}1050 \\
1050\end{array}$ & 20 & $\begin{array}{l}11100.0 \\
1\end{array}$ & 10.5 & $\begin{array}{l}11150.0 \\
1150\end{array}$ & 67 & 1200.0 & & & \\
\hline & 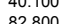 & 1050 & & 1 & $\begin{array}{l}1.16 \\
30.6\end{array}$ & & & & $\begin{array}{l}4.1 \\
7.1\end{array}$ & & 2.6 \\
\hline & 29.400 & 1050 & & 1100.0 & 9.2 & 1150.0 & 56 & 1200 & 3.6 & 1250 & \\
\hline & 86.300 & 1050 & 49.5 & 1100.0 & 28.5 & 1150.0 & 17.3 & 1200.0 & 10.9 & 1250.0 & \\
\hline & 43.70 & 1050 & 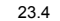 & 1100.0 & 13.5 & 11500 & 8.2 & & 52 & & \\
\hline & 45.400 & 1050 & 2.8 & 1100.0 & & 1150.0 & 7.6 & 1200. & 4.8 & & \\
\hline & 12.700 & 1050 & & 1100.0 & 4.7 & 1150.0 & 3.1 & 1200.0 & 2.2 & 1250.0 & \\
\hline
\end{tabular}

$<1.0 \quad 0.004 \quad 0.009 \quad 0.02 \quad 0.026$

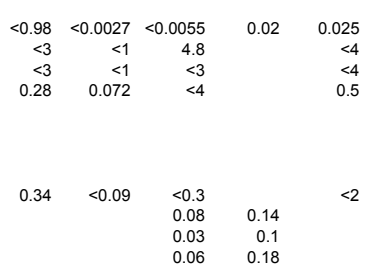




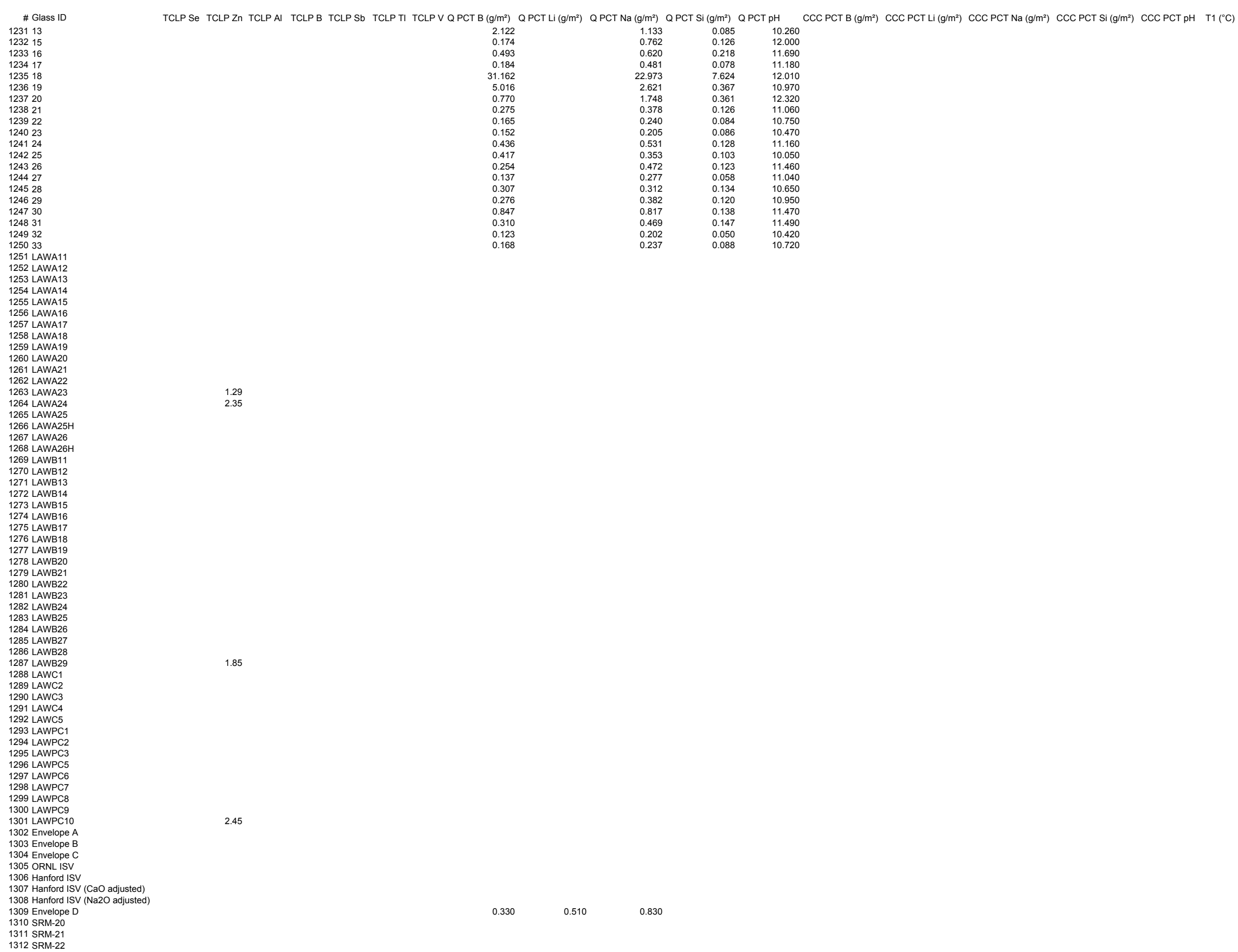




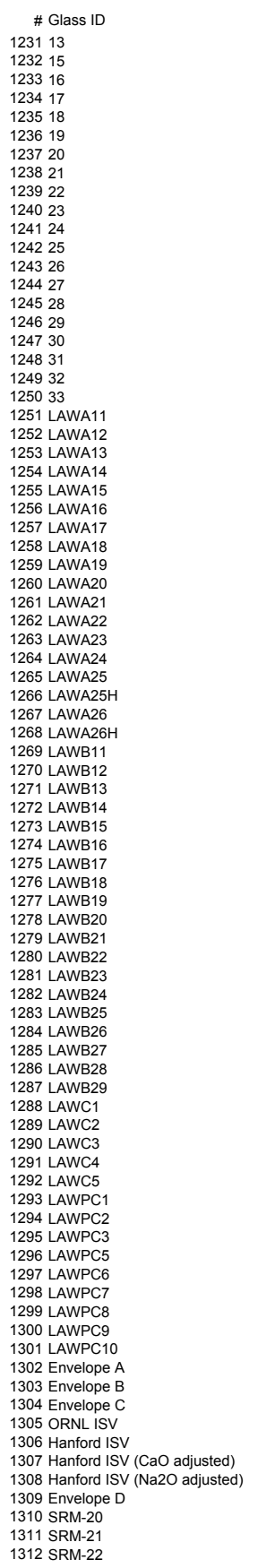




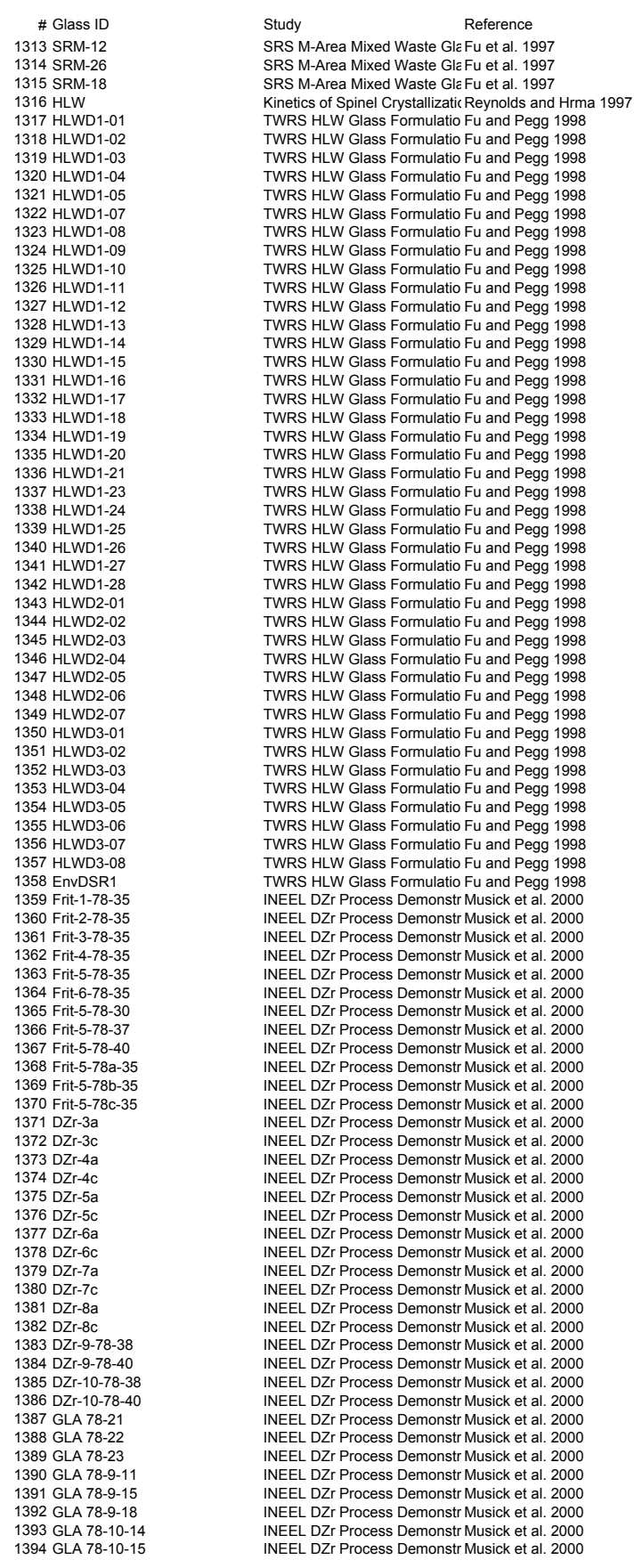

Al2O3-t B2O3-t CaO-t Fe2O3-t FeO-t $\begin{array}{llll}0.0900 & 0.1300 & 0.0050 & 0.0030 \\ 0.0900 & 0.1600 & 0.0060 & 0.0020\end{array}$ $\begin{array}{lllll}0.0900 & 0.1600 & 0.0060 & 0.0020 \\ 0.0900 & 0.1300 & 0.0060 & 0.0020\end{array}$ $\begin{array}{llll}0.0833 & 0.0500 & 0.0132 & 0.0705 \\ 0.0760 & 0.0842 & 0.0288 & 0.1191\end{array}$ $\begin{array}{llllll}0.0753 & 0.1345 & 0.0285 & 0.1181\end{array}$ $\begin{array}{llll}0.0753 & 0.0835 & 0.0796 & 0.1181\end{array}$ $\begin{array}{lllll}0.0753 & 0.0835 & 0.0285 & 0.11181 \\ 0.0328 & 0.0679 & 0.0000 & 0.1897\end{array}$ $\begin{array}{lllll}0.0320 & 0.0969 & 0.0000 & 0.1855\end{array}$ $\begin{array}{llll}0.0320 & 0.0485 & 0.0000 & 0.1855 \\ 0.0301 & 0.0921 & 0.0000 & 0.1756\end{array}$ $\begin{array}{llll}0.0301 & 0.0921 & 0.0000 & 0.1756 \\ 0.1177 & 0.0607 & 0.0163 & 0.2626\end{array}$ $\begin{array}{llll}0.1332 & 0.0665 & 0.0052 & 0.2185\end{array}$ $\begin{array}{llll}0.0382 & 0.0668 & 0.0763 & 0.2289 \\ 0.0378 & 0.1606 & 0.0000 & 0.0756\end{array}$ $\begin{array}{llll}0.0378 & 0.1606 & 0.0000 & 0.0756 \\ 0.0661 & 0.1134 & 0.0756 & 0.2362 \\ 0.0378 & 0.1606 & 0.000 & 0.2362\end{array}$ $\begin{array}{llll}0.1606 & 0.0661 & 0.0189 & 0.0567 \\ 0.0378 & 0.1606 & 0.0000 & 0.2362 \\ 0.034 & 0.156 & 0.075 & 0.072\end{array}$ $\begin{array}{llll}0.0378 & 0.1606 & 0.0000 & 0.2362 \\ 0.1604 & 0.1510 & 0.0000 & 0.0472\end{array}$ $\begin{array}{lllll}0.0378 & 0.0661 & 0.0756 & 0.2079\end{array}$ $\begin{array}{llll}0.0378 & 0.0661 & 0.0756 & 0.0472 \\ 0.0997 & 0.1526 & 0.0055 & 0.2212\end{array}$ $\begin{array}{lllll}0.0603 & 0.1549 & 0.0132 & 0.1431\end{array}$ $\begin{array}{lllll}0.0750 & 0.1117 & 0.0164 & 0.1779\end{array}$ $\begin{array}{llll}0.0692 & 0.1111 & 0.0152 & 0.1642 \\ 0.0607 & 0.1321 & 0.0100 & 0.1201\end{array}$ $\begin{array}{llll}0.0606 & 0.11107 & 0.0634 & 0.1437 \\ 0.0948 & 0.1036 & 0.0607 & 0.157\end{array}$ $\begin{array}{lllll}0.0846 & 0.0669 & 0.0231 & 0.1587\end{array}$ $\begin{array}{llll}0.0174 & 0.0599 & 0.0000 & 0.2414 \\ 0.1606 & 0.0661 & 0.0756 & 0.0472\end{array}$ $\begin{array}{lllll}0.0850 & 0.0661 & 0.0000 & 0.2362\end{array}$ $\begin{array}{lllll}0.0378 & 0.0661 & 0.0756 & 0.0850 \\ 0.0017 & 0.119 & 0.054 & 0.1059\end{array}$ $\begin{array}{llll}0.0917 & 0.1199 & 0.0564 & 0.1703 \\ 0.0446 & 0.1480 & 0.053 & 0.1059\end{array}$ $\begin{array}{lllll}0.0832 & 0.0707 & 0.0204 & 0.0970\end{array}$ $\begin{array}{llll}0.0466 & 0.0714 & 0.0004 & 0.1175 \\ 0.1622 & 0.0763 & 0.0763 & 0.1049\end{array}$ $\begin{array}{lllll}0.0378 & 0.1606 & 0.0756 & 0.0472\end{array}$ $\begin{array}{llll}0.1606 & 0.1134 & 0.0000 & 0.1890 \\ 0.1708 & 0.0887 & 0.0147 & 0.1058\end{array}$ $\begin{array}{llll}0.0900 & 0.0686 & 0.0558 & 0.2255 \\ 0.0693 & 0.0634 & 0.1259 & 0.0300\end{array}$ $\begin{array}{llll}0.0693 & 0.0634 & 0.1259 & 0.0300 \\ 0.0693 & 0.1234 & 0.1259 & 0.0300\end{array}$ $\begin{array}{lllll}0.0693 & 0.123 & 0.1259 & 0.0300 \\ 0.0693 & 0.1234 & 0.1259 & 0.0600\end{array}$ $\begin{array}{llll}0.0693 & 0.1234 & 0.1259 & 0.0025 \\ 0.0693 & 0.0534 & 0.1259 & 0.0298\end{array}$ $\begin{array}{llll}0.0693 & 0.0534 & 0.1259 & 0.0298 \\ 0.0693 & 0.0613 & 0.1259 & 0.0610\end{array}$ $\begin{array}{llll}0.0693 & 0.0613 & 0.1259 & 0.0610 \\ 0.0594 & 0.0555 & 0.1079 & 0.0315\end{array}$ $\begin{array}{llll}0.0733 & 0.0526 & 0.1331 & 0.0291 \\ 0.0792 & 0.0514 & 0.1439 & 0.0280\end{array}$ $\begin{array}{llll}0.0693 & 0.0534 & 0.1260 & 0.0298 \\ 0.0859 & 0.0509 & 0.1132 & 0.0300\end{array}$ $\begin{array}{llll}0.0881 & 0.0522 & 0.1160 & 0.0307 \\ 0.0933 & 0.0631 & 0.1229 & 0.0308\end{array}$ $\begin{array}{llll}0.0958 & 0.0648 & 0.1229 & 0.0308 \\ 0.0316 & 0.0316\end{array}$ $\begin{array}{llll}0.0958 & 0.0457 & 0.1262 & 0.0475\end{array}$ $\begin{array}{llll}0.0933 & 0.0445 & 0.1229 & 0.0277\end{array}$ $\begin{array}{llll}0.0958 & 0.0457 & 0.1262 & 0.0284 \\ 0.0933 & 0.0569 & 0.1229 & 0.0525\end{array}$ $\begin{array}{llll}0.0958 & 0.0584 & 0.1262 & 0.0539 \\ 0.0933 & 0.0631 & 0.1229 & 0.0029\end{array}$ $\begin{array}{llll}0.0933 & 0.0631 & 0.1229 & 0.0029 \\ 0.0958 & 0.0648 & 0.1262 & 0.0030\end{array}$ $\begin{array}{llll}0.0933 & 0.0569 & 0.1229 & 0.0215 \\ 0 & 0.0594 & 0.1262 & 0.0221\end{array}$ $\begin{array}{llll}0.0958 & 0.0584 & 0.1262 & 0.0221 \\ 0.0933 & 0.0724 & 0.1229 & 0.0029\end{array}$ $\begin{array}{llll}0.0933 & 0.0724 & 0.1229 & 0.0029 \\ 0.0982 & 0.0707 & 0.1294 & 0.0031\end{array}$ $\begin{array}{llll}0.0933 & 0.0724 & 0.1229 & 0.0031 \\ 0.0982 & 0.0707 & 0.1294 & 0.0271\end{array}$

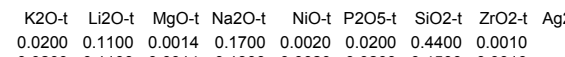

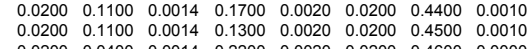
$\begin{array}{llllllll}0.0014 & 0.0186 & 0.0014 & 0.2200 & 0.00020 & 0.0200 & 0.4600 & 0.00000 \\ 0.00161 & 0.1621 & 0.0145 & 0.0302 & 0.3960 & 0.0454\end{array}$ $\begin{array}{lllllllll}0.0047 & 0.0009 & 0.0101 & 0.0788 & 0.0087 & 0.0116 & 0.3629 & 0.0582 & 0.0000 \\ 0.0046 & 0.0009 & 0.0100 & 0.1292 & 0.0086 & 0.0115 & 0.3086 & 0.0576 & 0.0000\end{array}$

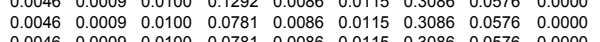
$\begin{array}{lllllllll}0.0046 & 0.0009 & 0.0100 & 0.0781 & 0.0086 & 0.0115 & 0.3086 & 0.0576 & 0.0000 \\ 0.0046 & 0.0315 & 0.0100 & 0.0781 & 0.0086 & 0.0115 & 0.3290 & 0.0576 & 0.0000\end{array}$ $\begin{array}{lllllllllll}0.0001 & 0.0291 & 0.0000 & 0.0709 & 0.0009 & 0.0165 & 0.3202 & 0.0000 & 0.0000\end{array}$

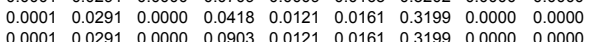
$\begin{array}{lllllllll}0.0001 & 0.0291 & 0.0000 & 0.0903 & 0.0121 & 0.0161 & 0.3199 & 0.0000 & 0.0000 \\ 0.0873 & 0.0000 & 0.0000 & 0.0396 & 0.0114 & 0.0152 & 0.3005 & 0.0000 & 0.0000\end{array}$

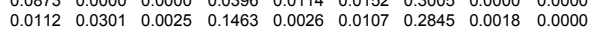
$\begin{array}{lllllllll}0.0053 & 0.0402 & 0.0014 & 0.1110 & 0.0079 & 0.0002 & 0.2731 & 0.0662 & 0.0000\end{array}$

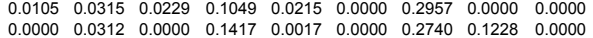
$\begin{array}{llllllllll}0.0000 & 0.0312 & 0.0000 & 0.0945 & 0.0017 & 0.0000 & 0.2362 & 0.1228 & 0.0000\end{array}$ $\begin{array}{lllllllll}0.0126 & 0.0312 & 0.0000 & 0.0945 & 0.0017 & 0.0000 & 0.2929 & 0.1228 & 0.0047 \\ 0.0000 & 0.0312 & 0.0000 & 0.0945 & 0.0017 & 0.0000 & 0.2835 & 0.0000 & 0.0000\end{array}$ $\begin{array}{lllllllll}0.0105 & 0.0311 & 0.0229 & 0.0944 & 0.0214 & 0.0000 & 0.2359 & 0.1227 & 0.0000 \\ 0.0126 & 0.0312 & 0.0000 & 0.1890 & 0.0017 & 0.0000 & 0.2362 & 0.0000 & 0.0047\end{array}$ $\begin{array}{lllllllll}0.0126 & 0.0312 & 0.0000 & 0.1890 & 0.0017 & 0.0000 & 0.2740 & 0.1228 & 0.0047 \\ 0.0001 & 0.0410 & 0.0022 & 0.1127 & 0.0021 & 0.0078 & 0.3071 & 0.0015 & 0.0035\end{array}$ $\begin{array}{lllllllll}0.0044 & 0.0403 & 0.0044 & 0.1360 & 0.0044 & 0.0088 & 0.32200 & 0.0009 & 0.00021 \\ 0.0055 & 0.0202 & 0.0055 & 0.1691 & 0.0055 & 0.0109 & 0.2654 & 0.0012 & 0.0022\end{array}$ $\begin{array}{llllllllll}0.0051 & 0.0200 & 0.0051 & 0.1561 & 0.0051 & 0.0101 & 0.3121 & 0.0000 & 0.0024 & 0.0005 \\ 0.0033 & 0.0375 & 0.0033 & 0.1027 & 0.0033 & 0.0066 & 0.4239 & 0.0139 & 0.0016 & 0.0003 \\ 0.0044 & 0.0200 & 0.0044 & 0.1366 & 0.0044 & 0.0088 & 0.3324 & 0.0009 & 0.0021 & 0.0004\end{array}$ $\begin{array}{lllllllll}0.0038 & 0.0308 & 0.0010 & 0.0795 & 0.0056 & 0.0002 & 0.3511 & 0.0471 & 0.0000\end{array}$

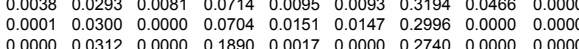

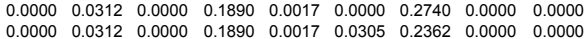
$\begin{array}{lllllllllll}0.0104 & 0.0312 & 0.0227 & 0.1890 & 0.0213 & 0.0000 & 0.2362 & 0.0000 & 0.0000 \\ 0.0000 & 0.000\end{array}$ $\begin{array}{lllllllllll}0.0022 & 0.0299 & 0.0222 & 0.1136 & 0.0116 & 0.0043 & 0.3380 & 0.0005 & 0.0010 & 0.0002 \\ 0.033 & 0.0297 & 0.0033 & 0.1007 & 0.0376 & 0.0065 & 0.3648 & 0.0007 & 0.0016 & 0.0003\end{array}$ $\begin{array}{llllllllll}0.0210 & 0.0006 & 0.0070 & 0.1947 & 0.0060 & 0.0188 & 0.3018 & 0.0400 & 0.0000 \\ 0.0000\end{array}$ $\begin{array}{lllllllll}0.0153 & 0.0000 & 0.0000 & 0.1584 & 0.0075 & 0.0195 & 0.4004 & 0.0001 & 0.0000 \\ 0.0000 & 0.0315 & 0.0000 & 0.0954 & 0.0017 & 0.0308 & 0.2957 & 0.0000 & 0.0000\end{array}$ $\begin{array}{lllllllll}0.0000 & 0.0315 & 0.0000 & 0.0954 & 0.0017 & 0.0308 & 0.2957 & 0.0000 & 0.0000 \\ 0.0126 & 0.0312 & 0.0000 & 0.0945 & 0.0017 & 0.0000 & 0.2362 & 0.0000 & 0.0047\end{array}$

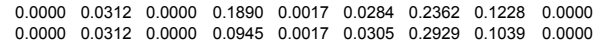
$\begin{array}{lllllllll}0.0000 & 0.0312 & 0.0000 & 0.1890 & 0.0017 & 0.0000 & 0.2929 & 0.0000 & 0.0000\end{array}$

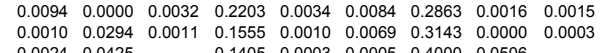

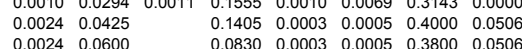
$\begin{array}{llllllll}0.0024 & 0.0600 & & 0.0830 & 0.0003 & 0.0005 & 0.3800 & 0.0506 \\ 0.0024 & 0.0300 & & 0.0630 & 0.0003 & 0.0005 & 0.4000 & 0.0506\end{array}$ $\begin{array}{lllllll}0.0024 & 0.0600 & 0.06304 & 0.0003 & 0.0005 & 0.4000 & 0.0506 \\ 0.0024 & 0.0600 & 0.132 & 0.00003 & 0.0005 & 0.4000 & 0.0506 \\ 0.0024 & 0.0005 & 0.4200 & 0.00506\end{array}$ $\begin{array}{llllllll}0.0024 & 0.0600 & & 0.1132 & 0.0003 & 0.0005 & 0.4200 & 0.0506 \\ 0.0024 & 0.0325 & & 0.1317 & 0.0003 & 0.0005 & 0.3900 & 0.0506\end{array}$ $\begin{array}{llllllll}0.0024 & 0.0325 & & 0.1317 & 0.0003 & 0.0005 & 0.3900 & 0.0506 \\ 0.0021 & 0.0646 & & 0.1186 & 0.0002 & 0.0005 & 0.4524 & 0.0433\end{array}$ $\begin{array}{llllllll}0.0026 & 0.0581 & & 0.1110 & 0.0003 & 0.0006 & 0.4070 & 0.0535 \\ 0.0028 & 0.0554 & & 0.1077 & 0.0003 & 0.0006 & 0.3877 & 0.0578\end{array}$

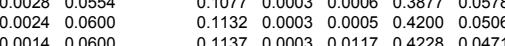
00150.0615 0.00150 .0602 $0.0016 \quad 0.062$ 0.00160 .0573 0.00150 .074 $\begin{array}{ll}0.0016 & 0.0764 \\ 0.0015 & 0.0558\end{array}$ $0.0016 \quad 0.0573$ 0.00150 .0605 0.00150 .055 $\begin{array}{ll}0.0016 & 0.0573 \\ 0.0015 & 0.0620 \\ 0.0015 & 0.000\end{array}$ $\begin{array}{ll}0.0016 & 0.0600 \\ 0.0015 & 0.0620\end{array}$ $\begin{array}{ll}0.0015 & 0.0620 \\ 0.0016 & 0.0600\end{array}$

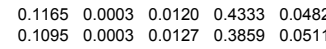

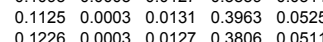
$\begin{array}{lllll}0.1259 & 0.0003 & 0.0131 & 0.3909 & 0.0525 \\ 0.1033 & 0.0003 & 0.0127 & 0.3999 & 0.0511\end{array}$

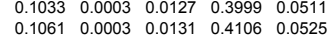

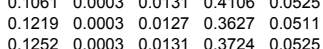
$\begin{array}{lllll}0.1252 & 0.0003 & 0.0131 & 0.3724 & 0.0525 \\ 0.1095 & 0.0003 & 0.0127 & 0.3859 & 0.0511\end{array}$

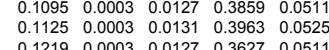

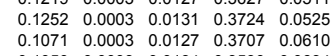

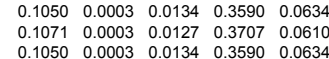

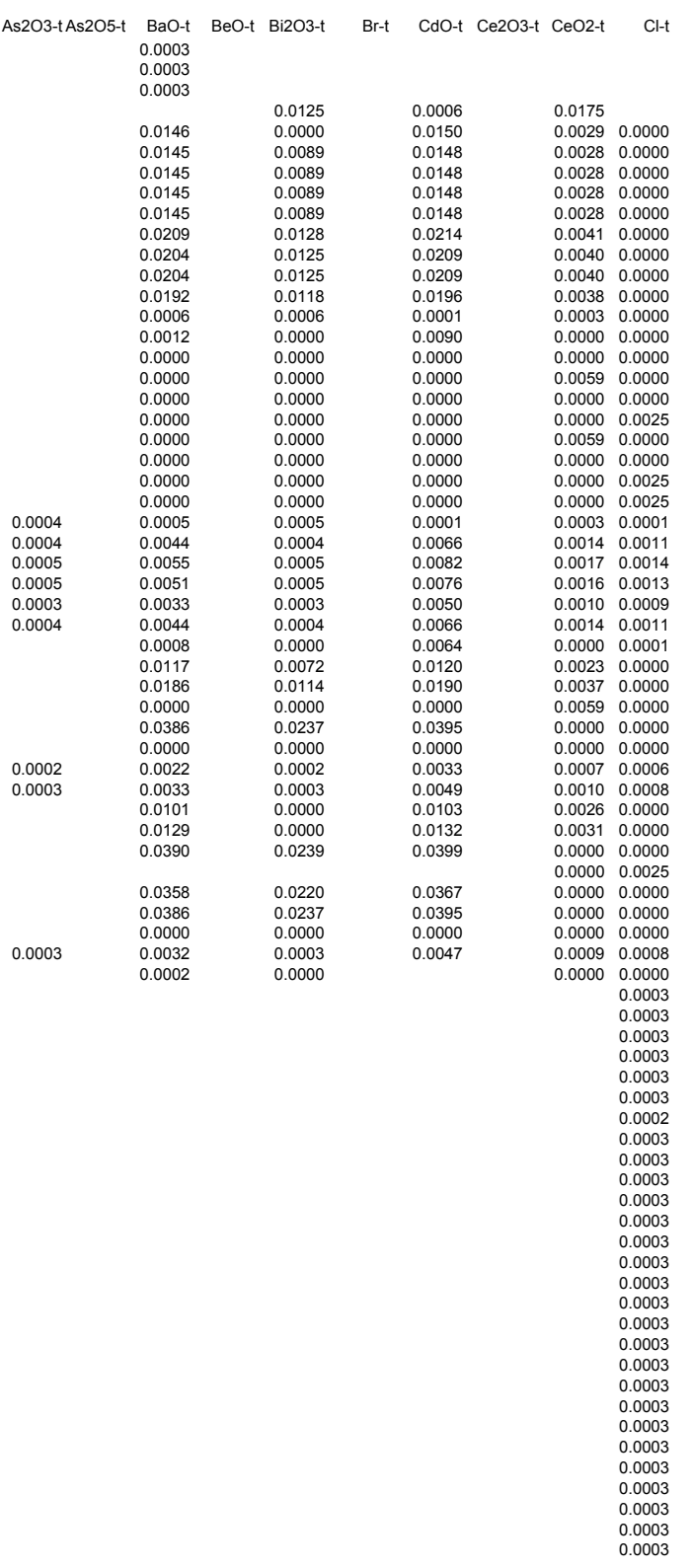


\# Glass ID 1313 SRM-12
1314 SRM-26

316 HLW

1316 HLW
1317 HLWD1-01
1318 HLWD1-02

1318 HLWD1-02
1319 HLWD1-03
1320 HLWD1-04

321 HLWD1-05
1322 HWWD1-07

1323 HLWD1-08

1324 HLWD1-09
1325 HLWD1-10

1327 HLWD1-12

328 HLWD1-13

1330 HLWD1-15

331 HLWD1-16

1332 HLWD1-17

1334 HLWD1-19
1335 HLWD1-20

1336 HLWDD-121
1337 HLW1-23

1338 HLWD1-24

1340 HLWD1-26

1341 HLWD1-27

1343 HLWD2-01
1344 HLWD2-02

1345 HLWD2-03

1346 HLWD2-04
347 HLWD2-05

1348 HLWD2-06
1349 HLWD2-07

1350 HLWD3-01

1351 HLWD3-02

353 HLWD3-04

1355 HLWD3-06

1356 HLWD3-07
1357 HLWD3-08

1358 EnvDSR1

1359 Frit-1-78-35
1360 Frit-2-78-35

362 Frit-4-78-35

1363 Frit-5-78-35

1364 Frt-6-78-35

1366 Frit-5-78-37

1368 Frit-5-78a-35

$-5-78 c-3$

1371 DZr-3a

373 DZr-4a

1374 DZr-4c
1375 DZr-5a

$376 \mathrm{DZZ}-5 \mathrm{c}$

1378 DZr-6c

1379 DZr-7a
1380 DZr-7C

1381 DZr-8a

1382 DZr-8c
1383 DZr-9-78-38

1384 DZr-9-78-40

1385 DZr-10-78-38
1386 DZr-10-78-40

1387 GLA 78-2

389 GLA 78-23
130 GLA 78-9-11

1391 GLA 78-9-1

1393 GLA 78-10-14
1394 GLA 78-10-15
CoO-t Co2O3-t Cr2O3-t Cs2O-t CuO-t Dy203-t Eu2O3-t F-t Ga203-t Gd2O3-t HiO2-t HgO-t 0.0002
0.0002
0.002

\begin{tabular}{|c|c|c|c|}
\hline & 0.0002 & & \\
\hline & 0.0029 & & \\
\hline 0.0017 & $\begin{array}{l}0.0029 \\
0.0028\end{array}$ & $\begin{array}{l}0.0018 \\
0.0018\end{array}$ & 0.0017 \\
\hline 0.0017 & 0.0028 & 0.0018 & 0.0017 \\
\hline 0.0017 & 0.0028 & 0.0018 & 0.0017 \\
\hline $\begin{array}{l}0.00017 \\
0.0024\end{array}$ & 0.0028 & 0.0018 & 0.0017 \\
\hline $\begin{array}{l}0.0024 \\
0.0023\end{array}$ & $\begin{array}{l}0.0041 \\
0.0040\end{array}$ & $\begin{array}{l}0.0026 \\
0.0025\end{array}$ & $\begin{array}{l}0.0025 \\
0.0024\end{array}$ \\
\hline 0.0023 & $\begin{array}{l}0.0040 \\
0.0040\end{array}$ & $\begin{array}{l}0.0025 \\
0.0025\end{array}$ & $\begin{array}{l}0.00242 \\
0.0024\end{array}$ \\
\hline 0 & & 0.0023 & $\begin{array}{l}0.0024 \\
0.0023\end{array}$ \\
\hline 0.0000 & 0.0014 & 0.0000 & \\
\hline 0.0000 & 0.0015 & 0.0001 & 0.0008 \\
\hline 0.0038 & 0.0065 & 0.0014 & 0.0000 \\
\hline & 0.0000 & 0.0014 & 0.0000 \\
\hline 0.00000 & 0.0000 & 0.0014 & 0.0000 \\
\hline 0.0000 & $\begin{array}{l}0.00000 \\
0.0000\end{array}$ & $\begin{array}{l}0.0063 \\
0.0014\end{array}$ & $\begin{array}{l}0.0048 \\
0.0000\end{array}$ \\
\hline 0.0038 & $\begin{array}{l}0.00000 \\
0.0065\end{array}$ & $\begin{array}{l}0.00144 \\
0.0014\end{array}$ & $\begin{array}{l}0.00000 \\
0.0000\end{array}$ \\
\hline & 0.0065 & $\begin{array}{l}0.00014 \\
0.0063\end{array}$ & $\begin{array}{l}0.00000 \\
0.0048\end{array}$ \\
\hline 0.0000 & 0.0000 & 0.0063 & $\begin{array}{l}0.00488^{\circ} \\
0.000\end{array}$ \\
\hline 0.0000 & 0.0012 & 0.0005 & 0.0002 \\
\hline 0.0013 & 0.0031 & 0.0015 & 0.0004 \\
\hline 0.0016 & 0.0038 & 0.0018 & 0.0005 \\
\hline 0.0015 & 0.0035 & 0.0017 & 0.0005 \\
\hline $\begin{array}{l}0.0010 \\
0.00113\end{array}$ & $\begin{array}{l}0.0023 \\
0.0031\end{array}$ & $\begin{array}{l}0.0011 \\
0.0015\end{array}$ & $\begin{array}{l}0.0003 \\
0.0004\end{array}$ \\
\hline 0.0000 & $\begin{array}{l}0.0031 \\
0.0011\end{array}$ & $\begin{array}{l}0.0015 \\
0.0001\end{array}$ & $\begin{array}{l}0.00004 \\
0.0006\end{array}$ \\
\hline 0.0013 & 0.0046 & $\begin{array}{l}0.0001 \\
0.0014\end{array}$ & $\begin{array}{l}0.00006 \\
0.0014\end{array}$ \\
\hline 0.0021 & 0.0073 & $\begin{array}{l}0.00274 \\
0.0023\end{array}$ & $\begin{array}{l}0.0004 \\
0.0022\end{array}$ \\
\hline 0.0000 & 0.0000 & 0.0014 & 0.0000 \\
\hline 0.0000 & 0.0000 & 0.0014 & 0.0000 \\
\hline 0.0037 & 0.0065 & 0.0014 & 0.0000 \\
\hline 0.0007 & 0.0056 & 0.0007 & 0.0002 \\
\hline 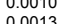 & & 0.0011 & 0.0003 \\
\hline 0.0015 & $\begin{array}{l}0.0020 \\
0.0025\end{array}$ & $\begin{array}{l}0.00013 \\
0.0016\end{array}$ & $\begin{array}{l}0.0012 \\
0.0015\end{array}$ \\
\hline 0.0000 & 0.0000 & $\begin{array}{l}0.0016 \\
0.0014\end{array}$ & $\begin{array}{l}0.001515 \\
0.0000\end{array}$ \\
\hline 0.0000 & 0.0000 & 0.0063 & 0.0048 \\
\hline 0.0000 & 0.0000 & 0.0014 & 0.0000 \\
\hline 0.0000 & 0.0000 & 0.0014 & 0.0000 \\
\hline 0.0000 & 0.0000 & 0.0014 & 0.0000 \\
\hline & 0.0077 & 0.0010 & 0.0003 \\
\hline 0.0000 & 0.0004 & & 0.0000 \\
\hline & $\begin{array}{l}0.0014 \\
0.0014\end{array}$ & & \\
\hline & 0.0014 & & \\
\hline & 0.0014 & & \\
\hline & $\begin{array}{l}0.0014 \\
0.0014\end{array}$ & & \\
\hline & 0.0012 & & \\
\hline & 0.0015 & & \\
\hline & 0.0016 & & \\
\hline & 0.0014 & & \\
\hline & 0.0013 & & \\
\hline & 0.0014 & & \\
\hline & 0.0014 & & \\
\hline & 0.0014 & & \\
\hline & 0.0015 & & \\
\hline & 0.0014 & & \\
\hline & $\begin{array}{l}0.0015 \\
0.0014\end{array}$ & & \\
\hline & 0.0015 & & \\
\hline & 0.0014 & & \\
\hline & 0.0015 & & \\
\hline & $\begin{array}{l}0.0014 \\
0.0015\end{array}$ & & \\
\hline & 0.0014 & & \\
\hline & 0.0015 & & \\
\hline & $\begin{array}{l}0.0014 \\
0.0015\end{array}$ & & \\
\hline
\end{tabular}

0.0036

0.008
0.0080
0.0080

.0152
.0152
0.0152

0.0152
0.0152

0.0014

0.0000
0.0000
0.0000

0.0280

0.0000

0.028

0.0000
0.0009
0.0011

0.0010

0.0007
0.0009
0.0010

0.0082

0.013

0.0000
0.0000
0.0000

0.0004

0.007

0.0091

0.0000

0.0000

0.0000

0.0599

0.0599
0.0599
0.0599

${ }_{0.0599}^{0.0599}$

0.0684

0.0599

0.0510

0.0510
0.0255
0.0255

0.0255

0.0255
0.0510
0.0255
0.0510
0.055

0.0510
0.0255
0.0510
0.0537

0.0537
0.0510
0.0537
I-t La203-t MnO2-t MnO-t MoO-t MoO2-t MoO3-t Nb205-t Nd203-t Np02-t PbO-t PdO2-t PdO-t Pr203-t Pr6011-t Rb2O-t ReO-t 0.0006
0.0006
0.0006

0.0027

0.0095

0.00870 .0296

$0.0087 \quad 0.0296$

$\begin{array}{ll}0.0087 & 0.0296 \\ 0.0125 & 0.0426 \\ 0.0122 & 0.0415\end{array}$

$\begin{array}{lll}0.0122 & 0.0415 \\ 0.0122 & 0.0415\end{array}$

0.01150 .0391

$0.0061 \quad 0.0068$

$0.0000 \quad 0.0672$

$0.0000 \quad 0.0000$

$\begin{array}{ll}0.0000 & 0.0000 \\ 0.0179 & 0.0000 \\ 0.0000 & 0.0672\end{array}$

$0.0000 \quad 0.0672$

$\begin{array}{ll}0.0000 & 0.0000 \\ 0.0000 & 0.0000\end{array}$

0.00010 .0048

$\begin{array}{ll}0.0018 & 0.0044 \\ 0.0022 & 0.0055\end{array}$

$\begin{array}{ll}0.0020 & 0.0051 \\ 0.0013 & 0.0033\end{array}$

$0.0018 \quad 0.0044$

$\begin{array}{lll}0.00070 & 0.0240 \\ 0 & 0.0212 & 0.030\end{array}$

$\begin{array}{ll}0.0112 & 0.0379 \\ 0.0179 & 0.0000\end{array}$

0.00000 .0000

$\begin{array}{lll}0.00009 & 0.0666 \\ 0.00022 & 0.0003\end{array}$

$0.0013 \quad 0.0033$

$\begin{array}{ll}0.0061 & 0.0210 \\ 0.0077 & 0.0265\end{array}$

$\begin{array}{ll}0.0000 & 0.0000 \\ 0.0000 & 0.0000\end{array}$

$0.0000 \quad 0.0000$

$0.0000 \quad 0.0000$

$\begin{array}{ll}0.0000 & 0.0000 \\ 0.0013 & 0.0066 \\ 0.0000 & 0.0039\end{array}$

0.0005
0.0005

0.0022
0.0022
0.0022

0.0005

0.0560
0.0058
0.0057
0.0057
0.0057
0.0057
0.0083
0.0081
0.0081
0.0076
0.0003
0.0000
0.0000
0.0118
0.0000
0.0000
0.0118
0.0000
0.0000
0.0000
0.0003
0.0000
0.0000
0.0000
0.00000
0.0000
0.0000
0.0046
0.0074
0.0118
0.0000
0.0000
0.0000
0.0000
0.0040
0.0051
0.00000
0.0000
0.0000
0.0000
0.0000
0.0000
0.0000

0.023
0.0034
0.0034

$\begin{array}{ll}0.0000 & 0.0000 \\ 0.0000 & 0.0000\end{array}$

$\begin{array}{ll}0.0000 & 0.0000 \\ 0.0000 & 0.0000 \\ 0\end{array}$

$\begin{array}{ll}0.00000 & 0.00000 \\ 0.0000 & 0.0000\end{array}$

$\begin{array}{lll}0.00000 & 0.00000 \\ 0.0000 & 0.0000\end{array}$

$0.0000 \quad 0.0000$

$\begin{array}{ll}0.0000 & 0.0000 \\ 0.0000 & 0.0000\end{array}$

\begin{tabular}{ll}
0.0000 & 0.0026 \\
0.0000 & 0.0000 \\
\hline
\end{tabular}

$0.0000 \quad 0.0000$

$\begin{array}{ll}0.0000 & 0.0026 \\ 0 & 0.000\end{array}$

$\begin{array}{ll}0.0000 & 0.0000 \\ 0.0000 & 0.0000\end{array}$

$\begin{array}{ll}0.0000 & 0.0000 \\ 0.0007 & 0.0000\end{array}$

$\begin{array}{ll}8 & 0.0000 \\ 8 & 0.0000\end{array}$

$\begin{array}{ll}0.0005 & 0.0000 \\ 0.0007 & 0.0000\end{array}$

$\begin{array}{ll}0.0000 & 0.0000 \\ 0.0000 & 0.0000 \\ 0\end{array}$

$\begin{array}{ll}0.0000 & 0.0000 \\ 0.0000 & 0.0026\end{array}$

$\begin{array}{lll}0.0000 \quad 0.0000 & 0\end{array}$

$\begin{array}{ll}0.0000 & 0.0000 \\ 0.0003 & 0.0000\end{array}$

$0.0000 \quad 0.0000$

$0.0000 \quad 0.0000$

$\begin{array}{ll}0.0000 & 0.0000 \\ 0.0000 & 0.0000\end{array}$

$\begin{array}{ll}0.0000 & 0.0000 \\ 0.0000 & 0.0000 \\ 0.000 & 0.000\end{array}$

$\begin{array}{ll}0.0000 & 0.00000 \\ 0.00000 & 0.00000\end{array}$ 
\# Glass ID 1313 SRM-12
1314 SRM-26
1315 SRM-18

1316 HLW
1317 HLWD1-01
1318 HLWD1-02

1319 HLWD1-03
1320 HLWD1-04

321 HLWD1-05
1322 HWWD1-07

1323 HLWD1-08

1324 HLWD1-09
1325 HLWD1-10

1328 HLWD1-13

1330 HLWD1-15

1331 HLWD1-16

1332 HLWD1-17

1334 HLWD1-19
1335 HLWD1-20

1336 HLWDD-121
1337 HLWD1-23

1338 HLWD1-24

1340 HLWD1-26

1341 HLWD1-27

1343 HLWD2-01

345 HLWD2-03

1346 HLWD2-04
347 HLWD2-05

1348 HLWD2-06
1349 HLWD2-07

1350 HLWD3-01

1351 HLWD3-02
1352 HLWD3-03
1353 HLWD3-04

1354 HLWD3-05

1355 HLWD3-06
1356 HLWD3-07

357 HLWD3-08
358 EnvDSR1

1359 Frit-1-78-35

1360 Frit-2-78-35

362 Frit-4-78-35
1363 Frit-5-78-35

1364 Fritt-6-78-35

1366 Frit-5-78-37

1368 Frit-5-78a-35

1369 Frit-5-78b-35

1371 DZr-3a

372 DZr-3c

1374 DZr-4c
1375 DZr-5a

1376 DZr-5c

1378 DZr-6C

1379 DZr-7a

1381 DZZ-8a

1382 DZr-8c
1383 DZr-9-78-38

1385 DZZ-10-78-38

1386 DZr-10-78-40

1387 GLA 78-21

1389 GLA $87-23$
1390 GLA 78-9-11

1390 GLA 78-9-11

1393 GLA 78-10-14
1394 GLA 78-10-1

ReO2-t Re207-t Rh203-t RhO2-t RuO2-t Ru2O3-t Sb203-t Sb205-t SeO2-t Sm203-t SnO-t SnO2-t SO3-t SrO-t Tc207-t TeO2-t ThO2-t TiO2-t T12O-t T1203-t U308-t UO2-t U03-t V205-t wO3-t Y203-t ZnO-t Sum-t Al203-a 0.0001

0.0007

$\begin{array}{ll}0.9953 & 0.0900 \\ 0.9953 & 0.1000\end{array}$

$0.0000 \quad 0.0000 \quad 0.0000 \quad 0.0000$

$\begin{array}{ll}0.0022 & 0.0027 \\ 0.0047 & 0.0018 \\ 0.0046 & 0.0018\end{array}$

$\begin{array}{ll}0.0046 & 0.0018 \\ 0.0046 & 0.0018\end{array}$

$\begin{array}{ll}0.0046 & 0.0018 \\ 0.0067 & 0.0307\end{array}$

$0.0065 \quad 0.0306$

$\begin{array}{lll}0.0065 & 0.0306 \\ 0 & 0.0615 & 0.0305\end{array}$

$\begin{array}{lll}0.0005 & 0.0257 \\ 0.0000 & 0.0290\end{array}$

$\begin{array}{lll}0.0000 & 0.0182 \\ 0.0000 & 0.0180\end{array}$

$\begin{array}{lll}0.0000 & 0.0180 \\ 0.0000 & 0.0180\end{array}$

$\begin{array}{lll}0.0127 & 0.0228 \\ 0.0000 & 0.0180\end{array}$

$\begin{array}{ll}0.0000 & 0.0180 \\ 0.0127 & 0.026\end{array}$

$\begin{array}{ll}0.0127 & 0.0228 \\ 0.0004 & 0.0206\end{array}$

0.00440 .0275

$\begin{array}{lll}0.0051 & 0.0341 \\ 0.00533 & 0.0315 \\ 0.0034 & 0.0207\end{array}$

$\begin{array}{lll}0.0051 & 0.0315 \\ 0.0033 & 0.0207 \\ 0.044 & 0.0276\end{array}$

$\begin{array}{ll}0.0044 & 0.0276 \\ 0.0000 & 0.0240 \\ 0.0074 & 0.0313\end{array}$

$0.0047 \quad 0.0292$

$\begin{array}{ll}0.0074 & 0.0313 \\ 0.0000 & 0.0180\end{array}$

$\begin{array}{ll}0.0000 & 0.0180 \\ 0.0000 & 0.0180\end{array}$

$\begin{array}{lll}0.00057 & 0.0180 \\ 0.0095 & 0.0135 \\ 0.0049 & 0.0203\end{array}$

$\begin{array}{ll}0.0039 & 0.0249\end{array}$

$\begin{array}{ll}0.0047 & 0.0195 \\ 0.0000 & 0.0182\end{array}$

$0.0000 \quad 0.0180$

$\begin{array}{ll}0.0000 & 0.0180 \\ 0.0000 & 0.0180 \\ 0 & 0032\end{array}$

$\begin{array}{lll}0.0000 & 0.0180 \\ 0.0032 & 0.0227\end{array}$

$\begin{array}{lll}0.0032 & 0.0227 \\ 0.0000 & 0.0412\end{array}$

0.0124

0.0124
0.0124
0.0124

0.0142

$\begin{array}{ll}0.0105 & 0.0024 \\ 0.0107 & 0.0025\end{array}$

$\begin{array}{lll}0.0107 & 0.0025 \\ 0.0114 & 0.0026 \\ 0.0117 & 0.0027\end{array}$

$\begin{array}{lll}0.0117 & 0.0027 \\ 0.0114 & 0.0026 \\ 0.0117 & 0.0027\end{array}$

$\begin{array}{lll}0.0117 & 0.0027 \\ 0.0114 & 0.0026\end{array}$

$\begin{array}{lll}0.0117 & 0.0027 \\ 0.0114 & 0.0026 \\ 0.0117 & 0.0027\end{array}$

$0.0114 \quad 0.0026$

$\begin{array}{lll}0.0117 & 0.0027 \\ 0.0114 & 0.0026\end{array}$

$\begin{array}{ll}0.0114 & 0.0026 \\ 0.0120 & 0.0027\end{array}$

$\begin{array}{llllll} & 0.0011 & & & 1.0001 & \\ 0.0000 & 0.0000 & 0.0000 & 0.0000 & 1.0002 & 0.062 \\ 0.0000 & 0.0000 & 0.0000 & 0.0000 & 0.9998 & 0.0677\end{array}$ $\begin{array}{lll}0.0000 & 0.0000 & 0.00 \\ 0.0000 & 0.0000 & 0.00 \\ 0.0000 & 0.0000 & 0.0062\end{array}$

0.0000
0.0000
0.0000

0.04

$\begin{array}{lll}0.0000 & 0.00000 & 0.0062 \\ 0.0062\end{array}$

$\begin{array}{lll}0.0000 & 0.0000 & 0.0087\end{array}$

$\begin{array}{lll}0.0000 & 0.00000 & 0.0082 \\ 0 & 0.0000 & 0\end{array}$

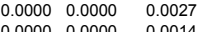

$\begin{array}{lll}0.0000 & 0.0000 & 0.0014 \\ .0000 & 0.0000 & 0.0000 \\ 0.0000 & 0.005 & 0.000\end{array}$

$\begin{array}{lll}0.0000 & 0.0035 & 0.0000 \\ 0 & 0.000 & 0.0000\end{array}$

$\begin{array}{lll}0.0000 & 0.0000 & 0.0170 \\ 0.0000 & 0.0035 & 0.0000\end{array}$

$\begin{array}{lll}0.0000 & 0.0035 & 0.0000 \\ 0.0000 & 0.0000 & 0.0000\end{array}$

$\begin{array}{lll}0.0000 & 0.00000 & 0.0170 \\ 0.0110\end{array}$

$\begin{array}{lll}0.0000 & 0.0000 & 0.0023 \\ 0.0013 & 0.0011 & 0.0014\end{array}$

$\begin{array}{lll}0.016 & 0.0013 & 0.0017 \\ 0.000 & 0.0000 & 0.0000\end{array}$

$\begin{array}{lll}0.0010 & 0.00008 & 0.00010 \\ 0.0013 & 0.0011 & 0.0014\end{array}$

$\begin{array}{rrr}0.0019 & 0.0000 & 0.0010 \\ 0.000 & 0.0000 & 0.0057\end{array}$

$\begin{array}{lll}0.0000 & 0.0000 & 0.000 \\ 0.0000 & 0.0005 & 0\end{array}$

$0.0000 \quad 0.0000 \quad 0.0000$

$\begin{array}{lll}0.0000 & 0.0000 & 0.0000 \\ 0.0007 & 0.0005 & 0.0007 \\ 0.0000 & 0.0000 & 0.0043\end{array}$

$\begin{array}{lll}0.0010 & 0.0008 & 0.0010\end{array}$

$\begin{array}{lll}0.0000 & 0.0000 & 0.0043 \\ 0.000 & 0.0015 & 0.0055\end{array}$

$\begin{array}{lll}0.0000 & 0.0015 & 0.0005 \\ 0.0000 & 0.0000 & 0.0000 \\ 0.0000 & 0.0000 & 0.000\end{array}$

$\begin{array}{llll}0.0000 & 0.0000 & 0.0000\end{array}$

$\begin{array}{lll}0.0000 & 0.0000 & 0.0000\end{array}$

$\begin{array}{lll}0.0009 & 0.0008 & 0.0010 \\ 0.0000 & 0.0000 & 0.0019\end{array}$

0.0300

0.0046
0.0055

0.0033
0.0044
0.0007

0.0000
0.0000

0.0022

0.0040

0.0032

0.0032
0.0442

0.0637
0.0621
0.0621
0.0584

0.0584

0.0087

0.0009

0.0000

0.0909

0.0000

0.0000
0.0022
0.0264

0.0264
0.0328
0.0000
0.0199

0.0000
0.0199
0.0265

0.0265
0.0062
0.0567

0.0357
0.0567

0.0909
0.0000

0.0000
0.0000
0.0130

0.0196

0.0308
0.0093
0.0000
0.0000

0.0000

0.0000

0.0189 $\begin{array}{llllllll}0.0000 & 0.0000 & 0.0000 & 0.0000 & 0.9997 & 0.0677 \\ 0.0673\end{array}$ $\begin{array}{llllll}0.0000 & 0.0000 & 0.0000 & 0.0000 & 0.9998 & 0.0704\end{array}$ $\begin{array}{lllll}0.0000 & 0.0000 & 0.0000 & 0.9955 & 0.0324 \\ 0.0000 & 0.0000 & 0.0000 & 0.9952 & 0.034 \\ 0.0000 & 0.000 & 0.000 & 0.955 & 0.336\end{array}$ $\begin{array}{lllll}0.0000 & 0.0000 & 0.0000 & 0.9953 & 0.0336\end{array}$ $\begin{array}{llllll}0.0000 & 0.0000 & 0.00000 & 0.0098 & 0.0309 & 0.0300\end{array}$ $\begin{array}{lllll}0.0000 & 0.0000 & 0.0000 & 0.9956 & \end{array}$ $\begin{array}{lllll}0.0020 & 0.0000 & 0.0000 & 0.9966 & 0.0391 \\ 0.0000 & 0.0000 & 0.0000 & 0.9974 & 0.0389\end{array}$ $\begin{array}{llll}0.0000 & 0.0000 & 0.0000 & 0.9971 \\ 0.0000 & 0.0000 & 0.0041 & 0.9906\end{array}$ $\begin{array}{lllll}0.0000 & 0.0000 & 0.00000 & 0.9975 & 0.0414 \\ 0.0020 & 0.0000 & 0.0000 & 0.9967 & 0.0139\end{array}$ $\begin{array}{llllll}0.0020 & 0.0000 & 0.0000 & 0.9967 & 0.139 \\ 0.0000 & 0.0000 & 0.0041 & 1.0000 & 0.0395\end{array}$ $\begin{array}{llllll}0.0000 & 0.0000 & 0.0041 & 0.9898 & 0.0395\end{array}$

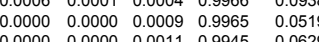
$\begin{array}{llllll}0.0000 & 0.0000 & 0.0000 & 0.9543 & 0.0561\end{array}$ $\begin{array}{lllll}0.0000 & 0.0000 & 0.0007 & 0.9965 & \end{array}$ $\begin{array}{llllll}0.0000 & 0.0000 & 0.0005 & 0.9937 & 0.0520\end{array}$ \begin{tabular}{llllll}
0.0000 & 0.0000 & 0.0000 & 0.9955 & 0.0785 \\
\hline
\end{tabular} $\begin{array}{lllll}0.0000 & 0.0000 & 0.0000 & 0.9953 & 0.0220 \\ 0.0000 & 0.0000 & 0.9974 & 0.1565\end{array}$ $\begin{array}{llllll}0.0000 & 0.0000 & 0.0000 & 0.9971 & 0.0749\end{array}$ $\begin{array}{lllll}0.0020 & 0.0000 & 0.0000 & 0.9966 & 0.0416 \\ 0.0000 & 0.0000 & 0.0004 & 1.0003 & 0.0770\end{array}$ $\begin{array}{llllll}0.0000 & 0.0000 & 0.0000 & 0.9963 & 0.0736\end{array}$ $\begin{array}{lllll}0.0000 & 0.0000 & 0.0000 & 0.9972 & 0.048\end{array}$ $\begin{array}{lllll}0.0000 & 0.0000 & 0.0000 & 0.9972 & 0.1407 \\ 0.0000 & 0.0000 & 0.0041 & 0.9946 & 0.1490\end{array}$

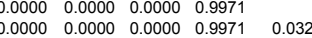

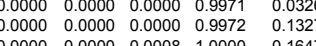
$\begin{array}{lllll}0.0000 & 0.0000 & 0.0008 & 1.0000 & 0.1647 \\ 0.0000 & 0.0000 & 0.0001 & 0.9999 & 0.0757\end{array}$

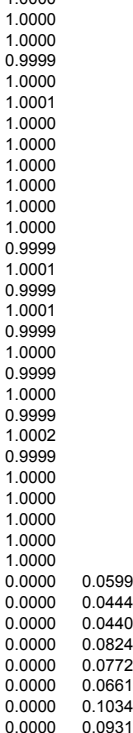


1378 DZr-6C

1380 DZr-7c

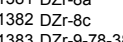

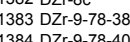

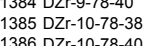

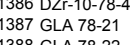

1389 GLA 78-23

1390 GLA 78-9.911

1391 GLA 78-9-15
1392 GLA 78-9-18

1393 GLA 78-10-14
1394 GLA 78-10-15
B2O3-a CaO-a $\mathrm{Fe} 2 \mathrm{O} 3-\mathrm{a} \quad \mathrm{FeO}-\mathrm{a}$ $\begin{array}{llll}0.1500 & 0.0050 & 0.00330\end{array}$

$\begin{array}{lll}0.1300 & 0.0060 & 0.0030\end{array}$

$\begin{array}{lll}0.0803 & 0.0267 & 0.1016 \\ 0.0786 & 0.0286 & 0.1048 \\ 0.1255 & 0.280 & 0.1058\end{array}$

$\begin{array}{llll}0.1235 & 0.0280 & 0.1036 \\ 0 & 0.0233 & 0.0747 & 0.138\end{array}$

$\begin{array}{lll}0.0883 & 0.0747 & 0.1038 \\ 0.0833 & 0.0269 & 0.1030\end{array}$

$\begin{array}{lll}0.0958 & 0.0007 & 0.1621\end{array}$

$\begin{array}{lll}0.0503 & 0.0007 & 0.1672 \\ 0.0908 & 0.0006 & 0.1501\end{array}$

$\begin{array}{lll}0.0615 & 0.016 & 0.2011 \\ 0.4472 & 0.0015 & 0.0609\end{array}$

$\begin{array}{lll}0.1650 & 0.0006 & 0.1812 \\ 0.1444 & 0.0013 & 0.0522\end{array}$

$\begin{array}{lll}0.1444 & 0.0013 & 0.0522 \\ 0.0607 & 0.0638 & 0.1911\end{array}$

$\begin{array}{lll}0.0675 & 0.0675 & 0.0466 \\ 0.1522 & 0.0066 & 0.1840 \\ 0.133 & 0.371 & 0.164\end{array}$

$\begin{array}{llll}0.1531 & 0.0139 & 0.1354\end{array}$

$\begin{array}{lll}0.113 & 0.0171 & 0.1695 \\ 0.1100 & 0.0157 & 0.1479\end{array}$

$\begin{array}{lll}0.1177 & 0.0612 & 0.1391\end{array}$

$\begin{array}{llll}0.0622 & 0.0229 & 0.1416\end{array}$

$\begin{array}{lll}0.0578 & 0.0014 & 0.2155 \\ 0.0720 & 0.0823 & 0.0515\end{array}$

$0.0624 \quad 0.0024 \quad 0.2076$

$\begin{array}{llll}0.0631 & 0.0631 & 0.0729 \\ 0.1214 & 0.0496 & 0.1499\end{array}$

$\begin{array}{lll}0.1452 & 0.0540 & 0.0903\end{array}$

$\begin{array}{lll}0.0672 & 0.0193 & 0.0864 \\ 0.0737 & 0.0004 & 0.1212 \\ 0.0763 & 0.0666 & 0.0917\end{array}$

$\begin{array}{lll}0.1466 & 0.0666 & 0.0510 \\ 0.1152 & 0.0008 & 0.1756\end{array}$

$\begin{array}{lll}0.0898 & 0.0155 & 0.0959 \\ 0.0687 & 0.0529 & 0.2046\end{array}$

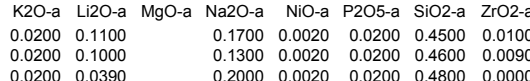

$\begin{array}{llllllll}0.0052 & 0.0017 & 0.0100 & 0.0637 & 0.0077 & 0.0330 & 0.3374 & 0.0310\end{array}$ $\begin{array}{lllllllll}0.0047 & 0.0015 & 0.0100 & 0.0674 & 0.0042 & 0.0100 & 0.2920 & 0.0294\end{array}$ $\begin{array}{lllllllll}0.0047 & 0.0018 & 0.0109 & 0.0699 & 0.0075 & 0.0125 & 0.3092 & 0.0451\end{array}$

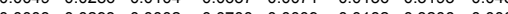
$\begin{array}{lllllllll}0.0005 & 0.0280 & 0.0002 & 0.0417 & 0.0107 & 0.0184 & 0.3229 & 0.0002\end{array}$ $\begin{array}{llllllll}0.0004 & 0.0302 & 0.0002 & 0.0816 & 0.0110 & 0.0185 & 0.3186 & 0.0001 \\ 0.0909 & 0.0005 & 0.0001 & 0.0397 & 0.0090 & 0.0166 & 0.2976 & 0.0001\end{array}$

$\begin{array}{llllllll}0.0099 & 0.0290 & 0.0201 & 0.0799 & 0.0195 & 0.0007 & 0.3274 & 0.0003 \\ 0.0006 & 0.0299 & 0.0006 & 0.1061 & 0.0019 & 0.0010 & 0.2968 & 0.1013\end{array}$

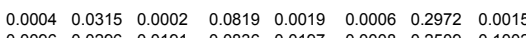
$\begin{array}{lllllllll}0.0096 & 0.0296 & 0.0191 & 0.0836 & 0.019 & 0.00000 & 0.2509 & 0.1002\end{array}$ $\begin{array}{lllllllll}0.0126 & 0.0338 & 0.0006 & 0.1583 & 0.0017 & 0.0013 & 0.2994 & 0.0735 & 0.0026 \\ 0.0007 & 0.0388 & 0.0023 & 0.1009 & 0.0019 & 0.0084 & 0.3204 & 0.0016 & 0.0032\end{array}$ $\begin{array}{lllllllllll}0.0007 & 0.0388 & 0.0023 & 0.1009 & 0.0019 & 0.0084 & 0.3204 & 0.0016 & 0.0032\end{array}$ $\begin{array}{lllllllll}0.0054 & 0.004 & 0.0048 & 0.1297 & 0.0044 & 0.0100 & 0.3375 & 0.0015 & 0.002 \\ 0.0063 & 0.0208 & 0.0058 & 0.1574 & 0.0054 & 0.0126 & 0.2802 & 0.0014 & 0.0025\end{array}$

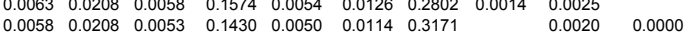

$\begin{array}{lllllllll}0.0050 & 0.0213 & 0.0049 & 0.1260 & 0.0045 & 0.0099 & 0.3504 & 0.0012 & 0.0021\end{array}$ $\begin{array}{llllllll}0.0038 & 0.0292 & 0.0082 & 0.0664 & 0.0078 & 0.0111 & 0.3172 & 0.0421 \\ 0.0007 & 0.0271 & 0.0003 & 0.0605 & 0.0129 & 0.0160 & 0.2954 & 0.0007\end{array}$

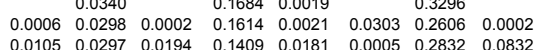

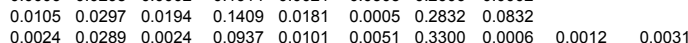

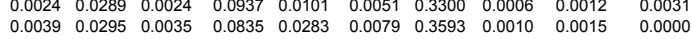
$\begin{array}{llllllllll}0.0175 & 0.0013 & 0.0074 & 0.1648 & 0.0055 & 0.0202 & 0.3078 & 0.0323\end{array}$ $\begin{array}{llllllll}0.0158 & & & 0.1349 & 0.0078 & 0.0201 & 0.4130 & 0.0001 \\ 0.0010 & 0.0325 & 0.0006 & 0.0810 & 0.0016 & 0.0279 & 0.3010 & 0.0006\end{array}$

\section{$\begin{array}{llllllll}0.0005 & 0.0221 & 0.0006 & 0.0822 & 0.0029 & 0.0299 & 0.3038 & 0.0868 \\ 0.0005 & 0.0312 & 0.00002 & 0.1617 & 0.0016 & 0.00055 & 0.3040 & 0.0004\end{array}$}

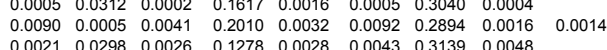

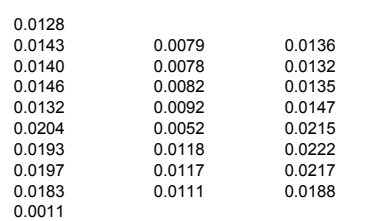

0.0001

0.0002

0.0001
0.0001
0.0004

0.0004
0.0007
0.0051
0.0004

0.0051
0.0064
0.0061

0.0054

0.0011
0.0111
0.0166

0.0166

0.0336

0.0001
0.0025
0.0039
0.0099
0.0133

0.0099
0.0133
0.0348

0.034

0.0341
0.0002
0.0034

0.0034

0.0007

0.0008
0.0008
0.0009

0.0008

0.0003
0.0075
0.0140

0.0220

0.0006
0.0008
0.0007

0.0237

0.0219

0.0006 (
0.0018
0.0017

0.0011

0.0018

0.0024
0.0026
0.0022

0.0034

0.0040

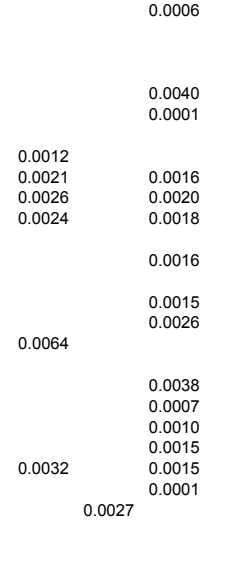

$\begin{array}{lll}0.0022 & 0.0022 & \\ 0.0022 & 0.0016 & 0.0016\end{array}$ $\begin{array}{llll}0.0021 & 0.0016 & 0.0016 \\ 0.0017 & 0.0018 & 0.0017 \\ 0.016 & 0.016\end{array}$ $\begin{array}{lll}0.0021 & 0.0016 & 0.016 \\ 0.0016 & 0.0016 & 0.0018 \\ 0.022 & 0.024 & 0.025\end{array}$ $\begin{array}{lll}0.0022 & 0.0023 & 0.0024 \\ 0.0030 & 0.024 & 0.0024\end{array}$ $\begin{array}{lll}0.0030 & 0.0224 & 0.0024 \\ 0.0029 & 0.0022 & 0.0023 \\ 0.0011 & & \end{array}$ $\begin{array}{ll}0.0077 & 0.0013 \\ 0.0002 & 0.0015\end{array}$

$\begin{array}{ll}0.0001 & 0.0016 \\ 0.0068 & 0.0014 \\ 0.0014 & \end{array}$ 0.0068 $\begin{array}{lll}0.0001 & & 0.0048 \\ 0.0011 & 0.0001 & 0.0004\end{array}$

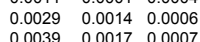
$\begin{array}{llll}0.0035 & 0.0016 & 0.0006\end{array}$

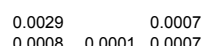
$\begin{array}{lll}0.0026 & 0.00014 & 0.0007 \\ 0.0026 & 0.0014 & 0.0014 \\ 0.0047 & 0.0020 & 0.0025\end{array}$ $\begin{array}{ll}0.0015 \\ 0.0016 & 0.0013\end{array}$ $\begin{array}{ll}0.0016 & 0.0013 \\ 0.0061 & \end{array}$

$\begin{array}{lll}0.00641 & 0.0009 & 0.0004 \\ 0.0049 & 0.0012 & 0.005\end{array}$ $\begin{array}{llll}0.0049 & 0.0012 & 0.0005 \\ 0.0017 & 0.0011 & 0.0017\end{array}$ $\begin{array}{llll}0.0017 & 0.0011 & 0.0017 & \\ 0.0026 & 0.0016 & 0.0016 & 0.0094 \\ 0.0001 & 0.0000 & & \end{array}$ $0.0066 \quad 0.0051$ $\begin{array}{ll}0.0024 & 0.0013 \\ 0.0001 & 0.0014 \\ 0.0027 & \end{array}$

$\begin{array}{lll}0.0001 & 0.0014 & \\ 0.0058 & 0.0009 & 0.0004 \\ 0.0027 & & \end{array}$

\begin{tabular}{|c|c|c|c|c|c|c|c|c|c|}
\hline & 0.1199 & 0.0233 & 0.0003 & 0.0633 & 0.1205 & .0002 & 0.0295 & 0.4075 & \\
\hline & & & 0.0003 & 0.0643 & 0.1267 & & 0.0273 & 0.4043 & \\
\hline & 0.1220 & 0.0237 & 0.0003 & 0.0627 & 0.1207 & 0.0001 & 0.0329 & 0.4064 & 0.0597 \\
\hline & 0.1149 & 0.0075 & 0.0011 & 0.0601 & 0.1122 & 0.0002 & 0.0312 & 0.3698 & 0.0565 \\
\hline & 11114 & 0.0033 & 0.0009 & 0.0569 & 0.1057 & 0.0003 & 0.0290 & 0.3627 & 0.0547 \\
\hline $\begin{array}{l}0.00777 \\
0.0808\end{array}$ & $\begin{array}{l}0.1269 \\
0.0695\end{array}$ & $\begin{array}{l}0.0032 \\
0.0170\end{array}$ & $\begin{array}{l}0.00000 \\
0.0032\end{array}$ & $\begin{array}{l}0.0558 \\
0.0597\end{array}$ & $\begin{array}{l}0.1077 \\
0.1176\end{array}$ & $\begin{array}{l}0.00000 \\
0.0001\end{array}$ & $\begin{array}{l}0.0315 \\
0.0382\end{array}$ & $\begin{array}{l}0.3679 \\
0.4047\end{array}$ & $\begin{array}{l}0.0570 \\
0.0677\end{array}$ \\
\hline & 0.0688 & 0.0175 & 0.0029 & 0.0550 & 0.1208 & 0.0002 & 0.0362 & 0.4110 & 0.06 \\
\hline
\end{tabular}

0.0003
0.0003
0.0003

0.0003
0.0004

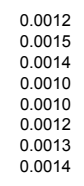

0.0545
0.0567
0.0630
0.0600
0.0649
0.0724
0.0292
0.0248 
1314 SRM-26

1317 HLWD1-01

319 HLWD103

320 HLWD1-04

322 HLWD1-07

1323 HLWD1-08

1324 HLWD1-09

327 HLWD1-12

1328 HLWD1-13

1330 HLWD1-14

331 HLWD1-16

1332 HLWD1-17

1334 HLWD1-19

1335 HLWD1-20

1336 HLWD1-21
1337 HLWD1-23

1338 HLWD1-24
1339 HLWD1-25

1340 HLWDD1-26
1341 HLWD1-27

1342 HWWD1-28

1343 HLWD2-01

1344 HLWD2-02
1345 HLWD2-03

346 HLWD2-04

1347 HLWD2-05

1348 HLWD2-06
1349 HLWD2-07

350 HLWD3-01

1351 HLWD3-02

1352 HLWD3-03

1354 HLWD3-05

1355 HLWD3-06
1356 HLWD3-07

1358 EnvDSR1

1359 Friti-178-35

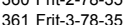

1362 Frit-478-35

1363 Frit:-578-35

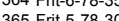

1366 Frit-5-78-37

1367 Frit:-578-40

1369 Frit-5-78b-35

371 DZr-3a

$372 \mathrm{DZr}-3 \mathrm{C}$

1374 DZr-4c

1375 DZr-5a

1377 DZZ-6a

1378 DZr-6C

1379 DZr-7a

1381 DZr-8a

1382 DZr-8c
1383 DZr-9-78-38
1384 DZr-9-78-40

1385 DZr-10-78-38

1387 GLA 78-21

389 GLA 78-23

390 GLA 78-9-11

(391 GLA 78-9-15

1393 GLA 78-10-14

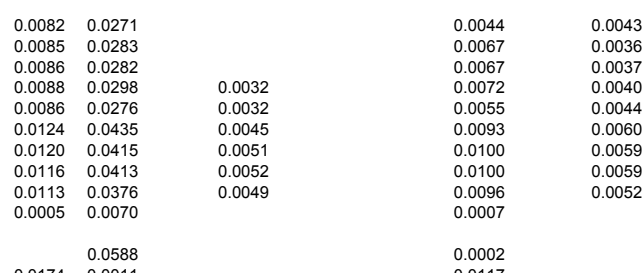

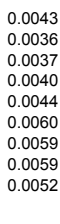

\begin{tabular}{l}
$0.0174 \quad 0.0011$ \\
\hline
\end{tabular}

0.0002
0.0010

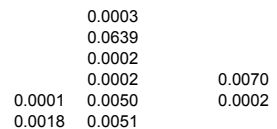

$0.0023 \quad 0.0063$

$0.0022 \quad 0.0058$

$\begin{array}{ll}0.0023 & 0.0053 \\ 0.0041 & 0.0051\end{array}$

$\begin{array}{lll}0.0067 & 0.0235 & 0.0019 \\ 0.0110 & 0.0346 & 0.0029\end{array}$

0.0002

$\begin{array}{ll}0.0009 & 0.0024 \\ 0.0011 & 0.0024\end{array}$

$0.0060 \quad 0.0204$

$\begin{array}{ll}0.0080 & 0.0273 \\ & 0.0004\end{array}$

0.0020

0.0059

$0.0001 \quad 0.0006$

0.0001

$\begin{array}{ll}0.0013 & 0.0064 \\ & 0.0076\end{array}$

0.0001

0.0004
0.0002
0.0002
0.0002
0.0005
0.0002
0.0049
0.0069
0.0128

0.0002
0.0041
0.0053



0.0001

0.0099

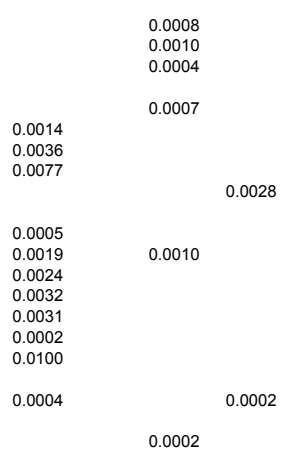

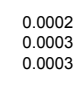

0.0006

0.0003

$0.0084 \quad 0.0060$

$\begin{array}{ll}0.0002 & 0.0007 \\ 0.0001 & 0.0005 \\ 0.0002 & 0.0005 \\ 0.0246 & 0.0009 \\ 0.0277 & 0.0007 \\ 0.0282 & 0.0004 \\ 0.0035 & 0.0010 \\ 0.0032 & 0.0009\end{array}$

0.0001
0.0000
0.0001
0.0000
0.0000
0.0001
0.0000
0.0000 
1337 HLWD1-23
1338 HLWD1-24
1339 HLWD1-25

1340 HLWD1-26

342 HLWD1-28

1343 HLWD2-01

345 HLWD2-03

1346 HLWD2-04
1347 HLWD2-05

1348 HLWD2-06
1349 HLWD2-07

350 HLWD3-01

1351 HLWD3-02

1352 HLWD $3-03$

1354 HLWD3-05
1355 HLWD3-06

1356 HLWD3-07

358 EnvDSR1

1359 Frit-1-78-35

Frit-4-78-35

1363 Frit-5-78-35

1364 Frt-6-78-35

1366 Frit-5-78-37

1367 Frit-5-78-40

1369 Frit-5-78b-35

$371 \mathrm{DZr}-3 \mathrm{a}$

$372 \mathrm{DZZ}-3 \mathrm{C}$

1374 DZr-4c
1375 DZr-5a

DZr-5c

1378 DZr-6c

1379 DZr-7a

1381 DZr-8a

1382 DZr-8c

385 DZr-10-78-38

1386 DZr-10-78-40

1387 GLA 78-21

389 GLA 78-23

1390 GLA 78-9-11

391 GLA 78-9-15

1393 GLA 78-10-14

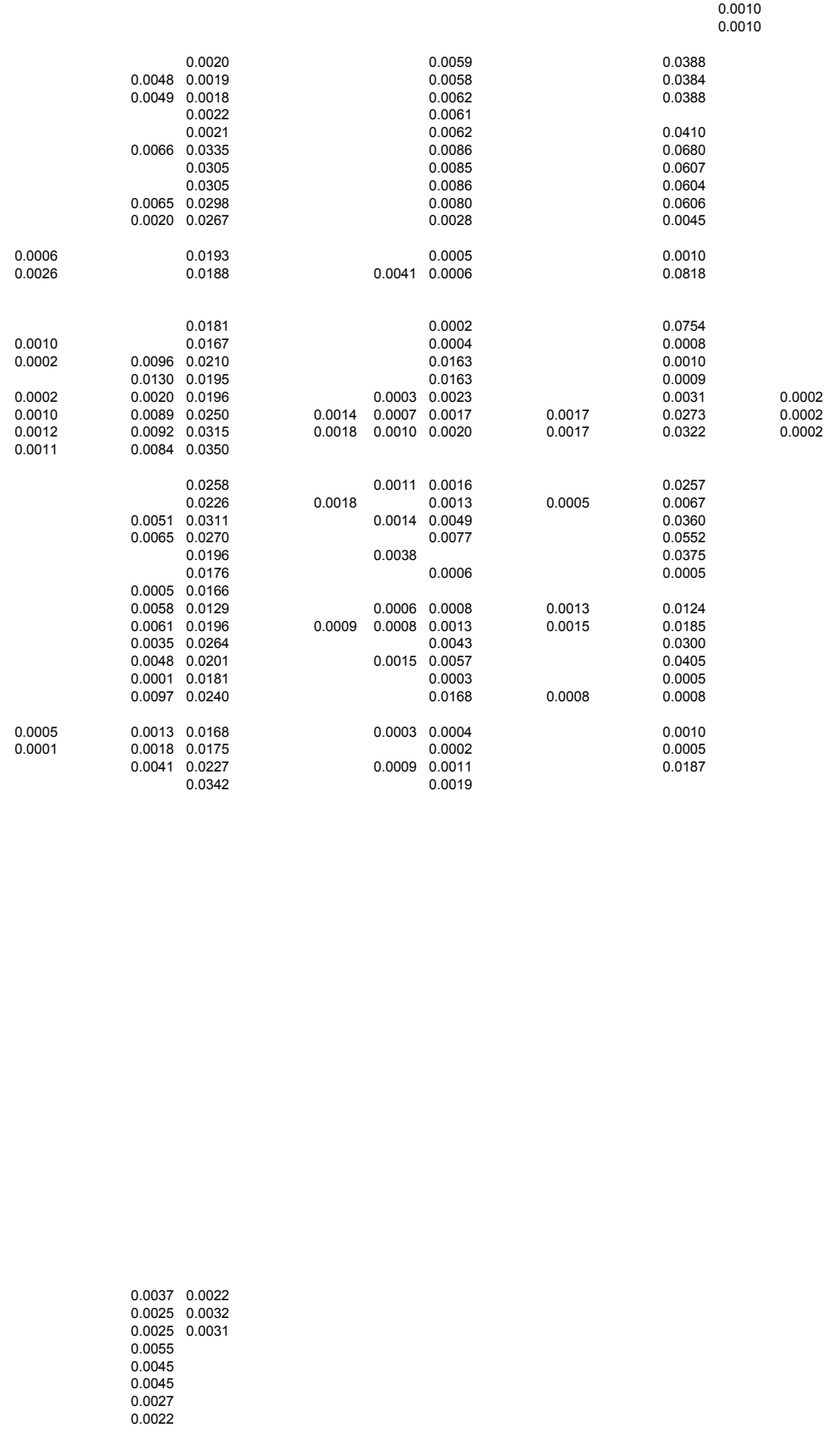

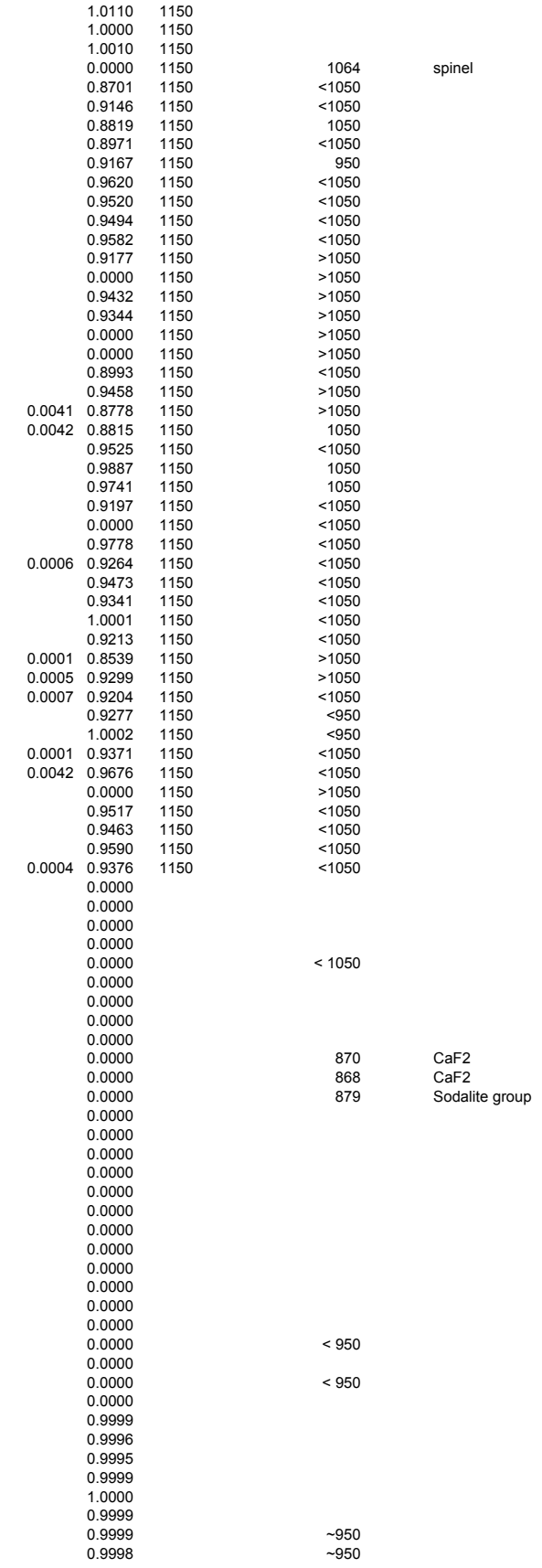


1314 SRM-26
1315 SRM-18

$1316 \mathrm{HLW}$

1317 HLWD1-01

1319 HLWD1-03
1320 HLWD1-04

1321 HLWD1-05

1323 HLWD1-08

1324 HLWD1 109
1325 HLWD1-10
1320 HLWD 111

1327 HLWD1-12

1328 HLWD1-13
1329 HLWD1-14

330 HLWD1-15
331 HLWD1-16

1332 HLWD1-17

1334 HLWD1-19
1335 HLWD1-20

1336 HLWD1-21

1337 HLWD1-23
1338 HLWD1-24

1339 HLWD1-25
1340 HLWD1-26

341 HLWD1-27
342 HLWD1-28

1343 HLWD2-01
1344 HLWD2-02

1346 HLWD2-04

1347 HLWD2-05

1348 HLWD2-06

1350 HLWD3-01

1351 HLWD3-02

1352 HLWD3-03
1353 HLWD3-04

1354 HLWD3-05
1355 HLWD3-06

1356 HLWD3-07
1357 HLWD3-08

1358 EnvDSR1

1359 Frit-1-78-35
1360 Frit-2-78-35
1301 F Fit- $78-35$

Frit-3-78-35

363 Frit-5-78-35

1364 Frit-6-78-35

1366 Frit-5-78-37

1367 Frit-5-78-40

1369 Frit-5-78b-35

1371 DZr-3a

1373 DZZ-A

$1374 \mathrm{DZr}-4 \mathrm{c}$

D DZr-5c

1378 DZr-6C

1379 DZr-7a

1381 DZr-8a

$1382 \mathrm{DZZ-8 \textrm {C }}$
$1383 \mathrm{DZ}-\mathrm{-}-78-38$
$1384 \mathrm{DZ}-9-78-40$

1385 DZr-10-78-38

1386 DZr-10-78-40

1387 GLA 78-21
1388 GLA 78-22

1389 GLA 78-23

1390 GLA 78-9-11

1391 GLA 78-9-1

1393 GLA 78-10-14
1394 GLA $78-10-15$ clear dark brown glass
clear dark brown glass

clear dark brown glass

clear dark brown glass
clear dark brown glass

glassy brown

glassy brown

glassy brown
glassy brown

glassy brown
opaque brown

opaque brown

top and bottom but crystallized bulk

heavily crystallized crystallized

clear top and bottom with middle section crystallized

brown glass

aque green $W / w$ chite on top of brown glass

opaque brown glass w/ brown spots on bottom
ons

glassy brown but crucible is hetrogeneous

glassy brown but crucible is heterogeneous -

brown glass with opaque brown \& yellow stre
glassy brown but crucible is heterogeneous

glassy brown opaue dark brown glass

glassy brown
clear light brown

glascy brown

brown glass w/ blue metallic coating

clear brown glass

glassy brown

glassy brown

opaque brown $w /$ white spots on

opaque yellowish green glass $\mathrm{w} / \mathrm{white} \mathrm{spo}$

clear light brown

glassy brown

glassy brown

Homogeneous

Some crystals

Homogeneous

Some crystals

Homogeneris

thognews

-5 vol\% crystallinity

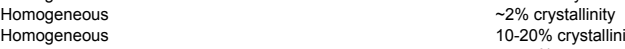

$\begin{array}{ll}\text { Homogeneous } & 10-20 \% \text { crystall } \\ \text { Homogeneous } & 10-20 \% \text { crrstallinity } \\ \text { Horystallinity }\end{array}$

Homogeneous $\quad 5-10 \%$ crystallinity

Homogeneous
Homogeneo

Homogeneous
Homogeneous

-

homogenous on top, crystallization on bottom, $-20 \%$ overall crystallization $-30 \%$ overall crystalization

some mottling, likely crystalliziz

lization on bottom, $\sim 30 \%$ overall crystallization

-1 vol\% (buk) crystalliting

Homogeneous
Homogeneous

Homogeneous
small $(-1 \mathrm{~mm})$ black particles $<0.5 \%$ crystallinity $<0.5 \%$ crystallinity
$r<1$ vol\% cuspidin

multiple phases and a transparer $\sim 1.3$ vol\% cuspidine and $\sim 3.1$ vol $-1-2$ vol\% dendritic crystals with distorted edges

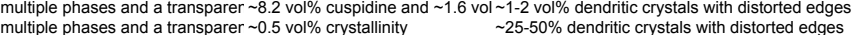

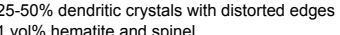

-1 vol\% hematite and spinel

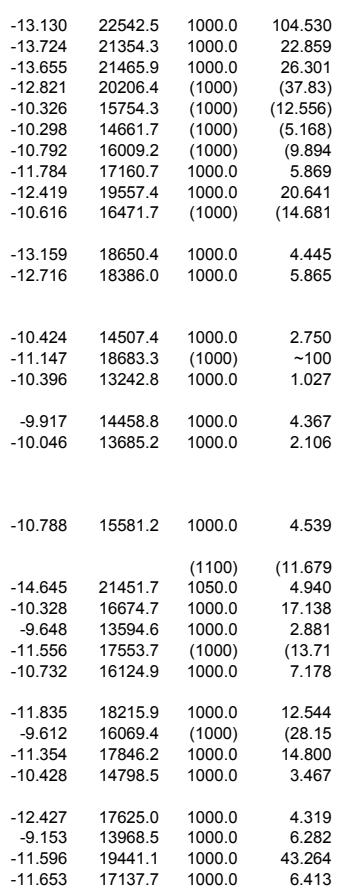

$\begin{array}{rrrr}-11.596 & 19441.1 & 1000.0 & 43.264 \\ -11.653 & 17137.7 & 1000.0 & 6.413\end{array}$ 


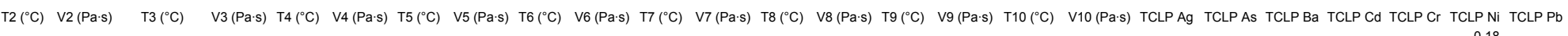
1313 SRM-12
1314 SRM-26
1315 SRM-18 $316 \mathrm{HLW}$ 1317 HLWD1-01 1319 HLWD1-03 1320 HLWD1-04 1321 HLWD1-05 1323 HLWD1-08 1324 HLWD1-09 1326 HLWD1-11 1327 HLWD1-12 1328 HLWD1-13

1330 HLWD1-14

1331 HLWD1-16

1332 HLWD1-17

1334 HLWD1-19

1335 HLWD1-20

1336 HLWD1-21

1338 HLWD1-24

1339 HLWD1-25

1341 HLWD1-27

1343 HLWD2-01

1344 HLWD2-02
1345 HLWD2-03

1346 HLWD2-04

347 HLWD2-05

1348 HLWD2-06

350 HLWD3-01

1351 HLWD3-02

1352 HLWD3-03

354 HLWD3-05

1356 HLWD3-07

357 HLWD3-08

1359 Frit-1-78-35

1360 Frit-2-78-35

362 Frit-4-78-35

1363 Frit-5-78-35

1364 Frit-6-78-35

366 Frit-5-78-37

1367 Fit-5-78-40

1369 Frit-5-78b-35

1371 DZr-3a

$372 \mathrm{DZZ}-3 \mathrm{C}$

$1374 \mathrm{DZZ}-4 \mathrm{C}$

$1375 \mathrm{DZZ}-5 \mathrm{a}$

377 DZr-6a

1378 DZr-6C

1379 DZr-7a

381 DZr-8a

1382 DZr-8C

1383 DZr-9-978-38
1384 DZr-9-98-40

385 DZr-10-78-38

386 DZr-10-78-40

1387 GLA 78-21

389 GLA 78-23

1390 GLA 78-9-11

1392 GLA 78-9-9-18

1393 GLA $78-10-14$

\begin{tabular}{|c|c|c|c|c|c|c|c|c|c|c|c|}
\hline $\begin{array}{l}1050 \\
1050\end{array}$ & 49.278 & 1100 & 25.5 & 1150.0 & 14.3 & 1200.0 & 8.5 & 1250.0 & 5.4 & 1300.0 & 3.5 \\
\hline $\begin{array}{l}1050 \\
1050\end{array}$ & $\begin{array}{l}11.066 \\
11\end{array}$ & 1100 & 5.9 & $\begin{array}{l}1150.0 \\
11500\end{array}$ & 3.4 & 1200.0 & 2.1 & 1250.0 & 1.4 & 1300.0 & 0.9 \\
\hline (1050) & $\begin{array}{r}12.9292 \\
-\end{array}$ & $\begin{array}{l}1100 \\
1100\end{array}$ & 6.9 & $\begin{array}{l}11550.0 \\
11500\end{array}$ & $\begin{array}{l}4.0 \\
3.8\end{array}$ & $\begin{aligned} 12000 \\
1200\end{aligned}$ & $\begin{array}{l}2.4 \\
2.3 \\
\end{array}$ & $\begin{array}{l}1250.0 \\
1250\end{array}$ & $\begin{array}{l}1.6 \\
1.5\end{array}$ & $\begin{array}{l}1300.0 \\
13000\end{array}$ & \\
\hline (1050) & $(5.922$ & 1100 & $\begin{array}{l}3.0 \\
3.3\end{array}$ & 115000 & 2.1 & 12000 & $\begin{array}{l}2.3 \\
1.4\end{array}$ & 12500 & $\begin{array}{l}1.5 \\
1.0\end{array}$ & 1300.0 & 0.8 \\
\hline (1050) & $(2607$ & 1100 & 15 & 11500 & 10 & 12000 & 07 & 12500 & 0.5 & 1000 & 0.4 \\
\hline (1050) & (4.554 & 1100 & 25 & 1150.0 & 1.5 & 12000 & 10 & 12500 & 0.7 & 13000 & 0.6 \\
\hline 105 & 3.237 & 1100 & 1.9 & 1150.0 & 1.2 & 12000 & 0.8 & 1250.0 & 0.6 & 1300.0 & 0.4 \\
\hline 1050 & 10.475 & 1100 & 5.9 & 1150.0 & 3.5 & 1200.0 & 2.3 & 1250.0 & & 1300.0 & 1.1 \\
\hline (1050) & $(7.294$ & 1100 & 4.1 & 1150.0 & 2.6 & 1200.0 & 1.7 & 1250.0 & 1.2 & 1300.0 & 0.9 \\
\hline 1050 & 2.555 & 1100 & 1.5 & 1150.0 & 0.9 & 1200.0 & 0.6 & 1250.0 & 0.4 & 1300.0 & 0.3 \\
\hline 1050 & 3.236 & 1100 & 1.9 & 1150.0 & 1.2 & 1200.0 & 0.8 & 1250.0 & 0.5 & 1300.0 & 0.4 \\
\hline 1050 & 1.708 & 1100 & 1.1 & 1150.0 & 0.8 & 1200.0 & 0.6 & 1250.0 & 0.4 & 1300.0 & 0.3 \\
\hline $\begin{array}{r}(1050) \\
1050\end{array}$ & -40 & (1100) & $\sim 20$ & (1150) & $\sim 10$ & 1200.0 & 4.7 & $\begin{array}{l}1250.0 \\
0\end{array}$ & 3.0 & 1300.0 & $\begin{array}{l}2.1 \\
0.1\end{array}$ \\
\hline & 2776 & 1110 & 18 & & 12 & 1200 & 0 & 1250 & 07 & & 0.5 \\
\hline 1050 & 1.338 & 1100 & 0.9 & 1150.0 & 0.6 & 1200.0 & 0.5 & 1250.0 & 0.3 & 1300.0 & 0.3 \\
\hline 1050 & 2.660 & 1100 & 1.7 & 1150.0 & 1.1 & 1200.0 & 0.8 & 12500 & 0.6 & 13000 & 04 \\
\hline$(1050)$ & $(4.941)$ & $(1200)$ & $(3.71)$ & $(1250)$ & $(3.214$ & & & & & & \\
\hline 1100 & 2.615 & 1150 & 1.5 & 1200.0 & & 1250.0 & 0.6 & & & & \\
\hline 1050 & 9.612 & 1100 & 5.9 & 1150.0 & 3.8 & 1200.0 & 2.6 & 1250.0 & 1.9 & 1300.0 & 1.4 \\
\hline $\begin{array}{l}1050 \\
1050\end{array}$ & $\begin{array}{r}1.866 \\
\end{array}$ & 1100 & 1.3 & $\begin{array}{l}1150.0 \\
11500\end{array}$ & 0.9 & 1200.0 & 0.7 & $\begin{array}{l}1250.0 \\
\end{array}$ & 0.5 & 1300.0 & 0.4 \\
\hline $\begin{array}{r}(1050) \\
1050\end{array}$ & $\begin{array}{l}(6.5513 \\
4.266\end{array}$ & 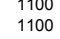 & $\begin{array}{l}3.5 \\
2.7\end{array}$ & $\begin{array}{l}\begin{array}{l}11550.0 \\
1150.0\end{array}\end{array}$ & $\begin{array}{l}2.1 \\
1.8\end{array}$ & $\begin{array}{l}12000.0 \\
1200.0\end{array}$ & $\begin{array}{l}1.4 \\
1.2\end{array}$ & $\begin{array}{l}1250.0 \\
1250.0\end{array}$ & $\begin{array}{l}1.0 \\
0.9\end{array}$ & $\begin{array}{l}13000.0 \\
1300 .\end{array}$ & $\begin{array}{l}0.7 \\
0.6\end{array}$ \\
\hline & 6.780 & 1100 & 4.0 & 1150.0 & 2.5 & 1200.0 & 1.7 & 1250.0 & 1.2 & & \\
\hline (1050) & (14.472 & 1100 & 8.4 & 1150.0 & 5.3 & 1200.0 & 3.6 & 1250.0 & 2.5 & 1300.0 & 1.9 \\
\hline & & 1100 & 5.1 & 1150.0 & 3.2 & 1200.0 & 2.1 & 1250.0 & & 1300.0 & \\
\hline 1050 & 2.117 & 1100 & 1.4 & 1150.0 & 0.9 & 1200.0 & 0.7 & 1250.0 & 0.5 & 1300.0 & 0.4 \\
\hline 1050 & 2.430 & 1100 & 1.5 & 1150.0 & 0.9 & 120 & 0.6 & 1250.0 & 0.4 & 1300.0 & 0.3 \\
\hline 1050 & $\begin{array}{r}4.065 \\
1\end{array}$ & $\begin{array}{l}1100 \\
1100\end{array}$ & 2.7 & $\begin{array}{l}1150.0 \\
11500\end{array}$ & 1.9 & 1200 & 1.4 & $\begin{array}{l}1250.0 \\
1250\end{array}$ & 1.0 & ${ }^{1300.0}$ & \\
\hline 1050 & 3.641 & 1100 & $\begin{array}{c}1.2 \\
2.2\end{array}$ & 1150.0 & $\begin{array}{l}1.4 \\
1.4\end{array}$ & 1200.0 & $\begin{array}{l}4.8 \\
1.0\end{array}$ & 1250.0 & $\begin{array}{l}3.3 \\
0.7\end{array}$ & 1300.0 & 0.5 \\
\hline
\end{tabular}

$\begin{array}{rrrr}5.43 & 5.24 & <0.006 & 0.04 \\ 0.76 & 0.04 & 0.02 & 0.76 \\ 8.45 & 9.22 & <0.006 & 0.07 \\ 2.19 & 2.62 & 2.32 & 1.02 \\ 0.08 & <0.003 & <0.006 & 0.12\end{array}$


1377 DZr-6a

1378 DZr-6C

1379 DZr-7a
1380 Dr $-7 \mathrm{Ca}$

138 Dr-8a

\begin{tabular}{|c|c|c|c|c|}
\hline & & 0.133 & 0.102 & \\
\hline 0.395 & & 0.339 & 0.125 & \\
\hline 0.179 & & 0.175 & 0.095 & 9.200 \\
\hline 0.175 & & 0.191 & 0.068 & $\begin{array}{r}9.800 \\
\end{array}$ \\
\hline 0.330 & 0.305 & 0.273 & 0.128 & 10.300 \\
\hline 0.526 & 0.529 & 0.512 & 0.243 & $\begin{array}{r}10.000 \\
0.300\end{array}$ \\
\hline $\begin{array}{l}0.327 \\
0.0997\end{array}$ & 0.338 & 0.290 & $\begin{array}{l}0.188 \\
0.267\end{array}$ & $\begin{array}{r}9.300 \\
1040\end{array}$ \\
\hline $\begin{array}{l}0.499 \\
0.368\end{array}$ & & 0.498 & $\begin{array}{l}0.267 \\
0.157\end{array}$ & $\begin{array}{r}10.400 \\
9.300\end{array}$ \\
\hline $\begin{array}{l}0.3000 \\
0.801\end{array}$ & 0.666 & 0.633 & 0.308 & 11.300 \\
\hline & & 0.263 & & \\
\hline 1.330 & 0.980 & 0.840 & 0.140 & 10.100 \\
\hline 1.221 & 1.009 & 0.792 & 0.218 & 10.700 \\
\hline 0.648 & 0.536 & 0.477 & 0.176 & 9.600 \\
\hline 1.470 & 1.400 & 1.890 & 0.490 & $\begin{array}{r}11.800 \\
\end{array}$ \\
\hline $\begin{array}{l}0.612 \\
0.608\end{array}$ & $\begin{array}{l}0.565 \\
0.513\end{array}$ & $\begin{array}{l}0.739 \\
0.432\end{array}$ & $\begin{array}{l}0.229 \\
0.198\end{array}$ & $\begin{array}{l}11.600 \\
10.10\end{array}$ \\
\hline $\begin{array}{l}0.608 \\
2.493\end{array}$ & $\begin{array}{l}0.513 \\
2.006\end{array}$ & $\begin{array}{l}0.432 \\
1.694\end{array}$ & $\begin{array}{l}0.198 \\
0.380\end{array}$ & $\begin{array}{l}10.100 \\
10.820\end{array}$ \\
\hline 1.961 & 1.558 & 1.524 & 0.374 & 10.300 \\
\hline 1.050 & 0.980 & 1.120 & 0.280 & 11.100 \\
\hline 0.232 & 0.253 & 0.213 & 0.111 & 10.000 \\
\hline 0.475 & 0.439 & 0.432 & 0.286 & \\
\hline 0.227 & 0.342 & 0.526 & 0.185 & 11.200 \\
\hline $\begin{array}{l}3.570 \\
5810\end{array}$ & $\begin{array}{r}2.870 \\
2\end{array}$ & 3.010 & $\begin{array}{l}0.630 \\
\end{array}$ & $\begin{array}{l}11.900 \\
112\end{array}$ \\
\hline $\begin{array}{l}5.810 \\
0.210\end{array}$ & $\begin{array}{l}\begin{array}{r}4.200 \\
0.210\end{array} \\
000\end{array}$ & $\begin{array}{l}4.690 \\
0.280\end{array}$ & $\begin{array}{l}.050 \\
0.140\end{array}$ & $\begin{array}{l}12.200 \\
10.200\end{array}$ \\
\hline 0.770 & 0.700 & 0.910 & 0.210 & $\begin{array}{r}11.300 \\
\end{array}$ \\
\hline $\begin{array}{l}0.420 \\
.0 .109\end{array}$ & 0.148 & $\begin{array}{l}0.490 \\
0.150\end{array}$ & $\begin{array}{l}0.210 \\
0.066\end{array}$ & $\begin{array}{l}10.200 \\
10.300\end{array}$ \\
\hline 0.279 & 0.298 & 0.282 & 0.071 & \\
\hline 0.561 & 0.512 & 0.535 & 0.144 & \\
\hline .691 & 0.465 & 0.582 & 0.254 & \\
\hline 4.656 & & 2.493 & 0.223 & 11.500 \\
\hline 0.630 & 0.700 & & & 11.300 \\
\hline
\end{tabular}

$\begin{array}{llll}0.098 & 0.168 & 0.033 & 0.034 \\ 0.180 & 0.280 & 0.162 & 0.087 \\ 0.098 & 0.155 & 0.015 & 0.027 \\ 0.078 & 0.146 & 0.015 & 0.021\end{array}$

$\begin{array}{lllll}0.199 & & 0.418 & 0.065 & \\ 0.190 & & 0.320 & 0.061 & \\ 0.208 & & 0.339 & 0.065 & \\ 0.169 & & 0.295 & 0.058 & \\ 0.228 & 0.348 & 0.395 & 0.091 & 11.200 \\ 0.180 & 0.304 & 0.330 & 0.077 & 11.150 \\ 0.169 & 0.293 & 0.336 & 0.089 & 11.110 \\ 0.188 & 0.281 & 0.302 & 0.076 & 10.930 \\ 0.173 & 0.304 & 0.349 & 0.100 & 10.790 \\ 0.222 & 0.284 & 0.338 & 0.079 & 10.900 \\ 0.260 & 0.369 & 0.326 & 0.113 & 10.860 \\ 0.231 & 0.403 & 0.326 & 0.112 & 10.930\end{array}$


1314 SRM-26

$1315 \mathrm{SRM}-18$
$1316 \mathrm{HLW}$

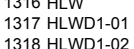

1318 HLWD1-02
1319 HLWD1-03

1320 HLWD1-04

1322 HLWD1-07

1323 HLWD1-08

1324 LLWD1-09
1325 HLWD1-10
1

1327 HWDD1-12

1328 HLWD1-1 13

1330 HLWD1-15

1331 HLWD $1-16$

1332 HLWD1-17

(1)

1335 HLWD $1-20$

1336 HLWD1-21
1337 HLWD1-23

1338 HLWD1-24
1339 HLWD1-25

1340 HLWD1-26
1341 HWWD-27

1342 HWDD-2.28

1343 HLWD2-01

$1344 \mathrm{HLWD2}-02$
$1345 \mathrm{HWDD2}-03$

1346 HLWD2-04

1347 HLWD2-05

1348 LLWD2-06
1349 HLWD2-07

1350 HLWD3-01

1351 HLWD3-02

1352 HLWD3-03
1353 HWDD3-04

1354 HLWD3-05

1356 HLWD3-07

1358 EnvDSR1

1359 Frit-1-78-35

1360 Frit-2-78-35
1361 Frit-3-78-35

1362 Frit-478-35

1363 Frit-5-78-35

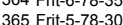

1366 Frit-5-78-37

1367 Frit:-5-78-40

1369 Frit-5-58b-35

1370 Frit-5-78c-35

$1371 \mathrm{DZr}-3 \mathrm{a}$

$372 \mathrm{DZZ}-3 \mathrm{C}$
$373 \mathrm{DZ}-4 \mathrm{a}$

${ }_{1375}^{1374 \mathrm{DZZ}-4 \mathrm{C}}$

1377 DZr-6a

1378 DZr-6C

1379 DZF-7a
1380 Dr-7C

1381 DZr-8a

1382 DZr-8C

1383 DZZ-9.9-78-38
1384 DZ-9.9-48.40

1385 DZr-10-7-78-38
1386 DZr-10-78-40

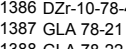

1389 GLA 78-23

390 GLA 78-9-11

1391 GLA 78-9.15
1392 GLA 78-9-18

1393 GLA 78-10-14
1394 GLA 78-10-15 


\begin{tabular}{|c|c|c|}
\hline \multirow{2}{*}{\multicolumn{3}{|c|}{$\begin{array}{l}\text { \# Glass ID } \\
395 \text { GLA 78-10-16 }\end{array}$}} \\
\hline & & \\
\hline 1397 P2-0Ca-1F & INEEL CVS 2a & Peeler et al. 1999 \\
\hline 1398 P2-OCa-2F & INEEL CVS 2a & Peeler et al. 1999 \\
\hline 1399 P2-OCa-3F & INEEL CVS 2a & Peeler et al. 1999 \\
\hline $\begin{array}{l}1400 \text { P2-OCa-4F } \\
1400 \text { P2-CCa-5F }\end{array}$ & $\begin{array}{l}\text { INEEL CVS 2a } \\
\text { INFI }\end{array}$ & Peeler et al. 1999 \\
\hline $\begin{array}{l}1401 \text { 12-2-Ca-5F } \\
1402 \text { P2-3Ca-0F }\end{array}$ & $\begin{array}{l}\text { INEEL CVS } 2 a \\
\text { INEEL CVS 2a }\end{array}$ & $\begin{array}{l}\text { Peeler et al. } 1999 \\
\text { Peeler et al. } 1999\end{array}$ \\
\hline $\begin{array}{l}1402 \text { P2-3Ca--oF } \\
1403 \text { P2-3Ca-1F }\end{array}$ & $\begin{array}{l}\text { NEEL CVS 2a } \\
\text { INEEL CVS 2a }\end{array}$ & $\begin{array}{l}\text { Peeler et al. } 1999 \\
\text { Peeler et al. } 1999\end{array}$ \\
\hline 1404 P2-3Ca-2F & $\begin{array}{l}\text { INEEL CVS } 2 a \\
\text { INEE }\end{array}$ & Peeler et al. 1999 \\
\hline 5 P2-3Ca-3F & INEEL CVS 2a & Peeler et al. 1999 \\
\hline 1406 P2-3Ca-4F & INEEL CVS 2a & Peeler et al. 1999 \\
\hline 1407 P2-3Ca-5F & INEEL CVS 2a & Peeler et al. 1999 \\
\hline 1408 P2-9Ca-OF & INEEL CVS 2a & Peeler et al. 1999 \\
\hline 1409 P2-9Ca-1F & INEEL CVS 2a & Peeler et al. 1999 \\
\hline 1410 P2-9Ca-2F & INEEL CVS 2a & Peeler et al. 1999 \\
\hline $\begin{array}{l}4111 \text { P2-9C-3F-3F } \\
412 \text { P2-9G-4F }\end{array}$ & INEEL CVS 2a & $\begin{array}{l}\text { Peeler et al. } 1999 \\
\end{array}$ \\
\hline 1413 P2-9Ca-5F & INEEL CVS 2a & $\begin{array}{l}\text { Peeler et al. } 1999 \\
\text { Peeele et al } 1999\end{array}$ \\
\hline 1414 SRL 165 HiFe & SRL 165 Glasses & $\begin{array}{l}\text { Peeler et al. } 1999 \\
\text { Pye } 1985\end{array}$ \\
\hline 1415 SRL 165 TDS & SRL 165 Glasses & Pye 1985 \\
\hline 1416 SRL 165 HiAl & SRL 165 Glasses & Pye 1985 \\
\hline 1417 HLW98-01 & RPP-WTP HLW Formulation & Kot and Pegg 2001 \\
\hline 1418 HLW98-02 & RPP-WTP HLW Formulation & Kot and Pegg 2001 \\
\hline 1419 HLW98-03 & RPP-WTP HLW Formulation & Kot and Pegg 2001 \\
\hline $\begin{array}{l}1420 \text { HLW98--04 } \\
1421 \text { HLW98-05 }\end{array}$ & RPPPWTP HLW Formulation & Kot and Pegg 2001 \\
\hline & & \\
\hline $\begin{array}{l}1422 \text { HLW989-06 } \\
4223 \text { HLW98-07 }\end{array}$ & RPP-WTP HLW Formulation & Kot and Pegg 2001 \\
\hline $\begin{array}{l}23 \text { HLW98-07 } \\
24 \text { HLW9-08 }\end{array}$ & 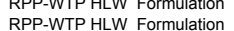 & Kot and Pegg 2001 \\
\hline 425 HLW98-09 & $\begin{array}{l}\text { RPP-WTP HLW Formulation } \\
\text { RPT }\end{array}$ & Kot and Pegg 2001 \\
\hline 426 HLW98-10 & RPP-WTP HLW Formulation & $\begin{array}{l}\text { Kot and Pegg } 2001 \\
\text { Kot and Pega } 2001\end{array}$ \\
\hline 427 HLW98-11 & RPP-WTP HLW Formulation & Kot and Pegg 2001 \\
\hline 28 HLW98-12 & RPP-WTP HLW Formulation & Kot and Pegg 2001 \\
\hline 1429 HLW98-13 & RPP-WTP HLW Formulation & Kot and Pegg 2001 \\
\hline 1430 HLW98-14 & RPP-WTP HLW Formulation & Kot and Pegg 2001 \\
\hline 1431 HLW98-15 & RPP-WTP HLW Formulation & Kot and Pegg 2001 \\
\hline 1432 HLW98-16 & RPP-WTP HLW Formulation & Kot and Pegg 2001 \\
\hline $\begin{array}{l}1433 \text { HLW98-17 } \\
4334 \text { HLW98-18 }\end{array}$ & & Kot and Pegg 2001 \\
\hline $\begin{array}{l}1444 \text { HLW98-18 } \\
1435 \text { HLW98-19 }\end{array}$ & 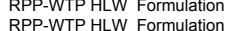 & $\begin{array}{l}\text { Kot and Pegg } 2001 \\
\text { Kot and Peg } 2001\end{array}$ \\
\hline $\begin{array}{l}14356 \text { HLWY88-199 } \\
1436-20\end{array}$ & $\begin{array}{l}\text { RPP-WTP HLW Formulation } \\
\text { RPT }\end{array}$ & Kot and Pegg 2001 \\
\hline 1437 HLW98-21 & RPP-WTP HLW Formulation & Kot and Pegg 2001 \\
\hline 1438 HLW98-22 & RPP-WTP HLW Formulation & Kot and Pegg 2001 \\
\hline 1439 HLW98-23 & RPP-WTP HLW Formulation & Kot and Pegg 2001 \\
\hline 1440 HLW98-24 & RPP-WTP HLW Formulation & Kot and Pegg 2001 \\
\hline 1441 HLW98-25 & RPP-WTP HLW Formulation & Kot and Pegg 2001 \\
\hline 1442 HLW98-26 & RPP-WTP HLW Formulation & Kot and Pegg 2001 \\
\hline $\begin{array}{l}1443 \text { HLW98-27B } \\
4444 \text { HLW98-28 }\end{array}$ & 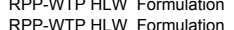 & $\begin{array}{l}\text { Kot and Pegg } 2001 \\
\text { Kot and Pega } 2001\end{array}$ \\
\hline 1445 HLW98-29 & $\begin{array}{l}\text { RPP-WTP HLW Formulation } \\
\text { RPP H. }\end{array}$ & Kot and Pegg 2001 \\
\hline 1446 HLW98-30 & RPP-WTP HLW Formulation & Kot and Pegg 2001 \\
\hline 1447 HLW98-31 & RPP-WTP HLW Formulation & Kot and Pegg 2001 \\
\hline 1448 HLW98-32A & RPP-WTP HLW Formulation & Kot and Pegg 2001 \\
\hline 1449 HLW98-33 & RPP-WTP HLW Formulation & Kot and Pegg 2001 \\
\hline 1450 HLW98-34 & & Kot and Pegg 2001 \\
\hline 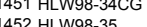 & RPP-WTP HLW Formulation & Kot and Pegg 2001 \\
\hline $\begin{array}{l}1452 \text { HLWGod-35 } \\
445 \text { HLW98-36 }\end{array}$ & RPP-WTP HLW Formulation & $\begin{array}{l}\text { Kot and Pegg } 2001 \\
\text { Kot and Peg } 2001\end{array}$ \\
\hline 454 HLW98-37 & RPP-WTP HLW Formulation & Kot and Pegg 2001 \\
\hline 5 HLW98-38 & RPP-WTP HLW Formulation & Kot and Pegg 2001 \\
\hline 66 HLW98-39 & RPP-WTP HLW Formula & Kot and Pegg 2 \\
\hline 1457 HLW98-40 & RPP-WTP HLW Formulation & egg 2001 \\
\hline 1458 HLW98-41 & RPP-WTP HLW Formulation & egg 2001 \\
\hline $99 \mathrm{HLW}$ 9 $88-42$ & RPP-WTP HLW Formulati & Kot and Pegg 2001 \\
\hline 年 & RPD-WTP HW Formulat & Kot and Pegg 2001 \\
\hline $\begin{array}{l}1462 \mathrm{HLW} 98-45 \\
1468\end{array}$ & RPP-WTP HLW Formulation & $\begin{array}{l}\text { Kot and Pegg } 2001 \\
\text { Kot and Pegg } 2001\end{array}$ \\
\hline 1463 HLW98-46 & RPP-WTP HLW Formulation & egg 2001 \\
\hline HLW98-47 & RPP-WTP HLW Formulation & \\
\hline HLW98-48 & RPP-WTP HLW Forn & Kot and $\mathrm{Y}-\mathrm{C}$ \\
\hline 1466 HLW98-49 & RPP-WTP HLW Formula & Kot and $\mathrm{F}$ \\
\hline HLW98-50 & RPP-WTP HLW Formulatior & egg 2001 \\
\hline $8.51 R$ & Formul: & egg 2001 \\
\hline 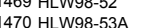 & P.NTP HIV & egg 2001 \\
\hline 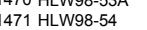 & RPP-WTP HLW Formulation & $\begin{array}{l}\text { Kot and Pegg 2001 } \\
\text { Kot and Pegg } 2001\end{array}$ \\
\hline HLW98-55 & RPP-WTP HLW Formulation & Kot and Pegg 2001 \\
\hline HLW98-56 & RPP-WTP HLW Formulation & Kot and Pegg 2001 \\
\hline $\begin{array}{l}474 \text { HLW98-57 } \\
475 \text { HIW98-58 }\end{array}$ & 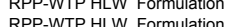 & Kot and Pegg 2001 \\
\hline $\begin{array}{l}475 \text { HLW98-58 } \\
476 \text { HLW98-59 }\end{array}$ & RPP-WTP HLW Formulation & Kot and Peg 2001 \\
\hline
\end{tabular}

$\begin{array}{llll}0.0772 & 0.1096 & 0.0000 & 0.0243\end{array}$

$\begin{array}{llll}0.0772 & 0.1096 & 0.0000 & 0.0243 \\ 0.0094 & 0.0986 & 0.0000 & 0.0219 \\ 0.091 & 0.0981 & 0.0000 & 0.0219\end{array}$

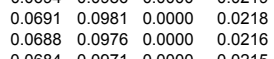
$\begin{array}{lllll}0.0679 & 0.0964 & 0.0000 & 0.0215 \\ 0.0749 & 0.0214 \\ 0.0776 & 0.1093 & 0.0300 & 0.0236\end{array}$ $\begin{array}{llll}0.0749 & 0.1063 & 0.0300 & 0.0236 \\ 0.0676 & 0.0959 & 0.0270 & 0.0213\end{array}$ $\begin{array}{llll}0.0676 & 0.0959 & 0.0270 & 0.0213 \\ 0.0672 & 0.0954 & 0.0269 & 0.0212\end{array}$ $\begin{array}{llll}0.0667 & 0.09448 & 0.0268 & 0.02211 \\ 0.0664 & 0.0943 & 0.0266 & 0.0210\end{array}$ $\begin{array}{llll}0.0664 & 0.0943 & 0.0266 & 0.0210 \\ 0.0660 & 0.0937 & 0.0264 & 0.0209\end{array}$ $\begin{array}{lllll}0.0630 & 0.0894 & 0.0943 & 0.0203 \\ 0 & 0.0199\end{array}$

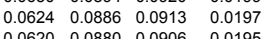
$\begin{array}{llll}0.0620 & 0.0880 & 0.0906 & 0.0195 \\ 0.0616 & 0.0875 & 0.0901 & 0.195\end{array}$ $\begin{array}{llll}0.0616 & 0.0875 & 0.0900 & 0.0194 \\ 0.0177 & 0.0684 & 0.0113 & 0.1454\end{array}$ $\begin{array}{llll}0.0418 & 0.0692 & 0.0153 & 0.1202 \\ 0.0928 & 0.0739 & 0.0114 & 0.0635 \\ 0.971 & 0.02 & 0.097 & 0.129\end{array}$ $\begin{array}{llll}0.1071 & 0.1062 & 0.0097 & 0.1169 \\ 0.1106 & 0.1096 & 0.0101 & 0.1207\end{array}$ $\begin{array}{llll}0.1106 & 0.1096 & 0.01101 & 0.12207 \\ 0.1179 & 0.1000 & 0.0142 & 0.0943 \\ 0.179 & 0.1300 & 0.0437 & 0.1217\end{array}$ $\begin{array}{lllll}0.1179 & 0.1300 & 0.0142 & 0.0943\end{array}$ $\begin{array}{llll}0.1146 & 0.1300 & 0.0137 & 0.1217 \\ 0.0851 & 0.1000 & 0.0043 & 0.1276 \\ 0.0782 & 0.1000 & 0.044 & 0.123\end{array}$ $\begin{array}{llll}0.0882 & 0.1000 & 0.0044 & 0.1323 \\ 0.0743 & 0.1200 & 0.0153 & 0.1226\end{array}$ $\begin{array}{llll}0.0797 & 0.1200 & 0.0040 & 0.1196\end{array}$ $\begin{array}{llll}0.0701 & 0.1200 & 0.0144 & 0.1157 \\ 0.0729 & 0.1200 & 0.0150 & 0.1202\end{array}$ $\begin{array}{lllll}0.0749 & 0.1500 & 0.0027 & 0.1267\end{array}$ $\begin{array}{lllll}0.0666 & 0.1500 & 0.0024 & 0.1126\end{array}$ $\begin{array}{lllll}0.0870 & 0.1200 & 0.0190 & 0.0876 \\ 0.0870 & 0.1500 & 0.0190 & 0.0876\end{array}$ $\begin{array}{llll}0.0720 & 0.1200 & 0.0148 & 0.1187\end{array}$ $\begin{array}{llll}0.0703 & 0.1200 & 0.0145 & 0.1154 \\ 0.0772 & 0.0500 & 0.028 & 0.1296\end{array}$ $\begin{array}{llll}0.0772 & 0.0500 & 0.0028 & 0.1296 \\ 0.0915 & 0.1200 & 0.0200 & 0.0921\end{array}$ $\begin{array}{llll}0.0891 & 0.1200 & 0.0195 & 0.0978 \\ 0.0677 & 0.0700 & 0.024 & 0.1136\end{array}$ $\begin{array}{llll}0.0077 & 0.0700 & 0.0024 & 0.1136 \\ 0.0535 & 0.1200 & 0.0117 & 0.1462 \\ 0.0677 & 0.12000 & 0.0024 & 0.1136\end{array}$ $\begin{array}{llll}0.0677 & 0.1100 & 0.0024 & 0.1136 \\ 0.0664 & 0.1000 & 0.0024 & 0.1114\end{array}$ $\begin{array}{llll}0.0664 & 0.1000 & 0.0024 & 0.1114 \\ 0.0781 & 0.0700 & 0.0026 & 0.1305\end{array}$ $\begin{array}{llll}0.0781 & 0.0700 & 0.026 & 0.134 \\ 0.0781 & 0.0400 & 0.0026 & 0.1305 \\ 0 & 0.1305\end{array}$ $\begin{array}{llll}0.0781 & 0.0200 & 0.0026 & 0.1305 \\ 0.0781 & 0.1289 & 0.0026 & 0.1305\end{array}$ $\begin{array}{llll}0.0781 & 0.12800 & 0.02026 & 0.13097 \\ 0.0740 & 0.1000 & 0.0025 & 0.1039\end{array}$ $\begin{array}{llll}0.1368 & 0.0900 & 0.0024 & 0.1110 \\ 0.1003 & 0.0700 & 0.0067 & 0.1092 \\ 0.1978 & 0.0700 & 0.071 & 0.1173\end{array}$ $\begin{array}{lllll}0.11003 & 0.0700 & 0.0067 & 0.11092 \\ 0.1078 & 0.0700 & 0.0071 & 0.1173 \\ 0.1078 & 0.0700 & 0.071 & 0.173\end{array}$ $\begin{array}{llll}0.1034 & 0.0700 & 0.0176 & 0.1206 \\ 0.0939 & 0.0900 & 0.0281 & 0.0382\end{array}$ $\begin{array}{llll}0.0443 & 0.0505 & 0.0045 & 0.0561 \\ 0.0336 & 0.0500 & 0.0064 & 0.0663\end{array}$ $\begin{array}{llll}0.0370 & 0.0300 & 0.0070 & 0.0730 \\ 0.0823 & 0.0660 & 0.0026 & 0.1189\end{array}$ $\begin{array}{llll}0.0823 & 0.0600 & 0.0026 & 0.1189 \\ 0.0343 & 0.0300 & 0.0065 & 0.0677\end{array}$ $\begin{array}{llll}0.0343 & 0.030 & 0.0065 & 0.0677 \\ 0.0797 & 0.0850 & 0.0025 & 0.1152 \\ 0.0325 & 0.0500 & 0.002 & 0.041\end{array}$ $\begin{array}{lllll}0.0325 & 0.0500 & 0.0062 & 0.0641 \\ 0.0325 & 0.0700 & 0.0062 & 0.0641\end{array}$ $\begin{array}{llll}0.0314 & 0.0700 & 0.0060 & 0.0620 \\ 0.0300 & 0.0750 & 0.0057 & 0.0591\end{array}$ $\begin{array}{lllll}0.0287 & 0.0750 & 0.0055 & 0.0567 \\ 0.0449 & 0.0900 & 0.0047 & 0.0492\end{array}$ $\begin{array}{llll}0.0449 & 0.0900 & 0.00047 & 0.0492 \\ 0.0588 & 0.0700 & 0.0045 & 0.0470\end{array}$ $\begin{array}{llll}0.0250 & 0.1100 & 0.0043 & 0.0448 \\ 0.02357 & 0.0900 & 0.045 & 0.0470\end{array}$ $\begin{array}{llll}0.0238 & 0.0900 & 0.0045 & 0.0470 \\ 0.0797 & 0.0700 & 0.0025 & 0.1152\end{array}$ $\begin{array}{llll}0.0811 & 0.0725 & 0.0025 & 0.1172 \\ 0.0797 & 0.1000 & 0.0025 & 0.1152\end{array}$ $\begin{array}{lllll}0.0797 & 0.0700 & 0.0025 & 0.1152 \\ 0.0756 & 0.0700 & 0.0058 & 0.1179\end{array}$ $\begin{array}{llll}0.0763 & 0.0400 & 0.0061 & 0.1208 \\ 0.0800 & 0.0400 & 0.0025 & 0.1156\end{array}$ $\begin{array}{lllllllll}0.0015 & 0.0365 & 0.0012 & 0.0684 & 0.0003 & 0.0002 & 0.6235 & 0.0538 \\ 0.0013 & 0.0329 & 0.0011 & 0.1556 & 0.0003 & 0.0001 & 0.5612 & 0.0485 \\ 0.00033 & 0.0326 & 0.0011 & 0.150 & 0.003 & 0.001 & 0.5652 & 0.0479\end{array}$

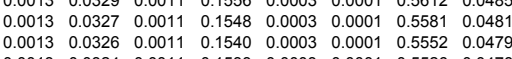
$\begin{array}{lllllllll}0.0014 & 0.0354 & 0.0012 & 0.0664 & 0.0003 & 0.0001 & 0.6048 & 0.0522\end{array}$

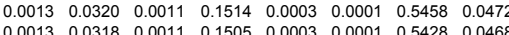
$\begin{array}{llllllllll}0.0013 & 0.0316 & 0.0011 & 0.1495 & 0.0003 & 0.0001 & 0.5392 & 0.0465\end{array}$ $\begin{array}{lllllllll}0.0013 & 0.0314 & 0.0011 & 0.1487 & 0.0003 & 0.0001 & 0.5363 & 0.0463 \\ 0.0013 & 0.0312 & 0.0010 & 0.1478 & 0.0003 & 0.0001 & 0.5330 & 0.0460\end{array}$ $\begin{array}{lllllllll}0.0013 & 0.0312 & 0.0010 & 0.1478 & 0.0003 & 0.0001 & 0.5330 & 0.0440 \\ 0.0012 & 0.0305 & 0.0010 & 0.1273 & 0.0002 & 0.0001 & 0.5211 & 0.0450\end{array}$ $\begin{array}{llllllll}0.0012 & 0.0298 & 0.0010 & 0.1411 & 0.00002 & 0.0001 & 0.52187 & 0.04439\end{array}$ $\begin{array}{lllllllll}0.0012 & 0.0295 & 0.0010 & 0.1398 & 0.0002 & 0.0001 & 0.5043 & 0.0435 \\ 0.0012 & 0.0294 & 0.0010 & 0.1388 & 0.0002 & 0.0001 & 0.5007 & 0.0432\end{array}$ $\begin{array}{llllllll}0.0012 & 0.0292 & 0.0010 & 0.1380 & 0.0002 & 0.0001 & 0.4977 & 0.0430 \\ 0.0012 & 0.0291 & 0.0074 & 0.130 & 0.0002 & & \end{array}$

$$
\begin{array}{lrrrrrr}
0.0291 & 0.0010 & 0.1380 & 0.0002 & 0.0001 & 0.4975 & 0.0430 \\
0.0476 & 0.0074 & 0.1154 & 0.0183 & 0.4991 & 0.0091 \\
0.0479 & 0.0081 & 0.1049 & 0.0000 & & 0.5509 & 0.0122 \\
0.0514 & 0.0080 & 0.1176 & 0.0068 & & 0.5429 & 0.0101
\end{array}
$$

$\begin{array}{llllllllll}0.0043 & 0.0195 & 0.0009 & 0.1305 & 0.00043 & 0.0085 & 0.34213 & 0.0604 & 0.0019 & 0.0005\end{array}$ $\begin{array}{llllllllllll}0.0044 & 0.0201 & 0.0101 & 0.1347 & 0.0044 & 0.0087 & 0.3318 & 0.0302 & 0.0020 & 0.0005 \\ 0.0024 & 0.0300 & 0.0094 & 0.1179 & 0.0024 & 0.0094 & 0.3943 & 0.0377 & 0.0009 & 0.0005 \\ 0.0024 & & 0.0092 & 0.1146 & 0.0023 & 0.0092 & 0.417 & & 0.0009 & 0.0005\end{array}$

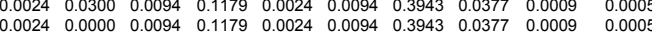
$\begin{array}{llllllllll}0.0023 & & 0.0092 & 0.1146 & 0.0023 & 0.0092 & 0.4117 & & 0.0009 & 0.0005 \\ 0.0043 & 0.0300 & 0.0085 & 0.1106 & 0.0043 & 0.0064 & 0.4276 & 0.0213 & 0.0009 & 0.0009\end{array}$ $\begin{array}{llllllllll}0.0044 & 0.0100 & 0.0088 & 0.1146 & 0.0044 & 0.0066 & 0.4323 & 0.0220 & 0.0009 & 0.0000 \\ 0.0044 & 0.0300 & 0.0087 & 0.0918 & 0.0044 & 0.0066 & 0.4093 & 0.0219 & 0.0009 & 0.0000\end{array}$ $\begin{array}{llllllllll}0.0040 & 0.0300 & 0.0080 & 0.0837 & 0.0040 & 0.0060 & 0.4496 & 0.0199 & 0.0008 & 0.0008 \\ 0.0041 & 0.0300 & 0.0084 & 0.0867 & 0.0041 & 0.0062 & 0.4332 & 0.0206 & 0.0008 & 0.0008\end{array}$ $\begin{array}{llllllllll}0.0041 & 0.0300 & 0.0084 & 0.0867 & 0.0041 & 0.0062 & 0.4332 & 0.0206 & 0.0008 & 0.0008 \\ 0.0043 & 0.0300 & 0.0086 & 0.0901 & 0.0043 & 0.0064 & 0.4173 & 0.0215 & 0.0009 & 0.0009\end{array}$

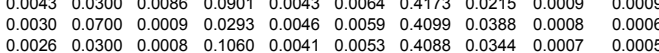
$\begin{array}{llllllllll}0.0019 & 0.03300 & 0.0114 & 0.1404 & 0.0016 & 0.00056 & 0.4169 & 0.00031 & 0.00011 \\ 0.0019 & 0.0000 & 0.0114 & 0.1404 & 0.0016 & 0.0065 & 0.0169 & 0.0031 & 0.011\end{array}$

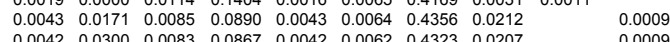

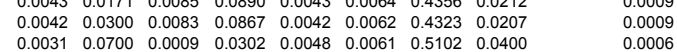
$\begin{array}{lllllllll}0.0031 & 0.0700 & 0.0009 & 0.0302 & 0.0048 & 0.0061 & 0.5102 & 0.0400 & \\ 0.0020 & 0.0000 & 0.0120 & 0.1477 & 0.0017 & 0.0068 & 0.4256 & 0.0033 & 0.0012 \\ 0.0020 & 0.0150 & 0.0117 & 0.143 & 0.0000\end{array}$ $\begin{array}{llllllllll}0.0020 & 0.0150 & 0.0117 & 0.1438 & 0.0016 & 0.0066 & 0.4360 & 0.0032 & 0.0000 & \\ 0.0027 & 0.0600 & 0.0008 & 0.0664 & 0.0042 & 0.0053 & 0.5089 & 0.0350 & & 0.000\end{array}$ $\begin{array}{llllllllll}0.0012 & 0.0300 & 0.0070 & 0.0863 & 0.0010 & 0.0040 & 0.4026 & 0.0019\end{array}$

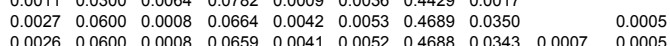

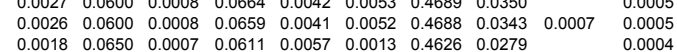
$\begin{array}{lllllllll}0.0018 & 0.0650 & 0.0007 & 0.0611 & 0.0057 & 0.0013 & 0.4626 & 0.0279 & 0.0004 \\ 0.0018 & 0.0450 & 0.0007 & 0.1435 & 0.0057 & 0.0013 & 0.4302 & 0.0279 & 0.0004\end{array}$

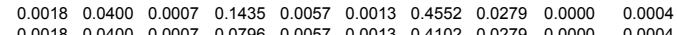

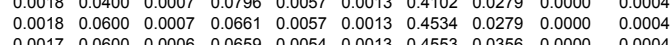
$\begin{array}{llllllllll}0.0005 & 0.0700 & 0.0007 & 0.0358 & 0.0067 & 0.0005 & 0.4550 & 0.0146 & 0.0000 & 0.0004 \\ 0.0003 & 0.0300 & 0.0020 & 0.0879 & 0.0014 & 0.0014 & 0.3773 & 0.0011 & 0.0022 & 0.0000\end{array}$ $\begin{array}{llllllllll}0.0003 & 0.0300 & 0.0020 & 0.0879 & 0.0014 & 0.0014 & 0.3773 & 0.0011 & 0.0022 & 0.004 \\ 0.0003 & 0.0400 & 0.0022 & 0.0936 & 0.0015 & 0.0015 & 0.4089 & 0.0012 & 0.0024 & \end{array}$

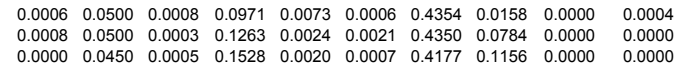

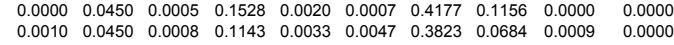
$\begin{array}{llllllllll}0.0011 & 0.0400 & 0.0009 & 0.1223 & 0.0036 & 0.0051 & 0.3696 & 0.0753 & 0.0010 & 0.0000 \\ 0.0006 & 0.0450 & 0.0008 & 0.1419 & 0.0072 & 0.0006 & 0.4353 & 0.0156 & 0.0004 & 0.0004\end{array}$

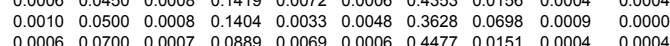
$\begin{array}{lllllllllll}0.0010 & 0.0600 & 0.0008 & 0.1275 & 0.0032 & 0.00045 & 0.3666 & 0.0661 & 0.0009 & 0.0000\end{array}$ $\begin{array}{llllllllll}0.0010 & 0.0600 & 0.0008 & 0.1025 & 0.0032 & 0.0045 & 0.3716 & 0.0661 & 0.0009 & 0.0000 \\ 0.0010 & 0.0600 & 0.0007 & 0.1033 & 0.0031 & 0.0044 & 0.3834 & 0.0639 & 0.0009 & 0.0000\end{array}$ $\begin{array}{llllllllllll}0.0009 & 0.0700 & 0.0007 & 0.0984 & 0.0029 & 0.0042 & 0.3899 & 0.0610 & 0.0008 & 0.00000\end{array}$ $\begin{array}{llllllllll}0.0009 & 0.0700 & 0.0007 & 0.1014 & 0.0028 & 0.0040 & 0.4016 & 0.0584 & 0.0008 & 0.0000 \\ 0.0008 & 0.0500 & 0.0006 & 0.1103 & 0.0024 & 0.0035 & 0.4216 & 0.0507 & 0.0007 & 0.0000\end{array}$ $\begin{array}{llllllllllll}0.0007 & 0.0400 & 0.0006 & 0.1385 & 0.0023 & 0.0033 & 0.4208 & 0.0485 & 0.0007 & 0.0000\end{array}$ $\begin{array}{llllllllll}0.0002 & 0.0400 & 0.0006 & 0.1359 & 0.0026 & 0.0035 & 0.4544 & 0.0546 & 0.0007 & 0.0000 \\ 0.0007 & 0.0500 & 0.0006 & 0.0860 & 0.0023 & 0.0033 & 0.4783 & 0.0485 & 0.0007 & 0.0000\end{array}$

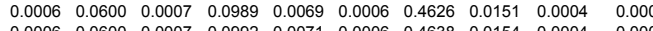
$\begin{array}{lllllllllll}0.0006 & 0.0600 & 0.0007 & 0.0564 & 0.0069 & 0.0006 & 0.4752 & 0.0151 & 0.0004 & 0.00004\end{array}$ $\begin{array}{llllllllll}0.0006 & 0.0700 & 0.0007 & 0.0914 & 0.0069 & 0.0006 & 0.4886 & 0.0151 & 0.0004 & 0.0004 \\ 0.0000 & 0.0700 & 0.0012 & 0.0954 & 0.0077 & 0.0024 & 0.4972 & 0.0165 & 0.0002 & 0.0000\end{array}$

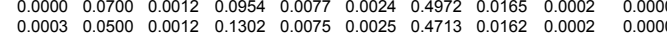

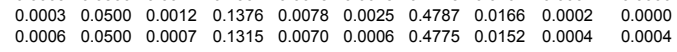

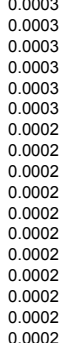

\begin{tabular}{|c|c|c|c|c|c|}
\hline 0.0043 & 0.0004 & 0.0063 & & 0.0049 & \\
\hline & 0.0004 & 0.0065 & & 0.0051 & \\
\hline 0.0024 & $\begin{array}{l}0.0005 \\
0.0005\end{array}$ & $\begin{array}{l}0.0024 \\
0.0024\end{array}$ & & 0.0024 & \\
\hline $\begin{array}{l}0.0024 \\
0.0023\end{array}$ & $\begin{array}{l}0.00050 \\
0.0005\end{array}$ & $\begin{array}{l}0.0024 \\
0.0046\end{array}$ & & $\begin{array}{l}0.0024 \\
0.0023\end{array}$ & $\begin{array}{l}0.0024 \\
0.0023\end{array}$ \\
\hline 0.0043 & 0.0004 & 0.0034 & & 0.0013 & 0.0009 \\
\hline 0.00044 & 0.0004 & $\begin{array}{l}0.0035 \\
0.0035\end{array}$ & & 0.0013 & \\
\hline $\begin{array}{l}0.0044 \\
0.00040\end{array}$ & $\begin{array}{l}0.0004 \\
0.0004\end{array}$ & $\begin{array}{l}0.0035 \\
0.0032\end{array}$ & & $\begin{array}{l}0.0013 \\
0.0012\end{array}$ & $\begin{array}{l}0.0009 \\
0.0008\end{array}$ \\
\hline & $\begin{array}{l}0.0004 \\
0.0004\end{array}$ & $\begin{array}{l}0.00032 \\
0.0033\end{array}$ & & & 0.00008 \\
\hline 0.0043 & 0.0004 & 0.0034 & & & \\
\hline 0.0006 & & $\begin{array}{l}0.00035 \\
0.0047\end{array}$ & & $\begin{array}{l}0.0012 \\
0.0011\end{array}$ & $\begin{array}{l}0.0001 \\
0.0001\end{array}$ \\
\hline 0.0059 & 0.0006 & $\begin{array}{l}0.0004 \\
0.0\end{array}$ & & & $\begin{array}{l}0.0001 \\
0.000\end{array}$ \\
\hline 0.0059 & 0.0006 & 0.0004 & & & \\
\hline $\begin{array}{l}0.00043 \\
0.0042\end{array}$ & 0.0004 & 0.0034 & & & \\
\hline $\begin{array}{l}0.0042 \\
0.00006\end{array}$ & & $\begin{array}{l}0.0033 \\
0.0054\end{array}$ & & $\begin{array}{l}0.0013 \\
0.0012\end{array}$ & $\begin{array}{l}0.0009 \\
0.0001\end{array}$ \\
\hline 0.0062 & 0.0006 & 0.0004 & & & \\
\hline 0.0061 & 0.0006 & 0.0004 & & & \\
\hline $\begin{array}{l}0.0005 \\
0.00036\end{array}$ & & $\begin{array}{l}0.0004 \\
0.0002\end{array}$ & & 0.0011 & 0.0001 \\
\hline 0.0033 & $\begin{array}{l}0.0044 \\
0.0003\end{array}$ & $\begin{array}{l}0.0002 \\
0.002\end{array}$ & & & $\begin{array}{l}0.0004 \\
0.0004\end{array}$ \\
\hline 0.0005 & & 0.0048 & & 0.0011 & \\
\hline & & & & 0.0011 & 0.0001 \\
\hline 0.0004 & & $\begin{array}{l}0.00039 \\
0.0039\end{array}$ & & 0.00009 & $\begin{array}{l}0.0001 \\
0.0001\end{array}$ \\
\hline 0.0004 & & 0.0039 & & 0.0009 & $\begin{array}{l}0.0001 \\
0.0001\end{array}$ \\
\hline 0.0004 & & 0.0039 & & & \\
\hline 004 & & 0.0039 & & 0.0009 & \\
\hline 0.0004 & & 0.0037 & & 0.0008 & 0.000 \\
\hline 0.0003 & & 0.0078 & & 0.0004 & 0.0001 \\
\hline 0.0006 & & 0.0004 & & 0.0003 & 0.0003 \\
\hline 0.0007 & & 0.0004 & 0.0003 & & \\
\hline $\begin{array}{l}0.000 \% \\
0.0003\end{array}$ & & 0.0004 & 0.0003 & & $\begin{array}{l}0.0002 \\
0.0002\end{array}$ \\
\hline 0.0000 & & $\begin{array}{l}0.00000 \\
0.0006\end{array}$ & & 0.00000 & $\begin{array}{l}0.0002 \\
0.0001\end{array}$ \\
\hline 0.0000 & & 0.0009 & & 0.0000 & \\
\hline 0.0003 & & 0.0009 & & 0.0012 & 0.0000 \\
\hline $\begin{array}{l}0.0003 \\
0.0003\end{array}$ & & $\begin{array}{l}0.00090 \\
0.0084\end{array}$ & & 0.0013 & $\begin{array}{l}0.0000 \\
0.0000\end{array}$ \\
\hline $\begin{array}{l}0.00033 \\
0.0003\end{array}$ & & $\begin{array}{l}0.0084 \\
0.0009\end{array}$ & & & $\begin{array}{l}0.0000 \\
0.0000\end{array}$ \\
\hline 0.0003 & & 0.0081 & & 0.000 & 0.0000 \\
\hline 0.0003 & & 0.0008 & & 0.0011 & 0.000 \\
\hline 0.000 & 0.0000 & 0.0008 & & 0.0011 & \\
\hline 3 & 0.0000 & 0.0008 & & 0.0011 & \\
\hline 0.0002 & 0.0000 & 0.0008 & & 0.0011 & \\
\hline & 0.00000 & 0.0007 & & 0.0010 & 0.0000 \\
\hline & 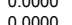 & 0 & & 0.000 & $\begin{array}{l}0.0000 \\
0.0000\end{array}$ \\
\hline 0.0002 & $\begin{array}{l}0.00000 \\
0.00\end{array}$ & 0.00007 & & 0.00008 & \\
\hline & 0.0000 & 0.0006 & & & \\
\hline & & 0.0081 & & 0.0005 & \\
\hline & & 0.0083 & & 0.0005 & \\
\hline & & 0.0081 & & 0.0005 & \\
\hline & & 0.0081 & & 0.0005 & 0.000 \\
\hline & 0.0000 & 0.0133 & & 0.0000 & \\
\hline & 0.0000 & 0 & & & \\
\hline & & 0008 & & & \\
\hline
\end{tabular}


\# Glass ID

1395 GLA 78-10-16
1396 P2-OCa-0F

1397 P2-OCa-1F

1398 P2-OCa-2F

1400 P2-0Ca-4F

1402 P2-3Ca-0F

1403 P2-3Ca-1F

$1405 \mathrm{P} 2-3 \mathrm{Ca}-3 \mathrm{~F}$

1407 P2-3Ca-5F

1410 P2-9Ca-2F

412 P2-9Ca-4F

1414 SRL 165 HiFe

1415 SRL 165 TDS
1416 SRL 165 HiAl

1417 HLW98-01

1419 HLW98-03

1421 HLW98-05

1422 HLW98-06
1423 HLW98-07

1423 HLW98-07
1424 HLW98-08

1425 HLW98-09
1426 HLW98-10

1428 HLW98-12

1429 HLW98-13

1431 HLW98-15
1432 HWW

1433 HLW98-17

1434 HLW98-18
1435 HLW98-19

1436 HLW98-20

1438 HLW88-22

439 HLW98-23

1440 HLW98-24
1441 HLW98-25

1442 HLW98-26
1443 HLW98-27日

1444 HLW98-28

1445 HLW98-29

1447 HLW98-31

1448 HLW68-32A
1449 HLW98-33

1450 HLW98-34
1451 HLW98-34CC

1452 HLW98-35

1454 HLW98-37

1455 HLW98-38

1457 HLW W8-40

1459 HLW98-42

1460 HLW98-43

1462 HLW98-45

1463 HLW98-46
1464 HLW98-47

1465 HLW98-48
1466 HLW98-49

1467 HLW98-50
1468 HLW98-51R

1469 HLW98-52

1472 HLW98-55

1473 HLW98-56
1474 HLW98-57

1475 HLW98-58
1476 HLW98-59
CoO-t Co203-t Cr2O3-t Cs2O-t CuO-t Dy2O3-t Eu2O3-t

F-t Ga2O3-t Gd2O3-t HfO2-t HgO-t

1-t La203-t MnO2-t MnO-t MoO-t MoO2-t MoO3-t Nb205-t Nd2O3-t NpO2-t PbO-t PdO2-t PdO-t Pr203-t Pr6011-t Rb2O-t ReO-t

0.0010

0.0013
0.0013
0.0009

0.0013
0.0009

0.0009
0.0009

$\begin{array}{lll}0.0029 & 0.0014 & 0.0010 \\ 0.0030 & 0.0014 & 0.001\end{array}$

$\begin{array}{lll}0.0017 & 0.0017 & 0.0009 \\ 0.0018 & 0.0018 & 0.0009\end{array}$

$0.0017 \quad 0.0063 \quad 0.0009$

$\begin{array}{llll}0.0016 & 0.0032 & 0.0008 \\ 0.0017 & 0.0059 & 0.0008 \\ 0.017 & 0.002 & 0.009\end{array}$

$0.0017 \quad 0.0062 \quad 0.0009$

$\begin{array}{lll}0.0009 & 0.0048 & 0.0004 \\ 0.0008 & 0.0042 & 0.0004\end{array}$

$\begin{array}{lll}0.0018 & 0.0078 \\ 0.0018 & 0.0078 & 0\end{array}$

$\begin{array}{lll}0.0017 & 0.0061 & 0.0009 \\ 0.0017 & 0.0060 & 0.0009 \\ 0.0010 & 0.0044 & 0.0004\end{array}$

.00190 .0082

$\begin{array}{lll}0.0018 & 0.0038 & \\ 0.0009 & 0.0039 & 0.0004\end{array}$

$\begin{array}{lll}0.0011 & 0.0023 \\ 0.0010 & 0.0021\end{array}$

$\begin{array}{lll}0.0009 & 0.0039 & 0.0004 \\ 0.0008 & 0.0038 & 0.0004 \\ 0.0005 & 0.0008 & 0.0003\end{array}$

$\begin{array}{lll}0.0005 & 0.0008 & 0.0003\end{array}$

$\begin{array}{lll}0.0005 & 0.0008 & 0.0003 \\ 0.0005 & 0.0008 & 0.0003 \\ 0.005 & 0.008 & 0\end{array}$

$\begin{array}{llll}0.0005 & 0.0008 & 0.0003 \\ 0.0005 & 0.0008 & 0.0003 \\ 0 & 0.004 & 0.0008 & 0.0003\end{array}$

$\begin{array}{llll}0.0008 & 0.0009 & 0.0002 \\ 0.0015 & 0.0009 & 0.0000\end{array}$

$\begin{array}{lll}0.0015 & 0.0009 \\ 0.0016 & 0.0010 \\ 0.0016 & 0.0010\end{array}$

$\begin{array}{llll}0.0008 & 0.0010 & 0.0002 \\ 0.0014 & 0.0000 & 0.0000\end{array}$

$\begin{array}{lll}0.0014 & 0.0010 & 0.0002 \\ 0.0011 & 0.0000 & 0.0000 \\ 0.0011 & 0.0011 & 0.0000\end{array}$

$0.0019 \quad 0.0000 \quad 0.0004$

$\begin{array}{lll}0.0021 & 0.0000 & 0.0004 \\ 0.0008 & 0.0010 & 0.0002 \\ 0.002 & 0.000 & 0.004\end{array}$

$\begin{array}{llll}0.0020 & 0.00000 & 0.00004 \\ 0.000 & 0.0009 & 0.002\end{array}$

$\begin{array}{lll}0.0008 & 0.0009 & 0.0002 \\ 0.0019 & 0.0000 & 0.0004 \\ 0.0019 & 0.0000 & 0.0004\end{array}$

$\begin{array}{llll}0.0019 & 0.0000 & 0.0004 \\ 0.0018 & 0.0000 & 0.0003 \\ 0.0017 & 0.000 & 0.003\end{array}$

$\begin{array}{llll}0.0017 & 0.0000 & 0.0003\end{array}$

$\begin{array}{llll}0.0017 & 0.0000 & 0.0003 \\ 0.0014 & 0.0000 & 0.0003\end{array}$

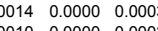

\begin{tabular}{llll} 
& 0.0014 & 0.0000 & 0.00002 \\
0.0014 & 0.0000 & 0.0003 \\
\hline
\end{tabular}

$\begin{array}{llll}0.0008 & 0.0009 & 0.0002 \\ 0.0008 & 0.0010 & 0.0002\end{array}$

$\begin{array}{llll}0.0008 & 0.0009 & 0.0002\end{array}$

$\begin{array}{llll}0.0008 & 0.00009 & 0.00002\end{array}$

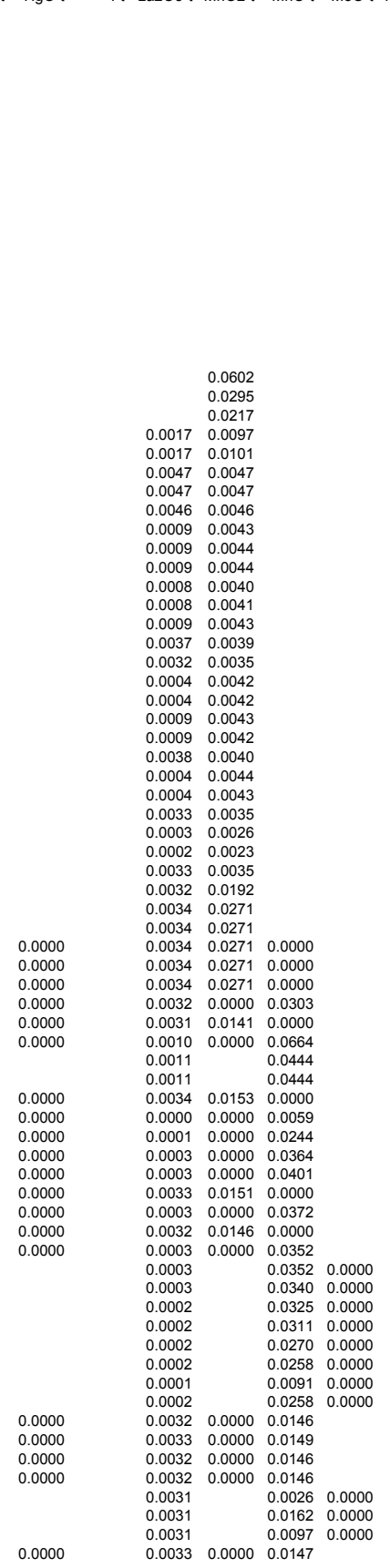

0.0000

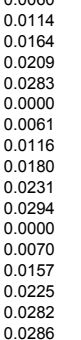

0.0001

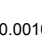

0.0010
0.0010
0.005

0.0005
0.0005
0.0005

0.0005
0.0009
0.0009

0.0008

0.0008
0.0009
0.0005

0.0005
0.0005

0.0009
0.0009
0.0006

0.0005

0.0005
0.0005
0.0004
0.0004

0.0004
0.0004
0.0004
0.0004

0.0004
0.0004
0.0001

0.0001
0.0003

0.0002
0.0002
0.0002

0.0001
0.0000
0.0000

0.0000

0.0000

0.00000
0.0000

0.0000
0.0000
0.000

0.0000
0.0000
0.0000

0.0000

0.0000
0.0002
0.0002

0.0002

0.0001
0.0001
0.0001

0.0002

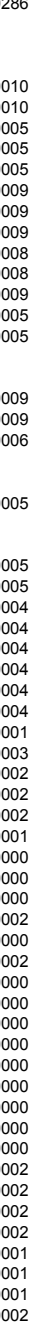

(

0.004

0.0047
0.0046

0.0044

0.0040
0.0041
0.0043

0.0043
0.0042

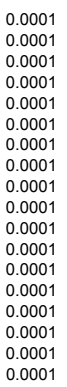

0.0022

0.0000

0.0000
0.0000

0.0000
0.0000

0.0000

0.0000
0.0000
0.0000 
$0.0007 \quad 0.0003 \quad 0.0010$

$\begin{array}{lll}0.0006 & 0.0002 & 0.0009 \\ 0.0006 & 0.0002 & 0.0009\end{array}$

1398 P2-OCa-2F
1399 P2-OCa-3F
1400 P2-OCa-4F

1400 P2-OCa-4F
1401 P2-OCa-5F
1402 2 2-3-3a-0F

1402 P2-3Ca-OF

$1405 \mathrm{P} 2-3 \mathrm{Ca}-3 \mathrm{~F}$

1406 P2-3Ca-4F

1407 P2-3Ca-5F

1409 P2-9Ca-1F

411 P2-9Ca-3F

1414 SRL 165 HiF

1415 SRL 165 TD
1416 SRL 165 Hi

1417 HLW98-01

1419 HLW98-03

1421 HLW98-05

1422 HLW98-06

1424 HLW88-08

1425 HLW98-09
1426 HLW98-10

1427 HLW98-11

428 HLW $48-12$
429 HLW

1430 HLW98-14
1431 HLW98-15

1432 HLW98-16

1433 HLW98-17

1434 HLW98-18

1436 HLW98-20

1437 HLW98-21

1438 HLW98-22

1440 HLW98-24
1441 HLW98-25

1442 HLW98-26
1443 HLW98-27日

1444 HLW98-28

1446 HLW98-30

1448 HLW98-32A

1449 HLW98-33

1451 HLW98-34CG

1452 HLW98-35

1454 HLW98-37

HLW W8 -38
HLW98-39

1457 HLW98-40

1459 HLW98-42

1460 HLW98-43

1461 HLW98-44
1462 HLW98-45

463 HLW98-46
464 HLW98-47

1465 HLW98-48

1467 HLW98-50

1469 HLW98-52

HLW98-53A

1471 HLW98-54
1472 HLW98-55

1473 HLW988-56
1474 HLW98-57

1475 HLW98-58
1476 HLW98-59

$\begin{array}{llll}0.0006 & 0.0002 & 0.0009 \\ 0 & 0.0006 & 0.0002 & 0.009\end{array}$

$\begin{array}{lll}0.0006 & 0.0002 & 0.0009 \\ 0.0007 & 0.0003 & 0.0010\end{array}$

$\begin{array}{llll}0.0006 & 0.0002 & 0.0009 \\ 0.0006 & 0.0002 & 0.0009\end{array}$

$\begin{array}{lll}0.0006 & 0.0002 & 0.0009 \\ .0006 & 0.0002 & 0.0009 \\ 0.000 & 0.002\end{array}$

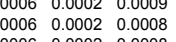

$\begin{array}{lll} & 0.0002 & 0.000 \\ 0.006 & 0.00002 & 0.00008 \\ 0.0008\end{array}$

$\begin{array}{lll}0.0006 & 0.0002 & 0.0008\end{array}$

$\begin{array}{lll}0.0006 & 0.0002 & 0.0008 \\ 0.0006 & 0.0002 & 0.0008\end{array}$

$\begin{array}{ll}0.0019 & 0.0019 \\ 0.0020 & 0.0020 \\ 0.0005 & 0.0005\end{array}$

$\begin{array}{ll}0.0005 & 0.0005 \\ 0.0005 & 0.0005 \\ 0.0005 & 0.0005 \\ 0.0053 & 0.0026\end{array}$

$\begin{array}{ll}0.0005 & 0.0005 \\ 0.0043 & 0.0026 \\ 0.0044 & 0.0026\end{array}$

$0.0020 \quad 0.0024$

0.00210 .0025

$\begin{array}{ll}0.0030 & 0.0021 \\ 0.0027 & 0.0019\end{array}$

$\begin{array}{ll}0.0021 & 0.0025\end{array}$

$\begin{array}{ll}0.0021 & 0.0025 \\ 0.0021 & 0.0025 \\ 0.0031 & 0.0022\end{array}$

$0.0027 \quad 0.0019$

$0.0027 \quad 0.0019$

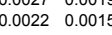

$0.0022 \quad 0.0015$

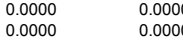

0.0000
0.0000
0.0000
0.0000

$\begin{array}{ll}0.0000 & 0.0000 \\ 0.0000 & 0.0000 \\ 0.0000 & 0.0000\end{array}$

$0.0022 \quad 0.0015$

$\begin{array}{ll}0.0034 & 0.0006 \\ 0.0000 & 0.0000\end{array}$

$\begin{array}{ll}0.0000 & 0.0000 \\ 0.0000 & 0.0000\end{array}$

$\begin{array}{ll}0.0037 & 0.0007 \\ 0.0000 & 0.0000 \\ 0.000 & 0.0000\end{array}$

$.0000 \quad 0.0000$

$0.0000 \quad 0.0000$

$0.0037 \quad 0.0006$

0.00360 .0006

$0.0000 \quad 0.0000$

$\begin{array}{ll}0.0000 & 0.0000 \\ 0.0000 & 0.0000\end{array}$

$0.0000 \quad 0.0000$

$\begin{array}{ll}0.0000 & 0.0000 \\ 0.0000 & 0.0000 \\ 0.0000 & 0.0000\end{array}$

.00000 .0000

0.00360 .0006

$0.0036 \quad 0.0006$

$0.0036 \quad 0.0006$

$\begin{array}{ll}0.0000 & 0.0000 \\ 0.0000 & 0.0000 \\ 0.0000 & 0.0000\end{array}$

$\begin{array}{ll}0.0000 & 0.0000 \\ 0.0036 & 0.0006\end{array}$

0.00430 .0229

$\begin{array}{lll}0.0022 & 0.011 \\ 0.0024 & 0.0118 \\ 0.0023 & 0.0115 & 0\end{array}$

0.0023
0.0021
0.0 .00055
0.0222
0.0088

0.002220 .0008
0.0022
0.0229
0.02020
0.0104

$\begin{array}{lll}0.00222 & 0.02104 \\ 0.0021 & 0.021 \\ 0.021 & 0.0225 \\ 0.0030 & 0.015\end{array}$

$\begin{array}{ll}0.0021 & 0.0225 \\ 0.0038 & 0.0175 \\ 0.0033 & 0.0156 \\ 0.0013 & 0.0275\end{array}$

$\begin{array}{ll}0.0013 & 0.0156 \\ 0.0013 & 0.0275 \\ 0.0013 & 0.0275\end{array}$

$\begin{array}{ll}0.0021 & 0.0222 \\ 0.0021 & 0.0217\end{array}$

$\begin{array}{lll}0.0021 & 0.0222 \\ 0.0039 & 0.02143 \\ 0.0014 & 0.0290\end{array}$

$\begin{array}{lll}0.0014 & 0.0116 \\ 0.0034 & 0.0125\end{array}$

$\begin{array}{ll}0.0034 & 0.0125 \\ 0.0008 & 0.1013 \\ 0.0007 & 0.0917\end{array}$

$\begin{array}{ll}0.0034 & 0.0125 \\ 0.0033 & 0.0117\end{array}$

$\begin{array}{ll}0.0033 & 0.0117 \\ 0.0026 & 0.0245\end{array}$

$\begin{array}{rr}0.0026 & 0.0245 \\ 0.0026 & 0.0225 \\ 0.000 & 0.026 \\ 0 & 0.0245\end{array}$

$\begin{array}{lll}0.0000 & 0.0026 & 0.0245 \\ 0.0000 & 0.0026 & 0.0245\end{array}$

$\begin{array}{lll}0.0000 & 0.0026 & 0.0225 \\ 0.0000 & 0.0025 & 0.0232 \\ 0.0000 & 0.0002 & 0.126\end{array}$

$\begin{array}{lll}0.0000 & 0.0002 & 0.0226 \\ 0.0000 & 0.0003 & 0.1156\end{array}$

$\begin{array}{ll}0.0002 & 0.0735 \\ 0.0002 & 0.0735 \\ 0.0003 & 0.0245\end{array}$

$\begin{array}{lll}0.0000 & 0.0003 & 0.0245 \\ 0.0000 & 0.0001 & 0.0000 \\ 0.00010 & 0.0000 & 0.0290\end{array}$

$\begin{array}{lll}0.0000 & 0.0000 & 0.029 \\ 0.0010 & 0.0000 & 0.0480\end{array}$

$\begin{array}{lll}0.0011 & 0.0000 & 0.0529 \\ 0.0000 & 0.0003 & 0.0242\end{array}$

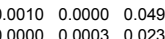

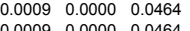

$\begin{array}{llllllll} & 0.0000 & 0.0000 & 0.0541 & 0.0002 \\ 0\end{array}$

$\begin{array}{llllllll}0.0008 & 0.0000 & 0.0410 & 0.00000 & 0.0000 & 0.04494 & 0.00002 \\ 0.0007 & 0.0000 & 0.0356 & 0.0000 & 0.0000 & 0.0429 & 0.0002\end{array}$

$\begin{array}{lllllllll}0.0007 & 0.0000 & 0.0340 & 0.0000 & 0.0000 & 0.0410 & 0.0002\end{array}$

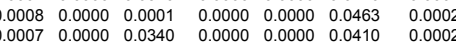

$\begin{array}{lllllll}0.0000 & 0.0003 & 0.0234 & & 0.0002 & 0.0000 & 0.0000 \\ 0.0000 & 0.0003 & 0.0238 & & 0.0002 & 0.0000 & 0.0000\end{array}$

$\begin{array}{llllll}0.0000 & 0.0003 & 0.0234 & 0.0002 & 0.0000 & 0.00000 \\ 0 & & 0.00000\end{array}$

$\begin{array}{lllllll}0.0000 & 0.0003 & 0.0234 & & 0.0002 & 0.0000 & 0.0000\end{array}$

$\begin{array}{lllllll}0.0000 & 0.0002 & 0.0000 & 0.0000 & 0.0000 & 0.0000 & 0.000 \\ 0.0000 & 0.0003 & 0.0262 & 0.0000 & 0.0000 & 0.0001 & 0.0001\end{array}$

$\begin{array}{lllllll}0.0000 & 0.0003 & 0.0136 & 0.0000 & 0.0000 & 0.0001 & 0.0001 \\ 0.0000 & 0.0003 & 0.0235 & & 0.0002 & 0.0000 & 0.0000\end{array}$

$0.0195 \quad 0.9999$

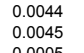

0.0005
0.0005
0.0005

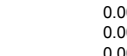

0.0005

$\begin{array}{ll}0.0005 & 0.0000 \\ 0.0009 & 0.0009\end{array}$

$\begin{array}{lll}0.0009 & 0.0009 \\ 0.0009 & 0.0009 \\ 0.0008 & 0.0009\end{array}$

$\begin{array}{ll}0.0008 & 0.0008 \\ 0.0008 & 0.0008\end{array}$

0.0050
0.0044

0.00090 .0009

$\begin{array}{lll}0.0200 & 1.0003 & 0.1085 \\ 0.1070\end{array}$

$\begin{array}{lll}0.0200 & 1.0007 & 0.1048 \\ 0.0200 & 1.0008 & 0.0819 \\ 0.0200 & 1.0003 & 0.0723\end{array}$

$\begin{array}{lll}0.0200 & 1.0003 & 0.0723 \\ 0\end{array}$

$\begin{array}{lll}0.0200 & 1.0001 & 0.0839 \\ 0.0200 & 1.0000 & 0.0715 \\ 0.0200 & 1.0003 & 0.065\end{array}$

$\begin{array}{lll}0.0200 & 1.0003 & 0.0665\end{array}$

$\begin{array}{lll}0.0200 & 1.0003 & 0.0749 \\ 0.0200 & 1.0000 & \\ 0.02000 & 0.0958\end{array}$

$0.02000 .9998 \quad 0.0835$

$\begin{array}{lllllll}0.0000 & 0.0009 & 0.0009 & & 0.0200 & 1.0002 & 0.0749 \\ & 0.0009 & 0.0009 & & 0.0200 & 1.0000 & 0.066\end{array}$

$\begin{array}{lll}0.0200 & 1.0000 & 0.066 \\ 0.0200 & 1.0002 & -1\end{array}$

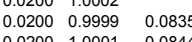

$\begin{array}{lll}0.0200 & 1.0001 & 0.0844 \\ 0.0200 & 1.0000 & 0.0623 \\ 0.0200 & 1.0000 & 0.059\end{array}$

$\begin{array}{lll}0.0200 & 1.0000 & 0.0559 \\ 0.0200 & 1.0000 & 0.048\end{array}$

$\begin{array}{lll}0.0200 & 1.0000 \\ 0.0200 & 1.0000\end{array}$

.

0.0200
0.0200
1.0001

$0.0200 \quad 1.0001$

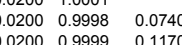

$\begin{array}{lll}0.0200 & 1.0000 & 0.1040 \\ 0.0200 & 1.0000 & 0.104\end{array}$

$\begin{array}{lll}0.0200 & 1.0002 \quad 0.0947\end{array}$

$\begin{array}{lll}.0200 & 1.0000 & 0.0453 \\ 0.0200 & 1.0002 & 0.0340\end{array}$

$\begin{array}{lll}.0100 & 0.9998 & 0.040 \\ 0.0200 & 1.0001 & 0.0767 \\ 0.0200 & 1.0000 & 0.04\end{array}$

$0.0200 \quad 09999 \quad 0.048$

0.032

0.0000

0.00000
0.0000

$\begin{array}{lllll}0.0000 & 0.0200 & 1.0001 & 0.0313\end{array}$

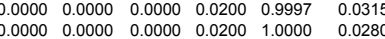

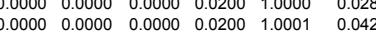

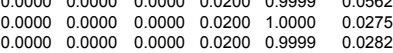
$\begin{array}{lll}0.0200 & 1.0002 & 0.0756 \\ 0.0200 & 1.0000 & 0.0577\end{array}$ $\begin{array}{lll}0.0002 & 0.9999 & 0.077\end{array}$ 
$\begin{array}{lll}0.1025 & 0.0183 & 0.0897 \\ 0.1269 & 0.0151 & 0.087\end{array}$ $\begin{array}{lll}0.1309 & 0.0148 & 0.1108 \\ 0.0988 & 0.0051 & 0.1171\end{array}$ $\begin{array}{lll}0.1003 & 0.0056 & 0.1248 \\ 0.1264 & 0.0163 & 0.1168 \\ 0.120 & 0.058 & 0.143\end{array}$ $\begin{array}{lll}0.1049 & 0.0135 & 0.1110 \\ 0.158 & 0.0170 & 0.1061 \\ 0.1466 & 0.0037 & 0.1194\end{array}$ $\begin{array}{lll}0.1260 & 0.0196 & 0.0853 \\ 0.1579 & 0.0199 & 0.0850 \\ 0.1248 & 0151 & 0.1072\end{array}$ $\begin{array}{lll}0.1579 & 0.0199 & 0.0850 \\ 0.1141 & 0.0151 & 0.1072 \\ 0.1238 & 0.0134 & 0.1065\end{array}$ $\begin{array}{lll}0.1200 & 0.0200 & 0.0821\end{array}$ $\begin{array}{lll}0.1200 & 0.0200 & 0.0821 \\ 0.1245 & 0.0203 & 0.0922 \\ 0.0728 & 0.0033 & 0.1058 \\ 0.1168 & 0.117 & 0.124\end{array}$ $\begin{array}{lll}0.0728 & 0.0033 & 0.1058 \\ 0.1176 & 0.0129 & 0.1274 \\ 0.1133 & 0.117 & 0.105\end{array}$ $\begin{array}{lll}0.1158 & 0.0117 & 0.1189 \\ 0.1133 & 0.0032 & 0.1056\end{array}$

\section{$0.0388 \quad 0.0040 \quad 0.1238$} $\begin{array}{lll}0.0950 & 0.0029 & 0.0964 \\ 0.0900 & 0.0029 & 0.1014\end{array}$ $\begin{array}{lll}0.0678 & 0.0086 & 0.1101\end{array}$ $\begin{array}{lll}0.0671 & 0.0163 & 0.1095 \\ 0.0868 & 0.0279 & 0.0376\end{array}$ $\begin{array}{lll}0.0868 & 0.0279 & 0.0376 \\ 0.0519 & 0.0053 & 0.0537\end{array}$ $\begin{array}{lll}0.0519 & 0.0053 & 0.0537 \\ 0.0529 & 0.0075 & 0.0656 \\ 0.023 & 0.0087 & 0.0711\end{array}$ $\begin{array}{lll}0.0233 & 0.0087 & 0.0711 \\ 0.0603 & 0.0035 & 0.1123 \\ 0.0359 & 0.0120 & \end{array}$ $0.0598 \quad 0.0066 \quad 0.0610$ $\begin{array}{lll}0.0705 & 0.0071 & 0.0599 \\ 0.0716 & 0.0070 & 0.060\end{array}$ $\begin{array}{lll}0.0730 & 0.0067 & 0.0568\end{array}$ $\begin{array}{lll}0.0749 & 0.0070 & 0.0532 \\ 0.0903 & 0.0056 & 0.0470 \\ 0.1177 & 0.051 & 0.0474\end{array}$ $\begin{array}{lll}0.0717 & 0.0052 & 0.0457 \\ 0.1117 & 0.0051 & 0.0444 \\ 0.0952 & 0.0055 & 0.0473\end{array}$

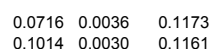
$\begin{array}{lll}0.0745 & 0.0069 \quad 0.1128\end{array}$ $\begin{array}{lll}0.0397 & 0.0075 & 0.1086 \\ 0.0427 & 0.0033 & 0.1055\end{array}$ $0.1220 \quad 0.0058 \quad 0.1143$ $\begin{array}{llllllllll}0.0032 & 0.0289 & 0.0089 & 0.1124 & 0.0022 & 0.0108 & 0.3883 & 0.0324 & 0.0010 & 0.0003 \\ 0.0032 & 0.0003 & 0.0084 & 0.1052 & 0.02020 & 0.0105 & 0.3764 & 0.0193 & 0.0009 & 0.0004\end{array}$

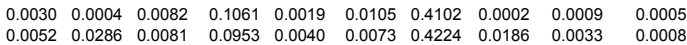

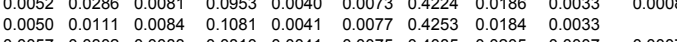
$\begin{array}{llllllllll}0.0057 & 0.0302 & 0.0083 & 0.0810 & 0.0041 & 0.0075 & 0.4085 & 0.0205 & 0.0007 & 0.0007 \\ 0.0053 & 0.0268 & 0.0084 & 0.0762 & 0.0038 & 0.0070 & 0.4336 & 0.0199 & 0.0008 & 0.0005\end{array}$ $\begin{array}{llllllllll}0.0039 & 0.0243 & 0.0073 & 0.0682 & 0.0051 & 0.0081 & 0.3799 & 0.0162 & 0.0018 & 0.0004 \\ 0.0050 & 0.0308 & 0.0090 & 0.0848 & 0.0042 & 0.0069 & 0.4215 & 0.0207 & 0.0006 & 0.0005 \\ 0.0042 & 0.0615 & 0.0012 & 0.0290 & 0.0046 & 0.0095 & 0.4074 & 0.0359 & 0.0007 & 0.0006\end{array}$ $\begin{array}{lllllllllll}0.0031 & 0.0280 & 0.0118 & 0.1274 & 0.0018 & 0.0104 & 0.4214 & 0.0044 & 0.0010 & \\ 0.0029 & 0.0007 & 0.0115 & 0.1237 & 0.0016 & 0.0088 & 0.4107 & 0.0041 & 0.0010 & 0.0001\end{array}$ $\begin{array}{llllllllll}0.0029 & 0.0007 & 0.0115 & 0.1237 & 0.0016 & 0.0088 & 0.4107 & 0.0041 & 0.0010 & 0.0001 \\ 0.0034 & 0.0164 & 0.0106 & 0.0986 & 0.0042 & 0.0055 & 0.4265 & 0.0121 & 0.0001 & 0.0007 \\ 0.0031 & 0.0294 & 0.0092 & 0.0893 & 0.0038 & 0.0049 & 0.4226 & 0.0109 & & 0.0007\end{array}$

$\begin{array}{lllllllll}0.0027 & 0.0006 & 0.0111 & 0.1205 & 0.0016 & 0.0079 & 0.3933 & 0.0032 & 0.0011\end{array}$

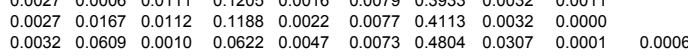
$\begin{array}{lllllllll}0.0019 & 0.0301 & 0.00067 & 0.00775 & 0.0047 & 0.0010 & 0.0048 & 0.43734 & 0.0018\end{array}$

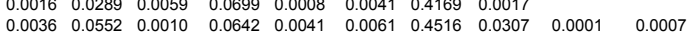

$\begin{array}{llllllllll}0.0035 & 0.0403 & 0.0011 & 0.1267 & 0.0054 & 0.0019 & 0.4232 & 0.0241 & 0.0001 & 0.0003\end{array}$

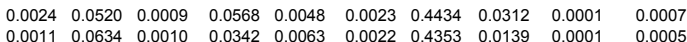

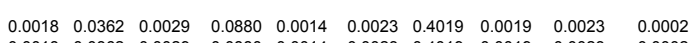

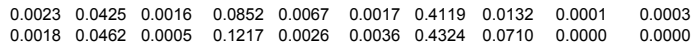

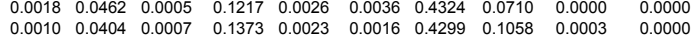

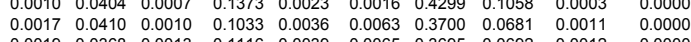

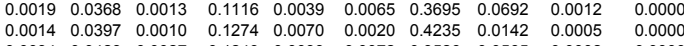

$\begin{array}{llllllllll}0.0013 & 0.0539 & 0.0010 & 0.1035 & 0.0033 & 0.0055 & 0.3383 & 0.0560 & 0.0011 & 0.0003\end{array}$

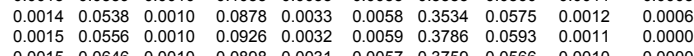

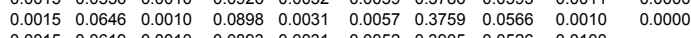

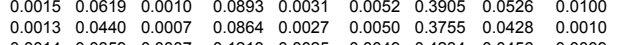

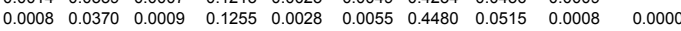

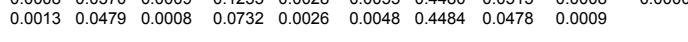

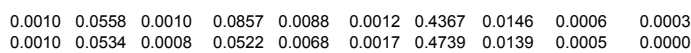

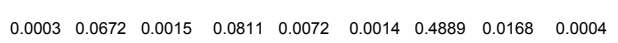

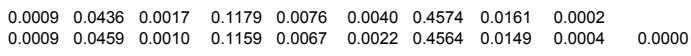

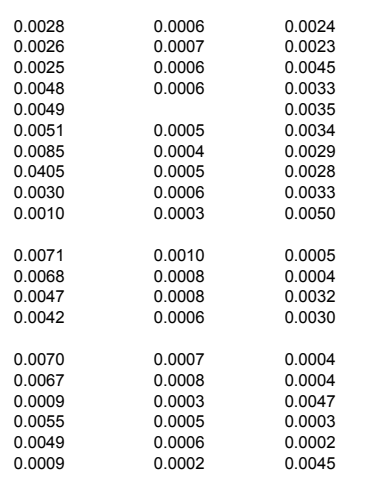


$0.0000 \quad 0.0415$

$\begin{array}{ll}0.0000 & 0.0415 \\ 0.0146 & 0.0000 \\ 0.0000 & 0.0060\end{array}$

$0.0000 \quad 0.0060$

$\begin{array}{ll}0.0000 & 0.0246 \\ 0.0000 & 0.0353\end{array}$

$\begin{array}{ll}0.0000 & 0.0367 \\ 0.0160 & 0.0000\end{array}$

$0.0000 \quad 0.0326$

0.0000
0.0326
0.0413
0.0347
0.0317
0.0260
0.0261
0.0100
0.0271

$\begin{array}{ll}0.0000 & 0.0159 \\ 0.0000 & 0.0157\end{array}$

0.0028

$\begin{array}{ll}0.0000 & 0.0106 \\ 0.016\end{array}$

$\begin{array}{ll}0.0028 & 0.0000 \\ 0.0028 & 0.0000\end{array}$

$\begin{array}{ll}0.0028 & 0.0000 \\ 0.0012 & 0.0000 \\ 0.0011 & 0.0000 \\ 0.0030 & 0.0004\end{array}$

$\begin{array}{ll}0.0012 & 0.0002 \\ 0.0013 & 0.0004 \\ 0.030 & 0.0005\end{array}$

$\begin{array}{ll}0.0036 & 0.0005 \\ 0.0013 & 0.0000\end{array}$

$0.0028 \quad 0.0002$

0.0029
0.0030 $\quad \begin{aligned} & 0.0002 \\ & 0.0004 \\ & 0.0025\end{aligned}$

$\begin{array}{ll}0.0028 & 0.0004 \\ 0.0027 & 0.0003 \\ 0.000 & 0.0002\end{array}$

$\begin{array}{ll}0.00019 & 0.0002 \\ 0.0017 & 0.000\end{array}$

$0.0011 \quad 0.0001$

0.0004

0.0005 


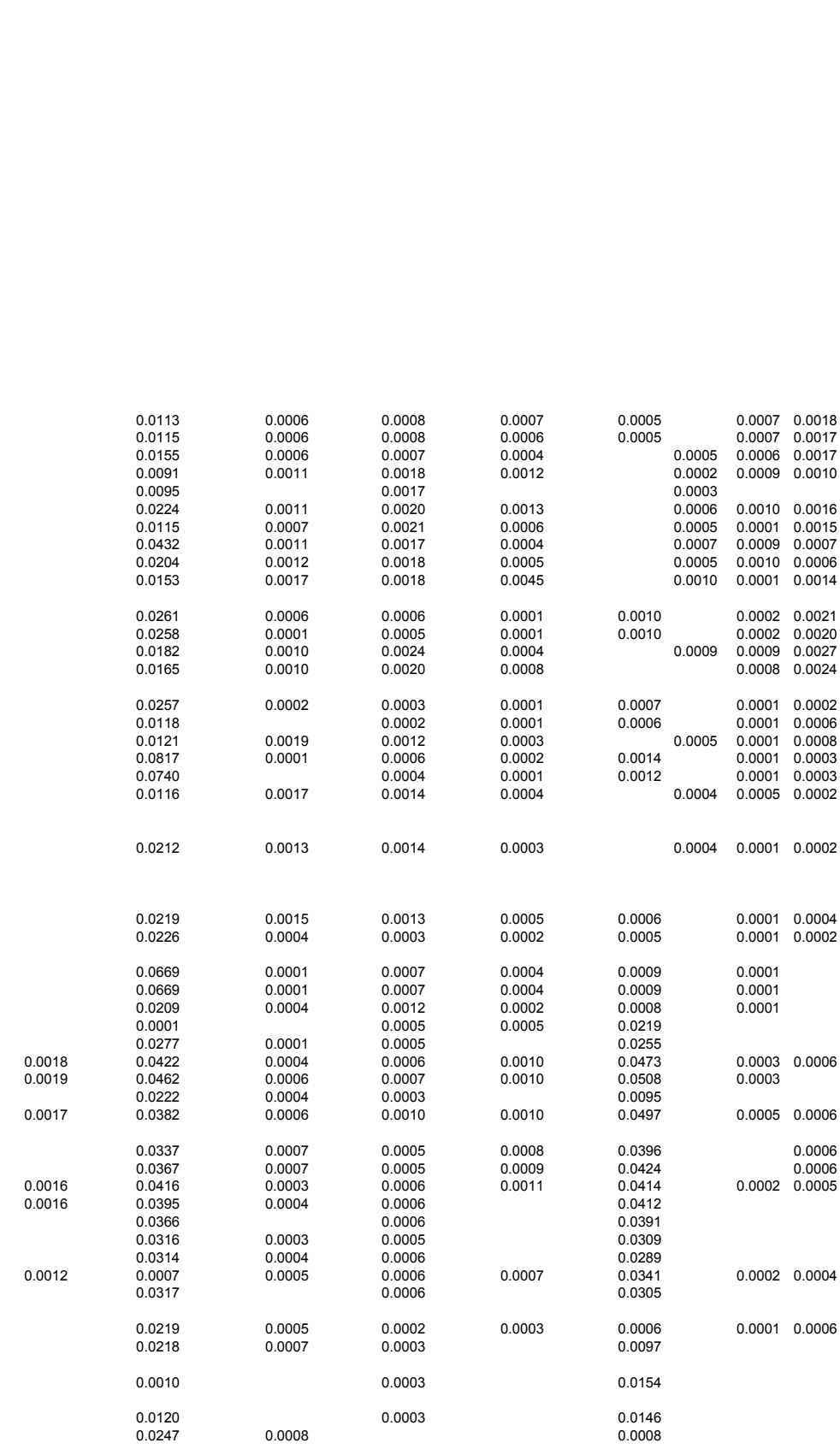

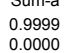

0.0000 
Homogeneous glass with a few undissolved inclusions

Homogeneous glass

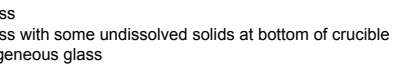

Dark brown homogeneous glas

Mostly homogeneous glass with some multicolor swirls

Inhomogeneous glass with brown swirls. Undissolved secondary phase

Dull looking brown glass
Homogeneous dark brown glass

Homogeneous dark brown glass

Inhomogeneous glass shows iridescent swirls
Inhomogeneous glass with greenish brown crystallization

Homogeneous dark brown glass
Homogeneous dark brown glass

Homogeneous dark brown glass

Homogeneous brown glass

Homogeneous brown glass
Homogeneous dark brown glas

Homogeneous dark brown glass
Homogeneous dark brown glass

Homogeneous glass with some undissolved solids

Homogeneous dark brown glass
Homogeneous dark brown glass

Dark brown homogeneous glass

Inhomogeneous glass shows multicolor swirls

Homogeneous dark brown glass
Homogeneous dark brown glas

Homogeneous dark brown glass

Homogeneous amber tinted glass
Homogeneous dark brown glass

Homogeneous dark brown glass

Mostly homogeneous with small amount of undissolved solids

Homogeneous dark brown glass
Homogeneous dark brown glass

glass with metallic appearance Homogeneous dark brown glass with bluish metallic app

Inhomogeneous glass with multicolor swirls

Homogeneous dark brown glass
Homogeneous dark brown glass

Homogeneous dark brown glass

Homogeneous dark brown glass
Homogeneous dark brown glass

Dark brown glass with very small amount of undissolved solids

Homogeneous dark brown glass

Clear, homogeneous dark brown gias

Homogeneous dark brown glass

Inhomogeneous brown opaque glass with light color swirls

Inhomogeneous brown glass with
Inhomogeareneous brown glass with multicolor swirls

Inhomogeneous glass with many brown and green streaks

Homogeneous dark brown ngass

inhomogeneous brown glass with swirling patterns throughout
2.801
2.662
2.622

$\begin{array}{ll}1100.0 & 4.600 \\ 1100.0 & 7.700 \\ 1100 & 6.700\end{array}$

$\begin{array}{llll}-12.081 & 20002.5 & 1000.0 & 37.953\end{array}$

$\begin{array}{rrrr}-11.363 & 18962.8 & 1000.0 & 34.228 \\ -13.517 & 23522.8 & 1000.0 & 142.840 \\ -11.691 & 18832.3 & 1000.0 & 22.380\end{array}$

$\begin{array}{llll}-11.723 & 18293.2 & 1000.0 & 14.450\end{array}$

$\begin{array}{llll}-10.742 & 16937.1 & 1000.0 & 13.128 \\ -12.045 & 201703 & 1000 & 4000\end{array}$

$\begin{array}{llll}-11.278 & 19350.5 & 1000.0 & 51.088\end{array}$

$\begin{array}{llll}-10.746 & 17369.6 & 1000.0 & 18.481 \\ -10.833 & 17819.0 & 1000.0 & 24.045\end{array}$

2.750

2830

$\begin{array}{llll}-11.094 & 17871.5 & 1000.0 & 19.199\end{array}$

$\begin{array}{llll}-11.803 & 18764.9 & 1000.0 & 19.235\end{array}$

$\begin{array}{lll}-10.165 & 15695.5 & 1000.0\end{array}$

$\begin{array}{llll}-10.165 & 15695.5 & 1000.0 & 8.833\end{array}$

$\begin{array}{llll}-11.392 & 16326.0 & 1000.0 & 4.251\end{array}$ 


\begin{tabular}{|c|c|c|c|c|c|c|c|}
\hline 1050 & 20.793 & 1100 & 12.0 & 1150.0 & 7.2 & 1200.0 & 4.5 \\
\hline 1050 & 19.493 & 1100 & 11.6 & 1150.0 & 7.1 & 1200.0 & 4.5 \\
\hline $\begin{array}{l}1050 \\
1050\end{array}$ & $\begin{array}{l}71.050 \\
12686\end{array}$ & $\begin{array}{l}1100 \\
11100\end{array}$ & $\begin{array}{r}37.2 \\
75\end{array}$ & $\begin{array}{l}1150.0 \\
11500\end{array}$ & 20.4 & 1200.0 & $\begin{array}{c}11.6 \\
30\end{array}$ \\
\hline 1050 & 8.072 & 1100 & 4.8 & 1150.0 & 3.1 & 1200.0 & 2.1 \\
\hline 1050 & 7.782 & 1100 & 4.9 & 1150.0 & 3.2 & 1200.0 & 2.2 \\
\hline 1050 & 24.311 & 1100 & 13.8 & 1150.0 & 8.4 & 1200.0 & 5.4 \\
\hline 1050 & 28.278 & 1100 & 16.5 & 1150.0 & 10.1 & 1200.0 & 6.5 \\
\hline 1050 & 10.717 & 1100 & 6.6 & 1150.0 & 4.3 & 1200.0 & 2.9 \\
\hline 1050 & 13.813 & 1100 & 8.4 & 1150.0 & 5.4 & 1200.0 & 3.6 \\
\hline 1050 & 11.114 & 1100 & 6.8 & 1150.0 & 4.3 & 1200.0 & 2.9 \\
\hline 1050 & 10.676 & 1100 & 6.3 & 1150.0 & 4.0 & 1200.0 & 2.6 \\
\hline
\end{tabular}

$\begin{array}{llllllll}1050 & 5.420 & 1100 & 3.5 & 1150.0 & 2.4 & 1200.0 & 1.7 \\ 1050 & 5.420 & 1100 & 3.5 & 1150.0 & 2.4 & 1200.0 & 1.7 \\ 1050 & 2.558 & 1100 & 1.6 & 1150.0 & 1.1 & 1200.0 & 0.7 \\ & & & & & & & \\ 1050 & 9.850 & 1100 & 5.9 & 1150.0 & 3.8 & 1200.0 & 2.5\end{array}$

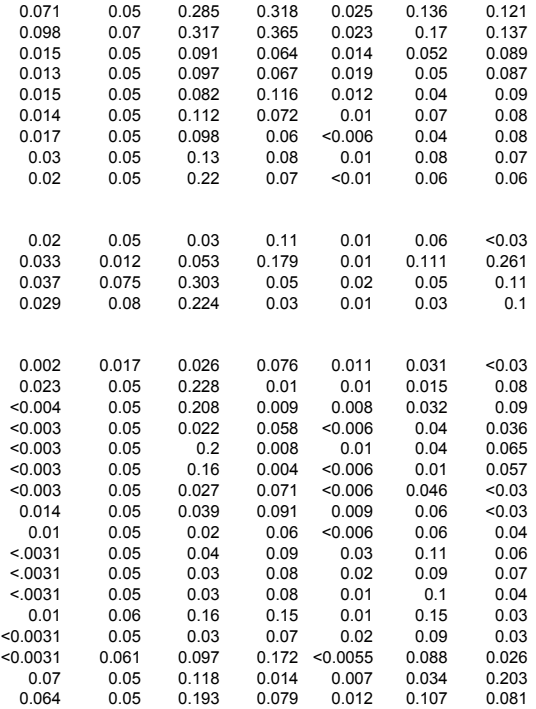

$\begin{array}{lrrrrrr}0.02 & 0.05 & 0.126 & 0.082 & 0.024 & 0.291 & 0.162 \\ 0.02 & \text { NA } & 0.09 & 0.484 & 0.011 & 0.179 & 0.635 \\ 0.06 & \text { NA } & 0.122 & 0.077 & 0.017 & 0.213 & 0.242\end{array}$

$\begin{array}{lllllll}0.061 & N A & 0.106 & 0.066 & 0.011 & 0.183 & 0.232\end{array}$

$\begin{array}{lllllll}0.025 & 0.05 & 0.038 & 0.029 & 0.02 & 0.247 & 0.101\end{array}$

$\begin{array}{lllllll}0.014 & 0.05 & 0.041 & 0.203 & 0.014 & 0.187 & 0.06\end{array}$

$\begin{array}{rrrrrrr}<0.0031 & 0.05 & 0.465 & 0.268 & <0.0055 & 0.11 & 0.081 \\ 0.007 & 0.118 & 0.469 & 0.157 & <0.0055 & 0.095 & <0.0243\end{array}$ 
\# Glass ID

$1395 \mathrm{GLA} 78-10-16$
1396 P2-0Ca-0F

1397 P2-0Ca-1F

1398 P2-OCa-2F

$1402 \mathrm{P} 2-3 \mathrm{Ca}-0 \mathrm{~F}$

(403

405 P2-3Ca-3F

$1406 \mathrm{P2}-3 \mathrm{Ca}-4 \mathrm{~F}$

(407 $22-3 \mathrm{Ca}-5 \mathrm{~F}$

1409 P2-9Ca-1F

(411 $\mathrm{P2}$ -

1412 P2-9Ca-4F

1414 SRL 165 HiFe

1417 HLW98-01

1419 HLW98-0

1420 HLW98-04

422 HLW98-06
1423 HLW98-07

1424 HLW88-08

1425 HLW98-09

1426 HLW98-10
1427 HLW98-11

1428 HLW98-12

1430 HLW98-14
1431 HLW98-15

1432 HLW98-16

1433 HLW98-17

1434 HLW98-18

1436 HLW98-20

1437 HLW98-21

1438 HLW98-22

1440 HLW98-24

1441 HLW98-25

1443 HLW88-278

1444 HLW98-28

1445 HLW98-29

1447 HLW88-31

1448 HLW98-32A

1449 HLW98-33

1450 HLW98-34
1451 HLW98-34CG

1452 HLW98-35

1453 HLW98-36

1456 HLW98-39

1457 HLW98-40
1458 HLW98-41

1459 HLW98-42
1460 HLW98-43

1461 HLW98-44

463 HLW98-46

1464 HLW98-47

(6) HLW98-50

1468 HLW98-51R

1471 HLW98-54

1472 HLW98-55

1476 HLW98-59
TCLPSE TCLP Zn TCLPAI TCLP B TCLPSB TCLP TI TCLPV QPCT B $\left(g / \mathrm{m}^{2}\right)$ QPCT Li $\left(g / \mathrm{m}^{2}\right)$ QPCT Na $\left(g / \mathrm{m}^{2}\right)$ QPCT Si $\left(g / \mathrm{m}^{2}\right)$ QPCT pH

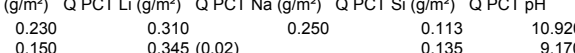

CCC PCT B $\left(g / \mathrm{m}^{2}\right) \quad \operatorname{CCC~PCT~Li~}\left(g / \mathrm{m}^{2}\right) \quad \operatorname{CCC}$ PCT Na $\left(g / \mathrm{m}^{2}\right) \quad \operatorname{CCC}$ PCT Si $\left(g / \mathrm{m}^{2}\right) \quad \operatorname{CCC~PCT~pH~T1~}\left({ }^{\circ} \mathrm{C}\right)$

(1)

$\begin{array}{llll}0.345 & & 0.135 & \\ 0.205 & 0.270 & 0.135 & 9.170 \\ 0.205 & 0.280 & 0.125 & 10.120 \\ 0.200 & 0.280 & 0.120 & 10.080 \\ 0.215 & 0.300 & 0.115 & 9.050 \\ 0.220 & 0305 & 0.11 & 9.930\end{array}$

$0.215 \quad 0.30$

$0.275(0.03)$

0.220
0.200
0.195

0.290

0.285
0.270
0.270

0.280

0.120
0.380
0.360

0.380
0.360
0.405
0.390

0.115
0.9 .830

$0.240 \quad 0.195$

$\begin{array}{ll}0.105 & 0.210 \\ .225 & 0.325\end{array}$

$\begin{array}{ll}0.260 & 0.290 \\ 0.285 & 0.325 \\ 0.300 & 0.320 \\ 0.280 & 0.290\end{array}$

$\begin{array}{lr}0.0532 & 0.894 \\ 0.0532 & 1.02 \\ 0\end{array}$

0.390

0.115

$\begin{array}{ll}0.110 & 10.08 \\ 0.110 & 10.100 \\ 0.050 & 1.000\end{array}$

$\begin{array}{ll}0.050 & 10.010 \\ 0.105 & 10.900\end{array}$

$\begin{array}{ll}0.105 & 10.8010 \\ 0.120 & 10.830\end{array}$

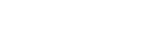

0.215

0.370

10.800
10.710

$\begin{array}{ll}0.0532 & 0.488 \\ 0.0532 & 0.505 \\ 0.0532 & 0.45\end{array}$

$\begin{array}{ll}0.0532 & 0.45 \\ 0.0532 & 0.44\end{array}$

$\begin{array}{ll}0.0532 & 0.35 \\ 0.0532 & 0.46 \\ 0.0532 & 0.42\end{array}$

$\begin{array}{cc}0.0532 & 0.401 \\ 0.038 & 0.77 \\ 0.0532 & 0.54\end{array}$

$\begin{array}{ll}0.0532 & 0.79 \\ 0.0532 & 0.54\end{array}$

$\begin{array}{llll}0.0532 & 0.007 & & \\ 0.0532 & 0.529 & 0.745 & 1.43 \\ 0.0532 & 0.531 & 0.525 & 1.4\end{array}$

$\begin{array}{ll}0.0532 & 0.531 \\ 0.0532 & 0.246 \\ 0.0532 & 0.51\end{array}$

$\begin{array}{ll}0.0532 & 0.59 \\ 0.0532 & 0.51 \\ 0.052 & 0.9\end{array}$

$\begin{array}{llll}0.0532 & 0.29 & 0.511 & 0.892\end{array}$

$\begin{array}{lr}0.0532 & 0.343 \\ 0.0532 & 0.34\end{array}$

$0.0532 \quad 0.45$

$\begin{array}{ll}0.0532 & 0.37 \\ 0.0532 & 0.41\end{array}$

$\begin{array}{llll}0.0532 & 0.41 & 0.394 & 0.642\end{array}$

\begin{tabular}{ll}
0.09 & 0.33 \\
0.0532 & 0.337 \\
0.0532 & 0.607 \\
\hline
\end{tabular}

$0.0532 \quad 0.547$

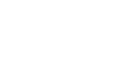

$<0.0338<0.0417$

\begin{tabular}{rr}
0.0532 & 1.475 \\
NA & 0.692 \\
\hline & 1.481
\end{tabular}

$\begin{array}{ll}\text { NA } & 0.692 \\ \text { NA } & 1.581 \\ \text { NA } & 1.449\end{array}$

NA 1.636

$0.068 \quad 3.23$

$0.062 \quad 1.479$

$\begin{array}{lrll}0.0532 & 0.13 & & \\ 0.0532 & 0.422 & 0.507 & 0.404\end{array}$
0.280

0.310

0.150

0.135

9.510

$\begin{array}{lllll}0.295 & 0.225 & 0.250 & 0.145 & 9.520 \\ 0.280 & 0.290 & 0.135 & 0.165 & 9.810 \\ 0.215 & 0.230 & 0.215 & 0.100 & 9.670 \\ 0.220 & 0.235 & 0.205 & 0.110 & 9.510\end{array}$

0.235

0.260
0.215

0.235

$\begin{array}{lll}0.190 & 0.160 & 9.840 \\ 0.075 & 0.155 & 9.540 \\ 0.205 & 0.115 & 9.750\end{array}$

0.139

0.180

0.171

$\begin{array}{lllll}1.765 & 1.265 & 1.095 & 0.450 & 10.510 \\ 1.355 & 1.080 & 0.915 & 0.405 & 10.550 \\ 0.425 & 0.405 & 0.355 & 0.225 & 10.430 \\ & & & & \\ 0.695 & 0.445 & 0.455 & 0.265 & 10.610 \\ & & & \\ 0.475 & 0.550 & 0.670 & 0.330 & 11.320 \\ 0.695 & 0.445 & 0.455 & 0.265 & 10.610\end{array}$


1397 P2-0Ca-1

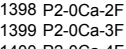

1401 P2-0Ca-5F

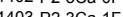

1044 P2-3C2-25

$1405 \mathrm{P} 2-3 \mathrm{CC}-3 \mathrm{~F}$
$1406 \mathrm{P2}-3 \mathrm{CO}-4 \mathrm{~F}$

1407 P2-30.-5F

1409 P2-9Ca-1F

$1410 \mathrm{P} 2-9 \mathrm{CC}-2 \mathrm{~F}$

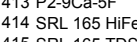

1416 SLL 165 HiA

1417 HLW98-01

1418 HLW98-02

1420 HLW98-04

1421 HLW98-05

1422 HLW98-06

1424 H. W98-08

1425 HLW98-09

1426 HLW98-10

1428 HWW98-12

1429 HLW98-13

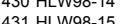

1432 HLW W98-16

1433 HLW98-17

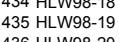

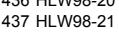

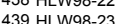

1440 HLW98-24

1441 HLW W98-25

1442 HLW98-26
1443 HLW98-278

1444 HLW98-28

1445 HLW98-29

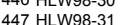

1448 HLW98-32A

${ }_{1449}^{14 L W 98-33}$

1450 HLW998-34

1452 HLW98-35

1453 HLW98-36

1456 HLW98-39

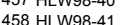

1459 HLW $198-42$
1408

1460 HLW98-43

1461 HLW98-44

1463 HLW98-46

1464 HLW $198-47$

1465 HLW98-48

1467 HLW98.50

1468 HLW W98-51R

1469 HLW98-52
1470 HLW98-53A

1471 HLW $98-54$

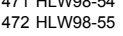

1473 HLW98-56
1474 HLW98-57

1475 HLW98-58

1476 HLW98-59 
\# Glass ID

1477 HLW98-60
1478 HLWS88-61
1479 HLWSE-61

1480 HLW98-62

1481 HLWB8-63

1482 HLW98-64
1483 HLWMS-01
1484 HLWMS-02

1485 HLWMS-03

1486 HLWMS-04
1487 HLWMS-05

1488 HLWMS-06

1489 HLWMS-07

1491 HLWMS-09

1492 HLWMS-10

1494 HLWMS-12

1495 HLWMS-13

1497 HLWMS-15

1499 HLW99-02

1500 HLW99-03

HLW99-05
HLW99-06

1504 HLW99-07

1505 HLW99-08
1506 HLW99-09

1507 HLW99-10
1508 HLW99-11

509 HLW99-12
510 HLW99-13

1511 HLW99-14

1512 HLW99-15
1513 HLW9-16
1514 HLW9-17

515 HLW99-18

1516 HLW99-19
1517 HLW99-20

1518 HLW99-21

1519 HLW99-22
1520 HLW99-23

1522 HLW99-25
1523 HLW99-26

1524 HLW99-27

1526 HLW99-29

1528 HLW99-31

1530 HLW99-33

1531 HLW99-34

1532 HLW99-35

1534 HLW99-37
1535 HLW99-38

He HLW99-39

1538 HLW99-41

1539 HLW99-42

541 HLW99-44
542 HLW99-45

1543 HLW99-46
1544 HLW99-47

1545 HLW99-48

1546 HLW99-49
1547 HLW99-50

1550 HLW99-53

1551 HLW99-54

1553 HLW99-56R

1554 HLW99-57

1555 HLW99-58
556 HLW99-59

1557 HLW99-60
1558 HLW99-61
Study

Reference

RPP-WTP HLW Formulation Kot and Pegg 2001 -WTP HLW Formulation Kot and RPP-WTP HLW Formulation Kot and Pegg 2001 RPP-WTP HLW Formulation Kot and Pegg 2001 RPP-WTP HLW Formulation Kot and Pegs RPP-WTP HLW Formulation Kot and Pegg 2001 RPP-WTP HLW Formulation Kol and Pegg 2001 RPP-WTP HLW Formulation Kot and Pegg 200 RPP-WTP HLW Formulation Kot and Pegg 200 RPP-WTP HLW Formulation Kot and Pegg 2001 RPP-WTP HLW Formulation Kot and Pegg 2001 RPP-WTP HLW Formulation Kot and Pegg 2001 RPP-WTP HLW Formulation Kot and Pegg 2001 RPP-WTP HLW Formulation Kot and Pegg 2001 RPP-WTP HLW Formulation Kot and Pegg 2001 RPP-WTP HLW Formulation Ko and Pegg 2001 RPP-WTP HLW Formulation Kot and Pegg 2001 RPP-WTP HLW Formulation Kot and Pegg 2001 WTP HLW Formulation Kot and Pegg 2000 RPP-WTP HLW Formulation Kot and Pegg 2001 RPP-WTP HLW Fornulation Kol and Pegg 201 KPP-WTP HLW Formulation Kot and Pegg 2001 RPP-WTP HLW Formulation Kot and Pegg 201 RPP-WTP HLW Formulation Kot and Pegg 2001 PPP-WTP HLW Formulation Kot and Pegg 2001 PWTP HLW Formulation Kot and Pegg 200 Tot RPP-WTP HLW Formulation Kot and Pegg 2001 RPP-WTP HLW Formulation Kot and Pegg 2001 作P-WTP HLW Formulation Kot and Pegg 2001 PPP-WTP HLW Formulation Kot and Pegg 200 Kot RPP-WTP HLW Formulation Kot and Pegg 2001 RPP-WTP HLW Formulation Kot and Pegg 2001 RPP-WTP HLW Formulation Kot and Pegg 2001 RPP-WTP HLW Formulation Kot and Pegg 200 P P-WTP HLW Formulation Kot and Pegg 2001 PP.WTP HLW Formulation Kot and Pegg 2001 Pot and Pegg 2001 RPP-WTP HLW Formulation Kot and Pegg 2001
RPP-WTP HLW Formulation Kot and Pegg 2001 (1) Kon and Pegg 2000 lation Kot and Pegg 2001 RPP-WTP HLW Formulation Kot and Pegg 200 RPP-WTP HLW Formulation Kot and Pegg 200 RPP-WTP HLW Formulation Kot and Pegg 2001 RPP-WTP HLW Formulation Kot and Pegg 200 RPP-WTP HLW Formulation Kot and Pegg 2001 Frmulation Kot and Pegg 2001 RPP-WTP HLW Formulation Kot and Pegg 200 RPP-WTP HLW Formulation Kot and Pegg 2001 RPP-WTP HLW Formulation Kot and Pegg 2001 RPP-WTP HLW Formulation Kol and Pegg 2001 RPP-WTP HLW Formulation Kot and Pegg 2001
Al2O3-t B2O3-t CaO-t Fe2O3-t FeO-t $\begin{array}{llll}0.0760 & 0.0400 & 0.0059 & 0.1194 \\ 0.0769 & 0.0400 & 0.0049 & 0.1223\end{array}$ $\begin{array}{llll}0.0769 & 0.0400 & 0.0049 & 0.1223\end{array}$

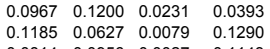
$\begin{array}{lllll}0.0814 & 0.0956 & 0.0027 & 0.1143\end{array}$ $0.0500 \quad 0.0600$ $\begin{array}{ll}0.0100 & 0.0560 \\ 0.0400 & 0.0500\end{array}$ $\begin{array}{ll}0.0400 & 0.0500 \\ 0.0600 & 0.0100 \\ 0.0400\end{array}$ $\begin{array}{ll}0.0100 & 0.0400 \\ 0.0300 & 0.1300\end{array}$ 0.1200
0.1100
0300
0.1100 $\begin{array}{ll}0.0300 & 0.1100 \\ 0.0500 & 0.1000 \\ 0.1120 & 0.1000\end{array}$ $0.1120 \quad 0.1000$ 0.11200 .1000 $0.0000 \quad 0.0500 \quad 0.0700 \quad 0.0200$

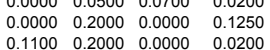
$\begin{array}{llll}0.1100 & 0.0500 & 0.0000 & 0.0200 \\ 0 & 0.1000 & 0.0500 & 0.0000\end{array}$ $\begin{array}{llll}0.1100 & 0.0500 & 0.0700 & 0.0200\end{array}$ $\begin{array}{llll}0.0000 & 0.2000 & 0.0700 & 0.0200 \\ 0.0000 & 0.0500 & 0.0700 & 0.1250\end{array}$ $\begin{array}{llll}0.0000 & 0.2000 & 0.0700 & 0.0200\end{array}$ $\begin{array}{llll}0.0000 & 0.0500 & 0.0630 & 0.0200 \\ 0.11000 & 0.200 & 0.000 & 0.020\end{array}$ $\begin{array}{llll}0.1100 & 0.2000 & 0.0700 & 0.0200 \\ 0.0100 & 0.2000 & 0.0700 & 0.1250\end{array}$ $\begin{array}{llll}0.0000 & 0.0500 & 0.0000 & 0.02200 \\ 0.0000 & 0.2000 & 0.0000 & 0.1250\end{array}$ $\begin{array}{llll}0.0000 & 0.2000 & 0.0350 & 0.0200 \\ 0.0000 & 0.0500 & 0.0000 & 0.1250\end{array}$ $\begin{array}{lllll}0.0000 & 0.0500 & 0.0700 & 0.0200\end{array}$ $\begin{array}{llll}0.1100 & 0.0700 & 0.0700 & 0.0200\end{array}$ $\begin{array}{llll}0.0850 & 0.2000 & 0.0000 & 0.1250 \\ 0.0530 & 0.0900 & 0.0000 & 0.0200\end{array}$

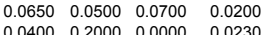

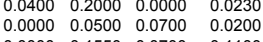
$\begin{array}{llll}0.1100 & 0.0900 & 0.0700 & 0.11000 \\ 0.0000 & 0.0500 & 0.0000 & 0.130\end{array}$ $\begin{array}{llll}0.0000 & 0.1600 & 0.00000 & 0.07730 \\ 0.1250\end{array}$

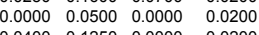
$\begin{array}{llll}0.0400 & 0.1250 & 0.0000 & 0.0200 \\ 0.1100 & 0.1830 & 0.0000 & 0.0200 \\ 0.000 & 0.050 & 0.000 & 0.125\end{array}$ $\begin{array}{llll}0.1100 & 0.1830 & 0.0000 & 0.0200 \\ 0.0000 & 0.0500 & 0.0000 & 0.1250\end{array}$ $\begin{array}{llll}0.0000 & 0.2000 & 0.0700 & 0.0220 \\ 0.0000 & 0.0500 & 0.0000 & 0.0297\end{array}$ $\begin{array}{llll}0.0914 & 0.0500 & 0.0400 & 0.0700 \\ 0.0000 & 0.2000 & 0.0133 & 0.1250 \\ 0.1200 & 0.070 & 0.000 & 0.020\end{array}$ $\begin{array}{llll}0.1100 & 0.0700 & 0.0000 & 0.0200 \\ 0.0000 & 0.0500 & 0.0700 & 0.0200\end{array}$

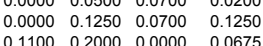
$\begin{array}{llll}0.0516 & 0.1171 & 0.0303 & 0.0614 \\ 0.1100 & 0.1775 & 0.0450 & 0.0200\end{array}$

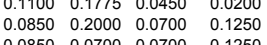
$\begin{array}{lllll}0.1067 & 0.1600 & 0.0700 & 0.0200\end{array}$ $\begin{array}{llll}0.0000 & 0.1158 & 0.0700 & 0.0200 \\ 0.0500 & 0.0500 & 0.0350 & 0.1250\end{array}$ $\begin{array}{llll}0.1100 & 0.0650 & 0.0000 & 0.0850\end{array}$ $\begin{array}{llll}0.0425 & 0.0650 & 0.0000 & 0.1250 \\ 0.0500 & 0.0600 & 0.0000 & 0.0200\end{array}$ $\begin{array}{lllll}0.0000 & 0.2000 & 0.0700 & 0.0200\end{array}$

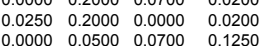
$\begin{array}{llll}0.0000 & 0.0500 & 0.0700 & 0.1250 \\ 0.1100 & 0.0500 & 0.0000 & 0.0200\end{array}$ $\begin{array}{llll}0.0400 & 0.0500 & 0.0700 & 0.0700 \\ 0.0367 & 0.0500 & 0.0000 & 0.1250\end{array}$ $\begin{array}{llll}0.0000 & 0.0500 & 0.0000 & 0.1250 \\ 0.1100 & 0.2000 & 0.0700 & 0.0200 \\ 0.1000 & 0.135 & 0.000 & 0.1250\end{array}$ $\begin{array}{llll}0.0000 & 0.1350 & 0.0000 & 0.1250 \\ 0.0250 & 0.2000 & 0.0000 & 0.0200\end{array}$
K2O-t Li2O-t MgO-t Na2O-t NiO-t P2O5-t SiO2-t ZrO2-t Ag2O-t As203-tAs205-t BaO-t BeO-t Bi2O3-t $\begin{array}{lllllllllll}0.0003 & 0.00500 & 0.0012 & 0.1325 & 0.0077 & 0.0024 & 0.4765 & 0.0166 & 0.0002 & 0.0000\end{array}$

$\begin{array}{llllllllll}0.0003 & 0.0500 & 0.0012 & 0.1390 & 0.0076 & 0.0046 & 0.4704 & 0.0147 & 0.0002\end{array}$

$\begin{array}{llllllllll}0.0008 & 0.0500 & 0.0003 & 0.0964 & 0.0025 & 0.0022 & 0.4255 & 0.0808 & 0.0000 & 0.0000 \\ 0.0003 & 0.0358 & 0.0024 & 0.1010 & 0.0017 & 0.0017 & 0.3783 & 0.0013 & 0.0026 & 0.0000\end{array}$

$\begin{array}{lllllllllll} & 0.0574 & 0.0007 & 0.0639 & 0.0059 & 0.0014 & 0.4354 & 0.0392 & 0.0000 & 0.0004\end{array}$ 0.0004
0.0004
0.0004
0.0000
0.0007

CdO-t Ce2O3-t CeO2-t Cl-t $\begin{array}{llll}0.0137 & 0.000 & 0.0007 & 0.0005 \\ 0.0139 & 0.0006 & 0.0003\end{array}$ 0.01390 .0006 0.0006 $\begin{array}{lll}0.0000 & 0.0002 \\ 0.0003 & 0.0000 \\ 0.0009 & 0.0001 & 0\end{array}$

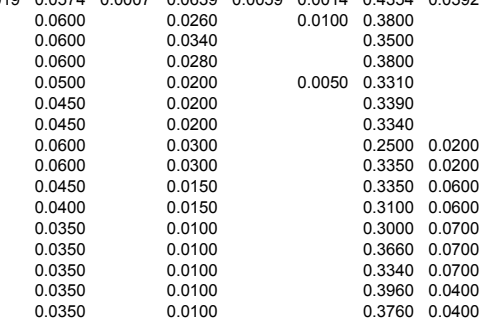

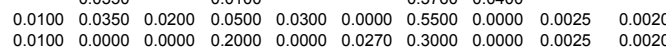

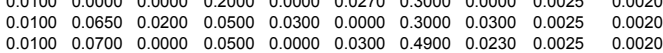
$\begin{array}{llllllllll}0.0100 & 0.0700 & 0.0000 & 0.0500 & 0.0000 & 0.03300 & 0.4900 & 0.0230 & 0.0025 & 0.0020 \\ 0.0100 & 0.0100 & 0.0200 & 0.22000 & 0.0300 & 0.0184 & 0.3100 & 0.1000 & 0.0025 & 0.0020\end{array}$ $\begin{array}{llllllllll}0.0100 & 0.0000 & 0.0000 & 0.2000 & 0.0000 & 0.0000 & 0.4800 & 0.0050 & 0.0025 & 0.0020 \\ 0.0100 & 0.0350 & 0.0000 & 0.1500 & 0.0000 & 0.0037 & 0.3450 & 0.1000 & 0.0025 & 0.0020\end{array}$

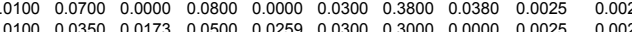
$\begin{array}{llllllllll}0.0100 & 0.0000 & 0.0000 & 0.1300 & 0.00000 & 0.0300 & 0.5500 & 0.0000 & 0.0025 & 0.0020 \\ 0.0100 & 0.0070 & 0.0000 & 0.1060 & 0.0000 & 0.0300 & 0.3000 & 0.0000 & 0.0025 & 0.0022\end{array}$

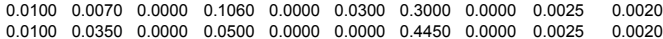

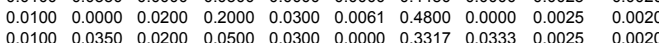

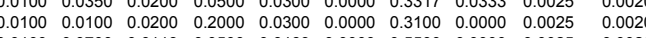
$\begin{array}{lllllllll}0.0113 & 0.0500 & 0.0169 & 0.0000 & 0.5500 & 0.0000 & 0.025 & 0.0022\end{array}$ $\begin{array}{llllllllll}0.0100 & 0.0100 & 0.0156 & 0.2000 & 0.0234 & 0.0000 & 0.3000 & 0.0300 & 0.0025 & 0.0020 \\ 0.0100 & 0.0530 & 0.0000 & 0.0500 & 0.0000 & 0.0300 & 0.3000 & 0.0000 & 0.0025 & 0.0020\end{array}$

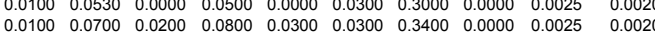
$\begin{array}{llllllllll}0.0100 & 0.0700 & 0.0000 & 0.0750 & 0.0000 & 0.0000 & 0.5350 & 0.0500 & 0.0025 & 0.0020 \\ 0.0100 & 0.0000 & 0.0000 & 0.1500 & 0.0000 & 0.0300 & 0.3000 & 0.1000 & 0.0025 & 0.0020\end{array}$

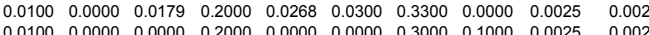

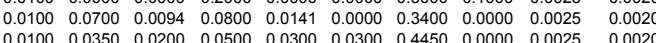

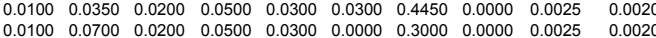
$\begin{array}{llllllllll}0.01100 & 0.0700 & 0.02000 & 0.0550 & 0.0300 & 0.0000 & 0.33000 & 0.0000 & 0.0025 & 0.0002 \\ 0.00000 & 0.0200 & 0.1200 & 0.0300 & 0.0000 & 0.3000 & 0.0800 & 0.0025 & 0.0022\end{array}$ $\begin{array}{llllllllll}0.0100 & 0.030 & 0.0123 & 0.1300 & 0.0184 & 0.0000 & 0.5200 & 0.0867 & 0.0025 & 0.0020\end{array}$

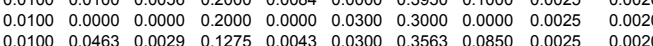
$\begin{array}{llllllllll}0.0100 & 0.0700 & 0.0000 & 0.0800 & 0.00000 & 0.0300 & 0.3730 & 0.0000 & 0.0025 & 0.0020 \\ 0.0100 & 0.0700 & 0.0000 & 0.0800 & 0.0000 & 0.0300 & 0.5300 & 0.0533 & 0.0025 & 0.0020\end{array}$ $\begin{array}{llllllllll}0.0100 & 0.0700 & 0.0000 & 0.0800 & 0.0000 & 0.0300 & 0.5300 & 0.0533 & 0.0025 & 0.0020 \\ 0.0100 & 0.0536 & 0.0000 & 0.0729 & 0.0000 & 0.0000 & 0.5086 & 0.0486 & 0.0025 & 0.0020\end{array}$

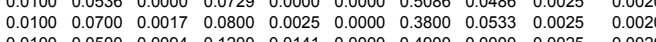
$\begin{array}{llllllllll}0.0100 & 0.0500 & 0.0094 & 0.1200 & 0.0141 & 0.0000 & 0.4900 & 0.0000 & 0.0025 & 0.0020 \\ 0.0100 & 0.0700 & 0.0137 & 0.0500 & 0.0206 & 0.0300 & 0.4100 & 0.0333 & 0.0025 & 0.0020\end{array}$ $\begin{array}{llllllllll}0.0100 & 0.0400 & 0.0013 & 0.1400 & 0.0019 & 0.0000 & 0.4250 & 0.0000 & 0.0025 & 0.0020\end{array}$ $\begin{array}{llllllllll}0.0100 & 0.0317 & 0.0062 & 0.1129 & 0.0094 & 0.0124 & 0.3973 & 0.0322 & 0.0025 & 0.0020 \\ 0.0100 & 0.0350 & 0.0000 & 0.0500 & 0.0000 & 0.0000 & 0.4675 & 0.0300 & 0.0025 & 0.0020\end{array}$ $\begin{array}{llllllllll}0.0100 & 0.0350 & 0.0000 & 0.0500 & 0.0000 & 0.0000 & 0.4675 & 0.032 & 0.0025 & 0.020 \\ 0.0100 & 0.0675 & 0.0000 & 0.0850 & 0.0000 & 0.0000 & 0.3025 & 0.0000 & 0.0025 & 0.002020\end{array}$ $\begin{array}{llllllllll}0.0100 & 0.0700 & 0.0004 & 0.0500 & 0.0006 & 0.0300 & 0.3000 & 0.0333 & 0.0025 & 0.0020 \\ 0.0100 & 0.0700 & 0.0200 & 0.0800 & 0.0300 & 0.0000 & 0.3658 & 0.0533 & 0.0025 & 0.0022\end{array}$ $\begin{array}{llllllllll}0.0100 & 0.0700 & 0.0200 & 0.0800 & 0.0300 & 0.0000 & 0.3658 & 0.0533 & 0.0025 & 0.0020 \\ 0.0100 & 0.0300 & 0.0000 & 0.0950 & 0.0000 & 0.0000 & 0.5500 & 0.0000 & 0.0025 & 0.0020\end{array}$

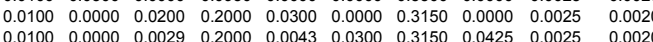

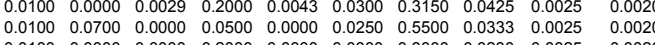
$\begin{array}{llllllllll}0.0100 & 0.0000 & 0.0000 & 0.2000 & 0.0000 & 0.0300 & 0.3000 & 0.0230 & 0.0025 & 0.0022 \\ 0.0100 & 0.0000 & 0.0200 & 0.1600 & 0.0300 & 0.0000 & 0.3700 & 0.0000 & 0.0025 & 0.0022\end{array}$ $\begin{array}{llllllllll}0.0100 & 0.0000 & 0.0124 & 0.1200 & 0.0186 & 0.0150 & 0.4100 & 0.0000 & 0.0025 & 0.0020 \\ 0.0100 & 0.0350 & 0.0138 & 0.1200 & 0.0206 & 0.0000 & 0.4900 & 0.0000 & 0.0025 & 0.0022\end{array}$ $\begin{array}{llllllllll}0.0100 & 0.0100 & 0.0094 & 0.2000 & 0.0141 & 0.0000 & 0.3200 & 0.1000 & 0.0025 & 0.0020\end{array}$

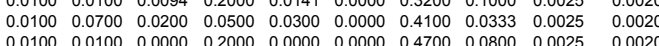

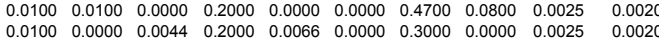
$\begin{array}{llllllllll}0.0100 & 0.0700 & 0.0200 & 0.0500 & 0.0300 & 0.0000 & 0.4100 & 0.0000 & 0.0025 & 0.020\end{array}$

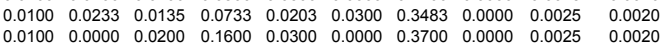

\begin{tabular}{|c|c|c|}
\hline 0.0400 & 0.0200 & 0.0300 \\
\hline & 0.0000 & 0.0000 \\
\hline 0.0400 & 0.0200 & 0.0300 \\
\hline $\begin{array}{l}0.0000 \\
0.0400\end{array}$ & $\begin{array}{l}0.0000 \\
0.0200\end{array}$ & $\begin{array}{l}0.0000 \\
0.0300\end{array}$ \\
\hline $\begin{array}{l}0.00000 \\
0.000\end{array}$ & 0.02000 & 0.0000 \\
\hline & 0.0000 & 0.0000 \\
\hline 0.0000 & 0.0000 & 0.0000 \\
\hline 0.0345 & 0.0173 & $\begin{array}{l}0.0259 \\
0.000\end{array}$ \\
\hline 0.0000 & 0.0000 & $\begin{array}{l}0.0000 \\
0.000\end{array}$ \\
\hline $\begin{array}{l}0.00000 \\
0.0000\end{array}$ & $\begin{array}{l}0.00000 \\
0.000\end{array}$ & $\begin{array}{l}0.0000 \\
0.0000\end{array}$ \\
\hline 0.0400 & 0.0200 & 0.0300 \\
\hline 0.0400 & 0.0200 & 0.0300 \\
\hline 0.0400 & 0.0200 & 0.0300 \\
\hline 0.0225$)$ & 0.0113 & 0.0169 \\
\hline $\begin{array}{l}0.00000 \\
0.0313\end{array}$ & $\begin{array}{l}0.0000 \\
0.0156\end{array}$ & $\begin{array}{l}0.0000 \\
0.0234\end{array}$ \\
\hline $\begin{array}{l}0.0000 \\
0.003\end{array}$ & 0.0000 & $\begin{array}{l}0.0234 \\
0.0000\end{array}$ \\
\hline 0.0400 & 0.0200 & 0.0300 \\
\hline 0.0000 & 0.0000 & 0.0000 \\
\hline 0.0000 & 0.0000 & 0.0000 \\
\hline 0.0358 & 0.0179 & 0.0268 \\
\hline 0.0000 & 0.0000 & 0.0000 \\
\hline $\begin{array}{l}0.0188 \\
0.0480\end{array}$ & $\begin{array}{l}0.0094 \\
0.020\end{array}$ & $\begin{array}{l}0.00141 \\
0.0300\end{array}$ \\
\hline $\begin{array}{l}0.04400 \\
0.040\end{array}$ & 0.0200 & 0.0300 \\
\hline 0.0400 & 0.0200 & 0.0300 \\
\hline 0.0246 & 0.0123 & 0.0184 \\
\hline 0.0113 & 0.0056 & 0.0084 \\
\hline $\begin{array}{l}0.0000 \\
0.0058\end{array}$ & $\begin{array}{l}0.0000 \\
0.0029\end{array}$ & $\begin{array}{l}0.0000 \\
0.0043\end{array}$ \\
\hline $\begin{array}{l}0.00000 \\
0.000\end{array}$ & $\begin{array}{l}0.0029 \\
0.0000\end{array}$ & $\begin{array}{l}0.0043 \\
0.0000\end{array}$ \\
\hline 0.0000 & 0.0000 & $\begin{array}{l}0.0000 \\
0.0000\end{array}$ \\
\hline 0.0000 & 0.0000 & 0.0000 \\
\hline 0.0033 & 0.0017 & 0.0025 \\
\hline 0.0188 & 0.0094 & 0.0141 \\
\hline 0.0274 & 0.0137 & 0.0200 \\
\hline 0.0025 & 0.0013 & 0.0019 \\
\hline $\begin{array}{l}0.00000 \\
0.0125\end{array}$ & $\begin{array}{l}0.0000 \\
0.0062\end{array}$ & $\begin{array}{l}0.0000 \\
0.0094\end{array}$ \\
\hline 0.0000 & 0.0002 & $\begin{array}{l}0.0094 \\
0.0000\end{array}$ \\
\hline 0.0000 & 0.0000 & 0.00000 \\
\hline 0.0000 & 0.0000 & 0.0000 \\
\hline 0.0008 & 0.0004 & 0.0000 \\
\hline 0.0400 & 0.0200 & 0.0300 \\
\hline 0.0000 & 0.0000 & 0.0000 \\
\hline 0.0400 & 0.0200 & 0.0300 \\
\hline 0.0058 & $\begin{array}{l}0.0029 \\
0.0000\end{array}$ & $\begin{array}{l}0.0043 \\
0.0000\end{array}$ \\
\hline 0.0000 & 0.0000 & $\begin{array}{l}.0 .0000 \\
0.0000\end{array}$ \\
\hline 0.0400 & 0.0200 & \\
\hline 0.0248 & 0.0124 & \\
\hline 0.0275 & 0.0138 & \\
\hline 0.0188 & 0.0094 & 0.0141 \\
\hline 0.0400 & 0.0200 & $\begin{array}{l}0.0300 \\
0\end{array}$ \\
\hline 0.0000 & 0.0000 & \\
\hline $\begin{array}{l}0.0080 \\
0.0400\end{array}$ & $\begin{array}{l}0.0044 \\
0.0200\end{array}$ & \\
\hline 0.0270 & 0.0135 & 0.0203 \\
\hline 0.0400 & 0.0200 & 0.0300 \\
\hline
\end{tabular}
$\begin{array}{ll}0.0020 & 0.002 \\ 0.0020 & 0.002 \\ 0.0020 & 0.002\end{array}$ $\begin{array}{lll}0.0020 & 0.0020 \\ 0.0020 & 0.0020 & 0\end{array}$ $\begin{array}{ll}0.0020 & 0.0020 \\ 0.0020 & 0.0020\end{array}$ .00200 .0022 $0.0020 \quad 0.0020$ $\begin{array}{lll}0.0020 & 0.002 \\ 0.0020 & 0.002\end{array}$ $\begin{array}{ll}0.0020 & 0.0020 \\ 0.0020 & 0.0020\end{array}$ 0.00200 .0022 $0.0020 \quad 0.0020$ $0.0020 \quad 0.0020$ $\begin{array}{lll}0.0020 & 0.0020 \\ 0.0020 & 0.0020 \\ 0.020 & 0.0020\end{array}$ $\begin{array}{ll}0.0020 & 0.0020 \\ 0.0020 & 0.0020 \\ 0.0020 & 0.0020\end{array}$ 0.00200 .0022 $0.0020 \quad 0.0020$ $0.0020 \quad 0.0020$ $\begin{array}{lll}0.0020 & 0.0020 \\ 0.0020 & 0.0020 & 0\end{array}$ $0.0020 \quad 0.0020$ $\begin{array}{ll}0.0020 & 0.0020 \\ 0.0020 & 0.0020 \\ 0.0020 & 0.0020\end{array}$ $0.0020 \quad 0.0022$ $\begin{array}{ll}0.0020 & 0.0020 \\ 0.0020 & 0.0020\end{array}$ $\begin{array}{ll}0.0020 & 0.0020 \\ 0.0020 & 0.0020 \\ 0.0020 & 0.0020\end{array}$ $\begin{array}{ll}0.0020 & 0.0020 \\ 0.0020 & 0.0020\end{array}$ $0.0020 \quad 0.0020$ $\begin{array}{lll}0.0020 & 0.0020 \\ 0.0020 & 0.0020\end{array}$ $0.0020 \quad 0.0020$ $\begin{array}{ll}0.0020 & 0.0020 \\ 0.0020 & 0.0020\end{array}$ $0.0020 \quad 0.0020$ $\begin{array}{ll}0.0020 & 0.0020 \\ 0.0020 & 0.0020\end{array}$ $\begin{array}{lll}0.0020 & 0.0022 \\ 0.0020 & 0.0020\end{array}$ $\begin{array}{lll}0.0020 & 0.0020 \\ 0 & 0.02020 & 0.0020\end{array}$ $\begin{array}{lll}0.0020 & 0.0020 \\ 0.0020 & 0.0020\end{array}$ $0.0020 \quad 0.0020$ $\begin{array}{ll}0.0020 & 0.0020 \\ 0.0020 & 0.0020\end{array}$ $\begin{array}{ll}0.0020 & 0.0020 \\ 0.0020 & 0.0020\end{array}$ $\begin{array}{ll}0.0020 & 0.0020 \\ 0.0020 & 0.0020\end{array}$ 
\# Glass ID

\# Hass ID
1477 HLW98-60
1478 HLWW8-61

1479 HLW98-61CG

1480 HLW98-62

1482 HLW98-64

1483 HLWMS-01
1484 HLWMS-02

1485 HLWMS-03

487 HLWMS-05

1488 HLWMS -06

490 HLWMS- 08
HLWMS-09

1492 HLWMS-10

1493 HLWMS-11

1495 HLWMS-13

1496 HLWMS-14

1498 HLW99-01
1499 HLW99-02

1499
1500 HLW $H 99-03$

1501 HLW99-04

1503 HLW99-06
1504 HLW99-07

1505 HLW99-08
1506 HLW99-09

1507 HLW99-10

1508 HLW99-11
1509 HLW99-12

1510 HLW99-13

1511 HLW99-14

1513 HLW99-16

1515 HLW99-18

1516 HLW99-19

1518 HLW99-21

1520 HLW99-23

1522 HLW99-25
1523 HLW99-26

1524 HLW99-27

1526 HLW99-29

1527 HLW99-30

1528 HLW99-31
1529 HLW99-32

1530 HLW99-33

1531 HLW99-34

1532 HLW99-35

1534 HLW99-37

1536 HLW99-39

1537 HLW99-40

1539 HLW99-42

1540 HLW99-43

1542 HLW99-45
1543 HLW99-46

1545 HLW99-48

1546 HLW99-49
1547 HLW99-50

$9-53$

1551 HLW99-54

1553 HLW99-56R

1554 HLW99-57

1555 HLW99-58

1557 HLW99-60
1558 HLW99-61
CoO-t Co203-t Cr2O3-t Cs2O-t CuO-t Dy203-t Eu2O3-t

$\begin{array}{llll}0.0001 & 0.0010 & 0.0000 & 0.0000\end{array}$

$\begin{array}{llll}0.0001 & 0.0011 & 0.0003 \\ 0.0005 & 0.0014 & 0.0007 & 0.0000 \\ 0.0000 & 0.0018 & 0.0011 & 0.0000 \\ 0.0000 & 0.0005 & 0.0009 & 0.0003\end{array}$

0.0000

0.0000
0.000

0.0001
0.0002
0.0003
0.0004

0.0200

\begin{tabular}{|c|c|c|c|}
\hline \multicolumn{4}{|c|}{$\begin{array}{ll}0.0020 & 0.0020\end{array}$} \\
\hline & 0.0045 & 0.0020 & 0.0020 \\
\hline & 0.0000 & 0.0020 & 0.0020 \\
\hline & & 0.0020 & 0.0020 \\
\hline & 0.0031 & 0.0020 & 0.0020 \\
\hline & $\begin{array}{l}0.00000 \\
0.0006\end{array}$ & $\begin{array}{l}0.0020 \\
\end{array}$ & $\begin{array}{l}0.0020 \\
0.0020\end{array}$ \\
\hline 0.0020 & $\begin{array}{l}0.0006 \\
0.0050\end{array}$ & $\begin{array}{l}0.00020 \\
0.020\end{array}$ & $\begin{array}{l}0.002020 \\
0.0020\end{array}$ \\
\hline & 0.0050 & 0.0020 & 0.0020 \\
\hline & 0.0050 & 0.0020 & $\begin{array}{l}0.0020 \\
0 \\
0.0220\end{array}$ \\
\hline $\begin{array}{l}0.00020 \\
0.0022\end{array}$ & $\begin{array}{l}0.00050 \\
0.0000\end{array}$ & $\begin{array}{l}0.0000 \\
0.0020\end{array}$ & $\begin{array}{l}0.002020 \\
0.0020\end{array}$ \\
\hline 0.0020 & 0.0010 & 0.0020 & 0.0020 \\
\hline & 0.0000 & 0.0020 & 0.0020 \\
\hline $\begin{array}{l}0.0020 \\
0.0022\end{array}$ & $\begin{array}{l}0.00000 \\
0.0000\end{array}$ & $\begin{array}{l}0.0002 \\
0.020\end{array}$ & $\begin{array}{l}0.0020 \\
0.0020\end{array}$ \\
\hline 0.0020 & 0.0050 & 0.0020 & 0.0020 \\
\hline 0.0020 & 0.0000 & 0.0020 & 0.0020 \\
\hline $\begin{array}{l}0.0020 \\
0.0020\end{array}$ & 0.0050 & 0.0020 & $\begin{array}{l}0.0020 \\
00020\end{array}$ \\
\hline $\begin{array}{l}0.00020 \\
0.00220\end{array}$ & $\begin{array}{l}0.00050 \\
0.0000\end{array}$ & $\begin{array}{l}0.0000 \\
0.0020\end{array}$ & $\begin{array}{l}0.002020 \\
0.0020\end{array}$ \\
\hline 0.0020 & 0.0050 & 0.0020 & 0.0020 \\
\hline 0.0020 & 0.0050 & 0.0020 & 0.0020 \\
\hline 0.0020 & 0.0000 & 0.0020 & 0.0020 \\
\hline $\begin{array}{l}0.00020 \\
0.0022\end{array}$ & $\begin{array}{l}0.0000 \\
0.0050\end{array}$ & $\begin{array}{l}0.0020 \\
0.0020\end{array}$ & $\begin{array}{l}0.002020 \\
0.0020\end{array}$ \\
\hline 0.0020 & 0.0000 & 0.0020 & 0.0020 \\
\hline 0.0020 & 0.0000 & 0.0020 & 0.0020 \\
\hline $\begin{array}{l}0.0020 \\
0.0020\end{array}$ & $\begin{array}{l}0.00000 \\
0.0000\end{array}$ & $\begin{array}{l}0.0020 \\
0.020\end{array}$ & $\begin{array}{l}0.0020 \\
0.0020\end{array}$ \\
\hline $\begin{array}{l}0.0020 \\
0.0020\end{array}$ & $\begin{array}{l}0.0000 \\
0.0050\end{array}$ & $\begin{array}{l}0.0020 \\
0.0020\end{array}$ & $\begin{array}{l}0.002020 \\
0.0020\end{array}$ \\
\hline 0020 & 0.0050 & 0.0020 & 0.0020 \\
\hline & 0.0050 & 0.0020 & 0.0020 \\
\hline & 0.0050 & 0.0020 & 0.0020 \\
\hline & 0.0000 & 0.0020 & 0.0020 \\
\hline .0020 & $\begin{array}{l}0.00000 \\
0.0000\end{array}$ & 0.0020 & 0.0020 \\
\hline 020 & $\begin{array}{l}0.0000 \\
0.0050\end{array}$ & $\begin{array}{l}0.0020 \\
0.0020\end{array}$ & $\begin{array}{l}0.002020 \\
0.0020\end{array}$ \\
\hline & 0.0000 & 0.0020 & 0.0020 \\
\hline & 0.0000 & 0.0020 & 0.0020 \\
\hline & 0.0021 & & 0.0020 \\
\hline & 0.0000 & 0.0020 & 0.0020 \\
\hline & $\begin{array}{l}0.00000 \\
0.0031\end{array}$ & $\begin{array}{l}0.00020 \\
0.0020\end{array}$ & $\left\{\begin{array}{l}0.02020 \\
0.0020\end{array}\right.$ \\
\hline & 0.0050 & 0.0020 & 0.0020 \\
\hline 0.00 & 0.0000 & 0.0020 & 0.0020 \\
\hline & 0.00000 & $\begin{array}{l}0.0020 \\
0\end{array}$ & 0.0020 \\
\hline 0020 & $\begin{array}{l}0.0000 \\
0.0050\end{array}$ & $\begin{array}{l}0.0020 \\
0.0020\end{array}$ & $\begin{array}{l}0.0020 \\
0.02020\end{array}$ \\
\hline & & & 0.0020 \\
\hline 0.002 & 0.0050 & 0.0020 & 0.0020 \\
\hline & 0.0000 & 0.0020 & 0.0020 \\
\hline & 0.0025 & 0.0020 & 0.0020 \\
\hline & 0.00000 & 0.0020 & 0.0020 \\
\hline & $\begin{array}{l}0.0000 \\
0.0000\end{array}$ & $\begin{array}{l}0.0020 \\
0.020\end{array}$ & $\begin{array}{l}0.00020 \\
0.0020\end{array}$ \\
\hline & $\begin{array}{l}0.00000 \\
0.0000\end{array}$ & $\begin{array}{l}0.0020 \\
0.020\end{array}$ & $\begin{array}{l}0.0020 \\
0.0020\end{array}$ \\
\hline & 0.0000 & 0.0020 & 0.0020 \\
\hline & 0.0000 & 0.0020 & 0.0020 \\
\hline & 0.0050 & 0.0020 & 0.0020 \\
\hline & & & \\
\hline
\end{tabular}

Ga2O3-t Gd2O3-t HfO2-t HgO-t

I-t La2O

La203-t MnO2-t MnO-t MoO-t

$0.0030 \quad 0.0061$

$\begin{array}{lll}0.0030 & 0.0103 \\ 0.0000 & 0.0000 & 0.0171\end{array}$

$\begin{array}{lll}0.0012 & 0.0000 & 00 \\ 0.0036 & 0.0333 & 0\end{array}$

0.0488
0.0000
0.1200
0.1300

2-t PbO-t PdO2-t PdO-t Pr2O3-t Pr6011-t Rb2O-t ReO-t

0.0000
0.0000

(1) 0.1200
0.1300
0.1300
0.1510

0.1610

0.1500
0.1360
0.1360
0.1360

0.1360
0.1360
0.0960

$\begin{array}{ll}0.0020 & 0.0020 \\ 0.0020 & 0.0020\end{array}$

0.00200 .0020

$\begin{array}{ll}0.0020 & 0.0020 \\ 0.0020 & 0.0020\end{array}$

$\begin{array}{ll}0.0020 & 0.0020 \\ 0.0020 & 0.0020\end{array}$

$0.0020 \quad 0.0020$

$0.0020 \quad 0.0020$

$0.0020 \quad 0.0020$

$\begin{array}{ll}0.0020 & 0.0020 \\ 0.0020 & 0.0020\end{array}$

$0.0020 \quad 0.0020$

$\begin{array}{ll}0.0020 & 0.0020 \\ 0.0020 & 0.0020\end{array}$

$0.0020 \quad 0.0020$

$\begin{array}{ll}0.0020 & 0.0020 \\ 0.0020 & 0.0020\end{array}$

$0.0020 \quad 0.0020$

$0.0020 \quad 0.0020$

$0.0020 \quad 0.0020$

$0.0020 \quad 0.0020$

$\begin{array}{lll}0.0020 & 0.0020 \\ 0.0020 & 0.0020\end{array}$

$0.0020 \quad 0.0020$

$\begin{array}{ll}0.0020 & 0.0020 \\ 0.0020 & 0.0020 \\ 0.0020 & 0.0020\end{array}$

$\begin{array}{ll}0.0020 & 0.0020 \\ 0.0020 & 0.0020 \\ 0.0020 & 0.0020\end{array}$

$\begin{array}{ll}0.0020 & 0.0020 \\ 0.0020 & 0.0020\end{array}$

$\begin{array}{lll}0.0020 & 0.0020 \\ 0.0020 & 0.0020\end{array}$

$\begin{array}{ll}0.0020 & 0.0020 \\ 0.0020 & 0.0020 \\ 0.0020 & 0.0020\end{array}$

$\begin{array}{ll}0.0020 & 0.0020 \\ 0.0020 & 0.0020\end{array}$

$\begin{array}{ll}0.0020 & 0.0020 \\ 0.0020 & 0.0020\end{array}$

$\begin{array}{ll}0.0020 & 0.0020 \\ 0.0020 & 0.0020 \\ 0.0020 & 0.0020\end{array}$

$\begin{array}{ll}0.0020 & 0.0020 \\ 0.0020 & 0.0020\end{array}$

$0.0020 \quad 0.0020$

$0.0020 \quad 0.0020$

$\begin{array}{ll}0.0020 & 0.0020 \\ 0.0020 & 0.0020\end{array}$

$\begin{array}{ll}0.0020 & 0.0020 \\ 0.0020 & 0.0020\end{array}$

$\begin{array}{lll}0.0020 & 0.0020 \\ 0.0020 & 0.0020\end{array}$

$\begin{array}{ll}0.0020 & 0.0020 \\ 0.0020 & 0.0020 \\ 0.0020 & 0.0020\end{array}$

$\begin{array}{lll}0.0020 & 0.0020 \\ 0 & 0.0020 & 0\end{array}$

$\begin{array}{ll}0.0020 & 0.0020 \\ 0.0020 & 0.0020 \\ 0.0020 & 0.0020\end{array}$

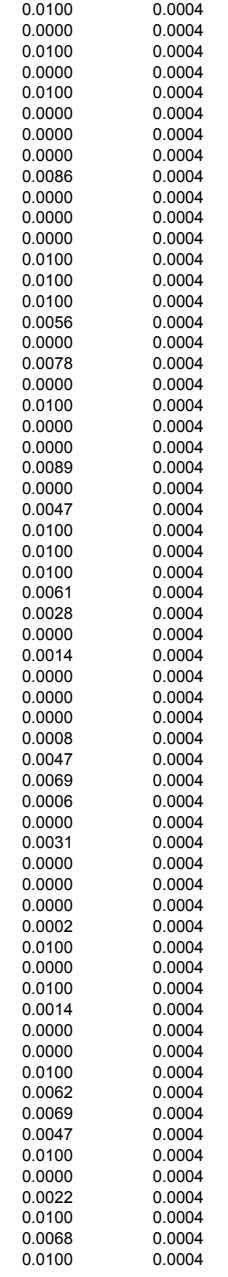


\# Glass ID

1477 HLWG8-60
1478 HLLV8-61

1479 HLW988-61CG
1480 HLW98-62

1480 HLW98-62
1481 HLW98-63

1482 HLW98-64
1483 HLWMS-01
1484 HLWMS-02

1485 HLWMS-03

1486 HLWMS-04
1487 HLWMS-05

1488 HLWMS-06

1490 HLWMS-08

1491 HLWMS-09

1493 HLWMS-11

495 HLWMS-13

1496 HLWMS-14

1498 HLW99-01
1499 HLW99-02

1500 HLW99-03

HLW99-05

1503 HLW99-06
1504 HLW99-07

1505 HLW99-08

1507 HLW99-10

509 HLW99-12

列 1 HLW99-14 14

1512 HLW99-15

列 15 HLW99-18

1516 HLW99-19

518 HLW99-21

1520 HLW99-23

522 HLW99-25

1523 HLW99-26

1524 HLW99-27
1525 HLW99-28

1526 HLW99-29
1527 HLW99-30

1528 HLW99-31

1530 HLW99-33

532 HLW99-35

1533 HLW99-36

1534 HLW99-37
1535 HLW99-38

1536 HLW99-39

1538 HLW99-41
1539 HLW99-42

1540 HLW99-43

1542 HLW99-45

1543 HLW99-46
1544 HLW99-47

HLW9-49

1547 HLW99-50

549 HLW99-52

1550 HLW99-53

1552 HLW99-55

1553 HLW99-56R
1554 HLW99-57

1555 HLW99-58

1557 HLW99-60
1558 HLW99-61

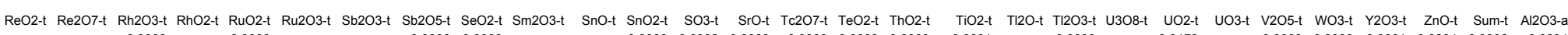
$0.0000 \quad 0.0000 \quad 0.0000 \quad 0.0000$

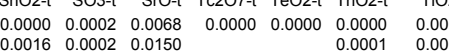

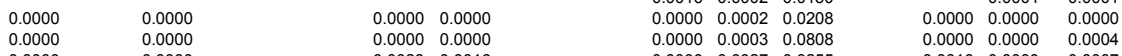

$\begin{array}{lll}0.0016 & 0.0000 & 0.0007\end{array}$

0.0171

$\begin{array}{llll}0.0001 & 0.0204 & 0.9996 & 0.0694 \\ 0.0001 & 0.0004 & 1.0000 & 0.0665\end{array}$

$\begin{array}{lll}0.2640 \\ 0.0000 & 0.0027 & 0.0255 \\ 0 & 0.02860\end{array}$

0.2860
0.2860
0.2330
0.3550

2990

0.2990

0.2330
0.2110

\begin{tabular}{|c|c|c|c|}
\hline 0008 & 00013 & 00020 & 00020 \\
\hline 0.0008 & 0.0013 & $\begin{array}{l}0.0020 \\
0.0020\end{array}$ & $\begin{array}{l}0.0020 \\
0.0020\end{array}$ \\
\hline 0.0008 & 0.0013 & 0.0020 & \\
\hline 0.0008 & 0.0013 & 0.0020 & \\
\hline $\begin{array}{l}0.0008 \\
0.0008\end{array}$ & $\begin{array}{l}0.0013 \\
0.0013\end{array}$ & $\begin{array}{l}0.0020 \\
0.0020\end{array}$ & $\begin{array}{l}0.0020 \\
0.0020\end{array}$ \\
\hline 0.0008 & $\begin{array}{l}0.0013 \\
0.0013\end{array}$ & $\begin{array}{l}0.0020 \\
0.0020\end{array}$ & \\
\hline & & $\begin{array}{l}0.00<20 \\
0.0020\end{array}$ & $\begin{array}{l}0.0020 \\
0.0020\end{array}$ \\
\hline 0.0008 & 0.0013 & 0.0020 & \\
\hline 0.0008 & 0.0013 & 0.0020 & \\
\hline 0.0008 & 0.0013 & 0.0020 & 0.0020 \\
\hline 0.0008 & 0.0013 & 0.0020 & 0.0020 \\
\hline 0.0008 & 0.0013 & 0.0020 & 0.0020 \\
\hline $\begin{array}{l}0.0008 \\
0.0008\end{array}$ & $\begin{array}{l}0.0013 \\
0.0013\end{array}$ & $\begin{array}{l}0.0020 \\
0.0020\end{array}$ & $\begin{array}{l}0.0020 \\
0.0020\end{array}$ \\
\hline $\begin{array}{l}0.0008 \\
0.0008\end{array}$ & $\begin{array}{l}0.0013 \\
0.0013\end{array}$ & $\begin{array}{l}0.0020 \\
0.0020\end{array}$ & $\begin{array}{l}0.0020 \\
0.0020\end{array}$ \\
\hline & $\begin{array}{l}0.000 \\
0.001\end{array}$ & 0.0020 & \\
\hline 0.0008 & 0.0013 & 0.0020 & 0.0020 \\
\hline 0.0008 & 0.0013 & 0.0020 & 0.0020 \\
\hline 0.0008 & 0.0013 & 0.0020 & 0.0020 \\
\hline 0.0008 & 0.0013 & 0.0020 & 0.0020 \\
\hline 0.0008 & 0.0013 & 0.0020 & 0.0020 \\
\hline 0.0008 & 0.0013 & 0.0020 & 0.0020 \\
\hline $\begin{array}{l}0.0008 \\
0.0008\end{array}$ & $\begin{array}{l}0.0013 \\
0.0013\end{array}$ & $\begin{array}{l}0.0020 \\
0.0020\end{array}$ & $\begin{array}{l}0.0020 \\
0.0020\end{array}$ \\
\hline & $\begin{array}{l}0.0013 \\
0.0013\end{array}$ & $\begin{array}{l}0.0020 \\
0.0020\end{array}$ & $\begin{array}{l}0.0020 \\
0.0020\end{array}$ \\
\hline 0.0008 & 0.0013 & 0.0020 & 0.0020 \\
\hline & 0.0013 & 0.0020 & 0.0020 \\
\hline 0.0008 & 0.0013 & 0.0020 & 0.0020 \\
\hline 0.0008 & 0.0013 & 0.0020 & 0.0020 \\
\hline 0.0008 & 0.0013 & 0.0020 & 0.0020 \\
\hline 0.0008 & 0.0013 & 0.0020 & 0.0020 \\
\hline 0.0008 & 0.0013 & 0.0020 & 0.0020 \\
\hline 0.0008 & $\begin{array}{l}0.0013 \\
0.0013\end{array}$ & $\begin{array}{l}0.0020 \\
0.0020\end{array}$ & $\begin{array}{l}0.0020 \\
0.0020\end{array}$ \\
\hline 0.0008 & 0.0013 & 0.0020 & $\begin{array}{l}0.0020 \\
0.0020\end{array}$ \\
\hline 0.0008 & & 0.0020 & 0.0020 \\
\hline 0.0008 & 0.0013 & 0.0020 & 0.0020 \\
\hline 0.0008 & 0.0013 & 0.0020 & 0.0020 \\
\hline 0.0008 & 0.0013 & 0.0020 & 0.0020 \\
\hline 0.0008 & 0.0013 & 0.0020 & 0.0020 \\
\hline $\begin{array}{l}0.0008 \\
0.0008\end{array}$ & $\begin{array}{l}0.0013 \\
0.0013\end{array}$ & $\begin{array}{l}0.0020 \\
0.0020\end{array}$ & $\begin{array}{l}0.0020 \\
0.0020\end{array}$ \\
\hline $\begin{array}{l}0.00008 \\
0.000\end{array}$ & & 0.0020 & \\
\hline 0.0008 & 0.0013 & 0.0020 & 0.0020 \\
\hline 0.0008 & 0.0013 & 0.0020 & 0.0020 \\
\hline 0.0008 & 0.0013 & 0.0020 & 0.0020 \\
\hline 0.0008 & 0.0013 & 0.0020 & 0.0020 \\
\hline 0.0008 & 0.0013 & 0.0020 & 0.0020 \\
\hline 0.0008 & 0.0013 & 0.0020 & 0.0020 \\
\hline 0.0008 & 0.0013 & 0.0020 & $\begin{array}{l}0.0020 \\
0.0020\end{array}$ \\
\hline 0 & 0.0013 & $\begin{array}{l}0.0020 \\
0.0020\end{array}$ & $\begin{array}{l}0.0020 \\
0.0020\end{array}$ \\
\hline 0.0008 & 0.0013 & 0.0020 & $\begin{array}{l}0.002020 \\
0.002\end{array}$ \\
\hline 0.0008 & 0.0013 & 0.0020 & 0.0020 \\
\hline 0.0008 & 0.0013 & 0.0020 & 0.0020 \\
\hline 0.0008 & 0.0013 & 0.0020 & 0.0020 \\
\hline & 0.0013 & 0.0020 & 0.0020 \\
\hline $\begin{array}{l}0.0008 \\
0.00008\end{array}$ & $\begin{array}{l}0.0013 \\
0.0013\end{array}$ & $\begin{array}{l}0.0020 \\
0.0020\end{array}$ & $\begin{array}{l}0.0020 \\
0.0020\end{array}$ \\
\hline 0.0008 & 0.0013 & 0.0020 & 0.0020 \\
\hline
\end{tabular}

$\begin{array}{lll}0.0020 & 0.0000 \quad 0.0100\end{array}$

$\begin{array}{lll}0.0020 & 0.0020 & 0.00000 \\ 0.0020 & 0.0022 & 0.0000\end{array}$

$\begin{array}{lll}0.0020 & 0.0000 & 0.0000 \\ 0.020 & 0.0002 & 0\end{array}$

$\begin{array}{lll}0.0020 & 0.0020 & 0.00000 \\ 0 & 0.0000\end{array}$

$\begin{array}{lll}0.0020 & 0.0020 & 0.0086 \\ 0.0020 & 0.0020 & 0.0000\end{array}$

$\begin{array}{lll}0.0020 & 0.0020 & 0.0000 \\ 0.0020 & 0.0000 & 0.0000\end{array}$

$\begin{array}{lll}0.0020 & 0.0000 & 0.0000 \\ 0.0020 & 0.0004 & 0.01100 \\ 0.020 & 0.0000 & 0.0100 \\ 0.020 & 0.0000 & 0.100\end{array}$

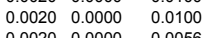

$\begin{array}{lll}0.0020 & 0.00000 & 0.0056 \\ 0.0020 & 0.0020 & 0.0000 \\ 0.0020 & 0.0000 & 0.078\end{array}$

$\begin{array}{lll}0.0020 & 0.0000 & 0.0078 \\ 0.0020 & 0.0020 & 0.0000 \\ 0.020 & 0.0000 & 0.000\end{array}$

$\begin{array}{lll}0.0020 & 0.0020 & 0.0100 \\ 0.0020 & 0.0000 & 0.0000\end{array}$

$\begin{array}{lll}0.0020 & 0.00000 & 0.0000 \\ 0.0020 & 0.0020 & 0.0000 \\ 0.020 & 0.0020 & 0.000\end{array}$

$\begin{array}{lll}0.02020 & 0.00000 & 0.0000 \\ 0.0020 & 0.00000 & 0.0077 \\ 0.020 & 0.0020 & 0.0100\end{array}$

$\begin{array}{lll}0.00020 & 0.0020 & 0.0117 \\ 0.0020 & 0.0000 & 0.0100 \\ 0.020 & \end{array}$

$\begin{array}{lll}0.0020 & 0.0000 & 0.0100 \\ 0.0020 & 0.0000 & 0.0061\end{array}$

$\begin{array}{lll}0.0020 & 0.0000 & 0.0028 \\ 0.0020 & 0.0020 & 0.0000 \\ 0.020 & 0.020 & 0\end{array}$

$\begin{array}{lll}0.0020 & 0.0020 & 0.0014 \\ 0.0220 & 0.0220 & 0.00000\end{array}$

$\begin{array}{lll}0.0020 & 0.0020 & 0.0000 \\ 0.0020 & 0.0000 & 0.0000\end{array}$

$\begin{array}{lll}0.0020 & 0.00000 & 0.0008 \\ 0.0002 & 0.0047\end{array}$

$\begin{array}{lll}0.0020 & 0.0020 & 0.00069 \\ 0.0020 & 0.0000 & 0.0006\end{array}$

$\begin{array}{lll}0.0020 & 0.0000 & 0.0000 \\ 0.020 & 0.0008 & 0.0031\end{array}$

$\begin{array}{lll}0.0020 & 0.00000 & 0.00000 \\ 0.0020 & 0.0000 & 0.000\end{array}$

$\begin{array}{lll}0.02020 & 0.0000 & 0.0000 \\ 0.0020 & 0.0012 & 0.00000 \\ 0.0020 & 0.0020 & 0.0002\end{array}$

$\begin{array}{lll}0.0020 & 0.0020 & 0.0002 \\ 0.0020 & 0.0000 & 0.0100\end{array}$

$\begin{array}{lll}0.0020 & 0.0000 & 0.0000 \\ 0.0020 & 0.0000 & 0.0100 \\ 0.0020 & 0.020 & 0.014\end{array}$

$\begin{array}{lll}0.0020 & 0.0020 & 0.0014 \\ 0.0020 & 0.0017 & 0.0000 \\ 0.0220 & 0.020 & 0.000\end{array}$

$\begin{array}{lll}0.0020 & 0.0020 & 0.0000\end{array}$

$\begin{array}{lll}0.0020 & 0.0000 & 0.0100 \\ 0.020 & 0.0010 & 0.0062\end{array}$

$\begin{array}{lll}0.0020 & 0.0010 & 0.0002 \\ 0.0000 & 0.0069\end{array}$

$\begin{array}{lll}0.0020 & 0.0000 & 0.0047 \\ 0.0020 & 0.0000 & 0.0100\end{array}$

$\begin{array}{lll}0.0020 & 0.0000 & 0.0000\end{array}$

$\begin{array}{lll}0.0020 & 0.0000 & 0.0022 \\ 0.0020 & 0.0000 & 0.0100\end{array}$

$\begin{array}{lll}0.0020 & 0.0020 & 0.0068 \\ 0.0020 & 0.0000 & 0.0100\end{array}$ $\begin{array}{lll}0.0020 & 0.0020 & 0.00089 \\ 0.0020 & 0.0000 & 0.0000\end{array}$
0.0000
0.0000
0.0000

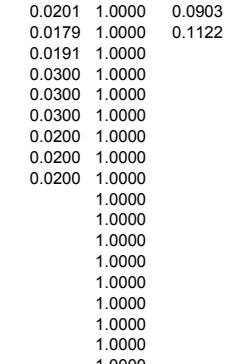

0.0020
0.0020
0.0020
0.0020
0.0020
0.0020
0.0020
0.0020
0.0020
0.0020
0.0020
0.0020
0.0020
0.0020
0.0020
0.0020
0.0020
0.0020
0.0020
0.0020
0.0020
0.0020
0.0020
0.0020
0.0020
0.0020
0.0020
0.0020
0.0020
0.0020
0.0020
0.0020
0.0020
0.0020
0.0020
0.0020
0.0020
0.0020
0.0020
0.0020
0.0020
0.0020
0.0020
0.0020
0.0020
0.0020
0.0020
0.0020
0.0020
0.0020
0.0020
0.0020
0.0020
0.0020
0.0020
0.0020
0.0020
0.0020
0.0020
0.0020
0.0020

\subsection{0721}

\subsection{0}

0.0492
0.0000
0.0098
0.0800

0.0800
0.0800
0.000

0.0000
0.0800
0.0800
0.0800
0.0000
0.014

0.0000
0.0164
0.0000

0.0000

0.0000
0.0800
0.000

0.0800
0.0000
0.0800

0.0800
0.0800
0.0000

0.0000
0.0800
0.0800

0.0800
0.0000

0.08000
0.00000
0.0800
0.0000

0.0000

0.0000
0.0000
0.0000
0.0800
0.0800

0.0800
0.0800

0.0800
0.0800
0.0000
0.000

0.0000
0.0000
0.0800

0.0800

0.0000
0.0332
0.0000

0.0000
0.0000
0.0492

0.0492
0.0800
0.0000

0.0000
0.0000
0.0000
0.0800
0.0607

0.0667

0.0800
0.0000

0.0000
0.0400
0.0000
0.0000

0.0000

0.0000
0.0000

0.0000
0.0000
0.0800

0.0800
0.0000

$\begin{array}{lll}0.0200 & 1.00000 & 0.0182 \\ 0.0200 & 0.9999 & 0.0157 \\ 0.0200 & 1.000 & 0.105\end{array}$

$\begin{array}{lll}0.0200 & 1.0000 & 0.1052 \\ 0.0200 & 1.0000 & 0.1040\end{array}$ $\begin{array}{lll}0.0200 & 1.0000 & 0.1044 \\ 0.0200 & 1.0000 & 0.0103 \\ 0.2200 & 1.0000 & 0.1037\end{array}$

$\begin{array}{lll}0.0200 & 1.0000 & 0.1037 \\ 0.0200 & 0.9999 & 0.0178\end{array}$ $0.02001 .0001 \quad 0.0229$ $\begin{array}{lll}0.0200 & 1.0000 & 0.0081\end{array}$ $0.0200 \quad 1.0000 \quad 0.0248$ $\begin{array}{lll}0.0200 & 0.9999 & 0.0065 \\ 0.0200 & 1.0000 & 0.0249\end{array}$ $\begin{array}{lll}0.0200 & 1.0000 & 0.0173 \\ 0.0200 & 110001 & 0.0137\end{array}$ $\begin{array}{lll}0.0200 & 1.0000 & 0.0080 \\ 0.0200 & 0.9999 & 0.1074 \\ 0.0200 & 1.000 & 0.05\end{array}$ $\begin{array}{lll}0.02000 & 1.09000 & 0.1074 \\ 0.0200 & 1.0000 & 0.0797 \\ 0.02200 & 1.0000 & 0.0551\end{array}$

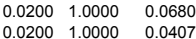
$\begin{array}{lll}0.02200 & 1.00000 & 0.0400 \\ 0.0200 & 0.0086\end{array}$ $\begin{array}{lll}0.0200 & 1.0002 & 0.1126\end{array}$ $\begin{array}{lll}0.0200 & 1.0000 & 0.0051 \\ 0.0200 & 1.0000 & 0.0297\end{array}$ $\begin{array}{lll}0.0200 & 1.0000 & 0.0297 \\ 0.0200 & 1.0000 & 0.0397\end{array}$ $0.0200 \quad 0.9999 \quad 0.0046$ $\begin{array}{lll}0.0200 & 1.0000 & 0.0118 \\ 0 & 0200 & 1.0000 \\ 0.0200 & 0.0000\end{array}$

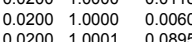
$\begin{array}{lll}0.0200 & 1.0001 & 0.0895 \\ 0.0200 & 0.9999 & 0.0140\end{array}$ $\begin{array}{lll}0.0200 & 1.0002 & 0.1076 \\ 0.0200 & 1.0001 & 0.0086\end{array}$

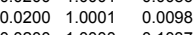
$0.0200 \quad 1.0000 \quad 0.0521$ $\begin{array}{lll}0.0200 & 1.0000 & 0.1060 \\ 0.0200 & 1.0000 & 0.0913\end{array}$ $\begin{array}{lll}0.0200 & 1.0000 & 0.0913 \\ 0.0200 & 1.0000 & 0.0835\end{array}$ $\begin{array}{lll}0.0200 & 1.0002 & 0.1003 \\ 0.0200 & 0.9999 & 0.0152\end{array}$ $0.0200 \quad 1.0000 \quad 0.1050$ $\begin{array}{lll}0.0200 & 1.0000 & 0.0404 \\ 0.0200 & 1.0001 & 0.0486\end{array}$ $\begin{array}{lll}0.0200 & 1.0000 & 0.0098 \\ 0.0200 & 1.0000 & 0.0301\end{array}$ $\begin{array}{lll}0.0200 & 1.00002 & 0.00017 \\ 0.02000 & 0.0073\end{array}$ $\begin{array}{lll}0.0200 & 1.0001 & 0.1043 \\ 0.0200 & 1.0002 & 0.0443\end{array}$ $\begin{array}{lll}0.0200 & 1.0000 & 0.0415 \\ 0.0200 & 1.0000 & 0.0060\end{array}$ $\begin{array}{lll}0.0200 & 1.0000 & 0.0060 \\ 0.0200 & 1.0002 & 0.1043\end{array}$ $\begin{array}{lll}0.0200 & 1.0000 & 0.1073\end{array}$ $\begin{array}{lll}0.0200 & 1.0001 & 0.0115 \\ 0.0200 & 1.0000 & 0.0285\end{array}$ $\begin{array}{lll}0.0200 & 0.9999 & 0.0437 \\ 0.0000 & 0.1018 \\ 0.0200 & 10001 & .016\end{array}$ 
\# Glass ID

1477 HLWG8-60
1478 HLLV8-61

1479 HLW98-61CG
1480 HLW98-62

1481 HLW98-63
1482 HLW98-64

1482 HLW98-64
1483 HLWMS-01
1484 HLWMS-02

1485 HLWMS-03

1486 HLWMS-04
1487 HLWMS-05

1488 HLWMS-06

1490 HLWMS-08

491 HLWMS-09

1493 HLWMS-11

494 HLWMS-12
195 HLWMS-13

1496 HLWMS-13

1497 HLWMS-15
1498 HLW99-01

1499 HLW99-02

1501 HLW9-0

(

1503 HLW99-06
1504 HLW99-07

1505 HLW99-08
1506 HLW9-09

1507 HLW99-10

1508 HLW99-11

510 HLW99-13

(511 HLW99-14

513 HLW99-16

514 HLW99-17
515 HLW99-18

1516 HLW99-19

518 HLW99-21

1519 HLW99-22

1520 HLW99-23

1522 HLW99-25

1523 HLW99-26

1524 HLW99-27

1526 HLW99-29

1528 HLW99-31

W99-32

1531 HLW99-34

1532 HLW99-36

1534 HLW99-37

1536 HLW99-39

W99-40

1539 HLW99-42

1541 HLW99-44

1543 HLW99-46

1545 HLW99-48

1546 HLW99-49

1547 HLW99-50
1548 HLW99-51R

549 HLW99-52

1550 HLW99-53

1551 HLW99-54

1553 HLW99-56R

1554 HLW99-57

1556 HLW99-59

1557 HLW99-60
1558 HLW99-61
B2O3-a CaO-a Fe2O3-a FeO-a K2O-a Li2O-a MgO-a Na2O-a NiO-a P2O5-a SiO2-a Zro2-a Ag2O-a As203-a As205-a BaO-a BeO-a Bi2O3-a Bra $0.0084 \quad 0.0904$

$\begin{array}{llllllllll}0.0008 & 0.0433 & 0.0015 & 0.1143 & 0.0081 & 0.0037 & 0.4603 & 0.0160 & 0.0003 & \\ 0.0019 & 0.0383 & 0.0018 & 0.1015 & 0.0061 & 0.0067 & 0.4961 & 0.0132 & 0.0003 & 0.0002\end{array}$ 0.0008
0.0008

0.000 0.012

$\begin{array}{lll}0.1180 & 0.0231 & 0.0436 \\ 0.0651 & 0.0095 & 0.1129\end{array}$

$\begin{array}{llllllllll}0.0012 & 0.0483 & 0.0007 & 0.0905 & 0.0034 & 0.0047 & 0.4224 & 0.0876 & 0.0001 & 0.0002 \\ 0.0010 & 0.0330 & 0.0028 & 0.0937 & 0.0015 & 0.0030 & 0.3702 & 0.0018 & 0.0028 & 0.0000\end{array}$

0.0005

0.00000

0.0006
0.0005
CoO-a Co2O3-a Cr203-a Cs2O-a CuO-a

0.0004

0.0010

$\begin{array}{ll}0.0008 & 0.0027 \\ 0.0002 & 0.0016\end{array}$

0.0001
0.0001 $\begin{array}{lll}0.0488 & 0.0633 & 0.0206\end{array}$ $\begin{array}{lll}0.1860 & 0.0012 & 0.1101 \\ 0.2003 & 0.0013 & 0.0201\end{array}$ $\begin{array}{lll}0.0529 & 0.0008 & 0.0241 \\ 0.0564 & 0.0010 & 0.0200\end{array}$ $\begin{array}{lll}0.0504 & 0.0658 & 0.0203 \\ 0.1947 & 0.0652 & 0.0209\end{array}$ $\begin{array}{lll}0.0487 & 0.0643 & 0.1131 \\ 0.1771 & 0.0610 & 0.0194\end{array}$ $0.0506 \quad 0.0620 \quad 0.0206$ $\begin{array}{lll}0.1838 & 0.0644 & 0.0218 \\ 0.1858 & 0.0618 & 0.1136\end{array}$ $0.0511 \quad 0.0030 \quad 0.0199$ $\begin{array}{lll}0.1885 & 0.0008 & 0.1138\end{array}$ $\begin{array}{lll}0.1924 & 0.0353 & 0.0214 \\ 0.0516 & 0.0025 & 0.1228\end{array}$ $\begin{array}{lll}0.0488 & 0.0641 & 0.0197 \\ 0.0412 & 0.128\end{array}$ $\begin{array}{lll}0.0708 & 0.0698 & 0.0214\end{array}$ $\begin{array}{lll}0.1924 & 0.0028 & 0.1127 \\ 0.0970 & 0.0014 & 0.0227\end{array}$ $\begin{array}{lll}0.0501 & 0.0667 & 0.0202\end{array}$ $\begin{array}{lll}0.1856 & 0.0006 & 0.0229 \\ 0.0615 & 0.0037 & 0.0201\end{array}$ $\begin{array}{lll}0.0615 & 0.0637 & 0.0201 \\ 0.1502 & 0.0676 & 0.1022\end{array}$ $\begin{array}{lll}0.0899 & 0.0686 & 0.0943\end{array}$ $\begin{array}{lll}0.0559 & 0.0041 & 0.0628 \\ 0.1457 & 0.0442 & 0.096\end{array}$ $\begin{array}{lll}0.1457 & 0.0642 & 0.1096 \\ 0.1490 & 0.0630 & 0.0205\end{array}$ $\begin{array}{lll}0.0532 & 0.0012 & 0.0210\end{array}$ $\begin{array}{lll}0.1218 & 0.0009 & 0.0204 \\ 0.1756 & 0.0011 & 0.0223\end{array}$ $\begin{array}{lll}0.1756 & 0.0011 & 0.0223 \\ 0.0641 & 0.0008 & 0.1156 \\ 0.1503 & 0.051 & 0.025\end{array}$ $\begin{array}{lll}0.1903 & 0.0631 & 0.0259\end{array}$ $\begin{array}{lll}0.0622 & 0.0038 & 0.0297\end{array}$ $\begin{array}{lll}0.0500 & 0.0387 & 0.0686 \\ 0.1927 & 0.0137 & 0.1170\end{array}$ $\begin{array}{lll}0.0707 & 0.0009 & 0.0198 \\ 0.013 & 0.051 & 0.007\end{array}$

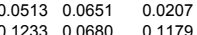
$\begin{array}{lll}0.1944 & 0.0003 & 0.0645\end{array}$ $\begin{array}{lll}0.1124 & 0.0292 & 0.0587 \\ 0.1631 & 0.426 & 0.0195\end{array}$ $\begin{array}{lll}0.1631 & 0.0426 & 0.0195 \\ 0.1901 & 0.0666 & 0.1153\end{array}$ $\begin{array}{lll}0.0693 & 0.0658 & 0.1169\end{array}$ $\begin{array}{lll}0.1584 & 0.0664 & 0.0208 \\ 0.132 & 0.0657 & 0.0206\end{array}$ $\begin{array}{lll}0.1132 & 0.0657 & 0.0206 \\ 0.0466 & 0.0338 & 0.1127\end{array}$ $\begin{array}{lll}0.0624 & 0.0008 & 0.0808\end{array}$ $\begin{array}{lll}0.0628 & 0.0002 & 0.1126 \\ 0.0603 & 0.0007 & 0.0208\end{array}$ $\begin{array}{lll}0.1876 & 0.0644 & 0.0205 \\ 0.1852 & 0.0008 & 0.0193\end{array}$ $0.0526 \quad 0.0598 \quad 0.1037$ $\begin{array}{lll}0.0491 & 0.0010 & 0.0187 \\ 0.0523 & 0.0666 & 0.0675\end{array}$ $0.0531 \quad 0.0012 \quad 0.1153$ $\begin{array}{lll}0.0516 & 0.0006 & 0.1184 \\ 0.1881 & 0.0654 & 0.0230\end{array}$ $\begin{array}{lll}0.1881 & 0.0654 & 0.0230 \\ 0.0533 & 0.0617 & 0.0209\end{array}$ $\begin{array}{lll}0.1289 & 0.0012 & 0.1029 \\ 0.1873 & 0.0008 & 0.0191\end{array}$

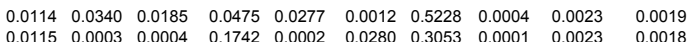
$\begin{array}{llllllllll}0.0115 & 0.0003 & 0.0004 & 0.1742 & 0.0002 & 0.0280 & 0.3053 & 0.0004 & 0.00023 & 0.0018 \\ 0.0109 & 0.0576 & 0.0192 & 0.0471 & 0.0284 & 0.0013 & 0.3035 & 0.0253 & 0.0025 & 0.0019\end{array}$ $\begin{array}{llllllllll}0.0114 & 0.0604 & 0.0003 & 0.0407 & 0.02003 & 0.00309 & 0.47705 & 0.0153 & 0.0025 & 0.0020 \\ 0.0111 & 0.0114 & 0.0178 & 0.1807 & 0.0278 & 0.0196 & 0.3185 & 0.0821 & 0.0024 & 0.0020\end{array}$ $\begin{array}{llllllllll}0.0116 & 0.0009 & 0.0003 & 0.1748 & 0.0001 & 0.0013 & 0.4527 & 0.0050 & 0.0017 & 0.0019 \\ 0.0106 & 0.0368 & 0.0006 & 0.1338 & 0.0006 & 0.0056 & 0.3588 & 0.0862 & 0.0020 & 0.0019\end{array}$

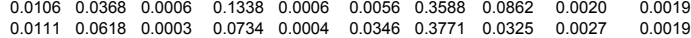
$\begin{array}{lllllllllllll}0.0106 & 0.0322 & 0.0154 & 0.0449 & 0.0229 & 0.0293 & 0.3122 & 0.0020 & 0.0021 & 0.0014\end{array}$ $\begin{array}{llllllllll}0.0109 & 0.0012 & 0.0003 & 0.1140 & 0.0004 & 0.0351 & 0.5336 & 0.0023 & 0.0026 & 0.0021 \\ 0.0109 & 0.0099 & 0.0002 & 0.0924 & 0.0003 & 0.0330 & 0.2956 & 0.0011 & 0.0026 & 0.0019\end{array}$

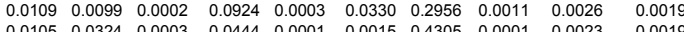
$\begin{array}{llllllllll}0.0109 & 0.0004 & 0.0177 & 0.1879 & 0.0276 & 0.0080 & 0.4713 & 0.0004 & 0.0020 & 0.0020\end{array}$ $\begin{array}{lllllllllll}0.0198 & 0.0328 & 0.0177 & 0.0487 & 0.0253 & 0.0010 & 0.3466 & 0.0312 & 0.0022 & 0.0019 \\ 0.0116 & 0.0116 & 0.195 & 0.1808 & 0.0272 & 0.0004 & 0.3256 & 0.0004 & 0.0017 & 0.0018\end{array}$

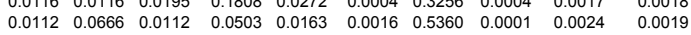
$\begin{array}{lllllllllll}0.0106 & 0.0104 & 0.0005 & 0.1829 & 0.0009 & 0.0258 & 0.3647 & 0.1009 & 0.0028 & 0.0019\end{array}$ $\begin{array}{llllllllll}0.0111 & 0.0123 & 0.0157 & 0.1859 & 0.0234 & 0.0012 & 0.3083 & 0.0285 & 0.0018 & 0.0020 \\ 0.0111 & 0.0462 & 0.0002 & 0.0485 & 0.0004 & 0.0354 & 0.3177 & 0.0032 & 0.0025 & 0.0020\end{array}$ $\begin{array}{lllllllllllll} & 0.0635 & 0.0176 & 0.0737 & 0.0276 & 0.0354 & 0.3387 & 0.0007 & 0.0024 & 0.0021\end{array}$ $\begin{array}{llllllllll}0.0107 & 0.0648 & 0.0004 & 0.0757 & 0.0002 & 0.0012 & 0.4989 & 0.0483 & 0.0021 & 0.0020 \\ 0.0111 & 0.0026 & 0.0007 & 0.1355 & 0.0011 & 0.0337 & 0.3043 & 0.0878 & 0.0023 & 0.0023\end{array}$

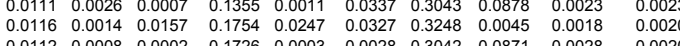
$\begin{array}{llllllllll}0.0114 & 0.0659 & 0.0100 & 0.0797 & 0.0132 & 0.0005 & 0.3496 & 0.0020 & 0.0022 & 0.0019 \\ 0.0106 & 0.0301 & 0.0171 & 0.0530 & 0.0260 & 0.0329 & 0.4072 & 0.0008 & 0.0025 & 0.0019\end{array}$

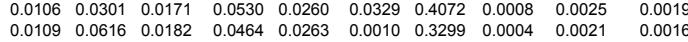
$\begin{array}{lllllllllll}0.0106 & 0.0016 & 0.0185 & 0.1065 & 0.0268 & 0.0017 & 0.3204 & 0.00696 & 0.0024 & 0.0019\end{array}$ $\begin{array}{llllllllll}0.0099 & 0.0315 & 0.0122 & 0.1156 & 0.0187 & 0.0017 & 0.5209 & 0.0858 & 0.0026 & 0.0021 \\ 0.0117 & 0.0115 & 0.0059 & 0.1840 & 0.0078 & 0.0012 & 0.3965 & 0.0898 & 0.0024 & 0.0022\end{array}$

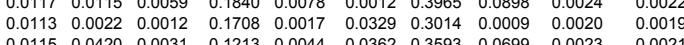
$\begin{array}{lllllllllll}0.0117 & 0.0645 & 0.0005 & 0.0761 & 0.0007 & 0.0325 & 0.3727 & 0.0048 & 0.0024 & 0.0022\end{array}$ $\begin{array}{lllllllllll}0.0113 & 0.0652 & 0.0003 & 0.0763 & 0.0004 & 0.0368 & 0.4976 & 0.0432 & 0.0026 & 0.0022\end{array}$

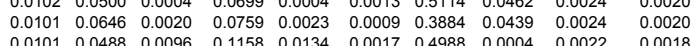
$\begin{array}{lllllllllll}0.0101 & 0.0488 & 0.0096 & 0.1158 & 0.0134 & 0.0017 & 0.4988 & 0.0004 & 0.0022 & 0.0018\end{array}$

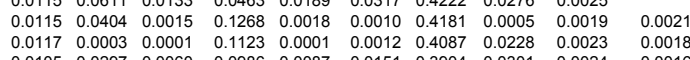

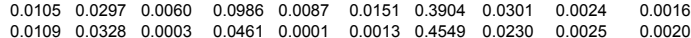
$\begin{array}{lllllllllll}0.0112 & 0.0617 & 0.00003 & 0.0789 & 0.0001 & 0.0010 & 0.4010 & 0.3117 & 0.00002 & 0.00023 & 0.0020 \\ 0.0022 & 0.0023 & 0.0025\end{array}$

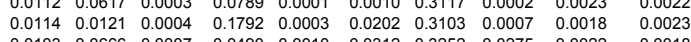

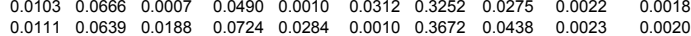
$\begin{array}{llllllllllll}0.0111 & 0.0272 & 0.0003 & 0.0834 & 0.0003 & 0.0008 & 0.5352 & 0.00005 & 0.0022 & 0.0021\end{array}$ $\begin{array}{llllllllll}0.0118 & 0.0007 & 0.0187 & 0.1799 & 0.0281 & 0.0012 & 0.316 & 0.0175 & 0.0017 & 0.0020 \\ 0.0110 & 0.0029 & 0.1765 & 0.0044 & 0.0313 & 0.3077 & 0.0394 & 0.0019 & 0.0013\end{array}$ $\begin{array}{llllllllll}0.0116 & 0.0607 & 0.0002 & 0.0484 & 0.0005 & 0.0280 & 0.5334 & 0.0262 & 0.0025 & 0\end{array}$ $\begin{array}{lllllllllll}0.0105 & 0.0005 & 0.0182 & 0.1471 & 0.0276 & 0.0012 & 0.3728 & 0.0001 & 0.0024 & 0.0018\end{array}$ $\begin{array}{llllllllll}0.0093 & 0.0045 & 0.0112 & 0.0973 & 0.0157 & 0.0161 & 0.3849 & 0.0037 & 0.0024 & 0.0020 \\ 0.0106 & 0.0330 & 0.0133 & 0.1100 & 0.0195 & 0.0004 & 0.4768 & 0.0003 & 0.0027 & 0.0015\end{array}$ $\begin{array}{lllllllllll}0.0117 & 0.0129 & 0.0095 & 0.1612 & 0.0129 & 0.0009 & 0.3245 & 0.0808 & 0.0020\end{array}$ $\begin{array}{llllllllll}0.0105 & 0.0638 & 0.0183 & 0.0470 & 0.0275 & 0.0015 & 0.04093 & 0.00292 & 0.0024 & 0.0020 \\ 0.0118 & 0.0138 & 0.007 & 0.1743 & 0.0009 & 0.0017 & 0.4646 & 0.0654 & 0.0027 & 0.0020\end{array}$

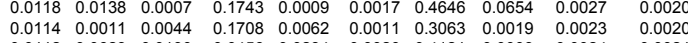
$\begin{array}{llllllll} & 0.00003 & 0.022\end{array}$ $\begin{array}{llllllllll}0.0106 & 0.0209 & 0.0129 & 0.0640 & 0.0154 & 0.0333 & 0.3278 & 0.0004 & 0.0026 & 0.0020 \\ 0.0103 & 0.0003 & 0.0182 & 0.1456 & 0.0277 & 0.0002 & 0.3710 & 0.0002 & 0.0024 & 0.0020\end{array}$

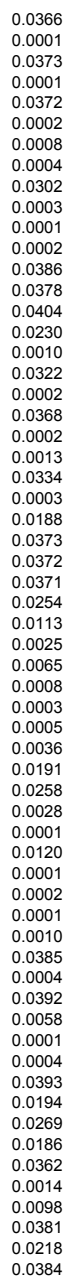

$\begin{array}{ll}0.0200 & 0.0276 \\ 0.0004 & 0.0001 \\ 0.0201 & 0.0283 \\ 0.0003 & 0.0000 \\ 0.0192 & 0.0268 \\ 0.0000 & 0.0000 \\ 0.0005 & 0.0005 \\ 0.0005 & 0.0002 \\ 0.0149 & 0.0225 \\ 0.0006 & 0.0002 \\ 0.0003 & 0.0000 \\ 0.0002 & 0.0001 \\ 0.0190 & 0.0274 \\ 0.0186 & 0.0268 \\ 0.0196 & 0.0278 \\ 0.0115 & 0.0162 \\ 0.0006 & 0.0005 \\ 0.0159 & 0.0229 \\ 0.0002 & 0.0001 \\ 0.0199 & 0.0278 \\ 0.0000 & 0.0001 \\ 0.0009 & 0.0008 \\ 0.0183 & 0.0245 \\ 0.0000 & 0.0001 \\ 0.0093 & 0.0135 \\ 0.0200 & 0.0279 \\ 0.0189 & 0.0270 \\ 0.0198 & 0.0276 \\ 0.0124 & 0.0182 \\ 0.0056 & 0.0080 \\ 0.0014 & 0.0016 \\ 0.0034 & 0.0044 \\ 0.0006 & 0.0005 \\ 0.0004 & 0.0002 \\ 0.0003 & 0.0002 \\ 0.0016 & 0.0023 \\ 0.0093 & 0.0137 \\ 0.0013 & 0.0180 \\ 0.0018 & 0.0018 \\ 0.0002 & 0.00000 \\ 0.0065 & 0.00089 \\ 0.0003 & 0.00000 \\ 0.0006 & 0.00000 \\ 0.0003 & 0.0001 \\ 0.0008 & 0.00006 \\ 0.0216 & 0.0301 \\ 0.0010 & 0.00002 \\ 0.0204 & 0.0290 \\ 0.0031 & 0.0041 \\ 0.0006 & 0.0001 \\ 0.0195 & 0.0003 \\ 0.0110 & 0.0282 \\ 0.0133 & 0.0165 \\ 0.0192 & 0.0126 \\ 0.0192 & 0.0268 \\ 0.0006 & 0.00008 \\ 0.0044 & 0.00061 \\ 0.0199 & 0.0279 \\ 0.0127 & 0.0181 \\ 0.0197 & 0.0269 \\ & \\ & \end{array}$

$\begin{array}{ll}0.00000 & 0.0020 \\ 0.0038 & 0.0023 \\ 0.00000 & 0.0021 \\ 0.0031 & 0.0023 \\ 0.0027 & 0.0025 \\ 0.0000 & 0.0022 \\ 0.0007 & 0.0023 \\ 0.0003 & 0.0025 \\ 0.0039 & 0.0021 \\ 0.0043 & 0.0024 \\ 0.0030 & 0.0024 \\ 0.0000 & 0.0021 \\ 0.0010 & 0.0023 \\ 0.0000 & 0.0021 \\ 0.00000 & 0.0021 \\ 0.0000 & 0.0022 \\ 0.0042 & 0.0021 \\ 0.0000 & 0.0023 \\ 0.0033 & 0.0023 \\ 0.0038 & 0.0024 \\ 0.0001 & 0.0023 \\ 0.0044 & 0.0023 \\ 0.0046 & 0.0023 \\ 0.0001 & 0.0023 \\ 0.0000 & 0.0022 \\ 0.0032 & 0.0022 \\ 0.0001 & 0.0021 \\ 0.0001 & 0.0022 \\ 0.0002 & 0.0020 \\ 0.0000 & 0.0022 \\ 0.0046 & 0.0024 \\ 0.0045 & 0.0023 \\ 0.0045 & 0.0022 \\ 0.0044 & 0.0023 \\ 0.0001 & 0.0021 \\ 0.0001 & 0.0019 \\ 0.0001 & 0.0021 \\ 0.0047 & 0.0020 \\ 0.0001 & 0.0020 \\ 0.0000 & 0.0020 \\ 0.0018 & 0.0021 \\ 0.00000 & 0.0021 \\ 0.0001 & 0.0023 \\ 0.0029 & 0.0022 \\ 0.0043 & 0.0023 \\ 0.0001 & 0.0020 \\ 0.0000 & 0.0018 \\ 0.0000 & 0.0021 \\ 0.0007 & 0.0022 \\ 0.0040 & 0.0031 \\ 0.0044 & 0.0031 \\ 0.0000 & 0.0022 \\ 0.0020 & 0.0021 \\ 0.00000 & 0.0023 \\ 0.0002 & 0.0021 \\ 0.0001 & 0.0021 \\ 0.0001 & 0.0022 \\ 0.0001 & 0.0022 \\ 0.0001 & 0.0022 \\ 0.0021 & 0.0042 \\ 0.0001 & 0.0022 \\ & \end{array}$


\# Glass ID

1477 HLW98-60
1478 HLW98-61

1478 HLW98-61

1480 HLW98-62

1481 HLW98-63
1482 HLW98-64

1483 HLWMS-01

1484 HLWMS-02

486 HLWMS-04

1487 HLWMS-05

1488 HLWMS-06

1400 HWWS- 08

1491 HLWMS-09

1492 HLWMS-10

1493 HLWMS-11

1495 HLWMS -13

1496 HLWMS-14

1498 HLW99-01

1499 HLW99-02

1501 HLW99-04

1503 HLW99-06
1504 HLW99-07

1505 HLW99-08

1508 HLW99-11
1509 HLW99-12

1510 HLW99-13

1511 HLW99-14

1513 HLW99-1 6

14 HLW99-17

1515 HLW99-18

1517 HLW99-20

518 HLW99-21

1519 HLW99-22

1520 HLW99-23

1522 HLW99-25

1523 HLW99-26

1525 HLW99-28

1526 HLW99-29

527 HLW99-30

1529 HLW99-32

1530 HLW99-33

1531 HLW99-34

1534 HLW99-37

1536 HLW-38

1537 HLW99-40

1538 HLW99-41
1539 HLW99-42

1540 HLW99-43
1541 HLW99-44

1542 HLW99-45

1543 HLW99-46

1545 HLW99-48

546 HLW99-49

1547 HLW99-50

549 HLW99-52

1550 HLW99-53

1551 HLW99-54

1553 HLW99-56R

1554 HLW99-57

1556 HLW99-59

1558 HLW99-61
Ga2O3-a Gd2O3-a

I-a La203-a MnO2-a 0.0072
0.0112

$\begin{array}{ll}0.0000 & 0.0210 \\ 0.0000 & 0.0562\end{array}$

0.0019

PdO-a
0.0000
0.0000

0.0009
0.0030

0.0000

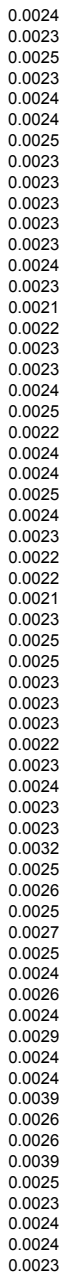

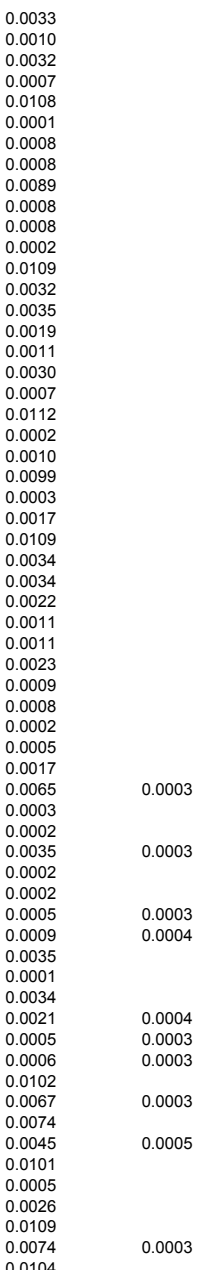

$\begin{array}{ll}0.0022 & 0.0007 \\ 0.0020 & 0.0006 \\ 0.0021 & 0.007\end{array}$

$\begin{array}{ll}0.0020 \quad 0.0007 \\ 0.0021 & 0.0009\end{array}$

$\begin{array}{ll}0.0021 & 0.0009 \\ 0.0023 & 0.0013\end{array}$

$\begin{array}{ll}0.0022 & 0.0013 \\ 0.00020 & 0.000\end{array}$

$0.0024 \quad 0.001$

$\begin{array}{ll}0.0023 & 0.0009 \\ 0.0019 & 0.0010 \\ 0.0019 & 0.0002\end{array}$

$\begin{array}{ll}0.0023 & 0.0011 \\ 0.0019 & 0.0006\end{array}$

$\begin{array}{ll}0.0019 & 0.0006 \\ 0.0021 & 0.0010\end{array}$

$0.0023 \quad 0.0011$

$0.0021 \quad 0.0017$

$\begin{array}{ll}0.0021 & 0.0005 \\ 0.0023 & 0.0014\end{array}$

$\begin{array}{ll}0.0019 & 0.0013 \\ 0.0023 & 0.0008\end{array}$

$\begin{array}{ll}0.0022 & 0.0013 \\ 0.0018 & 0.0006\end{array}$

$\begin{array}{ll}0.0020 & 0.0013 \\ 0.0023 & 0.0007\end{array}$

\begin{tabular}{ll}
0.0023 & 0.0007 \\
0.0020 & 0.0009 \\
\hline 0.0020 & 0.0012
\end{tabular}

$0.0022 \quad 0.0010$

$\begin{array}{ll}0.0022 & 0.0012 \\ 0.0024 & 0.0013 \\ 0.0025 & 0.0015\end{array}$

$0.0023 \quad 0.0010$

$0.0023 \quad 0.001$

$\begin{array}{ll}0.0019 & 0.000 \\ 0.0019 & 0.0014\end{array}$

$\begin{array}{llll} & & & 0.0003 \\ & 0.0003 & 0.0019 & 0.0014 \\ & & 0.0004 & 0.0014\end{array}$

$\begin{array}{ll}0.0020 & 0.0006 \\ 0.0030 & 0.0010 \\ 0.020 & 0.0006\end{array}$

\begin{tabular}{lll}
0.0020 & 0.0000 \\
0.0018 & 0.0000 \\
\hline
\end{tabular}

$\begin{array}{llll}0.0003 & 0.0004 & 0.0035 & 0.0010 \\ 0.0005 & 0.0003 & 0.0039 & 0.0011\end{array}$

$0.0020 \quad 0.0006$

$0.0019 \quad 0.0016$

$\begin{array}{llll}0.0003 & 0.0003 & 0.0030 & 0.0017 \\ 0.0002 & 0.0002 & 0.0026 & 0.0012 \\ 0.0003 & 0.0004 & 0.0022 & 0.0006\end{array}$

$\begin{array}{lll}0.00033 & 0.0022 & 0.0011 \\ 0.0004 & 0.0022 & 0.0012 \\ 0 & 0.00001 & 0.0017\end{array}$

\begin{tabular}{ll}
0.0025 & 0.0017 \\
0.0020 & 0.0007 \\
\hline
\end{tabular}

$0.0022 \quad 0.0007$

$0.0022 \quad 0.0010$

$\begin{array}{ll}0.0022 & 0.0010 \\ 0.0021 & 0.0007\end{array}$ 
\# Glass ID

1477 HLWG8-60
1478 HLLV8-61

1479 HLW98-61CG1
1480 HLW98-62

1480 HLW98-62
1481 HLW88-63

482 HLW98-64
483 HLWMS-01

1484 HLWMS-02

1486 HLWMS-04
1487 HLWMS-05

1488 HLWMS-06

1489 HLWMS-07

1491 HLWMS-09

1492 HLWMS-10

494 HLWMS-12

1495 HLWMS-13
1496 HLWMS-14

1497 HLWMS-15
1498 HLW99-01

1499 HLW99-02

501 HLW-03

1503 HLW99-06
1504 HLW99-07

1505 HLW99-08

1507 HLW99-10

1508 HLW99-11

1510 HLW99-13

1511 HLW99-14

1513 HLW99-16

1515 HLW99-18

1516 HLW99-19

1518 HLW99-21

1519 HLW99-22
1520 HLW99-23

1523 HLW99-26

1524 HLW99-27
1525 HLW99-28

1526 HLW99-29

1527 HLW99-30

1529 HLW99-32

1530 HLW99-33

1531 HLW99-34

1533 HLW99-36
534 HLW99-37

1534 HLW99-37

536 HLW99-39

1538 HLW99-41

540 HLW99-43

1541 HLW99-44

1542 HLW99-45
1543 HLW99-46

1545 HLW99-48

1546 HLW99-49
1547 HLW99-50

1549 HLW99-52
1550 HLW99-53

1551 HLW99-54

1553 HLW99-56R

1554 HLW99-57

1556 HLW99-59

1558 HLW99-61

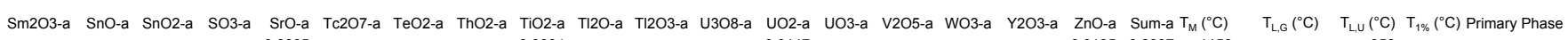

$\begin{array}{ll}0.0018 & 0.0085 \\ 0.0109\end{array}$

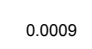

0.0006

0.0003

0.0147

0.000

$\begin{array}{ll}0.0001 & 0.0005 \\ 0.0001 & 0.0003\end{array}$

\begin{tabular}{|c|c|c|}
\hline 0.0026 & 0.0021 & \\
\hline 0.0019 & 0.0019 & \\
\hline 0.0025 & 0.0023 & 0.0109 \\
\hline $\begin{array}{l}0.0019 \\
0.00224\end{array}$ & $\begin{array}{l}0.00211 \\
0.0224\end{array}$ & $\begin{array}{l}0.0006 \\
0.006\end{array}$ \\
\hline $\begin{array}{l}0.0024 \\
0.0024\end{array}$ & $\begin{array}{l}0.0024 \\
0.0022\end{array}$ & $\begin{array}{l}0.0106 \\
0.0003\end{array}$ \\
\hline 0.0023 & 0.0021 & \\
\hline 0.0021 & 0.0020 & 0.0006 \\
\hline $\begin{array}{l}0.0023 \\
0.0022\end{array}$ & $\begin{array}{l}0.0019 \\
0.0021\end{array}$ & $\begin{array}{l}0.0090 \\
0.0004\end{array}$ \\
\hline 0.0020 & 0.0022 & $\begin{array}{l}0.00004 \\
0.0004\end{array}$ \\
\hline 0.0021 & 0.0020 & \\
\hline $\begin{array}{l}0.0024 \\
0.0027\end{array}$ & $\begin{array}{l}0.0020 \\
0.0021\end{array}$ & $\begin{array}{l}0.0101 \\
0.0111\end{array}$ \\
\hline 0.0025 & 0.0020 & 0.0107 \\
\hline 0.0023 & 0.0020 & 0.0061 \\
\hline 0.0021 & 0.0020 & \\
\hline $\begin{array}{l}0.0024 \\
0.0021\end{array}$ & $\begin{array}{l}0.0020 \\
0.0018\end{array}$ & $\begin{array}{l}0.0085 \\
0.0005\end{array}$ \\
\hline $\begin{array}{l}0.0021 \\
0.0025\end{array}$ & $\begin{array}{l}0.0018 \\
0.0022\end{array}$ & $\begin{array}{l}0.0005 \\
0.00103\end{array}$ \\
\hline & $\begin{array}{l}0.0022 \\
0.0021\end{array}$ & $\begin{array}{l}.00103 \\
0.0006\end{array}$ \\
\hline 0.0021 & 0.0020 & 0.0007 \\
\hline 0.0025 & 0.0020 & 0.0089 \\
\hline 0.0024 & 0.0022 & 0.0006 \\
\hline 0.0025 & 0.0017 & 0.0056 \\
\hline $\begin{array}{l}0.0003 \\
0.0024\end{array}$ & $\begin{array}{l}0.0021 \\
0.0019\end{array}$ & $\begin{array}{l}0.00999 \\
0.0115\end{array}$ \\
\hline 0.0023 & 0.0020 & 0.0110 \\
\hline 0.0022 & 0.0020 & 0.0066 \\
\hline 0.0022 & 0.0045 & 0.0031 \\
\hline 0.0022 & 0.0020 & 0.0009 \\
\hline $\begin{array}{l}0.00222 \\
0.00222\end{array}$ & $\begin{array}{l}0.00020 \\
0.0020\end{array}$ & $\begin{array}{l}0.0020 \\
0.0007\end{array}$ \\
\hline 0.0021 & $\begin{array}{l}0.00020 \\
0.0020\end{array}$ & $\begin{array}{l}0.0007 \\
0.0005\end{array}$ \\
\hline 0.0021 & 0.0018 & $\begin{array}{l}.00005 \\
0.0005\end{array}$ \\
\hline 0.0020 & 0.0017 & 0.0016 \\
\hline 0.0022 & 0.0021 & 0.0049 \\
\hline 0.0024 & 0.0024 & 0.0072 \\
\hline 0.0023 & 0.0021 & 0.0011 \\
\hline & 0.0020 & 0.0003 \\
\hline $\begin{array}{l}0.0030 \\
0.0021\end{array}$ & $\begin{array}{l}0.0026 \\
0.0017\end{array}$ & $\begin{array}{l}0.0037 \\
0.0004\end{array}$ \\
\hline 0.0022 & 0.0019 & 0.0008 \\
\hline & & 0.0005 \\
\hline 0.00255 & 0.0021 & 0.0007 \\
\hline $\begin{array}{l}0.00027 \\
0.0022\end{array}$ & 0.0021 & 0.0112 \\
\hline $\begin{array}{l}0.0022 \\
0.0026\end{array}$ & $\begin{array}{l}0.00020 \\
0.0022\end{array}$ & $\begin{array}{l}0.0003 \\
0.0112\end{array}$ \\
\hline 0.0021 & 0.0024 & \\
\hline 0.0027 & 0.0022 & 0.0004 \\
\hline 0.0023 & 0.0017 & \\
\hline 0.0024 & 0.0021 & 0.0104 \\
\hline 0.0034 & 0.0025 & 0.0057 \\
\hline 0.00222 & 0.0021 & 0.0071 \\
\hline $\begin{array}{l}0.000636 \\
0.0034\end{array}$ & $\begin{array}{l}0.0021 \\
0.0018\end{array}$ & $\begin{array}{l}0.0055 \\
0.0105\end{array}$ \\
\hline 0.0024 & $\begin{array}{l}0.0019 \\
0.0019\end{array}$ & 0.0007 \\
\hline 0.0024 & 0.0020 & \\
\hline 0.0028 & 0.0021 & \\
\hline 0.00 & 0.0026 & \\
\hline 0023 & 0.0023 & 0.0100 \\
\hline
\end{tabular}

0.0007
0.0009
0.0010
0.0013
0.0018
0.0007
0.0018
0.0017
0.0014
0.0014
0.0019
0.0099
0.0010
0.0013
0.0005
0.0009
0.0029
0.0008
0.0012
0.0015
0.0010
0.0021
0.0009
0.0015
0.0006
0.0015
0.0006
0.0016
0.0013
0.0015
0.0012
0.0021
0.0012
0.0018
0.0012
0.0009
0.0010
0.0005
0.0015
0.0015
0.0013
0.0006
0.0009
0.0018
0.0008
0.0007
0.0012
0.0017
0.0012
0.0008
0.0010
0.0011
0.0013
0.0013
0.0007
0.0005
0.0012
0.0008

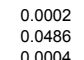

0.0002
0.0486
0.0004

0.0026
0.0023
0.0026

0.0412
0.0004
0

0.0107
0.0617
0.056

0.0617
0.0556
0.0753

0.0753
0.0695
0.0006

0.0006
0.0167
0.0001

0.0001
0.0001

0.0001
0.0853
0.0003

0.0003
0.0680
0.0634
0.0635

0.0006
0.0565

0.0632
0.0008
0.005

0.0008
0.0003
0.0712
0.0004
0.0006

0.0006
0.0006
0.0003
0.0454

0.0454
0.0686
0.080

0.06060
0.0774
0.0006

0.0006
0.0005
0.0004
0.0669

0.0004
0.0668
0.0004
0.025

0.0004
0.0002
0.0294

0.0294
0.0004
0.0005
0.0225

0.0005
0.0422
0.0698
0.0005

0.0005
0.0003
0.002

0.0002
0.0611
0.0576
0.0658
0.0324

0.0658
0.0002

0.0002
0.0344
0.0001

.00001
0.0010
0.0003
0.0003

0.0003
0.0003
0.0005
0.0004

0.0683
0.0004 $\begin{array}{lll}0.0195 & 0.9397 & 1150 \\ 0.0013 & 0.9315 & 1150\end{array}$

0.0000

" 950
$<950$

$\begin{array}{lll}0.0196 & 1.0060 & 1150 \\ 0.0171 & 0.9685 & 1150 \\ & 0.0000 & 1150\end{array}$

$\begin{array}{ll}0.0000 & 1150 \\ 0.0000 & 1150 \\ 0.0000 & 1150\end{array}$

$\begin{array}{ll}0.0000 & 1150 \\ 0.0000 & 1150 \\ 0.0000 & 1150 \\ 0.0000 & 1150\end{array}$ $\begin{array}{ll}0.0000 & 1150 \\ 0.0000 & 1150 \\ 0.0000 & 1150\end{array}$ $\begin{array}{ll}0.0000 & 1150 \\ 0.0000 & 1150\end{array}$ $\begin{array}{ll}0.0000 & 1150 \\ 0.0000 & 1200\end{array}$ $\begin{array}{ll}0.00000 & 12200 \\ 0.0000 & 1200 \\ 0.0000 & 1200\end{array}$

$\begin{array}{ll}0.0000 & 1200 \\ 0.0000 & 1200\end{array}$

$\begin{array}{ll}0.0000 & 1200 \\ 0.0000 & 1200 \\ 0.9274 & 1150\end{array}$

$\begin{array}{lll}0.0196 & 0.9533 & 1150 \\ .0192 & 0.9274 & \end{array}$

$\begin{array}{lll}0.0198 & 0.9625 & 1150 \\ 0.0189 & 0.9372 & \end{array}$

$\begin{array}{lll}0.0200 & 0.9500 & \\ 0.0193 & 0.9283 & 1150 \\ 0.0124 & 0.989 & \end{array}$

$\begin{array}{lll}0.0204 & 0.9897 \\ 0.0192 & 0.9393\end{array}$

$\begin{array}{ll}0.0192 & 0.9393 \\ 0.0184 & 0.9356\end{array}$

$\begin{array}{lll}0.0203 & 0.9653 & 1150 \\ 0.0191 & 0.9340 & 1150 \\ 0.0196 & 0.9706 & \end{array}$

$\begin{array}{lll}0.0186 & 0.9884 & 1150 \\ 0.0190 & 0.9841 & 1150\end{array}$

$\begin{array}{lll}0.0190 & 0.9841 & 1150 \\ 0.0199 & 0.9838 & 1150\end{array}$

$\begin{array}{llll}0.0199 & 0.98509 & 1150 \\ 0.020 & 0.967 & 1150\end{array}$

$\begin{array}{lll}0.0195 & 0.94481 & 1150 \\ 0.0211 & 0.9373 & 1150\end{array}$

$\begin{array}{lll}0.0198 & 0.9506 & \\ 0.0200 & 0.9513 & 1200 \\ 0.0200 & 0.9884 & 1150 \\ 0.0190 & 0.9284 & 1150\end{array}$

$\begin{array}{lll}0.0190 & 0.9284 & 1150 \\ 0.0193 & 0.9820 & 1150 \\ 0.0235 & 0.982 & 1150\end{array}$

$\begin{array}{lll}0.0190 & 0.9692 & 1150 \\ 0.0235 & 0.9875 & 1250 \\ 0.0199 & 0.9692 & 1150\end{array}$

$\begin{array}{lll}0.0199 & 0.9692 & 1150 \\ 0.0203 & 0.9241 & \end{array}$

$\begin{array}{lll}0.0199 & 0.9739 & 1150\end{array}$

$\begin{array}{lll}0.0193 & 0.9542 & \\ 0.0281 & 0.9882 & 1150\end{array}$

$\begin{array}{lll}0.0281 & 0.9882 & 1150 \\ 0.0218 & 0.9772 & 1150 \\ 0.0229 & 0.9909 & 1150\end{array}$

$\begin{array}{llll}0.0191 & 0.9421 & \\ 0.0203 & 0.969 & 1150\end{array}$

$\begin{array}{lll}0.0203 & 0.9689 & 1150 \\ 0.0194 & 0.9613 & 1200\end{array}$

$\begin{array}{lll}0.0191 & 0.9522 & \\ 0.0204 & 0.9435 & 1150\end{array}$

$\begin{array}{lll}0.0207 & 0.9725 & 1150 \\ 0.0133 & 0.9609 & \end{array}$

0.01990 .9828

0.0210
0.0003 0.94996

$\begin{array}{lll}0.0188 & 0.9158 & 1150 \\ 0.0194 & 0.9393 & \end{array}$

$\begin{array}{lll}0.0194 & 0.9615 & 1150\end{array}$

0.01890 .9070

$\begin{array}{lll}0.0197 & 0.9530 & \\ 0.0193 & 0.9237 & 1150 \\ 0.0201 & 0.9654 & 1150\end{array}$

$\begin{array}{llll}0.0207 & 0.9571 & 1150\end{array}$

$\begin{array}{lll}0.0198 & 0.9506 & 1150 \\ 0.0197 & 0.9745 & 1150\end{array}$

$\begin{array}{lll}0.0203 & 0.9568 & 1150\end{array}$ $\begin{array}{lll}0.0182 & 0.9637 & 1150 \\ 0.0202 & 0.9619 & 1150\end{array}$

$\begin{array}{lll}0.0203 & 0.9689 & 1150 \\ 0.0210 & 0.9435 & \end{array}$

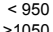




\section{Homogeneous dark brown glas
Homogeneous dark brown glas}

\section{Homogeneous dark brown glass}

Homogeneous dark brown glass

Brown glass wish mathe appear-ance. Trace amount of secondary phas

Homogeneous glass with metallic appearance

Metallic blue glass with extensive surface crystallization

Homogeneous glas with metallic appearance

Homogeneous dark glass

Homogeneous dark glass
Homogeneous dark brown

Homogeneous dark brown glass

Homogeneous dark brown glass

Homogeneous reddish brown glass

Homogeneous dark brown glass with a tinge of blue. Undis-solved materials left in crucible

Mostly homogeneous dark brown glass with some cloudiness on surface

Blue glass with metallic like fragments and brown regions

Mostly clear brown//lue glass with dull gray lumps on surface. Some gray-colored swirls

tion appears uniform Dark green glass with black patches on top and bottom. Cross section appears uniform

Homogeneous black glass with silvery patches left in crucible

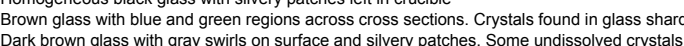
Dark brown glass with gray swirls on surface and silvery patches. Some undissolved crystals
Blue glass with green regions on surface and d ross sections
Mostly uniform dark brown glass with gray pathes on surface and small amount of undissolved crys

Mostly uniform dark brown glass with gray patches on surface and small amount of undissolved crys

Brown glass with multicolored regions and undissolved
Brown glass with streaks and siver patches on surface

Brown glass with streaks and silver patches on surface
Brown gith green areas in cross section. Glass shards with crystals

Brown glass with swirls on surface. IInhomogeneous cross section with undissolved materia
Mostly homogenenous black lass with red tinge Trace amount fol undissolved matrial

Inhomogeneous glass with green and gray regions and siver patches. Trace amount of undissolved material
Inter

Mostly homogeneous black glass with brown streaks on surface

Dark green glass with cross section fragments of white and green

Inhomogeneous glass with green and black regions. Silvery crystals and undis-solved materia Mostly uniform black glass with green and silver patches
Mostly homogeneous black glass with grayish cross section

Brownish green glass with multicolored regions. Cross section appears uniform

Homogeneous black glass with a reddish yellow tings

Homogeneous black glass

Green glass with multicolored patches on surface. Uniform cross sectio

Dark brown glass with small amount of a yellow non-glassy phase. Yellow phase deposit on crucible

Homogeneous black glass with trace amount of gray precipitate

Dark brown glass with patches of metallic looking precipitates on surface. Crystal-lization throughout bulk

Cloudy brown glass with traces of metallic precipitate on bottom

Cloudy green glass with multicolored bottom

Mostly uniform brown glass with small areas of light brown swirls

Homogeneous red glass 
1482 HLW98-64

1483 HLWMS-01
1484 HLWMS-02

1485 HLWMS-03

1486 HLWMS-04
1487 HLWMS-05

1487 HLWMS-05
1488 HLWMS-06

WMS-08

491 HLWMS-09

1492 HLWMS-10

494 HLWMS-12
195 HLWMS-13

496 HLWMS-14

1497 HLWMS-15

1499 HLW99-02

1501 HLW9-0

1503 HLW99-06

1505 HLW99-08

1507 HLW99-10

1508 HLW99-11
1509 HLW99-12

1510 HLW99-13

1511 HLW99-14

513 HLW99-16
514 HLW99-17

515 HLW99-18

1516 HLW99-19

1518 HLW99-21

1519 HLW99-22

1520 HLW99-23

522 HLW99-25

1523 HLW99-26

1524 HLW99-27

526 HLW99-29

1528 HLW99-30

530 HLW99-33

1531 HLW99-34

1534 HLW99-37

1536 HLW99 39

1539 HLW99-42

1541 HLW99-44

1542 HLW99-45

1544 HLW99-47

1545 HLW99-48

1546 HLW99-49
1547 HLW99-50

1550 HLW99-53

1553 HLW99-56R
1554 HLW99-57

1558 HLW99-61

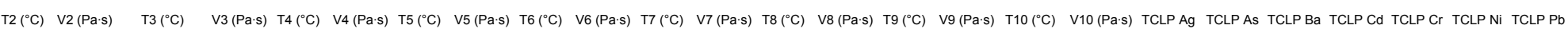

$\begin{array}{llllllll}1050 & 34.638 & 1100 & 20.4 & 1150.0 & 12.6 & 1200.0 & 8.1\end{array}$

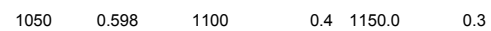

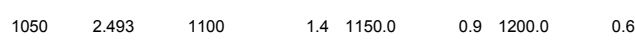

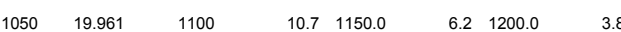

$\begin{array}{llllllll}1050 & 47.915 & 1100 & 26.9 & 1150.0 & 15.9 & 1200.0 & 9.9\end{array}$

$1050 \quad 0.662 \quad 1100-0511500=0312000=030$

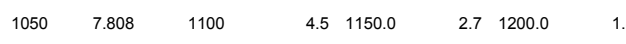


1496 HLWMS-14

1498 HLW99-01

1499 HLW99-02

501 HLW90

1503 HLW99-06
1504 HLW99-07

1505 HLW99-08
1506 HLW99-09

1507 HLW99-10

1508 HLW99-11
1509 HLW99-12

1510 HLW99-13

1511 HLW99-14

1512 HLW99-15
1513 HLW99-16

514 HLW99-17

1515 HLW99-18

1516 HLW99-19

518 HLW99-21

1519 HLW99-22

1520 HLW99-23

522 HLW99-25

1523 HLW99-26

1524 HLW99-27

526 HLW99-29

1527 HLW99-30

1528 HLW99-31

530 HLW99-33

1531 HLW99-34

1534 HLW99-37

1535 HLW99-38

1538 HLW99-41

1539 HLW99-42
1540 HLW99-43

541 HLW99-44

1542 HLW99-45

1543 HLW99-46
1544 HLW99-47

1545 HLW99-48

546 HLW99-49

1547 HLW99-50

549 HLW99-52

1550 HLW99-53

1551 HLW99-54

1553 HLW99-56R

1554 HLW99-57

1555 HLW99-58
1556 HLW99-59
TCLP Se TCLP Zn TCLPAI TCLP B TCLPSb TCLP TI TCLPV QPCT B $\left(g / \mathrm{m}^{2}\right)$ Q PCT Li $\left(g / \mathrm{m}^{2}\right)$ QPCT Na $\left(g / \mathrm{m}^{2}\right)$ Q PCT Si $\left(g / \mathrm{m}^{2}\right)$ Q PCT pH

$<0.0532 \quad 0.207$

$<0.0338<0.0417$

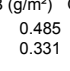

0.374

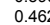

0.275

0.28

0.305

0.230

10.160

0.28

0.286

0.359

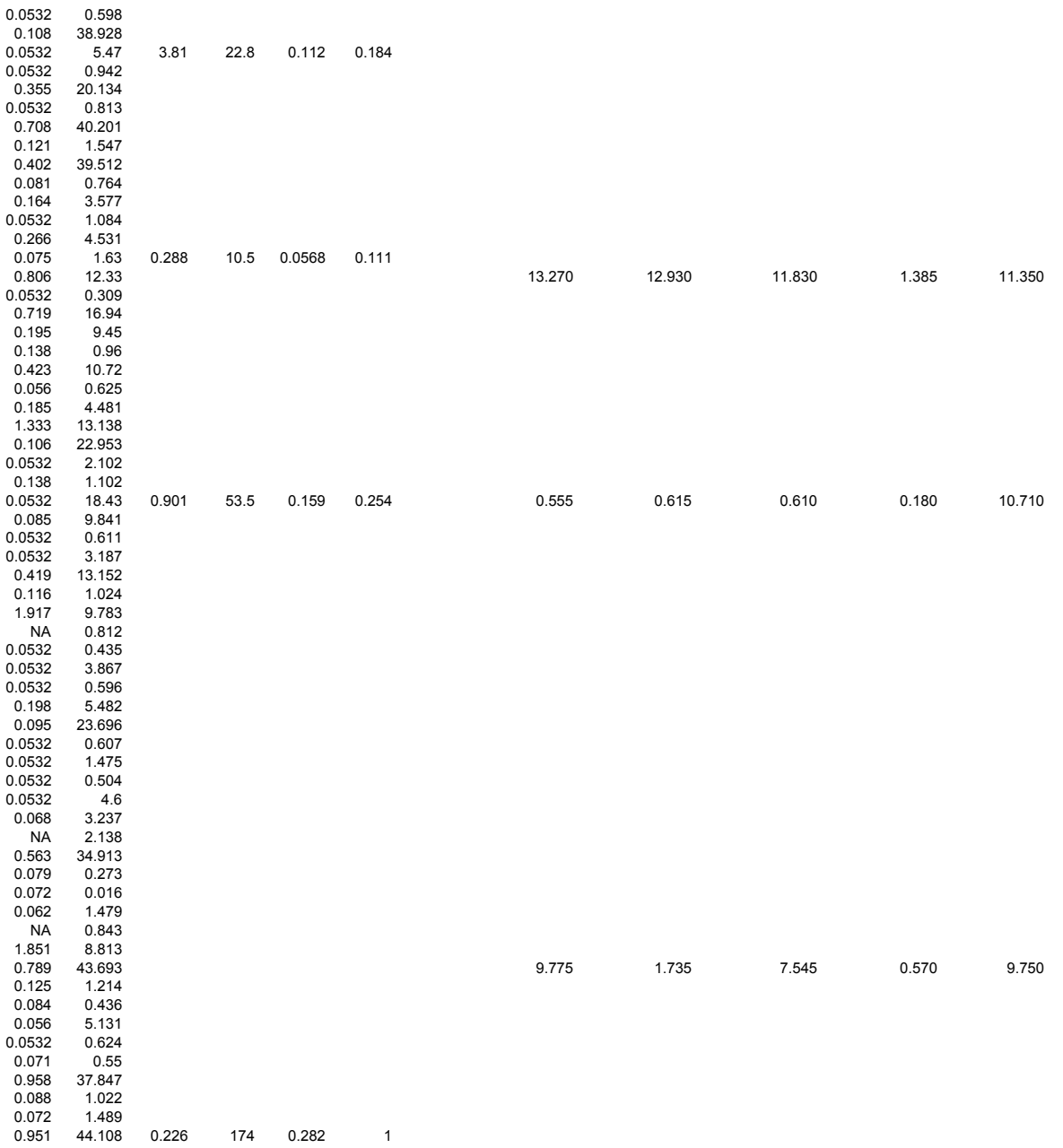


1481 HLW988-63

1482 HLW98-64
1483 HLWMS-01

1483 HLWMS-01
1484 HLWMS-02

1485 HLWMS-03
1486 HLWMS-04

1487 HLWMS-05

1488 HLWMS-06

1489 HLWMS-07

1491 HLWMS-09

1492 HLWMS-10

1494 HLWMS-12

1495 HLWMS-13

1496 HLWMS-14

1497 HLWMS-15

1499 HLW99-02

1501 HLW9-0

1502 HLW99-05

1503 HLW99-06

1505 HLW99-08

506 HLW99-09

1507 HLW99-10

1509 HLW99-12

510 HLW99-13

(11)

515 HLW99-18

1516 HLW99-19

1518 HLW99-21

1519 HLW99-22

1521 HLW99-24R

1522 HLW99-25

1523 HLW99-26

525 HLW99-28

1526 HLW99-29

1527 HLW99-30

1528 HLW $1599-31$
1529 HLW99-32

1530 HLW99-33

531 HLW99-34

533 HLW99-36

1534 HLW99-37

535 HLW99-38

1538 HLW99-41

1539 HLW99-42

1540 HLW99-43
1541 HLW99-44

542 HLW99-45

1543 HLW99-46
1544 HLW99-47

1545 HLW99-48

1546 HLW $159-49$

1547 HLW99-50

549 HLW99-52

1550 HLW99-53

1551 HLW99-54

1553 HLW99-56R

1554 HLW99-57

1555 HLW99-58
1556 HLW99-59

1558 HLW99-61 


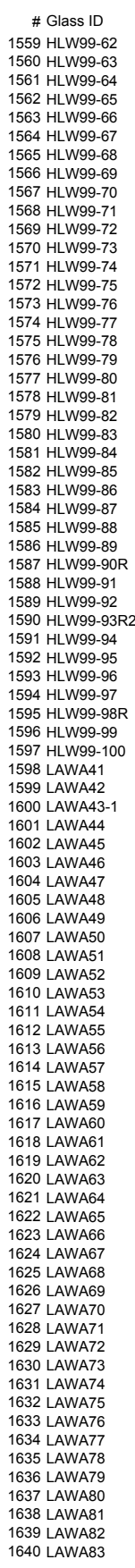

Study

Reference

RPP-WTP HLW Formulation Kot and Pegg 2001 PP-WTP HLW Formulation Kot and Pegg 2001 RPP-WTP HLW Formulation Kot and Pegg 2001 RPP-WTP HLW Formulation Kot and Pegg 2001 RPP-WTP HLW Formulation Kot and Pegg 2001 RPP-WTP HLW Formulation Kot and Pegg 2001 RPP-WTP HLW Formulation Kol and Pegg 201 RPP-WTP HLW Formulation Kot and Pegg 2001 RPP-WTP HLW Formulation Kot and Pegg 200 RPP-WTP HLW Formulation Kot and Pegg 2001 RPP-WTP HLW Formulation Kot and Pegg 2001 RPP-WTP HLW Formulation Kot and Pegg 200 RPP-WTP HIW Formulation Ko and Pegg 2001 RPP-WTP HLW Formulation Kot and Pegg 2001 RPP-WTP HLW Formulation Kot and Pegg 2001 RPP-WTP HLW Formulation Kot an Pegg 2001 RPP-WTP HLW Formulation Kot and Pegg 2001 RPP-WTP HLW Formulation Kot and Pegg 2001 RPP-WTP HLW Formulation Kot and Pegg 2001 RPP-WTP HLW Formulation Kot and Pegg 2001 RPP-WTP HLW Formulation Kot and Pegg 2001 RPP-WTP HLW Formulation Kot and Pegg 2001 RPP-WTP HLW Formulation Kot and Pegg 2001 RPP-WTP HLW Formulation Kot and Pegg 2001 RPP-WTP HLW Formulation Kot and Pegg 2001 RPP-WTP HLW Formulation Kot and Pegg 2001 RPP-WTP HLW Formulation Kot and Pegg 2001 RPP-WTP HLW Formulation Kot and Pegg 2001 P-WTP LAW Formulation, Muller et al. 2001 RPP-WTP LAW Formulation, Muller et al. 2001 RPP-WTP LAW Formulation, Muller et al. 2001 RPP-WTP LAW Formulation Muller et al. 2001 RPP-WTP LAW Formulation Muller et al. 2001 RPP-WTP LAW Formulation Muller et al. 2001 RPP-WTP LAW Formulation, Muller et al. 2001 RPP-WTP LAW Formulation, Muller et al. 2001 RPP-WTP LAW Formulation, Muller et al. 2001 RPP-WTP LAW Formulation MUlo el. 2001 RPP-WTP LAW Formulation Muller et al. 2001 RPP-WTP LAW Formulation, Muller et al. 2001 RPP-WTP LAW Formulation Muller et al. 2001 RPP-WTP LAW Formulation, Muller et al. 2001 RPP-WTP LAW Formulation Muller et al. 2001 RPP-WTP LAW Formulation Muller et al. 200 RPP-WTP LAW Formulation Muller et al. 200 RPP-WTP LAW Formulation Muller et al. 200 RPP-WTP LAW Formulation Muller et al. 2001 RPP-WTP LAW Formulation Muller et al. 2001 RPP-WTP LAW Fomlatlon Muller et al. 200 RPP-WTP LAW Formulation Muller et al. 2001 RPP-WTP LAW Formulation Muller et al. 2001 RPP-WTP LAW Formulation Muller et al. 2001 RPP-WTP LAW Formulation Muller et al. 2001 RPP-WTP LAW Formulation Muller et al. 200 RPP-WTP LAW Formulation Muller et al. 2001 RPP-WTP LAW Formulation, Muller et ala. 2001
Al2O3-t B2O3-t CaO-t Fe2O3-t FeO-t $\begin{array}{llll}0.0000 & 0.0500 & 0.0000 & 0.0200 \\ 0.0516 & 0.1171 & 0.0303 & 0.0614\end{array}$ $\begin{array}{llll}0.0530 & 0.0900 & 0.0000 & 0.0200\end{array}$ $\begin{array}{llll}0.1100 & 0.1830 & 0.0000 & 0.0200 \\ 0.0735 & 0.0525 & 0.0210 & 0.0210\end{array}$ $\begin{array}{lllll}0.0735 & 0.0525 & 0.0210 & 0.1176\end{array}$ $\begin{array}{llll}0.0735 & 0.0525 & 0.0210 & 0.1313 \\ 0.0735 & 0.0525 & 0.0210 & 0.0210\end{array}$ $\begin{array}{llll}0.0735 & 0.0525 & 0.0210 & 0.0210 \\ 0.0735 & 0.0525 & 0.0210 & 0.1313\end{array}$

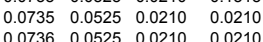
$\begin{array}{llll}0.0736 & 0.0525 & 0.0210 & 0.0210 \\ 0.0735 & 0.0525 & 0.0210 & 0.1313\end{array}$ $\begin{array}{llll}0.0735 & 0.0525 & 0.0210 & 0.1313 \\ 0.0735 & 0.0525 & 0.0210 & 0.0210 \\ 0.0735 & 0.025 & 0.020 & 0.131\end{array}$ $\begin{array}{llll}0.0735 & 0.0525 & 0.0210 & 0.1313\end{array}$ $\begin{array}{llll}0.0735 & 0.0525 & 0.0210 & 0.0562 \\ 0.0735 & 0.0525 & 0.0210 & 0.0210\end{array}$ $\begin{array}{llll}0.0700 & 0.1000 & 0.02200 & 0.0725 \\ 0.0736 & 0.0525 & 0.210 & 0.0210\end{array}$ $\begin{array}{llll}0.0736 & 0.0525 & 0.0210 & 0.0210\end{array}$ $\begin{array}{llll}0.0700 & 0.1000 & 0.02200 & 0.0560 \\ 0.0700 & 0.1000 & 0.0200 & 0.0804 \\ 0.0700 & 0.1000 & 0.2200 & 0.0725\end{array}$ $\begin{array}{llll}0.0700 & 0.1000 & 0.0220 & 0.0200 \\ 0.0700 & 0.1000 & 0.0200 & 0.0725\end{array}$ $\begin{array}{llll}0.0700 & 0.1000 & 0.0200 & 0.0200 \\ 0.0700 & 0.1000 & 0.0200 & 0.1250\end{array}$ $\begin{array}{llll}0.0700 & 0.1000 & 0.0200 & 0.1250\end{array}$ $\begin{array}{llll}0.0700 & 0.1000 & 0.02200 & 0.1250 \\ 0.0700 & 0.1000 & 0.0200 & 0.1250\end{array}$ $\begin{array}{llll}0.0700 & 0.1000 & 0.0200 & 0.1250 \\ 0.1120\end{array}$ $\begin{array}{llll}0.0700 & 0.1000 & 0.0200 & 0.0200 \\ 0.0700 & 0.1000 & 0.0200 & 0.0200 \\ 0 & 0700 & 0.0200\end{array}$ $\begin{array}{llll}0.0700 & 0.1000 & 0.0200 & 0.0200 \\ 0.0700 & 0.0200\end{array}$ $\begin{array}{llll}0.0700 & 0.1000 & 0.0200 & 0.02200 \\ .0 .0700 & 0.1000 & 0.0200 & 0.1250\end{array}$ $\begin{array}{llll}0.0700 & 0.1000 & 0.02200 & 0.1250 \\ 0 & 0700 & 0.1000 & 0.0200 \\ 0\end{array}$ $\begin{array}{llll}0.0700 & 0.1000 & 0.0200 & 0.0200\end{array}$ $\begin{array}{llll}0.0700 & 0.1000 & 0.0200 & 0.0200\end{array}$ $\begin{array}{llll}0.0620 & 0.0750 & 0.0200 & 0.0698 \\ 0.0620 & 0.0903 & 0.0240 & 0.0841 \\ 0 & \end{array}$

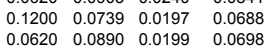
$\begin{array}{lll}0.0620 & 0.1190 & 0.0698\end{array}$

$\begin{array}{ll}0.0620 & 0.089 \\ 0.0620 & 0.0890\end{array}$

$\begin{array}{ll}0.0620 & 0.0890 \\ 0.0620 & 0.0890\end{array}$ $\begin{array}{llll}0.0620 & 0.1197 & 0.07700 \\ 0.0618 & 0.0619 & 0.0788 & 0.0751\end{array}$ $\begin{array}{llll}0.0618 & 0.0619 & 0.0788 & 0.0751 \\ 0.0609 & 0.0611 & 0.0777 & 0.0740 \\ 0 & 06090 & 0.0611 & \end{array}$ $\begin{array}{llll}0.0609 & 0.0611 & & 0.0740 \\ 0.0609 & 0.1193 & 0.0195 & 0.0740\end{array}$ $\begin{array}{llll}0.0609 & 0.1193 & 0.0195 & 0.0740 \\ 0.0609 & 0.0611 & 0.0284 & 0.0740\end{array}$ $\begin{array}{llll}0.0609 & 0.0611 & 0.0284 & 0.0770 \\ 0.0609 & 0.0611 & 0.0284 & 0.0740 \\ 0 & 0.011 & 0.0625 & 0.0740\end{array}$ $\begin{array}{llll}0.0609 & 0.0611 & 0.0482 & 0.0740 \\ 0.0053 & 0.1123 & 0.0432 & \end{array}$ $\begin{array}{llll}0.0609 & 0.0611 & 0.0477 & 0.0740\end{array}$ $\begin{array}{llll}0.0609 & 0.0611 & 0.0477 & 0.0740 \\ 0.0618 & 0.0619 & & 0.0751\end{array}$ $\begin{array}{llll}0.0618 & 0.0619 & & 0.0751 \\ 0.0609 & 0.0611 & 0.0326 & 0.0740\end{array}$ $\begin{array}{llll}0.0609 & 0.0611 & 0.0326 & 0.0740 \\ 0.0609 & 0.0611 & 0.0477 & 0.0740 \\ 0009 & 0.0611 & 00477 & 0.740\end{array}$ $\begin{array}{llll}0.0609 & 0.0611 & 0.0477 & 0.0740 \\ 0.0609 & 0.0611 & 0.0316 & 0.0740\end{array}$ $\begin{array}{lll}0.0609 & 0.1088 & 0.0740\end{array}$ $\begin{array}{llll}0.0609 & 0.0611 & 0.0577 & 0.0740 \\ 0.0609 & 0.1085 & 0.0777 & 0.0740\end{array}$ $\begin{array}{llll}0.0609 & 0.1085 & 0.077 & 0.0740 \\ 0.0609 & 0.0611 & 0.0777 & 0.0740\end{array}$

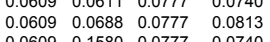
$\begin{array}{llll}0.0609 & 0.1580 & 0.0777 & 0.0740 \\ 0.0609 & 0.1085 & 0.0777 & 0.0740 \\ 0.1005 & 0.105 & 0.1273 & 0.0740\end{array}$

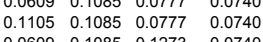
$\begin{array}{llll}0.0609 & 0.1085 & 0.1273 & 0.0740 \\ 0.1105 & 0.0611 & 0.0777 & 0.0740\end{array}$ $\begin{array}{llll}0.1105 & 0.0611 & 0.0777 & 0.0245 \\ 0.0620 & 0.0890 & 0.0399 & 0.0698\end{array}$

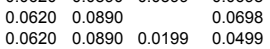

K2O-t Li2O-t MgO-t Na2O-t NiO-t P2O5-t SiO2-t ZrO2-t Ag2O-t As203-tAs2O $\begin{array}{llllllllll}0.0100 & 0.0300 & 0.0123 & 0.1300 & 0.0184 & 0.0000 & 0.5200 & 0.0867 & 0.0025 & 0.0022 \\ 0.0100 & 0.0317 & 0.0062 & 0.1129 & 0.0094 & 0.0124 & 0.3973 & 0.0322 & 0.0025 & 0.0022\end{array}$ $\begin{array}{lllllllllll}0.0100 & 0.0700 & 0.0200 & 0.0800 & 0.0300 & 0.0300 & 0.3400 & 0.0000 & 0.0025 & 0.0020\end{array}$ $\begin{array}{llllllllll}0.0100 & 0.0000 & 0.0000 & 0.2000 & 0.0000 & 0.0300 & 0.3000 & 0.0000 & 0.0025 & 0.0020 \\ 0.0105 & 0.0315 & 0.00000 & 0.0992 & 0.0000 & 0.0013 & 0.5008 & 0.0315 & 0.0026 & 0.0021\end{array}$ $\begin{array}{lllllllllll}0.0105 & 0.0315 & 0.0131 & 0.0704 & 0.0197 & 0.0013 & 0.3145 & 0.0315 & 0.0026 & 0.0021\end{array}$ $\begin{array}{llllllllll}0.0105 & 0.0315 & 0.0131 & 0.0525 & 0.0197 & 0.0013 & 0.3146 & 0.0315 & 0.0026 & 0.0021 \\ 0.0105 & 0.0315 & 0.0000 & 0.1139 & 0.0000 & 0.0013 & 0.3495 & 0.0315 & 0.0026 & 0.0022\end{array}$ $\begin{array}{llllllllllll}0.0105 & 0.0315 & 0.0000 & 0.0771 & 0.0000 & 0.0013 & 0.4127 & 0.0315 & 0.0026 & 0.0021\end{array}$ $\begin{array}{llllllllll}0.0105 & 0.0315 & 0.0131 & 0.0781 & 0.0197 & 0.0013 & 0.4169 & 0.0315 & 0.0026 & 0.0022\end{array}$

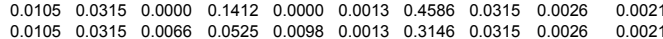
$\begin{array}{llllllllll}0.0105 & 0.0315 & 0.0088 & 0.1051 & 0.0132 & 0.0013 & 0.3142 & 0.0315 & 0.0026 & 0.0021 \\ 0.0105 & 0.0315 & 0.0131 & 0.1051 & 0.0197 & 0.0013 & 0.3142 & 0.0315 & 0.0026 & 0.0021\end{array}$ $\begin{array}{llllllllll}0.0105 & 0.0315 & 0.0000 & 0.1051 & 0.0000 & 0.0013 & 0.3142 & 0.0315 & 0.0026 & 0.0021 \\ 0.0105 & 0.0315 & 0.0044 & 0.0788 & 0.0066 & 0.0013 & 0.3144 & 0.0315 & 0.0026 & 0.0021\end{array}$

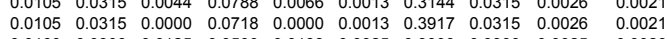
$\begin{array}{lllllllllll}0.0100 & 0.0300 & 0.0125 & 0.0500 & 0.0188 & 0.0025 & 0.3000 & 0.0300 & 0.0025 & 0.0020 \\ 0.0105 & 0.0315 & 0.0000 & 0.1276 & 0.0000 & 0.0013 & 0.4040 & 0.0315 & 0.026 & 0.0021\end{array}$

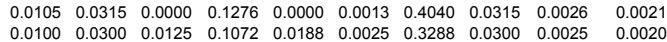
$\begin{array}{lllllllllll}0.0100 & 0.0300 & 0.0000 & 0.0672 & 0.0000 & 0.0025 & 0.3690 & 0.0300 & 0.0025 & 0.0020\end{array}$

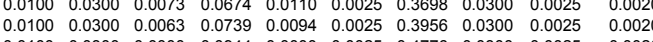
$\begin{array}{llllllllll}0.0100 & 0.0300 & 0.0000 & 0.0944 & 0.0000 & 0.0025 & 0.4776 & 0.03300 & 0.0025 & 0.0022 \\ 0.0100 & 0.0300 & 0.0042 & 00750 & 0.0063 & 0.025 & 0.4300 & 0.0300 & 0.025 & 0.0022\end{array}$ $\begin{array}{lllllllllll}0.0100 & 0.0300 & 0.0125 & 0.0670 & 0.0188 & 0.0025 & 0.3000 & 0.0300 & 0.0025 & 0.0020 \\ 0.0100 & 0.0300 & 0.0000 & 0.0734 & 0.0000 & 0.0025 & 0.3936 & 0.0300 & 0.0025 & 0.0020\end{array}$ $\begin{array}{llllllllll}0.0100 & 0.0300 & 0.0000 & 0.0734 & 0.0000 & 0.0025 & 0.3936 & 0.0300 & 0.0025 & 0.0020 \\ 0.0100 & 0.0300 & 0.0000 & 0.1000 & 0.0000 & 0.0025 & 0.3000 & 0.0300 & 0.0025 & 0.0020\end{array}$ $\begin{array}{lllllllllll}0.0100 & 0.0300 & 0.0000 & 0.0500 & 0.0000 & 0.0025 & 0.3000 & 0.0300 & 0.0025 & 0.0020\end{array}$ $\begin{array}{llllllllll}0.0100 & 0.0300 & 0.0125 & 0.0500 & 0.0188 & 0.0025 & 0.3000 & 0.0300 & 0.0025 & 0.0020 \\ 0.0100 & 0.0300 & 0.0000 & 0.1084 & 0.0000 & 0.0025 & 0.3336 & 0.0300 & 0.0025 & 0.0020\end{array}$

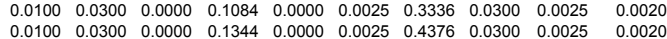

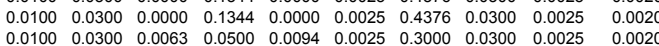

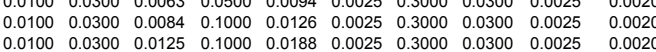

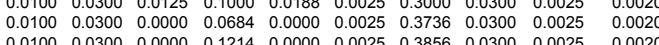
$\begin{array}{lllllllllll}0.0100 & 0.0300 & 0.0000 & 0.1214 & 0.0000 & 0.0025 & 0.3856 & 0.0300 & 0.0025 & 0.0020\end{array}$

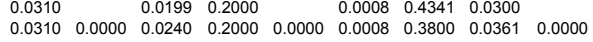

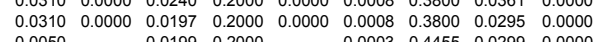

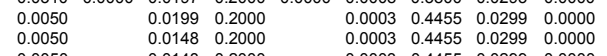

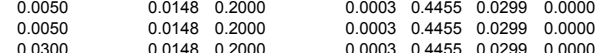

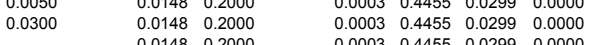
0.01480 .2000

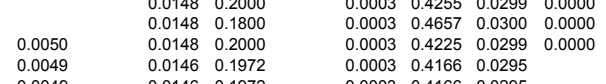

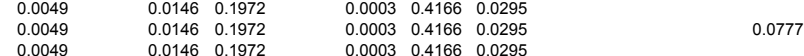

$\begin{array}{lll}0.0049 & 0.0146 & 0.1972 \\ 0.0049 & 0.0146 & 0.1972 \\ 0.0049 & 0.0146 & 0.1972\end{array}$

$\begin{array}{llll}0.0049 & 0.0146 & 0.1972 \\ 0.0049 & 0.0146 & 0.1972\end{array}$ $\begin{array}{lll}0.0003 & 0.4166 & 0.029 \\ 0.0003 & 0.4166 & 0.029\end{array}$ $\begin{array}{lll}0.0003 & 0.4166 & 0.0295 \\ 0.0003 & 0.4166 & 0.0295\end{array}$ $\begin{array}{llll}0.0003 & 0.4166 & 0.0295 & \\ 0.0003 & 0.4455 & 0.0299 & 0.0000\end{array}$ $\begin{array}{lll}0.0049 & 0.0146 & 0.1972 \\ 0.0050 & 0.0199 & 0.2000 \\ 0.0049 & 0.0146 & 0.1972 \\ 0.0049 & 0.146 & 0.1972\end{array}$ $\begin{array}{lll}0.0049 & 0.0146 & 0.1972 \\ 0.0049 & 0.0146 & 0.197 \\ 0.0050 & 0.0148 & 0.2000 \\ 0 & 0.0196 & 0.972\end{array}$

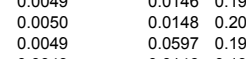

$\begin{array}{lll}0.0049 & 0.0597 & 0.1972 \\ 0.0049 & 0.0146 & 0.19 \\ 0.0049 & 0.0146 & 0.1972 \\ 0.0049 & 0.0146 & 0.1972\end{array}$

$\begin{array}{llll}0.0049 & 0.0146 & 0.1972 \\ 0.0049 & 0.0146 & 0.1972 \\ 0.0049 & 0.0146 & 0.1972 \\ 0.0049 & 0.146 & 0.172\end{array}$

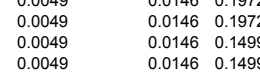

$\begin{array}{llll}0.0049 & 0.0146 & 0.1499 \\ 0.0049 & 0.0146 & 0.1972\end{array}$

$\begin{array}{llll}0.0049 & 0.0219 & 0.1972 \\ 0.0049 & 0.0146 & 0.1003\end{array}$

$\begin{array}{llll}0.0049 & 0.0495 & 0.0146 & 0.1003 \\ 0.0049 & & 0.0146 & 0.1003\end{array}$

$\begin{array}{llll}0.0049 & & 0.0146 & 0.1003 \\ 0.0049 & & 0.0146 & 0.1003 \\ 0.049 & & 0.0146 & 0.1972 \\ 0.049 & & 0.145\end{array}$

$\begin{array}{llll}0.0049 & 0.0146 & 0.1972 \\ 0.0049 & 0.0146 & 0.1972\end{array}$

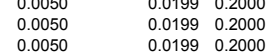
$0003 \quad 0.4166 \quad 0.0295$ $\begin{array}{lllll}0.0003 & 0.4166 & 0.0295 & \\ 0.0003 & 0.4225 & 0.0299 & 0.0000\end{array}$ $\begin{array}{llll}0.0003 & 0.4166 & 0.0295\end{array}$ $\begin{array}{llll}0.0003 & 0.4166 & 0.0295\end{array}$ $\begin{array}{lll}0.0003 & 0.4327 & 0.0295 \\ 0.0303 & 0.4166 & 0.0295\end{array}$ $\begin{array}{llll}0.0003 & 0.4166 & 0.0295\end{array}$ $\begin{array}{lll}0.0003 & 0.4166 & 0.0295 \\ 0.0003 & 0.4166 & 0.0295\end{array}$ $\begin{array}{llll}0.0003 & 0.4166 & 0.029 \\ 0.0003 & 0.4166 & 0.029\end{array}$ $\begin{array}{llll}0.0003 & 0.4166 & 0.0295\end{array}$ $\begin{array}{llll}0.0003 & 0.4166 & 0.0295 \\ 0.0003 & 0.4166 & 0.0295 \\ 0.0003 & 0.4166 & 0.029\end{array}$ $\begin{array}{llll}0.0003 & 0.4166 & 0.0295 \\ 0.0003 & 0.3670 & 0.0295 \\ 0.003 & 0.4455 & 0.259\end{array}$ $\begin{array}{llll}0.0003 & 0.4166 & 0.0295 & \\ 0.0003 & 0.4455 & 0.0299 & 0.0000\end{array}$ $\begin{array}{llll}0.0003 & 0.4455 & 0.0299 & 0.0000 \\ 0.0203 & 0.4455 & 0.0299 & 0.0000\end{array}$

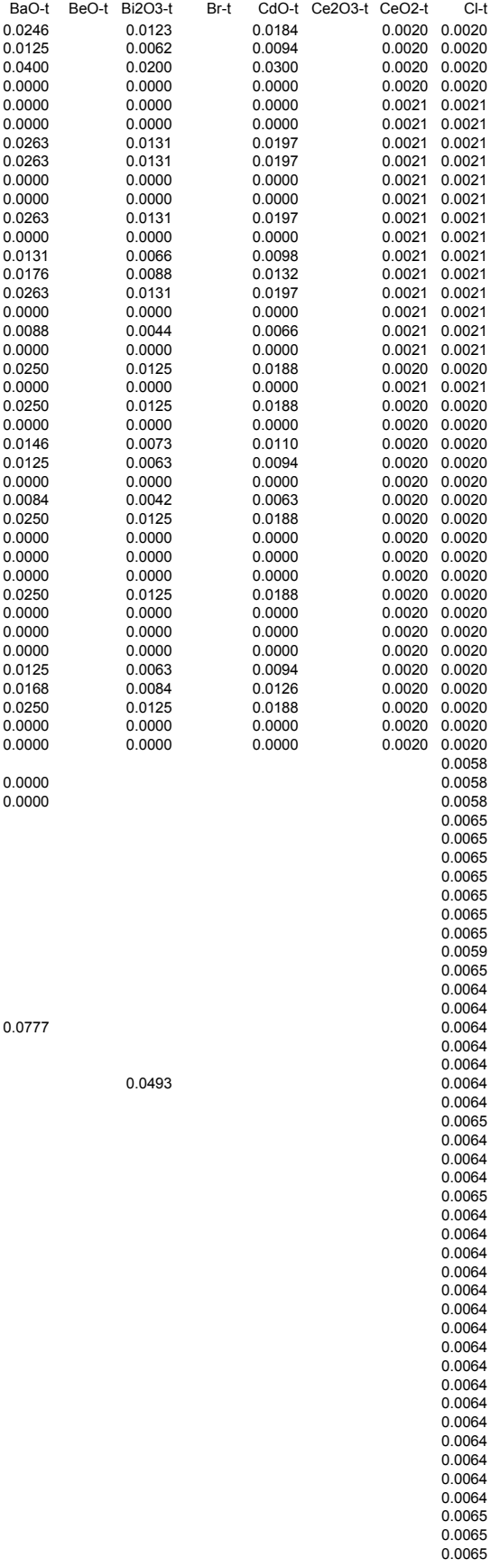


\# Glass ID

\# Glass ID
1559 HLW99-62
1560 HLW99-63

1560 HLW99-63
1561 HLW99-64

1562 HLW99-65

1565 HLW99-68

1567 HLW99-70

1569 HLW99-72
1570 HLW99-73

1571 HLW99-74
1572 HLW99-75

1573 HLW99-76

1574 HLW99-77

1575 HLW99-78
1576 HLW99-79

1577 HLW99-80

1579 HLW9-81

1580 HLW99-83
1581 HLW99-84

1582 HLW99-85

1583 HLW99-86
1584 HLW99-87

1585 HLW99-88
1586 HLW99-89

1587 HLW99-90R
1588 HLW99-91

1589 HLW99-92 1590 HLW99-93R2

1591 HLW99-94

1592 HLW99-95
1593 HLW99-96

1594 HLW99-97

1596 HLW99-99

1597 HLW99-10
1598 LAWA41

599 LAWA42

1601 LAWA44

1602 LAWA4

1604 LAWA47

1605 LAWA48

1606 LAWA49
1607 LAWA50

1608 LAWA5

1609 LAWA52

1610 LAWA53

1612 LAWA55
1613 LAWA56

1614 LAWA57

1616 LAWA59

1617 LAWAGO

1618 LAWA61
1619 LAWA62

1620 LAWA63

1622 LAWA65

1625 LAWA68

1627 LAWA70

1628 LAWA71

1629 LAWA72

1631 LAWA74

1632 LAWA75

1633 LAWA76

1635 LAWA7

1636 LAWA79

1638 LAWAB

1639 LAWA82
CoO-t Co2O3-t Cr2O3-t Cs2O-t CuO-t Dy203-t Eu2O3-t

$\begin{array}{llll}0.0020 & 0.0000 & 0.0020 & 0.0020 \\ 0.0020 & 0.0050 & 0.0020 & 0.0020\end{array}$

$0.0050 \quad 0.0020 \quad 0.0020$

$\begin{array}{lll}0.0050 & 0.0020 & 0.0020 \\ 0.0021 & 0.0021 & 0.0021\end{array}$

$\begin{array}{lll}0.0021 & 0.0021 & 0.0021 \\ 0.0021 & 0.0021 & 0.0021 \\ 0.0021 & 0.022 & 0.021\end{array}$

0.002110 .00210 .0021

$\begin{array}{llll}0.0021 & 0.0021 & 0.0021\end{array}$

$0.00210 .0021 \quad 0.0021$

$\begin{array}{lll}0.0021 & 0.0021 & 0.0021 \\ 0.0021 & 0.0021 & 0.0021\end{array}$

$\begin{array}{lll}0.0021 & 0.0021 & 0.002\end{array}$

$\begin{array}{lll}0.0021 & 0.0021 & 0.0021 \\ 0.0021 & 0.0021 & 0.0021\end{array}$

00210.00210 .0021

$\begin{array}{lll}0.0020 & 0.0020 & 0.0021 \\ 0.0021 & 0.0021 & 0.002\end{array}$

$0.0020 \quad 0.0020 \quad 0.0020$

$\begin{array}{lll}0.0020 & 0.0020 & 0.0020 \\ 0.0020 & 0.0020 & 0.0020\end{array}$

$0.0020 \quad 0.0020 \quad 0.0020$

$0.00200 .0020 \quad 0.0020$

$0.0020 \quad 0.0020 \quad 0.0020$

$0.0020 \quad 0.0020 \quad 0.0020$

$\begin{array}{lll}.002020 & 0.0020 & 0.0020 \\ .0020 & 0.0020 & 0.0020 \\ 0.0020 & 0.020 & \end{array}$

$\begin{array}{lll}0.0020 & 0.0020 & 0.0020 \\ 0.0020 & 0.0020 & 0.020\end{array}$

$\begin{array}{llll}0.0020 & 0.0020 & 0.0020 \\ 0 & 0.00220 & 0.020 & 0.0020\end{array}$

$\begin{array}{llll}0.0020 & 0.0020 & 0.0020\end{array}$

$0.0002 \quad 0.0000$

$\begin{array}{lll}0.0002 & 0.0000 \\ 0.0002 & 0.0000 \\ 0.0002 & 0.0000\end{array}$

$0.0002 \quad 0.0000$

$0.0002 \quad 0.0000$

0.00020 .00000

$0.0002 \quad 0.0000$

$\begin{array}{ll}0.0002 & 0.0000 \\ 0.0002 & 0.0000 \\ 0.0002 & \end{array}$

$\begin{array}{ll}0.0002 & 0.0000 \\ 0.0002 & \end{array}$

0.0002

0.0002

0.0002

$\begin{array}{ll}0.0002 & \\ 0.0002 & 0.0000\end{array}$

0.0002

$\begin{array}{ll}0.0002 & \\ 0.0002 & 0.0300\end{array}$

$0.0002 \quad 0.0000$

0.0002
0.0002

0.0002

0.0002
0.0002

0.0002
0.0002
0.002

0.0002
0.0002
0.0002

0.0002
0.0002

0.0002
0.0002
0.0002

0.0002

$\begin{array}{ll}0.0002 & 0.0000 \\ 0.0002 & 0.0000\end{array}$

$\begin{array}{lll}0.0002 & 0.0000 \\ 0.0002 & 0.0000\end{array}$

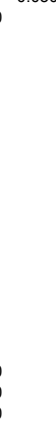

Ft+

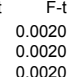

Ga2O3-t Gd2O3-t HiO2-t HgO-t

1-t La203-t MnO2-t MnO-t MoO-t MoO2-t MoO3-t Nb205-t Nd203-t NpO2-t PbO-t PdO2-t PdO-t Pr203-t Pr6011-t Rb2O-t ReO-t

$\begin{array}{ll}0.0020 & 0.0020 \\ 0.0020 & 0.0020 \\ 0.020 & 0.020\end{array}$

$0.0020 \quad 0.0020$

0.00210 .0373

$\begin{array}{lll}0.0021 & 0.0912 \\ 0.0021 & 0.0373\end{array}$

$0.0021 \quad 0.0878$

$\begin{array}{lll}0.0021 & 0.0912 \\ 0.0021 & 0.0373\end{array}$

$\begin{array}{ll}0.0021 & 0.0373 \\ 0.0021 & 0.0373\end{array}$

$0.0021 \quad 0.0651$

$\begin{array}{ll}0.0021 & 0.0373 \\ 0.0021 & 0.0672\end{array}$

0.00210 .0651

0.00210 .0912

$0.0020 \quad 0.0629$

$\begin{array}{lll}0.0021 & 0.0643 \\ 0.0020 & 0.0355\end{array}$

0.00200 .0355

$\begin{array}{ll}0.0020 & 0.0657 \\ 0.0020 & 0.0355 \\ 0.020 & 0\end{array}$

$\begin{array}{ll}0.0020 & 0.0355 \\ 0.0020 & 0.0868\end{array}$

$\begin{array}{ll}0.0020 & 0.0355 \\ 0.0020 & 0.0355\end{array}$

$0.0020 \quad 0.0620$

$\begin{array}{ll}0.0020 & 0.0868 \\ 0.0020 & 0.0337 \\ 0 & 0.020\end{array}$

$0.0020 \quad 0.0868$

$\begin{array}{ll}0.0020 & 0.0355 \\ 0.0020 & 0.0620\end{array}$

$0.0020 \quad 0.0355$

$\begin{array}{ll}0.0020 & 0.0639 \\ 0.0020 & 0.0868 \\ 0.0020 & 0.0612\end{array}$

0.0000
0.0000

0.0300

0.0050

0.0050

0.0001

0.0000

0.0001

0.000

0.0300

$\begin{array}{ll}0.0061 & 0.0004 \\ 0.0031 & 0.0004\end{array}$

0.0031

$\begin{array}{ll}0.0000 & 0.0004 \\ 0.0000 & 0.0004 \\ 0.0066 & 0.0004\end{array}$

$\begin{array}{ll}0.0066 & 0.0004 \\ .0066 & 0.0004 \\ 0.0000 & 0.0004\end{array}$

$\begin{array}{ll}0.0000 & 0.0004 \\ 0.0066 & 0.0004 \\ 0.0000 & 0.004\end{array}$

.0000

$\begin{array}{ll}0.0044 & 0.0004 \\ .0066 & 0.0004 \\ 0.0022 & 0.0004\end{array}$

$\begin{array}{ll}0.0000 & 0.0004 \\ 0.0022 & 0.0004 \\ 0.0000 & 0.004\end{array}$

0.0004
0.0004

0.0004

0.0000
0.0037
0.0031

0.0000
0.0021
0.0637

$\begin{array}{ll}0.0063 & 0.0004 \\ .0000 & 0.0004 \\ 0.0000 & 0.004\end{array}$

.0000

$\begin{array}{ll}0.0063 & 0.0004 \\ 0.0000 & 0.0004 \\ 0.0000 & 0.004\end{array}$

$\begin{array}{ll}0.0000 & 0.0004 \\ 0.031 & 0.0004 \\ 0.042 & 0.04\end{array}$

0.0042

$\begin{array}{ll}0.0042 & 0.0004 \\ 0.0000 & 0.0004 \\ 0.0000 & 0.0004\end{array}$

0.0000

0.0000

.0000
0.0000
0.0000

0.0000
0.0000
0.0000
0.0000

0.0000
0.0000

0.0001
0.0001
0.0001

0.000

0.000

0.000

0.0001

0.000

0.0000 
\# Glass ID 1559 HLW99-62 1560 HLW99-63
1561 HLW99-64 1562 HLW99-65 1563 HLW99-66
1564 HLW9 67 1565 HLW99-68 1566 HLW99-69 1567 HLW99-70 1569 HLW99-71 1570 HLW99-73 1571 HLW99-74 573 HLW99-76 1574 HLW99-77 1576 HuW99-79 1577 HLW99-80 579 -1
$-99-83$
$9-84$ 1582 HLW99-85 1584 HLW99-87 1585 HLW99-88 1587 HLW99-90R 1589 HLW99-92
1590 HLW99-93R 591 HLW99-94 1594 HLW99-97 596 HLW99-99 1597 HLW99-100 1598 LAWA41 1600 LAWA43-1 1601 LAWA44 1603 LAWA4 1605 LAWA4 1606 LAWA49
1607 LAWA50 1608 LAWA51
1609 LAWA52 1610 LAWA5 1612 LAWA55 1613 LAWA5 1615 LAWA5 1617 LAWA6
1618 LAWAG 1618 LAWAG 1620 LAWA63 1622 LAWA65 1623 LAWAA6
624 LAWA67 1625 LAWA68 1628 LAWA7 1631 LAWA74
1632 LAWA75 1633 LAWA76 1635 LAWA78 1637 LAWA8 1639 LAWA82
ReO2-t Re207-t Rh2O3-t RhO2-t RuO2-t Ru2O3-t Sb2O3-t Sb205-t SeO2-t Sm2O3-t SnO-t SnO2-t

\begin{tabular}{|c|c|c|c|}
\hline .0008 & & 00020 & \\
\hline & 0.0013 & & \\
\hline 0008 & 0.0011 & 0.0020 & \\
\hline 0.0008 & 0.0013 & & \\
\hline 0.0008 & 0.0014 & 0.0021 & 0.0021 \\
\hline & 0.0014 & & \\
\hline 0.0008 & 0.0014 & 0.0021 & 0.0021 \\
\hline $\begin{array}{l}0.0008 \\
0.0008\end{array}$ & $\begin{array}{l}0.0014 \\
0.0014\end{array}$ & $\begin{array}{l}0.0021 \\
0.0221\end{array}$ & 0.0021 \\
\hline 0.0008 & 0.0014 & & $\begin{array}{l}0.0021 \\
0.0021\end{array}$ \\
\hline & & & \\
\hline 0.0008 & 0.0014 & 0.0021 & 0.0021 \\
\hline .0008 & 0.0014 & 0.0021 & 0.0021 \\
\hline .0008 & 0.0014 & 0.0021 & 0.0021 \\
\hline 0.0008 & 0.0014 & 0.0021 & 0.0021 \\
\hline $\begin{array}{l}0.0008 \\
0.0008\end{array}$ & 0.0014 & 0.0021 & 0.0021 \\
\hline $\begin{array}{l}0.0008 \\
0.0008\end{array}$ & $\begin{array}{l}0.00014 \\
0.0014\end{array}$ & 0.0021 & 0.0021 \\
\hline 0.00008 & $\begin{array}{l}0.014 \\
0.0013\end{array}$ & $\begin{array}{l}0.0027 \\
0.0020\end{array}$ & $\begin{array}{l}0.0021 \\
0.0020\end{array}$ \\
\hline .0008 & & & \\
\hline .0008 & 0.0013 & 0.0020 & 0.0020 \\
\hline .0008 & 0.0013 & 0.0020 & 0.0020 \\
\hline 0.0008 & 0.0013 & 0.0020 & 0.0020 \\
\hline 0.0008 & 0.0013 & 0.0020 & 0.0020 \\
\hline $\begin{array}{l}0.0008 \\
0.0008\end{array}$ & 0.0013 & 0.0020 & 0.0020 \\
\hline $\begin{array}{l}0.0008 \\
0.0008\end{array}$ & 0.0013 & 0.0020 & 0.0020 \\
\hline 0.0008 & $\begin{array}{l}0.00013 \\
0.0013\end{array}$ & $\begin{array}{l}0.0020 \\
0.0020\end{array}$ & $\begin{array}{l}0.0020 \\
0.0020\end{array}$ \\
\hline .0008 & 0.0013 & 0.0020 & $\begin{array}{l}0.0020 \\
0.0020\end{array}$ \\
\hline .0008 & 0.0013 & 0.0020 & 0.0020 \\
\hline .0008 & 0.0013 & 0.0020 & 0.0020 \\
\hline 0.0008 & 0.0013 & 0.0020 & 0.0020 \\
\hline 0.0008 & 0.0013 & 0.0020 & 0.0020 \\
\hline $\begin{array}{c}0.0008 \\
0.0008\end{array}$ & 0.0013 & 0.0020 & 0.0020 \\
\hline .00008 & 0.0013 & 0.00220 & 0.0020 \\
\hline .0008 & $\begin{array}{l}0.00733 \\
0.0013\end{array}$ & $\begin{array}{l}0.0020 \\
0.0020\end{array}$ & 0.0020 \\
\hline 0.0008 & 0.0013 & 0.0020 & 0.0020 \\
\hline 0.0008 & 0.0013 & & 0.0020 \\
\hline & & & $\begin{array}{l}0.0001 \\
0.0001\end{array}$ \\
\hline
\end{tabular}

0.0010
0.0010

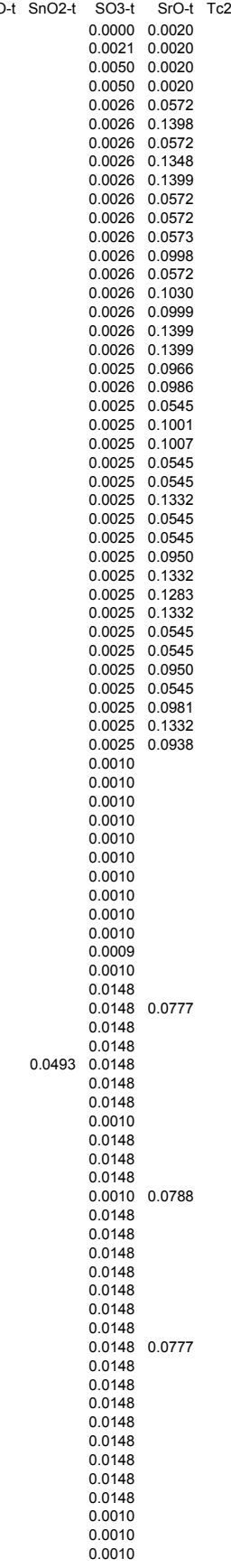

$\begin{array}{ccc}\text { TeO2-t } & \text { ThO2-t } & \text { TiO2-t } \\ 0.0020 & 0.0000 & 0.0061\end{array}$

$\begin{array}{lll}0.0020 & 0.0008 & 0.0031\end{array}$

$\begin{array}{lll}0.0020 & 0.00020 & 0.0000 \\ 0.0021 & 0.0000 & 0.0000\end{array}$

$\begin{array}{lll}0.0021 & 0.0000 & 0.0000 \\ 0 & 0.021 & 0.0000\end{array}$

$\begin{array}{lll}0.0021 & 0.0000 & 0.0066 \\ 0.0021 & 0.0000 & 0.0000\end{array}$

$\begin{array}{lll}0.0021 & 0.0000 & 0.0000 \\ 0.0021 & 0.0000 & 0.0066\end{array}$

$\begin{array}{lll}0.0021 & 0.00000 & 0.0000 \\ 0.0021 & 0.0000 & 0.0033\end{array}$

$\begin{array}{lll}0.021 & 0.0000 & 0.0044 \\ 0.0021 & 0.0000 & 0.0066\end{array}$

$\begin{array}{lll}0.0021 & 0.0000 & 0.0000 \\ 0.0021 & 0.0000 & 0.0022 \\ 0.021 & 0.000 & 0.000\end{array}$

$\begin{array}{llll}0.0021 & 0.0000 & 0.0000\end{array}$

$\begin{array}{lll}0.0020 & 0.00000 & 0.0000 \\ 0 & 0.00037\end{array}$

$\begin{array}{lll}0.0020 & 0.0000 & 0.0031 \\ 0.0020 & 0.0000 & 0.0000 \\ 0.0020 & 0.0000 & 0.0063\end{array}$

$\begin{array}{lll}0.0020 & 0.0000 & 0.0021 \\ 0.0063 & 0.0000 & 0.000\end{array}$

$\begin{array}{lll}0.0020 & 0.0000 & 0.0000 \\ 0.0020 & 0.0000 & 0.0000\end{array}$

$\begin{array}{lll}0.0020 & 0.0000 & 0.0000 \\ 0.0020 & 0.0000 & 0.0063\end{array}$

$\begin{array}{lll}0.0020 & 0.0000 & 0.0000 \\ 0.0020 & 0.0000 & 0.0000\end{array}$

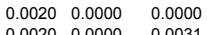

$\begin{array}{lll}0.0020 & 0.0000 & 0.0031 \\ 0.0020 & 0.0000 & 0.0042 \\ 0.020 & \end{array}$

$0.0020 \quad 0.00000 .0 .0063$

0.00200 .0000

$\begin{array}{lll}0.0063 & 0.0020 & 0.0000 \\ 0.0000 & 0.0020 & 0.0000 \\ 0.0000 & 0.0020 & 0.0000 \\ 0.0020 & 0.0000\end{array}$

0.0000
0.0000

$\begin{array}{lr}\text { TT2O3-t U3O8-t } & \text { UO2-t } \\ 0.0020 & 0.0000 \\ 0.020 & 0.0332\end{array}$

0.0000
0.0332

0.0800
0.0800
0.0000

0.0000
0.0000
0.0000

0.0000
0.0000
0.0000

0.0000
0.0000
0.0000
0.0000

0.0000

0.0000
0.0000
0.0000
0.0000

0.0021

0.0021

0.0021
0.0020
0.0021
0.0020

0.0020
0.0020
0.0020

0.0020
0.0020
0.0020

0.0020
0.0020
0.0020

0.0020
0.0020

0.0020
0.0020

0.0020
0.0020

0.0000
0.0000
0.0000

0.0000
0.0000
0.0000

0.0000

0.0000

0.0000
0.0000

0.0000
0.0000

0.0000

0.0000

0.0000
0.0000

0000

0.0240
0.0197

0.0199
0.0199
6099

0.0199
0.0199
0.0199

0.0199
0.0199

0.0200
0.0211
0.011

0.0111
0.0109
0.0909

0.0109
0.0109
0.109

0.0119
0.0109
0.009

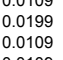

0.0109
0.0109
0.0109
0.0109

0.011
0.01109
00109

0.0109
0.0109
0.109

0.0109
0.0109
0.0109

0.0109
0.0109

0.0404
0.0182
0.019

0.0109
0.0109

0.0199
0.0109
0.0109

0.0109

0.0399
0.0199
WO3-t Y2O3-t ZnO-t Sum-t Al2O3-a

$\begin{array}{llll}0.0020 & 0.0200 & 0.9999 & 0.0062 \\ 0.0020 & 0.0200 & 10000 & 0.0499\end{array}$ $\begin{array}{llll}0.0200 & 1.0000 & 0.0499 \\ 0.0200 & 1.0000 & 0.0541\end{array}$

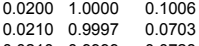
$\begin{array}{lll}0.0210 & 1.0000 & 0.0742\end{array}$ $\begin{array}{lll}0.0210 & 1.0000 & 0.0746 \\ 0.0210 & 0.9997 & 0.0158\end{array}$ $\begin{array}{lll}0.0210 & 0.9998 & 0.0758 \\ 0.0210 & 0.9998 & 0.751\end{array}$ $\begin{array}{lll}0.0210 & 0.9997 & 0.0599 \\ 0.0210 & 1.0000 & 0.0661\end{array}$ $\begin{array}{lll}0.0210 & 0.9997 & 0.0599 \\ 0.0210 & 0.9998 & 0.0692\end{array}$ $\begin{array}{lll}0.0210 & 0.9998 & 0.0679 \\ 0.0210 & 0.9999 & 0.0712\end{array}$ $\begin{array}{lll}0.0210 & 0.9999 & 0.0718 \\ 0.0210 & 0.9998 & 0.0726\end{array}$

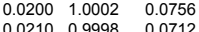
$\begin{array}{lll}0.0210 & 0.9998 & 0.0712 \\ 0.0200 & 10002 & 0.068\end{array}$ $\begin{array}{lll}0.0220 & 1.0000 & 0.07707 \\ 0.020 & 10002 & 0.0654\end{array}$ $\begin{array}{lll}0.0200 & 1.0002 & 0.0654 \\ 0.0200 & 1.0001 & 0.0737 \\ 0.0200 & 1.0000 & 0.0635\end{array}$ $\begin{array}{lll}0.0200 & 1.0000 & 0.0635 \\ 0.0200 & 1.0001 & 0.0784\end{array}$ $\begin{array}{lll}0.0200 & 1.0002 & 0.0674\end{array}$ $\begin{array}{lll}0.0200 & 1.0000 & 0.0717 \\ 0.0200 & 1.0000 & 0.0680\end{array}$ $0.0200 \quad 1.0000 \quad 0.0712$ $\begin{array}{lll}0.02200 & 1.0002 & 0.0742 \\ 0.0200 & 1.0000 & 0.075\end{array}$ $\begin{array}{lll}0.0200 & 1.0000 & 0.0795 \\ 0.0200 & 1.0000 & 0.0687\end{array}$ $0.0200 \quad 1.0000 \quad 0.0680$ $\begin{array}{lll}0.0200 & 1.00001 & 0.0751\end{array}$ $\begin{array}{lll}0.0200 & 1.0002 & 0.0714 \\ 0.0200 & 1.0002 & 0.0702\end{array}$ $\begin{array}{lll}0.0200 & 1.0000 & 0.0731\end{array}$ $\begin{array}{lll}0.0200 & 1.0000 & 0.0675 \\ 0.0299 & 0.9999 & 0.0592\end{array}$ $\begin{array}{lll}0.0360 & 1.0000 & 0.0582 \\ 0.0295 & 1.0000 & 0.1118\end{array}$ $\begin{array}{llll}0.0297 & 0.9999 & 0.0586 \\ 0.0248 & 0.9999 & 0.0568\end{array}$ $\begin{array}{lll}0.0248 & 0.9999 & 0.0590\end{array}$

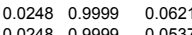
$\begin{array}{lll}0.0248 & 0.9999 & 0.0537 \\ 0.0248 & 0.9999 & 0.0588\end{array}$ $\begin{array}{lll}0.0249 & 0.9999 & 0.0592 \\ 0.0299 & 1.0000 & 0.0595\end{array}$ 0.0295
0.929998

$0.0295 \quad 0.9988$ 0.0295
0.0295
0.99988 $\begin{array}{lll}0.0295 & 0.9988 & \\ 0.0297 & 0.9999 & 0.0824\end{array}$ $\begin{array}{lll}0.0297 & 0.9999 & 0 \\ 0.0295 & 0.9988 & \end{array}$ $\begin{array}{lll}0.0295 & 0.9988 \\ 0.0295 & 0.9988\end{array}$ $\begin{array}{lll}0.0295 & 0.9988 & \\ 0.0299 & 0.9999 & 0.0576\end{array}$ $\begin{array}{lll}0.0295 & 0.9998 \\ 0.0295 & 0.9988\end{array}$

$\begin{array}{lll}0.0295 & 0.9988 \\ 0.0295 & 09988\end{array}$

$\begin{array}{ll}0.0295 & 0.9988 \\ 0.0295 & 0.9989 \\ 0.0295 & 0.9989\end{array}$ $\begin{array}{ll}0.0295 & 0.9989 \\ 0.9988 & \end{array}$

$0.0295 \quad 0.9988$ $\begin{array}{ll}0.0295 & 0.9998 \\ 0.0295 & 0.9989\end{array}$ 0.02950 .9988 $0.0297 \quad 0.9999 \quad 0.0619$ $\begin{array}{lll}0.0297 & 0.9999 & 0.0619 \\ 0.0297 & 0.9999 & 0.0614 \\ 0.0297 & 0.9999 & 0.0633\end{array}$ 
\# Glass ID

1559 HLW99-62

1561 HLW99-64

1562 HLW99-65
1563 HLW99-66

564 HLW99-67

1565 HLW99-68
1566 HLW9

1567 HLW99-70

1569 HLW99-72

1570 HLW99-73

1572 HLW99-75
1573 HLW99-76

1574 HLW99-77

1575 HLW99-78
1576 HLW99-79

1577 HLW99-80

1578 HLW99-81

580 HLW99-83

1582 HLW99-85

1583 HLW99-86
1584 HLW99-87

1585 HLW99-88
1586 HLW99-89

1587 HLW99-90R

1589 HLW99-92

1590 HLW99-93R
1591 HLW99-94

1592 HLW99-95

1593 HLW99-96

1594 HLW99-97
1595 HLW99-98R

1596 HLW99-99

1598 LAWA41

1600 LAWA43-

1601 LAWA44

1602 LAWA4

1604 LAWA47

1605 LAWA48

1606 LAWA49
1607 LAWA50

1608 LAWA51

1609 LAWA52

1610 LAWA53

1612 LAWA55
1613 LAWA56

1614 LAWA5
1615 LAW55

1616 LAWA59

1617 LAWA60

1618 LAWA6
1619 LAWA62

1620 LAWA63

1622 LAWA65

1624 LAWA67

1625 LAWA68
1626 LAWA69

1627 LAWA7

1628 LAWA71

1630 AWA73

1632 LAWA75

1633 LAWA7

1635 LAWA78

1636 LAWA79

(637 LAWA8

1639 LAWA82
B2O3-a CaO-a Fe2O3-a FeO-a

$\begin{array}{lll}0.0521 & 0.0014 & 0.020 \\ 0.1151 & 0.0279 & 0.05\end{array}$

$\begin{array}{lll}0.0890 & 0.0017 & 0.0211\end{array}$

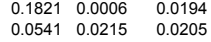

$\begin{array}{llll}0.0542 & 0.0225 & 0.1062\end{array}$

$\begin{array}{lll}0.0553 & 0.0209 & 0.1172 \\ 0.0568 & 0.0217 & 0.0209\end{array}$

$\begin{array}{lll}0.0558 & 0.0217 & 0.0209 \\ 0.0533 & 0.0219 & 0.0264\end{array}$

$\begin{array}{lll}0.0567 & 0.0240 & 0.0232\end{array}$

$\begin{array}{lll}0.0575 & 0.0235 & 0.0233 \\ 0.543 & 0.0228 & 0.119\end{array}$

$\begin{array}{lll}0.0543 & 0.0228 & 0.1191 \\ 0.0548 & 0.0291 & 0.1220\end{array}$

$\begin{array}{lll}0.0541 & 0.0222 & 0.0212\end{array}$

$\begin{array}{lll}0.0558 & 0.0235 & 0.1161 \\ 0.0544 & 0.0223 & 0.0546\end{array}$

$\begin{array}{lll}0.0544 & 0.0223 & 0.0546 \\ 0.0553 & 0.0224 & 0.0194\end{array}$

$\begin{array}{lll}0.1035 & 0.0216 & 0.0677\end{array}$

$\begin{array}{lll}0.0543 & 0.0212 & 0.0213\end{array}$

$\begin{array}{llll}0.1054 & 0.0210 & 0.05777 \\ 0.107 & 0.078\end{array}$

$\begin{array}{lll}0.1007 & 0.0198 & 0.0189 \\ 0.1125 & 0.0219 & 0.0655\end{array}$

$\begin{array}{lll}0.1000 & 0.0195 & 0.0190 \\ 0.1047 & 0.0225 & 0.0504\end{array}$

$\begin{array}{lll}0.1038 & 0.0199 & 0.1124 \\ 0.1036 & 0.0213 & 0.1090 \\ 0.091 & 0.920 & 0.1022\end{array}$

$\begin{array}{lll}0.0985 & 0.0193 & 0.1129 \\ 0.0991 & 0.0206 & 0.1022\end{array}$

$\begin{array}{lll}0.1000 & 0.0195 & 0.0201\end{array}$

$\begin{array}{lll}0.1032 & 0.0215 & 0.0201 \\ 0.1022 & 0.197 & 0.019\end{array}$

$\begin{array}{lll}0.0996 & 0.0198 & 0.0199\end{array}$

$\begin{array}{lll}0.1030 & 0.0209 & 0.1169\end{array}$

$\begin{array}{lll}0.1026 & 0.0214 & 0.1151 \\ 0.0992 & 0.0209 & 0.0202\end{array}$

$\begin{array}{lll}0.1032 & 0.0219 & 0.0208\end{array}$

$\begin{array}{lll}0.1002 & 0.0195 & 0.0193 \\ 0.0735 & 0.0218 & 0.0950\end{array}$

$\begin{array}{lll}0.0735 & 0.0218 & 0.0650 \\ 0.0849 & 0.0248 & 0.0783\end{array}$

$\begin{array}{lll}0.0733 & 0.0208 & 0.0642\end{array}$

$\begin{array}{lll}0.0881 & 0.0203 & 0.0659\end{array}$

$\begin{array}{lll}0.1183 & 0.0006 & 0.0661 \\ 0.0914 & 0.0009 & 0.0664\end{array}$

$\begin{array}{lll}0.0935 & 0.0007 & 0.0663 \\ 0.0554 & 0.0006 & 0.0868 \\ 0.006\end{array}$

$\begin{array}{lll}0.0886 & 0.0006 & 0.1091\end{array}$

$\begin{array}{lll}0.1179 & 0.0006 & 0.0660 \\ 0.0640 & 0.0769 & 0.0774\end{array}$

$\begin{array}{lll}0.1172 & 0.0432 \quad 0.0011\end{array}$

$\begin{array}{lll}0.0593 & 0.0009 & 0.0751\end{array}$

$\begin{array}{llllllll}0.0054 & 0.0005 & 0.0193 & 0.1823 & 0.0000 & 0.0017 & 0.4322 & 0.0283\end{array}$

BaO-a BeO-a Bi203-a

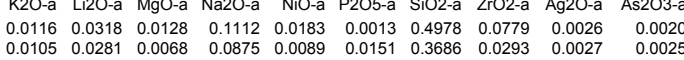

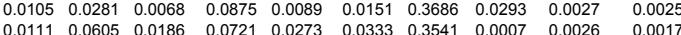

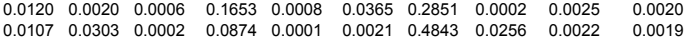

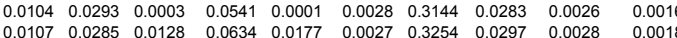

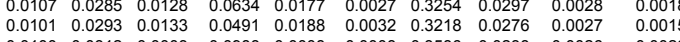

$\begin{array}{llllllllllll}0.0105 & 0.0307 & 0.0002 & 0.0754 & 0.0001 & 0.0025 & 0.4012 & 0.0313 & 0.0028 & 0.0022\end{array}$

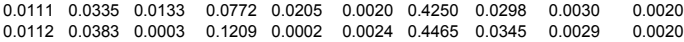

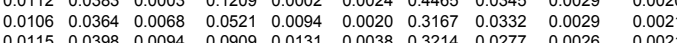

$\begin{array}{lllllllllll}0.0115 & 0.0366 & 0.0140 & 0.0898 & 0.0194 & 0.0024 & 0.3295 & 0.0271 & 0.0029 & 0.0019\end{array}$

$\begin{array}{llllllllll}0.0115 & 0.0388 & 0.0003 & 0.0849 & 0.0001 & 0.027 & 0.3115 & 0.03300 & 0.029 & 0.0021 \\ 0.0115 & 0.0322 & 0.0048 & 0.0768 & 0.0069 & 0.0029 & 0.3260 & 0.0279 & 0.0029 & 0.0021\end{array}$

$\begin{array}{llllllllll}0.0102 & 0.0281 & 0.00121 & 0.0697 & 0.04001 & 0.0024 & 0.3910 & 0.0227 & 0.0029 & 0.021 \\ 0 & 0.0179 & 0.0030 & 0.3021 & 0.0256 & 0.0025 & 0.0015\end{array}$

$\begin{array}{lllllllllll}0.0116 & 0.0305 & 0.0002 & 0.1116 & 0.0001 & 0.0028 & 0.4005 & 0.0274 & 0.0027 & 0.0022 \\ 0.0107 & 0.020 & 0.0128 & 0.0026 & 0.0178 & 0.043 & 0.303 & 0.0274 & 0.026 & 0.025\end{array}$

$\begin{array}{llllllllll}0.0289 & 0.0003 & 0.0637 & 0.0001 & 0.0037 & 0.3673 & 0.0244 & 0.0025 & 0.0023\end{array}$

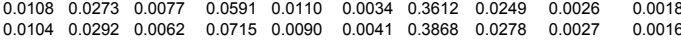

$\begin{array}{llllllllll}0.0111 & 0.02270 & 0.00002 & 0.08088 & 0.0001 & 0.0035 & 0.4773 & 0.0285 & 0.0026 & 0.0021 \\ 0.0100 & 0.0201 & 0.0041 & 0.0752 & 0.0060 & 0.034 & 0.3013 & 0.0277 & 0.025 & 0.0017\end{array}$

$\begin{array}{llllllllll}0.0110 & 0.0280 & 0.0121 & 0.0600 & 0.0171 & 0.0028 & 0.3030 & 0.0261 & 0.0024 & 0.0020 \\ 0.0107 & 0.0289 & 0.0001 & 0.0714 & 0.0001 & 0.0040 & 0.3889 & 0.0252 & 0.0025 & 0.0020\end{array}$

$\begin{array}{lllllllllll}0.0109 & 0.0269 & 0.0002 & 0.0838 & 0.0001 & 0.00031 & 0.2948 & 0.0246 & 0.0027 & 0.0019\end{array}$

$\begin{array}{llllllllll}0.0105 & 0.0290 & 0.0117 & 0.0459 & 0.00171 & 0.0037 & 0.30606 & 0.0229 & 0.0025 & 0.0016 \\ 0 & 0.0278 & 0.0023 & 0.0021\end{array}$

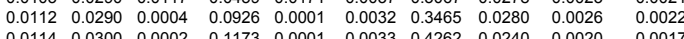

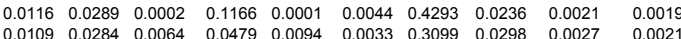

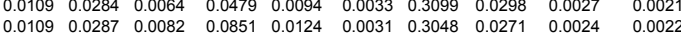

$\begin{array}{llllllllll}0.0274 & 0.0111 & 0.0858 & 0.0172 & 0.0033 & 0.3359 & 0.0257 & 0.0025 & 0.0022\end{array}$

$\begin{array}{lllllllllll}0.0112 & 0.0286 & 0.0002 & 0.1063 & 0.0002 & 0.0042 & 0.3935 & 0.0217 & 0.0021 & 0.0021\end{array}$

$\begin{array}{lllllllll}0.0003 & 0.0227 & 0.1694 & 0.0001 & 0.0025 & 0.4460 & 0.0217 & 0.025 & 0.0021\end{array}$

$\begin{array}{lllllllll}0.0291 & 0.0003 & 0.0190 & 0.1778 & 0.0001 & 0.0020 & 0.3576 & 0.0260 & 0.0001\end{array}$

$\begin{array}{lllllllll}0.0060 & 0.0003 & 0.0185 & 0.1792 & 0.0001 & 0.0017 & 0.4202 & 0.0254 & 0.0001 \\ 0.006151 & 0.1803 & 0.0001 & 0.0020 & 0.4274 & 0.0266 & 0.0001\end{array}$

$\begin{array}{llllllllll}0.0002 & 0.0160 & 0.1858 & 0.0001 & 0.0017 & 0.4280 & 0.0256 & 0.000\end{array}$

$\begin{array}{llllllll}0.0002 & 0.0145 & 0.1772 & 0.0001 & 0.0014 & 0.4124 & 0.0251 & 0.0001 \\ 0.0002 & 0.0151 & 0.1832 & 0.0001 & 0.0019 & 0.4161 & 0.0254 & 0.0001\end{array}$

$\begin{array}{lllllllll} & 0.0002 & 0.0149 & 0.1675 & 0.0001 & 0.0015 & 0.4161 & 0.0254 & 0.0001 \\ 0.0053 & 0.0006 & 0.0144 & 0.1900 & 0.0004 & 0.0007 & 0.4188 & 0.0272 & 0.0001\end{array}$

$\begin{array}{llllllll}0.0049 & 0.0008 & 0.0138 & 0.1747 & 0.0002 & 0.0007 & 0.4080 & 0.0306\end{array}$

0.000

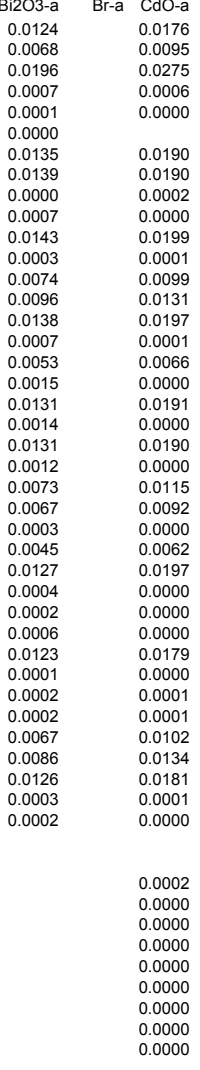

-a CoO-a Co2O3-a Cr2O3-a Cs2O-a CuO-a

$\begin{array}{lll}0.0021 & 0.0000 & 0.0023 \\ 0.0022 & 0.0018 & 0.023\end{array}$

0.0113

0.0010
0.0012
0

0.0024
0.0225
0.0268

0.0012
0.0151

0.0273
0.0019
0.0115

0.0247
0.0001
0.001

0.0014
0.01435

0.0001

0.0001

0.0001
0.0246
0.0024

0.0001
0.0010
0.0142

0.0177

0.0024
0.0016
0.0000

0.0000
0.0000

0.0000
0.0000
0.0000

0.0000
0.000
0.000

0.0000
0.0000

.

0.0095
.0275

.0006
0.0000

0.0190
0.0190
0.000

.0000
.0199
0.000

0.00099
0.0131
0.0197

0.0197
0.0001

0.0066
0.0000
0.0191

0.0000

.0000
0.0115

.0000
.0062

0.0000

0000
0.179

0001

.0102
0.0134

0.0000

.0002
.0000

0.0000
0.0000
0.0000

0.0000
0.0000

.

$\begin{array}{lll}0.0022 & 0.0018 & 0.0023 \\ 0.0022 & 0.0035 & 0.0021 \\ 0.0026 & 0.0046 & 0.0019 \\ 0.0065 & 0.0020 & 0.0021 \\ 0.0060 & 0.0019 & 0.0024 \\ 0.0058 & 0.0017 & 0.0022 \\ 0.0061 & 0.0018 & 0.0020 \\ 0.0058 & 0.0031 & 0.0025 \\ 0.0060 & 0.0018 & 0.0023 \\ 0.0065 & 0.0018 & 0.0023 \\ 0.0062 & 0.0019 & 0.0023 \\ 0.0059 & 0.0019 & 0.0024 \\ 0.0064 & 0.0019 & 0.0023 \\ 0.0064 & 0.0020 & 0.0022 \\ 0.0061 & 0.0019 & 0.0025 \\ 0.0064 & 0.0019 & 0.0021 \\ 0.0064 & 0.0019 & 0.0024 \\ 0.0020 & 0.0018 & 0.0022 \\ 0.0064 & 0.00220 & 0.0031 \\ 0.0021 & 0.0019 & 0.0021 \\ 0.0022 & 0.0018 & 0.0022 \\ 0.0021 & 0.0017 & 0.0026 \\ 0.0020 & 0.0018 & 0.0036 \\ 0.0022 & 0.0018 & 0.0019 \\ 0.0020 & 0.0017 & 0.0022 \\ 0.0020 & 0.0017 & 0.0022 \\ 0.0021 & 0.0016 & 0.0023 \\ 0.0020 & 0.0018 & 0.0021 \\ 0.0020 & 0.0017 & 0.0022 \\ 0.0021 & 0.0019 & 0.0020 \\ 0.0020 & 0.0018 & 0.0021 \\ 0.0021 & 0.0021 & 0.0022 \\ 0.0023 & 0.0020 & 0.0021 \\ 0.0022 & 0.0018 & 0.0024 \\ 0.0021 & 0.0018 & 0.00222 \\ 0.0020 & 0.0019 & 0.0023 \\ 0.0019 & 0.0016 & 0.0026 \\ 0.0021 & 0.0020 & 0.0021 \\ & 0.0002 & \end{array}$

.0001

0.0003

0.0002
0.0002
0.0002

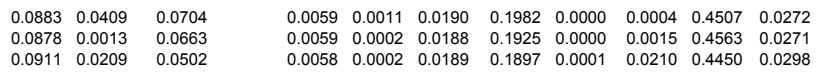


\# Glass ID 1559 HLW99-62 1561 HLW99-64 1562 HLW99-65 1563 HLW99-66 565 HLW99-68 1566 HLW99-69 1567 HLW99-70 1569 HLW99-72 1570 HLW99-73 572 HLW99-74 1573 HLW99-76 1574 HLW99-77 1576 HLW99-79 1577 HLW99-80 1578 HLW99-81 1581 HLW99-84 1582 HLW99-85

1584 HLW99-87

1585 HLW99-88
1586 HLW99-89 587 HLW99-90R 1589 HLW99-92 1590 HLW99-93R
1591 HLW99-94 592 HLW99-95 1593 HLW99-96 1594 HLW99-97 1596 HLW99-99 1597 HLW99-100 1598 LAWA41 1600 LAWA43-1 1601 LAWA44 1602 LAWA45 1604 LAWA47 1605 LAWA4A 1606 LAWA49 1608 LAWA51 1609 LAWA52 1610 LAWA55
1611 LAWA54 1612 LAWA55 1613 LAWA5 16145 LAWA5 1616 LAWA59 1618 LAWA61 1620 LAWA63 1621 LAWA64 1623 LAWA66 1624 LAWA67 1625 LAWA68 1627 LAWA70 1628 LAWA71 1629 LAWA72 1631 LAWA74 1632 LAWA75 1633 LAWA76 1635 LAWA7 1636 LAWA79 1637 LAWA8 1639 LAWA82
1640 LAWA83
I-a La2O3-a MnO2-a MnO-a MoO-a MoO3-a Nb2O5-a Nd2O3-a NpO2-a 0.0023

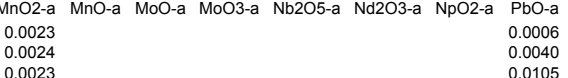<smiles>C1CC[PbH]CC1</smiles>

0.0023
0.0024
0.0376
0.0861

0.086

0.0364
0.0859
0.0908

0.0392

0.0401

0.066

0.0675

0.0900

0.0633

0.0362

0.0624
0.0380

0.0889

0.0375

0.0829

0.0816

0.0359
0.0619

0.0368

0.0864

0.0001

0.0000

0.0001
0.0000
0

0.0000

0.00001
0.0002

0.0002
0.0003
0.0002

0.0003
0.0005
0.0005

0.0005
0.0005
0.004

0.0004
0.0003
0.0003

0.0003

0.0004
0.0005

0.0004

0.0003

.0005

.0003

.0004

.0004

0.0004

0.0005

0.0003

$\begin{array}{llll}0.0003 & 0.0002 & 0.0021 & 0.0009 \\ 0.0002 & 0.0004 & 0.021 & 0.0009\end{array}$

$\begin{array}{llll}0.0002 & 0.0002 & 0.0023 & 0.0008 \\ 0.0001 & 0.00004 & 0.0021 & 0.001 \\ 0.0022 & 0.0012\end{array}$

$\begin{array}{llll}0.0002 & 0.0001 & 0.0048 & 0.0009 \\ 0.0009 & 0.0004 & 0.0042 & 0.0005 \\ 0.0009 & 0.0002 & 0.0044 & 0.0005\end{array}$

$\begin{array}{ll}0.0049 & 0.0011 \\ 0.0044 & 0.0005\end{array}$

$\begin{array}{ll}0.0025 & 0.0012 \\ 0.0025 & 0.0011 \\ 0.0028 & 0.0011\end{array}$

\begin{tabular}{lll}
0.0027 & 0.0006 \\
\hline
\end{tabular}

$\begin{array}{ll}0.0030 & 0.0015 \\ 0.0029 & 0.0003\end{array}$

$\begin{array}{ll}0.0023 & 0.0007 \\ 0.0025 & 0.0009\end{array}$

$\begin{array}{ll}0.0029 & 0.0003 \\ 0.0024 & 0.0007 \\ 0.0025 & 0.006\end{array}$

$\begin{array}{ll}0.0025 & 0.0006 \\ 0.0019 & 0.0003\end{array}$

$0.0025 \quad 0.0011$

$0.024 \quad 0.0005$

$\begin{array}{ll}0.0021 & 0.0007 \\ 0.0019 & 0.0008 \\ 0.0022 & 0.005\end{array}$

$\begin{array}{ll}0.0022 & 0.0005 \\ 0.0020 & 0.0010\end{array}$

$0.0025 \quad 0.0008$

$\begin{array}{ll}0.0024 & 0.0006 \\ 0.0022 & 0.0002\end{array}$

$\begin{array}{ll}0.0026 & 0.000 \\ 0.0022 & 0.0000\end{array}$

$\begin{array}{ll}0.0022 & 0.0004 \\ 0.0021 & 0.0003\end{array}$

$0.0020 \quad 0.0008$

$\begin{array}{lll}0.0023 & 0.0005 \\ 0.0023 & 0.0008\end{array}$

$\begin{array}{ll}0.0023 & 0.0008 \\ 0.0021 & 0.0010\end{array}$

$\begin{array}{llll}0.0003 & 0.0001 & 0.0024 & 0.0003 \\ 0.0004 & 0.0001 & 0.0021 & 0.0007 \\ & & & 0.000\end{array}$ 
581 HLW99-84

1582 HLW99-85

1584 HLW99-87

1585 HLW99-88
1586 HLW99-89

1587 HLW99-90R

1589 HLW99-92

1590 HLW99-93R2
1591 HLW99-94

1592 HLW99-95

1593 HLW99-96

1595 HLW99-98R

1596 HLW99-99

1598 LAWA41

1600 LAWA43-

1601 LAWA44

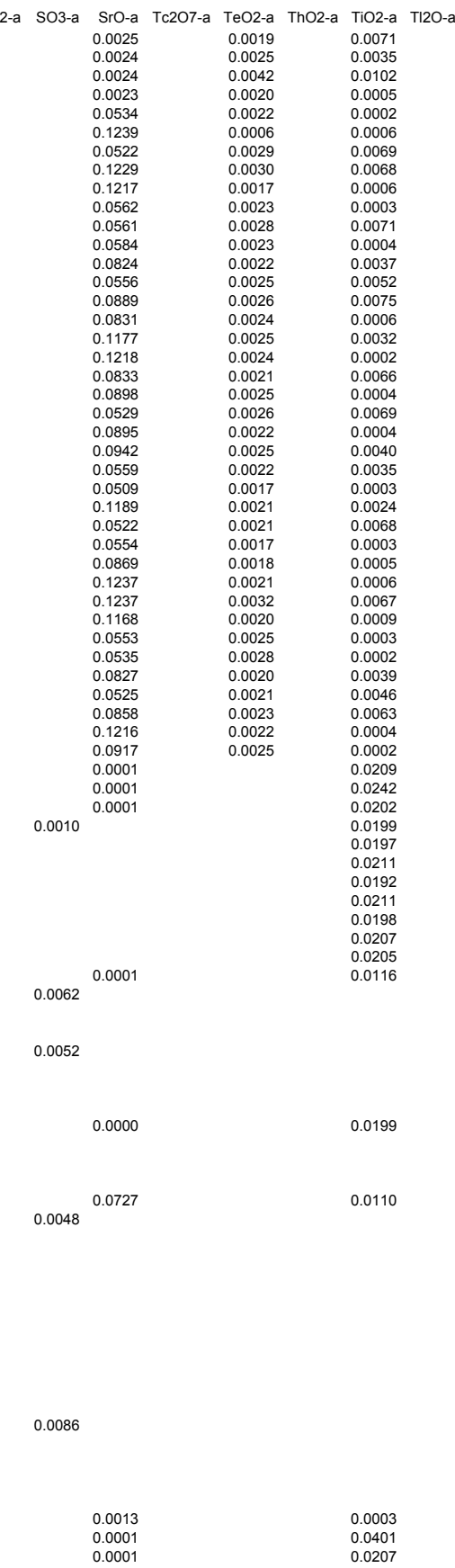

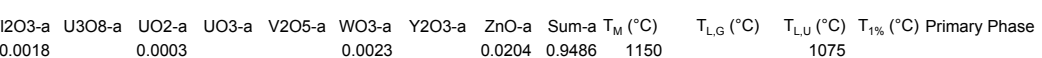

$\mathrm{C}_{650^{\circ} \mathrm{C}} \mathrm{C}_{700^{\circ} \mathrm{C}} \mathrm{C}_{750^{\circ} \mathrm{C}}$

$\begin{array}{lll}0.0204 & 0.0966 \\ 0.0184 & 0.9162 \\ 0.0188 & 0.9607\end{array}$

$\begin{array}{ll}0.0201 & 0.9666 \\ .0207 & 0.9818\end{array}$

$\begin{array}{ll}0.0212 & 0.9757 \\ 0.0218 & 0.9715 \\ 0.0222 & 1.0132\end{array}$

$\begin{array}{ll}0.0222 & 1.0132 \\ 0.0216 & 0.9701 \\ 0.0213 & 0.9680\end{array}$

$\begin{array}{ll}0.0213 & 0.9680 \\ 0.0216 & 0.9798 \\ 0.0210 & 0.9797\end{array}$

$\begin{array}{lll}0.0215 & 0.9474 \\ 0.0204 & 0.924\end{array}$

$\begin{array}{ll}0.0209 & 0.9834 \\ 0.0214 & 0.9663\end{array}$

0.02020 .9764

$\begin{array}{lll}0.0201 & 0.9762\end{array}$

$\begin{array}{ll}0.0206 & 0.9770 \\ 0.0201 & 0.9500\end{array}$

$\begin{array}{ll}0.0210 & 0.9940 \\ 0.0202 & 0.9545\end{array}$

$\begin{array}{lll}0.0202 & 0.9545 \\ 0.0199 & 0.9903\end{array}$

$\begin{array}{ll}0.0200 & 0.9667 \\ 0.0200 & 0.9711\end{array}$

$\begin{array}{lll}0.0198 & 0.9310 \\ 0.0196 & 0.9592\end{array}$

$\begin{array}{ll}0.0181 & 0.9864 \\ 0.0204 & 0.9866\end{array}$

0.01820 .9574

$\begin{array}{ll}0.0187 & 0.9568 \\ 0.0203 & 0.9915 \\ 0.0205 & 0.9739\end{array}$

$\begin{array}{ll}0.0205 & 0.9739 \\ 0.0200 & 0.9903\end{array}$

$0.0204 \quad 0.9978$

$\begin{array}{lll}0.0186 & 0.9715 \\ 0.0283 & 0.9320 \\ 0.038 & 0.989\end{array}$

$\begin{array}{lll}0.0293 & 0.9352 \\ 0.0243 & 0.9452\end{array}$

$\begin{array}{lll}0.0247 & 0.9518 \\ 0.0240 & 0.9153\end{array}$

$\begin{array}{ll}0.0247 & 0.936 \\ 0.029 & 0.936\end{array}$

0.02290 .906

$0.0243 \quad 0.937$

0.0291

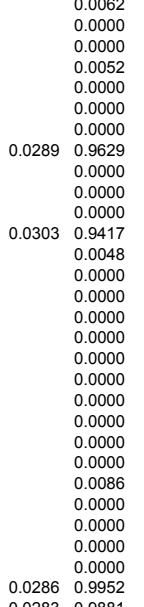

$\begin{array}{ll}0.0286 & 0.9952 \\ 0.0283 & 0.9881 \\ 0.0284 & 0.9852\end{array}$ 
1561 HLW99-64
1562 HLW99-65

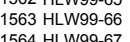

1565 HLW 199.68

1566 HLW999.69

1567 HLW99-70
1568 HLW99-71

1569 HLW99-72

1570 HLW99-73

1572 HLW $1599-75$

1573 HLW99-7

1574 HLW999-77

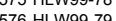

1577 HLW99-80

1578 HLW99-81

1581 HLW99-84

1582 HLW

1584 HLW

1585 HLW99-88

1587 HLW99-900

1588 HLW99-91

1589 HLW999-92
1500 HLW99-93R

${ }_{1593}$ HLW99-96

1594 HLW99-97
$1595 \mathrm{HLW}$.

1596 HLW99-99

1597 HLW999-100

1598 LAWA41

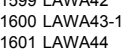

2.680

2.670

1604 LAWA47

1605 LAWA48

1606 LAWA49
1607 LAWA50

1608 LAWA51

1609 LAWA52

1610 LAWA5
1611 LAWA5

1612 LAWA55

1613 LAAA5
1614 LWANA5

1614 LAWAS7

1616 LAWA59

1617 LWWA60

1618 LAWA61
1619 LAWA62

1620 LAWA63

1621 LAWA64

1622 LAWA65
1623 LANAG6

1622 LANA66
1624 LAWA67

1625 LAWA68
1626 LAWA69

1627 LAWA70

1628 LAWA7

1629 LAWA72

1631 LAWA74

1632 LAWA75

1633 LAWA76
1634 LAWA77

1635 LAWA78

1636 LAWA79

1637 LAWAB
1638 LAWAB

1639 LAWAB82
1640 LAWAB3

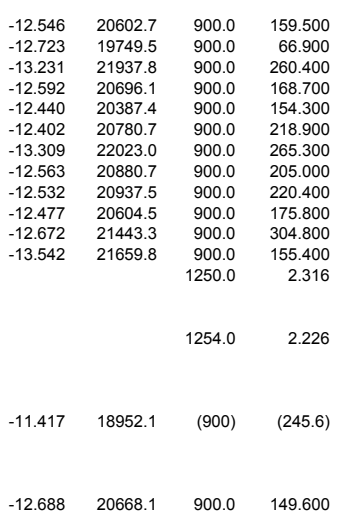
$\begin{array}{llll}-13.3822 & 21825.3 & 900.0 & 197.800 \\ -12.527 & 21089.4 & 900.0 & 250.300 \\ -12.882 & 21519.6 & 900.0 & 254.800\end{array}$ 


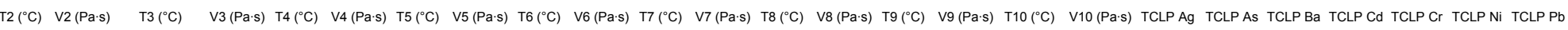
1559 HLW99-62

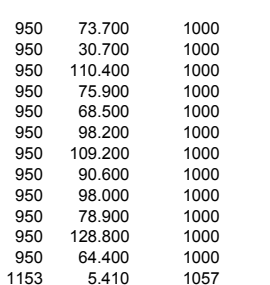

$\begin{array}{rrrr}36.9 & 1055.0 & 19.9 & 1100.0 \\ 15.5 & 1050.0 & 8.5 & 1100.0 \\ 53.0 & 1050.0 & 26.7 & 1100.0 \\ 37.5 & 1050.0 & 20.0 & 1100.0 \\ 38.4 & 11050\end{array}$

$\begin{array}{rr}11.3 & 1150.0 \\ 5.0 & 1150.0 \\ 14.8 & 1150.0 \\ 11.4 & 11500\end{array}$ $\begin{array}{rr}14.8 & 1150.0 \\ 11.4 & 1150.0 \\ 10.5 & 1150.0 \\ 14.7 & 1150.0\end{array}$

$\begin{array}{lll}3 & \end{array}$

$\begin{array}{llll}50.7 & 1050.0 & 25.9 & 1100.0 \\ 44.3 & 1050.0 & 23.6 & 1100.0\end{array}$

$\begin{array}{ll}14.7 & 1150.0 \\ 14.4 & 1150.0 \\ 13.4 & 1150.0 \\ 14.5 & 1150.0\end{array}$

$\begin{array}{ll}14.5 & 1150.0 \\ 12.0 & 1150.0 \\ 17.8 & 1150.0\end{array}$

$\begin{array}{rl}14.0 & 1150.0 \\ 8.7 & 1150.0\end{array}$

$\begin{array}{llll}61.0 & 1050.0 & 31.8 & 1100.0 \\ 30.1 & 1050.0 & 15.5 & 1100.0 \\ 15.8 & 961.0 & 64.5 & \end{array}$

$\begin{array}{lll}13.4 & 961.0 \quad 48.9\end{array}$

$\begin{array}{lll}1156 & 4.829 & 1058\end{array}$

$\begin{array}{lll}(950) & (82.3) \quad 1000\end{array}$

$35.1 \quad 1050.0$

$17.7 \quad 1100.0$

$950 \quad 67.300 \quad 1000$

33.31050 .0

17.81100 .0

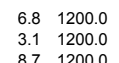

$\begin{array}{ll}4.3 & 1250.0 \\ 2.0 & 1250.0 \\ 5.4 & 1250.0 \\ 4.4 & 1250.0\end{array}$

$\begin{array}{ll}5.4 & 1250.0 \\ 4.4 & 1250.0\end{array}$

$\begin{array}{ll}4.2 & 1250.0 \\ 5.6 & 1250.0\end{array}$

$\begin{array}{ll}5.6 & 1250.0 \\ 5.1 & 1250.0\end{array}$

$\begin{array}{ll}5.5 & 1250.0 \\ 46 & 1250.0\end{array}$

$\begin{array}{ll}4.6 & 1250.0 \\ 6.8 & 1250.0 \\ 3.3 & 1250.0\end{array}$

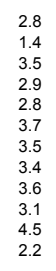

$\begin{array}{ll}7.0 & 1200.0 \\ 9.7 & 1200.0 \\ 9.2 & 1200.0\end{array}$ $\begin{array}{lllllll}0.054 & 0.056 & 0.699 & 0.451 & <0.0055 & 0.352 & 0.148 \\ 0.129 & 0.049 & 1.238 & 0.524 & 0.017 & 0.381 & 0.143\end{array}$ $\begin{array}{lllllll}0.129 & 0.049 & 1.238 & 0.524 & 0.017 & 0.381 & 0.143 \\ 0.448 & 0.201 & 1.056 & 1.492 & 0.328 & 0.22 & 0.126\end{array}$

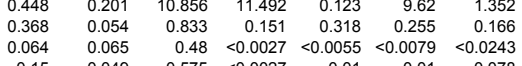

$\begin{array}{rrrrrrr}0.15 & 0.049 & 0.575 & <0.0027 & 0.01 & 0.01 & 0.078 \\ 0.137 & 0.049 & 1.571 & 0.956 & <0.0055 & 0.147 & 0.311\end{array}$

$\begin{array}{ll}0.309 & 0.049 \\ 0.519 & 0.099\end{array}$

$\begin{array}{ccccccc}0.069 & 0.099 & 1.097 & 0.006 & 0.0102 & 0.026 & 0.416 \\ 0.105 & 0.061 & 0.46 & <.00027 & <0.0055 & 0.008 & <0.0243 \\ 0.11 & 1.511 & 0.848 & 0.015 & 0.584 & 0.368\end{array}$

$\begin{array}{lllllll}0.187 & 0.063 & 0.883 & <.80 .027 & 0.015 & 0.584 & 0.268 \\ 0.041 & 0.016 & 0.042 \\ 0 & 0.0212 & 2.95 & 1.102 & 0.032 & 0374 & 0.29\end{array}$

$\begin{array}{llrrrrr}0.148 & 0.063 & 1.164 & 0.562 & 0.028 & 0.134 & 0.203 \\ 0.208 & 0.212 & 2.195 & 1.102 & 0.032 & 0.374 & 0.29 \\ 0.273 & 0.149 & 1132 & 10328 & 0.127 & 7.274 & 1.554\end{array}$

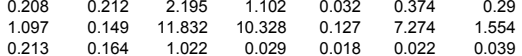

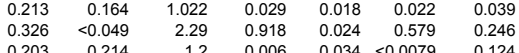

$\begin{array}{llllllll}0.249 & 0.128 & 3.673 & 2.343 & 0.027 & 1.287 & 0.641 \\ 0.254 & 0.049 & 1.69 & 0.101 & 0.019 & 0.039 & 0.124\end{array}$

$\begin{array}{lllllll}0.254 & 0.049 & 1.169 & 0.101 & 0.019 & 0.039 & 0.124 \\ 0.334 & 0.049 & 4.458 & 2.875 & 0.02 & 2.089 & 0.688 \\ 0 & 0.105 & 1.19 & 0.16 & 0.0055 & 0.079 & 0.044\end{array}$

$\begin{array}{lllllll}0.111 & 0.105 & 1.019 & 0.016 & <0.0055 & <.0079 & 0.044 \\ 0.231 & 0.172 & 2.512 & 1.2 & <.0055 & 0.937 & 0.388 \\ 0.081 & 0.238 & 1.373 & 0.337 & 0.036 & 0.27 & 0.115\end{array}$

$\begin{array}{rrrrrrr}0.081 & 0.238 & 1.373 & 0.337 & 0.036 & 0.27 & 0.115 \\ 0.07 & 0.049 & 0.816 & 0.011 & 0.035 & 0.039 & <0.0243\end{array}$

$\begin{array}{llllllll}0.579 & 0.122 & 2.737 & 1.64 & 0.073 & 1.171 & 0.583 \\ 0.233 & 0.055 & 2.559 & 1.508 & 0.0055 & 0.399 & 0.416\end{array}$

$\begin{array}{rrrrrrr}0.233 & 0.055 & 2.659 & 1.508 & <.0055 & 0.399 & 0.416 \\ 0.105 & 0.049 & 0.807 & 0.017 & 0.015 & <0.0079 & <0.0243 \\ 0.201 & 0.049 & 0.767 & <0.0027 & 0.02 & 0.015 & 0.085\end{array}$

$\begin{array}{rrrrrrr}0.201 & <.049 & 0.767 & <.0027 & 0.02 & 0.015 & 0.085 \\ 0.18 & <0.049 & 0.819 & 0.011 & <0.0055 & <0.0079 & 0.066 \\ 0.57 & 0.063 & 6.268 & 4.021 & 0.04 & 3913 & 1.016\end{array}$

$\begin{array}{rrrrrrr}0.527 & 0.063 & 6.268 & 4.021 & 0.04 & 3.913 & 1.016 \\ 0.739 & 0.224 & 1.415 & <0.0027 & 0.081 & 0.019 & 0.233\end{array}$

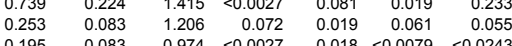

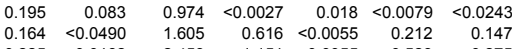
$\begin{array}{rrrrrrr}0.225 & 0.0182 & 2.453 & 1.151 & <.0055 & 0.523 & 0.275 \\ 0.605 & 0.072 & 10.716 & 8.643 & 0.081 & 7.903 & 1.292\end{array}$ $\begin{array}{rrrrrrr}0.267 & <0.0490 & 1.262 & 0.018 & 0.024 & 0.009 & 0.077 \\ 0.795 & 0.186 & 1.627 & 0.02 & 0.072 & 0.022 & 0.227\end{array}$

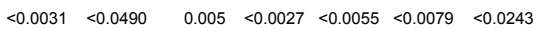


\# Glass ID

1559 HLW99-62

1560 HLW99-63
1561 HLW99-64

1562 HLW99-65

1563 HLW99-66

1566 HLW99-69

1567 HLW99-70

1569 HLW99-72

1570 HLW99-73

1572 HLW9-74

1573 HLW99-7

1574 HLW99-77

1576 HLW9-78

1577 HLW99-80

1578 HLW99-81

(50)

1581 HLW99-84

1582 HLW99-85

1584 HWW99-87

1585 HLW99-88
1586 HLW99-89

1588 HLW99-91

1589 HLW99-92

1590 HLW99-93R
1591 HLW99-94

1592 HLW99-95

593 HLW99-96

1594 HLW99-97
1595 HLW99-98R

596 HLW99-99

1597 HLW99-100

1598 LAWA41

1600 LAWA43-

1600 LAWA43-

1602 LAWA45

1604 LAWA47

1605 LAWA48

16067 LAWA45

1608 LAWA5

1609 LAWA52

1610 LAWA53
1611 LAWA54

1612 LAWA55

1613 LAWA56

1015 LAWA58

1616 LAWA59
1617 LAWA60

1618 LAWA6 1
1619 LAWA62

1620 LAWA63

1621 LAWA64

1623 LAWA66

1624 LAWA67

1625 LAWA68
1626 LAWA69

1627 LAWA70

1628 LAWA71

1629 LAWA72

1631 LAWA74

1632 LAWA75

1633 LAWA76

1635 LAWA78

1636 LAWA79

1638 LAWA8

1639 LAWA82

TCLP Se TCLP Zn TCLPAl TCLPB TCLP Sb TCLP TI TCLPV QPCT B $\left(g / \mathrm{m}^{2}\right) \quad$ QPCT Li $\left(g / \mathrm{m}^{2}\right) \quad$ QPCT Na $\left(g / \mathrm{m}^{2}\right)$ QPCT Si $\left(g / \mathrm{m}^{2}\right) \quad$ QPCT pH

$\begin{array}{lllllll}0.0532 & 0.459 & 0.59 & 4.97 & 0.0373 & 0.0332\end{array}$

$\begin{array}{ll}0.338 & 6.956 \\ 0.27 & 8.745\end{array}$

$\begin{array}{ll}0.175 & 0.614 \\ 0.111 & 1.145\end{array}$

$\begin{array}{ll}0.0532 & 1.01 \\ 0.094 & 2521\end{array}$

$\begin{array}{ll}0.094 & 2.521 \\ 0.124 & 5.859 \\ 0.065 & 0.591\end{array}$

$\begin{array}{ll}0.0532 & 0.871 \\ 0.0532 & 1.622\end{array}$

\begin{tabular}{rr}
0.062 & 1.105 \\
0.057 & 1.606 \\
0.248 & 11.135 \\
\hline
\end{tabular}

$\begin{array}{rr}0.105 & 1.473 \\ <0.0532 & 2.427\end{array}$

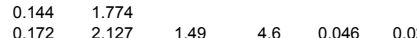

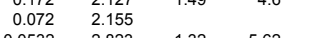

$0.045 \quad 0.0371$

$\begin{array}{ll}0.0532 & 1.097 \\ 0.055 & 2.171 \\ 0.0121 & 0.073\end{array}$

$\begin{array}{rr}0.0532 & 0.0779 \\ 0.121 & 0.73 \\ 0.5522 & 4.27\end{array}$

$\begin{array}{ll}0.0532 & 4.627 \\ 0.0532 & 1.376 \\ 0.0532 & .049\end{array}$

$\begin{array}{ll}0.0532 & 4.376 \\ 0.0532 & 0.649 \\ 0.0532 & 1.714\end{array}$

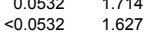

\begin{tabular}{rr}
$<0.0532$ & 4.434 \\
0.081 & 8.326 \\
\hline & 0032
\end{tabular}

$\begin{array}{lll}0.064 & 1.812 \\ 0.075 & 1.151\end{array}$

$\begin{array}{rr}0.081 & 9.125 \\ 0.125 & 2.486 \\ 0.12 & 8.829\end{array}$

$<0.0532 \quad 1.121$

0.470
0.780
0.380
0.370
0.770
0.430
0.380
0.390
0.310
0.310
0.350
0.430
0.402

0.290

0.380
0.705

0.710

0.390
0.340
0.310

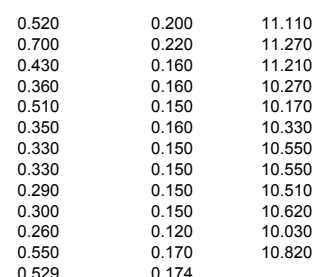




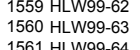

1561 HLW99.64
1562 HLW99-65

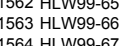

1565 HLW99.68

1566 HLW99.69

1567 HLW99-70
1568 HLW99-71

1569 HLW99-7/2

1570 HLW99-73

1572 H.w99-74

1573 HLW99-7

1574 HLW99-77

1576

1577 HLW99-80

1578 HLW99-81

1581 HLW99-84

1582 HLW

1584 H-W99-87

1585 HLW99-88

1587 HLW99-900

1588 HLW99-91

1589 HLW999-92
1590 HLW99-93R2

${ }_{1593}^{15 L W 99-96}$

15944 LWW99-97
1595 HWW

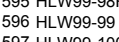

1597 HLW99-100

1598 LAWA4

1600 LAWA43-1

1601 LANA44

1602 LAWA45

1604 LAWA47

1605 LAWA48

1606 LAWA49
1607 LAWA5

1608 LAWA5

1609 LAWA52

1610 LAWA53

1612 LAWA55

1613 LAWA56

1615 LAMA5

1616 LAWA59

1617 LAWA60

1618 LAWA61

1620 LAWA63

1621 LAWA64

1622 LAWA66

1624 LAWA67

1625 LAWA68

1627 LAWA70

1628 LAWA7 1

1022 LAWA72

1631 LAWA74

1632 LAWA75

1633 LAWA76

1635 LAWA78

1636 LAWA79

1638 LANAB

1639 LAAAB
1640 LAWAB3

$\begin{array}{lllllll}0.713 & 1154 & 0.529 & 1052 & 0.369 & 951 & 0.238 \\ 0.71 & 1152 & 0.561 & 1054 & 0.393 & 956 & 0.248 \\ 0.704 & 1153 & 0.537 & 1054 & 0.364 & 957 & 0.254 \\ 0.643 & 1149 & 0.527 & 1050 & 0.366 & 948 & 0.242 \\ & & & & & & \\ & & & & & & \\ 0.683 & 1150 & 0.521 & 1050 & 0.369 & 951 & 0.242 \\ 0.657 & 1151 & 0.514 & 1051 & 0.38 & 953 & 0.248 \\ 0.55 & 1150 & 0.408 & 1049 & 0.29 & 951 & 0.186 \\ 0.568 & 1142 & 0.426 & 1045 & 0.262 & 946 & 0.163 \\ 0.568 & 1142 & 0.426 & 1045 & 0.262 & 946 & 0.163 \\ & & & & & & \\ 0.567 & 1142 & 0.426 & 1044 & 0.285 & 946 & 0.178 \\ & & & & & & \\ 0.543 & 1148 & 0.415 & 1050 & 0.293 & 950 & 0.197\end{array}$

$\begin{array}{lllllll}0.637 & 1138 & 0.538 & 1039 & 0.32 & 942 & 0.211 \\ 0.705 & 1139 & 0.499 & 1044 & 0.358 & 944 & 0.244 \\ 0.613 & 1142 & 0.45 & 1043 & 0.315 & 944 & 0.201\end{array}$ 


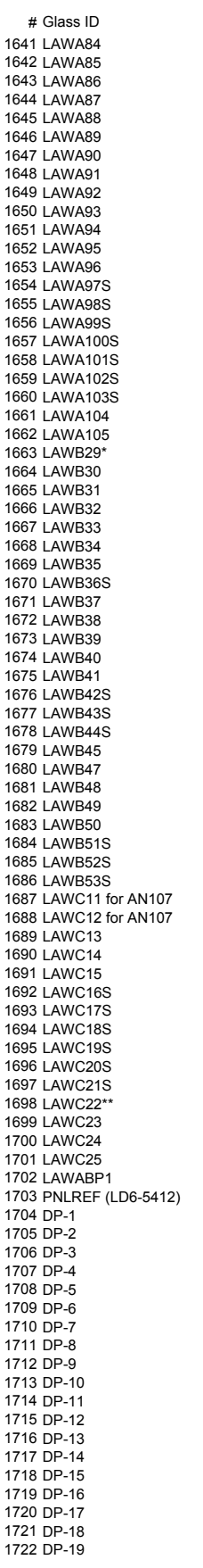

Study

Reference

RPP-WTP LAW Formulation, Muler et al. 2001 RPP-WTP LAW Formulation Muller 2001 RPP-WTP LAW Formulation, Muller et al. 2001 RPP-WTP LAW Formulation, Muller et al. 2001 RPP-WTP LAW Formulation, Muller et al. 2001 RPP-WTP LAW Formulation Muller et al. 2001 2001 RPP-WTP LAW Formulation Muller et al. 2001 RPP-WTP LAW Formulation Muller et al. 2001 RPP-WTP LAW Formulation MUle el. 200 RPP-WTP LAW Formulation Muller et al. 2001 RPP-WTP LAW Formulation Muller et al. 2001 2001 RPP-WTP LAW Formulation Muller et al. 200 RPP-WTP LAW Formulation Muller et al. 2001 2001 RPP-WTP LAW Formulation Muller et al. 2001 RPP-WTP LAW Formulation, Muller et al. 2001 RPP-WTP LAW Formulation, Muller et at al. 2001 RPP-WTP LAW Formulation, Muller et al. 2001 RPP-WTP LAW Formulation, Muller et al. 2001 RPP-WTP LAW Formulation, Mule al. 200 RPP-WTP LAW Formulation, Muller et al. 2001 RPP-WTP LAW Formulation, Muller et al. 2001 RPP-WTP LAW Formulation, Muller et al. 2001 RPP-WTP LAW Formulation, Muller et al. 2001 RPP-WT LAW Formulation Muller et al. 2001 RPP-WTP LAW Formulation Muller et al. 2001 RPP-WTP LAW Formulation Muller et al. 2001 RPP-WTP LAW Formulation Muller et al. 2001 RPP-WTP LAW Formulation Muller et al. 2001 RPP-WTP LAW Formulation Muller et al. 2001 RPP-WTP LAW Formulation Muller et al. 2001 RPP-WTP LAW Formulation Muller et al. 2001 RPP-WTP LAW Formulation Muller et al. 2001 RPP-WTP LAW Fom RPP-WTP LAW Formulation Muller et al. 2001 RPP-WTP LAW Formulation, Muller et al. 2001 RPP-WTP LAW Formulation Muller et al. 2001 RPP-WTP LAW Formulation Muller et al. 2001 RPP-WTP LAW Formulation Muller et al. 2001 RPP-WTP LAW Formulation Muller et al. 2001 RPP-WTP LAW Formulation, Muller et al. 2001 RPP-WTP LAW Formulation Muller et al. 2001 RPP-WTP LAW Formulation Muller et al. 2001 DP Glasses for INEEL HLW Pitman et al. 2001 DP Glasses for INEEL HLW Pittman et al. 2001 DP Glasses for INEEL HLW Pittman et al. 2001 DP Glasses for INEEL HLW Pittman et al. 2001 DP Glasses for INEEL HLW Pittman et al. 2001 2001 $\begin{array}{ll}\text { DP Glasses for INEEL HLW } & \text { Pittman et al. } 2001 \\ \text { DP Glasses for INEEL HLW Pittman et al. } 200\end{array}$ DP Glasses for INEEL HLW Pittman et al. 2001 DP Glasses for INEEL HLW Pittman et al. 2000 DP Glasses for INEEL HLW Pittman et al. 2001 DP Glasses for INEEL HIW Pittman etal. 201 DP Glasses for INEEL HLW Pittman et al. 200
Al2O3-t B2O3-t CaO-t Fe2O3-t FeO-t $\begin{array}{llll}0.0620 & 0.0890 & 0.0199 & 0.0299 \\ 0.0620 & 0.0890 & & 0.0499\end{array}$

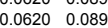
$\begin{array}{llll}0.0448 & 0.0887 & 0.0199 & 0.0697 \\ 0.0608 & 0.0970 & 0.0199 & 0.0553\end{array}$ $\begin{array}{llll}0.0608 & 0.0970 & 0.0553\end{array}$

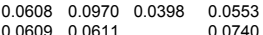
$\begin{array}{lll}0.0609 & 0.0611 & 0.0 \\ 0.0609 & 0.0611\end{array}$

$\begin{array}{llll}0.0618 & 0.1110 & 0.0788 & 0.0770 \\ 0.0751\end{array}$ $\begin{array}{llll}0.0609 & 0.0611 & 0.0632 & 0.0740 \\ 0.0609 & 0.0611 & 0.0559 & 0.0370\end{array}$ $\begin{array}{lllll}0.0620 & 0.0791 & 0.0399 & 0.0299\end{array}$ $\begin{array}{lllll}0.0603 & 0.1074 & 0.0508 & 0.0750\end{array}$ $\begin{array}{llll}0.0603 & 0.1074 & 0.0613 & 0.0452 \\ 0.0603 & 0.1074 & 0.0613 & 0.075\end{array}$ $\begin{array}{lllll}0.0603 & 0.1074 & 0.0613 & 0.0750 \\ 0.0603 & 0.1074 & 0.0710 & 0.0750\end{array}$ $\begin{array}{llll}0.0606 & 0.1000 & 0.0507 & 0.0541\end{array}$ $\begin{array}{llll}0.0606 & 0.1000 & 0.0507 & 0.0500\end{array}$ $\begin{array}{llll}0.0703 & 0.0828 & 0.0185 & 0.06649 \\ 0.0030 & 0.0607 & 0.0619\end{array}$ $\begin{array}{llll}0.0803 & 0.0807 & 0.0701 & 0.0802 \\ 0.0860 & 0.1004 & 0.0723 & 0.0827\end{array}$ $\begin{array}{llll}0.0616 & 0.1209 & 0.0403 & 0.0717\end{array}$ $\begin{array}{lllll}0.0616 & 0.1209 & 0.0403 & 0.0515\end{array}$ $\begin{array}{llll}0.0616 & 0.1209 & 0.0605 & 0.0515\end{array}$ $\begin{array}{llll}0.0616 & 0.1209 & 0.0403 & 0.0515\end{array}$ $\begin{array}{llll}0.0616 & 0.1209 & 0.0470 & 0.0515\end{array}$ $\begin{array}{llll}0.0616 & 0.1209 & 0.0475 & 0.0515 \\ 0.0616 & 0.1209 & 00470 & 0.0515\end{array}$

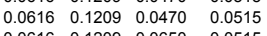
$\begin{array}{llll}0.0616 & 0.1209 & 0.0650 & 0.0515 \\ 0.0607 & 0.1192 & 0.0463 & 0.0605\end{array}$ $\begin{array}{lll}0.1192 & 0.0463 & 0.1033 \\ 0.1192 & 0.0463 & 0.1320\end{array}$ $\begin{array}{lllll}0.0613 & 0.1234 & 0.0663 & 0.0526\end{array}$ $\begin{array}{llll}0.0613 & 0.1234 & 0.0601 & 0.0526 \\ 0.0613 & 0.1234 & 0.0540 & 0.0526\end{array}$ $\begin{array}{llll}0.0613 & 0.1234 & 0.0480 & 0.0526 \\ 0616 & 0.1209 & 0.0649 & 0.0515\end{array}$ $\begin{array}{llll}0.0616 & 0.1209 & 0.0649 & 0.0515 \\ 0.0609 & 0.1254 & 0.0674 & 0.0534\end{array}$ $\begin{array}{lll}0.0674 & 0.0534\end{array}$ $\begin{array}{llll}0.1197 & 0.0862 & 0.0160 & 0.0571\end{array}$ $\begin{array}{llll}0.1197 & 0.0913 & 0.0159 & 0.0571\end{array}$ $\begin{array}{llll}0.0612 & 0.0616 & 0.0550 & 0.0347 \\ 0.0623 & 0.0905 & 0501 & 0.0702\end{array}$ $\begin{array}{llll}0.0623 & 0.0895 & 0.0201 & 0.0772 \\ 0.0613 & 0.1010 & 0.0637 & 0.0752 \\ 0.0613 & 0.1010 & 0.0736 & 0.045\end{array}$ $\begin{array}{lllll}0.0613 & 0.1010 & 0.0736 & 0.0752\end{array}$ $\begin{array}{lllll}0.06613 & 0.1010 & 0.0836 & 0.0752\end{array}$

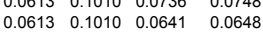
$\begin{array}{llll}0.0607 & 0.1005 & 0.0511 & 0.0542 \\ 0.0612 & 0.1008 & 0.0640 & 0.0647\end{array}$

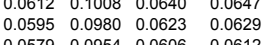
$\begin{array}{llll}0.1000 & 0.0925 & 0.000 & 0.0250 \\ 0.1200 & 0.0500 & 0.0400 & 0.0000\end{array}$ $\begin{array}{llll}0.1200 & 0.0500 & 0.0400 & 0.0000 \\ 0.1250 & 0.0600 & 0.1500 & 0.0000 \\ 0.1250 & 0.0600 & 0.050 & 0.050\end{array}$ $\begin{array}{llll}0.1250 & 0.0600 & 0.1550 & 0.0000 \\ 0.1250 & 0.0600 & 0.1500 & 0.0100\end{array}$ $\begin{array}{llll}0.1250 & 0.0600 & 0.0950 & 0.0500 \\ 0.1250 & 0.0600 & 0.0950 & 0.0000\end{array}$ $\begin{array}{llll}0.1250 & 0.0600 & 0.0950 & 0.0500 \\ 0.1250 & 0.0600 & 0.050 & 0.000\end{array}$ $\begin{array}{llll}0.1250 & 0.1350 & 0.0950 & 0.0000\end{array}$ $\begin{array}{llll}0.0700 & 0.1500 & 0.0950 & 0.0000\end{array}$ $\begin{array}{llll}0.0700 & 0.1500 & 0.1000 & 0.0000 \\ 0.0000 & 0.15000 & 0.1100 & 0.0550\end{array}$ $\begin{array}{llll}0.0700 & 0.1500 & 0.1000 & 0.0500 \\ 0.0700 & 0.0600 & 0.1500 & 0.0500\end{array}$ $\begin{array}{llll}0.0700 & 0.0600 & 0.1500 & 0.0000 \\ 0.0700 & 0.0600 & 0.1500 & 0.0500 \\ 0.0700 & 0.060 & 0.1500 & 0.000\end{array}$

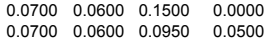
$\begin{array}{llll}0.0700 & 0.0600 & 0.0950 & 0.0500 \\ 0.0700 & 0.0600 & 0.0950 & 0.0500\end{array}$

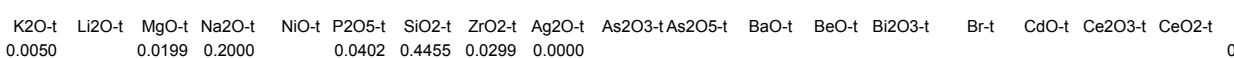
0.0050

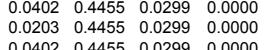

$\begin{array}{lll}0.0007 & 0.4446 & 0.0299 \\ 0.0007 & 0.4399 & 0.0299\end{array}$ $\begin{array}{lll}0.0007 & 0.4399 & 0.0299\end{array}$ $\begin{array}{lll}0.0007 & 0.4399 & 0.0299 \\ 0.0003 & 0.4166 & 0.029\end{array}$ $\begin{array}{llll}0.0003 & 0.4166 & 0.0295 & \\ 0.0003 & 0.4225 & 0.0299 & 0.0000\end{array}$ $\begin{array}{llll}0.0003 & 0.4166 & 0.0441 & 0.0000 \\ 0.0295 & 0.4166 & 0.0295 & 0.000\end{array}$ $\begin{array}{llll}0.0295 & 0.4166 & 0.0295 & \\ 0.0402 & 0.4356 & 0.0299 & 0.0000\end{array}$

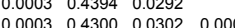
$\begin{array}{llll}0.0003 & 0.4300 & 0.0302 & 0.0000\end{array}$ $\begin{array}{llllllll}0.0003 & 0.4300 & 0.0302 & 0.0000\end{array}$ $\begin{array}{lll}0.0013 & 0.4660 & 0.0302 \\ 0.0013 & 0.4551 & 0.0302\end{array}$ $\begin{array}{llll}0.0013 & 0.4551 & 0.0302 & \\ 0.0004 & 0.4299 & 0.0289 & 0.0000\end{array}$ $\begin{array}{llll}0.0026 & 0.0250 & 0.0150 & 0\end{array}$ $\begin{array}{llll}0.0026 & 0.0250 & 0.0150 & 0.1449\end{array}$ $\begin{array}{llll}0.0038 & 0.0408 & 0.0298 & 0.1000\end{array}$ $\begin{array}{lllll}0.0032 & 0.0407 & 0.0307 & 0.0790\end{array}$ $\begin{array}{llll}0.0032 & 0.0296 & 0.0224 & 0.0790 \\ 0.0032 & 0.0296 & 0.0224 & 0.0790\end{array}$ $\begin{array}{llll}0.0032 & 0.0296 & 0.0224 & 0.0790 \\ 0.0032 & 0.0296 & 0.0224 & 0.0790\end{array}$ $\begin{array}{llll}0.0032 & 0.0296 & 0.0224 & 0.0790 \\ 0.0032 & 0.0296 & 0.0426 & 0.0790\end{array}$ $\begin{array}{llll}0.0032 & 0.0385 & 0.0224 & 0.0790\end{array}$ $\begin{array}{llll}0.0032 & 0.0296 & 0.0291 & 0.0790 \\ 0.0032 & 0.0381 & 0.0224 & 0.0790\end{array}$ $\begin{array}{llll}0.0032 & 0.0296 & 0.0291 & 0.0790\end{array}$ $\begin{array}{llll}0.0032 & 0.0632 & 0.0291 & 0.0790 \\ 0.0032 & 0.0452 & 0.0291 & 0.0790\end{array}$ $\begin{array}{lll}0.0031 & 0.0287 & 0.0779 \\ 0.0031 & 0.0287 & 0.0779\end{array}$ $\begin{array}{lrl}0.0031 & 0.0287 & 0.079 \\ 0.0779\end{array}$ $\begin{array}{llll}0.0026 & 0.0462 & 0.0297 & 0.0650 \\ 0.0026 & 0.0409 & 0.0297 & 0.0650 \\ 0.026 & 0.0302 & 0.0257 & 0.050\end{array}$ $\begin{array}{llll}0.0026 & 0.0409 & 0.0297 & 0.0650 \\ 0.0026 & 0.0356 & 0.0297 & 0.0650\end{array}$ $\begin{array}{llll}0.0026 & 0.0302 & 0.0297 & 0.0650 \\ 0.032 & 0.0452 & 0.029 & 0.0790\end{array}$ $\begin{array}{llll}0.0032 & 0.0452 & 0.0291 & 0.0790 \\ 0.0023 & 0.0473 & 0.0300 & 0.0500\end{array}$ $\begin{array}{llll}0.0023 & 0.0585 & 0.0300 & 0.0500 \\ 0.0023 & 0.0585 & 0.0300 & 0.0500\end{array}$

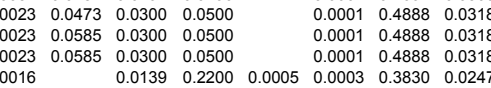
$\begin{array}{lllllllll}0.0014 & 0.0139 & 0.22000 & 0.0005 & 0.00003 & 0.3830 & 0.02247 & 0.00000 \\ 0.00003 & 0.3933 & 0.0246 & 0.0000\end{array}$ $\begin{array}{lllllllll}0.0025 & 0.0154 & 0.2000 & 0.0003 & 0.0019 & 0.4234 & 0.0308\end{array}$

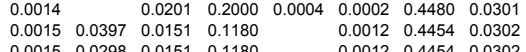
$\begin{array}{lllllllll}0.0015 & 0.0298 & 0.0151 & 0.1180 & & 0.0012 & 0.4454 & 0.0302 \\ 0.0015 & 0.0199 & 0.0151 & 0.1180 & 0.0012 & 0.4454 & 0.0302\end{array}$ $\begin{array}{lllllllll}0.0015 & 0.0199 & 0.0151 & 0.1180 & & 0.0012 & 0.4454 & 0.0302 \\ 0.0015 & 0.0151 & 0.1180 & & 0.0012 & 0.4454 & 0.0302\end{array}$ $\begin{array}{lllllllll}0.0015 & 0.0274 & 0.0151 & 0.1180 & 0.1158 & 0.0012 & 0.4445 & 0.0302 \\ 0.0018 & 0.0012 & 0.4677 & 0.0302\end{array}$ $\begin{array}{llllllll}0.0008 & 0.0274 & 0.0151 & 0.1188 & & 0.0012 & 0.4454 & 0.032 \\ 0.0288 & 0.0151 & 0.1440 & 0.0003 & 0.0007 & 0.4667 & 0.0302 \\ 0.1186 & 0.00303 & 0.0012 & 0.4677 & 0.0302\end{array}$

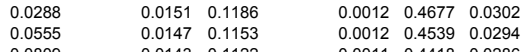
$\begin{array}{llllllll}0.0220 & 0.0100 & 0.2000 & 0.0008 & 0.4189 & 0.0525\end{array}$ $\begin{array}{llllllll}0.0146 & & 0.0000 & 0.2000 & & 0.0019 & 0.5591 & \\ & 0.0700 & & 0.0800 & & 0.0000 & 0.3650 & 0.0800\end{array}$ $\begin{array}{lll}0.0000 & 0.3650 & 0.0800 \\ 0.0000 & 0.3600 & 0.0400 \\ 0.0000 & 0.3750 & 0.0400\end{array}$ $\begin{array}{lll}0.0000 & 0.3750 & 0.0400 \\ 0.0250 & 0.3600 & 0.0800\end{array}$ $\begin{array}{llll}0.0250 & 0.3600 & 0.080 \\ 0.0250 & 0.3600 & 0.0400\end{array}$ $\begin{array}{lll}0.0000 & 0.3600 & 0.0400 \\ 0.0250 & 0.3600 & 0.0800\end{array}$ $\begin{array}{lll}0.0250 & 0.3650 & 0.0400 \\ 0.0000 & 0.3600 & 0.0400\end{array}$ $\begin{array}{llll}0.0000 & 0.3600 & 0.0400 \\ 0.0250 & 03000 & 0.0400\end{array}$ $\begin{array}{lll}0.0250 & 0.3600 & 0.0800 \\ 0.0250 & 0.3600 & 0.0400\end{array}$ $\begin{array}{lll}0.0000 & 0.4500 & 0.0400 \\ 0.0000 & 0.450 & 0.000\end{array}$ $\begin{array}{lll}0.0000 & 0.4650 & 0.0400 \\ 0.0250 & 0.4150 & 0.0400 \\ 0.0000 & 0.3550 & 0.080\end{array}$ $\begin{array}{lll}0.0000 & 0.3650 & 0.0800 \\ 0.0000 & 0.3750 & 0.0800\end{array}$ 


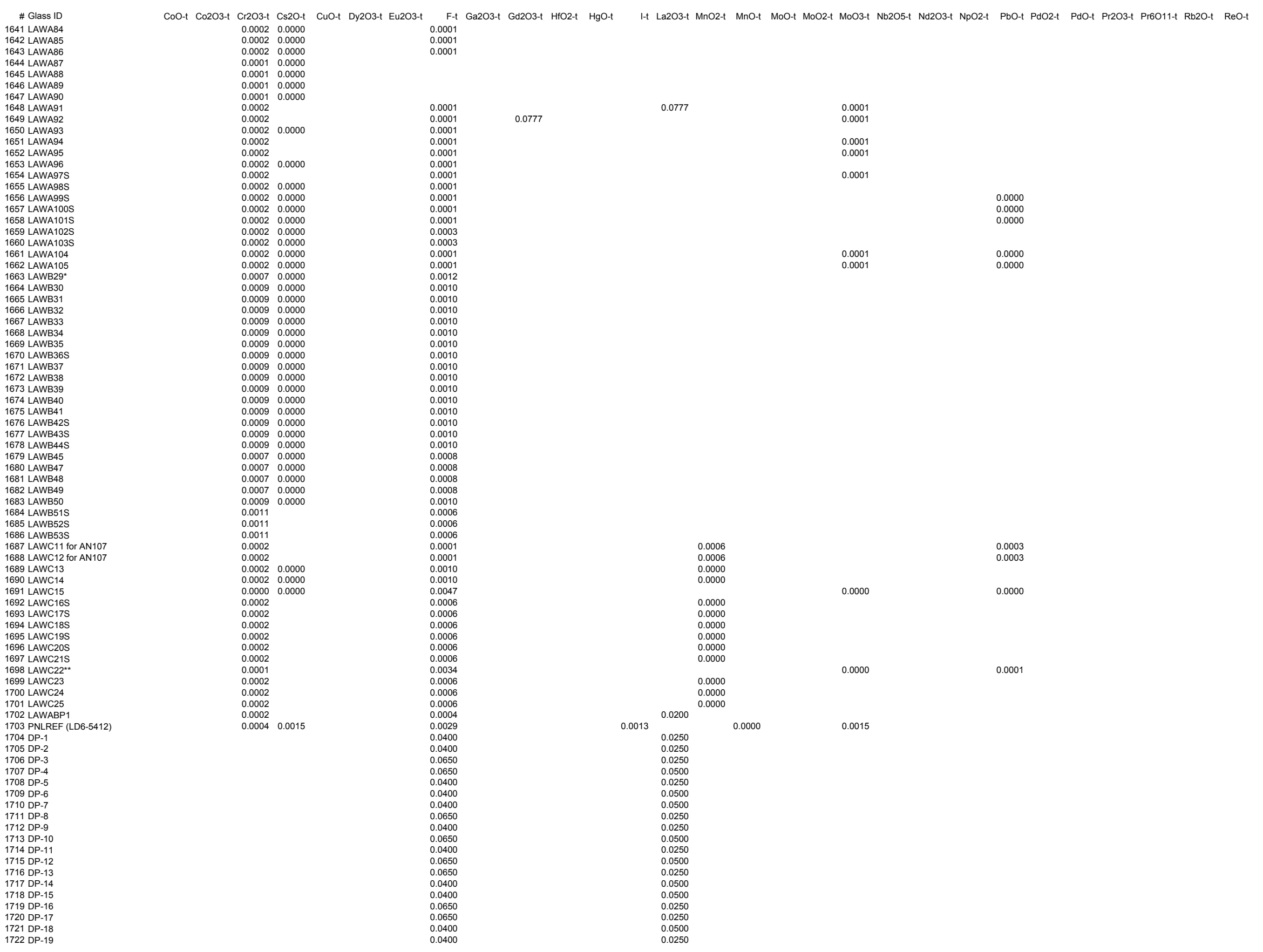




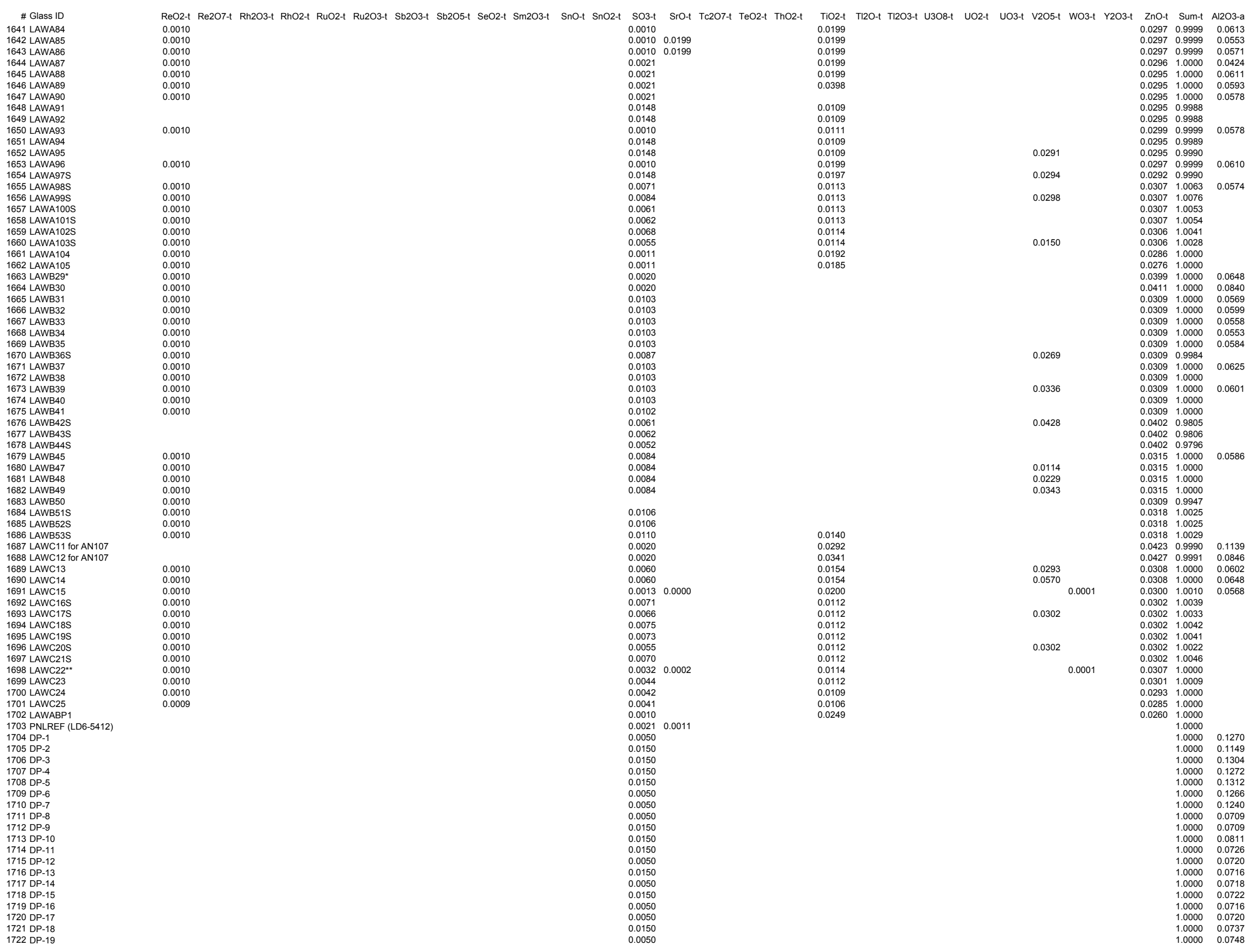


\# Glass ID

1641 LAWA84

1642 LAWAB
1643 LAAAB

1644 LAWA8

1645 LAWA88

1647 LAWA99
1648 LAWA99

1649 LAWA92

1651 LAWA94

1652 LAWA95

1653 LAWA96

1655 LAWA998
1656 LAWA99S

1656 LAWA99S
1657 LAWA100S

1659 LAWA102S

1663 LAWB29

1664 LAWB30

1666 LAWB32

1667 LAWB33

1668 LAWB34
1669 LAWB35

1670 LAWB36S

1671 LAWB37

1672 LAWB38

1674 LAWB40

1675 LAWB41

1676 LAWB42S
1677 LAWB43S

1678 LAWB445

1680 LAWB47
1681 LAWB48

1682 LAWB49
1683 LAWB50
1684 LAWB51S

1684 LAWB51S
1685 LAWB52S

1687 LAWC11 for AN107

1687 LAWC11 for AN107
1688 LAWC12 for AN107
1689 LWWC13

1690 LAWC14

1691 LAWC15

1692 LAWC16S
1693 LAWC17S

1694 LAWC18S

1695 LAWC19S

1697 LAWC21S

1698 LAWC22

1700 LAWC24

1701 LAWC2

1703 PNLREF (LD6-5412)
(1)

$1705 \mathrm{DP}-2$

1706 DP-3

1707 DP-4

1709 DP-

1710 DP-7

1711 DP-8

1713 DP-10

1714 DP-11

(1715 DP-12

1717 DP-14

1718 DP-15

1719 DP-16

1721 DP-18
B203-a CaO-a Fe2O3-a FeO-a K2O-a Li2O-a MgO-a Na2O-a NiO-a P2O5-a SiO2-a Zro2-a Ag2O-a As203-a As205-a

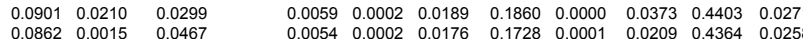
$0.08620 .0008 \quad 0.0286$ $0.0870 \quad 0.0203 \quad 0.0653$ $\begin{array}{lll}0.09660 & 0.0205 & 0.0546 \\ 0.0940 & 0.0007 & 0.0487 \\ 0.0948 & 0.0380 & 0.0516\end{array}$

$0.1087 \quad 0.0757 \quad 0.0710$

$0.0773 \quad 0.0394 \quad 0.0288$

$0.1080 \quad 0.0482 \quad 0.0790$

$\begin{array}{lllllllll}0.0225 & 0.0003 & 0.0180 & 0.1840 & 0.0001 & 0.0022 & 0.4385 & 0.0253\end{array}$

$\begin{array}{llllllll}0.0235 & 0.0004 & 0.0143 & 0.1735 & 0.0002 & 0.0026 & 0.4164 & 0.0275 \\ 0.0230 & 0.0004 & 0.0150 & 0.1691 & 0.0001 & 0.0016 & 0.4217 & 0.026\end{array}$

$\begin{array}{llllllll}0.0061 & 0.0465 & 0.0153 & 0.0864 & 0.0001 & 0.0017 & 0.4022 & 0.0278\end{array}$

$\begin{array}{llllllll}0.0055 & 0.0006 & 0.0182 & 0.1921 & 0.0001 & 0.0403 & 0.4038 & 0.028\end{array}$

$\begin{array}{llllllll}0.0039 & 0.0282 & 0.0157 & 0.1094 & 0.0013 & 0.0023 & 0.4194 & 0.0298\end{array}$

$\begin{array}{lll}0.0778 & 0.0700 & 0.0813 \\ 0.0986 & 0.0700 & 0.0793\end{array}$

$0.1500 \quad 0.0395 \quad 0.0399$

$\begin{array}{lll}0.1174 & 0.0371 & 0.0469 \\ 0.1143 & 0.0550 & 0.0476\end{array}$

$\begin{array}{lll}0.1145 & 0.0451 \quad 0.0470\end{array}$

$0.1163 \quad 0.0446 \quad 0.0487$

$0.1296 \quad 0.0620 \quad 0.0500$

$\begin{array}{llll}0.0023 & 0.0426 & 0.0263 & 0.0541\end{array}$

$\begin{array}{lll}0.0882 & 0.0185 & 0.0525 \\ 0.0972 & 0.0192 & 0.0546\end{array}$

$\begin{array}{lll}0.0553 & 0.0530 & 0.0334 \\ 0.0917 & 0.0201 & 0.0725\end{array}$

0.002

$\begin{array}{llll}0.050 & 0.0391 & 0.0275 & 0.0869 \\ 0.0033 & 0.0283 & 0.0750\end{array}$

$\begin{array}{lll}0.0021 & 0.4540 & 0.0276 \\ 0.0019 & 0.4092 & 0.0309\end{array}$

$0.0270 \quad 0.4322 \quad 0.0275$

$\begin{array}{lll}0.0438 & 0.4383 & 0.0246 \\ 0.269 & 0.4552 & 0.0247\end{array}$

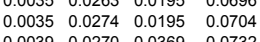

$\begin{array}{llll}0.0035 & 0.0285 & 0.0253 & 0.0764\end{array}$

$0.0340 \quad 0.4728 \quad 0.0265$

$0.0014 \quad 0.4670 \quad 0.0276$

Cr2O3-a Cs2O-a CuO-a F-a

$\begin{array}{lll}0.0583 & 0.1440 & 0.0002 \\ 0.0544 & 0.1345 & 0.0176\end{array}$

$\begin{array}{llll}0.0602 & 0.1015 & 0.0523\end{array}$

$\begin{array}{lll}0.0576 & 0.0970 & 0.0001 \\ 0.0596 & 0.1000 & 0.0513\end{array}$

$\begin{array}{lll}0.0556 & 0.0990 & 0.0001 \\ 0.1301 & 0.0966 & 0.0005 \\ 0.142 & 0.000\end{array}$

$0.1462 \quad 0.0980 \quad 0.0003$

$\begin{array}{lll}0.1465 & 0.0980 & 0.0000 \\ 0.1658 & 0.1078 & 0.0003 \\ 0.1517 & 0.046 & 0.0009\end{array}$

$\begin{array}{lll}0.1517 & 0.1046 & 0.0509 \\ 0.0663 & 0.1442 & 0.0499 \\ 0.059 & 0.142 & 0.051\end{array}$

$\begin{array}{llll}0.0663 & 0.1442 & 0.049 \\ 0.0605 & 0.1442 & 0.0001 \\ 0.052 & 0.156 & 0.051\end{array}$

$\begin{array}{lll}0.0592 & 0.1456 & 0.0513 \\ 0.0583 & 0.1442 & 0.0000 \\ 0.0589 & 0.098 & 0.051\end{array}$

$\begin{array}{lll}0.0583 & 0.1442 & 0.0000 \\ 0.0583 & 0.0998 & 0.0511\end{array}$

$\begin{array}{lll}0.0592 & 0.1009 & 0.0004 \\ 0.0615 & 0.0998 & 0.0521 \\ 0.0586 & 0.1019 & 0.0518\end{array}$ \begin{tabular}{llllllll}
0.0133 & 0.1963 & 0.0005 & 0.00192 & 0.3756 & 0.0196 & 0.0000 \\
\hline & 0.0028 & 0.3855 & 0.0219 & 0.0001
\end{tabular}

$\begin{array}{llllllll}0.0026 & 0.0005 & 0.0160 & 0.1814 & 0.0012 & 0.0021 & 0.4032 & 0.0289 \\ 0.0018 & 0.0004 & 0.0187 & 0.1789 & 0.0012 & 0.0024 & 0.4382 & 0.0281\end{array}$ $\begin{array}{llllll}0.1679 & 0.0006 & 0.0297 & 0.4124 & 0.0303 \\ 0.174 & 0.0289\end{array}$

\subsection{0}

0.0002
0.0000

0.0000

0.0000

0.0000

0.0001

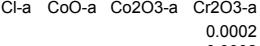

0.0002
0.0002
0.0002
0.0002
0.0002

0.0003

0.0002

0.0027

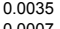

0.0010
0.0009
0.0008

0.0008

0.0008

0.0005

$\begin{array}{ll}0.0000 & 0.0002 \\ 0.0000 & 0.0001\end{array}$

0.0001

0.0003

0.0004
0.0002
0.0002
0.0010
0.0018 


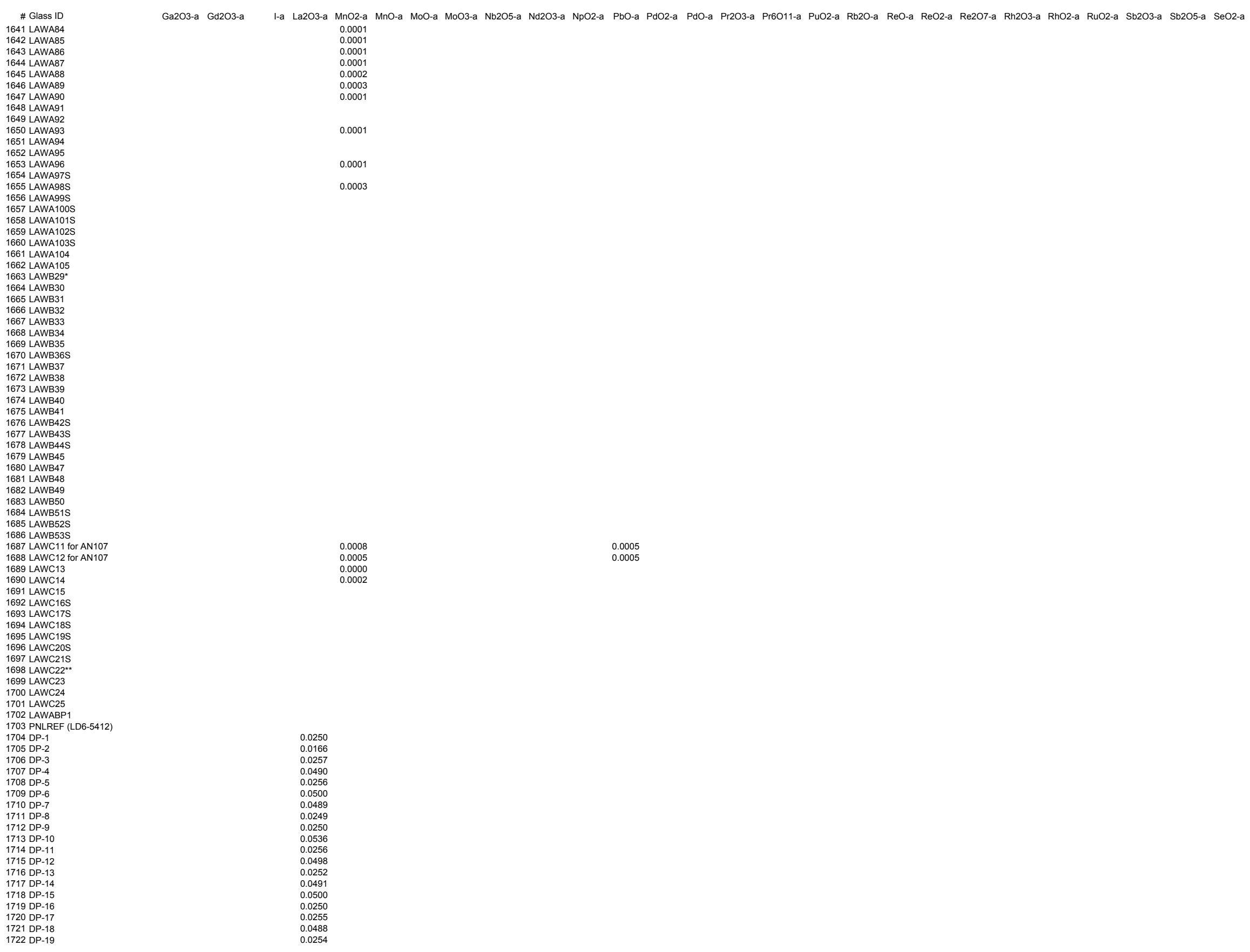




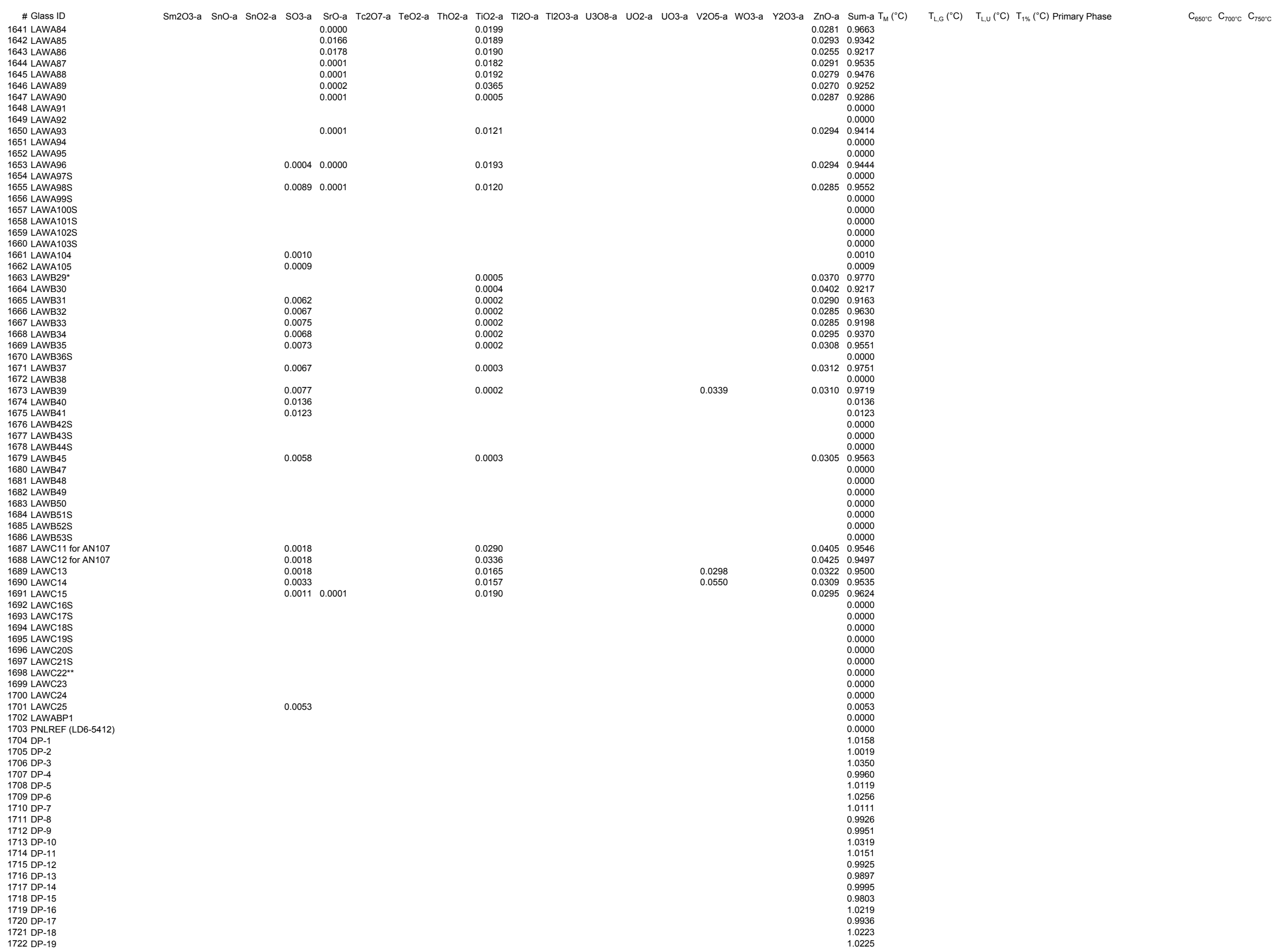




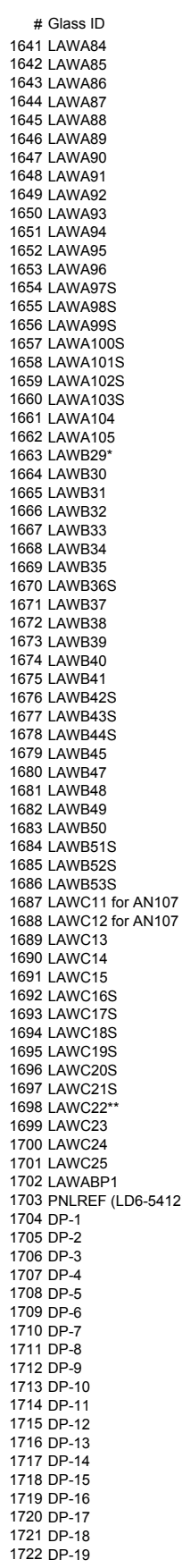

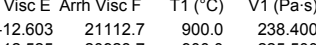

2.670

2.670

2.610

2.630
2.640

2.610

2.650

2.690
2.690
2.670
2.680

2.660

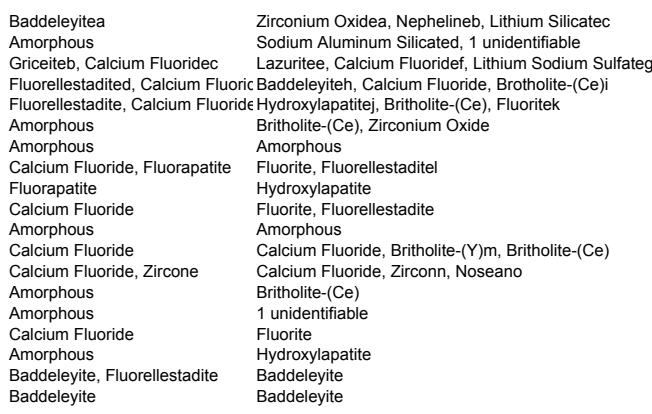

$\begin{array}{rrrr}-11.869 & 19176.9 & 900.0 & 94.500 \\ -11.891 & 19487.1 & 900.0 & 119.900\end{array}$ $\begin{array}{rrrr}-12.550 & 20577.0 & 9000.0 & 163.900 \\ -12.201 & 20053.1 & 900.0 & 143.900\end{array}$

$-11.341 \quad 168311-9000-21.000$

$\begin{array}{llll}-12.791 & 21146.3 & 900.0 & 210.400\end{array}$ $\begin{array}{llll}-10.909 & 17329.9 & 900.0 & 51.000\end{array}$ $\begin{array}{llll}-12.393 & 19647.8 & 900.0 & 84.100 \\ -12.265 & 19509.2 & 900.0 & 83.100 \\ -1.263 & 229.2 & 90.0 & 17.000\end{array}$ $\begin{array}{llll}-12.888 & 20915.2 & 900.0 & 151.800 \\ -12.037 & 19589.0 & 900.0 & 117.000\end{array}$ $\begin{array}{rrrr}-12.240 & 19811.5 & 900.0 & 112.300 \\ -11.682 & 18560.1 & 9000 & 67.300\end{array}$ $\begin{array}{llll}-12.879 & 19959.4 & 900.0 & 71.500\end{array}$

$\begin{array}{llll}-13.262 & 21953.9 & 900.0 & 253.300\end{array}$ $\begin{array}{llll}-12.737 & 21299.0 & 900.0 & 243.400 \\ -12.102 & 19910.6 & 900.0 & 139.800 \\ -12495 & 20494.0 & 900.0 & 156.100\end{array}$ $\begin{array}{llll}-11.338 & 17384.8 & 900.0 & 36.600 \\ -12255 & 1929.7 & 9000 & 240200\end{array}$ $\begin{array}{rrrr}-12.255 & 19259.7 & 900.0 & 69.200 \\ -15.794 & 27581.7 & 900.0 & 2490.200\end{array}$

$\begin{array}{lllr}-12.549 & 19966.7 & 900.0 & 96.400 \\ -12.234 & 20101.0 & 900.0 & 145.100\end{array}$ $\begin{array}{llll}-12.234 & 20101.0 & 9000.0 & 145.100 \\ -13.247 & 21931.3 & 900.0 & 260.400 \\ -12.819 & 21371.8 & 900.0 & 239.000\end{array}$ \begin{tabular}{lllll}
-13.034 & 21298.8 & 900.0 & 191.500 \\
\hline-1114 & 19508 & 9000 & 102900
\end{tabular} $\begin{array}{llll}-12.114 & 1950.8 & 900.0 & 102.900 \\ -12.528 & 20045.7 & 900.0 & 102.800 \\ -12483 & 203165 & 9000 & 135000\end{array}$ $\begin{array}{llll}-12.483 & 20316.5 & 900.0 & 135.500 \\ -12.560 & 20343.6 & 900.0 & 131.900\end{array}$ $\begin{array}{llll}-13.823 & 22785.2 & 900.0 & 321.200\end{array}$ $\begin{array}{llll}-13.517 & 21544.8 & 900.0 & 143.200 \\ -12432 & 20561.6 & 900.0 & 176.100\end{array}$ $\begin{array}{lllll}-11.926 & 18581.6 & 9000 & 53.700\end{array}$ $\begin{array}{rrrr}-12.437 & 19884.8 & 900.0 & 98.400 \\ -12.822 & 20494.0 & 900.0 & 111.100\end{array}$ $\begin{array}{llll}-12.398 & 20130.8 & 900.0 & 129.300\end{array}$

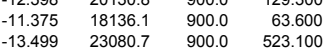
$\begin{array}{llll}-13.494 & 22857.8 & 900.0 & 434.600 \\ -13.437 & 22364.5 & 900.0 & 301.200 \\ -14.107 & 23557.5 & 9000 & 554.300\end{array}$ 


\begin{tabular}{|c|c|c|c|c|c|c|c|c|c|c|c|c|c|c|}
\hline \# Glass ID & $\mathrm{T} 2\left({ }^{\circ} \mathrm{C}\right)$ & V2 (Pa-s) & T3 $\left({ }^{\circ} \mathrm{C}\right)$ & V3 (Pa:s) & $\mathrm{T} 4\left({ }^{\circ} \mathrm{C}\right)$ & V4 (Pa:s) & T5 $\left({ }^{\circ} \mathrm{C}\right)$ & V5 (Pa:s) & T6 $\left({ }^{\circ} \mathrm{C}\right)$ & V6 (Pa:s) & $\mathrm{T} 7\left({ }^{\circ} \mathrm{C}\right)$ & V7 (Pa-s) & $\mathrm{T} 8\left({ }^{\circ} \mathrm{C}\right)$ & V8 (Pa:s) \\
\hline 1641 LAWA84 & 950 & 105.500 & 1000 & 51.4 & 1050.0 & 27.1 & 1100.0 & 15.3 & 1150.0 & 9.2 & 1200.0 & 5.7 & 1250.0 & 3.8 \\
\hline 1642 LAWA85 & & 97.500 & & 47.1 & 1050.0 & 24.9 & 1100.0 & 14.2 & 1150.0 & 8.6 & 1200.0 & 5.5 & 1250.0 & 3.7 \\
\hline $\begin{array}{l}\text { 16434 LAWA868 } \\
\text { 1644 LAWA }\end{array}$ & 950 & 45.100 & 1000 & 23.5 & 1050.0 & 13.2 & 1100.0 & 7.8 & 1150.0 & 4.9 & 1200.0 & 3.2 & 1250.0 & 2.2 \\
\hline 1645 LAWA88 & 950 & 56.900 & 1000 & 29.4 & 1050.0 & 16.3 & 1100.0 & 9.6 & 1150.0 & 6.0 & 1200.0 & 3.9 & 1250.0 & 2.6 \\
\hline 1646 LAWA89 & 950 & 71.200 & 1000 & 34.7 & 1050.0 & 18.5 & 1100.0 & 10.7 & 1150.0 & 6.5 & 1200.0 & 4.2 & 1250.0 & 2.9 \\
\hline $\begin{array}{l}\text { 1667 LAWA90 } \\
\text { 1648 LAWA91 }\end{array}$ & 950 & 66.300 & 1000 & 33.4 & 1050.0 & 18.2 & 1100.0 & 10.6 & 1150.0 & 6.5 & 1200.0 & 4.2 & 1250.0 & 2.8 \\
\hline 1649 LAWA92 & & & & & & & & & & & & & & \\
\hline 1650 LAWA93 & 950 & 11.300 & 1000 & 6.3 & 1050.0 & 3.8 & 1100.0 & 2.4 & 1150.0 & 1.6 & 1200.0 & 1.1 & 1250.0 & 0.8 \\
\hline $\begin{array}{l}\text { 1651 LAWA94 } \\
1652 \text { LAWA95 }\end{array}$ & & & & & & & & & & & & & & \\
\hline 1653 LAWA96 & 950 & 89.500 & 1000 & 42.8 & 1050.0 & 22.5 & 1100.0 & 12.8 & 1150.0 & 7.7 & 1200.0 & 4.9 & 1250.0 & 3.3 \\
\hline 1654 LAWA97S & 950 & 86.100 & 1000 & 41.2 & 1050.0 & 21.7 & 1100.0 & 12.4 & 1150.0 & 7.6 & 1200.0 & 4.9 & 1250.0 & 3.3 \\
\hline 1655 LAWA98S & 950 & 26.100 & & 14.4 & 1050.0 & 8.5 & 1100.0 & 5.3 & 1150.0 & 3.5 & 1200.0 & 2.4 & 1250.0 & 1.7 \\
\hline 1656 LAWA99S & 950 & 39.300 & 1000 & 20.1 & 1050.0 & 11.1 & 1100.0 & 6.5 & 1150.0 & 4.0 & 1200.0 & 2.6 & 1250.0 & 1.8 \\
\hline 1657 LAWA100S & $\begin{array}{l}950 \\
950\end{array}$ & 39.800 & $\begin{array}{l}1000 \\
1000\end{array}$ & $\begin{array}{l}20.7 \\
33.1\end{array}$ & $\begin{array}{l}1050.0 \\
10500\end{array}$ & $\begin{array}{l}11.5 \\
17.5\end{array}$ & $\begin{array}{l}1100.0 \\
1100.0\end{array}$ & $\begin{array}{l}6.8 \\
9.9\end{array}$ & $\begin{array}{l}1150.0 \\
11500\end{array}$ & $\begin{array}{l}4.2 \\
6.0\end{array}$ & $\begin{array}{l}1200.0 \\
12000\end{array}$ & 2.7 & $\begin{array}{l}1250.0 \\
12500\end{array}$ & $\begin{array}{l}1.8 \\
2.5\end{array}$ \\
\hline $\begin{array}{l}1658 \text { LAWA1015 } \\
1659 \text { LAWA102S }\end{array}$ & $\begin{array}{l}950 \\
950\end{array}$ & $\begin{array}{l}67.600 \\
53.300\end{array}$ & $\begin{array}{l}1000 \\
1000\end{array}$ & $\begin{array}{l}33.1 \\
27.0\end{array}$ & $\begin{array}{l}1050.0 \\
1050.0\end{array}$ & $\begin{array}{l}17.5 \\
14.9\end{array}$ & $\begin{array}{l}110.0 \\
1100.0\end{array}$ & $\begin{array}{l}9.9 \\
8.8\end{array}$ & $\begin{array}{l}1150.0 \\
1150.0\end{array}$ & $\begin{array}{l}6.0 \\
5.5\end{array}$ & $\begin{array}{l}1200.0 \\
1200.0\end{array}$ & $\begin{array}{l}3.8 \\
3.6\end{array}$ & $\begin{array}{l}1250.0 \\
1250.0\end{array}$ & $\begin{array}{l}2.5 \\
2.5\end{array}$ \\
\hline $\begin{array}{l}\text { 1660 LAWA1003S } \\
\text { 1661 LAWA104 }\end{array}$ & 950 & 52300 & & 267 & & & 11000 & 8.6 & & & & 34 & 12500 & \\
\hline 1662 LAWA105 & 950 & 32.900 & 1000 & 17.5 & 1050.0 & 10.0 & 1100.0 & 6.0 & 1150.0 & 3.8 & 1200.0 & 2.5 & 1250.0 & 1.8 \\
\hline $\begin{array}{l}1663 \text { LAWB29* } \\
1664 \text { LAWB30 }\end{array}$ & 950 & 30.900 & 1000 & 15.2 & 1050.0 & 8.3 & 1100.0 & 49 & 1150.0 & 3.1 & 12000 & 20 & 1250.0 & 1.4 \\
\hline 1665 LAWB31 & & & & & & & & & & & & & & \\
\hline 1666 LAW & & & & & & & & & & & & & & \\
\hline $\begin{array}{l}1667 \text { LAWB33 } \\
1668 \text { LAWB34 }\end{array}$ & 950 & 108.500 & 1000 & 51.4 & 1050.0 & 26.4 & 1100.0 & 14.6 & 1150.0 & 8.5 & 1200.0 & 5.3 & 1250.0 & 3.4 \\
\hline $\begin{array}{l}1669 \text { LAWB35 } \\
1670 \text { LAWB36S }\end{array}$ & & & & & & & & & & & & & & \\
\hline 1671 LAWB37 & 950 & 107.200 & 1000 & 52.0 & 1050.0 & 27.3 & 1100.0 & 15.4 & 1150.0 & 9.2 & 1200.0 & 5.7 & 1250.0 & 3.7 \\
\hline 1672 LAWB38 & 950 & 65.100 & 1000 & & 1050.0 & 18.2 & 1100.0 & 10.6 & 1150.0 & 6.5 & 1200.0 & 4.2 & 1250.0 & 2.8 \\
\hline 1673 LAWB39 & 950 & 70.800 & 1000 & 35.3 & 1050.0 & 19.0 & 1100.0 & 10.9 & 1150.0 & 6.6 & 1200.0 & 4.2 & 1250.0 & 2.8 \\
\hline 1674 LAWB40 & 950 & 17.600 & 1000 & 9.5 & 1050.0 & 5.6 & 1100.0 & 3.5 & 1150.0 & 2.4 & 1200.0 & 1.6 & 1250.0 & 1.2 \\
\hline 1675 LAWB41 & $\begin{array}{l}950 \\
950\end{array}$ & $\begin{array}{r}32.900 \\
859030\end{array}$ & 1000 & 17.1 & 1050.0 & 9.5 & 1100.0 & 5.6 & $\begin{array}{l}1150.0 \\
\end{array}$ & $\begin{array}{l}3.5 \\
3.5\end{array}$ & $\begin{array}{l}1200.0 \\
1200\end{array}$ & 2.3 & $\begin{array}{l}1250.0 \\
1250\end{array}$ & 1.6 \\
\hline $\begin{array}{l}1766 \text { LAWBA2S } \\
1677 \text { LAWB43S }\end{array}$ & & 859.300 & 1000 & 336.0 & & 145.9 & 1100.0 & 69.1 & 1150.0 & 35.3 & 1200.0 & 19.2 & 1250.0 & 11.1 \\
\hline 1678 LAWB44S & & & & & & & & & & & & & & \\
\hline & 950 & 43.400 & 1000 & 21.8 & 1050.0 & 11.9 & 1100.0 & 7.0 & 1150.0 & 4.3 & 1200.0 & 2.8 & 1250.0 & \\
\hline $80 \mathrm{LA}$ & 950 & 66.700 & 10 & 33.6 & 1050.0 & 18.3 & 1100.0 & 10.6 & 1150.0 & 6.5 & 1200.0 & 4.2 & 1250.0 & \\
\hline 1681 LAWB48 & 950 & 107.900 & 1000 & 50.3 & 1050.0 & 25.8 & 1100.0 & 14.3 & 1150.0 & 8.5 & 1200.0 & 5.3 & 1250.0 & 3.5 \\
\hline $\begin{array}{l}1682 \text { LAWB49 } \\
1683 \text { LAWB50 }\end{array}$ & 950 & 104.900 & 1000 & 50.7 & 1050.0 & 26.6 & 1100.0 & 14.9 & 1150.0 & 8.9 & 1200.0 & 5.5 & 1250.0 & 3.6 \\
\hline 1684 LAW & 950 & 79.500 & 100 & 37.5 & 1050.0 & 19.5 & 1100.0 & 11.1 & 1150.0 & 6.7 & 1200.0 & 4.3 & & \\
\hline s & 95 & 48.4 & & 24.8 & 1050 & 13.7 & 1100 & 8.1 & 1150 & 5.0 & 120 & 3.3 & 125 & 2 \\
\hline 1080 LAV & & 47.4 & & 24.0 & 1050 & 13.1 & & 7.6 & & 4.7 & 120 & 3.0 & 125 & 2. \\
\hline LA & & 62.0 & & 31.1 & & 16.8 & & 9.7 & & 5.9 & & 3.8 & & 2.5 \\
\hline & $\begin{array}{l}95 \\
95 \\
95\end{array}$ & & & $\begin{array}{l}28.9 \\
53.4\end{array}$ & & $\begin{array}{l}15.6 \\
26.6\end{array}$ & & $\begin{array}{r}9.0 \\
146\end{array}$ & & $\begin{array}{l}5.6 \\
86\end{array}$ & & $\begin{array}{l}3.6 \\
54\end{array}$ & & 23 \\
\hline & $\begin{array}{l}95 \\
95 \\
\end{array}$ & $\begin{array}{l}121.100 \\
60200\end{array}$ & & $\begin{array}{l}53.4 \\
28.4\end{array}$ & To5.0 & 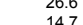 & & $\begin{array}{l}14.6 \\
14.6\end{array}$ & & $\begin{array}{l}8.6 \\
49\end{array}$ & & S.4 & & \\
\hline & 95 & . & & $\begin{array}{l}20.4 \\
39.6\end{array}$ & 10500 & 214. & 11000 & $\begin{array}{l}0.2 \\
12.2\end{array}$ & & $\begin{array}{l}4.9 \\
7.4\end{array}$ & 1200 & 47 & & 2.1 \\
\hline 1692 LAWC16S & 950 & 26.200 & 1000 & $\begin{array}{l}39.6 \\
13.9\end{array}$ & 1050.0 & $\begin{array}{r}21.2 \\
7.9\end{array}$ & 1100.0 & $\begin{array}{r}1.2 \\
4.8\end{array}$ & 1150.0 & 3.1 & 1200.0 & $\begin{array}{l}4.1 \\
2.0\end{array}$ & 1250.0 & $\begin{array}{l}3.1 \\
1.4\end{array}$ \\
\hline & & & & & & & & & & & & & & \\
\hline $1694 \angle \mathrm{LA}$ & 950 & 45.600 & & 23.1 & 1050.0 & 12.7 & 1100.0 & 7.4 & 1150 . & 4.6 & 1200 & 2.9 & 1250.0 & 2.0 \\
\hline & 950 & 51.100 & 1000 & 25.6 & 1050.0 & 13.8 & 1100.0 & 7.9 & 1150.0 & 4.8 & 1200.0 & 3.0 & 1250.0 & 2.0 \\
\hline 1697 LAWC21S & & 57.9 & 1000 & & & & & & & 5.6 & & 3.6 & & \\
\hline 1698 LAWC22** & 950 & 31.6 & 10 & 17.0 & 1050.0 & 9.8 & & 6.0 & & 3.9 & & 2.6 & & \\
\hline & 950 & 214.9 & 10 & 98.0 & 105 & 48.8 & & 26.1 & & 14.9 & & 9.0 & & 5. \\
\hline & 950 & 180.100 & & 82.7 & 1050.0 & 41.5 & & 22.3 & & 12.8 & 1200 & 7.7 & $125 c$ & 4. \\
\hline & 950 & 127.200 & 1000 & 59.5 & 1050.0 & 30.3 & 1100.0 & 16.5 & 1150.0 & 9.6 & 1200.0 & 5.9 & 1250.0 & 3.7 \\
\hline 2 LAWABP1 & 950 & 220.900 & 1000 & 98.1 & 1050.0 & 47.7 & 1100.0 & 25.0 & 1150.0 & 14.0 & 1200.0 & 8.3 & 1250.0 & 5.1 \\
\hline
\end{tabular}




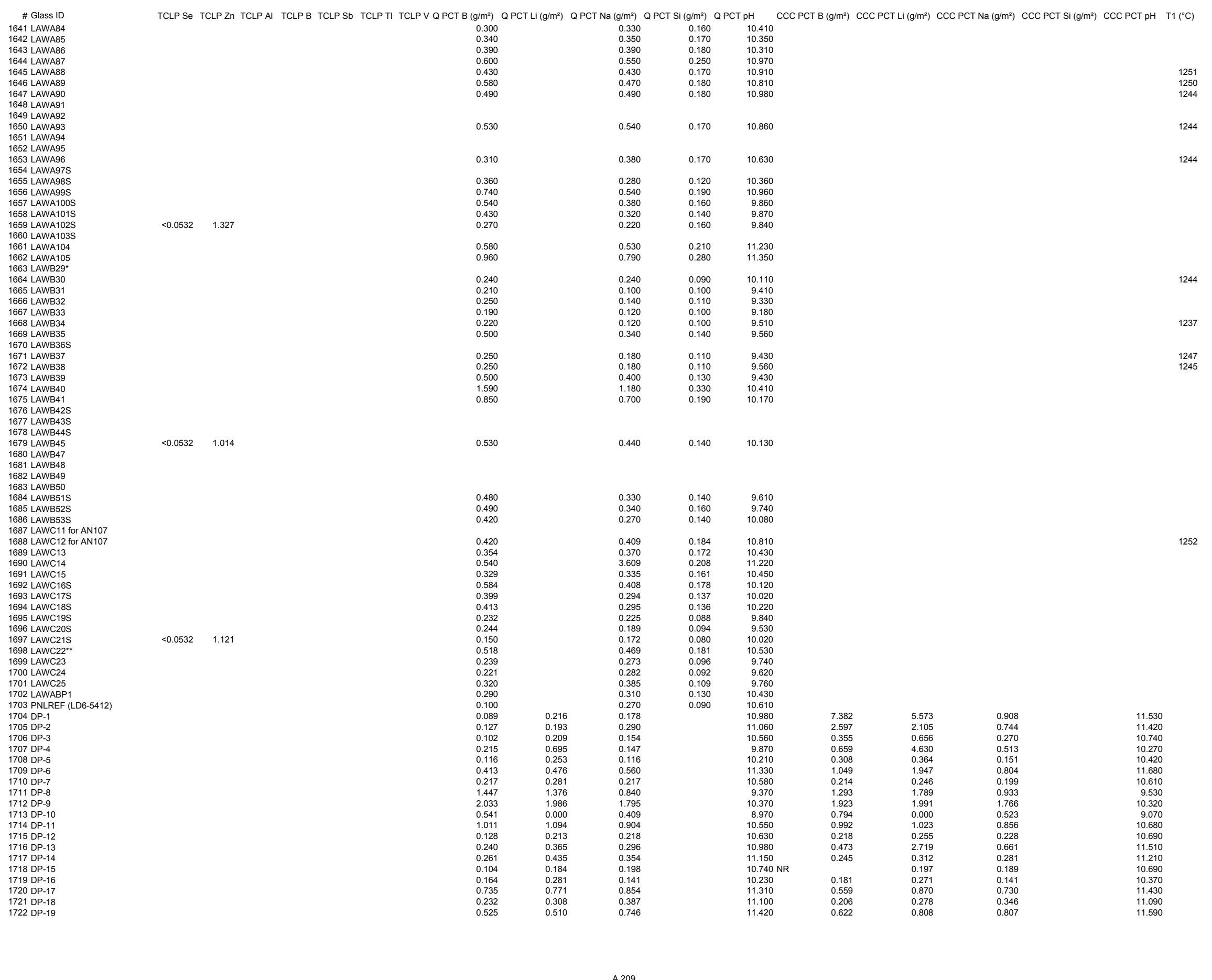


1642 LAWA85

1643 LAWA86
1644 LAWA87

645 LAWA88

1647 LAWA90

1648 LAWA99

1649 LAWA92

1651 LAWA94

1651 LAWA94

1653 LAWA96

1655 LAWA98S
1656 LAWA99S

1657 LAWA100S

1659 LAWA102S

1660 LANA1025

1661 LAWA104

1663 LAWB29*

1665 LAWB3

1667 LAWB33

1668 LAWB34
1669 LAWB35

1669 LAWB35

1671 LAWB37

1672 LAWB38

1673 LAWB39
1674 LAWB40
1675 LAWB41

1676 LAWB42

1678 LAWB44

1679 LAWB45

1680 LAWB47

1682 LAWB49
1683 LAWB50

1684 LAWB51S

1685 LAWB52S
686 LAWB53S

1687 LAWC11 for AN107

1688 LAWC12 for AN107
1689 LAWC13

1690 LAWC14

1691 LAWC15

1692 LAWC16S
1693 LAWC17S

1694 LAWC18S

1695 LAWC19S

1697 LAWC21S

1698 LAWC22*

1699 LAWC23

17001 LAWC24

1702 LAWABP1

1703 PNLREF (LD6-541

$1704 \mathrm{DP}-1$
$1705 \mathrm{DP}-2$

1706 DP-3

(707 DP-4

$1709 \mathrm{DP}-6$

1710 DP-7

1711 DP-8

713 DP-10

$1714 \mathrm{DP}-11$

$1715 \mathrm{DP}-12$
$1716 \mathrm{DP}-13$

1717 DP-14

$1718 \mathrm{DP}-15$

1719 DP-16
1720 DP-17

$1721 \mathrm{DP}-18$

$\begin{array}{lllllll}0.765 & 1143 & 0.554 & 1044 & 0.37 & 949 & 0.248 \\ 0.697 & 1147 & 0.542 & 1046 & 0.39 & 947 & 0.26 \\ 0.653 & 1150 & 0.511 & 1051 & 0.35 & 955 & 0.233 \\ & & & & & & \\ 0.558 & 1139 & 0.432 & 1041 & 0.297 & 947 & 0.183 \\ & & & & & & \\ 0.748 & 1162 & 0.533 & 1052 & 0.307 & 951 & 0.204\end{array}$

$\begin{array}{llllll}0.424 & 1137 & 0.276 \quad 1040 & 0.163 & 925\end{array}$

$\begin{array}{lllllll}0.361 & 1143 & 0.218 & 1045 & 0.141 & 947 & 0.07\end{array}$

$\begin{array}{lllllll}0.297 & 1139 & 0.229 & 1042 & 0.144 & 947 & 0.083 \\ 0.386 & 1141 & 0.283 & 1043 & 0.163 & 944 & 0.10\end{array}$

$\begin{array}{lllllll}0.793 & 1150 & 0.6 & 1050 & 0.409 & 950 & 0.260\end{array}$

1722 DP-19 


\begin{tabular}{|c|c|c|c|c|c|c|}
\hline \# Glass ID & Study & Reference & & B2O3-t & & $\mathrm{Fe} 2 \mathrm{O}-\mathrm{t}$ \\
\hline 1723 DP-20 & DP Glasses for INEEL HLW & $\begin{array}{l}\text { Pittman et al. } 2001 \\
\text { Putman }\end{array}$ & 0.0700 & 0.0600 & 0.1000 & 0.0000 \\
\hline $\begin{array}{l}1724 \text { DP-21 } \\
1725 \text { DP-22 }\end{array}$ & $\begin{array}{l}\text { P G Glasses for INEEE HLW } \\
\text { DP Glasses for INELE HLW }\end{array}$ & $\begin{array}{l}\text { Pittman et al. } 2001 \\
\text { Pifttman et al. } 2001\end{array}$ & $\begin{array}{l}0.0700 \\
0.0700\end{array}$ & $\begin{array}{l}0.1450 \\
0.050\end{array}$ & 0.1500 & \\
\hline 1726 DP-23 & DP Glasses for INEEL HLW & Pittman et al. 2001 & 0.1200 & 0.0600 & 0.1500 & 0.0000 \\
\hline 1727 DP-24 & DP Glasses for INEEL HLW & Pittman et al. 2001 & 0.0800 & 0.0600 & 0.0950 & 0.0000 \\
\hline 1728 DP-BL1 & DP Glasses for INEEL HLW & Pittman et al. 2001 & 0.0900 & 0.0900 & 0.1200 & 0.0250 \\
\hline 1729 DP-BL2 & DP Glasses for INEEL HLW & Pittman et al. 2001 & 0.0859 & 0.0509 & 0.1132 & 0.0300 \\
\hline $\begin{array}{l}1730 \text { DD-centroid } \\
1731 \text { DZr-CV-1 }\end{array}$ & $\begin{array}{l}\text { DP Glassess for INEEL HLW } \\
\text { INEEL DZZCV Glasses }\end{array}$ & $\begin{array}{l}\text { Pittman et ala. } 2001 \\
\text { Riley et al. } 2001\end{array}$ & $\begin{array}{l}0.0890 \\
0.0700\end{array}$ & $\begin{array}{l}0.0819 \\
0.0518\end{array}$ & $\begin{array}{l}0.1140 \\
0.1152\end{array}$ & $\begin{array}{l}0.0183 \\
0.0305\end{array}$ \\
\hline $\begin{array}{l}17 / 31 \text { DLrCVV-1 } \\
1732 \text { DZr-CV-2 }\end{array}$ & $\begin{array}{l}\text { INEEL Dr-CV Glasses } \\
\text { INEEL DZr-CV Glasses }\end{array}$ & $\begin{array}{l}\text { Riley et al. } 2001 \\
\text { Riley et a. } 2001\end{array}$ & & & & $\begin{array}{l}0.0305 \\
0.0287\end{array}$ \\
\hline 1733 DZr-CV-3 & $\begin{array}{l}\text { INEEL DVR-CV G Gasses } \\
\text { INEEL DZr-CV Glasses }\end{array}$ & $\begin{array}{l}\text { Riley et al. } 2001 \\
\text { Riley et al. } 2001\end{array}$ & $\begin{array}{l}0.1250 \\
0.0851\end{array}$ & $\begin{array}{l}0.048 / \\
0.0600\end{array}$ & $\begin{array}{l}0.1084 \\
0.1121\end{array}$ & $\begin{array}{l}0.0287 \\
0.0297\end{array}$ \\
\hline 1734 DZr-CV-4 & INEEL DZr-CV Glasses & 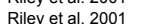 & & & 0.1014 & \\
\hline 1735 DZr-CV-5 & INEEL DZr-CV Glasses & Riley et al. 2001 & 0.0877 & 0.0519 & 0.0950 & 0.0306 \\
\hline 1736 DZr-CV-6 & INEEL DZr-CV Glasses & Riley et al. 2001 & 0.0824 & 0.0488 & 0.1500 & 0.0287 \\
\hline 1737 DZr-CV-7 & INEEL DZr-CV Glasses & Riley et al. 2001 & 0.0866 & 0.0513 & 0.1140 & 0.0302 \\
\hline $\begin{array}{l}1738 \text { DZr-CV-8 } \\
1739 \text { DZr-CV-9 }\end{array}$ & INEEL DZr-CV Glasses & Riley et al. 2001 & 0.0843 & 0.0499 & 0.1111 & 0.0294 \\
\hline 1740 DZr-CV-10 & $\begin{array}{l}\text { INEELL DZZ-CV G Gasses } \\
\text { INEEL DZr-CV Glasses }\end{array}$ & $\begin{array}{l}\text { Riliyey et ala.2001 } \\
\text { Riley et al. } 2001\end{array}$ & $\begin{array}{l}0.0886 \\
0.0842\end{array}$ & $\begin{array}{l}0.0525 \\
0.0498\end{array}$ & $\begin{array}{l}0.1167 \\
0.1109\end{array}$ & $\begin{array}{l}0.0000 \\
0.0500\end{array}$ \\
\hline 1741 DZr-CV-11 & INEEL DZr-CV Glasses & Riley et al. 2001 & 0.0838 & & & 0.0292 \\
\hline $1742 \mathrm{DZr}-\mathrm{CV}-12$ & INEEL DZr-CV Glasses & Riley et al. 2001 & 0.0816 & 0.0483 & 0.1075 & 0.0285 \\
\hline 1743 DZr-CV-13 & INEEL DZr-CV Glasses & Riley et al. 2001 & 0.0873 & 0.0517 & 0.1150 & 0.0304 \\
\hline 1744 DZr-CV-14 & INEEL DZr-CV Glasses & Riley et al. 2001 & $\begin{array}{l}0.0850 \\
0.0892\end{array}$ & $\begin{array}{l}0.0504 \\
0.0528\end{array}$ & $\begin{array}{l}0.1120 \\
0.1175\end{array}$ & \\
\hline $\begin{array}{l}1745 \text { DZr-CV-15 } \\
1746 \text { br-CV-16 }\end{array}$ & INEEL DZR-CV Glasses & Riley et al 2001 & 0.0844 & 0.0500 & $\begin{array}{l}0.11111 \\
0.1111\end{array}$ & $\begin{array}{l}0.0331 \\
0.0294\end{array}$ \\
\hline 1747 DZr-CV-17 & INEEL DZr-CV Glasses & Riley et al. 2001 & 0.0870 & 0.0515 & 0.1146 & $\begin{array}{l}.0 .0394 \\
0.0303\end{array}$ \\
\hline 1748 DZr-CV-18 & $\begin{array}{l}\text { INEEL DZR-CV Glasses } \\
\text { INEL DZS }\end{array}$ & $\begin{array}{l}\text { Riley et al. } 2001 \\
\text { Rile }\end{array}$ & 0.0848 & 0.0502 & 0.1117 & \\
\hline 1749 DZr-CV-19 & INEEL DZr-CV Glasses & Riley et al. 2001 & 0.0864 & 0.0512 & 0.1138 & 0.0301 \\
\hline 1750 DZr-CV-20 & INEEL DZr-CV Glasses & Riley et al. 2001 & 0.0856 & 0.0507 & 0.1127 & 0.0298 \\
\hline 1751 DZr-CV-21 & INEEL DZr-CV Glasses & Riley et al. 2001 & 0.0953 & 0.0564 & 0.1256 & 0.0332 \\
\hline 1752 DZr-CV-22 & INEEL DZr-CV Glasses & Riley et al. 2001 & 0.0789 & 0.0467 & 0.1039 & 0.0275 \\
\hline 1753 DZr-CV-23 & INEEL DZr-CV Glasses & $\begin{array}{l}\text { Riley et al. } 2001 \\
\text { Riley }\end{array}$ & 0.0866 & 0.0513 & 0.1140 & 0.0302 \\
\hline $\begin{array}{l}1754 \text { DLP-CV-24 } \\
1755 \text { HLP-01 }\end{array}$ & $\begin{array}{l}\text { INEEL DZr-CV Glasses } \\
\text { HLP glasses }\end{array}$ & $\begin{array}{l}\text { Riley et al. } 2001 \\
\text { Vienna et al } 2001\end{array}$ & $\begin{array}{l}0.0830 \\
0.0700\end{array}$ & $\begin{array}{l}0.0491 \\
0.1000\end{array}$ & $\begin{array}{l}0.1093 \\
0.0001\end{array}$ & $\begin{array}{l}0.0289 \\
0.0550\end{array}$ \\
\hline 1756 HLP-02 & $\begin{array}{l}\text { HLP glasses } \\
\text { HLPses }\end{array}$ & Vienna et al. 2001 & 0.0879 & 0.1257 & 0.0002 & 0.0692 \\
\hline 1757 HLP- -03 & HLP glasses & $\begin{array}{l}\text { Vienna et al. } 2001 \\
\text { Vent }\end{array}$ & 0.0660 & 0.0943 & 0.0001 & $\begin{array}{l}0.0519 \\
0.052\end{array}$ \\
\hline 1758 HLP-04 & HLP glasses & Vienna et al. 2001 & 0.0824 & 0.1178 & 0.0001 & 0.0648 \\
\hline 1759 HLP-05 & HLP glasses & Vienna et al. 2001 & 0.0400 & 0.1032 & 0.0001 & 0.0568 \\
\hline 1760 HLP-06 & HLP glasses & Vienna et al. 2001 & 0.1194 & 0.0947 & 0.0001 & 0.0521 \\
\hline 1761 HLP-07 & HLP glasses & Vienna et al. 2001 & 0.0900 & 0.0979 & 0.0001 & 0.0538 \\
\hline $\begin{array}{ll}1762 \\
1763\end{array}$ & $\begin{array}{l}\text { HLP glasses } \\
\text { HLP lasses }\end{array}$ & Vienna et al. 2001 & 0.0731 & 0.0600 & 0.0001 & 0.0575 \\
\hline $1764=4 P-10$ & $\begin{array}{l}\text { HLP glasses } \\
\text { HLP lasses }\end{array}$ & $\begin{array}{l}\text { Vienna et al. } 2001 \\
\text { Viennat at al } 2001\end{array}$ & $\begin{array}{l}0.0684 \\
0.0715\end{array}$ & $\begin{array}{l}0.1280 \\
0.0800\end{array}$ & 0.0001 & 0.0563 \\
\hline 1765 HLP-11 & $\begin{array}{l}\text { HLF g glassses } \\
\text { HLP }\end{array}$ & Vienna et al. 2001 & 0.0741 & 0.1058 & 0.0001 & 0.0000 \\
\hline $1766 \mathrm{HLP}-12$ & $\begin{array}{l}\text { HLP glasses } \\
\text { HLises }\end{array}$ & $\begin{array}{l}\text { Vienna et al al. } 2001 \\
\text { nan }\end{array}$ & 0.0674 & & 0.0001 & 0.0900 \\
\hline 1767 HLP-13 & HLP glasses & Vienna et al. 2001 & 0.0718 & 0.1027 & 0.0001 & 0.0300 \\
\hline 1768 HLP-14 & HLP glasses & Vienna et al. 2001 & 0.0721 & 0.1031 & 0.0001 & 0.0567 \\
\hline 1769 HLP-15 & HLP glasses & Vienna et al. 2001 & 0.0678 & 0.0969 & 0.0001 & 0.0533 \\
\hline (7770 HLP-16 & HLP glasses & Vienna et al. 2001 & 0.0710 & 0.1015 & 0.0001 & 0.0559 \\
\hline $\begin{array}{l}17711 \text { HLP-17 } \\
1772 \text { HLP-18 }\end{array}$ & $\begin{array}{l}\text { HLP glasses } \\
\text { HLP glasses }\end{array}$ & $\begin{array}{l}\text { Vienna et al. } 2001 \\
\text { Vienna et al. } 2001\end{array}$ & $\begin{array}{l}0.0682 \\
0.0710\end{array}$ & $\begin{array}{l}0.0975 \\
0.1015\end{array}$ & $\begin{array}{l}0.0001 \\
0.0001\end{array}$ & $\begin{array}{l}0.0536 \\
0.0559\end{array}$ \\
\hline 1773 HLP-19 & $\begin{array}{l}\text { MLF g gassses } \\
\text { HLP }\end{array}$ & $\begin{array}{l}\text { Vienna et al. } 2001 \\
\text { Vienna et al. } 2001\end{array}$ & 0.0668 & & $\begin{array}{l}0.00001 \\
0.0001\end{array}$ & 0.0525 \\
\hline 1774 HLP-20 & HLP glasses & Vienna et al. 2001 & 0.0710 & 0.1015 & 0.0001 & 0.0559 \\
\hline 1775 HLP-21 & HLP glasses & Vienna et al. 2001 & 0.0682 & & 0.0001 & 0.0536 \\
\hline 1776 HLP-22 & HLP glasses & Vienna et al. 2001 & 0.0737 & 0.1053 & 0.0001 & 0.0579 \\
\hline 1777 HLP-23 & HLP glasses & Vienna et al. 2001 & 0.0672 & 0.0961 & 0.0001 & 0.0529 \\
\hline 1778 HLP-24 & & & & & 0.0001 & 0.0565 \\
\hline $1779 \mathrm{HLP}-25$ & HLP glasses & Vienna et al. 2001 & 0.0700 & 0.1000 & 0.0001 & 0.0550 \\
\hline $\begin{array}{l}1780 \text { HLP-26 } \\
1781 \text { HLP-27 }\end{array}$ & $\begin{array}{l}\text { HLP g gasses } \\
\text { HLP lasses }\end{array}$ & $\begin{array}{l}\text { Vienna et al. } 2001 \\
\text { Vienna et al. } 2001\end{array}$ & $\begin{array}{l}0.0700 \\
0.1194\end{array}$ & $\begin{array}{l}0.1000 \\
0.1200\end{array}$ & $\begin{array}{l}0.0001 \\
0.0001\end{array}$ & $\begin{array}{l}0.0550 \\
0.0000\end{array}$ \\
\hline 1782 HLP-28 & $\begin{array}{l}\text { HLF g galasses } \\
\text { HLP lasses }\end{array}$ & Vienna et al. 2001 & 0.1194 & 0.1200 & 0.0001 & \\
\hline 1783 HLP-29 & HLP glasses & & 0.1194 & 0.0600 & 0.0001 & 0.0254 \\
\hline 1784 HLP-30 & HLP glasses & Vienna et al. 2001 & 0.1194 & 0.0600 & 0.0001 & 0.0564 \\
\hline 1785 HLP-31 & HLP glasses & Vienna et al. 2001 & 0.0400 & 0.1200 & 0.0001 & 0.0336 \\
\hline 1786 HLP-32 & HLP glasses & Vienna et al. 2001 & 0.0400 & 0.1200 & 0.0001 & 0.0646 \\
\hline 1787 HLP-33 & HLP glasses & Vienna et & 0.0400 & 0.0600 & & \\
\hline 1788 HLP-34 & HLP glasses & & 0.0400 & 0.0600 & 0.00 & 0.0900 \\
\hline $\begin{array}{l}1789 \mathrm{HLP}-35 \\
1790 \mathrm{HP}-36\end{array}$ & $\begin{array}{l}\text { HLP g gasses } \\
\text { HLP lasses }\end{array}$ & $\begin{array}{l}\text { Vienna et al. } 2001 \\
\text { Vijenna ta }\end{array}$ & $\begin{array}{l}0.1194 \\
0.1194\end{array}$ & 0.12200 & 0.0001 & $\begin{array}{l}0.0677 \\
0.0987\end{array}$ \\
\hline $\begin{array}{l}1791 \text { HLP-37 } \\
1790\end{array}$ & $\begin{array}{l}\text { HLF glassses } \\
\text { HLP }\end{array}$ & Vienna et al. 2001 & 0.1194 & $\begin{array}{l}0.1200 \\
0.0600\end{array}$ & 0.0001 & 0.0931 \\
\hline & HLP glasses & & & & & \\
\hline 1793 HLP-39 & HLP glasses & Vienna et al. 2001 & 0.0400 & 0.1200 & 0.0001 & \\
\hline 1794 HLP-4C & HLP glasses & $\begin{array}{l}\text { Vienna et al. } 2001 \\
\text { Ven }\end{array}$ & 0.0400 & 0.1200 & 0.0001 & 0.1323 \\
\hline 1795 HLP-40Q & HLP glasses & Vienna et al. 2001 & 0.0400 & 0.1200 & 0.0001 & 0.1323 \\
\hline & es & na et al. 2001 & 0.04 & 0.0600 & 0.0001 & 0.1267 \\
\hline & LP glass & & 0.0400 & & & 0.1577 \\
\hline $\begin{array}{l}1798 \mathrm{HLP}-42 \mathrm{Q} \\
1799 \mathrm{HIP}-43\end{array}$ & $\begin{array}{l}\text { P g glasses } \\
\text { P glasses }\end{array}$ & Vienna et al. 2001 & 0.0400 & 6000 & 0.0001 & \\
\hline HLP.-44 & 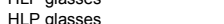 & - & 0.0700 & 0.1000 & 0.0001 & \\
\hline & & & & & & \\
\hline ALP-46 & LP glasses & al. 2001 & 0.1200 & 0.0500 & & \\
\hline 3 HLP-47 & LP glasses & Vienna et al. 2001 & 0.1000 & 0.0800 & 0.0050 & 00 \\
\hline 1804 HLP-48 & HLP glasses & Vienna et al. 2001 & 0.1197 & 0.0885 & 0.0000 & 0.0577 \\
\hline
\end{tabular}

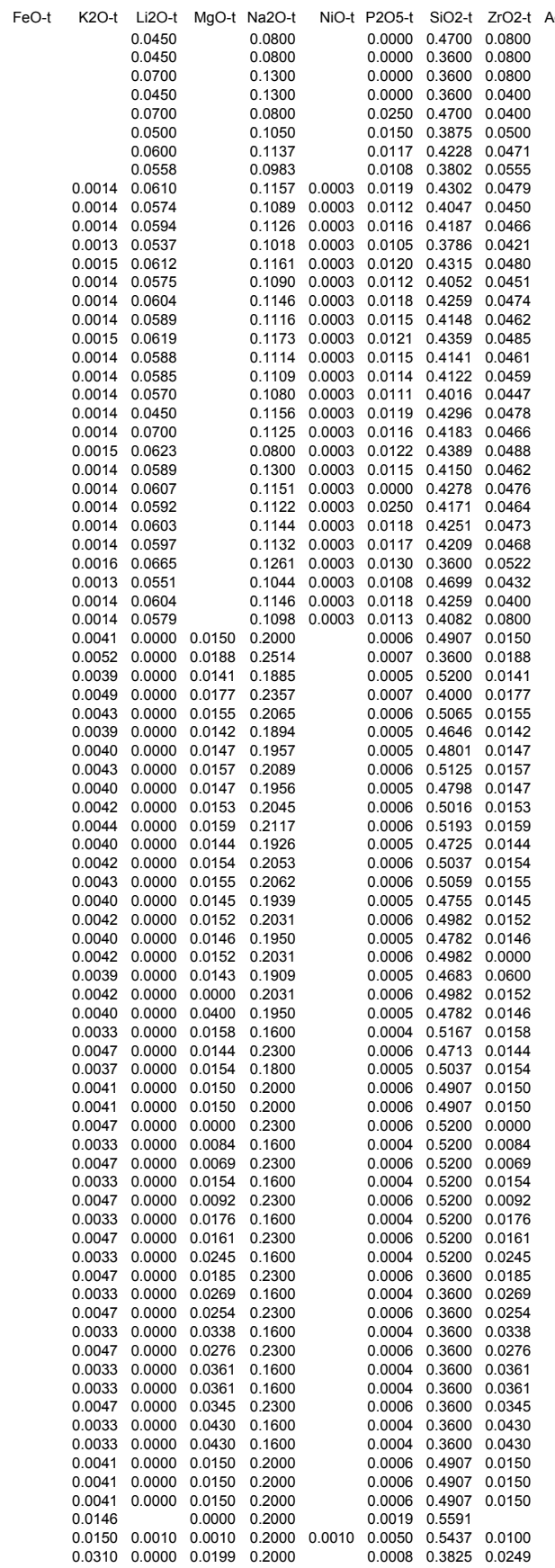

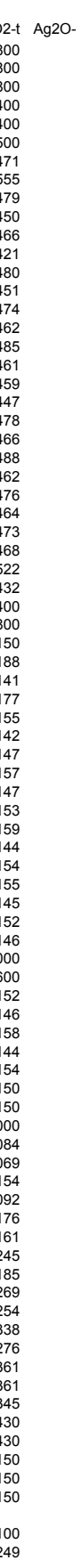




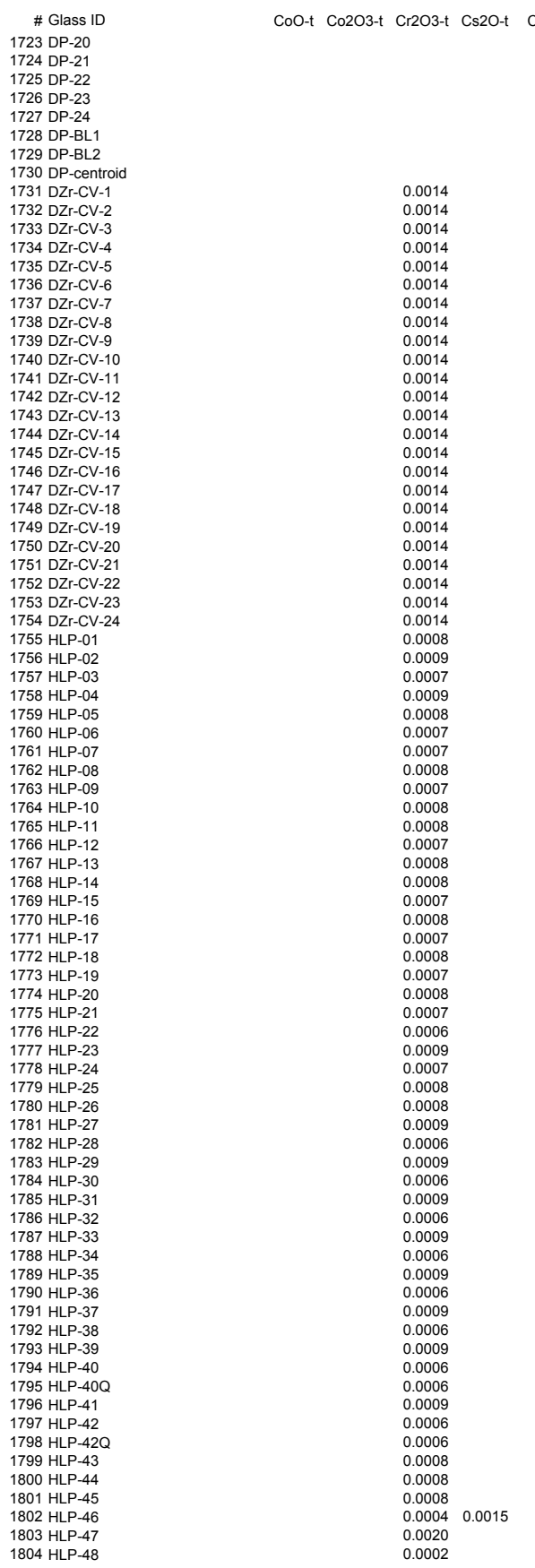

F-t Ga2O3-t Gd2O3-t HiO2-t HgO-t

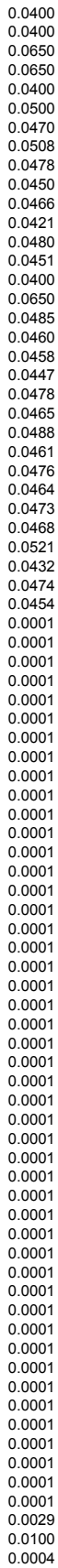

1-t La203-t MnO2-t MnO-t MoO-t MoO2-t MoO3-t Nb205-t Nd203-t NpO2-t PbO-t PdO2-t PdO-t Pr203-t Pr6011-t Rb2O-t ReO-t 0.0500

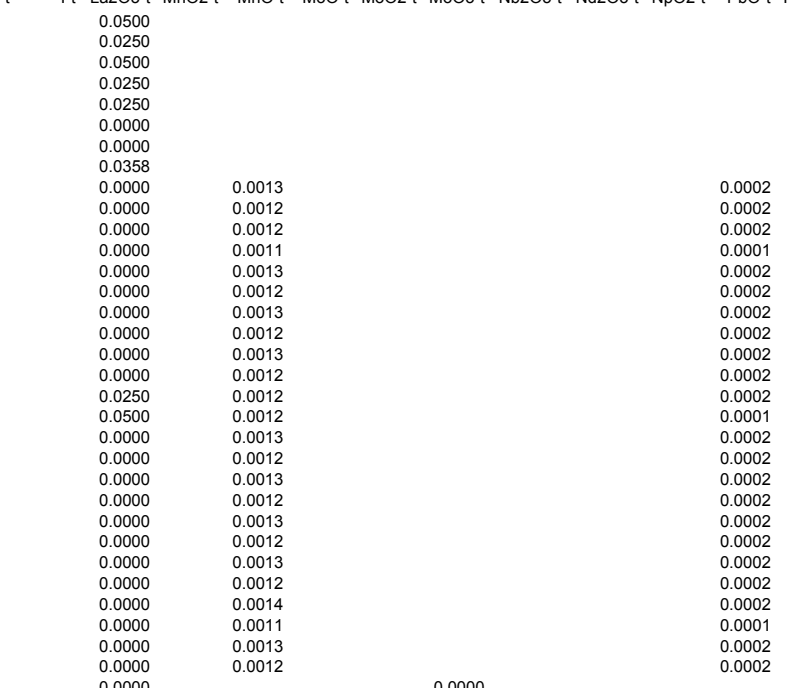

\begin{tabular}{l}
0.0001 \\
0.0001 \\
0.0001 \\
0.0001 \\
0.0001 \\
0.0001 \\
0.0001 \\
0.0001 \\
0.0001 \\
0.0001 \\
0.0001 \\
0.0001 \\
0.0001 \\
0.0001 \\
0.0001 \\
0.0001 \\
0.0001 \\
0.0001 \\
0.0001 \\
0.0001 \\
0.0001 \\
0.0001 \\
0.0001 \\
0.0001 \\
0.0001 \\
0.0001 \\
0.0001 \\
0.0001 \\
0.0001 \\
0.0001 \\
0.0001 \\
0.0001 \\
0.0001 \\
0.0001 \\
0.0001 \\
0.0001 \\
0.0001 \\
0.0001 \\
0.0001 \\
0.0001 \\
0.0001 \\
0.0001 \\
0.0001 \\
0.0001 \\
0.0001 \\
0.0001 \\
0.0001 \\
0.00001 \\
0.0000 \\
\hline
\end{tabular} 

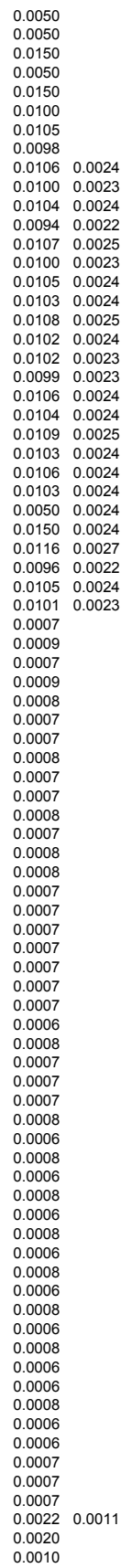

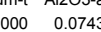
$1.0000 \quad 0.0718$ $\begin{array}{ll}1.0000 & 0.072 \\ 1.0000 & 0.122 \\ 1.0000 & 0.0818\end{array}$ $\begin{array}{ll}1.0000 & 0.0818 \\ 0.9925 & 0.0932 \\ 0.9928 & 0.0896\end{array}$ $\begin{array}{ll}0.9925 & 0.0932 \\ 0.9928 & 0.0886 \\ 1.0002 & 0.0903\end{array}$

1.0000
1.0000

$0.0150 \quad 1.0000 \quad 0.0704$ $\begin{array}{lll}0.0188 & 0.9999 & 0.0895 \\ 0.0141 & 0.9999 & 0.070 \\ 0.0177 & 11001 & 0.044\end{array}$ $\begin{array}{lll}0.0177 & 1.0001 & 0.0874 \\ 0.0155 & 1.0001 & 0.0424\end{array}$ $\begin{array}{lll}0.0142 & 0.9999 & 0.123\end{array}$ $\begin{array}{lll}0.0147 & 0.9998 & 0.0979\end{array}$ $\begin{array}{lll}0.0157 & 1.0001 & 0.079 \\ 0.0147 & 0.9999 & 0.0712\end{array}$ $\begin{array}{lll}0.0153 & 0.9998 & 0.0704 \\ 0.0159 & 10001 & 0.069\end{array}$ $\begin{array}{lll}0.0159 & 1.0001 & 0.0691 \\ 0.0144 & 0.9998 & 0.0644\end{array}$ $\begin{array}{lll}0.0144 & 0.9908 & 0.0644 \\ 0.0154 & 1.0000 & 0.0644 \\ 0.0155 & 1.0002 & 0.0723\end{array}$ $\begin{array}{lll}0.0155 & 1.0002 & 0.0743 \\ 0.0145 & 0.9998 & 0.0652 \\ 0.0400 & 0.998 & 0.0684\end{array}$ $\begin{array}{lll}0.0000 & 0.9999 & 0.0679 \\ 0.0400 & 0.998 & 0.0684\end{array}$ $\begin{array}{llll}0.0152 & 0.9999 & 0.0745\end{array}$

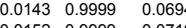
$\begin{array}{lll}0.0152 & 0.9999 & 0.0719 \\ 0.0146 & 0.9998 & 0.068\end{array}$ $\begin{array}{lll}0.0158 & 1.0000 & 0.0767 \\ 0.0144 & 1.0000 & 0.0692\end{array}$ $\begin{array}{llll}0.0154 & 1.0001 & 0.0744\end{array}$ $\begin{array}{lll}0.0150 & 1.0000 & 0.0676 \\ 0.0150 & 1.0000 & 0.0668 \\ 0 & 0000 & 0.128\end{array}$ $\begin{array}{llll}0.0000 & 0.9999 & 0.1200 \\ 0 & 0004 & 0.999 & 0.1210\end{array}$ $\begin{array}{lll}0.0084 & 0.9999 & 0.120 \\ 0.0069 & 0.9998 & 0.1180\end{array}$ $\begin{array}{lll}0.0154 & 1.0001 & 0.1220 \\ 0.0092 & 1.0000 & 0.0420\end{array}$ $\begin{array}{lll}0.0176 & 1.0000 & 0.0421 \\ 0.0161 & 0.9999 & 0.0416 \\ 0.0245 & 0.999 & 0.097\end{array}$ $\begin{array}{llll}0.0245 & 0.9999 & 0.0397\end{array}$ $\begin{array}{lll}0.0185 & 1.0000 & 0.1150 \\ 0.0269 & 1.0000 & 0.1200\end{array}$ $\begin{array}{llll}0.0254 & 0.9999 & 0.1160\end{array}$ $\begin{array}{lll}0.0338 & 0.9999 & 0.1200 \\ 0.0276 & 0.9998 & 0.0399\end{array}$ $\begin{array}{lll}0.0361 & 1.0001 & 0.042 \\ 0.0361 & 1.0001 & 0.0401 \\ 0.035 & 0.9997 & 0.091\end{array}$ $\begin{array}{lll}0.0345 & 0.9097 & 0.0391 \\ 0.0430 & 0.9900 & 0.040 \\ 0.0430 & 1.0000 & 0.0496\end{array}$ $\begin{array}{lll}0.0430 & 1.0000 & 0.0413 \\ 0.0430 & 1.0000 & 0.0409\end{array}$ $\begin{array}{lll}0.0150 & 1.0000 & 0.0669 \\ 0.0150 & 1.0000 & 0.0693\end{array}$ $\begin{array}{lll}0.0150 & 1.0000 & 0.0710 \\ 0.0000 & 0.1170\end{array}$ $\begin{array}{lll}0.0000 & 0.9989 & 0.0942 \\ 0.0427 & 1.0000 & 0.1150\end{array}$ 
\# Glass ID

1723 DP-20

$1725 \mathrm{DP}-22$

1726 DP-23

1728 DP.BL1

1730 DP-centroid

1731 DZr-CV-1

1733 DZr-CV-3
1734 DZr-CV-4

1735 DZR-CV-5

1738 DZr-CV-8

740 DZCV-9

1741 DZr-CV-11
1742 DZr-CV-12

1744 DZr-CV-14

1745 DZR-CV-15
1746 DZr-CV-16
1777 Dz- 17

1748 DZr-CV-18

1749 DZr-CV-19
1750 DZr-CV-20

1753 DZr-CV-23
1754 DZr-CV-24

1755 HLP-01

1757 HLP-03

1758 HLP-P 04

1760 HLP-06

1761 HLP- 07

1762 HLP-08

764 HLP-10

1766 HLP-12

767 HLP-13

1769 HLP-15

1770 HLP-16

1772 HLP-18

1773 HLP-19

1774 HLP.-20

1776 HLP-22

(1776 HLP-23

1779 HLP-25

1781 HLP-27

$1782 \mathrm{HLP}-28$
$1783 \mathrm{HLP}-29$

1784 HLP- 30

(784 HLP.30

1787 HLP-33

1789 HLP-35

791 HLP-37

192 HLP-38

1794 HLP-40

列

1797 HLP-42

1799 HLP-43

1800 HLP-44

1803 HLP-47
1804 HLP-48
B2O3-a CaO-a Fe2O3-a FeO-a K2O

$\begin{array}{lll}0.0599 & 0.1043 & 0.0017 \\ 0.1417 & 0.1442 & 0.0000\end{array}$

$\begin{array}{lll}0.0657 & 0.1008 & 0.0004 \\ 0.0596 & 0.1414 & 0.0017\end{array}$

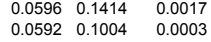

$\begin{array}{lll}0.1001 & 0.1224 & 0.0256 \\ 0.0538 & 0.1133 & 0.0330 \\ 0.0802 & 0.1165 & 0.0192\end{array}$

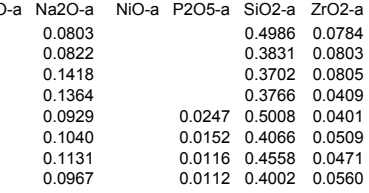

$\begin{array}{llll}0.0112 & 0.4002 & 0.056\end{array}$

\begin{tabular}{|c|c|c|c|c|c|c|c|c|}
\hline 0.0892 & 0.0001 & 0.0652 & 0.0040 & & 0.0141 & 0.1840 & 0.4880 & \\
\hline 0.1140 & & 0.0763 & 0.0059 & & 0.0181 & 0.2580 & 0.3520 & 0.0188 \\
\hline 0.0909 & & 0.0606 & 0.0041 & & & 0.1790 & & \\
\hline $\begin{array}{l}0.12440 \\
0.1230\end{array}$ & & 0.0772 & 0.0052 & & $\begin{array}{l}0.0168 \\
0.0145\end{array}$ & $\begin{array}{l}0.2220 \\
0\end{array}$ & 0.4040 & 0.0174 \\
\hline $\begin{array}{l}0.1080 \\
0.0889\end{array}$ & & $\begin{array}{l}0.0680 \\
0.0596\end{array}$ & $\begin{array}{l}0.0050 \\
0.0042\end{array}$ & & $\begin{array}{l}0.0145 \\
0.0134\end{array}$ & $\begin{array}{l}0.2030 \\
0.1910\end{array}$ & & $\begin{array}{l}0.0145 \\
0.0135\end{array}$ \\
\hline 0.0945 & & 0.0632 & 0.0043 & & 0.0146 & 0.1930 & 0.5030 & \\
\hline 0.0594 & & 0.0584 & 0.0056 & & 0.0131 & 0.1950 & 0.4890 & 0.0144 \\
\hline $\begin{array}{l}0.1180 \\
0.0892\end{array}$ & & $\begin{array}{l}0.0640 \\
0.0633\end{array}$ & $\begin{array}{l}0.0041 \\
0.0048\end{array}$ & & $\begin{array}{l}0.0129 \\
0.0136\end{array}$ & $\begin{array}{l}0.1860 \\
\end{array}$ & 0.4330 & 0.0122 \\
\hline $\begin{array}{l}0.08922 \\
0.10660\end{array}$ & & $\begin{array}{l}0.0633 \\
0.0055\end{array}$ & $\begin{array}{l}0.0048 \\
0.0040\end{array}$ & & $\begin{array}{l}0.0176 \\
0.0123\end{array}$ & $\begin{array}{l}. .1840 \\
0.1820\end{array}$ & $\begin{array}{l}0.4 / 90 \\
0.5080\end{array}$ & $\begin{array}{l}0.0155 \\
0.0138\end{array}$ \\
\hline & & $\begin{array}{l}0.00520 \\
0.00\end{array}$ & $\begin{array}{l}0.004040 \\
0.0046\end{array}$ & & $\begin{array}{l}0.0123 \\
0.0123\end{array}$ & $\begin{array}{l}.11820 \\
0.1800\end{array}$ & $\begin{array}{l}0.5080 \\
0.4480\end{array}$ & $\begin{array}{l}0.0138 \\
0.0134\end{array}$ \\
\hline 0.1020 & 0.0006 & 0.0380 & 0.0041 & & $\begin{array}{l}0.0153 \\
0.0151\end{array}$ & 0.1850 & $\begin{array}{l}0.4990 \\
0.490\end{array}$ & $\begin{array}{l}0.0134 \\
0.0134\end{array}$ \\
\hline 0.1010 & & 0.0631 & 0.0045 & & 0.0153 & 0.1940 & 0.4730 & \\
\hline 0.0972 & 0.0007 & 0.0669 & 0.0038 & & 0.0154 & 0.1860 & 0.4780 & \\
\hline 0.1000 & 0.0008 & 0.0684 & 0.0041 & & 0.0161 & 0.1900 & 0.5340 & \\
\hline 0.0986 & & 0.0636 & 0.0036 & & 0.0153 & 0.1840 & 0.4830 & 0.0130 \\
\hline 0.1020 & & $\begin{array}{l}0.0622 \\
0.0642\end{array}$ & $\begin{array}{l}0.0043 \\
0.0043\end{array}$ & & $\begin{array}{l}0.0154 \\
0.0147\end{array}$ & $\begin{array}{l}0.1920 \\
0.1780\end{array}$ & 0.4620 & \\
\hline $\begin{array}{l}0.0963 \\
0.1010\end{array}$ & & $\begin{array}{l}0.0640 \\
0.0626\end{array}$ & $\begin{array}{l}0.0043 \\
0.0040\end{array}$ & & & $\begin{array}{l}0.1780 \\
0.1930\end{array}$ & $\begin{array}{l}0.4820 \\
0.4930\end{array}$ & $\begin{array}{l}0.0564 \\
0.0151\end{array}$ \\
\hline $\begin{array}{l}0.1020 \\
0.1020\end{array}$ & & $\begin{array}{l}0.0626 \\
0.0671\end{array}$ & $\begin{array}{l}0.004030 \\
0.0037\end{array}$ & & & $\begin{array}{l}.11330 \\
0.1830\end{array}$ & $\begin{array}{l}0.4930 \\
0.4590\end{array}$ & $\begin{array}{l}0.0151 \\
0.0134\end{array}$ \\
\hline 0.1100 & & 0.0702 & 0.0030 & & $\begin{array}{l}0.0166 \\
0.016\end{array}$ & $\begin{array}{l}.11530 \\
0.1540\end{array}$ & $\begin{array}{l}0.45390 \\
0.5330\end{array}$ & $\begin{array}{l}0.0134 \\
0.0126\end{array}$ \\
\hline 0.0965 & & 0.0591 & 0.0042 & & 0.0147 & 0.2150 & 0.4410 & 0.0138 \\
\hline 0.1000 & & 0.0659 & 0.0040 & & 0.0163 & 0.1720 & 0.4890 & \\
\hline 0.1010 & 0.0001 & 0.0627 & 0.0041 & & 0.0154 & 0.1850 & 0.4720 & \\
\hline 0.0943 & & 0.0597 & 0.0043 & & 0.0145 & 0.1820 & 0.4680 & 0.0150 \\
\hline $\begin{array}{l}0.1230 \\
0.1300\end{array}$ & & $\begin{array}{l}0.0060 \\
0.038\end{array}$ & 0.0044 & & 00080 & $\begin{array}{l}0.2170 \\
0 \\
0.1710\end{array}$ & $\begin{array}{l}0.5040 \\
0.5180\end{array}$ & \\
\hline 0.0651 & & $\begin{array}{l}0.0382 \\
0.0312\end{array}$ & 0.0042 & & $\begin{array}{l}0.0000 \\
0.0069\end{array}$ & $\begin{array}{l}.01510 \\
0.2150\end{array}$ & $\begin{array}{l}0.51800 \\
0.4950\end{array}$ & $\begin{array}{l}0.0075 \\
0.0064\end{array}$ \\
\hline 0.0656 & & 0.0638 & 0.0029 & & 0.0150 & 0.1540 & $\begin{array}{l}0.4730 \\
0.4730\end{array}$ & $\begin{array}{l}0.0064 \\
0.0143\end{array}$ \\
\hline 0.1230 & & 0.0430 & 0.0040 & & 0.0084 & 0.2200 & 0.4890 & \\
\hline 0.1290 & & 0.0705 & $\begin{array}{l}0.0031 \\
0.0045\end{array}$ & & $\begin{array}{l}0.0166 \\
0.01157\end{array}$ & 0.1570 & 0.4600 & $\begin{array}{l}.0160 \\
0131\end{array}$ \\
\hline $\begin{array}{l}0.06644 \\
0.0624\end{array}$ & & $\begin{array}{l}0.0692 \\
0.1020\end{array}$ & $\begin{array}{l}0.0045 \\
0.0030\end{array}$ & & $\begin{array}{l}0.0157 \\
0.0210\end{array}$ & $\begin{array}{l}0.2200 \\
0.1430\end{array}$ & $\begin{array}{l}0.5230 \\
0.5100\end{array}$ & $\begin{array}{l}0.0131 \\
0.0200\end{array}$ \\
\hline $\begin{array}{l}0.0624 \\
0.1210\end{array}$ & & $\begin{array}{l}.1020 \\
0.0747\end{array}$ & 0.0050 & & $\begin{array}{l}0.0<10 \\
0.0168\end{array}$ & $\begin{array}{l}.112160 \\
0.2160\end{array}$ & $\begin{array}{l}0.51700 \\
0.3600\end{array}$ & $\begin{array}{l}0.02000 \\
0.0173\end{array}$ \\
\hline 0.1260 & & 0.1030 & 0.0027 & & 0.0241 & 0.1550 & 0.3560 & 0.0244 \\
\hline 0.0678 & & 0.0971 & 0.00 & & 0.0219 & 0.2180 & 0.3560 & \\
\hline 0.0609 & & 0.1260 & & & 0.0315 & 0.1530 & 0.3540 & \\
\hline 0.1260 & & 0.1040 & 0.0045 & & 0.0259 & 0.2150 & 0.3440 & 0.0262 \\
\hline 0.1240 & & 0.1340 & & & 0.0365 & 0.1630 & 0.3510 & \\
\hline 0.1270 & & 0.1340 & & & $\begin{array}{l}0.0346 \\
0.0320\end{array}$ & 0.1540 & $\begin{array}{l}0.3410 \\
03500\end{array}$ & 0.0316 \\
\hline $\begin{array}{l}0.0471 \\
0.064\end{array}$ & & $\begin{array}{l}0.1300 \\
0.1580\end{array}$ & 0.0043 & & 0.0320 & $\begin{array}{l}0.2160 \\
0\end{array}$ & $\begin{array}{l}0.3500 \\
0.3400\end{array}$ & $\begin{array}{l}0.0319 \\
0.0378\end{array}$ \\
\hline 0.0632 & & $\begin{array}{l}0.1580 \\
0.1680\end{array}$ & $\begin{array}{l}0.0042 \\
0.0037\end{array}$ & & $\begin{array}{l}0.0499 \\
0.0405\end{array}$ & $\begin{array}{l}.11500 \\
0.1550\end{array}$ & $\begin{array}{l}0.3420 \\
0.3510\end{array}$ & $\begin{array}{l}0.0378 \\
0.0327\end{array}$ \\
\hline 0.0985 & 0.0001 & 0.0623 & 0.0047 & & $\begin{array}{l}0.0133 \\
0.013\end{array}$ & 0.1850 & 0.4840 & \\
\hline 0.0997 & & 0.0607 & 0.0035 & & 0.0146 & 0.1860 & 0.4830 & \\
\hline 0.1010 & & 0.0606 & 0.0034 & & 0.0155 & 0.1850 & 0.4860 & \\
\hline 0.0550 & 0.0392 & 0.0049 & 0.0028 & & & 0.1800 & 0.5770 & \\
\hline $\begin{array}{l}0.07644 \\
0.08993\end{array}$ & & $\begin{array}{l}0.0204 \\
0.0637\end{array}$ & $\begin{array}{l}0.0140 \\
0.0275\end{array}$ & 0012 & $\begin{array}{l}0.00111 \\
0.0183\end{array}$ & $\begin{array}{l}0.1890 \\
0.1850\end{array}$ & $\begin{array}{l}0.5510 \\
0.3640\end{array}$ & $\begin{array}{l}0.0094 \\
0.0204\end{array}$ \\
\hline
\end{tabular}

0.025
0.0019
0.0024
0.0027
0.0014
0.0020
0.0009
0.0021
0.0022
0.0021
0.0023
0.0024
0.0024
0.0026
0.0026
0.0023
0.0026
0.0025
0.0026
0.0025
0.0027
0.0027
0.0024
0.0021
0.0021
0.0026
0.0022
0.0025
0.0023
0.0024
0.0025
0.0030
0.0020
0.0022
0.0017
0.0019
0.0017
0.0023
0.0018
0.0020
0.0022
0.0017
0.0019
0.0023
0.0021
0.0022
0.0016
0.0032
0.0014 
\# Glass ID

1723 DP-20
1724 DP-21

$1724 \mathrm{DP}-21$

1726 DP-23

1728 DP-BL1

1729 DP-BL2
1730 DP-centrold

1731 DZr-CV-1

1733 DZR-CV-3

1734 DZr-CV-4

1736 DZr-CV-6
1737 DZr-CV-7

1737 DZr-CV-7

1740 DZr-CV-10

1741 DZr-CV-11

1742 DZr-CV-12

1744 DZr-CV-14

1745 DZR-CV-15
1746 DZr-CV-16
1777 DrC-17

1748 DZr-CV-18

1749 DZr-CV-19

1750 DZr-CV-20

1752 DZr-CV-22

1753 DZr-CV-23
1754 DZr-CV-24

1755 HLP- 01

1756 HLP-02
1757 HLP-03

1758 HLP. 04

1760 HLP-06

1761 HLP- -07

$1762 \mathrm{HLP}-08$

1764 HLP-10

1765 HLP-11

1766 HLP-12
1767 HP-13

1768 HLP. -14

1769 HLP-15

1770 HLP-16

1772 HLP. 18

1723 HPL-19
1774 LP 19

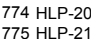

1776 HLP. 22

1777 HLP-23

178 HLP-24
1779 HLP-25
178 HP.

1780 HLP-26

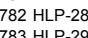

1784 HLP-30

1785 HLP-31

(7)

1788 HLP-34

1789 HLP-35
1790 HP 36

1791 HLP-37

$192 \mathrm{HLP}-38$

1793 HLP-39

1795 HLP-400

175 HLP-400
1796 HPL-41
1779 HLP-42
$1772-420$

1799 HLP-43

1800 HLP-44

1801 HLP-45

1803 HLP. 47
1804 HLP-48
Ga203-a Gd203-a

I-a La203-a MnO2-a MnO-a MoO-a MoO3-a Nb2O5-a Nd2O3-a NpO2-a PbO-a PdO2-a PdO-a Pr2O3-a Pr6011-a PuO2-a Rb2O-a ReO-a ReO2-a Re207-a Rh203-a RhO2-a RuO2-a Sb203-a Sb205-a SeO2-a

0.0515

0.0256

0.0012

0.0002

0.0001

0.0005

0.0002

0.0010 
1769 HLP-15
1770 HLP -16
171 - 17

1772 HLP-18

1733 HLP-19

1774 HLP-20
1755 HP 21

1776 HLP. 22

1777 HLP-23

1780 HLP-26
1781 HLP-27

1782 HLP-28
1783 HLP-29

184 HLP-30

785 HLP. 31

(786 HLP 32

788 HLP. 34

1789 HLP-35

1794 HLP.40

1795 HLP-400

1797 HLP-42

(799 HLP-43

1800 HLP-44

801 HLP-45

1803 HLP-47

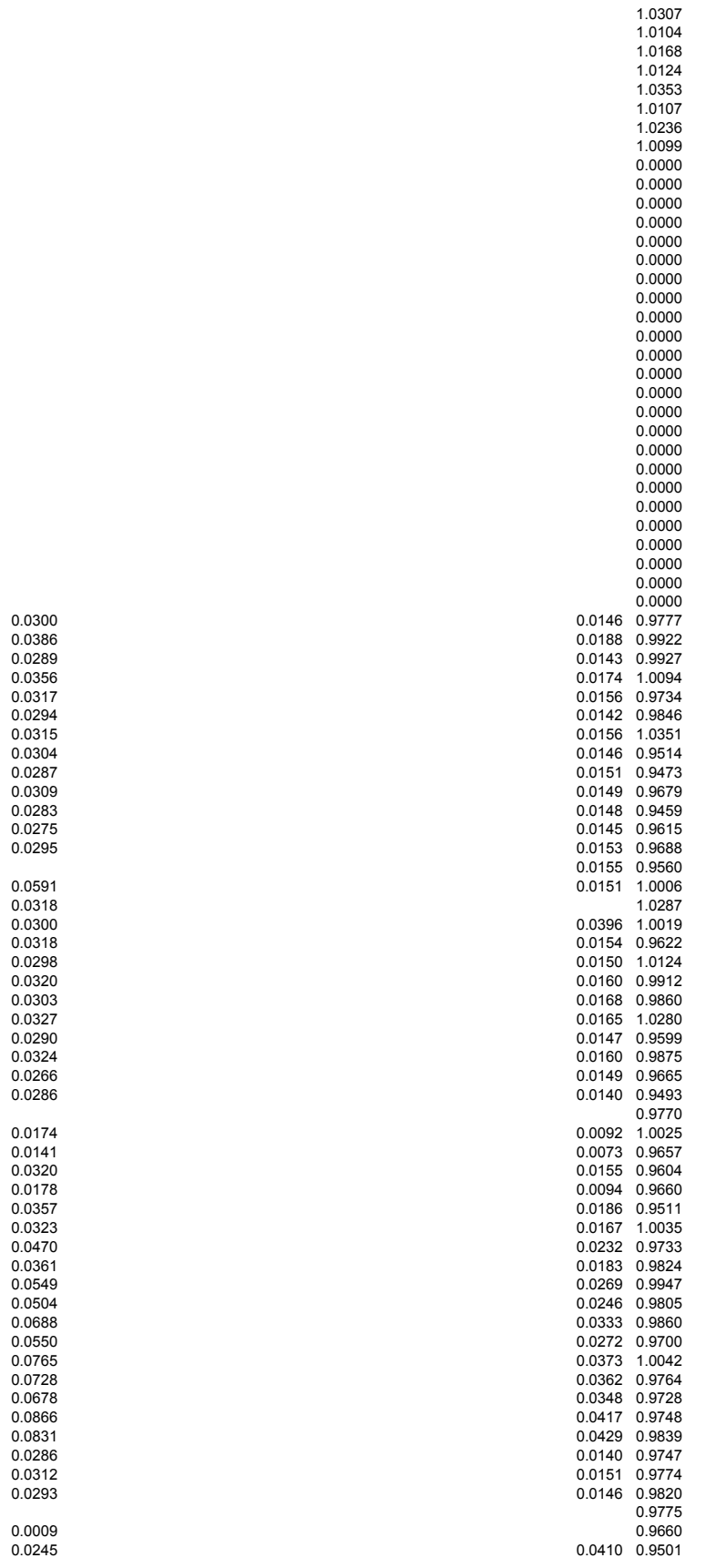




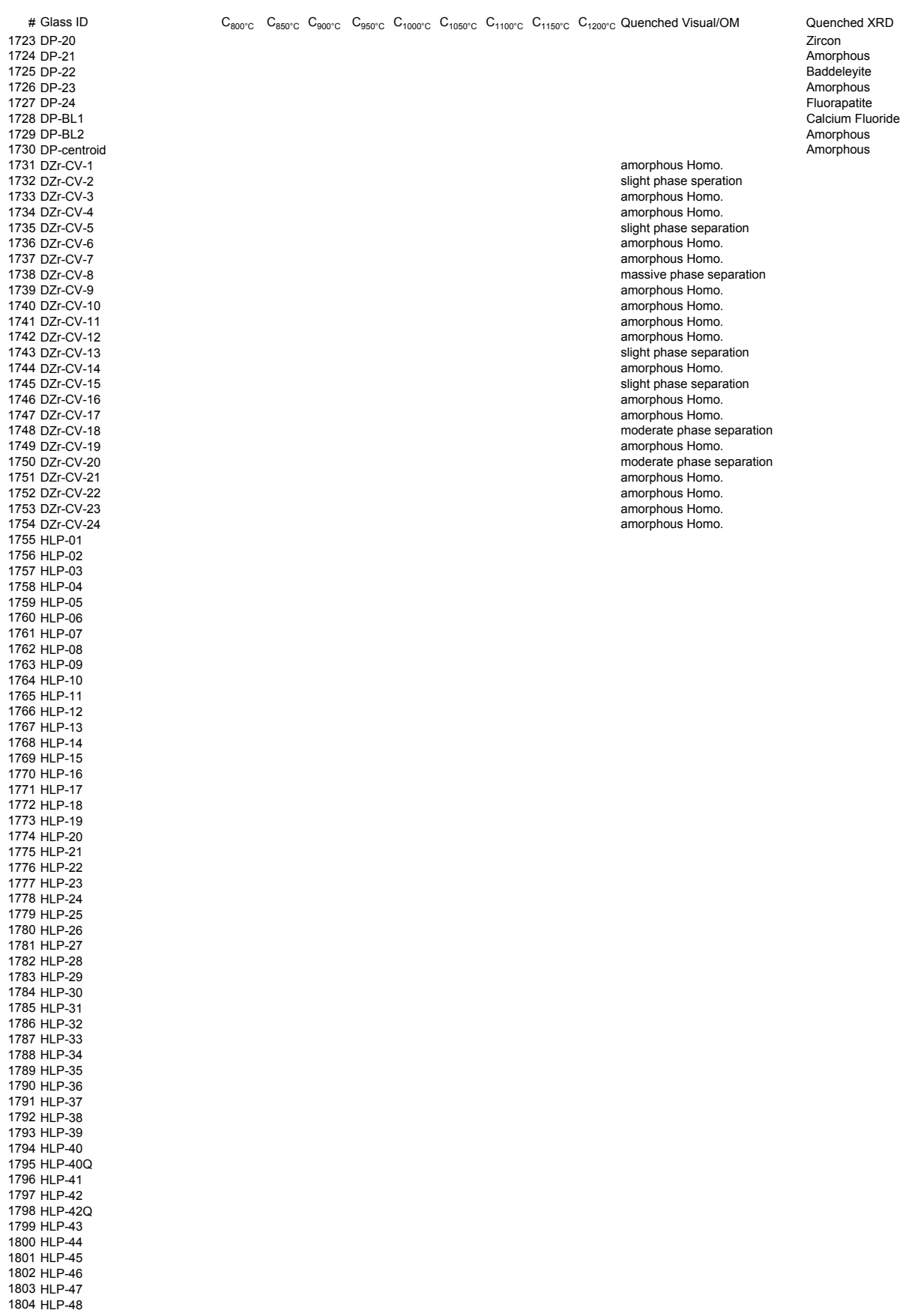

CCC XRD

Nospean, Baddeleyite

unidentifiable

Baddeleyite, Hydroxylapatite

Amorphous

no crystal hiortdahlite, 3.5 wt\% nepheline, $13.1 \mathrm{wt} \%$ sodium aluminum silicate suffice

6.2 wt\% cuspidine, 2.1 wt\% sodium aluminum silicate sulfide
1.7 wto fluorazatite

no crystal

0.3 wto fluorapatite, $3.8 \mathrm{w}$ w\% fluorite

$2.1 \mathrm{wt} \%$ fluorapatite
$3.0 \mathrm{wt} \%$ cuspidine

$1.6 \mathrm{wt} \%$ cuspidine

$5.5 \mathrm{wt} \%$ apatite

oxide fluoride phosphate

no crystal
2.4 wit fluorite

no crystal

$1.3 \mathrm{wt} \%$ cuspidine
$4.8 \mathrm{wt} \%$ fluorapatite

no crystal
8.7 wt\% sodalite

$3.7 \mathrm{wt} \%$ cuspidine, $12.1 \mathrm{wt} \%$ nosean
$0.6 \mathrm{wt} \%$ fluorapatite, 06 wto fluorite

1.0 wt\% cuspidine
0.6 wt\% fluorite, 15.3 wt\% hiortdahlite

${ }_{2.702}^{2649}$

2.636
2.683

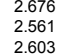

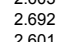

2.663

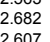

2.588
2.283
2.208

2.592
2655
205

2.600
2.669
2.690

2.626
2615
2609

2.590
2.259
2.291

2.609
2641
251

2.642
2.505
2505

2517
2569
2509

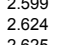

2.625
2.635
2.673

2.635
2.639
2.657
2.539
2.523
2.687

2.635
2.639
2.657
2.539
2.523
2.687 
1741 DZr-CV-11
1742 DZr-CV-12

1743 DZr-CV-12 12

1745 DZrCCV-15

1746 DZFCCV-16
747 Dr-CV 17

1748 DZZCCV

1749 DZr-CV-19

1750 DZF-CC-20
1751 Z7r-CV-21

1752 DZrCC-22
1753 DZC-CV-23

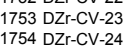

1755 HLP- -1

1756 HLP-02
1757 HLP-03

1758 HPL-DA

1760 HLP. -06

1761 HLP-06
$1712-07$

1762 HLP- -08
1763 HP

1764 HLP-10
1765 HLP- 11

1766 HLP-12

1768 HLP-14

1799 HLP-15

1770 HLP.-16
1771 HLP-17

1772 HLP-18

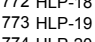

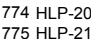

1776 HLP. 22

1777 HLP-23

1778 HLP-24

1780 HLP-26

1782 HLL-28
1783 HLP-29

1784 HLP-30

1785 HLP-31

1787 HPP.33

1788 HLP-34

1789 HLP-35

1791 HLP.-37

1792 HLP-38

1793 HLP-39

1795 HLP. 400

1956 LLP-41

1797 HLP. 42

1799 HLP-43

800 HLP-44

1801 HLP. 45
1802 HP - 46
$19604 H P-47$

803 HLP-47

1804 HLP-48 
TCLP Se TCLP Zn TCLPAI TCLP B TCLP Sb TCLP TI TCLPV Q PCT B $\left(g / \mathrm{m}^{2}\right)$ Q PCT Li $\left(g / \mathrm{m}^{2}\right)$ Q PCT Na $\left(g / \mathrm{m}^{2}\right)$ QPCT Si $\left(g / \mathrm{m}^{2}\right) \quad$ QPCT pH

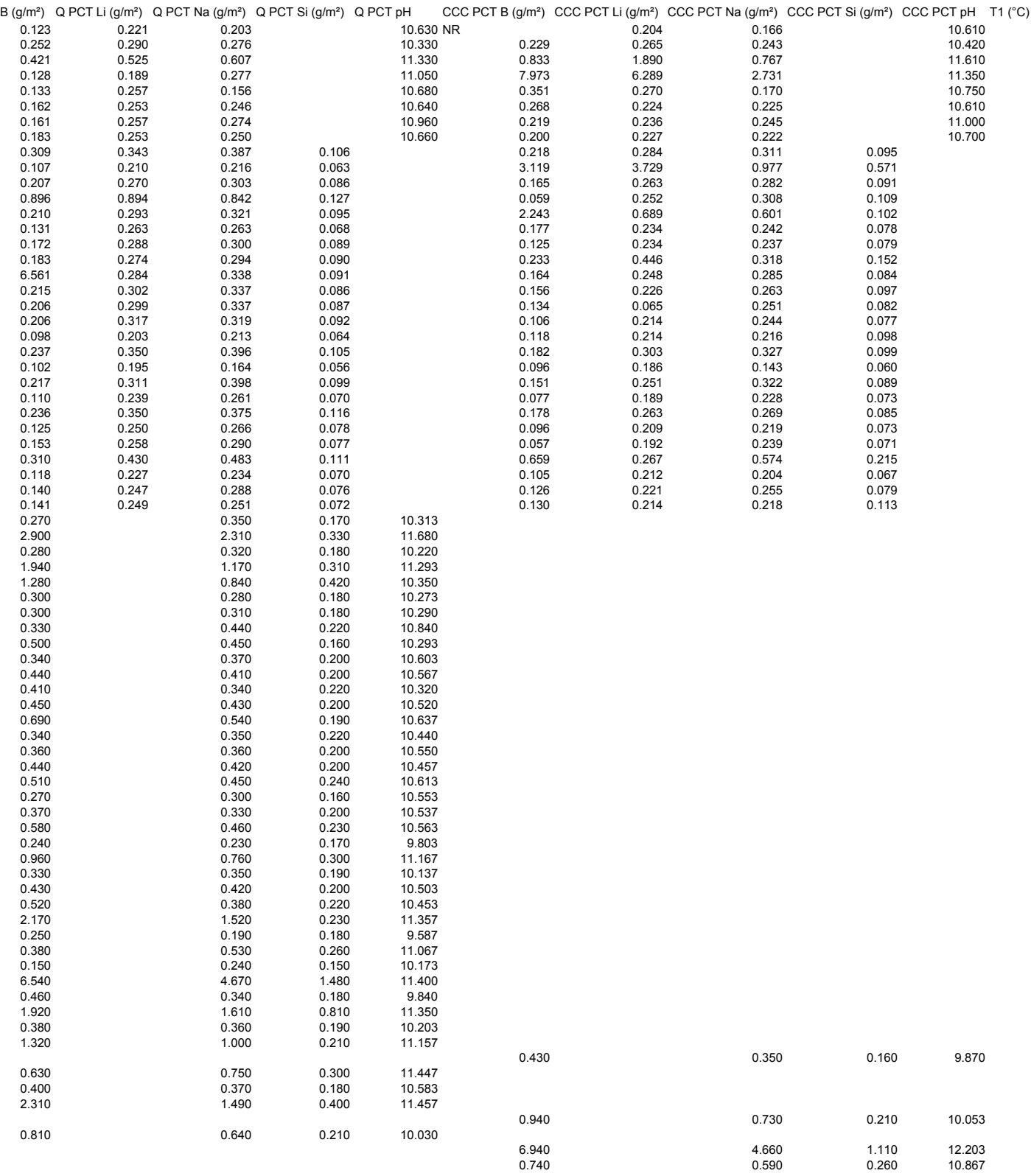


1724 DP-21

1726 DP-23

1727 DP-24

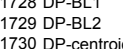

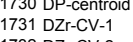

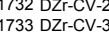

1734 DZr-CV-4

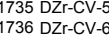

$1737 \mathrm{DZF}-\mathrm{CV}-7$

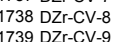

1740 Dzr-CV-10

1741 DZr-CV-11

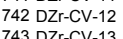

1744 DZr.CV -14
1745 Drr.CV-15

1746 DZr.CV 16

1748 Dr.cV - 18

1749 DZr-CV-19

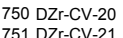

1752 DZR-CV 22
1753 DZrCVV-23

1754 DZr-CV-24

1756 HLP- 02

1756 HLP-02
157 HLP-03

1758 HLP-04

1760 HLP. -06

1761 HLP-06
$1712-07$

1762 HLP- -88

764 HLP-10
1765 HLP-11

1766 HLP-12

1768 HLP. 14

1799 HLP-15

1770 HLP-16
1771 HLP-17

1772 HLP-18
1773 HLP-19

1774 HLP-20
1755 HP -21
171 P

1776 HLP. 22

1777 HLP-23

1778 HLP-24

1780 HLP-26

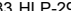

(784

1786 HP 32

1790 HLP-36

1792 HLP.

1794 HLP-40

1795 HLP-400

195

1798 HIP.420

1799 HLP-43

1500 hrp. 44

1802 HLP-46

1803 HLP-47
1804 HLP-48 


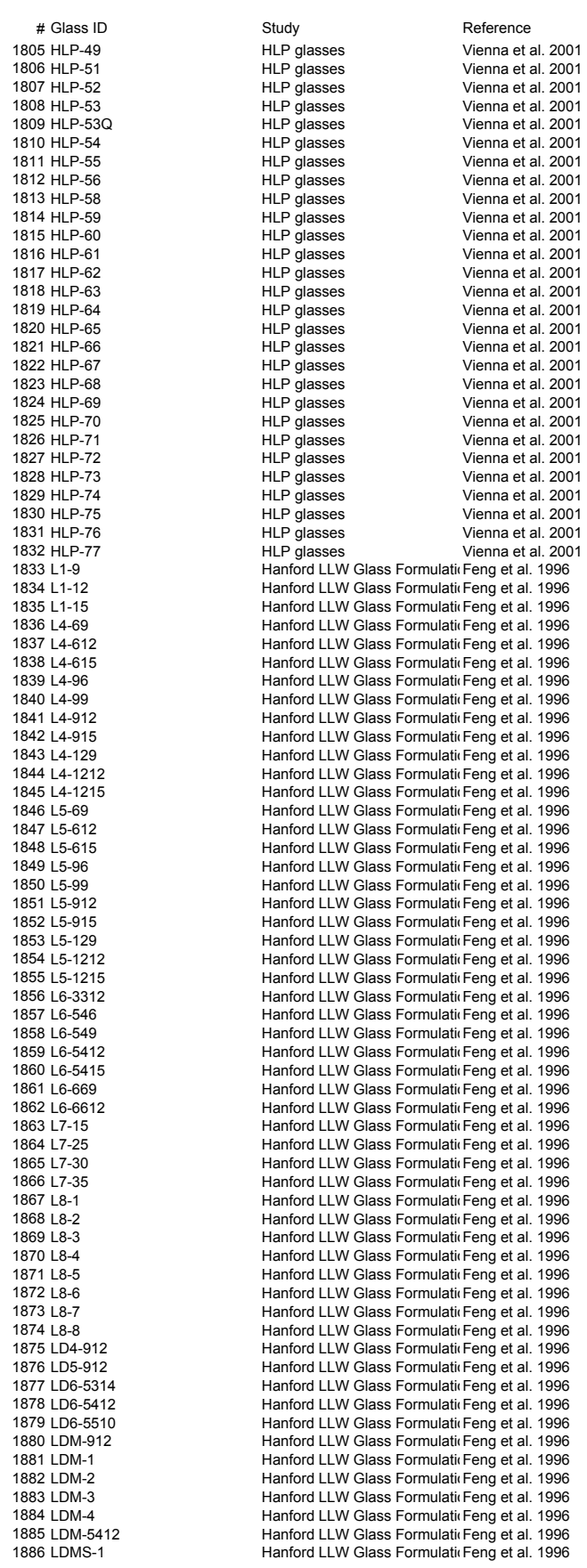

Al2O3-t B2O3-t CaO-t Fe2O3-t FeO-t $\begin{array}{llll}0.0803 & 0.0807 & 0.07703 & 0.0803 \\ 0.1000 & 0.0925 & 0.0000 & 0.0250\end{array}$ K2O-t Li2O-t MgO-t Na2O-t NiO$\begin{array}{llll}0.1019 & 0.0000 & 0.0260 & 0.0254\end{array}$ $\begin{array}{llll}0.0986 & 0.0423 & 0.0438 & 0.0730 \\ 0.0986 & 0.0423 & 0.0438 & 0.0730 \\ 0.0000 & 0.050 & 0.000 & 0.000\end{array}$ $\begin{array}{lllll}0.0900 & 0.0900 & 0.0000 & 0.0000\end{array}$ $\begin{array}{llll}0.0620 & 0.0890 & 0.0199 & 0.0698 \\ 0.0686 & 0.0980 & 0.0200 & 0.0539\end{array}$ $\begin{array}{llll}0.0686 & 0.0980 & 0.0200 & 0.0539\end{array}$ $\begin{array}{llll}0.1194 & 0.1257 & 0.0001 & 0.05171 \\ 0.0400 & 0.1257 & 0.0500 & 0.0000\end{array}$ $\begin{array}{llll}0.0400 & 0.1257 & 0.0500 & 0.0000 \\ 0.0400 & 0.1257 & 0.0500 & 0.0000\end{array}$ $\begin{array}{llll}0.04400 & 0.1257 & 0.0500 & 0.0000 \\ 0.0400 & 0.0600 & 0.0001 & 0.1504 \\ 0 & 0.0000\end{array}$ $\begin{array}{llll}0.0400 & 0.0600 & 0.0001 & 0.0000 \\ 0.0400 & 0.0605 & 0.0500 & 0.0982\end{array}$ $\begin{array}{llll}0.0400 & 0.0600 & 0.0500 & 0.0982 \\ 0.0400 & 0.1257 & 0.0001 & 0.0536\end{array}$ $\begin{array}{lllll}0.0400 & 0.1257 & 0.0001 & 0.0443 \\ 0.0964 & 0.0600 & 0.0500 & 0.1577\end{array}$ $\begin{array}{lllll}0.1194 & 0.0600 & 0.00001 & 0.0024 \\ 0.1194 & 0.0000 & 0.050\end{array}$ $\begin{array}{llll}0.1194 & 0.0600 & 0.0500 & 0.0040\end{array}$ $\begin{array}{llll}0.0400 & 0.0600 & 0.0001 & 0.1577 \\ 0.1194 & 0.1257 & 0.0001 & 0.1359\end{array}$ $\begin{array}{llll}0.1194 & 0.1257 & 0.0001 & 0.1359 \\ 0.1194 & 0.1257 & 0.0500 & 0.1131\end{array}$ $\begin{array}{llll}0.0743 & 0.0893 & 0.0231 & 0.0554\end{array}$ $\begin{array}{llll}0.1000 & 0.0800 & 0.0050 & 0.0100 \\ 0.1000 & 0.0800 & 0.0050 & 0.0100 \\ 0.090 & & & 0.000\end{array}$ 0.0900 0.1500 $\begin{array}{lll}0.1500 & & 0.0000 \\ 0.0900 & 0.0600 & 0.0000 \\ 0.1200 & 0.0600 & 0.0000 \\ .0000 & 0.9000 & 0.0000\end{array}$ $\begin{array}{lll}0.1500 & 0.0600 & 0.0000 \\ 0.0600 & 0.0900 & 0.000 \\ 0 & 0.0000\end{array}$ $\begin{array}{lll}0.0900 & 0.0900 & 0.0000 \\ 0.1200 & 0.0900 & 0.0000 \\ 0 & \end{array}$ $0.1500 \quad 0.0900$

$\begin{array}{ll}0.0900 & 0.1200 \\ 0.1200 & 0.1200 \\ 0.150 & 0.120\end{array}$

0.0000
0.0000
0.06000
0.0000

$\begin{array}{lll}0.0900 & 0.0600 & 0.0000 \\ 0.1200 & 0.0600 & 0.0000 \\ 0 & 0.0000\end{array}$ $\begin{array}{lll}0.1200 & 0.0600 & 0.0000 \\ 0.1500 & 0.0600 & 0.0000 \\ 0.0600 & 0.0000 & 0.0000 \\ 0 & & 0.0000\end{array}$

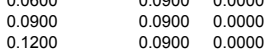
$\begin{array}{lll}0.1500 & 0.0900 & 0.0000 \\ 0.0900 & 0.1200 & 0.0000 \\ 0.12000 & 0.1200 & 0.0000\end{array}$ $\begin{array}{lll}0.1200 & 0.1200 & 0.0000 \\ 0.1500 & 0.1200 & 0.0000\end{array}$ $\begin{array}{llll}0.1500 & & 0.1200 & 0.0000 \\ 0.1200 & 0.0300 & 0.0300 & 0.0000 \\ 0.0600 & 0.0500 & 0.0400 & 0.000\end{array}$ $\begin{array}{llll}0.0900 & 0.0500 & 0.0400 & 0.0000 \\ 0.1200 & 0.0500 & 0.400 & 0.0000\end{array}$ $\begin{array}{llll}0.1200 & 0.0500 & 0.0400 & 0.0000 \\ 0.1500 & 0.0500 & 0.0400 & 0.0000\end{array}$ $\begin{array}{llll}0.0900 & 0.0600 & 0.0600 & 0.00000 \\ 0\end{array}$ $\begin{array}{llll}0.1200 & 0.0600 & 0.0660 & 0.00000 \\ 0.1245 & 0.0539 & 0.0431 & 0.0000 \\ 0.1155 & 0.049 & 0.0039\end{array}$ $\begin{array}{llll}0.1155 & 0.0461 & 0.0369 & 0.0000 \\ 0.1110 & 0.0422 & 0.0338 & 0.0001\end{array}$ $\begin{array}{llll}0.1065 & 0.0384 & 0.0307 & 0.0001 \\ 0.0900 & 0.0600 & 0.0000 & 0.000\end{array}$

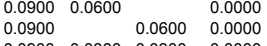
$\begin{array}{llll}0.0900 & 0.000 & 0.0600 & 0.00000 \\ 0.0900 & 0.0300 & 0.0300 & 0.0000 \\ 0.0900 & 0.0600 & & 0.0600\end{array}$

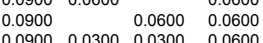
$\begin{array}{llll}0.0900 & 0.0300 & 0.0300 & 0.0660 \\ 0 & 0900 & 0.0600 & \end{array}$

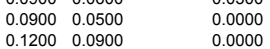
$\begin{array}{lll}0.1200 & 0.0900 \\ 0.1200 & 0.0900 & 0.0000 \\ 0 & 0.0000\end{array}$ $\begin{array}{llll}0.1200 & 0.000 & 0.0900 & 0.00000 \\ 0.1400 & 0.0500 & 0.0300 & 0.0000 \\ 0.1200 & 0.0500 & 0.0400 & 0.0000\end{array}$ $\begin{array}{llll}0.1000 & 0.0500 & 0.0500 & 0.0000\end{array}$

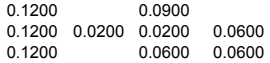
$\begin{array}{llll}0.1200 & 0.020 & 0.0600 & 0.0600 \\ 0.1200 & 0.0600 & & \end{array}$ $\begin{array}{llll}0.1200 & 0.0600 \\ 0.1000 & 0.0600 & 0.0600 & 0.0600\end{array}$ $\begin{array}{llll}0.1200 & 0.0500 & 0.0400 & \\ 0.1200 & 0.0200 & 0.0200 & 0.0600\end{array}$

\begin{tabular}{|c|c|c|c|c|c|c|}
\hline 0.0036 & & 0.0300 & 0.1000 & 0.000 & 0.439 & 0.0304 \\
\hline & 0.0000 & 0.0100 & 0.2000 & 0.0008 & 0.4189 & $\begin{array}{l}0.0525 \\
0.0504\end{array}$ \\
\hline & 0.0000 & 0.0118 & 0.2874 & 0.0191 & 0.4464 & 0.0502 \\
\hline 0310 & $\begin{array}{l}0.0204 \\
0.0204\end{array}$ & $\begin{array}{l}0.02204 \\
0.0204\end{array}$ & $\begin{array}{l}0.2000 \\
0.2000\end{array}$ & $\begin{array}{l}0.0008 \\
0.0008\end{array}$ & $\begin{array}{l}0.4015 \\
0.4015\end{array}$ & $\begin{array}{l}0.0299 \\
0.0299\end{array}$ \\
\hline 0033 & 0.0000 & & 0.2000 & & & \\
\hline 0033 & 0.0000 & 0.0000 & 0.2000 & 0.0119 & 0.5978 & \\
\hline & 0.0000 & 0.0199 & 0.2000 & 0.0003 & 0.4455 & 0.0299 \\
\hline & 0.0000 & $\begin{array}{l}0.0147 \\
0.0143\end{array}$ & $\begin{array}{l}0.1960 \\
0\end{array}$ & $\begin{array}{l}0.0006 \\
0.0006\end{array}$ & 0.4809 & 0.0147 \\
\hline & $\begin{array}{l}0.0000 \\
0.0000\end{array}$ & $\begin{array}{l}0.0043 \\
0.0430\end{array}$ & $\begin{array}{l}0.1900 \\
0.1600\end{array}$ & $\begin{array}{l}0.0006 \\
0.0200\end{array}$ & $\begin{array}{l}0.4662 \\
0.3600\end{array}$ & $\begin{array}{l}0.0143 \\
0.0600\end{array}$ \\
\hline & 0.0000 & 0.0430 & 0.1600 & & 0.3965 & \\
\hline & .0000 & 0.0000 & 0.1600 & 0.0004 & 0.4692 & 0.06 \\
\hline & 0.0000 & 0.0430 & 0.2514 & 0.0004 & 0.3600 & 0.0000 \\
\hline & 0.0000 & 0.0430 & 0.1600 & 0.0200 & 0.5200 & 0.0600 \\
\hline & 0.0000 & 0.0430 & 0.1600 & 0.0200 & 0.5200 & $\begin{array}{l}0.0000 \\
0.0000\end{array}$ \\
\hline .0500 & $\begin{array}{l}0.0000 \\
0.0000\end{array}$ & $\begin{array}{l}0.0000 \\
0.0000\end{array}$ & $\begin{array}{l}0.2214 \\
0.2514\end{array}$ & $\begin{array}{l}0.0004 \\
0.0200\end{array}$ & $\begin{array}{l}0.52200 \\
0.3600\end{array}$ & $\begin{array}{l}0.00000 \\
0.0600\end{array}$ \\
\hline .0500 & 0.0000 & 0.0000 & 0.1600 & 0.0004 & 0.3600 & 0.0600 \\
\hline 0.0033 & 0.0000 & 0.0000 & 0.1600 & 0.0004 & 0.5200 & \\
\hline & 0000 & & & & & \\
\hline 033 & 0.0000 & 0.0430 & 0.2514 & 0.0004 & 0.3600 & 0.0600 \\
\hline 0.0500 & 0.0000 & 0.0430 & $\begin{array}{l}0.11600 \\
0.1600\end{array}$ & $\begin{array}{l}0.0200 \\
0.0004\end{array}$ & $\begin{array}{l}0.3645 \\
0.3600\end{array}$ & $\begin{array}{l}0.06000 \\
0.0000\end{array}$ \\
\hline 0.0033 & 0.0000 & & & & 0.3600 & \\
\hline .0249 & 0.0000 & 0.0200 & 0.1984 & & 0.4155 & 0.0270 \\
\hline $\begin{array}{l}.0150 \\
0.150\end{array}$ & 0.0010 & 0.0010 & $\begin{array}{ll}0.2000 & 0.0010\end{array}$ & & 0.5437 & 0.0100 \\
\hline $\begin{array}{l}0.0030 \\
0.0033\end{array}$ & & & $\begin{array}{l}0.2000 \\
0.2000\end{array}$ & $\begin{array}{l}0.0050 \\
0.0119\end{array}$ & $\begin{array}{l}0.5437 \\
0.6878\end{array}$ & $\begin{array}{l}0.0100 \\
0.0000\end{array}$ \\
\hline .0033 & & & & & & \\
\hline .0033 & & & 0.2000 & 0.0119 & 0.6278 & 0.0000 \\
\hline & & & $\begin{array}{l}0.2000 \\
02000\end{array}$ & $\begin{array}{l}0.0119 \\
0.0119\end{array}$ & $\begin{array}{l}0.6278 \\
0.578\end{array}$ & $\begin{array}{l}0.0000 \\
0\end{array}$ \\
\hline .0033 & & & 0.2000 & 0.0119 & 0.5678 & 0.0000 \\
\hline & & & 0.2000 & & & \\
\hline & & & 0.2000 & 0.0119 & 0.5978 & 0.0000 \\
\hline & & & $\begin{array}{l}0.2000 \\
0.2000\end{array}$ & $\begin{array}{l}0.0119 \\
0.0119\end{array}$ & $\begin{array}{l}0.5678 \\
0.5788\end{array}$ & $\begin{array}{l}0.00000 \\
0.0000\end{array}$ \\
\hline & & & & & 0.5678 & \\
\hline & & & & 0.0119 & & 0.0000 \\
\hline & & & 0.2000 & 0.0119 & 0.5078 & \\
\hline & & & 0.2000 & 0.0119 & 0.6278 & 0.0000 \\
\hline & & & 0.2000 & 0.0119 & 0.5978 & 0.0000 \\
\hline & & & & & & \\
\hline & & & & & $\begin{array}{l}0.6278 \\
0.5978\end{array}$ & $\begin{array}{l}0.0000 \\
0.0000\end{array}$ \\
\hline 0.0033 & & & 0.2000 & 0.0119 & 0.5678 & 0.0000 \\
\hline & & & & & & \\
\hline & & & & & & \\
\hline 3 & & & 0.2000 & 0.0119 & 0.5378 & 0.0000 \\
\hline & & & & 0.0119 & $\begin{array}{l}0.5078 \\
0.5978\end{array}$ & 0.0000 \\
\hline & & & & & $\begin{array}{l}.56278 \\
0.6278\end{array}$ & 0.0000 \\
\hline & & & & & & \\
\hline & & & & & & \\
\hline & & & 0.20 & & 0.5378 & 0.0000 \\
\hline & & & 0.2000 & & 0.5678 & \\
\hline & & & & 0.0119 & & 0.0000 \\
\hline & & & & 0.0148 & 0.5238 & $\begin{array}{l}0.0000 \\
0.0001\end{array}$ \\
\hline 0.00 & & & & & & 0.0001 \\
\hline & & & & & & \\
\hline & & & & 0.0119 & & 1.0600 \\
\hline & & & $\begin{array}{l}0.2000 \\
0.2000\end{array}$ & & & \\
\hline & & & & & & \\
\hline & & & & & & \\
\hline & & & & & & 0.0000 \\
\hline & & ก0400 & & 0.0119 & 0.5678 & 0.0300 \\
\hline 0.003 & & & 年 & 0.0119 & $\begin{array}{l}0.5799 \\
05591\end{array}$ & \\
\hline & & 0.00 & 0.2 & 0.0019 & & \\
\hline & & & & & & \\
\hline & & & & & & \\
\hline & & 0.0000 & & & & \\
\hline & & & & & & \\
\hline & & & & & & \\
\hline & & & & & & \\
\hline & & & & & & 0.0400 \\
\hline & & & 0.2000 & & & \\
\hline & & & & 0019 & 5014 & \\
\hline
\end{tabular}

\begin{tabular}{|c|c|c|c|}
\hline $\begin{array}{l}0.0001 \\
0.0000\end{array}$ & $\begin{array}{l}0.0001 \\
0.0001 \\
0.0001 \\
0.0001 \\
0.0001 \\
0.0001 \\
0.0001 \\
0.0001 \\
0.0001 \\
0.0001 \\
0.0001 \\
0.0001 \\
0.0001 \\
0.0001 \\
0.0001 \\
0.0001 \\
0.0001 \\
0.0001 \\
0.0001 \\
0.0001 \\
0.0001 \\
0.0001 \\
0.0001 \\
0.0001 \\
0.0001 \\
0.0001 \\
0.0001 \\
0.0001 \\
0.0001 \\
0.0001 \\
0.0001 \\
0.0002 \\
0.0002 \\
0.0002 \\
0.0001 \\
0.0001 \\
0.0001 \\
0.0001 \\
0.0001 \\
0.0001 \\
0.0001 \\
0.00001\end{array}$ & $\mathrm{Br}-\mathrm{t} \quad \mathrm{CdO}-\mathrm{t} \quad \mathrm{Ce} 2 \mathrm{O}-\mathrm{t} \mathrm{CeO} 2-\mathrm{t}$ & 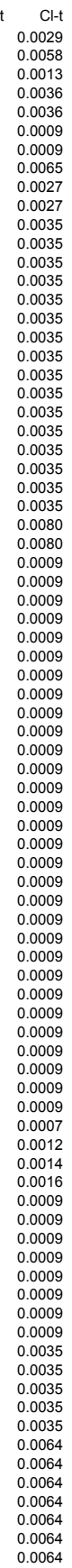 \\
\hline
\end{tabular}




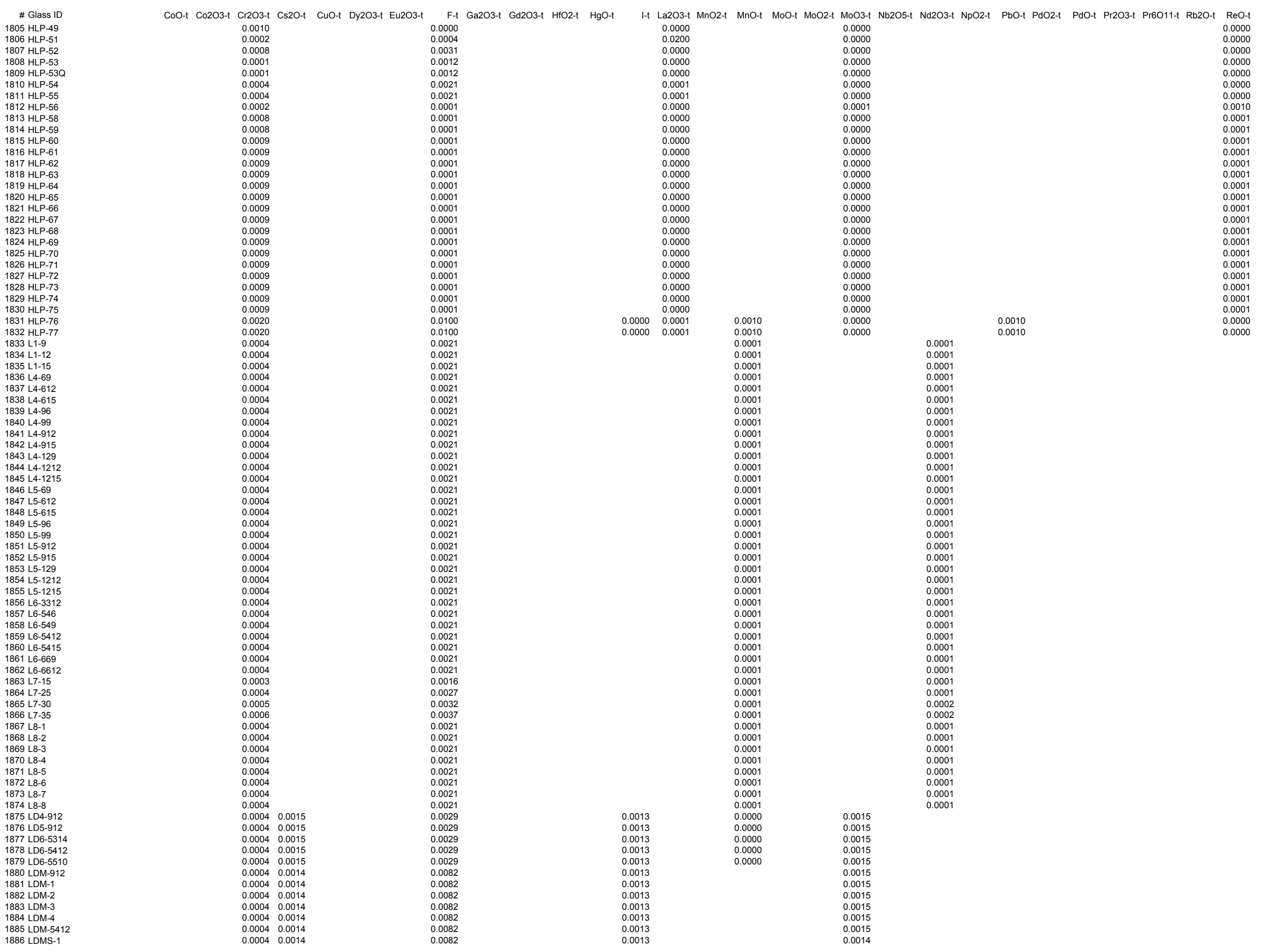


TiO2-t T12O-t T1203-t U3O8-t UO2-t UO3-t V2O5-t WO3-t Y2O3-t

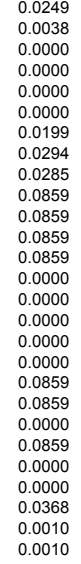
$\begin{array}{lll}\text { ZnO-t } & \text { Sum-t } \\ 0.0399 & 0.9998 & 0.073 \\ 0.0200 & 0.077\end{array}$ $0.02601 .0000 \quad 0.0968$ $\begin{array}{llll}0.0000 & 1.0000 \\ 0.0328 & 0.90998 & 0.00939 \\ 0.0931\end{array}$ $\begin{array}{lll}0.0328 & 0.9998 \\ 0.0000 & 0.9997 & 0.0979 \\ 0.117 & 0.9095\end{array}$ $0.00000 .9997 \quad 0.0887$ $\begin{array}{llll}0.0296 & 0.9997 & 0.0633\end{array}$

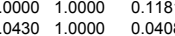

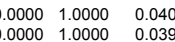
0.04141 .00000 .00404

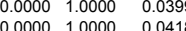
0.033010000000041 0.000001 .000000 .0094 $\begin{array}{lll}0.0430 & 1.0000 & 0.113 \\ 0.0000 & 1.0000 & 0.1179\end{array}$

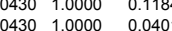
0.0000
0.00000
0 $\begin{array}{lll}0.0430 & 1.0000 & 0.1199 \\ 0.0200 & 1.0001 & 0.0762\end{array}$ $\begin{array}{lll}0.0000 & 0.9989 & 0.0997\end{array}$ 1.0000 1.0000 1.0000 1.0000
1.0000 1.0000
1.0000
1 1.0000
1.0000
1.0000 1.0000
1.0000 1.0000 1.0000
1.0000
1.0000
1.000 1.0000 
\# Glass ID 1805 HLP-49 1807 HLP-52 1808 HLP-53
1809 HLP-53Q
1810 HLP.54 1811 HLP- 55 1812 HLP-56 1813 HLP-58 1815 HLP-60 1816 HLP-61

817 HLP-62

1819 HLP-64

1820 HLP-65

1821 HLP-66

1823 HLP-68

1824 HLP-69

1826 HLP-71

1827 HLP.72
1828 HLP-73

1829 HLP-74

1831 HLP-76

1832 HLP. 77

1834 L1-12
1835 L1-15

1835 L L-1 15
1836 L4-69

1839 L4-96

1840 L4 L-99
1841 L4-912

$1842 \angle 4-915$

1843 L4-129
1844 L4-1212

1846 L5-69

1847 L5-612

1850 L5-99

1852 L5-915
$115355-129$

1854 L5-1212

1855 L5-121

1856 L6-3312

1858 L6-549

1860 L6-5415

1861 L6-669

1865 L7-30

1866 L7-35

1867 L8-1

1868 L8-2

1870 L8-4

1871 L8-5

1873 L8-7

1874 L8-8
1875 LD4-912

877 LD6-5314

1878 LD6-5412

(1800 DM-912

1881 LDM-1

1883 LDM-

1885 LDM-5412
B203-a CaO-a Fe203-a FeO-a K2O-a Li2O-a MgO-a Na2O-a NiO-a P2O5-a SiO2-a Zro2-a Ag2O-a As203-a As205-a BaO-a BeO-a Bi2O3-a Br-a CdO-a Ce203-a CeO2-a Cl-a CoO-a Co203-a Cr2O3-a Cs2O-a CuO-a F-a

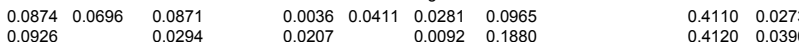

$0.0010 \quad 0.0251 \quad 0.0300$

$0.0546 \quad 0.0421 \quad 0.0767$

0.0892

$\begin{array}{ll}0.4580 & 0.0254 \\ 0.3950 & 0.0276 \\ 0.4080 & 0.0282\end{array}$

$\begin{array}{lllll}0.0275 & 0.0229 & 0.0193 & 0.1860 \\ 0.0302 & 0.0213 & 0.0184 & 0.2040 & 0\end{array}$

$\begin{array}{ll}0.4080 & 0.0282 \\ 0.5640 & 0.0007\end{array}$

$\begin{array}{lll}0.0931 & 0.0206 & 0.0705 \\ 0.0998 & 0.0202 & 0.0545\end{array}$

$0.12250 .0002 \quad 0.0185$

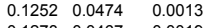

$\begin{array}{lll}0.1278 & 0.0467 & 0.0019 \\ 0.0600 & 0.0001 & 0.1452\end{array}$

$\begin{array}{lll}0.0609 & 0.0016\end{array}$

$\begin{array}{lll}0.0602 & 0.0466 & 0.0990 \\ 0.1257 & 0.0004 & 0.0544\end{array}$

$\begin{array}{llll}0.1232 & 0.0002 & 0.0443\end{array}$

$\begin{array}{lll}0.0624 & 0.0470 & 0.1590 \\ 0.0611 & 0.0038 & \end{array}$

$\begin{array}{lll}0.0616 & 0.0456 & 0.0023\end{array}$

$\begin{array}{llll}0.0615 & 0.0017 & 0.1533\end{array}$

$\begin{array}{lll}0.1220 & 0.0004 & 0.1539 \\ 0.1245 & 0.0473 & 0.1108\end{array}$

$\begin{array}{lll}0.1245 & 0.0473 & 0.1340 \\ 0.0943 & 0.0238 & 0.0585\end{array}$

$\begin{array}{lll}0.0785 & 0.0054 & 0.0157 \\ 0.0790 & 0.0049 & 0.0167\end{array}$

$\begin{array}{llll}0.0032 & & 0.17 \\ 0.0212 & 0.0204 & 0.18 \\ 0 & 0.000\end{array}$

$\begin{array}{ll}0.6060 & 0.01 \\ 0.4690 & 0.0135\end{array}$

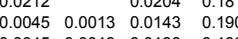

$\begin{array}{llll}0.0008 & 0.4622 & 0.0135 \\ 0.0005 & 0.4482 & 0.0146\end{array}$

$\begin{array}{llll}0.0037 & 0.0012 & 0.0387 & 0.15\end{array}$

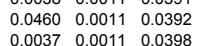

$\begin{array}{llllllll}0.0037 & 0.0011 & 0.0398 & 0.1599 & & 0.0106 & 0.5296 & 0.0424 \\ 0.048 & & 0.0198 & 0.5340 & 0.024\end{array}$

$0.0464 \quad 0.0012$

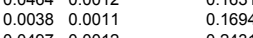

$\begin{array}{llll}0.0037 & 0.0013 & 0.0391 & 0.2447 \\ 0.0037 & 0.0015 & 0.0004 & 0.1472 \\ 0.0478 & 0.013 & 0.1691 & 0.156\end{array}$

$\begin{array}{llll}0.0478 & 0.0013 & 0.00391 & 0.1652 \\ 0.0042 & 0.0014\end{array}$

$\begin{array}{llll}0.0264 & 0.0028 & 0.0195 & 0.1925\end{array}$

$\begin{array}{llll}0.0164 & 0.0018 & 0.0009 & 0.1938 \\ 0.0156 & 0.0019 & 0.0006 & 0.2029\end{array}$

$\begin{array}{llll}0.0009 & 0.5389 & 0.0007 \\ 0.0193 & 0.3799 & 0.0005\end{array}$

$\begin{array}{llll}0.0004 & 0.3579 & 0.0612 \\ .0087 & 0.3701 & 0.0385\end{array}$

$\begin{array}{lll}0.0005 & 0.3532 & 0.000 \\ 0.0201 & 0.3584 & 0\end{array}$

$\begin{array}{lll}0.0080 & 0.4225 & 0.0253\end{array}$

$\begin{array}{lll}0.0052 & 0.5546 & 0.0111 \\ 0.0052 & 0.5612 & 0.0101\end{array}$

$\begin{array}{lll} & 0.0024 & \\ & 0.00014 & \\ & 0.0021 & \\ & 0.0014 & \\ & 0.0016 & \\ & 0.0017 & \\ 0.0027 & 0.0002 & \\ 0.0031 & 0.0008 & 0.0005 \\ 0.0031 & 0.0008 & 0.0005 \\ 0.0032 & 0.0010 & 0.0011 \\ 0.0032 & 0.0010 & 0.0005 \\ 0.0036 & 0.0007 & 0.0015 \\ 0.0033 & 0.0008 & 0.0005 \\ 0.0032 & 0.0007 & 0.0005 \\ 0.0034 & 0.0008 & 0.0005 \\ 0.0035 & 0.0009 & 0.0005 \\ 0.0029 & 0.0009 & 0.0005 \\ 0.0030 & 0.0008 & 0.0005 \\ 0.0037 & 0.0007 & 0.0005 \\ 0.0036 & 0.0007 & 0.0005 \\ 0.0033 & 0.0009 & 0.0005 \\ 0.0032 & 0.0008 & 0.0005 \\ 0.0034 & 0.0009 & 0.0005 \\ 0.0028 & 0.0008 & 0.0005 \\ 0.0011 & 0.0009 & 0.0005 \\ 0.0010 & 0.0019 & 0.0086 \\ & 0.0018 & 0.0086\end{array}$

\begin{tabular}{|c|c|c|c|c|c|c|c|c|c|}
\hline 0.0505 & 0.0412 & 0.0011 & $0.0166 \quad 0.0001$ & & 0.2041 & 0.0003 & 0.0022 & 0.5544 & 0.0008 \\
\hline & 0. & 0.00 & 0.0167 & 0.0010 & 0.1992 & & 0.0024 & 0.5530 & \\
\hline 0.0201 & 0.0178 & 0.0621 & 0.0146 & & 0.2105 & & 0.0046 & 0.5006 & 0.0402 \\
\hline & & $\begin{array}{l}0.0619 \\
0.0005\end{array}$ & $\begin{array}{l}0.0179 \\
0.0230\end{array}$ & & $\begin{array}{l}0.2030 \\
0.1885\end{array}$ & 0.0005 & $\begin{array}{l}0.0028 \\
0.0028\end{array}$ & $\begin{array}{l}0.5187 \\
0.5350\end{array}$ & $\begin{array}{l}0.0001 \\
0.0527\end{array}$ \\
\hline & 0.0648 & 0.0614 & 0.0202 & & 0.2036 & 0.0003 & 0.0027 & 0.4370 & 0.0382 \\
\hline & 0.0394 & 0.0006 & 0.0213 & 0.0015 & 0.2122 & & 0.0062 & 0.5356 & \\
\hline & 0.0205 & 0.0607 & 0.02500 & 0.0011 & 0.2083 & 0.0002 & 0.0028 & 0.4803 & \\
\hline
\end{tabular}


\# Glass ID

1805 HLP-49

1807 HLP-52

1808 HLP-53

1809 HLP-530
1810 HLP-54

1810 HLP-54
1811 HLP-55

1812 HLP-56

1813 HLP-58

1815 HLP-60

1816 HLP-61

1817 HLP- 62

1819 HLP-64

$1820 \mathrm{HLP}-65$

1821 HLP. 66

1823 HLP-68

1824 HLP-69

1828 HLP-73

1831 HLP-76

1832 HLP.77

1833 L1-9
1834 L1-12
1835 L1-15

1836 L4-69

1837 L4-612
1838 L4-615

1839 L4-96

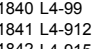

1842 L4-915
1843 L L-129

1844 L4-12121
$18454-1215$

1846 L5-69

$18475-5612$

1844 L5-615

1850 L5-99

1855 L5-912

$1852 \quad$ L5.915
1853 L5-129

1854 L5-1212

1855 L5-1215

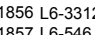

1858 L6-549

1859 L6-5412

860 L6-5415
$86166-669$

1861 L6-669
1862 L6-6612

1863 L7-15

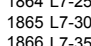

1867 L8-1

1868 L8-2
$186998-3$

1869 L8-3
1870 L L8-4

$1871<8$

1874 L.8
1875 LD4.912
1876 .

1877 L66-53.4

1878 LD6-5412

(1890.55

1881 LDM-1

$882 \mathrm{LDM}-2$

1883 LDM-3

1885 LDM-5412
1886 LDMS-1
Ga2O3-a Gd2O3-a

1-a La203-a MnO2-a MnO-a MoO-a MoO3-a Nb205-a Nd203-a NpO2-a PbO-a PdO2-a PdO-a Pr2O3-a Pr6011-a PuO2-a Rb2O-a ReO-a ReO2-a Re207-a Rh203-a RhO2-a RuO2-a Sb203-a Sb205-a SeO2-a 0.0194

$\begin{array}{ll}0.0001 & 0.0002 \\ 0.0001 & 0.0002 \\ 0.0001 & 0.0002 \\ 0.0001 & 0.0002 \\ 0.0001 & 0.0002 \\ 0.0001 & 0.0002 \\ 0.0001 & 0.0002 \\ 0.0001 & 0.0002 \\ 0.0001 & 0.0002 \\ 0.0001 & 0.0002 \\ 0.0001 & 0.0002 \\ 0.0001 & 0.0002 \\ 0.0001 & 0.0002 \\ 0.0001 & 0.0002 \\ 0.0001 & 0.0002 \\ 0.0001 & 0.0002 \\ 0.0001 & 0.0002 \\ 0.0001 & 0.0002 \\ 0.0001 & 0.0009 \\ 0.0001 & 0.0008\end{array}$

$\begin{array}{lll}0.0001 & 0.0013 & 0.0006 \\ & 0.0016 & \\ 0.0001 & 0.0015 & 0.0005 \\ & 0.0015 & 0.0006 \\ & 0.0016 & 0.0007 \\ 0.0001 & 0.0015 & 0.0008 \\ 0.0001 & 0.0016 & 0.0006 \\ & 0.0013 & 0.0008\end{array}$


0.00180 .0009

0.00260 .0012

0.002310 .0011 0.0026
0.0009
0.0012
0.02611
0.0009

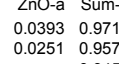

0.0309

0.03241 .0049

0.9573
0.9716
0.09937
0.9937

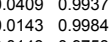

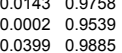

0.0399 .0 .085

0.03730 .09742

0.00010 .0950

0.0395
0.030 .138
$0.0394 \quad 1.0250$
0.0356

0.0393
0.0386
0.098742

0.0386
0.0001
0.9779

0.0001
0.0401
0.09894
0.0197
0.0116

0.00010 .9991

政

0.0000

0.0000

0.0000
0.0000
0

0.0000
0.0000
0

0.0000
0.0000

0.0000
0.00000
0.0000

0.0000
0.0000

0.0000
0.0000
0.0000

0.0000
0.0000

0.0000
0.0000
0

0.0000

0.0000
0.0000
0.000

0.0000

0.0000
0.0000
0.0000
0.0000

0.0000

0.0000
0.0000
0

0.0000

0.0000
0.0000

0.0000

$\begin{aligned} & 0.0000 \\ & 0.00000 \\ & 0.0000 \\ & 0.0000 \\ & 0.0000 \\ & 0.0014 \\ & 0.99998 \\ & 0.00000 \\ & 0.0971\end{aligned}$

0.011
0.99971
0.00997
0.0000
0.09942
0.008
0.0995

0.00140 .9994

0.0016
0.0006
0.99894 
$\begin{array}{llll}-11.479 & 21915.7 & 1089.0 & 108.800\end{array}$ $\begin{array}{rrrr}-11.864 & 22949.3 & 1139.0 & 85.200 \\ -9.784 & 20688.7 & 1188.0 & 86.900 \\ -9.868 & 192965 & 10090 & 78500\end{array}$ $\begin{array}{llll}-9.858 & 1929.65 & 1089.0 & 78.600 \\ -9.899 .0 & 95.200\end{array}$ $\begin{array}{llll}-9.845 & 19822.99 & 1139.0 & 71.300 \\ -9.945 & 203035 & 11300 & \end{array}$

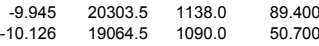

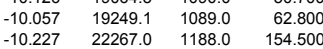

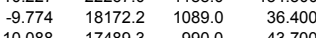

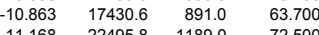

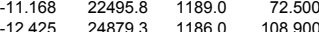

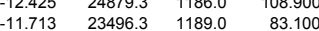
$\begin{array}{llll}-12.055 & 23160.4 & 1094.0 & 77.300 \\ -10915 & 21619 & 100 & \end{array}$

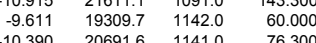
$\begin{array}{lllll}-9.780 & 20032.8 & 1190.0 & 54.500\end{array}$

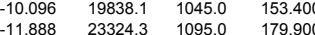
$\begin{array}{lllll}-10.564 & 21253.2 & 1070.0 & 198.300\end{array}$

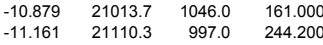

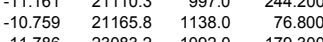

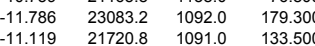
$\begin{array}{llll}-11.119 & 21720.8 & 1099.0 & 133.500 \\ -11.395 & 22585.1 & 1138.0 & 11.100 \\ -12435 & 2122.1 & 1910 & 157000\end{array}$

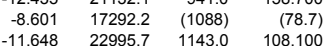




\begin{tabular}{|c|c|c|c|c|c|c|c|c|c|c|c|c|c|c|c|c|c|}
\hline $\begin{array}{l}1264 \\
1189\end{array}$ & $\begin{array}{l}50.100 \\
25.500\end{array}$ & $\begin{array}{l}1314 \\
1239\end{array}$ & $\begin{array}{l}32.9 \\
16.4\end{array}$ & $\begin{array}{l}1363.0 \\
1288.0\end{array}$ & $\begin{array}{r}18.9 \\
9.5\end{array}$ & $\begin{array}{l}\begin{array}{l}1412.0 \\
1337.0\end{array}\end{array}$ & $\begin{array}{r}13.9 \\
6.8\end{array}$ & $\begin{array}{l}1413.0 \\
1337.0\end{array}$ & $\begin{array}{r}13.5 \\
6.9\end{array}$ & $\begin{array}{l}1462.0 \\
1387.0\end{array}$ & $\begin{array}{l}9.5 \\
4.8\end{array}$ & $\begin{array}{l}1462.0 \\
1387.0\end{array}$ & $\begin{array}{r}10.1 \\
5.0\end{array}$ & $\begin{array}{l}1464.0 \\
1388.0\end{array}$ & $\begin{array}{r}11.1 \\
5.6\end{array}$ & $\begin{array}{l}(1516) \\
1437.0\end{array}$ & $\begin{array}{r}(9.7) \\
3.7\end{array}$ \\
\hline 1189 & 40.600 & 1239 & 26.4 & 1289.0 & 14.3 & 1338.0 & 10.2 & 1338.0 & 10.4 & 1388.0 & 7.2 & 1388.0 & 7.6 & 1388.0 & 8.9 & 1438.0 & 6.0 \\
\hline 1139 & 24.900 & 1189 & 15.8 & 1238.0 & 9.5 & 1287.0 & 6.6 & 1287.0 & 6.7 & 1336.0 & 4.7 & 1337.0 & 4.9 & 1338.0 & 5.3 & 1387.0 & 3.7 \\
\hline 1189 & 27.600 & 1239 & 18.4 & 1288.0 & 10.5 & 1337.0 & 7.3 & 1338.0 & 7.6 & 1387.0 & 5.2 & 1388.0 & 5.8 & 1388.0 & 6.6 & 1437.0 & 4.4 \\
\hline 1189 & 43.000 & 1238 & 26.0 & 1287.0 & 16.5 & 1287.0 & 16.6 & 1336.0 & 11.2 & 1337.0 & 11.2 & 1338.0 & 11.4 & 1387.0 & 7.6 & 1437.0 & 5.5 \\
\hline 1139 & 58.000 & 1189 & 32.0 & 1238.0 & 19.5 & 1238.0 & 19.7 & 1287.0 & 12.8 & 1287.0 & 12.9 & 1288.0 & 12.9 & 1337.0 & 8.4 & 1387.0 & 5.8 \\
\hline 1189 & 46.600 & 1238 & 27.1 & 1287.0 & 16.5 & 1287.0 & 16.5 & 1336.0 & 10.8 & 1337.0 & 10.8 & 1338.0 & 10.8 & 1387.0 & 7.3 & 1437.0 & 4.9 \\
\hline $\begin{array}{l}1238 \\
1138\end{array}$ & $\begin{array}{l}53.200 \\
45.100\end{array}$ & $\begin{array}{l}1287 \\
11188\end{array}$ & $\begin{array}{l}29.8 \\
256\end{array}$ & $\begin{array}{l}1336.0 \\
1237\end{array}$ & $\begin{array}{l}19.9 \\
167\end{array}$ & $\begin{array}{l}1336.0 \\
1238\end{array}$ & $\begin{array}{l}20.3 \\
16.4\end{array}$ & $\begin{array}{l}1386.0 \\
12860\end{array}$ & $\begin{array}{l}13.6 \\
1115\end{array}$ & $\begin{array}{l}1386.0 \\
1287\end{array}$ & $\begin{array}{l}14.4 \\
110\end{array}$ & $\begin{array}{l}1388.0 \\
128.0\end{array}$ & $\begin{array}{l}15.5 \\
122\end{array}$ & $\begin{array}{l}1436.0 \\
1377\end{array}$ & $\begin{array}{c}10.1 \\
8.0\end{array}$ & $\begin{array}{l}1487.0 \\
1387.0\end{array}$ & 7.4 \\
\hline $\begin{array}{l}\begin{array}{l}1138 \\
1138\end{array} \\
\text { a }\end{array}$ & $\begin{array}{l}455.100 \\
55.000\end{array}$ & $\begin{array}{l}\text { 1188 } \\
1187\end{array}$ & $\begin{array}{l}25.6 \\
29.5\end{array}$ & $\begin{array}{l}1237.0 \\
1236.0\end{array}$ & $\begin{array}{l}16.7 \\
19.6\end{array}$ & $\begin{array}{l}1238.0 \\
1237.0\end{array}$ & $\begin{array}{l}16.4 \\
19.0\end{array}$ & $\begin{array}{l}1286.0 \\
1286.0\end{array}$ & $\begin{array}{l}11.5 \\
12.8\end{array}$ & $\begin{array}{l}1288.0 \\
1286.0\end{array}$ & $\begin{array}{l}\begin{array}{l}11.0 \\
13.6\end{array} \\
\text { a }\end{array}$ & $\begin{array}{l}1288.0 \\
1287.0\end{array}$ & $\begin{array}{l}12.2 \\
14.8\end{array}$ & $\begin{array}{l}1337.0 \\
1336.0\end{array}$ & $\begin{array}{l}8.0 \\
9.4\end{array}$ & $\begin{array}{l}1387.0 \\
1386.0\end{array}$ & $\begin{array}{l}5.9 \\
6.9\end{array}$ \\
\hline 1188 & 43.100 & 1238 & 24.6 & 1287.0 & 16.0 & 1287.0 & 16.5 & 1337.0 & 10.9 & 1337.0 & 11.7 & 1337.0 & 12.6 & 1387.0 & 8.4 & 1437.0 & 6.2 \\
\hline 1188 & $\begin{array}{r}53.200 \\
\end{array}$ & $\begin{array}{l}1237 \\
11189\end{array}$ & $\begin{array}{l}31.7 \\
172\end{array}$ & $\begin{array}{l}1287.0 \\
{ }_{11230}\end{array}$ & 20.5 & 1287.0 & 21.0 & $\begin{array}{l}1336.0 \\
11388\end{array}$ & 13.6 & 1336.0 & 14.5 & 1337.0 & 15.0 & 1386.0 & 10.0 & 1437.0 & 7.3 \\
\hline $\begin{array}{l}1140 \\
1138\end{array}$ & $\begin{array}{l}29.400 \\
36220\end{array}$ & $\begin{array}{l}1189 \\
1188\end{array}$ & $\begin{array}{l}17.2 \\
21.3\end{array}$ & $\begin{array}{l}1239.0 \\
12380\end{array}$ & $\begin{array}{l}11.5 \\
114.2\end{array}$ & $\begin{array}{l}1239.0 \\
1238\end{array}$ & $\begin{array}{l}11.6 \\
14.6\end{array}$ & $\begin{array}{l}1288.0 \\
128.0\end{array}$ & $\begin{array}{l}7.7 \\
94\end{array}$ & $\begin{array}{l}1288.0 \\
\end{array}$ & $\begin{array}{l}8.0 \\
97\end{array}$ & $\begin{array}{l}1289.0 \\
\end{array}$ & 8.3 & $\begin{array}{l}1338.0 \\
1327\end{array}$ & 5.6 & $\begin{array}{l}1388.0 \\
13890\end{array}$ & 4.1 \\
\hline $\begin{array}{l}1138 \\
1237\end{array}$ & $\begin{array}{l}36.200 \\
91.300\end{array}$ & $\begin{array}{l}\text { 1188 } \\
1287\end{array}$ & $\begin{array}{l}21.3 \\
56.3\end{array}$ & $\begin{array}{l}1238.0 \\
1336.0\end{array}$ & $\begin{array}{l}14.2 \\
35.8\end{array}$ & $\begin{array}{l}1238.0 \\
1386.0\end{array}$ & $\begin{array}{l}14.3 \\
23.6\end{array}$ & $\begin{array}{l}1287.0 \\
1386.0\end{array}$ & $\begin{array}{r}9.4 \\
25.0\end{array}$ & $\begin{array}{l}1287.0 \\
1436.0\end{array}$ & $\begin{array}{c}9.7 \\
15.7\end{array}$ & $\begin{array}{l}1288.0 \\
1436.0\end{array}$ & $\begin{array}{r}9.9 \\
17.7\end{array}$ & $\begin{array}{l}1337.0 \\
1436.0\end{array}$ & $\begin{array}{r}6.9 \\
16.7\end{array}$ & 1387.0 & 4.9 \\
\hline 1138 & 22.600 & 1188 & 13.8 & 1237.0 & 9.4 & 1237.0 & 9.5 & 1286.0 & 6.4 & 1286.0 & 6.6 & 1287.0 & 6.7 & 1336.0 & 4.6 & 1387.0 & 3.3 \\
\hline 1040 & 25.400 & 1090 & 15.4 & 1139.0 & 9.7 & 1188.0 & 6.5 & 1189.0 & 6.5 & 1238.0 & 4.4 & 1238.0 & 4.4 & 1239.0 & 4.5 & 1288.0 & 3.1 \\
\hline 941 & 32.400 & 991 & 18.3 & 1040.0 & 11.0 & 1089.0 & 6.8 & 1089.0 & 6.8 & 1139.0 & 4.4 & 1139.0 & 4.4 & 1140.0 & 4.4 & 1189.0 & 3.0 \\
\hline 1239 & 42.100 & 1288 & 26.1 & 1338.0 & 14.4 & 1388.0 & 10.0 & 1389.0 & 9.7 & 1438.0 & 6.9 & 1438.0 & 7.5 & 1438.0 & 7.7 & 1488.0 & 5.7 \\
\hline 1236 & 60.100 & 1286 & 32.6 & 1336.0 & 19.8 & 1336.0 & 20.0 & 1386.0 & 12.9 & 1386.0 & 12.9 & 1386.0 & 13.1 & 1436.0 & 8.4 & 1486.0 & 5.7 \\
\hline 1239 & $\begin{array}{l}47.700 \\
{ }^{2}\end{array}$ & 1288 & 26.7 & 1338.0 & 16.5 & 1338.0 & 17.0 & 1388.0 & 10.5 & 1388.0 & 11.3 & 1388.0 & 11.9 & 1438.0 & 7.8 & 1488.0 & 5.5 \\
\hline $\begin{array}{l}\begin{array}{l}1145 \\
1141\end{array} \\
\text { a }\end{array}$ & 39.600 & $\begin{array}{l}1194 \\
1191\end{array}$ & $\begin{array}{l}21.8 \\
456\end{array}$ & $\begin{array}{l}1244.0 \\
124.0\end{array}$ & $\begin{array}{l}13.3 \\
28.2\end{array}$ & $\begin{array}{l}1244.0 \\
129.0\end{array}$ & $\begin{array}{l}13.4 \\
17.8\end{array}$ & $\begin{array}{l}1294.0 \\
134.0\end{array}$ & $\begin{array}{l}8.1 \\
120\end{array}$ & $\begin{array}{l}1299.0 \\
13910\end{array}$ & 8.4 & 1294.0 & 8.5 & 1344.0 & 5.4 & 1344.0 & 5.7 \\
\hline 1192 & $\begin{array}{l}38.000 \\
35.800\end{array}$ & 1242 & $\begin{array}{l}\begin{array}{l}43.10 \\
21.9\end{array} \\
2\end{array}$ & $\begin{array}{l}124.0 .0 \\
1292.0\end{array}$ & $\begin{array}{l}28.2 \\
14.9\end{array}$ & 1292.0 & $\begin{array}{l}11.8 \\
14.6\end{array}$ & 1342.0 & $\begin{array}{l}12.0 \\
10.0\end{array}$ & $\begin{array}{l}339.0 .0 \\
1342 .\end{array}$ & $\begin{array}{l}0.4 \\
10.4\end{array}$ & 1343.0 & 10.7 & 1392.0 & 7.3 & 1443.0 & 5.5 \\
\hline 1191 & 45.300 & 1240 & 24.1 & $\begin{array}{l}1290.0 \\
\end{array}$ & 15.7 & 1290.0 & $\begin{array}{l}15.9 \\
\end{array}$ & 1340.0 & 10.6 & 1340.0 & 11.2 & 1341.0 & 12.4 & 1390.0 & 7.9 & 1442.0 & 6.0 \\
\hline $\begin{array}{l}1239 \\
1144\end{array}$ & $\begin{array}{r}33.100 \\
4850\end{array}$ & 1289 & 19.6 & $\begin{array}{l}1339.0 \\
1302\end{array}$ & 13.3 & 1339.0 & 13.4 & 1388.0 & 9.3 & 1389.0 & 9.0 & 1390.0 & 10.2 & 1439.0 & 7.1 & 1489.0 & 5.4 \\
\hline $\begin{array}{l}1144 \\
1145\end{array}$ & $\begin{array}{l}48.500 \\
94.600\end{array}$ & $\begin{array}{l}1243 \\
1244\end{array}$ & $\begin{array}{l}19.4 \\
322\end{array}$ & $\begin{array}{l}1342.0 \\
13430\end{array}$ & $\begin{array}{r}7.4 \\
123\end{array}$ & $\begin{array}{l}1343.0 \\
13430\end{array}$ & $\begin{array}{r}9.2 \\
126\end{array}$ & $\begin{array}{l}1392.0 \\
1390.0\end{array}$ & $\begin{array}{l}5.8 \\
8.2\end{array}$ & $\begin{array}{l}1442.0 \\
14430\end{array}$ & $\begin{array}{l}4.4 \\
5.8\end{array}$ & 1492.0 & & & & & \\
\hline $\begin{array}{l}1145 \\
1119\end{array}$ & $\begin{array}{c}94.600 \\
109.400\end{array}$ & $\begin{array}{l}\text { 1244 } \\
1220\end{array}$ & $\begin{array}{l}32.2 \\
39.6\end{array}$ & $\begin{array}{l}1343.0 \\
1318.0\end{array}$ & $\begin{array}{l}12.3 \\
14.4\end{array}$ & $\begin{array}{l}1343.0 \\
1319.0\end{array}$ & $\begin{array}{l}12.6 \\
16.9\end{array}$ & $\begin{array}{l}1398.0 \\
1368.0\end{array}$ & $\begin{array}{r}8.2 \\
10.6\end{array}$ & $\begin{array}{l}1444.0 \\
1418.0\end{array}$ & $\begin{array}{l}5.8 \\
8.1\end{array}$ & & & & & & \\
\hline 1145 & 49.700 & 1244 & 19.1 & 1342.0 & 8.7 & & & & & & & & & & & & \\
\hline 1096 & 69.400 & 1195 & 23.7 & 1294.0 & 9.6 & 1294.0 & 10.3 & 1344.0 & 7.1 & & & & & & & & \\
\hline $\begin{array}{l}1186 \\
1140\end{array}$ & 40.9 & $\begin{array}{l}1236 \\
1191\end{array}$ & $\begin{array}{l}24.8 \\
5.8\end{array}$ & 123 & 25.9 & $\begin{array}{l}1285.0 \\
\end{array}$ & $\begin{array}{l}15.6 \\
\end{array}$ & 1285.0 & 16.5 & 1286.0 & 17.1 & 1336.0 & 11.2 & 1385.0 & 7.6 & 1436.0 & 5.3 \\
\hline 1142 & $\begin{array}{r}96.100 \\
7777\end{array}$ & $\begin{array}{l}1191 \\
11900\end{array}$ & 50.8 & 1241.0 & 29.8 & $\begin{array}{r}1241.0 \\
\end{array}$ & $\begin{array}{l}31.1 \\
23.1\end{array}$ & $\begin{array}{l}1290.0 \\
\end{array}$ & $\begin{array}{l}17.9 \\
1157\end{array}$ & 1290.0 & 19.3 & 1292.0 & 19.8 & 1343.0 & 13.0 & $\begin{array}{l}1392.0 \\
12002\end{array}$ & 8.6 \\
\hline 1187 & $\begin{array}{l}72.700 \\
62800\end{array}$ & $\begin{array}{l}1190 \\
1236\end{array}$ & $\begin{array}{l}38.6 \\
31.8\end{array}$ & $\begin{array}{l}1239.0 \\
11280\end{array}$ & $\begin{array}{l}24.7 \\
2.7\end{array}$ & $\begin{array}{l}21240.0 \\
128.0\end{array}$ & $\begin{array}{l}23.7 \\
20.8\end{array}$ & $\begin{array}{l}1289.0 \\
13320\end{array}$ & $\begin{array}{l}15.7 \\
1125\end{array}$ & $\begin{array}{l}21290.0 \\
\end{array}$ & $\begin{array}{l}14.9 \\
139\end{array}$ & $\begin{array}{l}21290.0 \\
13320\end{array}$ & $\begin{array}{l}16.1 \\
150\end{array}$ & $\begin{array}{l}1340.0 \\
138.0\end{array}$ & $\begin{array}{c}10.8 \\
9.7\end{array}$ & $\begin{array}{l}1392.0 \\
14330\end{array}$ & 7.8 \\
\hline 992 & $\begin{array}{l}0.28000 \\
72.600\end{array}$ & 1041 & $\begin{array}{l}31.8 \\
35.9\end{array}$ & $\begin{array}{l}128 \\
109\end{array}$ & $\begin{array}{l}20.0 \\
20.1\end{array}$ & $\begin{array}{l}1288.0 \\
1091.0\end{array}$ & 20.1 & $\begin{array}{l}11330.0 \\
1140.0\end{array}$ & $\begin{array}{l}17.5 \\
12.2\end{array}$ & $\begin{array}{l}1353.0 \\
1141.0\end{array}$ & $\begin{array}{l}13.9 \\
11.9\end{array}$ & $\begin{array}{l}1353.0 .0 \\
1141.0\end{array}$ & $\begin{array}{l}15.0 \\
12.4\end{array}$ & $\begin{array}{l}1380.0 \\
1190.0\end{array}$ & $\begin{array}{l}9.1 \\
7.5\end{array}$ & $\begin{array}{l}\begin{array}{l}4253.0 \\
1240.0\end{array}\end{array}$ & $\begin{array}{l}\text {. } \\
5.2\end{array}$ \\
\hline 1138 & 40.000 & 1187 & 24.9 & 1187.0 & 25.9 & 1236.0 & 15.8 & 1237.0 & 17.0 & 1237.0 & 18.8 & 1286.0 & 12.0 & 1336.0 & 8.6 & 1386.0 & 6.3 \\
\hline 1193 & 60.300 & 1243 & 30.1 & 1292.0 & 19.9 & 1293.0 & 18.8 & 1342.0 & 12.2 & 1342.0 & 13.4 & 1344.0 & 14.4 & 1393.0 & 8.8 & 1444.0 & 6.3 \\
\hline
\end{tabular}


828 HLP-73

1830 HLP-75

1831 HLP-76
1832 HLP-77

1835 L1-15

1836 L4-69

1838 L4-615

$839\llcorner 4-96$
1830

1840 L4-99
1841 L4-912

1842 L4-915
1843 L4-129

844 L4-1212
1845 - 12121

184615.59

1847 L5-612

1848 L5-615

1850 L5-99

1851 L5-9912

1852 L5-915

1854 L5-1212

1855 L5-1215
1856 L6-3312

1856 L6-3312
1857.5546

1858 L6-549

1860 L-6-5415

1861 L6-669

1862 L6-6612
1863 L7-15

1865 L L7-30
1866 L7-35

1867 L8-1

1868 L8-2

1870 L8-4

1873 L8-7

1874 L8-8

1877 LD6-5314

1878 LD6-5412

1879 LD6-5510

1881 LDM-1

1882 LDM-2

1883 LDM-3

1885 LDM-5412
1886 LDMS-1

TCLP Se TCLP Zn TCLPAI TCLPB TCLPSb TCLP TI TCLPV QPCT B $\left(g / \mathrm{m}^{2}\right)$ QPCT Li $\left(g / \mathrm{m}^{2}\right) \quad$ QPCT Na $\left(g / \mathrm{m}^{2}\right) \quad$ QPCT Si $\left(g / \mathrm{m}^{2}\right) \quad$ Q PCT pH

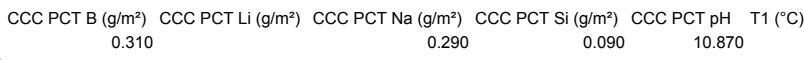

0.340

0.380
5.570

$\begin{array}{ll}0.150 & 12.680 \\ 0.650 & 10.330\end{array}$

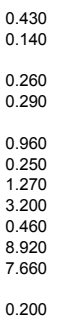

$\begin{array}{lll}0.450 & 0.190 & 10.323 \\ 0.250 & 0.110 & 11.363\end{array}$

$\begin{array}{lll}0.310 & 0.140 & 10.463 \\ 0.370 & 0.150 & 10737\end{array}$

$\begin{array}{lll}0.330 & 0.240 & 10.573 \\ 0.270 & 0.120\end{array}$

$\begin{array}{lll}0.330 & 0.240 & 10.573 \\ 0.270 & 0.120 & 9.970 \\ 3.730 & 0.990 & 11.860 \\ 2.400 & 0.580 & 11.193 \\ 0.490 & 0.230 & 10.570\end{array}$

$\begin{array}{lll}6.500 & 2.340 & 11.540 \\ 5.620 & 0.910 & 12.197\end{array}$

0.200

0.210

0.140

10.29

0.320

0.330
0.230

$\begin{array}{lll}0.430 & 0.170 & 10.967 \\ 0.300 & 0.170 & 10.760\end{array}$

0.310
42.680
0.290
2.060
0.250

$\begin{array}{rrr}0.430 & & \\ 0.140 & 0.130 & 12.277 \\ 11.10 & 0.430 & 12.467 \\ 0.300 & 0.120 & 9.893 \\ 1.240 & 0.140 & 1.267 \\ 0.290 & 0.100 & 10.183\end{array}$

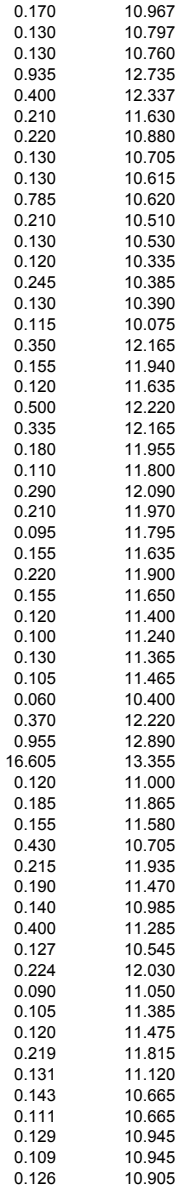


1812 HLP.-56
1813 HLP-58

1814 HLP-59
1815 HLP-60

$1816 \mathrm{HLP}-61$
$1017 \mathrm{H}-61$

1817 HLP.-62
1818 HLP.63

1819 HLP-64

1820 HLP.-65

1821 HLP- -66

1823 HLP- 68

1824 HLP-69

1827 HLP-72

1828 HLP-73

1830 HP. -75

1831 HLP. 76
1832 HLP-77

$1835 \mathrm{~L} L 1-15$
1836
$14-69$

1837 L4-612

$1838 \mathrm{~L} 4-615$
1839 L-966

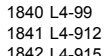

1842 L4.915
1843 L4-129

1844 L4-12121
1845 L4-1215
$1045-59$

1846 L5-69

1847 L5-6612

184755.612
$1845.5-615$
1849

1850 L5-99

1855 L5-912

$18525.5-15$
$185355-129$
1545

1854 L5-1212

1855 L5-1215

1856 L6-3312
$18576-546$

$18576-6-546$
1858 L-549

1859 L6-5412

1861 L6-669

1862 L6-6612

10547725

1865 L7-3
1866 L7-35

1867 L8-1

1868 L8-2
1869 L8.3

1870 L8-4

1871 L8-5

1873 L8-7

1874 L8-8

1875 LD4-912

1877.065314

1877 LD6-5314
$1878 \mathrm{LD}-544212$

1890 LDD-551

1880 LDM-912
1881 LDM-1

$1882 \mathrm{LDM}-2$

1883 LDM-3

885 LDM-54

1885 LLM-54
1886 LDMS-1 


\begin{tabular}{|c|c|c|c|c|c|c|}
\hline \# Glass ID & & Reference & Al2O3-t & B2O3-t & CaO-t & $\mathrm{Fe} 2 \mathrm{O}-\mathrm{t}$ \\
\hline 37 LRM-912 & Hanford LLW Glass Formulatii & Feng et al. 1996 & 0.1200 & & 0.0900 & \\
\hline 1888 LRM-1 & Hanford LLW Glass Formulatit, & & 0.1200 & 0.0200 & 0.0200 & 0.0600 \\
\hline 1889 LRM-2 & Hanford LLW Glass Formulatit & & & & 0.0600 & 0.0600 \\
\hline 1890 LRM-3 & Hanford LLW Glass Formulatit & & 0.1200 & 0.0600 & & \\
\hline 1891 LRM-4 & Hanford LLW Glass Formulati & & 0.1000 & 0.0600 & 0.0600 & 0.0600 \\
\hline 1892 LRM- 5412 & Hanford LLW Glass Formulati & Feng et al. 1996 & 0.1200 & 0.0500 & 0.0400 & \\
\hline & $\begin{array}{l}\text { Hanford LLW Glass Formulatiti } \\
\text { Hanfordd }\end{array}$ & Feng et al. 1996 & 0.1200 & 0.0200 & 0.0200 & 0.0600 \\
\hline $\begin{array}{l}1894 \text { SSHTM-3 } \\
1895 \text { B1G9-0IIC4 }\end{array}$ & $\begin{array}{l}\text { Hanford LW Glass Forrmulatit } \\
\text { Hanford LW Glass Formulatiu }\end{array}$ & $\begin{array}{l}\text { Feng et al. } 1996 \\
\text { Feng en al } 1996\end{array}$ & $\begin{array}{l}0.1200 \\
0.1000\end{array}$ & $\begin{array}{l}0.05500 \\
0.0500\end{array}$ & $\begin{array}{l}0.0400 \\
0.0500\end{array}$ & $\begin{array}{l}0.0000 \\
0.0000\end{array}$ \\
\hline 1896 B1G9-013C5 & Hafford LW Glass Forrmulatiti & & 0.1000 & & & \\
\hline $1897 \mathrm{~B} 1 \mathrm{Gg}-014 \mathrm{C}$ & 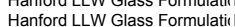 & Fengetal 19906 & 0.1000 & & 0.0000 & 0.0000 \\
\hline 1898 D1G4-022P2 & Hafford LW Glass Forrmulatiti & Feng et ala 19996 & 0.0614 & 0.0615 & 0.0780 & 0.0750 \\
\hline 1899 D1G4-023P3 & Hanford LLW Glass Formulatit & Feng et al. 1996 & 0.0614 & & 0.0780 & 0.0750 \\
\hline 1900 Duratek & Hanford LLW Glass Formulatit & iFeng et al. 1996 & 0.0614 & 0.0615 & 0.0780 & 0.0750 \\
\hline 1901 PEI & Hanford LLW Glass Formulatiti & Feng et al. 1996 & 0.0600 & & 0.0973 & 0.0100 \\
\hline 1902 M1G1-008P & Hanford LLW Glass Formulatiti & Feng et al. 1996 & 0.1000 & 0.0500 & 0.0500 & 0.0000 \\
\hline $\begin{array}{l}1903 \text { M1G1-011P } \\
9904 \text { V1M2 6 32011P1 }\end{array}$ & $\begin{array}{l}\text { Hanford LLW Glass Forrmulatiti } \\
\text { Hanfford WW Glass Formulatit }\end{array}$ & $\begin{array}{l}\text { Feng et al. } 1996 \\
\text { Feeng en }\end{array}$ & $\begin{array}{l}0.1000 \\
0.1000\end{array}$ & $\begin{array}{l}0.0500 \\
0.0800\end{array}$ & $\begin{array}{l}0.0500 \\
0.0290\end{array}$ & $\begin{array}{r}0.0000 \\
-1000\end{array}$ \\
\hline $\begin{array}{l}1904 \mathrm{~V} 1 \mathrm{M} 2632011 \mathrm{P} 1 \\
1905 \mathrm{~V} 1 \mathrm{M} 2632040 \mathrm{P} 2\end{array}$ & $\begin{array}{l}\text { Hantord LLW Glass Formulatiti } \\
\text { Hanford LLW Glass Formulatii }\end{array}$ & $\begin{array}{l}\text { Feng et al. } 1996 \\
\text { Fieng en }\end{array}$ & $\begin{array}{l}0.1000 \\
0.1000\end{array}$ & 0.00800 & 0.0290 & 0.0100 \\
\hline 1906 V1M3 632059 P1 & $\begin{array}{l}\text { Hanfirord LLW Glass Sormulaut } \\
\text { Hanford LLW Glass Formulatii }\end{array}$ & & $\begin{array}{l}0.1000 \\
0.1000\end{array}$ & $\begin{array}{l}0.0000 \\
0.0800\end{array}$ & $\begin{array}{l}0.0290 \\
0.0290\end{array}$ & $\begin{array}{l}0.0100 \\
0.0100\end{array}$ \\
\hline $1907 \mathrm{~V} 1 \mathrm{M} 3632075 \mathrm{P} 2$ & Hanford LLW Glass Formulatii & Feng et al. 1996 & 0.1000 & 0.0800 & 0.0290 & 0.0100 \\
\hline 1908 V1M4 632088 P1 & Hanford LLW Glass Formulatii & Feng et al. 1996 & 0.1000 & 0.0800 & 0.0290 & 0.0100 \\
\hline 1909 V1M4 632096 P2 & Hanford LLW Glass Formulatiti & Feng et al. 1996 & 0.1000 & 0.0800 & 0.0290 & 0.0100 \\
\hline 1910 Vectra & Hanford LLW Glass Formulatii & Feng et al. 1996 & 0.1000 & 0.0800 & 0.0290 & 0.0100 \\
\hline 1911 WSTC & $\begin{array}{l}\text { Hanford LLW Glass Formulatii } \\
\text { SBW }\end{array}$ & IFeng et al. 1996 & 0.1822 & 0.0945 & 0.0465 & 0.0000 \\
\hline $\begin{array}{ll}1912 \text { SBW1-01 } \\
\end{array}$ & $\begin{array}{l}\text { SBW CVS Phase } 1 \\
\text { SBW CVS Phase } 1\end{array}$ & $\begin{array}{l}\text { Scholes et al. } 2002 \\
\text { Scholes et } 2012002\end{array}$ & 0.1003 & 0.0900 & 0.0218 & $\begin{array}{l}0.0412 \\
0.0608\end{array}-2$ \\
\hline & $\begin{array}{l}\text { SBWCVS Phase } 1 \\
\text { SPW }\end{array}$ & $\begin{array}{l}\text { Shchoses et all. } 2002 \\
\text { Chon }\end{array}$ & 0.0980 & & 0.0218 & 0.0608 \\
\hline 1915 SBW1-04 & SBW CVS Phase 1 & $\begin{array}{l}\text { Scholes et al. } 2002 \\
\text { Scholes et al. } 2002\end{array}$ & $\begin{array}{l}0.0980 \\
0.0980\end{array}$ & $\begin{array}{l}0.0990 \\
0.1100\end{array}$ & $\begin{array}{l}0.0218 \\
0.0218\end{array}$ & $\begin{array}{l}0.0412 \\
0.0412\end{array}$ \\
\hline 1916 SBW1-05 & SBW CVS Phase 1 & Scholes et al. 2002 & 0.1270 & 0.0900 & 0.0218 & $\begin{array}{l}0.0412 \\
0.0412\end{array}$ \\
\hline 1917 SBW1-06 & SBW CVS Phase 1 & Scholes et al. 2002 & 0.1171 & 0.0900 & 0.0218 & 0.0608 \\
\hline 1918 SBW1-07 & SBW CVS Phase 1 & Scholes et al. 2002 & 0.0980 & 0.0900 & 0.0218 & 0.0608 \\
\hline 1919 SBW1-08 & SBW CVS Phase 1 & Scholes et al. 2002 & 0.1270 & 0.0904 & 0.0300 & 0.0412 \\
\hline 1920 SBW1-09 & $\begin{array}{l}\text { SBW CVS Phase } 1 \\
\text { S }\end{array}$ & Scholes et al. 2002 & 0.0980 & 0.1100 & 0.0218 & 0.0412 \\
\hline $\begin{array}{l}1921 \text { SBW1-10 } \\
1922 \text { SBW1-11 }\end{array}$ & $\begin{array}{l}\text { SBW CVS Phase } 1 \\
\text { SBW CVS Phase } 1\end{array}$ & $\begin{array}{l}\text { Scholes et al. } 2002 \\
\text { Sctoles et }\end{array}$ & 0.0980 & $\begin{array}{l}0.0900 \\
0.1100\end{array}$ & 0.0300 & 0.0412 \\
\hline $\begin{array}{ll}11922 \\
1923\end{array}$ & 作 & $\begin{array}{l}\text { Scholose ex ala. } 2002 \\
\text { Schen }\end{array}$ & 0.1029 & 0.1000 & 0.0218 & 0.0472 \\
\hline 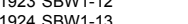 & 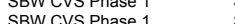 & 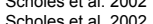 & 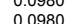 & 0.0000 & 0.0030 & 0.0412 \\
\hline 1925 SBW1-14 & SBW CVS Phase 1 & Scholes et al. 2002 & 0.0980 & 0.0908 & 0.0218 & 0.0412 \\
\hline 1926 SBW1-15 & SBW CVS Phase 1 & Scholes et al. 2002 & 0.0980 & 0.1100 & 0.0300 & 0.0595 \\
\hline 1927 SBW1-16 & SBW CVS Phase 1 & Scholes et al. 2002 & 0.0690 & 0.1300 & 0.0124 & 0.0216 \\
\hline 1928 SBW1-17 & SBW CVS Phase 1 & Scholes et al. 2002 & 0.0690 & 0.0700 & 0.0400 & 0.0804 \\
\hline & SBW CVS Phase 1 & Scholes et al. 2002 & 0.1560 & 0.0700 & 0.0400 & 0.0804 \\
\hline & $\begin{array}{l}\text { SBW CVS Phase } 1 \\
\text { SBW CVS Phase }\end{array}$ & Scholes et al. 2002 & 0.0690 & 0.0700 & 0.0124 & 0.0216 \\
\hline 1932 SBW1-21 & SBW CVS Phase 1 & 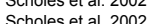 & 0.0090 & & 0.0014 & $\begin{array}{l}0.0804 \\
0.0804\end{array}$ \\
\hline $\begin{array}{l}1 \\
1\end{array}$ & 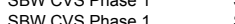 & 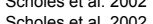 & 年 & 0.070 & 列 & 0.08046 \\
\hline 1934 SBW1-23 & $\begin{array}{l}\text { SEW CVS Phase } 1 \\
\text { SBW Phe }\end{array}$ & Shchose et al 2002 & 0.1110 & 0.0700 & 0.024 & $\begin{array}{l}0.0216 \\
0.0216\end{array}$ \\
\hline 1935 SBW1-24 & SBW CVS Phase 1 & Scholes et al. 2002 & 0.1560 & & 0.0124 & 0.0804 \\
\hline 1936 SBW1-25 & SBW CVS Phase 1 & Scholes et al. 2002 & 0.0690 & 0.1300 & 0.0400 & 0.0216 \\
\hline 1937 SBW1-26 & SBW CVS Phase 1 & Scholes et al. 2002 & 0.0690 & 0.0700 & 0.0124 & 0.0804 \\
\hline 1938 SBW1-27 & SBW CVS Phase 1 & Scholes et al. 2002 & 0.1560 & 0.1300 & 0.0124 & 0.0216 \\
\hline 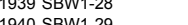 & $\begin{array}{l}\text { SBW CVS Phase } 1 \\
\text { SBW CVS Phase } 1\end{array}$ & Scholes et al. 2002 & 0.0938 & 0.1300 & 0.0124 & 0.0804 \\
\hline 1941 SBW1-30 & $\begin{array}{l}1 \\
\text { SRW CYS Phase 1 }\end{array}$ & 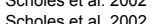 & 列 & 0.1300 & 0.0400 & 0.0216 \\
\hline 作 & 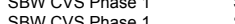 & 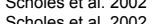 & 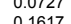 & 0.11300 & 0.0124 & 0.08044 \\
\hline 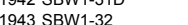 & 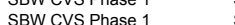 & $\begin{array}{l}\text { Sholes et al } 2002 \\
\text { Sher }\end{array}$ & 0.0400 & $\begin{array}{l}.11534 \\
0.150\end{array}$ & $\begin{array}{l}.00030 \\
0.030\end{array}$ & $\begin{array}{l}0.0852 \\
0.0020\end{array}$ \\
\hline 1944 SBW1-33A & $\begin{array}{l}\text { SBW CVS Phase } 1 \\
\text { SB P }\end{array}$ & Scholes et al. 2002 & 0.0400 & 0.1500 & 0.0500 & 0.1000 \\
\hline 1945 SBW1-34 & SBW CVS Phase 1 & Scholes et al. 2002 & 0.0400 & 0.1289 & 0.0030 & 0.0020 \\
\hline 1946 SBW1-35 & SBW CVS Phase 1 & Scholes et al. 2002 & 0.1799 & 0.1500 & 0.0500 & 0.0020 \\
\hline 1947 SBW1-36 & SBW CVS Phase 1 & Scholes et al. 2002 & 0.1850 & 0.0500 & 0.0030 & 0.0020 \\
\hline 1948 SBW1-37 & $\begin{array}{l}\text { SBW CVS Phase } 1 \\
\text { SBW CVS Phase } 1\end{array}$ & Scholes et al. 2002 & 0.0400 & 0.0500 & 0.0030 & 0.1000 \\
\hline 1949 SBW1-38B & SBW CVS Phase 1 & $\begin{array}{l}\text { Scholes et al. } 2002 \\
S\end{array}$ & 0.1617 & 0.1354 & 0.0085 & $\begin{array}{l}0.0854 \\
0.951\end{array}$ \\
\hline 1951 SBW1-40 & $\begin{array}{l}\text { SBWCVS Phase } 1 \\
\text { SBW CVS Pase } 1\end{array}$ & $\begin{array}{l}\text { Scholose et al. } 2002 \\
\text { Scholes et } 2002\end{array}$ & $\begin{array}{l}0.1592 \\
0.1523\end{array}$ & $\begin{array}{l}0.01356 \\
0.1356\end{array}$ & $\begin{array}{l}0.0048 \\
0.0030\end{array}$ & 0.0950 \\
\hline 1952 SBW1-41 & SBW CVS Phase 1 & Shchose et al 2002 & 0.0400 & 0.1500 & 0.0030 & 0.1000 \\
\hline 1953 SBW1-42 & $\begin{array}{l}\text { SBW CVS Phase } 1 \\
\text { SB }\end{array}$ & Scholes et al. 2002 & 0.0400 & 0.1500 & 0.0500 & 0.1000 \\
\hline 1954 SBW1-43 & SBW CVS Phase 1 & Scholes et al. 2002 & 0.1650 & 0.1500 & 0.0030 & 0.0020 \\
\hline 1955 SBW1-44B & SBW CVS Phase 1 & Scholes et al. 2002 & 0.0602 & 0.0654 & 0.0085 & 0.0854 \\
\hline 1956 SBW1-45 & SBW CVS Phase 1 & Scholes et al. 2002 & 0.0400 & 0.1500 & 0.0030 & 0.0020 \\
\hline 1957 SBW1-46 & SBW CVS Phase 1 & Scholes et al. 2002 & 0.1072 & 0.1014 & 0.0214 & 0.0513 \\
\hline 1958 SBW1-47 & SBW CVS Phase 1 & $\begin{array}{l}\text { Scholes et al. } 2002 \\
\text { Scon }\end{array}$ & 0.1072 & 0.1014 & 0.0214 & 0.0513 \\
\hline $\begin{array}{l}1599 \mathrm{SBW}-48 \\
1006\end{array}$ & 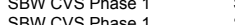 & 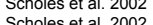 & 0.0904 & 0.1736 & 0.0133 & 0.00994 \\
\hline 列 & 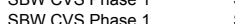 & 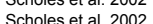 & 列 & 0.0198 & 0.0053 & 0.0054 \\
\hline SBW1-51 & $\begin{array}{l}\text { SBW CVS Phase } 1 \\
\text { SB }\end{array}$ & $\begin{array}{l}\text { Scholes et al } 2002 \\
\text { Scher }\end{array}$ & 0.0904 & 0.0700 & 0.0103 & $\begin{array}{l}0.0894 \\
0.0894\end{array}$ \\
\hline 1963 SBW1-52 & SBW CVS Phase 1 & Scholes et al. 2002 & 0.0904 & 0.1236 & 0.0453 & 0.0367 \\
\hline SBW1-53 & SBW CVS Phase 1 & Scholes et al. 2002 & & 0.0700 & 0.0112 & 0.0894 \\
\hline SBW1-54 & SBW CVS Phase 1 & Scholes et al. 2002 & & 0.1125 & & 0.0795 \\
\hline 1966 SBW1-55 & BW CVS Phase 1 & Scholes et al. 2002 & 0.0870 & 0.1050 & 0.0453 & 0.0754 \\
\hline 1967 SBW1-56 & SBW CVS Phase 1 & Scholes et al. 2002 & 0.1014 & 0.0975 & 0.0445 & 0.0713 \\
\hline & & & & & & \\
\hline
\end{tabular}

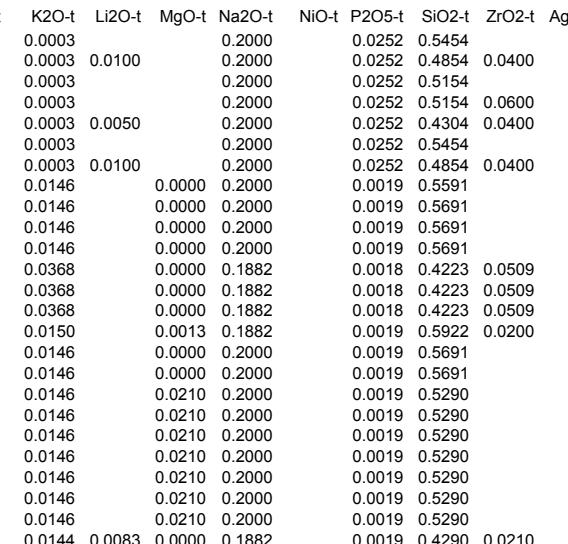

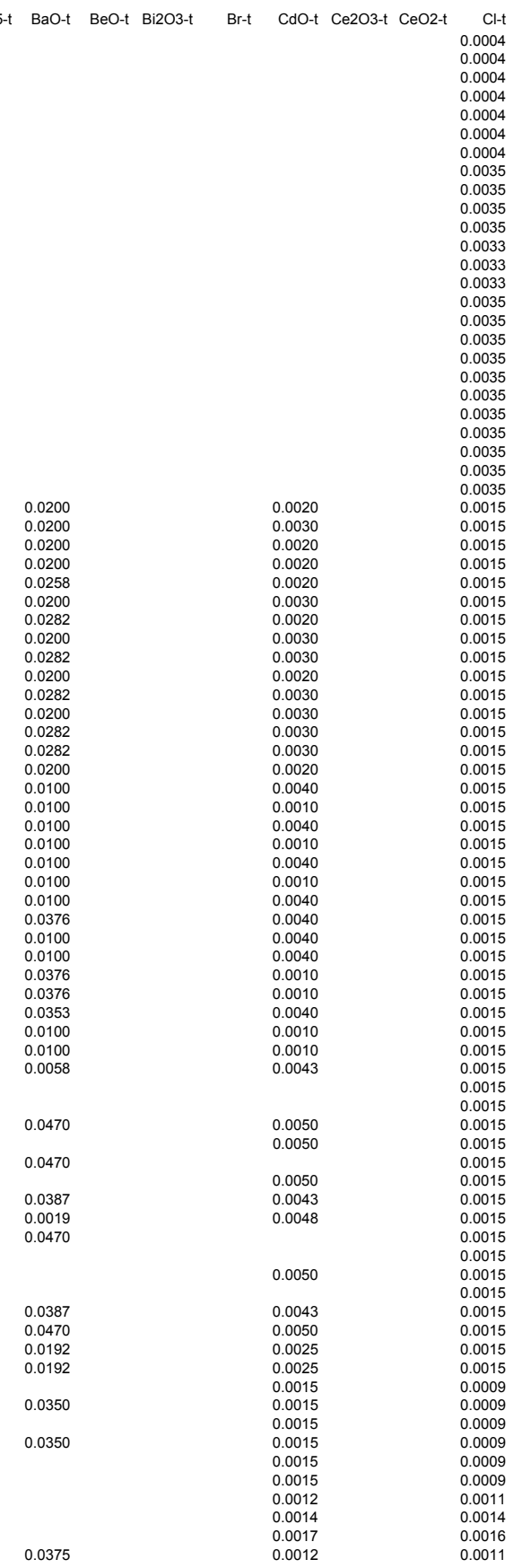




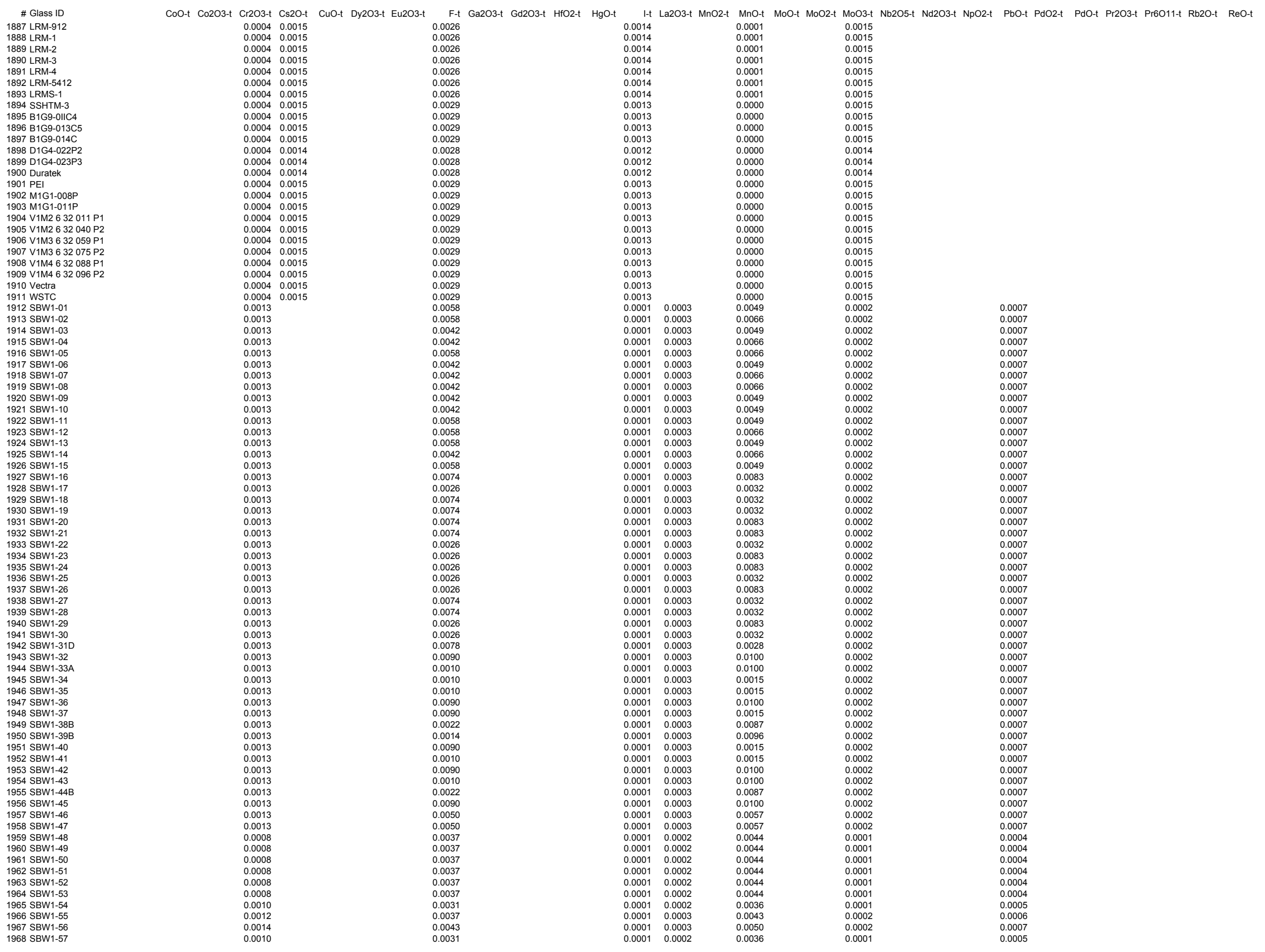


TiO2-t T12O-t T1203-t U308-t UO2-t UO3-t V2O5-t WO3-t Y203-t ZnO-t Sum-t Al203-a 1888 LRM-1 


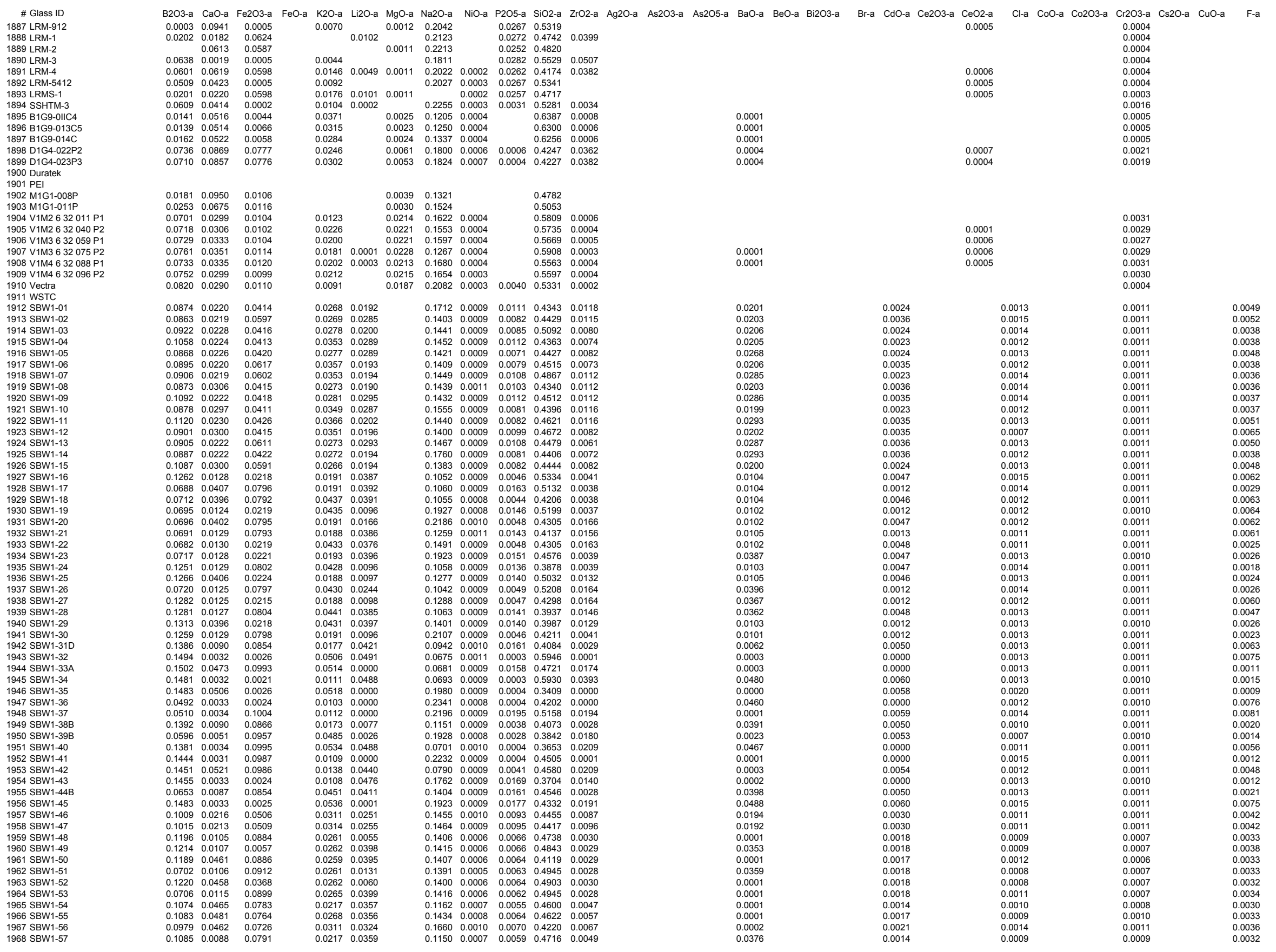


I-a La203-a MnO2-a MnO-a MoO-a MoO3-a Nb205-a Nd203-a NpO2-a PbO-a PdO2-a PdO-a Pr2O3-a Pr6011-a PuO2-a Rb2O-a ReO-a ReO2-a Re207-a Rh203-a RhO2-a RuO2-a Sb203-a Sb205-a SeO2-a 1888 LRM-1

1889 LRM-2

1890 LRM-3

1893 LRMS-1

1894 SSTTM-3

1895 B1G9-0IIC4
1896 B1G9-013C5

$1897 \mathrm{~B} 1 \mathrm{G} 9-014 \mathrm{C}$

1898 D1G4-022P 2

899 D1G4-023P3

1901 PEI

1902 M1G1-008P

1904 V1M1-01P

1905 V1M2 63204040 P2
1906 V1M3 632059 P1

1907 V1M 632075 P2

1908 V1M4 632088 P1

1910 Vectra

1911 WSTC
1912 SBW1-0

1913 SBW1-02

1914 SBW1-03
1915 SBW1-04

1916 SBW1-05

1917 SBW1-06

1918 SBW1-07
1919 SBW1-0

1920 SBW1-09

1921 SBW1-10

1922 SBW1-11

1924 SBW1-13

1925 SBW1-14

1926 SBW1-1

1928 SBW1-17

1930 SBW1-19

1933 SBW1-22

1934 SBW1-23

1936 SBW1-25

1937 SBW1-26

1938 SBW1-27

1940 SBW1-29

1941 SBW1-30

1942 SBW1-31D

1944 SBW1-33A

1945 SBW1-34

1946 SBW1-35

1948 SBW1-37

1949 SBW1-38B

1951 SBW1-40

1952 SBW1-41

1953 SBW1-42
1954 SBW1-43

1955 SBW1-44B

1956 SBW1-45

1957 SBW1-46
1958 SBW1-47

1959 SBW1-48

1960 SBW1-49

1961 SBW1-50
1962 SBW1-5

1963 SBW1-52

1964 SBW1-53

1965 SBW1-54

967 SBW1-5

1968 SBW1-57

$\begin{array}{lll}0.0001 & 0.0016 & 0.0009 \\ 0.0002 & 0.0015 & 0.0003 \\ 0.0002 & 0.0016 & 0.0003 \\ 0.0001 & 0.0017 & 0.0004 \\ 0.0002 & 0.0015 & 0.0009 \\ 0.0001 & 0.0016 & 0.0008 \\ 0.0001 & 0.0015 & 0.0010 \\ 0.0001 & 0.0014 & 0.0002 \\ 0.0002 & 0.0006 & 0.0008 \\ 0.0002 & 0.0006 & 0.0011 \\ 0.0002 & 0.0007 & 0.0013 \\ 0.0002 & 0.0015 & 0.0005 \\ 0.0002 & 0.0015 & 0.0003 \\ & & \\ & 0.0007 & \\ & 0.0009 & \\ 0.0001 & 0.0022 & 0.0002 \\ 0.0001 & 0.0019 & 0.0004 \\ 0.0001 & 0.0016 & 0.0010 \\ 0.0002 & 0.0016 & 0.0009 \\ 0.0003 & 0.0013 & 0.0009 \\ 0.0001 & 0.0013 & 0.0006 \\ & 0.0015 & 0.0003\end{array}$

0.0035

?ocos

0.0046

然

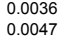

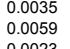

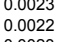

0.0069
0.0059
0.059

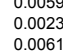

$\underset{\substack{20000 \\ 0.0022}}{0.020}$

0.0000

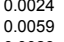

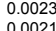

0.007
0.0007
0.0071

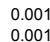

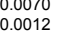

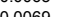

然

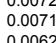

$\underset{20002}{0.002}$

吕004

0.0033
0.0032
0.032

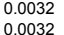

000022

0.0032
0.0037

0.0026 

0.0010
0.0021
0.09997
0.0997

\subsection{2}

$\begin{array}{lll}0.0074 & 0.0012 \\ 0.0059 & 0.0013\end{array}$

$\begin{array}{ll}0.0093 & 0.0012 \\ 0.0087 & 0.0013\end{array}$

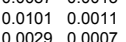

0.0029
0.0007
0.0010

0.0010
0.0010
$0.0021 \quad 0.0010$

$\begin{array}{ll}0.0021 & 0.0010 \\ 0.0021 & 0.0010\end{array}$

0.0002

0.0002
0.0007
0.0019

0.0017
0.0017
0.0089
0.001

$\begin{array}{ll} & \\ & 0.0068 \\ 0.0006 & 0.0035 \\ 0.0006 & 0.0001 \\ 0.0008 & 0.0001 \\ 0.0008 & 0.0001 \\ 0.0009 & 0.0002 \\ 0.0008 & 0.0002 \\ 0.0001\end{array}$

1911 WSTC

1913 SBW1-02

1915 SBW1-04

1917 SBW1-06

1918 SBW1-07

1920 SBW1-09

1920 SBW1-09

1922 SBW1-11
1923 SBW1-12

1924 SBW1-1
1925 SBW1-14

1926 SBW1-15

1928 SBW1-17

1929 SBW1-18

1930 SBW1-19
1931 SBW1-20

1932 SBWW1-21
1933 SBW1-22

1934 SBW1-23

1937 SBW1-26

1938 SBW1-27

1940 SBW1-29

1941 SBW1-30

1942 SBW1-31D
1943 SBW1-32

1944 SBW1-33A
1945 SBW1-34

1946 SBW1-35

1949 SBW1-38B

1950 SBW1-39B
1951 SBW1-40

1952 SBW1-41

1953 SBW1-42
1954 SBW1-43

1955 SBW1-44B

1956 SBW1-45

1957 SBW1-46

1959 SBW1-48

1960 SBW1-49

1961 SBW1-50
1962 SBW1-51
1963 SBW1 52

1963 SBW1-52

1964 SBW1-53

1965 SBW1-54

1967 SWW1-56
1968 SBW1-57

0.0056
0.0065

0.0054

0.0054

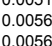

0.005

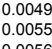

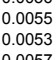

0.0005

0.0055
0.0053

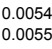

0.0033
0.0052
0.0030

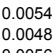

0.0056
0.0055
0.0058

0.0055

0.0007
0.0048
0.0049
0.049

0.0055

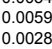

0.0054

0.0057

0.0055
0.055

$\underset{\substack{0.00057 \\ 0.0054}}{0.0005}$

0.0055
0.0034

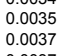

0.0037
0.0037
0.0037
0.037

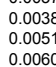

$\begin{array}{ll}0.0021 & 0.9997 \\ 0.0018 & 0.9767\end{array}$

$\begin{array}{ll}0.0024 & 1.0220 \\ 0.0009 & 0.9994\end{array}$

$\begin{array}{lll}0.0011 & 0.999 \\ .0006 & 0.7628\end{array}$

0.00261 .0000

0.00140 .998

$0.0019 \quad 0.9987$

0.0027

0.0000
0.0000
0.8952

$\begin{array}{ll}0.0008 & 0.9981 \\ 0.0006 & 0.9978\end{array}$

$\begin{array}{ll}0.0006 & 0.9981 \\ 0.0007 & 1.0978 \\ 0.0000 & 0.099\end{array}$

$\begin{array}{ll}0.0008 & 0.9997 \\ 0.0008 & 0.9997\end{array}$

0.0028

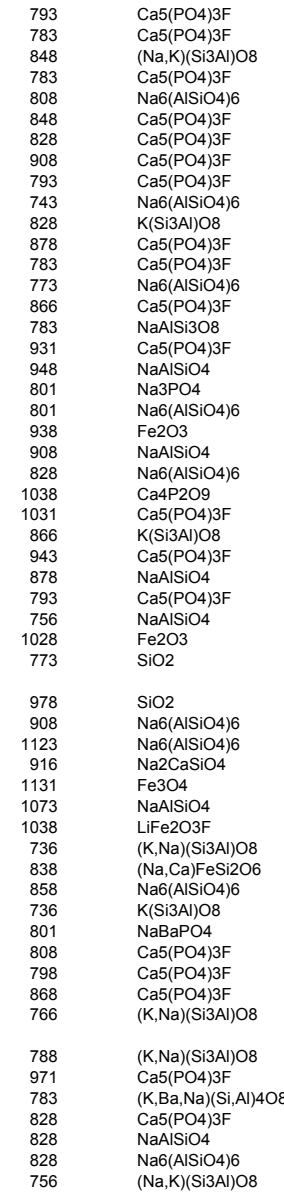




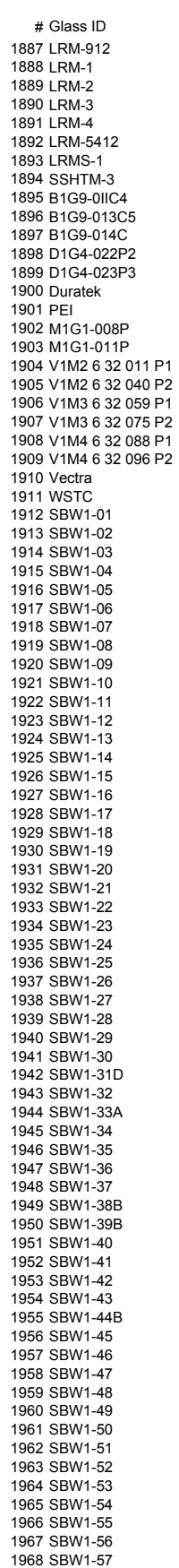

Clear dark brown glass with blact Amorphous Clear dark brown glass with blact Amorphow Clear dark reddish brown glass $n$ Amorphous Clear dark brown glass with blact Amorphous Clear dark brown glass with blact Amorphous Clear dark brown glass with a fev Amorphos Clear dark brown glass with blacl Amorphous Clear dark brown gass with a fer Anophocs Clear dark brown glass with a fev Amorphous Clear dark brown glass with blacl Amorphous Clear dark brown glass with blact Amorphous Clear reddish brown glass with bl Amorphous Clear brown glass with few black Amorphous
Clear dark reddish brown glass A Amornhous Clear dark brown glass with a fev Amorphous
Clear brown glass with a few blac Amorphous Clear dark reddish brown glass 4 Amorohous
Clear dark brown glass with blact $\mathrm{Ca} 5 \mathrm{PO}(\mathrm{PO}) 3(\mathrm{OH})$ Clear dark brown glass with blact $\mathrm{Na3Ca6}(\mathrm{PO} 4)$ Clear dark brown glass with blact Amorphous
Clear brown glass with black spe Amorphous Clear very dark brown glass with Amorphous Clear dark brown glass with few $I$ Amorphous Clear very dark reddish brown gl: Amorphous Clear brown glass with some blar Amorpho Clear dark reddish brown glass w Amorphous Clear brown glass with black spe Amorphous Clear dark brown nabss sing glass Amorphous Clear dark brown glass with air b.Amorphous Clear brown glass with a few blac Amorphous Clear dark black glass with some Anpors Clear very dark purplish brown g/ Amorphous
Clear dark brown glass with blact Amorphous Clear brown glass with few black Amorohous Clear reddish brown glass with bi Amorphous Clear brown glass with black spe Amorpho Clear brown glass with black spe Amorphous Clear brown glass with black spe Amorphous Clear light brown glass with black Amorphous

\begin{tabular}{|c|c|}
\hline norphous & \\
\hline & \\
\hline riphous & 5859 \\
\hline $\begin{array}{l}\text { orphous } \\
\text { orphous }\end{array}$ & 2.5893 \\
\hline $\begin{array}{l}\text { orphous } \\
\text { orphous }\end{array}$ & 2.6900 \\
\hline & 626 \\
\hline OO3, LiOH, NaHF2, and Na2C & 2.584 \\
\hline $\begin{array}{l}\text { orphous } \\
\text { orronous }\end{array}$ & 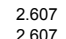 \\
\hline orphous & 2.607 \\
\hline $\begin{array}{l}\text { M33F7 7and ZZOO2 } 20.000 \text { vol\% } \mathrm{c} \\
\text { Mn3F7 (0.05 vol\% crystallinity) }\end{array}$ & $\begin{array}{l}2.602 \\
2.595\end{array}$ \\
\hline $\begin{array}{l}\text { orphous } \\
\text { ons }\end{array}$ & 2.626 \\
\hline $5 \mathrm{Si} 6016(\mathrm{OH}) 2(0.03$ vol\% cry & 2.618 \\
\hline 25Si6016(OH)2 (0.11 vol\% cry & 2.610 \\
\hline $\begin{array}{l}\text { torphous } \\
\text { 5(PO4) (0.20 vol\% crystallir }\end{array}$ & 2.593 \\
\hline orphous & 2.605 \\
\hline orphous & 2.546 \\
\hline iphous & \\
\hline $\begin{array}{l}\text { or (0.15 vol\% crystallinity) } \\
\text { yphous }\end{array}$ & 594 \\
\hline $\begin{array}{l}\text { orphous } \\
\text { orphous }\end{array}$ & 2.607 \\
\hline $5(\mathrm{PO} 4) 3 \mathrm{FF}$ and Fe304 $(0.76 \mathrm{vc}$ & 2.522 \\
\hline $\begin{array}{l}\text { (PO4) } 3 F \text { and CaF2 (0.42 vol' } \\
\text { (rohous }\end{array}$ & 2.557 \\
\hline $\begin{array}{l}\text { prphous } \\
\text { prphous }\end{array}$ & \\
\hline r(BO3)2 and $\mathrm{AlF}(\mathrm{PO} 3) 6(0 . \mathrm{l}$ & 2.638 \\
\hline $\begin{array}{l}\text { horphous } \\
\text { horphous }\end{array}$ & 576 \\
\hline OP 30.88 vol\% crystallinity) & $\begin{array}{l}616 \\
532\end{array}$ \\
\hline $\begin{array}{l}\text { rphous } \\
\text { rpol\% crystallinity) }\end{array}$ & 442 \\
\hline $5(\mathrm{PO} 4) 3(\mathrm{OH})(0.40 \mathrm{vol} \%$ cryst & 2.551 \\
\hline & \\
\hline norphous & \\
\hline Alsio7 & \\
\hline $\begin{array}{l}\text { O4, KFeO2, Ba2FeF6, and N } \\
\text { renous }\end{array}$ & \\
\hline rohous & 627 \\
\hline is & 606 \\
\hline & 2.652 \\
\hline & \\
\hline & \\
\hline & $\begin{array}{l}2.640 \\
2.600\end{array}$ \\
\hline & \\
\hline & \\
\hline & \\
\hline $\begin{array}{l}\text { s } \\
\text { s }\end{array}$ & \\
\hline & \\
\hline & \\
\hline & \\
\hline & \\
\hline & .629 \\
\hline
\end{tabular}

\begin{tabular}{|c|c|c|c|}
\hline 9.500 & 17677.5 & 1090.0 & 33.400 \\
\hline & 18288.5 & 1040.0 & 54.700 \\
\hline $\begin{array}{r}-9.866 \\
-10.105\end{array}$ & 16504.0 & $\begin{array}{l}1155.4 \\
1157 .\end{array}$ & 5.259 \\
\hline $\begin{array}{r}-10.105 \\
-9.996\end{array}$ & $\begin{array}{l}16909.0 \\
17920\end{array}$ & $\begin{array}{l}1157.1 \\
1155.1\end{array}$ & $\begin{array}{r}5.516 \\
\end{array}$ \\
\hline & 16145.0 & $\begin{array}{l}115.8 \\
1157.0\end{array}$ & $\begin{array}{r}12.149 \\
3.902\end{array}$ \\
\hline & 16730.0 & 1155.7 & 7.563 \\
\hline $\begin{array}{r}-9.904 \\
-10.333\end{array}$ & $\begin{array}{l}17477.0 \\
175910\end{array}$ & 1155.8 & 10.009 \\
\hline & $\begin{array}{l}18249.0 \\
\end{array}$ & $\begin{array}{l}1160.2 \\
1156.5\end{array}$ & $\begin{array}{r}6.961 \\
10.768\end{array}$ \\
\hline-9.967 & 16451.0 & 1153.7 & 4.661 \\
\hline & $\begin{array}{l}16250.0 \\
1720\end{array}$ & $\begin{array}{l}1156.6 \\
1156\end{array}$ & \\
\hline-10.243 & $\begin{array}{l}1 / 2229.0 \\
17669.0\end{array}$ & $\begin{array}{l}1156.1 \\
1159\end{array}$ & $\begin{array}{l}6.179 \\
7.889\end{array}$ \\
\hline-9.824 & 16351.0 & 1154.1 & $\begin{array}{l}7.889 \\
5.011\end{array}$ \\
\hline & 16323.0 & 1155.1 & \\
\hline & & 1156.0 & \\
\hline & 17309.0 & 1155.3 & 7.488 \\
\hline & 18108.0 & 1156.2 & 9.623 \\
\hline & 18071.0 & 1155.1 & \\
\hline & $\begin{array}{l}16081.0 \\
15870\end{array}$ & 1755.8 & \\
\hline & 186830 & & \\
\hline & $\begin{array}{l}176273.0 \\
1727.0\end{array}$ & $\begin{array}{l}1156.6 \\
115.6\end{array}$ & $\begin{array}{r}10.838 \\
9.640\end{array}$ \\
\hline & 15119.0 & 11553 & \\
\hline & 20624.0 & 1154.2 & 28.332 \\
\hline & 20246.0 & 1156.9 & 12.822 \\
\hline & 19202.0 & 1160.1 & 14.707 \\
\hline & 19604.0 & 1157.0 & 21.673 \\
\hline & & 1159.7 & 2.393 \\
\hline & & 1154.1 & $\begin{array}{r}2.072 \\
1.096\end{array}$ \\
\hline & 170030 & 115.5 & $\begin{array}{l}\text {. } \\
7.8807\end{array}$ \\
\hline-10.601 & 17980.0 & 1155.3 & 6.908 \\
\hline & & & \\
\hline & $\begin{array}{l}107455.0 \\
18745\end{array}$ & $\begin{array}{l}11566.6 \\
1157.0\end{array}$ & 23.670 \\
\hline & 19060.0 & 1161.7 & 10.183 \\
\hline & 20392.0 & 1254.8 & 11.102 \\
\hline & 20554.0 & 1260.0 & 9.175 \\
\hline & 16274.0 & 1157.4 & 3.062 \\
\hline & 15888.0 & 1158.9 & 1.849 \\
\hline & $\begin{array}{l}16330 \\
1497\end{array}$ & 1155. & $\begin{array}{l}1.836 \\
0.077\end{array}$ \\
\hline & 150580 & 10154 & \\
\hline .520 & 175890 & 11540 & $\begin{array}{l}3.150 \\
3.129\end{array}$ \\
\hline & & & $\begin{array}{l}5.1261 \\
5.561\end{array}$ \\
\hline & 1678 & 11591 & \\
\hline & 19308.0 & 1154 & \\
\hline & 15609.0 & 1155 & \\
\hline & 14557.0 & 1155.9 & 1. \\
\hline & 10876 & 1155 & \\
\hline & 1650 & & \\
\hline-10.144 & 164450 & $\begin{array}{l}1155,6 \\
\end{array}$ & \\
\hline & 15968.0 & 1157.5 & \\
\hline & & & \\
\hline & & 1156.5 & \\
\hline
\end{tabular}




\begin{tabular}{|c|c|c|c|c|c|c|c|c|c|c|c|c|c|c|c|c|c|c|}
\hline & T2 $\left({ }^{\circ} \mathrm{C}\right)$ & V2 (Pa:s) & $\mathrm{T} 3\left({ }^{\circ} \mathrm{C}\right)$ & V3 (Pa:s) & $\mathrm{T} 4\left({ }^{\circ} \mathrm{C}\right)$ & $\mathrm{V} 4$ (Pa:s) & $\mathrm{T} 5\left({ }^{\circ} \mathrm{C}\right)$ & V5 (Pa:s) & T6 $\left({ }^{\circ} \mathrm{C}\right)$ & V6 (Pa.s) & $\mathrm{T} 7\left({ }^{\circ} \mathrm{C}\right)$ & V7 (Pa:s) & $\mathrm{T} 8\left({ }^{\circ} \mathrm{C}\right)$ & V8 (Pa:s) & $\mathrm{T9}\left({ }^{\circ} \mathrm{C}\right)$ & V9 (Pa:s) & $\mathrm{T} 10\left({ }^{\circ} \mathrm{C}\right)$ & V10 (Pa:s) TCLP Ag TCLP As TCLP Ba TCLP Cd TCLP Cr TCLP Ni TCLP Pb \\
\hline & 1237 & 35.300 & 1286 & & 1336.0 & 14.0 & 1336.0 & 14.2 & 1386.0 & 9.6 & 1386.0 & 9.7 & 1386.0 & 9.7 & 1436.0 & 6.6 & 1487.0 & 4.7 \\
\hline $\begin{array}{l}1887 \text { LRM-912 } \\
1888 \text { LRM-1 } \\
1889 \text { LRM-2 }\end{array}$ & 1192 & 38.300 & 1242 & 22.6 & 1291.0 & 14.2 & 1292.0 & 14.0 & 1341.0 & 9.2 & 1341.0 & 9.5 & 1342.0 & 9.5 & 1392.0 & 6.3 & 1442.0 & 4.3 \\
\hline \multirow[b]{2}{*}{$\begin{array}{l}1890 \text { LRM-3 } \\
1891 \text { LRM-4 }\end{array}$} & 1241 & 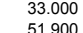 & 1291 & 20.9 & 1291.0 & 21.0 & $\begin{array}{r}1340.0 \\
13860\end{array}$ & $\begin{array}{l}13.7 \\
117\end{array}$ & 1340.0 & 13.9 & 1342.0 & 13.6 & 1392.0 & 9.4 & 1441.0 & 6.2 & 1492.0 & 4.3 \\
\hline & $\begin{array}{r}1237 \\
990\end{array}$ & $\begin{array}{l}51.900 \\
89.400\end{array}$ & $\begin{array}{l}1286 \\
1040\end{array}$ & $\begin{array}{l}31.3 \\
41.9\end{array}$ & $\begin{array}{l}1336.0 \\
1089.0\end{array}$ & $\begin{array}{l}17.9 \\
22.4\end{array}$ & $\begin{array}{l}1386.0 \\
1090.0\end{array}$ & $\begin{array}{l}11.7 \\
21.3\end{array}$ & $\begin{array}{l}1386.0 \\
1139.0\end{array}$ & $\begin{array}{l}12.2 \\
12.7\end{array}$ & $\begin{array}{l}1435.0 \\
1140.0\end{array}$ & $\begin{array}{r}7.6 \\
12.8\end{array}$ & $\begin{array}{l}1446.0 \\
1140.0\end{array}$ & $\begin{array}{c}8.2 \\
13.1\end{array}$ & $\begin{array}{l}1436.0 \\
1189.0\end{array}$ & $\begin{array}{l}8.5 \\
7.9\end{array}$ & $\begin{array}{l}1485.0 \\
1240.0\end{array}$ & $\begin{array}{l}6.0 \\
5.4\end{array}$ \\
\hline $\begin{array}{l}1890 \text { LRMM-3 } \\
1891 \text { LRM-4 } \\
1892 \text { LRM-5412 }\end{array}$ & 1237 & $\begin{array}{l}89.400 \\
23.600\end{array}$ & 1286 & $\begin{array}{l}41.9 \\
15.8\end{array}$ & 1287.0 & $\begin{array}{l}22.4 \\
15.3\end{array}$ & 1336.0 & 10.3 & $\begin{array}{l}1336.0 \\
1336.0\end{array}$ & $\begin{array}{l}11.1 \\
11.1\end{array}$ & 1337.0 & $\begin{array}{l}72.8 \\
11.9\end{array}$ & 1386.0 & $\begin{array}{r}13.1 \\
8.0\end{array}$ & $\begin{array}{l}1899.0 \\
1437.0\end{array}$ & $\begin{array}{l}.9 \\
5.9\end{array}$ & $\begin{array}{l}1488.0 \\
1488.0\end{array}$ & $\begin{array}{l}5.4 \\
4.8\end{array}$ \\
\hline 1893 LRMS-1 & 1192 & 38.500 & 1243 & 22.5 & 1292.0 & 14.1 & 1293.0 & 14.0 & 1341.0 & 9.4 & 1342.0 & 9.6 & 1343.0 & 9.8 & 1392.0 & 6.6 & 1443.0 & $\begin{array}{l}4.8 \\
4.6\end{array}$ \\
\hline 1894 SSHTM-3 & 1245 & 22.400 & 1294 & 14.2 & 1295.0 & 13.7 & 1344.0 & 8.6 & 1345.0 & 9.1 & 1345.0 & 9.0 & 1395.0 & 5.8 & 1446.0 & 3.9 & & \\
\hline 1895 B1G9-0IIC4 & & & & & & & & & & & & & & & & & & \\
\hline $\begin{array}{l}1896 \mathrm{~B} 1199-013 \mathrm{CSC} \\
1897 \mathrm{~B} 1 \mathrm{Gg}-014 \mathrm{C}\end{array}$ & & & & & & & & & & & & & & & & & & \\
\hline $1898 \mathrm{D} 1 \mathrm{G} 4-022 \mathrm{P}$ & & & & & & & & & & & & & & & & & & \\
\hline $\begin{array}{l}1899 \mathrm{D} \text { D } 14-0233 P^{2} \\
1900 \text { Dratek }\end{array}$ & 992 & 35.000 & 1041 & 17.3 & 1091.0 & 9.8 & 1091.0 & 9.9 & 1140.0 & 6.0 & 1141.0 & 5.9 & 1141.0 & 6.2 & 1190.0 & 3.9 & & 29 \\
\hline $1901 \mathrm{PEI}$ & 1140 & 64.700 & 1188 & 35.0 & 1238.0 & 21.3 & 1238.0 & 21.4 & 1287.0 & 13.6 & 1287.0 & 13.7 & 1288.0 & 13.9 & 1337.0 & 9.1 & 1387.0 & $\begin{array}{l}2.9 \\
6.3\end{array}$ \\
\hline $\begin{array}{l}1902 \text { M1G1-008P } \\
1903 \text { M1G1-011P }\end{array}$ & & & & & & & & & & & & & & & & & & \\
\hline 1904 V1M2 6320 & & & & & & & & & & & & & & & & & & \\
\hline & & & & & & & & & & & & & & & & & & \\
\hline $\begin{array}{l}1906 \text { V1M3 } 6320 \\
1907\end{array}$ & & & & & & & & & & & & & & & & & & \\
\hline $\begin{array}{l}1907 \text { V11336 } 6320 \\
1908 \text { V1M4 } 6320\end{array}$ & & & & & & & & & & & & & & & & & & \\
\hline 1909 V1M4 6320 & & & & & & & & & & & & & & & & & & \\
\hline 1910 Vectra & 1144 & 20.600 & 1190 & 12.6 & 1240.0 & 8.4 & 1240.0 & 8.4 & 1289.0 & 5.9 & 1290.0 & 6.0 & 1291.0 & 6.4 & 1344.0 & 4.4 & 1390.0 & 3.2 \\
\hline 1911 WSTC & 1090 & 30.800 & 1090 & 31.3 & & 18.6 & 1139.0 & 18.8 & 1140.0 & 20.1 & 1188.0 & 12.1 & 1237.0 & 8.2 & 1238.0 & 8.6 & 1287.0 & 5.8 \\
\hline $\begin{array}{l}1912 \text { SBW1-01 } \\
1913 \text { SBW1-02 }\end{array}$ & $\begin{array}{r}1079.5 \\
1081345\end{array}$ & $\begin{array}{r}9.915 \\
10474\end{array}$ & $\begin{array}{c}1004.245 \\
100643\end{array}$ & $\begin{array}{l}21.0 \\
22.1\end{array}$ & $\begin{array}{l}954.5 \\
956.8\end{array}$ & $\begin{array}{l}37.2 \\
393\end{array}$ & 1252.6 & 2.7 & $\begin{array}{l}1153.2 \\
11559\end{array}$ & $\begin{array}{l}5.5 \\
57\end{array}$ & & & & & & & & \\
\hline $\begin{array}{l}1913 \text { SBW1-02 } \\
1914 \text { SBW1-03 }\end{array}$ & $\begin{array}{r}1081.345 \\
1080\end{array}$ & $\begin{array}{l}10.474 \\
24.098\end{array}$ & $\begin{array}{l}1006.643 \\
1004.62\end{array}$ & $\begin{array}{l}22.1 \\
54.2\end{array}$ & $\begin{array}{l}956.8 \\
954.8\end{array}$ & $\begin{array}{r}39.3 \\
101.3\end{array}$ & $\begin{array}{l}1255.0 \\
1253.7\end{array}$ & $\begin{array}{l}2.7 \\
5.9\end{array}$ & $\begin{array}{l}1155.9 \\
1153.9\end{array}$ & $\begin{array}{r}5.7 \\
12.6\end{array}$ & & & & & & & & \\
\hline 1915 SBW1-04 & 1081.585 & $\begin{array}{r}24.098 \\
7.193\end{array}$ & $\begin{array}{l}1000.46 \\
1006.76\end{array}$ & $\begin{array}{l}54.2 \\
14.8\end{array}$ & $\begin{array}{l}954.8 \\
957.2\end{array}$ & $\begin{array}{r}101.3 \\
26.0\end{array}$ & $\begin{array}{l}1253.7 \\
1255.3\end{array}$ & $\begin{array}{l}5.9 \\
2.0\end{array}$ & 1156.1 & $\begin{array}{r}2.0 \\
4.0\end{array}$ & & & & & & & & \\
\hline 1916 SBW1-05 & 1079.895 & 14.474 & 1004.635 & 31.0 & 955.0 & 55.6 & 1252.9 & 3.9 & 1153.6 & 7.9 & & & & & & & & \\
\hline 1917 SBW1-06 & 1080.345 & 19.455 & 1005.29 & 42.7 & 955.5 & 78.3 & 1253.6 & 4.9 & 1154.2 & 10.2 & & & & & & & & \\
\hline 1918 SBW1-07 & 1083.05 & 13.227 & 1007.38 & 29.1 & 960.7 & 53.4 & 1254.6 & 3.3 & 1158.4 & 7.1 & & & & & & & & \\
\hline 1919 SBW1-08 & 1081.1 & 21.495 & 1006.28 & 48.8 & 956.6 & 91.1 & 1255.2 & 5.0 & 1155.5 & 11.1 & & & & & & & & \\
\hline 1920 SBW1-09 & $\begin{array}{l}1079.255 \\
1080.855\end{array}$ & 8.670 & 1003.655 & 18.3 & $\begin{array}{l}954.1 \\
0565\end{array}$ & 32.4 & 1252.0 & 2.4 & 1152.6 & 4.8 & & & & & & & & \\
\hline $\begin{array}{l}1921 \text { SBW1-10 } \\
1922 \text { SBW1-1 }\end{array}$ & $\begin{array}{l}1080.855 \\
1081.135\end{array}$ & $\begin{array}{r}7.254 \\
11872\end{array}$ & $\begin{array}{l}1006.075 \\
1005995\end{array}$ & $\begin{array}{l}14.9 \\
25.9\end{array}$ & $\begin{array}{l}956.5 \\
956.2\end{array}$ & $\begin{array}{l}26.2 \\
474\end{array}$ & $\begin{array}{l}1253.3 \\
12552\end{array}$ & 2.0 & $\begin{array}{l}1154.3 \\
11563\end{array}$ & $\begin{array}{l}4.1 \\
6.4\end{array}$ & & & & & & & & \\
\hline 1923 SBW1-12 & $\begin{array}{l}1081.135 \\
1082.765\end{array}$ & $\begin{array}{l}11.8 / 2 \\
15.535\end{array}$ & $\begin{array}{l}1005.995 \\
1007.765\end{array}$ & $\begin{array}{l}25.9 \\
34.3\end{array}$ & $\begin{array}{l}956.2 \\
958.2\end{array}$ & $\begin{array}{l}47.4 \\
63.4\end{array}$ & $\begin{array}{l}1255.2 \\
1255.9\end{array}$ & $\begin{array}{l}3.1 \\
3.9\end{array}$ & $\begin{array}{l}1156.3 \\
1156.7\end{array}$ & $\begin{array}{l}6.4 \\
8.2\end{array}$ & & & & & & & & \\
\hline 1924 SBW1-13 & 1078.835 & $\begin{array}{r}15.530 \\
9.339\end{array}$ & 1004.105 & $\begin{array}{l}19.4 \\
19.4\end{array}$ & $\begin{array}{l}954.5 \\
954.2\end{array}$ & $\begin{array}{l}03.4 \\
34.1\end{array}$ & $\begin{array}{l}1253.9 \\
1252.6\end{array}$ & $\begin{array}{l}3.9 \\
2.5\end{array}$ & 1153.3 & $\begin{array}{l}8.2 \\
5.1\end{array}$ & & & & & & & & \\
\hline 1925 SBW1-14 & 1079.69 & 8.393 & 1005.39 & 17.4 & $\begin{array}{l}955.3 \\
95.0\end{array}$ & 30.7 & 1253.6 & 2.3 & 1154.2 & 4.7 & & & & & & & & \\
\hline 1926 SBW1-15 & 1079.735 & 11.736 & 1004.87 & 25.6 & 955.3 & 46.9 & 1253.7 & 3.0 & 1154.2 & 6.3 & & & & & & & & \\
\hline 1927 SBW1-16 & 1079.57 & 14.619 & 1004.42 & 32.4 & 954.6 & 60.6 & 1253.2 & 3.9 & 1153.8 & 7.8 & & & & & & & & \\
\hline 1928 SBW1-17 & 1080.47 & 18.767 & 1005.325 & 41.6 & 955.6 & 76.7 & 1154.8 & 9.7 & & & & & & & & & & \\
\hline 1929 SBW1-18 & 1079.64 & 15.742 & 1004.67 & 34.6 & 954.9 & 63.1 & 1154.2 & 8.0 & & & & & & & & & & \\
\hline 1930 SBW1-19 & $\begin{array}{r}1079.9 \\
\end{array}$ & $\begin{array}{l}16.231 \\
4390\end{array}$ & $\begin{array}{l}1004.555 \\
1025\end{array}$ & 35.5 & 954.9 & $\begin{array}{l}64.7 \\
15.1\end{array}$ & $\begin{array}{l}1252.8 \\
\end{array}$ & 4.4 & $\begin{array}{l}1153.6 \\
1153.5\end{array}$ & 9.0 & & & & & & & & \\
\hline $\begin{array}{l}1931 \text { SBW1-20 } \\
1932 \text { SBW1-21 }\end{array}$ & $\begin{array}{l}1080.435 \\
1078.335\end{array}$ & $\begin{array}{r}4.390 \\
21817\end{array}$ & $\begin{array}{l}1005.435 \\
100353\end{array}$ & $\begin{array}{r}89.9 \\
490\end{array}$ & $\begin{array}{l}955.8 \\
953.9\end{array}$ & $\begin{array}{l}15.1 \\
91.3\end{array}$ & $\begin{array}{l}1253.0 \\
11027\end{array}$ & $\begin{array}{l}1.2 \\
7.0\end{array}$ & $\begin{array}{l}1153.5 \\
11529\end{array}$ & $\begin{array}{r}2.5 \\
106\end{array}$ & & & & & & & & \\
\hline 1933 SBW1-22 & 1080.6 & $\begin{array}{l}21.811 \\
18.791\end{array}$ & $\begin{array}{l}1005.535 \\
100535\end{array}$ & $\begin{array}{l}49.0 \\
40.5\end{array}$ & $\begin{array}{l}953.9 \\
955.9\end{array}$ & $\begin{array}{l}91.3 \\
72.1\end{array}$ & $\begin{array}{l}1202.7 \\
1254.0\end{array}$ & $\begin{array}{l}7.0 \\
4.6\end{array}$ & $\begin{array}{l}1152.9 \\
1154.5\end{array}$ & $\begin{array}{l}10.6 \\
10.0\end{array}$ & & & & & & & & \\
\hline 1934 SBW1-23 & 1080.01 & 6.878 & 1004.95 & 13.5 & 955.3 & 22.4 & 1253.7 & 2.0 & 1154.2 & 3.9 & & & & & & & & \\
\hline 1935 SBW1-24 & 1079.415 & 60.128 & 1004.655 & 147.8 & 954.8 & 295.9 & & & & & & & & & & & & \\
\hline 1936 SBW1-25 & 1080.985 & 27.433 & 1005.8 & 69.2 & 956.0 & 146.3 & 1254.4 & 5.9 & 1154.7 & 13.3 & & & & & & & & \\
\hline 1937 SBW1-26 & 1084.345 & 29.521 & 1009.2 & 68.2 & 959.4 & 130.8 & 1157.7 & 14.8 & & & & & & & & & & \\
\hline 1938 SBW1-27 & 1081.845 & 45.233 & 1005.935 & 110.3 & 956.3 & 215.5 & 1254.9 & 9.7 & 1155.3 & 21.8 & & & & & & & & \\
\hline 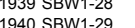 & $\begin{array}{r}1083.59 \\
1078835\end{array}$ & $\begin{array}{l}4.367 \\
3.726\end{array}$ & 1008.395 & 8.8 & & $\begin{array}{l}15.1 \\
125\end{array}$ & 1255.4 & & $\begin{array}{l}1157.6 \\
11528\end{array}$ & 2.5 & & & & & & & & \\
\hline 19401 SBW1-29 & $\begin{array}{l}1078.835 \\
1081.915\end{array}$ & $\begin{array}{l}3.726 \\
3508\end{array}$ & $\begin{array}{l}1003.38 \\
100705\end{array}$ & 7.4 & 953.7 & $\begin{array}{l}12.5 \\
118\end{array}$ & $\begin{array}{l}1251.9 \\
12558\end{array}$ & 1.1 & $\begin{array}{l}1152.8 \\
1156.9\end{array}$ & 2.1 & & & & & & & & \\
\hline 1942 SBW1-31D & 1080.105 & $\begin{array}{r}3.508 \\
15.421\end{array}$ & $\begin{array}{l}1007.2049 \\
1004.92\end{array}$ & $\begin{array}{l}6.9 \\
33.6\end{array}$ & $\begin{array}{l}957.5 \\
955.2\end{array}$ & $\begin{array}{l}11.8 \\
60.4\end{array}$ & $\begin{array}{l}1255.8 \\
1204.5\end{array}$ & $\begin{array}{l}1.1 \\
5.4\end{array}$ & $\begin{array}{l}T 156.9 \\
1154.1\end{array}$ & $\begin{array}{l}2.0 \\
8.0\end{array}$ & & & & & & & & \\
\hline 1943 SBW1-32 & 1079.78 & $\begin{array}{l}15.427 \\
13.866\end{array}$ & 1004.685 & $\begin{array}{l}30.0 \\
31.6\end{array}$ & $\begin{array}{l}950.2 \\
955.0\end{array}$ & $\begin{array}{l}0.4 \\
60.2\end{array}$ & $\begin{array}{l}1253.7 \\
1253.7\end{array}$ & $\begin{array}{l}.4 .4 \\
3.5\end{array}$ & 1154.4 & $\begin{array}{l}.00 \\
7.3\end{array}$ & & & & & & & & \\
\hline 1944 SBW1-33A & & 10.000 & 10004.000 & & 9000.0 & & (1200.1 & & 11104.4 & & & & & & & & & \\
\hline 1945 SBW1-34 & & & & & & & & & & & & & & & & & & \\
\hline 1946 SBW1-35 & 1080.32 & 6.891 & 1004.795 & 14.2 & 955.0 & 25.0 & 1253.2 & 1.9 & 1153.9 & 3.9 & & & & & & & & \\
\hline 1947 SBW1-36 & 1081.19 & 47.480 & 1006.3 & 108.7 & 956.6 & 206.7 & 1254.7 & 10.4 & 1155.4 & 24.0 & & & & & & & & \\
\hline 1948 SBW1-37 & 1085.76 & 20.740 & 1010.76 & 48.9 & $\begin{array}{r}961.2 \\
10557\end{array}$ & 93.6 & 1258.1 & 4.6 & 1159.9 & 10.7 & & & & & & & & \\
\hline 1949 SBW1-38B & $\begin{array}{r}1180.25 \\
1184.255\end{array}$ & $\begin{array}{l}22.0 \\
185\end{array}$ & $\begin{array}{r}1106 \\
1109\end{array}$ & $\begin{array}{l}47.5 \\
404\end{array}$ & $\begin{array}{l}1055.7 \\
1060.3\end{array}$ & 85.1 & $\begin{array}{l}1351.9 \\
1356.9\end{array}$ & 5.2 & $\begin{array}{l}1253.0 \\
12580\end{array}$ & 11.3 & & & & & & & & \\
\hline 1951 SBW1-40 & $\begin{array}{r}1184.255 \\
1082.48\end{array}$ & $\begin{array}{r}18.5 / 5 \\
5.741\end{array}$ & $\begin{array}{l}1100.911 \\
1007.43\end{array}$ & $\begin{array}{l}40.4 \\
11.8\end{array}$ & $\begin{array}{r}1060.3 \\
957.7\end{array}$ & $\begin{array}{l}11.4 \\
20.5\end{array}$ & $\begin{array}{l}3556.9 \\
1256.5\end{array}$ & $\begin{array}{l}4.3 \\
1.6\end{array}$ & $\begin{array}{l}1258.0 \\
11572\end{array}$ & $\begin{array}{l}9.8 \\
32\end{array}$ & & & & & & & & \\
\hline 1952 SBW1-41 & $\begin{array}{r}108.48 \\
1083.2\end{array}$ & $\begin{array}{l}5.141 \\
3.336\end{array}$ & $\begin{array}{l}1000.7 .43 \\
1005\end{array}$ & $\begin{array}{l}71.8 \\
6.9\end{array}$ & 958.0 & $\begin{array}{l}20.5 \\
12.3 \\
\end{array}$ & $\begin{array}{l}1256.5 \\
1255.6\end{array}$ & $\begin{array}{l}1.6 \\
1.0\end{array}$ & 1156.9 & 1.9 & & & & & & & & \\
\hline 1953 SBW1-42 & 1079.7 & $\begin{array}{l}.0 .000 \\
3.400\end{array}$ & 1004.86 & $\begin{array}{l}0.9 \\
7.1\end{array}$ & $\begin{array}{l}954.8 \\
954.0\end{array}$ & 12.7 & $\begin{array}{l}1254.2 \\
125.2\end{array}$ & 0.9 & 1153.8 & 1.9 & & & & & & & & \\
\hline 1954 SBW1-43 & 1082.58 & 4.001 & 1007.635 & 7.7 & 958.0 & 12.8 & 1255.4 & 1.2 & 1157.1 & 2.3 & & & & & & & & \\
\hline 1955 SBW1-44B & 1079.26 & 5.604 & 1004.295 & 10.9 & 954.6 & 18.2 & 1252.8 & 1.7 & 1153.1 & 3.2 & & & & & & & & \\
\hline 1956 SBW1-45 & 1078.9 & 4.123 & 1004.31 & 9.3 & 954.7 & 17.7 & 1252.2 & 1.1 & 1152.9 & 2.3 & & & & & & & & \\
\hline 1957 SBW1-46 & 1080.15 & 10.540 & 1004.6 & 22.4 & 954.8 & 40.3 & 1253.3 & 2.8 & 1154.0 & 5.7 & & & & & & & & \\
\hline $\begin{array}{l}1958 \text { SBW1-47 } \\
1959 \text { SBW1-48 }\end{array}$ & $\begin{array}{l}1083.405 \\
1178.805\end{array}$ & $\begin{array}{r}9.956 \\
30.158\end{array}$ & $\begin{array}{l}1008.245 \\
\quad 10035\end{array}$ & $\begin{array}{l}21.0 \\
70.7\end{array}$ & $\begin{array}{l}958.7 \\
9543\end{array}$ & $\begin{array}{r}37.3 \\
137.8\end{array}$ & 1254.7 & 2.6 & $\begin{array}{l}1156.9 \\
11529\end{array}$ & $\begin{array}{r}5.5 \\
147\end{array}$ & & & & & & & & \\
\hline 1960 SBW1-48 & $\begin{array}{l}1078.805 \\
1080.095\end{array}$ & $\begin{aligned} 30.158 \\
6.747\end{aligned}$ & $\begin{array}{l}1003.95 \\
100552\end{array}$ & $\begin{array}{l}70.7 \\
13.6\end{array}$ & $\begin{array}{l}954.3 \\
9558\end{array}$ & $\begin{array}{r}137.8 \\
237\end{array}$ & $\begin{array}{l}1202.4 \\
1254.1\end{array}$ & $\begin{array}{l}9.7 \\
2.0\end{array}$ & $\begin{array}{l}1152.9 \\
11546\end{array}$ & $\begin{aligned} 14.1 \\
38\end{aligned}$ & & & & & & & & \\
\hline 1961 SBW1-50 & $\begin{array}{r}1080.095 \\
1079.82\end{array}$ & $\begin{array}{l}6.147 \\
2.868\end{array}$ & $\begin{array}{l}1000.552 \\
1004.655\end{array}$ & $\begin{array}{r}13.6 \\
5.4\end{array}$ & $\begin{array}{l}953.8 \\
954.6\end{array}$ & $\begin{array}{r}2.1 \\
9.0\end{array}$ & $\begin{array}{l}1254.1 \\
1252.9\end{array}$ & $\begin{array}{l}2.0 \\
0.9\end{array}$ & $\begin{array}{l}1154.6 \\
1153.5\end{array}$ & $\begin{array}{l}.00 \\
1.7\end{array}$ & & & & & & & & \\
\hline 1962 SBW1-51 & $\begin{array}{l}1079.635 \\
1079.635\end{array}$ & 32.506 & $\begin{array}{l}1004.485 \\
1004.055\end{array}$ & $\begin{array}{l}0.4 \\
73.5\end{array}$ & $\begin{array}{l}954.6 \\
954.5\end{array}$ & 139.4 & $\begin{array}{l}1202.9 \\
1154.0\end{array}$ & $\begin{array}{l}0.9 \\
16.2\end{array}$ & 1103.5 & & & & & & & & & \\
\hline 1963 SBW1-52 & 1082.09 & 22.229 & 1006.96 & 53.6 & 957.3 & 107.9 & 1255.4 & 5.1 & 1155.5 & 11.1 & & & & & & & & \\
\hline 1964 SBW1-53 & 1079.005 & 11.287 & 10 & 23.1 & & 39.5 & & 3.1 & & 6.2 & & & & & & & & \\
\hline 1965 SBW1-54 & 1079.83 & 7.236 & 04.78 & 15.1 & 955.2 & 26.4 & 1253.6 & 1.9 & 1154.0 & 3.9 & & & & & & & & \\
\hline 1966 SBW1-55 & 1082.835 & 5.5 & 1008.045 & 11.2 & 958 & 19.5 & 125 & 1.6 & 1157.2 & 3.1 & & & & & & & & \\
\hline 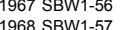 & $\begin{array}{r}1078.04 \\
1080825\end{array}$ & $\begin{array}{l}4.508 \\
8.311\end{array}$ & $\begin{array}{l}1003.085 \\
100523\end{array}$ & $\begin{aligned} 8.8 \\
17.2\end{aligned}$ & $\begin{array}{l}953.5 \\
9557\end{array}$ & $\begin{array}{l}14.9 \\
302\end{array}$ & $\begin{array}{l}1251.9 \\
12534\end{array}$ & $\begin{array}{l}1.3 \\
2.3\end{array}$ & $\begin{array}{l}1152.7 \\
11539\end{array}$ & 2.5 & & & & & & & & \\
\hline 1968 SBW1-57 & 1080.825 & 8.311 & 1005.23 & 17.2 & 955.7 & 30.2 & 1253.4 & 2.3 & 1153.9 & 4.6 & & & & & & & & \\
\hline
\end{tabular}


TCLPSe TCLP Zn TCLPAI TCLP B TCLPSb TCLP TI TCLPV QPCT B $\left(g / \mathrm{m}^{2}\right)$ QPCT Li $\left(g / \mathrm{m}^{2}\right)$ QPCT Na $\left(g / \mathrm{m}^{2}\right)$ QPCT Si $\left(g / \mathrm{m}^{2}\right) \quad$ QPCT pH

CCC PCT B $\left(g / \mathrm{m}^{2}\right) \quad$ CCC PCT Li $\left(g / \mathrm{m}^{2}\right) \quad \operatorname{CCC}$ PCT Na $\left(g / \mathrm{m}^{2}\right) \quad \operatorname{CCC}$ PCT Si $\left(g / \mathrm{m}^{2}\right) \quad \operatorname{CCC~PCT~pH~T1~}\left({ }^{\circ} \mathrm{C}\right)$ 1887 LRM-912
1888 LRM-1

1889 LRM-2

1891 LRM-4
1892 LRM-5412

1892 LRM-5412
1893 LRMS-1
1894 SSHTM-3

1894 SSHTM-3
1895 B1G9-0IIC4

1895 B1G9-011C4
1896 B1G9-013C5

$1898 \mathrm{D} 1 \mathrm{G} 4-022 \mathrm{P} 2$

1899 D1G4-023P

1901 PEI

1902 M1G1-008P
1903 M1G1-011P

$1903 \mathrm{M} 1 \mathrm{G} 1-011 \mathrm{P}$
$1904 \mathrm{~V} 1 \mathrm{M} 2632011 \mathrm{P} 1$

1905 V1M2 632040 P2
1906 V1M3 632059 P1

1907 V1M3 632075 P 2

$1908 \mathrm{~V} 1 \mathrm{M} 4632088 \mathrm{P} 1$
$1909 \mathrm{~V} 1 \mathrm{M} 4632096 \mathrm{P} 2$

1910 Vectra

1912 SBW1-01
1913 SBW1-02

1913 SBW1-02

1915 SBW1-04

1917 SBW1-06

1918 SBW1-07

1920 SBW1-09

1921 SBW1-10

1922 SBW1-11

1924 SBW1-13

1925 SBW1-14

1926 SBW1-15
1927 SBW1-16

1928 SBW1-17

1930 SBW1-19

1932 SBW1-21

1934 SBW1-23

1937 SBW1-26

1939 SBW $1-28$

1940 SBW1-29

1941 SBW1-31D

1943 SBW1-32
1944 SBW1-33A

1945 SBW1-34

1947 SBW1-36

1948 SBW1-37

1949 SBW1-38B

1951 SBW1-40

1952 SBW1-41

1953 SBW1-42

1955 SBW1-44B

1956 SBW1-45

1958 SBW1-47

1959 SBW1-48
1960 SBW1-4

1961 SBW1-50
1962 SBW1-51

1963 SBW1-52

1964 SBW1-53

1965 SBW1-54

1967 SBW1-5

1968 SBW1-57

\begin{tabular}{|c|c|c|c|c|}
\hline & & & & \\
\hline 0.205 & 0.105 & $\begin{array}{l}0.462 \\
0.762\end{array}$ & $\begin{array}{l}0.154 \\
0.185\end{array}$ & $\begin{array}{l}11.225 \\
11.495\end{array}$ \\
\hline 0.164 & & 0.359 & 0.118 & 10.855 \\
\hline 0.287 & 0.134 & 0.470 & 0.149 & 11.190 \\
\hline $\begin{array}{l}0.154 \\
0.205\end{array}$ & ก & 0.391 & 0.134 & $\begin{array}{l}10.975 \\
1123\end{array}$ \\
\hline $\begin{array}{l}0.205 \\
0.174\end{array}$ & 0.096 & $\begin{array}{l}0.445 \\
0.439\end{array}$ & $\begin{array}{l}0.159 \\
0.119\end{array}$ & $\begin{array}{l}11.230 \\
11.325\end{array}$ \\
\hline 0.049 & & 0.125 & 0.050 & 10.365 \\
\hline 0.065 & & 0.158 & 0.052 & $\begin{array}{l}10.620 \\
0\end{array}$ \\
\hline $\begin{array}{l}0.045 \\
0.538\end{array}$ & & $\begin{array}{l}0.124 \\
0.620\end{array}$ & $\begin{array}{l}0.049 \\
0\end{array}$ & $\begin{array}{l}10.445 \\
11.45\end{array}$ \\
\hline $\begin{array}{l}0.538 \\
0.538\end{array}$ & & $\begin{array}{l}0.620 \\
0.608\end{array}$ & $\begin{array}{l}0.168 \\
0.167\end{array}$ & $\begin{array}{l}11.455 \\
11.455\end{array}$ \\
\hline & & 1.696 & 0.258 & 12.140 \\
\hline 0.080 & & 0.220 & 0.060 & 10.535 \\
\hline 0.100 & & 0.320 & 0.080 & 11.060 \\
\hline 0.134 & & 0.213 & 0.094 & $\begin{array}{r}10.295 \\
\end{array}$ \\
\hline 0.129 & & 0.190 & 0.087 & 10.215 \\
\hline 0.147 & & $\begin{array}{l}0.223 \\
0263\end{array}$ & 0.096 & $\begin{array}{l}10.340 \\
\end{array}$ \\
\hline $\begin{array}{l}0.130 \\
0.142\end{array}$ & & 0.262 & 0.008 & $\begin{array}{l}10.315 \\
1037\end{array}$ \\
\hline 0 & & 0 & 0.094 & $\begin{array}{l}10.375 \\
10.370\end{array}$ \\
\hline 0.461 & & 0.549 & 0.185 & 11.115 \\
\hline 0.151 & 0.079 & 0.244 & 0.085 & 10.815 \\
\hline 0.630 & 0.409 & 0.591 & 0.165 & 11.010 \\
\hline 0.361 & 0.353 & 0.528 & 0.154 & 10.510 \\
\hline 0.247 & 0.262 & 0.307 & 0.122 & 10.240 \\
\hline $\begin{array}{l}1.139 \\
292\end{array}$ & 0.938 & $\begin{array}{l}1.007 \\
0.330\end{array}$ & $\begin{array}{l}0.199 \\
\end{array}$ & $\begin{array}{l}11.230 \\
1\end{array}$ \\
\hline $\begin{array}{l}0.292 \\
0.238\end{array}$ & $\begin{array}{l}0.309 \\
0 \\
0.257\end{array}$ & $\begin{array}{l}0.330 \\
0.254\end{array}$ & $\begin{array}{l}0.142 \\
0.111\end{array}$ & $\begin{array}{l}10.440 \\
1028\end{array}$ \\
\hline & $\begin{array}{l}0.251 \\
0.324\end{array}$ & $\begin{array}{l}0.254 \\
0.383\end{array}$ & $\begin{array}{l}0.111 \\
0.125\end{array}$ & $\begin{array}{l}10.285 \\
10.630\end{array}$ \\
\hline 0.182 & $\begin{array}{l}0.209 \\
0.209\end{array}$ & 0.325 & 0.100 & $\begin{array}{l}10.630 \\
10.005\end{array}$ \\
\hline 0.738 & $\begin{array}{l}0.622 \\
0.62\end{array}$ & 0.598 & 0.164 & 10.0605 \\
\hline 0.663 & 0.567 & 0.770 & 0.187 & 11.220 \\
\hline 0.667 & 0.526 & 0.665 & 0.133 & 10.220 \\
\hline 0.315 & 0.316 & 0.469 & 0.135 & 10.570 \\
\hline 0.374 & 0.353 & 0.411 & 0.156 & 10.635 \\
\hline 0.852 & $\begin{array}{l}0.578 \\
0\end{array}$ & $\begin{array}{l}0.902 \\
0.380\end{array}$ & $\begin{array}{l}0.207 \\
0\end{array}$ & $\begin{array}{l}11.370 \\
1\end{array}$ \\
\hline $\begin{array}{l}0.442 \\
1490\end{array}$ & $\begin{array}{l}0.379 \\
1.355\end{array}$ & $\begin{array}{l}0.380 \\
0.950\end{array}$ & $\begin{array}{l}0.138 \\
0.267\end{array}$ & $\begin{array}{r}10.190 \\
999\end{array}$ \\
\hline $\begin{array}{r}.490 \\
0.243\end{array}$ & 0.389 & $\begin{array}{l}0.950 \\
0.277\end{array}$ & $\begin{array}{l}0.267 \\
0.136\end{array}$ & $\begin{array}{r}9.905 \\
10.165\end{array}$ \\
\hline 0.180 & 0.290 & 0.175 & 0.099 & $\begin{array}{l}10.105 \\
10.250\end{array}$ \\
\hline 3.514 & 2.529 & 2.952 & 0.957 & 11.595 \\
\hline 1.197 & 0.804 & 1.424 & 0.370 & 11.695 \\
\hline 0.229 & 0.309 & 0.215 & 0.161 & 10.215 \\
\hline & 0.368 & 0.613 & 0.173 & 10.770 \\
\hline 1.139 & 0.832 & 1.372 & 0.372 & 11.575 \\
\hline 0.409 & 0.451 & 0.395 & 0.120 & 9.200 \\
\hline 0.907 & 0.791 & 0.815 & 0.160 & 9.630 \\
\hline 0.224 & 0.333 & 0.251 & 0.114 & 10.330 \\
\hline 0.339 & $\begin{array}{l}0.333 \\
\end{array}$ & 0.377 & 0.099 & 8.890 \\
\hline $\begin{array}{l}1.445 \\
1.643\end{array}$ & $\begin{array}{r}1.218 \\
1428\end{array}$ & $\begin{array}{l}0.949 \\
1275\end{array}$ & $\begin{array}{l}0.146 \\
0.220\end{array}$ & $\begin{array}{l}10.605 \\
11.235\end{array}$ \\
\hline $\begin{array}{r}.643 \\
4.192\end{array}$ & $\begin{array}{l}1.48 \\
290\end{array}$ & 3.240 & $\begin{array}{l}.220 \\
0.42\end{array}$ & $\begin{array}{l}11.235 \\
11.795\end{array}$ \\
\hline $\begin{array}{l}4.192 \\
0.392\end{array}$ & 0.514 & $\begin{array}{l}0.040 \\
0.073\end{array}$ & $\begin{array}{l}0.186 \\
0.186\end{array}$ & 9.340 \\
\hline 4. & 3.722 & $\begin{array}{l}3.3 .142 \\
\end{array}$ & 0.065 & $\begin{array}{r}9.340 \\
10.045\end{array}$ \\
\hline 1.143 & & 0.761 & 0.147 & 8.885 \\
\hline 1.386 & & 1.256 & 0.173 & 11.090 \\
\hline 0.413 & & 0.674 & 0.215 & 11.42 \\
\hline 1.466 & & 1.366 & 0.505 & 11.305 \\
\hline 0.116 & 0.241 & 0.093 & 0.044 & 8.795 \\
\hline 0.412 & 0.172 & 0.631 & 0.217 & 10.945 \\
\hline 0.799 & 0.761 & $\begin{array}{l}0.372 \\
5509\end{array}$ & 0.122 & 9.990 \\
\hline 6.724 & & 5.099 & 0.917 & 11.295 \\
\hline $\begin{array}{l}1.478 \\
3638\end{array}$ & $\begin{array}{l}1.398 \\
2.588\end{array}$ & $\begin{array}{l}1.066 \\
2.192\end{array}$ & $\begin{array}{l}0.263 \\
0\end{array}$ & $\begin{array}{r}9.880 \\
11775\end{array}$ \\
\hline 3.031 & $\begin{array}{l}2.508 \\
0.090\end{array}$ & $\begin{array}{l}2.192 \\
1078\end{array}$ & 0.239 & $\begin{array}{l}11.15 \\
1130\end{array}$ \\
\hline 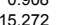 & & $\begin{array}{l}14.260 \\
142\end{array}$ & $\begin{array}{l}0.000 \\
1701\end{array}$ & $\begin{array}{l}11.3071 \\
10.7\end{array}$ \\
\hline 0.520 & 0.422 & 0.461 & 0.162 & 10.525 \\
\hline 0.516 & 0.435 & 0.478 & 0.131 & 10.765 \\
\hline 0.602 & 0.462 & 0.392 & 0.131 & 9.330 \\
\hline 2.587 & 2.233 & 2.028 & 0.418 & 11.360 \\
\hline 0.961 & 0.899 & 0.893 & 0.240 & 10.935 \\
\hline 0.191 & 0.234 & 0.289 & 0.114 & 10.230 \\
\hline 0.461 & 0.508 & 0.325 & 0.105 & 9.890 \\
\hline 0.402 & 0.435 & 0.469 & 0.222 & 10.780 \\
\hline 0.449 & 0.491 & 0.389 & 0.166 & 10.340 \\
\hline 0.455 & 0.461 & 0.583 & 0.168 & 10.880 \\
\hline 0.569 & $\begin{array}{l}0.492 \\
0.62\end{array}$ & 0.597 & $\begin{array}{l}0.203 \\
0.173\end{array}$ & $\begin{array}{r}10.905 \\
10.385\end{array}$ \\
\hline
\end{tabular}


1887 LRM-912
1888 LRM-1
1889 LRM-2

1889 LRM-2

1891 LRM-4

1892 LRM-5412

1894 SSHTM-3

1895 B1G9--IIC4 4
1896 B1G9-013C5

$1897 \mathrm{~B} 1 \mathrm{G} 9-014 \mathrm{C}$

1898 D164-022P2

1900 Duratek

1900 Duratek

$1902 \mathrm{M} 1 \mathrm{G1}-008 \mathrm{P}$
$1903 \mathrm{M} 1 \mathrm{G1}-011 \mathrm{P}$

$1904 \mathrm{~V} 1 \mathrm{M} 2632011 \mathrm{P} 1$
$1905 \mathrm{~V} 1 \mathrm{M} 2632040 \mathrm{P2}$

$1905 \mathrm{~V} 1 \mathrm{M} 2632040 \mathrm{P} 2$
$1906 \mathrm{~V}$ VM $632059 \mathrm{P} 1$

(907) $\mathrm{V} M 3332$

1908 V1M4 632088 P1
1909 V1M4 632096 P2

1910 vectra

1911 WSTC

1913 SBW1-02

1915 SBW1-04

1917 SBW1-06

1918 SBW1-07
1919 SBW1-08

1920 SBW1-09

1921 SBW1-10

1922 SBW1-1
1923 SBW1-1

1924 SBW1-13

1925 SBW1-14

1926 SBW1-15
1927 SBW1-16
1928 SBW1-17

1928 SBW1-17

1930 SBW1-19

1932 SBW1-21

1933 SBW1-22
1934 SBW1-23

1934 SBW1-23
1935 SBW1-24

1936 SBW1-25

1937 SBW1-26

1938 SBW1-27

1940 SBW1-29

1941 SBW1-30

1942 SBW1-31D
1943 SBW1-32

1944 SBW1-33A

1945 SBW1-34

1946 SBW1-35
1947 SBW1-36

1948 SBW1-37

1949 SBW1-38B

1950 SBW1-398
1951 SBW1-40

1952 SBW1-41

1953 SBW1-42

1955 SBW1-44B

1956 SBWW-45

1957 SBW1-4

1959 SBW1-4

1960 SBW1-49

1961 SBW1-50
1962 SBW1-51

1963 SBW1-52

1964 SBW1-53

1965 SBW1-54
1966 SBW1-55

1967 SBW1-5

1968 SBW1-57 


\begin{tabular}{|c|c|c|}
\hline $\begin{array}{l}\text { \# Glass ID } \\
\text { nat }\end{array}$ & Study & Reference \\
\hline $\begin{array}{l}1969 \text { SBW1-58 } \\
1970 \text { SBW1-59 }\end{array}$ & $\begin{array}{l}\text { SBW CVS Phase } 1 \\
\text { SBW CVS Phase } 1\end{array}$ & $\begin{array}{l}\text { Scholes et al. } 2002 \\
\text { Scholes et ala } 20002\end{array}$ \\
\hline 1971 SBW1-60 & $\begin{array}{l}\text { SBW CVS Phase } 1 \\
\text { SB P }\end{array}$ & Scholes et al. 2002 \\
\hline 1972 SBW1-61 & SBW CVS Phase 1 & Scholes et al. 2002 \\
\hline 1973 SBW1-62 & SBW CVS Phase 1 & Scholes et al. 2002 \\
\hline 1974 SBW1-63 & SBW CVS Phase 1 & Scholes et al. 2002 \\
\hline 1975 SBW1-64 & SBW CVS Phase 1 & Scholes et al. 2002 \\
\hline $\begin{array}{l}1976 \text { SBW-111-18.5 } \\
1977 \text { SBW-11-30 }\end{array}$ & $\begin{array}{l}\text { SBW Melter G lass Formulatiol } \\
\text { SBW Melte Glass Formulat }\end{array}$ & $\begin{array}{l}\text { ivienna et al. } 2002 \\
\text { ivienna et al } 2002\end{array}$ \\
\hline $\begin{array}{l}1977 \text { SBW-11-30 } \\
1978 \text { SBW-12-18.5 }\end{array}$ & 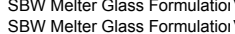 & \\
\hline $\begin{array}{l}1998 \text { SBW-12-18.5 } \\
1979 \text { SBW-13-18.5 }\end{array}$ & 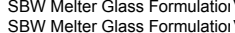 & \\
\hline 1980 SBW-14-18.5 & SBW Melter Glass Formulatioi & Vienna et al. 2002 \\
\hline 1981 SBW-15-18.5 & SBW Melter Glass Formulatioi & \\
\hline 1982 SBW-16-18.5 & SBW Melter Glass Formulatioi & \\
\hline & SBW Melter Glass Formulatiol & \\
\hline 1984 SBW-18-18.5 & SBW Melter GGass Formulatio & Vienna et al. 2002 \\
\hline 1985 SBW-19-18.5 & SBW Melter Glass Formulatio & \\
\hline 1986 SBW-20-18.5 & SBW Melter Glass Formulatio & \\
\hline $\begin{array}{l}1987 \text { SBW-21-18.5 } \\
1988 \text { SBW-22-15 }\end{array}$ & $\begin{array}{l}\text { SBW Metter G Gass Formulatiol } \\
\text { SWW Melte Glass Formulat }\end{array}$ & IVienna et al. 2002 \\
\hline 1989 SBW-22-18.5 & SBW Melter Glass Formulatioi & \\
\hline 1990 SBW-22-20 & SBW Melter Glass Formulatioi & IVienna et al. 2002 \\
\hline 1991 SBW-22-25 & SBW Melter Glass Formulatiol & \\
\hline 1992 SBW-23-15 & SBW Melter Glass Formulatiol & Vienna et al. 2002 \\
\hline 1993 SBW-23-18.5 & SBW Melter Glass Formulatio & Vienna et al. 2002 \\
\hline 1994 SBW-23-20 & SBW Melter Glass Formulatio & \\
\hline 1995 SBW-23-25 & SBW Melter G Gass Formulatio & IVienna et al. 2002 \\
\hline 1996 SBW-24-18.5 & & \\
\hline 997 SBW-25-15 & SBW Melter Glass Formulatio & Vienna et al. 2002 \\
\hline 998 SBW-25-18.5 & SBW Melter Glass Formulatio & IVienna et al. 2002 \\
\hline $\begin{array}{l}999 \text { SBW-25-20 } \\
0000 \text { SBW-25-25 }\end{array}$ & 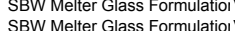 & IVienna et al. 2002 \\
\hline 0001 SBW-25-25 & SBW Melter Glass Formulatiol & $\begin{array}{l}\text { Vivenna et al. } 2002 \\
\text { Vienna et al } 2002\end{array}$ \\
\hline 2002 SBW-27-18.5 & SBW Melter Glass Formulatioi & \\
\hline 2003 HTB651 & Waste Loading Maximization & Bailey et al. 1995 \\
\hline 2004 HTB651Cr0.6 & Waste Loading Maximization & Bailey et al. 1995 \\
\hline 2005 HTB651Cr0.8 & Waste Loading Maximization & Bailey et al. 1995 \\
\hline 2006 НTB651P5 & Waste Loading Maximization & Bailey et al. 1995 \\
\hline 2007 HTB651P7 & Waste Loading Maximization & Bailey et al. 1995 \\
\hline 2008 HTB651P9 & Waste Loading Maximization & Bailey et al. 1995 \\
\hline 009 HTB651Ti1 & & \\
\hline 10 HTB651Ti3 & Waste Loading Maximization & Bailey et al. 1995 \\
\hline 11 HTB651Ti5 & Waste Loading Maximization & Bailey et al. 1995 \\
\hline $12 \mathrm{NPPL}$ & Nepheline Precipitation Studd & Liet al 1997 \\
\hline $\begin{array}{l}13 \text { NPPSI-3 } \\
14 \text { NP-Si-4 }\end{array}$ & $\begin{array}{l}\text { Nepheline Precepititition Studd } \\
\text { Nepheline Precipitation Study }\end{array}$ & $\begin{array}{l}\text { Li et al } 1997 \\
\text { Lietal } 1997\end{array}$ \\
\hline $\begin{array}{l}1 \\
14 \text { NP-Al-4-1 }\end{array}$ & 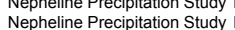 & $\begin{array}{l}\text { Li et al } 1997 \\
\text { Liet al } 1997\end{array}$ \\
\hline 16 NP-Al-2 & Nepheline Precipitation Study & $\begin{array}{l}\text { Let al 1997 } \\
\text { Liet al } 1997\end{array}$ \\
\hline 017 NP-Al-3 & Nepheline Precipitation Study & Liet al 1997 \\
\hline 2018 NP-Al-4 & & \\
\hline 2019 NP-Na-1 & Nepheline Precipitation Study & \\
\hline 2020 NP-Na-2 & Nepheline Precipitation Study & \\
\hline $\begin{array}{l}2021 \text { NP-Li-1 } \\
2022 \text { NP-li-2 }\end{array}$ & 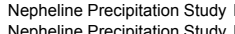 & Liet al 1997 \\
\hline 2022 NP-Li-2 & & Liet al 1997 \\
\hline 023 NP-B-1 & 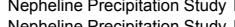 & Lietalal 1997 \\
\hline $\begin{array}{l}022 \text { NP-B-2 } \\
025 \text { NP-K-1 }\end{array}$ & $\begin{array}{l}\text { Nepheline Precipitation Study } \\
\text { Nepheline Precipitation Study }\end{array}$ & Liet al 1997 \\
\hline $\begin{array}{l}025 \text { NP-K-1 } \\
0256 \text { NP-K-2 }\end{array}$ & Nepheline Prectiptatiton Study & \\
\hline $\begin{array}{l}026 \text { NPF-K-2 } \\
027 \text { NP-Ca-1 }\end{array}$ & $\begin{array}{l}\text { Nepheline Precipititation Study } \\
\text { Nenheline Precinitation Study }\end{array}$ & Liet al 1997 \\
\hline 2028 NP-Ca-1 & $\begin{array}{l}\text { Nepheline Precipititation Study } \\
\text { Nenhelline Precinitation Study }\end{array}$ & \\
\hline 2028 NP-Ca-2 & Nepheline Precipitatition Study & Lietalal 1997 \\
\hline $\begin{array}{l}\begin{array}{l}\text { NP-Fe-1 } \\
\text { NPFe-2 }\end{array} \\
\text { NPe }\end{array}$ & 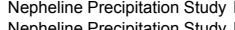 & $\begin{array}{l}\text { Liet al } 1997 \\
\text { Liet al } 1997\end{array}$ \\
\hline $\begin{array}{l}2030 \text { NP-Fe-2 } \\
2031 \text { NP-Fe-3 }\end{array}$ & $\begin{array}{l}\text { Nepheline Precipititition Stuc } \\
\text { Nepheline Precipitation St }\end{array}$ & $\begin{array}{l}\text { Lietal } 19997 \\
\text { Liet al } 1997\end{array}$ \\
\hline 2032 WVCM30 & $\begin{array}{l}\text { CUA data } \\
\text { Colpun }\end{array}$ & Peters et al. 2003 \\
\hline 2033 WVCM32 & CUA data & Peters et al. 2003 \\
\hline 2034 WVCM34 & CUA data & Peters et al. 2003 \\
\hline 2035 WVCM39 & CUA data & Peters et al. 2003 \\
\hline 2036 WVCM40 & $\begin{array}{l}\text { CUA data } \\
\text { CUA data }\end{array}$ & $\begin{array}{l}\text { Peters et al. } 2003 \\
\text { Peter }\end{array}$ \\
\hline $\begin{array}{l}2037 \text { WVCM41 } \\
2038 \text { WVCM51 }\end{array}$ & $\begin{array}{l}\text { CUA data } \\
\text { CUA data }\end{array}$ & \\
\hline 2039 WVCM52 & $\begin{array}{l}\text { CUA data } \\
\text { CUA ata }\end{array}$ & $\begin{array}{l}\text { Peters et al. } 2003 \\
\text { Peters et al. } 2003\end{array}$ \\
\hline 2040 WVCM54 & $\begin{array}{l}\text { CUA data } \\
\text { Cuta }\end{array}$ & 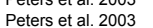 \\
\hline 2041 WVCM62 & CUA data & Peters et al. 2003 \\
\hline 2042 WVUTH9 & CUA data & Peters et al. 2003 \\
\hline 2043 WVUTH16 & CUA data & Peters et al. 2003 \\
\hline 2044 WVUTH20 & CUA data & Peters et al. 2003 \\
\hline 2045 WVUTH27 & CUA data & 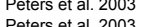 \\
\hline - & $\begin{array}{l}\text { CUA data } \\
\text { CUA data }\end{array}$ & Peters et al. 2003 \\
\hline WVUTH 60 & $\begin{array}{l}\text { CUAdata } \\
\text { Cund a }\end{array}$ & $\begin{array}{l}\text { Peters et al. } 20033 \\
\text { Peters et al. } 2003\end{array}$ \\
\hline $\begin{array}{l}\text { WVUTH } 76 \\
\text { WUIH }\end{array}$ & CUA data & Peters et al. 2003 \\
\hline 2050 WVUTH 85 & CUA data & Peters et al. 2003 \\
\hline
\end{tabular}

Al2O3-t B2O3-t CaO-t Fe2O3-t FeO-t $\begin{array}{llll}0.0870 & 0.1050 & 0.0103 & 0.0754 \\ 0.1014 & 0.0975 & 0.0120 & 0.0713\end{array}$ $\begin{array}{lllll}0.0725 & 0.1125 & 0.0273 & 0.0795 \\ 0\end{array}$ $\begin{array}{llll}0.0870 & 0.1050 & 0.0278 & 0.0754 \\ 0.1014 & 0.0975 & 0.0282 & 0.0713\end{array}$ $\begin{array}{llll}0.1014 & 0.0975 & 0.0282 & 0.0713 \\ 0.1501 & 0.1501 & 0.0008 & 0.0005 \\ 0 & 0.055 & 0.049 & 0\end{array}$ $\begin{array}{llll}0.0375 & 0.1250 & 0.0009 & 0.0005 \\ 0.0127 & 0.097 & 0.0049 & 0.0150\end{array}$ $\begin{array}{llll}0.0510 & 0.0997 & 0.0449 & 0.0150 \\ 0.0827 & 0.0861 & 0.0416 & 0.0149\end{array}$

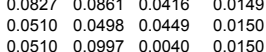
$\begin{array}{lllll}0.0510 & 0.1184 & 0.0449 & 0.0150\end{array}$ $\begin{array}{llll}0.0510 & 0.0577 & 0.0692 & 0.0678 \\ 0.0510 & 0.0498 & 0.049 & 0.0150\end{array}$ $\begin{array}{lllll}0.0510 & 0.0984 & 0.0203 & 0.1004\end{array}$ $\begin{array}{llll}0.0510 & 0.0984 & 0.0203 & 0.1004 \\ 0.0510 & 0.0984 & 0.0203 & 0.1004 \\ 0.0510 & 0.982 & 0.036 & 0.104\end{array}$ $\begin{array}{llll}0.0510 & 0.0984 & 0.0203 & 0.1004 \\ 0.0510 & 0.0984 & 0.0366 & 0.1004\end{array}$ $\begin{array}{llll}0.0510 & 0.0821 & 0.0366 & 0.1004 \\ 0.0414 & 0.0518 & 0.0459 & 0.0150\end{array}$ $\begin{array}{llll}0.0414 & 0.0518 & 0.0459 & 0.0150 \\ 0.0510 & 0.0498 & 0.0449 & 0.0150\end{array}$ $\begin{array}{llll}0.0551 & 0.0490 & 0.0445 & 0.0150 \\ 0.0689 & 0.0461 & 0.0430 & 0.0149\end{array}$ $\begin{array}{llll}0.0414 & 0.1038 & 0.0032 & 0.0150\end{array}$ $\begin{array}{llll}0.0510 & 0.0997 & 0.0040 & 0.0150 \\ 0.0551 & 0.0979 & 0.0043 & 0.0150\end{array}$ $\begin{array}{llll}0.0689 & 0.0920 & 0.0054 & 0.0149 \\ 0.0510 & 0.0911 & 0.0449 & 0.0150\end{array}$ $\begin{array}{lllll}0.0414 & 0.1038 & 0.0459 & 0.1041\end{array}$ $\begin{array}{lllll}0.0510 & 0.0997 & 0.0449 & 0.1004\end{array}$ $\begin{array}{llll}0.0551 & 0.0979 & 0.0445 & 0.0988 \\ 0.0689 & 0.0920 & 0.0430 & 0.0935 \\ 0.0510 & 0.0498 & 0.0967 & 0.0150\end{array}$ $\begin{array}{llll}0.0689 & 0.0920 & 0.0430 & 0.0935 \\ 0.0510 & 0.0498 & 0.1196 & 0.0150\end{array}$

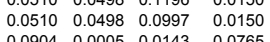
$\begin{array}{llll}0.0510 & 0.0498 & 0.0997 & 0.0150 \\ 0.0094 & 0.0005 & 0.0143 & 0.06765 \\ 0.0901 & 0.0005 & 0.0143 & 0.0763\end{array}$ $\begin{array}{llll}0.0901 & 0.0005 & 0.0143 & 0.0763 \\ 0.0899 & 0.0005 & 0.0143 & 0.0761\end{array}$ $\begin{array}{lllll}0.0887 & 0.0005 & 0.0141 & 0.0751\end{array}$ $\begin{array}{llll}0.0869 & 0.0005 & 0.0138 & 0.0735 \\ 0.0869 & 0.0005 & 0.0138 & 0.0735 \\ 0.0895 & 0.005 & 0.0142 & 0.0757\end{array}$ $\begin{array}{llll}0.0876 & 0.0005 & 0.0139 & 0.0742 \\ 0.0958 & 0.0005 & 0.0136 & 0.0727\end{array}$ $\begin{array}{llll}0.0858 & 0.0005 & 0.0136 & 0.0727 \\ 0.1376 & 0.0800 & 0.0112 & 0.0994 \\ 0.1175 & 0.0683 & 0.0096 & 0.0049\end{array}$ $\begin{array}{llll}0.1175 & 0.0683 & 0.0096 & 0.0849 \\ 0.0630 & 0.0869 & 0.122 & 0.1080\end{array}$ $\begin{array}{llll}0.0630 & 0.0869 & 0.0122 & 0.1080 \\ 0.0775 & 0.0856 & 0.0120 & 0.1063\end{array}$

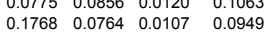
$\begin{array}{llll}0.1929 & 0.0749 & 0.0105 & 0.0930 \\ 0.1496 & 0.0770 & 0.122 & 0.1081\end{array}$

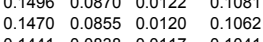
$\begin{array}{llll}0.1326 & 0.0771 & 0.0108 & 0.0958\end{array}$ $\begin{array}{llll}0.1496 & 0.0000 & 0.0122 & 0.0980 \\ 0.1197 & 02000 & 0.097 & 0.143\end{array}$ $\begin{array}{llll}0.1197 & 0.2000 & 0.0097 & 0.0864 \\ 0.1336 & 0.0777 & 0.0109 & 0.0965\end{array}$ $\begin{array}{llll}0.1295 & 0.0753 & 0.0105 & 0.0935 \\ 0.1392 & 0.0809 & 0.0000 & 0.1005\end{array}$ $\begin{array}{llll}0.1252 & 0.0728 & 0.01000 & 0.1005 \\ 0.1430 & 0.0905\end{array}$ $\begin{array}{llll}0.1408 & 0.0819 & 0.0115 & 0.0795 \\ 0.1331 & 0.0774 & 0.0108 & 0.1295\end{array}$

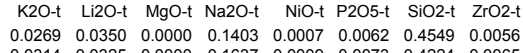

$\begin{array}{llllllllll}0.0224 & 0.0375 & 0.0000 & 0.1169 & 0.0006 & 0.0052 & 0.4873 & 0.0047\end{array}$

$\begin{array}{llllllll}0.0269 & 0.0350 & 0.0000 & 0.1403 & 0.0007 & 0.0062 & 0.4549 & 0.0056 \\ 0.0314 & 0.0325 & 0.0000 & 0.1637 & 0.0009 & 0.0073 & 0.4224 & 0.0065\end{array}$

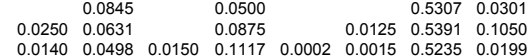

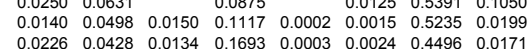
$\begin{array}{llllllll}0.0226 & 0.0428 & 0.0134 & 0.11693 & 0.0003 & 0.0024 & 0.4446 & 0.0191 \\ 0.0140 & 0.0498 & 0.0150 & 0.1531 & 0.0002 & 0.0015 & 0.5320 & 0.0199\end{array}$

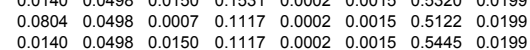
$\begin{array}{llllllll}0.0140 & 0.0498 & 0.0150 & 0.1117 & 0.0002 & 0.0015 & 0.5445 & 0.0199 \\ 0.0140 & 0.0489 & 0.0007 & 0.1532 & 0.0002 & 0.0015 & 0.5216 & 0.0000\end{array}$ $\begin{array}{lllllllll}0.0140 & 0.0498 & 0.0150 & 0.1312 & 0.0002 & 0.0002 & 0.0015 & 0.5216 & 0.0000 \\ 0.0015 & 0.5538 & 0.0199\end{array}$ $\begin{array}{lllllllll}0.0140 & 0.0326 & 0.0007 & 0.0962 & 0.0002 & 0.0015 & 0.5705 & 0.0000\end{array}$ $\begin{array}{llllllll}0.0140 & 0.0245 & 0.0007 & 0.1288 & 0.0002 & 0.0015 & 0.5216 & 0.0082 \\ 0.0140 & 0.0245 & 0.0007 & 0.1288 & 0.0002 & 0.0015 & 0.5053 & 0.0082\end{array}$ $\begin{array}{lllllllll}0.0140 & 0.0245 & 0.0007 & 0.1288 & 0.0002 & 0.0015 & 0.4890 & 0.0082 \\ 0.0140 & 0.025 & 0.0155 & 0.1145 & 0.000 & 0.0015 & 0.5053 & 0.0082\end{array}$ $\begin{array}{llllllll}0.0140 & 0.0245 & 0.0007 & 0.1288 & 0.0002 & 0.0015 & 0.5053 & 0.0082 \\ 0.0113 & 0.0519 & 0.0155 & 0.1145 & 0.0001 & 0.0012 & 0.5776 & 0.0208\end{array}$ $\begin{array}{llllllllll}0.0151 & 0.0489 & 0.0148 & 0.1384 & 0.0002 & 0.0015 & 0.5538 & 0.019 \\ 0.00136 & 0.0195 & 0.0136 & 0.0195\end{array}$ $\begin{array}{llllllll}0.0189 & 0.0458 & 0.0141 & 0.1622 & 0.0002 & 0.0020 & 0.5096 & 0.0183 \\ 0.0480 & 0.0519 & 0.0006 & 0.0941 & 0.0001 & 0.0012 & 0.5668 & 0.0208\end{array}$ $\begin{array}{llllllllll}0.0492 & 0.0498 & 0.00007 & 0.1117 & 0.0002 & 0.0015 & 0.5435 & 0.0199\end{array}$ $\begin{array}{lllllllll}0.0492 & 0.0498 & 0.0007 & 0.1117 & 0.0002 & 0.0015 & 0.5435 & 0.0199 \\ 0.0496 & 0.0489 & 0.0008 & 0.1192 & 0.0002 & 0.0016 & 0.5335 & 0.0195\end{array}$ $\begin{array}{llllllll}0.0512 & 0.0458 & 0.0010 & 0.1442 & 0.0002 & 0.0020 & 0.5001 & 0.0183 \\ 0.0140 & 0.0498 & 0.0150 & 0.1117 & 0.0002 & 0.0015 & 0.5718 & 0.0199\end{array}$ $\begin{array}{llllllll}0.0140 & 0.0498 & 0.0150 & 0.1117 & 0.0002 & 0.0015 & 0.5718 & 0.0199 \\ 0.0113 & 0.0292 & 0.0155 & 0.0780 & 0.0001 & 0.0012 & 0.4958 & 0.0208\end{array}$ $\begin{array}{lllllllll}0.0140 & 0.0280 & 0.0150 & 0.0962 & 0.0002 & 0.0015 & 0.4753 & 0.0199\end{array}$ $\begin{array}{llllllll}0.0151 & 0.0275 & 0.0148 & 0.1040 & 0.0002 & 0.0016 & 0.4666 & 0.0195 \\ 0.0189 & 0.0258 & 0.0141 & 0.1300 & 0.0002 & 0.0020 & 0.4374 & 0.0183\end{array}$ $\begin{array}{llllllll}0.0189 & 0.0258 & 0.0141 & 0.1300 & 0.0002 & 0.0020 & 0.4374 & 0.0183 \\ 0.0140 & 0.0287 & 0.0150 & 0.1117 & 0.0002 & 0.0015 & 0.5197 & 0.0199\end{array}$ $\begin{array}{llllllll}0.0140 & 0.0333 & 0.0150 & 0.1117 & 0.0002 & 0.0015 & 0.5152 & 0.0199\end{array}$ $\begin{array}{llllllll}0.0016 & 0.0005 & 0.1759 & 0.0158 & 0.0327 & 0.4185 & 0.0492 \\ 0.0016 & 0.0005 & 0.1754 & 0.0157 & 0.0327 & 0.4173 & 0.0491\end{array}$ $\begin{array}{llllllll}0.0016 & 0.0005 & 0.1754 & 0.0157 & 0.0327 & 0.4173 & 0.0499 \\ 0.0016 & 0.0005 & 0.1750 & 0.0157 & 0.0326 & 0.4165 & 0.0490 \\ 0 & 0.005 & 0.129 & 0.0151 & 0.0700 & 0.4024 & 0.0473\end{array}$ $\begin{array}{llllllll}0.0015 & 0.0005 & 0.1728 & 0.0155 & 0.0500 & 0.4111 & 0.0483\end{array}$ $\begin{array}{llllllll}0.0015 & 0.0005 & 0.1691 & 0.0151 & 0.0700 & 0.4024 & 0.0473 \\ 0.0015 & & 0.0005 & 0.1691 & 0.0151 & 0.0700 & 0.4024 & 0.0473\end{array}$ $\begin{array}{llllllll}0.0015 & 0.0005 & 0.1706 & 0.0153 & 0.0318 & 0.4060 & 0.0477\end{array}$ $\begin{array}{lllllllll}0.0015 & 0.0005 & 0.1671 & 0.0150 & 0.0311 & 0.3976 & 0.0468\end{array}$

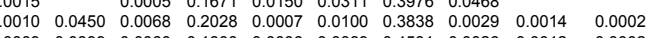
$\begin{array}{lllllllllllll}0.0009 & 0.0384 & 0.0058 & 0.1731 & 0.0006 & 0.0085 & 0.4740 & 0.0025 & 0.0012 & 0.0002\end{array}$ $\begin{array}{lllllllllllll}0.0011 & 0.0489 & 0.0074 & 0.2203 & 0.0008 & 0.0108 & 0.4470 & 0.0032 & 0.0015 & 0.00002\end{array}$

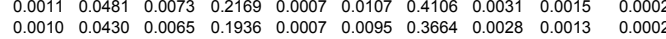
$\begin{array}{llllllllll}0.0009 & 0.0421 & 0.0064 & 0.1898 & 0.0007 & 0.0094 & 0.3592 & 0.0027 & 0.0013 & 0.0002\end{array}$

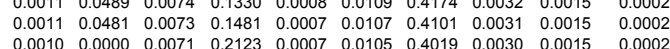
$\begin{array}{llllllllll}0.0010 & 0.0800 & 0.0066 & 0.1953 & 0.0007 & 0.0096 & 0.3698 & 0.0028 & 0.0013 & 0.0002 \\ 0.0011 & 0.0489 & 0.0074 & 0.2204 & 0.0008 & 0.0108 & 0.4172 & 0.0032 & 0.0015 & 0.0002\end{array}$ $\begin{array}{lllllllllll}0.0011 & 0.0489 & 0.0074 & 0.22204 & 0.0008 & 0.0108 & 0.4172 & 0.0032 & 0.0015 & 0.0002 \\ 0.0009 & 0.0391 & 0.0059 & 0.1763 & 0.0006 & 0.0087 & 0.3338 & 0.0025 & 0.0012 & 0.0002\end{array}$ $\begin{array}{llllllllllll}0.0300 & 0.0437 & 0.0066 & 0.1969 & 0.0007 & 0.0097 & 0.3727 & 0.0028 & 0.0014 & 0.0002\end{array}$ $\begin{array}{llllllllll}0.0600 & 0.0423 & 0.0064 & 0.1908 & 0.0007 & 0.0094 & 0.3612 & 0.0027 & 0.0013 & 0.0002 \\ 0.0010 & 0.0455 & 0.0069 & 0.2051 & 0.0007 & 0.0101 & 0.3882 & 0.0029 & 0.0014 & 0.0002\end{array}$

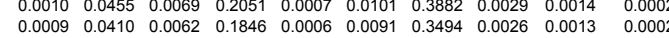
$\begin{array}{llllllllll}0.0009 & 0.0410 & 0.0062 & 0.1846 & 0.0006 & 0.0091 & 0.3494 & 0.0026 & 0.0013 & 0.0002 \\ 0.0010 & 0.0470 & 0.0071 & 0.2120 & 0.0007 & 0.0102 & 0.4012 & 0.0030 & 0.0014 & 0.0002\end{array}$

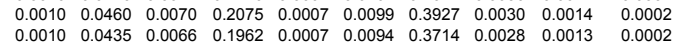

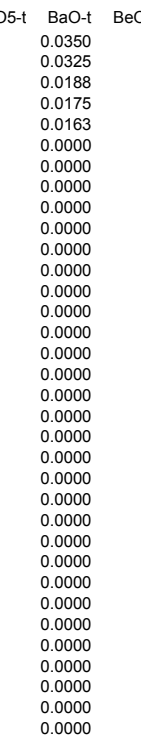
$\begin{array}{lll}0.0136 & 0.0000 \\ 0.0136 & 0.0000 \\ 0.0135 & 0.0000 & \end{array}$ $\begin{array}{lll}0.0131 & 0.0000 & 0.0006 \\ 0.0131 & 0.0000 & 0.0006 \\ 0.013 & 0.0000\end{array}$ $\begin{array}{lll}0.0132 & 0.0000 \\ 0.0129 & 0.0000 & 0.0\end{array}$

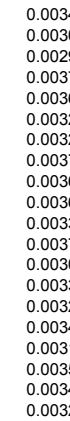

0.0034
0.0030
0.0029
0.0037
0.00036
0.0032
0.0032
0.0037
0.0036
0.0036
0.0033
0.0037
0.0030
0.0333
0.0032
0.0334
0.0031
0.035
0.0034
0.0032 $\begin{array}{lr}\text { CdO-t } & \text { Ce2O3-t } \text { CeO2-t } \\ 0.0014 & 0.0014 \\ 0.0017 & 0.0016 \\ 0.0012 & 0.0011\end{array}$ 0.0014 0.0014
0.0016

$\begin{array}{ll}0.0001 & 0.0016 \\ 0.0002 & 0.0026 \\ 0.0001 & 0.0016 \\ 0.0001 & 0.0016 \\ 0.0001 & 0.0016 \\ 0.0001 & 0.0016 \\ 0.0001 & 0.0016 \\ 0.0001 & 0.0016 \\ 0.0001 & 0.0016 \\ 0.0001 & 0.0016 \\ 0.0001 & 0.0016 \\ 0.0001 & 0.0016 \\ 0.0001 & 0.0013 \\ 0.0001 & 0.0016 \\ 0.0002 & 0.0017 \\ 0.0002 & 0.0022 \\ 0.0001 & 0.0013 \\ 0.0001 & 0.0016 \\ 0.0002 & 0.0017 \\ 0.0002 & 0.0022 \\ 0.0001 & 0.0016 \\ 0.0001 & 0.0013 \\ 0.0001 & 0.0016 \\ 0.0002 & 0.0017 \\ 0.0002 & 0.0022 \\ 0.0001 & 0.0016 \\ 0.0001 & 0.0016 \\ 0.0007 & 0.0190 \\ 0.0006 & 0.0190 \\ 0.0006 & 0.0189 \\ 0.0006 & 0.0187 \\ 0.0006 & 0.0183 \\ 0.0006 & 0.0183 \\ 0.0006 & 0.0188 \\ 0.0006 & 0.0184 \\ 0.0006 & 0.0181 \\ 0.0002 & \end{array}$

0.0002
0.0002
0.0002
0.0002
0.0002
0.0002
0.0002
0.0002
0.0002
0.0002
0.0002
0.0002
0.0002
0.0002
0.0002
0.0002
0.000 


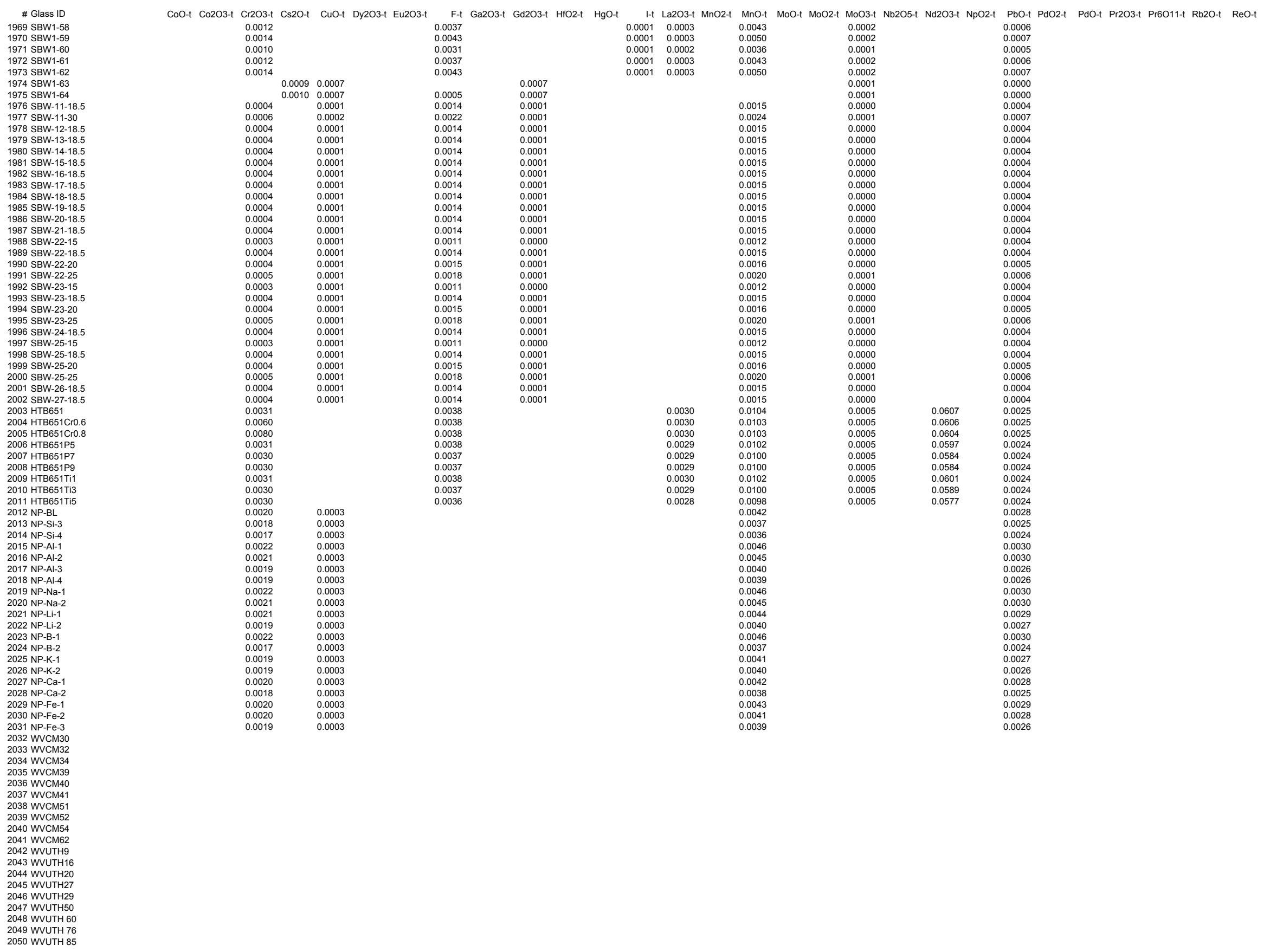


\# Glass ID 1969 SBW1-58

1970 SBW1-59

1972 SBW1-61
1973 SBW1-62

1974 SBW1-63
1975 SBW1-64

1976 SBW-11-18.5

1978 SBW-12-18.

1980 SBW-14-18

1981 SBW-15-18.5

1983 SBW-17-18

1984 SBW-18-18.5

(986 SBW-20-18

1987 SBW-21-18.5

1989 SBW-22-18.5

1990 SBW-22-20

1992 SBW-23-15

1993 SBW-23-18.5

1995 SBW-23-25
1996 SBW-24-18.5

$1997 \mathrm{SBW}-25-15$

1999 SBW-25-20

2001 SBW-26-18.5

2002 SBW-27-18
2003 HTB651

2004 HTB651Cr0.6

2006 HTB651P5

2008 HTB651P9

2010 HTB651Ti3

2011 HTB651

$2014 \mathrm{NP}-\mathrm{Si}-4$

2015 NP-Al-1

2016 NP-Al-2

2018 NP-Al-4

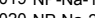

2022 NP-L-1

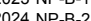

2027 NP-Ca-1

2028 NP-Ca-2

(2030 NP-Fe-2

2034 WVCM33

2035 WVCM 39

2037 WVCM4

2038 WVCM5

2040 WVCM54

2041 WVCME

2043 WVUTH16

2045 WVUTH27

2046 WVUTH29

2047 WVUTH50

2049 WVUTH 76
ReO2-t Re207-t Rh203-t RhO2-t RuO2-t Ru203-t Sb203-t Sb205-t SeO2-t Sm203-t SnO-t SnO2-t SO3-t SrO-t To207-t TeO2-t ThO2-t TiO2-t T12O-t T1203-t U308-t U02-t U03-t V205-t wO3-t Y203-t ZnO-t Sum-t Al203-a 0.0000

0.0000

0.0045

0.0002

0.0084

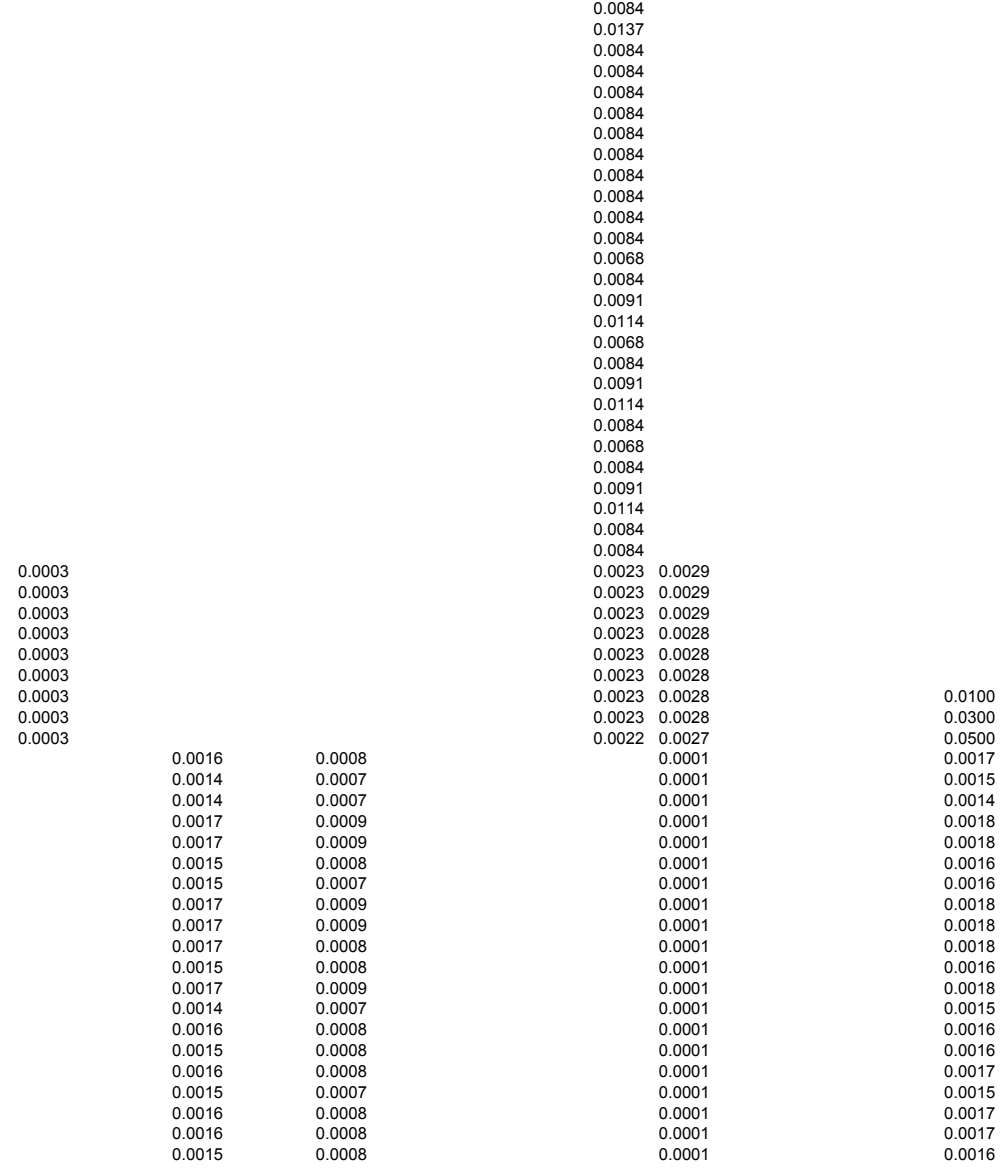

0.0006

0.0399
0.0344
0.0399
0.0399
0.000
0.000
0.039
0.000
0.01
0.0327
0.0327
0.0327
0.04
0.039
0.039
0.03
0.04
0.0399
0.039
0.03
0.000
0.04
0.0399
0.0392
0.039
0.05

$\begin{array}{ll}1.0000 & 0.032 \\ 1.0000 & 0.1056\end{array}$

$\begin{array}{ll}1.0000 & 0.0744 \\ 1.0000 & 0.0896 \\ 1.0000 & 0.146\end{array}$

$\begin{array}{ll}1.0000 & 0.1067 \\ 1.0000 & 0.1496 \\ 1.0000 & 0.0389 \\ 1.000 & 0.0533\end{array}$

$\begin{array}{lll}0.0000 & 1.0000 & 0.0533\end{array}$

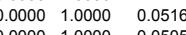

$\begin{array}{lll}.00000 & 1.0000 & 0.0512 \\ 0\end{array}$

$\begin{array}{lll}0.0000 & 1.0000 & 0.0512\end{array}$

$\begin{array}{lll}0.0000 & 1.0000 & 0.0523 \\ 0.0000 & 1.0000 & 0.0524 \\ 0\end{array}$

$\begin{array}{lll}0.0000 & 1.0000 & 0.0524 \\ 0.0000 & 1.0000 & 0.053\end{array}$

$\begin{array}{lll}0.0000 & 1.0000 & 0.0530\end{array}$

$\begin{array}{lll}0.0000 & 1.0000 & 0.0542 \\ 0.0000 & 1.0000 & 0.0427\end{array}$

$\begin{array}{lll}.0000 & 1.0000 & 0.0503 \\ 0.0003 & 0.0003\end{array}$

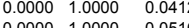

$\begin{array}{llll}0.0000 & 1.0000 & 0.0554\end{array}$

$\begin{array}{lll}0.0000 & 1.0000 & 0.0675\end{array}$

$\begin{array}{lll}0.0000 & 1.0000 & \\ 0.0000 & 1.0000 & 0.041\end{array}$

$\begin{array}{lll}0.0000 & 1.0000 & 0.0418 \\ 0.0000 & 1.0000 & 0.0516 \\ 0.0000 & 1.0000 & 0.0559\end{array}$

$\begin{array}{lll}0.0000 & 1.0000 & 0.0559 \\ 0.0000 & 1.0000 & 0.0686\end{array}$

$\begin{array}{ll}0.0000 & 1.0000 \\ 0.0000 & 1.0000\end{array}$

0.0012

1.0000

1.0000
1.0000

1.0000

1.0000
1.0000

.00011 .0000

$0.0001 \quad 1.0000$

0.000111 .0000

0.00011 .0000

0.00011 .0000

$\begin{array}{ll}0.0001 & 1.0000 \\ 0.0001 & 1.0000 \\ 0.0001 & 1.0000\end{array}$

0.00011 .0000

$\begin{array}{lll}0.0001 & 1.0000 \\ 0.0001 & 1.0000\end{array}$

0.00011 .0000

$\begin{array}{ll}0.0001 & 1.0000 \\ 0.0001 & 1.0000\end{array}$

$\begin{array}{ll}0.0001 & 1.0000 \\ 0.0001 & 1.0000\end{array}$

$\begin{array}{ll}0.0001 & 1.0000 \\ 0.0001 & 1.0000\end{array}$

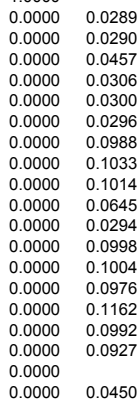


B2O3-a CaO-a Fe2O3-a FeO-a K2O-a Li2O-a MgO-a Na2O-a NiO-a P2O5-a SiO2-a ZrO2-a Ag2O-a As2O3-a As205-a BaO-a BeO-a Bi2O3-a

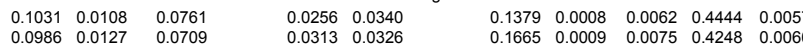

$\begin{array}{lll}0.0986 & 0.0127 & 0.0709 \\ 0.1108 & 0.0274 & 0.0797\end{array}$ $\begin{array}{lll}0.0313 & 0.0326 \\ 0.0224 & 0.0366\end{array}$

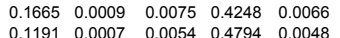

$\begin{array}{lll}0.026 & 0.0282 & 0.0744 \\ 0.0749 & 0.0300 & 0.0718 \\ 0.1260 & 0.0012 & 0.0008\end{array}$

$\begin{array}{lll}0.1474 & 0.0010 & 0.0009 \\ 0.1260 & 0.0012 & 0.0008 \\ 0.1060 & 0.0465 & 0.0163\end{array}$

0.03060 .0315

$\begin{array}{lllll}0.1399 & 0.0008 & 0.0063 & 0.4371 & 0.0055\end{array}$

0.02410 .0619

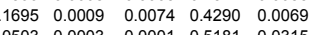

$\begin{array}{lll}0.0486 & 0.0456 & 0.0150 \\ 0.0921 & 0.0039 & 0.0144\end{array}$

$\begin{array}{ll}0.5233 & 0.0011 \\ 0.5410 & 0.0189\end{array}$

$\begin{array}{lll}0.1130 & 0.0456 & 0.0150 \\ 0.0554 & 0.0701 & 0.0683\end{array}$

$\begin{array}{lll}0.0580 & 0.0619 & 0.0031\end{array}$

$\begin{array}{lll}0.1000 & 0.0180 & 0.0959 \\ 0.0989 & 0.0177 & 0.0969\end{array}$

$\begin{array}{lll}0.0989 & 0.0177 & 0.0969 \\ 0.0986 & 0.0180 & 0.0989 \\ 0.0829 & 0.0782 & 0.1010\end{array}$

$\begin{array}{lll}0.0985 & 0.0371 & 0.1000 \\ 0.0829 & 0.0382 & 0.1010 \\ 0.0028 & 0.023 & 0.015\end{array}$

$\begin{array}{lll}0.0528 & 0.0423 & 0.0176\end{array}$

$\begin{array}{lll}0.0466 & 0.0407 & 0.0147 \\ 0.0455 & 0.0400 & 0.014\end{array}$

$\begin{array}{lll}0.1020 & 0.0400 & 0.0149 \\ 0.0167\end{array}$

$\begin{array}{lll}0.0972 & 0.0017 & 0.0163 \\ 0.0955 & 0.0015 & 0.0167 \\ 0.0884 & 0.0025 & 0.0166\end{array}$

$\begin{array}{lll}0.1010 & 0.0494 & 0.1030 \\ 0.0959 & 0.0473 & 0.0998\end{array}$

$\begin{array}{lllllll}0.0148 & 0.0488 & 0.0150 & 0.1540 & 0.0003 & 0.5410 & 0.0197\end{array}$

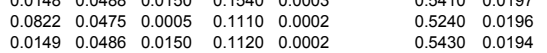

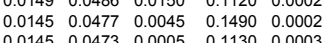

$\begin{array}{ll}0.5360 & 0.0000 \\ 0.5140 & 0.0074\end{array}$

0.0124
0.0127
0.0321
0.0218

$\begin{array}{ll}0.5140 & 0.0074 \\ 0.5090 & 0.0076 \\ 0.5170 & 0.0076\end{array}$

$0.0127 \quad 0.0210$

$\begin{array}{ll}0.4990 & 0.0078 \\ 0.5170 & 0.0076\end{array}$

$\begin{array}{llllllll}0.0125 & 0.0497 & 0.0161 & 0.1129 & 0.0002 & 0.0013 & 0.5842 & 0.0196\end{array}$ $\begin{array}{llllllllll}0.0166 & 0.0462 & 0.0151 & 0.1350 & 0.0002 & 0.0017 & 0.5500 & 0.0182\end{array}$ $\begin{array}{llllllll}0.0210 & 0.0430 & 0.0146 & 0.1620 & 0.0002 & 0.0021 & 0.5222 & 0.0176 \\ 0.0427 & 0.0544 & 0.0004 & 0.0932 & 0.0002 & 0.0017 & 0.5630 & 0.0201\end{array}$ $\begin{array}{llllllll}0.0438 & 0.0528 & 0.0006 & 0.1110 & 0.0002 & 0.0020 & 0.5240 & 0.0192\end{array}$ $\begin{array}{lllllllll}0.0446 & 0.0485 & 0.0009 & 0.1410 & 0.0003 & 0.0024 & 0.4990 & 0.0182\end{array}$ $\begin{array}{lll}0.0966 & 0.0484 & 0.1000 \\ 0.0911 & 0.0473 & 0.0978\end{array}$

$\begin{array}{lllllllll}0.0112 & 0.0295 & 0.0155 & 0.0760 & 0.0001 & 0.0017 & 0.4860 & 0.0201\end{array}$ $\begin{array}{llllllll}0.0145 & 0.0275 & 0.0149 & 0.1060 & 0.0002 & 0.0021 & 0.4240 & 0.0200 \\ 0.0184 & 0.0258 & 0.0144 & 0.1240 & 0.0002 & 0.0025 & 0.4560 & 0.02178\end{array}$

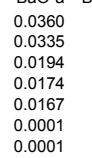

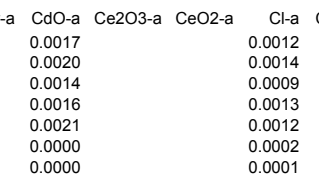

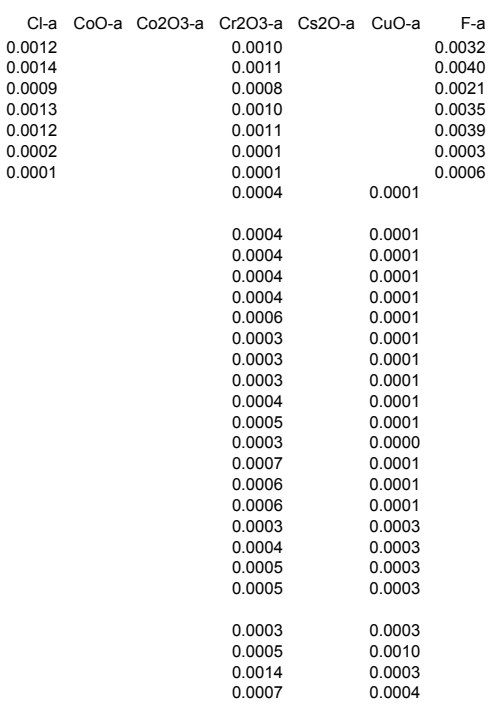

$\begin{array}{lll}0.1015 & 0.0063 & 0.1241 \\ 0.1423 & 0.0063 & 0.1247 \\ 0.1212 & 0.032 & 0.093\end{array}$ $\begin{array}{lll}0.1012 & 0.0312 & 0.0983 \\ 0.0978 & 0.0099 & 0.1247\end{array}$ $\begin{array}{lll}0.0983 & 0.0097 & 0.1228 \\ 0.0887 & 0.0089 & 0.1240\end{array}$ $\begin{array}{lll}0.1228 & 0.0082 & 0.1193 \\ 0.1272 & 0.0084 & 0.1240 \\ 0\end{array}$ $\begin{array}{lll}0.1247 & 0.0050 & 0.1287 \\ 0.1289 & 0.0068 & 0.1202\end{array}$ $\begin{array}{lll}0.0963 & 0.0095 & 0.1203 \\ 0.1241 & 0.0073 & 0.1234\end{array}$ $\begin{array}{lll}0.1251 & 0.0073 & 0.1234 \\ 0.1251 & 0.0083 & 0.1216 \\ 0.1203 & 0.0071 & 0.136\end{array}$ $\begin{array}{lll}0.1124 & 0.0071 & 0.1356 \\ 0.1203 & 0.0080 & 0.1169 \\ 0.121 & 0.080\end{array}$ $\begin{array}{lll}0.121 & 0.0080 & 0.1191 \\ 0.1154 & 0.0077 & 0.1721\end{array}$

$0.1066 \quad 0.0082 \quad 0.1506$

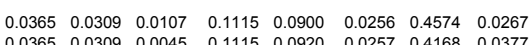
$\begin{array}{llllllll}0.0188 & 0.0303 & 0.0142 & 0.1147 & 0.0108 & 0.0306 & 0.4430 & 0.0193\end{array}$ $\begin{array}{llllllll}0.0201 & 0.0271 & 0.0096 & 0.1327 & 0.0040 & 0.0268 & 0.4333 & 0.0056\end{array}$ $\begin{array}{lllllllll}0.0354 & 0.0299 & 0.0129 & 0.0980 & 0.0034 & 0.0248 & 0.4635 & 0.0042\end{array}$ $\begin{array}{llllllll}0.0160 & 0.0222 & 0.0079 & 0.0980 & 0.0030 & 0.0248 & 0.3961 & 0.0039 \\ 0.0164 & 0.0236 & 0.0081 & 0.1017 & 0.0031 & 0.0261 & 0.3738 & 0.0040\end{array}$ $\begin{array}{llllllllll}0.0016 & 0.0180 & 0.0016 & 0.1142 & 0.0025 & 0.0260 & 0.4077 & 0.0019\end{array}$

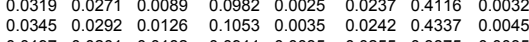

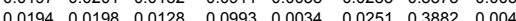
$\begin{array}{llllllllll}0.0191 & 0.0220 & 0.0126 & 0.0964 & 0.0034 & 0.0247 & 0.3814 & 0.0044\end{array}$ $\begin{array}{llllllll}0.0157 & 0.0217 & 0.0078 & 0.0960 & 0.0029 & 0.0242 & 0.3882 & 0.0038 \\ 0.0157 & 0.0227 & 0.0078 & 0.0976 & 0.0029 & 0.0250 & 0.3588 & 0.0038\end{array}$ $\begin{array}{llllllll}0.0368 & 0.0315 & 0.0090 & 0.1140 & 0.0030 & 0.0236 & 0.3992 & 0.0039\end{array}$

0.0000
0.0000
0.0000
0.0023
0.0023
0.0021
0.0019
0.0020
0.0016
0.0022
0.0017
0.0017
0.0017
0.0019
0.0019
0.0018
0.0020

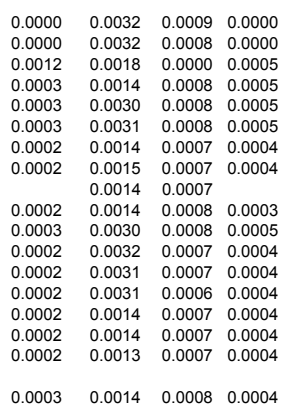


\# Glass ID
1969 SBW1-58

1970 SBW1-5

1972 SBW1-61
1973 SBW1-62

1974 SBW1-63

1976 SBW-11-18.5

1977 SBW-11-30

1979 SBW-13-18.5

1980 SBW-14-18.5

1981 SBW-15-18.5

1983 SBW-17-18.5

1984 SBW-18-18.5

1986 SBW-20-18.5

1987 SBW-21-18.5

1988 S SW-22-15

989 SBW-22-18.5

991 SBW-22-25

1993 SBW-23-18.5

1995 SBW-23-25

1996 SBW-24-18.5
1997 SBW-25-15

1998 SBW-25-18.5

1999 SBW-25-20

2001 SBW-26-18.5

2002 SBW-27-18

2004 HTB655Cr0.6

2006 HTB651P5

2007 HTB651P7

2008 HTB651P9

2010 HTB651Ti3

2011 HTB651
2012 NP-BL
2013 NPPS-3

2014 NP-Si-4

2015 NP-Al-1

2016 NP-Al-2

2018 NP-Al-4

2019 NP-Na-1

$2020 \mathrm{NP}-\mathrm{Na}-2$

2022 NP-Li-2

2024 NP-B-2

2025 NP-K-1

2027 NP-Ca-

2028 NP-Ca-2

$2030 \mathrm{NP}-\mathrm{Fe}-2$

2032 WVCM 30

2033 WVCM3
2034 WVCM33

2035 WVCM3

2037 WVCM 4

2038 WVCM51

2040 WVCM54

2041 WVCM62

2043 WVUTH16

2045 WVUTH27

2046 WVUTH29

2047 WUTTH50
2048 WVUTH 60

2049 WVUTH 76

2050 WVUTH 85
Ga2O3-a Gd2O3-a

I-a La203-a MnO2-a MnO-a MoO-a MoO3-a Nb205-a Nd203-a NpO2-a PbO-a PdO2-a PdO-a Pr203-a Pr6011-a PuO2-a Rb2O-a ReO-a ReO2-a Re207-a Rh203-a RhO2-a RuO2-a Sb203-a Sb205-a SeO2-a 0.0031

0.000

0.0001

0.000

0.0001
0.0001
0.0001

0.000

0.000

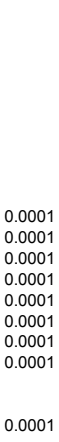

0.0015
0.0015
0.0015

0.0015
0.0014
0.0014

0.0014
0.0014
0.0014

$\begin{array}{ll}0.0014 & 0012 \\ 0.0014 & 0.0010 \\ 0.0015 & 0.0010\end{array}$

$\begin{array}{ll}0.0014 & 0.0010 \\ 0.0015 & 0.0010 \\ 0.0020 & 0.0009\end{array}$

0.0012
0.0015
0.0016

0.0001

$\begin{array}{ll}0.0012 & \\ 0.0015 & 0.0001 \\ 0.0016 & 0.0001 \\ 0.001 & 0.001\end{array}$

$\begin{array}{ll}0.00021 & 0.0001 \\ 0.0021 & 0.0001\end{array}$

0.0005
0.0004
0.0004
0.0005
0.0004
0.0004
0.0004
0.0004
0.0004
0.0004
0.0004
0.0004
0.0005
0.0006
0.0003
0.0004
0.0005
0.0006
0.0004
0.0007
0.0005
0.0006

$\begin{array}{lll}.0001 & 0.0006 & 0.0134 \\ .0002 & 0.0012 & 0.0134 \\ & 0.005 & 0.0233\end{array}$

$\begin{array}{ll}0.0012 & 0.0134 \\ 0.0005 & 0.0233 \\ 0.0003 & 0.0147 \\ 0.0003 & 0.0132\end{array}$

$\begin{array}{ll}0.0003 & 0.0147 \\ 0.0003 & 0.0144 \\ 0.0003 & 0.132\end{array}$

$\begin{array}{ll}0.0003 & 0.0144 \\ 0.0003 & 0.0132 \\ 0.0004 & 0.0121 \\ 0.0004 & 0.124\end{array}$

$\begin{array}{ll}0.0004 & 0.0121 \\ 0.0004 & 0.0124 \\ 0.0004 & 0.0101\end{array}$

$0.0004 \quad 0.0101$

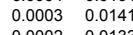

$\begin{array}{ll}0.0002 & 0.0133 \\ 0.0002 & 0.0131 \\ 0.0002 & 0.0128\end{array}$

$\begin{array}{ll}0.0002 & 0.0128 \\ 0.0004 & 0.0119 \\ 0 & 0004\end{array}$

$\begin{array}{ll}0.0004 & 0.0119 \\ 0.0004 & 0.0114\end{array}$

$0.0005 \quad 0.0122$

0.0004
0.0004
0.0004

0.0004
0.0004

0.0004
0.0004

0.0004

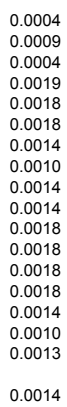

$0.0002 \quad 0.0008$

$0.0002 \quad 0.0003$

$\begin{array}{ll}0.0002 & 0.0004\end{array}$

$0.0002 \quad 0.0004$
0.0004

$0.0002 \quad 0.0003$ 
\# Glass ID
1969 SBW1-58

1970 SBW1-5
1971 SBW1-60

1972 SBW1-61

1974 SBW1-63

1976 SBW-11-18.5

1977 SBW-11-30

1979 SBW-13-18.5

1980 SBW-14-18.5

1981 SBW-15-18.5

1983 SBW-17-18.5

1984 SBW-18-18.5

1985 SBW-19-18.5

1987 SBW-21-18.5

(988 SBW-22-15

1993 SBW-23-18.5

1996 SBW-24-18.5

1998 SBW-25-18.5

1999 SBW-25-20
2000 SBW-25-25

2002 SBW-27-18.5

2003 HTB651

2004 HTB655Cro. 6

2006 HTB651P5

2007 HTB651P7
2008 HTB651P9

2010 HTB651Ti3

2011 HTB651
2012 NP-BL

2014 NP-Si-4

2015 NP-Al-1

2016 NP-Al-2

2018 NP-Al-4

2019 NP-Na-1

2020 NP-Na-2

2022 NP-Li-2

2024 NP-B-2

2025 NP-K-1

2027 NP-Ca-1

2028 NP-Ca-2

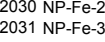

2032 WVCM 30

2034 WVCM34

2035 WVCM3

2037 WVCM4

2038 WVCM5

2040 WVCM5

2041 WVCM62

2043 WVUTH16
2044 WVUTH20
2045 WVUTH27

2045 WVUTH27

2046 WVUTH29

2048 WVUTH 60

2049 WVUTH 76

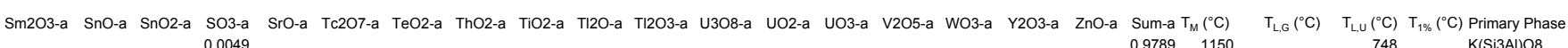
$\begin{array}{ll}0.9789 & 1150 \\ 1.0096 & 1150\end{array}$

Nab(AlISio4)6

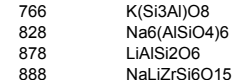

0.0047
0.0050
0.0058
0.0001

$\begin{array}{ll}0.9927 & 1150 \\ 0.9772 & 1150 \\ 0.9171 & 1150\end{array}$

$\begin{array}{ll}0.9813 & 1150 \\ 0.9763 & 1150\end{array}$

0.0396

0.0082

0.008

0.0048
0.0060
0.005

0.0057

0.0059

0.0074

0.008
0.0107

0.007

0.0051
0.0058
0.0059

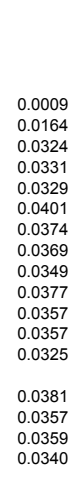

0.0000

0.000

.034

0.0329

$\begin{array}{ll}0.0000 & 0.9901 \\ 0.0000 & 1.0012 \\ 0.0002 & 0.9818\end{array}$

$\begin{array}{ll}0.0002 & 0.9818 \\ 0.0001 & 0.9668\end{array}$

$\begin{array}{ll}0.0001 & 0.9774 \\ 0.0001 & 0.9773\end{array}$

$\begin{array}{lll}0.0381 & 0.0002 \quad 0.0809 \\ 0.0357 & 0.0000\end{array}$

$\begin{array}{lll}0.0359 & 0.0004 & 0.9720 \\ 0.0359 & 0.0001 & 0.9560\end{array}$

0.00021 .0104

0.000

0.0000
0.0000

0.0000
0.0000
0

0.0000

0.0000

0.0000

0.0000

0.0000
0.0000

$\begin{array}{lll}0.0002 \quad 0.0000 & 1.081\end{array}$

$\begin{array}{ll}0.0000 & 1.0831 \\ 0.0005 & 1.0001\end{array}$

0.00041 .0001

$\begin{array}{ll}0.0000 \\ 0.000098 \\ 0.0363 & 0.0099\end{array}$

$\begin{array}{lll}0.0303 \\ 0.0357 & 0.00998 \\ 0.0098 & 0.0098\end{array}$

$\begin{array}{lll}0.0355 & 0.0097 \\ 0.0352 & 0.0082\end{array}$

0.0352
0.0366
0.00084

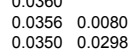

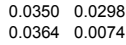

$\begin{array}{ll}0.0358 & 0.0073 \\ 0.0404 & 0.0072\end{array}$

0.0345
0.0352
0.0081

0.0352
0.0331
0.00071

0.03560 .0083

$0.0023 \quad 0.0002$ 


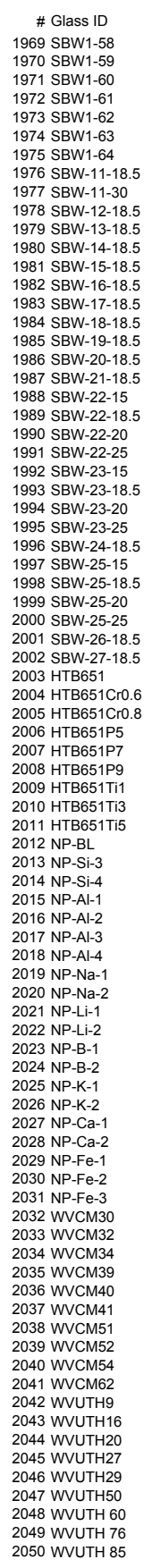
$\mathrm{C}_{800^{\circ} \mathrm{C}} \mathrm{C}_{850^{\circ} \mathrm{C}} \mathrm{C}_{900^{\circ} \mathrm{C}} \mathrm{C}_{950^{\circ} \mathrm{C}} \mathrm{C}_{1000^{\circ} \mathrm{C}} \mathrm{C}_{1050^{\circ} \mathrm{C}} \mathrm{C}_{1100^{\circ} \mathrm{C}} \mathrm{C}_{1150^{\circ} \mathrm{C}} \mathrm{C}_{1200^{\circ} \mathrm{C}}$ Quenched Visual//OM Quenched XRD
Clear brown glass with black spe Amorphous
Clear brown glass with black spe Amorpho
Clear brown glass with black spe Amorphow
Clear blue glass with hots of air b A Amorphous
Clear blue glass with lots of air bi Amorphous

CCC XRD

Amorphous

Amorphou
Amorphous

Amorphous
Amorphous

Amorphous
Amorphous

0.4 vol\% crystalline (85\% nepheline, $5 \%$ Na(Si3A) O8, 10\% Mn3B206) 0.01 vol\% crystallin

0.01 vol\% crystalline $(\mathrm{Ag})$

\subsection{1 vol\% crystalline $(\mathrm{Ag})$}

.5 vol\% crystalline (80\% nepheli, $5 \%$ Na(Si3A) $) 08,10 \%$ Mn3B2206 0.2 vol\% crystalline $(75 \%$ nepheline, $5 \%$ Sio2, $15 \%$ spinel, $5 \%$ Mn 38206$)$
0.4 vol\% crystalline $(90 \%$ nepheline, $1 \%$ SiO2, $<5 \%$ spinel, $<5 \%$ Mn3B2O6) 0.01 vol\% crystalline ( $45 \%$ nepheline, $55 \%$ Ag) 0.6 vol\% crystalline ( $55 \%$ nepheline, $35 \%$ L Li2SiO3, <5\% Li8SiO6, 10\% spinel) 0.6 vol\% crystalline (10\% nepheline, $90 \%$ NaAlSiO4)

0.45 vol\% crystalline $(>95 \%$ nepheline, $<5 \% \mathrm{SiO}, 1 \% \mathrm{Li} 2 \mathrm{SiO} 3)$ 0.5 vol\% crystalline (95\% nepheline, $5 \%$ Li2SiO3) 35 vol\% crystalline ( $75 \%$ nepheline, $25 \%$ spinel)

35 vol\% crystilline (90\% nepheline. $5 \%$ Li2SiO3. $5 \%$ unknown)

0.3 vol\% crystalline (90\% nepheline, $5 \%$ Li2SiO3, 5\% unknown)
0.45 vol\% crystalline (70\% nepheline, $5 \%$ Li2SiO3, 15\% spinel, $10 \%$ Fe2O3) 
\# Glass ID

1969 SBW1-58

1970 SBW1-59

1972 SBW1-61

1974 SBW1-63

1976 SBW-11-18.5

1977 SBW-11-30

1979 SBW-13-18.5

1980 SBW-14-18.5

1982 SBW-16-18.5

1984 SBW-18-18.5

1985 SBW-19-18.5

1987 SBW-21-18.5

1988 SBW-22-15

1989 SBW-22-20

1991 SBW-22-25

1992 SBW-23-15

1993 SBW-23-18.5

$1995 \mathrm{SBW}-23-25$
$1996 \mathrm{SBW}-22-18.5$

1998 SBW-25-18.5

1999 SBW-25-20

2001 SBW-26-18.5

2002 SBW-27-18.

2003 HTBB51

2006 HTB651P5

2007 HTB651P

2008 HTB651P9
2009 HTB651Ti1

2010 HTB651Ti3
2011 HTB651Ti5

2012 NP-BL

$2014 \mathrm{NP}-\mathrm{Si}-4$

2015 NP-Al-1

2016 NP-Al-2

2018 NP-Al-4

2019 NP-Na-1

2020 NP-Na-2

2022 NP-Li-2

2025 NP-K-1

2026 NP-K-2

2027 NP-Ca- 1

2029 NP-Fe- 1

2030 NP-Fe-2

(2)

2033 WVCM32

2035 WVCM 39
2036 WVCM 40

2037 WVCM4

2038 WVCM51

2040 WVCM54

2041 WVCME2

2043 WVUTH1 16

2045 WVUTH27

2046 WVUTH29

2047 WUUTH50
2048 WVUTH 60

2050 WVUTH 85

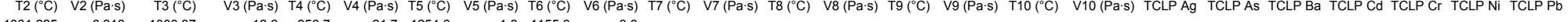

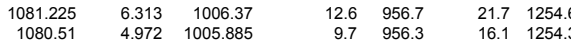

$\begin{array}{lllllll}1082.38 & 7.662 & 1007.505 & 15.9 & 957.8 & 280 & 1254.3\end{array}$

$1080.095 \quad 5.89331005 .125 \quad 11.9 \quad 955.4 \quad 20.6 \quad 1254.4$

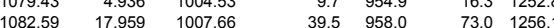

$\begin{array}{rrrrrrr}1082.645 & 15.251 & 1007.69 & 38.4 & 958.1 & 78.7 & 1256.0 \\ 1095.5 & 5.776 & 1043 & 8.7 & 1150.5 & 3.9 & 1206.0\end{array}$

$1.51155 .2-2.9$

$\begin{array}{ll}2.1 & 1156.5 \\ 1.7 & 1155.5 \\ 1.4 & 1153.4\end{array}$

$\begin{array}{lll}1.4 & 1153.4 & 2.8 \\ 4.5 & 1157.1 & 9.4\end{array}$

$\begin{array}{lll}3.1 & 1156.4 & 7.2 \\ 2.6 & 1151.1 & 3.9\end{array}$

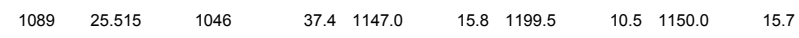

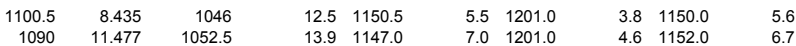

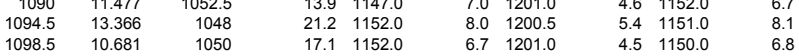

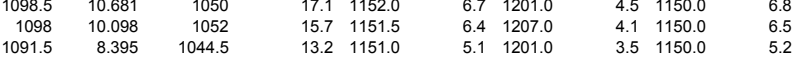

$\begin{array}{rrrrrrrrrr}1094.5 & 10.507 & 1045.5 & 17.3 & 1150.0 & 6.3 & 1207.0 & 3.9 & 1151.5 & 6.2 \\ 1094.5 & 9.345 & 1043.5 & 15.5 & 1150.0 & 5.7 & 1216.0 & 3.5 & \\ 1097 & 6.399 & 1037 & 10.9 & 1152.0 & 4.3 & 1108.0 & 2.7 & 1149.5 & 4.4 \\ 1082 & 12804 & 10475 & 1187 & 11510 & 65 & 12025 & 4.3 & 1150 . & 65\end{array}$

$5.6 \quad 1204.0$

3.71151 .0

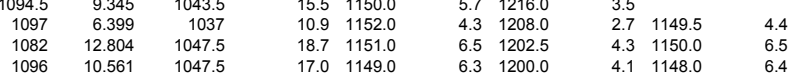

$\begin{array}{rr}1100 & 12.500 \\ 1100 & 4.600 \\ 1100 & 7.500\end{array}$

\begin{tabular}{rr}
1100 & 7.600 \\
1100 & 5.500 \\
\hline & 6.1000
\end{tabular}

$\begin{array}{rr}1100 & 6.700 \\ 1100 & 11.000\end{array}$

$1100 \quad 6.205$ 


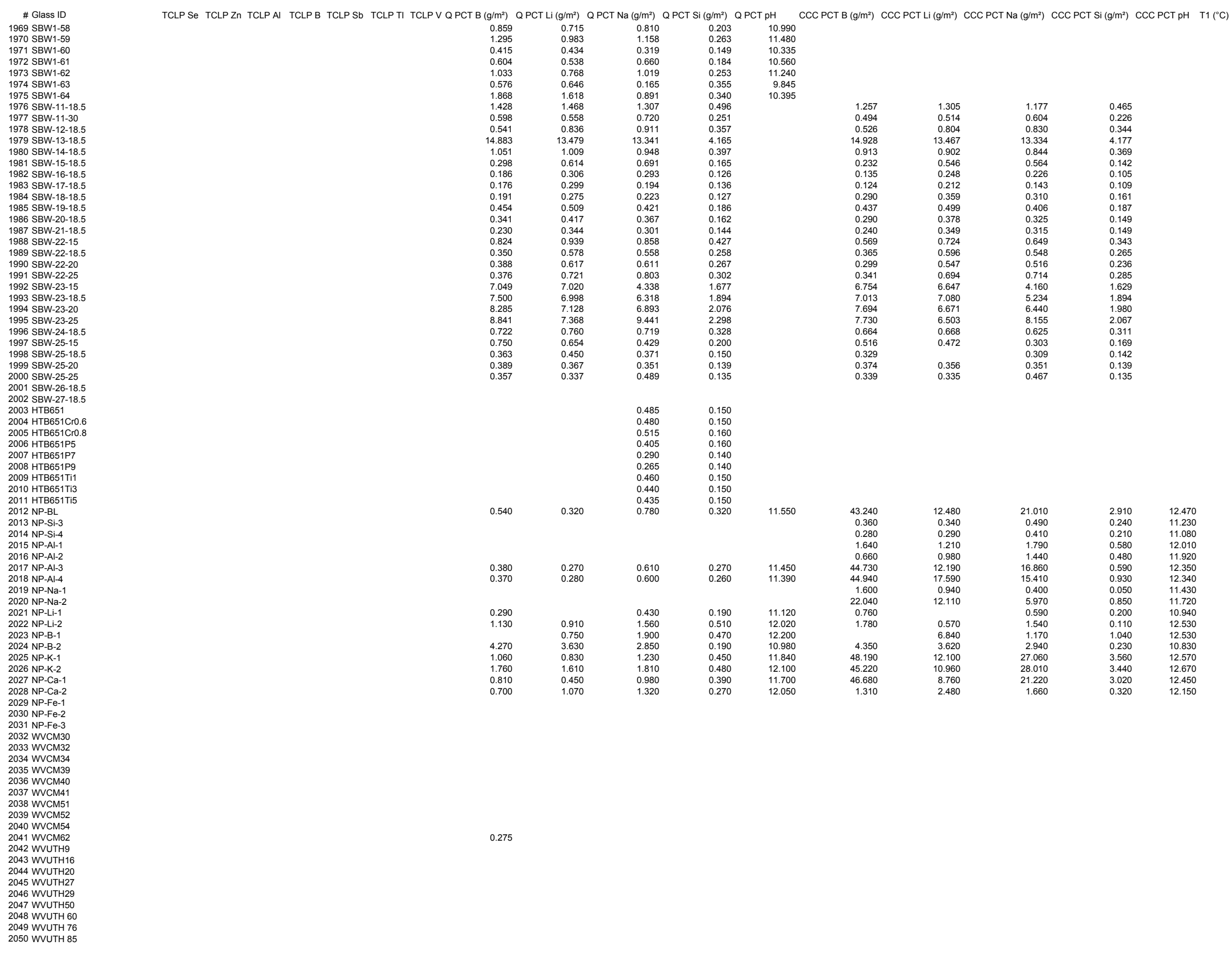


1969 SBW1-58
1970 SBW1-59
1971 SBW1-60

1972 SBW1-61

1973 SBW1-62
1974 SBW1-63

1975 SBW1-64

1976 SBW-11-18.5

1977 SBW-11-30
1978 SBW-12-18.5

1979 SBW-13-18.5

1980 SBW-14-18.5

1981 SBW-15-18.5
1982 SBW-16-18.5

1983 SBW-17-18.5

1984 SBW-18-18.5

1985 SBW-19-18.5

1987 SBW-21-18.5

1988 SBW-22-15

1989 SBW-22-18

1991 SBW-22-25

1992 SBW-23-15

1993 SBW-23-18.5

1995 SBW-23-25

1997 SBW-25-15

1998 SBW-25-18.

1999 SBW-25-20

2001 SBW-26-18.5

2002 SBW-27-18.5

2003 HTB651

2004 HTB6551Cr0.6
2005 HTB651Cr0.8

2006 HTB651P5

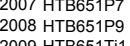

$2010 \mathrm{HTTS655TT}$
2011 HTB651T
2215

2012 NP-BL

2014 NP-Si-4

2015 NP-A-1

2016 NPPA-A-2

2018 NP-Al-4

2019 NP-Na-1

2020 NP-Na-2

2022 NPP-L-B-1

2025 NP-K-1

2026 NP-K-2

$2027 \mathrm{NP}-\mathrm{Ca}-1$

2028 NP-CE- 1

2030 NP-Fe-2

2031 NP.Fe-3

2032 WVCM 30
2033 WVCM32

2034 WVCM 34

2035 WVCM3
2036 WVCM4

2037 WVCM4

2038 WVCM5

2039 WCML52

2041 WCM 62

2042 WVUTHS

2043 WUUTH16

2045 WVUTH 27

2046 WVUTH29

2047 WWUTH50

2049 WVUTH 76

2050 WVUTH 85 


\begin{tabular}{|c|c|c|}
\hline \# Glass ID & Study & Reference \\
\hline 2051 WVUTH 88 & CUA data & Peters et al. 2003 \\
\hline 2052 WVUTH 106 & CUA data & 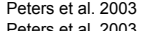 \\
\hline $\begin{array}{l}2053 \text { WVUTH } 107 \\
2054 \text { WVUTH } 108\end{array}$ & $\begin{array}{l}\text { CUA data } \\
\text { CUA data }\end{array}$ & $\begin{array}{l}\text { Peters et al. } 2003 \\
\text { Peters et al } 2000\end{array}$ \\
\hline $\begin{array}{l}2054 \text { WVUTH } 108 \\
2055 \text { WVTH } 109\end{array}$ & $\begin{array}{l}\text { CUA data } \\
\text { CUA data }\end{array}$ & $\begin{array}{l}\text { Peters et al. } 2003 \\
\text { Peters et al } 2003\end{array}$ \\
\hline 2056 WVUTH110 & $\begin{array}{l}\text { CUA data } \\
\text { CUata }\end{array}$ & $\begin{array}{l}\text { Peters et al. } 2003 \\
\text { Peters et al. } 2003\end{array}$ \\
\hline & CUA data & Peters et al. 2003 \\
\hline 2058 WVUTH112 & CUA data & $\begin{array}{l}\text { Peters et al. } 2003 \\
\end{array}$ \\
\hline 2059 WVUTH113 & CUA data & Peters et al. 2003 \\
\hline 2060 WVUTH114 & CUA data & Peters et al. 2003 \\
\hline 2061 WVUTH115 & CUA data & Peters et al. 2003 \\
\hline 2062 WVUTH116 & $\begin{array}{l}\text { CUA data } \\
\text { CUA dat }\end{array}$ & Peters et al. 2003 \\
\hline $\begin{array}{l}2063 \text { WVUTH117 } \\
2064 \text { WVUTH119 }\end{array}$ & $\begin{array}{l}\text { CUA data } \\
\text { CUA data }\end{array}$ & $\begin{array}{l}\text { Peters et al. } 2003 \\
\text { Peters et al } 2003\end{array}$ \\
\hline $\begin{array}{l}2064 \text { WVUTH118 } \\
2065 \text { WVUTH119 }\end{array}$ & $\begin{array}{l}\text { CUA d data } \\
\text { CUA data }\end{array}$ & $\begin{array}{l}\text { Peters et al. } 2003 \\
\text { Peters et al. } 2003\end{array}$ \\
\hline 2066 WVUTH 120 & $\begin{array}{l}\text { CUA data } \\
\text { CUata }\end{array}$ & Peters et al. 2003 \\
\hline 2067 WVUTH121 & $\begin{array}{l}\text { CUA data } \\
\text { CAta }\end{array}$ & Peters et al. 2003 \\
\hline 2068 WVUTH122 & CUA data & Peters et al. 2003 \\
\hline 2069 WVUTH 123 & CUA data & Peters et al. 2003 \\
\hline 2070 WVUTH124 & CUA data & Peters et al. 2003 \\
\hline 2071 WVUTH125 & CUA data & Peters et al. 2003 \\
\hline $\begin{array}{l}2072 \text { WVUTHH126 } \\
2073 \text { WVUTH127 }\end{array}$ & $\begin{array}{l}\text { CUA data } \\
\text { CUA data }\end{array}$ & $\begin{array}{l}\text { Peters et al. } 2003 \\
\text { Peters et al } 2003\end{array}$ \\
\hline $\begin{array}{l}2073 \text { WVUUT127 } 27 \\
2074 \text { WVUTH } 128\end{array}$ & $\begin{array}{l}\text { CUA data } \\
\text { CUA data }\end{array}$ & $\begin{array}{l}\text { Peters et al. } 2003 \\
\text { Peters tal al. } 2003\end{array}$ \\
\hline 2075 WVUTH129 & $\begin{array}{l}\text { CUA data } \\
\text { CUA data }\end{array}$ & $\begin{array}{l}\text { Peters et al. } 2003 \\
\text { Petrs et al. } 2003\end{array}$ \\
\hline 2076 WVUTH 130 & $\begin{array}{l}\text { CUA data } \\
\text { CAta }\end{array}$ & Peters et al. 2003 \\
\hline 2077 WVUTH131 & CUA data & Peters et al. 2003 \\
\hline 2078 WVUTH 132 & CUA data & Peters et al. 2003 \\
\hline 2079 WVUTH133 & CUA data & Peters et al. 2003 \\
\hline 2080 WVUTH134 & CUA data & Peters et al. 2003 \\
\hline $\begin{array}{l}2081 \text { WVUTTH135 } \\
2082 \text { WVUTH135 }\end{array}$ & $\begin{array}{l}\text { CUA data } \\
\text { CUA data }\end{array}$ & $\begin{array}{l}\text { Peters et al. } 2003 \\
\text { Peters te al } 2003\end{array}$ \\
\hline $\begin{array}{l}2082 \text { WVUUTH136 } \\
2083 \text { WVUTH137 }\end{array}$ & $\begin{array}{l}\text { CUA data } \\
\text { CUA data }\end{array}$ & $\begin{array}{l}\text { Peters et al. } 2003 \\
\text { Peters tal al. } 2003\end{array}$ \\
\hline 2084 WVUTH 138 & $\begin{array}{l}\text { UA data } \\
\text { CUA data }\end{array}$ & $\begin{array}{l}\text { Peters et al. } 2003 \\
\text { Petrs al. } 2003\end{array}$ \\
\hline 2085 WVUTH 139 & CUA data & $\begin{array}{l}\text { Peters et al. } 2003 \\
\end{array}$ \\
\hline 2086 WVUTH140 & CUA data & Peters et al. 2003 \\
\hline 2087 WVUTH141 & CUA data & Peters et al. 2003 \\
\hline 2088 WVUTH142 & CUA data & Peters et al. 2003 \\
\hline 2089 WVUTH143 & CUA data & Peters et al. 2003 \\
\hline 2090 WVUTH144 & CUA data & 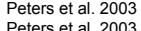 \\
\hline $\begin{array}{l}2001 \text { WVUTTH145 } \\
2092 \text { WVUTH146 }\end{array}$ & $\begin{array}{l}\text { CUA data } \\
\text { CUA data }\end{array}$ & $\begin{array}{l}\text { Peters et al. } 2003 \\
\text { Peters tal } 2003\end{array}$ \\
\hline 2093 WVUTH 147 & $\begin{array}{l}\text { CUA data } \\
\text { CUA data }\end{array}$ & $\begin{array}{l}\text { Peters et al. } 2003 \\
\text { Peters et al. } 2003\end{array}$ \\
\hline 2094 WVUTH 148 & CUA data & $\begin{array}{l}\text { Peters et al. } 2003 \\
\end{array}$ \\
\hline 2095 WVUTH149 & CUA data & Peters et al. 2003 \\
\hline 2096 WVUTH 150 & CUA data & Peters et al. 2003 \\
\hline 2097 WVUTH151 & CUA data & Peters et al. 2003 \\
\hline 2098 WVUTH152 & CUA data & Peters et al. 2003 \\
\hline 2099 WVUTH153 & CUA data & $\begin{array}{l}\text { Peters et al. } 2003 \\
\end{array}$ \\
\hline $\begin{array}{l}2100 \text { WVUUTH154 } \\
2101 \text { WVUTH155 }\end{array}$ & $\begin{array}{l}\text { CUA data } \\
\text { CUA data }\end{array}$ & $\begin{array}{l}\text { Peters et al. } 2003 \\
\text { Peters et al } 2003\end{array}$ \\
\hline 2102 WVUTH155 & $\begin{array}{l}\text { CUA data } \\
\text { CUA data }\end{array}$ & $\begin{array}{l}\text { Peters et al. } 2003 \\
\text { Petrs et al. } 2003\end{array}$ \\
\hline 2103 WVUTH 157 & $\begin{array}{l}\text { CUA data } \\
\text { CUta }\end{array}$ & Peters et al. 2003 \\
\hline 2104 WVUTH158 & CUA data & Peters et al. 2003 \\
\hline 2105 WVUTH159 & CUA data & Peters et al. 2003 \\
\hline 2106 WVUTH160 & CUA data & Peters et al. 2003 \\
\hline 2107 WVUTH161 & CUA data & Peters et al. 2003 \\
\hline 2108 WVUTH162 & CUA data & Peters et al. 2003 \\
\hline 2109 WVUTH 163 & CUA data & Peters et al. 2003 \\
\hline 2110 WVUTH 164 & CUA data & Peters et al. 2003 \\
\hline 21111 & HWVP & Bates March 1987 \\
\hline 21122 & HWVP & Bates March 1987 \\
\hline 21133 & $\begin{array}{l}\text { HWVP } \\
\text { HWWVP }\end{array}$ & Bates March 1987 \\
\hline $\begin{array}{l}21144 \\
21155\end{array}$ & $\begin{array}{l}\text { HWVP } \\
\text { HWWVP }\end{array}$ & $\begin{array}{l}\text { Bates March } 1987 \\
\text { Bartes } 1987\end{array}$ \\
\hline 21166 & $\begin{array}{l}\text { HWVYP } \\
\text { HWVP }\end{array}$ & $\begin{array}{l}\text { Bates March } \\
\text { Bates March } 1987\end{array}$ \\
\hline 21177 & HWYP & Bates March 1987 \\
\hline 21188 & HWVP & Bates March 1987 \\
\hline 21199 & HWVP & Bates March 1987 \\
\hline 21201 & HWVP & Bates March 1987 \\
\hline 21212 & HWVP & Bates March 1987 \\
\hline 21223 & HWVP & Bates March 1987 \\
\hline 21234 & HWVP & Bates March 1987 \\
\hline 21245 & HWVP & Bates March 1987 \\
\hline 21256 & HWVP & Bates March 1987 \\
\hline 267 & HWVP & Bates March 1987 \\
\hline 1282 & $\begin{array}{ll}\text { HWWP } \\
\text { HWWP }\end{array}$ & Bates March 1987 \\
\hline 109 & nove & 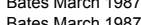 \\
\hline 1304 & HWYP & 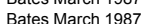 \\
\hline 21315 & HWYP & Bates March 1987 \\
\hline 21326 & HWVP & Bates March 1987 \\
\hline
\end{tabular}

$\begin{array}{llll}0.0000 & 0.1084 & 0.0319 & 0.0000 \\ 0.0266 & 0.1084 & 0.0323 & 0.0687\end{array}$

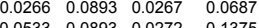
$0.0266 \quad 0.0988 \quad 0.0225 \quad 0.0687$

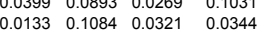
$\begin{array}{llll}0.0399 & 0.0988 & 0.0298 & 0.1034 \\ 0.0133 & 0.0988 & 0.0293 & 0.0344\end{array}$

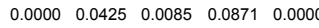

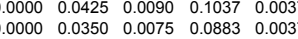
$\begin{array}{llllll}.0000 & 0.0350 & 0.0079 & 0.1049 & 0.0074\end{array}$

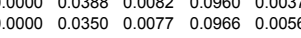
$\begin{array}{lllll}0.0000 & 0.0425 & 0.0087 & 0.0954 & 0.0019 \\ 0.0000 & 0.0388 & 0.0084 & 0.1043 & 0.0056 \\ 0.0000 & 0.0388 & 0.0080 & 0.0877 & 0.0019\end{array}$ $\begin{array}{ll}0.5716 & 0.1500 \\ 0.5763 & 0.0000\end{array}$

$4754 \quad 0.1500$
0.5250

$0.5258 \quad 0.0750$

$\begin{array}{ll}0.4777 & 0.0750 \\ 0.5739 & 0.0750 \\ 0.5282 & 0.000\end{array}$

$\begin{array}{ll}0.5282 & 0.0000 \\ 0.5235 & 0.1500\end{array}$

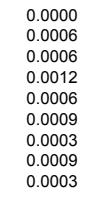

0.0000
0.0011
0.0011
0.0022
0.0011
0.016
0.0005
0.0016
0.0005 
\# Glass ID

2051 WVUTH 88

2052 WVUTH 106

2054 WVUTH 108

2056 WVUTH110

2057 WVUTH111

2059 WVUTH113

2060 WVUTH114
2061 WVUTH115

2062 WVUTH116

2063 WVUTH117

2065 WVUTH 119

2006 W WUTH 120
2067 WVUTH 121

2068 WVUTH 122

2069 WVUTH 123

(2071. WVUTH125

2072 WVUTH126

年 2074 WUUTH 128

2075 WVUTH122
2076 WVUTH 139

2077 WVUTH131

2078 WVUTH 132

2079 WVUTH 133
2080 WVUTH 134

2081 WVUTH 135

2082 WVUTH 136
2083 WVUTH 137

2084 WVUTH138

2085 WVUTH 139

2086 WUUTH 140

2087 WVUTH 141

2088 WUTH1142
2029 WWUTH143

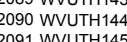

2092 WVUTH 146

2092 WUUTH146
2039 WWUTH147

2094 WUUTTH 148
2095 WVUTH

2096 WVUTH 150

2097 WUUTH1151
2098 WWUTH152

2098 WUUTH1152
2099 WVUTH153

2100 WVUTH 154

210 WWUTH155

2102 WVUTH 156
2103 WVUTH 157

2104 WVUTH 158

2105 WUUTH159

2106 WVUTH1160
2107 WVUTH161

2108 WVUTH162

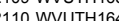

21111

2113

(115

21166
21177

21188

21199
21201

21212

21223

21256
21267

2127

21282

2129
2130
2131

21326

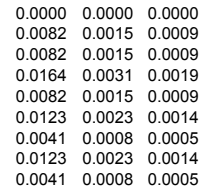

0.0000
0.0019
0.0019
0.0037
0.0019
0.0028
0.0009
0.0028
0.0009

$\begin{array}{ll}0.0000 & 0.0000 \\ 0.0034 & 0.0011 \\ 0.034 & 0.0011 \\ 0.0068 & 0.0022 \\ 0.0034 & 0.0011 \\ 0.0051 & 0.0016 \\ 0.0017 & 0.0005 \\ 0.0051 & 0.0016 \\ 0.0017 & 0.0005\end{array}$

0.0000
0.0019
0.0019
0.0037
0.0019
0.0028
0.0009
0.0028
0.0009

0.0000
0.0033
0.0033
0.0065
0.0033
0.0049
0.0016
0.0049
0.0016
0.0000
0.0000
0.0000
0.0000
0.0000
0.0000
0.0000
0.0000
0.0000 
2110 WVUTH164

21111

21133

21144

2116

21177

21188
21199

\begin{tabular}{l}
21201 \\
21212 \\
\hline
\end{tabular}

21223

21245

2126
2127

2128
-1282 


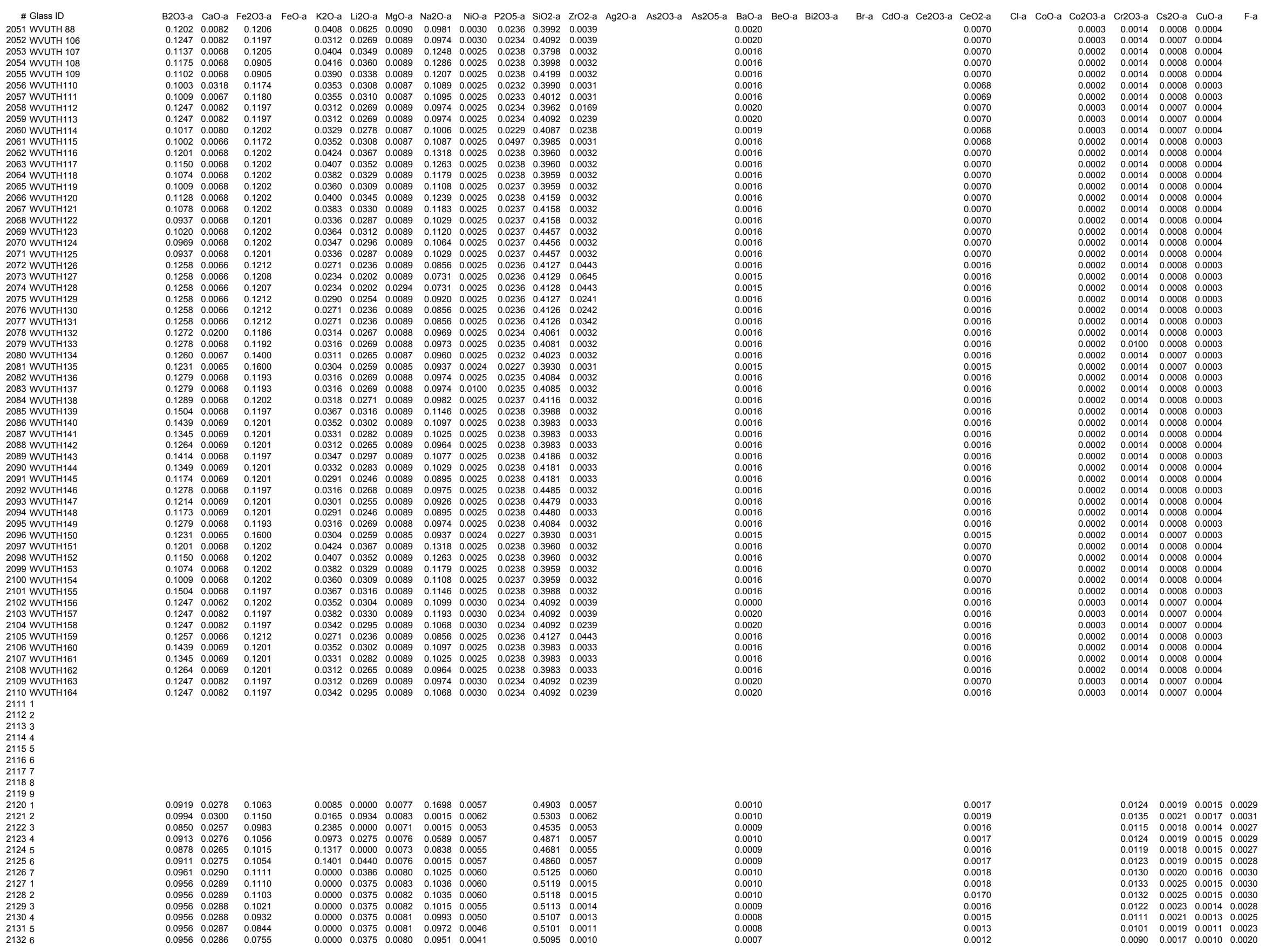


\# Glass ID
2051 WVUTH

2052 WVUTH 106

2054 WVUTH 108
2055 WVUTH 109

2056 WVUTH 110

2057 WVUTH111

2060 WVUTH1114

2061 WVUTH115

2063 WVUTH117

2065 WVUTH119

2066 WVUTH120

2070 WVUTH124

2072 WVUTH126

2074 WVUTH128

2075 WVUTH129

2077 WVUTH131

2079 WVUTH133

W WVUTH 13

2082 WVUTH136

2084 WVUTH138

2085 WVUTH139

2086 WVUTH1140

2089 WVUTH143

2090 WVUTH144

2092 WVUTH146

2094 WVUTH148

2096 WVUTH150

2097 WVUTH151

2099 WVUTH153

101 WVUTH155

2102 WVUTH15

2104 WVUTH158

2106 WVUTH16

2108 WVUTH162

2110 WVUTH164

21133

2114

21155
21166
21177
21188

21188

21201
21212

21223

21245

21267

21282

2131

1-a La203-a MnO2-a MnO-a MoO-a MoO3-a Nb2O5-a Nd2O3-a NpO2-a PbO-a PdO2-a PdO-a Pr203-a Pr6011-a PuO2-a Rb2O-a ReO-a ReO2-a Re207-a Rh2O3-a RhO2-a RuO2-a Sb203-a Sb2O5-a SeO2-a

$\begin{array}{lll}0.0005 & 0.0122 & \\ 0.0005 & 0.0121 & 0.0004 \\ 0.005 & 0.0122 & 0.004\end{array}$

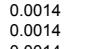

0.000330 .0004

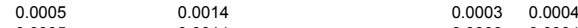

$\begin{array}{ll}0.0005 & 0.0102 \\ 0.0005 & 0.0102 \\ 0.0004 & 0.0098\end{array}$

$0.0004 \quad 0.0099$

$\begin{array}{ll}0.0005 & 0.0121 \\ 0.0005 & 0.0121\end{array}$

$\begin{array}{ll}0.0005 & 0.0118 \\ 0.0004 & 0.0098\end{array}$

$0.0004 \quad 0.0101$

$\begin{array}{ll}0.0004 & 0.0101 \\ 0.0004 & 0.011 \\ 0.0004 & 0.0101\end{array}$

$\begin{array}{lll}0.0004 & 0.0101 \\ 0.0004 & 0.010\end{array}$

$\begin{array}{ll}0.0004 & 0.010 \\ 0.0004 & 0.010\end{array}$

$\begin{array}{ll}0.0004 & 0.0101 \\ 0.0004 & 0.0101 \\ 0.0004 & 0.0101\end{array}$

\begin{tabular}{ll}
0.00004 & 0.0101 \\
\hline
\end{tabular}

$0.0004 \quad 0.0099$

$\begin{array}{lll}0.0004 & 0.0099\end{array}$

$0.0004 \quad 0.0100$

$\begin{array}{ll}0.0004 & 0.0100 \\ 0.0004 & 0.0100\end{array}$

$0.0004 \quad 0.0100$

$0.0004 \quad 0.009$

$\begin{array}{lll}0.0004 & 0.0100 \\ 0.0004 & 0.0100\end{array}$

$0.0004 \quad 0.0306$

$\begin{array}{ll}0.0004 & 0.0101 \\ 0.0004 & 0.0102\end{array}$

$0.0004 \quad 0.0102$

$0.0004 \quad 0.010$

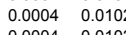

$0.0004 \quad 0.0101$

$\begin{array}{ll}0.0004 & 0.0102 \\ 0.0004 & 0.0102\end{array}$

$\begin{array}{lll}0.0004 & 0.0102 \\ 0.0004 & 0.0097\end{array}$

$\begin{array}{ll}0.0004 & 0.001 \\ 0.0004 & 0.0101 \\ 0.0004 & 0.0101\end{array}$

$0.0004 \quad 0.0101$

$0.0004 \quad 0.010$
0

$\begin{array}{ll}0.0004 & 0.0101\end{array}$

$\begin{array}{ll}0.0005 & 0.012 \\ 0.0005 & 0.012\end{array}$

\begin{tabular}{ll}
0.0005 & 0.0121 \\
0.0004 & 0.0100 \\
\hline
\end{tabular}

$\begin{array}{ll}0.0004 & 0.0102 \\ 0.0004 & 0.0102\end{array}$

$\begin{array}{ll}0.0004 & 0.0102 \\ 0.0005 & 0.012\end{array}$

0.0005
0.0005
0.0004

0.0004
0.0004
0.0004

0.0014

0.0014
0.0014
0.00014

$\begin{array}{ll}0.0003 & 0.0004 \\ 0.0003 & 0.0004 \\ 0.0003 & 0.004\end{array}$

$\begin{array}{ll}0.0003 & 0.0004\end{array}$

$\begin{array}{ll}0.0003 & 0.0004 \\ 0.0003 & 0.0004\end{array}$

\begin{tabular}{ll}
0.0003 & 0.0004 \\
\hline
\end{tabular}

$\begin{array}{ll}0.0003 & 0.0004 \\ 0.0003 & 0.0004\end{array}$

$\begin{array}{ll}0.0003 & 0.0004 \\ 0.0003 & 0.0004 \\ 0.0003 & 0.0004\end{array}$

$\begin{array}{ll}.0003 & 0.0004 \\ .0003 & 0.0004 \\ 0\end{array}$

$\begin{array}{ll}0.0003 & 0.0004 \\ 0.0003 & 0.0004\end{array}$

$\begin{array}{ll}0.0003 & 0.0004 \\ 0.0003 & 0.0004 \\ 0.003 & 0.004\end{array}$

$\begin{array}{ll}0.0003 & 0.0004 \\ 0.0003 & 0.0004\end{array}$

$\begin{array}{ll}0.0003 & 0.0004 \\ 0.0003 & 0.0004 \\ 0.0003 & 0.0004\end{array}$

$\begin{array}{ll}0.0003 & 0.0004 \\ 0.0003 & 0.0004\end{array}$

$\begin{array}{ll}0.0003 & 0.0004 \\ 0.0003 & 0.0004 \\ 0.0003 & 0.0004\end{array}$

$\begin{array}{ll}0.0003 & 0.0004 \\ 0.0003 & 0.0004\end{array}$

$\begin{array}{ll}0.0003 & 0.0004 \\ 0.0003 & 0.0004 \\ 0\end{array}$

\begin{tabular}{ll}
0.0003 & 0.0004 \\
0.0003 & 0.0004 \\
\hline
\end{tabular}

$0.0003 \quad 0.0004$

$\begin{array}{ll}0.0003 & 0.0004 \\ 0 & 0.0003\end{array}$

$\begin{array}{ll}0.0003 & 0.0004 \\ 0.0003 & 0.0004\end{array}$

$0.0003 \quad 0.0004$

\begin{tabular}{ll}
0.0003 & 0.0004 \\
0.0003 & 0.0004 \\
\hline
\end{tabular}

$\begin{array}{ll}0.0003 & 0.0004 \\ 0.0003 & 0.0004 \\ 0.003\end{array}$

$\begin{array}{ll}0.0003 & 0.0004 \\ 0.0003 & 0.0004\end{array}$

$\begin{array}{ll}0.0003 & 0.0004 \\ 0.0003 & 0.0004 \\ 0.000 & 0.004\end{array}$

$\begin{array}{ll}0.0003 & 0.0004 \\ 0.0003 & 0.0004 \\ 0.0003 & 0.004\end{array}$

\begin{tabular}{ll}
0.0003 & 0.0004 \\
0.0003 & 0.0004 \\
\hline & 0.003
\end{tabular}

$\begin{array}{ll}0.0003 & 0.0004 \\ 0.0003 & 0.0004 \\ 0.003\end{array}$

$\begin{array}{ll}0.0003 & 0.0004 \\ 0.0003 & 0.0004 \\ 0.0003 & 0.0004\end{array}$

$\begin{array}{ll}0.0003 & 0.0004 \\ 0.0003 & 0.0004\end{array}$

$\begin{array}{lll}0.0003 & 0.0004 \\ 0.0003 & 0.0004\end{array}$

\begin{tabular}{ll}
0.0003 & 0.0004 \\
0.0003 & 0.0004 \\
\hline
\end{tabular}

$0.0003 \quad 0.0004$

$\begin{array}{ll}0.0002 & 0.0008 \\ 0.0002 & 0.0008\end{array}$

$\begin{array}{ll}0.0002 & 0.0008 \\ 0.0002 & 0.0008\end{array}$

$0.0002 \quad 0.0008$

$0.0002 \quad 0.0006$

$0.0002 \quad 0.0006$

$\begin{array}{lll}0.0002 & 0.0006\end{array}$

$\begin{array}{ll}0.0002 & 0.0008 \\ 0.0002 & 0.0008\end{array}$

$\begin{array}{ll}0.0002 & 0.0008 \\ 0.0002 & 0.0008\end{array}$

$\begin{array}{ll}0.0002 & 0.0008 \\ 0.0002 & 0.0008\end{array}$

$0.0002 \quad 0.0008$

$0.0002 \quad 0.0007$

$\begin{array}{ll}0.0002 & 0.0008 \\ 0.0002 & 0.0008\end{array}$

$\begin{array}{lll}0.0002 & 0.0008 \\ 0.0002 & 0.0008\end{array}$

$\begin{array}{ll}0.0002 & 0.0008 \\ 0.0002 & 0.0008\end{array}$

$\begin{array}{ll}0.0002 & 0.0008\end{array}$

\begin{tabular}{lll}
0.0002 & 0.0008 \\
0.0002 & 0.0008 \\
\hline & 0.002 & 0.008
\end{tabular}

$\begin{array}{lll}0.0002 & 0.0008 \\ 0.0002 & 0.0008\end{array}$

$\begin{array}{lll}0.0002 & 0.0008 \\ 0.0002 & 0.0008\end{array}$

$0.0002 \quad 0.0007$

$0.0002 \quad 0.0006$

$0.0002 \quad 0.0006$

\begin{tabular}{lll}
0.0002 & 0.0000 \\
0.0002 & 0.0008 \\
\hline
\end{tabular}

$0.0002 \quad 0.0008$
0.0002

$\begin{array}{lll}0.0002 & 0.0008 \\ 0.0002 & 0.0008\end{array}$

$\begin{array}{ll}0.0002 & 0.0008 \\ 0.0002 & 0.0008\end{array}$

\begin{tabular}{|c|c|c|c|}
\hline 0.0057 & 0.0017 & 0.0031 & 0.0048 \\
\hline 0.0062 & 0.0019 & 0.0033 & \\
\hline 0.0053 & 0.0016 & 0.0028 & \\
\hline 0.0057 & $\begin{array}{l}0.0017 \\
0.0016\end{array}$ & $\begin{array}{l}0.0030 \\
0.0029\end{array}$ & $\begin{array}{l}0.00 \\
0.00\end{array}$ \\
\hline 0.0057 & 0.0017 & 0.0030 & \\
\hline 0.0060 & 0.0018 & 0.0032 & \\
\hline 0.0055 & 0.0018 & 0.0030 & \\
\hline 0.0055 & 0.0017 & 0.0030 & \\
\hline 0.0051 & 0.0016 & 0.0028 & 48 \\
\hline 0.0046 & 0.0015 & 0.0025 & 44 \\
\hline 0.0032 & 0.0013 & 0.0023 & \\
\hline
\end{tabular}

0.0010
0.0010
0.0009
0.0010
0.0009
0.0009
0.0010
0.00000
0.0000
0.0000
0.0000
0.0000
0.0000

0.0015
0.0017
0.0014
0.0015
0.0015
0.0015
0.0016
0.0015
0.0015
0.0014
0.0013
0.0011
0.0010 
\# Glass ID

\# Glass ID
2051 WVUTH 88
2052 WVUTH 106

2052 WVUTH 106

2054 WVUTH 108

2056 WVUTH 110

2057 WVUTH111

2059 WVUTH113

2061 WVUTH115

2063 WVUTH117

2065 WVUTH119

2066 WVUTH120

2068 WVUTH122

2069 WVUTH123

2070 WVUTH124

2072 WVUTH126

2074 WVUTH 128

2075 WVUTH129

2077 WVUTH131

2079 WVUTH133

1 WVUTH135

2082 WVUTH 136
2083 WVUTH137

2084 WVUTH 138

2085 WVUTH139

2086 WVUTH140
2087 WVUTH141

2088 WVUTH142

2090 WVUTH144

2092 WVUTH146

2093 WVUTH147

2094 WVUTH 48

096 WVUTH150

2097 WVUTH151

2099 WVUTH 153

101 WVUTH155

2102 WVUTH156
2103 WVUTH157

2104 WVUTH158

2105 WVUTH159

2107 WVUTH161

2109 WVUTH163

(113

\begin{tabular}{l}
21155 \\
21166 \\
21177 \\
21188 \\
\hline 219
\end{tabular}

21188
21199
21201
21212
21223
2123

21201
21212
21223
21234
21245

21234

21267

-128
-127

21293

21315
Sm203-a SnO-a SnO2-a SO3-a SrO-a Tc207-a TeO2-a ThO2-a TiO2-a T12O-a T1203-a U308-a UO2-a $\begin{array}{lll}0.0023 & 0.0002 \\ 0.0022 & 0.0002\end{array}$ $\begin{array}{ll}0.0356 & 0.0083 \\ 0.0353 & 0.0082\end{array}$

$\begin{array}{lll}0.0022 & 0.0002 \\ 0.0023 & 0.0002\end{array}$

$\begin{array}{lll}0.0353 & 0.0082 \\ 0.0351 & 0.0077\end{array}$

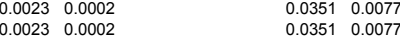

$\begin{array}{llll}0.0022 & 0.0002 & 0.0351 & 0.0075 \\ 0.0023 & 0.0002 & 0.0551 & 0.0075\end{array}$

$0.0022 \quad 0.0002$

$\begin{array}{ll}0.0022 & 0.0002 \\ 0.0022 & 0.0002\end{array}$

0.00220 .0002

$\begin{array}{ll}0.0022 & 0.0002 \\ 0.0022 & 0.0002\end{array}$

$\begin{array}{lll}0.0022 & 0.0002 \\ 0.0022 & 0.0002\end{array}$

$\begin{array}{ll}0.0022 & 0.0002 \\ 0.0022 & 0.0002 \\ 0.0022 & 0.0002\end{array}$

$\begin{array}{ll}0.0022 & 0.0002 \\ 0.0022 & 0.0002\end{array}$

$\begin{array}{ll}0.0022 & 0.0002 \\ 0.0023 & 0.0002\end{array}$

$0.0023 \quad 0.0002$

$\begin{array}{ll}0.0023 & 0.0002 \\ 0.0023 & 0.0002\end{array}$

$\begin{array}{ll}0.0023 & 0.0002 \\ 0.022 & 0.002\end{array}$

$\begin{array}{ll}0.0022 & 0.0002 \\ 0.0002 & 0.002\end{array}$

$0.0022 \quad 0.0002$

$0.0022 \quad 0.0002$

$\begin{array}{ll}0.0023 & 0.0002 \\ 0.0023 & 0.0002 \\ 0.0023 & 0.0002\end{array}$

$\begin{array}{lll}0.0023 & 0.0002 \\ 0.0023 & 0.0002\end{array}$

$\begin{array}{ll}0.0023 & 0.0002\end{array}$

$\begin{array}{ll}0.0023 & 0.0002 \\ 0.0023 & 0.0002 \\ 0.0023 & 0.0002\end{array}$

$\begin{array}{lll}0.0023 & 0.0002\end{array}$

$\begin{array}{ll}0.0023 & 0.0002 \\ 0.0023 & 0.0002 \\ 0.0100 & 0.0002\end{array}$

$0.0022 \quad 0.0002$

$\begin{array}{ll}0.0022 & 0.0002 \\ 0.0022 & 0.0002\end{array}$

$0.0022 \quad 0.0002$

$0.0023 \quad 0.0002$

$\begin{array}{lll}0.0022 & 0.0003 \\ 0.0022 & 0.0003\end{array}$

$0.0022 \quad 0.0003$

$0.0023 \quad 0.0002$

$\begin{array}{ll}0.0023 & 0.0002 \\ 0.0023 & 0.0002\end{array}$

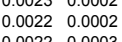

$\begin{array}{lll}0.03553 & 0.00082 \\ 0.0353 & 0.002\end{array}$

$\begin{array}{ll}0.0346 & 0.0080 \\ 0.0350 & 0.0075\end{array}$

0.03550 .0076

0.03550 .0076

$\begin{array}{lll}0.0355 & 0.0076 \\ 0.0355 & 0.0076\end{array}$

$\begin{array}{lll}0.0355 & 0.0076 \\ 0.0355 & 0.0076\end{array}$

$0.0355 \quad 0.0076$

0.03550 .0076

0.03550 .0076

$\begin{array}{ll}0.0357 & 0.0080 \\ 0.0357 & 0.0080\end{array}$

$\begin{array}{ll}0.0358 & 0.0080 \\ 0.0358 & 0.0080\end{array}$

$\begin{array}{ll}0.0358 & 0.0080 \\ 0.0351 & 0.0079 \\ 0.0352 & 0.0079\end{array}$

$\begin{array}{lll}0.0352 & 0.0079 \\ 0.0348 & 0.0078\end{array}$

0.03390 .0076

$\begin{array}{lll}0.0353 & 0.0079\end{array}$

$0.0356 \quad 0.0080$

$\begin{array}{lll}0.0356 & 0.0080 \\ 0.0357 & 0.0080\end{array}$

$0.0357 \quad 0.0080$

0.03570 .0080

$\begin{array}{lll}0.0356 & 0.0080 \\ 0.0357 & 0.0080\end{array}$

$\begin{array}{ll}0.0357 & 0.0080 \\ 0.0357 & 0.0080\end{array}$

$\begin{array}{lll}0.0356 & 0.0080 \\ 0.0357 & 0.0080\end{array}$

0.03570 .0080

$\begin{array}{ll}0.0339 & 0.0076 \\ 0.0355 & 0.0076\end{array}$

$0.0355 \quad 0.0076$

$\begin{array}{lll} & \\ 355 & 0.0076 \\ 0355 & 0.0076\end{array}$

$\begin{array}{lll}0.0356 & 0.0080 \\ 0.0355 & 0.0082\end{array}$

$\begin{array}{lll}0.0355 & 0.0082 \\ 0.0353 & 0.0082\end{array}$

$\begin{array}{ll}0.0353 & 0.0082\end{array}$

$\begin{array}{lll}0.0358 & 0.0080 \\ 0.0357 & 0.0080\end{array}$

$\begin{array}{lll}0.0357 & 0.0080 \\ 0.0357 & 0.0080\end{array}$

$\begin{array}{ll}0.0357 & 0.0080 \\ 0.0353 & 0.0082 \\ 0.0353 & 0.0082\end{array}$

$\begin{array}{ll}0.0038 & 0.0010 \\ 0.0041 & 0.0010 \\ 0.0035 & 0.0009\end{array}$

$\begin{array}{ll}0.0035 & 0.0009 \\ 0.0038 & 0.0010\end{array}$

$0.0037 \quad 0.0009$

$\begin{array}{ll}0.0040 & 0.0010 \\ 0.0045 & 0.0010\end{array}$

$\begin{array}{lll}0.0045 & 0.0010 \\ 0.0041 & 0.0009\end{array}$

$0.0038 \quad 0.0008$

$\begin{array}{ll}0.0034 & 0.0008 \\ 0.0031 & 0.0007\end{array}$
UO2-a
0.0058
0.0058
0.0058

$\begin{array}{lll}0.002 & 0.0003 & 1.0017\end{array}$

$\begin{array}{llll}0.002 & 0.0003 & 1.0016 \\ 0.0002 & 0.00003 & 1.0017 \\ 0.002 & 0.002 & 1\end{array}$

$\begin{array}{llll}0.0002 & 0.0002 & 1.0003\end{array}$

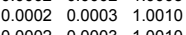

$\begin{array}{lll}.0000 & 0.0003 & 1.001 \\ .0002 & 0.0002 & 1.0002 \\ 0.002 & .0 .003 & 0.096\end{array}$

$\begin{array}{lll}0.0002 & 0.0003 & 0.9996 \\ 0.0002 & 0.0003 & 0.9997\end{array}$

$\begin{array}{lll}0.002 & 0.0003 & 0.9997 \\ 0.0002 & 0.0003 & 0.9996 \\ 0.002 & 0.003 & 0.006\end{array}$

$\begin{array}{lll}0.0002 & 0.0003 & 0.9997 \\ 0.0002 & 0.0003 & 0.9996\end{array}$

$\begin{array}{lll}0.0002 & 0.0003 & 0.9997 \\ 0.0002 & 0.0003 & 09988 \\ 0.002 & 0.003 & 0.996\end{array}$

$\begin{array}{lll}0.0002 & 0.0003 & 0.0989 \\ 0.0002 & 0.0003 & 0.9996 \\ 0.002 & 0.0003 & 0.9999\end{array}$

$\begin{array}{llll}0.0002 & 0.0002 & 0.9998\end{array}$

$\begin{array}{lll}0.0002 & 0.0002 & 0.9999 \\ 0.0002 & 0.0002 & 0.9999 \\ 0.002 & 0.002 & \end{array}$

$\begin{array}{llll}0.0002 & 0.0002 & 1.0394 \\ 0.0002 & 0.0002 & 0.9998\end{array}$

$\begin{array}{llll}0.0002 & 0.0002 & 0.9999\end{array}$

$\begin{array}{llll}0.0002 & 0.0002 & 1.0001 \\ 0.0002 & 0.0002 & 1.0000 \\ 0.0002 & 0.002 & 0.999\end{array}$

$\begin{array}{lll}0.0002 & 0.0002 & 0.9999 \\ 0.0002 & 0.0002 & 0.9997 \\ 0.002 & 0.002 & 10205\end{array}$

$\begin{array}{lll}0.002 & 0.0002 & 1.0205 \\ 0.002 & 0.0002 & 0.9999 \\ 0.002 & 0.0003 & 1009\end{array}$

$\begin{array}{lll}0.0002 & 0.0003 & 1.0000 \\ 0.0002 & 0.0003 & 1.0001 \\ 0.002 & 0.003 & 1.002\end{array}$

$\begin{array}{llll}0.0002 & 0.0003 & 1.0002\end{array}$

$\begin{array}{lll}0.0002 & 0.0002 & 0.9999\end{array}$

$\begin{array}{lll}0.002 & 0.00033 & 1.0001 \\ 0002 & 0.0003 & 1.000 \\ 0.002 & 0.0022 & 1.000\end{array}$

$\begin{array}{lll}0.0002 & 0.0002 & 1.0000 \\ .0002 & 0.0003 & 1.0002\end{array}$

$\begin{array}{lll}0.0002 & 0.0003 & 1.000 \\ 0.0002 & 0.0002 & 1.0002\end{array}$

$\begin{array}{llll}0.0002 & 0.0002 & 1.00000\end{array}$

$\begin{array}{lll}0.0002 & 0.0003 & 0.9996 \\ 0.0002 & 0.0003 & 0.9997\end{array}$

0.002 000003 0.0997

$\begin{array}{lll}0.0002 & 0.0003 & 0.9996\end{array}$

$\begin{array}{llll}0.0002 & 0.0003 & 0.9929\end{array}$

$\begin{array}{llll}0.0002 & 0.0003 & 0.9962\end{array}$

$\begin{array}{lll}0.0002 & 0.0002 & 0.999\end{array}$

$0.002 \quad 0.0003 \quad 1.0001$

$\begin{array}{lll}0.0002 & 0.0003 & 1.001 \\ 0.0002 & 0.0003 & 1 . \\ 0.0002 & 0.0003 & 0.9\end{array}$

0.0000
0.0000

0.0000

$\begin{array}{ll} & 0.0000 \\ 0.0005 & 1.000 \\ 0.0005 & 1.000\end{array}$

$\begin{array}{ll}0.0005 & 1.000 \\ 0.0004 & 1.0000 \\ 0.0005 & 1.0909\end{array}$

$\begin{array}{ll}0.0005 & 1.0000 \\ 0.0005 & 0.9999\end{array}$

0.0005
0.0005

0.0005

$\begin{array}{ll}0.0004 & 0.9947 \\ 0.0004 & 0.994\end{array}$

$\begin{array}{ll}0.0004 & 0.9954 \\ 0.0003 & 0.995\end{array}$

58


2029 WVUTH133

2081 WVUTH 135

2082 W WUTHTH 136
2083 WVUTH

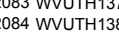

2084 WUUTH 138
2025 WWUTH 139

2086 WWUTH 140

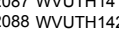

2089 WVUTH 143

2090 WUUTHTH4
2091 WWUTH

2092 WVUTH 146

2093 WVUTH 147

2094 WUUTH148
2095 WVUTH 149

2096 WVUTH 150

2097 WUUTH1151
2098 WWUTH152

2098 WVUTH152
2099 WVUTH153

2100 WVUTH 154
2101 WVUTH155

2102 WVUTH156
2103 WUTH 157

2103 WVUTH1157
2104 WVUTH158

2105 WVUTH 159

2106 WVUTH1160

2107 WUTTH16
2108 WWUTH162
2109 WUTH 169

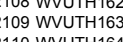

2110 WVUTH164

2112

21133
21144

2114

2116
2116

21177
2118

21188
21199

2120

21212

21223
21234
21245
2125

21256
21267

2127

21271
21282
2128

21293
21304
21315 
\# Glass ID

2051 WVUTH 88

2052 WVUTH 106

2054 WVUTH 108
2055 WVUTH 109

2056 WVUTH110

2057 WVUTH111

2059 WVUTH113
2060 WVUTH114

2060 WVUTH114
2061 WVUTH115

2061 WVUTH115
2062 WVUTH116
2063 WVUTH117

2063 WVUTH117
2064 WVUTH118

2066 WVUTH120

2068 WVUTH122

2069 WVUTH123

2070 WVUTH124

2072 WVUTH 126

2073 WUUTH 127

2075 WVUTH 129

2077 WVUTH1313

2078 WUUTH 132

2079 WVUTH 133
2080 WVUTH 134

2081 WVUTH 135

2082 WUUTH 136
2083 WVUTH137

2084 WVUTH 138

2085 WVUTH139

2086 WUUTH 14
2087 WWUTH 141
2012

2088 WVUTH142

2089 WVUTH 143

2090 WWUTH144
2091 WWUTH 145

2092 WVUTH146

2092 WUUTH 146
2093 WVUTH 147
2029 WVUTH 148

2094 WUUTH1 148
2095 WVUTH 149

2096 WVUTH 150

2097 WUUTH1151
2098 WWUTH152

2098 W WUTH1152
2099 WUUTH153

2100 WVUTH 154

210 WWUTH155

2102 WUUTH1156
2103 WUUTH157

2104 WVUTH158

2105 WWUTH1159
2106 WVUTH 1160

2106 WVUTH1160

2108 WUUTH162

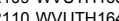

21111
21122

2113

2116

21177

21188

2120

21223

21245
21256

2126
2127

2128

21293
21304
21315
21326

21326

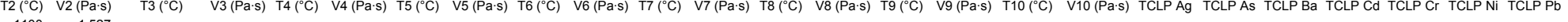

$\begin{array}{ll}1100 & 2.079 \\ 1100 & 2.360 \\ 1100 & 4.431\end{array}$

1100

1100
1100 $\quad 5.930$

$\begin{array}{ll}1100 & 4.629 \\ 1100 & 6.131 \\ 1100 & 3.623 \\ 1100 & \end{array}$

$1100 \quad 1.75$

$\begin{array}{lr}1100 & 7.250 \\ 1100 & 11.746 \\ 1100 & 9.194 \\ 1100 & 5.457 \\ 1100 & 7.979 \\ 1100 & 7.879 \\ 1100 & 5.872 \\ 1100 & 6.808 \\ 1100 & 6.335 \\ 1100 & 6.544 \\ 1100 & 6.976 \\ 1100 & 6.226 \\ 1100 & 6.105 \\ 1100 & 2.002 \\ 1100 & 2.957 \\ 1100 & 4.754 \\ 1100 & 6.106 \\ 1100 & 3.183 \\ 1100 & 4.322 \\ 1100 & 11.1354 \\ 1100 & 5.362 \\ 1100 & 7.523 \\ 1100 & 8.464\end{array}$

$\begin{array}{ll}1100 & 4.283 \\ 1100 & 2.712 \\ 1100 & 3.630\end{array}$ 
2052 WUUTH 106

2053 WUTH 107
2054 WUUTH 108

2055 WVUTH 109
2056 WVUTH110

2056 WUUTH110
2058 WVUTH111
2052

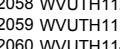

2006 WVUTH1115

2002 WWUTH116

2063 WWUTH1117

2065 WVUTH119

2066 W WUTH 120
2067 WVUTH 121

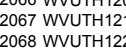

2069 WVUTH 123

2070 WVUTH1124
2071 WVUTH 125

2071 WVUTH 125
2072 WVUTH 126

2073 WVUTH 127

2074 WUUTH128
2075 WWUTH 129

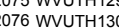

2077 WVUTH13

2078 WUUTH 132
2079 WVUTH 133

2080 WVUTH 133

2081 WVUTH 135

2082 WVUTH 136
2083 WVUTH 137

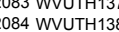

2085 WVUTH 139

2086 WUUTH 140
2087 WUUTH141

2088 WVUTH142

2089 WVUTH 143

2090 WWUTH144
2091 WUUTH 145

2092 WVUTH 146

2093 WVUTH 147

2094 WVUTH148
2095 WVUTH149

2096 WVUTH150

2097 WVUTH15
2098 WVUTH 152

2098 WWUTH1152

2100 WVUTH 154

2101 WVUTH155

2102 WUUTH1156
2103 WVUTH157

2104 WVUTH158

2105 WVUTH1159

2106 WVUTH1160

2108 WVUTH162

2109 WUUTH163
2110 WUTHH164

2112

(1133

(115

21166

21177

21188

(120

2122

2124

21256
21267

(127

21293
21304

21326

0.353
0.351
0.284
0.284
0.348
0.353
0.295
0.398
0.330
0.351
0.351
0.329
0.378
0.347
0.365
0.378
0.282
0.282
0.282
0.282
0.282
0.282
0.278
0.277
0.289
0.288
0.277
0.277
0.275
0.235
0.246
0.263
0.280
0.250
0.263
0.302
0.277
0.292
0.302
0.277
0.288
0.308
0.330
0.351
0.235
0.284
0.284
0.284
0.282
0.246
0.263
0.280
0.284
0.284 
2052 WUUTH 106

2053 WUUTH 107
2054 WUTH 108
2055 WUTH

2055 WWUTH 109

2056 WUUTH110
2058 WVUTH111
2052

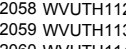

2060 WVUTH114
2061 WVUTH115

2062 WWUTH116

2063 WWUTH1117

2065 WVUTH 119

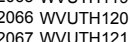

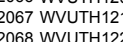

2069 WVUTH 123

2070 WWUTH 124
2071 WUTH

2071 WVUTH 125
2072 WVUTH 126

2073 WVUTH 127

2074 WVUTH1128
2075 WVUTH 29

2075 WVUTH122
2076 WVUTH 139

2077 WVUTH13

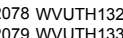

2079 WVUTH 133
2080 WVUTH 134

2081 WVUTH 135

2082 WUUTH 136

2083 WVUTH137
2084 WVUTH 138

2084 WUUTH 138
2025 WWUTH 139

2086 WUUTH 140

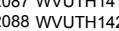

2089 WVUTH 143

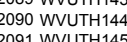

2092 WUUTH 146
2093 WWUTH 147
205 WUH

2094 WVUTH 148

2096 WVUTH 150

2097 WUUTH1151
2098 WWUTH152

2098 WVUTH 152
2099 WUTTH153

2100 WVUTH 154

210 WWUTH155

2102 WVUTH 156
2103 WVUTH 157

2104 WVUTH 158

2105 WUUTH159

2106 WVUTH1160

2107 WUTH161
2108 WVTHH162

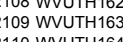

1110 WVUTH164

2112

(1133

2114

2116

21177

21188

2120

2122

21245

21256
21267

21293

21315
21326 


\begin{tabular}{|c|c|c|}
\hline \# Glass ID & Study & Reference \\
\hline 21331 & HWVP & Bates March 1987 \\
\hline 21342 & $\begin{array}{ll}\text { HWVP } \\
\text { HWVP }\end{array}$ & $\begin{array}{l}\text { Bates March } 1987 \\
\text { Rat }\end{array}$ \\
\hline $\begin{array}{l}21353 \\
21364\end{array}$ & $\begin{array}{ll}\text { HWVP } \\
\text { HWYP }\end{array}$ & $\begin{array}{l}\text { Bates March } 1987 \\
\text { Bates March } 1987\end{array}$ \\
\hline $\begin{array}{l}21364 \\
21375\end{array}$ & $\begin{array}{l}\text { HWVP } \\
\text { HWVP }\end{array}$ & $\begin{array}{l}\text { Bates March } 1987 \\
\text { Bates March } 1987\end{array}$ \\
\hline 2138 Low Al & $\begin{array}{l}\text { HWVP } \\
\text { HWVP }\end{array}$ & $\begin{array}{l}\text { Bales Marcn } \\
\text { Bates March } 1987\end{array}$ \\
\hline 2139 High Zr & & \\
\hline 140 High Na & HWVP & Bates March 1987 \\
\hline 2141 Low Fe2O3 & HWVP & Bates March 1987 \\
\hline $21420.3 \% \mathrm{~F}$ & HWVP & Bates July 1987 \\
\hline $21432 \% \mathrm{~F}$ & HWVP & Bates July 1987 \\
\hline $\begin{array}{l}21443 \% \mathrm{~F} \\
21454 \% \mathrm{~F}\end{array}$ & HWVP & Bates July 1987 \\
\hline 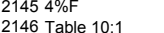 & $\begin{array}{l}\text { HWVP } \\
\text { HWVP }\end{array}$ & $\begin{array}{l}\text { Bates July } 1987 \\
\text { Bates }\end{array}$ \\
\hline $\begin{array}{l}2146 \text { Table 10; } \\
2147 \text { Table 10;2 }\end{array}$ & $\begin{array}{l}\text { HWVP } \\
\text { HWVP }\end{array}$ & $\begin{array}{l}\text { Bates July } 1987 \\
\text { Bates July } 1987\end{array}$ \\
\hline 2148 Table 10;3 & HWVP & $\begin{array}{l}\text { Bates July } 1987 \\
\text { Bates }\end{array}$ \\
\hline 2149 Table $10: 4$ & HWVP & Bates July 1987 \\
\hline 2150 Table 10;5 & HWVP & Bates July 1987 \\
\hline 2151 Table 10;6 & HWVP & Bates July 1987 \\
\hline 2152 Table 10;7 & HWVP & Bates July 1987 \\
\hline 2153 Table 10;8 & HWVP & Bates July 1987 \\
\hline 2154 Table 10;9 & $\begin{array}{ll}\text { HWVP } \\
\text { HWYP }\end{array}$ & Bates July 1987 \\
\hline $\begin{array}{l}2155 \text { Table 10;10 } \\
2156 \text { HWW3 }\end{array}$ & $\begin{array}{l}\text { HWVP } \\
\text { HWVP }\end{array}$ & $\begin{array}{l}\text { Bates July } 1987 \\
\text { Bates Sept. 1987 }\end{array}$ \\
\hline $\begin{array}{l}2156 \text { HWW3 } \\
2157 \text { HW44 }\end{array}$ & $\begin{array}{l}\text { HWVP } \\
\text { HWVP }\end{array}$ & $\begin{array}{l}\text { Bates Sept. } 1987 \\
\text { Bates Sept. } 1987\end{array}$ \\
\hline $2158 \mathrm{HW} 45$ & HWVP & Bates Sept. 1987 \\
\hline 2159 Low Al2O3 & HWVP & Bates Sept. 1987 \\
\hline 2160 High ZrO2 & HWVP & Bates Sept. 1987 \\
\hline 2161 High Na2O & HWVP & Bates Sept. 1987 \\
\hline 2162 Low Fe2O3 & HWVP & Bates Sept. 1987 \\
\hline $\begin{array}{l}2163 \text { AWV-407 } \\
2164 \text { AWV-416 }\end{array}$ & $\begin{array}{l}\text { West Vaalley Studides } \\
\text { West Valley Studies }\end{array}$ & Bowan et al. 1985 \\
\hline $\begin{array}{l}2164 \text { AWV-416 } \\
2165 \text { AWV-417 }\end{array}$ & $\begin{array}{l}\text { West Valllly Studides } \\
\text { West Valley Studies }\end{array}$ & $\begin{array}{l}\text { Bowan et al. } 1985 \\
\text { Bowan et al. } 1985\end{array}$ \\
\hline $\begin{array}{l}2165 \text { AWV-417 } \\
2166 \text { AWV-418 }\end{array}$ & $\begin{array}{l}\text { West Valley Studides } \\
\text { West Valley Studies }\end{array}$ & $\begin{array}{l}\text { Bowan et al. } 1985 \\
\text { Bowan et al. } 1985\end{array}$ \\
\hline 2167 AWV-419 & $\begin{array}{l}\text { West Valley Studies } \\
\text { Wes }\end{array}$ & Bowan et al. 1985 \\
\hline 2168 AWV-420 & West Valley Studies & Bowan et al. 1985 \\
\hline 2169 AWV-421 & West Valley Studies & Bowan et al. 1985 \\
\hline $21705 \% \mathrm{Ba}$ & HWVP Comp. Variability S & Brouns et al. 1988 \\
\hline $21715 \% \mathrm{Mn}$ & HWVP Comp. Variability S & B Brouns et al. 1988 \\
\hline $\begin{array}{l}21122 \% \mathrm{Mo} \\
2173 \\
22 \% \mathrm{Ni}\end{array}$ & HWVP Comp. Variability S & OBrouns et al. 1988 \\
\hline $\begin{array}{l}21732 \% \mathrm{Ni} \\
2174 \mathrm{PG}-\mathrm{WN} 1\end{array}$ & HWVP Comp. Variability S & $\begin{array}{l}\text { Brouns et al. } 1988 \\
\text { Brouns et al } 1988 \mathrm{a}\end{array}$ \\
\hline $\begin{array}{l}\text { 2174 DG-WV1 } \\
2175 \text { DG-WV2 }\end{array}$ & $\begin{array}{l}\text { Comp. Varaiablity Study } \\
\text { Comp. Variablity Study }\end{array}$ & $\begin{array}{l}\text { Brouns et al, } 1988 \mathrm{a} \\
\text { Brouns et al, 1988a }\end{array}$ \\
\hline 2176 DG-WV3 & $\begin{array}{l}\text { Comp. Variablity Study } \\
\text { Stmp }\end{array}$ & $\begin{array}{l}\text { Brouns et al., } 1988 \mathrm{a} \\
\text { Bro }\end{array}$ \\
\hline 2177 DG-WV4 & Comp. Variablity Study & Brouns et al., 1988a \\
\hline 2178 DG-WV5 & Comp. Variablity Study & Brouns et al., 1988a \\
\hline 2179 DG-WV6 & Comp. Variablity Study & Brouns et al., 1988a \\
\hline 2180 DG-WV7 & Comp. Variablity Study & Brouns et al., 1988a \\
\hline 2181 DG-WV8 & Comp. Variablity Study & $\begin{array}{l}\text { Brouns et al., 1988a } \\
\text { nol }\end{array}$ \\
\hline $\begin{array}{l}2182 \text { DG-WW15 } \\
2183 \text { DG-WV16 }\end{array}$ & Comp. Variablity Study & $\begin{array}{l}\text { Brouns et al., 1988a } \\
\text { Brouns setal } 1988 \mathrm{a}\end{array}$ \\
\hline $\begin{array}{l}2183 \text { DG-WV16 } \\
2184 \text { DG-WV17 }\end{array}$ & $\begin{array}{l}\text { Comp. Variablity Study } \\
\text { Comp. Variablity Study }\end{array}$ & $\begin{array}{l}\text { Brouns et al., 1988a } \\
\text { Brouns et al,. 1988a }\end{array}$ \\
\hline $2185 \mathrm{DG}-\mathrm{WV} 18$ & Comp. Variablity Study & Brouns et al., 1988a \\
\hline 186 DG-WV19 & Comp. Variablity Study & Brouns et al., 1988a \\
\hline 2187 DG-WV20 & Comp. Variablity Study & Brouns et al., 1988a \\
\hline 2188 DG-WV21 & Comp. Variablity Study & Brouns et al., 1988a \\
\hline 2189 DG-WV22 & Comp. Variablity Study & Brouns et al., 1988a \\
\hline 2190 DG-WV23 & Comp. Variablity Study & $\begin{array}{l}\text { Brouns et al., 1988a } \\
\text { nal }\end{array}$ \\
\hline 19191 DG-WV24 & $\begin{array}{l}\text { Comp. Variabbity Study } \\
\text { Comp. Variablity Study }\end{array}$ & $\begin{array}{l}\text { Brouns et al., 1988a } \\
\text { Brons }\end{array}$ \\
\hline 2193 DG-WV26 & Comp. Variablity Study & $\begin{array}{l}\text { Brouns et al,., 1988a } \\
\text { Brouns et al, 1988a }\end{array}$ \\
\hline 194 DG-WV27 & Comp. Variablity Study & $\begin{array}{l}\text { Drouns etal, } \\
\text { Brouns et al,, 1988a }\end{array}$ \\
\hline DG-WV28 & Comp. Variablity Study & Brouns et al., 1988a \\
\hline 2196 DG-WV29 & Comp. Variablity Study & Brouns et al., 1988a \\
\hline 2197 DG-WV30 & Comp. Variablity Study & Brouns et al., 1988a \\
\hline BAZ-R & Italian HLW & Cantale et al. 1991 \\
\hline BA- & Italian HLW & Cantale et al. 1991 \\
\hline TSS & Low Leach Rate Glasses & $\begin{array}{l}\text { Chick } 1980 \\
\text { Chick } 1980\end{array}$ \\
\hline 2 TDS/211 & Low Leach Rate Glassses & $\begin{array}{l}\text { Chick } 1980 \\
\text { Chick } 1980\end{array}$ \\
\hline RHO Purex & Low Leach Rate Glasses & $\begin{array}{l}\text { Cnick 1980 } \\
\text { Chick } 1980\end{array}$ \\
\hline RHO Res.Liq. & Low Leach Rate Glasses & Chick 1980 \\
\hline & Low Leach Rate Glasses & Chick 1980 \\
\hline 2206 77-260 & Low Leach Rate Glasses & Chick 1980 \\
\hline 79-417 & Low Leach Rate Glasses & Chick 1980 \\
\hline $79-418$ & Low Leach Rate Glasses & Chick 1980 \\
\hline Vitreous & Evaluation of HLW Forms & $\begin{array}{l}\text { Chick, et al } 1986 \\
\text { nas }\end{array}$ \\
\hline & $\begin{array}{l}\text { INEL IRU Study } \\
\text { INEL TRU Study }\end{array}$ & $\begin{array}{l}\text { Fllnn et al. } 1981 \\
\text { Flinn et al. } 1981\end{array}$ \\
\hline $2212 \mathrm{~A} 40$ & NEL TRU Study & Flinn et al. 1981 \\
\hline & & \\
\hline & INEL TRU Study & Flinn et al. 1981 \\
\hline
\end{tabular}

Al2O3-t B2O3-t CaO-t Fe2O3-t FeO-t K2O-t Li2O-t MgO-t Na2O-t NiO-t P2O5-t SiO2-t ZrO2-t Ag2O-t As203-tAs205-t BaO-t BeO-t Bi2O3-t Br-t CdO-t Ce203-t CeO2-t CAt

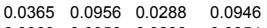

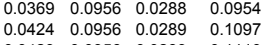
$0.0805 \quad 0.1208 \quad 0.0060 \quad 0.1220$ $0.0804 \quad 0.1207 \quad 0.0060 \quad 0.1219$ $0.06500 .09250 .0056 \quad 0.1130$ $\begin{array}{llll}0.0600 & 0.1235 & 0.0061 & 0.1241 \\ 0.0797 & 0.1196 & 0059 & 0.1210\end{array}$ $0.07970 .1196 \quad 0.0059 \quad 0.1208$ $0.07490 .0730 .0059 \quad 0.1198$ $\begin{array}{llll}0.0900 & 0.1297 & 0.0137 & 0.0900\end{array}$

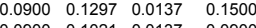
$\begin{array}{llll}0.0900 & 0.1021 & 0.0137 & 0.0900 \\ 0.0900 & 0.1095 & 0.0046 & 0.1500\end{array}$ $\begin{array}{llll}0.1200 & 0.1035 & 0.0137 & 0.1500\end{array}$ $\begin{array}{llll}0.1200 & 0.1106 & 0.0137 & 0.0900\end{array}$ $\begin{array}{llll}0.1200 & 0.1182 & 0.0046 & 0.1500\end{array}$ $\begin{array}{llll}0.0942 & 0.1248 & 0.0050 & 0.1424 \\ 0.1232 & 0.1322 & 0.0048 & 0.0867\end{array}$ $\begin{array}{lllll}0.0930 & 0.1090 & 0.0050 & 0.0867\end{array}$ $\begin{array}{llll}0.1227 & 0.1338 & 0.0134 & 0.0867\end{array}$ $\begin{array}{lllll}0.1217 & 0.1258 & 0.0049 & 0.0868 \\ 0.0968 & 0.1066 & 0.0048 & 0.1211\end{array}$ $\begin{array}{llll}0.0928 & 0.1145 & 0.0133 & 0.0874 \\ 0.1007 & 0.1188 & 0.0084 & 0.1148\end{array}$

\section{$\begin{array}{llll}0.0648 & 0.1133 & 0.0104 & 0.0000\end{array}$} $\begin{array}{llll}0.0562 & 0.0984 & 0.0095 & 0.1422 \\ 0.0319 & 0.0798 & 0.0501 & 0.1426\end{array}$ $\begin{array}{llll}0.0548 & 0.1047 & 0.0086 & 0.1324 \\ 0.0682 & 0.1020 & 0.0000 & 0.0000\end{array}$ $\begin{array}{llll}0.0000 & 0.0980 & 0.0209 & 0.1018\end{array}$ $\begin{array}{llll}0.0202 & 0.0921 & 0.0104 & 0.0119 \\ 0.0459 & 0.0939 & 0.0029 & 0.0144\end{array}$

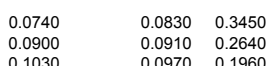

$\begin{array}{lll}0.1030 & 0.0970 & 0.1960 \\ 0.1140 & 0.1030 & 0.1390\end{array}$

$\begin{array}{llllll}0.0375 & 0.0083 & 0.0994 & 0.0049 & 0.5106 & 0.0049\end{array}$ $\begin{array}{lllllll}0.0375 & 0.0083 & 0.0996 & 0.0050 & & 0.5107 & 0.0050 \\ 0.0750 & 0.0084 & 0.1031 & 0.0057 & & 0.5116 & 0.0057\end{array}$ $\begin{array}{llllllll}0.0129 & 0.0203 & 0.0133 & 0.0926 & 0.0028 & 0.0252 & 0.4255 & 0.0029\end{array}$ $\begin{array}{llllllll}0.0333 & 0.0282 & 0.0121 & 0.1016 & 0.0032 & 0.0233 & 0.4487 & 0.0027 \\ 0.0125 & 0.0197 & 0.0129 & 0.1220 & 0.0027 & 0.0244 & 0.4122 & 0.0022\end{array}$ $\begin{array}{lllllllll}0.0132 & 0.0208 & 0.0136 & 0.0948 & 0.0029 & 0.0258 & 0.4354 & 0.0030\end{array}$ $\begin{array}{llllllll}0.0128 & 0.0221 & 0.0132 & 0.0918 & 0.0028 & 0.0350 & 0.4216 & 0.0029\end{array}$

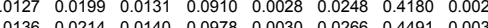
$\begin{array}{lllllllll}0.0172 & 0.0237 & 0.0132 & 0.1046 & 0.0053 & 0.0150 & 0.3900 & 0.0069\end{array}$ $\begin{array}{llllllll}0.0138 & 0.0191 & 0.0132 & 0.0842 & 0.0016 & 0.0350 & 0.3900 & 0.0021\end{array}$ $\begin{array}{llllllll}0.0172 & 0.0237 & 0.0132 & 0.1046 & 0.0058 & 0.0150 & 0.3900 & 0.00015 \\ 0.0184 & 0.0254 & 0.0044 & 0.1122 & 0.0017 & 0.0150 & 0.4248 & 0.0022\end{array}$ $\begin{array}{llllllll}0.0156 & 0.0216 & 0.0132 & 0.0953 & 0.0016 & 0.0150 & 0.3665 & 0.0021\end{array}$ $\begin{array}{lllllllll}0.0148 & 0.0204 & 0.0132 & 0.0902 & 0.0058 & 0.0350 & 0.3940 & 0.0075\end{array}$

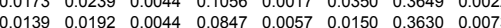
$\begin{array}{llllllll}0.0186 & 0.0180 & 0.0054 & 0.0823 & 0.0018 & 0.0330 & 0.3931 & 0.0024 \\ 0.0252 & 0.0205 & 0.0056 & 0.0956 & 0.0017 & 0.0141 & 0.4210 & 0.0022\end{array}$ $\begin{array}{llllllllll}0.0252 & 0.0205 & 0.0056 & 0.0956 & 0.0017 & 0.0141 & 0.4210 & 0.0022\end{array}$ $\begin{array}{lllllllllll}0.0264 & 0.0200 & 0.0135 & 0.0909 & 0.0019 & 0.0162 & 0.4095 & 0.0025\end{array}$ $\begin{array}{llllllll}0.0245 & 0.0228 & 0.0062 & 0.1014 & 0.0062 & 0.0348 & 0.3672 & 0.0080 \\ 0.0135 & 0.0205 & 0.0062 & 0.0886 & 0.0059 & 0.0153 & 0.4338 & 0.0077\end{array}$

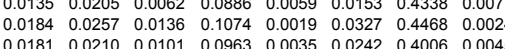

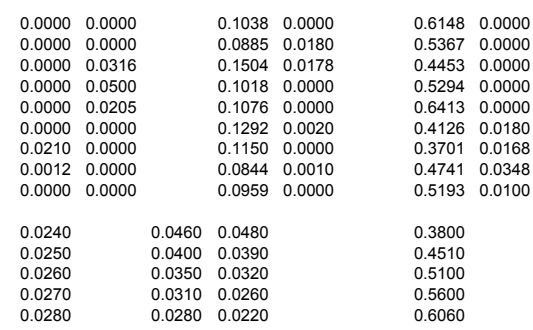

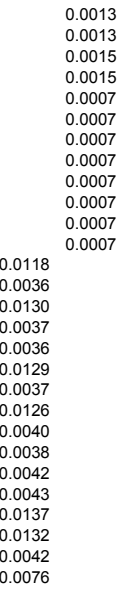




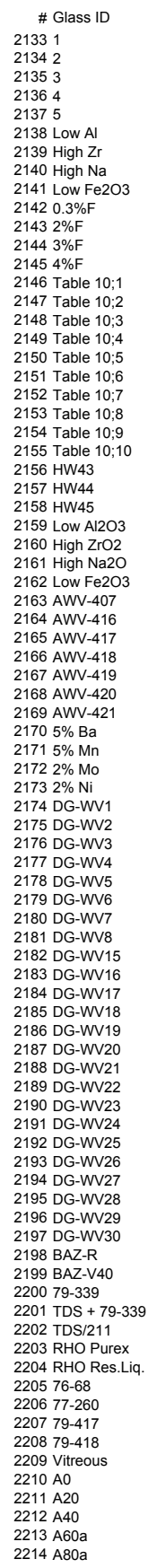

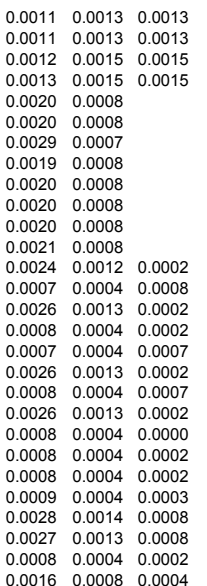

$0.0000 \quad 0.0000 \quad 0.0000$

\begin{tabular}{llll}
\hline .0000 & 0.0080 & 0.000 \\
0.0000 & 0.0084 & 0.000
\end{tabular}

$\begin{array}{llll}0.0000 & 0.0000 & 0.0000 \\ 0.0024 & 0.0045 & 0.0000 \\ 0.0040 & 0.012 & 0.000\end{array}$

$0.0040 \quad 0.0112 \quad 0.0000$

$\begin{array}{llll}0.00039 & 0.0018 & 0.0315 \\ 0.0039 & 0.0000\end{array}$

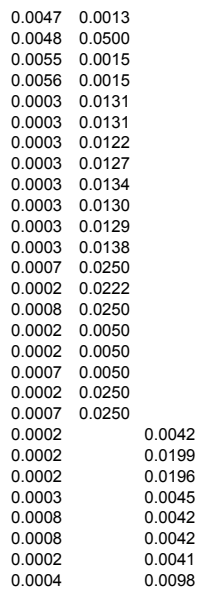

0.0026
0.0026
0.0200
0.0030
0.0001
0.0001
0.0000
0.0001
0.0001
0.0001
0.0001
0.0001
0.0007
0.0002
0.0008
0.0002
0.0002
0.0007
0.0002
0.0007
0.0002
0.0002
0.0002
0.0003
0.0008
0.0008
0.0002
0.0004

0.0000
0.0000
0.0000
0.0000
0.0000
0.0191
0.0160
0.0407
0.0117

0.0148
0.0381
0.0375
0.0120
0.0000
0.0000
0.0011
0.0011
0.0000

.0000
.0000
.0011

0.0000

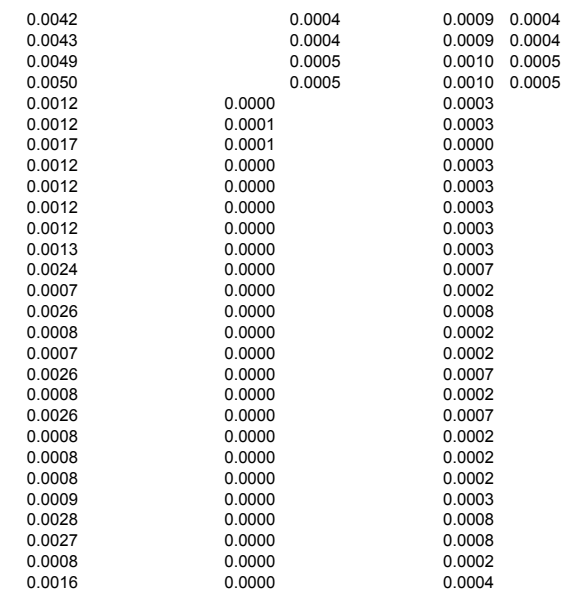

0.0000
0.0000
0.0000
0.0000
0.0000
0.0000
0.1076
0.0000
0.0000

0.0016

0.0000

0.0002 
2186 DG-WV19

2188 DG-WV21

2190 DG-WV23

2191 DG-WV24

2192 DG-WV25

2194 DG-WV27

2196 DG-WV29

2198 BAZ-R

2199 BAZ-V40
$220079-39$
2201

2201 TDS + 79-33

2202 TDS/211
2203 RHO Purex

2206 77-260

2207 79-417

2209 Vitreous

2211 A20

2213 A60a
2214 A80a

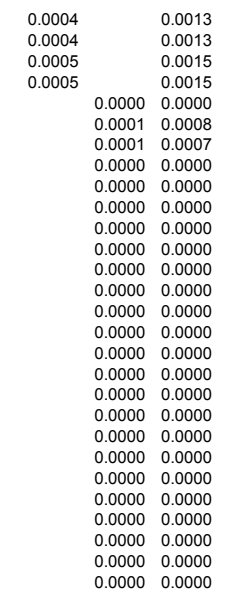

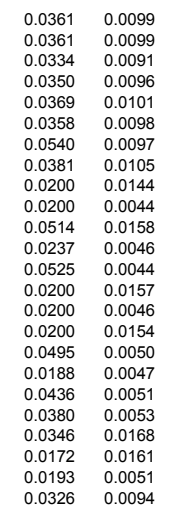

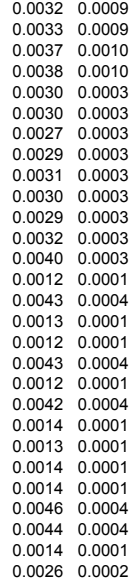

0.0000
0.0044
0.0046
0.0064
0.0000
0.0041
0.0026
0.0080
0.0028 $\begin{array}{ll}0.0000 & 0.0436 \\ 0.0000 & 0.0433 \\ 0.000 & 0.0431\end{array}$

$0.0000 \quad 0.0431$

$\begin{array}{ll}0.0000 & 0.0430 \\ 0.0000 & 0.0427 \\ 0.0000 & 0.0279\end{array}$

$\begin{array}{ll}0.0000 & 0.004 \\ 0.0000 & 0.0279\end{array}$

$\begin{array}{ll}0.00000 & 0.04700 \\ 0.00000 & 0.006\end{array}$

$0.0000 \quad 0.0444$

0.00000 .044

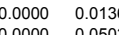

$\begin{array}{ll}0.00000 & 0.0503 \\ 0.00000 & 0.0272 \\ 0.0000 & 0.0000 \\ 0.0000 & 0.0194\end{array}$

$\begin{array}{ll}0.0000 & 0.0194 \\ 0.0000 & 0.000\end{array}$

$\begin{array}{ll}0.0000 & 0.0000 \\ 0.0000 & 0.0252\end{array}$

$0.0000 \quad 0.0194$

\begin{tabular}{ll}
0.0000 & 0.0000 \\
0.0000 & 0.025 \\
\hline
\end{tabular}

$\begin{array}{ll}0.0000 & 0.0310 \\ 0.0000 & 0.0362\end{array}$

$0.0000 \quad 0.0040$

$\begin{array}{ll}0.0000 & 0.0398 \\ 0.0000 & 0.0579 \\ 0.000\end{array}$

0.0000
0.0000
0.0000

0.0000
0.0000
0.0000

0.0004

0.0000

$\begin{array}{ll}0.0004 & 1.0014 \\ 0.0005 & 1.0374 \\ 0.0005 & 0.999\end{array}$

$\begin{array}{lll}0.0005 & & 0.9999 \\ 0.0002 & 0.9999 & 0.0874 \\ 0.0002 & 0.9999 & 0.086\end{array}$

$\begin{array}{llll}0.0002 & 1.0000 & 0.0861 \\ 0.0002 & 1.009 & 0.0030\end{array}$

$\begin{array}{lll}0.0002 & 1.0001 & 0.0838 \\ 0.0002 & 0.9991 & 0.0090 \\ 0.0002 & 1.0001 & 0.0855 \\ 0 & 1.001 & \end{array}$

$\begin{array}{lll}0.0002 & 1.0001 & 0.0884\end{array}$

$\begin{array}{llll}0.0003 & 0.0002 & 0.9971 & 0.0960\end{array}$ $\begin{array}{llll}0.0001 & 0.0002 & 1.0031 & 0.0943 \\ 0.0004 & 0.0006 & 0.9982 & 0.0943\end{array}$ $\begin{array}{llll}0.0004 & 0.0006 & 0.9982 & 0.0943 \\ 0.0001 & 0.0000 & 1.0026 & 0.0947\end{array}$ $\begin{array}{llll}0.0001 & 0.0000 & 1.0111 & 0.1223\end{array}$ $\begin{array}{llll}0.0004 & 0.0002 & 1.0064 & 0.1224 \\ 0.0001 & 0.0006 & 1.0622 & 0.1251\end{array}$ $\begin{array}{llll}0.0004 & 0.0002 & 0.9966 & 0.1250\end{array}$ $\begin{array}{llll}0.0001 & 0.0002 & 0.9990 \\ 0.0001 & 0.0002 & 0.9954\end{array}$ $\begin{array}{llll}0.0001 & 0.0000 & 0.9950 \\ 0.0001 & 0.0002 & 0.9989\end{array}$ $\begin{array}{llll}0.0004 & 0.0006 & 0.9984 \\ 0 & 0.004 & 0.0006 & 0.9897\end{array}$ $\begin{array}{llll}0.0004 & 0.0006 & 0.9987 \\ 0.0001 & 0.0002 & 0.9985 \\ 0.0002 & 0.0003 & 0.996\end{array}$ $\begin{array}{lll}0.0001 & 0.0002 & 0.998 \\ 0.0002 & 0.0003 & 0.9976\end{array}$ $\begin{array}{ll}0.0000 & 0.1375 \\ 0.0000 & 0.1347\end{array}$ $\begin{array}{ll}0.0000 & 1.0000 \\ 0.0000 & 1.0001 \\ 0.0509 & 0.9036\end{array}$ $\begin{array}{lll}0.0000 & 1.0000 \\ 0.0519 & 0.9036\end{array}$ $\begin{array}{lll}0.0519 & 0.9036 \\ 0.0000 & 0.8856 \\ 0.0021 & 0.8265\end{array}$ $\begin{array}{ll}0.0021 & 0.826 \\ 0.0011 & 0.948\end{array}$ 


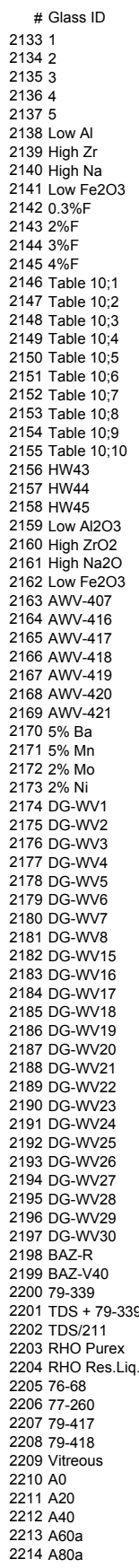

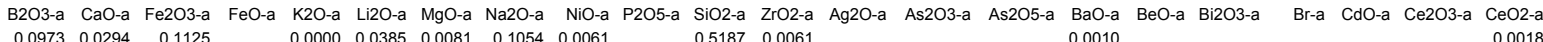

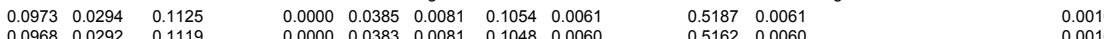

0.0010

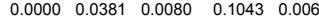

$\begin{array}{lll}0.09963 & 0.0290 & 0.1110 \\ 0.0953 & 0.0288 & 0.1102 \\ 0.0560 & 0.0286 & 0.1720\end{array}$

$\begin{array}{lll}0.0956 & 0.0286 & 0.0720 \\ 0.0556 & 0.0283 & 0.0230\end{array}$

$\begin{array}{lll}0.0956 & 0.0288 & 0.1030 \\ 0.0956 & 0.0293 & 0.0550\end{array}$

$\begin{array}{lll}0.0956 & 0.0293 & 0.0550 \\ 0.0953 & 0.0288 & 0.1173 \\ 0.0939\end{array}$

$\begin{array}{lll}0.0942 & 0.0285 & 0.1159 \\ 0.039 & 0.1158 & 0.115\end{array}$

$0.0927 \quad 0.0280 \quad 0.1142$

$\begin{array}{lll}0.0921 & 0.1552 & 0.1102 \\ 0.0921 & 0.0279 & 0.1302\end{array}$

$\begin{array}{lll}0.0921 & 0.1554 & 0.0703 \\ 0.0920 & 0.1550 & 0.1502\end{array}$

$0.09210 .0914 \quad 0.1572$

$\begin{array}{lll}0.0920 & 0.0271 & 0.2781 \\ 0.0921 & 0.0275 & 0.2042\end{array}$

$\begin{array}{lll}0.0921 & 0.0914 & 0.152 \\ 0.0921 & 0.1552 & 0.1102 \\ 0.021 & 0.1550 & 0.1502\end{array}$

$\begin{array}{lll}0.0921 & 0.1550 & 0.1502 \\ 0.0959 & 0.0289 & 0.0723\end{array}$

$0.0896 \quad 0.0272 \quad 0.0868$

$0.09560 .0291 \quad 0.1408$

$\begin{array}{lll}0.0956 & 0.0286 & 0.0720 \\ 0.0956 & 0.0288 & 0.1029\end{array}$

0.0956
0.0765

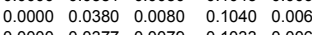

$\begin{array}{llll}0.0375 & 0.0085 & 0.1110 & 0.0076 \\ 0.0375 & 0.0080 & 0.0944 & 0.0039\end{array}$

$\begin{array}{llll}0.0375 & 0.0082 & 0.1319 & 0.0055\end{array}$

$\begin{array}{lll}0.0085 & 0.1047 & 0.0062\end{array}$

$\begin{array}{llll}0.0369 & 0.0084 & 0.1034 & 0.006 \\ 0.0369 & 0.0084 & 0.1032 & 0.006\end{array}$

$\begin{array}{llll}0.0364 & 0.0082 & 0.1019 & 0.0006 \\ 0.0361 & 0.0075 & 0.0824 & 0.0019\end{array}$

$\begin{array}{llll}0.0361 & 0.0082 & 0.1051 & 0.0070\end{array}$

$\begin{array}{llll}0.0361 & 0.0077 & 0.0908 & 0.0038 \\ 0.0361 & 0.0072 & 0.0740 & 0.0000\end{array}$

$\begin{array}{llll}0.0361 & 0.0076 & 0.0860 & 0.0027\end{array}$

$\begin{array}{llll}0.0361 & 0.0072 & 0.0740 & 0.0000 \\ 0.0361 & 0.0077 & 0.0896 & 0.0035\end{array}$

$\begin{array}{llll}0.0361 & 0.0075 & 0.0824 & 0.0027 \\ 0.0361 & 0.0019\end{array}$

$\begin{array}{llll}0.0361 & 0.0072 & 0.0824 & 0.0079 \\ 0.0375 & 0.0084 & 0.0121 & 0.0000 \\ 0.035 & 0.0059\end{array}$

$\begin{array}{llll}0.0350 & 0.0081 & 0.1221 & 0.0059 \\ 0.1260 & 0.0071\end{array}$

$\begin{array}{llll}0.0325 & 0.0078 & 0.1299 & 0.0083 \\ 0.0375 & 0.0087 & 0.1105 & 0.0074 \\ 0.0355 & 0.0083 & 0.1318 & 0.054\end{array}$

$\begin{array}{llll}0.0375 & 0.0081 & 0.094 & 0.0038 \\ 0.0375 & 0.0883 & 0.1318 & 0.054\end{array}$

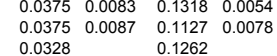

$\begin{array}{ll}0.5162 & 0.0060 \\ 0.5136 & 0.0060\end{array}$

$\begin{array}{ll}0.5118 & 0.0060 \\ 0.5084 & 0.0060\end{array}$

$\begin{array}{lll}.5140 & 0.0076 \\ 0.5093 & 0.1000 \\ 0.514 & 0.055\end{array}$

0.51140 .0055

$\begin{array}{ll}0.5106 & 0.0062 \\ 0.5044 & 0.0061\end{array}$

$\begin{array}{ll}0.5032 & 0.0061 \\ 0.4967 & 0.0060\end{array}$

$\begin{array}{ll}0.4878 & 0.0019 \\ 0.4942 & 0.0070 \\ 0.402 & 0.0000\end{array}$

$\begin{array}{ll}0.4942 & 0.0070 \\ 0.4902 & 0.0038 \\ 0.4855 & 0.0000\end{array}$

$\begin{array}{ll}0.4855 & 0.0000 \\ 0.4888 & 0.0027\end{array}$

$\begin{array}{ll}0.4855 & 0.0000 \\ 0.4898 & 0.0035\end{array}$

$\begin{array}{ll}0.4888 & 0.0027 \\ 0.4878 & 0.0019 \\ 0.4855 & 0.000\end{array}$

$\begin{array}{ll}0.4878 & 0.0019 \\ 0.4855 & 0.0000 \\ 0.5276 & 0.0387\end{array}$

$0.4986 \quad 0.0465$

$\begin{array}{lll}4697 \quad 0.0542 \\ 5137 & 0.0074\end{array}$

$0.5091 \quad 0.1000$

$\begin{array}{ll}0.5143 & 0.0078 \\ 0.3938 & \end{array}$

$\begin{array}{lll}0.1217 & 0.0083 & 0.1174 \\ 0.0933 & 0.0063 & 0.1081 \\ 0.127 & 0.0038 & 0.1191\end{array}$

$\begin{array}{lll}0.1241 & 0.0068 & 0.1191\end{array}$

$\begin{array}{lll}0.1215 & 0.0069 & 0.1155 \\ 0.1215 & 0.0065 & 0.1155\end{array}$

$\begin{array}{lll}0.1215 & 0.0065 & 0.1155 \\ 0.0751 & 0.0067 & 0.1229\end{array}$

$\begin{array}{lll}0.1258 & 0.0136 & 0.0880 \\ 0.1261 & 0.0137 & 0.1442 \\ 0.1628 & 0.434 & 0.144\end{array}$

$\begin{array}{llll}0.1261 & 0.0137 & 0.1442 \\ 0.0978 & 0.0143 & 0.0887 \\ 0.1008 & 0.054 & 0.144\end{array}$

$0.1008 \quad 0.0131 \quad 0.1420$

$\begin{array}{lll}0.1062 & 0.0134 & 0.0875 \\ 0.1005 & 0.0050 & 0.1467\end{array}$

$\begin{array}{llllllll}0.0116 & 0.0174 & 0.0132 & 0.0913 & 0.0031 & 0.0258 & 0.4325 & 0.0059\end{array}$ $\begin{array}{llllllll}0.0091 & 0.0176 & 0.0130 & 0.0904 & 0.0027 & 0.0260 & 0.4360 & 0.0056 \\ 0.0192 & 0.0234 & 0.0172 & 0.0982 & 0.0036 & 0.0241 & 0.4546 & 0.0064\end{array}$ $\begin{array}{llllllll}0.0129 & 0.1147 & 0.0027 & 0.0254 & 0.4194 & 0.0054\end{array}$ $\begin{array}{llllllll}0.0150 & 0.0176 & 0.0135 & 0.0910 & 0.0030 & 0.0263 & 0.4443 & 0.006\end{array}$ $\begin{array}{lllllllll}0.0127 & 0.0171 & 0.0128 & 0.0868 & 0.0028 & 0.0251 & 0.4303 & 0.0062\end{array}$ $\begin{array}{lllll}0.0184 & 0.0139 & 0.0938 & 0.0030 & 0.4\end{array}$

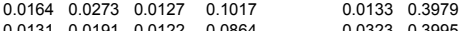
$\begin{array}{lllllll}0.0207 & 0.0234 & 0.0129 & 0.0164 & 0.01057 & 0.0323 & 0.3995 \\ 0 & 0.0124 & 0.3957\end{array}$ $\begin{array}{llllll}0.0244 & 0.0044 & 0.1114 & 0.0155 & 0.4320\end{array}$ $\begin{array}{lllllll}0.0197 & 0.0207 & 0.0128 & 0.0988 & 0.0135 & 0.3601 \\ 0.0223 & 0.0204 & 0.0142 & 0.0863 & 0.0334 & 0.3995\end{array}$

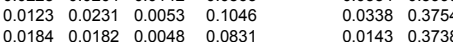

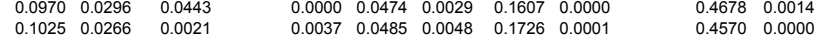

$0.0019 \quad 0.0005$

$0.0029 \quad 0.0006$

$\begin{array}{ll}0.0019 & 0.0005 \\ 0.0020 & 0.0004\end{array}$

$0.0019 \quad 0.0014$

$\begin{array}{ll}0.0000 & 0.0042 \\ 0.0012 & 0.0000\end{array}$

$0.0120 \quad 0.0095 \quad 0.3180 \quad 0.0060$

0.0019

0.0009 


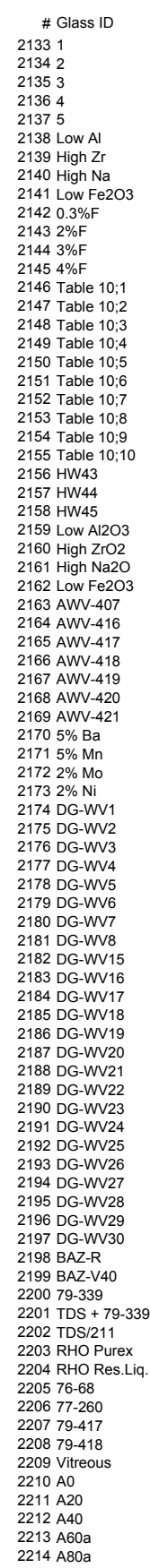

I-a La203-a MnO2-a MnO-a MoO-a MoO3-a Nb205-a Nd203-a NpO2-a PbO-a PdO2-a PdO-a Pr2O3-a Pr6011-a PuO2-a Rb2O-a ReO-a ReO2-a Re207-a Rh203-a RhO2-a RuO2-a Sb203-a Sb205-a SeO2-a

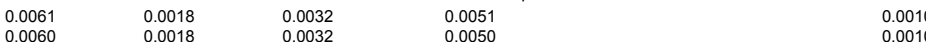

\begin{tabular}{|c|c|c|}
\hline & & \\
\hline & 0.0018 & 0.0032 \\
\hline 0.0060 & 0.0018 & 0.0032 \\
\hline $\begin{array}{l}0.0060 \\
0.0070\end{array}$ & & \\
\hline 0.0036 & $\begin{array}{l}0.0023 \\
0.0012\end{array}$ & $\begin{array}{l}0.0038 \\
0.0020\end{array}$ \\
\hline 0.0051 & 0.0017 & 0.0028 \\
\hline 0.0085 & 0.0028 & 0.0047 \\
\hline 0.0059 & 0.0016 & \\
\hline 0.0058 & 0.0016 & 0.0032 \\
\hline $\begin{array}{l}0.0058 \\
0.0057\end{array}$ & $\begin{array}{l}0.0016 \\
0.0016\end{array}$ & $\begin{array}{l}0.0031 \\
0.031\end{array}$ \\
\hline & 0.00005 & $\begin{array}{l}0.00031 \\
0.0009\end{array}$ \\
\hline .0064 & 0.0017 & $\begin{array}{l}0.0039 \\
0.003\end{array}$ \\
\hline .0035 & 0.0009 & 0.0019 \\
\hline & 0.0000 & \\
\hline $\begin{array}{c}0.0025 \\
0.0000\end{array}$ & 0.0007 & 0.0013 \\
\hline $\begin{array}{l}0.00000 \\
0.00032\end{array}$ & $\begin{array}{l}0.0000 \\
0.0009\end{array}$ & $\begin{array}{l}0.00000 \\
0.0017\end{array}$ \\
\hline 0.0025 & 0.0007 & $\begin{array}{l}0.0017 \\
0.00\end{array}$ \\
\hline & 0.0005 & 0.0009 \\
\hline 0.0000 & 0.0000 & 0.0000 \\
\hline b.0052 & 0.0015 & 0.0031 \\
\hline $\begin{array}{l}.0062 \\
.0072\end{array}$ & & \\
\hline $\begin{array}{l}.0072 \\
.0070\end{array}$ & $\begin{array}{l}0.0022 \\
0.0019\end{array}$ & $\begin{array}{l}0.000434 \\
0.0038\end{array}$ \\
\hline .0036 & 0.0010 & 0.0020 \\
\hline 0051 & 0.0014 & 0.0028 \\
\hline
\end{tabular}

$$
\begin{aligned}
& 0.0010 \\
& 0.0010 \\
& 0.0010
\end{aligned}
$$

0.0010

$\begin{array}{rr} & 0.0016 \\ 0.0016 \\ 0.0016\end{array}$

0.0016
0.0016
0.0016
0.0016
0.0016

.0034

0.0034
0.0089
0.0053

.0052

0.0061

0.0000

00031

.0017
.0000
.0061

.0065

0.0005
0.0005
0.0005
0.0005
0.0001
0.0005
0.0003
0.0000
0.0002
0.0000
0.0003
0.0002
0.0001
0.0000
0.0005
0.0006
0.0007
0.0006
0.0003
0.0005
0.0007

$$
\begin{aligned}
& 0.0007 \\
& 0.0010
\end{aligned}
$$

0.0016

$\begin{array}{ll}0.0011 & 0.0005 \\ 0.0011 & 0.0005 \\ 0 & 0.011\end{array}$

$\begin{array}{ll}0.0011 & 0.0005 \\ 0.0011 & 0.0005 \\ 0.0011 & 0.0005 \\ 0 & 0.0000\end{array}$

0.0003
0.0010

0.0000
0.0006
0.0000
0

0.0002
0.0007
0.0004
0.0000
0.0003

0.0000
0.0003
0.0000

0.0000
0.0003
0.0003

0.0004
0.0003

0.0003
0.0002
0.0000
0.0005

$\begin{array}{ll}0.0010 & 0.0005 \\ 0.0012 & 0.0006 \\ 0.0014 & 0.0007\end{array}$

0.0014
0.0013
0.0007
0.0009

0.0007
0.0006
0.0003
0.0005

0.0014

0.0007

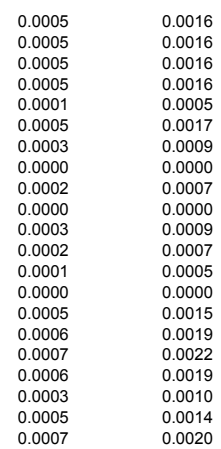
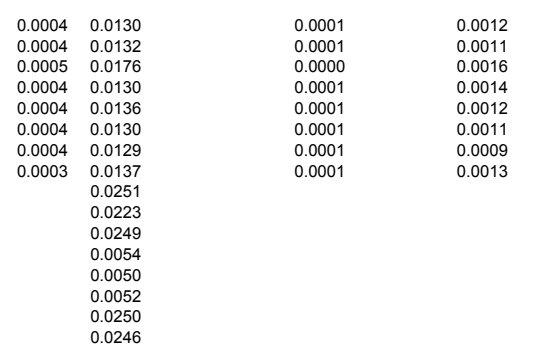

$\begin{array}{ll}0.0000 & 0.0003 \\ 0.0001 & 0.0003 \\ 0.0001 & 0.0000 \\ 0.0000 & 0.0003 \\ 0.0000 & 0.0003 \\ 0.0000 & 0.0003 \\ 0.0000 & 0.0003 \\ 0.0000 & 0.0003\end{array}$

$\begin{array}{ll}0.0000 & 0.0000 \\ 0.0001 & 0.0008 \\ 0.0000 & 0.000\end{array}$

\begin{tabular}{ll}
0.0001 & 0.0007 \\
\hline & 0.0000 \\
\hline
\end{tabular}

$0.0000 \quad 0.00000$

$\begin{array}{ll}0.0000 & 0.0000 \\ 0.0000 & 0.0000\end{array}$

$0.0000 \quad 0.0000$ 


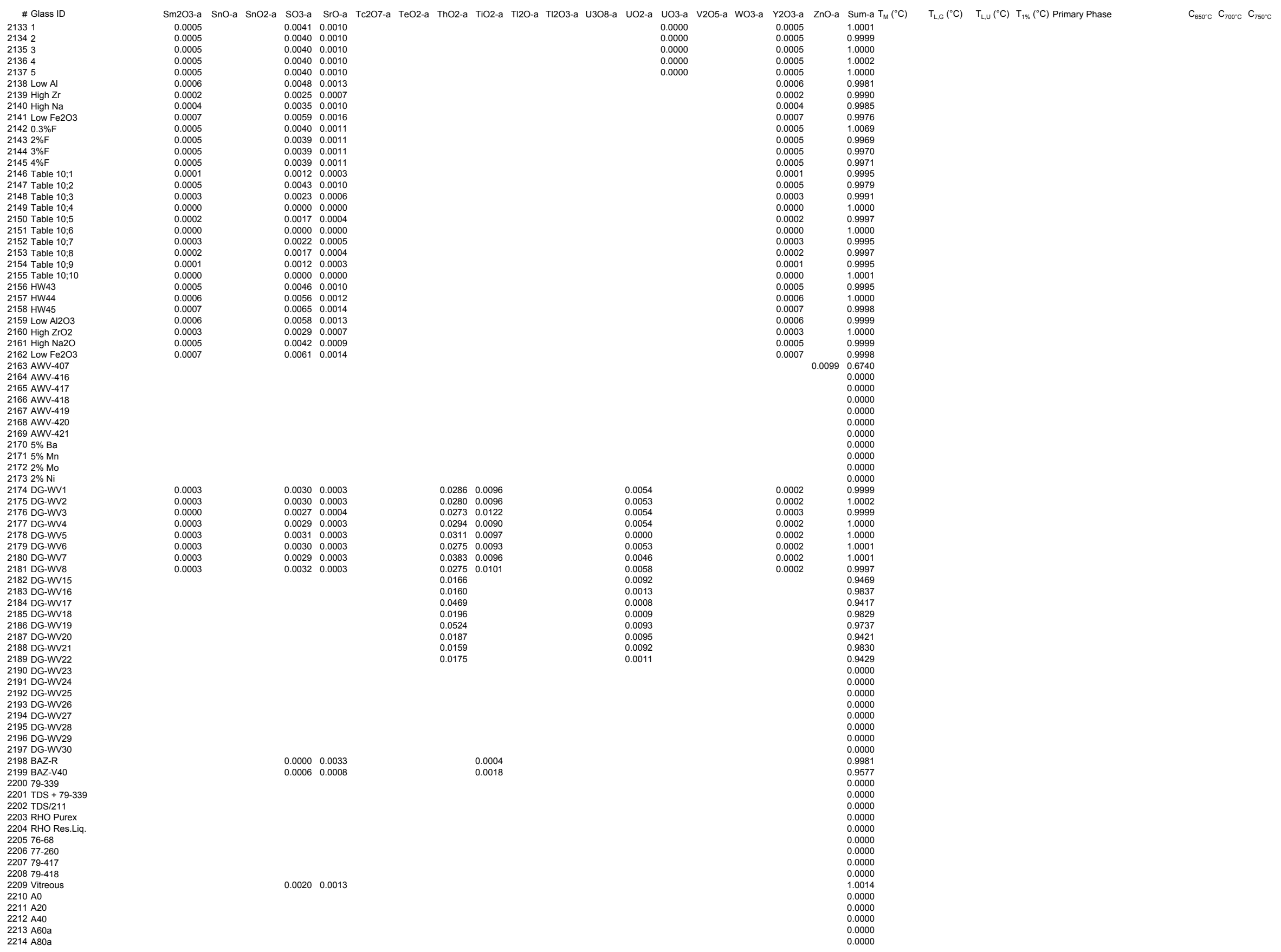


645.031623 .000

$\begin{array}{rr}1200.0 & 87.900 \\ 1200.0 & 0.800 \\ 1200.0 & 6.400 \\ 1200.0 & 30.000 \\ 1200.0 & 2.080 \\ & \\ & \\ & \\ 870.0 & 31.000 \\ 1450.0 & 4.500 \\ 1350.0 & 25.200 \\ 1350.0 & 41.500 \\ 1300.0 & 283.300 \\ 1350.0 & 1037.400\end{array}$




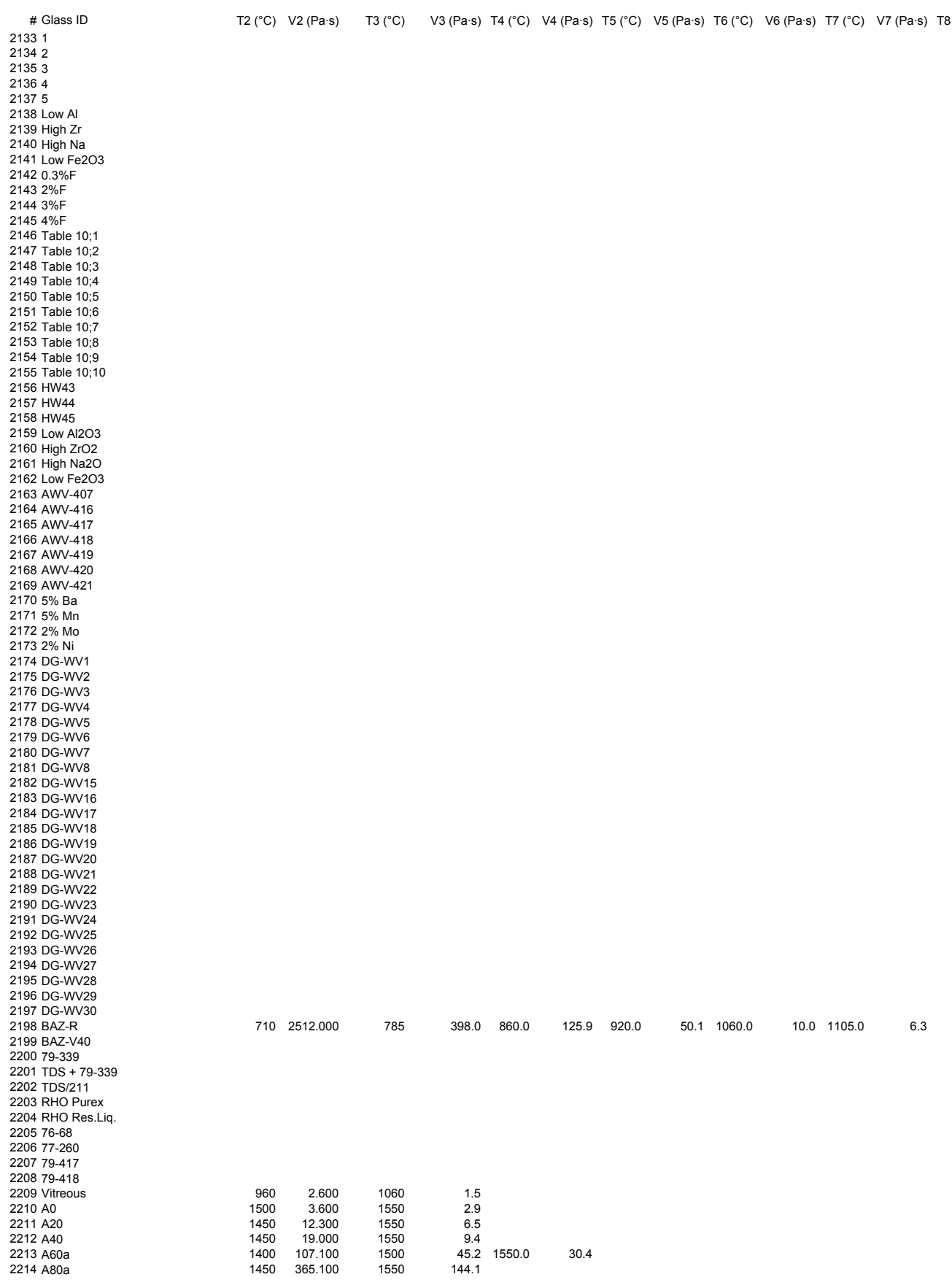


21342

21353
21364
21375

2138 Low

2139 High Zr
$2140 \mathrm{High} \mathrm{Na}$

2141 Low Fe2

$21432 \% \mathrm{~F}$

$2145 \% \mathrm{~F}$

2146 Table 10;1
2147 Table 10:2

2148 Table $10 ; 3$

2149 Table 10;4

2151 Table 10;6

2152 Table $10 ; 7$

2154 Table 10;9
2155 Table 10:10

$2156 \mathrm{HW} 43$

$2157 \mathrm{HW} 4$
$2158 \mathrm{HW} 4$

2159 Low Al2O3

2160 High ZrO2

2161 High Na2O

$2163 \mathrm{AWV}-407$

2164 AWV-416
2165 AWV-417

2166 AWV-418

2167 AWV-419

2168 AWV-420
2169 AWV-421

$21705 \% \mathrm{Ba}$

$21715 \% \mathrm{Mn}$

$21722 \% \mathrm{Mo}$

2174 DG-WV1

2175 DG-WV2

2176 DG-WW3

2178 DG-WV5

2179 DG-WV6

2181 DG-WV8

2182 DG-WV15

2183 DG-WV16
2184 DG-WV17

$2184 \mathrm{DG}-\mathrm{W} 17$
$2185 \mathrm{DO}-\mathrm{WW} 18$

2186 DG-WV19

$2187 \mathrm{DG}-\mathrm{WW} 20$
$2188 \mathrm{DG}-\mathrm{W} 2121$

$2189 \mathrm{DG}-\mathrm{W} 222$

(290 DG-WV23

2192 DG-WV25

2193 DG-WV26
2194 DG-WV27

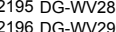

2197 DG-WV30

2198 BAZ-R

$220079-339$
2201

2201 TDS + 79-339
2202 TDS/211

2202
2203 R PHS 1211
220 Purex

$22200676-68$
22260

$220779-417$
2208
$20-418$

2209 Vitreous

2210 AD
2211 O

$2211 \mathrm{~A} 20$
$2212 \mathrm{~A} A 0$

2213 A60a
2214 A 80 a 


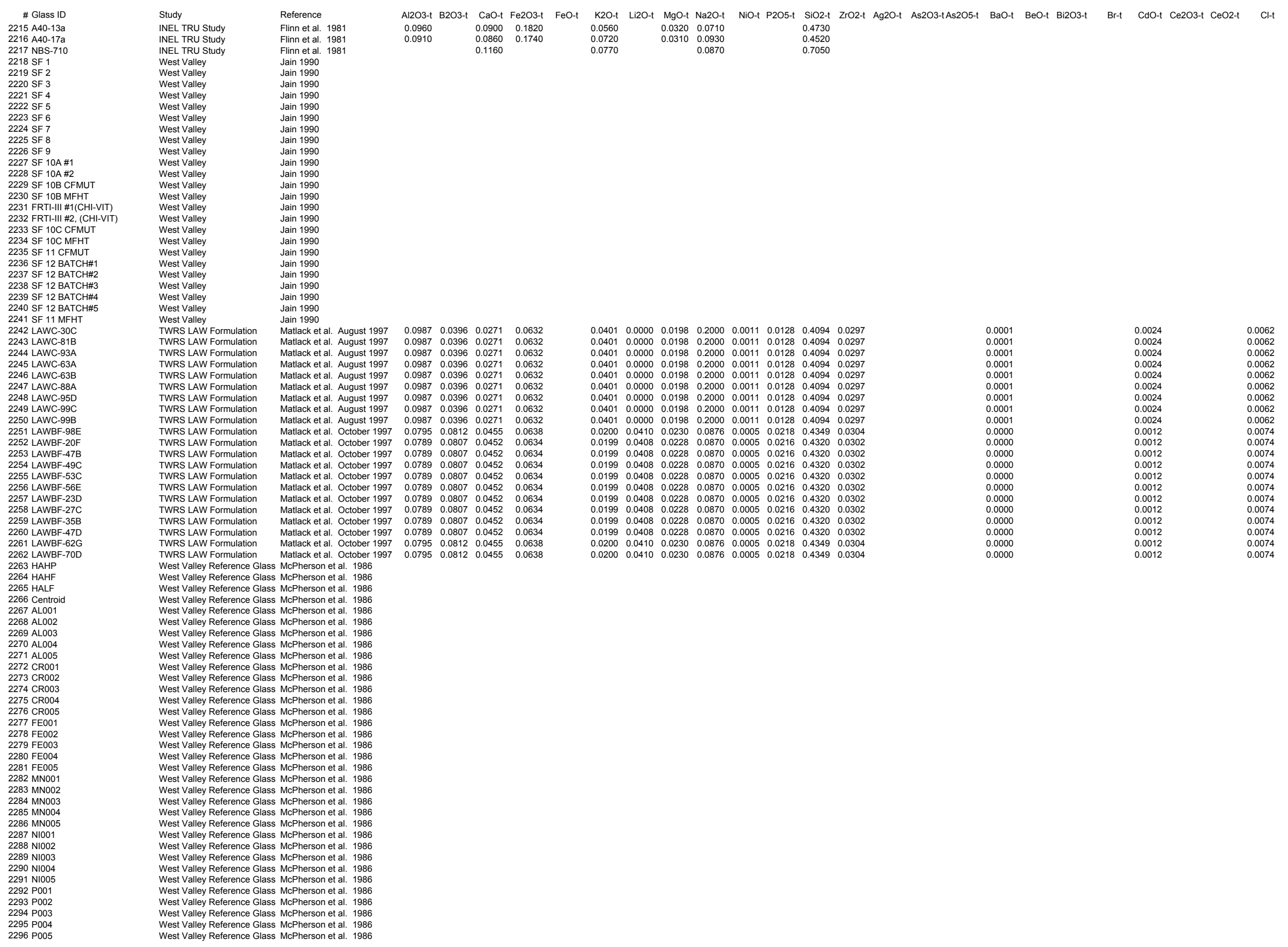


2237 SF 12 BATCH\#2

2238 SF 12 BATCH\#3

$2240 \mathrm{SF} 12 \mathrm{BATCHA}$

2241 S F 11 MFHT
2242 LAWC-30C

2243 LAWC-81B

2244 LAWC-93A

2246 LAWC-63B

2247 LAWC-88A
2248 LAWC-95D

2248 LAWC-95D
2249 LAWC-99C

2250 LAWC-99B
2251 LAWBF-98E

2252 LAWBF-20F

2253 LAWBF-47B

2254 LAWBF-49C

2255 LAWBF-53C

2257 LAABFF-23D
2258 LAWBF-27C

2259 LAWBF-35B
2260 LAWBF-47D

2261 LAWBF-62G

2262 LAWBE-70D
2263 HAHP

2264 HAHF

2265 HALF
2266 Centroid
2267 AL001

2267
2268 AL001

2268 AL002
2269 AL003
2270 AL004

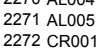

2272 CR001

2274 CR003
2275 CR004

2276 CR005

2277 FEO01
2278 FE002
2279 FEO03

2280 FEO0

2821 FED05
2282 MNO0

$2282 \mathrm{MNO01}$
$2283 \mathrm{MNO00}$

$2284 \mathrm{MNON0}$
$2285 \mathrm{MNO} 04$

$2288 \mathrm{~N} 1002$

$2289 \mathrm{~N} N 1003$
$2290 \mathrm{~N} 1004$

229011004
2291

$2292 \mathrm{P} 00$

2293 P 002
2294 P003

$2295 \mathrm{P} 004$

$\begin{array}{ll}0.0025 & 0.0008 \\ 0.0025 & 0.0008\end{array}$

$\begin{array}{ll}0.0025 & 0.0008 \\ 0.0025 & 0.0008 \\ 0.0025 & 0.0008\end{array}$

$\begin{array}{ll}0.0025 & 0.0008 \\ 0.0025 & 0.0008\end{array}$

$0.0025 \quad 0.0008$

$\begin{array}{ll}0.0025 & 0.0008 \\ 0.0025 & 0.0008\end{array}$

$\begin{array}{llll}0.0025 & 0.0000 \\ 0.0036 & 0.0008\end{array}$

0.00360 .0008

0.00360 .0008

$\begin{array}{lll}0.0036 & 0.00008 \\ 0.0036 & 0.0008\end{array}$

$0.0036 \quad 0.0008$

$0.0036 \quad 0.0008$

$\begin{array}{ll}0.0036 & 0.0008 \\ 0.0036 & 0.0008\end{array}$

$\begin{array}{lll}0.0036 & 0.0008 \\ 0.0036 & 0.0008\end{array}$

0.0001
0.0001

0.0007
0.0007
0.007

0.0082

0.0082
0.0082

0.0082
0.0082
0.0082

0.0082

0.000
0.000
0.009

0.0001
0.000
0.001

0.0001
0.0001 
2237 SF 12 BATCH\#2

2238 SF 12 BATCH\#3

2240 SF 12 BATCH H

$2241 \mathrm{SF} 11 \mathrm{MFHT}$
2242 LAWC-30C

$0.0037 \quad 0.0011$

$0.0037 \quad 0.0011$

2244 LAWC-93A

2245 LAWC-63A
2246 LAWC-63B

2247 LAWC-88A

2249 LAWC-99C

2250 LAWC-99B
2251 LAWBF-98E

2252 LAWBF-20F

2253 LAWBF-47B

2254 LAWBF-49C
2255 LAWBE-53C

2256 LAWBF-56E

2256 LAWBF-56
2257 LAWBF-23D

2258 LAWBF-27C

2260 LAWBF-47D

2261 LAWBF-62G

2262 LAWBE-70D
2263 HAHP

$2264 \mathrm{HAHF}$

2265 HALF
2266 Centroid
2267 AL001

2268 ALO02

2269 AL003

2271 AL005

2272 CR001

$2274 \mathrm{C} 80023$

$2275 \mathrm{CR} 004$
$2276 \mathrm{CR} 005$

$2277 \mathrm{FE} E 00$
$2278 \mathrm{FE} 002$

2278 FEO02
2279 FE003

$2280 \mathrm{FE} 004$

2281 MN001
2283 MN002

2283 MN002
22824 MN003

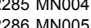

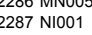

2288 N1000

$2289 \mathrm{~N} N 1003$
2290
$\mathrm{~N} 1004$

-2291 N1004
22905

$2292 \mathrm{POO}$

2223 P P02
2224 P003

22255 P POOA
2296 P005

$\begin{array}{lll}0.0037 & 0.0011 \\ 0.0037 & 0.0011\end{array}$

$0.0037 \quad 0.0011$

$\begin{array}{lll}0.0037 & 0.001 \\ 0.0037 & 0.001\end{array}$

$0.0037 \quad 0.001$

$0.0131 \quad 0.0008$

0.01310 .0008

0.01310 .0008

0.01310 .0008

$\begin{array}{llll}0.0066 & 0.00008\end{array}$

$\begin{array}{lll}0.0066 & 0.0008 \\ 0.0066 & 0.0008\end{array}$ 
2218 SF 1

$2219 \mathrm{SF} 2$

2220 SF 3
2221 SF 4
2222 SF 5

$2222 \mathrm{SF} 5$
$2223 \mathrm{SF} 6$

$2224 \mathrm{SF} 7$

$2225 \mathrm{SF} 8$

2227 SF 10A \#

2229 SF $10 \mathrm{~B}$ CFMUT

2230 SF 10B MFHT

2232 FRTI-III \#2, (CHI-VIT)

2233 SF $10 C$ CFMUT
2234 SF 10C MFHT

(235 SF 11 CFMUT

$2237 \mathrm{SF} 12$ BATCH\#2

2238 SF 12 BATCH\#3

2240 SF 12 BATCH

2241 SF 11 MFH

2243 LAWC-81B
2244 LAWC-93A

2245 LAWC-63A
2246 LAWC-63B

列

2248 LAWC-95D
2249 LAWC-99C

2250 LAWC-99B
2251 LAWBE-98E

2252 LAWBF-20F

2253 LAWBF-47B

2254 LAWBF-49C
2255 LAWBE-53C

2256 LAWBF-56E

2257 LAWBF-23D

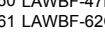

2262 LAWBF-70D

265 HALF

2266 Centroid

2268 AL002

2269 AL003

270 AL004

2272 CR001
2273 CR002

2274 CR003

2276 CR005

2278 FE002

2280 FE004

2281 FEN005

$2283 \mathrm{MNO02}$

$2825 \mathrm{MN} 004$
$2286 \mathrm{MNO05}$

$2288 \mathrm{~N} 1002$

$2290 \mathrm{~N} 1004$

2293 P002

$2296 \mathrm{P} 005$ $\begin{array}{lll}0.1390 & 0.0073 & 0.1221 \\ 0.1120 & 0.0083 & 0.126 \\ 0.1103 & 0.0080 & 0.1096\end{array}$

$\begin{array}{lll}0.1206 & 0.0087 & 0.1180 \\ 0 & 0.1120 & 0\end{array}$

$\begin{array}{lll}0.1024 & 0.0049 & 0.1151\end{array}$

$\begin{array}{lll}0.1050 & 0.0041 & 0.1150 \\ 0.1073 & 0.0060 & 0.1168\end{array}$

$\begin{array}{llll}0.1049 & 0.0047 & 0.1270\end{array}$

$\begin{array}{lll}0.0995 & 0.0060 & 0.1169 \\ 0 & 0.1099 & 0.0066\end{array}$

$\begin{array}{lll}0.1099 & 0.0066 & 0.1217 \\ 0.1099 & 0.0066 & 0.1217\end{array}$

$\begin{array}{lll}0.1144 & 0.0048 & 0.1226 \\ 0.1170 & 0.008 & 0.122\end{array}$

$\begin{array}{lll}0.1170 & 0.0048 & 0.1222 \\ 0.1107 & 0.0050 & 0.1129 \\ 0.107 & 0.005 & 0.129\end{array}$

$\begin{array}{lll}0.1056 & 0.0053 & 0.1127\end{array}$

$\begin{array}{lll}0.1107 & 0.0044 & 0.1280 \\ 0.1081 & 0.0050 & 0.1161 \\ 0.163 & 0.002 & 0\end{array}$

$\begin{array}{lll}0.1096 & 0.0052 & 0.1212 \\ 0.1053 & 0.055 & 0.1250\end{array}$

$\begin{array}{lll}0.1053 & 0.0051 & 0.1250 \\ 0.1057 & 0.0051 & 0.1234 \\ 0.1035 & 0.050 & 0.1200\end{array}$

$\begin{array}{lll}0.1057 & 0.0055 & 0.1234 \\ 0.1035 & 0.0050 & 0.12200\end{array}$

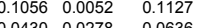

$\begin{array}{lll}0.0430 & 0.0278 & 0.0636 \\ 0.0450 & 0.0314 & 0.0663 \\ 0.0463 & 0.0326 & 0.0644\end{array}$

$\begin{array}{lll}0.0472 & 0.0334 & 0.0663\end{array}$

$\begin{array}{lll}0.0491 & 0.0351 & 0.0697 \\ 0.0453 & 0.0317 & 0.0650\end{array}$

$\begin{array}{lll}0.0450 & 0.0306 & 0.0661\end{array}$

$\begin{array}{lll}0.0786 & 0.0419 & 0.0574\end{array}$

$\begin{array}{lll}0.0807 & 0.0460 & 0.0611\end{array}$

$\begin{array}{lll}0.0803 & 0.0471 & 0.0621 \\ 0.0810 & 0.0417 & 0.0583\end{array}$

$\begin{array}{lll}0.0816 & 0.0428 & 0.0576 \\ 0.0820 & 0.035 & 0.0583\end{array}$

$\begin{array}{lll}0.0787 & 0.0457 & 0.0639\end{array}$

$\begin{array}{lll}0.0794 & 0.0457 & 0.0608 \\ 0.0766 & 0.0477 & 0.0032\end{array}$

$\begin{array}{lll}0.0766 & 0.0477 & 0.0632 \\ 0.1030 & 0.0043 & 0.1061 \\ 0.1026 & 0.0022 & 0.174\end{array}$

$\begin{array}{lll}0.1026 & 0.0022 & 0.1774\end{array}$

$\begin{array}{lll}0.1033 & 0.0063 & 0.1039 \\ 0.131 & 0.0054 & 0.1406\end{array}$

$\begin{array}{lll}0.1031 & 0.0054 & 0.1406 \\ 0.1032 & 0.0057 & 0.1488\end{array}$

$\begin{array}{lll}0.1032 & 0.0056 & 0.1447 \\ 0.1031 & 0.0054 & 0.1406\end{array}$

$\begin{array}{ll}0.0050 & 0.1267 \\ 0.0055 & 0.1417\end{array}$

$0.10310 .0055 \quad 0.1412$

$\begin{array}{lll}0.1131 & 0.0054 & 0.1406 \\ 0.1031 & 0.0054 & 0.1388\end{array}$

$\begin{array}{lll}0.1031 & 0.0054 & 0.1370 \\ 0.1034 & 0.0072 & 0.1039\end{array}$

$0.10310 .0054 \quad 0.1406$

$\begin{array}{lll}0.1032 & 0.0057 & 0.1478 \\ 0 & 0.1322 & 0.0056\end{array}$

$\begin{array}{lll}0.1031 & 0.0054 & 0.1406 \\ 0.1031 & 0.0053 & 0.1348\end{array}$

$0.10310 .0051 \quad 0.1290$

$\begin{array}{lll}0.1031 & 0.0055 & 0.1418\end{array}$

$\begin{array}{lll}0.1031 & 0.0054 & 0.1406 \\ 0.1031 & 0.0054 & 0.1387\end{array}$

$\begin{array}{lll}0.1031 & 0.0054 & 0.1368\end{array}$

$\begin{array}{lll}0.1032 & 0.0056 & 0.1452 \\ 0.1032 & 0.0055 & 0.1429\end{array}$

$\begin{array}{lll}0.1032 & 0.0055 & 0.1429 \\ 0.1031 & 0.0054 & 0.1406\end{array}$

$\begin{array}{lll}0.1031 & 0.0052 & 0.1340 \\ 0.1031 & 0.0050 & 0.1274\end{array}$ $\begin{array}{llllllll}0.0059 & 0.0071 & 0.0031 & 0.1352 & 0.0088 & 0.0290 & 0.4592 & 0.0270\end{array}$ $\begin{array}{lllllllll}0.0300 & 0.0281 & 0.0114 & 0.1055 & 0.0064 & 0.0238 & 0.4358 & 0.0278\end{array}$ $\begin{array}{llllllll}0.0228 & 0.0202 & 0.0082 & 0.1311 & 0.0073 & 0.0232 & 0.4666 & 0.0293 \\ 0 & 0.0235 & 0.029 & 0.029\end{array}$ $\begin{array}{lllllllll}0.0390 & 0.0342 & 0.0090 & 0.1131 & 0.0073 & 0.0255 & 0.4460 & 0.028\end{array}$ $\begin{array}{llllllllll}0.0439 & 0.0329 & 0.0105 & 0.1261 & 0.0069 & 0.0263 & 0.4245 & 0.0239\end{array}$ $\begin{array}{lllllll}0.0322 & 0.0294 & 0.0126 & 0.1062 & 0.0040 & 0.4447 & 0.0222\end{array}$ $\begin{array}{llllllll}0.0347 & 0.0306 & 0.0083 & 0.1160 & 0.024 & 0.0263 & 0.4473 & 0.0216\end{array}$ $\begin{array}{llllllll}0.0321 & 0.0294 & 0.0085 & 0.1201 & 0.0026 & 0.0225 & 0.4325 & 0.0228\end{array}$ $\begin{array}{llllllll}0.0321 & 0.0298 & 0.0082 & 0.1185 & 0.0026 & 0.0229 & 0.4316 & 0.0228 \\ 0.0368 & 0.0306 & 0.0099 & 0.1196 & 0.0022 & 0.0233 & 0.4339 & 0.0229\end{array}$ $\begin{array}{llllllll}0.0378 & 0.0316 & 0.0098 & 0.1168 & 0.0028 & 0.0292 & 0.4248 & 0.0193\end{array}$ $\begin{array}{llllllll}0.0351 & 0.0329 & 0.0093 & 0.1118 & 0.023 & 0.0248 & 0.4442 & 0.0191\end{array}$ $\begin{array}{lllllllll}0.0395 & 0.0308 & 0.0096 & 0.1160 & 0.0024 & 0.0249 & 0.4208 & 0.0243\end{array}$

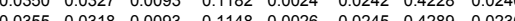

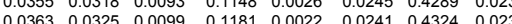
$\begin{array}{llllllll}0.0378 & 0.0316 & 0.0098 & 0.1168 & 0.0028 & 0.0292 & 0.4250 & 0.0193\end{array}$

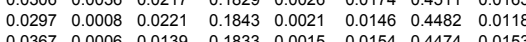
$\begin{array}{llllllll}0.0297 & 0.0011 & 0.0166 & 0.1843 & 0.0021 & 0.0151 & 0.4460 & 0.0154\end{array}$ $\begin{array}{llllllll}0.0392 & 0.0006 & 0.0183 & 0.2048 & 0.0017 & 0.0180 & 0.3916 & 0.0142 \\ 0.0361 & 0.0006 & 0.0189 & 0.1821 & 0.0017 & 0.0153 & 0.4454 & 0.0187\end{array}$ $\begin{array}{llllllll}0.0312 & 0.0008 & 0.0184 & 0.1843 & 0.0020 & 0.0148 & 0.4391 & 0.0173\end{array}$ $\begin{array}{llllllll}0.0233 & 0.0394 & 0.0205 & 0.0927 & 0.0010 & 0.0209 & 0.4173 & 0.0269\end{array}$ $\begin{array}{llllllll}0.0234 & 0.0388 & 0.0225 & 0.0896 & 0.0009 & 0.0225 & 0.4208 & 0.0281\end{array}$ $\begin{array}{llllllll}0.0231 & 0.0399 & 0.0214 & 0.0874 & 0.0012 & 0.0231 & 0.4262 & 0.0284 \\ 0.0224 & 0.0404 & 0.0214 & 0.0906 & 0.0010 & 0.0215 & 0.4160 & 0.0272\end{array}$ $\begin{array}{llllllll}0.0224 & 0.0404 & 0.0214 & 0.0906 & 0.0010 & 0.0215 & 0.4160 & 0.0272 \\ 0.0224 & 0.0398 & 0.0152 & 0.0920 & 0.0010 & 0.0207 & 0.4156 & 0.0274\end{array}$ $\begin{array}{llllllll}0.0384 & 0.0137 & 0.0947 & 0.0011 & 0.0219 & 0.4205 & 0.0269\end{array}$ $\begin{array}{llllllll}0.0229 & 0.0371 & 0.0235 & 0.0894 & 0.0017 & 0.0228 & 0.4101 & 0.0276 \\ 0.0194 & 0.0410 & 0.0224 & 0.0845 & 0.0010 & 0.0238 & 0.4262 & 0.0263\end{array}$

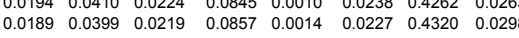
$\begin{array}{lllllll}0.0314 & 0.0135 & 0.1113 & 0.0029 & 0.0253 & 0.4538 & 0.018\end{array}$ $\begin{array}{llllllll}0.0362 & 0.0314 & 0.0130 & 0.1059 & 0.0011 & 0.0022 & 0.4522 & 0.0068\end{array}$ $\begin{array}{lllllllll}0.0367 & 0.0314 & 0.0137 & 0.1161 & 0.0045 & 0.00090 & 0.4552 & 0.0298 \\ 0.03141 & 0.038 & 0.0076 & 0.4546 & 0.0253\end{array}$ $\begin{array}{llll}0.0039 & 0.0078 & 0.4547 & 0.0260\end{array}$ $\begin{array}{lllllllll}0.0367 & 0.0314 & 0.0137 & 0.1141 & 0.0038 & 0.0076 & 0.4546 & 0.0253\end{array}$ $\begin{array}{llllllll}0.0367 & 0.0314 & 0.0136 & 0.1130 & 0.034 & 0.0069 & 0.4444 & 0.0242\end{array}$

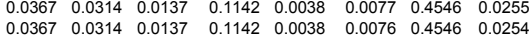
$\begin{array}{llllllllll}0.0367 & 0.0314 & 0.0137 & 0.1141 & 0.0038 & 0.0076 & 0.4546 & 0.0253\end{array}$ $\begin{array}{lllllll}0.0147 & 0.1140 & 0.0038 & 0.0075 & 0.4545 & 0.024\end{array}$ $\begin{array}{lllllllll}0.037 & 0.0314 & 0.0137 & 0.1138 & 0.0037 & 0.0074 & 0.4545 & 0.0246 \\ 0.0370 & 0.0314 & 0.0141 & 0.1185 & 0.0053 & 0.0105 & 0.4558 & 0.0352\end{array}$ $\begin{array}{lllllllll}0.0369 & 0.0314 & 0.0139 & 0.1163 & 0.00045 & 0.0091 & 0.4552 & 0.0302\end{array}$ $\begin{array}{llllllll}0.0367 & 0.0314 & 0.0137 & 0.1141 & 0.0038 & 0.0076 & 0.4546 & 0.0253 \\ 0.0364 & 0.0344 & 0.333 & 0.197 & 0.032 & 0.0047 & 0.4539 & 0.0203\end{array}$ $\begin{array}{lllllllll}0.0364 & 0.0314 & 0.0133 & 0.1119 & 0.0031 & 0.0002 & 0.4439 & 0.023 \\ 0.0023 & 0.0047 & 0.4533 & 0.015\end{array}$ $\begin{array}{llll}0.0078 & 0.4546 & 0.0259\end{array}$ $\begin{array}{llllllll}0.0367 & 0.0314 & 0.0137 & 0.1141 & 0.0038 & 0.0076 & 0.4546 & 0.0253 \\ 0.0367 & 0.0314 & 0.0137 & 0.1136 & 0.0036 & 0.0073 & 0.4544 & 0.0242\end{array}$ $\begin{array}{llllllll}0.0367 & 0.0314 & 0.0137 & 0.1132 & 0.0035 & 0.0070 & 0.4543 & 0.023\end{array}$ \begin{tabular}{lllllll}
0.0314 & 0.0137 & 0.1143 & 0.0001 & 0.00077 & 0.4546 & 0.025 \\
\hline & 0.0137 & 0.142 & 0.0019 & 0.0077 & 0.4546 & 0.025
\end{tabular} $\begin{array}{lllllllll}0.0367 & 0.0314 & 0.0137 & 0.1141 & 0.00038 & 0.0076 & 0.4546 & 0.0253\end{array}$ $\begin{array}{lllllllll}0.0367 & 0.0314 & 0.0137 & 0.1138 & 0.0101 & 0.0074 & 0.4545 & 0.0246\end{array}$ $\begin{array}{lllllllll}0.0368 & 0.0314 & 0.0138 & 0.1145 & 0.0039 & 0.0003 & 0.4547 & 0.026\end{array}$

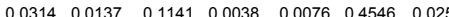
$\begin{array}{llllllll}0.0367 & 0.0314 & 0.0137 & 0.1136 & 0.0036 & 0.0184 & 0.4544 & 0.0240 \\ 0.0367 & 0.0314 & 0.0136 & 0.1130 & 0.0034 & 0.0293 & 0.4542 & 0.0228\end{array}$

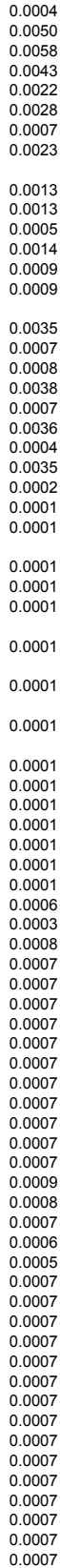

$\begin{array}{lll}0.0012 & 0.0012 & 0.0007\end{array}$ $\begin{array}{lll}0.0014 & 0.0009 & 0.0007 \\ 0.0009 & 0.0008 & 0.0000\end{array}$ \begin{tabular}{lll}
0.0010 & 0.0010 & 0.0005 \\
\hline & 00010 & 0.0006
\end{tabular} $\begin{array}{lll}0.0012 & 0.0010 & 0.000 \\ 0.0010 & 0.0009 & 0.0006\end{array}$ $\begin{array}{lll}0.0011 & 0.0010 & 0.000 \\ 0.0012 & 0.0012 & 0.0000\end{array}$ $\begin{array}{ll}0.0039 & 0.0004 \\ 0.0031 & 0.0004 \\ 0.0037 & 0.0005\end{array}$

$\begin{array}{ll}0.0034 & 0.0005 \\ 0.0043 & 0.0006\end{array}$ $\begin{array}{ll}0.0043 & 0.0006 \\ 0.0031 & 0.0006\end{array}$

$\begin{array}{ll}0.0034 & 0.0005\end{array}$

$0.0011 \quad 0.0003$

$\begin{array}{ll}0.0010 \quad 0.0007 \\ 0.0014 & 0.0004\end{array}$

$\begin{array}{ll}0.0014 & 0.0004 \\ 0.0028 & 0.0004 \\ 0.0010 & 0.005\end{array}$

$\begin{array}{ll}0.0004 & 0.0004 \\ 0.0022 & 0.0000 \\ 0.002 & 0.005\end{array}$

$\begin{array}{ll}0.0010 & 0.0005 \\ 0.0022 & 0.0005 \\ 0.0010 & 0.0005 \\ 0.0024 & 0.004\end{array}$ $\begin{array}{ll}0.0010 & 0.0005 \\ 0.0024 & 0.004\end{array}$ 
\# Glass ID 2215 A40-13a
2216 A40-17a

(2217 NBS-7 1

$2219 \mathrm{SF} 2$

$2220 \mathrm{SF} 3$

$2222 \mathrm{SF} 5$

$2224 \mathrm{SF}$

2226 SF 9

2228 SF $10 \mathrm{AA} \# 2$

$2230 \mathrm{SF}$ 10B MFHT

2231 FRTI-III \#1(CHI-VIT)

2233 SF 10C CFMUT

$2235 \mathrm{SF} 11$ CFMUT

2236 SF 12 BATCH\#

2238 SF 12 BATCH\#3

2240 SF 12 BATCH\#5

2241 SF 11 MFH

2245 LAWC-63A
2246 LAWC-63B

2247 LAWC-88A

2248 LAWC-95D

2250 LAWC-998
2251 LAWBF-98E

2252 LAWBF-20F

2253 LAWBF-47B

2254 LAWBF-49C

2256 LAWBF-56E

2257 LAWBF-23D

2258 LAWBF-27C

2260 LAWBF-47D

2261 LAWBF-62G

2263 HAHP

2264 HAHF

2266 Centroid

2268 AL002

2269 AL003

2271 ALO005
2272 CR001

27273 CR001
227302

2274 CR003

2276 CR005

2278 FE002

2280 FE004

(2282 MN001

$2283 \mathrm{MN002}$

$2285 \mathrm{MNO} 004$

$2287 \mathrm{~N} 1001$

$2290 \mathrm{N1003}$

$2291 \mathrm{~N} 1005$

$2292 \mathrm{P} 001$

2293 P0003

$2296 \mathrm{P} 005$
Ga203-a Gd2O3-a

I-a La203-a MnO2-a MnO-a MoO-a MoO3-a Nb205-a Nd203-a NpO2-a PbO-a PdO2-a PdO-a Pr203-a Pr6011-a PuO2-a Rb2O-a ReO-a ReO2-a Re207-a Rh203-a RhO2-a RuO2-a Sb203-a Sb205-a SeO2-a

\begin{tabular}{|c|c|c|c|}
\hline 0.0033 & & & \\
\hline & 0.0123 & 0.0007 & 0.0004 \\
\hline 0.0004 & 0.0133 & 0.0007 & 0.0004 \\
\hline & & & \\
\hline 0.0009 & 0.018 & & 0.0008 \\
\hline 0.0012 & 0.0135 & 0.00000 & 0.0000 \\
\hline 0.0009 & $\begin{array}{l}0.0237 \\
0.0133\end{array}$ & $\begin{array}{l}0.0000 \\
0.000\end{array}$ & 0.00000 \\
\hline 0.0002 & $\begin{array}{l}0.0116 \\
0.0001\end{array}$ & 0.0000 & \\
\hline 0.0002 & 0.0091 & 0.0000 & 0.0012 \\
\hline 0.0003 & 0.0044 & 0.0000 & 0.0015 \\
\hline 0.0003 & 0.0032 & 0.0000 & 0.0016 \\
\hline $\begin{array}{l}0.0005 \\
0.0005\end{array}$ & 0.0104 & 0.0005 & 0.0020 \\
\hline & & & \\
\hline 0.0004 & 0.0100 & 0.0064 & 0.0012 \\
\hline $\begin{array}{l}.0005 \\
0.0003\end{array}$ & 0.0105 & 0.0007 & 0.0012 \\
\hline 0.00002 & 0.0099 & 0.0005 & 0.0016 \\
\hline 0.0002 & 0.0100 & $\begin{array}{l}0.0000 \\
0.0005\end{array}$ & $\begin{array}{l}0.0013 \\
0.0011\end{array}$ \\
\hline 0.0004 & 0.0101 & 0.0006 & 0.0014 \\
\hline 0.0002 & 0.0099 & 0.0004 & 0.0010 \\
\hline & & & \\
\hline
\end{tabular}

$\begin{array}{ll}0.0003 & 0.0086 \\ 0.0001 & 0.0031 \\ 0.0005 & 0.0114\end{array}$

$0.0005 \quad 0.0135$

$\begin{array}{ll}0.0004 & 0.011 \\ 0.0005 & 0.012 \\ 0.0004 & 0.0118 \\ 0.0004 & 0.0114\end{array}$

$\begin{array}{ll}0.0004 & 0.0118 \\ 0.0004 & 0.0114 \\ 0.0004 & 0.0110\end{array}$

$\begin{array}{ll}0.0004 & 0.0110 \\ 0.0004 & 0.0103 \\ 0.0004 & 0.0115\end{array}$

$\begin{array}{ll}0.0004 & 0.0115 \\ 0.0004 & 0.0115 \\ 0.0004 & 0.114\end{array}$

$0.0004 \quad 0.0114$

$0.0004 \quad 0.0111$

$0.0006 \quad 0.015$

$\begin{array}{ll}0.0005 & 0.0137 \\ 0.0004 & 0.0114 \\ 0.0004 & 0.0092\end{array}$

$\begin{array}{ll}0.0004 & 0.0092 \\ 0.0003 & 0.0070\end{array}$

$0.0005 \quad 0.000$

$\begin{array}{ll}0.0004 & 0.0114 \\ 0.0004 & 0.0208\end{array}$

$\begin{array}{lll}0.0004 & 0.0208 \\ 0.0004 & 0.0301\end{array}$

$0.0004 \quad 0.0116$

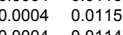

$\begin{array}{ll}0.0004 & 0.0113 \\ 0.0004 & 0.011\end{array}$

$\begin{array}{ll}0.0004 & 0.0111 \\ 0.0004 & 0.0118 \\ 0.004 & 0.011\end{array}$

$\begin{array}{ll}0.0004 & 0.011 \\ 0.0004 & 0.011 \\ 0.0004 & 0.011\end{array}$

$\begin{array}{ll}0.0004 & 0.0109 \\ 0.0004 & 0.0103\end{array}$
0.0110

$\begin{array}{ll}0.0002 & 0.0007 \\ 0.0001 & 0.0002 \\ 0.0003 & 0.0011\end{array}$

$\begin{array}{lll}0.0003 & 0.0009 & 0.0001 \\ 0.0003 & 0.0010 & 0.0001\end{array}$

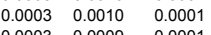

$\begin{array}{lll}0.0003 & 0.0009 & 0.001 \\ 0.0003 & 0.0009 & 0.0001\end{array}$

$\begin{array}{lll}.0002 & 0.0008 & 0.0001 \\ 0.003 & 0.0009 & 0.0001\end{array}$

$\begin{array}{lll}0.0003 & 0.0010 & 0.0001 \\ 0.0003 & 0.0009 & 0.001\end{array}$

$\begin{array}{lll}0.0003 & 0.0009 & 0.0001 \\ 0 & 0.0001\end{array}$

$\begin{array}{lll}0.0003 & 0.0009 & 0.0001 \\ 0.0004 & 0.0013 & 0.001\end{array}$

$\begin{array}{lll}0.0004 & 0.0013 & 0.001 \\ 0.0003 & 0.0011 & 0.0001\end{array}$

$\begin{array}{lll}0.0003 & 0.0009 & 0.0001 \\ 0.0002 & 0.0007 & 0.0001 \\ 0.002 & 0.006 & 0.001\end{array}$

$\begin{array}{lll}0.0002 & 0.0006 & 0.0001 \\ 0.0003 & 0.0010 & 0.0001 \\ 0.003 & 0.009 & 0.001\end{array}$

$\begin{array}{lll}0.0003 & 0.0009 & 0.0001\end{array}$

$\begin{array}{lll}0.0003 & 0.0009 & 0.0001 \\ 0.0003 & 0.0009 & 0.0001\end{array}$

$\begin{array}{lll}0.0002 & 0.0008 & 0.0001\end{array}$

$\begin{array}{lll}0.0003 & 0.0009 & 0.0001 \\ 0.0003 & 0.0009 & 0.0001\end{array}$

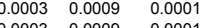

$\begin{array}{lll}0.0003 & 0.0009 & 0.0001\end{array}$

$\begin{array}{lll}0.0003 & 0.0010 & 0.0001 \\ 0.0003 & 0.0009 & 0.001\end{array}$

$\begin{array}{lll}0.0003 & 0.0009 & 0.0001 \\ 0.0002 & 0.0008 & 0.000\end{array}$ 
$\begin{array}{ll}0.0024 & 0.0001 \\ 0.0010 & 0.0001\end{array}$ 0.0000

$2220 \mathrm{SF} 3$

$2222 \mathrm{SF} 5$
$2223 \mathrm{SF} 6$

$2224 \mathrm{SF} 7$
2225 SF 8

$226 \mathrm{SF} 9$

2227 SF 10A \#1

2228 SF 1OA \#2
2229 SF 10B CFMUT
2230 SF 10B MFHT

2231 FRTI-III \#1(CHI-VIT)

2232 FRTI-III \#2, (CHI-VTT)

2234 SF $10 C$ MFHT

2236 SF 12 BATCH\#

2238 SF 12 BATCH\#3

2220 SF 12 BATCH

$2241 \mathrm{SF} 11 \mathrm{MFHT}$

2242 LAWC-30C

2244 LAWC-93A

2245 LAWC-63A
2246 LAWC-63B

2247 LAWC-88A

2248 LAWC-95D

2250 LAWC-998
2251 LAWBF-98E

2252 LAWBF-20F

2253 LAWBF-47B

2254 LAWBF-49C

2256 LAWBF-56E

2257 LAWBF-23D

2259 LAWBF-35

2261 LAWBF-62C

2262 LAWBF-7
2263 HAHP

2264 HAHF

2265 HALF

2267 AL001

2268 AL002
2269 AL003

2270 AL004

2272 CR001

22274 CR003

2275 CR004
2276 CR005

2277 FE001

2278 FE002

$2280 \mathrm{FE} 004$

2282 MNNO1

$2284 \mathrm{MNO03}$

2286 MN005

2287 N1001

(2288 $\mathrm{N} 1002$

$22901 \mathrm{~N} 1004$

$2292 \mathrm{P} 00$

$2293 \mathrm{P} 000$

$2295 \mathrm{P} 004$

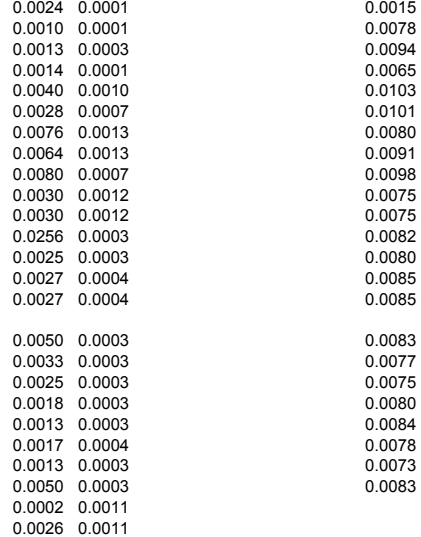

$\begin{array}{ll}0.0001 & 0.9997 \\ 0.0001 & 0.9990 \\ 0.00001 & 0.9955\end{array}$

0.9665

0.00020 .9983

$\begin{array}{ll}0.0001 & 0.9971 \\ 0.0012 & 1.0078 \\ 0.0008 & 0.9981\end{array}$

$\begin{array}{ll}0.9652 \\ 0.0049 & 1.0001 \\ 0.0049 & 1.0001\end{array}$

$0.0003 \quad 1.0230$

0.00031 .0038

\begin{tabular}{ll}
0.0006 & 1.0000 \\
0.0006 & 1.0000 \\
\hline & 0.0000
\end{tabular}

$\begin{array}{lll}0.0004 \quad 1.0056 & 0.000\end{array}$

$\begin{array}{ll}0.0038 & 0.9999 \\ 0.0010 & 0.9999\end{array}$

$0.0002 \quad 0.9997$

$0004 \quad 0.9999$

$\begin{array}{lll}0.0038 & 0.9999 \\ 0.004 \quad 0.9999\end{array}$

0.02991 .0034

$\begin{array}{lll}0.0364 & 1.0054\end{array}$

$\begin{array}{ll}0.0359 & 0.0000 \\ 0.0374 & 1.0046\end{array}$

$\begin{array}{lll}0.0374 & 1.0034 \\ 0.0353 & 1.0064\end{array}$

$\begin{array}{ll}0.0026 & 0.0012\end{array}$

$\begin{array}{lll}0.0010 & 0.0012\end{array}$

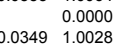

$\begin{array}{lll}0.0352 \quad 0.9390 & 0.0000 \\ 0.0353 & 0.0574\end{array}$

$0.0067 \quad 0.0003$

$0.0074 \quad 0.0008$

$\begin{array}{ll}0.0000 \\ 0.0353 \quad 0.9574 \\ 0.0554 & 0.9620\end{array}$

$0.0354 \quad 0.9620$

0.03540 .9436

0.03390 .9645

$\begin{array}{lll}0.0075 & 0.0008\end{array}$

$\begin{array}{lll}0.0038 & 0.0009 \\ 0.010 & 0.009\end{array}$

$\begin{array}{ll}0.0028 & 0.0003 \\ 0.0010 & 0.0001\end{array}$

.0102
.0102

$\begin{array}{lll}0.0002 & 0.0200 & 1.0196 \\ 0.0000 & 0.0001 & 0.9993 \\ 0.002 & 0.004 & 0.96\end{array}$

$\begin{array}{lll}0.0000 & 0.0001 & 0.9993 \\ 0.0002 & 0.0004 & 0.9996 \\ 0.0001 & 0.0003 & 0.9998\end{array}$

$\begin{array}{lll}0.0001 & 0.0003 & 0.9998 \\ 0.0002 & 0.0003 & 1.0002\end{array}$

$\begin{array}{lll}0.0002 & 0.0003 & 0.9999 \\ 0.0001 & 0.0003 & 0.9998 \\ 0.002 & 0.003 & 1.0000\end{array}$

(1)

$\begin{array}{llll}0.0002 & 0.0003 & 0.9998 \\ 0.0002 & 0.0003 & 1.0002 \\ 0.0002 & .0 .003 & 1.000\end{array}$

$\begin{array}{lll}0.001 & 0.0003 & 0.9998 \\ 0.002 & 0.0003 & 1.0000\end{array}$

$\begin{array}{llll}0.0002 & 0.0003 & 1.0002\end{array}$

$\begin{array}{llll}0.0003 & 0.0004 & 0.9999 \\ 0.0002 & 0.0004 & 0.9998\end{array}$

$\begin{array}{llll}0.0002 & 0.0004 & 0.9998 \\ 0.0001 & 0.0003 & 0.9998\end{array}$

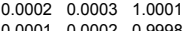

$\begin{array}{llll}0.0002 & 0.0003 & 1.0003\end{array}$

$\begin{array}{lll}0.0002 & 0.0003 & 0.9999 \\ .0001 & 0.0003 & 0.9998\end{array}$

$\begin{array}{lll}0.0002 & 0.0003 & 1.0001 \\ 0.0002 & 0.0003 & 1.0001\end{array}$

$\begin{array}{llll}0.0002 & 0.0003 & 1.0001 \\ 0.0002 & 0.0003 & 0.9999\end{array}$

$\begin{array}{lll}0.0002 & 0.0003 & 0.9999 \\ 0.0001 & 0.0003 & 0.9998\end{array}$

$\begin{array}{lll}.0002 & 0.0003 & 1.0000 \\ 0.002 & 0.0003 & 1.000\end{array}$

$\begin{array}{lll}0.0002 & 0.0003 & 1.0002\end{array}$

$\begin{array}{llll}0.0001 & 0.0003 & 0.9998\end{array}$

$\begin{array}{lll}0.0002 & 0.0003 & 1.000 \\ 0.0002 & 0.0003 & 0.999\end{array}$ 


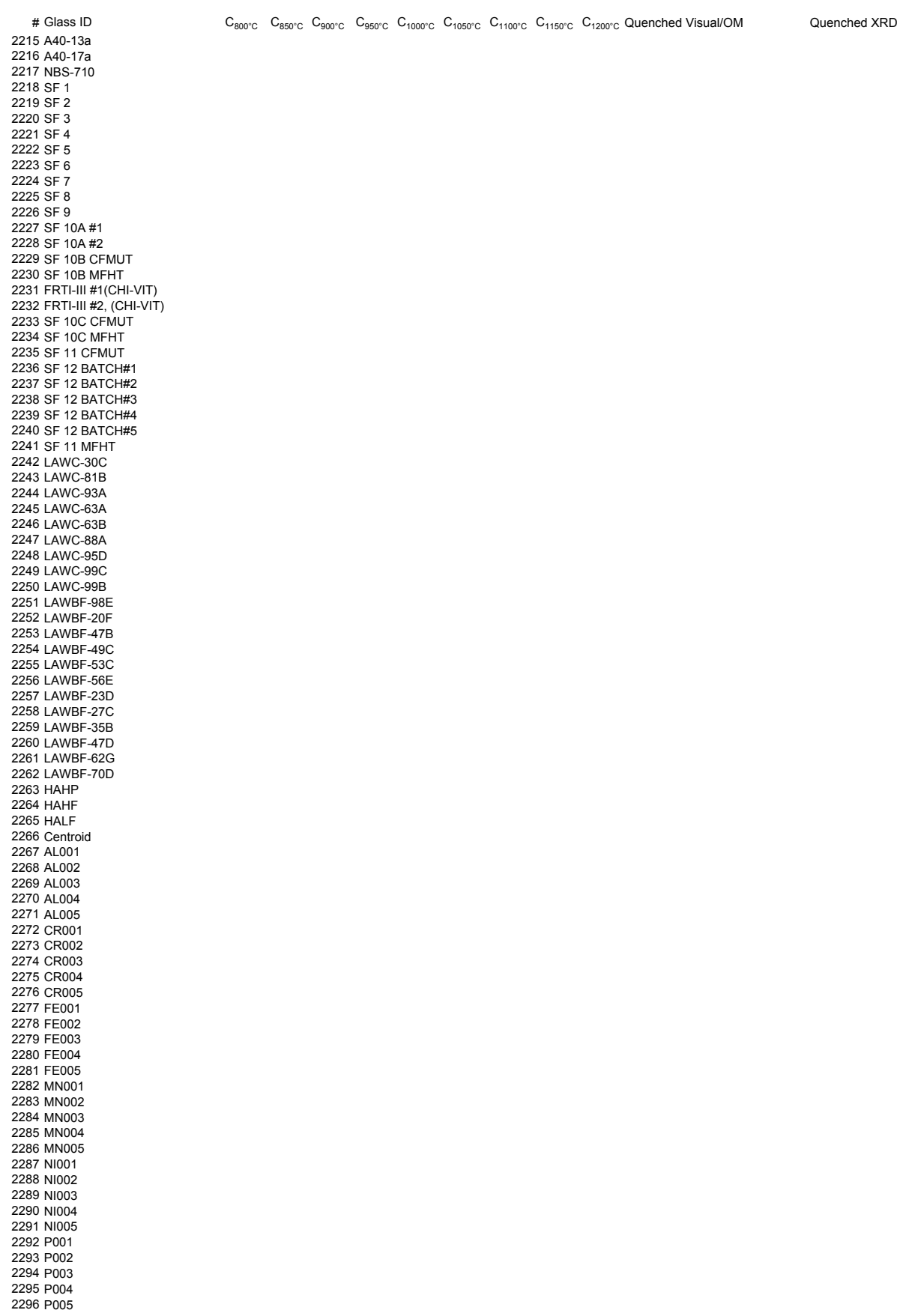

\begin{tabular}{|c|c|c|c|c|}
\hline $\begin{array}{l}2.740 \\
2.740 \\
2.730\end{array}$ & $\begin{array}{l}-3.420 \\
-3.370 \\
-1.520\end{array}$ & $\begin{array}{l}5556.2 \\
5556.2 \\
2500.0\end{array}$ & $\begin{array}{l}233.7 \\
169.1 \\
509.8\end{array}$ & $\begin{array}{l}1100.0 \\
110.0 \\
1100.0\end{array}$ \\
\hline $\begin{array}{l}2.7 .700 \\
2.2900 \\
2.770 \\
2.710\end{array}$ & $\begin{array}{l}-3.040 \\
-3.120 \\
0.440 \\
0.4460\end{array}$ & $\begin{array}{l}5556.0 \\
5566.2 \\
427.7 \\
447.5\end{array}$ & $\begin{array}{l}148.8 \\
179.1 \\
957.6 \\
944.5\end{array}$ & $\begin{array}{l}1100.0 \\
1100.0 \\
1100.0 \\
11100.0\end{array}$ \\
\hline $\begin{array}{l}2.720 \\
2.7720 \\
2.7700 \\
2.760\end{array}$ & $\begin{array}{l}-1.840 \\
0.440 \\
-3.04040 \\
-3.1 .100\end{array}$ & $\begin{array}{r}3337.0 \\
393.8 \\
558.0\end{array}$ & $\begin{array}{l}385.0 \\
955.5 \\
148.2 \\
133.3\end{array}$ & 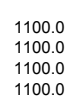 \\
\hline $\begin{array}{l}2.700 \\
2.760 \\
2.730 \\
2.720\end{array}$ & $\begin{array}{l}-.397 \\
-2.949 \\
-2.9990 \\
-3.050\end{array}$ & $\begin{array}{l}383.1 \\
5556.0 \\
5550.0 \\
5556.0\end{array}$ & $\begin{array}{l}951.7 \\
155.5 \\
99.1 \\
137.7\end{array}$ & $\begin{array}{l}1100.0 \\
1100.0 \\
110.0 \\
1100.0\end{array}$ \\
\hline $\begin{array}{l}2.730 \\
2.7780 \\
2.690 \\
2.720\end{array}$ & $\begin{array}{l}-3.040 \\
-3.020 \\
-0.340 \\
-0.430\end{array}$ & $\begin{array}{r}5556.0 \\
5555.0 \\
1111.8 \\
117.0\end{array}$ & $\begin{array}{l}144.6 \\
147.3 \\
775.5 \\
759.3\end{array}$ & $\begin{array}{l}110.0 .0 \\
110.0 \\
Z 110.0 \\
1100.0\end{array}$ \\
\hline $\begin{array}{l}2.720 \\
2.700 \\
2.7101 \\
2.720\end{array}$ & $\begin{array}{l}-0.425 \\
-0.510 \\
-\begin{array}{l}-.8 .890 \\
-3.080\end{array}\end{array}$ & $\begin{array}{l}1116.0 \\
11640.0 \\
3337.0 \\
5556.0\end{array}$ & $\begin{array}{l}761.0 \\
777.5 \\
384.6 \\
139.2\end{array}$ & 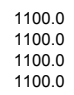 \\
\hline $\begin{array}{l}2.720 \\
27200\end{array}$ & $\begin{array}{r}-1.850 \\
-2.060\end{array}$ & $\begin{array}{r}3337.0 \\
40000\end{array}$ & $\begin{array}{l}368.9 \\
2187\end{array}$ & $\begin{array}{l}1100.0 \\
1000\end{array}$ \\
\hline
\end{tabular}




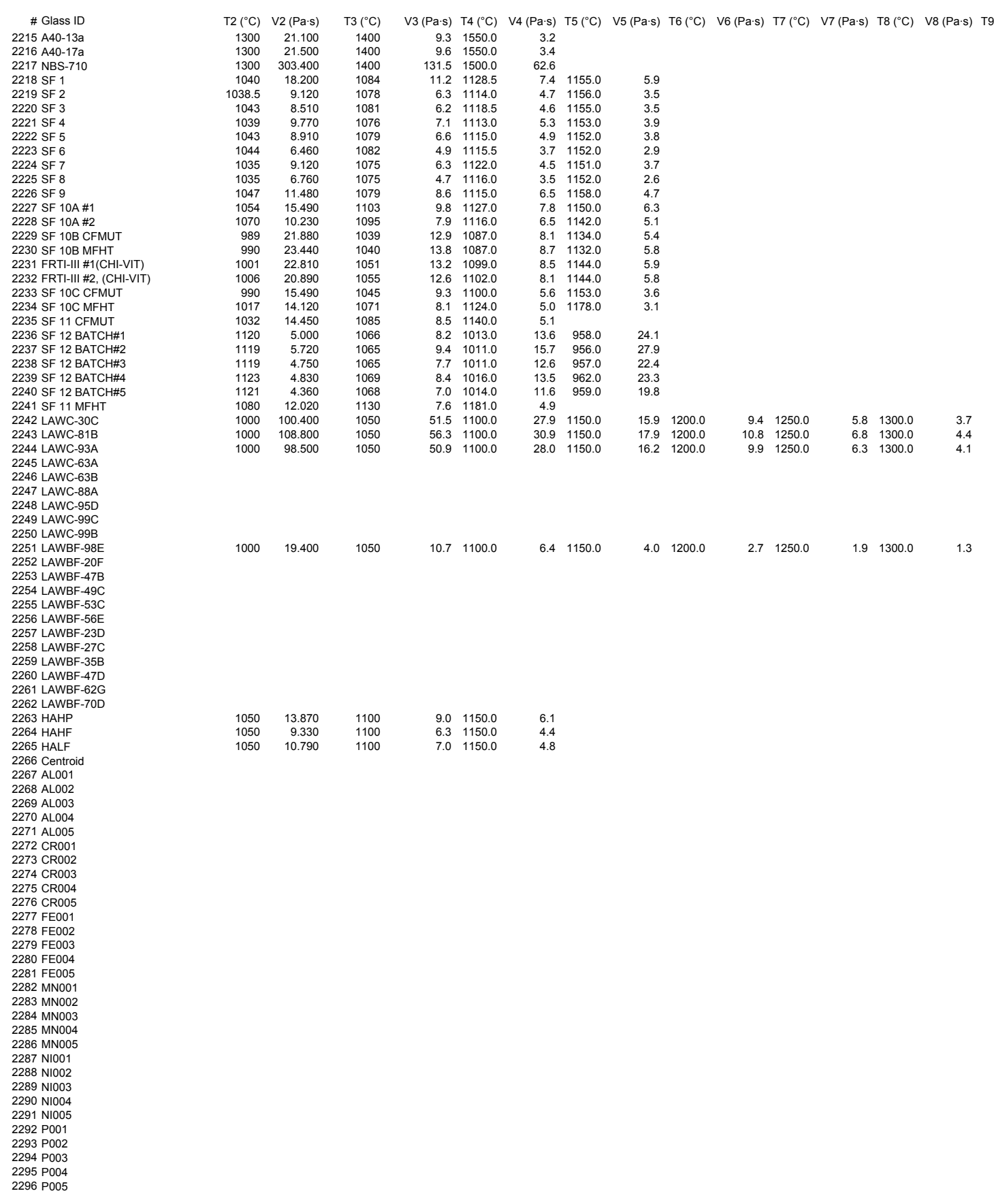


$2227 \mathrm{SF} 10 \mathrm{~A} \#$

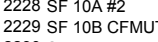

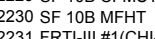

2231 RRT-1IIII(CHI-VIT)

2233 SF $10 \mathrm{C}$ CFMUT
2234 SF $10 \mathrm{CMFHT}$

2235 SF 11 CFMUT

2237 SF 12 BATCH 2

2238 SF 12 BACCHA3
2239 SF 12 BATCHHA

$2240 \mathrm{SF} 12 \mathrm{BATCH}$

2241 SF 11 MFHT

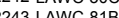

2244 LAWC-93A
2245 LAWC-63A

2245 LAWC-63A
22244 LAAC- -638
-227

2247 AAWC-88A
2248 LAWC-950

2248 AAWC-95D
2249 LACC.99C
2255 LACG

2250 LAWC-998
2251 LAWBF-985E

2252 LAWBF-20\%

2252 LAWBF-20F
22535 LAWBF-47B

2255 LANBFF-49C
2255 LAWBE-53C

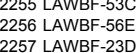

\begin{tabular}{l}
2255 LAABFF-27 \\
2259 LAWB- 258 \\
\hline
\end{tabular}

2260 LAWBF-47D

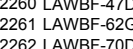

2262 LAWBF-70
2263 AAAP

2264 HAHF

2265 A ALF
226 Centroid
2267 A 20101

2267 ALO01
2268 ALL02

22689 ALLO02
22603

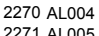

2272 CRO01

$2274 \mathrm{C} 8003$

$2275 \mathrm{CR} 004$
$2276 \mathrm{CR} 005$

2277 FE001
2278 FE002

2278 FEE002
2279 FE003

2280 FE004

$2282 \mathrm{MNO0}$

$2283 \mathrm{MN002}$

$2284 \mathrm{MNO03}$
$2225 \mathrm{MNN04}$

2287 N1001

2288 N1000

$2289 \mathrm{~N} N 1003$
2290
$\mathrm{~N} 1004$

2290 NN1004
22291 N1005

2291 N N1005
2922001
2293

$22239 \mathrm{P} 002$
2294
22003

2295 P 004
2296 P P005

1.78
4.37

1.9
1.18

1.314
1.88

1.381
1.293
1.448
1.38

1.448
1.328 $\begin{array}{lll}0.000 & 0.039 & 0.005 \\ 0.076 & 0.039 & 0.005 \\ 0.030 & 0.031 & 0.006\end{array}$

$0.016-0.030-0.007$

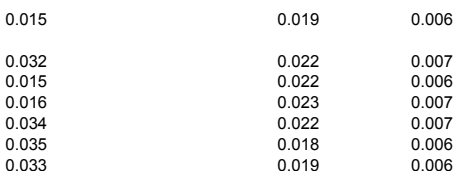


2216 A40-17a

2217 NBS-710

$2219 \mathrm{SF} 2$

2220 SF 3
2221 SF 4
2222 SF 5

$2223 \mathrm{SF} 6$

$2224 \mathrm{SF} 7$
$2225 \mathrm{SF} 8$
$2226 \mathrm{SF} 9$

2226 SF 9

2228 SF 10A \#2

$2229 \mathrm{SF}$ 10B CFMUT
$2230 \mathrm{SF} 10 \mathrm{~B} \mathrm{MFHT}$
$2231 \mathrm{FRTH}-\mathrm{III} \# 1$ (CHI

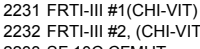

2233 SF $10 C$ CFMUT
2234 SF 10C MFHT

2235 SF 11 CFMUT

2236 SF 12 BATCHA

2238 SF 12 BATCH

2239 SF 12 BATCH\#

2241 SF 11 MFHT

2242 LAWC-30C

2244 LAWC-93A

2245 LAWC-63A

2246 LAWC-63B

2248 LAWC-95D

2249 LAWC-99C

2250 LAWC-99B

2252 LAWBF-20F

2253 LAWBF-47B

2254 LAWBF-49C

2256 LAWBFF-56E

2256 AAWBF-56
2255 LAWBF-23D

2258 LAWBF-27C
2259 LAWBF-35B

2260 LAWBF-47D

2261 LAWBF-62C

2262 LAWBF-70D

$2264 \mathrm{HAHF}$

2265 HALF

2266 Centroid

2268 AL002

22689 ALLO02
22603

2270 AL004
2271 AL 005

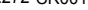

$2273 \mathrm{CRO02}$
$2274 \mathrm{CRO03}$

$2275 \mathrm{CR} 004$
$2276 \mathrm{CR} 005$

2277 FEE001
2278 FE002

2278 FE002
2279 FE 003

2280 FE004

2282 MNO01

2283 MNoor

2285 MNO04

2288 N1000

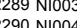

2291 N1005

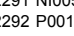

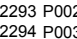

$2295 \mathrm{P} 004$

2296 P005 


\begin{tabular}{|c|c|c|c|}
\hline \# Glass ID & Study & \multicolumn{2}{|l|}{ Reference } \\
\hline 2297 Frit 18 & Savannah River & \multirow{2}{*}{\multicolumn{2}{|c|}{$\begin{array}{l}\text { Plodinec } 1978 \\
\text { Plodinec } 1978\end{array}$}} \\
\hline 2298 Frit $18,25 \mathrm{HI}$ & Savannah River & & \\
\hline $\begin{array}{l}2299 \text { Frit } 21 \\
230 \text { F rit } 21 \\
25 \mathrm{HI}\end{array}$ & Savannah River & \multicolumn{2}{|l|}{ Plodinec 1978} \\
\hline $\begin{array}{l}2300 \text { Frit } 21,25 \mathrm{HI} \\
2301 \text { Frit } 21,3 \mathrm{HI}\end{array}$ & Savannah River & \multicolumn{2}{|l|}{$\begin{array}{l}\text { Plodinec } 1978 \\
\text { Pldinec } 1978\end{array}$} \\
\hline $\begin{array}{l}23002 \text { Frit 21, } 21,10 \mathrm{HI} \\
23 \mathrm{HA}\end{array}$ & $\begin{array}{l}\text { Savannahh River } \\
\text { Savannah River }\end{array}$ & \multicolumn{2}{|l|}{$\begin{array}{l}\text { Plodinec } 1978 \\
\text { Plodinec } 1978\end{array}$} \\
\hline 2303 Frit 21, 15 HA & $\begin{array}{l}\text { Savannann River } \\
\text { Savannah River }\end{array}$ & \multicolumn{2}{|l|}{$\begin{array}{l}\text { Plodinec } 1978 \\
\text { Plodinec } 1978\end{array}$} \\
\hline 304 Frit 21, $20 \mathrm{HA}$ & Savannah River & \multicolumn{2}{|l|}{$\begin{array}{l}\text { Plodinec } 1978 \\
\text { Plodinec } 1978\end{array}$} \\
\hline 05 Frit 21, $25 \mathrm{HA}$ & Savannah River & \multicolumn{2}{|l|}{$\begin{array}{l}\text { Plodinec } 1978 \\
\text { Plof }\end{array}$} \\
\hline 2306 Frit $21,35 \mathrm{HA}$ & Savannah River & \multicolumn{2}{|l|}{ Plodinec 1978} \\
\hline 2307 Frit 18, $25 \mathrm{HA}$ & Savannah River & \multicolumn{2}{|l|}{ Plodinec 1978} \\
\hline $\begin{array}{l}2308 \text { Frit } 18,25 \text { SA } \\
230 \text { F rit } 1835 \text { SA }\end{array}$ & Savannah River & Plodinec 1978 & \\
\hline $\begin{array}{l}2309 \text { F rit } 18,35 \text { SA } \\
2310 \text { Frit } 21,25 \mathrm{SA}\end{array}$ & Savannah River & Plodinec 1978 & \\
\hline 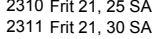 & $\begin{array}{l}\text { Savannahh River } \\
\text { Savannah River }\end{array}$ & $\begin{array}{l}\text { Plodinec } 1978 \\
\text { Plodinec } 1978\end{array}$ & \\
\hline 2312 Frit 18,25 SC & $\begin{array}{l}\text { Savannannah River } \\
\text { Savanah }\end{array}$ & Plodinec 1978 & \\
\hline 2313 Frit 21,20 SC & Savannah River & Plodinec 1978 & \\
\hline 314 Frit $21,25 \mathrm{SC}$ & & Plodinec 1978 & \\
\hline 315 Frit $21,30 \mathrm{SC}$ & Savannah River & Plodinec 1978 & \\
\hline 316 HT-S-1 & WVNS Production Glass & Reimus et al. & 1988 \\
\hline 2317 HT-S-2 & WVNS Production Glass & Reimus et al. & 1988 \\
\hline $\begin{array}{l}2318 \text { HT-S-3 } \\
2319 \text { HTS-S-4 }\end{array}$ & WVNS Production Glass & Reimus et al. & \\
\hline $\begin{array}{l}2319 \text { HTTS-S } \\
2320 \text { HT }-\mathrm{S}-5\end{array}$ & $\begin{array}{l}\text { WVNS Production Glass } \\
\text { WVNS Production Glass }\end{array}$ & $\begin{array}{l}\text { Reimus et al. } \\
\text { Reimus et al }\end{array}$ & $\begin{array}{l}1988 \\
1988\end{array}$ \\
\hline 2321 HT-S-6 6 & $\begin{array}{l}\text { WVNS Production Glass } \\
\text { WVNS Production Glass }\end{array}$ & $\begin{array}{l}\text { Reimus et al. } \\
\text { Reimus t al }\end{array}$ & $\begin{array}{l}1988 \\
1988\end{array}$ \\
\hline 2322 AL1TC34 & WVNS Production Glass & Reimus et al. & $\begin{array}{l}1988 \\
1988\end{array}$ \\
\hline 2323 AL1TC36 & WVNS Production Glass & Reimus et al. & 1988 \\
\hline 2324 AL4TC34 & WVNS Production Glass & Reimus et al. & 1988 \\
\hline 2325 AL5TC34 & WVNS Production Glass & Reimus et al. & 1988 \\
\hline 2326 AL9TC27 & WVNS Production Glass & Reimus et al. & 1988 \\
\hline $\begin{array}{l}2327 \text { HTS1 } \\
2328 \text { HTS? }\end{array}$ & 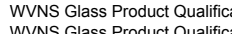 & : Reimus et al. & 1988 \\
\hline $\begin{array}{l}2328 \text { HTS2 } \\
2329 \text { HTS } 3\end{array}$ & 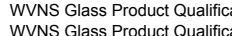 & $\begin{array}{l}\text { Reimus et al. } \\
\text { Reimus et al }\end{array}$ & 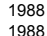 \\
\hline $\begin{array}{ll}23293 \mathrm{HTS} 3 \\
230 \mathrm{HTS} 4\end{array}$ & $\begin{array}{l}\text { WNS Glass Product Qualifici } \\
\text { wWNS Glass Product }\end{array}$ & $\begin{array}{l}\text { Reimus et al. } \\
\text { Reimus et al. }\end{array}$ & $\begin{array}{l}1988 \\
1988\end{array}$ \\
\hline 2331 HTS5 & 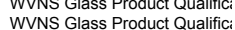 & $\begin{array}{l}\text { CiReemus et al. } \\
\text { CiReimus et al. }\end{array}$ & $\begin{array}{l}1988 \\
1988\end{array}$ \\
\hline $\begin{array}{l}2332 \text { HTS6 } \\
23150\end{array}$ & WVNS Glass Product Qualifici & & 1988 \\
\hline 2333 HTS7 & WVNS Glass Product Qualifici & : Reimus et al. & 1988 \\
\hline 2334 HTS8 & WVNS Glass Product Quallific & & 1988 \\
\hline 2335 HTS9 & WVNS Glass Product Qualifici & Reimus et al. & 1988 \\
\hline 2336 HTS10 & WVNS Glass Product Qualifici & ci Reimus et al. & 1988 \\
\hline $\begin{array}{l}2337 \text { HTS11 } \\
2338 \text { HTS12 }\end{array}$ & 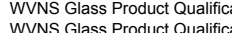 & c: Reimus et al. & 1988 \\
\hline $\begin{array}{l}2338 \text { HTS } 12 \\
2339 \text { HTS13 }\end{array}$ & $\begin{array}{l}\text { WNS Glass Product Qualificici } \\
\text { WVNS G Gass Product Qualia }\end{array}$ & ci Reimus et al. & 1988 \\
\hline $\begin{array}{l}2399 \mathrm{HIST} 13 \\
2340 \mathrm{HTS} 14\end{array}$ & $\begin{array}{l}\text { WNS Glass Product } \\
\text { WNalfificics }\end{array}$ & $\begin{array}{l}\text { CiReimus et al. } \\
\text { ci Reimus et al. }\end{array}$ & $\begin{array}{l}1988 \\
1988\end{array}$ \\
\hline 2341 HTS15 & WVNS Glass Product Qualifici & & 1988 \\
\hline $2342 \mathrm{HTS} 16$ & WVNS Glass Product Qualifici & ci Reimus et al. & 1988 \\
\hline 2343 HTS17 & WVNS Glass Product Quallific & & 1988 \\
\hline $2344 \mathrm{HTS} 18$ & WVNS Glass Product Qualifici & c: Reimus et al. & 1988 \\
\hline 2345 HTS 19 & WVNS Glass Product Qualifici & c: Reimus et al. & 1988 \\
\hline 2346 HTS20 & WWNS Glass Product Qualifici & ci Reimus et al. & 1988 \\
\hline $\begin{array}{l}2347 \text { HTS21 } \\
2348 \text { HTS22 }\end{array}$ & $\begin{array}{l}\text { WNS Glass Product Qualificici } \\
\text { WVNS G Glass Product Qualici }\end{array}$ & $\begin{array}{l}\text { ciReimus et al. } \\
\text { cReimus etal. }\end{array}$ & $\begin{array}{l}1988 \\
1988\end{array}$ \\
\hline $\begin{array}{l}2349 \text { WVRefGlass } \\
2349\end{array}$ & 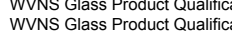 & $\begin{array}{l}\text { CiReimus et al. } \\
\text { ciReimus tal. }\end{array}$ & $\begin{array}{l}1988 \\
1988\end{array}$ \\
\hline 2350 SM513FR & Pamela Testing & Scheiwer 198 & \\
\hline 351 SM513LW08 & Pamela Testing & Scheiwer 198 & \\
\hline 352 SM513LW11 & Pamela Testing & Scheiwer 198 & \\
\hline 2353 SM513LW14 & Pamela Testing & Scheiwer 198 & \\
\hline 2354 SRL-202-G & $\begin{array}{l}\text { SRS } \\
\text { SPS }\end{array}$ & Shade 1991 & \\
\hline 2355 SRL-202-P & SRS & Shade 1991 & \\
\hline 2356 W-Al/131 & $\begin{array}{l}\text { Savannah River S Site } \\
\text { Savannah River Site }\end{array}$ & $\begin{array}{l}\text { Soper et al. } 1 \\
\end{array}$ & 1982 \\
\hline $\begin{array}{l}2357 \text { W-A-A/1/141 } \\
2358 \text { W-A//142 }\end{array}$ & $\begin{array}{l}\text { Savannanh Rviver sile } \\
\text { Savannah River Site }\end{array}$ & $\begin{array}{l}\text { Sopere te al. } 1 \\
\text { Soperet al. } 1\end{array}$ & 9822 \\
\hline $\begin{array}{l}2358 \text { W-A//1/142 } \\
2359 \text { W-Al/143 }\end{array}$ & $\begin{array}{l}\text { Savannann Rvererste } \\
\text { Savannah River Site }\end{array}$ & $\begin{array}{l}\text { Sopere et al. } \\
\text { Soper al. } 1\end{array}$ & 1982 \\
\hline 2360 W-Al/144 & Savannah River Site & Soper et al. & 1982 \\
\hline 2361 W-Al/145 & Savannah River Site & Soper et al. 1 & 1982 \\
\hline 2362 W-Al/146 & Savannah River Site & Soper et al. 1 & 1982 \\
\hline 2363 W-Al/147 & Savannah River Site & Soper et al. 1 & 1982 \\
\hline 2364 W-Al/148 & Savannah River Site & Soper et al. 1 & 1982 \\
\hline $\begin{array}{l}2365 \text { WWA//1/49 } \\
2366 \text { W-A/150 }\end{array}$ & $\begin{array}{l}\text { Savannah River S Site } \\
\text { Savannah River Site }\end{array}$ & $\begin{array}{l}\text { Soper et al. } 1 \\
\text { Soneretal }\end{array}$ & 1982 \\
\hline $\begin{array}{l}2366 \text { W-A } 23 / 150 \\
2367 \text { W-A/151 }\end{array}$ & $\begin{array}{l}\text { Savannahn River Site } \\
\text { Savannah River Sitee }\end{array}$ & $\begin{array}{l}\text { Sopere te al. } 1 \\
\text { Soperet al } 1 \\
1\end{array}$ & 1982 \\
\hline $\begin{array}{l}236 / \text { W-A//1151 } \\
2368 \text { W-A//152 }\end{array}$ & $\begin{array}{l}\text { Savannahn River Sste } \\
\text { Savannah River Site }\end{array}$ & $\begin{array}{l}\text { Sopere et al. } 1 \\
\text { Soperet al } 1 \\
1\end{array}$ & 1982 \\
\hline 2369 W-Al/154 & Savannah River Site & $\begin{array}{l}\text { Soper et al. } 1 \\
\text { Sol }\end{array}$ & 1982 \\
\hline 2370 W-Al/155 & Savannah River Site & Soper et al. 1 & 1982 \\
\hline 2371 W-A//156 & Savannah River Site & Soper et al. 1 & 1982 \\
\hline 2372 W-Al/157 & Savannah River Site & Soper et al. 1 & 1982 \\
\hline 2373 W-A A//158 & Savannah River Site & Soper et al. 1 & 1982 \\
\hline $\begin{array}{l}2374 \text { W-A A/1/59 } \\
2375\end{array}$ & $\begin{array}{l}\text { Savannah River Site } \\
\text { Savannab River Sito }\end{array}$ & Soper et al. 1 & 1982 \\
\hline 2375 W-Al/160 & $\begin{array}{l}\text { Savannahn River S Ste } \\
\text { Savannah River Site }\end{array}$ & $\begin{array}{l}\text { Sopere et al. } \\
\text { Sooper tal } \\
1\end{array}$ & 1982 \\
\hline 2377 W-A//161 & Savannah River Site & Soperetal 1 & 1982 \\
\hline 2378 W-Al/163 & Savannah River Site & Soper et al. 1 & 1982 \\
\hline
\end{tabular}

\begin{tabular}{|c|c|c|c|c|c|c|c|c|c|c|c|c|c|c|c|c|c|c|c|c|c|c|}
\hline $\begin{array}{l}\text { Al2O3-t } \\
0.0000\end{array}$ & $\begin{array}{l}\text { B2O3-t } \\
0.1000\end{array}$ & $\begin{array}{c}\mathrm{CaO}-\mathrm{t} \\
0.0500\end{array}$ & $\begin{array}{c}\mathrm{Fe} 2 \mathrm{O}-\mathrm{t} \\
0.0000\end{array}$ & $\mathrm{FeO}-\mathrm{t}$ & K2O-t & $\begin{array}{c}\text { Li2O-t } \\
0.0000\end{array}$ & MgO-t & $\begin{array}{l}\mathrm{Na} 2 \mathrm{O}-\mathrm{t} \\
0.2250\end{array}$ & $\begin{array}{c}\text { NiO-t } \\
0.0000\end{array}$ & P2O5-t & $\begin{array}{l}\text { sio2-t } \\
0.5250\end{array}$ & $\mathrm{ZrO2-t}$ & Ag2O-t & As2O3-t As2O5-t & BaO-t & BeO-t & Bi2O3-t & Br-t & CdO-t & Ce2O3-t & CeO2-t & Cl-t \\
\hline & 0.0750 & & & & & & & & & & & & & & & & & & & & & \\
\hline & 0.1000 & 0.0500 & 0.0000 & & & 0.0400 & & 0.1850 & 0.0000 & & 0.5250 & & & & & & & & & & & \\
\hline $\begin{array}{l}0.0140 \\
0.0168\end{array}$ & $\begin{array}{l}0.0750 \\
0.0700\end{array}$ & $\begin{array}{l}0.0480 \\
0.0476\end{array}$ & $\begin{array}{l}0.1535 \\
0.1842\end{array}$ & & & 0.0300 & & 0.1387 & 0.0263 & & 0.3937 & & & & & & & & & & & \\
\hline $\begin{array}{l}0.0168 \\
0.0863\end{array}$ & $\begin{array}{l}0.0700 \\
0.0900\end{array}$ & $\begin{array}{l}0.0476 \\
0.0454\end{array}$ & $\begin{array}{l}0.1842 \\
0.0060\end{array}$ & & & $\begin{array}{l}0.0280 \\
0.0360\end{array}$ & & $\begin{array}{l}0.1295 \\
0.1665\end{array}$ & $\begin{array}{l}0.0315 \\
0.0009\end{array}$ & & $\begin{array}{l}0.3675 \\
0.4725\end{array}$ & & & & & & & & & & & \\
\hline $\begin{array}{l}0.0835 \\
0.1294\end{array}$ & $\begin{array}{l}0.0850 \\
0.0900\end{array}$ & $\begin{array}{l}0.454 \\
0.0431\end{array}$ & $\begin{array}{l}0.0060 \\
0.0090\end{array}$ & & & $\begin{array}{l}0.03360 \\
0.0340\end{array}$ & & $\begin{array}{l}0.1665 \\
0.1572\end{array}$ & $\begin{array}{l}0.0009 \\
0.0014\end{array}$ & & $\begin{array}{l}0.4 / 25 \\
0.4462\end{array}$ & & & & & & & & & & & \\
\hline 0.1726 & 0.0800 & 0.0408 & 0.0120 & & & $\begin{array}{l}0.0320 \\
0.030\end{array}$ & & 0.1480 & 0.0018 & & 0.4200 & & & & & & & & & & & \\
\hline 0.2157 & 0.0750 & 0.0385 & 0.0150 & & & 0.0300 & & 0.1387 & 0.0023 & & 0.3937 & & & & & & & & & & & \\
\hline 0.3020 & 0.0650 & & 0.0210 & & & & & 0.1202 & & & 0.3412 & & & & & & & & & & & \\
\hline 0.2157 & 0.0750 & 0.0385 & 0.0150 & & & 0.0000 & & 0.1687 & 0.0023 & & 0.3937 & & & & & & & & & & & \\
\hline 0.0645 & 0.0750 & 0.0450 & 0.1087 & & & 0.0000 & & 0.1687 & 0.0125 & & 0.3937 & & & & & & & & & & & \\
\hline 0.0903 & 0.0650 & & & & & & & & & & & & & & & & & & & & & \\
\hline $\begin{array}{l}0.0645 \\
0.0774\end{array}$ & $\begin{array}{l}0.0750 \\
0.0700\end{array}$ & $\begin{array}{l}0.0450 \\
0.0440\end{array}$ & $\begin{array}{l}0.1087 \\
0.1305\end{array}$ & & & & & 0.1387 & & & 0.3937 & & & & & & & & & & & \\
\hline $\begin{array}{l}0.07140 \\
0.1160\end{array}$ & $\begin{array}{l}0.0700 \\
0.0750\end{array}$ & $\begin{array}{l}0.0440 \\
0.0457\end{array}$ & $\begin{array}{l}0.1305 \\
0.0790\end{array}$ & & & $\begin{array}{l}0.0280 \\
0.0000\end{array}$ & & $\begin{array}{l}0.1295 \\
0.1687\end{array}$ & $\begin{array}{l}0.0150 \\
0.0058\end{array}$ & & $\begin{array}{l}0.3675 \\
0.3937\end{array}$ & & & & & & & & & & & \\
\hline 0.0928 & $\begin{array}{l}0.0750 \\
0.0800\end{array}$ & $\begin{array}{l}0.045 / \\
0.0466\end{array}$ & $\begin{array}{l}0.0632 \\
0.0690\end{array}$ & & & $\begin{array}{l}0.0000 \\
0.0320\end{array}$ & & $\begin{array}{l}0.1687 \\
0.1480\end{array}$ & $\begin{array}{l}0.0058 \\
0.0046\end{array}$ & & $\begin{array}{l}0.3931 \\
0.4200\end{array}$ & & & & & & & & & & & \\
\hline 0.1160 & 0.0750 & 0.0457 & 0.0790 & & & 0.0300 & & 0.1387 & 0.0058 & & 0.3937 & & & & & & & & & & & \\
\hline 0.1392 & 0.0700 & 0.0449 & 0.0948 & & & 0.0280 & & 0.1295 & 0.0069 & & 0.3675 & & & & & & & & & & & \\
\hline 0.0999 & . 1069 & & 0.1219 & 0.0000 & 0.0244 & 0.0263 & 0.0133 & 0.0958 & 0.0028 & 0.0252 & 0.3996 & 0.0029 & 0.0000 & 0.0000 & 0.0005 & & 0.0000 & & 0.0000 & 0.0000 & 0.0007 & 0.0000 \\
\hline 0.0999 & 0.1069 & 0.0060 & 0.1219 & 0.0000 & 0.0244 & 0.0263 & 0.0133 & 0.0958 & 0.0028 & 0.0252 & 0.3996 & 0.0029 & 0.0000 & 0.0000 & 0.0005 & & 0.0000 & & 0.0000 & 0.0000 & 0007 & 0.0000 \\
\hline & 0.1069 & 0.0060 & 0.1219 & 0.0000 & 0.0244 & & & & 0.0028 & & & & & 0.000 & 0.008 & & & & & 0.0000 & 0007 & \\
\hline 0.0999 & 0.1069 & $\begin{array}{l}0.0060 \\
0.0060\end{array}$ & 0.1219 & 0.0000 & 0.0244 & 0.0263 & 0.0133 & 0.0958 & 0.0028 & 0.0252 & 0.3996 & 0.0029 & 0.0000 & 0 & 0.0005 & & 0.006 & & 0.0000 & 0.0000 & 0.0007 & 0.0000 \\
\hline $\begin{array}{l}0.09999 \\
0.0999\end{array}$ & $\begin{array}{l}.01069 \\
.1069\end{array}$ & $\begin{array}{l}0.0060 \\
0.0060\end{array}$ & $\begin{array}{l}0.1219 \\
0.1219\end{array}$ & 0.00000 & $\begin{array}{l}0.0244 \\
0.0244\end{array}$ & $\begin{array}{l}0.0263 \\
0.0263\end{array}$ & $\begin{array}{l}0.0133 \\
0.0133\end{array}$ & $\begin{array}{l}0.0958 \\
0.0958\end{array}$ & $\begin{array}{l}0.0028 \\
0.0028\end{array}$ & $\begin{array}{l}0.0252 \\
0.0252\end{array}$ & $\begin{array}{l}0.3996 \\
0.3996\end{array}$ & $\begin{array}{l}0.0029 \\
0.0029\end{array}$ & $\begin{array}{l}0.00000 \\
0.0000\end{array}$ & $\begin{array}{l}0.00000 \\
0.0000\end{array}$ & $\begin{array}{l}0.0005 \\
0.0005\end{array}$ & & $\begin{array}{l}0.0000 \\
0.0000\end{array}$ & & $\begin{array}{l}0.0000 \\
0.0000\end{array}$ & 0.00000 & $\begin{array}{l}0.0007 \\
0.0007\end{array}$ & $\begin{array}{l}0.0000 \\
0.0000\end{array}$ \\
\hline 0.0000 & 0.0000 & 0.0000 & 0.0000 & 0.0000 & $\begin{array}{l}0.0244 \\
0.0000\end{array}$ & 0.0000 & 0.0000 & $\begin{array}{l}0.00000 \\
0.000\end{array}$ & 0.0000 & 0.0000 & 0.0000 & 0.0000 & 0.0000 & 0.0000 & $\begin{array}{l}0.0000 \\
0.0000\end{array}$ & & $\begin{array}{l}0.0000 \\
0.0000\end{array}$ & & 0.0000 & 0.0000 & 0.0000 & 0.00000 \\
\hline 0.0000 & 0.0000 & 0.0000 & 0.0000 & 0.0000 & 0.0000 & 0.0000 & 0.0000 & 0.0000 & 0.0000 & 0.0000 & 0.0000 & 0 & 00000 & 0.0000 & 0.00 & & & & & 0.0000 & 0.0000 & .0000 \\
\hline 0.0000 & 0.0000 & 0.0000 & 0.0000 & 0.0000 & 0.0000 & 0.0000 & 0.0000 & 0.0000 & 0.0000 & 0.0000 & 0.0000 & 0.0000 & 0.0000 & 0.0000 & 0.0000 & & 0.0000 & & 0.0000 & 0.0000 & 0.0000 & .0000 \\
\hline & 0.0000 & & 0.0000 & 0.0000 & 0.0000 & 0.0000 & 0.0000 & 0.0000 & 0.0000 & 0.0000 & & 0.0000 & 0.0000 & 0.0000 & 0.0000 & & 0.0000 & & 0.0000 & 0.0000 & 0.0000 & \\
\hline 1.0000 & .0000 & 0.0000 & 0.0000 & 0.0000 & 0.0000 & 0.0000 & 0.0000 & 0.0000 & 0.0000 & 0.0000 & 0.0000 & 0.0000 & 0.0000 & 0.0000 & 0.0000 & & 0.0000 & & 0.0000 & 0.0000 & 0.0000 & b000 \\
\hline
\end{tabular}

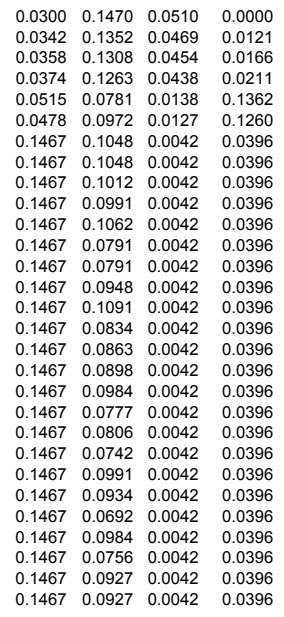

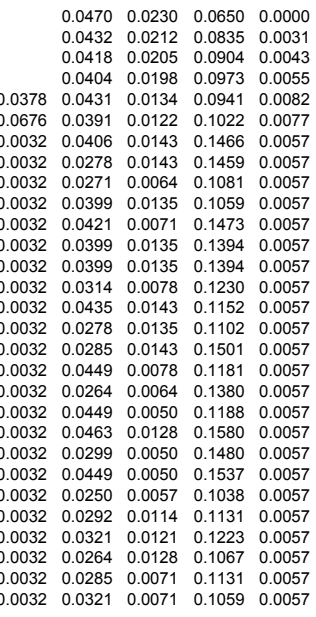

$\begin{array}{lll}0.5860 & 0.0000\end{array}$

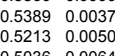

$\begin{array}{lll}4.4650 & 0\end{array}$

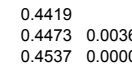

0.5050
0.0905
0.0 .0000
0.000

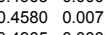

496550.0000
48430.0000
0.000

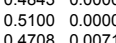

5933007

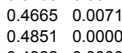

0.4922
0.5164
0.000000
0.0000

$\begin{array}{lll}0.4644 & 0.0000 \\ 0.4979 & 0.0000\end{array}$

$\begin{array}{ll}0.4508 & 0.0000 \\ 0.5228 & 0.0029\end{array}$

$\begin{array}{ll}0.4508 & 0.000 \\ 0.5285 & 0.0000 \\ 0.5029 & 0.0000\end{array}$

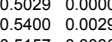

0.5157
0.5193
0.0036
0.0000

$\begin{array}{ll}0.0000 & 0.0000 \\ 0.0006 & 0.0037 \\ 0.0009 & 0.0050 \\ 0.0011 & 0.0064 \\ 0.0016 & \\ 0.0022 & \end{array}$


2319 HTT-S-4
2320 HT-S-5

2321 HTS-S-6
2322 AL1TC34

2322 AL1TC34
2323 AL1TC36

2324 AL4TC34

2326 AL9TC27

2327 HTS1
2328 HTS2

2339 HTS

2330 HTS4
2331 HTS5

2332 HTS

2334 HTS8

2335 HTS9

2337 HTS1

2338 HTS 12
2339 HTS 13
2340 HTS 14

2340 HTS 14

2342 HTS16

2343 HTS17

$2345 \mathrm{HTS} 19$
$2346 \mathrm{HTS20}$

2337 HTS21

2350 WMRefGlas

2350 SM513FR
2351 SM513LW0

2353 SM513LW14

2355 SRL-202-G

2357 W-Al/141

2358 W-Al/142

2359 W-Al/143

2361 W-Al/145

2362 W-Al/146

2363 W-Al/147

2365 W-A//149

2366 W-Al/150

2367 W-Al/151

2369 W-Al/154

2370 W-Al/155

2371 W-Al/156

2373 W-Al/155

2374 W-Al/159

2375 W-Al1160

2377 W-Al/162
2378 W-Al/163
CoO-t Co2O3-t Cr2O3-t Cs2O-t CuO-t Dy2O3-t Eu2O3-t

F-t Ga2O3-t Gd2O3-t HfO2-t HgO-t

.0000

0.0000
0.0103
0.0123
0.0049

0.0292

0.0257

$\begin{array}{llll}0.0000 & 0.0018 & 0.0008 & 0.0000\end{array}$

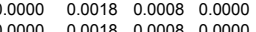

$\begin{array}{llll}0.0000 & 0.0018 & 0.0008 & 0.0000\end{array}$

$\begin{array}{lllllll}0.00000 & 0.0018 & 0.0008 & 0.0000\end{array}$

$0.0000 \quad 0.0000 \quad 0.0000$

$\begin{array}{llll}0.0000 & 0.0000 & 0.0000 & 0.0000\end{array}$

$\begin{array}{llll}0.0000 & 0.0000 & 0.0000 & 0.0000 \\ 0.0000 & 0.0000 & 0.0000 & 0.000\end{array}$

$\begin{array}{ll}0.0000 & 0.0000 \\ 0.0000 & 0.0000 \\ 0.0000 & 0.0000 \\ 0.0000 & 0.0000 \\ 0.0000 & 0.0000 \\ 0.0000 & 0.0000 \\ 0.0000 & 0.0000 \\ 0.0000 & 0.0000 \\ 0.0000 & 0.0000 \\ 0.0000 & 0.0000 \\ 0.0000 & 0.0000\end{array}$

$\begin{array}{llll}0.0000 & 0.0003 & 0.0309 & 0.0131 \\ 0.00000\end{array}$

$\begin{array}{llll}0.0000 & 0.0003 & 0.0131 & 0.0000\end{array}$

$\begin{array}{llll}0.0000 & 0.0003 & 0.0131 & 0.0000 \\ .00000 & 0.0003 & 0.0131 & 0.0000 \\ 0 & 0.0000\end{array}$

$\begin{array}{llll}0.0000 & 0.0003 & 0.0131 & 0.0000 \\ 0.0000 & 0.0000 & 0.0000 & 0.0000\end{array}$

$\begin{array}{llll}0.0000 & 0.0000 & 0.0000 & 0.0000\end{array}$

$\begin{array}{llll}0.0000 & 0.0000 & 0.0000 & 0.0000 \\ 0.0000 & 0.0000 & 0.00000 & 0.0000\end{array}$

$\begin{array}{lll}0.0001 & 0.0000 & 0.0012 \\ 0.0001 & 0.0000 & 0.0012 \\ 0.0001 & 0.000 & 0.0012\end{array}$

$\begin{array}{lll}0.0001 & 0.0000 & 0.0012\end{array}$

$\begin{array}{lll}0.0001 & 0.0000 & 0.0012 \\ 0.0001 & 0.0000 & 0.0012\end{array}$

$\begin{array}{lll}0.0001 & 0.0000 & 0.0012 \\ 0.0000 & 0.0000 & 0.0000\end{array}$

$\begin{array}{lll}0.0000 & 0.0000 & 0.0000 \\ 0.0000 & 0.0000 & 0.0000\end{array}$

$\begin{array}{lll}0.00000 & 0.0000 & 0.00000 \\ 0.0000 & 0.0000 & 0.0000 \\ 0.0000 & 0.0000 & 0.0000\end{array}$

$\begin{array}{llll}0.0000 & 0.0000 & 0.0000 & 0.0000\end{array}$

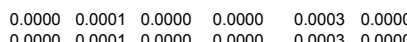
$\begin{array}{llllll}0.0000 & 0.0001 & 0.0000 & 0.0000 & 0.0003 & 0.0000\end{array}$ $\begin{array}{llllll}0.0000 & 0.0001 & 0.0000 & 0.0000 & 0.0003 & 0.0000 \\ 0\end{array}$ $\begin{array}{llllll}0.0000 & 0.0001 & 0.000 & 0.000 & 0.003 & 0.0000\end{array}$ $\begin{array}{lllllll}0.0000 & 0.0000 & 0.0000 & 0.0000 & 0.0000 & 0.0000\end{array}$ \begin{tabular}{lllllll}
\hline .0000 & 0.0000 & 0.0000 & 0.0000 & 0.0000 & 0.0000
\end{tabular}

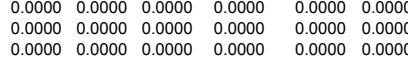

$\begin{array}{ll}0.0000 & 0.0000 \\ 0.0031 & 0.0006 \\ 0.0045 & 0.0009\end{array}$

$\begin{array}{lll}0.0043 & 0.0009 \\ 0.0055 & 0.0011\end{array}$

$\begin{array}{lll}0.0011 & 0.0000 & 0.0043 \\ 0.0011 & 0.0000 & 0.0074\end{array}$
0.0000
0.0054
0.0075
0.0095

$\begin{array}{llll}0.0000 & 0.0000 & 0.0000 & 0.0000 \\ 0.0032 & 0.0020 & 0.0036 & 0.0005 \\ 0.0043 & 0.0027 & 0.0050 & 0.0007 \\ 0.0055 & 0.0035 & 0.0064 & 0.0010 \\ 0.0223 & & & \end{array}$

$\begin{array}{lll}0.00000 & 0.0324 \\ 0 & 0.00000 & 0.0324\end{array}$

$\begin{array}{ll}0.0000 & 0.0324 \\ 0.0000 & 0.0324\end{array}$

$0.0000 \quad 0.0324$

0.00710 .0324

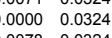

$0.0064 \quad 0.0324$

$0.0000 \quad 0.0324$

$\begin{array}{lll}0.0057 & 0.0324 \\ 0.0000 & 0.0324\end{array}$

$0.0000 \quad 0.0324$

$\begin{array}{lll}0 & 036 & 0.0324\end{array}$

$\begin{array}{ll}0.0000 & 0.0324 \\ 0.0036 & 0.0324 \\ 0.0000 & 0.0324\end{array}$

$\begin{array}{ll}0.0000 & 0.0324 \\ 0.0000 & 0.0324\end{array}$ 
2318 HT S-S 3

2320 HTTS-5

2321 HT-S-6
2322 ALITC34
2323 AL1TC36

2323 AL1TC36
2324 AL4TC34

2325 AL5TC34
2326 AL9TC27

2327 HTS1

2329 HTS3

2330 HTS

2332 HTS

$2334 \mathrm{HTS}$

2335 HTS9

2337 HTS

2338 HTS 12
2339 HTS 13

2340 HTS 14

2342 HTS16

2343 HTS17

2345 HTS 19

2337 HTS21
2348 HTS22

2349 WVRefGlas
2350 SM513FR

2350 SM513FR
2351 SM513LW0

2352 SM513LW1
2353 SM513LW1

2354 SRL-202-G

(356 W-A//131

2358 W-Al/142

2359 W-Al/143

2361 W-A A//145

2362 W-Al/146

2363 W-Al/147
2364 W-Al/148

(365 W-Al/149

2366 W-Al/150

2368 W-A//152

2369 W-All154

2370 W-Al/155

2372 W-Al/155

2373 W-Al/155

2374 W-Al/159

2376 W-Al/166

2377 W-Al/162

ReO2-t Re207-t Rh203-t RhO2-t RuO2-t Ru203-t Sb2O3-t Sb2O5-t SeO2-t Sm203-t SnO-t SnO2-t SO3-t SrO-t Tc207-t TeO2-t ThO2-t

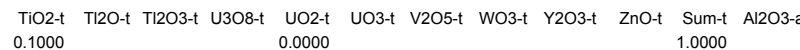

0.1070
0.1500
0.0750
0.0700
0.0990
0.0850
0.0800
0.0750
0.0650
0.0750
0.0750
0.0650
0.0570
0.0700
0.0750
0.0800
0.0750
0.0700
0.0099
0.0099
0.0099
0.0099
0.0099
0.0099
0.0000
0.0000
0.0000
0.0000
0.0000

$\begin{array}{llll}0.0000 & 0.0000 & 0.0001 & 0.0008 \\ 0.0000 & 0.0000 & 0.0001 & 0.00000\end{array}$

0.0008
0.0008

$\begin{array}{llll}0.0000 & 0.0001 & 0.0008\end{array}$

0.00000 .00010 .0008

$\begin{array}{lll}0.0000 & 0.0000 & 0.0000 \\ 0.0000 & 0.0000 & 0.0000\end{array}$

$\begin{array}{lll}0.0000 & 0.0000 & 0.0000 \\ 0.0000 & 0.0000 & 0.0000\end{array}$

\subsection{0 \\ $0.0000 \quad 0.0003$}

$\begin{array}{ll}0.0000 & 0.0003 \\ 0.0000 & 0.0003 \\ 0.000 & 0.0003\end{array}$

$\begin{array}{ll}0.0000 \\ 0.0000 \\ 0.0000 & 0.00003 \\ 0.0000 & 0.003\end{array}$

$\begin{array}{ll}0.0000 & 0.0003 \\ 0.0000 & 0.0000 \\ 0\end{array}$

$0.0000 \quad 0.0000$

$\begin{array}{ll}0.0000 & 0.0000 \\ 0.0000 & 0.0000\end{array}$

$\begin{array}{lll}0.0000 & 0.0030 & 0.0003 \\ 0.0000 & 0.0030 & 0.0003\end{array}$

$\begin{array}{lll}0.0000 & 0.0030 & 0.0003 \\ 0.0000 & 0.0030 & 0.0003\end{array}$

$\begin{array}{lll}.0000 & 0.0030 & 0.0003 \\ .0000 & 0.0030 & 0.0003\end{array}$

$\begin{array}{lll}0.0000 & 0.0030 & 0.0003 \\ 0.0000 & 0.0000 & 0.0000\end{array}$

$\begin{array}{lll}0.0000 & 0.0000 & 0.0000 \\ 0.0000 & 0.0000 & 0.0000 \\ 0.0000 & 0.0000 & 0.0000\end{array}$

0.0000
0.0000

$\begin{array}{ll}0.0000 & 0.0000 \\ 0.0006 & 0.0006 \\ 0.00011 & 0.00011\end{array}$

$\begin{array}{ll}0.0008 & 0.0008 \\ 0.0011 & 0.0011\end{array}$

$\begin{array}{ll}0.0000 & 0.0000 \\ 0.0039 & 0.0006 \\ 0.0054 & 0.0009\end{array}$

$\begin{array}{ll}0.0054 & 0.0009 \\ 0.0069 & 0.0011\end{array}$

$\begin{array}{lll}0.0000 & 0.0361 \\ 0.0000 & 0.0361 & 0 \\ 0.0000 & 0.0361 & 0\end{array}$

$\begin{array}{lll}0.0000 & 0.0361 & 0.0099\end{array}$

$0.0000 \quad 0.0000 \quad 0.0000$

$\begin{array}{lll}0.0000 & 0.0000 & 0.0000 \\ 0.0000 & 0.0000 & 0.0000 \\ 0.0000 & 0.0000 & 0.0000\end{array}$

$0.02-t$
0.0000
0.0355

1.0000

0.0000
0.0355
0.0426
0.0015

0.0015
0.0023

0.0023
0.0030
0.0038
0.0053

0.0053
0.0038
0.0255

0.0275
0.0385

0.0275
0.0330

0.0153

0.0183

$\begin{array}{lll}0.0000 & 0.0056 & 0.0000 \\ 0.0000 & 0.0056 & 0.0000\end{array}$

$\begin{array}{llllll} & & & 1.0000 & \\ 0.0000 & 0.0002 & 0.0000 & 1.0002 & 0.1060 \\ 0.0000 & 0.0002 & 0.0000 & 1.0002 & 0.1032 \\ 0.0000 & 0.002 & .0000 & 1.002 & 0.1047\end{array}$

$\begin{array}{lll}0.0000 & 0.0056 & 0.0000 \\ .0000 & 0.0056 & 0.0000\end{array}$

$\begin{array}{llll}0.00000 & 0.0056 & 0.0000 \\ 0.00000 & 0.0056 & 0.0000 \\ 0.0000 & 0.056 & 0.0000\end{array}$

$0.0000 \quad 0.0000 \quad 0.0000$

$0.0000 \quad 0.0000 \quad 0.0000$

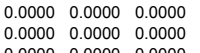

$\begin{array}{lllll}0.00000 & 0.0002 & 0.00000 & 1.0002 & 0.1032 \\ 0.1041\end{array}$ $\begin{array}{llllll}0.0000 & 0.0002 & 0.0000 & 1.0002 & 0.1037 \\ 0.0000 & 0.0002 & 0.0000 & 1.0002 & 0.1033\end{array}$ $\begin{array}{lllll}0.0000 & 0.0002 & 0.0000 & 1.00002 & 0.1035 \\ 0.0000 & 0.0000 & 0.0000 & 0.0000 & 0.0534\end{array}$ $\begin{array}{lllll}0.0000 & 0.00000 & 0.0000 & 0.00000 & 0.0611 \\ 0.0000 & 0.0000 & 0.0000 & 0.0000 & 0.0520 \\ 0.0000 & 0.0000 & 0.0000 & 0.0000 & 0.0529\end{array}$ $\begin{array}{llllll}0.0000 & 0.0000 & 0.0000 & 0.0000 & 0.0638\end{array}$

$\begin{array}{ll}0.0000 & 0.1060 \\ 0.0000 & 0.1032 \\ 0.0000 & 0.1041 \\ 0.0000 & 0.1037 \\ 0.0000 & 0.1033 \\ 0.0000 & 0.1035 \\ 0.0000 & 0.0999 \\ 0.0000 & 0.0999 \\ 0.0000 & 0.0999 \\ 0.0000 & 0.0999 \\ 0.0000 & 0.0999 \\ 0.00000 & 0.0999 \\ 0.0000 & 0.0999 \\ 0.00000 & 0.0999 \\ 0.0000 & 0.0999 \\ 0.00000 & 0.0999 \\ 0.0000 & 0.0999 \\ 0.00000 & 0.0999 \\ 0.0000 & 0.0999 \\ 0.00000 & 0.0999 \\ 0.0000 & 0.0999 \\ 0.0000 & 0.0999 \\ 0.0000 & 0.1132\end{array}$

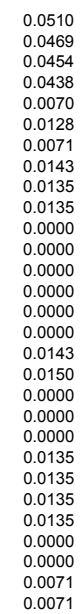

0.0000
0.0025
0.0034

0.0034
0.0044 
2319 HT-S-4

2321 HTTS-6 6
2322 AL 1TC34

2323 AL1TC36

2324 AL4TC34

2326 AL9TC27

2327 HTS1

2329 HTS

2330 HTS4
2331 HTS5

2332 HTS6
2333 HTS7

$2334 \mathrm{HTS} 8$

2335 HTS9

2338 HTS 12

2339 HTS13

2342 HTS 16

2343 HTS17

2344 HTS 18
2345 HTS19

2346 HTS20

2348 HTS22

2349 WVRefGlass
2350 SM513FR

2350 SM513FR
2351 SM513LW0
2352 SM513LW11

$\begin{array}{lll}0.10893 & 0.0064 & 0.11 \\ 0.1095 & 0.1167 \\ 0.1182 & 0.0066 & 0.1176\end{array}$

$\begin{array}{lll}0.1087 & 0.0062 & 0.1181\end{array}$

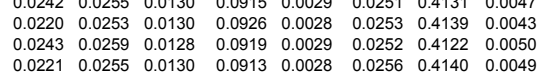

$0.1090 \quad 0.0063 \quad 0.1174$

0.1027
0.0997

0.1053

0.1128

$\begin{array}{lll}0.1081 & 0.0064 & 0.1185\end{array}$

$\begin{array}{lll}0.1093 & 0.0064 & 0.1167 \\ 0.1082 & 0.0066 & 0.1176\end{array}$

$\begin{array}{lll}0.1087 & 0.0062 & 0.1181\end{array}$

$\begin{array}{llll}0.1096 & 0.0064 & 0.1158\end{array}$

$\begin{array}{lll}0.1090 & 0.0063 & 0.1174 \\ 0.1069 & 0.0060 & 0.1219\end{array}$

$\begin{array}{llll}0.1069 & 0.0060 & 0.1219\end{array}$

$\begin{array}{lll}0.1069 & 0.0060 & 0.1219\end{array}$

$\begin{array}{lll}0.1069 & 0.0060 & 0.1219 \\ 0.1069 & 0.0060 & 0.1219\end{array}$

$\begin{array}{lll}0.1069 & 0.0060 & 0.1219 \\ 0.1069 & 0.0060 & 0.1219 \\ 0.1009 & 0.0060 & 0.121\end{array}$

$\begin{array}{lll}0.1069 & 0.0060 & 0.1219 \\ 0.1069 & 0.0060 & 0.1219\end{array}$

$\begin{array}{lll}0.1069 & 0.0060 & 0.1219\end{array}$

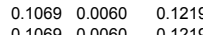

$\begin{array}{lll}0.1069 & 0.0060 & 0.1219 \\ 0.1069 & 0.0060 & 0.1219\end{array}$

$\begin{array}{llll}0.1069 & 0.0060 & 0.1219\end{array}$

$\begin{array}{lll}0.1609 & 0.0060 & 0.129 \\ 0.1069 & 0.0060 & 0.1219 \\ 0.1072 & 0.0058 & 0.1152\end{array}$ $\begin{array}{lll}0.0252 & 0.4122 & 0.0050 \\ 0.0256 & 0.4140 & 0.0049\end{array}$ $\begin{array}{lll}0.0237 & 0.4399 \\ 0.02311 & 0.425\end{array}$

0.02480 .4493

$\begin{array}{lll} & 0.439 \\ 0 & 0.4391\end{array}$

$\begin{array}{llllllll}0.0230 & 0.0257 & 0.0129 & 0.0906 & 0.0029 & 0.0254 & 0.4115 & 0.0052 \\ 0.0233 & 0.0256 & 0.0129 & 0.0923 & 0.0027 & 0.0254 & 0.4141 & 0.0046\end{array}$ $\begin{array}{lllllllll}0.0233 & 0.0256 & 0.0129 & 0.0923 & 0.0027 & 0.0254 & 0.415 & 0.0052 \\ 0.0242 & 0.0255 & 0.0130 & 0.0915 & 0.0029 & 0.0251 & 0.4131 & 0.0047\end{array}$

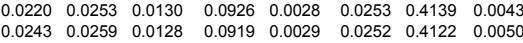
$\begin{array}{llllllll}0.0221 & 0.0255 & 0.0130 & 0.0913 & 0.0028 & 0.0256 & 0.4140 & 0.0049\end{array}$ $\begin{array}{lllllllll}0.0244 & 0.0263 & 0.0133 & 0.0958 & 0.0028 & 0.0252 & 0.3996 & 0.0029\end{array}$ $\begin{array}{llllllll}0.0244 & 0.0263 & 0.0133 & 0.0958 & 0.0028 & 0.0252 & 0.3996 & 0.0029 \\ 0.0244 & 0.0263 & 0.0133 & 0.0958 & 0.0028 & 0.0252 & 0.3996 & 0.0029\end{array}$ $\begin{array}{llllllllll}0.0244 & 0.0263 & 0.0133 & 0.0958 & 0.0028 & 0.0252 & 0.3996 & 0.0029\end{array}$ $\begin{array}{llllllll}0.0244 & 0.0263 & 0.0133 & 0.0958 & 0.0028 & 0.0252 & 0.3996 & 0.0029\end{array}$ $\begin{array}{llllllll}0.0244 & 0.0263 & 0.0133 & 0.0958 & 0.0028 & 0.0252 & 0.3996 & 0.0029 \\ 0.0244 & 0.0263 & 0.0133 & 0.0958 & 0.0028 & 0.0252 & 0.3996 & 0.0029\end{array}$ $\begin{array}{llllllll}0.0263 & 0.0133 & 0.0958 & 0.0028 & 0.0252 & 0.3996 & 0.0029\end{array}$

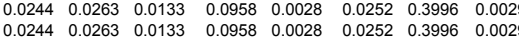
$\begin{array}{llllllll}0.0244 & 0.0263 & 0.0133 & 0.0958 & 0.0028 & 0.0252 & 0.3996 & 0.0029\end{array}$ $\begin{array}{llllllll}0.0244 & 0.0263 & 0.0133 & 0.0958 & 0.0028 & 0.0252 & 0.3996 & 0.0029\end{array}$ $\begin{array}{lllllllll}0.0244 & 0.0263 & 0.0133 & 0.0958 & 0.0028 & 0.0252 & 0.3996 & 0.0029 \\ 0.0244 & 0.0263 & 0.0133 & 0.0958 & 0.0028 & 0.0252 & 0.3996 & 0.0029\end{array}$

$\begin{array}{ll}0.0019 & 0.0008 \\ 0.0018 & 0.0007 \\ 0.000 & 0.007\end{array}$

0.00190 .0007 $\begin{array}{ll}0.0017 & 0.00007 \\ 0.0019 & 0.00007\end{array}$

2355 SRL-202-P

2357 W-Al/1/14

2358 W-Al/142

2359 W-Al/143

2361 W-Al/145

2362 W-Al/146

$2363 \mathrm{~W}-\mathrm{A} / 1 / 147$
$2364 \mathrm{~W}-\mathrm{Al} / 148$

2365 W-A//149

2366 W-Al/150

2367 W-Al/151

2369 W-Al/154

$2370 \mathrm{~W}-\mathrm{A} / 1 / 155$

2372 W-A/14156

2373 W-A//15

2374 W-Al/159

2375 W-Al/160

2377 W-Al/162

0.0004
0.0005
0.0004
0.0005
0.0005
0.0005
0.0005
0.0005
0.0005
0.0005
0.0005
0.0005
0.0005
0.0005
0.0005
0.0005
0.0005
0.0005
0.0005
0.0005
0.0005
0.0005
0.0005

0.0007
0.0007
0.0007
0.0007
0.0007
0.0007
0.0007
0.0007
0.0007
0.0007
0.0007
0.0007
0.0007
0.0007
0.0007
0.0007
0.0007
0.0007
0.0007
0.0007
0.0007
0.0007
0.0007

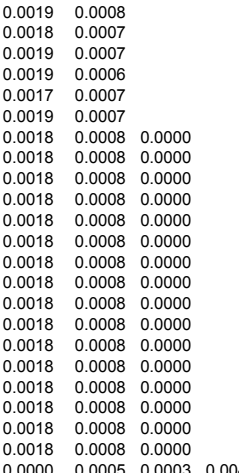


2318 HT-S-3

2320 HT-S-5 5

2321 HT-S-6
2322 ALITC34
2323 AL1TC36

2323 AL1TC36
2324 AL4TC34

2325 ALLTC3

2327 HTS 1

2328 HTS2

2329 HTS3
2330 HTS

2331 HTS5

$2332 \mathrm{HTS6}$

2334 HTS8

2335 HTS9

(337 HTS

2338 HTS12

$2340 \mathrm{HTS} 14$

HTS16

$2343 \mathrm{HTS17}$
$2344 \mathrm{HTS} 18$

$2344 \mathrm{HTS} 18$
$2345 \mathrm{HTS} 19$

$2346 \mathrm{HTS} 20$
$234 \mathrm{HTS2}$

2348 HTS22

2349 WVRefGlass
2350 SMI135R

2351 SM513LW

2353 SM513LW14

2354 SRL-202-G
2355 SRL-202-P

2358 W-A-A/14143

2359 W-All/43
2360 W.Al/144

2361 W-AAl/145

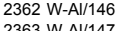

2363 W-All147
2364 W-Al/148

2365 W-Al/149

2366 W-All150

2367 W-All/151

2369 W-All154

2370 W-All1155

2371 W-All156

2373 W-A/1/58

2374 W-Al/159

2375 W-All160
2376 W-All161

2378 W-AAl/163

\begin{tabular}{|c|c|c|c|c|c|c|c|}
\hline & & & & & & & \\
\hline 0.0004 & 0.0133 & 0.0001 & 0.0013 & 0.0001 & 0.0003 & 0.0001 & $\begin{array}{l}0.0012 \\
0.0013\end{array}$ \\
\hline 0.0003 & 0.0136 & 0.0001 & 0.0011 & 0.0001 & 0.0003 & 0.0001 & $\begin{array}{l}0.0012 \\
0.012\end{array}$ \\
\hline & 0.0135 & 0.0001 & 0.0013 & & 0.0003 & 0.0001 & \\
\hline & $\begin{array}{l}0.0133 \\
0.0136\end{array}$ & $\begin{array}{l}0.0001 \\
0.00001\end{array}$ & $\begin{array}{l}0.0011 \\
0.0013\end{array}$ & $\begin{array}{l}0.0001 \\
0.0001\end{array}$ & 0.0003 & 0.0001 & \\
\hline & $\begin{array}{l}0.0136 \\
0.0128 \\
0\end{array}$ & & 0.0013 & & & & \\
\hline & $\begin{array}{l}0.0112 \\
0.0122\end{array}$ & & & & & & \\
\hline & $\begin{array}{l}0.0123 \\
0.0123\end{array}$ & & & & & & \\
\hline 0.0004 & 0.0134 & 0.0001 & 0.0011 & 0.0001 & 0.0003 & 00001 & \\
\hline & & 0.0001 & 00013 & 00001 & 0.0000 & ת & \\
\hline 0.0003 & 0.0136 & 0.0001 & 0.0011 & 0.0001 & 0.0003 & 0.0001 & \\
\hline 0.0003 & 0.0135 & & 0.0013 & 0.0001 & 0.0003 & 0.0001 & \\
\hline 0.0004 & 0.0133 & 0.0001 & 0.0011 & 0.0001 & 0.0003 & 0.0001 & 0.0011 \\
\hline 0.0004 & 0.0136 & 0.0001 & 0.0013 & 0.0001 & 0.0003 & 0.0001 & 0.0013 \\
\hline 0.0003 & 0.0131 & 0.0001 & 0.0012 & 0.0001 & 0.0003 & 0.0001 & 0.0008 \\
\hline $\begin{array}{l}0.0003 \\
0.0003\end{array}$ & 0.0131 & 0.0001 & 0.0012 & 0.0001 & 0.00033 & 0.0001 & $\begin{array}{l}0.0008 \\
0.0008\end{array}$ \\
\hline $\begin{array}{l}0.0003 \\
0.0003\end{array}$ & 0.0131 & 0.00001 & 0.0012 & 0.00001 & 0.0003 & 0.0001 & $\begin{array}{l}0.0008 \\
0.0008\end{array}$ \\
\hline $\begin{array}{l}0.0003 \\
0.0003\end{array}$ & 0.0131 & Q & 0.012 & 0.000 & 0 & 0 & $\begin{array}{l}0.0008 \\
0.0008\end{array}$ \\
\hline $\begin{array}{l}0.0003 \\
0.0003\end{array}$ & 0.0131 & 0.0001 & 0001 & 0.000 & 0.000 & 0.0001 & 0.0008 \\
\hline 0.0003 & 0.0131 & 0.0001 & 0.001 & 0.0001 & 0.0003 & 0.0001 & 0.0008 \\
\hline 0.0003 & 0.0131 & 0.0001 & 0.0012 & 0.0001 & 0.0003 & 0.0001 & \\
\hline 0.0003 & 0.0131 & 0.0001 & 0.0012 & 0.0001 & 0.0003 & 0.0001 & 0.0008 \\
\hline 0.0003 & 0.0131 & 0.0001 & 0.0012 & 0.0001 & 0.0003 & 0.0001 & 0.0008 \\
\hline 0.0003 & 0.0131 & & & 0.0001 & 0.00003 & 0.0001 & 0.0008 \\
\hline $\begin{array}{l}0.0003 \\
0.0003\end{array}$ & 0.0131 & 0.00001 & 0.0012 & 0.00001 & 0.00033 & 0.0001 & $\begin{array}{l}0.0008 \\
0.00008\end{array}$ \\
\hline 0.0003 & 0 & 1 & 0.0012 & 0.000 & 0 & 0.001 & $\begin{array}{l}0.00008 \\
0.00008\end{array}$ \\
\hline . & 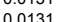 & 0.00 & 0 & 0.000 & 8000 & 0.000 & \\
\hline & 0.0131 & 00001 & 00012 & 0.0001 & 00000 & 0.0001 & 0.0008 \\
\hline 0.0003 & 0.0121 & 0.001 & 00012 & 0.0001 & 0.0003 & 0000 & 0.0006 \\
\hline
\end{tabular}

2378 W-Al/163 
2318 HTS-S-3

2320 HT-S-5

2321 HT-S-6
2322 ALITC34
2323 AL1TC36

2323 AL1TC36
2324 AL4TC34

2325 AL5TC34

2326 AL9T 127

2329 HTS3

2330 HTS

2332 HTS6

2334 HTS8

2335 HTS9

2337 HTS

2339 HTS13

2340 HTS14

2342 HTS 16

2343 HTS17

2345 HTS19 19

2346 HTS20
2347 HTS21

2348 HTS22

2350 SM513FR

2351 SM513LW08

2353 SM513LW1

2354 SRL-202-G

2358 W-Al/142

2359 W-Al/143

2361 W-Al/145

2362 W-Al/146

2363 W-Al/147
2364 W-Al/148

2365 W-Al/149

2366 W-Al/150

2367 W-Al/151

3369 W-Al/154

2370 W-Al/155

2372 W-Al/155

2373 W-Al/155

2374 W-Al/159

2376 W-Al/166

2377 W-Al/162
2378 W-Al/163

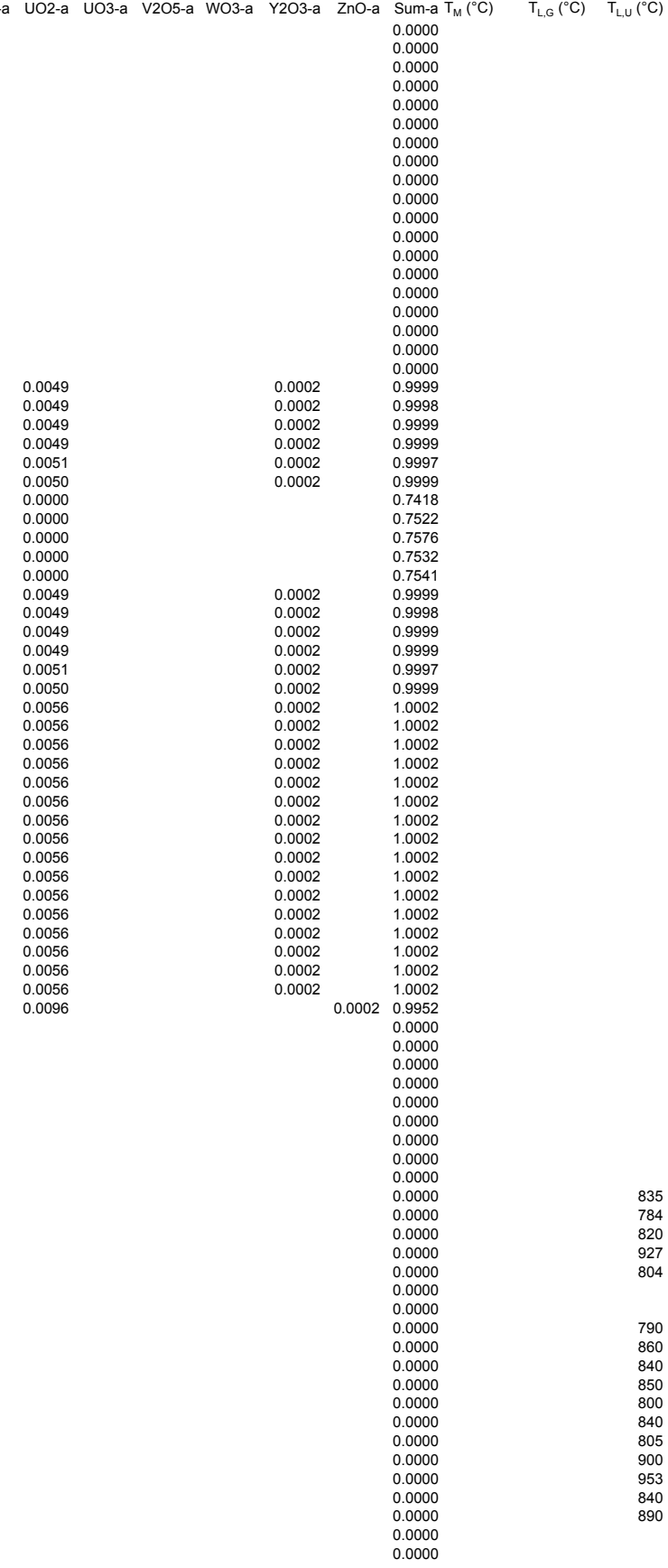


2319 HT-S- 4

$2320 \mathrm{HTTS}-\mathrm{S}$
$2321 \mathrm{HT}-\mathrm{S}-6$

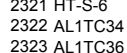

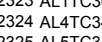

2325 AL5TC34

23267 ALTS1
$2322 \mathrm{TTS}$

$2328 \mathrm{HTS} 2$
$2329 \mathrm{HTS3}$

2329
$2330 \mathrm{HTS}$
$23 \mathrm{SS}$

2330 HTS4
2333 HTSS

$2332 \mathrm{HTS6}$
$2333 \mathrm{HTS7}$

$2334 \mathrm{HTSB}$

$2335 \mathrm{HTS9}$

2336 HTS10

$2338 \mathrm{HTS} 12$

$2339 \mathrm{HTSS} 13$
$2340 \mathrm{HTS14}$

$2342 \mathrm{HTS15}$

$2343 \mathrm{HTS17}$

$2344 \mathrm{HTS} 18$
$2345 \mathrm{HTS} 19$

2346 HTS20

2348 HTS22

2349 WVRReflass
2350 SMS13rR

2351 SM513LR

2352 SM513LW1
2353 SM513LW1

2354 SRL-202-C

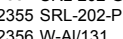

2356 W-Al/131

2358 W-All142
2359

2359 W-Al/143

2360 W-A//144
2361 W-Al/145

2362 W-Al/146

2363 W W-All147
2364 W-All 148

2365 W-AAl/149

2366 W-Al/150

2367 W-All15
2368 W-Al/152

2369 W-Al1154

2370 W-All1155

2371 W-Al/156
2372 W-Al/157

2373 W-Al/158

2377 W-All159

2375 W-Al1/160

2377 W-All162

2378 W-Al/163

$15.75 \%$ spinel, $1.75 \%$ f fluorite, $<2.87 \%$ hematitit
s.

$14 \%$ spinel, $3.5 \%$ fluorite, $<0.87 \%$ hematite

$14.88 \%$ spinel, $1.75 \%$ fluorite, $<0.87 \%$ hematio 
2317 HT-S-S-2
2318 HT-S-3

2319 HT.S-4

$2320 \mathrm{HTTS}-5$
$2321 \mathrm{HT}-\mathrm{S}-6$

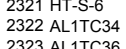

2323 ALLTC36

2325 ALLTC34
2326 A ATOTC27

2326 AL9T 1271
$2327 \mathrm{HTS} 1$

$2328 \mathrm{HTS}$
$2329 \mathrm{HTS}$

2329 HTS3
2330 HTS4

2330 HTS4
2333 HTSS

$2332 \mathrm{HTS}$
$2333 \mathrm{HTS}$

2334 HTS8

$2335 \mathrm{HTS9}$

2336 HTS10

2338 HTS 1

2338 HTS 12
$2339 \mathrm{HTSS}$
2235

2344 HTS15

$2342 \mathrm{HTS16}$
$2333 \mathrm{HTS} 17$

$2344 \mathrm{HTS18}$
$2355 \mathrm{HTS} 19$

2346 HTS20

$234 \mathrm{HTS21}$
238 HST22

2349 WVRefGlass

2350 SM513FR

2351 SM513LW0

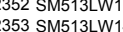

2354 SRL-202-C

2355 SRL-202-P

2357 W-Al/14

2358 W-All142

2360 W-All144
2361 W.Al

2361 W-All145
2362 W-All14

2363 W-All147
2364 W-All 148

2364 W-Al/148
2365 W-AA/149

$2366 \mathrm{~W}-\mathrm{A} / 150$

2367 W-AAl/15
2368 W-Al/152

2369 W-Al1154

2370 W-All1155

2371 W-Al1156

2373 W-Al/158

2374 W-Al/159

2375 W-All160
2376 W-Al116

2377 W-Al/162

2378 W-Al/163

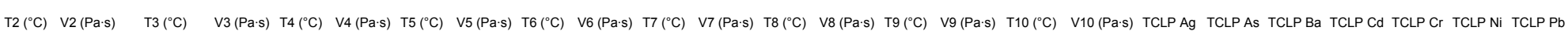


2318 HT-S- 3

2321 HT-S- 6

2322 AL1TC34

2324 AL4TC34

2325 AL5TC34

2327 HTS

2328 HTS2

2339 HTS3

2330 HTS4
2331 HTS5

2332 HTS

2334 HTS8

2335 HTS9

2336 HTS10

$2338 \mathrm{HTS} 12$

2340 HTS14

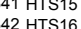

2343 HTS17

$2344 \mathrm{HTS18}$
$2355 \mathrm{HTS} 19$

2346 HTS20

$2347 \mathrm{HTS2} 1$
$238 \mathrm{HTS22}$

2348 HTS22
2349 WVRefG

2350 SMRi13rR

2351 SM513LW

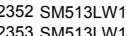

2354 SRL-202-G

2355 SRL-202-P

2357 W-AAl/141

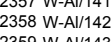

2350 W-A-A/1/44

2360 W-Al/144
2361 W-Al/145

2362 W-Al/146

2363 W-AA/1/47
2364 W-Al/148

2365 W-All149

2366 W-Al/150

2367 W-Al/1/51
2368 W-Al/152

2369 W-All154

2370 W-Al1155

2371 W-Al/156

2373 W-Al/158

2374 W-Al/159

2375 W-Al1/160

2377 W-Al/162

2378 W-Al/163

$\begin{array}{lllll}0.246 & 1.030 & 0.265 & 0.142 & 10.140 \\ 0.204 & 0.581 & 0.217 & 0.124 & 9.890 \\ 0.192 & 0.408 & 0.206 & 0.117 & 9.810 \\ 0.175 & 0.295 & 0.118 & 0.199 & 9.770 \\ 0.228 & 1.000 & 0.249 & 0.141 & 10.200 \\ 0.218 & 0.930 & 0.237 & 0.136 & 10.140\end{array}$

$\begin{array}{lllll}36.100 & 32.800 & 108.050 & 139.500 & 10.700 \\ 83.960 & 46.240 & 194.670 & 163.750 & 11.200\end{array}$


2310 Frit 21,25 SA

2311 Frit $21,30 \mathrm{SA}$
2312 Fit $18,25 \mathrm{SC}$

2313 Frit 21, $20 \mathrm{SC}$
2314 Frit $21,25 \mathrm{SC}$

2315 Frit 21,30 sC

2316 HT-S-1

$2319 H T$ T.S.3
$2319 H-S-4$

2320 HT.S-5

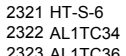

$2323 \mathrm{ALLTC36}$
$2324 \mathrm{AL} 4 \mathrm{LTC} 34$

2325 ALLTCC34
2326 ALTTC27

2327 HTS 1

2328 HTS2
2329 HTS 3

2329 HTS3
$2330 \mathrm{HTS}$

2330 HTS4
2333 HTSS

$2332 \mathrm{HTS6}$
$2333 \mathrm{HTS7}$

2334 HTS8

2335 HTS

2336 HTS10

2338 HTS 12

$2339 \mathrm{HTS13}$

$2342 \mathrm{HTS} 16$

234t

2347 HTS21

2348 HTS22

2349 WRReflass
2355 SMISFrR
2251535

2351 SM513LW0

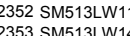

2354 SRL-202-G

2356 W-Al/131

2357 W-Al/141

2358 W-All142

2360 W-Al/144

2361 W-All145
2362 W-All14

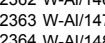

2364 W-Al/148
2365 W-Al/149

$2366 \mathrm{~W}-\mathrm{A} / 150$

2367 W-AAl/15
2368 W-Al/152

2369 W-Al1154

2370 W-Al1155

2371 W-All156
2372 W-All157

2373 W-Al/158

2374 W-Al/159

2375 W-All1160
2376 WA116

2377 W-All162

2378 W-Al/163

(2) 


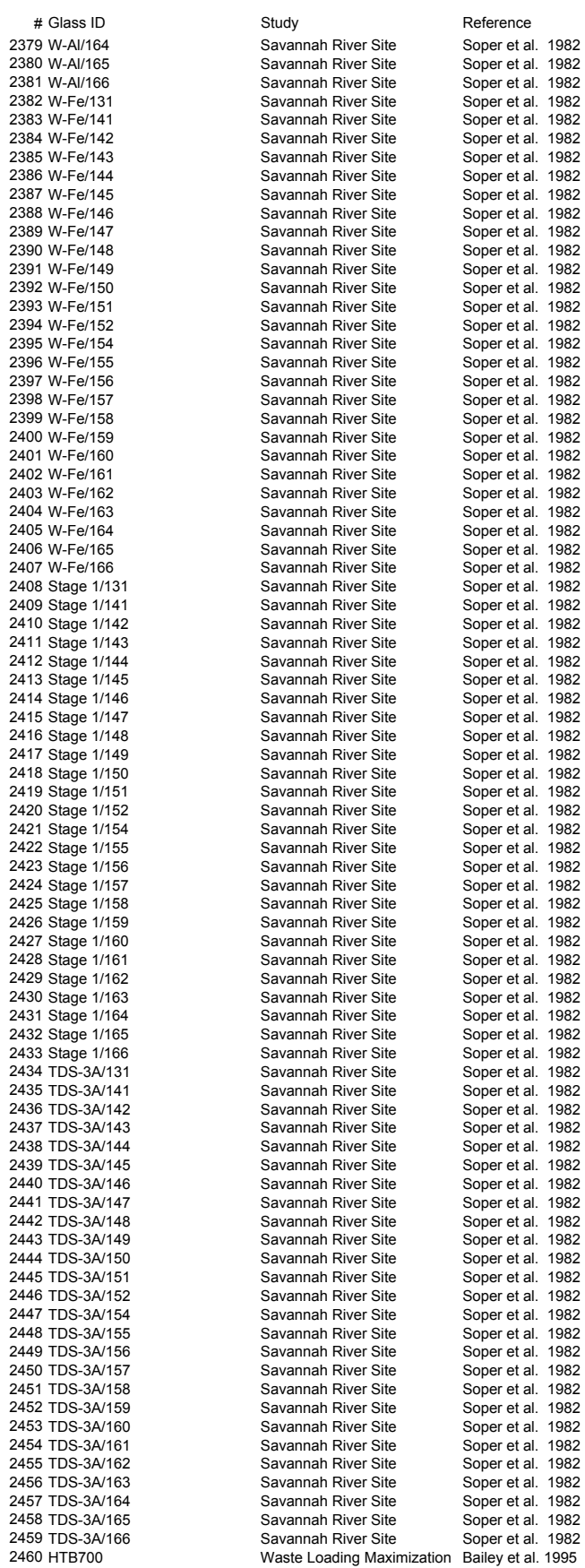

Al2O3-t B2O3-t CaO-t Fe2O3-t FeO-t $\begin{array}{llll}0.1467 & 0.0856 & 0.0042 & 0.0396 \\ 0.1467 & 0.0713 & 0.0042 & 0.0396\end{array}$ $\begin{array}{lllll}0.1467 & 0.0856 & 0.0042 & 0.0396\end{array}$ $\begin{array}{llll}0.0080 & 0.1032 & 0.0164 & 0.1761 \\ 0.0080 & 0.1032 & 0.0164 & 0.1761\end{array}$

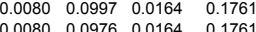
$\begin{array}{llll}0.0080 & 0.0976 & 0.0164 & 0.1761 \\ 0.0080 & 0.1046 & 0.0164 & 0.1761\end{array}$ $\begin{array}{llll}0.0080 & 0.0779 & 0.0164 & 0.1761\end{array}$ $\begin{array}{lllll}0.0080 & 0.0934 & 0.0164 & 0.1761\end{array}$ $\begin{array}{llll}0.0080 & 0.1074 & 0.0164 & 0.1761 \\ 0.0080 & 0.0821 & 0.0164 & 0.1761\end{array}$ $\begin{array}{lllll}0.0080 & 0.0821 & 0.0164 & 0.1761 \\ 0.0080 & 0.0849 & 0.0164 & 0.1761\end{array}$ $\begin{array}{llll}0.0080 & 0.0885 & 0.0164 & 0.1761\end{array}$ $\begin{array}{llll}0.0080 & 0.0969 & 0.0164 & 0.1761 \\ 0.0080 & 0.0765 & 0.0164 & 0.1761\end{array}$ $\begin{array}{lllll}0.0080 & 0.0765 & 0.0164 & 0.1761 \\ 0.0080 & 0.0793 & 0.0164 & 0.1761\end{array}$ $\begin{array}{llll}0.0080 & 0.0730 & 0.0164 & 0.1761 \\ 0.0080 & 0.0976 & 0.164 & 0.1761\end{array}$ $\begin{array}{llll}0.0080 & 0.0976 & 0.0164 & 0.1761 \\ 0.0080 & 0.0920 & 0.0164 & 0.1761\end{array}$ $\begin{array}{llll}0.0080 & 0.0920 & 0.0164 & 0.1761 \\ 0.0080 & 0.0681 & 0.0164 & 0.1761 \\ 0 & 0.0908 & 0.0164 & 0.1761\end{array}$ $\begin{array}{llll}0.0080 & 0.0969 & 0.0164 & 0.1761 \\ 0.0080 & 0.0744 & 0.0164 & 0.1761\end{array}$ $\begin{array}{llll}0.0080 & 0.0913 & 0.0164 & 0.1761 \\ 0.0080 & 0.0913 & 0.164 & 0.1761\end{array}$ $\begin{array}{lllll}0.0080 & 0.0842 & 0.0164 & 0.1761\end{array}$ $\begin{array}{llll}0.0080 & 0.0702 & 0.0164 & 0.1761\end{array}$ $\begin{array}{llll}0.0498 & 0.1020 & 0.0168 & 0.1264\end{array}$ $\begin{array}{llll}0.0498 & 0.1020 & 0.0168 & 0.1264\end{array}$ $\begin{array}{llll}0.0498 & 0.0985 & 0.0168 & 0.1264 \\ 0.0498 & 0.095 & 0.0168 & 0.124\end{array}$

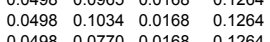
$\begin{array}{lllll}0.0498 & 0.0770 & 0.0168 & 0.1264 \\ 0.0498 & 0.0770 & 0.0168 & 0.1264\end{array}$ $\begin{array}{lllll}0.0498 & 0.0923 & 0.0168 & 0.1264\end{array}$ $\begin{array}{lllll}0.0498 & 0.1062 & 0.0168 & 0.1264\end{array}$ $\begin{array}{llll}0.0498 & 0.0812 & 0.0168 & 0.124 \\ 0.0498 & 0.0840 & 0.0168 & 0.1264 \\ 0.0498 & 0.074 & 0.0188 & 0.1264\end{array}$ $\begin{array}{llll}0.0498 & 0.0958 & 0.0168 & 0.1264\end{array}$ $\begin{array}{llll}0.0498 & 0.0756 & 0.0168 & 0.1264\end{array}$ $\begin{array}{llll}0.0498 & 0.0784 & 0.0168 & 0.1264 \\ 0.0498 & 0.0722 & 0.0168 & 0.1264\end{array}$

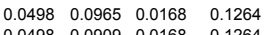

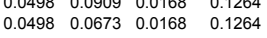
$\begin{array}{ll}.0168 & 0.1264 \\ .0168 & 0.1264\end{array}$ $\begin{array}{lllll}0.0498 & 0.0902 & 0.0168 & 0.1264\end{array}$ $\begin{array}{llll}0.0498 & 0.0902 & 0.0168 & 0.1264 \\ 0.0498 & 0.0833 & 0.0168 & 0.1264\end{array}$ $\begin{array}{llll}0.0498 & 0.0694 & 0.0168 & 0.1264 \\ 0.0498 & 0.0833 & 0.0168 & 0.1264\end{array}$ $\begin{array}{lllll}0.0315 & 0.1032 & 0.0152 & 0.1410\end{array}$ $\begin{array}{llll}0.0315 & 0.1032 & 0.0152 & 0.1410 \\ 0.0315 & 0.0997 & 0.0152 & 0.1410\end{array}$ $\begin{array}{llll}0.0315 & 0.0976 & 0.0152 & 0.1410 \\ 0.0315 & 0.1046 & 0.52 & 0.140\end{array}$ $\begin{array}{llll}0.0315 & 0.1046 & 0.0152 & 0.1410 \\ 0.0315 & 0.0779 & 0.0152 & 0.1410\end{array}$ $\begin{array}{llll}0.0315 & 0.0779 & 0.0152 & 0.1410 \\ 0.0315 & 0.0934 & 0.0152 & 0.1410 \\ 0.0315 & 0.084 & 0.052 & 0.1410\end{array}$ $\begin{array}{llll}0.0315 & 0.1074 & 0.0152 & 0.1410 \\ 0.0315 & 0.021 & 0.152 & 0.140\end{array}$ $\begin{array}{lllll}0.0315 & 0.0849 & 0.0152 & 0.1410\end{array}$ $\begin{array}{llll}0.0315 & 0.0885 & 0.0152 & 0.1410 \\ 0.0315 & 0.0969 & 0.152 & 0.1410\end{array}$ $\begin{array}{llll}0.0315 & 0.0765 & 0.0152 & 0.1410\end{array}$ $\begin{array}{llll}0.0315 & 0.0793 & 0.0152 & 0.1410 \\ 0.0315 & 0.0730 & 0.0152 & 0.1410\end{array}$ $\begin{array}{llll}0.0315 & 0.0920 & 0.0152 & 0.1410 \\ 0 & 0.1410\end{array}$ $\begin{array}{llll}0.0315 & 0.0681 & 0.0152 & 0.1410 \\ 0.0315 & 0.0969 & 0.0152 & 0.1410\end{array}$ $\begin{array}{llll}0.0315 & 0.0969 & 0.0152 & 0.1410 \\ 0.0315 & 0.0744 & 0.0152 & 0.1410 \\ 0 & 0.01510\end{array}$ $\begin{array}{llll}0.0315 & 0.0913 & 0.0152 & 0.1410 \\ 0.0315 & 0.0913 & 0.152 & 0.1410\end{array}$ $\begin{array}{llll}0.0315 & 0.0913 & 0.0152 & 0.1410 \\ 0.0315 & 0.0842 & 0.0152 & 0.1410\end{array}$ $\begin{array}{llll}0.0315 & 0.0842 & 0.0152 & 0.1410 \\ 0.0972 & 0.0006 & 0.0154 & 0.0822\end{array}$
K2O-t Li2O-t MgO-t Na2O-t NiO-t P2O5-t SiO2-t Zro2-t Ag2O-t As203-tAs205-t BaO-t BeO-t Bi2O3-t Br-t CdO-t Ce2O3-t CeO2-t Cl-t

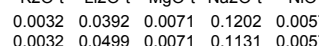
$\begin{array}{llllllllll}0.0032 & 0.0499 & 0.0037 & 0.12131 & 0.0057\end{array}$

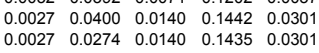
$\begin{array}{llllll}0.0027 & 0.0267 & 0.0063 & 0.1063 & 0.030\end{array}$

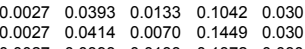
$\begin{array}{lllll}0.0027 & 0.0393 & 0.0133 & 0.1372 & 0.030 \\ 0.0027 & 0.0393 & 0.0133 & 0.1372 & 030\end{array}$ $\begin{array}{lllllll}0.0027 & 0.0309 & 0.0077 & 0.1210 & 0.0301\end{array}$

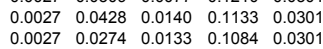
$\begin{array}{llllll}0.0027 & 0.0274 & 0.0133 & 0.1084 & 0.030 \\ 0.0027 & 0.0281 & 0.0140 & 0.147 & 0.030\end{array}$ $\begin{array}{llllll}0.0027 & 0.0442 & 0.0077 & 0.1161 & 0.030\end{array}$ $\begin{array}{lllll}0.0027 & 0.0442 & 0.0077 & 0.147 & 0.0301 \\ 0.0027 & 0.0260 & 0.0063 & 0.1538 & 0.0301 \\ 0.0027 & 0.0442 & 0.0049 & 0.1168 & 0.0301 \\ 0.0301\end{array}$ $\begin{array}{lllll}0.0027 & 0.0442 & 0.0049 & 0.1168 & 0.0301 \\ 0.0027 & 0.0456 & 0.0126 & 0.1554 & 0.0301\end{array}$ $\begin{array}{lllll}0.0027 & 0.04295 & 0.00249 & 0.1545 & 0.0301 \\ 0.0027 & 0.00442 & 0.0049 & 0.156 & 0.0301 \\ 0.0027 & 0.0301 & 0.0301\end{array}$ $\begin{array}{lllll}0.0027 & 0.0442 & 0.0049 & 0.1512 & 0.0301 \\ 0.0027 & 0.0246 & 0.0056 & 0.1021 & 0.0301 \\ 0.027 & 0.0336 & 0.112 & 0.120 & 0301\end{array}$ $\begin{array}{llllll}0.0027 & 0.02268 & 0.005 & 0.1521 & 0.1121 & 0.030 \\ 0.0227 & 0.0288 & 0.0112 & 0.112 & 0.0301\end{array}$ $\begin{array}{lllll}0.0027 & 0.0316 & 0.0119 & 0.1203 & 0.030 \\ 0.0027 & 0.0260 & 0.0126 & 0.1049 & 0.0301\end{array}$ $\begin{array}{lllll}0.0027 & 0.0260 & 0.0126 & 0.1049 & 0.0301 \\ 0.0027 & 0.0281 & 0.0070 & 0.1112 & 0.0301\end{array}$ $\begin{array}{llllll}0.0027 & 0.0281 & 0.0070 & 0.1112 & 0.0301 \\ 0.0027 & 0.0316 & 0.0070 & 0.1042 & 0.030\end{array}$ $\begin{array}{llllll}0.0027 & 0.0386 & 0.0070 & 0.1182 & 0.0301 \\ 0.0027 & 0.0491 & 0.0070 & 0.1112 & 0.030\end{array}$ $\begin{array}{llllll}0.0027 & 0.0491 & 0.0070 & 0.1112 & 0.030 \\ 0.0027 & 0.0386 & 0.0070 & 0.1182 & 0.0301\end{array}$ $\begin{array}{lllllll}0.0019 & 0.0396 & 0.0139 & 0.1329 & 0.0107\end{array}$ $\begin{array}{lllll}0.0019 & 0.0271 & 0.0139 & 0.1322 & 0.0107 \\ 0.0019 & 0.0264 & 0.0062 & 0.0954 & 0.0107\end{array}$ $\begin{array}{lllll}0.0019 & 0.0264 & 0.0062 & 0.0954 & 0.0107 \\ 0.0019 & 0.0389 & 0.0132 & 0.0933 & 0.0107\end{array}$ $\begin{array}{lllll}0.0019 & 0.0409 & 0.0069 & 0.1336 & 0.0177\end{array}$ $\begin{array}{lllll}0.0019 & 0.0389 & 0.0132 & 0.1260 & 0.0107 \\ 0.0019 & 0.0389 & 0.0132 & 0.1260 & 0.0107\end{array}$ $\begin{array}{lllll}0.0019 & 0.0389 & 0.0132 & 0.1260 & 0.0107 \\ 0.0019 & 0.0305 & 0.0076 & 0.1100 & 0.0107\end{array}$ $\begin{array}{llllll}0.0019 & 0.0423 & 0.0139 & 0.1024 & 0.0107\end{array}$ $\begin{array}{llllll}0.0019 & 0.0271 & 0.0132 & 0.0975 & 0.0107 \\ 0.0019 & 0.0278 & 0.0139 & 0.1364 & 0.0107\end{array}$ $\begin{array}{llllll}0.0019 & 0.0278 & 0.0139 & 0.1364 & 0.0117 \\ 0.0019 & 0.0437 & 0.0076 & 0.1051 & 0.0107 \\ 0.0019 & 0.0437 & 0.004 & 0.1058 & 0.0107\end{array}$ $\begin{array}{lllll}0.0019 & 0.0257 & 0.0062 & 0.1246 & 0.0107 \\ 0.0019 & 0.0437 & 0.0049 & 0.1058 & 0.0107\end{array}$ $\begin{array}{lllll}0.0019 & 0.0451 & 0.0125 & 0.1440 & 0.0107 \\ 0.0019 & 0.0291 & 0.0049 & 0.1343 & 0.010\end{array}$ $\begin{array}{lllll}0.0019 & 0.0437 & 0.0049 & 0.1398 & 0.0107\end{array}$ $\begin{array}{llllll}0.0019 & 0.0243 & 0.0056 & 0.0913 & 0.0107 \\ 0.0019 & 0.0285 & 0.0110 & 0.1003 & 0.0107\end{array}$ $\begin{array}{llllll}0.0019 & 0.0285 & 0.0110 & 0.1003 & 0.0107 \\ 0.0019 & 0.0312 & 0.0118 & 0.1093 & 0.0107\end{array}$ $\begin{array}{lllll}0.0019 & 0.0257 & 0.0125 & 0.0940 & 0.0107\end{array}$ $\begin{array}{lllll}0.0019 & 0.0278 & 0.0069 & 0.1003 & 0.0107 \\ 0.0019 & 0.0312 & 0.0069 & 0.0933 & 0.0107\end{array}$ $\begin{array}{lllll}0.0019 & 0.0312 & 0.0069 & 0.0933 & 0.0107 \\ 0.0019 & 0.0382 & 0.0069 & 0.1072 & 0.0107\end{array}$

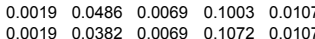
$\begin{array}{llllll}0.0019 & 0.0382 & 0.0069 & 0.1072 & 0.0107 \\ 0.0033 & 0.0400 & 0.0140 & 0.1358 & 0.0173\end{array}$ $\begin{array}{llllll}0.0033 & 0.0267 & 0.0063 & 0.0979 & 0.013 \\ 0.0033 & 0.0393 & 0.0133 & 0.0958 & 0.0173 \\ 0.033 & 0.0443 & 0.0133 & 0.128 & 0.0173\end{array}$ $\begin{array}{lllll}0.0033 & 0.0414 & 0.0070 & 0.1365 & 0.0173 \\ 0.0033 & 0.0393 & 0.0133 & 0.1288 & 0.0173\end{array}$ $\begin{array}{llllll}0.0033 & 0.0393 & 0.0133 & 0.1288 & 0.0173 \\ 0.0033 & 0.0309 & 0.0077 & 0.1127 & 0.0173\end{array}$ $\begin{array}{llllll} & 0.0033 & 0.0309 & 0.0077 & 0.1127 & 0.0173 \\ 0.0033 & 0.0428 & 0.0140 & 0.1050 & 0.0173\end{array}$ $\begin{array}{llllll}0.0033 & 0.0428 & 0.0140 & 0.11050 & 0.0173 \\ 0.0033 & 0.0274 & 0.0133 & 0.1000 & 0.0173\end{array}$ $\begin{array}{lllll}0.0033 & 0.0281 & 0.0140 & 0.1394 & 0.0173 \\ 0.0033 & 0.0442 & 0.0077 & 0.1078 & 0.0173\end{array}$ $\begin{array}{llllll}0.0033 & 0.0260 & 0.0063 & 0.1274 & 0.0173\end{array}$ $\begin{array}{llll}0.0033 & 0.0456 & 0.00426 & 0.1085 \\ 0.0033 & 0.1471 & 0.0173\end{array}$ $\begin{array}{llllll}0.0033 & 0.0442 & 0.0049 & 0.1429 & 0.0173\end{array}$ $\begin{array}{lllll}0.0033 & 0.0246 & 0.0056 & 0.0937 & 0.0173 \\ 0.0033 & 0.0288 & 0.0112 & 0.1028 & 0.0173\end{array}$ $\begin{array}{llllll}0.0033 & 0.0288 & 0.0112 & 0.1028 & 0.0173 \\ 0.0033 & 0.0316 & 0.0112 & 0.1120 & 0.0173\end{array}$ $\begin{array}{lllll}0.0033 & 0.0260 & 0.0126 & 0.0965 & 0.0173 \\ 0.0033 & 0.0281 & 0.0070 & 0.1028 & 0.0173\end{array}$ $\begin{array}{lllll}0.0033 & 0.0316 & 0.0070 & 0.0958 & 0.0173 \\ 0.0033 & 0.0386 & 0.0070 & 0.1099 & 0.0173\end{array}$ $\begin{array}{lllll}0.0033 & 0.0386 & 0.0070 & 0.1099 & 0.0173 \\ 0.0033 & 0.0491 & 0.0070 & 0.1028 & 0.0173\end{array}$

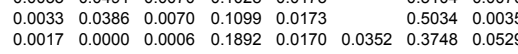

$\begin{array}{lll}0.5122 & 0.0036 \\ 0.5193 & 0.0071\end{array}$

$\begin{array}{ll}0.5122 & 0.0036 \\ 0.4348 & 0.0035\end{array}$

$\begin{array}{lll}0.4411 & 0.0000 \\ 0.4916 & 0.0063\end{array}$

$0.4832 \quad 0.0000$

$\begin{array}{ll}0.4453 & 0.0070 \\ 0.4832 & 0.0000 \\ 0.4713 & 0.0000\end{array}$

$\begin{array}{lll}0.4773 & 0.0000 \\ 0.4965 & 0.0000\end{array}$

0.45790 .0070

$\begin{array}{lll}0.5057 & 0.0070 \\ 0.4537 & 0.0070 \\ 0.4720 & 0.0000\end{array}$

$\begin{array}{ll}0.4720 & 0.0000 \\ 0.4790 & 0.0000 \\ 0.5029 & 0.0000\end{array}$

$\begin{array}{lll}0.4516 & 0.0000 \\ 0.4846 & 0.0000 & 0\end{array}$

$\begin{array}{ll}0.4846 & 0.0000 \\ 0.4383 & 0.0000\end{array}$

$\begin{array}{lll}0.5092 & 0.0028 \\ 0.5148 & 0.0000 & 0\end{array}$

0.48950 .0000

$0.5022 \quad 0.0035$

0.49870 .0035

$0.5057 \quad 0.0070$

$\begin{array}{lll}0.4554 & 0.0035 \\ 0.4616 & 0.0000 & 0\end{array}$

$\begin{array}{ll}0.4616 & 0.0000 \\ 0.5116 & 0.0062\end{array}$

$\begin{array}{ll}0.4658 & 0.0069 \\ 0.5033 & 0.0000\end{array}$

$\begin{array}{ll}0.4015 & 0.0000 \\ 0.49164 & 0.0000\end{array}$

$0.4783 \quad 0.006$

$\begin{array}{ll}0.5255 & 0.0069 \\ 0.4741 & 0.0069\end{array}$

0.49910 .0000

0.5270 .00000

$\begin{array}{lll}0.4527 & 0.0000 \\ 0.4720 & 0.0000 \\ 0.5047 & 0.0000\end{array}$

$\begin{array}{ll}0.4588 & 0.0000 \\ 0.5289 & 0.0028\end{array}$

$\begin{array}{lll}0.5345 & 0.0000 \\ 0.5095 & 0.0000 \\ 0.556 & 0.002\end{array}$

$0.5456 \quad 0.0028$

$\begin{array}{ll}0.5220 & 0.0035 \\ 0.5255 & 0.0035 \\ 0.5185 & 0.0035\end{array}$

$0.5255 \quad 0.0069$

$\begin{array}{lll}0.5185 & 0.0035\end{array}$

$\begin{array}{lll}4459 & 0.0000 \\ 4964 & 0.0063\end{array}$

$0.4880 \quad 0.0000$

$\begin{array}{ll}0.4501 & 0.0070 \\ 0.4880 & 0.0000\end{array}$

$0.4760 \quad 0.0000$

0.46270 .0070

$\begin{array}{ll}0.5104 & 0.0070 \\ 0.4585 & 0.0070 \\ 0.476 & 0.0000\end{array}$

$0.4838 \quad 0.0000$

\begin{tabular}{ll}
5.5076 & 0.0000 \\
\hline $4564 \quad 0.0000$
\end{tabular}

$0.4430 \quad 0.0000$

.

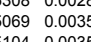

0.0035
0.54

$\begin{array}{ll}0.5034 & 0.0035 \\ 0.3748 & 0.0529\end{array}$
0.0146

0.0007 
I-t La2O3-t MnO2-t MnO-t MoO-t MoO2-t MoO3-t Nb205-t Nd2O3-t NpO2-t PbO-t PdO2-t PdO-t Pr2O3-t Pr6011-t Rb2O-t ReO-t

2380 W-Al/165

2381 W-Al/1/166
2382 W-Fe/131
2333
W-Fe/114

$2383 \mathrm{~W}-\mathrm{F} / 1414$
$2384 \mathrm{~W}-\mathrm{F} / 142$

$2385 \mathrm{~W}$-Fe//43

2387 W-Fe/ 145

$2388 \mathrm{~W}-\mathrm{Fe} / 14$

$2390 \mathrm{~W}-\mathrm{Fe} / 148$

2392 W-Fer/150

2393 W-Fe/15

$2395 \mathrm{~W}$-Fe/154

2397 W-Fe/156 2398 W-Fe/15

2399 W-Fe/1

2401 W-Fe/ 160

2403 W-Fe/162

2404 W-Fe/16

2406 W-Fe/ 16

2408 Stage $1 / 13$

2409 Stage $1 / 141$

2410 Stage $1 / 142$

2412 Stage $1 / 144$

2413 Stage 1/145

2414 Stage $1 / 146$

2416 Stage $1 / 148$

2417 Stage 1/149

2418 Stage $1 / 150$
2419 Stage $1 / 151$

2420 Stage $1 / 1152$

2421 Stage $1 / 154$
2422 Stage $1 / 155$

2422 Stage 1/155
2423 Stage $1 / 156$
2424 Stage $1 / 157$

2424 Stage $1 / 157$
2425 Stage $1 / 158$

2426 Stage 1/159

2428 Stage 1/16

2429 Stage 1/162

2430 Stage $1 / 16$

2432 Stage $1 / 165$

2433 Stage 1/166

2436 TDS-3A/142

2437 TDS-3A14 143

2438 TDS-3A 144

2440 TDS-3A 14

2441 TDS-3A/147
2442 TDS-3A148
2443 TDS-3A/149

2444 TDS-3A/15

2445 TDS 3 -3A115
2446 TDS-3A15 152

447 TDS-3A/15

2448 TDS-3A/15

2449 TDS-3A 15

2451 TDS-3A/15

2452 TDS-3A 155

2453 TDS-3A/160

2455 TDS-3A162

2456 TDS-3A163

2458 TDS-3A/16

2459 TDS-3A/166
2460 HTB700 


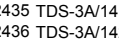

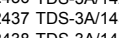

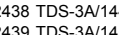

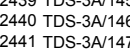

2442 TDS-3A 3148
2434

2444 TDS $3 A 15$

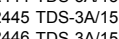

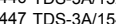

2448 TDS-3A155
2449 TDS-3A 156

2450 TDS-3A157

2451 TDS-3A158
2452 TDS- $3 A$ A 159 
2380 W-Al/165

2382 W-Fe/ 131

2383 W-Fe/141

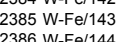

2387 W-Fe/ 145

$2388 \mathrm{~W}-\mathrm{Fe} / 146$
2389
$\mathrm{~W}-\mathrm{Fe} / 142$

3900 W-Fel/148

2391 W-Fel/149

2393 W-Fel/151

2394 W-Fe/l152
2355 W

2395 W-Fer/154

2397 W-Fe/156

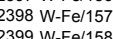

2399 W-Fer/15
2400 WF-eet150

2401 W-Fe/160

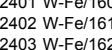

2403 W-Fel/162

2405 W-Fel/164

2406 W-Fel/165
2407 W-Fe/ 166

2407 W.Fel/166
2408 Stage $1 / 1 / 13$

2409 Stage $1 / 1 / 14$

2410 Stage $1 / 1442$

2412 Stage $1 / 1 / 44$

2413 Stage $1 / 145$

\begin{tabular}{l}
2414 Stage 1/146 \\
2415 Stage $1 / 147$ \\
\hline
\end{tabular}

2416 Stage 1/148
2417 Stage $1 / 149$

2418 Stage $1 / 1150$
2419 Stang 1151

2420 Stage $1 / 152$

2421 Stage 1154
2422 Stage $1 / 155$
(2)

\begin{tabular}{l}
2425 Stage 1155 \\
2423 Stage $1 / 156$ \\
\hline
\end{tabular}

2424 Stage $1 / 1157$

2425 Stage $1 / 158$
2426

2426 Stage $1 / 159$
2427 Stage $1 / 160$

2428 Stage 1/161

2429 Stage $1 / 1162$
24305

2430 Stage $1 / 163$
2431 stage $1 / 164$

2432 Stage 1/165

2433 Stage $1 / 1166$
2434 TDS $3 A 1131$

2435 TDS-3A $1 / 14$

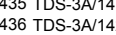

2437T DDS-3A144

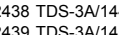

2440 TDS-3A 14

2441 TDS.-3A14

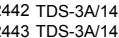

2444 TDS-3A15

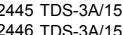

2477 TDS-3A154

2448 TDS-3A155

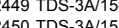

2451 TDS-3A 15

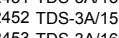

2453 TDS-3A166
2454 TDS-3A146

2455 TDS-3A162

2456 TDS-3A163

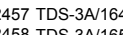

2459 TDS-3A 3166
2460 HTB700 
2417 Stage $1 / 149$
2418 stage $1 / 150$

2419 Stage $1 / 115$
2420 Stage $1 / 152$
2.225

2420 Stage $1 / 1152$
2421 Stage $1 / 154$

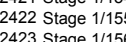

2423 Stage $1 / 1156$
2424 stage $1 / 157$
2253

2425
2 tage $1 / 1155$
22255

2426 tage 111159
2427 stage 11160
2

2428 stage $1 / 116$

2429 Stage 11162

2430 Stage 1/1163
2431 Stage 116464

2432 Stage $1 / 165$
2433 Stage $1 / 166$
2434 Tag $3 A 1131$

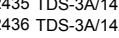

2437 TDS 3 A 143

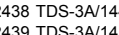

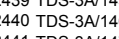

2441 TDS-3A 14

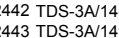

2444 TDS-3A 15

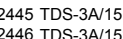

2447 TDS-3A 154

2448 TDS-3A 155

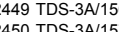

2451 TDS-3A158

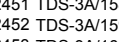

2453 SDS-3A166
254 TDS

2455 TDS-3A 162

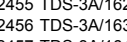

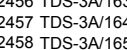

2459 TDS-3A166 
2413 Stage $1 / 145$
2414 Stage 11146

2414 Stage $1 / 146$
2415 Stage $1 / 147$

2416 Stage $1 / 1 / 48$

2417 Stage 1/149

2418 Stage 1/150
2419 Stage $1 / 151$

2420 Stage $1 / 152$

2421 Stage $1 / 1154$
24225

2422 Stage $1 / 155$
2423 Stage $1 / 156$

2424 Stage $1 / 1157$

2425 Stage 111158
2226
227

2426 Stage $1 / 155$
2427 Stage $1 / 160$

2428 Stage 1/116

2229 Stage 1/162

2430 Stage $1 / 1163$
2431 Stage $1 / 164$

2432 Stage 1/165

2433 Stage 1/1466

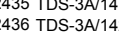

2437 TDS 3 A 143

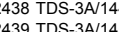

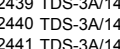

2441 DDS-3A 147

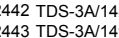

2444 TDS-3A 15

2445 TDS $-3 A 15$

(4) TDS-3A154

2448 PDS-3A1155

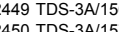

2451 TDS-3A 15

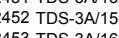

2453 SDS-3A166
254 TDS

2455 TDS-3A 162

2456 TDS-3A163

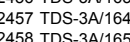

2459 DDS-3A11166
2460 HTB700

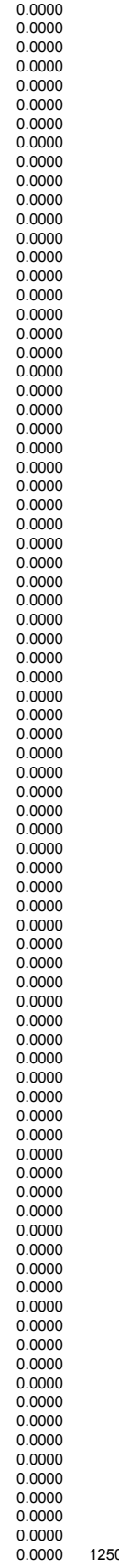


2417 Stage $1 / 149$

2418 stage $1 / 1 / 50$
2419 Stage 1115
25

2420 Stage $1 / 152$

2421 stage $1 / 1154$

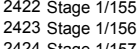

2424 Stage 1/157

2425 Stage $1 / 1158$

2426 Stage 1/1159
2427 Stage 11160

2428 stage $1 / 161$

2429 stage $1 / 162$

2430 Stage 1/1163
2431 Stage 116464

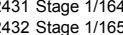

2433 stage $1 / 166$
-2445

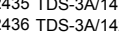

2437 TDS 3 A 143

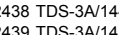

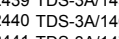

年

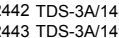

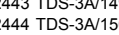

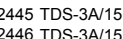

2447 TDS-3A154

2448 TDS-3A1155

2449 TDS -3 A 155

2451 TDS-3A158

2451
2455 TDS-3A
TAA 1155

2453 SDS-3A166
254 TDS

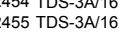

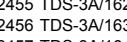

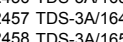


2417 Stage $1 / 149$

2418 Stage 1/150
2419 stage $1 / 151$

2420 Stage $1 / 152$

2421 stage $1 / 1154$

2422 Stage $1 / 155$
2423 stage $1 / 156$

2424 Stage $1 / 1157$

2425 Stage $1 / 1158$

2426 stage $1 / 1155$
2427 Stage $1 / 160$
245

2428 Stage $1 / 166$

2429 Stage 11162

2430 Stage 1/1163
2431 Stage 116464

2432 Stage $1 / 165$
2433 Stage $1 / 166$
2434 Tag $3 A 1131$

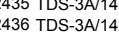

2437 TDS 3 A 143

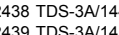

2440 TDS-3A 14

2441 TDS-3A 14

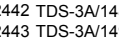

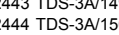

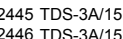

2447 TDS-3A 154

2448 TDS-3A1155

2449 TDS-3A15

2451 TDS-3A158

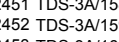

2453 SDS-3A166
254 TDS

2455 TDS-3A 162

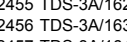

2457 TDS-3A1616
2458 TDS-3A165

2459 TDS-3A166 
2417 Stage $1 / 149$

2418 Stage $1 / 150$
2419 Stage $1 / 151$

2420 Stage $1 / 152$

2421 stage $1 / 1154$

2422 Stage $1 / 155$
2423 stage $1 / 156$

2424 Stage $1 / 1157$

2425 Stage $1 / 1158$

2426 Stage $1 / 1159$
2427 Stage $1 / 160$

2428 Stage $1 / 166$

2429 Stage 11162

2430 Stage 1/1163
2431 Stage 116464

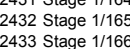

2434 TDS-3A 1131

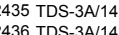

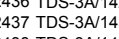

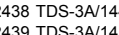

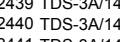

2441 TDS-3A 14

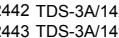

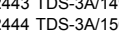

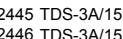

2447 TDS-3A154

2448 TDS-3A1155

2449 TDS -3 A 155

2451 TSS-3A1158

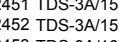

2453 DDS-3A146
2454 TDS-3A146

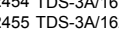

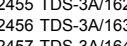

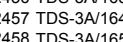


2380 W-Al/165

2382 W-Fel/1361

$2383 \mathrm{~W}-\mathrm{Fe} / 141$

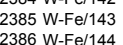

2387 W-Fel/45

2388 W-Fell146

2300 W-Fe/ 148

2391 W-Fel/149

2393 W-Fe/151

2395 W-Fell152

2395 W-Fer/154

2397 W-Fe/156

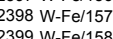

2399 W-Fer/15
2400 WF-eet150

$2400 \mathrm{~W}-\mathrm{eel} 159$
2401
$20 \mathrm{~F}-\mathrm{F} / 160$

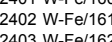

2403 W-Fel/162

2405 W-Fel/164

2406
207 - Fel/165
20 -Fe/ 166

2407 W-Fel/166
2408 Stage $1 / 131$

2409 Stage $1 / 1 / 14$

2410 Stage $1 / 1424$

2411 Stage $1 / 1433$
2412 Stage $1 / 1 / 14$

2413 Stage $1 / 145$

2414 Stage $1 / 146$
2415 Stage $1 / 147$

2416 Stage 1/148

2417 Stage 1/149

2418 Stage 1/150
2419 Stage $1 / 1151$

2420 Stage $1 / 152$

2421 Stage 1154
2422 Stage $1 / 155$
(2)

2422 Stage $1 / 155$
2423 Stage $1 / 156$

2424 Stage $1 / 1157$

2425 Stage 111158
2226
227

2426 Stage $1 / 159$
2427 Stage $1 / 160$

2428 Stage 1/161

2429 Stage $1 / 1162$
24305

2430 Stage 111163
2431 stage 11164

2432 stage $1 / 165$

2433 Stage $1 / 1166$
2434 TDS $3 A 1131$

2435 TDS-3A $1 / 14$

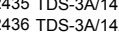

2437T DDS-3A144

2438 DDS-3B 144
2439
$20 S-3 A 144$

2440 TDS-3A 146

2441 TDS-3A 14

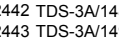

2444 TDS-3A15

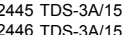

2477 TDS-3A154

2448 PDS-3A1155

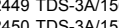

2451 TDS-3A 15

2452 TDS -3A A 150

2453 TDS-3A166
2454 TDS-3A146

2455 TDS-3A 162

2456 TDS-3A163

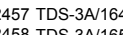

2459 TDS-3A166 


\begin{tabular}{|c|c|c|c|}
\hline \# Glass ID & Study & Reference & \\
\hline 2461 HTB725 & Waste Loading Maximization & Bailey et al. 1 & 1995 \\
\hline 2462 HTB750 & Waste Loading Maximization & & 1995 \\
\hline 2463 HTB775 & Waste Loading Maximization & Bailey et al. 1 & 1995 \\
\hline $\begin{array}{l}2464 \text { HTB800 } \\
2465 \text { HTB825 }\end{array}$ & Waste Loading Maximization & Bailey et al. 1 & 1995 \\
\hline $\begin{array}{l}2465 \text { HTB825 } \\
2466 \text { HTB850 }\end{array}$ & $\begin{array}{l}\text { Waste Loading Maximization } \\
\text { Waste Loading Maximization }\end{array}$ & $\begin{array}{l}\text { Bailey et al. } 1 \\
\text { Baley tal }\end{array}$ & 1995 \\
\hline 2467 PFP-OP & $\begin{array}{l}\text { Waste Laading Maximization } \\
\text { PFP Phosphorous Study }\end{array}$ & $\begin{array}{l}\text { Balily et al. } 1 \\
\text { Heckle et al. } 1\end{array}$ & 1995 \\
\hline 68 PFP-3.3P & $\begin{array}{l}\text { PFP Phosphorous Study } \\
\text { PFos }\end{array}$ & $\begin{array}{l}\text { Heckle et al. } 1 \text {. } \\
\text { Hecle ent }\end{array}$ & $\begin{array}{l}.1994 \\
.1994\end{array}$ \\
\hline 469 PFP-5.3P & PFP Phosphorous Study & Heckle et al. 1 & \\
\hline 2470 PFP-7.3P & PFP Phosphorous Study & Heckle et al. 1 & \\
\hline 2471 PFP 5.3P/no Cr & PFP Phosphorous Study & Heckle et al. 1 & 1994 \\
\hline 2472 PFP 3.3P/no Cr & PFP Phosphorous Study & Heckle et al 1 & \\
\hline 2473 PFP 3.3P/no Li & 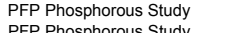 & Heckle et al. 1 & \\
\hline 2474 PFP 3.3P/3 Li & PFP Phosphorous Study & Heckle et al. 1 & .1994 \\
\hline $\begin{array}{l}2445 \text { HTBB51 } \\
2476 \text { HTB651 }\end{array}$ & $\begin{array}{l}\text { Waste Laading Maximization } \\
\text { Waste Loading Maximization }\end{array}$ & $\begin{array}{l}\text { Baliey et al. } 1 \\
\text { Baleyetal }\end{array}$ & 1995 \\
\hline $\begin{array}{l}24767 \text { HTB651 } \\
247651\end{array}$ & Waste Loading Maximization & $\begin{array}{l}\text { Balley ex al. } 1 \\
\text { Bailey et al. } 1\end{array}$ & $\begin{array}{l}1995 \\
1995\end{array}$ \\
\hline 2478 HTB651Cro. 6 & $\begin{array}{l}\text { Waste Loading Maximization } \\
\text { Waste Lation }\end{array}$ & $\begin{array}{l}\text { Bailey et al. } 1 \\
\text { Balley }\end{array}$ & 1995 \\
\hline 2479 HTB651Cro. 8 & Waste Loading Maximization & $\begin{array}{l}\text { Baley et al. } 1 \\
\text { Ball }\end{array}$ & 1995 \\
\hline 2480 HTB651P5 & Waste Loading Maximization & Bailey et al. 1 & 1995 \\
\hline 2481 HTB651P7 & Waste Loading Maximization & Bailey et al. 1 & 1995 \\
\hline 2482 HTB651P9 & Waste Loading Maximization & Bailey et al. 1 & \\
\hline $\begin{array}{l}2483 \text { HTB651Ti1 } \\
\text { 2484 HTB651Ti }\end{array}$ & $\begin{array}{l}\text { Waste Loading Maximization } \\
\text { Waste Lagding Maximization }\end{array}$ & $\begin{array}{l}\text { Bailey et al. } 1 \\
\end{array}$ & 1995 \\
\hline $\begin{array}{l}2484 \text { HTB651Ti3 } \\
\text { 2485 HTB651Ti5 }\end{array}$ & $\begin{array}{l}\text { Waste Laading Maximization } \\
\text { Waste Loading Maximization }\end{array}$ & $\begin{array}{l}\text { Balily et al. } 1 \\
\text { Baley tal al } 1\end{array}$ & 1995 \\
\hline $\begin{array}{l}2485 \text { HTB651Ti5 } \\
2486 \text { HLW98-01 }\end{array}$ & $\begin{array}{l}\text { Waste Loading Maximization } \\
\text { BNFL HLW98 }\end{array}$ & $\begin{array}{l}\text { Baliey et al. } 1 \text {. } \\
\text { Fu and Pegg }\end{array}$ & $\begin{array}{l}1995 \\
91998\end{array}$ \\
\hline 2487 HLW98-02 & BNFL HLW98 & Fu and Pegg & $\begin{array}{l}91998 \\
\text { g } 1998\end{array}$ \\
\hline 2488 HLW98-03 & $\begin{array}{l}\text { BNFL HLW98 } \\
\text { BLW }\end{array}$ & Fu and Pegg & $\begin{array}{l}g 1998 \\
g\end{array}$ \\
\hline 2489 HLW98-04 & BNFL HLW98 & Fu and Pegg & g 1998 \\
\hline 2490 HLW98-05 & BNFL HLW98 & Fu and Pegg & g 1998 \\
\hline 2491 HLW98-06 & $\begin{array}{l}\text { BNFL HLW98 } \\
\text { BNFI HIV8 }\end{array}$ & Fu and Pegg & g 1998 \\
\hline 2492 HLW98-07 & $\begin{array}{l}\text { BNFL HLW98 } \\
\text { BNFL HLW98 }\end{array}$ & Fu and Pegg & $g 1998$ \\
\hline $\begin{array}{l}24993 \text { HLW98-08 } \\
2494 \text { HLW98-09 }\end{array}$ & $\begin{array}{l}\text { BNFL HLW98 } \\
\text { BNFL HLW98 }\end{array}$ & $\begin{array}{l}\text { Fu and Pegg } \\
\text { Fu and Pegg }\end{array}$ & $\begin{array}{l}g 1998 \\
g \\
q\end{array} 1998$ \\
\hline $\begin{array}{l}24944 \text { HLWG8-09 } \\
2495 \text { HLW }-10\end{array}$ & $\begin{array}{l}\text { BNFL HLW98 } \\
\text { BNFL HLW98 }\end{array}$ & $\begin{array}{l}\text { Fu and Pegg } \\
\text { Fu and Pegg }\end{array}$ & $\begin{array}{l}g 1998 \\
g \\
g\end{array} 1998$ \\
\hline 2496 HLW98-11 & BNFL HLW98 & $\begin{array}{l}\text { Fu and Pegg } \\
\text { Fu and Pegg }\end{array}$ & $\begin{array}{l}9 \\
g 1998\end{array}$ \\
\hline 2497 HLW98-12 & BNFL HLW98 & Fu and Pegg & $\begin{array}{l}91990 \\
g\end{array}$ \\
\hline 2498 HLW98-13 & BNFL HLW98 & Fu and Pegg & g 1998 \\
\hline 2499 HLW98-14 & BNFL HLW98 & Fu and Pegg & g 1998 \\
\hline 2500 HLW98-15 & BNFL HLW98 & Fu and Pegg & g 1998 \\
\hline 2501 HLW98-16 & BNFL HLW98 & Fu and Pegg & g 1998 \\
\hline $\begin{array}{l}2502 \text { HLW98-17 } \\
2503 \text { HLW98-18 }\end{array}$ & $\begin{array}{l}\text { BNFL HLW98 } \\
\text { BNFL HLW98 }\end{array}$ & $\begin{array}{l}\text { Fu and Pegg } \\
\text { Fu and Pegg }\end{array}$ & $\begin{array}{l}g \\
g\end{array}$ \\
\hline $\begin{array}{l}2553 \text { HLW98-18 } \\
2504 \text { HLW98-19 }\end{array}$ & $\begin{array}{l}\text { BNFL HLW98 } \\
\text { BNFL HLW98 }\end{array}$ & $\begin{array}{l}\text { Fu and Pegg } \\
\text { Fu and Pegg }\end{array}$ & $\begin{array}{l}g \\
g\end{array}$ \\
\hline $\begin{array}{l}2504 \text { HLWYS HLW -19 } \\
250598-20\end{array}$ & BNFL HLW98 & $\begin{array}{l}\text { Fu and Pegg } \\
\text { Fu and Pegg }\end{array}$ & (1998 \\
\hline 2506 HLW98-21 & BNFL HLW98 & Fu and Pegg & $\begin{array}{l}91990 \\
g\end{array}$ \\
\hline 2507 HLW98-22 & BNFL HLW98 & Fu and Pegg & g 1998 \\
\hline 2508 HLW98-23 & BNFL HLW98 & Fu and Pegg & g 1998 \\
\hline 2509 HLW98-24 & BNFL HLW98 & Fu and Pegg & g 1998 \\
\hline 2510 HLW98-25 & BNFL HLW98 & Fu and Pegg & $g 1998$ \\
\hline 2511 HLW98-26 & $\begin{array}{l}\text { BNFLL HLW98 } \\
\text { BNFL HLW98 }\end{array}$ & Fu and Pegg & g 1998 \\
\hline $\begin{array}{l}2512 \text { HLW } 988-27 B \\
2513 \text { HLW98-28 }\end{array}$ & $\begin{array}{l}\text { BNFL HLW98 } \\
\text { BNFL HLW98 }\end{array}$ & $\begin{array}{l}\text { Fu and Pegg } \\
\text { Fu and Pegg }\end{array}$ & $\begin{array}{l}g \\
g\end{array}$ \\
\hline 2514 HLW98-29 & $\begin{array}{l}\text { BNFL HLV98 } \\
\text { BNFL HLW98 }\end{array}$ & $\begin{array}{l}\text { Fu and Pegg } \\
\text { Fu and Pegg }\end{array}$ & $\begin{array}{l}91998 \\
91998\end{array}$ \\
\hline 2515 HLW98-30 & BNFL HLW98 & $\begin{array}{l}\text { Fu and Pegg } \\
\text { Fu }\end{array}$ & $\begin{array}{l}91998 \\
g 1998\end{array}$ \\
\hline 2516 HLW98-31 & BNFL HLW98 & Fu and Pegg & g 1998 \\
\hline 2517 HLW98-32A & BNFL HLW98 & Fu and Pegg & g 1998 \\
\hline 2518 HLW98-33 & BNFL HLW98 & Fu and Pegg & g 1998 \\
\hline 2519 HLW98-35 & BNFL HLW98 & Fu and Pegg & g 1998 \\
\hline 2520 HLW98-36 & $\begin{array}{l}\text { BNFL HLW98 } \\
\text { BNFI H98 }\end{array}$ & Fu and Pegg & g 1998 \\
\hline $\begin{array}{l}2521 \text { HLWG98-37 } \\
2522 \text { HLW98-38 }\end{array}$ & $\begin{array}{l}\text { BNFL HLW98 } \\
\text { BNFL HLW98 }\end{array}$ & $\begin{array}{l}\text { Fu and Pegg } \\
\text { Fu and Pegg }\end{array}$ & g 1998 \\
\hline 2523 HLW988-39 & BNFL HLW98 & $\begin{array}{l}\text { Fu and Pegg } \\
\text { Fu and Pega }\end{array}$ & $\begin{array}{l}91998 \\
91998\end{array}$ \\
\hline 2524 HLW98-40 & BNFL HLW98 & $\begin{array}{l}\text { Fu and Pegg } \\
\text { Fu Page reg }\end{array}$ & $\begin{array}{l}91990 \\
g\end{array}$ \\
\hline 2525 HLW98-41 & BNFL HLW98 & Fu and Pegg & 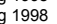 \\
\hline 2526 HLW98-42 & BNFL HLW98 & Fu and Pegg & g 1998 \\
\hline 2527 HLW98-43 & BNFL HLW98 & Fu and Pegg & g 1998 \\
\hline 2528 HLW98-44 & BNFL HLW98 & Fu and Pegg & g 1998 \\
\hline 2529 HLW98-45 & $\begin{array}{l}\text { BNFL HLW98 } \\
\text { BNFI }\end{array}$ & Fu and Pegg & $g 1998$ \\
\hline 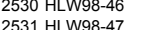 & $\begin{array}{l}\text { BNFL HLW98 } \\
\text { BNFL HLW98 }\end{array}$ & $\begin{array}{l}\text { Fu and Pegg } \\
\text { Fu and Pega }\end{array}$ & $\begin{array}{l}g 1998 \\
g\end{array}$ \\
\hline 2532 HLW98-48 & $\begin{array}{l}\text { BNFL HLW98 } \\
\text { BNFL HLW98 }\end{array}$ & $\begin{array}{l}\text { Fu and Pegg } \\
\text { Fu and Pega }\end{array}$ & $\begin{array}{l}g 1998 \\
9\end{array}$ \\
\hline 2533 HLW98-49 & BNFL HLW98 & 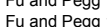 & (1998 \\
\hline 2534 HLW98-50 & $\begin{array}{l}\text { BNFL HLW98 }\end{array}$ & Fu and Pegg & g 1998 \\
\hline 2535 HLW98-51R & BNFL HLW98 & Fu and Pegg & g 1998 \\
\hline 2536 HLW98-52 & BNFL HLW98 & Fu and Pegg & g 1998 \\
\hline 2537 HLW98-53A & BNFL HLW98 & Fu and Pegg & g 1998 \\
\hline 2538 HLW98-54 & $\begin{array}{l}\text { BNFL HLW98 } \\
\text { BNFI HIS }\end{array}$ & Fu and Pegg & $g 1998$ \\
\hline 2540 HLW98-56 & BNFL HLW98 & $\begin{array}{l}\text { Fu and Pegg } \\
\text { Fu and Pegg }\end{array}$ & 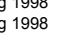 \\
\hline & BNFL HLW98 & Fu and Pegg & \\
\hline HLW98-58 & BNFL HLW98 & Fu and Pegg & g 1998 \\
\hline
\end{tabular}

A12O3-t B2O3-t CaO-t Fe2O3-t FeO-t $\begin{array}{llll}0.1007 & 0.0006 & 0.0160 & 0.0852 \\ 0.1041 & 0.0006 & 0.0165 & 0.0881 \\ 0.1076 & 0.0006 & 0.0171 & 0.0911\end{array}$ $\begin{array}{llll}0.1111 & 0.0006 & 0.0176 & 0.0940 \\ 0.1146 & 0.0007 & 0.0182 & 0.0969 \\ 0.1068 & 0.141 & 0.088 & 0.0371\end{array}$ $\begin{array}{llll}0.1180 & 0.0007 & 0.0187 & 0.0999 \\ 0.0898 & 0.1411 & 0.0088 & 0.0371 \\ 0.0079 & 0.136 & 0.0085 & 0.0359\end{array}$ $\begin{array}{llll}0.0867 & 0.1363 & 0.0085 & 0.0359 \\ 0.0849 & 0.1334 & 0.0083 & 0.0351\end{array}$ $\begin{array}{llll}0.0849 & 0.1334 & 0.0083 & 0.0351 \\ 0.0831 & 0.1306 & 0.0081 & 0.0344\end{array}$ $\begin{array}{llll}0.0870 & 0.1367 & 0.0085 & 0.0360 \\ 0.0889 & 0.1396 & 0.0087 & 0.0368\end{array}$ $\begin{array}{llll}0.0889 & 0.1396 & 0.0087 & 0.0368 \\ 0.0867 & 0.1382 & 0.0084 & 0.0359\end{array}$ $\begin{array}{llll}0.0867 & 0.1382 & 0.0084 & 0.0359 \\ 0.0867 & 0.1382 & 0.0084 & 0.0359\end{array}$ $\begin{array}{llll}0.0910 & 0.0144 & 0.0770 \\ 0.0910 & 0.0144 & 0.0770\end{array}$

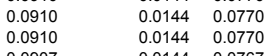
$\begin{array}{lll}0.0905 & 0.0143 \quad 0.0766\end{array}$ $\begin{array}{llll}0.0894 & 0.0142 & 0.0756 \\ 0.0875 & 0.0139 & 0.070\end{array}$ $\begin{array}{lll}0.0875 & 0.0139 & 0.0740 \\ 0.0856 & 0.0136 & 0.0724 \\ 0.0802 & 0.143 & 0.0762\end{array}$ $\begin{array}{lll}0.0900 & 0.0143 & 0.0762 \\ 0.0882 & 0.0140 & 0.0747\end{array}$ $\begin{array}{llll}0.0864 & & 0.0137 & 0.0731 \\ 0.1074 & 0.1064 & 0.0097 & 0.1172\end{array}$ $\begin{array}{llll}0.1109 & 0.1099 & 0.0101 & 0.1210 \\ 0.1180 & 0.1001 & 0.0142 & 0.0944 \\ 0.1147 & 0.130 & 0.437 & 0.1248\end{array}$ $\begin{array}{llll}0.1180 & 0.1001 & 0.0142 & 0.0944 \\ 0.1180 & 0.1301 & 0.0142 & 0.0944 \\ 0.042 & 0.1001 & 0.0043 & 0.1277\end{array}$ $\begin{array}{llll}0.1147 & 0.1301 & 0.0137 & 0.1218\end{array}$ $\begin{array}{llll}0.0852 & 0.1001 & 0.0043 & 0.1277 \\ 0.0883 & 0.1001 & 0.0044 & 0.1325\end{array}$ $\begin{array}{llll}0.0798 & 0.1201 & 0.0040 & 0.127\end{array}$

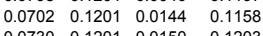
$\begin{array}{llll}0.0749 & 0.1502 & 0.0027 & 0.1268\end{array}$ $\begin{array}{llll}0.0667 & 0.1502 & 0.0024 & 0.1127 \\ 0 & 0.1201 & 0.127 & 0.0877\end{array}$ $\begin{array}{llll}0.0871 & 0.1201 & 0.0190 & 0.0877 \\ 0.0871 & 0.1501 & 0.0190 & 0.0877\end{array}$

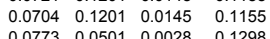
$\begin{array}{llll}0.0773 & 0.0501 & 0.0028 & 0.1298 \\ 0.0916 & 0.1201 & 0.0200 & 0.0922\end{array}$ $\begin{array}{llll}0.0678 & 0.0701 & 0.0024 & 0.1137\end{array}$

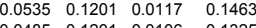

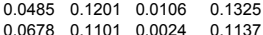
$\begin{array}{llll}0.0667 & 0.1004 & 0.0024 & 0.1118 \\ 0.0785 & 0.0704 & 0.026 & 0.1312\end{array}$ $\begin{array}{llll}0.0785 & 0.0704 & 0.0026 & 0.1132 \\ 0.0785 & 0.0402 & 0.0026 & 0.1312\end{array}$ $\begin{array}{llll}0.0785 & 0.0402 & 0.026 & 0.132 \\ 0.0785 & 0.0201 & 0.0026 & 0.1312\end{array}$ $\begin{array}{llll}0.0785 & 0.1295 & 0.0026 & 0.1312 \\ 0.0785 & 0.1005 & 0.0026 & 0.1102\end{array}$ $\begin{array}{llll}0.0740 & 0.1000 & 0.0025 & 0.1039 \\ 0.1368 & 0.0900 & 0.024 & 0.1110\end{array}$ $\begin{array}{llll}0.1003 & 0.0700 & 0.0067 & 0.1092 \\ 0.1034 & 0.0700 & 0.0176 & 0.1206\end{array}$ $\begin{array}{llll}0.0938 & 0.0899 & 0.0281 & 0.0381 \\ 0.0442 & 0.0504 & 0.0045 & 0.0560\end{array}$ $\begin{array}{llll}0.0335 & 0.0499 & 0.0064 & 0.0661 \\ 0.0369 & 0.0299 & 0.0070 & 0.0728\end{array}$ $\begin{array}{llll}0.0823 & 0.0600 & 0.0026 & 0.1189 \\ 0.0342 & 0.0299 & 0.0065 & 0.0675\end{array}$ $\begin{array}{llll}0.0797 & 0.0850 & 0.0025 & 0.1152 \\ 0.0324 & 0.0499 & 0.0062 & 0.0639\end{array}$ $\begin{array}{llll}0.0324 & 0.0499 & 0.0062 & 0.0639 \\ 0.0324 & 0.0698 & 0.0062 & 0.0639 \\ 0.029 & 0.0748 & 0.0057 & 0.0590\end{array}$ $\begin{array}{llll}0.0313 & 0.0698 & 0.0060 & 0.0618\end{array}$ $\begin{array}{llll}0.0299 & 0.0748 & 0.0057 & 0.0590 \\ 0.0286 & 0.0748 & 0.0055 & 0.0566\end{array}$ $\begin{array}{llll}0.0448 & 0.0898 & 0.0047 & 0.0491 \\ 0.0587 & 0.0699 & 0.0045 & 0.0469\end{array}$ $\begin{array}{llll}0.0249 & 0.1097 & 0.0043 & 0.0457 \\ 0.0238 & 0.0898 & 0.0045 & 0.0469\end{array}$ $\begin{array}{llll}0.0238 & 0.0898 & 0.0045 & 0.0469 \\ 0.0797 & 0.0700 & 0.0025 & 0.1152 \\ 0.0797 & 0.1250 & 0.025 & 0.1172\end{array}$ $\begin{array}{llll}0.0811 & 0.0725 & 0.0025 & 0.1172 \\ 0.0797 & 0.1000 & 0.0025 & 0.1152 \\ 0 & 0797 & 0.0700 & 0.025\end{array}$ $\begin{array}{llll}0.0797 & 0.1000 & 0.0025 & 0.1122 \\ 0.0797 & 0.0700 & 0.0025 & 0.11152 \\ 0.0755 & 0.0690 & 0.057 & 0.1178\end{array}$ $\begin{array}{llll}0.0743 & 0.0400 & 0.0062 & 0.1194 \\ 0.0763 & 0.0399 & 0.0061 & 0.1207\end{array}$

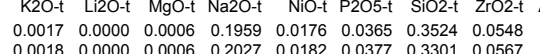

$\begin{array}{lllllllllllllllllll}0.0018 & 0.0000 & 0.0006 & 0.2094 & 0.0188 & 0.0390 & 0.3078 & 0.0586\end{array}$

$\begin{array}{llllllll}0.0019 & 0.0000 & 0.0007 & 0.2162 & 0.0194 & 0.0402 & 0.2854 & 0.0605 \\ 0.0020 & 0.0000 & 0.0007 & 0.2229 & 0.0200 & 0.0415 & 0.2631 & 0.0624 \\ 0.0020 & 0.0000 & 0.0007 & 0.229 & 0.0200 & 0428 & 0.2404 & 0.003\end{array}$ $\begin{array}{llllllll}0.0744 & 0.0031 & 0.0436 & 0.0000 & 0.5404 & 0.0006 \\ 0.0719 & 0.0030 & 0.0421 & 0.0008 & 0.0333 & 0.5220 & 0.0006 \\ 0.064 & 0.025 & 0.042 & 0.0008 & 0.0733 & 0.5002 & 0.0006\end{array}$

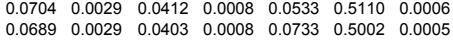
$\begin{array}{llllllll}0.0721 & 0.0030 & 0.0422 & 0.0009 & 0.0546 & 0.5234 & 0.0000\end{array}$ $\begin{array}{lllllll}0.0736 & 0.0031 & 0.0431 & 0.0009 & 0.0342 & 0.5347 & 0.0006 \\ 0.0000 & 0.0030 & 0.079 & 0.0008 & 0.0333 & 0.4258 & 0.0006\end{array}$ $\begin{array}{lllllll}0.0000 & 0.0030 & 0.2079 & 0.0008 & 0.0333 & 0.4258 & 0.0006 \\ 0.0302 & 0.0030 & 0.1777 & 0.0008 & 0.0333 & 0.4258 & 0.0006\end{array}$

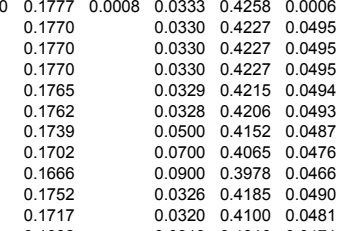

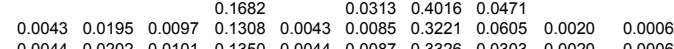

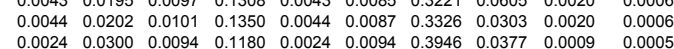

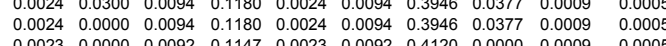

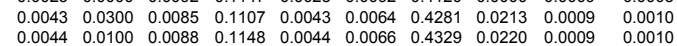
$\begin{array}{llllllllll}0.0044 & 0.0100 & 0.0088 & 0.1148 & 0.0044 & 0.0066 & 0.4329 & 0.0220 & 0.0009 & 0.0010 \\ 0.0044 & 0.0300 & 0.0087 & 0.0919 & 0.0044 & 0.0066 & 0.4097 & 0.0219 & 0.0009 & 0.0010\end{array}$

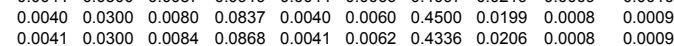
$\begin{array}{llllllllll}0.00043 & 0.03300 & 0.00086 & 0.00902 & 0.00413 & 0.00064 & 0.44177 & 0.0215 & 0.0009 & 0.0010 \\ 0.0030 & 0.0701 & 0.0009 & 0.0293 & 0.0046 & 0.0059 & 0.4103 & 0.0388 & 0.0008 & 0.0007\end{array}$ $\begin{array}{llllllllll}0.0026 & 0.0300 & 0.0008 & 0.1061 & 0.0041 & 0.0053 & 0.4092 & 0.0344 & 0.0007 & 0.0006\end{array}$

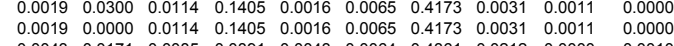
$\begin{array}{llllllllll}0.0042 & 0.0300 & 0.0083 & 0.0868 & 0.0042 & 0.0062 & 0.4328 & 0.0207 & 0.0000 & 0.0010\end{array}$ $\begin{array}{llllllllll}0.0031 & 0.0701 & 0.0009 & 0.0302 & 0.0048 & 0.00061 & 0.5109 & 0.0401 & 0.0000 & 0.0007 \\ 0.0020 & 0.0000 & 0.0120 & 0.1479 & 0.0017 & 0.0068 & 0.4260 & 0.0033 & 0.0012 & 0.0000\end{array}$

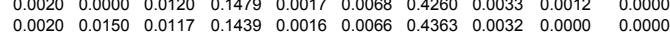
$\begin{array}{llllllllll}0.0027 & 0.0601 & 0.0008 & 0.0665 & 0.0042 & 0.0053 & 0.5093 & 0.0350 & 0.0000 & 0.0006\end{array}$ $\begin{array}{llllllllll}0.00012 & 0.0300 & 0.0070 & 0.0863 & 0.0010 & 0.0040 & 0.4028 & 0.00019 & 0.0000 & 0.00000 \\ 0.0011 & 0.0300 & 0.0064 & 0.0782 & 0.0009 & 0.0036 & 0.4431 & 0.0017 & 0.0000 & 0.0000\end{array}$ $\begin{array}{llllllllll}0.0026 & 0.0602 & 0.0008 & 0.0661 & 0.0041 & 0.00052 & 0.4706 & 0.0344 & 0.0007 & 0.00006 \\ 0.0018 & 0.0653 & 0.0007 & 0.0614 & 0.0057 & 0.0013 & 0.04650 & 0.0280 & 0.0000 & 0.0005\end{array}$ $\begin{array}{llllllllll}0.0018 & 0.0653 & 0.0007 & 0.0614 & 0.0057 & 0.0013 & 0.4650 & 0.0280 & 0.0000 & 0.0005 \\ 0.0018 & 0.0452 & 0.0007 & 0.1442 & 0.0057 & 0.0013 & 0.4324 & 0.0280 & 0.0000 & 0.0005\end{array}$

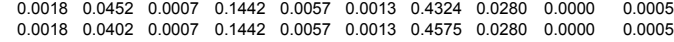
$\begin{array}{llllllllll}0.0018 & 0.0402 & 0.0007 & 0.0800 & 0.0057 & 0.0013 & 0.4123 & 0.0280 & 0.0000 & 0.0005 \\ 0.0018 & 0.0603 & 0.0007 & 0.0664 & 0.0057 & 0.0013 & 0.4557 & 0.0280 & 0.0000 & 0.0005\end{array}$

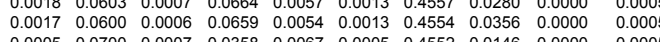
$\begin{array}{llllllllll}0.0003 & 0.03300 & 0.0020 & 0.0879 & 0.0014 & 0.0014 & 0.3773 & 0.0011 & 0.00022 & 0.0000 \\ 0.0006 & 0.0500 & 0.0008 & 0.0971 & 0.0073 & 0.0006 & 0.4355 & 0.0158 & 0.0000 & 0.0005\end{array}$

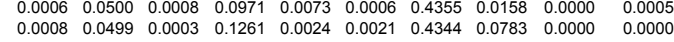
$\begin{array}{llllllllll}0.0008 & 0.0499 & 0.0003 & 0.126 & 0.0024 & 0.0021 & 0.4344 & 0.0783 & 0.0000 & 0.0000 \\ 0.0000 & 0.0449 & 0.0005 & 0.1525 & 0.0020 & 0.0007 & 0.4169 & 0.1154 & 0.0000 & 0.0000\end{array}$ $\begin{array}{llllllllll}0.0010 & 0.0449 & 0.0008 & 0.1139 & 0.0033 & 0.0047 & 0.3811 & 0.0682 & 0.0009 & 0.0000 \\ 0.0011 & 0.0399 & 0.0009 & 0.1219 & 0.0036 & 0.0051 & 0.3684 & 0.0751 & 0.0010 & 0.0000\end{array}$ $\begin{array}{llllllllll}0.0006 & 0.0450 & 0.00008 & 0.1419 & 0.0072 & 0.00006 & 0.4352 & 0.0156 & 0.0004 & 0.0005\end{array}$

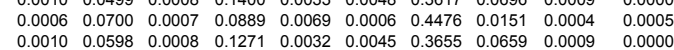
$\begin{array}{llllllllll}0.0010 & 0.0598 & 0.0008 & 0.1271 & 0.0032 & 0.0045 & 0.3655 & 0.0659 & 0.0009 & 0.0000 \\ 0.0010 & 0.0598 & 0.0008 & 0.1022 & 0.0032 & 0.0045 & 0.3705 & 0.0659 & 0.0009 & 0.0000\end{array}$

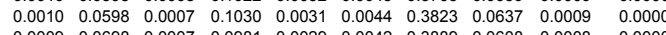

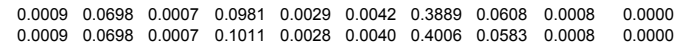
$\begin{array}{llll}0.4206 & 0.0506 & 0.0007 & 0.0000 \\ 0.4200 & 0.0484 & 0.0007 & 0.0000\end{array}$

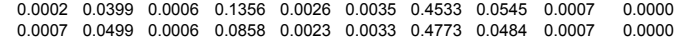
$\begin{array}{lllllllllll}0.0006 & 0.0600 & 0.0000 & 0.0989 & 0.00699 & 0.0006 & 0.4626 & 0.0151 & 0.0004 & 0.00005\end{array}$

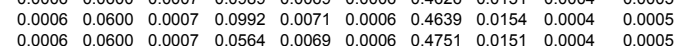
$\begin{array}{llllllllll}0.0006 & 0.0600 & 0.0007 & 0.0564 & 0.0069 & 0.0006 & 0.4751 & 0.0151 & 0.0004 & 0.0005 \\ 0.0006 & 0.0700 & 0.0007 & 0.0914 & 0.0069 & 0.0006 & 0.4888 & 0.0151 & 0.0004 & 0.0005\end{array}$ $\begin{array}{lllllllllll}0.0699 & 0.0012 & 0.0953 & 0.0077 & 0.0024 & 0.4967 & 0.0165 & 0.0002 & 0.000\end{array}$

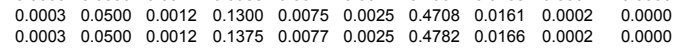

0.0000
0.0000
0.0000
0.0000
0.0000
0.0000
0.0000

0.0043
0.0045
0.0024

0.0045
0.0024
0.0024
0.0023

0.0023

0.0044

0.0044
0.0040
0.0041

0.0043

0.0006
0.0005
0.0059

0.0059
Br-t CdO-t Ce2O3-t CeO2-t Cl-t

20

$\begin{array}{ll}0.0035 & 0.0012 \\ 0.0032 & 0.0011 \\ 0.0033 & 0.0011\end{array}$

$\begin{array}{ll}0.0033 & 0.0011 \\ 0.0034 & 0.0012\end{array}$

$\begin{array}{ll}0.0034 & 0.0012 \\ 0.0053 & 0.0011 \\ 0.0047 & 0.0010\end{array}$

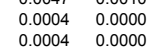

$0.0034 \quad 0.0012$

$\begin{array}{ll}0.0033 & 0.0012 \\ 0.0054 & 0.0011\end{array}$

$\begin{array}{ll}0.0054 & 0.001 \\ 0.0004 & 0.0000 \\ 0.0004 & 0.0000\end{array}$

$\begin{array}{ll}0.0004 & 0.00010 \\ 0.00002 & 0.0000 \\ 0.0002 & 0.000\end{array}$

\begin{tabular}{ll}
0.0002 & 0.0000 \\
0.0002 & 0.0000 \\
\hline 0.0048 & 0.001
\end{tabular}

$\begin{array}{ll}0.0048 & 0.0001 \\ 0.0047 & 0.0011 \\ 0.0039 & 0.0009\end{array}$

$\begin{array}{ll}0.0039 & 0.0009 \\ 0.0039 & 0.0009 \\ 0.0039 & 0.009\end{array}$

\begin{tabular}{ll}
0.0039 & 0.0009 \\
0.0039 & 0.0009 \\
\hline
\end{tabular}

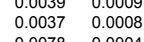

\begin{tabular}{ll}
0.0078 & 0.0004 \\
0.0004 & 0.0003 \\
0.0085 & 0.0005 \\
\hline
\end{tabular}

\begin{tabular}{ll}
\hline .0085 & 0.0005 \\
\hline 0.0006 & 0.0000 \\
\hline
\end{tabular}

\begin{tabular}{ll}
0.0009 & 0.001 \\
0.0009 & 0.0012 \\
\hline
\end{tabular}

$\begin{array}{ll}0.00094 & 0.0012 \\ 0.0084 & 0.0005 \\ 0.0009 & 0.0011\end{array}$

$\begin{array}{ll}0.0009 & 0.0011 \\ 0.0081 & 0.0005 \\ 0.0008 & 0.0010 \\ 0.008 & 0.010\end{array}$

$\begin{array}{ll}0.0008 & 0.0010 \\ 0.0008 & 0.0010 \\ 0.0008 & 0.0010\end{array}$

$\begin{array}{ll}0.0008 & 0.0010 \\ 0.0008 & 0.0010\end{array}$

$\begin{array}{ll}0.0008 & 0.0010 \\ 0.0007 & 0.0010 \\ 0.0006 & 0.0009\end{array}$

\begin{tabular}{ll}
0.0006 & 0.0008 \\
0.0007 & 0.0008 \\
\hline
\end{tabular}

$\begin{array}{ll}0.0007 & 0.0008 \\ 0.0006 & 0.0008 \\ 0.0081 & 0.0005\end{array}$

$\begin{array}{ll}0.0081 & 0.000 \\ 0.0083 & 0.0005 \\ 0.0081 & 0.0005\end{array}$

$\begin{array}{ll}0.0081 & 0.0005 \\ 0.0081 & 0.0005 \\ 0.0137 & 0.000\end{array}$

$\begin{array}{ll}0.0133 & 0.0008 \\ 0.0137 & 0.0007\end{array}$ 


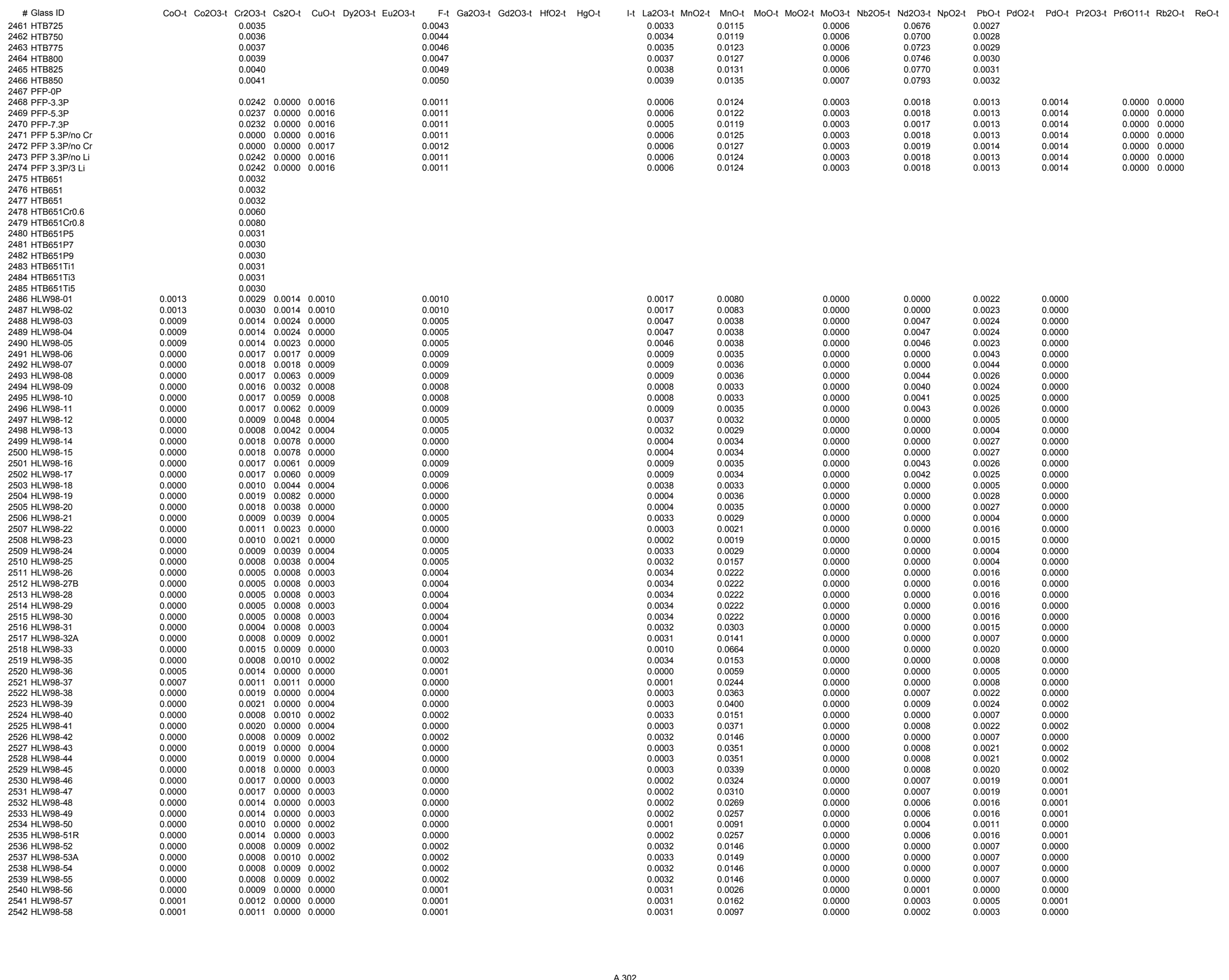




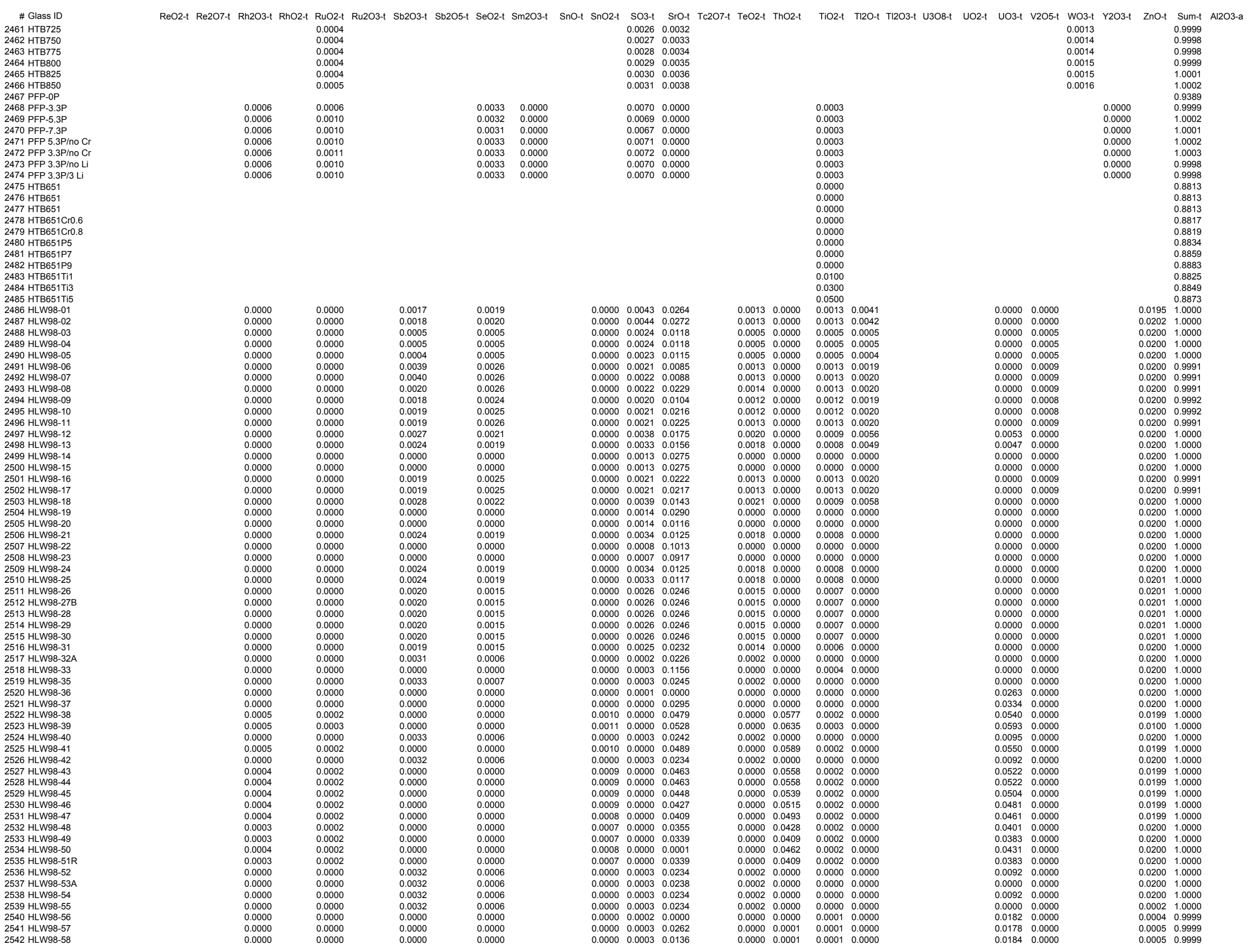


(2476

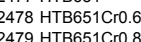

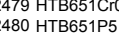

2481 HTB65

$2483 \mathrm{HTB}$
$24551 T \mathrm{TT}$

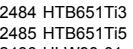

2486 HLWG8-01
2487 HLW98-02

2488 HLW99-03
2489 HLW $W 8-04$

2489 HLWG8-04
2490 HLW98-05

$2491 \mathrm{HLWW8}-06$

2492 HLW98-07
2493 HLW98-08

2494 HLW98-09

2495 HLWS8-10

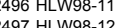

2498 HLW $998-13$

2999 HLW98-14

2550 HLW98-15
2501 HLW98-16

2502 HLW $98-17$

2503 HLW $98-18$

(2504 Hitwo-19

2506 HLW98-21

2507 HLW $98-22$

2508 HLW $998-22$
2509

2510 HLW98-25

2511 HLW98-26

2512 HLWG9-278
2513 HUW

2514 HLW98-29

2515 HLW $998-30$

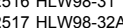

2518 HLW98-33

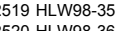

2520 HLWG8-36
2521 HLW $38-37$

2522 HLW98-38

2523 HLW HL8-39
2524 HLW8-40
2525 HLW98-41

2526 HLWM8-42

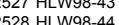

2529 HLW $998-45$

$2530 \mathrm{HLW}$ W9-46

(53) HLW98-47

2533 HLW $198-49$

2534 HLW98.50

2535 HLW98-51

2537 HLW $98-53 \mathrm{~A}$

2538 HLW W98.54
2530 H

(2540

2541 HLW98.57

2542 HLW $98-58$ 
2462 HTB 750

2463 HTB775

$2465 \mathrm{HTBR25}$

2467 PFP-0P

2467 PPP

2469 PFP-5.3P

247112 FPP 5 .3P/RO C C

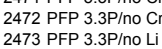

2473 PFP 3.3PP/no
2474 PFP $3.3 P$ L L

$2475 \mathrm{HTRB65}$
$2476 \mathrm{HTR655}$

$2477 \mathrm{HTBB51}$
$2478 \mathrm{HTB651} C \mathrm{Cr} .0$.

2479 HTB651Cro.

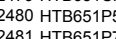

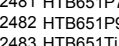

2483 HTBG51T11
2484 HTB65TTi3

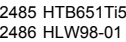

2487 HLW98-02

2488 HLW99-03
2489 HLWS-04

2490 HLW

2491 HLW98-06

$2492 \mathrm{HLW}$ W98-07
$2493 \mathrm{HLW} 98-08$

2494 HLW $98-09$

$2495 \mathrm{HLW}$ W98-10

$2496 \mathrm{HLW}$ W8-11
$2497 \mathrm{HW}=98-12$

2498 HLW $998-13$

2999 HLW98-14

2500 HLW98-15
2501 HLW98-16
250

2502 HLW $98-17$

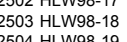

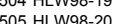

2506 HLW98-21

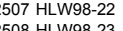

2508 HLW $998-22$
2509

2510 HLW98-25

2511 HLW98-26

2512 HLWG9-278
2513 HUW

2514 HLW98-29

2515 HLW $998-30$

2516 HLWG8-31
2517 HWW $98-32 \mathrm{~A}$

2518 HLW98-33

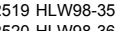

2520 HLWG8-36
2521 HLW $38-37$

2522 HLW98-38

2523 HLW HL8-39
2524 HLW8-40
2525 HLW98-41

$2526 \mathrm{HLW} 98 \mathrm{~B}-42$

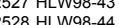

2529 HLW $998-45$

$2530 \mathrm{HLW}$ W9-46

(52)

2533 HLW $198-49$

2534 HLW98.50

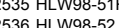

2537 HLW $98-53 \mathrm{~A}$

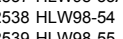

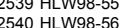

2541 HLW W98-57

2542 HLW $98-58$ 
2480 HTB651P5

2481 HTB651P7
2482 HTB651P9
2483 HTB65T1

2483 HTB651Ti1

2485 HTB651Ti5
2486 HLW98-01

2487 HLW98-02

2488 HLW98-03
2489 HLW98-04

2490 HLW88-05

$2491 \mathrm{HLW}$ W98-06

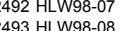

2494 HLW W98-09

2495 HLW98-10

2496
$2497-W 98-11$

2498 HLW98-13

2499 HLW98-14

2500 HLW98-15
2501 L 2 W98-16

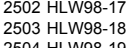

2504 HLW98-19

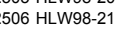

2507 HLW98-22

2508 HLW98-23
2509 HLW 24

2510 HLW98-25

2511 HLW $198-26$

2512 HLWG8-278
2513 HLW98-28

(514 HLW98-29

(251

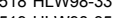

2520 HLW $98-36$

2522 HLW98-38

2523 HLW

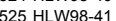

2526 HLW98-42

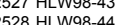

2529 HLW $98-45$

$2530 \mathrm{HLW}$ W9-46

2532 HLWW8-48

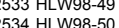

2535 HLWV88-51R

2537 HLW W98-53A

2538 HLW98-54

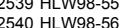

2542 HLW $98-58$

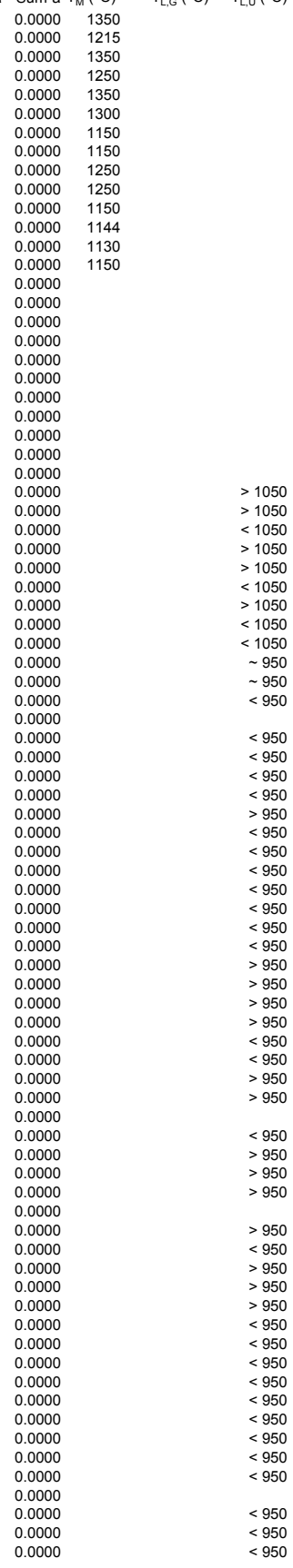


2480
HTB

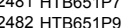

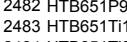

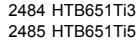

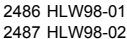

2487 HLL98-02
2488 HLWS-03

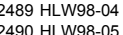

2491 HLW98-06

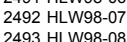

2493 HLWG8.08

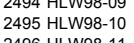

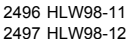

2497 HLW98-12
2498 HLWS8-13

2499 HLWW8-14

2500 HLW $198-15$
2501 HLW $W 9-16$

2502 HLW

2503 HLW98-18

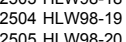

2506 HLW $98-21$

2507 HLW $98-22$

2508 HLW98-23
2509 HWW

2510 HLW98-25

2511 HLW98-26

2512 HLW98-27
2513 HLW

2514 HLW98-29

2515 HLW98-30

2517 H HW

2518 HLW98-33

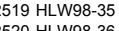

2520 HLWG8-36
2521 HLW

2522 HLW98-38

2523 HWW $198-39$
2524

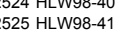

2526 HLW98-42

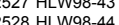

2529 HLW $98-45$

2530 HLW

2532 HLW W98-48

2533 HLW $198-49$

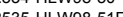

2536 HW08.52

2537 HLW98-53A

2538 HLW98-54

2540 HLW W98-56

2542 HLW $98-58$ 


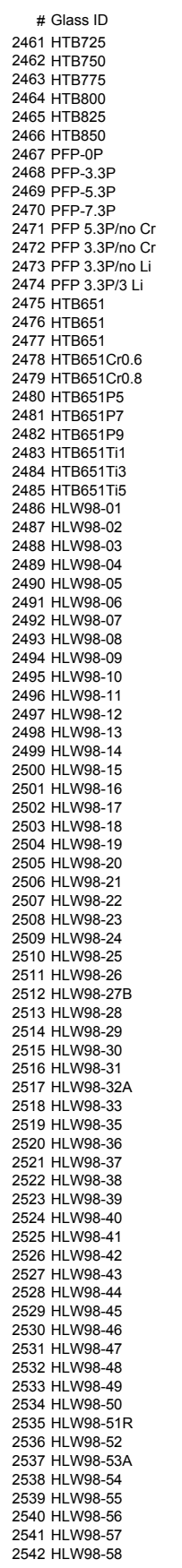

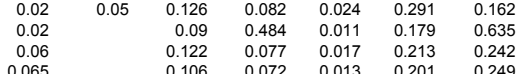
$\begin{array}{llllll}0.061 & 0.106 & 0.066 & 0.011 & 0.183 & 0.232\end{array}$ $\begin{array}{lllllll}0.025 & 0.05 & 0.038 & 0.029 & 0.02 & 0.247 & 0.101\end{array}$

$\begin{array}{lllllll}0.014 & 0.05 & 0.041 & 0.203 & 0.014 & 0.187 & 0.06\end{array}$

$\begin{array}{lllllll}<.0031 & 0.05 & 0.465 & 0.268 & <0.0055 & 0.11 & 0.081\end{array}$ 


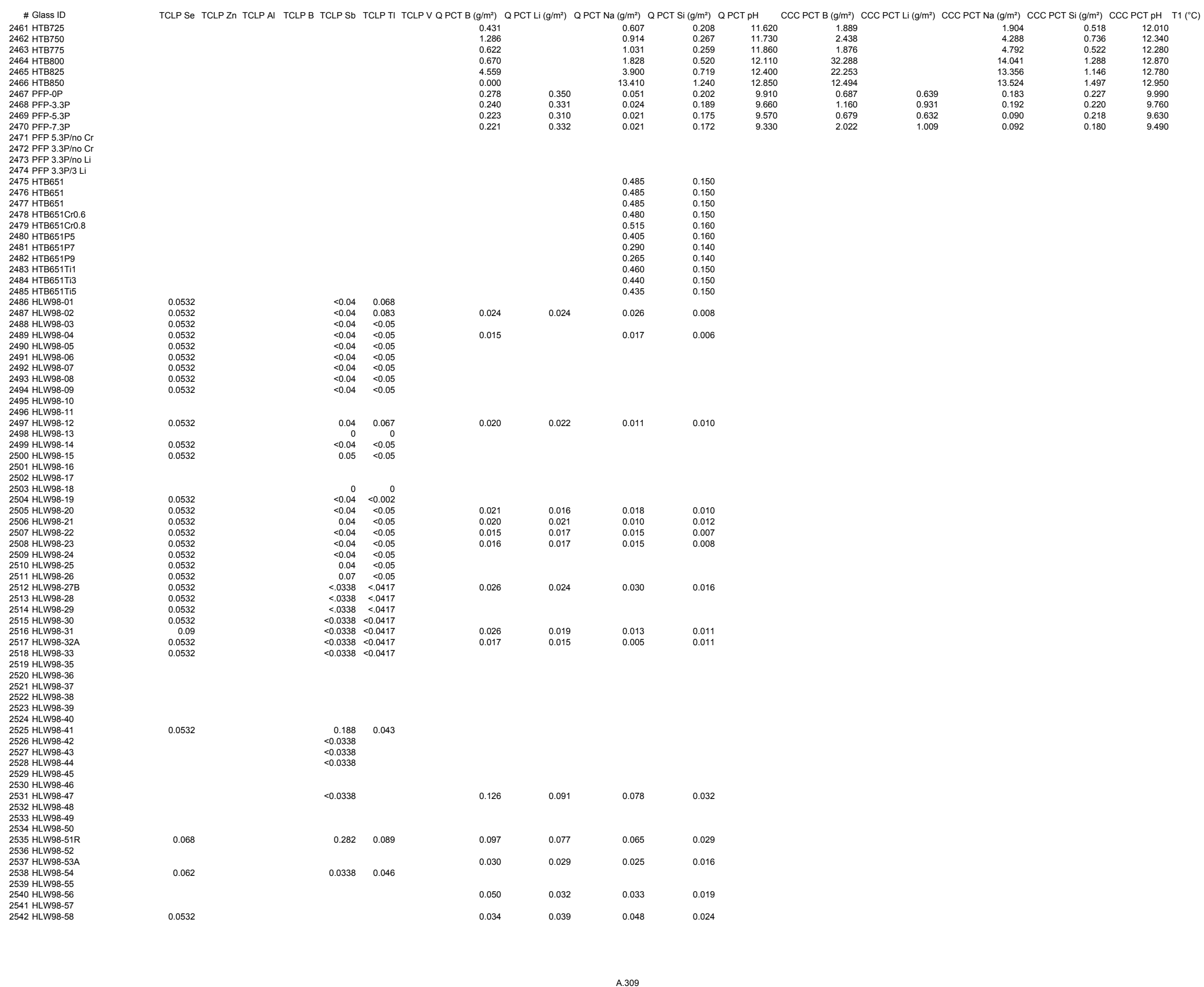


2462 HTB 750

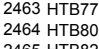

2465
2466 TTBB825

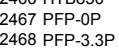

2469 PFP-5.5.3P

2471 PFP $5.3 P /$ Ino $^{-1}$

2472 PFP $3.3 \mathrm{PP} / \mathrm{MOC} C$

2473 PFP $3.3 \mathrm{PP} / 10 \mathrm{~L}$
$2474 \mathrm{PFP} 3.3 \mathrm{P} / 3 \mathrm{Li}$

$2476 \mathrm{HTRB55}$

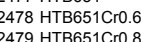

$2480 \mathrm{HTB651P5}$

2481 H

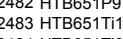

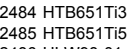

2486 HLWG8-01
2487 HLW98-02

2488 HLW98-03
2489 HLW98-04

2489 HLW98-04
2400 HLW98-05

$2491 \mathrm{HLWW8}-06$

2492 HLW98-07
2493 HLW98-08

2494 HLW98-09

2495 HLW98-10

2497 HLWG8-12
2498 HLW98-13

24988 HLW98-13
2499 HLWS-14

2500 HLWG8-15
2501 HLW98-16
2.16

2502 HLW $98-17$

2503 HLW W98-18
2504 H

2504 HLWG6-19

2506 HLW98-21

2557 HLW98-22
2508 HLW $198-23$

2508 HLW989-2

2510 HLW98-25

2511 HLW98-26

2512 HLWG9-278
2513 HLW

2514 HLW98-29

2515 HLW98-30
2516 HWW $38-31$

2517 H HW

2518 HLW98-33

2519 HLW98-35
$2520 \mathrm{HLW}-368$ - 36

2520 HLWG8-36
2521 HLW $38-37$

2522 HLW98-38

2524 HLWS8-40

$252 \mathrm{HLW} 98 .-41$
$2526 \mathrm{HLWS}-42$

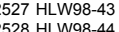

2529 HLW $998-45$

$2530 \mathrm{HLW}$ W9-46

2532 HLWW8-48

2533 HLW98-49
2534 HLW98-50

$2535 \mathrm{HLW}$ W8-512

2537 HLW98-53A

2538 HLW $198-54$
254

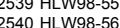

2541 HLW W98-57

2542 HLW $98-58$ 


\begin{tabular}{|c|c|c|c|c|c|c|}
\hline \# Glass ID & Study & Reference & Al2O3-t & B2O3-t & $\mathrm{CaO}-\mathrm{I}$ & Fe203-t \\
\hline 2543 HLW98-59 & BNFL HLW98 & Fu and Pegg 1998 & 0.0800 & 0.0400 & 0.0025 & 0.1156 \\
\hline 2544 HLW98-60 & BNFL HLW98 & Fu and Pegg 1998 & 0.0760 & 0.0400 & 0.0059 & 0.1193 \\
\hline $\begin{array}{l}2545 \text { HLW98-62 } \\
2546 \text { HLW98-63 }\end{array}$ & $\begin{array}{l}\text { BNFL HLW } 98 \\
\text { BNFL HWW98 }\end{array}$ & $\begin{array}{l}\text { Fu and Pegg } 1998 \\
F\end{array}$ & 0.0967 & 0.1200 & 0.0231 & 0.0393 \\
\hline $\begin{array}{l}2546 \text { HLW } 258-63 \\
2547 \text { HLW8-64 }\end{array}$ & $\begin{array}{l}\text { BNLFLW98 } \\
\text { BNFL HLW98 }\end{array}$ & $\begin{array}{l}\text { Fu and Pegg } 1998 \\
\text { Fu and Pegg } 1998\end{array}$ & $\begin{array}{l}0.01185 \\
0.0814\end{array}$ & $\begin{array}{l}0.0627 \\
0.0956\end{array}$ & $\begin{array}{l}0.0079 \\
0.0027\end{array}$ & $\begin{array}{l}0.1290 \\
0.1143\end{array}$ \\
\hline $2548 \mathrm{C} 4 \mathrm{~A}-03$ & Fernald & Gimpel 1996 & 0.0589 & 0.1289 & 0.0508 & 0.0398 \\
\hline $549 C 4 C-03$ & Fernald & Gimpel 1996 & 0.1235 & 0.1223 & 0.0943 & 0.0572 \\
\hline $\begin{array}{l}2550 \mathrm{C} 4 \mathrm{C}-08 \\
2551 \mathrm{CA}-0.4\end{array}$ & $\begin{array}{l}\text { Fernald } \\
\text { Fermald }\end{array}$ & $\begin{array}{l}\text { Gimpel } 1996 \\
\text { Gimpen } 1996\end{array}$ & 0.1443 & 0.0600 & 0.0861 & $\begin{array}{l}0.0770 \\
0.0533\end{array}$ \\
\hline $\begin{array}{l}2551 \text { C4LD-04 } \\
2552 \text { C4D-15 }\end{array}$ & $\begin{array}{l}\text { Ferrald } \\
\text { Fernald }\end{array}$ & $\begin{array}{l}\text { Gimpel } 1996 \\
\text { Gimpel } 1996\end{array}$ & $\begin{array}{l}0.0963 \\
0.1513\end{array}$ & $\begin{array}{l}0.1496 \\
0.1006\end{array}$ & $\begin{array}{l}0.0697 \\
0.0770\end{array}$ & $\begin{array}{l}0.0533 \\
0.0558\end{array}$ \\
\hline $\begin{array}{l}2552 \text { C4LD-15 } \\
2553 \text { C4D-1 }\end{array}$ & $\begin{array}{l}\text { Fermala } \\
\text { Fernald }\end{array}$ & $\begin{array}{l}\text { Gimpen l996 } \\
\text { Gimpel } 1996\end{array}$ & $\begin{array}{l}0.1513 \\
0.1398\end{array}$ & 0.1004 & 0.1388 & $\begin{array}{l}0.0588 \\
0.0433\end{array}$ \\
\hline 2554 S12-04 (C4A-03 mod.) & Fernald & Gimpel 1996 & 0.0615 & 0.1051 & 0.1331 & 0.0360 \\
\hline 55 SB4-NEPH-01cCC & DWPF Nepheline -1 & Peeler et al. 2005 & 0.1258 & 0.0468 & 0.0089 & 0.0844 \\
\hline 2556 SB4-NEPH-02ccc & DWPF Nepheline -1 & Peeler et al. 2005 & 0.1189 & 0.0457 & 0.0081 & 0.0791 \\
\hline 2557 SB4-NEPH-03ccC & DWPF Nepheline -1 & Peeler et al. 2005 & 0.0858 & 0.0475 & 0.0085 & 0.0924 \\
\hline 2558 SB4-NEPH-04ccC & DWPF Nepheline -1 & Peeler et al. 2005 & 0.1279 & 0.0475 & 0.0087 & 0.0837 \\
\hline $\begin{array}{l}2559 \text { SBB-NEPH-05ccC } \\
2560 \text { SB4-NEPH-06ccc }\end{array}$ & $\begin{array}{l}\text { DWFF Nepheine - } 1 \\
\text { DWPFE Neheline - } 1\end{array}$ & $\begin{array}{l}\text { Peeler et al. } 2005 \\
\text { Peepler et al } 2005\end{array}$ & $\begin{array}{l}0.0922 \\
0.0855\end{array}$ & $\begin{array}{l}0.0474 \\
0.0464\end{array}$ & $\begin{array}{l}0.0090 \\
0.0074\end{array}$ & $\begin{array}{l}0.0967 \\
0.0639\end{array}$ \\
\hline 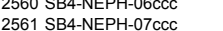 & $\begin{array}{l}\text { WWFF Nephenine - } \\
\text { DWPF Nepheline -1 }\end{array}$ & $\begin{array}{l}\text { Peeler et al. } 2005 \\
\text { Peeler et al. } 2005\end{array}$ & $\begin{array}{l}0.0855 \\
0.0934\end{array}$ & $\begin{array}{l}0.0464 \\
0.0472\end{array}$ & $\begin{array}{l}0.0074 \\
0.0074\end{array}$ & $\begin{array}{l}0.0889 \\
0.0887\end{array}$ \\
\hline 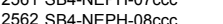 & DWPF Nenheline - -1 & Pelaretal. 2005 & 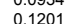 & 0.046 & 0.0082 & 0.076 \\
\hline 2563 SB4-NEPH-09ccC & DWPF Nepheline -1 & Peeler et al. 2005 & 0.1123 & 0.0509 & 0.0076 & 0.0762 \\
\hline 2564 SB4-NEPH-10ccC & DWPF Nepheline -1 & Peeler et al. 2005 & 0.0826 & & & 0.0941 \\
\hline 2565 SB4-NEPH-11ccc & DWPF Nepheline -1 & Peeler et al. 2005 & 0.0889 & 0.0474 & 0.0089 & 0.0974 \\
\hline 2566 SB4-NEPH-12ccc & DWPF Nepheline - 1 & Peeler et al. 2005 & 0.1043 & 0.0503 & 0.0073 & 0.0691 \\
\hline 2567 NEPH2-13 & DWPF Nepheline -2 & $\begin{array}{l}\text { Peeler et al. } 20066 \\
\text { Pan }\end{array}$ & 0.1021 & & & 0.0865 \\
\hline $\begin{array}{l}2568 \text { NEPH2-14 } \\
2569 \text { NEPH2-15 }\end{array}$ & DWFF Nephenine - 2 & Peeler et al. 2006 & 0.1700 & 0.0464 & 0.0084 & 0.0931 \\
\hline 2570 NEPH2-16 & $\begin{array}{l}\text { DWPF Nepheline -2 } \\
\text { DWFF N }\end{array}$ & Peleretal 2006 & 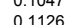 & 0.046 & 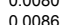 & $\begin{array}{l}0.0887 \\
0.0954\end{array}$ \\
\hline $\begin{array}{l}2571 \mathrm{NEPH}-17 \\
\end{array}$ & $\begin{array}{l}\text { DWPF Nepheline - } \\
\text { DWF }\end{array}$ & 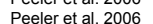 & 0.1178 & & 0.0090 & $\begin{array}{l}0.0998 \\
0.098\end{array}$ \\
\hline 2572 NEPH2-18 & DWPF Nepheline -2 & Peeler et a a. 20006 & 0.1073 & & 0.0082 & $\begin{array}{l}0.0909 \\
0.096\end{array}$ \\
\hline 2573 NEPH2-19 & DWPF Nepheline -2 & Peeler et al. 2006 & 0.1152 & 0.0448 & .0088 & 0.0976 \\
\hline 2574 NEPHZ2-20 & DWPF Nepheline -2 & Peeler et al. 2006 & 0.1230 & 0.0424 & 0.0094 & 0.1042 \\
\hline 2575 NEPH2-21 & DWPF Nepheline -2 & Peeler et al. 2006 & 0.1100 & 0.0464 & 0.0084 & 0.0931 \\
\hline $\begin{array}{l}2576 \text { NEPH2-22 } \\
2577 \text { NEPH2.23 }\end{array}$ & $\begin{array}{l}\text { DWPF Nepheline - } 2 \\
\text { DWPFE Nenheline -2 }\end{array}$ & $\begin{array}{l}\text { Peeler et al. } 2006 \\
\text { Peeler et }\end{array}$ & $\begin{array}{l}0.1204 \\
0.1283\end{array}$ & $\begin{array}{l}0.0432 \\
0.048\end{array}$ & $\begin{array}{l}0.0092 \\
0.0098\end{array}$ & $\begin{array}{l}0.1020 \\
0.1087\end{array}$ \\
\hline 2578 NEPHH2-24 & $\begin{array}{l}\text { WWFF Nepheneine -2 } \\
\text { DWPF Nepheline -2 }\end{array}$ & $\begin{array}{l}\text { Peeler et al. } 2006 \\
\text { Peeler et al. } 2006\end{array}$ & $\begin{array}{l}0.1283 \\
0.1126\end{array}$ & $\begin{array}{l}0.0488 \\
0.0456\end{array}$ & $\begin{array}{l}0.00986 \\
0.0086\end{array}$ & $\begin{array}{l}0.1087 \\
0.0954\end{array}$ \\
\hline 2579 NEPH2-25 25 & DWPF Nepheline -2 & Peeler et al. 2006 & 0.1230 & $\begin{array}{l}0.0456 \\
0.0424\end{array}$ & $\begin{array}{l}0.0096 \\
0.0094\end{array}$ & $\begin{array}{l}.0 .054 \\
0.1042\end{array}$ \\
\hline 2580 NEPH2-26 & DWPF Nepheline -2 & Peeler et al. 2006 & 0.1335 & 0.0392 & 0.0102 & 0.1131 \\
\hline 2581 NEPH2-27 & DWPF Nepheline -2 & Peeler et al. 2006 & 0.1164 & 0.0488 & 0.0075 & 0.0833 \\
\hline & DWPF Nepheline -2 & Peeler et al. 2006 & 0.1313 & 0.0448 & 0.0085 & $\begin{array}{l}0.0940 \\
\end{array}$ \\
\hline $\begin{array}{l}2583 \text { NEPH2-29 } \\
2584 \text { NEPH2-36 }\end{array}$ & $\begin{array}{l}\text { DWFF Nepheine - } 2 \\
\text { DWPFE Neheline - }\end{array}$ & $\begin{array}{l}\text { Peeler et al. } 2006 \\
\text { Peeperet al } 2006\end{array}$ & $\begin{array}{l}0.1463 \\
0.1194\end{array}$ & $\begin{array}{l}0.0408 \\
0.048\end{array}$ & $\begin{array}{l}0.0094 \\
0.0077\end{array}$ & $\begin{array}{l}0.1047 \\
0.0854\end{array}$ \\
\hline $\begin{array}{l}2584 \text { NEPH2-30 } \\
\text { 2585 NEPH-23 }\end{array}$ & $\begin{array}{l}\text { WWFF Nephenine - - } \\
\text { DWPF Nepheline -2 }\end{array}$ & $\begin{array}{l}\text { Peeler et al. } 2006 \\
\text { Peeler et al. } 2006\end{array}$ & $\begin{array}{l}0.1194 \\
0.1313\end{array}$ & $\begin{array}{l}0.0480 \\
0.0448\end{array}$ & $\begin{array}{l}0.007 / 7 \\
0.0085\end{array}$ & $\begin{array}{l}0.0894 \\
0.0940\end{array}$ \\
\hline $\begin{array}{l}2585 \text { NEPHH2-31 } \\
2586 \text { NEPH2-32 }\end{array}$ & DWPF Nepheline -2 & Peeler et al. 2006 & 0.1433 & $\begin{array}{l}0.0416 \\
0.0416\end{array}$ & 0.0092 & 0.1025 \\
\hline 2587 NEPH2-33 & DWPF Nepheline -2 & Peeler et al. 2006 & 0.1224 & 0.0472 & 0.0079 & 0.0876 \\
\hline 2588 NEPH2-34 & DWPF Nepheline -2 & Peeler et al. 2006 & 0.1343 & 0.0440 & 0.0087 & 0.0961 \\
\hline 2589 NEPH2-35 & DWPF Nepheline -2 & Peeler et al. 2006 & 0.1433 & 0.0416 & 0.0092 & 0.1025 \\
\hline 2590 NEPH2-36 & DWPF Nepheline -2 & Peeler et al. 2006 & 0.1254 & 0464 & 0.0081 & 0.0897 \\
\hline 2591 NEPH2-37 & DWPF Nepheline -2 & Peeler et al. 2006 & 0.1343 & 0.0440 & 0.0087 & 0.0961 \\
\hline $\begin{array}{l}2592 \text { NEPRL-38 } \\
2593 \text { NEPH2-39 }\end{array}$ & $\begin{array}{l}\text { DWFF Nepheneline - }-2 \\
\text { DWPF Neline }\end{array}$ & $\begin{array}{l}\text { Peeeler et a. } 2006 \\
\text { Peeler et al. } 2006\end{array}$ & $\begin{array}{l}0.1403 \\
0.1284\end{array}$ & $\begin{array}{l}0.0424 \\
0.0456\end{array}$ & $\begin{array}{l}0.0090 \\
0.0083\end{array}$ & $\begin{array}{l}0.1704 \\
0.0918\end{array}$ \\
\hline 2594 NEPH2-40 & $\begin{array}{l}\text { DWPF Nepheline }-2 \\
\text { DWF }\end{array}$ & Peeler et al. 2006 & $\begin{array}{l}0.1284 \\
0.1373\end{array}$ & & 0.0088 & 0.0983 \\
\hline 2595 NEPH3-41 & DWPF Nepheline -3 & Fox et al. 2006 & 0.0868 & 0.0520 & 0.0084 & 0.0930 \\
\hline 2596 NEPH3-42 & DWPF Nepheline -3 & Fox et al. 2006 & 0.0992 & 0.0480 & 0.0096 & 0.1063 \\
\hline 2597 NEPH3-43 & DWPF Nepheline -3 & Fox et al. 2006 & 0.1141 & 0.0432 & 0.0110 & 0.1222 \\
\hline 2598 NEPH3-44 & DWPF Nephelin & Fox et al. 2006 & 0.1240 & 0.0400 & 0.0119 & 0.1328 \\
\hline & & & & & & 0.0930 \\
\hline $\begin{array}{l}2600 \text { NEPH3-46 } \\
2601 \text { NEPH3-47 }\end{array}$ & $\begin{array}{l}\text { WWF Nephe } \\
\text { DWPF Nephe }\end{array}$ & 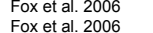 & $\begin{array}{l}0.0992 \\
0.1166\end{array}$ & $\begin{array}{l}0.0540 \\
0.0477\end{array}$ & $\begin{array}{l}0.0096 \\
0.0112\end{array}$ & $\begin{array}{l}0.1063 \\
0.1249\end{array}$ \\
\hline 2602 NEPH3-48 & DWPF Nepheline -3 & Fox et al. 2006 & 0.12 & 0.0441 & 0.0122 & $\begin{array}{l}0.1355 \\
0\end{array}$ \\
\hline 2603 NEPH3-49 & & Fox et al. 2006 & & & & 0.0930 \\
\hline & & & & & & 0.1063 \\
\hline-51 & DWPF Nephe & 06 & 0.1092 & 0.0448 & 0.0105 & 0.1169 \\
\hline 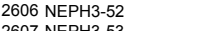 & DWPF Nepheline - 3 & 006 & & & & 0.1275 \\
\hline $2607 \mathrm{NEPH}$ & DWPF Neph & tal. 2006 & & & 0.0084 & 0.0930 \\
\hline & DW & & & & & \\
\hline $\begin{array}{l}55 \\
56\end{array}$ & $\begin{array}{l}\text { DWPF Nephe } \\
\text { DWPF Nephe }\end{array}$ & $x$ et al 2 & & $\begin{array}{l}0.041 \\
0.039\end{array}$ & & $\begin{array}{l}0.1275 \\
0.1355\end{array}$ \\
\hline $\begin{array}{l}\text { HLW98-77 } \\
\text { HLW }\end{array}$ & HLW Compar & ot et al. 2005a & 0.05 & 0.11 & 0.0028 & 0.1222 \\
\hline 2612 HLW98-77R2 & HLW Compar & ot et al. $2005 a$ & & 0.1191 & 0.0028 & 0.1222 \\
\hline & & & & & & \\
\hline 4-07RE39 & HLW Compari: & 105a & & & 0.0046 & 0.1400 \\
\hline $\begin{array}{l}9 \mathrm{CCCC} \\
99\end{array}$ & HLW Compa & $05 a$ & & & & 0.14 \\
\hline 99 & ILW Co & tal. 2005a & & & 0.0046 & 0.1401 \\
\hline $\mathrm{CCCC}$ & Idy & 105a & & 0.1037 & & $\begin{array}{l}0.1401 \\
0.1221\end{array}$ \\
\hline & LW Comparison St & $005 a$ & $\begin{array}{l}0.05 \\
0.03\end{array}$ & $\begin{array}{l}0.1190 \\
0.1081\end{array}$ & & 0.0954 \\
\hline 1AW & ison Study & tet al. 2005a & 55 & 0.0923 & 0.0068 & \\
\hline S1AWCG & ison Study & & & & 0.0068 & \\
\hline & & 2008 & 0617 & & & 0.1090 \\
\hline & & & 0400 & & & \\
\hline 102-03 & HLW02 Initial M & Piepel et al. 2008 & 0.0400 & 0.1400 & 0.0050 & 0.0800 \\
\hline
\end{tabular}

K2O-t Li2O-t MgO-t Na2O-t NiO-t P2O5-t SiO2-t ZrO2-t Ag2O-t As2O3-tAs205-t BaO-t BeO-t Bi2O3-t

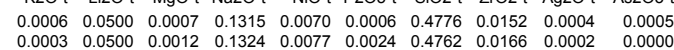

$\begin{array}{llllllllll}0.0008 & 0.0500 & 0.0003 & 0.0964 & 0.0025 & 0.0022 & 0.4255 & 0.0808 & 0.0000 & 0.0000 \\ 0.0003 & 0.0358 & 0.0024 & 0.1010 & 0.0017 & 0.0017 & 0.3783 & 0.0013 & 0.0026 & 0.0000\end{array}$

$\begin{array}{lllllllll}0.0019 & 0.0574 & 0.0007 & 0.0639 & 0.0059 & 0.0014 & 0.4355 & 0.0392 & 0\end{array}$

$\begin{array}{lllll} & 0.0000 & 0.0082 & 0.0005 & 0.0002 \\ 0.0004 & 0.0000 & 0.0137 & 0.0007 & 0.0005 \\ 0.0000 & 0.0000 & 0.0006 & 0.0000 & 0.0002 \\ 0.0007 & 0.0000 & 0.0005 & 0.0003 & 0.0003 \\ 0.0005 & 0.0000 & 0.0041 & 0.0009 & 0.0001\end{array}$

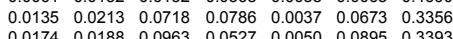

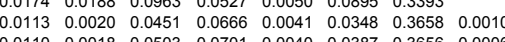

$\begin{array}{lllllll}0.0079 & 0.0108 & 0.0276 & 0.0709 & 0.00010 & 0.0246 & 0.356\end{array}$

$\begin{array}{llllllll}0.0058 & 0.0128 & 0.0123 & 0.0733 & 0.0056 & 0.0056 & 0.4338\end{array}$

$\begin{array}{lllllllll}0.0073 & 0.0460 & 0.0069 & 0.1709 & 0.0050 & 0.0000 & 0.4311 & 0.0009 & 0.0000\end{array}$

$\begin{array}{lllllllllllll}0.0043 & 0.0469 & 0.0067 & 0.1709 & 0.0122 & 0.0000 & 0.4327 & 0.0010 & 0.0000\end{array}$

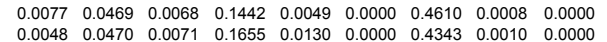

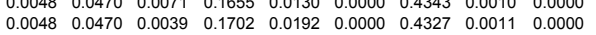

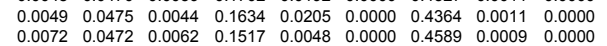

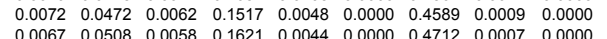

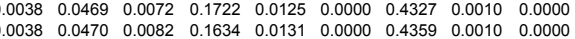

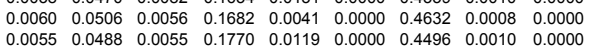

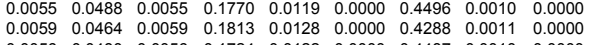

$\begin{array}{ccccccccc}0.0056 & 0.0480 & 0.0056 & 0.1724 & 0.0122 & 0.00000 & 0.4487 & 0.0010 & 0.0000 \\ 0.0060 & 0.0456 & 0.0060 & 0.1771 & 0.0131 & 0.0000 & 0.4276 & 0.0011 & 0.0000\end{array}$

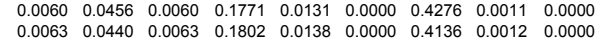

$\begin{array}{llllllllll}0.0057 & 0.0472 & 0.0058 & 0.1681 & 0.0125 & 0.0000 & 0.4476 & 0.0011 & 0.0000\end{array}$

$\begin{array}{lllllllll}0.0062 & 0.0448 & 0.0062 & 0.1731 & 0.0135 & 0.0000 & 0.4262 & 0.0011 & 0.0000 \\ 0.0066 & 0.0424 & 0.0066 & 0.1780 & 0.0144 & 0.0000 & 0.4048 & 0.0012 & 0.0000\end{array}$

$\begin{array}{lllllllll}0.0066 & 0.0424 & 0.0066 & 0.1780 & 0.0144 & 0.00000 & 0.4048 & 0.0012 & 0.0000 \\ 0.0059 & 0.0464 & 0.0059 & 0.1639 & 0.0128 & 0.0000 & 0.4462 & 0.0011 & 0.0000\end{array}$

$\begin{array}{lllllllll}0.0064 & 0.0432 & 0.0065 & 0.1710 & 0.0141 & 0.00000 & 0.4173 & 0.0012 & 0.00000 \\ 0.0069 & 0.0408 & 0.0069 & 0.1763 & 0.0150 & 0.0000 & 0.3956 & 0.0013 & 0.0000\end{array}$

$\begin{array}{lllllllll}0.0060 & 0.0456 & 0.0060 & 0.1600 & 0.0131 & 0.0000 & 0.4447 & 0.0011 & 0.00000 \\ 0.0066 & 0.0424 & 0.0066 & 0.1674 & 0.0144 & 0.0000 & 0.4154 & 0.0012 & 0.0000\end{array}$

$\begin{array}{lllllllllll}0.0071 & 0.0392 & 0.0072 & 0.1749 & 0.0156 & 0.0000 & 0.3861 & 0.0013 & 0.0000\end{array}$

$\begin{array}{lllllllll}0.0067 & 0.0488 & 0.0042 & 0.1651 & 0.0136 & 0.0000 & 0.4499 & 0.0011 & 0.0000 \\ 0.0076 & 0.0448 & 0.0047 & 0.1709 & 0.0153 & 0.0000 & 0.4152 & 0.0012 & 0.0000\end{array}$

$\begin{array}{lllllllll}0.0069 & 0.0480 & 0.0043 & 0.1603 & 0.0139 & 0.0000 & 0.4489 & 0.0011 & 0.0000\end{array}$

$\begin{array}{lllllllll}0.0076 & 0.0448 & 0.0047 & 0.1653 & 0.0153 & 0.00000 & 0.44208 & 0.0012 & 0.00000 \\ 0.0082 & 0.0416 & 0.0051 & 0.1704 & 0.0167 & 0.0000 & 0.3927 & 0.0013 & 0.0000\end{array}$

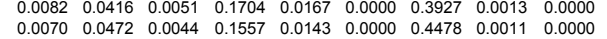

$\begin{array}{lllllllll}0.0077 & 0.0440 & 0.0048 & 0.1611 & 0.0157 & 0.0000 & 0.4193 & 0.0012 & 0.0000 \\ 0.0082 & 0.0416 & 0.0051 & 0.1652 & 0.0167 & 0.0000 & 0.3979 & 0.0013 & 0.0000\end{array}$

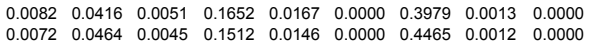

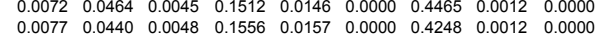
$\begin{array}{llllllllll}0.00081 & 0.0424 & 0.0050 & 0.1585 & 0.0164 & 0.00000 & 0.4104 & 0.0013 & 0.00000 \\ 0.0074 & 0.0256 & 0.0046 & 0.1470 & 0.0150 & 0.0000 & 0.4450 & 0.012 & 0.0000\end{array}$ $\begin{array}{lllllllll}0.0074 & 0.0456 & 0.0046 & 0.1470 & 0.0150 & 0.0000 & 0.4450 & 0.0012 & 0.0000 \\ 0.0079 & 0.0432 & 0.0049 & 0.1516 & 0.0160 & 0.0000 & 0.4230 & 0.0013 & 0.0000 \\ 0.0012 & 0.0520 & 0.0087 & 0.1293 & 0.0055 & 0.0000 & 0.5084 & 0.0008 & 0.0000\end{array}$ $\begin{array}{lllllllll}0.0014 & 0.0480 & 0.0100 & 0.1363 & 0.0063 & 0.0000 & 0.4725 & 0.0010 & 0.0000 \\ 0.0016 & 0.0432 & 0.0115 & 0.1448 & 0.0073 & 0.0000 & 0.4293 & 0.0011 & 0.0000\end{array}$ $\begin{array}{lllllllll}0.0016 & 0.0432 & 0.0115 & 0.1448 & 0.0073 & 0.0000 & 0.4293 & 0.0011 & 0.00000 \\ 0.0017 & 0.0400 & 0.0125 & 0.1504 & 0.0079 & 0.0000 & 0.4006 & 0.0012 & 0.0000\end{array}$ $\begin{array}{lllllllll}0.0017 & 0.040650 & 0.0087 & 0.1098 & 0.0055 & 0.00000 & 0.5084 & 0.00008 & 0.00000\end{array}$ $\begin{array}{lllllllll}0.0014 & 0.0600 & 0.0100 & 0.1183 & 0.0063 & 0.0000 & 0.4725 & 0.0010 & 0.0000 \\ 0.0016 & 0.0530 & 0.0117 & 0.1303 & 0.0074 & 0.0000 & 0.4221 & 0.0011 & 0.0000\end{array}$

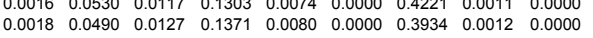
$\begin{array}{lllllllll}0.0018 & 0.0490 & 0.0127 & 0.1371 & 0.0080 & 0.0000 & 0.3934 & 0.0012 & 0.0000 \\ 0.0012 & 0.0520 & 0.0087 & 0.1423 & 0.0055 & 0.0000 & 0.4954 & 0.00008 & 0.00000\end{array}$ $\begin{array}{lllllllll}0.0014 & 0.0480 & 0.0100 & 0.1483 & 0.0063 & 0.0000 & 0.4605 & 0.0010 & 0.0000 \\ 0.0015 & 0.0448 & 0.0110 & 0.1532 & 0.0069 & 0.0000 & 0.4325 & 0.0010 & 0.0000\end{array}$ $\begin{array}{lllllllll}0.0015 & 0.0448 & 0.0110 & 0.1532 & 0.0069 & 0.0000 & 0.4325 & 0.0011 & 0.0000 \\ 0.0017 & 0.0416 & 0.0120 & 0.1580 & 0.0076 & 0.0000 & 0.4045 & 0.0011 & 0.0000\end{array}$ $\begin{array}{lllllllll}0.0017 & 0.0416 & 0.0120 & 0.1580 & 0.0076 & 0.0000 & 0.4045 & 0.0011 & 0.0000 \\ 0.0012 & 0.0715 & 0.0087 & 0.1098 & 0.0055 & 0.00000 & 0.5884 & 0.00008 & 0.00000 \\ 0.0047 & 0.0572 & 0.0120 & 0.1320 & 0.0076 & 0.0000 & 0.4725 & 0.0010 & 0.0000\end{array}$ $\begin{array}{lllllllll}0.0014 & 0.0660 & 0.0100 & 0.1183 & 0.0063 & 0.0000 & 0.4725 & 0.0010 & 0.0000 \\ 0.0017 & 0.0572 & 0.0120 & 0.1320 & 0.0076 & 0.0000 & 0.4149 & 0.0011 & 0.0000\end{array}$ $\begin{array}{lllllllll}0.0017 & 0.0572 & 0.0120 & 0.1320 & 0.0076 & 0.0000 & 0.4149 & 0.0011 & 0.0000 \\ 0.0018 & 0.0539 & 0.0127 & 0.1371 & 0.0080 & 0.0000 & 0.3934 & 0.0012 & 0.0000\end{array}$ $\begin{array}{lllllllllll}0.0003 & 0.0353 & 0.0011 & 0.1166 & 0.0061 & 0.0000 & 0.4745 & 0.0381 & 0.0002\end{array}$ $\begin{array}{llllllllll}0.0003 & 0.0353 & 0.0011 & 0.1166 & 0.0061 & 0.0000 & 0.4745 & 0.0381 & 0.0002 & 0.00000 \\ 0.0003 & 0.0353 & 0.0011 & 0.1166 & 0.0061 & 0.0000 & 0.4745 & 0.0381 & 0.0002 & 0.0000\end{array}$ $\begin{array}{llllll}0.0041 & 0.0056 & 0.4745 & 0.0046 & 0.0019\end{array}$

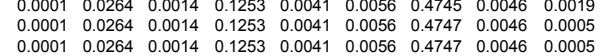
$\begin{array}{llllllll}0.0001 & 0.0264 & 0.0014 & 0.1253 & 0.004 & 0.0056 & 0.4747 & 0.0046 \\ 0.0003 & 0.0352 & 0.0011 & 0.1165 & 0.0061 & & 0.4740 & 0.0380\end{array}$

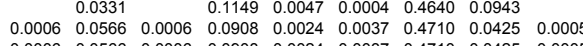

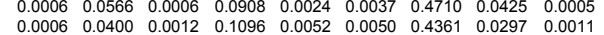

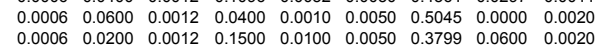

0.0003 0.0246
0.0245
0.0266 .0266 .0004 0.0004
0.0006
0.0004 0.0006 .0008 0.0004 0.0003 .0006 0.0005 .0005 0.0006
0.0006

0.0007

0.0006
0.0006
0.0006

0.0005

0.0006

0.0007
0.0000

0.0008
0.0008
0.0008
0.0008

0.0008

.0009

0.0008
0.0009
0.0009

0.0008
0.0009
0.0010

0.0008

0.0009
0.0010

0.0008
0.0009
0.0009

0.000

0.0008

0.0009
0.0009
0.0008
0.0009

0.0009
0.0005

0.0006
0.0007

0.0008
0.0005

.0007

0.0008
0.0005
0.0006

0.0006
0.0007
0.0007
0.0005

0.0006

0.0000
0.0000

\begin{tabular}{ll} 
& 0.0008 \\
.0006 & 0.0000 \\
.0006 & 0.0000 \\
\hline
\end{tabular}

$\begin{array}{ll}0.0006 & 0.0000 \\ 0.0001 & 0.0010 \\ 0.0001 & 0.0010\end{array}$

$\begin{array}{ll}0.0001 & 0.0010 \\ .0001 & 0.0010 \\ .0001 & 0.0010 \\ 0.000 & 0.001\end{array}$

$\begin{array}{ll}0.0001 & 0.0010 \\ 0.0001 & 0.0010\end{array}$

$\begin{array}{ll}0.0006 & 0.0008 \\ 0.0006 & 0.0008 \\ 0.007 & 0.005\end{array}$

\begin{tabular}{ll}
\hline 0.0006 & 0.0008 \\
\hline 0.0077 & 0.0005 \\
\hline
\end{tabular}

0.0001
0.0001

$\begin{array}{ll}0.0150 & 0.0005 \\ 0.0150 & 0.000\end{array}$ 
\# Glass ID

H Glass ID
2534 HLW98-59
2544 HLW98-60

2544 HLW98-60

2546 HLW98-63
2547 HLW98-64

$2548 \mathrm{CAA}-03$

$2599 \mathrm{C} 4 \mathrm{C}-03$
$2550 \mathrm{C} C \mathrm{C}-08$

$2551 \mathrm{C} 4 \mathrm{D}-04$
$2552 \mathrm{C}-15$
$2553 \mathrm{C}-15 \mathrm{D}-01$

2554 S12-04 (C4A-03 mod.)

2555 SB4-NEPH-01cCC

2557 SB4-NEPH-03cCC

2558 SB4-NEPH-04cCC

2560 SB4-NEPH-06cCC

2562 SB4-NEPH-08CCCC

2565 SB4-NEPH-11ccC

2567 NEPH2-13
2568 NEPH2-14

2569 NEPH2-15

2570 NEPH2-16

2572 NEPH2-18

2573 NEPP2-19

2576 NEPH2-22

2577 NEPH2-23

2579 NEPH2-25

2581 NEPP2-27

2582 NEPH2-28
2583 NEPH2-29

2584 NEPH2-30

2585 NEPH2-31

2587 NEPH2-33

2588 NEPH2-34

2589 NEPH2-35

(592 NEPH2-38

2503 NEPH2-30

2595 NEPH3-41

2597 NEPH3-43

2598 NEPH3-44

2600 NEPH3-46

2602 NEPH3-48

2603 NEPH3-49

2605 NEPH3-51

2607 NEPH3-53

2608 NEPH3-54

2610 NEPH3-56
2611 HLW98-77

2612 HLW98-77R2

2614 HLW04-07RE39

2615 HLW04-07RE39CCC

2616 HLW04-07NRE9

2618 A12-G-122A

2620 HLW98-51AW

2622 HLW02-01

2623 HLW02-02
CoO-t Co203-t Cr2O3-t Cs2O-t CuO-t Dy203-t Eu2O3-t

$0.0000 \quad 0.0000000000002$

$\begin{array}{lll}0.0010 & 0.0000 & 0.0000\end{array}$

$\begin{array}{llll}0.0000 & 0.0018 & 0.0007 & 0.0000 \\ 0.0000 & 0.0005 & 0.00009 & 0.0000 \\ & & & 0.0003\end{array}$

$\begin{array}{lll}0.033 & 0.0147 \\ 0.031 & 0.001\end{array}$

O3-t Nb205-t Nd203-t NpO2-t PbO-t PdO2-t PdO-t Pr203-t Pr6011-t Rb2O-t ReO-t

0.0002

$\begin{array}{lll}0.0031 & 0.0061 & 0.000 \\ 0.0000 & 0.0171 & 0.0000 \\ 0.0012 & 0.0030 & 0\end{array}$

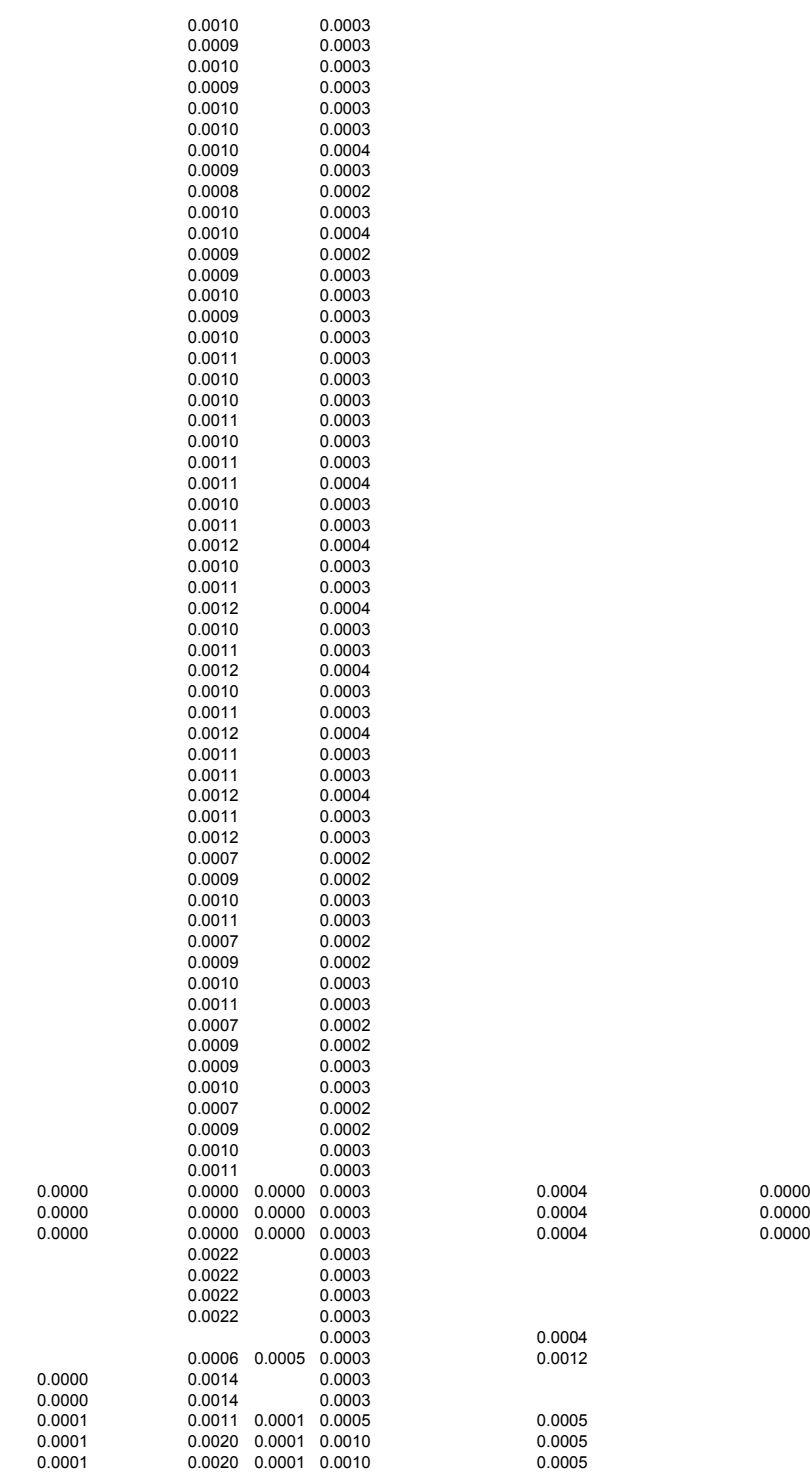

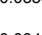

0.0048

$\begin{array}{llll}0.0000 & 0.0000 & 0.0007 & 0.0000 \\ 0.0000 & 0.0002 & 0.0001 & 0.0000 \\ 0.0000 & 0.0000 & 0.0024 & 0.0000\end{array}$

$\begin{array}{llll}0.0000 & 0.0000 & 0.0005 & 0.0000 \\ 0.0000 & 0.0000 & 0.0024 & 0.0000 \\ 0.0000 & 0.0000 & 0.0017 & 0.0000\end{array}$

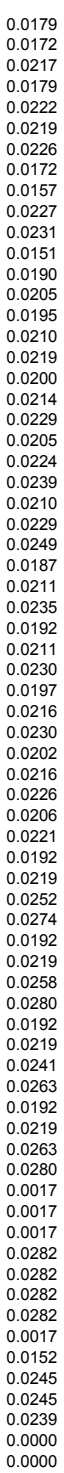

0.0000

0.0003
0.0003
0.0003

0.0003

0.0000

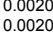

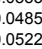

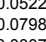

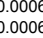

0.0007
0.0006
0.0006
0.006

10000

$\underbrace{0.0009}_{0.0000}$

10.000

0.0007
0.0007
0.0008

.00008
.0007
0

0.00008
0.0008
0.0007

0.0007
0.0008
0.0009

0.0009
0.0008
0.0008

0.0009
0.0008
0.0000

0.0008
0.0009
0.0010

0.0008
0.0009

0.0009
0.0008
0.0009

0.0009
0.0008
0.0009

0.0009
0.0008
0.0009
0.0003

0.00004

0.0004
0.0004
0.0005

0.0005
0.0003
0.0004

.0 .0004
.0000
0.005

0.0005
0.0003
0.0004

0.0004

0.0004
0.0003

0.0004

$0.0003-0.0000 \quad 0.0000$

0.0010

0.0015
0.00152
0.00002
0.0030
0.0030

0.0017
0.0152
0.0245
0.0245
0.0239
0.0239

0.0000 
\# Glass ID

\# Glass ID
2543 HLW98-59
2544 HLW98-60

2545 HLW98-62

2546 HLW W98-63
2547 HLW98-64

$2548 \mathrm{C} 4 \mathrm{~A}-03$

$2549 \mathrm{CAC}-03$
$2550 \mathrm{CC}-08$

2551 C4D-04
2552 C4D-15
2553 C4D-01

2554 S12-04 (C4A-03 mod.)

2555 SBA-NEPH-01 1 CCC

2557 SB4-NEPH-03c

2559 SBA $\mathrm{NEPH}-050 \mathrm{COCC}$

2561 SB4-NEPH-07CCCC

2562 SBA-NEPH- $080 \mathrm{CCC}$

S

2566 SB4-NEPH-12cCC

2568 NEPH2-14

2569 NEPH2-15

2571 NEPH2-17

2573 NEPH2-19

2575 NEPH2-21

2576 NEPH2-22

2578 NEPH2-24

2581 NEPP2-27

2582 NEPH2-28

2584 NEPH2-30

2586 NEPH2-32

2588 NEPH2-34

2589 NEPH2-35

2590 NEPH2-36

2592 NEPH2-38

2594 NEPH2-40

2597 NEPH3-43

2599 NEPH3-45

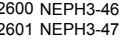

2602 NEPH3-48
2603 NEPH3-49

2604 NEPP3-50

2608 NEPH3-54

2610 NEPH3-56
2611 HLW98-77

2612 HLW98-77R2

2615 HLW04-07RE39CCC

2616 HLW04-07NRE9

2618 A12-G-122A
2619 BLF-G-87A

2620 HLW98-51AW

2622 HLW02-01

2623 HLW02-02
2624 HLW02-03
Re02-t Re207-t Rh2O3-t RhO2-t RuO2-t Ru2O3-t Sb203-t Sb205-t SeO2-t Sm203-t SnO-t SnO2-t SO3-t SrO-t Tc207-t Te02-t ThO2-t TiO2-t T12O-t T1203-t U308-t U02-t U03-t V205-t W03-t Y203-t ZnO-t Sum-t Al203-a

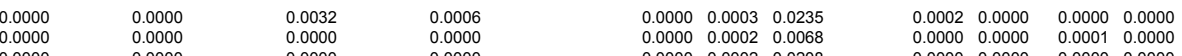

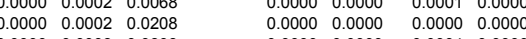

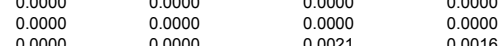

0.0000

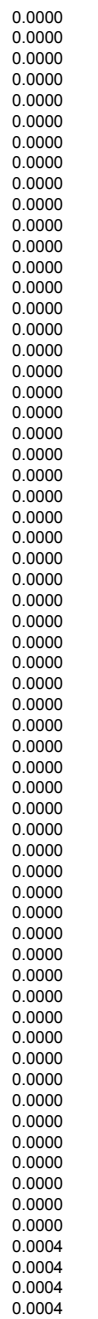

0.0000

0.0000

0.0000

0.0000

0.0000

0.00000

0.0000

0.0000
0.0000
0.0000
0.0000

0.0000

0.0000

0.0000
0.0000
0.0000
0.000

0.0000
0.0000

0.0000
0.0000

0.0000
0.0000
0.0000

0.0000

0.0000
0.0000

0.0000

0.0000
0.0000

0.0000

0.0000
0.0000
0.0000

0.0000

0.0000

0.0000
0.0000
0.000

0.0000

0.0000
0.0000

0.0000
0.0000
0.0000

0.0000
0.0000
0.0000

0.0000
0.0000
0.0000

$\begin{array}{ll}0.0000 & 0.0007 \\ 0.0000 & 0.0007\end{array}$

0.0004

$\begin{array}{ll}0.0003 & 0.0002 \\ 0.0003 & 0.0002 \\ 0.0005 & 0.0008 \\ 0 & 0.0000\end{array}$

$\begin{array}{ll}0.0005 & 0.0008 \\ 0.0005 & 0.0008 \\ 0.0005 & 0.0008\end{array}$

0.0011
0.0020
0.0002

0.0011
0.0002
0.0020

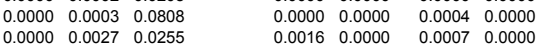

0.0197

0.0033
0.0035
0.0039$$
\begin{aligned}
& 0.0039 \\
& 0.0033 \\
& 0.0033
\end{aligned}
$$$$
\begin{aligned}
& 0.0033 \\
& 0.0037 \\
& 0.0033
\end{aligned}
$$$$
\begin{aligned}
& 0.0033 \\
& 0.0036 \\
& 0.0028
\end{aligned}
$$$$
\begin{aligned}
& 0.0028 \\
& 0.0038 \\
& 0.0032
\end{aligned}
$$$$
\begin{aligned}
& 0.0032 \\
& 0.0035 \\
& 0.0037 \\
& 0.0038
\end{aligned}
$$

$$
\begin{aligned}
& 0.0037 \\
& 0.0036 \\
& 0.0038 \\
& 0.0040
\end{aligned}
$$$$
\begin{aligned}
& 0.0040 \\
& 0.0037
\end{aligned}
$$$$
\begin{aligned}
& 0.0039 \\
& 0.0042
\end{aligned}
$$$$
\begin{aligned}
& 0.0037 \\
& 0.0041
\end{aligned}
$$$$
\begin{aligned}
& 0.0041 \\
& 0.0044 \\
& 0.0038
\end{aligned}
$$$$
\begin{aligned}
& 0.0038 \\
& 0.0042 \\
& 0.0046
\end{aligned}
$$$$
\begin{aligned}
& 0.0036 \\
& 0.0038 \\
& 0.0042
\end{aligned}
$$$$
\begin{aligned}
& 0.0038 \\
& 0.0042 \\
& 0.0034
\end{aligned}
$$$$
\begin{aligned}
& 0.0034 \\
& 0.0038 \\
& 0.0041
\end{aligned}
$$$$
\begin{aligned}
& 0.0041 \\
& 0.0035 \\
& 0.0039
\end{aligned}
$$$$
\begin{aligned}
& 0.0039 \\
& 0.0041 \\
& 0.0036
\end{aligned}
$$$$
\begin{aligned}
& 0.0036 \\
& 0.0039 \\
& 0.0040
\end{aligned}
$$$$
\begin{aligned}
& 0.0040 \\
& 0.0037
\end{aligned}
$$$$
\begin{aligned}
& 0.0040 \\
& 0.0039 \\
& 0.0045
\end{aligned}
$$$$
\begin{aligned}
& 0.0039 \\
& 0.0045 \\
& 0.0051
\end{aligned}
$$$$
\begin{aligned}
& 0.0056 \\
& 0.0039 \\
& 0.0055
\end{aligned}
$$$$
\begin{aligned}
& 0.0045 \\
& 0.0052
\end{aligned}
$$$$
\begin{aligned}
& 0.0057 \\
& 0.0039 \\
& 0.0045
\end{aligned}
$$$$
\begin{aligned}
& 0.0045 \\
& 0.0049
\end{aligned}
$$$$
\begin{aligned}
& 0.0054 \\
& 0.0039 \\
& 0.0045
\end{aligned}
$$$$
\begin{aligned}
& 0.0045 \\
& 0.0054 \\
& 0.057
\end{aligned}
$$

$\begin{array}{lll}0.0000 & 0.0057 & \\ 0.0008 & 0.0003\end{array}$

$\begin{array}{lll}0.0000 & 0.0008 & 0.0003 \\ 0.0000 & 0.0008 & 0.0003 \\ 0.0000 & 0.0008 & 0.0003\end{array}$

$\begin{array}{lll}0.0006 & 0.0019 & 0.0017 \\ 0.0006 & 0.0019 & 0.0017\end{array}$

$\begin{array}{lll}0.0006 & 0.0019 & 0.0017 \\ 0.0006 & 0.0019 & 0.0017 \\ 0.0006 & 0.0019 & 0.0017\end{array}$

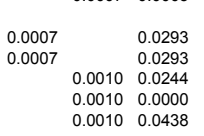

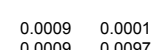

$\begin{array}{ll}0.0009 & 0.0009 \\ 0.0013 & 0.0091 \\ 0.0009 & 0.001\end{array}$

$\begin{array}{ll}0.0013 & 0.0091 \\ 0.0009 & 0.0001 \\ 0.0014 & 0.0001\end{array}$

$\begin{array}{ll}0.0015 & 0.009 \\ 0.0017 & 0.000\end{array}$

$\begin{array}{ll}0.0017 & 0.0001 \\ 0.0009 & 0.0092 \\ 0.0006 & 0.001\end{array}$

$\begin{array}{ll}0.0008 & 0.0001 \\ 0.0013 & 0.0093 \\ 0.0015 & 0.0002 \\ 0.000 & 0.002\end{array}$

$\begin{array}{ll}0.0008 & 0.0081 \\ 0.0002 & 0.0001\end{array}$

$\begin{array}{ll}0.0002 & 0.0001 \\ 0.0002 & 0.0001 \\ 0.0002 & 0.0001\end{array}$

\begin{tabular}{ll}
0.0002 & 0.0001 \\
0.0002 & 0.0001 \\
\hline
\end{tabular}

$\begin{array}{ll}0.0002 & 0.0001 \\ 0.0002 & 0.0001 \\ 0.0002 & 0.0001\end{array}$

$\begin{array}{ll}0.0002 & 0.0001 \\ 0.0002 & 0.0001 \\ 0.0002 & 0.001\end{array}$

$\begin{array}{ll}0.0002 & 0.0001 \\ 0.0002 & 0.0001 \\ 0.0002 & 0.0001\end{array}$

$\begin{array}{ll}0.0002 & 0.0001 \\ 0.0002 & 0.0001\end{array}$

\begin{tabular}{ll}
0.0002 & 0.0001 \\
0.0002 & 0.0001 \\
\hline .002 & 0.0001
\end{tabular}

$\begin{array}{ll}0.0002 & 0.000 \\ 0.0002 & 0.000\end{array}$

$\begin{array}{ll}0.0002 & 0.0001 \\ 0.0002 & 0.0001\end{array}$

$\begin{array}{ll}0.0002 & 0.0001 \\ 0.0002 & 0.0001 \\ 0.0002 & 0.0001\end{array}$

$\begin{array}{ll}0.0002 & 0.0001 \\ 0.0002 & 0.0001\end{array}$

$\begin{array}{ll}0.0002 & 0.0001 \\ 0.0002 & 0.0001 \\ 0.0002 & 0.001\end{array}$

\begin{tabular}{ll}
0.0002 & 0.0001 \\
0.0002 & 0.0001 \\
\hline
\end{tabular}

$\begin{array}{ll}0.0003 & 0.0001 \\ 0.0003 & 0.0001 \\ 0.0003 & 0.0001\end{array}$

$\begin{array}{ll}0.0003 & 0.0001 \\ 0.0003 & 0.0001 \\ 0.0002 & 0.0001\end{array}$

$\begin{array}{ll}0.0003 & 0.0001 \\ 0.0002 & 0.0001\end{array}$

$\begin{array}{ll}0.0003 & 0.0001 \\ 0.0003 & 0.0001 \\ 0.0002 & 0.000\end{array}$

$\begin{array}{ll}0.0002 & 0.0001 \\ 0.0003 & 0.0001 \\ 0.000 & 0.001\end{array}$

$\begin{array}{ll}0.0003 & 0.0001 \\ 0.0003 & 0.0001\end{array}$

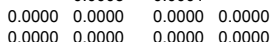

$\begin{array}{llll}0.0000 & 0.0000 & 0.0000 & 0.0000 \\ 0.0000 & 0.0000 & 0.0000 & 0.000\end{array}$

$\begin{array}{ll}0.0000 & 0.0000 \\ 0.0000 & 0.0000 \\ 0.0003 & \\ 0.0003 & \\ 0.0003 & \\ 0.0003 & \end{array}$

0.0380
0.0380

0.0002
0.0003
0.0003

0.0001
0.0001

$\begin{array}{ll}0.0003 & 0.0010 \\ 0.0003 & 0.0002 \\ 0.0003 & 0.0019\end{array}$

$\begin{array}{ll}0.0003 & 0.0002 \\ 0.0003 & 0.0019\end{array}$ \begin{tabular}{ll}
0.0002 & 0.0001 \\
0.0002 & 0.0001 \\
0.0002 & 0.0001 \\
\hline .0002 & 0.0001
\end{tabular}

$\begin{array}{ll}0.0003 & 0.0001 \\ 0.0002 & 0.0001 \\ 0.0003 & 0.001\end{array}$ $\begin{array}{lll}0.0000 & 0.0000 \\ 0.0183 & 0.0000 \\ 0.000 & 0.0000\end{array}$

$0.0000 \quad 0.0000$

$\begin{array}{ll}0.0000 & 0.0000 \\ 0.0000 & 0.0000\end{array}$

0.0036
0.0048

$\begin{array}{ll}0.0200 & 1.0000 \\ 0.0204 & 0.9999\end{array}$

$\begin{array}{ll}0.0201 & 1.0000 \\ 0.0179 & 1.0000\end{array}$

10003

$\begin{array}{ll}1.0003 & 0.0608 \\ 0.9998 & 0.1198 \\ 0.9999 & 0.1375 \\ 1.0000 & 0.0934 \\ 0.9998 & 0.1269 \\ 1.0002 & 0.1337\end{array}$

$\begin{array}{ll}1.0056 \\ 0.0004 \quad 0.9799 \\ 0.004 & 0.9753\end{array}$

$0.0007 \quad 0.9824$

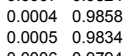

$0.0006 \quad 0.9891$

$0.0004 \quad 0.9842$

$\begin{array}{ll}.0005 & 0.9840 \\ .0005 & 0.9841\end{array}$

$\begin{array}{lll}0.0004 & 0.977 \\ 0.0004 & 0.9993\end{array}$

0.00050 .9993

$\begin{array}{lll}0.0004 & 0.9993 \\ 0.0005 & 0.9992\end{array}$

0.00040 .9993

$0.0005 \quad 0.9992$

0.00050 .9993

$\begin{array}{ll}0.0005 & 0.9992 \\ 0.0005 & 0.9992\end{array}$

$0.0005 \quad 0.9992$

0.00060 .9991

$0.0004 \quad 0.9993$

0.00050 .9992

$0.0004 \quad 0.9993$

0.00050 .9992

$\begin{array}{lll}0.0005 & 0.9993 \\ 0.0005 & 0.9992\end{array}$

0.00050 .9993

0.00050 .9992

$0.0005 \quad 0.9993$

$\begin{array}{ll}0.0005 & 0.9992 \\ 0.0003 & 0.9992\end{array}$

0.00040 .9991

$0.0005 \quad 0.9989$

0.00050 .9990

0.00030 .9992

$\begin{array}{lll}0.0004 & 0.9991 \\ 0.0004 & 0.9990 \\ 0 & 0003 & 0.9992\end{array}$

$\begin{array}{lll}0.0005 & 0.9989 \\ 0.0003 & 0.9992\end{array}$

$\begin{array}{ll}0.0004 & 0.9991 \\ 0.0005 & 0.9989\end{array}$

$\begin{array}{lllll}0.0000 & 0.0000 & 0.0002 & & 1.9989 \\ 0.0000 & 0.0000 & 0.0202 & 1.0000 & 0.0528 \\ 0.0000 & 0.0000 & 0.0202 & 1.0000 & 0.0510 \\ & 0.0202 & 1.0000 & 0.0517\end{array}$

$0.0148 \quad 1.0000$

0.01481 .0000

$\begin{array}{lll}0.0201 & 0.9998 & 0.0538\end{array}$

$\begin{array}{ll}0.0410 \quad 0.0000 \\ 0.0410 & 0.0000\end{array}$

0.0005
0.0005

0.0010
0.0010

$\begin{array}{ll}0.0200 & 1.0000 \\ 0.0200 & 1.0000\end{array}$ 
$2544 \mathrm{HLW}$ H98-60

2546 HLW98-63

2557 HLW98-04

$2548 \mathrm{CAA}-03$

$2550 \mathrm{C} 4 \mathrm{C}-08$

2551 C4D-04

2553 S12-04 (C4A-03 mod.)

2555 SBB-NEPH-01CCC

2557 SB4-NEPH-03cCC

2558 SBA-NEPH-04CCC

2559 SB4-NEPH-05cCC

2561 SB4-NEPH-07CCC

2562 SB4-NEPH-08cCC

2564 SB4-NEPH-10cC

2566 SB4-NEPH-12cC

2567 NEPH2-13

2569 NEPH2-15

2570 NEPH2-16

2572 NEPH2 18

2573 NEPH2-19

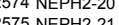

2576 NEPH2-22

2577 NEPH2-23

2578 NEPH2-24
2579 NEPH2-25

2580 NEPH2-26

2581 NEPH2-27

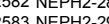

2584 NEPH2-30

2555 NEPH2-31

2586 NEPH2-32

2588 NEPH2-34

2589 NEPP2-35

2590 NEPH2-36
2591 NEPH 237

2592 NEPH2-38

2593 NEPP2 39
254 N

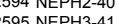

2596 NEPH3.42

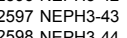

2598 NEP 3 344
2599 NEPH $3-45$

2000 NEPH3 3-46

(2602 NEPH3-48

2603 NEPH3-49

2604 NEPH 3.50

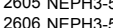

2607 NEPH3.53

(2008

2610 NEPH3.-56
2611 HLW98-77

2612 HLW98-77R2

2613 HLW98-77CG
2614 HLW04-07RE39

2615 HLWO4-07RE $390 C C$

2616 HLW04-07NRE9

2618 A12-G-G122A

2619 BLF-G-87A

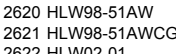

2623 HLW02-O2

2623 HLWW2-02
2624 HLW02-03

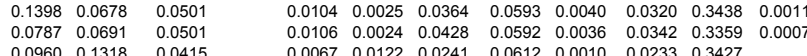

0.0411
0.0004

0.0211
0.0230
0.0232

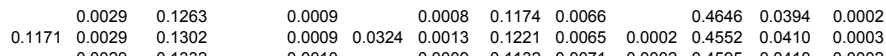

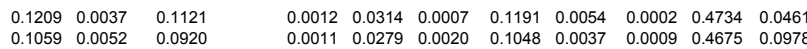

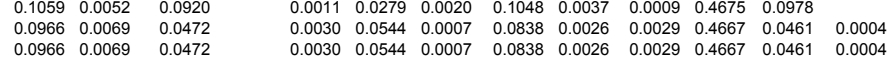

0.0007
0.0009
0.0007

$\begin{array}{ll}0.0007 & 0.0001 \\ 0.0003 & 0.0000\end{array}$

0.0009
0.0004

0.0005

$0007<0.0001$

$\begin{array}{ll}0.0006 & 0.0008 \\ 0.0006 & 0.0008\end{array}$

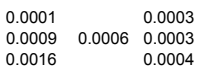

$\begin{array}{ll}0.0016 & 0.0004 \\ 0.0016 & 0.0004 \\ 0\end{array}$ 
\# Glass ID

2543 HLW98-59

2544 HLW98-60
2545 HLW98-62

2546 HLW98-63

$2548 \mathrm{C} 4 \mathrm{~A}-03$

$2549 \mathrm{C} 4 \mathrm{C}-03$
$2550 \mathrm{C} 4 \mathrm{C}-08$

2551 C4D- 04

2553 C4D- 01

2554 S12-04 (C4A-03 mod.)

2555 SB4-NEPH-01 CCC
2556 SB4-NEPH-O2CC

2557 SB4-NEPH-03cCC

2558 SBA-NEPH-04cCC
2559 SBA-NEPH-05cCC

S560

2561 SB4-NEPH-07CCC

2562 SBA-NEPH-08cCC

2564 SB4-NEPH-10cC

2565 SB4-NEPH-11cCC
2566 SB4-NEPH-12cCC

2567 NEPH2-13

$2569 \mathrm{NEPH} 2-14$

2570 NEPH2-16

2572 NEPH2-18

2573 NEPH2 219

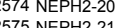

2576 NEPH2.22

2577 NEPH2-23

2578 NEPH2-24

2580 NEPH2-26

2581 NEPH2-27

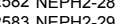

2554 NEPH2-30
2585 NEPP2 -3

2556 NEPH2-32
2587 NEPH2 233

2588 NEPH2-34

2589 NEP 2235
2500
NEP -36

$2590 \mathrm{NEPH} 2-3$
2591 NEPH2-33

2592 NEPH2-38

2593 NEPH2-39

2594 NEPH2-40
2595 NEPH3-41

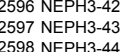

2598 NEP 3 344
2599 NEPH $3-45$

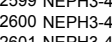

(2)

2603 NEPH3

2604 NEPH3-50

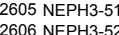

(2607 NEPH3.53

2608 NEPH3-54

2609 NEPP3 355
2610 NEPH3.56

2611 HLW98-77

2612 HLW98-77R2
2613 HLW

2615 HLWO4-07RE39CCCC

2616 HLW04-07NRE9

2617 HLW04-07NRE9CCCC

2619 BLF-G-87A

2620 HLW98-51AW
2621 HLW98-51AWCG

2621 HLW98-51AW
2622 HLW02-01

2623 HLW02-02

2623 HLWW2-02
2624 HLW02-03
Ga2O3-a Gd2O3-a

I-a La203-a MnO2-a MnO-a MoO-a MoO3-a Nb205-a Nd203-a Np02-a PbO-a PdO2-a PdO-a Pr2O3-a Pr6011-a PuO2-a Rb2O-a ReO-a ReO2-a Re207-a Rh203-a Rh02-a RuO2-a Sb203-a Sb205-a Se02-a

0.0053

0.078

0.0502

0.054

$\begin{array}{llll}0.0042 & 0.0018 & 0.0032 & 0.0003 \\ 0.0005 & 0.0019 & 0.0033 & 0.0005 \\ 0.0040 & 0.0019 & 0.0032 & 0.0004 \\ & & & \end{array}$

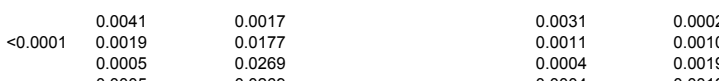


0.0156

2569 NEPH2-15

2570 NEPH2-16

2572 NEPH2-18

2573 NEPH2-19

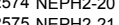

2576 NEPH2-22

2577 NEPH2-23

2578 NEPH2 24
2579 NEPH2 25
250 N

2580 NEPH2-26

2581 NEPH2-27

2582 NEPH2-28

2584 NEPH2 -30
2585 NEPP 231
2531

2556 NEPH2-32

2588 NEPH2-34

2589 NEPP2-35

2590 NEPH2-36
2591 NEPH 237

2592 NEPH2-38

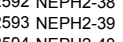

2594 NEPH2-40
2595 NEPH3-41
2

2596 NEPH3-42

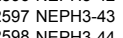

2599 NEPH3-45

2000 NEPH3-46

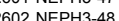

2603 NEPH3 -49
2604 NEPH 350

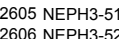

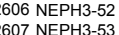

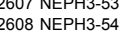

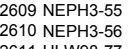

2611 HLWG8-77
26212 HLWS8-77R2

2613 HLWG8-77CG
2614 HLWO- $07 R E 39$

2615 HLWW4-07RE $399 C C$

2616 HLWO4-07NREE

2618 A12-G-G122A
2619 BLF-G-87A

2620
2621
HLWW89-51AW

2621 HLW98-51AWC
2622 HLWO2-01

2623 HLW02.

2624 HLWO2-03

0.0007
0.0000
0.00003

0.0003
0.0002

$\begin{array}{rrr}0.0008 & 0.0003 \\ 0.0003 & 0.0013 \\ 0.0008 & 0.0334 \\ 0.0008 & 0.0334\end{array}$

0.0009
0.0014

$\begin{array}{lll}0.0340 & 0.0014 \\ 0.0340 & 0.0003\end{array}$

0.0377
0.0377

$$
\begin{array}{r}
r 950 \\
<950 \\
<1500 \\
>1050 \\
<100 \\
<1000 \\
<1050 \\
<100 \\
<1050 \\
<1050
\end{array}
$$

$\begin{array}{ll}0.0001 & 0.0192 \\ 0.0193 & 0.9 \\ 0.0208\end{array}$

0.8432
0.0941
0.8443
0.0000

0.0443
0.00000
0.0000

$\begin{array}{lll}0.0174 & 0.00981 \\ 0 & 0.9985 & 0.9915\end{array}$

$\begin{array}{ll}0.0185 & 0.9945 \\ 0.0187 & 1.0030\end{array}$ 
2569 NEPH2-1

2570 NEPH2-16

572 NEPH2-18

2573 NEPH2-19

2574 NEPP $2-20$

2576 NEPH2-22

2577 NEPH12-23

2578 NEPH2-24
2579 NEPH 225

2580 NEPH2-26

2581 NEPH2-27

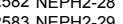

2584 NEPH2 200

2585 NEPH2-31

2586 NEPH2 232
2587 N N PPH2-33

2588 NEPH2-34

2589 NEPH2-35

$2590 N E P P 2-36$
2501

2592 NEPH2-38

2593 NEPH2-39

2596 NEPH3-42

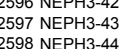

2598 NEPH3 344
2599 NEPH $3-45$

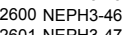

2602 NEPH3

2602 NEPP3 -48
2603 NEPH $3-49$

2604 NEPH 350

2605 NEPH3-51
2606 NEPH3-52

2607 NEPH 3.53

2608 NEPH3-54

2609 NEPP3 355
2610 NEPH3.56

2611
HEW $W 98-77$

2612 HLW98-77R2
2613 HLW $98-77 C G$

2615 HLW04-07RE39CCCC

2616 HHWO4-07TRRE

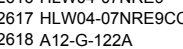

2619 BLF-G-87A

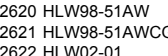

2623 HLW02-02

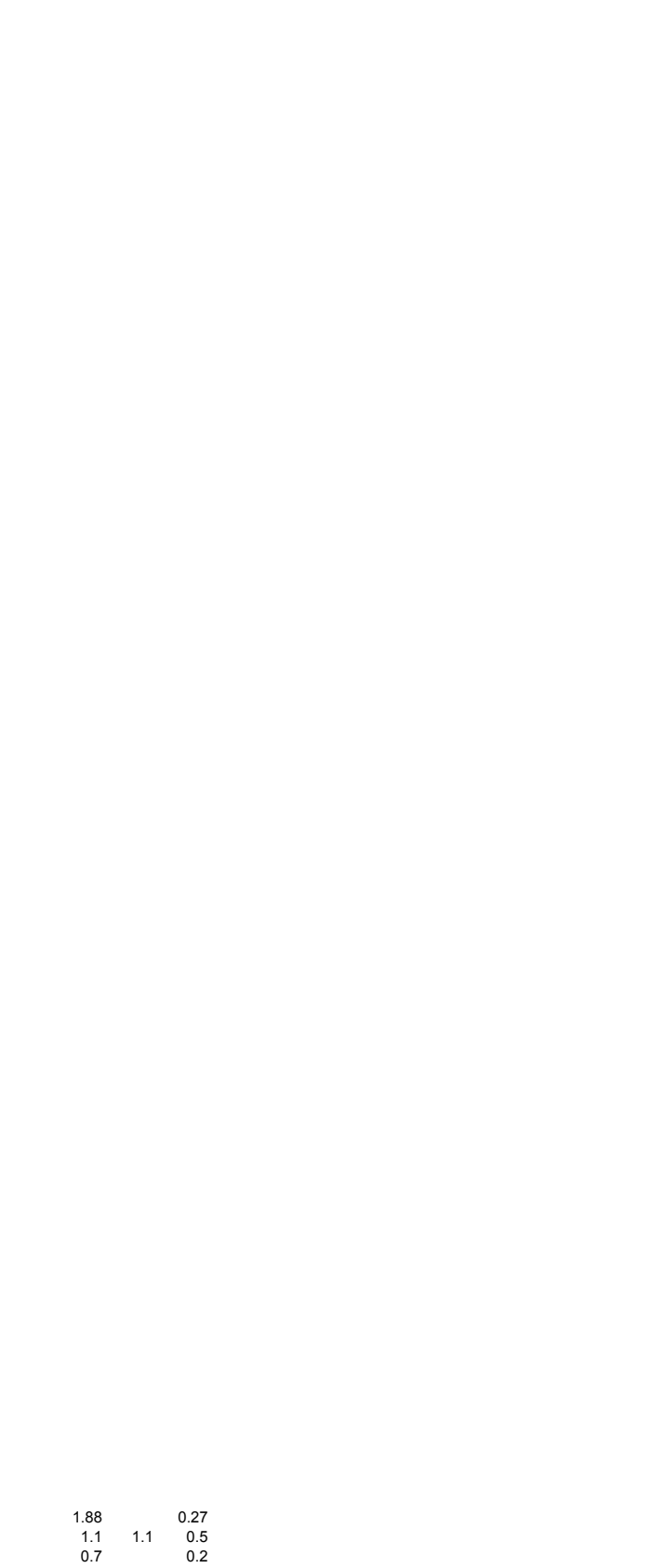

2.640

2.760
2.490
2.700
2.710

Nepheline / Homogeneous

Trevorite / Homogeneo

Carnegeite / Homogeneous

Homogeneous
Homogeneous

Homogeneous

Homogeneous
Homogeneous/ Homogeneous

Homegeneos

Homogeneous

amorphous

morphous

Trevorite, Nepheline, Lithium Silicate

Trevorite, Nepheline
Trevorite, Nepheline, Lithium Silicate

Trevorite, Nepheline
Trevorite, Nepheline, Lithium Silicate amorphous
Nepheline, Trevorite

Nepheline, Trevorite, Lithium Silicate amorphous

Trevorite Trevorite, Nepheline, Lithium Silicate Trevonte, Nephir Trevorite, Nepheline Lithium Silicate Trevorite, Nepheline, Lithium Silicate

Trevorite, Nepheline
Trevorite, Nepheline, Lithium Silicate Trevorite
Trevorite 
2545 HLW98-62

2546 HLW98-63
2547 HLW98-64

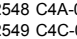

2550 C4C-08

2551 C4D-04
2552 C4D-15

253 C4D-01

2555 SBA-NEPH-01cCC

2557 SB4-NEPH-03cCC

2558 SB4-NEPH-04cCC

2560 SB4-NEPH-06cCC

2561 SB4-NEPH-07cCC

(563 SB4NEPH $1000 \mathrm{CCC}$

2566 NEA-NEPH-1200C
$25672-13$

$2568 \mathrm{NEPH} 2-14$

2569 NEPH2-14

2571 NEPH2-17

2573 NEPP2-19
2574 NEPH2-20

(

2577 NEPH2-23

2578 NEPHL-24
2579 NEPH 225

2580 NEPH2-26

2581 NEPH2-27

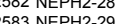

2584 NEPH2-30

2585 NEPH2-31

2586 NEPH2-32

2588 NEPH2-34

2589 NEPP2-35

2590 NEPH2 236
2591 NEPH2-37

2592 NEPH2-38

$2593 N E P P 2-39$
$254 N$ N

2594 NEPH2-40
2595 NEPH3-41

2596 NEPH3.42

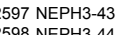

2599 NEPH3-45

2000 NEPH3-46

(2602 NEPH3-4

2603 NEPH3-49

2004 NEPA $3-50$

2006 NEPH3-52

2607 NEPH3.5

2008 NEPH3-54

2610 NEPH3.56

2611 HLW98-77

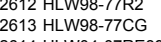

2615 HLWW4-07RE39CCC

2616 HLW04-07NRE9

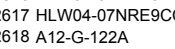

2619 BLF-G-87A

2620 HLWG8-51AW
2621 HLW98-51AWCC

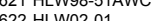

2623 HLWW2-02
2624 HLW02-03

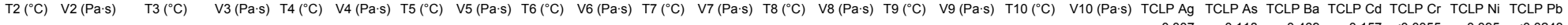

$\begin{array}{lrlllllllllll}1200 & 12.310 & 1250 & 7.6 & 1300.0 & 5.3 & 1350.0 & 3.9 & & & & \\ 1200 & 2.521 & 1250 & 1.7 & 1300.0 & 1.2 & 1350.0 & 0.9 & & & & \\ 12200 & 3.496 & 1250 & 2.4 & 130.0 & 1.6 & 1350.0 & 1.1 & & & & \\ 1150 & 11.492 & 1200 & 7.0 & 1250.0 & 4.5 & 1300.0 & 3.1 & 1350.0 & 2.2 & & \\ 1150 & 16.130 & 1200 & 9.3 & 1250.0 & 5.8 & 1300.0 & 3.9 & 1350.0 & 2.7 & & \\ 1150 & 5.316 & 1200 & 3.5 & 1250.0 & 2.4 & 1300.0 & 1.7 & 1350.0 & 1.3 & & \\ 1150 & 14.000 & 1200 & 8.4 & 1250.0 & 5.3 & 1300.0 & 3.5 & 1350.0 & 2.3 & 1400.0 & 1.7\end{array}$

2.06
0.24
0.88
1.16
1.2
1.88

$\begin{array}{ll}0.11 & 2.6 \\ 0.47 & 0.55 \\ 0.71 & 0.07 \\ 0.32 & 2.43 \\ 0.12 & .44 \\ 0.1 & 3.15\end{array}$

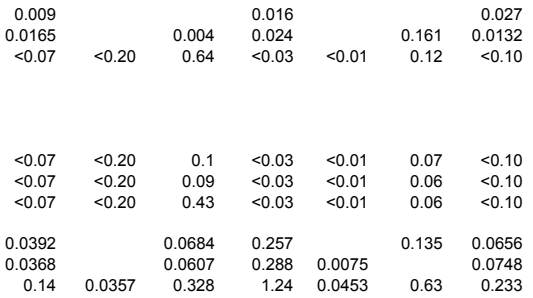


\# Glass ID

2543 HLW98-59

2544 HLW98-60

2546 HLW98-63

$254 \mathrm{C} 4 \mathrm{~A}-03$

$2549 \mathrm{C} 4 \mathrm{C}-03$
$2550 \mathrm{C} 4 \mathrm{C}-08$

2551 C4D-04

$2552 \mathrm{C} 4 \mathrm{D}-15$

2554 S12-04 (C4A-03 mod.)

2555 SBA-NEPH-01cCC

2557 SB4-NEPH-03cCC

2558 SB4-NEPH-04cCC

(2560)

2561 SB4-NEPH-07CCCCC

源

2564 SB4-NEPH-10CC

2566 SBA-NEPH-12CC

2567 NEPH2-13

2569 NEPH2-1

2570 NEPH2-16

572 NEPH2-18

2573 NEPH2-19

(5)

2577 NEPH2-23

2579 NEPH2-25

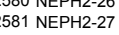

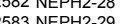

2584 NEPH2-30

2585 NEPH2-31

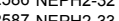

2588 NEPH2-34

2589 NEPH $2-35$
250
2

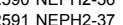

2592 NEPH2-38

2593 NEPH2-39

(2595 NEPH3.11

2596 NEPH3-42

2597 NEPP3-43

2599 NEPH3-45

2000 NEPH3-46

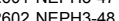

2603 NEPH3 -49
2604 NEPH 350

2605
2006 NEH33.5

2006 N NPPH -52
2602 NEPH-53

2608 NEPH3.54

2609 NEPP3.-55
2610 NEPH3-56

$2612 \mathrm{HWW} 988-77 \mathrm{TR} 2$

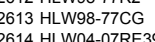

2615 HLW04-07RE $39 \mathrm{CCC}$

2616 HLW04-07NRE9

2618 A A2-G-122A
2619 BLF-G

2620 HLW98-51AW

2621 HLW98-51AWCG

2623 HLW02-02
TCLP Se TCLP Zn TCLPAl TCLP B TCLP Sb TCLP TI TCLP V Q PCT B $\left(g / \mathrm{m}^{2}\right) \quad$ Q PCT Li $\left(g / \mathrm{m}^{2}\right) \quad$ Q PCT Na $\left(g / \mathrm{m}^{2}\right) \quad$ QPCT Si $\left(g / \mathrm{m}^{2}\right) \quad$ Q PCT pH

$\begin{array}{llll}0.035 & 0.036 & 0.042 & 0.021 \\ 0.020 & 0.022 & 0.017 & 0.010\end{array}$

CCC PCT B $\left(g / \mathrm{m}^{2}\right) \quad \operatorname{CCC}$ PCT Li $\left(g / \mathrm{m}^{2}\right) \quad \operatorname{CCC~PCT~Na~}\left(g / \mathrm{m}^{2}\right) \quad$ CCC PCT Si $\left(g / \mathrm{m}^{2}\right)$ CCC PCT pH T1 ( $\left.{ }^{\circ} \mathrm{C}\right)$

$0.022-0.017-0.010$

.

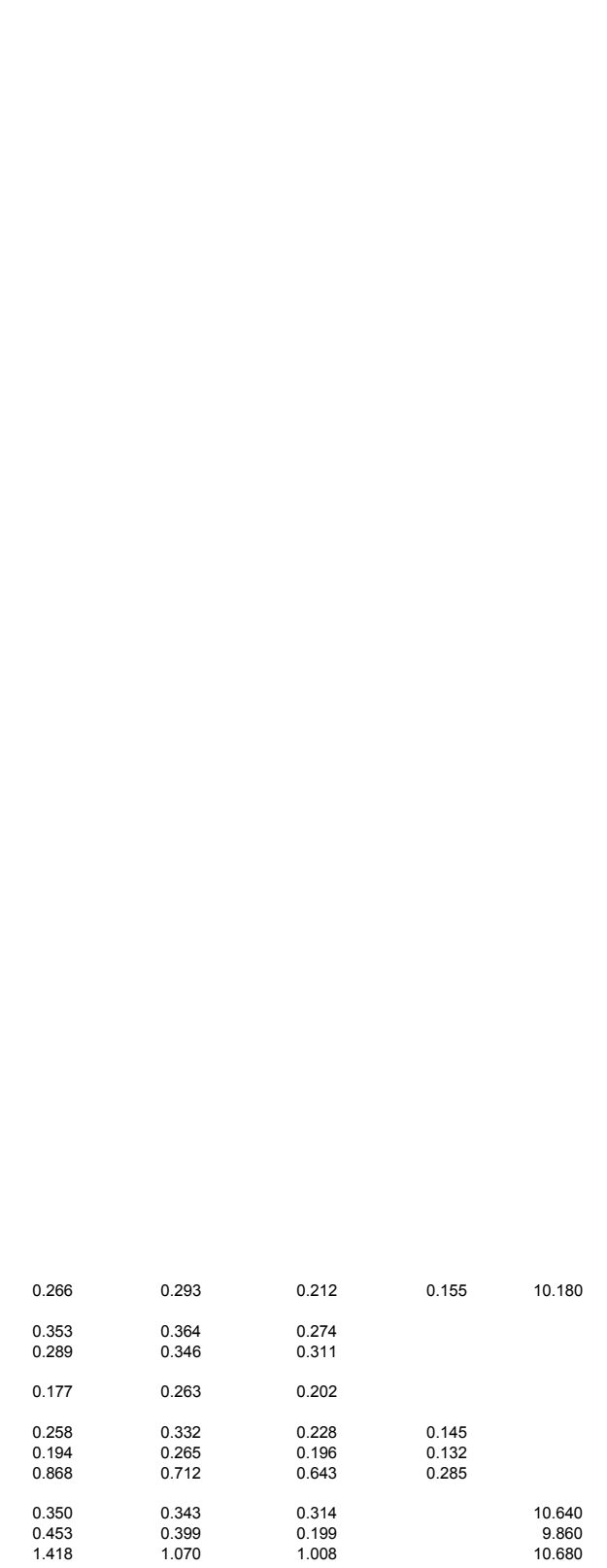


2544 HLW98-60

2546 HLW $198-63$

2547 HLW $W 88-64$

$2549 \mathrm{C} 4 \mathrm{C}-03$

$2550 \mathrm{C} 4 \mathrm{C}-03$

$2551 \mathrm{C} 4 \mathrm{D}-04$
$2552 \mathrm{C} 4 \mathrm{D}-15$
$2553 \mathrm{C}-\mathrm{D}-01$

2553 S12-04 (C4A-03 mod.)

2555 SB4-NEPH-01ccC

2557 SB4-NEPH-03CCC
2558 SB4-NEPH-04CCC

2559 SB4-NEPH-05CCC

2560 SB4-NEPH-06cCC

2562 SB4-NEPH-O8CCC

2564 SB4-NEPH-10cCC

2565 SB4-NEPH-111cCC

2567 NEPH2-13

2569 NEPH2-15

2570 NEPH2-16

572 NEPH2-18

2573 NEPH2-19

2575 NEPH2-21

2576 NEPH2-22

2577 NEPH2-23

2578 NEPH2-24
2579 NEPH2-25

2580 NEPH2-26

2581 NEPH2-27

2582 NEPH2-28

2584 NEPH2 200

2585 NEPH2-31

2556 NEPH2-32

2588 NEPH2-34

2589 NEPP2-35

2590 NEPH2-36
2591 NEPH 237

2592 NEPH2-38

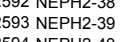

2594 NEPH2-40

2596 NEPH3.42

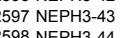

2598 NEPH3-44
2599 NEPH $3-45$

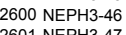

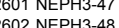

2602 NEPP3 -48
2603 NEPH $3-49$

2604 NEPH3.50

2605 NEPH3 -51
2006 NEP $3-52$

2607 NEPH 3.53

2608 NEPH3.54

2609 NEPH3 355
2610 NEPH 356

2610 NEPH3.56
2611 HLW $98-77$

2612 HLW98-77R2
2613 HLW98-77CG

2614 HLW04-07RE 39

2615 HLWW4-07R $399 \mathrm{CCC}$

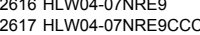

2618 A $12-G-122 A$
2619 BLF-G-87A

2620 HLW98-51AW

2621 HLW98-51AWCG

2623 HLW02-02

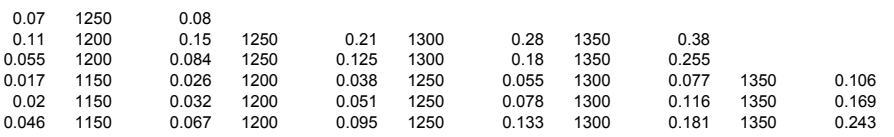

2624 HLW02-03

$0.454 \quad 1131$

$0.351 \quad 1036$

$0.219 \quad 942$

$2.1863 \quad-2918.65 \quad 240$

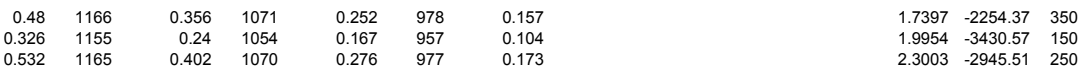




\begin{tabular}{|c|c|c|c|c|c|c|}
\hline \# Glass ID & Study & Reference & Al2O3-t & B2O3-t & CaO-t & $\mathrm{Fe} 203-\mathrm{t}$ \\
\hline 2625 HLW02-04 & HLW02 Initial M & Piepel et al. 2008 & 0.0400 & & 0.0050 & \\
\hline 2626 HLW02-05 & HLW02 Initial M & Piepel et al. 2008 & 0.0400 & & 0.0050 & 0.0800 \\
\hline 2627 HLW02-06 & HLW02 Initial M & Piepel et al. 2008 & 0.0850 & 0.0500 & 0.0050 & 0.0800 \\
\hline $\begin{array}{l}2628 \text { HLW02-07 } \\
2629 \text { HLW02-08 }\end{array}$ & HLW02 Initial M & Piepel et al. 2008 & 0.0850 & 0.0500 & 0.0050 & 0.0800 \\
\hline $\begin{array}{l}\text { 2629 HLW02-28 } \\
2630 \text { HLW02-09 }\end{array}$ & $\begin{array}{l}\text { HLW02 nitial M } \\
\text { HLW02 Initial M }\end{array}$ & $\begin{array}{l}\text { Piepel et all. } 2008 \\
\text { Piepel et al. } 2008\end{array}$ & $\begin{array}{l}0.0400 \\
0.0850\end{array}$ & $\begin{array}{l}0.1400 \\
0.1159\end{array}$ & $\begin{array}{l}0.0050 \\
0.0050\end{array}$ & $\begin{array}{l}0.0921 \\
0.1400\end{array}$ \\
\hline 2631 HLW02-10 & HLW02 Initial M & $\begin{array}{l}\text { Piepel et al al. } 20088 \\
\text { Piepel al } 2008\end{array}$ & 0.0850 & 0.1400 & 0.0050 & $\begin{array}{l}0.1400 \\
0.0800\end{array}$ \\
\hline & HLW02 Initial M & Piepel et al. 2008 & 0.0400 & 0.1400 & & $\begin{array}{l}0.0800 \\
0.0800\end{array}$ \\
\hline 2633 HLW02-12 & HLW02 Initial M & Piepel et al. 2008 & 0.0400 & 0.0500 & 0.0050 & 0.1138 \\
\hline 2634 HLW02-13 & HLW02 Initial M & Piepel et al. 2008 & 0.0400 & & 0.0050 & 0.1400 \\
\hline 2635 HLW02-14 & HLW02 Initial M & Piepel et al. 2008 & 0.0400 & 0.0500 & 0.0050 & 0.0800 \\
\hline 2636 HLW02-15 & HLW02 Initial M & Piepel et al. 2008 & 0.0850 & 0.1400 & 0.0050 & 0.1400 \\
\hline 2637 HLW02-16 & HLW02 Initial M & Piepel et al. 2008 & 0.0850 & 0.0500 & 0.0050 & 0.0800 \\
\hline $\begin{array}{l}2638 \text { HLW02-17 } \\
2639 \text { HLW02-18 }\end{array}$ & $\begin{array}{l}\text { HLW02 Intital M } \\
\text { HLW02 Intial M }\end{array}$ & $\begin{array}{l}\text { Piepel et al. } 2008 \\
\text { Piepel et al } 2008\end{array}$ & $\begin{array}{l}0.0850 \\
0.0400\end{array}-2$ & $\begin{array}{l}0.05500 \\
0.0500\end{array}$ & 0.0050 & $\begin{array}{l}0.1384 \\
0.1113\end{array}-1$ \\
\hline $\begin{array}{l}2639 \text { HLW02-18 } \\
2640 \text { HLW02-19 }\end{array}$ & $\begin{array}{l}\text { HLW02 Intilal M } \\
\text { HLW02 Initial M }\end{array}$ & $\begin{array}{l}\text { Piepel et al. } 2008 \\
\text { Piepel et al. } 2008\end{array}$ & 0.0400 & $\begin{array}{l}0.00500 \\
0.1400\end{array}$ & $\begin{array}{l}0.0050 \\
0.0050\end{array}$ & $\begin{array}{l}0.1113 \\
0.1078\end{array}$ \\
\hline 2641 HLWO2-20, H & HLW02 Initial M & $\begin{array}{l}\text { Piepel et al. } 2008 \\
\text { Piepel et al. } 2008\end{array}$ & $\begin{array}{l}0.0400 \\
0.0400\end{array}$ & $\begin{array}{l}0.1400 \\
0.1400\end{array}$ & $\begin{array}{l}0.0050 \\
0.0050\end{array}$ & $\begin{array}{l}0.1078 \\
0.1400\end{array}$ \\
\hline 2642 HLW02-21, HLWO2-21R1 & HLW02 Initial M & Piepel et al. 2008 & 0.0850 & 0.1400 & 0.0050 & 0.0800 \\
\hline 2643 HLW02-22 & HLW02 Initial M & Piepel et al. 2008 & 0.0850 & 0.1400 & 0.0050 & \\
\hline 2644 HLW02-23, HLW02-23R 1 & HLW02 Initial M & Piepel et al. 2008 & 0.0850 & 0.1400 & 0.0050 & 0.0800 \\
\hline 2645 HLW02-24 & HLW02 Initial M & Piepel et al. 2008 & 0.0850 & 0.0500 & 0.0050 & 0.0800 \\
\hline 2646 HLW02-25, HLW02-25R1 & HLW02 Initial M & Piepel et al. 2008 & 0.0850 & 0.1400 & 0.0050 & 0.0950 \\
\hline $\begin{array}{l}2647 \text { HLW02-26 } \\
2648 \text { HLW02-27 }\end{array}$ & $\begin{array}{l}\text { HLW02 Intital M } \\
\text { HLW02 Intial M }\end{array}$ & $\begin{array}{l}\text { Piepel et al. } 2008 \\
\text { Piepelet tal } 2008\end{array}$ & $\begin{array}{l}0.0400 \\
0.0400\end{array}-2$ & 0.0500 & 0.0050 & $\begin{array}{l}0.1250 \\
0.1250\end{array}$ \\
\hline $\begin{array}{l}2648 \text { HLWU2-27 } \\
2649 \text { HLW02-28 }\end{array}$ & $\begin{array}{l}\text { HLW02 Intilal M } \\
\text { HLW02 Initial M }\end{array}$ & $\begin{array}{l}\text { Piepel et al. } 2008 \\
\text { Piepel et al } 2008\end{array}$ & $\begin{array}{l}0.0400 \\
0.0750\end{array}$ & $\begin{array}{l}0.1400 \\
0.0800\end{array}$ & $\begin{array}{l}0.0050 \\
0.0050\end{array}$ & $\begin{array}{l}0.1250 \\
0.1045\end{array}$ \\
\hline 2650 HLWO2-29. HLWO2-29R1 & HLW02 Initial M & Piepel et al. 2008 & 0.0750 & & 0.0050 & $\begin{array}{l}0.1045 \\
0.1000\end{array}$ \\
\hline 2651 HLW02-30 & HLW02 Initial M & Piepel et al. 2008 & 0.0550 & 0.0972 & 0.0050 & 0.1000 \\
\hline 2652 HLW02-31 & HLW02 Initial M & Piepel et al. 2008 & 0.0550 & 0.1300 & 0.0050 & 0.1000 \\
\hline 2653 HLW02-32, HLW02-32R1 & HLW02 Initial M & Piepel et al. 2008 & 0.0750 & 0.0800 & 0.0050 & 0.1000 \\
\hline 2654 HLW02-33 & HLW02 Initial M & Piepel et al. 2008 & 0.0634 & 0.0800 & 0.0050 & 0.1200 \\
\hline 2655 HLW02-34, HLW02-34R1 & HLW02 Initial M & Piepel et al. 2008 & 0.0550 & 0.1300 & 0.0050 & 0.1200 \\
\hline 2656 HLW02-35 & HLW02 Initial M & Piepel et al. 2008 & 0.0649 & 0.1300 & 0.0050 & 0.1200 \\
\hline $\begin{array}{l}2657 \text { HLW02-36 } \\
2658 \text { HLW02-37 }\end{array}$ & $\begin{array}{l}\text { HLW02 Intitial M } \\
\text { HLW02 nitial M }\end{array}$ & $\begin{array}{l}\text { Piepel et al. } 2008 \\
\text { Piepel et al } 2008\end{array}$ & $\begin{array}{l}0.0550 \\
0.0676\end{array}$ & $\begin{array}{l}0.0867 \\
0.1300\end{array}$ & $\begin{array}{l}0.0050 \\
0.0050\end{array}$ & $\begin{array}{l}0.1200 \\
0.1000\end{array}$ \\
\hline $\begin{array}{l}2658 \text { HLWO2-38 } \\
2659\end{array}$ & $\begin{array}{l}\text { HLW02 Initial M } \\
\text { HLW02 Initial M }\end{array}$ & $\begin{array}{l}\text { Piepel et al. } 2008 \\
\text { Piepel et al. } 2008\end{array}$ & $\begin{array}{l}0.0689 \\
0.0689\end{array}$ & 0.1300 & 0.0050 & $\begin{array}{l}0.1000 \\
0.1200\end{array}$ \\
\hline 2660 HLWO2-39 & HLW02 Initial M & Piepel et al. 2008 & 0.0750 & 0.0899 & 0.0050 & 0.1000 \\
\hline 2661 HLW02-40 & HLW02 Initial M & Piepel et al. 2008 & 0.0750 & & 0.0050 & 0.1000 \\
\hline 2662 HLW02-41 & HLW02 Initial M & Piepel et al. 2008 & 0.0650 & 0.1300 & 0.0050 & 0.1000 \\
\hline 2663 HLW02-42 & HLW02 Initial M & Piepel et al. 2008 & 0.0550 & 0.1300 & 0.0050 & 0.1000 \\
\hline $\begin{array}{l}2664 \text { HLW02-43 } \\
2665 \text { HLW02-44 }\end{array}$ & $\begin{array}{l}\text { HLW02 Initial M } \\
\text { HLW02 nitial M }\end{array}$ & $\begin{array}{l}\text { Piepel et al. } 2008 \\
\text { Piepel en tal } 2008\end{array}$ & $\begin{array}{l}0.0650 \\
0.0550\end{array}$ & $\begin{array}{l}0.1300 \\
0.097\end{array}$ & $\begin{array}{l}0.0050 \\
0.0050\end{array}$ & $\begin{array}{l}0.1000 \\
0.01000\end{array}$ \\
\hline $\begin{array}{l}2665 \text { HLWU2-44 } \\
2666 \text { HLW02-45 }\end{array}$ & $\begin{array}{l}\text { HLWO22 Intilal M } \\
\text { HLW02 nitial M }\end{array}$ & $\begin{array}{l}\text { Piepel et al. } 2008 \\
\text { Piepel et al. } 2008\end{array}$ & $\begin{array}{l}0.0550 \\
0.0550\end{array}$ & $\begin{array}{l}0.0097 \\
0.0800\end{array}$ & $\begin{array}{l}0.0050 \\
0.0050\end{array}$ & $\begin{array}{l}0.1000 \\
0.1100\end{array}$ \\
\hline 2667 HLW02-46 & HLW02 Initial M & Piepel et al. 2008 & 0.0550 & 0.0915 & 0.0050 & 0.1000 \\
\hline 2668 HLW02-47 & HLW02 Initial M & Piepel et al. 2008 & 0.0550 & 0.0981 & 0.0050 & 0.1000 \\
\hline 2669 HLW02-48 & HLW02 Initial M & Piepel et al. 2008 & 0.0600 & 0.0800 & 0.0050 & 0.1200 \\
\hline $\begin{array}{l}2670 \text { HLW02-49 } \\
2671 \text { HLW02-50 }\end{array}$ & $\begin{array}{l}\text { HLW02 Intialal M } \\
\text { HLW02 Intial M M }\end{array}$ & $\begin{array}{l}\text { Piepel et al. } 2008 \\
\text { Piepelet tal } 2008\end{array}$ & $\begin{array}{l}0.0750 \\
0.0600\end{array}$ & 0.0874 & 0.0050 & 0.1200 \\
\hline 2672 HLW02-51, HLW02-51R1 & $\begin{array}{l}\text { HLWO2 Intial M } \\
\text { HLW02 Initial M }\end{array}$ & $\begin{array}{l}\text { Piepel et al. } 2008 \\
\text { Piepel et al. } 2008\end{array}$ & $\begin{array}{l}0.0600 \\
0.0750\end{array}$ & $\begin{array}{l}0.1300 \\
0.0800\end{array}$ & $\begin{array}{l}0.0050 \\
0.0050\end{array}$ & $\begin{array}{l}0.12200 \\
0.1050\end{array}$ \\
\hline 2673 HLW02-52 & HLW02 Initial M & & & 0.0993 & 0.0050 & 0.1090 \\
\hline $02-53$ & nitial M & peel et al. 2008 & 0.0850 & 0.0500 & 0.0050 & 0.1384 \\
\hline 2675 HLW02-54 & HLW02 Initial M & Piepel et al. 2008 & 0.0400 & 0.0500 & 0.0050 & 0.1400 \\
\hline 2676 HLW02-55 & HLW02 Initial M & Piepel et al. 2008 & 0.0550 & 0.1300 & 0.0050 & 0.1000 \\
\hline 2677 HLW02-56 & & Piepel et al. 2008 & & 0.0907 & 0.0050 & 0.1000 \\
\hline & & t tal. 2008 & & & & 21 \\
\hline $\begin{array}{l}2679 \text { HLW03-01 } \\
2680 \text { HLW03-02 }\end{array}$ & HWw3 Augmentat & elet al. 24 & & & 0.0050 & 0.1373 \\
\hline $\begin{array}{l}2680 \text { HLW03-02 } \\
2681 \text { HLW03-03 }\end{array}$ & HWW3 Aumentartio & 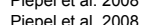 & 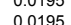 & 0.1080 & 列 0050 & $\begin{array}{l}0.1366 \\
0.1368\end{array}$ \\
\hline $\begin{array}{l}2682 \text { HLW03-04 } \\
2681\end{array}$ & $\begin{array}{l}\text { HLW03 Augmentation M } \\
\text { HLW }\end{array}$ & Piepel et al al 2008 & 0.0195 & & 0.0050 & $\begin{array}{l}0.1368 \\
0.1368\end{array}$ \\
\hline 2683 HLWO3-05 & HLWO3 Augmentation M & et al. 2008 & 0.0818 & 0.0481 & 0.0050 & $\begin{array}{l}0.0192 \\
0.01968\end{array}$ \\
\hline 2684 HLW03-06 & HLW03 Augmentation M & Piepel et al. 2008 & 0.08 & 0.04 & 0.0050 & 0.0196 \\
\hline 2685 HLW03-07 & HLV & tal. 20 & & & & 0.1389 \\
\hline 2686 HLW03-08 & 103 Augmentati & tal. 2008 & 0.0803 & 483 & 0.0050 & 0.0193 \\
\hline & Augnematu & al. 24 & & & & 0.03 \\
\hline $6688 \mathrm{H}$ & HLWO3 Augn & Piepel et al. 20 & 0.0837 & 0.0492 & 0.0050 & 0.09 \\
\hline & HLWwo3 Augmentatiton in & Piepel et al. 20 & 0.0198 & 0.0446 & & $\begin{array}{l}0.0954 \\
0.0198\end{array}$ \\
\hline & HLW03 Augmentation M & Prepel et al. 24 & & 0.0496 & 0.0050 & $\begin{array}{l}0.0198 \\
0.0863\end{array}$ \\
\hline & & & & & & \\
\hline & & al. 2 & & & & \\
\hline & & al. 2 & & & & \\
\hline & HLW & al. $26>>2$ & & 0.137 & 0.0050 & 0.0197 \\
\hline & & 88 & & & & $\begin{array}{l}0.1392 \\
0.0963\end{array}$ \\
\hline & $\begin{array}{l}M \\
M\end{array}$ & $x^{2}+2$ & & & & \\
\hline & HLV & t al. 2 & & & & \\
\hline & & 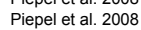 & & & & \\
\hline & & & & & & \\
\hline & & & & & & \\
\hline & tation $\mathrm{M}$ & 08 & 0.0296 & & & \\
\hline & 3 Augmentation M & l et al. 2008 & 0.0492 & 0.0689 & 0.0050 & 0.04 \\
\hline $\begin{array}{l}\text { HLW03-27 } \\
\text { HLW03-28 }\end{array}$ & HLW03 Augmentation M & $\begin{array}{l}\text { Piepel et al. } 2008 \\
\text { Pienel }\end{array}$ & 0.0493 & 0.0690 & 0.0050 & $\begin{array}{l}0.0936 \\
0.0933\end{array}$ \\
\hline HLW03-28 & & & & & & \\
\hline
\end{tabular}

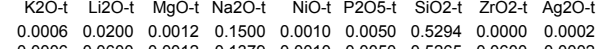

$\begin{array}{lllllllll}0.0006 & 0.0600 & 0.0012 & 0.1379 & 0.0010 & 0.0050 & 0.5265 & 0.0060 & 0.0002 \\ 0.0006 & 0.0474 & 0.0012 & 0.1500 & 0.0100 & 0.0050 & 0.4050 & 0.0600 & 0.0002\end{array}$

$\begin{array}{lllllllll}0.0006 & 0.0600 & 0.0012 & 0.1170 & 0.0010 & 0.0050 & 0.5299 & 0.0153 & 0.0020 \\ 0.0006 & 0.0331 & 0.0012 & 0.1500 & 0.0010 & 0.0050 & 0.3874 & 0.0600 & 0.0002\end{array}$

$\begin{array}{lllllllll}0.0006 & 0.0227 & 0.0012 & 0.1500 & 0.0100 & 0.0050 & 0.3800 & 0.0000 & 0.0002\end{array}$

$\begin{array}{lllllllll}0.0006 & 0.0600 & 0.0012 & 0.1051 & 0.0010 & 0.0050 & 0.3800 & 0.0600 & 0.0002 \\ 0.0006 & 0.0600 & 0.0012 & 0.1086 & 0.0100 & 0.0050 & 0.4515 & 0.0600 & 0.0002\end{array}$

$\begin{array}{lllllllll}0.0006 & 0.0600 & 0.0012 & 0.1276 & 0.0100 & 0.0050 & 0.4140 & 0.0600 & 0.0022 \\ 0.0006 & 0.0600 & 0.0012 & 0.1043 & 0.0010 & 0.0050 & 0.4311 & 0.0118 & 0.0020\end{array}$

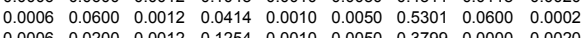
$\begin{array}{lllllllll}0.0006 & 0.0200 & 0.0012 & 0.1254 & 0.0010 & 0.0050 & 0.3799 & 0.0000 & 0.0020 \\ 0.0006 & 0.0600 & 0.0012 & 0.0965 & 0.0010 & 0.0050 & 0.3911 & 0.0600 & 0.0020\end{array}$ $\begin{array}{lllllllll}0.0006 & 0.0600 & 0.0012 & 0.0965 & 0.0010 & 0.0050 & 0.3911 & 0.0600 & 0.00020 \\ 0.0006 & 0.0600 & 0.0012 & 0.0769 & 0.0010 & 0.0050 & 0.5296 & 0.0000 & 0.0002\end{array}$ $\begin{array}{lllllllll}0.0006 & 0.0221 & 0.0012 & 0.1500 & 0.01100 & 0.0050 & 0.4985 & 0.0660 & 0.0020\end{array}$ $\begin{array}{lllllllll}0.0006 & 0.0200 & 0.0012 & 0.0885 & 0.0010 & 0.0050 & 0.3800 & 0.0600 & 0.0002 \\ 0.0006 & 0.0200 & 0.0012 & 0.0645 & 0.0010 & 0.0050 & 0.5173 & 0.0000 & 0.0002\end{array}$ $\begin{array}{lllllllll}0.0006 & 0.0220 & 0.0012 & 0.0645 & 0.0010 & 0.0050 & 0.5173 & 0.0000 & 0.0002 \\ 0.0006 & 0.0200 & 0.0012 & 0.1500 & 0.0010 & 0.0050 & 0.4644 & 0.0150 & 0.0002\end{array}$

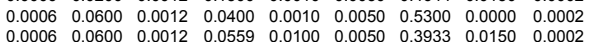
$\begin{array}{lllllllll}0.0006 & 0.0600 & 0.0012 & 0.0559 & 0.0100 & 0.0050 & 0.3933 & 0.0150 & 0.0002 \\ 0.0006 & 0.0200 & 0.0012 & 0.1219 & 0.0010 & 0.0050 & 0.4705 & 0.0150 & 0.0015\end{array}$ $\begin{array}{llllllllll}0.0006 & 0.0200 & 0.0012 & 0.0688 & 0.0010 & 0.0050 & 0.4705 & 0.0150 & 0.00015 \\ 0.00000 & 0.4740 & 0.0000 & 0.0002\end{array}$ $\begin{array}{lllllllll}0.0006 & 0.0200 & 0.0012 & 0.1500 & 0.0010 & 0.0050 & 0.3799 & 0.0600 & 0.0020 \\ 0.0006 & 0.0600 & 0.0012 & 0.0427 & 0.0010 & 0.0050 & 0.3995 & 0.0600 & 0.0002\end{array}$ $\begin{array}{lllllllll}0.0006 & 0.0600 & 0.0012 & 0.0427 & 0.0010 & 0.0050 & 0.3995 & 0.0600 & 0.0002 \\ 0.0006 & 0.0250 & 0.0012 & 0.1500 & 0.0030 & 0.0050 & 0.4100 & 0.0450 & 0.0004\end{array}$ $\begin{array}{lllllllll}0.0006 & 0.0250 & 0.0012 & 0.1500 & 0.00030 & 0.0050 & 0.4100 & 0.0450 & 0.0004 \\ 0.0006 & 0.0375 & 0.0012 & 0.0900 & 0.0030 & 0.0050 & 0.4099 & 0.0450 & 0.0013\end{array}$ $\begin{array}{lllllllll}0.0006 & 0.0500 & 0.0012 & 0.1123 & 0.0030 & 0.0050 & 0.4100 & 0.0450 & 0.0004 \\ 0.0006 & 0.0250 & 0.0012 & 0.1498 & 0.0030 & 0.0050 & 0.4100 & 0.0437 & 0.0004\end{array}$ $\begin{array}{lllllllll}0.0006 & 0.0250 & 0.0012 & 0.1498 & 0.0030 & 0.0050 & 0.4100 & 0.0437 & 0.0004 \\ 0.0006 & 0.0500 & 0.0012 & 0.1073 & 0.0030 & 0.0050 & 0.4612 & 0.0150 & 0.0004\end{array}$ $\begin{array}{llllllllllll}0.0006 & 0.0250 & 0.0012 & 0.1500 & 0.0080 & 0.0050 & 0.4100 & 0.0201 & 0.0004\end{array}$ $\begin{array}{lllllllll}0.0006 & 0.0396 & 0.0012 & 0.1089 & 0.0080 & 0.0050 & 0.4099 & 0.0150 & 0.0013 \\ 0.0006 & 0.0500 & 0.0012 & 0.0916 & 0.0080 & 0.0050 & 0.4100 & 0.0150 & 0.0013\end{array}$ $\begin{array}{lllllllll}0.0006 & 0.0500 & 0.0012 & 0.0916 & 0.0080 & 0.0050 & 0.4100 & 0.0150 & 0.0013 \\ 0.0006 & 0.0250 & 0.0012 & 0.1500 & 0.0030 & 0.0050 & 0.4507 & 0.0150 & 0.0013\end{array}$

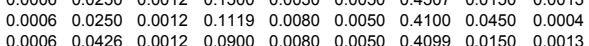
$\begin{array}{lllllllll}0.0006 & 0.0426 & 0.0012 & 0.0900 & 0.0080 & 0.0050 & 0.4099 & 0.0150 & 0.0013 \\ 0.0006 & 0.0250 & 0.0012 & 0.1106 & 0.0080 & 0.0050 & 0.4900 & 0.0150 & 0.0004\end{array}$ $\begin{array}{lllllllll}0.0006 & 0.0250 & 0.0012 & 0.1106 & 0.0080 & 0.0050 & 0.4900 & 0.0150 & 0.0004 \\ 0.0006 & 0.0263 & 0.0012 & 0.0968 & 0.0030 & 0.0050 & 0.4900 & 0.0150 & 0.0013\end{array}$ $\begin{array}{lllllllll}0.0006 & 0.0250 & 0.0012 & 0.0900 & 0.0030 & 0.0050 & 0.4895 & 0.0150 & 0.0004\end{array}$

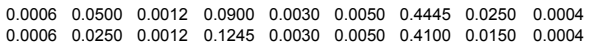
$\begin{array}{lllllllll}0.0006 & 0.0250 & 0.0012 & 0.0962 & 0.0030 & 0.0050 & 0.4883 & 0.0150 & 0.0004 \\ 0.0006 & 0.0370 & 0.0012 & 0.0900 & 0.0080 & 0.0050 & 0.4900 & 0.0250 & 0.0004\end{array}$

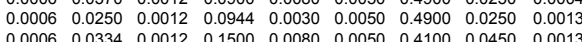
$\begin{array}{lllllllll}0.0006 & .0 .500 & 0.0012 & 0.0900 & 0.00030 & 0.0050 & 0.44421 & 0.0300 & 0.00004 \\ 0.0006 & 0.0250 & 0.0012 & 0.0982 & 0.0030 & 0.0050 & 0.4353 & 0.0450 & 0.0004\end{array}$ $\begin{array}{lllllllll}0.0006 & 0.0250 & 0.0012 & 0.0982 & 0.0030 & 0.00050 & 0.44353 & 0.0450 & 0.0004 \\ 0.0006 & 0.0459 & 0.0012 & 0.0900 & 0.0030 & 0.0050 & 0.4516 & 0.0450 & 0.0004\end{array}$ $\begin{array}{lllllllll}0.0006 & 0.0459 & 0.0012 & 0.0900 & 0.0030 & 0.0050 & 0.4516 & 0.0450 & 0.0004 \\ 0.0006 & 0.0400 & 0.0012 & 0.1096 & 0.0052 & 0.0050 & 0.4361 & 0.0297 & 0.0011\end{array}$

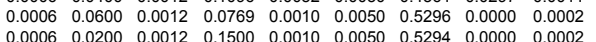
$\begin{array}{lllllllll}0.0006 & 0.0200 & 0.0012 & 0.1500 & 0.0010 & 0.0050 & 0.5294 & 0.0000 & 0.0002 \\ 0.0006 & 0.0500 & 0.0012 & 0.0900 & 0.0030 & 0.0050 & 0.4445 & 0.0250 & 0.0004\end{array}$ $\begin{array}{lllllllll}0.0006 & 0.0500 & 0.0012 & 0.0900 & 0.0030 & 0.0050 & 0.4445 & 0.0250 & 0.0004 \\ 0.0006 & 0.0250 & 0.0012 & 0.1500 & 0.0030 & 0.0050 & 0.4367 & 0.0250 & 0.0013\end{array}$ $\begin{array}{llllllllll}0.0006 & 0.0331 & 0.0012 & 0.1500 & 0.0010 & 0.0050 & 0.3874 & 0.0600 & 0.0002 \\ 0.0006 & 0.0588 & 0.0012 & 0.0392 & 0.0010 & 0.0050 & 0.3530 & 0.0298 & 0.0020\end{array}$ $\begin{array}{lllllllll}0.0006 & 0.0588 & 0.0012 & 0.0392 & 0.0010 & 0.0050 & 0.3530 & 0.0298 & 0.0020 \\ 0.0006 & 0.0247 & 0.0012 & 0.1118 & 0.0098 & 0.0050 & 0.3513 & 0.0909 & 0.0002\end{array}$ $\begin{array}{lllllllllll}0.00006 & 0.0268 & 0.0012 & 0.1076 & 0.0098 & 0.0050 & 0.3517 & 0.06440 & 0.0002\end{array}$ $\begin{array}{lllllllll}0.0006 & 0.0586 & 0.0012 & 0.0444 & 0.0010 & 0.0050 & 0.4242 & 0.0293 & 0.0020 \\ 0.0006 & 0.0192 & 0.0012 & 0.1443 & 0.0010 & 0.0050 & 0.4784 & 0.0000 & 0.0020\end{array}$

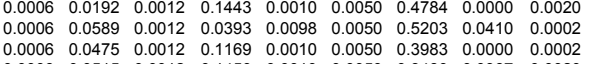
$\begin{array}{lllllllll}0.0006 & 0.0515 & 0.0012 & 0.1450 & 0.0010 & 0.0050 & 0.3480 & 0.0967 & 0.0020 \\ 0.0006 & 0.0198 & 0.0012 & 0.1485 & 0.0010 & 0.0050 & 0.5246 & 0.0000 & 0.0002\end{array}$ $\begin{array}{lllllllll}0.0006 & 0.0198 & 0.0012 & 0.1485 & 0.0010 & 0.0050 & 0.5246 & 0.0000 & 0.0002 \\ 0.0006 & 0.0591 & 0.0012 & 0.1285 & 0.0099 & 0.0050 & 0.3545 & 0.0725 & 0.0020\end{array}$ $\begin{array}{lllllllll}0.0006 & 0.0591 & 0.0012 & 0.1285 & 0.0099 & 0.0050 & 0.3545 & 0.0725 & 0.0020 \\ 0.0006 & 0.0198 & 0.0012 & 0.1488 & 0.0010 & 0.0050 & 0.5259 & 0.00000 & 0.0002\end{array}$

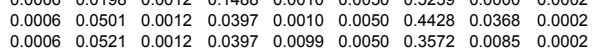
$\begin{array}{lllllllll}0.0006 & 0.0521 & 0.0012 & 0.0397 & 0.0099 & 0.0050 & 0.3572 & 0.0085 & 0.0002 \\ 0.0006 & 0.0250 & 0.0012 & 0.1043 & 0.0099 & 0.0050 & 0.3581 & 0.0651 & 0.0002\end{array}$ $\begin{array}{lllllllll}0.0006 & 0.0195 & 0.0012 & 0.0525 & 0.0010 & 0.0050 & 0.5172 & 0.0000 & 0.00002\end{array}$ $\begin{array}{lllllllll}0.0006 & 0.0286 & 0.0012 & 0.0391 & 0.0098 & 0.0050 & 0.4424 & 0.0100 & 0.0020 \\ 0.0006 & 0.0197 & 0.0012 & 0.0446 & 0.0029 & 0.0050 & 0.4609 & 0.0164 & 0.0020\end{array}$ $\begin{array}{lllllllll}0.0006 & 0.0197 & 0.0012 & 0.0446 & 0.0029 & 0.0050 & 0.4609 & 0.0164 & 0.0022 \\ 0.0006 & 0.0592 & 0.0012 & 0.1053 & 0.0098 & 0.0050 & 0.5248 & 0.0000 & 0.0002\end{array}$

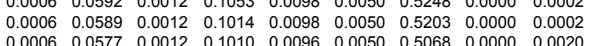

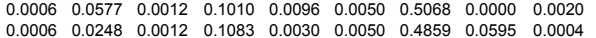
$\begin{array}{llllllllll}0.00006 & 0.0246 & 0.0012 & 0.01083 & 0.1180 & 0.0030 & 0.0050 & 0.4859 & 0.0595 & 0.0004 \\ 0.00030 & 0.0050 & 0.4030 & 0.0590 & 0.0013\end{array}$

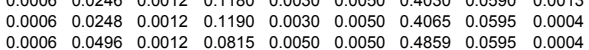
$\begin{array}{lllllllll}0.0006 & 0.0496 & 0.0012 & 0.0815 & 0.0050 & 0.0050 & 0.4859 & 0.0595 & 0.0004 \\ 0.0006 & 0.0246 & 0.0012 & 0.1182 & 0.0049 & 0.0050 & 0.4209 & 0.0591 & 0.0013\end{array}$

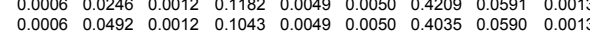

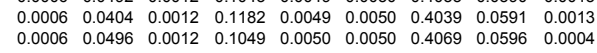

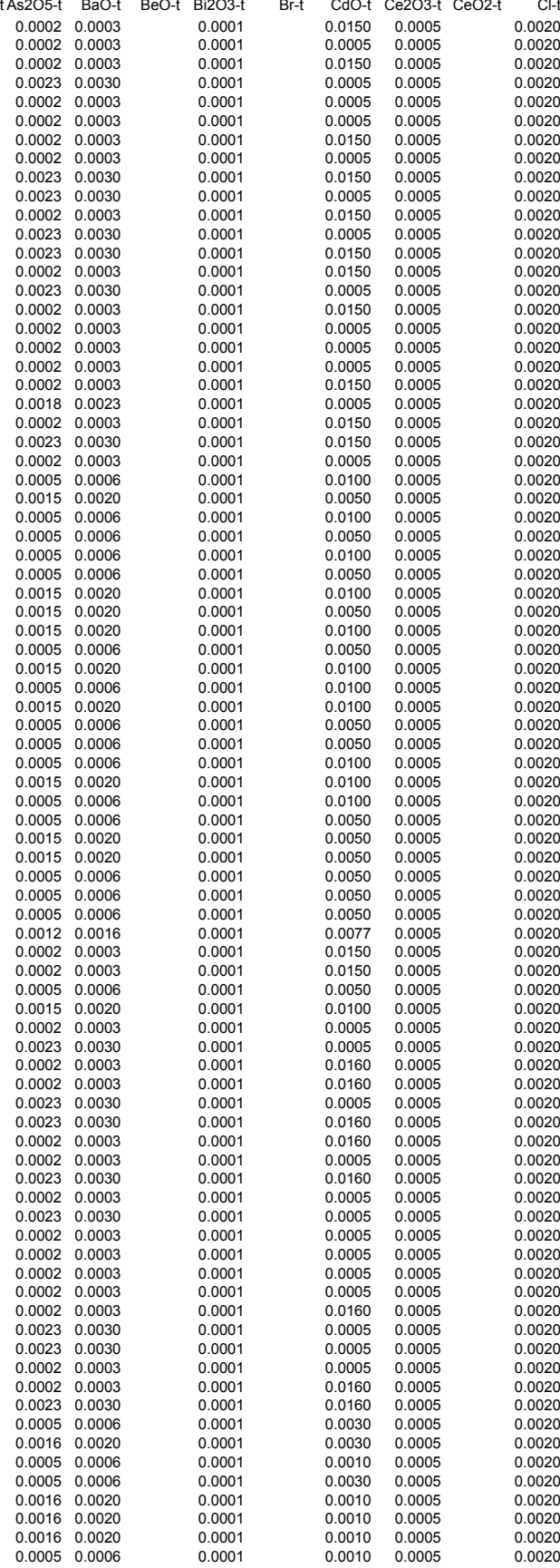


\# Glass ID

2625 HLWW02-04
2626 HLW02-05

2626 HLW02-05
2627 HLW02-06

2628 HLW02-07
2629 HLW02-08

2630 HLW202-09

2631 HLW02-10

2633 HLWO2-12

2635 HLW2-14

2636 HLW02-15
2637 HLW02-16

(638 HLW02-17

2640 HLW02-19

2641 HLW02-20, HLW02-20R1

2643 HLW02-22

2644 HLW02-23, HLW02-23R1

2645 HLW02-24 HLW HLW2-25, HLW02-25R1

2647 HLW02-26
2648 HLW02-27

2650 HLW02-29, HLW02-29R 1

2651 HLW02-30

2653 HLW02-32, HLW02-32R1

HLW02-33

$2656 \mathrm{HLW02-35}$

2659 HLW02-38

2661 HLW02-40

2662 HLW02-4
2633 HLW02-42

2664 HLW02-43
2665 HLWO2-44

2666 HLW02-45

2667 HLWO2-46
2668 HLWO2-47

2669 HLW02-48

2671 HLW02-50

2672 HLW02-51, HLW02-51R1

2673 HLW02-52
2674 HLW02-53

2675 HLW02-54

2676 HLWO2-55
2677 HLWO2-56

2678 HLW02-57

2680 HLW03-02

2682 HLWO3-04

(2684 HLW3-05

2685 HLW03-07
2686 HLW03-08

2687 HLW03-09

2689 HLWO-11
6990 HLW -12

(2691 HLW03-13

2693 HLW03-15

2694 HLW03-16

2696 HLW03-18

(697 HLW03-19

(2699 HLW03-21

2701 HLW03-23

2702 HLW03-24

2706 HLW03-28
CoO-t Co2O3-t Cr2O3-t Cs2O-t CuO-t Dy203-t Eu2O3-t

$\begin{array}{lllllll}0.0001 & 0.0002 & 0.0001 & 0.0001 & 0.000\end{array}$

0.0001

$0.0020 \quad 0.0001 \quad 0.0010$

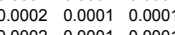

$\begin{array}{lll}0.0002 & 0.0001 & 0.0001 \\ 0.0002 & 0.0001 & 0.0001\end{array}$

$\begin{array}{lll}0.0020 & 0.0001 & 0.0010 \\ 0.0020 & 0.0001 & 0.0010\end{array}$

$0.0002 \quad 0.0001 \quad 0.0001$

$\begin{array}{lll}0.0020 & 0.0001 & 0.0010 \\ 0.0020 & 0.0001 & 0.0010\end{array}$

$\begin{array}{lll}0.0002 & 0.0001 & 0.000 \\ 0.0020 & 0.0001 & 0.0010 \\ 0.002 & 0.001 & 0\end{array}$

$\begin{array}{llll}0.0002 & 0.0001 & 0.000 \\ 0.0002 & 0.0001 & 0.000\end{array}$

$\begin{array}{lll}0.0002 & 0.0001 & 0.0001 \\ 0.0002 & 0.0001 & 0.0001 \\ 0.0022 & 0.0001 & 0.0001\end{array}$

$0.0002 \quad 0.00010 .000$

$\begin{array}{lll}0.0002 & 0.0001 & 0.0001 \\ 0.0015 & 0.0001 & 0.0008\end{array}$

$\begin{array}{lll}.0002 & 0.0001 & 0.0001 \\ .0020 & 0.0001 & 0.0010\end{array}$

$\begin{array}{lll}0.0020 & 0.0001 & 0.001 \\ 0.0002 & 0.0001 & 0.0001 \\ 0.00013 & 0.001 & 0\end{array}$

$\begin{array}{lll}0.0004 & 0.0001 & 0.0002 \\ 0.0013 & 0.0001 & 0.0007\end{array}$

$\begin{array}{lll}0.0004 & 0.0001 & 0.0002 \\ 0.0004 & 0.0001 & 0.0002\end{array}$

$0.0004 \quad 0.0001 \quad 0.0002$

$\begin{array}{llll}0.00013 & 0.0001 & 0.0007 \\ 0.0013 & 0.001 & 0.0007\end{array}$

$\begin{array}{lll}0.0013 & 0.0001 & 0.0007 \\ 0.0013 & 0.0001 & 0.0007\end{array}$

$\begin{array}{rrrr}0.0004 & 0.0001 & 0.0002 \\ 0.0013 & 0.0001 & 0.0007 \\ 0.004 & 0.001 & 0.0007\end{array}$

$\begin{array}{llll}0.0004 & 0.0001 & 0.0002 \\ 0.0013 & 0.0001 & 0 & 0 \\ 0 & 0.0007\end{array}$

$0.0004 \quad 0.0001 \quad 0.0002$

$\begin{array}{llll}0.0004 & 0.0001 & 0.0002 \\ 0.0004 & 0.0001 & 0.0002\end{array}$

$\begin{array}{lll}0.0004 & 0.0001 & 0.0002 \\ 0.0013 & 0.0001 & 0.0007\end{array}$

$\begin{array}{lll}0.0004 & 0.0001 & 0.0002 \\ 0.0004 & 0.0001 & 0.0002\end{array}$

$\begin{array}{lll}0.0013 & 0.0001 & 0.0007 \\ 0.0013 & 0.0001 & 0.0000\end{array}$

$\begin{array}{lll}0.0004 & 0.0001 & 0.0002\end{array}$

$\begin{array}{lll}0.0004 & 0.0001 & 0.0002 \\ 0.0004 & 0.0001 & 0.0002\end{array}$

$\begin{array}{lll}0.0004 & 0.0001 & 0.0002 \\ 0.0011 & 0.0001 & 0.0005\end{array}$

$0.0002 \quad 0.0001 \quad 0.000$

$\begin{array}{llll}0.0002 & 0.0001 & 0.000 \\ 0.0004 & 0.0001 & 0.0002\end{array}$

$\begin{array}{llll}0.0004 & 0.0001 & 0.0002 \\ 0.0013 & 0.0001 & 0.0007\end{array}$

$\begin{array}{llll}0.0002 & 0.0001 & 0.0001\end{array}$

$\begin{array}{llll}0.0002 & 0.0001 & 0.0010\end{array}$

$\begin{array}{llll}.0049 & 0.0001 & 0.000 \\ 0.0049 & 0.0001 & 0.0010\end{array}$

$0.00480 .0001 \quad 0.0010$

$\begin{array}{lll}0.0049 & 0.0001 & 0.000 \\ 0.0050 & 0.0001 & 0.0001\end{array}$

$\begin{array}{llll}0.0048 & 0.0001 & 0.0010\end{array}$

$\begin{array}{llll}0.0002 & 0.0001 & 0.00010 \\ 0 & 0002 & 0.001 & 0001\end{array}$

$\begin{array}{lll}0.0002 & 0.0001 & 0.000 \\ 0.050 & 0.0001 & 0.000 \\ 0.000 & 00001 & 0.001\end{array}$

$\begin{array}{llll}0 & 0.0052 & 0.0001 & 0.001 \\ 0.0050 & 0.0001 & 0.0001 \\ 0.0002 & 0.001 & 0 & 0\end{array}$

$\begin{array}{lll}0.0002 & 0.0001 & 0.000\end{array}$

$\begin{array}{llll}0.0002 & 0.0001 & 0.0010 \\ 0.0002 & 0.0001 & 0.0010\end{array}$

$\begin{array}{llll}0.00050 & 0.0001 & 0.00001 \\ 0 & 0.0009 & 0.0001 & 0.0001\end{array}$

$\begin{array}{lll}.0049 & 0.0001 & 0.000 \\ 0.002 & 0.0001 & 0.001 \\ 0.000 & 0.001 & 0\end{array}$

$\begin{array}{llll}0.0008 & 0.0001 & 0.0002 \\ 0.0008 & 0.0001 & 0.0007\end{array}$

$0.0020 \quad 0.0001 \quad 0.0002$

$\begin{array}{llll}0.0008 & 0.0001 & 0.0002\end{array}$

$\begin{array}{lll}0.0020 & 0.0001 & 0.0007\end{array}$

$\begin{array}{llll}0.0020 & 0.0001 & 0.0007 \\ 0.0008 & 0.0001 & 0.0002\end{array}$
F-t Ga2O3-t Gd203-t HFO2-t HgO-t 0.0005
0.0005

\subsection{5}

0.0005
0.0005
0.0005

0.0005
0.0005
0.0005

0.0005

0.0005
0.0005
0.0005

0.0005

0.0005
0.0005

0.0005

0.0005

0.0005

0.0005
0.0005
0.0005

0.0005
0.0005
0.005

0.0005

0.0005

0.0005

0.0005

0.0005

0.0005

0.0005

0.0005

0.0005

0.0005

0.0005

0.0005
0.0005

0.0005

0.0005
0.0005
0.005

0.0005
0.0005

0.0005

0.0005

0.0005

0.0005

0.0005

0.0005

0.0005
0.0005

0.0005

0.0005

0.0005 $\begin{array}{ll}\text { La2O3-t MnO2-t } & \text { MnO-t } \\ 0.0030 & 0.0052 \\ 0.0030 & 0.0000 \\ 0.030 & 0.0500\end{array}$

0.0030
0.0030

0.003
0.003

0.0030
0.0030

0.0030
0.0030
0.030

0.0030
0.0030
0.030

0.0030
0.0030
0.030

0.0030
0.0030
0.030

0.0030

0.0030

0.0030
0.0030
0.0030

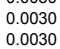

0.030
0.0030
0.0030

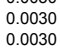

0.0030
0.0030
0.0300
00030

0.0030
0.030
0.030

0.00030
0.030
0.030
0.030

0.0030

0.0030

0.0030

然.0033

0.0030

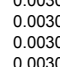

0.0030
0.0030

.0.0033

0.0030
0.030
0.030
0

0.003

0.0030
0.0030
0.030

0.0030
0.030
0.0030
0.030

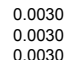

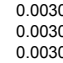

0.0030
0.030
0.0030
0.030

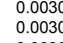

0.0000
0.0030
0.0030

0.00030
0.030
0.030

0.0030
0.0030
0.0030

0.0030
0.0030

0.0030
0.0030
0.0030
0.0030

0.0030
0.0030
0.0030

0.0030

0.0149
PbO-t PdO2-t PdO-t Pr2O3-t Pr6011-t Rb2O-t ReO-t

$\begin{array}{ll}0.0004 & 0.0012 \\ 0.0004 & 0.0012\end{array}$

$\begin{array}{ll}0.004 & 0.0012 \\ 0.0004 & 0.0012 \\ 0.0004 & 0.0012\end{array}$

$\begin{array}{ll}0.0004 & 0.0012 \\ 0.0004 & 0.0012 \\ 0.004 & 0.0012\end{array}$

$\begin{array}{ll}0.0040 & 0.0012 \\ 0.0004 & 0.0012\end{array}$

$\begin{array}{ll}0.0040 & 0.0012 \\ 0.0040 & 0.0012\end{array}$

$\begin{array}{ll}0.0004 & 0.0012 \\ 0.0040 & 0.0012\end{array}$

$\begin{array}{ll}0.0004 & 0.0012 \\ 0.0004 & 0.0012 \\ 0.0004 & 0.0012\end{array}$

$\begin{array}{ll}0.0004 & 0.0012 \\ 0.0004 & 0.0012\end{array}$

$\begin{array}{ll}0.0031 & 0.0012 \\ 0.0004 & 0.0012 \\ 0.0040 & 0.0012\end{array}$

$\begin{array}{ll}0.0004 & 0.0012 \\ 0.0008 & 0.0012 \\ 0 & 0.028\end{array}$

$\begin{array}{ll}0.0027 & 0.0012 \\ 0.0008 & 0.0012 \\ 0.0008 & 0.0012\end{array}$

$0.0008-0.0012$

$\begin{array}{ll}0.0027 & 0.0012 \\ 0.0027 & 0.0012\end{array}$

$\begin{array}{ll}0.0027 & 0.0012 \\ 0.0027 & 0.0012 \\ 0.0027 & 0.0012\end{array}$

$\begin{array}{ll}0.0008 & 0.0012 \\ 0.0027 & 0.0012 \\ 0 & 0.0008\end{array}$

$\begin{array}{ll}0.0008 & 0.0012 \\ 0.0008 & 0.0012\end{array}$

0.0012

$\begin{array}{ll}0.00008 & 0.0012 \\ 0.00008 & 0.0012 \\ 0.002 & 0.0012\end{array}$

$\begin{array}{ll}0.0027 & 0.0012 \\ 0.0008 & 0.0012 \\ 0 & 0.000\end{array}$

0.0008
0.0008 $\quad 0.0012$

$0.0004-0.0012$

$\begin{array}{ll}0.00004 \\ 0.0008 \\ 0.0027 & 0.0012 \\ 0.0012 & 0.0012 \\ 0 & 0.0012\end{array}$

\begin{tabular}{ll}
0.0004 & 0.0012 \\
0.0040 & 0.0012 \\
\hline
\end{tabular}

$\begin{array}{lll}0.00004 & 0.00012 \\ 0.0004 & 0.0012\end{array}$

\begin{tabular}{ll}
0.00040 & 0.00012 \\
0.0004 & 0.000 \\
\hline
\end{tabular}

$\begin{array}{ll}0.00004 & 0.0012 \\ 0.0004 & 0.0012 \\ 0.0004 & 0.012\end{array}$

$\begin{array}{ll}0.00004 & 0.0012 \\ 0 & 0.0004 \\ 0 & 0.0002\end{array}$

$\begin{array}{ll}0.0040 & 0.0012 \\ 0.0004 & 0.0012 \\ 0.0004 & 0.0012\end{array}$

$0.0004 \quad 0.0012$

$0.0004 \quad 0.0012$

$\begin{array}{ll}0.0040 & 0.0012 \\ 0.0040 & 0.0012 \\ 0.0004 & 0.00012\end{array}$

$\begin{array}{ll}0.0004 & 0.0012 \\ 0.0004 & 0.0012 \\ 0.0040 & 0.0012\end{array}$

$\begin{array}{ll}0.0040 & 0.0012 \\ 0.0008 & 0.0012 \\ 0.0027 & 0.0012\end{array}$

$\begin{array}{ll}0.0027 & 0.0012 \\ 0.0008 & 0.0012 \\ 0.0008 & 0.0012 \\ 0.0027 & 0.0012\end{array}$

$\begin{array}{ll}0.0027 & 0.0012 \\ 0.0027 & 0.0012 \\ 0.027 & 0.0012\end{array}$ 


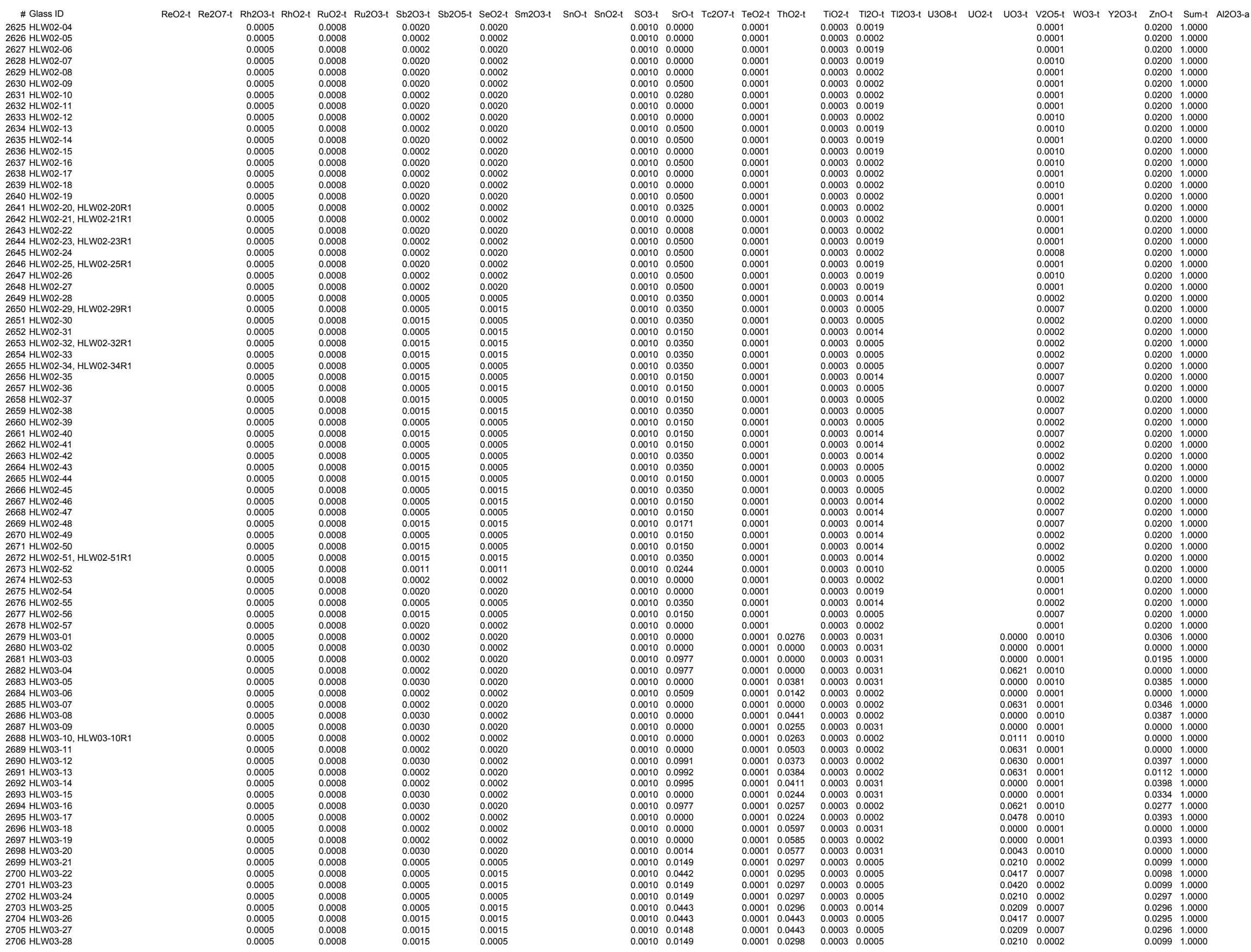


2626 HLW02-05

2628 HLW02-07

2639 HLW02-08
2630

2631 HLW02-10

2633 HLW02-12

2634 HLWO2-13

2636 HLW02-1

2638 HLW02-17

2639 HLW02-19

2640 HLWO2-19

2641 HLWW2-20, HLW02-20R

2444 HLW02-23, HLW02-23R 1

2646 HLW02-22
2647 HWWO2-20

$\begin{array}{ll}2 & -1\end{array}$

650 HLW02-29, HLW02-29R1

2651 HLW02-30

2652 HLW02-31 HLW02-32R1

2655 HLW02-34, HLW02-34R

2658 HLW02-37

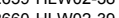

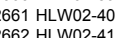

2662 HLWW2-41
263 HLWO2-42

2664 LLWO2-43
2655 H WWO2-44

$2666 \mathrm{HLW}$ W2-45

(2667 HLWD2-46

2670 HLWO2-49

2671 HLWW2-50
2672 HLWO2-51, HLW02-51R1

2672 HLWW2.59,
2673 HLW02.52

2674 HLW02.53

2675 HLW02-54

2676
267 HLW02-5

2678 HLW02.57

2679 HLW03-0

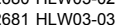

$2682 \mathrm{H}$ LW03-04

2084

2685 HLW03-07

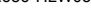

2688 HLW03-10, HLW03-10R1

2689 HLW03-11

(2090

2692 HLW03-14

2693 HLWO3-15

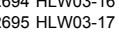

2697 HLW03-19

2698 HLWO3-20

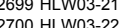

2701 HLWVO-23.

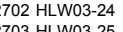

-1
0

2705 HLWO3-27

2706 HLW $13-28$ 


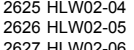

2628 HLW02-07

2628 HLWW2-07

2630 HLLW02-09

2632 HLWO2-11

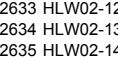

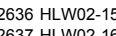

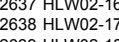

2639 HLW02-18

2640 HLW02-19
2641 HLWO2-20, HLW02-20R

2641 HLWO2-20, HLWO2-20R1
2642 HLWO2-21, HLWO2-21R1

2643 HLWV2-22
2644 HWW02-23, HLW02-23R1

2644 HLWO2-23, HLW02-23R1
2645 HLWO2-24

2646 HLW02-25, HLW02-25R

2647 HLW02-26
2648 HLWO2-27

2650 HLW02-29. HLW02-2981

2651 HLW02-30

2652 HLW02-31

2653 HLW02-32, HLW02-32R1

2655 HLW02-34, HLW02-34A

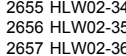

2658 HLW02-3

2659 HLW02-38

2660 HLW02-39

2662 HLW02-41

2663 HLW02-42

2664 HLWO2-4
2665 HLWO2-44

2666 HLW02-45

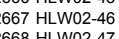

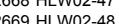

2670 HLWO2-49

2671 HLWW2-50
2672 HLWO2-51, HLW02-51R1

(2672 HLWD2-59,

2674 HLW02-53

2675 HLW02-54

2676
2 HLWO2-25

2678 HLW02.57

2679 HLW03-0

2681 HLW03-03

2682 HLW03-04

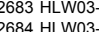

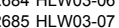

2686 HLW03-08

2688 HLW03-10, HLW03-10R

2688
2689
HLW

2690 HLWO3-12

2691 HLW03-13

2093 HLWNo3-15

2694 HLWO3-16

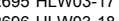

2697 HLW03-19

2698 HLWO3-20

(2699 HLW

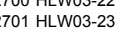

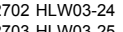

2704 HLW03-26

2706 HLW $13-28$ 
2653 HLWO2-31 262 , HLW02-32R1

2654 HLW02-33

2655 HLW02-34

2656 HLW02-35
2657 HLWO2-36

2658 HLW02-37

2659 HLW02-38

2660 HLW02-39
2661 HLW02-40

2662 HLW02-41

2663 HLW02-42

2665 HLW02-44

2666 HLW02-45

2667 HLW22-46

2671 HLW02-50

2672 HLW02-51,

2674 HLW02-53

2675 HLW02-54

2676 HLW02-55
2677 HLW02-56

2678 HLW02-57

2679 HLW03-0

2662 HLW03-04
2683 HLWW3-05

2685 HLWO3-07

2688 HLW03-08

2688 HLW03-10, HLW03-10R1

2690 HLW03-12

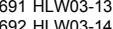

2693 HLW03-15

$2694 \mathrm{HLWO3}-16$
$2695 \mathrm{HLW}=3 \mathrm{~B}-17$

2696 HLW03-18

2697 HLWO3-19
2698 HLWO3-20

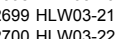

2701 HLW03-23.

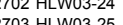

$2705 \mathrm{HLW}=3-27$
$2706 \mathrm{HLW}=32.28$

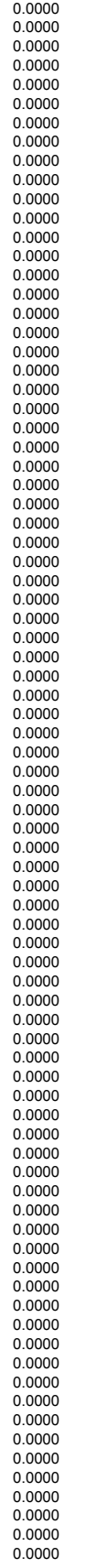

941 Spinel

938 Spinel
946 Spinel

922 Spin

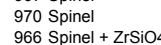

966 Spinel + ZrSiO4
925 Spinel

925 Spinel

898 Spinel

797 Spinel
801 Spinel
947 Spinel

941 Spinel

980 Spinel
870 Spinel

980 Spinel

987 Spininel
990 Spinel
9080

990 Spinel
958 Spinel
936 Spinel

936 Spine
821 Spine

916 Spinel
814 Spinel

920 Spinel

963 Spinel

847 Spinel
960 Spinel

960 Spinel
987 Spinel

975 Spinel

906 Spinel

949 Spinel

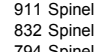

741 Spine

1074 Spinel
1157 Spinel +ZrO2
1145 Spinel

946 Spinel

963 Spinel

$1248 \mathrm{ZrO} 2$

ND Noble Metal
1190 Spinel + Zro2 (ThO2)

811 Spinel

1215 Spinel + ThO2 (UO2)

1069 Spinel + Zro2
1052 Spinel + ThO2

1021 Spinel
1005 Spinel

952 Spinel

875 Spine

866 Spinel
906 Spinel

831 Spininel
825 Sinel

796 Spinel
797 Spinel + ThO2

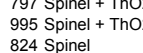




\begin{tabular}{|c|c|c|c|c|c|c|c|c|c|}
\hline \# Glass ID & $\mathrm{C}_{8000^{\circ} \mathrm{C}}$ & $\mathrm{C}_{850^{\circ} \mathrm{C}} \mathrm{C}$ & $c_{9000^{\circ} \mathrm{C}}$ & $C_{950^{\circ} \mathrm{C}}$ & $\mathrm{C}_{1000^{\circ} \mathrm{C}}$ & $\mathrm{C}_{1050^{\circ} \mathrm{C}} \mathrm{C}$ & $c_{1100^{\circ} \mathrm{C}}$ & $\mathrm{C}_{1150^{\circ} \mathrm{C}} \mathrm{C}$ & $\mathrm{C}_{1200^{\circ} \mathrm{C}}$ Quenched Visual/OM \\
\hline 2625 HLW02-04 & 14.2 & & & & & & & & \\
\hline 2626 HLW02-05 & & & 0.1 & 0.1 & & & & & \\
\hline 2627 HLW02-06 & & 5.1 & 3.2 & 2.8 & & 0.8 & & & \\
\hline $\begin{array}{l}2628 \text { HLWW2-07 } \\
2629 \text { HWW02-08 }\end{array}$ & & 0.8 & 0.5 & 0.4 & & & & & \\
\hline $\begin{array}{l}2629 \text { HLW202-08 } \\
2630 \text { HLW02-09 }\end{array}$ & & $\begin{array}{l}0.6 \\
2.9\end{array}$ & 4.4 & $\begin{array}{l}0.1 \\
12\end{array}$ & 0.2 & & & & \\
\hline 2631 HLW02-10 & & $\begin{array}{l}2.9 \\
0.1\end{array}$ & $\begin{array}{l}4.4 \\
0.1\end{array}$ & $\begin{array}{l}1.2 \\
0.1\end{array}$ & 0.2 & & & & \\
\hline & & 1 & & 0.1 & & & & & \\
\hline 2633 HLW02-12 & & 4.4 & 3.3 & & & 1 & & & \\
\hline 2634 HLW02-13 & & 5.6 & 0.2 & & 1 & 0.3 & & & \\
\hline 2635 HLW02-14 & & 1.7 & & 1.4 & 0.2 & & & & \\
\hline 2636 HLW02-15 & & 3.7 & 3.1 & 2.7 & & 0.9 & & & \\
\hline 2637 HLW02-16 & & 4 & 3.6 & 3.1 & 0.7 & & & & \\
\hline $\begin{array}{l}2638 \text { HLW02-17 } \\
2639 \text { HLW02-18 }\end{array}$ & & 18 & 2.07 & 0.9 & 0.2 & 0.4 & & & \\
\hline $\begin{array}{l}2640 \text { HLWO2-19 } \\
26402-18\end{array}$ & & $\begin{array}{l}1.8 \\
2.4\end{array}$ & 1.2 & 0.8 & & & & & \\
\hline 2641 HLW02-20, HLW02-20R1 & & $\begin{array}{l}2.4 \\
6.4\end{array}$ & 1.65 & $\begin{array}{ll}0.8 \\
0.8\end{array}$ & & & & & \\
\hline 2642 HLW02-21, HLW02-21R1 & 0.2 & & 0.2 & & & & & & \\
\hline 2643 HLW02-22 & & 1.6 & 0.7 & 1.5 & & 0.2 & & & \\
\hline 2644 HLW02-23, HLW02-23R1 & & 2.4 & 1.7 & 1.1 & & & & & \\
\hline 2645 HLW02-24 & & 2.3 & 1.24 & 0.61 & & & & & \\
\hline $\begin{array}{l}2646 \text { HLW02-25, HLW02-25R1 } \\
2667 \text { HLW02-26 }\end{array}$ & & 2 & 0.5 & 1 & & & & & \\
\hline $\begin{array}{l}2667 \text { HLW02-26 } \\
2648 \text { HLW02-27 }\end{array}$ & & $\begin{array}{l}3.9 \\
2.5\end{array}$ & $\begin{array}{l}2.47 \\
128\end{array}$ & 0.9 & $\begin{array}{l}0.8 \\
0.5\end{array}$ & & & & \\
\hline $\begin{array}{l}2648 \text { HLWL2-27 } \\
2649 \text { HLW02-28 }\end{array}$ & & $\begin{array}{l}2.5 \\
1.9\end{array}$ & 1.1 & & $\begin{array}{l}0.5 \\
0.1\end{array}$ & 0.2 & & & \\
\hline 2650 HLW02-29, HLW02-29R1 & & $\begin{array}{l}3.9 \\
3.5\end{array}$ & 1.86 & 0.7 & 0.1 & 0.2 & & & \\
\hline 2651 HLW02-30 & & 1.1 & & & & & & & \\
\hline 2652 HLW02-31 & & 0.99 & & & & & & & \\
\hline $\begin{array}{l}2653 \text { HLW02-32, HLW02-32R1 } \\
2654 \text { HLW02-33 }\end{array}$ & 1.4 & $\begin{array}{l}0.267 \\
58\end{array}$ & 0.44 & 0.14 & & & & & \\
\hline 2655 HLW02-34, HLW02-34R1 & & 6.2 & 2.46 & 0.11 & & & & & \\
\hline 2656 HLW02-35 & & 7.2 & & 1.7 & 0.8 & & & & \\
\hline 2657 HLW02-36 & & 1.4 & 0.3 & 0.8 & & & & & \\
\hline 2658 HLW02-37 & & 2.9 & 2.1 & 0.8 & & 0.6 & & & \\
\hline 2659 HLW02-38 & & 2.7 & 1.25 & 1.61 & & 0.2 & & & \\
\hline 2660 HLWO2-39 & & 2.5 & 1.79 & 1.95 & & 0.2 & & & \\
\hline $\begin{array}{l}2661 \text { HLW02-40 } \\
2662 \text { HLW02-41 }\end{array}$ & & $\begin{array}{l}1.95 \\
24\end{array}$ & 104 & $\begin{array}{l}1.6 \\
0.6\end{array}$ & 0.4 & 01 & & & \\
\hline 2663 HLW02-42 & & $\begin{array}{l}2.4 \\
0.66\end{array}$ & $\begin{array}{l}1.04 \\
0.3\end{array}-35$ & $\begin{array}{l}0.6 \\
0.5\end{array}$ & & 0.1 & & & \\
\hline 2664 HLWO2-43 & & 2.6 & 0.58 & 0.31 & & & & & \\
\hline 2665 HLWO2-44 & 1.1 & 0.8 & 0.57 & & & & & & \\
\hline 2666 HLW02-45 & & 2.4 & 0.95 & 0.8 & & & & & \\
\hline 2667 HLW02-46 & & 2.9 & 1.6 & 1.07 & & 0.2 & & & \\
\hline 2668 HLW02-47 & 2.4 & 0.8 & & & & & & & \\
\hline 2669 HLW02-48 & & 1.5 & & 1.4 & 0.7 & & & & \\
\hline $\begin{array}{l}2670 \text { HLW02-49 } \\
2671 \text { HLW02-50 }\end{array}$ & & $\begin{array}{l}3.68 \\
53\end{array}$ & $\begin{array}{l}1.73 \\
1.96\end{array}$ & $\begin{array}{r}2.1 \\
1.52\end{array}$ & & 0.8 & & & \\
\hline 2672 HLW02-51, HLW02-51R1 & & $\begin{array}{r}5.3 \\
2\end{array}$ & $\begin{array}{l}1.96 \\
0.8\end{array}$ & $\begin{array}{l}1.52 \\
0.8\end{array}$ & & & & & \\
\hline $\begin{array}{l}2673 \text { HLWO2-52 } \\
2 \text { L }\end{array}$ & & 1.3 & 2.1 & $\begin{array}{l}0.0 \\
1.2\end{array}$ & & 0.4 & & & \\
\hline 2674 HLWO2-53 & & 2.1 & 1.4 & 1 & 0.4 & & & & \\
\hline 2675 HLW02-54 & & 2.6 & 1.1 & 0.1 & & & & & \\
\hline 2676 HLW02-55 & & 1.4 & & 0.2 & & & & & \\
\hline 2677 HLW02-56 & & 1 & 0.4 & 0.5 & & & & & \\
\hline $\begin{array}{l}2678 \text { HLW02-57 } \\
2679 \text { HLW0-01 }\end{array}$ & & $\begin{array}{c}0.3 \\
10\end{array}$ & & 14 & & & & 07 & \\
\hline 2680 HLW03-02 & & 7.3 & & 4.6 & & 2 & 3.3 & 3.3 & 4 \\
\hline 2681 HLW03-03 & & & & 2.6 & & 2.5 & & 2 & 0.1 \\
\hline 2682 HLW03-04 & 8.3 & 2.4 & & 0.6 & & 0.4 & & & \\
\hline $\begin{array}{l}2683 \text { HLWW3-05 } \\
2684 \text { HLW03-06 }\end{array}$ & & 2.1 & ${ }_{2}^{1.2}$ & $\begin{array}{r}0.5 \\
1\end{array}$ & 0.9 & & & & \\
\hline 2685 HLW03-07 & & 6.2 & & 6 & & 4.2 & & 2.9 & 1 \\
\hline 2686 HLW03-08 & & & & 12.2 & & 6.6 & 7.3 & 9.8 & 4.2 \\
\hline 2687 HLW03-09 & & 0.2 & & & & & & & \\
\hline $\begin{array}{l}\text { 26888 HLW03-10, HLW03-10R1 } \\
2689 \text { HLW03-11 }\end{array}$ & & $\begin{array}{l}12.5 \\
0.3\end{array}$ & & $\begin{array}{l}8.7 \\
0.2\end{array}$ & & 3.7 & 2.9 & 3.5 & 1.4 \\
\hline 2690 HLW03-12 & & 0.7 & 0.8 & 0.6 & & & & & \\
\hline 2691 HLW03-13 & & 7.9 & & 5.3 & & 5 & & 3.5 & 0.6 \\
\hline 2692 HLW03-14 & & 4.6 & & 3.5 & 1.5 & 2.2 & 0.5 & & \\
\hline $\begin{array}{l}2693 \text { HLWO3-15 } \\
2694 \text { HLW03-16 }\end{array}$ & & & & $\begin{array}{l}2.7 \\
19\end{array}$ & & 0.9 & 0.3 & & \\
\hline $\begin{array}{l}26945 \text { HLWO3- } \\
2695-1\end{array}$ & & $\begin{array}{l}2.8 \\
5.7\end{array}$ & & 2.4 & & 0.5 & & & \\
\hline 2696 HLW03. & & 2.4 & & 0.5 & & 0.5 & & & \\
\hline 2697 HLW03-19 & & 1.5 & & 0.8 & & 0.6 & & & \\
\hline 2698 HLW03-20 & & & 1 & 0.3 & & & & & \\
\hline 2699 HLW03- & 2 & 1 & 0.3 & 0.2 & & & & & \\
\hline 2700 HLW03-22 & & 2.3 & 0.6 & 0.5 & & & & & \\
\hline 2700 & $\begin{array}{l}1.3 \\
1.6\end{array}$ & 0.9 & & & & & & & \\
\hline LW03- & 0.2 & 0.2 & & 0.1 & & & & & \\
\hline 2704 HLWWO3-26 & 1 & 0.8 & 0.4 & 0.3 & & & & & \\
\hline $\begin{array}{l}705 \text { HLWW03-27 } \\
2706 \text { HWW03-28 }\end{array}$ & 13 & $\begin{array}{l}1.7 \\
1\end{array}$ & & $\begin{array}{l}1.6 \\
0.2\end{array}$ & & 0.6 & & & \\
\hline
\end{tabular}

Quenched XRD CCC XRD

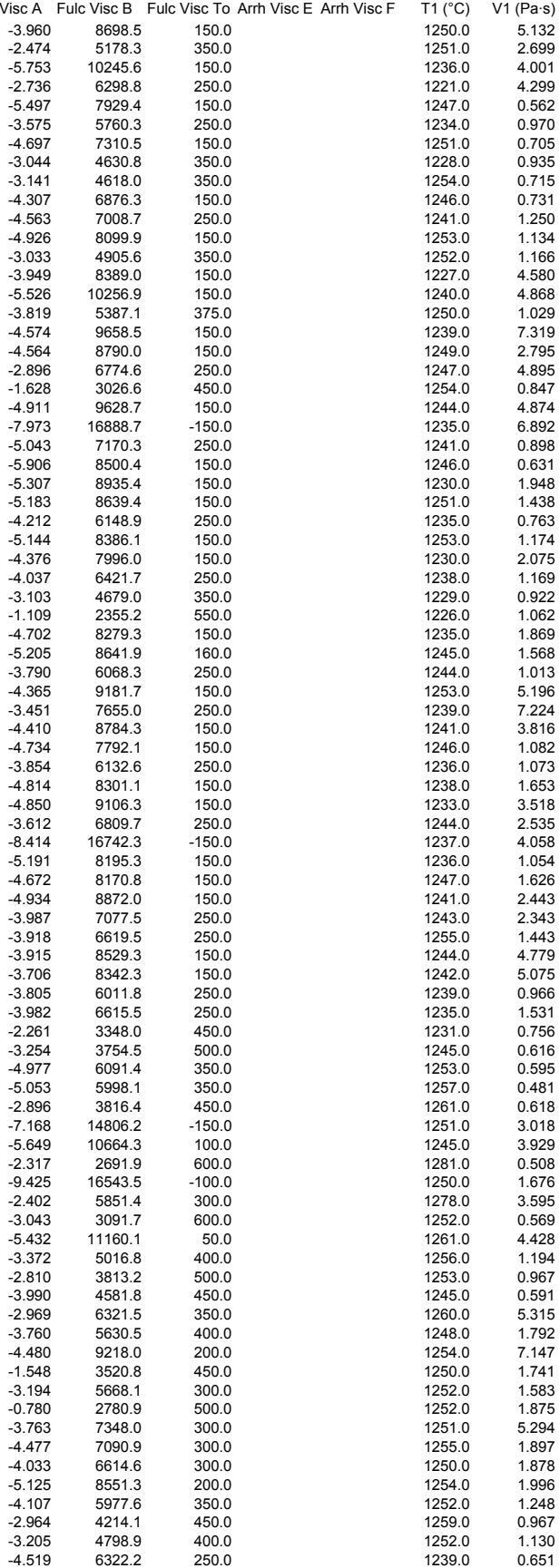




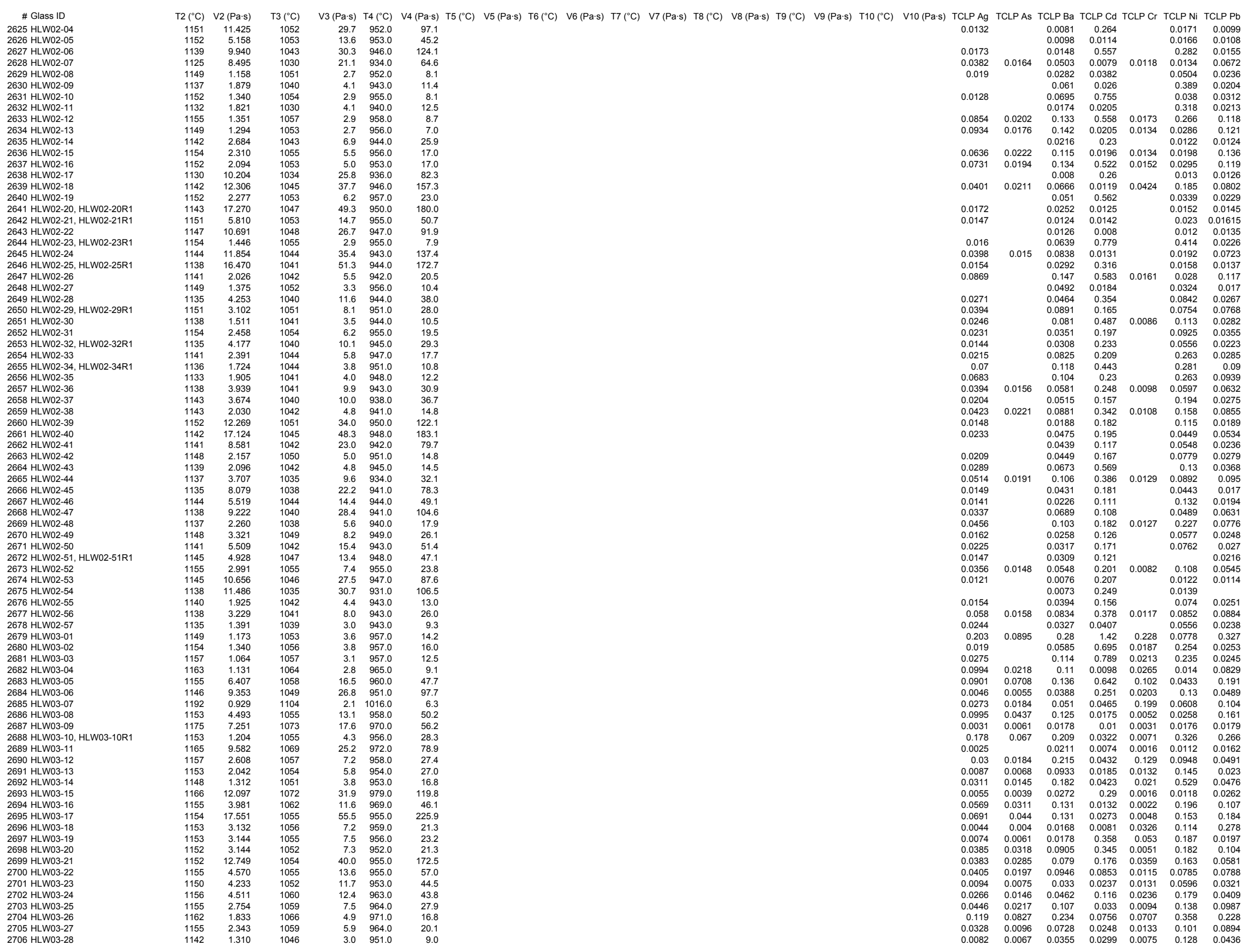




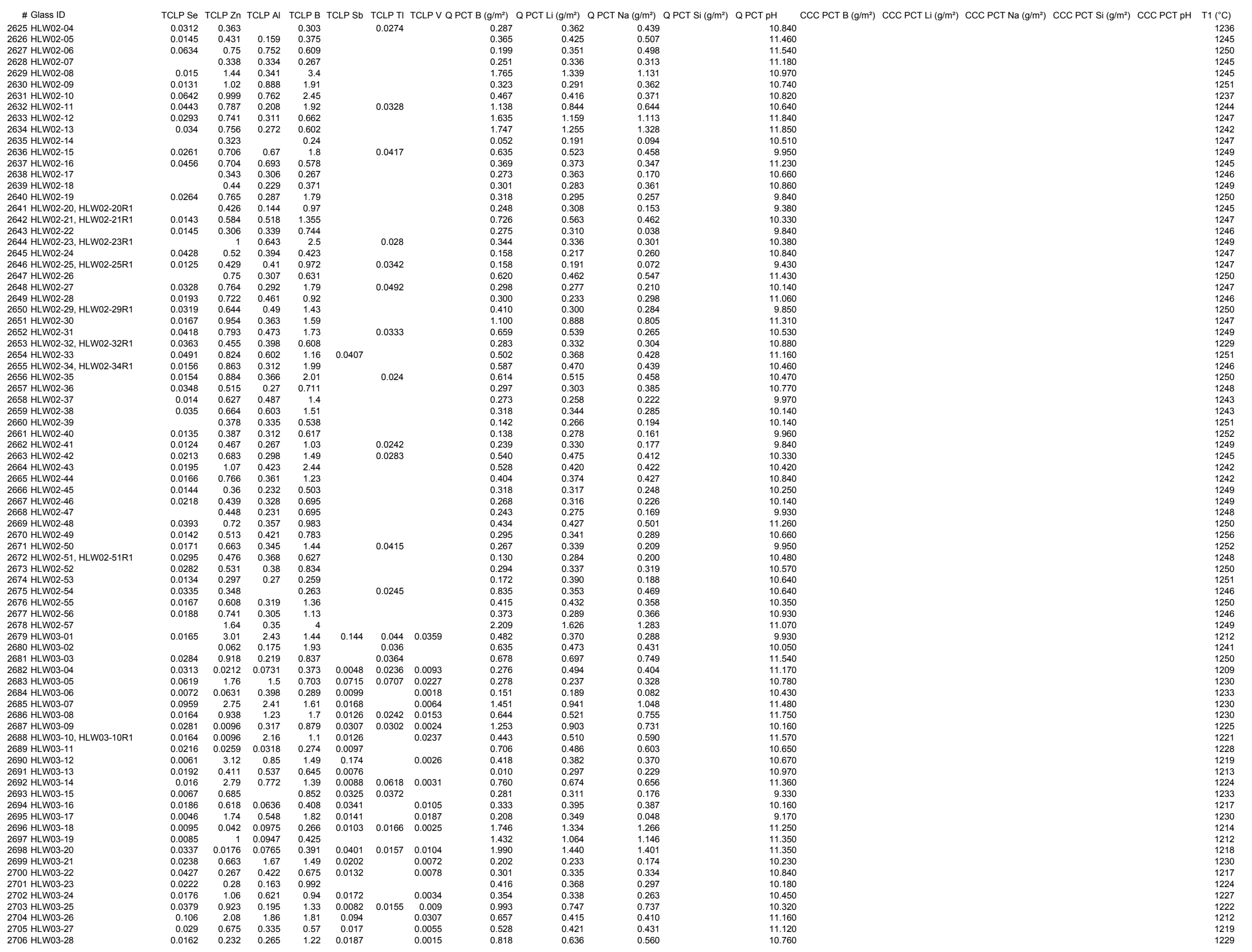




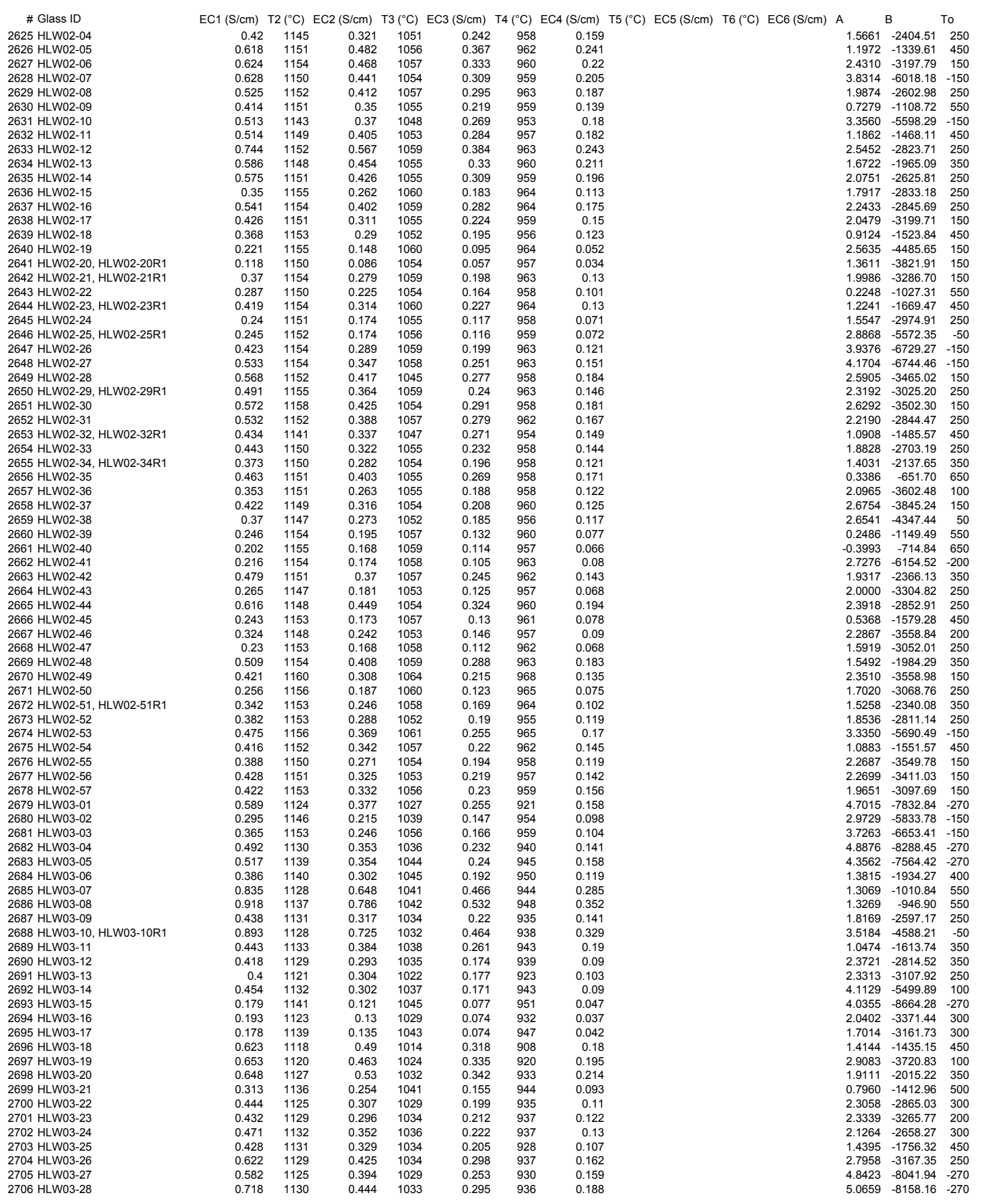




\begin{tabular}{|c|c|c|c|c|c|c|}
\hline \# Glass ID & Study & Reference & & & & \\
\hline 07 HLW03-29 & & Piepel et al. 2008 & 0.0297 & 0.069 & 0.0050 & 0.0495 \\
\hline & HLW03 Augmentation M & Piepel et al. 2008 & 0.0297 & 0.1189 & 0.0050 & 0.0495 \\
\hline 2709 HLW03-31 & & Piepel et al. 2008 & 0.0495 & 0.0693 & & \\
\hline $\begin{array}{l}2710 \text { HLW03-32 } \\
271 \text { HLW03-33 }\end{array}$ & HLW03 Augmentation M & Piepel et al. 2008 & 0.0492 & 0.0689 & 0.0050 & 0.0492 \\
\hline $\begin{array}{l}2771 \text { HLW03-33 } \\
2712 \text { HLW0-34 }\end{array}$ & $\begin{array}{l}\text { HLW03 Augmentation M } \\
\text { HLW3 Augmentation M }\end{array}$ & $\begin{array}{l}\text { Piepelel te al. } 2008 \\
\text { Piepel tel al } 2008\end{array}$ & 0.0296 & 0.1790 & 0.0050 & $\begin{array}{l}0.0092 \\
0.0985\end{array}$ \\
\hline $\begin{array}{l}2713 \text { HLW03-35 } \\
27124\end{array}$ & HLW03 Augmentation M & Piepel et al. 2008 & 0.0496 & 0.1040 & & 0.0496 \\
\hline 2714 HLWO3-36 & HLW03 Augmentation M & Piepel et al. 2008 & 0.0492 & 0.0689 & & 0.0951 \\
\hline 2715 HLW03-37 & HLW03 Augmentation M & Piepel et al. 2008 & 0.0295 & 0.1181 & 0.0050 & 0.0722 \\
\hline 2716 HLW03-38 & HLW03 Augmentation M & Piepel et al. 2008 & 0.0491 & 0.0688 & 0.0050 & 0.0983 \\
\hline 2717 HLWO3-39 & & Piepel et al. 2008 & 0.0295 & 0.0688 & 0.0050 & 0.0850 \\
\hline $\begin{array}{l}2718 \text { HLW03-40 } \\
2719 \text { HLW03-41 }\end{array}$ & HLW03 Augmentation M & $\begin{array}{l}\text { Piepel et al. } 2008 \\
\text { PPiepeletel al } 2008\end{array}$ & 0.0494 & 0.0692 & 0.0050 & $\begin{array}{l}0.0939 \\
0.0944\end{array}$ \\
\hline $\begin{array}{l}2719 \text { HLW03-41 } \\
2720 \text { HLW03-42 }\end{array}$ & $\begin{array}{l}\text { HLW03 Augmentation M } \\
\text { HLW3 Augmentation M }\end{array}$ & $\begin{array}{l}\text { Piepel et al. } 2008 \\
\text { Piepel tet al. } 2008\end{array}$ & $\begin{array}{l}0.0389 \\
0.0550\end{array}$ & $\begin{array}{l}0.0873 \\
0.0915\end{array}$ & $\begin{array}{l}0.0050 \\
0.0050\end{array}$ & 0.0694 \\
\hline $\begin{array}{l}2721 \text { HLW03-43 } \\
2720\end{array}$ & HLW03 Augmentation M & $\begin{array}{l}\text { Piepele te al. } 2008 \\
\text { Piepel et al. } 2008\end{array}$ & $\begin{array}{l}0.0550 \\
0.0834\end{array}$ & $\begin{array}{l}0.0915 \\
0.0491\end{array}$ & $\begin{array}{l}0.0050 \\
0.0050\end{array}$ & $\begin{array}{l}0.1000 \\
0.0196\end{array}$ \\
\hline 2722 HLW03-44 & HLW03 Augmentation M & Piepel et al. 2008 & $\begin{array}{l}0.0198 \\
0.0034\end{array}$ & & 0.0050 & 0.0954 \\
\hline 2723 HLW03-45 & HLW03 Augmentation M M & $\begin{array}{l}\text { Pipel et al. } 2008 \\
\text { Pens }\end{array}$ & 0.0492 & 0.0689 & 0.0050 & \\
\hline 2724 HLW04-01 & HLW04 C106 actual & Kot and Pegg 2004 & 0.0478 & 0.1126 & 0.0036 & 0.1309 \\
\hline 2725 HLW04-02 & HLW04 C106 actual & Kot and Pegg 2004 & 0.0478 & 0.1251 & & 0.1309 \\
\hline 2726 HLW04-03 & HLW04 C106 actual & Kot and Pegg 2004 & 0.0492 & 0.1109 & 0.0037 & 0.1346 \\
\hline $\begin{array}{l}2727 \text { HLW04-04 } \\
2728 \text { HIOS4 }\end{array}$ & HLW04 C106 actual & Kot and Pegg 2004 & 0.0458 & 0.1076 & 0.0035 & 0.1255 \\
\hline $\begin{array}{l}2728 \text { HLW04-06 } \\
2729 \text { HLW04-07 }\end{array}$ & $\begin{array}{l}\text { HLW04 C106 actual } \\
\text { HLW04 C106 actual }\end{array}$ & $\begin{array}{l}\text { Kot and Pegg } 2004 \\
\text { Kot and Pego } 2004\end{array}$ & 0.0492 & $\begin{array}{l}0.1036 \\
0.103\end{array}$ & 0.0037 & $\begin{array}{l}0.1346 \\
0.1401\end{array}$ \\
\hline $\begin{array}{l}2729 \mathrm{HLVU}-\mathrm{O} T \\
2730 \mathrm{HLW}-08\end{array}$ & HLW04 C106 actual & $\begin{array}{l}\text { Kot and Pegg } 2004 \\
\text { Kot and Pegg } 2004\end{array}$ & $\begin{array}{l}0.0488 \\
0.0478\end{array}$ & $\begin{array}{l}0.1037 \\
0.1036\end{array}$ & $\begin{array}{l}0.0046 \\
0.0045\end{array}$ & $\begin{array}{l}0.1401 \\
0.1371\end{array}$ \\
\hline 2731 HLW04-09 & HLW04 C106 actual & Kot and Pegg 2004 & 0.0488 & 0.1027 & 0.0046 & 0.1401 \\
\hline 2732 HLW05-01 & HLW05 ThZr M & Kot et al. 2005 & 0.0600 & 0.1200 & 0.0075 & 0.0606 \\
\hline 2733 HLW05-02 & HLW05 ThZr M & Kot et al. 2005 & 0.1000 & 0.1200 & 0.0075 & 0.0500 \\
\hline 2734 HLW05-03 & HLW05 ThZr M & Kot et al. 2005 & 0.0600 & 0.1130 & 0.0075 & 0.0500 \\
\hline 2735 HLW05-04 & HLW05 ThZr M & Kot et al. 2005 & 0.0600 & 0.0700 & 0.0075 & 0.0500 \\
\hline $\begin{array}{l}2736 \text { HLW05-05 } \\
2737 \text { HUW05-06 }\end{array}$ & HLW05 ThZr M & Kot et al. 2005 & 0.0600 & 0.0700 & 0.0075 & 0.0700 \\
\hline $\begin{array}{l}2737 \text { HLW05-06 } \\
2738 \text { HLW05-07 }\end{array}$ & $\begin{array}{l}\text { HLW05 ThrzM } \\
\text { HLW05 Thr M }\end{array}$ & Kot et al. 2005 & $\begin{array}{l}0.0600 \\
0.1000\end{array}$ & 0.0706 & 0.0075 & 0.0800 \\
\hline 2738 HLWO5-08 & HLW05 ThZr M & Kot et al. 2005 & $\begin{array}{l}0.0600 \\
0.0600\end{array}$ & & $\begin{array}{l}0.0075 \\
0.0075\end{array}$ & \\
\hline 2740 HLWO5-09 & HLW05 ThZr M & Kot et al. 2005 & 0.0600 & 0.1200 & 0.0075 & 0.0767 \\
\hline 2741 HLW05-10 & $\begin{array}{l}\text { HLWW05 ThZr M M } \\
\text { HLW }\end{array}$ & $\begin{array}{l}\text { Kot etal. } 2005 \\
\text { Kot al }\end{array}$ & 0.1000 & 0.0700 & 0.0075 & 0.0500 \\
\hline 2742 HLW05-11 & HLW05 ThZr M & Kot et al. 2005 & 0.0600 & 0.1200 & 0.0075 & 0.0800 \\
\hline 2743 HLW05-12 & HLW05 ThZr M & Kot et al. 2005 & 0.0600 & 0.0706 & 0.0075 & 0.0800 \\
\hline 2744 HLWO5-13 & HLW05 ThZr M & Kot et al. 2005 & 0.0977 & 0.0700 & 0.0075 & 0.0763 \\
\hline 2745 HLWO5-14 & HLW05 ThZr M & Kot et al. 2005 & 0.0600 & 0.1130 & 0.0075 & 0.0800 \\
\hline $\begin{array}{l}2746 \text { HLW05-15 } \\
2747 \text { HLW05-16 }\end{array}$ & $\begin{array}{l}\text { HLW05 TLZr M } \\
\text { HLW05 ThZr M }\end{array}$ & $\begin{array}{l}\text { Kot et al. } 2005 \\
\text { Kot tet al. } 2005\end{array}$ & $\begin{array}{l}0.0200 \\
0.0511\end{array}$ & $\begin{array}{l}0.0450 \\
0.1400\end{array}$ & $\begin{array}{l}0.0075 \\
0.0075\end{array}$ & $\begin{array}{l}0.12200 \\
0.0150\end{array}$ \\
\hline 2748 HLWO5-17 & HLW05 ThZr M & Kot et al. 2005 & 0.1187 & $\begin{array}{l}0.1400 \\
0.1400\end{array}$ & 0.0075 & \\
\hline 2749 HLWO5-18 & HLW05 ThZr M & Kot et al. 2005 & 0.0200 & 0.1400 & 0.0075 & 0.0646 \\
\hline 2750 HLWO5-19 & HLW05 ThZr M & & 0.1300 & 0.0450 & 0.0075 & 0.0592 \\
\hline 2751 HLW05-20 & HLW05 ThZr M & Kot et al. 2005 & 0.1300 & 0.1400 & 0.0075 & 0.0150 \\
\hline 2752 HLW05-21 & HLW05 ThZr M & Kot et al. 2005 & 0.0200 & 0.1400 & 0.0075 & 0.1200 \\
\hline 2753 HLW05-22 & HLW05 ThZr M & Kot et al. 2005 & 0.1300 & 0.1400 & 0.0075 & 0.0250 \\
\hline $2754 \mathrm{HLW} 05-23$ & HLW05 ThZr M & Kot et al. 2005 & 0.1273 & 0.0450 & 0.0075 & 0.0150 \\
\hline 2755 HLW05-24 & The & Kot et al. 2005 & 0.0217 & $\begin{array}{l}0.0450 \\
\end{array}$ & 0.0075 & 0.0894 \\
\hline $\begin{array}{l}2756 \text { HLWO5-25 } \\
2757 \text { HLWO5-26 }\end{array}$ & $\begin{array}{l}\text { HLWU5 ThrM M } \\
\text { HLW05 Thr M }\end{array}$ & $\begin{array}{l}\text { Kot et al. } 2005 \\
\text { Kot et al. } 2005\end{array}$ & $\begin{array}{l}0.0200 \\
0.0500\end{array}$ & $\begin{array}{l}0.0433 \\
0.0450\end{array}$ & $\begin{array}{l}0.0075 \\
0.0075\end{array}$ & \\
\hline 2758 HLWO5-27 & HLW05 ThZr M & Kot et al. 2005 & 0.0200 & 0.0450 & 0.0075 & 0.1200 \\
\hline 2759 HLW05-28 & HLW05 ThZr M & Kot et al. 2005 & & & 0.0075 & 0.0646 \\
\hline 2760 HLW05-29 & HLW05 ThZr M & Kot et al. 2005 & 0.0500 & 0.0450 & 0.0075 & 0.0150 \\
\hline 2761 HLW05-30 & HLW05 ThZr M & Kot et al. 2005 & 0.0661 & 0.0986 & 0.0075 & 0.0625 \\
\hline 2762 HLW06-01 & HLW06 Spinel M & Kot et al. 2005 & 0.1100 & 0.0650 & 0.0100 & 0.0700 \\
\hline 2763 HLW06-02 & HLW06 Spinel M & Kot et al. 2005 & 0.1039 & 0.1100 & 0.0100 & 0.0700 \\
\hline $\begin{array}{l}2764 \text { HLW06-03 } \\
2765 \text { HLW06-04 }\end{array}$ & $\begin{array}{l}\text { HLW06 Spinel M } \\
\text { HLW06 Spinel M }\end{array}$ & $\begin{array}{l}\text { Kot et al. } 2005 \\
\text { Kot et al } 2005\end{array}$ & $\begin{array}{l}0.1100 \\
0.0800\end{array}$ & $\begin{array}{l}0.0650 \\
0.0650\end{array}$ & $\begin{array}{l}0.0047 \\
0.0076\end{array}$ & $\begin{array}{l}0.1440 \\
0.0700\end{array}$ \\
\hline 2766 HLWO6-05 & HLW06 Spinel M & Kot et al. 2005 & $\begin{array}{l}0.08000 \\
0.0800\end{array}$ & $\begin{array}{l}0.0650 \\
0.0650\end{array}$ & $\begin{array}{l}0.0016 \\
0.0067\end{array}$ & \\
\hline 2767 HLW06-06 & HLW06 Spinel M & Kot et al. 2005 & 0.11 & $\begin{array}{l}0.0650 \\
0.0650\end{array}$ & 0.0094 & 0.0700 \\
\hline 2768 HLW06-07 & HLW06 Spinel M & Kot et al. 2005 & 0.0800 & 0.0650 & 0.0100 & 0.1400 \\
\hline 2769 HLW06-08 & HLW06 Spinel M & Kot et al. 2005 & 0.0800 & 0.0650 & 0.0061 & 0.0700 \\
\hline 2770 HLWO6-09 & HLW06 Spinel M & Kot et al. 2005 & 0.0800 & 0.0650 & 0.0017 & 0.1400 \\
\hline 2771 HLW06-10 & HLW06 Spinel M & Kot et al. 2 & 0.08 & 0.1100 & 0.0047 & 0.0700 \\
\hline 2772 HLW06-11 & HLW06 Spinel M & Kot et al. 2005 & 0.0800 & $\begin{array}{r}0.1100 \\
\end{array}$ & 0.0055 & 0.1400 \\
\hline 2774 HLWO6-13 & HLW06 Spinel M & $\begin{array}{l}\text { Kotet al. } 2005 \\
\text { Kot et al. } 2005\end{array}$ & & $\begin{array}{l}0.1100 \\
0.0650\end{array}$ & $\begin{array}{l}0.0017 \\
0.0094\end{array}$ & $\begin{array}{l}0.0700 \\
0.0700\end{array}$ \\
\hline 2775 HLWOG-14 & HLW06 Spinel M & Kot et al. 2005 & & 0.1100 & $\begin{array}{l}0.0100 \\
0.0100\end{array}$ & 0.0700 \\
\hline HLW06-15 & HLW06 Spinel M & Kot et al. 2005 & 0.0800 & & 0.0017 & 0.0700 \\
\hline HLW06-16 & HLW06 Spinel M & Kot et al. 2005 & 0.1000 & 0.0650 & 0.0032 & 0.0700 \\
\hline 2778 HLW06-17 & HLW06 Spinel M & Kot et al. 2005 & 0.1100 & 0.0650 & 0.0100 & 0.0700 \\
\hline 2779 HLW06-18 & HLW06 Spinel M & Kot et al. 2005 & & 0.1100 & 0.0028 & 0.0700 \\
\hline & & tet al. 2005 & & 0.0650 & & 0.1206 \\
\hline & & & & & & 0.0729 \\
\hline $\begin{array}{l}\text { HLW06-21 } \\
\text { HLWO6-22 }\end{array}$ & HLW06 Spinel M & $\begin{array}{l}\text { Kot et al. } 2005 \\
\text { Kot et al. } 2005\end{array}$ & & $\begin{array}{l}0.0430 \\
0.1500\end{array}$ & $\begin{array}{l}0.0000 \\
0.0000\end{array}$ & $\begin{array}{l}0.0140 \\
0.0140\end{array}$ \\
\hline & 106 Spinel M & Kot et al. 2005 & & 0.0430 & & 0.1500 \\
\hline & & & 0.1300 & & & 0.0140 \\
\hline & 106 Spinel M & tet al. 2005 & 0.1300 & & & 0.0140 \\
\hline HLW06-26 & HLW06 Spinel M & Kot et al. 2005 & 0.0188 & 0.0430 & 0.000 & 0.01 \\
\hline 88 HLW06-27 & HLW06 Spinel M & Kot et al. 2005 & 0.0188 & 0.0982 & 0.0000 & 0.0140 \\
\hline
\end{tabular}

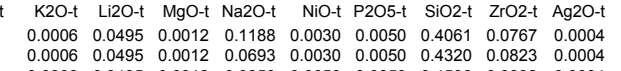

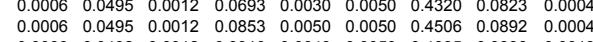

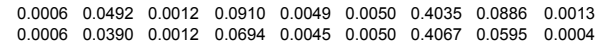
$\begin{array}{lllllllllll}0.0006 & 0.0246 & 0.0012 & 0.0954 & 0.0049 & 0.0050 & 0.4828 & 0.0591 & 0.0013\end{array}$ $\begin{array}{lllllllll}0.0006 & 0.0248 & 0.0012 & 0.0695 & 0.0050 & 0.0050 & 0.4864 & 0.0596 & 0.0004 \\ 0.0006 & 0.0269 & 0.0012 & 0.0796 & 0.0049 & 0.0050 & 0.4823 & 0.0591 & 0.0013\end{array}$ $\begin{array}{lllllllll}0.0006 & 0.0486 & 0.0012 & 0.0689 & 0.0049 & 0.0050 & 0.4035 & 0.0733 & 0.000\end{array}$ $\begin{array}{llllllllll}0.0006 & 0.0485 & 0.0012 & 0.0688 & 0.0029 & 0.0050 & 0.4813 & 0.0708 & 0.00013\end{array}$

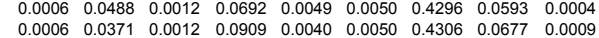
$\begin{array}{lllllllll}0.0006 & 0.0371 & 0.0012 & 0.0909 & 0.0040 & 0.0050 & 0.4306 & 0.0677 & 0.0009 \\ 0.0006 & 0.0370 & 0.0012 & 0.0900 & 0.0080 & 0.0050 & 0.4900 & 0.0250 & 0.0004\end{array}$ $\begin{array}{lllllllll}0.0006 & 0.0589 & 0.0012 & 0.0393 & 0.0098 & 0.0050 & 0.5203 & 0.0410 & 0.0002\end{array}$ $\begin{array}{lllllllll}0.0006 & 0.0198 & 0.0012 & 0.1488 & 0.0010 & 0.0050 & 0.5259 & 0.0000 & 0.0002 \\ 0.0006 & 0.0492 & 0.0012 & 0.1043 & 0.0049 & 0.0050 & 0.4035 & 0.0590 & 0.0013\end{array}$ $\begin{array}{lllllllll}0.0006 & 0.0492 & 0.0011 & 0.1043 & 0.0049 & 0.0050 & 0.4035 & 0.0590 & 0.0001 \\ 0.0000 & 0.0281 & 0.0011 & 0.1225 & 0.0038 & 0.0042 & 0.4851 & 0.0000 & 0.0007\end{array}$ $\begin{array}{lllllllll}0.00000 & 0.0281 & 0.0011 & 0.1225 & 0.00038 & 0.0042 & 0.4726 & 0.0000 & 0.0007 \\ 0.0000 & 0.0277 & 0.0012 & 0.1231 & 0.0040 & 0.0043 & 0.4802 & 0.0000 & 0.0007\end{array}$

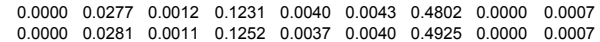

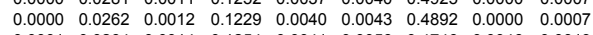
$\begin{array}{llllllllll}0.0001 & 0.0264 & 0.0014 & 0.1254 & 0.00041 & 0.00056 & 0.4474 & 0.00046 & 0.00019 \\ 0.0001 & 0.0264 & 0.0014 & 0.1241 & 0.0040 & 0.0055 & 0.4812 & 0.0045 & 0.0018\end{array}$ $\begin{array}{lllllllll}0.0001 & 0.0264 & 0.0014 & 0.1241 & 0.0040 & 0.0055 & 0.4812 & 0.0045 & 0.0018 \\ 0.0001 & 0.0264 & 0.0014 & 0.1253 & 0.0041 & 0.0056 & 0.4777 & 0.0046 & 0.0019\end{array}$ $\begin{array}{llllllllll}0.0020 & 0.0300 & 0.0010 & 0.0800 & 0.0013 & 0.0030 & 0.4376 & 0.0608 & 0.0010\end{array}$ $\begin{array}{lllllllll}0.0020 & 0.0300 & 0.0010 & 0.1168 & 0.0025 & 0.0030 & 0.4112 & 0.0500 & 0.0010 \\ 0.0020 & 0.0300 & 0.0010 & 0.1600 & 0.0013 & 0.0030 & 0.3800 & 0.0700 & 0.0010\end{array}$ $\begin{array}{lllllllll}0.0020 & 0.0300 & 0.0010 & 0.1600 & 0.0013 & 0.0030 & 0.3800 & 0.0700 & 0.0010 \\ 0.0020 & 0.0502 & 0.0010 & 0.1075 & 0.0025 & 0.0030 & 0.4400 & 0.0815 & 0.0010\end{array}$ $\begin{array}{lllllllll}0.0020 & 0.0325 & 0.0010 & 0.1600 & 0.0013 & 0.0030 & 0.4400 & 0.0500 & 0.0010\end{array}$

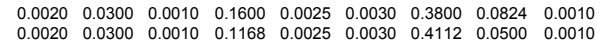

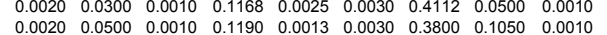

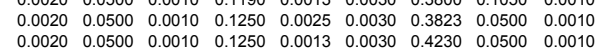
$\begin{array}{lllllllll}0.0022 & 0.0500 & 0.0010 & 0.0830 & 0.0013 & 0.0030 & 0.4400 & 0.0500 & 0.0010\end{array}$ $\begin{array}{llllllllll}0.0020 & 0.0300 & 0.0010 & 0.1600 & 0.0013 & 0.030 & 33800 & 0.0500 & 0.0010\end{array}$ $\begin{array}{lllllllll}0.0020 & 0.05500 & 0.0010 & 0.1250 & 0.0025 & 0.0030 & 0.3800 & 0.0500 & 0.0010 \\ 0.0020 & 0.0000 & 0.0010 & 0.1667 & 0.0005 & 0.0030 & 0.4900 & 0.0400 & 0.0010\end{array}$

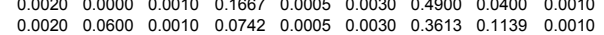
$\begin{array}{lllllllll}0.0020 & 0.0600 & 0.0010 & 0.1075 & 0.0005 & 0.0030 & 0.3400 & 0.0795 & 0.0010\end{array}$ $\begin{array}{lllllllll}0.0020 & 0.0000 & 0.0010 & 0.1142 & 0.0050 & 0.0030 & 0.4203 & 0.0954 & 0.0010 \\ 0.0020 & 0.0145 & 0.0010 & 0.2000 & 0.0005 & 0.0030 & 0.3438 & 0.0250 & 0.0010\end{array}$ $\begin{array}{llllllllll}0.0020 & 0.0000 & 0.0010 & 0.2000 & 0.0050 & 0.0030 & 0.3775 & 0.0350 & 0.0010\end{array}$ $\begin{array}{lllllllll}0.0020 & 0.0000 & 0.0010 & 0.1971 & 0.0005 & 0.0030 & 0.3400 & 0.0534 & 0.0010 \\ 0.0020 & 0.0370 & 0.0010 & 0.0650 & 0.0005 & 0.0030 & 0.4405 & 0.0250 & 0.0010\end{array}$ $\begin{array}{lllllllll}0.0020 & 0.0600 & 0.0010 & 0.1000 & 0.0050 & 0.0030 & 0.4675 & 0.0377 & 0.0010\end{array}$

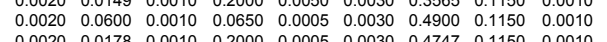
$\begin{array}{lllllllll}0.0020 & 0.0178 & 0.0010 & 0.2000 & 0.0005 & 0.0030 & 0.4747 & 0.1150 & 0.0010 \\ 0.0020 & 0.0595 & 0.0010 & 0.1039 & 0.0050 & 0.0030 & 0.4561 & 0.0400 & 0.0010\end{array}$ $\begin{array}{lllllllll}0.0020 & 0.0595 & 0.0010 & 0.1039 & 0.0050 & 0.0030 & 0.4561 & 0.0400 & 0.0010 \\ 0.0020 & 0.0000 & 0.0010 & 0.1142 & 0.0050 & 0.0030 & 0.4203 & 0.0954 & 0.0010\end{array}$ $\begin{array}{lllllllll}0.0020 & 0.0178 & 0.0010 & 0.2000 & 0.0005 & 0.0030 & 0.4747 & 0.1150 & 0.0010 \\ 0.0020 & 0.0278 & 0.0010 & 0.1471 & 0.0025 & 0.0030 & 0.4097 & 0.0600 & 0.0010\end{array}$ $\begin{array}{lllllllll}0.0020 & 0.0278 & 0.0010 & 0.1471 & 0.0025 & 0.0030 & 0.4097 & 0.0600 & 0.0010 \\ 0.0164 & 0.0300 & 0.0117 & 0.1400 & 0.0100 & 0.0050 & 0.4000 & 0.0150 & 0.0010\end{array}$

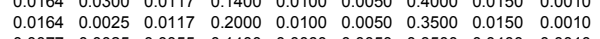
$\begin{array}{lllllllll}0.0077 & 0.0025 & 0.0055 & 0.1400 & 0.0060 & 0.0050 & 0.3500 & 0.0400 & 0.0010 \\ 0.0124 & 0.0025 & 0.0089 & 0.2000 & 0.0060 & 0.0050 & 0.3500 & 0.0400 & 0.0010\end{array}$

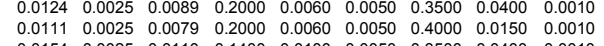

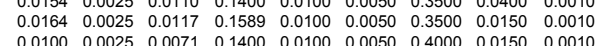
$\begin{array}{lllllllll}0.0100 & 0.0025 & 0.0071 & 0.1400 & 0.0100 & 0.0050 & 0.4000 & 0.0150 & 0.0010 \\ 0.0027 & 0.0300 & 0.0019 & 0.1400 & 0.0100 & 0.0050 & 0.3500 & 0.0400 & 0.0010\end{array}$ $\begin{array}{lllllllll}0.0027 & 0.0300 & 0.0019 & 0.1400 & 0.0100 & 0.0050 & 0.3500 & 0.04400 & 0.0010 \\ 0.0077 & 0.0025 & 0.0055 & 0.1400 & 0.0060 & 0.0050 & 0.4000 & 0.0400 & 0.0010\end{array}$

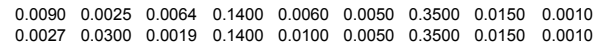
$\begin{array}{lllllllll}0.0027 & 0.0300 & 0.0019 & 0.1400 & 0.01100 & 0.0050 & 0.3550 & 0.0150 & 0.0010 \\ 0.0154 & 0.0025 & 0.0110 & 0.1400 & 0.0100 & 0.0050 & 0.3500 & 0.0400 & 0.0010\end{array}$ $\begin{array}{lllllllll}0.0164 & 0.02300 & 0.0117 & 0.1400 & 0.0060 & 0.0050 & 0.3500 & 0.0329 & 0.0010 \\ 0.0027 & 0.0300 & 0.0019 & 0.2000 & 0.0100 & 0.0050 & 0.3500 & 0.0400 & 0.0010\end{array}$

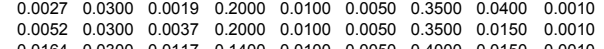

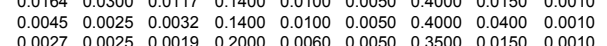
$\begin{array}{lllllllll}0.0027 & 0.0025 & 0.0019 & 0.2000 & 0.0060 & 0.0050 & 0.3500 & 0.0150 & 0.0010 \\ 0.0164 & 0.0300 & 0.0117 & 0.1400 & 0.0060 & 0.0050 & 0.3500 & 0.0150 & 0.0010\end{array}$

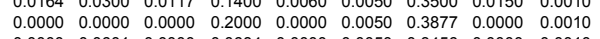

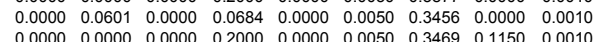
$\begin{array}{lllllllll}0.0000 & 0.0000 & 0.0000 & 0.2000 & 0.0000 & 0.0050 & 0.3469 & 0.1150 & 0.0010 \\ 0.0164 & 0.0000 & 0.0117 & 0.1625 & 0.0000 & 0.0050 & 0.3300 & 0.0000 & 0.0010\end{array}$ $\begin{array}{llllllllll} & 0.0000 & 0.0050 & 0.4224 & 0.0000 & 0.0010\end{array}$

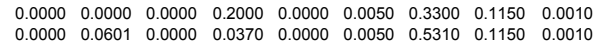

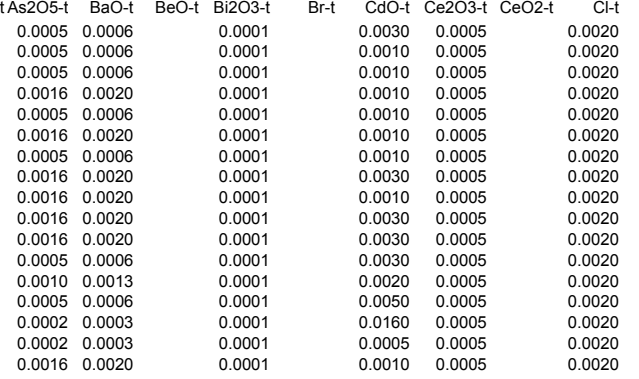


\# Glass ID 2707 HLW03-29
2708 HLW03-30 2700 HLWOS-3-31
2710 HLW03-32

2710 HLWO3-32
2711 HLWO3-33

2712 HLWOS-334
2713 HLWo3-35

2714 HLW03-36

2716 HLWO3-38
2717 HLWO3-39

2718 HLW03-40

2720 HLW03-42
2721 HLW03-43

2722 HLW03-44

2724 HLW04-01

2725 HLW04-02
2726 HLW04-03

2727 HLW04-04

2729 HLW04-07
2730 HLW04-08

2731 HLW04-09

2733 HLW05-02

2735 HLW05-04

2736 HLW05-05
2737 HLW05-06

2738 HLWW5-07

2740 HLW05-09

2742 HLW05-11

2744 HLW05-13

2745 HLW05-14

2747 HLW05-16
2748 HLW05-17

2749 HLW05-18

2750 HLW05-19

2752 HLW05-21

2753 HLW05-22

2755 HLW05-24

2756 HLW05-25
2757 HLW05-26

2758 HLWO5-27

2760 HLW05-29

2761 HLW05-30

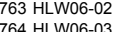

2765 HLW06-04

2767 HLW06-06

2768 HLW06-07

2770 HLW06-09

2771 HLW06-10
2772 HLWO6-11
273 H

2773 HLW06-12
2774 HLW06-13

2775 HLW06-14

2776 HLW06-15
2777 HLW06-16

2779 HLW06-18

2780 HLW06-19

2782 HLW06-21

2783 HLW06-22

2784 HLW06-23
2785 HLW06-24

2786 HLW06-25

2788 HLW06-27
CoO-t Co2O3-t Cr2O3-t Cs2O-t CuO-t Dy203-t Eu2O3-t

$\begin{array}{lll}0.0020 & 0.0001 & 0.0002\end{array}$

0.000
0.000
0.000
0.000
0.000
0.000
0.000
0.000
0.000
0.000
0.000
0.000
0.000
0.000
0.000
0.000
0.000
0.000
0.00

0.0001

$$
\begin{array}{llll}
0.0020 & 0.0001 & 0.0002
\end{array}
$$

$\begin{array}{lll}0.0008 & 0.0001 & 0.0007 \\ 0.0020 & 0.0001 & 0.0002\end{array}$

$\begin{array}{llll}0.0020 & 0.0001 & 0.0007\end{array}$

$\begin{array}{llll}0.0008 & 0.0001 & 0.0002 \\ 0.0020 & 0.0001 & 0.0007 \\ 0.008 & 0.001 & 0.007\end{array}$

$\begin{array}{llll}0.0008 & 0.0001 & 0.0007 \\ 0.0022 & 0.0001 & 0.0007\end{array}$

$\begin{array}{lll}0.0020 & 0.0001 & 0.0007 \\ 0.0008 & 0.0001 & 0.0002\end{array}$

$\begin{array}{llll}0.0008 & 0.0001 & 0.0002 \\ 0.0014 & 0.0001 & 0.0004\end{array}$

$\begin{array}{lll}0.0004 & 0.0001 & 0.0002 \\ 0.0049 & 0.0001 & 0.0001 \\ 0.000 & 0.001 & 0.001\end{array}$

$\begin{array}{llll}0.0002 & 0.0001 & 0.0001 \\ 0.0020 & 0.0001 & 0.007\end{array}$

$\begin{array}{llll}0.0021 & 0.0000 & 0.0003\end{array}$

$\begin{array}{lll}0.0021 & 0.0000 & 0.0003 \\ 0.0022 & 0.0000 & 0.0003 \\ 0.0020 & 0.0000\end{array}$

$\begin{array}{lll}0.0022 & 0.0000 & 0.0003 \\ 0.0022 & 0.0000 & 0.0003 \\ 0.022 & 0.000 & 0.003\end{array}$

$\begin{array}{lrl}0.0022 & 0.0000 & 0.0003 \\ 0.0022 & 0.0003 \\ 0.022 & 0.0003 \\ 0 & 0.022\end{array}$

$\begin{array}{ll}0.0022 & 0.0003 \\ 0.0022 & 0.0003\end{array}$

0.0020

0.0020
0.0010
0.0010

0.0010
0.0020

0.0020
0.0010

0.0010
0.0010
0.0020

0.0020
0.0020

0.0010

0.0025

0.0005

0.0005

0.0025
0.0025

0.0025
0.0005
0.0025

0.0025
0.0025

0.0025
0.0005
0.0005
0.005

0.0005
0.0014
0.0055

0.0050
0.0025

0.0025
0.0025
0.0025

0.0025
0.0050

0.0025
0.0025

0.0025
0.0050

0.0055
0.0025
0.0025

0.0050
0.0025

0.0025
0.0050
0.0025

0.0050
0.0025

0.0055
0.0050
0.0025

0.0000
0.0060
0.0000

0.0060
0.0060
0.0000

0.0060
0.0060

0.0000

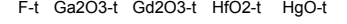
0.0005

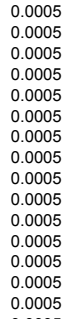

It La2O3-t MnO2-t MnO-t MoO-t MoO2-t MoO3-t Nb205-t Nd2O3-t Np02-t PbO-t PdO2-t PdO-t Pr203-t Pr6011-t Rb2O-t ReO-t

$\begin{array}{ll}0.0030 \\ 0.0030 & 0.0352 \\ 0 & 0.04456\end{array}$
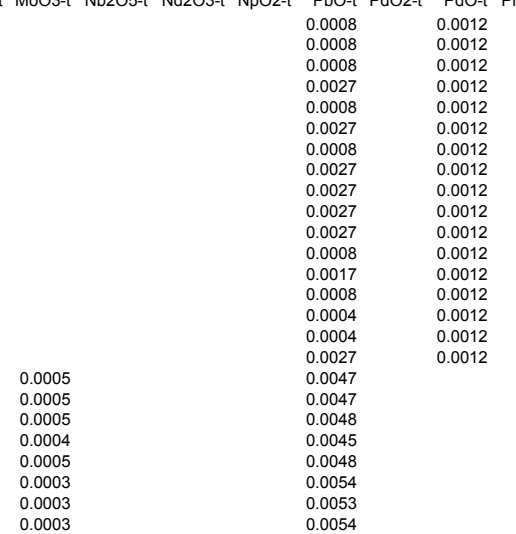

0.0000
0.0000

0.0000
0.0000
0.0000
0.0000
0.0000
0.0000
0.0000
0.0001

0.0005

0.0001

0.0005
0.0005
0.0005
0.0005
0.0005
0.0005
0.0005
0.0005
0.0005
0.0005
0.0005
0.0005
0.0005
0.0005
0.0005
0.0005
0.0005
0.0005
0.0005
0.0005
0.0005
0.0005
0.0005
0.0005
0.0005
0.0005
0.0005
0.0005
0.0005
0.0044
0.0044
0.0044
0.0044
0.0044
0.0044
0.0044
0.0044
0.0044
0.0044
0.0044
0.0044
0.0044
0.0044
0.0044
0.0044
0.0044
0.0044
0.0044
0.0044
0.0044
0.0044
0.0044
0.0044
0.0044
0.0044
0.0044
0

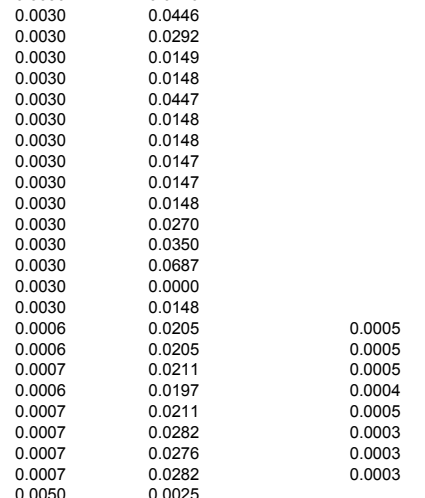

2

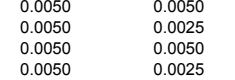

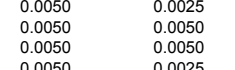

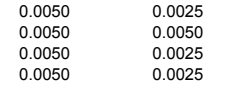

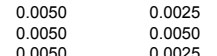

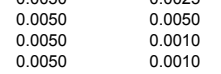

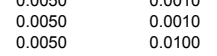

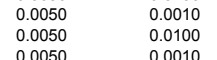

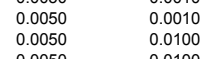

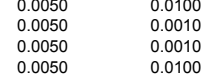

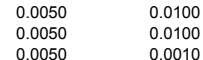

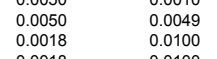

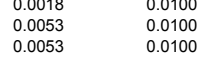

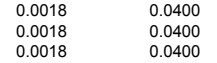

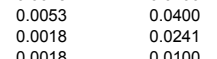

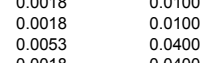

0.0018
0.0018
0.0018
0.0078
0.0000
0.0201

0.0018
0.018
0.0018
0.018

0.01100
0.000
0.0000
0.0018
0.0018
0.0018

0.0018
0.0018
0.023

0.0000
0.0123
0.0000
0.0123

0.0000
0.0123
0.0000

0.0800
0.0000
0.00000
0.0000
0.0000
0.0073
0.0000

\begin{tabular}{|c|c|c|}
\hline & 0.0015 & \\
\hline & 0.0015 & 0.0002 \\
\hline & 0.0015 & 0.0002 \\
\hline & $\begin{array}{l}0.0015 \\
0.0015\end{array}$ & $\begin{array}{l}0.0002 \\
0.0000\end{array}$ \\
\hline & $\begin{array}{l}0.00015 \\
0.0015\end{array}$ & 0.0002 \\
\hline & 0.0015 & \\
\hline & 0.0015 & 0.0002 \\
\hline & 0.0015 & 0.0002 \\
\hline & 0.0015 & 0.0002 \\
\hline & $\begin{array}{l}0.0015 \\
0.0015\end{array}$ & $\begin{array}{l}0.0002 \\
0.0000\end{array}$ \\
\hline & $\begin{array}{l}0.0015 \\
0.0015\end{array}$ & $\begin{array}{l}0.0002 \\
0.0002\end{array}$ \\
\hline & 0.0015 & $\begin{array}{l}0.0002 \\
0.0002\end{array}$ \\
\hline & & 0.00002 \\
\hline & 0.0015 & 0.0002 \\
\hline & 0.0015 & 0.0002 \\
\hline & 0.0015 & 0.0002 \\
\hline & 0.0015 & 0.0002 \\
\hline & 0.0015 & $\begin{array}{l}0.0002 \\
0.002\end{array}$ \\
\hline & $\begin{array}{l}0.0015 \\
0.0015\end{array}$ & $\begin{array}{l}0.0002 \\
0.000\end{array}$ \\
\hline & 0 & $\begin{array}{l}0.00002 \\
0.0002\end{array}$ \\
\hline & $\begin{array}{l}0.0015 \\
0.0\end{array}$ & 0 \\
\hline & 0.0015 & 0.0002 \\
\hline & 0.0015 & 0.0002 \\
\hline & 0.0015 & 0.0002 \\
\hline & & 0.0002 \\
\hline & 0.0015 & $\begin{array}{l}0.0002 \\
0.002\end{array}$ \\
\hline 0.0033 & $\begin{array}{l}0.0091 \\
0\end{array}$ & $\begin{array}{l}0.0012 \\
0.0012\end{array}$ \\
\hline 0.0033 & 0.0091 & 0.0012 \\
\hline 0.0049 & 0.0042 & 0.0012 \\
\hline 0.0049 & $\begin{array}{l}0.0069 \\
0.006\end{array}$ & $\begin{array}{l}0.0012 \\
0.0012\end{array}$ \\
\hline $\begin{array}{l}0.00333 \\
0.03033\end{array}$ & $\begin{array}{l}0.0061 \\
0.0086\end{array}$ & $\begin{array}{l}0.0012 \\
0.0012\end{array}$ \\
\hline 0.00033 & 0.0086 & $\begin{array}{l}0.0012 \\
0.0012\end{array}$ \\
\hline 0.0049 & 0 & 0.0012 \\
\hline 0.0033 & 0.0015 & 0.0012 \\
\hline 0.0033 & 0.0042 & 0.0012 \\
\hline 0.0033 & 0.0050 & 0.0012 \\
\hline 0.0049 & 0.0015 & 0.0012 \\
\hline 0.0033 & 0.0086 & 0.0012 \\
\hline 0.00033 & $\begin{array}{l}0.0091 \\
0.0015\end{array}$ & $\begin{array}{l}0.0012 \\
0.0012\end{array}$ \\
\hline $\begin{array}{l}0.035 \\
0.033\end{array}$ & $\begin{array}{l}0.0025 \\
0\end{array}$ & $\begin{array}{l}0.0012 \\
0.0012\end{array}$ \\
\hline $\begin{array}{l}0.0033 \\
\end{array}$ & 0.0091 & $\begin{array}{l}0.00012 \\
0.0012\end{array}$ \\
\hline 0.0033 & 0.0025 & 0.0012 \\
\hline 0.0033 & 0.0015 & 0.0012 \\
\hline 0.0033 & 0.0091 & 0.0012 \\
\hline & 0.0000 & 0.0012 \\
\hline 0.0080 & 0.00000 & 0.0012 \\
\hline 0.0080 & $\begin{array}{l}0.0000 \\
0.0091\end{array}$ & $\begin{array}{l}0.0012 \\
0.0012\end{array}$ \\
\hline & 0.0091 & 0.0012 \\
\hline 0.0080 & 0.0000 & 0.0012 \\
\hline 0.0025 & 0.0000 & 0.0012 \\
\hline
\end{tabular}




\begin{tabular}{|c|c|c|c|}
\hline 0.0005 & & & 0 \\
\hline & 0.0008 & $\begin{array}{l}0.00005 \\
0.0015\end{array}$ & $\begin{array}{l}0.0005 \\
0.0015\end{array}$ \\
\hline 0005 & 0.0008 & 0.0015 & 0.0015 \\
\hline & 0.0008 & 0.0015 & 0.0005 \\
\hline 0.0005 & 0.0008 & 0.0015 & 0.0005 \\
\hline & 0.0008 & 0.0005 & 0.0015 \\
\hline & 0.0008 & 0.0005 & 0.0005 \\
\hline $\begin{array}{l}.00005 \\
.0005\end{array}$ & $\begin{array}{l}0.0008 \\
0.0008\end{array}$ & $\begin{array}{l}0.00015 \\
0.0005\end{array}$ & $\begin{array}{l}0.00055 \\
0.0015\end{array}$ \\
\hline 0.0005 & 0.0008 & $\begin{array}{l}0.0005 \\
0.0005\end{array}$ & \\
\hline & 0.0008 & 0.0015 & \\
\hline .0005 & 0.0008 & 0.0015 & 0.0015 \\
\hline .0005 & 0.0008 & 0.0010 & 0.0010 \\
\hline .0005 & 0.0008 & 0.0005 & 0.0015 \\
\hline 0.0005 & 0.0008 & 0.0002 & 0.0002 \\
\hline $\begin{array}{l}0.0005 \\
0.0005\end{array}$ & $\begin{array}{l}0.0008 \\
0.0008\end{array}$ & $\begin{array}{l}0.0002 \\
0.0015\end{array}$ & $\begin{array}{l}0.0020 \\
0.0015\end{array}$ \\
\hline & & 0.0000 & \\
\hline & & $\begin{array}{l}0.0000 \\
0.0000\end{array}$ & \\
\hline & & 0.0000 & \\
\hline & & & \\
\hline & & & \\
\hline & & & \\
\hline
\end{tabular}

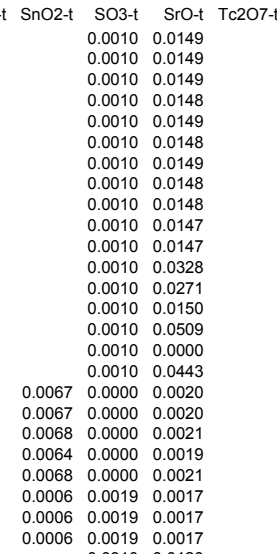

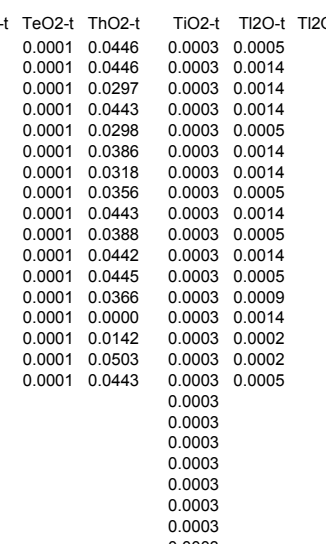

$\begin{array}{lll}0.0010 & 0.0120 \\ 0.0010 & 0.0120 \\ 0.0010 & 0.0120 \\ 0.0010 & 0.02120\end{array}$

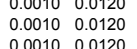

$\begin{array}{ll}0.0010 & 0.0120 \\ 0.0010 & 0.0120\end{array}$

$\begin{array}{ll}0.0010 & 0.0120 \\ 0.0010 & 0.0120 \\ 0.0010 & 0.0120\end{array}$

$\begin{array}{lll}0.0010 & 0.0120 \\ 0.0010 & 0.0120 \\ 0.0010 & 0.012\end{array}$

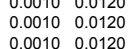

$\begin{array}{ll}0.0010 & 0.0120 \\ 0.0010 & 0.0120 \\ 0.0010 & 0.0120\end{array}$

$\begin{array}{ll}0.0010 & 0.0120 \\ 0.0010 & 0.0120\end{array}$

$\begin{array}{lll}0.0010 & 0.012 \\ 0.0010 & 0.0120\end{array}$

$\begin{array}{ll}0.0010 & 0.0120 \\ 0.00010 & 0.0120 \\ 0.0010 & 0.0120\end{array}$

$\begin{array}{ll}0.0010 & 0.0120 \\ 0.0010 & 0.0120 \\ 0.0120 & 0.120\end{array}$

$0.0010 \quad 0.0120$

$\begin{array}{ll}0.0010 & 0.0120 \\ 0.0010 & 0.0120\end{array}$

$\begin{array}{ll}0.0010 & 0.0120 \\ 0.0010 & 0.0120\end{array}$

$\begin{array}{ll}0.0010 & 0.0120 \\ 0.0004 & 0.0147\end{array}$

0.00040 .0147

$\begin{array}{ll}0.0012 & 0.044 \\ 0.0012 & 0.044 \\ 0.0004 & 0.0147\end{array}$

$\begin{array}{ll}0.0004 & 0.0147 \\ 0.0004 & 0.0147\end{array}$

$\begin{array}{ll}0.0004 & 0.0147 \\ 0.0012 & 0.0441\end{array}$

$\begin{array}{lll}0.0012 & 0.0441 \\ 0.0004 & 0.0147 \\ 0.004 & 0.0147\end{array}$

$0.0004 \quad 0.0147$

$\begin{array}{ll}0.0012 & 0.0441 \\ 0.0004 & 0.0147\end{array}$

$\begin{array}{lll}0.0004 & 0.0147 \\ 0.0004 & 0.0147\end{array}$

$\begin{array}{ll}0.0004 & 0.0147 \\ 0.0004 & 0.0147\end{array}$

$\begin{array}{lll}0.0004 & 0.0147\end{array}$

$\begin{array}{lll}0.0004 & 0.0147 \\ 0.0004 & 0.0147\end{array}$

$\begin{array}{ll}0.0004 & 0.0147 \\ 0.0000 & 0.0000 \\ 0.0028 & 0.1029\end{array}$

$0.0000 \quad 0.0000$

$\begin{array}{ll}0.0028 & 0.1029 \\ 0.0000 & 0.0000\end{array}$

\begin{tabular}{|c|c|c|}
\hline & 0.0000 & \\
\hline 0.0450 & 0.0002 & 0.0005 \\
\hline $\begin{array}{l}0.0300 \\
0.0300\end{array}$ & $\begin{array}{l}0.0002 \\
0.0002\end{array}$ & $\begin{array}{l}0.0005 \\
0.0005\end{array}$ \\
\hline 0.0300 & & 0.0005 \\
\hline 0.0300 & & \\
\hline $\begin{array}{l}0.0450 \\
0.030\end{array}$ & 0.0002 & 0.0005 \\
\hline $\begin{array}{l}0.0300 \\
0.0300\end{array}$ & $\begin{array}{l}0.0002 \\
0.0002\end{array}$ & $\begin{array}{l}0.0005 \\
0.0005\end{array}$ \\
\hline 0.0450 & $\begin{array}{l}0.0002 \\
.000\end{array}$ & 0.0005 \\
\hline 0.0450 & 0.0002 & 0.0005 \\
\hline $\begin{array}{l}0.0300 \\
0.0450\end{array}$ & 0.0002 & 0.0005 \\
\hline $\begin{array}{l}0.0450 \\
0.0300\end{array}$ & $\begin{array}{l}0.0002 \\
0.0002\end{array}$ & $\begin{array}{l}0.00055 \\
0.0005\end{array}$ \\
\hline 0.0300 & 0.0002 & \\
\hline $\begin{array}{l}0.0150 \\
0.0600\end{array}$ & $\begin{array}{l}0.0002 \\
0.0002\end{array}$ & $\begin{array}{l}0.0005 \\
0.000\end{array}$ \\
\hline 0.0150 & 0.0002 & 0.0005 \\
\hline 0.0600 & 0.0002 & 0.0005 \\
\hline 0.0600 & 0.0002 & \\
\hline $\begin{array}{l}0.0150 \\
0.0600\end{array}$ & 0.0002 & 0.0005 \\
\hline $\begin{array}{l}0.0600 \\
0.0150\end{array}$ & $\begin{array}{l}0.0002 \\
0.0002\end{array}$ & $\begin{array}{l}0.0005 \\
0.0005\end{array}$ \\
\hline 0.0600 & 0.0002 & 0.0005 \\
\hline 0.0150 & 0.0002 & 0.0005 \\
\hline $\begin{array}{l}0.0150 \\
0.0150\end{array}$ & $\begin{array}{l}0.0002 \\
0.0002\end{array}$ & $\begin{array}{l}0.0005 \\
0.0005\end{array}$ \\
\hline 0.0150 & 0.0002 & 0.0005 \\
\hline 0.0600 & 0.0002 & 0.0005 \\
\hline $\begin{array}{l}0.0150 \\
0.0320\end{array}$ & $\begin{array}{l}0.0002 \\
0.0002\end{array}$ & $\begin{array}{l}0.0005 \\
0.000\end{array}$ \\
\hline 0.0150 & 0.0100 & \\
\hline 0.0025 & 0.0100 & \\
\hline $\begin{array}{l}0.0025 \\
0.015\end{array}$ & $\begin{array}{l}0.0047 \\
0.0076\end{array}$ & \\
\hline 0.0150 & 0.0067 & \\
\hline 0.0150 & 0.0094 & \\
\hline 0.0025 & 0.0100 & \\
\hline 0.0025 & 0.0061 & \\
\hline $\begin{array}{l}0.0025 \\
0.0025\end{array}$ & $\begin{array}{l}0.0017 \\
0.0047\end{array}$ & \\
\hline 0.0150 & 0.0055 & \\
\hline 0.0150 & 0.0017 & \\
\hline $\begin{array}{l}0.0150 \\
0.0150\end{array}$ & $\begin{array}{l}0.0094 \\
0.0100\end{array}$ & \\
\hline 0.0025 & 0.0017 & \\
\hline & & \\
\hline $\begin{array}{l}0.0150 \\
0.0150\end{array}$ & $\begin{array}{l}0.0100 \\
0.0028\end{array}$ & \\
\hline 0.0025 & 0.0017 & \\
\hline 0.0025 & 0.0100 & \\
\hline 0.0594 & 0.0000 & \\
\hline $\begin{array}{l}0.0000 \\
0.0594\end{array}$ & $\begin{array}{l}0.0000 \\
0.0000\end{array}$ & \\
\hline 0.0594 & 0.0100 & \\
\hline 0.0594 & 0.0100 & \\
\hline 0 & 0.0000 & \\
\hline & & \\
\hline
\end{tabular}

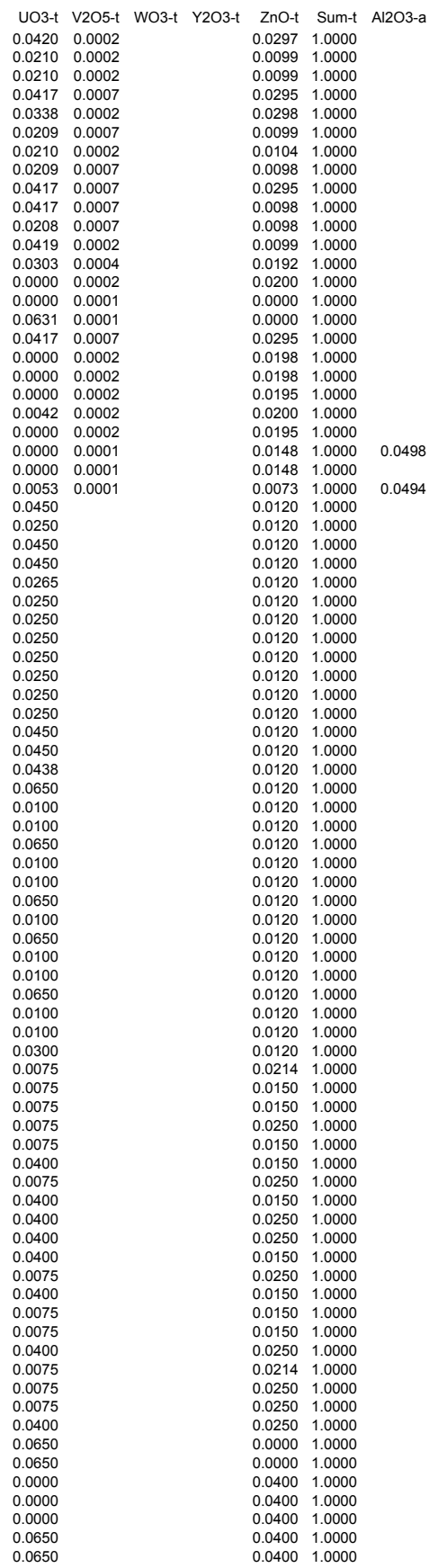


2708 HLW03-30

$2710+L W 03-32$
2711 HLWo3-33

2712 HLW03-34

2713 HLW03-35

2715 HLWO3-37

2716 HLWO3-38
2717 HLW03-39

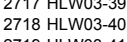

2720 HLWO3-42 272

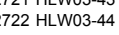

2724 HWos.401

2725 HLW04.02

2726 HLW04-03

2728 HLWW4.06
2729 HLW04-07

2730 H HWW 64.08

2773 HLW04-09

2733 HLW50.-02
2734 HLWOS-03

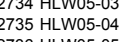

2736 HLWW5-05
2237 HLWW5-06

2738 HLLO55-07
2739 HLW05-08

2779 HLWW5.-08
2740 HLWOS-09

2741 LLWOS-10

2772 HWW5:-11
2743 HLWOS-12

2744 HLW05-13

2745 HLWOS-14

2746 HLW05-15
2747 HLW05-16
2748 HLW05-17

2749 HLWOS-18

2750 HLWO5-5
27519

2751 HLWW5-20
2752 HLWO5-2

2554

2755 HWW05-24

2756 HLW05-25

$2758+4$ W05-27

2759 HLW05-28

25ro

2762 HW06-01

2764 HLW

2766 HUW06

2767 HLW06-00

2270 H

2771 HLW06-10

2772

2774 HLW06-13

2775 HLW06-14

277

2778 HW60-17

27200

2782 HLW06-21

2783 HLW06-22

2784 HLW06-23

2786 HWWO- 25

2787 HLW66-26
2788 HLW06-27 $\begin{array}{lll}0.1059 & 0.0048 & 0.1512\end{array}$

$\begin{array}{lll}0.1041 & 0.0049 \quad 0.1473\end{array}$

$\begin{array}{lllllllll}0.0007 & 0.0225 & 0.0019 & 0.1200 & 0.0047 & 0.0054 & 0.4597 & 0.0052 & 0.0024\end{array}$

$\begin{array}{llllllllll}0.0007 & 0.0222 & 0.0014 & 0.1290 & 0.0045 & 0.0047 & 0.4599 & 0.0051 & 0.0023\end{array}$
$0.0000 \quad 0.0011$

$0.0002 \quad 0.0011$
$0.0024 \quad 0.0000 \quad 0.0005$

0.0026
0.0005 
\# Glass ID

2707 HLW03-29
2708 HLW03-30

2708 HLW03-30

2710 HLWO3-32
2711 HLWOS-33

2712 HLWO3-34

2713 HLW03-35
2714 H WWO3-36

2715 HLWO3-37

2716 HLWO3-38
2717 HLW03-39

2717 HLWON-39
270 -

2720 HLWOO-42
2721 HLWOS-43

2722 HLWO3-44

2724 HLW $04-01$

2725 HLW04-02
2726 HLW04-03

2727 HLW04-04

2728 HLWW4-06
2729 HLW0

2730 HLWO4-.08

2731 HLWW4-09
2732 HLW05-01

2733 HLW05-02
2734 HLWW5-03

$2736 \mathrm{HL}$

2737 HLW05-06

2738 HLW05-07
2739 HLW05-08

2740 HLW05-09

2741 HLWO5-10

2742 HLWOS-11
2743 HWW W5-12

2744 HLWOS-13

2745 HLW05-14

2746 HLWOS-15
277 HLWW5-16

2748 HLW05-17

2749 HLW05-18

2750 HLW05-19
2751 HWW-20

2752 HLW05-2 2

2754 HW05-23

2756 HLW05-25

2758 HW05.52

2759 HLW

2772

2764 HLW

2766 HUW06

2767 HLW06-0.0

2270 H

2771 HLW06-11

2772 HLW0o-11

2774 HLW06-13

2775 HLW06-14

2777

2778 HWW6-17

2782 HLW06-21

2783 HLW06-22

2505

2786 HWWO- 25

2787 HLW66-26
2788 HLW06-27 
$\mathrm{C}_{650^{\circ} \mathrm{C}} \mathrm{C}_{700^{\circ} \mathrm{C}} \mathrm{C}_{750^{\circ} \mathrm{C}}$

1072 Spinel + Zro2

1087 Spinel + Z ZrO2
1109 rriou + ThO2 + Spine

1133 ZrSiO4 + ThO2 2 + Zro2

902 Spinel (ThO2)

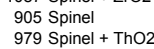

354 Spinel
978 spinel

947 Spinel

Noble Metal
848 Spinel 1 ThO2

$<950$

$<950$

$\begin{array}{llll}0.0009 & 0.0018 & 0.0022\end{array}$

0.0004

$\begin{array}{llll}0.0008 & 0.0015 & 0.0022\end{array}$

0.0004

0.0050

0.0002

0.0153

0.0002

$<950$
$<950$
$<950$
$<92$

$<25$
822 ThO2 + Spinel

757 Spinel
723 spinel
703 Spinel

Spinel
$1064 \mathrm{ZrO2}+$ ThO2

$\begin{aligned} 732 & \text { Spinel } \\ 1065 \text { Zro2 } & \end{aligned}$

698 Spinel
909 ThO2 + Spine

$\begin{aligned} 690 & \text { Spinel } \\ 1069 & \text { ZrO2 + ThO2 }\end{aligned}$

741 Spinel + ThO2

685 Spinel + Na-U-Sr Oxide
Spinel + Noble Metal

$1307 \mathrm{ZrO} 2$

$1240 \mathrm{ZrSiO} 4$

754 Sodium Aluminum silicate

Spinel + Noble
694 Uranium Oxide

$1036 \mathrm{ThO} 2$
$1240 \mathrm{ZrO}+\mathrm{Na} 2 \mathrm{ZrS} 2 \mathrm{O}$

1243 rrSiO4
$1197 \mathrm{Na2ZrSi207}$

$852 \mathrm{Spinel}$
$1207 \mathrm{ZrSiO} 4$

1203 Na2Zrsi2O7

Spine
1022 Spine
(234 Spine

934 Spinel
1212 Spinel + Zro2

$844 \mathrm{Spi}$

896 Spinel
1231 Spinel + ZrO2

1191 Spinel
1045 Spinel

1193 Spinel
1012 Spinel

1126 Spinel
1014 Spinel
116 Splat

1176 Spinel

969 Spinel
934 Spinel

962 Spinel
1033 Spinel

1070 Spinel

1137 Spinel
1127 Th02

650 Unidentified $\mathrm{Cr}-\mathrm{Fe}$ Oxide $1226 \mathrm{ZrO} 2$
$1227 \mathrm{ThO} 2$

1048 Spinel + ThO2

1048 Spinel + ThO2
1481 Na2ZrSi2O + ZrO2
1388 ZrSiO4 
\# Glass ID

2707 HLWO3-29
2708 HLW03-30

2709 HLWO3-31

2710 HLW03-32

2712 HLW03-34
2713 HLW03-35

2714 HLW03-36

2716 HLW03-38

2718 HLW03-40

(279

2721 HLW03-43

2722 HLWO3-44
2723 HLW03-45

2724 HLW04-01

2726 HLW04-03

2728 HLW04-06
2729 HLW04-07

2730 HLW04-08
2731 HLW04-09

2732 HLWW50-01

2733 HLWO5-02
2734 HLW05-03

2736 HLW05-05

2737 HLW05-06

2738 HLW05-07
2739 HLW05-08

2740 HLW05-09

2741 HLW05-10

2743 HLW05-12

2745 HLWO5-14

2747 HLW50-16

2751 HLW05-20

2752 HLW05-21

2753 HLWO5-22

2756 HLW05-25

2758 HLW05-27

2761 HLW05-30

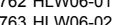

2764 HLW60-03
2765 HLW06-04

2767 HLW06-06

2768 HLW06-07

2770 HLW06-09

2771 HLW06-10

2773 HLW06-12
2774 HLW06-13

2775 HLW06-14

2776 HLW06-15

列

(2781 HLW06- 19

2782 HWW06-21

2783 HLW06-22

2784 HLW06-23

2787 HLW06-26

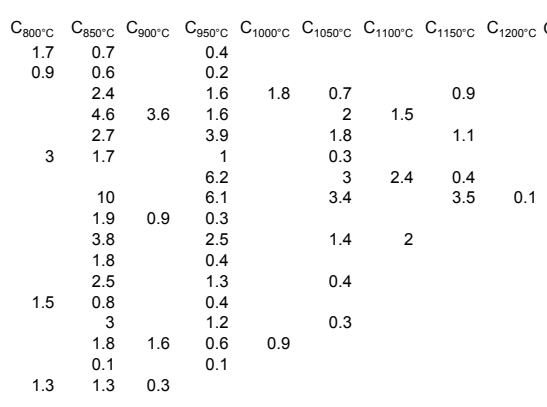

Quenched XRD

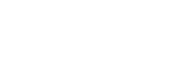

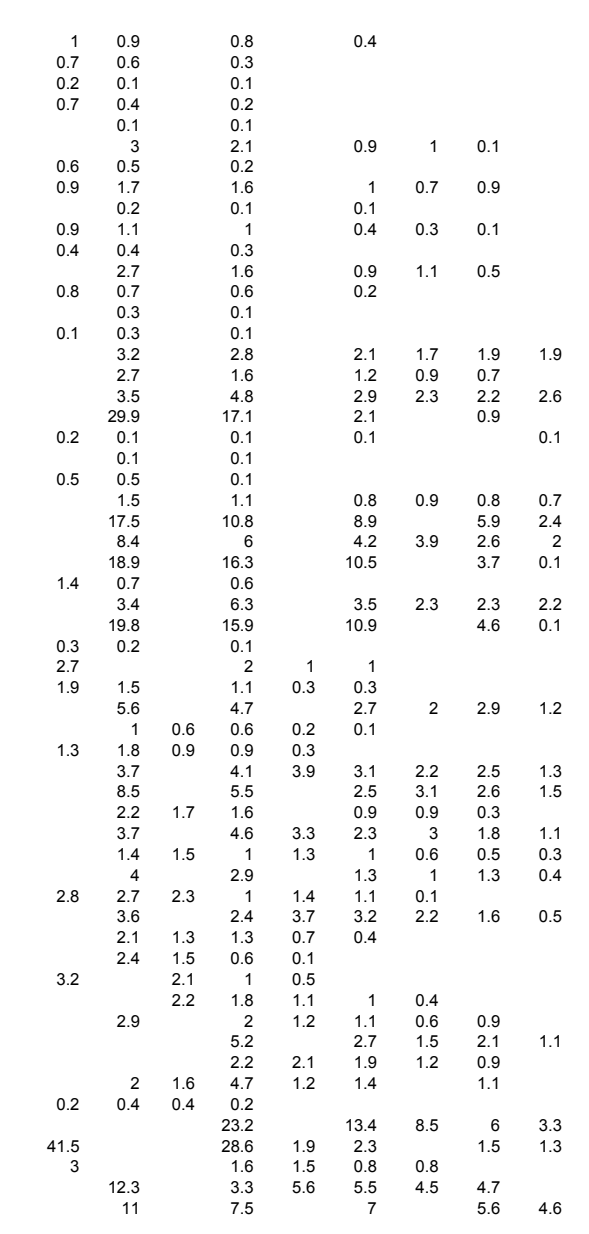

CCC XRD

$\rho\left(g / \mathrm{cm}^{3}\right)$ Fulc Visc A Fulc Visc B Fulc Visc To Arrh Visc E Arrh Visc $F$

\begin{tabular}{|c|c|c|}
\hline 4.282 & 5788.0 & 350.0 \\
\hline & 6748.2 & 300.0 \\
\hline 3.394 & 6104.8 & \\
\hline & $\begin{array}{l}5060.1 \\
6781.4\end{array}$ & $\begin{array}{r}\begin{array}{l}550.0 \\
300.0\end{array}\end{array}$ \\
\hline 4.503 & 8326.5 & 250.0 \\
\hline & 7680.8 & 300.0 \\
\hline 206 & 7986.1 & 300.0 \\
\hline & 6896.2 & 250.0 \\
\hline 4.009 & 6947.2 & 350.0 \\
\hline 4.041 & 7004.5 & 300.0 \\
\hline $\begin{array}{l}3.674 \\
3.8850\end{array}$ & 5897.9 & 350.0 \\
\hline $\begin{array}{l}3.8 .950 \\
.4945\end{array}$ & 5985.4 & 350.0 \\
\hline $\begin{array}{l}.4945 \\
4.603\end{array}$ & $\begin{array}{l}9125.1 \\
8358.1\end{array}$ & $\begin{array}{l}150.0 \\
250.0\end{array}$ \\
\hline .620 & 62453 & $\begin{array}{l}250.0 \\
300.0\end{array}$ \\
\hline .807 & 2860.8 & $\begin{array}{l}50.0 \\
550.0\end{array}$ \\
\hline .909 & 5753.7 & 275.0 \\
\hline 2.648 & 5336.3 & 350.0 \\
\hline
\end{tabular}

$0.1 \%$ of spinel crystals

$\begin{array}{rr}9831.3 & 50.0 \\ 7987.4 & 150.0\end{array}$

$0.1 \%$ of spinel crystals and siver $\quad 2.778$ 
\# Glass ID 2707 HLW03-29
2708 HLW03-30
2709 HLW03-31 2710 HLW03-32
2711 HLW03-33 2712 HLWO3-34

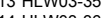
2715 HLW03-37 2716 HLW03-38 2718 HLW03-40 2721 HLW03-43 2722 HLW03-44 2724 HLW04-45 2725 HLW04-02 2726 HLW04-03 2729 HLW04-07 2731 HLW04-09 2732 HLW05-01
2733 HLW05-02 2734 HLW05-03 2736 HLW05-05 2737 HLW05-06 2738 HLW05-07
2739 HLW05-08 2740 HLW05-09 2741 HLW05-10 2742 HLW05-11 2744 HLW05-13 2745 HLW05-14 2746 HLW05-15
2747 HLW05-16 2748 HLW05-17 2749 HLW05-18 2750 HLW05-19 2751 HLW05-20
2752 HLWW5-21 2753 HLW05-22

2754 HLW05-23
2755 HLW05-24

2756 HLW05-25

2757 HLW05-26

2759 HLW05-28

2761 HLW05-30

2762 HLW06-01

2764 HLW06-03

2766 HLW06-05

2767 HLW06-06

2770 HLW06-09

2772 HLWO6-11

2774 HLW06-13

2775 HLW06-14

2777 HLWO-15

2778 HLW06-17

2779 HLW06-18

2781 HLW06-20

2783 HLW06-22

2784 HLW06-23

2786 HLW06-25

2788 HLW06-27

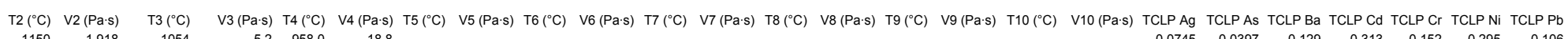

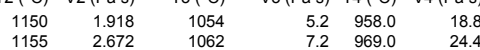

$\begin{array}{rrrrrr}1155 & 2.672 & 1062 & 7.2 & 969.0 & 24.4 \\ 1147 & 5.382 & 1051 & 15.8 & 954.0 & 63.5 \\ 1149 & 3.119 & 1052 & 8.9 & 955.0 & 29.8 \\ 1153 & 3.197 & 1056 & 9.0 & 960.0 & 32.9 \\ 1151 & 11.297 & 1054 & 35.3 & 957.0 & 143.6 \\ 1146 & 17.742 & 1049 & 56.7 & 952.0 & 264.6 \\ 1148 & 18.437 & 1049 & 63.6 & 951.0 & 316.6 \\ 1147 & 2.271 & 1049 & 6.1 & 950.0 & 20.2 \\ 1151 & 10.495 & 1053 & 35.2 & 956.0 & 174.2 \\ 1153 & 6.455 & 1056 & 18.5 & 959.0 & 72.8 \\ 1156 & 3.728 & 1058 & 10.6 & 959.0 & 40.8 \\ 1147 & 3.920 & 1049 & 11.1 & 952.0 & 44.3 \\ 1148 & 6.699 & 1052 & 17.2 & 956.0 & 59.5 \\ 1161 & 9.467 & 1066 & 28.6 & 971.0 & 108.2 \\ 1161 & 10.435 & 1063 & 25.7 & 965.0 & 87.7 \\ 1155 & 1.870 & 1054 & 4.8 & 952.0 & 20.1 \\ 1139 & 4.159 & 1041 & 10.1 & 941.0 & 30.8 \\ 1144 & 5.874 & 1046 & 15.2 & 948.0 & 53.0 \\ 114 & & & & & \\ 1142 & 5.927 & 1045 & 14.5 & 947.0 & 42.1 \\ 1149 & 4.424 & 1050 & 11.0 & 952.0 & 32.2 \\ & & & & & \end{array}$

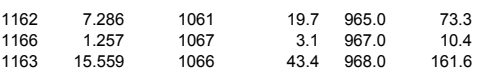

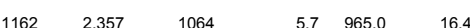

$1163 \quad 2.572 \quad 1066$

$1166 \quad 3079$

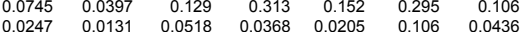

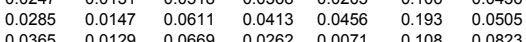
$\begin{array}{lllllll}0.0365 & 0.0129 & 0.0669 & 0.0262 & 0.0071 & 0.108 & 0.0823 \\ 0.0073 & 0.0092 & 0.033 & 0.0197 & 0.0061 & 0.0652 & 0.0264\end{array}$ $\begin{array}{llllllll}0.042 & 0.017 & 0.057 & 0.0173 & 0.0176 & 0.0856 & 0.0573\end{array}$ $\begin{array}{lllllll}0.0068 & 0.0267 & 0.0142 & 0.005 & 0.0592 & 0.0214 \\ 0.0198 & 0.0112 & 0.0483 & 0.043 & 0.0067 & 0.0486 & 0.0791\end{array}$ $\begin{array}{llllllll}0.0163 & 0.0156 & 0.0462 & 0.0368 & 0.0015 & 0.06152 & 0.0402 \\ 0.0253 & 0.0108 & 0.059 & 0.153 & 0.0131 & 0.182 & 0.0505\end{array}$ $\begin{array}{llllllll}0.0138 & 0.0108 & 0.0619 & 0.536 & 0.0771 & 0.296 & 0.0268 \\ 0.017 & & 0.0214 & 0.0067 & & 0.11 & 0.0186\end{array}$ $\begin{array}{llllllll}0.0017 & 0.0175 & 0.0214 & 0.09067 & 0.00674 & 0.0183 & 0.0123 & 0.0186 \\ 0.0423 & 0.0872\end{array}$ $\begin{array}{lllll}0.1 & <0.03 & <0.01 & 0.07 & <0.10\end{array}$

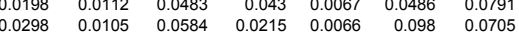
$\begin{array}{lllllll}0.0347 & 0.0198 & 0.0912 & 0.0688 & 0.0176 & 0.0523 & 0.0745 \\ 0.0203 & 0.002 & 0.034 & 0.058 & 0.0045 & 0.064 & 0.021\end{array}$ $\begin{array}{lllllll}0.0063 & 0.0069 & 0.0504 & 0.0357 & 0.0083 & 0.0284 & 0.0446 \\ 0.0343 & 0.0486 & 0.0045 & 0.0679 & 0.021\end{array}$ 
\# Glass ID 2707 HLW03-29 2708 HLW03-30
2709 HLW03-31

2711 HLWOS-32

2712 HLW03-34

2713 HLW03-35

2715 HLW03-37

2716 HLW03-38

2718 HLW03-40

2720 HLW03-42

2721 HLW03-43

2722 HLW03-44
2723 HLW03-45

2724 HLW04-01

2726 HLW04-03

2728 HLW04-06
2729 HLW04-07

2730 HLW04-08
2731 LLW04-09

2731 HLW04-09
2732 HLW05-01

2733 HLW05-02
2734 HLW5-03

2735 HLW05-04

2737 HLW05-06

2738 HLW05-07
2739 HLW05-08

2740 HLW05-09

2741 HLW05-10

2743 HLW05-12

2745 HLW05-14

2747 HLW55-16

2749 HLW05-18

2750 HLW05-19
2751 HLW05-20

2752 HLW05-21

2753 HLWO5-22

2755 HLW05-24

2756 HLW05-25

2757 HLW05-26

2759 HLW05-28

2761 HLWO5-30

2762 HLW06-01

2764 HLW06-03

2765 HLW06-04
2766 HLW06-05

2767 HLW06-06

2768 HLW06-07

2770 HLW06-09

2771 HLW06-10

2772 HLW06-11

2774 HLWO6-13

2776 HLW06-15

2778 HLW06-17

2779 HLW06-18

2780 HLW06-19

2782 HLW06-21

2784 HLW06-23
2785 HLW06-24

2788 HLWO6-27

TCLP Se TCLP Zn TCLP Al TCLP B TCLP Sb TCLP TI TCLP V QPCT B $\left(\mathrm{g} / \mathrm{m}^{2}\right)$ Q PCT Li $\left(g / \mathrm{m}^{2}\right)$ Q PCT Na $\left(\mathrm{g} / \mathrm{m}^{2}\right)$ Q PCT Si $\left(\mathrm{g} / \mathrm{m}^{2}\right) \quad$ QPCT pH

$0.467 \quad 0.485$

$\begin{array}{lrrrrrrrrrr}0.033 & 2.99 & 1.74 & 2.54 & 0.0491 & 0.017 & 0.013 & 0.548 & 0.467 & 0.485 & 11.230 \\ 0.0261 & 0.346 & 0.543 & 1.55 & 0.0389 & 0.017 & 0.033 & 0.460 & 0.366 & 0.245 & 160 \\ 0.0468 & 0.389 & 1.08 & 1.03 & 0.0468 & 0.0308 & 0.0046 & 0.551 & 0.305 & 0.208 & 10.660\end{array}$

$\begin{array}{llllll}0.0161 & 0.704 & 0.322 & 0.62 & 0.0172 & 0.0058 \\ .0137 & 0.545 & 0.253 & 0.805 & 0.0208 & 0.0017 \\ 0.003 & 0.156 & 0.18 & 0.419 & 0.0105 & 0.004\end{array}$

$\begin{array}{lllllll}0.0099 & 0.184 & 0.165 & 0.523 & 0.0103 & 0.0159 & 0.004\end{array}$

$0.0128 \quad 0.154$

$\begin{array}{llllllll}0.0228 & 0.217 & 0.41 & 0.852 & 0.0081 & 0.0102 & 0.00068 \\ 0.0053 & 0.053 & 0.005 & 0.0078\end{array}$

$\begin{array}{llllllll}0.0133 & 0.148 & 0.12 & 0.3 & 0.0075 & 0.0095 & 0.0031\end{array}$

$\begin{array}{lllll}0.0179 & 0.196 & 0.135 & 0.414 & 0.0148 \\ 0.0123 & 0.418 & 0.01 & 0\end{array}$

$\begin{array}{lllllll}0.0207 & 0.524 & 0.378 & 0.78 & 0.0106 & 0.0033 \\ 0 & 0.0207 & 0.0189\end{array}$

$\begin{array}{rrrrrr}0.0207 & 0.524 & 0.378 & 0.78 & & 0.0188 \\ 0.009 & 0.0359 & 1.49 & 0.637 & 0.0105 & 0.0021 \\ 0.0243 & 0.0121 & 0.0554 & 0.259 & 0.0049 & \\ 0.0359 & 1.02 & 0.0267 & 0.731 & 0.019 & \end{array}$

0.0078

0.44

$\begin{array}{ll}0.305 & 0.208 \\ 0.330 & 0.275 \\ 0.295 & 0.173 \\ 0.390 & 0.237 \\ 0.311 & 0.113 \\ 0.332 & 0.120 \\ 0.940 & 0.478 \\ 0.334 & 0.198 \\ 0.325 & 0.148 \\ 0.255 & 0.185 \\ 0.281 & 0.232 \\ 0.236 & 0.171 \\ 0.191 & 0.076 \\ 0.483 & 0.569 \\ 0.377 & 0.388\end{array}$

10.160
10.660
11.140

CCC PCT B $\left(\mathrm{g} / \mathrm{m}^{2}\right) \quad$ CCC PCT Li $\left(g / \mathrm{m}^{2}\right) \quad \operatorname{CCC}$ PCT Na $\left(g / \mathrm{m}^{2}\right) \quad$ CCC PCT Si $\left(\mathrm{g} / \mathrm{m}^{2}\right) \quad$ CCC PCT pH T1 $\left({ }^{\circ} \mathrm{C}\right)$

0.419

0.758

0.29
0.24

$<0.50$
$<0.50$

$\begin{array}{ll}0.343 & 0.397 \\ 0.419 & 0.401 \\ 0.274 & 0.304\end{array}$

0.368
0.361
0.277

10.230
10.160
10.330

\begin{tabular}{|c|c|c|c|}
\hline & 0.254 & 0.330 & \\
\hline 0.987 & 0.483 & 0.714 & \\
\hline 0.160 & & & \\
\hline & 0.397 & 1.173 & \\
\hline 0.386 & 0.268 & 0.520 & 11.330 \\
\hline 0.162 & & & \\
\hline 0.293 & 0.208 & 0.498 & 11.110 \\
\hline 0.350 & 0.268 & 0.451 & 10.710 \\
\hline 0.764 & 0.705 & 0.888 & 11.320 \\
\hline & 0.158 & 0.223 & 9.950 \\
\hline 0.225 & 0.186 & 0.263 & \\
\hline & 0.610 & 0.724 & 10.940 \\
\hline 0.162 & 0.139 & 0.266 & 10.510 \\
\hline 0.709 & 0.588 & 0.555 & 11.020 \\
\hline 1.636 & 0.991 & 1.153 & 11.720 \\
\hline 0.600 & 0.380 & 0.823 & 11.750 \\
\hline 0.232 & 0.265 & 0.351 & 11.220 \\
\hline 0.163 & 0.161 & 0.179 & 9.810 \\
\hline 0.357 & 0.113 & 0.514 & 11.460 \\
\hline 0.334 & 0.330 & 0.427 & 11.330 \\
\hline $\begin{array}{l}0.668 \\
0.1334\end{array}$ & 0.175 & $\begin{array}{l}0.766 \\
0.132\end{array}$ & $\begin{array}{l}10.360 \\
1022\end{array}$ \\
\hline $\begin{array}{l}0.144 \\
1.116\end{array}$ & & $\begin{array}{l}0.921 \\
0.921\end{array}$ & $\begin{array}{l}0.220 \\
11.910\end{array}$ \\
\hline 0.124 & & 0.534 & \\
\hline 0.230 & 0.235 & 0.094 & 9.810 \\
\hline 0752 & & 1.446 & 11.790 \\
\hline 0.218 & 0.273 & 0.025 & 9.950 \\
\hline
\end{tabular}


\# Glass ID 2707 HLW03-29
2708 HLW03-30
2709 HLWO3-31

2709 HLW03-31

2710 HLW03-32
2711 HLW03-33

2712 HLW03-34

2713 HLW03-35

2715 HLWO3-37

2716 HLW03-38

2718 HLW03-40

2720 HLW03-42

2722 HLW03-44

2724 HLW04-01

2725 HLW04-02
2726 HLW04-03

2728 HLW04-06

2729 HLW04-07

2731 HLW04-09

$2732 \mathrm{HLW} 05-1$

2733 HLW55-02

2735 HLWO5-04
2736 HLW05-05

2737 HLW05-06

2738 HLW05-07
2739 HLW05-08

2740 HLW05-09

2741 HLW05-10

2743 HLW05-12

2745 HLW05-14

2749 HLW $05-18$

2751 HLW05-20

2752 HLW05-21

2753 HLWO5-22

2756 HLW05-25

277 HLW05-26

2760 HLW05-29

2762 HLW06-01

2764 HLW06-03

2766 HLW06-0

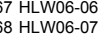

2769 HLW06-08

2771 HLW06-10

2772 HLW06-11

2774 HLW06-13

2775 HLW06-14

2777 HLWO-15

2778 HLW06-17

2779 HLW06-18

2781 HLW06-20

2783 HLW06-22

2784 HLW06-23

2786 HLW06-25

2788 HLW06-27
EC1 $(\mathrm{S} / \mathrm{cm})$ T2 $\left({ }^{\circ} \mathrm{C}\right)$ EC2 $(\mathrm{S} / \mathrm{cm})$ T3 $\left({ }^{\circ} \mathrm{C}\right)$ EC3 $(\mathrm{S} / \mathrm{cm})$ T4 $4\left({ }^{\circ} \mathrm{C}\right)$ EC4 $(\mathrm{S} / \mathrm{cm})$ T5 $\left({ }^{\circ} \mathrm{C}\right)$ EC5 $(\mathrm{S} / \mathrm{cm})$ T6 $\left({ }^{\circ} \mathrm{C}\right)$ EC6 $(\mathrm{s} / \mathrm{cm})$ A

\begin{tabular}{|c|c|c|c|c|c|c|c|c|}
\hline & 1134 & 0.552 & 1037 & 0368 & 941 & & & \\
\hline 0.406 & 1127 & 0.316 & 1024 & 0.0213 & 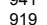 & 0.105 & 0.2803 & -68.77 \\
\hline 0.425 & 1121 & 0.327 & 1022 & 0.194 & 923 & & 1.0609 & -1261.42 \\
\hline 0.523 & 1121 & 0.394 & 1022 & 0.248 & 920 & 0.133 & 1.8211 & $\begin{array}{l}-1995.70 \\
\end{array}$ \\
\hline 0.339 & $\begin{array}{l}1125 \\
1125\end{array}$ & 0.247 & & & 941 & 0.096 & $\begin{array}{r}1.1493 \\
3.0355\end{array}$ & $\begin{array}{l}-1710.99 \\
-551143\end{array}$ \\
\hline & $\begin{array}{l}1129 \\
1133\end{array}$ & $\begin{array}{l}0.199 \\
0.136\end{array}$ & $\begin{array}{l}1039 \\
1039\end{array}$ & $\begin{array}{l}0.128 \\
0.088\end{array}$ & $\begin{array}{l}940 \\
942\end{array}$ & $\begin{array}{r}0.08 \\
0.049\end{array}$ & $\begin{array}{l}3.0355 \\
1.6879\end{array}$ & $\begin{array}{l}-5511.43 \\
-3242.83\end{array}$ \\
\hline $\begin{array}{l}0.189 \\
0.231\end{array}$ & $\begin{array}{l}11133 \\
1132\end{array}$ & $\begin{array}{l}0.136 \\
0.179\end{array}$ & $\begin{array}{l}10399 \\
1034\end{array}$ & $\begin{array}{l}0.088 \\
0.111\end{array}$ & $\begin{array}{l}942 \\
938\end{array}$ & $\begin{array}{l}0.049 \\
0.071\end{array}$ & $\begin{array}{l}1.6879 \\
1.8929\end{array}$ & $\begin{array}{r}-3244.838 \\
-3586.29\end{array}$ \\
\hline 0.443 & 1122 & 0.227 & 1026 & 0.207 & 927 & 0.122 & 2.4577 & -3317.93 \\
\hline 0.368 & 1128 & 0.288 & 1032 & 0.195 & 935 & 0.107 & 0.7047 & -1129.36 \\
\hline 0.302 & 1131 & 0.223 & 1036 & 0.124 & 934 & 0.079 & 4.5156 & -8520.04 \\
\hline $\begin{array}{l}0.405 \\
0.344\end{array}$ & $\begin{array}{l}1132 \\
1120\end{array}$ & $\begin{array}{r}0.31 \\
0.278\end{array}$ & $\begin{array}{l}1031 \\
1019\end{array}$ & $\begin{array}{l}0.198 \\
0.181\end{array}$ & $\begin{array}{l}937 \\
921\end{array}$ & $\begin{array}{l}0.116 \\
0.111\end{array}$ & $\begin{array}{l}1.2355 \\
1\end{array}$ & -1653.05 \\
\hline $\begin{array}{l}0.344 \\
0.261\end{array}$ & $\begin{array}{l}71120 \\
1152\end{array}$ & $\begin{array}{l}0.218 \\
0.182\end{array}$ & $\begin{array}{l}1009 \\
1050\end{array}$ & $\begin{array}{r}0.181 \\
0.12\end{array}$ & $\begin{array}{l}921 \\
953\end{array}$ & $\begin{array}{l}0.111 \\
0.073\end{array}$ & $\begin{array}{l}1.2866 \\
3.3637\end{array}$ & $\begin{array}{l}-1999.291 \\
-6593.01\end{array}$ \\
\hline 0.407 & 1132 & 0.283 & 1038 & 0.185 & 943 & $\begin{array}{l}0.119 \\
0.079\end{array}$ & 4.4150 & -7953.29 \\
\hline 465 & 1133 & 0.413 & 1038 & 0.281 & 941 & 0.17 & 0.3255 & -610.35 \\
\hline & 1137 & 0.474 & 1043 & 0.335 & 945 & 0.191 & 0.8800 & \\
\hline . 48 & 1146 & 0.36 & 1047 & 0.261 & & 0.148 & 0.6179 & -881.45 \\
\hline
\end{tabular}

$\begin{array}{llllll}0.419 & 1139 & 0.338 & 1042 \quad 0.243 \quad 942 & 0.158\end{array}$

$\begin{array}{lllllll}0.504 & 1132 & 0.463 & 1029 & 0.285 & 928 & 0.174 \\ 0.638 & 1137 & 0.533 & 1041 & 0.369 & 943 & 0.225 \\ 0.261 & 1133 & 0.186 & 1037 & 0.134 & 938 & 0.08\end{array}$

$\begin{array}{lllllll}0.712 & 1128 & 0.478 & 1032 & 0.364 & 933 & 0.228\end{array}$

$\begin{array}{lllllll}0.697 & 1136 & 0.499 & 1040 & 0.363 & 939 & 0.244\end{array}$

$\begin{array}{lllllll}0.687 & 1132 & 0.491 & 1033 & 0.385 & 932 & 0.268\end{array}$ 


\begin{tabular}{|c|c|c|}
\hline \# Glass ID & Study & Reference \\
\hline 2789 HLW06-28 & HLW06 Spinel M & Kot et al. 2005 \\
\hline 2790 HLW06-29 & HLW06 Spinel M & Kot et al. 2005 \\
\hline $\begin{array}{l}2791 \text { HLW06-30 } \\
2792 \text { HLW60-31 }\end{array}$ & $\begin{array}{l}\text { HLW06 Spinel M } \\
\text { HLW06 Sninel M }\end{array}$ & $\begin{array}{l}\text { Kot et al. } 2005 \\
\text { Kotetal } 2005\end{array}$ \\
\hline $\begin{array}{l}\text { 2792 HLW606-31 } \\
2793 \text { HLWO6-32 }\end{array}$ & $\begin{array}{l}\text { HLW66 Spinel M } \\
\text { HLW06 Spinel M }\end{array}$ & $\begin{array}{l}\text { Kot et al. } 2005 \\
\text { Kot et al. } 2005\end{array}$ \\
\hline 2794 HLW06-33 & HLW06 Spinel M & Kot et al. 2005 \\
\hline 2795 HLW06-34 & HLW06 Spinel M & \\
\hline 2796 HLW06-35 & HLW06 Spinel M & Kot et al. 2005 \\
\hline 2797 HLW98-66 & HLW98 & Piepel et al. 2008 \\
\hline 2798 HLW98-67 & HLW98 & Piepel et al. 2008 \\
\hline 2799 HLW98-68 & HLW98 & Piepel et al. 2008 \\
\hline 2800 HLW98-69 & $\begin{array}{ll}\text { HLW98 } \\
\text { HLW98 }\end{array}$ & Piepel et al. 2008 \\
\hline $\begin{array}{l}\begin{array}{l}2801 \text { HLW W98-70 } \\
2802 \text { HLW88-71 }\end{array}\end{array}$ & $\begin{array}{l}\text { HLW98 } \\
\text { HLW98 }\end{array}$ & $\begin{array}{l}\text { Piepel et al. } 2008 \\
\text { Piepel et a. } 2008\end{array}$ \\
\hline 2803 HLW98-72 & $\begin{array}{l}\text { HLW98 } \\
\text { HLW98 }\end{array}$ & $\begin{array}{l}\text { Piepelet at. } 2008 \\
\text { Piepel et al. } 2008\end{array}$ \\
\hline 2804 HLW98-73 & $\begin{array}{l}\text { HLW98 } \\
\text { HLW98 }\end{array}$ & $\begin{array}{l}\text { Piepel et al. } 2008 \\
\text { Ping }\end{array}$ \\
\hline 2805 HLW98-74 & $\begin{array}{l}\text { HLW98 } \\
\text { HLW98 }\end{array}$ & Piepel et al. 2008 \\
\hline 2806 HLW98-75 & HLW98 & Piepel et al. 2008 \\
\hline 2807 HLW98-76 & HLW98 & Piepel et al. 2008 \\
\hline 2808 HLW98-78 & HLW98 & Piepel et al. 2008 \\
\hline 2809 HLW98-79 & HLW98 & Piepel et al. 2008 \\
\hline $\begin{array}{l}2810 \text { HLW989-80, HLW98-80R1 } \\
2811 \text { HLW98-80CG }\end{array}$ & $\begin{array}{l}\text { HLW98 } \\
\text { HLW98 }\end{array}$ & $\begin{array}{l}\text { Piepel et al. } 2008 \\
\text { Piepel et a. } 2008\end{array}$ \\
\hline $\begin{array}{l}2811 \text { HLW88-80CG } \\
2812 \text { HLW98-81 }\end{array}$ & $\begin{array}{l}\text { HLW98 } \\
\text { HLW98 }\end{array}$ & $\begin{array}{l}\text { Piepel et al. } 2008 \\
\text { Piepel et al. } 2008\end{array}$ \\
\hline $\begin{array}{l}2813 \text { HLW98-82 } \\
2812\end{array}$ & $\begin{array}{ll}\text { HLW98 } \\
\text { HLW98 }\end{array}$ & $\begin{array}{l}\text { Plepel et al. } 2008 \\
\text { Piepel et al. } 2008\end{array}$ \\
\hline 2814 HLW98-83 & HLW98 & Piepel et al. 2008 \\
\hline 2815 HLW98-84, HLW98-84R1 & HLW98 & Piepel et al. 2008 \\
\hline 2816 HLW98-84CG & HLW98 & Piepel et al. 2008 \\
\hline 2817 HLW98-85 & HLW98 & Piepel et al. 2008 \\
\hline 2818 HLW98-86, HLW98-86R2 & HLW98 & Piepel et al. 2008 \\
\hline $\begin{array}{l}2819 \text { HLW698-86AG } \\
2820 \text { HLW98-87 }\end{array}$ & $\begin{array}{l}\text { HLW98 } \\
\text { HLW98 }\end{array}$ & $\begin{array}{l}\text { Piepel et al. } 2008 \\
\text { Pienele }\end{array}$ \\
\hline $\begin{array}{l}2820 \text { HLWS98-87 } \\
2821 \text { HLW98-88 }\end{array}$ & $\begin{array}{l}\text { HLW98 } \\
\text { HLW98 }\end{array}$ & $\begin{array}{l}\text { Piepel et al. } 2008 \\
\text { Piepel et al. } 2008\end{array}$ \\
\hline $\begin{array}{l}2827 \text { HLW98-88 } \\
2822 \text { HLW88-89 }\end{array}$ & $\begin{array}{l}\text { HLW98 } \\
\text { HLW98 }\end{array}$ & $\begin{array}{l}\text { Prepelet al. } 2008 \\
\text { Piepel et al. } 2008\end{array}$ \\
\hline 2823 HLW98-90 & HLW98 & Piepel et al. 2008 \\
\hline 2824 HLW98-91 & HLW98 & Piepel et al. 2008 \\
\hline 2825 HLW98-92 & HLW98 & Piepel et al. 2008 \\
\hline 2826 HLW98-93 & HLW98 & Piepel et al. 2008 \\
\hline 2827 HLW98-94 & HLW98 & Piepel et al. 2008 \\
\hline $\begin{array}{l}2828 \text { HLLW89-95, HLW98-95R1 } \\
2829 \text { HLW98-95CG }\end{array}$ & $\begin{array}{l}\text { HLW98 } \\
\text { HLW98 }\end{array}$ & $\begin{array}{l}\text { Piepel et al. } 2008 \\
\text { Pieene et al } 2008\end{array}$ \\
\hline 2830 HLW98-96, HLW98-96R1 & $\begin{array}{l}\text { HLW98 } \\
\text { HLW98 }\end{array}$ & $\begin{array}{l}\text { Piepel et al. } 2008 \\
\text { Piepel et al. } 2008\end{array}$ \\
\hline & $\begin{array}{l}\text { HLW98 } \\
\text { HLW98 }\end{array}$ & $\begin{array}{l}\text { Piepelet at. } 2008 \\
\text { Piepel et al. } 2008\end{array}$ \\
\hline 2832 HLW98-96NRE4 & HLW98 & 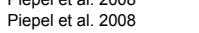 \\
\hline 2833 HLW98-96NRE4CCC & HLW98 & Piepel et al. 2008 \\
\hline 2834 HLW98-96RE7 & HLW98 & Piepel et al. 2008 \\
\hline 2835 HLW98-96RE7CCC & HLW98 & Piepel et al. 2008 \\
\hline 2836 HLW98-96A & HLW98 & Piepel et al. 2008 \\
\hline $\begin{array}{l}2837 \text { HLW988-96B } \\
2838 \text { HLW98-96C }\end{array}$ & $\begin{array}{l}\text { HLW98 } \\
\text { HLW98 }\end{array}$ & Piepel et al. 2008 \\
\hline $\begin{array}{l}2838 \text { HLW98-96C } \\
2839 \text { HLW98-960 }\end{array}$ & $\begin{array}{l}\text { HLW98 } \\
\text { HLW98 }\end{array}$ & $\begin{array}{l}\text { Piepel et al. } 2008 \\
\text { Piepel et al. } 2008\end{array}$ \\
\hline $\begin{array}{l}2839 \text { HLLVG8-96D } \\
2840 \text { HLW98-96DR2 }\end{array}$ & $\begin{array}{l}\text { HLW98 } \\
\text { HLW98 }\end{array}$ & $\begin{array}{l}\text { Piepelet at. } 20008 \\
\text { Piepel et al. } 2008\end{array}$ \\
\hline 8841 HLW98-97 & HLW98 & Piepel et al. 2008 \\
\hline 842 LRM & Round Robin Standard & Ebert and Wolf 1999 \\
\hline 2843 DWPF Startup Frit & Round Robin Standard & Edwards et al. 2009 \\
\hline 2844 ARM-1 & Round Robin Standard & Mellinger and Daniel 1984 \\
\hline 2845 ATM-1a & Round Robin Standard & Wald 1985 \\
\hline $\begin{array}{l}2846 \text { ATM-1b } \\
2847 \text { ATM-1c }\end{array}$ & Round Robin Standard & Wald 1985 \\
\hline $\begin{array}{l}2847 \text { ATM-1c } \\
2848 \text { ATM-11 }\end{array}$ & Round Robin Standard & $\begin{array}{l}\text { Wald } 1985 \\
\text { Waniel } 1986\end{array}$ \\
\hline $\begin{array}{l}\text { 2848 ATM-11 } \\
2849 \text { ARG-1 }\end{array}$ & $\begin{array}{l}\text { Round Robin Standard } \\
\text { Round Robin Standard }\end{array}$ & $\begin{array}{l}\text { Wald and Daniel } 1986 \\
\text { Smith } 1993\end{array}$ \\
\hline $\begin{array}{l}2850 \text { DWPF-EA } \\
2850 \text { - }\end{array}$ & Round Robin Standard & $\begin{array}{l}\text { Simtin } \\
\text { Jantzen et al. } 1993\end{array}$ \\
\hline $2851 \mathrm{Hal}-01$ & Russian High Al & $\begin{array}{l}\text { Riley et al. } 2009 \\
\text { Reg }\end{array}$ \\
\hline $2852 \mathrm{Hal}-02$ & Russian High Al & Riley et al. 2009 \\
\hline $2853 \mathrm{Hal}-03$ & Russian High Al & Riley et al. 2009 \\
\hline $2854 \mathrm{Hal}-04$ & Russian High Al & Riley et al. 2009 \\
\hline $\begin{array}{ll}2855 & 28 \mathrm{Hal}-05 \\
2856 & \end{array}$ & $\begin{array}{l}\text { Russian High Al } \\
\text { Pan }\end{array}$ & Riley et al. 2009 \\
\hline $\begin{array}{l}28567 \mathrm{Hal}-06 \\
2857\end{array}$ & $\begin{array}{l}\text { Russian High AI } \\
\text { Russian High AI }\end{array}$ & $\begin{array}{l}\text { Riley et al. } 2009 \\
\text { Riley to tal } 2009\end{array}$ \\
\hline $\begin{array}{ll}2858 \mathrm{Hal}-08 \\
2858\end{array}$ & $\begin{array}{l}\text { Russsann Hign Al } \\
\text { Russian High Al }\end{array}$ & $\begin{array}{l}\text { Riley et al. } 2009 \\
\text { Riley et al } 2009\end{array}$ \\
\hline $2859 \mathrm{Hal}-09$ & $\begin{array}{l}\text { Russian High Al } \\
\text { Rut }\end{array}$ & Riley et al. 2009 \\
\hline $2860 \mathrm{Hal}-10$ & Russian High Al & Riley et al. 2009 \\
\hline $2861 \mathrm{Hal}-11$ & Russian High Al & Riley et al. 2009 \\
\hline $2862 \mathrm{Hal}-12$ & Russian High Al & Riley et al. 2009 \\
\hline $2863 \mathrm{Hal}-13$ & Russian High Al & Riley et al. 2009 \\
\hline $2864 \mathrm{Hal}-14$ & Russian High Al & Riley et al. 2009 \\
\hline $\begin{array}{l}8865 \mathrm{Hal}-15 \\
8866 \mathrm{Hal}-16\end{array}$ & $\begin{array}{l}\text { Russian High Al } \\
\text { Russiann Hiab Al }\end{array}$ & $\begin{array}{l}\text { Riley et al. } 2009 \\
\text { Riley }\end{array}$ \\
\hline $\begin{array}{l}8866 \mathrm{Hal}-16 \\
8867 \\
\mathrm{Hal}-17\end{array}$ & $\begin{array}{l}\text { Russian High AA } \\
\text { Russian High Al }\end{array}$ & 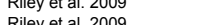 \\
\hline $2868 \mathrm{Hal}-18$ & $\begin{array}{l}\text { Russian High Al } \\
\text { Rugh }\end{array}$ & Riley et al. 2009 \\
\hline KRI-01 & Russian High Alur & $\begin{array}{l}\text { Fox et al. } 20008 \\
\text { Fox }\end{array}$ \\
\hline KRI-02 ( & Russian High Alumir & Fox et al. 2008 \\
\hline
\end{tabular}

Al2O3-t B2O3-t CaO-t Fe2O3-t FeO-t $\begin{array}{llll}0.0188 & 0.1500 & 0.0100 & 0.1148 \\ 0.0188 & 0.0430 & 0.0100 & 0.1500\end{array}$ $\begin{array}{llll}0.1300 & 0.0430 & 0.00000 & 0.15500 \\ 0.1500\end{array}$ $\begin{array}{llll}0.1300 & 0.0430 & 0.0000 & 0.1500 \\ 0.0188 & 0.1500 & 0.0000 & 0.1500\end{array}$ $\begin{array}{llll}0.0188 & 0.1500 & 0.0000 & 0.0140\end{array}$

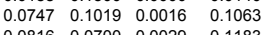
$\begin{array}{llll}0.0816 & 0.0700 & 0.0029 & 0.1183 \\ 0.0700 & 0.0450 & 0.0069 & 0.1257\end{array}$ $\begin{array}{llll}0.0756 & 0.0458 & 0.00031 & 0.1285 \\ 0.0766 & 0.0700 & 0.0037 & 0.1136\end{array}$ $\begin{array}{lllll}0.0766 & 0.0700 & 0.0037 & 0.1136 \\ 0.0256 & 0.0898 & 0.0049 & 0.0505 \\ 0.051 & 0.089 & 0.0028 & 0.129\end{array}$ $\begin{array}{lllll}0.0256 & 0.0898 & 0.0049 & 0.0505 \\ 0.0270 & 0.0798 & 0.0051 & 0.0533\end{array}$

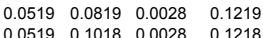
$\begin{array}{llll}0.0519 & 0.1018 & 0.0028 & 0.1218 \\ 0.0550 & 0.0819 & 0.0029 & 0.1290\end{array}$ $\begin{array}{llll}0.0550 & 0.0669 & 0.0029 & 0.1290 \\ 0.052 & 0.0028 & 0\end{array}$ $\begin{array}{llll}0.0519 & 0.0818 & 0.028 & 0.1218 \\ 0.0520 & 0.1041 & 0.0028 & 0.1222\end{array}$ $\begin{array}{llll}0.0550 & 0.1166 & 0.0030 & 0.1225 \\ 0.0559 & 0.1253 & 0.023 & 0.125\end{array}$ $\begin{array}{llll}0.0559 & 0.1253 & 0.0023 & 0.1253 \\ 0.0588 & 0.1205 & 0.024 & 0.1317\end{array}$ $\begin{array}{llll}0.0588 & 0.1205 & 0.0024 & 0.1317 \\ 0.0588 & 0.1180 & 0.0024 & 0.1317\end{array}$ $\begin{array}{llll}0.0588 & 0.1180 & 0.0024 & 0.1317 \\ 0.0143 & 0.1191 & 0.0067 & 0.1275\end{array}$ $\begin{array}{llll}0.0518 & 0.1116 & 0.0067 & 0.1275 \\ 0.0518 & 0.1116 & 0.0067 & 0.1275\end{array}$ $\begin{array}{llll}0.0504 & 0.0914 & 0.0030 & 0.1256 \\ 0.0529 & 0.0939 & 0.03030 & 0.1256\end{array}$ $\begin{array}{llll}0.0530 & 0.0941 & 0.0030 & 0.1258 \\ 0.0769 & 0.1011 & 0.0044 & 0.1177\end{array}$ $\begin{array}{llll}0.0769 & 0.1011 & 0.0044 & 0.1177 \\ 0.0745 & 0.1060 & 0.0043 & 0.1141\end{array}$

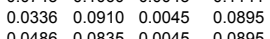

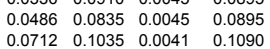
$\begin{array}{llll}0.0712 & 0.1035 & 0.0041 & 0.1090 \\ 0.0334 & 0.0760 & 0.0045 & 0.0888\end{array}$ $\begin{array}{llll}0.0334 & 0.0510 & 0.0045 & 0.0888\end{array}$ $\begin{array}{llll}0.0938 & 0.1151 & 0.0024 & 0.1052 \\ 0.0732 & 0.1063 & 0.0042 & 0.1121\end{array}$ $\begin{array}{llll}0.0336 & 0.1010 & 0.0045 & 0.0895\end{array}$

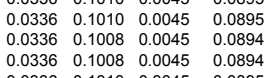
$\begin{array}{llll}0.0336 & 0.1010 & 0.0045 & 0.0895 \\ 0.0336 & 0.1010 & 0.0045 & 0.0895 \\ 0.0345 & 0.106 & 0.0046 & 0.098\end{array}$ $\begin{array}{llll}0.0336 & 0.1010 & 0.0045 & 0.0895 \\ 0.0345 & 0.1036 & 0.0046 & 0.0918\end{array}$ $\begin{array}{llll}0.0345 & 0.1036 & 0.0046 & 0.0918 \\ 0.0342 & 0.1029 & 0.0046 & 0.0912\end{array}$ $\begin{array}{llll}0.0336 & 0.1011 & 0.0045 & 0.0896 \\ 0.0360 & 0.1081 & 0.0048 & 0.0958 \\ 0.0360 & 0.101 & 0.045 & 0.058\end{array}$

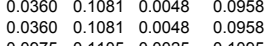
$\begin{array}{llll}0.0360 & 0.1081 & 0.0048 & 0.0958 \\ 0.0975 & 0.1105 & 0.0025 & 0.1095 \\ 0.0470 & 0.0800 & 0.0120 & 0.105\end{array}$ $\begin{array}{llll}0.1000 & 0.0800 & 0.0050 & 0.0100 \\ 0.0470 & 0.0880 & 0.0120 & 0.1350\end{array}$ $\begin{array}{llll}0.0057 & 0.0881 & 0.0230 & 0.0929\end{array}$ $\begin{array}{llll}0.0084 & 0.0860 & 0.0228 & 0.0903 \\ 0.0049 & 0.0917 & 0.0230 & 0.0895\end{array}$ $\begin{array}{llll}0.0432 & 0.0672 & 0.0163 & 0.1149 \\ 0.0470 & 0.0880 & 0.0120 & 0.1350\end{array}$ $\begin{array}{lllll}0.0367 & 0.1112 & 0.0113 & 0.0808 \\ 0.2663 & 0.2021 & 0.0320 & 0.0655\end{array}$ $\begin{array}{llll}0.2663 & 0.2521 & 0.0120 & 0.0655 \\ 0.2663 & 0.2021 & 0.0370 & 0.0655 \\ 0.2563 & 0.2221 & 0.520 & 0.555\end{array}$ $\begin{array}{llll}0.2663 & 0.2521 & 0.0520 & 0.0655 \\ 0.2557 & 0.1580 & 0.0323 & 0.0629\end{array}$ $\begin{array}{llll}0.2397 & 0.1519 & 0.0608 & 0.0590 \\ 0.2500 & 0.1467 & 0.0595 & 0.0615\end{array}$ $\begin{array}{llll}0.2500 & 0.1500 & 0.0600 & 0.0615 \\ 0.2500 & 0.1500 & 0.1000 & 0.0615 \\ 0.02500 & 0.150 & 0.060 & 0.0615\end{array}$ $\begin{array}{llll}0.2500 & 0.1500 & 0.0600 & 0.0615 \\ 0.2500 & 0.1500 & 0.0600 & 0.0615 \\ 0.2501 & 0.180 & 0.600 & 0.065\end{array}$

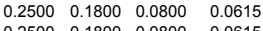
$\begin{array}{llll}0.2664 & 0.1800 & 0.01400 & 0.06615 \\ 0.06 & 0.060\end{array}$ $\begin{array}{llll}0.2664 & 0.1800 & 0.1400 & 0.0615 \\ 0.2263 & 0.1521 & 0.0650 & 0.0655 \\ 0.2589 & 0.1614 & 0.0733 & 0.0637\end{array}$ $\begin{array}{llll}0.2589 & 0.1614 & 0.0733 & 0.0637 \\ 0.2589 & 0.1614 & 0.0733 & 0.0377 \\ 0.159 & 0.1205 & 0.300\end{array}$ $\begin{array}{llll}0.1519 & 0.0956 & 0.0000 & 0.0975 \\ 0.1447 & 0.1440 & 0.0070 & 0.0823\end{array}$
K2O-t Li2O-t MgO-t Na2O-t NiO-t P2O5-t SiO2-t Zro2-t Ag2O-t As2O3-tAs2O5-t BaO-t BeO-t Bi2O3-t

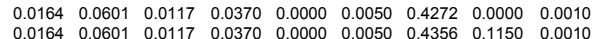
$\begin{array}{llllllllll}0.0000 & 0.0000 & 0.0000 & 0.2000 & 0.0000 & 0.0050 & 0.3511 & 0.0000 & 0.0010\end{array}$

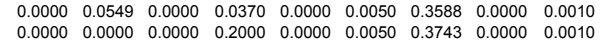
$\begin{array}{llllllllllll}0.0164 & 0.0473 & 0.0117 & 0.0370 & 0.0000 & 0.0050 & 0.3300 & 0.0878 & 0.0010\end{array}$

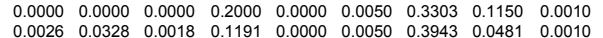

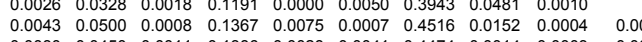

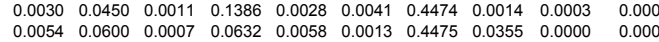

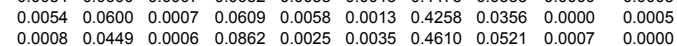

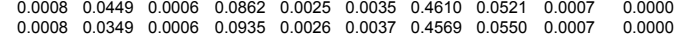
$\begin{array}{llllllllll}0.0006 & 0.0553 & 0.0011 & 0.1345 & 0.0062 & 0.0000 & 0.4798 & 0.0379 & 0.0002 & 0.0000\end{array}$

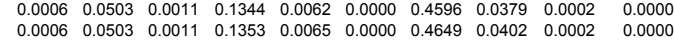
$\begin{array}{llllllllll}0.0006 & 0.0503 & 0.0011 & 0.1353 & 0.0065 & 0.0000 & 0.4649 & 0.0402 & 0.0002 & 0.0000 \\ 0.0006 & 0.0503 & 0.0011 & 0.1353 & 0.0065 & 0.0000 & 0.4799 & 0.0402 & 0.0002 & 0.0000\end{array}$

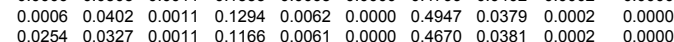

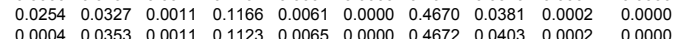

$\begin{array}{lllllllll}0.0003 & 0.0326 & 0.0007 & 0.1203 & 0.0045 & 0.0002 & 0.4831 & 0.0177 & 0.0003\end{array}$

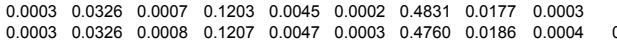
$\begin{array}{llllllllll}0.0003 & 0.0326 & 0.0008 & 0.1207 & 0.0047 & 0.0003 & 0.4760 & 0.0186 & 0.0004 & 0.0000 \\ 0.0003 & 0.0276 & 0.0008 & 0.1332 & 0.0047 & 0.0003 & 0.4710 & 0.0186 & 0.0004 & 0.0000\end{array}$ $\begin{array}{lllllllll}0.0000 & 0.0276 & 0.0056 & 0.1174 & 0.0037 & 0.0010 & 0.5094 & 0.0013 & 0.0015 \\ 0.0000 & 0.0276 & 0.0056 & 0.1199 & 0.0037 & 0.0010 & 0.4768 & 0.0013 & 0.0015\end{array}$

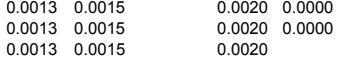
$0.4782 \quad 0.0026 \quad 0.0015 \quad 0.0019$ $\begin{array}{lllllllllllll}00000 & 0.0301 & 0.0117 & 0.1186 & 0.0017 & 0.0009 & 0.4714 & 0.0026 & 0.0000 & 0.0019 & 0.0000 & 0.000 & 0.0000\end{array}$

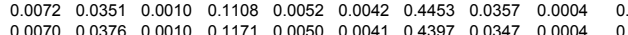

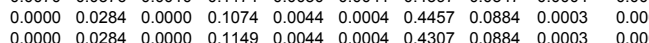
$\begin{array}{llllllllll}0.0000 & 0.0284 & 0.00000 & 0.1149 & 0.0044 & 0.00004 & 0.44307 & 0.00884 & 0.0003 & 0.0000 \\ 0.0066 & 0.0401 & 0.0010 & 0.1179 & 0.0048 & 0.0039 & 0.4516 & 0.0331 & 0.0004 & 0.0000\end{array}$

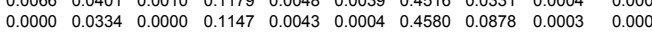
$\begin{array}{llllllllll}0.0000 & 0.0409 & 0.0000 & 0.1172 & 0.0043 & 0.0004 & 0.4755 & 0.0878 & 0.0003 & 0.0000\end{array}$ $\begin{array}{llllllllll}0.0041 & 0.0387 & 0.0009 & 0.1185 & 0.0060 & 0.0000 & 0.4395 & 0.0324 & 0.0000 & 0.0000 \\ 0.0014 & 0.0376 & 0.0010 & 0.1192 & 0.0049 & 0.0040 & 0.4469 & 0.0340 & 0.0004 & 0.0000\end{array}$

\section{$\begin{array}{lllllll}0.0309 & 0.1074 & 0.00044 & 0.0004 & 0.4332 & 0.0884 & 0.0003 \\ 0.0309 & 0.1074 & 0.0044 & 0.0004 & 0.4332 & 0.0884 & 0.0003\end{array}$}

$\begin{array}{lllllll}0.0309 & 0.1074 & 0.0044 & 0.0004 & 0.4332 & 0.0884 & 0.0003 \\ 0.0309 & 0.1072 & 0.0044 & 0.0004 & 0.4327 & 0.0883 & 0.0013\end{array}$

$\begin{array}{lllllll}0.0309 & 0.1074 & 0.0044 & 0.0004 & 0.4332 & 0.08884 & 0.00003 \\ 0.0309 & 0.1074 & 0.0044 & 0.0004 & 0.4332 & 0.0884 & 0.0003\end{array}$

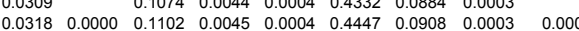

$\begin{array}{lllllllllll}0.0315 & 0.0000 & 0.1094 & 0.0045 & 0.0004 & 0.4415 & 0.0901 & 0.0003 & 0.00000\end{array}$

$\begin{array}{lllllllll}0.0310 & 0.0000 & 0.1075 & 0.0044 & 0.0004 & 0.4338 & 0.0886 & 0.0003 & 0.0000 \\ 0.0331 & 0.0000 & 0.1150 & 0.0047 & 0.0004 & 0.4639 & 0.0947 & 0.0003 & 0.0000\end{array}$

$\begin{array}{llllllllll} & 0.0331 & 0.1150 & 0.0047 & 0.0004 & 0.4639 & 0.0947 & 0.0003 & \\ 0.0043 & 0.0388 & 0.0009 & 0.1218 & 0.0062 & 0.0000 & 0.4300 & 0.0337 & 0.0000 & 0.0000\end{array}$ $\begin{array}{llllllll}0.0150 & 0.0010 & 0.0010 & 0.2000 & 0.0010 & 0.0050 & 0.5500 & 0.0100 \\ 0.0260 & 0.0350 & 0.0070 & 0.1150 & 0.0110 & 0.4900 & \end{array}$

$\begin{array}{llllllll}<0.0006 & 0.0016 & 0.1242 & 0.0022 & 0.0078 & 0.4083 & 0.0184 \\ 0.0009 & & 0.0018 & 0.1153 & 0.0025 & 0.0056 & 0.4011 & 0.0232\end{array}$

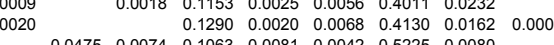
$\begin{array}{llllllll}0.0475 & 0.0074 & 0.1063 & 0.0001 & 0.0042 & 0.5225 & 0.0162 & \end{array}$

$\begin{array}{ll}0.0030 & 0.0030 \\ 0.0030 & 0.0030 \\ 0.0000 & 0.0030 \\ 0.0000 & 0.0030 \\ 0.0000 & 0.0030 \\ 0.0030 & 0.0030 \\ 0.0000 & 0.0030 \\ 0.0005 & 0.0030 \\ 0.0003 & 0.0000 \\ 0.0004 & 0.0000 \\ 0.0005 & 0.0000 \\ 0.0005 & 0.0000 \\ 0.0002 & 0.0000 \\ 0.0002 & 0.0000 \\ 0.0000 & 0.0000 \\ 0.0000 & 0.0000 \\ 0.0000 & 0.0000 \\ 0.0000 & 0.0000 \\ 0.0000 & 0.0000 \\ 0.0000 & 0.0000 \\ 0.0000 & 0\end{array}$

CdO-t Ce2O3-t CeO2-t 0.0000
0.0000
0.000

0.0000
0.0000

0.0165
0.0000
0.0165

0.0000

0.0021

$\begin{array}{ll}0.0102 & 0.0005 \\ 0.0001 & 0.0000 \\ 0.0037 & 0.000\end{array}$

$\begin{array}{lr}0.00037 & 0.00009 \\ 0.00037 & 0.0010 \\ 0.0007 & 0.0009\end{array}$

$\begin{array}{ll}0.0037 & 0.0010 \\ 0.0007 & 0.0009\end{array}$

$\begin{array}{ll}0.0007 & 0.0009 \\ 0.0006 & 0.0000 \\ 0\end{array}$

$\begin{array}{ll}0.0006 & 0.0000 \\ 0.0007 & 0.0000 \\ 0.0006 & 0.0000\end{array}$

$\begin{array}{ll}0.0007 & 0.0000 \\ 0.0007 & 0.0000 \\ 0.0006 & 0.0000\end{array}$

$\begin{array}{ll}0.00006 & 0.0000 \\ 0.0006 & 0.0000 \\ 0.0007 & 0.000\end{array}$

$\begin{array}{ll}0.0006 & 0.0000 \\ 0.0007 & 0.0000 \\ 0.011 & 0.000\end{array}$

$0.0011 \quad 0.0000$

0.0000

0.0011
0.0012

0.0000
0.0000
0.0000

0.0000

0.0000
0.0000
0.0000

0.0000

0.0000
0.0000
0.0000

0.0000

0.0000
0.0000

0.0000
0.0000
0.0000

$\begin{array}{ll}0.0012 & 0.0000 \\ 0.0000 & 0.0000 \\ 0.0000 & 0.000\end{array}$

$0.0000 \quad 0.0000$

$\begin{array}{ll}0.0000 & 0.0000 \\ 0.0000 & 0.0000 \\ 0.0005 & 0.025\end{array}$

$\begin{array}{ll}0.0067 & 0.0025 \\ 0.0065 & 0.0024\end{array}$

$\begin{array}{ll}0.0000 & 0.0000 \\ 0.0000 & 0.0000 \\ 0.0000 & 0.0000\end{array}$

$0.0062 \quad 0.0023$

$0.0000 \quad 0.0000$

$0.0064 \quad 0.0024$

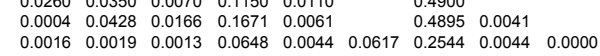

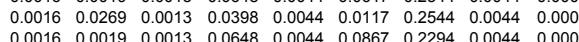
$\begin{array}{lllllllll}0.0016 & 0.0019 & 0.0013 & 0.0648 & 0.0004 & 0.0117 & 0.22294 & 0.0044 & 0.00000\end{array}$ $\begin{array}{lllllllll}0.0171 & 0.0018 & 0.0012 & 0.0382 & 0.0043 & 0.1152 & 0.262 & 0.0042 & 0.00000 \\ 0.0014 & 0.0357 & 0.0012 & 0.0958 & 0.0040 & 0.0105 & 0.3050 & 0.0039 & 0.0000\end{array}$ $\begin{array}{lllllllll}0.0015 & 0.0346 & 0.0012 & 0.0952 & 0.0042 & 0.0110 & 0.2981 & 0.0041 & 0.0000 \\ 0.0015 & 0.0350 & 0.0012 & 0.0950 & 0.0042 & 0.0110 & 0.2941 & 0.0041 & 0.0000\end{array}$

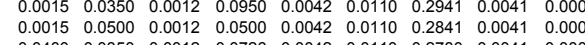

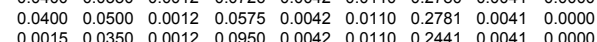
$\begin{array}{lllllllll}0.0015 & 0.0350 & 0.0012 & 0.0950 & 0.0042 & 0.0110 & 0.2441 & 0.0041 & 0.0000 \\ 0.0015 & 0.0350 & 0.0012 & 0.0650 & 0.0042 & 0.0110 & 0.2741 & 0.0041 & 0.0000\end{array}$ $\begin{array}{lllllllll}0.0370 & 0.0000 & 0.0012 & 0.0500 & 0.0042 & 0.0110 & 0.2121 & 0.0041 & 0.0000 \\ 0.0016 & 0.0404 & 0.0013 & 0.0898 & 0.0044 & 0.0117 & 0.2629 & 0.0044 & 0.0000\end{array}$ $\begin{array}{llllllll}0.0272 & 0.0275 & 0.0013 & 0.0875 & 0.0043 & 0.0114 & 0.2456 & 0.0043\end{array}$

$\begin{array}{lllllllllll}0.0272 & 0.0400 & 0.0013 & 0.0607 & 0.0043 & 0.014 & 0.2456 & 0.0043 \\ 0.0272 & 0.0275 & 0.0013 & 0.0666 & 0.0043 & 0.0114 & 0.2412 & 0.0043\end{array}$

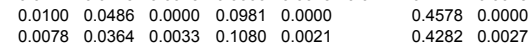

$\begin{array}{lllll}0.0000 & 0.0000 & 0.0000 & 0.0259 & 0.0000 \\ 0.0000 & 0.0000 & 0.0000 & 0.0000 & 0.0000 \\ 0.0000 & 0.0000 & 0.0000 & 0.00000 & 0.00000 \\ 0.0000 & 0.0000 & 0.0000 & 0.0000 & 0.0000 \\ 0.0007 & 0.0000 & 0.0068 & 0.0041 & 0.0000 \\ 0.0001 & & 0.0020 & & \\ & & & & \\ 0.0053 & & 0.0005 & & 0.0089 \\ 0.0062 & & 0.0005 & & 0.0100 \\ 0.0053 & & 0.0003 & 0.0100 \\ 0.0015 & & & & 0.0009\end{array}$

$\begin{array}{llll}0.0006 & 0.0127 & 0.0003 & 0.0000 \\ 0.0006 & 0.0127 & 0.0003 & 0.0000 \\ 0.0006 & 0.0127 & 0.0003 & 0.0000 \\ 0.0006 & 0.0127 & 0.0003 & 0.0000 \\ 0.0006 & 0.0122 & 0.0003 & 0.0000 \\ 0.0005 & 0.0114 & 0.0002 & 0.00000 \\ 0.0006 & 0.0119 & 0.0003 & 0.0000 \\ 0.0006 & 0.0119 & 0.0003 & 0.0000 \\ 0.0006 & 0.0119 & 0.0003 & 0.0000 \\ 0.0006 & 0.0119 & 0.0003 & 0.0000 \\ 0.0006 & 0.0119 & 0.0003 & 0.00000 \\ 0.0006 & 0.0119 & 0.0003 & 0.0000 \\ 0.0006 & 0.0119 & 0.0003 & 0.0000 \\ 0.0006 & 0.0119 & 0.0003 & 0.0000 \\ 0.0006 & 0.0127 & 0.0003 & 0.0000 \\ 0.0006 & 0.0124 & 0.0003 & \\ 0.0006 & 0.0124 & 0.0003 & \\ 0.0006 & 0.0124 & 0.0003 & \end{array}$


\# Glass ID
2789 HLW06-28
2790 HLW06-29

2791 HLW06-30

2792 HLW06-31
2793 HLW6-32

2794 HLW06-33
2795 HLW06-34

2796 HLW06-35
2797 HLW98-66

2798 HLW98-67
2799 HLW98-68

2800 HLW $988-69$

2801 HLW989-70

2803 HLW98-72

2804 HLWW98-73
2805 HLW $98-74$

2806 HLW98-75

2808 HLW W98-78

2810 HLW98-80, HLW98-80R

2811 HLW98-80,

2813 HLW98-82

2815 HLW98-84, HLW98-84R1

2816 HLW98-84CG
2817 HLW98-85

2818 HLW98-86, HLW98-86R2

2819 HLW98-86AG
2820 HLW98-87

2821 HLW98-88

2822 HLW W88-89
2823 HLW98-90

2824 HLW98-91

2826 HLW98-93

2827 HLWW89-94 2828 HLW98- HLW8-95R1

2830 HLW98-96, HLW98-96R

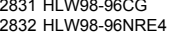

2833 HLW98-96NRE4CCC

2834 HLW98-96RE7
2835 HLW98-96RE7CCC

2836 HLW98-96A

2837 HLW98-96B
2838 HLW98-96C

2839 HLW98-96D

2841 HLW98-

2842 LRM 28 PWF Startup Frit

2844 ARM- 1

2846 ATM- -16
2847 ATM-1C

2848 ATM-11

2850 DWPF-EA

$2851 \mathrm{Hal}-01$

2853 Hal-03

$2854 \mathrm{Hal}-04$

$2856 \mathrm{Hal}-00$

$2858 \mathrm{Hal}-0 \mathrm{O}$

(2860 Hal-10

$2861 \mathrm{Hal}-1$

2863 Hal-1

2865 Hal-15

$2867 \mathrm{Hal}-17$

2869 KRI-01 (repl. US-08)

2869 KRR-01 (repl. US-08)
CoO-t Co2O3-t Cr2O3-t Cs2O-t CuO-t Dy203-t Eu2O3-t 0.0060 0.0044

\begin{tabular}{|c|c|c|c|c|}
\hline & 0.0000 & & & \\
\hline & $\begin{array}{l}0.00060 \\
0.0060\end{array}$ & & & \\
\hline & $\begin{array}{l}0.0060 \\
0.0000\end{array}$ & & & \\
\hline & $\begin{array}{l}0.0000 \\
0.00660\end{array}$ & & & \\
\hline & & & & \\
\hline 0.00000 & $\begin{array}{l}0.0011 \\
0.0018\end{array}$ & & $\begin{array}{l}0.0007 \\
0.0006\end{array}$ & \\
\hline 0.0000 & $\begin{array}{l}0.0018 \\
0.00009\end{array}$ & & & \\
\hline 0.0000 & 0.0013 & & & \\
\hline 0.0000 & 0.0015 & 0.0000 & & \\
\hline 0.0000 & 0.0016 & 0.0000 & & \\
\hline 0.0000 & 0.0000 & 0.0001 & 0.0004 & \\
\hline $\begin{array}{l}0.00000 \\
0.00000\end{array}$ & $\begin{array}{l}0.00000 \\
0.00000\end{array}$ & $\begin{array}{l}0.0001 \\
0.0001\end{array}$ & $\begin{array}{l}0.00044 \\
0.0004\end{array}$ & \\
\hline 0.0000 & $\begin{array}{l}0.00000 \\
0.0000\end{array}$ & 0.0001 & 0.0004 & \\
\hline 0.0000 & 0.0000 & 0.0001 & 0.0004 & \\
\hline 0.0000 & 0.0000 & 0.0000 & 0.0003 & \\
\hline & 0.0000 & 0.0000 & 0.0003 & \\
\hline 0.0000 & 0.0000 & 0.0000 & 0.0000 & \\
\hline 0.0000 & 0.0002 & 0.0000 & 0.0000 & \\
\hline 0.0000 & 0.0002 & 0.0000 & 0.0000 & \\
\hline 0.0000 & 0.0004 & 0.0000 & 0.0005 & \\
\hline 0.0000 & 0.0004 & 0.0000 & $\begin{array}{l}0.0005 \\
0.00005\end{array}$ & \\
\hline 0.0000 & $\begin{array}{l}0.0004 \\
0.0008\end{array}$ & 0.0000 & $\begin{array}{l}0.0005 \\
0.0004\end{array}$ & \\
\hline 0.0000 & 0.0008 & 0.0000 & 0.0004 & \\
\hline 0.0000 & 0.0008 & 0.0000 & 0.0004 & \\
\hline 0.0000 & 0.0014 & 0.0000 & 0.0004 & \\
\hline 0.0000 & 0.0014 & 0.0000 & 0.0004 & \\
\hline $\begin{array}{l}0.00000 \\
0.00000\end{array}$ & $\begin{array}{l}0.00066 \\
0.00006\end{array}$ & $\begin{array}{l}0.00000 \\
0.0000\end{array}$ & $\begin{array}{l}0.0003 \\
0.00003\end{array}$ & \\
\hline 0.0000 & $\begin{array}{l}0.00006 \\
0.0013\end{array}$ & $\begin{array}{l}0.00000 \\
0.000\end{array}$ & $\begin{array}{l}.000033 \\
0.0004\end{array}$ & \\
\hline 0.0000 & 0.0006 & 0.0000 & 0.0003 & \\
\hline 0.0000 & 0.0006 & 0.0000 & 0.0003 & \\
\hline 0.0000 & 0.0003 & 0.0006 & 0.0010 & \\
\hline 0.0000 & 0.0014 & 0.0000 & 0.0003 & \\
\hline 0.0000 & 0.0014 & 0.0000 & 0.0003 & \\
\hline & 0.0006 & 0.0000 & 0.0003 & \\
\hline & $\begin{array}{l}0.00066 \\
0.0006\end{array}$ & & $\begin{array}{l}0.00033 \\
0.0003\end{array}$ & \\
\hline & 0.0006 & & 0.0003 & \\
\hline & 0.0006 & & 0.0003 & \\
\hline & & & $\begin{array}{l}.00003 \\
0.0003\end{array}$ & \\
\hline 0.0000 & 0.0006 & 0.0000 & 0.0003 & \\
\hline 0.0000 & 0.0006 & 0.0000 & 0.0003 & \\
\hline 0.0000 & 0.0006 & 0.0000 & 0.0003 & \\
\hline 0.0000 & $\begin{array}{l}0.0006 \\
0.0000\end{array}$ & 0.0006 & & \\
\hline & $\begin{array}{l}0.0020 \\
0.0010\end{array}$ & & & \\
\hline & & & & \\
\hline & 0.0050 & 0.0120 & & 0.0001 \\
\hline
\end{tabular}

$\begin{array}{rrr} & \\ 0.044 & 0.0000 & 0.0000 \\ 0.0000 & 0.0000 \\ 0 & 0.0000 & 0.0000\end{array}$

0.0044
0.0044

0.0044

0.0044
0.0044
0.0002

0.0000
0.0004
0.0004

0.0000
0.0000
0.0004

0.0004
0.0004
0.0004
0.0004

0.0004
0.0004
0.0004

0.0000
0.0000

0.0000

0.0000
0.0000
0.0000
0.0000

0.0000

0.0000
0.0000
0.0000
0.0000

0.0000

0.0000
0.0000
0.0000
0.0000

0.0000
0.0011
0.0011
0.0000

0.0000
0.0011
0.0011

0.0004
0.0002
0.0002

0.0002
0.0011
0.0011

0.0011
0.0011
0.0011

0.0011
0.0011
0.0011

0.0011

0.0012
0.0012
0.004

0.0100

$0.0050 \quad 0.0120$

$0.0004 \quad 0.0002$

0.0058
0.0058
0.058
0.0058
0.056
0.0052
0.054
0.0054
0.054
0.0054
0.054
0.0054
0.054
0.0054
0.0558
0.0056
0.0056
0.0056
0.0000
0.0007

$\begin{array}{ll}0.0000 & 0.0124 \\ 0.0000 & 0.0074 \\ 0.0000 & 0.0074 \\ 0.0000 & 0.0174 \\ 0.0000 & 0.0071 \\ 0.00000 & 0.0067 \\ 0.0000 & 0.0070 \\ 0.00000 & 0.0070 \\ 0.0000 & 0.0070 \\ 0.0000 & 0.0070 \\ 0.0000 & 0.0070 \\ 0.0000 & 0.0070 \\ 0.00000 & 0.0070 \\ 0.0000 & 0.0070 \\ 0.00000 & 0.0074 \\ & 0.0072 \\ & 0.0072 \\ & 0.0072\end{array}$

0.0124
0.0074
0.0074
0.0174
0.0071
0.0067
0.0070
0.0070
0.0070
0.0070
0.0070
0.0070
0.0070
0.0070
0.0074
0.0072
0.0072
0.0072

0.0000
0.0000

0.0000
0.0000

0.0000

0.0000
0.0000
0.0000
0.0000
0.0000

0.0000

0.0000
0.0000
0.0000
0.0000

0.0000

0.0000
0.0000
0.0000

0.0000

0.0000

0.0329
0.0406
0.0000

0.0000

$\begin{array}{ll}0.0123 & 0.0000 \\ 0.0000 & 0.0800 \\ 0.0123 & 0.0000 \\ 0.0000 & 0.0800\end{array}$

$\begin{array}{ll}0.0016 & 0.0029 \\ 0.0033 & 0.0083 \\ 0.0002 & 0.0351\end{array}$

$\begin{array}{ll}0.0002 & 0.0351 \\ 0.0033 & 0.0338 \\ 0.0033 & 0.0467 \\ 0.0002 & 0.0277\end{array}$

$\begin{array}{ll}0.0002 & 0.0293 \\ 0.0041 & 0.0017 \\ 0.0041 & 0.0017 \\ 0.0043 & 0.0018\end{array}$

$\begin{array}{ll}0.0043 & 0.0018 \\ 0.0041 & 0.0017 \\ 0.0045 & 0.0017\end{array}$

$\begin{array}{ll}0.0041 & 0.0017 \\ 0.0041 & 0.0018\end{array}$

$\begin{array}{ll}0.0038 & 0.0036 \\ 0.0038 & 0.0036 \\ 0.0040 & 0.0038\end{array}$

0.0040

$\begin{array}{ll}0.0025 & 0.0304 \\ 0.0025 & 0.0304 \\ 0.0025 & 0.0304\end{array}$

$\begin{array}{ll}0.0024 & 0.0399 \\ 0.0024 & 0.0399 \\ 0.0024 & 0.0400 \\ 0.0028 & 0.0028\end{array}$

$\begin{array}{ll}0.0028 & 0.0028 \\ 0.0027 & 0.0033 \\ 0.0015 & 0.0142 \\ 0.0015 & 0.0142\end{array}$

$\begin{array}{ll}0.0015 & 0.0142 \\ 0.0015 & 0.0142 \\ 0.0026 & 0.0032\end{array}$

$\begin{array}{ll}0.0015 & 0.0141 \\ 0.0015 & 0.0141 \\ 0.0034 & 0.0012\end{array}$

$\begin{array}{ll}0.0034 & 0.0012 \\ 0.0026 & 0.0033 \\ 0.0026 & 0.0033\end{array}$

$\begin{array}{ll}0.0026 & 0.0013 \\ 0.0015 & 0.0142 \\ 0.0015 & 0.0142 \\ 0.0015 & 0.0142 \\ 0.0015 & 0.0142\end{array}$

$\begin{array}{ll}0.0015 & 0.0142 \\ 0.0015 & 0.0142 \\ 0.0015 & 0.0146 \\ 0.0015 & 0.0145\end{array}$

$\begin{array}{ll}0.0015 & 0.0145 \\ 0.0015 & 0.0143 \\ 0.0016 & 0.0153 \\ 0.0016 & 0.0153 \\ 0.036 & 0.0013\end{array}$

$0.0000 \quad 0.000$

0.0230

0.0000
0.0001
0.0000
0.0000
0.0000
0.0000
0.0000
0.0000
0.0000
0.0000
0.0000
0.0000
0.0000
0.0000
0.0000
0.0000
0.0000
0.0000
0.0000
0.0000
0.0000
0.0000
0.0000
0.0000
0.0000
0.0000
0.0000
0.0000
0.0000
0.0000
0.0000
0.0000

$\begin{array}{lll}0.0404 & 0.0006 & 0.0192\end{array}$

0.0000

0.0000
0.0000

0.0010

MoO3-t Nb205-t Nd2O3-t NpO2-t PbO-t Pd02-t PdO-t Pr203-t Pr6011-t Rb2O-t ReO-t

$\begin{array}{ll}0.0404 & 0.0006 \\ 0.0486 & 0.0006 \\ 0.0185 & 0.0008\end{array}$

0.0192

0.0216
0.0178

0.0134

$\begin{array}{ll}0.0000 & 0.0000 \\ 0.0250 & 0.0000 \\ 0.0000 & 0.0000\end{array}$

$\begin{array}{ll}0.0250 & 0.0000 \\ 0.0000 & 0.0000 \\ 0.0156 & 0.000\end{array}$

$\begin{array}{ll}0.0000 & 0.0000 \\ 0.0000 & 0.0000\end{array}$

$\begin{array}{ll}0.0000 & 0.0000 \\ 0.0000 & 0.0000 \\ 0.000 & 0.0000\end{array}$

0.0000

0.0000
0.0000
0.0000
0.0000

0.0000
0.0000
0.0000
0.0000
0.0000

$\underset{0.0199}{0.024}$
0.0012

0.0012

0.0012

0.0012
0.0012

0.0000
0.0000
0.000

0.0002

$\begin{array}{ll}0.0000 & 0.0000 \\ 0.0000 & 0.0000 \\ 0.0000 & 0.0000\end{array}$

\begin{tabular}{ll}
0.0000 & 0.0000 \\
0.0000 & 0.0000 \\
\hline 0.0000 & 0.0000
\end{tabular}

$0.0000 \quad 0.0000$

$\begin{array}{ll}0.0000 & 0.0000 \\ 0.0000 & 0.0000\end{array}$

$\begin{array}{ll}0.0000 & 0.0000 \\ 0.0000 & 0.0000\end{array}$

$\begin{array}{ll}0.0000 & 0.0000 \\ 0.0000 & 0.0000\end{array}$

0.00000 .00000

$0.0000 \quad 0.0000$

$\begin{array}{ll}0.0011 & 0.0000 \\ 0.0010 & 0.0000 \\ 0.000 & 0.000\end{array}$

$\begin{array}{ll}0.0000 & 0.0000 \\ 0.0000 & 0.0000\end{array}$

$0.0010 \quad 0.0000$

$0.0000 \quad 0.0000$

$0.0000 \quad 0.0005$

$0.0000 \quad 0.0000$

0.0000

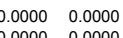

$0.0000 \quad 0.0000$

$0.0000 \quad 0.0005$

0.0137

0.0164
0.0130
0.029

0.0057
0.0001

0.0001

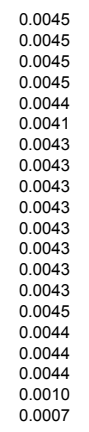


\# Glass ID

2789 HLWW6-28

2790 HLW06-29
2791 HLW06-30

2792 HLW06-31
2793 HLWO6-32

2794 HLWO6-33

2795 HLWO6-34
2796 HLWO6-35

2797 HLW W98-66
2798 HW

2799 HLW98-68

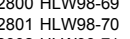

2802 HLW989-71

2804 HLW98-73
2805 HLW98-74

2806 HLW98-75

2807 HLW W $98-76$
2808 HLW98-78

(2809 HLW98-79

2811 HLW98-80CG

2813 HLW98-82

2815 HLW98-84, HLW98-84R1

2816 HLW $288-8484 \mathrm{HCG}$
2817 HLW98-85

2818 HLW98-86, HLW98-86R2

2819 HLW98-86AG

(2821 HLW98

2822 HLW W88-89
2823 HLW98-90

2824 HLW98-91

2826 HLW98-93

2828 HLW89-95, HLW98-95R1

2830 HLW98-96, HLW98-96R1

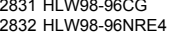

2833 HLW98-96NRE4CCC

2834 HLW98-96RE7
2835 HLW98-96RE7CCC

$2836 \mathrm{HLW} 988-96 \mathrm{~A}$
$-2837 \mathrm{HLCO}$

2838 HLW98-96C

2839 HLW98-96D

2840 HLW98-96DR
2841 HLW98-97

2842 LRM

2845 ATM-1a

2846 ATM- $1 \mathrm{~b}$

2848 ATM- 11

2850 DWPF-EA

$2851 \mathrm{Hal}-0$

2853 Hal-03

$2854 \mathrm{Hal}-04$

$2856 \mathrm{Hal}-06$

2858 Hal-

2862 Hal-12

2863 Hal-13

2865 Hal-15

$2867 \mathrm{Hal}-17$

2869 KRL-01 (repl. US-08)
2870 KRl-02 (repl. US-19)
Re02-t Re207-t Rh203-t RhO2-t RuO2-t Ru2O3-t Sb203-t Sb205-t SeO2-t Sm203-t SnO-t SnO2-t SO3-t SrO-t Tc207-t TeO2-t ThO2-t TiO2-t T12O-t T1203-t U308-t U02-t U03-t V205-t WO3-t Y2O3-t ZnO-t Sum-t A1203-a $\begin{array}{ll}0.0005 & 0.0013 \\ 0.0005 & 0013 \\ 0.0005 & 0.013\end{array}$

\begin{tabular}{|c|c|c|c|}
\hline 0.0005 & 0.0013 & & \\
\hline 0.0005 & 0.0013 & & \\
\hline $\begin{array}{l}0.0005 \\
0.0005\end{array}$ & $\begin{array}{l}0.0013 \\
0.0013\end{array}$ & & \\
\hline $\begin{array}{l}0.00055 \\
0.0005\end{array}$ & $\begin{array}{l}0.0013 \\
0.0013\end{array}$ & & \\
\hline & $\begin{array}{l}0.0013 \\
0.0013\end{array}$ & & \\
\hline & 0.0013 & & \\
\hline & 0.0000 & 0.0000 & 0.0007 \\
\hline $\begin{array}{l}0.00000 \\
0.00000\end{array}$ & $\begin{array}{l}0.00000 \\
0.00000\end{array}$ & $\begin{array}{l}0.0000 \\
0.0019\end{array}$ & $\begin{array}{l}0.0000 \\
0.001\end{array}$ \\
\hline $\begin{array}{l}0.0000 \\
0.0000\end{array}$ & $\begin{array}{l}0.00000 \\
0.0000\end{array}$ & $\begin{array}{l}0.0019 \\
0.0019\end{array}$ & $\begin{array}{l}0.0015 \\
0.0015\end{array}$ \\
\hline 0.0003 & $\begin{array}{l}0.00002 \\
0.000\end{array}$ & $\begin{array}{l}0.0019 \\
0.0000\end{array}$ & $\begin{array}{l}0.0015 \\
0.0000\end{array}$ \\
\hline 0.0004 & 0.0002 & 0.0000 & $\begin{array}{l}0.000000 \\
0.000\end{array}$ \\
\hline 0.0000 & 0.0000 & 0.0000 & 0.0000 \\
\hline & 0.0000 & 0.0000 & 0.0000 \\
\hline 0.0000 & 0.0000 & 0.0000 & 0.0000 \\
\hline 0.0000 & 0.0000 & 0.00000 & 0.0000 \\
\hline 0.0000 & 0.0000 & 0.0000 & 0.0000 \\
\hline $\begin{array}{l}0.00000 \\
0.0002\end{array}$ & 0.0000 & 0.0000 & 0.0000 \\
\hline $\begin{array}{l}0.0000 \\
0.000\end{array}$ & $\begin{array}{l}0.00000 \\
0.00000\end{array}$ & $\begin{array}{l}0.00000 \\
0.0000\end{array}$ & $\begin{array}{l}0.00000 \\
0.0000\end{array}$ \\
\hline 0.0000 & 0.0000 & 0.0000 & 0.0000 \\
\hline $\begin{array}{l}0.00000 \\
0.00000\end{array}$ & 0.0000 & 0.0000 & $\begin{array}{l}0.0000 \\
0.0020\end{array}$ \\
\hline 0.0000 & 0.0000 & 0.0011 & 0.0021 \\
\hline & & $\begin{array}{l}0.00011 \\
0.0026\end{array}$ & $\begin{array}{l}0.00211 \\
0.0037\end{array}$ \\
\hline 0.0000 & 0.0000 & 0.0026 & 0.0037 \\
\hline 0.0000 & 0.0000 & 0.0026 & 0.0037 \\
\hline 0.0003 & 0.0008 & 0.0000 & 0.0000 \\
\hline $\begin{array}{l}0.0002 \\
0.0000\end{array}$ & 0.0008 & 0.0000 & 0.0000 \\
\hline $\begin{array}{l}0.0000 \\
0.0000\end{array}$ & $\begin{array}{l}0.00000 \\
0.00000\end{array}$ & $\begin{array}{l}0.00000 \\
0.00000\end{array}$ & $\begin{array}{l}0.00000 \\
0.0000\end{array}$ \\
\hline 0.0002 & 0.0007 & 0.0000 & 0.0000 \\
\hline 0.0000 & 0.0000 & 0.0000 & 0.0000 \\
\hline & $\begin{array}{l}0.00000 \\
0.0000\end{array}$ & 0.0000 & 0.0000 \\
\hline & $\begin{array}{l}0.00000 \\
0.0000\end{array}$ & $\begin{array}{l}0.00000 \\
0.0000\end{array}$ & $\begin{array}{l}0.00000 \\
0.00000\end{array}$ \\
\hline 0.0002 & 0.0008 & 0.0000 & 0.0000 \\
\hline & & & \\
\hline
\end{tabular}

0.0002

0.0001

0.0000

0.0000
0.0000
0.0000

0.0000

0.0000
0.0000
0.0000
0.0000

0.0000

$\begin{array}{ll}0.0000 & 0.0000 \\ 0.0000 & 0.0000 \\ 0.0000 & 0.0000 \\ 0.0000 & 0.0000 \\ & \end{array}$

0.0000

\begin{tabular}{|c|c|c|c|c|c|c|}
\hline & & & & & & \\
\hline & 0.00000 & 0.00000 & & 0.0594 & 0 & \\
\hline & 0.0028 & 0.1029 & & $\begin{array}{l}0.0000 \\
0.05994\end{array}$ & $\begin{array}{l}0.0000 \\
0.0000\end{array}$ & \\
\hline & 0.0000 & 0.0000 & & 0.0000 & 0.0000 & \\
\hline & 0.0028 & 0.1029 & & 0.0000 & 0.0100 & \\
\hline & $\begin{array}{l}0.00000 \\
0.0004\end{array}$ & $\begin{array}{l}0.0000 \\
0.0133\end{array}$ & & $\begin{array}{l}0.00000 \\
0.0325\end{array}$ & $\begin{array}{l}0.0000 \\
0.0016\end{array}$ & \\
\hline & $\begin{array}{l}0.0004 \\
0.0003\end{array}$ & 0.0119 & 0.0002 & $\begin{array}{l}0.0325 \\
0.0000\end{array}$ & $\begin{array}{l}0.00000 \\
0.0000\end{array}$ & 0.0000 \\
\hline 0.0000 & 0.0000 & 0.0662 & 0.0000 & 0.0000 & 0.0012 & 0.0000 \\
\hline $\begin{array}{l}0.00000 \\
0.0000\end{array}$ & $\begin{array}{l}0.0025 \\
\end{array}$ & $\begin{array}{l}0.0255 \\
0.0504\end{array}$ & $\begin{array}{l}0.00144 \\
0.0014\end{array}$ & 0.0000 & 0.0006 & 0.0000 \\
\hline & $\begin{array}{l}0.0025 \\
0.0000\end{array}$ & $\begin{array}{l}0.0004 \\
0.0365\end{array}$ & $\begin{array}{l}0.00044 \\
0.0000\end{array}$ & $\begin{array}{l}0.0000 \\
0.0441\end{array}$ & $\begin{array}{l}0.0006 \\
0.0002\end{array}$ & $\begin{array}{l}0.0000 \\
0.0000\end{array}$ \\
\hline & 0.0000 & 0.0386 & 0.0000 & 0.0465 & 0.0002 & 0.0000 \\
\hline 0.0000 & 0.0007 & 0.0000 & 0.0000 & 0.0000 & 0.0000 & 0.0000 \\
\hline 000 & 0.0007 & 0.0003 & 0.00000 & 0.0000 & 0.0000 & 0.0000 \\
\hline & 0.0008 & 0.0004 & 0.0000 & 0.00000 & 0.0000 & 0.0000 \\
\hline 0000 & 0.0008 & 0.0004 & & & 0.0000 & 0.0000 \\
\hline & 0.0007 & 0.0003 & 0.00000 & 0.00000 & & \\
\hline & $\begin{array}{l}0.0008 \\
\end{array}$ & 0.00033 & 0.00000 & 0.00000 & & 0.0000 \\
\hline $\begin{array}{l}0.0000 \\
0.0000\end{array}$ & $\begin{array}{l}0.0008 \\
0.0004\end{array}$ & $\begin{array}{l}0.0003 \\
0.0000\end{array}$ & $\begin{array}{l}0.0000 \\
0.0000\end{array}$ & $\begin{array}{l}0.00000 \\
0.00000\end{array}$ & $\begin{array}{l}0.00000 \\
0.0000\end{array}$ & \\
\hline & 0.0004 & م & & & & \\
\hline & 0.0004 & 0.0000 & 0.0000 & 0.0000 & 0.0000 & 0.0000 \\
\hline & 0.0000 & & & 0.0000 & & \\
\hline 0.0000 & 0.0000 & 0.0000 & 0.0000 & 0.0000 & 0.0003 & 0.0000 \\
\hline & 0.0000 & 0.0092 & 0.0000 & 0.0000 & & 0.0000 \\
\hline & 0.0000 & 0.0092 & $\begin{array}{l}0.00000 \\
0.0000\end{array}$ & $\begin{array}{l}0.00000 \\
0.0000\end{array}$ & 0.0014 & $\begin{array}{l}0.00000 \\
0.0000\end{array}$ \\
\hline 0.0000 & 0.0008 & $\begin{array}{l}.0092 \\
0.0016\end{array}$ & $\begin{array}{l}0.0000 \\
0.000\end{array}$ & 0.0000 & $\begin{array}{l}0.0000 \\
0.0000\end{array}$ & $\begin{array}{l}0.00000 \\
0.000\end{array}$ \\
\hline & 0.0008 & 0.0016 & 0.0000 & & & 0.0000 \\
\hline 0.0000 & 0.0000 & $\begin{array}{l}0.0000 \\
0 \\
0.0000\end{array}$ & 0.0000 & 0.0405 & 0.0002 & $\begin{array}{l}0.0000 \\
0.0000\end{array}$ \\
\hline $\begin{array}{l}0.00000 \\
0.0000\end{array}$ & $\begin{array}{l}0.0000 \\
0.0008\end{array}$ & $\begin{array}{l}0.0000 \\
0.0015\end{array}$ & $\begin{array}{l}0.0000 \\
0.0000\end{array}$ & $\begin{array}{l}0.04050 \\
0.0000\end{array}$ & $\begin{array}{l}0.0002 \\
0.0000\end{array}$ & $\begin{array}{l}0.00000 \\
0.0000\end{array}$ \\
\hline 0.0000 & 0.0000 & 0.0000 & 0.0000 & 0.0402 & & 0.0000 \\
\hline 0.0000 & 0.0000 & 0.0000 & 0.0000 & 0.0402 & & 0.0000 \\
\hline $\begin{array}{l}0.00000 \\
0.0000\end{array}$ & $\begin{array}{l}0.0013 \\
\end{array}$ & 0.0003 & 0.00000 & 0.0000 & 0.0000 & 0.0000 \\
\hline & $\begin{array}{l}0.0012 \\
0.012\end{array}$ & $\begin{array}{l}0.0016 \\
0.016\end{array}$ & $\begin{array}{l}0.0000 \\
0.0000\end{array}$ & $\begin{array}{l}0.00000 \\
0.0000\end{array}$ & $\begin{array}{l}0.0001 \\
0.0001\end{array}$ & $\begin{array}{l}0.00000 \\
0.0000\end{array}$ \\
\hline 0.0000 & 0.0000 & 0.0000 & 0.0000 & 0.0405 & & 0.0000 \\
\hline & & & & 0.0405 & $\begin{array}{l}0.0002 \\
0.002\end{array}$ & \\
\hline & & & & 0.0405 & & \\
\hline & & & & 0.0 & 0.0002 & \\
\hline & & & & 0.0405 & 0.0002 & \\
\hline & 0.000 & 0.0000 & & 0.0000 & 0.0002 & \\
\hline & 0.000 & 0.0000 & 0.0000 & 0.0000 & 0.0002 & 0.0000 \\
\hline 0.0000 & 0.6 & 0.0000 & 0.0000 & 0.0000 & 0.0002 & 0.0000 \\
\hline & 000 & 0.0000 & 0.0000 & 0.0000 & & 0.0000 \\
\hline 0000 & & 0.0003 & 0.0000 & 0.0000 & & 0.0000 \\
\hline & & & & & $\begin{array}{l}0.0010 \\
0.0100\end{array}$ & \\
\hline
\end{tabular}

0.0650

$\begin{array}{rr}\text { ZnO-t } & \text { Sum-t } \\ 0.0400 & 1.0000 \\ 00000 & 1.0000\end{array}$

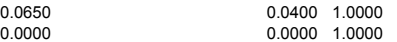

0.0000

0.0329

$\begin{array}{ll}0.0000 & 0.0000 \\ 0.0000 & 0.0000 \\ 0.0000 & 0.0000\end{array}$

$\begin{array}{lll}0.00000 & 0.00000 \\ 0.0000 & 0.0000 \\ 0.0412 & 0.0000\end{array}$

$\begin{array}{ll}0.0000 & 0.0000 \\ 0.0412 & 0.0000 \\ 0.0435 & 0.0000\end{array}$

$0.0000 \quad 0.0000$

$0.0000 \quad 0.0000$

0.00000 .0000

$\begin{array}{ll}0.0000 & 0.0000 \\ 0.0000 & 0.0000\end{array}$

$\begin{array}{ll}0.0000 & 0.0000 \\ 0.0000 & 0.0000\end{array}$

$0.0000 \quad 0.0000$

$\begin{array}{ll}0.0000 & 0.0000 \\ 0.0000 & 0.0000 \\ 0.0000 & 0.0000\end{array}$

$0.0000 \quad 0.0000$

$\begin{array}{ll}0.0000 & 0.0000 \\ 0.0000 & 0.0000 \\ 0.0004 & 0.0000\end{array}$

$\begin{array}{lll}0.0097 & 0.0000 \\ 0.0094 & 0.0000\end{array}$

$\begin{array}{ll}0.0094 & 0.0000 \\ 0.0257 & 0.0000 \\ 0.0257 & 0.0000\end{array}$

$\begin{array}{lll}0.0257 & 0.00000 \\ 0.0005 & 0.0000\end{array}$

$\begin{array}{ll}0.0255 & 0.0000 \\ 0.0255 & 0.0000\end{array}$

$0.0000 \quad 0.0000$

$\begin{array}{ll}0.0093 & 0.0000 \\ 0.0093 & 0.0000 \\ 0.0257 & 0.0000\end{array}$

$0.0257 \quad 0.0000$

0.0257

0.0257
0.0257
0.0000

$\begin{array}{ll}0.0257 & \\ 0.0000 & 0.0000\end{array}$

$\begin{array}{lll}0.0000 & 0.0000 \\ 0.0000 & 0.0000\end{array}$

$\begin{array}{ll}0.0000 & 0.0000 \\ 0.0000 & 0.0000\end{array}$

$0.0000 \quad 0.0000$

$\begin{array}{ll}0.0000 & 1.0000 \\ 0.0000 & 1.0000 \\ 0.0000 & 1.0000 \\ .0000 & 1.0000\end{array}$

$\begin{array}{ll}0.0000 & 1.0000 \\ 0.0031 & 1.0000\end{array}$

$\begin{array}{ll}0.0031 & 1.0000 \\ 0.0200 & 1.0000 \\ 0.0020 & 1.0000\end{array}$

0.0002
0.0200
0.00000

$\begin{array}{ll}0.0200 & 1.0000 \\ 0.0200 & 1.0000\end{array}$

$\begin{array}{ll}0.0200 & 1.0000 \\ 0.0202 & 1.0000\end{array}$

$\begin{array}{ll}0.0202 & 1.0000 \\ 0.0202 & 1.0000\end{array}$

$\begin{array}{ll}0.0202 & 1.0000 \\ 0.0202 & 1.0000\end{array}$

$\begin{array}{ll}0.0202 & 1.0000 \\ 0.0202 & 1.0000 \\ 0.0202 & 1.0000\end{array}$

$\begin{array}{lll}0.0201 & 1.0000 & 0.0542\end{array}$

0.02011 .0000

(

0.02011 .0000

0.02021 .0000
0.0202
0.0000

$\begin{array}{ll}0.0202 & 1.0000 \\ 0.0207 & 1.0000\end{array}$

$\begin{array}{lll}0.0207 & 1.0000 & 0.0527\end{array}$

$0.0207 \quad 1.0000$
0.0202
0.02000

$\begin{array}{ll}0.0202 & 1.0000 \\ 0.0201 & 1.0000\end{array}$

$\begin{array}{ll}0.0202 & 1.0000 \\ 0.0202 & 1.0000\end{array}$

0.0201
0.0127
0.0000
0.0000

$0.0102 \quad 1.0000$

$0.0206 \quad 1.0000$

$\begin{array}{lll}0.0201 & 1.0000 & 0.0643 \\ 0.0201 & 1.0000 & 0.0727\end{array}$

$\begin{array}{ll}0.0202 & 1.0000 \\ 0.0202 & 1.0000\end{array}$

0.02011 .0000

0.02021 .0000

$\begin{array}{ll}0.0202 & 1.0000 \\ 0.0207 & 1.0000\end{array}$

$\begin{array}{ll}0.0206 & 1.0000 \\ 0.0202 & 1.0000 \\ 0.0216 & 0.0999\end{array}$

$\begin{array}{ll}0.0202 & 1.0000 \\ 0.0216 & 1.0000 \\ 0.0216 & 0.9999\end{array}$

$\begin{array}{ll}0.0216 & 0.9999 \\ 0.0206 & 1.0000\end{array}$

$\begin{array}{ll}0.9982 & 0.0951 \\ 1.0000 & 0.0460 \\ 0.0000 & 0.055\end{array}$

0.0398
0.0400
0.040

$\begin{array}{lll}0.0473 & 1.0000 & 0.0085 \\ 0.0445 & 1.0000 & 0.0058 \\ 0.0007 & 1.0000 & 0.0484\end{array}$

$\begin{array}{lll}0.0049 & & \\ 0.0040 & & 0.0028 \\ 0.0002 & 0.0002 & 0.000\end{array}$

0.0299
0.0285
0.0295
0.0019

$0.009 \quad 0.9999$
0.0009

$\begin{array}{lll}0.0009 & 10999 \\ 0 & 0.999 & 0.999 \\ 0.009 & 0.9999\end{array}$

$\begin{array}{lll}0.0009 & 0.9999\end{array}$

0.00091 .0000

0.00091 .0002

0.00091 .0002

0.00091 .0002

$\begin{array}{ll}0.0009 & 1.0002 \\ 0.0009 & 1.0002\end{array}$

$\begin{array}{lll}0.0009 & 1.0002 \\ 0.0009 & 1.000\end{array}$

0.00091 .0000

$\begin{array}{ll}0.0009 & 1.0000 \\ 0.0009 & 1.0000\end{array}$

.0009 1.0000

0.0000
0.0000

$\begin{array}{ll}0.0000 & 1.0000 \\ 0.0007 & 1.0000\end{array}$ 
2790 HLWO6-29

2792 HLW06-31

2793 HLWO6-32
2794 HLW06-33

2795 HLWO6-34

2795 HLW06-34
2796 HLW06-35
2797 HLW98-66

2797 HLW98-66
2798 HLW98-67

2799 HLW98-68

2800 HLW98-69

2801 HLW98-70

2803 HLW98-72

2804 HLW98-73
2805 HLW98-74

2806 HLW98-

$2807 \mathrm{HLW} 98 \mathrm{8}-76$

(2808

2810 HLW98-80, HLW98-80R1

2811 HLW988-80CG
2812 HLW98-81

2813 HLW98-82
2814 HLW98-83

2815 HLW98-84, HLW98-84R

2816 HLW98-84CG
2817 HLW98-85

2818 HLW98-86, HLW98-86R2

2819 HLW98-86AG

2820 HLWW8-87

2822 HLW988-89

2823 HLW W98-90

2824 HLW98-91

2826 HLW98-93

2827 HLW98-94
2828 HLW98-95, HLW98-95R1

2830 HLW98-96, HLW98-96R 1

2830 HLW988-96, HLL
2831 LLW98-966G

2832 HLWB8-96NRE4

2834 HLWG8-96RET
2835 HLW98-96RE7CCC

2835 HLW98-96RE7CCC
2836 HLW98-96A

2836 HLW98-96A
2837 HLW98-96B

2838 HLW98-96C

2839 HLW98-96D

2840 HLW98-96DR2
2841 HLW98-97

2842 LRM

2843 DWPF Startup Frit

2844 ARM- 1
2845 ATM-1a

2846 ATM-1b

2847 ATM-1C

2849 ARG-1

2850 DWPF-EA

2851 Hal-01
2852 Hal- 02

$2852 \mathrm{Hal}-02$
$2853 \mathrm{Hal}-03$

2853
$2854 \mathrm{Hal}-04$
255
-1204

2855 Hal-0
2856 Hal-0

$2856 \mathrm{Hal}-06$

$2858 \mathrm{Hal}-08$

$2860 \mathrm{Hal}-10$

2861 Hal-11

$2862 \mathrm{Hal}-12$

2864 Hat-14

2865 Hal-1

$2867 \mathrm{Hal}-17$

2869 KRI-01 (repl. US-08) $\begin{array}{llllllllllll}0.1242 & 0.0028 & 0.1259 & 0.0008 & 0.0306 & 0.0008 & 0.1249 & 0.0049 & 0.0003 & 0.4730 & 0.0197 & 0.0004\end{array}$

$\begin{array}{lllllllll}0.0278 & 0.0117 & 0.1255 & 0.0021 & 0.0010 & 0.4503 & 0.0031 & 0.0020 & 0.0020\end{array}$

$0.0962 \quad 0.0032 \quad 0.1291$

$\begin{array}{lllllllllllll} & 0.0042 & 0.1275 & & 0.0012 & 0.0009 & 0.1182 & 0.0064 & 0.0037 & 0.4250 & 0.0391 & 0.0004 \\ 0.1055 & 0.0040 & 0.1144 & & 0.0019 & 0.0327 & 0.0006 & 0.1254 & 0.0035 & 0.0036 & 0.4401 & 0.0344 & 0.0005\end{array}$

$\begin{array}{lll}0.0785 & 0.0054 & 0.0138 \\ 0.0851 & 0.0147 & 0.1420 \\ 0.0857 & 0.025 & 0.081\end{array}$

$\begin{array}{lll}0.1155 & 0.0226 & 0.1420 \\ 0.0847 & 0.0199 & 0.08\end{array}$

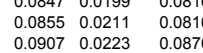

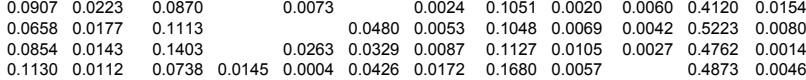

$\begin{array}{llllllll}0.0148 & 0.0011 & 0.0010 & 0.2003 & 0.0019 & 0.0054 & 0.5420 & 0.0093 \\ 0.0270 & 0.0325 & 0.0084 & 0.1153 & 0.0111 & & 0.4790 & 0.0011\end{array}$

$\begin{array}{lllllll}0.0030 & 0.1014 & 0.0018 & 0.0063 & 0.3873 & 0.0146\end{array}$

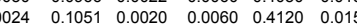

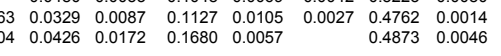

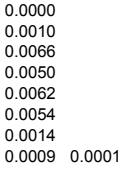

0.0016

0.0004
0.0004
0.0004

$\begin{array}{llll}0.0019 & & & 0.0086 \\ 0.0009 & & & \\ & 0.0113 & & \\ 0.0039 & 0.0071 & & \\ 0.0045 & 0.0077 & & \\ 0.0042 & 0.0078 & & \\ 0.0010 & 0.0004 & 0.0002 & \\ 0.0010 & & 0.0001 & \end{array}$


2789 HLW $06-28$
2790 HLW06-29

2791 HLW06-30

2792 HLW06-31
2793 HLW06-32

2794 HLWO6-33

2795 HLW06-34
2796 HLW06-35

2797 HLW98-66

2799 HLW98-68

2800 HLW988-69

2801 HLW988-70

2803 HLW98-72

2804 HLW988-73

2805 HLW98-

2807 HLW98-76

2808 HLW W98-78

2809 HLW98-79 HLW8-80R

2811 HLW98-80CG

2812 HLW988-81
2813 HLWG8-82

2814 HLL98-83

2816 HLW $288-84 \mathrm{CH}$
$2817 \mathrm{HLW}$

2818 HLW98-86, HLW98-86R2

2819 HLW98-86AG

2820 HLW98-87

2822 HLW988-89

2823 HLW W98-90

2824 HLW98-91
2825 HLW98-92

2825 HLWo8-93
2827 HLW98-94
2827

2827 HLW98-94
2828 HLW98-95, HLW98-95R

2830 HLW98-96, HLW98-96

2831 HLW98-96CG

2832 HLW98-96NRE4

2834 HLW98-96RE7

2835 HLW98-96RE7CCC

2836 HLW98-96A
2837 HLW98-96B

2838 HLW98-96C

2839 HLW989-96D

2840 HLW688-96D
2841 HLW98-97

2842 LRM

2843 DWPF Startup Frit

2844 ARM-1

2846 ATM-1b

2847 ATM-1C

2849 ARG-1

2850 DWPF-EA

$2851 \mathrm{Hal}-01$
$2852 \mathrm{Hal}-02$

$2852 \mathrm{Hal}-02$

$2854 \mathrm{Hal}-04$

$2855 \mathrm{Hal}-\mathrm{-}-5$
$2856 \mathrm{Hal}-06$

$2857 \mathrm{Hal}-07$

2858 Hal-0

2861 Hal-11

$2864 \mathrm{Hal}-14$

2865 Hal-1 15

$2866 \mathrm{Hal}-16$
$2867 \mathrm{Hal}-17$
$2868 \mathrm{Hal}-18$

2869 KRI-101 (repl. US-08)
2870 KRl-02 (repl. US-19)
0.0040

0.0027

0.0449

0.0018

0.0047

$0.0000 \quad 0.000$

$0.0237^{0.0008}$

$<0.0003$
$<0.0003$

$\begin{array}{ll}0.0400 & 0.0003 \\ 0.0507 & 0.0003 \\ 0.0439 & 0.0003 \\ & 0.0257\end{array}$

$\begin{array}{lll} & 0.0257 & \\ 0.0231 & \\ 0.0042 & 0.0134\end{array}$
0.0019

0.000

$0.0016 \quad 0.0014$

0.0031

0.0011 $\begin{array}{lll}0.0021 & 0.0007 & 0.0007 \\ 0.0023 & 0.0006 & 0.0007\end{array}$

0.0010

\begin{tabular}{|c|c|}
\hline 0.0178 & \\
\hline 0.0172 & 0.0134 \\
\hline 0.0199 & \\
\hline 0.0182 & 0.0142 \\
\hline
\end{tabular}

$0.0001 \quad 0.0001$

0.0010 
\# Glass ID

2789 HLW06-28
2790 HLWO6-29

2791 HLW06-30

2793 HLWO6-32

2794 HLW06-33
2795 HLW06-34

2796 HLWO6-35

2797 HLW98-66
2798 HLW98-67

2799 HLW98-68

2800 HLW98-69

(2801 HLW98-70

2803 HLW98-72

2804 HLW98-73

2807 HLW98-75

(2808

2811 HLW98-80CC

2813 HLW98-82

2815 HLW98-84, HLW98-84R1

2816 HLW98-84CG
2817 HLW98-85

2818 HLW98-86, HLW98-86R2

2819 HLW98-86AG

2821 HLW98-88

2822 HLW98-89

(2823

2825 HLW W89-92
2826 HWW

2827 HLW98-94

2828 HLW988-95, HLW98-95R

2830 HLW98-96, HLW98-96R

2831 HLW98-96CG

2833 HLW98-96NRE4CCC

2834 HLW98-96RE7

2837 HLW98-96B

2838 HLW98-96C

2841 HLW98-97

2842 LRM

2845 ATM-1

2846 ATM-1

2848 ATM-11

2849 ARG-1

2851 Hal- 01

2852 Hal-02

2853
$2854 \mathrm{Hal}-04$
255
-125

2855 Hal- 05
$2856 \mathrm{Hal}-00$

$2857 \mathrm{Hal}-07$

2858 Hal-0

2861 Hal-1

2863 Hal-13

2865 Hal-15

$2866 \mathrm{Hal}-16$
$2867 \mathrm{Hal}-17$

2870 KRI-02 (repl. US-19)
Sm2O3-a SnO-a SnO2-a SO3-a SrO-a TC2O7-a TeO2-a ThO2-a TiO2-a T12O-a T12O3-a U308-a UO2-a U03-a V2O5-a WO3-a Y2O3-a ZnO-a Sum-a $T_{M}\left({ }^{\circ} \mathrm{C}\right) \quad T_{L, G}\left({ }^{\circ} \mathrm{C}\right) \quad T_{L, U}\left({ }^{\circ} \mathrm{C}\right) T_{1 \%}\left({ }^{\circ} \mathrm{C}\right)$ Primary Phase

965 Spinel + Na-Ca-U oxide
1253 ZrSiO4 + ZrO2

$\mathrm{C}_{650^{\circ} \mathrm{C}} \mathrm{C}_{700^{\circ} \mathrm{C}} \mathrm{C}_{750^{\circ} \mathrm{C}}$

1299 Spinel + ThO2

$1278 \mathrm{Zro2}$

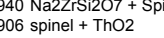

2.5
19.1
2.8
2.7

0.0113

0.0019

0.0005

0.0201

0.0000
0.0000

0.0000
0.9951
0.0000

963
875

$0.0216 \quad 0.0988$

0.0030

0.0010
0.0118
0.0332
0.0262
0.0260
0.0282
0.0021
0.0116
0.0070

0.0123
0.0079

$\begin{array}{ll}0.0001 & 0.0191 \\ 0.0188\end{array}$

0.0000
0.8530
0.9932

$\begin{array}{ll}0.0010 & 0.0019 \\ 0.0009 & 0.0019\end{array}$

0.0046

$\begin{array}{llll}0.0040 & & & \\ 0 & 0002 & 0.0001 & 0.000\end{array}$

0.0000

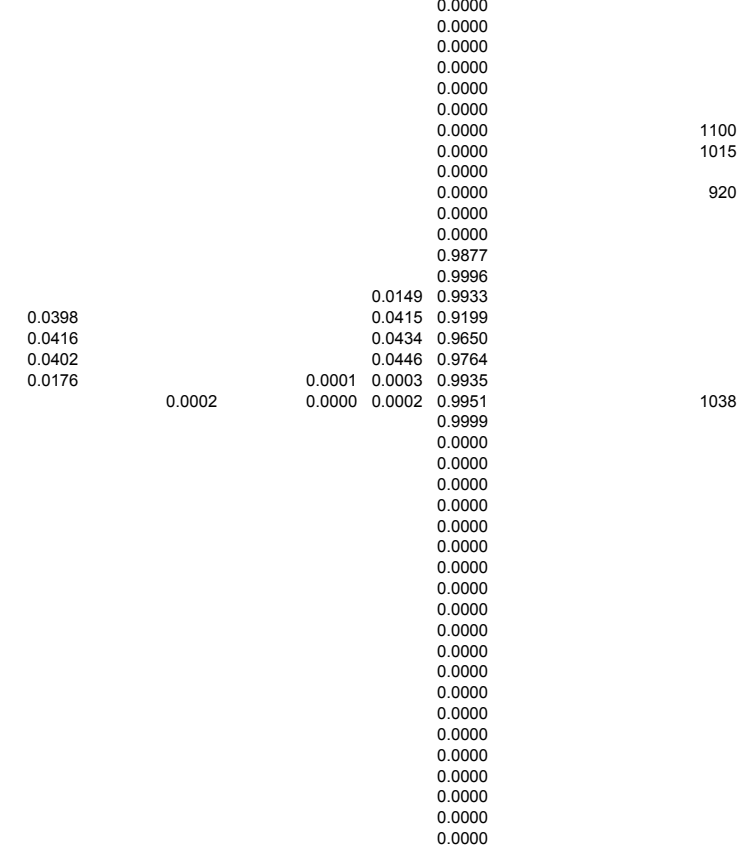



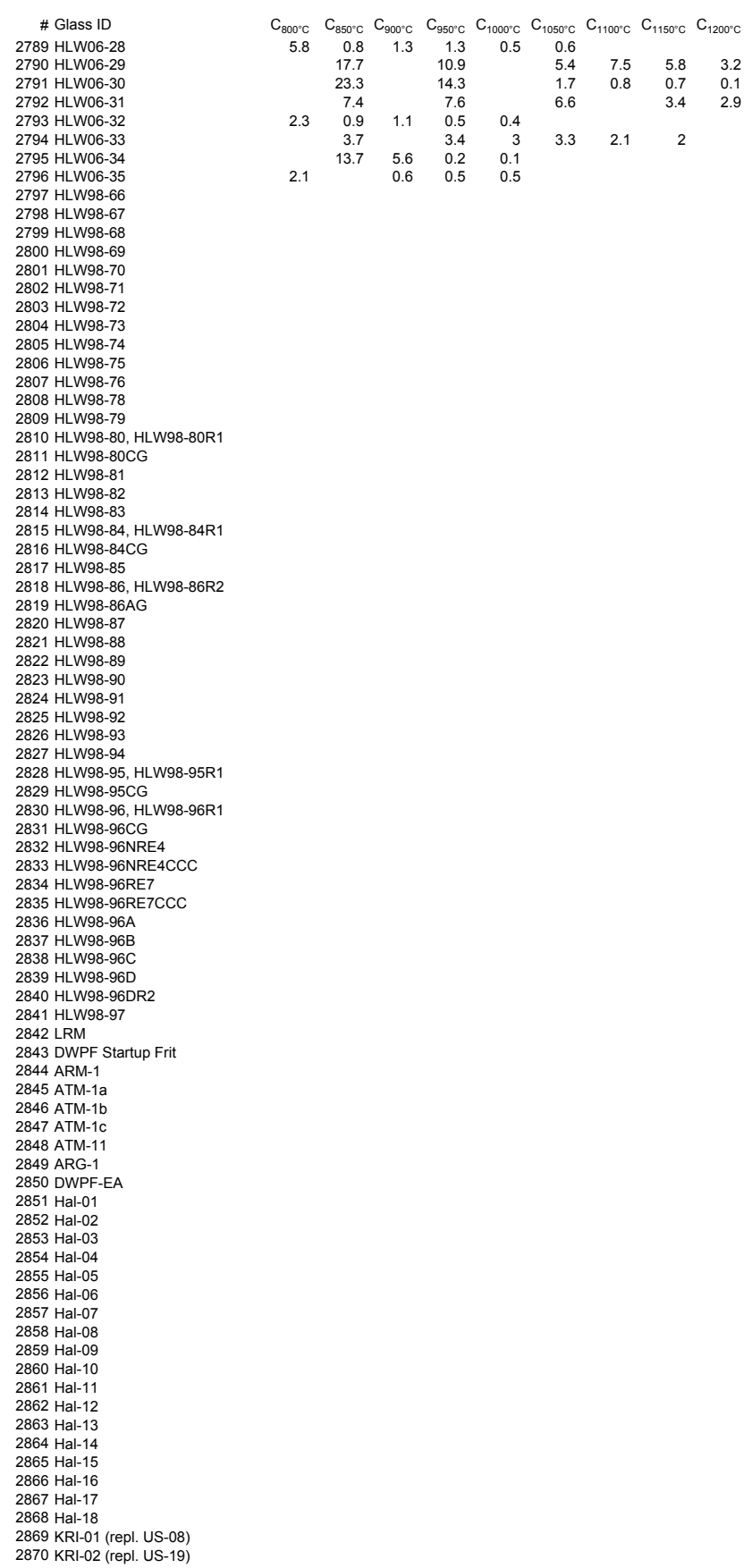

2811 HLW98-80CG
2812 HLW98-81

2824 HLW98-91

2828 HLW98-95, HLW98-95R1

2830 HLW98-96, HLW98-96R 1

2832 HLW98-96NRE

2837 HLW98-96B
2838 HLW98-96C

2839 HLW98-96D

2848 ATM-

$2852 \mathrm{Hal}-02$

2870 KRl-02 (repl. US-19)
$12460 \quad 0385$

$1256.0 \quad 0.569$

$1277.0 \quad 1.206$

$1261.0 \quad 2.278$

$\begin{array}{ll}1264.0 & 1.682 \\ 1261.0 & 2.232\end{array}$

$1264.0 \quad 1.862$

$\begin{array}{ll}1266.0 & 1.850 \\ 1265.0 & 2.410\end{array}$

$261.0 \quad 2.251$

$1250.0 \quad 2.306$

$\begin{array}{ll}1236.0 & 2.104 \\ 1235.0 & 2605\end{array}$

$1255.0 \quad 2.590$

$1253.0 \quad 1.942$

$450.0 \quad 4.09 \mathrm{E}+12$

$830.0 \quad 37.442$

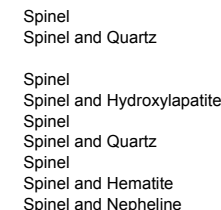




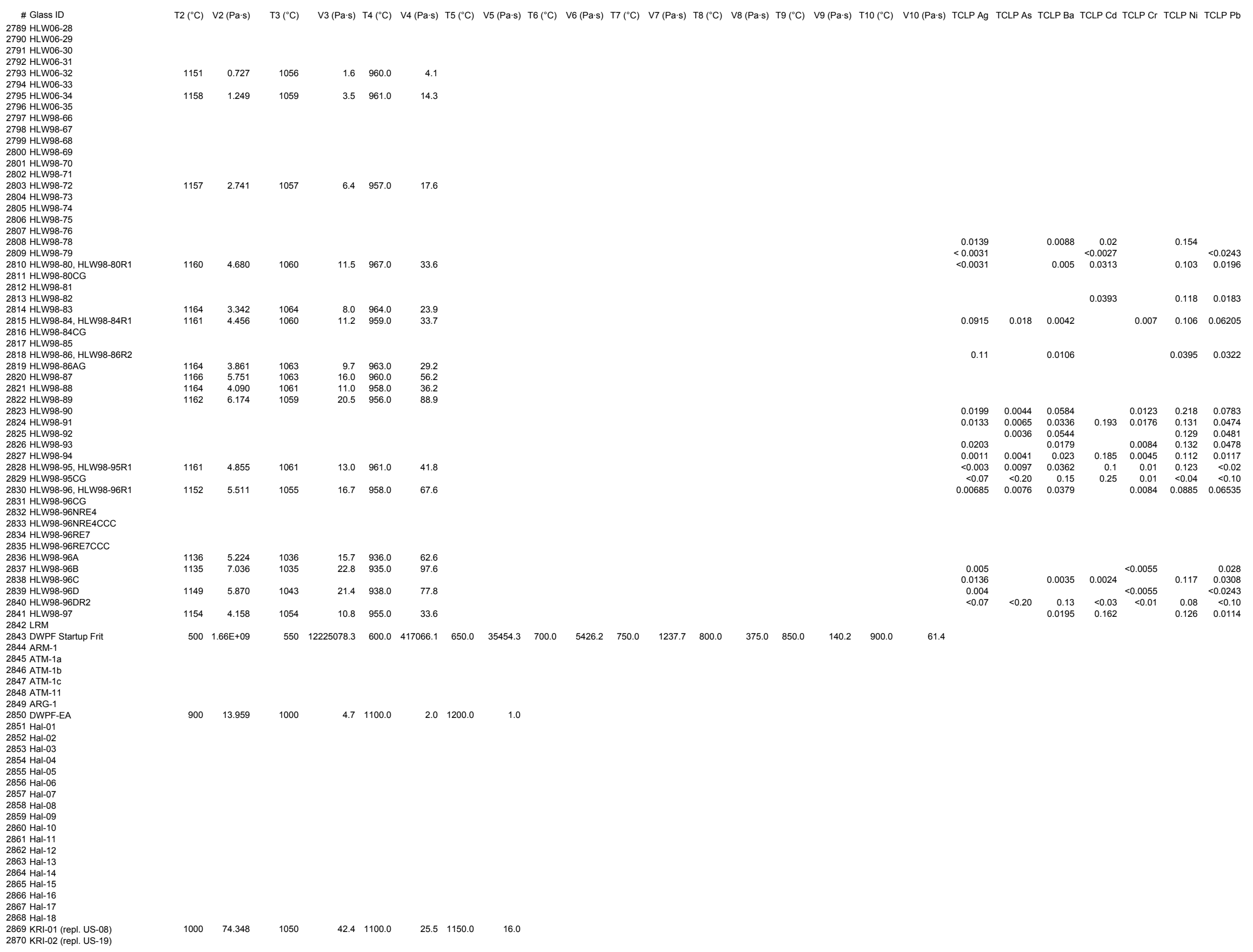




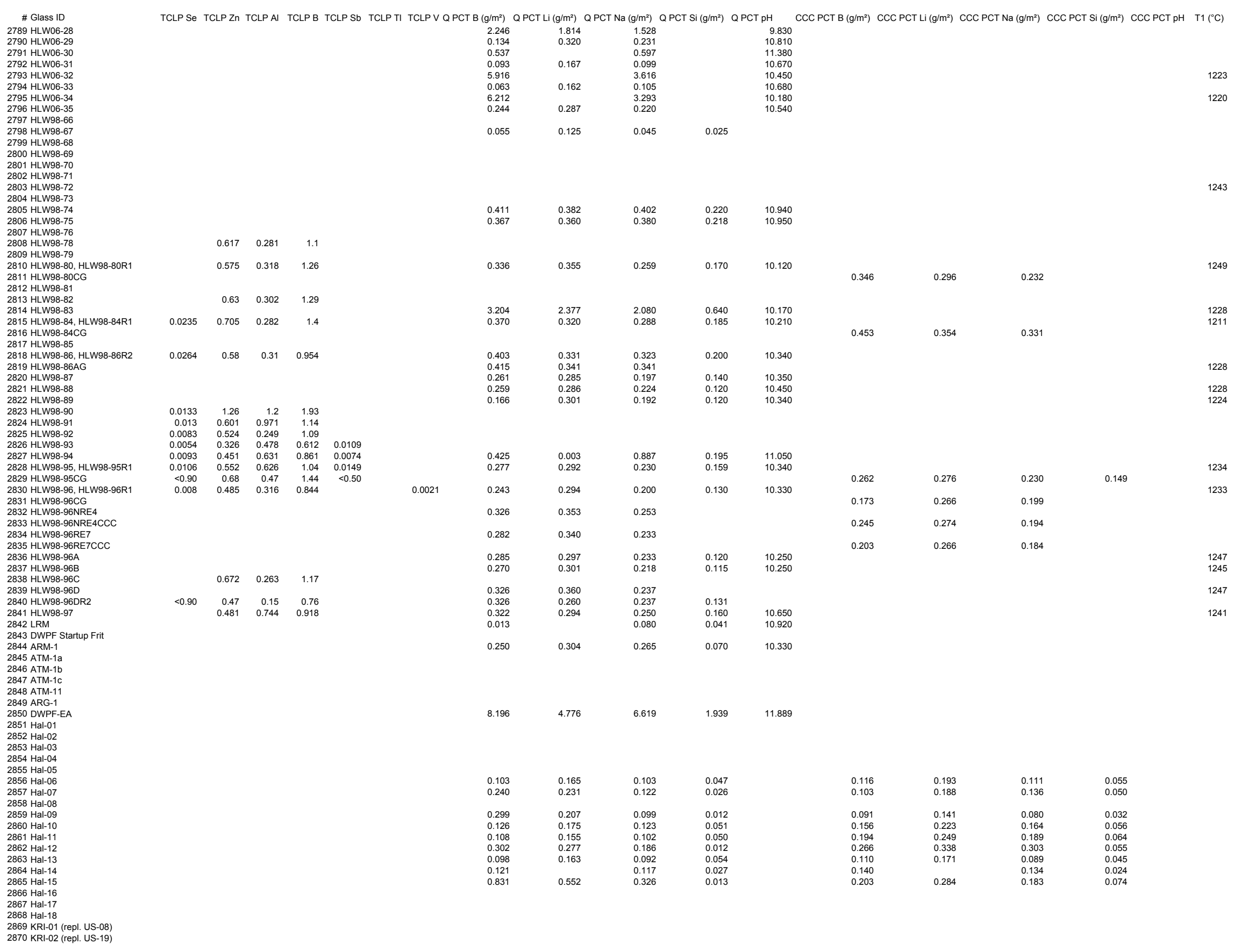


2790 HLW06-29

2791 HLW06-30
2792 HLW06-31

2793 HLWO66-32
2794 HLW06-33

2794 HLW06-33

2796 HLWO6-35

2797 HLW98-66
2798 HLW98-67

2799 HLW98-68

2800 HLWW8-69

2801 HLW98-70
2802 HLW98-71

2803 HLW98-72

2804 HLW98-73

2807 HLW98-76

(2808

2811 HLW98-80CG
2812 HLW98-81

2813 HLW98-82

2815 HLW98-84, HLW98-84R1

2816 HLW98-84CG
2817 HLW98-85

2818 HLW98-86, HLW98-86R2

2819 HLW98-86AG

2820 HLW98-87

2821 HLW98-88
2822 HLW98-89

2823 HLW W98-90

2824 HLW98-91
2825 HLW98-92

2826 HLW98-93

2827 HLW98-94

2828 HLW98-95, HLW98-95R 1

2830 HLW98-96, HLW98-96R1

2831 HLW98-96CG

2832 HLWG8-96NRE4

2834 HLW98-96RET
2835 HLW98-96RE7CCC

2835 HLW98-96RETCCC
2836 HLW98-96A

2837 HLW98-96B

2839 HLW98-96D

2840 HLW98-960R2
2841 HLW98-97

2842 LRM

2843 DWPF Startup Frit

2844 ARM-1

2846 ATM- 1 b

2847 ATM-1C

2848 ATM-11

2850 DWPF-EA

2851 Hal- 01

$2852 \mathrm{Hal}-02$

$2854 \mathrm{Hal}-04$

2855 Hal-0
2856 Hal-06

$2857 \mathrm{Hal}-07$
$2858 \mathrm{Hal}-08$

$2859 \mathrm{Hal}-09$

$2861 \mathrm{Hal}-11$

-1
$2802 \mathrm{Hal}-12$

2864 Hal-14

2865 Hal-

2867 Hal-17

2869 KRl-01 (repl. US-08)
2870 KRl-02 (repl. US-19) $\begin{array}{lllllll}0.699 & 1133 & 0.557 & 1043 & 0.398 & 954 & 0.277 \\ 0.65 & 1130 & 0.475 & 1033 & 0.333 & 932 & 0.214\end{array}$

$\begin{array}{lllllll}0.827 & 1142 & 0.582 & 1046 & 0.467 & 950 & 0.27\end{array}$

$\begin{array}{lllllll}0.491 & 1151 & 0.37 & 1054 & 0.242 & 958 & 0.146\end{array}$

$\begin{array}{lllllll}0.425 & 1135 & 0.323 & 1041 & 0.223 & 947 & 0.138 \\ 0.479 & 1118 & 0.348 & 1026 & 0.238 & 933 & 0.149\end{array}$

$\begin{array}{lllllll}0.462 & 1134 & 0.348 & 1040 & 0.239 & 952 & 0.158\end{array}$

$\begin{array}{lllllll}0.441 & 1135 & 0.329 & 1037 & 0.223 & 943 & 0.138 \\ 0.451 & 1129 & 0.332 & 1037 & 0.224 & 942 & 0.138\end{array}$

$\begin{array}{lllllll}0.472 & 1137 & 0.35 & 1043 & 0.247 & 948 & 0.156\end{array}$

$\begin{array}{lllllll}0.353 & 1138 & 0.266 & 1044 & 0.19 & 950 & 0.109\end{array}$

$\begin{array}{rrrrrrr}0.5 & 1153 & 0.312 & 1059 & 0.237 & 964 & 0.145 \\ 0.527 & 1152 & 0.4 & 1057 & 0.262 & 963 & 0.152\end{array}$

$\begin{array}{lllllll}0.64 & 1154 & 0.479 & 1058 & 0.316 & 964 & 0.189\end{array}$

$\begin{array}{lllllll}0.502 & 1147 & 0.365 & 1052 & 0.257 & 958 & 0.159\end{array}$
$1.4438-1232.99 \quad 500$

$1.8577-2299.62 \quad 350$

$\begin{array}{lll}1.5382 & -2099.29 & 350 \\ 2.5534 & -3492.39 & 150\end{array}$

$2.3695 \quad-3379.61 \quad 150$

$\begin{array}{lll}2.0099 & -2763.68 & 250 \\ 21236 & -2842.45 & 250\end{array}$

$1.9474 \quad-2655.35 \quad 250$

$\begin{array}{lll}1.0349 & -1618.69 \quad 450\end{array}$

$\begin{array}{lll}4.4488 & -7871.34 & -270 \\ 2.5539 & -3153.77 & 250\end{array}$

$\begin{array}{lll}2.2210 & -2386.40 \quad 350\end{array}$

$\begin{array}{lll}4.2899 & -7506.06 & -270\end{array}$ 


\begin{tabular}{|c|c|c|}
\hline \# Glass ID & Study & Reference \\
\hline 2871 KRI-03 (repl. US-20) & Russian High Alumina - Rus & Fox et al. 2008 \\
\hline 2872 KRI-04 (repl. US-24) & Russian High Alumina - Rus & Fox et al. 2008 \\
\hline 2873 KRI-05 (repl. US-35) & Russian High Alumina - Rus & $\begin{array}{l}\text { Fox et al. } 2008 \\
\text { Foxetal } 2008\end{array}$ \\
\hline 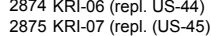 & $\begin{array}{l}\text { Russian High Alumina - Rus } \\
\text { Russian High Alumina - Rus }\end{array}$ & $\begin{array}{l}\text { Fox et al. } 2008 \\
\text { Fox tal al } 2008\end{array}$ \\
\hline 2876 KRl-08 08 (repl. (US-45) & $\begin{array}{l}\text { Russian High Alumina - Rus } \\
\text { Russian High Alumina - Rus }\end{array}$ & $\begin{array}{l}\text { Fox et al. } 2008 \\
\text { Fox et al. } 2008\end{array}$ \\
\hline 2877 KRl-09 & $\begin{array}{l}\text { Russiann Hign numunan - Rus } \\
\text { Russian High Alumina - Rus }\end{array}$ & Fox et al. 2008 \\
\hline $8 \mathrm{KRl}-10$ & ussian High Alumina - Rus & Fox et al. 2008 \\
\hline 79 KRl-11 & Russian High Alumina - Rus & Fox et al. 2008 \\
\hline $2880 \mathrm{KRl}-12$ & Russian High Alumina - Rus & Fox et al. 2008 \\
\hline $2881 \mathrm{KRl}-13$ & Russian High Alumina - Rus & Fox et al. 2008 \\
\hline $\begin{array}{ll}2882 \mathrm{KR}-14 \\
2283\end{array}$ & Russian High Alumina - Rus & Fox et al. 2008 \\
\hline $\begin{array}{l}2883 \text { KRR-15 } \\
2284 \text { KR }-16\end{array}$ & $\begin{array}{l}\text { Russian High Alumina - Rus } \\
\text { Russian High Alumina - Rus }\end{array}$ & $\begin{array}{l}\text { Fox et al. } 2008 \\
\text { Fox ex al } 2008\end{array}$ \\
\hline $\begin{array}{l}2884 \text { KRR-16 } \\
2885 \text { KRI-17 }\end{array}$ & $\begin{array}{l}\text { Russiann High Alumina - Rus } \\
\text { Russian High Alumina - Rus }\end{array}$ & $\begin{array}{l}\text { Fox et al. } 2008 \\
\text { Fox et al. } 2008\end{array}$ \\
\hline $\begin{array}{l}2885 \text { KRR-17 } \\
2886 \text { KRI-18 }\end{array}$ & $\begin{array}{l}\text { Russian High Alumina - Rus } \\
\text { Russian Alumina - Rus }\end{array}$ & $\begin{array}{l}\text { Fox et al. } 2008 \\
\text { Fox et al. } 2008\end{array}$ \\
\hline $2887 \mathrm{KRl}-19$ & Russian High Alumina - Rus & Fox et al. 2008 \\
\hline $2888 \mathrm{KRl}-20$ & Russian High Alumina - Rus & Fox et al. 2008 \\
\hline 2889 KRl-21 & Russian High Alumina - Rus & Fox et al. 2008 \\
\hline $2890 \mathrm{KRl}-22$ & Russian High Alumina - Rus & Fox et al. 2008 \\
\hline $2891 \mathrm{KRl}-23$ & Russian High Alumina - Rus & Fox et al. 2008 \\
\hline $\begin{array}{ll}2892 \text { KRR-24 } \\
2893 \\
\text { KR } 25\end{array}$ & $\begin{array}{l}\text { Russian High Alumina - Rus } \\
\text { Russian High Alumina - Rus }\end{array}$ & $\begin{array}{l}\text { Fox et al. } 2008 \\
\text { F }\end{array}$ \\
\hline $\begin{array}{l}2893 \text { KRl-25 } \\
2894 \text { KR-26 }\end{array}$ & $\begin{array}{l}\text { Russian High Alumina - Rus } \\
\text { Russian High Alumina - Rus }\end{array}$ & $\begin{array}{l}\text { Fox et al. } 2008 \\
\text { Fox et al. } 2008\end{array}$ \\
\hline $\begin{array}{l}28994 \mathrm{KRR}-26 \\
2895\end{array}$ & $\begin{array}{l}\text { Russlan High Alumina - Rus } \\
\text { Russian High Alumina - Rus }\end{array}$ & $\begin{array}{l}\text { Fox et al. } 2008 \\
\text { Fox et al. } 2008\end{array}$ \\
\hline $\begin{array}{ll}2896 \mathrm{KRl}-28 \\
2896\end{array}$ & $\begin{array}{l}\text { Russian High Alumina - Rus } \\
\text { Rustan }\end{array}$ & Fox et al. 2008 \\
\hline $2897 \mathrm{KRl}-29$ & Russian High Alumina - Rus & Fox et al. 2008 \\
\hline $2898 \mathrm{KRl}-30$ & Russian High Alumina - Rus & Fox et al. 2008 \\
\hline 2899 US-01 & Russian High Alumina - US & Riley et al. 2009 \\
\hline 2900 US-02 & Russian High Alumina - US & Riley et al. 2009 \\
\hline 2901 US -03 & Russian High Alumina - US & Riley et al. 2009 \\
\hline $\begin{array}{l}2902 \text { US }-04 \\
2903 \text { US }-05\end{array}$ & $\begin{array}{l}\text { Russian High A Alumina - US } \\
\text { Russian High Alumina - US }\end{array}$ & $\begin{array}{l}\text { Riliey et al. } 2009 \\
\text { Riley et al. } 2009\end{array}$ \\
\hline 2904 US -06 & $\begin{array}{l}\text { Russlan High A Aumina - US } \\
\text { Russian High Alumina - SS }\end{array}$ & $\begin{array}{l}\text { Riley et al. } 2009 \\
\text { Riley et al. } 2009\end{array}$ \\
\hline 2905 US-07 07 & $\begin{array}{l}\text { Russian High Alumina - US } \\
\text { Rosh }\end{array}$ & Riley et al. 2009 \\
\hline 2906 US-08 (repl. KRI-01) & Russian High Alumina - US & Riley et al. 2009 \\
\hline 2907 US-09 & Russian High Alumina - US & Riley et al. 2009 \\
\hline 2908 US-10 & Russian High Alumina - US & Riley et al. 2009 \\
\hline 2909 US-11 & Russian High Alumina - US & Riley et al. 2009 \\
\hline 2910 US -12 & Russian High Alumina - US & Riley et al. 2009 \\
\hline $\begin{array}{l}2911 \text { US }-13 \\
2912 \text { US-14 }\end{array}$ & $\begin{array}{l}\text { Russian High Alumina - US } \\
\text { Russian High Alumina - US }\end{array}$ & $\begin{array}{l}\text { Riliey et al. } 20099 \\
\text { Riley et al } 2009\end{array}$ \\
\hline 2913 US -15 & $\begin{array}{l}\text { Russian High A Alumina - US } \\
\text { Russian High Alumina - SS }\end{array}$ & $\begin{array}{l}\text { Riley et al. } 2009 \\
\text { Riley et al. } 2009\end{array}$ \\
\hline 2914 US -16 & 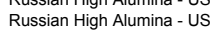 & Riley et al. 2009 \\
\hline 2915 US-17 & Russian High Alumina - US & Riley et al. 2009 \\
\hline 2916 US-18 & Russian High Alumina - US & Riley et al. 2009 \\
\hline 2917 US-19 (repl. KRl-02) & Russian High Alumina - US & Riley et al. 2009 \\
\hline 2918 US-20 (repl. KRI-03) & Russian High Alumina - US & Riley et al. 2009 \\
\hline 2919 US 212 & Russian High Alumina - US & Riley et al. 2009 \\
\hline 2920 US 222 & $\begin{array}{l}\text { Russian High Alumina - US } \\
\text { Russian High Alumina - US }\end{array}$ & $\begin{array}{l}\text { Riliey et al. } 20099 \\
\text { Rilev et }\end{array}$ \\
\hline 2921 US 2324 Us 24 & $\begin{array}{l}\text { Russian High Alumina - US } \\
\text { Russian High Alumina - US }\end{array}$ & $\begin{array}{l}\text { Rley et al. } 2009 \\
\text { Riley et al. } 2009\end{array}$ \\
\hline 2923 US-25 (IRPI. RRI-U4) & $\begin{array}{l}\text { Russian Hign Alumina- } \\
\text { Russian Alumina - }\end{array}$ & Riley et al. 2009 \\
\hline 2924 US-26 & Russian High Alumina - US & $\begin{array}{l}\text { al. } 2009 \\
\text { al. }\end{array}$ \\
\hline 2925 US- & Russian High Alumina - US & Riley et al. 2009 \\
\hline 2926 US & Russian High Alumina - US & Riley et al. 2009 \\
\hline 2927 US & Russian High Alumina - US & Riley et al. 2009 \\
\hline 2928 US & Russian High Alumina - US & et al. 2009 \\
\hline 2929 US -31 & Russian High Alumina - US & $\begin{array}{l}\text { yet al. } 2009 \\
\text { y. }\end{array}$ \\
\hline 2930 US 232 & $\begin{array}{l}\text { Russian High Alumina - US } \\
\text { Russian High Alumina - US }\end{array}$ & $\begin{array}{l}\text { ey et al. } 2009 \\
\text { ey et al. } 2009\end{array}$ \\
\hline $\begin{array}{l}2933 \text { US-33 } \\
2932-34\end{array}$ & $\begin{array}{l}\text { Russian High A Alumina - US } \\
\text { Russian High Alumina - }\end{array}$ & $\begin{array}{l}\text { Riley yetal. } 2009 \\
\text { Riley et al. } 2009\end{array}$ \\
\hline 2933 US-35 (repl. KRI-05) & Russian High Alumina - US & Riley et al. 2009 \\
\hline 2934 US-36 & Russian High Alumina - US & Riley et al. 2009 \\
\hline 35 us & Russian High Alumina - US & Riley et al. 2009 \\
\hline 293 & Russian High Alumina - US & et al. 2009 \\
\hline 2937 US & Russian High Alumina - US & et al. 2009 \\
\hline 2938 US -40 & Russian High Alumina - US & y et al. 2009 \\
\hline 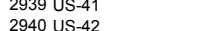 & $\begin{array}{l}\text { Russian High Alumina - US } \\
\text { Russian High Alumina - US }\end{array}$ & $\begin{array}{l}\text { Riliey et al. } 2009 \\
\text { Riley et al. } 2009\end{array}$ \\
\hline 2941 US- & $\begin{array}{l}\text { Russlan Hign AAumina- - SS } \\
\text { Russian High Alumina - }\end{array}$ & Riley et al. 2009 \\
\hline 2942 US-4 & Russian High Alumina - US & Riley et al. 2009 \\
\hline KRI-07) & Russian High Alumina - US & Riley et al. 2009 \\
\hline $44 \mathrm{HLV}$ & T series & Kot et al. 2003b \\
\hline HLW & T se & Kot et al. 2003b \\
\hline & T series & 003b \\
\hline & T series & o3b \\
\hline & $\begin{array}{l}\text { T series } \\
\text { T series }\end{array}$ & to et al. 20036 \\
\hline & $\begin{array}{l}1 \text { series } \\
\text { T series }\end{array}$ & $\begin{array}{l}\text { of tetal. } 2003 \mathrm{~b} \\
\text { ot et al } 2003 \mathrm{~b}\end{array}$ \\
\hline & T series & $\begin{array}{l}\text { tal. } 2003 \mathrm{~b} \\
\text { al. } 2003 \mathrm{~b}\end{array}$ \\
\hline HLW98-T09 & T series & Kot et al. 2003b \\
\hline
\end{tabular}

A1203-t B2O3-t CaO-t Fe2O3-t $\begin{array}{llll}0.1447 & 0.1440 & 0.0070 & 0.0823 \\ 0.1450 & 0.1225 & 0.0075 & 0.0779\end{array}$ $\begin{array}{llll}0.1450 & 0.1425 & 0.0075 & 0.0779 \\ 0.1002 & 0.1996 & 0.0100 & 0.1135\end{array}$ $\begin{array}{llll}0.1280 & 0.1259 & 0.0056 & 0.0886 \\ 0.1280 & 0.1259 & 0.0056 & 0.0886\end{array}$ $\begin{array}{llll}0.1269 & 0.1625 & 0.0025 & 0.0800 \\ 0.1331 & 0.0025 & 0.0800\end{array}$ $\begin{array}{lllll}0.1331 & 0.1434 & 0.025 & 0.0800 \\ 0.1250 & 0.0938 & 0.0075 & 0.0800\end{array}$ $\begin{array}{llll}0.1579 & 0.1399 & 0.0025 & 0.0991\end{array}$ $\begin{array}{llll}0.1250 & 0.1097 & 0.0025 & 0.08800 \\ 0.132 & 0.1546 & 0.025 & 0.0800\end{array}$ $\begin{array}{llll}0.1312 & 0.1546 & 0.0025 & 0.0800 \\ 0.1623 & 0.1368 & 0.0025 & 0.0800\end{array}$ $\begin{array}{llll}0.1250 & 0.0875 & 0.0025 & 0.00800 \\ 0.0800 & 0.0025\end{array}$ $\begin{array}{llll}0.1286 & 0.1240 & 0.0075 & 0.0859 \\ 0.1338 & 0.1483 & 00075 & 0.0991\end{array}$ $\begin{array}{llll}0.1338 & 0.1483 & 0.0075 & 0.0891 \\ 0.1354 & 0.1112 & 0.0025 & 0.0981\end{array}$ $\begin{array}{lllll}0.1440 & 0.1479 & 0.0075 & 0.0864\end{array}$ $\begin{array}{llll}0.1352 & 0.1183 & 0.0075 & 0.0906 \\ 0.1574 & 0.1152 & 0.0025 & 0.0800\end{array}$ $\begin{array}{llll}0.1626 & 0.1260 & 0.0025 & 0.0800\end{array}$ $\begin{array}{lllll}0.1506 & 0.1351 & 0.0028 & 0.1123\end{array}$ $\begin{array}{llll}0.11048 & 0.1019 & 0.0100 & 0.1114 \\ 0.044 & 0.1720 & 0.000 & 0.058\end{array}$ $\begin{array}{llll}0.1044 & 0.1720 & 0.0100 & 0.0987 \\ 0.1835 & 0.0613 & 0.0000 & 0.0500\end{array}$ $\begin{array}{llll}0.1427 & 0.1138 & 0.0000 & 0.0565 \\ 0.2000 & 0.1530 & 0.0075 & 0.0551 \\ 0.1400 & 0.168 & 0.000 & 0.059\end{array}$ $\begin{array}{llll}0.2000 & 0.1530 & 0.0075 & 0.0551 \\ 0.1493 & 0.0789 & 0.0000 & 0.0859\end{array}$ $\begin{array}{llll}0.1600 & 0.168 & 0.0000 & 0.0659 \\ 0.1731 & 0.00100 & 0.0653\end{array}$ $\begin{array}{llll}0.1600 & 0.1731 & 0.0100 & 0.0653 \\ 0.1600 & 0.1129 & 0.0100 & 0.0827\end{array}$ $\begin{array}{llll}0.1600 & 0.1129 & 0.0100 & 0.0827 \\ 0.1599 & 0.1670 & 0.0070 & 0.0816\end{array}$ $\begin{array}{llll}0.1600 & 0.1406 & 0.0100 & 0.08815 \\ 0.1600 & 0.1349 & 0.0100 & 0.0730\end{array}$ $\begin{array}{llll}0.1500 & 0.1349 & 0.0100 & 0.0730 \\ 0.1519 & 0.0956 & 0.0000 & 0.0975\end{array}$ $\begin{array}{lllll}0.1337 & 0.1505 & 0.0100 & 0.1088\end{array}$ $\begin{array}{lllll}0.1412 & 0.1099 & 0.0100 & 0.1069 \\ 0.1370 & 0.1998 & 0.0100 & 0.0776\end{array}$ $\begin{array}{llll}0.1324 & 0.1608 & 0.0100 & 0.0874 \\ 0.1600 & 0.1457 & 0.0100 & 0.0730\end{array}$ $\begin{array}{llll}0.1302 & 0.1790 & 0.0040 & 0.0941 \\ 0.1000 & 0.1780 & 0.0050 & 0.0500\end{array}$ $\begin{array}{llll}0.11000 & 0.1780 & 0.0050 & 0.0500 \\ 0.1332 & 0.1881 & 0.0100 & 0.0903 \\ 0.1600 & 0.1171 & 0.0100 & 0.035\end{array}$ $\begin{array}{llll}0.1600 & 0.0913 & 0.0100 & 0.0022 \\ 0.1447 & 0.1440 & 0.0070 & 0.0823\end{array}$ $\begin{array}{llll}0.1447 & 0.1440 & 0.0070 & 0.0823 \\ 0.1447 & 0.1440 & 0.0070 & 0.0823 \\ 0.1334 & 0.115 & 0.035 & 0.0947\end{array}$ $\begin{array}{lllll}0.1447 & 0.1440 & 0.0007 & 0.0823 \\ 0.1422 & 0.1375 & 0.0038 & 0.0845 \\ 0 & 0.145\end{array}$ $\begin{array}{llll}0.1334 & 0.1169 & 0.0075 & 0.0947 \\ 0.1450 & 0.1332 & 0.0075 & 0.0762 \\ 0.450 & 0.125 & 0.075 & 0.079\end{array}$ $\begin{array}{llll}0.1430 & 0.1332 & 0.075 & 0.0972 \\ 0.1450 & 0.1225 & 0.0075 & 0.0779 \\ 0.1158 & 0.1625 & 0.075 & 0.0794\end{array}$ $\begin{array}{llll}0.1883 & 0.1069 & 0.0100 & 0.0500 \\ 0.1040 & 0.0648 & 0.0000 & 0.1518 \\ 0.1747 & 0.1796 & 0.000 & 0.0572\end{array}$ $\begin{array}{llll}0.1747 & 0.1796 & 0.0000 & 0.0572 \\ 0.1000 & 0.0873 & 0.0100 & 0.0500\end{array}$ $\begin{array}{llll}0.1265 & 0.1819 & 0.0100 & 0.0533 \\ 0.1267 & 0.1452 & 0.0000 & 0.1545 \\ 0.1500 & 0.150 & 0.000 & 0.50\end{array}$ $\begin{array}{llll}0.1000 & 0.1901 & 0.0100 & 0.1545 \\ 0.1134 & 0.0500 & 0.0000 & 0.050\end{array}$ $\begin{array}{llll}0.1000 & 0.1369 & 0.0000 & 0.0639 \\ 0.1002 & 0.1996 & 0.0100 & 0.1135 \\ 0.1265 & 0.159 & 0.021 & 0.076\end{array}$ $\begin{array}{llll}0.1002 & 0.1996 & 0.0100 & 0.1135 \\ 0.1286 & 0.1551 & 0.0021 & 0.0976\end{array}$ $\begin{array}{llll}0.1286 & 0.1551 & 0.0021 & 0.0956 \\ 0.1000 & 0.0577 & 0.0100 & 0.1700 \\ 0.155 & 0.135 & 0.070 & 0.050\end{array}$ $\begin{array}{llll}0.1000 & 0.0731 & 0.0079 & 0.0500 \\ 0.1155 & 0.1635 & 0.0000 & 0.0750 \\ 0 & 0.1500\end{array}$ $\begin{array}{llll}0.1000 & 0.1555 & 0.0100 & 0.1553\end{array}$ $\begin{array}{llll}0.2000 & 0.0619 & 0.0100 & 0.0500 \\ 0.1717 & 0.0864 & 0.0100 & 0.0886\end{array}$ $\begin{array}{llll}0.1717 & 0.0864 & 0.01100 & 0.0886 \\ 0.1280 & 0.1259 & 0.0056 & 0.0886 \\ 0 & 0.259\end{array}$ $\begin{array}{llll}0.1280 & 0.1259 & 0.0056 & 0.0886 \\ 0.0529 & 0.1206 & 0.0027 & 0.1230\end{array}$

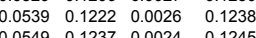
$\begin{array}{lllll}0.0549 & 0.1219 & 0.0034 & 0.1258\end{array}$ $\begin{array}{llll}0.0539 & 0.1185 & 0.0045 & 0.1264 \\ 0.0528 & 0.1150 & 0.056 & 0.1209\end{array}$

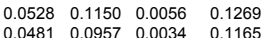
$\begin{array}{llll}0.0432 & 0.0974 & 0.0038 & 0.1075 \\ 0.0385 & 0.0992 & 0.0041 & 0.0985\end{array}$
K2O-t Li2O-t MgO-t Na2O-t NiO-t P2O5-t SiO2-t ZrO2-t Ag2O-t As203-tAs205-t BaO-t BeO-t Bi203-t Br-t CdO-t Ce203-t CeO2-t Cl$\begin{array}{llllll}0.0078 & 0.0364 & 0.0033 & 0.1080 & 0.002 \\ 0.0075 & 0.0300 & 0.0037 & 0.1157 & 0.0050\end{array}$ $0.0050-0.4444 \quad 0.0038$ $\begin{array}{llllll}0.0064 & 0.0405 & 0.0025 & 0.1273 & 0.0003\end{array}$

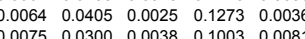
$\begin{array}{lllll}0.0025 & 0.0300 & 0.0038 & 0.1121 & 0.0075\end{array}$ $\begin{array}{lllll}0.0075 & 0.0311 & 0.0013 & 0.1280 & 0.0075\end{array}$ 0.00450 .04080 .00130 .10660 .0075 $\begin{array}{lllll}0.0075 & 0.0300 & 0.0013 & 0.1180 & 0.0075\end{array}$ $\begin{array}{lllll}0.0025 & 0.0500 & 0.0038 & 0.0876 & 0.0110\end{array}$ $0.0025 .00492 \quad 0.0013 \quad 0.1036 \quad 0.0075$ $\begin{array}{lllll}0.0037 & 0.0388 & 0.0038 & 0.1033 & 0.0075\end{array}$

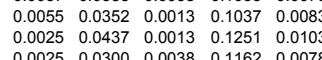
$\begin{array}{lllll}0.0044 & 0.0329 & 0.0021 & 0.1259 & 0.0105 \\ 0.0025 & 0.0500 & 0.0013 & 0.11108 & 0.0075 \\ 0.0025 & 0.0300 & 0.0035 & 0.1220 & 0.075\end{array}$ $\begin{array}{lllllll}0.0075 & 0.0377 & 0.0013 & 0.1024 & 0.0075\end{array}$ $\begin{array}{lllll}0.0068 & 0.0375 & 0.0013 & 0.1151 & 0.0075 \\ 0.0000 & 0.0600 & 0.0000 & 0.1354 & 0.030\end{array}$ $\begin{array}{llllll}0.0000 & 0.0600 & 0.0000 & 0.1354 & 0.0300 \\ 0.0000 & 0.0573 & 0.0050 & 0.1023 & 0.0047\end{array}$ $\begin{array}{lllll}0.0000 & 0.0573 & 0.0050 & 0.1023 & 0.0047 \\ 0.0000 & 0.0572 & 0.0000 & 0.1852 & 0.0000\end{array}$ $\begin{array}{llllll}0.0100 & 0.0200 & 0.0050 & 0.2000 & 0.0300 \\ 0.0000 & 0.0579 & 0.0050 & 0.0724 & 0.0000\end{array}$ $\begin{array}{lllll}0.0000 & 0.0579 & 0.0050 & 0.0724 & 0.0000 \\ 0.0100 & 0.0562 & 0.0050 & 0.1237 & 0.0006\end{array}$ $\begin{array}{lllllllllllllll}0.0000 & 0.0529 & 0.0000 & 0.1233 & 0.0000\end{array}$ $\begin{array}{lllll}0.0100 & 0.0407 & 0.0050 & 0.0788 & 0.0029 \\ 0.0100 & 0.0374 & 0.0050 & 0.1033 & 0.0000\end{array}$ $\begin{array}{lllll}0.0100 & 0.0374 & 0.0050 & 0.1033 & 0.0000 \\ 0.0100 & 0.0200 & 0.0050 & 0.0958 & 0.0000\end{array}$ $\begin{array}{lllll}0.0100 & 0.0247 & 0.00050 & 0.1078 & 0.0000 \\ 0.0100 & 0.0266 & 0.0050 & 0.1067 & 0.00020\end{array}$ $\begin{array}{lllll}0.0100 & 0.0266 & 0.0050 & 0.1067 & 0.00020 \\ 0.0100 & 0.0486 & 0.0000 & 0.0981 & 0.0000\end{array}$ $\begin{array}{lllll}0.0100 & 0.0486 & 0.0000 & 0.0981 & 0.0000 \\ 0.0000 & 0.0200 & 0.0050 & 0.1411 & 0.0000\end{array}$ $\begin{array}{llllll}0.0100 & 0.0200 & 0.0050 & 0.1267 & 0.00000\end{array}$ $\begin{array}{ccccc}0.0100 & 0.0200 & 0.0050 & 0.0669 & 0.0000 \\ 0.0100 & 0.0600 & 0.0000 & 0.1116 & 0.0184\end{array}$ $\begin{array}{lllll}0.0100 & 0.0600 & 0.0000 & 0.1116 & 0.0184 \\ 0.0100 & 0.0215 & 0.0050 & 0.1153 & 0.0028\end{array}$ $\begin{array}{lllll}0.0100 & 0.0574 & 0.0050 & 0.1122 & 0.0000\end{array}$ $\begin{array}{lllll}0.0100 & 0.02200 & 0.0000 & 0.1500 & 0.00000 \\ 0.0100 & 0.0256 & 0.0000 & 0.0630 & 0.0000\end{array}$ $\begin{array}{lllll}0.0100 & 0.0256 & 0.0000 & 0.06030 & 0.0000 \\ 0.0000 & 0.0536 & 0.0050 & 0.0858 & 0.0108\end{array}$ $\begin{array}{lllll}0.0000 & 0.0495 & 0.0000 & 0.1341 & 0.0000\end{array}$ $\begin{array}{llllll}0.0078 & 0.0364 & 0.0033 & 0.1080 & 0.002\end{array}$ $\begin{array}{lllll}0.0078 & 0.0364 & 0.0033 & 0.1080 & 0.002\end{array}$ $\begin{array}{lllllll}0.0075 & 0.0500 & 0.0037 & 0.1178 & 0.0092\end{array}$ $\begin{array}{lllllllllllllllll}0.0025 & 0.0350 & 0.0012 & 0.1168 & 0.005\end{array}$ $\begin{array}{lllll}0.0075 & 0.0300 & 0.0037 & 0.1157 & 0.005 \\ 0.0029 & 0.0500 & 0.0037 & 0.1088 & 0.014\end{array}$ $\begin{array}{llllll}0.0000 & 0.0600 & 0.0050 & 0.1102 & 0.0000\end{array}$ $\begin{array}{llllll}0.0100 & 0.0600 & 0.0050 & 0.1687 & 0.0000\end{array}$ $\begin{array}{lllll}0.0100 & 0.0200 & 0.0000 & 0.1336 & 0.000 \\ 0.0100 & 0.0600 & 0.0050 & 0.1868 & 0.0000\end{array}$

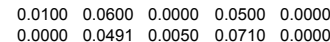
$\begin{array}{lllll}0.0000 & 0.0491 & 0.0050 & 0.0710 & 0.0000 \\ 0.0000 & 0.0600 & 0.0000 & 0.0750 & 0.0000 \\ 0.0000 & 0.0200 & 0.0050 & 0.1976 & 0.0097\end{array}$ $\begin{array}{lllll}0.0100 & 0.0242 & 0.0050 & 0.2000 & 0.0000 \\ 0.0000 & 0.0200 & 0.0050 & 0.0794 & 0.0000\end{array}$ $\begin{array}{llllll}0.0052 & 0.02242 & 0.0050 & 0.0794 & 0.0000 \\ 0.0005 & 0.1252 & 0.0056\end{array}$ $\begin{array}{lllll}0.0052 & 0.0242 & 0.0005 & 0.1252 & 0.005 \\ 0.0000 & 0.0200 & 0.0000 & 0.1889 & 0.0000\end{array}$ $\begin{array}{lllll}0.0100 & 0.0200 & 0.0000 & 0.1972 & 0.0291 \\ 0.0100 & 0.0600 & 0.0050 & 0.0500 & 0.0183\end{array}$ $\begin{array}{llllll}0.0100 & 0.0251 & 0.0000 & 0.1013 & 0.0000\end{array}$ $\begin{array}{lllll}0.0100 & 0.0372 & 0.0000 & 0.0652 & 0.0000 \\ 0.0100 & 0.0600 & 0.0000 & 0.1882 & 0.0000\end{array}$ $\begin{array}{lllll}0.0100 & 0.0490 & 0.0050 & 0.1029 & 0.0022 \\ 0.0064 & 0.0405 & 0.0025 & 0.1273 & 0.0003\end{array}$ $\begin{array}{llllll}0.0064 & 0.0405 & 0.0025 & 0.1273 & 0.00036 \\ 0.0064 & 0.0405 & 0.0025 & 0.1273 & 0.0036\end{array}$

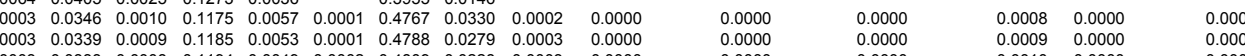

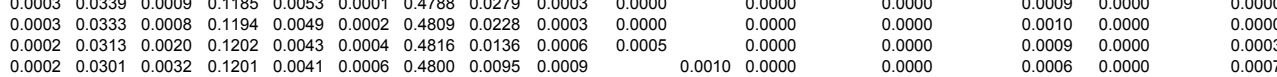

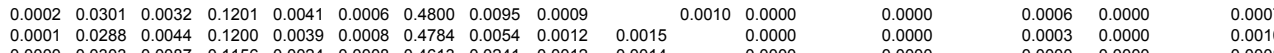

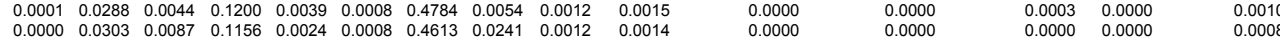

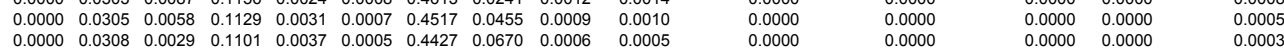


\# Glass ID

$2871 \mathrm{KRI}-03$ (repl. US-20)

2873 KRR-05 (repl. US-35)

2874 KRI-06 (repl. US-44)

$2876 \mathrm{KRI}-08$

2877
$2878 \mathrm{KRl}-10$
288

$2879 \mathrm{KRI}-11$
$2880 \mathrm{KRl}-12$
$2881 \mathrm{KRl}-13$

2881 KRl-13

2883 KRl-15

$2884 \mathrm{KRl}-16$
$2885 \mathrm{KRl}-17$

$2886 \mathrm{KRl}-18$

$2888 \mathrm{KRl}-20$

$2889 \mathrm{KRR}-21$

$2891 \mathrm{KRl}-23$

$2892 \mathrm{KRl}-24$

$2894 \mathrm{KRR}-26$

$2895 \mathrm{KRl}-27$
$2896 \mathrm{KR}-28$

$2897 \mathrm{KRI}-29$

2901 US-03

2902 US-04

2904 US- 06

2905 US-07

2906 US-08 (repl. KRI-0)

2908 US-10

2009 US-11

2912 US-14

2914 US- 1

2916 US-18

2917 US-19 (repl. KRL-02)

2918 US-20 (repl. KRI-03)

2920 US-22

2922 US-24 (repl. KRI-04)

2923 US -25

2925 US-22

2926 US-28

2928 US-30

2930 US-32

2931 US-33

2933 US-35 (repl. KRI-05)

2934 US-36

2936 US-38

2937 US-39

2939 US- 4

2940 US-4

2942 US-44 (repl. KRI-06)

2943 US-45 (repl. KRI-07)

2945 HLW98-TO2

2946 HLW98-TO3

2948 HLW98-T05

(2949 HLW98-T06
CoO-t Co2O3-t Cr2O3-t Cs2O-t CuO-t Dy203-t Eu2O3-t

1-t La2O3-t MnO2-t MnO-t MoO-t MoO2-t MoO3-t Nb2O5-t Nd2O3-t NpO2-t 0.0007

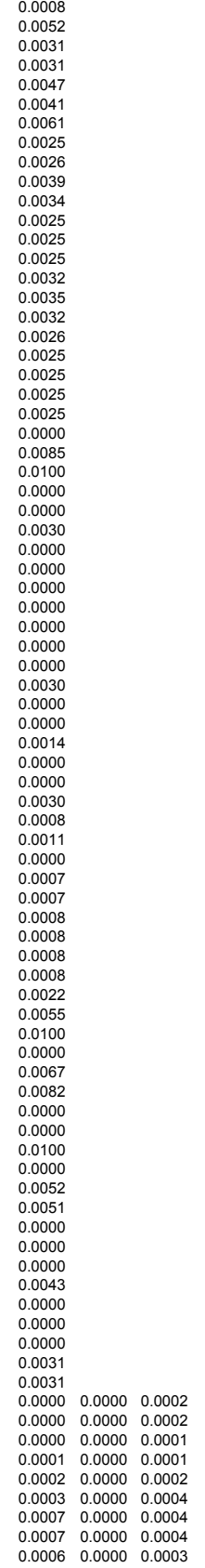

0.0000

0.0000

0.0000
0.0000

0.00000

0.0000
F-t Ga2O3-t Gd2O3-t HFO2-t HgO-t

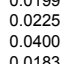

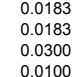

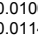

20.0300

$\underset{20.0300}{0.011}$

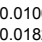

$\underset{\substack{0.0230 \\ 0.0000}}{0.020}$

10127

00001

o.

0.0250

0.0000

$\underset{\substack{0.0300 \\ 0.0300}}{0.030}$

docos

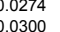

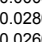

$\underset{\substack{0.000 \\ 0.024}}{0.024}$

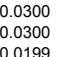

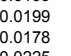

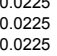

0.0000

0.0000

0.0000
0.0350

0.0400

0.0400

0.012

0.00000

0.0000
0.0400
0

0.0183

0.0040
0.0039
0.0038
0.0034
0.0031
0.0028
0.0022
0.0020
0.0017

0.0000
0.0000
0.0000
0.0000
0.0000
0.0000
0.0000
0.0000
0.0000

0.0000
0.0007

2-t PdO-t Pr203-t Pr6011-t Rb2O-t ReO-t

0.0025
0.0075
0.0075

.0075

0.0038
0.0075
0.0025

0.0047

0.0025

0.0075
0.025
0.025

0.0100

0.0100

0.0010

0.0010

0.0000
0.0010

0.0010
0.0010

0.0010
0.0010

0.0000
0.0010
0.0007

0.00010

0.0000
0.0010
0.0007

0.0007

0.0008
0.0003
0.0008

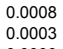

.00000

0.0100

0.0100

.0000

0.0100
0.0100

.0100

0.0100
0.0100

0.0067
0.0067

0.0004
0.0005
0.0006
.0002

0.0003
0.0002
0.0001
0.0000
0.0000
0.0000
0.0003
0.0006
0.0008

0.0026
0.0031
0.0103
0.0170
0.0237
0.0335
0.0271
0.0207

0.0000
0.0000

0.0000

0.0000
0.0000
0.0000

0.0000 $\begin{array}{ll}0.0000 & 0.0000\end{array}$

0.00000 .00000

\begin{tabular}{ll}
0.0000 & 0.0000 \\
0.0000 & 0.0000 \\
\hline
\end{tabular}

\begin{tabular}{lll}
0.0000 & 0.0000 \\
0.0000 & 0.0000 \\
\hline
\end{tabular}

$\begin{array}{ll}0.0000 & 0.0000 \\ 0.0000 & 0.0000\end{array}$ 
$\begin{array}{ll}\text { so3-t } & \text { sro-t } \\ 0.0028 & 0.0006 \\ 0.0037 & 0.0008\end{array}$

$0.0000 \quad 0.0270$

$0.0022 \quad 0.0162$

$\begin{array}{ll}0.0013 & 0.0075 \\ 0.0013 & 0.0075\end{array}$

0.00130 .0179

$\begin{array}{lll}0.0013 & 0.0225 \\ 0.0038 & 0.0075 \\ 0.030 & 0.0075\end{array}$

$\begin{array}{lll}0.0038 & 0.0075 \\ 0.0033 & 0.0077\end{array}$

$\begin{array}{ll}0.0033 & 0.0077 \\ 0.0038 & 0.0225 \\ 0.0038 & 0.0225\end{array}$

$\begin{array}{ll}0.0038 & 0.0225 \\ 0.0038 & 0.0075 \\ 0.0013 & 0.0225\end{array}$

$\begin{array}{ll}0.0013 & 0.0225 \\ 0.0013 & 0.0225\end{array}$

$0.0038 \quad 0.0082$

0.00380 .0075

$\begin{array}{ll}0.0038 & 0.0118 \\ 0.0019 & 0.0075 \\ 0.0050 & 0.0000\end{array}$

0.00500 .0300

$\begin{array}{lll}0.0050 & 0.0000 \\ 0.0000 & 0.0220 \\ 0 & 0.000000 & 0.0300\end{array}$

$\begin{array}{ll}0.0000 & 0.0220 \\ 0.0050 & 0.0300\end{array}$

$\begin{array}{ll}0.0000 & 0.0010 \\ 0.0000 & 0.0000\end{array}$

$\begin{array}{lll}0.00000 & 0.00010 \\ 0.0050 & 0.0010\end{array}$

0.00500 .0010

$\begin{array}{ll}0.0050 & 0.0010 \\ 0.0050 & 0.0010\end{array}$

0.00500 .00000

$0.0050 \quad 0.0010$

$\begin{array}{ll}0.0050 & 0.0000 \\ 0.0050 & 0.0010 \\ 0.0000 & 0.0000\end{array}$

$0.0000 \quad 0.0000$

$\begin{array}{lll}0.00000 & 0.00010 \\ 0.0000 & 0.0000\end{array}$

$0.0000 \quad 0.001$

$\begin{array}{ll}0.0000 & 0.0010 \\ 0.0028 & 0.0006 \\ 0.0028 & 0.0006\end{array}$

$\begin{array}{lll}0.0028 & 0.000 \\ 0.0012 & 0.0008 \\ 0.000 & 0\end{array}$

0.00120 .0008

$\begin{array}{lll}0.0037 & 0.0003 \\ 0.0037 & 0.0008 \\ 0.0037 & 0.0008\end{array}$

$0.0000 \quad 0.0300$

$\begin{array}{ll}0.0000 & 0.0300 \\ 0.0050 & 0.0000 \\ 0.0050 & 0.0300\end{array}$

$\begin{array}{lll}0.0000 & 0.011 \\ 0.0050 & 0.030\end{array}$

$0.0050 \quad 0.0300$

$\begin{array}{ll}0.0050 & 0.0000 \\ 0.0050 & 0.0300 \\ 0.0000 & 0.0200\end{array}$

$0.0000 \quad 0.0270$

0.00000 .0001

$\begin{array}{lll}0.0000 & 0.0000 \\ 0.0050 & 0.0300 \\ 0 & 0.000 & 0.0000\end{array}$

$\begin{array}{lll}0.00000 & 0.03080 \\ 0.0000 & 0.000000 & 0.0000\end{array}$

$\begin{array}{lll}0.0000 & 0.0000 \\ 0.0000 & 0.0300\end{array}$

$\begin{array}{ll}0.0000 & 0.0000 \\ 0.0050 & 0.0047\end{array}$

$\begin{array}{ll}0.0022 & 0.0162 \\ 0.0022 & 0.0162\end{array}$

$\begin{array}{lll}0.0000 & 0.0007 & 0.0002 \\ 0.0000 & 0.0006 & 0.0002\end{array}$ $\begin{array}{lll}0.0000 & 0.0006 & 0.0002 \\ 0.0000 & 0.0005 & 0.0001 \\ 0 & 0.000 & 0.003\end{array}$ $\begin{array}{llll}0.0000 & 0.0005 & 0.0001 \\ 0.0000 & 0.0003 & 0.0000 \\ 0.0000 & 0.0002 & 0.000\end{array}$ $\begin{array}{llll}0.0000 & 0.0002 & 0.0000 \\ 0.0000 & 0.0001 & 00000\end{array}$ $0.0000 \quad 0.0000 \quad 0.0069$ $\begin{array}{lll}0.0000 & 0.0000 & 0.0046 \\ 0.0000 & 0.0000 & 0.0023\end{array}$

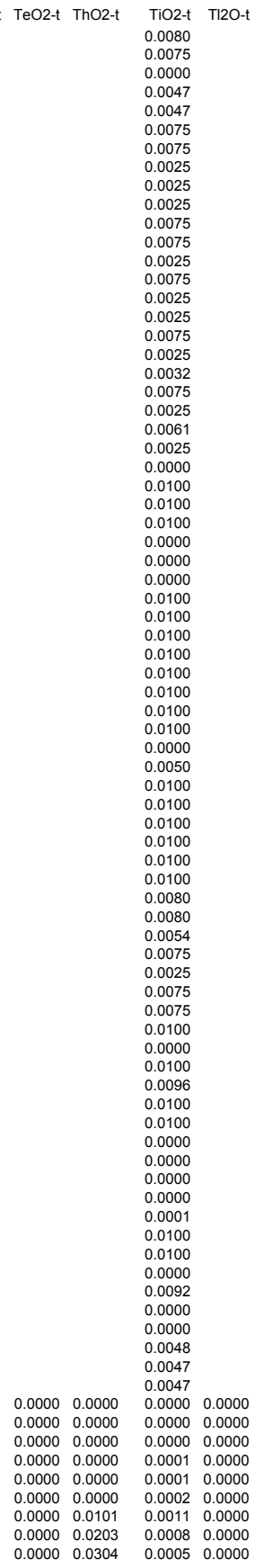

U03-t V2O5-t WO3-t Y2O3-t ZnO-t Sum-t Al2O3-a 0.0000

$\begin{array}{ll}0.0007 & 1.0000 \\ 0.0008 & 1.0000 \\ 0.0200 & 1.0000\end{array}$

$\begin{array}{ll}0.0102 & 1.0000 \\ 0.0102 & 1.0000\end{array}$

$\begin{array}{lll}0.0102 & 1.00000 \\ 0.0150 & 1.0000\end{array}$

$\begin{array}{ll}.0150 & 1.0000 \\ .00107 & 1.0000 \\ 0.0050 & 1.0000\end{array}$

0.00501 .0000

$0.0150 \quad 1.0000$

$\begin{array}{lll}0.0142 & 1.0000 \\ .0070 & 1.0000\end{array}$

$\begin{array}{ll}0.0070 & 1.0000 \\ 0.0150 & 1.0000 \\ 0.0050 & 1.0000\end{array}$

$\begin{array}{ll}0.0050 & 1.0000 \\ 0.0050 & 1.0000 \\ 0.0000 & 1.000\end{array}$

$0.0050 \quad 1.0000$

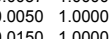

$\begin{array}{ll}0.0120 & 1.0000 \\ 0.0050 & 1.0000\end{array}$

0.00001 .0000

$\begin{array}{lll}0.0000 & 1.0000 \\ 0.0200 & 1.0000\end{array}$

0.00001 .0000

$\begin{array}{ll}0.0010 & 1.0000 \\ 0.000 & 1.0000\end{array}$

0.00101 .0000

$.0000 \quad 1.0000$

$\begin{array}{lll}0.0000 & 1.0000 \\ 0.0010 & 1.0000 \\ 0.0000 & 1.0000\end{array}$

D.0000 1.0000

$\begin{array}{ll}0.0010 & 1.0000 \\ 0.0010 & 1.0000 \\ 0.001 & 1.0000\end{array}$

0.00101 .0000

0.00101 .0000

$0.0010 \quad 1.0000$

0.00001 .0000

$.0010 \quad 1.0000$

$\begin{array}{ll}0.0007 & 1.0000 \\ 0.007 & 1.0000\end{array}$

$0.0003 \quad 1.0000$

$0.0008 \quad 1.0000$

$0.0008 \quad 1.0000$

$\begin{array}{ll}0.0008 & 1.0000 \\ 0.0008 & 1.0000\end{array}$

$0.0200 \quad 1.0000$

0.02001 .0000

$\begin{array}{ll}.0000 & 1.0000 \\ .0048 & 1.0000\end{array}$

0.00001 .0000

0.0000110000

$0.0000 \quad 1.0000$

$0.0001 \quad 1.0000$

$\begin{array}{lll}.0200 & 1.0000 \\ .0200 & 1.0000\end{array}$

0.02001 .0000

$0.0000 \quad 1.0000$

$0.0149 \quad 1.0000$

$0.0102 \quad 1.0000$

$0.0102 \quad 1.0000$

$\begin{array}{lll}0.0000 & 0.0000 \\ 0 & 00000 & 0.0000\end{array}$

$\begin{array}{llll}0.0000 & 0.00000 \\ 0.0000 & 0.00000\end{array}$

$0.0000 \quad 0.00000$

0006500000

$\begin{array}{ll}0.0129 & 0.0000 \\ 0.0193 & 0.0000\end{array}$ 
2872 KRI-04 (repl. US-24)

2873 KRl-05 (repl. US-35)

2875 KRl-07 (repl. (US-45)

$2876 \mathrm{KRL}-08$

$2878 \mathrm{KRl}-10$

2879 KRl-11

$2881 \mathrm{KRR}-13$

$2882 \mathrm{KRI}-14$

$2883 \mathrm{KRl}-15$

2885 KRI-17

2886 KRI-18
2887

$2887 \mathrm{KRl}-19$

$2889 \mathrm{KR}-21$

$2892 \mathrm{KR} l-24$
$2893 \mathrm{KRl}-25$

$2894 \mathrm{KRl}-26$

2896 KRI-28

$2897 \mathrm{KRl}-29$

2008 KR-1.30

2900 US-12

(2901 US-03

(2)

2904 US-06

2906 US-08 (repl. KRI-01)

2909 Us-11

2910 US -12
2911 Us -13

2912 US-14

2913 US-15
2914 US-16

2916 US-18

2917 US-19 (repl. KRR-02

2918 US-20 (repl. KRI-03)

2920 US-22

2921 US-23 2922 US-24 (repl. KRI-04)

2923 US 24

2924 US-26

2925 US -27

2926 US- 28

2928 US-30

2930 US-32

2931 US-33

2933 US-35 (repl. KRl-05)

2936 US-38

2937 US-39

2939 US-4

2940 US-42

2944 US-43

2942 US-44 (repl. KRI-06)

2944 HLWB8-T01

2947 HLW98-T04

2948 HLWW8-T05

2949 HLW98-TO6
2950 HLW98-T07

2951 HLW98-T08

2952 HLW98-To9 
2878 KRR-10

2879 KRI-11

$2880 \mathrm{KR}-12$

$2882 \mathrm{KRl}-14$

2883 KRI-15
2884 KRI-16
2885 KRl-17

$2885 \mathrm{KRl}-17$

2887 KRI-19

2889 KRl-21

(2200

$2892 \mathrm{KR}-24$
$2893 \mathrm{KR}-25$

$2894 \mathrm{KR}-26$

$2896 \mathrm{KR}-28$

$2897 \mathrm{KR}-29$
$2898 \mathrm{KR}-30$

2901 Us-03

2092
2003
US--04

2903 US-05
2904 US -06

2905 US-07

2906 Us-08 (repl. KRl-01)

2908 US -10

2909 US-11

2912 US-14

2914 US-11

2916 US-18

2917 US-19 (repl. KR:-02

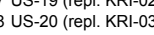

2920 US-22

2921 US-23
2922 US-24 (repl. KRR-04)

2923 US 25

2924 US-26

2925 US-27
2226 U- 28

2926 US-28

2928 Us-30

2929 US-31

$\begin{array}{ll}2930 & \text { US }-32 \\ 2031 & \text { US }-33\end{array}$

(932 US 34

2933 US-35 (repl. KRI-05)

2934 US-36

(936 US-38

2937 US-39

2939 US-4

2940 US- 4

2941 US-43
2242 US-44 (repl. KRl-0e

2943 US-45 (rep) KRl- KR-07)

2944 HLWW8-T01

2947 HLW98-T04

2948 HLWW8-TO5

2949 HLW98-TOO
2050 HLW

2951 HLW98-T08

2952 HLW98-TOS 
2878 KRl-10
2870

$2879 \mathrm{KRR}-11$

$2880 \mathrm{KRR}-12$

2882 KRI-14

$2883 \mathrm{KRI}-15$
$2884 \mathrm{KRI}-16$
$2885 \mathrm{KRl}-17$

$2886 \mathrm{KRl}-17$

2887 KRR-19
2888 KR-120

$2889 \mathrm{KR}-21$

2.201

$2892 \mathrm{KR}-24$
$2893 \mathrm{KR}-25$

$2894 \mathrm{KRl}-26$

$2896 \mathrm{KR}-28$

$2897 \mathrm{KRl}-29$

${ }_{2}^{2898} \mathrm{KRl}-30$

2900 US-0

2901 Us-03

2902 US-04

2903 US.05
2900 US-06
2905 US-07

2006 US-08 (repl. KRL-1)

2908 US 10

2909 Us-11

2910 US -12
2911 US 13

2912 US-14

$\begin{array}{ll}2914 & \text { US-1 } \\ 2 & 15 \text { SS. } 17\end{array}$

2916 US-18

2917 US-19 (repl. KR:-02

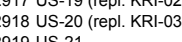

2920 US-22

2921
2222
US-2-24 (repl. KRI-04)

2923 US -25

2924 US -26

2020

2927 US-29

2928 US-30

2930 Ss-32

2932 US -34

2933 US-35 (repl. KRl-05)

2936 US-38

2937 US-39

2939 US-4

2940 US-4

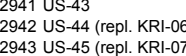

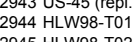

2947 HLW $998-T 04$

2948 HLWW8-TO5

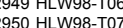

2951 HLW98-To8

2952 HLW98-TO9

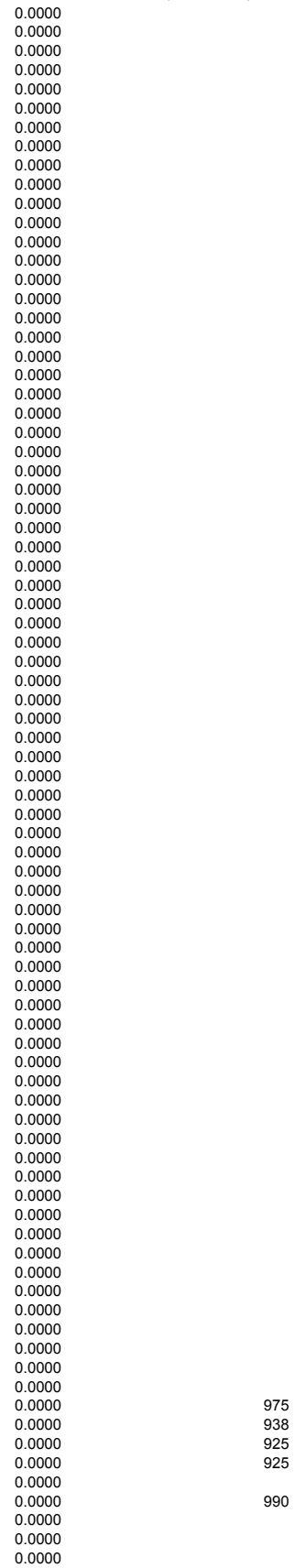

$\mathrm{C}_{650^{\circ} \mathrm{C}} \mathrm{C}_{700^{\circ} \mathrm{C}} \mathrm{C}_{750^{\circ} \mathrm{C}}$ 
\# Glass ID

$2871 \mathrm{KRI}-03$ (repl. US-20)

2887 KRR-04 (repl. US-24)

2874 KRI-06 (repl. US-44)
$2875 \mathrm{KRI}-07$ (repl. (US-45)
$2876 \mathrm{KRL}-08$

$2877 \mathrm{KRI}-09$

$2878 \mathrm{KR}-10$
2870

$2879 \mathrm{KRI}-1$

$2881 \mathrm{KRl}-13$

2882 KRl-14

$2883 \mathrm{KRR}-15$
$2884 \mathrm{KR}-16$
2055

$2885 \mathrm{KRR}-17$
$2886 \mathrm{KR}-18$

2887 KRR-19
2888 KR-120

$2889 \mathrm{KR}-21$

$2090 \mathrm{KR}-22$

$2892 \mathrm{KRR}-24$
$2893 \mathrm{KR}-25$

$2894 \mathrm{KRl}-26$

$2896 \mathrm{KR}-28$

$2897 \mathrm{KRl}-29$

$2898 \mathrm{KR} 1-30$

2900 US-0

2901 US-03

29022
US-04

2904 US-06

2905 US-07

2906 Us-08 (repl. KRl-01)

2908 US-10

2909 US-11

2912 US -14
2913 US-15

2914 US-11

2916 US-18

2917 US-19 (repl. KRR-02)
2918 US-20 (repl. KRL-03)

2920 US-22

2921 US-23

2923 US-25

2924 US-26

2925 US-27
2026 US 28

2927 US-29

2928 US-30

2929 US-31

$\begin{array}{ll}2930 \\ 2931 \\ 295-35 & -32 \\ 2 & \end{array}$

2932 US-34 (repl. KRL-05)

2936 US-38

2937 US -39

2939 US-4

2940 US-42

2941 US-43
2922 US-44 (repl. KRR-00

2942 US-44 (repl. KRR-06)
2943 US-45 (repl. KR-107)

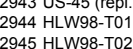

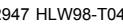

2948 HLWW8-T.T5
2949 HLW

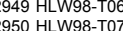

2951 HLW99-Tog
2952 HLW98--09
$\mathrm{C}_{800^{\circ} \mathrm{C}} \mathrm{C}_{850^{\circ} \mathrm{C}} \mathrm{C}_{900^{\circ} \mathrm{C}} \mathrm{C}_{950^{\circ} \mathrm{C}} \mathrm{C}_{1000^{\circ} \mathrm{C}} \mathrm{C}_{1050^{\circ} \mathrm{C}} \mathrm{C}_{1100^{\circ} \mathrm{C}} \mathrm{C}_{1150^{\circ} \mathrm{C}} \mathrm{C}_{1200^{\circ} \mathrm{C}}$ Quenched Visual/OM

Quenched XFD

CCC XRD

$\rho\left(g / \mathrm{cm}^{3}\right)$ Fulc Visc A Fulc Visc B Fulc Visc To Arrh Visc E Arrh Visc F

$\begin{array}{rr}\mathrm{T} 1\left({ }^{\circ} \mathrm{C}\right) & \mathrm{V} 1(\mathrm{~Pa} \cdot \mathrm{s}) \\ 860.0 & 257.249\end{array}$

$8200 \quad 170.477$

900.0223011

$950.0 \quad 156.066$

nepheline

nepheline
nepheline

nepheline

nepheline
nepheline 
\# Glass ID

2871 KRI-03 (repl. US-20

2873 KRI-05 (repl. US-35)

2874 KRl-06 (repl. US-44)

$2876 \mathrm{KRI}-08$

$2879 \mathrm{KRl}-11$

$2882 \mathrm{KRl}-14$

$2884 \mathrm{KRl}-16$

$2885 \mathrm{KRl}-17$

$2886 \mathrm{KRl}-18$

$2887 \mathrm{KRl}-19$
$2888 \mathrm{KR}-20$

$2889 \mathrm{KRRl}-22$

$2893 \mathrm{KRl}-25$

$2894 \mathrm{KRl}-26$

2896 KRl-28

$2897 \mathrm{KRI}-29$
$2898 \mathrm{KRI}-30$

2901 US-03

2902 US- 04

2904 US-06

2905 US-07

2906 US-08 (repl. KRI-01)

2908 US-10

2909 Us- 11

$2910 \mathrm{US}-12$
2211 US 11

2912 SS-14

2914 US-16

2916 US-18

2917 US-19 (repl. KRR-02)

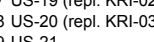

2920 US-22

2921 US-23 2922 US-24 (repl. KRI-04)

2924 Us-22

2925 US-27

2927 US-29

2928 US-30

2929 US-31

2930 US- 32

2933 US-35 (repl. KRl-05)

2935 US-37

2936 US-38

2937 US-39

2939 US-4

2940 US-4

2941 US.43
2942 US-44 (repl. KR-00

2942 US-44 (repl. KRR-06)
2943 US-45 (repl. KR-107)

2944 HLWW8-T01

2947 HLW98-T04

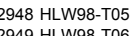

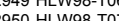

2951 HLWW98-To8
2952 HLW98-To9

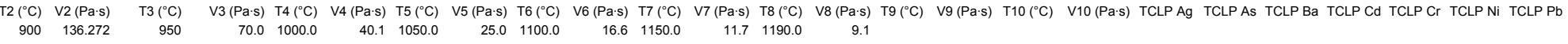

$\begin{array}{lll}850 & 88.507 & 900\end{array}$

$36.9 \quad 950.0$

$18.7 \quad 1000.0$

10.91050 .0

7.01100 .0

$950 \quad 88.624 \quad 1000$

$43.7 \quad 1050.0$

$25.0 \quad 1100.0$

15.9 
2879 KRl-11

2880 KRI-12
2881 KRI-13

$2882 \mathrm{KRI}-14$
$2883 \mathrm{KRI}-15$

$2884 \mathrm{KRl}-16$

2886 KRI-18
2887 KRl-19

$2887 \mathrm{KRl}-19$

$2889 \mathrm{KRR}-21$

$2891 \mathrm{KRl}-23$

$2894 \mathrm{KR}-26$

$2895 \mathrm{KRl}-27$
$2896 \mathrm{KRI}-28$

2897 KRI-29

(2909

2902 US-04

2904 US-06

2906 US-07 (repl. KRI-01)

2908 US-10

2909 US-11

2910 US-12

2912 US-14

2914 US-16

2916 US-1 18

2917 US-19 (repl. KRI-02)
2918 US-20 (repl. KRI-03)
2919 US-21

2920 US-22

2922 US-24 (repl. KRl-04)

2923 US-25

2924 US-26

2927 US-29

2929 US-31

2930 US-32

2932 US-34
2933 US-35 (repl. KRI-05)

2935 US-37

2936 US-38

2937 US-39

2939 US- 4

2940 US-4

2944 US-43 (repl. KRI-06)

2943 US-45 (repp. KRPL-07)

2944 HLW98-TO1

2945 HLW98-T02

2947 HLW98-T04

2948 HLW98-T05

2950 HLW98-T07

2952 HLW98-T09

0.235
0.867
0.178
0.175
0.170
0.168
0.177
0.217
0.217
0.178
0.165
0.636
0.176
1.562
2.327
0.320
0.247
0.262
0.222
0.223
0.208
0.283
0.225
0.198
0.573
0.210
0.625
0.854
0.914
0.288
0.249
1.784
0.181
2.078
0.401
0.325
0.363
0.395
0.248
0.547
0.163
0.649
0.203
0.262
0.237

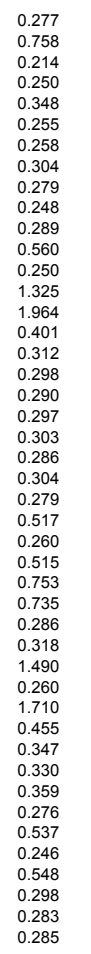

$\begin{array}{ll}0.267 & 0.202 \\ 0.447 & 0.172 \\ 0.122 & 0.135 \\ 0.143 & 0.126 \\ 0.099 & 0.120 \\ 0.145 & 0.114 \\ 0.147 & 0.117 \\ 0.163 & 0.190 \\ 0.201 & 0.140 \\ 0.195 & 0.122 \\ 0.127 & 0.097 \\ 0.448 & 0.178 \\ 0.165 & 0.121 \\ 0.918 & 0.170 \\ 1.314 & 0.109 \\ 0.155 & 0.126 \\ 0.144 & 0.196 \\ 0.291 & 0.202 \\ 0.172 & 0.160 \\ 0.177 & 0.156 \\ 0.159 & 0.180 \\ 0.258 & 0.180 \\ 0.181 & 0.176 \\ 0.169 & 0.145 \\ 0.390 & 0.171 \\ 0.209 & 0.133 \\ 0.782 & 0.415 \\ 0.525 & 0.133 \\ 1.044 & 0.386 \\ 0.195 & 0.114 \\ 0.148 & 0.143 \\ 0.933 & 0.171 \\ 0.449 & 0.178 \\ 1.483 & 0.235 \\ 0.317 & 0.104 \\ 0.211 & 0.113 \\ 0.466 & 0.199 \\ 0.542 & 0.217 \\ 0.110 & 0.137 \\ 0.320 & 0.150 \\ 0.104 & 0.106 \\ 0.828 & 0.356 \\ 0.180 & 0.140 \\ 0.250 & 0.141 \\ 0.247 & 0.143 \\ & \end{array}$

$\begin{array}{ll}0.231 & \\ 0.271 \\ 0.699 & 0.613 \\ 0.162 & 0.238 \\ 0.166 & 0.229 \\ 0.245 & 0.340 \\ 0.154 & 0.224 \\ 0.161 & 0.226 \\ 0.204 & 0.267 \\ 0.221 & 0.266 \\ 0.174 & 0.240 \\ 0.399 & 0.457 \\ 0.873 & 0.748 \\ 0.149 & 0.217 \\ 1.309 & 1.114 \\ 1.825 & 1.550 \\ 0.432 & 0.470 \\ 0.241 & 0.282 \\ 5.330 & 4.106 \\ 0.207 & 0.267 \\ 0.209 & 0.275 \\ 0.198 & 0.280 \\ 0.266 & 0.274 \\ 0.219 & 0.282 \\ 0.204 & 0.274 \\ 0.707 & 0.619 \\ 1.801 & 1.273 \\ 8.226 & 3.571 \\ 0.689 & 0.629 \\ 0.768 & 0.687 \\ 0.320 & 0.311 \\ 0.154 & 0.247 \\ 1.410 & 1.181 \\ 0.172 & 0.285 \\ 1.923 & 1.599 \\ 1.980 & 1.773 \\ 0.401 & 0.394 \\ 1.064 & 0.830 \\ 0.400 & 0.407 \\ 0.219 & 0.259 \\ 0.770 & 0.694 \\ 0.140 & 0.245 \\ 0.463 & 1.001 \\ 0.878 & 0.890 \\ 0.256 & 0.284 \\ 0.226 & 0.274 \\ & \end{array}$

$\begin{array}{ll}0.253 & 0.197 \\ 0.381 & 0.172 \\ 0.082 & 0.128 \\ 0.142 & 0.122 \\ 0.179 & 0.137 \\ 0.137 & 0.112 \\ 0.135 & 0.113 \\ 0.162 & 0.178 \\ 0.131 & 0.122 \\ 0.190 & 0.119 \\ 0.256 & 0.115 \\ 0.548 & 0.192 \\ 0.146 & 0.112 \\ 0.784 & 0.169 \\ 1.050 & 0.116 \\ 0.226 & 0.133 \\ 0.156 & 0.188 \\ 1.664 & 0.431 \\ 0.171 & 0.149 \\ 0.172 & 0.150 \\ 0.154 & 0.150 \\ 0.286 & 0.208 \\ 0.179 & 0.161 \\ 0.177 & 0.148 \\ 0.462 & 0.212 \\ 0.666 & 0.165 \\ 3.732 & 1.152 \\ 0.454 & 0.139 \\ 0.874 & 0.371 \\ 0.221 & 0.127 \\ 0.101 & 0.125 \\ 0.754 & 0.169 \\ 0.426 & 0.185 \\ 1.360 & 0.224 \\ 1.259 & 0.110 \\ 0.247 & 0.119 \\ 0.707 & 0.261 \\ 0.562 & 0.247 \\ 0.091 & 0.138 \\ 0.418 & 0.146 \\ 0.090 & 0.103 \\ 1.822 & 0.098 \\ 0.263 & 0.172 \\ 0.247 & 0.146 \\ 0.230 & 0.142 \\ & \end{array}$

$\begin{array}{rrrrr}0.145 & & & & \\ 0.0186 & 0.364 & 0.197 & 0.726 & 0.013 \\ <0.0532 & 0.5 & 0.381 & 1.09 & \end{array}$

0.342 
2871 KRI-03 (repl. US-20)

2873 KRl-05 (repl. US-35)

2874 KRI--06 (repl. US -44)
2875 RRI-07 (repl. (US-45)

$2876 \mathrm{KRl}-08$

$2878 \mathrm{KRl}-10$

$2879 \mathrm{KRI}-11$

$2880 \mathrm{KRR}-12$

$2882 \mathrm{KRl}-14$

$2883 \mathrm{KRl}-15$

$2885 \mathrm{KRl}-17$

2886 KRI-18

2888 KRI-20

$2889 \mathrm{KR}-21$

$2892 \mathrm{KRl}-24$

$2894 \mathrm{KRI}-26$

$2896 \mathrm{KRl}-28$

$2897 \mathrm{KRl}-29$

$2898 \mathrm{KRI}-30$

2900 US-02

2901 US-03

2902 US-04

2904 US-06

2905 US-07

2906 US-08 (repl. KRI-01)

2908 US-10

2909 US 11

2910 US -12

2912 US-14

2914 Us- 16

2916 Us-18

2917 US-19 (repl. KRl-02)

8 US-20 (repl. KRI-03)

2920 US-22

2921 US-23 2922 US-24 (repl. KRI-04)

(2023 US 25

2924 US-26

2925 US 27

2926 US-28
2927 US-29

2928 US-30

2929 US-31

2930 US-32

US-34

2933 US-35 (repl. KRI-05)

(2934 US-36

2936 US-38

2937 US-39

2939 US-41

2940 US-4

2944 US-43

2942 US-44 (repl. KRI-06)

2944 HLW $W 8$-TO 1

2945 HLW98-T02

2947 HLW98-T04

2948 HLW98-T05

2949 HLW98-T0
2950 HLW98-T0

2951 HLW98-T08 


\begin{tabular}{|c|c|c|c|c|c|c|}
\hline \# Glass ID & Study & Reference & Al2O3-t & B2O3-t & $\mathrm{CaO}-\mathrm{t}$ & Fe2O3-t \\
\hline 2953 HLW98-V01 & V series & Kot et al. 2003b & 0.0611 & 0.1124 & 0.0033 & 0.1437 \\
\hline 2954 HLW98-V02 & V series & Kot et al. 2003b & 0.0576 & 0.1150 & 0.0031 & 0.1354 \\
\hline 2955 HLW98-V03 & $\checkmark$ series & Kot et al. 2003b & 0.0539 & 0.1177 & 0.0029 & 0.1267 \\
\hline $\begin{array}{l}2956 \text { 6LW98-V04 } \\
2957 \text { HLW98-V05 }\end{array}$ & $\begin{array}{l}\text { V series } \\
\text { V series }\end{array}$ & $\begin{array}{l}\text { Kot tet al. } 20003 \mathrm{~b} \\
\text { Kot et al. 2003b }\end{array}$ & $\begin{array}{l}0.0500 \\
0.0459\end{array}$ & $\begin{array}{l}0.1205 \\
0.1235\end{array}$ & $\begin{array}{l}0.0027 \\
0.0025\end{array}$ & $\begin{array}{l}0.1176 \\
0.1080\end{array}$ \\
\hline 2958 HLW98-V06 & $\begin{array}{l}\text { V series } \\
\text { V series }\end{array}$ & Kot et al. $2003 b$ & 0.0416 & $\begin{array}{l}0.1267 \\
0.1230\end{array}$ & 0.0022 & 0.0978 \\
\hline & V series & Kot et al. 2003b & 0.0659 & 0.1194 & 0.0027 & 0.1477 \\
\hline 2960 HLW98-V08 & $\begin{array}{l}V \text { series } \\
\text { V serices }\end{array}$ & Kot et al. 2003b & 0.0620 & 0.1217 & 0.0026 & 0.1391 \\
\hline $\begin{array}{l}29611 \text { HLW98-V09 } \\
2962 \text { HLW98-V10 }\end{array}$ & $\begin{array}{l}\text { V series } \\
\text { V series }\end{array}$ & $\begin{array}{l}\text { Kotet al. } 2003 \mathrm{~b} \\
\text { Kot et al. 2003b }\end{array}$ & $\begin{array}{l}0.0580 \\
0.0538\end{array}$ & $\begin{array}{l}0.1240 \\
0.1266\end{array}$ & $\begin{array}{l}0.0024 \\
0.0022\end{array}$ & $\begin{array}{l}0.1300 \\
0.1205\end{array}$ \\
\hline $\begin{array}{l}2962 \text { HLW98-V10 } \\
2963 \text { HLW98-V11 }\end{array}$ & 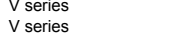 & $\begin{array}{l}\text { Kot et ala.2003b } \\
\text { Kot et al. 2003b }\end{array}$ & $\begin{array}{l}0.0538 \\
0.0493\end{array}$ & $\begin{array}{l}0.1266 \\
0.1292\end{array}$ & $\begin{array}{l}0.0022 \\
0.0021\end{array}$ & $\begin{array}{l}0.1205 \\
0.1105\end{array}$ \\
\hline 2964 HLW98-V12 & V series & Kot et al. 2003b & 0.0446 & 0.1320 & 0.0019 & 0.1000 \\
\hline 2965 HLW98-V13 & V series & Kot et al. 2003b & 0.0523 & 0.1058 & 0.0079 & 0.1505 \\
\hline 2966 HLW98-V14 & V series & Kot et al. 2003b & 0.0521 & 0.1081 & 0.0074 & 0.1416 \\
\hline 2967 HLW98-V15 & V series & Kot et al. 2003b & 0.0519 & 0.1104 & 0.0069 & 0.1323 \\
\hline 2968 HLW98-V16 & V series & Kot et al. $2003 \mathrm{~b}$ & 0.0517 & 0.1129 & 0.0064 & 0.1225 \\
\hline 2969 HLW98-V17 & $\begin{array}{l}V \text { series } \\
\text { V serics }\end{array}$ & Kot et al. 2003b & 0.0515 & 0.1155 & 0.0059 & 0.1123 \\
\hline $\begin{array}{l}2970 \text { HLW98-V18 } \\
2971 \text { HLW98-V19 }\end{array}$ & $\begin{array}{l}\text { V series } \\
\text { V series }\end{array}$ & $\begin{array}{l}\text { Kot et al. } 20003 b \\
\text { Kot et al. 2003b }\end{array}$ & $\begin{array}{l}0.0513 \\
0.0577\end{array}$ & $\begin{array}{l}0.1182 \\
0.0882\end{array}$ & $\begin{array}{l}0.0053 \\
0.0035\end{array}$ & $\begin{array}{l}0.1015 \\
0.1469\end{array}$ \\
\hline 2972 HLW98-V20 & 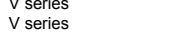 & $\begin{array}{l}\text { Kot et ala. 20033b } \\
\text { Kot et al. 2003b }\end{array}$ & $\begin{array}{l}0.05 / 1 \\
0.0559\end{array}$ & $\begin{array}{l}0.0882 \\
0.0904\end{array}$ & $\begin{array}{l}0.0035 \\
0.0033\end{array}$ & $\begin{array}{l}0.1469 \\
0.1387\end{array}$ \\
\hline 2973 HLW98-V21 & $\begin{array}{l}\text { V serres } \\
V \text { series }\end{array}$ & Kot et al. $2003 \mathrm{~b}$ & 0.0539 & 0.0927 & 0.0031 & 0.1301 \\
\hline 2974 HLW98-V22 & V series & Kot et al. 2003b & 0.0518 & 0.0952 & 0.0029 & 0.1210 \\
\hline 2975 HLW98-V23 & V series & Kot et al. 2003b & 0.0496 & 0.0978 & 0.0027 & 0.1114 \\
\hline 2976 HLW98-V24 & V series & Kot et al. 2003b & 0.0473 & 0.1005 & 0.0024 & 0.1012 \\
\hline 2977 HLW98-V25 & V series & Kot et al. 2003b & 0.0386 & 0.0931 & 0.0052 & 0.1029 \\
\hline 2978 HLW98-V26 & $\begin{array}{l}V \text { series } \\
\text { Vesices }\end{array}$ & Kot et al. 2003b & 0.0368 & 0.0961 & 0.0049 & 0.0978 \\
\hline $\begin{array}{l}2979 \text { HLW98-V27 } \\
2980 \text { HLW98-V28 }\end{array}$ & 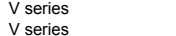 & $\begin{array}{l}\text { Kot tet al. } 20003 b \\
\text { Kot et al. 2003b }\end{array}$ & $\begin{array}{l}0.0347 \\
0.0325\end{array}$ & $\begin{array}{l}0.0993 \\
0.1027\end{array}$ & $\begin{array}{l}0.0047 \\
0.0044\end{array}$ & $\begin{array}{l}0.0924 \\
0.0865\end{array}$ \\
\hline $\begin{array}{l}2980 \mathrm{HLW} 98-\mathrm{V} 28 \\
2981 \text { HLW98-V29 }\end{array}$ & 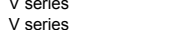 & $\begin{array}{l}\text { ot et al. } 2003 \mathrm{~b} \\
\text { Kot et al. 2003b }\end{array}$ & $\begin{array}{l}0.0325 \\
0.0302\end{array}$ & $\begin{array}{l}0.1127 \\
0.1064\end{array}$ & $\begin{array}{l}0.0044 \\
0.0041\end{array}$ & $\begin{array}{l}0.0865 \\
0.0802\end{array}$ \\
\hline 2982 HLW98-V30 & $\begin{array}{l}V \text { seres } \\
V \text { series }\end{array}$ & Kot et al. $2003 \mathrm{~b}$ & 0.0276 & $\begin{array}{l}0.1103 \\
0.1103\end{array}$ & 0.0037 & 0.0735 \\
\hline 2983 HLW-E-Cr-10 & VSL Enhanced HLW & Matlack et al. 2007 & 0.1106 & 0.1444 & 0.0107 & 0.0569 \\
\hline 2984 HLW-E-Cr-M & VSL Enhanced HLW & Matlack et al. 2007 & 0.0898 & 0.1617 & 0.0087 & 0.0462 \\
\hline 2985 HLW-E-Bi-6 & VSL Enhanced HLW & Matlack et al. 2007 & 0.1166 & 0.1130 & 0.0084 & 0.0696 \\
\hline 2986 HLW-E-Al-27 & VSLL Enhanced HLW & Matlack et al. 2007 & 0.2397 & 0.1519 & 0.0608 & 0.0590 \\
\hline 2987 HLW-E-Al-01 & VSL Enhanced HLW & Matlack et al. 2007 & 0.2663 & 0.1021 & 0.0120 & 0.0655 \\
\hline 2988 HLW-E-AL-02 202 & $\begin{array}{l}\text { VSL Enhanced HLW } \\
\text { vSL Enhanced HLW }\end{array}$ & $\begin{array}{l}\text { Matlack et al. } 2007 \\
\text { Mattack etal } 2007\end{array}$ & $\begin{array}{l}0.2663 \\
0.2663\end{array}$ & $\begin{array}{l}0.0321 \\
0.0821\end{array}$ & $\begin{array}{l}0.1420 \\
0.1420\end{array}$ & $\begin{array}{l}0.0655 \\
0.0655\end{array}$ \\
\hline 29990 HLW-W-E-AA-03 2904 & $\begin{array}{l}\text { VSL Enhanced HLW } \\
\text { VSL Enhanced HLW }\end{array}$ & $\begin{array}{l}\text { antallack et all.2007 } \\
\text { Matlack et al. } 2007\end{array}$ & $\begin{array}{l}0.2663 \\
0.2663\end{array}$ & $\begin{array}{l}0.0821 \\
0.1021\end{array}$ & $\begin{array}{l}0.1420 \\
0.1620\end{array}$ & $\begin{array}{l}0.0655 \\
0.0655\end{array}$ \\
\hline 2991 HLW-E-Al-05 & VSL Enhanced HLW & Matlack et al. 2007 & 0.2663 & 0.0821 & 0.1120 & \\
\hline 2992 HLW-E-Al-06 & VSL Enhanced HLW & Matlack et al. 2007 & 0.2663 & 0.1221 & 0.1820 & 0.0655 \\
\hline 2993 HLW-E-Al-07 & VSL Enhanced HLW & Matlack et al. 2007 & 0.2663 & 0.1521 & 0.2020 & 0.0655 \\
\hline 2994 HLW-E-Al-08 & VSL Enhanced HLW & Matlack et al. 2007 & 0.2663 & 0.1821 & 0.1820 & 0.0655 \\
\hline 2995 HLW-E-Al-09 & VSL Enhanced HLW & Matlack et al. 2007 & 0.2663 & 0.1821 & 0.1620 & 0.0655 \\
\hline 2996 HLW-E-Al-10 & VSL Enhanced HLW & Matlack et al. 2007 & 0.2663 & 0.2021 & 0.1420 & 0.0655 \\
\hline 2997 HLW-E-Al-11 & $\begin{array}{l}\text { VSL Enhanced HLW } \\
\text { vSL Enhanced HLW }\end{array}$ & $\begin{array}{l}\text { Matlack et al. } 2007 \\
\text { Matalack etal } 2007\end{array}$ & $\begin{array}{l}0.2663 \\
0.2397\end{array}$ & $\begin{array}{l}0.1621 \\
0.2019\end{array}$ & $\begin{array}{l}0.1420 \\
0.1408\end{array}$ & $\begin{array}{l}0.0655 \\
0.0590\end{array}$ \\
\hline 2999 HLW-E-Al-12 & $\begin{array}{l}\text { vSL Enhananced HLd HLW } \\
\text { vSt }\end{array}$ & $\begin{array}{l}\text { atatlack et al. } 2007 \\
\text { Matlack et al. } 2007\end{array}$ & $\begin{array}{l}0.2397 \\
0.2530\end{array}$ & $\begin{array}{l}0.2019 \\
0.1929\end{array}$ & $\begin{array}{l}0.1408 \\
0.1355\end{array}$ & $\begin{array}{l}0.0590 \\
0.0623\end{array}$ \\
\hline 3000 HLW-E-Al-14 & $\begin{array}{l}\text { VSL Enhanced HLW } \\
\text { VEnced }\end{array}$ & $\begin{array}{l}\text { Matalack et al. } 2007 \\
\text { attack tal. } 2007\end{array}$ & $\begin{array}{l}0.2563 \\
0.2663\end{array}$ & $\begin{array}{l}0.1929 \\
0.1821\end{array}$ & 0.0120 & $\begin{array}{l}0.0623 \\
0.0655\end{array}$ \\
\hline 3001 HLW-E-Al-15 & VSL Enhanced HLW & Matlack et al. 2007 & 0.2530 & 0.2020 & 0.0114 & 0.0623 \\
\hline 3002 HLW-E-Al-16 & VSL Enhanced HLW & Matlack et al. 2007 & 0.2397 & 0.2019 & 0.0108 & 0.0590 \\
\hline 3003 HLW-E-Al-17 & VSL Enhanced HLW & Matlack et al. 2007 & 0.2930 & 0.1823 & 0.0132 & 0.0721 \\
\hline 3004 HLW-E-Al-18 & VSL Enhanced HLW & Matlack et al. 2007 & 0.2397 & 0.1919 & 0.1308 & 0.0590 \\
\hline 3005 HLW-E-Al-19 & VSL Enhanced HLW & Matlack et al. 2007 & 0.2663 & 0.1441 & 0.0120 & 0.0655 \\
\hline 3006 HLW-E-Al-20 & VSL Enhanced HLW & Matlack et al. 2007 & 0.2530 & 0.1440 & 0.0114 & 0.0623 \\
\hline 3008 HLW-E-Al-22 & $\begin{array}{l}\text { vSL Enhananced HLd HLW } \\
\text { vSL Enad }\end{array}$ & $\begin{array}{l}\text { attlack et at al. } 2007 \\
\text { atlat }\end{array}$ & $\begin{array}{l}0.2530 \\
0.2530\end{array}$ & $\begin{array}{l}.11400 \\
0.1400\end{array}$ & 0.0114 & $\begin{array}{l}0.0023 \\
0.0623\end{array}$ \\
\hline 3009 HLW-E-Al-23 & $\begin{array}{l}\text { VSL Enhanced HLW } \\
\text { VEnced }\end{array}$ & Matlack et al. 2007 & 0.2397 & 0.1419 & 0.0108 & 0.0590 \\
\hline 3010 HLW-E-Al-24 & VsL Enhanced HLW & Matlack et al. 2007 & 0.1864 & 0.1515 & 0.0084 & 0.0459 \\
\hline 3011 HLW-E-Al-25 & VSL Enhanced HLW & Matlack et al. 2007 & 0.2397 & 0.1419 & 0.0608 & 0.0590 \\
\hline 3012 HLW-E-Al-26 & VSL Enhanced HLW & Matlack et al. 2007 & 0.2397 & 0.1619 & 0.0108 & 0.0590 \\
\hline 3013 HLW-E-Al-27 & VSL Enhanced HLW & Matlack et al. 2007 & 0.2397 & 0.1519 & 0.0608 & 0.0590 \\
\hline $\begin{array}{l}\text { EA-A-28 } \\
\text { EA-20 }\end{array}$ & VSL Enhanced HLW & Matlack et al. 2007 & & 0.1440 & 0.0614 & 0.0623 \\
\hline 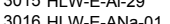 & 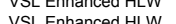 & 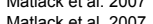 & 0.2530 & 0.1444 & 0.0914 & 0.0623 \\
\hline 3017 & vSL Enhanced HLW & Mathack at al 2007 & $\begin{array}{l}0.2907 \\
0.2134\end{array}$ & $\begin{array}{l}0.1433 \\
0.1437\end{array}$ & $\begin{array}{l}0.0065 \\
0.0072\end{array}$ & $\begin{array}{l}0.0252 \\
0.0282\end{array}$ \\
\hline 3018 HLW-E-ANa-003 & VSL Enhanced HLW & 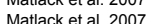 & $\begin{array}{ll}0.234 \\
0.2361\end{array}$ & 0.141 & 0.0080 & $\begin{array}{l}0.0282 \\
0.0311\end{array}$ \\
\hline E-ANa-04 & VSL Enhanced HLW & Matlack et al. 20 & 0.2134 & 0.1437 & 0.0072 & 0.0282 \\
\hline $3020 \mathrm{HL}$ & VSL Enhanced HLW & Matlack et al. 2007 & 0.2361 & 0.1441 & 0.0080 & 0.0311 \\
\hline 3021 HLW-E-ANa-06 & VSL Enhanced HLW & Matlack et al. 2007 & 0.2588 & 0.1444 & 0.0088 & 0.0341 \\
\hline 3022 HLW-E-ANa-07 & VSL Enhanced HLW & Matlack et al. 2007 & 0.2724 & 0.1447 & 0.0092 & 0.0359 \\
\hline & VSLL Enhanced HLW & keta & & & & 0.0341 \\
\hline 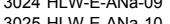 & d HLW & (at) & & 0.1444 & 10088 & 0.0341 \\
\hline $3026 \mathrm{HL}$ & $\begin{array}{l}\text { VSLEnhan } \\
\text { VSL Enhan }\end{array}$ & ick et al. 2 & $\begin{array}{l}0.2588 \\
0.258\end{array}$ & 0.1344 & $\begin{array}{l}0.0088 \\
0.008\end{array}$ & \\
\hline E-ANa-12 & d HLW & 007 & & & 0.0100 & \\
\hline & & Matlack et al. 2007 & 0.2134 & & & \\
\hline-14 & VSL Enhanced HLW & Matlack et al. 2007 & 0.2361 & 0.1441 & & \\
\hline W-E-ANa-15 & VSL Enhanced HLW & Matlack et al. 2007 & 0.2361 & 0.1541 & 0.0580 & 0.0311 \\
\hline E-ANa-16 & VSL Enhanced HLW & ick et al. 2007 & 0.2361 & 0.1441 & 0.0580 & 0.0311 \\
\hline Na-17 & $\begin{array}{l}\text { Enhanced HLW } \\
\text { Enhanced HLW }\end{array}$ & $\begin{array}{l}\text { itlack et al. } 2007 \\
\text { tlack et al 201 }\end{array}$ & $\begin{array}{l}0.2043 \\
0.2043\end{array}$ & 0.1535 & 0.0569 & $\begin{array}{l}0.0270 \\
0.0270\end{array}$ \\
\hline 3034 HLW-EA-ANa-1 & vSL Enhanced HLW & 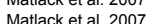 & 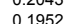 & 作 & 0.0566 & 0258 \\
\hline
\end{tabular}

K2O-t Li2O-t MgO-t Na2O-t NiO-t P2O5-t SiO2-t Zro2-t Ag2O-t As2O3-tAs205-t BaO-t BeO-t Bi2O3-t $\begin{array}{llllllllll}0.0004 & 0.0332 & 0.0013 & 0.1124 & 0.0072 & 0.0000 & 0.4474 & 0.0448 & 0.0003 & 0.0000 \\ 0.0004 & 0.0340 & 0.0012 & 0.1140 & 0.0068 & 0.0000 & 0.4578 & 0.0422 & 0.0002 & 0.0000\end{array}$

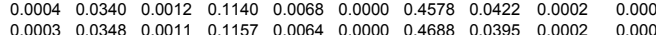

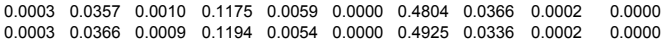
$\begin{array}{lllllllllll}0.0003 & 0.0376 & 0.0009 & 0.1214 & 0.0049 & 0.0000 & 0.5053 & 0.0305 & 0.0002 & 0.0000\end{array}$

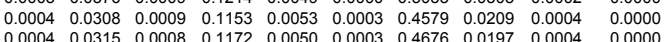

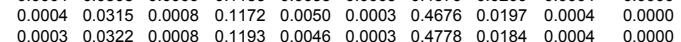
$\begin{array}{llllllllll}0.0003 & 0.0330 & 0.0007 & 0.1214 & 0.0043 & 0.0002 & 0.4885 & 0.0170 & 0.0003 & 0.0000 \\ 0.0003 & 0.0338 & 0.0006 & 0.1237 & 0.0039 & 0.0002 & 0.4997 & 0.0156 & 0.0003 & 0.0000\end{array}$ $\begin{array}{llllllllll}0.0003 & 0.0347 & 0.0006 & 0.1260 & 0.0036 & 0.0002 & 0.5115 & 0.0141 & 0.0003 & 0.0000\end{array}$ $\begin{array}{llllllllll}0.0000 & 0.0260 & 0.0067 & 0.1144 & 0.0044 & 0.0012 & 0.4555 & 0.0015 & 0.0018 & 0.0024 \\ 0.0000 & 0.0266 & 0.0063 & 0.1165 & 0.0042 & 0.0011 & 0.4638 & 0.0014 & 0.0017 & 0.0022\end{array}$ $\begin{array}{llllllllll}0.0000 & 0.0272 & 0.0059 & 0.1187 & 0.0039 & 0.0010 & 0.4724 & 0.0013 & 0.0016 & 0.0021\end{array}$ $\begin{array}{llllllllll}0.0000 & 0.0279 & 0.0054 & 0.1211 & 0.0036 & 0.0009 & 0.4815 & 0.0012 & 0.0015 & 0.0019 \\ 0.0000 & 0.0286 & 0.0050 & 0.1235 & 0.0033 & 0.0009 & 0.4908 & 0.0011 & 0.0013 & 0.0018\end{array}$ $\begin{array}{llllllllllll}0.0000 & 0.0293 & 0.0045 & 0.1261 & 0.0030 & 0.0008 & 0.5007 & 0.0010 & 0.0012 & 0.0016 & 0.00000\end{array}$ $\begin{array}{llllllllllll}0.0000 & 0.0281 & 0.0136 & 0.1121 & 0.0020 & 0.0011 & 0.4450 & 0.0030 & 0.0017 & 0.0022 & 0.00000\end{array}$

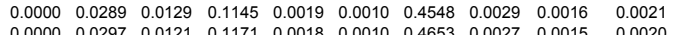

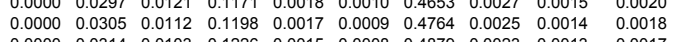
$\begin{array}{lllllllllllll}0.0000 & 0.0314 & 0.0103 & 0.1226 & 0.0015 & 0.0008 & 0.4879 & 0.0023 & 0.0013 & 0.0017 & 0.0000 \\ 0.0000 & 0.023 & 0.000 & 0.122 & 0.0050 & 0.0004 & 0.403 & 0.1017 & 0.0003 & 0.0000 & 0.0000\end{array}$

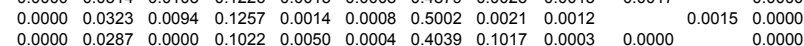

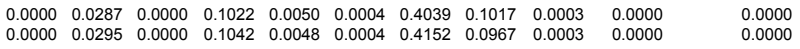
$\begin{array}{lllllllllll}0.0000 & 0.0305 & 0.0000 & 0.1063 & 0.0045 & 0.0004 & 0.4270 & 0.0913 & 0.0003 & 0.0000 & 0.0000\end{array}$ $\begin{array}{lllllllllll}0.0000 & 0.0315 & 0.0000 & 0.1085 & 0.0042 & 0.0004 & 0.4397 & 0.0855 & 0.0003 & 0.0000 & 0.0000\end{array}$ $\begin{array}{llllllllll}0.00000 & 0.0337 & 0.0000 & 0.1135 & 0.0036 & 0.0003 & 0.4681 & 0.0793 & 0.0002 & 0.0000\end{array}$ $\begin{array}{lllllllll}0.0542 & 0.0331 & 0.0007 & 0.0870 & 0.0046 & 0.0145 & 0.4194 & 0.0005 \\ 0.0605 & 0.0368 & 0.0006 & 0.0707 & 0.0037 & 0.0118 & 0.4576 & 0.0004\end{array}$ $\begin{array}{llllllll}0.0605 & 0.0368 & 0.0006 & 0.0707 & 0.0037 & 0.0118 & 0.4576 & 0.0004 \\ 0.0046 & 0.0016 & 0.0043 & 0.1574 & 0.0193 & 0.0499 & 0.3626 & 0.0021\end{array}$ $\begin{array}{llllllll}0.0014 & 0.0357 & 0.0012 & 0.0958 & 0.0040 & 0.0405 & 0.3026 & 0.0021 \\ 0.016 & 0.0019 & 0.0039\end{array}$ $\begin{array}{llllllll}0.0016 & 0.0019 & 0.0913 & 0.1098 & 0.0044 & 0.0117 & 0.2944 & 0.0044 \\ 0.0156 & 0.0019 & 0.0013 & 0.0958 & 0.0044 & 0.0117 & 0.3244 & 0.0044\end{array}$ $\begin{array}{llllllll}0.0156 & 0.0019 & 0.0013 & 0.0958 & 0.0044 & 0.0117 & 0.324 & 0.0044 \\ 0.0016 & 0.0019 & 0.0013 & 0.0898 & 0.0044 & 0.0117 & 0.2944 & 0.0044\end{array}$ $\begin{array}{lllllllll}0.0016 & 0.0019 & 0.0013 & 0.0698 & 0.0044 & 0.0117 & 0.2744 & 0.0044\end{array}$ $\begin{array}{llllllll}0.0016 & 0.0019 & 0.0013 & 0.1198 & 0.0044 & 0.0117 & 0.2944 & 0.0044 \\ 0.0016 & 0.0019 & 0.0013 & 0.0498 & 0.0044 & 0.0117 & 0.2544 & 0.0044\end{array}$ $\begin{array}{llllllll}0.0016 & 0.0019 & 0.0013 & 0.0498 & 0.0044 & 0.0117 & 0.2544 & 0.0044 \\ 0.0016 & 0.0119 & 0.0013 & 0.0398 & 0.0044 & 0.0117 & 0.2044 & 0.0044\end{array}$ $\begin{array}{llllllll}0.0016 & 0.0119 & 0.0013 & 0.0398 & 0.0044 & 0.0117 & 0.1944 & 0.0044\end{array}$ $\begin{array}{lllllllll}0.0016 & 0.0319 & 0.0013 & 0.0398 & 0.0044 & 0.0117 & 0.1944 & 0.0044 \\ 0.0016 & 0.0519 & 0.0013 & 0.0398 & 0.0044 & 0.0117 & 0.1744 & 0.0044\end{array}$ $\begin{array}{llllllll}0.0016 & 0.0519 & 0.0013 & 0.0398 & 0.0044 & 0.0117 & 0.1744 & 0.0044 \\ 0.0016 & 0.0519 & 0.0013 & 0.0398 & 0.0044 & 0.0117 & 0.2144 & 0.0044\end{array}$ $\begin{array}{llllllll}0.0014 & 0.0517 & 0.0012 & 0.0358 & 0.0040 & 0.0105 & 0.2190 & 0.0039\end{array}$ $\begin{array}{llllllll}0.0015 & 0.0495 & 0.0012 & 0.0378 & 0.0042 & 0.0111 & 0.2139 & 0.0042 \\ 0.0016 & 0.0719 & 0.0013 & 0.0398 & 0.0044 & 0.0117 & 0.3044 & 0.0044\end{array}$ $\begin{array}{llllllll}0.0016 & 0.0719 & 0.0013 & 0.0398 & 0.0044 & 0.0117 & 0.3044 & 0.0004 \\ 0.0015 & 0.0768 & 0.0012 & 0.0378 & 0.0042 & 0.0111 & 0.3017 & 0.0042\end{array}$ $\begin{array}{llllllll}0.0014 & 0.0767 & 0.0012 & 0.0358 & 0.0040 & 0.0335 & 0.2990 & 0.0039\end{array}$ $\begin{array}{llllllll}0.0017 & 0.0771 & 0.0014 & 0.0438 & 0.0049 & 0.0129 & 0.2548 & 0.0048 \\ 0.0014 & 0.0517 & 0.0012 & 0.0358 & 0.0040 & 0.0105 & 0.2190 & 0.0239\end{array}$ $\begin{array}{llllllll}0.0014 & 0.0517 & 0.0012 & 0.0358 & 0.0040 & 0.0105 & 0.2190 & 0.0239 \\ 0.0016 & 0.0349 & 0.0013 & 0.1398 & 0.0044 & 0.0117 & 0.2794 & 0.0044\end{array}$ $\begin{array}{llllllll}0.0015 & 0.0418 & 0.0012 & 0.1378 & 0.0042 & 0.0111 & 0.2947 & 0.0042 \\ 0.0015 & 0.0503 & 0.0012 & 0.1478 & 0.0042 & 0.0111 & 0.2802 & 0.0042\end{array}$ $\begin{array}{llllllll}0.0515 & 0.0503 & 0.0012 & 0.0978 & 0.0042 & 0.0111 & 0.2802 & 0.0042\end{array}$ $\begin{array}{llllllll}0.0011 & 0.0353 & 0.0009 & 0.1478 & 0.0031 & 0.0082 & 0.3581 & 0.0291 \\ 0.0014 & 0.0357 & 0.0012 & 0.0958 & 0.0040 & 0.0105 & 0.3150 & 0.0039\end{array}$ $\begin{array}{lllllllll}0.0014 & 0.0357 & 0.0012 & 0.1308 & 0.0040 & 0.0105 & 0.3100 & 0.0039 \\ 0.0014 & 0.0357 & 0.0012 & 0.058 & 0.040 & 0.005 & 0.3050 & 0.0030\end{array}$ $\begin{array}{llllllll}0.0015 & 0.0368 & 0.0012 & 0.0978 & 0.0042 & 0.0111 & 0.2897 & 0.0042 \\ 0.0015 & 0.0268 & 0.0012 & 0.0978 & 0.0042 & 0.0111 & 0.2697 & 0.0042\end{array}$

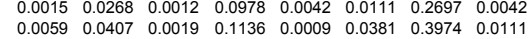
$\begin{array}{llllllll}0.0059 & 0.0407 & 0.0019 & 0.1136 & 0.0009 & 0.0381 & 0.3974 & 0.0111 \\ 0.0066 & 0.0308 & 0.0022 & 0.1271 & 0.0010 & 0.0202 & 0.3906 & 0.0012\end{array}$ $\begin{array}{llllllll}0.0073 & 0.0258 & 0.0024 & 0.1406 & 0.0011 & 0.0224 & 0.3589 & 0.0014 \\ 0.0066 & 0.0408 & 0.0022 & 0.1271 & 0.0010 & 0.0202 & 0.3806 & 0.0012\end{array}$ $\begin{array}{llllllll}0.0066 & 0.0408 & 0.0022 & 0.1271 & 0.0010 & 0.0202 & 0.3806 & 0.0012 \\ 0.0073 & 0.0358 & 0.0024 & 0.1406 & 0.0011 & 0.0224 & 0.3389 & 0.0014\end{array}$

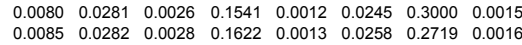
$\begin{array}{llllllll}0.0080 & 0.0359 & 0.0026 & 0.1541 & 0.0012 & 0.0245 & 0.2922 & 0.0015\end{array}$ $\begin{array}{llllllllll}0.0080 & 0.0359 & 0.0026 & 0.1541 & 0.0012 & 0.0245 & 0.2772 & 0.0015\end{array}$ $\begin{array}{llllllll}0.0080 & 0.0509 & 0.0026 & 0.1541 & 0.0012 & 0.0245 & 0.2872 & 0.0015\end{array}$

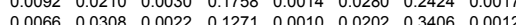
$\begin{array}{llllllllll}0.0073 & 0.0258 & 0.0024 & 0.1406 & 0.0011 & 0.0224 & 0.2989 & 0.0014\end{array}$ $\begin{array}{llllllll}0.0073 & 0.0258 & 0.0024 & 0.1406 & 0.0011 & 0.0224 & 0.2889 & 0.0014\end{array}$

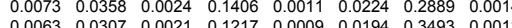

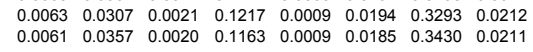

Br-t CdO-t Ce2O3-t CeO2-t Cl-t $\begin{array}{lll}0.0008 & 0.0000 & 0.0000 \\ 0.0007 & 0.0000 & 0.0000\end{array}$ $0.0007 \quad 0.0000$ $\begin{array}{ll}0.0006 & 0.0000 \\ 0.0006 & 0.0000 \\ 0.0005 & 0.0000\end{array}$ $0.0013 \quad 0.0000$ $0.0013 \quad 0.0000$ $\begin{array}{lll}0.0011 & 0.0000 & 0.00000\end{array}$ $\begin{array}{lll}0.0010 & 0.0000 & 0.0000 \\ 0.0009 & 0.0000 & 0.0000\end{array}$ $\begin{array}{ll}0.0000 & 0.0000 \\ 0.0000 & 0.0000\end{array}$ $\begin{array}{lll}0.0000 & 0.0000 & 0.0015 \\ 0.0000 & 0.0014 \\ 0.0000 & 0.0000 & 0.0000\end{array}$ $\begin{array}{lll}0.0000 & 0.0000 & 0.0013 \\ 0.0000 & 0.0000 & 0.0012\end{array}$ $\begin{array}{lll}0.0000 & 0.0000 & 0.0011\end{array}$ $\begin{array}{lll}0.0000 & 0.0000 & 0.0013 \\ 0.0000 & 0.0000 & 0.0012\end{array}$ $\begin{array}{lll}0.0000 & 0.0000 & 0.0012 \\ 0.0000 & 0.0000 & 0.0011\end{array}$ $\begin{array}{lll}0.0000 & 0.0000 & 0.0011 \\ 0.0000 & 0.0000 & 0.0010 \\ 0 & 0.0000\end{array}$ $\begin{array}{lll}0.0000 & 0.0000 & 0.0010 \\ 0.0000 & 0.0000 & 0.0009 \\ 0 & 0.0000 & 0.0000\end{array}$ $\begin{array}{lll}0.0000 & 0.0000 & 0.0000 \\ 0.00000 & 0.00000 & 0.0000 \\ 0.0000 & 0.0000 & 0.000\end{array}$

$<0.000$

0.0002
0.0003
0.0003

0.0003
0.0003

0.0003
0.0003
0.003

0.0003
0.0003
0.0003
0.0003

0.0003
0.0003

0.0003
0.0003
0.002

0.0002
0.0003
0.0003

0.0003
0.0003
0.0002

0.0000
0.0003

0.0002
0.0003
0.0003

0.0003
0.0003
0.0002

0.0003
0.0002
0

0.0002
0.0002
0.0002

0.0002
0.0002
0.0003

0.0003
0.0003

0.0001
0.0001

0.0001
0.0001
0.0001

0.0001
0.0001

0.0001
0.0001

0.0001
0.0001

0.0001

0.0001
0.0001

0.0001
0.0001
0.0001

0.0001
0.0001
0.0001

0.0001
0.0001 
\# Glass ID

2953 HLW98-VO

2954 HLW98-V02

2956 HLW98-V04

2958 HLWB8-VOG

2960 HLW98-V0

2961 HLW98-V09
2962 HLW98-V10

2963 HLW98-V11

2964 HLWB8-V12

2966 HLW98-V14

2968 HLW98-V16

2969 HLW98-V17

2971 HLW98-V19

2972 HLW98-V20
2973 HLW98-V21

2974 HLW98-V22
2975 HLW98-V23

2976 HLW W98-V24

2977 HLW98-V25
2978 HLW98-V26
2979 HLW98-V27

2980 HLW98-V28

2981 HLW98-V29

2983 HLW-E-Cr-10
2984 HLW-E-Cr-M

2985 HLW-E-Bi-6

987 HLW-E-Al-0

2988 HLW-E-Al-02

2990 HLW-E-Al-04

2992 HLW-E-Al-00

2994 HLW-E-Al-0

2996 HLW-E-Al-10

2997 HLW-E-Al-11

2999 HLW-E-Al-13

(3001 HLW-AA-15

3002 HLW-E-Al-16

3004 HLW-E-Al-18

HLW-E-Al-19

3006 HLW-E-Al-20

3008 HLW-E-Al-22

3010 HLW-E-Al-24

3012 HLW-E-AL-26

3014 HLW-E-Al-28

3016 HLW-E-ANa-01

3018 HLW-E-ANa-03

3020 HLW-E-ANa-05
3021 HLW-E-ANa-06

3022 HLW-E-ANa-07

3023 HLW-E-ANa-08

3025 HLW-E-ANa-10

3026 HLW-E-ANa-11
3027 HLW-E-ANa-12

3030 HLW-E-ANa-15

(3031 HLW-E-ANa-16

3033 HLW-E-ANa-18
3034 HLW-E-ANa-19
CoO-t Co2O3-t Cr2O3-t Cs2O-t CuO-t Dy203-t Eu2O3-t

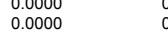

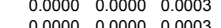

$\begin{array}{llll}0.0000 & 0.0000 & 0.0000 & 0.0003 \\ 0.0000 & 0.0000 & 0.0000 & 0.0003\end{array}$

$\begin{array}{lll}0.0000 & 0.0000 & 0.0002 \\ 0 & 0.0000 & 0\end{array}$

$\begin{array}{lll}0.0000 & 0.0000 & 0.0000\end{array}$

$\begin{array}{lll}0.0000 & 0.00000 & 0.0000 \\ 0.0000 & 0.0000 & 0.0000\end{array}$

$\begin{array}{lll}0.0000 & 0.0000 & 0.0000 \\ 0 & 0.0005 & 0.0000\end{array}$

$\begin{array}{lll}0.0004 & 0.0000 & 0.0005 \\ 0 & .0000 & 0.0000\end{array}$

0.0004

$\begin{array}{lll}0.0009 & 0.0000 & 0.0005 \\ 0.0009 & 0.0000 & 0.0005\end{array}$

$\begin{array}{llll}0.0007 & 0.0000 & 0.00004 \\ 0 & 0.0000 & 0\end{array}$

$\begin{array}{lll}0.0007 & 0.0000 & 0.0004 \\ 0.0006 & 0.0000 & 0.0004\end{array}$

$0.0007 \quad 0.0000 \quad 0.0003$

$\begin{array}{lll}0.0006 & 0.0000 & 0.0003\end{array}$

$\begin{array}{lll}0.0006 & 0.0000 & 0.0003 \\ 0.0005 & 0.0000 & 0.0002\end{array}$

$\begin{array}{llll}0.0005 & 0.0000 & 0.0002\end{array}$

0.052

0.0052
0.0058
0.0058

0.0058
0.0058

0.0058
0.0058
0.0058

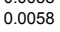

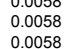

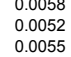

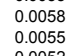

0.0052
0.0064
0.052
0.058

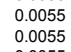

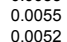

0.0001
0.0052
0.0522
5.052

0.0052
0.0052
0.0055

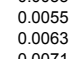

0.0079

0.0071
0.0079
0.0069

0.0086
0.0091
0.0086

0.0086
0.0086
0.0086

0.0086
0.0098
0.0071

0.0071
0.0079

0.079

0.0068
0.0068

0.0065
F-t Ga203-t Gd2O3-t HFO2-t HgO-t 0.0004

$\begin{array}{ll}0.0004 & 0.0000 \\ 0.0004 & 0.0000 \\ 0.0004 & 0.0000 \\ 0.0004 & 0.0000 \\ 0.00003 & 0.0000 \\ 0.0003 & 0.0000 \\ 0.00000 & 0.0000 \\ 0.00000 & 0.0000 \\ 0.00000 & 0.0000 \\ 0.0000 & 0.0000 \\ 0.0000 & 0.0000 \\ 0.00000 & 0.0000 \\ 0.0000 & 0.0000 \\ 0.00000 & 0.0000 \\ 0.00000 & 0.0000 \\ 0.00000 & 0.0000 \\ 0.00000 & 0.0000 \\ 0.0000 & 0.0000 \\ 0.00000 & 0.0000 \\ 0.0000 & 0.0000 \\ 0.00000 & 0.0000 \\ 0.00000 & 0.0000 \\ 0.00000 & 0.0000 \\ 0.0000 & 0.0000 \\ 0.0013 & 0.0000 \\ 0.0012 & 0.0000 \\ 0.0011 & 0.0000 \\ 0.0011 & 0.0000 \\ 0.0010 & 0.0000 \\ 0.00009 & 0.0000\end{array}$

I-t La2 $\quad 0.0048$

$\begin{array}{lllllll}\text { La2O3-t MnO2-t } & \text { MnO-t } & \text { MoO-t MoO2-t MoO3-t Nb205-t Nd2O3-t NpO2-t } & \text { PbO-t PdO2-t PdO-t Pr2O3-t Pr6011-t Rb2O-t ReO-t } \\ 0.0048 & 0.0020 & 0.0000 & 0.0036 & 0.0004 & 0.0000 & 0.0000\end{array}$

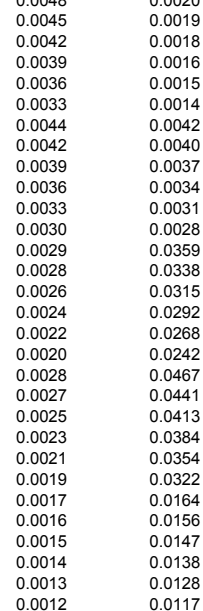

0.0000

0.0034

0.0004

$\begin{array}{ll}0.0000 & 0.0000 \\ 0.0000 & 0.0000 \\ 0.0000 & 0.0000\end{array}$

0.0018
0.0015

0.0042

0.0037

0.0031
0.0028
0.0359

0.0338
0.0315
0.0292

0.0268
0.0242

0.0441
0.0413

0.0354
0.0322
0.0164
0.0156

0.0147
0.0138
0.0128
0.0117

0.0000
0.0000

0.0000
0.0000
0.0000

0.0000

0.0000
0.0000
0.0000
0.0000

0.0000

0.0000
0.0000
0.0000

0.0000

0.0000
0.0000
0.0000

0.0000
0.0000
0.0000

0.0000

0.0000
0.0000
0.0000

0.0000
0.0000
0.0000

0.0030
0.0027
0.0025
0.0019
0.0018
0.0017
0.0016
0.0015
0.0013
0.0019
0.0018
0.0016
0.0015
0.0014
0.0013
0.0018
0.0017
0.0016
0.0014
0.0013
0.0012
0.0012
0.0011
0.0010
0.0010
0.0009
0.0008

$\begin{array}{lll}0.0004 & 0.0000 & 0.0000 \\ 0.0003 & 0.0000 & 0.0000 \\ 0.0003 & 0.0000 & 0.00000 \\ 0.0003 & 0.00000 & 0.0000 \\ 0.0008 & 0.00000 & 0.0000 \\ 0.0008 & 0.0000 & 0.0000 \\ 0.0007 & 0.0000 & 0.0000 \\ 0.0007 & 0.0000 & 0.0000 \\ 0.0006 & 0.00000 & 0.0000 \\ 0.0006 & 0.0000 & 0.0000 \\ 0.0030 & 0.00000 & 0.0000 \\ 0.0028 & 0.0000 & 0.0000 \\ 0.0026 & 0.00000 & 0.0000 \\ 0.0024 & 0.0000 & 0.00000 \\ 0.0022 & 0.00000 & 0.0000 \\ 0.0020 & 0.0000 & 0.00000 \\ 0.0017 & 0.00000 & 0.0000 \\ 0.0016 & 0.00000 & 0.0000 \\ 0.0015 & 0.00000 & 0.0000 \\ 0.0014 & 0.00000 & 0.0000 \\ 0.0013 & 0.00000 & 0.0000 \\ 0.0012 & 0.00000 & 0.0000 \\ 0.0013 & 0.0000 & 0.0000 \\ 0.0013 & 0.00000 & 0.0000 \\ 0.0012 & 0.0000 & 0.0000 \\ 0.0011 & 0.00000 & 0.00000 \\ 0.0010 & 0.0000 & 0.0000 \\ 0.0009 & 0.0000 & 0.0000\end{array}$

0.0070

0.0067
0.0074

0.0074

0.0074

0.0074

0.0074
0.0074

0.0074
0.0074

0.0067

0.0074
0.0070

0.006
0.0082

0.0074

0.0070

0.006

0.0067
0.0067
0.0067

0.007

0.0020

0.0025
0.0023

0.0025

0.0027
0.0029
0.0027
0.0027

0.0027
0.0027
0.0027

0.0023
0.0025
0.0025

0.002

$\begin{array}{lll}0.0009 & 0.0000 & 0.00000 \\ 0.0000 & 0.0000\end{array}$

(

(2)

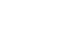


\# Glass ID 2953 HLW98-VO 2954 HLW98-V02
2955 HLW98-V03 2956 HLW98-V04
2957 HLW88-V05 2958 HLW98-V06 2960 HLW98-V08 2961 HLW 28 -V09 2963 HLW98-V11 2964 HLW98-V12 2965 HLW98-V13 2967 HLW98-V1 2968 HLW98-V16 2969 HLW98-V17
2970 HLW98-V1 2971 HLW98-V19 2972 HLW98-V20 2974 HLW98-V22 2976 HLW98-V24 2978 HLW88-V26 2979 HLW98-V2 2981 HLWW8-V29 2983 HLW-E-Cr-10 2985 HLW-E-Bi-6 2986 HLW-E-Al-27 2987 HLW-E-Al-01 2991 HLW-E-Al-0 (992 HLW-E-Al-06 2994 HLW-E-Al-08 2996 HLW-E-Al-10 2998 HLW-E-Al-12 2999 HLW-E-Al-13 3001 HLWEAT-45

3002 HLW-E-Al-16 3004 HLW-E-Al-1 05 HLW-E-Al-19

3007 HLW-E-AA-21 3008 HLW-E-Al-22 3010 HLW-E-Al-24 3011 HLW-E-Al-25 3013 HLW-E-Al-27 3014 HLW-E-AA-28
3015 HLW-E-Al-29 3017 HLW-E-ANa-02 3018 HLW-E-ANa-03 020 HLW-E-ANa-05 3022 HLW-E-ANa-07 3024 HLW-E-ANa-09
3025 HLW-E-ANa-10 3026 HLW-E-ANa-11 3027 HLW-E-ANa-12 3029 HLW-E-ANa-14 3030 HLW-E-ANa-15 (3031 HLW-E-ANa-16 3033 HLW-E-ANa-18

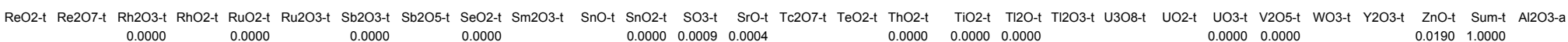

\begin{tabular}{|c|c|c|c|}
\hline & & & \\
\hline $\begin{array}{l}0.00000 \\
0.0000\end{array}$ & $\begin{array}{l}0.0000 \\
0.0000\end{array}$ & 0.0000 & 0.0000 \\
\hline & $\begin{array}{l}0.00000 \\
0.00000\end{array}$ & $\begin{array}{l}0.00000 \\
0.0000\end{array}$ & $\begin{array}{l}0.00000 \\
0.0000\end{array}$ \\
\hline 0.0000 & 0.0000 & 0.0000 & 0.0000 \\
\hline & & & \\
\hline $\begin{array}{l}0.00000 \\
0.0000\end{array}$ & $\begin{array}{l}0.00000 \\
0.0000\end{array}$ & $\begin{array}{l}0.00000 \\
0.0000\end{array}$ & $\begin{array}{l}0.0000 \\
0.00000\end{array}$ \\
\hline $\begin{array}{l}0.0000 \\
0.0000\end{array}$ & $\begin{array}{l}0.0000 \\
0.0000\end{array}$ & $\begin{array}{l}0.0000 \\
0.0000\end{array}$ & $\begin{array}{l}0.00000 \\
0.00000\end{array}$ \\
\hline 0.0000 & 0.0000 & 0.0000 & 0.0000 \\
\hline & $\begin{array}{l}0.00000 \\
0.0000\end{array}$ & 0.0000 & 0.0000 \\
\hline $\begin{array}{l}0.0000 \\
0.0000\end{array}$ & $\begin{array}{l}0.0000 \\
0.0000\end{array}$ & $\begin{array}{l}0.0000 \\
0.0000\end{array}$ & $\begin{array}{l}0.00000 \\
0.00000\end{array}$ \\
\hline 0.0000 & 0.0000 & 0.0013 & 0.0025 \\
\hline 0.0000 & 0.0000 & 0.0012 & 0.0024 \\
\hline 0.0000 & 0.0000 & 0.0011 & 0.0022 \\
\hline $\begin{array}{l}0.00000 \\
0.0000\end{array}$ & $\begin{array}{l}0.0000 \\
0.0000\end{array}$ & $\begin{array}{l}0.001010 \\
0.0010\end{array}$ & $\begin{array}{l}0.0020 \\
0.0019\end{array}$ \\
\hline 0.0000 & 0.0000 & 0.0009 & 0.0017 \\
\hline & 0.0000 & 0.0030 & 0.0043 \\
\hline 0.0000 & 0.0000 & 0.0028 & 0.0041 \\
\hline $\begin{array}{l}0.00000 \\
0.0000\end{array}$ & $\begin{array}{l}0.0000 \\
0.0000\end{array}$ & $\begin{array}{l}0.0026 \\
0.0025\end{array}$ & $\begin{array}{l}0.0038 \\
0.0036\end{array}$ \\
\hline & $\begin{array}{l}0.00000 \\
0.0000\end{array}$ & 0.0023 & 0.0033 \\
\hline 0.0000 & 0.0000 & 0.0021 & 0.0030 \\
\hline 0.0000 & 0.0000 & 0.0000 & 0.0000 \\
\hline 0.0000 & 0.0000 & 0.0000 & 0.0000 \\
\hline 0.0000 & 0.0000 & 0.0000 & 0.0000 \\
\hline 0.0000 & 0.00000 & 0.0000 & 0.0000 \\
\hline & 0 & 0.000 & \\
\hline
\end{tabular}

$0.0000 \quad 0.000900 .0004$

$\begin{array}{lll}0.0000 & 0.0008 & 0.0003 \\ 0000 & 0.0007 & 0.0003\end{array}$

$\begin{array}{llll}0.0000 & 0.0007 & 0.00003 \\ 0 & 0000 & 0.0006 & 0.0003\end{array}$

$\begin{array}{lll}0.0000 & 0.0005 & 0.0000 \\ 0.0000 & 0.0005 & 0.0000\end{array}$

$\begin{array}{lll}0.0000 & 0.0004 & 0.0000 \\ 0.0000 & 0.0004 & 0.0000\end{array}$

$\begin{array}{llll}0.0000 & 0.0004 & 0.0000 \\ 0.0000 & 0.0003 & 0.0000\end{array}$

$\begin{array}{llll}0.0000 & 0.0000 & 0.0000\end{array}$

$\begin{array}{llll}0.0000 & 0.0000 \quad 0.0000\end{array}$

$\begin{array}{llll}0.0000 & 0.0000 & 0.0000\end{array}$

0.0000
0.0107

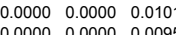

$\begin{array}{lll}0.0000 & 0.0000 & 0.0088 \\ 0.0000 & 0.0000 & 0.0081 \\ 0 & 0.000 & 0.003\end{array}$

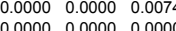

$\begin{array}{llll}0.0000 & 0.00000 & 0.0000 \\ 0.000 & 0.0000 & 0.0000\end{array}$

$\begin{array}{lll}0.00000 & 0.0000 & 0.0000 \\ 0.00000 & 0.0000 & 0\end{array}$

$\begin{array}{lll}0.0000 & 0.0000 & 0.0000 \\ 0.0000 & 0.0000 & 0.0000\end{array}$

0.0066

$0.0022 \quad 0.0000$

$\begin{array}{ll}0.0022 & 0.0000 \\ 0.0022 & 0.0000\end{array}$

$\begin{array}{ll}0.0002 & 0.0000 \\ 0.0022 & 0.0000 \\ 0.002 & 00000\end{array}$

$\begin{array}{ll}0.0022 & 0.0000 \\ 0.0022 & 0.0000\end{array}$

$\begin{array}{ll}0.0022 & 0.0000 \\ 0.0022 & 0.0000 \\ 0.0022 & 0.0000\end{array}$

$\begin{array}{ll}0.0022 & 0.0000 \\ 0.0022 & 0.0000\end{array}$

$\begin{array}{ll}0.0020 & 0.0000 \\ 0.0021 & 0.0000 \\ 0.0022 & 0.0000\end{array}$

$\begin{array}{ll}0.0022 & 0.0000 \\ 0.0021 & 0.0000 \\ 0.0020 & 0.0000\end{array}$

$\begin{array}{ll}0.0020 & 0.0000 \\ 0.0024 & 0.0000\end{array}$

$\begin{array}{ll}0.0024 & 0.0000 \\ 0.0020 & 0.0000 \\ 0.0022 & 0.0000\end{array}$

0.00210 .0000

0.00210 .0000

$0.0016 \quad 0.0000$

$0.0020 \quad 0.0000$

$\begin{array}{lll}0.0021 & 0.0000 \\ 0.021 & 0.0000\end{array}$

$0.0019 \quad 0.0000$

$\begin{array}{ll}0.0022 & 0.0000 \\ 0.0024 & 0.0000 \\ 0.0022 & 0.0000\end{array}$

$\begin{array}{lll}0.0024 & 0.0000 \\ 0.0024 & 0.0000 & 0.000\end{array}$

$\begin{array}{lll}0.0028 & 0.0000 \\ 0.026 & 0.0000\end{array}$

$\begin{array}{lll}0.0026 & 0.0000 \\ 0.026 & 0.0000\end{array}$

$\begin{array}{lll}0.0026 & 0.0000 \\ 0.0030 & 0.0000\end{array}$

$\begin{array}{lll}0.0022 & 0.0000 \\ 0.0024 & 0.0000\end{array}$

$0.0024 \quad 0.0000$

$0.0020 \quad 0.0000$

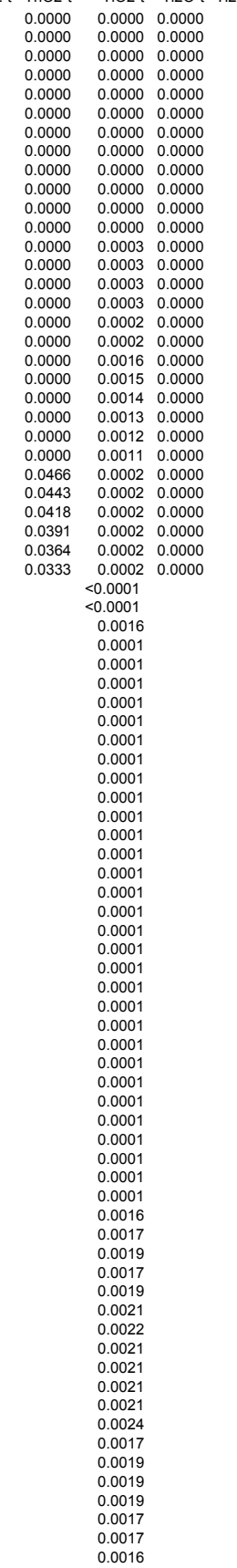

$0.0000 \quad 0.0000$

$\begin{array}{ll}0.0194 & 1.0000 \\ 0.0199 & 1.0000\end{array}$

$\begin{array}{lll}0.0000 & 0.0000 \\ 0.0000 & 0.0000\end{array}$

$\begin{array}{ll}0.0000 & 0.0000 \\ 0.0000 & 0.0000\end{array}$

$0.0000 \quad 0.0000$

$0.0000 \quad 0.0000$

$0.0000 \quad 0.0000$

$\begin{array}{ll}0.0000 & 0.0000 \\ 0.0000 & 0.0000\end{array}$

$\begin{array}{lll}0.0000 & 0.0000 \\ 0.0000 & 0.0000\end{array}$

0.00000 .0000

$0.0000 \quad 0.0000$

$0.0000 \quad 0.0000$

$\begin{array}{lll}0.0296 & 0.0000 \\ 0.0281 & 0.0000\end{array}$

$\begin{array}{ll}0.0266 & 0.0000\end{array}$

$\begin{array}{ll}0.0231 & 0.0000 \\ 0.0212 & 0.0000\end{array}$

$\begin{array}{ll}0.0204 & 1.0000 \\ 0.0209 & 1.0000 \\ 0.0215 & 1.0000\end{array}$

$\begin{array}{ll}0.0215 & 1.0000 \\ 0.0190 & 1.0000\end{array}$

$\begin{array}{lll}0.0194 & 1.0000 \\ 0.0199 & 1.0000\end{array}$

$\begin{array}{ll}.0204 & 1.0000 \\ .0209 & 1.0000\end{array}$

$0.0214 \quad 1.0000$

.

0.02001 .0000

$\begin{array}{ll}0.0204 & 1.0000 \\ 0.0209 & 1.0000 \\ 0.0214 & 1.0000\end{array}$

$0.0195 \quad 1.0000$

$0.0200 \quad 1.0000$

$\begin{array}{ll}0.0210 & 1.0000 \\ 0.0215 & 1.0000\end{array}$

0.02211 .0000

$\begin{array}{ll}0.0192 & 1.0000 \\ 0.0199 & 1.0000 \\ 0 & 1.025\end{array}$

$\begin{array}{ll}0.0199 & 1.0000 \\ 0.0205 & 1.0000 \\ 0.0213 & 1.0000\end{array}$

$0.0221 \quad 1.0000$

.0016 1.0005

$\begin{array}{ll}0.0008 & 0.9999 \\ 0.0009 & 1.0000\end{array}$

$\begin{array}{ll}0.0009 & 1.0000 \\ 0.0009 & 1.0000\end{array}$

0.00091 .0000

0.00091 .0000

0.00091 .0000

0.00091 .0000

0.00091 .0000

0.00091 .0000

$0.0008 \quad 1.0000$

$\begin{array}{ll}0.0009 & 1.0000 \\ 0.0009 & 1.0000\end{array}$

$0.0009 \quad 1.0000$

$0.0008 \quad 1.0000$

0.00101 .0000

$\begin{array}{ll}0.0008 & 1.0000 \\ 0.0009 & 1.0000\end{array}$

$0.0009 \quad 1.0000$

$\begin{array}{lll}0.00009 & 1.0000 \\ 0.0009 & 1.0000\end{array}$

$\begin{array}{lll}0.0009 & 1.0000 \\ 0.0008 & 1.0000\end{array}$

\begin{tabular}{lll}
0.0006 & 1.0000 \\
0.0008 & 1.0000 \\
\hline & .0000 & 1.0000
\end{tabular}

$\begin{array}{ll}0.0008 & 1.0000 \\ 0.0008 & 1.0000\end{array}$

0.00091 .0000

$\begin{array}{ll}0.0009 & 1.0000 \\ 0.0016 & 1.0000 \\ 0.0018 & 1.0000\end{array}$

0.00201 .0000

$\begin{array}{lll}0.0018 & 1.0000 \\ 0.0020 & 1.0000\end{array}$

$0.0022 \quad 1.0000$

$\begin{array}{ll}0.0023 & 1.0000 \\ 0.0022 & 1.0000\end{array}$

$0.0022 \quad 1.0000$

0.00221 .0000

0.00181 .0000

$0.0020 \quad 1.0000$

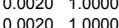

0.00171 .0000

$\begin{array}{ll}0.0017 & 1.0000 \\ 0.0016 & 1.0000\end{array}$ 
2954 HLW98-V02

2955 HLW98-V03
2956 HLW98-V04

2957 HLW98-V05
2958 HLW98-V06

2958 HLLW88-V07

2960 HLWB8-V08

2961 HLW98-V09

2963 HLW98-V11

2964 HLW98-V12

2965 HLW98-V13

2967 HLW98-V15

2968 HLW98-V16

(969) HLW98-V18

2971 HLW98-V19

2972 HLW98-V20

(9)

2975 HLW98-V23

2976 HLW98-V24

2977 HLW98-V25

2979 HLW98-V2

2980 HLW98-V28
2981 HLW98-V29

2981 HLW98-V29

2983 HLW-E-Cr-10
2984 HLW-E-Cr-M

2985 HLW-E-Bi-6

2987 HLW-E-Al-01

2988 HLW-E-Al-02
2989 HLW-E-Al-03

2990 HLW-E-Al-04

2991 HLW-E-Al-05

2992 HLW-E-Al-0

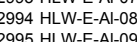

2995 HLW-E-Al-09

2997 HLW-E-Al-1 1

2999 HLW-E-Al-13

3000 HLW-E-Al-14
3001 HLW-E-Al-15

3002 HLW-E-Al-16

3003 HLW-E-Al-17

3004 HLW-E-Al-18
3005 HLW-E-Al-19

3006 HLW-E-Al-20

3007 HLW-E-Al-21

3009 HLW-E-Al-23

3010 HLW-E-Al-24

2017 HLW-E-Al-25

3012 HLW-E-Al-26

3014 HLW-E-Al-28

3016 HLW-E-ANa-01

3018 HLW-E-ANa-03

3019 HLW-E-ANa-04

3021 HLW-E-ANa-06

3022 HLW-E-ANa-07

3023 HLW-E-ANa-08

3025 HLW-E-ANa-10

3026 HLW-E-ANa-11

3027 HLW-E-ANa-12

3029 HLW-E-ANa-14

3030 HLW-E-ANa-15

3032 HLW-E-ANa-17

3033 HLW-E-ANa-18

3034 HLW-E-ANa-19 
29545 HWB8-V02

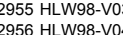

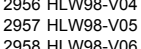

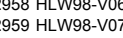

2960 HLW98-V08

2961 HLW98-V09
2962 HLW98-V10

2963 HLW W98-V11

2964 HLW98-V12

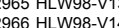

2967 HLW 98 -V15

2968 HLW98-V16
2069 HLW

(1)

2971 HLW98-VV19

2972 HLWW88VV2

2973 HLW98-V22
2974 HLW $198-$ V22

2975 HLW98-VV2

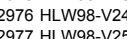

2977 HLW98-V25
2978 HLW98-V26

2999 HLW $18-$ V 22

2980 HLWG98.V22
2081 HLW98-V29

29891 HLWG8-V29
2982 HLW98-V30

2983 HLW-E-Cr-10
2984 HLW-E-Cr-M

2985 HLW-E-Bi-6

2986 HLW-E-A-27
2987 HLW-EAl-0

2988 HLW-EA-A-O2
2989 HLW-EAL-OS

2990 HLW-E-AL-04

2991 HLW-EAA-0

2992 HLW-E-AL-OC

2994 HLWE-AAl-O8

2995 HLW-E-AL-09
2996 HLW-EAl-11

2999 HLW-E-ALA-10
2997 HWWE-A-Al1
2099

2998 HLW-E-AA-12
2999 HLW-EA-A-1

3000 HLW-E-AA-1
3001 HWWE-AL-15

3002 HLW-E-AL-16

3003 HLW-E-AL-17

3004 HLW-E-Al-18
3005 HLW-E-AL-19

3006 HLW-E-Al-20

3007 HLW-E-Al-2

3009 HLW-E-A-AL-22

3010 HLW-E-Al-22

3011 HLW-E-A-25

3012 HLW-E-Al-26

3014 HLW-E-AL-28
3015 HLW-EA-29

3018 HLW-E-ANa-03

3019 HLW-E-ANa-04

3021 HLW-E-ANa-06

3022 HLW-E-ANa-07

3023 HLW-E-ANa-08
3024 HLW-E-ANa-09

3025 HLW-E-ANa-10

3026 HLW-E-ANa-11

3027 HLW-E-ANa-12

3029 HLW-E-ANa-14

3030 HLW-E-ANa-15

3032 HLW-E-ANa-17

3033 HLW-E-ANa-1

3034 HLW-E-ANa-19 
2954 HLW98-V02 2955 HLW98-V03

Sm203-a SnO-a SnO2-a SO3-a SrO-a Tc207-a TeO2-a ThO2-a TiO2-a T12O-a T1203-a U308-a UO2-a UO3-a V2O5-a WO3-a Y2O3-a ZnO-a Suna

1087
1025

1025
1000
925

2968 HLW98-V16

2970 HLW9-V

2971 HLW98-V19

(2973

2974 HLW98-V22
2975 HLW98-V23

2976 HLWG8-V24

2977 HLW98-V25
2978 HLW98-V26

2979 HLW98-V2

2980 HLW98-V28

2981 HLW98-V29
2982 HLW98-V30

2983 HLW-E-Cr-10

2984 HLW-E-Cr-M

2985 HLW-E-Bi-6

2987 HLW-E-Al-01

2988 HLW-E-Al-02
2989 HLW-E-Al-03

2990 HLW-E-Al-04

2991 HLW-E-Al-05

2992 HLW-E-Al-0

2994 HLW-E-AL-08

2995 HLW-E-Al-09

2996 HLW-E-Al-10
2997 HLW-E-Al-11

2998 HLW-E-Al-12

2999 HLW-E-Al-13

3000 HLW-E-Al-14

3002 HLW-E-Al-16

3003 HLW-E-Al-17

3004 HLW-E-Al-18
3005 HLW-E-Al-19

3006 HLW-E-Al-20

3007 HLW-E-Al-2

3009 HLW-E-Al-23

3010 HLW-E-Al-24

3011 HLW-E-Al-25

3013 HLW-E-Al-27

3014 HLW-E-A-28
3015 HLW-E-Al-29

3018 HLW-E-ANa-03

3020 HLW-E-ANa-05

3021 HLW-E-ANa-06

3022 HLW-E-ANa-07

3025 HLW-E-ANa-10

3026 HLW-E-ANa-11

3027 HLW-E-ANa-1

3029 HLW-E-ANa-14

3030 HLW-E-ANa-15

3031 HLW-E-ANa-16

3033 HLW-E-ANa-18 
2.890
2.860
2.820

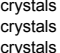

34\% (R-Al-Silicate+Apatite+Spinel) $32 \%$ (R-Al-Silicate+Apatite+Spinel)
$6.7 \%$ (Apatite+R-Al-silicate+Spinel) 6.8\% (Spinel+R-A-A-Silicate) $4 \%$ (Spinel+Ap
0.021 $2.9 \%$ (Spinel+Apatite)
$1.6 \%$ (Spinel) $1.6 \%$ (Spinel)

$1.8 \%$ (Spinel+ZrO2)

$0.3 \%$ (Cr-Fe oxide)

$0.5 \%$ (Spinel)

$0.5 \%$ (Spinel)
$0.3 \%$ (Spinel)

$0.3 \%$ (Spinel)
$\sim 0.4$ vol $\%$
$0.8 \%$ (Spinel)

$0.8 \%$ (Spinel)
crystals
$0.4 \%$ (Spinel)

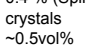

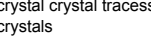

constal crysat tracess

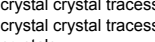

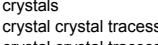

crystal crystal tracess
crysta crystat racess
crysta crystal tracess
crystals

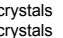

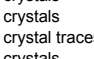

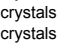

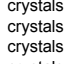

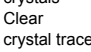

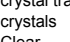

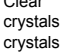

crystal traces (Spine//Cr2O3)

Clear
crystal traces (Spinel)

clear
crystat racess Spinel|

crystat traess (spinel)

Hetergogenous
Heterogenous

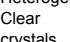

Heterogeneous
Minor (Ca phosphatet +Nephline)

Minor (Ca-phosphate+Nepheline)

crystal traces (Ca-phosphate+Nepheline)

Minor (Ca phosphate)
crystal traces (Ca phosp

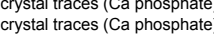


\# Glass ID

\# Glass ID
2953 HLW98-V0
2954 HLW98-V02

2954 HLW98-V02

2956 HLW98-VO

2957 HLW98-V05
2958 HLW98-V06

2959 HLW98-V07

2960 HLW98-V08

2961 HLW98-V09

2963 HLW98-V11

2964 HLW98-V12

2965 HLW98-V13

2967 HLW98-V15

2968 HLW98-V1

2969 HLW98-V 17

2971 HLW98-V19

2972 HLW W98-V2O

2973 HLW98-V22

2975 HLW98-V23

2976 HLW98-V24
2977 HLW98-V25

2977 HLW98-V25

2979 HLW98-V27

2981 HLW98-V29

2982 HLW98-V30

2983 HLW-E-Cr-10
2984 HLW-E-Cr-M

2985 HLW-E-Bi-6

2987 HLW-E-Al-01

2988 HLW-E-Al-02
2989 HLW-E-Al-03

2990 HLW-E-Al-04

2991 HLW-E-Al-05

2992 HLW-E-Al-OO
2993 HLW-E-Al-07

2994 HLW-E-A-Al-18

2995 HLW-E-Al-09

2996 HLW-E-Al-10

2998 HLW-E-Al-12

2999 HLW-E-Al-13

3000 HLW-E-Al-14
3001 HLW-E-Al-15

3002 HLW-E-Al-16

3003 HLW-E-Al-17

3004 HLW-E-Al-18

3006 HLW-E-Al-20

3007 HLW-E-Al-2

3009 HLW-E-Al-23

3010 HLW-E-Al-24

30112 HLW-E-Al-25

3012 HLW-E-Al-26

3014 HLW-E-Al-28

3015 HLW-E-Al-29

3017 HLW-E-ANa-02

3018 HLW-E-ANa-03

3019 HLW-E-ANa-04

3021 HLW-E-ANa-06

3022 HLW-E-ANa-07

3023 HLW-E-ANa-08
3024 HLW-E-ANa-09

3025 HLW-E-ANa-10

3026 HLW-E-ANa-11

3027 HLW-E-ANa-12

3029 HLW-E-ANa-14

3030 HLW-E-ANa-15

3031 HLW-E-ANa-16

3033 HLW-E-ANa-18

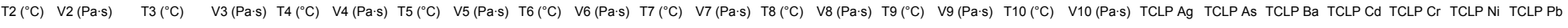

\begin{tabular}{|c|c|c|c|c|c|}
\hline $\begin{array}{r}0.0031 \\
0.005\end{array}$ & & & $\begin{array}{r}0.01 \\
<0.0027\end{array}$ & & $\begin{array}{l}\quad 0.0243 \\
<0.0243\end{array}$ \\
\hline 0.0139 & & & 0.0326 & & \\
\hline 0.0133 & & 0.0038 & 0.0312 & 0.1 & 0.0149 \\
\hline 0.0054 & 0.0038 & 0.0147 & 0.0248 & & 0.0107 \\
\hline & 0.182 & & & & 0.056 \\
\hline
\end{tabular}

$0.043<0.049$

0.007

0.037

$1153 \quad 4.275 \quad 1048 \quad 10.7 \quad 945.0 \quad 32.6$ 
\# Glass ID 2953 HLW98-V0 2954 HLW98-V02 2956 HLW98-V04

2957 HLW88-V05
2958 HLW98-V06 2959 HLW98-V07 2960 HLW98-V08 2961 HLW98-V09 2963 HLW98-V11 2964 HLW98-V12 2965 HLW98-V13 2967 HLW98-V15 2968 HLW98-V16

2970 HLW98-V18

2971 HLW98-V19

2972 HLW98-V2O

2974 HLW98-V2

2975 HLW98-V23

2976 HLW98-V24

2978 HLW98-V26

2979 HLW98-V27

2981 HLWW8-V29

2983 HLW-E-Cr-10

2984 HLW-E-Cr-M

2986 HLW-E-Al-27

2987 HLW-E-Al-01

2988 HLW-E-Al-02
2989 HLW-E-Al-0 03

2990 HLW-

2991 HLW-E-Al-0

2993 HLW-E-Al-O

2994 HLW-E-Al-0

2996 HLW-E-Al-11

2998 HLW-E-Al-12

2999 HLW-E-Al-13

3000 HLW-E-Al-14

3002 HLW-E-Al-16

3003 HLW-E-Al-17

3004 HLW-E-Al-18

3006 HLW-E-Al-20

3007 HLW-E-Al-21

3008 HLW-E-Al-22

3010 HLW-E-Al-24

3011 HLW-E-Al-2

3013 HLW-E-Al-27

3014 HLW-E-Al-28

3015 HLW-E-Al-29

3017 HLW-EANa 22

3018 HLW-E-ANa-03

3019 HLW-E-ANa-04

3021 HLW-E-ANa-06

3022 HLW-E-ANa-07

3023 HLW-E-ANa-08

3025 HLW-E-ANa-10

3026 HLW-E-ANa-11

3027 HLW-E-ANa-12

3029 HLW-E-ANa-14

3030 HLW-E-ANa-15

3031 HLW-E-ANa-16
3032 HLW-E-ANa-17

3033 HLW-E-ANa-18

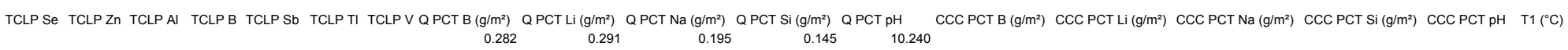

$$
0.282
$$

$\begin{array}{lllll}0.286 & 0.300 & 0.210 & 0.155 & 10.840\end{array}$

$\begin{array}{lll}0.571 & 0.258 & 1.28\end{array}$

$\begin{array}{llll}0.0056 & 0.575 & 0.149 & 1.08\end{array}$

0.095

$\begin{array}{lllll} & 0.367 & 0.331 & 0.205 & 10.350 \\ & & & & \\ & & & & \\ 0.394 & 0.378 & 0.368 & 0.205 & 10.240\end{array}$

0.245

10.32

$\begin{array}{lll}1.450 & 1.000 & 0.750 \\ 0.245 & 0.090 & 0.265 \\ 0.135 & 0.220 & 0.150\end{array}$

$\begin{array}{lll}0.885 & 0.920 & 0.930 \\ 0.225 & 0.275 & 0.260 \\ 0.260 & 0.255 & 0.270\end{array}$

$\begin{array}{lll} & & \\ \text { Pass } & \text { Pass } & \text { Pass } \\ \text { Pass } & \text { Pass } & \end{array}$

Pass Pass Pass

\begin{tabular}{|c|c|c|}
\hline Pas & Pass & \\
\hline & 0.330 & \\
\hline 210 & $\begin{array}{l}0.300 \\
0.300\end{array}$ & \\
\hline 320 & 0.410 & \\
\hline Pas. & $\begin{array}{l}0.600 \\
\text { Pass }\end{array}$ & \\
\hline .36 & $\begin{array}{l}0.760 \\
1.010\end{array}$ & \\
\hline & 0.855 & \\
\hline & & \\
\hline
\end{tabular}

0.590

0.23

40.550

24.650

9.200 
2954 LLW98-V02

2955 HLW98-V03

2957 HLW98-V05
2958 HLW98-V06

2958 HLWB8-V06

2960 HLW98-V08

2962 HLW98-V10

2963 HLW98-V1
2964 HLW98-V12
2965 HLW98-V13

2965 HLW98-V13 2966 HLW98-V14

2966 HLW98-V14
2967 HLW98-V15

2968 HLW89-VV16
2969 HLW8-V17

2969 HLW98-V17
2970 HLW98-V18

2971 HLW98-V19

2971 HLW HLW8-V19-V20
2973 HLW88-V21

2973 HLW98-V2

2974 HLW98-V22
2975 HLW8-V23
2976 HLW98-V24

2977 HLW98-V25

2979 HLW98-V27

2980 HLW89--272
2981 HLW88-V29

2981 HLW98-V29

2983 HLW-E-Er-10
2984 HLW-E-Cr-M

2985 HLW-E-Bi-6

2987 HLW-E-AA-01

2988 HLW-E-EA-OA

2990 HLW-E-Al-04

2991 HLW-E-Al-05

2992 HLW-E-Al-06

29944 LLW-E-EA-A-D8
2995 HLW-E-ALA 09

2996 HLW-E-Al-10

2997 HLW-E-Al-11

2999 HLW-E-Al-13

3000 HLW-E-Al-14
3001 HLW-E-Al-15

3002 HLW-E-Al-16

3003 HLW-E-Al-17

3004 HLW-E-Al-18
3005 HLW-E-Al-19

3006 HLW-E-Al-20

2007 HLW-E-Al-21

3010 HLW-E-Al-24
3011 HLW-E-AA-25

3012 HLW-E-Al-26
3013 HLW-E-Al-27

3014 HLW-E-Al-28

3016 HLW-E-ANa-01

3017 HLW-E-ANa-02

3019 HLWE-ANa-04

3020 HLW-E-ANa-05
3021 HLW-E-ANa-06

3022 HLW-E-ANa-07

3023 HLW-E-ANa-08

3025 HLW-E-ANa-10

3026 HLW-E-ANa-11

3028 HLW-E-ANa-13

3029 HLW-E-ANa-14

3030 HLW-E-ANa-15

3031 HLW-E-ANa-16

3033 HLW-E-ANa-18

3034 HLW-E-ANa-19

$\begin{array}{lllllll}0.448 & 1153 & 0.354 & 1059 & 0.241 & 966 & 0.145 \\ & & & & & & \\ 0.508 & 1148 & 0.378 & 1054 & 0.273 & 962 & 0.169\end{array}$

$\begin{array}{lll}1.3208 & -1674.12 \quad 450\end{array}$

0.37
0.2
0.47

0.03

0.32
0.25 


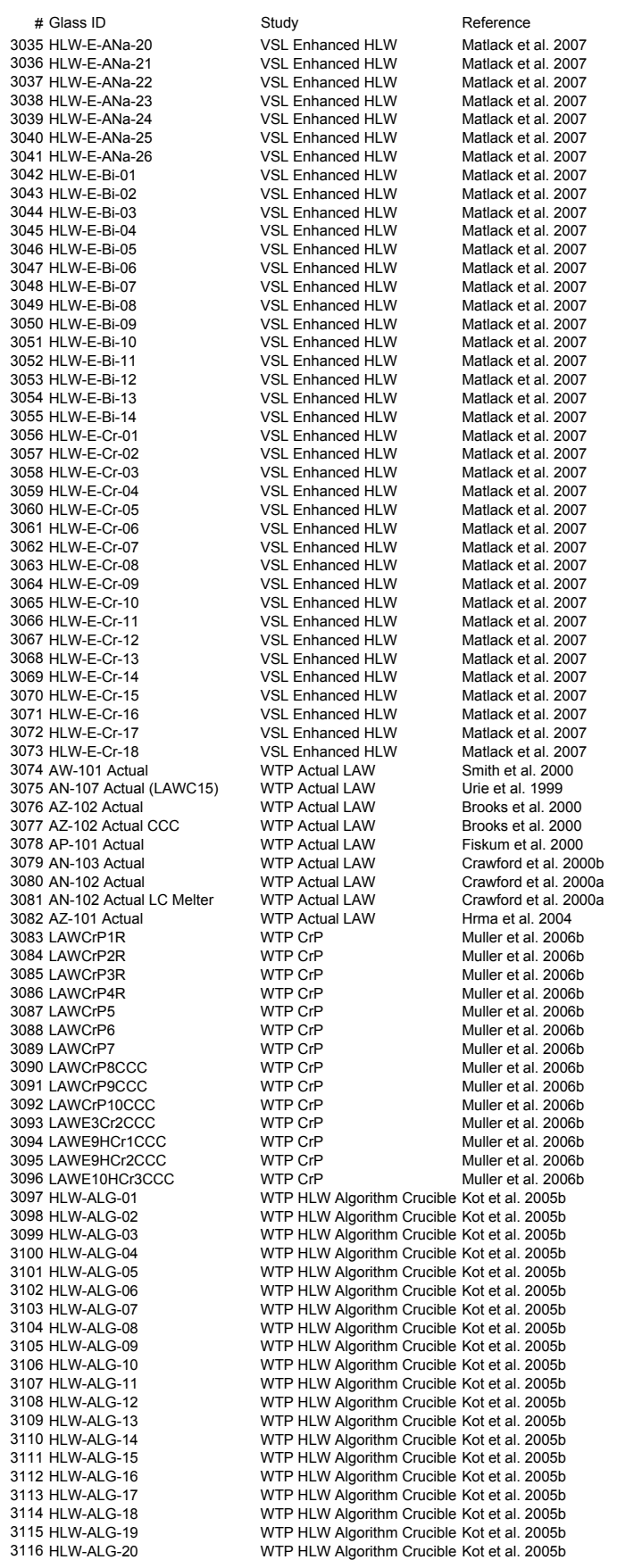
$\begin{array}{cccc}\text { Al2O3-t } & \text { B2O3-t } & \text { CaO-t } & \text { Fe2O3-t } \\ 0.2134 & 0.1437 & 0.0372 & 0.0282 \\ 0.2134 & 0.1537 & 0.0372 & 0.0282 \\ 0.2134 & 0.1837 & 0.0072 & 0.0282 \\ 0.2234 & 0.1377 & 0.072 & 0.028\end{array}$ $\begin{array}{llll}0.2134 & 0.1837 & 0.0072 & 0.0282 \\ 0.2270 & 0.1839 & 0.0077 & 0.0300 \\ 02270 & 0.1939 & 0.0077 & 0.0300\end{array}$ $\begin{array}{llll}0.2361 & 0.1941 & 0.0080 & 0.0311 \\ 0.0932 & 0.1374 & 0.0067 & 0.0557\end{array}$

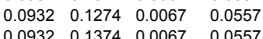

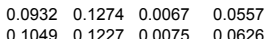
$\begin{array}{llll}0.1049 & 0.1227 & 0.0075 & 0.0626 \\ 0.1166 & 0.1130 & 0.0084 & 0.0696 \\ 0.12025 & 0.1427 & 0.095 & 0.626\end{array}$ $\begin{array}{llll}0.1166 & 0.1130 & 0.0084 & 0.0696 \\ 0.1282 & 0.1033 & 0.0092 & 0.0766\end{array}$

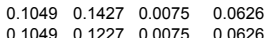
$\begin{array}{llll}0.1049 & 0.1227 & 0.0075 & 0.0626 \\ 0.1049 & 0.1227 & 0.0075 & 0.0626\end{array}$

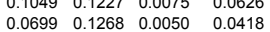
$\begin{array}{llll}0.1399 & 0.1136 & 0.0100 & 0.0835 \\ 0.1282 & 0.1033 & 0.0092 & 0.0766\end{array}$ $\begin{array}{llll}0.1282 & 0.1033 & 0.0092 & 0.0766 \\ 0.1515 & 0.1039 & 0.0109 & 0.0905\end{array}$

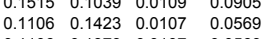

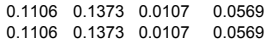
$\begin{array}{llll}0.1106 & 0.1373 & 0.0107 & 0.0569 \\ 0.1106 & 0.1373 & 0.0107 & 0.0569\end{array}$ $\begin{array}{llll}0.1106 & 0.1373 & 0.0117 & 0.0599 \\ 0.1106 & 0.1373 & 0.0107 & 0.0569 \\ 0.1106 & 0.1373 & 0.0107 & 0.059\end{array}$ $\begin{array}{llll}0.1106 & 0.1373 & 0.0107 & 0.0569 \\ 0.1106 & 0.1373 & 0.0107 & 0.0569\end{array}$ $\begin{array}{llll}0.1106 & 0.1373 & 0.0107 & 0.0569 \\ 0.1106 & 0.1373 & 0.0107 & 0.0569 \\ 0.1106 & 0.144 & 0.0107 & 0.059\end{array}$

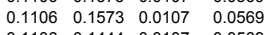
$\begin{array}{llll}0.1106 & 0.1444 & 0.0107 & 0.0569 \\ 0.1106 & 0.1444 & 0.0107 & 0.0569\end{array}$ $\begin{array}{llll}0.1106 & 0.1444 & 0.0147 & 0.0569 \\ 0.1244 & 0.1447 & 0.0120 & 0.0640 \\ 0.0640\end{array}$

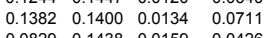
$\begin{array}{llll}0.0129 & 0.1438 & 0.0159 & 0.0426 \\ 0.1244 & 0.1447 & 0.0120 & 0.0640 \\ 0.1175 & 0.1445 & 0.014 & 0.0604\end{array}$ $\begin{array}{llll}0.1175 & 0.1445 & 0.0114 & 0.0604\end{array}$ $\begin{array}{llllllllllllll}0.1175 & 0.1445 & 0.0317 & 0.0604 & & 0.0543 & 0.0317 & 0.0007 & 0.0924 & 0.0049 & 0.0154 & 0.3786 & 0.0005 & \\ 0.0608 & 0.0971 & 0.0199 & 0.0554 & 0.0000 & 0.0258 & 0.0000 & 0.0148 & 0.2000 & 0.00000 & 0.0007 & 0.4405 & 0.0299 & 0\end{array}$ \begin{tabular}{llllllllllllll} 
& 0.0000 & 0.0214 & 0.0000 & 0.0201 & 0.2000 & 0.00004 & 0.00002 & 0.4478 & 0.0299 & 0.0000 & 0.0000 \\
\hline
\end{tabular}

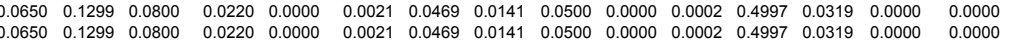

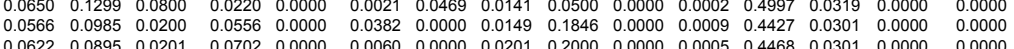
$\begin{array}{lllllllllllllll}0.0615 & 0.1013 & 0.0642 & 0.0649 & 0.00000 & 0.0009 & 0.0274 & 0.0152 & 0.1180 & 0.0001 & 0.0013 & 0.4675 & 0.0303 & 0.00000 & 0.00000 \\ 0.0615 & 0.1010 & 0.0443 & 0.0650 & 0.0000 & 0.0007 & 0.0275 & 0.152 & 0.1180 & 0.001 & 0.0008 & 04678 & 0.0304 & 0.0000 & 0.0000\end{array}$

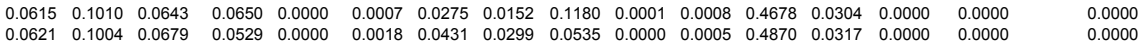
$\begin{array}{llllll}0.0621 & 0.1004 & 0.0679 & 0.0529 & 0.0000 \\ 0.0610 & 0.1000 & 0.0276 & 0.0550 & 0 & 0\end{array}$

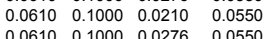

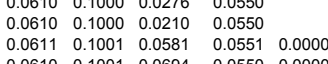
$\begin{array}{lllll}0.0610 & 0.1001 & 0.0694 & 0.0550 & 0.00000 \\ 0.0610 & 0.1001 & 0.0698 & 0.0550 & 0.0000\end{array}$ $\begin{array}{llll}0.0610 & 0.1000 & 0.0694 & 0.0550 \\ 0.0610 & 0.1000 & 0.0698 & 0.0550\end{array}$ $\begin{array}{llll}0.0610 & 0.1000 & 0.0698 & 0.0550 \\ 0.0610 & 0.1000 & 0.0202 & 0.0550\end{array}$ $\begin{array}{llll}0.0610 & 0.1000 & 0.0202 & 0.0550 \\ 0.0605 & 0.0992 & 0.0686 & 0.0545\end{array}$ $\begin{array}{llll}0.0605 & 0.0992 & 0.0686 & 0.0545 \\ 0.0605 & 0.0992 & 0.0686 & 0.0545\end{array}$ $\begin{array}{llll}0.0607 & 0.0995 & 0.0696 & 0.0548 \\ 0.1109 & 0.1056 & 0.0033 & 0.0962\end{array}$ $\begin{array}{llll}0.1109 & 0.1056 & 0.0033 & 0.0962 \\ 0.0800 & 0.1109 & 0.0035 & 0.0714\end{array}$ $\begin{array}{llll}0.0800 & 0.1109 & 0.0035 & 0.0714 \\ 0.0193 & 0.0862 & 0.0034 & 0.1265 \\ 0.003 & 0.1113 & 0.022 & 0.1264\end{array}$ $\begin{array}{llll}0.0193 & 0.1113 & 0.0022 & 0.1264 \\ 0.0193 & 0.0481 & 0.0052 & 0.1254\end{array}$ $\begin{array}{llll}0.0193 & 0.0481 & 0.0052 & 0.1254 \\ 0.0967 & 0.0481 & 0.0058 & 0.1062\end{array}$

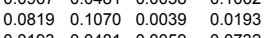
$\begin{array}{llll}0.0193 & 0.0481 & 0.0059 & 0.0732 \\ 0.0548 & 0.0481 & 0.0056 & 0.1393\end{array}$ $\begin{array}{llll}0.0193 & 0.0945 & 0.0033 & 0.1098 \\ 0.1189 & 0.1219 & 0.0057 & 0.0193\end{array}$

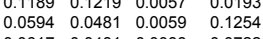
$\begin{array}{llll}0.0617 & 0.0481 & 0.0088 & 0.0722 \\ 0.0354 & 0.0481 & 0.0051 & 0.1256 \\ 0\end{array}$

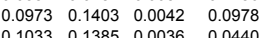
$\begin{array}{llll}0.1033 & 0.1385 & 0.00036 & 0.0440 \\ 0.0193 & 0.1034 & 0.0033 & 0.1252 \\ 0 & 0 & 0\end{array}$ $\begin{array}{llll}0.0193 & 0.0965 & 0.0034 & 0.1265 \\ 0.0193 & 0.0775 & 0.0034 & 0.1265\end{array}$

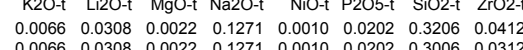

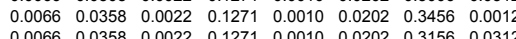

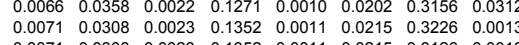

$\begin{array}{llllllll}0.0073 & 0.0308 & 0.0024 & 0.1406 & 0.0011 & 0.0224 & 0.2939 & 0.00014\end{array}$ $\begin{array}{llllllll}0.0037 & 0.0013 & 0.0034 & 0.1739 & 0.0154 & 0.0239 & 0.3950 & 0.0017 \\ 0.0037 & 0.0013 & 0.0034 & 0.1639 & 0.0154 & 0.0399 & 0.3950 & 0.0117\end{array}$ $\begin{array}{lllllllll}0.0037 & 0.0013 & 0.0034 & 0.1639 & 0.0154 & 0.0399 & 0.3900 & 0.0067\end{array}$ $\begin{array}{llllllll}0.0041 & 0.0014 & 0.0038 & 0.1606 & 0.0173 & 0.0449 & 0.3763 & 0.0119\end{array}$ $\begin{array}{lllllllll}0.0051 & 0.0018 & 0.0047 & 0.1541 & 0.0212 & 0.0548 & 0.3388 & 0.0023\end{array}$ $\begin{array}{llllllll}0.0041 & 0.0014 & 0.0038 & 0.1606 & 0.0173 & 0.0449 & 0.3663 & 0.0019\end{array}$ $\begin{array}{llllllll}0.0041 & 0.0014 & 0.0038 & 0.1806 & 0.0173 & 0.0449 & 0.3663 & 0.0019 \\ 0.0241 & 0.0014 & 0.0038 & 0.1606 & 0.0173 & 0.0449 & 0.3663 & 0.0019\end{array}$ $\begin{array}{llllllll}0.0055 & 0.0010 & 0.0026 & 0.1804 & 0.0116 & 0.0299 & 0.4425 & 0.0213\end{array}$

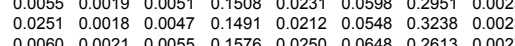
$\begin{array}{lllllllllll}0.1116 & 0.0016 & 0.0007 & 0.0870 & 0.0046 & 0.0145 & 0.3907 & 0.0005\end{array}$ $\begin{array}{llllllll}0.1016 & 0.0116 & 0.0007 & 0.0870 & 0.0046 & 0.0145 & 0.3907 & 0.0005 \\ 0.0766 & 0.0266 & 0.0007 & 0.0870 & 0.0046 & 0.0145 & 0.3907 & 0.0005\end{array}$ $\begin{array}{llllllll}0.0266 & 0.0316 & 0.0007 & 0.0870 & 0.0046 & 0.0145 & 0.4457 & 0.0005 \\ 0.0516 & 0.0216 & 0.0007 & 0.087 & 0.046 & 0.045 & 0.457 & 0.0005\end{array}$ $\begin{array}{llllllll}0.0366 & 0.0316 & 0.0007 & 0.0870 & 0.0046 & 0.0145 & 0.4257 & 0.0005\end{array}$ $\begin{array}{lllllllll}0.0516 & 0.0316 & 0.0007 & 0.0870 & 0.0046 & 0.0145 & 0.4007 & 0.0005 \\ 0.0766 & 0.0266 & 0.0007 & 0.0870 & 0.0046 & 0.0145 & 0.3807 & 0.0005\end{array}$ $\begin{array}{lllllllll}0.0516 & 0.0316 & 0.0007 & 0.0870 & 0.0046 & 0.0145 & 0.3807 & 0.0005\end{array}$ $\begin{array}{llllllll}0.0542 & 0.0331 & 0.0007 & 0.0870 & 0.0046 & 0.0145 & 0.4194 & 0.0005 \\ 0.0542 & 0.0331 & 0.0007 & 0.0870 & 0.0046 & 0.0145 & 0.4094 & 0.0105\end{array}$

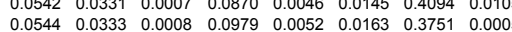
$\begin{array}{lllllllll}0.0520 & 0.0220 & 0.0009 & 0.1087 & 0.0057 & 0.0181 & 0.3500 & 0.0006\end{array}$ $\begin{array}{lllllllll}0.0612 & 0.0412 & 0.0005 & 0.0652 & 0.0034 & 0.0108 & 0.4543 & 0.0304 \\ 0.0544 & 0.0333 & 0.0008 & 0.0979 & 0.0052 & 0.0163 & 0.3601 & 0.0005\end{array}$ $\begin{array}{llllllll}0.0544 & 0.0333 & 0.0008 & 0.0979 & 0.0052 & 0.0163 & 0.3601 & 0.0005\end{array}$ $\begin{array}{llllllll}0.0543 & 0.0331 & 0.0007 & 0.0924 & 0.0049 & 0.0154 & 0.3975 & 0.0005\end{array}$

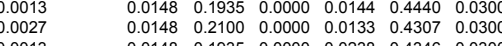
$\begin{array}{lllllllll}0.013 & 0.0148 & 0.1935 & 0.0000 & 0.0238 & 0.4346 & 0.0300 \\ 0.027 & 0.0148 & 0.2099 & 0.0000 & 0.0238 & 0.4201 & 0.0300\end{array}$ $\begin{array}{llllllllll}0.0027 & 0.0148 & 0.2099 & 0.0000 & 0.0238 & 0.4201 & 0.0300 & & \\ 0.0009 & 0.0264 & 0.0149 & 0.1440 & 0.0001 & 0.0133 & 0.4351 & 0.0300 & 0.0000 & 0.0000\end{array}$

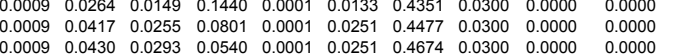

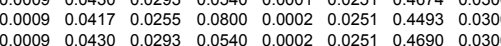
$\begin{array}{llllllll}0.0009 & 0.0430 & 0.0293 & 0.0540 & 0.0002 & 0.0133 & 0.4818 & 0.0300 \\ 0.0499 & 0.0000 & 0.0148 & 0.1821 & 0.0001 & 0.0012 & 0.4163 & 0.0300\end{array}$ $\begin{array}{llllllll}0.0499 & 0.0000 & 0.0148 & 0.1821 & 0.0001 & 0.0012 & 0.4163 & 0.0300 \\ 0.0054 & 0.0408 & 0.0236 & 0.0893 & 0.0001 & 0.0012 & 0.4627 & 0.0297\end{array}$ $\begin{array}{lllllllll}0.0054 & 0.0408 & 0.0236 & 0.0893 & 0.0001 & 0.0012 & 0.4627 & 0.0297 \\ 0.0054 & 0.0408 & 0.0236 & 0.0893 & 0.0001 & 0.0012 & 0.4642 & 0.0297\end{array}$ $\begin{array}{lllllllll}0.0054 & 0.0426 & 0.0294 & 0.0572 & 0.0001 & 0.0012 & 0.4865 & 0.0299 & \\ 0.0012 & 0.0333 & 0.0010 & 0.1728 & 0.0000 & 0.0010 & 0.4529 & 0.0000 & 0.0005\end{array}$

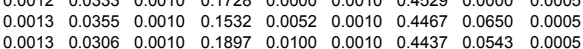

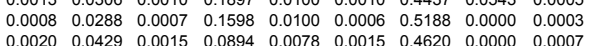

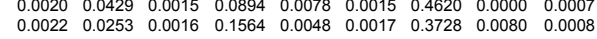
$\begin{array}{llllllllll}0.0015 & 0.0326 & 0.0011 & 0.1178 & 0.0000 & 0.0012 & 0.4411 & 0.0626 & 0.0005\end{array}$

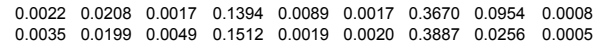
$\begin{array}{lllllllllll}0.0012 & 0.0202 & 0.0010 & 0.1741 & 0.0100 & 0.0010 & 0.4711 & 0.0016 & 0.0005\end{array}$ $\begin{array}{lllllllll}0.0021 & 0.0601 & 0.0016 & 0.0606 & 0.0000 & 0.0017 & 0.3508 & 0.0913 & 0.0008 \\ 0.0022 & 0.0275 & 0.0017 & 0.1590 & 0.0081 & 0.0017 & 0.4035 & 0.0239 & 0.0008\end{array}$

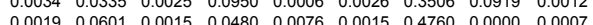
$\begin{array}{llllllllllll}0.0016 & 0.0193 & 0.0012 & 0.1758 & 0.0064 & 0.0012 & 0.3745 & 0.0685 & 0.0006\end{array}$ $\begin{array}{lllllllll}0.0014 & 0.0202 & 0.0010 & 0.1741 & 0.0075 & 0.0011 & 0.4377 & 0.0376 & 0.0005 \\ 0.0012 & 0.0202 & 0.0010 & 0.1741 & 0.0100 & 0.0010 & 0.4587 & 0.0016 & 0.0005\end{array}$ $\begin{array}{llllllllllllllllllll}0.0013 & 0.0344 & 0.0010 & 0.1481 & 0.0000 & 0.0010 & 0.4599 & 0.0000 & 0.00005\end{array}$

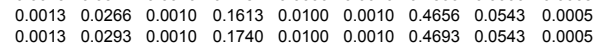

\begin{tabular}{|c|c|c|c|c|c|}
\hline $\begin{array}{l}\text { BaO-t } \\
0.0003\end{array}$ & $\begin{array}{ll} & B=0116-t\end{array}$ & Dil- & 0.0001 & 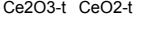 & \\
\hline & & & & & \\
\hline & 0.0116 & & 0.0001 & & \\
\hline $\begin{array}{l}0.0003 \\
0.0003\end{array}$ & $\begin{array}{l}0.0116 \\
0.0123\end{array}$ & & $\begin{array}{l}0.0001 \\
0.0001\end{array}$ & & \\
\hline 0.0003 & 0.0123 & & 0.0001 & & \\
\hline 0.0003 & 0.0128 & & 0.0001 & & \\
\hline $\begin{array}{l}0.0001 \\
0.0001\end{array}$ & 0.0536 & & 0.0000 & & \\
\hline $\begin{array}{l}0.0001 \\
0.0001\end{array}$ & $\begin{array}{l}0.0536 \\
0.0536\end{array}$ & & $\begin{array}{l}0.0000 \\
0.0000\end{array}$ & & \\
\hline $\begin{array}{l}0.0001 \\
0.0001\end{array}$ & $\begin{array}{l}0.0536 \\
0.0536\end{array}$ & & $\begin{array}{l}0.00000 \\
0.0000\end{array}$ & & \\
\hline & 0.0603 & & 0.0000 & & \\
\hline 0.0001 & 0.0671 & & 0.0000 & & \\
\hline 0.0001 & 0.0738 & & 0.0000 & & \\
\hline 0.0001 & 0.0603 & & 0.0000 & & \\
\hline 0.0001 & 0.0603 & & 0.0000 & & \\
\hline & $\begin{array}{l}0.0603 \\
0.0402\end{array}$ & & 0.0000 & & \\
\hline $\begin{array}{l}0.0001 \\
0.0001\end{array}$ & $\begin{array}{l}0.0402 \\
0.0805\end{array}$ & & $\begin{array}{l}0.0000 \\
0.0000\end{array}$ & & \\
\hline 0.0001 & $\begin{array}{l}0.0805 \\
0.0738\end{array}$ & & $\begin{array}{l}0.00000 \\
0.0000\end{array}$ & & \\
\hline 0.0001 & 0.0872 & & 0.0000 & & \\
\hline 0.0001 & 0.0316 & & 0.0000 & & \\
\hline & 0.0316 & & 0.0000 & & \\
\hline 0.0001 & 0.0316 & & 0.0000 & & \\
\hline 0.0001 & 0.0316 & & 0.0000 & & \\
\hline 0.0001 & 0.0316 & & 0.0000 & & \\
\hline $\begin{array}{l}0.0001 \\
0.0001\end{array}$ & $\begin{array}{l}0.0316 \\
0.0316\end{array}$ & & $\begin{array}{l}0.0000 \\
0.0000\end{array}$ & & \\
\hline $\begin{array}{l}0.0001 \\
0.0001\end{array}$ & $\begin{array}{l}0.0316 \\
0.0316\end{array}$ & & $\begin{array}{l}0.0000 \\
0.0000\end{array}$ & & \\
\hline 0.0001 & $\begin{array}{l}0.0316 \\
0.0316\end{array}$ & & 0.0000 & & \\
\hline 0.0001 & 0.0316 & & 0.0000 & & \\
\hline 0.0001 & 0.0316 & & 0.0000 & & \\
\hline 0.0001 & 0.0355 & & 0.0000 & & \\
\hline 0.0002 & 0.0395 & & 0.0001 & & \\
\hline $\begin{array}{l}0.0001 \\
0.0001\end{array}$ & $\begin{array}{l}0.0237 \\
0.0355\end{array}$ & & 0.0000 & & \\
\hline $\begin{array}{l}0.0001 \\
0.0002\end{array}$ & 0.0355 & & 0.0000 & & \\
\hline $\begin{array}{l}0.0001 \\
0.0001\end{array}$ & 0.0335 & & $\begin{array}{l}0.0001 \\
0.0000\end{array}$ & & \\
\hline 0.0001 & 0.0335 & & 0.0000 & & \\
\hline 0.0000 & 0.0000 & 0.0000 & 0.0000 & $\begin{array}{lll}0.0000 & 0.0000\end{array}$ & 0.0008 \\
\hline $\begin{array}{l}0.0002 \\
0.0000\end{array}$ & $\begin{array}{l}0.0000 \\
0.0000\end{array}$ & $\begin{array}{l}0.0008 \\
0.0000\end{array}$ & $\begin{array}{l}0.00000 \\
0.0000\end{array}$ & $\begin{array}{ll}0.0000 & 0.0000 \\
0\end{array}$ & 0.0008 \\
\hline 0.0000 & 0.0000 & $\begin{array}{l}0.0000 \\
0.000\end{array}$ & 0.0000 & $0.0000 \quad 0.0000$ & 0.0001 \\
\hline 0.0000 & 0.0000 & 0.0000 & 0.0000 & $0.0000 \quad 0.0000$ & 0.0017 \\
\hline 0.0000 & 0.0000 & 0.0000 & 0.0000 & $0.0000 \quad 0.0000$ & 0.0031 \\
\hline $\begin{array}{l}0.0000 \\
0.0000\end{array}$ & 0.0000 & 0.0000 & 0.0000 & $0.0000 \quad 0.0000$ & 0.0012 \\
\hline & $\begin{array}{l}0.00000 \\
0.0000\end{array}$ & 0.0000 & & $\begin{array}{lll}0.0000 & 0.0000\end{array}$ & \\
\hline & & & & & $\begin{array}{l}0.0000 \\
0.0012\end{array}$ \\
\hline & & & & & 0.0019 \\
\hline & & & & & 0.0019 \\
\hline 0.0000 & 0.0000 & 0.0000 & 0.0000 & $\begin{array}{lll}0.0000 & 0.0000\end{array}$ & 0.0014 \\
\hline 0.0000 & 0.0000 & 0.0000 & 0.0000 & $\begin{array}{ll}0.0000 & 0.00000\end{array}$ & 0.0014 \\
\hline \multirow[t]{5}{*}{0.0000} & 0.0000 & 0.0000 & 0.0000 & & $\begin{array}{l}0.0014 \\
0.0014\end{array}$ \\
\hline & & & & & 0.0014 \\
\hline & & & & & 0.0014 \\
\hline & & & & & $\begin{array}{l}0.0020 \\
0.0020\end{array}$ \\
\hline & & & & & 0.0020 \\
\hline & & & & & \\
\hline .0004 & 0.0000 & & $\begin{array}{l}0.00000 \\
0.0000\end{array}$ & 0.0005 & 0.00000 \\
\hline .0004 & & & $\begin{array}{l}0.00000 \\
0.0000\end{array}$ & 0.0005 & 0.0000 \\
\hline 0.0000 & 0.0000 & & 0.0000 & 0.0003 & 0.0000 \\
\hline 0.0006 & 0.0000 & & 0.0120 & 0.0008 & 0.0000 \\
\hline 0.0006 & 0.0000 & & 0.0132 & 0.0009 & 0.0000 \\
\hline 14 & 0.0000 & & 0.0000 & 0.0006 & 0.0000 \\
\hline 0.00 & 0.0000 & & 0.0135 & 0.0009 & 0.0000 \\
\hline 0.0008 & 0.0000 & & 0.0008 & 0.0012 & 0.0005 \\
\hline 0.00 & 0.0000 & & 0.0085 & 0.0005 & 0.0000 \\
\hline 6 & 0.0000 & & 0.00041 & (2) & 0.0000 \\
\hline 0.0010 & 0.0003 & & 0.0006 & 0.0013 & $\begin{array}{l}0.0000 \\
0.0003\end{array}$ \\
\hline & 0.0000 & & 0.0149 & 0.0008 & 0.0000 \\
\hline & 0.0000 & & 0.0000 & 0.0006 & \\
\hline & 0.0000 & & 0.0003 & 0.0006 & 0.0000 \\
\hline & 0.0000 & & 0.0000 & 0.0005 & 0.0000 \\
\hline 0.0004 & 0.0000 & & 0.0000 & 0.0005 & 0.0000 \\
\hline 0.0004 & 0.00000 & & 0.0000 & 0.00005 & 0.00000 \\
\hline 0.0004 & 0.0000 & & & & 0.0000 \\
\hline
\end{tabular}


\# Glass ID

3035 HLW-E-ANa-20

3037 HLW-E-ANa-22

3038 HLW-E-ANa-23
3039 HLW-E-ANa-24

3040 HLW-E-ANa-25

3041 HLW-E-ANa-26

3042 HLW-E-Bi-02

3045 HLW-E-Bi-04

3047 HLW-E-Bi-06

3048 HLW-E-Bi-07

SO HLW-E-Bi-09

3051 HLW-E-Bi-10

3052 HLW-E-Bi-1

3053 HLW-E-Bi-12
3054 HLW-E-Bi-13

3054 HLW-E-B-Bi-13

3056 HLW-E-Cr-01

3057 HLW-E-Cr-02
3058 HLW-E-Cr-03

3059 HLW-E-Cr-04

3061 HLW-E-Cr-06

3063 HLW-E-Cr-08

WW-E-Cr-09

30656 HLW-E-CC-10
3066 HLW-E-CC-11

3068 HLW-E-Cr-1

3069 HLW-E-Cr-14

3070 HLW-E-Cr-15
3071 HLW-E-Cr-16

3072 HLW-E-Cr-1 1

075 AN-107 Actual (LAWC15)

3076 AZ-102 Actual

3078 AP-101 Actual

3080 AN-102 Actual

3082 AZ-101 Actual

3084 LAWCrP2R
3085 LAWCrP3R

3086 LAWCrP4R

-17
0

3089 LAWCFP7

3092 LAWCRP 10CCC
3093 LAWE3Cr2CCC

3095 LAWE9HCrC2CCC

3096 LLW-ALG-01

098 HLW-ALG-02

(100 HLW-ALG-04

3101 HLW-ALG-05

3103 HLW-ALG-07

3105 HLW-ALG-09

3107 HLW-ALG-11

3108 HLW-ALG-12

3110 HLW-ALG-14

3111 HLW-ALG-15

3112 HLW-ALG-16

3114 HLW-ALG-18

3116 HLW-ALG-20
CoO-t Co2O3-t Cr2O3-t Cs2O-t CuO-t Dy203-t Eu2O3-t 0.0071
0.0071

$$
\begin{aligned}
& 0.0071 \\
& 0.0071 \\
& 0.0071
\end{aligned}
$$

0.0071
0.0076
0.0076

0.0079

0.0042

0.0042

0.0047

0.0057

0.0047

0.0031

0.0062

0.0068
0.0133
0.0133

0.0133

0.0133

0.0133
0.0133
0.0133

0.0133

0.0150
0.0166

0.0166
0.0100
0.0150

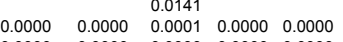

$\begin{array}{llllll}0.0000 & 0.0000 & 0.0006 & 0.0000 & 0.0000\end{array}$

$\begin{array}{lllll}0.0000 & 0.0000 & 0.0006 & 0.0000 & 0.0000\end{array}$

$0.0000 \quad 0.0000 \quad 0.0001 \quad 0.0000 \quad 0.0000$

$\begin{array}{lllll}0.0000 & 0.0000 & 0.0002 & 0.0000 & 0.0000\end{array}$

$\begin{array}{lllll}0.0000 & 0.0000 & 0.0002 & 0.0000 & 0.0000 \\ 0.0000 & 0.0000 & 0.0003 & 0.0000 & 0.0000\end{array}$

0.0033
0.0059

0.0033

$\begin{array}{llllll}0.0000 & 0.0000 & 0.0059 & 0.0059 & 0.0000 & 0.0000\end{array}$

$\begin{array}{lllll}0.0000 & 0.0000 & 0.0063 & 0.0000 & 0.0000 \\ 0.0000 & 0.0000 & 0.0063 & 0.0000 & 0.0000\end{array}$

0.004

0.0140

0.0060
0.0045

$\begin{array}{lll}0.00550 & 0.0000 & 0.0000\end{array}$

$\begin{array}{lll}0.0028 & 0.0000 & 0.000 \\ 0.0050 & 0.0000 & 0.0000 \\ 0.0050 & .0 .000 & 0.0000\end{array}$

$\begin{array}{llll}0.0050 & 0.0000 & 0.0000\end{array}$

$\begin{array}{llll}0.004 & 0.0000 & 0.0000 \\ 0.0020 & 0.0003 & 0.0003\end{array}$

-10
0

$\begin{array}{llll}0.0047 & 0.0003 & 0.0003 \\ 0.0021 & 0.0000 & 0.0000 \\ 0.0000 & 0.0000 & \end{array}$

$\begin{array}{llll}.0000 & 0.0000 & 0.0000\end{array}$

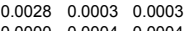

$\begin{array}{lll}0.0000 & 0.0000 & 0.0000 \\ 0.0054 & 0.0000 & 0.0000\end{array}$

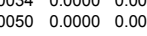

$\begin{array}{llll}0.0050 & 0.0000 & 0.0000 \\ 0.0000 & 0.0000 & 0.0000\end{array}$

$\begin{array}{lll}0.0050 & 0.0000 & 0.0000 \\ 0.0050 & 0.0000 & 0.0000\end{array}$
0.0023

0.0023
0.0023
0.0023

0.0024

0.0025

0.0066
0.0066

0.0074

0.0090

0.0074

0.0090

0.008

0.0087

0.0087

0.0087

0.008

0.0106
0.0065

0.0092

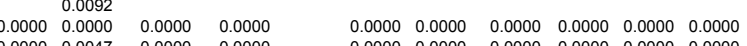

$\begin{array}{lllll}0.0000 & 0.0005 & 0.0000 & 0.0000\end{array}$

$\begin{array}{llll}0.0000 & 0.0005 & 0.0000 & 0.0000\end{array}$

$\begin{array}{llll}0.0000 & 0.0002 & 0.0000 & 0.0000\end{array}$

$\begin{array}{llll}0.00000 & 0.0006 & 0.0000 & 0.00000 \\ 0.000 & 0.0005 & 0.0000 & 0.0000\end{array}$

$\begin{array}{llll}0.0000 & 0.0005 & 0.0000 & 0.0000 \\ 0.0000 & 0.0008 & 0.0000 & 0.0000\end{array}$

0.001
0.0010

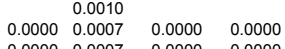

$\begin{array}{llll}0.0000 & 0.0007 & 0.0000 & 0.0000 \\ 0.0000 & 0.0007 & 0.0000 & 0.0000\end{array}$

$\begin{array}{llllll}0.0000 & 0.0000 & 0.0000 & 0.00000 & 0.0000 & 0.0000\end{array}$

$\begin{array}{lllllll}0.0000 & 0.0000 & 0.0000 & 0.00000 & 0.0000 & 0.00000\end{array}$

$\begin{array}{lllllllllll}0.0000 & 0.0000 & 0.0000 & 0.0000 & 0.0000\end{array}$

$\begin{array}{llllll}0.0000 & 0.0000 & 0.0000 & 0.0000 & 0.0000 & 0.0000 \\ 0.0000 & 0.0000 & 0.0000 & 0.0000 & 0.0000 & 0.0000\end{array}$

$\begin{array}{rrr}0.0000 & 0.0000 \quad 0.0000 \\ 0.0000 & 0.000 & 0.0000\end{array}$ $\begin{array}{lll}0.0000 & 0.0000 & 0.0000\end{array}$ $\begin{array}{lll}0.0000 & 0.0000 & 0.0000 \\ 0.0000 & 0.0000 & 0.0000\end{array}$

$\begin{array}{lll}0.0000 & 0.0000 & 0.0000 \\ 0.0000 & 0.0000 & 0.0000\end{array}$

$\begin{array}{lll}0.0000 & 0.0000 & 0.0000 \\ 0.0000 & 0.0000 & 0.0000\end{array}$

$\begin{array}{lll}0.0000 & 0.0000 & 0.0000 \\ 0.0000 & 0.0000 & 0.0000\end{array}$

$\begin{array}{llllll}0.0000 & 0.0000 & 0.0000 & 0.0000 & 0.0000 & 0.0000\end{array}$ $\begin{array}{llllll}0.0000 & 0.0000 & 0.0000 & 0.0000 & 0.0000 & 0.0000 \\ 0.0000 & 0.0000 & 0.0000 & 0.0000 & 0.0000 & 0.0000\end{array}$

$0.0000 \quad 0.0000 \quad 0.0000$ $\begin{array}{lll}0.0000 & 0.0000 & 0.0000 \\ 0.0000 & 0.0000 & 0.0000\end{array}$

0.0007
0.0007
0.0007
0.0008
0.0008
0.0008
0.0008
0.0003
0.0003
0.0003
0.0000
0.0005
0.0006
0.0000
0.0006
0.0008
0.0003
0.0005
0.0006
0.0008
0.0005
0.0004
0.0003
0.0003
0.0003
0.0003
0.0003

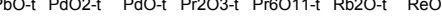
0.0000

0.0010

0.0020

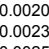

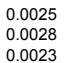

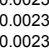

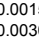

(1)

0.0071

0.0222
0.0212
0.021

20.022

(20321

0.0021

0.0026
0.0016

$\begin{array}{lllllll}0.0022 & & & & & & \\ 0.0000 & 0.0000 & 0.0000 & 0.0000 & 0.0000 & 0.0000 & 0.0000\end{array}$ $\begin{array}{llllllll}0.0000 & 0.0000 & 0.0000 & 0.0000 & 0.0000 & 0.0000 & 0.0000\end{array}$

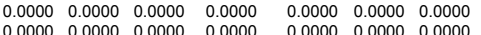
$\begin{array}{llllllllll}0.0000 & 0.0000 & 0.0000 & 0.0000 & 0.0000 & 0.0000 & 0.0000\end{array}$ $\begin{array}{lllllll}0.0000 & 0.0000 & 0.0000 & 0.0000 & 0.0000 & 0.0000 & 0.0000 \\ 0.0000 & 0.0000 & 0.0000 & 0.0000 & 0.0000 & 0.0000 & 0.0000\end{array}$ $\begin{array}{llllllll} & 0.00000 & 0.0000 & 0.0000 & 0.0000\end{array}$

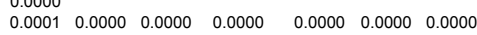
$\begin{array}{llllllll}. .0001 & 0.0000 & 0.0000 & 0.0000 & 0.0000 & 0.0000 & 0.0000\end{array}$ $\begin{array}{lllllll}0.0001 & 0.0000 & 0.0000 & 0.0000 & 0.0000 & 0.0000 & 0.0000\end{array}$ 0.0001

0.0001

0.0001

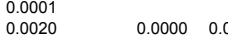

\begin{tabular}{|c|c|c|}
\hline & & \\
\hline & & \\
\hline $\begin{array}{l}0.0021 \\
0.0014\end{array}$ & $\begin{array}{l}0.00000 \\
0.00000\end{array}$ & \\
\hline 0.0032 & 0.0000 & \\
\hline & 0.0000 & 0.00 \\
\hline & 0.0000 & \\
\hline $\begin{array}{l}.0036 \\
.0041\end{array}$ & 0.0000 & 0.00 \\
\hline $\begin{array}{l}.00041 \\
.0020\end{array}$ & $\begin{array}{l}0.0000 \\
0.0000\end{array}$ & $\begin{array}{l}0.00 \\
0.00\end{array}$ \\
\hline $\begin{array}{l}0.02000 \\
0.0035\end{array}$ & 0.0000 & \\
\hline 0.0036 & & \\
\hline 0.0055 & 0.0000 & \\
\hline 0.0031 & 0.0000 & \\
\hline $\begin{array}{l}0.02026 \\
.00022\end{array}$ & 0.0000 & \\
\hline .0020 & 0.00000 & \\
\hline .002 & 0.0000 & \\
\hline . & 0.0000 & \\
\hline
\end{tabular}

0.0000
0.0000
0.0000
0.0000
0.0000
0.0000
0.0000
0.0000
0.0000
0.0000
0.0000
0.0000
0.0000
0.0000
0.0000
0.0000
0.0000
0.0000
0.0000
0.0000 
3036 HLW-E-ANa-2

3038 HLW-E-ANa-23
3039 HLW-E-ANa-24

3040 HLW-E-ANa-25

3041 HLW-E-ANa-26
3042 HLW-E-Bi-01

3043 HLW-E-Bi-02

3045 HLW-E-Bi-04
3046 HLW-E-Bi-05

3047 HLW-E-Bi-06
3048 HLW-E-Bi-07

049 HLW-E-Bi-08

3050 HLW-E-Bi-09

3052 HLW-E-Bi-11
3053 HLW-E-Bi-12

3053 HLW-E-Bi-12

3055 HLW-E-Bi-14

3059 HLW-E-Cr-04

3062 HLW-E-Cr-O

3063 HLW-E-Cr-0

3065 HLW-E-Cr-10

3068 HLW-E-Cr-1

3069 HLW-E-Cr-14

3071 HLW-E-Cr-16

3072 HLW-E-CC-17
3073 HLW-E-Cr-18

3074 AW-101 Actual
3075 AN-107 Actual (LAWC15)

3076 AZ-102 Actual

3078 AP-101 Actual

3080 AN-102 Actua

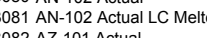

3082 AZ-101 Actualc
3083 LAWCrP1R

3084 LAWCrP2R

3085 LAWCrP3R

(2086 LAWCP4R

3088 LAWCrP6

3089 LAWCrP7

(3)

3092 LAWCrP10CCC

3094 LAWE9HCrCCCC
3095 LAWEHHCRCCCC

3096 LAWE10HCr3C
3097 HLW-ALG-01

3098 HLW-ALG-02

3100 HLW-ALG-04

3101 HLW-ALG-05

3103 HLW-ALG-07

3104 HLW-ALG-08
3105 HLW-ALG-09

3107 HLW-ALG-1

3108 HLW-ALG-12

3109 HLW-ALG-13

3111 HLW-ALG-15

3112 HLW-ALG-16

(113 HLW-ALG-17

3115 HLW-ALG-19
3116 HLW-ALG-20

$\begin{array}{lllll}0.0000 & 0.0000 & 0.0000 & 0.0000 & 0.0000 \\ 0.0000 & 0.0000 & 0.0000 & 0.0000 & 0.0000\end{array}$ $\begin{array}{llllll}0.0000 & 0.0000 & 0.0000 & 0.0000 & 0.0000\end{array}$ $\begin{array}{lllll}0.0000 & 0.0000 & 0.0000 & 0.0000 & 0.0000\end{array}$ $\begin{array}{lllllll}0.0000 & 0.0000 & 0.0000 & 0.0000\end{array}$ $\begin{array}{lllll}0.0000 & 0.0000 & 0.0000 & 0.0000 & 0.0000 \\ 0.0000 & 0.0000 & 0.0000 & 0.0000 & 0.0000\end{array}$

$0.0000 \quad 0.0000$

$\begin{array}{lllll}0.0000 & 0.0000 & 0.0000 & 0.0000 & 0.0000\end{array}$ $\begin{array}{lllll}0.0000 & 0.0000 & 0.0000 & 0.0000 & 0.0000 \\ 0.0000 & 0.0000 & 0.0000 & 0.0000 & 0.0000\end{array}$

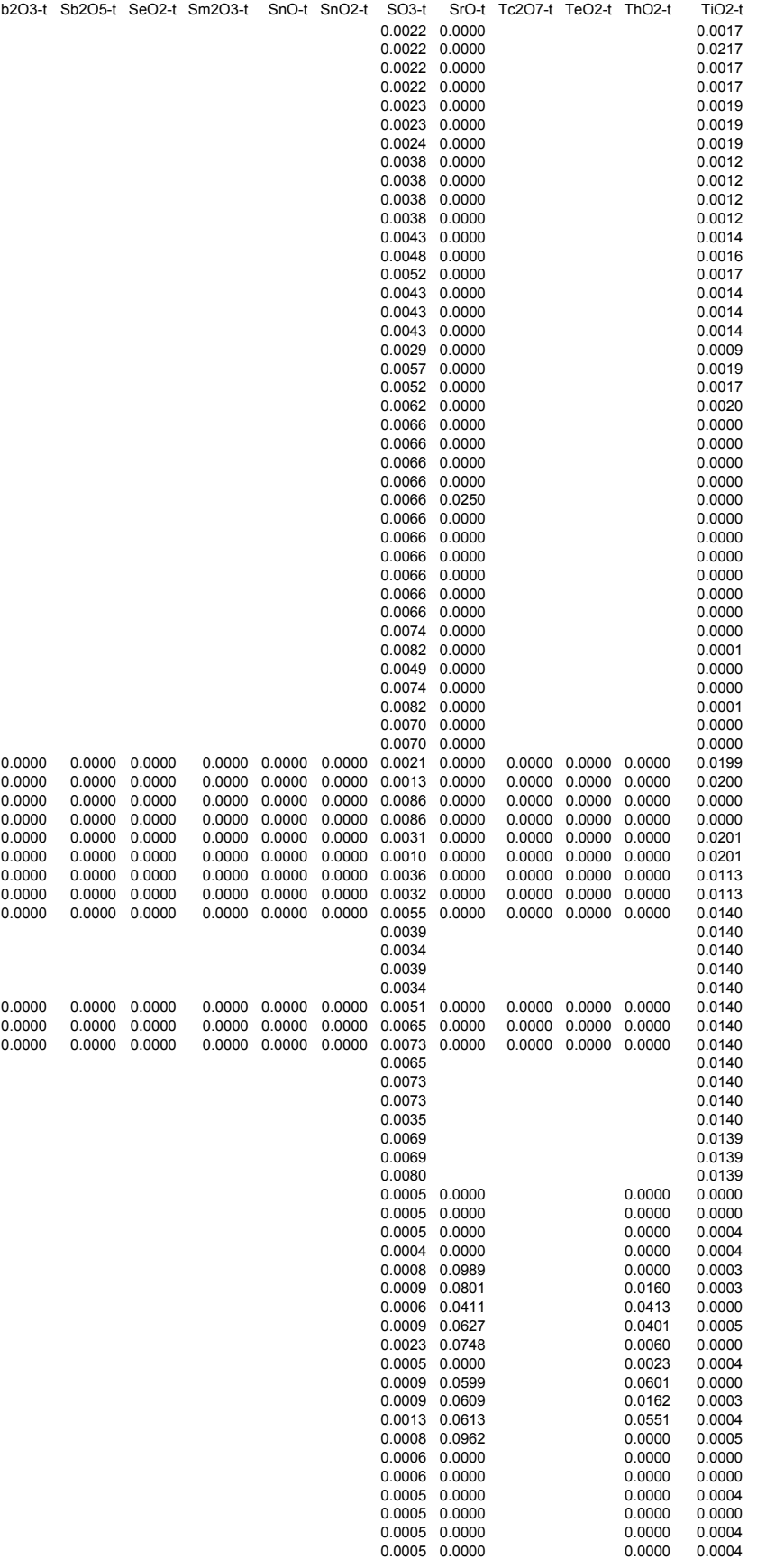

TiO2-t T12O-t T12O3-t U3O8-t UO2-t UO3-t V2O5-t WO3-t Y2O3-t ZnO-t Sum-t A12O3-a<smiles>[101In]</smiles>

0.0000

$0.0018 \quad 1.0000$

$\begin{array}{ll}0.0018 & 1.0000 \\ 0.0018 & 1.0000\end{array}$

$\begin{array}{ll}0.0018 & 1.0000 \\ 0.0019 & 1.0000 \\ 0.0010 & 1.0000\end{array}$

$0.0020 \quad 1.0000$

$0.0013 \quad 1.0000$

$\begin{array}{ll}0.0113 & 1.0000 \\ 0.0003 & 1.0000\end{array}$

$\begin{array}{ll}0.0063 & 1.0000 \\ 0.0014 & 1.0000\end{array}$

0.00161 .0000

$\begin{array}{ll}.0018 & 1.0000 \\ .0014 & 1.0000\end{array}$

0.00141 .0000

$\begin{array}{lll}0.0014 & 1.0000 \\ 0.0110 & 1.0000\end{array}$

0.00191 .0000

0.00181 .0000

$\begin{array}{ll}0.0011 & 1.0000 \\ 0.0011 & 1.0000\end{array}$

0.00111 .0000

0.00111 .0000

0.00111 .0000

$\begin{array}{ll}0.0011 & 1.0000 \\ 0.0011 & 1.0000\end{array}$

0.00111 .0000

0.00111 .0000

0.00111 .0000

0.00141 .000

$\begin{array}{ll}0.0008 & 1.0000 \\ 0.0012 & 1.0000 \\ 0.0014 & 1.0000\end{array}$

$\begin{array}{lll}0.0012 & 1.0000\end{array}$

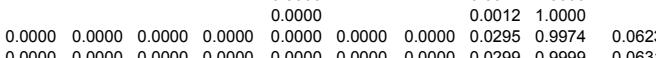
$\begin{array}{lllllllllll}0.0000 & 0.0000 & 0.0000 & 0.0000 & 0.0000 & 0.0000 & 0.0000 & 0.0487 & 1.0001 & 0.0584\end{array}$

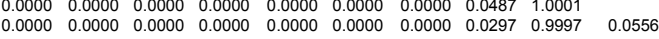
$\begin{array}{llllllllllll}0.0000 & 0.0000 & 0.0000 & 0.0000 & 0.0000 & 0.0000 & 0.0000 & 0.0300 & 1.0000 & 0.0652\end{array}$

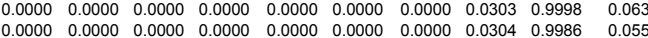
$\begin{array}{lllllllllll}0.0000 & 0.0000 & 0.0000 & 0.0000 & 0.0000 & 0.0000 & 0.0000 & 0.0304 & 0.9986 & 0.0554\end{array}$

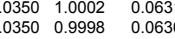
$\begin{array}{lll}0.0350 & 1.0001 & 0.0616 \\ 0.0350 & 0.9997 & 0.0633\end{array}$

$\begin{array}{llllllllllll}0.0000 & 0.0000 & 0.0000 & 0.0000 & 0.0000 & 0.0000 & 0.0000 & 0.0350 & 1.0013 & 0.0622\end{array}$ $\begin{array}{llllllllll}0.0000 & 0.0000 & 0.0000 & 0.0000 & 0.0000 & 0.0000 & 0.0000 & 0.0350 & 1.0007 & 0.0627 \\ 0.0000 & 0.0000 & 0.0000 & 0.0000 & 0.0000 & 0.0000 & 0.0000 & 0.0350 & 1.0007 & 0.0627\end{array}$

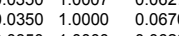
$\begin{array}{lll}.0350 & 1.0000 & 0.0523 \\ 0.0347 & 1.0000 & 0.0632\end{array}$ $\begin{array}{lll}0.0350 & 1.0000 & 0.0632 \\ 0.0347 & 1.0000 & 0.0668\end{array}$ $\begin{array}{lll}0.0347 & 1.0000 & 0.0594\end{array}$ $\begin{array}{lll}0.0348 & 1.0000 & 0.0677 \\ 0.0106 & 1.0000 & 0.1109\end{array}$ $\begin{array}{lll}0.0106 & 1.0000 & 0.1109 \\ 0.0158 & 1.0000 & 0.0800 \\ 0.00123 & 1.0000 & 0.0193\end{array}$ $\begin{array}{lll}0.0158 & 1.0000 & 0.0800 \\ 0.0003 & 1.0000 & 0.0193 \\ 0.0055 & 110000 & 0.0193\end{array}$ $\begin{array}{lll}0.0005 & 1.0000 & 0.0193 \\ 0 & 0.0006 & 0\end{array}$ $\begin{array}{lll}0.0167 & 1.0000 & 0.0819\end{array}$ $\begin{array}{lll}0.0006 & 1.0000 & 0.0193 \\ 0.0006 & 1.0000 & 0.0548 \\ 0.0003 & 1.0000 & 0.093\end{array}$ $\begin{array}{lll}0.0006 & 1.0000 & 0.0548 \\ 0.0003 & 1.0000 & 0.0193 \\ 0.005 & 1\end{array}$ $0.0005 \quad 1.0000 \quad 0.1189$ $0.0006 \quad 1.0000 \quad 0.0594$ $\begin{array}{lll}0.0005 & 1.0000 & 0.0354\end{array}$ $\begin{array}{lll}0.0004 & 1.0000 & 0.0973 \\ 0.079 & 1.000 & 0.10393\end{array}$ $\begin{array}{lll}0.0179 & 1.0000 & 0.1033 \\ 0.0003 & 1.0000 & 0.0193\end{array}$ $\begin{array}{lll}0.0162 & 1.0000 & 0.1136 \\ 0.003 & 1.000 & 0.0003\end{array}$ $\begin{array}{lll}0.0003 & 1.0000 & 0.0193 \\ 0.0003 & 1.0000 & 0.0193\end{array}$ 
$\begin{array}{lll}0.0943 & 0.0184 & 0.050 \\ 0.0858 & 0.0180 & 0.0645\end{array}$ $\begin{array}{lll}0.0858 & 0.0180 & 0.0645 \\ 0.1245 & 0.0666 & 0.0229\end{array}$ $\begin{array}{lll}0.0993 & 0.0200 & 0.0573\end{array}$ $\begin{array}{lll}0.0880 & 0.0216 & 0.063 \\ 0.1059 & 0.0625 & 0.0705 \\ 0.0955 & 0.0578 & 0.0652\end{array}$ $\begin{array}{lll}0.0985 & 0.0578 & 0.0652 \\ 0.1004 & 0.0655 & 0.0526\end{array}$ $\begin{array}{lll}0.1000 & 0.0220 & 0.0550 \\ 0 & 0.0270 & 0\end{array}$ $\begin{array}{lll}0.0965 & 0.0279 & 0.0531\end{array}$ $\begin{array}{lll}0.1010 & 0.0218 & 0.0541 \\ 0.1074 & 0.0614 & 0.0598 \\ 0.0093 & 0.063 & 0.055\end{array}$ $\begin{array}{lll}0.1004 & 0.0663 & 0.0557 \\ 0.0993 & 0.0663 & 0.0549 \\ 0.1008 & 0.0668 & 0.051\end{array}$ $\begin{array}{lll}0.1008 & 0.0666 & 0.0511 \\ 0.1008 & 0.0668 & 0.0552\end{array}$ $\begin{array}{lll}0.1020 & 0.06669 & 0.0601 \\ 0.1011 & 0.0204 & 0.0557\end{array}$ $\begin{array}{lll}0.1095 & 0.0661 & 0.0540 \\ 0.1101 & 0.0661 & 0.0519\end{array}$ $\begin{array}{lll}0.1105 & 0.0667 & 0.0545 \\ 0.1056 & 0.0033 & 0.0962 \\ 0.1106 & 0.035 & 0.0756\end{array}$ $\begin{array}{lll}0.1109 & 0.0035 & 0.0715 \\ 0.0862 & 0.0034 & 0.1265\end{array}$ $\begin{array}{lll}0.1113 & 0.0022 & 0.1264 \\ 0.041 & 0.0052 & 0.1254\end{array}$ $\begin{array}{lll}0.0481 & 0.0052 & 0.1254 \\ 0.0481 & 0.058 & 0.1062\end{array}$ $\begin{array}{lll}0.1070 & 0.0039 & 0.0193 \\ 0\end{array}$ $\begin{array}{lll}0.0481 & 0.0059 & 0.0732 \\ 0.0481 & 0.0056 & 0.1393\end{array}$

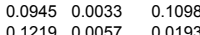
$\begin{array}{lll}0.0481 & 0.0059 & 0.1254 \\ 0.0481 & 0.0088 & 0.0722\end{array}$ $\begin{array}{lll}0.0481 & 0.0051 & 0.1256 \\ 0.1403 & 0.0042 & 0.0978 \\ 0 & 0.034\end{array}$ $\begin{array}{lll}0.1335 & 0.036 & 0.0440 \\ 0.1034 & 0.0033 & 0.1252 \\ 0.053 & 0.252\end{array}$ $\begin{array}{lll}0.10956 & 0.0034 & 0.01265 \\ 0.0775 & 0.0034 & 0.1265\end{array}$

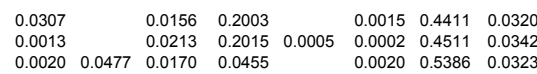
$\begin{array}{llllll}0.0283 & 0.0159 & 0.1862 & 0.0012 & 0.4722 & 0.0273 \\ 0.0071 & 0.0202 & 0.1930 & 0.012006 & 0.02328 & 0.0306\end{array}$

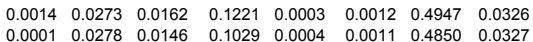

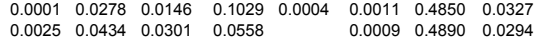

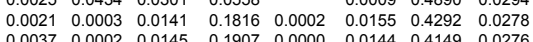

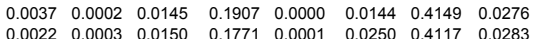

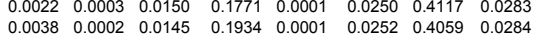

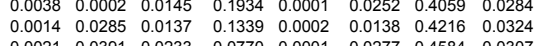

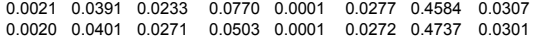

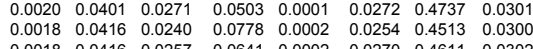

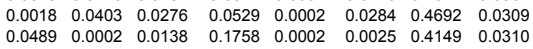

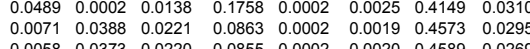

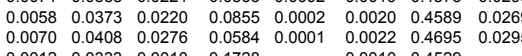

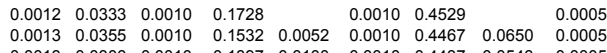

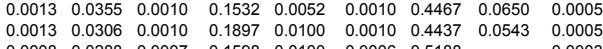

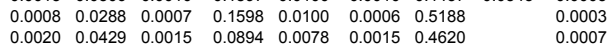

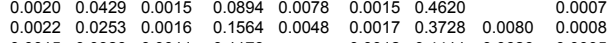
$\begin{array}{lllllllll}0.0015 & 0.0326 & 0.0011 & 0.1178 & 0.0012 & 0.4411 & 0.0626 & 0.0005\end{array}$

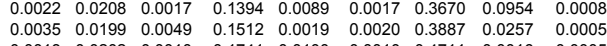

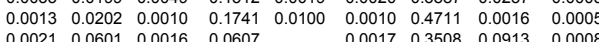

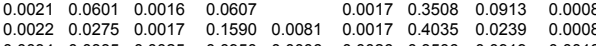

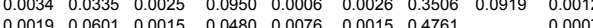

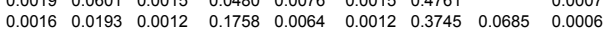

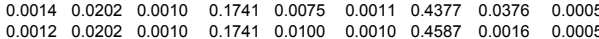

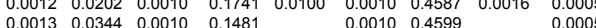

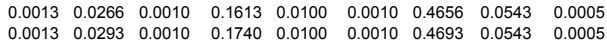

0.0008

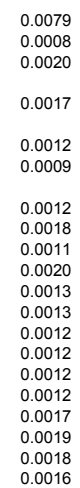

0.0004
0.0004
0.00004
0.0006
0.0006
0.00004
0.0006
0.0008
0.00004
0.0006
0.0006
0.00010
0.00005
0.0005
0.0004
0.0004
0.0004
0.0004
0.0004

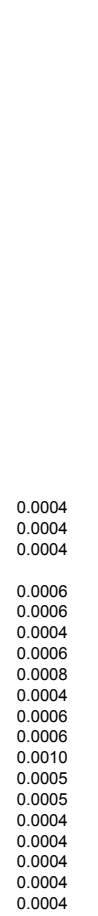

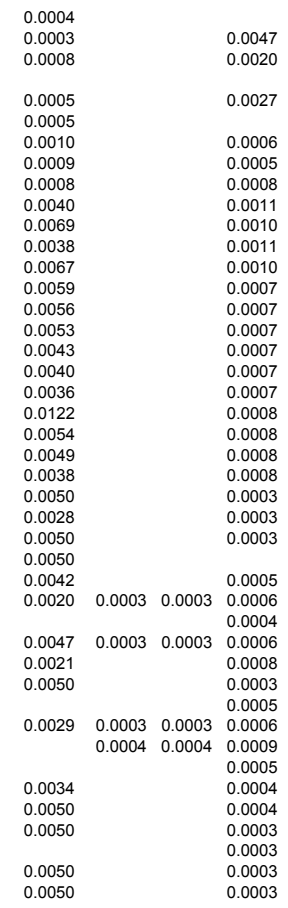


\# Glass ID

3035 HLW-E-ANa-20

3036 HLW-E-ANa-21

3038 HLW-E-ANa-23
3039 HLW-E-ANa-24

3040 HLWE-ANa-25

3041 HLW-E-ANa-26
3042 HLW-E-Bi-01

3043 HLW-E-Bi-02

3046 HLW-E-Bi-0

3048 HLW-E-Bi-07

3049 HLW-E-Bi-08

3050 HLW-E-Bi-09

3053 HLW-E-Bi-12

3054 HLW-E-Bi-13

3056 HLW-E-B-14

3057 HLW-E-Cr-02

3058 HLW-E-Cr-03

3060 HLW-E-Cr-05

3061 HLW-E-Cr-06

3063 HLW-E-CC-D

3065 HLW-E-Cr-10

3067 HLW-E-Cr-12

3068 HLW-E-Cr-13

3069 HLW-E-Cr-14

3071 HLW-E-Cr-16

3072 HLW-E-Cr-17
3073 HLW-E-Cr-18

3073 HLW-E-C-17

3075 AN-107 Actual (LAWC15)

3076 AZ-102 Actual

3078 AP-101 Actual

3080 AN-102 Actual

3081 AN-102 Actual LC Melter

3082 AZ-101 Actual
3083 LAWCrP1R

3084 LAWCrP2R

3085 LAWCrP3R

3087 LAWCrP5

3088 LAWCrPG

3089 LAWCrP7
3090 LAWCrPBCCC

3092 LAWCPP 10CCC

3093 LAWE3Cr2CCC
3094 LAWESHCrICCC

3095 LAWE9HCr2CCC

3097 HLW-ALG-01

3099 HLW-ALG-03

3100 HLW-ALG-04

3101 HLW-ALG-05

3103 HLW-ALG-07

3105 HLW-ALG-09

3107 HLW-ALG-11

3108 HLW-ALG-12

3109 HLW-ALG-13

3111 HLW-ALG-15

3112 HLW-ALG-16

3113 HLW-ALG-17

3116 HLW-ALG-2

HLW-ALG-20
Ga2O3-a Gd2O3-a

1-a La203-a MnO2-a MnO-a MoO-a MoO3-a Nb2O5-a Nd2O3-a NpO2-a PbO-a PdO2-a PdO-a Pr2O3-a Pr6011-a PuO2-a Rb2O-a ReO-a ReO2-a Re207-a Rh2O3-a RhO2-a RuO2-a Sb203-a Sb2O5-a SeO2-a

$\begin{array}{ll} & \\ & \\ & \\ & \\ & \\ & \\ & \\ & \\ & \\ 0.0010 & \\ 0.0011 & \\ 0.0010 & \\ 0.0007 & 0.0210 \\ 0.0016 & 0.0691 \\ 0.0018 & 0.0401 \\ 0.0012 & 0.0234 \\ 0.0018 & 0.0356 \\ 0.0039 & 0.0392 \\ 0.0010 & 0.0701 \\ 0.0017 & 0.0341 \\ 0.0018 & 0.0203 \\ 0.0027 & 0.0306 \\ 0.0016 & 0.0672 \\ 0.0013 & \\ 0.0011 & \\ 0.0010 & 0.0691 \\ 0.0010 & \\ 0.0010 & 0.0210 \\ 0.0010 & 0.0210 \\ & \end{array}$



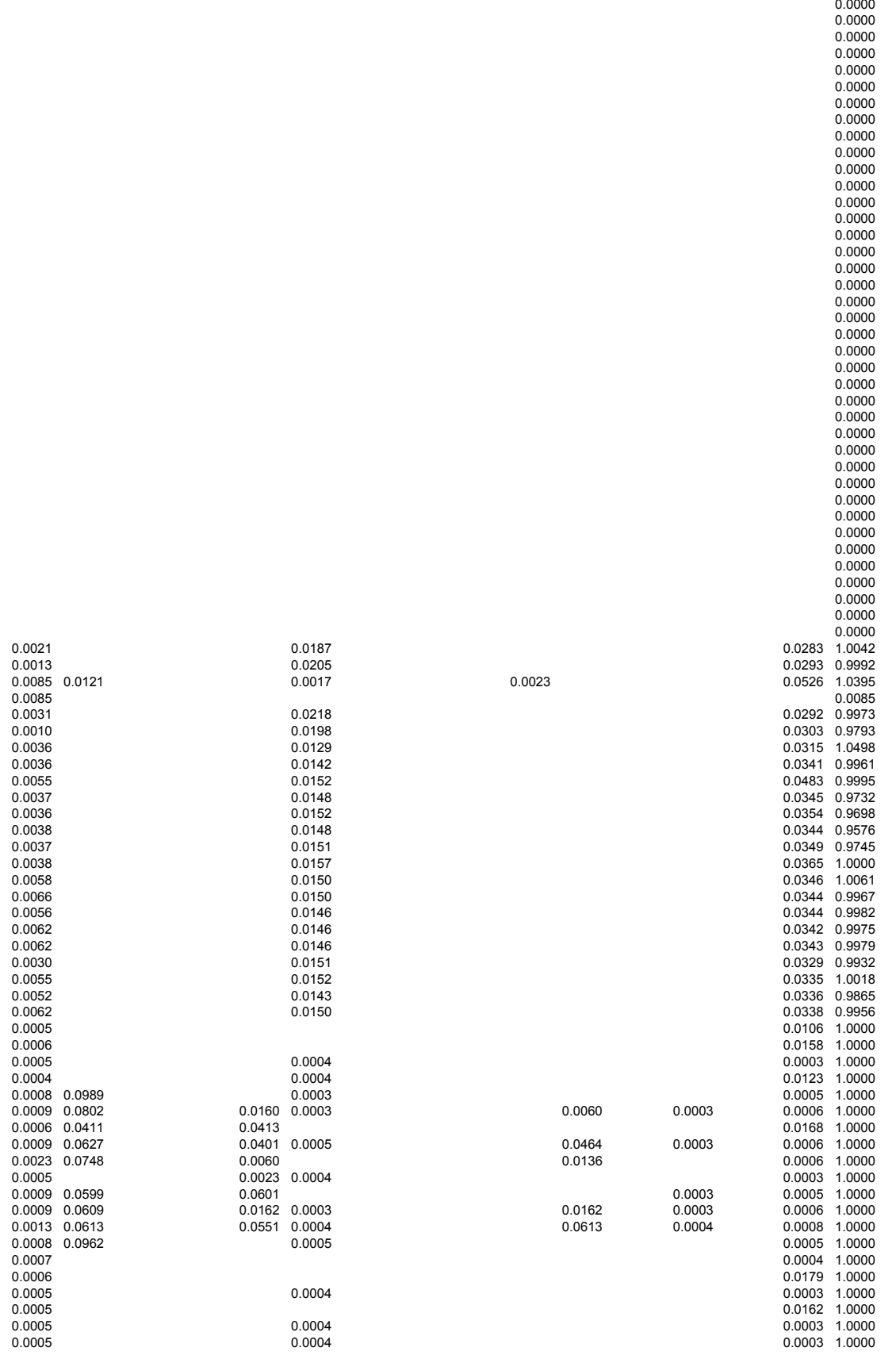

759 Spinel

949 Spinel

$1171 \mathrm{ZrO2}$
$801 \mathrm{Spinel}$

$0.0060 \quad 0.0003$

0.0003

$\begin{array}{lll}0.0413 & \\ 0.0401 & 0.0005 \\ 0.0000 & \end{array}$

$\begin{array}{ll}0.0401 & 0.0005 \\ 0.0060 & \end{array}$

$\begin{array}{ll}0.0023 & 0.0004 \\ 0.0601 & \\ 0.0162 & 0.0003\end{array}$

$\begin{array}{rr}0.0551 & 0.0004 \\ 0.0005\end{array}$

0.0004

0.0004
0.0004

\section{Spinel
775 Spinel
1182 ZrO2 + ThO2 \\ 880 Spinel
$1293 \mathrm{ZrO2}+$ ThO2 \\ 992 Spinel
797 Spinel}

$820 \mathrm{Spinel}+\mathrm{Na}(\mathrm{Mn}, \mathrm{Fe}, \mathrm{Ni})$ Silicate 750 Spinel
638 Spinel $\begin{array}{lll}0.1 & 0.1 & 0.1\end{array}$

$\begin{array}{lll}0.5 & 0.1 \\ 2.25 & 2.2\end{array}$ $\begin{array}{rr}2.3 & 2.7 \\ 25.6 & 24.6 \\ 0.1 & 0.1\end{array}$

$\begin{array}{rrr} & 1.1 & 1 \\ & 3.5 & 0.5 \\ 0.7 & & 1.2 \\ & & 0.4\end{array}$

$\begin{array}{lll} & 0.2 & 0.5 \\ 0.1 & 0.1 & 0.1 \\ 0 & 0.1 & 0.1\end{array}$

$\begin{array}{lll}0.11 & 0.1 & 0.3 \\ 0.8 & 0.1 & 0.1 \\ & 0.3 & 0.1 \\ & 0.4 & 0.6\end{array}$ 
\# Glass ID

3035 HLW-E-ANa-20

3037 HLW-E-ANa-22

3038 HLW-E-ANa-23
3039 HLW-E-ANa-24

3040 HLW-E-ANa-25

3041 HLW-E-ANa-26
3042 HLW-E-Bi-01

3043 HLW-E-Bi-02

3045 HLW-E-Bi-04
3046 HLW-E-Bi-05

3046 HLW-E-Bi-05
3047 HLW-E-Bi-06

3048 HLW-E-Bi-07

3050 HLW-E-Bi-09

(2051 HLWE-BM-

3053 HLW-E-Bi-12

3054 HLW-E-Bi-1

(205

3057 HLW-E-Cr-02

3059 HLW-E-Cr-04

3061 HLW-E-Cr-06

3063 HLW-E-Cr-08

3066 HLW-E-Cr-11

3068 HLW-E-Cr-13

3069 HLW-E-Cr-14

3070 HLW-E-Cr-15
3071 HLW-E-Cr-16

3072 HLW-E-Cr-17

3073 HLW-E-Cr-18

3074 AW-101 Actual
3075 AN-107 Actual (LAWC15)

3076 AZ-102 Actual

3077 AZ-102 Actual CCC

3079 AN-103 Actul

3080 AN-102 Actual LC Mel

082 AZ-101 Actual

3084 LAWCrP2R

3085 LAWCPP3R

(3086 LAWCPP4R

3088 LAWCrP6

3089 LAWCrP7

3092 LAWCrP 10CCC

3094 LAWE9HCrICCC

3095 LAWE9HCr2CCC

3097 HLW-ALG-0

3099 HLW-ALG-03

3100 HLW-ALG-04

3101 HLW-ALG-05

3103 HLW-ALG-07

3104 HLW-ALG-08

3105 HLW-ALG-09

107 HLW-ALG-11

3108 HLW-ALG-12

3110 HLW-ALG-14

3111 HLW-ALG-15

3112 HLW-ALG-16

3113 HLW-ALG-17

3116 HLW-ALG-20

Minor

Quenched XRD

CCC XRD

T1 ( $\left.{ }^{\circ} \mathrm{C}\right) \quad \mathrm{V} 1$ (Pa.s)

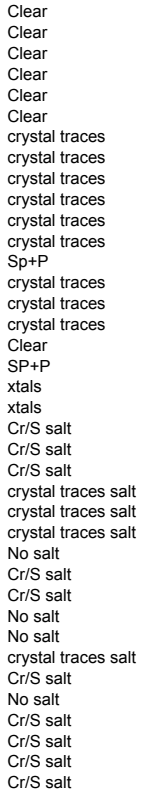

$-0.1 \mathrm{v} 01 \%$
$\sim 0.1 \mathrm{vol} \%$

$-0.1 \mathrm{vol} \%$
$\sim 0.1 \mathrm{vol} \%$
$\sim 0.4 \mathrm{vol} \%$

$-1.8 v 01 \%$

$\sim 2.7 \mathrm{vol} \%$
$\sim 0.5 \mathrm{vol} \%$
$\sim 0.5 \mathrm{vol} \%$
$\sim 0.00$

$-0.4 \mathrm{vol} \%$
$\sim 0.1 \mathrm{vol} \%$
$-4.2 \mathrm{vol} \%$

$-3.2 \mathrm{vol} \%$

$\sim 0.500 \% \%$
$\sim 0.3 v 01 \%$

$\sim-0.3 v 01 \%$

$-0.9 \mathrm{vol} \%$

$\sim 0.5 \mathrm{vol} \%$

$0.7 v 01 \%$

$-0.7 \mathrm{vol} \%$
$-1.2 \mathrm{vol} \%$
$\sim 0.1 \mathrm{vol} \%$

2.68

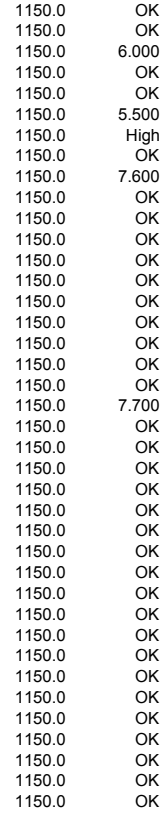

Clear glass with no secondary phase
Few Cr-rich spinels $\sim 0.1$ vol. $\%$.

Clear glass with no secondary phase

Few small $(\sim 1 \mu \mathrm{m})$ Cr-rich ZnFeeT spinels $~ 02$ vol.

Few small Cr-rich ZnFeTi spinels $\sim 0.2$ vol.\%. Small residue in the crucible of $52 \%$ silica-15\% phosphate glassy nodule.

Few small Cr-ricic ZnFFetis spinels $\sim 0.2$ vol. \%.

Few small Cr-rich ZnFeTi spinel <0.1 vol.\% - Remaining glass shows $\sim 0.3 w 1 \%$ Cr2O3.

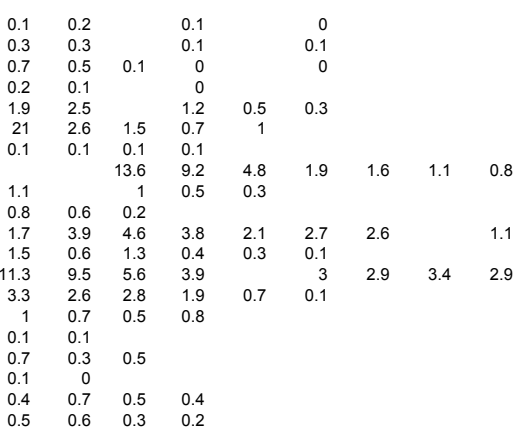

$<0.1$ vol\% of Cr-rich spinel and 0.2 vol\% of $\mathrm{Na}(\mathrm{Al}, \mathrm{Fe})$ Silicate

$<0.1$ vol\% of spine

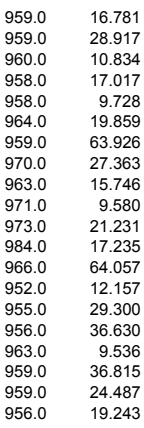

1.9 vol\% crystals $(70 \%$ spinel $+30 \%$ zirconia with trace amount of thoria

4 vol\% crystals $(80 \%$ zironia $+20 \%$ thoria)

1.2 vol\% of spinel 


\begin{tabular}{|c|c|c|c|c|}
\hline & 6.084 & 1156 & 29 & \\
\hline 59 & 8.847 & 1160 & & \\
\hline $\begin{array}{l}1061 \\
1060\end{array}$ & $\begin{array}{l}3.631 \\
5.935\end{array}$ & $\begin{array}{l}1161 \\
1161\end{array}$ & $\begin{array}{l}1.6 \\
27\end{array}$ & 1261.0 \\
\hline 1058 & 3.306 & 1159 & 1.5 & $\begin{array}{l}1258.0 \\
\end{array}$ \\
\hline & 6.252 & 1170 & $\begin{array}{l}2.0 \\
2.6\end{array}$ & 1273.0 \\
\hline 1058 & 17.210 & 1156 & 5.9 & 1255.0 \\
\hline $\begin{array}{l}1065 \\
1055\end{array}$ & $\begin{array}{l}6.477 \\
4656\end{array}$ & $\begin{array}{l}1159 \\
11153\end{array}$ & $\begin{array}{l}2.1 \\
2.1\end{array}$ & $\begin{array}{l}1254.0 \\
1240\end{array}$ \\
\hline $\begin{array}{l}1057 \\
1070\end{array}$ & $\begin{array}{l}\begin{array}{r}4.656 \\
3.623\end{array} \\
\end{array}$ & $\begin{array}{l}1153 \\
1168\end{array}$ & $\begin{array}{l}2.1 \\
1.7\end{array}$ & $\begin{array}{l}1249.0 \\
1287.0\end{array}$ \\
\hline 1073 & 5.933 & 1172 & 2.2 & 1272.0 \\
\hline 1071 & 5.212 & 1160 & 2.3 & 1249.0 \\
\hline 1059 & 15.679 & 1154 & 4.2 & 1249.0 \\
\hline 1055 & 4.099 & 1158 & 1.8 & 1261.0 \\
\hline $\begin{array}{l}1054 \\
1053\end{array}$ & $\begin{array}{r}8.645 \\
11.076\end{array}$ & $\begin{array}{l}\begin{array}{l}1153 \\
1155\end{array}\end{array}$ & $\begin{array}{l}3.3 \\
4.3\end{array}$ & $\begin{array}{l}12251.0 \\
1248.0\end{array}$ \\
\hline $\begin{array}{l}1003 \\
1063\end{array}$ & 3.568 & 1163 & $\begin{array}{l}\text {.3 } \\
1.8\end{array}$ & $\begin{array}{l}264.0 \\
126\end{array}$ \\
\hline 1060 & 13.116 & 1161 & 5.7 & 1263.0 \\
\hline & 7.346 & 1164 & 2.9 & 1265.0 \\
\hline & & & & \\
\hline
\end{tabular}


Appendix B

Scatterplot Matrices 


\section{Appendix B: Scatterplot Matrices}

\section{B-1 Viscosity at $950^{\circ} \mathrm{C}$}

Figure B.1 shows the scatterplot matrix of the compositions used in fitting the $\ln \left[\eta_{950}\right]$ model. The scatterplot matrix, although difficult to see, does show relatively even coverage of each of the components across their validity regions. For the most part, the two-dimensional coverage is adequate, with the exceptions of:

- $\mathrm{BaO}$ with: $\mathrm{CaO}, \mathrm{F}, \mathrm{La}_{2} \mathrm{O}_{3}, \mathrm{MgO}, \mathrm{MnO}, \mathrm{PbO}, \mathrm{SrO}, \mathrm{UO}_{3}, \mathrm{ZnO}, \mathrm{ZrO}_{2}$, and Others

- $\mathrm{CaO}$ with: $\mathrm{MnO}, \mathrm{PbO}$, and $\mathrm{UO}_{3}$

- F with: $\mathrm{MgO}, \mathrm{MnO}, \mathrm{PbO}, \mathrm{UO}_{3}$, and $\mathrm{ZnO}$

- $\mathrm{K}_{2} \mathrm{O}$ with: $\mathrm{La}_{2} \mathrm{O}_{3}, \mathrm{MnO}$, and $\mathrm{UO}_{3}$

- $\mathrm{La}_{2} \mathrm{O}_{3}$ with: $\mathrm{MnO}, \mathrm{PbO}, \mathrm{UO}_{3}$, and $\mathrm{ZnO}$

- $\mathrm{MgO}$ with: $\mathrm{MnO}, \mathrm{SrO}$, and $\mathrm{UO}_{3}$

- $\mathrm{P}_{2} \mathrm{O}_{5}$ with: $\mathrm{PbO}, \mathrm{SrO}$, and $\mathrm{UO}_{3}$.

On the other hand, no strong component correlations are evident from the data shown in this plot. 


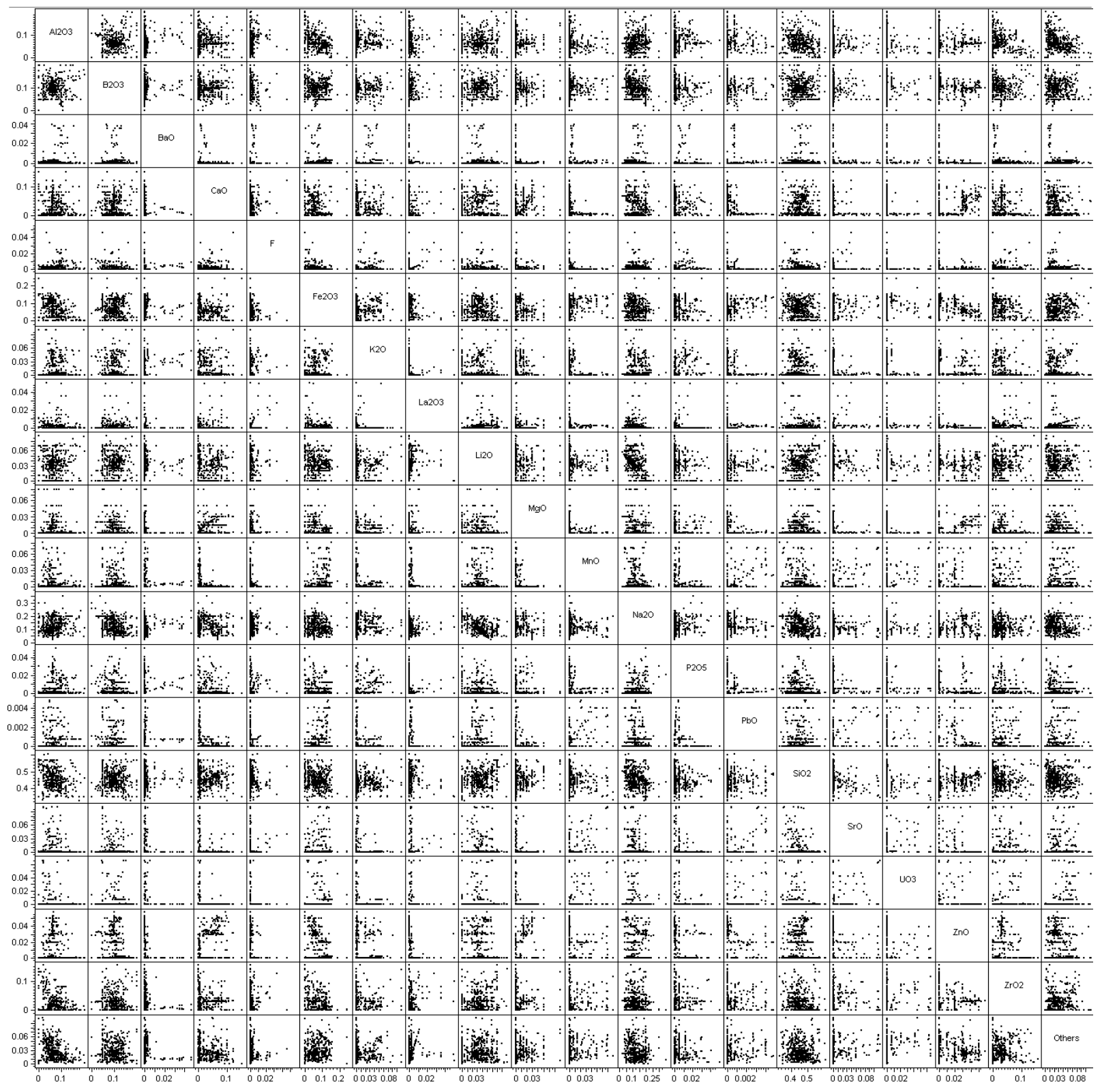

Figure B.1. Scatterplot Matrix of $\ln \left[n_{950}\right]$ Data Component Concentrations (in mass fractions)

\section{B-2 Viscosity at $1150^{\circ} \mathrm{C}$}

Figure B.2 shows the scatterplot matrix of the compositions used in fitting the $\ln \left[n_{1150}\right]$ model. The scatterplot matrix, although difficult to see, does show relatively even coverage of each of the components across their validity region. For the most part, the two-dimensional coverage is adequate, with the exceptions of:

- $\mathrm{BaO}$ with: $\mathrm{CaO}, \mathrm{F}, \mathrm{La}_{2} \mathrm{O}_{3}, \mathrm{MgO}, \mathrm{MnO}$, and $\mathrm{ZnO}$

- $\mathrm{CaO}$ with: $\mathrm{MnO}$ and $\mathrm{SrO}$

- $\mathrm{K}_{2} \mathrm{O}$ with: $\mathrm{La}_{2} \mathrm{O}_{3}, \mathrm{SrO}$, and $\mathrm{UO}_{3}$

- $\mathrm{La}_{2} \mathrm{O}_{3}$ with: $\mathrm{MnO}, \mathrm{PbO}$, and $\mathrm{ZnO}$ 
- $\mathrm{MgO}$ with: $\mathrm{MnO}, \mathrm{SrO}$, and $\mathrm{UO}_{3}$

- $\mathrm{P}_{2} \mathrm{O}_{5}$ with SrO.

On the other hand, no strong component correlations are evident from the data shown in this plot.

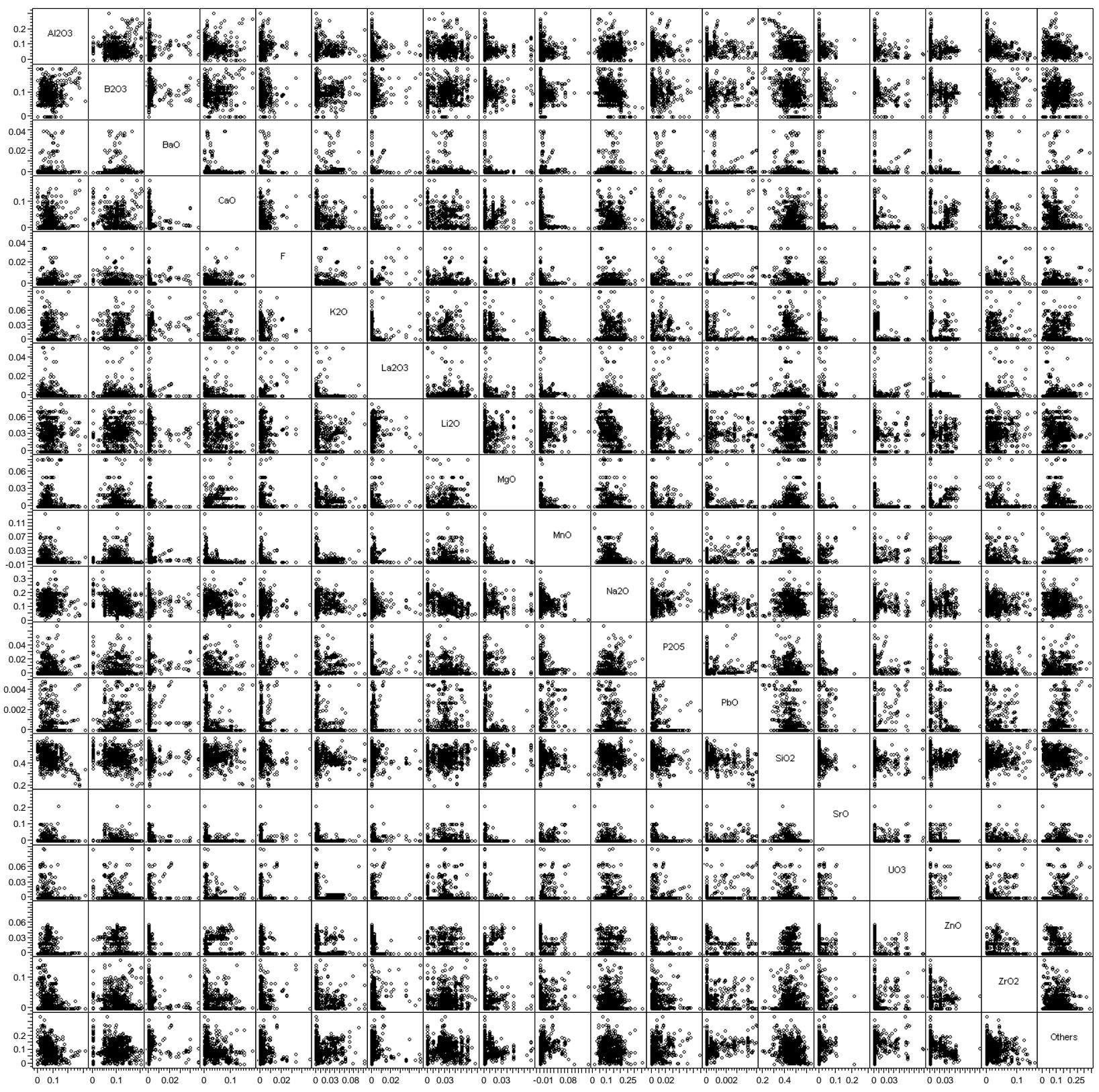

Figure B.2. Scatterplot Matrix of $\operatorname{In}\left[\eta_{1150}\right]$ Data Component Concentrations (in mass fractions)

\section{B-3 Viscosity at $1250^{\circ} \mathrm{C}$}

Figure B.3 shows the scatterplot matrix of the compositions used in fitting the $\ln \left[\eta_{1250}\right]$ model. The scatterplot matrix, although difficult to see, does show relatively even coverage of 
each of the components across their validity region. For the most part, the two-dimensional coverage is adequate, with the exceptions of:

- $\mathrm{BaO}$ with: $\mathrm{CaO}, \mathrm{F}, \mathrm{La}_{2} \mathrm{O}_{3}, \mathrm{MgO}, \mathrm{MnO}, \mathrm{PbO}, \mathrm{SrO}, \mathrm{UO}_{3}, \mathrm{ZnO}, \mathrm{ZrO}_{2}$, and Others

- $\mathrm{CaO}$ with: $\mathrm{MnO}, \mathrm{PbO}$, and $\mathrm{UO}_{3}$

- F with: $\mathrm{MgO}, \mathrm{MnO}, \mathrm{PbO}, \mathrm{UO}_{3}$, and $\mathrm{ZnO}$

- $\mathrm{K}_{2} \mathrm{O}$ with: $\mathrm{La}_{2} \mathrm{O}_{3}, \mathrm{MnO}$, and $\mathrm{UO}_{3}$

- $\quad \mathrm{La}_{2} \mathrm{O}_{3}$ with: $\mathrm{MnO}, \mathrm{PbO}, \mathrm{UO}_{3}$, and $\mathrm{ZnO}$

- $\mathrm{MgO}$ with: $\mathrm{MnO}, \mathrm{SrO}$, and $\mathrm{UO}_{3}$

- $\mathrm{P}_{2} \mathrm{O}_{5}$ with: $\mathrm{PbO}, \mathrm{SrO}$, and $\mathrm{UO}_{3}$.

On the other hand, no strong component correlations are evident from the data shown in this plot. 


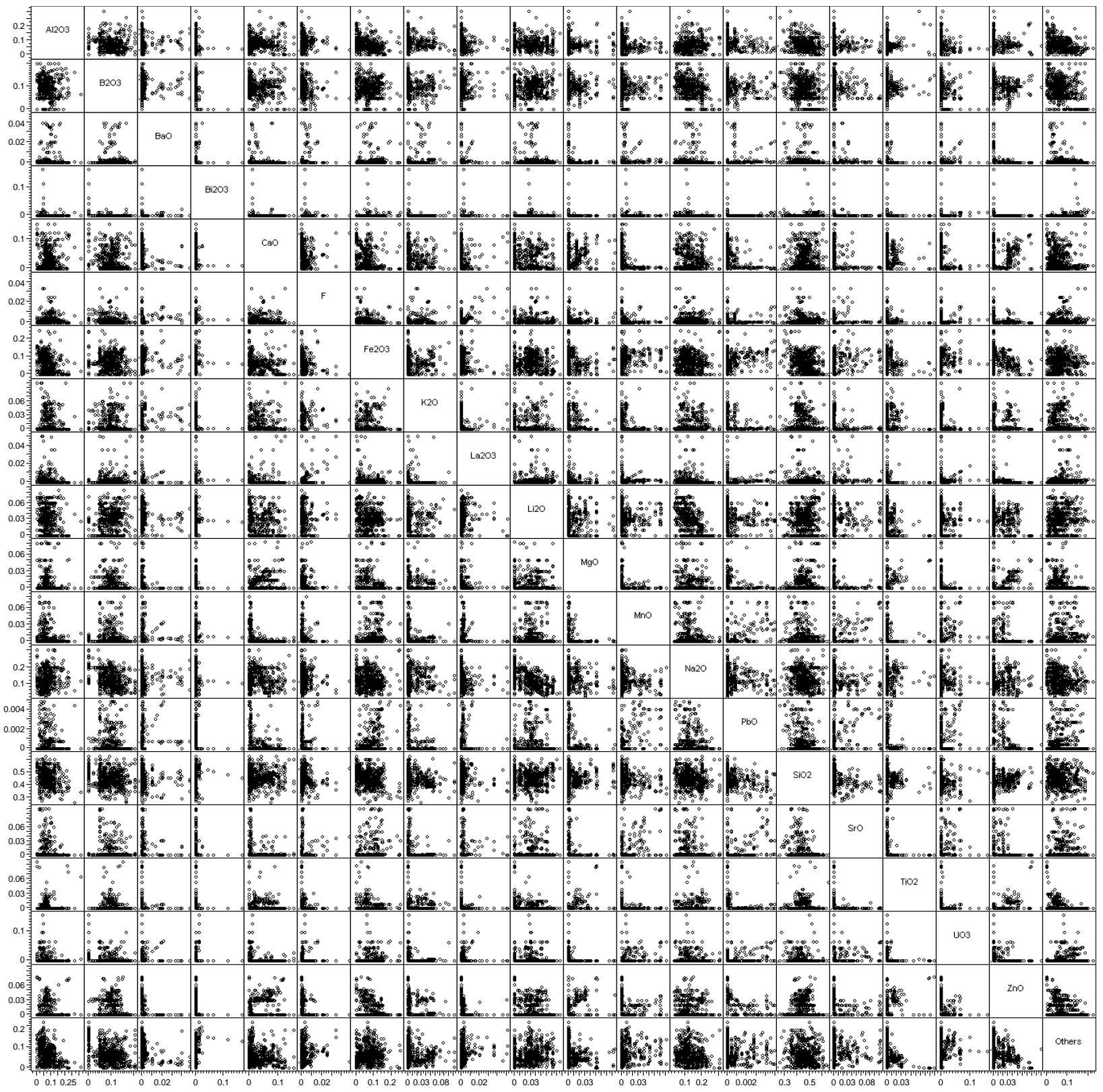

Figure B.3. Scatterplot Matrix of $\ln \left[\eta_{1250}\right]$ Data Component Concentrations (in mass fractions)

\section{B-4 Electrical Conductivity at $1000^{\circ} \mathrm{C}$}

Figure B.4 shows the scatterplot matrix of the compositions used in fitting the $\ln \left[\varepsilon_{1000}\right]$ model. The scatterplot matrix shows relatively even coverage of each of the components across their validity region. For the most part, the two-dimensional coverage is adequate, with a few exceptions. No strong component correlations are evident from the data shown in this plot. 


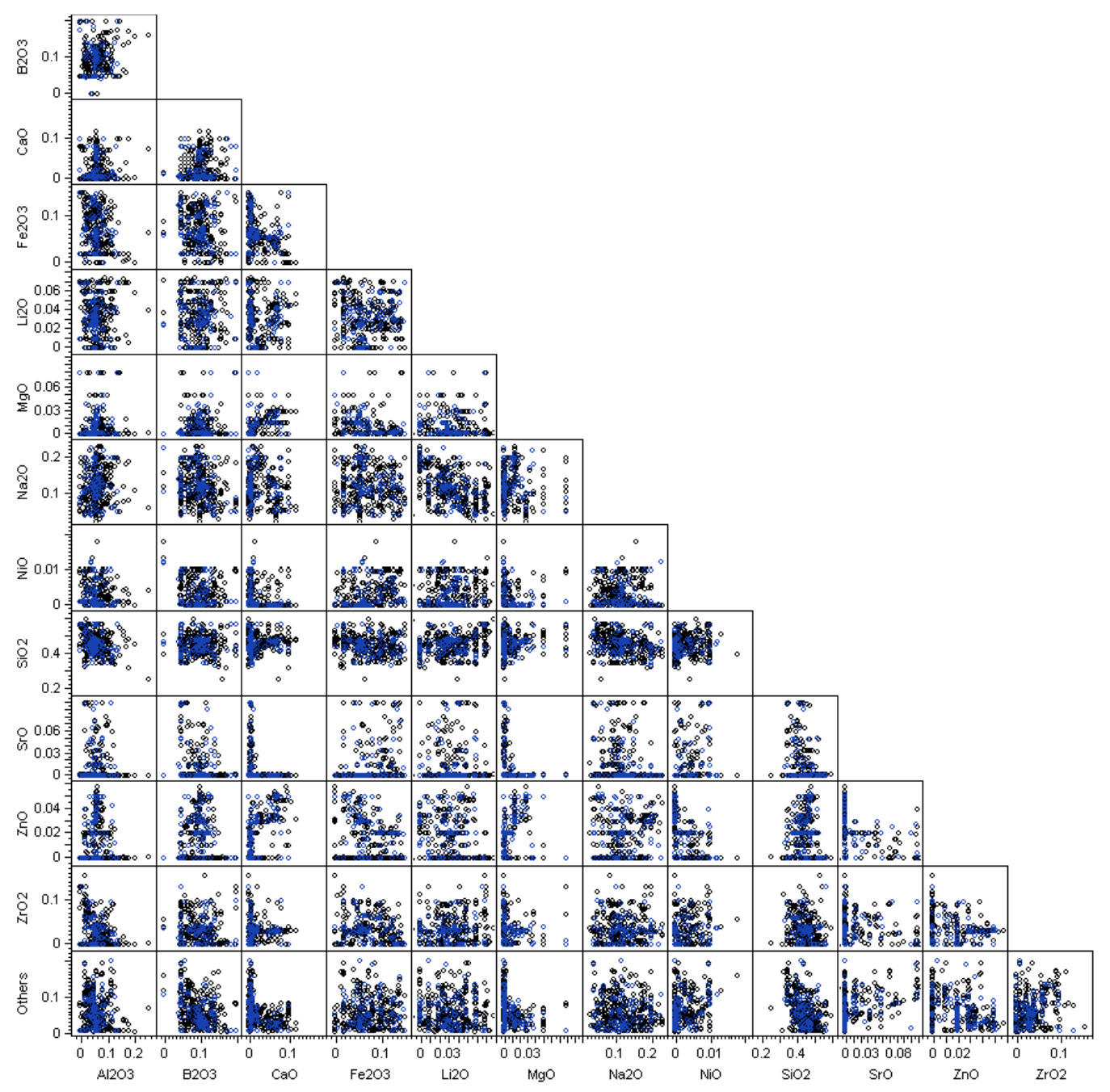

Figure B.4. Scatterplot Matrix of $\ln \left[\varepsilon_{1000}\right]$ Data Component Concentrations (in mass fractions)

\section{B-5 Electrical Conductivity at $1100^{\circ} \mathrm{C}$}

Figure B.5 shows the scatterplot matrix of the compositions used in fitting the $\ln \left[\varepsilon_{1100}\right]$ model. The scatterplot matrix shows relatively even coverage of each of the components across their validity region. For the most part, the two-dimensional coverage is adequate, with a few exceptions. No strong component correlations are evident from the data shown in this plot. 


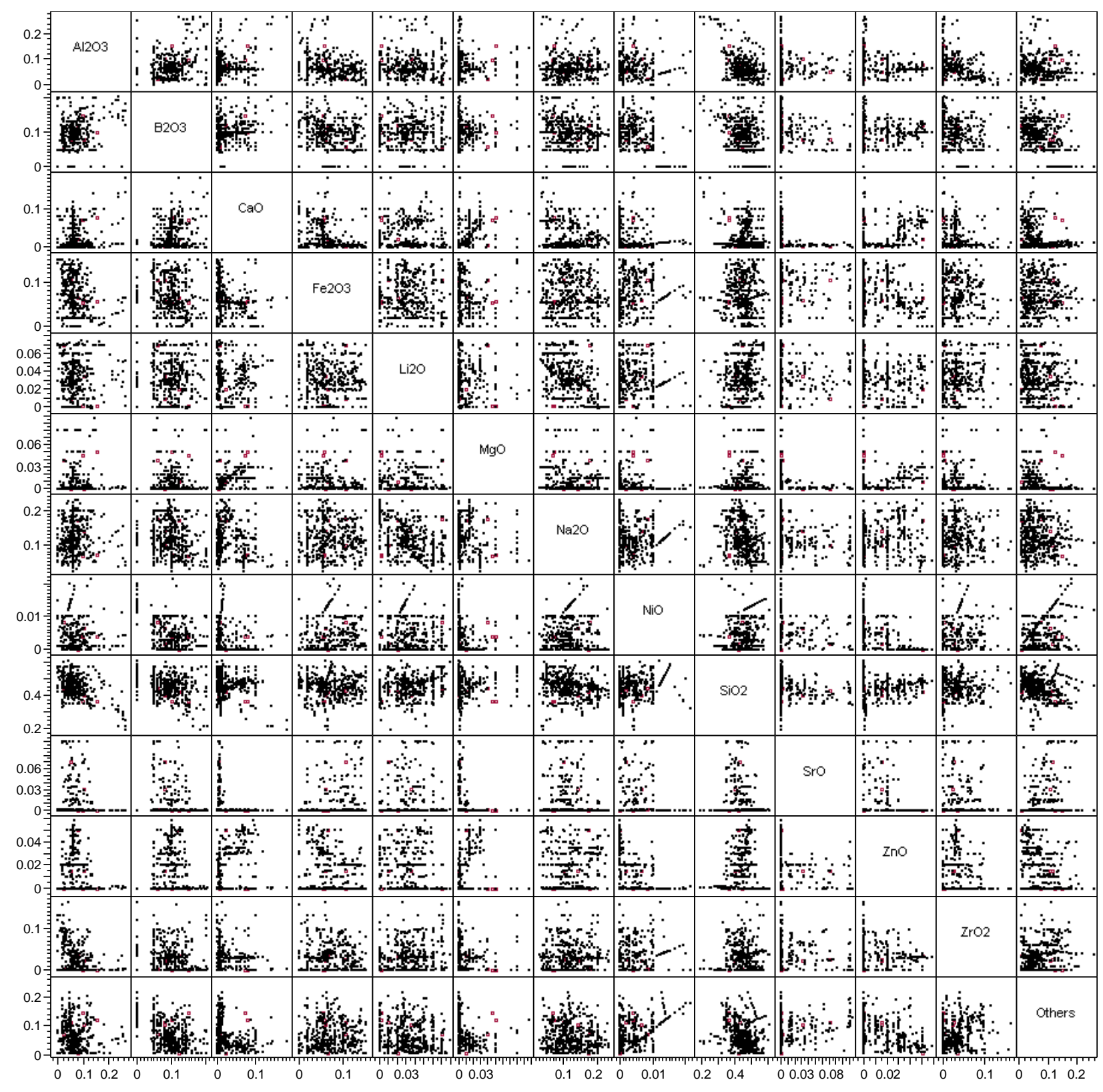

Figure B.5. Scatterplot Matrix of $\ln \left[\varepsilon_{1100}\right]$ Data Component Concentrations (in mass fractions)

\section{B-6 Electrical Conductivity at $1150^{\circ} \mathrm{C}$}

Figure B.6 shows the scatterplot matrix of the compositions used in fitting the $\ln \left[\varepsilon_{1150}\right]$ model. The scatterplot matrix shows relatively even coverage of each of the components across their validity region. For the most part, the two-dimensional coverage is adequate, with a few exceptions. No strong component correlations are evident from the data shown in this plot. 


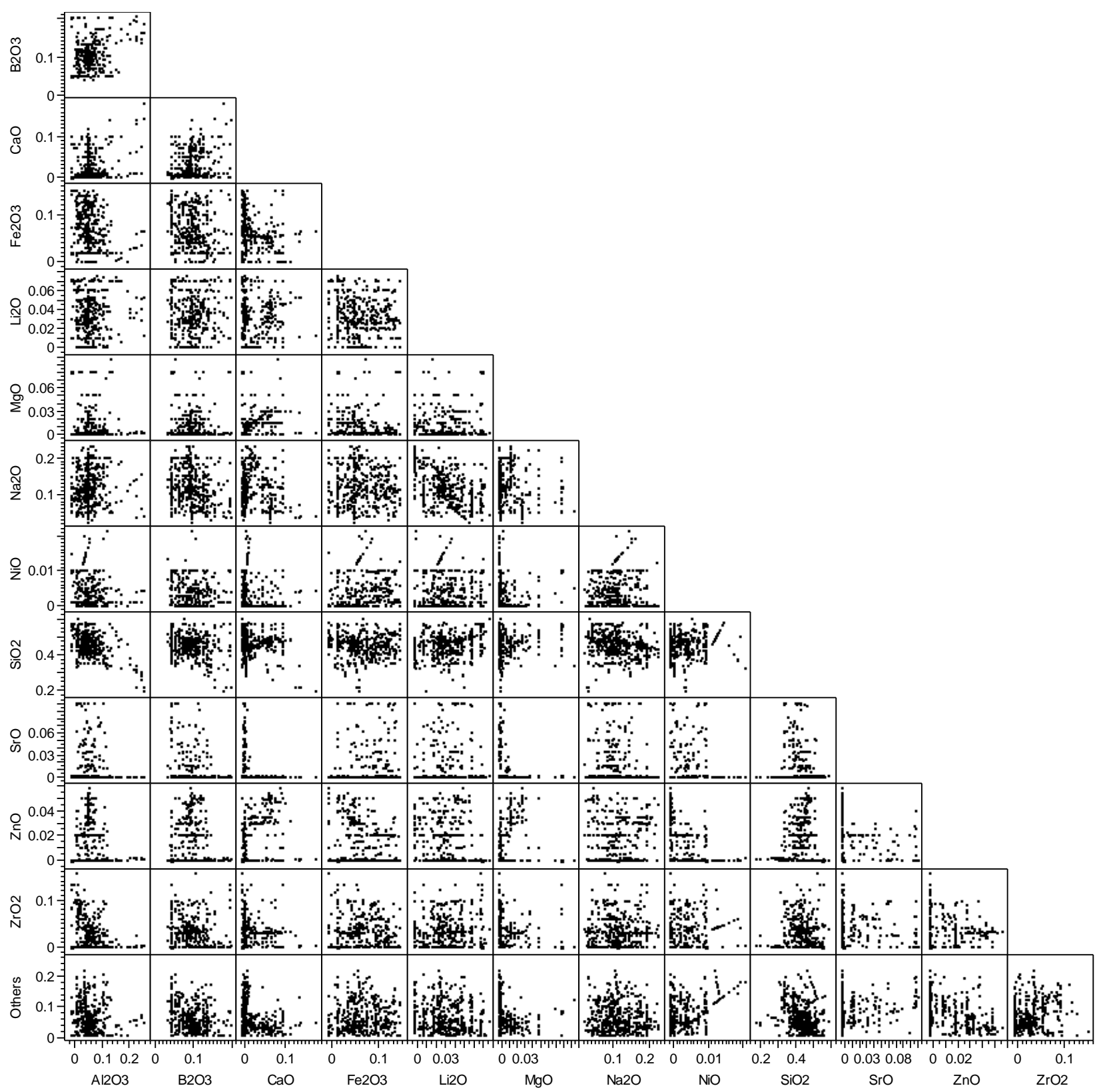

Figure B.6. Scatterplot Matrix of $\ln \left[\varepsilon_{1150}\right]$ Data Component Concentrations (in mass fractions)

\section{B-7 Electrical Conductivity at $1200^{\circ} \mathrm{C}$}

Figure B.7 shows the scatterplot matrix of the compositions used in fitting the $\ln \left[\varepsilon_{1200}\right]$ model. The scatterplot matrix shows relatively even coverage of each of the components across their validity region. For the most part, the two-dimensional coverage is adequate, with a few exceptions. No strong component correlations are evident from the data shown in this plot. 


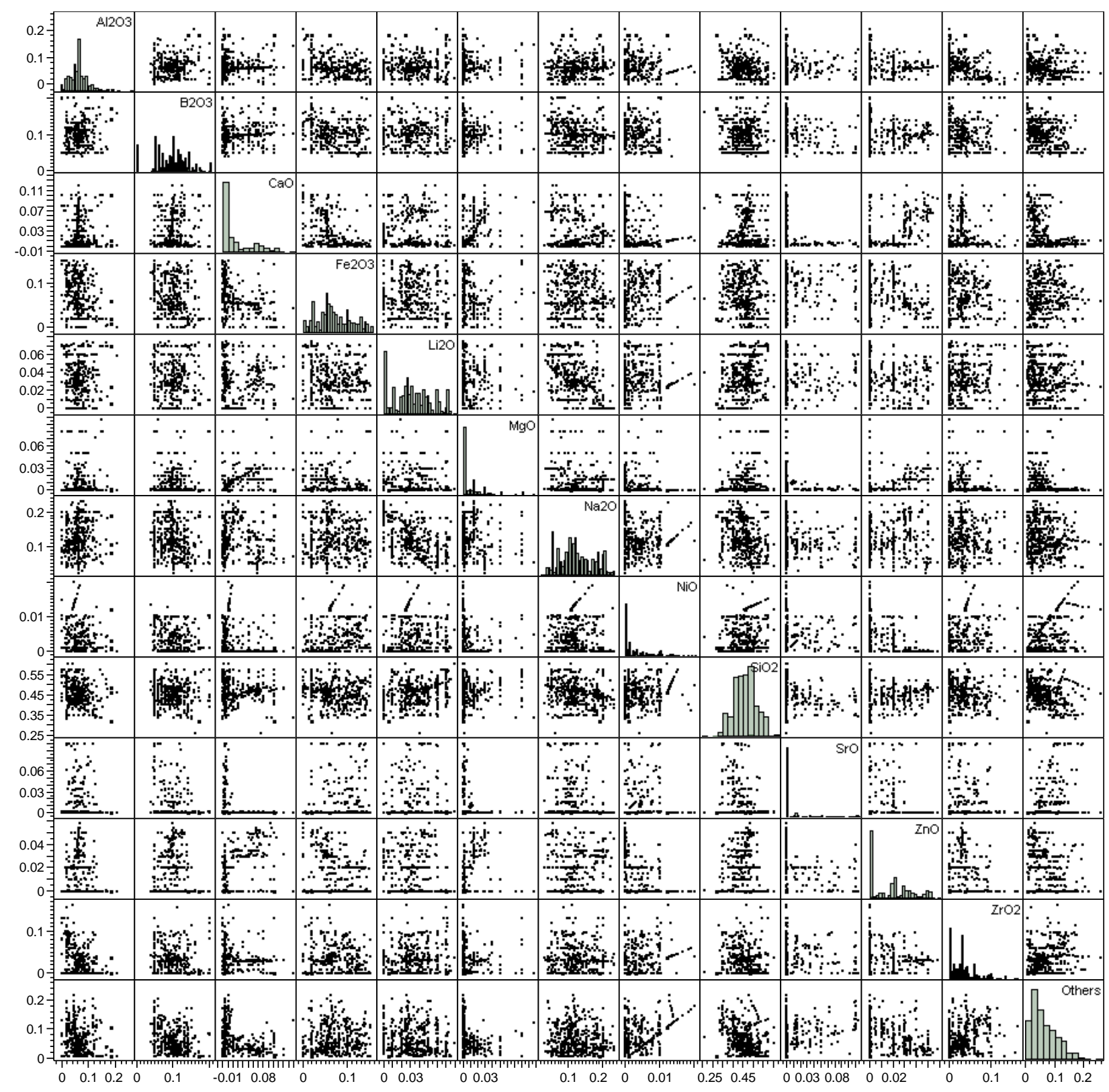

Figure B.7. Scatterplot Matrix of $\ln \left[\varepsilon_{1200}\right]$ Data Component Concentrations (in mass fractions)

\section{B-8 Product Consistency Test Boron Response}

Figure B.8 shows the scatterplot matrix of the compositions used in fitting $\ln \left[\mathrm{PCT}_{\mathrm{B}}\right]$ model. The scatterplot matrix, although difficult to see, does show relatively even coverage of each of the components across their validity region. For the most part, the two-dimensional coverage is adequate, with some exceptions. No strong component correlations are evident from the data shown in this plot. 


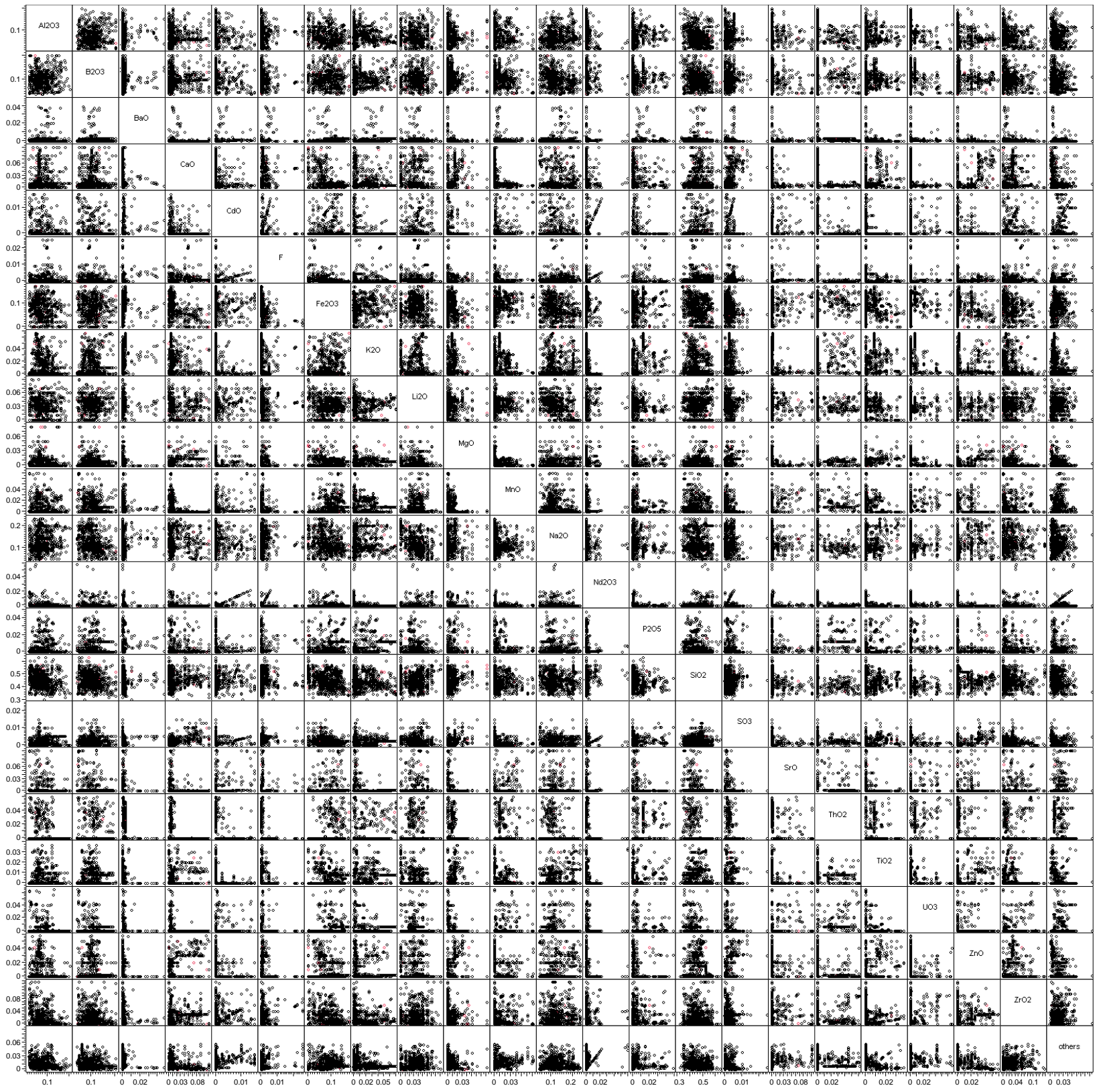

Figure B.8. Scatterplot Matrix of $\ln \left[\mathrm{PCT}_{\mathrm{B}}\right]$ Data Component Concentrations (in mass fractions)

\section{B-9 Product Consistency Test Sodium Response}

Figure B.9 shows the scatterplot matrix of the compositions used in fitting the $\ln \left[\mathrm{PCT}_{\mathrm{Na}}\right]$ model. The scatterplot matrix, although difficult to see, does show relatively even coverage of each of the components across their validity region. For the most part, the two-dimensional coverage is adequate, with a few exceptions. No strong component correlations are evident from the data shown in this plot. 


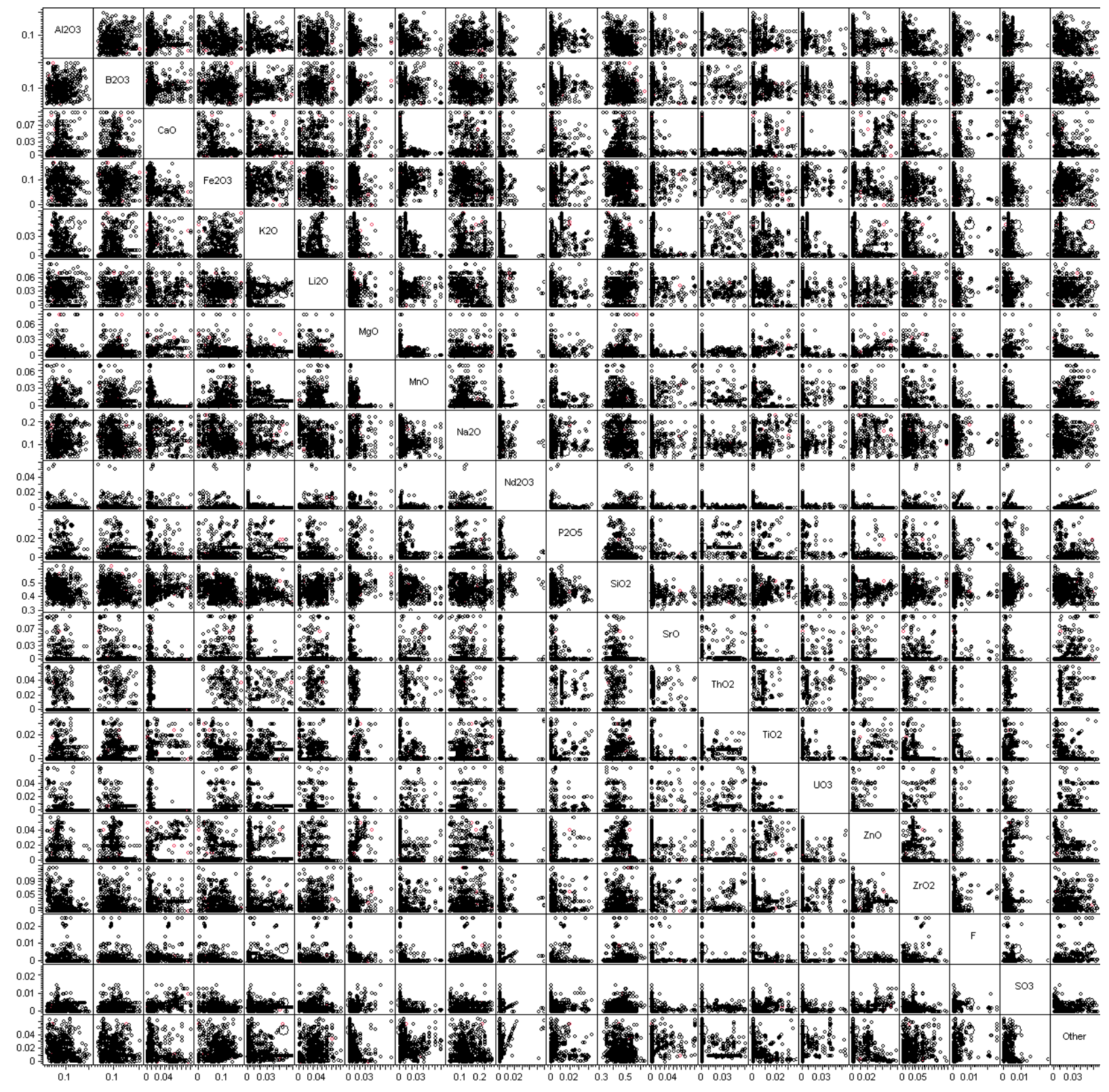

Figure B.9. Scatterplot Matrix of $\ln \left[\mathrm{PCT}_{\mathrm{Na}}\right]$ Data Component Concentrations (in mass fractions)

\section{B-10 Product Consistency Test Lithium Response}

Figure B.10 shows the scatterplot matrix of the compositions used in fitting the $\ln \left[\mathrm{PCT}_{\mathrm{Li}}\right]$ model. The scatterplot matrix, although difficult to see, does show relatively even coverage of each of the components across their validity region. For the most part, the two-dimensional coverage is adequate, with a few exceptions. No strong component correlations are evident from the data shown in this plot. 


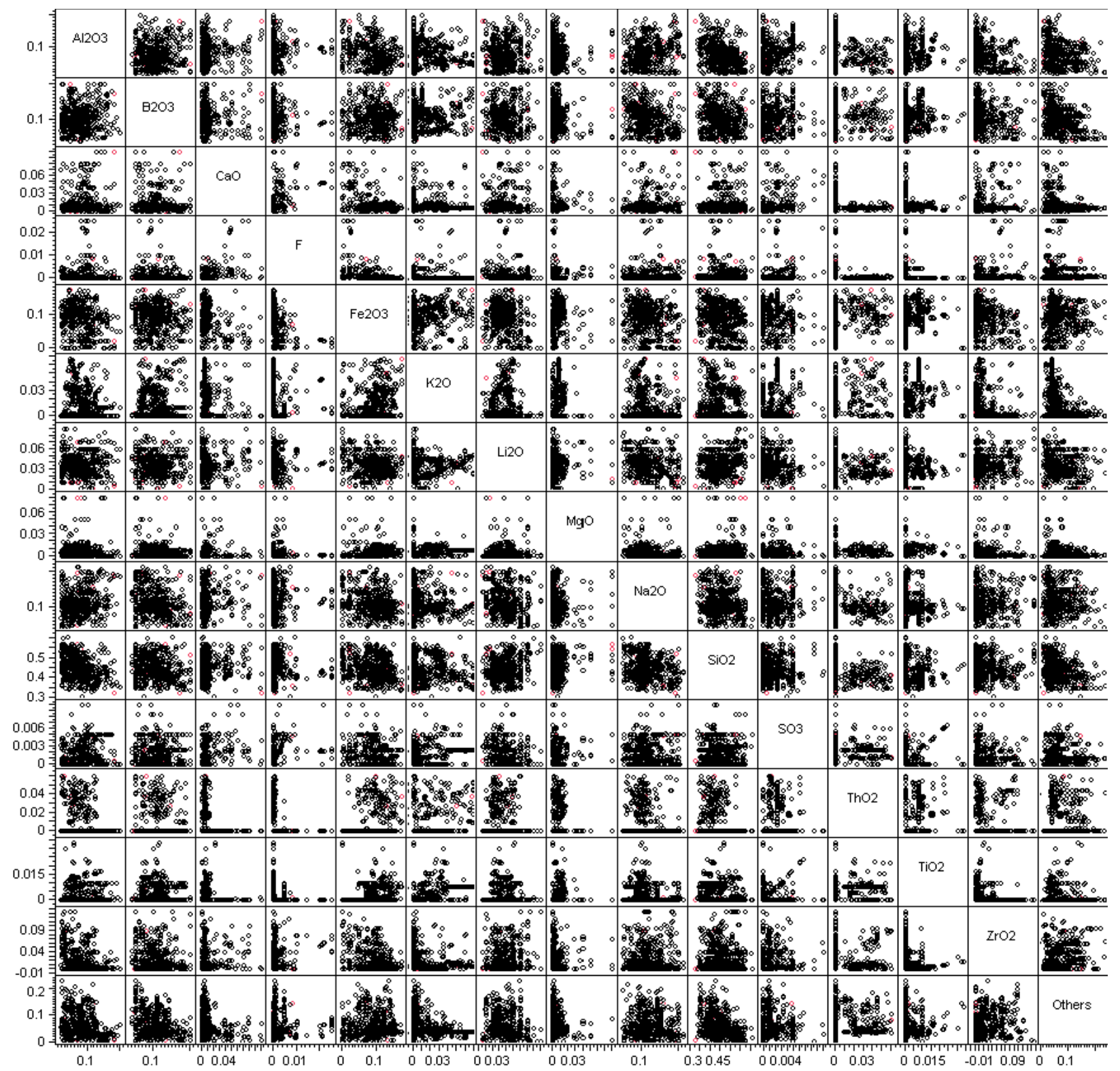

Figure B.10. Scatterplot Matrix of $\ln \left[\mathrm{PCT}_{\mathrm{Li}}\right]$ Data Component Concentrations (in mass fractions)

\section{B-11 Toxicity Characteristic Leaching Procedure Response}

Figure B.11 shows the scatterplot matrix of the compositions used in fitting the $\ln \left[\mathrm{N}_{\text {TCLP }}\right]$ model. The scatterplot matrix, although difficult to see, does show relatively even coverage of each of the components across their validity region. For the most part, the two-dimensional coverage is adequate, with some exceptions. No strong component correlations are evident from the data shown in this plot. 


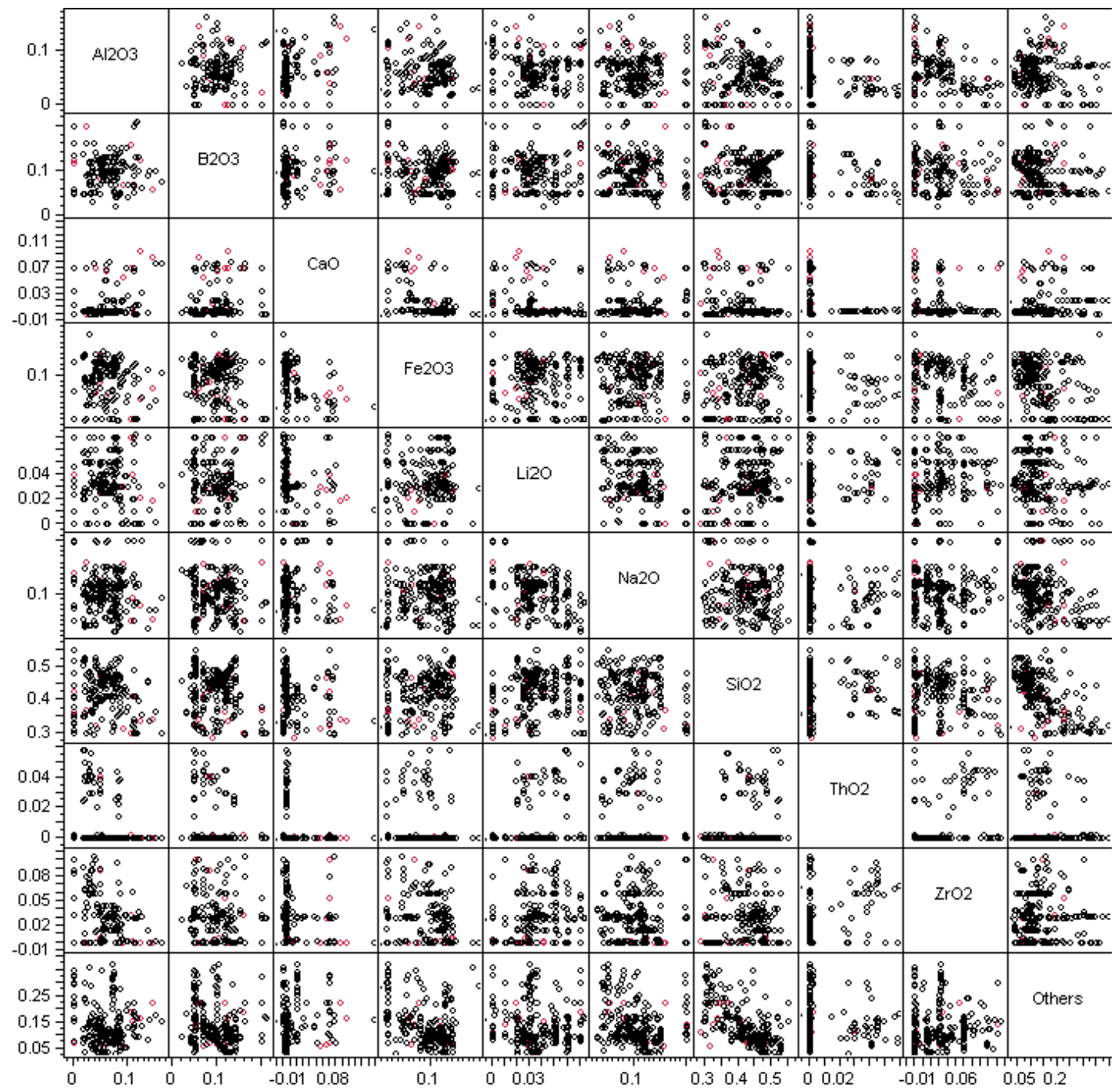

Figure B.11. Scatterplot Matrix of $\ln \left[N_{T C L P}\right]$ Data Component Concentrations (in mass fractions)

\section{B-12 Spinel One Percent Crystal Temperature}

Figure B.12 shows the scatterplot matrix of the compositions used in fitting the $T_{1 \%, s p}$ model. The scatterplot matrix, although difficult to see, does show relatively even coverage of each of the components across their validity region with the exception of $\mathrm{MgO}$. Removing the two high $\mathrm{MgO}$ glasses changed the fit very little (statistics and $\mathrm{MgO}$ coefficient), so they were retained. For the most part, the two-dimensional coverage is adequate, with some exceptions. No strong component correlations are evident from this plot. 


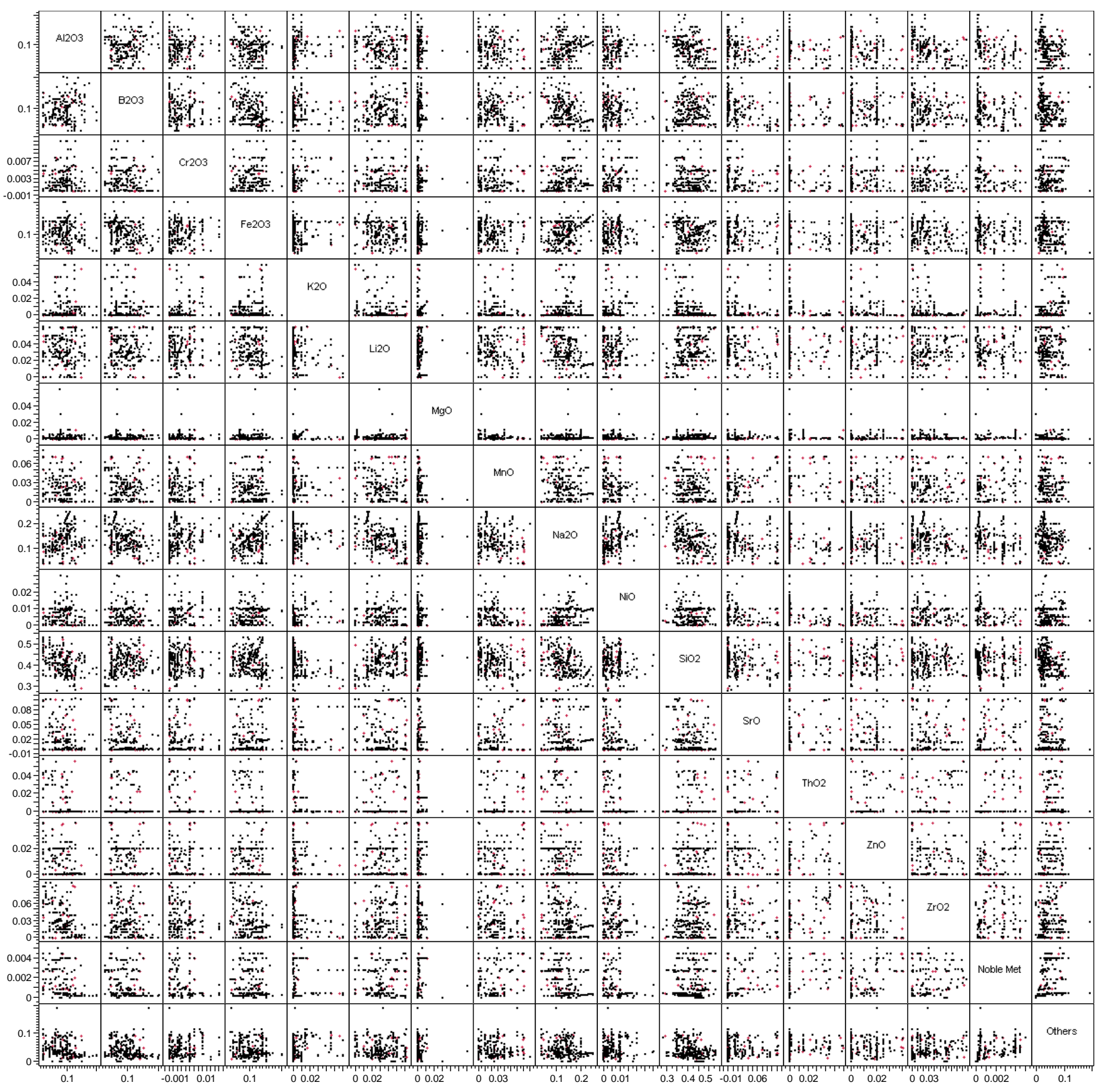

Figure B.12. Scatterplot Matrix of $T_{1 \%}$ Data Component Concentrations (in mass fractions)

\section{B-13 Zirconia Containing Phases Liquidus Temperature}

Figure B.13 shows the scatterplot matrix of the compositions used in fitting the $T_{L, z s}$ model. The scatterplot matrix shows relatively even coverage of each of the components across their validity region with the exception of SrO. Removing the two high $\mathrm{SrO}$ glasses significantly improves validation statistics, but not the coefficient values, so they were retained to allow for prediction of high $\mathrm{SrO}$ glasses. For the most part, the two-dimensional coverage is adequate. No strong component correlations are evident from this plot. 


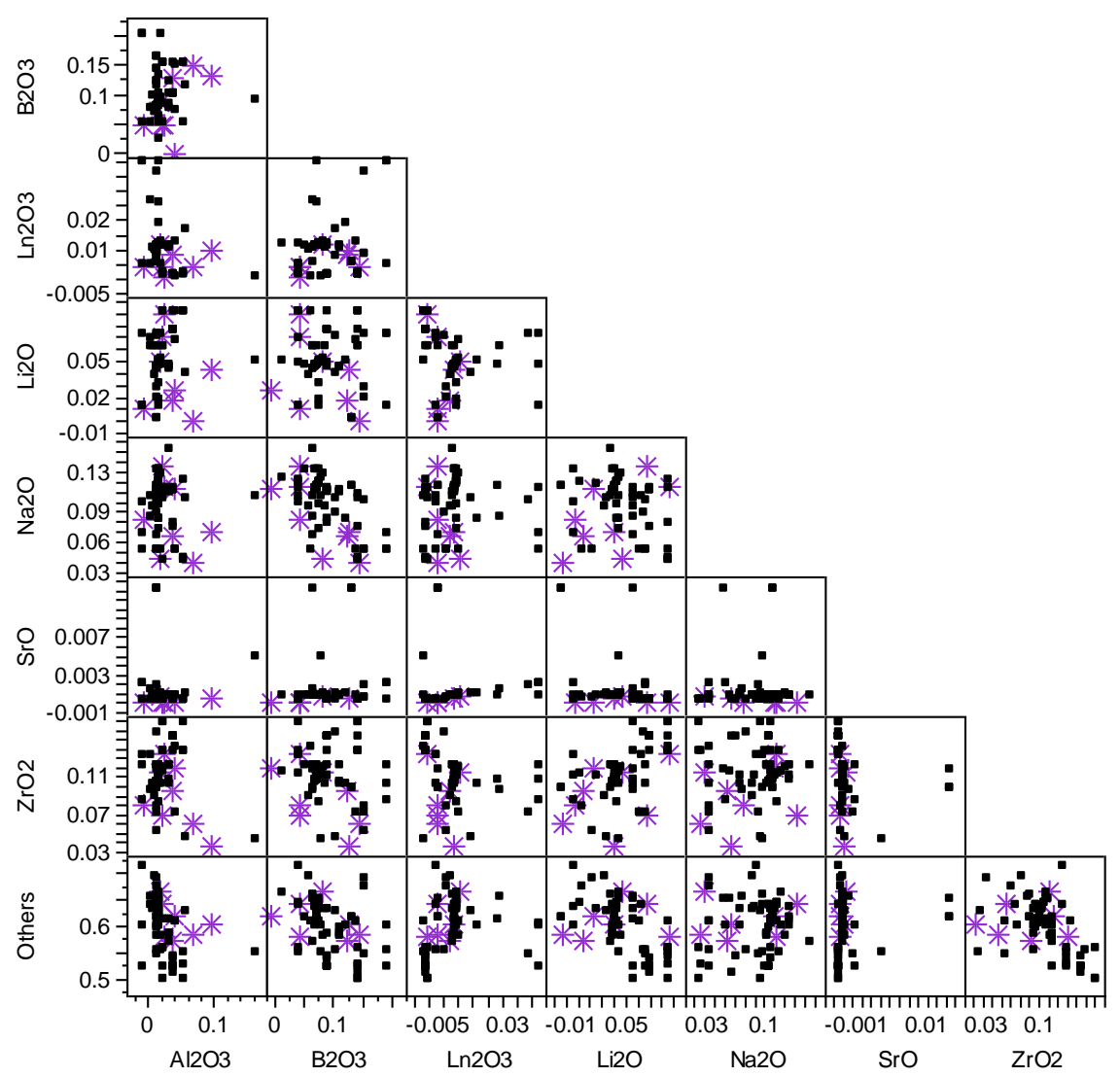

Figure B.13. Scatterplot Matrix of $T_{L}$ Data Component Concentrations (in mass fractions)

\section{B-14 Density and Specific Volume}

Figure B.14 shows the scatterplot matrix of the compositions used in fitting the $v$ model. The scatterplot matrix shows relatively even coverage of each of the components across their validity region with the exception of $\mathrm{Bi}_{2} \mathrm{O}_{3}$ and $\mathrm{ThO}_{2}$. Removing the high $\mathrm{Bi}_{2} \mathrm{O}_{3}$ and $\mathrm{ThO}_{2}$ glasses does not significantly affect the coefficient values, so they were retained to allow for a broader region of glasses. For the most part, the two-dimensional coverage is adequate. No strong component correlations are evident from this plot. 


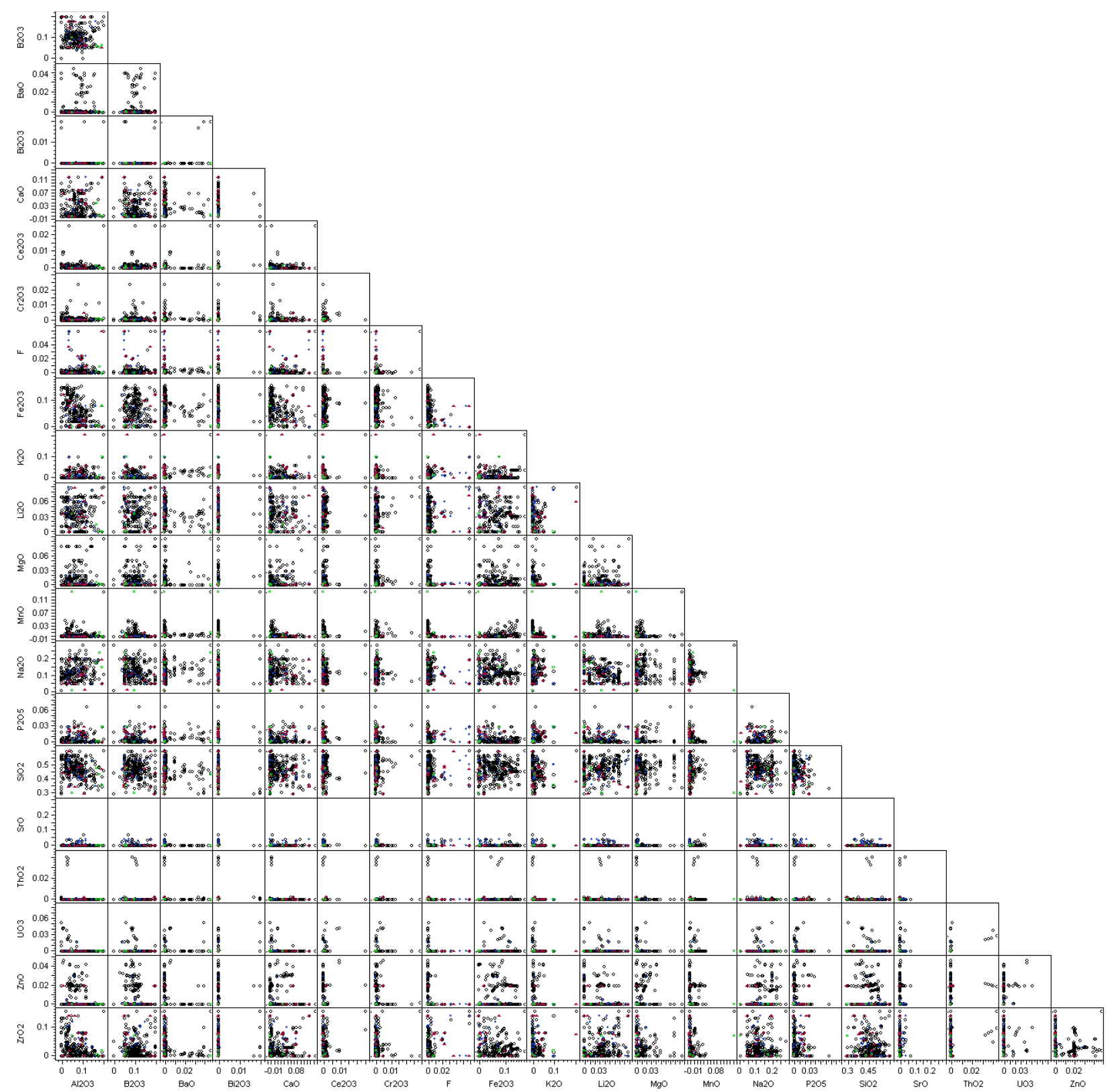

Figure B.14. Scatterplot Matrix of $\rho$ and $v$ Data Component Concentrations (in mass fractions) 
Appendix C

Variance-Covariance Matrices 


\section{Appendix C: Variance-Covariance Matrices}

\section{C.1 Viscosity at $950^{\circ} \mathrm{C}$}

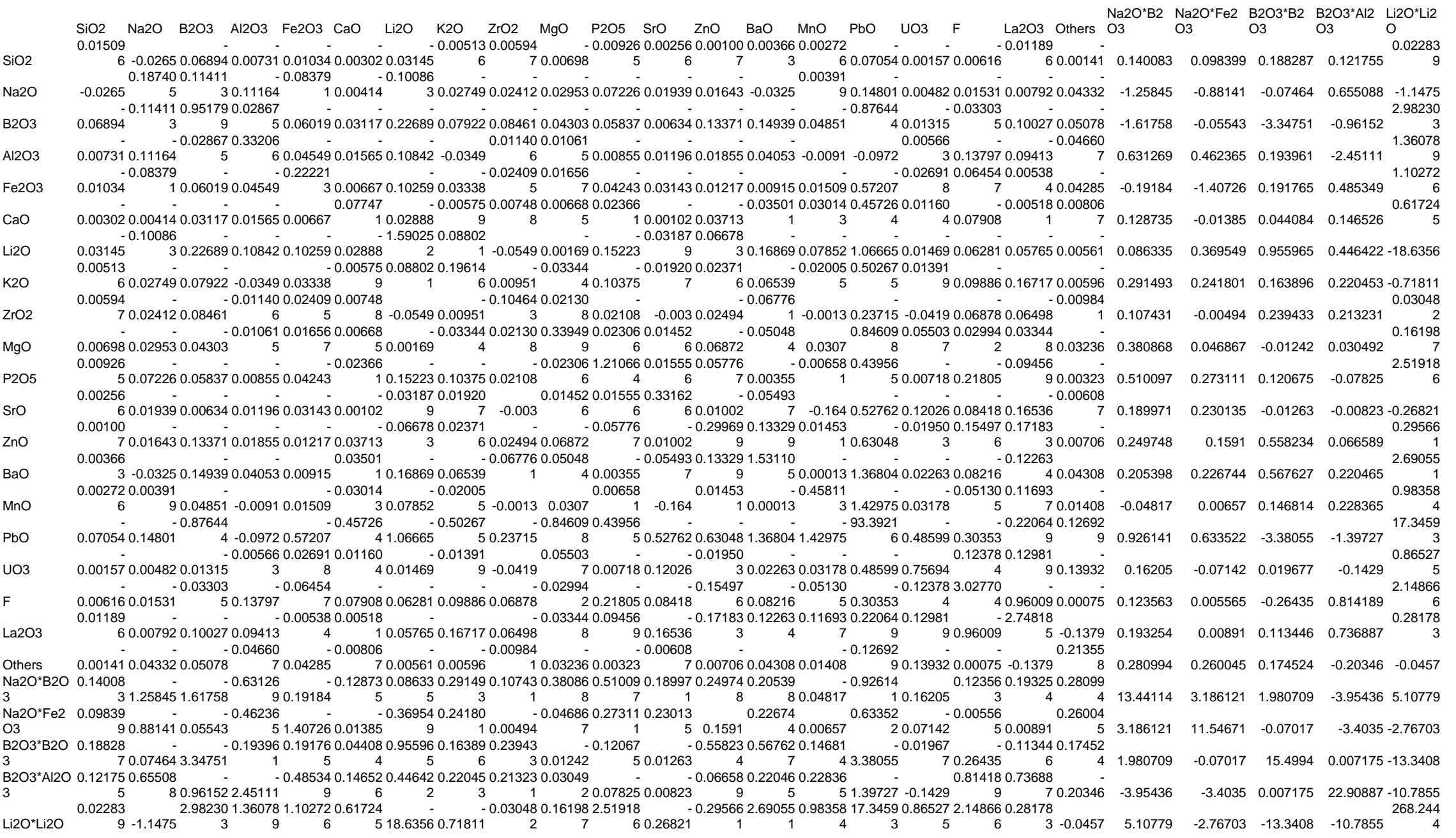




\section{C.2 Viscosity at $1150^{\circ} \mathrm{C}$}

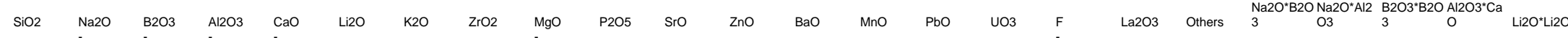

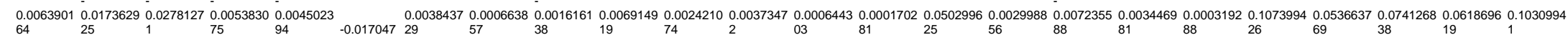

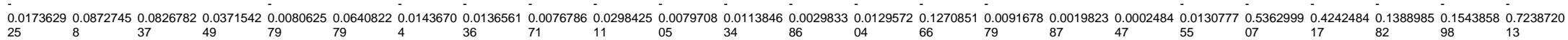

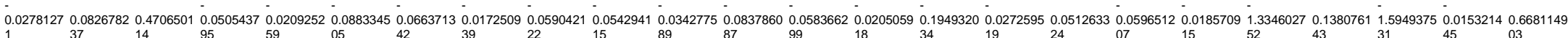

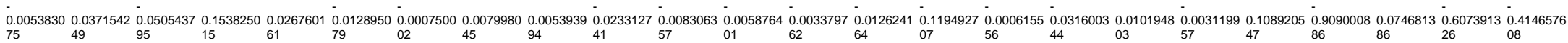

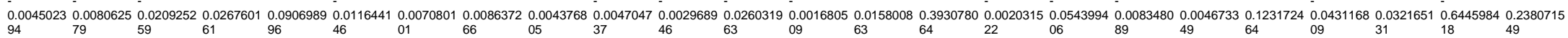

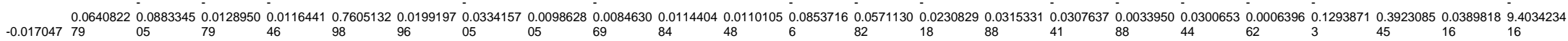

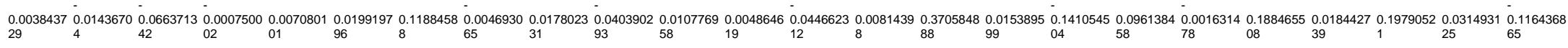

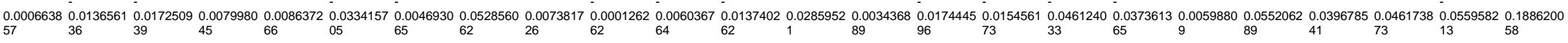

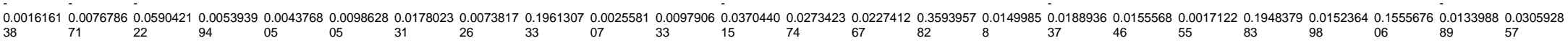

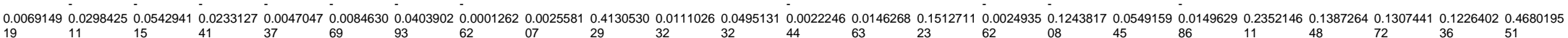

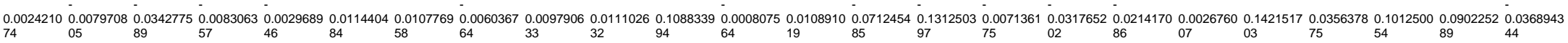

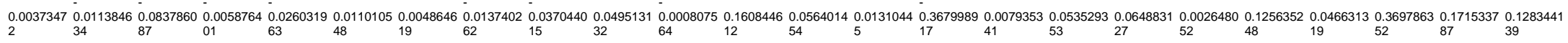

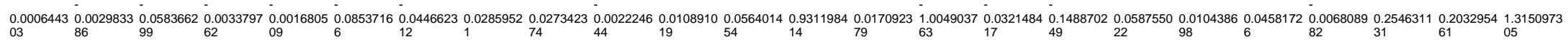

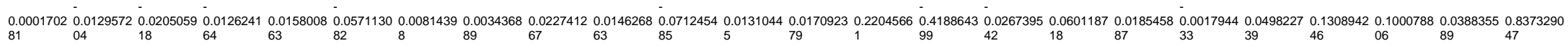

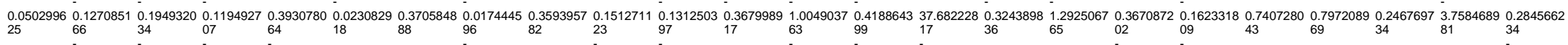

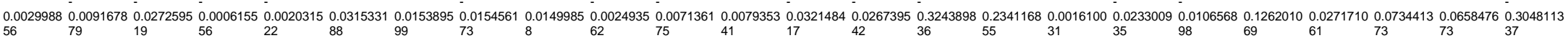

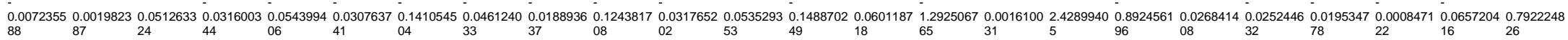

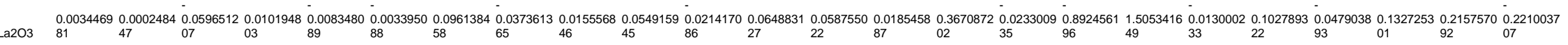

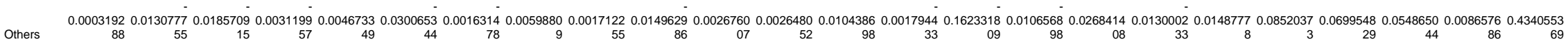

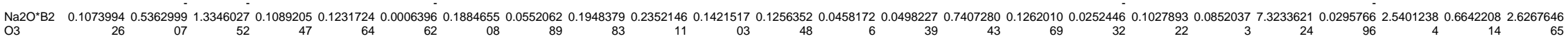

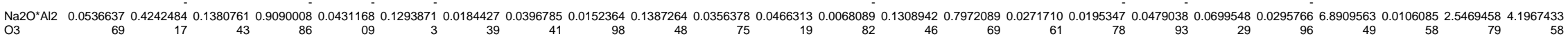

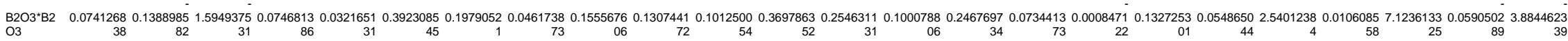

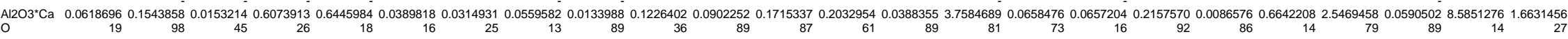

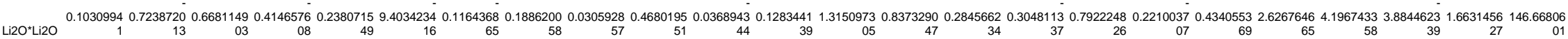




\section{C.3 Viscosity at $1250^{\circ} \mathrm{C}$}

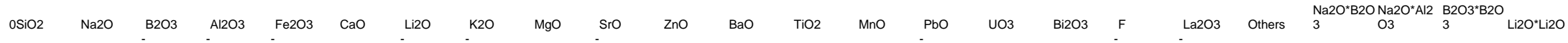

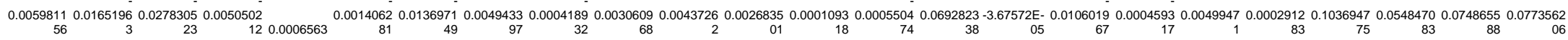

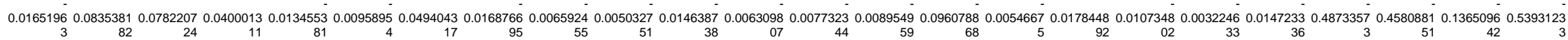

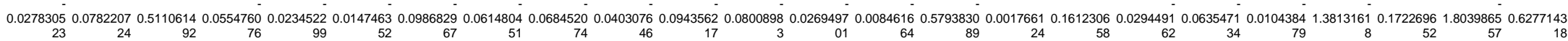

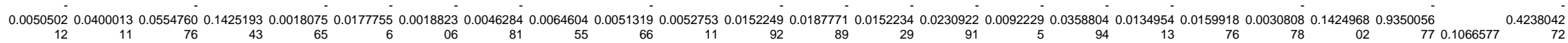

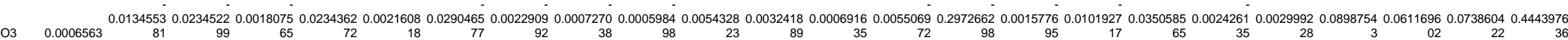

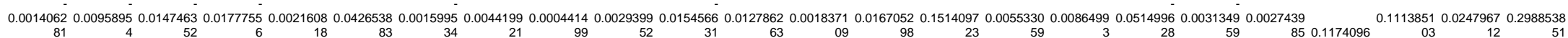

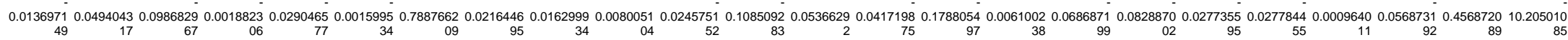

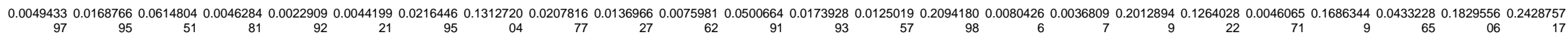

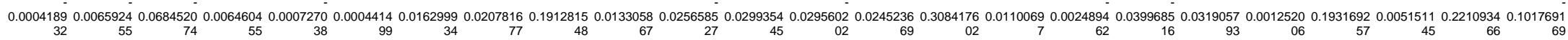

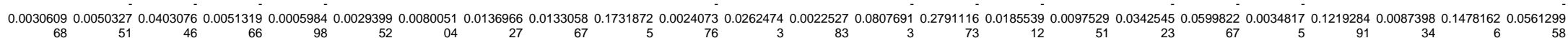

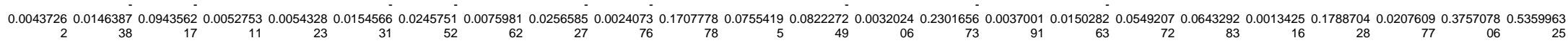

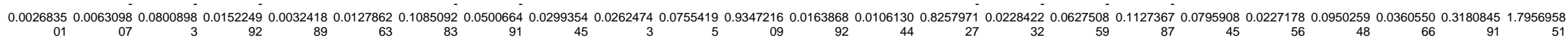

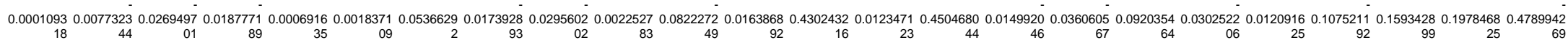

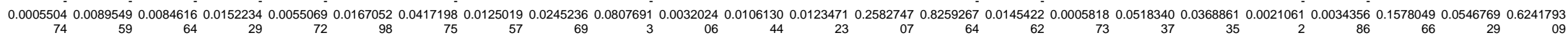

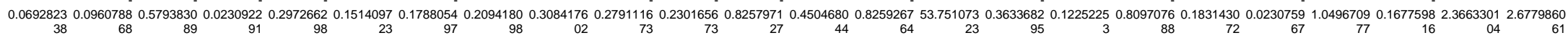

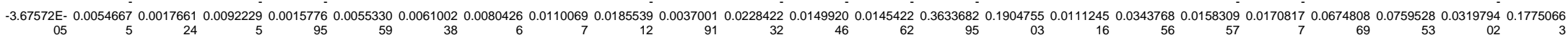

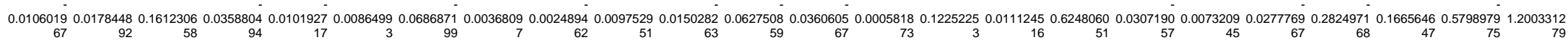

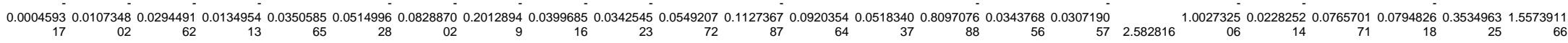

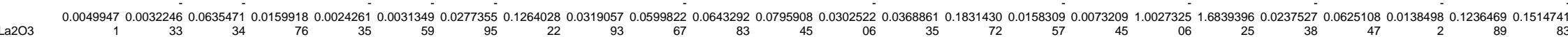

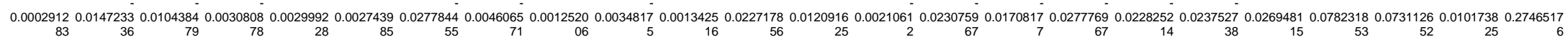

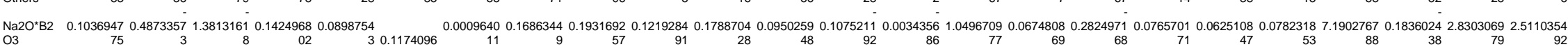

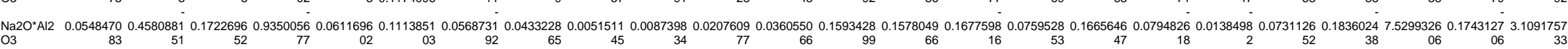

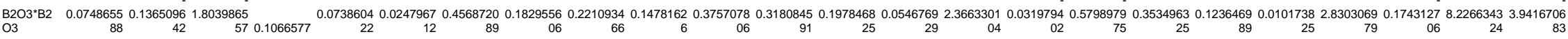

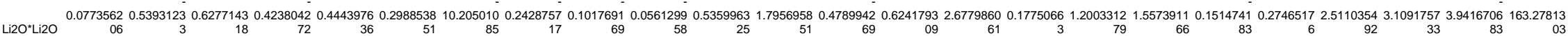




\section{C.4 Electrical Conductivity at $1000^{\circ} \mathrm{C}$}
$\mathrm{Al} 2 \mathrm{O} 3$
B2O3
$\mathrm{CaO}$
$\mathrm{Fe} 2 \mathrm{O} 3$

Li2O

$\mathrm{MgO}$

$\mathrm{Na} 2 \mathrm{O}$

$\mathrm{NiO}$

$\mathrm{SiO} 2$

$\mathrm{SrO}$

$\mathrm{ZnO}$

ZrO2

Others

$\mathrm{Na} \times \mathrm{Li}$

Al2O3 $\quad 0.063840405-0.014816908-0.0039843380 .012863283-0.0762221380 .014060859-0.0262035280 .062570004 \quad 0.001383404-0.017403708 \quad-0.00226075 \quad 0.024877602-0.003059366 \quad 0.310824589$

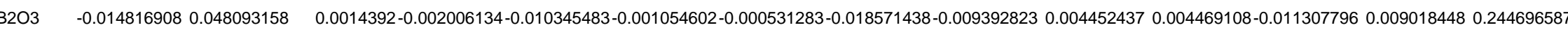

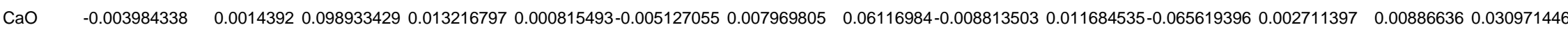
Fe2O3 $\quad 0.012863283-0.0020061340 .013216797 \quad 0.050115614-0.017864504 \quad 0.030505543-0.009337631-0.101135164-0.006763237-0.018159256-0.015679338 \quad 0.01424065 \quad 0.001087882 \quad 0.105442049$ $\begin{array}{lllllll}-0.076222138-0.010345483 & 0.000815493-0.017864504 & 1.03531116-0.031746463 & 0.223634378 & 0.027314042 & -0.06115983-0.007361267-0.031986168-0.060805106-0.020885511-6.576827199\end{array}$ $0.014060859-0.001054602-0.0051270550 .030505543-0.0317464630 .270110552-0.008395917-0.007238135-0.0137885420 .009231098-0.017037347 \quad 0.016305490 .0165752220 .360899876$ $\begin{array}{lllllll}-0.026203528-0.000531283 & 0.007969805-0.009337631 & 0.223634378-0.008395917 & 0.070631346 & 0.017170465-0.017036598 & 0.010188954-0.014926494-0.020565355-0.007695928-1.383109812\end{array}$ $\begin{array}{lllllll}0.062570004-0.018571438 & 0.06116984-0.101135164 & 0.027314042-0.007238135 & 0.017170465 & 7.63330829-0.027350883-0.020679696 & 0.492830024-0.015903831-0.174835694-2.283445706\end{array}$ $0.001383404-0.009392823-0.008813503-0.006763237 \quad-0.06115983-0.013788542-0.017036598-0.0273508830 .011722568 \quad 0.001283913-0.0057146760 .001578036-0.004072103 \quad 0.25048017$

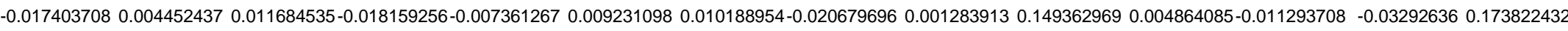
$-0.002260750 .004469108-0.065619396-0.015679338-0.031986168-0.017037347-0.014926494 \quad 0.492830024-0.0057146760 .0048640850 .315458509-0.018946721-0.0124583010 .646786119$

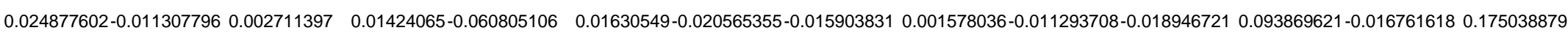

Others $\begin{array}{lllllllllll}-0.003059366 & 0.009018448 & 0.00886636 & 0.001087882-0.020885511 & 0.016575222-0.007695928-0.174835694-0.004072103 & -0.03292636-0.012458301-0.016761618 & 0.052569081 & 0.267313176\end{array}$

$\mathrm{Na} \times \mathrm{Li} \quad 0.3108245890 .244696587 \quad 0.030971446 \quad 0.105442049-6.576827199 \quad 0.360899876-1.383109812-2.283445706 \quad 0.25048017 \quad 0.1738224320 .6467861190 .1750388790 .26731317655 .58502616$

$\Omega_{n}$

\section{C.5 Electrical Conductivity at $1100^{\circ} \mathrm{C}$}

$\mathrm{Al} 2 \mathrm{O} 3 \quad \mathrm{~B} 2 \mathrm{O}$

$\mathrm{Fe} 2 \mathrm{O} 3$

Li20

$\mathrm{MgO}$

$\mathrm{Na} 2 \mathrm{O}$

$\mathrm{SiO} 2$

$\mathrm{ZnO}$

Others

$\mathrm{Na} \times \mathrm{Li}$ В2О3 $-0.011902272 \quad 0.040002850 .002546097-0.001345376-0.018529899-0.000758682-0.0030646840 .034134902-0.007148095-0.000941705 \quad 0.001502946-0.0076039090 .009217904 \quad 0.235106857$ $\begin{array}{llllllllllllllll}\mathrm{CaO} & -0.006154117 & 0.002546097 & 0.086025257 & 0.011441372 & 0.008859866 & -0.004881599 & 0.009314008 & 0.044067861 & -0.008429751 & 0.013585581-0.054464217 & 0.000763082 & 0.00585899-0.000353413\end{array}$

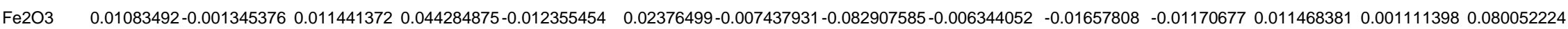
Li2O $\quad-0.065174969-0.0185298990 .008859866-0.0123554540 .930813213-0.0217010780 .199038125 \quad 0.081287837-0.052983441-0.016094259-0.047678739-0.046107534-0.016819255-6.049096548$ $\begin{array}{lllllllll}\mathrm{MgO} & 0.011084302-0.000758682-0.004881599 & 0.02376499-0.021701078 & 0.238159547-0.005402227 & 0.022972769-0.012603048 & 0.009108846-0.011226855 & 0.012293254 & 0.01496421 & 0.292549437\end{array}$ $\mathrm{Na2O} \quad-0.022819372-0.0030646840 .009314008-0.0074379310 .199038125-0.005402227 \quad 0.0632023550 .030975679-0.0147199590 .006841218-0.018669081-0.016362238-0.006458031-1.267939588$

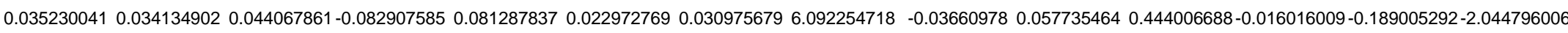
$\mathrm{SiO} 2 \quad 0.00091908-0.007148095-0.008429751-0.006344052-0.052983441-0.012603048-0.014719959-0.036609780 .010097706 \quad 0.002386894-0.003779179 \quad-2.9442 \mathrm{E}-05-0.003629456 \quad 0.229413263$ SrO $\quad-0.013136852-0.0009417050 .013585581 \quad-0.01657808-0.0160942590 .009108846 \quad 0.0068412180 .0577354640 .0023868940 .131503575 \quad 0.002226539-0.009523811-0.027238626 \quad 0.171017238$ $\begin{array}{lllllll}0.000897855 & 0.001502946-0.054464217 & -0.01170677-0.047678739-0.011226855-0.018669081 & 0.444006688-0.003779179 & 0.002226539 & 0.28147995-0.011565227-0.010446257 & 0.661782155\end{array}$

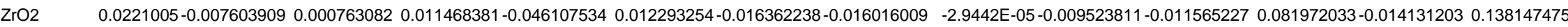
Others $\quad-0.0044738090 .009217904 \quad 0.005858990 .001111398-0.016819255 \quad 0.01496421-0.006458031-0.189005292-0.003629456-0.027238626-0.010446257-0.014131203 \quad 0.0457131680 .239465785$ $\mathrm{Na} x \mathrm{Li} \quad 0.274341231 \quad 0.235106857-0.000353413 \quad 0.080052224-6.049096548 \quad 0.292549437-1.267939588-2.044796006 \quad 0.229413263 \quad 0.1710172380 .6617821550 .138147475 \quad 0.239465785 \quad 51.50820402$ 


\section{C.6 Electrical Conductivity at $1150^{\circ} \mathrm{C}$}
$\mathrm{Al} 2 \mathrm{O} 3$
B2O3
$\mathrm{CaO}$
$\mathrm{Fe} 2 \mathrm{O} 3$

Li2O

$\mathrm{MgO}$

$\mathrm{Na} 2 \mathrm{O}$

$\mathrm{NiO}$

$\mathrm{SiO} 2$

$\mathrm{SrO}$

$\mathrm{ZnO}$

ZrO2

Others

$\mathrm{Na} \times \mathrm{Li}$

Al2O3 $0.037529339-0.014538019-0.0137532210 .005512233-0.0536717920 .005421377-0.0186778670 .0079266710 .004183445-0.0052559810 .0114099730 .017756924-0.0073907290 .203081292$

B2O3 $-0.0145380190 .034931815-0.000141546-0.002852716-0.022188624-0.000863047-0.003759436 \quad 0.0471703-0.004978271-0.000598384 \quad 0.003405756-0.005792242 \quad 0.007663845 \quad 0.234948979$

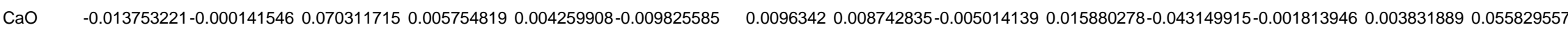
Fe2O3 $\quad 0.005512233-0.0028527160 .0057548190 .039258569-0.0115447290 .015445234-0.006387325-0.075367257-0.004399802-0.015676935 \quad-0.00555435 \quad 0.008435966 \quad 0.000706175 \quad 0.076803504$ Li2O $\quad-0.053671792-0.022188624 \quad 0.004259908-0.0115447290 .868387054-0.0078608380 .1842262550 .073454999-0.04843871-0.024382519-0.055395643-0.042109288-0.010440608-5.688288549$ $\begin{array}{llllllllllll}\mathrm{MgO} & 0.005421377-0.000863047-0.009825585 & 0.015445234-0.007860838 & 0.204003108-0.001251387 & 0.043934371-0.010794766 & 0.010519777-0.001024928 & 0.008185678 & 0.011249661 & 0.213429839\end{array}$ $\mathrm{Na2O} \quad-0.018677867-0.003759436 \quad 0.0096342-0.006387325 \quad 0.184226255-0.001251387 \quad 0.0591130090 .029060792-0.0138950290 .003952095-0.022516327-0.014688466-0.004462139-1.187619942$ $\mathrm{NiO} \quad \begin{array}{llllllllll}0.007926671 & 0.0471703 & 0.008742835 & -0.075367257 & 0.073454999 & 0.043934371 & 0.029060792 & 5.199882036-0.033679982 & 0.091629932 & 0.4370174-0.017258401-0.192143077-1.438777309\end{array}$ $\mathrm{SiO2} \quad 0.004183445-0.004978271-0.005014139-0.004399802 \quad-0.04843871-0.010794766-0.013895029-0.033679982 \quad 0.00826283 \quad 0.001890088-0.005825745 \quad 0.00019005-0.003185216 \quad 0.210165313$ SrO $\quad-0.005255981-0.0005983840 .015880278-0.015676935-0.0243825190 .0105197770 .003952095 \quad 0.0916299320 .0018900880 .124087692-0.002652093-0.009009109-0.023272298 \quad 0.198627874$ $\mathrm{ZnO} \quad 0.0114099730 .003405756-0.043149915-0.00555435-0.055395643-0.001024928-0.022516327 \quad 0.4370174-0.005825745-0.002652093 \quad 0.264852877-0.007480735-0.006635173 \quad 0.687526057$ ZrO2 $\quad 0.017756924-0.005792242-0.001813946 \quad 0.008435966-0.0421092880 .008185678-0.014688466-0.017258401 \quad 0.00019005-0.009009109-0.007480735 \quad 0.07534482-0.012012007 \quad 0.132144937$ Others $\quad-0.0073907290 .0076638450 .0038318890 .000706175-0.0104406080 .011249661-0.004462139-0.192143077-0.003185216-0.023272298-0.006635173-0.012012007 \quad 0.040877071 \quad 0.200518599$ $\mathrm{Na} \times \mathrm{Li} \quad 0.2030812920 .2349489790 .055829557 \quad 0.076803504-5.6882885490 .213429839-1.187619942-1.4387773090 .2101653130 .1986278740 .687526057 \quad 0.1321449370 .20051859948 .69830299$

\section{C.7 Electrical Conductivity at $1200^{\circ} \mathrm{C}$}
$\mathrm{Al} 2 \mathrm{O} 3$
$\mathrm{B} 2 \mathrm{O} 3$
$\mathrm{CaO}$
$\mathrm{Fe} 2 \mathrm{O} 3$

$\mathrm{MgO}$

Na20

$\mathrm{NiO}$

$\mathrm{SiO} 2$

SrO

$\mathrm{ZnO}$

$\mathrm{ZrO} 2$

Others

$\mathrm{Na} \times \mathrm{Li}$

$\begin{array}{lllllllllll}\text { Al2O3 } & 0.048313941 & -0.008658852 & -0.008488968 & 0.007780878-0.053171996 & 0.006107298-0.018628434 & 0.013458006 & 0.000675666-0.009168168 & 0.005147291 & 0.017328243-0.004779446 & 0.238314317\end{array}$

В2O3 $-0.008658852 \quad 0.032903571 \quad 0.00203488 \quad-0.00170395-0.022113385-0.001438719-0.0047161180 .063013105-0.005056948-0.003901822-0.001026565-0.0044268910 .008058063 \quad 0.20656585$

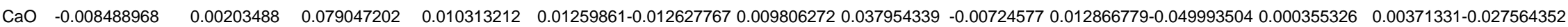
Fe2O3 $0.007780878 \quad-0.00170395 \quad 0.010313212 \quad 0.038195293 \quad-0.006877520 .013234146-0.005458067-0.060476956-0.005245589-0.015323877-0.009252374 \quad 0.008635180 .0005549520 .044664659$ Li2O $\quad-0.053171996 \quad-0.022113385 \quad 0.01259861 \quad-0.006877520 .849788831-0.003342646 \quad 0.1787039230 .128544052-0.047687309-0.0229688009-0.060275422-0.036074753-0.014312755-5.603411058$

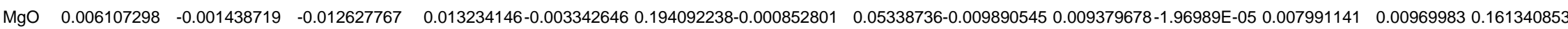
$\mathrm{Na2O}-0.018628434 \quad-0.004716118 \quad 0.009806272 \quad-0.005458067 \quad 0.178703923-0.0008528010 .0562746080 .043723271-0.0130278010 .003870589-0.021247403-0.012721313-0.005597006-1.164667819$

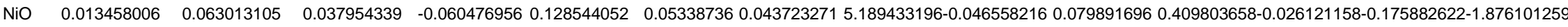
$\mathrm{SiO} 2 \quad 0.000675666 \quad-0.005056948 \quad-0.00724577 \quad-0.005245589-0.047687309-0.009890545-0.013027801-0.0465582160 .0084932450 .003247711-0.002610215-0.000688795-0.0028592020 .221273171$ SrO $\quad-0.009168168-0.003901822 \quad 0.012866779-0.015323877-0.0229688090 .0093796780 .0038705890 .0798916960 .0032477110 .1171868270 .000614711-0.006665347-0.0238385430 .175080369$ $\mathrm{ZnO} \quad 0.005147291 \quad-0.001026565 \quad-0.049993504 \quad-0.009252374-0.060275422-1.96989 \mathrm{E}-05-0.0212474030 .409803658-0.0026102150 .000614711 \quad 0.255870856-0.007168483-0.008170774 \quad 0.68284315$ ZrO2 $0.017328243 \quad-0.004426891 \quad 0.000355326 \quad 0.00863518-0.036074753 \quad 0.007991141-0.012721313-0.026121158-0.000688795-0.006665347-0.007168483 \quad 0.068150864-0.011484496 \quad 0.123353114$ Others-0.004779446 $\quad 0.008058063 \quad 0.00371331 \quad 0.000554952-0.014312755 \quad 0.00969983-0.005597006-0.175882622-0.002859202-0.023838543-0.008170774-0.011484496 \quad 0.039570427 \quad 0.208849719$ $\mathrm{Na} \times$ Li $0.238314317 \quad 0.20656585 \quad-0.027564352 \quad 0.044664659-5.6034110580 .161340853-1.164667819-1.8761012590 .2212731710 .175080369 \quad 0.682843150 .1233531140 .20884971947 .59124249$ 


\section{C.8 Product Consistency Test Normalized Boron Response}

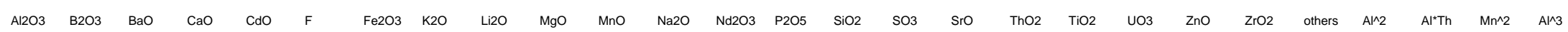

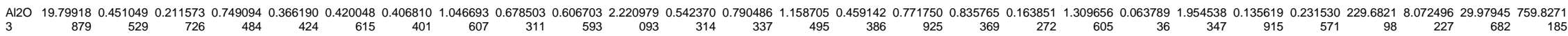

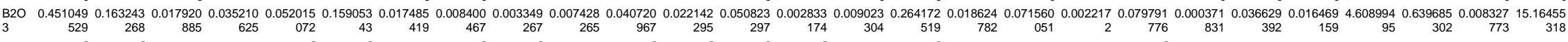

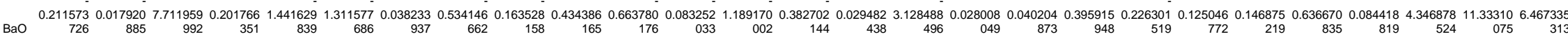

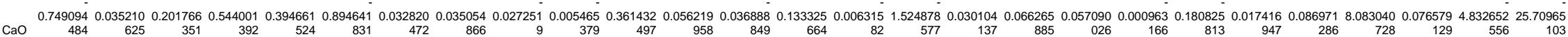

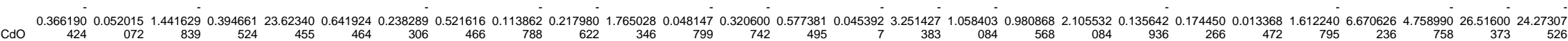

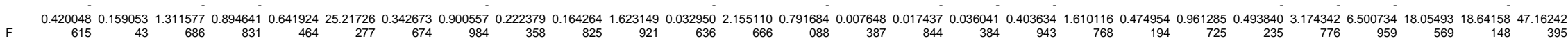

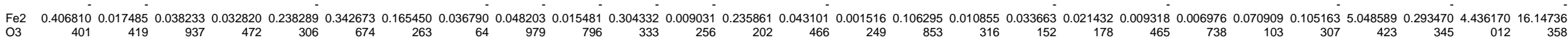

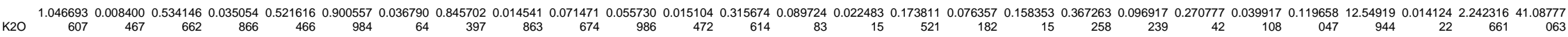

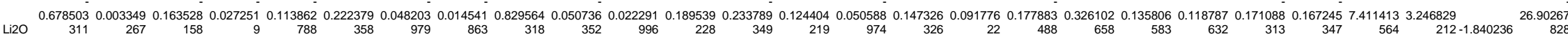

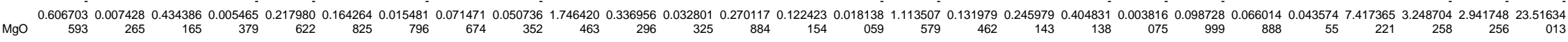

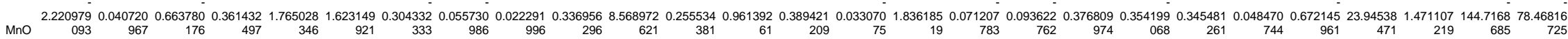

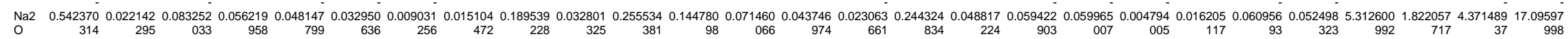

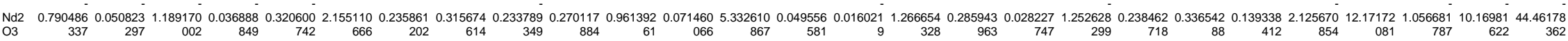

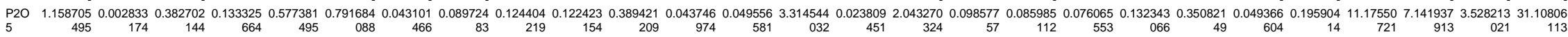

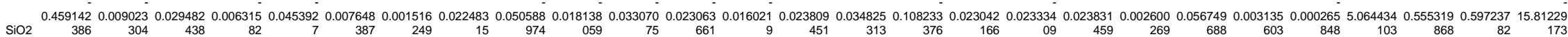

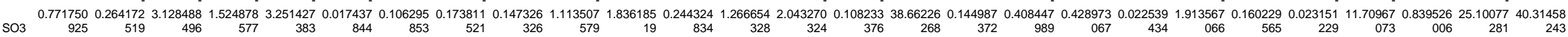

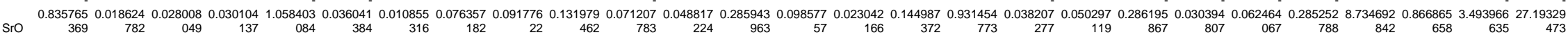

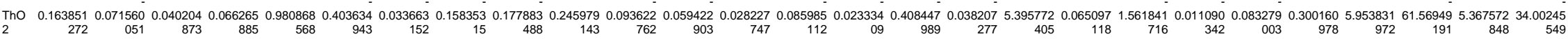

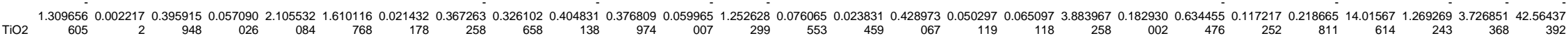

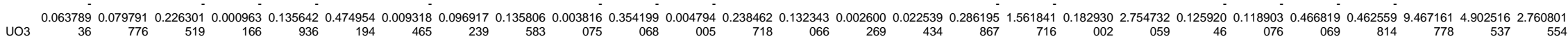

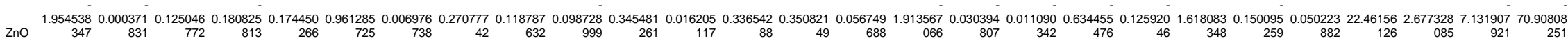

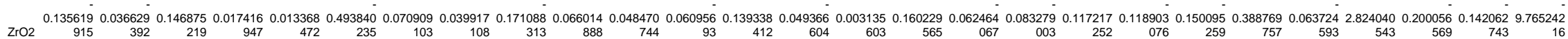

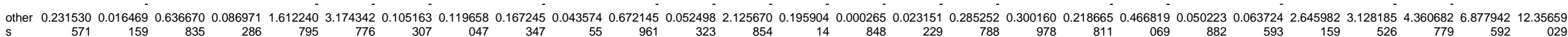

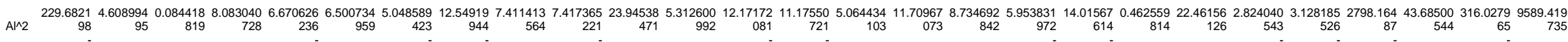

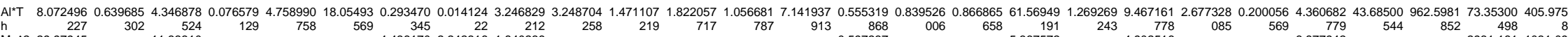

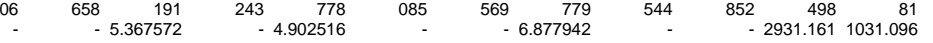




\section{C.8 Product Consistency Test Normalized Boron Response}

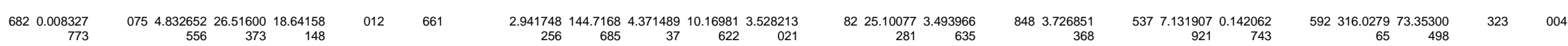

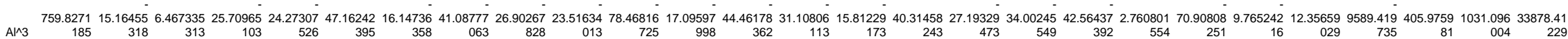

\section{C.9 Product Consistency Test Normalized Sodium Response}

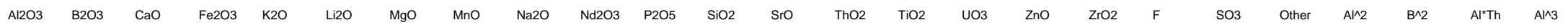

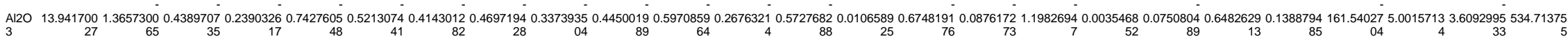

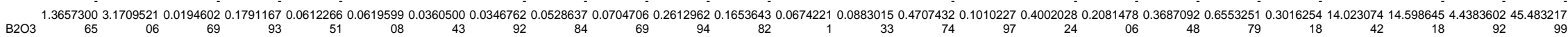

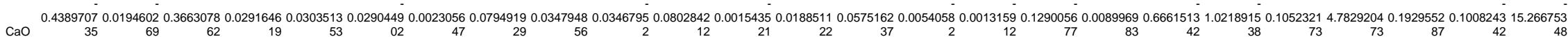

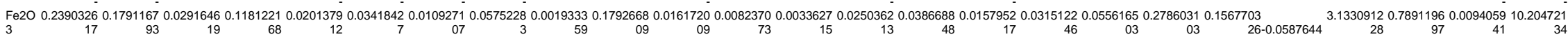

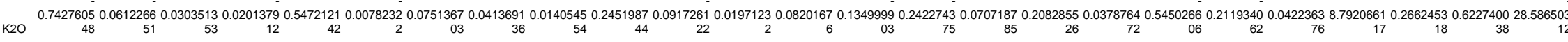

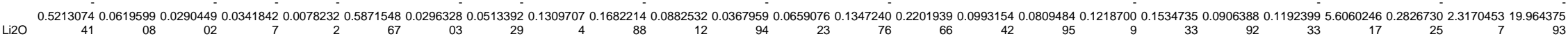

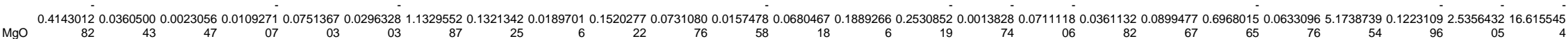

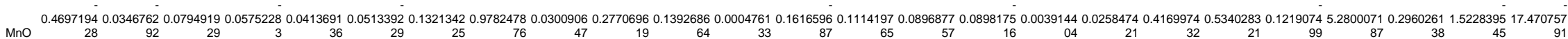

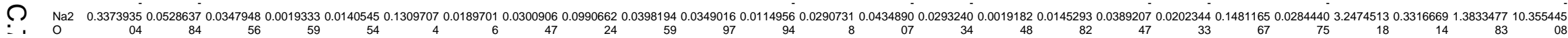

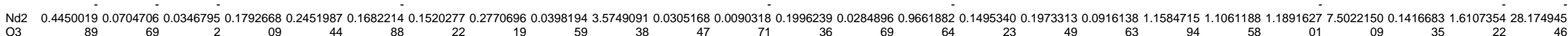

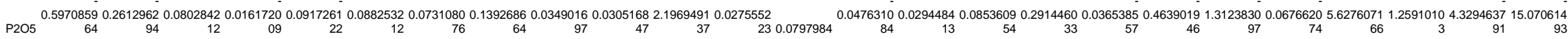

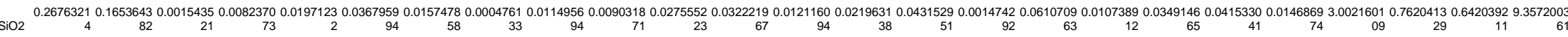

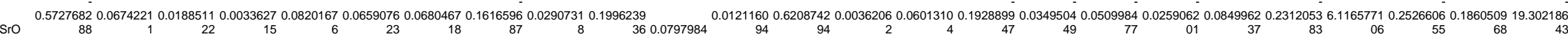

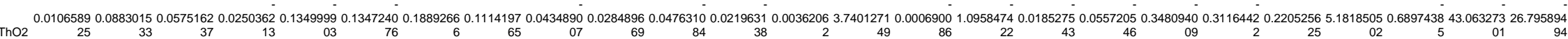

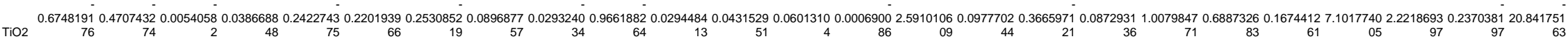

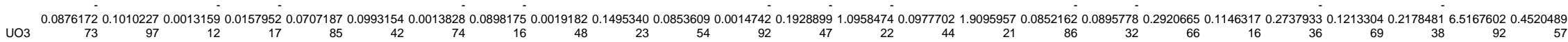

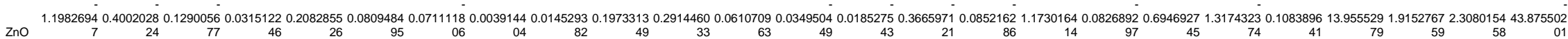

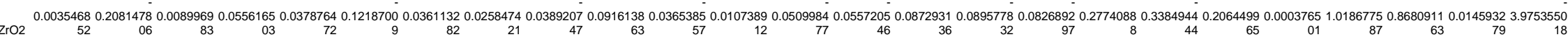

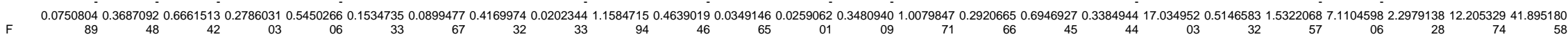

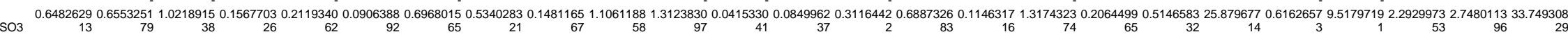

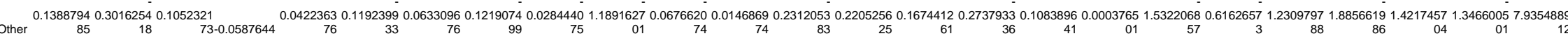

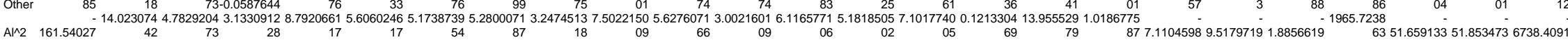




\section{C.9 Product Consistency Test Normalized Sodium Response}

$\mathrm{B}^{\wedge} 2$

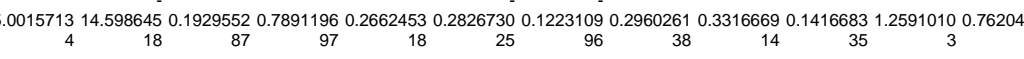

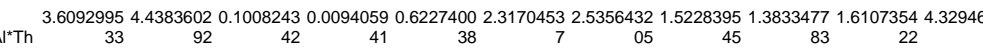

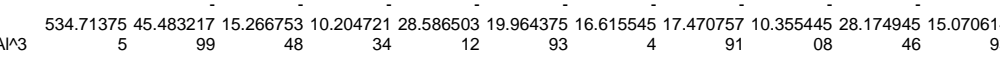

\section{C.10 Product Consistency Test Normalized Lithium Response}

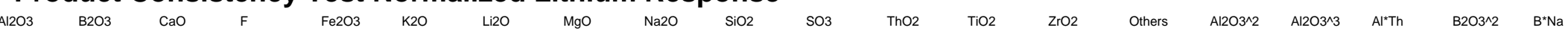

Al2O3 16.52926973-1.545384204-0.610031381 0.927419971-0.337983498-1.114259507-0.484725577 -0.16223294-0.360717032-0.314305902 4.112911975-0.299209617-0.400256056-0.051931087-0.544400505-194.7404169 647.4804645 1.332595892 5.251907131 0.937437706

В2О3 - -1.545384204 6.343420389-0.226823979-1.238430378-0.423657707-0.207020135 $\quad 0.0610148-0.351284543$ 1.137597715-0.458785122-2.732316657-0.040762989-1.584357515 -0.51918204-0.288722681 14.07548411-48.71450792-6.787155721-22.16599762-14.90805121 $\mathrm{CaO} \quad-0.610031381-0.226823979$ 1.093173231-2.017698972 0.104442424 0.092629792-0.007582304 0.135966085-0.160190427 0.008144728-0.579817021 $0.025600518-0.1263451430 .0657457160 .0977591746 .005216079-15.78337849 \quad 1.134325108 \quad 0.27071816 \quad 1.97580709$

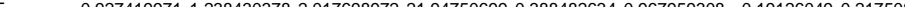
Fe2O3 $\quad-0.337983498-0.4236577070 .1044424240 .3884826340 .182453126-0.0396274170 .0541344860 .013237113-0.1312676310 .0200741150 .3370559120 .0113628820 .2591497390 .1084155540 .0093609984 .317361397-13.27789657-0.2633630431 .2823042491 .409196739$

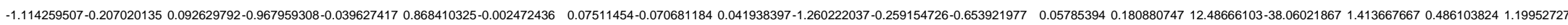

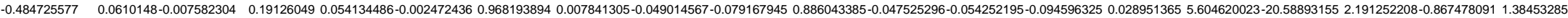

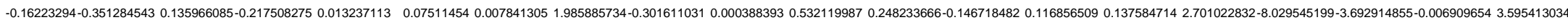
$-0.3607170321 .137597715-0.160190427-0.068384186-0.131267631-0.070681184-0.049014567-0.3016110310 .923842673-0.123954599-0.936633994-0.237386208-0.065222231 \quad-0.1885064-0.125045995$ 2.187352236-8.488729843 2.094900153-1.151248205-8.889915105

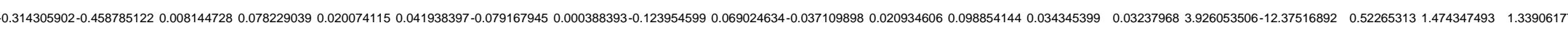
$4.112911975-2.732316657-0.579817021-5.910512411$ 0.337055912-1.260222037 0.886043385 0.532119987-0.936633994-0.037109898 70.54873124-0.900200217 1.903204288 0.702569663-0.084044223 -52.7995788 180.8837 1.403773545 6.049338655 8.272128562 $-0.299209617-0.0407629890 .0256005180 .3357584510 .011362882-0.259154726-0.0475252960 .248233666-0.2373862080 .020934606-0.9002002174 .006399071$ 0.413875817-0.092924957-0.0888638339 10.68036606-48.45207429-49.86142014-0.909524403 1.954531382 (6)

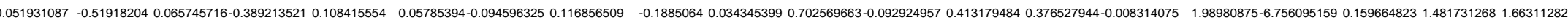
Others $\quad-0.544400505-0.2887226810 .097759174-0.260558130 .0093609980 .1808807470 .0289513650 .137584714-0.125045995 \quad 0.03237968-0.084044223-0.088638339-0.205509051-0.0083140750 .1737901016 .016317725-17.70980690 .901196391 \quad 0.778056579$ 1.48355978 Al2O3^2 -194.7404169 14.07548411 6.005216079-18.74567618 4.317361397 12.48666103 5.604620023 2.701022832 2.187352236 3.926053506 -52.7995788 10.68036606 6.595514674 $1.989808756 .0163177252417 .106334-8331.991402-142.7436615-51.428850770 .917027155$ Al2O3^3 $3647.4804645-48.71450792-15.78337849 \quad 80.8180439-13.27789657-38.06021867-20.58893155-8.029545199-8.488729843-12.37516892 \quad 180.8837-48.45207429-24.31011104-6.756095159$-17.7098069-8331.991402 29625.42363 723.2265267 172.7528199 19.66122049

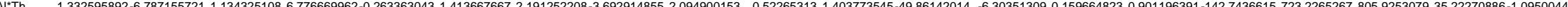

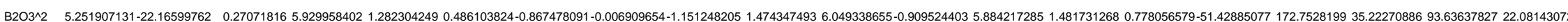

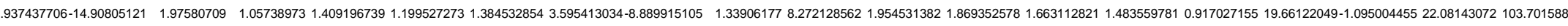




\section{C.11 Toxicity Characteristic Leaching Procedure Response}

\begin{tabular}{|c|c|c|c|c|c|c|c|c|c|c|}
\hline & $\mathrm{Al} 2 \mathrm{O} 3$ & $\mathrm{~B} 2 \mathrm{O} 3$ & $\mathrm{CaO}$ & $\mathrm{Fe} 2 \mathrm{O} 3$ & $\mathrm{Li} 2 \mathrm{O}$ & $\mathrm{Na} 2 \mathrm{O}$ & $\mathrm{SiO} 2$ & ThO2 & $\mathrm{ZrO} 2$ & Others \\
\hline $\mathrm{Al} 2 \mathrm{O} 3$ & 1.042843388 & -0.101700415 & -0.133314732 & 0.029642566 & -0.597683355 & -0.271942061 & -0.020906321 & 0.168189368 & 0.284568086 & -0.614675728 \\
\hline $\mathrm{B} 2 \mathrm{O} 3$ & -0.101700415 & 0.481678862 & -0.15319744 & -0.137164869 & 0.1545264 & 0.048039652 & -0.052050133 & 0.135894338 & -0.149822776 & -0.415167246 \\
\hline $\mathrm{aO}$ & -0.133314732 & -0.15319744 & 3.322292293 & 0.185206441 & -0.121354167 & -0.081204232 & -0.017073241 & -0.361574746 & 0.072570032 & 1.82204859 \\
\hline $\mathrm{Fe} 2 \mathrm{O} 3$ & 0.029642566 & -0.137164869 & 0.185206441 & 0.660777028 & -0.270820463 & -0.104928376 & -0.093516047 & 0.308784107 & 0.162342896 & -0.304099467 \\
\hline Li2O & -0.597683355 & 0.1545264 & -0.121354167 & -0.270820463 & 3.585674248 & 0.838832423 & -0.280085632 & -0.340258452 & -0.6470695 & -0.265116378 \\
\hline $\mathrm{Na} 2 \mathrm{O}$ & -0.271942061 & 0.048039652 & -0.081204232 & -0.104928376 & 0.838832423 & 0.620850908 & -0.115904298 & -0.077143084 & -0.327142481 & -0.087832236 \\
\hline $\mathrm{SiO} 2$ & -0.020906321 & -0.052050133 & -0.017073241 & -0.093516047 & -0.280085632 & -0.115904298 & 0.110643497 & -0.132076613 & 0.021460489 & -0.008371581 \\
\hline ThO2 & 0.168189368 & 0.135894338 & -0.361574746 & 0.308784107 & -0.340258452 & -0.077143084 & -0.132076613 & 5.028373315 & -0.653831521 & 0.124932575 \\
\hline $\mathrm{ZrO} 2$ & 0.284568086 & -0.149822776 & 0.072570032 & 0.162342896 & -0.6470695 & -0.327142481 & 0.021460489 & -0.653831521 & 1.370624484 & -0.264642659 \\
\hline Others & -0.614675728 & -0.415167246 & 1.82204859 & -0.304099467 & -0.265116378 & -0.087832236 & -0.008371581 & 0.124932575 & -0.264642659 & 13.81402636 \\
\hline
\end{tabular}

\section{C.12 One Percent Spinel Temperature}




\section{C.13 Zircon Liquidus Temperature}

\begin{tabular}{|c|c|c|c|c|c|c|c|c|}
\hline & Al2O3 & B2O3 & Ln2O3 & Li2O & $\mathrm{Na} 2 \mathrm{O}$ & $\mathrm{SrO}$ & ZrO2 & Others \\
\hline $\mathrm{Al} 2 \mathrm{O} 3$ & 36866.26138 & -5799.888564 & 44567.34341 & -26798.29741 & -13555.8839 & -36355.71736 & 17890.9687 & -363.7241761 \\
\hline B2O3 & -5799.888564 & 9277.83125 & -19508.58441 & 2450.367392 & 7865.096334 & -23166.55394 & -6514.830864 & -1173.851483 \\
\hline Ln2O3 & 44567.34341 & -19508.58441 & 224350.8801 & -29383.63569 & -26904.43604 & 60874.75505 & 42864.82322 & -3878.190823 \\
\hline Li2O & -26798.29741 & 2450.367392 & -29383.63569 & 58010.59476 & 16883.04038 & 102908.803 & -32737.16866 & -369.6373168 \\
\hline $\mathrm{Na} 2 \mathrm{O}$ & -13555.8839 & 7865.096334 & -26904.43604 & 16883.04038 & 26584.49275 & 6667.999084 & -16736.7845 & -2897.093652 \\
\hline $\mathrm{SrO}$ & -36355.71736 & -23166.55394 & 60874.75505 & 102908.803 & 6667.999084 & 2095713.065 & -9353.337303 & -7281.735614 \\
\hline $\mathrm{ZrO} 2$ & 17890.9687 & -6514.830864 & 42864.82322 & -32737.16866 & -16736.7845 & -9353.337303 & 33140.18983 & -1150.325916 \\
\hline Others & -363.7241761 & -1173.851483 & -3878.190823 & -369.6373168 & -2897.093652 & -7281.735614 & -1150.325916 & 1042.8734 \\
\hline
\end{tabular}

$?$

$\overrightarrow{0}$ 
PNNL-18501

\section{Distribution List}

\begin{tabular}{|c|c|c|c|}
\hline $\begin{array}{l}\text { No. of } \\
\text { Copies }\end{array}$ & $\begin{array}{c}\text { With } \\
\text { Appen- } \\
\text { dices }\end{array}$ & Recipient & Hanford Mail Stop \\
\hline 1 & no & Paul Bredt & $\mathrm{K} 6-24$ \\
\hline 1 & no & Tom Brouns & K9-69 \\
\hline 1 & yes & Paul Certa & B1-55 \\
\hline 3 & no & Paul Certa & B1-55 \\
\hline 1 & no & Jarrod Crum & K6-24 \\
\hline 1 & no & Rob Gilbert & $\mathrm{H} 6-60$ \\
\hline 1 & no & Fred Hidden & $\mathrm{H} 6-60$ \\
\hline 1 & no & Pavel Hrma & K6-24 \\
\hline 1 & no & Gary Josephson & K9-69 \\
\hline 1 & no & Dong Kim & $K 6-24$ \\
\hline 1 & no & Albert Kruger & $\mathrm{H} 6-60$ \\
\hline 1 & no & Pepa Matyas & $K 6-24$ \\
\hline 1 & no & Billie Mauss & $\mathrm{H} 6-60$ \\
\hline 1 & no & Loni Peurrung & K9-09 \\
\hline 1 & no & Brian Riley & $K 6-24$ \\
\hline 1 & no & Joe Ryan & $K 6-24$ \\
\hline 1 & no & Mike Schweiger & $\mathrm{K} 6-24$ \\
\hline 1 & no & Mike Thien & B1-55 \\
\hline 2 & yes & John Vienna & K6-24 \\
\hline 10 & no & John Vienna & K6-24 \\
\hline $\begin{array}{l}\text { No. of } \\
\text { Copies }\end{array}$ & $\begin{array}{c}\text { With } \\
\text { Appen- } \\
\text { dices }\end{array}$ & Recipient & Offsite Address \\
\hline 1 & yes & Tommy Edwards & Savannah River National Laboratory, 999-W, Aiken, SC 29808 \\
\hline 3 & no & Kurt Gerdes & $\begin{array}{l}\text { U.S. Department of Energy, EM-31, } 19901 \text { Germantown Road, } \\
\text { Germantown, MD } 20874\end{array}$ \\
\hline 1 & no & Rod Gimpel & $\begin{array}{l}\text { Waste Treatment and Immobilization Plant, } 2435 \text { Stevens Center } \\
\text { Place, Richland, WA } 99354\end{array}$ \\
\hline 1 & no & Vijay Jain & $\begin{array}{l}\text { Waste Treatment and Immobilization Plant, } 2435 \text { Stevens Center } \\
\text { Place, Richland, WA } 99354\end{array}$ \\
\hline 1 & no & Carol Jantzen & Savannah River National Laboratory, 773-A, Aiken, SC 29808 \\
\hline 1 & yes & Wing Kot & $\begin{array}{l}\text { Catholic University of America, Vitreous State Laboratory, } 620 \\
\text { Michigan Ave., N.E., Washington, D. C. } 20064\end{array}$ \\
\hline 1 & no & Ernie Lee & $\begin{array}{l}\text { Waste Treatment and Immobilization Plant, } 2435 \text { Stevens Center } \\
\text { Place, Richland, WA } 99354\end{array}$ \\
\hline 1 & no & Sharon Marra & Savannah River National Laboratory, 773-A, Aiken, SC 29808 \\
\hline 1 & no & David Peeler & Savannah River National Laboratory, 999-W, Aiken, SC 29808 \\
\hline 1 & no & lan Pegg & $\begin{array}{l}\text { Catholic University of America, Vitreous State Laboratory, } 620 \\
\text { Michigan Ave., N.E., Washington, D. C. } 20064\end{array}$ \\
\hline 1 & no & Bill Wilmarth & Savannah River National Laboratory, 773-A, Aiken, SC 29808 \\
\hline
\end{tabular}

Distr. 1 


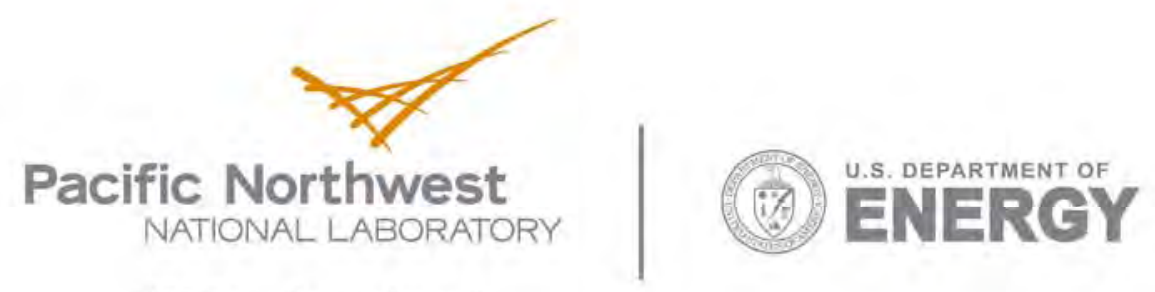

902 Battelle Boulevard

P.O. Box 999

Richland, WA 99352

1-888-375-PNNL (7665)

www.pnl.gov 